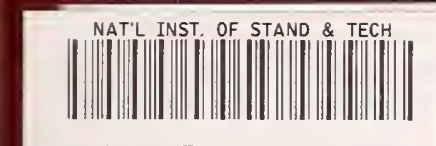

\title{
REFERENCE
}

\section{Alliob 04b6?}

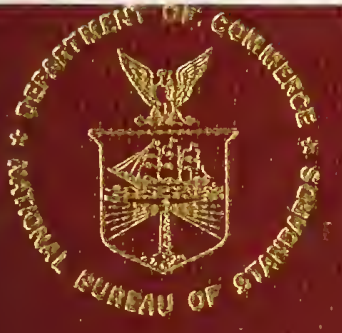

NBS SPECIAL PUBLICATION 329

\section{SUPPLEMENT 2}

US. DEPARTMENT OF COMMERCESANational Bureau of Standards

\section{An Index of \\ U.S. Voluntary}

Engineering Standards 



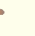



National Bureau of Stondards

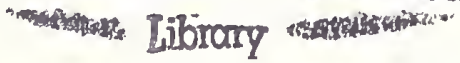

\section{An Index of U.S. Voluntary \\ Engineering Standards}

AUG 41975

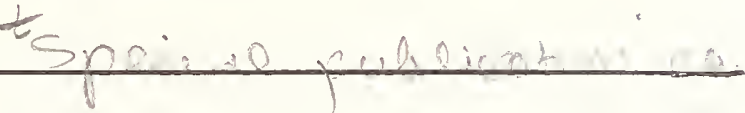

Covering those Standards, Specifications,

Test Methods, and Recommended Practices

Issued by National Standardization Organizations

in the United States

William J. Slattery, Editor

Engineering and Product Standards Division

Institute for Applied Technology

National Bureau of Standards

Washington, D.C. 20234

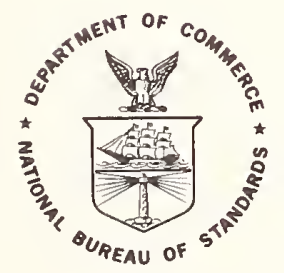

U.S. DEPARTMENT OF COMMERCE, Frederick B. Dent, Secretary

NATIONAL BUREAU OF STANDARDS, Richard W. Roberts, Director

Issued May 1975 


\section{Library of Congress Catalog Card Number: 77-607150}

\section{National Bureau of Standards Special Publication 329 Supplement 2}

Nat. Bur. Stand. (U.S.), Spec. Publ. 329, Suppl. 2, 472 pages (May 1975)

CODEN: XNBSAV 


\section{Contents}

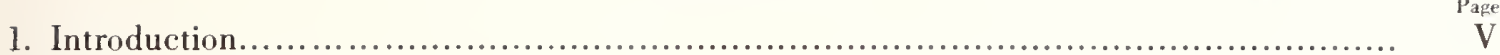

2. List of organizations.......................................................................... VI

2.1. Alphabetical by acronym....................................................... VI

2.2. Alphabetical by organization with addresses..................................... VII

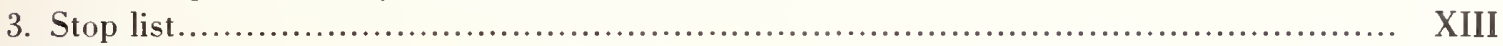

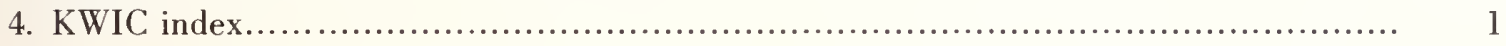


$$
*
$$ 


\title{
An Index of U.S. Voluntary Engineering Standards Supplement 2
}

\author{
William J. Slattery, Editor
}

\begin{abstract}
This supplement contains the permuted titles of more than 5.700 voluntary enginering standards. specifications. test methods. condes and recommended practices publisted by 164 [i.s. technical socielies, professionat organizations and trade associations. Earh title can be found under all the significant key words which it contains. These keys words are arranged alphabetically down the center of parh page logether with their surmonding context. The date of publication or last revision. Hes standard number and an acronym designating the standards-issuing organization appear as part of each entry.
\end{abstract}

Key words: Engineering Standards. index of: Index of standards. recommended practices. sperifications.

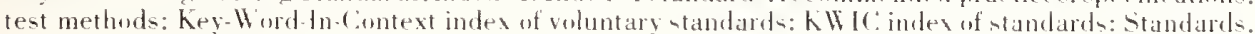
voluntary, index of.

\section{Introduction}

This supplement to NBS Special Publication 329 contains the permuted titles of more than 5.700 voluntary engineering standards. specifications, test methods, codes and recommended practices published by 164 U.S. technical societies. professional organizations, and trade associations. The titles listed fall into three categories: (1) those pul, lished between January 1, 1972 and July 1, 1974: (2) those erroneously omitted from SP 329 and Supplement No. 1. and (3) those published prior to 1972 but not on file witl the Standards Information and Analysis Section at the time of publication of SP 329.

Standards Information Services, Engineering and Product Standards Division continues to serve as a technical tibrary and referral activ. ity. Insuirers may write or visit the Center. Which is forated in the Technotery Buitding. Roums B151-B159, NBS, Gaithersburg. Maryland, about 20 miles northwest of Washington. D.C.: or call on Area Code 301-921-2587. Comments or sugrestions concerning this supplement of SP 329 are welcome. and should be sent to the editor. Stantardo Information Serviess. Texhnotogy Blde. BI63. National Bureau of Standards, Washington. D.C. 20234. To be placed on the announcement list of future supplements, please fill out the tear sheert at the end of this Index.

This supplement, which like supplement No. 1 is noncumulative. shoutd be used with both the basic index (NBS SP 329) and supplement No. 1. 


\section{List of Organizations}

\subsection{Alphabetical by Acronym}

AA

$\mathrm{AACCH}$

AAFCO

AAMA

AAPCC

AATCC

ABS

ABYC

ACGIH

ACI

ACPA

ADA

AFBMA

AGMA

AHA

AHAM

AI

AIOA

AISC

AISI

AITC

ALCA

ALI

AMCA

ANS

ANSI

AOSA

APA

API

ARI

ASAE

ASE

ASHRA

ASHTO

ASME

ASPA

ASQC

ASSE

ASSFE

ASTM

ATAA

ATI

AWI

AWPA

AW PI

AWS

AWWA

BAMI

BCA

BCI

BHMA

BIA

BMA
Aluminum Association

American Association of Cereal Chemists

Association of American Feed Control Officials. Inc.

Architectural Aluminum Manufacturers Association

American Association of Poison Control Centers

American Association of Textile Chemists and Colorists

American Bureau of Shipping

American Boat \& Yacht Council, Inc.

American Conference of Governmental Industrial Hygienists

American Concrete Institute

American Concrete Paving Association

American Dental Association

Anti-Friction Bearing Manufacturers Association, Inc.

American Gear Manufacturers Association

American Hardboard Association

Association of Home Appliance Manufacturers

Asphalt Institute

American Institute of Architects

American Institute of Steel Construction, Inc.

American Iron and Steel Institute

American Institute of Timber Construction

A merican Leather Chemists Association

American Ladder Institute

Air Moving \& Conditioning Association, Inc.

American Nuclear Society

American National Standards Institute

Association of Official Seed Analysts

American Plywood Association

American Petroleum Institute

Air Conditioning and Refrigeration Institute

American Society of Agricultural Engineers

American Society of Enologists

American Society of Heating, Refrigerating and

Air Conditioning Engineers

American Association of State Highway and

Transportation Officials

American Society of Mechanical Engineers

American Sod Producers Association, Inc.

American Society for Quality Control

American Society of Sanitary Engineering

American Society of Safety Engineers, Inc.

American Society for Testing and Materials

Air Transport Association of America

Asbestos Textile Institute

Architectural Woodwork Institute

American Wood-Preservers Association

American Wood Preservers Institute

American Welding Society, Inc.

American Water Works Association. Inc.

Barley and Malt Institute

Billiard Congress of America

Battery Council International

Builders Hardware Manufacturers Association

Brick Institute of America

Bicycle Manufacturers Association of America
BMI Book Manufacturers Institute

CCTI Composite Can and Tube Institute

CDA Copper Development Association

CEMA Conveyor Equipment Manufacturers Association

CFTMA Caster and Floor Truck Manufacturers Association

CGA

CHI

CISCA

Compressed Gas Association

Chlorine Institute, Inc.

Ceilings and Interior Systems Contractors Association

CISPI Cast Iron Soil Pipe Institute

CLFMI Chain Link Fence Manufacturers Institute

CMAA Crane Manufacturers Association of America, Inc.

CPAI

CR

CRA

CRSI

CSI

CSMA

CTI

DCDMA

DEMA

DFISA

EASA

EEI

EIA

EOA

FCI

FHDA

FI

FMS

GT

HMI

HPMA

IAPMO

Canvas Products Association, International

Corn Refiners Association

California Redwood Association

Concrete Reinforcing Steel Institute

Construction Specifications Institute

Chemical Specialties Manufacturers Association

Cooling Tower Institute

Diamond Core Drill Manufacturers Association

Diesel Engine Manufacturers Association

Dairy and Food Industries Supply Association, Inc.

Electrical Apparatus Service Association, Ine.

Edison Electric Institute

Electronic Industries Association

Essential Oil Association of U.S.A., Ine.

Fluid Controls Institute, Inc.

Fir and Hemlock Door Association

Fertilizer Institute

Factory Mutual System

Gravure Technical Association, Inc.

Hoist Manufacturers Institute

Hardwood Plywood Manufacturers Association

International Association of Plumbing and Mechanical Officials

IATA

ICBO

IEEE

IES

IFI

IMACA

IME

IPA

IPC

IPCEA

ISA

ISIS

ITA

ITE

JIC

JPMA

LBI

MCA

MMA

MSS

International Air Transport Association

International Conference of Building Officials

Institute of Electrical and Electronics Engineers, Inc.

Illuminating Engineering Society

Industrial Fasteners Institute

International Mobile Air Conditioning Association. Inc.

Institute of Makers of Explosives

Industrial Perforators Association

lnstitute of Printed Circuits

Insulated Power Cable Engineers Association

Instrument Suciety of America

Institute of Scrap Iron and Steel, Inc.

Industrial Truck Association

Institute of Traffic Engineers

Joint Industrial Council

Juvenile Products Manufacturers Association. Inc.

Library Binding Institute

Manufacturing Chemists Association

Monorail Manufacturers Association

Manufacturers Standardization Society of the Valve and Fittings Industry

NAAMM National Association of Architectural Metal Manufacturers 
NACE

NAPCA

NAPHC

National Association of Corrosion Engineers

National Association of Pipe Coating Applicators

NAR

NARM

NBBPV

NBHA

NCPA

NCRPM

NCTA

NEA

NEMA

NESCA

NFLDP

NFORP

NFP

NFPA

NGPA

NHLA

NIOP

NMA

NMTBA

NPA

NRA

NRCA

NRMCA

NSA

NSF

NSPA

NSPI

NWHA

NWMA

National Association of Plumbing-Heating Cooling Contractors

National Association of Rocketry

National Association of Relay Manufacturers

National Board of Boiler and Pressure Vessel Inspectors

National Builder's Hardware Association

National Cottonseed Products Association

National Council on Radiation Protection and Measurements

National Cable Television Association

National Education Association

National Electrical Manufacturers Association

National Environmental Systems Contractors Association

National Fluid Power Association

National Forest Products Association

National Flaxseed Processors Association

National Fire Protection Association

Natural Gas Processors Association

National Hardwood Lumber Association

National Institute of Oilseed Products

National Microfilm Association

National Machine Tool Builders Association

National Particleboard Association

National Rifle Association of America

National Roofing Contractors Association

National Ready Mixed Concrete Association

National Standards Association

National Sanitation Foundation

National Soybean Processors Association

National Swimming Pool Institute

National Wholesale Hardware Association

National Woodwork Manufacturers Association
PPC

PSA

PSTC

RTCA

RTI

$\mathrm{SAE}$

SAMA

SBCC

SDCE

SIGMA

SJI

SMACN

SMI

SMPTE

SNAME

SPI

SSI

STDI

SWA

SWl

TAPPI

TCA

TRA

TTMA

$\mathrm{UL}$

USC

USGA

VESC

VRCI

WCF

WRI

WSC

WSFI

WSTI

WWPA
Paperboard Packaging Council

Photographic Society of America

Pressure Sensitive Tape Council

Radio Technical Commission for Aeronautics

Resilient Tile Institute

Society of Automotive Engineers, lnc.

Scientific Apparatus Makers Association

Southern Building Code Congress

Society of Die Casting Engineers

Sealed Insulating Glass Manufacturers Association

Steel Joist Institute

Sheet Metal and Air Conditioning Contractors

National Association, Inc.

Spring Manufacturers Institute

Society of Motion Picture and Television Engineers

Society of Naval Architects and Marine Engineers

Society of the Plastics Industry, lnc.

Scaffolding and Shoring Institute

Steel Door Institute

Specialty Wire Association

Steel Window Institute

Technical Association of the Pulp and Paper lndustry

Tile Council of America, Inc.

Tire and Rim Association, Inc.

Truck Trailer Manufacturers Association

Underwriters Laboratories, lnc.

U.S. Department of Commerce

U.S. Golf Association

Vehicle Equipment Safety Commission

Variable Resistive Components Institute

Water Conditioning Foundation

Wire Reinforcement Institute

Water Systems Council

Wood and Synthetic Flooring Institute of America

Welded Steel 'Tube Institute, Inc.

Western Wood Products Association

\subsection{Alphabetical by Organization with Addresses}

Air Conditioning and Refrigeration Institute (ARI)

1815 North Fort Myer Drive

Arlington, Virginia 22209

Air Moving and Conditioning Association, Inc. (AMCA)

30 West University Drive

Arlington Heights, Illinois 60004

Air Transport Association of America (A'TAA)

1709 New York Avenue, NW

Washington, D.C. 20006

The Aluminum Association (AA)

750 Third Avenue

New York, New York 10017

American Association of Cereal Chemists (AACCH)

3340 Pilot Knob Road

St. Paul, Minnesota 55121

American Association of Poison Control Centers (AAPCC)

Nebraska Master Poison Control Center

Children's Memorial Hospital

Omaha, Nebraska 68105
American Association of State Highway and

Transportation Officials (ASHTO)

341 National Press Building

Washington, D.C. 20004

American Association of Textile Chemists and Colorists (AATCC)

P.O. Box 12215

Research Triangle Park

North Carolina 27709

American Boat \& Yacht Council, Inc. (ABYC)

15 East 26th Street, Room 1603

New York, New York 10010

American Bureau of Shipping (ABS)

45 Broad Street

New York, New York 10004

American Concrete Institute (ACl)

Box 4754, Redford Station

22400 West Seven Mile Road

Detroit, Michigan 48219 
American Concrete Paving Association (ACPA)

1211 West 22nd Street

Oak Brook, Illinois 60523

American Conference of Governmental Industrial Hygienists (ACGIH)

P.O. Box 1937

Cincinnati, Ohio 45201

American Dental Association (ADA)

211 East Chicago Avenue

Chicago, Illinois 60611

American (jear Manufacturers Association (AGMA) 1330 Massachusetts Avenue, NW.

Washington, D.C. 20005

American Hardboard Association (AHA)

20 North Wacker Drive

Chicago, Illinois 60606

American Institute of Architects (AIOA)

1735 New York Avenue, NW.

Washington, D.C. 20006

American Institute of Steel Construction, Inc. (AISC)

1221 Avenue of the Americas

New York, New York 10020

American Institute of Timber Construction (AITC)

333 West Hampden Avenue

Englewood, Colorado 80110

American Iron \& Steel Institute (AISI)

$100016 \mathrm{th}$ St. NW.

Washington, D.C. 20036

American Ladder Institute (ALI)

111 East Wacker Drive

Chicago, Illinois 60601

American Leather Chemists Association (ALCA)

Tanners Council Research Laboratory

University of Cincinnati

Cincinnati, Ohio 45221

American National Standards Institute, Inc. (ANSI)

1430 Broradway

New York, New York 10018

American Nuclear Society (ANS)

244 East Ogden Avenue

Hinsdale, Illinois 60521

American Petroleum Institute (API)

$1801 \mathrm{~K}$ Street, NW.

Washington, D.C. 20006

American Plywood Association (APA)

1119 A Street

Tacoma, Washington 98401

American Society for Quality Control (ASQC)

161 West Wisconsin Avenue

Milwaukee. Wisconsin 53203
American Society for Testing and Materials (ASTM) 1916 Race Street

Philadelphia, Pennsylvania 19103

American Society of Agricultural Engineers (ASAE)

P.O. Box 229, 2950 Niles Road

St. Joseph, Michigan 49085

American Society of Enologists (ASE)

Box 411

Davis, California 95616

American Suciety of Heating, Refrigerating

and Air Conditioning Engineers (ASHRA)

345 East 47 th Street

New York, New York 10017

American Society of Mechanical Engineers (ASME)

345 East 47 th Street

New York, New York 10017

American Society of Safety Engineers (ASSFE)

850 Busse Highway

Park Ridge, Illinois 60068

American Society of Sanitary Engineering (ASSE)

960 Illuminating Building

Cleveland, Ohio 44113

American Sod Producers Association, Inc. (ASPA)

Garmo Inc., Assuciation Building

9th \& Minnesota

Hastings, Nebraska 68901

American Water Works Assuciation. Inc. (AWWA)

6666 West Quincy Avenue

Denver, Colorado 80235

American Welding Society, Inc. (AWS)

2501 NW., 7th Street

Miami, Florida 33125

American Wood-Preservers Association (AWPA)

1625 Eye Street, NW.

W ashington, D.C. 20006

American Wood Preservers Institute (AWPI)

1651 Old Meadow Road

McLean, Virginia 22101

Anti-Friction Bearing Manufacturers Association, Inc. (AFBMA) 60 East 42 nd Street

New York. New York 10017

Architectural Aluminum Manufacturers Association (AAMA)

410 N. Michigan Avenue, Ste. 960

Chicago, Illinois 60611

Architectural Woodwork Institute (AWI)

5055 South Chesterfield Road

Arlington, Virginia 22206 
Asbestos Textile Institute (ATI)

P.O. Box 471

131 N. York Road

Willow Grove. Pennsylvania 19090

The Asphalt Institute (AI)

Asphalt Building

College Park, Maryland 20740

Association of American Feed Control Officials, Inc. (AAFCO) Box 3160

College Station, Texas 77840

Association of Home Appliance Manufacturers (AHAM)

20 North Wacker Drive

Chicago, Illinois 60606

Association of Official Analytical Chemists (AOAC)

P.O. Box 540

Benjamin Franklin Station

Washington, D.C. 20044

Barley and Malt Institute (BAMI)

P.O. Box 308

Crystal Lake. Illinois 60014

Battery Council International (BCI)

1801 Murchison Drive

Burlingame, California 94010

Bicycle Manufacturers Association of America (BMA)

122 East 42nd Street

New York, New York 10017

Billiard Congress of America (BCA)

717 N. Michigan Avenue

Chicago, Illinois 60611

Book Manufacturers Institute (BMI)

904 Ethan Allen Highway

Ridgefield, Connecticut 06877

Brick Institute of America (BIA)

1750 Old Meadow Road

McLean, Virginia 22101

Builders Hardware Manufacturers Association (BHMA)

60 East 42nd Street

New York, New York 10017

California Redwood Association (CRA)

617 Montgomery Street

San Francisco, California 94111

Canvas Products Association International (CPAI)

600 Endicott Building

St. Paul, Minnesota 55101

Cast Iron Soil Pipe Institute (CISPI)

2029 K Street. NW.

Washington, D.C. 20006

Caster and Floor Truck Manufacturers Association (CFTMA)

1717 Howard Street

Evanston, Illinois 60202
Ceilings and Interior Systems Contractors Association (CISCA)

1201 Waukegan Road

Glenview, Illinois 60025

Chain Link Fence Manufacturers Institute (CLFMI)

One Stone Place, Box 515

Bronxville, New York 10708

Chemical Specialities Manufacturers Association (CSMA) 50 East 41 st Street

New York, New York 10017

Chlorine Institute, Inc. (CHI)

342 Madison Avenue

New York, New York 10017

Composite Can and Tube Institute (CCTI)

1725 Eye Street, NW.

Washington, D.C. 20006

Compressed Gas Association, Inc. (CGA)

500 Fifth Avenue

New York, New York 10036

Concrete Reinforcing Steel Institute (CRSI)

228 North LaSalle Street

Chicago, Illinois 60601

Construction Specifications Institute (CSI)

1150 17th Street, NW., Ste. 300

W ashington, D.C. 20036

Conveyor Equipment Manufacturers Association (CEMA) 1000 Vermont Avenue, NW.

Washington, D.C. 20005

Cooling Tower Institute (CTI)

3003 Yale Street, Suite 107

Homston, Texas 77018

Copper Development Association (CDA)

57th Floor, Chrysler Building

405 Lexington Avenue

New York, New York 10017

Corn Refiners Association (CR)

1001 Connecticut Avenue, NW.

Washington, D.C. 20036

Crane Manufacturers Association of America, Inc. (CMAA) 1326 Freeport Road

Pittsburgh, Pennsylvania 15238

Dairy and Food Industries Supply Association, Inc. (DFISA) Office of the Secretary

$5530 \mathrm{Wisconsin}$ Avenue

Washington, D.C. 20015

Diamond Core Drill Manufacturers Association (DCDMA)

53 East Main Street

Moorestown, New Jersey 08057

Diesel Engine Manufacturers Association (DEMA)

2130 Keith Building

Cleveland. Ohio 44115 
Edison Electric Institute (EEI)

90 Park Avenue

New York, New York 10016

Electrical Apparatus Service Association, Inc. (EASA) 7710 Carondelet Avenue

St. Louis, Missouri 63105

Electronic Industries Association (EIA)

2001 Eye Street, NW

Washington, D.C. 20006

Essential Oil Association of U.S.A., Inc. (EOA)

60 East 42nd Street

New York, New York 10017

Factory Mutual System (FMS)

1151 Boston-Providence Turnpike

Norwood, Massachusetts 02062

The Fertilizer Institute (FI)

1015 18th Street, NW

Washington, D.C. 20036

Fir and Hemlock Door Association (FHDA)

Yeon Building

Portland, Oregon 97204

Fluid Controls Institute, Inc. (FCI)

12 Bank Street

Summit. New Jersey 07901

Gravure Technical Association, Inc. (G'T)

60 East 42 nd Street

New York, New York 10017

Hardwood Plywood Manufacturers Association (HPMA)

P.O. Box 6246

2310 South Walter Reed Drive

Arlington, Virginia 22206

Hoist Manufacturers Institute (HMI)

1326 Freeport Road

Pittsburgh, Pennsylvania 15238

Illuminating Engineering Society (IES)

345 East 47 th Street

New York, New York 10017

Industrial Fasteners Institute (IFI)

1505 East Ohio Building

1717 East Ninth Street

Cleveland, Ohio 44114

Industrial Perforators Association (IPA)

739 Second Street

Milwaukee, Wisconsin 53203

Industrial Truck Association (ITA)

1326 Freeport Road

Pittsburgh, Pennsylvania 15238

Institute of Electrical and Electronics Engineers, Inc. (IEEE)

345 East 47 th Street

New York, New York 10017
Institute of Makers of Explosives (IME)

420 Lexington Avenue

New York, New York 10017

Institute of Printed Circuits (IPC)

1717 Howard Street

Evanston, Illinois 60202

Institute of Scrap Iron and Steel, Inc. (ISIS)

1729 H Street, NW.

Washington, D.C. 20006

Institute of Traffic Engineers (ITE)

1815 N. Ft. Myer Drive Ste. 905

Arlington, Virginia 22209

Instrument Society of America (ISA)

400 Stanwix Street

Pittsburgh, Pennsylvania 15222

Insulated Power Cable Engineers Association (IPCEA)

192 Washington Street

Belmont, Massachusetts 02178

International Air Transport Association (IATA)

500 Fifth Avenue

New York, New York 10036

International Association of Plumbing \& Mechanical Officials (IAPMO)

5032 Alhambra Avenue

Los Angeles, California 90032

International Conference of Building Officials (ICBO)

5360 South Workman Mill Road

Whittier, California 90601

International Mobile Air Conditioning Association, Inc. (IMACA)

6116 North Central Expressway

Dallas, Texas 75206

Joint Industrial Council (JIC)

7901 Westpark Drive

Mclean, Virginia 22101

Juvenile Products Manufacturers Association, Inc. (JPMA)

53 East Main Street

Moorestown, New Jersey 08057

Library Binding Institute (LBI)

50 Congress Street

Boston, Massachusetts 02109

Manufacturers Standardization Society of the

Valve \& Fittings Industry (MSS)

1815 North Fort Myer Drive

Arlington, Virginia 22209

Manufacturing Chemists' Association (MCA)

1825 Connecticut Avenue, NW.

Washington, D.C. 20009

Monorail Manufacturers Association (MMA)

1326 Freeport Road

Pittsburgh, Pennsylvania 15238 
National Association of Architectural Metal Manufacturers (NAAMII)

1010 West Lake Street, Ste. 500

Oak Park, Illinois 60301

National Association of Corrosion Engineers (NACE)

2400 West Loop South

Houston, Texas 77027

National Association of Pipe Coating Applicators (NAPCA) 2504 Flournoy-Lucas Road

Shreveport, Louisiana 71108

National Association of Plumbing-Heating Cooling Contractors (NAPHC)

101620 th Street, NW.

W ashington. D.C. 20036

National Association of Relay Manufacturers (NARM) P.O. Box 1649

Scottsdale, Arizona 85252

National Association of Rocketry (NAR)

1891 Massachusetts Avenue

McLean. Virginia 22101

National Board of Boiler and Pressure Vessel

Inspectors (NBBPV)

1155 North High Street

Columbus, Ohio 43201

National Builder's Hardware Association (NBHA)

1815 N. Fort Myer Drive, Ste. 412

Rosslyn, Virginia 22209

National Cable Television Association (NCTA)

918 16th Street, NW.

Washington. D.C. 20006

National Cottonseed Products Association (NCPA)

P.O. Box 12023

2400 Poplar Avenue

Memphis, Tennessee 38112

Vational Council on Radiation Protection \&

Measurements (NCRPM)

7910 Woodmont Avenue

Suite 1016

Washington, D.C. 20014

National Education Association (NEA)

1201 16th Street, NW.

Washington, D.C. 20036

National Electrical Manufacturers Association (NEMA)

155 East 44 th Street

New York, New York 10017

National Environmental Systems Contractors Association

(NESCA)

1501 Wilson Boulevard

Suite 1101

Arlington, Virginia 22209
National Fire Protection Association (NFPA)

470 Atlantic Avenue

Boston, Massachusetts 02110

National Flaxseed Processors Association (NFP)

P.O. Box 246

Littleton, North Carolina 27850

National Fluid Power Association (NFLDP)

P.O. Box 49

Thiensville, Wisconsin 53092

National Forest Products Association (NFORP)

1619 Massachusetts Avenue, NW.

Washington, D.C. 20036

National Hardwood Lumber Association (NHLA)

59 East Van Buren St.

Chicago, Illinois 60605

National lnstitute of Oilseed Products (NIOP)

111 Sutter Street

San Francisco, California 94104

National Machine Tool Builders Association (NMTBA)

7901 Westpark Drive

McLean, Virginia 22101

National Microfilm Association (NMA)

8728 Colesville Road

Suite 1101

Silver Spring, Maryland 20910

National Particleboard Association (NPA)

2306 Perkins Place

Silver Spring. Maryland 20910

National Ready Mixed Concrete Association (NRMCA)

900 Spring Street

Silver Spring, Maryland 20910

National Rifle Association of America (NRA)

1600 Rhode lsland Avenue, NW.

Washington, D.C. 20036

National Roofing Contractors Association (NRCA)

1515 North Harlem Avenue

Oak Park, lllinois 60302

National Sanitation Foundation (NSF)

NSF Building

Ann Arbor, Michigan 48105

National Soybean Processors Association (NSPA) 1730 Pennsylvania Avenue, NW.

Washington, D.C. 20006

National Standards Association (NSA)

1321 14th Street, NW.

Washington. D.C. 20005

National Swimming Pool Institute (NSPI)

$2000 \mathrm{~K}$ Street, NW.

W ashington, D.C. 20006 
National Wholesale Hardware Association (NWHA)

1900 Arch Street

Philadelphia, Pennsylvania 19103

National Woodwork Manufacturers Assuciation (NWMA)

400 West Madison Street

Chicago. Illinois 60606

National Gas Processors' Association (NGPA)

1812 First Place

Tulsa, Oklahoma 74.103

Paperboard Packaging Council (PPC)

$1800 \mathrm{~K}$ Street, NW., Ste. 600

W ashington, D.C. 20036

Photographic Society of America (PHA)

2005 Walnut Street

Philadelphia, Pennsylvania 19103

Pressure Sensitive Tape Council (PSTC)

1201 Waukegan Road

Glenview, Illinois 60025

Radio Technical Commission for Aeronautics (RTCA)

1771 H Street, NW., Ste. 655

Washington, D.C. 20006

Resilient Tile Institute (RTI)

26 Washington Street

East Orange, New Jersey 07017

Scaffolding and Shoring Institute (SSI)

2130 Keith Building

Cleveland, Ohio 44115

Scientific Apparatus Makers Association (SAMA)

1140 Connecticut Avenue, NW.

Washington, D.C. 20036

Sealed Insulating Glass Manufacturers Association (SIGMA)

202 South Cook Street, Ste. 209

Barrington, Illinois 60010

Sheet Metal \& Air Conditioning Contractors National

Association, Inc. (SMACN)

1611 North Kent Street

Arlington, Virginia 22209

Society of Automotive Engineers, Inc. (SAE)

Two Penusylvania Plaza

New York. New York 10001

Society of Die Casting Engineers, Inc. (SDCE)

16007 West 8 Mile Road

Detroit, Michigan 48235

Society of Motion Picture and Television Engineers (SMPTE)

862 Scarsdale Avenue

Scarsdale, New York 10583

Society of NavaI Architects and Marine Engineers (SNAME)

74. Trinity Place

New York, New York 10006
Society of the Plastics Industry, Inc. (SPI)

250 Park Avenue

New York, New York 10017

Southern Building Code Congress (SBCC)

3617 8th Avenue South

Birmingham, Alabama 35222

Specialty Wire Association (SWA)

1625 Eye Street, NW.

W ashington, D.C. 20006

Spring Manufacturers Institute (SMI)

P.O. Box 959

24. Stearns Street

Bristol, Connecticut 06010

Steel Door Institute (STDI)

2130 Keith Building

Cleveland, Ohio 44115

Steel Joist Institute (SJI)

2001 Jefferson Davis Highway

Arlington, Virginia 22202

Steel Window Institute (SWI)

2130 Keith Building

Cleveland, Ohio 44115

Technical Association of the Pulp and

Paper Industry (TAPPI)

One Dunwoody Park

Atlanta, Georgia 30341

Tile Council of America, Inc. (TCA)

Box 326

Princeton, New Jersey 08540

Tire and Rim Association, Inc. (TRA)

3200 West Market Street

Akron, Ohio 44313

Truck Trailer Manufacturers Association (TTMA)

2430 Pennsylvania Avenue, NW

W ashington, D.C. 20037

Underwriters' Laboratories, Inc. (UL)

207 East Ohio Street

Chicago, Illinois 60611

U.S. Department of Commerce (USC)

Standards Development Services Section

National Bureau of Standards

Washington, D.C. 20234

U.S. Golf Association (USGA)

Golf House

Far Hills, New Jersey 07931

Variable Resistive Components Institute (VRCI)

1717 Howard Street

Evanston, Illinois 60202 
Vehicle Equipment Safety Commission (VESC) 1030 15th Street, NW.

Washington, D.C. 20005

Water Conditioning Foundation (WCF)

1780 Maple Street

Northfield, Illinois 60093

Water Systems Council (WSC)

221 North LaSalle Street

Chicago, Illinois 60601

Western Wood Products Association (WWPA) 1500 Yeon Building

Portland, Oregon 97204
Welded Steel Tube Institute, Inc. (WSTI) 522 Westgate Tower

Cleveland, Ohio 44116

Wire Reinforcemént Institute (WRI)

7900 Westpark Drive

McLean, Virginia 22101

Wood and Synthetic Flooring Institute of America (WSFI) 1201 Waukegan Road

Glenview, Illinois 60025

\section{Stop List}

ABILITY

ABOVE

ABSENCE

ACCEPTING

ACCOMMODATE

ACCORDANCE

ACCORDING

ACCUMULATE

ACQUIRED

ACROSS

ACT

ADD

ADDENDA

ADJACENT

ADJUST

AFFECTING

AFFORD

AFTER

AFTER-FINISHED

AGAINST

AGENCIES

ALL

ALONG

ALPHA

ALSO

ALUMINIUM

AMOUNT

AND/OR

ANSI

ANY

APPENDICES

APPENDIX

APPLICABLE

APPLIED

APPLYING

APPRAISE

APPROXIMATE

APPROXIMATELY

APPROXIMATION

AROUND

ASSIGNMENT

ASSIGNMENTS

ASSOCIATED

ASSURE

ASTM

AVAILABLE

AWAY
BACKWARDLY

BASED

BEFORE

BEHIND

BEING

BELOW

BETWEEN

BLENDED

BODIED

BOTH

BROKEN

BTU

BURNED

BUT

CANNOT

CAPABILITIES

CAPABILITY

CAUSED

CENT

CENTIGRADE
CENTIMETER
CENTIMETERS
CENTRE
CENTRES

CERTAIN

CHANGED

CHIEFLY

CHOICE

CHOOSING

CLASS

CLASSES

CLEANED

COINCIDENT

COLOUR

COLOURS

COMBINED

COMMITTEE

COMMONLY

COMPARABLE

COMPARING

COMPARISON

COMPLEMENT

COMPLIANCE

COMPOUNDED

CONFORMING

CONJUNCTION

CONSIDERED
CONSISTING

CONTEN'T

CON'TENTS

CONVENTIONALLY

COVERAGE

CUBIC

DEFINED

DEFINITE

DEG

DEGREE

DEGREES

DELAYED

DEPLETED

DERIVED

DESIGNATE

DESIGNATED

DESIRABLE

DESIRED

DETAILED

DETERMINE

DETERMINING

DIFFERENT

DIFFICULT

DILUTE

DIRECTLY

DISCUSSION

DISSIMILAR

DIVIDED

DUPLICATE

DURING

EASILY

EDGED

EDGEWISE

EFFECT

EFFECTIVENESS

EFFECTS

EITHER

ENTRY

ENTERING

EQUIVALENT

ESTABLISHMENT

ESTABLISHMENTS

EVACUATE

EVIDENCE

EXAMINE 
EXCEEDING

EXCEP'T

EXCLUDING

EXIS'TEN'T

EXPENDABLE

EXPERIENCE

EXPERIENCES

EXPLANATION

EXPOSED

EX'TAN'T

EXTENDED

EXTENDING

EXTERNALLY

EX'TRA

EXTRACTABLE

EX'TRAC'TED

EXTRAC'TING,

EX'TREME

FLATWISE

FOR

FORMERLY

FOR'TY

FORWARN

FOUR'TH

F' .

FULLY

GENERAL

GIVEN

GIVING

GRAM

GREAT

GREATER

GROUPING

GROUPS

GROW'TH

HALF

HANDLED

HAVE

IIAVINO

HEAVILY

HEIGHT

HEIGHTS

HELD

HIGHLY

HOW

IMMEDIA'TE

IMPERMEABLE

IMPERVIOUS

IMPOR'TANCE

IMPROVED

IMPROVEMEN'T

INCH

IN

IN.

INCLIDE

INCLIDED

INCLUDES

INCIUDING,

INCLUSION

INCI.USIONS

INCLUUSIVE

INCORPORATE

INCREASE

INDIVIDUAL

INFLUENCE
INHIBITED

INNER

INSIDE

INSPEC' $T$

IN'TRINSICALLY

IN'TERIM

INVOLVING;

ISO

ITS

KGF

$\mathrm{KGF} / \mathrm{M}$

KILOCYCLE

KILOCYCLES

KILOHERTZ

KILOVOLT

KILOVOLTS

KILOWA'T'

KILOWATTS

KIND

KVA

K.TWO

LADEN

LAID

LB

LEASED

LEAVE

LESS

LFW

LICENSED

LIKE

LOCA'TED

LOWER

MADE

MAKE

MA'T'TERS

MAXIMUM

MAY

MEDIU Y

MEGAHERTZ

METH.

METH

ME'THOD

ME'THODS

MEV

MHZ

MIL

MILLED

MILLIAMPERE

MIILIME'TER

MIIS

MIN.

MINIMUM

MINIMIZATION

MINIMIZE

MINIMIZING

MODE

MODERATE

MORE

MOST

$\mathrm{MPH}$

NATURE

NEAR

NEEDED

NOT

NOTE

NOTIFICATION
OBSOLE'TE

OBTAIN

OBTAINED

OB'TAINING

$\mathrm{OFF}$

OHMS

ONE-FOUR'TH

ONE-HALF

ONLY

OP'TIONAL

ORDER

ORDERS

ORGANIZED

ORIGIN

OTHER

OUNCE

OUT

OVER

OWN

O.D.

PAR

PAR'TICULAR PAR'TICULARLY

PAST

PAYABLE

PENALTIES

PER

PERCEIVED

PERCENT

PERCENTAGE

PERIOD

PERMANEN'TLY

PERMIT

PER'TAINING

PLEASURE

PLUS

POUND

POUNDS

PRAC'TICE

PRACTICES

PRECAUTIONS

PRECISE

PREMIUM

PREPARED

PRESENCE

PRESENT

PRESENTINC

PREVAILING

PREVIOUS

PRICE

PRIMARILY

PRIMARY

PRINCIPAL

PRIOR

PROGRESS

PROMO'TE

PROPER

PROPERLY

PROPOSAL

PROPOSALS

PROPOSED

PROVIDE

PROVIDED

PSI

PULL

PURPOSE

PURPOSES 
QUADDED

QUADRUPLE

QUARTERING

QUICK

QUICKLY

QUOTATION

REACH

REBOUND

REBUTTED

RECEIVABLE

RECEIVE

RECEIVED

RECKONING

RECOMMENDED

RECOMMENDING

REGARD

REGARDING

RELATED

RELATING

RELATION

REMAINING

\section{REMOTELY}

REMOVABILITY

REPEATED

REPRESENTA'TION

RESIST

RESPECT

RESPONSIBILITIES

RESPONSIBILITY

RESTRAINED

RESULTANT

RESULTS

RE'TAINED

REQUIRE

REQUIRED

SAME

SEPARATED

SEVENTY

SEVERAL

SHOWN

SIMILAR

SINGLY

SIXTEEN'TH

SMALL

SMALLER
SPEC.

SPECIALLY

'TIGH'TLY

SPECIFIC

SPECIFICA'TION

SPECIFICATIONS

SPECIFIED

SPEN'T

STAND

STANDARD

STANDARDIZE

STANDARDS

STATE

STD

STDS

STEPPED

STUDY

STYLE

STYLES

SUBJECTED

SUCH

SUFFICIENTLY

SUGGESTED

SUITABILITY

SUI'TABLE

SUPPLEMENT

SUPPLIED

TAKEN

TAKING

TECH

TENDENCIES

TENTATIVE

TENT

THAN

THAT

THEIR

THEREIN

THEREAFTER

THEREFROM

THESE

THIRD

THIS

THOSE

THROUGH

THRU
TOGE'THER

TONS

TO'TAL

TO'TALLY

TOWARD

TRIPLE

TYPE

TYPES

TYPICAI

UI

UNATTENDED

UNCER'TAIN'TY

UNDER

UNDERS'TANDINC

UNI'T

UPON

USA

USE

USED

USING

USUAL

UNW ANTED

VARIATION

VARIATIONS

VARIOUS

VERY

VERSA

VICE

WATT

WAY

WHEN

WHERE

WHICH

WITH

WHO

WHOLESALE

WITHIN

WITHOUT

WORKED

YD.

ZERO 

r the Pest Control Chemical 4-Chloro5-(Methylamino) -2Std. Meth. of Measurement of the

I Switches for Use in Hazardous Locations: Class I, Groups enclosures for Use in Hazardous Locations, Class I, Groups Equipment for Use in Hazardous Locations, Class I, Groups ilitate, Demolish) (1973) (Cereal Chemistry) (1967) ust Ventilation Systems (19/ edules and Specs. (1961) $\mathrm{g}$ the District of Colu/ xt (1972) Uniform Code for the Index of Refraction, with Rec. Pract. for Definitions,

ber) (1973)

Std. for Identification Codes and Std. for

Uniform Building Code: Definitions and Western Woods Use Book: Std. Rec. Pract. for Internal Temperature Measurements in Liquids (1972)

h. for Determining the Resistance of Photogra he Test Mer Determining Res eum Wax or Wax Based Blends Appli/ 74)

Std. Meth. of Test for Std. Meth. of Test for Std. Meth. of Test for

perature (1972)

eels (1968) ANSI J2.26

Std. Meth. of Test for Std. Meth. of Test for Test Meth. for le MateriaIs: Accelerator Meth. (1970) Std. Meth. of Test for

) (19) Test Meth. for Color Change in Fabrics Due to Flat stnes/ Test Meth. for Color Change of Fabrics Due to Flat of Test for Resistance of Transparent Plastics to Surface Std. Meth. of Chemical Analysis of Aluminum Oxide Std. Meth. of Chemical Analysis of Silicon Carbide Std for Checking the Size of Diamond Std. Test Meth. for Bulk Density of Diamond Std. Spec. for Grading of

Std. for Surfaces of New Steel Airblast Cleaned with Sand Std. Spec for Acrylonitrile-Butadiene-Styrene lvent Cements for Joining Acrylonitrile Butadiene Styrene 1973/ Std. Spec, for Acrylonitrile-Butadiene-Styrene or Solvent Cement for Acrylonitrile-Butadiene-Styrene spec. for Socket Type Acrylonitrile-Butadiene-Styrene d. Spec. for Threaded Acrylonitrile-Butadiene-Styrene spec. for Socket Type Acrylonitrile-Butadiene-Styrene Std. Spec. for Acrylonitrile-Butadiene-Styrene Std. Spec. for Acrylonitrile-Butadiene-Styrene Std Spec, for Acrylonitrile-Butadiene-Styrene K65.57 Std. Spec. for Acrylonitrile Butadiene Styrene 72.4 Std. Spec. for Rigid Acrylonitrile-Butadiene-Styrene Std. Meth. for

and Gamma Rays (196I)

ic Sulfate Dosimeter (1971) ANSI/ rous Sulfate Cupric Sulfate Dosim/ 1972)

Std. Meth of Test for Rec. for Measurement of Std. Meth. of Test for Std. Meth. of Test for Std. Test Meth. for Procedure for Wate Test Meth. for Meth. for Ink

\section{Is (Packaging) (1964) ANSI /}

s (1972)

ups in Rubber Extender and Processing Oils by the Clay Gel i K90.I0 Std. Meth

n Mixes, Beadlets, Oils, and Emulsions (Cere) Ultraviole

Ultraviolet eth. of Test for Trace Metals in Gas Turbine Fuels (Atomic nstructions (1970) Std. Meth. of Test for Specific Gravity and Std. Test Meth. for Water Std. Meth. of Test for Specific Gravity and Std. Meth of Test for Oi (2) Std. Meth. of Test for W ater Std. Meth. of Test for Lead in Gasoline by Atomic Std. Meth. of Test for Aluminium in lron Ores by Atomic of Test for Calcium and Magnesjum in Iron Ores by Atomic $\operatorname{dex}(j \quad$ Std. Rec. Pract. for Identification of Material by Std. for Gas Fired

test Meth. for Water Repellency of Textile Fabrics: Static

or Water Repellency of Textile Fabrics: Tumble Jar Dynamic Std. Meth. of Painting Ceiling Materials for Acoustical I962) Table for Converting ts: Approximate Corrections for Changing as-is Farinograph Std. Meth. of Test for Water

Meth. for Evaluation of White Mineral Oils by Ulraviolet of Burn/ Std. Meth. of Test for Apparent Porosity, Water nt Specific Gravity of Fire/

b Test) (1973) Std. Meth. of Test for $W$ ater Std. Meth. of Test for Water Std. for Writing

ation of Uranium and Plutonium Concentrations and Isotopic y Stranded Aluminum Conductors, Aluminum Alloy Reinforced
(a,a,a-Trifluoro-m-tolyl) $\cdot 3$ (2H)Pyridazinone Norfluraz

A.D Dimension of Aerosol Valve Dip Tubes (I972)

A-D, and Class Il, Groups E-G (I972) ANSI C33.29

A.D, and Class II, Groups E-G (1973)

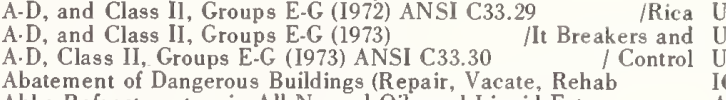

Abbe Refractometer, in All Normal Oils and Liquid Fats

Abbreviations and General Principles of Industrial Exha

Abbreviations and Symbols Used in Builders Hardware Sch

Abbreviations for States of the United States (Includin

Abbreviations for Use on Drawings and in Text (1972) Te

Abbreviations (1973)

Abbreviations, Symbols, and Terminology (Lumber and Tim

Ablation (1972)

Ablative Materials (1968)

Aboveground Storage Tanks for Flammable and Combustible

Abrasion During Processing (1972)

Abrasion of Automotive Bodycloth (1972)

Abrasion Resistance of Glossy Smooth Coatings of Petrol

Abrasion Resistance of Horizontal Concrete Surfaces (19

Abrasion Resistance of Pipeline Coatings (1972)

Abrasion Resistance of Refractory Materials at Room Tem

Abrasion Resistance of Rubber Compounds for Soles and $\mathrm{H}$

Abrasion Resistance of Textile Fabrics and Other Flexib

Abrasion Resistance of Textile Fabrics (1971)

Abrasion (Frosting): Every Meth. (Textile Colorfastness

Abrasion (Frosting): Screen Wire Meth. (Textile Colorfa

Abrasion (1973) ANSI K65.72

Abrasive Grain and Crude (1971)

Abrasive Grain and Crude (1971)

Abrasive Grain (1971)

Abrasive Grains (1973)

Abrasive Microgrits (1972)

Abrasive (I970)

(ABS) Composite Sewer Piping (I974)

(ABS) Pipe and Fittings to Poly(vinyl Chloride) (PVC) Pi

(ABS) Plastic Drain, Waste, and Vent Pipe and Fittings (

(ABS) Plastic Pipe and Fittings (1973) ANSI B 72.23

(ABS) Plastic Pipe Fittings, Schedule 40 (1973) ANSI K65

(ABS) Plastic Pipe Fittings, Schedule 80 (1973) ANSI K65

(ABS) Plastic Pipe Fittings, Schedule 80 (1973) ANSI K65

(ABS) Plastic Pipe (SDR-PR) (1973) ANSI B72.3

(ABS) Plastic Pipe, Schedules 40 and 80 (1973)

(ABS) Plastic Sewer Pipe and Fittings (1973) ANSI K65.59

(ABS) Plastic Utilities Conduit and Fittings (1972) ANSI

(ABS) PIastics (1973) ANSI K65.205

Absolute Calibration of Reflectance Std. (1971) ANSI Z1

Absolute Viscosity of Asphalts (1966)

Absorbed Dose of Neutrons, and of Mixtures of Neutrons

Absorbed Gamma and Electron Radiation Dose with the Cer

Absorbed Gamma and Electron Radiation Dose with the Fer

Absorbed Gamma Radiation Dose in the Fricke Dosimeter (

Absorben cy of Bibulous Papers (1972)

Absorbency of Bleached Woven Cloth (1972) ANSI L14.115

Absorbency of Blotting Paper (1972)

Absorbing Characteristics of Package Cushioning Materia

Absorbing Properties of (Sports) Playing Surface System

Absorption Chromatographic Meth. (1973) ANSI Z1I.180

Absorption Cross Section of Nuclear Graphite (1971) Ans Absorption Meth. of Analysis of Vitamin A in Concentrat Absorption Meth. of Analysis of Vitamin A in Dry Vitami Absorption Meth) (1972) ANSI ZII 3I7

Absorption of Coarse Aggregate (1973) ANSI A37.5

Absorption of Core Materials for Structural Sandwich Co

Absorption of Fine Aggregate (1973) ANSI A37.6

Absorption of Pigments by Gardner-Coleman Meth. (1968

Absorption of Rigid Cellular Plastics (1969E) ANSI K65.

Absorption Spectrometry (1973)

Absorption Spectroscopy (1973)

Absorption Speciroscopy (1973)

Absorption Spectroscopy, Using the ASTM Punched Card in ASTM

Absorption Summer Air Conditioning Appliances (1973)

Absorption Test (1972) ANSI L14.61, ASTM D583

Absorption Test (1972) ANSI L14.87, ASTM D583

Absorption Tests (1973)

Absorption to $14.0 \%$ Moisture Basis (Cereal Chemistry) (

Absorption to $14.0 \%$ Moisture Basis (Constant Dough Weig

Absorption (Static) of Leather (1970) ALCA E30

Absorption (1973) ANSI Z11.210

Absorption (1974)

Absorption, Apparent Specific Gravity, and Bulk Density

Absorption, Bulk Density, Apparent Porosity, and Appare

Absorptiveness of Nonbibulous Paper and Paperboard (Cob

Abstracts (1971)

Abundances (1970) ANSI N115

(Acar, EC/6201) (1972) ANSI C7.65

Eth of Test for Determin IC. for Concentric La ASTM
ANSl

ASTM

698

UBC*6

ACGlH *1

NBHA

ANSI

ANSI

WWPA

ASTM

UL

ANSI

SAE

ASTM

ASTM

ASTM

ASTM

AATCC

ASTM

AATCC

AATCC

ASTM

ANSI

ANSI

NACE

ASTM

ASTM

ASTM

1. F ASTM

ASTM

ASTM

ASTM

ASTM

ASTY

ASTM

STM

NCRPM

ASTM

ASTM

TAPPI

AATCC

ASTM

ASTM

ASTY

ASTM

AACCH

std. M ASTM

ASTM

ASTM

ASTM

ANSI

Meth. F AATCC

70

AACCH 82.21

AACCH $54-29$

ASTM D 1815

Std. ASTM D2269

ASTM D235?

ASTM

ASTM

ASTM

C20

C373

Z39.14

E267

B524 
National Bureau of Standards KWIC Index of Engineering Standards

14.1 (1967) ANSI J5.1 essure Meth. (1973) ANSI J4.1 973) est Specimens (1974) ing (1972) A/ Test Meth for Colorfastness of Textiles to Services by Means of Small Size Specimens/ of Woods (1970) ide to Be Used Std. Specs, and Tests for Strain Gage Linear rec. for Operation and Maintenance of the Reliable Model B Rec. for Shielding for High Energy Electron esistance of Textile Fabrics and Other Flexible Materials: Rec. for Operation of Grimes AB Dry Pipe Valve for Linear, Single Axis, Pendulous, Analog Torque Balance Chromium Compounds (Air Contamination-Occupa/ luorocarbon 12) (1974)

lkyl Organo Compounds (1972) places (1974)

uorocarbon 11) (1973)

luoroethane (Fluorocarbon 1!3) (1973)

nt as Received from Manufacturer (1969) ANSI C/ Guide for Steel Door and Frame Assemblies (19/ Std. Test Proc. and and Frame Assemblies (1972) Construction (1974) ity Sampling (1969) ANSI Z1.12 grade (Nonreflux Me) Interim Guideline for Protection and Std. for Std. Std. for Std. for Std. for Std. Std. 西 Std. Meth. of Test for Nonamine Acid Std. Definitions and Symbols for Rec. Std. Minimum Test Meth. for Carpet (Rug) Soiling; mechanical Security Surveillance Sys/ Std. for Safety for power Cylinders (1972) ANS1 B93.29 Std. Dimensions for

code Std. for Metal, Wire and Wire Fabric Laths, and Metal Std. for Electrical Feeder and Plug in Busways and de for High Voltage Air Switches, Bus Supports. and Switch td. for Safety for Refrigerant-Containing Components and and Containers (1972) Billiard Tournament Table and Rec. Pract. for Sid. Spec. for pecial Signs (Symbols) Complementary to ANSI Z35.1-1972. ata Derived from the lnvestigation of $\mathrm{Scl}$ Std. Underwater ision Deformation (Damage) Classification (Highway Vehicle meth. of Recording and Measuring the Off-The-Job Disabling ion of Data Derived from the Investigation of Scuba Diving Guide Spec. for Std. for Analytical Procedures for Std. for Analytical Procedures for Architect's td. for Auditing Nuclear Materials Statements and Reports igurement of Paint Films by Fungal Growth or Soil and Dir ater/ Std. Rec. Pract. for Use of the Terms Precision and Std. Meth. of Test for Spectral Bandwidth and Wavelength

972) Std. for Sid. Spec. for Glass Reinforced and Feedstuffs (Cereal Chemistry) (1962) (1973) ANSI B72.12 ANS1 B72.4 gs (1973) A/ Colorimetric Std. Spec. for Cellulose Std. Spec. for Cellulose Meth of Test. Cellulose Acetate Propionate and Cellulose Std. Spec. for A myl Std. for Sodium Determination by the Uranyl Zinc Std. Meth. of Test. Cellulose Safe Handling and Use of Ethyl Std. Meth. of Test. Cellulose Std. Spec. for Normal Propyl Sid. Spec. for Normal Butyl Spec, for lsopropy Rapid (Magnesium Std. Meth. of mistry) (1962) ies and Essential lnformation for Safe Handling and Use of de in Flour Bleaching Experiments in Laboratories (Cerea
Meth. of Analysis for chemistry) (1962) Van Dame Meth. of Analysis for Formic, ic Acids (Cereal) Std. for cereal Chemistry) (1967) Meth. of Analysis of ) (1971) Quality of Extruded Poly (Vinyl Chloride) Plastic Pipe by Std. for Acceptable Concentrations of Std. Spec. for

age and Handling of Flammable Liquids (Gasoline, Kerosene, Std. Analytical Meth. for Determining Safety Std. for and Cutting) (1973) Safety Std. for
Accelerated Aging of Sulfur Dyed Textiles (1972) ANSI L Accelerated Aging of Vulcanized Rubber by the Oven Meth Accelerated Aging of Vulcanized Rubber by the Oxygen $\mathrm{Pr}$ Accelerated Aging of Wood Sash Face Glazing Compound (1 Accelerated Curing, and Test. of Concrete Compression T Accelerated Domestic and Commercial Washing and Launder Accelerated Evaluation of Wood Preservatives for Marine Accelerated Laboratory Test of Natural Decay Resistance (Accelerated Meth.) (1974)

Acceleration of Concrete, Curing of Concrete, and Other Acceleration Transducers (Instrumentation) (1971)

Accelerator for Dry Pipe Systems (Fire Protection) (197

Accelerator lnstallations (1964)

Accelerator Meth. (1970)

Accelerator (1973)

Accelerometer (1972)

Test Meth. for Abrasion

Acceptable Concentration of Chromic Acid and Hexavalent

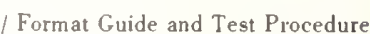

Format Guide and Test Procedure

Acceptable Concentrations of Acetone (1971)

Acceptable Concentrations of Dichlorodifluoromethane ( $F$

Acceptable Concentrations of Hydrogen Sulfide (1972)

Acceptable Concentrations of Inorganic Mercury and Nona

Acceptable Concentrations of Toluene in the Air of Work

Acceptable Concentrations of Trichlorofluoromethane (Fl

Acceptable Concentrations of 1,1,2-Trichloro-1,2,2trif

Acceptance and Maintenance of lnsulating Oil in Equipme

Acceptance Criteria for Rate of Air Flow Through Closed

Acceptance Criteria for Water Resistance on Steel Door

Acceptance of Concrete Pavements Exposed to Rain During

Acceptance of Evidence Based on the Results of Probabil

Acceptance of Halogenated Organic Solvents, Degreasing

Acceptance Sampling by Attributes (1971) ANSI Z1.6

Acceptance Values for Steel Doors and Frames (1972)

Accerated Meth. (1973) ANS1 L14.217

Access Control System Units (Electronic / Electrical /

Accessories for Cataloged Square Head Industrial Fluid

Accessories (Designed for Use as a Plaster Base) (1973)

Accessories (1972)

Accessories (1972)

Accessories, Nonelectrical (1973)

Accessory Equipment Specs. (1971)

Accident Prevention Signs for Display on Tank Trailers

Accident Prevention Signs (1972)

Accident Prevention Signs (1973)

ccident Report Form (Acquisition and Codification of D Rec. Pract. for Coll SAE

Accident) (1972)

Accidental Injury

Accidents) (1973)
Accordion Folding Partitions (1972)

Accountability of Uranium Hexafluoride (1972)

Accounting Forms (1972)

(Accounting) (1973)

Accumulation (1973)

Accuracy as Applied to Measurement of a Property of a M

Accuracy of Fluorescence Spectrometers (1972)

Acephate (Common Name for the Pest Control Chemical) (1

Acetal Plastics for Molding and Extrusion (1972)

Acetate Ashing Meth. of Analysis of Phosphorus in Feeds

Acetate Butyrate (CAB) Plastic Pipe (SDR-PR) and Tubing

Acetate Butyrate (CAB) Plastic Pipe. Schedule 40 (1973)

Acetate Butyrate (CAB) Plastic Pipe, Tubing, and Fittin

Acetate Butyrate (1972)

Acetate Made from Fusel Oil (85 to 88\% Grade) (1972)

Acetate Meth. Pulp and Paper) (1973)

Acetate Propionate and Cellulose Acetate Butyrate (1972

Acetate (Chemical Safety) (1972)

Acetate (1972) ANS1 K65.24

Acetate (90 to $92 \%$ Grade) (1972)

Acetate (98\% Grade) (1972)

Acetate (98\% Grade) (1972)

Acetate) Meth. of Analysis for Ash in Flour (Cereal Che

Acetic-Acid Salt Spray (Fog) Test. (1974) ANSI Z118.2

Acetic Acid (1973)

Acetic Anhydride Meth. for Generation of Chlorine Dioxi

Acetic, Butyric, and Lactic Acids in Rye Flour (Cereal

Acetic, Propionic, Butyric, Valeric. Succinic, and Lact

Acetochlor (Herbicide) (1971)

Acetone-Insoluble Lecithin in Soybean and Corn Oils (

Acetone Immersion) (1967) ANSl B72.9

Acetone Peroxides in Milling Premixes (Cereal Chemistry

Acetone (1971)

Acetone (99.5 (Grade) (1972)

Acetone, Alcohol, Cleaning Fluids, Flammable Aerosols,

Acetyl Content of Corn Starch (1968)

Acetylene Generators, Portable, Medium Pressure (1973)

Acetylene Generators, Stationary, Low Pressure (Welding
AATCC 26

ASTM D573

ASTM D572

ASTM C741

ASTM C684

AATCC 61

ASTM D2481

ASTM D2017

ASTM D2274

ASTM D98

ISA $\quad$ S 37.5

FMS 2.55

NCRPM R3

AATCC 9.3

$\begin{array}{ll}\text { FMS } & 2.56\end{array}$

IEEE 337

ANSI $\quad$ Z37.7

ANS1 Z37.32

ANSl Z37.38

ANS1 Z37.2

ANSl Z37.8

ANSI Z37.12

ANS1 Z37.36

ANSl $\quad$ Z37.35

IEEE 64

STD1 116

STD1 115

ACPA TBl7

ASTM E141

ASTM D2942

ASQC A2

ACC 123

UL 294

NFLDP T3.6.8

ICBO UBCS47-4

NEMA BU1

ANS1 C 37.32

UL 207

BCA *1

TTMA RP35

ANS1 Z35.1

Z35.4

Z86. 2

$\mathrm{J} 224 \mathrm{~A}$

Z16.3

786.2

CSl 10623

ANSl N15.7
ANSI

AlOA F700

ANSI N15.11

ASTM D3274

ASTM E177

ASTM E388

ANSI K62.120

ASTM D2948

AACCH 40.55

ASTM D2446

ASTM D1503

ASTM D2560

Std. ASTM

ASTM D554

TAPPI T623

ASTM D817

MCA SD5

ASTM D871

ASTM D3130

ASTM D3126

ASTM D3131

$\mathrm{AACCH} \quad 08.02$

ASTM B287

Propert MCA SD4l

A ACCH $48-30$

AACCH $\quad 04-20$

A ACCH 04-23

ANSI K62.108

A ACCH $58-35$

ASTM D2152

AACCH 48.05

ANSI $\quad$ Z37.32

ASTM D329

1CBO UBCS10-1

CR C.2

UL 297 
ing and Cutting) (1973) niform Fire Code: Welding and Cutting, Calcium Carbide and sing Grade (Nonreflux Me Std. Meth. of Test for Nonamin earing Materials When Suitable Amounts of Sample, Sulfuric earing Materials When Suitable Amounts of Sample, Sulfuric earing Materials When Suitable Amounts of Sample, Sulfuric on-Occupal Whentration of Chromic Std. Analytical Meth. for Determining Lactic Std. Analytical Meth. for Determining Lactic Std. Meth. for Analysis of Sulfuri y) (1973)

atter in Fats and Shortenings (Butter Oleomargarine, High and Rodent Hairs in Flour (Cereal Chemistry) (1962) Std. Spec. fo Analysis of Starch in Flour and Semolina (With Subsequent (1962) (1962)

(1962)

(1962)

Std. Meth. of Test for Isophthali Qualitative Meth. of Analysis for Phosphoric Quantitative Meth. of Analysis for Phosphoric Meth. of Analysis for Sulfuric Meth. of Analysis for Tartaric Meth. of Analysis for Total Phosphoric Meth. of Analysis of Lipoid Phosphoric Meth of Analysis for Lactic

Meth. for Determination of Arsanilic ntitative Determination of Both Nitrophenide and Arsanilic Meth. of Analysis for Benzoic Meth. of Analysis for Lactic ualitative Meth. of Analysis for Free or Combined Tartaric Tent. Meth. of Analysis for Volatile Official Meth. of Analysis for Tota ice (1974) Std. for Determination of Capacity of Lead sodium Acid Pyrophosphate in Prepared Mixes (/ Perchloric $s$ of Crude Protein in Wheat and Flour Mill Products (Boric

r Determining Chromic Oxide Content in Leather (Perchloric td. Meth. of Test for Chromic Oxide in Leather (Perchloric d Meth. of Analysis of Monocalcium Phosphate and/or Sodium Meth. of Analysis for Neutralizing Value of Sodium emistry) (1) Meth. of Analysis for Neutralizing Value of erating Performance of Anion Exchange Materials for Strong ry) (1962)

Type Glass (1972T) Std. Meth. of Acetic Meth. for Colorimeter or Spectrophotometer Analysis o

Meth. for Preparation and Standardization of Hydrochloric

Meth. for Preparation and Standardization of Lactic Meth. for Preparation and Standardization of Sulfuric or Weight Loss of Sheet Steel During Immersion in Sulfuric Std Analytical Meth for Determining Sulfuric Number of Petroleum Products by Potentiometric Perchloric ats, Tractors, Automotive and Industrial A/ Std. for Lead Meth. of Analysis of Pantothenic

dophenol-Xylene Extraction Meth of Analysis of Ascorbic

Ethylenediaminetetraacetic (Ethylenedinitrilotetraacetic)

Essential Information for Safe Handling and Use of Acetic

d. Including Guarantee Policy for Motor Vehicle Type (Lead Std. Spec. for Photographic Grade Hydrochloric for Automated Separation and Collection of Particulate and their Admixtures (1974) ducts Obtained Fro/ Seth. Of Test for Std. Analytical Meth. for Determining Std. Analytical Meth. for Determining Std. Analytical Meth. for Determining Tent. Meth. of Test for Total Rapid Meth. of Analysis for Fat General Meth. of Analysis for Fat Rapid Meth. of Analysis for Fat Colorimetric Meth. of Analysis for Fat Meth. of Analysis for Titratable and Similar Industrial Aromatic / Std. Meth. of Test for

5) roducts) (1955) rine $\mathrm{Oi} /$ td. Analytical Meth. for Determining Std. Analytical Meth. for Determining Test Meth. for Colorfastness of Yarn or Fabric to Std. Meth. of Test for Total Rosin meth. for Preparation of Methyl Esters of Long Chain Fatty Meth. of Analysis of Free Fatty
Acetylene Generators, Stationary, Medium Pressure (Weld Acetylene (1973)

Acid Acceptance of Halogenated Organic Solvents, Degrea Acid and Catalyst Are Employed (1960)

Acid and Catalyst Are Employed (1962)

Acid and Catalyst Are Employed (1962)

Acid and Hexavalent Chromium Compound

Acid and Salts Content in Feedstuffs (Corn) (1964)

Acid and Salts Content in Steepwater (Corn) (1962)

Acid as Used in Pulp and Paper Manufacture (1973)

Acid Coconut Oil, etc.) (Cereal Chemistry) (1962)

Acid Composition by Gas Chromatography (Cereal Chemistr

Acid Copper Chromate (1971)

Acid Hydrolysis Meth. of Analysis for Insect Fragments

Acid Hydrolysis) (Cereal Chemistry) (1962)

Acid in Alkyd and Polyester Resins (1973)

Acid in Baking Powders and Chemicals (Cereal Chemistry)

Acid in Baking Powders and Chemicals (Cereal Chemistry)

Acid in Baking Powders and Chemicals (Cereal Clemistry)

Acid in Baking Powders and Chemicals (Cereal Chemistry)

Acid in Dried Eggs (Cereal Chemistry) (1962)

Acid in Dried Eggs (Cereal Chemistry) (1962)

Acid in Dried Geeg (Cereal Chemistry) (1962)

Acid in Feeds (Cereal Chemistry) (1962)

Acid in Feeds (Cereal Chemistry) (1962)

Acid in Flour (Cereal Che mistry) (1962)

Acid in Nonfat Dry Milk (Cereal Chemistry) (1962)

Acid in Presence of Phosphates (Cereal Chemistry) (1962

Acid in Wine (Enology) (1972)

Acid in Winc (Enology) (1972)

Acid Industrial Storage Batteries for Motive Power Sery

Acid Meth. of Analysis of Monocalcium Phosphate and/or

Acid Modification) (Cereal Chemistry) (1962)

Acid Oxidation) (1969) ASTM D2807

Acid Oxidation) (1972)

Acid Pyrophosphate in Prepared Mixes (Cereal Chemistry)

Acid Pyrophosphate (Cereal Chemistry) (1962)

Acid Reacting Materials Other Than Phosphates (Cereal C

Acid Removal (1972)

Std. Meth. of Test for Op

Acid Residues in Grains and Dried Foods (Cereal Chemist

Acid Resistance of Ceramic Decorations on Arrchitectural

Acid Resistance of Ceramic Decorations on Returnable Be

Acid Salt Spray (Fog) Test. (1974) ANSI Z118.2

Acid Soluble Manganese (Cereal Chemistry) (1962)

Acid Solution (Cereal Chemisiry) (1962)

Acid Solution (Cereal Chemistry) (1962)

Acid Solution (Cereal Chemistry) (1962)

Acid Solution (1971) ANSI Z167.25

Acid Solutions Content of Reagents and Indicators (197

Acid Type Storage Batteries for Motor Vehicles, Motorbo

Acid (Cereal Chemistry) (1962)

Acid (Cereal Chemistry) (1962)

Acid (EDT A) and Its Salts $\left(\mathrm{C}_{10} \mathrm{H}_{16} \mathbf{N}_{2} \mathrm{O}_{\mathrm{K}}(1973)\right.$

Acid (1973)

Acid) Storage Batteries (1972)

Acid, HCI (1972)

Acidic Gaseous Fluoride in the Atmosphere (Double Paper Acidity Alkalinity of Halogenated Organic Solvents and

Acidity Content in Steepwater and Most Other Liquid Pro

Acidity Content of Corn Sugar (Crude and Refined) (1958

Acidity Content of Corn Syrup and Starch Hydrolyzates (

Acidity in Aviation Turbine Fuel (1973)

Acidity in Corn (Cereal Chemistry) (1962)

Acidity in Grain and Flour (Cereal Chemistry) (1962)

Acidity in Grain (Cereal Chemistry) (1962)

Acidity in Grain (Cereal Chemistry) (1962)

Acidity in Nonfat Dry Milk (Cereal Chemistry) (1962)

Acidity of Benzene, Toluene, Xylenes, Solvent Naphthas,

Acidity (Extractable) Content in Corn Starch (1955)

Acidity (Extractable) Content in Feedstuffs (Corn) (195

Acidity (Paste) Content in Corn Starch (Gelatinizable P

Acids and Alkalis (1972) ANSI L 14.2

Acids Content in Corn Oil and to Other Vegetable and $\mathrm{Ma}$ Acids Content of Coating Vehicles (1973)

Acids in Common Fats, Oils, and Fatty Acids (Cereal Che

Acids in Fats (Cereal Chemistry) (1967)

Acids in Fruit and Its Products (Cereal Chemistry) (196

Acids in Rye Flour (Cereal Chemistry) (1962)

Acids in Solvent Type Paints (1972)

Acids Used in Protective Coatings (1972)

Acids (Cereal Chemistry) (1962)

Acids (Cereal Chemistry) (1962)

Acids (Cereal Chemistry) (1973)

Acids (Wood, Pulp, etc.) (1973)

Acids (1972) ANSI Z107.4
UL

IC BO

ASTM

CR

CR

ANS

CR

44.30

AACCH 58.18

ASTM D 1624

AACCH 28-41

ACCH 76-10

ASTM D2690

AACCH $04-10$

$A A C C H$ 04-11

AACCH 04-14

AACCH 04.27

AACCH $04-13$

AACCH 20-02

AACCH $04-25$

$\mathrm{AACCH} \quad 18-20$

$\mathrm{AACCH} \quad 18.26$

$\mathrm{AACCH}$ 04.-21

$\mathrm{AACCH} \quad 04-26$

$\mathrm{AACCH} \quad 04.28$

ASE *11

ASE

NEMA IB2

AACCH 40-51

Analysi AACCH 46-12

Meth. Fo ALCA D10

ASTM D2807

$\mathrm{AACCH} 40-51$

AACCH $02-34$

$A A C C H \quad 02.32$

DSTM 3087

AACCH $60-20$

ASTM C724

ASTM C735

ASTM B287

AACCH $40-45$

$\triangle A C C H \quad 70-20$

$\triangle A C C H \quad 70-30$

AACCH $70-80$

ASTM C694

CR R-30

ASTM D2896

SAE J537H

AACCH $86-60$

in $\mathrm{AACCH} 86.10$

ANSI PH4.185

$\mathrm{MCA}$ SD41

BCI *1.15

ANSI PH4.104

ASTM D3266

ASTM D2989

$\mathrm{J}$-4

F-2

CR E-2

ASTM D3242

$\mathrm{AACCH} \quad 02-03$

$\triangle A C C H \quad 02-01$

$\mathrm{AACCH} \quad 02-02$

$\mathrm{AACCH} \quad 02-04$

$A A C C H \quad 02-31$

ASTM D847

CR B. 2

CR G-2

CR B-4

AATCC 6

CR H-22

ASTM D 1469

$\mathrm{AACCH}$ 58-17

AACCH $58-16$

AACCH $04-22$

AACCH $04-20$

ASTM D2245

ASTM Dl467

Meth. of Ana AACCH 04.24

/ Analysis for Formic, AACCH 04-23

/Tion of Methyl Esters AACCH 58-17

$\begin{array}{ll}\text { TAPPI } & \text { UM- } 243 \\ \text { ASTM } & \text { D } 2748\end{array}$ 
Std. Meth. of Test for Gel Time of Tar i.Cr.Fe Alloys to Stress Corrosion Cracking in Polythionic pract. for Handling and Proper Usage of Inhibited Oilfield (1962) Meth. of Analysis of Apparent Viscosity of Std. for Std. for Stub

Std. Dimensions for Flareless Std. for Procedures for Calibration of Underwater Electro Std. Meth. of Painting Ceiling Materials for test. Duct Liner Materials and Prefabricated Silencers for Spec. for

semblies (1972)

Std. Symbols and Definitions for Mechanical and Std. Meth. of Test for Airflow Resistance of

s (1973)

Std. Definitions of Terms Relating to niform Building Code Std. for MetaI Suspension Systems for on for Determination of Sound Transmission Class (Building g. Wall) (1972) Western Woods Use Book: Sound Control meth. for the Measurement of Sound Pressure Levels in Air ound Power Levels of Small Sources in Reverberation Rooms Uniform Building Code: Sound Transmission Control Std. for Psychoacoustical Terminology Structural Clay

nvestigation of $\mathrm{Sc} /$

Std. Underwater Accident Report Form (1972) ANSI S6./

Rec. Pract. for Qualifying a Sound Data

head, Close Tolerance, Short Thread, TWI Wing Recess, A286 st for Methyl Ether of Hydroquinone in Colorless Monomeric er Piping (1974) Waste, and Vent Pipe and Fittings (1973/ Std. Spec. for and Fittings (1973) An/

(SDR.PR) (1973) ANSI B72.3

fittings, Schedule 80 (1973) Ans!

fittings, Schedule 40 (1973) /

fittings, Schedule 80 (1973) /

Schedules 40 and $80(1973)$

Pipe and Fittings (1973) ANSI K65.59

3) ANSI K65.205

ipe and Fittings (1971)

$d$ Vent Pipe and Fitting/

to Poly(Viny/

Std. Spec. for Solvent Cement for Std. Spec. for

Std. Spec, for Threaded Std. Spec. for Socket Type Std. Spec. for Socket Type Std. Spec. for Std. Spec, for Std. Spec. for Rigid Std. for lnstallation for Conduit and Fittings (1972) ANSI K65.57 Meth. for Evaluation of and Test Formula for Butadiene Safe Handling and Use of steel Core Wire for Aluminum Conductors, Steel Reinforced nded Steel Core for Aluminum Conductors, Steel Reinforced num Conductors, Concentric Lay Stranded Steel Reinforced, steel Core Wire for Aluminum Conductors, Steel Reinforced Std. for Pin, Quick Release, Positive Locking, Single Std. for Pin, Quick Release, Positive Locking, Double

1972)

8)

Std. Meth. for Determining the Rec. Pract. for the Safe Safety Std. for Dry Pipe, Deluge, and Pre Std. Definitions of Terms Relating to

d. Meth. of Test for Oxygen Content Using a $14 \mathrm{MeV}$ Neutron Std. Guide for Selection of Neutron rocedure (1974) Std. Meth. of Test for Synthetic A nionic ) Meth. of Analysis of Proteolytic Activity of Flour and rophotometric Meth. of Analysis of Proteolytic Activity in Colorimetric Meth. of Analysis of Proteolytic Activity in f Test for Fast Neutron Flux by Analysis of Molybdenum .99 istr/ Spectrophotometric Meth. of Analysis of Proteolytic istry) (19/ Colorimetric Meth. of Analysis of Proteolytic 1 Chemistry) (1962) $\quad \begin{array}{r}\text { Meth. of Thiosulfate-Maltose (Diastatic } \\ \text { Meth. of Analysis of Residual Urease }\end{array}$ Rec. Pract. for Measurement of Low Level Pressuremeter Meth of Analysis of Diastatic Volumetric Meth. of Analysis of Diastatic 25 Test Meth. for Detection of Antibacterial ereal Chemistry) (1962) Meth. of Analysis of Proteolytic ) (1962)

Meth. of Analysis of Diastatic Meth. of Analysis of Diastatic Meth. of Analysis of Lipoxidase Meth. of Analysis of Diastatic Meth. of Analysis of Alpha Amylase Meth. of Analysis of Proteolytic Meth. of Analysis of Lipoxidase

62) Electrometric Meth. of Analysis for Hydrogen lon Colorimetric Meth of Analysis for Hydrogen Ion Meth. of Analysis of Alpha Amylase Safety Requirements for Powder n, Mechanically Operated Type for I/ Safety Std. for Heat Std. for Safety for Manually Std. for Safety for Electrically t Products (Wheat, Flour, Bran, Shorts, etc.) to Determine Qualitative Meth of Analysis of
Acids (1972) ANS1 Z78.23

Acids (1973) Acids (1973) API Bul D15

Acidulated Flour-Water Suspensions (Cereal Chemistry)

ACME Screw Threads (1973)

ACME Screw Threads (1973)

Acorn Fitting End (1974)

Acoustic Transducers (1972)

Acoustical Absorption Tests (1973)

Acoustical and Airflow Performance (1973)

Acoustical Ceiling Systems (1972)

Acoustical Elements as Used in Schematic Diagrams (1972

Acoustical Materials (1973)

Acoustical Performance Test for Steel Door and Frame as

Acoustical Tests of Building Constructions and Material

Acoustical Tile and for Lay in Panel Ceilings (1973)

Acoustics Design and Construction) (1973)

Acoustics) in Buildings, Walls, and Floors (1973)

(Acoustics) in Wood Building Construction (Floor, Ceilin

Acoustics) (1971)

Acoustics) (1972)

lor

(Acoustics, Audiometry) (1973)

Acoustile (Tech Notes) (1961)

Acquisition and Codification of Data Derived from the I

Acquisition System for Vehicle Noise Sound Level Meters

Acres, Self Locking and Nonlocking (1972)

Acrylate Esters (1972)

Acrylonitrile-Butadiene-Styrene (ABS) Composite Sew

Acrylonitrile-Butadiene-Styrene (ABS) Plastic Drain

Acrylonitrile-Butadiene-Styrene (ABS) Plastic Pipe

Acrylonitrile-Butadiene-Styrene (ABS) Plastic Pipe

Acrylonitrile-Butadiene-Styrene (ABS) Plastic Pipe

Acrylonitrile--Butadiene-Styrene (ABS) PIastic Pipe

Acrylonitrile-Butadiene-Styrene (ABS) Plastic Pipe

Acrylonitrile-Butadiene-Styrene (ABS) Plastic Pipe.

Acrylonitrile-Butadiene-Styrene (ABS) Plastic Sewer

Acrylonitrile-Butadiene-Styrene (ABS) Plastics (197

Arrylonitrile Butadiene Styrene Drain, Waste and Vent P

Acrylonitrile Butadiene Styrene Plastic Drain, Waste an

Acrylonitrile Butadiene Styrene (ABS) Pipe and Fittings

Acrylonitrile Butadiene Styrene (ABS) Plastic Utilities

Acrylonitrile Rubbers (NBR) (1973)

Acrylonitrile (1974)

ACSR) (1972) ANSI C 7.47

(ACSR) (1972) ANSI C 7.61

ANSI C7.60

/AIuminum Coated (Aluminized) ASTM

/Inum Coated (Aluminized) Stra ASTM

Std Spec for Alumi ASTM

Acting (1973)

Acting (1973)

Actinity or Photographic Effectiveness of Illuminants

Action and Title Areas for Television Transmission (196

Action Valves for Fire Protection Service (1970)

Activated Carbon (1974)

Activation and Direct Counting Technique (1973)

Activation Detector Materials (1973)

Active Ingredient in Detergents by Cationic Titration P

Active Proteinase Preparations (Cereal Chemistry) (1962

Active Proteinase Preparations (Cereal Chemistry) (1962

Active Proteinase Preparations (Cereal Chemistry) (1968

Activity from Uranium -238 Fission (1972)

/Td.

Activity in Active Proteinase Preparations (Cereal Chem

Activity in Active Proteinase Preparations (Cereal Chem

Activity in Flour) Conversion (Cereal Chemistry) (1962)

Activity in Soybean Meals, Soy Flour, Mill Feeds (Cerea

Activity in Water (1972T)

Activity of Doughs (Cereal Chemistry) (1962)

Activity of Doughs (Cereal Chemistry) (1962)

Activity of Fabrics: Agar PIate Meth. (1970) ANSI L14.1

Activity of Flour and Active Proteinase Preparations (C

Activity of Flour and Semolina (Cereal Chemistry) 11962

Activity of Flour with the Amylograph (Cereal Chemistry

Activity of Ground Wheat (Cereal Chemistry) (1962)

Activity of Malt Flour (Cereal Chemistry) (1962)

Activity of Malt (Cereal Chemistry) (1962)

Activity of MaIt (Cereal Chemistry) (1962)

Activity of Semolina and Flour (Cereal Chemistry) (1962

Activity (PH) in Flour and Bread (Cereal Chemistry) (19

Activity (PH) in Flour (Cereal Chemistry) (1962)

Activity, by Beta Amylase GBI (Cereal Chemistry) (1962)

Actuated Fastening Systems (1972)

Actuated Fire Alarm Devices, Single and Multiple Statio

Actuated Signaling Boxes for Use with Fire Protective S

Actuated Transmitters (1974)

Added Amounts of Malathion in Range of $0.2 \cdot 2.0$ p.p.m. (

Added Iron in Flour (Cereal Chemistry) (1962)
ASTM

ASTM

NACE

ANS

ANSI

NSA

ANSI,

ASTM

Meth. of ASTM

CSI

ASTM

STDI

ASTM

ICBO

ASTM

WWPA

Std. ANSI

ANSI

ICBO

ANSI

BIA

ANSI

SAE

D. for Pan NSA

ASTM

ASTM

ASTM

ASTM

ASTM

ASTM

A STM

ASTM

ASTM

APMO

ASTM

ASTM

ASTM

ASTN

ASTM

NSA

NSA

SMPTE

UL

ASTM

St ASTM

ASTM

ASTM

CCH $22-60$

AACCH 22-62

AACCH $22-63$

Meth. O ASTM E343

AACCH 22.62

AACCH 22.63

AACCH $22-17$

AACCH 22.90

ASTM D3085

$\mathrm{AACCH} 22.11$

AACCH 22-14

AATCC 90

AACCH $22-60$

AACCH 22-15

AACCH 22.10

AACCH 22-41

AACCH 22.16

AACCH 22.01

AACCH 22-61

AACCH 22.40

AACCH 02.52

$\triangle A C C H$ 02.51

AACCH 22.02

ANSl Al0.3

UL

UL

UL

539

AACCH $60-30$

$\mathrm{AACCH} \quad 40.40$ 
Meth. of Analysis of Std. Spec. for Noncarbonized, Single Ply, ductivity of Aviation Fuels Containing a Static Dissipator Std. Meth. of Test. Anti Leak Antifreezes and Stopeak r Chemical Analysis of Ferrovanadium and Vanadium Alloying Std. Meth. of Test for er Cyclic Movement (1972)

to Solution Coil Coatings and $\mathrm{S}$

Std. Meth. of Test for Spec. for Measurement of Std. Meth. of Test for Extension Recovery and Std. Meth. of Test for Std. Meth. of Test for Deg. Angle and Constant Stress (/ itized Microfilm Carrier (Ape) 1)

Std. Meth. for Determining Std. Meth. of Test for Strap Peel Std. Meth. of Test for Std. Meth. of Test for si J3. 1 Test Meth. for Determination of Film everse lmpacting $(\mathrm{C})$ (1969) ANSI Z167.22 Std. Meth. for 3) Rec. Pract. for Preparation of Bar and Rod Specimens for er, Board, Cloth, etc.) (1973) Test Meth. for sion Test for Resistance to Blistering, Wrinkling, Loss of 24 sion Test for Resistance to Blistering, Wrinkling, Loss of
Std. Rec. Pract. for Atmospheric Exposure of (1970) Std. Test Meth. for Permanence of 0 Std. Test Meth. for Flexural Strength of nsi Z197.1/ ec. Pract. for Preparation of Surfaces of Plastic Prior to Std. Meth. of Test for Strength Properties of Std. Meth. of Test for Resistance of Std. Test. Meth. for Impact Strength of std. Meth. of Test for Cleavage Strength of Metal to Metal d. Meth. of Test for Effect of Moisture and Temperature on Std. Test Meth. for Tensile Properties of 974) ANS1 C59.136 Std. Meth. of Test. Pressure Sensitive Std. Test Meth. for Hydrogen lon Concentration of Dry Std. Rec. Pract. for Determining Durability of 971) Ansi/ Std. Rec. Pract. for Determining Durability of for Determining Adhesion of Protection Sheet to Aperture
Spec. for Mosaic Wood Parquet Flooring Set in d. Meth. of Test for Applied Weight Per Unit Area of Dried N141

Meth. of Test for Applied Weight Per Unit Area of Liquid Std. Spec. for a Liquid Optical dation for Materials for Setting Ceramic Tile with Mortar Mixing Operations (Paint, Varnish, Lacquer, Printing Ink shear $\mathrm{B} /$ Std. Rec. Pract. for Determining the Strength of ar by $\mathrm{T}$ ! Std. Rec. Pract. for Determining the Strength of Std. Meth. of Test for Apparent Viscosity of Hot Mel Std. Rec. Pract. for Preparation of Metal Surfaces for d. Meth. of Test for Strength Properties of Metal to Metal Z197.3 Rec. Pract. for Flexibility Determination of Hot Melt Std. Meth. of Test for Tensile Strength of Std. Meth. of Test for Tack and Pressure Sensitive Fiberboa/ Std. Spec. for Water or Solvent Soluble Liquid g (1973) g (1973) Std. Spec. for Uniform Building Code Std. for 1) Interior (Dry Use) Exposu/ Std. Ppec. for Protein Base e Under Exterior (Wet Use) Exposure Condi/ Std. Spec. for 1969) Std. Test Meth. for Apparent Viscosity of Std. Test Meth. for Density of ion Loadin/
al) (1969) ratures $(\mathrm{M} /$ e Range $\mathrm{Fr} /$ mblies (19) (1972) Ans/ (1969) 1973) ion Loading (/ n (1970) ansi Z197.19 1972) 197.32 Std. Meth. of Test for Strength Properties of Std. Test Meth. for Creep Properties of Std. Meth. of Test for Strength Properties of Std. Meth. of Test for Strength Properties of Std. Meth. of Test for Strength Properties of Std. Meth. of Test for Strength Properties of Std. Test Meth. for Creep Properties of Std. Meth. of Test for Fatigue Properties of Std. Test Meth. for Sirength Properties of Std. Meth. of Test.

Std. Test Meth. for Resistance of Std. Spec. for Std. Meth. of Test for Pressure Sensitive Tack of Sid. Test Meth. for Viscosity of Std. Meth. of Test for Peel Resistance of Std. Meth. for Climbing Drum PeeI Test for Std. Test Method for Penetration of

Std. Test Meth. for Flow Properties of

Std. Test Meth. for Amylaceous Matter in th. for Filler Content of Phenol, Resorcinol, and Melamine or NonvoIatile Content of Phenol, Resorcinol, and Melamine Std. Meth. of Test for Volume Resistivity of Conductive
of Testing Cross Lap Specimens for Tensile Properties of
Added Lysine in Wheat and Bulgur (Cereal Chemistry) (19 Added Salt in Feeds and Feedstuffs (Cereal Chemistry) ( Adding Machine Paper Rolls (1973) Additive (1971) ANSI Z11.281

Additives for Engine Coolants (1972) Additives (1974)

Adhering Vinyl Asbestos Floor Tile (1972)

I Test for Electrical Con Std. Meth. Fo

Adhesion After lmpact of Preformed Sealing Tapes (1973)

Adhesion and Cohesion of Elastomeric Joint Sealants Und

Adhesion and Flexibility by the Wedge Bend (Applicable

Adhesion by Cross Hatch Tape Test (Coil Coating) (1974)

Adhesion of Latex Sealing Compound (1972)

Adhesion of Metallic Coatings (1972)

Adhesion of Pressure Sensitive Tape to Fiberboard at 90

Adhesion of Protection Sheet to Aperture Adhesive of Un

Adhesion of Reinforcing F abrics or Cords to Rubber (197

Adhesion of Vulcanized Rubber to Metal (1973)

Adhesion of Vulcanized Rubber to Steel Cord (1973)

Adhesion of Vulcanized Rubber (Friction T est) (1970) an

Adhesion on All Metals by Cross Hatch Tape Test After R

Adhesion or Cohesive Strength of Flame Sprayed Coatings

Adhesion Tests (1969)

Adhesion to Non Porous Flexible Substrates (Paper) (197

Adhesion to Porous Substrates (Subjective Ratings) (Pap

Adhesion, or Other Failure of Paint, Varnish, Lacquer,

Adhesive Bonded Joints and Structures (1970) ANS1 Z197.

Adhesive Bonded Joints in Plywood Under Mold Conditions

Adhesive Bonding (1969) ANSI Z197.25

Adhesive Bonds in Shear by Compression Loading (1949) a

Adhesive Bonds to Chemical Reagents (1966) ANS] Z197.7

Adhesive Bonds (1972) ANS1 Z197.14

Adhesive Bonds (1972) ANSI Z197.16

Adhesive Bonds (1972) ANSI Z197.18

Adhesive Bonds (1972) ANSI Z197.8

Adhesive Coated Tapes Used for Electrical Insulation (1

Adhesive Films (1970)

Adhesive Joints Stressed in Peel (1971) ANSI Z197.30

Adhesive Joints Stressed in Shear by Tension Loading (1

Adhesive of Unitized Microfilm Carrier (A perture Card)

Adhesive Over Concrete Slab or Wood Subfloors (1962)

Adhesive Solids (1969) ANSI Z197.9

Adhesive Specimens to High Energy Radiation (1970) ANSI

Adhesive (1951) ANSI Z197.10

Adhesive (1970)

Adhesive, and Mastic (1973)

Adhesive, etc.) (1974)

Adhesively Bonded Plastic Lap Shear Sandwich Joints in

Adhesives and Coating Materials (1973)

Adhesives Bonding (1967) ANS1 Z197.28

Adhesives by Compression Loading (Disk Shear) (1972) an

Adhesives by Mandrel Bend Test Meth. (1972T)

Adhesives by Means of Bar and Rod Specimens (1972) ANSI

Adhesives by Rolling Ball (1973)

Adhesives for Automatic Machine Sealing of Top Flaps of

Adhesives for Fastening Gypsum Wallboard to Wood Framin

Adhesives for Fastening Gypsum Wallboard to Wood Framin

Adhesives for Field Gluing Plywood to Wood Framing (197

Adhesives for Structural Laminated Products for Use Und

Adhesives for Structural Laminated Wood Products for Us

Adhesives Having Shear Rate Dependent Flow Properties (

Adhesives in Fluid Form (1969)

Adhesives in Plywood Type Construction in Shear by Tens

Adhesives in Shear by Compression Loading (Metal to Met

Adhesives in Shear by Tension Loading at Elevated Tempe

Adhesives in Shear by Tension Loading in the Temperatur

Adhesives in Shear by Tension Loading of Laminated Asse

Adhesives in Shear by Tension Loading (Metal to Metal)

Adhesives in Shear by Tension Loading (Metal to Metal)

Adhesives in Shear by Tension Loading (Metal / Metal) (

Adhesives in Two Ply Wood Construction in Shear by Tens

Adhesives Relative to Their Use as Electrical Insulatio

Adhesives to Cyclic Laboratory Aging Conditions (1970)

Adhesives Used in Nonstructural Glued Lumber Products (

Adhesives Using an lnverted Probe Machine (1971) ANSI Z

Adhesives (R 1970)

Adhesives (T-Peel Test) (1972) ANSl Z197.2

Adhesives (1962) ANS1 Z197.23

Adhesives (1969)

Adhesives (1969)

Adhesives (1970)

Adhesives (1970)

Adhesives (1970)

Adhesives (1972)

Adhesives (1972) ANS1 Z197.21
AACCH $46-40$

$\triangle A C C H \quad 40.60$

ANSI $\quad \mathrm{X} 4.8$

D2624

ASTM E

RT1

ASTM

ASTM

NCCA

NCCA

ASTM

ASTM

ASTM

NMA

ASTM

ASTM

ASTM

ASTM

NCCA

ASTM

ASTM

TAPP1

TAPPI

NCCA

ASTM

ASTM

ASTM

td. R ASTM

ASTM

ASTM

ASTM

ASTM

St ASTM

ASTM

ASTM

ASTM

ASTM

ASTM

NMA

WSFl

St ASTM

ASTM

Std ASTM

ASTM

TCA

ASTM

ASTM

ASTM

ASTM

ASTM

ASTM

ASTM

ASTM

ASTM

ASTM

ICBO

APA

ASTM

ASTM

ASTM

(

ASTM

ASTM

ASTM

ASTM

A STM

ASTM

ASTM

ASTM

ASTM

ASTM

ASTM

ASTM

ASTM

ASTM

STM

ASTM

ASTM

ASTM

ASTM

Std. Test Me ASTM

Std. Test Meth. F ASTM

ASTM

Std. Meth ASTM

E36

C.766

C719 
Rec. Pract. for Conducting Creep Tests of Metal to Metal tent. Meth. of Test for Floating Roller Peel Resistance of Std. Definitions of Terms Relating to Uniform Building Code Std for Rec. Pract. Meth. of Tests for Automotive Type Sealers. tion) (1971) Duct Liner. olumns, Joints, Screws, Bolts, Connectors, Nails, Staples, (1970)

r and Semolina: Bread; Feedstuffs, Ground Grains and Their

Std. for Clevis-Engine Control Rod. Std. for Machine Bolt, Crowned Hexagon Head.

Std. for

Std. for

ortation (DOT) Special Permits for Radioactive

Std. for

ioactive Materials (Safety) (1973)

Std. Meth. for Spectrochemical Analysis of ract. for Combustion Chambers, Fuel Oil Injection, and Gas 962)

Trifructosan Meth. of Analysis for Rec. for

Uniform Building Code Std. for Std. Spec. for Air Entrainin Rec. Guide for Utse of

e Due To/ Std. Meth. of Test for Effectiveness of Mineral idity Alkalinity of Halogenated Organic Solvents and Their luble Halide lon in Halogenated Organic Solvents and Their Std. for water and Wastewater Treatment (Septic, Holding, Settling, graphic Film in Rolls, Including Leaders and Trailers, for Std. for Dimensions of

ts and Tows (1973) Std. for Safety Requirements for for Dimensions of Containers for Packaging and Storage of Std. for in Dividual of Analysis of Thermophilic Spore Counts (Flat Sour, Total t Flying Insects/

Std. Meth. of Test for Effectiveness of Std. Meth. of Test for Pressure in Glass Meth of Drop Test of Glass

Std. Spec. for Tin Plate Fabricated Std. Rec. Pract. for Labeling

Std. Meth. of Test for Solids Content of Std. Spec. for Safe Fill or

Std. Rec. Pract. for Inspection of Glass

Meth. of Test for Pressure in Metal

Rec. Pract for Reporting Laboratory Results When Checking $f$ Test for Effective Crimping on Outside Crimped Valves of Std. Meth. of Test for Effectiveness of Std. Meth. of Test for Pickup Efficiency of Residual Std. Spec. for Simple Can Puncturing Device for Use in Std. Meth of Test. Std. Definitions of Terms and Nomenclature Relating to Std. Meth. of Test for Flammability of Std. Meth. of Test for Delivery Rate of Std. Meth. of Test for Volatile Content of Std. Rec. Pract. for Storage of

Std. Meth. of Test for Seepage Rate of Std Meth of Measurement of the A.D Dimension of Std. Meth. of Test for Overrun of Food ne. Kerosene, Acetone, Alcohol, Cleaning Fluids, Flammable Meth of Test for Explosive Reactivity of Lubricants with trasonic Inspection of Aluminum Alloy Wrought Products for or Nonvolatile Residue of Halogenated Solvent Extract from

for Identification of Metallic and Fibrous Contaminants in d Lay) (1973)

Std. for Gear Tooth Surface Texture for

SI Metric Units (1973)

d. Semi Finished and Rough Items (1971)

1974) Std. Fastener Code Std. for National

Std. Analytical Meth. Determining Iodine rability Requirements for Both Permanently and Temporarily TLC Meth. of Analysis of 2) Meth. for Detection of Antibacterial Activity of Fabrics: nd Shapes (1/ Std. Spec. for Hot Rolled and Cold Finished $g$ Weight Determination for Chromate Conversion Coatings on for Coating Weight Determination for Conversion Coating on Rec. for Halogenated Fire Extinguishing Spec. for Tetrasodium Pyrophosphate (Anhydrous) (Cleaning 4.79 Chemistry) (1962) Test Meth. for Evaluation of Commercial Rewetting Meth. for Preparation of Sample: Leavening Test Meth. for Evaluation of Wetting Uniform Fire Code: Explosives and Blasting orm Building Code Std. for Exterior Plaster Liquid Bonding ransportation. Storage, and Use of Explosives and Blasting Rec for Preplaced Rec. Guide for Structural Lightweight Uniform Building Code Std. for Rec. Spec. for Optimum Size Coarse
Adhesives (1972) ANSI Z197.22

Adhesives (1973)

Adhesives (1973)

Adhesives (1973)

Adhesives, and Deadeners (1971)

Adhesives, and Fasteners Application Std. (Air Distribu

Adhesives, Fiberboard, Particleboard) (1973)

Adjuncts (Cereal Chemistry) (1962)

Adjustable Face Vehicular Traffic Control Signal Heads

Adjustable (1973)

Adjusting (1973)

Administrative Controls for Nuclear Power Plants (1972)

Administrative Guide for Obtaining Department of Transp

Administrative Guide for Packaging and Transporting Rad

Admiralty Metal by the Cast Pin and Point to Plane Tech

Admission Systems (1972)

Admixture of Rye and Wheat Flours (Cereal Chemistry) (1

Admixtures for Concrete (1960)

Admixtures for Concrete (1973)

Admixtures for Concrete (1973) ANSI A37.132

Admixtures in Concrete (1968)

Admixtures in Preventing Excessive Expansion of Concret

Admixtures (1974)

Admixtures (1974)

Std. Meth of Test for AC

Advertising of Books (1971)

Std. Meth of Test for Water So

Aeration Chambers, Chemical

Aerial and Related Uses (1973)

Aerial Film Spools (1972)

Aerial Passenger Tramways lncluding Ski and Surface Lif

Aerial Photographic Film on Spools (1972)

Aerobic Wastewater Treatment Plants (1970)

Aerobic, Anaerobic) in Cereal Products and Food Ingredi

Aerosol and Pressurized Space Spray Insecticides Agains

Aerosol Bottles (1972)

Aerosol Bottles (1972)

Aerosol Cans (1972)

Aerosol Cans (1972)

Aerosol Coatings (1972)

Aerosol Containers (1972)

Aerosol Containers (1972)

Aerosol Containers (1972)

Aerosol Containers (1972)

Aerosol Containers (1972)

Aerosol Insecticides Against Cockroaches (1972)

Aerosol Insecticides (1972)

Aerosol Laboratories (1972)

Aerosol Products for Delivered Mass (1972)

Aerosol Products (1972)

Aerosol Products (1972)

Aerosol Products (1972)

Aerosol Products (1972)

Aerosol Products (1972)

Aerosol Products (1972)

Aerosol Valve Dip Tubes (1972)

Aerosols (1972)

Aerosols, etc. with a Flash Point Below 200 Deg. F) (19

Aerospace Alloys Under High Shear (1972) ANS1 Z11.323

Aerospace Applications (1974)

Aerospace Components (Using Rotary Flash Evaporator) (1

Aerospace Fluids (1970E)

Std. Meth.

Aerospace Gearing (Surface Roughness, W

Aerospace Std. Documents Preparation and Maintenance in

Aerospace Std. ldentification Marking Meths. on Finishe

Aerospace Stds. Documents Preparation and Maintenance (

Affinity of Corn Starch (1973)

Affixed Safety Signs for Construction and lndustrial Eq

Aflatoxins in Corn and Soybeans (Cereal Chemistry) (197

Agar Plate Meth. (1970) ANSI L14.125

Age Hardening Stainless and Heat Resisting Steel Bars a

Aged or Backed Aluminum Alloy Panels (Coil) (1974)

Aged or Baked Aluminized Steel (Coil) (1974)

Agent Systems (1973)

Agent) (1970) ANS1 K60.20

Agents in Caustic Soda for Mercerization (1971) ANSI L1

Agents in Flour: Reaction with Potassium lodide (Cereal

Agents on Cotton Textile Fabrics (1971) ANSI L14.106

Agents (Cereal Chemistry) (1962)

Agents (Cotton Test Skein Meth.) (1971) ANSl L14.11

Agents (1973)

Agents (1973)

Agents (1973) ANSI Z271.1

Code for the Manufacture,

Aggregate Concrete for Structural and Mass Concrete (19

Aggregate Concrete (1964)

Aggregate for Masonry Mortar (1973)

Aggregate for Portland Cement Concrete Paving (1972)
Sid ASTM

ASTM

ASTM

$\mathrm{CBO}$

$\mathrm{SAE}$

SMACN

paced $\mathrm{C}$ ICBO

$\mathrm{AACCH}$

ITE

NSA

NSA

ANSl

ANSI

ANSI

ASTM

Std. P DEMA

$* 1-2$

AACCH $\quad 06-10$

$\mathrm{ACI}$

ICBO

ASTM

$\mathrm{ACl}$

ASTM

ASTM

ASTM

ANSI

ANS1

ANSl

ANSI

Std. ANS

NSF

AACC

ASTM

ASTM

ASTM

ASTM

ASTM

ASTM

ASTM

ASTM

ASTM

Std. ASTM

Meth. O ASTM

ASTM

ASTM

ASTM

ASTM

ASTM

ASTM

ASTM

ASTM

ASTM

ASTM

ASTM

ASTM

ICBO

ASTM

ASTM

ASTM

ASTM

A GMA

NSA

NSA

SAE

NSA

CR

$\mathrm{SAE}$

Test AATCC ASTM

in NCCA

Spec NCCA

FMS

Std. ASTM

AATCC

$\mathrm{AACCH}$

AATCC

AACCH

AATCC

ICBO
ICBO

IFBO

$\mathrm{ACl}$

$\mathrm{ACl}$

ICBO

ACPA

D1780

D3167

J243

170

428

N18.7

60-64

$\mathrm{C} 260$

68-56

C441

D2989

D2988

Z39. 13

UBCS 25.19

UBC $* 3.25$

N14.10.2

N14.10.1

UBCS26.9 
Std. Rec. Pract. for Classification of Soils and Their enting Excessive Expansion of Concrete Due to the Alkali Std. Meth. of Test for Surface Moisture in Fine Concrete eth. of Test for Specific Gravity and Absorption of Coarse Meth. of Test for Specific Gravity and Absorption of Fine $r$ Use in Waterproofing (Asphalt Cement, Mineral Filler and e (1973) AASHO T104,/ Std. Meth. of Test for Soundness of Rec. for Selection and Use of

Uniform Building Code Std for iform Building Code Std. for Perlite, Vermiculite and Sand Std. Spec. for Std. Descriptive Nomenclature of Constituents of Uniform Building Code Std. for Lightweigh Std. Meth. of Test for Potential Reactivity of iron Blast Furna/ Uniform Building Code Std. for Concrete Std. Definitions of Terms Relating to Concrete and Its

$t$ for Sieve or Screen Analysis of Fine and Coarse Concrete Std. Meth. of Test for Insoluble Residue in Carbonate Uniform Building Code Std. for Mineral Roofing Std. Spec. for Concrete

Prepared Roofings, and Plast/ Spec. for Roof Membranes $s$ Applied Over Film Insul/

Std. Meth. of Test for Thermal est Meth. for Resistance of Adhesives to Cyclic Laboratory Compounds (1972)

Std Meth. of Test for Std. Meth. of Test for Weight Loss After Heat Std. Test Meth. for Laboratory

Test Meth. for Accelerated i J5.1 Std. Meth. of Test for Accelerated Std. Meth of Test for Accelerated Std. Meth. of Test for Accelerated (1973) ANSI J4.1 Std. Meth. of Test for Extrudability, After Package an Normal Engineering Services (1972) Truck Mixer and Std. Form of Std. Form of Std. Form of Std. for

gn Services (1972)

ces (1972) Std. Form of Std. Form of Std. Form of Joint Venture

ruction Contracts Where Basis of Payment Is/

Std. for Safety for

rill) Press, and Gage Wheel,/ Std. for Tires and Rims for

Std for Power Takeoff Definitions and Terminology for and Wheel Stds. for Motorcycles, Passenger Cars, Off Road, Uniform Building Code Std. for Lighting and Marking of Std. for Operator Controls on Rec. Safety Alert Symbol for Std. for T-Hook Slots for Securement in Shipment of Rec. Pract. for Safety for Std. for Safety for

72) ties $(1968)$ Specific Gravity, and Weight-Moisture Relationships of

ty on Enclosed Mobile Tanks for Transture Relationships of 971 Rec. Safety Sign for Front End

Rec. Pract. for Liquid Ballast Table for Drive Tires of Std. Liquid Ballast Table for Drive Tires of

(1971) Std. for ) (1971) 71)

972) Std. for Area Classification in Hazardous Dust Locations
ion of Electrical lnstruments in Hazardous Dust Locations
Std. for SAE J720

Std. for Rim Contours for Rec. Pract. for

Std. for

for Application of Hydraulic Remote Control Cylinders to Application of Remote Hydraulic Motors to t Procedure for Measuring Hydraulic Lift Force Capacity on $t$ Procedure for Measuring Hydraulic Lift Force Capacity on cedures and Performance R/ Std, for Protective Frame (For requirements) (1972) SAE J/ requirements) (1972) / Sid. Protective Frame (For Std. for Protective Enclosures for Std for Operator Protection for Wheel Type nsi B114.2, Sa/ Std. for Symbols for Operator Controls on ng Coupler for Thre/ Std. for Attachment of Implements to or 3 Point Free Link Attachment for Hitching Implements to d. for 3 Point Free Link Hitch Attachment of Implements to mp and Slow Mo/ Std. for Mounting Brackets and Socket for for Flashing Warning Lamp (Light) for Remote Mounting on
Aggregate Mixtures for Highway Construction Purposes (1 Aggregate Reaction (1969) ANSI A37.119 Aggregate (1973) AASHO T142, ANSI A37.21 Aggregate (1973) ANSI A37.5

Aggregate (1973) ANSl A37.6

Aggregate) (1941) ANSI A 109.17

Aggregates by Use of Sodium Sulfate or Magnesium Sulfat Aggregates for Concrete (1958)

Aggregates for Grout (1973)

Aggregates for Gypsum Plaster (1973)

Aggregates for Radiation Shielding Concrete (1973)

Aggregates for Radiation Shielding Concrete (1973)

\section{Xture:}

ASTM

ASTM

ASTM

Std. M ASTM

Std. ASTM

ASTM

$\mathrm{ACI}$

ICBO

Aggregates for Structural Concrete (1973)

Aggregates (Chemical Meth.) (1971) ANSI A37.133

Aggregates (Sand, Crushed Stone, Gravel and Air Cooled

Aggregates (1968) ANSI A37.76

Aggregates (1971) AASHO T27, ANSI A37.8 / Meth. of Tes

Aggregates (1972)

Aggregates (1973)

Aggregates (1974) ANSI A37.124

Aggregates, Saturated Felts, Saturated and Coated Felts

Aging Characteristics of Electrical Insulating Varnishe

Aging Conditions (1970) ANSI Z197.19

Aging Effects of Artificial Weathering on Latex Sealing

Aging of Preformed Sealing Tapes (1974)

Aging of Sandwich Constructions (1970)

Aging of Sulfur Dyed Textiles (1972) ANSI L14.1

Aging of Vulcanized Rubber by the Oyen Meth. (1967) Ans

Aging of Vulcanized Rubber by the Oxygen Pressure Meth.

Aging of Wood Sash Face Glazing Compound (1973)

Aging, of Latex Sealing Compounds (1972)

Agitator Stds. (Concrete Plant Equipment) (1973)

Agreement Between Architect and Consultant for Other Th

Agreement Between Architect and Engineer (1974)

Agreement Between Owner and Architect for Interior Desi

Agreement Between Owner and Architect for Special Servi

Agreement Between Owner and Architect (1974)

Agreement Between Owner and Construction Manager (1973)

Agreement Between Owner and Contractor (For Small Const

Agreement (Form) for Design Professionals (1972)

Agricultrual Auger Conveying Equipment (1973)

Agricultural and Industrial Steering, Drive, Planter (D

Agricultural and Light Industrial Tractors (1972) SAE J

Agricultural and Log Skidder, Truck, Bus, Bead Seat, in

Agricultural Buildings (1973)

Agricultural Equipment on Highways (1971)

Agricultural Equipment (1972)

Agricultural Equipment (1972)

Agricultural Equipment (1972)

Agricultural Equipment (1972)

Agricultural Equipment (1973) ANSl Bl14.3

Agricultural Grain and lts Products (Flour, Starch) (19

Agricultural Grain and Seed for Storage (1972)

Agricultural Grain Storage Loads. Pressures, and Capac

Agricultural Grains (1968)

Agricultural Liquids and Slurry (1973)

Agricultural Loaders (1972)

Agricultural Machinery Data and Management Practices (1

Agricultural Machines (1971)

Agricultural Machines (1972)

Agricultural Planter Press Wheel Tires (Farm Machinery)

(Agricultural Plastics, Chemicals, Metal Dusts) (1973)

Agricultural Plastics, Chemicals, Metal Dusts) (1973)

Agricultural Press and Gage Wheel Tires (Farm Machinery

Agricultural Press and Gage Wheels (Farm Machinery) (19

Agricultural Tractor Auxiliary Power Take Off Drives (1

Agricultural Tractor Belt Speed and Pulley Width (1971)

Agricultural Tractor Test Code (1972)

Agricultural Tractor Test Code (1972) SAE J708C

Agricultural Tractors and Trailing Type Agricultural (F

Agricultural Tractors and Trailing Type (Farm) lmplemen

Agricultural Tractors Equipped with 3 Point Hitch (1972

Agricultural Tractors Equipped with 3 Point Hitch (1972

Agricultural Tractors for Overhead Protection (Test Pro

Agricultural Tractors (Test Procedures and Performance

Agricultural Tractors (Test Procedures and Performance

Agricultural Tractors (1972)

Agricultural Tractors (1972) SAE J333

Agricultural Tractors, Machinery and Equipment (1971) a

Agricultural Wheel Tractors Equipped with Quick Attachi

Agricultural Wheel Tractors (1972) SAE J715

Agricultural Wheeled Tractors (1972)

Agricultural (Farm) and Industrial Equipm ent Warning La Agricultural (Farm) Equipment (1972

Agricultural (Farm) Grain Wagons (1972)

Agricultural (Farm) Implements and Tractors (1972) SAE
Un $1 \mathrm{CBO}$

ASTM

ASTM

ICBO

ASTM

ICBO

ASTM

ASTM

ASTM

$\mathrm{ICBO}$

ASTM

NRCA

ASTM

Std. T ASTM

ASTM

A STM

ASTM

A A TCC

ASTM

ASTM

ASTM

ASTM

NRMC

AIOA

AIOA

AlOA

AlOA

AlOA

AlOA

AIOA

ASAE

TRA

ASAE

TRA

ICBO

SAE

ASAE

ASAE

ASAE

SAE

ASAE

ASAE

/Ensity ASAE

ASAE

ASAE

ASAE

ASAE

ASAE

SAE

ASAE

ASAE

ISA

ISA

ASAE

ASAE

SAE

ASAE

SAE

A.SAE

ASAE

ASAE

ASAE

SAE

ASAE

ASAE

ASAE

REC.

ASAE

ASAE

ASAE

Std. F ASAE

St SAE

ASAE

Std ASAE

ASAE

ASAE

D3282

C441

C70

C12?

C128

D491

C88

UBCS24-24

UBCS47.3

C637

C638

UBCS26-3

C289

UBCS26-2

$\mathrm{Cl} 25$

Cl36

D3042 


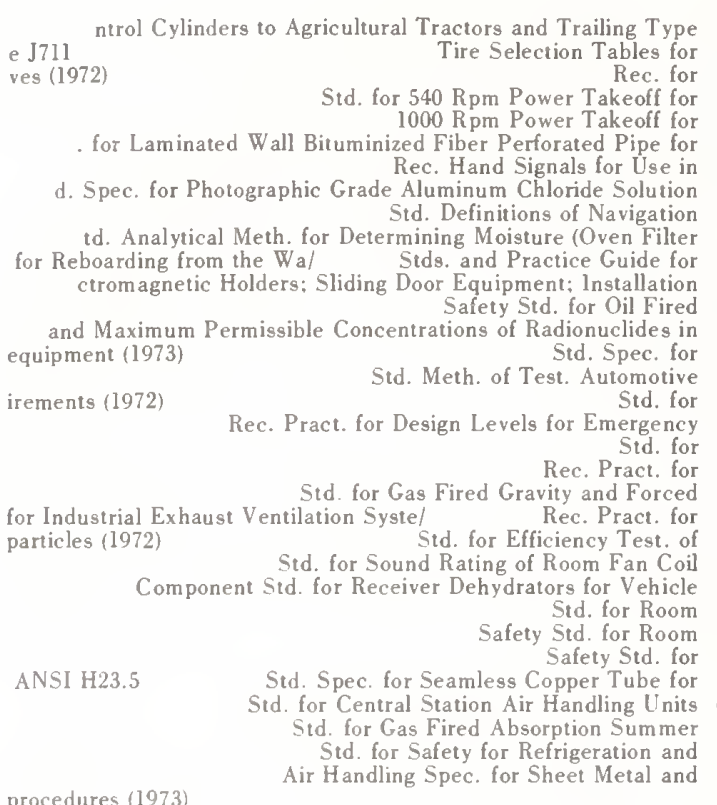

th. of Testing for Sound Rating Heating, Refrigerating and Sid. for Unitary Std. for Recreational Vehicle Std. Meth. of Testing Automotive Std. for Recreational Vehicle What Makes a Good

and Test Equipment and $\mathrm{R}$ conditioning System) (1966)

a Good Air Conditioning System (Information on Year Round Std. for Liquid Line Driers in Refrigerating and

Fibrous Glass Duct Construction Std. for Ventilating and

Minimum Installation Stds. for Residential Heating and Std. for Installation of Central Warm Air Heating and uction Line Filters and Filter Driers in Refrigeration and icating and Regulating Electrical Equipment for Control of eir Combination Duct System/ ystems) (1972)

(1970) Std. for the Installation of Safety Std. for Limit Controls Std. for

tration of Chromic Acid and Hexavalent Chromium Compounds eth. (1972T) ANSI A37.70 Tent. Meth. of Test for for Concrete Aggregates (Sand, Crushed Stone, Gravel and asolin/ Rec. Pract. and Std. Covering the Installation of Std. for Forced Circulation
Std. for Std. for

res (1966)

Std for Air Outlets and Inlets Used in Duct Liner, Adhesives, and Fasteners Application Std. Std. for Safety for Factory Made Std. for Central Forced

132

4)

electric Fans (Blower. Humidifier. Evaporative Cooler, and Ventilation Syste/ Rec. Pract. for Air Cleaning Devices ne Bleach) 190 Deg. F, Colors 160 Deg. F Maximum, Press or (19) Sid. Test Proc. and Acceptance Criteria for Rate of

Contractors (1973)

(1974) Rec. Pract. for Make Up and Recirculated

Ducted Electric Heat Guide for Std. for Central Station Std. for Direct Gas Fired Make Up Sid. for Meth. of Test. for Rating Nonresidential Warm Std. for Safety for Electric 44.3 Std. for Installation of Central W arm Std. for Forced Circulation Air Cooling and Safety Std. for Electric Central ignition Properties (Temperatures) of Plastics Using a Hot for Low and Medium Speed Stationary Diesel and Gas Engine Std. Operating Limits for Central Station Units Std. Coil Face Areas for Central Station Units Sid. for Certified Ratings Program Std. Test Code for sical Measurements (19) Std. Meth. of Test for Density in Std. for Acceptable Concentrations of Toluene in the Std. Meth. of Test for Effect of Heat and
Agricultural (Farm) lmplements (1971) SAE J716

Agricultural (Farm) Machines of Future Design (1972) Sa

Agricultural (Farm) Tractor Auxiliary Power Takeoff Dri

Agricultural (Farm) Tractors (1973) SAE J718

Agricultural (Farm) Tractors (1973) SAE J719

Agricultural, Land, and General Drainage (1973) ANSI AI

Agriculture (1972)

$\mathrm{AICI}_{31}(1972)$

Aid Terms (1971)

Aid) Content of Corn Syrup Unmixed (1954)

Aids to Security (Safety) of Personnel on Boat Deck and

Aids (1969) /Ers, Stops, S

Air and in Water for Occupational Exposure (1959)

Air Brake and Vacuum Brake Hose (1973) ANSI S2.10

Air Brake Reservoir Performance and Identification Requ

Air Brake Systems (1971)

Air Brake Tubing and Pipe (1970)

Air Carbon Arc Gouging and Cutting (1974)

Air Central Furnaces (1973)

Air Cleaning Devices (Air Filters and Dust Collectors)

Air Cleaning Systems Containing Devices for Removal of

Air Conditioners Delivering Less Than 1500 cfm (1971)

Air Conditioners (1967)

Air Conditioners (1972) ANSI Z234.1

Air Conditioners (1973) ANSI C33.14

Air Conditioners, Central Cooling (1972) ANSI B 144.1

Air Conditioning and Refrigeration Field Service (1974)

(Air Conditioning and Refrigeration) (1974)

Air Conditioning Appliances (1973)

Air Conditioning Condensing and Compressor Units (1973)

Air Conditioning Contractors (1973)

Air Conditioning Equipment Selection and System Design

Air Conditioning Equipment (1972)

Air Conditioning Equipment (1974)

Air Conditioning Equipment (1974)

Air Conditioning Hose (1968E) ANSI J2.27

Air Conditioning System Rating Definitions, Test. Meth.

Air Conditioning System (Information on Year Round Air

Air Conditioning System) (1966)

Air Conditioning Systems (1971)

Air Conditioning Systems (1972)

Air Conditioning Systems (1973)

Air Conditioning Systems (1973) ANSI B144.3

Air Conditioning Systems (1974)

Air Conditioning, Heating, Cooking, Refrigeration, and
Air Conditioning, Heating, Exhaust, Ventilating, and Th

(Air Conditioning, Heating, Ventilating and Processing S

Air Containers for Entranceways in Food Establishments

(Air Contamination-Occupational Health and Safety) (19

Air Content of Freshly Mixed Concrete by the Pressure M

Lote Co ASAE S201.4

ASAE R220.3

ASAE R R R33.1

ASAE S203.7

ASAE S204.6

ASTM D2417

ASAE R351

St ANSI PH4.154

IEEE $\quad 172$

CR E-42

$\begin{array}{ll}\text { ABYC } & \text { A18 }\end{array}$

NBHA 8

UL

733

INs NCRPM R22

ASTM D3283

ASTM D622

SAE J10B

SAE J263

SAE J844C

AWS C5.3

ANSI Z21.47

ACGIH *1.11

ANSI N10l.

ARI

IMACA

AHAM

UL

UL

ASTM

ARI

ANS

UL

SMACN

NESCA MANK

ASHRA 36

ARI 210

ARI 250

ASTM D1680

IMACA 400

NESCA MAN C

NESCA MAN C

ARI $\quad 710$

SMACN *6

SMACN $* 10$

NFPA $90 \mathrm{~B}$

ARI 730

UL 873

NFPA $\quad 90 \mathrm{~A}$

UL $\quad 353$

NSF $\quad 37$

ANSI Z37.7

ASTM C231

Air Cooled Iron Blast Furnace Slag) (1973) /G Cod / Dir Cooled Marine Engines and Auxiliaries (Diesel and G

Air Cooling and Air Heating Coils (1972)

Air Data Computers Mps (1970)

Air Density Ratios-At Various Altitudes and Temperatu

Air Distribution Systems (1973)

(Air Distribution) (1971)

Air Duct Materials and Connectors (1974)

Air Electric Heating Equipment (1974)

Air Entraining Admixtures for Concrete (1973) ANSI A37.

Air Exchange Coolers for Packaged Compressor Units (197

Air Filtering Appliances Rated at 600 Volts or Less) (1

(Air Filters and Dust Collectors) for Industrial Exhaust

Air Finish (1973) /Mmercial Wash-Whites (With Chlor

Air Flow Through Closed Steel Door and Frame Assemblies

Air for Industrial Exhaust Ventilation Systems (1974)

Air Handling Spec. for Sheet Metal and Air Conditioning

Air Handling Systems (1971)

Air Handling Units (Air Conditioning and Refrigeration)

Air Heaters (1972)

Air Heaters (1972)

Air Heaters (1973)

Air Heating and Air Conditioning Systems (1973) ANSI BI

Air Heating Coils (1972)

Air Heating Equipment (1973) ANSI C33.104

Air Ignition Furnace) (Fire Safety) (1973)

Air lntake and Exhaust Systems (1972)

(Air Moving and Conditioning) (1966)

(Air Moving and Conditioning) (1966)

Air Moving Devices (Performance) (1970)

Air Moving Devices (1967)

Air of Manufactured Carbon and Graphite Articles by Phy

Air of Workplaces (1974)

Air on Asphaltic Materials (Thin Film Oven Test) (1973) 

edstuffs (Cereal Chemistry) (1962) Modified Two Stage chemistry) (1962)

eg. C (Cereal Chemistry) (1962)

in Flour and Semolina (Cereal Chemistry) (1962)

Std. Test Meth. for Polynuclear Aromatic Hydrocarbons in Std. Meth. of Test for Fineness of Portland Cement by Std. Meth. of Test for Std. Meth. or Test for

of Test for Average Particle Size of Alumina and Silica by Rec. Pract. for Exhaust Emission Controls
Std. for n Systems (Instrumentation) (1970)

Std. for

n Systems (1967)

fiers, Nebulizers, Gas Therapy and Suction Equipment, Room ) Std. Meth. of Test for Modulus of Rupture of Std. Meth. of Test for Cold Bonding Strength of Uniform Fire Code: Tents and anufacturing Spec., and Application Guide for High Voltage Specifying Duct, Sheet Metal, Low Pressure, tems Designed to Streamline Supply Transactions Within the 3) Std. Meth. of Test for Percent std. Meth. for the Measurement of Sound Pressure Levels in
Std. Performance Requirements for Oil Powered Forced Std. Meth of Test for Resistance to Paper to Passage of equipment and Pipe Operating at Temperatures Above Ambient Std. for Driving and Spindle Ends for Portable Hand,
m, Hydrogen, Krypto/ Uniform Fire Code: Cryogenic Fluids Spec, for Cabin Duct, Test Meth. Visual Std. for Surfaces of New Steel xtile) (1971) Measurement of Minimum Performance Stds. R/ Minimum Performance Std. for Rec. Guide for the Prediction of the Dispersion of Rec. for

th Emergency Locator Tran

I. Ceiling, Floor, and $\mathrm{Fl} /$ ery (1973)

Rec. Basic Characteristics for Uniform Building Code Std. for Std. for Test Procedure for (1970) Code Std. for Laboratory Determination and Measurement of Std. Rec. Pract. for Laboratory Measurement of Minimum Operational Characteristics tional Characteristics Vertical Guidance Equipment Used in adio Frequency Range of 108/ Minimum Performance Std. for Uniform Fire Code: Airports, Heliports, Helistops and ology, Environment and Design Stds for (1972)

ic Particle Inspection Procedure) (197/ Spec. for Premium Rec. Pract. for Spark Plug, Std. for Tires, Tubes, Rims and Valves for Std. for Tire Valves for All Vehicles Except Steel Machine Screw (1974)

Std. for

000 psi Alloy Steel Machine Screw (1974) Std. for

r Materials and Prefabricated Silencers for Acoustical and Rec. Pract. for Automotive Engine Carburetor Std. Meth. of Test for

ec. for Bearings, Ball and Roller, Rod End, Anti Friction. Std. for Tires. Tubes, Rims and Valves for Aircraft for Laminated Wall Bituminized Fiber Perforated Pipe for 73) eous Perforated Bituminous Fiber Pipe Systems for Highway,

ment, Room Air Purifiers, Filters, Compressors, Artificial oods Use Book: Fire Protection and Safety (Exit, Stairway, Inspection of Laminators Quality Control by the

ntral Station Protective Signaling Systems for Guard, Fire oprietary Protective Signalling Systems for Watchman, Fire Safety Std. for Central Stations for Watchman, Fire y Operated Type for I/ Safety Std. for Heat Actuated Fire and Use of Auxiliary Protective Signaling Systems for Fire Safety Std. for Household Burglar Std. for Safety for Proprietary Burglar d. for Construction, Operation, and Maintenance of Vehicle Uniform Building Code Std. for Fire and Bank Burglar or Safety for Connectors and Switches for Use with Burglar Safety Std. for Holdup fire Protection Service (1973) ANSI /
tective Signalling Systems for Fire Protection and Safety tective Signalling Systems for Fire Protection and Safety
of Local Protective Signaling Systems for Watchman, Fire Safety Std. for Key Locks (Burglar Std. for Safety for Antitheft and Maintenance of Systems and Appliances (Extinguishers, , Combustible and Flammable Materials; Reporting and False ermal Process (Western Red Cedar, Northern White Cedar and al Process Preservative Treatment of Western Red Cedar and Std. Meth. for Determination of Dichloromethane and ater in Engine Antifreeze-Coolant Concentrate (Glycol or Chemistry) (1962)
Air Oven Meth. of Analys is of Moisture in Flour, Farina

Air Oven Meth. of Analys is of Moisture in Grains and Fe Air Oven Meth. of Analys is of Moisture in Malt (Cereal Air Oven Meth. of Analys is of Moisture, Drying at 135 D Air Oven (Aluminum Plate) Meth. of Analysis of Moisture Air Particulate Matter (1971)

Air Permeability Apparatus (1973) AASHO T153, ANSI A1.1 Air Permeability of Asbestos Fibers (1972)

Air Permeability (1972)

Air Pollution) for Powered Industrial Trucks (1973)

Air Pressures for Pneumatic Controllers and Transmissio

Air Pressures for Pneumatic Controllers and Transmissio

Air Purifiers, Filters, Compressors, Artificial Airways

Air Purifying Respirator Canisters and Cartridges (1973

Air Setting Plastic Refractories (1972)

Air Setting Refractory Mortar (Wet Type) (1972) ANSI Al

Air Supported Structures (1973)

Air Switches, Bus Supports, and Switch Accessories (197

Air Transmission (1967)

Air Transport Industry (1974)

Ted Data Processing

Air Voids in a Compacted Bituminous Paving Mixture (197

Air (Acoustics) (1971)

Air (Domestic and Light Commercial) Central Furnaces)

Air (1958) TAPPI T460

Air (1972)

Air, and Electric Tools (1973)

Air, Argon, Carbon Monoxide, Deuterium, Fluorine, Heliu

Air, Flexible and Semi Rigid (1972)

Airblast Cleaned with Sand Abrasive (1970)

Airborne Asbestos Fiber by the IIembrane Filter Meth. (Te

Airborne ATC Transponder Equipment (1972)

Airborne Distance Measuring Equipment (DME) Operating W

Airborne Effluents (1968)

Airborne Pulp Driers (1973)

Airborne Radio Homing and Alerting Equipment for Use Wi

Airborne Sound Insulation Field Test of Partitions (Wal

Airborne Sound Measurements on Rotating Electric Machin

Airborne Sound Transmission Class (Stc) of Building Par

Airborne Sound Transmission Loss of Building Partitions

Airborne VHF Omnirange (VOR) Systems (1972)

Airborne Volumetric Navigation Systems (1972)

Airborne VOR Receiving Equipment Operating Within the

Aircraft Hangers (1973)

Aircraft Instrument Stds. (Wording, Terminology, Phrase

Aircraft Quality Steel Cleanliness Requirements (Magnet

Aircraft Reciprocating Engine (1971)

Aircraft (Airplane, Helicopter) (1974)

Aircraft (1974)

Aircraft, Pan Head, Phillips Recess Full Threaded, Allo

Aircraft, Pan Head, Phillips Recess, Short Thread, 160

Airflow Performance (1973)

Airflow Reference Std. (1971)

Airflow Resistance of Acoustical Materials (1973)

Airframe (1974)

Airplane, Helicopter) (1974)

Airport and Roadwork Drainage (1973)

Airport, and Similar Drainage (1973)

Airways. Appurtenances) (1973)

A isle, Corridor, Building Const

AlTC Inspection Bureau (1962)

Alarm and Supervisory Service (1972) ANSI SE3.2

Alarm and Supervisory Service (1972) ANSI SE3.6

Alarm and Supervisory Services (1972) ANSl Se 3.1

Alarm Devices. Single and Multiple Station, Mechanicall

Alarm Service (1972) ANSI SE3 4

Alarm System Units (1972) ANSl Se2.4

Alarm System Units (1974) ANSI Se2.9

Alarm Systems and Units for Protection Against Holdup a

Alarm Systems for High-Rise Buildings (1973)

Alarm Systems (1972) ANSI Se2.3

Alarm Systems (1973) ANSI Se2.6

Alarm Units and Systems (1973) ANSI Se2.7

Alarm Valves for Use in Wet Pipe Sprinkler Systems for

Alarm) (1973)

Alarm, and Supervisory Service (1972) ANSI SE3.

Alarm, Door. Deposit and Collection Safes) (1973)

Alarms and Devices (Burglary Protection) (1974)

Alarms, Sandpipes), Building Construction, Basement Pip

Alarm; Use of Equipment, Appliances, Devices and Vacant

/Ole Butts by the Th AWP

Alaska Yellow Cedar Poles) (1973)

Alaska Yellow Cedar Timber Poles (1973)

Alcohol-Benzene Solubles in Wood (1973)

Length Therm AWPA

TAPPI

Alcohol Meth. of Analysis of Ergot in Rye Flour (Cereal

ASTM

IT A

SAMA

NSF

ANSI

ASTM

ICBO

ANS

ATA

ASTM

ANSI

ANSI

ASTM

ANSI

ICBO

NSA

NACE

ATI

RTCA

ASME

FMS

RTCA

ICBO

IEEE

ASTM

RTCA

RTCA

RTCA

$S A E$

SAE

TRA

TRA

NSA

ASTM

SAE

NSA

TRA

ASTM

ICBO

NSF

WW PA

AITC

lof Pr NFPA

UL

UL

UL

UL

UL

UL

UL

UL

D Us NFPA

UL

ICBO

ICBO

ARI

AACCH 44-15A

AACCH 44-18

AACCH 44.20

ASTM D2682

ASTM C204

ASTM D2752

C721

6F1

PMC2.5

48

K13.1

C491

UFC 2 ART 30

C 37.32

15836

D3203

51.13

Z91.1

D726

C667

B107.4

LFC*2ART 36

1369

TM-01-70

DO. 150

D0.151

$7-20$

D0-154

UBC $\$ 35.3$

85

UBC $\$ 35-1$

E90

D0.149

DO- 152

DO-153

$\mathrm{UFC} * 2 \mathrm{ART} 33$

AIR818B

AMS2300B

ARP590B 
lcohol, Versene, and Sodium Phosphate Solutions; lsopropyl Tent. Ebulliometer Meth. for Analysis of Tent. Hydrometer Meth. of Analysis for Tent. Pycnometer Meth. of Analysis for Std. Spec, for Methyl Properties, etc. of Isopropyl Std. Spec. for Diacetone andling of Flammable Liquids (Gasoline, Kerosene, Acetone, Std. Meth. for Chemical Analysis of formaldehyde; Gasoline; Herwig's, Pancreatin, Tween 80-60\% for Extraneous Matter Meth. of Analysis (Cereal Chemistry)

Rec. Basic Characteristics for Airborne Radio Homing and Test Meth. for Shive Content of Mechanical Pulp (Von Meth. of Test for ksi Ftu, 450 Deg. F and 800 Deg. F (1/ Std. for Nut, Self tape Recording (1969) Std. for Nut, Washer, Self Rec. Pract. for Video

opic Examination of Baked Goods, Ready.To-Eat Cereals, and Hard to Hydrate in Baked Goods, Ready-To-Eat Cereals, and and Rodent Filth in Baked Goods, Ready-To-Eat Cereals, and

std. Meth. of Test for Total Bromine Number of Unsaturated $s$ in Preventing Excessive Expansion of Concrete Due to the 72) ANSI L14.129 Test Meth. for L14.1/ Std. Meth, of Test for Vegetable Matter and Other Std. Meth. of Test for Meth. of Assay for

s (1972) ANSI D 14.2 mixtures (1974) Std. Meth. of Test for Reserve Std. Meth. of Test for Acidity Std. Meth of Test for

est Meth. for Colorfastness of Yarn or Fabric to Acids and Std. Meth. of Test for lsophthalic Acid in h. for Quantitiative Determination of Cellulose Nitrate in Std. Rec. Pract. for Test. Std. Meth. of Test for Uniform Fire Code: Bowling allic Gas Valves in Gas Distribution Systems Whose Maximum 974) Std. Meth. for Evaluating uide (Rec. for Garden Grades, Application and Design, Span Std. for Undrilled and Drilled, Plain and Self Locking, mperature Services (1974) A NSl G38. )

Spec, for Aluminum and lis Spec for Copper and lts Std. Spec. for Columbium and lts Std. Spec. for Molybdenum and Molybdenum 4. Std. Spec. for Titanium and Titanium iron Base-13.3Cr-38 Ni-5.5Mo-0.85Cb-2.5Ti-1.6/ nickel Base $19.5 \mathrm{Cr}-13.5 \mathrm{Co}-4.3 \mathrm{Mo}-3.0 \mathrm{Ti}-1.4 \mathrm{Al}$, Con $(6061-0))(1973)$ nickel Base-19.5 $\mathrm{Cr}-13.5 \mathrm{Co}-4.3 \mathrm{Mo}-3.0 \mathrm{Ti}-1.4 \mathrm{AI}, \mathrm{C} /$ (6061-T6) (1973) Spec. for Aluminum Base Spec. for Aluminum stant, Nickel Base $\left(19.5 \mathrm{Cr}_{\mathrm{r}}-13.5 \mathrm{Co}-4.3 \mathrm{Mo}-1\right.$ Spec, for ature Serv/ Std. Spec. for Precipitation Hardening Nickel Std. Spec. for Precipitation Hardening Cobalt Containing heat Treated, 15.1 Spec. for Corrosion and Heat Resistant istant, Nickel Base-15.5Cr-8.0Fe (1973)

istant, Nickel Base $-15.8 \mathrm{Cr}-15.2 \mathrm{Mo}-0.30 \mathrm{Al}-0.05 \mathrm{La} /$ istant, Nickel Base $-5.0 \mathrm{Cr}-24.5 \mathrm{Mo}-5.5 \mathrm{Fe}$, Solution $\mathrm{H}$ ase $-35.5 \mathrm{Ni}-18.5 \mathrm{Cr}-1.1 \mathrm{Sl}$ (1973)

Spec. for Hot Rolled and Cold Finished Zirconium and lts Std. Spec. for Aluminum

e and Other Structural Uses/ Sid Spec for Rolled Copper aded (1973) Std. Spec. for Molybdenum and Molybdenum
Spec. for Titanium ted, Roll Threaded (1973) Spec. for Titanium
Spec. for Titanium istant Iron Chromium, Iron Chromium Nickel and Nickel Base es (1973) A/ Std. Spec. for lron, Cobalt, and Nickel Base iographs for High Strength Copper Base and Nickel-Copper Std. Spec. for Titanium and Its Sid Spec for Chromium Nickel ce (1974) ANSI G/ Std. Spec. for Chromium-Nickel-lron Std. Spec. for Copper Base Std. Spec. for Copper and Its Std. Spec. for Lead and Lead

19674 ANSl C7.15

) ANSI G54.16 Std. Spec. for Alloy Steel Wire, 1 Sheet and Strip, Hot and Cold Rolled, High Strength, Low 1972) ANSl G41.6 Std. Spec. for High Strength Low 4.42 Std. Spec for Seamless Nickel and Nickel $s$ and Heat Exchangers (1973) Std. Spec. for Copper Std. Spec. for Concentric Lay Stranded 5005-H19 Aluminum
Alcohol Saturated with Gasoline: Methyl Blue and Green;

Alcohol (Ethanol) in Wine (Enology) (1972)

Alcohol (Ethanol) in Wine (Enology) (1972)

Alcohol (Ethanol) in Wine (Enology) (1972)

Alcohol (Methanol (99.850) (1972)

Alcohol (1972)

Alcohol (1973)

Alcohol, Cleaning Fluids, Flammable Aerosols, etc. with

Alcohol, Poly(vinyl Butyral) (1973)

Alcohol, Versene, and Sodium Phosphate Solutions; lsopr

Alcohol; Carbon Tetrachloride; Chloral Hydrate; Chlorof

Alert Symbol for Agricultural Equipment (1972)

Alerting Equipment for Use with Emergency Locator Trans Alfthan Shive Analyzer) (1973)

Algal Resistance of Plastic Films (1972T)

Aligning, Self Locking, Plate-Two Lug, Floating, 125

Aligning, 450 and 800 Deg. F (1973)

Alignment Signal Spec. for a Quadruplet Video Magnetic

Alignment Tests-NAS 900 Series Equipment Specs. (1972

Alimentary Pastes for Detection of Rodent Excreta, Inse

Alimentary Pastes (Cereal Chemistry) (1962)

Alimentary Pastes (Cereal Chemistry) (1962)

Aliphatic Chemicals (1972) ANSI Z159.2

Alkali-Aggregate Reaction (1969) ANSI A37.119

Alkali in Bleach Baths Containing Hydrogen Peroxide (19

Alkali Insoluble lmpurities in Scoured Wool (1972) ANSl

Alkali Resistance of Porcelain Enamels (1974) ANSI Z167

Alkaline Protease (1972T)

Alkalinity of Engine Antifreeze, Antirusts, and Coolant

Alkalinity of Halogenated Organic Solvents and Their Ad

Alkalinity of Hollow Glass Microspheres (1972)

Alkalis (1972) ANSI Ll4.2

Alkyd and Polyester Resins (1973)

Alkyd Lacquers by Infrared Spectrophotometry (1972)

Alkyd Resins (1973)

Alkyl Benzene Sulfonate in Water (1968)

Alleys (1973)

Allowable Operating Pressure Does Not Exceed 60 or 125

Allowable Properties for Grades of Structural Lumber (1

Allowances, Storage, Finishes and Nails and Fastenings)

Alloy and Corrosion Resisting Steel, UNJC-3A Thread, Ca

Alloy and Stainless Steel Bolting Materials for High Te

Alloy and Temper Designation Systems for Aluminum (1972

Alloy and Temper Designation Systems for Aluminum (1972

Alloy Arc Welding Electrodes (1969) ANSI W3.3

Alloy Arc Welding Electrodes (1969) ANS1 W3.6

Alloy Bar, Rod, and Wire (1964) ANSl Z179.19

Alloy Bar, Rod, and Wire (1974)

Alloy Bar, Rod, Wire, and Forging Stock (Rolled or Draw

Alloy Bare Welding Rods and Electrodes (1969) ANSI W3.1

Alloy Bare Welding Rods and Electrodes (1970) ANSI W3.1

Alloy Bars and Billets (1972) ANSI Z179.2

Alloy Bars and Forgings, Corrosion and Heat Resistant,

Alloy Bars and Forgings, Corrosion and Heat Resistant,

Alloy Bars and Forgings, Corrosion and Heat Resistant,

Alloy Bars and Rings $(1.0 \mathrm{Mg}-0.60 \mathrm{SI}-0.28 \mathrm{Cu}-0.20 \mathrm{Cr}$

Alloy Bars and Rings $(1.0 \mathrm{Mg}-0.60 \mathrm{Sl}-0.28 \mathrm{Cu}-0.20 \mathrm{Cr}$

Alloy Bars, Forgings and Rings, Corrosion and Heat Resi

Alloy Bars, Forgings, and Forging Stock for High Temper

Alloy Bars, Forgings, and Forging Stock for High Temper

Alloy Bars, Forgings, and Rings (Nickel Base, Solution

Alloy Bars, Forgings, and Rings, Corrosion and Heat Res

Alloy Bars, Forgings, and Rings, Corrosion and Heat Res

Alloy Bars, Forgings, and Rings, Corrosion and Heat Res

Alloy Bars, Forgings, and Rings, Heat Resistant, Iron B

Alloy Bars, Rod, and Wire for Nuclear Application (1973

Alloy Bars, Rods and Wires (1974) ANSl H38.4

Alloy Bearing and Expansion Plates and Sheets for Bridg

Alloy Billets for Reforging (1974)

Alloy Bolts and Screws, $6 \mathrm{Al}-4 \mathrm{~V}$, Heat Treated, Roll Thre

Alloy Bolts and Screws, $6 \mathrm{Al}-4 \mathrm{~V}$, Upset Headed, Heat Trea

Alloy Castings for General Application (I972) ANSI G81.

Alloy Castings for High Strength at Elevated Temperatur

Alloy Castings (1967) ANS1 Z166.17

Alloy Castings (1969) ANS1 Z179.16

Alloy Castings (1974)

Alloy Castings (25-12 Class) for High Temperature Servi

Alloy Centrifugal Castings (1973)

Alloy Clad Steel Plate (1973)

Alloy Coated Soft Copper Wire for Electrical Purposes (

Alloy Cold Heading Quality for Hexagon Head Bolts (1972

Alloy Columbium and/or Vanadium (1970) ANSI G24.32

Alloy Columbium Vanadium Steels of Structural Quality

Alloy Condenser and Heat Exchanger Tubes (1972) ANSI H3

Alloy Condenser Rolled Tube Plates in Surface Condenser

Alloy Conductors (1972) ANS1 C7.50
AACCH 28-91

ASE

ASE

ASE

ACTM

ASTM

ICBO

ASTM

$\mathrm{AACCH}$

RTCA DO-154

TAPPI UM-24

G29

NSA $\quad 1765$

NSA 1727

SMPTE RPI0

NSA 985

A ACCH 28.30

Materials AACCH 28.32

R Insect AACCH 28-31

ASTM E234

Xture ASTM C441

AATCC 98

ASTM D1113

ASTM C614

ASTM D3048

ASTM DI121

ASTM D2989

ASTM D3100

A ATCC 6

ASTM D2690

/T ASTM D3133

ASTM D2689

ASTM D2330

ICBO UFC*2ART4

ANSI Bl6.33

ASTM D2915

CRA 3A5

NSA 1352

ASTM A 193

ANSl H35.1

SAE J993

AWS A5.3

$A W S \quad A 5.6$

ASTM B392

ASTM B387

SAE

A WS

AWS

ASTM

SAE

SAE

SAE

SAE

SAE

SAE

ASTM

ASTM

$S A E$

SAE

SAE

SAE

SAE

A STM

ASTM

ASTM

ASTM

SAE

SAE

ASTM

ASTM

ASTM

ASTM

ASTM

ASTM

ASTM

ASTM

ASTM

/Ee ASTM

ASTM

ASTM

ASTM

AMS2201J

A5. 14

A5. 16

B348

AMS5633A

AMS5709B

A M $55738 \mathrm{C}$

AMS4115C

AMS4117D

AMS5707D

A637

A639

AMS5750B

AM $\$ 5665 \mathrm{H}$

AMS5711

AMS5755B 
National Bureau of Standards KWIC Index of Engineering Standards

Std. Spec. for Concentric Lay Stranded 6201-T 81 Aluminum Std. Spec. for Copper Base Spec. for Nickel and Its Std. Spec. for Aluminum Std. Spec. for Lead and Tin Std. Spec, for Zinc Std. Spec. for Magnesium Std. Spec. for Aluminum

Std. Spec. for Copper and Its Std. Spec. for Aluminum Std. Spec. for Aluminum Std. Spec. for Aluminum . angers (1973) ANSI H38.6

3) ANSI H38.17

Spec. for Tolerances of Aluminum Std. Spec. for Gold Copper Std. Spec. for Aluminum r Electrical Purposes (Bus Condu/ Tolerances Applicable to Titanium and Its Base

i $\mathrm{H} 38.5$ Std. Spec. for Aluminum 70) ANSI H45.6 pplications (1973) ANSI H38.18 Std. Spec. for Magnesium Std. Spec. for Aluminum Std. Spec. for Aluminum Spec. for Tolerances of Aluminum and Magnesium Base Std. for Welded Aluminum Std. Spec. for Annealed Aluminum Std. Spec. for Steel Castings, Heavy Walled, Carbon and Std. Spec. for Copper and Its 1967) ANSI N125

Std. Spec. for Zirconium and It Std. Spec. for Copper Beryllium Std. Spec. for Nicke Std. Spec. for Magnesium Std. Spec. for Molybdenum and Molybdenum ) (1973) Spec. for Aluminum 736) Solution and Precipitation Heat Treated (/ Aluminum 20 Std, Spec. for Aluminum (1965) ANSI H38.23 Std. for Hardenability Bands for Std. Spec. for Aluminum $\operatorname{ded}(1973)$ Std. Spec. for U-Bend Seamless Copper and Its Bolts and Screws, Steel, Low Std. Spec. for Zirconium and lts Std. Spec. for Magnesium

truction Manual Sect. 1) (1/

Spec for Design of Aluminum Std. Spec. for Copper-Cobalt-Beryllium Alloy (Copper ge for Heat Exchanger Tubing (1972/ Std. Spec. for Coppe (1973) Spec. for Self-Locking Corrosion Resistan or Chromate Conversion Coatings on Aged or Backed Aluminum Std. Spec. for Magnesium Std. Spec. for Aluminum ed on the Composition and Cryst/ Std. Meth. for Assigning
hromium Nickel Iron Molybdenum Copper Columbium Stabilized hromium Nickel Iron Molybdenum Copper Columbium Stabilized
nickel-Iron-Molydenium-Copper-Columbium Stabilized h52.1 Std. Spec. for Copper Nicke Std. Spec for Copper Silicon Std. Spec. for Nickel-Molybdenum Std. Spec. for Nickel-Molybdenum-Chrom ium Std. Meth. for Ultrasonic Inspection of Aluminum Std. Spec. for Nickel Chromium Molybdenum Columbium Spec. for Nickel-Chromium-Iron-Molybdenum-Copper ice (1972) Std. Spec. for Precipitation Hardening Nickel Sid. Spec. for Nickel-Chromium-Iron Std. Spec. for Nickel Copper

Std. Spec. for Low Carbon Nickel Molybdenum Chromium Std. Spec. for Nickel Iron Chromium Molybdenum Copper pec. for Nickel-Iron-Chromium-Manganese-Molybdenum hromium Nickel Iron Molybdenum Copper Columbium Stabilized Std. Spec. for Nickel-lron-Chromium Std. Spec. for Copper-Beryllium Std Spec for Copper Iron

Std. Spec. for Copper-Zinc-Aluminum-Cobal -Nickel-Zinc Alloy (Nickel Silver) and Copper-Nickel ec. for General Requirements for Wrought Copper and Copper of Tension Test. Wrought and Cast Aluminum and Magnesium Std. Spec. for Seamless Copper and It

for Concentric Lay Stranded Aluminum Conductors, Aluminum 38.12

Std. Meth. of Shear Testing of Aluminum and Aluminum Std. Spec. for Nickel Chromium Iron Std. Spec. for Copper Beryllium

Std. Spec. for Nickel lron Chromium Molybdenum Coppe Std. Spec. for Nickel Chromium Molybdenum Columbium Std. Spec. for Copper-Nickel-Silicon Std. Spec. for Nickel-Iron-Chromium tid. Spec. for Nickel-Molybdenum Std. Spec. for Nickel-Molybdenum-Chromium Std. Spec. for Nickel Chromium Molybdenum Iron Std. Spec. for Nickel Molybdenum Chromium Iron

Std. Spec. for Low Carbon Nickel Molybdenum Chromium . Spec. for Nickel-Chromium-lron-Molybdenum-Copper
Alloy Conductors (1972) ANSI C7.52

Alloy Continuous Castings (1973)

Alloy Covered Welding Electrodes (1969) ANSI W3.11

Alloy Die and Hand Forgings (1973) ANSl H38.8

Alloy Die Castings (1952) ANSI H48.

Alloy Die Castings (1971) ANSI H47.1

Alloy Die Castings (1972)

Alloy Die Castings (1972) ANSI H38.22

Alloy Die Forgings (Hot Pressed) (1974) ANSl H7.10

Alloy Drawn Seamless Tubes for Condensers and Heat Exch

Alloy Drawn Seamless Tubes (1974) ANSI H38.3

Alloy Drawn Tubes for General Purpose Applications (197

Alloy Drawn Tubing (1973)

Alloy Electrical Contact Material (1973)

Alloy Extruded Bar, Rod, Pipe, and Structural Shapes Fo

Alloy Extruded Bars, Rods, and Shapes (1973)

Alloy Extruded Bars, Rods, Shapes, and Tubes (1973) Ans

Alloy Extruded Bars, Rods, Shapes, Tubes, and Wires (19

Alloy Extruded Round Coiled Tubes for General Purpose a

Alloy Extruded Structural Pipe and Tube (1973) ANSI H38

Alloy Extrusions (1973)

Alloy Field Erected Storage Tanks (1973)

Alloy Foil for Flexible Barrier Applications (1973)

Alloy for Pressure Vessels (1974)

Alloy Forging Rod, Bar and Shapes (1974) ANSI H7.1

Alloy Forgings and Extrusions for Nuclear Application(

Alloy Forgings and Extrusions (1972)

Alloy Forgings (1972)

Alloy Forgings (1972)

Alloy Forgings (1974)

Alloy Forgings $(5.6 \mathrm{Zn}-2.5 \mathrm{Mg}-1.6 \mathrm{Cu}-0.26 \mathrm{Cr}$ (7075. T6

Alloy Forgings, $5.6 \mathrm{Zn}-2.5 \mathrm{Mg}-1.6 \mathrm{Cu}-0.26 \mathrm{Cr}$ (7075-

Alloy Formed and Arc Welded Round Tube (1974) ANSI H38.

Alloy H Steels (1971)

Alloy Hardeners Used in Making Zinc Die Casting Alloys

Alloy Heat Exchanger and Condenser Tubes (1973)

Alloy Heat Resistant, Hardened and Tempered, Roll Threa

Alloy Ingots for Nuclear Application (1973) ANSI G53.28

Alloy Investment Castings (1972)

Alloy Load Carrying (Members) Structures (Aluminum Cons

Alloy No. 175), Plate, Sheet, Strip and Rolled Bar (197

Alloy No. 260 Brass Strip in Narrow Widths and Light Ga

Alloy Nuts, High Strength, Prevailing Torque, All Metal

Alloy Panels (Coil) (1974) /Ing Weight Determination F

Alloy Permanent Mold Castings (1972) ANSI H45.2

Alloy Permanent Mold Castings (1974) ANSI H38.26

Alloy Phase Designations (Code) in Metallic Systems Bas

Alloy Pipe (1973) /Td. Spec. for Seamless and Welded C

Alloy Pipe (1974)

Alloy Plate and Sheet for Pressure Vessels (1969) ANSI

Alloy Plate and Sheet for Pressure Vessels (1973)

Alloy Plate and Sheet (1971) ANSI H34.11

Alloy Plate and Sheet (1971) ANSI H34.12

Alloy Plate for Pressure Vessels (1971) ANSI H38.21

Alloy Plate, Sheet and Strip (1972) ANSI H34.19

Alloy Plate, Sheet and Strip (1973)

Alloy Plate, Sheet, and Strip for High Temperature Serv

Alloy Plate, Sheet, and Strip (1970) ANSI H34.10

Alloy Plate, Sheet, and Strip (1970) ANSI H34.6

Alloy Plate, Sheet, and Strip (1972)

Alloy Plate, Sheet, and Strip (1972) ANSI H34.17

Alloy Plate, Sheet, and Strip (1973)

Alloy Plate, Sheet, and Strip (1973) ANSI H34.37

Alloy Plate, Sheet, and Strip (1973) ANSI H34.40

Alloy Plate, Sheet, Strip, and Rolled Bar (1973)

Alloy Plate, Sheet, Strip, and Rolled Bar (1973)

Alloy Plate. Sheet, Strip, and Rolled Bar (1973)

Alloy Plate, Sheet, Strip, and Rolled Bar (1973)

Alloy Plate, Sheet, Strip, and Rolled Bar (1974)

Alloy Products (1973) ANSl H46.1

Alloy Rectangular Waveguide Tube (1974) ANSI H37.1

Alloy Reinforced (Acar, EC/6201) (1972) ANSI C7.65

Alloy Rivet and Cold Heading Wire and Rod (1973) ANSI H

Alloy Rivets and Cold Heading Wire and Rods (1972)

Alloy Rod and Bar (1970) ANSI H34.4

Alloy Rod and Bar (1971A) ANSI H7.7

Alloy Rod and Bar (1972) ANSI H34.18

Alloy Rod and Bar (1972) ANSI H34.22

Alloy Rod and Bar (1973)

Alloy Rod and Bar (1973) ANSI H34.39

Alloy Rod (1971) ANSI H34.13

Alloy Rod (1971) ANSI H34.14

Alloy Rod (1972)

Alloy Rod (1972)

Alloy Rod (1972)

Alloy Rod (1973)
ASTM

ASTM

AWS

ASTM

ASTM

ASTM

ASTM

ASTM

ASTM

ASTM

ASTM

ASTM

SAE

ASTM

ASTM

SAE

ASTM

ASTM

ASTM

ASTM

SAE

ANSI

ASTM

ASTM

ASTM

ASTM

ASTM

ASTM

ASTM

ASTM

SAE

SAE

ASTM

SAE

ASTM

ASTM

SAE

ASTM

ASTM

AA

ASTM

A STM

SAE

NCCA

A STM

ASTM

ASTM

ASTM

ASTM

ASTM

ASTM

A STM

ASTM

ASTM

ASTM

Std ASTM

ASTM

ASTM

ASTM

ASTM

ASTM

Std. S ASTM

lor C ASTM

ASTM

ASTM

ASTM

ASTM

/Pper ASTM

1. Sp ASTM

Std. Meth ASTM

ASTM

/C. ASTM

ASTM

ASTM

ASTM

ASTM

ASTM

ASTM

ASTM

ASTM

ASTM

ASTM

ASTM

ASTM

ASTM

Std ASTM

B399

5505

A5. 11

B247

B86

B85

B283

B234

B210

B483

MS2203G

B596

B317

AMS 2245

B221

$\mathrm{B} 107$ 
Spec. for General Requirements for Wrought Copper and Its Std. Spec. for Copper Silicon Std. Spec for Aluminum Std. Spec. for Copper Std. Spec. for Magnesium

Std. Spec, for Aluminum Spec. for Magnesium solution and Precipitation Treated (/ Std. Spec. for Nickel lron Chromium Silicon 71) ANSI N124 Std. Spec. for Wrought Zirconium and Its Std. Spec. for Columbium and Its Std. Spec. for Copper and Its integral Fins (1974)

ANSI H23.14

ransmission and Distribution Pip/ Std. Spec. for Copper and Its Std. Spec. for Aluminum Std. Spec. for Aluminum Std. Spec. for Nickel Chromium Iron Spec. for Nickel-Iron-Chromium-Molybdenum-Copper Sid Spec for Nickel Chromium Molybdenum Columbium ec. for Nickel Chromium Iron Columbium Molybdenum Tungsten Std. Spec. for Copper Silicon with Integral Fins (1973) Std. Spec. for Nickel-Iron-Chromium Std. Spec. for Aluminum Std. Spec. for Magnesium Std. Spec. for Nickel-Molybdenum-Chromium-Iron Spec. for Tolerances of Aluminum and Magnesium Base Std. Spec. for Aluminum ckel Base-15.5Cr-2.4Ti-0.70Al-7.0Fe (1973)

1973) ANSI N123 Std. Spec. for Zirconium and Its tant, Nickel Base, $20 \mathrm{Cr}-20 \mathrm{Co}-5.9 \mathrm{Mo}-2.2 \mathrm{Ti} /$ tant. Nickel Base-15.8Cr-15.2Mo-0.30Al-0.05La (1/ ling, $53 \mathrm{Fe}-29 \mathrm{Ni}-17 \mathrm{Co}$ (1973)

Titanium Unhardened Steel and Standard for Wrought Copper and Its ec. Pract. for Conducting Bending Fatigue Tests for Copper Spec. for Tolerances of Aluminum

ANSI H38.10 Std Spec for Aluminum

Std, for Undrilled and Drilled, Plain and Self Locking, ures (1971) Std. Spec. for Hot, Hot Cold, and Cold Worked Std Spec for Carbon and Std. Spec. for Cold Finished Spec. for Tolerances of Low 25.9 Std. Spec. for Seamless Carbon Molybdenum td Spec for Electric Resistance Welded Carbon Molybdenum es $(1973 /$ Std. Spec. for Seamless Ferritic and Austenitic ves, Flanges, and Fittings for Low Temper/ si $(1,345 \mathrm{MPa})$ Tensile Strength, Hardened / ed (1973)

si G24.19 Std. Spec. for Spec. for Low Spec. for Low meth. for Spectrochemical Analysis of Plain Carbon and Low able for High T/

Std Spec for Martensitic Stainless and Std. Spec. for Heavy $\mathbb{W}$ alled Carbon and Low e Containing Parts Suitable for High Temp/ 4) ANSI G52.5 Std. Spec. for

Longitudinal Beam Ultrasonic lnspection of Carbon. For Low o (0.38-0.46C) (SAE 4340 Modified) (1973) Low -0.35 C) (1973)

w3.5

Low

w Temperature

Spec, for Low .2 Std. Spec. for Piping Fittings of W rought Carbon and 3) / Std. Spec, for Piping Fittings of W rought Carbon and id. Spec. for Hot Worked, Hot Cold Worked, and Cold Worked r Generators (1970) ANSI G55.6

n Gears (1972) ANSI G55.8 Std. Spec. for 73) Ansil Sid Spec for Quenched and Tpec. for Carbon an Spec. for Quenched and Tempered Vacuum Treated Carbon and er Pressure Vessel Components (1970) Ansi/ Std. Spec. for (1970) ANSI G55.15

4) ANSI G55.I4 nsi B125/ Sid. Spec. for Seamless Cold Drawn lntermediate td. for Aircraft, Pan Head, Phillips Recess Full Threaded, raft, Pan Head, Phillips Recess, Short Thread, 160,000 Ps Std. Spec. for Seamless Carbon and ature Service (1973) ANS1 E38.l Std. Spec. for Carbon and nd Parts for High Temper Std. Spec. for Forged or Rolled spec, for Welded Large Diameter Austenitic Chromium Nicke spec. for Electric Fusion Welded Autenitic Chromium Nicke Std. Spec. for Centrifugally Cast Ferritic si B125.24 Std. Spec. for Seamless Ferritic

Spec. for General Requirements for Specialized Carbon and
Std. Spec. for Hot, Hot Cold, and Cold Worked t Elevated/ Std. Spec. for Hot, Hot Cold, and Cold Worked
Std. Spec. for High Yield Strength, Quenched and Tempered Std. Spec. for Manganese Vanadium Std. Spec. for Nickel
Alloy Rod, Bar, and Shapes (1973)

Alloy Rod, Bar, and Shapes (1973) ANSI H7.3

Alloy Round Welded Tubes (1973) ANSI H38.11

Alloy Sand Castings for General Applications (1973)

Alloy Sand Castings (1972) ANSI H45.1

Alloy Sand Castings (1974) ANSI H38.24

Alloy Sand Castings (5.75Zn-2.5Re-0.70Zr (Ze63.T6)

Alloy Seamless and Welded Pipe (1972)

Alloy Seamless and Welded Tubes for Nuclear Service (19

Alloy Seamless and Welded Tubes (1970) ANSI H53.1

Alloy Seamless Condenser and Heat Exchanger Tubes with

Alloy Seamless Condenser Tubes and Ferrule Stock (1974)

Alloy Seamless Extruded Tube and Pipe for Gas and Oil T

Alloy Seamless Pipe and Seamless Extruded Tube (1973) a

Alloy Seamless Pipe and Tube (1970) ANSI H34.3

Alloy Seamless Pipe and Tube (1972) ANSI H34.16

Alloy Seamless Pipe and Tube (1972) ANSI H34.20

Alloy Seamless Pipe and Tube (1972) ANSI H34.21

Alloy Seamless Pipe and Tube (1974) ANSI H26.3

Alloy Seamless Pipe Tube (1973) ANSI H34.41

Alloy Seamless Round Condenser and Heat Exchanger Tubes

Alloy Sheet and Plate (1970) ANSI H45.3

Alloy Sheet and Plate (1971) ANSI H34.44

Alloy Sheet and Plate (1973)

Alloy Sheet and Plate (1973) ANSI H38.2

Alloy Sheet and Strip, Corrosion and Heat Resistant, Ni

Alloy Sheet, Strip, and Plate for Nuclear Application (

Alloy Sheet, Strip, and Plate, Corrosion and Heat Resis

Alloy Sheet, Strip, and Plate, Corrosion and Heat Resis

Alloy Sheet, Strip, and Plate, Low Expansion, Glass Sea

Alloy Sheet, Strip, and Plate, 6A1-4V Annealed (1973)

Alloy Silver Brazing Joints (1973)

Alloy Solder Joint Drainage Fittings (1973)

Alloy Spring Materials (1973)

Alloy Std. Structural Shapes (1973)

Alloy Std. Structural Shapes, Rolled or Extruded (1973)

Alloy Steel and Corrosion Resisting Steel, UNJF.3A Thre

Alloy Steel Bar (1973) ANSI G24.5 ID. Spec. for

Alloy Steel Bars for High Strength at Elevated Temperat

Alloy Steel Bars for Springs (1974)

Alloy Steel Bars (1964) ANSl G24.12

Alloy Steel Bars (1973)

Alloy Steel Boiler and Superheater Tubes (1973) ANSI B 1

Alloy Steel Boiler and Superheater Tubes (1973) ANSI BI

Alloy Steel Boiler, Superheater, and Heat Exchanger Tub

Alloy Steel Bolting Materials for Pressure Vessels, Val

Alloy Steel Bolts and Screws, Heat Resistant, 195,000 P

Alloy Steel Bolts and Screws, Heat Treated, Roll Thread

Alloy Steel Bolts for Structural Steel Joints (1971) an

Alloy Steel by the Point to Plane Technique Using an $O_{p}$

Alloy Steel Castings for Pressure Containing Parts Suit

Alloy Steel Castings for Steam Turbines (1974)

Alloy Steel Castings Specially Heat Treated for Pressur

Alloy Steel Castings Suitable for Pressure Service (197

Alloy Steel Castings (1970) ANSI G52.7

Alloy Steel Castings, Investment $0.80 \mathrm{Cr}-1.8 \mathrm{Ni}-0.35 \mathrm{M}$

Alloy Steel Castings, Investment, $0.95 \mathrm{Cr}-0.20 \mathrm{Mo}(0.25$

Alloy Steel Covered Arc Welding Electrodes (1969) ANSI

Alloy Steel Flanges, Fittings, Valves, and Parts for Lo

Alloy Steel for Boron by the Point to Plane Arc Techniq

Alloy Steel for Low Temperature Service (1973) ANSI G46

Alloy Steel for Moderate and Elevated Temperatures (197

Alloy Steel Forgings and Forging Billets for High Stren

Alloy Steel Forgings for Nonmagnetic Retaining Rings Fo

Alloy Steel Forgings for Pinions and Gears for Reductio

Alloy Steel Forgings for Pressure Vessel Components (19

Alloy Steel Forgings for Pressure Vessels (1974) ANSI G

Alloy Steel Forgings for Seamless Drums, Heads, and Oth

Alloy Steel Forgings for Turbine Rotor Disks and Wheels

Alloy Steel Forgings for Turbine Rotors and Shafts (197

Alloy Steel H.Piles and Sheet Piling for Use in Marine

Alloy Steel Heat Exchanger and Condenser Tubes (1973) a

Alloy Steel Machine Screw (1974)

Alloy Steel Machine Screw (1974)

Alloy Steel Mechanical Tubing (1973) ANSI G62.4

Std. for Airc

Alloy Steel Nuts for Bolts for High Pressure and Temper

Alloy Steel Pipe Flanges, Forged Fittings, and Valves a

Alloy Steel Pipe for Corrosive or High Temperature Serv

Alloy Steel Pipe for High Temperature Service (1972)

Alloy Steel Pipe for High Temperature Service (1973)

Alloy Steel Pipe for High Temperature Service (1973) an

Alloy Steel Pipe (1972) ANSI B125.20

Alloy Steel Plate, Sheet, and Strip for High Strength a

Alloy Steel Plate, Suitable for Welding (1970E) ANS1 G2

Alloy Steel Plates for Pressure Vessels (1972)

Alloy Steel Plates for Pressure Vessels (1972) ANSI G33

\section{ID. $\mathrm{Sp}$}

Std. AST

ASTM

ASTM

ASTM

ASTM

ASTM

SAE

ASTM

ASTM

ASTM

ASTM

ASTM

ASTM

ASTM

ASTM

Std ASTM

ASTM

ASTM

ASTM

ASTM

ASTM

ASTM

SAE

ASTM

SAE

ASTM

SAE

SAE

SAE

SAE

SAE

ANSI

Std. R ASTM

SAE

ASTM

NSA

ASTM

ASTM

ASTM

SAE

ASTM

ASTM

ASTM

ASTM

SAE

SAE

ASTM

ASTM

ASTM

ASTM

ASTM

ASTM

ASTM

SAE

SAE

AWS

ASTM

ASTM

ASTM

ASTM

ASTM

ASTM

ASTM

ASTM

ASTM

ASTM

ASTM

ASTM

ASTM

ASTM

NSA

NSA

ASTM

ASTM

ASTM

ASTM

ASTM

ASTM

ASTM

Std. ASTM

ASTM

ASTM

ASTM

ASTM 
1

15 Std. Spec. for Manganese Molybdenum and Its Nickel Pressure Vessels (1/ Std. Spec. for Chromium Molybdenum, Vessels (1972) Std. Spec. for Nickel Chromium Molybdenum Std. Spec. for Chromium-Copper-Nickel-Aluminum Std. Spec. for Regular Quality Hot and Cold Rolled Std. Spec. for Drawing Quality Hot and Cold Rolled i B125.23 Std. Spec. for Seamless Intermediate red, Roll Threaded (135,000 psi (931 MPa) / Spec. for Low nsi B125.1/ Std. Spec. for Seamless and Welded Carbon and al Requirements for Carbon, Ferritic Alloy, and Austenitic Spec. for Seamless Ferritic Austenitic Treated for High Temperature Service (1/ Sid. Spec. for Std. Meth. for Spectrochemical Analysis of Carbon and Low n Head Bolts (1972) ANSI G54.16

5.16 Std. Spec, for Pressure Vessel Plates, Rec. Pract. for High Strength, Low td. for Screw, Machine, 100 Deg., Flat Head Full Threaded, 2.1 Std. Spec, for Pressure Vessel Plates, Spec, for Fasteners, Std. for Rod End, Threaded, Std. for Screw, Hex Head, Tri Wing Recess, 1973) Std. for Screw, 100 Deg. Head, Tri Wing Recess, a) ANSI G35.11 Std. Spec. for Pressure Vessel Plates, for Bolt, 100 Deg. Head, Tri Wing Recess, Close Tolerance, 1972) ckel (1972A)

ckel (1972A) Ansi/

lybdenum-Chromi/ Std. for Bolt, Hex Head, Close Tolerance, Std. Spec. for Pressure Vessel Plates, Std. Spec. for Pressure Vessel Plates. Std. Spec. for Pressure Vessel Plates,

2A) ANSI G35.20 Std. Spec. for Pressure Vessel Plates,
manganese-Molybdenum and Manganese-Molybdenum-Nickel

Pan Head, Close Tolerance, Short Thread, TWI Wing Recess, crew, Flat Fillister Head, Full Threaded, Tri Wing Recess, (1972) 100 Deg. Reduced Head, Tri Wing Recess, Close Tolerance, Std. for Bolt, Hex Head, Close Tolerance, for Bolt, 100 Deg. Head, Tri Wing Recess, Close Tolerance, Std. Spec. for Pressure Vessels Plates, 5\% Nickel d. Part Bolt, 100 Deg. Flush Tension Head Hi Torque Recess Std. Spec. for Pressure Vessel Plates, 1972A) ANSI G35.3 Std. Spec. for Pressure Vessel Plates, k) Spec. for Quality Assurance Sampling of Carbon and Low
Std. for Chemical Compositions of Sae Analysis of Tool Steels and Other Similar Medium and High Std. Spec. for Copper Spec. for Titanium and Titanium Std. Spec. for Columbium and Its git. Spec. for Molybdenum and Molybdenum
Std. Spec. for High Strength Low Std. Spec. for High Strength Low Std Spec, for Normalized High Strength Low spec. for Hot Formed Welded and Seamless High Strength Low Std. for Titanium

(1973) Std Spec, for Wrought Std. Spec. for Welded Copper and Its
eneral Requirements for $W$ rought Seamless Copper and Copper Sid. Spec. for Copper nickel-Iron-Molybdenum-Copper-Columbium Stabilized spec, for Centrifugally Cast Iron-Chromium-Nickel High Std. Spec. for Tantalum and Its Std. Spec. for Welded Copper h3/ Std. Spec. for Factory Made Wrought Nickel and Nickel
Std. Spec. for Factory Made Wrought Aluminum and Its 0 Spec. for Aluminum and lts Std. Spec. for Copper Silicon Std. Spec. for General Requirements for Wrought Copper It Base-25.5 Cr $-10.5 \mathrm{Ni}-7.5 \mathrm{~W}(1973)$ el Base-19.5 $\mathrm{Cr}-18 \mathrm{Co}-2.5 \mathrm{Ti}-1.5 \mathrm{Al}$ (1973)

) nsi C7.66 Std. Meth. for Ultrasonic Inspection of Aluminum i C7.49

Sid Spec. for Aluminum

Close Tolerance, Short Thread, Tri Wing Recess, Titanium i $C 7.51$ olled Bar (19)

sheet, Strip, and / Std. Spec. for Aluminum

1973)

td. Meth. Test for Electrodeposited Coatings of Tin-Lead Std. Spec. for Gold, Silver, Nickel Electrical Contact

Std. for Bolt, Hex Head, Close Tolerance, Titanium Std. Spec. for Gold Electrical Contact

Std. Procurement Spec. for Fasteners, Titanium

Bolt Lock, Tension, Protruding Head, Stump Type, Titanium
Alloy Steel Plates for Pressure Vessels (1972) ANSI G34 Alloy Steel Plates for Pressure Vessels (1972) ANSI G35 Alloy Steel Plates for Pressure Vessels (1973) ANSI G35 Alloy Steel Plates, Quenched and Tempered Chromium, for Alloy Steel Plates, Quenched and Tempered, for Pressure Alloy Steel Pressure Vessel Plates (1972) ANSI G33.3

Alloy Steel Sheet and Strip (1973) ANSI G24.24

Alloy Steel Sheet and Strip (1973) ANSI G24.25

Alloy Steel Still Tubes for Refinery Service (1972) Ans

Alloy Steel Studs, Heat Resistant, Normalized and Tempe

Alloy Steel Studs, Heat Treated, Roll Threaded (1973)

Alloy Steel Tubes for Low Temperature Service (1972A) a

Alloy Steel Tubes (1971A) ANSI B125.34 /Pec. for Gener

Alloy Steel Tubes (1972)

Alloy Steel Turbine Type Bolting Material Specially Hea

Alloy Steel Using a Vacuum Spectrometer (1971) ANSl Z12

Alloy Steel Wire, Alloy Cold Heading Quality for Hexago

Alloy Steel 5\% Chromium, 0.5\% Molybdenum (1972) ANSI G3

Alloy Steel (1970) ANSI G88.3

Alloy Steel (1973)

Alloy Steel, Chromium Manganese Silicon (1972A) ANSI G3

Alloy Steel, Externally Threaded (1972)

Alloy Steel, Flash Welding Type (1973)

Alloy Steel, Full Thread, Nonlocking (1973)

Alloy Steel, Full Thread, Self-Locking and Nonlocking

Alloy Steel, High Strength, Quenched and Tempered (1972

Alloy Steel, Long Thread, Self Locking and Nonlocking (

Alloy Steel, Long Thread, Self Locking and Nonlocking

Alloy Steel, Precipitation Hardening (Maraging), $12 \% \mathrm{Ni}$

Alloy Steel, Precipitation Hardening (Maraging), 18\% Ni

Alloy Steel, Quenched and Tempered Nickel-Cobalt-Mo

Alloy Steel, Quenched and Tempered 8 and 9\% Nickel (197

Alloy Steel, Quenched and Tempered, for Pressure Vessel

Alloy Steel, Self Locking and Nonlocking (1972)

Alloy Steel, Self-Locking and Nonlocking (1973)

Alloy Steel, Short Thread, Nonlocking (1973)

Alloy Steel, Short Thread, Self Locking and Nonlocking

Alloy Steel, Short Thread, Self-Locking and Nonlocking

Alloy Steel, Specially Heat Treated (1972A)

Alloy Steel, 160,000 psi (1973)

Alloy Steel, 36\% Nickel (1972)

Alloy Steel, 9\% Nickel Double Normalized and Tempered

Alloy Steels (Wrought Products Except Forgings and Stoc

Alloy Steels (1970) ANSI G88.2

Alloy Steels (1973)

Alloy Strip for Flexible Metal Hose (1973)

Alloy Strip, Sheet, and Plate (1972) ANSI Z179.1

Alloy Strip, Sheet, Foil, and Plate (1974)

Alloy Structural Manganese Vanadium Steel (1970A) ANSI

Alloy Structural Steel (1970A) ANSI G41.2

Alloy Structural Steel (1973)

Alloy Structural Tubing (1973)

Alloy Stump Type Protruding Head Shear Bolt Lock (1974)

Alloy Stump Type 100 Deg. Head Shear Bolt Lock (1974)

Alloy Tool Steel Products (Including Dies and Fixtures)

Alloy Tube (1973)

Alloy Tube (1974) ANSI H23.4

Alloy Tubes for Pressure Applications (1972)

Alloy Tubes (1973) for Seamless and Welded Chromium-

Alloy Tubing for Pressure Application at High Temperatu

Alloy Tubing (1972) ANSI H54.1

Alloy Water Tube (1973)

Alloy Welding Fittings for Pressure Piping (1972) ANSI

Alloy Welding Fittings (1973) ANSI H38.19

Alloy Welding Rods and Bare Electrodes (1969) ANSI W 3.1

Alloy Welding Rods and Bare Electrodes (1969) ANSI W3.1

Alloy Welding Rods (1969) ANSI W3.7

Alloy Wire for General Purposes (1973) ANSI H30.1

Alloy Wire (1974) ANSI H 30.2

Alloy Wire, Welding, Corrosion and Heat Resistant, Coba

Alloy Wire, Welding, Corrosion and Heat Resistant, Nick

Alloy Wrought Products for Aerospace Applications (1974

Alloy 5005 Rolled Rods for Electrical Purposes (1972) a

Alloy 5005-H 19 Wire for Electrical Purposes (1972A) Ans

Alloy 6Al-4V, Self Locking and Nonlocking (1972)

Alloy 6201 T 81 Wire for Electrical Purposes (1972) Ans

Alloy (Copper Alloy No. 175), Plate, Sheet, Strip and R

Alloy (Nickel Silver) and Copper-Nickel Alloy Plate,

Alloy (Nickel Silver) and Copper-Nickel Rod and Bar (

Alloy (Solder Plate) (1973)

Alloy (1972)

Alloy (1972)

Alloy (1973)

Alloy (1973)

Alloy (1973)
Bolt, NSA

Ine S NSA

for Bolt NSA

NSA

NSA

ASTM

St NSA

ASTM

ASTM

SAE

ASTM

ASTM

ASTM

ASTM

ASTM

ASTM

STM

STM

STM

STM

STM

STM

AE

STM

STM

STM

STM

SA

STM

SA

SA

SA

STM

STM

STM

TM

ASTM

ASTM

ASTM

Std. ASTM

NSA

NSA

ASTM

ASTM

STM

ASTM

ASTM

ASTM

ASTM

AWS

AWS

AWS

ASTM

ASTM

$S A E$

SAE

ASTM

ASTM

ASTM

/Head NSA

ASTM

ASTM

ASTM

ASTM

ASTM

ASTM

NSA

ASTM

NSA

Std. for NSA

A204

A302

A387

A542

506

A200

AMS 7458C

AMS7456G

A334.

A450

A669

A 437

E415

A5 47

357

$\mathrm{J} 4.10 \mathrm{C}$

514

A202

5900-03

5600-06

517

4104-16

6604-20

A590

538

A655

A533

$5000-6$

5300-06

4703-16

6203-20

$4400-16$

A645

583

A658

A353

AMS2370A

J404G

(352 
t Lock, Tension, 100 Deg. Crown Head, Stump Type, Titanium ock, Tension, 100 Deg. Head (MS24694), Pull Type, Titanium ck, Tension, 100 Deg. Head (MS24694), Stump Type, Titanium ck, Tension, $100 \mathrm{Deg}$. Head (MS20426), Stump Type, Titanium ear, 100 Deg. Head, Std. and Oversize, Pull Type, Titanium r, Protruding Head, Std. and Oversize, Pull Type, Titanium $n$, Protruding Head, Std. and Oversize, Pull Type, Titanium 00 Deg. Crown Head, Std. and Oversize, Pull Type, Titanium eg. Head (MS20426), Std. and Oversize, Pull Type, Titanium Irons (/ Std. Spec. for General Requirements for Carbon, Ferritic Std. Meth. for Chemical Analysis of Carbon, Low Spec, for Fasteners, 6Al.4V Titanium Std. Spec. for Steel Forgings, Carbon and std. for Screw, Hex Head, Tri Wing Recess, 6Al-4V Titanium for Screw, 100 Deg. Head Tri Wing Recess, 6Al-4V Titanium $0.50 \mathrm{C}) \mathrm{V} / \quad$ Spec. for Steel Bars, Forgings, and Tubing. Low $6 \mathrm{Al}-4 \mathrm{~V}$ Titanium

Std. for Bolt, Hex Head, Close Tolerance, 6Al-4V Titanium Std. for Pin, Swage Locking, Aluminum Std. for Pin, Swage Locking, Aluminum Tolerance, $6 \mathrm{Al}-4 \mathrm{~V}$ Titanium

100 Deg. Tri Wing Recess, Close Tolerance, 6Al-4V Titanium std. for Bolt, Hex Head, Close Tolerance, $6 \mathrm{Al}-4 \mathrm{~V}$, Titanium Std. for Pin, Swage Locking, Aluminum $\begin{array}{ll}\text { 3) } & \text { Std. for Pin, Swage Locking, Aluminum } \\ \text { 3) } & \text { Std. for Pin, Swage Locking, Aluminum } \\ 73) & \text { Std. for Pin, Swage Locking, Aluminum } \\ & \text { Std. for Pin, Swage Locking, Aluminum }\end{array}$ $\begin{array}{ll}\text { 3) } & \text { Std. for Pin, Swage Locking, Aluminum } \\ \text { 3) } & \text { Std. for Pin, Swage Locking, Aluminum } \\ 73) & \text { Std. for Pin, Swage Locking, Aluminum } \\ & \text { Std. for Pin, Swage Locking, Aluminum }\end{array}$ $\begin{array}{ll}\text { 3) } & \text { Std. for Pin, Swage Locking, Aluminum } \\ \text { 3) } & \text { Std. for Pin, Swage Locking, Aluminum } \\ 73) & \text { Std. for Pin, Swage Locking, Aluminum } \\ & \text { Std. for Pin, Swage Locking, Aluminum }\end{array}$ 73) lat Fillister Head, Full Thread, Tri Wing Recess, Titanium Meth. for Chemical Analysis of Ferrovanadium and Vanadium d. Meth. for Spectrochemical Analysis of Thermionic Nickel

Std. Meth. for Spectrochemical Analysis of Nickel-Base

Pract. for Preparation of and Electroplating on Aluminum Std. Spec. for Zinc

castings (1973) ANSI H38.l castings (1966) ANSI H45.8

spec. for Electrodeposited Coatings of Lead and Lead-Tin Std. Spec. for Copper-Zinc-Tin

he Susceptibility of Stainless Steels and Related Ni-Cr.Fe test for Explosive Reactivity of Lubricants with Aerospace n Susceptibility in $7 \mathrm{XXX}$ Series Copper Containing Aluminum Spec. for Conversion Coating of Titanium as/ Std. Meth. of Preparation of Micrographs of Metals and (1972) Std. Designations for Copper and Copper r Aluminum Alloy Hardeners Used in Making Zinc Die Casting oatings of Lead and Lead-Tin Alloys on Steel and Ferrous Std for Cast Coppe Std. for Magnesium Casting Std. for Magnesium Wrough Std. Spec. for Modern Pewte tric Meth. for Chemical Analysis of Copper and Copper Base Limits and Dimensional Tolerances of SAE Wrought Aluminum ergranular Attack in Wrought Nickel Rich, Chromium Bearing td. Spec. for Fire Refined Copper for Wrought Products and analysis of Nickel-Chromium and Nickel-Chromium-Iron Magnetic, and Other Similar Iron, Nickel, and Cobalt Base uate the Stress Corrosion Cracking Susceptibility of $\mathrm{Cu}-\mathrm{Zn}$ ting, Maraging, and Other Similar Chromium-Nickel-Iron Std. Spec. for White Metal Bearing

Std. Meth. of Mercurous Nitrate Test for Copper and It Std. Rec. Pract. for Heat Treatment of Aluminum emical Analysis of Nuclear Grade Silver-Indium-Cadmium

Pract. for Codification of Certain Nonferrous Metals and Safe Handling and Use of std. Rec. Pract. for Compression Molding Test Specimens of

Iding Code: Existing Buildings: Minimum Safety Requirement for Colorfastness of Textile Fabrics to Water and Light:

ess of Textile Fabrics to Water (High Humidity) and Light: for Colorfastness of Textiles to Light: Carbon Arc Lamp, astness of Textiles to Light: Water Cooled Xenon Arc Lamp, Meth. for Colorfastness of Textiles to Light and Washing: ors (1972) ractional and Integral Horsepowe/ ractional and Integral $\mathrm{Hor} /$ ntegral Horsepower) (1/ al and Integral Horsepower) (1972) Constant (Permittivity) of Solid/ osures (1973) ANSI C37.13 Std. for Frame Assignments for Std. for Dimensions for Std for Application Data for it. for Tests and Performance of Std. for Ratings for Std. Meth. of Test for Sid. for Low Voltage Std. for Surge (Lightning) Arresters for Line Sectionalizers and Oil Filled Capacitor Switches for eth. of Test for Magnetic Shield Efficiency in Attenuating Std. Air Density Ratios-At Various chromium Potassium Sulfate $\mathrm{CrK}\left(\mathrm{SO}_{4}\right)_{2}: 12 \mathrm{H}_{2} \mathrm{O}$ (Chrome minum Potassium Sulfate AlK(SO $)_{2}$ : $12 \mathrm{H}_{2} \mathrm{O}$ (Potassium Std Meth. of Test for Average Particle Size of Uniform Building Code Std. for Machine Made and High Std. Classification of Fireclay and High
Alloy (1973)

Alloy (1973)

Alloy (1973)

Alloy (1973)

Alloy (1973)

Alloy (1973)

Alloy (1973)

Alloy (1973)

Alloy (1973)

Alloy, and Austenitic Alloy Steel Tubes (1971A) ANSI B 1 , ASTM

Alloy, and Silicon Electrical Steels, Ingot and Wrought

Alloy, Externally Threaded (1972)

Alloy, for General Industrial Use (1972)

Alloy, Full Thread, Nonlocking (1972)

Alloy, Full Thread, Self Locking and Nonlocking (1972)

Alloy, Heat Resistant $(0.95 \mathrm{Cr}-0.55 \mathrm{Mo}-0.30 \mathrm{~V}(0.40-$

Alloy, Long Thread, Self Locking and Nonlocking (1972)

Alloy, Long Thread, Self Locking and Nonlocking (1972)

Alloy, Protruding Head, Tension, Pull Type (1973)

Alloy, Protruding Head, Tension, Stump Type (1973)

Alloy, Short Thread, Nonlocking (1972)

Alloy, Short Thread, Self Locking and Nonlocking (1972)

Alloy, Short Thread, Self Locking and Nonlocking (1972)

Alloy, 100 Deg. Head (MS20426), Tension, Pull Type (197

Alloy, 100 Deg. Head (Ms20426, Tension, Stump Type (197

Alloy, 100 Deg. Head (MS24694), Tension, Pull Type (197

Alloy, 100 Deg. Head (MS24694), Tension, Stump Type (19

Alloy, 6Al-4V, Self-Locking and Nonlocking (1973)

Alloying Additives (1974)

Alloys by the Powder Direct Current Arc Technique (1961

Alloys by the Silver Chloride-Lithium Fluoride Carrie

Alloys by Zincate Process (1973) ANSI G53.12

Alloys in Ingot Form for Die Castings (1964) ANSI H47.2

Alloys in Ingot Form for Sand, Permanent Mold, and Die

Alloys in Ingot Form for Sand, Permanent Mold, and Die

Alloys on Steel and Ferrous Alloys (1970) ANSI G53.8

Alloys Plate, Sheet, Strip, and Rolled Bar (1973)

Alloys to Stress Corrosion Cracking in Polythionic Acid

Alloys Under High Shear (1972) ANSI Zll 323

Alloys (Exco Test) (1972)

Alloys (Fluoride-Phosphate Type) (1973)

St for Exfoliation Corrosio

Alloys (Including Recommended Practice for Photography

Alloys (Wrought Products Only); Application Data Sheet

Alloys (1965) ANSI H38.23

Alloys (1970) ANSI G53.8

Alloys (1971)

Alloys (1971)

Alloys (1971)

Alloys (1972)

Alloys (1972)

Alloys (1972)

Alloys (1972) ANSI G80.

Alloys (1972) ANSI H23.12

Alloys (1973)

Alloys (1973)

Alloys (1973)

Alloys (1973)

Alloys (1973) ANSI H39.1

Alloys (1973) ANSI H39.3

Alloys (1974)

Alloys (1974)

Alloys, Cast and Wrought (1973)

Allyl Chloride (Chemical Safety) (1973)

Allyl Molding Materials (1972)

Alterations (1973)

Alternate Exposure (1971) ANSI L14.227

Alternate Exposure (1972) ANSI L14.228

Alternate Light and Darkness (1971) ANSI L14. 16

Alternate Light and Darkness (1971) ANSI L14.171

Alternate Sunlight Exposure (1965) ANSI L14.118

Alternating-Current Integral Horsepower Induction Mot

Alternating and Direct Current Motors and Generators (F

Alternating and Direct Current Motors and Generators (F

Alternating and Direct Current Motors (Fractional and I

Alternating Current and Direct Current Motors (Fraction

Alternating Current Loss Characteristics and Dielectric

Alternating Current Power Circuit Breakers Used in EncI

Alternating Current Power Circuits (1972) ANSI C62.1

Alternating Current Systems (1972)

Alternating Magnetic Fields (1974)

Altitudes and Temperatures (1966)

/Closers, Automatic

Alum) (1972)

Alum) (1972)

Std. Spec. for Photographic Grade

Alumina and Silica by Air Permeability (1972)

Alumina Fireclay Refractory Bricks (1973)

Alumina Refractory Brick (1970) ANSI A111.5 d. for Bol NSA NSA NSA NSA NSA NSA NSA

NSA ASTM

NSA

ASTM

NSA

NSA

SAE

NSA

NSA

NSA

NSA

NSA

NSA

NSA

NSA

NSA

NSA

NSA

1, F NSA Sid. ASTM

ASTM

ASTM

ASTM

ASTM

ASTM

ATTM

AST

ASTM

SAE

ASTM

CDA

td. Spec. Fo

ASTM

ASTM

SAE

SAE

SAE

ASTM

id. Photome

ASTM

SAE

ASTM

ASTM

ASTM

ASTM

ASTM

ASTM

ASTM

ASTM

ASTM

ASTM

ASTM

MCA

Uniform Bui ICBO

Test Meth AATCC

Colorfastn AATCC

/Meth AATCC

Lorf AATCC

Test AATCC

NEMA

NEMA

NEMA

NEMA

NEMA

ASTM

IEEE

ASTM

AMCA

ANSI

ANSI

ASTM

ICBO

ASTM

2325

2105

2306

2506

2406

2125

2115

A450

E.350

4004

A668

5800.6

AMS6305

4304. 16

6804-20

1525

4903-16

4600-16 
ight Determination for Conversion Coating on Aged or Baked el Reinforced (ACSR) (1972)

Spec for Aluminum Coated d. Spec. for Zinc Coated (Galvanized) and Aluminum Coated ations (1973) ANSI H38.17 Bar (1973) EEI Tdj/

Std. for Connectors for Use Between Aluminum or NEMA C $c /$ Std. for Connectors for Use Between Aluminum or $-0.20 \mathrm{Cr}(6061-\mathrm{T} 6))(1973)$

Spec. for

Sid. Spec. for

Std. Spec. for Concentric Lay Stranded 5005-H19 Sid. Spec. for Concentric Lay Stranded 6201-T 81 Std. Spec. for Std. Spec. for Std. Spec. for Std. Spec. for

heat Exchangers (1973) ANSI H38.6

Spec. for Tolerances of Std. Spec. for Std. Spec. for Std. Spec. for Std. Spec. for 1973) ANSI H38.5

purpose Applications (1973) ANSI H38.18 ANSI H38.16

Std. for Welded

1973) Std. Spec, for Annealed (7075-T6) (1973) Spec. for (7075-T736) Solution and Precipitation Heat Treated $(V$ ansi $\mathrm{H} 38.20$ g Alloys (1965) ANSI H38.23

inum Construction Manual Sect. 1) (1/ Std. Spec. for 26 Construction Manual Sect. 1) (1) Coatings on Aged or Backed 8.21 Std. Meth. for Ultrasonic Inspection of td. Spec. for Concentric Lay Stranded Aluminum Conductors, 3) ANSI H38.12 972)

Std. Meth. of Shear Testing of Aluminum and Std. Spec for Std. Spec. for Std. Spec. for Std. Spec. for Std. Spec. for Std. Spec, for

and Oil Transmission and Distribution Pip/ (1973) ANSI H38.7 ger Tubes with Integral Fins (1973)

Spec. for Tolerances of ed (1973) ANSI H38. 10 G33.3 ons (1974)

(1972) ANSI C7.66

972A) ANSI C7.49

1972) ANSI C7.51

73)

973)

type (1973)

type (1973)

type (1973)

Type (1973) Std. for Pin, Swage Locking, and Die Castings (1973) ANSI H38.

Property Limits and Dimensional Tolerances of SAE Wrought Std. Rec. Pract. for Heat Treatment of si W3.3 k (Rolled or Drawn) (1973) Spec. for Tolerances of .19 Factory Made Wrought (1969) ANSI W/3.10 Std. Meth. of Tension Test. Wrought and Cast Spec. for Tolerances of ) Spec. for Tolerances of

2166.8 Std. Reference Radiographs for Inspection of Rec. Pract. for

Meth. for Cavitation Erosion Corrosion Characteristics of 973) ANSI C7.27

$28 \mathrm{Cu}-0.20 \mathrm{Cr}(6061-0))(1973)$

Std. Spec. for Spec. for Std. Spec. for

si G53.21

Std. Spec. for Photographic Grade um Conductors, Steel Reinforced (ACSR) (1972) / Std. Spec. for uminum Condu/ Std. Spec. for Zinc Coated (Galvanized) and

Operating Storm Windows for External Applicat/ Specs. for Spec. for Compact Round Concentric Lay Stranded EC Grade lid Dielectric Power Cable $15 \mathrm{kV}$ Through $35 \mathrm{kV}$ (Copper and c/6201) (1972) An/ Std. Spec. for Concentric Lay Stranded forced, (ACSR) (1972A) ANSI C7.22

Std. Spec. for Std. Spec. for spec. for Aluminum Coated (Aluminized) Steel Core Wire for and Aluminum Coated (Aluminized) Stranded Steel Core for td. Spec. for Zinc Coated (Galvanized) Steel Core Wire for
Aluminized Steel (Coil) (1974)

Spec. for Coating We NCCA

Aluminized) Steel Core Wire for Aluminum Conductors, St

(Aluminized) Stranded Steel Core for Aluminum Conductors Aluminum-Alloy Drawn Tubes for General Purpose Applic Aluminum-Cobalt Alloy Plate, Sheet, Strip, and Rolled

Aluminum-Copper Overhead Electrical Conductors (1973)

Aluminum-Copper Overhead Electrical Connectors (1973)

Aluminum Alloy Bars and Rings $(1.0 \mathrm{Mg}-0.60 \mathrm{SI}-0.28 \mathrm{Cu}$

Aluminum Alloy Bars, Rods and Wires (1974) ANSI H38.4

Aluminum Alloy Conductors (1972) ANSI C7.50

Aluminum Alloy Conductors (1972) ANSl C7.52

Aluminum Alloy Die and Hand Forgings (1973) ANSI H38.8

Aluminum Alloy Die Castings (1972) ANSI H38.22

Aluminum Alloy Drawn Seamless Tubes for Condensers and

Aluminum Alloy Drawn Seamless Tubes (1974) ANS1 H38.3

Aluminum Alloy Drawn Tubing (1973)

Aluminum Alloy Extruded Bar, Rod, Pipe, and Structural

Aluminum Alloy Extruded Bars, Rods, Shapes, and Tubes (

Aluminum Alloy Extruded Round Coiled Tubes for General

Aluminum Alloy Extruded Structural Pipe and Tube (1973)

Aluminum Alloy Field Erected Storage Tanks (1973)

Aluminum Alloy Foil for Flexible Barrier Applications

Aluminum Alloy Forgings $(5.6 \mathrm{Zn}-2.5 \mathrm{Mg}-1.6 \mathrm{Cu}-0.26 \mathrm{Cr}$

Aluminum Alloy Forgings, $5.6 \mathrm{Zn}-2.5 \mathrm{Mg}-1.6 \mathrm{Cu}-0.26 \mathrm{Cr}$

Aluminum Alloy Formed and Arc Welded Round Tube (1974)

Aluminum Alloy Hardeners Used in Making Zinc Die Castin

Aluminum Alloy Load Carrying (Members) Structures (Alum

Aluminum Alloy Panels (Coil) (1974) /Ing Weight D

Aluminum Alloy Plate for Pressure Vessels (1971) ANSI H

Aluminum Alloy Reinforced (Acar, EC/6201) (1972) ANSI C

Aluminum Alloy Rivet and Cold Heading Wire and Rod (197

Aluminum Alloy Rivets and Cold Heading Wire and Rods (1

Aluminum Alloy Round Welded Tubes (1973) ANS1 H38.11

Aluminum Alloy Sand Castings (1974) ANSI H38.24

Aluminum Alloy Seamless Extruded Tube and Pipe for Gas

Aluminum Alloy Seamless Pipe and Seamless Extruded Tube

Aluminum Alloy Seamless Round Condenser and Heat Exchan

Aluminum Alloy Sheet and Plate (1973) ANSl H38.2

Aluminum Alloy Std. Structural Shapes (1973)

Aluminum Alloy Std. Structural Shapes, Rolled or Extrud

Aluminum Alloy Steel Pressure Vessel Plates (1972) ANSI

Aluminum Alloy Wrought Products for Aerospace Applicati

Aluminum Alloy 5005 Rolled Rods for Electrical Purposes

Aluminum Alloy 5005.H19 Wire for Electrical Purposes (1

Aluminum Alloy 6201 T 81 Wire for Electrical Purposes (

Aluminum Alloy, Protruding Head, Tension, Pull Type (19

Aluminum Alloy, Protruding Head, Tension, Stump Type (1

Aluminum Alloy, 100 Deg. Head (MS20426), Tension, Pull

Aluminum Alloy, 100 Deg. Head (Ms20426, Tension, Stump

Aluminum Alloy, 100 Deg. Head (MS24694), Tension, Pull

Aluminum Alloy, $100 \mathrm{Deg}$. Head (MS24694), Tension, Stump

Aluminum Alloys by Zincate Process (1973) ANSI G53.12

Aluminum Alloys in lngot Form for Sand. Permanent Mold.

Aluminum Alloys (Exco Test) (1972) /St for Exfoliation

Aluminum Alloys (1972)

ISt for Exfoliation

Aluminum Alloys (1974)

Aluminum and Aluminum Alloy Rivets and Cold Heading Wir

Aluminum and Its Alloy Arc Welding Electrodes (1969) an

Aluminum and Its Alloy Bar, Rod. Wire, and Forging Stoc

Aluminum and Its Alloy Welding Fittings (1973) ANSI H38

Aluminum and Its Alloy Welding Rods and Bare Electrodes

Aluminum and Magnesium Alloy Products (1973) ANSI H46.1

Aluminum and Magnesium Base Alloy Extrusions (1973)

Aluminum and Magnesium Base Alloy Sheet and Plate (1973

Aluminum and Magnesium Castings, Series II (1974) ANSI

Aluminum and Zinc Protection of Iron and Steel (1967)

Aluminum Applications for Boats and Yachts (1971)

Aluminum Automotive Water Pumps with Coolants (1972)

Aluminum Bar for Electrical Purposes (Bus Connector) (1

Aluminum Base Alloy Bars and Rings (1.0Mg-0.60SI -0

Aluminum Bronze Rod, Bar, and Shapes (1974) ANSI H7.6

Aluminum Chloride Solution $\mathrm{AICI}_{3}$ (1972)

Aluminum Coated Steel Chain Link Fence Fabric (1971) an

Aluminum Coated (Aluminized) Steel Core Wire for Alumin

Aluminum Coated (Aluminized) Stranded Steel Core for Al

Aluminum Combination Storm Doors (1971)

Aluminum Combination Vertically Sliding or Horizontally

Aluminum Conductors (1972) ANSI C7.53

Aluminum Conductors) (1972)

is for Single Conductor So

Aluminum Conductors, Aluminum Alloy Reinforced (Acar, E

Aluminum Conductors, Concentric Lay Stranded Steel Rein

Aluminum Conductors, Concentric Lay Stranded (1972)

Aluminum Conductors, Steel Reinforced (ACSR) (1972) Ans

Aluminum Conductors, Steel Reinforced (ACSR) (1972) Ans

Aluminum Conductors, Steel Reinforced (ACSR) (1974) Ans
ASTM

ASTM

ASTM

ASTM

NEMA

EEl

SAE

ASTM

ASTM

ASTM

ASTM

ASTM

ASTM

SAE

ASTM

ASTM

ASTM

ASTM

ANSI

ASTM

$\mathrm{SAE}$

SAE

ASTM

ASTM

AA

NCCA

ASTM

ASTM

ASTM

ASTM

AST

ASTM

ASTM

ASTM

ASTM

ASTM

SAE

ASTM

ASTM

ASTM

ASTM

ASTM

ASTM

NSA

NSA

NSA

NSA

NSA

ASTM

ASTM

ASTM

SAE

ASTM

AWS

SAE

ASTM

AWS

ASTM

SAE

$S A E$

ASTM

AWS

$A B Y C$

ASTM

AST

SAE

ASTM

ANSI

ASTM

ASTM

ASTM

AAMA

AAMA

id ASTM

IPCEA

ASTM

ASTM

ASTM

ASTM

ASTM

TB. 11.8

B341

B500

B483

C33

TDJ-162

AMS 4117D

B211

B397

B399

B85 
re and Cable Inst/

Std. for Safety for Flexible Steel and sign of Aluminum Alloy Load Carrying (Members) Structures or the Design of Aluminum Formed Sheet Building Sheathing c. for Aluminum Sheet Metal Work in Building Construction let Boxes, Device Boxes, Covers and Box Supports, and Cast Rec. Procedure for Processing Hardware for Custom Code Words for Std. Spec, for nsi H38.25 truction Manual) (1969) Std. Spec. for ereal Chemistry) (1962) Guide for the Design of Stds. for Forced Entry Resistant Qualitative Meth. of Analysis of Std. Meth. of Test for Minimum Std. for Design, Manufacture and Test of Std. for Dimensional Tolerances for Std. for Connectors for Use Between ctors (1973) EEI Tdj/ ctors (1973) NEMA Cel Std. for Connectors for Use Between Std. Meth. of Chemical Analysis of 73)

Std. Meth. for Chemical Analysis for Std. Meth. of Test for Air Oven and Semolina (Cereal Chemistry) (1962) Std. Spec. for Photographic Grade

Spec. for assium Alum) (1972) minum Construction Manual, Sect. 5) (1971) Spec. for Spec. for Spec, for Installation of Residential Std. Meth. of Analysis of Std. for Forced Entry Spec. for Std. Spec. for Sintered rical Power Cable Specs.) (1973) Uniform Building Code Std. for

Std. Spec. for

Std. Spec, for Copper Clad Std. Spec. for nsi C 7.20 eth. for Measuring Fast Neutron Flux by Radioactivation of rior Perimeter Sealing Compound for Use with Architectural ectrochemical Analysis of High Purity lron for Silicon and Std. for Alloy and Temper Designation Systems for Std. for Alloy and Temper Designation Systems for th. of Test for Spalling Resistance of Porcelain Enameled Meth. of Test for Stain Resistance of Anodic Coatings on sulfochromate Etch Solution Used in Surface Preparation of Uniform Building Code: on Aluminum (1973) Std. Spec. for Anodic Oxide Coatings on Aluminum (1973) Std. Spec. for Anodic Oxide Coatings on Test Meth. for Measurment of Impact Resistance of Painted secondary Cable (1966) IPCEA S-64-474 1973) Minimum Std. for ems for Equipment and Pipe Operating at Temperatures Above Spur, Helical, Double Helical and Rack) (1971)

d. for Graphic Representation of the Control Characters of Rec. Pract. for Units (Measurement-Metric and British . hylamino)4-(Isopropylamino)-6-(Methylthio)-S-Triazine Std. Meth. of Test for Solvent Tolerance of

istry) (1962)

istry) (1962)

istry) (1962)

fety Relief) (1/ Linderstrom-Lang Meth. of Analysis of Modified Sorensen Meth. of Analysis of Van Slyke Meth. of Analysis of Std. Spec. for Photographic Grade P. Std. for Safety for Valves for Anhydrous Safety Std. for Pumps for Anhydrous afety for Liquid Level Gauges and Indicators for Anhydrous Safety Std. for Safety Relief Valves for Anhydrous

ry) (1962) Meth. of Analysis of Definition and Test Procedures for Std. Analytical Meth. for Determining er Latex (1972A) ANSI J8.12 Std. Spec. for Concentrated,
ined Strainer or Filter for Flammable Fluids and Anhydrous ogen and Fertilizer Solutions Containing More Than $2 \%$ Free Safety Requirements for Storage and Handling of Anhydrous Std. for Driver Attended Loading of Anhydrous Operational Safety Manual for Anhydrous or Photographic Grade Ammonium Hydroxide, Nh, $\mathrm{OH}$ (Aqueous Std. Spec. for

Std. Meth. for Chemical Analysis of Meth. of Analysis of Urea and Std. Spec. for Photographic Grade Meth. for Preparation of

y) (1962)

Rec. for Safe Use of Mixed Fertilizers Containing Qualitative lodide Test Meth. for Quantitative Meth. of Analysis of Qualitative Meth of Analysis of Qualitative Meth. of Analysis for Nature of Quantitative Meth. of Analysis of
Aluminum Conduit for Safe Use as a Metal Raceway for Wi

(Aluminum Construction Manual Sect. 1) (1971)

(Aluminum Construction Manual) (1969)

(Aluminum Construction Manual, Sect. 5) (1971)

1971)

Aluminum Covers (1973)

Aluminum Doors (1972)

Aluminum Electrical Conductor (1972)

Aluminum Foil for Capacitors (1974) ANSI C116.1

Aluminum for Use in Iron and Steel Manufacture (1960) a

Aluminum Formed Sheet Building Sheathing (Aluminum Cons

Aluminum Horizontal Sliding Doors (1971)

Aluminum in Baking Powder, in Presence of Phosphates (C

Aluminum in Water (1969E)

Aluminum Irrigation Tubing (1972)

Aluminum Mill Products (1973)

Aluminum or Aluminum-Copper Overhead Electrical Condu

Aluminum or Aluminum-Copper Overhead Electrical Conne

Aluminum Oxide Abrasive Grain and Crude (1971)

Aluminum Oxide Content of Titanium Dioxide Pigments (19

Aluminum Oxide in Iron Ores (1972)

(Alumirum Plate) Meth. of Analysis of Moisture in Flour

Aluminum Potassium Sulfate A1K $\left(\mathrm{SO}_{4}\right)_{2}: 12 \mathrm{H}_{2} \mathrm{O}$ (Pot

Aluminum Prime Windows (1972) ANSI A 134.1

Aluminum Redraw Rod for Electrical Purposes (1974)

Aluminum Sheet Metal Work in Building Construction (Alu

Aluminum Siding (1970)

Aluminum Siding (1971)

Aluminum Silicate Pigment (1973)

Aluminum Sliding Glass Doors (1971)

Aluminum Sliding Glass Doors (1972) ANSI A 134.2

Aluminum Structural Parts (1973)

Aluminum Structures (1973)

Aluminum Underground Distribution Reference Book (Elect

Aluminum Wire for Communication Cable (1969) ANSl C7.40

Aluminum Wire (1972)

Aluminum Wire, EC-H19, for Electrical Purposes (1972) a

Aluminum (1970) ANSI N114

Aluminum (197I)

Aluminum (7971) ANSI Z128.35

Aluminum (1972)

Aluminum (1972)

Aluminum (1972)

Aluminum (1972) ANSI G53.30

Aluminum (1972) ANSI Z197.29

Aluminum (1973)

Aluminum (1973) Std. Spec. for Anodic Oxide Co

Aluminum, Steel and Gavanized Steel (Coil Coating) (197

Aluminum. Triplex, Neutral Supported, Service Drop and

Amateur Radio Antenna: Part l-Base or Fixed Station (

Ambient Air (1972)

/Ricated Reflective Insulatior

Spec, for Exte

Std. M

Std. Meth. for $S_{p}$

American Drafting Manual (Gear Drawing Stds. Part l for

American National Std. Code for Information Interchange

American) in Published Scientific and Technical Work (1

Ametryn (1973)

/E for the Pest Control Chemical 2-(Et

Amine Resins (1973)

Amino Nitrogen in Wheat Flour and Semolina (Cereal Chem

Amino Nitrogen in Wheat Flour and Semolina (Cereal Chem

Amino Nitrogen in Wheat Flour and Semolina (Cereal Chem

Aminophenol Hydrochloride $\mathrm{HOC}_{6} \mathrm{H}_{4} \mathrm{Nh}_{2} \mathrm{HCI}$ (1972)

Ammonia and Liquefied Petroleum (LP) Gas (Other Than Sa

Ammonia and Liquefied Petroleum (LP) Gas (1972)

Ammonia and Liquefied Petroleum (LP) Gas (1973)

Ammonia and LP (Liquid Petroleum) Gas (1973)

Ammonia Compressors and Units (1973)

Ammonia in Baking Powders and Chemicals (Cereal Chemist

Ammonia Nitrate (1973)

Ammonia Nitrogen Content in Steepwater (Corn) (1962)

Ammonia Preserved, Creamed and Centrifuged Natural Rubb

Ammonia (Fertilizer Grade) in Residential and Commerica

Ammonia (1970)

Ammonia (1972)

Ammonia (1972)

Ammonia (1973)

Ammonia) (1972)

Ammoniacal Copper Arsenite (1971) ANSI 011.8

Ammoniacal Copper Arsenite (1971) ANS1 011.23

Ammonical Nitrogen (Cereal Chemistry) (1965)

Ammonium Hydroxide, $\mathrm{Nh}_{4} \mathrm{OH}$ (Aqueous Ammonia) (1972)

Ammonium Molybdate Solution (Cereal Chemistry) (1962)

Ammonium Nitrate (1972)

Ammonium Persulfate in Flour Improvers (Cereal Chemistr

Ammonium Persulfate in Flour (Cereal Chemistry) (1962)

Ammonium Salts in Yeast Foods (Cereal Chemistry) (1962)

Ammonium Salts in Yeast Foods (Cereal Chemistry) (1962)

Ammonium Salts in Yeast Foods (Cereal Chemistry) (1962)

UL

AA

AA

AA

AA

AA

itd. M ASTM

EEI

EIA

UL

/for S UL

UL

UI

UL

FI

FI

FI

$A \mathrm{AC}$

NEMA

NBHA

ASTM

ASTM B37

AA $* 11$

AAMA 1302.3

AACCH $40-10$

ASTM D857

ASAE S263.2

ANSI H35.2

NEMA C 33

EEI TDJ-162

ANSI B74.14

ASTM D2701

ASTM E464

AACCH 44-16

ANSI PH4.150

AAMA 302.8

ASTM B233

AAMA 1402.1

AAMA 1403.1

ASTM D718

AAMA 1303.3

AAMA 4028

ASTM B595

ICBO UBCS28-1

ASTM

ASTM

ASTM

52

B314

B566

B230

E266

808.2

$\begin{array}{ll}\text { ASTM } & \text { E421 } \\ \text { ANSI } & \text { H35.1 }\end{array}$

SAE

Std ASTM

J993

C703

B136

D2674

UBC *3-28

ICBO

ASTM

ASTM

NCCA

B580

B580

TDJ-180

RS409

C667

Y14.7.1

ANSI Y14.7.

ANSI X3.3

IEEE

268

K62.142

ASTM D1198

AACCH $46-30$

$\mathrm{AACCH} \quad 46-31$

AACCH 46.32

ANSl $\mathrm{PH} 4.129$

125

51

ARI 510

AACCH $40-15$

FI $\quad$ P40

CR J-12

ASTM D1076

ANSI

ASTM

ASTM

$A A C C$

ANSI 
Spec. for Photographic Grade international and Domestic-Trap, Clay Pigeon, and Skeet: Std. Meth. of Test for Trace ucts (Wheat, Flour, Bran, Shorts, etc.) to Determine Added starches and Other Protein Bearing Materials When Suitable starches and Other Protein Bearing Materials When Suitable starches and Other Protein Bearing Materials When Suitable

uctor Solid Dielectric Power Cable 15 kV Through 35/ Std. s of Plugs and Receptacles: Specific Purpose 250 Volts, 20 locking Grounding Type, Specific Purpose, 277 Volts Ac, I5 sions of Plugs and Receptacles, Locking Type I25 Volts, 15 ions of Plugs and Receptacles, Locking Type, 250 Volts, 20 $s$ of Plugs and Receptacles: Specific Purpose 125 Volts, 30 s of Plugs and Receptacles: Specific Purpose 250 Volts, 20 $\mathrm{s}$ of Plugs and Receptacles: Specific Purpose 125 Volts, 20 cles, Midget Locking Type, Specific Purpose, 125 Volts, 15 $\mathrm{s}$ of Plugs and Receptacles: Specific Purpose 600 Volts, 30 $\mathrm{s}$ of Plugs and Receptacles: Specific Purpose I25 Volts, 20 s of Plugs and Receptacles: Specific Purpose 125 Volts, 30 $\mathrm{s}$ of Plugs and Receptacles: Specific Purpose 250 Volts, 15 $\mathrm{s}$ of Plugs and Receptacles: Specific Purpose 250 Volts, 20 $\mathrm{s}$ of Plugs and Receptacles: Specific Purpose 250 Volts, 30 f Plugs and Receptacles: Specific Purpose 277 Volts Ac, 20 f Plugs and Receptacles: Specific Purpose 277 Volts Ac, 30 $s$ of Plugs and Receptacles: Specific Purpose 480 Volts, 20 $s$ of Plugs and Receptacles: Specific Purpose 480 Volts, 30 s of Plugs and Receptacles: Specific Purpose 600 Volts, 20 Plugs and Receptacles: Specific Purpose 347/600 Volts, 20 Plugs and Receptacles: Specific Purpose 347/600 Volts, 30 Plugs and Receptacles: Specific Purpose 120/208 Volts, 20 Plugs and Receptacles: Specific Purpose 120/208 Volts, 30 Plugs and Receptacles: Specific Purpose 277/480 Volts, 20 Plugs and Receptacles\& Specific Purpose 277/480 Volts, 30 Plugs and Receptacles: Specific Purpose 120/208 Volts, 20 Plugs and Receptacles: Specific Purpose 120/208 Volts, 30 Plugs and Receptacles: Specific Purpose 277/480 Volts, 20 Plugs and Receptacles: Specific Purpose 277/480 Volts, 30 Plugs and Receptacles: Specific Purpose $347 / 600$ Volts, 20 Plugs and Receptacles: Specific Purpose 347/600 Volts, 30 $s$ of Plugs and Receptacles: Specific Purpose 250 Volts, 30 s of Plugs and Receptacles: Specific Purpose 480 Volts, 20 $s$ of Plugs and Receptacles: Specific Purpose 480 Volts, 30 s of Plugs and Receptacles: Specific Purpose 600 Volts, 30 s of Plugs and Receptacles: Specific Purpose 250 Volts, 15 $\$$ of Plugs and Receptacles: Specific Purpose 250 Volts, 20 s of Plugs and Receptacles: Specific Purpose 250 Volts, 30 s of Plugs and Receptacles: Specific Purpose 480 Volts, 20 $s$ of Plugs and Receptacles: Specific Purpose 480 Volts, 30 $s$ of Plugs and Receptacles: Specific Purpose 600 Volts, 30 Plugs and Receptacles: Specific Purpose 125/250 Volts, 20 Midget Locking Type, Specific Purpose, 125/250 Volts, 15 Plugs and Receptacles: Specific Purpose 125/250 Volts, 30 Plugs and Receptacles: Specific Purpose 125/250 Volts, 20 Plugs and Receptacles: Specific Purpose 125/250 Volts, 30 nsions of Receptacles Off Center Boss Type, 30, 50, and 60 Std. Test Procedure for Toroidal Magnetic io-Visual Equipment Use/ n Detectors (1969) ANSI N42.2 Std. Meth. for Measuring Audio Std. Test Procedure for Std. for Safety for Vending and Std. Spec. for Std. Test Meth. for Meth. of Analysis of Alpha Meth. of Analysis of Alpha ) $(1962$ Test Meth. for Assay of Bacterial Alpha . Meth. of Analysis of Alpha Amylase Activity, by Beta
Arations Which Are Susceptible to Hydrolysis by Alpha Preparations Which Are Susceptible to Hydrolysis by Alpha
Meth. of Analysis of Diastatic Activity of Flour with the is of Thermophilic Spore Counts (Flat Sour, Total Aerobic, Std. for Requirements for Electrical ide and Test Procedure for Linear, Single Axis, Pendulous, real Chemistry) (1967)

Std. Meth. for Calculating Coal and Coke content of the Atmosphere (196/ Std. Meth. for Continuous

fferential Thermal Analysis, Thermogravimetry, Evolved Gas Std. Meth. for Obtaining Natural Gas Samples for ibution of Particulate Substances Of/ Std. Rec. Pract for nsi B93.30 Std. Meth. of Reporting Contamination or Radiation Safety for X-Ray Diffraction and Fluorescence lour (Cereal Chemistry) (1962) chemistry) (1962)

Meth. of Trifructosan Meth. of Tent. Hydrometer Meth. of Tent. Pycnometer Meth. of Direct Trapping Meth. of
Std. Meth. for Chemical cts and Piecrust (Cereal Chemis/
Ammonium Thiosulfate Solution $\left(\mathrm{NH}_{4}\right)_{2} \mathrm{~S}_{3} \mathrm{O}_{3}$ (I973)

Ammunition and Target Spec. (1973)

$1 \mathrm{G}$

Amounts of Lead in Gasoline (1972) ANSI ZI1.324

Amounts of Malathion in Range of $0.2-2.0$ p.p.m. (Cereal

Amounts of Sample, Sulfuric Acid and Catalyst Are Emplo

Amounts of Sample, Sulfuric Acid and Catalyst Are Emplo

Amounts of Sample, Sulfuric Acid and Catalyst Are Emplo

Ampacities Cables in Open Top Cable Trays (1972)

Ampacities with Effect of Shield Losses for Single Cond

Amperes 3 Phase, 3 Pole, 4 Wire Locking, Grounding Type

Amperes, 2 Pole 3 Wire (1973)

Amperes, 2 Pole, 1 Wire (1973)

Amperes, 2 Pole, 2 Wire (1973)

Amperes, 2 Pole, 2 Wire (1973)

Amperes, 2 Pole, 2 Wire (1973)

Amperes, 2 Pole, 2 Wire (1973)

Amperes, 2 Pole, 2 Wire (1973)

Amperes, 2 Pole, 3 Wire Locking, Grounding Type 1973)

Amperes, 2 Pole, 3 Wire Locking, Grounding Type (1973)

Amperes, 2 Pole, 3 Wire Locking, Grounding Type (1973)

Amperes, 2 Pole, 3 Wire Locking, Grounding Type (1973)

Amperes, 2 Pole, 3 Wire Locking, Grounding Type (1973)

Amperes, 2 Pole, 3 Wire Locking, Grounding Type (I973)

Amperes, 2 Pole, 3 Wire Locking, Grounding Type (1973)

Amperes, 2 Pole, 3 Wire Locking, Grounding Type (1973)

Amperes, 2 Pole, 3 Wire Locking, Grounding Type (1973)

Amperes, 2 Pole, 3 Wire Locking, Grounding Type (1973)

Amperes, 3 Phase Wye, 4 Pole, 4 Wire Locking Type (1972

Amperes, 3 Phase Wye, 4 Pole, 4 Wire Locking Type (1972

Amperes, 3 Phase Wye, 4 Pole, 4 Wire Locking Type (1973

Amperes, 3 Phase Wye, 4 Pole, 4 Wire Locking Type (1973

Amperes, 3 Phase Wye, 4 Pole, 4 Wire Locking Type (1973

Amperes, 3 Phase Wye, 4 Pole, 4 Wire Locking Type (I973

Amperes, 3 Phase Wye, 4 Pole, 5 Wire Locking, Grounding

Amperes, 3 Phase Wye, 4 Pole, 5 Wire Locking, Grounding

Amperes, 3 Phase Wye, 4 Pole, 5 Wire Locking, Grounding

Amperes, 3 Phase Wye, 4 Pole, 5 Wire Locking, Grounding

Amperes, 3 Phase Wye, 4 Pole, 5 Wire Locking, Grounding

Amperes, 3 Phase Wye, 4 Pole, 5 Wire Locking, Grounding

Amperes, 3 Phase, 3 Pole, 3 Wire Locking Type (I973)

Amperes, 3 Phase, 3 Pole, 3 Wire Locking Type (1973)

Amperes, 3 Phase, 3 Pole, 3 Wire Locking Type (1973)

Amperes, 3 Phase, 3 Pole, 3 Wire Locking Type (1973)

Amperes, 3 Phase, 3 Pole, 3 Wire Locking Type (1973)

Amperes, 3 Phase, 3 Pole, 3 Wire Locking Type (1973)

Amperes, 3 Phase, 3 Pole, 4 Wire Locking, Grounding Typ

Amperes, 3 Phase, 3 Pole, 4 Wire Locking, Grounding Typ

Amperes, 3 Phase, 3 Pole, 4 Wire Locking, Grounding Typ

Amperes, 3 Phase, 3 Pole, 4 Wire Locking, Grounding Typ

Amperes, 3 Pole, 3 Wire Locking Type (1973)

Amperes, 3 Pole, 3 Wire (1973)

Amperes, 3 Pole, 3 Wire (1973) / Plugs and Re
Amperes, 3 Pole, 3 Wire, Locking Type (1973)
Amperes, 3 Pole, 4 Wire Locking, Grounding Type (1973)

Nsions of ANSI

Amperes, 3 Pole, 4. Wire Locking, Grounding Type (1973)

Amperes, 4 Wire (1966) IT andard Mounting

Amplifier Cores (lncluding Material Data) (1972)

Amplifier Single Frequency Output for Institutional Aud

Amplifiers and Preamplifiers for Semiconductor Radiatio

Amplifiers Used in Home Equipment (197I)

Amusement Machines (1974)

Amyl Acetate Made from Fusel Oil (85 to $88 \%$ Grade) (197

Amylaceous Matter in Adhesives (1970)

Amylase Activity of Malt (Cereal Chemistry) (1962)

Amylase Activity, by Beta Amylase GBI (Cereal Chemistry

Amylase Enzymes Used in Textile Desizing (1973) ANSI L1

Amylase GBI (Cereal Chemistry) (1962)

Amylase) (Cereal Chemistry) (1969)

Amylograph (Cereal Chemistry) (I962)

Anaerobic) in Cereal Products and Food Ingredients (Cer

Analog Indicating Instruments (1972)

Analog Torque Balance Accelerometer (1972)

in Flour or Starch

Analsis of Benzoyl Peroxide with Diaminophenylamine ( $\mathrm{Ce}$

Analyses from As-Determined to Different Bases (1974)

Analysis and Automatic Recording of the Sulfur Dioxide

Analysis and Detection (1973)

Analysis by Gas Chromatography (1968)

/ Reporting Data from Di

Analysis by Microscopical Meth. for Particle Size Distr

Analysis Data of Hydraulic Fluid Power Systems (1973) a

Analysis Equipment (1971) NBS HB111

Analysis for Acetic, Butyric, and Lactic Acids in Rye F

Analysis for Admixture of Rye and Wheat Flours (Cereal

Analysis for Alcohol (Ethanol) in Wine (Enology) (1972)

Analysis for Alcohol (Ethanol) in Wine (Enology) (1972)

Analysis for All Baked Materials Except Chocolate Produ

Analysis for Aluminum Oxide Content of Titanium Dioxide
ANSI

NRA

ASTM

CR

CR

CR

IPCEA

ANS1

ANSl

ANSI

ANS

ANS

ANSI

ANSI

ANSI

ANSI

ANSI

ANSI

ANS

ANS

ANSI

ANSI

ANSI

ANSI

ANSI

ANSl

ANS

ANSI

ANSI

ANSI

ANSI

ANSI

ANS

ANSI

ANS

ANS1

ANSI

ANSI

ANS

ANSI

ANSI

ANSI

ANSI

ANSI

ANSI

ANSI

IEEE

ANSI

IEEE

ElA

ASTM

ASTM

$\mathrm{AACCH}$

$\mathrm{AACCH}$

A ATCC

103

AACCH $22-02$

AACCH $76-30 A$

$\mathrm{AACCH} \quad 42-40$

AACC

IEEE 337

AACCH 48-06A

ASTM D3180

ASTM D 1355

ASTM E472

NGPA 2166

NFLDP T2.9.3

Std. F ANSI N43.2

AACCH $04-20$

AACCH 06.10

ASE $* 2$

ASE *3

AACCH 28-33

$\begin{array}{ll}A S T M & \text { D2701 }\end{array}$ 
1962)

) (1962)

гу) (1962)

(Cereal Chemistry) (1962)

1 Chemistry) (1962)

Products (Cereal Chemistry) (1962)

2)

istry) (1962)

stry) (1962)

mistry) (1962)

chemistry) (1962)

try) (1962)

chemistry) (1962)

r (Cereal Chemistry) (1962)

l Chemistry) (1962)

ucts (Cereal Chemistry) (1962)

(1973)

1962)

Std. Meth. for Measuring Fast Neutron Flux for

s Products (Cereal Chemistry) (1962)

ation of Carbon Dioxide (Cereal Chemistry) (196/ ers, Wood Fibers, Splinters, etc. in St/

62)

mistry) (1962

962)

962)

nt Tissues (Manual Procedures) (1973)

nt Tissues (Semiautomated Meth.) (1973)

c, Succinic, and Lactic Acids (Cereal/

of Phosphates (Cereal Chemistry) (/ 2)

rtaric Acids (Cereal Chemistry) (1962)

ead (Cereal Chemistry) (1962)

1 Chemistry) (1962)

ady-To-Eat Cereals, and Alimentary Past/

rina, and Semolina (Cereal Chemistry)/

(Cereal Chemistry) (1962)

r (Cereal Chemistry) (1962)

lour (1962)

(Cereal Chemistry) (1962)

lour (Cereal Chemistry) (1962)

lour (Cereal Chemistry) (1962/

y) (1962)

mistry) (1962)

ready-To-Eat Cereals, and Alimentary $\mathrm{Pa}$

ereal Chemistry) (1962)

Qualiat
als Other Than Phosphates (Cereal Chemistry) (1/
e: Monohydrate and Anhydrous (Cereal Chemistry)/

phate (Cereal Chemistry) (1962)

ed Self Rising Flour (Cereal Chemistr/

icals (Cereal Chemistry) (1962)

icals (Cereal Chemistry) (1962)

cereal Chemistry) (1962)

(1962)

na, and Semolina (Cereal Chemistry)/

als (Cereal Chemistry) (1962)

als (Cereal Chemistry) (1962)

eal Chemistry) (1962)

Self Rising Flours (Cereal Chemistry) (1962)

1 Chemistry) (1962)

y) (1962)

72)

g Powder (Cereal Chemistry) (1962)

Special Techniques for Extraneous Matter Meth. of Corn Oils (Cereal Chemistry) (1967)

al Chemistry) (1971)

1962)

2)

emistry) (1973)

chemistry) (1962)

o Plane Techniques (1973)

mistry) (1972)

Meth. for Colorimeter or Spectrophotom eter

Meth. of

Gustafson Meth. of

Qualitative Meth. of

Quantitative Meth. of Meth of

Decantation Meth. of

Decantation Meth. of Meth. of Meth. of

Official Meth. of Meth. of Meth. of Meth. of

Tent. Meth. of Meth. of

Meth. of

Meth. of

Meth. of

Std. Meth. for Spectrochemical

TLC Meth. of

Tent. Ebulliometer Meth. for Std. Meth. for Chemical

Meth. of
Meth. of

istry) (1962)

(Cereal Chemistry) (1962)

hosphates (Cereal Chemistry) (1962)

71)

Meth. of

Qualitative Meth. of Std. Meth. of
Analysis for Ash in Cacao Products (Cereal Chemistry) ( Analysis for Ash in Dry Milk Products (Cereal Chemistry Analysis for Ash in Farina and Semolina (Cereal Chemist Analysis for Ash in Feedstuffs (Cereal Chemistry) (1962 Analysis for Ash in Flour (Cereal Chemistry) (1962) Analysis for Ash in Flour, Feeds, Feedstuffs, and Bread Analysis for Ash in Molasses, Sugars, and Syrups (Cerea Analysis for Ash in Soy Flour (Cereal Chemistry) (1962) Analysis for Ash in Starch, Dextrin, and Other Modified Analysis for Ash in Yeast Foods (Cereal Chemistry) (196 Analysis for Baking Quality of Bread Flour (Cereal Chem Analysis for Baking Quality of Cake Flour (Cereal Chemi Analysis for Baking Quality of Cookie Flour (Cereal Che Analysis for Baking Quality of Nonfat Dry Milk (Cereal Analysis for Baking Quality of Pie Flour (Cereal Chemis Analysis for Baking Quality of Rye Bread Flour (Cereal Analysis for Baking Quality of Self Rising Biscuit Flou Analysis for Baking Quality of Wheat Bread Flour (Cerea Analys is for Baking Test (Quality) for Sweet Yeast Prod Analysis for Barium 140 Produced by Uranium 238 Fission Analysis for Benzoic Acid in Flour (Cereal Chemistry) ( Analysis for Brix (Balling) of Wine (Enology) (1972) Analysis for Citric and Isocitric Acids in Fruit and It Analysis for Copper in Wine (Enology) (1972)

Analysis for Correction Factors for Gasometric Determin Analysis for Dirt, Sand, Insect Fragments, Hairs, Feath Analysis for Fat Acidity in Corn (Cereal Chemisiry) (19 Analysis for Fat Acidity in Grain and Flour (Cereal Che Analysis for Fat Acidity in Grain (Cereal Chemistry) (1 Analysis for Fat Acidity in Grain (Cereal Chemistry) (1 Analysis for Fluoride Content of the Atmosphere and Pla Analysis for Fluoride Content of the Atmosphere and $\mathrm{Pl}$ Analysis for Formic, Acetic, Propionic, Butyric, Valeri Analysis for Free or Combined Tartaric Acid in Presence Analysis for Free Sulfur Dioxide in Wine (Enology) (197 Analysis for Fumaric, Pyruvic, Lactic, Succinic, and Ta Analysis for Hydrogen Ion Activity (PH) in Flour and $\mathrm{Br}$ Analysis for Hydrogen Ion Activity $(\mathrm{PH})$ in Flour (Cerea Analysis for Insect and Rodent Filth in Baked Goods, Re Analysis for Insect and Rodent Filth in Meal, Grits, Fa Analysis for Insect and Rodent Filth in Popped Popcorn Analysis for Insect Eggs in White, Wheat, and Corn Flou Analysis for Insect Excreta in White, Wheat, and Corn F Analysis for Insect Fragments and Rodent Hairs in Flour Analysis for Insect Fragments and Rodent Hairs in Rye F Analysis for Insect Fragments and Rodent Hairs in Soy F Analysis for Iron in Wine (Enology) (1972)

Analysis for Lactic Acid in Dried Geeg (Cereal Chemistr Analysis for Lactic Acid in Nonfat Dry Milk (Cereal Che Analysis for Materials Hard to Hydrate in Baked Goods, Analysis for Nature of Ammonium Salts in Yeast Foods (C Analysis for Neutralizing Value of Acid Reacting Materi Analysis for Neutralizing Value of Monocalcium Phosphat Analysis for Neutralizing Value of Sodium Acid Pyrophos Analysis for Original Ash in Phosphated and Freshly Mix Analysis for Phosphoric Acid in Baking Powders and Chem Analysis for Phosphoric Acid in Baking Powders and Chem Analysis for Residual Carbon Dioxide in Baking Powder ( Analysis for Rodent Excreta in Flour (Cereal Chemistry) Analysis for Rodent Excreta in Meal, Grits, Flour, Fari Analysis for Sulfuric Acid in Baking Powders and Chemic Analysis for Tartaric Acid in Baking Powders and Chemic Analysis for Titratable Acidity in Nonfat Dry Milk (Cer Analysis for Total Acid in Wine (Enology) (1972) Analysis for Total Carbon Dioxide in Prepared Mixes and Analysis for Total Phosphoric Acid in Dried Eggs (Cerea Analysis for Total Reducing Substances (Cereal Chemistr Analysis for Total Sulfur Dioxide in Wine (Enology) (19 Analysis for Total (Gasometric) Carbon Dioxide in Bakin Analysis for Volatile Acid in Wine (Enology) (1972) Analysis in Cereal Chemistry (1962) Analysis of Acetone-Insoluble Lecithin in Soybean and Analysis of Acetone Peroxides in Milling Premixes (Cere Analysis of Acid Soluble Manganese (Cereal Chemistry) ( Analysis of Added lron in Flour (Cereal Chemistry) (196 Analysis of Added Lysine in Wheat and Bulgur (Cereal Ch Analysis of Added Salt in Feeds and Feedstuffs (Cereal Analysis of Admiralty Metal by the Cast Pin and Point T Analysis of Aflatoxins in Corn and Soybeans (Cereal Che Analysis of Alcohol (Ethanol) in Wine (Enology) (1972) Analysis of Alcohol. Poly(vinyl Butyral) (1973)

Analysis of Alpha Amylase Activity of Malt (Cereal Chem Analysis of Alpha Amylase Activity, by Beta Amylase GBI Analysis of Aluminum in Baking Powder, in Presence of P

Analysis of Aluminum Oxide Abrasive Grain and Crude (19 Analysis of Aluminum Silicate Pigment (1973)
AACCH 08.10

$\mathrm{AACCH}$ 08-11

$\mathrm{AACCH} \quad 08.12$

$\mathrm{AACCH} \quad 08.03$

AACCH $08-02$

$\mathrm{AACCH} 08.01$

AACCH 08-14

AACCH 08.16

AACCH 08.17

AACCH 08.18

AACCH 10.11

AACCH 10.90

AACCH $\quad 10-50 B$

$\mathrm{AACCH} \quad 10.85$

AACCH 10.60

$\mathrm{AACCH} \quad 10.70$

AACCH $10.31 \mathrm{~A}$

AACCH $10-10$

$\mathrm{AACCH} \quad 10-20$

ASTM E393

AACCH 04.21

$\mathrm{ASE} * 4$

AACCH 04-22

ASE $* 9$

AACCH 12.29

$\mathrm{AACCH} \quad 28.75$

AACCH 02.03

\begin{tabular}{l}
$\mathrm{AACCH} \quad 02.01$ \\
\hline
\end{tabular}

AACCH $\quad 02.02$

A ACCH 02-04

ASTM D3269

ASTM D3270

AACCH 04.23

AACCH $04-28$

ASE $* 8$

A ACCH 04.24

$\triangle A C C H \quad 02.52$

$\begin{array}{ll}A A C C H & 02.51\end{array}$

$\mathrm{AACCH} 28.31$

A ACCH 28-5]

AACCH $28-80$

$\mathrm{AACCH} 28.4$

$\mathrm{AACCH} \quad 28-43$

AACCH 28.41

AACCH 28.60

AACCH 28.70

ASE $* 10$

AACCH $04-25$

AACCH $04-26$

AACCH 28.32

AACCH $40-17$

$\begin{array}{ll}A A C C H & 02-32\end{array}$

$\mathrm{AACCH} \quad 02.33$

AACCH $\quad 02-34$

AACCH 08-15

$\mathrm{AACCH} 04-10$

$\mathrm{AACCH}$ 04-11

AACCH $12-10$

AACCH $28-42$

$\mathrm{AACCH} \quad 28-50$

$\mathrm{AACCH} 04.14$

AACCH $04-27$

AACCH 02-31

$\mathrm{ASE}$ *5

$\mathrm{AACCH}$ 12-21

AACCH 04-13

$\mathrm{AACCH} \quad 10.01$

ASE $* 7$

AACCH $\quad 12-20$

ASE *11

$\mathrm{AACCH} \quad 28.92$

$\mathrm{AACCH} \quad 58.35$

$\mathrm{AACCH} \quad 48.05$

$\mathrm{AACCH} \quad 40.45$

$\mathrm{AACCH} \quad 40.40$

AACCH 46.40

AACCH $40-60$

ASTM E486

AACCH $45-05$

ASE *1

ASTM D1396

AACCH 22-01

AACCH $22-02$

AACCH $40-10$

ANSI B74.14

ASTM D718 
National Bureau of Standards KWIC Index of Engineering Standards

(Cereal Chemistry) (1962) (Cereal Chemistry) (196?) (Cereal Chemistry) (1962) real Chemistry) (1962) 23 try) (1962) stry) (1962) stry) (1962) ter Suspensions (Cereal Chemistry) (1962)

Linderstrom-Lang Meth. of Modified Sorensen Meth. of Van Slyke Meth. of Meth. of

Std. Meth. for Chemical Quantitative Meth. of Qualitative Meth. of Quantitative Meth. of Meth. of Meth. for Screen Indophenol-Xylene Extraction Meth. of Sampling Procedure for Semiquantitative Meth. of ) (1968) chnique Using an Optical / real Chemistry) (1962) stry) (1962) Std. Meth. for Spectrochemical Qualitative Meth. of Qualitative Meth. of liquid Chromatography (1973) Std. Rec. Pract. for Sampling and Std. Meth. for Meth. for Meth. of Meth. of Meth. of y) (1962) 962) (1962)

aining Monoglyceride, Salts, Soy F/

Quantitative Meth, of Qualitative Meth of Std. Meth. for Chemical pectrometer (1971) ANSI Z/ teels, Ingot and Wrought lrons (/ Std. Meth. for Spectrochemical Std. Meth. for Chemical Meth. for Std. Meth. for Chemical Std. Meth. of by Colorimetry (1972) ) (1962)

) (1962)

(1962)

(1962)

Gravimetric Meth. of Volumetric Meth. of Qualitative Meth. of Meth. of Std. Meth. of Chemical Std. Meth. for Chemical Counting Meth. of

re (1970) ANSI A139.2

Chemistry) (1962)

Chemistry) (1962)

ble Fats (Cereal Chemistry) (1967)

Products (Cereal Chemistry) (1973)

Gravimetric Meth. of Meth. of Meth. of Std Meth of Laboratory Sampling and Std. Meth. of Test for Proximate

eal Chemistry) (1962)

y) (1962)

y) (1962)

Meth. of

Disk Meth. of

h Congealor Splidif Wectrophotometric Meth. of

io System (Cereal Chesi, try) (1962)

Meth. of Meth. of

Std Photometric Meth for Chemical

71) ANSI Z12 ts (1973)

Std. Meth. for Spectrochemical Std. Meth. for Chemical Std. Rec. Pract. for Applying Statistics to
Meth. of 973) ry) (1962) Std. Rec. Pract. for Applying Statistics to
Meth. of Meth. of Meth. of Meth. of istry) (1962)

cereal Chemistry) (1962)

Products Not Containing Fruit (Cereal Chemistr/ Meth. of Meth. of Meth of Meth. of Meth. of Meth. of Meth. of

(1962)

Cooked Feeds (Cereal Chemistry) (1962)

Chemistry) (1962)

from Cottonseed, Soybeans, and Peanuts (Cereal ins/ Improved Kjeldahl Meth. for Nitrate Free Samples for egumes, Forages, and Animal and Dairy P/ UDy Dye Meth. of al Chemistry) (1962) cts (Boric Acid Modification) (Cereal/ Improved Kjeldahl Meth. of Kjeldahl Meth. of Micro Kjeldahl Meth. of Preparations Which Are Susceptible to Hydrolys/ Meth. of try) (1962)

try) (1962)

ereal Chemistry) (1962)

raph (Cereal Chemistry) (1962)

emistry) (1962)

Pressuremeter Meth. of

Volumetric Meth. of Meth. of Meth. of Meth. of

mistry) (1962)

henyls (1974)

cereal Chemistry) (1962) on of Cereal Grains, Unpopped Popcorn, Dried Pe/ Meth. of nd Flour of Whe at, Rye, Barley, Other Grains, A/ ons of Terms
Analysis of Amino Nitrogen in Wheat Flour and Semolina Analysis of Amino Nitrogen in Wheat Flour and Semolina Analysis of Amino Nitrogen in Wheat Flour and Semolina Analysis of Ammonia in Baking Powders and Chemicals (Ce Analysis of Ammoniacal Copper Arsenite (1971) ANSI 011. Analysis of Ammonium Persulfate in Flour (Cereal Chemis Analysis of Ammonium Salts in Yeast Foods (Cereal Chemi Analysis of Ammonium Salts in Yeast Foods (Cereal Chemi Analysis of Apparent Viscosity of Acidulated Flour-Wa Analysis of Asbestos Fibers (1971T)

Analysis of Ascorbic Acid (Cereal Chemistry) (1962)

Analysis of Bacteria (Cereal Chemistry) (1962)

Analysis of Benzoyl Peroxide in Flour (Cereal Chemistry Analysis of Blast Furnace lron by the Point to Plane Te Analysis of Bromates and lodates in Flour Improvers (Ce Analysis of Bromates and Iodates in Flour (Cereal Chemi Analysis of Built Up Roofs (1972)

Analysis of Calcium and Barium Petroleum Sulfonates by Analysis of Calcium Chloride Types I and II (1972T)

Analysis of Calcium in Bread and Flour (Cereal Chemistr Analysis of Calcium in Feedstuffs (Cereal Chemistry) (1 Analysis of Calcium in Mineral Feeds (Cereal Chemistry) Analysis of Calcium Peroxide in Dough Conditioners Cont Analysis of Calcium Peroxide (Cereal Chemistry) (1962 Analysis of Carbon and Graphite (1969) ANSI K90.3 Analysis of Carbon and Low Alloy Steel Using a Vacuum S Analysis of Carbon, Low Alloy, and Silicon Electrical S Analvsis of Casein (1972)

Ane sis of Cast Iron-All Types (1974)

Analysis of CCA Treating Solutions and CCA Treated Wood Analysis of Chlorides in Ash of Bread (Cereal Chemistry Analysis of Chlorides in Ash of Bread (Cereal Chemistry Analysis of Chlorides in Yeast Foods (Cereal Chemistry) Analysis of Chlorides in Yeast Foods (Cereal Chemistry) Analysis of Choline (Cereal Chemistry) (1962)

Analysis of Chromated Zinc Chloride (1970) ANSI 011.19 Analysis of Chrome Containing Refractories and Chrome $\mathrm{O}$ Analysis of Cinder and Sand Particles in Farina (Cereal Analysis of Cinder and Sand Particles in Farina (Cereal Analysis of Cloud Point in All Edible Animal and Vegeta Analysis of $r$ agulase Positive Staphylococcus in Cereal Analysis of coal and Coke (1970) ANSI K18.1

Analysis of Coal and Coke (1973)

Analysis of Coliform Bacteria in All Food Products (Cer Analysis of Color of Macaroni Products (Cereal Chemistr Analysis of Color of Macaroni Products (Cereal Chemistr Analysis of Congeal Point in Fats and Fat Mixtures Whic Analysis of Cooking Characteristics of Macaroni-Boras Analysis of Cooking Characteristics of Macaroni (1962) Analysis of Copper and Copper Base Alloys (1972)

Analysis of Copper by the Briquet D-C Arc Technique (19 Analysis of Copper Phthalocyanine Blue and Green Pigmen Analysis of Corrosion Data (1971) ANSI G80.3

Analysis of Cracking Flotation Test for Internal Insect Analysis of Creosote and Oil Type Wood Preservatives (1 Analysis of Crude Fat in Baked Dog Food (Cereal Che mist Analysis of Crude Fat in Cocoa (Cereal Chemistry) (1962 Analysis of Crude Fat in Dry Milk Products (Cereal Chem Analysis of Crude Fat in Egg Yolk and Dried Whole Egg( Analysis of Crude Fat in Flour, Bread, and Baked Cereal Analysis of Crude Fat in Grain and Stock Feeds (Cereal Analysis of Crude Fat in Soy Flours (Cereal Chemistry) Analysis of Crude Fat in Wheat and Soy Flour, Feeds and Analysis of Crude Fiber in Feeds and Feedstuffs (Cereal Analysis of Crude Fiber in Soy Flour for Cake and Meal Analysis of Crude Protein in Bread, Wheat and Other Gra Analysis of Crude Protein in Cereal Grains, Oilseeds, L Analysis of Crude Protein in Feeds and Feedstuffs (Cere Analysis of Crude Protein in Wheat and Flour Mill Produ Analysis of Crude Protein (Cereal Chemistry) (1962) Analysis of Damaged Starch (Granules in Flour or Starch Analysis of Diastatic Activity of Doughs (Cereal Chemis Analysis of Diastatic Activity of Doughs (Cereal Chemis Analysis of Diastatic Activity of Flour and Semolina (C Analysis of Diastatic Activity of Flour with the Amylog Analysis of Diastatic Activity of Malt Flour (Cereal Ch Analysis of Dried Egg Solids (Cereal Chemistry) (1962) Analysis of Egg Solids in Macaroni Products (Cereal Che Analysis of Environmental Materials Polychlorinated Bip Analysis of Ergot in Rye and Wheat Flour and Semolina ( Analysis of Ergot in Rye Flour (Cereal Chemistry) (1962 Analysis of External Insect and Rodent Hair Contaminati Analysis of Falling Number Determination of Both Meal a Analysis of Fatigue Data (1972) ANSI 292.2

Analysis of Fecal Streptococci in Food Products (Cereal Analysis of Ferroalloys (1973)

AACCH $46 \cdot 30$

$\mathrm{AACCH}$ 46-31

AACCH 46.32

AACCH 40.15

ASTM D1326

AACCH $\$ 8.62$

AACCH $40-16$

AACCH 40.18

AACCH 56.80

$\begin{array}{ll}\text { ASTM } & \text { D } 2947\end{array}$

AACCH 86.10

AACCH $42-10$

AACCH 48-07

ASTM E485

AACCH $48-40$

AACCH 48-41

ASTM D2829

ASTM D2894

ASTM E449

AACCH $40-20$

$\mathrm{AACCH} \quad 40.21$

AACCH $\quad 40.26$

$\mathrm{AACCH}$ 48-51

AACCH 48.50

ASTM C560

ASTM E415

ASTM E350

TAPPI T607SL

ASTM E351

AWPA A10.72

AACCH $40-30$

AACCH 40-31

AACCH $40-32$

AACCH $40-33$

AACCH 86-45

ASTM D1033

ASTM $\quad$ C572

AACCH 28-01

AACCH 28-02

AACCH $56-10$

AACCH $42-30 \mathrm{~A}$

ASTM D271

ASTM D3172

AACCH 42.15

AACCH $\quad 14-20$

AACCH 14-21

$\mathrm{AACCH} \quad 58-12 \mathrm{~A}$

AACCH 16-51

AACCH $\quad 16-50$

ASTM E62

ASTM E414

ASTM D3256

ASTM G16

AACCH $28-22$

AWPA Al

A ACCH $30-14$

AACCH $30-12$

AACCH 30.16

AACCH $30-18$

AACCH $30-10$

A ACCH 30.20

AACCH $30-26$

AACCH $30-25$

AACCH 32.15

AACCH 32.17

AACCH 46-11

AACCH 46-14

$\mathrm{AACCH} \quad 46.10$

AACCH 46-12

AACCH 46-13

$\mathrm{AACCH} \quad 76-30 \mathrm{~A}$

AACCH 22.11 
Std. Meth. for Chemical Std. Meth. for Chemical Tent. Meth. for Chemical Std. Meth. for Chemical Std. Meth. for Chemical Std. Meth. for Chemical Std. Meth. for Chemical

es (1974)

aasho T27, ANSI A/

1.20

cts (Cereal Chemistry) (1962)

Std. Meth. of Test for Sieve or Screen

Std. Meth. for Chemical

Meth. of

Meth. of

ry) $(1962)$

Std. Meth. for High lonizing Voltage Mass Spectrometric Tent. Meth of Test for Aromatic Types Std. Rec. Pract. for Sampling Atmospheres for Std. Meths. for Chemical Hand Washing Meth. of try) (1967)

try) (1962)

Machine Washing Meth of Std. Meth. for Chemical

1971) ANSI Z128.35 Std. Meth. for Spectrochemical llary Gas Chromatography (1971) ANSI Z11/ Other Similar I es and Cereal Products (Cereal Chemistry) (1968/ ed Foods (Cereal Chemistry) (1962)

eal Products (Cereal Chemistry) (1962) stry) (1962)

cereal Chemistry) (1967)

Chemistry) (1962)

d (Cereal Chemistry) (1962)

72) ANSI K67.3

I Chemistry) (1962)

Chemistry) (1962)

cereal Chemistry) (1962

gas Chromatography (1973)

dy-To-Eat Cereals, and Alimentary Pastes for De/

npopped Popcorn, Dried Peas and Beans for Roden

\section{Meth. of}

Meth. of

Meth. o

Meth. of Meth. of

Quantitative Meth. of Meth. of Meth. of

Std. Meth. of Chemical Meth. of Meth. of Meth. of Std. Meth. for Meth. of Meth. of Std. Meth. of (Wheat, Flour, Bran, Shorts, etc.) table Fats (Cereal Chemistry) (1967)

e Fats (Cereal Chemistry) (1967) Colorimetric Meth. of Capillary Meth. o

Wiley Meth. of r Apparatus, Reagents, and Safety Precautions for Chemical istry) (1962)

chemistry) (1968)

Low Blank Meth. of Meth. of tification and Qualitative (Including Optical Microscopic) al Chemistry) (1968) Std. Meth. of Test for Sieve ortenings (Butter, Oleomargarine, High Acid Coc/ Meth. of Meth. of (Cereal Chemistry) (1962) (Cereal Chemistry) (1962) ation with Toluene (Cereal C/ ation with Toluene (Cereal $\mathrm{C}$ istry) (1962) stuffs, Ground Grains and Grain, Soybeans, Rice, Beans, Peas, L Vacuum Oven Meth. of Bidwell-Sterling Meth. of Bidwell-Sterling Meth. of Modified Vacuum Oven Meth. of ) Distillation Meth. (Brown-Duvel) of ice, and Other Commodities For/ Dielectric Meter Meth. of $r$ Liquid and Semiliquid Products (Drying Upon Q/ Oven Meth. of ry) (1962) (1962)

istry) (1962)

eal Chemistry) (1962) Meth. of ission (1972, Std. Meth. of Test for Fast Neutron Flux by rophosphate in Prepared Mixes (/ Perchloric Acid Meth. of ils and Shortenings (Cereal Chemistry) (1967) graphy (1973) ANSI Zl1.238 graphy (1968)

(Cereal Chemistry) (1967)

concentrates Used to Enrich Cereals (Cereal Che/ Std. Meth. for
Std. Meth. for Meth. of Meth. of

- Lithium Fluoride Carri/ ron Alloys (1973)

Microbiological Meth. of

Std. Meth. for Spectrochemical Std. Meth. for Chemica

revention of Cecal and Intestinal Coccidiosis $\mathrm{l} /$ Meth. of eth. for Chemical, Mass Spectrometric, and Spectrochemical eth. for Chemical, Mass Spectrometric, and Spectrochemical spectrometric, Spectrochemical, Nuclear and Radio Chemical spectrometric, Spectrochemical, Nuclear, and Radiochemical oys (1974) Std. Meth. for Chemical and Spectrochemical Sys/ th. for Chemical, Mass Spectrometric, and Spectrochemical Trial Use Guide: General Principles for Reliability Std. Meth. for

Preconcentration Spark T/ Std. Meth. for Spectrochmeical tassium lodide (Cereal Chemistry) (1962)
Analysis of Ferroboron (1974)

Analysis of Ferrochrome-Silicon (1974)

Analysis of Ferrochromium and Chromium Metal (1971T)

Analysis of Ferromanganese and Spiegeleisen (1974)

Analysis of Ferromolybdenum (1974)

Analysis of Ferrosilicon and Silicon Metal (1974)

Analysis of Ferrovanadium and Vanadium Alloying Additiv Analysis of Fine and Coarse Concrete Aggregates (1971) Analysis of Fluor Chrome Arsenate Phenol (1971) ANSI Ol Analysis of Food Poisoning Staphylococcus in Food Produ Analysis of Free Fatty Acids in Fats (Cereal Chemistry) Analysis of Fungicide Residues in Grain (Cereal Chemist Analysis of Gas-Oil Saturate Fractions (1971) ANSI Zl Analysis of Gas Oil Aromatic Fractions by Mass Spectrom Analysis of Gases and Vapors (1967) ANSI Z257.2

Analysis of Glass Sand (1972)

Analysis of Gluten in Flour and Semolina (Cereal Chemis Analysis of Gluten in Flour and Semolina (Cereal Chemis Analysis of Gypsum and Products (1972)

Analysis of High Purity lron for Silic on and Aluminum ( Analysis of High Purity N-Heptane and lsooctane by Cap Analysis of High Temperature, Electrical. Magnetic, and Analysis of Hydration Capacity of Pregelatinized Starch Analysis of Hydrocyanic Acid Residues in Grains and Dri Analysis of Inorganic Bromide Residues in Grain and Cer Analysis of lodine in Mineral Mixed Feeds (Cereal Chemi Analysis of lodine Number in All Edible Fats and Oils ( Analysis of Iron in Flour (Cereal Chemistry) (1962)

Analysis of Lactose (Milk Sugar) in Mixed Feeds (Cereal Analysis of Lactose (Sugar) and Nonfat Dry Milk in Brea Analysis of Limestone, Quicklime, and Hydrated Lime (19 Analysis of Lipoid Phosphoric Acid in Dried Eggs (Cerea Analysis of Lipoxidase Activity of Ground Wheat (Cerea Analysis of Lipoxidase Activity of Semolina and Flour ( Analysis of Liquefied Petroleum Gases (LPG) by Process Analysis of Macroscopic Examination of Baked Goods, Rea Analysis of Macroscopic Examination of Cereal Grains, U Analysis of Magnesium Silicate Pigment (1973) Analysis of Malathion Residues in Milled Wheat Products Analys is of Melting Point of All Normal Animal and Vege Analysis of Melting Point of Normal Animal and Vegetabl Analys is of Metals (1973) Std. Rec. Pra Analysis of Methoxychlor Residues in Foods (Cereal Chem Analysis of Micro Sedimentation Test for Wheat (Cereal Analysis of Mineral Filler and Coating of Paper (1973) Analysis of Mineral Filler (1972) AASHO T37, ANSI A37. Analysis of Modified Sedimentation Test for Wheat (Cere Analysis of Moisture and Volatile Matter in Fats and Sh Analysis of Moisture and Volatile Matter in Soy Flours Analysis of Moisture and Volatile Matter in Yeast Foods Analysis of Moisture in Fats and Shortenings by Distill Analysis of Moisture in Feeds and Feedstuffs by Distil Analysis of Moisture in Flour and Semolina (Cereal Chem Analysis of Moisture in Flour and Semolina; Bread; Feed Analysis of Moisture in Flour, Farina, Semolina, Bread, Analysis of Moisture in Grains and Feedstuffs (Cereal C Analysis of Moisture in Grains (Cereal Chemistry) (1962 Analysis of Moisture in Grains, Beans, Peas, Lentils, R Analysis of Moisture in Malt (Cereal Chemistry) (1962) Analysis of Moisture in Massecuites, Molasses, and Othe Analysis of Moisture in Nonfat Dry Milk (Cereal Chemist Analysis of Moisture in Whole Grain (Cereal Chemistry) Analysis of Moisture, Drying at 135 Deg. C (Cereal Chem Analysis of Mold and Yeast Counts in Food Products (Cer Analysis of Molybdenum -99 Activity from Uranium -238 F Analysis of Monoglycerides and Free Glycerol in Fats, $O$ Analysis of Natural Gas Liquid Mixtures by Gas Chromato Analysis of Natural Gas Liquid Mixtures by Gas Chromato Analysis of Niacin and Nicotinamide in Cereal Products Analysis of Niacin in Enrichment (Vitamin and Mineral) Analysis of Niacin (Cereal Chemistry) (1962) Analysis of Nickel-Base Alloys by the Silver Chloride Analysis of Nickel-Chromium and Nickel-Chromium-l Analysis of Nitrophenide in Poultry Mashes (Feed) for P Analysis of Nuclear Grade Mixed Oxides $\left((\mathrm{U}, \mathrm{Pu}) 0_{2}\right)(1$ Analysis of Nuclear Grade Plutonium Dioxide Powders and Analysis of Nuclear Grade Plutonium Metal (1973)

Analysis of Nuclear Grade Plutonium Nitrate Solutions Analysis of Nuclear Grade Silver-Indium-Cadmium All Analysis of Nuclear Grade Uranium Dioxide Powders and P Analysis of Nuclear Power Generating Station Protection Analysis of Oil Borne Wood Preservatives (1973) Analysis of Ores, Minerals, and Rocks by the Fire Assay Analysis of Oxidizing Agents in Flour: Reaction with $\mathrm{Po}$ Analysis of Pantothenic Acid (Cereal Chemistry) (1962)

ASTM

ASTM

E364

ASTM E363

ASTM E361

ASTM E368

ASTM E360

ASTM E365

ASTM C136

ASTM D 1035

AACCH $42-30$

AACCH 58.16

$\mathrm{AACCH} \quad 60.01$

ASTM D2786

ASTM D3239

ASTM Dl605

ASTM C146

A ACCH 38.10

AACCH 38-11

ASTM C471

ASTM E42]

ASTM D2268

ASTM E354

AACCH $56-20$

AACCH $\quad 60-20$

$\mathrm{AACCH} \quad 60-10$

AACCH $\quad 40.35$

AACCH $58-30$

AACCH 40.4

AACCH $80-30$

AACCH 80-3]

ASTM C25

AACCH 20.02

A ACCH 22-41

AACCH $22-40$

ASTM D2888

AACCH $28-30$

AACCH 28.10

ASTM D717

A ACCH $60-30$

A ACCH $58-40$

A ACCH 58-4l

ASTM E50

AACCH 60.40

AACCH 56.63

TAPPI T42]

ASTM D546

AACCH $56-62$

AACCH $44-30$

AACCH 44-3]

$\mathrm{AACCH} \quad 44-32$

$\mathrm{AACCH}$ 44.51

AACCH $44-50$

AACCH 44-16

$\mathrm{AACCH} \quad 44-40$

AACCH 44-15A

AACCH 44-18

AACCH 44.53

$\mathrm{AACCH}$ 44-1]

AACCH 44.20

$\mathrm{AACCH} \quad 44.60$

$\mathrm{AACCH} \quad 44.52$

$\mathrm{AACCH} \quad 44-10$

AACCH 44-19

A ACCH $42-50$

ASTM E343

AACCH 40-5l

AACCH 58.45

ASTM D2597

NGPA 2165

A ACCH $86-50$

AACCH 86.49 
Meth. of Std. Meth. for Chemical Volumetric Bromine Meth. of Xylene Partition Meth. of Chemistry) (1969) Meth. of meth of Test for Carbon Distribution and Structural Group stry) (1962)

chemistry) (1962)

chemistry) (1962)

chemistry) (1962) 2) Meth. of Colorimetric Acetate Ashing Meth. of Gravimetric Meth. of

Volumetric Meth. of Colorimetric Modified Fiske-Subbarow Meth. of Std. Meth. for

stry) (1962)

Structural Relaxation Meth. of Extensigraph

heat (1962) Meth. of

nt to Plane Technique Usi/

by the Point to Plane Arc/

flour (Cereal Chemistry) (1962)

Std. Meth. for Spectrochemical Std. Meth. for Spectrochemical

Quantitative Meth. of reparations (Cereal Chemistr) reparations (Cereal Chemistry) (19/ Spectrophotometric Meth. of oteinase Preparations (Cereal Chemistry) (1962)

try) (1962) Meth. of Meth. of ts-Emergency Monitoring (Cereal Chemistry) (1/

Test Meth. for Sieve (1) Meth. of Std. General Meth. for

i Z258.1

d Semolina (Cereal Chemistry) (1962) Std. Meth. for Chemical Meth. of Schoorl Meth. of Meth. of soy Flour, Mill Feeds (Cereal Chemistry) (1962)

Farinograph Meth. for

Mixograph Meth. for Chemistry) (1962)

Chemistry) (1962)

meal, Flaked and Puffed Cereals, F/

Fluorometric Meth. of Microbiological Meth. of Meth. of

Chemistry) (1962)

Std. Meth. of Chemical Meth. of Meth. of Meth. of Meth. of Meth. of

ds and Feedstuffs (Cereal Chemistry) (1962)

Oils (Cereal Chemistry) (1967)

chemistry) (1962)

try) (1965)

try) (1962)

try) (1962)

try) (1962)

962)

962)

Disk Meth. of

Macro Scale Meth. of

Micro Scale Meth. of Harland and Ashworth Meth. of

Rowland Meth. of

(1974)

971)

962)

62)

)

Std. Meth. for Chemical Std. Meth. for Chemical Std. Meth. of Chemical Sedimentation Meth of

Microscopic Meth. of

Std. Meth. of Chemical Std. for

eal Chemistry) (1962)

stry) (1962)

1962)

ormed Deposits (Chemical Cleaning of Indus/

962)

Baume of Sugar Solutions (Cereal Chemistry) (1/

Spindle Meth. of

Refractometer Meth. of Meth. of

Std. Meth. of Meth. of Meth. of ereal Chemistry) (1962)

ngs (Cereal Chemistry) (1967)

her Similar Chromium-Nickel-/

nt Acid Hydrolysis) (Cereal Chemistry)/

ight Modification, to Corn, Rye, B/

on (1973)

real Chemistry) (1962)

(1962)

(1962)

Preparation of Aluminum (1972) ANSI Z197./ Oxygen-Bomb Meth. of Meth. of Std. Meth. for Chemical Diastase Meth, of

Polarimetric Meth. of Std. Meth. for Chemical Meth. of Bomb Meth. of Gravimetric Meth. of Std. Meth. of Std. Meth. for ufacture (1973) ne Butadiene Copolymers (SBR) (1/ mistry) (1962)

ansi L14.132

Std. Meth. for Chemical Meth. of

Test Meth. for Ouantitative

Std. Meth. for Quantitative Guide for Statistical

ct Current Arc Technique / Std. Meth. for Spectrochemical food Products (Cereal Chemistry) (1962)

A robic, Anaerobic) in Cereal Products and Fool hole Grain Products, Corn Grits, Cor/ gh Alloy Steels (1973) mistry) (1962) Meth. of Thiochrome Meth. of Std. Meth. for Chemical Std. Plate Count Meth. of Std. Meth. for Chemical Std. Meth. for Chemical

Meth. of

Dry Milk (Cereal Chemistry) (1962)

(Cereal Chemistry) (1962)

arrier D-C Arc Technique /

ry) (1965)

rials (Cereal Chemistry) (1/

Meth. of

Std. Meth. for Spectrochemical

Meth. of Xanthydrol Test for
Analysis of Pelshenke Test (Wheat Meal Fermentation Tim Analysis of Pentachlorophenol (1971) ANSI 011.22

Analysis of Pentosans (Cereal Chemistry) (1962)

Analysis of Pentosans (Cereal Chemistry) (1962)

Analysis of Pesticide Residues in Food Products (Cereal

Analysis of Petroleum Oils by the n-d-M Meth. (1973)

A nalysis of Phosphates in Flour Improvers (Cereal Chemi

Analysis of Phosphorus in Feeds and Feedstuffs (Cereal

Analysis of Phosphorus in Feeds and Feedstuffs (Cereal

Analysis of Phosphorus in Feeds and Feedstuffs (Cereal

Analysis of Phosphorus in Yeast (Cereal Chemistry) (196

Analysis of Phosphorus-32 (1962) ANSI N 149

Analysis of Physical Dough Tests of Flour (Cereal Chemi

Analysis of Pigments in Flour. Semolina, Macaroni and W

Analysis of Plain Carbon and Low Alloy Steel by the Poi

Analysis of Plain Carbon and Low Alloy Steel for Boron

Analysis of Potassium Bromate in White and Whole Wheat

Analysis of Proteolytic Activity in Active Proteinase P

Analysis of Proteolytic Activity in Active Proteinase P

Analysis of Proteolytic Activity of Flour and Active Pr

Analysis of Proteolytic Activity of Malt (Cereal Chemis

Analysis of Pulpwood Chips (Pulp and Paper) (1973)

Analysis of Radioactivity (Beta Gamma) in Cereal Produc

Analysis of Radioisotopes (1968) ANSI N 148

Analysis of Reactor and Commercial Columbium (1968) Ans

Analysis of Reducing and Nonreducing Sugars in Flour an

Analysis of Reducing Sugars in Prepared Bakery Mixes (C

Analysis of Residual Urease Activity in Soybean Meals,

A nalysis of Resistance of Flour Dough to Mixing (Cereal

Analysis of Resistance of Flour Dough to Mixing (Cereal

Analysis of Riboflavin in Whole Grain Products, Grits,

Analysis of Riboflavin (Cereal Chemistry) (1962)

Analysis of Rope Spore Count in Cereal Products (Cereal

Analysis of Rubber Products (1972A) ANS1 J10.1

Analysis of Salmonella Bacteria in Food Products (Cerea

Analysis of Salt Calculated from Total Chlorides in Fee

Analysis of Saponification Value of All Normal Fats and

Analysis of Sedimentation Test for Wheat Flour (Cereal

Analysis of Sedimentation Test for Wheat (Cereal Chemis

Analysis of Semolina Quality in Macaroni (Cereal Chemis

Analysis of Semolina Quality in Macaroni (Cereal Chemis

Analysis of Semolina Quality in Macaroni (Cereal Chemis

Analysis of Serum Protein in Milk (Cereal Chemistry) (1

Analysis of Serum Protein in Milk (Cereal Chemistry) (1

Analysis of Silica Refractories (1970) ANSI A 139.5

Analysis of Silicomanganese and Ferrosilicon Manganese

Analysis of Silicon Carbide Abrasive Grain and Crude (1

Analysis of Smut in Wheat and Rye (Cereal Chemistry) (1

Analysis of Smut in Wheat or Rye (Cereal Chemistry) (19

Analysis of Soaps Containing Synthetic Detergents (1973

Analysis of Soda Ash (1972)

Analysis of Solids in Juices, Syrups, and Molasses (Cer

Analysis of Solids in Syrups and Molasses (Cereal Chemi

Analysis of Soluble Mineral Matter (Cereal Chemistry) (

Analysis of Solvent Systems Used for Removal of Water F

Analysis of Soy Flour in Macaroni (Cereal Chemistry) (1

Analysis of Specific Gravity, Degrees Brix, and Degrees

Analysis of Stability in Fats, Oils, and Shortenings (C

Analysis of Stability (AOM) of Fats, Oils, and Shorteni

Analysis of Stainless, Heat Resisting, Maraging, and Ot

Analysis of Starch in Flour and Semolina (With Subseque

Analysis of Starch in Flour and Semolina; Also, with SI

Analysis of Steel and Cast, Open Hearth, and Wrought Ir

Analysis of Sucrose (Sugar) in Feeds and Feedstuffs ( $\mathrm{Ce}$

Analysis of Sulfates in Yeast Foods (Cereal Chemistry)

Analysis of Sulfates in Yeast Foods (Cereal Chemistry)

Analysis of Sulfochromate Etch Solution Used in Surface

Analysis of Sulfuric Acid as Used in Pulp and Paper Man

Analysis of Synthetic Elastomers for Use on Solid Styre

Analysis of Test Weight Per Bushel of Grain (Cereal Che

Analysis of Textiles (Moisture and Composition) (1971)

Analysis of Textiles (1972) ANSI L14.132, AATCC 20A

Analysis of Thermal Life Test Data (1972)

Analysis of Thermionic Nickel Alloys by the Powder Dire

Analysis of Thermophilic and Psychrophilic Bacteria in

Analysis of Thermophilic Spore Counts (Flat Sour, Total

Analysis of Thiamine in Bread, Wheat, Rice, and Other W

Analysis of Tool Steels and Other Similar Medium and Hi

Analysis of Total Bacteria in Food Products (Cereal Che

Analysis of Tribasic Lead Phosphosilicate (1973)

Analysis of Tungsten (1973)

Analysis of Undenatured Whey Protein Nitrogen in Nonfat

Analysis of Unsaponifiable Matter in Macaroni Products

Analysis of Uranium Oxide $\left(\mathrm{U}_{3} \mathrm{O}_{k}\right)$ by Gallium Oxide $\mathrm{C}$

Analysis of Urea and Ammonical Nitrogen (Cereal Chemist

AACCH 56.50

ASTM D1274

AACCH $52-10$

$\mathrm{AACCH} 52.11$

$\mathrm{AACCH} 60.50$

ASTM D3238

$\mathrm{AACCH} \quad 40.50$

AACCH 40.55

$\mathrm{AACCH} \quad 40.57$

AACCH $40-58$

$\mathrm{AACCH} \quad 40-56$

ASTM E182

AACCH 54-II

$\mathrm{AACCH} \quad 14.50$

ASTM E 403

ASTM E404

AACCH $48-42$

$\mathrm{AACCH} 22.62$

AACCH $22-63$

AACCH $22-60$

AACCH 22.6I

TAPPI UM.2

AACCH $28-99 A$

ASTM E181

ASTM E195

AACCH $80-60$

$\mathrm{AACCH} \quad 80-68$

AACCH 22.90

AACCH $54-21$

AACCH 54.40

AACCH 86.70

$\mathrm{AACCH} 86.72$

AACCH $42-20$

ASTM D297

$\mathrm{AACCH} \quad 42.25$

$\mathrm{AACCH}$ 40-6 I

AACCH $58-50$

$\mathrm{AACCH} 56-60$

AACCH 56-6IA

$\mathrm{AACCH} \quad 66.40$

AACCH 66-4l

$\mathrm{AACCH} \quad 66-42$

$\mathrm{AACCH} 46.2 \mathrm{I}$

AACCH $46-22$

ASTM C575

ASTM E362

ANSI B74.15

AACCH $42-71$

$\mathrm{AACCH} \quad 42-70$

ASTM D820

TAPPI T612

AACCH $\quad 68-62$

AACCH $\quad 68-60$

$\mathrm{AACCH} \quad 40-01$

ASTM D2790

$\triangle \mathrm{ACCH}$ 06-11 
nd Cereal/ Meth. of Urease Bromthymol Blue Test Paper for ) Mixed Feeds (Cereal Chemistry) (1962) Meth. of ) (1962) ils, and Emulsions (Cere/ ry) (1967) d Dry Milks (Cereal Chemistry) (19/

Ultraviolet Absorption Meth. of Ultraviolet Absorption Meth. of Meth. of Microbiological Meth. of Sid. Meth. of Reporting Results of Std. Meth. for Chemical Std. Meth. of Chemical Std. Meth. for Std. Meth. for Particle Size tation in Cereal Grains, Unpopped Popcorn, Drie/ Meth. of (1962) Meth. of Std. Meth. of Chemical eth. for Chemical, Mass Spectrometric, and Spectrochemica Std. Meth. of Test for Moisture in the Std. Meth. of Test for Ash in the Std. Meth. of Test for Volatile Matter in the Std. Meth. of Test for Carbon and Hydrogen in the Std. Meth. of Test for Nitrogen in the Std. Meth. of Test for Total Sulfur in the de; Chloral Hydr/ Reagents for Extraneous Matter Meth. of Pekar Color Test (Slick) Meth. of Flour Test Meth. for Chip Length Test Meth. for Chip Length Std Deviations for Specific Meth. of Cereal Chemistry pract. for Using Controlled Atmospheres in Spectrochemical Rec. Pract. for Meth. of Sampling Steel for Chemical Std. Meth. of Test for Nickel on Steel by Photometric Std. Meth, of Preparing Coal Samples for tandardization, and Storage of Std. Solutions for Chemical Std. Definitions of Terms Relating to Thermal Std. Meth. for Sampling Pressurized Gas for Gas Definitions of Terms Relating to Atmospheric Sampling and of Assay of Di-T-Butyl Peroxide by Gas Chromatographic Calibration of Temperature Scale for Differential Thermal rties of Liquefied Petroleum (LP) Gases from Compositional ydraulic Fluid Power System (For Particulate Contamination $\operatorname{arch}(1973)$

Rec. Pract. for Reporting Data from Differential Thermal

oups in Hydroxyethyl and Hydroxypropyl Starch Ether/ $\mathrm{n}$ Starch Which Is Gelatinized When Heated in a Boil/ $\mathrm{rn}$ Starch Which Is Substantially Insoluble in Water/ $\mathrm{n}$ Starch, Syrup and Sugar Obtained from the Corn We/ Starch (1968)

epwater and Most Other Liquid Products Obtained Fro/ n Syrup and Starch Hydrolyzates (1952)

n Sugar (Crude and Refined) (1958)

content in Corn Starch (1955)

content in Feedstuffs (Corn) (1955)

$t$ in Corn Starch (Gelatinizable Products) (1955)

nt in Steepwater (Corn) (1962)

$t$ of Corn Syrup, Refined and Crude Sugar (1967)

n Syrup Unmixed and Corn Sugar (1954)

n Sugar (Crude and Refined) (1958)

arch, Dextrines and Other Modified Starch Products / 957)

ffs (Corn) (1955)

ter (Corn) (1964)

gar (Crude and Refined) (1958)

rup, Finished Sugar and Other Starch Hydrolyzates (/ syrup Unmixed and lts Syrups (1959)

in and Corn Starch Products (1971)

ntent in Corn Starch (1968)

ator, $1 \%$ Content of Reagents and Indicators (1971)

n Corn Starch (1963)

n Feedstuffs (Corn) and Other Wet Milled Solid Frod n Starch (1960)

n Syrup, Sugar and Other Carbohydrates (1957)

etric) Content in Corn Starch (1973)

etric) Content of Corn Syrup and Other Starch Hydro/

etric) Content of Corn Sugar (Crude and Refined), S/

ion Content of Corn Syrup ['nmixed (1952)

rn Starch (1966)

in Industrial Waste Waters and Most Organic Compou/ rn Syrup Unmixed, Finished Sugar and Other Clarifie/ rn Syrup Unmixed, Finished Sugar and Other Clarifie rn Sugar (Crude and Refined), S:rups and Other Star/ orn Oil (1965)

t of Corn Syrup Unmixed (1954)

ric) Content in Corn Starch (1957)
Analysis of Urea in Cloth or Sack Fibers, Whole or Grou Analysis of Vital Wheat Gluten (Cereal Chemistry) (1962 Analysis of Vitamin A and Carotene in Enriched Cereals Analysis of Vitamin A in Concentrates (Cereal Chemistry Analysis of Vitamin A in Dry Vit tmin Mixes, Beadlets, O Analysis of Vitamin A in Enriched Flour (Cereal Chemist Analysis of Vitamin A in Nonfat Dry Milk and Instantize Analysis of Vitamin B12 (Cereal Chemistry) (1962)

Analysis of Vitamin B6 Complex (Cereal Chemistry) (1962 Analysis of Vitamin B6 Complex (Cereal Chemistry) (1962 Analysis of Water (1969E)

Analysis of White Lead Pigments (1973)

Analysis of White Pigments (1973) ANSI K 15.]

Analysis of White Zinc Pigments (1973)

Analysis of Whiteware Clays (1974)

Analy sis of X-Ray Examination for Internal Insect Infes

Analysis of Yeast Strength of Doughs (Cereal Chemistry) Analy sis of Zirconia Refractories (1972)

Analysis Of, and Physical Tests On, Beryllium Oxide Pow

Analysis Sample of Coal and Coke (1973)

Analysis Sample of Coal and Coke (1973)

Analysis Sample of Coal and Coke (1973)

Analysis Sample of Coal and Coke (1973)

Analysis Sample of Coal and Coke (1973)

Analysis Sample of Coal and Std. Meih. Coke (1973)

Analysis (Cereal Cremistry) Alcohol; Carbon Tetrachlori

Analy sis (Cereal Chemistry) (Bolting Cloth, Filter Pape

Analy sis (Cereal Chemistry) (1962)

Analysis (Measurement) (Pulp and Paper) (1973)

Analy sis (Two Screen) (Pulp and Paper) (1973)

Analysis (1962)

Analysis (1970) ANSI Z128.30

Analysis (1971)

Analysis (1972)

Analysis (1972) ANSI K20.17

Analysis (1972) ANSI Z159.]

Analysis (1973)

Analysis (1973)

Analysis (1973)

Analysis (1973)

Analysis (1973)

Analysis (1973) ANSI Z11.239

Analysis) (1972) ANSI B93.19

Analysis, Thermogravimetry, Evolved Gas Analysis and De

Analytical Meth. Determining Iodine Affinity of Corn St

Std.

Std.

Sid.

Std.

Std.

Sid.

Std.

Std.

Std.

Std

Std.

Std.

Std.

Std.

Std.

Std.

Std.

Sid.

Sid.

Std.

Std.

Std.

Std.

Std.

Std.

Std.

Analutical Meth for Determining Calcium Content in Cor

Std. Analytical Meth. for Determining Calcium Content of Cor

Std. Analytical Meth. for Determining Calcium (Egta-Titrim

Std. Analytical Meth. for Determining Calcium (Egta-Titrim

Analytical Meth for Determining Calcium (Egta-Titrim

Std. Analytical Meth. for Determining Candy Color and Invers

Std. Analytical Meth. for Determining Carboxyl Content of Co

Std. Analytical Meth. for Determining Chemical Oxygen Demand

Std. Analytical Meth. for Determining Chloride Content of Co

Std. Analytical Meth. for Determining Chloride Content of $\mathrm{Co}$

Std. Analytical Meth. for Determining Chloride Content of $\mathrm{Co}$

Std. Analytical Meth. for Determining Cold Test Content in C

Std. Analytical Meth. for Determining Color Stability Conten

Std. Analytical Meth. for Determining Color (Spectrophotomet
AACCH $28-87$

AACCH 38.20

AACCH $86-05$

AACCH 86-0]

AACCH 86.01A

AACCH 86.03

AACCH 86-02

AACCH 86.40

AACCH 86.30

AACCH 86-31

ASTM D596

ASTM D1301

ASTM D34

ASTM D3280

ASTM C775

AACCH 28-21

AACCH 22-13

ASTM C705

ASTM C699

ASTM D3173

ASTM D3174

ASTM D3175

ASTM D3178

ASTM D3179

ASTM D3177

AACCH 28.91

AACCH $28-90$

$\mathrm{AACCH} \quad 14-10$

TAPPI UM-5

TAPPI UM.6

AACCH 78.50

Std. Rec. ASTM E406

SAE J408C

ASTM C715

ASTM D2013

ASTM E200

ASTM E 473

Std. ASTM D1356

Std. Meth. ASTM E475

d. Meth. for ASTM E474

E474

T2.9.1

$\mathrm{E} 472$

$\begin{array}{ll}\mathrm{CR} & \mathrm{B} \cdot 28\end{array}$

CR C.30

CR C. 42

CR

CR

CR

CR

CR

CR

$\mathrm{CR}$

CR

CR

CR

CR

CR

CR

CR

CR

CR

CR

CR

CR

CR

CR

CR

CR

CR

CR

CR

CR

CR

CR

CR

CR

CR

CR 
ric) Content of Corn Syrup (1957)

ric) Content in Feedstuffs and All Other Natural Dr/ ric) Content in Corn Oil (1964)

on) Content of Corn Syrup (1954)

ectrophotometric) Content of Corn Sugar (Crude and /

trophotometric) Content of Corn Sugar (Crude and Re/

Syrup Unmixed and Finished Sugars (1954)

Sugar (Crude and Refined), Syrup, and Other Starch/

$0.1 \%$ Content of Reagents and Indicators (1971)

actables) Content in Corn (1958)

actables) Content in Feedstuffs (Corn) (1954)

les) Content in Corn Starch, Gluten, Germand Finished Corn and Other Grains, Foodstuffs Containing Veget/

Feedstuffs (Corn) Containing Vegetable Material, C/ rn Syrup and All Starch Hydrolyzates (1967)

ntent of Corn Syrup and All Starch Hydrolyzates (19)

ntent of Corn Sugar (Crude and Refined) (1972)

ontent of Corn Syrup, Crude and Refined Sugar and O/

f Corn Syrup, Sugars, and Starch Hydrolyzates (1957)

tation Content in Corn (1954)

nt in Corn Oil and to Other Vegetable and Marine Oi/

$f$ of Corn Syrup Unmixed and Dextrose (1956)

f Corn Sugar (Crude and Refined), Syrups and Other /

of Corn Starch and Ethers (1967)

e Point) Content in Corn Starch (1963)

tent (Radiographic Inspection) in Corn and Other Wh/

th.) Content in Corn, Grain Sorghum and Other Veget/

t of Reagents and Indicators (1971)

tarch Hydrolzates Including Syrups and Sugars, and /

ugar (Crude and Refined) (1961)

ine) Content of Corn Syrup and Starch Hydrolyzates /

Corn Syrup (1970)

content in Feedstuffs (Corn) (1964)

content in Steepwater (Corn) (1962)

r. $0.1 \%$ Content of Reagents and Indicators (I971)

or, $1 \%$ Content of Reagents and Indicators (1971)

istillation) Content in Corn (1955)

istillation) Content in Corn Starch (1956)

istillation) Content of Corn Syrup Unmixed (1952)

istillation) Content in Feedstuffs (Corn) (1956)

) Content in Corn Starch (Unmodified) (196I)

Content of Corn Syrup (1966)

) Content of Corn Sugar (Crude and Refined) (1966)

) Content in Steepwater (Corn) (1966)

aid) Content of Corn Syrup Unmixed (1954)

paper) Content of Corn Syrup Unmixed and Unbleaches $\mathrm{t}$ in Corn Starch (1956)

t of Corn Sugar (Crude and Refined) (Crystalline) (/

$t$ in Commercial Feedstuffs (Corn) (1957)

fs (Corn) (1955)

er and Most Other Liquid Products Obtained from Cor/ ar (Crude and Refined), Syrups and Other Starch Hyd/ up, Starch Hydrolyzates and a Variety of Other Mate/

corn Starch (1958)

Corn Starch (1956)

tor, 1\% Content of Reagents and Indicators (1971)

corn Starch (1957)

corn Starch (I969)

corn Syrup (1957)

Solution Content of Reagents and lndicators (1971)

nt in Corn, Other Grains and Plant Materials of $\mathrm{Com} /$

nt in Corn Starch, Sugars, Syrups and Other Protein/

nt of Corn Syrup, Sugars, Starches and Other Protei/

nt of Corn Sugar (Crude and Refined), Syrups, Starc/

nt in Feedstuffs (Corn), Syrups, Sugars, Starches A/

nt in Steepwater, (Corn) Feedstuffs, Syrups, Sugars/

$t$ in Dextrin (1970)

$t$ of Corn Sugar (Crude and Refined) (1972)

$t$ in Steepwater (Corn) (1964)

nt of Corn Syrup, Sugar and Dextrose (1956)

raphic) Content of Corn Syrup (1957)

d Chromatography) Content of Corn Syrup, Sugar, And/

d Chromatography) Content of Corn Sugar, Starch Mol/

ions Content of Reagents and Indicators (1971)

ution Content of Reagents and Indicators (1971)

in Starch (1956)

$\mathrm{xtrin}(\mathrm{I} 970)$

edstuffs (Corn) (1954)

ent of Corn Syrup, Dextrose and Starch Hydrolyzates/

ent of Corn Sugar (Crude and Refined), Dextrose, Sy

(1964)

stuffs (Corn) (1954)

ontent of Reagents and Indicators (1971)

olution Content of Reagents and Indicators (1971)

n Syrup Unmixed and Finished Corn Sugars (1954)

in Corn Starch (1956)
Std. Analytical Meth. for Determining Color (Spectrophotomet

Std. Analytical Meth. for Determining Color (Spectrophotomet

Std. Analytical Meth. for Determining Color (Spectrophotomet

Std. Analytical Meth. for Determining Color (Visual Comparis

Std. Analytical Meth. for Determining Color, Reflectance (Sp

Std. Analytical Meth. for Determining Color, Solutions (Spec

Std. Analytical Meth. for Determining Copper Content of Corn

Std. Analytical Meth. for Determining Copper Content of Corn

Std. Analytical Meth. for Determining Cresol Red Indicator.

Std. Analytical Meth. for Determining Crude Fat (Ccl, Extr

Std. Analytical Meth. for Determining Crude Fat (CcI, Extr

Std. Analytical Meth. for Determining Crude Fat (Ccl, Prod

Std. Analytical Meth. for Determining Crude Fiber Content in

Std. Analytical Meth. for Determining Crude Fiber Content in

Std. Analytical Meth. for Determining Dextrose Content of $\mathrm{Co}_{0}$

Std. Analytical Meth. for Determining Dextrose Equivalent Co

Std. Analytical Meth. for Determining Dextrose Equivalent Co

Std. Analytical Meth. for Determining Extraneous Materials C

$S$ td. Analytical Meth. for Determining Fermentables Content $O$

Std. Analytical Meth. for Determining Filth and Insect Infes

Std. Analytical Meth. for Determining Free Fatty Acids Conte

Std. Analytical Meth. for Determining Heavy Metals Content O

Std. Analytical Meth. for Determining Heavy Metals Content O

Std. Analytical Meth. for Determining Hydroxyalkoxyl Content

Std. Analytical Meth. for Determining Inherent Viscosity (On

Std. Analytical Meth. for Determining Insect Infestation Con

Std. Analytical Meth. for Determining Iodine Number (Wijs Me

Std. Analytical Meth. for Determining Iodine Solution Conten

Std. Analytical Meth. for Determining Iron Content in Corn S

Std. Analytical Meth. for Determining Iron Content of Corn S

Std. Analytical Meth. for Determining Iron (Orthophenanthrol

Std. Analytical Meth. for Determining Iron (TPTZ) Content of

Std. Analytical Meth. for Determining Lactic Acid and Salts

Std. Analytical Meth. for Determining Lactic Acid and Salts

Std. Analytical Meth. for Determining Methyl Orange Indicato

Std. Analytical Meth. for Determining Methylene Blue Indicat

Std. Analytical Meth. for Determining Moisture (Azeotropic D

Std. Analytical Meth. for Determining Moisture (Azeotropic D

Std. Analytical Meth. for Determining Moisture (Azeotropic D

Std. Analytical Meth. for Determining Moisture (Azeotropic D

Std. Analytical Meth. for Determining Moisture (Karl Fischer

Std. Analytical Meth. for Determining Moisture (Karl Fischer

Std. Analytical Meth. for Determining Moisture (Karl Fischer

Std. Analytical Meth. for Determining Moisture (Karl Fischer

Std. Analytical Meth. for Determining Moisture (Oven Filter

Std. Analytical Meth. for Determining Moisture (Oven Filter

Std. Analytical Meth. for Determining Moisture (Oven) Conten

Std. Analytical Meth. for Determining Moisture (Oven) Conten

Std. nalytical Meth. for Determining Moisture (Oven) Conten

Std. Analytical Meth. for Determining $\mathrm{pH}$ Content in Feedstuf

Std. Analytical Meth. for Determining pH Content in Steepwat

Std. Analytical Meth. for Determining $\mathrm{pH}$ Content of Corn Sug

Std. Analytical Meth. for Determining $\mathrm{pH}$ Content of Corn Syr

Std. Analytical Meth. for Determining pH (Paste) Content in

Std. Analytical Meth. for Determining $\mathrm{pH}$ (Slurry) Content in

Std. Analytical Meth. for Determining Phenolphthalein Indica

Std. Analytical Meth. for Determining Phosphorus Content in

Std. Analytical Meth. for Determining Phosphorus Content of

Std. Analytical Meth. for Determining Phosphorus Content of

Std. Analytical Meth. for Determining Potassium Permanganate

Std. Analytical Meth. for Determining Protein Nitrogen Conte

Std. Analytical Meth. for Determining Protein Nitrogen Conte

Std. Analytical Meth. for Determining Protein Nitrogen Conte

Std. Analytical Meth. for Determining Protein Nitrogen Conte

Std. Analytical Meth. for Determining Protein Nitrogen Conte

Std. Analytical Meth. for Determining Protein Nitrogen Conte

Std. Analytical Meth. for Determining Reducing Sugars Conten

Std. Analytical Meth. for Determining Reducing Sugars Conten

Std. Analytical Meth. for Determining Reducing Sugars Conten

Std. Analytical Meth. for Determining Refractive Index Conte

Std. Analytical Meth. for Determining Saccharides (Chromatog

Std. Analytical Meth. for Determining Saccharides ( $G$ as Liqui

Std. Analytical Meth. for Determining Saccharides (Gas Liqui

Std. Analytical Meth. for Determining Sodium Hydroxide Solut

Std. Analytical Meth. for Determining Sodium Thiosulfate Sol

$\mathrm{Std}$. Analytical Meth. for Determining Solubles Content in $\mathrm{Co}$

Std. Analytical Meth. for Determining Solubles Content in De

Std. Analytical Meth. for Determining Solubles Content in Fe

Std. Analytical Meth. for Determining Specific Rotation Cont

Std. Analytical Meth. for Determining Specific Rotation Cont

Std. Analytical Meth. for Determining Starch Content in Corn

$\mathrm{S}$ td. Analytical Meth. for Determining Starch Content in Feed

Std. Analytical Meth. for Determining Starch Indicator, I\% C

Std. Analytical Meth. for Determining Std. Arsenious Oxide S

Std. Analytical Meth. for Determining Sulfate Content of Cor

Std. Analytical Meth. for Determining Sulfur Dioxide Content
$\mathrm{C}$

CR

CR

CR

$\mathrm{CR}$

CR

CR

CR

CR

CR

$\mathrm{CR}$
$\mathrm{CR}$

CR

CR

CR

CR
CR

CR

CR
CR

CR

CR

CR

CR
CR

CR

CR

CR
CR

CR

CR

CR

CR

CR

CR

CR

$\mathrm{CR}$

CR
CR

CR

CR

CR

CR

CR

CR
CR

CR

CR

CR

CR

CR

CR

CR

CR

CR
CR

CR

CR

CR

CR
CR

CR

CR

CR
CR

CR

CR

CR

CR

CR

CR

CR

CR

CR

CR
CR

CR

CR

CR

CR

CR

CR
CR

E-16

G-6

H. 12
E. 18

F.16

F.I4

E. 22

F-18

R. 60

A. 6

G. 10

B. 18

A- 8

G. 12

E-24

E-26

F-22

E-27

E-28

A-10

H. 22

E-30

F. 26

C. 20

B-6I

A-1I

H-32

R-10

B. 30

F. 28

E-32

G-I3

J-36

R-70

R. 65

A-12

B-34

E- 40

G-14

B-36

E.46

F-32

J-44

E- 42

E-44

B-38

F-34 
of Corn Syrup (1952)

of Corn Sugar (Crude and Refined) (1961)

$s$ Content of Reagents and Indicators (1971) orn Starch (1955)

f Corn Sugars (Crude and Refined), Starch Molasses / content in Corn (1959)

nt in Corn and Grain Sorghum, and Their Mixtures (1) nt in Corn Starch (1960)

$n$ Feedstuffs (Corn) (1964)

afluoride (1972)

rafluoride (1972)

stry) (1962)

Using the Mass Spectrometer Leak Detector or Residual Gas

h. for Shive Content of Mechanical Pulp (Von Alfthan Shive

for Research and Motor Meth. Octane Ratings Using on Line

d. Meth. for Validation of Results of Process Distillation

r Operations Terminology Including Terms for Reporting and

:1) Aspect Ratio (1971) Std. Meth. for Std. Dimensions of Projectable Std. Meth. for Ultimate Std Meth for Chemical Structural Clay Differential Movement (Flexible onstruction and lnstallation of Fittings and Equipment for es, Washers, Pins, Dowels, Joints, Split Rings and Framing Std, for 974) y Conductive Equipment and Materials for Use in Flammable hesion of Pressure Sensitive Tape to Fiberboard at 90 Deg. Std. Spec. for Ultrasonic, Std. for Punches-Variable, Std. for Punches-Basic, Combination Std for Punches: Basic hickness of Epitaxial or Diffused Layers in Silicon by the Rec. Pract. for Radius of Load and Boom h. for Wrinkle Recovery of Woven Textile Fabrics: Recovery cel Bars Including Rounds, Squares, Hexagons, and Shapes. lour Bleaching Experiments in Laboratories (Cerea/ Acetic er Than Safety Relief) (1/ Std. for Safety for Valves for 2)

Safety Std. for Pumps for std. for Safety for Liquid Level Gauges and lndicators for Safety Std. for Safety Relief Valves for self Contained Strainer or Filter for Flammable Fluids and Safety Requirements for Storage and Handling of Operational Safety Manual for Spec. for Photographic Grade Sodium Sulfate, utralizing Value of Monocalcium Phosphate: Monohydrate and Spec. for Ph. Spec. for Tetrasodium Pyrophosphate Std. Spec. for Photographic Grade Sodium Sulfite, Std. for Driver Attended Loading of Tent. Spec for

Protein in Cereal Grains, Oilseeds, Legumes, Forages, and Meth. of Analysis of Cloud Point in All Edible capillary Meth. of Analysis of Melting Point of All Normal Wiley Meth. of Analysis of Melting Point of Normal ent in Corn Oil and to Other Vegetable and Marine Oils and ers (Also Includes Terms and Defini/ Std. Spec. for Limestone for

Std. Meth. of Test for Diameter of Wool and Other Sid. Meth. of Test for Operating Performance of ration Procedure (1974) Std. Meth. of Test for Synthetic Meth. of Test for Synthetic

cations (1973)

i C7.1 Std. Spec. for

i C 7.4 Std. Spec. for Soft or Std. Spec. for Tinned Soft or Std. Spec. for Silver Coated Soft or Std. Spec. for Nickel Coated Soft or Std. for Seamless Low Carbon Steel Tubing Std. for Welded and Cold Drawn Low Carbon Steel Tubing Titanium Alloy Sheet, Strip, and Plate, 6Al-4V 17-0.23C), Premium Quality, Consumable Electrode Melted, s, Corrosion and Moderate Heat Resistant, $17 \mathrm{Cr}$ (SAE 51430) Std. Spec. for Seamless Copper Tube, Bright

c. for Steel Bars, Forgings, Rings, and Mechanical Tubing ng (1972) gation (1971) Std. Rec. Guide for in Service
Std. Meth. of Test for Std. Meth. of Test for

Std. for Cast and Wrought Galvanic Zinc Std. Meth. of Test for Stain Resistance of or Anodic Oxide Coatings on Aluminum (1973) Std. Spec. for Anodic Oxide Coatings on Aluminum (1973) Std. Spec. for

erence Meth. for Making Potentiostatic and Potentiodynamic Safety Std. Structural Std. for Steel Std. for Electrical and Mechanical Characteristics of Std. Definitions of Terms for
Analytical Meth. for Determining Sulfur Dioxide Content Analytical Meth. for Determining Sulfur Dioxide Content Analytical Meth. for Determining Sulfuric Acid Solution Analytical Meth. for Determining Total Fat Content in C Analytical Meth. for Determining Viability (Sprouting) Analytical Meth. for Determining Waxy and Nonwaxy Conte Analytical Meth. for Determining Waxy and Nonwaxy Conte Analytical Meth. for Determining Xanthophylls Content 1

Analytical Procedures for Accountability of Uranium Hex Analytical Procedures for Accountability of Uranium Tet Analytical Values to $14.0 \%$ Moisture Basis (Cereal Chemi Analyzer in the Tracer Probe Mode (1973)

Analyzer) (1973)

Analyzers (1970T)

Analyzers (1973)

Analyzing Outage of Electrical Transmission and Distrib

Analyzing Stress in Glass (1968)

Anamorphic Image Area on $5 \mathrm{~mm}$ Motion Picture Film (2.35

Anaylsis of Coal and Coke (1974)

Anaylsis of Ferrocolumbium (1974)

Anchorage) (Tech. Notes) (1963)

Anchoring, Mooring, Docking, Towing and Lifting Boats

Anchors) (1973)

IS. Lag Screws, Plates, Nails and Spik

Ancymidol (Plant Growth Regulator) (1971)

Anesthetizing Locations (Bonding Appliances, Casters, F

Anf Protection Rec. for lndoor Storage of Roll Paper (1

Angle and Constant Stress (1972)

Meth. of Test

Angle Beam Examination of Steel Plates (1973) ANSl G35.

Angle Head Type and Related Quill Bushings (1972)

Angle Head Type and Related Quill Bushings (1972)

Angle Head Type and Related Quill Bushings (1972)

Angle Lapping and Staining Technique (1972) ANSI Z173.4

Angle Measuring System (1972)

Angle Meth. (1972) ANSI L14.110, ASTM D1295

Angles, TEES and Channels (1972) ANSl G81.18

Anhydride Meth. for Generation of Chlorine Dioxide in F

Anhydrous Ammonia and Liquefied Petroleum (LP) Gas (Oth

Anhydrous Ammonia and Liquefied Petroleum (LP) Gas (197

Anhydrous Ammonia and Liquefied Petroleum (LP) Gas (197

Anhydrous Ammonia and LP (Liquid Petroleum) Gas (1973)

Anhydrous Ammonia (Fertilizer Grade) in Residential and

Anhydrous Ammonia (1972)

Anhydrous Ammonia (1973)

Anhydrous $\mathrm{Na}_{2} \mathrm{SO}_{3}$ (1973)

Anhydrous (Cereal Chemistry) (1962)

Anhydrous $\left(\mathrm{Na}_{2} \mathrm{~S}_{2} \mathrm{O}_{3}\right)$ and Crystalline ( $\mathrm{Na}_{2} \mathrm{~S}_{2} \mathrm{O} / 3$

Anhydrous) (Cleaning Agent) (1970) ANSI K60.20

Anhydrous, $\mathrm{Na}_{2} \mathrm{SO}_{3}$ (1972)

Anlydrous Ammonia (1972

Aniline.62 (1973)

Animal and Dairy Products (Cereal Chemistry) (1968)

Animal and Vegetable Fats (Cereal Chemistry) (1967)

Animal and Vegetable Fats (Cereal Chemistry) (1967)

Animal and Vegetable Fats (Cereal Chemistry) (1967)

Animal Fats (1965)

/ Determining Free Fatty Acid

Animal Feed and Food

Animal Fibers by Microprojection (1972) ANSI L14.143

Anion Exchange Materials for Strong Acid Removal (1972)

Anionic Active Ingredient in Detergents by Cationic Tit

Anionic Ingredient by Cationic Titration (1972T)

Annealed Aluminum Alloy Foil for Flexible Barrier Appli

Annealed Copper Wire for Electrical Purposes (1974) Ans

Annealed Copper Wire for Electrical Purposes (1974) Ans

Annealed Copper Wire (1974) ANSI C7.38

Annealed Copper Wire (1974) ANSI C7.48

Annealed for Bending and Flaring (1972)

Annealed for Bending and Flaring (1972)

Annealed (1973)

Annealed (1973)

Annealed (1973)

Annealed (1973) ANSI H23.2

R $-9.0 \mathrm{Ni}-4.5 \mathrm{Co}-1.0 \mathrm{Mo}-0.09 \mathrm{~V}(0$.

(Annealed) $(0.48 \mathrm{Cr}-8.0 \mathrm{Ni}-4.0 \mathrm{Co}-0.48 \mathrm{Mo}-0.09 \mathrm{~V}(0.2$

Annealing of Water Cooled Nuclear Reactor Vessels (1974

Annealing Point and Strain Point of Glass by Beam Bendi

Annealing Point and Strain Point of Glass by Fiber Elon

Anodes for Use in Saline Electrolytes (1973) ANSI H44.1

Anodic Coatings on Aluminum (1972) ANSl G53.30

Anodic Oxide Coatings on Aluminum (1973)

Anodic Oxide Coatings on Aluminum (1973)

Anodic Polarization Measurements (1972)

Antenna Discharge Units (1972) ANSI C33.39

Antenna Towers and Supporting Structures (1972)

Antennas for Satellite Earth Stations (1973)

Antennas (1973)

\section{/T. for}

Test Met

CR E-66

CR

$\mathrm{CR}$
$\mathrm{CR}$

CR

CR

CR

CR

ANSI

ANSI N15.6

AACCH 82.22

$82-22$

UM-241

D2885

D2891

$\begin{array}{ll}\text { IEEE } & 346 \\ \text { ASTM } & \text { F218 }\end{array}$

$\begin{array}{ll}\text { ASTM } & \text { F218 } \\ \text { ANSI } & \text { PH22.106 }\end{array}$

ASTM D3176

ASTM E367

BIA 18B

ABYC A5

WWPA *31-1

ANS1 K62.109

UL 1067

FMS

8-21

D2860

ANS]

ANSI

ANSl

ASTM

SAE

Test Met AATCC

A577

B94.38

B94.39

B94.41

F110

J $375 \mathrm{~A}$

429

AACCH $\quad 48-30$

UL 125

UL 51

UL 565

UL 132

UL 331

Fl Pllo

ANSI PH4.175

AACCH $02-33$

ANSI PH4.250

ASTM D595

ANSI PH4.275

FI P135

ASTM D3264

IE AACCH 46-14

AACCH 56.10

$\mathrm{AACCH} \quad 58-40$

$\mathrm{AACCH}$ 58.41

CR

AAFCO *

ASTM C706

ASTM D2130

ASTM D3087

ASTM D 1681

ASTM D3049

ASTM B479

ASTM B3

ASTM B33

ASTM B298

ASTM B355

$\mathrm{SAE} \quad J 524 \mathrm{~B}$

$\mathrm{SAE} \quad \mathrm{J} 525 \mathrm{~B}$

SAE

AMS4911C

AMS6523A

AMS5627B

SAE

ASTM

SAE

ASTM

ASTM

ASTM

ASTM

ASTM

B68

MS6540B

E509

C598

C 336
B 418

B136
B580

B580

C5

/ for Std. Ref ASTM

UL

ElA

ElA 
Minimum Std. for Land-Mobile Communication Minimum Std. for Amateur Radio Std. Gravel Sand and

e Coolants (1972)

\section{0) ANSI L14.125}

Spec. for Bearings, Ball and Roller, Rod End, Std. Meth. of Test.

Performance Requirements for Individual Test Meth. for Detection of Test Meth. for Evaluation of Std. for Rec.

) for Poison Control Centers (1972) Std. Meth. for Test for Water in Engine Std. Meth. of Test for Detecting Glycol Base Std. Meth. of Test for Reserve Alkalinity of Engine (1972) Std. Meth. of Test. Anti Leak th. for Sampling and Preparing Aqueous Solutions of Engine std. Meth for Corrosion Test on Metal Specimens for Engine
Std. Meth. of Test for Boiling Point of Engine Std. Spec. for Metallic

$\mathrm{g}$ and Preparing Aqueous Solutions of Engine Antifreezes or meth. of Test for Reserve Alkalinity of Engine Aneifreeze,

4)

(1967) Std. for Safety for Meth. of Analysis of Stability std. Meth. for Determining Adhesion of Protection Sheet to Sheet to Aperture Adhesive of Unitized Microfilm Carrier Std. Dimensions of $35 \mathrm{~mm}$ Motion Picture Camera Std. for Dimensions for Unitized Microfilm Carriers Thickness of Buildup Area on Unitized Microfilm Carriers pbic Lenses (197) Std. Meth. of Designating and Measuring
Std. for Designation of 3) tronics) (1973) Std. for Industrial Electrical Power Control Std. for lndustrial Control Std. for Industrial Control Std. for Industrial Electrical Power Control Std. for Industrial Electrical Power Control Safety Std. for Fire Extinguisher and Std. Meth. of Test for Combustible Gases in Electrical nalysis (Cereal Chemistry) (Bolting Cloth, Filter Paper,/ filled and Dry Transformers, Hand Po/ Std. for Electrical $\mathrm{g}$ the Thermal Conductivity of Leather with the Cenco Fitch tion and Cohesion of Bituminous Mixtures by Means of Hveem or Relays and Relay Systems Associated with Electric Power Std for Safety for Mechanical Power Transmission Meth. of Test for Flash Point of Liquids by Tag Open Cup Test for Flash Point of Cutback Asphalt with Tag Open Cup Iary Moisture Relationships for Soils by Pressure Membrane relative Stiffness of Leather by Means of a Torsional Wire bility of Attached Organic Coatings with Impact Wedge Bend ance to Plastic Flow of Bituminous Mixtures Using Marshall f Test for Fineness of Portland Cement by Air Permeabilit Std. for Magnetic Wire for Use in Electrical ber and Rubber Like Materials by Means of a Torsional Wire ne Drive) (1972)

Std. for Induction Motors (Large Std for Synchronous Motors (Large Std. for Direct Current Motors (Large Std. for Direct Current Generators (Large Std. Wet Process Porcelain Insulators Std. Wet Process Porcelain Insulator

1 Analysis of Metals (1973) Std. Rec. Pract. for pearance, and Shrinkage Propensity of Bonded and Laminated Std Spec. for Performance of Bonded and Laminated Std. for Body Measurements for the Sizing of Girls 972) Std. Meth. of Test for Std. Meth. of Test for Sid. Meth. of Test for Std. Meth. of Test for Std. Meth. of Test for the Scott Volumeter (1970) ANSI H/ inforced Plastics by Short Beam M/ Std. Meth. of Test for Water Absorption, Bulk Density, gravity, and Bulk Density of Burn

Std. Meth. of Test for for Water Absorption, Bulk Density, Apparent Porosity, and td. Meth. of Test for Apparent Porosity, Water Absorption, e Sugar (1967) Std. Analytical Meth. for Determinin ons (Cereal Chemistry) (1962)

dent Flow Properties (1969)

ng the Brookfield Viscometer (197)

terials (1973)

11.72

ng the Cold Spanking Simulator (1/ office Building, Manufacturer, Restaurant, and Dwelling or Test Meth. for Wrinkle Recovery of Textile Fabrics: After Home Laundering (1973) ANSI L14.17) Test Meth. for press) After Repeated Home Laundering/ Std. Definitions of Terms Relating to (1973) Ans/ Test Meth. for Std. Meth. for Grading Cotton Yarns for Meth. of Analysis of Std. Test Meth. for Std. Meth. of Test for Std. Meth of Test for Std. Meth. of Test for Std. Meth. of Test for
Antennas: Part II-Vehicular (1972)

Antenna: Part 1-Base or Fixed Station (1973)

Anthrante Filtering Material (1972)

Anti Friction, Airframe (1974)

Anti Leak Antifreezes and Stop Leak Additives for Engin

Anti Scaid Type Water Shower Control Valves (1973)

Antibacterial Activity of Fabrics: Agar Plate Meth. (19

Antibacterial Finishes on Fabrics (1970) ANSI L14.144

Antidotes, Supplies (Packaging), and References (Dosage

Antifreeze-Coolant Concentrate (Glycol or Alcohol Bas

Antifreeze in Used Lubricating Oils (1973)

Antifreeze, Antirusts, and Coolants (1972) ANSI D 14.2

Antifreezes and Stop Leak Additives for Engine Coolants

Antifreezes or Antirusts for Test. Purposes (1971) ANSl

Antifreezes Tested in Glassware (1970) ANSI D14.9

Antifreezes (1972) ANSI D 14.I

Antimony (1972) ANSI H4I.1

Antirusts for Test. Purposes (1971) ANSI D 14.4

Antirusts, and Coolants (1972) ANSI D 14.2

Antitheft Alarms and Devices (Burglary Protection) (197

AOM) of Fats, Oils, and Shortenings (Cereal Chemistry)

Aperture Adhesive of Unitized Microfilm Carrier (Apertu

Aperture Card) (1973) ANSI PH5. 14

Aperture lmages (Photography) (1974)

Esion of Protection

Aperture, Camera, Copy and lmage Cards) (1972)

Aperture, Camera, Copy, and Image Cards) (1973) ANSI pH

Apertures and Related Quantities Pertaining to Photogra

Apertures of Photographic Lenses (1973)

Apparatus-Control Circuit Devices and Assemblies (197

Apparatus-Controllers and Controller Assemblies (Elec

Apparatus-Enclosures (Electronic) (1973)

Apparatus-General (1973)

Apparatus-Switching and Controlling Devices (1973)

Apparatus Booster Hose (1973)

Apparatus in the Field (1973)

Apparatus or Materials for Extraneous Matter Meth. of a

Apparatus Sales and Service Industry (Rotating, Liquid

Apparatus (1970) ALCA E60

Apparatus (1971) ANSI A37.137

Apparatus (1971) ANSI C 37.90

Apparatus (1972)

Apparatus (1972)

Apparatus (1972)

Apparatus (1972)

Apparatus (1972) ALCA E63

Apparatus (1973)

Std. Meth. of Test for Resist
Apparatus (1973)

Apparatus (1973) ANSI C9.100

Apparatus (1973) ANS1 J2.2

Apparatus) (Excl

Apparatus) (1972

Apparatus) (1972)

Apparatus) (1972)

Apparatus) (1972)

(Apparatus, Cap and Pin Type) (1971)

(Apparatus, Post Type) (1971)

Apparatus, Reagents, and Safety Precautions for Chemica Apparel Fabrics (Textile) After Dry Cleaning and Launde

Apparel Fabrics (1972) ANSI L14.291

Apparel (Clothing) (1972)

Apparent Density of Industrial Aromatic Hydrocarbons (1

Apparent Density of Leather (1968) ALCA E62

Meth. of Test for Estimatin

Sid. F IEEE

ANS1

Std ASTM

Std. Meth. of ASTM

Std. Meth. of Test for Capil ASTM

D. Meth for Measuring the ASTM

Std. Meth. of Test for Forma ASTM

Al.19 of Test for Resist ASTM

Perature Stiffening of Rub

ASTM

NEMA

NEMA

NEMA

NEMA

NEMA

ANSl

ANSl

ASTM

ASTM

ASTM

USC

Apparent Density of Metal Powders (1948) ANSI H9.1

A pparent Density of Refractory Metals and Compounds by

Apparent Horizontal Shear Strength of Parallel Fiber Re

Apparent Porosity, and Apparent Specific Gravity of Fir

Apparent Porosity, Water Absorption, Apparent Specific

Apparent Specific Gravity of Fired Whiteware Products (

Apparent Specific Gravity, and Bulk Density of Burned R

Apparent Starch Content of Corn Syrup, Refined and Crud

Apparent Viscosity of Acidulated Flour-Water Suspensi

Apparent Viscosity of Adhesives Having Shear Rate Depen

Apparent Viscosity of Gear Oils at Low Temperatures Usi

Apparent Viscosity of Hot Melt Adhesives and Coating Ma

Apparent Viscosity of Lubricating Greases (1973) ANSI Z

Apparent Viscosity of Motor Oils at Low Temperature Usi

Appartment House (1973) /Tor

Appearance of Creases in Wash and Wear Items (Clothing)

Appearance of Durable Press Textile Fabrics (Permanent

Appearance of Materials (1971) ANSI Z138.I

Appearance of Seams in Wash and Wear Items (Textile / C

Appearance Point of Distillate Fuels (1972) ANSI Z11.32

Appearance (1972) ANSI L14.164

Appearance (1973)

RS329.1

RS409

B100

661

D3147

1016

100

* 1

D 1123

D2982

D 1121

D3147

D1176

D 1384

D1120

B237

D1176

D1 121

1037

58.54

MSI0

MSI0

PH22.59

PH5.8

MS9

PH 3.29

$\mathrm{PH} 3.33$

C 19.6

C19.7

C19.4

C19.3

C19.5

92

D3284

28-90

D2214

D 1560

313

B15.1

D1310

D3143

D3152

D2821

D3281

D1559

C204

MW 1000

D1053

MG1.22

MGI.20

MGI-21

MGI.23

MGl-24

C 29.8

C29.9

E50

D2724

D3135

PS54

ASTM D2935

ASTM D2346

ASTM B212

ASTM B329

ASTM D2344

ASTM C373

ASTM C20

ASTM C373

ASTM C20

$\mathrm{AACCH} \quad 56.80$

ASTM D2556 
Std. Meth. of Test. Delamination, Strengih of Bond, Std. for Performance of Std. for Gas Std. for Gas

Std. for Safety for Thermal Cutoffs for Use in Electrical Safety Std. for Electric Motors for Std. for Safety for Sheathed Heating Elements for afety for High Voltage Components for Television Receiving afety for Special Fuses for Radio and Television Receiving e Ray Tubes (Picture and Display) for Television Receiving Std. for Decorative Gas

1973) ANSI Al47/ Safety Std. for Liquid Fuel Burning Heat (Blower, Humidifier, Evaporative Cooler, and Air Filtering t Plates and Griddles, etc.) (1973) Std. for Gas Counter way Clearance, Installation and Maintenance of Systems and e / Std. for Safety for Cord Connected Electric Gardening less) (1972) C33.11 Safety Std. for Electric Heating std. for Draft Equipment for Combustion Chamber in Heating std. for Gas Hose Connectors for Portable Indoor Gas Fired Std. for Gas Fired Illuminating td. for Gas Hose Connectors for Portable Outdoor Gas Fired le Connectors of Other Than All Metal Construction for Gas Sed. for Household Cooking Gas Std. for Recreational Vehicle Cooking Gas Std. for Outdoor Cooking Gas Safety Std. for Commercial Electric Cooking f Terms Relating to Chimneys, Gas Vents and Heat Producing fety for Household Electric Coffee Makers and Brewing Type Safety Std. for Motor Operated

Std. for Leakage Current (Electricity) for Std. for Gas Fired Absorption Summer Air Conditioning Std. for Vented Decorative Gas

flammability of Plastic Materials for Parts in Devices and olymeric Enclosures for Portable Cord Connected Electrical for Safety for Household Electric Skillets and Frying Type Std. for Safety for Hair Clipping and Shaving
Std. for Automatic Valves for Gas

Std. for Safety for Pumps for Oil Burning Sid. for Metal Connectors for Gas ation Systems, Incinerators and Other Misc. Heat Producing ials for Use in Flammable Anesthetizing Locations (Bonding le Materials; Reporting and False Alarm; Use of Equipment, hes And/ Redwood Landscape Guide (Rec. for Garden Grades, 73) st Iron-Chromium-Nickel High Alloy Tubing for Pressure Iniform Building Code Std. for Veneer ors and Generators (Fractional and Integral Hor/Std. for ions for Copper and Copper Alloys (Wrought Products Only); Construction (1974) Stds., Guide Specs., and Materials AC High Voltage Circuit Breakers Rated on a Symmet/ Std

r Schedules of Preferred Ratings, Manufacturing Spec,, and Insulators (1974)

High Voltage Circuit Breakers Rated on a Symmetric/ Std. Sid. for Redwood Lumber Spec. for Std. for Douglas Fir Lumber Spec, for Std. for Southern Pine Lumber Spec. for to Electromagnet Iron (EMI) Culd Rolled Strip for Magnetic uer, Stain, etc.) (1973)

Uniform Fire Code

tuds, Runners (Track) and Rigid Furring Channels for Screw I Studs (1972) Std. Spec, for Steel Drill Screws for the ricultural Tractors and Trailing Type Agricultu/ eans of Spray Gun or Fluidized Bed (Fire Protec/

tractors and Trailing Type (Farm) Implements (1971)

(1973)

n Refrigeration and $\mathrm{Ai} /$

Std. Rec. Pract. for power Generating Station Protect/ or Low Voltage Power Circuit/ Std. for Flow Capacity Rating and Trial Use Guide for the Std. for Preferred Ratings, Duct Liner, Adhesives, and Fasteners Pressure Sensitive Tape

Std. Rec. Pract for Paving Vises and Brick in Landscape Architecture Miscellaneous irconium and Its Alloy Forgings and Extrusions for Nuclear c. for Nickel Coated Copper Clad Steel Wire for Electronic iding or Horizontally Operating Storm Windows for External chromium Nickel and Nickel Base Alloy Castings for General Std. Spec. for Zirconium Ingots for Nonnuclear spec. for Zirconium Forgings and Extrusions for Nonnuclear Std. Spec for Primary Zirconium for Nonnuclear ec. for Seamless and Welded Zirconium Tubes for Nonnuclear Mild to Medium Strength Carbon Steel Castings for General

Std. Spec, for Zirconium and Its Alloy Ingots for Nuclear rconium Sponge and Other Forms of Virgin Metal for Nuclear ed Zirconium and Its Alloy Bars, Rod, and Wire for Nuclear
Appearance, and Shrinkage Propensity of Bonded and Lami Appliance Humidifier (1972) ANSI Z235.1

Appliance Pressure Regulators (1973)

Appliance Thermostats (1971)

Appliances and Components (1972) ANSI C33.88

Appliances and Equipment (1972)

Appliances and Equipment (1973)

Appliances and Other Electronic Equipment (Flyback Tran

Appliances and Other Electronic Equipment (1974)

Appliances and Other Electronic Equipment (1974)

Appliances for Installation in Vented Fireplaces (1973)

Appliances for Mobile Homes and Recreational Vehicles (

Appliances Rated at 600 Volts or Less) (1972) ANSI C33.

Appliances (Coffee Pots and Urns, Food Dish Warmers, Ho

Appliances (Extinguishers, Alarms, Sand pipes), Building

Appliances (Lawn Mower, Cultivator, Edger-Trimmer, Hedg

Appliances (Portable Fixed and Stationary 600 Volts or

Appliances (Regulators, Automatic Damper Controls, Fans

Appliances (1971)

Appliances (1971)

Appliances (1971)

Appliances (1971)

Appliances (1971)

Appliances (1972)

Appliances (1972)

Appliances (1972)

Appliances (1972)

Appliances (1972)

Appliances (1972) ANSI C 33.11.1

Appliances (1972) ANSI C 33.36

Appliances (1973)

Appliances (1973)

Appliances (1973)

Appliances (1973)

Appliances (1973)

Appliances (1973) ANSI C 33.11.2

Appliances (1973) ANSI C 33.36.2

Appliances (1974)

Appliances (1974) ANSI B 130.1

Appliances (1967)

Appliances) (1973) / $/ \mathrm{Ng}$, Ventilating, Cooling, Refriger

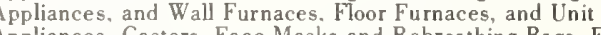

Appliances, Devices and Vacant Buildings) (1973)

Application and Design, Span Allowances, Storage, Finis

Application and Removal Torque of Threaded Closures (19

Application and Use of Radial Lip Type Oil Seals (1972)

Application at High Temperatures (1970) ASTM G82.1

Application Dampers (1973)

Application Dara for Alternating and Direct Current Mot Application Data Sheet (1972)

Application for Thermoplastic (Polyvinyl Chloride) Duct

Application Guide for Capacitance Current Switching for

Application Guide for High Voltage Air Switches, Bus Su

Application Guide for Porcelain Suspension (Power Line)

Application Guide for Transient Recovery Voltage for AC

Application in Industrial Water Cooling Towers (1971)

Application in Industrial Water Cooling Towers (1971)

Application in Industrial Water Cooling Towers (1971)

Application in Relays and Solenoids (1972)

Application of Flammable Finishes (Paint, Varnish, Lace

Application of Gypsum Board (1974)

Application of Gypsum Sheet Material to Light Gage Stee

Application of Hydraulic Remote Control Cylinders to Ag

Application of Plastic Polymers in Dry Powder Form by

Application of Remote Hydraulic Motors to Agricultural

Application of Spray Aplied Fibrous Thermal Insulation of Suction Iine Filters and Filter Driers I

Application of the Single Failure Criterion to Nuclear

Application Recommendations, and Related Requirements F

Application Std. (Air Distribution) (1971)

Application Stds. for Fibrous Glass Duct (1973)

Application Temperatures for Road Tars (1972)

Application (Tech. Notes) (1967)

Application (1967) ANSI N 125

Application (1972)

Application (1972) ANSI A 134.3

Application (1972) ANSI G81.10

Application (1972) ANSI Z179.10

Application (1972) ANSI Z179.8

Application (1972) ANSI Z179.9

Application (1972) ANSI Z212.1

Application (1973) ANSI G50.1

Application (1973) ANSI G53.28

Application (1973) ANSI N 12.1

Application (1973) ANSI NI22
(L) Bearin

ASTM

AHAM

ANSI

ANSI

UL

UL

UL

lor S UL

Thod UL

ANSI

UL

UL

ANSI

UL

UL

ANSI

ANSI

ANSI

ANSI

ANS

ANSI

ANSI

ANSI

UL

NFPA

Std. for Sa UL

UN

ANSI

ANSI

UL

for $P$ UL

Std. UL

UL

ANSI

ICBO

ICB

UL

/Mmab ICBO

CRA

ASTM

SAE

/Ca ASTM

ICBO

NEMA

Designat $C D A$

SMACN

IEEE

ANSI

NEMA

IEEE

CTI

CTI

$\begin{array}{ll}\text { Pplicable } & \text { NAI } \\ & \text { ICBO }\end{array}$

ICBO

ASTM

ASTM

ASAE

FMS

ASAE

ASTM

ARI

IEEE

ANSI

SMACN

SMACN

ASTM

BIA

Std. Spec. for Z ASTM

Spe ASTM

Bination Vertically SI AAMA

/T Iron Chromium, Iron ASTM

ASTM

Std. ASTM

ASTM

Std Sp ASTM

Std. Spec. for ASTM

ASTM

Std Spec, for $\mathrm{Zi}$ ASTM

/T Rolled and Cold Finish ASTM

D2724

HU.1

Z21.18

21.23

1020

1004

1030

492.3

492.7

4.92 .8

Z21.60

(

07

Z21.31

(

378

21.12

21.2

221.42

Z21.54

1.45

21.1

21.57

Z21.58

$97 \mathrm{M}$

73

C101.1 
irconium and Its Alloy Sheet, Strip, and Plate for Nuclear r Precision Coaxial Connectors for CATV (Cable Television) ' Hardware on Std. Steel Doors and Frames (Reinforcement ervice (1972)

d Grade, Panel Characteristics, Material Composition, Use, rcuit Reclosers (1973) ANSI C37.61 Sid. Guide for the tires for Wet Traction in Cornering Without Driving Torque Rec. Pract. for Aluminum nt of Neutron Flux and Spectra for Phy sical and Biological $\mathrm{K}$ Thermocouples for Nuclear or for Other High Reliability

Meth. of Test. Vitrified Ceramic Materials for Electrical Std. Spec. for Hand Operated Chain Hoists for Industrial nic Examination of Plain and Clad Steel Plates for Special Std Spec for Copper Alloy Tubes for Pressure ly(Vinyl Chloride) (PVC) Pipe and Fittings for Nonpressure

Vehicles, Golf Car, and Other Diversified Off the Highway Std. for Dry Type Transformers for General

$\mathrm{d}$ and Cold Finished Zirconium Bars and Wire for Nonnuclear Spec. for Beryllia Ceramics for Electronic and Electrical Std. Spec. for Copper Alloy Sand Castings for General pec. for Annealed Aluminum Alloy Foil for Flexible Barrier Quenched and Tempered Carbon Steel Plates for Structura r/Film, Film Dielectric Capacitors for Power Semiconducto

Vehicles, Motorboats, Tractors, Automotive and Industrial spec. for Aluminum-Alloy Drawn Tubes for General Purpos inum Alloy Extruded Round Coiled Tubes for General Purpose n System for Elastomeric Materials (Rubber) for Automotiv d Hair for Cushioning Materials for Upholstery and Bedding Std. Spec. for Flat Rolled Electrical Steels for Magnetic

bars, Carbon, Hot Rolled, Special Quality, for Fluid Power nspection of Aluminum Alloy Wrought Products for Aerospace Spec. for Hard Drawn Copper Capillary Tube for Restrictor
Std. for Viewing Conditions for the c Arts $(1972)$ designed to Open Automatically in Case of Fire (1974)

m Air Purifiers, Filters, Compressors, Artificial Airways,

Spec. for Photographic Grade Ammonium Hydroxide, $\mathrm{Nh}_{4} \mathrm{OH}$

of the Refractometer for Determining the Freezind Point of r Test. Purposes (/ Std. Meth. Ior Sampling and reparing
T200 Std. Meth. of Test or $\mathrm{pH}$ of

Std. Meth. for Colorimetric Determination of Uranium in Std. for System for Romanization of Std, for Milling Machine Molybdenum Sheet, Strip, and Plate, Rec. Pract. for Plasma

Std. Rec. Pract. for Designing a Hig's Current Std. Meth. Measuring Plasma Rec. Pract. for Air Carbon resistance of Textile Materials and Coated Fabrics: Carbon

Test Meth. for Weather Resistance of Textiles: Carbon

Test Meth. for Colorfastness of Textiles to Light: Carbon for Colorfastness of Textiles to Light: Water Cooled Xenon for Colorfastness of Textiles to Light: Water Cooled Xenon

Test Meth. for Colorfastness of Textules to Light, Carbon t Resistant $(0.95 \mathrm{Cr}-0.55 \mathrm{Mo}-0.30 \mathrm{~V}(0.40-0.50 \mathrm{C}) \mathrm{Vacuum}$ $\mathbf{r}-4.5 \mathrm{Ni}-3.5 \mathrm{Cu}-(\mathrm{Cb}+\mathrm{Ta})$, Consumable Electrode Vacuum Std. Meth. of Test for High Voltage, Low Current, Dry loys by the Silver Chloride-Lithium Fluoride Carrier D-C $s$ of Thermionic Nickel Alloys by the Powder Direct Current $\mathrm{s}$ of Uranium Oxide $\left(\mathrm{U}_{3} \mathrm{O}_{k}\right)$ by Gallium Oxide Carrier D-C carbon and Low Alloy Steel for Boron by the Point to Plane for Spectrochemical Analysis of Copper by the Briquet D.C Std. Spec. for Aluminum Alloy Formed and rvice (1971) ANSI G36.49 Std. Spec. for Metal Spec, for Mild Steel Covered Spec. for Tungsten Spec. for Aluminum and lis Alloy Spec. for Low Alloy Steel Covered Spec. for Copper and Its Alloy Safety Std. for Transformer Type c. for Bare Mild Steel Electrodes and Fluxes for Submerged Spec. for Mild Steel Electrodes for Gas Metal Spec. for Mild Steel Electrodes for Flux Cored Rec. Pract. for Plasm Std. for Manual Gas Metal Std. for Manual Gas Tungsten Std Spec for Reinforced Concrete Structural Design of Semicircular Brick Masonry Structural Design of Brick Masonry Std. Form of Agreement Between Std. Form of Agreement Between ing Services (1972)

Std. Form of Agreement Between Owner and Std. for Agreement Between Owner and Std. Form of Agreement Between Owner and

spec. for Exterior Perimeter Sealing Compound for Use with
Application (1973) ANSI N123

Application (75 Ohms) (1973)

A pplication) (1972)

Application, Care, and Use of Wire Rope for Oil Field S

Application, Finishes and Other Technical Data) (1973)

Application, Using Highway Jehicles (1973)

Applications for Boats and Yachts (1971)

Applications (1960)

Applications (1967) ANSI N142

Applications (1969) ANSI C59.73

Applications (1971)

Applications (1971) ANSI G35.25

Applications (1972)

Applications (1972)

Applications (1972)

Applications (1972) ANSI C89.2

Applications (1972) ANSI Z179.11

Applications (1972T)

Applications (1973)

Applications (1973)

Applications (1973)

Applications (1973)

Applications (1973)

A pplications (1973) ANSI H38.17

Applications (1973) ANSI 38.18

Applications (1973) ANSI J8.15, SAE J200

Applications (1973) ANSI J8.8

Applications (1974)

Applications (1974)

Applicat ons (1974)

Applicá ns (1974) ANSl H23.8

/Ene (ABS) Pipe and Fittings to Po

Rec. for Measureme NCRPM

/Pec. for Sheathed, Type ASTM

Std. ASTM

HMI

/Straight Beam Ultraso ASTM

ASTM

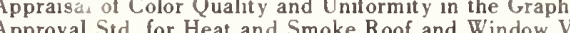

A ppurtenances) (1973)

Aqueous Ammonia) (1972)

Aqueous Engine olants (1974)

Aqueous Solution of Engine Antifreezes or Antirusts Fo

Aqueous Solutions with the Glass Electro

Arabic Written L iguage (Transliteration) (1972)

Arbor Assemblie (1972)

Arc Cast, Stres. Relieved (1973)

Arc Cutting (1973)

Arc Erosion Test for Electrical Contact Materials (1973

Arc Gas Enthalpy by Energy Balance (1973)

Arc Gouging and Cutting (1974)

Arc Lamp Exposure with Wetting (1972) ANSI L14.224.

Arc Lamp Exposure Without Wetting (1972) ANSI L14.225

Arc Lamp, Alternate Light and Darkness (1971) ANSl L14.

Arc Lamp, Alternate Light and Darkness (1971) ANS1 L14.

Arc Lamp, Continuous Light (1971) ANSI L14.170

Arc Lamp. Continuous Light (1971) ANSI L14.53

Arc Remelted (1973)

Arc Remelted (1973)

/Gings, and Tubing, Low Alloy, Hea

Arc Resistance of Solid Electrical Insulation (1973)

Arc Technique Using an Optical Emission Spectrograph (1

Arc Technique (1961) ANSI Z128.2

Arc Technique (1970) ANSI Z128.27

Arc Technique (1970) ANSI Z128.29

/Ctrochemical Analysi

/Trochemical Analysi

Analysis of Plain

Arc Technique (1971) ANSI Z128.32

Arc Welded Round Tube (1974) ANSI H38.20 Std. Meth.

Arc Welding Electrodes (1969) ANSI A5.1

Arc Welding Electrodes (1969) ANSI W 3.12

Arc Welding Electrodes (1969) ANSI W 3.3

Arc Welding Electrodes (1969) ANSI W3.5

Arc Welding Electrodes (1969) ANSI W3.6

Arc Welding Machines (1972) ANSl C33.2

Arc Welding (1969) ANSI W3.17

Arc Welding (1969) ANSI W3.18

Arc Welding (1969) ANSI W3.20

Arc Welding (1973)

Arc (Gma) Welding Equipment (1974)

Arc (Gta) Fusion Welding Equipment (1974)

Arch Culvert, Storm Drain, and Sewer Pipe (1971)

Arches (Tech. Note) (1971)

Arches (Tech. Notes) (1967)

Architect and Consultant for Other Than Normal Engineer

Architect and Engineer (1974)

Architect for Interior Design Services (1972)

Architect for Special Services (1972)

Architect (1974)

Architect's Accounting Forms (1972)

Architectural Aluminum (1971)

Code for Architectural Design Competitions (1972)
ASTM

NEM

ASTM

ASTM

ASTM

Std. S ASTM

ASTH

ASTM

ASTM

STM

Sid. ASTM
Sid.

ANSl

FMS

Std. ANSl

ASTM

ASTM

ASTM

ASTM

ANSI

ANSl

SAE

AWS

AATM

ASTM

AWS

/R AATCC

AATCC

AATCC

AATCC

AATCC

AATC

SAE

SAE

STM

STM

ASTM

ASTM

ASTM

ASTM

AWS

AWS

AWS

AWS

AWS

UL

Spe AWS

AWS

AWS

AWS

NSA

NSA

ASTM

BIA

BIA

AlOA

AIOA

AlOA

A IOA

AlOA

AlOA

AAMA

AlOA
Motor SAE

IST

B352

RS403

107

$9 \mathrm{~B}$

3 A 9

321

376

S1

R23

235

200
A5
B 689

B469

D3138

1.48

ST 20

B550

F 356

B584

B479

RS401

$\mathrm{J} 537 \mathrm{H}$
$\mathrm{B} 483$

B491

D2000

D2 128

A 345

A 695

B594.

B360

PH2.32

4430

48

PH4.232

D3321

D 1176

E70
E318

Z39.12

B5.47

AMS7801A

C5.2

B576

E341

C 5.3 
National Bureau of Standards' KWIC Index of Engineering Standards

Std. for Architectural Door Trim (1972)

Std. of Architectural Ethical Practice (1972)

Glossary of Architectural Metal Terms (1973)

Architectural Sheet Metal Spec. (1973)

er (1972)

Rec. Architectural Spec, for Structural Glued Laminated Timb

eth. of Test for Acid Resistance of Ceramic Decorations on Architectural Type Glass (1972T)

Quality Stds. for Lumber Grades (Architectural Woodwork) (1973)

Quality Stds. for Exterior Sash (Architectural Woodwork) (1973)

Quality Stds. for Flush Solid and Hollow Core Doors (Architectural Woodwork) (1973)

Quality Stds. for Stile and Rail Doors (Architectural Woodwork) (1973)

Quality Stds, for Factory Finishing (Architectural Woodwork) (1973)

Quality Sids. for Plywood and Particleboard Grades (Architectural Woodwork) (1973)

Quality Stds. for Standing and Running Trim (Architectural Woodwork) (1973)
Quality Stds. for Flush Wall Paneling (Architectural Woodwork) (1973)

Quality Stds. for Misc. Ornamental ltems (Architectural Woodwork) (1973)
Quality Stds. for Stairwork and Handrails (Architectural Woodwork) (1973)

Quality Stds. for Exterior Frames (Architectural Woodwork) (1973)

ality Stds. for Wood Screens for Window and Door Openings (Architectural Woodwork) (1973)

stds. for High Pressure Laminate Flush Type Wall Paneling (Architectural Woodwork) (1973)

for Blinds and Shutters Using Stile and Rail Construction (Architectural Woodwork) (1973)

rk: Cabinets, Cases, Counters and Enclosures of All Kinds (Architectural Woodwork) (1973)

Symbols for Electrical Wiring and Layout Diagrams Used in

967)

Brick in Landscape Brick in Landscape
Brick in Landscape

er Base (1973)

e (1973)

Glossary of Construction Industry Terms Spec. for Photographic Film for Std. Spec. for Photographic Film for Std, for Motor Vehicle Brake Fluid for Use Under ltural Plastics, Chemicals, Metal Dusts) (1973) Std for motion Picture Film/ Std. for Location of Super 8 Printed ure Film, Perforate/ Std. for Location of Super 8 Printed perforated 1.4 (1971)

Sid. for Dimensions for Printed Std. Meth. of Test for Surface Std. Meth. of Test for Applied Weight Per Unit Std. Meth. of Measuring

Std. Meth. of Test for Applied Weight Per Unit Std. Meth. for Measuring Thickness of Buildup Std. for Location of Super 8 Printed copy, and / (1971)

Std. Dimensions of Projectable Anamorphic Image I Instrument Panel Laboratory Impact Test Procedure-Head (1973) oning) (1966) Std. Dimensions of lmage Std. Coil Face

Petroleum Production /

Std. Wheel Diameters and Outlet

Rec. Pract. for Classification of Rec. Pract. for the Safe Action and Title strial Trucks (1973) ANSI B5/ Std. for Type Designations,

Rec. Pract. for Homes and Camps in Forest Uniform Fire Code: Suppression and Control of Hazardous construction of Surface Drainage Systems on Farms in Humid drogen, Krypto/ Uniform Fire Code: Cryogenic Fluids (Air. verters for Motor Drives: Part l-Converters for DC Motor Std. for Flash Points, Closed Cup, tent. Meth. of Test for Aromatic Types Analysis of Gas Oil nsi Z78.15 Std. Meth. of Test for Bromine Index of

Meth. of Test for Nonaromatic Hydrocarbons in Monocyclic Std. Test Meth. for Polynuclear $\begin{array}{ll}\text { ical Insulating Liquid) (1973/ } & \text { Std. Spec. for Chlorinated } \\ \text { Std. Spec. for Chlorinated }\end{array}$

toluene, Xylenes, Solvent Naphthas, and Similar Industrial ide and Sulfur Dioxide Content (Qualitative) of Industrial Std. Meth. of Test for Apparent Density of Industrial

- Meth. for Calculation of Volume and Weight of Industrial chromatography (1972) ANSI Z11.27/ y Mass Spectrometry (1973)

Std. Meth. of Test for Tent. Meth. of Test for Rec. Safeguards for Std. for Typewriter Keyboard Std. Drive Std. Drive

Test for Length and Length Distribution of Cotton Fibers Std. Meth. of Test for Linear Density of Cotton Fibers ing Resistivity of Silicon Slices with a Collinear 4 Probe istance of Thin Metallic Films with a Collinear Four Probe ance of Silicon Epitaxial Layers Using a Collinear 4 Probe Rec. Pract. for Spark Rec. for Lightning ANSI C62.1 2). Std. for Surge (Lightning) Rec. Pract. for Spark Std. for Safety for Flame Surge

Meth. for Determination of th. of Quantitative Determination of Both Nitrophenide and Std. Spec. for Fluor Chrome Std. Meth. for Chemical Analysis of Fluor Chrome Std. Analytical Meth. for Determining
Architectural Woodwork: Standing and Running Trim, Case

Architecture and Building Construction (1972)

Architecture Garden Walls (Tech. Notes) (1968)

Architecture Miscellaneous Application (Tech. Notes) (1

Architecture Terraces and Walks (Tech. Notes) (1966)

Architecture) (1970)

Archival Records, Silver Gelatin Type, on Cellulose Est

Archival Records, Silver Gelatin Type, on Polyester Bas Arctic Almospheric Conditions (1972)

Area Classification in Hazardous Dust Locations (Agricu

Area in Optical Reduction or Contact Printing on $35 \mathrm{~mm}$

Area in Optical Reduction Printing on $35 \mathrm{~mm}$ Motion Pict

Area in Super 8 Printing on $16 / 8 \mathrm{Mm}$ Motion Picture Film

Area of Carbon Black (1973)

Area of Dried Adhesive Solids (1969) ANSI Z197.9

Area of Leather Test Specimens (1968) ALCA E2

Area of Liquid Adhesive (1951) ANSl Z197.10

Area on Unitized Microfilm Carriers (Aperture, Camera,

Area on $16 \mathrm{~mm}$ Motion Picture Film, Perforated Super 8 (

Area on $5 \mathrm{~mm}$ Motion Picture Film (2.35:1) Aspect Ratio

Area $(1971)$

Areas and Mounts for Slides and Opaques for Television

Areas for Central Station Units (Air Moving and Conditi

Areas for Centrifugal Fans (1966)

Areas for Electrical Installations at Drilling Rigs and

Areas for Television Transmission (1968)

Areas of Use, Maintenance and Operation of Powered Indu

Areas (1972)

Areas (1973)

Areas (1973)

Rec. for Design and

Argon, Carbon Monoxide, Deuterium, Fluorine, Helium, Hy

Armature Supplies (1973) ANSI C 34.3

Aromatic Chemicals and Isolates (1973)

/for Thyristor Con

Aromatic Fractions by Mass Spectrometry (1973)

Aromatic Hydrocarbons by Coulometric Titration (1974) a

Aromatic Hydrocarbons by Gas Chromatography (1968) ANSI

Aromatic Hydrocarbons in Air Particulate Matter (1971)

Aromatic Hydrocarbons (Askarels) for Capacitors (Electr

Aromatic Hydrocarbons (Askarels) for Transformers (1973

Aromatic Hydrocarbons (1947) ANSI Z78.9

Aromatic Hydrocarbons (1971) ANSI Z78.13

Y Benzene,

Aromatic Hydrocarbons (1972)

Aromatic Hydrocarbons (1973) ANSI Z106.1

Aromatic Traces in Light Saturated Hydrocarbons by Gas

Aromatic Types Analysis of Gas Oil Aromatic Fractions B

Arrangement of Carpet Storage Racks (1972)

Arrangement (1973)

Arrangements for Centrifugal Fans (1966)

Arrangements for Tubular Centrifugal Fans (1966)

(Array Meth.) (1972) ANSI L 14.91

(Array Sample) (1973) ANSI L14.136

Array (1973)

Array (1973)

Array (1974)

Tent. Meth Std. Meth. for Measur

Arrester Test Procedure for Medium Size Engines (1972)

Arresters and Grounds (1974)

Arresters for Alternating Current Power Circuits (1972)

Arresters Used on Multiposition Small Size Engines (197

Arresters (Fire) for Use on Vents of Storage Tanks for

Arresters (1972)

Arsanilic Acid in Feeds (Cereal Chemistry) (1962)

Arsanilic Acid in Feeds (Cereal Chemistry) (1962)

Arsenate Phenol (1971)

Arsenate Phenol (1971) ANS1 O11.20

Arsenic Content of Corn Sugar (Crude and Refined) (1958

ANSI A156.6

AIOA J330

NAAMM $* 35$

SMACN *1

AITC 6.5/A!

ASTM C724

AWI $* 1.100$

$A W 1 * 1.1000$

$A W 1 \quad * 1.1300$

AW1 *1-1400

AWI $* 1.1500$

$A W I \quad * 1-200$

$A W 1 \quad * 1.300$

$A W I * 1-500 A$

AWI $* 1.700$

AWI *1-800

AWI $\quad * 1-900$

Ou AWl

Quality AWI

$* 1.1100$

$* 1-500 \mathrm{~B}$

$* 1.1200$

* 1.400

*1-1

Y32.9

$29 \mathrm{~A}$

ANSI

BIA

BIA
BIA

AIOA

ANSI

SAE

ISA

ANS ]

ANSI

ANSl

ASTM

ASTM

ASTM

ASTM

NMA

ANSI

ANSI

SAE

ANSI

AMCA

$A M C$

API

SMPTE

NFPA

NFPA

CBO

ASAE

ICBO

IEEE

EOA

ASTM

ASTM

ASTM

ASTM

ASTM

ASTM

29B

29

PH1.28

PH1.41

J1702D

S12.10

PH22.180

PH22.179

PH22, 153

D3037

D898

D2347

D899

MSo

PH22.181

PH22.106

J921B

PH22.94

1402

2401

RP500B

RP8

505

$\mathrm{UFC} * 2 \mathrm{E}$

R302.2

UFC *2ART 36

444

D.3239

D. 3239
D 1492

D2360

D2682

D2233

D2283

D847

D853

$\begin{array}{ll}\text { ASTM } & \text { D853 } \\ \text { ASTM } & \text { D2935 }\end{array}$

Std ASTM D1555

ASTM D2600

ASTM D3239

FMS LPD8-30

ANSI $\quad \mathrm{X} 4.7$

AMCA 2404

AMCA 2410

ASTM D 1440

ASTM Dl769

F84

F390

F374

SAE J350A

FMS 5-11

$\begin{array}{ll}\text { IEEE } & 28 \\ \text { SAE } & \text { J } 335 A\end{array}$

UL 525

NEMA LA

AACCH $18-20$

/ Me AACCH 18-26

ASTM D 1034

ASTM D1035

CR 
954)

Std. Analytical Meth. for Determining oluminescence and the Corresponding Composition of Gallium tors (1971)

Std. Analytical Meth. for Determining Std. Std. Spec. for Ammoniacal Copper Std. Meth. for Chemical Analysis of Ammoniacal Copper Rec. for Crayons and Related electrical Resistivity of Manufactured Carbon and Graphite est for Density in Air of Manufactured Carbon and Graphite nnest Spot in a Zinc (Galvanized) Coating on lron or Steel End Loaders and Dozers/ Std. for Steering Frame Lock for ction Equipment, Room Air Purifiers, Filters, Compressors,
Spec. for Base Plate

one Vibrators (1972) Spec. for Base Plate Std. Meth. of Test for Aging Effects of w Temperature Flexibility of Latex Sealing Compounds After Std. Dimensions for Graphic e Appraisal of Color Quality and Uniformity in the Graphic Std. Meth. for Calculating Coal and Coke Analyses from physical Dough Tests: Approximate Corrections for Changing Rec. Installation Spec. for Vinyl
No Wax Maintenance of Vinyl No Wax Maintenance of Vinyl utional and Industrial Build/ strial and Commercial Buil/ Std. Definitions of Terms Relating to 1973) ANSI A 165.4

a 165.2 Std. Spec. for Linings for Std. Spec. for

72) ds (1972) or Water and Fire Service (1973) Uniform Building Code Std. for Std. Spec. for Corrugated Std. Spec. for Flat Std. Spec. for Corrugated weathering Tests of Latex and Emulsion Ex/ Std. Spec. for Std. Spec. for Std. Spec. for Std. Spec. for Woven Std. Meth. of Test for

nsi L14.141 for Special Purpose Roofs (Wood Shakes and Shingles with 1971)

Pitch, Coal-Tar Saturated.Organic Felt, Asphalt-Saturated

Measurement of Airborne Std. Meth. of Sampling td. Meth. of Test for Bauer-McNett Wet Classification of

Std. Meth. of Test for Air Permeability of Spec. for Self Adhering Vinyl Spec. for Vinyl

Std. Meth. of Test for Asbestos Content of Rec. Health Safety Practices for Handling and Fabricating or Water Emulsion Floor Polish for Use on Asphalt or Vinyl Spec. for Cleaners for Use on Asphalt and Vinyl r Coverings (Asphalt, Cork, Linoleum, Rubber, Vinyl, Vinyl Indophenol-Xylene Extraction Meth. of Analysis of dstuffs (Cereal Chemistry) (1962)

c.) (1973) Colorimetric Acetate

spec. for AsphaIt for Damproofing and Waterproofing (1973) , and Other Road Conditioning Purposes (1974) ANSI A37.37. rficial Hardness of Metallic Materials (1974) ANSI Z115.6,

r Autoclave Expansion of Portland Cement (1971) ANSl A 1.8, or Specific Gravity of Hydraulic Cement (1972) ANS1 A1.12, cement Content of Soil Cement Mixtures (1974) ANSI A37.58, ssive Strength of Bituminous Mixtures (1974) ANS1 A37.103,

or $\mathrm{pH}$ of Aqueous Solutions with the Glass Electrode (1974)

td. Meth. of Test. Emulsified Asphalts (1974) ANS1 A37.42, real Chemistry) (1962) Harland and ty of Transformer lnsulation and Construction Materials in

Boiling Homologues of Chlorinated Biphenyls for Capacitor (1973) Std. Spec. for Chlorinated Aromatic Hydrocarbons
Std. Spec. for Chlorinated Aromatic Hydrocarbons s; Fruit Tree Grades; Small Fruits (Berry, Currant, Grape, anamorphic Image Area on $5 \mathrm{~mm}$ Motion Picture Film (2.35:1) nstruction of Built Up Roof Coverings: Hot Mopped Asphalt, Spec for Cleaners for Use on

Std. Spec. for Asphalt Mastic for Use in Waterproofing and Coal Tar Saturated Organic Felts, Coal Tar Pitch, and ec. for Enamel Coating System Designations for Coal Tar or Std. Meth. of Test for Specific Gravity of Solid Pitch Spec. for

Std. Spec. for
Std. Spec. for Std. Spec. for Std. Spec. for Std. Spec. for Std. Spec, for

Arsenic Content of Corn Syrup Unmixed and Corn Sugar (1 Arsenide Phosphide Wafers (1973) /Elength of Peak Arsenite (1971) ANS1 O11.8 Arsenite (1971) ANSI 011.23

Art Materials for School Use (1963)

Articles at Room Temperatures (1969) ANSI K90.7

Articles by Physical Measurements (1969) ANSI K90.2

Articles by the Preece Test (Copper Sulfate Dip) (1973)

Articulated Vehicles (Construction and Industrial Front

Artificial Airways, Appurtenances) (1973)

(Artificial Dentures) Wax (1971)

Artificial Headbone for the Calibration of Audiometer B

Artificial Weathering on Latex Sealing Compounds (1972)

Artificial Weathering (1972)

Arts Sheet Films (photography) (1973)

Td. Meth. of Test for Lo

Arts (1972)

Std. for Viewing Conditions for Th

As-Determined to Different Bases (1974)

s-is Farinograph Absorption to 14.0\% Moisture Basis (C

Asbestos and Asphalt Tile Flooring (1973)

Asbestos and Asphalt Tile Floors for Commercial, Instit

Asbestos and Asphalt Tile Floors in lnstitutional, Indu

Asbestos Cement and Related Products (1972) ANSl Al30.1

Asbestos Cement Fiberboard Insulating Panels (1972)

Asbestos Cement Nonpressure Sewer Pipe (1973) ANS1 A165

Asbestos Cement Nonpressure Small Diameter Sewer Pipe

Asbestos Cement Perforated Underdrain Pipe (1973) ANSl

Asbestos Cement Pipe (1973)

Asbestos Cement Pipe (1973) ANSI A 165.1

Asbestos Cement Plastic Foam Core Insulating Panels (19

Asbestos Cement Pressure Pipe for Water and Other Liqui

Asbestos Cement Pressure Pipe (1973) ANSl A165.5

Asbestos Cement Pressure Pipe, Couplings, and Gaskets F

Asbestos Cement Roofing Shingles (1973)

Asbestos Cement Sheets for Bulkheading (1974)

Asbestos Cement Sheets (1972) ANSI A124.1

Asbestos Cement Sheets (1974) ANSI AI25.1

Asbestos Cement Shingle Blanks to Be Used as Panels in

Asbestos Cement Storm Drain Pipe (1973)

Asbestos Cement Transmission Pipe (1973)

Asbestos Cloth (1973) ANSI L14.133

Asbestos Content of Asbestos Textile Materials (1972) a

Asbestos Felt or Gypsum Board Underlayment) (1973)

Asbestos Felt, Asphalt-Coated Glass Fiber Mat (1973)

Asbestos Fiber by the Membrane Filter Meth. (Textile)(

Asbestos Fiber for Test. (1970) ANSI K91.2

Asbestos Fiber (1970) ANSI K9l.1

Asbestos Fibers (1971T)

Asbestos Fibers (1972)

Asbestos Floor Tile (1972)

Asbestos Floor Tile (1972)

Asbestos Textile Materials (1972) ANSI L14.141

Asbestos Textile Products (1971)

Asbestos Tile as a Wall Covering (1973)

Asbestos Tile Floors (1969)

Asbestos Tile Floors (1970)

Asbestos, Polymeric Poured Seamless, etc.) (1973)

Ascorbic Acid (Cereal Chemistry) (1962)

Ashing Meth. of Analysis of Phosphorus in Feeds and Fee

Ashing with Nitric and Perchloric Acids (Wood, Pulp, Et

Ashto M115, ANSI A 109.16

/Celeration of Concrete, Curing of Concrete ASTM

Ashto M144

Ashto T080

Ashto T107

/St for Rockwell Hardness and Rockwell Sup Std Meth of Test Fo A Std. Meth. of Test $\mathrm{F}$

Ashto T133 Std. Meth. of Test for

Ashto T167

Ashto T200 h. of Test for Compre

Ashto T59

Std. Meth. of Test F

Ashworth Meth. of Analysis of Serum Protein in Milk (Ce

Askarels (1973)

Askarels (1973)

Askarels (1974) ANSI C59.62

Th. for lnvestigating the Compatibili

Chromatographic Estimation

Askarels) for Transformers (1973)

Asparagus); Lining Out Stock; Seedling Trees and Shrubs

Aspect Ratio (197I)

Asphalt and Coal Tar Sat urated Organic Felts, Coal Tar

Asphalt and Vinyl Asbestos Tile Floors (1970)

(Asphalt Cement, Mineral Filler and Aggregate) (1941) an

Asphalt Coated Glass Fiber Mat (1973)

A sphalt Coatings (1972)

Asphalt Displacement M

Asphalt Floor Tile (1972)
Asphalt for Damproofing and Waterproofing (1973) Ashto

Asphalt for Use in Constructing Built Up Roof Coverings
CR

AST

ASTM

ASTM

USC

for ASTM

IT ASTM

ASTM

SAE

NSF

ADA

ASTM

ASTM

ANSI

ASTM

$\mathrm{AACCH}$

RT1

RTl

ASTM

ASTM

ASTM

ASTM

ASTM

ASTM

ASTM

ASTM

$A$ WW

ASTM

UL

ICBO

ASTM

ASTM

ASTM

ASTM

ASTM

ASTM

ASTM

ASTM

Td ICBO

UL

ATl

ASTM

ASTM

ASTM

ASTM

RTI

RTI

ASTM

ATl

RTl
RTl

/Loo ASTM

F141

AACCH $86-10$

AACCH $40-55$

TAPPI

ASTM

ASTM

ASTM

ASTM

ASTM

ASTM

AACCH

ASTM

ASTM

ASTM

ASTM

ASTM

ANSI

ANSI

ICBO

RTl

ASTM

/Sphalt, Asphalt ICBO

Rec. Sp

NAPCA

ASTM

RTI

ASTM
ASTM

E. 4

R.5

D 1325

D 1326

R 192

C611

A239
A239

J276

48

S 3.13

C732

C734

D3180

54

PHI.16

PH2.32 
m 116, ANSl Al09.1

s (1970) ANS1 A 109.18

Std. Spec. for Primer for Use with Std. Spec. for

,Mineral Filler and Aggregate) (1941) An/ Std. Spec. for
Spec, for Water Emulsion Floor Polish for Use on Spec. for Water Emulsion Floor Polish for Use on
Std. spec. for .48 ention Data on Elastomer Roof Coverings (Multiply Felt and 73)

Tent. Meth. of Test for Wind Resistance of Rec. Installation Spec. for Vinyl Ast - stos and No Wax Maintenance of Vinyl Asbestos and

ndustrial Build/ mmercial Buil/ Sid. for Maintenance of Vinyl Asbestos and ines (Wrapped Systems) (1972) Tent. Rec. Spec. for Tent. Meth. of Test for Viscosity of Std. Meth. of Test for Flash Point of Cutback Sid. Spec. for Liquid Std. Spec. for Liquid Std. Spec. for Liquid Std Spec for Cationic Emulsified Uniform Building Code Std. for Roofing Std. Spec. for Emulsified use in Construction of Built Up Roof Coverings: Hot Mopped td. for Materials for Built. Up Roof Coverings: Hot Mopping Std. Nomenclature Relating to Resilient Floor Coverings or Solubility of Bituminous Road Materials (Tar, Petroleum r Saturated Organic Felt, Asphalt-Saturated Asbestos Felt. nic Felt, Coal Tar Pitch, Coal.Tar Saturated Organic Felt, aterials for Built-Up Roof Coverings: Hot Mopping Asphalt. Std. Meth. of Test for Effect of Heat and Air on a 37.45 Std. Meth. of Test for Distillation of Cut Back

Std. Meth. of Test for Specific or API Gravity of Liquid Std. Meth. of Test for Kinematic Viscosity of Std. Meth. of Test for Absolute Viscosity of Std. Meth. of Test. Emulsified Meth. of

ile Desizing (1973) ANSI L14.147 Test Meth. for Std. Meth. of analysis (1973)

chmeical Analysis of Ores, Minerals, and Rocks by the Fire requirements for Household Dishwashers (1971) ANSI A 197.1, equipment (washer, Dryer, Combination) (1971) ANSI A 197.2. or Household Food Waste Disposer Units (1971) ANS1 A 197.3,

Std. Meth. of Test for Compressive Strength of Masonry Std. Spec. for Zinc Coating (Hot Dip) on ferred) (1974) Std. for Pan Head Screw and

Power Generating Statio/ Std. for Electrical Penetration Industrial Control Apparatus-Controllers and Controller t Meth. for Flexural Strength of Adhesive Bonded Laminated and Procedures for SAE $100 \mathrm{R}$ Series Hydraulic Hose and Hose Std. for Milling Machine Arbor Sid. Meth. of Fire Tests of Door

Std. Thermal Performance Test for Steel Door and Frame Std. Acoustical Performance Test for Steel Door and Frame Safety Sid for Semiautomatic Fire Hose Rack

Std. for Seismic Restraint Direct Hung Suspended Ceiling ance Criteria for Water Resistance on Steel Door and Frame a for Rate of Air Flow Through Closed Steel Door and Frame Uniform Building Code Std. for Fire Tests of Door

Uniform Building Code Std. for Fire Tests of Window Safety Std. for Oil Fired Boiler

ical Power Control Apparatus-Control Circuit Devices and

ties of Adhesives in Shear by Tension Loading of Laminated Sid. Meth. of Fire Tests of Door Std. for Safety for Fire Tests of Door

t of Partitions (Wall, Ceiling, Floor, and Floor-Ceiling

stc) of Building Partitions Such as W alls, Floor-Ceiling Std. for Electrical Harness icle Manufacturing Plants (Fire and Wind Hazards at Plants Std. for Strap

for Low and Medium Speed Stationary Diesel and Gas Engine les lncluding Tests for Static Load, Frame, Fork, Steering Std. for Forged Carbon Steel, Shoulder Nut

Std. for Fuel

Design, Manufacture, Inspection and ement Data; Group Numbers, Dimensional Spec., and Ratings; chers, etc.) (1973) Uniform Fire Code: Places of

Rec. Pract. for Diesel Fuel lnjection Nozzle and Holder

dle, Latching lnternal and External Release Components and Std. Consumer Safety Spec. for Components,

General Requirements for Regular Bicycles Including Wheel Rod Ends (1973)

Std. for Tube

Std. for Tube

Std. for Tube

Std. for Tube

d Rod Ends (1973)

threaded Rod Ends (1973)

stness to Light (1972) Ansi/ dividual Observed Values) (1974) ystems Based on the Composition and Cryst/

Std. for Streame

Test Meth. for Detection and

Std. Meth. for
Asphalt in Dampproofing and Waterproofing (1973) AASHO

Asphalt Insulating Siding Surfaced with Mineral Granule

Asphalt Mastic for Use in Waterproofing (Asphalt Cement Asphalt or Vinyl Asbestos Tile Floors (1969)

Asphalt Plank as Used for Bridge Floors (1970) ANS1 A37

Asphalt Preferably with Gravel or Slag Surface) (1968)

Asphalt Roll Roofing Surfaced with Mineral Granules (19

Asphalt Shingles (1972T)

Asphalt Tile Flooring (1973)

Asphalt Tile Floors for Commercial, Institutional and 1

Asphalt Tile Floors in Institutional, Industrial and $\mathrm{C}_{0}$

Asphalt Type Protective Coatings for Underground Pipe L

Asphalt with Cone and Plate Viscometer (1973)

Asphalt with Tag Open Cup Apparatus (1972)

Asphalt (Medium Curing Type) (1972)

Asphalt (Rapid Curing Type) (1972)

Asphalt (Slow Curing Type) (1972)

Asphalt (1973)

Asphalt (1973)

Asphalt (1973) ANS1 A37.55

Asphalt. Asphalt and Coal Tar Saturated Organic Felts,

Asphalt, Asphalt.Saturated Organic Felt, Coal Tar Pitch

Asphalt, Cork, Linoleum. Rubber, Vinyl, Vinyl Asbestos,

Asphalt, etc.) in Organic Solvents (1966) ANSI A37.12

Asphalt-Coated Glass Fiber Mat (1973)

Asphalt. Saturated Asbestos Felt. Asphalt. Coated Glass F

Asphalt-Saturated Organic Felt, Coal Tar Pitch. Coal.Ta

Asphaltic Materials (Thin Film Oven Test) (1973)

Asphaltic (Bituminous) Products (1973) AASHO T78, ANSI

Asphalts by Hydrometer Meth. (1973)

Asphalts (Bitumens) (1974) AASHO T201

Asphalts (1966)

Asphalts (1974) ANS1 A37.42. ASHTO T 59

Assay for Alkaline Protease (1972T)

Assay of Bacterial Alpha-Amylase Enzymes Ulsed in Text

Assay of Di-T-Butyl Peroxide by Gas Chromatographic

Assay Preconcentration Spark Technique (1971) ANS1 Z128

Std. and Test Procedures for Plumbing

Asse 1006

Asse 1008

Assemblages (1972)

Std. and Test Procedures for Home Laundry Test Procedures for Plumbing Requirements F

Assembled Steel Products (1973) ANSI G8.18

Assembled Washers-Electrical Terminal Attachment (Pre

Assemblies in Containment Structures for Nuclear Fueled

Assemblies (Electronics) (1973)

Assemblies (1969) ANSI Z 197.20

Assemblies (1970)

Assem blies (1972

Assemblies (1972)

Assemblies (1972)

Assem blies (1972)

Assemblies (1972)

Assemblies (1972)

Assemblies (1972)

Assemblies (1972)

Assemblies (1973)

Assemblies (1973)

Assemblies (1973)

Assemblies (1973)

Assemblies (1973)

Assemblies (1973) ANSI A2.2

Assem blies (1974) ANSl A.2.2

Assemblies) in Existing Buildings (1973)

Assemblies, Doors, and Other Space Dividing Elemer

Assembling Mobile Ho

Assembly and Equipment (1972)

Assembly and Tire Blowout (1972) /Ts for Regula
Assembly Eyebolt (For Ground Support Equipment) (1974)

Assembly Manual for Fine Pitch Gearing (1973)

Assembly Numbers, Cell Layouts and Terminals (For Forei

Assembly (Theaters, Reviewing Stands, Grandstands, Blea

Assembly (17Mm Nominal Diameter) (1971)

Assembly (1972)

Assembly, and Use of a Trampoline (1974)

Assembly, Chainguards, Saddle Braces, Pedal Clearance.

Assembly, Control Steel with Welded Clevis and Threaded

Assembly, Control Steel with Velded Threaded Rod Ends

Assembly, Control, Steel, wi Flash Welded and Threade

Assembly, Control, Steel, with Flash Welded Clevis and

Assembly, Warning (1972)

Assessment of Colored Textiles to Photochromism Colorfa Assessment of the Assumption of Normality (Employing in Assigning Alloy Phase Designations (Code) in Metallic S
Asse 1007

ASTM

ASTM

ASTM

RTI

ASTM

FMS

ASTM

ASTM

RTI

RTI

NACE

ASTM

ASTM

ASTM

ASTM

ASTM

ICBO

ASTM

ICBO

UL

ASTM

ASTM

UL

UL

UL

ASTM

ASTM

ASTM

ASTM

ASTM

ASTM

AATCC

ASTM

AHAM

AHAM

AHAM

ASTM

ASTM

NSA

IEEE

Std. for ANSI

Std. Tes ASTM

td. for Tests

SAE

ANPI

STDI

STDl

UL

Rec CISCA

D41

D 1226

D491

D 617

1.47

D249

D3161

$* 8$

*10

RS*1

D3205

D3143

D2027

D2028

D2026

D2397

UBCS32-2

D977

(5)

141

$55 \mathrm{~A}$

$55 \mathrm{~A}$

$55 \mathrm{~A}$

D1754

D 402

D3142

D2170

D2171

D244

D3048

E475

E400

DW.2PR

HLW-2PR

FWD-2PR

E447

A.386

720
317

C19.7

D) 184

J343A

B5.47

113

114

115 
values) (1974)

cilities (1972)

er Plants-Terms and Definitions (I973/ (197I)

ght Products Except Forgings and Stock/

Std. Assessment of the Standard for Quality Std. for Quality Std. for Quality Minimum Performance Stds. Airborne

Tent. Meth. for Analysis for Fluoride Content of the 3)

Tent. Meth. of Analysis for Flucride Content of the Meth. of Test for Lead in the d. Meth. of Test for $C_{1}$ Through $C_{5}$ Hydrocarbons in the Std. for Gas

Test Meth. for Colorfastness of Textiles to Ozone in the meth. for Colorfastness of Textile Fabrics to Ozone in the rared Spel Tent. Meth. of Test for Carbon Monoxide in the llection of Particulate and Acidic Gaseous Fluoride in the of Particulate and Water Soluble Gaseous Fluorides in the and Collection of Particulate and Gaseous Fluorides in the Std. Rec. Pract. for Planning the Sampling of the d Automatic Recording of the Sulfur Dioxide Content of the i 2257.2 Std. Rec. Pract. for Sampling 8.30 Std. Rec. Pract. for Using Controlled f Dry Solid Film Lubricants in Vacuum and Other Controlled Std. Spec. for Electric Fusion Welded Steel Pipe for Std. for Motor Vehicle Brake Fluid for Use Under Arctic ecimens (1972) ctures (1970) ANSI Z197.24 Std. Rec. Pract. for Recording Data from Std. Rec. Pract. for ANSI Li4.54 Test Meth. for Colorfastness of Textiles to Std. Definitions of Terms Relating to Performance Requirements for Pipe Applied

er Fuel (1973) Safety Std for ackflow Preventers in Water Supply Lines with Intermediate s Spectrometric Meth.) (1969) Ans/ dymium-148 Meth.) (1969) ANSI N11/ Std. Meth. of Test for Std. Meth. of Test for Std. Meth. of Test for h.) (I969) ANSl N107 Std. Meth. of Test for Lead in Gasoline by Std. Meth. of Test for Aluminium in lron Ores by d. Meth. of Test for Calcium and Magnesium in lron Ores by for Installation of Steel Framing Members to Receive Screw ucts and Rec. Pract. for Use of These Terms on Permanently tus (1973)

Std. Meth. of Test for Formability of lements to Agricultural Wheel Tractors Equipped with Quick
I Tractors (1972) SAE J715 iding Tractors (1972) Std. for 3 Point Free Link Equipped with Quick Attaching Coupler for Thre/ Std. for rs (1972) Std. for 3 Point Free Link Hitch
Std. on Dimensions of Std. for Safety for Electrical

pan Head Screw and Assembled Washers-Electrical Terminal Std. for H Type Mill Chains

Std. for Double Pitch Conveyor Roller Chains. Sid. Malleable Iron Detachable Link Chain and 972)

Hook Work, Clamshell, Magnet, Grapple, or Concrete Bucket Std. for 700 Class Pintle Chains,

Std. for Steel Detachable Link Chains, Std. Meth. of Detecting Susceptibility to Intergranular th. of Test for Resistance of Glass Containers to Chemical

Tentative Manual for Driver

Std. Meth. of Test for Magnetic Shield Efficiency in Spec. and Test Meth. for Fixed and Variable

Std. for Sampling Procedures and Tables for Inspection by Std. Definitions and Symbols for Acceptance Sampling by

Audio Amplifier Single Frequency Output for Institutional al Audio-Visual Equipment Use/ Std. Meth. for Measuring tic Tape Quadruplex Recorded A/ Std. Meth. of Measurement for Std. Dimensions of Video,

Rec. Pract. and Std. for Electronic Equipment on Boats Std. for Power Output Ratings of Packaged Cable Connectors for Std. Spec. for an

Video Magnetic Tape Recorders Operati/ Spec. for an

ponse and Operating Level of Recorders and Reproducers for
Magnetic Tape Cartridge Type Cp II (Compact Cassette) for or Safety for Hospital Signaling and Nurse Call Equipment Std. for Artificial Headbone for the Calibration of Std. for Psychoacoustical Terminology (Acoustics, $\mathrm{g}$ of the Product (Receiving and Source Inspection, Product unting) (1973) Std. for

ing Code: Minimum Facilities Required for School, Theater. changer Tubes (1973/ Std. Spec. for Seamless Ferritic and for General Requirements for Carbon, Ferritic Alloy, and Spec, for Seamless Ferritic ve or High Temperat/Std. Spec. for Welded Large Diameter ry Service (1972) ANSI B36.39 Std. Spec. for Seamless
Assumption of Normality (Employing Individual Observed Assurance for Protective Coatings Applied to Nuclear Fa Assurance Practices for the Construction of Nuclear Pow Assurance Program Requirements for Nuclear Power Plants Assurance Sampling of Carbon and Low Alloy Steels (Wrou ATC Transponder Equipment (1972)

Atmosphere and Plant Tissues (Manual Procedures) (1973)

Atmosphere and Plant Tissues (Semiautomated Meth.) (I97

Atmosphere by Colorimetric Dithizone Procedure (1972T)

Atmosphere by Gas Chromatrography (1972)

Atmosphere Generators (1971)

Atmosphere Under High Humidities (I972) ANSI LI4.238

Atmosphere Under Low Humidities (1972) ANSI L14.174

Atmosphere (Continuous Measurement by Nondispersive Inf

Atmosphere (Double Paper Tape Sampler Meth.) (1973)

Atmosphere (Filter and Impinger Meth.) (1973) Atmosphere (Sodium Bicarbonate Coated Glass Tube and a
Atmosphere (1957) ANSI Z257.1

Atmosphere (1967)

7D. Meth for Continuous Analys

Atmospheres for Analysis of Gases and Vapors (1967) Ans

Atmospheres in Spectrochemical Analysis (1970) ANSI Z12

Atmospheres (1971) ANSI Z11.303 /E

Atmospheric Conditions (1972)

Atmospheric Corrosion Tests of Metallic Coated Steel Sp

Atmospheric Exposure of Adhesive Bonded Joints and Stru

Atmospheric Oxides of Nitrogen (Burnt Gas Fumes) (1972)

Atmospheric Sampling and Analysis (I973)

Atmospheric Type Vacuum Breakers (1970)

Atmospheric Type Welded Steel Inside Tanks for Oil Burn

Atmospheric Vent (1972)

/Erformance Requirements

Atom Percent Fission in Uranium and Plutonium Fuel (Mas

Atom Percent Fission in Uranium and Plutonium Fuel (Neo

Atom Percent Fission in Uranium Fuel (Radiochemical Met

(Atomic A bsorption Meth.) (1972) ANSI Z11.317

Atomic A bsorption Spectrometry (1973)

Atomic A bsorption Spectroscopy (1973)

Atomic Absorption Spectroscopy (1973)

Attached Gypsum Wallboard and Backing Board (1971)

Attached Labels (1972)

Attached Organic Coatings with Impact Wedge Bend Appara

Attaching Coupler for Three Point Free Link Hitch (1971

Attachment for Hitching Implements to Agricultural Whee

Attachment for Hitching Implements to Lawn and Garden R

Attachment of Implements to Agricultural Wheel Tractors

Attachment of Implements to Agricultural Wheeled Tracto

Attachment Plugs and Receptacles (1973)

Attachment Plugs and Receptacles (1974)

Attachment (Preferred) (1974)

Attachments and Sprocket Teeth (197I)

Attachments and Sprockets (1972)

Attachments (1971)

Attachments (1971)

Attachments

Attachments, and Sprockets (1972)

Attack in Wrought Nickel Rich, Chromium Bearing Alloys

Attack (1973)

Attended Loading of Anhydrous Ammenia (1972)

Attended Loading of Liquefied Petroleum Gas (1966)

Attenuating Alternating Magnetic FieIds (1974)

Attenuators, DC to $40 \mathrm{GHz}$ (1973)

Aitributes (MIL Std. 105D) (1971)

Attributes (1971) ANSI Z1.6

Audio-Visual Equipment Used Primarily for Speech (197

Audio Amplifier Single Frequency Output for Institution

Audio Amplifiers Used in Home Equipment (1971)

Audio and Tracking Control Records on 2 In. Video Magne

Audio Device, Automatic Pilot, Depth Determination Devi

Audio Equipment for Home Use (1967)

Audio Facilities for Radio Broadcasting (1970)

Audio Level and Multifrequency Test Tape for Quadruplex

Audio Record One for 2.In. Quadruplex Video Magnetic Ta

Audio Recording and Reproducing (1974)

(Audio / Visual Communication) (1974)

Audiometer Bone Vibrators (1972)

Audiometry) (1973)

Audit) (1969)

C. Pract. for Quality Control Monitorin

Auditing Nuclear Materials Statements and Reports (Acco

Auditorium, Dormatory, Public or Office Building, Manuf

Auger Conveying Equipment (1973)

Austenitic Alloy Steel Boiler, Superheater, and Heat Ex

Austenitic Alloy Steel Tubes (1971 A) ANSl B125.34

Austenitic Alloy Steel Tubes (1972)

Austenitic Chromiun Nickel Alloy Steel Pipe for Corrosi

Austenitic Chromium Nickel Steel Still Tubes for Refine Austenitic Gray Iron Castings (1972A)
ANSI

ANSI

ANSI

ANSI

SAE

RTCA

ASTM

ASTM

St ASTM

ANSI

AATCC

AATCC

ASTM

/O ASTM

ASTM

ASTM

ASTM

ASTM

ASTM

AST

ASTM

SAE

ASTM

ASTM

AATCC

ASTM

ASSE

UL

ASSE

ASTM

ASTM

ASTM

ASTM

ASTM

ASTM

ASTM

ASTM

ASAE

ASAE

ASAE

ASAE

SAE

ANSI

UL

Std. for NSA

ANSI

ANSI

ANSl

SAE

ANSI

ANSI

ASTM

Std Me ASTM

FI

NGPA

ASTM

IEEE

ANSI

ASQC

ANSl

ANSI

ElA

ANSI

$A B Y C$

EIA

EIA

ANSI

ANSI

ElA

UL

ANSI

ASQC

ANSI

ICBO

ASAE

ASTM

Pec ASTM

ASTM

ASTM

ASTM

ASTM

N15. I5

N10I. 4

N45.2.10

N45. 2

AMS $2370 \mathrm{~A}$

DO. I50

D3269

D3270

D3112

D2820

Z83.2

129

I09

D3162

D3266

D3267

D3268

D1357

D1355 
37.8

b36. 38

1973) ANSI B 125.15

nd Condenser Tubes

(1972) ANSI G52.2

ion Service (1973) ANSl B125.25

2) ANSI B125.39 perature Service (/ Std. Spec. for Electric Fusion Welded er, Road, Hydrant, Drivew/ Uniform Fire Code: Protection, , Ashto T107

1972)

acidic Gaseous Fluoride in the Atmospher/ Std. Meth. of Test for Std. for

Guide for the Application, Operation, and Maintenance of ers and Oil Filled Capacitor Switches for Alter/

for Combustion Chamber in Heating Appliances (Regulators. Std for Household

hazards and Protection Rec, for Low Water Fuel Cutoffs and Uniform Building Code Std. for Installation of Std. for

ems (1972)

woven or Knit Text/

switches for Alter/

d. Spec. for Water or Solvent Soluble Liquid Adhesives for Std for Automatic Circ Std. for Safety for

72) ANSl B93.28 Std. Meth. for Calibration of Liquid
St. Meth. for

wn Systems and Components for Particulate Contamination by and Std. for Electronic Equipment on Boats (Audio Device, e Atmosphere (196) Std. Meth. for Continuous Analysis and han 75,000 Btu Per Hr.) ( Vol Std. for Gas Water Heaters ers: Volume 111, Circulating Tank, Instantaneous and Large Safety Std. for Rec. Pract. for Std. for

(1974) Std. for Safety for Use of Shears (Mechanical, Hydraulic, Pneumatic, Semi and for Heat and Smoke Roof and Window Vents Designed to Open Guide for Centralized Control and $t$ of Stopping Distance on Paved Surfaces Using a Passenger for Stopping Distance on Paved Surfaces Using a Passenger Uniform Fire Code: ndling Plants (1973) Uniform Fire Code: $\$ 2.10$ vehicular, lgnition, Lighting, and Starting) for Passenger Std. Meth. of Test. Std. Meth. of Testing torage Batteries for Motor Vehicles, Motorboats, Tractors, ity Requirements for Externally Threaded Fasteners Used in cal and Quality Requirements for Machine Screws for Use in assification System for Elastomeric Materials (Rubber) for th. for Determining Resistance to Snagging and Abrasion of n (1972) Rec. Pract. for pract. for Determination of Brake Fluid Temperature in the ract. for Determination of Brake Fluid Temperature in the Std. for the Test Meth. for Wicking of
Safety Std. for Rec. Pract. for Std. for (1971)

\section{Emergency Std. Spec. for} Safety Std. for Std. Meth. of Test. h. (1972)

rs (Course of lnstruction) (1972)

s (Course of lnstruction) (1972)

Rec. Pract. for Flammability of Std. for Training of Std. for Training of for Electroplating of Nickel and Chromium on Metal Parts Rec. Pract. for

Rec Pract, for Life Test for Rec. Pract. Spec. for Cast lron Sealing Rings for Rec. Pract. for $\operatorname{ology}(1970)$ Rec. Pract. for est Procedure (1972)

Rec. Pract. for SAE No. pecimen for Fabrics, Vinyls, Coated Fiberboards, and Other ning Blocking Resistance and Associated Characteristics of Std. for

Rec Pract, Meth. of Tests for Std. for Trailer Couplings and Hitches: Mobile Radio Telephone for Rec. Pract. for Cornering Lamps (Light) for r Cavitation Erosion Corrosion Characteristics of Aluminum pression lgnited) Inboard Liquid Cooled Marine Engines and
Austenitic Manganese Steel Castings (1973) ANSI G52.4 Austenitic Stainless Steel Feedwater Heater Tubes (1973 Austenitic Stainless Steel Pipe (1972A) ANSI B 125.16 Austenitic Stainless Steel Piping Fittings (1973) ANSI Austenitic Stainless Steel Sanitary Tubing (1973) ANSI Austenitic Stainless Steel Tubing for General Service ( Austenitic Steel Boiler, Superheater, Heat Exchanger, a Austenitic Steel Castings for High Temperature Service Austenitic Steel Pipe for High Temperature Central Stat Austenitic Steel Pipe for High Temperature Service (19? Austenitic Steels (1970) A NSI G38.12 /20 KSI Yield Str

Autenitic Chromium Nickel Alloy Steel Pipe for High Tem

Authority, Departmental Regulations and Controls, Barri

Autoclave Expansion of Portland Cement (1971) ANSI A 1.8

Automated Machine Lay Up Composite Filament Tape Reel (

Automated Separation and Collection of Particulate and

Automatic Circuit Reclosers (1973) ANSI C37.61

Automatic Circuit Reclosers, Automatic Line Sectionaliz

Automatic Damper Controls, Fans) (1973)

Automatic Electric Storage Type Water Heaters (1972)

Automatic Feedwater Regulators for Boilers (1974)

Automatic Fire Extinguishing Systems (1973)

Automatic Gas Ignition Systems and Components (1971)

Automatic Gas Shutoff Devices for Hot Water Supply Syst

Automatic Home Laundering of Durable (Permanent) Press

Automatic Ice Makers (600 Volts or Less Rating) (1974)

Automatic Line Sectionalizers and Oil Filled Capacitor

Automatic Machine Sealing of Top Flaps of Fiberboard Sh

Automatic Particle Counter Size Setting (1972)

Automatic Particle Counters Using AC Fine Test Dust (19

Automatic Particle Monitor Meth. (1972)

/G Gas Blo

Automatic Pilot, Depth Determination Device and Fish Fi

Automatic Recording of the Sulfur Dioxide Content of Th

Automatic Storage Type Water Heaters with Inputs Less T

Automatic Storage Type (1974)

Automatic Transfer Switches (1972)

Automatic Transmission Functions Terminology (1969)

Automatic Valves for Gas Appliances (1974)

Automatic Water Sprinklers for Fire Protection Service

Automatic, and Manually Powered) (1973)

Automatically in Case of Fire (1974)

Automation of Ship's Steam Propulsion Plant (1970)

Automobile Equipped with Full Scale Tires in the Diagon

Automobile Equipped with Full Scale Tires (1971T)

Automobile Tire Rebuilding Plants (1973)

Automobile Wrecking and Junk Yards or Waste Material $\mathrm{Ha}$

Automobiles, Commercial Vehicles, Golf Car, and Other D

Automotive Air Brake and Vacuum Brake Hose (1973) ANSI

Automotive Air Conditioning Hose (1968E) ANSl J2.27

Automotive and Industrial Applications (1973)

Automotive and Related Industries (1971)

Automotive and Related Industries (1972)

Automotive Applications (1973) ANSI J8.15, SAE J200

Automotive Bodycloth (1972)

Automotive Brake Definitions and Nomenclature (1971)

/ Type S SAE for Mechani SAE

Automotive Brake Disc and Drum Thermocouple lnstallatio

Automotive Braking System of Vehicles Equipped with Dis

Automotive Engine Carburetor Airflow Reference Std. (19

Automotive Fabrics and Fibrous Materials (1972)

Automotive Fuel Tanks (1973)

Automotive Gasoline Performance and Information System

Automotive Gasoline (1973)

Automotive Gasoline (1974)

Automotive Glass Tube Fuses (1973)

Automotive Gray lron Castings (1972)

Automotive Hydraulic Brake Hose (1972) ANSl J2.9

Automotive Interior Trim Materials: Horizontal Test Met

Automotive Mechanics for Buses, Heavy Trucks and Traile

Automotive Mechanics for Passenger Cars and Light Truck

Automotive Ornamentation and Hardware (1970)

Automotive Printed Circuits (1967)

Automotive Storage Batteries (1972)

Automotive Transmission and Hydraulic Systems (1972)

Automotive Transmission Hydraulic Control System Termin

Automotive Transmission Terminology (1969)

Automotive Transmission) Clutch Friction Test Machine $\mathrm{T}$

/L ASTM

Test $\mathrm{Me}$

Automotive Trim Materials (1972)

Automotive Trim Materials (1972)

/ Matched to Master S

Automotive Tube Fittings (1971)

/St Meth. for Determi

Automotive Type Sealers, Adhesives, and Deadeners (1971

Automotive Type (1972)

Automotive Use (HS 27) (1968)

Automotive Vehicles (1965)

Automotive Water Pumps with Coolants (1972) /Onstruction of Diesel (Com ABYC

ASTM

ASTM

ASTM

ASTM

ASTM

ASTM

ASTM

ASTM

ICBO

ASTM

NSA

ASTM

Std IEEE NEM

ANSI

/Re FMS

AAT

UL

NEMA

ASTM

ASTM

NFL DP

ASTM

ABYC

ASTM

SAE

ANSl

ANSI
UL
SAE

ANS!

UL

UL

ASTM

ASTM

SAE

ANSI

ANSI

$S A E$

SAE

SAE

$S A E$

SAE

SAE

UBCS38-1

ANSI Z21.22A

135

563

SGl3

D1874

F321

T2.9.6

D1355

J258

Z21.10.1

$\mathrm{Z} 21.10 .3$

1008

J649B

Z21.21

199

B11.4

4430

SNAME $3-23$

ASTM E503

Est ASTM E445

ICBO UFC*2ART2

ICBO UFC *2ART3

BCI $* 1.48$

ASTM D622

D 1680

$\mathrm{J} 537 \mathrm{H}$

$\mathrm{J} 429 \mathrm{~F}$

J82

D2000

J948A

$\mathrm{J} 656 \mathrm{~F}$

J79

J29]

J228

J913A

395

J282

ASTM D439

ASTM D439

ES1

A159

D571

J369 A

D18.2

D18.1

J207

J240A

J281

J648A

J645B

J286

J361 A 
covering the Installation of Air Cooled Marine Engines and e Fueled Inboard, Liquid Cooled Marine Engines (Boats) and Thyristor Power Supplies for MetaI Rolling Mill Std. for Safety for Wireways, Std. for Safety for Wireways, Sid. for Std. for wiring) (1973) ANSI C33.45 or Safe Installation of Exhaust Systems for Propulsion and Rec. Pract. for Agricultural Tractor ervice (19/ Rec. for Agricultural (Farm) Tractor by Radio/ Std. Meth. of Test for Neutron Flux Density and eability (1972) Std. Meth. of Test for atd. Rec. Pract. for Choice of Sample Size to Estimate the Facilities for Motor Vehicles (1971) Rec. Pract. for (1971)/ Std. Meth. of Test for Electrical Conductivity of Std. Meth. of Test for Water Reaction of Tent. Meth. of Test for Total Acidity in Tent. Meth. of Test for Flash Point of Std. Meth. of Test for Smoke Point of Std. Meth. of Test for Thermal Stability of ) Test for Water Separation Characteristics of Tent. Meth. of Test for Undissolved Water in Guidance Material for Std. Spec. for

Std. Meth. of Test for Particulate Contaminant in Meth. of Test for Mercaptan Sulfur in Gasoline, Kerosine, coa/ Std. Performance Requirements for Cotton Woven Tent, d. Performance Requirements for Woven Man Made Fiber Tent, Light Diffusers in Ceilings, Partitions, Exterior Veneer, Reinforced Brick Masonry Std. for Reel Packaging of Components with

Code Std. for Wood Members Subjected to Both Flexural and d Cores (1974) Spec. Format Guide and Test Procedure for Linear, Single ement (1972)

G57.12 se (1973) ANSI G57.11 Std. Spec. for Carbon Steel Std. Analytical Meth. for Determining Moisture Std. Analytical Meth. for Determining Moisture 1956) Std. Analytical Meth. for Determining Moisture (1952) Std. Analytical Meth. for Determining Moisture hemistry) (1971) rformance, Quality, Description, and Grade of Palmkernels; ansi A37.45 Std. Meth. of Test for Distillation of Cut Test Meth. for Rug ply/ Performance Requirements for Double Check Valve Type Std. for

Determination for Chromate Conversion Coatings on Aged or 974) Std. Spec. for Tetrafluoroethylene
nce Requirements for Double Check Valve Type Back Pressure iate Atmospheric Vent (1972/ Performance Requirements for rs, Camp Cars and Trailer Coaches (1970)

rmining the SAE Rating and Struck Capacity for Hoe Dipper

ing Members to Receive Screw Attached Gypsum Wallboard and Std. Spec. for Gypsum

Uniform Building Code Std. for Water Resistant Gypsum Uniform Building Code Std. for Gypsum Std. for Dimensions of 126 Size Cartridges, Film, and Std. Meth. of Test for Coating Thickness by the Beta Std. for

Meth of Analysis of Coliform Std. Plate Count Meth of Analysis of Total Meth. of Analysis of Salmonella Meth. of Analysis of Thermophilic and Psychrophilic Std. Meth. of Test for Iron Sampling Procedure for Analysis of Test Meth. for Assay of

ing (1973) ANSI L14.147 Test Meth. for Assay of
8 Watt T5 Preheat Start and Electrical Characteristics of 8 Watt T5 Preheat Start nd Electrical Characteristics of 30 Watt, T8 Preheat Start Std. for Criteria for Film Std, for Minimum Safety and Health Requirements for Paper Test Meth. for Determination of Useful Fiber in nal Type Vehicles, Counter Balanced Fork Lift Truck, Boat, raviolet Light Examination for Presence of Rodent Urine on

Meth. of Xanthydrol Test for Analysis of Urea in Cloth

Test Meth. for Drop Tests for Paper Shipping Sacks and Std. Meth. of Drop Test for

s (Bonding Appliances, Casters, Face Masks and Rebreathing ing Weight Determination for Conversion Coating on Aged or istr/

Meth. of Analysis of Crude $F$ at in Flour, Bread, and Meth. of Analysis of Crude Fat in
Auxiliaries (Diesel and Gasoline) (Boats) (1972)

ITd. ABYC

Auxiliaries (1972)

\section{$/ \mathrm{De}$}

Auxiliary Gutters and Associated

Auxiliary Gutters, and Associated Fittings (Electrical

Auxiliary Locks (Hardware) (1971)

Auxiliary Low Beam Lamp (Headlamp-Light) (1973)

Auxiliary Machinery (Boats) (1973)

Auxiliary Power Take Off Drives (1972)

Auxiliary Power Takeoff Drives (1972)

Auxiliary Protective Signaling Systems for Fire Alarm S

Average Energy from ${ }_{3} \mathrm{H}(\mathrm{D}, \mathrm{N})$, He Neutron Generators

Average Particle Size of Alumina and Silica by Air Perm

Average Quality of a Lot or Process (1972) ANSI Z1.11

Average Vehicle Dimensions for Use in Designing Docking

Aviation Fuels Containing a Static Dissipator Additive

Aviation Fuels (1972) ANSI Z11.82

Aviation Turbine Fuel (1973)

Aviation Turbine Fuels by Setaflash Closed Tester (1973

Aviation Turbine Fuels (1972) ANSI 211.137

Aviation Turbine Fuels (1972) ANSI Z11.148

Aviation Turbine Fuels (1972) ANSI Z11.301

Aviation Turbine Fuels (1973)

Aviation Turbine Fuels (1973)

Aviation Turbine Fuels (1973) ANSI Z11.204

Aviation Turbine Fuels (1973) ANSI Z11.264

Aviation Turbine, and Distillate Fuels (Potentiometric

Awning, and Canopy Fabrics, Painted, Printed, or Vinyl

Awning, and Canopy Fabrics, Vinyl Coated or Laminated

Awnings and Patio Covers, Greenhouses, Canopies) (1973)

(Axial Design) (Tech. Notes) (196.3)

Axial Leads (1972) ANSI C83.55

Axial Loading Combined (1973)

Axial (End to End) Compression Composite Cans, Tubes an

(Axial) Bearing Steel Studs, Runners (Track) and Rigid F

Axis, Pendulous, Analog Torque Balance Accelerometer (1

Axle Steel Deformed and Plain Bars for Concrete Reinfor

Axles for Export and General Industrial Use (1971) ANSI

Axles (1973)

Axles, Non Heat Treated and Heat Treated, for Railway U

(Azeotropic Distillation) Content in Corn Starch (1956)

Azeotropic Distillation) Content in Corn (1955)

Azeotropic Distillation) Content in Feedstuffs (Corn) (

Azeotropic Distillation) Content of Corn Syrup Unmixed

Azodicarbonamide Content in Commercial Premix (Cereal C

Babassu; Flaxseed; Castor, Sesame, Safflower, Oleic, an

Back Asphaltic (Bituminous) Products (1973) AASHO T78,

Back Floor Staining on Vinyl Tile (1973)

Back Pressure Backflow Preventers for Potable Water Sup

Back Window Location for Roll Film Cameras (1973)

Backed Aluminum Alloy Panels (Coil) (1974)

Backed Paint Film (Coil Coating) (1974)

Backed Pressure Sensitive Electrical Insulating Tape

Backflow Preventers for Potable Water Supply Lines (197

Ing Weight

Backflow Preventers in W ater Supply Lines with Intermed

Backflow Preventers) and Relief Valves in Travel Traile

Backhoe Bucket) (1972)

Std. for Uniform Meth. of Dete

Backing Board (1971)

Backing Board (1972)

Backing Board (1973)

Backing Board (1973)

Backing Paper (Photography) (1972)

Backscatter Principle (1972)

Backwater Valves (1971)

Bacteria in All Food Products (Cereal Chemistry) (1962)

Bacteria in Food Products (Cereal Chemistry) (1962)

Bacteria in Food Products (Cereal Chemistry) (1962)

Bacteria in Food Products (Cereal Chemistry) (1962)

Bacteria in Water and Water Formed Deposits (1972)

Bacteria (Cereal Chemistry) (1962)

Bacterial Alpha-Amylase Enzymes Used in Textile Desiz

Bactericidal Lamp (1971)

Bactericidal Lamp (1971)

Bactericidal Lamp (1971)

Badge Performance (Nuclear Radiation) (1972)

$\mathrm{Bag}$ and Sack Manufacture (Packaging) (1974)

Bagasse (Pulp and Paper) (1973)

Baggage, Special Type Trailer, Mobile Home, Garden Trac

Bags and Other Packaging Materials (Cereal Chemistry)

Bags and Other Packaging Materials (Cereal Chemistry) (

Bags (Containers) (1973)

Bags (Packaging) (1950) A NSI MH12.6

Bags, Footwear, Hose and Tubing, Mattresses and Pads, S

Baked Alu minized Steel (Coil) (1974)

Baked Cereal Products Not Containing Fruit (Cereal Chem Std. Dimensional Std. Dimensional Std. Dimen

Baked Dog Food (Cereal Chemistry) (1962)
P10

P4

PV 2

370

870

501

J582A

P1

J717C

R333.1

72B

E496

C721

E122

J699A

D2624

D1094

D3242

D3243

D 1322

D1660

D2550

D3240

*1

D1655

D2276

D3227

L24.1.1

L24. 1.10

UBC *3-52

17B

RS296C

UBCS25-16

CT107

C645

337

A617

A 383

11

A21

B-34

A-12

G. 14

$\begin{array}{ll}\text { CR } & \text { G.14 } \\ \text { CR } & \text { E.40 }\end{array}$

AACCH $48-71 \mathrm{~A}$

NIOP *1

ASTM D402

AATCC 137

ASSE 1015

ANSI PH3.1

TB-Il-2

TB-Il-18

D2686

1015

ASSE $\quad 1012$

IAPMO TSC 8

SAE J296

ANSI A97.2

ASTM C442

ICBO UBCS47-13

ICBO UBCS47-6

ANSI PHI.40

ASTM B567

IAPMO PS8

AACCH 42.15

A ACCH 42.11

AACCH $42-25$

A ACCH $42-45$

ASTM D932

AACCH $42-10$

AATCC 103

ANSI C78.1200

C78.1200
C78.1201

C78.1202

N13.7

$\begin{array}{ll}\text { ANSI } & \text { N13.7 } \\ \text { ANSI } & \mathrm{Z} 259.1\end{array}$

$\begin{array}{ll}\text { ANSI } & \text { Z259. } \\ \text { TAPPI } & \text { UM.3 }\end{array}$

TRA

AACCH 28.85

AACCH $28-86$

TAPPI UM.806

ASTM D959

$\begin{array}{lll}\mathrm{UL} & 1067\end{array}$

NCCA TB-II.8

AACCH 30.10

$\begin{array}{lll}\mathrm{AACH} & 30.14\end{array}$ 
s for Del Meth. of Analysis of Macroscopic Examination of Sieving Meth of Analysis for Insect and Rodent Filth in sieving Meth. of Analysis for Materials Hard to Hydrate in (Cereal Chemis/ mistry) (1962) Direct Trapping Meth. of Analygis for All Meth. of Compression Test with Std. Safety Requirements for Schoorl Meth. of Analysis of Reducing Sugars in Prepared Glossary of Terms Used in Uniform Fire Code: Industria Std. for Commercial Gas Meth of Analysis for Residual Carbon Dioxide in meth. of Analysis for Total (Gasometric) Carbon Dioxide in Qualitative Meth. of Analysis of Aluminum in Qualitative Meth. of Analysis for Phosphoric Acid in Quantitative Meth. of Analysis for Phosphoric Acid in Meth. of Analysis for Sulfuric Acid in Meth of Analysis for Tartaric Acid in Meth. of Analysis of Ammonia in Sponge Dough, Pound Loaf Meth. of Analysis for Meth. of Analysis fo Meth. of Analysis fo

962)

962) mistry) (1962)

(1962)

chemistry) (1962) 4) Meth. of Analysis for Meth. of Analysis fo Meth. of Analysis for Straight Dough Meth. of Analysis for
Meth. of Analysis for Meth. of Analysis for
dulous, Analog Torque Std. for Marine Steam Power Plant Hea Std. Rec. Pract. for Solar Simulation for Therma Std. Meth. Measuring Plasma Arc Gas Enthalpy by Energy ndustrial, Utility and Recreational Type Vehicles, Counter Test Meth. for Fiber Bundles in al, Flax, Synthetic Fibers, Wool) (1974) s) Conform/ Rec. for ) Std. Shaft and Housing Fits for Metric Radia Spec. for Bearings, Std. Load Ratings and Fatigue Life for Std. and Test Procedure for Water Closet Flush Tank 72) Std. Meth. of Test for Pneumatic rvice (1972) of Extreme Pressure Properties of Lubricating Fluids (Fou Test for Tack and Pressure Sensitive Adhesives by Rolling 974) (1972) Spec. for Steel Gate, Plug Std. Liquid (1971)

Rec. Pract for Liquid Std. Definitions for High Intensity Discharge Lamp Std. Spec. for Mercury Lamp Reference Specs. for Fluorescent Lamp

Std. Meth of Measurement of Fluorescent Lamp Std. Spec. for Fluorescent Lamp Reference Tent. Meth. of Analysis for Brix or Eastern White and Red Pine, Eastern Spruce and Hemlock, d. for Eastern Spruce, White Pine, and Hemlock-Tamarack; Uniform Fire Code: Fruit Ripening Process Std. for Low Power Wide Std. for Hardenabilit Rec. Pract for Hardenability rometers (1972) Std. Meth. of Test for Spectra

Mercantile and Safety Std. for Surveillance Cameras for Std. Spec, for Cartridge Brass Sheet, Strip, Plate Uniform Building Code Std. for Stee Rec. Pract. for Preparation of eth. of Test for Tensile Strength of Adhesives by Means of Std. Spec, for Copper and Its Alloy Forging Rod, C7.27 Std. Spec, for Aluminum Rec. Pract, for Draw Std. Meth. for Notched Std. Pract. for Fabrication of Reinforcing Steel Std. Pract. for Estimating Reinforcing Stee Rec. for Concrete Reinforcing Spec. for Placing Reinforcing Stee Spec. Steel Wire

(1970)

Std. Spec. for Nickel Chromium Iron Alloy Rod and Std. Spec. for Nickel Rod and Std. Spec. for Copper Beryllium Alloy Rod and for Nickel Iron Chromium Molybdenum Copper Alloy Rod and ec. for Nickel Chromium Molybdenum Columbium Alloy Rod and td. Spec. for Leaded Brass Plate, Sheet, Strip, and Rolled Std. Spec. for Brass Plate, Sheet, Strip, and Rolled

Std. Spec. for Copper-Nickel-Silicon Alloy Rod and pec. for Copper Iron Alloy Plate, Sheet, Strip, and Rolled r Copper-Beryllium Alloy Plate, Sheet, Strip, and Rolled
Baked Goods, Ready-To-Eat Cereals, and Alimentary Paste Baked Goods, Ready.To.Eat Cereals, and Alimentary Paste Baked Goods, Ready.To-Eat Cereals, and Alimentary Paste Baked Materials Except Chocolate Products and Piecrust Baker Compressimeter for Staleness of Bread (Cereal Che Bakery Equipment (1971)

Bakery Mixes (Cereal Chemistry) (1962)

Baking and Cereal Chemistry (1962)

Baking and Drying Ovens (1973)

Baking and Roasting Ovens (1971)

Baking Powder (Cereal Chemistry) (1962)

Baking Powder (Cereal Chemistry) (1962)

Baking Powder, in Presence of Phosphates (Cereal Chemis

Baking Powders and Chemicals (Cereal Chemistry) (1962)

Baking Powders and Chemicals (Cereal Chemistry) (1962)

Baking Powders and Chemicals (Cereal Chemistry) (1962)

Baking Powders and Chemicals (Cereal Chemistry) (1962)

Baking Powders and Chemicals (Cereal Chemistry) (1962)

Baking Quality of Bread Flour (Cereal Chemistry) (1962)

Baking Quality of Cake Flour (Cereal Chemistry) (1962)

Baking Ouality of Cookie Flour (Cereal Chemistry) (1962

Baking Quality of Nonfat Dry Milk (Cereal Chemistry) (1

Baking Quality of Pie Flour (Cereal Chemistry) (1962)

Baking Quality of Rye Bread Flour (Cereal Chemistry) (1

Baking Quality of Self Rising Biscuit Flour (Cereal Che

Baking Quality of Wheat Bread Flour (Cereal Chemistry)

Baking Test (Quality) for Sweet Yeast Products (Cereal

Balance Accelerometer (1972) / Format Guide

Balance Practices (1972)

Balance Test. of Spacecraft (1973)

Balance (1973)

Balanced Fork Lift Truck, Boat, Baggage, Special Type T

Baled Flash Dried Pulp (1973)

Baled Textile Fiber Storage (Cotton, Jute, Hemp and Sis

Ball and Roller Bearing Steel (1970) ASTM G56.2

Ball and Roller Bearings (Except Tapered Roller Bearing

Ball and Roller, Rod End, Anti Friction, Airframe (1974

Ball Bearings (1972) ANSI B3.15

Ball Cocks (1968)

Ball Impact Resistance of Plastic Film and Sheeting (19

Ball Meth.) (1971) ANSI Z11.309

Eth. for Measurement

Balves with Flanged or Butt Welding for General Se

Ball (1973)

Ball, and Check Valves for Petroleum Pipeline Valves (1

Ballast Table for Drive Tires of Agricultural Machines

Ballast Table for Drive Tires of Agricultural Machines

Ballasts and Transformers (1971)

Ballasts (1971)

Ballasts (1972)

Ballasts (1972)

Ballasts (1972)

(Balling) of Wine (Enology) (1972)

Balsam Fir and Tamarack Lumber (1973)

Balsam Fir; Northern Pine and White Cedar Lumber (1973)

(Banana, Citrus Fruit) (1973)

Band Transformers (Less Than 100 Watts Power) (1971)

Bands for Alloy $\mathrm{H}$ Steels (1971)

Bands for Carbon H Steels (1971)

Bandwidth and Wavelength Accuracy of Fluorescence Spect

Bank Burglar Alarm Systems (1972) ANS1 Se2.3

Bank Safes and Vaults (1971) ANS1 Se 2.1

Banks and Mercantile Premises (1973) ANSI Se2.5

Bar and Disks (1973)

Bar and Rod Mats (Sheets) for Concrete Reinforcement (1

Bar and Rod Specimens for Adhesion Tests (1969)

Bar and Rod Specimens (1972) ANSI Z197.3

Bar and Shapes (1974) ANS1 H7.l

Bar for Electrical Purposes (Bus Connector) (1973) ANSI

Bar for Forestry Tractors (1970)

Bar Impact Test. of Metallic Materials (1972)

Bar Materials and Service (1968)

Bar Materials and Service (1970)

Bar Splices (1971)

Bar Supports in Concrete Construction (1970)

Bar Supports Used to Position Reinforcement in Concrete

Bar (1970) ANSI H34.4

Bar (1970) ANSI H34.7

Bar (1971A) ANSI H7.7

Bar (1972) ANSI H34.18

Bar (1972) ANSI H34.22

Bar (1973)

Bar (1973)

Bar (1973)

Bar (1973)

Bar (1973)
AACCH 28.30

$\mathrm{AACCH} 28.31$

AACCH 28.32

AACCH 28.33

AACCH 74.10

ANSI Z50.1

AACCH $80-68$

AACCH $36.01 \mathrm{~A}$

ICBO UFC $* 2 A R T 25$

ANSI Z21.28

AACCH 12.10

AACCH 12.20

AACCH $\quad 40.10$

AACCH $04-10$

$\mathrm{AACCH}$ 04-11

$\mathrm{AACCH} \quad 04-14$

$\mathrm{AACCH} \quad 04.27$

AACCH $\quad 40.15$

$\mathrm{AACCH} 10.11$

$\mathrm{AACCH} \quad 10.90$

$\mathrm{AACCH} \quad 10-50 \mathrm{~B}$

$\mathrm{AACCH} \quad 10.85$

AACCH $\quad 10.60$

AACCH $\quad 10.70$

$\mathrm{AACCH} \quad 10.31 \mathrm{~A}$

AACCH 10.10

$\mathrm{AACCH} \quad 10.20$

IEEE 337

AGMA 515.01

SNAME 3.11

ASTM E491

ASTM E341

TRA 5

TAPPI UM-239

FMS 8.7

ASTM A29.5

AFBMA 7

NSA 661

AFBMA 9

ASSE $\quad 1002$

ASTM D3099

ASTM D2783

MSS SP72

ASTM D3121

API 6D

ASAE $\$ 346$

SAE J884B

ANS1 $\quad$ C82.9

ANSI C82.5

ANSI

ANSI C82.2

ANSI $\quad$ C82.3

ASE

C82.

UBC $\$ 25.5$

ICBO UBCS25.8

UFO UF 2 ART16

IEEE 111

SAE J407C

SAE J776D

ASTM E388

UL 681

UL 609

UL 983

ASTM B19

ICBO UBCS26.5

ASTM D2094

Std. M ASTM D2095

ASTM B124

ASTM B236

SAE J194

ASTM E23

CRSI $\$ 1.5 .3$

CRSI $\quad$ 1.5.1

CRSI

CRSI $\quad 1.3 .5$

CRSI $* 1.3 .1$

ASTM B166

ASTM B160

ASTM B196

Std. Spec ASTM B425

Std. Sp ASTM

ASTM B121

ASTM B36

ASTM B411

Std. S ASTM

Std. Spec. Fo ASTM B194 
copper-Zinc-Tin Alloys Plate, Sheet, Strip, and Rolled Bar (1973) osphor Bronze (Copper Tin) Plate, Sheet, Strip, and Rolled Bar (1973) -Aluminum-Cobalt Alloy PIate, Sheet, Strip, and Rolled l-Zinc Alloy (Nickel Silver) and Copper-Nickel Rod and loy (Copper Alloy No. 175), Plate, Sheet, Strip and Rolled and Copper-Nickel Alloy Plate, Sheet, Strip, and Rolled ts for Hot Rolled and Cold Finished Carbon and Alloy Stee Std. Spec. for Nickel-lron-Chromium Alloy Rod and Std. Spec. for Copper Sheet, Strip, Plate and Rolled Std. Spec. for Hand Scrubbing Soap (Grit Soap) Paste and ht Copper and Copper Alloy Plate, Sheet, Strip, and Rolled steel and Iron (Shape, Plate, Sheet, Strip, Connector, and Std. Spec. for Free Cutting Brass Rod general Requirements for Wrought Copper and Its Alloy Rod, Std. Spec. for Copper Silicon Alloy Rod, Std. Spec. for Copper Rod, Std. Spec. for Manganese Bronze Rod Std. Spec. for Aluminum Bronze Rod, Std. Spec. for Copper Bu Std. Spec. for Columbium and Its Alloy

Std. Spec. for Molybdenum and Molybdenum Alloy rposes (Bus Condu/ Std. Spec. for Aluminum Alloy Extruded 73) Spec. for Tolerances of Aluminum and Its Alloy ilding Code: Masonry or Concrete Chimneys, Fireplaces, and under the Uniform Building Code: Chimneys, Fireplaces, and Std. Spec. for Zinc Coated (Galvanized) Steel

Spec. for Aluminum and Its Alloy Welding Rods and Spec. for Magnesium Alloy Welding Rods and tlng Chromium and Chromium-Nickel Steel Welding Rods and
Welding (I969) ANSI W3.17 972)

Std. for Consumable Electric Covered Spec. for Nlckel and Its Alloy Spec. for Titanium and Its Alloy

3)

Tent. Meth. of Test for Sulfur Oxides im Flue Gases ield, Getter Gas Content, and Getter Sorption Capacity for Std. Meth. of Test for Radioactive Meth. for Preparation and Standardization of

$973)$ Std. Meth. for Analysis of Calcium and

apacity for Barium Flas/ Std. Rec. Pract. for Determinin td. Meth. for Measuring Fast Neutron Flux for Analysis for number Determination of Both Meal and Flour of Wheat, Rye,

nd Semolina; Also, with Slight Modification, to Corn, Rye, Std. for Retainer-Floating Std. for Flashing and Steady Burn

Std. Spec. for Annealed Aluminum Alloy Foil for Flexible Spec. for Fire

Std. Meth. of Test for Residual Solvents in Flexible Rec. Pract. for Electrostatic Charge Mobility on Flexible inology)/

Std for Safety for Bullet Resisting Equipmen tection, Authority, Departmental Regulations and Controls, Std. Rec. Pract. for Selection of Vapor Std. Spec. for Titanium and Titanium Alloy

ase-13.3Cr-38Ni-5.5Mo-0.85C b-2.5Ti-1.6/ Spec. for Steel

Alloy

Alloy

Alloy

Base-19.5Cr-13.5Co-4.3Mo-3.0Ti-1.4Al, C/

t, 12.5Cr Low Carbon (SAE 5l4l6F), Free Machining /

$\mathrm{t}, 15.5 \mathrm{Cr}-4.5 \mathrm{Ni}-2.9 \mathrm{Mo}-0.10 \mathrm{~N}$, Solution Heat $\mathrm{T} /$

t, 15.5 $\mathrm{Cr}-4.5 \mathrm{Ni}-2.9 \mathrm{Mo}-0.10 \mathrm{~N}$, Heat Treated, 1

$\mathrm{t}, 16.5 \mathrm{Cr}-4.5 \mathrm{Ni}-2.9 \mathrm{Mo}-0.10 \mathrm{~N}$, Equalized and $\mathrm{T} /$

Stee

Stee

Stee

Steel

0)) (1973)

t6)) (1973)

Spec. for Aluminum Base Alloy essels/ Spec. for Aluminum Alloy

Std. Spec. for Stainless and Heat Resisting Steel Finished Age Hardening Stainless and Heat Resisting Stee Std. Spec. for Hot Worked and Cold Finished Zirconium Std. Spec. for Deformed and Plain Billet Steel Std. Spec. for Rail Steel Deformed

Std Spec for Axle Steel Deformed and Plain Std. Spec. for Hot, Hot Cold, and Cold Worked Alloy Steel - Spec. for Stainless and Heat Resisting Steel Billets and
Std. Spec. for Carbon and Alloy Steel delivery of Rolled Steel Plates, Shapes, Sheet Piling, and ainless and Heat Resisting Chromium Nickel Manganese Steel Std. Spec. for Merchant Quality Hot Rolled Carbon Steel Std. Gravure Color

Spec. for Steel Std. Spec. for Cold Finished Alloy Steel

Std Rec Pract for Macroetch Test of Tool Steel

ld Finished Bars; Hot Rolled Deformed Concrete Reinforcing Std. Spec. for Std. Quality Cold Finished Carbon Steel Std. Spec. for Merchant Quality Hot Rolled Carbon Steel Std. Spec. for Machined Flat and Square Tool Stee Uniform Building Code Std. for Concrete Reinforcement Spec. for Tolerances of Low Alloy Steel copper Wire Bars, Cakes, Slabs, Billets, Ingots, and Ingot
Bar (1973)

Bar (1973)

Bar (1973)

Bar (1973)

Bar (1973) ANSI G24.5

Bar (1973) ANSI H34.39

Bar (1973) ANSI H7.9

Bar (1974)

Bar (1974)

Bar) (1973)

1. Spec. for General Requirements for Wroug

Bar, and Shapes for Use in Screw Machines (1972)

Bar, and Shapes (1973)

Bar, and Shapes (1973) ANSI H7.3

Bar, and Shapes (1973) ANSI H7.4

Bar, and Shapes (1974) ANSI H7.5

Bar, and Shapes (1974) ANSI H7.6

Bar, Rod, and Shapes (1974) ANSl C7.25

Bar, Rod, and Wire (1964) ANSI Z179.19

Bar, Rod, and Wire (1974)

Bar, Rod, Pipe, and Structural Shapes for Electrical Pu

Bar, Rod, Wire, and Forging Stock (Rolled or Drawn) (19

Barbecues (1973)

Barbecues (1973)

Barbed Wire (1973) ANSI G8.10

Bare Electrodes (1969) ANSl W3.10

Bare Electrodes (1969) ANSl W3.19

Bare Electrodes (1969) ANSI W3.9

Bare Mild Steel Electrodes and Fluxes for Submerged Aro

Bare Welding Rods and Electrodes (I969) ANSI W3.I4

Bare Welding Rods and Electrodes (1970) ANSI W3.16

(Barium Chloranilate Controlled Condensation Meth.) (I97

Barium Flash Getters (1972) ANSI Z173.50

Dwelling Construction

Barium in Water (1973) ANSI N155

Barium Lactate Solution (Cereal Chemistry) (1962)

Barium Petroleum Sulfonates by Liquid Chromatography (1

Barium Yield, Getter Gas Content, and Getter Sorption C

Barium 140 Produced by Uranium 238 Fission (1973)

Barley, Other Grains, and Malted Cereals (Cereal Chemis

Barley, Rice, Grain Sorghum, and Buckwheat (Cereal Chem

Barrel Nut (1973)

Barricade Warning Lights (1971)

Barrier Applications (1973)

Barrier Gypsum Board Membrane (1972T)

Barrier Materials (1972)

Barrier Materials (1973)

Barrier) for Protection Against Robbery or Holdup (Crim

Barrier, Road, Hydrant, Driveway Clearance, Installatio

Barriers for Thermal lnsulations (1973)

Bars and Billets (1972) ANSI Z179.2

Bars and Forgings (0.18-0.23C) (SAE 1022) (1973)

Bars and Forgings, Corrosion and Heat Resistant, Iron B

Bars and Forgings, Corrosion and Heat Resistant, Nickel

Bars and Forgings, Corrosion and Heat Resistant, Nickel

Bars and Forgings, Corrosion and Moderate Heat Resistan

Bars and Forgings, Corrosion and Moderate Heat Resistan

Bars and Forgings, Corrosion and Moderate Heat Resistan

Bars and Forgings, Corrosion and Moderate Heat Resistan

Bars and Rings $(1.0 \mathrm{Mg}-0.60 \mathrm{SI}-0.28 \mathrm{Cu}-0.20 \mathrm{Cr}(606 \mathrm{l}-$

Bars and Rings $(1.0 \mathrm{Mg}-0.60 \mathrm{SI}-0.28 \mathrm{Cu}-0.20 \mathrm{Cr}(606 \mathrm{I}-$

Bars and Shapes for Use in Boilers and Other Pressure V

/ Spec. for Hot Rolled and Cold

Bars and Wire for Nonnuclear Applications (1972) ANSI Z

Bars for Concrete Reinforcement (1972)

Bars for Concrete Reinforcement (1972)

Bars for Concrete Reinforcement (1972)

Bars for High Strength at Elevated Temperatures (1971)

Bars for Reforging (1972) ANS1 G81.13

Bars for Springs (1974)

Bars for Structural Use (1972) ANSI G24.4

Bars Including Rounds, Squares, Hexagons, and Shapes

Bars Subject to Mechanical Property Requirements (1972)

Bars (Printing) (1973)

Bars $(0.95 \mathrm{Cr}-0.20 \mathrm{Mo}-0.05 \mathrm{Te}(0.38-0.45 \mathrm{C}))(1973)$

Bars $(0.95 \mathrm{Cr}-0.20 \mathrm{Mo}-0.05 \mathrm{Te}(0.40-0.53 \mathrm{C}))(1973)$

Bars (1964) ANSI G24.12

Bars (1971) ANSI G60.9

Bars (1972)

Bars (1973)

Bars (1973)

/Mifinished for Forging; Hot Rolled and $C_{0}$

Bars (1973)

Bars (1973)

Bars (1973)

Bars (1973) ANSI H17.1
B591

B 103

B592

B 151

B534

$\mathrm{B} 122$

A29

B408

B152

D2955

B248

UBCS27.1

B16

B249

B98

B133

B138

B 150

B187

B392

B387

B317

AMS2201J

$\mathrm{UBC} * 3.37$

$\mathrm{UBC} * 8.6$

A 121

A5. 10

A5.19

A5.9

A5.1

EW

A5.14

A5. 16

D3226

F111

D2038

70-10

ASTM D2894

$\begin{array}{ll}\text { ASTM } & \text { F111 } \\ \text { ASTM } & \text { E393 }\end{array}$

$\mathrm{AACCH} \quad 56-81 \mathrm{~B}$

AACCH $\quad 76.20$

NSA 578

1TE

ASTM

ASTM

Tent. ASTM

UL

ICBO

ASTM

ASTM

SAE

SAE

SAE

SAE

SAE

SAE

SAE

SAE

SAE

SAE

ASTM

ASTM

ASTM

ASTM

ASTM

ASTM

ASTM

Std ASTM

ASTM

ASTM

ASTM

ASTM

GT

SAE

SAE

ASTM

ASTM

AlSI

ASTM

ASTM

ASTM

ICBO

SAE

Std. Spec. for Lake ASTM

578

B 479

C707

F151

F365

UFC *2ART13

C 755

B348

AMS5070D

AMS5633A

AMS5709B

AMS5738C

AMS5610H

AMS5743D

AMS5744A

AMS5745A

AMS4115C

AMS4117D 
copper Wire Bars, Cakes, Slabs, Billets, Ingots, and Ingot copper Wire Bars, Cakes, Slabs, Billets, Ingots, and Ingot opper Zinc Lead (Leaded Red Brass or Hardware Bronze) Rod, Std. Spec. for Stainless and Heat Resisting Steel Std. Spec. for Phosphorus Deoxidized Copper Wire Std. Spec. for Oxygen Free Electrolytic Copper Wire 73) ANSI H17.1 73) ANSI $\mathrm{H} /$ Std. Spec. for Lake Copper Wire 73) ANSI H 17.2 Std. Spec. for Chemically Refined Copper Wire Std. Spec. for Electrolytic Copper Wire lity, for Pressure Piping Component/ wer Applications (1974)

Nickel Base (19.5 $\mathrm{Cr}-13.5 \mathrm{Co}_{0}-4.3 \mathrm{Mo}^{-}$ Std. Spec. for Steel Std. Spec. for Steel Spec, for Alloy

serv/ Std Spec for Precipitation Hardening Nickel Alloy

td. Spec. for Precipitation Hardening Iron Base Superalloy Spec. for Precipitation Hardening Cobalt Containing Alloy $-1.0 \mathrm{Mo}-0.12 \mathrm{~V}(0.43-0.49 \mathrm{C}))(1973)$

$-1.82 \mathrm{Ni}-0.40 \mathrm{Mo}-0.07 \mathrm{~V}(0.41-0.46 \mathrm{C})$ I

Spec. for Steel

reated, 15.1 Spec. for Corrosion and Heat Resistant Alloy

Nickel Base-15.5Cr-8.0Fe (1973)

Nickel Base-15.8Cr-15.2 Mo-0.30Al-0.05La /

Nickel Base $-5.0 \mathrm{Cr}-24.5 \mathrm{Mo}-5.5 \mathrm{Fe}$, Solution $\mathrm{H} /$

$35.5 \mathrm{Ni}-18.5 \mathrm{Cr}-1.1 \mathrm{SI}(1973)$

$49-0.55 \mathrm{C}))(1973)$

$-4.0 \mathrm{Mo}-1.2 \mathrm{~V}(1.10-1.20 \mathrm{C})$, Premium Bearing $\mathrm{Qu} /$ $0.95 \mathrm{Cr}-0.55 \mathrm{Mo}-0.30 \mathrm{~V}(0.40-0.50 \mathrm{C}) \mathrm{V} /$

$.11-0.17 \mathrm{C})(\mathrm{SAE} 8615)(1973)$

$0.04 \mathrm{~V}-\mathrm{B}(0.38-0.43 \mathrm{C}$ ) (Modified 98Bv40) (1973)

38-0.43C) (SAE 4340), Premium Quality, Consumabl/

38-0.43C) (SAE 4340) (1973)

07-0.13C) (SAE 9310), Premium Quality, Vacuum Co/

07-0.13C) (SAE 9310), Premium Quality (1973)

gs $(1.3 \mathrm{Mn}-1.5 \mathrm{Sl}-0.30 \mathrm{Cr}-1.8 \mathrm{Ni}-0.4 /$

$(0.48 \mathrm{Cr}-8.0 \mathrm{Ni}-4.0 \mathrm{Co}-0.48 \mathrm{Mo}-0.09 /$

esistant $(15 \mathrm{Cr}-26 \mathrm{Ni}-1.3 \mathrm{Mo}-2.1 \mathrm{Ti}-1$

$5 \mathrm{Mo}-0.20 \mathrm{~V}(0.32-0.38 \mathrm{C})$, Special Grade (1973)

$(0.28-0.33 \mathrm{C})(1973)$

for Hot Rolled and Cold Finished Zirconium and Its Alloy Std. Spec. for Aluminum Alloy

erances Applicable to Titanium and Its Base Alloy Extruded Std. Spec. for Aluminum Alloy Extruded Std. Spec. for Magnesium Alloy Extruded stant, $12 \mathrm{Cr}-8.5 \mathrm{Ni}-2.0 \mathrm{Cu}-1.1 \mathrm{Ti}$, Vacuum Induct Steel

el: Semifinished for Forging: Hot Rolled and Cold Finished

lloy Bars and Forgings, Corrosion and Heat Resistant, Iron lloy Sheet and Strip, Corrosion and Heat Resistant, Nickel Forgings, and Rings, Corrosion and Heat Resistant, Nickel Forgings, and Rings, Corrosion and Heat Resistant, Nickel et, Strip, and Plate, Corrosion and Heat Resistant, Nickel oy Bars and Forgings, Corrosion and Heat Resistant, Nickel Alloy Wire, Welding, Corrosion and Heat Resistant, Nickel Alloy Wire, Welding, Corrosion and Heat Resistant, Cobalt Alloy Bars, Forgings, and Rings. Heat Resistant, lron

Forgings, and Rings, Corrosion and Heat Resistant, Nickel e Under Interior (Dry Use) Exposu/

Std. Spec. for Protein $20 \mathrm{Cr}(6061-0))$ (1973) Spec. for Aluminum

n Resistant Iron Chromium, Iron Chromium Nickel and Nickel ratures (1973) A/ Std. Spec. for lron, Cobalt, and Nickel Std. Spec. for Copper Std. Spec. for Copper Tolerances Applicable to Titanium and Its Spec. for Tolerances of Aluminum and Magnesium Spec for Tolerances of Aluminum and Magnesium arri/ Std. Meth. for Spectrochemical Analysis of Nickel . otometric Meth. for Chemical Analysis of Copper and Copper ical, Magnetic, and Other Similar Iron, Nickel, and Cobalt

6.17 Std. Reference Radiographs for High Strength Copper Std. Meth. of Test for Detecting Glycol Std. Spec. for Oil and Resin

hed Stone, Slag, and Gravel for Dry or Water Bound Macadam n Lamps (1972) Std. for Medium Prefocus Std. Meth. of Test. Bituminous 1 Std. Meth. of Evaluating the Properties of Wood and Industrial Filament Yarns, Made from Man Made Organic d. Test Meth. for Simulated Service Test. of Wood and Wood Std. for

Uniform Building Code Sid. for Gypsum Std for R-40 Bulb (Hard Glass) Mogul Screw Std. for Strip-High Temperature Terminal Block, Std. Meth. of Test for Slump of an Oil Std. for Physical Characteristics of T.2 Telephone Slide Std. Test Meth. for Specific Gravity of Wood and Wood Std. Meth. of Conducting Machining Tests of Wood and Wood afety Code for Cranes, Derricks, Hoists, Jacks and Slings:

ectric Wire Rope Hoists (Lug, Hook, Trolley Suspended, and chloric Acid Titration (197/ Std. Meth, of Test for Total
Bars (1973) ANSI H17.2

Bars (1973) ANSI H23.12

Bars, and Shapes (1972) ANSI H33.

Bars, and Shapes (1973) ANSI G24.31

Std. Spec. for Electrolytic ASTM

Bars, Billets, and Cakes (1972)

Bars, Billets, and Cakes (1972) ANSI H23.11

Bars, Cakes, Slabs, Billets, Ingots, and Ingot Bars (19

Bars, Cakes, Slabs, Billets, Ingots, and Ingot Bars (19

Bars, Cakes, Slabs, Billets, Ingots, and Ingot Bars (19 Std

Bars, Carbon, Hot Rolled and Cold Finished, Special Qua

Bars, Carbon, Hot Rolled, Special Quality, for Fluid Po

Bars, Forgings and Rings, Corrosion and Heat Resistant,

Bars, Forgings, and Forging Stock for High Temperature

Bars, Forgings, and Forging Stock for High Temperature

Bars, Forgings, and Forging Stock for High Temperature

Bars, Forgings, and Mech anical Tubing $(1.05 \mathrm{Cr}-0.55 \mathrm{Ni}$

Bars, Forgings, and Mechanical Tubing $(1.62 \mathrm{SI}-0.82 \mathrm{Cr}$

Bars, Forgings, and Rings (Nickel Base, Solution Heat T

Bars, Forgings, and Rings, Corrosion and Heat Resistant

Bars, Forgings, and Rings, Corrosion and Heat Resistant

Bars, Forgings, and Rings, Corrosion and Heat Resistant

Bars, Forgings, and Rings, Heat Resistant, Iron Base-

Bars, Forgings, and Tubing $(0.80 \mathrm{Cr}-1.8 \mathrm{Ni}-0.25 \mathrm{Mo}(0$

Bars, Forgings, and Tubing, Corrosion Resistant, $14.5 \mathrm{Cr}$

Bars, Forgings, and Tubing, Low Alloy, Heat Resistant (

Bars, Forgings, and Tubing, $0.50 \mathrm{Cr}-0.55 \mathrm{Ni}-0.20 \mathrm{Mo}(0$

Bars, Forgings, and Tubing, $0.80 \mathrm{Cr}-0.85 \mathrm{Ni}-0.20 \mathrm{Mo}-$

Bars, Forgings, and Tubing, $0.80 \mathrm{C}-1.8 \mathrm{Ni}-0.25 \mathrm{Mo}(0$.

Bars, Forgings, and Tubing, $0.80 \mathrm{Cr}-1.8 \mathrm{Ni}-0.25 \mathrm{Mo}(0$.

Bars, Forgings, and Tubing, $1.2 \mathrm{Cr}-3.25 \mathrm{Ni}-0.12 \mathrm{Mo}(0$.

Bars, Forgings, and Tubing, $1.2 \mathrm{Cr}-3.25 \mathrm{Ni}-0.12 \mathrm{Mo}(0$.

Bars, Forgings, Mechanical Tubing, and Flash Welded Rin

Bars, Forgings, Rings, and Mechanical Tubing (Annealed)

Bars, Forgings, Tubing, and Rings, Corrosion and Heat $\mathrm{R}$

Bars, Forgings, Tubing, and Rings, Corrosion and Modera

Bars, Forgings, Tubing, and Rings, $0.78 \mathrm{C} r-1.8 \mathrm{Ni}-0.3$

Bars, Forgings, Tubing, $0.88 \mathrm{Cr}-1.8 \mathrm{Ni}-0.42 \mathrm{Mo}-0.08 \mathrm{~V}$

Bars, Rod, and Wire for Nuclear Application (1973) ANSI

Bars, Rods and Wires (1974) ANSI H38.4

Bars, Rods, and Shapes (1973)

Bars, Rods, Shapes, and Tubes (1973) ANSI H38.5

Bars, Rods, Shapes, Tubes, and Wires (1970) ANSI H45.6

Bars, Wire, Forgings, and Forging Stock, Corrosion Resi

Bars: Hot Rolled Deformed Concrete Reinforcing Bars (19

Base $-13.3 \mathrm{Cr}-38 \mathrm{Ni}-5.5 \mathrm{Mo}-0.85 \mathrm{Cb}-2.5 \mathrm{Ti}-1.6 \mathrm{Al}-$

Base-15.5 Cr $-2.4 \mathrm{Ti}-0.70 \mathrm{~A} 1-7.0 \mathrm{Fe}$ (1973)

Base-15.5 Cr-8.0Fe (1973)

Base-15.8Cr-15.2Mo-0.30Al-0.05La (1973)

Base-15.8Cr-15.2Mo-0.30Al-0.05La (1973)

Base-19.5 Cr-13.5Co-4.3Mo-3.0Ti-1.4A1, Consuma

Base-19.5Cr-18Co-2.5Ti-1.5Al (1973)

Base-25.5 $\mathrm{Cr}-10.5 \mathrm{Ni}-7.5 \mathrm{~W}(1973)$

Base-35.5 Ni-18.5 $\mathrm{Cr}-1.1 \mathrm{SI}(1973)$

Base-5.0C r-24.5Mo-5.5Fe, Solution Heat Treated (1

Base Adhesives for Structural Laminated Products for Us

Base Alloy Bars and Rings $(1.0 \mathrm{Mg}-0.60 \mathrm{SI}-0.28 \mathrm{Cu}-0$.

Base Alloy Castings for General Application (1972) ANSI

Base Alloy Castings for High Strength at Elevated Tempe

Base Alloy Centrifugal Castings (1973)

Base Alloy Continuous Castings (1973)

Base Alloy Extruded Bars, Rods, and Shapes (1973)

Base Alloy Extrusions (1973)

Base Alloy Sheet and Plate (1973)

Base Alloys by the Silver Chloride-Lithium Fluoride C

Base Alloys (1972)

Base Alloys (1973)

Std. $\mathrm{pH}$
(Alysis of High Temperature, Electr

Base and Nickel-Copper Alloy Castings (1967) ANSI Z16

Base Antifreeze in Used Lubricating Oils (1973)

Base Caulking Compound for Building Construction (1972)

Base Courses (1971) ANSI A37

Base Spec.

Base Emulsions for Use as Protective Coatings (1973)

Base Fiber and Particle Panel Materials (1972) ANSI O8.

Th. of Testing Tire Cords, Fabrics

Base Fibers (1972)

Base Finish Flooring (1969)

1971)

Base for Veneer Plaster and Gypsum Veneer Plaster (1973

Base Incandescent (1966)

Base Insulating (1973)

Base Knife Grade Channel Glazing Compound (1972)

Base Lamp (1973)

Base Materials (1969)

Base Materials (1970)

Base Mounted Drum Hoists (1971)

Base Mounted Types (1971)

Base Number of Petroleum Products by Potentiometric Per
ASTM

ASTM

ASTM

ASTM

ASTM

ASTM

ASTM

ASTM

SAE

ASTM

ASTM

ASTM

SAE

SAE

SAE

SAE

$S A E$

$S A E$

SAE

SAE

SAE

SAE

SAE

SAE

SAE

SAE

SAE

$S A E$

SAE

SAE

SAE

ASTM

ASTM

Tol SAE

ASTM

ASTM

SAE

AISI

$S A E$

SAE

toy Bars, SAE

/Bars,

SAE

SAE

SAE

SAE

SAE

ASTM

SAE

ASTM

ASTM

ASTM

ASTM

SAE

SAE

ASTM

ASTM

ASTM

ASTM

ASTM

ASTM

ANSI

ASTM

ASTM

t ASTM

ANSI

ICBO

ANSI

NSA

ASTM

ANSI

ASTM

ASTM

ANSI

HMI

ASTM

B5

B442

B1 140
A2 76

B379

B170

B4

B442

B5

A696

A695

AMS5707D

A637

A638

A639

AMS6432A

AMS6416A

AMS5750B

AMS5665H

AMS5711

AMS5755B

AMS5716A

AMS6424

AMS5749

A 6305

AMS6270J 
Minimum Std. for Amateur Radio Antenna: Part 1 Tent. Rec. Pract. for Direct Injection of Solvent c. Pract. for Vacuum Distillation of Solvents from Solvent Spec. for for Hygroscopic Properties of Fire Retardant Wood and Its Uniform Building Code Std. for Fiberboard Nail

3.4 .27

igh Temperat/ Std. Spec. for Precipitation Hardening lron oy Bars and Forgings, Corrosion and Heat Resistant, Nickel Forgings and Rings, Corrosion and Heat Resistant, Nickel Im for Archival Records, Silver Gelatin Type, on Polyester Archival Records, Silver Gelatin Type, on Cellulose Este engine Antifreeze-Coolant Concentrate (Glycol or AIcoho Std. Spec. for Cellulosic Fiber (W ood aths, and Metal Accessories (Designed for Use as a Plaster std. Spec. for Hot Mixed, Hot Laid Tar Paving Mixtures for and Heat Resistant Alloy Bars, Forgings, and Rings (Nicke et, Strip, and Plate, Corrosion and Heat Resistant, Nickel or Less (1973) ANSI C33.95 Safety Std. for Electric Reclamation, Curtain and Building Foundation Drain, Under (Extinguishers, Alarms, Sandpipes), Building Construction, nuclear Power $\mathrm{Ge} /$ Std. Criteria for Preparation of Design Std. Meth. of Test for Pyridine

ing Coal and Coke Analyses from As-Determined to Differen

Rec. Pract. for Construction of Concrete Pavements and Std. for Punches:

er Bearings (Except Tapered Roller Bearings) Conforming to

; Knobs and HandIes; Exit Devices, Pulls, Push and Kick / rting Equipment for Use with Emergency Locator Tran/ Rec. projectors (1973)

devices Used in Treating Wastewater (1970)

buttress Inch Screw Threads (7/45 Deg. Form with 0.6 Pitch uffs, and Bread (CereaI Chemistry) (1962)

ic Fluid Power Pumps / Std. Meth. of Test, and Presenting Std for shings (1972)

(1972) spec. for Concrete Masonry Units for Construction of Catch verlay Projects (1973)

t. AC High Voltage Circuit Breakers Rated on a Symmetrical Table for Converting Absorption to $14.0 \%$ Moisture eth. for Correction of Analytical Values to $14.0 \%$ Moisture plication Factors for Correcting Weights to a 14\% Moisture or Changing as.is Farinograph Absorption to $14.0 \%$ Moisture gh Voltage Circuit Breakers Rated on a Symmetrical Current ircuit Breakers Rated on a Symmetrical and a Total Curren or AC High Voltage Circuit Breakers Rated on a Symmetrical circuit Breaker ExternaI Insulation Rated on a Symmetrical gh Voltage Circuit Breakers Rated on a Symmetrical Current gh Voltage Circuit Breakers Rated on a Symmetrical Curren gh Voltage Circuit Breakers Rated on a Symmetrical Curren gb Voltage Circuit Breakers Rated on a Symmetrical Curren or AC High Voltage Circuit Breakers Rated on a Symmetrical Std. Meth. of Test. Twine Made from wheats (Cereal Chemistry) (1962)

real Chemistry) (1962)

Tent. Spec. for Concrete Made by Volumetric Safety Std. for Electric Dry Std. Performance Requirements for Woven Std. Spec. for Ceramic Tile Panels for starch Which Is Gelatinized When Heated in a Boiling Wate Test Meth. for Alkali in Bleach

Test Meth. for Hydrogen Peroxide in Bleach Recommendation for Installation of Ceramic Tile 70)

or Nonmetallic Shower Receptors, Stall Showers Lavatories, Rec. Pract. for Storage Determination of Capacity of Lead Acid Industrial Storage omotive and Industrial A/ Std. for Lead Acid Type Storage

Safety and Health Rec. for Changing and Charging Storage Spec. for Dry Cells and Std. Test Proc. for Storage

Rec. Pract for Life Test for Automotive Storage uarantee Policy for Motor Vehicle Type (Lead Acid) Storage meth. for Determination of Specific Volume of Creams, Cake and Std. for the Design, Construction and Installation of Safety Std. fo and Starting) (1972) Uniform Plumbing Code: ner and Contractor (For Small Construction Contracts Where gh Voltage Circuit Breakers Rated on a Symmetrical Curren
Base or Fixed Station (1973)

Base Paints into a Gas Chromatograph (1973)

Base Paints (1973)

Base Plate (Artificial Dentures) Wax (I971)

Base Products (1973)

Base Products (1973)
Base Sheathing and Structural Insulating Board (1973)

Base Sintered Bearings (Oil Impregnated) (1973) ANSI H9

Base Superalloy Bars, Forgings, and Forging Stock for H

Base 19.5 $\mathrm{Cr}-13.5 \mathrm{Co}-4.3 \mathrm{Mo}-3.0 \mathrm{Ti}-1.4 \mathrm{AI}$, Consumabl

Base $(19.5 \mathrm{Cr}-13.5 \mathrm{Co}-4.3 \mathrm{Mo}-3.0 \mathrm{Ti}-1.4 \mathrm{Al})$ Consumab

Base (1973)

Base (1973)

Base) by the Iodine Reagent Meth. (1973)

Base) (I973)

hotographic $\mathrm{F}$

Tent. Re ASTM

Base) (1973)

Insulation (1973)

or Water in

Base, Binder, Leveling, and Surface Courses (1967)

ase, Solution Heat Treated, $15.5 \mathrm{Cr}-16 \mathrm{M}_{0}-3.8 \mathrm{~W}-5.5$

Base, $20 \mathrm{Cr}-20 \mathrm{Co}-5.9 \mathrm{Mo}-2.2 \mathrm{Ti}-0.45 \mathrm{Al}$ Solution Hea

Baseboard Heating Equipment (Portable and Fixed) $600 \mathrm{~V}$

Basement Floor, Sump Conductor, Downspout Runoff, Swimm

Basement Pipe InIets (1973)

F Systems and Appliances

Bases for Systems That Perform Protective Functions in

Bases in Crude and Refined Tar Acids (1972) ANSI Z107.4 Std. Meth. for Calculat

Bases (1974)

Basic Angle Head Type and Related Quill Bushings (1972)

Basic Boundary Plans (1972) ANSI B3.17 / Ball and Roll

Basic Builders' Hardware: Hands of Doors; Hinges; Locks

Basic Characteristics for Airborne Radio Homing and Ale

Basic Construction Requirements for Photographic Slide

Basic Criterial for Evaluation of Special Processes or

Basic Hardboard (1973)

Basic Height of Thread Engagement (1973)

Basic Lead Silico Chromate (1974)

Basic Meth. of Analysis for Ash in Flour, Feeds, Feedst

Basic Performance Data for Positve Displacement Hydraul

Basic Requirements for Cargo Containers (1971)

Basic Shapes (1974)

Basic, Combination Angle Head Type and Related Quill Bu

Basic, Cylindrical Head Type and Related Quill Bushings

Basins and Manholes (1973) ANSI A73.1

Basis of Payment Is a Stipulated Sum) (1974)

Basis of Payment on Portand Cement Concrete Pavement 0 -

Basis When Rated for Out of Phase Switching (1973) ANSI

Basis (Cereal Chemistry) (1962)

Basis (Cereal Chemistry) (1962

Basis (Cereal Chemistry) (1965)

Basis (Constant Dough Weight Meth. Only) (Cereal Chemis

Basis (1971) ANSI C 37.072

/ Recovery Voltage for AC Hi AAC

Basis (1972)

Basis (1972)

Basis (1972)

Electrical Control of AC High Vor AC

Ings for Capacitance Current Switching F ANSI

Basis (1972) ANSI C37.073

Basis (1972) ANSI C37.076

Basis (1972) ANSI C37.078

Basis (1973) ANSI C37.0731 for Outdoor AC High Voltage for Outdoor AC High Voltage ANSI
/Current Switching for AC Hi IEEE

/Surized Components of AC Hi IEEE

/Nsulation for Outdoor AC Hi IEEE

Urrent Switching for AC Hi IEEE

Basis ( $362 \mathrm{kV}$ and Above) (197.

Batch Meth. of Experimental Milling for Bread and Soft

Batch Meth. of Experimental Milling for Durum Wheat ( $\mathrm{Ce}$

Batching and Continuous Mixing (1973)

Bath Heaters (1972) ANSI C33.75

Bath Mats (InstitutionaI Textile) (1973)

Bath Tub Recess (1972)

Bath (1973)

Baths Containing Hydrogen

Baths (1972) ANSI L14.145

Bathtub Walls (1973)

Bathtubs and Combination Tank and Shower Receptor Units

Batteries for Construction and Industrial Machinery (19

Batteries for Motive Power Service (1974)

Batteries for Motor Vehicles, Motorboats, Tractors, Aut

Batteries in Motive Power Service (For Electric Trucks)

Batteries (1972)

Batteries (1972)

Batteries (1972)

Batteries (1972)

Batters, and Icings (Cereal Chemistry) (1962)

Battery Chargers on Boats (1973)

Battery Chargers (1972)

Battery Drainage System (1973)

Battery Materials Spec. (Vehicular, Ignition, Lighting

(Battery O perated) (Photography) (1972)

Battery Powered Emergen cy Lighting Equipment (Indoor) (
Basis (1971) ANSI C37.0721
EIA

ASTM

ADA

ASTM

ICBO

ASTM

ASTM

ASTM

$S A E$

ANSI

ANSI

ICBO

RS409

D3271

D3272

D3201

UBCS25-24

B439

B438

A638

AMS5709B

AMS5707D

PH1.41

PH1.28

D1123

UBCS 47.4

D1 753

AMS5750B

AMS5872

1042

D2311

UFC*2ART13

N18.8

D2748

D3180

316

B94.41

7

8

DO-154

PH3.65

C. 9

PS58

B1.9

D1 648

AACCH 08-01

$\begin{array}{ll} & \\ & \end{array}$

ANSI MH5.1

B94.44

C139

A 107

TM-6

417

ACCH 82.2

AACCH 82.22

54.29

327

C 37.11

C37.0732

C37.0781

341

340

343

342

339

D 1233

AACCH $26-21$

AACCH $26-30$

ASTM C685

UL

ANSI

TCA 8701

C. 42

AATCC 102

TCA 150

IAPMO TSCl

SAE J930A

$\mathrm{J} 930 \mathrm{~A}$
$\mathrm{IB} 2$

$\mathrm{SAE} \quad \mathrm{J} 537 \mathrm{H}$

ITA $8 \mathrm{Kl}$

ANSI C18.1

BCI *1-66

SAE J240A

*1. I5

AACCH $\quad 72.10$

ABYC A20

UL 1236

ICBO UPC*1.H

BCI *1.41

ANSI PH3.51

UL

924 
National Bureau of Standards KWIC Index of Engineering Standards

Std. for Safety for Electric ec, and Ratings: Assembly Numbers, Cell Layouts and Ter/ earance Dimensions on the Mating Plates (Patterns) Used in

ing) for Passenger Automobiles, Commercial Vehic/ Storage
70) ANSI K91.1 Meth. of Test for 70) ANSI K91.l

Std. Analytical Meth. for Determinin of Analysis of Specific Gravity, Degrees Brix, and Degree and Double Screened Ground Refractory Materials (Calcined Cars, Off Road, Agricultural and Log Skidder, Truck, Bus n Steel Tubing Normalized for Bending, Double Flaring, and ption Meth. of Analysis of Vitamin A in Dry Vitamin Mixes. of Test for Annealing Point and Strain Point of Glass b Std. Test. Procedure for End Supported Rec. Horizontal Shorin Std. Spec. for Ultrasonic, Angle building Code Std. for Structural Floor and Roof Plank and Std, for Dimensional Spec, for Sealed ec. Pract. for Service Performance Requirements for Sealed rent or Opaque Metal Oxide and Metallic Coatings by Double on Silicon Wafers and Metallization Thickness by Multiple Std. for Auxiliary Low ar Strength of Parallel Fiber Reinforced Plastics by Short Rec. Horizontal Shoring

Std. Rec. Pract. for Preparation of Bituminous Mixture to. Test. Procedure for End Supported Std. Meth. for Preparation and Use of Ben Rec. Pract. for Semiautomatic Headlamp (Light) tes for Special Applications (19/ Std. Spec. for Straigh e Vessels (1973) ANSI

l Castings (1970) ANSI G52.7

Std. Meth. and Spec. for Straight Std. Spec. for Longitudinal

h. of Test for Flexural Strength of Concrete (Using Simple of Test for Stiffness of Plastics by Means of a Cantileve and Stiffness Computations (1973)

Rec, for Wood Concrete Construction Design Handbook Tent. Rec. for Design of Concrete Composite Plywood Lumber Design Spec. for Plywood uilt Up Members-Plywood Stressed Skin and Curved Panels, ination of Cereal Grains, Unpopped Popcorn, Dried Peas and ion in Cereal Grains, Unpopped Popcorn, Dried Peas and an ination of Cereal Grains, Unpopped Popcorn, Dried Peas and insects in Cereal Grains, Unpopped Popcorn, Dried Peas and

in Flour, Farina, Semolina, Bread, Grain, Soybeans, Rice,

Dielectric Meter Meth of Analysis of Moisture in Grains, y to Intergranular Attack in Wrought Nickel Rich, Chromium Std. Spec. for White Metal

other Structural Uses Std. Spec. for Rolled Copper Alloy
atic Load on Spread Footings (1972) Std. Meth. of Test for
Stal Uses ings (1972) Std. Meth. of Test $\mathrm{F}_{0} /$ Std. Spec. for Hollow Load Std. Spec. for Hollow Nonload Sid. Spec for Solid Load Uniform Building Code Std. for Hollow Load Uniform Building Code Std. for Solid Load Uniform Building Code Std. for Hollow NonIoad ar (Crude and Refined), Syrups, Starches and Other Protein dstuffs (Corn), Syrups, Sugars, Starches and Other Protein rn) Feedstuffs, Syrups, Sugars, Starches and Other Protein Content of Corn Syrup, Sugars, Starches and Other Protein

$n$ Content in Corn Starch, Sugars, Syrups and Other Protein

n Resistant, $14.5 \mathrm{Cr}-4.0 \mathrm{Mo}-1.2 \mathrm{~V}(1.10-1.20 \mathrm{C})$, Premium channels for Screw Applic/ Std. Spec, for Nonload (Axial) Std. Spec. for Carbon-Chromium Ball and Rolle Fire Resistance Requirements Relating to Brick Contemporary Contemporary (1964) Contemporary

stru/ Uniform Building Code Std. for Structural Clay Load Uniform Building Code Std. for Structural Clay Nonload Contemporar Contemporar Engineered Design of the Contemporary Std. for Cam Follower, Needle

Shaft and Housing Fits for Metric Radial Ball and Roller Std. Spec. for Iron Base Sintered Std. Spec. for Copper Base Sintered Std. Load Ratings and Fatigue Life for Rolle Std. Load Ratings and Fatigue Life for Ball Std, for In, and Metric Radial Needle Rolle sintered Powder MetaI Structural Parts and Oil Impregnated rame (1974)

ric Radial Ball and Roller Bearings (Except Tapered Roller Std. for Design of Components: Enclosed Gear Drives Test Meth. for
Battery Powered Industrial Truck8 (1972) ANSI B56.3

attery Replacement Data; Group Num

Battery Sockets (1974) ANSI C83.53

Battery Spec. (Vehicular, Ignition, Lighting, and Start

Baume Content of Corn Syrup Unmixed and Irs Syrups (195

Baume of Sugar Solutions (Cereal Chemistry) (1962)

Bauxite, Calcine, Chrome Ore, Clays, Diaspore, Ganister

Bead Seat, Industrial and Bicycle Tires (1974)

for Welded Flash Controlled Low

Beadlets, Oils, and Emulsions (Cereal Chemistry) (1973)

Beam Bending (1972)

Beam Deflection Composite Tubes and Cores (1972)

Beam Erection Procedure (1972)

Beam Examination of Steel Plates (1973) ANSI G35.24

Beam Framing (1973)

Beam Headlamp Units (1972)

Beam Headlamp (Light) Units for Motor Vehicles (1973)

Beam Interference Microscope Technique (1973)

Beam Interference (Tolansky Meth.) (1973)

Beam Lamp (Headlamp-Light) (1973)

lof Test for Apparent Horizontal She

Beam Meth. (1972)

Beam Safety Rules (1972)

Beam Specimens by Means of the California Kneading Comp

Beam Strength Fibre Tubes and Cores (1972)

Beam Stress Corrosion Metal Specimens (1973)

Beam Switching Devices (1972)

Beam Ultrasonic Examination of Plain and Clad Steel Pla

Beam Ultrasonic Examination of Steel Plates for Pressur

Beam Ultrasonic Inspection of Carbon and Low Alloy Stee

Beam with Center Point Loading) (1968) ANSI A37.84

Beam (1970) ANSI K65.13

Beam, Joint and Rafter Span, Load, Deflection, Strength

Beam, Slab, Footing, Column and Other Members) (1973)

Beams and Girders for Building (1957)

Beams Fabrication Spec. (1971)

Beams (1972)

Beams, and Components (1973)

Beans for Rodent and/or Insect

Beans (Cereal Chemistry) (1962)

Beans (Cereal Chemistry) (1962)

Beans (Cereal Chemistry) (1962)

Beans, Peas, Lentils, Corn Meal,

Beans, Peas, Lentils, Rice, and O

Bearing Alloys (1972) ANSI G80.4

Bearing Alloys (1973) ANSI H39.1

Bearing and Expansion Plates and Sheets for Bridge and

Bearing Capacity of Soil for Static Load on Spread Foot

Bearing Capacity of Soil for Static Load on Spread Foot

Bearing Concrete Masonry Units (1970) ANSI A79.1

Bearing Concrete Masonry Units (1971) ANSI A80.1

Bearing Concrete Masonry Units (1971) ANSI A81.1

Bearing Concrete Masonry Units (1973)

Bearing Concrete Masonry Units (1973)

Bearing Concrete Masonry Units (1973)

Bearing Materials When Suitable Amounts of Sample, Sulf

Bearing Materials When Suitable Amounts of Sample, Sulf

Bearing Materials When Suitable Amounts of Sample, Sulf

Bearing Materials (1952)

Bearing Materials (1956)

Determining Protein Nitrogen

Bearing Quality, Consumable Electrode Vacuum Melted (19

Bearing Steel Studs, Runners (Track) and Rigid Furring

Bearing Steel (1970) ASTM G56.2

Bearing Tile (1971) ANSI A76.1

Bearing Wall Buildings (Tech. Notes) (1969)

Bearing Wall Detailing ( $T$ ech. Notes) (1968)

Bearing Wall Detailing (Tech. Notes) (1968)

Bearing Wall Example of Shear Wall Design (Tech. Notes)

Bearing W all Tile and Std. Meth. of Sampling and Test.

Bearing Wall Tile (1973)

Bearing Wall Types and Properties (Tech. Notes) (1969)

Bearing Wall (For High Buildings) (Tech. Notes) (1970)

Bearing Wall (Tech. Notes) (1966)

Bearing, High Strength Stud (1973)

Bearings (Except Tapered Roller Bearings) Conforming to

Bearings (Oil Impregnated) (1970) ANSI G63.4

Bearings (Oil Impregnated) (1973) ANSI H9.27

Bearings (1972)

Bearings (1972) ANSI B3.15

Bearings (1973) ANSI B3. 18

Bearings (1973) ANSI H9.14

Bearings) Conforming to Basic Boundary Plans (1972) Ans

Bearings, Bolting Keys and Shafting (1974)

Beating Control (Simons Stain) (Pulp and Paper) (1973)

Lication of Plastic Poly

UL

$\mathrm{BCI}$

EIA RS156. B

BCI $* 1.48$

D2589

CR E.8

1. AACCH 80.75

ASTM C316

SSenger TRA

SAE

AACCH $356 \mathrm{~A}$

J356A

ASTM C598

CCTI T.113

SSI

ASTM A577

Uniform ICBO UBCS25.22

$\mathrm{SAE} \quad \mathrm{J} 7 \mathrm{CH}$

SAE J32

Transpa ASTM B588

F588

SAE J582A

ASTM D2344

SSI *7

ASTM D3202

CCTI Tl14

ASTM G39

SAE J565C

ASTM A578

ASTM A435

ASTM A609

/Et ASTM C293

d. Meth. ASTM D747

WWPA $\quad$ 30

ACI SP17

ACI $\quad 57.29$

APA BB-8

APA *3PDS2

ICEO

UBCS25. 18

AACCH $28-10$

AACCH 28-21

AACCH 28.20

AACCH 28-22

AACCH 44-15A

AACCH 44-11

ASTM G28

ASTM $\quad$ B23

ASTM D1194

ASTM D1194

ASTM C90

ASTM C129

Cl45

ICBO UBCS24-4

ICBO UBCS24-5

ICBO UBCS24-6

CR F.44

CR $\mathrm{G} .22$

CR J.56

$\mathrm{CR}$

CR

SAE

ASTM

ASTM

BIA

BIA

BIA

BIA

ICBO

ICBO

BIA

BIA

BIA

NSA

AFBMA

ASTM

ASTM B438

AFBMA 11

AFBMA 9

AFBMA 18

B-48

AMS5749

C645

$16 \mathrm{~A}$

$24 \mathrm{G}$

$24 \mathrm{G}$

D

UBCS 24.8

$24 \mathrm{H}$

24.

$24 \mathrm{~B}$

62

ASTM

AFBMA

NSA

AGMA 7

661 Bed (Fire Protection) (1973) 
ed Curled Hair for Cushioning Materials for Upholstery and Std. Meth. of Test for Slump of Face Glazing and Std. Nomenclature for

Std. Performance Requirements for Woven Text/ Std. Performance Requirements tor Woven Decorative t for Acid Resistance of Ceramic Decorations on Returnable
2) ANSI / Std. Meth. of Test for Differential Dyeing (1972) ANSI 2.20 Std. Spec. for Compression Joints for Vitrified Clay Rec. for Fire Protection for Std. Motor Positions for Agricultural Tractor 73) Spec. for Lineman's Climbing Equipment (Body Test for Heavy Cotton Fabrics for Manufacture of Hose and ng Rules (1974) Std. Procedure for Implants) (1973) Construction Spec. for Rifle formability of Attached Organic Coatings with Impact Wedge flexibility Determination of Hot Melt Adhesives by Mandrel Std. Rec. Pract, for Static Std. Rec. Pract. for Static - for Measurement of Adhesion and Flexibility by the Wedge Std. Meth. of Test for

Std. for Seamless Low Carbon Steel Tubing Annealed for welded and Cold Drawn Low Carbon Steel Tubing Annealed for (1973)

Std. Rec. Pract. for Conducting

Std for Fiberboard Crease

test for Annealing Point and Strain Point of Glass by Beam

Casing Joint Strength with Combined Internal Pressure and Std. Test Meth. for Determining Stiffness (Modulus of

ed Flash Controlled Low Carbon Steel Tubing Normalized for Test Meth. for Smoothness of Paper and Paperboard Std. Meth. for Preparation and Use of

72)

972)

ansi 011.13 Meth. for Determination of Dichloromethane and Alcohol.

Std. Meth. of Test for Alkyl Std. Spec. for Refined r Industrial Aromatic /

Std. Spec. for Industrial Grade Std. Meth. of Test for Acidity of Meth. of Analysis for Std. Spec. for Photographic Grade Semiquantitative Meth. of Analysis of

try) (1967)

Qualitative Meth. of Analsis of Vines and Ground Covers; Fruit Tree Grades; Small Fruits
Spec. for tions (1972T) Sid. Spec. for Copper Std. Spec. for Copper . Std. Spec. for Copper trip and Rolled Bar (19/ Std. Spec. for Copper-Cobalt . c, and Spectrochemical Analysis Of, and Physical Tests On Std. Spec. for Nuclear Grad t, Varnish, Lacquer and Related Products --Film Thickne/ Spec. for Steel Spring Wire, Meth. of Analysis of Alpha Amylase Activity, by Std. Meth. of Test for Coating Thickness by the cereal Chemistry) (1/ ustrial Waste Watel ron Volts (1954)

Meth. of Analysis of Radioactivity Std. Meth. of Test for Measurement of Rec. for Protection Agains e Measurement for Enclosed Helical, Herringbone and Spiral ling Tower Fan Drives (1972) Info Sheet for Spiral d Resistance of Ceramic Decorations on Returnable Beer and ec. Pract. for Determining Effect of Packaging on Food and Conditions for Laboratory Sensory Evaluation of Foods and Rec. Spec. for Steel Std. for Double Race or station Wagons, and Multipurpose Passenger Vehi/ Sid. for c Substrates (1974) Tentative Meth. of Test for -Pr) Based on Controlled Outside Diameter/ Std. Spec. for Procedure for Water Absorbency of articulate and Gaseous Fluorides in the Atmosphere (Sodium Flour and Semolina (Cereal Chemistry) (1962) Ether. ral and Log Skidder, Truck, Bus, Bead Seat, Industrial and or Tires and Rims for Motorcycle, Side Car, Mini Bike, and intenance and Rules of the Road/ Safety Std. for Regular stee/ Safety Std. for Structural Requirements for Regular braces,/ Safety Std. for General Requirements for Regular Test Procedures for Regula Safety Std. Definitions for Regula Test Procedures for the Safety Std. for Regular topping Power, Traction and Steering Stability for Regular ghts (1972) nary Dies/ Safety Std. for Lighting for Regula Std. Pract. for Preparation of Invitations for 8 and Shortenings by Distillation with Toluene (Cereal C) $\mathrm{ds}$ and Feedstuffs by Distillation with Toluene (Cereal C)
Bedding Applications (1973) ANS1 J8.8

Bedding Compounds on Metal Sash (1974)

/C. for Rubberiz ASTM

Bedding Sizes (Mattresses and Sheets) (1971)

Bedspreads (Institutional Textile) (1973)

Bedspreads, Pillows, and Bolster Fabrics (Institutional

Beer and Beverage Glass Containers (1972T)

Behavior of Cotton (Raw Fiber, Yarn and Textile Fabric)

/Th.

Bell End Poly(vinyl Chloride) (PVC) Pipe (1973) ANSI B7

Belt Conveyors (1972)

Belt or Chain Drive Centrifugal Fans (1966)

Belt Speed and Pulley Width (1971) SAE J720

Belt, Safety Strap, Lanyard, Pole and Tree Climber) (19

Belts (1942) ANSI L14.283

Std. Spec, and

Bench Calibration of Tank Level Gaging Tapes and Soundi

Bench Rest (Portable and Permanent) (1967)

Bend and Torsion Test. of Intramedullary Rods (Surgical

Std. Meth. of Test for Rec. Pract. for

Bend Tpparatus (1973)

Bend Test. of Bone Plates (Surgical Implants) (1973)

Bend Test. of Nail Plates (Surgical Implants) (1973)

Bend (Applicable to Solution Coil Coatings and Single C

Bendability of Pipeline Coatings (1972)

Bending and Flaring (1972)

Bending and Flaring (1972)

Bending F atigue Tests for Copper Alloy Spring Material

Bending Test (1972)

Bending (1972)

Bending (1972)

Bending) of Fiberboards (1972)

Bending, Double Flaring, and Beading (1972)

Bendtsen Tester) (1973)

Bent Beam Stress Corrosion Metal Specimens (1973)

Bentazon (Common Name for the Pest Control Chemical (19

Benzene Content of Cyclohexane by Gas Chromatography (1

Benzene Insoluble Matter in Creosotc (1973) AASHO T81,

Benzene Solubles in Wood (1973)

Benzene Sulfonate in Water (1968)

Benzene 485 (Nitration Grade) (1971) ANSI Z78.2

Benzene (1971) ANSI Z78.3

Benzene, Toluene, Xylenes, Solvent Naphthas, and Simila

Benzoic Acid in Flour (Cereal Chemistry) (1962)

Benzotriazole, $\mathrm{C}_{13} \mathrm{H}_{1} \mathrm{NHN}$ : N $(1,2,3)(1972)$

Benzoyl Peroxide in Flour (Cereal Chemistry) (1968)

Benzoyl Peroxide with Diaminophenylamine (Cereal Chemis

Berry, Currant, Grape, Asparagus); Lining Out Stock; Se

Beryllia Ceramics for Electronic and Electrical Applica

Beryllium Alloy Forgings and Extrusions (1972)

Beryllium Alloy Plate, Sheet, Strip, and Rolled Bar (19

Beryllium Alloy Rod and Bar (1971A) ANSI H7.7

Beryllium Alloy (Copper Alloy No. 175), Plate, Sheet, S

Beryllium Oxide Powder (1972) ANSI N140, / Spectrometri

Beryllium Oxide Powder (1972A)

Best Meth. for Measurment of Dry Film Thickness of Pain

Best Quality Music (1973)

Beta Amylase GBI (Cereal Chemistry) (1962)

Beta Backscatter Principle (1972)

(Beta Gamma) in Cereal Products-Emergency Monitoring (

Beta Particle Radioactivity of Industrial $W$ ater and Ind

Betatron Synchrotron Radiations Up to 100 Million Elect

Bevel Gear Drives (1973)

Std. for Sound

Bevel, Helical and Herringbone Gear Units for Water Coo

Beverage Glass Containers (1972T)

Beverage Products During Storage (1972)

/Th. of Test for

Std. Rec.

Beverages (1973)

Bi-Fold Closet Doors and Frames (1972)
Bi-Level Swivel and Rigid Industrial Ca

Pers (1973)

for Passenger Cars,

Biaxially Oriented Polyethylene (PEO) Plastic Pipe (SDR Bibulous Papers (1972)

Bicarbonate Coated Glass Tube and a Particulate Filter

Bicarbonate Meth. of Analysis of Ergot in Rye and Wheat

Bicycle Tires (1974)

Bicycle (1974)

Bicycles Including Selection, Purchase, Preparation, Ma

Bicycles Including Tests for Static Load, Frame, Fork

Bicycles Including Wheel Assembly, Chainguards, Saddle

Bicycles (1972)

Bicycles (1972)

Bicycles (1972)

Bicycles (1972)

Bicycles, Including Reflectivity, Headlights and Tailli

Bids and Detailed Spec. for Low and Medium Speed Statio

Bidwell-Sterling Meth. of Analysis of Moisture in Fat

Bidwell-Sterling Meth. of Analysis of Moisture in Fee
ASTM

ANSI

ANS

ANSI

ASTM

ASTM

ASTM

ASTM

FMS

AMCA

ASAE

EEI

ASTM

NRA

ASTM

ASTM

ASTM

ASTM

NCCA

ASTM

Std. for $S A E$

ASTM

SAE

.d. Meth. of

API

SAE

TAPP

ASTM

ANSI

ASTM

ASTM

Std TAPPI

ASTM

ASTM

ASTM

ASTM

AACC

ANSI

AACCH

ANSI

ASTM

ASTM

ASTM

ASTM

ASTM

ASTM

ASTM

NCCA

SAE

$\mathrm{AACCH}$

ASTM

AACCH

NCRPM

ACMA

AGMA

ASTM Std. R ASTM

ASTM

STDI

ANSI

TRA

ASTM

ASTM

TAPPI

ASTM

AACCH

TRA

Std. F TRA

BMA

BMA

BMA

BMA

BMA

BMA

BMA

BMA $6 / 6$

DEMA *1.24

AACCH 44.51

AACCH 44.50

D2376

C425

D2672

7.11

2407

AP. 2

D 181

B88.2

F 983

D3281

D3111

D2128

24.2 .8

C735

D 1464

\$210.2 
l Chemical Methyl 5(2,4-Dichlorophenoxy)-2-Nitrobenzoate Std. for Tires and Rims for Motorcycle, Side Car, Mini Std. Spec. for Deformed and Plain Std. Spec. for Stainless and Heat Resisting Steel d Worked, and Cold Worked Alloy Steel Forgings and Forging Std. Spec. for Molybdenum and Molybdenum Alloy Std. Spec. for Titanium and Titanium Alloy Bars and Std. Spec. for Phosphorus Deoxidized Copper Wire Bars, Sid. Spec. for Oxygen Free Electrolytic Copper Wire Bars, Std. Spec. for Lake Copper Wire Bars, Cakes, Slabs, td Spec, for Electrolytic Copper Wire Bars, Cakes, Slabs,

ec. for Chemically Refined Copper Wire Bars, Cakes, Slabs, (1971)

forms and Information Flow Used in Ordering, Shipping, and Rec. for Deep, Cast in Place Concrete (Storage) Rec. for Indoor General Storage (Solid, Palletized and
cal Control and Data Terminal Equipment Employing Parallel pec. for Hot Mixed, Hot Laid Tar Paving Mixtures for Base, Std. for

Handbook for Library Binding (Rec. for Good Book Handbook for Library

th. of Test for Density and Specific Gravity of Liquids by r Measurement of Neutron Flux and Spectra for Physical and s (1972) Std. Rec. Pract. for Experimental Test. for

packaging and Transportation of Radioactively Contaminated Std. for Program for
Std. for Industrial Engineering Terminology: hic Estimation of Higher Boiling Homologues of Chlorinated h. for Analysis of Environmental Materials Polychlorinated on Name for the Pest Control Chemical 2-(Ethylthio) -4,6(197) Std. Common Name for the Pest Control Chemical 2,4etry/ Std. Common Name for the Pest Control Chemical 2,4-

2) Summary of Meth. for

Meth. of Analysis for Baking Quality of Self Rising

Std. Spec. for Photographic Grade Sodium Meta .

Rec. Pract. for Hole Placement on Bulldozer (Dozer) End Std. Part

Std. Meth. of Test for Quantitative Extraction of Std. Meth of Test for Std. Meth. of Test for Kinematic Viscosity of Asphalts Std. Spec. for Homogeneous .1 2 and General Drainage ( 1 ) ork Drainage (1973) (1973) ANSI A 176.4 (1973) ANSI A17/ s (1973) Std. Spec. for Laminated Wall Std. Spec. for Homogeneous Perforated Sid. Spec. for Laminated Wall Perforated Std Meth of Test. Std. for Safety for Septic Tanks, Std. Meth. of Test for Density of Rec. Pract. for Reinforce Similar Drainage / Std. Spec. for Homogeneous Perforated for Inspection and Test. Agencies for Concrete, Steel and Std. Meth. of Test for Penetration of ornia Kneading Compa/ Std. Rec. Pract. for Preparation of
pactor (/ $\begin{array}{cc}\text { pactor (/ } & \text { Std. Meth. for Preparation of Test Specimens of } \\ \text { eth. of Test for Resistance to Deformation and Cohesion of }\end{array}$ Std. Meth. of Test for Resistance to Plastic Flow of Std. Meth of Test for Compressive Strength of Std. Meth. of Test for Percent Air Voids in a Compacted Std. Spec. for Mineral Filler for

for Requirements for Mixing Plants for Hot Mixed, Hot Laid Meth. of Test for Quantitative Extraction of Bitumen from Std. Meth. of Test for Steam Distillation of Std. Meth. of Test for Solubility of

) in Organic Solven/ and Woven Fabrics Saturated with sampling and Test. Felted and Woven Fabrics Saturated with $\mathrm{ng}$ and Structural Construction (Nonextruding and Resilient std. Meth. of Test for Distillation of Cut Back Asphaltic terproofing, and $\mathrm{R} /$ Sid. Definitions of Terms Relating to b]25. 1 Std. Spec. for Welded and Seamless d Seamless Steel Pipe for Ordinary Uses (/ Std. Spec. for
Rec. Pract. for the Density and Contrast Range of

Rec. Pract. for the Density and Contrast Range of

Std. Meth. of Test for Solvent Extractable Material in ansi C8.32 Sid. Spec. for Extra Heavy Duty Std. Meth. of Test for Relative Density of Sid. Test Meth. for Heating Loss of Carbon stating the Precision of ASTM Test Meth. Related to Carbon f Test for Discoloration of Orthodichlorobenzene by Carbon Std. Meth. of Test for Surface Area of Carbon Std. Meth. of Test for Tint Strength of Carbon Std. Spec. for Slate

Std. Meth. for Evaluation of and Test Formula for Carbon
Bifenox (1973) Bike, and Bicycle (1974)

Billet Steel Bars for Concrete Reinforcement (1972)

Billets and Bars for Reforging (1972) ANSI G81.13
Billets for High Strength at Elevated Temperatures (197

Billets for Reforging (1974)

Billets (1972) ANSI Z179.2

Billets, and Cakes (1972)

Billets, and Cakes (1972) ANSI H23.11

Billets, Ingots, and Ingot Bars (1973) ANSI H17.

Billets, Ingots, and Ingot Bars (1973) ANSI H17.2

Billets, Ingots, and Ingot Bars (1973) ANSI H23.12

Billiard Terms (1971)

Billiard Tournament Table and Accessory Equipment Specs

Billing of Merchandise Within the Hardgoods (Hardware)

Bin and Silo Wall Design and Construction (1965)

Bin Box Storage Up to $30 \mathrm{Ft}$. and Shelf Storage Up to 15

Binary Data Interchange (1973)

Binder, Leveling, and Surface Courses (1967)

Binding Post-Terminal Board, Electrical (1973)

Binding Practices) (1971)

Binding (Rec. for Good Book Binding Practices) (1971)

Bingham Pycnometer (1968) ANSI Z11.96

Biological Applications (1960)

Biological Compatibility of Metals for Surgical Implant

Biological Materials (Safety) (1973)

Biological Shielding in Nuclear Reactor Plants (1972)

Biomechanics (1972)

Biphenyls for Capacitor Askarels (1974)

Biphenyls (1974)

bis (Isopropylamino) Striazine) (1973)

bis (Isopropylamino)-6-Methoxy-S-Triazine-Prometon

bis (Isopropylamino)-6-(Methylthio)-S-Triazine-Prom

Biscuit and Cracker Flour Test. (Cereal Chemistry) (196

Biscuit Flour (Cereal Chemistry) (1962)

Bisulfite $\mathrm{Na}_{2} \mathrm{~S}_{2} 0_{5}$ (1972)

Bit Supports (1972)

Bit, Screwdriver, Tri Wing Recess (1972)

Bit, Screwdriver, Tri Wing, Spec. for (1972)

Bitumen from Bituminous Paving Mixtures (1972)

Bitumen (1970) ANSI A37.3

Bitumens) (1974) AASHO T201

Bituminized Fiber Drain and Sewer Pipe (1973) ANSI A176

Bituminized Fiber Drain and Sewer Pipe (1973) ANSI A176

Bituminized Fiber Perforated Pipe for Agricultural, Lan

Bituminized Fiber Perforated Pipe for Airport and Roadw

Bituminized Fiber Pipe for Septic Tank Disposal Fields

Bituminized Fiber Pipe for Septic Tank Disposal Fields

Bituminous Base Emulsions for Use as Protective Coating

Bituminous Coated Metal (1974) ANSI A 62.1

Bituminous Concrete in Place by Nuclear Meth. (1974)

Bituminous Concrete Overlays (Welded Wire Fabric) (1962

Bituminous Fiber Pipe Systems for Highway, Airport, and

Bituminous Materials as Used in Construction (1972) Ans

Bituminous Materials (1973) ANSI A37.1

Bituminous Mixture Beam Specimens by Means of the Calif

Bituminous Mixtures by Means of California Kneading Com

Bituminous Mixtures by Means of Hreem Apparatus (1971)

Bituminous Mixtures Using Marshall Apparatus (1973)

Bituminous Mixtures (1974) ANSI A37.103, Ashto T167

Bituminous Paving Mixture (1973)

Bituminous Paving Mixtures (1970) ANSI A37.41

Bituminous Paving Mixtures (1971) ANSI A37.102

Bituminous Paving Mixtures (1972)

Bituminous Protective Coatings (1970) ANSI A 109.11

Bituminous Road Materials (Tar, Petroleum Asphalt, etc.

Bituminous Substances for Use in Waterproofing and Roof

(Bituminous Type) (1971) ANSI A37.101

Bituminous Types) (1973) ANSI A37.113

(Bituminous) Products (1973) A ASHO T78, ANSI A37.45

Bituminous, and Other Organic Materials for Roofing, W

Black and Hot Dipped Galvanized Steel Pipe (1973) ANSI

Black and Hot Dipped Zinc Coated (Galvanized) Welded an

Black and White Films and Slides for Television (1970)

Black Oxide Treatment (Part Coating) (1973)

Black Pigments (1972)

Black Polychloroprene Jacket for Wire and Cable (1973)

Black Polychloroprene Jacket for Wire and Cable (1973)

Black Smoke (Ringelmann Meth.) (1973)

Black (1972)

Black (1972)

Black (1972A)

Black (1973)

Black (1973)

Blackboards (1967)

Blacks in Natural Rubber (Nr) (1973)
/ Chromatograp ASTM

Std. Met ASTM

ANSI

ASTM

ASTM

ASTM

ASTM

ASTM

ASTM

ASTM

ASTM

ISp ASTM

BCA

NWHA

FMI

EIA

Std. S ASTM

NSA

LBI

LBI

Std. Me ASTM
Rec. Fo NCRPM

ASTM

K6

A615

A314

A477

B385

B348

B379

B170

B4

B442

$* 2$

65.37

$8 \cdot 25 \mathrm{~N}$

RS408

D1753

192

$* 2$

D 1217

R23

F36 1

N14.3

N 18.9

Z94.1

D3303

D3304

K62.135

ANSI K62.144

ANSI K62.143

AACCH $\quad 10.30$

AACCH $10.31 \mathrm{~A}$

ANSI PH4.276

SAE

NSA

NSA

ASTM

ASTM

ASTM

ASTM

ASTM

ASTM

ASTM

ASTM

ASTM

ASTM

UL

ASTM

WRI

ASTM

ASTM

ASTM

ASTM

ASTM

ASTM

ASTM

ASTM

ASTM

ASTM

/Spec. ASTM

itd. ASTM

ASTM

ASTM

ASTM

Std ASTM

ASTM

ASTM

ASTM

SMPTE

SAE

ASTM

ASTM

ASTM

ASTM

ASTM

Std. Rec. Pract. for ASTM Sid. Metl., O

ASTM

ASTM

ASTM

ASTM

ASTM

J63

4005

4001

D2172

D2170

D 1861

D2417

D2418

D2312 
National Bureau of Standards KWIC Index of Engineering Standards

Std. Meth. for Evaluation of and Test Formula for Carbon Std. Classification System for Carbon ds (Cereal Chemistry) (1962) Std. Spec. for Mineral Fiber struction Buildings (1970)/ Std. Spec. for Mineral Fiber ion of the Thermal Resistance of Low Density Mineral Fiber Roller Turner Type (1972) Std. Performance Requirements for Woven
Meth. of Test for Resiliency and Thickness of Std. for Carbide $x$ and Emulsion Ex/ Std. Spec. for Asbestos Cement Shingle $\mathrm{g}$ an Optical / Std. Meth. for Spectrochemical Analysis of ggregates (Sand, Crushed Stone, Gravel and Air Cooled Iron Uniform Fire Code: Explosives and acture, Transportation, Storage, and Use of Explosives and t Radio Frequency Radiation Hazards in the Use of Electric Rec. Safe Pract. for Thermal Spraying and 14.129 ace Preparation of Steel and Other Hard Materials by Water Test Meth. for Alkali in

Test Meth. for Hydrogen Peroxide in Drapery (Curtain) Fabrics Handwashable at 105 Deg. F-No lass Fiber Curtain Fabrics Handwashable at 105 Deg. F-No or Blend Fabrics, Commercial Wash-Whites (With Chlorine Test Meth. for Ash Content of

Test Meth. for Fluidity of Dispersions of Cellulose Form Test Meth. for Noncotton Content of Test Meth for Reflectance, Blue, and Whiteness of Test Meth. for $\mathrm{pH}$ of the Water Extracted from Test Meth. for Absorbency of rs, Exits and Occupant Loads (Buildings, Reviewing Stands, aces of Assembly (Theaters, Reviewing Stands, Grandstands, nhydride Meth. for Generation of Chlorine Dioxide in Flour Test Meth. for Cotton and Linen Textile Colorfastness to Test Meth. for Colorfastness of Textiles to

Sample Meth. of Textile Tensile Loss by Retained Chlorine Std. Meth. of Test for Oil Migration or Plasticizer 1 and Industrial Use Garments (Clothing), 100\% Man Made or $\mathrm{I}$ and Industrial Use Garments (Clothing), 100\% Man Made or Std. Spec. for Petroleum for ce of Glossy Smooth Coatings of Petroleum Wax or Wax Based Std. Meth. of Test for Compatibility of Fuel Oil

for Measuring Flat Spring Test for Hot Tack of Wax Polymer pose, Flush Head, Self Locking) (1972/ Std. for 450 Deg. F Self Locking Serrated Head

Std. for 450 Deg. F Closed End Self Locking Serrated Head Std. for 450 Deg. F Self Locking Elliptical Head Std. for Protruding Head, Locked Spindle td. for Bulbed Mechanically Locked Spindle Protruding Head Std. for Bulbed Mechanically Locked Spindle Self Plugging td. for Bulbed Spindle Mechanically Locked Protruding Head for Bulbed Mechanically Locked Spindle 100 Deg. Flush Head for Bulbed Mechanically Locked Spindle 100 Deg. Flush Head Std. for Drive Pin Sid. for Break Mandrel Closed End std. for Structural Flush Break Pull Mandrel Self Plugging Sandwich Panel Lightweight Selflocking and Nonselflocking Sandwich Panel Lightweight Selflocking and Nonselflocking ight Floating, Sand wich Pane/

rpose, Protruding Head Self Locking)/ rature, Protruding Head, (1972) rature, Flush Head, Self Locking) (1972) ht, Protruding Head, Self Locking) (1/ ht, Millable Head Self Locking) (1972/ ng) (1972) Std. for Insert, Molded In, Std. for Fastener Std. for Fastener Std. for Fastener Spec, for Fastener Std. for

architectural Woodwork) (1973)

Quality Stds. for

tairwork and Handrails, Exterior Frames and Sash, Screens, (1972)

for Douglas Fir, Western Hemlock, Sitka Spruce Doors and rel Meth. for Measuring Std. Meth. for Water Immersion Test for Resistance to Std. Meth. of Test. Wood Preservatives by Laboratory Soil Std. for Terminal Std. Spec. for Creosoted End Grain Wood Std. for Laminated Hardwood g Nume/ Std. for Interchangeable Perforated Tape Variable 4) Interchangeable Perforated Tape Std., Variable a-Dielectric Capacitors (1971) / i C59.27 oints $(1970)$ Std. Spec. for Natural Std. Spec. for Natural Muscovite erties of Oil Soluble Wood Preservatives by Using W/ Soil Std. Meth. of Test. Cellular Glass Insulating Uniform Building Code Std. for Gypsum Partition Tile or Std. for Strip-High Temperature Terminal utomotive Trim Materials/ Std. Test Meth. for Determining Coil Co/ Test Meth. for Determining Pressure Marking and Interior Floors Exposed to W/
Blacks in Styrene-Butadiene Rubber (SBR) (1973)

Blacks Used in Rubber Products (1973) ANSI J8.6

Blank Meth. of Analysis of Methoxychlor Residues in Foo

Blanket Thermal Insulation for Wood Frame and Light Con

Blanket Type Building Insulation (1970) ANSI Z98.35

Blankets (Institutional Textile) (1973)

Blankets (Textile) (1973)

Blanks and Cutting Tools, Single Point, Carbide Tipped,

Blanks for Tipping Circular Saws (1972)

Blanks to Be Used as Panels in Weathering Tests of Late

Blast Furnace Iron by the Point to Plane Technique Usin

Blast Furnace Slag) (1973)

Blasting Agents (1973)

Blasting Agents (1973) ANSI Z271.1

Blasting Caps (1971) ANSI C95.4

Blasting Equipment (1973)

Blasting Prior to Coating or Recoating (1972)

Bleach Baths Containing Hydrogen

Bleach (Institutional Textile) (1973)

Bleach (InstitutionaI Textiles) (1973)

G Code Std. for Concrete a ICBO

/Code for the Manuf NFPA Safeguards Agains IME

Bleach) 190 Deg. F, Colors 160 Deg.

Bleached Cellulosic Textiles (1971) ANSI L 14.11

Bleached Cotton Textiles (1972) ANSI L14.128

Bleached Fabric (1972) ANSI L 14.175

Bleached Textiles (1969) ANSI L14.116

Bleached Woven Cloth (1972) ANSI L14.115

Bleachers, and Grandstands) (1973)

Bleachers, etc.) (1973)

Bleaching Experiments in Laboratories (Cerea

Bleaching with Chlorine (1972) ANSI L14.57

Bleaching with Peroxide (1972) ANSI L14.146

Bleaching (1971) ANSl Ll 4.180

Bleed Out of Preformed Sealing Tapes (1974)

Blend Fabrics, Commercial Wash-Whites (With Chlori

Blend Fabrics, Drycleanable Only (1973)

Blending with Creosote (1971)

Blends Applied to Paper and Paperboard (1973)

Blends by Spot Test (1972) ANS! Z11.315

Blends (1972)

Blind Internally Threaded, External Sleeve, General Pur

Blind Nut (1974)

Blind Nut (1974)

Blind Nut (1974)

Blind Rivet (1972)

Blind Rivet (1974)

Blind Rivet (1974)

Blind Rivet (1974)

Blind Rivet (1974)

Blind Rivet (1974)

Blind Rivets (1973)

Blind Rivets (1973)

Blind Rivets, Type 2A (1973)

Blind Threaded Molded in Insert (1974)

Blind Threaded Molded in Insert (1974)

Blind Threaded, Self Locking, Nonself Locking, Light We

(Blind, Internally Threaded, External Sleeve, General Pu

(Blind, Internally Threaded, External Sleeve, High Tempe

(Blind, Internally Threaded, External Sleeve, High Tempe

(Blind, Internally Threaded, External Sleeve, Light Weig

(Blind, Internally Threaded, External Sleeve, Light Weig

(Blind, Internally Threaded, External Sleeve, Self Locki

Blind, 100 Deg. Flush Head, Locked Spindle Rivet (1972)

Blinds and Shutters Using Stile and Rail Construction (

Blinds and Shutters, Flush, Stile, and Rail Doors, Fact

Blinds (1972)

Blister Resistance of Coated Paper in Heatset Printing

Blistering, Wrinkling, Loss of Adhesion, or Other Failu

Block and Pipe Thermal Insulation (1973)

Block Cultures (1970)

Block Electrical Stud (1974)

Block Flooring for Interior Use (1970) ANSI 010.1

Block Flooring (1971)

Block Format for Contouring and Contouring-Positionin

Block Format, for Numerically Controlled Equipment (197

Block Mica and Mica Films Suitable for Use in Fixed Mic

Block Mica and Thins Based on Visual Quality (1971) Ans

Block Test for Quality Control of Glue Bonds in Scarf J

Block Test: Std. Meths. for Test. the Preservative Prop

Block (1972)

Block (1973)

Block, Base Insulating (1973)

Blocking Resistance and Associated Characteristics of a

Blocking Resistance of Organic Coatings When Applied to

Blocks for Exposed Platforms, Pavements, Driveways, and
ICBO

AWS

ASTM

ASTM

ASTM

T ASTM

ANSI

ANS

ANSI

ASTM

TTM

BO

NACE

AATCC

AATCC

ANSI

ANSI

ANS1

AATCC

AATCC

AATCC

AATCC 110

AATCC 81

AATCC

ICBO

ICBO

AACCH

AATCC

AATCC

ANSl

ASTM

/Esistan ASTM

ASTM

Meth. TAPPI

NSA

NSA

NSA

NSA

NSA

NSA

NSA

NSA

Std. NSA

Std. NSA

IFI

IFI

Std. for NSA

Std. for NSA

NSA

NSA

NSA

NSA

NSA

NSA

NSA

NSA

A WI

FHDA

TAPPI

NCCA

ASTM

ASTM

NSA

ASTM

HPMA

EIA

NSA

ASTM

ASTM

ASTM

NWMA

ASTM

ICBO

NSA

SAE

NCCA

ASTM

D3191

D1765

$60-40$

665

653

L24.2.9

4

4.42

E485

UBCS $26 \cdot 2$

UFC *2ART II

495

(

RP.01.72

98

L24.1.9

L24.1.3

L24.4.13

97

UBC $* 3 \cdot 33$

UFC $* 2 A R T 26$

8-30

101 
Std. for Precision In. Gage Std. Meth. of Test for Polystyrene Std. for Fire Doors, Windows, Shutters, and Glass Meth. for Ink Absorbency of Std Tooling Nomenclature for Solid Die Two ination by Manual Meth. (197/ Std. Meth. for Sampling Gas ination by Automatic Particle/ Std. Meth. of Sampling Gas Meth. of Test for Drop Impact Resistance of Polyethylene

Std. Test. Procedure for the emoval or Conveying (1973) / Sid. for the Installation of Safety Std. for Vacuum Cleaning Machines and Rec. Pract. for Electric Std. for or Removal (1973) ng Appliances Rated at $60 /$ Uniform Building Code Std. for Safety Std. for Electric Fans for Compatible Operation of Forage Harvesters, Wagons and $s$ for Static Load, Frame, Fork, Steering Assembly and Tire Std. Meth. for Chemical Analysis of Copper Phthalocyanine lutions; Isopropyl Alcohol Saturated with Gasoline; Methyl 1971) Sid. Analytical Meth. for Determining Bromophenol 1971) Std. Analytical Meth. for Determining Methylene metric Meth. for Measuring Residual C/ Std. for Methylene th. of Test for Hydrogen Sulfide in Natural Gas (Methylene ibers, Whole or Ground Cereal/ Meth. of Urease Bromthymol 175 Test Meth for Reflectance, of Test for Penetration Resistance of Pipeline Coatings Meth. for Measuring Color of Paper and Paper gid Boards (1970) ANSI C83/ Std. Spec. for Printed Wiring a Std. General Document for Printed Wiring
(National

(National

Spec. for Fire Barrier Gypsum Suggested Guide Lines for Printed Wiring ofs (Wood Shakes and Shingles with Asbestos Felt or Gypsum Design Guide for Wood Composition Std. Spec. for Insulating

72) 72) ers to Receive Screw Attached Gypsum Wallboard and Backing Std. Spec. for Gypsum Backing Std. Spec. for Mineral Fiber Roof Insulation Std. Spec. for Perlite Thermal Insulation Uniform Building Code Std. for Gypsum Backing Uniform Building Code Std. for Gypsum Sheathing form Building Code Std. for Water Resistant Gypsum Backing r Fiberboard Nail Base Sheathing and Structural Insulating Std, for Cellulosic Fiber Insulating and Rigid Furring Channels for Screw Application of Gypsum adhesion to Porous Substrates (Subjective Ratings) (Paper, Std. for Binding Post-Terminal

design Values, Framing Rec., Properties, Weight, Stress of board Dimensions and Tolerances Single and Two Sided Rigid Std. for Safety for Printed Wiring

$\mathrm{n}$ of End Product Description Data (Tooling, Printed Wiring actice Guide for Aids to Security (Safety) of Personnel on td. and Rec. Pract. for Buoyancy in the Event of Swamping Rec. Pract. for Sid. for

Rec. Pract, and Std Covering Non Commercial Rec. Pract. for Outboard Motor reational Type Vehicles, Counter Balanced Fork Lift Truck, d Rec. Meth. of Identifying AC and DC Electrical Wiring on Rec. Pract. for Aluminum Applications for ation of Power Transmission Units Penetrating the Hull for Std. and Rec. Pract. for Level Flotation of on Devi/ Rec. Pract. and Std. for Electronic Equipment on Std. for Electrical Bonding of Direct Current Systems on Rec. Pract. and Std. for Cathodic Protection Systems on sign, Construction and Installation of Battery Chargers on

, Size, and lnstallation of Propeller Shafting Systems for ipment for Anchoring, Mooring, Docking, Towing and Liftin ly Installed Fuel Systems for Inboard and Outboard Powered r Outboard Motor Boat Transom and Motor Well Dimensions on

of Gasoline Fueled Inboard, Liquid Cooled Marine Engines Rec. Pract, and Stds. for Marine Engine Mountings Rec. Pract. for Outboard Motor Dimensions nd Stds. for Location and Visibility of Navigation Lights covering Design and Construction of Cockpits and Scupper stating the Horsepower Rating of Inboard Gasoline Engines

Holding and/or Treatment Devices for Marine Toilet Waste uel Systems and Portable Containers for Flammable Liquids

s, Outboard Water Strainers, Water Scoops and Drain Plugs ted) Inboard Liquid Cooled Marine Engines and Auxiliaries

oled Marine Engines and Auxiliaries (Diesel and Gasoline)

ncluding Their lnstallation, Operation and Maintenance (On

of Exhaust Systems for Propulsion and A uxiliary Machinery d Installation of Plumbing. Heati/ Std. for Mobile Homes:
Blocks for Length Measurement (Thru 20 In.) (1973)

Blocks in Sbr Rubbers and Latices (1974)

Blocks (1973) ANSI A2.7

Blotting Paper (1972)

Blow Cold Headers (1972)

Blow Down Systems and Components for Particulate Contam

Blow Down Systems and Components for Particulate Contam

Blow Molded Containers (1970)

Blow Off Pressure of Composite Cans (1973)

Blower and Exhaust Systems for Dust, Stock, and Vapor $\mathbf{R}$

Blower Cleaners (1973) ANSI C 33.36.1

Blower Motor Switch for Vehicles (1971)

Blower Pipe and Connecting Flange Dimensions (1972)

Blower, Fan, and Exhaust System for Dust, Stock and Vap

(Blower, Humidifier, Evaporative Cooler, and Air Filteri

Std. Dimensions A

Blowers (1972)

Blowout (1972)

Blue and Green Pigments (1973) Ts for Regular Bicycles Including Test

Blue and Green; Mineral Oil; Urease Tablets; Xanthyrol)

Blue Indicator, $1 \%$ Content of Reagents and Indicators (

Blue Indicator, 1\% Content of Reagents and Indicators (

Blue Meth. for Measuring Thiosulfate and Silver Densito

Blue Meth.) (1970) ANSI Z77.10

Blue Test Paper for Analysis of Urea in Cloth or Sack F

Blue, and Whiteness of Bleached Fabric (1972) ANSI L14

Blunt Rod) (1972)

Board by Hunter L, A, B Colorimetry (1972)

Board Dimensions and Tolerances Single and Two Sided Ri

Board Electric Connectors (1974) ANSI C83.88, Ipc C-405

Board Inspection Code) Manual for Boiler and Pressure V

Board Membrane (1972T)

Board Plants (1972)

Board Repairs (1973)

Board Underlayment) (1973)

Board Wall Panels (1973)

/Td. for Special Purpose Ro

Board (Cellulosic Fiber), Structural and Decorative (19

Board (Cellulosic Fiber), Structural and Decorative (19

Board (1971)

Board (1972)

Board (1972)

Board (1972)

Board (1973)

Board (1973)

Board (1973)

Board (1973)

Board (1973) ANSI A 149.1

Board (1974)

/. for Installation of Steel Framing Memb

Board, Cloth, etc.) (1973)

Board, Electrical (1973)

Board, Timber, Lumber, Siding, Framing, Decking, Floori

Boards (1970) ANSl C83.98

Boards (1973) ANSI C 33.46

Boards) in Numeric (Digital) Form on Punched Cards, Mag

Boat Deck and for Reboarding from the Water (1973)

Boat Flotation) (1973)

Boat Hull Identification Numbers (1973)

Boat Load Capacity (Under $26 \mathrm{Ft}$. in Length) (1973)

Boat Trailer (1972)

Boat Transom and Motor Well Dimensions on Boats (1974)

Boat, Baggage, Special Type Trailer, Mobile Home, Garde

Boats and Engines (Color Code) (1973)

Requirements an

Boats and Yachts (1971)

Boats Equipped with Outdrives, Jet Drives and/or Bow Th

Boats When Swamped (1973)

Boats (Audio Device, Automatic Pilot, Depth Determinati

Boats (1972)

Boats (1972)

Boats (1973)

Boats (1973)

Boats (1973)

Boats (1973)

Boats (1974)

(Boats) and Auxiliaries (1972)

(Boats) (1971)

(Boats) (1972)

(Boats) (1972)

(Boats) (1972)

(Boats) (1972)

(Boats) (1972)

(Boats) (1972)

(Boats) (1972)

(Boats) (1972)

(Boats) (1972)

Boats) (1973)

(Boats) (1973)

/Pract, and Stds, for Safe Installation

Rec. Pract, and Std. for the De

/Guide to Design, Construction, Materials

IIon and Installation of Fittings and Equ

Nstruction and Installation of Permanent Rec. Pract. Fo

/Design and Construction

Rec. Pract. a

Rec. Pract and Std

Rec. Pract for Determining and A

Rec. Pract. and Std. Covering Sewage / Construction and Stowage of Portable F lon of Seacocks. Through Hull Connection

/Onstruction of Diesel (Compression Igni

$/ \mathrm{Td}$. Covering the Installation of Air Co

Lammable (Combustible) Gas Indicators I

Pract. and Stds. for Safe Installa
Construction Requirements, an

ANSI

ASTM

NFPA

TAPPI

IFI

ASTM

ASTM

ASTM

CCTI

NFPA

UL

ASAE

ICBO

UL

ASAE

BMA

ASTM

AAC

CR

CR

ANSI

B89.I.9

D3314

T431SL

118

308

F327
D2463

91

1017

J235

S347T

UBCS 10.3

507

S328.1T

$6 / 4$

D3256

28.91

R.55

R. 65

ASTM D2725

AACCH $28-87$

AATCC 110

ASTM G17

TAPPI

IPC

EIA

NBBPV

ASTM

FMS

IPC

ICBO

ASTM

ASTM

ANSI

ASTM

ASTM

ASTM

ICBO

ICBO

ni ICBO

ICBO

USC

ASTM

NSA

WWPA

IPC

UL

IPC

ABYC

$A B Y C$

$A B Y C$

ABYC

ABYC

TRA

$A B Y C$

ABYC

$A B Y C$

ABYC

$A B Y C$

$A B Y C$

ABYC

$A B Y C$

$A B Y C$

ABYC

$A B Y C$

ABYC

$A B Y C$

$A B Y C$

$A B Y C$

ABYC

$A B Y C$

$A B Y C$ 
) (1973) Spec, for Lineman's Climbing Equipment radionuclides Rec. for Maximum Permissible hing) (1972) Std. for ermining Resistance to Snagging and Abrasion of Automotive (National Board Inspection Code) Manual for

(l Std. Spec. for Electric Resistance Welded Carbon Steel Std. Spec. for Seamless Medium Carbon Stee r Electric Resistance W'elded Carbon Molybdenum Alloy Steel Std. Spec. for Seamless Carbon Molybdenum Alloy Steel Safety Std, for Oil Fired

Temperature Service Conforming to ISO Recommendations for ace Explosions in Fuel Oil and Natural Gas Fired Watertube urnace Explosions in Pulverized Coal Fired Multiple Burner

.7 Std Spec for Seamless Carbon Steel

Std. Spec. for Electric Resistance Welded Carbon Steel td. Spec. for Seamless Ferritic and Austenitic Alloy Steel s (1973) ANSl B 12/ Std. Spec. for Welded Austenitic Steel inless and Heat Resisting Steel Bars and Shapes for Use in Std. for Gas Fired High Pressure Steam and Hot Water Std. for Gas Utilization Equipment in Large Std. for Gas Fired Single Firebox

Std. for Gas Fired Low Pressure Steam and Hot Water sting Procedures and Maintenance of Steam Safety Valves on Std. Spec. for Refractories for lncinerators and Water Fuel Cutoffs and Automatic Feedwater Regulators for Meth. for Rapid Gas Chromatographic Estimation of Higher (1973) Sid. Spec, for Low servative Std. Spec. for for High
. Tracking Tests in A ve Solutions Such as Pent/ pract. for Performing Stress Corrosion Cracking Tests in A

8 Chromatography (1973) Tent. Meth, of Test for aste) of Corn Starch Which Is Gelatinized When Heated in $A$ requirements for Woven Decorative Bedspreads, Pillows, and hread (1973) 1973)

Std. for Std. for Std. for Sid. for Std. for istant (1973) Std. for Std. for Std. for Std. for

Std. for Titanium Alloy Stump Type Protruding Head Shear Std. for Titanium Alloy Stump Type 100 Deg. Head Shear ull Type, Titanium Alloy (1973)

1 Type, Titanium Alloy (1973)

Pull Type, Titanium Alloy (1973) um Alloy (1973)

ize, Pull Type, Titanium Alloy (1973)

tanium Alloy (1973)

versize, Pull Type, Titanium Alloy (1973)

, Titanium Alloy (1973)

Titanium Alloy (1973)

, Titanium Alloy (1973) Std. for Hexagon Head Modified Short Thread Shear Std. for Full Threaded, $160 \mathrm{KSI}$ Steel, Drilled Head Std for Shear Hexagon Head Std. for Self Locking, Hexagon Head Shear 73)

Close Tolerance Head and Shank, 160,000 psi Short Thread Std. for

ksi (1973)

ue, 180 KSI (1973)

ad, Self Locking and Nonlocking (1972)

ead, Self Locking and Nonlocking (1972)

s, Self Locking and Nonlocking (1972)

d, Self Locking and Nonlocking (1972)

Long Thread, Self Locking and Nonlocking (1972/ Short Thread, Self Locking and Nonlocking (19/ Recess, Titanium Alloy $6 \mathrm{Al}-4 \mathrm{~V}$, Self Locking An/ Recess, Alloy Steel, Self Locking and Nonlocki/

\section{ze (1973)}

(1973)

y Steel, 160,000 psi (1973)

alloy Steel, Long Thread, Self Locking and Nonl/

alloy Steel, Short Thread, Self-Locking and Non/ a286 Cres, Short Thread, Self Locking and Nonlo/ erance, Alloy Steel, Short Thread, Nonlocking (/ erance, A286 Cres, Short Thread, Nonlocking (19/ Titanium Alloy, Short Thread, Self Locking

Std. for Machine
(Body Belt, Safety Strap, Lanyard, Pole and Tree Climber Body Burdens and Maximum Permissible Concentrations of Body Measurements for the Sizing of Girls' Apparel (Clo Bodycloth (1972) Boiler and Pressure Vessel Inspectors (1973)

Boiler and Superheater Tubes for High Pressure Service

Boiler and Superheater Tubes (1973) ANSI B125.10

Boiler and Superheater Tubes (1973) ANSI B125.13

Boiler and Superheater Tubes (1973) ANSI B125.9

Boiler Assemblies (1973)

Boiler Construction (1972)

Tubular Products for High

Boiler Furnaces with One Burner (1972) /Ention of Furn

Boiler Furnaces (Fire Protection) (1973) /Vention of F

Boiler Tubes for High Pressure Service (1973) ANSI Bl 25

Boiler Tubes (1973) ANSI B125.6

Boiler, Superheater, and Heat Exchanger Tubes (1973) an

Boiler, Superheater, Heat Exchanger, and Condenser Tube

Boilers and Other Pressure Vessels (1972) ANSI G81.29

Boilers (Inputs Not Over 400,000 Btu Per Hr.) (1972)

Boilers (1971)

Boilers (1971)

Boilers (1972)

Boilers (1972)

Boilers (1972) ANSI A111.8

Boilers (1974)

/Re Hazards and Protection Rec. for Low

Boiling Homologues of Chlorinated Biphenyls for Capacit

Boiling Hydrocarbon Solvent for Oil Borne Preservatives

Boiling Hydrocarbon Solvent for Preparing Oil Borne Pre

Boiling Magnesium Chloride Solution (1973)

Boiling Point of Engine A thtifreezes (1972) ANS1 D14.1

Boiling Range Distribution of Petroleum Fractions by $\mathrm{Ga}$

Boiling W ater Bath (1973)

$/$ tor $\mathrm{D}$

Bolt-Close Tolerance, Hexagon Head, Titanium, Short T

Bolt-Internal Wrenching, Steel 1/4-28 Thru 1-1/8.12(

Bolt-lnternal Wrenching, Steel, 1.2500-12 (1973)

Bolt-Internal Wrenching, Steel, 1.3750-12 (1973)

Bolt-Internal Wrenching, Steel, 1.5000.12 (1973)

Bolt-Machine, Hexagon Head, Nonmagnetic, and Heat Res

Bolt-Shear, Close Tolerance (1973)

Bolt-TEE Head (1973)

Bolt-100 Deg. Close Tolerance, High Strength (1973)

Bolt Lock (1974)

Bolt Lock (1974)

td. for Bolt Lock, Shear, Protruding Head, Std. and Oversize, $P$

Std. for Bolt Lock, Shear, $100 \mathrm{Deg}$. Head, Std. and Oversize, Pul

Std. for Bolt Lock, Tension, Protruding Head, Std. and Oversize,

Std. for Bolt Lock, Tension, Protruding Head, Stump Type, Titani

Std. for Bolt Lock, Tension, $100 \mathrm{Deg}$. Crown Head, Std. and Overs

Std. for Bolt Lock, Tension, $100 \mathrm{Deg}$. Crown Head, Stump Type, Ti

Std. for Bolt Lock, Tension, 100 Deg. Head (MS20426), Std. and O

Std. for Bolt Lock, Tension, $100 \mathrm{Deg}$. Head (MS20426), Stump Type

Std. for Bolt Lock, Tension, 100 Deg. Head (MS24694), Pull Type,

Bolt Lock, Tension, 100 Deg. Head (MS24694), Stump Type

Bolt (1972)

Bolt (1972)

Bolt (1973)

Bolt (1974)

Bolt (1974)

Bolt, Brazier Head, Torq Set and Hi Torque, $180 \mathrm{KSI}$ (19

Std. for

Std. for Bolt, Flat, 100 Deg. Head, Torq Set and Hi Torque, 180

Std. for Bolt, Flat, 100 Deg. Reduced Head, Torq Set and Hi Torq

Std. for Bolt, Hex Head, Close Tolerance, Alloy Steel, Long Thre

Std. for Bolt, Hcx Head, Close Tolerance, Alloy Steel, Short Thr

Std. for Bolt, Hex Head, Close Tolerance, A286 Cres, Long Thread

Std. for Bolt, Hex Head, Close Tolerance, A286 Cres, Short Threa

Std. for Bolt, Hex Head, Close Tolerance, Titanium Alloy (1972)

Std. for Bolt, Hex Head, Close Tolerance, $6 \mathrm{Al}-4 \mathrm{~V}$ Titanium Alloy,

Std. for Bolt, Hex Head, Close Tolerance, 6Al-4V, Titanium Alloy

Std. for Bolt, Pan Head, Close Tolerance, Short Thread, Tri Win

Std. for Bolt, Pan Head, Close Tolerance, Short Thread, TWl Wing

Std. for Bolt, Shear, Hexagon Head, 180 KSI (1973)

Std. for Bolt, Taper Shank, Shear Type, Protruding Head-Oversi

Std. for Bolt, Taper Shank, Shear Type, Protruding Head (1973)

Std. for Bolt, Taper Shank, Shear Type, 100 Deg. Head-Oversize

Std. for Bolt, Taper Shank, Shear Type, 100 Deg. Head (1973)

Std. Part Bolt, 100 Deg. Flush Tension Head Hi Torque Recess Allo

Std. for Bolt, 100 Deg. Head, Tri Wing Recess, Close Tolerance.

Std, for Bolt, 100 Deg. Head, Tri Wing Recess, Close Tolerance, (1972)

Std. for Bolt, 100 Deg. Head, Tri Wing Recess, Close Tolerance, (1972)

Std. for Bolt, 100 Deg. Head, Tri Wing Recess, Close Tolerance,

Std. for Bolt, 100 Deg. Head, Tri Wing Recess, Close Tolerance,

Std. for Bolt, 100 Deg. Reduced Head, Tri Wing Recess, Close Tol

Std. for Bolt, 100 Deg. Reduced Head, Tri Wing Recess, Close Tol

Std. for Bolt, $100 \mathrm{Deg}$. Reduced Head, Tri Wing Recess, Close To
onlocki/ Bolt, $100 \mathrm{Deg}$. Tri Wing Recess, Close Tolerance, 6Al-4V
EEl

USC

$\mathrm{SAE}$

NBBPV

ASTM A226

ASTM A210

1. Fo ASTM A250

ASTM A209

UL

726

A 520

85

6.2

A 192

A 178

A 213

A249

A 479

Z21.59

Z21.33

Z21.52

Z21.13

LPD 12.59

C64

12.37

D3303

D3225

D2604

G36

D1120

D2887

C. 42

L24.1.2

653

144

172

174

176

1003

464

28

333

2605

2705

2406

2506

2005

2206

2125

2325

2115

2315

2105

2306

1103.20

$563-572$

1303-20

1223

1202

1982

428

1972

1992

6604-20

6203-20

6704.20

6303.20

1266-70

6804. 20

6403.20

5200-06

5000.6

1953

1729

1728

1725

1724

583

$4104 \cdot 16$

4204. 16

4304. 16

$4400 \cdot 16$

$4500 \cdot 16$

4703.16

4803. 16

4600-16 
ght / Uniform Building Code Std. for Timber Connector and ode Std. for High Strength Steel Bolts, Nuts, Washers, and or Extraneous Matter Meth. of Analysis (Cereal Chemistry) for Design of Components: Enclosed Gear Drives-Bearings, ature Service (1/ Std. Spec. for Alloy Steel Turbine Type ansi $\mathrm{G} 38.4$ Std. Spec. for Alloy and Stainless Steel , and Fittings for Low Temper/ Std. Spec. for Alloy Steel d Strength, Materials with Expansion Coef/ Std. Spec. for a) Tensile Strength, Hardened / Spec. for Low Alloy Steel ened and Tempered, Roll Threaded (1973) 1973)

oll Threaded (1973)

in. Series (1971) Spec, for Low Alloy Steel

Spec. for Titanium Alloy Spec. for Titanium Alloy Std. for Design Parameters for ansi E38./ Std. Spec. for High Strength Nonheaded Steel Std. Spec. for Carbon and Alloy Steel Nuts for Std. Spec, for Quenched and Tempered Alloy Steel uts and Plain Hardene/ Std. Spec. for High Strength Steel Specifying Rock

Spec. for Structural Steel Joints Using ASTM A325 or A490 Medium High Carbon Cold Heading Quality for Hexagon Head oy Steel Wire, Alloy Cold Heading Quality for Hexagon Head Std. Fastener Code (A erospace Lockbolts, Rivets, ng Code Std. for Timber Connector and Bolted Joints, Drift

e Treatment, Poles, Piles, Spaced Columns, Joints, Screws, in/ Western Woods Use Book: Timber Fasteners (Connectors Uniform Building Code Std. for High Strength Steel bs and Handles; Exit Devices, Pulls, Push and Kick Plates;

oss Calorific Value of Solid Fuel by the Isothermal Jacket shortenings (Cereal Chemistry) (1962) al Chemistry) (1962)

nuity of Steam Turbine Oil Oxidation Stability by Rotating 1972) taining 2,6-Ditertiary-Butyl Para-Cresol by Rotating ) Test Meth. for Internal Test Meth. for ing Surface Strength of Coated Papers Using the Interfiber d Laminate/ Std. Meth. of Test. Delamination, Strength of Strength of Bond, Appearance, and Shrinkage Propensity of Std. Spec. for Performance of Test Meth. for Bond Strength of Std. Rec. Pract. for Atmospheric Exposure of Adhesive Std. Test Meth. for Permanence of Adhesive Std. Test Meth. for Flexural Strength of Adhesive id. Rec. Pract. for Determining the Strength of Adhesively td. Rec. Pract. for Determining the Strength of Adhesively 1) An/ Std. Meth. of Test for Measuring the Wear Life and Uniform Building Code Std. for Exterior Plaster Liquid nd Materials for Use in Flammable Anesthetizing Locations (1972) ANSI C33.8

type) (1972) ANSI A 111.29

ater Mass of Material (1970) ANSI Z Std. for Safety for Grounding and Std. for Electrical Std. Meth. of Test for Cold Std. Rec. Pract, for ec. Pract. for Preparation of Metal Surfaces for Adhesives for Preparation of Surfaces of Plastic Prior to Adhesive Loss Prevention Data on

of Conducting Shear Block Test for Quality Control of Glue Std. Meth. of Test for Strength Properties of Adhesive Std Meth. of Test for Resistance of Adhesive Std. Test. Meth. for Impact Strength of Adhesive of Test for Cleavage Strength of Metal to Metal Adhesive of Test for Effect of Moisture and Temperature on Adhesive Std. Test Meth. for Tensile Properties of Adhesive Std. Rec. Pract. for Static Bend Test. of for Dimensions and Tolerances for Screw Holes and SIots in for Artificial Headbone for the Calibration of Audiometer by Surgeons in the Treatment of Fractures of the Skeletal Handbook for Library Binding (Rec. for Good Std. for

Aluminum Underground Distribution Reference Std. for Title Leaves of $A$ Std. for Advertising of Manufacturing Std. and Spec. for Textbooks nd Timber) (1973)

Corridor, Building Construction and / d Floors (1973)

plates, Nails and Spikes, Washers, Pin/ n Properties and Design Loads, Timber,

Bolted Joints, Drift Bolts, and Wood and Lag Screws; Li
Bolted Parts (1973) Bolting CIoth, Filter Paper, Wide Field and Compound Mi Bolting Keys and Shafting (1974)

Bolting Material Specially Heat Treated for High Temper Bolting Materials for High Temperature Services (1974)

Bolting Materials for Pressure Vessels, Valves, Flanges

Bolting Materials, High Temperature, 50 to $120 \mathrm{KSI}$ Yiel

Bolts and Screws, Heat Resistant, 195,000 psi (1,345 Mp

Bolts and Screws, Heat Treated, Roll Threaded (1973)

Bolts and Screws, Steel, Low Alloy Heat Resistant, Hard

Bolts and Screws, 6A 1.4V, Heat Treated, Roll Threaded (

Bolts and Screws, $6 \mathrm{~A}$ l.4V, Upset Headed, Heat Treated, R

Bolts and Screws; External Wrenching, Unified Threaded

Bolts and Studs (1973)

Bolts for High Pressure and Temperature Service (1973)

Bolts for Structural Steel Joints (1971) ANSI G24.19

Bolts for Structural Steel Joints, Including Suitable N

Bolts (1966)

Bolts (1970)

Bolts (1972) ANSI G54.15

Bolts (1972) ANSI G54.16

Bolts) (1973)

Bolts, and Wood and Lag Screws; Light Metal Plate Conne

Bolts, Connectors, Nails, Staples, Adhesives, Fiberboar

Bolts, Lag Screws, Plates, Nails and Spikes, Washers, P

Bolts, Nuts, Washers, and Bolted Parts (1973)

Bolts; Closers; HospitaI a

Bomb Meth. of Analysis of Stability in Fats, Oils, and

Bomb Meth. of Analysis of Sulfates in Yeast Foods (Cere

Bomb (1967) ANSI Z11.230

Bomb (1973) ANSI C59.113

Sid. Meth. of Test for Conti

Bond Strength of Bonded and Laminated Textile Fabrics (

Bond Strength of Paperboard (Z-Direction Tensile) (1973

Bond Tester (1973)

or Coating Pick Tests for Dete

Bond, Appearance, and Shrinkage Propensity of Bonded an

Bonded and Laminated A pparel Fabrics (Textile) After Dr

Bonded and Laminated Textile Fabrics (1972)

Bonded Joints and Structures (1970) ANSI Z197.24

Bonded Joints in Plywood Under Mold Conditions (1970)

Bonded Laminated Assemblies (1969) ANSI Z197.20

Bonded Plastic Lap Shear Sandwich Joints in Shear by Te

Bonded Rigid Plastic Lap Shear Joints in Shear by Tensi

Bonded Solid Film Lubricants in Oscillating Motion (197

Bonding Agents (1973)

Bonding A ppliances, Casters, Face Masks and Rebreathing

Bonding Equipment (Internal Electrical Wiring Systems)

Bonding of Direct Current Systems on Boats (1972)

Bonding Strength of Air Setting Refractory Mortar (Wet

Bonding Thin Spectrochemical Samples and Stds. to a Gre

Bonding (1967) ANSI Z197.28

Bonding (1969) ANSI Z197.25

Bonds and Guarantees for Roof Installations (1973)

Bonds and Patterns in Brickwork (Tech. Notes) (1967)

Bonds in Scarf Joints (1970)

Bonds in Shear by Compression Loading (1949) ANSI Z197.

Bonds to Chemical Reagents (1966) ANSI Z197.7

Bonds (1972) ANSI Z197.14

Bonds (1972) ANSI Z197.16

Bonds (1972) ANSI Z197.18

Bonds (1972) ANSI Z197.8

Bone Plates (Surgical Implants) (1973)

Bone Plates (1973)

Bone Vibrators (1972)

Bone) System (1973)

Book Binding Practices) (1971)

Book Numbering (1973)

Book (Electrical Power Cable Specs.) (1973)

Book (1971)

Books (1971)

(Books) (1973)

Book: Abbreviations, Symbols, and Terminology (Lumber a Western Woods Use Western Woods Use Western Woods Use Western Woods Use Western Woods Use Western Woods Use

Rec. Pract. for Radius of Load and

All Mobile Construction Type Cranes Having Rope Supported rope Supporte/

Rec. Pract. for Performance Sid. for Crane Safety Std. for Fire Extinguisher and Apparatus

ow and Medium Speed Stationary Diesel and Gas Engine Gaug meth. of Analysis of Cooking Characteristics of Macaroni
Book: Fire Protection and Safety (Exit, Stairway, Aisle

Book: Lumber Std., Grading Rules, Design Values, Sectio

Book: Sound Control (Acoustics) in Buildings, Walls, an

Book: Timber Fasteners (Connectors, Bolts, Lag Screws,

Book: Wood Preservation (Preservative) (1973)

Book: Wood Tanks, Vats, and Pipe (1973)

Booms, Equipped for Hook Work, Clamshell, Magnet, Grapp

Boomstop of All Mobile Construction Type Cranes Having

Borads, Protective Devices, and Instrumentation (1972)

Borasio System (Cereal Chemistry) (1962)
Boom Angle Measuring System (1972) Booster Hose (1973)
ICBO

ICBO

$\mathrm{AACCH}$

Std. AGMA

ASTM

ASTM

ASTM

ASTM

SAE

SAE

$\mathrm{SAE}$

SAE

ASTM

ASTM

ASTM

ASTM

CSI

AISC

ASTM

ASTM

ICA

ICBO

WWPA

ICBO

NBHA

ASTM

AACCH $58-55$

$\mathrm{AACCH} \quad 40.65$

ASTM D2272

ASTM

AATCC

TAPPI

TAPP

ASTM

ASTM

ASTM

AATCC

ASTM

ASTM

ASTM

ASTM

ASTM

ASTM

ICBO

UL

ULYC

ASTM

ASTM

Std. R ASTM

ASTM

FMS

BIA

ASTM

ASTM

ASTM

ASTM

Std. Meth ASTM

Std. Meth. ASTM

ASTM

ASTM

Std. Rec. Pract. ASTM

Std. ANSI

ASTM

LBI

ANSI

AA

ANSI

ANSI

BMI

WWPA

WWPA

WWPA

WWPA

WWPA

WWPA

WWPA

SAE

SAE

SAE

DEMA

AACCH

$28-90$
260.02

A 437

320

A453

A687

A

UBCS25-17

BC 27.7

7459B

MS $7452 \mathrm{~K}$

AMS 7455D

AMS $7460 \mathrm{C}$

S $7461 \mathrm{~B}$

(1132A 
Std. Analytical Meth. for Determinin Spec. for Powdered Hand Cleaner (Soap with Dimensional Identification Code, and Mounting Dime/ Std. ion Code, and Mounting Dimensions for 3/4, l and $11 / 8 \mathrm{In}$ nalysis of Crude Protein in Wheat and Flour Mill Products td. for Numerically Controlled Horizontal and Vertical Jig Std. for Horizonta

Std. for Numerically Controlled Horizontal for for High Boiling Hydrocarbon Soivent for Preparing Oil Std. Spec. for Low Boiling Hydrocarbon Solvent for Oil Std. Meth. for Analysis of Oi

Std. for Hydrocarbon Solvents for Oil ochemical Analysis of Plain Carbon and Low Alloy Sieel for Tent. Spec. for Nuclear Grade Std. Spec. for evices $(1972)$

Meth. of Test for Std. Spec. for Type 58

ard Mounting and Face Dimensions of Receptacles Off Center Std. for Packing, Preformed, Straight Thread Tube Fitting Std. for Packing, Preformed, Straight Thread Tube Fitting Std. Meth. for Open Std. Meth. of Thermal Shock Test on Glass Containers Std. Meth. of Sampling Glass Container Std. Spec. for Dimensions and Tolerances of Plastic Std. Meth. of Test for Pressure in Glass Aerosol Meth. of Drop Test. of Glass Aerosol Std. Test. Procedure for Filled Cast Flat spec. for Crushed Stone, Slag, and Gravel for Dry or W ater rings (Except Tapered Roller Bearings) Conforming to Basic Hull for Boats Equipped with Outdrives, Jet Drives and/or r Vitreous China Plumbing Fixtures (Lavatory, Water Closet

72)

type Sealants (1972)

h. of Determining Interior Dimensions of Fiberboard Boxes s. for Post Tensioned Prestressed Concrete (Post Tensioned Std. Inlet

rec. for Indoor General Storage (Solid, Palletized and Bin ctrical Sheet Steel Outlet Boxes, Device Boxes, Covers and Std. for Safety for Electrical Outlet Safety Std. for Underwater Lighting Fixtures and Junction 974)

Std. for Safety for Manually Actuated Signaling td. Meth. of Determining Interior Dimensions of Fiberboard s (/ Std. for Electrical Sheet Steel Outlet Boxes, Device aluminum Covers (/ Std. for Electrical Sheet Steel Outlet Std. Analyrical Meth. for Determining 1973)

lar Bicycles Including Wheel Assembly, Chainguards, Saddle Std. for lntegrity and Test Spec. for Selected Rec. for Protection Against Radiation from Std. for Leak Test. Radioactive Spec. for Gamma-Ray ial Equipment Warning Lamp and Slow Mo/ Std. for Mounting oving Vehicle (SMV) Identification Emb/ Std. for Mounting Std. Meth. of Test. Automotive Air Rec. Pract. for Automotive lever Operated Chain Hoists for Ratchet and Pawl, and Load (1972) Rec. Pract. for Automotive Std. for Motor Vehicle m of Vehicles Equipped / Rec. Pract. for Determination of Std. for Motor Vehicle

Std. Meth. of Test. Automotive Hydraulic

Std. Meth. of Test. Automotive Air Brake and Vacuum Rec. Pract. for Gogan Hardness of requirements and Uniform Test Procedures for Motor Vehicle ents (1972) ar (1971)

uses, and Combination Vehicles $\mathbf{F} /$

Rec. Pract. for Service Rec Pract for Design Std. for Air

e Automotive Braking System of Vehicles Equipped with Disc equirements for Construction, Care, and Use of Power Press nguards, Saddle Braces, Pedal Clearance, Steering Control, Automobile Equipped with Full Scale Tires in the Diagonal determination of Brake Fluid Temperature in the Automotive malathion Residues in Milled Wheat Products (Wheat, Flour, ansi $\mathrm{H} 33.1$ Std. Spec. for Copper Zinc Lead (Leaded Red Std. Spec. for Seamless Red Std. Spec. for Leaded

972)

changer Tubing (1972) Std. Spec. for

Std. Spec. for Free Cutting Std. Spec. for Cartridge Std. Spec. for Copper Alloy No. 260 Std. Spec. for Sintered
Borax Content in Dextrin and Corn Starch Products (1971 Borax) (1972T)

Bore and Rod Size Combinations, Rod End Configurations, Bore Cataloged Square Head Tie Rod Type Industrial Flui (Boric Acid Modification) (Cereal Chemistry) (1962)

Boring Machines (1974)

Boring, Drilling and Milling Machine (1974)

Boring, Drilling and Milling Machine (1974)

Borne Preservative Solutions Such as Pentachlorophenol

Borne Preservatives (1973)

Borne Wood Preservatives (1973)

Borne Wood Preservatives (1973)

Boron by the Point to Plane Arc Technique (1970) ANSI Z Boron Carbide Powder (1973)

Boron Deoxidized Copper in Wrought Forms for Electron D

Boron in Water (1972T)

Borosilicate Sealing Glass (1972) ANSI Z173.47

Boss Type, 30, 50, and 60 Amperes, 4 Wire (1966)

Boss, MIL-R-25897 Rubber, 75 Shore, O-Ring (1973)

Boss, MIL·R·25897 Rubber, 90 Shore, O-Ring (1973)

Bottle Tap Sampling of Noncryogenic Fluid Systems (1972

(Bottle, Jar) (1971)

(Bottle, Jar, etc.) (1956)

Bottles (1972)

Bottles (1972)

Bottles (1972)

Bottom Drop Composite Motor Oil Cans (1974)

Bound Macadam Base Courses (1971) ANSl A37.99

Boundary Plans (1972) ANSI B3.1

/Ansmission Units Penetrating the

Bow Thrusters (1973)

Bowl, Urinal) (1973)

Bowling Alleys (1973)

Box Dimensions for Agricultural (Farm) Grain Wagons (19

Box Exposure of One Part, Elastomeric, Solvent Release

Box Gage Meth.) (Packaging) (1968) ANSI MH12.19

Box Girder Bridges Design and Construction) (1969)

Box Positions for Centrifugal Fans (1966)

Box Storage Up to $30 \mathrm{Ft}$. and Shelf Storage Up to $15 \mathrm{Ft}$.

Box Supports, and Cast Aluminum Covers (1973)

Boxes and Fittings (1974)

Boxes for Swimming Pools (1972)

Boxes for Use with Fire Protective Signaling Systems (1

Boxes (Box Gage Meth.) (Packaging) (1968) ANSI MH12.19

Boxes, Covers and Box Supports, and Cast Aluminum Cover

Boxes, Device Boxes, Covers and Box Supports, and Cast

Brabender Viscosity Content in Corn Starch (1968)

Braces, Pedal Clearance, Steering Control, Brakes, Tire

Brachytherapy Sources (Medical Radiation-Radiology) (

Brachytherapy Sources (1972)

Brachytherapy Sources (1973)

Brachytherapy Sources (1974)

Brackets and Socket for Agricultural (Farm) and Industr

Brackets and Socket for Warning Lamp (Light) and Slow M

Brake and Vacuum Brake Hose (1973) ANSI S2.10

Brake Definitions and Nomenclature (1971)

Brake Designs (1971)

Std. Spec. for Manually

Brake Disc and Drum Thermocouple Installation (1972)

Brake Fluid for Use Under Arctic Atmospheric Conditions

Brake Fluid Temperature in the Automotive Braking Syste

Brake Fluid (Used Under Nonarctic Conditions) (1972)

Brake Hose (1972) ANSI J2.9

Brake Hose (1973) ANSI S2.10

Brake Lining (1972)

Brake Linings (1966)

Brake Reservoir Performance and Identification Requirem

Brake Structural Integrity Test Procedure-Passenger C

Brake Structural Integrity Test Procedure for Trucks, B

Brake System (1972)

Brake Systems (1971)

Brake Tubing and Pipe (1970)

Brakes (1972)

Brakes (1973)

Brakes, Tires and Controls (1972)

1. Multipurpose Passenger Vehicles

Braking Mode (1973)

/Nation of Brake Fluid Temperature in Th Std. for Safety $R$

/ Paved Surfaces Using a Passenger Braking System of Vehicles Equipped with Disc Brakes (1

Bran, Shorts, etc.) to Determine Added A mounts of Malat

Brass Die Castings (1970) ANSI H49.1

Brass or Hardware Bronze) Rod, Bars, and Shapes (1972)

Brass Pipe, Std. Sizes (1972)

Brass Plate, Sheet, Strip, and Rolled Bar (1973)

Brass Plate, Sheet, Strip, and Rolled Bar (1973)

Brass Rod, Bar, and Shapes for Use in Screw Machines (1

Brass Sheet, Strip, Plate, Bar and Disks (1973)

Brass Strip in Narrow Widths and Light Gage for Heat Ex

Brass Structural Parts (1970) ANSI H9.8

Brass Tube (1971) ANSI H36.1

CR

ASTM D3046

NFLDP T3.6.11

NFLDP T3.6.11

a AACCH 46.12

NSA 963

NSA 910

NSA 954

ASTM D2604

ASTM D3225

AWPA A5

AWPA

ASTM

ASTM

ASTM

ASTM

ASTM

T Tand ANSI

NSA

NSA

ASTM

ASTM

ASTM

ASTM

ASTM

AFBMA

$A B Y C$

ANSI

ICBO

ASAE

ASTM

Met ASTM

EC CRSI

AMCA

FMS

E404

C750

F107

D3082

F105

C 73.67

1595

1596

F301

C149

D2911

D3071

C125. 1

D694

P16

A 112.19 .2

UFC $* 2 A R T$

R239

C718

D2658

$* 2$

2405

$8-25 \mathrm{~N}$

NEMA OS1

UL 514

UL $\quad 676$

ASTM D2658

NEMA OSI

NEMA OSI

CR

BMA $6 / 5$

ANSI N44.1

NCRPM R40

ANSI N 44.2

NCRPM R41

ASAE S277.2

SAE J725D

ASTM D622

SAE J656F

HMI 300

SAE J1702D

SAE J291

SAE J1703B

ASTM D57I

ASTM D622

SAE J379A

VESC V.3

SAE JI0B

SAE J229

SAE J294

SAE J299

SAE J263

SAE J844C

J291

B1 1.3

$6 / 5$

ASTM E503

SAE J291

AACCH $\quad 60-30$

ASTM B176

ASTM B140

ASTM B43 
National Bureau of Standards KWIC Index of Engineering Standards

Std. Spec. for Welded Std. Spec for

d, Hexagonal, Octagonal, and Flat (Rectangular and Square) Std. for Std. Spec. for Copper Std. for Bolt, Spec. for Std. Spec. for

Unhardened Steel and Alloy Silver for Safety for LP Gas Torches for Heating, Soldering, and Meth. of Analysis of Calcium in Buhler Meth. of Experimental Milling for Batch Meth. of Experimental Milling for White Pan

Meth. of Analysis for Baking Quality of Rye raight Dough Meth. of Analysis for Baking Quality of Wheat Dough, Pound Loaf Meth. of Analysis for Baking Quality of Gravimetric Meth. of Analysis of Chlorides in Ash of

Volumetric Meth. of Analysis of Chlorides in Ash of Meth. for Preparation of Sample:

Meth. of Sensory Perception Test for Staleness of eth. of Analysis of Lactose (Sugar) and Nonfat Dry Milk in meth. of Analysis for Ash in Flour, Feeds, Feedstuffs, and ompression Test with Baker Compressimeter for Staleness of

h. of Analysis for Hydrogen Ion Activity (PH) in Flour and cereal Chemistr/ Meth. of Analysis of Crude Fat in Flour, Products, Grits, Meal, Flaked and Puffed Cereals, Farina, Meth. of Analysis of Moisture in Flour, Farina, Semolina, for Nitrate Free Samples for Analysis of Crude Protein in n Grits, Cor/ Thiochrome Meth. of Analysis of Thiamine in Oven Meth. of Analysis of Moisture in Flour and Semolina; (1973) Std. Meth. of Measuring Std. for Structural Flush Std. Meth. of Measuring Break Pattern of Leather stivity of Silicon Epitaxial Layers by the 3 Probe Voltage ommercial Power Freque/ n Using VDE Electrodes/ n Under Impulse Condit/ Std. Meth. of Test for Dielectric Std. Meth. of Test for Dielectric Sid. Meth. of Test for Dielectric

d. for Safety for Molded Case Circuit Breakers and Circuit dielectric Test Values for Outdoor AC High Voltage Circuit

Rec. Pract. for Automatic Reset Internal Mounted Circuit ns, and Related Requirements for Low Voltage Power Circuit Std for Safety for Molded Case Circuit Class I, Groups A.D, and Class / Safety Std. for Circuit irements for Electrical Control of AC High Voltage Circuit t of Phase / Sid. Meth. for Test. AC High Voltage Circuit Capacitance Current Switching for AC High Voltage Circuit se Voltage Insulation Strength for AC High Voltage Circuit for Transient Recovery Voltage for AC High Voltage Circuit for Transient Recovery Voltage for AC High Voltage Circuit ents for Pressurized Components of AC High Voltage Circuit Capacitance Current Switching for AC High Voltage Circuit or External Insulation for Outdoor AC High Voltage Circuit Capacitance Current Switching for AC High Voltage Circuit Std. for Test Procedures for Low Voltage AC Power Circuit Std. for Low Voltage Alternating Current Power Circuit rs (1973) Std. for Carbide Chip

ance Requirements for Pipe Applied Atmospheric Type Vacuum Related Required Capabilities for AC High Voltage Circuit Rec. Pract. for Circuit Insulating Oil for Use in Transformers and in Oil Circuit ral Insulating Oil for Use in Transformers and Oil Circuit Corn Meal, Corn Grits, Rolled Oats, Bulgur, Rolled Wheat, tant Rate of Extension Instrument/ rs (Flat Bundle Meth.) (1972) Ans Std. Meth. of Test for Std. Meth. of Test for Std. Meth. of Test for Knot Configurations (1973) Tent. Meth. of Test for erapy and Suctio/ Std. for Respiratory Therapy Equipment Std. for Safety for Household Electric Coffee Makers and

old Crushing Strength and Modulus of Rupture of Refractory Sid. Meth. of Sampling and Test.

te Treatment Facilities (Tech. Notes) (1969) sanitary Structures (Tech. Notes) (1969) nt Facilities (Tech. Notes) (1969) t Facilities (Tech. Notes) (1969) Construction Details, 6 In Heat Transmission Coefficients of Moisture Control in clay (Properly Constructed Masonry) Insulated Cavity 6 In.
Brass Tube (1973)

Brass Wire (1971) ANSI H32.1

Uniform Building Code Std. for Roun ICBO

Brazed Double Wall Low Carbon Steel Tubing (1972)

Brazed Steel Tubing (1970) ANSI B125.33

Brazier Head, Torq Set and Hi Torque, 180 KSI (1973)

Brazing Filler Metal (1969) ANSI W3.8

Brazing Filler Metals for Electron Devices (1972)

Brazing Joints (1973)

Brazing Purposes (1973) ANSI Z250.1

Bread and Flour (Cereal Chemistry) (1962)

Bread and Soft Wheats (Cereal Chemistry) (1962)

Bread and Soft Wheats (Cereal Chemistry) (1962)

Bread Flour Spec. (Cereal Chemistry) (1962)

Bread Flour (Cereal Chemistry) (1962)

Bread Flour (Cereal Chemistry) (1962)

Bread Flour (Cereal Chemistry) (1962)

Bread (Cereal Chemistry) (1962)

Bread (Cereal Chemistry) (1962)

Bread (Cereal Chemistry) (1962)

Bread (Cereal Chemistry) (1962)

Bread (Cereal Chemistry) (1962

Bread (Cereal Chemistry) (1962)

Bread (Cereal Chemistry) (1962)

Bread (Cereal Chemistry) (1962)

Bread, and Baked Cereal Products Not Containiectrom

Bread, etc. (Cereal Chemistry) (1962)

Bread, Grain, Soybeans, Rice, Beans, Peas, Lentils, Cor

Bread, Wheat and Other Grains, and Yeast Foods (Cereal

Bread, Wheat, Rice, and Other Whole Grain Products, Cor

Bread; Feedstuffs, Ground Grains and Their Adjuncts (Ce

Break Mandrel Closed End Blind Rivets (1973)

Break Pattern of Leather (Break Scale) (1973) ALCA E64

Break Pull Mandrel Self Plugging Blind Rivets, Type $2 \mathrm{~A}$ (Break Scale) (1973) ALCA E64

Breakdown Meth. (1972) ANSI Z173.47

Breakdown Voltage and Strength of Insulating Cof Test

Breakdown Voltage of Insulating Oils of Petroleum Origi

Breakdown Voltage of Insulating Oils of Petroleum Origi

Breaker Enclosures (1972)

Breaker External Insulation Rated on a Symmetrical Basi

Breaker (1971)

Breakers and AC Power Circuit Protectors (1973)

Breakers and Circuit Breaker Enclosures (1972)

Breakers and Enclosures for Use in Hazardous Locations,

Breakers Rated on a Symmetrical and a Total Current Bas

Breakers Rated on a Symmetrical Basis When Rated for Ou

Breakers Rated on a Symmetrical Basis (1972)

Breakers Rated on a Symmetrical Basis ( $362 \mathrm{kV}$ and Above

Breakers Rated on a Symmetrical Current Basis (1971) an

Breakers Rated on a Symmetrical Current Basis (1971) an

Breakers Rated on a Symmetrical Current Basis (1972) an

Breakers Rated on a Symmetrical Current Basis (1972) an

Breakers Rated on a Symmetrical Current Basis (1972) an

Breakers Rated on a Symmetrical Current Basis (1973) an

Breakers Used in Enclosures (1973)

Breakers Used in Enclosures (1973) ANS1 C37.13

Breakers Used in Indexable Inserts for Clamp Type Holde

Breakers (1970)

Breakers (1971)

Breakers (1972)

Breakers (1973)

Breakers (1973)

/Ransient Recovery Voltage Ratings and

Std. Spec. for Uninhibited Mineral

Breakfast Cereals (Excep! Those Which Are Sugar Coated)

Breaking Load and Elongatoin of Elastomeric Yarns (Cons

Breaking Strength and Elongation of Cotton Textile Fibe

Breaking Strength of Ceramic Tile (1971) ANSI Al73.1

Breaking Tenacity of Man Made Textile Fibers in Loop or

Breathing Devices, Room Humidifiers, Nebulizers, Gas Th

Brewing Type Appliances (1972) ANSI C33.11.1

Brick and Shapes (1972) ANSI All1.17

Brick and Structural Clay Tile (1973)

Brick and Tile Cavity Walls (Tech. Notes) (1965)

Brick and Tile in Sanitation Structures, Industrial Was

Brick and Tile in Sanitation Structures, Miscellaneous

Brick and Tile in Sanitation Structures, Sewage Treatme

Brick and Tile in Sanitation Structures, Water Treatmen

Brick and Tile Lintels (Tech. Notes) (1964)

Brick and Tile Masonry (Tech. Note) (Part III Execution

Brick and Tile Metal Tied Walls (Tech. Notes) (1966)

Brick and Tile or Deviation from a Plane Surface (1972)

Brick and Tile Walls for 1 Story Buildings (Tech. Notes

Brick and Tile Walls (Tech. Notes) (1961)

Brick and Tile Walls (Tech. Notes) (1965)

Brick and Tile Walls (Tech. Notes) (1966)

Brick and Tile Walls, Condensation (Tech. Notes) (1965)

$\triangle A B$

ASTM

NSA

A WS

ASTM

SAE

Std. UL

IFI

ASTM

ASTM

ASTM

ASTM

ASTM

St UL

ANSI

SAE

UL

UNS

IEEE

Ings for ANSI

lEEE

IEEE

IEEE

IEEE

IEEE

IEEE

ANSI

IEEE

ANSI

ASSE

SAE

ASTM

ASTM

AACC.

ASTM

ASTM

ASTM

ASTM

$\mathrm{NSF}$

$\mathrm{UL}$

147

AACCH 40.20

AACCH 26.20

AACCH 26.21

AACCH 34.01

AACCH $10-70$

St AACCH 10.10

Sponge AACCH 10.11

AACCH $\quad 40-30$

AACCH 40.31

$\mathrm{AACCH} 62.05$

AACCH 74.30

AACCH 80.31

Basic AACCH 08.01

eth. of C AACCH 74.10

AACCH 02.52

AACCH $\quad 30.10$

AACCH 86.70

AACCH 44.15 A

AACCH 46.11

AACCH 86.80

AACCH 44.40

IFI 126

ASTM D294]

119

D2941

F 108

D2477

D1816

D3300

C 37.0781

J258

C 37.16

489

877

C37.11

(4)

C37.0732

339

327

328

340

341

343

342

C37.50

B94.47

1001

C39.0722

J553C

D 1040

D3146

44.15A

D2653

D1445

C648

D3217

48

1082

C133

ASTM $\quad$ 67

BIA $21 \mathrm{~A}$

BIA 34B

BIA $34 \mathrm{C}$

BIA 34A 
National Bureau of Standards KWIC Index of Engineering Standards

Std. Meth. of Drip Slag Test. Refractory Std. Meth. of Load Test. for Refractory Fire Resistance Requirements Relating to

es) (1968)

on (Tech. Notes) (1967)

h. Notes) (1966) eral Forces) (Tech. Notes) (1968)

Structural Design of Semicircular Structural Design of Reinforced

4 In. Reinforced Design Tables for Reinforced Reinforced Reinforced Reinforced Reinforced Reinforced

72)

Guide Specs. for Early Strength of Estimating Modular Guide Spec. for Engineered High Lift Grouted Reinforced Rec. Pract. for Engineered Std. Spec. for Portland Cement Lime Mortar for Rec. Spec, for Prefabricated

Notes) (1966)

Design and Construction of Flat and Sloped niform Building Code Std. for Building, Facing, and Hollow Std. Spec. for Sewer and Manhole Salvaged Std. Meth. of Panel Spalling Test for High Duty Fireclay Std. Meth. of Panel Spalling Test for Super Duty Fireclay or Size and Bulk Density of Refractory and lnsulating Fire Std. Classification of Insulating Fire td. Classification of Fireclay and High Alumina Refractory Std. Meth. of Test for Thermal Conductivity of Refractory Std. Spec, for Concrete Building Meth. of Test for Thermal Conductivity of Insulating Fire Std. Spec. for Vitrified Clay Paving

Uniform Building Code Std. for Sand-Lime Building

Uniform Building Code Std. for Sampling and Test. Uniform Building Code Std. for Concrete Building nt Specific Gravity, and Bulk Density of Burned Refractory

Ornamentation, Protectio/ Uniform Building Code: Veneer std. for Machine Made and High Alumina Fireclay Refractory ite; Gravel/ Bonds and Patterns in Uniform Building Code: Weights of Materials processes (1973) Std. for Lumber, Timbers, d Copper Alloy Bearing and Expansion Plates and Sheets for Rec. Pract. for Concrete Highway Std. Spec. for Asphalt Plank as Used for Tensioned Prestressed Concrete (Post Tensioned Box Girder Std. Specs. for W elding of Structural Steel Highway Std. Spec. for Steel Castings for Highway Std. Spec. for Seamless Copper Tube, ve Radiance of $P$ aper and $P$ aperboard Containing Fluorescent observation) (1972)

Meth. for Measuring

ardness Conversion Tables for Metals (Re Std. Meth. for Spectrochemical Analysis of Copper by the al Work / Rec. Pract. for Units (Measurement-Metric and est for Measuring Surface Frictional Properties Using the us Effects of Dyes, Finishes, and Finishings (Sublimation, mpact (1973) Std. Meth. for Determining the Std. Meth. of Test for

istry) (1) Meth. of Analysis of Specific Gravity, Degrees Cable Connectors for Audio Facilities for Radio

hade and Flowering Trees; Deciduous Shrubs; Coniferous and std. for Hotel and Restaurant Gas Ranges (Stoves) and Unit y) (1962) ry) $(1962$ Quantitative Meth. of Analysis of Potassium Qualitative Meth. of Analysis of Meth of A nalysis of Inorganic Std. Meth. of Test for Std. Meth. of Test for Volumetric

hemistry) (1962)

itration (1974) ANSI Z78.15

c Titration (1972) ANS1 Z11.248

y) $(1962)$

) ANSI Z159.2

indicators (1971) or the Pest Control Chemical lsopropyl 4,4'dibromobenzilate or Sack Fibers, Whole or Ground Cereal/ Meth. of Urease Std. Spec, for Steam or Valve

Std. Spec. for Composition Std. Spec. for Manganese
Brick at High Temperature (1973)

Brick at High Temperatures (1970) ANSl Alll.

Brick Bearing Wall Buildings (Tech. Notes) (1969)

Brick F acing for Exterior Walls (Tech. Notes) (1962)

Brick in Landscape Architecture Garden Walls (Tech. No

Brick in Landscape Architecture Miscellaneous Applicati

Brick in Landscape Architecture Terraces and Walks (Tec

Brick Masonry Arches (Tech. Note) (1971)

Brick Masonry Arches (Tech. Notes) (1967)

Brick Masonry Columns and Pilasters (Tech. Notes) (1965

Brick Masonry Curtain and Panel Walls (In Resisting Lat

Brick Masonry Flexural Members (Tech. Notes) (1967)

Brick Masonry Retaining Walls (Tech. Notes) (1965)

Brick Masonry Swimming Pools (Tech. Notes) (1967)

Brick Masonry (Axial Design) (Tech. Notes) (1963)

Brick Masonry (Flexural Design) (Tech Notes) (1964)

Brick Masonry (Inspectors Guide) (Tech. Notes) (1963)

Brick Masonry (Tech. Note) (Part I and II Products) (19

Brick Masonry (Tech. Notes) (1969)

Brick Masonry (Tech. Notes) (1971)

Brick Masonry (Tech. Notes) (1972)

Brick Masonry (Tech. Notes) (1972)

Brick Masonry (1969)

Brick Masonry (1972)

Brick Masonry (1974)

Brick Soffits (Tech. Notes) (1970)

Brick Veneer; New and Existing Frame Construction (Tech

Brick (Made from Clay or Shale) (1973)

Brick (Made from Clay or Shale) (1973) ANS1 Al00.1

Brick (Tech. Notes) (1962)

Brick (1970) ANSI All1.14

Brick (1970) ANSI All1.16

Brick (1970) ANSI All1.18

Brick (1970) ANSI All1.22

Brick (1970) ANSl All1.5

Brick (1971) ANSI All 1.32

Brick (1971) ANSI A75.1

Brick (1972) ANSI All1.28

Brick (1972) ANSI A37.15

Brick (1973)

Brick (1973)

Brick (1973)

Brick (1973) ANSI All1.3

Brick, Concre

Bricks (1973)

Brickwork (Tech. Notes) (1967)

Brick; Cast Iron; Cinders; Concrete; Earih; Glass; Gran

Bridge and Gantry Multiple Gerder Traveling Cranes (197

Bridge and Mine Tie Preservative Treatment by Pressure

Bridge and Other Structural Uses (1972) ANS1 H31.1

Bridge Deck Construction (1974)

Bridge Floors (1970) ANS1 A37.48

Bridges Design and Construction) (1969)

Bridges (1974)

Bridges (1974) ANSI G52.3

Bright Annealed (1973) ANSI H23.2

Brighteners (1973)

Brightness of Pulp (Diffuse Mlumination and Zero Deg.

Brinell, Vickers, Rockwell and Rockwell Superficial, an

Briquet D.C Arc Technique (1971) ANSI Z128.32

British-American) in Published Scientific and Technic

British Portable Tester (1974)

Brittleness and Chlorine Retention) (Institutional Text

Brittleness of Photographic Film (1973)

Brittleness Temperature of Plastics and Elastomers by

Brix (Balling) of Wine (Enology) (1972)

Brix, and Degrees Baume of Sugar Solutions (Cereal Chem

Broadcasting (1970)

Broadleaf Evergreens; Rose Grades; Vines and Ground Cov Broilers (1973)

Bromate in White and Whole Wheat Flour (Cereal Chemistr

Bromates and Iodates in Flour Improvers (Cereal Chemist

Bromates and lodates in Flour (Cereal Chemistry) (1962)

Bromide Residues in Grain and Cereal Products (Cereal

Bromine Index of Aromatic Hydrocarbons by Coulometric $T$

Bromine Index of Petroleum Hydrocarbons by Electrometri

Bromine Meth. of Analysis of Pentosans (Cereal Chemistr

Bromine Number of Unsaturated Aliphatic Chemicals (1972

Bromophenol Blue Indicator, $1 \%$ Content of Reagents and

Bromopropylate (1973)
Bromthymol Blue Test Paper for Analysis of Urea in Clot

Bronze Castings (1974)

Bronze Flanges and Flanged Fittings (150 and $300 \mathrm{Lb}$.) (

Bronze or Ounce Metal Castings (1974)

Bronze Rod, Bar, and Shapes (1974) ANSI H7.5
ASTM

ASTM

BIA

BIA

BIA

BIA

BIA

BIA

BIA

BIA

BlA

BIA

BIA

BIA

BIA

BIA

BIA

CSI

BIA

BIA

BIA

BIA

BIA

BIA

BIA

BIA

BIA

ICBO

ASTM

BIA

ASTM

ASTM

ASTM

ASTM

ASTM

ASTM

ASTM

Std. ASTM

ASTM

ICBO

ICBO

ICBO

ASTM

ICBO

ICBO

BIA

CMAA

$A W P A$

/Le ASTM

$\mathrm{ACl}$

ASTM

CRSI

ASHTO

ASTM

ASTM

TAPPI

TAPPI

ASTM

ASTM

ASTM

IEEE

ASTM

ANSI

ANSI

ASTM

ASE

$\mathrm{AACCH}$

EIA

ANS1

ANSI

$\mathrm{AACCH}$

$\mathrm{AACCH}$

$\mathrm{AACCH}$

AACCH

ASTM

ASTM

$\mathrm{AACCH}$

ASTM

CR

ANSI

$\mathrm{AACCH}$

ASTM

ANS1

ASTM

AST N

C768

$\mathrm{C} 16$

$6 \mathrm{~A}$

13

29B

29

$31 \mathrm{C}$

$31 \mathrm{~A}$

$7 \mathrm{~L}$

$17 \mathrm{E} \cdot \mathrm{G}$

$17 \mathrm{~K}$

17B

$17 \mathrm{~A}$

$17 \mathrm{C}$

$11 \mathrm{~A}$

11C.11D

17D

43

$\mathrm{Ml}$

(6A A

UBCS24.1 
per Water Tube (1973)

ms (1973)

(1973)
Std. Spec. for Aluminum Std. for Wrought Copper and Std. for Cast Std. for Cast Std. Spec. for Sintered Std. Cast

Std, Spec. for Std. Spec. for Phosphor Std. Spec. for Phosphor Std for Bushing-Clamp Up, Std. for Bushing-Flanged, Press Fit, Steel and Spec. for Copper Zinc Lead (Leaded Red Brass or Hardware arent Viscosity of Gear Oils at Low Temperatures Using the I Chemistry) (1962) Distillation Meth. ract. for Determining Hermeticity of Electron Devices by A alants (1972)

ped for Hook Work, Clamshell, Magnet, Crapple, or Concrete ruck Capacity for Shovel Dipper, Clam Bucket, and Dragline the SAE Rating and Struck Capacity for Hoe Dipper (Backhoe the SAE Rating and Struck Capacity for Shovel Dipper, Clam

dification, to Corn, Rye, Barley, Rice, Grain Sorghum, and Wheats (Cereal Chemistry) (1962)

Meth. for Preparation and Standardization of Std. for Std. for

Abbreviations and Symbols Used in Std. for Butts and Hinges for Doors

forcement-Application) (1972) Rec. Spec for Rec. Locations for $s$ and Handles; Exit Devices, Pulls, Push and Kick / Basic

uggested Noise Measurement Techniques for the Machine Too ssification for Determination of Sound Transmission Class
Rules for Std. Spec. for Concrete

Uniform Building Code Std. for Sand-Lime Uniform Building Code Std. for Concrete Southern Std. for

buildings and $O$ ther Structures (1972)

1) ANSI A 89.1

e (1972) ANSI A 169.1

e (1972) ACI 322

allboard to Wood Framing (1973)

73)

test of Partitions (W/all, Ceiling, Floor, and Fl/

of Unburned Clay Masonry Units (1973) (1973)

for Dust, Stock and Vapor Removal (1973)

ck (Made from Clay or ShaIe) (1973)

\section{g Systems (1973)}

Film (1973)

ing the Density of Smoke from the Burning or Dec/ ing Plants or Systems (1973)

meth. of Grading for AIl Species of Lumber (1973/

- Larch (North), Eastern Hemlock-Tamarack (Nor/ te Reinforcement (1973)

ire Extinguishment) (1973)

et (Roll) and Shingles) (1973)

ed Stone, Gravel and Air Cooled Iron Blast Furna/

3)

trodeposited Coatings of Copper, Nickel, Chromiu/ e Strength of MoIded Concrete Cylinders (1973) coast Hemlock; Western Red Cedar; White Fir; And/ onderosa, Idaho White, Sugar, and Lodgepole Pine/ hemlock-Tamarack; Balsam Fir; Northern Pine An/ ern Spruce and Hemlock, Balsam Fir and Tamarack / ation and Painting of Structural Steel (1973)

esults in Field Concrete Cylinders (1973)

973)

agents (1973)

nd Structural Insulating Board (1973)

(1973)

se Buildings (1973)

erials (1973)

ion and Materials (1973)

973)
Bronze Rod, Bar, and Shapes (1974) ANSI H7.6

ASTM

Bronze Solder-Joint Pressure Fitting for Use with Cop

Bronze Solder Joint Pressure Fittings (1972)

Bronze Structural Parts (1970) ANSI H9.6

Bronze Threaded Fittings (125 and 250 Lb.) (1971)

Bronze Trolley Wire (1974) ANSI C7.5

Bronze Wire for General and Spring Purposes (1972)

Bronze (Copper Tin) Plate, Sheet, Strip, and Rolled Bar

Bronze (1973)

Bronze (1973)

Bronze) Rod, Bars, and Shapes (1972) ANSI H33.1

Brookfield Viscometer (1972) ANSI Z11.321

(Brown-Duvel) of Analysis of Moisture in Grains (Cerea

Bubble Emission Techniques (1974)

Bubble Test (1972)

Bubbling of 1 Part, Elastomeric Solvent Release Type Se

Bucket (Construction and Industrial Machinery) (1972)

Bucket) (1972) Std. for Uniform Meth. of Dete

Bucket, and Dragline Bucket (Construction and Industria

Buckle, Web Strap, Light Duty (1972)

Buck wheat (Cereal Chemistry) (1962)

Buffer Solutions (Cereal Chemistry) (1962)

10 , with Slight Mo

Buhler Meth. of ExperimentaI Milling for Bread and Soft

Builders Cabinet Hardware (1971)

Builders Door Controls (Closers) (1971)

Builders Hardware Schedules and Specs. (1961)

(Builders Hardware) (1970)

Builders' Hardware on Std. Steel Doors and Frames (Rein

Builders' Hardware (1964)

Builders' Hardware: Hands of Doors; Hinges; Locks; Knob

Builders' Industry (1970)

Building Acoustics Design and Construction) (1973)

Building and Classing Steel Vessels (1972)

Building Brick (1971) ANSI A75.1

Building Brick (1973)

Building Brick (1973)

Building Code for Dwelling House Construction (1973)

Building Code Requirements for Minimum Design Loads in

Std. Building Code Requirements for Reinforced Concrete (197

Std. for Building Code Requirements for Structural Plain Concret

Std. for Building Code Requirements for Structural Plain Concret

Uniform Building Code Std. for Adhesives for Fastening Gypsum W

Uniform Building Code Std. for Adhesives (1973)

Uniform Building Code Std. for Admixtures for Concrete (1973)

Uniform Building Code Std. for Aggregate for Masonry Mortar (19

Uniform Building Code Std. for Aggregates for Grout (1973)

Uniform Building Code Std. for Airborne Sound Insulation Field

Uniform Building Code Std. for Aluminum Structures (1973)

Uniform Building Code Std. for and Meth. of Sampling and Test.

Uniform Building Code Sid. for As bestos Cement Roofing Shingles

Uniform Building Code Std. for Blower, Fan, and Exhaust System

Uniform Building Code Std. for Building, Facing, and Hollow Bri

Uniform Building Code Std. for California Redwood Lumber (1973)

Uniform Building Code Std. for Carbon Dioxide Fire Extinguishin

Uniform Building Code Std. for Cast Building Stone (1973)

Uniform Building Code Std. for Cellulose Nitrate Motion Picture

Uniform Building Code Std. for Chamber Meth. of Test for Measur

Uniform Building Code Std. for Class II and Class III Dry CIean

Uniform Building Code Std. for Classification, Definition, and

Uniform Building Code Std. for Coast Sitka Spruce, Douglas Fir

Uniform Building Code Std. for Cold Drawn SteeI Wire for Concre

Uniform Building Code Std. for Combination Standpipe Systems (F

Uniform Building Code Std. for Composition Roofing (Class C She

Uniform Building Code Std. for Concrete Aggregates (Sand, Crush

Uniform Building Code Std. for Concrete Building Brick (1973)

Uniform Building Code Std. for Concrete Reinforcement Bars (197

Uniform Building Code Std. for Concrete Tests (1973)

Uniform Building Code Std. for Corrosion Resistant Metals (EIec

Uniform Building Code Std. for Determining the Splitting Tensil

Uniform Building Code Std. for Douglas Fir, Coast Region; West

Uniform Building Code Std. for Douglas Fir; Larch; Hem-Fir; P

Uniform Building Code Std. for Eastern Spruce, White Pine, and

Uniform Building Code Std. for Eastern White and Red Pine, East

Uniform Building Code Std. for Erection, Fabrication, Identific

Uniform Building Code Std. for Evaluation of Compression Test $R$

Uniform Building Code Std. for Expansion Index Test for Soil (1

Uniform Building Code Std. for Exterior Plaster Liquid Bonding

Uniform Building Code Std. for Fiberboard Nail Base Sheathing a

Uniform Building Code Std. for FieId Tests for Grout and Mortar

Uniform Building Code Std. for Fire Alarm Systems for High-Ri

Uniform Building Code Std. for Fire Dampers (1973)

Uniform Building Code Std. for Fire Retardant Roof Covering Mat

Uniform Building Code Std. for Fire Tests of Building Construct

Uniform Building Code Std. for Fire Tests of Door Assemblies (1
ANSI

B16.22

ANSI B $\quad$ B 6.32

ANSI B16.18

ASTM B255

ANSI B16.15

ASTM B9

ASTM B159

ASTM B 103

NSA

NSA

Std ASTM

74

B I 40

D2983

$A \mathrm{CCH}-44.53$

$\begin{array}{ll}\text { ASTM } & \text { E515 } \\ \text { ASTM } & \text { F98 }\end{array}$

ASTM C712

J 712

$\mathrm{J} 220$

J296

J67

$\begin{array}{ll}\text { NSA } & 1284 \\ \text { AACCH } & 76-20\end{array}$

AACCH 70-15

AACCH 26.20

BHMA 201

BHMA 301

NBHA *3

ANSI A156.1

STDI 107

NBHA $* 9$

NBHA 8

/La ASTM E413

ABS

ASTM

ICBO

ICBO

SBCC

ANSI

ACI

ACI

ANSI

ICBO

ICBO

ICBO

$\mathrm{ICBO}$

ICBO

ICBO

ICBO

ICBO

ICBO

ICBO

ICBO

ICBO

ICBO

ICBO

$\mathrm{ICBO}$

ICBO

ICBO

ICBO

ICBO

ICBO

ICBO

ICBO

ICBO

ICBO

ICBO

ICBO

ICBO

ICBO

ICBO

ICBO

ICBO

ICBO

ICBO

ICBO

ICBO

ICBO

ICBO

ICBO

ICBO

ICBO

ICBO

ICBO

ICBO

107
8

*5 55

* 18

A58.1

318

322

A169. 1

UBCS24-2

UBCS24.3

UBCS 47.2

UBCS25-19

UBCS26-9

UBCS24-22

UBCS24-24

UBCS35-3

UBCS 28.1

UBCS24-15

UBCS 32.9

UBCS10-3

UBCS24.1

UBCS25-7

UBCS38-2

UBC $24-14$

UBC $\$ 48.2$

UBCS52-2

UBC 510.2

UBC S25-1

UBCS25.2

UBC $24-16$

UBC 538.3

UBCS32-3 
(1973)

azing (1973)

nd Gypsum Veneer Plaster (1973)

1973)

compound (1973)

Washers, and Bolted Parts (1973)

onry Units (1973)

masonry Units (1973)

es (1973)

xtinguishing Systems (1973)

indows (1973)

(Weatherproofing) (1973)

surement of Airborne Sound Transmission Class (S) tural Concrete (1973)

ity Pole Preservative Treatment by Pressure Proc/ reclay Refractory Bricks (1973)

s (1973)

973)

eel and Iron (Shape, Plate, Sheet, Strip, Connec/ $\mathrm{n}$ of Built Up Roof Coverings: Hot Mopped Asphalt ustical Tile and for Lay in Panel Ceilings (1973/

s, and Metal Accessories (Designed for Use as a / ry Units (1973)

)

ils (1973)

asonry Other Than Gypsum (1973)

3)

als Test. (1973)

3)

gregates for Gypsum Plaster (1973)

aulic Cements (1973)

e for Concrete Construction (1973)

1973)

purposes (1973)

recast Slabs, and Poured Gypsum Roof Diaphragms /

Flat (Rectangular and Square) Brass Wire (1973)

)

es (Temperatures) of Plastics Using a Hot Air lg/ or Composite Construction (1973)

ive Signalling Systems (1973)

ction and Industrial (1973)

ring Purposes (1973)

nry Units (1973)

g Joists, and Rafters (1973)

steel Structural Members (1973)

cold Formed Stainless Steel Structural Members (/ es and Shingles with Asbestos Felt or Gypsum Boa for Concrete Reinforcement (1973)

e Liquids (Gasoline, Kerosene, Acetone, Alcohol,

phic and X-Ray Nitrocellulose Films (1973)

sal Design of Structural Steel Members (1973)

1 Tile and Std. Meth. of Sampling and Test. Stru/ wall Tile (1973)

and Beam Framing (1973)

s-Plywood Stressed Skin and Curved Panels, Bea/ r (1973)

rs: Douglas and Hem Fir, Southern and Lodgepole /

d Wood Products (1973)

te (1973)

ated Lumber (1973)

ts, Drift Bolts, and Wood and Lag Screws; Light /
Uniform Building Code Std. for Fire Tests of Window Assemblics

Uniform Building Code Std. for Glass, lts Defects and Safety Gl

Uniform Building Code Std. for Gypsum Backing Board (1973)

Uniform Building Code Std. for Gypsum Base for Veneer Plaster a

Uniform Building Code Std. for Gypsum Lath (1973)

Uniform Building Code Std. for Gypsum Molding Plaster (1973)

Uniform Building Code Std for Gypsum Partition Tile or Block (

Uniform Building Code Std. for Gypsum Plastics (1973)

Uniform Building Code Std. for Gypsum Sheathing Board (1973)

Uniform Building Code Std. for Gypsum Wallboard Tape and Joint

Uniform Building Code Std. for Gypsum Wallboard (1973)

Uniform Building Code Std. for Gypsum (1973)

Uniform Building Code Std. for Hand Split Shakes (1973)

Uniform Building Code Std. for High Strength Steel Bolts, Nuts,

Uniform Building Code Std. for Hollow Load Bearing Concrete Mas

Uniform Building Code Std. for Hollow Nonload Bearing Concrete

Uniform Building Code Std. for Hydrated Lime for Masonry Purpos

Uniform Building Code Std. for Impact Sound lnsulation (1973)

Uniform Building Code Std. for in Place Density of Soils (1973)

Uniform Building Code Std. for Installation of Automatic Fire E

Uniform Building Code Std. for Installation of Fire Doors and W

Uniform Building Code Std. for Keene's Cement (1973)

Uniform Building Code Std. for Kraft Waterproof Building Paper

Uniform Building Code Std. for Laboratory Determination and Mea

Uniform Building Code Std. for Lightweight Aggregates for Struc

Uniform Building Code Std. for Lime (1973)

Uniform Building Code Std. for Lumber, Timber, Plywood and Util

Uniform Building Code Std. for Machine Made and High Alumina Fi

Uniform Building Code Std. for Masonry Cement for Use in Mortar

Uniform Building Code Std. for Mat Formed Wood Particleboard (1

Uniform Building Code Std. for Material Spec. for Structural St

Uniform Building Code Std. for Materials for Use in Constructio

Uniform Building Code Std. for Metal Suspension Systems for Aco

Uniform Building Code Std. for Metal, Wire and Wire Fabric Lath

Uniform Building Code Std. for Meth. of Test for Concrete Mason

Uniform Building Code Std. for Mineral Roofing Aggregates (1973

Uniform Building Code Std. for Moisture Density Relations of So

Uniform Building Code Std. for Mortar for Unit and Reinforced M

Uniform Building Code Std. for Natural Slate Roof Shingles (197

Uniform Building Code Std. for Noncombustible Elementary Materi

Uniform Building Code Std. for Open Web Steel Joist Design (197

Uniform Building Code Std. for Perlite, Vermiculite and Sand Ag

Uniform Building Code Std. for Plastic Materials (1973)

Uniform Building Code Std. for Portland Cement and Blended Hydr

Uniform Building Code Std. for Prestressed Steel Strand and Wir

Uniform Building Code Std. for Processed Pulverized Quicklime (

Uniform Building Code Std. for Proscenium Curtains-(1973)

Uniform Building Code Std. for Quicklime (Lime) for Structural

Uniform Building Code Std. for Ready Mixed Concrete (1973)

Uniform Building Code Std. for Reinforced Gypsum Concrete and P

Uniform Building Code Std. for Roofing Asphalt (1973)

Uniform Building Code Std. for Roofing Tile (1973)

Uniform Building Code Std. for Round Timber Piles (1973)

Uniform Building Code Std. for Round, Hexagonal, Octagonal, and

Uniform Building Code Std. for Sampling and Test. Brick (1973)

Uniform Building Code Std. for Sand-Lime Building Brick (1973

Uniform Building Code Std. for Sawn Wood Shingles (1973)

Uniform Building Code Std. for Self and Flash Ignition Properti

Uniform Building Code Std. for Shear Connectors (Steel Studs) F

Uniform Building Code Std. for Sheet Metals (1973)

Uniform Building Code Std. for Smoke Detectors for Fire Protect

Uniform Building Code Std. for Softwood Plywood Lumber, Constru

Uniform Building Code Std. for Soils Classification for Enginee

Uniform Building Code Std. for Solid Load Bearing Concrete Maso

Uniform Building Code Std. for Southern Pine Lumber (1973)

Uniform Building Code Std. for Spaced Column Design (1973)

Uniform Building Code Std. for Span Tables for Floor and Ceilin

Uniform Building Code Std. for Spec. and Design of Cold Formed

Uniform Building Code Std. for Spec, and Design of Light Gauge

Uniform Building Code Std. for Special Purpose Roofs (Wood Shak

Uniform Building Code Std. for Steel Bar and Rod Mats (Sheets)

Uniform Building Code Std. for Storage and Handling of Flammabl

Uniform Building Code Std. for Storage and Handling of Photogra

Uniform Building Code Std. for Stress Variation or Stress Rever

Uniform Building Code Std. for Structural Clay Floor Tile (1973

Uniform Building Code Std. for Structural Clay Load Bearing Wal

Uniform Building Code Std. for Structural Clay Nonload Bearing

Uniform Building Code Std. for Structural Floor and Roof Plank

Uniform Building Code Std. for Structural Glued Built Up Member

Uniform Building Code Std. for Structural Glued Laminated Timbe

Uniform Building Code Std. for Structural Glued Laminated Timbe

Uniform Building Code Std. for Structural Rivet Steel (1973)

Uniform Building Code Std. for Test for Glue Joints in Laminate

Uniform Building Code Std. for Test. Gypsum Plasters and Concre

Uniform Building Code Std. for Tests for Structural Glued Lamin

Unif orm Building Code Std. for Timber Connector and Bolted Join
ICBO

ICBO

ICBO

ICBO

ICBO

ICBO

ICBO

ICBO

ICBO
ICBO

ICBO

ICBO

ICBO

$1 \mathrm{CBO}$

ICBO

ICBO

$1 \mathrm{CBO}$

ICBO

ICBO

ICBO

ICBO

$\mathrm{ICBO}$

$\mathrm{ICBO}$
$\mathrm{IC} \mathrm{BO}$

ICBO

ICBO
ICBO

ICBO

ICBO

$1 \mathrm{CBO}$

ICBO

ICBO

ICBO

ICBO

ICBO

ICBO

ICBO

ICBO

$1 \mathrm{CBO}$

ICBO

$1 \mathrm{CBO}$

$1 \mathrm{CBO}$

ICBO

ICBO

ICBO

ICBO

ICBO

$1 \mathrm{CBO}$

$1 \mathrm{CBO}$

ICBO

$1 \mathrm{CBO}$

ICBO

ICBO

ICBO

ICBO

ICBO

ICBO

ICBO

ICBO

ICBO

ICBO

ICBO

$1 \mathrm{CBO}$

$1 \mathrm{CBO}$

ICBO

ICBO

ICBO

ICBO

ICBO

ICBO

ICBO

ICBO

ICBO

ICBO

ICBO

ICBO

ICBO

ICBO

ICBO

ICBO

ICBO

ICBO

ICBO

ICBO

$1 \mathrm{CBO}$

ICBO

UBC $\$ 43.4$

UBCS54. 1

UBC 47.6

UBC $\$ 47.14$

UBC $\$ 47.7$

UBCS47.12

UBC $\$ 24.11$

UBC $\$ 47.8$

UBC $\$ 47.9$

UBC $\$ 47.5$

UBC $\$ 47.10$

UBC $\$ 24.12$

UBC $\$ 32.8$

UBC $\$ 27.7$

UBCS24-4

UBC \$24-6

UBC 24.19

UBC $\$ 35.2$

UBC 570.2

UBC S38.1

UBC S43-5

UBC $47-11$

UBCS17. I

UBCS35. 1

UBC $26-3$

UBC $\$ 47.15$

UBC $525-12$

UBCS37.1

UBC S24-17

UBC $\$ 25.25$

UBCS27. I

UBCS32-I

UBC $47-16$

UBCS47-4

UBCS24-7

UBCS32-5

UBC 570.1

UBC $24-21$

UBCS32-10

UBC S4-1

UBCS27.4

UBC 547.3

UBC 5 52-1

UBCS26. I

UBCS26.7

UBC \$24-20

UBC 56-1

UBC $\$ 24.18$

UBC $\$ 26-13$

UBC S24-13

UBCS32-2

UBC S32-12

UBC $25-14$

UBC $32-13$

UBCS24-25

UBC 24.2

UBC S32-11

UBC $\$ 52.3$

UBC $527-8$

UBCS32-4 
(1973)

oard (1973)

ire Fabric for Concrete Reinforcement (1973)

Inserts and Connections in Reinforced Concrete /

lexural and Axial Loading Combined (1973)

a Preservative Treatment (1973)

cation of Building Materials (1973)

Dwelling Construction Under the Uniform

Southern Std.

Guide for Ceramic Tile in

Uniform

3)

Dwelling Construction Under the Uniform

Uniform

Uniform

Uniform

Uniform

Uniform

walls (1973)

rement Alterations (1973)

Dwelling Construction Under the Uniform and X-Ray Nitrocellulose) (1973)

Uniform

Uniform

Uniform

Uniform

Dwelling Construction Under the Uniform Dwelling Construction Under the Uniform Dwelling Construction Under the Uniform

Uniform

ec., Inspection)/ ning Detector (I)

, and Barbecues (1973)

Dwelling Construction Under the Uniform Dwelling Construction Under the' Uniform Dwelling Construction Under the Uniform

Uniform

Uniform

Uniform

Uniform

Uniform

Uniform

Uniform

Uniform

pire) (1973)

Lathing (1973)

Dwelling Construction Under the Uniform Dwelling Construction Under the Uniform itting Panels, Skylights, Monitors and Sawtooth / Uniform
Dwelling Construction Under the Uniform itting Panels, Skylights, Monitors and Sawtooth / Uniform
Dwelling Construction Under the Uniform d Projections Over Public Property (Pedestrian P/ Uniform 1973)

Uniform Uniform

Dwelling Construction Under the Uniform

Dwelling Construction Under the Uniform Uniform ow Unit, Cavity / Dwelling Construction Under the Uniform 1973) Uniform Uniform

ngs, Reviewing Stands, Bleachers, and Grandstand/ Uniform terproofing) (19/ Dwelling Construction Under the Uniform tal, Plastic, etc.) for Ornamentation, Protectio/ Under Uniform Dwelling Construction Under the Uniform 3)

Dwelling Construction Under the Uniform Dwelling Construction Under the Uniform cinders; Concrete; Earth; Glass; Granite; Gravel/ Uniform $r$, Plywood and Components, Timber, Preservative / Uniform ansi A10.21 Sid. for Safeguarding Against Fire During Std. Definitions of Terms Relating to Fire Tests of Uniform Building Code Std for Fire Tests of re Protection and Safety (Exit, Stairway, Aisle, Corridor, ct. 5) (1971) Spec. for Aluminum Sheet Metal Work in Rec. for Sound Control (Acoustics) in Wood re Processes) (19/ Std. for Round Poles and Posts Used in Std. Spec. for Oil and Resin Base Caulking Compound for
Std. Steel Doors and Frames for Modular Masonry Std. Steel Doors and Frames for Modular Masonry trical Wiring and Layout Diagrams Used in Architecture and Std. Definitions of Terms Relating to Acoustical Tests of Std. for Installation PVC Manual of Std. Pract. for Welded Wire d Pipe for General Drainage (Land Reclamation, Curtain and ermal Resistance of Low Density Mineral Fiber Blanket Type Test Meth. of Surface Burning Characteristics of
Building Code Std. for Tin Clad Fire Doors and Shutters Building Code Std. for Veneer Application Dampers (1973 Building Code Std. for Water Resistant Gypsum Backing B Building Code Std, for Welded Steel Wire and Deformed W Building Code Std. for W elding Reinforcing Steel, Metal Building Code Std. for Welding (1973)

Building Code Std. for Wood Members Subjected to Both F Building Code Std. for Wood Poles That Are to Be Given Building Code Std. Test. Meth. for Fire Hazard Classifi Building Code (1973)

Building Code (1973)

Building Code (1973)

Building Codes (1972)

Building Code: Agricultural Buildings (1973)

Building Code: Aluminum (1973)

Building Code: Chimneys, Fireplaces, and Barbecues (197

Building Code: Concrete (1973)

Building Code: Definitions and Abbreviations (1973)

Building Code: Elevators, Dumbwaiters, Escalators, and

Building Code: Excavation and Grading (1973)

Building Code: Excavations, Foundations, and Retaining

Building Code: Existing Buildings; Minimum Safety Requi

Building Code: Exits and Stairs (1973)

Building Code: Film Storage and Handling (Photographic

Building Code: Fire Extinguishing Systems (1973)

Building Code: Fire Resistive Std. (1973)

Building Code: Fire Zone Requirements (1973)

Building Code: Flooring (1973)

Building Code: Foundations and Retaining Walls (1973)

Building Code: Framing-General (1973)

Building Code: Glass and Glazing (1973)

Building Code: Interior Walls and Ceiling Finish (1973)

Building Code: Legal Requirements (Permit, Plans and Sp
Building Code: Light, Ventilation, Sanitation, Fire War

Building Code: Location on Lot (1973)

Building Code: Masonry or Concrete Chimneys, Fireplaces

Building Code: Masonry (1973)

Building Code: Motion Picture Projection Rooms (I973)

Building Code: Occupancy and General Requirement Classi

Building Code: Patio Covers (1973)

Building Code: Penthouses and Roof Structures (Tower, S

Building Code: Permanent Occupancy (1973)

Building Code: Permits and Inspections (1973)

Building Code: Plastering and Installation of Wallboard

Building Code: Plastering (I973)

Building Code: Plastics (General, Exterior Light Transm

Building Code: Prefabricated Construction (1973)

Building Code: Private Garages and Carports (I973)

Building Code: Regulations for Use of Public Streets an

Building Code: Regulations Governing Fallout Shelters (

Building Code: Roof Construction and Covering (1973)

Building Code: Roof Coverings (1973)

Building Code: Roof Framing (1973)

Building Code: Skylights (1973)

Building Code: Softwood Plywood Paneling (1973)

Building Code: Solid, Grouted, Reinforced Grouted, Holl

Building Code: Sound Transmission Control (Acoustics) (

Building Code: Stages and Platforms (1973)

Building Code: Stairs, Exits and Occupant Loads (Buildi

Building Code: Steel and Iron (1973)

Building Code: Valley Flashing (Sheet Metal for Roof Wa

Building Code: Veneer (Brick, Concrete, Stone, Tile, Me

Building Code: Veneered Walls (1973)

Building Code: Wall and Ceiling Coverings (1973)

Building Code: Wall Framing and Weather Protection (197

Building Code: Wallboard (1973)

Building Code: Weights of Materials (Brick; Cast Iron;

Building Code: Wood Members and Their Fastenings (Lumbe

Building Construction and Demolition Operations (1973)

Building Construction and Materials (1972)

Building Construction and Materials (1973)

Building Construction and Materials (1973)

Building Construction and Test. (1973)

Building Construction (Aluminum Construction Mse B

Building Construction (Floor, Ceiling, Wall) (1972)

Building Construction (Preservative Treatment by Pressu

Building Construction (1972)

Building Construction (1972)

Building Construction (1972)

Building Construction, Basement Pipe Inlets (1973)

Building Constructions and Materials (1973)

Building Drain Waste and
Building Fabric (1972)

Building Foundation Drain, Under Basement Floor Sump C

Building Insulation (1970) ANSI Z98.35

Building Materials (1972)
ICBO

ICBO

ICBO

ICBO

ICBO

ICBO

ICBO

ICBO

ICBO

ICBO

ICBO

SBCC

TCA

ICBO

ICBO

ICBO

ICBO

ICBO

ICBO

ICBO

ICBO

ICBO

ICBO

ICBO

ICBO

ICBO

ICBO

ICBO

ICBO

ICBO

ICBO

ICBO

ICBO

ICBO

ICBO

ICBO

C 30

ICBO

ICBO

ICBO

ICBO

ICBO

ICBO

ICBO

ICBO

ICBO

ICBO

ICBO

ICBO

ICBO

ICBO

ICBO

ICBO

ICBO

ICBO

ICBO

ICBO

ICBO

ICBO

ICBO

ICBO

ICBO

ICBO

ICBO

ICBO

ICBO

ICBO

NFPA

NFPA

ASTM

CBO

WWPA

AA

WWPA

AWPA

ASTM

STDI

ANSI

ASTM

IAPMO

WRI

ASTM

ASTM

NFPA

UBCS43-3

UBCS30. I

UBCS47.13

UBCS26.6

UBCS26. 8

UBCS27.6

UBCS25-16

BC 525.13

UBC 442.1

UBC $* 3$

UBC $* 8$

UBC $* 3.15$

$\mathrm{UBC} * 3.28$

UBC $* 8.6$

UBC * 3.26

$\mathrm{UBC} * 3.4$

$\mathrm{UBC} * 3.51$

UBC 3.70

UBC $* 3 \cdot 29$

UBC*3.13

UBC *8.13

UBC $* 3.48$

UBC *3.38

UBC $* 3.43$

UBC $* 3-16$

UBC $* 8-8$

$\mathrm{UBC} * 8.5$

UBC $* 8.7$

$\mathrm{UBC} * 3.54$ 
Std. Meth. of Test for Noncombustibility of Elementary ng Code Std. Test. Meth. for Fire Hazard Classification of Organic Materials for Roofing, Waterproofing, and Related Uniform Building Code Std. for Kraft Waterproof Measurement of Airborne Sound Transmission Class (Stc) of oratory Measurement of Airborne Sound Transmission Loss of Standard Specification for Std. Definitions of Terms Relating to Std. Spec. for Styrene Rubber Plastic Drain and Installation for Non Metallic

Guide for the Design of Aluminum Formed Sheet Uniform Building Code Std. for Cast Std. Definitions of Terms Relating to Natural ec. for Design of Concrete Composite Beams and Girders for Plywood Construction Guide for Residential Uniform Building Code Std. for hale) (1973) r School, Theater, Auditorium, Dormatory, Public or Office 73) Rec. for Prevention of Roof Collapse of for Building Code Requirements for Minimum Design Loads in vention of Collapse Due to Weak Construction and Design of Code for Safety to Life from Fire in Rec. Against Roof Leakage of

3)

Uniform Code for the Abatement of Dangerous nstruction Details, 6 In. Brick and Tile Walls for I Story ire Resistance Requirements Relating to Brick Bearing Wall Hardware for School

t Thermal Insulation for Wood Frame and Light Construction onstruction Systems (Specs.) for Commercial and Industrial h. of Test for Transparent Safety Glazing Material Used in

lt Tile Floors in Institutional, Industrial and Commercial Std. Meth. for Floor Measurement of Office

Rec. Against Weak Construction and Design of Medium Speed Stationary Diesel and Gas Engine Power Plant Rec. for Fire Safety in High Rise Uniform Building Code: Agricultural Building Code Std. for Fire Alarm Systems for High-Rise eiling, Floor, and Floor-Ceiling Assemblies) in Existing t Tile Floors for Commercial. Institutional and Indust rial Spec. for Structural Concrete for Contemporary Bearing Wall (For High

Uniform Housing Code (Residential se Alarm; Use of Equipment, Appliances, Devices and Vacant lndustrial Cost Trends for

Uniform Building Code: Stairs, Exits and Occupant Loads

Western Woods Use Book: Sound Control (Acoustics) in

) Uniform Building Code: Existing camera, Copy, and / Std. Meth. for Measuring Thickness of Std. for Particleboard Decking for Factory

els, Beal

Uniform Building Code Std. for Structural Glued Std. Spec. for Asphalt for Use in Constructing uilding Code Std. for Materials for Use in Construction of Std. Rec. Pract. for Sampling and Analysis of aturated Organic Felt, Coal Safety Std. for Materials for
Std. for R-40 Lamp (Light, Std. for Std. for Std. for Std. for Std. for

d Rivet (1974)

rivet (1974)

blind Rivet (1974)

blind Rivet (1974)

d Rivet (1974)

Lamps (1971)

Std. Nomenclature for Glass

, Asparagus); Lining Out Stock; Seedling Trees and Shrubs; Meth. of Analysis of Added Lysine in Wheat and

Beans, Peas, Lentils, Corn Meal, Corn Grits, Rolled Oats, rec. for Terminal Facilities for Pneumatic Transfer of Dry heres (1972) Std. Meth. of Test for

Std. Analytical Meth. for Determining porosity, Water Absorption, Apparent Specific Gravity, and
970) ANS1 A 111.18

gravity of Fire/ Std. Test Meth. for Std. Meth. of Test for Size and Std. Mer Classification and Definitions of std. for Inspection, Sampling and Measuring Procedures for Std. Spec. for Corrugated Asbestos Cement Sheets for

Std. for Hole Spacing for Scraper and Rec. Pract. for Hole Placement on inst Robbery or Holdup (Criminology)/ Construction of lndoor Rifle and Pistol Ranges and ts in Steel Pipe (1974)

Rec. Pract. for

) Spec. for Rope Lay Stranded Copper Conductors Having ing Strength and Elongation of Cotton Textile Fibers (Flat Test Meth. for Fiber 3)

r Safety Buoyant Devices (Vest, Jacket, Horseshoe and Ring
Building Materials (1973)

Building Materials (1973)

Building or Industrial Uses (1973) ANSl A109.29

Building Paper (Weatherproofing) (1973)

Building Partitions Such as Walls, Floor-Ceiling Asse

Std. Rec. Pract. for Lab

Partitions (1970)

Building Sandstone (1968) ANSI A192.1

Building Seals (1973)

Building Sewer Pipe and Fittings (1973)

Building Sewers (1971)

Building Sheathing (Aluminum Construction Manual) (1969

Building Stone (1973)

Building Stones (1974)

Building (1957)

Building (1971)

Building, Facing, and Hollow Brick (Made from Clay or S

Building, Manufacturer, Restaurant, and Dwelling or App

Buildings and Other Structures Caused by Snow Loads (19

Buildings and Other Structures (1972)

Buildings and Other Structures (1973)

Buildings and Structures (1970) ANSI A9.1

Buildings Not Due to Hail or Wind (1972)

Buildings (Repair, Vacate, Rehabilitate, Demolish) (197

Buildings (Tech. Notes) (1966)

Buildings (Tech. Notes) (1969)

Buildings (1957)

Buildings (1970) ANSI Z98.36

Buildings (1971)

Buildings (1971)

Buildings (1971)

Buildings (1972)

Buildings (1972)

Buildings (1972)

Buildings (1973)

Buildings (1973)

Buildings (1973)

Buildings (1973)

Buildings (1973)

Buildings (1973) ANSI A138.1

Buildings) (Tech. Notes) (1970)

Buildings) (1973)

Buildings) (1973)

Buildings, Machinery and Equipment (1974)

(Buildings, Reviewing Stands, Bleachers, and Grandstands

Buildings, Walls, and Floors (1973)

Buildings; Minimum Safety Requirement Alterations (1973

Buildup Area on Unitized Microfilm Carriers (Aperture

Built Housing (1972)

Built Up Members-Plywood Stressed Skin and Curved Pan

Built Up Roof Coverings (1971) ANS1 A 109.24

Built Up Roof Coverings: Hot Mopped Asphalt, Asphalt an

Built Up Roofs (1972)

Built-Up Roof Coverings: Hot Mopping Asphalt, Asphalt-S

Bulb (Hard Glass) Mogul Screw Base Incandescent (1966)

Bulb) (1973)

Bulbed Mechanically Locked Spindle Protruding Head Blin

Bulbed Mechanically Locked Spindle Self Plugging Blind

Bulbed Mechanically Locked Spindle 100 Deg. Flush Hlead

Bulbed Mechanically Locked Spindle 100 Deg. Flush Head

Bulbed Spindle Mechanically Locked Protruding Head Blin

Bulbs Intended for Use with Electron Tubes and Electric

Bulbs, Corms, and Tubers; Christmas Tree Std. (1973)

Bulgur (Cereal Chemistry) (1973)

Bulgur, Rolled Wheat, Breakfast Cereals (Except Those W

Bulk Chemicals and Plastics (1972)

Bulk Density and Packing Factor of Hollow Glass Microsp

Bulk Density Content in Corn Starch (1963)

Bulk Density Content in Feedstuffs (Corn) and Other Wet

Bulk Density of Burned Refractory Brick (1973) ANSI All

Bulk Density of Diamond Abrasive Grains (1973)

Bulk Density of Refractory and Insulating Fire Brick (1

Bulk Density, Apparent Porosity, and Apparent Specific

Bulk Materials (1970) ANSI MH4.8

Bulk Shipments and Transfers of Soybean Oil (1973)

Bulkheading (1974)

Bulldozer (Dozer) Cutting Edges (1972)

Bulldozer (Dozer) End Bit Supports (1972)

Bullet Resisting Equipment (Barrier) for Protection Aga Bullet Stops (1973)

Bulletin for Nondestructive Test. Terminology for Defec

Bumper Evaluation Test Procedure (1971)

Bunch Stranded Members, for Electrical Conductors (1971

Bundle Meth.) (1972) ANSI L14.96

Bundles in Baled Flash Dried Pulp (1973)

H. of Test for Break ASTM

Buoy) (1973) ANSI Z243.

/ Marine Special Purpose Wate

Buoyancy in the Event of Swamping (Boat Flotation) (197

ASTM

ICBO

ASTM

ICBO

ICBO

ASTM

ASTM

ASTM

IAPMO

AA

ICBO

ASTM

Tent. R ACI

APA

ICBO

ICBO

FMS

Std. ANSI

FMS

NFPA

FMS

ICBO

Co BIA

BIA

BHA

APA

ANSI

RT]

ANSI

FMS

FMS

ICBO

Uniform ICBO

NSI

ANSI

E136

UBCS42-1

D 1079

UBCS17.1

UBCS35. 1

E90

C616

C717

D2852

IS1

UBC 24.14

C119

57.29

UBCS24-1

UPC * $1 \cdot \mathrm{C}$

1.54

A58. 1

1.55

101

LPD 1.4

UBC $* 6$

$26 \mathrm{~A}$

$16 \mathrm{~A}$
.5

C665

*1

Z97.1

*10

Z65. 1

LPD 1.3

* $1-20$

$1-3$

UBC $* 3.15$

UBCS18-1

UBC. 35.3

*16

301

24

UBC $* 5$

UFC *2ART27

9-3

UBC $* 3-33$

$* 31.5$

$\mathrm{UBC} * 3.13$

MS9

2-70

UBC $525-18$

D312

UBC $532-1$

D2829

$55 \mathrm{~A}$

C78.255

C79. 255

1738

1740

1739

1769

1768

C79.1

260.1

CCH $\quad 46.40$

$\mathrm{AACCH} 44.15 \mathrm{~A}$ 
Tentative Meth. of Test for Density of Glass by

(19) Safety Std. for Marine Special Purpose Water Safety nuclides in Air and In/ Rec. for Maximum Permissible Body ction of Laminators Quality Control by the AITC Inspection Safety Std. for Household Std. for Safety for Proprietary and Bank

Std. for Safety for Connectors and Switches for Use with Safety Std. for Key Locks Safety Std. for Intrusion Detection Units for Std. for Safety for Antitheft Alarms and Devices
Std. for Safety for Safety Std.

as a Substitute for Plate Glass Show Wi/ Safety Std. for nerating Stations (Flood, Surface Water, Rigging Collapse, onding Characteristics of Pipeline Coatings by Direct Soi] Std. for Flashing and Steady on of Furnace Explosions in Pulverized Coal Fired Multiple td. for Atmospheric Type Welded Steel Inside Tanks for Oil I and Natural Cas Fired Watertube Boiler Furnaces with One Std. for Installation of Domestic Gas Conversion Safety Sid. for Oil Std. for Draft Hoods for Gas Std. for Safety for Pumps for Oil Test Meth. of Surface
Uniform Fire Code: Oil al Vehicles (1973) ANSI Al47/ Uniform Fire Code: Oil
Safety Std. for Liquid Fuel by / Std. Meth. of Test for Rate of Burning or Extent of Meth. of Test for Measuring the Density of Smoke from the a Supported Specimen by / Std. Meth. of Test for Rate of

Ceneral Precautions for Prevention (Incinerator and Open onia (Fertilizer Grade) in Residential and Commerical Fuel

lorfastness of Textiles to Atmospheric Oxides of Nitrogen ent (1972) ANSI / Std. Meth. for Verifying the Collapse / Std. Test. Procedure for lnternal Std. Spec. for Copper

Rod, Pipe, and Structural Shapes for Electrical Purposes Std. Spec. for Aluminum Bar for Electrical Purposes

Minimum Requirements for School Minimum Requirements for Type 11 School Std. Spec. for Seamless Copper Rec. Pract. for

Rec. Pract. for Proper Location of pec., and Application Cuide for High Voltage Air Switches, enger Cars, Off Road, Agricultural and Log Skidder, Truck, Std. for Nomenclature-Truck,
for Tires and Rims for Truck,

used in the Selection of Tires (Car, Station Wagon, Truck, Rec. Minimum Stds. for School

Rec. Pract. for Exterior Sound Level for Heavy Trucks and Std. for Inspection Procedures for School vice Brake Structural Integrity Test Procedure for Trucks, erminals (For Foreign and Domestic Passenger Cars, Trucks, ) (1972) Std. for Training of Automotive Mechanics for , and Demountable Rims Intended for Highway Use on Trucks, stance of Passenger Cars, Multipurpose Passenger Vehicles, Meth. of Analysis of Test Weight Per Std. for Sid. for Std. for Std. for Std. for Sleeve Std. for Punches: Basic Angle Head Type and Related Quill for Punches-Variable. Angle Head Type and Related Quill r Punches-Basic, Cylindrical Head Type and Related Quill hes-Basic, Combination Angle Head Type and Related Quill Std. for Jig

Std. Ferrous Pipe Plugs, Std. Rec. Pract. for Image Evaluation of Electrostatic
Std. for Electrical Feeder and Plug in Std. for Electrical Feeder and Plug in

Std. Meth. for Evaluation. Spec. for Acrylonitrile t Pipe and Fittings (1973) Std. Spec. for Acrylonitrile
Std. Spec. for Acrylonitrile 73) An/ le 80 (1973) Ans/ le $40(1973)$ le $80(1973)$ / ansi $\mathrm{B} 72.3$ d $80(1973)$ gs (1973) ANS1 K65.59

cable (1973) ANS1 C8.28 Soc. Solvent Cement for Acrylonitrile Std. Spec. for Socket Type Acrylonitrile Std. Spec. for Socket Type Acrylonitrile Sid. Spec. for Acrylonitrile Std. Spec. for Acrylonitrile Std. Spec. for Acrylonitrile Std. Spec. for Rigid Acrylonitrile. Std. Spec. for Styrene
Buoyancy (1971)

Buoyant Devices (Vest, Jacket, Horseshoe and Ring Buoy) Burdens and Maximum Permissible Concentrations of Radio Bureau (1962)

Burglar Alarm System Units (1972) ANSI Se2.4

Burglar Alarm System Units (1974) ANSI Se2.9

Burglar Alarm Systems (1972) ANSI Se2.3

Burglar Alarm Systems (1973) ANSI Se2.6

Burglar Alarm, Door, Deposit and Collection Safes) (197

Burglary Protection Systems (1969) ANSI C 33.70

Burglary Protection) (1974)

Burglary Resistant Electric Door Strikes (1974)

Burglary Resistant Safes (1972) ANSI Se 4.1

Burglary Resisting Glazing Material for Use Principally

Burglary, and Roof Collapse) (1974)

Burial (1972)

Burn Barricade Warning Lights (1971)

Burner Boiler Furnaces (Fire Protection) (1973)
Burner Fuel (1973)

id Meth of Teet for

Inspe

ASTM C693

1123

NCRPM R22

AITC 202

UL $\quad 1023$

UL

UL 68

UL

UL

UL

UL

UL

UL

Burner (1972)

Burners (1971)

Burners (1973)

Burning Appliances (1971)

Burning Appliances (1974) ANSI Bl30.1

Burning Characteristics of Building Materials (1972)

Burning Equipment (1973)

Burning Heat Appliances for Mobile Homes and Recreation

Burning of Cellular Plastics Using a Supported Specimen

Burning or Decomposition of Plastic Materials (1973)

Burning or Extent of Burning of Cellular Plastics Using

Burning Stoves (Room Heaters and Ranges) (1973)

Burning, Combustible and Flammable Materials; Reporting

Burning, Dispensing, and Handling Facilities (1974) Ans

Burnt Cas Fumes) (1972) ANSI L14.54 Test Met
Burst Resistance of a Hydraulic Fluid Power Filter Elem

Bursting of Composite Tubes and Cores (1974)

Bus Bar, Rod, and Shapes (1974) ANSI C7.25

Bus Conductor) (1973) ANSI C7.45

Bus Connector) (1973) ANSI C7.27

Bus Construction and Equipment (1972)

Bus Construction and Equipment (1973)

Bus Pipe and Tube (1974) ANSI C7.26

Bus Stops for Freeway Operations (1971)

Bus Stops (1967)

Bus Supports, and Switch Accessories (1972)

Bus Type Tires (1973)

Bus, Bead Seat, Industrial and Bicycle Tires (1974)

Bus, Trailer (1972)

Bus, Trailer, and Multipurpose Vehicle (1974)

Bus, Trailer, Multipurpose Passenger and Recreational V

Buses (1970)

Buses (1971)

Buses (1972)

Buses, and Combination Vehicles for Roadway Use Over 10

Buses; Coaches, Tractors, Industrial Road and Misc Equi

Buses, Heavy Trucks and Trailers (Course of Instruction

Buses, Trailers, and Multipurpose Passenger Vehicles (1

Buses, Trucks, and Combination Vehicles with Any Type 0

Bushel of Grain (Cereal Chemistry) (1962)

Bushing-Clamp Up, Bronze (1973)

Bushing-Clamp Up, Steel, Cadmium Plated (1973)

Bushing-Flanged, Press Fit-Undersize Inside Diamete

Bushing-Flanged, Press Fit, Steel and Bronze (1973)

Bushing-Plain, Press Fit, Steel (1973)

Bushing, Press Fit, Undersize Inside Diameter (1973)

Bushings (1972)

Bushings (1972)

Bushings (1972)

Bushings (1972)

Bushings (1974)

Bushings, and Locknuts with Pipe Threads (1971)

Business Copies (1972)

Busways and Accessories (1972)

Butadiene-Acrylonitrile Rubbers (NBR) (1973)

Butadiene-Styrene (ABS) Composite Sewer Piping (1974)

Butadiene-Styrene (ABS) Plastic Drain, Waste, and Ven

Butadiene-Styrene (ABS) Plastic Pipe and Fittings (19

Butadiene-Styrene (ABS) Plastic Pipe Fittings, Schedu

Butadiene-Styrene (ABS) Plastic Pipe Fittings, Schedu

Butadiene-Styrene (ABS) Plastic Pipe Fittings, Schedu

Butadiene-Styrene (ABS) Plastic Pipe (SDR.PR) (1973)

Butadiene-Styrene (ABS) Plastic Pipe, Schedules 40 an

Butadiene-Styrene (ABS) Plastic Sewer Pipe and Fittin

Butadiene-Styrene (ABS) Plastics (1973) ANSI K65.205

Butadiene-Synthetic Rubber (SBR) Jacket for Wire and Std. for Punc ANSI
/Venti FMS

Safety $S$ UL

NFPA 85

ANSI Z21.

UL 296

ANSI Z21.12

UL 343

ICBO UFC*2ART24

UL $307 \mathrm{~A}$

ASTM Dl692

ICBO UBCS52.2

ASTM D 1692

UL $\quad 896$

ICBO UFC $* 2 A R T 27$

UL

331

23

NFLDP

CCTI

ASTM

ASTM

ASTM

VESC

VESC

ASTM

ITE

ITE

/Cturing S ANSI

VESC

IS TRA

SAE

TRA

TRA

NEA

SAE

ANSI

SAE

$\mathrm{BCl}$

ANSI

$\mathrm{SAE}$

$S A E$

J299

NSA

NSA 74

NSA 538

NSA 77

NSA 75

NSA

ANSI

Std. ANSI

Std. Fo ANSl

ANSI

ANSI

ASTM

NEMA

ASTM

ASTM

ASTM

ASTM

ASTM

ASTM

ASTM

ASTM

ASTM

ASTM

ASTM

ASTM

10.8 .5

1112

B317

B236

V. 6
V. 10

V. 10
B188

$* 9$

$* 10$

C 37.32

V.9

J687C

2

$\mathrm{X} \cdot \mathrm{Xl}$

J 366

D7.4

J294

*1.56

D18.2

. 67

3

77

537

.

B94.38

B94.44

B94.39

B94.33

B16.14

F360 
Analysis of Synthetic Elastomers for Use on Solid Styrene matography (1973) Std. Meth. of Test for luation of and Test Formula for Carbon Blacks in Styrene h. for Evaluation of and Test Formulas for Solution Poly for Evaluation of and Test Formula for Pigmented Styrene evaluation of and Test Formulas for Nonpigmented Styrene d Fitting/ Materials and Property Stds. for Acrylonitrile Std. Spec. for Solvent Cements for Joining Acrylonitrile ittings (1972) ANSI K65.57

hedule 40 (1973) ANSI K65.160

hedule 80 (1973) ANSI K65.159

(PE) Plastic Pipe and Tubing (1973)

Std. Spec. for Acrylonitrile Safe Handling and Use of Sid. Spec, for Std. Spec. for Std. Spec. for olar

Std. Rec. Pract. for Making Reference Glass-Metal Std. for Steel

Std. Pract, for Ball Valves with Flanged or $\mathbf{s}$ of Moisture and Volatile Matter in Fats and Shortenings Std. for Safety for Std. Pract. for

h. of Test for Fusion Flow of Porcelain Enamel Frits (Flow for Symbols for Machine Tool lndicator Plates or Control tep Relief (1972)

tch Basic Height of Thread Engagement (1973) Std. for Die Std. for

Columns, Piers, Pilasters, and Std. for rn Whitel Std. for Preservative Treatment of Incised Pole Std. for

Std. Factory Made Wrought Stee Std. Spec. for Normal New Mineral Insulating Oils Containing 2,6-Ditertiary 8.37 Std. Meth. of Assay of Di-T

Std. Spec. for Ozone Resisting Std. Spec. for Para Tertiary for the Pest Control Ch/ Std. for Propargite 2-(P.Tert ansi $\mathrm{B} 72.12$

Std. Meth. for Chemical Analysis of Alcohol, Poly(Vinyl

2.4

) A/ y) $(1962)$ Std. Spec. for Solvent Cements for Cellulose Acetate Test. Cellulose Acetate Propionate and Cellulose Acetate Van Dame Meth. of Analysis for Formic, Acetic, Propionic, Std. Rec. Pract. for Making and Using the Std. Spec, for Cellulose Acetate Butyrate Std. Spec. for Cellulose Acetate Butyrate

Spec. for Solvent Cements for Cellulose Acetate Butyrate Sed for Builders

of Hydraulic Cements and Concretes/ std. Spec. for Moist

r Construction and Performance Std. for Kitchen and Vanity architectural Woodwork) (197) Quality Stds. for Casework: 23 Std. Meth. of Test for Gas Content of sting (1970)

al Energy (197) Std. for Thermoplastic Insulated Wire and ossed Linked Thermosetting Polyethylene Insulated Wire and Metal Floor Raceways and Fittings for Electrical Wire and minum Conduit for Safe Use as a Metal Raceway for Wire and td. for Safety for Electrical Metallic Tubing for Wire and

Underground Distribution Reference Book (Electrical Power $y$ of Quality of Electrical lnsulating Oil for Low Pressure of Quality of Electrical Insulating Oil for High Pressure Std. for Link,

Std. for Precision Coaxial Connectors for CATV Std. on Subscriber Visual Carrier Level Std. Ampacities Cables in Open Top 0/ Std. for Nonshielded Single Conductor Electrical Power quipment) (1974) Std for Wire

Shield Losses for Single Conductor Solid Dielectric Power um, Triplex, Neutral Supported, Service Drop and Secondary Std. Spec. for Aluminum Wire for Communication Std. Spec, for Polyethylene Insulated Wire and for Polyethylene Jacket for Electrical Insulated Wire and 7 Conductor Electrical Connector for Truck Trailer Jumper eral Purpose Ethylene Propylene Rubber Jacket for Wire and extra Heayy Duty Black Polychloroprene Jacket for Wire and Std. Spec. for Natural Rubber Jacket for W'ire and e-Butadiene-Synthetic Rubber (SBR) Jacket for Wire and c. for General Purpose Polychloroprene Jacket for Wire and for Heavy Duty Black Polychloroprene Jacket for Wire and td. Spec. for PolyvinyI Chloride (PVC) Jacket for Wire and und lnstallation for Communication and Electrical Wire and Std. Spec, for Ozone Resisting Insulation for Wire and for Ozone Resisting Butyl Rubber Insulation for Wire and Rubber Heat or Moisture Resisting Insulation for Wire and d Copper Concentric Neutrals (Underground Electrical Power

Std. Spec. for Synthetic Rubber Insulation for Wire and for Natural Rubber Heat Resisting Insulation for Wire and
Butadiene Copolymers (S BR) (1972)

Butadiene Purity and Hydrocar Butadiene Rubber (SBR) (1973)

Butadiene Rubbers (BR) (1973)

Butadiene Rubbers (SBR) (1973) Std. Meth. for

Butadiene Styrene Plastic Drain, Waste and Vent Pipe an

Butadiene Styrene (ABS) Pipe and Fittings to Poly(vinyl

Butadiene Styrene (ABS) Plastic Utilities Conduit and F

Butadiene (1974)

Butt Fusion Polyethylene (PE) Plastic Pipe Fittings, Sc

Butt Fusion Polyethylene (PE) Plastic Pipe Fittings, Sc

Butt Heat Fusion Polyethylene (PE) Plastic Fittings for

Butt Seals and Test. for Expansion Characteristics by $P$

Butt Welding End Valves (1973)

Butt Welding for General Service (1972)

Butter, Oleomargarine, High Acid Coconut Oil, etc.) (Ce

Butterfly Valves for Fire Protection Service (1974)

Butterfly Valves (1970)

Button Meth.) (1970) ANSI Z167.10

Buttons (1972)

Buttons, Variable, Press Fit, Headless and Head Type, S

Buttress Inch Screw Threads (7/45 Deg. Form with $0.6 \mathrm{Pi}$

Buttresses (Tech. Notes) (1962)

Butts and Hinges for Doors (Builders Hardware) (1970)

Butts by the Thermal Process (Western Red Cedar, Northe

Buttwelding Ends (1972)

Buttwelding Fittings (1971)

Butyl Acetate (98\% Grade) (1972)

Butyl Para-Cresol by Rolating Bomb (1973) ANSI C59.11

Butyl Peroxide by Gas Chromatographic Analysis (1973)

Butyl Rubber Insulation for Wire and Cable (1974) ANSI

Butylphenol 98 (1972) ANSI Z78.24

Butylphenoxy) Cyclohexyl 2Propynyl Sulfite (Common Name Butyral) (1973)

Butyrate (CAB) Plastic Pipe (SDR-PR) and Tubing (1973)

Butyrate (CAB) Plastic Pipe, Schedule 40 (1973) ANSI B7

Butyrate (CAB) Plastic Pipe, Tubing, and Fittings (1973 Butyrate (1972)

Butyric, and Lactic Acids in Rye Flour (Cereal Chemist

Butyric, Valeric, Succinic, and Lactic Acids (Cereal Ch

C-Ring Stress Corrosion Cracking Test Specimen (1973)

(CAB) Plastic Pipe (SDR-PR) and Tubing (1973) ANSI B72.1

CA B) Plastic Pipe, Schedule 40 (1973) ANSI B72.4

(CAB) Plastic Pipe, Tubing, and Fittings (1973) ANSI K65

Cabin Duct, Air, Flexible and Semi Rigid (1972)

Cabinet Hardware (1971)

Cabinets and (Specimen Storage) Rooms Used in the Test.

Cabinets (1973)

Cabinets, Cases, Counters and Enclosures of All Kinds

Cable and Capacitor (Insulating) Oils (R1969) ANSI C59.

Cable Connectors for Audio Facilities for Radio Broadca

Cable for the Transmission and Distribution of Electric

Cable for the Transmission and Distribution of Electric

Cable Installation (1973) ANSI C33.58

Cable Installation (1973) A NSI C33.92

Cable Installation (1973) ANSI C33.98

Cable Specs.) (1973)

Cable Systems (1972) ANSI C59.69

Cable Systems (1972) ANSI C59.70

Cable Take Up (1973)

Cable Television) Application (75 Ohms) (1973)

ITy for Cellular

/E Steel and Alu UL

Aluminum UL

Spec, for Continuit ASTM

ISpec. for Continuity ASTM

Cable Television) (1970)

Cable Trays (1972)

Cable with Ethylene-Propylene-Rubber Insulation, 20

Cable Wrought Steel Heavy Thimble (For Ground Support E

Cable $15 \mathrm{kV}$ Through $35 \mathrm{kV}$ (Copper and Aluminum Conducto

Cable (1966) IPCEA S-64-474

Cable (1969) ANSI C7.40

Cable (1972)

Cable (1972)

Cable (1972)

Cable (1972)

Cable (1973)

Cable (1973) ANSI C8.25

Cable (1973) ANSI C8.28

Cable (1973) ANSI C8.31

Cable (1973) ANSl C8.32

Cable (1974)

Cable (1974)

Cable (1974) ANSI C8.29

Cable (1974) ANSI C8.37

Cable (1974) ANSI C8.38

Cable) (1973)

Cable, 75 Deg. C Operation (1974) ANSI C8.24

Cable, 75 Deg. C Operation (1974) ANSI C8.27
Std. for Alumin

Std Spec. ASTM

Rec. Pract. for TTMA

Std. Spec for Gen ASTM

Std. Spec. for ASTM

ASTM

Std. Spec. for Styren ASTM

Std. Spe ASTM

Std. Spec ASTM

ASTM

/ for Plastic Utilities Duct for Undergro NEMA

ASTM

Std. Spec ASTM

Std. Spec. for Synthetic ASTM

Spec. for Tinne AA

ASTM

/D. Spec. ASTM

D1416

D2593

D3191

D3189

D3186

D3185

PS17

D3138

D2750

SD55

D2610

D2611

D3261

Fl 40

B16.34

SP72

44.30

1091

SP67

C374

B5.46

B 94.43

Bl.9

12

A 156.1

C7

B16.25

B16.9

D3126

D2112

E475

D1352

D2871

K62.131

D1396

D2446

D1503

D2560

D817

04.8

$04 \cdot 20$
$04-23$

G38

D2446

D1503

D2560

1369

201

C511

Al61.1

*1-400

D831

RS297.A

WC5

WC7

209

1

797

52

D1818

D 1819

511

RS403

001B-0670

P.54.440

W $\mathrm{C} 8 / 7$

1045

P. $53-426$

TDJ. 180

B314

D1351

D2308

RP40

D2768

D2819

D532

D866

D753

D752

D 1047

TC6 
rubber Heat and Moisture Resisting Insulation for Wire and Std. Spec. for Synthetic Rubber Insulation for Wire and Std. Ampacities ethylene Propylene Rubber Insu/ tant Ethylene-Propylene-Ru/ Std. for Electrical Power Std. for Electrical Power Std. for Mine Power 973) IPCEA \$68.516 or Portable Single and Multiple Conductor Electrical Power Safety Std. for Varnished Cloth Wires and all Thermoplastic Type MTW 600 Volt Machine Tool Wires and $r$ Conduit for Use as Raceway for Installation of Wires and ation Pract. for Underground Electrical Power Distribution Sid for Color Coding of Electrical Wires and ings for Underground Installations of Electrical Wires and Std. for Safety for Nonmetallic Sheathed Electrical Meth. of Analysis for Ash in pectrochemical Analysis of Nuclear Grade Silver-Indium Std. Meth. of Test for Lead and Std. for Bushing-Clamp Up. Steel, Std. for Plating. High Strength Steels, sited Coatings of Copper, Nickel, Chromium, Lead, Zinc, or Meth. of Analysis of Crude Fiber in Soy Flour for Meth. for Determination of Specific Volume of Creams, Meth. of Analysis for Baking Quality of Meth. for Use of Layer for Phosphorus Deoxidized Copper Wire Bars, Billets, and or Oxygen Free Electrolytic Copper Wire Bars, Billets, and si H17.l Std. Spec. for Lake Copper Wire Bars si H17.2 Std. Spec. for Chemically Refined Copper Wire Bars, le Screened Ground Refractory Materials (Calcined Bauxite. of Single and Double Screened Ground Refractory Materials oncrete (1973) ANS1 A31 Std. Spec. for Fly Ash and Raw or atography (1973) Spectroscopy (1973) Sid. Meth. for Analysis of Std. Meth. of Test for Uniform Fire Code: Welding and Cutting. on, lce Removal, Acceleration of Concrete/ Std. Spec. for Meth. for Analysis of rates $(1957)$ Std. Analytical Meth. for Determining Std. Analytical Meth. for Determining Meth. of Analysis of Meth. of Analysis of Meth. of Analysis of Std. Meth. of Test for

lyceride, Salts, Soy F/

73) de and Refined), S/ other Starch Hydro Meth. of Calculation of Crude Protein in Wheat and Flou (Cereal Chemistry) (1962) o Different Bases (1974) ure (1967) ANS1 B72.10

Petroleum (LP) Gases from Compositional / ool. Top, and Noil for Various Comm/ Quantitative Meth. of Analysis of Qualitative Meth. of Analysis of Std Spec for Prefaced Concrete and Std. Analytical Meth. for Determining Std. Analytical Meth. for Determining Std. Analytical Meth for Determinin culated from Percentage of Total Nitrogen) (C ated from Percentage of Total Nitrogen) (Cereal/ ematic Viscosity of Transparent and Opaque Liquids and the tes) (1969)

late Fuels (1971) ANSI Z11.313

2)

Hydrocarbons (1973) ANSI Z106.1 tional Units) (1972)

1971)

ntation) (1974)

ion of Machines (1974) ANSI Z1 15.2 Std. for Artificial Headbone for the Std. Meth. for Couple Sid. Meth. for

AC Fine Test Dust (1972) ANS1 B93.28 Std. Meth. for Verification and Std. Meth. for Absolute Std. Meth. for optical Pyrometer (1972) ANSI N144 es (1974)

al Analysis (1973)

(1972)

Spec and Rec. Pract for I Std. Procedure for Bench
Std. Meth. for
Pract. for Installation and Std. for Procedures for Std. Rec. Pract. for Rotameter ation of Test Specimens of Bituminous Mixtures by Means of ation of Bituminous Mixture Beam Specimens by Means of the $s$ and Hem Fir, Southern and Lodgepole Pine, Western Larch, Uniform Building Code Std. for 73)

Std. Rec. Pract. for Measuring bomb Calorimeter (1973)
Cable, 75 Deg. C Operation (1974) ANSI J8.4

Cable, 90 Deg. C Operation (1974) ANSl C8.40

Cables in Open Top Cable Trays (1972)

Cables Rated $0 \cdot 35,000$ Volts and Having Ozone Resistant

Ynthetic ASTM

Cales Rated 2000 Volts and Less and Having Ozone Resis

Cables with Ethylene-Propylene-Rubber Insulation (1

Cables with Ethylene-Propylene-Rubber Insulation (1

Cables (1971) ANSI C 33.73

Cables (1972)

Cables (1972) ANSI C33.37

Cables (1973)

Cables (1974)

Cables (1974)

Cables (1974) ANSI C33.56

Cacao Products (Cereal Chemistry) (1962)

Cadmium Alloys (1974)

Cadmium Extracted from Glazed Ceramic Surfaces (1972)
Cadmium Plated (1973)

Cadmium (1973)

Cadmium) (1973)

Cake and Meal from Cottonsosion Resistant Metals (Electr

Cake Batters, and lcings (Cereal Chemistry) (1962)

Cake Flour (Cereal Chem istry) (1962)

Cake Measuring Template (Cereal Chemistry) (1962)

Cakes (1972)

Cakes (1972) ANSI H23.11

Safety Std, for Std. for Safety for Fibe Install

Cakes, Slabs, Billets, Ingots, and Ingot Bars (1973) an

Cakes, Slabs, Billets, lngots, and lngot Bars (1973) an

Cakes, Slabs, Billets, Ingots, and Ingot Bars (1973) an

Calcine, Chrome Ore, Clays, Diaspore, Ganister Quarzite

Calcined Bauxite, Calcine, Chrome Ore, Clays, Diaspore,

Calcined Natural Pozzolans for Use in Pottland Cement C

Calcium and Barium Petroleum Sulfonates by Liquid Chrom

Calcium and Magnesium in Iron Ores by Atomic Absorption

Calcium Carbide and Acetylene (1973)

Calcium Chloride to Be Used for Dustlaying, Stabilizati

Calcium Chloride Types I and II (1972T)

Calcium Content in Corn Starch (1960)

Calcium Content of Corn Syrup, Sugar and Other Carbohyd

Calcium in Bread and Flour (Cereal Chemistry) (1962)

Calcium in Feedstuffs (Cereal Chemistry) (1962)

Calcium in Mineral Feeds (Cereal Chemistry) (1962)

Calcium or Zinc in Paint Driers by EDTA Meth. (1973)

Calcium Peroxide in Dough Conditioners Containing Monog

Calcium Peroxide (Cereal Chemistry) (1962)

Calcium Silicate Masonry Units (1973)

Calcium (Egta-Titrimetric) Content in Corn Starch (19

Calcium (Egta-Titrimetric) Content of Corn Sugar (Cru

Calcium (Egta-Titrimetric) Content of Corn Syrup and

(Calculated from Percentage of Total Nitrogen) (Cereal C

(Calculated from Percentage of Total Nitrogen) (Cereal $\mathrm{C}$

Calculated from Total Chlorides in Feeds and Feedstuffs

Calculating Coal and Coke Analyses from As-Determined T

Calculating Stress in Plastic Pipe Under Internal Press

Calculation of Certain Physical Properties of Liquefied

Calculation of Commercial Weight and Yield of Scoured W

Calculation of Crude Protein in Feeds and Feedstuffs ( $C$

Calculation of Crude Protein in Wheat and Flour (Calcu

Calculation of Dynamic Viscosity (1972) ANSI Z11.107

Calculation of Heat Gain Through Opaque Walls (Tech. No

Calculation of Liquid Heat Capacity of Petroleum Distil

Calculation of Percent Moisture (Cereal Chemistry) (196

Calculation of Volume and Weight of Industrial Aromatic

Calculations for Oil Sucker Rod Pumping Systems (Conven

Calendar and Ordinal Date for lnformation Interchange (

Calibration and Format for Nuclear Logs (Record. Docume

Calibration Instruments for Verifying Time Load Indicat

Calibration of Audiometer Bone Vibrators (1972)

Calibration of Earphones (1973)

Calibration of Liquid Automatic Particle Counters Using

Calibration of Liquid-ln-Glass Thermometers (1972) ANSl

Calibration of Pressure Transducers (1972)

Calibration of Reflectance Std. (1971) ANSI Z172.4

Calibration of Refractory Metal Thermocouples Using an

Calibration of Tank Level Gaging Tapes and Sounding Rul

Calibration of Temperature Scale for Differential Therm

Calibration of Turbine Flow meters (1972)

Calibration of Underwater Electro Acoustic Transducers

Calibration (1973)

California Kneading Compactor (1971) ANSI A37.138

California Kneading Compactor (1973)

California Redwood and Hardwood Timber (1973)

California Redwood Lumber (1973)

Caliper of Resilient Floor Covering with Foam Layer (19

Call Equipment (Audio / Visual Communication) (1974)

Calorific Value of Solid Fuel by the lsothermal Jacket
ASTM

APTM

NEMA

NEMA

D1679

D 1523

P-54.440

WC8

WC $8 / 1$

W $\mathrm{C} 8 / 5$

WC $8 / 6$

133

1063

543

$52 \cdot 2$

WC30

$\mathrm{TCl}$

$\begin{array}{ll}\text { UL } & 719 \\ \text { AACCH } & 08.10\end{array}$

ASTM C760

ASTM C738

NSA

NSA

ICBO

73
672

UBCS 32.6

AACCH 32.17

$\mathrm{AACCH} \quad 72-10$

AACCH $10-90$

A $\mathrm{CCH} \quad 10-9$

ASTM B379

ASTM B170

ASTM B442

ASTM B5

ASTM C316

ASTM C316

ASTM C618

ASTM D2894

ASTM E508

CBO UFC*2ART3I

ASTM D98

ASTM E449

CR B-10

CR E-10

$\mathrm{AACCH} \quad 40-20$

AACCH 40-21

$\mathrm{AACCH} \quad 40-26$

ASTM D2613

$\mathrm{AACCH}$ 48-51

$\mathrm{AACCH} \quad 48-50$

ASTM C744

CR B-11

CR F-8

CR E.11

AACCH 46.18

$\mathrm{AACCH} \quad 46-19$

$\mathrm{AACCH} \quad 40-61$

ASTM D3180

ASTM D2153

ASTM D2598

ASTM D2720

AACCH 46-18

$A A C C H$ 46-19

ASTM D445

BIA 4A

ASTM D2890T

AACCH 44-01

ASTM D1555

API $\quad 11 \mathrm{~L}$

ANSI $\quad \mathrm{X} 3.30$

API RP33

ASTM E74

ANSI \$3.13

ANSI $\quad \$ 3.7$

NFLDP T2.9.6

ASTM E77

ANSI B88.

ASTM E306

ASTM E452

ANSI B88.2

ASTM E474

ISA

ANSI

ASTM

RP31.1

S1. 20

D1561

D3202

ICBO UBCS25.11

$\mathrm{ICBO}$

UBC $\$ 25.7$

ASTM

UL

F387

ASTM D3286 
Std. Meth. for Design and Use of a Thin Skin ring Heat Transfer Rate Using a Thermal Capacitance (Slug) alorific Value of Solid Fuel by the Isothermal Jacket Bomb Std. for

Std. Dimensions of $35 \mathrm{~mm}$ Motion Picture s. Outline for Monochrome Cctv (Closed Circuit Television) etermining Speed of $16 \mathrm{Mm}, 8 \mathrm{~mm}$ and Super 8 Reversal Color for Dimensions for Unitized Microfilm Carriers (Aperture, of Buildup Area on Unitized Microfilm Carriers (Aperture, Safety Std. for Surveillance Std. for Shutter Tests for Still Pictur Std for Dimensions for Front Lens Mounts for Sizes for Roll, $35 \mathrm{~mm}$ Film, and $126 \mathrm{~mm}$ Film Still Picture markings for Variable Speed Shutters Used in Still Picture Recording Instruments and for Microfilm and Still Picture Std. for Back Window Location for Roll Film backflow Preventers) and Relief Valves in Travei Trailers, $50 \mathrm{Ft}$. Summe

Recreational Vehicles (Travel and Camping Trailers, Truck a Spec. for Flame Resistant Materials Used in le Homes, Modular Housing, Prefab Houses, Motor Homes, and tion of Plumb/ Std. for Recreational Vehicles (Travel and 1972) Rec. Pract. for Homes and Std. Spec. for Simple (I952) r Flexible Poly(viny! Chloride) Plastic Sheeting for Pond Std. Analytical Meth. for Determinin Std. for ldentification of Air Purifying Respirato 8, Exterior Veneer, Awnings and Patio Covers, Greenhouses, erformance Requirements for Cotton Woven Tent, Awning, and ce Requirements for Woven Man Made Fiber Tent, Awning, and

Std. for Loose Metal End Dimensions (6 Ounce Fibre Citrus Std. for Safety for Metal Waste (Trash, Garbage) Std. Spec. for Tin Plate Fabricated Aeroso Sid. Rec. Pract. for Labeling Aeroso

td. Test. Procedure for the Blow Off Pressure of Composite Std. Test. Proc. for Pressure Collapse Test of Composite General Rules and Formulae for Fibre

td. Test. Procedure for Oil Wicking of Composite Motor Oil Std. Test. Procedure for Filled Cast Flat Drop Composite edure for Filled Cast Flat Bottom Drop Composite Motor Oil Industry Std. for Loose Metal End Dimensions of Composite d. Test. Proc. for Measuring Inside Diameters of Composite Composite

est. Procedure for Measuring $\mathbb{W}$ all Thicknesses of Composite st. Procedure for Axial (End to End) Compression Composite 1. Procedure for Measuring Lengths or Heights of Composite std. Meth. of Test for Stiffness of Plastics by Means of A Std. Wet Process Porcelain Insulators (Apparatus,

Meth. of Measuring Water Vapor Transmission Through Screw king, Alloy and Corrosion Resisting Steel, UNJC-3A Thread, alloy Steel and Corrosion Resisting Steel, UNJF.3A Thread, it Breakers R/ Std. for Schedule of Preferred Ratings for it Breakers Rated on a Symmetrical/ it Breakers Rated on a Symmet/ Sid. Requirements for td. Meth. for Measuring Heat Transfer Rate Using a Thermal Sid. for Measurement of Smell Values of Transistor Agricultural Grain Storage Loads, Pressures, and Tower Silos: Unit Weight of Silage and Silo of Higher Boiling Homologues of Chlorinated Biphenyls for it Reclosers, Automatic Line Sectionalizers and Oil Filled Std. Meth. of Test for Gas Content of Cable and Line Compensation (Including Safety Req/ Std. for Series Std. for Paper, Paper/Film, Film Dielectric spec. for Chlorinated Aromatic Hydrocarbons (Askarels) for ronic lnstruments) (1/

Std. Requirements for Electrol ytic Std. for Molded and Dipped Mica and Mica Films Suitable for Use in Fixed Mica-Dielectric Requirements for Power Line Coupling Std. for Fixed Electrolytic Tantalum Std. for Fixed Composition Safety Std. for

Std. Spec, for Aluminum Foil for

ers (1972) Std. for ning Barium Yield, Getter Gas Content, and Getter Sorption for Uniform Meth. of Determining the SAE Rating and Struck for Uniform Meth. of Determining the SAE Rating and Struck Std. Meth. of Test for Load Carrying
Std. for Determination of

td. Meth for Calculation of Liquid Heat (Cereal Chemistry) (1968) Meth. of Analysis of Hydration Std. Meth. of Testing Flow d on Spread Footings (1972) Std. Meth. of Test for Bearing 72) Std. Meth. of Test Fol Std. Meth. of Test for Bearing I Std. Test Procedure for Measuring Hydraulic Lift Force std. for Test Procedure for Measuring Hydraulic Lift Force
Calorimeter for Measuring Heat Transfer Rate (1972)

Calorimeter (1972) Std. Meth. of Test for Gross C

ASTM

Calorimeter (1973)

Strength Stud (1973)

Cam Follower, Needle Bearing, High Strength

Camera Films Intended for Direct Projection in Motion P

(Ec.

Camera, Copy and Image Cards) (1972)

Camera, Copy, and Image Cards) (1973) ANSI PH5.12

Cameras for Banks and Mercantile Premises (1973) ANSI S

Cameras (Photography) (1972)

Cameras (1972)

Cameras (1972)

Cameras (1972)

Cameras (1972)

Cameras (1973)

Camp Cars and Trailer Coaches (1970)

Camp Rifle Range Plans (1972)

Campers, Motor Homes): Insta

Camping Tentage (Tent) (1974)

Camping Trailers) (1974)

Camping Trailers, Truck Cam

Can Puncturing Device for Use in Aerosol Laboratories (

Canal, and Reservoir Lining (1972T)

Candy Color and Inversion Con

Canopies) (1973)

Corn Syrup Unmixed

anopy Fabrics.

The Diffusers in Ceilings,

Std. Picture ANSI
Std. Exposure Time ANSI

Canopy Fabrics. Vinyl Coated or Laminated (Institutiona

Cans $202(52 \mathrm{Mm}$ ) Diameter) (I973)

Cans (For Combustible Materials) (1974) ANSI Z221.1

Cans (1972)

Cans (I972)

Cans (I973)

Cans (1973)

Cans (1973)

Cans (I974)

Cans (1974

Cans (1974)

Cans (211 (66 Mm) Diameter), Citrus Style (1974)

Cans, Tubes and Cores (1973)

Cans, Tubes and Cores (1974)

Cans, Tubes and Cores (1974)

Cans, Tubes and Cores (1974)

Cans, Tubes, and Cores (1974)

Cantilever Beam (1970) ANSI K65.13

Cap and Pin Type) (1971)

Cap Closure Liners (1973)

Cap Screw (1972)

Cap Screw (1972)

/Lled and Drilled, Plain and Self Loc /Nd Drilled, Plain and Self Locking,

Capacitance Current Switching for AC High Voltage Circu

apacitance Current Switching for AC High Voltage Circu

Capacitance Current Switching for AC High Voltage Circu

Capacitance (Slug) Calorimeter (1972)

Capacitance (1972)

Capacities (1968)

Capacities (1972)

Capacitor Askarels (1974)

Capacitor Switches for Alternating Current Systems (I9?

Capacitor (Insulating) Oils (R1969) ANSI C59.23

Capacitors for Electrical Transmission and Distribution

Capacitors for Power Semiconductor Applications (1973)

Capacitors (Electrical Insulating Liquid) (1973) ANSl C

Capacitors (For Use Primarily in Transmitters and Elect

Capacitors (Wire Lead Styles) (1972)

Capacitors (1971) ANSI C59.26

Capacitors (1972)

Capacitors (1972)

Capacitors (1972) ANSI C83.89

Capacitors (1973)

Capacitors (1974) ANSI C116.1

Capacity Designation for Fertilizer Hoppers and Contain

Capacity for Barium Flash Getters (1972) ANSI Z173.50

Capacity for Hoe Dipper (Backhoe Bucket) (1972)

Capacity for Shovel Dipper, Clam Bucket, and Dragline B

Capacity of Fluid Gear Lubricants (1973) ANSI Z11.161

Capacity of Lead Acid lndustrial Storage Batteries for

Capacity of Petroleum Distillate Fuels (1971) ANSI Z11.

Capacity of Pregelatinized Starches and Cereal Products

Capacity of Refrigerant Capillary Tubes (1972)

Capacity of Soil for Static Load on Spread Footings (19

Capacity of Soil for Static Load on Spread Footings (19

Capacity on Agricultural Tractors Equipped with 3 Point

Capacity on Agricultural Tractors Equipped with 3 Point
ASTM NSA ANSl EIA ANSI

Std. ANSl

Ess NMA

UL

ANS

ANSI

IAPM

NFPA

CPAI

FMS

NFPA

NFPA

ASTM

ASTM

CR

ANSI

ICBO

ANSI

Rec. CCT

UL

ASTM

AST

CCTI

CCTI

CCTI

CCT

CCTl

Rec. CCTI

St CCTI

CCTI

Std. T CCTI

Std. Te CCTI

Std. Tes CCTI

ASTM

ANS1

d. ASTM

NSA

NSA

IEEE

IEEE

ASTM

EIA

ASAE

ASAE

ASTM

NEMA

STA

ANSl

EIA

ASTM

EIA

EIA

ASTM

ANSI

ElA

EIA

UL

ASTM

ASAE

ASTM

Std. SAE

SAE

ASTM

NEMA

ASTM

$\mathrm{AACCH}$

ASHRA

ASTM

ASTM

ASAE

SAE

E459

E457

D3286

PH2 22.59

RS31 2

PH22.146

PH5.8

MS9

983

PH 3.48

PH3.14

PH 3.39

PH 3.32

PHI.33

PH3.1

TSC8

$-16$

(16

84
7.90

7.90
$501 \mathrm{C}$

224

D3058

D3083

E.12

K13.1 
and Filter Driers in Refrigeration and $\mathrm{A} i /$ ulent Flow (1974)

Std. for Control Valve Std. for Control Valve Std. for Boat Load

h. for Analysis of High Purity N-Heptane and Isooctane by Std. Spec. and Operating Instructions for Glass mal Animal and Vegetable Fats (Cereal Chemistry) (I967) membrane Apparatus (1972) h23.8

Std. Meth. of Test for Std Spec for Hard Drawn Copper Std. Meth. of Testing Flow Capacity of Refrigerant Std. for Nut. Std. Meth. of requency Radiation Hazards in the Use of Electric Blastin neral Purpose Semi-Tubular, Full Tubular, Split Rivets and etrachloroethyl) Thio)-4-Cyclohexene-1,2-Dicarboximide elated Documentation Practices: Chassis Frames-Passenge Rec. Pract. for Nomenclature for Passenger Rec. Pract. for Passenger Rec. Pract. for Passenge

echniques for Exhaust Gas Emissions Measurement (Passenge $r$ Test. Machines for Measuring the Uniformity of Passenge Tires Including Tire Treads Depth Requirements (Passenger Rec. Pract. for Passenger Rec. Pract. for Passenge Rec. Pract. for Passenger vice Brake Structural Integrity Test Procedure-Passenger ting) for Passenger Automobiles, Commercial Vehicles, Golf ng and Logging (Short Haul); Grader, Loader, Dozer, Mining Std. for Tires and Rims for Motorcycle, Side Definitions and Terms Used in the Selection of Tires me for the Pest Control Chemical Ethyl Mhydroxycarbanilate Std. Meth. of Chemical Analysis of Silicon Uniform Fire Code: Welding and Cutting, Calcium Tipped, Roller Turner Type (1972) Std. for Std. for mp Type Holders (1973) Std. for

e Holders (1973) Tent. Spec. for Nuclear Grade Boron Carbide Blanks and Cutting Tools, Single Po Meth. of Test for Transverse Rupture Strength of Cemented Std. Spec. for Methyl Isobuty determining Calcium Content of Corn Syrup, Sugar and Other astm G56.2 Std. Spec. for era/ Std. Spec. for Low and Intermediate Tensile Strength Std. Spec. for Steel Castings, Heavy Walled,

for General Requirements for Hot Rolled and Cold Finished Std. Spec. for

3) ANSI G46.2 atures (1973) / Std. Spec. for Piping Fittings of Wrought Std. Spec. for Piping Fittings of Wrought or Reduction Gears (1972) ANSI G55.8 Std. Spec, for ponents (1973) Ansi/ Std. Spec. for Quenched and Tempered 974)/ Std. Spec. for Quenched and Tempered Vacuum Treated shafts (1974) ANSI G55.I4 Std. Spec. for Vacuum Treated $2.4 \quad$ Sid. Spec. for Seamles and Temperature Service (1973) ANSI E38./ Std. Spec. for Std. Spec. for General Requirements for Specialized e (1972A) ANSI B125.1/ Std. Spec, for Seamless and Welded Std. Spec. for Steel Forgings, nery Service (1972) ANSI B12/ Std. Spec. for Seamless Low 19/. Meth. of Test for Electrical Resistivity of Manufactured i K90.12 Std. Meth. of Test for Thermal Diffusivity of
Std. Meth. for Chemical Analysis of

Std. Meth. of Test for Density in Air of Manufactured Std. Meth. for Chemical Analysis of
s of Terms Relating to Manufactured Std. Definitions of Terms Relating to Manufactured

coke (1973)

que Usi/ Std. Meth. for Spectrochemical Analysis of Plain Std. Spec. for Heavy Walled Std. Spec. for Longitudinal Beam Ultrasonic Inspection of ane Arcl Std. Meth. for Spectrochemical Analysis of Plain (1971) ANSI Z/ Std. Meth. for Spectrochemical Analysis of rgings and Stock/ Spec. for Quality Assurance Sampling of Rec. Pract. for Ai

eather Resistance of Textile Materials and Coated Fabrics:

si Ll/ Test Meth. for Weather Resistance of Textiles: Test Meth. for Colorfastness of Textiles to Light: Test Meth. for Colorfastness of Textiles to Light, Std. Test Meth. for Heating Loss of t. for Stating the Precision of ASTM Test Meth. Related to meth. of Test for Discoloration of Orthodichlorobenzene by Std. Meth. of Test for Surface Area of Std. Meth. of Test for Tint Strength of Std. Meth. for Evaluation of and Test Formula for Std. Meth. for Evaluation of and Test Formula for Std. Classification System for 2) ANSI G54.15 Std. Spec. for Steel Wire, Medium High
Capacity Rating and Application of Suction Line Filters Capacity Test Procedure for Compressible Fluids in Turb Capacity Test Procedure for Incompressible Fluids (1972 Capacity (Under $26 \mathrm{Ft}$. in Length) (1973)

Capillary Gas Chromatography (1971) ANSI Z11.262

Capillary Kinematic Viscometers (1972) ANSI Z11.275

Capillary Meth. of Analysis of Melting Point of All Nor

Capillary Moisture Relationships for Soils by Pressure

Capillary Tube for Restrictor Applications (1974) ANSI

Capillary Tubes (1972)

Capitive Washer, Shear Type, 450 and 800 Deg. F (1973)

Capping Cylindrical Concrete Specimens (1973)

Safeguards Against Radio F Std. for Ge

Caps (1972)

Captafol (1973)

Est Control Chemical cis- $N-((1,1,2,2 \mathrm{t}$

Car and Light Truck-Ground Vehicle Practices (1974)

Car and Light Truck Exhaust System Parts (1972)

Car and Light Truck Muffler Parts Nomenclature.(1971)

Car and Light Truck Resonator Parts Nomenclature (1971)

Car and Light Truck) (197I)

Car Tires (1972)

Car Type Tires) (1971)

Ory Instrumentation and $\mathrm{T}$

Rec. Pract. Fo

Safe Operating Condition of

Car Vehicle Identification Number Systems (1972)

Car Windshield Defrosting Systems (1971)

Car Windshield Washer Systems (1972)

Car (1971)

Car, and Other Diversified Off the Highway Applications

Car, Mini Bike, and Bicycle (1974)

(Car, Station Wagon, Truck, Bus, Trailer, Multipurpose P

Carbanilate (Ester) Desmedipham (1973)

Carbide Abrasive Grain and Crude (1971)

Carbide and Acetylene (1973)

Carbide Blanks and Cutting Tools, Single Point, Carbide

Carbide Blanks for Tipping Circular Saws (1972)

Carbide Chip Breakers Used in Indexable Inserts for Cla

Carbide Powder (1973)

Carbide Seats Used with Indexable Inserts for Clamp Typ

Carbide Tipped, Roller Turner Type (1972)

Carbides (1973) ANSI H9.23

Carbinol (1972)

Carbohydrates (1957)

Carbon-Chromium Ball and Roller Bearing Steel (I970)

Carbon-Silicon Steel Plates for Machine Parts
Carbon and Alloy for Pressure Vessels (1974)

Carbon and Alloy Steel Bar (1973) ANSI G24.5

Carbon and Alloy Steel Bars for Springs (1974)

Carbon and Alloy Steel for Low Temperature Service (197

Carbon and Alloy Steel for Moderate and Elevated Temper

Carbon and Alloy Steel Forgings for Pinions and Gears F

Carbon and Alloy Steel Forgings for Pressure Vessel Com

Carbon and Alloy SteeI Forgings for Pressure Vessels (1

Carbon and Alloy Steel Forgings for Turbine Rotors and

Carbon and Alloy Steel Mechanical Tubing (1973) ANSI G6

Carbon and Alloy Steel Nuts for Bolts for High Pressure

Carbon and Alloy Steel Pipe (1972) ANSI B125.20

Carbon and Alloy Steel Tubes for Low Temperature Servic

Carbon and Alloy, for General Industrial Use (1972)

Carbon and Carbon Molybdenum Steel Still Tubes for Refi

Carbon and Graphite Articles at Room Temperatures (1969

Carbon and Graphite Articles by Physical Measurements (

Carbon and Graphite by a Thermal Pulse Meth. (1972) Ans

Carbon and Graphite (1969) ANSI K90.3

Carbon and Graphite (1972)

Carbon and Hydrogen in the Analysis Sample of Coal and

Carbon and Low Alloy Steel by the Point to Plane Techni

Carbon and Low Alloy Steel Castings for Steam Turbines

Carbon and Low Alloy Steel Castings (1970) ANSI G52.7

Carbon and Low Alloy Steel for Boron by the Point to P]

Carbon and Low Alloy Steel Using a Vacuum Spectrometer

Carbon and Low Alloy Steels (Wrought Products Except Fo

Carbon Arc Gouging and Cutting (1974)

Carbon Arc Lamp Exposure with Wetting (1972) ANSI L14.2

Carbon Arc Lamp Exposure Without Wetting (1972) ANSI L1

Carbon Arc Lamp, Alternate Light and Darkness (1971) an

Carbon Arc Lamp, Contin uous Light (197I) ANSI L14.53

Carbon Black (1972)

Carbon Black (1972)

Carbon Black (1972A)

Std. Rec. Prac

Carbon Black (1973)

Carbon Black (1973)

Carbon Blacks in Natural Rubber (Nr) (1973)

Carbon Blacks in Styrene-Butadiene Rubber (SBR) (1973

Carbon Blacks Used in Rubber Products (1973) ANSI J8.6

Carbon Cold Heading Quality for Hexagon Head Bolts (197

Carbon Dioxide Fire Extinguishing Systems (1972)
ARI

ISA

ISA

ABYC

S39.4

$\mathrm{S} 39.2$

H5

D2268

ASTM D2515

AACCH 58.40

ASTM D3152

ASTM B360

ASHRA 28

NSA 1726

ASTM C617

$\mathrm{ME}$

20

B18. 7

K62.146

Y14.32.1

J97

AE

SAE

SAE

J261

$\mathrm{J} 262$

J332A

$\mathrm{V}-7$
$\mathrm{~J} 273$

$\mathrm{J} 273$

J902B

J942B

J229

*1.48

3

6

X.XI

K62.139

B74.15

UFC*2ART31

B94.37

B94.42

B94.47

$\begin{array}{ll}\text { ANSI } & \text { B94.4 } \\ \text { ASTM } & \text { C750 }\end{array}$

$\begin{array}{ll}\text { ASTM } & \text { C750 } \\ \text { ANSI } & \text { B94.46 }\end{array}$

ANSI B94.37

Std. ASTM B406

ASTM D2635

CR

ASTM

ASTM

ASTM

E-10

A 295

A284

A 643

A29

ASTM A689

ASTM A 420

ASTM A234

ASTM A291

ASTM A541

ASTM A508

ASTM A 470

ASTM A519

ASTM A194

ASTM A530

ASTM A334

ASTM A668

ASTM Al6I

ASTM C611

ASTM C559

ASTM C714

ASTM C560

ASTM C709

ASTM D3178

ASTM E403

ASTM A356

ASTM A609

ASTM E404

ASTM E415

SAE AMS2370A

AWS C5.3

AATCC 111A

AATCC $111 \mathrm{C}$

AATCC $16 \mathrm{D}$

AATCC $16 \mathrm{~A}$

ASTM D1509

$\begin{array}{ll}\text { Std. ASTM } & \text { D } 3051 \\ & \text { ASTM }\end{array}$

ASTM D3037

ASTM D3265

ASTM D3192

ASTM D3191

ASTM D1765

ASTM A546

NFPA 12 
(Cereal Chemistry) (1962) Meth of Analysis for Total sis for Correction Factors for Gasometric Determination of troleum Oils by the $\mathrm{n} \cdot \mathrm{d}-\mathrm{M}$ Meth. I

Tent. Meth. of Test for Std. Meth. of Tension Test. of Rec. Pract. for Hardenability Bands for

els (1972) ANSI G35.14 Std. Spec. for rvice (1972) Std. Spec. for Pressure Vessel Plates, Std. Spec. for Pressure Vessel Plates, Heat Treated, bes (1973) ANSI BI25.9 Std. Spec. for Seamless bes (1973) Ans/ Std. Spec. for Electric Resistance Welded e (1972) ANSI B12/ Std. Spec. for Seamless Low Carbon and nt by Nondispersive Infrared Spe/ Tent. Meth. of Test for Kryptol Uniform Fire Code: Cryogenic Fluids (Air, Argon, nd Strip (1972) Std. Spec. for Low Std. Spec. for Low therefrom (1972A) Std. Definitions of Terms Relating to Std. Meth. of Test for Thermal Conductivity of Steel Products Manual for Std. Spec. for for Railway Use (1973) ANSI G57.11 ality Hot Rolled ements (1972)

Std. Spec. for Merchant Quality Hot Rolled Std. Spec. for Merchant Quality Hot Rolled sure Service (/

I25. 10

Std. Spec. for Electric Resistance Welded

73) ANSI B 125.7

Std. Spec. for Seamless Medium Std. Spec. for Seamless

Std. Spec. for Electric Resistance Welded

si G50.1

Std. Spec. for Mild to Medium Strength igh Temperature Service (1974) ANSI G37.2 Std. Spec. for Std. Spec. for

steners (1968) ANSI G38.5

tting, Valve, etc.) (1973) ANSI G17.3

Std. Spec. for Weldless Std. Spec. for Std. Spec. for Std. Spec. for

ansi B125.30

Sid. Spec. for Centrifugally Cast Std. Spec. for Seamless Std. Spec. for Seamless

B125.38 Std. Spec. for Electric Welded Low 5.2 Std. Spec. for Low and Intermediate Tensile Strength Std. Spec. for Quenched and Tempered Std. Spec. for Structural 24.I Std. Spec. for Low and Intermediate Tensile Strength for Pressure Vessels (1972) ANSI G35.7

d Higher Temperature Service (1972) ANSI /

wer Temperature Service (I973) ANSI G35.1/

Std. Spec. for Std. Spec. for Std. Spec. for trial Use (1971) ANSI G57.12 Std. Spec. for Untreated Std. Spec. for Hot Rolled Std. Spec. for

(Steel Products Manual) Sid Spec for Cold Rolled 73) ANSI / Std. Spec. for Cold Formed Welded and Seamless Std. Spec. for Hot Formed Welded and Seamless sical Properties and Meth. of Test. for Helical Spring and

972)

972)

ing, and Beading (1/

Std for Welded and Cold Drawn Std. for Welded Flash Controlled Low y Requirements for Seamless and Electric Resistance Welded

\section{) ANSI G39.2}

71) ANSI G54.17

k91.3

1971) ANSI G54.1/

Service Piping (1969) ract. Meth. and Controls to Prevent in Service Cracking of Rivets,

lates, and Steel Sheet Piling (I/ (Steel Products Manual) ${ }^{8}$ Support Equipment) (1974) Std. Spec. for Pressure Vessel Plates, Rec. Pract. for Resistance Welding Coated Low Std. for Chemical Compositions of Sae cold Finished Bars; Hot Rolled D/ (Steel Products Manual) neous Matter Meth. of Analysis (Cereal Chemistry) Alcohol; Std. Spec. f

Std. Spec. for Steel Wire, Oil Tempered orgings, Corrosion and Moderate Heat Resistant, 12.5Cr Low ommercial Quality (1972) rip, Commercial Quality (1972) Spec. for Steel,
Std. Definitions of Terms Relating to Activated

Std. Spec, for Hot Rolled
Carbon Dioxide Fire Extinguishing Systems (1973)

Carbon Dioxide in Baking Powder (Cereal Chemistry) (196

Carbon Dioxide in Baking Powder (Cereal Chemistry) (I96

Carbon Dioxide in Prepared Mixes and Self Rising Flours Carbon Dioxide (Cereal Chemistry) (1962)

Carbon Distribution and Structural Group Analysis of PE Carbon Graphite Mechanical Materials (I971) ANSI K90.6 Carbon H Steels (1971)

Carbon Manganese Silicon Steel Plates for Pressure Vess Carbon Manganese Silicon (I972A)

Carbon Manganese, for Moderate and Lower Temperature Se

Carbon Molybdenum Alloy Steel Boiler and Superheater tu Carbon Molybdenum Alloy Steel Boiler and Superheater tu Carbon Molybdenum Steel Still Tubes for Refinery Scrvic Carbon Monoxide in the Atmosphere (Continuous Measureme Carbon Monoxide, Deuterium, Fluorine, Helium, Hydrogen, Carbon Nickel Molybdenum Chromium Alloy Plate, Sheet, a Carbon Nickel Molybdenum Chromium Alloy Rod (1972) Carbon Paper and Inked Ribbon Products and Images Made Carbon Refractories (1973)

Carbon Sheet Steel (coils and Cut Lengths) (1974)

Carbon Steel Axles, Non Heat Treated and Heat Treated,

Carbon Steel Bars Subject to Mechanical Property Requir Carbon Steel Bars (1973)

Carbon Steel Bars (1973)

Carbon Steel Boiler and Superheater Tubes for High Pres Carbon Steel Boiler and Superheater Tubes (1973) ANSI B Carbon Steel Boiler Tubes for High Pressure Service (19 Carbon Steel Boiler Tubes (1973) ANSI B 125.6

Carbon Steel Castings for General Application (1973) an

Carbon Steel Castings Suitable for Fusion Welding for $\mathrm{H}$

Carbon Steel Chain (1972) ANSI G61.2

Carbon Steel Chain (1973) ANSI G61.4

Carbon Steel Externally and Internally Threaded Std. Fa

Carbon Steel Forgings for Piping Components (Flange, Fi

Carbon Steel Nuts (1972) ANSI G24.30

Carbon Steel Pipe for High Temperature Service (1972)

Carbon Steel Pipe for High Temperature Service (1972A)

Carbon Steel Pipe for Process Piping (I972A)

Carbon Steel Pipe for the Chemical Industry (1971) ANSI

Carbon Steel Plates for Pressure Vessels (1972) ANSI G3

Carbon Steel Plates for Structural Applications (1973)

Carbon Steel Plates of Improved Toughness (1973)

Carbon Steel Plates of Structural Quality (1970) ANSI G

Carbon St eeI Plates with Improved Transition Properties

Carbon Steel Pressure Vessel Plates for Intermediate an

Carbon Steel Pressure Vessel Plates for Moderate and Lo

Carbon Steel Railway Axles for Export and General Indus

Carbon Steel Sheet and Strip, Structural Quality (1972)

Carbon Steel Sheets for Pressure Vessels (1971) ANSI G3

Carbon' Steel Strip (0.68-0.80C) (SAE 1074) (1973)

Carbon Steel Strip (0.90-1.04C) (SAE 1095) (1973)

Carbon Steel Strip (I971)

Carbon Steel Strip (1972A) ANSI G47.1

Carbon Steel Structural Tubing in Rounds and Shapes (19

Carbon Steel Structural Tubing (1973) ANSI G24.22

Carbon Steel Tooth Lock Washers (1972) IImensio

Carbon Steel Tubing Annealed for Bending and Flaring (1

Carbon Steel Tubing Annealed for Bending and Flaring (I

Carbon Steel Tubing Normalized for Bending, Double Flar

Carbon Steel Tubing (1972)

Carbon Steel Tubing (1972)

Carbon Steel Tubular Products for High Temperature Serv Carbon Steel Tubular Spring Pins (1973)

Carbon Steel Water Well Pipe (1972A)

Carbon Steel Wheels for Locomotives and Rail Cars (1971

Carbon Steel Wire for Machine Screws (1971) ANSI G54.14

Carbon Steel Wire for Tapping or Sheet Metal Screws (19

Carbon Steel Wire of Scrapless Nut Quality (1972) ANSI

Carbon Steel Wire Rods and Uncoated Coarse Round Wire

Carbon Steel Wrought Welding Fittings for High Pressure

Carbon Steel (P-I) Welds in Corrosive Petroleum Refinin

Carbon Steel (1973)

Carbon Steel' Plates, Structural Shapes, Rolled Floor P

Carbon Steel, High Strength Manganese (1972A) ANSI G35.

Carbon Steel, Shoulder Nut Assembly Eyebolt (For Ground

Carbon Steels (1970)

Carbon Steels (1970) ANSI G88. I

Carbon Steel: Semifinished for Forging; Hot Rolled and

Carbon Tetrachloride; Chloral Hydrate; Chloroform; Form

Carbon Tool Steels (1973)

Carbon Valve Spring Quality (1971) ANSI G54.4

Carbon (SAE 51416F), Free Machining (1973)

Carbon (0.15 Maximum, Percent) Steel Sheet and Strip. $C$

Carbon $(0.16$ to 0.25 Maximum \%, Hot Rolled Sheet and St

Carbon (1974)

ICBO

$\mathrm{AACC}$

UBCS 38.2

12-2I

AACCH $\quad 12.29$

ASTM D3238

ASTM C565

SAE J776D

ASTM A299

ASTM A537

ASTM A662

ASTM A209

ASTM A250

ASTM Al61

ASTM D3162

UFC*2ART36

ASTM B575

ASTM B574

C767

AISI

ASTM

ASTM

ASTM

ASTM

ASTM

ASTM

ASTM

ASTM

ASTM

ASTM

ASTM

ASTM

ASTM

ASTM

ASTM

ASTM

ASTM

ASTM

ASTM

ASTM

ASTM

ASTM

ASTM

ASTM

ASTM

ASTM

ASTM

ASTM

ASTM

SAE

SAE

AISI

ASTM

ASTM

ASTM

5

A2 I

A663

Al08

A575

A226

Al92

Al 78

A216

A413

A 466

A 307

A 105

A563

A 660

A524.

A587

A285

A678

A573

A283

A515

A516

A383

A570

AMS5120G

AMS5121D

A500

A501

B18.21.1

SAE

SAE

SAE

SAE

SAE

ASTM

SAE

ASTM

ASTM

ASTM

ASTM 
s (1971A) ANSI B/ Std. Spec. for General Requirements for for Pressure Piping Component/ Std. Spec. for Steel Bars, plications (1974) and Wrought Irons (/ ity (1973)

Std. Meth. for Chemical Analysis of Std. Spec. for Steel, Std. Spec, for Steel, Cold Rolled Sheet, Sid. Spec. for Steel Tubes, Low Rec. for the Disposal of Std. Meth. of Test for Insoluble Residue in Test Meth. for Test Meth. for Colorfastness of Dyed Wool Textiles to Std. Meth. of Test for Tensile Stress Strain o Std. Analytical Meth. for Determinin Std. Meth of Test. Sodium

Rec. Pract. for Automotive Engine aterial by Absorption Spectroscopy, Using the ASTM Punched Tent. Meth. of Test for Grading Cotton Aperture Adhesive of Unitized Microfilm Carrier (Aperture Std. for Magnetic Stripe Encoding (Code) for Credi tized Microfilm Carriers (Aperture, Camera, Copy and Image ized Microfilm Carriers (Aperture, Camera, Copy, and Image rinted Wiring Boards) in Numeric (Digital) Form on Punched

(1973)

e (Rtrp) (1972)

973)

se of These Terms /

2)

achin/

ic, Semi / Std. for Safety Requirements for the Construction,
Std. for Safety Requirements for the Construction, Std. for Safety Requirements for Construction, Std. Safety Requirements for the Construction, Rec. Pract, on Application, Std. for Basic Requirements for Std. for Requirements for Closed Van Type

s (1970)

ng) (1973)

emistry) (1962 Guide for Supplementary Ventilation in a Ship's Spec. for ile, and Rail Doors, Factery. Rec. Safeguards for Arrangement of Test Meth. for Visual Rating Meth. of Test Meth. for Test Meth. for for Shampooing: Washing of Textile Floor Coverings (Rug,
Test Meth. for Electrostatic Propensity of Test Meth. for Electrostatic Propensity of ction Under the Uniform Building Code: Private Garages and el-Base Alloys by the Silver Chloride-Lithium Fluoride ical Analysis of Uranium Oxide $\left(\mathrm{U}_{i} \mathrm{O}_{k}\right)$ by Gallium Oxide eady State Surface Pho/ ing-Materials Handling) (1973)

2 In. Quadruplex Video Magneti/ Tent. Meth, of Test for Minority Shipper-Motor Rec. Pract. for Reference Std. on Subscriber Visual rotection Sheet to Aperture Adhesive of Unitized Microfilm Std. for Forks and Fork Std. for Dimensions for Unitized Microfilm ness of Buildup Area on Unitized Microfilm Sid. Test Proc. for Determining the Load 211.161 Std. Meth. of Test for Load nual Sect. 1) (1/ Spec. for Design of Aluminum Alloy Load fuel Evaporative Emissions from Gasoline Powered Passenger Std for Training of Automotive Mechanics for Passenger fuel Evaporative Emissions from Gasoline Powered Passenger Std. for Sound Level for Passenger for New Tires for Passenger

Uniform Test Procedures for Retreaded Tires for Passenger low Preventers) and Relief Valves in Travel Trailers, Camp Hopper Std. Spec. for Structural Steel for Locomotives and

for Wrought Carbon Steel Wheels for Locomotives and Rail Std. for Tire and Rim Equipment for 1974 Passenger nd Combin/ Rec. Pract. for Stopping Distance of Passenger and Fill Pipes with Fuel Dispensing Facilities (Passenger s, Bead S/ Rim and Wheel Stds. for Motorcycles, Passenger Std. for Bias and Radial Ply Tires and Rims for Passenger Layouts and Terminals (For Foreign and Domestic Passenger

3) Std. Spec. for Std for Low Voltage Safety Std. for Class H Std. for Low Voltage Safety Std. for $21 / 2 \mathrm{Gal}$ $\operatorname{ding} \mathrm{An} /$ Std. for Dimensional Std. Coplanar Magnetic Tape 972)_Std. for Dimensions of 126 Size f Drilling Tapping Screws and Rec. Technique for Measuring
Carbon, Ferritic Alloy, and Austenitic Alloy Steel Tube Carbon, Hot Rolled and Cold Finished, Special Quality, Carbon, Hot Rolled, Special Quality, for Fluid Power Ap Carbon, Low Alloy, and Silicon Electrical Steels, Ingot Carbon, Strip, Cold Rolled Hard. Untempered Spring Qual Carbon, Structural (1972)

Carbon, Tapered for Structural Use (1973)

Carbon-14 Wastes (1953)

Carbonate Aggregates (1972)

Carbonates in Coated Paper (Qualitative) (1973)

Carbonizing (1972) ANSI L14.3

Carbons and Graphite (1973)

Carboxyl Content of Corn Starch (1966)

Carboxymethylcellulose (1972) ANSI K65.30

Carburetor Airflow Reference Std. (1971)

Card Index (1972)

Card Webs for Appearance (1973)

Card) (1973) ANSI PH5.14

Cards (1973)

Cards) (1972)

Cards) (1973) ANSI PH5.12

Rec. Pract, for Identification of $M$

Cards, Magnetic Tape and Punched Paper Tape (1972

Esion of Protection Sheet to

Std. for Dimensions for Uni

for Uni ANSI

Care and Use of Casing and Tubing (Petroleum Drilling)

Care and Use of Reinforced Thermosetting Resin Line Pip

Care Labeling Textile Products (Launder or Dryclean) (1

Care of Consumer Textile Products and Rec. Pract. for U

Care of Fire Hose (Including Couplings and Nozzles (197

Care, and Use of Die Casting Machines (1973)

Care, and Use of Packaging and Its Related Converting M

Care, and Use of Power Press Brakes (1973)

Care, and Use of Shears (Mechanical, Hydraulic, Pneumat

Care, and Use of Wire Rope for Oil Field Service (1972)

Cargo Containers (1971)

Cargo Containers (1971)

Cargo Hold During the Use of Internal Combustion Engine

Cargo Unit Load Devices (Materials Transport and Handli

Carotene in Enriched Cereals and Mixed Feeds (Cereal Ch

Carpentry, Flooring, Shingles, Exposed Roof Decking, Dr

Carpet Storage Racks (1972)

Carpet (Rug) Soiling (1973) ANSI L14.215

Carpet (Rug) Soiling; Accerated Meth. (1973) ANSI L14.2

Carpet (Rug) Soiling; Service Meth. (1973) ANSI L14.216

Carpet) (1972)

Carpets (1969)

Carports (1973)

Carr-Price Meth. of Analysis of Vitamin A in Nonfat D

Carrier D.C Arc Technique Using an Optical Emission Spe

Carrier D.C Arc Technique (1970) ANSI Z128.27

Carrier Diffusion Length in Silicon by Measurement of $S$

Carrier Dock Planning Manual (Truck Shipping and Receiv

Carrier Frequencies and Deemphasis Characteristics for

Carrier Level (Cable. Tele vision) (1970)

Carrier (Aperture Card) (1973) ANSI PH5.14

Carriers for Powered Industrial Fork Lift Trucks (1973)

Carriers (Aperture, Camera, Copy and Image Cards) (1972

Carriers (Aperture, Camera, Copy, and Image Cards) (19?

Carrying Ability of Farm Wagon Running Gear (1973)

Carrying Capacity of Fluid Gear Lubricants (1973) ANSI

Carrying (Members) Structures (Aluminum Construction Ma

Cars and Light Trucks Using the Enclosure Technique (19

Cars and Light Trucks (Course of lnstruction) (1972)

Cars and Light Trucks (1972) / for the Measurement of

Cars and Light Trucks (1972) ANSI \$6.3

Cars and Station Wagons (1965)

Cars and Station Wagons (1966)

Cars and Trailer Coaches (1970)

Cars for Malt and Grain Service (1964)

Cars (1970A) ANSI G39.1

Cars (1971) ANSI G39.2

Cars (1974)

Cars, Multipurpose Passenger Vehicles, Buses, Trucks, a

Cars, Multipurpose Vehicles, and Light Duty Trucks) (19

Cars, Off Road, Agricultural and Log Skidder, Truck, Bu

Cars, Station Wagons, and Multipurpose Passenger Vehicl

Cars, Trucks, Buses, Coaches, Tractors, Industrial Road

Cartridge Brass Sheet, Strip, Plate, Bar and Disks (197

Cartridge Fuses (1972)

Cartridge Fuses (1973)

Cartridge Fuses, 600 Volts or Less (1972)

Cartridge Operated Water Type Fire Extinguishers (1973)

Cartridge Type Cp 1 (Compact Cassette) for Audio Recor

Cartridges (1973)

Cartridges, Film, and Backing Paper (Photography) (1972

Case Circuit Breakers and Circuit Breaker Enclosures (1

Case Depth (1972)

ASTM

ASTM

ASTM

ASTM

ASTM

ASTM

ASTM

IP IPC

WSFI

API

API

ANSI

ASTM

NFPA

ANSI

ANS

ANSI

ANSI

API

ANSI

ICBO

SAE

ANSI

SAE

SAE

ESC

APMO

BAMI

ASTM

TRA

SAE

SAE

TRA

TRA

$\mathrm{BCl}$

ASTM

NEMA

UL

ANSI

UL

EIA

Std. Fo ANSI

R12

TAPPI UM.531

AATCC 11

ASTM C749

CR C-22

ASTM D1439

SAE J228

ASTM E204

ASTM D3216

MA MS10

$\begin{array}{ll}X & \\ & \end{array}$

PH5.8

MS9

D. 350

$5 \mathrm{C} 1$

RP5L4

L28.1

D3136

198

BI52.1

B]55.1

B]1.3

B 11.4

9B

SNAME 4.14

NSA 3610

$\mathrm{AACCH} \quad 86.05$

AWI 1.1

FMS LPD8.30

AATCC 121

AATCC 123

AATCC 122

AATCC 138

AATCC 134

UBC $* 8.4$

AACCH 86.02

ASTM E483

ASTM E402

ASTM F39]

AHSI

SMPTE RP6

NCTA 001B.0670

NMA MSlO

ANSI MHIl.4

ANSI PH5.8

NMA MS9

ASAE S360T

ASTM D1947

AA 10

J171A

D18.

$\mathrm{J} 170 \mathrm{~A}$

J986A

V-1

V.2

TSC 8

1

A504 
ke Roof and Window Vents Designed to Open Automatically in Meth. for Analysis of rmance Requirements for Woven Window Curtain Fabrics (Sash Std. Meth. of Test. Large Shipping omatic Machine Sealing of Top Flaps of Fiberboard Shipping ral Woodwork) (197/ Quality Stds, for Casework: Cabinets, cs. for Architectural Woodwork: Standing and Running Trim, I Kinds (Architectural Woodwork) (197) Quality Stds. for Std. Meth. of Test for Std. Spec for Maximum Std. Meth. of Test for Cashmere Coarse Hair Content in Std. Spec. for Maximum Cashmere Coarse Hair Content in Spec. for Grade C.75 and C.95 Rec. Pract. for Care and Use of Spec. for Oil Well

nd Bending (1972)

re Oil Well Service) (1972)

73)

Bul. on Round Thread Thread Compounds (For Spec. for

Spec. for High Strength

for Threading, Gaging, and Thread Inspection of Petroleum Rec. for Manufacture and Placement of Reinforced Concrete Std. Coplanar Magnetic Tape Cartridge Type Cp ll (Compact h46.1 Std. Meth. of Tension Test. Wrought and 1 Outlet Boxes, Device Boxes, Covers and Box Supports, and ANSI A21.11 Electrolytes (1973) ANSI H44.1 Std. for Rubber Gasket Joints for for Codification of Certain Nonferrous Metals and Alloys, (1972) ANSl B125.39 systems (1973) Std. Spec. for Centrifugally Std, for Std. for Std.

72)

Uniform Building Code Std. for Std. Spec. for Centrifugally Std. for Std. Spec. for Centrifugally Std. Spec, for Centrifugally vice (1973)

Std. Test. Procedure for Filled

Std. Test. Procedure for Filled Std. Sizes for lndustrial Centrifugal Fans with gn and Construction (1965) Rec, for Deep, Rec. for Precast Concrete Units Used as Forms for Specifying PortIand Cement Terrazzo, Rec. for

171.1 Spec. for

ssure Application at High T/

Std. Meth. for Chemical Analysis of Std. Spec. for Centrifugally n./S for Water and Other Liquids (1971) ANS1 A2/ Std. for Safety Std. for Gasketed Joints for c. Pract. for Polyethylene Encasement for Gray and Ductile i) Std. for Polyethylene Encasement for Gray and Ductile (1972)

Hydraulic Systems (1972) Std. for Enameled pec. Data for Hubless Rec. Pract. Spec. for Std. Spec, for

Std. Spec. for Neoprene Rubber Gaskets for Hub and Spigot Std. Spec. for Guide Spec. for Std.

Spec, for Welding Rods and Covered Electrodes for Welding std. Meth. of Impact Testing of avel/ Uniform Building Code: Weights of Materials (Brick; th. for Spectrochemical Analysis of Admiralty Metal by the Meth. and Definitions for MechanicaI Test. of Wrought and Std. Spec. for Std. for Ductile lron Pipe, Centrifugally Std. Meth. for Chemical Analysis of Steel and Molybdenum Sheet, Strip, and Plate, Arc Std. Spec. for Sizes for Threaded

d. for Double Race or Bi.Level Swivel and Rigid Industrial

Std. for Demountable Tired Wheels for Industrial Trucks, in Flammable Anesthetizing Locations (Bonding Appliances, spec. for Aluminum Alloy Hardeners Used in Making Zinc Die Std. for Magnesium Std. Spec. for Gypsum Std. Spec. for Fire Refined Die

ty Requirements for the Construction, $\mathrm{Care}$, and Use of Die Std, Meth. of Test for Linear Shrinkage of Thermosetting Iron Chromium, lron Chromium Nickel and NickeI Base Alloy Std. Spec. for Mild to Medium Strength Carbon Steel Std. Spec. for Copper Alloy Sand

73) A/

Std. Spec. for Iron, Cobalt, and Nickel Base Alloy Std. Spec. for Ferritic and Austenitic Steel Std. Spec, for Steel

d. Rec. Pract. for Production and Preparation of Gray Iron
Case of Fire (1974)

Casein (1972)

Approval Std. for Heat and Smo

Casement) (Excluding Glass Fibers) (Institutional Text

Cases and Crates (Packaging) (1953) ANSl MH12.11 Solvent Soluble Liquid Adhesives for Aut

ases, Counters and Enclosures of AlI Kinds (Architectu

Casework, Panelwork, Closet and Storage Shelving, Misc.

Casework: Cabinets, Cases, Counters and Enclosures of a

Cashmere Coarse Hair Content in Cashmere (1971)

Cashmere Coarse Hair Content in Cashmere (1971)

Cashmere (1971)

Cashmere (1971)

Casing and Tubing (Petroleum Drilling) (1973)

Casing and Tubing (Petroleum Drilling) (1973)

Casing Centralizers (1973)

Casing Joint Strength with Combined Internal Pressure a

Casing, Tubing and Line Pipe Connections in High Pressu

Casing, Tubing, and Drill Pipe (Petroleum Drilling) (19

Casing, Tubing, and Drill Pipe (Petroleum Drilling) (19

Casing, Tubing, and Line Pipe Threads (1974)

Casings for Irrigation Wells (1972)

Cassette) for Audio Recording and Reproducing (1974)

Cast Aluminum and Magnesium Alloy Products (1973) ANSI /for Electrical Sheet Stee

Cast Aluminum Covers (1973)

Fittings (1972)

Cast and Ductile Iron Pressure Pipe and Fittings (1972)

Cast and Wrought Galvanic Zinc Anodes for Use in Saline
Cast and Wrought (1973) Rec. Pract.

Cast Austenitic Steel Pipe for High Temperature Service

Cast Bronze Solder Joint Fittings for Solvent Drainage

Cast Bronze Solder Joint Pressure Fïtings (1972)

Cast Bronze Threaded Fittings (125 and 250 Lb.) (1971)

Cast Building Stone (1973)

Cast Carbon Steel Pipe for High Temperature Service (19

Cast Copper Alloys (1971)

Cast Dual Metal Cylinders (1972)

Cast Ferritic Alloy Steel Pipe for High Temperature Ser

Cast Flat Bottom Drop Composite Motor Oil Cans (1974)

Cast Flat Drop Composite Cans (1974)

Cast Housings (1966)

Cast in PIace Concrete (Storage) Bin and Silo Wall Desi

Cast in Place Concrete (1966)

Cast in PIace Floors (1967)

Cast in Place Nonreinforced Concrete Pipe (1966)

Cast in Place Nonreinforced Concrete Pipe (1970) ANSI a

Cast lron-All Types (1974)

Cast Iron-Chromium-Nickel High Alloy Tubing for Pre

Cast Iron and Ductile Iron Fittings, 2 ln. Through 48 I

Cast Iron Pipe and Fittings (1973)

Cast Iron Pipe for Water or Other Liquids (1972)

Cast Iron Piping for Water and Other Liquids (1972) Ans

Cast lron Plumbing Fixtures (1973)

Cast lron Sanitary System (Plumbing Pipe and Fittings)

Cast lron Sealing Rings for Automotive Transmission and

Cast Iron Soil Pipe and Fittings (1972)

Cast lron Soil Pipe and Fittings (1972)

Cast lron Soil Pipe and Fittings (1972) ANSI Al12.5.1

Cast lron Soil Pipe (1972)

Cast lron Threaded Drainage Fittings (1971)

Cast lron Threaded Fittings (125 and $250 \mathrm{Lb}$.) (1972)

Cast lron (1969) ANSI W 3.15

Cast lrons (1972)

Cast Iron; Cinders; Concrete; Earth; Glass; Granite; Gr

Cast Pin and Point to Plane Techniques (1973)

Cast SteeI Products (1973) ANSI G60.1

Cast Steel Wheels for Railway Service (1973)

Cast, in Metal Molds or Sand Lined Molds for Gas (1971)

Cast, Open Hearth, and Wrought Iron (1973)

Cast, Stress Relieved (1973)

Caster Stems (1965)

Casters (1973)

Casters, Conveyors, and Other Equipment (1966)

Casters, Face Masks and Rebreathing Bags, Footwear, Ho

Casting Alloys (1965) ANSl H38.23

Casting Alloys (1971)

Casting and Molding Plaster (1973) ANS1 A49.4

Casting Copper (1972) ANS1 H23.9

Casting Machine Stds. (1972)

Casting Machines (1973)

Casting Systems During Cure (1969) ANSI K65.181

Castings for General Application (1972) ANSI G81.10

Castings for General Application (1973) ANSI G50.1

Castings for General Applications (1973)

Castings for High Strength at Elevated Temperatures (19

Castings for High Temperature Service (1972) ANSI G52.2

Castings for Highway Bridges (1974) ANSl G52.3

Castings for Porcelain Enameling (1970) ANSI Z167.23
FMS

TAPP

ANSl

ASTM

ASTM

AW

$A$ WI

ASTM

ASTM

ASTM

ASTM

APl

APl

APl

APl

API

API

API

API

ASAE

ElA

ASTM

NEMA

$A W W A$

ASTM

ASTM

ASTM

ANSl

ANS

ANSI

ICBO

ASTM

SAE

ASTM

ASTM

CCTI

CCTl

AMCA

$\mathrm{ACl}$

$\mathrm{AC}$

CSI

ACI

$\mathrm{ACl}$

ASTM

ASTM

AWWA

UL

1. Re ASTM

$A W W A$

ANSI

CISPI

SAE

ClSPl

CISPI

ASTM

CSI

ANSI

ANSI

AWS

ASTM

ICBO

Std. Me ASTM

Std. ASTM

ASTM

ANSI

ASTM

SAE

CFTMA

St ANSI

CFTMA

UL

Std. ASTM

$\mathrm{SAE}$

ASTM

ASTM

SDCE

Std. for Safe ANSI

ASTM

IT ASTM

ASTM

ASTM

ASTM

ASTM

ASTM

ASTM

4430

7SU

24.14

D1083

D1874

400

* 1.400

D

D2816

D2817

$5 \mathrm{AC}$ 
h T/ Std. Spec, for Martensitic Stainless and Alloy Steel Temperature Service (1974) Std. Spec, for Heayy Walled Carbon and Low Alloy Stee Std. Spec. for High Strength Steel Std. Spec. for Gray Iron

Parts Suitable for High Temp/ re Service (1974) ANSI G37.2

4330 Modified) (1973)
Std. Spec, for Alloy Steel Std. Spec, for Carbon Steel

Std. Spec. for Low Alloy Steel Std. Reference Radiographs for Steel Spec. for Steel Sand Std. Spec. for Lead and Tin Alloy Die Std. Spec. for Zinc Alloys in Ingot Form for Die ium Alloys in Ingot Form for Sand, Permanent Mold, and Die hs for High Strength Copper Base and Nickel-Copper Alloy Std. Spec. for Titanium and Its Alloy l Beam Ultrasonic Inspection of Carbon and Low Alloy Steel Std. Spec. for Brass Die

Std. Spec. for Pearlitic Malleable Iron Std. Spec. for Zinc Alloy Die Spec. for Automotive Gray Iron Std. Spec. for Ductile Iron Std. Spec, for Magnesium Alloy Investment Std. Spec. for Magnesium Alloy Die ation of Procedures and Personnel for the Welding of Steel Std. Spec. for Aluminum Alloy Die Std. Spec. for Magnesium Alloy Sand Std. Spec, for Magnesium Alloy Permanent Mold rence Radiographs for Heavy Walled (4 1/2 to 12 In.) Steel Std. Spec. for Austenitic Gray Iron Std. Definitions of Terms Relating to Iron Std. Spec. for Copper Base Alloy Centrifugal Std. Spec. for Copper Base Alloy Continuous Std Spec for Austenitic Manganese Steel num Alloys in Ingot Form for Sand, Permanent Mold, and Die Std. Spec. for Chromium Nickel Alloy Std. Spec. for Steam or Valve Bronze Std. Spec. for Composition Bronze or Ounce Meta Std. Spec. for Aluminum Alloy Sand Std. Spec. for Aluminum Alloy Permanent Mold 74) ANSI G/ Std. Spec. for Chromium-Nickel-Iron Alloy d Precipitation Treated (/ Spec. for Magnesium Alloy Sand essels (1974) 36C) (SAE 4330 Modified) (1973) 46C) (SAE 4340 Modified) (1973) Std. Spec. for Stee Steel 973)

Low Alloy Stee Low Alloy Steel rence Radiographs for Inspection of Aluminum and Magnesium cluding Lacquers (1973)

Std. Spec. for Test Meth. for

Description, and Grade of Palmkernels; Babassu; Flaxseed; (1972) ANSI B93.29 Std. Dimensions for Accessories for ode, and Mounting Dimensions for 3/4, 1 and 1 1/8 In. Bore terials When Suitable Amounts of Sample, Sulfuric Acid and terials When Suitable Amounts of Sample, Sulfuric Acid and terials When Suitable Amounts of Sample, Sulfuric Acid and

Std. Spec. for Concrete Masonry Units for Construction of Std. Spec. for Electrolytic receiving Applia/ Std. for Safety for Implosion Protected Std. Meth. of Test for Rec. Pract. and Std. for td. Meth. of Test for Operating Performance of Particulate Std. Spec. for t for Synthetic Anionic Active Ingredient in Detergents by Meth of Test for Synthetic Anionic Ingredient by and Water Requirements of Farm Livestock (Poultry, Swine, Std. for Precision Coaxial Connectors for Std. Spec. for Oil and Resin Base Std. Meth. of Test for Slump of Std. Meth. of Test for Staining of Std. Meth. of Test for Tack Free Time of Structural Clay Differential Movemen Test Meth. for Evaluating Wetting Agents in ies and Essential Information for Safe Handling and Use of mating Maturity and Linear Density of Cotton Fibers by the std. Definitions of Terms Relating to Erosion of Solids by m Automotive Water Pumps with Coolan/ Std. Test Meth. for (1972) Std. Meth. of Vibratory Std. Recommendations for Die Rec for Stopping Powers for Use with ing Code: Solid, Grouted, Reinforced Grouted, Hollow Unit Structural Clay Insulated Brick and Tile

Structural Clay (Properly Constructed Masonry) Insulated welding Wire, Corrosion and Heat Resistant $(18 \mathrm{Cr}-11 \mathrm{Ni}-$ Std. Meth. of Analysis of CCA Treating Solutions and try (1972) Std. Meth. of Analysis of Rec. Std, for Engineering Specs. Outline for Monochrome
Castings for Pressure Containing Parts Suitable for Hig Castings for Pressure Containing Parts Suitable for Low Castings for Steam Turbines (1974)

Castings for Structural Purposes (1973) ANSI G52.1 Castings for Valves, Flanges, and Pipe Fittings (1973) Castings Specially Heat Treated for Pressure Containing Castings Suitable for Fusion Welding for High Temperatu Castings Suitable for Pressure Service (1974) ANSI G52. Castings Up to $2 \mathrm{In}$. in Thickness (1972) ANSI Z166.28 Castings $(0.80 \mathrm{Cr}-1.8 \mathrm{Ni}-0.35 \mathrm{Mo}(0.28-0.36 \mathrm{C}))(\mathrm{SAE}$ Castings (1952) ANSI H48.1 Castings (1964) ANSI H47.2 Castings (1966) ANSI H45.8 Castings (1967) ANSI Z166.17 Castings (1969) ANSI Z179.16 Castings (1970) ANSI G52.7 Castings (1970) ANSI H49.1 Castings (1971) ANSI G48.2 Castings (1971) ANSI H47.1

Castings (1972)

Castings (1972)

Castings (1972)

Castings (1972)

Castings (1972) ANSI G52.6

Castings (1972) ANSI H38.22

Castings (1972) ANSI H45.l

Castings (1972) ANSI H45.2

Castings (1972) ANSI Z166.19

Castings (1972A)

Castings (1972A)

Castings (1973)

Castings (1973)

Castings (1973) ANSI G52.4

Castings (1973) ANSI H38.1

Castings (1974)

Castings (1974)

Castings (1974)

Castings (1974) ANSI H38.24

Castings (1974) ANSI H38.26

Castings (25-12 Class) for High Temperature Service (19

Castings $(5.75 \mathrm{Zn}-2.5 \mathrm{Re}-0.70 \mathrm{Zr}$ (Ze63-T6) Solution an

Castings, Heavy Walled, Carbon and Alloy for Pressure V

Castings, Investment $0.80 \mathrm{Cr}-1.8 \mathrm{Ni}-0.35 \mathrm{Mo}(0.28-0$.

Castings, Investment $0.80 \mathrm{Cr}-1.8 \mathrm{Ni}-0.35 \mathrm{Mo}(0.38-0$.

Castings, Investment. $0.95 \mathrm{Cr}-0.20 \mathrm{Mo}(0.25-0.35 \mathrm{C})(1$

Castings, Series II (1974) ANSI Z166.8

Castor Oil for Use in Resins and Protective Coatings in

Castor Oil Penetration of Paper (1972)

Castor, Sesame, Saffower, Oleic, and Sunflower Seeds;

Cataloged Square Head Industrial Fluid Power Cylinders

Cataloged Square Head Tie Rod Type Industrial Fluid Pow

Catalyst Are Employed (1960)

Catalyst Are Employed (1962)

Catalyst Are Employed (1962)

Catch Basins and Manholes (1973)

Other Protein Bearing Ma

Other Protein Bearing Ma

Cathode Copper (1972) ANSI H23.10

Cathode Ray Tubes (Piclure and Display) for Television

Cathodic Disbonding of Pipeline Coatings (1972)

Cathodic Protection Systems on Boats (1972)

Cation Exchange Materials (1972) ANSI Z111.4

Cationic Emulsified Asphalt (1973)

Cationic Titration Procedure (1974)

Cationic Titration (1972T)

Cattle, Sheep) (1968)

/Heat and Moisture Loss and Feed

CATV (Cable Television) Application (75 Ohms) (1973)

Caulking Compound for Building Construction (1972)

Caulking Compounds and Sealants (1973)

Caulking Compounds and Sealants (1973)

Caulking Compounds and Sealants (1973)

(Cause and Effect) (Tech. Notes) (1963)

Caustic Soda for Mercerization (1971) ANSI L14.79

Caustic Soda (1974)

Causticaire Method (1971)

\section{Std. Meth. of Test for Esti}

Cavitation and Impingement by Liquid or Solid Particles

Cavitation Erosion Corrosion Characteristics of Aluminu

Cavitation Erosion Test (1972)

Cavity and Cutoff Die Sizes for Cold Forging Equipment

Cavity Chambers (1961)

Cavity Wall, and Stone Masonry (1973)

Cavity Walls (Tech. Notes) (1964)

Cavity Walls (Tech. Notes) (1965)

Cavity 6 In. Brick and Tile Walls (Tech. Notes) (1966)

/E Uniform Build

(Cb+Ta) (1973)

CCA Treated Wood by Colorimetry (1972)

Spec. for Steel

CCA Treating Solutions and CCA Treated Wood by Colorime

Cctv (Closed Circuit Television) Camera Equipment (1974

NIOP

NFLDP

NFLDP

CR

CR

ASTM

UL

ASTM

ABYC

ASTM

ASTM

ASTM

ASTM

ASAE

EIA

ASTM

ASTM

ASTM

ASTM

BIA

AATCC 43

MCA

ASTM

ASTM

ASTM

AST

IFI

NCRPM $R$ 
of Nitrophenide in Poultry Mashes (Feed) for Prevention of faquinoxaline in Poultry Feeds and Premixes for Control of by the Thermal Process (W estern Red Cedar, Northern White ngth Thermal Process Preservative Treatment of Western Red ite, Sugar, and Lodgepole Pines; Engelmann Spruce; W'estern nd Hemlock-Tamarack: Balsam Fir: Northern Pine and White (Western Red Cedar, Northern White Cedar and Alaska Yellow servative Treatment of Western Red Cedar and Alaska Yellow of Incised Pole Butts by the Thermal Process (Western Red rosa and Western White Pines, Spruce-Pine-Fir, Western douglas Fir, Coast Region; West Coast Hemlock; Western Red Rec. Std. for Seismic Restraint Direct Hung Suspended ield Test of Partitions (Wall, Ceiling, Floor, and Floor . Class (Stc) of Building Partitions Such as Walls, Floor Uniform Building Code: Wall and Uniform Building Code: Interior Walls and Uniform Building Code Std. for Span Tables for Floor and Std. Meth. of Paintin Spec. for Acoustical

board, Timber, Lumber, Siding, Framing, Decking, Flooring, Airborne Sound Insulation Field Test of Partitions (W all. Control (Acoustics) in Wood Building Construction (Floor Decorative and Protective Covering for Permanent $W$ all and uspension Systems for Acoustical Tile and for Lay in Panel skylights, Monitors and Sawtooth Roofs. Light Diffusers in ry: Mortar: Sand; Slag; Steel; Terra Cotta; Timber: W ater: numbers, Dimensional Spec., and Ratings; Assembly Numbers, Spec. for Dry Std. Spec. for

al Wire and Cable Installation (1973/ Sid. Meth of Test. Std. for Safety for Std. Spec. for Preformed

for Dielectric Constant and Dissipation Factor of Expanded Meth. of Test for Rate of Burning or Extent of Burning of Std. Meth. of Test for Water Absorption of Rigid Std. Meth. of Test for Tensile Properties of Rigid Std. Meth. of Test for Compressive Properties of Rigid Std. Meth. of Test for Flammability of Rigid Std. Spec. for Sponge and Expanded and Tubing (1973) ANSI B72.12 40 (1973) ANSl B72.4

and Fittings (1973) A/ Std. Spec. for Std. Spec, for Std. Spec. for Solvent Cements for rate (1972) Std. Meth. of Test. Cellulose Acetate Propionate and Std. Meth. of Test. Std. Meth. of Test. Std. Definitions of Terms Relating to Std. Definitions of Terms Relating to Cellulose and graphic Film for Archival Records, Silver Gelatin Type, on 7 Test Meth. for Fluidity of Dispersions of photometry/ Std. Meth. for Quantitiative Determination of Uniform Building Code Std. for Uniform Fire Code: Uniform Fire Code: Std. Spec. for Soluble Sid. for ion (1973) Std Spec for Std. Spec. for lnsulating Board Std. Meth. of Test. Insulating Board Std. Spec. for Structural Insulating Formboard Made from std. Meth. of Test for Moisture Content of Oil Impregnated Test Meth. for Ash Content of Bleached Uniform Building Code Std. for Portland Std. Spec. for Portland

d Interior (1971)

d Interi/

ANSI A 1.19

Std. Spec. for Lathing and Furring for Portland Std. Definitions of Terms Relating to Asbestos Std. Meth. of Test for Fineness of Portland Std. Meth. of Test for Fineness of Hydraulic Std. Meth. of Test for Fineness of Portland Recommendations for Basis of Payment on Portland Rec. Spec. for Optimum Size Coarse Aggregate for Portland and Raw or Calcined Natural Pozzolans for Use in Portland 58, Ashto Tl44 Std. Meth. of Test for Std. Spec. for Asbestos Uniform Building Std. Spec. for Solvent Std. Spec. for Portland (1971) Std. Spec. for Portland Cement and std. Spec. for Lathing and Furring for Portland Cement and Std. Meth. of Wetting and Drving Tests of Compacted Soil Std. Meth. of Test for Cement Content of Soi Std. Meth. of Test for Length Change of Hardened of Test for Length Change of Drilled or Sawed Specimens of ater Pipe (4 In. and Larger, Shop Applied) (197) Std. Spec. for Latex-Portland Std. Rec. Pract. for Use of Hydraulic Std. Meth. of Test for Compressive Strength of Hydraulic
Cecal and Intestinal Coccidiosis in Chickens (Cereal $\mathrm{Ch}$ Cecal and Intestinal Coccidiosis (Cereal Chemistry) (19 Cedar and Alaska Yellow Cedar Poles) (1973)

Cedar and Alaska Yellow Cedar Timber Poles (1973)

Cedar and Hemlock: Subal-Pine Fir; and Mountain Hemlo Cedar Lumber (1973)

Cedar Poles) (1973)

Cedar Timber Poles (1973)

/for Eastern Spruce, White Pine, a

/Ole Butts by the Thermal Process / Length Thermal Process Pre

Cedar, Northern White Cedar and Alaska Yellow Cedar Pol

Cedars (North) (Lumber) (1973) /M-Fir (Nor
Cedar: White Fir; and Sitka Spruce (Lumber) (1973)

Ceiling Assemblies (1972)

Ceiling Assemblies) in Existing Buildings (1973)

Ceiling Assemblies, Doors, and Other Space Dividing Ele

Ceiling Coverings (1973)

Ceiling Finish (1973)

Ceiling Joists, and Rafters (1973)

Ceiling Materials for Acoustical Absorption Tests (1973

Ceiling Systems (1972)

Ceiling, and Partition (1973)

Ceiling, Floor, and Floor-Ceiling Assemblies) in Exis

Ceiling, W all) (1972)

Ceilings (1969)

Ceilings (1973)

Rec. for Sound

Ceilings, Partitions, Exterior Veneer, Awnings and Pati

Ceiling: Partitions; Plaster: Roofing) and Earthquake

Cell Layouts and Terminals (For Foreign and Domestic Pa

Cells and Batteries (1972)

Cellular Glass Block and Pipe Thermal Insulation (1973)

Cellular Glass Insulating Block (1972)

Cellular Metal Floor Raceways and Fittings for Electric

Cellular Plastic Pressure Relief Joint Fillers (1973)

Cellular Plastics Used for Electrical Insulation (1973)

Cellular Plastics Using a Supported Specimen by a Horiz

Cellular Plastics (1969E) ANSI K65.122

Cellular Plastics (1972) ANSI K65.32

Cellular Plastics (1973) ANSI K65.31

Cellular Plastics (1974)

Cellular Rubber Products (1973) ANS1 J2.4

Cellulose Acetate Butyrate (CAB) Plastic Pipe (SDR-PR)

Cellulose Acetate Butyrate (CAB) Plastic Pipe, Schedule

Cellulose Acetate Butyrate (CAB) Plastic Pipe, Tubing,

Cellulose Acetate Butyrate (1972)

Cellulose Acetate Propionate and Cellulose Acetate Buty

Cellulose Acetate (1972) ANSI K65.24

Cellulose and Cellulose Derivatives (1972) ANS1 K64.6

Cellulose Derivatives (1972) ANSI K64.6

Cellulose Ester Base (1973)

Cellulose Form Bleached Cotton Cloth (1972) ANSI L14.11

Cellulose Nitrate in Alkyd Lacquers by Infrared Spectro

Cellulose Nitrate Motion Picture Film (1973)

Cellulose Nitrate Motion Picture Film (1973)

Cellulose Nitrate Plastics (Pyroxylin) (1973)

Cellulose Nitrate (1972) ANSI K65.22

Cellulosic Fiber lnsulating Board (1973) ANSl Al49.1

Cellulosic Fiber (Wood Base) Loose Fill Thermal Insulat

(Cellulosic Fiber), Structural and Decorative (1972)

(Cellulosic Fiber), Structural and Decorative (1972)

Cellulosic Fibers (1966)

Cellulosic Insulation (1973)

Cellulosic Textiles (1971) ANSI L14.114

Cement and Blended Hydraulic Cements (1973)

Cement and Cement Lime Plastering, Exterior (Stucco) an

Cement and Cement Lime Plastering, Exterior (Stucco) an

Cement and Related Products (1972) ANSI A 130.1

Cement by Air Permeability Apparatus (1973) AASHO T153,

Cement by No. 325 (45-?M) Sieve (1973)

Cement by the Turbidimeter (1973) ANSl Al.7

Cement Concrete Pavement Overlay Projects (1973)

Cement Concrete Paving (1972)

Cement Concrete (1973) ANSI A37.122

Cement Cor for

Cement Fiberboard Insulating Panels (1972)

Cement for Acrylonitrile-Butadiene-Styrene (ABS) Pl

Cement for Use in Mortars (1973)

Cement Lime Mortar for Brick Masonry (1972)

Cement Lime Plastering, Exterior (Stucco) and Interior

Cement Lime Plastering, Exterior (Stucco) and Interior

Cement Mixtures (1971) ANSI A37.5

Cement Mixtures (1974) ANSI A37.58, Ashto T144

Cement Mortar and Concrete (1974) ANSl A37.78

Cement Mortar and Concrete (1974T)

Cement Mortar Protective Lining and Coating for Steel W

Cement Mortar (1973) ANSI Al 18.4

Cement Mortars in Chemical Resistant Masonry (1965)

Cement Mortars (Using Portions of Prisms Broken in Flex

AACCH 18-25

AACCH $\quad 18.35$

AWPA C?

AWPA C8

ICBO

BO

AWA

AWPA

$A$ WPA

ICBO

ICBO

CISCA

Ion F ICBO

ICBO

$\mathrm{ICBO}$

ICBO

ICBO

ASTM

CSl

WWPA

ICBO

WWPA

CSl

ICBO

ICBO

$\mathrm{BCl}$

ANSI

ASTM

A STM

UL

ASTM

ASTM

ASTM

A STM

ASTM

ASTM

ASTM

ASTM

ASTM

ASTM

ASTM

ASTM

ASTM

ASTM

ASTM

ASTM

ANSI

AATCC

ASTM

ICBO

ICBO

ICBO

ASTM

USC

ASTM

ASTM

ASTM

ASTM

ASTM

A ATCC

ICBO

ANSI

ANSI

ASTM

ASTM

ASTM

ASTM

ACPA

ACPA

ASTM

ASTM

ASTM

ASTM

ICBO

BlA

ANSI

ANSI

ASTM

ASTM

ASTM

UBCS25.4 UBC $25-8$

$\mathrm{C} 7$

$\mathrm{C} 8$

C7

UBCS 25.2

UBCS 25.3

*3

UBC $\$ 35.3$

UBC 335.1

UBC $* 3.47$

UBC * 3.42

UBCS25.21

C643

09530

* 24

UBCS35-3

27

09951

UBCS47.16

UBC * 3.52

$\mathrm{BC} * 3-2$

$* 1.56$

C18.

C552 
Std Meth of Test for Compressive Strength of Hydraulic Std. Meth. of Test for Flexural Strength of Hydraulic Std. Spec. for Asbestos Std. Spec. for Asbestos Std. Spec. for Asbestos

i A 165.4 Std. Spec, for Linings for Asbestos Std. Meth. of Test. Asbestos Std. Spec. for Asbestos Std. for Ásbestos Sid. Spec. for Asbestos and Fire Service (1973)

Uniform Building Std. Spec. for Corrugated Asbestos Std. Spec. for Flat Asbestos Std. Spec. for Corrugated Asbestos g Tests of Latex and Emulsion Ex/ Std. Spec. for Asbestos Std. Spec. for Asbestos Specifying Portland Std. Spec. for Asbestos

Std. Meth of Test for False Set of Portland 1 Fiber Hydraulic Setting Thermal Insulating and Finishing Std. Definitions of Terms Relating to Hydraulic Std. Spec. for Masonry

Std. Meth. of Test for Autoclave Expansion of Portland Std. Meth. of Test for Optimum So3 in Portland Std. Meth. of Test for Specific Gravity of Hydraulic Std Spec for Portland Uniform Building Code Std. for Keene's Std. Meth. of Test for Normal Consistency of Hydraulic Sid. Meth of Test hor Heat of Hydration of Hydraulic Std. Speci for Natural

Std. Meth. of Sampling Hydraulic

spec. for Asphalt Mastic for Use in Waterproofing (Asphalt Std. Meth. of Test for Transverse Rupture Strength of fittings (1973) Ansi/ Std. Rec. Pract. for Making Solvent 71) Installation Std. for Solvent nd (Specimen Storage) Rooms Used in the Test. of Hydraulic pe, Tubing, and Fittings (1973) A/ s) Pipe and Fittings to Poly(Viny Fittings (1973) ANS1 B72.16 Std. Spec. for Solven Std Spec for Solven Std. Spec. for Solvent Std. Spec, for Solven

Std. Spec. for Blended Hydraulic

ilding Code Std. for Portland Cement and Blended Hydraulic or Estimating the Thermal Conductivity of Leather with the Standard Mounting and Face Dimensions of Receptacles Off Std. for System Design of Fine Pitch on for Flexural Strength of Concrete (Using Simple Beam with Std. for Directories of Libraries and Information es (Packaging), and References (Dosage) for Poison Control Safety Std. for Electric Safety Std. for Air Conditioners, Std. for

Std. for Gas Fired Gravity and Forced Air Safety Std for Oil Fired

for Oil Powered Forced Air (Domestic and Light Commercial) d Ref rigeration) (1974)

Fire Alar c. for Seamless Austenitic Steel Pipe for High Temperature 66) 66) Std. Operating Limits for Std. Coil Face Areas for Safety Std for

ry Services (1972) ANSl Se 3.1

Rec. Pract. Tests for Std. for Std. for Installation of

1973) ANSI B 144.3

ulsion Plant ( 1970

products (1974)

6)

Spec, for Oil Well Casing Sanitary Stds. for Std. Spec. for Copper Base Alloy

Std. Preparation of Std Sizes for Industrial Sid. Wheel Diameters and Outlet Areas for Std. Sizes for Industrial

Std. Drive Arrangements for Std. Inlet Box Positions for Std. Designations for Rotation and Discharge of Std Motor Positions for Belt or Chain Drive Std. Flue Gas Densities for Forced and lnduced Draft Std. Drive Arrangements for Tubular

Rec. for
74)

rature Service (1972) ANS1 B 125.39

re Service (1972)

emperature Service (1973)

tubing for Pressure Application at High T/ for Gas (1971)
Cement Mortars (Using $2 \mathrm{ln}$. (50 Mm) Cube Specimens) (19 Cement Mortars (1972)

Cement Nonpressure Sewer Pipe (1973) ANSI Al65.3

Cement Nonpressure Small Diameter Sewer Pipe (1973) Ans

Cement Perforated Underdrain Pipe (1973) ANSI A165.2

Cement Pipe (1973)

Cement Pipe (1973) ANSI Al65.1

Cement Plastic Foam Core Insulating Panels (1972)

Cement Pressure Pipe for Water and Other Liquids (1972)

Cement Pressure Pipe (1973) ANSI Al65.5

Cement Pressure Pipe, Couplings, and Gaskets for Water

Cement Roofing Shingles (1973)

Cement Sheets for Bulkheading (1974)

Cement Sheets (1972) ANSI A 124.1

Cement Sheets (1974) ANSI A125.1

Cement Shingle Blanks to Be Used as Panels in Weatherin

Cement Storm Drain Pipe (1973)

Cement Terrazzo, Cast in Place Floors (1967)

Cement Transmission Pipe (1973)

Cement (Paste Meth.) (1972) ANSI Al.27

Cement (1964) ANSl Z98.33

Cement (1971) ANSI A1.28

Cement (1971) ANSI Al.3

Cement (1971) ANSI A1.8, ASHTO T 107

Cement (1972)

Cement (1972) ANSI Al.12, ASHTO T 133

Cement (1973)

Cement (1973)

Cement (1973) AASHO T129, ANSI Al.11

Cement (1973) ANSI Al,10

Cemeht (1973) ANSI Al.18

Cement (1973) ANSI A1.2

Cement, Mineral Filler and Aggregate) (1941) ANSI Al09.

Cemented Carbides (1973) ANSl H9.23

Cemented Joints with Polyvinyl Chloride (PVC) Pipe and

Cemented PVC Pipe for Water Service and Yard Piping (19

$\begin{array}{ll}\text { Cements and Concretes (1973) } & \text { /Ec. for Moist C } \\ \text { Cements for Cellulose Acetate Butyrate (CAB) Plastic Pi }\end{array}$

Cements for Joining Acrylonitrile Butadiene Styrene (AB

Cements for Poly(vinyl Chloride) (PVC) Plastic Pipe and

Cements for Styrene Rubber (SR) Plastic Pipe and Fittin

Cements (1973)

Cements (1973)

Cenco Fitch Apparatus (1970) ALCA E60

th. of Test $F$

Center Boss Type, 30, 50, and 60 Amperes, 4 Wire (1966)

Center Face Gears for 20 Deg. Involute Spur Pinions (19

Center Point Loading) (1968) ANSt A37.84

Eth. of Test

Centers (1971)

Centers (1972)

Central Air Heating Equipment (1973) ANS1 C33.104

Central Cooling (1972) ANSl Bl44.1

Central Forced Air Electric Heating Equipment (1974)

Central Furnaces (1973)

Central Furnaces (1973) ANS1 Z96.1

Central Furnaces) (1972)

/D. Performance Requirements

Central Station Air Handling Units (Air Conditioning an

Central Station Protective Signaling Systems for Guard,

Central Station Service (1973) ANS1 B125.25

Central Station Units (Air Moving and Conditioning) (19

Central Station Units (Air Moving and Conditioning) (19

Central Stations for Watchman, Fire Alarm and Superviso

Central System Fluids (1970)

Central System Humidifiers (1974)

Central Warm Air Heating and Air Conditioning Systems (

Centralized Control and Automation of Ship's Steam Prop

Centralizers (1973)

Centrifugal and Positive Rotary Pumps for Milk and lts

Centrifugal Castings (1973)

Centrifugal Fans and Parts for Protective Coatings (196

Centrifugal Fans with Cast Housings (1966)

Centrifugal Fans (1966)

Centrifugal Fans (1966)

Centrifugal Fans (1966)

Centrifugal Fans (1966)

Centrifugal Fans (1966)

Centrifugal Fans (1966)

Centrifugal Fans (1966)

Centrifugal Fans (1966)
Centrifugal Fire (Water) Pumps (NFPA 20) (1974)

Std. for Centrifugal Water Chilling Packages (Refrigeration) (19

Std. Spec. for Centrifugally Cast Austenitic Steel Pipe for High Tempe

Std. Spec. for Centrifugally Cast Carbon Steel Pipe for High Temperatu

Std. Spec. for Centrifugally Cast Dual Metal Cylinders (1972)

Std. Spec. for Centrifugally Cast Ferritic Alloy Steel Pipe for High T

Std. Spec. for Centrifugally Cast Iron-Chromium-Nickel High Alloy Std. for Ductile lron Pipe,
ASTM

ASTM

ASTM

ASTM

ASTM

ASTM

ASTM

A

$A W W A$

ASTM

UL

ICBO

ASTM

ASTM

ASTM

ASTM

ASTM

CSI

ASTM

ASTM

ASTM

ASTM

ASTM

ASTM

ASTM

ASTM

ICBO

ASTM

ASTM

ASTM

ASTM

ASTM

ASTM

ASTM

1APMO
AS TM

ASTM

ASTM

ASTM

ASTM

ASTM

ICBO

ASTM

ANSl

AGMA

ASTM

AAPCC

UL

UL

ARI

ANS!

UL

ANS

ARI

Std. Spe ASTM

AMCA

AMC'A

UL

AE

ARI

NFPA

SNAME

API

DFISA

ASTM

AMCA

AMCA

AMCA

AMCA

AMCA

AMCA

$A M C A$

AMCA

AMCA

AMCA

FMS

ARI

ASTM

ASTM

ASTM

ASTM

ASTM

ANSI

C109

348

C428

C644

C508

C541

C500

(659

C400
C296

107

UBCS 32.9

C746

C220

C221

D1911

C663

09410

608

$\mathrm{C} 451$

C 449 
Meth. of Test for Sediment in Water Emulsion Polishes by td. Spec. for Concentrated, Ammonia Preserved, Creamed and Std. for Household Electric Ranges (Stove) with Glass Meth. of Test for Acid Resistance of Tentative Meth. of Test for Sulfide Resistance of Tent. Meth. of Test for Acid Resistance of ss Containers/ ic Constant (Permittivity) and Dissipation Factor of Solid otes) (1962) si C 59.73

Std. Meth. of Test. Vitrified std. Definitions of Terms Relating to Porcelain Enamel and Test for Biaxial Flexure Strength (Modulus of Rupture) of Meth. of Test for Lead and Cadmium Extracted from Glazed Recommendation for Installation of Recommendation for Installation of recommendation for Installation of Thresholds, Saddles for Recommendation for Installation Performance Levels of Recommendation for Installation of Interio Recommendation for Installation of Exterio Recommendation for Installation of Chemical Resistant Recommendation for Installation of Conductive Recommendation for Installation of Waterproof Guide for Std. Spec, for Recommendation for Installation of Recommendation for Installation of Recommendation for Installation of Recommendation for Installation of Std. Test Meth. for Resistance of

Recommendation for Installation of Recommendation for Installation of Interio Recommendation for Installation of Exterior Recommendation for Renovation of Interior

Definition and Recommendation for Materials for Settin

Std. Meth. of Test for Breaking Strength of Std. Meth. for Measuring Warpage of

Definition and Recommendation for Materials for Grouting

Expansion, Control, Contraction, and Isolation Joints for , and Definitions) (1961) Public Works Spec. for us, Shear Modulus, and Poisson's Ratio for Glass and Glass $72 \mathrm{~T})$

Std Meth of Flexure Test. of Definitions of Terms Relating to Surface Imperfections on Std. Deviations for Specific Meth. of Glossary of Terms Used in Baking and cial Techniques for Extraneous Matter Meth. of Analysis in Definitions of Statistical Terms Used in

l Hydr/ Reagents for Extraneous Matter Meth. of Analysis atus or Materials for Extraneous Matter Meth of Analysis

Rapid Meth. of Analysis for Fat Acidity in Grain

Rapid Meth. of Analysis for Fat Acidity in Corn

Colorimetric Meth. of Analysis for Fat Acidity in Grain meth. of Analysis for Total Phosphoric Acid in Dried Eggs Meth. of Analysis for Benzoic Acid in Flour Meth. of Analysis for Lactic Acid in Dried Geeg Meth. of Analysis for Lactic Acid in Nonfat Dry Milk

Meth. of Analysis of Soy Flour in Macaron Meth. of Analysis for Ash in Cacao Products

Meth. of Analysis for Ash in Dry Milk Products

Meth. of Analysis for Ash in Farina and Semolina meth. of Analysis for Ash in Molasses, Sugars, and Syrups Meth. of Analysis for Ash in Soy Flour Meth. of Analysis for Ash in Yeast Foods Meth. of Analysis for Total Reducing Substances

Summary of Meth. for Biscuit and Cracker Flour Test.

Meth. of Analysis for Baking Quality of Cookie Flour

Analys for Baking Quality of Rye Bread Flour (Cereal Chemistry) (1962)

Meth. of Analysis for Baking Quality of Nonfat Dry Milk (Cereal Chemistry) (1962)

Meth. of Analysis for Baking Quality of Cake Flour (Cereal Chemistry) (1962)

Meth. for Use of Layer Cake Measuring Template (Cereal Chemistry) (1962)

Pekar Color Test (Slick) Meth. of Flour Analysis (Cereal Chemistry) (1962)

Disk Meth. of Analysis of Color of Macaroni Products (Cereal Chemistry) (1962)

Meth. for Determination of Arsanilic Acid in Feeds (Cereal Chemistry) (1962)

Meth. for Determination of Nitrosal in Poultry Feeds (Cereal Chemistry) (1962)

meth of Analysis of Lipoid Phosphoric Acid in Dried Eggs (Cereal Chemistry) (1962)

Meth. of Analysis of Egg Solids in Macaroni Products (Cereal Chemistry) (1962)

Meth. of Analysis of Alpha Amylase Activity of Malt (Cereal Chemistry) (1962)

Meth. of Analysis of Yeast Strength of Doughs (Cereal Chemistry) (1962)

Meth of Analysis of Diastatic Activity of Malt Flour (Cereal Chemistry) (1962)

eth. of Ferricyanide-Maltose-Sucrose Conversion Table (Cereal Chemistry) (1962)

Meth. of Analysis of Lipoxidase Activity of Ground Wheat (Cereal Chemistry) (1962)

Meth. of Analysis of Proteolytic Activity of Malt (Cereal Chemistry) (1962)

Batch Meth. of Experimental Milling for Durum Wheat (Cereal Chemistry) (1962)

Meth. of Experimental Wheat Milling: Temper Table (Cereal Chemistry) (1962)

decantation. Meth. of Analysis for Rodent Excreta in Flour (Cereal Chemistry) (1962) Meth. of Analysis of Crude Fat in Cocoa (Cereal Chemistry) (1962)
Std ASTM

ASTM

AHAM

ASTM

ASTM

ASTM

ASTM

BIA

ASTM

ASTM

Tentative Meth. of ASTM

Std ASTM

TCA

TCA

TCA

TCA

TCA

TCA

TCA

TCA

TCA

TCA

TCA

TCA

TCA

TCA

ASTM

TCA

TCA

TCA

TCA

TCA

ASTM

ASTM

TCA

/Ecommendation for Installation of TCA F Test for Young's Modul ASTM

ASTM

Std. ASTM

F109

78.50

$\mathrm{AACCH} \quad 36.01 \mathrm{~A}$

Spe AACCH 28.92

$\mathrm{AACCH} \quad 78.40$

$\mathrm{AACCH} 28.91$

AACCH 28.90

AACCH 02.02

$\mathrm{AACCH} 02.03$

$\mathrm{AACCH} \quad 02.04$

$\mathrm{AACCH} \quad 04.13$

$\mathrm{AACCH}$ 04-21

AACCH 04.25

AACCH 04.26

AACCH 06.11

AACCH 08.10

AACCH 08.11

AACCH 08-12

AACCH 08.14

AACCH 08.16

AACCH 08.18

$\mathrm{AACCH} 10.01$

AACCH 10.30

$\mathrm{AACCH} 10.50 \mathrm{~B}$

AACCH 10.60

$\mathrm{AACCH} \quad 10.70$

AACCH 10.85

AACCH $10-90$

$\mathrm{AACCH} 10.91$

AACCH 14.10

AACCH $\quad 14.20$

$\mathrm{AACCH} \quad 18.20$

AACCH 18.27

AACCH 20.02

AACCH 20.10

AACCH 22.01

AACCH 22.13

AACCH 22.16

AACCH 22.18

AACCH 22.41

AACCH 22.61

$\mathrm{AACCH} 26.30$

AACCH 26-95

AACCH 28.42

$\mathrm{AACCH} \quad 30.12$ 
National Bureau of Standards KWIC Index of Engineering Standards

Meth. of Analysis of Crude Fat in Baked Dog Food (Cereal Chemistry) (1962)

Meth. of Analysis of Crude Fat in Dry Milk Products (Cereal Chemistry) (1962)

Meth. of Analysis of Crude Fat in Grain and Stock Feeds (Cereal Chemistry) (1962)

Meth. of Analysis of Crude Fat in Soy Flours (Cereal Chemistry) (1962)

Meth. of Analysis of Crude Fiber in Feeds and Feedst uffs (Cereal Chemistry) (1962)

White Pan Bread Flour Spec. (Cereal Chemistry) (1962)

Meth of Analysis of Vital Wheat Gluten (Cereal Chemistry) (1962)

Meth. of Analysis of Soluble Mineral Matter (Cereal Chemistry) (1962)

Meth. of Analysis of Calcium in Bread and Flour (Cereal Chemistry) (1962)

Meth. of Analysis of Calcium in Feedstuffs (Cereal Chemistry) (1962)

Meth. of Analysis of Calcium in Mineral Feeds (Cereal Chemistry) (1962) ravimetric Meth. of Analysis of Chlorides in Ash of Bread (Cereal Chemistry) (1962) volumetric Meth. of Analysis of Chlorides in Ash of Bread (Cereal Chemistry) (1962) qualitative Meth. of Analysis of Chlorides in Yeast Foods (Cereal Chemistry) (1962) uantitative Meth. of Analysis of Chlorides in Yeast Foods (Cereal Chemistry) (1962)

Meth. of Analysis of Iodine in Mineral Mixed Feeds (Cereal Chemistry) (1962)

Qualitative Meth. of Analysis of Added Iron in Flour (Cereal Chemistry) (1962)

Quantitative Meth. of Analysis of Iron in Flour (Cereal Chemistry) (1962)

Meth. of Analysis of Phosphates in Flour Improvers (Cereal Chemistry) (1962)

Meth. of Analysis of Added Salt in Feeds and Feedstuffs (Cereal Chemistry) (1962)

Bomb Meth. of Analysis of Sulfates in Yeast Foods (Cereal Chemistry) (1962)

Gravimetric Meth. of Analysis of Sulfates in Yeast Foods (Cereal Chemistry) (1962)

Sampling Procedure for Analysis of Bacteria (Cereal Chemistry) (1962)

Meth. of Analysis of Rope Spore Count in Cereal Products (Cereal Chemistry) (1962)

meth. of Analysis of Salmonella Bacteria in Food Products (Cereal Chemistry) (1962)

Meth. of Analysis of Fecal Streptococci in Food Products (Cereal Chemistry) (1962)

Microscopic Meth. of Analysis of Smut in Wheat or Rye (Cereal Chemistry) (1962)

Sedimentation Meth. of Analysis of Smut in Wheat and Rye (Cereal Chemistry) (1962)

Meth. of Calculation of Percent Moisture (Cereal Chemistry) (1962)

Electrical Meth. for Analysis of Moisture in Whole Grain (Cereal Chemistry) (1962)

Air Oven Meth. of Analysis of Moisture in Malt (Cereal Chemistry) (1962)

Meth. of Analysis of Moisture in Nonfat Dry Milk (Cereal Chemistry) (1962)

Micro Kjeldahl Meth. of Analysis of Crude Protein (Cereal Chemistry) (1962)

Rowland Meth. of Analysis of Serum Protein in Milk (Cereal Chemistry) (1962)

Qualitative Meth. of Analysis of Calcium Peroxide (Cereal Chemistry) (1962)

Meth. for Particle Size Distribution of Wheat Flour (Cereal Chemistry) (1962)

Volumetric Bromine Meth. of Analysis of Pentosans (Cereal Chemistry) (1962)

Xylene Partition Meth. of Analysis of Pentosans (Cereal Chemistry) (1962)

Meth. of Analysis of Sedimentation Test for Wheat Flour (Cereal Chemistry) (1962)

Meth. of Analysis of Fungicide Residues in Grain (Cereal Chemistry) (1962)

Meth. for Preparation of Sample: Bread (Cereal Chemistry) (1962)

Meth. for Preparation of Sample: Dried Eggs (Cereal Chemistry) (1962)

Meth. for Preparation of Sample: Feeds and Feedstuffs (Cereal Chemistry) (1962)

meth. for Preparation of Sample: Fruit and Fruit Products (Cereal Chemistry) (1962)

Meth. for Preparation of Sample: Leavening Agents (Cereal Chemistry) (1962)

Meth. for Preparation of Sample: Macaroni Products (Cereal Chemistry) (1962) Meth. for Preparation of Sample: Malt (Cereal Chemistry) (1962)

Meth. for Preparation of Sample: Semolina (Cereal Chemistry) (1962) Meth. for Preparation of Sample: Wheat (Cereal Chemistry) (1962)

Meth. for Sampling of Fats and Shortenings (Cereal Chemistry) (1962)

Meth. for Sampling of Solid Feeds and Feedstuffs (Cereal Chemistry) (1962)

Meth for Sampling of Wheat and Other Whole Grains (Cereal Chemistry) (1962)

Disk Meth. of Analysis of Semolina Quality in Miacaroni (Cereal Chemistry) (1962)

Indices of Refraction of Sucrose Solutions at $20 \mathrm{Deg}$. C (Cereal Chemistry) (1962)

Meth. for Preparation of Indicator Solutions (Cereal Chemistry) (1962)

Meth. for Preparation of Ammonium Molybdate Solution (Cereal Chemistry) (1962)

Meth. for Preparation of Potassium Thiocyanate Solution (Cereal Chemistry) (1962)

Meth. for Preparation of Silver Nitrate Solution (Cereal Chemistry) (1962)

Meth. of Sensory Perception Test for Staleness of Bread (Cereal Chemistry) (1962) emperature Corrections for Refractometer Readings (Sugar) (Cereal Chemistry) (1962) emperature Corrections for Saccharimeter Readings (Sugar) (Cereal Chemistry) (1962)

Meth. of Analysis of Lactose (Milk Sugar) in Mixed Feeds (Cereal Chemistry) (1962)

Table for Converting Absorption to $14.0 \%$ Moisture Basis (Cereal Chemistry) (1962)

Meth. of Analysis of Test Weight Per Bushel of Grain (Cereal Chemistry) (1962)

Chemical Meth. of Analysis of Vitamin B6 Complex (Cereal Chemistry) (1962)

Microbiological Meth. of Analysis of Vitamin B6 Complex (Cereal Chemistry) (1962)

Microbiological Meth. of Analysis of Vitamin Bl2 (Cereal Chemistry) (1962)

Meth. of Analysis of Choline (Cereal Chemistry) (1962)

Microbiological Meth. of Analysis of Niacin (Cereal Chemistry) (1962) Meth. of Analysis of Pantothenic Acid (Cereal Chemistry) (1962)

Microbiological Meth. of Analysis of Riboflavin (Cereal Chemistry) (1962) gitonin Cholesterol Meth. of Analysis of Dried Egg Solids (Cereal Chemistry) (1962) th. of Analysis for Titratable Acidity in Nonfat Dry Milk (Cereal Chemistry) (1962) th. of Analysis of Coliform Bacteria in All Food Products (Cereal Chemistry) (1962) th. of Analysis of Mold and Yeast Counts in Food Products (Cereal Chemistry) (1962)

Oven Meth. of Analysis of Moisture, Drying at 135 Deg. C (Cereal Chemistry) (1962)

oroform-Alcohol Meth. of Analysis of Ergot in Rye Flour (Cereal Chemistry) (1962)

eral Meth. of Analysis for Fat Acidity in Grain and Flour (Cereal Chemistry) (1962) eral Extensigraph Meth. for Physical Dough Tests of Flour (Cereal Chemistry) (1962)

h. of Analysis of Ammonia in Baking Powders and Chemicals (Cereal Chemistry) (1962)

h. of Analysis of Sucrose (Sugar) in Feeds and Feedstuffs (Cereal Chemistry) (1962)

id (Magnesium Acetate) Meth. of Analysis for Ash in Flour (Cereal Chemistry) (1962)

umetric Meth. of Analysis of Diastatic Activity of Doughs (Cereal Chemistry) (1962)

h Meth. of Experimental Milling for Bread and Soft Wheats (Cereal Chemistry) (1962)

blank Meth. of Analysis of Methoxychlor Residues in Foods (Cereal Chemistry) (1962)

o Scale Meth. of Analysis of Semolina Quality in Macaroni (Cereal Chemistry) (1962)

AACCH 30.14

AACCH 30.16

AACCH $30-20$

AACCH 30.26

AACCH 32.15

AACCH $34-01$

AACCH $38-20$

AACCH $40-01$

$\mathrm{AACCH} 40.20$

AACCH 40.21

AACCH 40.26

AACCH $40-30$

AACCH $40-31$

AACCH 40.32

AACCH $\quad 40-33$

AACCH 40.35

AACCH $40-40$

$\mathrm{AACCH} 40.41$

AACCH 40.50

AACCH $40-60$

$\mathrm{AACCH} \quad 40.65$

AACCH 40.66

AACCH $42 \cdot 10$

AACCH 42.20

AACCH 42.25

$\mathrm{AACCH} \quad 42-35$

AACCH $42-70$

AACCH $42-71$

AACCH 44.01

AACCH 44-10

$\mathrm{AACCH} \quad 44.20$

AACCH 44.52

AACCH 46.13

AACCH 46.22

AACCH 48.50

$\mathrm{AACCH} \quad 50.10$

$\triangle A C C H \quad 52.10$

AACCH 52.11

$\mathrm{AACCH} \quad 56.60$

$\mathrm{AACCH} 60.01$

AACCH 62.05

AACCH $62-15$

AACCH 62.20

AACCH 62.25

AACCH 62.30

AACCH $62-40$

AACCH $62-50$

AACCH 62.60

AACCH $62-70$

$\mathrm{AACCH} \quad 64.40$

AACCH 64.50

AACCH 64.70

$\mathrm{AACCH} \quad 66.40$

AACCH 68.61

AACCH $70-25$

A ACCH 70.35

AACCH $70-55$

$\mathrm{AACCH} \quad 70-60$

AACCH $74-30$

AACCH 80.20

$\mathrm{AACCH} \quad 80-21$

AACCH $80 \cdot 30$

AACCH $82-21$

AACCH 84.10

AACCH 86.30

AACCH 86.31

AACCH $86-40$

AACCH 86.45

$\mathrm{AACCH} 86.51$

$\mathrm{AACCH} \quad 86.60$

AACCH $86-72$

Di AACCH 20-01

Me AACCH 02-31

$\mathrm{Me}$ AACCH 42.15

Me AACCH 42.50

Air AACCH 44.19

Chl AACCH 42.55

Gen AACCH 02.01

Gen AACCH 54-10

Met AACCH 40.15

Met AACCH 80.50

Rap AACCH 08.02

Vol AACCH 22.14

Batc AACCH 26-21

Low AACCH 60.40

Macr AACCH 66.41 
of Analysis for Residual Carhon Dioxide in Baking Powder (Cereal Chemistry) (1962) of Analysis of Diastatic Activity of Flour and Semolina (Cereal Chemistry) (1962) for Preparation and Standardization of Buffer Solutions (Cereal Chemistry) (1962) - Scale Meth. of Analysis of Semolina Quality in Macaroni (Cereal Chemistry) (1962)

$r$ Meth. of Experimental Milling for Bread and Soft Wheats (Cereal Chemistry) (1962) of Analysis of Lipoxidase Activity of Semolina and Flour (Cereal Chemistry) (1962) of Analysis of Crude Fat in Egg Yolk and Dried Whole Egg (Cereal Chemistry) (1962) tative Meth. of Analysis of Ammonium Salts in Yeast Foods (Cereal Chemistry) (1962) tative Meth. of Analysis of Bromates and Iodates in Flour (Cereal Chemistry) (1962) itative Meth. of Analysis of Ammonium Persulfate in Flour (Cereal Chemistry) (1962) $\mathrm{d}$ and Ashworth Meth. of Analysis of Serum Protein in Milk (Cereal Chemistry) (1962) of Analysis of Unsaponifiahle Matter in Macaroni Products (Cereal Chemistry) (1962 of Analysis of Moisture and Volatile Matter in Soy Flours (Cereal Chemistry) (1962) remeter Meth. of Analysis of Diastatic Activity of Doughs (Cereal Chemistry) (1962) tative Meth. of Analysis of Ammonium Salts in Yeast Foods (Cereal Chemistry) (1962)

(2 Hr., 600 Deg.) Meth. of Analysis for Ash in Feedstuffs (Cereal Chemistry) (1962) or Determination of Phenothiazine in Feeds and Feedstuffs (Cereal Chemistry) (1962 or Preparation of Sample: Yeast Foods and Flour Improvers (Cereal Chemistry) (1962) f Analysis of Alpha Amylase Activity, hy Beta Amylase GBI (Cereal Chemistry) (1962) ometer Meth. of Analysis of Solids in Syrups and Molasses (Cereal Chemistry) (1962)

Meth. of Analysis of Cinder and Sand Particles in Farina (Cereal Chemistry) (1962) ol-Xylene Extraction Meth. of Analysis of Ascorbic Acid (Cereal Chemistry) (1962

washing Meth. of Analysis of Gluten in Flour and Semolina (Cereal Chemistry) (1962) r Preparation and Standardization of Lactic Acid Solution (Cereal Chemistry) (1962) r Correction of Analytical Values to $14.0 \%$ Moisture Basis (Cereal Chemistry) (1962) Analysis for Baking Quality of Self Rising Biscuit Flour (Cereal Chemistry) (1962 Analysis of Lactose (Sugar) and Nonfat Dry Milk in Bread (Cereal Chemistry) (1962 otometric Meth. of Analysis of Color of Macaroni Products (Cereal Chemistry) (1962) c Meth. of Analysis of Phosphorus in Feeds and Feedstuffs (Cereal Chemistry) (1962) c Meth. of Analysis of Phosphorus in Feeds and Feedstuffs (Cereal Chemistry) (1962) preparation and Standardization of Sulfuric Acid Solution (Cereal Chemistry) (1962) nalysis for Sulfuric Acid in Baking Powders and Chemicals (Cereal Chemistry) (1962) nalysis for Tartaric Acid in Baking Powders and Chemicals (Cereal Chemistry) (1962) nalysis of Food Poisoning Staphylococcus in Food Producfs (Cereal Chemistry) (1962) meth. for Analysis of Resistance of Flour Dough to Mixing

th. of Analysis of Solids in Juices, Syrups, and Molasses

n Meth. (Brown-Duvel) of Analysis of Moisture in Grains

Meth. of Analysis of Cinder and Sand Particles in Farin

reparation and Standardization of Barium Lactate Solution alysis for Acetic, Butyric, and Lactic Acids in Rye Flour alysis for Baking Test (Quality) for Sweet Yeast Products alysis of Diastatic Activity of Flour with the Amylograph $\mathrm{n}$ Meth. of Analysis for Admixture of Rye and Wheat Flours meth. for Analysis of Resistance of Flour Dough to Mixing lysis for Neutralizing Value of Sodium Acid Pyrophosphate of Analysis of Reducing Sugars in Prepared Bakery Mixes ount Meth. of Analysis of Total Bacteria in Food Product ahsorption Meth. of Analysis of Vitamin A in Concentrate f Analysis for Ash in Flour, Feeds, Feedstuffs, and Bread meth. of Analysis for Hydrogen Ion Activity $(\mathrm{PH})$ in Flour aration and Standardization of Hydrochloric Acid Solution sis of Pelshenke Test (Wheat Meal Fermentation Time Test) ulfate-Maltose (Diastatic Activity in Flour) Conversion Analysis for Insect Eggs in White, Wheat, and Corn Flou ration and Standardization of Sodium Thiosulfate Solution is for Total (Gasometric) Carhon Dioxide in Baking Powde is of Hydrocyanic Acid Residues in Grains and Dried Foods ide Test Meth. for Ammonium Persulfate in Flour Improvers

h. of Analysis of Bromates and Iodates in Flour Improver meth of Analysis for Baking Quality of Wheat Bread Flour

h. for Quality of Nonfat Dry Milk in Physical Dough Tests of Analysis for Insect and Rodent Filth in Popped Popcor

$s$ for Ash in Starch, Dextrin, and Other Modified Products

s of Cooking Characteristics of Macaroni-Borasio System $s$ of Undenatured Whey Protein Nitrogen in Nonfat Dry Milk

of Analysis for Nature of Ammonium Salts in Yeast Foods of Analysis of Amino Nitrogen in Wheat Flour and Semolina for Citric and Isocitric Acids in Fruit and Its Products of Reducing and Nonreducing Sugars in Flour and Semolina ion Test with Baker Compressimeter for Staleness of Bread of Analysis of Stahility in Fats, Oils, and Shortenings

eth. of Analysis of Crude Protein in Feeds and Feedstuft n for Weight of Flour to Give 100 Grams at $14.0 \%$ Moisture n and Standardization of Potassium Permanganate Solution or Fumaric, Pyruvic, Lactic, Succinic, and Tartaric Acids $f$ Inorganic Bromide Residues in Grain and Cereal Products

Loaf Meth. of Analysis for Baking Quality of Bread Flour f Analysis of Moisture and Volatile Matter in Yeast Foods late) Meth. of Analysis of Moisture in Flour and Semolina r or Spectrophotometer Analysis of Acid Soluhle Manganese Crude Fat in Wheat and Soy Flour. Feeds and Cooked Feeds

Thermophilic and Psychrophilic Bacteria in Food Products on of Specific Volume of Creams, Cake Batters, and Icings oxidizing Agents in Flour: Reaction with Potassium Iodide fiske-Subbarow Meth. of Analysis of Phosphorus in Yeas
pparent Viscosity of Acidulated Flour-Water Suspensions
Cereal Chemistry) (1962)

Cereal Chemistry) (1962)

Cereal Chemistry) (1962)

Cereal Chemistry) (1962

Cereal Chemistry) (1962)

Cereal Chemistry) (1962)

Cereal Chemistry) (1962

Cereal Chemistry) (1962)

Cereal Che mistry) (1962)

Cereal Chemistry) (1962)

Cereal Chemistry) (1962)

Cereal Chemistry) (1962)

Cereal Chemistry) (1962)

Cereal Chemistry) (1962)

Cereal Chemistry) (1962)

(Cereal Chemistry) (1962

(Cereal Chemistry) (1962

Cereal Chemistry) (1962)

Cereal Chemistry) (1962)

Cereal Chemistry) (1962)

Cereal Chemistry) (1962

Cereal Chemistry) (1962)

Cereal Chemistry) (1962)

Cereal Chemistry) (1962)

Cereal Chemistry) (1962)

Cereal Chemistry) (1962

Cereal Chemistry) (1962)

Cereal Chemistry) (1962)

(Cereal Chemistry) (1962)

(Cereal Chemistry) (1962)

(Cereal Chemistry) (1962)

(Cereal Chemistry) (1962)

(Cereal Chemistry) (1962)

(Cereal Chemistry) (1962)

Cereal Chemistry) (1962)

(Cereal Chemistry) (1962)

(Cereal Chemistry) (1962

(Cereal Chemistry) (1962)

(Cereal Chemistry) (1962)

Cereal Chemistry) (1962)

(Cereal Chemistry) (1962)

(Cereal Chemistry) (1962)

Cereal Chemistry) (1962)

(Cereal Chemistry) (1962)

(Cereal Chemistry) (1962)

(Cereal Chemistry) (1962

(Cereal Chemistry) (1962)
(Cereal Chemistry) (1962

Cereal Chemistry) (1962)

(Cereal Chemistry) (1962)

(Cereal Chemistry) (1962

(Cereal Chemistry) (1962)
Meth AACCH 12.10

Meth AACCH 22.15

Meth AACCH 70.15

Micr AACCH 66.42

Buhle AACCH 26.20

Meth. AACCH 22.40

Meth. AACCH 30.18

Quali AACCH 40.16

Quali AACCH 48.41

Quant AACCH 48.62

Harlan AACCH 46.21

Meth. AACCH $30-40$

Meth. AACCH 44.3

Pressu AACCH 22.1

Quanti AACCH 40.18

Rapid AACCH 08.03

Meth. F AACCH 18.30

Meth. F AACCH $62-80$

Meth. O AACCH 22-02

Refract AACCH 68-60

Counting AACCH 28-0

Indophen AACCH 86.10

Machine AACCH 38.1]

Meth. Fo AACCH 70.30

Meth. Fo AACCH 82-22

Meth. of AACCH $10.31 \mathrm{~A}$

Meth. of AACCH 80.3]

Spectroph AACCH 14.21

Volumetri AACCH 40.58

Gravimetri AACCH 40-5?

Meth. for AACCH 70.80

Meth. of a AACCH 04-1

Meth. of a AACCH $04-27$

Meth. of a AACCH 42.30

Mixograph AACCH $54-40$

Spindle Me AACCH $68-62$

Distillatio AACCH 44.53

Gravimetric AACCH 28-02

Meth. for P AACCH 70-10

Meth. of an AACCH 04-20

Meth. of an AACCH 10.20

Meth of an AACCH 22-10

Trifructosa AACCH 06-IO

Farinograph AACCH 54.21

Meth. of Ana AACCH 02.34

Schoorl Meth AACCH 80-68

Sid. Plate C AACCH 42-1]

Ultraviolet AACCH 86-01

Basic Meth. O AACCH 08-01

Colorimetric AACCH 02-51

Meth. for Prep AACCH $70-20$

Meth. of Analy AACCH 56-50

Meth. of Thios AACCH 22.17

Iodine Meth. of AACCH 28-44

Meth. for Prepa AACCH 70-75

Meth. of Analys AACCH $12-20$

Meth. of Analys AACCH 60.20

Qualitative Iod $\mathrm{AACCH} 48.61$

Oualitative Met AACCH $48-40$

Straight Dough AACCH 10-10

Extensigraph Met AACCH $54-12$

Flotation Meth. AACCH $28-80$

Meth. of Analysi AACCH 08.17

Meth. of Analysi AACCH 16-51

Meth. of Analysi AACCH 46-20

Qualitative Meth AACCH 40.17

Van Slyke Meth. AACCH 46.32

Meth. of Analysis AACCH $04-22$

Meth. of Analysis AACCH 80.60

Meth. of Compress AACCH 74.10

Oxygen-Bomh Meth AACCH 58-55

Improved Kjeldahl M AACCH 46.10

Meth. for Correctio AACCH $82-23$

Meth. for Preparati AACCH 70.50

Meth. of Analysis F AACCH 04.24

Meth. of Analysis O AACCH $60-10$

Sponge Dough, Pound AACCH 10-11

Vacuum Oven Meth. O AACCH 44.32

Air Oven (Aluminum P AACCH 44.16

Meth. for Colorimete AACCH $40-45$

Meth. of Analysis of AACCH $30-25$

Meth. of Analysis of AACCH 42.45

Meth. for Determinati AACCH 72-10

Meth. of Analysis of AACCH 48-02

Colorimetric Modified AACCH 40.56

Meth. of Analysis of a AACCH $56-80$ 
itamin a and Carotene in Enriched Cereals and Mixed Feeds (Cereal Chemistry) (1962) f Analysis for Insect Fragments and Rodent Hairs in Flour (Cereal Chemistry) (1962) nalysis for Hydrogen Ion Activity (PH) in Flour and Bread (Cereal Chemistry) (1962) Calculated from Total Chlorides in Feeds and Feedstuffs (Cereal Chemistry) (1962) of Analysis of Amino Nitrogen in Wheat Flour and Semolina (Cereal Chemistry) (1962) lysis for Phosphoric Acid in Baking Powders and Chemicals (Cereal Chemistry) (1962) of Analysis of Ergot in Rye and Wheat Flour and Semolina (Cereal Chemistry) (1962) of Analysis of Amino Nitrogen in Wheat Flour and Semolina (Cereal Chemistry) (1962) en Meth. of Analysis of Moisture in Grains and Feedstuffs (Cereal Chemistry) (1962) lysis for Phosphoric Acid in Baking Powders and Chemicals (Cereal Chemistry) (1962) lysis of Potassium Bromate in White and Whole Wheat Flour (Cereal Chemistry) (1962) alysis for Insect Fragments and Rodent Hairs in Rye Flour (Cereal Chemistry) (1962 $\mathrm{g}$ Meth. of Analysis of Phosphorus in Feeds and Feedstuffs (Cereal Chemistry) (1962) standardization of Sudium or Potassium Hydroxide Solution (Cereal Chemistry) (1962) 1 Carbon Dioxide in Prepared Mixes and Self Rising Flours (Cereal Chemistry) (1962)

Meth. for Sugar Determination: Schoorl's Reduction Table (Cereal Chemistry) (1962)

ytic Activity of Flour and Active Proteinase Preparations (Cereal Chemistry) (1962) 1 Urease Activity in Soybean Meals, Soy Flour, Mill Feeds (Cereal Chemistry) (1962)

sof Aluminum in Baking Powder, in Presence of Phosphates (Cereal Chemistry) (1962) of Extensigraph Analysis of Physical Dough Tests of Flour (Cereal Chemistry) (1962)

Propionic, Butyric, Valeric, Succinic, and Lactic Acids (Cereal Chemistry) (1962) $\mathrm{n}$ Wheat and Flour Mill Prnducts (Boric Acid Modification) (Cereal Chemistry) (1962)

sphate and/or Sodium Acid Pyrophosphate in Prepared Mixes (Cereal Chemistry) (1962) on and Classification in Cereal Grains and Grain Products (Cereal Chemistry) (1962) robic, Anaerobic) in Cereal Products and Food Ingredients (Cereal Chemistry) (1962) feedstuffs (Calculated from Percentage of Total Nitrogen) (Cereal Chemistry) (1962) lina, and Similar Products: Feeds and Feedstuffs in Sacks (Cereal Chemistry) (1962) its, Meal, Flaked and Puffed Cereals, Farina, Bread, etc. (Cereal Chemistry) (1962) nce of Rodent Urine on Bags and Other Packaging Materials (Cereal Chemistry) (1962) nal Ash in Phosphated and Freshly Mixed Self Rising Flour (Cereal Chemistry) (1962) mination of Both Nitrophenide and Arsanilic Acid in Feeds (Cereal Chemistry) (1962)

for Cake and Meal from Cottonseed, Soybeans, and Peanuts (Cereal Chemistry) (1962)

Corn Grits, Corn Meal, Puffed Cereals, Farina, and Flour (Cereal Chemistry) (1962)

revention of Cecal and Intestinal Coccidiosis in Chickens (Cereal Chemistry) (1962)

ommodities for Which Conversion Charts Have Been Prepared (Cereal Chemistry) (1962)

ng Value of Acid Reacting Materials Other Than Phosphates (Cereal Chemistry) (1962)

avity, Degrees Brix, and Degrees Baume of Sugar Solutions (Cereal Chemistry) (1962)

lysis of Urea in Cloth Bags and Other Packaging Materials (Cereal Chemistry) (1962

- 14.0\% Moisture Basis (Constant Dough Weight Meth. Only) (Cereal Chemistry) (1962)

ur, Bread, and Baked Cereal Products Not Containing Fruit (Cereal Chemistry) (1962) els, Rodent Excreta, Other Filth, and Extraneous Material (Cereal Chemistry) (1962) alysis for Insect Fragments and Rodent Hairs in Soy Flour (Cereal Chemistry) (1962) Breakfast Cereals (Except Those Which Are Sugar Coated) (Cereal Chemistry) (1962)

Hairs, Feathers, Wood Fibers, Splinters, etc. in Starch (Cereal Chemistry) (1962) ings (Butter, Oleomargarine, High Acid Coconut Oil, etc.) (Cereal Chemistry) (1962) $h$ in Flour and Semolina (With Subsequent Acid Hydrolysis) (Cereal Chemistry) (1962)

Liquid and Semiliquid Products (Drying Upon Quartz Sand) (Cereal Chemistry) (1962)

$s$ for Detection of Rodent Excreta, Insects, Webbing, etc. (Cereal Chemistry) (1962)

cereal Grains, Unpopped Popcorn, Dried Peas and and Beans (Cereal Chemistry) (1962)

value of Monocalcium Phosphate: Monohydrate and Anhydrous (Cereal Chemistry) (1962)

lina; Bread; Feedstuffs, Ground Grains and Their Adjuncts (Cereal Chemistry) (1962) onoglyceride, Salts, Soy Flour, and Other Cereal Products (Cereal Chemistry) (1962)

our Milling: Equipment, Sample Preparation, and Tempering (Cereal Chemistry) (1962)

Baked Goods, Ready.To.Eat Cereals, and Alimentary Pastes (Cereal Chemistry) (1962) protein in Bread, Wheat and Other Grains, and Yeast Foods (Cereal Chemistry) (1962) of Cereal Grains, Unpopped Popcorn, Dried Peas and Beans (Cereal Chemistry) (1962) ge Mixing Characteristics of Flour (Physical Dough Tests) (Cereal Chemistry) (1962) ture in Feeds and Feedstuffs by Distillation with Toluene (Cereal Chemistry) (1962) ture in Fats and Shortenings by Distillation with Toluene (Cereal Chemistry) (1962) to Corn, Rye, Barley, Rice, Grain Sorghum, and Buckwheat (Cereal Chemistry) (1962) ect and Rodent Filth in Meal, Grits, Farina, and Semolina (Cereal Chemistry) (1962) and Flour (Calculated from Percentage of Total Nitrogen) (Cereal Chemistry) (1962)

in Cereal Grains, Unpopped Popcorn, Dried Peas and Beans (Cereal Chemistry) (1962) of Proteolytic Activity in Active Proteinase Preparations (Cereal Chemistry) (1962)

Premixes for Control of Cecal and Intestinal Coccidiosis (Cereal Chemistry) (1962) 1l Baked Materials Except Chocolate Products and Piecrust (Cereal Chemistry) (1962) Baked Goods, Ready-To.Eat Cereals, and Alimentary Pastes (Cereal Chemistry) (1962) ine Added Amounts of Malathion in Range of 0.2-2.0 p.p.m. (Cereal Chemistry) (1962)

Free or Combined Tartaric Acid in Presence of Phosphates (Cereal Chemistry) (1962) ground Cereal Grains, Whole or Chopped Nuts, Spices, etc. (Cereal Chemistry) (1962) on Factors for Gasometric Determination of Carbon Dioxide (Cereal Chemistry) (1962) odent Excreta in Meal, Grits, Flour, Farina, and Semolina (Cereal Chemistry) (1962) Meth of Analysis of Urea and Ammonical Nitrogen (Cereal Chemistry) (1965) Meth. of Analysis of Sedimentatoon Test for Wheat (Cereal Chemistry) (1965) on Factors for Correcting Weights to a $14 \%$ Moisture Basis (Cereal Chemistry) (1965) Meth of Analysis of Free Fatty Acids in Fats (Cereal Chemistry) (1967) Meth of Analysis of Vitamin A in Enriched Flour (Cereal Chemistry) (1967) washing Meth. of Analysis of Gluten in Flour and Semolina (Cereal Chemistry) (1967) of Analysis of Iodine Number in All Edible Fats and Oils (Cereal Chemistry) (1967) of Analysis of Niacin and Nicotinamide in Cereal Products (Cereal Chemistry) (1967) nalysis of Stability (AOM) of Fats, Oils, and Shortenings (Cereal Chemistry) (1967)

lysis of Saponification Value of All Normal Fats and Oils (Cereal Chemistry) (1967) is of Cloud Point in All Edible Animal and Vegetable Fats (Cereal Chemistry) (1967)

h. of Analsis of Benzoyl Peroxide with Diaminophenylamine (Cereal Chemistry) (1967) of Acetone-Insoluble Lecithin in Soybean and Corn Oils (Cereal Chemistry) (1967) ysis of Melting Point of Normal Animal and Vegetable Fats (Cereal Chemistry) (1967)
Meth. of Analysis of V AACCH 86.05 Acid Hydrolysis Meth. O AACCH 28.41 Electrometric Meth. of a AACCH 02.52 Meth. of Analysis of Sal AACCH 40.61 Modified Sorensen Meth. AACCH 46.31 Qualitative Meth. of Ana AACCH 04.10 Ether-Bicarbonate Meth. AACCH 42.56 Linderstrom-Lang Meth. AACCH 46.30 Modified Two Stage Air Ov AACCH 44.18 Quantitative Meth. of Ana AACCH 04.11 Quantitative Meth. of Ana AACCH 48.42 Tween Versene Meth. of an AACCH 28.60 Colorimetric Acetate Ashin AACCH 40.55 Meth. for Preparation and AACCH 70.70 Meth. of Analysis for Tota AACCH 12.21

Volumetric Copper Reduction AACCH 80.69

Meth. of Analysis of Proteol AACCH 22.60 Meth. of Analysis of Residua AACCH 22.90 Qualitative Meth. of Analysi AACCH 40.10 Structural Relaxation Meth. AACCH 54.11

/ Analysis for Formic, Acetic AACCH 04.23 / Analysis of Crude Protein I AACCH 46.12

Analysis of Monocalcium Pho AACCH 40.5 / and Radiographic Illustrati AACCH 28.95 Counts (Flat Sour, Total Ae AACCH 42.40 Crude Protein in Feeds and AACCH 46.18

for Sampling of Flour, Semo AACCH 64.60 in Whole Grain Products, Gr AACCH 86.70

Light Examination for Prese AACCH 28.85

Meth. of Analysis for Origi AACCH 08.15

Meth. of Quantitative Deter AACCH 18.26 of Crude Fiber in Soy Flour AACCH 32.17

Other Whole Grain Products, AACCH 86.80

Poultry Mashes (Feed) for P AACCH 18.25

1. Lentils, Rice, and Other C AACCH 44.11

1. of Analysis for Neutralizi AACCH 02.32

1. of Analysis of Specific Gr AACCH 80.75

1. of Xanthydrol Test for Ana AACCH 28.86

/.Is Farinograph Absorption T AACCH 54.29

A Analysis of Crude Fat in Flo AACCH 30-10

land/or Insect Damage on Kern AACCH 28.10

Auting Digestion Meth. of an AACCH 28-70

D Oats, Bulgur, Rolled Wheat AACCH 44-15A

Dirt, Sand, Insect Fragments AACCH 28.75

E Matter in Fats and Shorten AACCH 44-30

E Meth of Analysis of Starc AACCH 76.10

/Ecuites, Molasses, and Other AACCH 44.60

Ereals, and Alimentary Paste AACCH 28-30

Ernal Insect Infestation in AACCH 28.21

F Analysis for Neutralizing AACCH 02.33

/F Moisture in Flour and Semo AACCH 44.40

Gh Conditioners Containing $\mathrm{M}$ AACCH 48.51

H. for Experimental Wheat Fl AACCH 26-10

Materials Hard to Hydrate in AACCH 28.32

Mples for Analysis of Crude AACCH 46.11

/Nd Rodent Hair Contamination AACCH 28.20

/Nfat Dry Milk Solids to Chan AACCH 54.20

$/ \mathrm{Ng}$ Meth. of Analysis of Mois AACCH 44.50

$/ \mathrm{N}_{\mathrm{g}}$ Meth. of Analysis of Mois AACCH 44.51

$/ 8$, with Slight Modification, AACCH 76.20

lon Meth. of Analysis for Ins AACCH 28.51

lon of Crude Protein in Wheat AACCH 46-19

lon Test for Internal Insects AACCH 28.22

Otometric Meth. of Analysis AACCH 22.62

Oxaline in Poultry Feeds and AACCH 18.35

Ping Meth. of Analysis for a AACCH 28.33

/R Insect and Rodent Filth in AACCH 28.31

/Ran, Shorts, etc.) to Determ AACCH 60.30

Tative Meth. of Analysis for AACCH 04-28

Th or Sack Fibers, Whole or AACCH 28.87

/Th. of Analysis for Correcti AACCH 12.29

Tion Meth. of Analysis for $R$ AACCH 28.50

AACCH 46.50

AACCH $56.61 \mathrm{~A}$

Meth. for Multiplicati AACCH 82.24

$\mathrm{AACCH} 58.16$

AACCH 86.03

Hand $\mathrm{AACCH} 38.10$

Meth. AACCH 58.30

Meth. AACCH 86.50

Meth. of a AACCH 58.54

Meth. of Ana AACCH 58.50

Meth. of Analys AACCH 56.10

Qualitative Met AACCH $48.06 \mathrm{~A}$

$\begin{array}{rr}\text { Meth. of Analysis AACCH } & 58-35 \\ \text { Wiley Meth. of Anal AACCH } & 58.41\end{array}$ 
th Abbe Refractometer, in All Normal Oils and Liquid Fats (Cereal Chemistry) (1967) lycerides and Free Glycerol in Fats, Oils and Shortenings (Cereal Chemistry) (1967) of Melting Point of All Normal Animal and Vegetable Fats (Cereal Chemistry) (1967) (Vitamin and Mineral) Concentrates Used to Enrich Cereals (Cereal Chemistry) (1967) eth of Analysis of Modified Sedimentation Test for Wheat (Cereal Chemistry) (1968) Meth. of Analysis of Micro Sedimentation Test for Wheat (Cereal Chemistry) (1968) h. of Triangle Test Taste Panel for Food Flavor Detection (Cereal Chemistry) (1968) antitative Meth. of Analysis of Benzoyl Peroxide in Flour (Cereal Chemistry) (1968) ilseeds, Legumes, Forages, and Animal and Dairy Products (Cereal Chemistry) (1968) $n$ Capacity of Pregelatinized Starches and Cereal Products (Cereal Chemistry) (1968) 1 Fat and Defatted Soy Flours and Grits, and Soybean Meal (Cereal Chemistry) (1968) 1 Fat and Defatted Soy Flours and Grits, and Soybean Meal (Cereal Chemistry) (1968) $\mathrm{r}$ of Wheat, Rye, Barley, Other Grains, and Malted Cereals (Cereal Chemistry) (1968) of Proteolytic Activity in Active Proteinase Preparations (Cereal Chemistry) (1968)

ne Dioxide in Flour Bleaching Experiments in Laboratories (Cereal Chemistry) (1968)

Meth. of Analysis of Pesticide Residues in Food Products (Cereal Chemistry) (1969)

ons Which Are Susceptible to Hydrolysis by Alpha Amylase) (Cereal Chemistry) (1969)

eth. of Analysis of Acetone Peroxides in Milling Premixes (Cereal Chemistry) (1971)

mination of Azodicarbonamide Content in Commercial Premix (Cereal Chemistry) (1971)

eal or Solidify Within Temperature Range of 25 to $45 \mathrm{Deg}$. (Cereal Chemistry) (1971)

of Vitamin A in Nonfat Dry Milk and Instantized Dry Milks (Cereal Chemistry) (1971)

TLC Meth. of Analysis of Aflatoxins in Corn and Soybeans (Cereal Chemistry) (1972)

Meth. of Analysis of Added Lysine in Wheat and Bulgur (Cereal Chemistry) (1973)

rmination of Fatty Acid Composition by Gas Chromatography (Cereal Chemistry) (1973)

$s$ of Coagulase Positive Staphylococcus in Cereal Products (Cereal Chemistry) (1973)

ty (Beta Gamma) in Cereal Products-Emergency Monitoring (Cereal Chemistry) (1973)

min a in Dry Vitamin Mixes, Beadlets, Oils, and Emulsions (Cereal Chemistry) (1973)

g Chain Fatty Acids in Common Fats, Oils, and Fatty Acids (Cereal Chemistry) (1973)

Hair, and Radiographic Illustration and Classification in nd Dairy $\mathrm{P}$

Dye Meth. of Analysis of Crude Protein in or Roden/ Meth. of Analysis of Macroscopic Examination of alysis of External Insect and Rodent Hair Contamination of nalysis of Cracking Flotation Test for lnternal Insects in Analysis of Urea in Cloth or Sack Fibers, Whole or Ground

y) (1/ Meth. of Analysis of Radioactivity (Beta Gamma) in ilic Spore Counts (Flat Sour, Total Aerobic, Anaerobic) in Meth. of Analysis of Crude Fat in Flour, Bread, and Baked Meth. of Analysis of Rope Spore Count in th. of Analysis of lnorganic Bromide Residues in Grain and ners Containing Monoglyceride, Salts, Soy Flour, and Other Meth of Analysis of Niacin and Nicotinamide in lysis of Hydration Capacity of Pregelatinized Starches and Meth. of Analysis of Coagulase Positive Staphylococcus in Meth. of Analysis of Vitamin A and Carotene in Enriched richment (Vitamin and Mineral) Concentrates Used to Enrich and Flour of Wheat, Rye, Barley, Other Grains, and Malted

, Corn Grits, Rolled Oats, Bulgur, Rolled Wheat, Breakfast is of Macroscopic Examination of Baked Goods, Ready.To-Eat $s$ for lnsect and Rodent Filth in Baked Goods, Ready.To-Eat for Materials Hard to Hydrate in Baked Goods, Ready. To. Eat Other Whole Grain Products, Corn Grits, Corn Meal, Puffed in in Whole Grain Products, Grits, Meal, Flaked and Puffed st for Absorbed Gamma and Electron Radiation Dose with the ce Requirements for Permanent Labels, Detachable Tags, and Requirements for the Construction and

olderless Wiring Devices-Recommended Procedures for User acth. for Determining GAWR and GVWR for Truck Trailer ce) $(1970)$ Std. for Std. Meth. of Test for Radioactive Std. Meth. for Radiochemical Determination of f Room Fan Coil Air Conditioners Delivering Less Than 1500 Std. Malleable Iron Detachable Link Spec. for Oil Field

Std. Motor Positions for Belt or

(Cereal) Meth. for Preparation of Methyl Esters of Long
Std. Spec. for Hand Operated ns (1971) Std. Spec. for Manually Lever Operated Std. Spec. for Electric Std. Spec. for Aluminum Coated Steel Std. Spec. for Zinc Coated Steel Spec. for Galvanized Steel Std. Spec. for Carbon Steel Std. Spec. for Steel Conveyor Std. Spec. for Machine and Sprocket Coil Std. Spec, for Weldless Carbon Steel nnecting Devices and Towing Meth. (Coupling, Hitch, Safety equirements for Regular Bicycles Including Wheel Assembly, Std. for $\mathrm{H}$ Type Mill

d. for Heavy Duty Offset Sidebar Power Transmission Roller Std. for Heavy Duty Roller Type Conveyor Std. for Double Pitch Conveyor Roller sion) (1972) Std. for 700 Class Pintle

Std, for Steel Detachable Link Voluntary Safety Std. for High
Cereal Grains and Grain Products (Cereal Chemistry) (19
Cereal Grains, Oilseeds, Legumes, Forages, and Animal a

Cereal Grains, Unpopped Popcorn, Dried Peas and and Bea

Cereal Grains, Unpopped Popcorn, Dried Peas and Beans F

Cereal Grains, Unpopped Popcorn, Dried Peas and Beans (

Cereal Grains, Unpopped Popcorn, Dried Peas and Beans

Cereal Grains, Whole or Chopped Nuts, Spices, etc. (Cer

Cereal Products-Emergency Monitoring (Cereal Chemistr

Cereal Products and Food Ingredients (Cereal Chemistry)

Cereal Products Not Containing Fruit (Cereal Chemistry)

Cereal Products (Cereal Chemistry) (1962)

Cereal Products (Cereal Chemistry) (1962)

Cereal Products (Cereal Chemistry) (1962)

Cereal Products (Cereal Chemistry) (1967)

Cereal Products (Cereal Chemistry) (1968)

Cereal Products (Cereal Chemistry) (1973)

Cereals and Mixed Feeds (Cereal Chemistry) (1962)

Cereals (Cereal Chemistry) (1967)

Cereals (Cereal Chemistry) (1968)

Cereals (Except Those Which Are Sugar Coaition of B

Cereals, and Alimentary Pastes for Detection of Rodent

Cereals, and Alimentary Pastes (Cereal Chemistry) (1962

Cereals, and Alimentary Pastes (Cereal Chemistry) (1962

Cereals, Farina, and Flour (Cereal Chemistry) (1962)

Cereals, Farina, Bread, etc. (Cereal Chemistry) (1962)

Ceric Sulfate Dosimeter (1.971) ANSI K65.230

Certification of Fabrics or Products (Institutional Tex

Certification of Freight Containers (1968)

Certification of (1962) ANS1 C83.79

Certification (1972)

Certified Ratings Program Air Moving Devices (Performan

Cesium in Water (1972)

Cesium. 137 in Nuclear Fuel Solutions (1970) ANSI N117

$\begin{array}{lll}\text { Me } & \text { AACCH } & 60.10 \\ & \text { AACCH } & 48-51\end{array}$

AACCH 86.50

Eth. of Ana AACCH 56.20

AACCH $42.30 \mathrm{~A}$

AACCH 86.05

$\begin{array}{rr}\text { Met AACCH } & 33.50 \\ & \end{array}$

rains, AACCH 46.14

cfm (1971)

Chain and Attachments (1971)

Chain and Sprockets (1970)

Chain Drive Centrifugal Fans (1966)

Chain Fatty Acids in Common Fats, Oils, and Fatty Acids

Chain Hoists for Industrial Applications (1971)

Chain Hoists for Ratchet and Pawl, and Load Brake Desig

Chain Hoists (1971)

Chain Link Fence Fabric (1971) ANSI G53.21

Chain Link Fence Fabric (1971A) ANSI G53.20

Chain Link Fence Fabric (1973)

Chain (1972) ANSI G61.2

Chain (1972) ANSI G61.3

Chain (1972) ANSI G61.5

Chain (1973) ANSl G61.4

Chain, Lighting) (1973)

Chainguards, Saddle Braces, Pedal Ch

Chains-Attachments and Sprocket Teeth (1971)

Chains and Sprocket Teeth (1972)

Chains and Sprocket Teeth (1973)

Chains, Attachments and Sprockets (1972)

Chains, Attachments, and Sprocket Teeth (Power Transmis

Chains, Attachments, and Sprockets (1972)

Chairs (Children's) (1972)
AACCH 86.49

AACCH $56.81 B$

AACCH $28-30$

AACCH 28-31

AACCH 28-32

AACCH $86-80$

AACCH $86-70$

D3001

ANSI L24.5.

ABS $* 17$

RS270

RP39

AMCA 211

ASTM D257

443

ARI

ANSI

API

B29.7

AMCA 240

A ACCH 58.17

HMI 200

HMl 300

HMl $\quad 400$

ASTM A491

ASTM A392

CLFM1 *5

ASTM A413

ASTM A454

ASTM A467

ASTM A466

V. 5

$6 / 5$

BMA

ANSI

St ANSI

ANSI

ANSI

ANS

ANSl

JPMA

B29.10

B29.4

B29.13

B29.6

1 
Std. Meth. for Measuring mper Cont/Safety Std. for Draft Equipment for Combustion e from the Burning or Dec/ Uniform Building Code Std. for Std. Meth. of Test for Effective Pumping Speed of Vacuum Id on the Surface of Interior Coatings in an Environmental om Temperature (1966) An/ Std. Spec. for Enclosures, Cold Rec. for Stopping Powers for Use with Cavity Aeration Chambers, Chemical Storage, Mixing, Filter, Grit

Wastewater Treatment (Septic, Holding, Settling, A eration (1972) (1973) Std. Pract. for Combustion y Meth. (Textile Colorfastness) (19/ Test Meth for Color Std. Meth of Test for meth. of Ability of Roller Dried Nonfat Dry Milk Solids to nd Concrete (1974T) Std. Meth. of Test for Length en Wire Meth. (Textile Colorfastnes/ Test Meth. for Color si A 37.78 Std. Meth. of Test for Reheat tile) $(197 /$ Std. Meth. of Test for Length Std. Meth. of Test for Relaxation Dimensional
Rec. Evaluation Procedure of Gray Scale for Color ent) Press Woven or Knit Text/ Test Meth. for Dimensiona ept Wool (1972) ANSI L14.138,/ Test Meth. for Dimensional 1972) - of Test tor Pilling Resistance and Other Reiated Surface Service (For Electric Trucks/ Safety and Health Rec. for meth. of Physical Dough Tests: Approximate Corrections for ough $30,000 / 40,000 / 50,000 /$ Std. Requirements for Load Tap

ec. for Large Power Transformers, with or Without Load Tap Std. Meth. of Test for Slump of an Oil Base Knife Grade

$f$ Test for Volatility of Oil and Resin Based, Knife Grade, al) Bearing Steel Studs, Runners (Track) and Rigid Furring g Rounds, Squares, Hexagons, and Shapes-Angles, TEES and Std. for Print Spec. for Magnetic lnk

ANSI C83.95 Oils by the Clay Gel Absorption 972)

Std. Meth. of Test for Alt (Du) Std. Performance Requirements and Claims for Specia ium Speed Stationary Diesel and Gas Engine Lubricating Oi medium Speed Stationary Diesel and Gas Engine Gaseous Fue reference Glass-Metal Butt Seals and Test. for Expansion erence Glass-Metal Sandwich Seal and Test. for Expansion equipment for Use with Emergency Locator Tran/ Rec. Basic

c. Pract. for Reference Carrier Frequencies and Deemphasis nt (1972) Ansi/ Coolan/ Std. Meth. for Verifying the Flow Fatigue s $(1973)$ Std. Test Meth, for Cavitation Erosion Corrosion Sid. for Electrical and Mechanical Meth. for Determining Blocking Resistance and Associated 11.301 Std. Meth. of Test for Water Separation Test Meth. of Surface Burning

(Electronics) (1974) Sid for the Electrical Std. Meth. of Test for Coefficient of Friction and Wear ied Over Film Insul/ Std. Meth. of Test for Thermal Aging 1.320 Std. Meth. of Test for Oxidation ty of Roller Dried Nonfat Dry Milk Solids to Change Mixing Std. for Dimensional and Electrical

124 Std. Meth. of Test for Water Washou Std. Meth. of Test for Foaming Meth. of Analysis of Cooking Meth. of A nalysis of Cooking emistry) (1962)

Std. Meth. of Test for Resistance Std. Meth. of Test for Knock Std. Meth. of Test for Knock tio (CR) Technique for Rese tio (CR) Technique for Moto/ ing) (1964) ANSl / Std. Meth. of Test for Shock Absorbing ial (1972) Std. Meth. of Test for Disbonding Std. for Guide for Describing the a Tempera/ Std. Test Meth. for Evaluating Low Temperature er (1972) An/ Std. Meth. of Test for Viscosity and Curing Std. for Physical

I Lamp (197I) al Lamp (1971)

Std. Dimensional and Electrical Lamp (1971) Std. Physical and Electrical Std Std. Dimensional and Electrical

Earthquakes (Genera and Medium Speed Stationary Diesel and Gas Engine Fuel Oi Redwood Plywood Guide (Spec. for Texture and Grade, Panel de on Solid State Devices: Varactor Measurements Part ll Test Meth. for Colorfastness to Washing: on lnterc/ Std. for Graphic Representation of the Control Tent. Rec. Pract. for Electrostatic Std. Meth. of Test for Electrostatic for the Design, Construction and lnstallation of Battery
Chalk Resistance of Exterior Coil Coatings (1974)

Chamber in Heating Appliances (Regulators, Automatic Da Chamber Meth. of Test for Measuring the Density of Smok Chamber Systems (1972)

Chamber (1973)

Chambers and Serving Units for Tests Above and Below Ro

Chambers (1961)

Chambers, and Flotation) (1973) /C. Holding, Settling,

Chambers, Chemical Storage, Mixing, Filter, Grit Chambe

Chambers, Fuel Oil Injection, and Gas Admission Systems

Change Characteristics of Photographic Films and Papers

Change in Fabrics Due to Flat Abrasion (Frosting

Change Mixing Characteristics of Flour (Physical Dough

Change of Drilled or Sawed Specimens of Cement Mortar a

Change of Fabrics Due to Flat Abrasion (Frosting): Scre

Change of Fireclay Nozzles and Sleeves (1972)

Change of Hardened Cement Mortar and Concrete (1974) an

Change (Shrinkage) of Stabilized Knit Wool Fabrics (Tex

Change (Textile Colorfastness) (1954) ISO 105.1

Changes in Automatic Home Laundering of Durable (Perman

Changes in Laundering of Woven and Knitted Textiles Exc

Changes of Textile Fabrics (1972) ANSl L 14.198

Changes (Shrinkage) of Woven and Knitted Wool Textiles

Changing and Charging Storage Batteries in Motive Power

Changing as-is Farinograph Absorption to $14.0 \%$ Moisture

Changing Transformers 138,000 Volts and Below, 3750 Thr

Changing (1971) Std. for Guide for Preparation of Sp

Channel Glazing Compound (1972)

Channel Glazing Compounds (1973)

Channels for Screw Application of Gypsum Board (1974)

Anese Steel Bars Includin

Characteristic Defining Dual in Line Type Sockets (1974

Characteristic Groups in Rubber Extender and Processing

Characteristics Airborne VHF Omnirange (VOR) Systems (1

Characteristics and Dielectric Constant (Permittivity)

Characteristics and Finishes of Institutional Textiles

Characteristics and General Spec. (1972)

Characteristics and General Spec. (1972)

Characteristics by Polarimetric Meth. (1973)

Characteristics by Polarimetric Meth. (1973)

Characteristics for Airborne Radio Homing and Alerting

Characteristics for $2 \mathrm{ln}$. Quadruplex Video Magnetic Tap

Characteristics of a Hydraulic Fluid Power Filter Eleme

Characteristics of Aluminum Automotive Water Pumps with

Characteristics of Antennas for Satellite Earth Station

Characteristics of Automotive Trim Materials (1972)

Characteristics of Aviation Turbine Fuels (1972) A NSI Z

Characteristics of Building Materials (1972)

Characteristics of Class a Closure lnterchange Circuits

Characteristics of Cotton Fibers (1972) ANSI L14.95

Characteristics of Dry Solid Filın Lubricants in Vacuum

Characteristics of Electrical lnsulating Varnishes Appl

Characteristics of Extreme Pressure Oils (1972) ANSI Z1

Characteristics of Flour (Physical Dough Tests) (Cereal

Characteristics of Fluorescent Lamps (1972)

Characteristics of Lubricating Greases (1973) ANS1 Z11.

Characteristics of Lubricating Oils (1972) ANSI Z11.78

Characteristics of Macaroni-Borasio System (Cereal Ch

Characteristics of Macaroni (1962)

Characteristics of Microcontacts (1972) A NSl Z55.15

Characteristics of Motor Fuels Using the Compression Ra

Characteristics of Motor Fuels Using the Compression Ra

Characteristics of Package Cushioning Materials (Packag

Characteristics of Photographic Films and Papers (1973)

Characteristics of Pipeline Coatings by Direct Soil Bur

Characteristics of Resilient Mountings (1972)

Characteristics of Rubber and Rubber Like Materials by

Characteristics of Rubber by the Shearing Disk Viscomet

Characteristics of T-2 Telephone Slide Base Lamp (1973)

Characteristics of 15 Watt T8 Preheat Start Bactericida

Characteristics of $30 \mathrm{~W}$ att, T8 Prehea

Characteristics of 50 Watt, H46 Mercury Lamps (1972)

Characteristics of $8 \mathrm{~W}$ att T5 Preheat Start Bactericidal

Characteristics Of, Rec. Construction and Water Sprinkl

Characteristics Vertical Guidance Equipment Used in Air

Characteristics (1972)

Characteristics, Material Composition, Use, Application

Characterization of Large Signal Devices (1972)

Characterization of Textile Colorants (1972)

Characters of American National Std. Code for lnformati

Charge Mobility on Flexible Barrier Materials (1973)

Charge (1973)

Chargers on Boats (1973)
Std. Std. Pract.

NCCA

UL

ICBO

ASTM

ASTM

ASTM

NSF 41

NSF 41

ANSI

ANSI 120

ASTM D471

$\mathrm{AACCH} \quad 54-20$

ASTM C 341

A ATC 119

ASTM C605

ASTM C 157

ASTM DI284

AATCC EPI

A TCC 135

A ATCC 96

1. Meth ASTM D1375

A ATCC 99

ITA $8 \mathrm{KI}$

$\mathrm{AACCH} \quad 54.29$

ANSl C57.12.30

C57.97

ASTM C713

ASTM C68

ASTM C645

ASTM

ANSI $\quad \mathrm{X} 3.2$

ElA RS415

D2007

RTSA DO-149

ASTM DI50

ANSl L24.7.1

* 1 . 12

$\begin{array}{ll}\text { Low and DEMA } & * 1-15 \\ \text { Making ASTM } & \text { Fl40 }\end{array}$

$\begin{array}{lll}\text { Making ASTM } & \text { F140 } \\ \text { King Ref ASTM } & \text { F144 }\end{array}$

RTCA DO-154

SMPTE RP6

NFLDP T3.10.8.7

ASTM D2809

ElA RS4II

is SAE

ASTM D2550

NFPA 255

ElA RS410

ASTM D1444

ASTM D2716

ASTM D2893

AACCH $\quad 54.20$

ANSI $\quad$ C78

ASTM DI264

ASTM D892

AACCH 16-5I

$\mathrm{AACCH} \quad 16.50$

ASTM B326

ASTM D2722

ASTM D2723

ASTM D1596

ANSl PHl.32

ASTM GI9

ANSl S2.8

ASTM DI329

ASTM D16

ANS1 C78.397

ANS1 C78.1201

ANSI C78.1202

C78.1323

ANSI C78.1324

ANSI C78.1200

FMS LPD 1.2

RTCA DO-152

DEMA *1.14

CRA $3 A 9$

1. Gui IEEE

AATCC 36

ANSl X3.32

ASTM

ASTM

Rec. Pract. and Std ABYC
F365 
fiber Electrometer Type Dosimeters and Companion Dosimeter Safety Std. for Battery for Division Services (Individuals, Clubs, and Exhibition Electric Trucks r Pressure Vessels (1972) Safety and Health Rec. for Changing and Std. Spec. for
Rec. Pract. for Lubrication valuation Procedure of Chromatic Transference Scale (Color Std for Operation and Flow Process Lentils, Rice, and Other Commodities for Which Conversion Microfont Std. Filler Metal Comparison tions, Symbols, Formulas, and Tables for (Quality) Control r Engineering Drawing and Related Documentation Practices: ment (Production Valves, Ring Joint, Segmented, Production potable Water Supply/ Performance Requirements for Double Spec. for Steel Gate, Plug, Ball, and Std. Rec. Pract for Reporting Laboratory Results When Std. for

Rec. Safe Pract. and Proc. (Ind ustrial Plant Safety m Dioxide Pigments (1973)

crude (1971)

ansi 011.23

0.3

ctrical Steels, Ingot and Wrought Irons (/

i 011.19

Chrome Ore (1970) ANSI Al39.2

2)

en Pigments (1973) Std. Meth. for Std. Meth. for Std. Meth. for Std. Meth. for Std. Meth. for 1971T)

974)

Tent. Meth. for Std. Meth. for Std. Meth. for Std. Meth. for Std. Meth. for Std. Meth. for Std. Meths. for Std. Meth. for Std. Meth. for Std. Meth. of

etic, and Other Similar lron, Nickel, And/

Lime (1972) ANSI K67.3 pract. for Apparatus, Reagents, and Safety Precautions for

omium-Iron Alloys (1973) Std. Meth. for 22 al, Mass Spectrometric, Spectrochemical, Nuclear and Radio
Std. Meth. for 1968) ANSI Z258.1 Std. Meth. for Std. Meth. of Std. Meth. for Std. Meth. for Std. Meth. of Std. Meth. of Std. Meth. for Std. Meth. for Std. Meth. for Std. Meth. for Std. Meth. for Std. Meth. for Std. Meth. for Std. Meth. of Std. Meth. of Rec. Pract. for Meth. of Sampling Steel for ration, Standardization, and Storage of Std. Solutions for Std. Meth. for Std. Meth. for silver-lndium-Cadmium Alloys (1974) Std. Meth. of Test for Resistance of Glass Containers to ohexene-1,2-Dicar/ Std. Common Name for the Pest Control solvent Systems Used for Removal of Water Formed Deposits Std. Test Proc. for the Locking Ability Performance of 88.2 Std. for g88.1 Std. for imensional Tolerances of SAE Wrought Aluminum A/ Std. for desmedipham (1/ Std. for Common Name for the Pest Control te Hydroprene/S Std. for Common Name for the Pest Control d. Spec. for Electric Welded Low Carbon Steel Pipe for the
Std. Common Name for the Pest Control e (1973) ate (1973) Std. Common Name for the Pest Control
Std. Common Name for the Pest Control

I Chemistry) (1962)

std. Meth. of Test for Potential Reactivity of Aggregates e Bifenox (197) Std. for Common Name for the Pest Control Std. Meth. for Examination of Water Formed Deposits by -N-Propyl-Ptoluid/ Std. Common Name for the Pest Control itro-N-Propyl-Pto/ Std. Common Name for the Pest Control 1973/ Std. for Ethoprop (Common Name for the Pest Control Std. for Methidathion (Common Name for the Pest Control
Chargers (1971)

Chargers (1972)
Charges) (1974)

Std. for Interrelationship of Quartz ANSI

UL

Uniform Pract. for Fee Determination PSA

Charging Storage Batteries in Motive Power Service (For

Charpy V-Notch Testing Requirements for Steel Plates Fo

Chart-Construction and Industrial Machinery (1971)

Chart / Textile Colorfastness) (1972)

Charts and Symbols (Mechanical Engineering) (1972)

Charts Have Been Prepared (Cereal Chemistry) (1962)

Charts (1969)

Charts (1971)

Charts (1971) ANSI Z1.5

Chassis Frames-Passenger Car and Light Truck-Ground
Check Flanges and Gaskets; Drilling Through, Multiple C

Check Valve Type Back Pressure Backflow Preventers for

Check Valves for Petroleum Pipeline Valves (1974)

Checking Aerosol Containers (1972)

Checking the Size of Diamond Abrasive Grain (1971)

Checklists) (1973)

Chemical Analysis for Aluminum Oxide Content of Titaniu

Chemical Analysis of Alcohol, Poly(vinyl Butyral) (1973

Chemical Analysis of Aluminum Oxide Abrasive Grain and

Chemical Analysis of Ammoniacal Copper Arsenite (1971)

Chemical Analysis of Carbon and Graphite (1969) ANSI K9

Chemical Analysis of Carbon, Low Alloy, and Silicon Ele

Chemical Analysis of Cast lron-All Types (1974)

Chemical Analysis of Chromated Zinc Chloride (1970) Ans

Chemical Analysis of Chrome Containing Refractories and

Chemical Analysis of Copper and Copper Base Alloys (197

Chemical Analysis of Copper Phthalocyanine Blue and Gre

Chemical Analysis of Ferroalloys (1973)

Chemical Analysis of Ferroboron (1974)

Chemical Analysis of Ferrochrome-Silicon (1974)

Chemical Analysis of Ferrochromium and Chromium Metal

Chemical Analysis of Ferromanganese and Spiegeleisen (1

Chemical Analysis of Ferromolybdenum (1974)

Chemical Analysis of Ferrosilicon and Silicon Metal (19

Chemical Analysis of Ferrovanadium and Vanadium Alloyin

Chemical Analysis of Fluor Chrome Arsenate Phenol (1971

Chemical Analysis of Glass Sand (1972)

Chemical Analysis of Gypsum and Products (1972)

Chemical Analysis of High Temperature, Electrical, Magn

Chemical Analysis of Limestone, Quicklime, and Hydrated

Chemical Analysis of Metals (1973) St

Chemical Analysis of Nickel-Chromium and Nickel-Chis

Chemical Analysis of Nuclear Grade Plutonium Metal (197

Chemical Analysis of Pentachlorophenol (1971) ANSI 011.

Chemical Analysis of Reactor and Commercial Columbium

Chemical Analysis of Rubber Products (1972A) ANSl J10.I

Chemical Analysis of Silica Refractories (1970) ANS1 Al

Chemical Analysis of Silicomanganese and Ferrosilicon M

Chemical Analysis of Silicon Carbide Abrasive Grain and

Chemical Analysis of Soaps Containing Synthetic Deterge

Chemical Analysis of Stainless, Heat Resisting, Maragin

Chemical Analysis of Steel and Cast, Open Hearth, and W

Chemical Analysis of Synthetic Elastomers for Use on So

Chemical Analysis of Tool Steels and Other Similar Med

Chemical Analysis of Tribasic Lead Phosphosilicate (197

Chemical Analysis of Tungsten (1973)

Chemical Analysis of White Lead Pigments (1973)

Chemical Analysis of White Pigments (1973) ANSI K15.1

Chemical Analysis of Zirconia Refractories (1972)

Chemical Analysis (1971)

Chemical Analysis (1972) ANSI Z159.1

Chemical Anaylsis of Ferrocolumbium (1974)

/ Meth. for Prepa

Chemical and Spectrochemical Analysis of Nuclear Grade

Chemical Attack (1973)

Chemical cis-N-((1,1,2,2tetrachloroethyl) Thio)-4-Cycl

Chemical Cleaning of Industrial Equipment) (1973)

Chemical Coated Lock Screws (1973)

Chemical Compositions of SAE Alloy Steels (1970) ANSI G

Chemical Compositions of SAE Carbon Steels (1970) ANS1

Chemical Compositions, Mechanical Property Limits and D

Chemical Ethyl Mhydroxycarbanilate Carbanilate (Ester)

Chemical Ethyl (E,E)3,7,1 1-Trimethyl-2,4-Dodecadienoa

Chemical Industry (1971) ANSI B 125.38

Chemical Isopropyl 4,4'dibromobenzilate-Bromopropylat

Chemical Isopropyl 4,4'd ichlorobenzilate-Chloropropyl

Chemical Meth. of Analysis of Vitamin B6 Complex (Cerea

Chemical Meth.) (1971) ANSl A37.133

Chemical Methyl 5(2,4-Dichlorophenoxy)-2-Nitrobenzoat Chemical Microscopy (1973)

Chemical N-(2Chloroethyl)-A, A, A-Trifluoro-2,6-Dinitro

Chemical N(Cyclopropylmethyl)-A, A, A-Trifluoro-2,6-Din

Chemical O-Ethyl S,S-Dipropyl Phosphorodithioate) (

Chemical O,O-Dimethyl Phosphorodithioate S-Ester W

\author{
Rec. E
}

ASTM

$\mathrm{SAE}$

AATCC EP3

ASME I01

1. $\mathrm{AACCH}$ 44.11

NMA MSlol

AWS

ASOC

ANSI

API

ASSE

API

ASTM

ANSI

MCA

ASTM

ASTM

ANSI

ASTM

ASTM

ASTM

ASTM

ASTM

ASTM

ASTM

ASTM

ASTM

ASTM

ASTM

ASTM

ASTM

ASTM

ASTM

ASTM

ASTM

ASTM

ASTM

ASTM

ASTM

ASTM

ASTM

ASTM

ASTM

ASTM

ASTM

ASTM

ASTM

ANSl

ASTM

ASTM

ASTM

ASTM

ASTM

ASTM

ASTM

ASTM

ASTM

ASTM

SAE

ASTM

ASTM

ASTM

ASTM

ANSI

tof ASTM

IFI

SAE

$S A E$

SAE

ANS1

ANSl

St ASTM

ANSI

ANS1

$\mathrm{AACCH}$

ASTM

ANSI

ASTM

ANSI

ANSI

ANSI

ANSI

11

Al

Y14.32.1

$6 \mathrm{~A}$

6D

D3067

B74.16

SG20

D2701

D1396

B74.14

$\mathrm{N} 42.6$

1236 
Dioxacarb (1973)

ost Organic Compou/

Std. Common Name for the Pest Control Std. Analytical Meth. for Determining Rec. for Std. for Tubular Heat Exchanges in Loss Prevention Data on Sight Glasses for Std Meth of Test for Physical and

(1972) Std. for Permissible Variations from Specified Ladle Detonation Protection) (1973) Manual of Hazardous Std. Meth of Test for Resistance of Adhesive Bonds to Sid. Test Meth for Recommendation for Installation of Std. Spec. for Resin Std. Spec for

Std. Rec. Pract. for Use of Hydraulic Cement Mortars in Std. Spec. for Chemically Setting Silicate and Silica Spec, to Std. Spec. for

Std. Rec. Pract, for Use of Rec. Pract. fo

Std. Rec. Pract, for Use of Chemically Settin 4-Dodecadienethi/ Std. Common Name for the Pest Contro
Safe Handling and Use of Ethyl Acetate
Safe Handling and Use of Allyl Chloride 4-Dodecadienethi/ Std. Common Name for the Pest Contro
Safe Handling and Use of Ethyl Acetate
Safe Handling and Use of Allyl Chloride 4-Dodecadienethi/ Std. Common Name for the Pest Contro
Safe Handling and Use of Ethyl Acetate
Safe Handling and Use of Allyl Chloride Treatment (Septic, Holding, Settling, Aeration Chambers, Std Test Meth. for Resistance of Ceramic Tile to Std. Meth. for Sampling Leather for Physical and

coating) (1974)

phosphorothioa/ ne-Prometon (I97) riazine-Prometry -Propazine (1973) adienoate Kino/ hio).S-Triazine $\cdot /$ Std. Common Name for the Pest Contro s-Triazine-Terb/ thio)-S-Triazine / o-M-Tolyl) $\cdot 3$ / Spec, for Detection of Mil

a Technical Guide for Landfill Disposal of Solid Std. for Common Name for the Pest Control Std. Common Name for the Pest Control Std. Common Name for the Pest Contro Sid Common Name for the Pest Control Std for Common Name for the Pest Control ryn (Common Name for the Pest Contro Std. Common Name for the Pest Contro Std. Common Name for the Pest Control Std. for Common Name for the Pest Control Std. for Bentazon (Common Name for the Pest Control

Sid. for Tetramethrin (Common Name for the Pest Control Std. for Fospirate (Common Name for the Pest Control Std. for Disugran (Common Name for the Pest Control Std. for Crufomate (Common Name for the Pest Control Std for Chlorbromuron (Common Name for the Pest Control Std. for Chlordimeform (Common Name for the Pest Control Std. for Fluorodifen (Common Name for the Pest Control Std. for Prynachlor (Common Name for the Pest Control Std for Glyphosine (Common Name for the Pest Control

Sid. for Mexacarbate (Common Name for the Pest Control Std. for Dinoseb (Common Name for the Pest Control Std. for Acephate (Common Name for the Pest Control Std, for Ethiolate (Common Name for the Pest Control Std. for Glyphosate (Common Name for the Pest Control Std. for Oxamyl (Common Name for the Pest Contro Std for Picloram (Common Name for the Pest Control Std. for Nitrapyin (Common Name for the Pest Control ohexyl 2Propynyl Sulfite (Common Name for the Pest Control

yl 2,4-Dodecadienoate) (Common Name for the Pest Control sis of Nuclear Grade Uranium Dioxide Powd/ sis of Nuclear Grade Plutonium Dioxide $\mathrm{Po} /$ sis of Nuclear Grade Mixed Oxides ((U, Pu) sis Of, and Physical Tests On, Beryllium and Radio Chemical Analysis of Nuclear Gr and Radiochemical Analysis of Nuclear $\mathrm{Gr}$ / ets, Ingots, and Ingot Bars (1973) ANSI H/ ca Mortars (1974) Std nt Mortars (1974)

Std. for Flash Points, Closed Cup, Aromatic for Terminal Facilities for Pneumatic Transfer of Dry Bulk Std Meth of Test for Flash Point of Rec. for Hazardous

fate and Silver Densitometric Meth. for Measuring Residua Meth. of Analysis for Sulfuric Acid in Baking Powders and Meth. of Analysis for Tartaric Acid in Baking Powders and Meth of Analysis of Ammonia in Baking Powders and eth. of Analysis for Phosphoric Acid in Baking Powders and eth. of Analysis for Phosphoric Acid in Baking Powders and of Test for Total Bromine Number of Unsaturated Aliphatic Uniform Fire Code: Hazardous cation in Hazardous Dust Locations (Agricultural Plastics, uments in Hazardous Dust Locations (A gricultural Plastics Std. Deviations for Specific Meth. of Cerea Glossary of Terms Used in Baking and Cereal chniques for Extraneous Matter Meth. of Analysis in Cereal Definitions of Statistical Terms Used in Cereal

Reagents for Extraneous Matter Meth. of Analysis (Cereal
Chemical 0-1,3-Dioxolan -2-Ylphenyl Methylcarbamate-

Chemical Oxygen Demand in Industrial Waste Waters and $M$

Chemical Plants (1974)

Chemical Process Service (1971)

Chemical Processing Equipment (Vessel) (1974)

Chemical Properties of Particulate lon Exchange Resins

Chemical Ranges and Limits for Steels (1972)

Chemical Reactions (Incandescence, Fire, Explosion, and

Chemical Reagents (1966) ANSI Z197.7

Chemical Resistance of Pipeline Coatings (1972)

Chemical Resistant Ceramic Tile Floors (1973)

Chemical Resistant Grouts (1974)

Chemical Resistant Masonry Units (1972) ANSI A103. I

Chemical Resistant Masonry (1965)

Chemical Resistant Mortars (1974)

Chemical Resistant Resin Monolithic Surfacings (1972T)

Chemical Resistant Resin Mortars (1974)

Chemical Resistant Resin Mortars (1974

Chemical Resistant Resin Tile Grouts (1972T) (1972T)

Chemical Resistant Silicate and Silica Mortars (1974)

Chemical S-Ethyl (E,E).11-Methoxy-3,7,11-Trimethyl-2

(Chemical Safety) (1972)

(Chemical Safety) (1973)

Chemical Storage, Mixing, Filter, Grit Chambers, and Fl

Chemical Substances (1971) ANSl A174

Chemical Tests (1971) ALCA J1

Chemical Treatments on Galvanized Steel Surfaces (Coil

Chemical Waste (1974)

Chemical 0,0 Dimethyl 0-(3,5,6-Trichloro-2-Pyridyl)

Chemical 2,4-Bis (Isopropylamino).6-Methoxy-S-Triazi

Chemical 2,4-Bis (Isopropylamino)-6-(Methylthio).S-T

Chemical 2-Chloro-4,6bis (Isopropylamino) -S-Triazine

Chemical 2-Propynyl (E,E)-3,7,11-Trimethyl-2,4-Dodec

Chemical 2-(Ethylamino)4-(Isopropylamino)-6-(Methylt

Chemical 2-(Ethylthio) $\cdot 4,6$-Bis (lsopropylamino) Stri

Chemical 2-(Tertbutylamino)-4-Chloro-6-(Ethylamino)-

Chemical 2-(Tertbutylamino)-4-(Ethylamino)-6-(Methyl

Chemical 4-Chloro5-(Methylamino) -2-(a,a,a- Trifluor

Chemical (1972)

Chemical) (1971)

Chemical) (1971)

Chemical) (1971)

Chemical) (1972)

Chemical) (1972)

Chemical) (1972)

Chemical) (1972)

Chemical) (1972)

Chemical) (1972)

Chemical) (1972)

Chemical) (1972)

Chemical) (1972)

Chemical) (1972)

Chemical) (1972)

Chemical) (1972)

Chemical) (1972

Chemical) (I972)

Chemical) (1973)

Chemical) (1973)

Rgite 2-(P-Tert-Butylphenoxy) Cycl

Chemical, Mass Spectrometric, and Spectrochemical Analy

Chemical, Mass Spectrometric, and Spectrochemical Analy

Chemical, Mass Spectrometric, and Spectrochemical Analy

Chemical, Mass Spectrometric, and Spectrochemical Analy

Chemical, Mass Spectrometric, Spectrochemical, Nuclear

Chemical, Mass Spectrometric, Spectrochemical, Nuclear,

Chemically Refined Copper Wire Bars, Cakes, Slabs, Bill

Chemically Setting Chemical Resistant Silicate and Sili

Chemically Setting Silicate and Silica Chemical Resista

Chemicals and Isolates (1973)

Chemicals and Plastics (1972)

Chemicals by Closed Up Meth. (1973)

Chemicals Data (Fire Protection and Safety) (1973)

Chemicals in Films, Plates, and Papers (1971)

Chemicals (Cereal Chemistry) (1962)

Chemicals (Cereal Chemistry) (1962)

Chemicals (Cereal Chemistry) (1962)

Chemicals (Cereal Chemistry) (1962)

Chemicals (Cereal Chemistry) (1962)

Chemicals (1972) ANSI Z159.2

Chemicals (1973)

Chemicals, Metal Dusts) (1973)

Chemicals, Metal Dusts) (1973)

Chemistry Analysis (1962)

Chemistry (1962)

Chemistry (I962)

Chemistry (1967)

Chemistry) Alcohol; Carbon Tetrachloride; Chloral Hydra
ANSI

CR

FMS

ANSI

FMS

ASTM

SAE

FMS

ASTM

ASTM

TCA

ASTM

ASTM

ASTM

ASTM

ASTM

A STM

ASTM

ASTM

ASTM

ANSI

$\mathrm{MCA}$

MCA

NSF

ASTM

ASTM

NCCA

MCA

A NSI

ANSI

ANSI

ANS

ANSI

ANSI

ANSI

NSI

ANSI

ANSI

ANSI

ANI

ANSI

ANSI

ANSI

ANSI

ANSI

ANSI

ANSI

ANS

ANSI

ANSI

ANS

ANSI

ANSI

ANS

ANS

ASI

ANSl

ASTM

A STM

ASTM

ASTM

ASTM

ASTM

ASTM

Rec. $\mathrm{MOA}$

ASTM

FMS

Thiosul ANSI

$\mathrm{ACCH} \quad 04.14$

$\triangle A C C H \quad 04.27$

A ACCH 40.15

Qualitative M AACCH 04-10

Quantitative M AACCH 04-11

Std. Meth. ASTM E234

ICBO UFC 2ART19

Std. for Area Classifi ISA

S 12.10

Ion of Electrical lnstr ISA S12.11

$\mathrm{AACCH} \quad 78.50$

$\mathrm{AACCH}$ 36.0IA

Special Te AACCH 28.92

$\mathrm{AACCH} \quad 78.40$

$\mathrm{AACCH}$ 28.9] 
Materials for Extraneous Matter Meth. of Analysis (Cereal Rapid Meth. of Analysis for Fat Acidity in Grain (Cereal Rapid Meth. of Analysis for Fat Acidity in Corn (Cereal Meth. of Analysis for Benzoic Acid in Flour (Cerea Meth of Analysis for Lactic Acid in Dried Geeg (Cerea Meth. of Analysis of Soy Flour in Macaroni (Cereal Meth. of Analysis for Ash in Cacao Products (Cereal Meth. of Analysis for Ash in Dry Milk Products (Cereal Meth. of Analysis for Ash in Farina and Semolina (Cereal Meth. of Analysis for Ash in Soy Flour (Cereal Meth. of Analysis for Ash in Yeast Foods (Cereal Meth. of Analysis for Total Reducing Substances (Cereal Meth. of Analysis for Baking Quality of Pie Flour (Cerea meth. of Analysis for Baking Quality of Cake Flour (Cereal

Meth. for U'se of Layer Cake Measuring Template (Cereal

Pekar Color Test (Slick) Meth. of Flour Analysis (Cereal Meth. for Determination of Arsanilic Acid in Feeds (Cereal eth. of Analysis of Alpha Amylase Activity of Malt (Cereal

Meth. of Analysis of Yeast Strength of Doughs (Cereal

Meth. of Analysis of Proteolytic Activity of Malt (Cereal atch Meth. of Experimental Milling for Durum Wheat (Cereal Meth. of Experimental Wheat Milling: Temper Table (Cereal Meth. of Analysis of Crude Fat in Cocoa (Cereal Meth. of Analysis of Crude Fat in Baked Dog Food (Cereal eth. of Analysis of Crude Fat in Dry Milk Products (Cerea) Meth. of Analysis of Crude Fat in Soy Flours (Cereal White Pan Bread Flour Spec. (Cereal Meth. of Analysis of Vital Wheat Gluten (Cereal

Meth of Analysis of Soluble Mineral Matter (Cerea Meth. of Analysis of Calcium in Bread and Flour (Cereal Meth. of Analysis of Calcium in Feedstuffs (Cereal Meth. of Analysis of Calcium in Mineral Feeds (Cereal meth. of Analysis of Iodine in Mineral Mixed Feeds (Cereal

Quantitative Meth. of Analysis of Iron in Flour (Cereal meth. of Analysis of Phosphates in Flour lmprovers (Cereal

Bomb Meth. of Analysis of Sulfates in Yeast Foods (Cereal

Sampling Procedure for Analysis of Bacteria (Cereal Meth. of Calculation of Percent Moisture (Cereal Air Oven Meth. of A nalysis of Moisture in Malt (Cereal Meth. of Analysis of Moisture in Nonfat Dry Milk (Cereal Micro Kjeldahl Meth. of Analysis of Crude Protein (Cereal rowland Meth. of Analysis of Serum Protein in Milk (Cereal Qualitative Meth. of Analysis of Calcium Peroxide (Cereal eth. for Particle Size Distribution of Wheat Flour (Cereal

Volumetric Bromine Meth. of Analysis of Pentosans (Cereal

Xylene Partition Meth. of Analysis of Pentosans (Cereal

Meth. of Analysis of Fungicide Residues in Grain (Cereal Meth for Preparation of Sample: Bread (Cereal

Meth. for Preparation of Sample: Dried Eggs (Cereal Meth. for Preparation of Sample: Leavening Agents (Cereal meth. for Preparation of Sample: Macaroni Products (Cereal Meth. for Preparation of Sample: Malt (Cereal

Meth. for Preparation of Sample: Semolina (Cereal Meth. for Preparation of Sample: Wheat (Cereal

Meth. for Sampling of Fats and Shortenings (Cereal Meth. for Sampling of Solid Feeds and Feedstuffs (Cereal meth. for Sampling of Wheat and Other Whole Grains (Cereal Meth. for Preparation of Indicator Solutions (Cereal

Meth. for Preparation of Silver Nitrate Solution (Cereal Chemical Meth. of Analysis of Vitamin B6 Complex (Cereal Microbiological Meth. of Analysis of Vitamin B12 (Cereal Meth of Analysis of Choline (Cereal

Microbiological Meth of Analysis of Niacin (Cereal Meth. of Analysis of Pantothenic Acid (Cereal Microbiological Meth. of Analysis of Riboflavin (Cereal sk Meth. of Analysis of Color of Macaroni Products (Cereal th. of Analysis for Lactic Acid in Nonfat Dry Milk (Cereal th. of Analys is for Baking Quality of Cookie Flour (Cereal th. for Determination of Nitrosal in Poultry Feeds (Cereal th. of Analysis of Egg Solids in Macaroni Products (Cereal

th. for Preparation of Ammonium Molybdate Solution (Cereal th. of Analysis of Test Weight Per Bushel of Grain (Cereal alitative Meth. of Analysis of Added Iron in Flour (Cereal mmary of Meth. for Biscuit and Cracker Flour Test. (Cereal h. of Analysis of Diastatic Activity of Malt Flour (Cereal h. for Preparation of Sample: Feeds and Feedstuffs (Cereal roscopic Meth. of Analysis of Smut in Wheat or Rye (Cereal

Meth. of Analysis of Semolina Quality in Macaroni (Cereal imetric Meth. of Analysis for Fat Acidity in Grain (Cereal es of Refraction of Sucrose Solutions at $20 \mathrm{Deg}$. C (Cereal of Analysis for Baking Quality of Rye Bread Flour (Cereal of Analysis for Baking Quality of Nonfat Dry Milk (Cereal of Analysis of Crude Fat in Grain and Stock Feeds (Cereal of Analysis of Added Salt in Feeds and Feedstuffs (Cereal of Analysis of Sedimentation Test for Wheat Flour (Cereal for Preparation of Potassium Thiocyanate Solution (Cereal
Chemistry) (Bolting Cloth, Filter Paper, Wide Field and Chemistry) (1962)

Chemistry) (1962)

Chemistry) (1962

Chemistry) (1962)

Chemistry) (1962)

Chemistry) (1962)

Chemistry) (1962)

Chemistry) (1962)

Chemistry) (1962)

Chemistry) (1962)

Chemistry) (1962)

Chem istry) (1962)

Chemistry) (1962)

Chemistry) (1962)

Chemistry) (1962)

Chemistry) (1962)

Chemistry) (1962)

Chemistry) (1962)

Chemistry) (1962)

Chemistry) (1962)

Chemistry) (1962)

Chemistry) (1962)

Chemistry) (1962)

Chemistry) (1962)

Chemistry) (1962)

Chemistry) (1962)

Chemistry) (1962)

Chemistry) (1962)

Chemistry) (1962)

Chemistry) (1962)

Chemistry) (1962)

Chemistry) (1962)

Chemistry) (1962)

Chemistry) (1962

Chemistry) (1962)

Chemistry) (1962)

Chemistry) (1962)

Chemistry) (1962)

Chemistry) (1962)

Chemistry) (1962)

Chemistry) (1962)

Chemistry) (1962)

Chemistry) (1962)

Chemistry) (1962)

Chemistry) (1962)

Chemistry) (1962)

Chemistry) (1962)

Chemistry) (1962)

Chemistry) (1962

Chemistry) (1962)

Chemistry) (1962)

Chemistry) (1962)

Chemistry) (1962)

Chemistry) (1962)

Chemistry) (1962)

Chemistry) (1962)

Chemistry) (1962

Chemistry) (1962)

Chemistry) (1962)

Chemistry) (1962)

Chemistry) (1962)

Chemistry) (1962)

Chemistry) (1962)

Chemistry) (1962)

Chemistry) (1962

Chemistry) (1962)

Chemistry) (1962)

Chemistry) (1962)

Chemistry) (1962)

Chemistry) (1962)

Chemistry) (1962)

Chemistry) (1962)

Chemistry) (1962

Chemistry) (1962)

Chemistry) (1962)

Chemistry) (1962)

Chemistry) (1962

Chemistry) (1962)

Chemistry) (1962)

Chemistry) (1962)

Chemistry) (1962)

Chemistry) (1962)

Chemistry) (1962

Chemistry) (1962)

Chemistry) (1962)
AACCH 28.90

AACCH 02.02

$\mathrm{AACCH} \quad 02.03$

$\mathrm{AACCH} \quad 04.21$

AACCH 04.25

$\mathrm{AACCH}$ 06.11

$\mathrm{AACCH} \quad 08.10$

$\mathrm{AACCH} \quad 08.11$

$\mathrm{AACCH} \quad 08.12$

AACCH 08.16

AACCH 08.18

AACCH 10.01

$\mathrm{AACCH} \quad 10.60$

AACCH 10.90

AACCH 10.91

AACCH 14.10

$\mathrm{AACCH} 18.20$

$\mathrm{AACCH} 22.01$

AACCH 22.13

A ACCH 22.61

AACCH 26.30

$\mathrm{AACCH} 26.95$

AACCH 30.12

AACCH 30.14

AACCH 30.16

AACCH 30.26

$\mathrm{AACCH} 34.01$

$\mathrm{AACCH} \quad 38.20$

$\mathrm{AACCH} 40.01$

AACCH $\quad 40-20$

$\mathrm{AACCH} \quad 40.21$

$\mathrm{AACCH} \quad 40.26$

$\mathrm{AACCH} \quad 40.35$

$\mathrm{AACCH} 40.41$

$\mathrm{AACCH} \quad 40.50$

$\mathrm{AACCH} \quad 40.65$

$\mathrm{AACCH} \quad 42.10$

$\mathrm{AACCH} \quad 44.01$

$\mathrm{AACCH} \quad 44.20$

$\mathrm{AACCH} \quad 44.52$

$\mathrm{AACCH}$ 46-13

A ACCH 46.22

$\mathrm{AACCH} \quad 48-50$

$\mathrm{AACCH} \quad 50-10$

$\mathrm{AACCH} \quad 52.10$

$\mathrm{AACCH} 52.11$

$\mathrm{AACCH} \quad 60-01$

AACCH 62.05

AACCH 62.15

$\mathrm{AACCH} \quad 62-30$

$\triangle A C C H \quad 62.40$

$\mathrm{AACCH} \quad 62.50$

$\mathrm{AACCH} \quad 62.60$

$\mathrm{AACCH} \quad 62-70$

$\mathrm{AACCH} \quad 64.40$

$\mathrm{AACCH} \quad 64.50$

AACCH $64-70$

AACCH 70.25

AACCH 70-60

AACCH 86.30

$\mathrm{AACCH} \quad 86-40$

$\mathrm{AACCH} 86.45$

$\mathrm{AACCH} \quad 86-51$

$\mathrm{AACCH} \quad 86.60$

$\mathrm{AACCH} \quad 86.72$

Di AACCH 14.20

$\mathrm{Me} A \mathrm{ACCH} \quad 04.26$

Me AACCH $10.50 \mathrm{~B}$

Me AACCH 18.27

$\mathrm{Me}$ AACCH 20.10

Me AACCH 70.35

$\mathrm{Me} A \mathrm{ACCH} \quad 84.10$

Qu AACCH 40.40

Su AACCH 10.30

Met AACCH 22-16

Met AACCH 62.20

$\mathrm{Mic} A \mathrm{AACH} \quad 42-70$

Disk AACCH 66.40

Color AACCH 02.04

Indic $\mathrm{AACCH} 68.61$

Meth. AACCH $10-70$

Meth. AACCH 10.85

Meth. AACCH 30.20

Meth. AACCH 40.60

Meth. AACCH 56.60

Meth. AACCH 70.55 
of Sensory Perecption Test for Staleness of Bread (Cereal biologieal Mcth, of Analysis of Vitamin B6 Complex (Cereal for Converting Absorption to $14.0 \%$ Moisture Basis (Cercal ical Metl. for Analysis of Moisture in Whole Grain (Cerea etric Metlo, of Analysis of Sulfates in Yeast Foods (Cereal of Analysis of Lipoxidase Activity of Gronnd Wheat (Cerea of Analysis of Crude Fiber in Feeds and Feedstuffs (Cereal of Analysis of Rope Spore Count in Cereal Products (Cerea of Analysis of Fecal Streptococei in Food Products (Cereal of Analysis of Laetose (Milk Sngar) in Mixed Feeds (Cerea ntation Meth. of Analysis of Smut in Wheat and Rye (Cereal tion Meth. of Analysis for Rorlent Exereta in Flour (Cereal or l'reparation of Sample: Fruit and Fruit Products (Cereal f Analysis for Total Phosphoric Acid in Dried Eggs (Ceresa f Aualysis for Ash in Molasse's, Sugars, and Syrups (Coreal f Analyais of lipoid Plosphorie Acid in loried Fighs (Cerea f Analysis of Sahmonclha Bacteria in Food l'roducts (Cerea tive Metlo of Analysis of Chlorides in Yeast Foods (Cereal ric Metl. of Analysis of Chlorisles in Asle of Bread (Cerea ric Metlo. of Analysis of Chlorides in Ash of Bread (Cereal

Ferricyande-Maltoge-Sucrose Conversion 'Tahle (Cereal tive Metl. of Analysis of Chlorides in Yeast Foods (Cereal ure Corrections for Refractoneter Readings (Sugar) (Cercal ure Corrections for Saccharimeter Realings (Sugar) (Cerea

Cloblesterol Mello of Analysis of Dried Erg Solids (Cereal anslysis for 'Titratable Acidity in Nonfat Dry Milk (Cereal analysis of Coliform Bacteria in All Food Prodncts (Cereal analysis of Mold and Yeast Counts in Food l'roducts (Cereal ctl. of Analysis of Moisture, Drying at 135 Der. C (Cerea - Alcohol Meth. of Aualysis of Ergot in Rye lilonr (Cerea unsigraph Metl. for l'hysical bough Tests of Flonr (Cereal tlı, of Analygis for Pat Arielity in Grain and Flour (Cerea nalysic of Ammonia in Baking Powders and Chemiculs (Cereal nalysis of Suctose (Sugar) in Feeds and Feedstuffs (Cereal nesium Acetate) Melh, of Analysis for Ash in Flonr (Cereal

Metlı, of Autlysis of Diastatic Activity of Jough (Cerea of Experimental Milling for Bread and Soft Wheats (Cereal - th. of Analysis of Mothoxychlor Residues in Foods (Cereal Meth. of Analysis of Semolina Quality in Macaroni (Cereal lysis for Residnal Carbon Dioxide in Baking l'owder (Cereal reparation and Standardization of Buffer Solutions (Cerea alysis of Diastatic Activity of Flonr and Scmolina (Cercal Meth. of Analysis of Semolina Quality in Macaroni (Cereal of Experimental Milling for Bread and Soft Wheats (Cerea Iysis of Lipoxidase Activity of Semolina and Flour (Cerea lysis of Crud. Fat in Egg Yolk and Dried Whole Egg (Cerea meill. of Analysis of Amuonium Salts in Yeast Foods (Cereal nueth of Analysis of Bromates and lodates in Flonr (Cercal Meth of Analysis of Ammonium l'ersulf ate in Flonr (Cerea shworth Meth. of Analysis of Serum l'rotein in Milk (Cereal ysis of Insaponifiable Matter in Macaroni P'roducts (Cereal

ysis of Moisture and Volatile Matter in Soy Flours (Cerea

Melh. of Analysis of Diastatic Activity of Doughs (Cereal nucth. of Analysis of Amnonium Salts in Yeast Foods (Cereal 600 Deg.) Meth. of Analysis for Ash in Feedstuffs (Cereal rmintion of Phenothiazine in Feeds and Feedstuffs (Cereal aration of Sample: Yeast Foods and Flour Improvers (Cereal sis of Alpha Amylase Activity, by Betal Amylase CBI (Cereal meth of Analysis of Solids in Syrups and Molasses (Cereal of Analysis of Cinder and Sand Particles in Farina (Cereal lene Extraction Meth. of Analysis of Ascorbic Aeid (Cereal Meth. of Analysis of Gluten in Flour and Semolina (Cereal ction of Analytical Values to $1.4 .0 \%$ Moisture Basis (Cereal ration and Standardization of Lactic Acid Solntion (Cereal

is for Baking Quality of Self Rising Bisenit Flour (Cerea is of Lactose (Nugar) and Nonfat Dry Milk in Bread (Cerca ic Mcth. of Analysis of Color of Macaroni Products (Cereal of Aualysis of l'hosphorns in Feeds and Feedstuffs (Cereal of Analysis of Phosphorus in Feeds and Feedstuffs (Cereal tion and Standardization of Sulfurie Aeid Solution (Cereal for Sulfurit Acid in Baking Powders and Chemieals (Cereal for Tartaric Acid in Baking Powders and Chemicals (Cereal of Food Poisoning Staphylococcus in Food Products (Cereal or Analysis of Resistance of Flour Dongl to Mixing (Cereal

analysis of Solids in Juiees. Syrups, and Molasses (Cereal

(Brown-Duvel) of Analysis of Moisture in Grains (Cereal of Analysis of Cinder and Sand Particles in Farina (Cereal ion and Staudardization of Barinm Lactate Solution (Cereal for Acelic, Butyric, and Lactic Acids in Rye Flour (Cereal for Baking Test (Quality) for Sweet Yeast Products (Cereal of Diastatic Activity of Flonr with the Amylograph (Cereal of Analysis for Admixume of Rye and Wheat Flours (Cereal or Analysis of Resistance of Flour Dough to Mixing (Cereal or Neutralizing Valıe of Sodium Acid Pyrophosphate (Cereal alysis of Reducing Sugars in Prepared Bakery Mixes (Cercal

th. of Analysis of Total Bacteria in Food Products (Cereal
Chemistry) (1962) Che mistry) (1962) Chemistry) (1962) Chemistry) (1962) Chemistry) (1962) Chemistry) (1962) Chemistry) (1962) Chemistry) (1962) Chemistry) (1962) Cliemistry) (1962) Chemistry) (1962) Chemistry) (1962) Chemistry) (1962) Chemistry) (1962) Chemistry) (1962) Chemistry) (1962) Chemistry) (1962) Chemistry) (1962) Chemistry) (1962) Chemistry) (1962) Chemistry) (1962) Chemistry) (I962) Chemistry) (1962) Chemistry) (1962) Chemistry) (1962) Chemistry) (1962) Clienistry) (1962) Chemistry) (1962) C.lemistry) (1962) Chemistry) (1962) Chemistry) (1962) Cliemistry) (1962)

Chemistry) (1962)

Chemistry) (1962) Chemistry) (1962) C.hemistry) (1962) Chemistry) (1962) Chenistry) (1962) Chemistry) (1962) Chemistry) (1962)

Chemistry) (1962)

Chemistry) (1962) Chemistry) (1962)

Chemistry) (1962)

Chemistry) (1962)

Chemistry) (1962)

Chemistry) (1962)

Chemistry) (1962)

Chem istry) $(1962)$

Chemistry) (1962)

Chemistry) (1962)

Chicmistry) (1962)

Chemistry) (1962)

Chem istry) (1962)

Chemistry) (1962)

Clemistry) (1962)

Chemistry) (1962)

Chemistry) (1962)

Chemistry) (1962)

Chemistry) (1962)

Chemistry) (1962)

Chemistry) (1962

Clicmistry) (1962)

Chemistry) (1962)

Chemistry) (1962)

Chem istry) (1962)

Chemistry) (1962)

Chemistry) (1962)

Chemistry) (1962)

Chemistry) (1962)

Chemistry) (1962)

Chemistry) (1962)

Chemistry) (1962)

Chemistry) (1962)

Chemistry) (1962)

Chemistry) (1962)

Chemistry) (1962)

Chemistry) (1962

Chemistry) (1962

Chemistry) (1962)

Chemistry) (1962)

Chemistry) (1962)

Chemistry) (1962)

Chemistry) (1962)

Cliemistry) (1962)

Chemistry) (1962)
Meth. AACCH 74.30

Micro AACCH 86.31

Table AACCH 82.21

Electr AACCH 44.10

Gravim AACCH 40.66

Meth. AACCH 22.41

Meth. AACCH 32.15

Meth. AACCH 42.20

Meth. AACCH 42.35

Meth. AACCH 80.30

Sedime AACCH 42.71

Decanta AACCH 28.42

Meth. F AACCH 62.25

Meih. O AACCH 04.13

Meth. O AACCH 08.14

Meth. O AACCH 20.02

Meth, O AACCH 42.25

Qualita AACCH 40.32

Volumet AACCH 40-31

Gravimet AACCH 40.30

Meth of AACCH 22.18

Ouantita AACCH 40.33

Temperat AACCH 80.20

Temperat AACCH 80.21

Digitonin AACCH 20-0I

Meth of AACCH 02.31

Meth. of AACCH 42.15

Meth. of AACCH 42.50

Air Oven M AACCH 44.19

Clıloroform AACCH 42.55

General Ex AACCH 54.10

General Me AACCH 02.01

Meth. of a $\triangle A C C H$ 40-15

Meth. of a AACCH 80.50

Rapid (Mag AACCH 08.02

Volumetric AACCH 22.14

Batch Meth AACCH 26-21

Low Blank M AACCH $60-40$

Macro Seale AACCH 66.41

Meth of Ana AACCH 12.10

Meth. for P AACCH 70.15

Mcth. of an AACCH 22-15

Micro Seale AACCH 66.42

Buhler Meth. AACCH 26-20

Meth of Ana AACCH 22.40

Meth. of Ana AACCH 30.18

Qualitative AACCH 40.16

Qualitative AACCH 48-41

Quantitative AACCH 48.62

Harland and a AACCH 46-21

Meth. of Anal AACCH $30-40$

Meth. of Anal AACCH 44-3I

P'ressuremeter AACCH 22-11

Quantitative AACCH $40-18$

Rapid (2 Hr., AACCH $08-03$

Metl for Dete AACCH 18-30

Meth. for Prep AACCH 62.80

Meth. of Analy AACCH $22-02$

Refractometer AACCH $68-60$

Counting Metl. AACCH 28-0] Indophenol-Xy $\mathrm{AACCH} 86.10$

Machine Washing AACCH 38.11

Metl. for Corre AACCH 82.22

Meth. for Prepa AACCH 70-30

Meth. of Analys AACCH 10-31A

Meth. of Analys AACCH 80-31

Spectrophotometr AACCH 14.21

Volumetrie Meth. AACCH 40.58

Gravimetric Meth. AACCH 40.57

Meth. for Prepara AACCH 70.80

Meth of Analysis AACCH 04-14

Metl, of Analys is AACCH 04-27

Meth. of Analysis A ACCH 42.30

Mixograpl Meth. F AACCH $54-40$

Spindle Meth. of AACCH $68-62$

Distillation Meth. AACCH 44.53

Gravimetric Meth. AACCH 28-02

Meth. for Preparat AACCH 70-10

Meth. of Analysis A ACCH 04-20

Meth of Analysis AACCH $10-20$

Meth. of Analysis AACCH 22.10

Trifrnetosan Meth. AACCH 06-10

Farinograph Meth. F AACCH 54.21

Meth. of Analysis F AACCH 02.34

Sehoorl Meth. of an AACCH 80.68

std. Plate Count Me AACCH 42.11 
ion Meth of Analysis of Vitamin A in Concentrates (Cereal Chemistry) (1962) sis for Ash in Flour, Feeds, Feedstuffs, and Bread (Cereal Chemistry) (1962) $f$ Analysis for Hydrogen Ion Activity (PH) in Flour (Cereal Chemistry) (1962) and Standardization of Hydrochloric Acid Solution (Cereal Chemistry) (1962) pelshenke Test (Wheat Meal Fermentation Time Test) (Cereal

-Maltose (Diastatic Activity in Flour) Conversion (Cereal is for Insect Eggs in White, Wheat, and Corn Flour (Cereal and Standardization of Sodium Thiosulfate Solution (Cereal total (Gasometric) Carbon Dioxide in Baking Powder (Cereal ydrocyanic Acid Residues in Grains and Dried Foods (Cereal t Meth. for Ammonium Persulfate in Flour Improvers (Cereal nalysis of Bromates and lodates in Flour Improvers (Cereal f Analysis for Baking Quality of Wheat Bread Flour (Cereal quality of Nonfat Dry Milk in Physical Dough Tests (Cereal ysis for Insect and Rodent Filth in Popped Popcorn (Cereal sh in Starch, Dextrin, and Other Modified Products (Cereal oking Characteristics of Macaroni-Borasio System (Cereal denatured Whey Protein Nitrogen in Nonfat Dry Milk (Cereal alysis for Nature of Ammonium Salts in Yeast Foods (Cereal ysis of Amino Nitrogen in Wheat Flour and Semolina (Cereal tric and Isocitric Acids in Fruit and Its Products (Cereal ucing and Nonreducing Sugars in Flour and Semolina (Cereal t with Baker Compressimeter for Staleness of Bread (Cereal alysis of Stability in Fats, Oils, and Shortenings (Cereal Analysis of Crude Protein in Feeds and Feedstuffs (Cereal eight of Flour to Give 100 Grams at $14.0 \%$ Moisture (Cereal standardization of Potassium Permanganate Solution (Cereal ric, Pyruvic, Lactic, Succinic, and Tartaric Acids (Cereal anic Bromide Residues in Grain and Cereal Products (Cereal eth. of Analysis for Baking Quality of Bread Flour (Cereal sis of Moisture and Volatile Matter in Yeast Foods (Cereal eth. of Analysis of Moisture in Flour and Semolina (Cereal ectrophotometer Analysis of Acid Soluble Manganese (Cereal fat in Wheat and Soy Flour, Feeds and Cooked Feeds (Cereal philic and Psychrophilic Bacteria in Food Products (Cereal pecific Volume of Creams, Cake Batters, and Icings (Cereal ng Agents in Flour: Reaction with Potassium Iodide (Cereal Subbarow Meth. of Analysis of Phosphorus in Yeast (Cereal

Viscosity of Acidulated Flour-Water Suspensions (Cereal a and Carotene in Enriched Cereals and Mixed Feeds (Cereal sis for Insect Fragments and Rodent Hairs in Flour (Cereal for Hydrogen Ion Activity (PH) in Flour and Bread (Cereal lated from Total Chlorides in Feeds and Feedstuffs (Cereal ysis of Amino Nitrogen in Wheat Flour and Semolina (Cereal or Phosphoric Acid in Baking Powders and Chemicals (Cereal lysis of Ergot in Rye and Wheat Flour and Semolina (Cereal ysis of Amino Nitrogen in Wheat Flour and Semolina (Cereal of Analysis of Moisture in Grains and Feedstuffs (Cereal or Phosphoric Acid in Baking Powders and Chemicals (Cereal f Potassium Bromate in White and Whole Wheat Flour (Cereal for Insect Fragments and Rodent Hairs in Rye Flour (Cereal of Analysis of Phosphorus in Feeds and Feedstuffs (Cereal dization of Sodium or Potassium Hydroxide Solution (Cereal $n$ Dioxide in Prepared Mixes and Self Rising Flours (Cereal for Sugar Determination: Schoorl's Reduction Table (Cereal tivity of Flour and Active Proteinase Preparations (Cereal e Activity in Soybean Meals, Soy Flour, Mill Feeds (Cereal uminum in Baking Powder, in Presence of Phosphates (Cereal nsigraph Analysis of Physical Dough Tests of Flour (Cerea onic, Butyric, Valeric, Succinic, and Lactic Acids (Cereal and Flour Mill Products (Boric Acid Modification) (Cereal

and/or Sodium Acid Pyrophosphate in Prepared Mixes (Cereal classification in Cereal Grains and Grain Products (Cerea anaerobic) in Cereal Products and Food Ingredients (Cereal ffs (Calculated from Percentage of Total Nitrogen) (Cereal nd Similar Products: Feeds and Feedstuffs in Sacks (Cereal al, Flaked and Puffed Cereals, Farina, Bread, etc. (Cereal rodent Urine on Bags and Other Packaging Materials (Cereal in Phosphated and Freshly Mixed Self Rising Flour (Cereal $n$ of Both Nitrophenide and Arsanilic Acid in Feeds (Cereal

ke and Meal from Cottonseed, Soybeans, and Peanuts (Cereal rits, Corn Meal, Puffed Cereals, Farina, and Flour (Cerea on of Cecal and Intestinal Coccidiosis in Chickens (Cerea ies for Which Conversion Charts Have Been Prepared (Cereal e of Acid Reacting Materials Other Than Phosphates (Cereal degrees Brix, and Degrees Baume of Sugar Solutions (Cereal $f$ Urea in Cloth Bags and Other Packaging Materials (Cereal Moisture Basis (Constant Dough Weight Meth. Only) (Cereal ad, and Baked Cereal Products Not Containing Fruit (Cereal dent Excreta, Other Filth, and Extraneous Material (Cereal for Insect Fragments and Rodent Hairs in Soy Flour (Cerea fast Cereals (Except Those Which Are Sugar Coated) (Cerea

, Feathers, Wood Fibers, Splinters, etc. in Starch (Cereal utter, Oleomargarine, High Acid Coconut Oil, etc.) (Cereal our and Semolina (With Subsequent Acid Hydrolysis) (Cerea and Semiliquid Products (Drying Upon Quartz Sand) (Cereal
Chemistry) (1962)

Chemistry) (1962) Che mistry) (1962) Chemistry) (1962) Chemistry) (1962)

Chemistry) (1962)

Chemistry) (1962) Chemistry) (1962) Chemistry) (1962)

Chemistry) (1962)

Chemistry) (1962) Chemistry) (1962) Chemistry) (1962) Chemistry) (1962) Chemistry) (1962) Chemistry) (1962) Chemistry) (1962) Chemistry) (1962) Chemistry) (1962) Chemistry) (1962) Chemistry) (1962) Chemistry) (1962) Chemistry) (1962) Chemistry) (1962) Chemistry) (1962) Chemistry) (1962) Chemistry) (1962) Chemistry) (1962) Chemistry) (1962) Chemistry) (1962) Chemistry) (1962) Che mistry) (1962)

Chemistry) (1962)

Chemistry) (1962) Chemistry) (1962) Chemistry) (1962) Chemistry) (1962) Chemistry) (1962) Chemistry) (1962) Chemistry) (1962) Chemistry) (1962) Chemistry) (1962) Chemistry) (1962) Chemistry) (1962) Chemistry) (1962) Chemistry) (1962) Chemistry) (1962) Chemistry) (1962) Chemistry) (1962) Chemistry) (1962) Chemistry) (1962) Chemistry) (1962) Chemistry) (1962) Chemistry) (1962) Chemistry) (1962) Chemistry) (1962) Chemistry) (1962) Chemistry) (1962) Chemistry) (1962) Chemistry) (1962) Chemistry) (1962) Chemistry) (1962) Chemistry) (1962) Chemistry) (1962) Chemistry) (1962) Chemistry) (1962) Chemistry) (1962) Chemistry) (1962) Chemistry) (1962) Chemistry) (1962) Chemistry) (1962) Chemistry) (1962) Chemistry) (1962) Chemistry) (1962) Chemistry) (1962) Chemistry) (1962) Chemistry) (1962) Chemistry) (1962) Chemistry) (1962) Chemistry) (1962) Chemistry) (1962) Chemistry) (1962)
Ultraviolet Absorpt AACCH 86.0I Basic Meth. of Analy AACCH 08-01 Colorimetric Meth. O AACCH 02.51 Meth. for Preparation AACCH 70.20 Meth. of Analysis of AACCH $56-50$ Meth. of Thiosulfate AACCH 22.17 lodine Meth. of Analys AACCH 28.44 Meth. for Preparation AACCH 70.75 Meth of Analysis for AACCH 12.20 Meth. of Analysis of $\mathrm{H}$ AACCH 60.20 Qualitative Iodide Tes AACCH 48.61 Qualitative Meth. of a AACCH 48.40 Straight Dough Meth. O AACCH 10.10 Extensigraph Meth. for AACCH 54.12 Flotation Meth. of Anal AACCH 28.80 Meth of Analysis for a AACCH 08.17 Meth. of Analysis of $\mathrm{Co}$ AACCH 16-51 Meth. of Analysis of Un AACCH 46.20 Qualitative Meth. of an AACCH 40.17

Van Slyke Meth of Anal AACCH 46-32 Meth. of Analysis for $\mathrm{Ci}$ AACCH $04-22$ Meth. of Analysis of Red AACCH $80-60$ Meth. of Compression Tes AACCH 74.10 Oxygen-Bomb Meth. of an AACCH 58-55 Improved Kjeldahl Meth. of AACCH 46.10 Meth. for Correction for W AACCH 82-23 Meth. for Preparation and AACCH 70.50

Meth of Analysis for Fuma AACCH 04-24 Meth. of Analysis of Inorg AACCH 60.10 Sponge Dough, Pound Loaf M AACCH 10.11 Vacuum Oven Meth. of Analy AACCH 44-32 Air Oven (Aluminum Plate) M AACCH 44-16

Meth. for Colorimeter or Sp AACCH 40-45

Meth. of Analysis of Crude AACCH 30-25

Meth. of Analysis of Thermo AACCH $42-45$

Meth. for Determination of S AACCH 72-10

Meth. of Analysis of Oxidizi AACCH 48-02 Colorimetric Modified Fiske- AACCH $40-56$

Meth. of Analysis of Apparent AACCH 56.80

Meth. of Analysis of Vitamin AACCH 86.05 Acid Hydrolysis Meth. of Analy AACCH 28-41 Electrometric Meth. of Analysis AACCH 02-52 Meth. of Analysis of Salt Calcu AACCH 40-61

Modified Sorensen Meth. of Anal AACCH 46-31 Qualitative Meth. of Analysis F AACCH 04.10 Ether-Bicarbonate Meth. of Ana AACCH 42.56 Linderstrom-Lang Meth of Anal AACCH 46.30 Modified Two Stage Air Oven Meth AACCH 44.18 Quantitative Meth. of Analysis F AACCH 04-11 Quantitative Meth. of Analysis O AACCH 48.42 Tween Versene Meth of Analysis AACCH 28-60 Colorimetric Acetate Ashing Meth. AACCH 40.55 Meth. for Preparation and Standar AACCH 70.70

Meth. of Analysis for Total Carbo AACCH 12-2]

Volumetric Copper Reduction Meth. AACCH 80.69 Meth. of Analysis of Proteolytic AC AACCH 22.60 Meth. of Analysis of Residual Ureas AACCH 22.90 Qualitative Meth. of Analysis of Al AACCH 40-10 Structural Relaxation Meth. of Exie AACCH 54.11

/ Analysis for Formic, Acetic, Propi AACCH 04-23

/ Analysis of Crude Protein in Wheat AACCH 46.12

Analysis of Monocalcium Phosphate AACCH 40.5]

I and Radiographic Illustration and AACCH 28-95 / Counts (Flat Sour, Total Aerobic, AACCH 42.40 / Crude Protein in Feeds and Feedstu AACCH 46.18

for Sampling of Flour, Semolina, a AACCH 64-60 / in Whole Grain Products, Grits, Me AACCH 86.70 / Light Examination for Presence of AACCH 28-85 Meth. of Analysis for Original Ash AACCH 08.15 Meth. of Quantitative Determinatio AACCH 18.26 of Crude Fiber in Soy Flour for Ca AACCH 32-17 / Other Whole Grain Products, Corn G AACCH 86-80

Poultry Mashes (Feed) for Preventi AACCH 18-25 1. Lentils, Rice, and Other Commodit AACCH 44.11

1. of Analysis for Neutralizing Valu AACCH 02-32 1. of Analysis of Specific Gravity, AACCH 80.75

1. of Xanthydrol Test for Analysis O AACCH $28-86$

1. Is Farinograph Absorption to 14.0\% AACCH 54.29 /Analysis of Crude Fat in Flour, Bre AACCH 30-10 /and/or Insect Damage on Kernels, Ro AACCH 28-10 Atting Digestion Meth. of Analysis AACCH 28-70 ID Oats, Bulgur, Rolled Wheat, Break AACCH 44-15A /Dirt, Sand, Insect Fragments, Hairs AACCH 28-75 /E Matter in Fats and Shortenings (B AACCH 44-30

E Meth of Analysis of Starch in Fl AACCH 76-10 /Ecuites, Molasses, and Other Liquid AACCH 44.60 
etection of Rodent Excreta, Insects, Webbing, etc. (Cereal grains, Unpopped Popcorn, Dried Peas and and Beans (Cereal f Monocalcium Phosphate: Monohydrate and Anhydrous (Cereal read; Feedstuffs, Ground Grains and Their Adjuncts (Cereal eride, Salts, Soy Flour, and Other Cereal Products (Cereal ling: Equipment, Sample Preparation, and Tempering (Cereal goods, Ready.To. Eat Cereals, and Alimentary Pastes (Cereal in Bread, Wheat and Other Grains, and Yeast Foods (Cereal eal Grains, Unpopped Popcorn, Dried Peas and Beans (Cereal ng Characteristics of Flour (Physical Dough Tests) (Cereal Feeds and Feedstuffs by Distillation with Toluene (Cereal Fats and Shortenings by Distillation with Toluene (Cereal n, Rye, Barley, Rice, Grain Sorghum, and Buckwheat (Cereal Rodent Filth in Meal, Grits, Farina, and Semolina (Cereal our (Calculated from Percentage of Total Nitrogen) (Cereal

eal Grains, Unpopped Popcorn, Dried Peas and Beans (Cereal eolytic Activity in Active Proteinase Preparations (Cereal es for Control of Cecal and Intestinal Coccidiosis (Cereal d Materials Except Chocolate Products and Piecrust (Cereal goods, Ready.To-Eat Cereals, and Alimentary Pastes (Cereal ed Amounts of Malathion in Range of $0.2 \cdot 2.0$ p.p.m. (Cereal r Combined Tartaric Acid in Presence of Phosphates (Cereal cereal Grains, Whole or Chopped Nuts, Spices, etc. (Cereal ors for Gasometric Determination of Carbon Dioxide (Cereal xcreta in Meal, Grits, Flour, Farina, and Semolina (Cereal Meth of Analysis of Urea and Ammonical Nitrogen (Cereal Meth. of Analysis of Sedimentatoon Test for Wheat (Cereal ors for Correcting Weights to a $14 \%$ Moisture Basis (Cereal Meth of Analysis of Free Fatty Acids in Fats (Cereal Meth of Analysis of Vitamin A in Enriched Flour (Cereal Meth. of Analysis of Gluten in Flour and Semolina (Cereal lysis of Iodine Number in All Edible Fats and Oils (Cereal ysis of Niacin and Nicotinamide in Cereal Products (Cereal of Stability (AOM) of Fats, Oils, and Shortenings (Cereal f Saponification Value of All Normal Fats and Oils (Cereal loud Point in All Edible Animal and Vegetable Fats (Cereal nalsis of Benzoyl Peroxide with Diaminophenvlamine (Cereal tone-Insoluble Lecithin in Soybean and Corn Oils (Cereal

Melting Point of Normal Animal and Vegetable Fats (Cereal Refractometer, in All Normal Oils and Liquid Fats (Cereal es and Free Glycerol in Fats, Oils and Shortenings (Cereal ting Point of All Normal Animal and Vegetable Fats (Cereal $\mathrm{n}$ and Mineral) Concentrates Used to Enrich Cereals (Cereal of Analysis of Micro Sedimentation Test for Wheat (Cereal Analysis of Modified Sedimentation Test for Wheat (Cereal riangle Test Taste Panel for Food Flavor Detection (Cereal ive Meth. of Analysis of Benzoyl Peroxide in Flour (Cereal

s. Legumes, Forages, and Animal and Dairy Products (Cereal ity of Pregelatinized Starches and Cereal Products (Cereal nd Defatted Soy Flours and Grits, and Soybean Meal (Cereal nd Defatted Soy Flours and Grits, and Soybean Meal (Cereal Sucrose, Mannose, Galactose, Dextrans, and Starch (Cereal eat, Rye, Barley, Other Grains, and Malted Cereals (Cereal eolytic Activity in Active Proteinase Preparations (Cereal ide in Flour Bleaching Experiments in Laboratories (Cereal of Analysis of Pesticide Residues in Food Products (Cereal ch Are Susceptible to Hydrolysis by Alpha Amylase) (Cereal

Analysis of Acetone Peroxides in Milling Premixes (Cereal

$n$ of Azodicarbonamide Content in Commercial Premix (Cereal solidify Within Temperature Range of 25 to 45 Deg. (Cereal min a in Nonfat Dry Milk and Instantized Dry Milks (Cereal th. of Analysis of Aflatoxins in Corn and Soybeans (Cereal h. of Analysis of Added Lysine in Wheat and Bulgur (Cereal on of Fatty Acid Composition by Gas Chromatography (Cereal

agulase Positive Staphylococcus in Cereal Products (Cereal a Gamma) in Cereal Products-Emergency Monitoring (Cereal

n Dry Vitamin Mixes, Beadlets, Oils, and Emulsions (Cereal

Fatty Acids in Common Fats, Oils, and Fatty Acids (Cereal eed) for Prevention of Cecal and Intestinal Coccidiosis in Voluntary Safety Std. for High Chairs Std for Centrifugal Water (1964) Design and Construction of Masonry Design and Construction of ec. for the Design and Construction of Reinforced Concrete Uniform Building Code: Masonry or Concrete Dwelling Construction Under the Uniform Building Code: Sid. for

rinal) (1973)

holders (1973)

3)

73)

3)
Chemistry) (1962) Chemistry) (1962)

Chemistry) (1962)

Chemistry) (1962)

Chemistry) (1962

Chemistry) (1962

Chemistry) (1962)

Chemistry) (1962)

Chemistry) (1962)

Chemistry) (1962)

Chemistry) (1962)

Chemistry) (1962)

Chemistry) (1962)

Chemistry) (1962)

Chemistry) (1962)

Chemistry) (1962)

Chemistry) (1962)

Chemistry) (1962)

Chemistry) (1962)

Chemistry) (1962)

Chemistry) (1962

Chemistry) (1962)

Chemistry) (1962

Chemistry) (1962)

Chemistry) (1962

Chemistry) (1965)

Chemistry) (1965

Chemistry) (1965)

Chemistry) (1967)

Chemistry) (1967)

Chemistry) (1967)

Chemistry) (1967)

Chemistry) (1967)

Chemistry) (1967)

Chemistry) (1967)

Chemistry) (1967)

Chemistry) (1967)

Chemistry) (I967

Chemistry) (1967)

Chemistry) (1967)

Chemistry) (1967)

Chemistry) (1967)

Chemistry) (1967)

Chemistry) (1968)

Chemistry) (1968)

Chemistry) (1968)

Chemistry) (1968

Chemistry) (1968)

Chemistry) (1968)

Chemistry) (1968)

Chemistry) (1968)

Chemistry) (1968)

Chemistry) (1968)

Chemistry) (1968)

Chemistry) (1968)

Chemistry) (1969)

Chemistry) (1969)

Chemistry) (1971)

Chemistry) (1971)

Chemistry) (1971)

Chemistry) (1971)

Chemistry) (1972)

Chemistry) (1973

Chemistry) (1973)

Chemistry) (1973)

Chemistry) (1973)

Chemistry) (1973)

Chemistry) (1973)

Chickens (Cereal
(Children's) (1972)

Chilling Packages (Refrigeration) (1974)

Chimneys (For Residential and lndustrial) (Tech. Notes)

Chimneys (Tech. Notes) (1964)

Chimneys (1969) ANSI A 158.

Chimneys, Fireplaces, and Barbecues (1973)

Chimneys, Fireplaces, and Barbecues (1973)

Chimneys, Fireplaces, and Vents (1972) A NSI A52.1

Chimneys, Gas Vents and Heat Producing Appliances (1972

China Plumbing Fixtures (Lavatory, Water Closet Bowl, U

Chip Breakers Used in Indexable Inserts for Clamp Type

Chip Classification (Hand Screen) (Pulp and Paper) (197

Chip Length Analysis (Measurement) (Pulp and Paper) (19

Chip Length Analysis (Two Screen) (Pulp and Paper) (197

Chip Resistance of Coatings (1973)

Chips from Conveyor (Pulp and Paper) (1973)

Chips (Moister Teller) (Pulp and Paper) (1973)
Test Meth. for

Test Meth. for Sid Meth of Test for

Test Meth. for Sampling of Wood Test Meth. for Moisture in
Ereals, and Alimentary Pastes for D AACCH 28.30 (1)

An Baked AACCH 28.32 Solids to Change Mixi AACCH 54.20 (n) Insect and AACCH 28.51 Internal Insects in Cer AACCH 28.22 Meth. of Analysis of Prot AACCH 22.62 AACCH 28.50 $\mathrm{AACCH} 46.50$ $\mathrm{AACCH} \quad 56.61 \mathrm{~A}$ $\mathrm{AACCH} \quad 82.24$ AACCH 58.16

AACCH 86.03

Hand Washing AACCH 38.10 Meth. of Ana AACCH 58.30 Meth of Anal AACCH 86.50 th of Analysis AACCH 58.54 Analysis of C AACCH $56-10$ W Analysis of Ace AACCH $58-35$ W Cot Analysis of Mel AACCH 58.40 Vitami AACCH 86.49 Meth. AACCH 56.63 Meth of $\mathrm{AACCH} 56.62$ Meth. of T AACCH 33.50 Semiquantitat AACCH 48.07

Grains, Oilseed AACCH 46.14

Flakes, Full Fat a AACCH 46.24

.

Meth AACCH 60.50 ons Whi AACCH 76-30A Meth. of AACCH 48-05

Meth. for Determinatio AACCH 48.71A

( $\begin{array}{lll}\text { TLC Me AACCH } & 46.02 \\ \end{array}$ Met AACCH 46.40

(5.18

Meth. of Analysis of $\mathrm{Co}$ AACCH $42.30 \mathrm{~A}$ 1. of Analysis of Radioactivity (Bet AACCH 28.99A

1. efters of Long Chain AACCH 58.17 $\begin{array}{ll}\text { JPAC } & 18.25 \\ & \text { JPMA }\end{array}$

ARI

BIA

p ACI

ICBO

ICBO

NFPA

NFPA

ANSI

ANSI

TAPPI

TAPPI

TAPPI

ASTM

TAPPI

TAPPI
550 $19 \mathrm{~A}$ 307 UBC $* 3-37$ $\mathrm{UBC} * 8.6$ 211 Al12.19.2

B 94.47 UM. 13 UM.5 UM.6 D3170 UM-4 
Test Meth. for Density and Moisture of Test Meth. for Dirt Count of Wood Test Meth. for Sieve Analysis of Pulpwood Test Meth. for Density of Wood Test Meth. for Moisture in Wood lp and Paper) (19/ Test Meth. for Density and Moisture of Test Meth. for Moisture in Wood round Tool Mountings (Farm Machinery) (1973) Std. for analysis (Cereal Chemistry) Alcohol; Carbon Tetrachloride; I) $(1972)$ ent. Meth. of Test for Sulfur Oxides in Flue Gases (Barium Std. for trochemical Analysis of Nickel-Base Alloys by the Silver ups and Other Star/ and Other Clarifie/ and Other Clarifie/ ns of Electrical W/

it and Electrical Metallic Tubing (19)

Std. Analytical Meth. for Determining Std. Analytical Meth. for Determining Std. Analytical Meth. for Determining Std. for Styrene Rubber and Polyvinyl Std. for Polyvinyl ng Tape (1973) ANSI C59.91 ng Tape (/ Std. Spec. for Low Temperature Resistant Vinyl . Meth. of Test for Compatibility of Plasticizers in Vinyl th. of Test for Volatile Matter (Including Water) of Vinyl Std. Spec. for Photographic Grade Aluminum ing Stress Corrosion Cracking Tests in a Boiling Magnesium removal, Acceleration of Concrete

td. Spec, for Calcium Meth. for Analysis of Calcium Safe Handling and Use of Allyl Sid. Spec. for Polyvinyl

Pract. for Making Solvent Cemented Joints with Polyvinyl Std. Meth. of Chemical Analy sis of Chromated Zinc Std. Spec. for Chromated Zinc sential Information for the Safe Handling and Use of Vinyl Std. Spec. for Sodium s., and Materials Application for Thermoplastic (Polyvinyl Std. Spec. for Crosslinked and Noncrosslinked Poly(Vinyl i) Std. Meth. of Test for Quality of Extruded Poly (Vinyl ir Lining (1972T) Sid. Spec for Poly(Vinyl (1973) Spec. for FIexible Poly(Vinyl Std. Spec. for Poly(Vinyl

(1973) Sid. Spec. for Chlorinated Poly(Vinyl le Butadiene Styrene (ABS) Pipe and Fittings to Poly(Vinyl fittings (1973) ANSI K65.56 Spec. for Bell End Poly(Vinyl
Std. Spec. for Poly(Vinyl 73) Std. Spec. for PolyrV inyl 72.16 Std. Spec. for Solvent Cements for Poly(Vinyl 3) Std. Spec. for Socket Type Poly(Vinyl 3) ANSI K65.166 3) (1973) ANSI B72.7 Std. Spec. for Socket Type Poly(Vinyl Std. Spec. for Poly(Vinyl Std. Spec. for Poly(Vinyl Std. for Rigid Polyvinyl Std. Spec for Poly(Vinyl Std. Spec. for Type PSP Poly(Vinyl Std. Spec. for Type PSM Poly(Vinyl Std. Spec. for Filled Poly (Vinyl oxylamine Hydrochloride, ( $\mathrm{Nh}_{2} \mathrm{OH}$. Hci) (Hydroxylammonium Std. Spec. for Photographic Grade Potassium Std. Spec. for Photographic Grade Sodium Gravimetric Meth. of Analysis of Volumetric Meth. of Analysis of 962) Meth. of Analysis of Salt Calculated from Total Qualitative Meth. of Analysis of

Quantitative Meth. of Analysis of tors.(Electrical Insulating Liquid) (1973/ Std. Spec. for ormers (1973) Std. Spec. for chromatographic Estimation of Higher Boiling Homologues of er Distribution Systems (1973) Std. Spec. for for Colorfastness of Colored Textile Yarns and Fabrics to Std. for Safety for Swimming Pool Pumps, Filters, and

Man Made or Blend Fabrics, Commercial Wash-Whites (W ith Multiple Sample Meth. of Textile Tensile Loss by Retained Std. Meth. of Test for Total Std. Meth. of Test for Hydrolyzable ratories (Cerea/ Acetic Anhydride Meth. for Generation of Std. Meth. of Test for Residual Maintenance Instructions for Container Procedure for Rec. for Electrolytic es, Finishes, and Finishings (Sublimation, Brittleness and Piping and Equipment for Use with Dry or Single Sample Meth. of Textile Tensile Loss by Retained r Cotton and Linen Textile Colorfastness to Bleaching with Std. Meth. of Test for Mercury in Liquid (1973/ Std. Common Name for the Pest Control Chemical 2.
Chips (Presoak Before Immersion) (Pulp and Paper) (1973 Chios (Pulp and Paper) (1973)

Chips (Pulp and Paper) (1973)

Chips (Sheet Machine Mold) (Pulp and Paper) (1973)

Chips (Steam Oven) (Pulp and Paper) (1973)

Chips (Submersion with Correction for Cracks; etc.) (Pu

Chips (Two Liter Graduate) (1973)

Chisel Plow, Field and Row Crop Cultivator Shafts and G

Chloral Hydrate; Chloroform; Formaldehyde; Gasoline; He

Chloranilate Controlled Condensation Meth.) (1973)

Chlorbromuron (Common Name for the Pest Control Chemica

Chlordimeform (Common Name for the Pest Control Chemica

Chloride-Lithium Fluoride Carrier D-C Arc Technique U

Chloride Content of Corn Sugar (Crude and Refined), Syr

Chloride Content of Corn Syrup Unmixed, Finished Sugar

Chloride Content of Corn Syrup Unmixed, Finished Sugar

Chloride Ducts and Fittings for Underground Installatio

Chloride Externally Coated Galvanized Rigid Steel Condu

Chloride Indicator) (1971) /Yrup Unmixed, Finished Sug

Chloride Plastic Pressure Sensitive Electrical Insulati

Chloride Plastic Pressure Sensitive Electrical Insulati

Chloride Plastics Under Compression (1974)

Chloride Resins (1972)

Chloride Solution $\mathrm{AICI}_{3}$ (1972)

Chloride Solution (1973)

Chloride to Be Used for Dustlaying

Chloride Types I and II (1972T)

Chloride (Chemical Safety) (1973)

Chloride (PVC) Jacket for Wire and Cable (1974)

Chloride (PVC) Pipe and Fittings (1973) ANSI K65.55

Chloride (1970) ANSI 011.19

Chloride (1971) ANS1 011.4

Chloride (1972)

Chloride (1972) ANSl A37.56

Chloride) Duct Construction (1974)

Chloride) Heat Shrinkable Tubing for Electricalds.

Chloride) Plastic Pipe by Acetone Immersion) (1967) An

Chloride) Plastic Pipe (SDR.PR) (1973) ANSI B72.2

Chloride) Plastic Sheeting for Pond, Canal, and Reservo

Chloride) Resins (1972) ANSI K65.207

Chloride) (CPVC) Plastic Hot Water Distribution System

Chloride) (PVC) Pipe and Fittings for Nonpressure Appli

Chloride) (PVC) Pipe (1973) ANSI B72.20

Chloride) (PVC) Plastic Drain, Waste and Vent Pipe and

Chloride) (PVC) Plastic Line Couplings, Socket Type (19

Chloride) (PVC) Plastic Pipe and Fittings (1973) ANSI B

Chloride) (PVC) Plastic Pipe Fittings, Schedule 40 (197

Chloride) (PVC) Plastic Pipe Fittings, Schedule 80 (197

Chloride) (PVC) Plastic Pipe Fittings, Schedule 80 (197

Chloride) (PVC) Plastic Pipe, Schedules 40, 80 and 120

Chloride) (PVC) Plastic Tubing (1973) ANSI B72.22

Chloride) (PVC) Plastic W all Siding (1972)

Chloride) (PVC) Sewer Pipe and Fittings (1972)

Chloride) (PVC) Sewer Pipe and Fittings (1973)

Chloride) (PVC) Sewer Pipe and Fittings (1973)

Chloride) (PVC) Sewer Pipe (1972)

Chloride) (1973)

Chloride, KCI (1972)

Chloride, Nacl (1972)

Chlorides in Ash of Bread (Cereal Chemistry) (1962)

Chlorides in Ash of Bread (Cereal Chemistry) (1962)

Chlorides in Feeds and Feedstuffs (Cereal Chemistry) (1

Chlorides in Yeast Foods (Cereal Chemistry) (1962)

Chlorides in Yeast Foods (Cereal Chemistry) (1962)

Chlorinated Aromatic Hydrocarbons (Askarels) for Capaci

Chlorinated Aromatic Hydrocarbons (Askarels) for Transf

Chlorinated Biphenyls for Capacitor Askarels (1974)

Chlorinated Poly(vinyl Chloride) (CPVC) Plastic Hot Wat

Chlorinated Pool Water (1972) ANSI L 14.149

Chlorinators (1972)

Chlorine Bleach) 190 Deg. F, Colors 160 Deg. F Maximum,

Chlorine Bleaching (1971) ANSI L 14.180

Chlorine Content of Epoxy Resins (1973)

Chlorine Content of Liquid Epoxy Resins (1973)

Test Meth. for

Chlorine Dioxide in Flour Bleaching Experiments in Labo

Chlorine in Tank Motor Vehicles (1973)

Chlorine in Water (1968)

Chlorine Institute Std. Excess Flow Valves (1972)

Chlorine Packaging (1973)

Chlorine Processes (Explosion Hazard During Manufacture

Chlorine Retention) (Institutional Textile) (1973)

Chlorine (1971)

Chlorine (1971) ANSI L14.126

Chlorine (1972) ANSI Ll4.57

Chlorine (1973)

Chloro-4,6bis (Isopropylamino) -S-Triazin e-Propazine

TAPPI

TAPPI

TAPP

TAPP

TAPPI

TAPPI UM-20

ASAE S225.1

AACCH $28-91$

ASTM D3226

ANSI K62.113

ANSI K62.114

ASTM E483

CR

CR

CR

NEMA

NEMA

CR

ASTM

Std ASTM

std. Me ASTM

ANS1

ASTM

ASTM

ASTM

MCA

ASTM

ASTM

ASTM

F-10

E-14

E-15

TCl

E.15

D2301

D3005

D3291

D3030

PH4. 154

G36

D98

E449
SD 99

D 1047

D2855

D1033

D 1032

SD56

D632

*11

ASTM D3150

ASTM D2152

ASTM D224]

ASTM D3083

ASTM D1755

D2846

ASTM D3138

ASTM D2672

ASTM D2665

D3036

ASTM D2564

ASTM D2466

ASTM

ASTM D2467

ASTM D1785

ASTM D2740

USC

ASTM

ASTM

ASTM

ASTM

PS55

D2729

D3033

D2836

PH4. 187

ANS1 PH4.202

ANSI PH4.203

AACCH $\quad 40-30$

AACCH $40-31$

AACCH 40-61

AACCH $40-32$

$\mathrm{AACCH} \quad 40-33$

ASTM D2233

ASTM D2283

ASTM D3303

AATCC 105

UL 1081

ANSI L24.4.13

AATCC 114

ASTM D1847

ASTM D1726

AACCH $48-30$

CHl 49

ASTM D1253

CHI 42

FMS 734

Dy ANS1 L24.7.2

$\mathrm{CHl} 6$

Test Meth. F AATCC 92 
Name for the Pest Control Chemical 2-(Tertbutylamino)-4flour (Cereal Chemistry) (1962)

ies and Essential Information for Safe Handling and Use of chemistry) Alcohol; Carbon Tetrachloride; Chloral Hydrate;

\section{Medium \\ Firm}

h. for Evaluation of and Test Formulas for General Purpose he Pest Control Chemical Isopropyl 4,4'dichlorobenzilate . Std. for Common Name for the Pest Control Chemical 4. dimethyl 0.(3,5,6-Trichloro-2-Pyridyl) Phosphorothioate Std. for

Trapping Meth. of Analysis for All Baked Materials Except water WeIlhead, and Christmas Tree Equipment; Flow Control al Chemistry) (1962) Digitonin Meth. of Analysis of Friction Coefficients of th or Sack Fibers, Whole or Ground Cereal Grains, Whole or I, Screwed and Flanged Wellhead, Underwater Wellhead, and tock; Seedling Trees and Shrubs; Bulbs, Corms, and Tubers; Alloy Panels/ Spec. for Coating Weight Determination for Spec. for Spot Test Solution for Detecting

d Sugar and Other Clarified Starch Hydrolyzates (Potassium Std. Spec. for Acid Copper Std. Spec. for Basic Lead Silico Std. Meth. of Chemical Analysis of Std. Spec, for

orfastness) (1972) Rec. Evaluation Procedure of ct. for Direct lneth. for Identification of Lignin (Paper Std. Meth of Assay of Di-T-Butyl Peroxide by Gas of Chlorinated Biphenyls for C/ Std. Meth. for Rapid Gas er Extender and Processing Oils by the Clay Gel Absorption Std. Analytical Meth. for Determining Saccharides Meth. for Determination of Fatty Acid Composition by Gas Meth. for Analysis of Natural Gas Liquid Mixtures by Gas eth. for Obtaining Natural Gas Samples for Analysis by Gas ic Hydrocarbons in Monocyclic Aromatic Hydrocarbons by Gas $\mathrm{s}$ of High Purity N-Heptane and Isooctane by Capillary Gas Test for Purity and Benzene Content of Cyclohexane by Gas for Aromatic Traces in Light Saturated Hydrocarbons by Gas analysis of Liquefied Petroleum Gases (LPG) by Process Gas est for Butadiene Purity and Hydrocarbon Impurities by Gas lysis of Calcium and Barium Petroleum Sulfonates by Liquid r Boiling Range Distribution of Petroleum Fractions by Gas Meth. for Analysis of Natural Gas Liquid Mixtures by Gas Analytical Meth. for Determining Saccharides (Gas Liquid Analytical Meth. for Determining Saccharides (Gas Liquid or $\mathrm{C}_{1}$ Through $\mathrm{C}_{2}$ Hydrocarbons in the Atmosphere by Gas Grade Chromium Potassium Sulfate $\mathrm{CrK}\left(\mathrm{SO}_{4}\right)_{2}: 12 \mathrm{H}_{n} \mathrm{O}$ Sid. Spec. for Fluor Std. Meth. for Chemical Analysis of Fluor

si A 139.2 Std Meth. for Chemical Analysis of or Chemical Analysis of Chrome Containing Refractories and ed Ground Refractory Materials (Calcined Bauxite, Calcine, tamination-Occupa/ tion) (1969) ASTM D2807 Std. for Acceptable Concentration of 972)

ure Vessel Plates (1972) ANSI G33.3 $t$ and Strip (1973)

Meth. for Determining Std. Meth. of Test for Std. Spec. for Std. Spec. for Nickel Std. Spec, for Nickel Std. Spec. for Nickel

si H34. 10 Nickel-Molybdenum 4

h. for Chemical Analysis of Nickel-Chromium and Nickel nd Strip (1973)

tube (1972) ANSI H34.16

ium Stabilized Alloy /

ium Stabilized All/ Std. Spec. for Nickel-Iron
Std. Spec. for Nickel-lron Std. Spec. for Seamless and Welded Std. Spec. for Electric Fusion Welded or High Temperature Service (1974) ANSI G/S Std. Spec. for of Stainless, Heat Resisting, Maraging, and Other Similar ation at High T/ 9) ANSl W 3.4 s (1969) Ansi/ Std. Spec. for Centrifugally Cast Iron Spec. for Corrosion Resisting Chromium and Spec. for Corrosion Resisting Chromium and Std. Spec. for Nickel-Molybdenum

Std. Spec. for Low Carbon Nickel Molybdenum Std. Spec. for Nickel-lron Std Spec for Nickel-Iron Std. Spec. for Nickel-Molybdenum Std. Spec. for Low Carbon Nickel Molybdenum ectrodes (1969) ANSI W 3.4 are Electrodes (1969) Ansi/ Std. Spec. for Nickel-lron Spec. for Corrosion Resisting Spec, for Corrosion Resisting Spec. for Flux Cored Corrosion Resisting 2 Std. Spec: for Carbon rip for Fusion Welded Unfil Std. Spec. for Heat Resisting
Chloro-6-(Ethylamino)-S-Triazine-Terbuthylazine (19 Chloroform-Alcohol Meth. of Analysis of Ergot in Rye Chloroform (1974)

Chloroform: Formaldehyde; Gasoline; Herwig's, Pancreati

Chloroprene-Rubber Sponge (1973)

Chloroprene-Rubber Sponge (1973)

Chloroprene Rubber Sponge (1973)

Chloroprene Rubbers (CR) (1973)

Chloropropylate (1973)

Chloro5-(Methylamino) -2

Chlorpyrifos MethyI (1973)

Chlorpyrifos (Insecticide) (1971)

Chocolate Products and Piecrust (Cereal Chemistry) (196

Chokes; and Water Sampling Devices) (1974)

Cholesterol Meth. of Analysis of Dried Egg Solids (Cer

Choline (Cereal Chemistry) (1962)

Chopped Forages (Grass, Corn, Hay, Straw) (1968)

Chopped Nuts, Spices, etc. (Cereal Chemistry) (1962)

Christmas Tree Equipment; Flow Control Chokes; and Wate

Christmas Tree Sid (1973)

1. Asparagus); Lining Out

Chromate Conversion Coatings on Aged or Backed Aluminum

Chromate Films on Galvanized Sheet (Coil Coating) (1974

Chromate Indicator) (1954)

Chromate (1971)

Chromate (1974)

Chromated Zinc Chloride (1970) ANSI 011.19

Orn Syrup Unmixed, Finishe

Chromated Zinc Chloride (1971) ANSI 011.19

Chromatic Transference Scale (Color Chart / Textile Col

Chromatogram) (1973)

Chromatograph (1973)

Chromatographic Analysis (1973)

Chromatographic Estimation of Higher Boiling Homologues

Chromatographic Meth. (1973) ANSI Z11.180

Chromatographic) Content of Corn Syrup (1957)

Chromatography (Cereal Chemistry) (1973)

Chromatography (1968)

Chromatography (1968)

Chromatography (1968) ANSl Z164.1

Chromatography (1971) ANSI Z11.262

Chromatography (1972)

Chromatography (1972) ANSI Z11.279, IP 262

Chromatography (1973)

Chromatography (1973)

Chromatography (1973)

Chromatography (1973)

Chromatography (1973) ANSI Z11.238

Chromatography) Content of Corn Sugar, Stareh Molasses

Chromatrography (1972)

Chrome Alum) (1972)

Chrome Arsenate Phenol (1971)

Std. Meth. of Test F

Chrome Arsenate Phenol (1971) ANSI O11.20

Test for Nonar. M

/ Meth. for Analysi ASTM

Std. Meth. of ASTM

1. of Test ASTM

Std. Meth. for ASTM

Std. Meth of T ASTM

Std. Meth. for Ana ASTM

Tent. Meth. of Test Fo ASTM

Std ASTM

CR

ASTM

Chrome Containing Refractories and Chrome Ore (1970) an

Chrome Ore (1970) ANSl Al39.2

Chrome Ore, Clays, Dias pore, Ganister Quarzite, Grog, K

Chromic Acid and Hexavalent Chromium Compounds (Air

Chromic Oxide in Leather (Perchloric Acid Oxidation) (1

Chromium-Copper-Nickel-Aluminum Alloy Steel Press

Chromium-lron-Molybdenum-Copper Alloy Plate, Shee

Chromium-lron-Molybdenum-Copper Alloy Rod (1973)

Chromium-Iron Alloy PIa te, Sheet, and Strip (1970) an

Chromium-Iron Alloy Sheet and Plate (1971) ANSI H34.4

Chromium-Iron Alloys (1973)

Chromium-Manganese-Molybdenum Alloy Plate, Sheet, a

Chromium-Molybdenum-Copper Alloy Seamless Pipe and

Chromium-Nickel-Iron-Molybdenum-Copper-Columb

Chromium-Nickel-lron-Molydenium-Copper-Columb

Chromium-Nickel-Iron Alloy Castings (25-12 Class) F

Chromium-Nickel-Iron Alloys (1973)

Mical Analysis

Chromium-Nickel High AIloy Tubing for Pressure Applic

Chromium-NickeI Steel Covered WeIding Electrodes (196

Chromium-Nickel SteeI Welding Rods and Bare Electrode

Chromium Alloy Plate and Sheet (1971) ANSI H34.12

Chromium Alloy Plate, Sheet, and Strip (1972)

Chromium Alloy Plate, Sheet, and Strip (1973) ANSI H34.

Chromium Alloy Rod and Bar (1973) ANSI H34.39

Chromium Alloy Rod (1971) ANSI H34.14

Chromium Alloy Rod (1972)

Chromium Alloy Seamless Pipe Tube (1973) ANSI H34.41

Chromium and Chromium-Nickel Steel Covered Welding El

Chromium and Chromium-Nickel Steel Welding Rods and B

Chromium and Chromium Nickel Steel Electrodes (1974)

Chromium and Chromium Nickel Steel Plate, Sheet, and St

Chromium and Nickel-Chromium-lron Alloys (1973)

Chromium Ball and Roller Bearing Steel (1970) ASTM G56. 
eptibility to Intergranular Attack in Wrought Nickel Rich, or Acceptable Concentration of Chromic Acid and Hexavalent r to Conversion $\mathrm{C} /$

Test Meth. for Detection of Hexavalent Std. Spec. for Nickel

Std. Spec. for Nickel Molybdenum

h34.3

ess Pipe and Tube (1972) ANSI H34./

Std. Spec. for Nickel Std. Spec. for Pressure Vessel Plates, Alloy Steel,

Tent. Meth. for Chemical Analysis of Ferrochromium and Std. Spec. for sels (1973) ANSI G35.4

mpered, for Pressure Vessels (1972) rip (1972) ANSI H34.19

ansi $\mathrm{H} 34.22$

ube (1972) ANSI H34.20

p (1972) ANSI H34.17

i H34.18

Std. Spec. for Std. Spec. for Nickel Std. Spec. for Nickel Std. Spec. for Nicke Std. Spec. for Nicke Std. Spec. for Nickel Iron

Std. Spec. for Nickel Iron Std. Spec. for Nickel $\begin{array}{ll}\text { empered Chromium, for Pressure Vessels (1/ } & \text { Std. Spec. for } \\ \text { Std. Spec. for }\end{array}$ $\begin{array}{ll}\text { empered Chromium, for Pressure Vessels (1/ } & \text { Std. Spec. for } \\ \text { Std. Spec. for }\end{array}$ temperat/ Std. Spec. for Welded Large Diameter Austenitic r/ Std. Spec. for Corrosion Resistant Iron Chromium, Iron ized Alloy Plate, Sheet, and Strip (1973)/ Std. Spec. for ized Alloy Pipe (1973/ Std. Spec. for Seamless and Welded

Hot Rolled and Cold Finished Stainless and Heat Resisting Spec. for Flux Cored Corrosion Resisting Chromium and $n$ Welded Unfi/ Std. Spec. for Heat Resisting Chromium and (1972) ANSI B36.39 Std. Spec. for Seamless Austenitic hardware (1970) Std. for Electroplating of Nickel and ome Alum) (1972) Std. Spec. for Photographic Grade Std. Spec. for Nickel Iron

81.6

Std. Spec. for Corrosion Resistin

2 Std. Spec. for Stainless and Heat Resisting teel, Quenched and Tempered Nickel-Cobalt-Molybdenum mium Molybdenum, Alloy Steel Plates, Quenched and Tempered stings for Gener/ Std. Spec. for Corrosion Resistant Iron stant Metals (Electrodeposited Coatings of Copper, Nickel, Std. Spec. for Pressure Vessel Plates, Alloy Steel 5\% Std. for Chucks and

(1962)

(1962) Std. for

Counting Meth. of Analysis of Gravimetric Meth. of Analysis of orm Building Code: Weights of Materials (Brick; Cast Iron; Std. for Safety for Molded Case Circuit Breakers and dule of Dielectric Test Values for Outdoor AC High Voltage Rec. Pract. for Automatic Reset Internal Mounted

mendations, and Related Requirements for Low Voltage Powe Std. for Safety for Molded Case cations, Class I, Groups A-D, and Class / Safety Std. for id. Requirements for Electrical Control of AC High Voltage d for Out of Phase/ Std. Meth. for Test. AC High Voltage ings for Capacitance Current Switching for AC High Voltage ng Impulse Voltage Insulation Strength for AC High Voltage rements for Transient Recovery Voltage for AC High Volt age n Guide for Transient Recovery Voltage for AC High Voltage requirements for Pressurized Components of AC High Voltage ents for Capacitance Current Switching for AC High Voltage uide for Capacitance Current Switching for AC High Voltage ements for External Insulation for Outdoor AC High Volt age Std. for Test Procedures for Low Voltage AC Power Std. for Low Voltage Alternating Current Powe ings and Related Required Capabilities for AC High Voltage Rec. Pract. for

Mineral Insulating Oil for Use in Transformers and in Oil ted Mineral Insulating Oil for Use in Transformers and Oi or Industrial Electrical Power Control Apparatus-Contro Safety Std. for Fused Powe Safety Std. for Ground Fault ements for Low Voltage Power Circuit Breakers and AC Power $r$ the Application, Operation, and Maintenance of Automatic I Filled Capacitor Switches for Alter/ Std. for Automatic

for Engineering Specs. Outline for Monochrome Cctv (Closed Performance Spec. for Flexible Multilayer Wiring (Printed ing Systems (1969) ANSI SE3.7

Std. for Interconnection Rec. for Protective Grounding of Electrical Electrical Characteristics of Class a Closure Interchange Rec. Pract. for Automotive Printe Surge (Lightning) Arresters for Alternating Current Power use Std. Criteria for Separation of Class Ie Equipment and
Design and Construction of Std. for Carbide Blanks for Tipping rage Type (1974) Std. for Gas Water Heaters: Volume III, Std. for Forced

Rec. for Thermoplastic Piping for Swimming Pool Water 2-Dicar/ Std. Common Name for the Pest Control Chemical
Chromium Bearing Alloys (1972) ANSI G80.4 /Ecting Susc

Chromium Compounds (A ir Contamination-Occupational He

Chromium Containing Treatments on Galvanized Steel Prio

Chromium Iron Alloy Rod and Bar (1970) ANSI H34.4

Chromium Iron Alloy Rod (1972)

Chromium Iron Alloy Seamless Pipe and Tube (1970) ANSI

Chromium Iron Columbium Molybdenum Tungsten Alloy Seaml

Chromium Manganese Silicon (1972A) ANSI G32.1

Chromium Metal (1971T)

Chromium Metal (1973) ANSI G75.1

Chromium Molybdenum Alloy Steel Plates for Pressure Ves

Chromium Molybdenum Alloy Steel Plates, Quenched and Te

Chromium Molybdenum Columbium Alloy Plate, Sheet and St

Chromium Molybdenum Columbium Alloy Rod and Bar (1972)

Chromium Molybdenum Columbium Alloy Seamless Pipe and T

Chromium Molybdenum Copper Alloy Plate, Sheet, and Stri

Chromium Molybdenum Copper Alloy Rod and Bar (1972) Ans

Chromium Molybdenum Iron Alloy Rod (1972)

Chromium Molybdenum, Alloy Steel Plates, Quenched and T

Chromium Nickel Alloy Castings (1974)

Chromium Nickel Alloy Steel Pipe for Corrosive or High

Chromium Nickel Alloy Steel Pipe for High Temperature S

Chromium Nickel and Nickel Base Alloy Castings for Gene

Chromium Nickel Iron Molybdenum Copper Columbium Stabil

Chromium Nickel Iron Molybdenum Copper Columbium Stabil

Chromium Nickel Manganese Steel Bars Including Rounds,

Chromium Nickel Steel Electrodes (1974)

Chromium Nickel Steel Plate, Sheet, and Strip for Fusio

Chromium Nickel Steel Still Tubes for Refinery Service

Chromium on Metal Parts-Automotive Ornamentation and

Chromium Potassium Sulfate $\mathrm{CrK}\left(\mathrm{SO}_{1}\right)_{2}: 12 \mathrm{H}_{2} \mathrm{O}(\mathrm{Chr}$

Chromium Silicon Alloy Seamless and Welded Pipe (1972)

Chromium Steel Clad Plate Sheet and Strip (1972) ANSI G

Chromium Steel Plate, Sheet, and Strip (1972) ANSI G81.

Chromium (1972) ANSI G35,

Re Vessel Plates,

Chromium, for Pressure Vessels (1972) ANSI G35.18

Chromium, Iron Chromium Nickel and Nickel Base Alloy Ca

Chromium, Lead, Zinc or Cadmium) (1973)

Chromium, 0.5\% Molybdenum (1972) ANSI G35.16

Chuck Jaw s (1972)

Chucks and Chuck Jaws (1972)

Cinder and Sand Particles in Farina (Cereal Chemistry)

Cinder and Sand Particles in Farina (Cereal Chemistry)

Cinders; Concrete; Earth; Glass; Granite; Gravel; Grani

Circuit Breaker Enclosures (1972)

Circuit Breaker External Insulation Rated on a Symmetri

Circuit Breaker (1971)

Circuit Breakers and AC Power Circuit Protectors (1973)

Circuit Breakers and Circuit Breaker Enclosures (1972)

Circuit Breakers and Enclosures for Use in Hazardous Lo

Circuit Breakers Rated on a Symmetrical and a Total Cur

Circuit Breakers Rated on a Symmetrical Basis When Rate

Circuit Breakers Rated on a Symmetrical Basis (1972)

Circuit Breakers Rated on a Symmetrical Basis $(362 \mathrm{kV}$ a

Circuit Breakers Rated on a Symmetrical Current Basis (

Circuit Breakers Rated on a Symmetrical Current Basis (

Circuit Breakers Rated on a Symmetrical Current Basis (

Circuit Breakers Rated on a Symmetrical Current Basis (

Circuit Breakers Rated on a Symmetrical Current Basis

Circuit Breakers Rated on a Symmetrical Current Basis (

Circuit Breakers Used in Enclosures (1973)

Circuit Breakers Used in Enclosures (1973) ANSI C37.13

Ransient Recovery Voltage Rat

Circuit Breakers (1971)

Circuit Breakers (1972)

Circuit Breakers (1973)

Círcuit Breakers (1973)

Circuit Devices and A Std. Spec. for Uninhibited
D. Spec. for Oxidation Inhibi

Circuit Interrupters (1972)

Circuit Protectors (1973)

Circuit Reclosers (1973) ANSI C37.6I

Std Guide Fo

Circuit) (1972)

Circuitry of Noncoded Remote Station Protective Signall

Circuits and Equipment (1974)

Circuits (Electronics) (1974)

Circuits (1967)

Circuits (1972) ANSI C62.1

Circuits (1974) ANSI N41.14

Circular Prestressed Concrete Structures (1967)

Circular Saws (1972)

Circulating Tank, Instantaneous and Large Automatic Sto

Circulation Air Cooling and Air Heating Coils (1972)

Circulation Systems (1972)

cis-N-((1,1,2,2tetrachloroethyl) Thio)-4-C yclohexene-1
ASTM

ANSI

NCCA

ASTM

ASTM

ASTM

ASTM

ASTM

ASTM

ASTM

ASTM

ASTM

ASTM

ASTM

ASTM

ASTM

ASTM

ASTM

ASTM

ASTM

ASTM

ASTM

ASTM

ASTM

AWS

ASTM

ATM

SAE

ANSI

ASTM

ASTM

ASTM

ASTM

/Hro ASTM

ASTM

ICBO

ASTM

ANSI

ANSI

AACCH

$\mathrm{AACCH}$

ICBO

UL

ANSI

SAE

ANSI

UL

UL

ANSI

IEEE

ANSI

IEEE

IEEE

EEE

IEEE

IEEE

IEEE

IEEF

ANSI

IEEE

ANSI

SAE

ASTM

ASTM

ANS

UL

ANSI

IEEE

NEMA

/Ec. Std. EIA

Std. IPC
NEM

FMS

FIA

$\begin{aligned} & \text { Std. for the EIA } \text { SAE } \\ & \text { Std. for IEEE }\end{aligned}$

Trial IEEE

$\mathrm{ACI}$

ANSI

ANSI

ARI

SPI

ANSI

G28

Z37.7

B-II-7

B 166

B573

B445

A 202

A481

A387

A543

B443

B444

B424 
ereal Chemistry) (1962) Std. Spec. for Photographic Grade Sodium Rec. Std. for Loose Metal End Dimensions (6 Ounce Fibre Uniform Fire Code: Fruit Ripening Process (Banana, 1 End Dimensions of Composite Cans (211 (66 Mm) Diameter), Std. Spec. for Copper Uniform Building Code Std. for Tin Safety Std. for Swinging Hardware for Std. Tin Std. for Safety for Tin

d. for Sliding Hardware for Std., Horizontally Mounted Tin Std. Spec, for Corrosion Resisting Chromium Steel Std. Spec. for Copper and Its Alloy pec. for Straight Beam Ultrasonic Examination of Plain and Spec, for Nickel Coated Coppe

Evaluating the Effect of Solar Radiation on Outdoor Meta
Std. Rec. Pract. for Examination of Fuel Elemen propert/ itutional Textiles (Du/ Std. Performance Requirements and ning the SAE Rating and Struck Capacity for Shovel Dipper Std for Carbide Seats Used with Indexable Inserts for d. for Carbide Chip Breakers Used in Indexable Inserts for Std. for Bushing Std. for Bushing. Std for Hose

ranes Having Rope Supported Booms, Equipped for Hook Work, de Content of Corn Syrup Unmixed, Finished Sugar and Other de Content of Corn Syrup Unmixed, Finished Sugar and Other ANSI MH4.8 971) Std. Meth. of Test for Flame Propagation Design and Construction) / Std. Uniform Building Code Std. for Soils Std. for Balance

erials (1973) Std. For Balance

Insects, Rodent Hair, and Radiographic Illustration and I Plastics. Chemicals, Metal Dusts) (1973)

Drilling Rigs and Petroleum Production

Std. for Area

Rec. Pract. for Std. Meth. of Test for Bauer-McNett Wet Uniform Building Code Std. Test. Meth. for Fire Hazard

brick (1970) ANSI All1.5

Std

1.22

quipment (1973) NEMA SH3

Lighting (1973) EEl TDJ.133 si L14.192

Radiographic Std. for

Std for Voltage Std. for Voltage Std. Tables of Std.

Std ractory Materials (Calcined Bauxite, Calcine, Chrom/ Std.
r Highway Construction Purposes (19/ Std. Rec. Pract. for 974) Std. Qualitative Std, for Std.

products (1973) ANSI J8.6

) for Automotive Applications (1973) ANSI J8.15, SAE J20/ ce in Ionizing Radiation (1971) ANSI N4.1

Test Meth. for Chip Rec. Pract. for Collision Deformation (Damage) Rec, for Flammable Liquid Drum Storage, Dispensing, and

Uniform Building Code: Occupancy and General Requirement l Species of Lumber (1973/ Uniform Building Code Std. for

Sid

Tent. Hazard

(1971) ASTM D2047, C/ Test. Std. (Procedure) for Products eir Hazardous Potentialities (Safety) (19) Std. Guide for $\mathrm{S}$ and Parts Made Therefrom (1970) ANSI K/ Rec. Pract. for Std. Rec. Pract. for Lighting Cotton Rules for Building and Structural

tes) (1963)

tes) (1963)

notes) (1963)

Std. Spec. for Compression Joints for Vitrified Structural Structural Structura Structura

Uniform Building Code Std. for Structura istic Groups in Rubber Extender and Processing Oils by the Structural Std. Spec. for Vitrified

and Test. Stru/

Uniform Building Code Std. for Structural

Principles of Structural

Code Std. for and Meth. of Sampling and Test. of Unburned Radiation Protection with

Painting

Flashing

Modular

Std Spec for Structural

Uniform Building Code Std. for Struct ural

ode Std. for Building. Facing, and Hollow Brick (Made from
Citrate, Dihydrate $\mathrm{Na}_{3} \mathrm{C}_{6 ;} \mathrm{H}_{5} \mathrm{O}_{\overline{7}}: 2 \mathrm{H}_{2} \mathrm{O}$ (1972)

Citric and Isocitric Acids in Fruit and lis Products (C

Citrus Cans $202(52 \mathrm{Mm})$ Diameter) (1973)

Citrus Fruit) (1973)

Citrus Style (1974)

Clad Aluminum Wire (1972)

Clad Fire Doors and Shutters (1973)

Clad Fire Doors (1973) ANSI A 133.1

Clad Fire Doors (1973) ANSI Al42.1

Clad Fire Doors (1973) ANSI A143.1

Clad Plate Sheet and Strip (1972) ANSI G81.6

Clad Steel Plate (1973)

Clad Steel Plates for Special Applications (1971) ANS1

Clad Steel Wire for Electronic Application (1972)

Clad Switchgear (1971) ANSl C37.24

Cladding Including the Determination of the Mechanical

Claims for Special Characteristics and Finishes of Inst

Clam Bucket, and Dragline Bucket (Construction and Indu

Clamp Type Holders (1973)

Clamp Type Holders (1973)

Clamp Up, Bronze (1973)

Clamp Up, Steel, Cadmium Plated (1973)

Clamps (1971)

Clamshell, Magnet, Grapple, or Concrete Bucket Attachme

Clarified Starch Hydrolyzates (Potassium Chromate Indic

Clarified Starch Hydrolzates (Silver-Silver Chloride

Classification and Definitions of Bulk Materials (1970)

Classification Flooring and Floor Covering Materials (1

Classification for Determination of Sound Transmission

Classification for Engineering Purposes (1973)

Classification for Flexible Gear Couplings (1974)

Classification for Metal Working Fluids and Related Mat

Classification in Cereal Grains and Grain Products /Cer

Classification in Hazardous Dust Locations (Agricultura

Classification of Areas for Electrical Installations at

Classification of Asbestos Fiber (1970) ANSI K91.1

Classification of Building Materials (1973)

Classification of Coppers (1973) ANSI H34.43

Classification of Fireclay and High Alumina Refractory

Classification of Fusion Weld Discontinuities (1972)

Classification of Insulating Fire Brick (1970) ANSI All

Classification of Luminaires Used in Roadway Lighting E

Classification of Luminaires I/sed in Street and Highway

Classification of Man Made and Natural Fibers (1971) an

Classification of Mullite Refractories (1972)

Classification of Pig Tin (1972) ANSl H42.1

Classification of Single and Double Screened Ground Ref

Classification of Soils and Their Aggregate Mixtures Fo

Classification of Surfactants by Infrared Absorption (1

Classification of Unirradiated Plutonium Scrap (1972)

Classification System for Carbon Blacks Used in Rubber

Classification System for Elastomeric Materials (Rubber

Classification System for Polymeric Materials for Servi

Classification (Hand Screen) (Pulp and Paper) (1973)

Classification (Highway Vehicle Accident) (1972)

Classification (1972)

Classification (1973)

Classification, Definition, and Meth. of Grading for Al

Classifications for Spark Resistant Construction (1966)

Classifications of Organic Peroxides (Plastic) (1974)

Classified as to Slip Resistant (Floor Polish and Wax)

Classifying and Labeling Epoxy Products According to Th

Classifying Visual Defects in Glass Reinforced Laminate

Classing Rooms for Color Grading (1971)

Classing Steel Vessels (1972)

Clay Acoustile (Tech Notes) (1961)

Clay Bell and Spigot Pipe (1972A)

Clay Differential Movement (Cause and Effect) (Tech. No

Clay Differential Movement (Expansion Joints) (Tech. No

Clay Differential Movement (Flexible Anchorage) (Tech.

Clay Facing Tile (Tech. Notes) (1965)

Clay Floor Tile (1973)

Clay Gel Absorption Chromatographic Meth. (1973) ANSI Z

Clay Insulated Cavity Walls (Tech. Notes) (1964)

Clay Liner Plates (1972)

Clay Load Bearing Wall Tile and Std. Meth. of Sampling

Clay Masonry Construction: Student's Manual (1960)

Clay Masonry Process (Tech. Notes) (1966)

Clay Masonry Units (1973)

Clay Masonry (Tech. Notes) (196I)

Clay Masonry (Tech. Notes) (1961)

Clay Masonry (Tech. Notes) (1962)

Clay Masonry (Tech. Notes) (1963)

Clay Nonload Bearing Tile (1971) ANSI A 76.l

Clay Nonload Bearing Wall Tile (1973)

Clay or Shale) (1973)
ANSI

AACCH

CCTI

ICBO

CCTI

ASTM

ICBO

$\mathrm{UL}$

UL

Safety St UL

ASTM

ASTM

ASTM

ASTM

IEEE

ASTM

ANS1

SAE

ANS]

St ANS!

NSA

NSA

SAE

SAE

CR

CR

CEMA

UL

ASTM

ICBO

AGMA

ASTM

AACC

ISA

API

ASTM

ICHO

ASTM

ASTM

NSA

ASTM

EEI

NEMA

ASTM

ASTM

ASTM

ASTM

ASTM

ASTM

ANSl

ASTM

ASTM

ASTM

SAE

FMS

ICBO

ICBO

AMCA

FMS

CSMA

ANS1

ASTM

ASTM

ABS

BIA

AST

BIA

BIA

BIA

BIA

ICBO

ASTM

BIA

ASTM

ICBO

BIA

BIA

ICBO

BIA

BIA

BIA

BIA

ASTM

ICBO

Uniform Building C ICBO

PH4.179

04.22

202.1

UFC*2ART 16

E211.1

B566

UBCS 43.3

I4C

$10 \mathrm{~A}$
$14 \mathrm{~B}$

A 263

B432

B 432
A578

B559

144

E453

L24.7. 1

J67 
Std. Spec. for Sewer and Manhole Brick (Made from Std. Spec, for Vitrified hooting Facility Plans: International and Domestic-Trap, Sid. Meth. of Test. Std. Spec. for Compression Couplings for Vitrified Cleaning Glossary of Terms Relating to Structura Std. Rec. Prsct. for Installing Vitrified Meth. for Viscosity of Coating

Guide Spec. for Std. Meth. of Sampling and Test. Brick and Structura Wall Tile and Std. Meth. of Sampling and Test. Structural in. Brick and Tile Walls (Tech. Notes) (1966) Structurs Structurs] Floors and Pavements of Siructural Std. Meth for Particle Size Analysis of Whiteware fractory Materials (Calcined Bauxite, Calcine, Chrome Ore, Sid. Meth. of Test for Spec, for Powdered Hand Spec. for Powdered Hand ors $(1970)$

Std. Definition of the Term Vacuum Spec. for Safoty Std. for Vacuum Cleaning Machines and Blower $f$ Bonded and Laminated Apparel Fabrics (Textile) After Dry

industrial Exhaust Ventilation Syste/

Std. Spec. for td. for Safety Requirements for Commercial Laundry and Dry f Flsmmable Liquids (Gasoline, Kerosene, Acetone, Alcohol,

6.1 Std. for Safety for Commercial Dry Std. Rec. Pract. for .33 ontainers / Std. Procedure for Qualifying and Controlling ing Construction Phase of Nuclear Power Plants / Std. for $\mathrm{ms}$ (1971) Rec. Pract. for Flushing and ystems Used for Removsl of Water Formed Deposits (Chemical en Pumps for Marine Ser/ Rec. Pract, for the Flushing and hydroelectric Equipment / Rec. Pract for the Flushing and Std. Household Electric Range Pyrolytic Self Uniform Building Code Std. for Class 11 and Class III Dry Std. Spec, for Hydrocarbon Dry

1972) ANSI G81.16

icles (1972)

ANSI G80.1

procedure) (197/

$r(1972 \mathrm{~T})$ Meth. for Measurement of Color of Low Colored Std. Meth. of Test for Volume Nonvolatile Matter in Std. Meth. for Establishing or Grain Sorghum Starch Which Are Substantially Optically ed in Battery Sockets (1974) ANSI C83.53 regulations and Controls, Barrier, Road, Hydrant, Driveway (1972)

ncluding Wheel Assembly, Chainguards, Saddle Braces, Pedal ice for Use by Vehicle Manufacturers in Designing for Tire 2) ANSI Z197.16 Std. Meth. of Test for Flash and Fire Points by

Std. for Tube Assembly, Control Steel with Welded Std, for Tube Assembly, Control, Steel, with Flash Welded equipment (Body Belt, Safety Strap, Lanyard, Pole and Tree 23 ole and Tree Climber) (1973) ) ices (1971) Std. Meth. for Spec, for Lineman's Test Meth. for Electrostatic Rec. Std. Practices for Std. for Safety for Hair Std, for Safety for Household Electric Std. for Screw-100 Deg. d Bolt (1974) Std. for Rivet-100 Deg. Countersunk Head Hi Shear Std. for $100 \mathrm{Deg}$ Sid. for Hi Shear 100 Deg. Flush Std. for Rivet-Flat Head, Hi Shear, Std. for Bolt-Shear,

Std, for Bolt, 100 Deg. Head, Tri W'ing Recess, Std. for Bolt, 100 Deg. Head, Tri Wing Recess, and Nonl/ Std. for Bolt, 100 Deg. Head, Tri Wing Recess, and Nonlocking (1972)

(1) $\mathrm{g}$ and Nonlocking (1972) Std. for Bolt Hex Head, $\mathrm{g}$ and Non/ Std. and Nonlocking (1972)

Std. for Bolt,

Clay or Shale) (1973) ANSI A100.1

Clay Paving Brick (1972) ANSI A37.15

Clay Pigeon, and Skeet: Ammunition and Target Spec. (19

Clay Pipe (1972) ANSI A 106.5

Clay Plain End Pipe (1973)

Clay Products Masonry (Tech. Notes) (1964)

Clay Products (Tech. Notes) (1961)

Clay Protective Construction (Tech. Notes) (1962)

Clay Sewer Pipe (1972) ANSI A 106.2

Clay Slurry (1972)

Clay Tile and Concrete Slabs (Tech. Notes) (1962)

Clay Tile Roofing (1972)

Clay Tile (1973)

Clay Tile (1973)

Clay W'all Tile (Tech. Notes) (1966)

Clay (Properly Constructed Masonry) Insulated Cavity 6

Clay (Tech. Notes) (1962)

Clays (1974)

Clays, Diaspore, Ganister Quarzite, Grog, Kyanite, Magn

Cleanability of Surface Finishes (1973)

Cleaner (Soap with Borax) (1972T)

Cleaner (Soap with Vegetahle Scrubber) (1972T)

Cleaner (1974)

Cleaners for Use on Asphalt and Vinyl Asbestos Tile Flo

Cleaners (1973) ANSI C 33.36.1

(Cleaning Agent) (1970) ANSI K60.20

Cleaning and Laundering (1972) ANSI L14.269

Cleaning Clay Products Masonry (Tech. Notes) (1964)

Cleaning Compound, General Purpose (1972)

Cleaning Devices (Air Filters and Dust Collectors) for

Cleaning Equipment and Operations (1972)

Cleaning Fluids, Flammable Aerosols, etc, with a Flash

Cleaning Machines and Blower Cleaners (1973) ANSI C33.3

Cleaning Machines (Class Iv) (1974)

Cleaning Metals Prior to Electroplating (1968) ANSI G53

Cleaning Meth. for Hydraulic Fluid Power Fluid Sample C

Cleaning of Fluid Systems and Associated Components Dur

Cleaning of Gas Turbine Generator Lubricating Oil Syste

Cleaning of Industrial Equipment) (1973) Jof S

Cleaning of Oil Systems for Lubrication and Control of

Cleaning Ovens (Stove) (1974)

Cleaning Plants or Systems (1973)

Cleaning Solvents (1971) ANSI Z11.42

Cleaning Stainless Steel Parts, Equipment and Systems (

Cleaning Systems Containing Devices for Removal of Part

Cleaning, and Evaluating Corrosion Test Specimens (1972

Cleanliness Requirements (Magnetic Particle Inspection

Clear and Pigmented Lacquers (1973)

Clear Liquids Using the Hunterlab Color Difference Mete

Clear or Pigmented Coatings (1973)

Clear Wood Strength Values (1973)

Clear (1959)

/Hydrolyzates Solutions Derived from Corn

Clearance Dimensions on the Mating Plates (Patterns) U

Clearance, Installation and Maintenance of Systems and

Clearance, Side Marker and ldentification Lamps (Light)

Clearance, Steering Control, Brakes, Tires and Controls

Clearances (1974) / Dimensions for Grown Tires in Serv

Cleavage Strength of Metal to Metal Adhesive Bonds (197

Cleveland Open Cup (1972) AASHO T48, ANSI Z11.6

Clevis-Engine Control Rod, Adjustable (1973)

Clevis and Threaded Rod Ends (1973)

Clevis and Threaded Rod Ends (1973)

Clevis Pins and Cotter Pins (1972)

Climber) (1973)

Spec. for Lineman's Climbing

Climbing Drum Peel Test for Adhesives (1962) ANSI Z197.

Climbing Equipment (Body Belt, Safety Strap, Lanyard, P

Clinging of Textile Fabric s Fabric to Metal Test) (1973

Clinical Research Protocol for Dental Materials and Dev

Clipping and Shaving Appliances (1973) ANSI C33.36.2

Clocks (1974) ANSI C33.47

Close Tolerance Flat Head 160,000 psi (1973)

Close Tolerance Head and Shank (1973)

Close Tolerance Head and Shank, 160,000 psi Short Threa

Close Tolerance Head Rivet (1972)

Close Tolerance Shank (1973)

Close Tolerance (1973)

Close Tolerance (1972)

Close Tolerance (1972)

Close Tolerance, Alloy Steel, Long Thread, Self Locking

Close Tolerance, Alloy Steel, Long Thread, Self Locking

Close Tolerance, Alloy Steel, Short Thread, Nonlocking

Close Tolerance, Alloy Steel, Short Thread, Self Lockin

Close Tolerance, Alloy Steel, Short Thread, Self-Lockin

Close Tolerance, A286 Cres, Long Threads, Self Locking

Close Tolerance, A286 Cres, Short Thread, Nonlocking (1
ASTM

ASTM

NRA

ASTM

ASTM

BlA

BlA

BlA

ASTM

TAPPI

BIA

CSI

ASTM

ICBO

BIA

BIA

BIA

ASTM

ASTM

ASTM

ASTM

ASTM

ASTM

RTI

UL

ASTM

Pensity O ASTM

BIA

ASTM

ACGIH

ANSI

$\mathrm{ICBO}$

UL

UL

ASTM

NFLDP

ANSI

ASME

ASTM

ASME

ASME

AHAM

ICBO

ASTM

ASTM

ANSI

ASTM

SAE

ASTM

ASTM

ASTM

ASTM

CR

EIA

ICBO

SAE

BMA

TRA

ASTM

ASTM

NSA

NSA

NSA

ANSI

EEI

AST

EEI

AATCC

ADA

UL

UL

NSA

NSA

NSA

NSA

NSA

NSA

NSA

NSA

NSA

NSA

NSA

NSA

NSA

NSA

NSA

C32

C7
.17

C301

C594

20

2

C. 12

T648SU

$14 \mathrm{~A}$

07350

C67

(

$22 \mathrm{~A}$

- 26

14

C 316

C756

D3046

D3047

F395

-5

D2724

20 
and Nonlo/ Std. for Bolt, 100 Deg. Head, Tri Wing Recess, and Nonlocking (1972)

1973)

\section{2)}

m Alloy $6 \mathrm{Al} \cdot 4 \mathrm{~V}$, Self Locking An/ teel, Self Locking and Nonlocki/ res, Self Locking and Nonlocking (197) Std. for Bolt, Hex Head, Std for Bolt. Std. for Bolt-100 Deg. Std. for Pan Head, Std. for Bolt, Pan Head, Std. for Bolt, Pan Head, Std. for Pan Head, Sid. for Bolt, Hex Head,

If Locking and Nonlocking (1972/ elf Locking and Nonlocki/ self Locking and Nonlocking (19/ Bolt, 100 Deg. Tri Wing Recess, Std. for Bolt, Hex Head, Std. for Engineering Specs. Outline for Monochrome Cctv Std. for Flash Points. Std. for Break Mandrel Sid. for $450 \mathrm{Deg}$. F proc. and Acceptance Criteria for Rate of Air Flow Through est for Flash Point of Aviation Turbine Fuels by Setaflash amel, Lacquer, Varnish, and Related Products) by Setaflash Sid. Meth. of Test for Flash Point by Pensky-Martens Std. Meth. of Test for Flash Point of Chemicals by Std. for Requirements for Std. for Door Controls . Std. for Builders Door Controls handles; Exit Devices, Pulls, Push and Kick Plates; Bolts; Quality Stds. for

Woodwork: Standing and Running Trim, Casework, Panelwork, std. for Vitreous China Plumbing Fixtures (Lavatory, Water Rec. Spec. for Steel Bi-Fold Std. and Test Procedure for W/ater Sid. for the Electrical Characteristics of Class A h. of Measuring Water Vapor Transmission Through Screw Cap h. of Measuring Application and Removal Torque of Threaded try) (1) Meth. of Xanthydrol Test for Analysis of Urea in Urease Bromthymol Blue Test Paper for Analysis of Urea in Std. Spec. for High Temperature Glass Std. Spec. for Wire Sid. for Laundering of

) (1973) Std. Performance Requirements for Terry
Safety Sid. for Varnished

Test Meth for Absorbency of Bleached Woven Fluidity of Dispersions of Cellulose Form Bleached Cotton Std. Spec. for Woven Asbestos n to Porous Substrates (Subjective Ratings) (Paper, Board, neous Matter Meth. of Analysis (Cereal Chemistry) (Bolting 81 Style Fabric, Self Extinguishing (1973)

d, 181 Style Fabric, Self Extinguishing (1973)

Ixtle, Jutes, Hemp, Tow, Cocoa Fibers, Oakum, Rags, Waste Std. for Volume 1, Type l, Gas Std. for Gas

Std. for Safety for Electric Coin Operated and Commercial Safety Std for Electric Home Laundry Equipment Std. for Safety for Electric Coin Operated and Commercial Performance Evaluation Procedure for Household (laundry, ANS1 L14.69, ASTM D1230 Test Meth. for Flammability of for Appearance of Seams in Wash and Wear ltems (Textile

st Meth. for Appearance of Creases in Wash and Wear ltems

d. for Body Measurements for the Sizing of Girls' A pparel

s (Textile) for lnstitutional and Industrial Use Garments $s$ (Textile) for lnstitutional and Industrial Use Garments

s (Textile) for Institutional and Industrial Use Garments

imation of Water Insoluble Impurities in Refined Phenol by real Chemistry) (1967) Meth. of Analysis of

for Fee Determination for Division Services (Individuals,

Rec. Pract. for SAE No. 2 (Automotive Transmission) Sid. for Industrial Power Take Offs for Overcenter

nd Relief Valves in Travel Trailers, Camp Cars and Trailer $\mathrm{s}$ (For Foreign and Domestic Passenger Cars, Trucks, Buses, ereal Chemistry) (1973) Meth. of Analysis of Std. Rec. Pract, for

bases (1974) Std. Meth. for Calculating Std. Meth. of Laboratory Sampling and Analysis of Std. Definitions of Terms Relating to Std. Meth. of Test for Proximate Analysis of

Sid. Meth of Test for Moisture in the Analysis Sample of Std. Meth. of Test for Ash in the Analysis Sample of Std. Meth. of Test for Nitrogen in the Analysis Sample of eth. of Test for Volatile Matter in the Analysis Sample of of Test for Carbon and Hydrogen in the Analysis Sample of Sid. Meth. for Ultimate Anaylsis of Meth. of Test for Total Sulfur in the Analysis Sample of

Rec. for Prevention of Furnace Explosions in Pulverized Safety Guide for Respiratory Protection Against es, Poles, and Timbers for Marine, Land, $/$ Std. Meth. of Preparing Rec. Pract. Meth. for Lining Lease Production Tanks with
Close Tolerance, A286 Cres, Short Thread, Self Locking

Close Tolerance, A286 Cres, Short Thread, Self Locking

Close Tolerance, Hexagon Head, Titanium, Short Thread (

Close Tolerance, High Strength (1973)

Close Tolerance, Short Thread, Torq Set Machine Screw

Close Tolerance, Short Thread, Tri Wing Recess, Titaniu

Close Tolerance, Short Thread, TWl Wing Recess, Alloy S

Close Tolerance, Short Thread, TWl Wing Recess, A286 AC

Close Tolerance, Titanium Alloy (1972)

Close Tolerance, 6Al-4V Titanium Alloy, Long Thread, Se

Close Tolerance, 6Al-4V Titanium Alloy, Short Thread, S

Close Tolerance, 6Al-4V, Titanium Alloy, Short Thread,

Closed Circuit Television) Camera Equipment (1974)

Closed Cup, Aromatic Chemicals and lsolates (1973)

Closed End Blind Rivets (1973)

Closed End Self Locking Serrated Head Blind Nut (1974)

Closed Steel Door and Frame Assemblies (1972)

Closed Tester (1973)

Closed Tester (1973)

Closed Tester (1973) ANS1 Z11.7

/Lash Point of Tent. Meth. of T

Closed Up Meth. (1973)

Closed Van Type Cargo Containers (1971)

Closers (1972)

Closers) (1971)

Closers; Hospital and Misc. ltems; Key Control Systems;

Closet and Storage Shelving (1973)

Closet and Storage Shelving, Misc. Ornamental ltems, St

Closet Bowl, Urinal) (1973)

Closet Doors and Frames (1972)

Closet Flush Tank Ball Cocks (1968)

Closure Interchange Circuits (Electronics) (1974)

Closure Liners (1973)

Closures (1973)

Cloth Bags and Other Packaging Materials (Cereal Chemis

Cloth or Sack Fibers, Whole or Ground Cereal Grains, Wh

Cloth Pressure Sensitive Electrical Tape (1973)

Cloth Sieves for Test. Purposes (1970) ANSI Z23.1

Cloth Toweling (1970)

Cloth Towels (Single and Double) (Institutional Textile

Cloth Wires and Cables (1971) ANSI C33.73

Cloth (1972) ANSl Ll4.115

Cloth (1972) ANSI Ll4.117

Cloth (1973) ANS1 L14.133

Cloth, etc.) (1973)

Cloth, Filter Paper, Wide Field and Compest Meth. for Ad

Cloth, Type E Glass, B Stage Epoxy Resin Impregnated, 1

Cloth, Type E Glass, B Stage Polyester Resin lmpregnat

Cloth, Wastepaper, Kapok, Hay, Straw, Spanish Moss, Exc

Clothes Dryers (1972)

Clothes Dryers, Volume 11, Type 2 (1974)

Clothes Drying Equipment (Laundry Dryer) (1974)

Clothes Washer and Dryer) (1972) ANSI C33.13

Clothes W ashing Equipment (Laundry W asher) (1974)

Clothes) Washer (1971) ANS1 Z224.1

Clothing Textiles (1962)

Clothing) After Home Laundering (1973) ANSI L14.172

Clothing) After Home Laundering (1973) ANSI L14.173

Clothing) (1972)

Clothing), Commercial Wash-Whites 190 Deg. F, Colors

Clothing), $100 \%$ Man Made or Blend Fabrics, Commercial W

Clothing), 100\% Man Made or Blend Fabrics, Drycleanable

Cloud Point Depression (1968) ANSI Z78.21 /lon and Est

Cloud Point in All Edible Animal and Vegetable Fats $(\mathrm{Ce}$

Clubs, and Exhibition Charges) (1974)

Clutch Friction Test Machine Test Procedure (1972)

Clutches (1971)

Coaches (1970)

Coaches, Tractors, Industrial Road and Mise Equipm

Coagulase Positive Staphylococcus in Cereal Products (C

Coagulation Flocculation Jar Test of Water (1974)

Coal and Coke Analyses from As-Determined to Different

Coal and Coke (1970) ANSI K18.1

Coal and Coke (1972) ANSI K20.6

Coal and Coke (1973)

Coal and Coke (1973)

Coal and Coke (1973)

Coal and Coke (1973)

Coal and Coke (1973)

Coal and Coke (1973)

Coal and Coke (1974)

Coal and Std. Meth. Coke (1973)

Coal Fired Multiple Burner Boiler Furnaces (Fire Protec

Coal Mine Dust (1972)

Coal Samples for Analysis (1972) ANSI K20.17

Coal Tar Creosote for the Preservative Treatment of Pil

Coal Tar Epoxy (1972)
NSA

NSA

NSA

NSA

NSA

NSA

NSA

NSA

NSA

NSA

NSA

/EC ElA

EOA

IFl

Test STD

ASTM

ASTM

ASTM

ANSI

ANSl

BHMA

NBHA

$A$ WI

AWI

ANSI

STDI

ASSE

EIA

Std. Met ASTM

to. Met ASTM

AACCH $28-86$

$\mathrm{AACCH} \quad 28-87$

ASTM D2754

ASTM El]

NSF 34

ANSl L24.2.5

UL

133

AATCC 79

AATCC 82

ASTM D1571

TAPPl UM-540

AACCH $28-90$

SAE AMS3821

SAE

ICBO

ANSI

ANS

UL

UL

UL

Std AHAM

AATCC

1. AATCC

IE AATC

St USC

ANS1

ANSl

ANSl

AMS 3827

UFC $* 2 A R T$

Z21.5.1

Z21.5.2

1240

1206

HLW-1

33

$88 \mathrm{~B}$

PS54

L24_4.12

L24.4.13

PSA

SAE

SAE

IAP

BCl

AACC

ASTM

ASTM

ASTM

ASTM

ASTM

ASTM

ASTM

ASTM

Std. M ASTM

Std. Meth. ASTM

ASTM

Std ASTM

FMS

ANSI

ASTM

ASTM

NACE 
Rec. Spec, for Enamel Coating System Designations for ped Asphalt, Asphalt and Coal Tar Saturated Organic Felts, ings: Hot Mopping Asphalt, Asphalt-Saturated Organic Felt, f Built Up Roof Coverings: Hot Mopped Asphalt, Asphalt and Std. Spec. for Creosote me and Specific Gravity Correction Tables for Creosote and Std. Meth. for Collection of a Gross Sample of Relating to Lithologic Classes and Physical Components of Determination of Volume Percent of Physical Components of ctance of the Organic Components in a Polished Specimen of 972) g Asphalt, Asphalt-Saturated Organic Felt, Coal Tar Pitch,

Std. Meth of Test for Specific. Spcc. for Optimum Size td. Meth of Test for Sieve or Screen Analysis of Fine and Std. Meth. of Test for Cashmere Std. Spec. for Maximum Cashmere

ANSI K42.1 Std. Meth. of Test for

neral Requirements for Carbon Steel Wire Rods and Uncoated

rs and Laminations Before Gluing of Southern Pine, Pacific orm Building Code Std. for Douglas Fir, Coast Region; West ite Fir; And/ Uniform Building Code Std. for Douglas Fir, n Hemlock-Tamarack (Nor/ Uniform Building Code Std. for (1972) edge Bend (Applicable to Solution Coil Coatings and Single overing for Permanent Wall and Ceilings / Spec. for Vinyl Std. Meth. of Test for Hydrogen Permeance of Rubber Std. Meth of Test.

test Meth. for Weather Resistance of Textile Materials and roof Membranes; Aggregates, Saturated Felts, Saturated and sual Color Matched to Master Specimen for Fabrics, Vinyls, tallic Tubing (19/ Std. for Polyvinyl Chloride Externally 1 Tar Saturated Organic Felts, Coal Tar Pitch, and Asphalt nd Gaseous Fluorides in the Atmosphere (Sodium Bicarbonate test Proc. for the Locking Ability Performance of Chemical Rec. Pract. for Resistance Welding ocking Resistance of Organic Coatings When Applied to Coil Std. for Safety for Septic Tanks, Bituminous Std. for Safety for Septic Tanks, Bituminous
ven Man Made Fiber Tent, Awning, and Canopy Fabrics, Vinyl Meth. for Measuring Blister Resistance of Test Meth. for Carbonates in Test Meth. for Sulfides in Test Meth. for Amount of Coating on Mineral for Coating Pick Tests for Determining Surface Strength of ansi C7.15 Std. Spec. for Lead and Lead Alloy Std. Spec. for Silver Std. Spec. for Nickel oil Co/ Meth. of Preperation and Evaluation of Painted or Std. Spec. for Aluminum Std. Spec. for Zine Meth. of Test. rrosion (1972T) ecording Data from Atmospheric Corrosion Tests of Metallic Std. Spec, for Zinc

C59. 136 Std. Meth. of Test. Pressure Sensitive Adhesive Std. Meth. of Low Temperature Impact Test for Fabrics tors, Steel Reinforced (ACSR) (1972)/ Spec. for Aluminum ndu/ Std. Spec, for Zinc Coated (Galvanized) and Aluminum randed Steel Core for Aluminum Condu/ Std. Spec. for Zinc Spec. for Steel Sheets for Culverts and Underdrains, Zinc eets of Structural Quality in Coils, and Cut Lengths, Zinc (1971) ANSI G8.21 Std. Spec. for Steel Sheet, Zinc

Std. Spec. for Steel Sheet of Lock Forming Quality, Zinc g8.21 Std. Spec. for Steel Sheet of Drawing Quality, Zinc Std. Spec. for General Requirements for Steel Sheet, Zinc quality (1971) ANSI G8./ Std. Spec. for Steel Sheet, Zinc ad Right of Way Wire Fencing (1973)/ Std. Spec. for Zinc Std. Spec. for Zinc tors, Steel Reinforced (ACSR) (1974)/ Std. Spec. for Zinc
ordinary Uses (/ Std. Spec. for Black and Hot Dipped Zinc nt, Awning, and Canopy Fabrics, Painted, Printed, or Vinyl

led Wheat, Breakfast Cereals (Except Those Which Are Sugar plied) (197) Std. for Cement Mortar Protective Lining and Spec. for E-CTFE- Fluoroplastic Molding, Extrusion, and of Test for Apparent Viscosity of Hot Melt Adhesives and Iuding Optical Microscopic) Analysis of Mineral Filler and

(1973) Spec. for Conversion Spec, for Coating Weight Determination for Conversion test for Locating the Thinnest Spot in a Zinc (Galvanized) Test Meth. for Amount of Steel and Other Hard Materials by Water Blasting Prior to coated Papers Using the Interfiber Bond T/ Test Meth. for Used for Electrical Insulation (1973) Std. Meth. of Test. insulation (1973) Std. Meth. of Test/ Std. Meth. of Test. tings (1972)
Coal Tar or Asphalt Coatings (1972)

Coal Tar Pitch, and Asphalt Coated Glass Fiber Mat (197 Coal Tar Pitch, Coal-Tar Saturated Organic Felt, Asphal Coal Tar Saturated Organic Felts, Coal Tar Pitch, and a Coal Tar Solution (1974) ANSI O11.2 Coal Tar (R1970)

Coal (1972)

Coal (1972)

Coal (1972)

Coal (1972)

Coal-Tar Saturated Organic Felt, Asphalt.Saturated Asbe

Coarse Aggregate (1973) ANSI A37.5

Coarse Concrete Aggregates (1971) AASHO T27, ANSI A37.8

Coarse Hair Content in Cashmere (I97I)

Coarse Hair Content in Cashmere (1971)

Coarse Particles in Pigments, Pastes, and Paints (1972)

Coarse Round Wire (1971) ANSI G54.12

Td. Spec. for Ge

Coarse Threaded Stud (1973)

Coast Douglas Fir and Western Hemlock by Pressure Proce Coast Hemlock: Western Red Cedar; White Fir; and Sitka

Coast Region; West Coast Hemlock; Western Red Cedar; Wh

Coast Sitka Spruce, Douglas Fir-Larch (North), Easter

Coat Organosols Only) (1974) land Flexibility by the W

Coated Copper Clad Steel Wire for Electronic Applicatio

Coated Fabric Coverings for Decorative and Protective C

Coated Fabrics (1973)

Coated Fabrics (1973) ANSI J2.12

Coated Fabrics: Carbon Arc Lamp Exposure with Wetting (

Coated Felts, Prepared Roofings, and Plastic Roll Mater

Coated Fiberboards, and Other Automotive Trim Materials

Coated Galvanized Rigid Steel Conduit and Electrical Me

Coated Glass Fiber Mat (1973) /Sphalt, Asphalt
Coated Glass Tube and a Particulate Filter Meth.) (1973

Coated Lock Screws (1973)

Coated Low Carbon Steels (1970)

Coated Metal (1974)
Coated Metal (1974) ANSI A 162.

Coated Metal (1974) ANSI A 162.1

Coated or Laminated (Institutional Textile) (1973)

Coated Paper in Heatset Printing (1972)

Coated Paper (Qualitative) (1973)

Coated Paper (Qualitative) (1973)

Coated Paper (1973)

Coated Papers Using the Interfiber Bond Tester (1973)

Coated Soft Copper Wire for Electrical Purposes (19674

Coated Soft or Annealed Copper Wire (1974) ANSI C7.38

Coated Soft or Annealed Copper Wire (1974) ANSI C7.48

Coated Specimens Subjected to Corrosive Environments (C

Coated Steel Chain Link Fence Fabric (1971) ANSI G53.21

Coated Steel Chain Link Fence Fabric (1971A) ANSI G53.2

Coated Steel Specimens Dynamically for Resistance to Co

Coated Steel Specimens (1972)
Coated Steel Wire Strand (1972A) ANS1 C7.46

Coated Steel (1972)

Coated Tapes Used for Electrical Insulation (1974) ANSI

Coated with Flexible Polymeric Materials (1973)

Coated (Aluminized) Steel Core Wire for Aluminum Conduc

Coated (Aluminized) Stranded Steel Core for Aluminum Co

Coated (Galvanized) and Aluminum Coated (Aluminized) St

Coated (Galvanized) by Hot Dip Process (1971) ANSI G8.2

Coated (Galvanized) by Hot Dip Process (1973) ANSI G8.2

Coated (Galvanized) by the Hot Dip Process for Roofing

Coated (Galvanized) by the Hot Dip Process (1971) ANSI

Coated (Galvanized) by the Hot Dip Process (1971) ANSI

Coated (Galvanized) by the Hot Dip Process (1973) ANSI

Coated (Galvanized) by the Hot Dip Process, Commercia

Coated (Galvanized) Iron or Steel Farm Field and Railro

Coated (Galvanized) Steel Barbed Wire (1973) ANSI G8.10

Coated (Galvanized) Steel Core Wire for Aluminum Conduc

Coated (Galvanized) Welded and Seamless Steel Pipe for

Coated (Institutional Textiles) (1973)

Coated) (Cereal Chemistry) (1962)

Coating Clay Slurry (I972)

Coating for Steel Water Pipe (4 In. and Larger, Shop Ap

Coating Materials (1973)

Coating Materials (1973)

Coating of Paper (1973)

Coating of Titanium Alion and Qua

Coating on Aged or Baked Aluminized Steel (Coil) (1974)

Coating on Iron or Steel Articles by the Preece Test (C

Coating on Mineral Coated Paper (1973)

Coating or Recoating (1972)

/for Surface Preparation of

Coating Pick Tests for Determining Surface Strength of

Coating Powders and Their Coatings Used for Electrical

Coating Powders and Their Coatings Used for Electrical

Coating System Designations for Coal Tar or Asphalt Coa

NAPCA BUL

ICBO UBCS32.

UL

ICBO

ASTM

ASTM

ASTM

UBC

D391

D347

D2234

D2796

D2799

ASTM

ASTM

UL

ACPA

ASTM

ASTM

ASTM

ASTM

A STM

ASTM

NSA

AW PA

ICBO

ICBO

ICBO

NCCA

ASTM

ATT

ASTM

A ATCC

NRCA

SAE

NEMA

lCBO

ASTM

IFI

AWS

NCCA

UL

/Wo ANSI

TAPPI

TAPPI

TAPPI

TAPPl

ASTM

ASTM

ASTM

NCCA

ASTM

ASTM

ASTM

ASTM

ASTM

ASTM

ASTM

ASTM

ASTM

ASTM

ASTM

ASTM

ASTM

ASTM

A STM

ASTM

ASTM

ASTM

ASTM

D2798

$55 \mathrm{~A}$

15

C 127

136

D2816

D2817

510

183

$\mathrm{C} 28$
$\mathrm{UBCS} 25.3$

UBC 525.3

UBCS 25.2

TB.11-10

B559

0995 I

D815

D751

$111 \mathrm{~A}$

*2.1V

J361 A

UBCS32-1

D3268

125

C1.3

TB. 11.17

70
L24. 1.10

T526SU

UM-531

UM-532

UM-542

UM.534

B 189

B298

B355

TB.11]-3

A491

A392

D2933

G33 
on and Preparation of Test Panels for U'se in Test. Organic uperstructure (1973) 72)

Std. Meth. of Test for Std. Meth. of Test for ross Secti/ Std. Meth. for Measurement of Metal and Oxide Std. Meth. of Test for Total Rosin Acids Content of atings on Aged or Backed Aluminum Alloy Panels/ Spec. for aged or Baked Aluminized Steel (Coil) (1974)

Spec, for zed Steel (Coil) (1/ Spec. for Determination of Phosphate Spec, for Determination of Phosphate taining Treatments on Galvanized Steel Prior to Conversion
Std. Spec. for Zinc Std. Meth. of Test for Thickness of Diffusion Black Oxide Treatment (Part mination of Relative Pencil Hardness of a Paint Film (Coil ermination of Film Adhesion by Cross Hatch Tape Test (Coil ill Chemical Treatments on Galvanized Steel Surfaces (Coil ion for Detecting Chromate Films on Galvanized Sheet (Coil film Thickness of Nonmagnetic Finishes on Mild Steel (Coil tance of Painted Aluminum, Steel and Gavanized Steel (Coil coated Specimens Subjected to Corrosive Environments (Coil als by Cross Hatch Tape Test After Reverse Impacting (Coil ted Products - Film Thickness of $0.3 \mathrm{MIL}$ or Greater (Coil (1971)

determination of Deg. of Cure of a Backed Paint Film (Coil Test Meth. for Exterior

flexibility by the Wedge Bend (Applicable to Solution Coil Standard for Quality Assurance for Protective - Meth. of Test for Disbonding Characteristics of Pipeline hickness of Transparent or Opaque Metal Oxide and Metallic Meth of Test for Moisture Vapor Permeability of Organic Tent. Rec. Spec. for Asphalt Type Protective or Resistance to Growth of Mold on the Surface of Interior std. Spec. for Castor Oil for Use in Resins and Protective code Std. for Corrosion Resistant Metals (Electrodeposited rous Alloys (1970) ANSI / Std. Spec. for Electrodeposited

td. Meth. of Test for Abrasion Resistance of Glossy Smooth Std. Meth. Test for Electrodeposited Std. Spec. for Electrodeposited

for Coating Weight Determination for Chromate Conversion Std. Meth of Test for Stain Resistance of Anodic de Coatings on Aluminum (1973) Std. Spec. for Anodic Oxide Coatings on Aluminum (1973) Std. Spec. for Anodic Oxide Std. Meth. for Condensation-Humidity Test. of Organic er Failure of Paint. Varnish, Lacquer, and Related Organic Spec. for Film Thickness Determination of Nonconductive

$r$ Nondestructive Measurement of Film Thickness of Pipeline

for Infrared Determination of the Temperature of Applied ation (1973) Std. Meth. of Test. Coating Powders and Their h. of Test/ Std. Meth. of Test. Coating Powders and Their mining Pressure Marking and Blocking Resistance of Organic Std. Meth. of Test for Formability of Attached Organic

Std. Meth of Test for Penetration Resistance of Pipeline Std. Meth. of Test for Impact Resistance of Pipeline Std. Meth. of Test for Impact Resistance of Pipeline ible Liquids A Uniform Fire Code: Manufacture of Organic inment Facilities (1972) Std Protective

Preparation of Centrifugal Fans and Parts for Protective Meth. for Adhesion or Cohesive Strength of Flame Sprayed h. of Test for Steam Distillation of Bituminous Protective Sid. for Protective Pipe Std. Meth. of Test for Adhesion of Metallic

Sid. Meth. of Testing Fatty Acids Used in Protective Std. Meth. of Test for Solids Content of Aerosol Std. Meth. of Test for Bendability of Pipeline

Std. Test Meth. for Chemical Resistance of Pipeline

Std. Meth. of Test for Abrasion Resistance of Pipeline

Std. Meth. of Test for Cathodic Disbonding of Pipeline

Std. Meth. of Test for Water Penetration into Pipeline eth. of Test for Effects of Outdoor Weathering on Pipeline enamel Coating System Designations for Coal Tar or Asphalt td. Meth. of Measurement of Microhardness of Electroplated

Std. Meth. of Test for Continuity of Porcelain Ename Std. Meth. of Test for Chip Resistance of

of Test. Bituminous Base Emulsions for Use as Protective

$f$ Test for Volume Nonvolatile Matter in Clear or Pigmented

Weathering Tests of Latex and Emulsion Exterior Paints or

Meth. of Test for Coefficient of Kinetic Friction for W ax

std. Meth. for Measuring Chalk Resistance of Exterior Coil

environment Test. of Spacecraft Thermal Control Materials ion (75 Ohms) (1973)

Sheet, Strip and Rolled Bar (19/ Std. for Precision yessel Plates, Alloy Steel, Quenched and Tempered Nickel. Std. Spec, for Copper-Zinc-Aluminum

Alloy Wire, Welding, Corrosion and Heat Resistant

Electrical, Magnetic, and Other Similar Iron, Nickel, and
Coating Systems (Coil) (1974)

Coating Systems (Paint) Guide for Ship Hull, Deck and S

Coating Thickness by the Beta Backscatter Principle (19

Coating Thickness by X.Ray Spectrometry (1972)

Coating Thicknesses by Microseopical Examination of a C

Coating Vehicles (1973)

Coating Weight Determination for Chromate Conversion Co

Coating Weight Determination for Conversion Coating on

Coating Weight on Hot Dip Galvanized and Electrogalvani

Coating Weights on Steel (Coil) (1974)

Coating (Coil) (1974)

Tion of Hexavalent Chromium Con

Coating (Hot Dip) on Assembled Steel Prc. cts (1973) an

Coating (1970) ANSI Z167.24

Coating) (1973)

Coating) (1974)

Coating) (1974)

Coating) (1974)

Coating) (1974)

Coating) (1974)

Coating) (1974)

Coating) (1974)

Coating) (1974

Coating) (1974)

Coating) (1974) /Ting a Solvent Hand Rub Test for the
Coatings and Overlays on DFPA Grade Trademarked Ply wood

Coatings and Overlays on DFPA Grade Trademarked Ply wood

Coatings and Single Coat Organosols Only) (1974)

Coatings Applied to Nuclear Facilities (1972)

Coatings by Direct Soil Burial (1972)

Coatings by Double Beam Interference Microscope Techniq

Coatings Films (1972)

Coatings for Underground Pipe Lines (Wrapped Systems) ( Std ASTM

Coatings in an Environmental Chamber (1973) /of Test F

Coatings Including Lacquers (1973)

Coatings of Copper, Nickel, Chromium, Lead, Zinc, or Ca

Coatings of Lead and Lead-Tin Alloys on Steel and Fer

Coatings of Petroleum $\mathbb{W}$ ax or $W$ ax Based Blends Applied T

Coatings of Tin-Lead Alloy (Solder Plate) (1973)

Coatings of Tin (1972)

Coatings on Aged or Backed Aluminum Alloy Panels (Coil)

Coatings on Aluminum (1972) ANSI G53.30

Coatings on Aluminum (1973)

Std. Spec. for Anodic Oxi

Coatings on Aluminum (1973) Std. Spec, for Anodic Oxide

Coatings on Metallic Surfaces (Coil) (1974)

Coatings on Metallic Surfaces (Coil) (1974)

Coatings on Nonferrous Substrate (Coil, Instrumentation

Coatings on Steel (1972)

Coatings on Wood Products During the Curing Cycle (1973

$\mathrm{N}$, Oth $\mathrm{NCCA}$

NCCA

Coatings Used for Electrical Insulation (1973)

Coatings Used for Electrical Insulation (1973) Std. Met

Coatings When Applied to Coil Coated Metal (1974)

Coatings with Impact Wedge Bend Apparatus (1973)

Coatings (Blunt Rod) (1972)

Coatings (Falling Weight Test) (1972)

Coatings (Limestòne Drop Test) (1972)

Coatings (Paint), and Handling of Flammable and Combust

Coatings (Paints) for Light Water Nuclear Reactor Conta

Coatings (1966)

Coatings (1969) ANS1 Z167.22

Coatings (1970) ANSI A 109.11

Coatings (1971)

Coatings (1972)

Coatings (1972)

Coatings (1972)

Coatings (1972)

Coatings (1972)

Coatings (1972)

Coatings (1972)

Coatings (1972)

Coatings (1972)

Coatings (1972)

Coatings (1973)

Coatings (1973)

Coatings (1973)

Coatings (1973)

Coatings (1973)

Coatings (1973)

Coatings (1973)

Meth. Fo ASTM
ASTM

ASTM

ICBO

ASTM

ASTM

ASTM

NCCA

ASTM

ASTM

ASTM

/Ter NCCA

ASTM

ASTM

ASTM

ASTM

ICBO

ANSI

Std AMCA

Std ASTM

Std. Met ASTM

IAPMO

ASTM

ASTM

ASTM

ASTM

ASTM

ASTM

ASTM

ASTM

Std. M ASTM

Rec. Spec. for

NAPCA

ASTM

ASTM

ASTM

Std. Meth ASTM

Std Meth O ASTM

(1974)

Std.

Coatings) with Electromagnetic and Particulate Radiatio

Coaxial Connectors for CATV (Cable Television) Applicat

Cobalt-Beryllium Alloy (Copper Alloy No. 175), Plate,

Cobalt-Molybdenum-Chromium (1972) ANSI G35.26

Cobalt Alloy Plate, Sheet, Strip, and Rolled Bar (1973)

Cobalt Base-25.5Cr-10.5 Ni-7.5W (1973)

/Alysis of High Temperature, ASTM

TB-IIII.5

$4 \cdot 10$

B567

B568

B 487

D 1469

TB. 11.2

TB.I1.8

TB. 11.3

TB.II.1

TB.II.7

A386

C664

AMS2485F

TB.II.12

TB. 11.5

TB-II. 11

TB.II.9

TB.II. 15

TB.II. 6

TB.III.3

TB.II. 16

TB.II. 4

TB.II. 18

4
-5

TB.II. 10

N101.4

G19

B588

D1653

RS*1

D3273

D960

UBCS 32.6

B200

D3234

B579

B545

TB-11.2

B 136

B580

B580

TB.III. 6

TB-III-1

TB.II. 14

G12

D3259

D3214

D3214

D3281

G17

G14

G13

UFC *2ART 34

N101.2

2601

C633

D255

PS22

B571

D 1467

D3062

G10

G20

G6

G9 
ck for High Tempe/

Std. Spec. for Precipitation Hardening
Std. Meth. of Test for Determination of $h$ at Elevated Temperatures (1973) A/ Std. Spec. for Iron, Water Absorptiveness of Nonbibulous Paper and Paperboard ultry Mashes (Feed) for Prevention of Cecal and Intestinal try Feeds and Premixes for Control of Cecal and Intestinal Rec. Pract. and Std. Covering Design and Construction of of Test for Effectiveness of Aerosol Insecticides Against Std. and Test Procedure for Water Closet Flush Tank Ball Fibres (Cotton, Sisal, Henequen, Ixtle, Jutes, Hemp, Tow, Meth. of Analysis of Crude Fat in in Fats and Shortenings (Butter, Oleomargarine, High Acid ic, and Sunflower Seeds; Ground Nuts (Peanuts); Vegetable, Std. for Engine Test U.S. Model Rocket Sporting and Safety Std. for Engine Rating Std. Test

se Mounted Drum Hoists (1971)

s (Electronics) (1973) ANSI C57.12.90

Safety

Std. Test

Std. Test

orms (1972)

Southern Building

tation of the Control Characters of American National Std. Std. Safety Color Std. for Lighting Inspection

Std. for

Sid.

on Systemis (1974)

cturës (1970) ANSI A9.1

Safety

vacate, Rehabilitate, Demolish) (1973)

use of Explosives and Blasting Agents (1973) ANSI Z/

Uniform

Std. aterials (Fire Protection) (1973)

Rec. Rec. Std. Safety and Comments on the National Electrical Code (NFPA 70) and and Other Structures (1972)

89.1

ansi A 169.1

aci 322

to Wood Framing (1973)

artitions (W all, Ceiling, Floor, and Fl/

ed Clay Masonry Units (1973)

Stock and Vapor RemovaI (1973)

from Clay or Shale) (1973)

(1973)

73)

ensity of Smoke from the Bürning or Dec $s$ or Systems (1973)

grading for All Species of Lumber (1973/

north), Eastern Hemlock-Tamarack (Nor/ rcement (1973)

guishment) (1973)

and Shingles) (1973)

Gravel and Air Cooled Iron Blast Furna/

ted Coatings of Copper, Nickel, Chromiu/ h of Molded Concrete Cylinders (1973)

lock; Western Red Cedar; White Fir; And/ Idaho White, Sugar, and Lodgepole Pine/ Tamarack: Balsam Fir; Northern Pine An/ $\mathrm{e}$ and Hemlock, Balsam Fir and Tamarack/ Painting of Structural Steel (1973)

FieId Concrete Cylinders (1973)

973)

ural Insulating Board (1973)

ngs (1973)

973)

aterials (1973)

d. for Building

Std. Building

Std. for Building

Std. for Building

Uniform Building

Uniform Building

Uniform Building

Uniform Building

Uniform Building

Uniform Building

Uniform Building

Uniform Building

Uniform Building

Uniform Building

Uniform Building

Uniform Building

Uniform Building

Uniform Building

Uniform Building

Uniform Building

Uniform Building

Uniform Building

Uniform Building

Uniform Building

Uniform Building

Uniform Building

Uniform Building

Uniform Building

Uniform Building

Uniform Building

Uniform Building

Uniform Building

Uniform Building

Uniform Building

Uniform Building

Uniform Building

Uniform Building

Uniform Building

Uniform Building

Uniform Building

Uniform Building

Uniform Building

Uniform Building

Uniform Building

Uniform Building

Uniform Building
Cobalt Containing Alloy Bars, Forgings, and Forging Sto

Cobalt in Paint Driers by EDTA Meth. (1973)

Cobalt, and Nickel Base Alloy Castings for High Strengt Std. Meth. of Test for ASTM

Coccidiosis in Chickens (Cereal Chemistry) (1962)

Coccidiosis (Cereal Chemistry) (1962)

Cockpits and Scuppers (Boats) (1972)

Cockroaches (1972)

Cocks (1968)

Cocoa Fibers, Oakum, Rags, Waste Cloth, Wastepaper, Kap
Cocoa (Cereal Chemistry) (1962)

Coconut Oil, etc.) (Cereal Chemistry) (1962)

Coconut, and Palm Oils) (1974)

Code-Spark Ignition and Diesel (1971)

Code and Engine Std. (1973)

Code Diesel (1971)

Code for Air Moving Devices (1967)

Code for Architectural Design Competitions (1972)

Code for Cranes, Derricks, Hoists, Jacks and Slings: Ba

/Oxalin
paper,
, Saffl
s: Ba

Cod

Code for Distribution, Power and Regulating Transformer

Code for Dwelling House Construction (1973)

Code for Fabricated Stage and Scaffold Planks and Platf

Code for Identification of Piping Systems Content (1973

Code for Information Interchange (1973)

Code for Marking Physical Hazards (1971)

Code for Motor Vehicles (1973)

Code for Power Piping (1973)

Code for Pressure Petroleum Refinery Piping (1973)

Code for Pressure Piping: Liquid Petroleum Transportati

Code for Safety to Life from Fire in Buildings and Stru

Code for Semiconductor Pöwer Converters (1973)

Code for Shipboard Hull Vibration Measurements (1969)

Code for the Abatement of Dangerous Buildings (Repair,

Code for the Manufacture, Transportation, Storage, and

Code for Transportation, Storage and Use of Explosive M

Code for Vertical Shoring (1968)

Code Requirements by OSHA (1973)

Code Requirements for Minimum Design Loads in Buildings

Uniform Building

Uniform Building
Coquirements for Reinforced Concrete (1971) ANSI a

Code Requirements for Structural Plain Concrete (1972)

Code Requirements for Structural Plain Concrete (1972)

Code Std. for Adhesives for Fastening Gypsum Wallboard

Code Std. for Adhesives (1973)

Code Std. for Admixtures for Concrete (1973)

Code Std. for Aggregate for Masonry Mortar (1973)

Code Std. for Aggregates for Grout (1973)

Code Std. for Airborne Sound Insulation Field Test of P

Code Std. for Aluminum Structures (1973)

Code Std. for and Meth. of Sampling and Test. of Unburn

Code Std. for Asbestos Cement Roofing Shingles (1973)

Code Std. for Blower, Fan, and Exhaust System for Dust,

Code Std. for Building, Facing, and Hollow Brick (Made

Code Std. for California Redwood Lumber (1973)

Code Std. for Carbon Dioxide Fire Extinguishing Systems

Code Std. for Cast Building Stone (1973)

Code Std. for Cellulose Nitrate Motion Picture Film (19

Code Std. for Chamber Meth. of Test for Measuring the D

Code Std. for Class II and Class III Dry Cleaning Plan

Code Std. for Classification, Definition, and Meth. of

Code Std. for Coast Sitka Spruce, Douglas Fir-Larch (

Code Std. for Cold Drawn SteeI Wire for Concrete Reinfo

Code Std. for Combination Standpipe Systems (Fire Extin

Code Std. for Composition Roofing (Class C Sheet (Roll)

Code Std. for Concrete Aggregates (Sand, Crushed Stone,

Code Std. for Concrete Building Brick (1973)

Code Std. for Concrete Reinforcement Bars (1973)

Code Std. for Concrete Tests (1973)

Code Std. for Corrosion Resistant Metals (Electrodeposi

Code Std. for Determining the Splitting Tensile Strengt

Code Std. for Douglas Fir, Coast Region; West Coast Hem

Code Std. for Douglas Fir; Larch; Hem-Fir; Ponderosa,

Code Std. for Eastern Spruce, White Pine, and Hemlock-

Code Std. for Eastern White and Red Pine, Eastern Spruc

Code Std. for Erection, Fabrication, Identification and

Code Std. for Evaluation of Compression Test Results in

Code Std. for Expansion Index Test for Soil (1973)

Code Std. for Exterior Plaster Liquid Bonding Agents (1

Code Std. for Fiberboard Nail Base Sheathing and Struct

Code Std. for Field Tests for Grout and Mortar (1973)

Code Std. for Fire Alarm Systems for High-Rise Buildi

Code Std. for Fire Dampers (1973)

Code Std. for Fire Retardant Roof Covering Materials (1

Code Std. for Fire Tests of Building Construction and M

Code Std. for Fire Tests of Door Assemblies (1973)

Code Std. for Fire Tests of Window Assemblies (1973)
SSI

Rec. FMS

ANSI

ACI

ACI

ANSI

ICBO

ICBO

ICBO

ICBO

ICBO

ICBO

ICBO

ICBO

ICBO

ICBO

IC BO

ICBO

ICBO

ICBO

ICBO

ICBO

ICBO

ICBO

ICBO

ICBO

ICBO

ICBO

ICBO

ICBO

ICBO

ICBO

IC BO

ICBO

ICBO

ICBO

ICBO

ICBO

ICBO

ICBO

ICBO

ICBO

ICBO

ICBO

ICBO

ICBO

ICBO

ICBO

ICBO

D328

Po AACCH 18.25

AACCH 18.35

$\begin{array}{ll}\text { ABYC } & \mathrm{H} 4 \\ \text { ASTM } & \text { D3095 }\end{array}$

ASSE 1002

ICBO UFC*2ART

AACCH 30.12

Matter AACCH 44.30

NIOP *1

SAE Ja16B

NAR *1

$\mathrm{J} 270$

AMCA $2 \mathrm{IO}$

$\begin{array}{ll}\text { AIOA } & \text { J331 } \\ \text { ANSI } & \text { B } 30.7\end{array}$

IEEE 113

IEEE 262

SBCC $* 18$

ALI *1

9.9

$\mathrm{X} 3.32$

Z53.1

J599C

B31.1

ANSI B31.3

ANSI B31.4

NFPA 101

NEMA PV 3

SNAME C.]

ICBO UBC $* 6$

NFPA 495 
73)

Veneer Plaster (1973)

(1973)

and Bolted Parts (1973)

s (1973)

nits (1973)

ing Systems (1973)

973)

roofing) (1973)

of Airborne Sound Transmission Class (S) crete (1973)

preservative Treatment by Pressure Proc/ fractory Bricks (1973)

ron (Shape, Plate, Sheet, Strip, Connec/ t Up Roof Coverings: Hot Mopped Asphalt/ ile and for Lay in Panel Ceilings (1973) tal Accessories (Designed for Use as a (1973)

her Than Gypsum (1973)

(1973)

for Gypsum Plaster (1973)

ents (1973)

crete Construction (1973)

(1973)

abs, and Poured Gypsum Roof Diaphragms /

ctangular and Square) Brass Wire (1973)

ratures) of Plastics Using a Hot Air Ig/ ite Construction (1973)

lling Systems (1973)

Industrial (1973)

oses (1973)

(1973)

and Rafters (1973)

uctural Members (1973)

ed Stainless Steel Structural Members (/ ingles with Asbestos Felt or Gypsum Boa/ ete Reinforcement (1973)

(Gasoline, Kerosene, Acetone, Alcohol,/

x-Ray Nitrocellulose Films (1973)

$\mathrm{n}$ of Structural Steel Members (1973)

d Std. Meth. of Sampling and Test. Stru/ (1973)

framing (1973)

od Stressed Skin and Curved Panels, Bea/

as and Hem Fir, Southern and Lodgepole /

oducts (1973)

er (1973)

Bolts, and Wood and Lag Screws; Light /
Uniform Building

Uniform Building

Uniform Building

Uniform Building

Uniform Building

Uniform Building

Uniform Building

Uniform Building

Uniform Building

Uniform Building

Uniform Building

Uniform Building

Uniform Building

Uniform Building

Uniform Building

Uniform Building

Uniform Building

Uniform Building

Uniform Building

Uniform Building

Uniform Building

Uniform Building

Uniform Building

Uniform Building

Uniform Building

Uniform Building

Uniform Building

Uniform Building

Uniform Building

Uniform Building

Uniform Building

Uniform Building

Uniform Building

Uniform Building

Uniform Building

Uniform Building

Uniform Building

Uniform Building

Uniform Building

Uniform Building

Uniform Building

Uniform Building

Uniform Building

Uniform Building

Uniform Building

Uniform Building

Uniform Building

Uniform Building

Uniform Building

Uniform Building

Uniform Building

Uniform Building

Uniform Building

Uniform Building

Uniform Building

Uniform Building

Uniform Building

Uniform Building

Uniform Building

Uniform Building

Uniform Building

Uniform Building

Uniform Building

Uniform Building

Uniform Building

Uniform Building

Uniform Building

Uniform Building

Uniform Building

Uniform Building

Uniform Building

Uniform Building

Uniform Building

Uniform Building

Uniform Building

Uniform Building

Uniform Building

Uniform Building

Uniform Building

Uniform Building

Uniform Building

Uniform Building

Uniform Building

Uniform Building

Uniform Building

Uniform Building
Code Std. for Glass, Its Defects and Safety Glazing (19

Code Std. for Gypsum Backing Board (1973)

Code Std. for Gypsum Base for Veneer Plaster and Gypsum

Code Std. for Gypsum Lath (1973)

Code Std. for Gypsum Molding Plaster (1973)

Code Std. for Gypsum Partition Tile or Block (1973)

Code Std. for Gypsum Plastics (1973)

Code Std. for Gypsum Sheathing Board (1973)

Code Std. for Gypsum Wallboard Tape and Joint Compound

Code Std. for Gypsum Wallboard (1973)

Code Std. for Gypsum (1973)

Code Std. for Hand Split Shakes (1973)

Code Std. for High Strength Steel Bolts, Nuts, Washers,

Code Std. for Hollow Load Bearing Concrete Masonry Unit

Code Std. for Hollow Nonload Bearing Concrete Masonry U

Code Std. for Hydrated Lime for Masonry Purposes (1973)

Code Std. for Impact Sound Insulation (1973)

Code Std. for in Place Density of Soils (1973)

Code Std. for Installation of Automatic Fire Extinguish

Code Std. for Installation of Fire Doors and Windows (1

Code Std. for Keene's Cement (1973)

Code Std. for Kraft Waterproof Building Paper (Weatherp

Code Std. for Laboratory Determination and Measurement

Code Std. for Lightweight Aggregates for Structural Con

Code Std. for Lime (1973)

Code Std. for Lumber, Timber, Plywood and Utility Pole

Code Std. for Machine Made and High Alumina Fireclay Re

Code Std. for Masonry Cement for Use in Mortars (1973)

Code Std. for Mat Formed Wood Particleboard (1973)

Code Std. for Material Spec. for Structural Steel and I

Code Std. for Materials for Use in Construction of Buil

Code Std. for Metal Suspension Systems for Acoustical T

Code Std. for Metal, Wire and Wire Fabric Laths, and Me

Code Std. for Meth. of Test for Concrete Masonry Units

Code Std. for Mineral Roofing Aggregates (1973)

Code Std. for Moisture Density Relations of Soils (1973

Code Std. for Mortar for Unit and Reinforced Masonry Ot

Code Std. for Natural Slate Roof Shingles (1973)

Code Std. for Noncombustible Elementary Materials Test.

Code Std. for Open Web Steel Joist Design (1973)

Code Std. for Perlite, Vermiculite and Sand Aggregates

Code Std. for Plastic Materials (1973)

Code Std. for Portland Cement and Blended Hydraulic Cem

Code Std. for Prestressed Steel Strand and Wire for Con

Code Std. for Processed Pulverized Quicklime (1973)

Code Std. for Proscenium Curtains (1973)

Code Std. for Quicklime (Lime) for Structural Purposes

Code Std. for Ready Mixed Concrete (1973)

Code Std. for Reinforced Gypsum Concrete and Precast SI

Code Std. for Roofing Asphalt (1973)

Code Std. for Roofing Tile (1973)

Code Std. for Round Timber Piles (1973)

Code Std. for Round, Hexagonal, Octagonal, and Flat (Re

Code Std. for Sampling and Test. Brick (1973)

Code Std. for Sand-Lime Building Brick (1973)

Code Std. for Sawn Wood Shingles (1973)

Code Std. for Self and Flash Ignition Properties (Tempe

Code Std. for Shear Connectors (Steel Studs) for Compos

Code Std. for Sheet Metals (1973)

Code Std. for Smoke Detectors for Fire Protective Signa

Code Std. for Softwood Plywood Lumber, Construction and

Code Std. for Soils Classification for Engineering Purp

Code Std. for Solid Load Bearing Concrete Masonry Units

Code Std. for Southern Pine Lumber (1973)

Code Std. for Spaced Column Design (1973)

Code Std. for Span Tables for Floor and Ceiling Joists,

Code Std. for Spec. and Design of Cold Formed Steel Str

Code Std. for Spec. and Design of Light Gauge Cold Form

Code Std. for Special Purpose Roofs (Wood Shakes and Sh

Code Std. for Steel Bar and Rod Mats (Sheets) for Concr

Code Std. for Storage and Handling of Flammable Liquids

Code Std. for Storage and Handling of Photographic and

Code Std. for Stress Variation or Stress Reversal Desig

Code Std. for Structural Clay Floor Tile (1973)

Code Std. for Structural Clay Load Bearing Wall Tile an

Code Std. for Structural Clay Nonload Bearing Wall Tile

Code Std. for Structural Floor and Roof Plank and Beam

Code Std. for Structural Glued Built Up Members-Plywo

Code Std. for Structural Glued Laminated Timber (1973)

Code Std. for Structural Glued Laminated Timbers: Dougl

Code Std. for Structural Rivet Steel (1973)

Code Std. for Test for Glue Joints in Laminated Wood Pr

Code Std. for Test. Gypsum Plasters and Concrete (1973)

Code Std. for Tests for Structural Glued Laminated Lumb

Code Std. for Timber Connector and Bolted Joints, Drift
Code Std. for Tin Clad Fire Doors and Shutters (1973)

ICBO

ICBO

ICBO

ICBO

$\mathrm{ICBO}$

ICBO

ICBO

ICBO

ICBO

ICBO

IC BO

IC BO

ICBO

IC BO

ICBO

IC BO

ICBO

ICBO

ICBO

IC BO

ICBO

ICBO

ICBO

ICBO

IC BO

ICBO

ICBO

ICBO

IC BO

ICBO

IC BO

ICBO

ICBO

ICBO

ICBO

IC BO

ICBO

ICBO

ICBO

IC BO

ICBO

ICBO

ICBO

ICBO

ICBO

IC BO

ICBO

ICBO

ICBO

ICBO

ICBO

ICBO

ICBO

ICBO

ICBO

ICBO

ICBO

ICBO

ICBO

IC BO

ICBO

ICBO

IC BO

ICBO

ICBO

ICBO

ICBO

ICBO

ICBO

ICBO

ICBO 
3)

c for Concrete Reinforcement (1973) and Connections in Reinforced Concrete /

nd Axial Loading Combined (1973)

ative Treatment (1973)

Building Materials (1973)

Uniform Building Code Std. for Veneer Application Dampers (1973) Rec. and Comments on the National Electrical aintenance of Heating, Ventilating, C/

Rec. Pract. for Lighting Identification

Flammable and Combustible Liquids

Std. for Agricultural Tractor Tes

Agricultural Tractor Test

National Electrical Safety

Uniform Swimming Pool

Dwelling Construction Under the Uniform Building

Std. for Instrumentation Symbols and Identification

Std. for Magnetic Stripe Encoding

cryst/

tion Intercha

Std. Merh. for Assigning Alloy Phase Designations (National Board Inspection

Std. for Structure for the Identification es for the Radio Installations (National Electrical Safety ric Supply Lines and Equipment (National Electrical Safety ng AC and DC Electrical Wiring on Boats and Engines (Color upply and Communications Lines (National Electrical Safety

ations, Rod End Configurations, Dimensional Identification e of Electrical Supply Statio/

(Including the District of Colu/

National Electrical Safety nstructions, and Conta/

Rec: Pract. for Symbols and Color Guide for Ceramic Tile in Building

Is (1973)

, Lacquer, Stain, etc.) (1973)

ial Handling Plants (1973)

Dwelling Construction Under the Uniform Building

uterium, Fluorine, Helium, Hydrogen, Krypto/

73)

terations (1973)

Dwelling Construction Under the Uniform Building

Dwelling Construction Under the Uniform Building Dwelling Construction Under the Uniform Building

73)

Dwelling Construction Under the Uniform Building Uniform Fire

Uniform Plumbing Uniform Fire Uniform Fire Uniform Fire Uniform Building Uniform Fire Uniform Fire cial Wastes (1973)
Uniform Building

Uniform Building

Uniform Building

Uniform Building

Uniform Building

Uniform Building

Uniform Building

Line

Std. Fastener

National Std. Plumbing

Uniform Mechanical

Uniform Housing

Std. Lifeboat

Structural Welding Uniform Buildin Uniform Sign Uniform Fire

Uniform Plumbin

Southern Std. Gas

Southern Std. Building

Std. for Identification Uniform Building Uniform Fire

Uniform Building Uniform Fire Uniform Fire Uniform Fire

Uniform Plumbin Uniform Fire Uniform Fire Uniform Fire Uniform Fire

Uniform Building Uniform Fire

Uniform Building

Uniform Plumbing

Uniform Building

Uniform Building

Uniform Building

Uniform Building Uniform Fire

Uniform Building

Uniform Building

Uniform Building

Uniform Building Uniform Fire

Uniform Plumbing

Uniform Fire

Code Std for Water Resistant Gypsum Backing Board (197

Code Std. for Welded Steel Wire and Deformed Wire Fabri

Code Std. for Welding Reinforcing Steel, Metal Inserts

Code Std for Welding (1973)

Code Std for Wood Members Subjected to Both Flexural a

Code Std. for Wood Poles That Are to Be Given a Preserv

Code Std. Test. Meth. for Fire Hazard Classification of

Code System for Welding Wire Identification (1973)

Code System for Welding Wire Identification (1973)

Code Words for Aluminum Electrical Conductor (1972)

Code (Aerospace Lockbolts, Rivets, Bolts) (1973)

Code (Including Definitions) (1973)

Code (NFPA 70) and Code Requirements by OSHA (1973)

Code (Requirements for the Installation, Control, and M

Code (Residential Buildings) (1973)

Code (1968)

Code (1971)

Code (1972)

Code $(1972)$

Code (1972)

Code (1972) SAE J708C

Code (1973)

Code (1973)

Code $(1973)$

Code (1973)

Code (1973)

Code (1973)

Code (1973)

Code (1973)

Code (1973)

Code (1973)

(Code) for Credit Cards (1973)

(Code) in Metallic Systems Based on the Composition and Code) Manual for Boiler and Pressure Vessel Inspectors

(Code) of the Countries of the United States for Informa

Code) (1940)

Code) (1973)

Code) (1973)

Rules for the Operation of Elect

Code, and Mounting Dimensions for $3 / 4,1$ and $11 / 8 \mathrm{In}$.

Requirements and Rec. Meth. of Identif y

Code, Part 1: Rules for the Installation and Maintenanc

Codes and Abbreviations for States of the United States

Codes for Lubricant and Fluid Requirement Maintenance I

Codes (1972)

Code: Agricultural Buildings (1973)

Code: Airports, Heliports, Helistops and Aircraft Hange

Code: Aluminum (1973)

Cade: Application of Flam mable Finishes (Paint, Varnish

Code: Automobile Tire Rebuilding Plants (1973)

Code: Automobile Wrecking and Junk Yards or Waste Mater

Code: Battery Drainage System (1973)

Code: Bow ling Alleys (1973)

Code: Cellulose Nitrate Motion Picture Film (1973)

Code: Cellulose Nitrate Plastics (Pyroxylin) (1973)

Code: Chimneys, Fireplaces, and Barbecues (1973)

Code: Compressed Gases (1973)

Code: Concrete (1973)

Code: Cryogenic Fluids (A ir, Argon, Carbon Monoxide, De

Code: Definitions and Abbreviations (1973)

Code: Drainage Systems (1973)

Code: Elevators, Dumbwaiters, Escalators, and Moving Wa

Code: Excavation and Grading (1973)

Code: Excavations, Foundations, and Retaining Walls (19

Code: Existing Buildings; Minimum Safety Requirement Al

Code: Exits and Stairs (1973)

Code: Explosives and Blasting Agents (1973)

Code: Film Storage and Handling (Photographic and X-Ray

Code: Fire Extinguishing Systems (1973)

Code: Fire Resistive Std. (1973)

Code: Fire Zone Requirements (1973)

Code: Fireworks (1973)

Code: Fixtures (1973)

Code: Flammable and Combustible Liquids (1973)

Code: Flooring (1973)

Code: Foundations and Retaining Walls (1973)

Code: Framing-General (1973)

Code: Fruit Ripening Process (Banana, Citrus Fruit) (19

Code: Fuel Gas Piping (1973)

Code: Fumigation and Thermal Insecticidal Fogging (1973

Code: Garage (1973)

Code: General Precautions for Prevention (Incinerator a

Code: Glass and Glazing (1973)

Code: Hazardous Chemicals (1973)

Code: High Piled Combustible Stock (Storage) (1973)

Uniform Plumbing Code: Indirect Waste Piping, Wet Vented Systems and Spe
ICBO

ICBO

ICBO

ICBO

ICBO

ICBO

ICBO

SAE

SAE

AA

NSA

$\mathrm{NAPH}$

FMS

IC BO

ICBO

SNA

SAE

AWS

NFPA

SAE

ASAE

ANSI

IAPMO

ICBO

ICBO

ICBO

ICBO

ICBO

ISA

SBCC

SBCC

A NSI

ASTM

NBBPV

ANSI

ul ANS

ANSI

ABYC

ANSI

NFLDP

ANSI

ANSI

SAE

TCA

ICBO

ICBO

ICBO

ICBO

ICBO

ICBO

$1 \mathrm{CBO}$

ICBO

$1 \mathrm{CBO}$

ICBO

ICBO

ICBO

CBO

ICBO

ICBO

ICBO

ICBO

ICBO

ICBO

ICBO

ICBO

ICBO

ICBO

ICBO

ICBO

ICBO

ICBO

ICBO

ICBO

ICBO

ICBO

ICBO

CBO

ICBO

ICBO

IC BO

ICBO

ICBO

ICBO

$1 \mathrm{CBO}$

ICBO

UBCS30-1

BC 4.7 .13

UBC $26-6$

UBCS26-8

UBCS27-6

UBC $25-16$

UBCS25-13

UBCS 42 -1

A.MS2815M

A.MS2816K

*31

523 
Uniform Fire Code: Industrial Baking and Drying Ovens (1973)

Uniform Building Code: Interior Walls and Ceiling Finish (1973)

Uniform Plumbing Code: Joints and Connections (1973)

ection)/Dwelling Construction Under the Uniform Building ctor (1) Dwelling Construction Under the Uniform Building

Dwelling Construction Under the Uniform Building Uniform Fire Uniform Fire Uniform Fire Uniform Fire

ling of Flammable and Combustible Liquids A/ becues (1973)

Uniform Building niform Building Uniform Fire Uniform Fire Uniform Plumbing Uniform Plumbing Uniform Plumbing

Uniform Building Uniform Fire

(1973)

Uniform Building Uniform Fire

Uniform Building

Uniform Building

Uniform Building

Uniform Building Uniform Fire

randstands, Bleachers, etc.) (1973) Dwelling Construction Under the Uniform Building Dwelling Construction Under the Uniform Building
Dwelling Construction Under the Uniform Building
nels, Skylights, Monitors and Sawtooth / Uniform Building Uniform Fire Dwelling Construction Under the Uniform Building Uniform Fire nd Controls, Barrier, Road, Hydrant, Drivew/

Uniform Plumbing Uniform Plumbing Uniform Fire 73) Corrosion for Underground Pipe, Fittings / ection and Tanks Containing Flammable or $\mathrm{Co}$ ions Over Public Property (Pedestrian P/ Uniform Fire Uniform Building Uniform Building d Wall Furnaces, Floor Furnaces, and /

Uniform Mechanical Uniform Fire

Uniform Building Dwelling Construction Under the Uniform Building Dwelling Construction Under the Uniform Building

Uniform Plumbing

Uniform Building Uniform Fire

cavity /

Dwelling Construction Under the Uniform Building Dwelling Construction Under the Uniform Building Uniform Building Uniform Building Uniform Building Uniform Building Uniform Fire Uniform Fire

ewing Stands, Bleachers, and Grandstand/

n, Sisal, Henequen, lxtle, Jutes, Hemp, Tow/

Uniform Plumbing Uniform Fire Uniform Plumbing ng) (19/ Dwelling Construction Under the Uniform Building tic, etc.) for Ornamentation, Protectio/ Uniform Building Dwelling Construction Under the Uniform Building

Uniform Plumbing

Uniform Building

Dwelling Construction Under the Uniform Building Dwelling Construction Under the Uniform Building Uniform Plumbing

Uniform Plumbing

Uniform Building Uniform Fire

concrete; Earth; Glass; Granite; Gravel/

e (1973)

d and Components, Timber, Preservative

Uniform Building

ast and Wrought (1973)

Std. Rec. Pract. for

scl Std. Underwater Accident Report Form (Acquisition and Industry Quality Std. Format and Std. for Color

Std. for Service Time Rec. Pract. for

e Fittings (1972) Rec. Std. Door Type Nomenclature (1973) Std. Meth. for Measuring Hall Mobility and Hall Solid Film Lubricants in Vacuum /

Std. Meth. of Test for Meth. of Test for

ANSI Z11.222

fiberboard (Horizontal Plane Meth/

fiberboard (Inclined Plane Meth.)/ Tent. Rec. Pract. for Measurement of Glass Stress Optical re, 50 to $120 \mathrm{KSl}$ Yield Strength, Materials with Expansion
Code: Legal Requirements (Permit, Plans and Spec., Insp

Code: Light, Ventilation, Sanitation, Fire Warning Dete

Code: Location on Lot (1973)

Code: Lumber Yards and Woodworking Plants (1973)

Code: Magnesium (1973)

Code: Maintenance of Exit Ways (1973)

Code: Manufacture of Organic Coatings (Paint), and Hand

Code: Masonry or Concrete Chimneys, Fireplaces, and Bar

Code: Masonry (1973)

Code: Matches (1973)

Code: Mechanical Refrigeration (1973)

Code: Medium Pressure Gas Piping Systems (1973)

Code: Minimum Facilities Required for School, Theater,

Code: Mobile Home (Trailer) Parks (1973)

Code: Motion Picture Projection Rooms (1973)

Code: Motion Picture Projection (1973)

Code: Occupancy and General Requirement Classification

Code: Oil Burning Equipment (1973)

Code: Patio Covers (1973)

Code: Penthouses and Roof Structures (Tower, Spire) (19

Code: Permanent Occupancy (1973)

Code: Permits and Inspections (1973)

Code: Places of Assembly (Theaters, Reviewing Stands, G

Code: Plastering and Installation of Wallboard Lathing

Code: Plastering (1973)

Code: Plastics (General, Exterior Light Transmitting $\mathrm{Pa}$

Code: Prefabricated Construction (1973)

Code: Prevention of Dust Explosions (1973)

Code: Private Garages and Carports (1973)

Code: Protection, Authority, Departmental Regulations a

Code: Rainwater Systems, Roof Drains and Piping (1973)

Code: Rec. Rules for Sizing the Water Supply System (19

Code: Recommended Guide to Safe Practice Protection Fro

Code: Recommended Safeguards and Practices for the Prot

Code: Regulations for Use of Public Streets and Project

Code: Regulations Governing Fallout Shelters (1973)

Code: Requirements for Vented Decorative Appliances, an

Code: Rifle Ranges (1973)

Code: Roof Construction and Covering (1973)

Code: Roof Coverings (1973)

Code: Roof Framing (1973)

Code: Sewers and Private Sewage Disposal Systems (1973)

Code: Skylights (1973)

Code: Smoking (1973)

Code: Softwood Plywood Paneling (1973)

Code: Solid, Grouted, Reinforced Grouted, Hollow Unit,

Code: Sound Transmission Control (Acoustics) (1973)

Code: Stages and Platforms (1973)

Code: Stairs, Exits and Occupant Loads (Buildings, Revi

Code: Steel and Iron (1973)

Code: Storage and Handling of Combustible Fibres (Cotto

Code: Suppression and Control of Hazardous Areas (1973)

Code: Swimming Pools (1973)

Code: Tents and Air Supported Structures (1973)

Code: Traps and Interceptors (1973)

Code: Valley Flashing (Sheet Metal for Roof Waterproofi

Code: Veneer (Brick, Concrete, Stone, Tile, Metal, Plas

Code: Veneered Walls (1973)

Code: Vents and Venting (1973)

Code: Wall and Ceiling Coverings (1973)

Code: Wall Framing and Weather Protection (1973)

Code: Wallboard (1973)

Code: Water Distribution (1973)

Code: Water Heaters and Vents (1973)

Code: Weights of Materials (Brick; Cast lron; Cinders;

Code: Welding and Cutting, Calcium Carbide and Acetylen

Code: Wood Members and Their Fastenings (Lumber, Plywoo

Codification of Certain Nonferrous Metals and Alloys, C

Codification of Data Derived from the lnvestigation of

Coding for Computer Output Microfilm (1971)

Coding of Electrical Wires and Cables (1974)

Coding of Propulsion Engine ltems (1971) ANSI B141.8

Coding System for ldentification of Tube, Pipe, and Hos

Coding) (1972)

Coefficient in Extrinsic Semiconductor Single Crystals

Coefficient of Friction and Wear Characteristics of Dry

Coefficient of Friction, Yarn to Metal (1972T)

Coefficient of Kinetic Friction for Wax Coatings (1973

Coefficient of Static Friction of Corrugated and Solid

Coefficient of Static Friction of Corrugated and Solid

Coefficient (1973)

Coefficients Comparable to Austenitic Steels (1970) Ans
Code: Liquefied Petroleum Gases (1973)
ICBO

ICBO

ICBO

$1 \mathrm{CBO}$

ICBO

ICBO

ICBO

ICBO

ICBO

ICBO

ICBO

ICBO

ICBO

ICBO

ICBO

ICBO

ICBO

ICBO

ICBO

ICBO

ICBO

ICBO

ICBO

ICBO

ICBO

ICBO

ICBO

ICBO

ICBO

ICBO

ICBO

ICBO

ICBO

ICBO

ICBO

ICBO

ICBO

ICBO

ICBO

$1 \mathrm{CBO}$

ICBO

ICBO

ICBO

ICBO

ICBO

ICBO

ICBO

ICBO

ICBO

ICBO

ICBO

ICBO

ICBO

ICBO

ICBO

ICBO

ICBO

ICBO

ICBO

ICBO

ICBO

ICBO

ICBO

ICBO

ICBO

ICBO

ICBO

ICBO

ICBO

ICBO

ICBO

ASTM

NMA

NEM

$S A E$

$S A E$

STDI

ASTM

ASTM

ASTM

ASTM

ASTM

A TTM

ASTM

ASTM

$\mathrm{UFC} * 2 \mathrm{ART} 25$

UBC $* 3.42$

UPC $* 1.8$

UBC $* 8 \cdot 1$

$\mathrm{UBC} * 8.3$

UFC $* 2 A R T 20$

UBC $* 8.2$

UFC *2ART21

UFC *2ART22

UFC *2ART 10

UFC *2ART34

$\mathrm{UBC} * 3.37$

UBC *3-24

UFC *2ART23

UFC *2ART28

UPC * 1 . F 
National Bureau of Standards KWIC Index of Engineering Standards

w) (1968)

33. II. 1

Griddles, etc.) (1973)

Std. for Safety for Household Electric atul Std. Meth. of Test for Resistance to Deformation and ement (1972) Std. Meth. of Test for Adhesion and Z167.22

71) Std. Meth. for Adhesion or Std. for Sound Rating of Room Fan Std. Meth. of Salt Spray (Fog) Test. for Spec. Purposes Std. Spec. for Machine and Sprocket nd Blocking Resistance of Organic Coatings When Applied to determination of Relative Pencil Hardness of a Paint Film r Determination of Film Adhesion by Cross Hatch Tape Test of Mill Chemical Treatments on Galvanized Steel Surfaces solution for Detecting Chromate Films on Galvanized Sheet t of Film Thickness of Nonmagnetic Finishes on Mild Steel resistance of Painted Aluminum, Steel and Gavanized Steel

d or Coated Specimens Subjected to Corrosive Environments 1 Metals by Cross Hatch Tape Test After Reverse Impacting Related Products - Film Thickness of 0.3 MIL or Greater the Determination of Deg. of Cure of a Backed Paint Film and Fle xibility by the $\mathbb{W}$ edge Bend (Applicable to Solution Std. Meth. for Measuring Chalk Resistance of Exterior nd Conditioning) (1966)

radation of Electrical Insulating Varnishes by the Helical Safety Std. for Room Type Fan . for Determination of Phosphate Coating Weights on Steel -Humidity Test. of Organic Coatings on Metallic Surfaces for Conversion Coating on Aged or Baked Aluminized Steel $n$ of Test Panels for Use in Test. Organic Coating Systems Weight on Hot Dip Galvanized and Electrogalvanized Steel nversion Coatings on Aged or Backed Aluminum Alloy Panels acquer, and Related Organic Coatings on Metallic Surfaces troleum) Gas Cyli Std. for Safety for Pigtail, Expansion ination of Nonconductive Coatings on Nonferrous Substrate
Std. for Smooth Wall si $\mathrm{H} 38.18$ Std. Spec. for Aluminum Alloy Extruded Round Std. for Consumable Electric Covered Bare Straight and Std. Part Pins, Spring, Slotted and Rec, for

Std. for Forced Circulation Air Cooling and Air Heating tems for AC Electric Machinery Employing Form Wound Stator Di/ Std. Spec. for Steel Sheets of Structural Quality in laundry Dryer) (1974)

(Laundry Washer) (1974)

Std. for Safety for Electric Meth. for Top Side of Filled Paper as Indicated by Silver 74) Std. Meth. for Calculating Coal and std. Meth. of Laboratory Sampling and Analysis of Coal and Std. Meth. of Drop Shatter Test for Std. Definitions of Terms Relating to Coal and Std. Meth. of Test for Proximate A nalysis of Coal and d. Meth. of Test for Specific Gravity and Porosity of Lump . Meth. of Test for Ash in the Analysis Sample of Coal and h. of Test for Moisture in the Analysis Sample of Coal and h. of Test for Nitrogen in the Analysis Sample of Coal and est for Volatile Matter in the Analysis Sample of Coal and

for Carbon and Hydrogen in the Analysis Sample of Coal and total Sulfur in the Analysis Sample of Coal and Std. Meth. Std. Meth. for Ultimate Anaylsis of Coal and

3) ANSl Al49.12

(Wet Type) (1972) ANSl A 111.29

ease Type Sealants (1972)

ow Room Temperature (1966) An/

tory Brick and Shapes (1972) Ansi/ condenser Tubes (1973) ANSl B 125 and Flaring (1972)

ANSI A 50.3

g Steel Bars and Shapes (1/ Std. Spec. for Code Std. for Std. Spec. for Hot Rolled and Std. Spec. for
Hot Rolled and Std. Spec. for General Requirements for Hot Rolled and Std. Spec. for Std. Quality

kel Manganese Steel Bars I/ re for Nuclear Application/ plications (1972) ANSI Z17) ponent Std. Spec, for Hot Worked and d. Recommet Rolled and

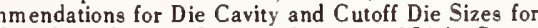
orm Building Code Std. for Spec, and Design of Light Gauge Uniform Building Code Std for Spec, and Design of Tubing in Rounds and Shapes (1973) ANSI / Std. Spec. for Std Tooling Nomenclature for Solid Die Two Blow ws (1971) ANSI G54.14
Std. Spec. for
Coefficients of Brick and Tile Walls (Tech. Notes) (196

Coefficients of Chopped Forages (Grass, Corn, Hay, Stra

Coefficients of Friction of Plastics (1972)

Coffee Makers and Brewing Type Appliances (1972) ANSI C (Coffee Pots and Urns, Food Dish Warmers, Hot Plates and Cohesion of Bituminous Mixtures by Means of Hveem Appar Cohesion of Elastomeric Joint Sealants Under Cyclic Mov Cohesive Strength of Flame Sprayed Coatings (1969) ANSI Coil Air Conditioners Delivering Less Than $1500 \mathrm{cfm}$ (19

(Coil Coating) (1974) ASTM Bl17

Coil Chain (1972) ANSI G61.5

Coil Coated Metal (1974)

(Coil Coating) (1974)

(Coil Coating) (1974)

(Coil Coating) (1974)

(Coil Coating) (1974)

(Coil Coating) (1974)

Coil Coating) (1974)

(Coil Coating) (1974)

(Coil Coating) (1974)

Coil Coating) (1974)

(Coil Coating) (1974)

Coil Coatings and Sing

Coil Coatings (1974)

Coil Meth. (1973)

Coil Units (

(Coil) (1974)

Coil) (1974)

(Coil) (1974)

Coil) (1974)

(Coil) (1974)

(Coil) (1974)

(Coil) (1974)

Coil, Instrumentation) (1974)

Coilable Polyethylene Electrical Plastic Duct (1972)

$R$ Film Thickness Determ

Coiled Welding Electrodes and Rods (1972)

Coiled, Heavy Duty (1973)

Coiling Polyethyle ne Plastic Pipe and Tubing (1972)

Coils (1972)

Coils (1972) ANSI C50.26

Coils, and Cut Lengths, Zinc Coated (Galvanized) by Hot

Coin Operated and Commercial Clothes Drying Equipment

Coin Operated and Commercial Clothes $\mathrm{W}$ ashing Equipment

Coin Test (1973)

Coke Analyses from As.Determined to Different Bases (19

Coke (1970) ANSI K18.1

Coke (1972) ANSI K20.24

Coke (1972) ANSI K20.6

Coke (1973)

Coke (1973)

Coke (1973)

Coke (1973)

Coke (1973)

Coke (1973)

Coke (1973)

Coke (1973)

Coke (1974)

Coking Value of Tar and Pitch (Modified Conradson) (197

Cold Bonding Strength of Air Setting Refractory Mortar

Cold Box Exposure of One Part, Elastomeric, Solvent Rel

Cold Chambers and Serving Units for Tests Above and Bel

Cold Crushing Strength and Modulus of Rupture of Refrac

Cold Drawn lntermediate Alloy Steel Heat Exchanger and

Cold Drawn Low Carbon Steel Tubing A nnealed for Bending

Cold Drawn Steel Wire for Concrete Reinforcement (1972)

Cold Drawn Steel Wire for Concrete Reinforcement (1973)

Cold Finished Age Hardening Stainless and Heat Resistin

Cold Finished Alloy Steel Bars (1964) ANSI G24.12

Cold Finished Bars; Hot Rolled Deformed Concrete Reinfo

Cold Finished Carbon and Alloy Steel Bar (1973) ANSI G2

Cold Finished Carbon Steel Bars (1973)

Cold Finished Stainless and Heat Resisting Chromium Nic

Cold Finished Zirconium and lis Alloy Bars, Rod, and Wi

Cold Finished Zirconium Bars and Wire for Nonnuclear Ap

Cold Finished, Special Quality, for Pressure Piping Com

Cold Forging Equipment (1972)

Cold Formed Stainless Steel Structural Members (1973)

Cold Formed Steel Structural Members (1973)

Cold Formed Welded and Seamless Carbon Steel Structural

Cold Formed Welded Structural Steel Tubing (I974)

Cold Headers (1972)

Cold Heading Quality Carbon Steel Wire for Machine Scre
BIA

ASAE

ASTM

UL

ANSI

ASTM

ASTM

ASTM

ARI

NCCA

AST M

NCCA pec. for NCCA

NCCA

NCCA

NCCA

NCCA

NCCA

NCCA
NCCA

NCCA

NCCA

NCCA

NCCA

AMCA

ASTM

UL

Spec NCCA

NCCA

NCCA

NCCA

NCCA

NCCA

NCCA

NCCA

UL

NCCA

NEMA

NSA

SPI

ARI

IEEE

ASTM

UL

UL

Test TAPP

ASTM

ASTM

ASTM

ASTM

ASTM

St ASTM

Std ASTM

tid. Met ASTM

Std. Met ASTM

ASTM

I ASTM

ASTM

ASTM

ASTM

ASTM

ASTM

ASTM

ASTM

ASTM

SAE

ASTM

ICBO

ASTM

ASTM

AISI

ASTM

ASTM

ASTM

ASTM

ASTM

ASTM

St IF

ICBO

ICBO

ASTM

WSTI

IFI

ASTM

4251

D3028

1082

Z21.31

1560

C719

C633

A 467

TB.II.17

TB.II. 12

TB.II.5

TB.[I.1]

TB. II.9

B.II.15

TB.II. 6

TB.III.3

TB.II.4

TB.II.18

TB.II. 10

TB.III.8

1402

D3145 
heet Metal Screws (1971) ANSI G.54.17

Std. Spec. for G54.15

G54.16

ansi G54.18

Std. Spec. for Steel Wire, Medium High Carbon Std. Spec, for Alloy Steel Wire, Alloy Std. Spec. for Std. Spec. for Aluminum Alloy Rivet and of Shear Testing of Aluminum and Aluminum Alloy Rivets and .24 Std. Spec. for Regular Quality Hot and Std. Spec. for

Std. Spec, for Steel, Carbon, Strip. Std Spcc for Steel d S/ General Spec. Applicable to Electromagnet Iron (EMl)
vanadium (/ Std. Spec. for Steel Sheet and Strip. Hot and arent Viscosity of Motor Oils at Low Temperature Using the

in.) (1971)

Std. Analytical Meth. for Determining Std. for Direct Reading Remote Registration Systems for

68) Rec. Pract for

notes) (1967)

ted Temperatures (1971) r High S/ Std. Spec. for Hot Worked, Hot Cold Worked, and $\mathrm{h}$ Strength at Elevated/ ent (1972) ANSl G45.2 orging Billets for High S/ Std. Spec, for Hot W orked, Hot
h at Elevated Temperatures (1971/ Std. Spec. for Hot, Hot Std. Spec. for Hot, Hot Cold, and Std. Spec. for ip for High Strength at Elevated/Std. Spec. for Hot. Hot y) (1962) Meth. of Test for Oil Absorption of Pigments by Gardner gs and Other Structures (1973) ow Loads (1973)

Meth. of Analysis of Rec for Prevention of Roof Std. Meth. of Test for Hydrostatic Sid. Test. Proc, for Pressure Std. Meth. for Verifying the

filter Element (1972) ANSI / flood, Surface $\mathbb{W}$ ater, Rigging Collapse, Burglary, and Roof
lectric Generating Stations (Flood, Surface $\mathbb{W}$ ater, Rigging bolts (1973) Std. for Gages, Pins and Std. for Std. for Pins and Rec. Pract. for Std. Meth. for 73) n the Atmospher/ Tent. Meth. for Automated Separation and
atmosphere (Sodium Bicarb/ Tent. Meth. for Separation and orides in the / Tent. Meth. for Continuous Separation and afety Std. for Key Locks (Burglar Alarm, Door, Deposit and rec. Pract. for Air Cleaning Devices (Air Filters and Dust Std. for Drill Drivers, Split Sleeve, of Test for Sheet Resistance of Thin Metallic Films with A Meth. for Measuring Resistivity of Silicon Slices with A I for Sheet Resistance of Silicon Epitaxial Layers Using A vehicle Accident) (1972) evaluating Confor/ Rec. Pract. for Rec. Pract for Selecting and Defining Std. Analytical Meth. for Determining Candy Std. Gravure Std. Meth. of Specifying

for Determining Speed of $16 \mathrm{Mm}, 8 \mathrm{~mm}$ and Super 8 Reversal Every Meth. (Textile Colorfastness) (19/ Screen Wire Meth. (Textile Colorfastnes/

Test Meth. for Rec. Evaluation Procedure of Gray Scale for rec. Evaluation Procedure of Chromatic Transference Scale Std. Safety

ntifying $A C$ and DC Electrical Wiring on Boats and Engines ance Instructions, and Conta/ Rec. Pract. for Symbols and h Permanentl/ Rec. Pract. for Signal Words, Letter Sizes,

of Color of Low Colored Clear Liquids Using the Hunterlab

Std. Rec. Pract. for Lighting Cotton Classing Rooms for (Stedical Une (1973) oated Fiberbo/ Std. for Test Meth. for Determining Visual
Whiteness and Yellowness of Near White, Opaque Materials Whiteness and Yellowness of Near White, Opaque Materials color Difference Meter (1972T) Meth. for Measurement of Disk Meth. of Analysis of Spectrophotometric Meth. of Analysis of metry (1972) Meth. for Measuring nant Wavelength and Excitation Purity/ Meth. of Measuring eserv/ Meth. for Spectral Reflectance, Transmittance, and Colorimeter (1971) Std. Meth. of Test for Std for Viewing Conditions for the Appraisal of ric Exposure and Evaluation Meth. for Determining Speed of istry) (1962) Std. Analytical Meth. for Determining Pekar 1 Other Natural Dr/ Std. Analytical Meth. for Determining Std. Analytical Meth. for Determining
Std. Analytical Meth. for Determining
Cold Heading Quality Carbon Steel Wire for Tapping or S Cold Heading Quality for Hexagon Head Bolts (1972) ANSI Cold Heading Quality for Hexagon Head Bolts (1972) ANS

Cold Heading Quality Steel Wire for Wood Screws (1971)

Cold Heading Wire and Rod (1973) ANSI H38.12

Cold Heading Wire and Rods (1972)

Cold Rolled Alloy Steel Sheet and Strip (1973) ANSI G24

Cold Rolled Alloy Steel Sheet and Strip (1973) ANSI G24

Cold Rolled Carbon Steel Strip (1972A) ANSI G47.1

Cold Rolled Hard, Untempered Spring Quality (1973)

Cold Rolled Sheet, Carbon, Structural (1972)

Cold Rolled Strip for Magnetic Application in Relays an

Cold Rolled. High Strength, Low Alloy Columbium and/or

Cold Spanking Simulator (1972) ANSI Z11.280

Cold Test Content in Corn Oil (1965)

Cold Water Meters (Displacement Type 5/8 In. Through 6

Cold Water Meters (1972)

Cold Weather Concreting (1966) ANSI A I44.1

Cold Weather Masonry Construction (Protection Rec.) (19

Cold Weather Masonry Construction, Introduction (Tech.

Cold Worked Alloy Steel Bars for High Strength at Eleva

Cold Worked Alloy Steel Forgings and Forging Billets Fo

Cold Worked Alloy Steel Plate, Sheet, and Strip for Hig

Cold Worked Deformed Steel Wire for Concrete Reinforcem

Cold Worked, and Cold Worked Alloy Steel Forgings and F

Cold, and Cold Worked Alloy Steel Bars for High Strengt

Cold, and Cold Worked Alloy Steel Plate, Sheet, and Str

Coleman Meth. (1968)

Coliform Bacteria in All Food Products (Cereal Chemistr

Collapse Due to Weak Construction and Design of Buildin

Collapse of Buildings and Other Structures Caused by Sn

Collapse Strength of Hollow Glass Microspheres (1972)

Collapse Test of Composite Cans (1973)

Collapse / Burst Resistance of a Hydraulic Fluid Power

Collapse) (1974)

Ls at Electric Generating

Collapse, Burglary, and Roof Collapse) (1974)

Collar Inspection (1973)

Collar, Swage Locking for Pull Type and Stump Type Lock

Collars, Swage Locking (1973)

Collection and Identification of Corrosion Products (19

Collection of a Gross Sample of Coal (1972)

Collection of Particulate and Acidic Gaseous Fluoride I

Collection of Particulate and Gaseous Fluorides in the

Collection of Particulate and Water Soluble Gaseous Flu

Collection Safes) (1973)

Collectors) for lndustrial Exhaust Ventilation Systems

Collet Type (1972)

Collinear Four Probe Array (1973)

Collinear 4 Probe Array (1973)

Collinear 4 Probe Array (1974)

Collision Deformation (Damage) Classificationtative M

Color and Gloss Tolerances of Opaque Materials and for

Color and Inversion Content of Corn Syrup Unmixed (1952

Color Bars (Printing) (1973)

Color by the Munsell System (1968)

Color Camera Films lntended for Direct Projection in Mo

Color Change in Fabrics Due to Flat Abrasion (Frosting)

Color Change of Fabrics Due to Flat Abrasion (Frosting)

Color Change (Textile Colorfastness) (1954) 1SO 105.1

(Color Chart / Textile Colorfastness) (1972)

Color Code for Marking Physical Hazards (1971)

Color Code System for Welding Wire Identification (1973

Color Code) (1973)

Requirements and Rec. Meth.

Color Codes for Lubricant and Fluid Requirement Mainten

Color Coding of Electrical Wires and Cables (1974)

Color Combinations, and Durability Requirements for Bot

Color Difference Meter (1972T)

Meth. for Measurement

Color Grading (1971)

Color Marking of Compressed Gas Cylinders Intended for

Color Mat ched to Master Specimen for Fabrics, Vinyls, C

(Color Measurement of Textiles, Paints, Plastics, etc.)

Color of High Gravity Glycerin (1973)

Color of Low Colored Clear Liquids Using the Hunterlab

Color of Macaroni Products (Cereal Chemistry) (1962)

Color of Macaroni Products (Cereal Chemistry) (1962)

Color of Paper and Paper Board by Hunter L, A, B Colori

Color of Paper and Paperboard in CIE Y; X, Y or Y, Domi

Color of Paper and Pulp (Using Spectrophotometer with R

Color of Raw Cotton Using the Nickerson-Hunter Cotton

Color Quality and Uniform ity in the Graphic Arts (1972)

Color Reversal Films for Still Photography (1972)

Color Stability Content of Corn Syrup Unmixed (1954)

Color Test (Slick) Meth. of Flour Analysis (Cereal Chem

Color (Spectrophotometric) Content in Corn Oil (1964)

Color (Spectrophotometric) Content in Corn Starch (1957

Color (Spectrophotometric) Content in Feedstuffs and Al

ASTM

ASTM

ASTM

ASTM

ASTM

ASTM

ASTM

ASTM

ASTM

ASTM

ASTM

NARM

ASTM

ASTM

$A W W$

AWWA

ACI

BIA

BIA

ASTM

ASTM

ASTM

ASTM

ASTM

ASTM

Std. ASTM

$\mathrm{AACCH}$

FMS

FUS

ASTM

CCTI

ations ( FMS

FMS

NSA

NSA

NACE

ASTM

ASTM

AST

UL

ACGIH

ANSl
ASTM

Std ASTM

ASTM

SAE

ASTM

GT

ASTM

ANSl

AATCC

A ATC

AATCC

AATC

ANSI

SAE

$A B Y C$

SAE

SAE

ASTM

CGA

SAE

ASTM

ASTM

ASTM

AACC

AACC

TAPP

TAPP

TAPPI

ASTM

ANSI

A 548

A546

A549

B316

B.565

A506

A507

A 109
A680

A611

121

A607

.

C706

306

A

A 458

A477

A 457

A 496 
Std. Analytical Meth. for Determining Std. Analytical Meth. for Determining Tent. Meth. for Grading Soybean Oil for Std. Analytical Meth. for Determining Sugar (Crude and / ugar (Crude and $\mathrm{Re}$ ) Std. Analytical Meth. for Determining for Colorfastness to Washing: Characterization of Textile nce Meter (1972T) Meth. for Measurement of Color of Low
y the AATCC Crockmeter M/ Test Meth. for Colorfastness of

Meth. for Measurement of Color of Low ater (1972) ANSI L14.149 Test Meth. for Colorfastness of 114.151

Test Meth. for Colorfastness of

Test Meth. for Colorfastness of t (1972) Ansi/ Test Meth. for Detection and Assessment of cking (Rubbing) by the AATCC Crockmeter M/ Test Meth. for hlorinated Pool Water (1972) ANSI L 14.149 ater (I972) ANSl Ll4.15]

) ANSI L 14.56

washing and Shrinkage: Rapid Control Test/

105 Deg. F: Rapid Control Test (1969)

toving (1972) ANSI L14.9

ing (1972) ANSI L 14.5

2) ANSI LI4.3

here Under Low Humidities (1972) ANSI L14/

ternate Exposure (1971) ANSI Ll4.227

2) ANSI L14.148

y) and Light: Alternate Exposure (1972) A/

ubbing) (Rotary Vertical Crockmeter Meth.

(1972) ANSI Ll 14.150

ommercial Washing and Laundering (1972) A/ ogen (Burnt Gas Fumes) (1972) ANSI L14.54

972) ANSI L14.146

ng) (1973) ISO 105-4

4.241

te Sunlight Exposure (1965) ANSI L14.118

si L14.168

1) ANSI L14.168

ntinuous Light (1971) ANSl Ll4.53

i L14.168

ternate Light and Darkness (1971) ANSI Ll/ are Lamp, Continuous Light (1971) ANSI L1/ arc Lamp, Alternate Light and Darkness (I

der High Humidities (1972) ANS1 LI4.238

L14.240, ISO I05.4

) (1973)

(1973)

972) ANSI L14.2

4.57

Test Meth. for . For Cotton and Linen Textile ection and Assessment of Colored Textiles to Photochromism L-7 (1971) ANSI L14.264 olorants (1972)

Test Meth. for Test Meth. for Evaluation Procedure of Gray Scale for Staining (Textile aluation Procedure of Gray Scale for Color Change (Textile ue to Flat Abrasion (Frosting): Screen Wire Meth. (Textile rics Due to Flat Abrasion (Frosting): Every Meth. (Textile ure of Chromatic Transference Scale (Color Chart / Textile le Manganese (Cereal Chemistry) (1962) Meth. for

or Color of Raw Cotton Using the Nickerson-Hunter Cotton orus in Feeds and Feedstuffs (Cereal Chemistry) (1962)

ons (1969) ANSI N116

Std. Meth. for

(Cereal Chemistry) (1962)

Meth. of Test for Lead in the Atmosphere by

y $(\mathrm{PH})$ in Flour (Cereal Chemistry) (1962)

Milled Wheat Products (Wheat, Flour, Bran, Shorts, etc.) in Active Proteinase Preparations (Cereal Chemistry) (19/ $\mathrm{s}$ of Phosphorus in Yeast (Cereal Chemistry) (1962)

measuring Color of Paper and Paper Board by Hunter L, A, B analysis of CCA Treating Solutions and CCA Treated Wood by Std. Meth. of Test for Methyl Ether of Hydroquinone in Garments (Clothing), Commercial Wash-Whites I90 Deg. F, ommercial Wash-Whites (With Chlorine Bleach) 190 Deg. F,

for States of the United States (Including the District of

20

z 179.19

) ANSI H53.1

1964) ANSI Z179.20 Std. Spec. for Nickel Chromium Molybdenum Std. Spec. for Nickel Chromium Molybdenum i Z179.17 ube (1972) ANSI H34. Std. Spec. for Nickel Chromiumary seamless and Welded Chromium Nickel lron Molybdeppor on Welded Chromium-Nickel-lron-Molydenium-Copper -

3)/ Std. Spec. for Chromium Nickel Iron Molybdenum Copper nd Welded Chromium-Nickel-lron-Molybdenum-Copper-
Color (Spectrophotometric) Content of Corn Syrup (I957) Color (Visual Comparison) Content of Corn Syrup (1954) Color (1973)

Color, Reflectance (Spectrophotometric) Content of Corn Color, Solutions (Spectrophotometric) Content of Corn S Colorants (1972)

Colored Clear Liquids Using the Hunterlab Color Differe Colored Textile Yarn and Fabric to Crocking (Rubbing) B Colored Textile Yarns and Fabrics to Chlorinated Pool W Colored Textile Yarns and Fabrics to Water (1972) ANSI Colored Textiles to Perspiration (I972) ANSI LI4.56 Colored Textiles to Photochromism Colorfastness to Ligh Colorfastness of Colored Textile Yarn and Fabric to Cro Colorfastness of Colored Textile Yarns and Fabrics to C Colorfastness of Colored Textile Yarns and Fabrics to W Colorfastness of Colored Textiles to Perspiration (1972 Colorfastness of Cotton and Linen Textiles to Combined Colorfastness of Dyed or Printed Textiles to Washing at Colorfastness of Dyed Textile Yarns to Sulfur Dioxide S Colorfastness of Dyed Wool Fabric and Yarn to Mill Full Colorfastness of Dyed Wool Textiles to Carbonizing (I97 Colorfastness of Textile Fabrics to Ozone in the Atmosp Colorfastness of Textile Fabrics to Water and Light: Al

Colorfastness of Textile Fabrics to Water Spotting (197

Colorfastness of Textile Fabrics to Water (High Humidit

Colorfastness of Textile Yarn and Fabric to Crocking ( $R$

Colorfastness of Textile Yarns and Fabrics to Sea Water

Colorfastness of Textiles to Accelerated Domestic and C

Colorfastness of Textiles to Atmospheric Oxides of Nitr

Colorfastness of Textiles to Bleaching with Peroxide (1

Colorfastness of Textiles to Dry Heat (Excluding Pressi

Colorfastness of Textiles to Drycleaning (1973) ANSI L1

Colorfastness of Textiles to Hot Pressing (1973)

Colorfastness of Textiles to Light and Washing: Alterna

Colorfastness of Textiles to Light (Daylight) (1971) an

Colorfastness of Textiles to Light (General Meth.) (197

Colorfastness of Textiles to Light, Carbon Arc Lamp, Co

Colorfastness of Textiles to Light, Sunlight (1971) Ans

Colorfastness of Textiles to Light: Carbon Arc Lamp, Al

Colorfastness of Textiles to Light: Water Cooled Xenon

Colorfastness of Textiles to Light: Water Cooled Xenon

Colorfastness of Textiles to Ozone in the Atmosphere Un

Colorfastness of Textiles to Steam Pleating (1971) ANSI

Colorfastness of Upholstery Fabrics to Solvent (Textile

Colorfastness of Upholstery Fabrics to Water (Textile)

Colorfastness of Yarn or Fabric to Acids and Alkalis (1

Colorfastness to Bleaching with Chlorine (I972) ANSI L1

Colorfastness to Degumming (1972) ANSI LI4.4

Colorfasiness to Light (1972) ANS1 L14 271

Colorfastness to Light: Determination of Fastness Above

Colorfastness to Washing: Characterization of Textile C

Colorfastness) (1954) ISO 105.1

Colorfastness) (1954) 1SO 105-1

Colorfastness) (1970)

Colorfastness) (1970)

Colorfastness) (1972)

Colorimeter or Spectro

Colorimeter (1971)

1. for Color Change of Fabrics

/T Meth. for Color Change in Fab Rec. Evaluation Proced

Colorimetric Acetate Ashing Meth. of Analysis of Phosph

Cotermination of Uranium in Aqueous Soluti

Colorimetric Dithizone Procedure (1972T)

Colorimetric Meth. of Analysis for Fat Acidity in Grain

Colorimetric Meth. of Analysis for Hydrogen Ion Activit

Colorimetric Meth. of Analysis of Malathion Residues in

Colorimetric Meth. of Analysis of Proteolytic Activity

Colorimetric Modified Fiske-Subbarow Meth. of Analysi

Colorimetry (1972)

Colorimetry (1972)

Colorless Monomeric Acrylate Esters (1972)

Colors I60 Deg. F Maximum Hot Head Press (300-330 Deg

Colors 160 Deg. F Maximum, Press or Air Finish (1973)

Columbia) for Information lnterchange (1972)

Columbium Alloy Plate, Sheet and Strip (1972) ANSI H34.

Columbium Alloy Rod and Bar (1972) ANS] H34.22

Columbium Alloy Seamless Pipe and Tube (1972) ANS1 H34.

Columbium and Its Alloy Bar, Rod, and Wire (I964) ANSI

Columbium and Its Alloy Seamless and Welded Tubes (1970

Columbium and Its Alloy Strip, Sheet, Foil, and Plate (

Columhium and/or Vanadium (I970) ANSI G24.32

Columbium Metal for Con solidation or Melting (1964) Ans

Columbium Molybdenum Tungsten Alloy Seamless Pipe and

Columbium Stabilized Alloy Pipe (1973)

Columbium Stabilized Alloy Pipe (1974)

Columbium Stabilized Alloy Plate, Sheet,

Columbium Stabilized Alloy Tubes (1973)

Columbium Vanadium Steels of Structural Quality (1972)
Teth for TAPP

td. Meth of AWPA

Eel Shee

CR

CR

CR

CR

AATCC

ASTM

AATCC

AATCC

AATCC

AATCC

AATCC

AATCC

AATCC

AATCC

AATCC

AATCC

AATCC

AATCC

AATCC

AATCC

AATCC

AATCC

AATCC 126

AATCC 116

AATCC 106

AATCC 61

AATCC 23

AATCC 101

AATCC 117

A TCC 132

AATCC 133

AATCC 83

AATCC $16 C$

AATCC 16

AATCC 16A

AATCC $16 \mathrm{~B}$

AATCC 16D

AATCC $16 \mathrm{E}$

AATCC $16 \mathrm{~F}$

AATCC 129

AATCC 131

ANSI L24T?

ANSI L24T6

AATCC 6

AATCC

AATCC

AATCC

AATCC

AATCC

AATCC

AATCC

AATCC

AATCC

AACCH

ASTM

ASTM

ASTM D3112

AACCH 02-04

AACCH 02-51

AACCH $60-30$

$\mathrm{AACCH} 22.63$

AACCH 40.56

A WPA

ANSl

ANSl

ANS1

ASTM

ASTM

ASTM

ASTM

ASTM

ASTM

ASTM

ASTM

ASTM Td. Spec. for ASTM Electric Fusi ASTM

ASTM

ASTM
T524SU

Al0-72

D3125

24. 13

$\mathrm{X} 3.38$

B443

B446

B444

B392

B394

B383

B464

B474

B 463

B468

A572 
std. Meth. for Chemical Analysis of Reactor and Commercial oncrete Construction Design Handbook (Beam, Slab, Footing, Uniform Building Code Std. for Spaced for Distillation of Crude Petroleum (15 Theoretical Plate Reinforced Brick Masonry (1962)

ents, Timber, Preservative Treatment, Poles, Piles, Spaced (1972)

Std for Punches-Basic,

air Conditioning, Heating, Exhaust, Ventilating, and Their 73)

Uniform Building Code Std, for Specs. for Aluminum

c Shower Receptors, Stall Showers Lavatories, Bathtubs and structural Integrity Test Procedure for Trucks, Buses, and Cars, Multipurpose Passenger Vehicles, Buses, Trucks, and g Storm Windows for External Applicat/ Spec. for Aluminum test Procedures for Home Laundry Equipment (washer, Dryer. C 33.97

anentl/ Safety Std. for Receptacle-Plug and Mounting Dimel Std. Bore and Rod Size (1973) ANSI Z240.1 Std. for Terminology for Safety Std. for Tests for

Precautions for Prevention (Incinerator and Open Burning. es, Hemp, Tow/ Uniform Fire Code: Storage and Handling of se (1972) Safety Std. for Tube Fittings for Flammable and (1973) Std. Meth. of Test for

of Organic Coatings (Paint), and Handling of Flammable and Flammable and tices for the Protection and Tanks Containing Flammable or afety Std. for Aboveground Storage Tanks for Flammable and Uniform Fire Code: Flammable and Safety Std. for Steel Underground Tanks for Flammable and ards and Protection Std. for Rack Storage of Flammable and std. for Safety for Metal Waste (Trash, Garbage) Cans (For Uniform Fire Code: High Piled $\mathrm{n}$, Operation and Mai/ utomatic Damper Cont/ ion Systems (1972) Rec. Pract. and Std. for Flammable Safety Std. for Draft Equipment for Std. Pract. for

tilation in a Ship's Cargo Hold During the Use of Internal of Total Sulfur in Volatile Organic Liquids (Oxy Hydrogen Std. for Gas Fired Gravity and Fan Type Sealed on) (1974)

code Requirements by OSHA (1973)

Std. Manual for Electric Safety Std. for

Plywood Construction Systems (Specs.) for Std. Meth. of Test for Moisture Present in Ordinary Industry Standard for Household Rec. Pract. and Std. Covering Non

74)

1974) $\mathrm{s}$ and Asphalt Tile Floors in Institutional, Industrial and Std for Safety for Electric Coin Operated and Std. for Safety for Electric Coin Operated and Std. Meth. for Chemical Analysis of Reactor and eight and Yield of Scoured Wool, Top, and Noil for Various Std. for Std. for Safety for Safety Std. for Std. for Safety for nalytical Meth. for Determining Moisture (Oven) Content in Std. for ty Std. for High Pressure Gas Manifolds for lndustrial and tions (1972) Std. for Safety Requirements for tric Breakdown Voltage and Strength of Insulating Gases at

Meth. for Determination of Azodicarbonamide Content in el Sheet, Zinc Coated (Galvanized) by the Hot Dip Process, lled Carbon (0.15 Maximum. Percent) Steel Sheet and Strip, arbon 0.16 to 0.25 Maximum \%, Hot Rolled Sheet and Strip, Safety Std. for

1971) ANS1 L14.106 Test Meth. for Evaluation of Std. Meth. of Test for Wool Content of Raw Wool. Std for Pot, Pan, and Utensil nition, Lighting, and Starting) for Passenger Automobiles, for Institutional and Industrial Use Garments (Clothing),

Use Garments (Clothing), 100\% Man Made or Blend Fabrics.

for Colorfastness of Textiles to Accelerated Domestic and oil for Various Comm/ Std. Rec. Pract. for Calculation of ple Fiber (1971) Std. Meth. of Test for 1 Rec. Pract. for Grounding of Industrial and equirements for Oil Powered Forced Air (Domestic and Light Maintenance of Vinyl Asbestos and Asphalt Tile Floors for

nd Anhydrous Ammonia (Fertilizer Grade) in Residential and

Moisture in Grains, Beans, Peas, Lentils, Rice, and Other

Preparation of Methyl Esters of Long Chain Fatty Acids in ,2tetrachloroethyl) Thio)-4-Cyclohexene-1,2-Dicar/ carbanilate Carbanilate (Ester) Desmedipham (1/ 711-Trimethyl-2,4-Dodecadienoate Hydroprene / 'dichlorobenzilate-Chloropropylate (1973) Rec, and

Columbium (1968) ANSI Z258.1

Column and Other Members) (1973)

Column Design (1973)

Column) (1973) ANSI Z11.314

Columns and Pilasters (Tech. Notes) (1965)

Columns, Joints, Screws, Bolts, Connectors, Nails, Stap
Columns, Piers, Pilasters, and Buttresses (Tech. Notes)

Combination Angle Head Type and Related Quill Bushings

Combination Duct Systems (1973) ANSI BI44.2

Combination Refrigerator-Freezers and Freezers (1974)

Combination Standpipe Systems (Fire Extinguishment) (I9

Combination Storm Doors (1971)

Combination Tank and Shower Receptor Units (1972)

Combination Vehicles for Roadway Use Over $10,000 \mathrm{Lb} .(4$

Combination Vehicles with Any Type of Brake System (197

Combination Vertically Sliding or Horizontally Operatin

Combination) (1971) ANSI A197.2, Asse I007

Combinations for Use in Hazardous Locations (1973) ANSI

Combinations, and Durability Requirements for Both Perm

Combinations, Rod End Configurations, Dimensional Ident

Combines and Grain Harvesting (1971)

Combustibility of Sweeping Compounds (Fire Protection)

Combustible and Flammable Materials; Reporting and Fals

Combustible Fibres (Cotton, Sisal, Henequen, Ixtle, Jut

Combustíble Fluids, Refrigeration Service, and Marine U

Combustible Gases in Electrical Apparatus in the Field

Combustible Liquids and Solids and Potential Dust Explo

Combustible Liquids Code (1972)

Combustible Liquids in Locations That May Be Flooded (1

Combustible Liquids (1972)

Combustible Liquids (1973)

Combustible Liquids (1973) ANSI B137.1

Combustible Materials (Fire Safety) (1974)

Combustible Materials) (1974) ANSI Z221.

Combustible Stock (Storage) (1973)

Combustible) Gas Indicators Including Their Installatio

Combustion Chamber in Heating Appliances (Regulators, a

Combustion Chambers, Fuel Oil Injection, and Gas Admiss

Guide for Supplementary Ven

Combustion Meth.) (1972)

Combustion System Wall Furnaces (1972)

F Test for Trace Quantitite

Comfort Conditioning (Heat Loss, Gain, Energy Consumpti

Comments on the National Electrical Code (NFPA 70) and

Commercial-Industrial Gas Heating Equipment (1973)

Commercial and lndustrial Buildings (1971)

Commercial and Industrial Samples of Wool by Oven Dryin

Commercial and Portable Exchange Water Softeners (1972)

Commercial Boat Trailer (1972)

Commercial Buildings (1971)

Commercial Clothes Drying Equipment (Laundry Dryer) (19

Commercial Clothes Washing Equipment (Laundry Washer)

Commercial Columbium (1968) ANS1 2258.1

Commercial Compositions (1972) ANSI L14.263

Commercial Cooking Equipment Exhaust Systems (1970)

Commercial Dry Cleaning Machines (Class Iv) (1974)

Commercial Electric Cooking Appliances (1972)

Commercial Electric Dishwashers (1973) ANSl C33.96

Commercial Feedstuffs (Corn) (1957)

Commercial Gas Baking and Roasting Ovens (1971)

Commercial Gas Cylinders (1972)

Commercial Laundry and Dry Cleaning Equipment and Opera

Commercial Power Frequencies (1974)

Commercial Premix (Cereal Chemistry) (1971)

Commercial Quality (1971) ANSI G8.24

Commercial Quality (1972)

Commercial Ouality (1972)

Commercial Refrigerators (1973)

Commercial Rewetting A

Commercial Spray Type Washing Machines (1970)

Commercial Vehicles, Golf Car, and Other Diversified of Commercial Wash-Whites 190 Deg. F, Colors 160 Deg. F Commercial Wash-Whites (With Chlorine Bleach) $190 \mathrm{Deg}$ Commercial Washing and Laundering (1972) ANSI L14.81 Commercial Weight and Yield of Scoured Wool, Top, and N

Commercial (Electrical) Power Systems (1972) ANSI C114.

Commercial) Central Furnaces) (1972)

Commercial, Institutional and Industrial Buildings (197

Commerical Fuel Burning, Dispensing, and Handling Facil

Commodities for Which Conversion Charts Have Been Prepa

Common Fats, Oils, and Fatty Acids (Cereal Chemistry) (

Common Name for the Pest Control Chemical cis-N-( $1,1,2$

Std. for Common Name for the Pest Control Chemical Ethyl Mhydrox

Std. for Common Name for the Pest Control Chemical Ethyl (E.E)3.

Sid. Common Name for the Pest Control Chemical Isopropyl 4,4

ASTM

ACI

ICBO

ASTM

BIA

ICBO

BIA

ANSI

tion of NFPA

AHAM

ICBO

AAMA

/Lli IAPMO

SAE

AAMA

d. and AHAM

UL

NFLD

ASAE

UL

ICBO

UL

UL

ICBO

NFPA

ICBO

UL

ICBO

UL

FMS

UL

ICBO

$\mathrm{ABYC}$

UL

DEMA

SNAME

ASTM

ANSI

NEMA

FMS

UL

ASTM

WCF

ABYC

RTI

UL

UL

ASTM

ASTM

NSF

UL

UL

Std. a CR

ANSI

Safe UL

ANSI

ASTM

AACCH

E195

SP17

UBCS25.15

D2892

17I

$\mathrm{UBC} * 3-25$

B94.39

$90 \mathrm{~A}$

HRF.2.ECFT

UBCS38.3

1102.5

TSC11

J294

J299

1002.8

HLW.2PR

1010
J115

3.6.1

$\mathrm{S} 343$

2ART 27

UFC $* 2 A R T$ ?

109

(3284

FC $* 2$ ART 34

UFC $\approx 2 \mathrm{~A}$

FC $\$ 2 A R T 15$

58

$8.33 \mathrm{~N}$

FC*2ART 35

A14

378

1.2

4-14

Z21.44A

$\mathrm{HEl}$

$5-7 \mathrm{~S}$ 
'dibromobenzilate-Bromopropylate (1973)

Dichlorophenoxy)-2-Nitrobenzoate Bifenox (197/

yl)-A, A, A-Trifluoro-2,6-Dinitro-N-Propyl-Ptoluid/ methyl).A, A, A-Trifluoro-2,6-Dinitro-N-Propyl-Pto/ -Dipropyl Phosphorodithioate) (1973/ I Phosphorodithioate S-Ester Wi/ Std. for
an -2-Ylphenyl Methylcarbamate-Dioxacarb (1973) )-11-Methoxy·3,7,11-Trimethyl-2,4-Dodecadienethi/ 0.(3,5,6-Trichloro-2-Pyridyl) Phosphorothioa/ propylamino)-6-(Methylthio)-S-Triazine-Prometry/ propylamino).6-Methoxy-S-Triazine-Prometon (197/ bis (Isopropylamino) -S-Triazine-Propazine (1973/ e, E)-3,7,11-Trimethyl-2,4-Dodecadienoate Kino/ o)4-(Isopropylamino).6-(Methylthio).S-Triazine ) $-4,6$-Bis (Isopropylamino) Stria/ amino)-4-(Ethylamino).6-(Methylthio)-S-Triazine / amino)-4-Chloro-6-(Ethylamino)-S-Triazine-Terb/ methylamino $) \cdot 2-(a, a, a-T$ rifluoro-m-tolyl $) \cdot 3$ / trd. for Dipropetry

Std. for Bentazo Std. for Tetramethrin

Std. for Fospirat

Std. for Disugran Std. for Crufomate Std. for Chlorbromuro

Std. for Chlordimeform

Std. for Fluorodife

Std. for Prynachlo

Std. for Glyphosin

Std. for Mexacarbat

Std, for Dinose

Std. for Acephate

Std. for Ethiolate

Std. for Glyphosate

Std. for Oxamyl

Std. for Picloram te 2-(P-Tert-Butylphenoxy) Cyclohexyl 2Propynyl Sulfite e)-11-Methoxy-3,7,11-Trimethyl -2,4-Dodecadienoate) tool Software (1972) Definitions of or Plastic Utilities Duct for Underground Installation for (R1970) Std. Spec. for Minimum Std. for Land-Mobile Std. Spec. for Aluminum Wire for y Between Data Terminal Equipment and Non-Synchronous Dat Minimum Stds. for Land Mobile or the Installation and Maintenance of Electric Supply and ospital Signaling and Nurse Call Equipment (Audio / Visual Minimum Std for Land Mobile

Ilation and Maintenance of Underground Electric Supply and ensional Std. Coplanar Magnetic Tape Cartridge Type Cp ll (1974) ANSI C7.59

Conductors (1972) ANSI C7.53

Std. Spec. for Std. Spec. for Std. Meth. of Test for Percent Air Voids in A Std. Meth. of Wetting and Drying Test s of ens of Bituminous Mixtures by Means of California Kneading mixture Beam Specimens by Means of the California Kneadin

ationship of Quartz Fiber Electrometer Type Dosimeters and Safety Std. for Tests for

Meth of Test for Density of Glass by the Sink Floa (1972) ANSI B93.23 Std. Meth. of Verifying the Materia si Z11.315

Intensity Meth.) (1973) Std. Meth. of Test for Std. Meth. of Test for

Std. Rec. Pract. for Experimental Test. for Biological s Under Compression (1974) n Materials in Askarels/ Std. Meth of Test for Rec. Pract. for Defining Conditions for Evaluating the pacitors for Electrical Transmission and Distribution Lin Code for Architectural Design

or Informational, Directional, and Special Signs (Symbols) tion Check Flanges and Gaskets; Drilling Through, Multiple ctrical Insulating Materials at Micr/ Std. Test Meth. for Chemical Meth. of Analysis of Vitamin B

Microbiological Meth of Analysis of Vitamin B6

Rec. Pract. for Instrumentation for Motor Vehicle and It Conditioners (1967) Std. Test Meth. for Passive Electronic

d. for Safety for Refrigerant-Containin ed and Cold Finished, Special Quality, for Pressure Piping lants / Sid for Cleaning of Fluid Systems and Associated article/ Std. Meth. of Sampling Gas Blow Down Systems and . (197) Std. Meth. for Sampling Gas Blow Down Systems and r Electronic Equipment / Std. for Safety for High Voltage rose ply and Installation Contract (1968) d. Common Name for the Pest Control Chemical Isopropyl 4,4 Common Name for the Pest Control Chemical Methyl 5(2,4Common Name for the Pest Control Che mical N.(2Chloroeth d. Common Name for the Pest Control Chemical N(Cyclopropyl (Common Name for the Pest Control Chemical O-Ethyl S,S (Common Name for the Pest Control Chemical O,O-Dimethy Common Name for the Pest Control Chemical O-1.3-Dioxol d. Common Name for the Pest Control Chemical S-Ethyl (E, E Common Name for the Pest Control Chemical 0,0 Dimethyl Common Name for the Pest Control Chemical 2,4-Bis (ISO . Common Name for the Pest Control Chemical 2,4-Bis (ISO Common Name for the Pest Control Chemical 2-Chloro-4,6 Common Name for the Pest Control Chemical 2-Propynyl ( Common Name for the Pest Control Chemical 2-(Ethylamin (Common Name for the Pest Control Chemical 2-(Ethylthio Common Name for the Pest Control Chemical 2-(Tertbutyl Common Name for the Pest Control Chemical 2-(Tertbutyl Common Name for the Pest Control Chemical 4-Chloro5-( (Common Name for the Pest Control Chemical (1972) (Common Name for the Pest Control Chemical) (1971) (Common Name for the Pest Control Chemical) (1971) (Common Name for the Pest Control Chemical) (1971) (Common Name for the Pest Control Chemical) (1972) (Common Name for the Pest Control Chemical) (1972)

(Common Name for the Pest Control Chemical) (1972)

(Common Name for the Pest Control Chemical) (1972)

Common Name for the Pest Control Chemical) (1972)

Common Name for the Pest Control Chemical) (1972)

(Common Name for the Pest Control Chemical) (1972)

(Common Name for the Pest Control Chemical) (1972)

(Common Name for the Pest Control Chemical) (1972)

(Common Name for the Pest Control Chemical) (1972)

(Common Name for the Pest Control Chemical) (1972)

(Common Name for the Pest Control Chemical) (1972)

(Common Name for the Pest Control Chemical) (1972)

(Common Name for the Pest Control Chemical) (1972)

(Common Name for the Pest Control Chemical) (1973)

(Common Name for the Pest Control Chemical) (1973)

Common Words Related to Numerically Controlled Machine

Communication and Electrical Wire and Cable (1974)

Communication and Signal Pin Type Lime Glass Insulators

Communication Antennas: Part II-Vehicular (1972)

Communication Cable (1969) ANSI C 7.40

Communication Equipment (1973)

Communication FM or PM Rec

Communication Lines (1960
Communication) (1974)

Art-Stop Signal Qualit

Communications FM or PM Transmitters $25-470 \mathrm{MHz}$ (1970)

Communications Lines (National Electrical Safety Code)

Compact Round Concentric Lay Stranded Copper Conouctors

Compact Round Concentric Lay Stranded EC Grade Aluminum

Compacted Bituminous Paving Mixture (1973)

Compacted Soil Cement Mixtures (1971) ANSI A37.51

Compactor (1971) ANS1 A37.138 /Paration of Test Specim

Compactor (1973) /Ract. for Preparation of Bituminous Companion Dosimeter Chargers (1971)

Comparative Flammability of Liquids (1972)

Comparator (1972T)

Compatibility of a Hydraulic Fluid Power Filter Element

Compatibility of Fuel Oil Blends by Spot Test (1972) an

Compatibility of Materials with Liquid Oxygen (Reaction

Compatibility of Metals for Surgical Implants (1972)

Compatibility of Plasticizers in Vinyl Chloride Plastic

Compatibility of Transformer Insulation and Constructio

Compatibility of Vehicle Fuel Tanks and Fill Pipes with

Compatible Operation of Forage Harvesters, Wagons and B

Compensation (Including Safety Requirements) (1973)

Competitions (1972)

Complementary to ANSI Z35.1-1972, Accident Prevention

Completion, and Flowline Valves; Drilling Through Contr

Complex Permittivity (Dielectric Constant) of Solid Ele

Complex (Cereal Chemistry) (1962)

Complex (Cereal Chemistry) (1962)

Component Impact Tests (1971)

Component Parts (1972)

Component Std. for Receiver Dehydrators for Vehicle Air

Components and Accessories, Nonelectrical (1973)

Components and Assembly (1972)

Components and Other Pressure Containing Parts (1974)

Components During Construction Phase of Nuclear Power P

Components for Particulate Contamination by Automatic P

Components for Particulate Contamination by Manual Meth Components for Television Receiving Appliances and Othe Components in a Polished Specimen of Coal (1972) Suggested . Components of a Concrete Reinforcing Steel Material Sup
ANSI

ANSI

ANSI

ANSI

ANSI

ANS

ANSI

ANSI

ANSI

ANSI

ANSI

ANSI

ANSI

ANSI

ANSI

ANSI

ANSI

ANSI

ANSI

ANSI

ANSI

ANSI

ANSl

ANS!

ANSl

ANSI

ANSI

ANSI

ANSI

ANSI

ANSI

ANSI

ANSI

ANSI

ANSI

ANSI

Ri ANSI

$/(\mathrm{E}$, ANSI NMTBA

F NEMA ASTM

EIA ASTM

EIA

EIA

ANSI

UL

EIA

ANSI

EIA

ASTM

ASTM

ASTM

ASTM

ASTM

ASTM

ANS1

A

ASTM

ASTM

ASTM

ASTM

ASTM

ASTM

SAE

ASAE

la ANSI

AIOA

ANSl

API

ASTM

$\mathrm{AACCH}$

SAE

IMACA

UL

NSA

ASTM

ANSI

ASTM

ASTM

UL

( Mic AST

CRSI

K62.151

62.138

K62.140

K62.152

K62.133

62.134

K62.147

K62.141

K62.130

K62.143

K62.144

K62.145

K62.137

K62.142

K62.135

K62.148

K62.149

K62.125

K62.129

K62.106

K62.111

K62.112

K62.110 
a Symmetrical Current/

Std. Requirements for Pressurized tions of Terms Relating to Lithologic Classes and Physical Microscopical Determination of Volume Percent of Physica Std. for Reel Packaging of tile Residue of Halogenated Solvent Extract from Aerospace d. Meth. of Test for Viscosity of Epoxy Resins and Related gings for Seamless Drums, Heads, and Other Pressure Vesse Std. for Automatic Gas lgnition Systems and rmance Requirements for Motor Vehicle Lighting Devices and

y for Thermal Cutoffs for Use in Electrical Appliances and bers-PIywood Stressed Skin and Curved Panels, Beams, and mpered Carbon and Alloy Steel Forgings for Pressure Vessel ransformer, Multiplier, Deflection Yoke, Picture Tube Neck Std. Consumer Safety Spec. for de: Wood Members and Their Fastenings (Lumber, Plywood and ys and Shafting (1974)

Std. for Design of Tent. Rec. for Design of Concrete Sid. Test. Procedure for the Blow Off Pressure of Std. Test. Proc, for Pressure Collapse Test of Std. Test. Procedure for Filled Cast Flat Drop Rec. Industry Std. for Loose Metal End Dimensions of Std. Test. Proc. for Measuring Inside Diameters of Std. Test. Procedure for Measuring Wall Thicknesses of Std. Test. Procedure for Axial (End to End) Compression

Std. Test. Procedure for Measuring Lengths or Heights of Building Code Std. for Shear Connectors (Steel Studs) for ntrolled (1973) Spec for Std. for Automated Machine Lay $U_{p}$ Std. Test. Procedure for Oil Wicking of Std. Test. Procedure for Filled Cast Flat Bottom Drop Std. Spec. for Acrylonitrile-Butadiene-Styrene (ABS) ansi W3.2 Spec. for

Std. Test. Procedure for End Supported Beam Deflectión Std. Test. Proc for Side to Side Crush Std. Test. Procedure for Internal Bursting of Std. Test. Procedure for Torque Strength of Spec. for Graphite Fibers for Structural

(483) Tensile $\mathrm{M}$ Meth of Test for Tensile Properties of Oriented Fiber Std. Meth. of Test for Fiber Content of Reinforced Resin phase Designations (Code) in Metallic Systems Based on the Design Guide for Wood Std. Spec, for Meth. for Determination of Fatty Acid Std. for Fixed

wavelength of Peak Photoluminescence and the Corresponding (1973)

Uniform Building Code Sid. fo rmic Temperature of Reacting Thermosetting Resins (Plastic

Meth. for Quantitative Analysis of Textiles (Moisture and ec. for Texture and Grade. Panel Characteristics, Material physical Properties of Liquefied Petroleum (LP) Gases from Sid. for Chemical Std. for Chemica

ield of Scoured Wool. Top, and Noil for Various Commercial 1 Tolerances of SAE Wrought Aluminum A/ Std. for Chemica ubber Products (1972) ANSl J1.l Std. Meth. of

Sid. Spec, for Oil and Resin Base Caulking Spec. for Exterior Perimeter Sealin

al Chemistry) (Bolting Cloth, Filter Paper, W'ide Field and Test for Slump of an Oil Base Knife Grade Channel Glazing

Test for Extension Recovery and Adhesion of Latex Sealing std. Meth. of Test for Degree of Set for Wood Sash Glazing orm Building Code Std. for Gypsum Wallboard Tape and Joint

h. of Test for Accelerated Aging of Wood Sash Face Glazing Sid. Spec, for Cleaning

of Test for Low Temperature Flexibility of Latex Sealing Std. Meth. of Test for Slump of Caulking Std. Meth. of Test for Staining of Caulking Std. Meth of Test for Tack Free Time of Caulking eth. of Test for Apparent Density of Refractory Metals and Std. Spec. for Liquid Membrane Forming

td. Materials, Equipment, and Procedures for Mixing Rubber Std. Meth of Test for Abrasion Resistance of Rubber Std. Meth. of Test for Slump of Face Glazing and Bedding able Concentration of Chromic Acid and Hexavalent Chromium Safety Std. for Tests for Combustibility of Sweeping in High Pressure Oil Well Service) (1972)

Thread Oxygen Demand in Industrial Waste Waters and Most Organic Std. Meth. of Test for Volume Shrinkage of Latex Sealing

e Concentrations of Inorganic Mercury and Nonalkyl Organo t for Extrudability, After Package Aging, of Latex Sealing ring the Spiral Flow of Low Pressure Thermosetting Molding or Aging Effects of Artificial Weathering on Latex Sealing eth of Test for Extrudability of Unvulcanized Elastomeric ility of Oil and Resin Based, Knife Grade, Channel Glazing
Components of $\mathrm{AC}$ High $\mathrm{V}$

Components of Coal (1972)

Components with Axial Leads (1972) ANSI C83.55

Components (Flange, Fitting, Valve, etc.) (1973) ANSI G

Components (Using Rotary Flash Evaporator) (1972)

Components (1968) ANSI K65.183

Components (1970) ANSI G38.7

Components (1971)

Components (1972)

Components (1972)
Components (1973)

for Structural Glued Built or Mem

Components (I973) ANSl G35.21

Components) (1974)

/Pec. for

ed on

lEEE

Std. Defini ASTM Std. Meth. for ASTM

EIA

ASTM

OOla ASTM

St ASTM

ASTM

ANSl

Rec. Pract. for Service Perfo SAE Std. for Safet UL
Built Up Mem ICBO

Ec. for Quenched and Te ASTM

Components, Assembly, and Use of a Trampoline (1974)

Components, Timber, Preservative Treatment, Poles, Pile

Components: Enclosed Gear Drives-Bearings, Bolting $\mathrm{Ke}$

Composite Beams and Girders for Building (1957)

Composite Cans (1973)

Composite Cans (1973)

Composite Cans (1974)

Composite Cans (211 (66 Mm) Diameter), Citrus Style (I9

Composite Cans, Tubes and Cores (1973)

Composite Cans, Tubes and Cores (1974)

Composite Cans, Tubes and Cores (1974)

Composite Cans, Tubes and Cores (1974)

Composite Cans, Tubes, and Cores (1974)

Composite Construction (1973)

Composite Filament Tape Laying Machine-Numerically C

Composite Filament Tape Reel (1972)

Composite Motor Oil Cans (1974)

Composite Motor Oil Cans (1974)

Composite Sewer Piping (1974)

Composite Surfacing Welding Rods and Electrodes (1970)

Composite Tubes and Cores (1972)

Composite Tubes and Cores (1973)

Composite Tubes and Cores (1974)

Composite Tubes and Cores (1974)

Composites (GF 220 (1517) Tensile Strength, 70,000,000

Composites (1972T)

Composites (1973)

Composition and Crystal Lattice of Each (I970) ANSl Z30

Composition Board Wall Panels (1973)

Composition Bronze or Ounce Metal Castings (1974)

Composition by Gas Chromatography (Cereal Chemistry) (1

Composition Capacitors (1972) ANSI C83.89

Composition of Gallium Arsenide Phosphide Wafers (1973)

Composition Roofing (Class C Sheet (Roll) and Shingles)

Composition) (1971) ANSI K65.182

Time and Peak Exothe

Composition. Ase, Application, Finishes and Other Techn

Compositional Analysis (1973) ANSI Z11.239

Compositions of SAE Alloy Steels (1970) ANSI G88.2

Compositions of SAE Carbon Steels (1970) ANSI G88.1

Compositions (1972) ANSl L14.263

Mercial Weight and $\mathrm{Y}$

Compositions, Mechanical Property Limits and Dimensiona

Compound and Sample Preparation for Physical Test. of R

Compound for Building Construction (1972)

Compound for Use with Architectural Aluminum (1971)

Compound Micros
Compound (1972)

Compound (I972)

Compound (1973)

Compound (1973)

Compound (1973)

Compound, General Purpose (1972)

Compounds After Artificial Weathering (1972)

Compounds and Sealants (1973)

Compounds and Sealants (1973)

Compounds and Sealants (1973)

Compounds by the Scott Volumeter (1970) ANSl H9.15

Compounds for Curing Concrete (1973) ANSI A37.87

Compounds for Preparing Vulcanized Test Sheets (1973)

Compounds for Soles and Heels (1968) A NSl J2.26

Compounds on Metal Sash (1974)

Compounds (Air Contamination-Occupational Health and

Compounds (Fire Protection) (1973) ANSI Z240.1

Compounds (For Casing, Tubing and Line Pipe Connections

Compounds (1966)

Compounds (1972)

Compounds (1972)

/Tical Meth. for Determining Chemical

\section{UL}

ICBO

AGMA

$\mathrm{ACI}$

CCTI

CCTI

CCTI

CCTI

CCTI

CCTI

CCT

CCT1

ICBO

NSA

NSA

CCTl

CCTl

ASTM

AWS

CCTI

CCTI

CCTI

CCTI

SAE

ASTM

ASTM

ASTM

HPMA

ASTM

AACC

EIA

ASTM

ICBO

Test AATCC

CRA

ASTM

SAE

SAE

ASTM

SAE

A AMA

$\mathrm{AACCH}$

Compounds (1972)

Compounds (1972)

Compounds (1972)

Compounds (1973)

Compounds (1973)
Std. Meth. of ASTM

Std. Meth. of ASTM

ASTM

Unif ICBO

Std. Met ASTM

ASTM

/Td. Meth ASTM

ASTM

ASTM

ASTM

/ M ASTM

ASTM

ASTM

ASTM

ASTM

ANSI

UL

APl

CR

Std for Acceptah AVSl

Std. Meth. of Tes ASTM

Std. Meth. of Measu ASTM

Std. Meth. of Test F ASTM

Std. M ASTM

Std. Meth. of Test for Volat ASTM

340

2796

D2799

RS296C

Al05

F33I

D2393

A 336

Z21.20

$\mathrm{J} 256 \mathrm{~A}$

1020

UBC 525.18

A541

492.3

F381

UBC*3-25

260.02

$57-29$

CI10

C125

E211.1

CT 102

CT101 
Rec. for Cutting Oils and o the Preparation of Precautionary Labeling and Marking of e United States (Safety) (I973) Safety Std. for Indicating Pressure Gauges for Rec. for Storage and Handling of Uniform Fire Code: Std. Meth. of Test for Std. for Control Valve Capacity Test Procedure for Std. Control Valve Sizing Equations for ) (I962) Meth. of Compression Test with Baker )$(1962)$ Std. Test. Procedure for Axial (End to End) Std. Test. Procedure for Axial (End to End)
Std. Spec. for
Pract. for Design and Construction of Diesel (1973) ipe (1972A) Stds. and Pract. for Design and Construction of Diesel
Std. Spec. for est for Strength Properties of Metal to Metal Adhesives by . Test Meth. for Creep Properties of Adhesives in Shear by erials $(1972)$

test for Strength Properties of Adhesive Bonds in Shear by of Test for Knock Characteristics of Motor Fuels Using the of Test for Knock Characteristics of Motor Fuels Using the Std. Spec. for Lubricant for Installation of Preformed

973) al46.1 Uniform Building Code Std. for Evaluation of meth. of Making, Accelerated Curing, and Test. of Concrete s of Bread (Cereal Chemistry) (1962) ture (R1973) Meth. of Std. Meth. of Rec. Procedure for Std. Groove Dimensions for Fluid Power Radial Meth. for

test for Elastic Moduli of Rock Core Specimens in Uniaxial atibility of Plasticizers in Vinyl Chloride Plastics Under Std. for Spherical Sleeve Handbook of Spring Design:

Tentative Meth of Test for ld Conditions (1974) or Mechanical Properties of Elastomeric Vulcanizates Under ) ANSI K65.31 A37.103, Ashto T167 (1972) AASHO T22, ANSI A37. 18 70) Std. Meth. of Test for Std. Meth. of Test for Std. Meth. of Test for Std. Test Meth. for Edgewise Sid. Meth. of Test for Std. Meth. of Test for Sid. Meth. of Test for Std. Test Meth. for Flatwise Std. Meth. of Test for Hydrostatic Tent. Meth. of Test for

K90.11 fety for Refrigeration and Air Conditioning Condensing and sheets for the Design of Air Exchange Coolers for Packaged Std. for Ammonia Safety Std. for Safety Std. for Sealed (Hermetic Type) Motor herapy and Suction Equipment, Room Air Purifiers, Filters, and Rafter Span, Load, Deflection, Strength and Stiffness Std. for the Protection of Electronic Industry Quality Std. for Industry Quality Std. Format and Coding for e Engine Steady State Performance Presentation for Digital Std. Meth. of Test for Wear Resistance of Inked guidelines for Describing Information Interchange Formats ons and Process Input-Output (1972/ Std. for Indust rial

c. for Location, Construction and Protection of Electronic Rec. for Fire, Heat, and Smoke Damage of Electronic Std. for Std. for Air Data

Rec. Pract. for Hardware Test. of Digital Process Rec. for d. Meth. for Test for Water in Engine Antifreeze-Coolant d Natural Rubber Latex (1972A) ANSI J8.12 Sid. Spec. for

of Analysis of Niacin in Enrichment (Vitamin and Mineral)

Ultraviolet Absorption Meth. of Analysis of Vitamin A in ompounds (Air Contamination-Occupa/ Std. for Acceptable Std. Test Meth. for Hydrogen Ion Std. Meth. for Determination of Low Meth. of Test for Determination of Uranium and Plutonium 12) (1974) Std. for Acceptable Std. Acceptable Std for Acceptable Std. for Acceptable Compounds (1972) r Maximum Permissible Body Burdens and Maximum Permissible 4)

11) (1973)

Std. for Acceptable Std. Acceptable Std. Acceptable Std. Spec. for Std. Spec. for lloy Reinforced (Acar, EC/6201) (1972) An Hard, or Soft (1972) ANSl C7.8 7.59 972) ANSI C7.53

) ANSI C7.22 Sid. Spec. for Compact Round
Std. Spec. for Compact Round Std. Spec. for Aluminum Conductors,
Compounds (1974)

Compressed Gas Containers (1971)

FMS

CGA

CGA

UL

FMS

ASTM

Compressed Gases in Cylinders (1974)

Compressed Gases (1973)

Compressibility of Leather (1970) ALCA E45

Compressible Fluids in Turbulent Flow (1974)

Compressible Fluids (1973)

Compressimeter for Staleness of Bread (Cereal Chemistry

Compression Composite Cans, Tubes and Cores (1974)

Compression Couplings for Vitrified Clay Plain End Pipe

Compression Ignited) Inboard Liquid Cooled Marine Engin

Compression Joints for Vitrified Clay Bell and Spigot P

Compression Loading (Disk Shear) (1972) ANSI Z197.4

Compression Loading (Metal to Metal) (1969)

Compression Loading (I949) ANSI Z197.11

Compression Molding Test Specimens of Allyl Molding Mat

Td. M

Compression Ratio (CR) Technique for Motor Meth. Rating

Compression Ratio (CR) Technique for Research Meth. Rat

Compression Seals in Concrete Pavements (1972)

Compression Test Results in Field Concrete Cylinders (1

Compression Test Results of Field Concrete (1965) ANSI

Compression Test Specimens (1974)

Compression Test with Baker Compressimeter for Stalenes

Compression Test. of Metallic Materials at Room Tempera

Compression Testing of Scaffolds and Shores (1967)

Compression Type Piston Rings (1973) ANSI B93.32

Compression Wood Identification in Pulpwood (1972)

Compression (1972)

Compression (1974)

Std. Meth. of
Std. Meth. of Test for Comp

Compression) Tube Fittings (1971)

Compression, Extension, Torsion, Flat, and Hot Wound (M

Compressive and Flexural Strength of Concrete Under Fie

Compressive or Shear Strains by the Mechanical Oscillog

Compressive Properties of Rigid Cellular PIastics (1973

Compressive Strength of Bituminous Mixtures (1974) ANSI

Compressive Strength of Cylindrical Concrete Specimens

Compressive Strength of Flat Sandwich Constructions (19

Compressive Strength of Hydraulic Cement Mortars (Using

Compressive Strength of Hydraulic Cement Mortars (Using

Compressive Strength of Masonry Assemblages (1972)

Compressive Strength of Sand wich Cores (1970)

Compressive Strength of Syntactic Foam (1972A)

Compressive (Crushing) Strength of Graphite (1971) ANS

Compressor Units (1973)

Compressor Units (1974)

Compressors and Units (1973)

Compressors for Process lndustries (1972)

Compressors (1972) ANSI Bl43.1

Compressors, Artificial Airways, Appurtenances) (1973)

Computations (1973)

Rec. for Wood Beam, Joint

Computer and Data Processing Equipment (1972)

Computer Output Microfilm (1971)

Computer Output Microfilm (1971)

Computer Programs (1970)

Computer Ribbons (1972)

Computer Software Documentation and Terminology) (I972)

Computer System FORTRAN Procedures for Executive Functi

Computer Systems (1972)

Computer Systems (1973)

Computer Type (Square Loop) Pulse Transformers (1970)

Computers Mps (1970)

Computers (1971)

Computing Electrical Demands for Farms (1972)

Concentrate (Glycol or Alcohol Base) by the Iodine Reag

Concentrated, Ammonia Preserved, Creamed and Centrifuge

Concentrates Used to Enrich Cereals (Cereal Chemistry)

Concentrates (Cereal Chemistry) (1962)

Concentration of Chromic Acid and Hexavalent Chromium C

Concentration of Dry Adhesive Films (1970)

Concentration of Lead in Paint (1973)

Concentrations and Isotopic Abundances (1970) ANSl N115

Concentrations of Acetone (1971)

Coricentrations of Dichlorodifluoromethane (Fluorocarbon

Concentrations of Hydrogen Sulfide (1972)

Concentrations of Inorganic Mercury and Nonalkyl Organo

Concentrations of Radionuclides in Air and in Water for

Concentrations of Toluene in the Air of Workplaces (197

Concentrations of Trichlorofluoromethane (Fluorocarbon

Concentrations of $1,1,2$-Trichloro-1,2,2trifluoroethane

Concentric Lay Stranded Aluminum Conductors, Aluminum a

Concentric Lay Stranded Copper Conductors, Hard, Medium

Concentric Lay Stranded Copper Conouctors (1974) ANSI C

Concentric Lay Stranded EC Grade Aluminum Conductors (1

Concentric Lay Stranded SteeI Reinforced, (ACSR) (1972A
ISA

ISA

AACC

CCTl

ASTM

ABYC

ASTM

T ASTM

Std ASTM

ASTM

ASTM

ASTM

ASTM

ASTM

CBO

ACI

Std. ASTM

AACC

ASTM

SSI

NFLDP

TAPP

ASTM

ASTM

SAE
SMI

ASTM

ASTM

ASTM

ASTM

ASTM

ASTM

ASTM

ASTM

ASTM

ASTM

ASTM

ASTM

d. for Sa LL

API

ARI

ANSI

UL

NSF

WWPA

NFPA

NMA

NMA

ASTM

ANSI

IS A

Re FMS

FMS

IEEE

SAE

ISA

ASAE

ASTM

ASTM

$\mathrm{AACCH}$

AACC

ANSI

ASTM

ASTM

ANSI

ANSI

ANS

ANSI

NCRPM

ANSI

ANSI

ANSI

ASTM

ASTM

ASTM

ASTM

ASTM

7.37

C7

404

7.50

UFC*2ART8

D2213

$\$ 39.4$

S 39.3

P15

$\mathrm{C} 425$ 
ors (1972) ANSI C7.50

tors (1972) ANSI C7.52

)$(1973)$

Std. Spec. for Std. Spec. for Aluminum Conductors, Spec. for Tinned Copper Std. for Glossary of Terms Rec.

Std. Meth. of Test for Surface Moisture in Fine r Cooled lron Blast Furnal Uniform Building Code Std. for h. of Test for Sieve or Screen Analysis of Fine and Coarse Std. Spec. for Std. Spec. for Prefaced Std. Definitions of Terms Relating to hragms / Uniform Building Code Std. for Reinforced Gypsum 1) Std. Spec. for Reinforced ms, Equipped for Hook Work, Clamshell, Magnet, Grapple, or Sid. Spec. for Uniform Building Code Std. for Tent. Meth. of Test for Air Content of Freshly Mixed Rec, for Manufacture and Placement of Reinforced Spec. for the Design and Construction of Reinforced Uniform Building Code: Masonry or Tent. Rec. for Design of

)

Std. Meth. of Making, Accelerated Curing, and Test. of ing, Column and Other Members) (1973)

Spec. for Placing Reinforcing Steel Bar Supports in ilding Code Std. for Prestressed Steel Strand and Wire for forcing Steel, Metal Inserts and Connections in Reinforced Std. Spec. for Reinforced e Std. for Evaluation of Compression Test Results in Field 973)

for Determining the Splitting Tensile Strength of Molded Std. Spec. for Reinforced Std. Spec. for e $(1972)$

of Mineral Admixtures in Preventing Excessive Expansion of Std. Spec. for Reinforced Rec. Pract. for Suggested Design Procedures for Combined Spec. for Structural Rec. for Preplaced Aggregate Rec. Safety Requirements for Shoring Suggested Design and Construction Procedures for Rec. Pract. for

Rec. for Erosion Resistance of Std. Meth. of Test for Density of Bituminous Rec. for Materials and Meth. for Durability of Rec. Guide for Making a Condition Survey of Std. Spec. for Nonreinforced
Std. Spec. for Reinforced

3)

ing (1973) and Manholes (1973) ANS1 A73. Tent. Spec. for Std Spec, for Std. Spec. for Hollow Load Bearing Std. Meth. of Sampling and Test.

Std. Spec for Hollow Nonload Bearing Std. Spec. for Solid Load Bearing Uniform Building Code Std. for Hollow Load Bearing Uniform Building Code Std. for Solid Load Bearing Uniform Building Code Std. for Hollow Nonload Bearing Uniform Building Code Std. for Meth. of Test for Tent. Rec. for

6) Std. Meth. of Test for Time of Setting of Design Criteria for Reinforced

Rec. Pract. for Reinforced Bituminous Guide Spec. for Specifying: Precast

Recommendations for Basis of Payment on Portland Cement Rec, Pract, for Construction of (1974/ Interim Guideline for Protection and Acceptance of bricant for lnstallation of Preformed Compression Seals in ng / Std. Spec. for Preformed Expansion Joint Fillers for pec. for Optimum Size Coarse Aggregate for Portland Cement Sid. Spec, for Std. Spec. for Porous Std. Meth. of Test.

Rec, for Cast in Place Nonreinforced Spec for Cast in Place Nonreinforced Std. Spec. for Steel Wire, Hard Drawn for Prestressing

Truck Mixer and Agitator Stds.

and Other Liquids (1972)

Std. for Prestressed Std. for

Uniform Building Code Std. for

Std. Spec, for Deformed and Plain Billet Steel Bars for Std. Spec. for Rail Steel Deformed Bars for Std. Spec. for Axle Steel Deformed and Plain Bars for
Concentric Lay Stranded 5005-H19 Aluminum Alloy Conduct

Concentric Lay Stranded 6201-T 81 Aluminum Alloy Conduc Concentric Lay Stranded (1972)

Concentric Neutrals (Underground Electrical Power Cable

Concerning Letter Symbols (1972)

Concerning Storage of Expanded Rubber (1972)

Concrete Aggregate (1973) AASHO T142. ANSI A37.21

Concrete Aggregates (Sand, Crushed Stone, Gravel and Ai

Concrete Aggregates (1971) AASHO T27, ANSI A37.8

Concrete Aggregates (1974) ANSI A37.124

Concrete and Calcium Silicate Masonry Units (1973)

Concrete and lts Aggregates (1968) ANSI A37.76

Concrete and Precast Slabs, and Poured Gypsum Roof Diap

Concrete Arch Culvert, Storm Drain, and Sewer Pipe (197

Concrete Bucket Attachments (1971)

Concrete Building Brick (1971) ANSI A75.1

/Rope Supported Boo

Concrete Building Brick (1973)

Concrete by the Pressure Meth. (1972T) ANSI A37.70

Concrete Casings for Irrigation Wells (1972)

Concrete Chimneys (1969) ANSl A 158.l

Concrete Chimneys, Fireplaces, and Barbecues (1973)

Concrete Composite Beams and Girders for Building (1957

Concrete Compression Test Specimens (1974)

Concrete Construction Design Handbook (Beam, Slab, Foot

Concrete Construction (1970)

Concrete Construction (1973)

Concrete Construction (1973)

/De Std. for Welding Rein

Concrete Culvert, Storm Drain, and Sewer Pipe (1973)

Uniform Bu lCBO

Concrete Cylinders (1973)

Concrete Cylinders (1973)

Uniform Building Code Std

Concrete D-Load Culvert, Storm Drain, and Sewer Pipe (1

Concrete Drain Tile (1972)

Concrete Due to the Alkali-Aggregate Reaction (1969)

Concrete Elliptical Culvert, Storm Drain, and Sewer Pip

Concrete Floor and Slab Construction (1969) ANSI A 157.1

Concrete Footings and Mats (Foundation) (1963)

Concrete for Buildings (1973) ANSI A 138.1

Concrete for Structural and Mass Concrete (1966)

Concrete Formwork (1972)

Concrete Foundation Piers (1969)

Concrete Highway Bridge Deck Construction (1974)

Concrete in Hydraulic Structures (1952)

Concrete in Place by Nuclear Meth. (1974)

Concrete in Service (1959)

Concrete in Service (1965)

Concrete Irrigation Pipe with Rubber Gasket Joints (197

Concrete Low Head Pressure Pipe (1973)

Concrete Made by Volumetric Batching and Continuous Mix

Concrete Manhole Sections (1973)

Concrete Masonry Units for Construction of Catch Basins

Concrete Masonry Units (1970) ANS1 A79.1

Concrete Masonry Units (1970) ANSI A84.]

Concrete Masonry Units (1971) ANSI A80.1

Concrete Masonry Units (1971) ANSI A81.1

Concrete Masonry Units (1973)

Concrete Masonry Units (1973)

Concrete Masonry Units (1973)

Concrete Masonry Units (1973)

Concrete Members Prestressed with Unbonded Tendons (196

Concrete Mixtures by Penetration Resistance (1970) ANSI

Concrete Nuclear Power Containment Structures (1969)

Concrete Overlays (Welded Wire Fabric) (1962)

Concrete Panels (1973)

Concrete Pavement Overlay Projects (1973)

Concrete Pavements and Bases (1974)

Concrete Pavements Exposed to Rain During Construction

Concrete Pavements (1965)

Concrete Pavements (1972)

Concrete Paving and Structural Construction (Nonextrod

Concrete Paving Jobs (1973)

Concrete Paving (1972)

Concrete Pipe for Irrigation or Drainage (1973)

Concrete Pipe for Use in Underdrains (1973)

Concrete Pipe or Tile (1973)

Concrete Pipe (1966)

Concrete Pipe (1970) ANSI A 171.1

Concrete Pipe (1973)

Concrete Plant Equipment Stds. (1973)

(Concrete Plant Equipment) (1973)

Concrete Plant Mixer (Equipment) Stds. (1973)

Concrete Pressure Pipe, Steel Cylinder Type, for Water

Concrete Radiation Shields (1972)

Concrete Reinforcement Bars (1973)

Concrete Reinforcement (1972)

Concrete Reinforcement (1972)

Concrete Reinforcement (1972)

ASTM

ASTM

ASTM

AA

ANSI

FMS

/ Met ASTM

ASTM

ASTM

ASTM

ICBO

ASTM

SAE

ASTM

ICBO

ASTM

ASAE

$\mathrm{ACl}$

ICBO

$\mathrm{ACl}$

ASTM

$\mathrm{ACl}$

ICBO

ASTM

ICBO

ICBO

ASTM

ASTM

ASTM

ASTM

$\mathrm{ACl}$

ACI

$\mathrm{ACl}$

$\mathrm{ACl}$

SSl .

$\mathrm{ACI}$

ACl

$\mathrm{ACl}$

ASTM

ACl
ACl

ASTM

ASTM

ASTM

ASTM

ASTM

ASTM

ASTM

ASTM

ASTM

ICBO

ICBO

ICBO

ICBO

ACI

A STM

$\mathrm{ACl}$

WRI

CSI

ACPA

ACI

ACPA

ACI

B397

B399

B231

2.1

Y 10.1

LPD8-2

UBCS26. 2

C 136

C33

C744

C 125

UBC $24 \cdot 13$

C506

$\mathrm{J} 220$

C55

UBCS24-3

C231

R266

07

UBC 3.37

57.29

C684

SP17

*1.3.5

UBCS26-7

UBCS26-8

C76

UBCS26-11

UBCS26-12

C655

C412

C441

63.49

301

66-65

*9

69-42

345

52-18

D2950 
Std. Spec, for Cold Drawn Steel Wire for Std. Spec. for Welded Steel Wire Fabric for Std. Spec. for Cold Worked Deformed Steel Wire for Std. Spec. for Welded Deformed Steel Wire Fabric for Uniform Building Code Std. for Cold Drawn Steel Wire for building Code Std. for Steel Bar and Rod Mats (Sheets) for de Std, for Welded Steel Wire and Deformed Wire Fabric for Rec, for ng; Hot Rolled and Cold Finished Bars; Hot Rolled Deformed tion Contract (1968)

Suggested Components of $A$ Spec. for Perlite Insulating oirs, etc. for Water and Waste Treatment) (1968/ Rec. for Std. Spec. for Rec. for

el Splined Continuous Strip Mastic Set Maple Flooring Over sleeper Construction with Nailers and Finish Flooring Over truction with Nailers, Subflooring or Finish Flooring Over ith Mastic Set Subflooring and Nailed Finish Flooring Over pec. for Mosaic Wood Parquet Flooring Set in Adhesive Over Clay Tile and Tent. Test Meth. for Critical Dilation of eth. of Test for Splitting Tensile Strength of Cylindrical std. Meth. of Test for Compressive Strength of Cylindrical Std. Meth. of Capping Cylindrical Stds. for Construction of Rec. Pract, for in Place

Design and Construction of Circular Prestressed 6) Rec. for Correct Preparation, Finishing and Testing of 37.81 Std. Meth. of Test for Abrasion Resistance of Horizontal Std. Meth, of Making and Curing Tent. Spec. for Molds for Forming Uniform Building Code Std. for Std Spec for Perforated ive Meth. of Test for Compressive and Flexural Strength of Guide Spec, for
Rec. for Precast (1966) construction)/ ction (1965) (1968) ANSl

Std. Spec. for Preformed Expansion Joint Filler for Std. Specs. for Post Tensioned Prestressed Rec. for Deep, Cast in Place Std. Meth. of Test for Flexural Strength of Rec. for Selection and Use of Aggregates for Rec. for Admixtures for Rec. Guide for Structural Lightweight Aggregate

Std. Rec. Pract. for Selecting Proportions for No Slump pract. for Evaluation of "Compression Test Results of Field for Preplaced Aggregate Concrete for Structural and Mass for Precast Concrete Units Used as Forms for Cast in Place Specifying Tests, Plastic

Rec. Guide for Use of Admixtures in ract. for Selecting Proportions for Structural Lightweight Steel Wire Bar Supports Used to Position Reinforcement in Rec. Pract. for Selecting Proportions for Normal Weight Rec. Pract. for Curing Std. for Preparation of Notation for Std. Building Code Requirements for Reinforced Std. Rec. Pract. for Curing Std. for Building Code Requirements for Structural Plain Std. for Building Code Requirements for Structural Plain Rec. Pract. for Consolidation of

Std. Meth. for Physical Test. of Gypsum Plasters and Std. Spec. for Aggregates for Radiation Shielding Uniform Building Code Std. for Test. Gypsum Plasters and Uniform Building Code Std. for Ready Mixed Uniform Building Code Std. for Admixtures for Uniform Building Code: Structural Deck (Roofing); Gypsum

c. Pract. for Measuring, Mixing, Transporting, and Placing ilding Code Std. for Lightweight Aggregates for Structural ture of Constituents of Aggregates for Radiation Shielding

Std. Meth. of Test for Organic lmpurities in Sands for w or Calcined Natural Pozzolans for Use in Portland Cement Std. Spec. for Air Entraining Admixtures for td. Spec. for Liquid Membrane Forming Compounds for Curing h. of Test for Length Change of Hardened Cement Mortar and Change of Drilled or Sawed Specimens of Cement Mortar and Rec. Pract. for Hot Weather Concreting lization, Ice Removal, Acceleration of Concrete, Curing of

or Dustlaying, Stabilization, lce Removal, Acceleration of 8/ Std. Rec. Pract. for Inspection and Test. Agencies for
ntation, Protectio/ Storage) Rooms Used in the Test. of Hydraulic Cements and ing Code: Weights of Materials (Brick; Cast Iron; Cinders; Rec. Pract. for Hot Weather Rec. Pract, for Hot Weather Rec. Pract, for Cold Weather Std. Rec. Pract. for Hot Weather
Concrete Reinforcement (1972) ANSl A50.3 Concrete Reinforcement (1972) ANSl G45.1

Concrete Reinforcement (1972) ANSI G45.2

Concrete Reinforcement (1972) ANSI G45.3

Concrete Reinforcement (1973)

Concrete Reinforcement (1973)

Concrete Reinforcement (1973)

Concrete Reinforcing Bar Splices (1971)

Concrete Reinforcing Bars (1972)

Concrete Reinforcing Steel

Concrete Sanitary Engineering Structures (Tanks, Reserv

Concrete Sewer, Storm Drain, and Culvert Pipe (1973)

Concrete Shell Structures (1973)

Concrete Slab Floors (1962)

Concrete Slab Floors (1962)

Concrete Slab Floors (1962)

Concrete Slab Floors (1962)

Concrete Slab or Wood Subfloors (1962)

Concrete Slabs (Tech. Notes) (1962)

Concrete Specimens Subject to Freezing (1972T)

Concrete Specimens (1971) ANSI A37.121

Concrete Specimens (1972) AASHO T22, ANSI A37.18

Concrete Specimens (1973)

Concrete Stave Farm Silos (1969)

Concrete Strength Evaluation (1970)

Concrete Structures (1967)

Concrete Subfloor Surfaces to Receive Wood Flooring (19

Concrete Surfaces (1974)

Concrete Test Specimens in the Laboratory (1969) ANSI a

Concrete Tests Cylinders Vertically (1973T) ANSI A37.95

Concrete Tests (1973)

Concrete Under Drainage Pipe (1973)

Concrete Under Field Conditions (1974)

Concrete Unit Masonry (1971)

Concrete Units Used as Forms for Cast in Place Concrete

Concrete (Bituminous Type) (1971) ANSI A37.101

Concrete (Post Tensioned Box Girder Bridges Design and

Concrete (Storage) Bin and Silo Wall Design and Constru

Concrete (Using Simple Beam with Center Point Loading)

Concrete (1958)

Concrcte (1960)

Concrete (1964)

Concrete (1965)

Concrete (1965) ANSI Al46.1

Concrete (1966)

Concrete (1966)

Concrete (1967)

Concrete (1968)

Concrete (1969) ANSI Al64.1

Concrete (1970)

Concrete (1970) ANS1 Al67.1

Concrete (1971) ANSl A168.1

Concrete (1971) ANSl A188.

Concrete (1971) ANSI A89.1

Concrete (1972) ACI 308

Concrete (1972) ACI 322

Concrete (1972) ANSl Al69.1

Concrete (1972) ANSl A185.1

Concrete (1973)

Concrete (1973)

Concrete (1973)

Concrete (1973)

Concrete (1973)

Concrete (1973)

Concrete (1973)

Concrete (1973)

Concrete (1973)

Concrete (1973)

Concrete (1973) AASHO T21, ANSl A37.19

Concrete (1973) ANSI A37.122

Concrete (1973) ANSI A37.132

Concrete (1973) ANSl A37.87

Concrete (1974) ANSI A37.78

Concrete (1974T)

Concrete) (1972) ANSI Al70.1

Concrete, and Other Road Conditioning Purposes (1974) a

Concrete, Curing of Concrete, and Other Road Conditioni

Concrete, Steel and Bituminous Materials as Used in Con

Concrete, Stone, Tile, Metal, Plastic, etc.) for Orname

Concretes (1973)

/Ec. for Moist Cabinets and

Class: Granite; Gravel; Granite, Limes

Concreting (Concrete) (1972) A.NSl A170.l

Concreting (1959)

Concreting (1966) ANSI A144.1

Concreting (1972) ACI 305
ASTM

ASTM

ASTM

ASTM

ICBO

Uniform ICBO

ICBO

CRSI

AISI

CRSI

CSI

$\mathrm{ACl}$

ASTM

ACI

Spec. for Ste WSF

WSFI

WSFI

WSFI

WSF

BIA

ASTM

Std. M ASTM

ASTM

ASTM

NSLO

NRMCA 133

ACl

WSFI

ASTM

ASTM

ASTM

ICBO

ASTM

Tentat ASTM

CSI

ACI

ASTM

CRSI

$\mathrm{ACl}$

ASTM

$\mathrm{ACl}$

ACl

$\mathrm{AC}$

$\mathrm{ACl}$

Rec. AC

Rec AC

Rec. AC

CSl

$\mathrm{ACI}$

Rec. P ACI

Spec. CRSI

ACI

ACI

$\mathrm{ACl}$

ACI

ANSl

ANS1

ACl

$\mathrm{ACl}$

ASTM

ASTM

ICBO

ICBO

ICBO

ICBO

NRCA

Re ACI

Uniform Bu ICBO td. Descriptive Nomencla ASTM ASTM

ASTM

ASTM

Std Met ASTM

ASTM

$\mathrm{ACl}$

ASTM

ASTM

ASTM

ICBO

ASTM

ICBO

$\mathrm{ACl}$

$\mathrm{ACl}$

$\mathrm{ACl}$

ANSI

A82

3

$\because 1.9 .1$

03521

68.50

Cl4

$* 4$
$* 8$

$* 6$

14A

$\mathrm{C} 496$

C39

67.40

$+1$

A 185

A 496

A497

UBCS24.16

UBCS26.5

BCS26.

C192 
tallic Surfaces (Coil) (1974) ulfur Oxides in Flue Cases (Barium Chloranilate Controlled Moisture Control in Brick and Tile Walls. Std. Meth. of Test for Thermal Instability of Confined

1974)

1973)

heat Exchangers (1973) Spec for Copper and lis Alloy Seamless Spec for Aluminum Alloy Seamless Round td. Spec. for Seamless Nickel and Nickel Alloy Std. Spec. for Copper Alloy Sto Alloy Heat Exchanger and Austenitic Steel Boiler, Superheater, Heat Exchanger, and ess Cold Drawn Intermediate Alloy Steel Heat Exchanger and Std. Spec for Seamless and Welded Titanium Tube for for Copper Alloy Condenser Rolled Tube Plates in Surface Sid. Spec. for Aluminum Alloy Drawn Seamless Tubes for Std. Meth. of Testing for Rating Water Cooled Refrigerant Sid for Safety for Refrigeration and Air Conditioning Safe Operating Safe Operating ents (Passenger Car Type Tires) (1971) Rec. Guide for Making A uantitative Meth. of Analysis of Calcium Peroxide in Dough Std for Sound Rating of Room Fan Coil Air Component Std. for Receiver Dehydrators for Vehicle Air Std. for Room Air

Safety Std. for Room Air Safety Std. for Air

i $\mathrm{H} 23.5$ Std. Spec. for Seamless Copper Tube for Air Std. for Central Station Air Handling Units (Air Std. for Gas Fired Absorption Summer Air Std. for Safety for Refrigeration and Air Air Handling Spec. for Sheet Metal and Air

edures (1973) of Testing for Sound Rating Heating, Refrigerating and Air Std. for Unitary Air Std. for Recreational Vehicle Air Std. Meth. of Testing Automotive Air Std. Meth. of

ppi T402

celeration of Concrete, Curing of Concrete, and Other Road d Test Equipment and R/ Std. for Recreational Vehicle Air itioning System) (1966 What Makes a Good Air ood Air Conditioning System (Information on Year Round Air Std. for Liquid Line Driers in Refrigerating and Air brous Glass Duct Construction Std. for Ventilating and Air minimum Installation Stds. for Residential Heating and Air Std. for Installation of Central Warm Air Heating and Air on Line Filters and Filter Driers in Refrigeration and Air 974) ANS1 L14.205 Std. Rec. Pract. for

Coil Face Areas for Central Station Units (Air Moving and operating Limits for Central Station Units (Air Moving and ing and Regulating Electrical Equipment for Control of Air combination Duct System/ Std. for the Installation of Air ms) (1972) fuel Tanks and Fill Pipes with / Rec. Pract. for Defining nd Beverages (1973) Std. Rec. Pract. for Establishing ance (1973) / Std. Rec. Pract. for Selection of Geometric rmity in the Graphic Arts (1972) Std. for Viewing permanence of Adhesive Bonded Joints in Plywood Under Mold

th. for Resistance of Adhesives to Cyclic Laboratory Aging nts, Driveways, and Interior Floors Exposed to Wet and Dry motor Vehicle Brake Fluid for Use Under Arctic Atmospheric of Direct Shear Test of Soils Inder Consolidated Drained

ed Wood Products for Use Under Exterior (Wet Use) Exposure minated Products for Use Under Interior (Dry Use) Exposure ombustible Liquids and Solids and Potential Dust Explosion Compressive and Flexural Strength of Concrete Under Field ltage of Insulating Oils of Petroleum Origin Under Impulse Std for Motor Vehicle Brake Fluid (Used Under Nonarctie s (1972) (Coil/ Packed Paint Film (Cor 2) ANSl 21

973)

als $(1970)$

Bonds in Scarf Joints (1970) Std. Rec. Pract. for Std. Rec. Pract. for Safety Std. for Hose for Std. Meth. of Std. Rec. Pract. for Std. Meth. of

Tent. Rec. Pract. for Std. Meth of Test for Volume Resistivity of Recommendation for Installation of Std. for Safety for Electrically Rec. for Electric ally

Anesthetizing Location/ Std. Meth. of Test for Electrical Resistance of
Std. Meth. of Test for Electrical Std. Meth. of Test for Electrical ipator Additive (1971)/ Meth of Test for D.C. Electrical
Condensation-Humidity Test. of Organic Coatings on Me Condensation Meth.) (1973) Condensation (Tech. Notes) (1965)

Condensed Phase System s (Confinement Test) (1973) Condenser and Heat Exchanger Tubes with Integral Fins condenser and Heat Exchanger Tubes with Integral Fins ( Condenser and Heat Exchanger Tubes (1972) ANSI H34.42 Condenser Rolled Tube Plates in Surface Condensers and Condenser Tubes and Ferrule Stock (1974) ANSl H23.14 Condenser Tubes (1973)

Condenser Tubes (1973) ANSI B125.32 Condenser Tubes (1973) ANSI Bl25.8 Condensers and Heat Exchangers (1965)

Condensers and Heat Exchangers (1973) / Spec. for Welded ASTM D. Spec. for Seaml ASTM

Condensers and Heat Exchangers (1973) ANSI H38.6 Condensers (1971)

Condensing and Compressor Units (1973)

Condition of Tires Including Tire Treads Depth Requirem

Condition of Truck, Trailer and Bus Type Tires (1973) Condition Survey of Concrete in Service (1965)

Conditioners Containing Monoglyceride, Salts, Soy Flour

Conditioners Delivering Less Than $1500 \mathrm{cfm}$ (1971)

Conditioners (1967)

Conditioners (1972) ANSI Z234.1

Conditioners (1973) ANSI C33.14

Conditioners, Central Cooling (1972) ANSI B144.

Conditioning and Refrigeration Field Service (1974) Ans

Conditioning and Refrigeration) (1974)

Conditioning Appliances (1973)

Conditioning Condensing and Compressor Units (1973)

Conditioning Contractors (1973)

Conditioning Equipment Selection and System Design Proc

Conditioning Equipment (1972)

Conditioning Equipment (1974)

Conditioning Equipment (1974)

Conditioning Hose (1968E) ANSI J2.27

Conditioning Paper and Its Products for Test. (1973) T

Conditioning Purposes (1974) ANSI A37.37, Ashto M144

Conditioning System Rating Definitions, Test. Meth., an

Conditioning System (Information on Year Round Air Cond

Conditioning System) (1966)

Conditioning Systems (1971)

Conditioning Systems (1972)

Conditioning Systems (1973)

Conditioning Systems (1973) ANSI B144.

Conditioning Systems (1974)

What Makes a

Conditioning Textiles and Textile Products for Test. (1

Conditioning) (1966)

Conditioning) (1966)

Conditioning, Heating, Cooking, Refrigeration, and Humi

Conditioning, Heating, Exhaust, Ventilating, and Their

Conditioning, Heating, Ventilating and Processing Syste

Conditions for Evaluating the Compatibility of Vehicle

Conditions for Laboratory Sensory Evaluation of Foods a

Conditions for Measurement of Reflectance and Transmitt

Conditions for the Appraisal of Color Quality and Unifo

ASTM

ASTM

ASHRA

$\mathrm{UL}$

VESC V-7

VESC V-9

ACl 65.67

AACCH 48.51

ARI 443

IMACA 309

AHAM RAC-1

UL

UL

ASTM B280

ARI 430

ANSl Z21.40.1

UL 303

$\mathrm{SMACN} * 3$

NESCA MAN K

Conditions (1970)

Conditions (1970) ANSl Z197.19

Conditions (1970) ANSI 09.1

Conditions (1972)

Conditions (1972)

Conditions (1972) ANSI Z197.27

Conditions (1972) ANSI Z197.33

Condition s (1973

Conditions (1974)

Conditions (1974)

Conditions) (1972)

(T), and Handling of Flammable and C

Tentative Meth. of Test for

Conducted Controlled Velocity Laboratory Corrosion Test

Conducting a Solvent Hand Rub Test for the Determinatio

Conducting Bending Fatigue Tests for Copper Alloy Sprin

Conducting Creep Tests of Metal to Metal Adhesives (197

Conducting Exterior Exposure Tests of Paints on Wood (1

Conducting Gasoline (1973)

Conducting Machining Tests of Wood and Wood Base Materi

Conducting Natural Light Exposures Under Glass (1973)

Conducting Shear Block Test for Quality Control of Glue

Conducting Wear Test on Textile Garments (1973)

Conductive Adhesives (1972)

Conductive Ceramic Tile Floors (1973)

Conductive Equipment and Materials for Use in Flammable

Conductive Floors (1973)

Conductive Resilient Flooring (1972)

Conductivity of Aviation Fuels Containing a Static Diss

Conductivity of Carbon Refractories (1973)

Conductivity of Hydrocarbons Fuels (1972) ANSI Z11.322
ASHRA

ARI

ASTM

AST M

ASTM

IMACA

NESCA

NESCA

ARI

SMACN *6

SMACN *10

NFPA

$\begin{array}{ll}\text { NFPA } & 90 \mathrm{~B} \\ \mathrm{ARI} & 730\end{array}$

NEMA $\mathrm{HEl}$

Std. AMCA 1402

Sid. AMCA 1401

IL

NFP

UL.

SAE

ASTM

ASTM

ANSI

ASTM

Test Me ASTM

Paveme ASTM

Std. for SAE

std. Meth ASTM

ASTM

ASTM

$1 \mathrm{CBO}$

ASTM

SAE

AWPA

NCCA

ASTM

ASTM

ASTM

UL

ASTM

ASTM

ASTM

ASTM

ASTM

TCA

UL.

FMS

ASTM

ASTM

ASTM

ASTM

210
ASTM D1776

171

234

A



Std. Meth. of Test for Thermal Std. Meth. of Test for Thermal Std. Meth. of Test for Thermal Rec. Pract. for 7

Cable (1972)

ene-Rubber Insulation, 200/

Std. for Nonshielded Single lene-Rubber lnsu/ Std. for Portable Single and Multiple Std. Meth. of Test for Resistivity of Electrical Std. Ampacities with Effect of Shield Losses for Single Code Words for Aluminum Electrical

Pipe, and Structural Shapes for Electrical Purposes (Bus and Building Foundation Drain, Under Basement Floor, Sump 1 Conductors (1971) Spec. for Rope Lay Stranded Copper r Conductors Having Bunch Stranded Members, for Electrical Spec. for Concentric Lay Stranded 5005-H19 Aluminum Alloy spec. for Concentric Lay Stranded 6201-T 81 Aluminum Alloy or Compact Round Concentric Lay Stranded AQGiAnarts tions of Terms Relating to Uninsulated Metallic Electrical Rec. Pract. for Determining Solderability of Thick Film

Between Aluminum or Aluminum-Copper Overhead Electrical ctric Power Cable $15 \mathrm{kV}$ Through $35 \mathrm{kV}$ (Copper and Aluminum 1972) An/ Std. Spec. for Concentric Lay Stranded Aluminum acsr) (1972A) ANSI C 7.22

Std. Spec. for Aluminum Std. Spec. for Aluminum

Std Spec for Concentric Lay Stranded Copper Aluminum Coated (Aluminized) Steel Core Wire for Aluminum minum Coated (Aluminized) Stranded Steel Core for Aluminum for Zinc Coated (Galvanized) Steel Core Wire for Aluminum olyvinyl Chloride Externally Coated Galvanized Rigid Steel or Acrylonitrile Butadiene Styrene (ABS) Plastic Utilities ble lnst/ Std. for Safety for Flexible Steel and Aluminum d Cables Std. for Safety for Rigid Nonmetallic

Tent. Meth. of Test for Viscosity of Asphalt with si Al11.4 Std. Meth. of Test for Pyrometric Test for Yield Stress of Heterogeneous Propellants by the Meth. for

Sid. for Wrenching

eaking Tenacity of Man Made Textile Fibers in Loop or Knot unting Dime/ Std. Bore and Rod Size Combinations, Rod End 73) Std. Meth. of Test for Thermal Instability of

Thermal Instability of Confined Condensed Phase Systems nd Gloss Tolerances of Opaque Materials and for Evaluating $f$ Analysis of Congeal Point in Fats and F at Mixtures Which Solidify Within Temperature Range / Meth. of Analysis of m (1971) ANSl Z11.61

m (1972) Std. Meth. of Test for Meth. for

ck: Deciduous Shade and Flowering Trees; Deciduous Shrubs: ltivator, Edger-Trimmer, Hedge / Std. for Safety for Cord safety for Test for Polymeric Enclosures for Portable Cord Safety Std. for Electric Cord

s, Drift Bolts, and Wood and Lag Screws; Light Metal Plate minimum Requirements for Motor Vehicle (Recreational Type) Std. for Blower Pipe and Std. for W ater

Thread Compounds (For Casing. Tubing and Line Pipe code Std. for Welding Reinforcing Steel, Metal lnserts and Std. for Metric Thread Fuel lnjection Tubing Uniform Plumbing Code: Joints and Drain P/ Std. for lnstallation of Seacocks, Through Hull lag Screws; Light / Uniform Building Code Std. for Timber Std. for Safety for Pigtail, Expansion Coil, and Flexible Rec. Pract. for 7 Conductor Electrical Std. for Receptacle Dummy Storage, Electrical

Std. Spec. for Aluminum Bar for Electrical Purposes (Bus for Structural Steel and lron (Shape, Plate, Sheet, Strip, ems (1973) ANSl Se2.6 (1970) ohms) (1973) Sid. for Safety for um) Gas (1972) UL 567

Std. for Precision Coaxial Std. for Supply Gas Safety Std. for Pipe Sid. for Metal 71) 971) Std. for Gas Hose
Std. for Gas Hose Overhead Electrical Connectors (1973) NEMA Cc/ Std. for r Overhead Electrical Conductors (1973) EEl Tdj/ Std. for Appliances (1971) Std. for Flexible ations (1973)

73)

\section{Std. for Electrical Plugs, Receptacles and} Uniform Building Code Std. for Shear Std. for Flexible Copper Water est Procedures for Low Frequency (Below $3 \mathrm{MHz}$.) Electrical Std. for Electric Power

Between Aluminum or Aluminum-Copper Overhead Electrical Std for Safety for Factory Made Air Duct Materials and Std. Test Proc. for Low Frequency (Below $3 \mathrm{MHz}$.) Electric
Conductivity of Insulating Fire Brick (1972) ANSI Alll. Conductivity of Leather with the Cenco Fitch Apparatus Conductivity of Liquids (1971) ANSI Z11.304

Conductivity of Refractory Brick (1971) ANSI All1.32

Conductivity of Unfired Monolithic Refractories (1972)

Conductor Electrical Connector for Truck Trailer Jumper Conductor Electrical Power Cable with Ethylene-Propyl

Conductor Electrical Power Cables with Ethylene-Propy

Conductor Materials (1972A) ANSI C7.24

Conductor Solid Dielectric Power Cable 15 kV Through 35

Conductor (1972)

Conductor) (1973) ANSI C7.45

Conductor Extruded B

Conductors Having Bunch Stranded Members, for Electrica

Conductors (1971)

Conductors (1972) ANS1 C 7.50

Conductors (1972) ANSI C7.52

Conductors (1972) ANSI C 7.53

Conductors (1972A)

Conductors (1972T)

Conductors (1973) EEI TDJ-162

Conductors) (1972) pec. for Rope Lay Stranded Coppe ASTM

Std. Spec, F ASTM

Sid. Defini ASTM

for Connectors for Use

Conductors, Aluminum Alloy Reinforced (Acar, EC/6201)

Conductors, Concentric Lay Stranded Steel Reinforced,

Conductors, Concentric Lay Stranded (1972)

Conductors, Hard, Medium Hard, or Soft (1972) ANSI C7.8

Conductors, Steel Reinforced (ACSR) (1972) ANSl C 7.47

Conductors, Steel Reinforced (ACSR) (1972) ANSI C 7.61

Conductors, Steel Reinforced (ACSR) (1974) ANSI C7.60

Conduit and Electrical Metallic Tubing (1974)

Conduit and Fittings (1972) ANSI K65.57

Conduit for Safe Use and $\mathrm{C}$

Conduit (1972) ANSI C 33.91

Cone and Plate Viscometer (1973)

Cone Equivalent (PCE) of Refractory Materials (1972) an

Cone Penetration Meth. (1972) ANSl Z11.319

Cone Penetration of Petrolatum (1972)

Configuration, Spline Drive Threaded Fasteners (1971)

Configurations (1973)

Tent. Meth of

Configurations, Dimensional ldentification Code, and Mo

Confined Condensed Phase
Confinement Test) (1973)

( Teth

Conformance (1972T)

for Selecting and Definin

Congeal or Solidify Within Temperature Range of 25 to 4

Congeal Point in Fats and Fat Mixtures Which Congeal or

Congealing Point of Petroleum Waxes Including Petrolatu

Congealing Point of Petroleum Waxes Including Petrolatu

Coniferous and Broadleaf Evergreens; Rose Grades; Vines

Connected Electric Gardening Appliances (Lawn Mower, Cu

Connected Electrical Appliances (1973)

Connected Indoor Signs (1971) ANSI C 33.35

Connected Wood Truss Design; and Nails and Staples (197

Connecting Devices and Towing Meth. (Coupling, Hitch, S

Connecting Flange Dimensions (1972)

Connection Flanges (1971)

Connections in High Pressure Oil Well Service) (1972)

Connections in Reinforced Concrete Construction (1973)

Connections (1971)

Connections (1973)

Connections, Outboard W ater Strainers, Water Scoops and

Connector and Bolted Joints, Drift Bolts, and Wood and

Connector Fittings for LP (Liquid Petroleum) Gas Cylind

Connector for Truck Trailer Jumper Cable (1972)

Connector (1972)

Connector) (1973) ANSI C7.27

Connector, and Bar) (1973)

Connectors and Switches for Use with Burglar Alarm Syst

Connectors for Audio Facilities for Radio Broadcasting

Connectors for CATV (Cable Television) Application (75

Connectors for Exterior Use on Mobile Homes (1972)

Connectors for Flammable Liquids and LP (Liquid Petrole

Connectors for Gas Appliances (1967)

Connectors for Portable Indoor Gas Fired Appliances (19

Connectors for Portable Outdoor Gas Fired Appliances (1

Connectors for Use Between Aluminum or Aluminum-Coppe

Connectors for Use Between Aluminum or Aluminum-Coppe

Connectors of Other Than All Metal Construction for Gas

Connectors of the Pin and Sleeve Type for Hazardous Loc

Connectors (Steel Studs) for Composite Construction (19

Connectors (1971)

Connectors (1971) ANSI C 83.63B

Connectors (1972)

Connectors (1973) NEMA CC3

Connectors (1974)

Connectors (1974) ANSI C 83.63C
Std. ASTM

Std. ASTM

ASTM

ASTM

ASTM

ASTM

TTMA

NEMA

ASTM

A A

ASTM

PCEA

ASTM

ASTM

ASTM

ASTM

ASTM

ASTM

NEMA

UL

UL

UL

STM

ASTM

ASTM

TAPPl

SAE

STM

NFLDP

ASTM

ASTM

STM

$\mathrm{AACCH}$

$\mathrm{AACCH}$

A STM

TAPP

ANS

UL

UL
ICBO

ICBO

VESC

ASAE

SAE

ICBO

$\mathrm{SAE}$

ICBO

$A B Y C$

ICBO

UL

TTMA

NSA

ASTM

CB

UL

ElA

IAPM

ANSI

ANSI

ANSI

ANSI

NEMA

ANSl

NEMA

ICBO

IAPMO

Std. T ElA

NEMA

EEl

UL

EIA

C182

D2214

02717

C202

RP40

W $\mathrm{C} 8 / 7$

W $\mathrm{C} 8 / 6$

B 193

P.53.426

*31

B317

D2311

B 172
B 172

B397

B399

B 400 
Std. General Document for Printed Wiring Board Electric Washers, Pin/ Western Woods Use Book: Timber Fasteners ment, Poles, Piles, Spaced Columns, Joints, Screws, Bolts, td. Spec. for Compact Round Concentric Lay Stranded Copper Meth. of Test for Coking Value of Tar and Pitch (Modified tion and Maintenance of Terrace (Erosion Control and Water A 1.11 of Fixed Partitions of Light Frame Type for the Purpose of Std. Meth. of Test for Normal Std. Meth. of Direct Shear Test of Soils Under Rec. Pract. for Std. Spec. for Primary Columbium Metal for astics Used for Electr/ Std. Meth. of Test for Dielectric Std. Spec. for Mercury Lamp Transformers, ging as.is Farinograph Absorption to $14.0 \%$ Moisture Basis Std. Meth. of Test for Time to Failure of Plastic Pipe Std. for Safety for est for Breaking Load and Elongatoin of Elastomeric Yarns meth. of Test for Elastic Properties of Elastomeric Yarns trength of Woven Fabrics by the Tongue (Single Rip) Meth. pressure Sensitive Tape to Fiberboard at $90 \mathrm{Deg}$. Angle and Ceramic Dielectrics A/ Std. Meth. of Test for Dielectric or Alternating Current Loss Characteristics and Dielectric icr/ Std. Test Meth. for Complex Permittivity (Dielectric rete (1973) Std. Descriptive Nomenclature of ing Plants (Fire and Wind Hazards at Pl/ Rec. for Factory ile Walls (Tech. Notes) (1966) 968)

Std. Spec, for Asphalt for Use in Requirements for the Uniform Building Code: Roof 21 Std. for Safeguarding Against Fire During Building Std for Safety Requircments for Personnel Hoists Rec. for Prevention of Collapse Due to Weak Rec. Against Weak es (1973)

Minimum Requirements for School Bus Minimum Requirements for Type II School Bus use of Eiectric Motors and Generators (1/ Safety Std. for Both Permanently and Temporarily Affixed Safety Signs for Rec. Pract. for Instrument Face Design and Location for nce Instructions, and Container and Filler Identification Std. for Steering Frame Lock for Articulated Vehicles Rec. Pract. for Storage Batteries for Rec. Pract. for Lubrication Chart. Std. for Dimensional Spec, for Sealed Lighting Unit for Rec. Pract. for Electrical System for acity for Shovel Dipper, Clam Bucket, and Dragline Bucket (ROPS) and Falling Object Protective Structures (FOPS) of Uniform Building Code Std. for Softwood Plywood Lumber, ats (1973) Rec. Pract. and Std. for the Design, for Anchori/

and Pract. Rec. for Selection. Design,

d. and Rec. Pract. for Requirements for Design, Materials, 1 and Water Conservation) (1972/ Rec. for Design, Layout, Std. Meth. of Fire Tests of Building Uniform Building Code Std. for Fire Tests of Building d. Definitions of Terms Relating to Fire Tests of Building y Cabinets (1973) ems (1972)

Rec. for

ortable Containers for Flam/

Rec. for Location, tion and Safety (Exit, Stairway, Aisle, Corridor, Building rotection / Earthquakes (General Characteristics Of, Rec

Fiber Blanket Thermal Insulation for Wood Frame and Ligh

Form of Agreement Between Owner and Contractor (For Small mn and Other Members) (1973)

story Buildings (Tech. Notes) (1966)

electrical Wiring Identification System for Industrial and Std. for Flexible Connectors of Other Than All Metal

Plywood

Test Meth. for Packaging Film and

Test for Strength Properties of Adhesives in Plywood Type meth. for Strength Properties of Adhesives in Two Ply Wood

Std. Form of Agreement Between Owner and

luminum Alloy Load Carrying (Members) Structures (Aluminum Laminators Quality Control System (Timber sign of Aluminum Formed Sheet Building Sheathing (Aluminum uminum Sheet Metal Work in Building Construction (Aluminum estigating the Compatibility of Transformer Insulation and Std. Page Format of the ed Lumber and Plywood) Foundation System: House Design and halt/ Uniform Building Code Std. for Materials for Use in 73.1 Std. Spec. for Concrete Masonry Units for Design and $s(1967)$ Design and Rec. Pract. and Std. Covering Design and Rec. Pract. for Stds. for quid Cooled Marine Engin/ Stds. and Pract. for Design and
Connectors (1974) ANSI C 83.88, Ipc C.405A

Connectors, Bolts, Lag Screws, Plates, Nails and Spikes

Connectors, Nails, Staples, Adhesives, Fiberboard, Part

Conouctors (1974) ANSI C 7.59

Conradson) (1973) ANS1 A 149.12

Conservation) (1972)

/Ec. for Design, Layout,

Conserving Their Sound Insulation Efficiency (1973)

Consistency of Hydraulic Cement (1973) AASHO T129, ANS1

Consolidated Drained Conditions (1972)

Consolidation of Concrete (1972) ANSI A 185.1

Consolidation or Melting (1964) ANSI Z179.17

Constant and Dissipation Factor of Expanded Cellular Pl

Constant Current (Series) Supply Type (1971)

Constant Dough Weight Meth. Only) (Cereal Chemistry) (1

Constant Internal Pressure (1974) ANSI B72.6

Constant Level Oil Valves (1974) ANSI B 127.1

Constant Rate of Extension Instruments) (1972) ANSI L14

(Constant Rate of Extension Machines) (1972) ANSI L14.28

Constant Rate of Traverse Tensile Test. Machine) (1971)

Constant Stress (1972) / Meth. of Test for Adh

Constant (Permittivity) and Dissipation Factor of Solid

Constant (Permittivity) of Solid Electrical Insulating

Constant) of Solid Electrical Insulating Materials at M

Constituents of Aggregates for Radiation Shielding Conc

Constructed Housing and Recreational Vehicle Manufactur

Constructed Masonry) Insulated Cavity 6 In. Brick and T

Constructing Built Up Roof Coverings (1971) ANSI A 109.2.

Construction and Certification of Freight Containers (1

Construction and Covering (1973)

Construction and Demolition Operations (1973) ANSI Al0.

Construction and Demolition Operations) (1973)

Construction and Design of Buildings and Other Structur

Construction and Design of Buildings (1972)

Construction and Equipment (1972)

Construction and Equipment (1973)

Construction and Guide for Selection, Installation and

Construction and Industrial Equipment (Machinery) (1971

Construction and Industrial Equipment (Machinery) (1971

Construction and Industrial Equipment) (1971)

Construction and Industrial Front End Loaders and Dozer

Construction and Industrial Machinery (1970)

Construction and Industrial Machinery (1971)

Construction and Industrial Machinery (1972)

Construction and Industrial Machinery (1972)

Construction and Industrial Machinery) (1972)

Construction and Industrial Vehicles and Equipment (197

Construction and Industrial (1973)

Construction and Installation of Battery Chargers on Bo

Construction and Installation of Fittings and Equipment

Construction and Installation of Permanently Installed

Construction and Maintenance of Terrace (Erosion Contro

Construction and Materials (1972)

Construction and Materials (1973)

Construction and Materials (1973)

Construction and Performance Std. for Kitchen and Vanit

Construction and Protection of Electronic Computer Syst

Construction and Stowage of Portable Fuel Systems and P

Construction and Test. (1973)

S Use Book: Fire

Construction and Water Sprinkler System Design-Fire P

Construction Buildings (1970) ANSI Z98.36

Construction Contracts Where Basis of Payment Is a Sti

Construction Design Handbook (Beam, SIab, Footing, Colu

Construction Details, 6 ln. Brick and Tile Walls for 1

Construction Equipment (Machinery) (1971)

Construction for Gas Appliances (1971)

Construction Guide for Residential Building (1971)

Construction ldentity Tests (Paper) (1973)

Construction in Shear by Tension Loading (1964) ANSI Z1

Construction in Shear by Tension Loading (1970)

Construction lndustry Terms (Architecture) (1970)

Construction Manager (1973)

Construction Manual Sect. 1) (1971)

Construction Manual) (1962)

Construction Manual) (1969)

Construction Manual, Sect. 5) (1971)

Construction Materials in Askarels (1973)

Construction Message (1974)

Construction Meth. (1972)

/Eather Wood (Pressure Treat

Construction of Built Up Roof Coverings: Hot Mopped A sp

Construction of Catch Basins and Manholes (1973) ANSI a

Construction of Chimneys (Tech. Notes) (1964)

Construction of Circular Prestressed Concrete Structure

Construction of Cockpits and Scuppers (Boats) (1972)

Construction of Concrete Pavements and Bases (1974)

Construction of Concrete Stave Farm Silos (1969)

Construction of Diesel (Compression Ignited) Inboard Li

Uck Cap

ElA

WWPA

ICBO

ASTM

Std. ASTM

ASAE

ASTM

ASTM

ACI

ASTM

ASTM

ANSI

AACCH

ASTM

UL

ASTM

ASTM

ASTM

ASTM

ASTM

ASTM

ASTM

ASTM

FMS

BIA

ASTM

ABS

ICBO

NFPA

ANSI

FMS

FMS

VESC

VESC

NEM

SAE

$\mathrm{SAE}$

$S A E$

SAE

SAE

SAE

SAE

SAE

$S A E$

ICBO

$A B Y C$

$A B Y C$

$A B Y C$

ASAE

NFPA

ICBO

St ASTM

ANSI

FMS

$A B Y C$

WWPA

FMS

ASTM

AIOA

$\mathrm{ACI}$

BIA

ANSI

APA

TAPPI

ASTM

S406

*31.1

UBC $* 3.25$

496

D2416

R268.1

E497

87

D3080

309

B383

1673

C82.7

54.29
D1598

352

D2653

D2731

D2262

D2860

D2149

DI50

C638

7.90

*26

D312

UBC $* 3.32$

241

A 10.4

1.55

LPDI. 3

V. 6

.10

$\mathrm{MG} 2$ 
tes) (1970)

marine Engines (/

Rec. Pract. and Std. for the Design and Safety Rules, Procedures For, and

et Stops (1973)

ndustrial) (Tech. Notes) (1964)

sive Service (1971) Ansi/ tions (1973)

i Al58.1 Std. for Quality Assurance Practices for the

mid Areas (1973)

Spec. for the Design and Rec. for Design and ections Over Public Property (Pedestrian Protection During

Std. for Housekeeping During the

cleaning of Fluid Systems and Associated Components During ns of Inspection, Examination, and Test. Personnel for the and Handling of Items for Nuclear Power Plants (During the th. for Establishing Rec. Design Stresses for Round Timber 1969) Suggest ed Design and Specifying fication of Soils and Their Aggregate Mixtures for Highway

tors (1973)

Std. B asic

tion Systems (1974)

ermanent) (1967)

systems (1972)

rial Buildings (1971)

Procedure (1971) act. for Performance Std. for Crane Boomstop of All Mobile

Fireplaces, and Barbecues (1973)

Stairs (1973)

(1973)

ns and Retaining Walls (1973)

General (1973)

uirements (Permit, Plans and Spec., Inspection)/

ntilation, Sanitation, Fire Warning Detector (1/

on Lot (1973)

$\mathrm{g}$ and Installation of Wallboard Lathing (1973)

g (1973)

arages and Carports (1973)

rings (1973)

ing (1973)

plywood Paneling (1973)

outed, Reinforced Grouted, Hollow Unit, Cavity /

ashing (Sheet Metal for Roof Waterproofing) (19/ walls (1973)

ing and Weather Protection (1973)

(1973)

ish Flooring Over Concrete Slab / rete Slab Floors (1962) Spec. for Rubber Cushion Sleepe
ng Over Concrete Slab Floors

Spec. Ior Mastic Naile 971) Spec, for Aluminum Sheet Metal Work in Buildin quality Stds. for Blinds and Shutters Using Stile and Rail Rec. for Sound Control (Acoustics) in Wood Building expansion Joint Fillers for Concrete Paving and Structura ses) $(19$ Rifle Range Std. for Round Poles and Posts Used in Building Cold Weather Masonry Structural Clay Protective Brick Veneer; New and Existing Frame $t$ in Place Concrete (Storage) Bin and Silo Wall Design and Std. Classifications for Spark Resistan Rec. Pract. for Concrete Floor and Slab ec. for Placing Reinforcing Steel Bar Supports in Concrete arbon-Silicon Steel Plates for Machine Parts and General Rec. Spec. for (Nonelectric) Farm Fence Std. Steel Doors and Frames for Modular Masonry Building Spec. for Golf Putting Green pec. for Oil and Resin Base Caulking Compound for Building ature Service Conforming to ISO Recommendations for Boiler ring and Layout Diagrams Used in Architecture and Build in es for Concrete, Steel and Bituminous Materials as Used in Std. for Pressure Treated Piles and Timbers in Marine Uniform Building Code: Prefabricated Southern Building Code for Dwelling House

vative Treatment by Pressure Processes of Wood for Highway code Std. for Shear Connectors (Steel Studs) for Composite de Std. for Prestressed Steel Strand and Wire for Concrete teel, Metal Inserts and Connections in Reinforced Concrete Rec. Pract. for Concrete Highway Bridge Deck Manual of Practice: Multiple Contract

Is Application for Thermoplastic (Polyvinyl Chloride) Duct nd Acceptance of Concrete Pavements Exposed to Rain During sed Concrete (Post Tensioned Box Girder Bridges Design and of Sound Transmission Class (Building Acoustics Design and hock Test. Machine for Lightweight/ Spec. for the Design, fety to Life-Property from Explosion and Fire in Design,
Construction of Flat and Sloped Brick Soffits (Tech. No Construction of Gasoline Fueled Inboard, Liquid Cooled Construction of High Power Rifle Ranges (1972) Construction of Indoor Rifle and Pistol Ranges and Bull Construction of Masonry Chimneys (For Residential and I Construction of Nonmetallic Enveloped Gaskets for Corro Construction of Nuclear Power Generative Stations (1971 Construction of Nuclear Power Plants-Terms and Defini Construction of Reinforced Concrete Chimneys (1969) Ans Construction of Surface Drainage Systems on Farms in Hu Construction or Demolition) (1973) /C Streets and Proj Construction Phase of Nuclear Power Plants (1973)

Construction Phase of Nuclear Power Plants (1973)

Construction Phase of Nuclear Power Plants (1973)

Construction Phase) (1972

pping, Receivint .

Construction Procedures for Concrete Foundation Piers (

Construction Progress Photographs (1967)

Construction Purposes (1973) AASHO M 145

Construction Requirements for Photographic Slide Projec

Construction Requirements, and Installation of Plumbing

Construction Spec. for Local Industrial Exhaust Ventila

Construction Spec. for Rifle Bench Rest (Portable and P

Construction Std. for Ventilating and Air Conditioning

Construction Systems (Specs.) for Commercial and Indust

Construction Type Crane Overload Indicating System Test

Construction Type Cranes Having Rope Supported Booms, E

Construction Under the Uniform Building Code (1973)

Construction Under the Uniform Building Code: Chimneys,

Construction Under the Uniform Building Code: Exits and

Construction Under the Uniform Building Code: Flooring

Construction Under the Uniform Building Code: Foundatio

Construction Under the Uniform Building Code: Framing-

Construction Under the Uniform Building Code: Legal Req

Construction Under the Uniform Building Code: Light, Ve

Construction Under the Uniform Building Code: Location

Construction. Under the Uniform Building Code: Plasterin

Construction Under the Uniform Building Code: Plasterin

Construction Under the Uniform Building Code: Private $G$

Construction Under the Uniform Building Code: Roof Cove

Construction Under the Uniform Building Code: Roof Fram

Construction Under the Uniform Building Code: Softwood

Construction Under the Uniform Building Code: Solid, Gr

Construction Under the Uniform Building Code: Valley Fl

Construction Under the Uniform Building Code: Veneered

Construction Under the Uniform Building Code: Wall Fram

Construction Under the Uniform Building Code: Wallboard

Construction with Mastic Set Subflooring and Nailed Fin

Construction with Nailers and Finish Flooring Over Conc

Construction with Nailers. Subflooring or Finish Floori

Construction (Aluminum Construction Manual, Sect. 5) (1

Construction (Architectural Woodwork) (1973)

Construction (Floor, Ceiling. Wall) (1972)

Construction (Nonextruding and Resilient Bituminous Typ

Construction (Part Time and Special) (1971)

Construction (Preservative Treatment by Pressure Proces

Construction (Protection Rec.) (1968)

Construction (Tech. Notes) (1962)

Construction (Tech. Notes) (1966)

Construction (1965)

Construction (1966)

Construction (1969) ANSI A 157.1

Construction (1970)

Construction (1970) ANSI G24.7

Construction (1972)

Construction (1972)

Construction (1972)

Construction (1972)

Construction (1972)

Construction (1972)

Construction (1972)

Construction (1973)

Construction (1973)

Construction (1973)

Construction (1973)

Construction (1973

Construction (1973)

Construction (1974)

Construction (1974)

Construction (1974)

Construction (1974)

Construction) (1969)

Construction) (1973)

Constrion, and Operation of Class Hi (High lmpact)

Tubular Products for High Temper Craphic Symbols for Electrical W.

ITion and Test. Agenci

Std. for Preser Uniform Building

Uniform Building Co

/De Std. for Welding Reinforcing S

Stds, Guide Specs, and Materia

Nterim Guideline for Protection a Ecs. for Post Tensioned Prestres

Lassification for Deter

d Product Standards Division
BIA

ABYC

NRA

NRA

AST

IEEE

ANSI

$\mathrm{ACl}$

ASAE

ICBO

ANSI

Tio ANSI

ANSI

ASTM

ACI

CSI

ANSI

NFPA

ACGIH

NRA

SMACN 6

$A P A$

$S A E$

$S A E$

ICBO

ICBO

ICBO

ICBO

ICBO

ICBO

ICBO

ICBO

ICBO

ICBO

ICBO

ICBO

ICBO

ICBO

ICBO

ICBO

ICBO

ICBO

ICBO

CBO

WSF]

WSFl

WSF

AA

AW 1

WWPA

ASTM

NRA

$A$ WPA

BIA

BIA

BIA

$\mathrm{ACI}$

ANCA

ACI

Sp CRSI

ASTM

TDI

USGA

ASTM

ASTM

ANSI

ASTM

$A$ W A

ICBO

$\triangle B C C$

AWPA

ICBO

CBO

CBO

CSI

SMACN

ACPA

CRSI

ASTM

ANSI

FMS

36 A

19B

F336

336

N45.2.10

R302.2

UBC*3.44

N45. 2.3

45.2.1

N45.2.6

N45.2.2 
Std. Guidelines for Thesaurus structure. nd Appliances (Extinguishern. Alarms. Sandpiges). Building 7.3) Std. for Safety. Requirements for the Converting Machin

Sher for tety Requirementh for the sitd. for safet, Reyuirementi for aulic. Pneumatic, semi/ Stil safet? Requirement for the Drving Farm Crops (1971) sid. for

smission linits Penetrating the $\mathrm{H} /$ Rec Guides for Design. eller shafting sysl std. and Rec. Pravt. Guide to Design. $\mathrm{rm}$ Sistems and I'nit for Protection Igail Safety Std. for initions of Terms Relating to Acoustical Test of Building std. Heth. of Tenmion Tent of Flat sanduich 1d. Neth, of Shear Test in Flatwise Plane of Flat -andwict. sid. Meth. of Flexure Test of Flat randujeth Sid. Teat Meth for Flexure (reep of sanduich

Std. Test Meth. for Laboratury Aging of sanduich t Meth. for Edgenise Compreanive trength of Flat rand wirh eth. for Density of Core Materials for - tructural sandwich water Absorption of Core Materials for structural Sandwich 1d. Weth of Test. Veneer. Plymood, and ()ther (,lued I eneer Principlen of Clas Manonry

1972) Sul. Furm of .

lding Electrodes and Risds (1972)

$12 \mathrm{Cr}-8.5 \mathrm{Ni}-2.0 \mathrm{Cu}-1.1 \mathrm{Ti}$. Varuum Induction Plun Vacuun $\mathrm{i}-1.560-1.0 \mathrm{Ho}-0.04 \mathrm{~S}(0.17-0.236)$. Premium Qualits. ant Nichel Bane $(19.50$ r $13.50 .0-1.3 .110-3.0 \mathrm{Ti}-1.1 \mathrm{Al}$ tant Nickel Base $19.50 \mathrm{r}-1.3 .5 \mathrm{Co}-1.3 \mathrm{M0}-3.0 \mathrm{Ti}-1 .+\mathrm{d}$ nt. Nirhel Band-19.5t.r-13.56o-1.3110-3.0Ti-1.+Al

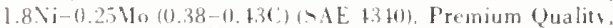
0. 12.110 (0.07-0.130 ) ( . AE 9310). Premium ()ualits. lacuum Corrosion Resiatant. $\left.1+80^{\circ} \mathrm{r}-1.5 \mathrm{Vj}-3.5 \mathrm{Cu}-\left(\mathrm{C}^{\circ} \mathrm{l}\right)+\mathrm{Ta}\right)$

er $-1.0110-1.21(1.10-1.206)$. Premium Bearing Qualit?. ecorler/ Std for himulated Shipping Test (l'ackaging) for of a Trampoline (197-1) Revide for Protective Relaving of Ctilit, ese Terms/ Gul. Definitions of Term Relating to 1 are of

Procedure to Determine the Frenzer Temperature and Energy

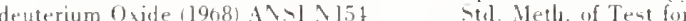
for Electric Comfort Conditoning (Heat Loss, (oain, Energs and spec. for Gold silver. Nickel Eletrical sul sper for Coslid Electrical ace atd. Weth. of Test for Diffusion stain of Rubber and ᄀed. Weth. for L'ltranonic ad. for Prescription Requirements for Firat Oualit tal spee for Gold (opper Allow Electrical general Requirement- for Wrought Precious Metal Electrical at Hetlu. of Teat for Hardneme of Electris'al r Denigning a High rument Are Eromion Test for Electrical std for Hedium Prefocus Base Doun Type, single and. Neeth for std. Spee. for Mank iscoratel for l'se in Photograplic $r$ Location of super 8 Printed Area in Optical Reduction or $r-(197)$

ber and Contat and Migration stain of Organir. Finiales in trl. for safets for Elevator loor Lonking Devices and $\rightarrow$ til. for self

pec. for ['etroleum [rilling Rig Packaging for Minimum self nhedrous Ammonia fertilizer Gri Gul for safets for self lers (1973) AN>l Al/ Std. for Drinking Fountain and solf std. for lerminolon for Pressure Relief Devices riant and Fluid Requirement Ifdintenanse lnstrustions, and 731 tul. for tites shipping

dot -

ste spee for 5 lal. Tizht Head L'nivernal Pails

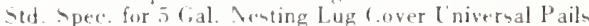

spec for 5 Gal. - traight vide Lug (.over I nivernal Pail Std. for 5 Cral. \esting Lug (over l'niversal Pail

spec for 5 Gal vraight >ide Lug (.over Iniversal Pail ting, Liquid Withdranal only/ Rec. Pract. for LP(yas Fur] Vertical Mounting). Liquil / Rec. Pract. for L1' Gas Fucl td. Neth. of Teut for $T$ ater lapor Tran-mission of Shipping

(4) the for Air truction and stondge of Portable Fuel siatemo and Portable. Sanitars sulm. for Fillers and healers of ringle Service phic Film on ypools (19-2) cul for Dimension of or Fire Protection of small Aonprewurized and Pressurized ati. Weth. of Tent for Renistance of Glass ation for Animal Feed and Fosd Labelling. Ingrediento and id Neth. of Thernal shock Test on Glase tid. Heth. of Sampling Glasis 8) A.VSI MH12.3. TAPP1 T800 Std. Neth. of Test Shipping std. Meth. of Drop Test for Cylindrical slupping

d. Meth of Test for Penetration of Liquid into submerged sid. Meth. of Drop Test for Shipping

td Meth. of Test for Water hapor Transmission of Shipping
Construction, and lise (197t)

Construction. Basenent Pipe Inlets (1973)

Construction. Banenent Pipe Inlets (1973) /

(19

Construction. Care, and lse of Power Press Brakes (1973

Construction. Care and I'se of Shears Mechanical, Hidr

Construction, Installation, and Rating of Equipment for

Cionatruction. Introduction (Terh. Votes) (1967)

Construction, Materials, and Installation of Power Tran

Cionstruetion. Materials, size, and Installation of Prop

Conatruction, Operation. and Maintenance of Vehicle Ald

Constructions and Materials (1973)

Constructions in Flatwise Plane (1970)

Cionatructions or Cores $(1970)$

Constructions (1970)

Constructions $(1970)$

Construitions (1970)

Constructions (1970)

Constructions $(1970)$

Cionstruction $(1970)$

Constructions $(1972)$ a $\mathrm{V}=107.1$

Cionstruetion: tudent - Manual (1960)

Consultant for ()ther Than Vormal Engineering Services

Consumable Electric Covered Bare Straight and roiled Die

Consumalsle Electrode Melted Solution Heat Treated (1973

Consumalle Electrode Multed Annealed

Consumalsle Electrode or Lacuum Induction Nelted 1825.19

Consumable Electrode or V'acuum Induction Melted. 1975 D

Consumable Electrode or Lacum Induction Melted. $1975 \mathrm{D}$

Consumable Electrode Remelted (1973)

/l bing, $0.80 \mathrm{Cr}$

(.onsumable Electrurto Renuelted $\left(10^{-3}\right)$

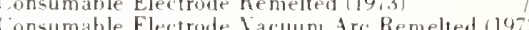

Consumalile Electrode Vacuum Veltet (lons)

Consumer Electronic. Produrtu iRadio

Consumer Interconnections (19-3)

Consumer

Conamer Textile Product and Rec. Pract. for I he of Th

Consumption of Household Refrigerators, Combination Ref

Consumption of Potasium Permanganate b) Impurities in

Consumption) (1971)

Contact Allot $(19-2)$

Contact Allow $\left(19^{-3}\right)$

Contact and Migration atain of Organdr. Finishes in Cont

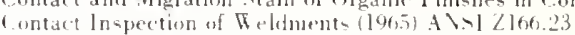

Contact Lensec $(19,2)$

Contact Naterial $(1473)$

Contact Materials $(19-2)$

Contact Material- $(1972)$ A 19.121 .50 .12

Contart Material $119^{-2} 3$

Contact Metion P'ieture

Contacl P'rintern $\left.199^{-}\right]$

Contaet Printing of Roll Film Negativen (19-2)

Contact Printing on 3.5 m n Motion Putur. Film. Perforat

Contact lniformit, Tent for Photograblac lontact Printe

Contact with Rubter (19-3) 1 $1.917 \% 3$

Contacts (1973)

Contained Humidifiers $(1974)$

Contained Platform (19-3)

Contained Strainer or Filter for Flammal, le Fluids and a

Containesl. Nechanically Refrigerated Drinking $\mathbb{D}$ ater Coo

Container and Equipment Protection) (1972)

Container and Filler ldentification (Construction and 1

Container Industry Clossars (l'akaging. Vocaloulary) (19

Contaner Procedure for C horine Packaging (1973)

Container) (Dot-17E. ['f*-Rule fo, \mfo-Item 260 ,

Contaner) (Dot-37A80. Dot-37Aa60. C'fe-Rule Ho. S

Container) (Dot-37.A80. Dot-37.160. ( fe-Rule $10 . \mathrm{Im}$

Container) (Dot-37C80) (1971)

(ontainer) (Dot-37C 80$)(1971$

Container. Quickly Demountable Tyge for Horizontal Moun Container. Quichly Demountable Lnivernal (Horizontal or Containers by Cicle Meth. Packaging) (1968) 1 I - I NH12

Containers for Entrancew as in Food Establishments 119 ?

Container for Flammable Liquids (Boats) (1972)

Containers for Nilk and is Fluisl Produrts (Packaging E

Containers for P'ackaging and storage of Aerial Photogra

Containers of Flammable Liquids (197.3)

Containers to $($ hemical Atlark (1973)

Containers (Also Includes Terms and Definitions) (197.

Containters (Bottle Jar) (1971)

Containers (Bottle. Jar etc.) (1956)

Containers (Pachaging) in Revolving Hexagonal Drum 1196

Containers (Packaging) (1950) ANSI MHI2.7

Containers (Packaging) (1951) ANSI MHI2.8

Containers (Packaging) (1961) A NSI MHI2.2

Containers (Packaging) $(196+4)$ A $\backslash S 1.11 \mathrm{H} 12.10$
A \\ड

ICBO

A $\backslash \leqslant 1$

A $\backslash$ SI

A $>1$

A $\backslash$ SI

$A S A E$

B1.

ABYC

ABYC

IL

Sul. Def A STII

AST M

A.T M

ASTM

ASTM

ASTM

td. Tes A.TI

A. $T, 1$

A.TM

BIA

AlOA

NE.MA

$\triangle \mathrm{AE}$

$S A E$

$\triangle \mathrm{AL}$

$\triangle \mathrm{AE}$

$\triangle A \mathrm{E}$

$\triangle \mathrm{AE}$

$\triangle A E$

Plate $A E$

$\triangle \mathrm{AE}$

EIA

IEEE

1. [ 11

A. 111

AHA

A T Y

NE II

AST!

А $T$ TI

\&ธT 1

A.T VI

A.1.1

A.T U

A.TH

A. -T

A. TI

4) 15

A. $\backslash \backslash 1$

A.>1

A $\backslash \mathrm{S}$

a $\backslash 51$

A.T11

II

ARI6

$\mathrm{APl}$

[L

ARI

ANS

$\mathrm{AE}$
$\mathrm{A} \mathrm{S}$

CHI

A. $\leq 1$

ANSI

A $\backslash 51$

ANSI

std ANS1

IT A

IT

A.T M

$\triangle \triangle F$

1 Cons ABYC

DFISA

ANS1

Rec, F FMS

A.T 11

A. $\mathrm{HCO}$

A.T.II

A.T M

1 $\triangle \mathrm{T} \mathrm{M}$

A. T. H

i A.T.M

A.T U

A.T H

239.19

CFC *2ARTI3

152.

B155. 1

Bl] 3

Bl1.4

5218.2

P16

P6

904

C63.1

C.297

C 273

C 393

C 180

C 181

C. 364

C.27!

C. 272

D 805

$* 11$

C. +31

ETh 2

A.11-561:A

A 11:6523.1 
Std. Meth. of Incline Impact Test for Shipping Std. Meth. of Vibration Test for Shipping irements for the Construction and Certification of Freight est for Drop Impact Resistance of Polyethylene Blow Molded Std. for Basic Requirements for Cargo

Std, for Requirements for Closed Van Type Cargo act. for Nomenclature and Terminology of Tank Trailers and on of Precautionary Labeling and Marking of Compressed Gas Spec. for International (Iso) Freight

Std. for Capacity Designation for Fertilizer Hoppers and Std. Spec. for Safe Fill or AerosoI

Std. Rec. Pract. for Inspection of Glass Aerosol Meth. of Test for Pressure in Metal Aerosol act for Reporting Laboratory Results When Checking Aerosol or Effective Crimping on Outside Crimped Valves of Aerosol accident Prevention Signs for Display on Tank Trailers and ling Cleaning Meth. for Hydraulic Fluid Power Fluid Sample Ceramic Decorations on Returnable Beer and Beverage Glass Rec. Pract. for Determining Permeability of Thermoplastic st Meth. for Drop Tests for Paper Shipping Sacks and Bags

for Water Leak Test for Paper Shipping and Refuse Sacks eth. of Test for Electrical Conductivity of Aviation Fuels high Tempe/ Std. Spec. for Precipitation Hardening Cobalt exfoliation Corrosion Susceptibility in 7XXX Series Copper

973) Rec, for Safe Use of Mixed Fertilizers Std. for Safety for Refrigerant . Std. for Efficiency Test. of Air Cleaning Systems on from Corrosion for Underground Pipe, Fittings and Tanks nded Safeguards and Practices for the Protection and Tanks ometric Test for Relative Radiance of Paper and Paperboard $f$ Crude Fat in Flour, Bread, and Baked Cereal Products Not Test Meth. for Alkali in Bleach Baths eth. of Analysis of Calcium Peroxide in Dough Conditioners Storage and Handling of Nitrogen and Fertilizer Solutions artensitic Stainless and Alloy Steel Castings for Pressure

c Alloy Steel Castings Specially Heat Treated for Pressure 1974/ Std. Spec. for Ferritic Steel Castings for Pressure .2 quality, for Pressure Piping Components and Other Pressure Std. Meth. for Chemical Analysis of Chrome Std. Meth of Chemical Analysis of Soaps ersion C/ Test Meth. for Detection of Hexavalent Chromium - for Determining Crude Fiber Content in Feedstuffs (Corn)

g Crude Fiber Content in Corn and Other Grains, Foodstuffs est for Oxidation Stability of New Mineral Insulating Oils otective Coatings (Paints) for Light Water Nuclear Reactor sts of Continuous Duty Class I Motors Installed Inside the ing Statio/ Std for Electrical Penetration Assemblies in Practice and Test Requirements for Leakage Rate Test, of Design Criteria for Reinforced Concrete Nuclear Power Std. Photographic Film Exposure Guide (Also

64

ulating Materials (1973) A/ Seth of Test for Particulate Std. Meth. of Test for Liquid - Meth. of Test for Identification of Metallic and Fibrous act. and Nomenclature for Identification of Structures and Std for Packaging and Transportation of Radioactively ion of Chromic Acid and Hexavalent Chromium Compounds (Air stems (1973) ANSI B93.30 Std. Meth. of Reporting

an Operating Hydraulic Fluid Power System (For Particulate pling Gas Blow Down Systems and Components for Particulate pling Gas Blow Down Systems and Components for Particulate Rec. for Control and Removal of Radioactive $\mathrm{Pe} /$ Meth. of Analysis of External Insect and Rodent Hair

(Tech. Notes) (1964)

otes) (1969)

otes) (1970)

Engineered Design of the Std. Meth. of Test for

Iow Pressure Cable Systems (1972) ANSI C5/ Std. Spec. for high Pressure Cable Systems (1972) ANSI C/ Std. Spec. for rotating Bomb (1967) ANSI Z11.230 Std. Meth. of Test for ur Dioxide Content of the Atmosphere (196/ Std. Meth. for

trode (1973) Std. Spec. for Copper Base Alloy

tainment of Nuclear Po/

Trial Use Guide for Type Tests of astness of Textiles to Light: Water Cooled Xenon Arc Lamp. for Colorfastness of Textiles to Light, Carbon Arc Lamp, tent. Meth. of Test for Carbon Monoxide in the Atmosphere Tent. Spec. for Concrete Made by Volumetric Batching and n (1971) ANSI L14.84

Water Soluble Gaseous Fluorides in the

Test Meth. for W ater Soluble Gas Floors (1962) Tent. Meth. for ning Speed of Photographic Negative Materials (Monochrome e Perforated Tape Variable Block Format for Contouring and std. for Milling Machine-Tracer Controlled Profiling and
Containers (Packaging) (1968) ANSI MH12.4, TAPPI T80

ASTM

ASTM

Containers (1968)

Containers (1970)

Containers (1971)

Containers (1971)

Containers (1971)

Containers (1971)

Containers (1972)

Containers (1972)

Containers (1972)

Containers (1972)

Containers (1972)

Containers (1972)

Containers (1972)

Containers (1972) ANSI B93.20

Containers (1972T)

Containers (1973)

(Containers) (1973)

Containers) (1973)

Containing a Static Dissipator Additive (1971) ANSI Z11

Containing Alloy Bars, Forgings, and Forging Stock for

Containing Aluminum Alloys (Exco Test) (1972)

Containing Ammonium Nitrate (1972)

Containing Components and Accessories, Nonelectrical (1

Containing Devices for Removal of Particles (1972)

ASTM

ANSI

ANSI

Guide to the Preparati CGA

ANSI

ASAE

ASTM

ASTM

ASTM

tec Pr AST

Meth. of Test F ASTM

Rec. Pract. for TTMA

Qualifying and Control NFLDP

ASTM

Std. ASTM

Test Meth TAPP

ASTM

ASTM

ISt for ASTM

FMS

UL

ANSI

Containing Flammable Liquids (1973)_Practice Protecti ICBO

Containing Flammable or Combustible Liquids in Location ICBO

Containing Fluorescent Brighteners (1973) /Spectrophot TAPPI

Containing Fruit (Cereal Chemistry) (1962

/Analysis O AACCH

(1972) ANSI L14.129

Containing Monoglyceride, Salts, Soy Flour, and Other C

Containing More Than $2 \%$ Free Ammonia (1970)

Containing Parts Suitable for High Temperature Service Std. for FI

ASTM

ASTM

Parts Suitable for High Temprature Service

Containing Parts Suitable for Low Temperature Service

D and Cold Finished, Special ASTM

Containing Refractories and Chrome Ore (1970) ANSI A139

Containing Synthetic Detergents (1973)

Containing Treatments on Galvanized Steel Prior to Conv Containing Vegetable Material, Corn and Other Grains, F Containing Vegetable Materials, Flours and Meals (1964)

Containing 2.6-Ditertiary-Butyl Para-Cresol by Ro

Containment Facilities (1972)

Containment of Nuclear Power Generating Stations (1971)

Containment Structures for Nuclear Fueled Power Generat

Containment Structures for Nuclear Reactors (1972)

Containment Structures (1969)

Contains Suggested Techniques for Photography) (1973)

Contaminant in Aviation Turbine Fuels (1973) ANSI Z11.2

Contaminant, Inclined Plane Tracking and Erosion of Ins

Contaminants in Aerospace Fluids (1970E)

Contaminants Seen on Specular Silicon Surfaces (1972T)

Contaminated Biological Materials (Safety) (1973)

Contamination-Occupational Health and Safety) (1973)

Contamination Analysis Data of Hydraulic Fluid Power Sy

Contamination Analysis) (1972) ANSI B93.19

Contamination by Manual Meth. (1973)

Contamination in Laboratories (1951)

D. Meth. for Sam

Contamination of Cereal Grains, Unpopped Popcorn, Dried

Contemporary Bearing Wall Detailing (Tech. Notes) (1968

Contemporary Bearing Wall Detailing (Tech. Notes) (1968

Contemporary Bearing Wall Example of Shear Wall Design

Contemporary Bearing Wall Types and Properties (Tech. N

Contemporary Bearing Wall (For High Buildings) (Tech. N

Contemporary Bearing W all (Tech. Notes) (1966)

Continuity of Porcelain Enamel Coatings (1973)

Continuity of Quality of Electrical Insulating Oil for

Continuity of Quality of Electrical Insulating Oil for

Continuity of Steam Turbine Oil Oxidation Stability by

Continuous Analysis and Automatic Recording of the Sulf

Continuous Castings (1973)

Continuous Determination of Sodium in Water by Ion Elec

Continuous Duty Class I Motors Installed Inside the Con

Continuous Light (1971) ANSI L14.170

Continuous Light (1971) ANSI L14.5

Continuous Measurement by Nondispersive Infrared Spect

Continuous Mixing (1973)

Continuous Scouring Wool Using Std. Raw Grease Wool Yar

Continuous Separation and Collection of Particulate and

Continuous Strip Mastic Set Maple Flooring Over Concret

Continuous Tone) (1972)

Std. Meth. for Determi

Contouring-Positioning Numerically Controlled Machine

Contouring-Vertical (1973)

CR

ASTM

IEEE

ANSI

$\mathrm{ACl}$

ANSI

ASTM

ASTM

Std ASTM

ASTM

ANSI

ANSI

NFLDP

BIA

BIA

BIA

BIA

BIA

BIA

ASTM

ASTM

ASTM

ASTM

D880

D2463

MH5.1

MH5.1.1

RP36

C7 5

5281.1

D3068

D3073

D3074

D3067

D3076

RP35

T2.9.2

C735

D2684

UM-529

D2624

A639

G34

LPD7-9

207

N101.1

$\mathrm{UFC} * 2 \mathrm{~B}$

UFC * $2 A$

UM- 460

30-10

$48-51$

P145

A 217

A389

A352

A696

C572

D820

TB-II-7

CR G-12

A-8

D2112

N10l.2

334

317

N45. 4

69-2

PH2.7

D2276

D2303

F314

F154

N14.3

Z37.7

$\mathrm{T} 2.9 .3$

T2.9.

ASTM F327

ASTM F308

NCRPM R8

AACCH $28-20$ 
Interchangeable Perforated Tape Variable Block Format for Std. for Numerically Controlled Profiling and Sid. for Rim
Manual of Practice: Multiple achinery) (1971) oncrete Reinforcing Steel Material Supply and Installation 3/ Recommendation for Installation of Expansion, Control,
8 of Payment Is/ Std. Form of Agreement Between Owner and Air Handling Spec. for Sheet Metal and Air Conditioning ement Between Owner and Contractor (For Small Construction television (1970)

(1970) Rec. Pract. for the Density and Guide for Centralized binary Data Interch/ oratories (1951)

Std. for Interface Between Numerical Layout, Construction and Maintenance of Terrace (Erosion ies (1973) es (Electronics) (1973) Std. for Industrial Electrical Power Std. for Industrial Std. for Industrial Std. for Industrial Electrical Power Std. for Industrial Electrical Power 1973) Std. for Symbols for Machine Tool Indicator Plates or
Inspection of Laminators Quality Std. for Symbols for Machine Tool Indicator Plates or
Inspection of Laminators Quality Supplies (Packaging), and References (Dosage) for Poison
Std. for Graphic Representation of the . Definitions, Symbols, Formulas, and Tables for (Quality) 4-Cyclohexene-1,2-Dicar/ (Ester) Desmedipham (1/ cadienoate Hydroprene/ propylate (1973)

ropropylate (1973)

obenzoate Bifenox (197) Dinitro.N-Propyl.Ptoluid/ 2.6-Dinitro.N-Propyl-Pto/ Std. Common Name for the Pest
hiop (Common Name for the Pest ester Wi/ Std. Common Name for the Pest
for. Methidathion (Common Name for the Pest Std. for Methidathion (Common Name for the Pest bamate-Dioxacarb (1973) methyl-2,4-Dodecadienethi/ y ridyl) Phosphorothioa/ -Triazine-Prometon (197) io).S-Triazine-Prometry/ triazine-Propazine (1973/ 4-Dodecadienoate Kino/ (Methylthio)-S-Triazine -/ Std. Common Name for the Pest Std. for Common Name for the Pest Std. for Common Name for the Pest Std. Common Name for the Pest Std. Common Name for the Pest Std. for Common Name for the Pest Std. Common Name for the Pest Std. Common Name for the Pest Std. Common Name for the Pest Std. for Common Name for the Pest Std. Common Name for the Pest Std. Common Name for the Pest Std. Common Name for the Pest Std. for Common Name for the Pest Std. Common Name for the Pest no) Stria/ Std. for Dipropetryn (Common Name for the Pest lamino).S-Triazine-Terb/ Std. Common Name for the Pest (Methylthio)-S-Triazine / trifluoro.M.Tolyl) -3 !

Std. Common Name for the Pest Std. for Common Name for the Pest Std. for Bentazon (Common Name for the Pest Std. for Tetramethrin (Common Name for the Pest Sid. for Fospirate (Common Name for the Pest Std. for Disugran (Common Name for the Pest Std. for Crufomate (Common Name for the Pest Std. for Chlorbromuron (Common Name for the Pest Std. for Chlordimeform (Common Name for the Pest Std. for Fluorodifen (Common Name for the Pest Std. for Prynachlor (Common Name for the Pest Std. for Glyphosine (Common Name for the Pest Std. for Mexacarbate (Common Name for the Pest Std. for Dinoseb (Common Name for the Pest Std. for Acephate (Common Name for the Pest Std. for Ethiolate (Common Name for the Pest Std. for Glyphosate (Common Name for the Pest

Std. for Oxamyl (Common Name for the Pest

Std. for Picloram (Common Name for the Pest

Std. for Nitrapyin (Common Name for the Pest xy) Cyclohexyl 2Propynyl Sulfite (Common Name for the Pest Trimethyl 2,4-Dodecadienoate) (Common Name for the Pest d, Underwater Wellhead, and Christmas Tree Equipment; Flow Std. for Industrial Electrical Power Control Apparatus Type Agricultu/ Std. for Application of Hydraulic Renıote I, Groups A.D. Class 1I, Gro/

Safety Std. for Industrial Moisture tes) (1965) Moisture d. Simulated Space Environment Test. of Spacecraft Thermal Inspection, Product Audit) (196/ Rec. Pract, for Quality symmetrical and a Total/ Std. Requirements for Electrical erature Indicating and Regulating Electrical Equipment for tion of Sulfaquinoxaline in Poultry Feeds and Premixes for ed Metallic Piping Systems (1972) Rec. Pract. for 72)

c. for Star Model F Hidromatic Deluge Valve for Water Flow Rec. Pract. Inspection and Quality Safety Std. for Electrically Operated Valves for the d (1972) ANSI Z270.1 Std. for Std. Meth. of Conducting Shear Block Test for Quality Uniform Fire Code: Suppression and t. for the Purification of Oil Systems for Lubrication and
Contouring and Contouring-Positioning Numerically Con Contouring Milling Machine (1974)

Contours for Agricultural Press and Gage Wheels (Farm M Contract Construction (1974) Contract (1968)

Contraction, and Isolation Joints for Ceramic Tile (197 Contractor (For Small Construction Contracts Where Basi Contractors (1973)

Contracts Where Basis of Payment Is a Stipulated Sum)( Contrast Range of Black and White Films and Slides for Control and Automation of Ship's Steam Propulsion Plant Control and Data Terminal Equipment Employing Parallel Control and Removal of Radioactive Contamination in Lab Control and Water Conservation) (1972) /Ec. for

Control A pparatus -Control Circuit Devices and Assembl Control Apparatus-Controllers and Controller Assembli Control A pparatus-Enclosures (Electronic) (1973)

Control A pparatus-General (1973)

Control Apparatus-Switching and Controlling Devices ( Control Buttons (1972)

Control by the AITC Inspection Bureau (1962)

Control Centers (1972) Std. for Rec. Antidotes Control Characters of American National Std. Code for I Control Charts (1971) ANSI Z1.5

Control Chemical cis. $\mathrm{N} \cdot(\{1,1,2,2$ tetrachloroethyl $)$ Thio $)$

Control Chemical Ethyl Mhydroxycarbanilate Carbanilate Control Chemical Ethyl (E, E)3,7,11-Trimethyl.2,4-Dode Control Chemical Isopropyl 4,4'dibromobenzilate-Bromo Control Chemical Isopropyl 4,4 'dichlorobenzilate-Chlo Control Chemical Methyl 5(2,4-Dichlorophenoxy)-2-Nitr Control Chemical N.(2Chloroethyl).A, A, A-Trifluoro:2,6Control Chemical N(Cyclopropylmethyl)-A. A. A-TrifluoroControl Chemical O-Ethyl S,S-Dipropyl Phosphorodith Control Chemical O,O-Dimethyl Phosphorodithioate SControl Chemical O-1,3-Dioxolan -2-Ylphenyl Methylcar Control Chemical S-Ethyl (E,E).11-Methoxy-3,7,11-Tri Control Chemical 0,0 Dimethyl 0*(3,5,6-Trichloro-2-P Control Chemical 2,4-Bis (Isopropylamino).6-Methoxy.S Control Chemical 2,4-Bis (lsopropylamino)-6-(Methylth Control Chemical 2-Chloro-4,6bis (Isopropylamino) -SControl Chemical 2-Propynyl (E,E)-3,7,11-Trimethyl-2, Control Chemical 2-(Ethylamino)4-(Isopropylamino).6Control Chemical 2-(Ethylthio) -4,6-Bis (Isopropylami Control Chemical 2-(Tertbutylamino)-4-Chloro-6-(Ethy Control Chemical 2-(Tertbutylamino).4-(Ethylamino).6Control Chemical 4-Chloro5-(Methylamino) -2-(a,a,aControl Chemical (1972)

Control Chemical) (1971)

Control Chemical) (1971)

Control Chemical) (1971)

Control Chemical) (1972)

Control Chemical) (1972

Control Chemical) (1972)

Control Chemical) (1972)

Control Chemical) (1972)

Control Chemical) (1972)

Control Chemical) (1972)

Control Chemical) (1972)

Control Chemical) (1972)

Control Chemical) (1972)

Control Chemical) (1972)

Control Chemical) (1972)

Control Chemical) (1972)

Control Chemical) (1972)

Control Chemical) (1973)

Control Chokes; and Water Sampling Devices) (1974)

Control Circuit Devices and Assemblies (1973)

Control Cylinders to Agricultural Tractors and Trailing

Control Equipment for Use in Hazardous Locations, Class

Control in Brick and Tile Walls (Tech. Notes) (1965)

Control in Brick and Tile Walls, Condensation (Tech. No

Control in Industrial Exhaust Systems (1974)

Control Materials (Coatings) with Electromagnetic and P

Control Monitoring of the Product (Receiving and Source

Control of AC High Voltage Circuit Breakers Rated on a

Control of Air Conditioning, Heating, Cooking, Refriger

Control of Cecal and Intestinal Coccidiosis (Cereal Che

Control of External Corrosion on Underground or Submerg

Control of Fire Extinguishing Systems (1973)

Control of First Generation Silver Habide Microfilm (19

Control of Fluids (1973)

Control of Gas Hazards on Vessels (Ships) to Be Repaire

Control of Glue Bonds in Scarf Joints (1970)

Control of Hazardous Areas (1973)

Control of Hydroelectric Equipment (1971)

Design

EIA

NSA

ASAE

CSI

CRSI

TCA

AIOA Al07

SMACN *3

AIOA AI0?

SMPTE RP?

SNAME 3.23

$\begin{array}{ll}\text { EIA } & \text { RS408 }\end{array}$

NCRPM R8

ASAE R268.1

ANSI C 19.6

Cl9.7

ANSI C 19.4

ANSI C19.3

ANSI C19.5

ANSI B5.46

AITC 202

ANSI X3.32

Sid ASQC Al

ANSI K62.146

ANSI K62.139

ANSI K62.136

K62.151

ANSI K62.150

ANSI K62.138

KNSI K62.140

ANSI K62.133

ANSI K62.134

ANS1 K62.147

ANSI K62.141

AN5I K62.130

ANSI K62.144

ANS1 K62.145

ANSI K62.137

ANSI K62.142

ANSI K62.135

ANSI K62.149

ANSI K62.148

ANSI K62.125

ANSI K62.129

ANSI K62.106

ANSI K62.111

ANSI K62.112

ANS1 K62.110

ANSI K62.113

ANSI K62.114

ANSI K62.115

ANSI K62.116

ANSI K62.117

ANSI K62.118

ANSI K62.119

ANSI K62.120

ANSI K62.123

ANSI K62.124

ANSI K62.126

ANSI K62.127

ANSI K62.128

K62.131

K62.132

$6 \mathrm{~A}$

ANSI $\quad$ C 19.6

ASAE S201.4

UL 698

BIA 7B

$\begin{array}{ll}\text { BIA } & 7 \mathrm{C} \\ \text { ACGIH } & * 1.3\end{array}$

ASTM E512

ASQC RP5

ANS1 C 37.11

UL 873

AACCH $\quad 18-35$

NACE RP.01.69

Re FMS 2.88

NMA MSl04

UL

NFPA

ASTM

429

ICBO 
e Flushing and Cleaning of Oil Systems for Lubrication and Pract. for the Design of Oil Systems for Lubrication and Rec. Pract, for Evaluating the Quality Recordings (1970) St. Dimensions of Video, A udio and Tracking
Recorded A/ (1973) e Preservative Treatment by Pressure Processes and Quality ons (1973) ANSI N41.17 Rec. Pract. for Automotive Transmission Hydraulic cal Security Surveillance Sys/

ractice) (1971) de to Practice) (1974) Std. for Residential Controls Direct Electric Ignition Std. Proposal for Solid State Load Switches for Traffic

kick Plates; Bolts; Closers; Hospital and Misc. Items; Key f Dyed or Printed Textiles to Washing at 105 Deg. F: Rapid nd Linen Textiles to Combined Washing and Shrinkage: Rapid ems (1972) ANSI C33.90

fluids in Turbulent Flow (1974)

e Fluids (1972)

(1973)

$s(1972)$

e Requirements for Individual Anti Scald Type Water Showe for 4 Way General Purpose Industrial Pneumatic Directiona r, Ceiling, W all) (1972)

Western Woods Use Book: Sound Uniform Building Code: Sound Transmission Efflorescence, Prevention and Rec. Pract. for Monitoring Special Process of Quality Rec. Pract. for Vendor Rating System for Quality

Rec. List of Terms and Definitions Related to Quality Pract. for Vendor Product Improvement Program for Quality Std. for Record and Reporting Units for Nuclear Materials Std. Glossary of General Terms Used in Quality

ndustrial Engineering Terminology: Production Planning and Rec. for Scrubbers for Pollution

Human Relations in Vendor-Vendee Relationships (Quality in the Radio Frequency Range of 960-1215 Megahertz (Flight niform Mechanical Code (Requirements for the Installation, bly, Chainguards, Saddle Braces, Pedal Clearance, Steering tile (1973) multiple Completion, and Flowline Valves; Drilling Through

(1973)

od Ends (1973)

0) ANSI Z128.30

teristics of Dry Solid Film Lubricants in Vacuum and Other Test for Sulfur Oxides in Flue Gases (Barium Chloranilate (1974) rforated Tape Std., Variable Block Format, for Numerically ine (1974)

ng, Double Flaring, and Beading ( $1 /$ Definitions of Common Words Related to Numerically at for Contouring and Contouring-Positioning Numerically oriented Polyethylene (PEO) Plastic Pipe (SDR.PR) Based on spec. for Polyethelene (PE) Plastic Pipe (SDR-PR) Based on Std. Meth. of Test for Uranium by Std. for Milling Machine-Trace

74) td. for Electrical Harness Assembling Machine, Numerically for Composite Filament Tape Laying Machine-Numerically Std. for Industrial Control Apparatus-Controllers and

73) (1970)

uid Sample Containers td. for Industrial Control Apparatus. Std. for Air Pressures for Pneumatic Std. for Air Pressures for Pneumatic Std. Procedure for Qualifying and Rec. for 166.7 ustrial Electrical Power Control Apparatus-Switching and Std. Meth. for Std. for Residential Std. for Door Spec. for Safety 72) as Fired Equipment (1972) (1972) Rec. Pract. for Quality Std. for Clevis-Engine Std. for Tube Assembly, Std. for Tube Assembly, Std. for Safety for Access Laminators Quality Std. for Nuclear Material Std. for Nuclear Material Test Meth. for Beating Std. for Resistance Welding Std for Numerically Std. for Numerically Std. for Numerically Sid. for Welded Flash Std. for Numerically

Control of Hydroelectric Equipment (1971)

Control of Hydroelectric Equipment (1972)

Control Program (1969)

Control Record for 2 In. Quadruplex Video Magnetic Tape

Control Records on 2 In. Video Magnetic Tape Quadruplex

Control Records System (1969)

Control Rod, Adjustable (1973)

Control Signal Heads (1970)

Control Std. (1973)

1. Timber, Plywood and Utility Pol ICE

Control Steel with Welded Clevis and Threaded Rod Ends

Control Steel with Welded Threaded Rod Ends (1973)

Control Switchboards for Nuclear Power Generating Stati

Control System Terminology (1970)

Control System Units (Electronic / Electrical / Mechani

Control System (Timber Construction Manual) (1962)

Control Systems for Conversion Facilities (A Guide to $P$

Control Systems for Fuel Reprocessing Facilities (A Guj

Control Systems for Gas Fired Equipment (1972)

Control Systems (1973)

Control Systems; Door Holders, Stops, Smoke and Heat De

Control Test (1969)

Control Test (1969)

Control Units for Indoor Fire Protective Colorfastness of

Control Valve Capacity Test Procedure for Compressible

Control Valve Capacity Test Procedure for Incompressibl

Control Valve Sizing Equations for Compressible Fluids

Control Valve Sizing Equations for Incompressible Fluid

Control Valves (1973)

Control Valves (1973) ANSI B93.33

Control (A coustics) in Buildings, Walls, and Floors (19

Control (Acoustics) in Wood Building Construction (Floo

Control (A coustics) (1973)

Control (Simons Stain) (Pulp and Paper) (1973)

Control (Tech. Notes) (1970)

Control (1969)

Control (1969)

Control (1969)

Control (1969)

Control (1971)

Control (1971) ANSI Zl.7

Control (1972)

Control (1974)

Control (1974)

Control) (1967)

Control) (1972)

Control, and Maintenance of Heating, Ventilating, Cooli

Control, Brakes, Tires and Controls (1972)

Control, Contraction, and Isolation Joints for Ceramic

Control, Screwed and Flanged Wellhead, Underwater Wellh

Control, Solid Steel (1973)

Control, Steel, with Flash Welded and Threaded Rod Ends

Control, Steel, with Flash Welded Clevis and Threaded R

Controlled Atmospheres in Spectrochemical Analysis (197

Controlled Atmospheres (1971) ANSI Z11.303

Controlled Condensation Meth.) (1973)

Controlled Drilling Machines (1974)

Controlled Equipment (1974)

Controlled Horizontal and Vertical Jig Boting

Controlled Horizontal Boring, Drilling and Milling Mach

Controlled Low Carbon Steel Tubing Normalized for Bendi

Controlled Machine Tool Software (1972)

Controlled Machines (Information Processing) (1974)

Controlled Outside Diameter (1973)

Controlled Outside Diameter (1974)

Controlled Potential Coulometry (1970) ANSI N106

Controlled Profiling and Contouring-Vertical (1973)

Controlled Profiling and Contouring Milling Machine (19

Controlled Spar Mill (1973)

Controlled Velocity Laboratory Corrosion Tests (1972)

Controlled (1973)

Controlled (1973)

Controller Assemblies (Electronics) (1973)

Controllers and Controller Assemblies (Electronics) (19

Controllers and Transmission Systems (Instrumentation)

Controllers and Transmission Systems (1967)

Controlling Cleaning Meth. for Hydraulic Fluid Power Fl

Controlling Cupping of Wood Floors (1962)

Controlling Devices (1973)

Controlling Quality of Radiographic Test. (1972) ANSI Z

Controls-Class 2 Transformers (1973)

Controls-Closers (1972)

Controls and Signaling Devices for Printing Presses (19

Std for Residential Controls Direct Electric Ignition Control Systems for $G$

Std. for Residential Controls for Low Voltage, Wall Mounted Room Thermostats Std. for Administrative
Std. for Operator
Controls for Nuclear Power Plants (1972)

Controls on Agricultural Equipment (1972) act for Th ASME

Rec ASME

ASQC

SMPTE

ANSI

ASQC

ASAC

ITE

NSA

NSA

IEEE

SAE

UL

AlTC

ANSI

ANSI

NEMA

NEMA

NBHA

AATCC

AATCC

UL

ISA

ISA

ISA

ISA

ASSE

NFLDP

WWPA

WWPA

ICBO

TAPP1

BlA

$A S O C$

ASQC

ASOC

Rec. ASOC

ANSI

ASQC

Std. for I ANSI

FMS

NEMA

ASQC

RTCA

ICBO

BMA

TCA

APl

NSA

NSA

NSA

ASTM

ASTM

/Ear Charac ASTM

NSA

NSA

NSA

NSA

SAE

NMTBA

/M EIA

ially ASTM

ASTM

ASTM

NSA

NSA

NSA

AWPA

NSA

Spec NSA

ANSl

ANSI

ISA

SAMA

NFLDP

WSFI

Std. for Ind ANSI

ASTM

NEMA

ANSI

ANSl

NEMA

NEMA

ANSI

ASAE

LOS. $5 \mathrm{Cl}$

LOS.5D 1

RP3

RP16

C98.6

TR1

UBCS25. 12

359

358

420

J648 A

294

N15.4 
ent (1971) ANSI Bl14.2, Sal

(P.1) Welds in Corrosive Petroleu/ ocessing Systems) (1972) (1973)

Std for Symbols for Operator Rec. Pract. Meth. and Safety Std. for Limit

Rec. Pract. for Exhaust Emission Std. for Builders Door Hot-Water Immersion aces. Pedal Clearance, Steering Control, Brakes, Tires and Code: Protection, Authority, Departmental Regulations and hamber in Heating Appliances (Regulators, Automatic Damper or Design Calculations for Oil Sucker Rod Pumping Systems ineering Drawing and Related Documentation Pract. for Line Std for Installation of Domestic Gas

eans, Peas, Lentils, Rice, and Other Commodities for Which hate Type) (1973) oil) (1974) Spec. for Spec. for Coating Weight Determination for hromium Containing Treatments on Galvanized Steel Prior to nels/ Spec. for Coating Weight Determination for Chromate Std. for Nuclear Material Control Systems for Meth. of Ferricyanide-Maltose-Sucrose ell, Vickers, Rockwell and Rockwell Superf/ Std. Hardness
th. of Thiosulfate-Maltose (Diastatic Activity in Flour th. of Thiosulfate-Maltose (Diastatic Activity in Flour)
Wound (Materials, Specs. Terminology, Gauge, Metric nd Hot Wound (Materials, Specs. Terminology, Gauge, Metric
ements for Thyristor Converters for Motor Drives: Part 1 . ements for Thyrist. Pract, and Requirements for Thyristor Motor Armatu/ Ris Phase Rural Distribution Service for Motors and Phase
ngle Std. for AC -DC Transfer Instruments and Safety Code for Semiconductor Powe

hemistry) (1962) Table for

e Construction, Care, and Use of Packaging and lts Related Std. for Safety for Agricultrual Auge and Exhaust Systems for Dust, Stock, and Vapor Removal or Std. Spec. for Steel Std. for Heavy Duty Roller Type
Std. for Double Pitch

Test Meth. for Sampling of Wood Chips from Safety Stds. for Sid. for Screw Std. for Screw

Rec for Fire Protection for Bel oning Std. and Terminology for Permanently Installed Screw $\mathrm{r}$ Demountable Tired Wheels for Industrial Trucks, Casters, of Analysis of Crude Fat in Wheat and Soy Flour, Feeds and Std. for Gas Fired Kettles, Steam Safety Std. for Household Type Pressure Meth. of Analysis for Baking Quality of Safety Std. for Commercial Electric

ereal Chemistry) (1962)

Meth. of Analysis of Meth. of Analysis of Std. for Commercial Std. for Household Std. for Recreational Vehicle Std. for Outdoor

for Household Electric Ranges (Stove) with Glass / Ceramic trical Equipment for Control of Air Conditioning, Heating. ine/ Std. Meth. for Test for Water in Engine Antifreeze
Rec. Pract. for Engine Preheaters Rec. Pract. for Engine Preheaters Std. for

layed Neutron Emitting Fission Products in Nuclear Reactor Std. Meth. of Test for Foaming Tendencies of Engine Anti Leak Antifreezes and Stop Leak Additives for Engine on Characteristics of Aluminum Automotive Water Pumps with

$h$. of Test for Simulated Service Corrosion Test. of Engine meter for Determining the Freezing Point of Aqueous Engine afety Related Systems, Structures, and Equipment for Water $r$ Concrete Aggregates (Sand, Crushed Stone, Gravel and Air onstruction of Diesel (Compression Ignited) Inboard Liquid Rec. Pract. and Std. Covering the Installation of Air

design and Construction of Gasoline Fueled Inboard, Liquid Std. Rec. Guide for in Service Annealing of Water Std. Meth. of Testing for Rating Water Test Meth. for Colorfastness of Textiles to Light: Water Test Meth. for Colorfastness of Textiles to Light: Water $r-4.5 \mathrm{Ni}-2.9 \mathrm{Mo}-0.10 \mathrm{~N}$, Solution Heat Treated, Sub Zero

ty Std. for Electric Fans (Blower, Humidifier, Evaporative

Engineering Data Sheets for the Design of Air Exchange Std. Meth. of Testing for Rating Liquid Safety Sid. for Drinking Water d Self Contained, Mechanically Refrigerated Drinking Water Std. for Forced Circulation Air spiral Bevel, Helical and Herringbone Gear Units for Water $r$ Redwood Lumber Spec. for Application in Industrial Water uglas Fir Lumber Spec. for Application in Industrial Water hern Pine Lumber Spec. for Application in Industrial Water sure Preservative Treatment of Lumber for Industrial Water for Low and Medium Speed Stationary Diesel and Gas Engine
Controls on Agricultural Tractors, Machinery and Equipm

Controls to Prevent in Service Cracking of Carbon Stee

Controls (Air Conditioning, Heating, Ventilating and Pr

Controls (Air Pollution) for Powered Industrial Trucks

Controls (Closers) (1971)

Controls (1972)

Controls (1972)

/Heel Assembly, Chainguards, Saddle Br

Controls, Barrier, Road, Hydrant, Driveway Clearance, I

Controls, Fans) (1973)

(Conventional Units) (1972)

Conventions and Lettering (1973)

Aft Equipment for Combustion C

Rec. Pract. F

Conversion Burners (1971)

Conversion Charts Have Been Prepared (Cereal Chemistry)

Conversion Coating of Titanium Alloys (Fluoride-Phosp

Conversion Coating on Aged or Baked Aluminized Steel (C

Conversion Coating (Coil) (1974)

ITion of Hexayalent $\mathrm{C}$

Conversion Coatings on Aged or Backed Aluminum Alloy P

Conversion Facilities (A Guide to Practice) (1971)

Conversion Table (Cereal Chemistry) (1962)

Conversion Tables for Metals (Relationship Between Brin

Conversion (Cereal Chemistry) (1962)

Conversions) (1972)

/Sion, Extension, Torsion, Flat, a

Converters for DC Motor Armature Supplies (1973) ANSI C

Converters for Motor Drives: Part 1-Converters for DC

Converters (1972)

Converters (1973)

Converters (1973)

Converting Absorption to 14.0\% Moisture Basis (Cereal C

Converting Machinery (1973)

Conveying Equipment (1973)

Conveying (1973) ANSI Z33.

Conveyor Chain (1972) ANSI G61.3

He lnstallation of Blower

Conveyor Chains and Sprocket Teeth (1973)

Conveyor Roller Chains, Attachments and Sprockets (1972

Conveyor (Pulp and Paper) (1973)

Conveyors and Related Equipment (1972)

Conveyors (Materials Handling) (1971) ANSI MH4.7

Conveyors (1971) ANSI MH4.7.1

Conveyors (1972)

Conveyors (1972)

Conveyors, and Other Equipment (1966)

Cooked Feeds (Cereal Chemistry) (1962)

Cookers and Generators (1971)

Cookers (1973)

Cookie Flour (Cereal Chc mistry) (1962)

Cooking Appliances (1972)

Cooking Characteristics of Macaroni-Borasio System (C

Cooking Characteristics of Macaroni (1962)

Cooking Equipment Exhaust Systems (1970)

Cooking Gas Appliances (1972)

Cooking Gas Appliances (1972)

Cooking G as Appliances (1972)

Cooking Tops (1974)

Cooking, Refrigeration, and Humidity (Humidistat. Therm

Coolant Concentrate (Glycol or Alcohol Base) by the Iod

(Coolant Heaters) (1971)

Coolant System Hoses (1971)

Coolant Water During Reactor Operation (1970) ANSI N 163

Coolants in Glassware (1973)

Coolants (1972)

Coolants (1972)

Coolants (1972) ANSI D14.2

Coolants (1973)

Coolants (1974)

Rec. Pract. for Use of the Refracto

Std. Meth. of Test

ASAE

UL

ITA

BHMA

NEMA

BMA

ICBO

UL

API

ANSI

$\mathrm{AACCH}$

$\mathrm{SAE}$

NCCA

NCCA

NCCA

ANSI

$\mathrm{AACCH} 22.18$

ASTM

AACCH 22.17

SMI $* 3$

IEEE $\mathbf{4 4 4}$

IEEE 444

ASAE R329

ANSI Cl00.4

NEMA PV 3

AACCH 82.21

ANSI B155.1

ASAE S36IT

NFPA 91

ASTM A454

ANSI B29.15

ANSI B29.4

TAPPI UM-4

ANSI B20.1

CEMA 350

CEMA 300

FMS 7.11

Dimensi ASAE S334T

id. Fo CFTMA W7

AACCH $30-25$

ANSI $\quad 221.46$

UL 136

AACCH $10-50 \mathrm{~B}$

UL 197

AACCH 16-5I

$\mathrm{AACCH} \quad 16-50$

NSF 33

ANSI Z21.]

ANSI Z21.57

ANSI 221.58

Std. AHAM ER.2

UL

ASTM

SAE

SAE

873

STM D2470

ASTM D1881

ASTM D3147

D2809

D1121

D2570

Cooled and Moderated Nuclear Power Generating Plants (1

Cooled and Moderaed Nuc lear Power G

Cooled Marine Engines and Auxiliaries (Boats) (1972)

Cooled Marine Engines and Auxiliaries (Diesel and Gasol

Cooled Marine Engines (Boats) and Auxiliaries (1972)

Cooled Nuclear Reactor Vessels (1974)

Cooled Refrigerant Condensers (1971)

Cooled Xenon Arc Lamp, Alternate Light and Darkness (19

Cooled, Equilized, and Over Tempered (1973)

Cooler, and Air Filtering Appliances Rated at 600 Volts

Coolers for Packaged Compressor Units (1974)

Coolers (1971)

Coolers (1972) ANSl C33.82

Coolers (1973) ANS1 A112.11.1

Cooling and Air Heating Coils (1972)

Cooling Tower Fan Drives (1972)

Cooling Towers (1971)

Cooling Towers (1971)

Cooling Towers (1971)

Cooling Towers (1971)

Cooling Water Systems (1972)
ANS

N 18.10

UBCS26.2

ABYC P15

ABYC P10

$A B Y C \quad P 4$

E509

ASHRA 22

AATCC $16 \mathrm{~F}$

C $16 \mathrm{E}$

UL

API

ASHRA

UL

AR

ARI

Info Sheet for AGMA

Std. Fo CTI

Std. for Do CTI

Std. for Sout CTI

Std. Spec, for Pres CTI

Std. Pract. DEMA
AMS5743D

507

$11 \mathrm{~K}$

1010

410

490.02

103

114

112

.1.10 
Safety Std. for Air Conditioners, Central Safety Std. for Room Type Fan Coil Units (Heating and llation, Control, and Maintenance of Heating, Ventilating, erical Designation (Identification System) of Modular Grid rec. Pract. for Image Evaluation of Electrostatic Business sette) for Audio Recording An/ Std. for Dimensional Std. of Synthetic Elastomers for Use on Solid Styrene Butadiene d Bar (1973)

). Plate, Sheet, Strip and Rolled Bar (19/ Std. Spec, for Std. Spec. for tric Fusion Welded Chromium-Nickel-Iron-Molydenium eamless and Welded Chromium-Nickel-Iron-Molybdenum plates (1972) ANSI G33.3 Std. Spec. for Chromium Std. Spec. for Std. Spec. for

-Nickel Alloy Plate, Sheet, Strip, and / Std. Spec. for

- Nickel Rod and Bar (1973) spec. for Copper-Nickel-Zinc Alloy (Nickel Silver) and spec. for Copper-Nicke-Zinc Alloy (Nickel Silver) and trip, and Rolled Bar (1973) Std. Spec. for led Bar (1973)

r Bridge and Other Structural Uses/ Std. Spec. for Std. Spec. for Rolled Std. Spec. for ar (19) Std. Spec, for Copper-Cobalt-Beryllium Alloy ight Gage for Heat Exchanger Tubing (1972/ Std. Spec. for Std. Spec. for Nickel-Chromium-Iron-Molybdenum . Std. Spec. for Nickel Std. Spec. for Nickel lron Chromium Molybdenum td. Spec. for General Requirements for Wrought Copper and Std. Spec. for Nickel Iron Chromium Molybdenum Std. Spec. for Nickel-Chromium-Iron-Molybdenum Std. Spec. for 73) Std. Spec, for Nickel-Iron-Chromium-MolybdenumStd. Rec. Pract. for Conducting Bending Fatigue Tests for Std. Spec. for

for General Requirements for Wrought Seamless Copper and Std. Spec. for Std. Spec. for Welded

Sheet (1972)

Std. Spec. for General Requirements for Wrought Copper and

onductor Solid Dielectric Power Cable $15 \mathrm{kV}$ Through $35 \mathrm{kV}$ se with Copper Water Tube (1973)

d. for Wrought

Bar (19) Std. Spec. for General Requirements for Wrought Std. Spec. for General Requirements for Wrought Seamless cation Data Sheet (1972)

Std. Designations for

Std. Photometric Meth. for Chemical Analysis of

W3.6 ansi $\mathrm{H} 7.10$

ANSI H7.l

(1973)

ansi $\mathrm{H} 37.1$
Spec. for

Std. Spec. for

Std. Spec. for

Std. Spec. for

Std. Spec. for U-Bend Seamless Std. Spec. for Seamless

Std. Spec. for General Requirements for Wrought ger Tubes with Integral Fins (1974)

le Stock (1974) ANSI H23.14 73)

Std. Spec. for Std. Spec, for Standard for Wrought Std. Spec. for Welded Spec. for

Std. Meth. of Mercurous Nitrate Test for Std. Spec. for Ammoniacal Std. Meth. for Chemical Analysis of Ammoniacal Std. Spec. for Std. Spec. for

std. Photometric Meth. for Chemical Analysis of Copper and nsi 2l66.17 Std. Reference Radiographs for High Strength ansi $\mathrm{H} 9.27$

8.32

) ANSI H23.8

Std. Meth. for Spectroc. for Seamless Std. Spec. for Seamless Std. Spec. for Std. Spec. for Std. Spec. for Std. Spec for Sectrochemicar Hard Drawn Std. Spec. for Acid Std. Spec. for )

Spec, for Nickel Coated c. for Seamless and Welded Chromium Nickel Iron Molybdenum ip (1973)/ Std. Spec. for Chromium Nickel Iron Molybden um r Cable) (1973)

ectrical Conductors (1971) Spec. for Tinned

si C7.8

Spec. for Rope Lay Stranded Std. Spec. for Compact Round Concentric Lay Stranded st for Exfoliation Corrosion Susceptibility in 7 XXX Series , and Other Starch/ s (1954)
Cooling (1972) ANSI B 144.1

UL

UL

Cooling, Refrigeration Systems, Incinerators and Other

Coordinates (1971)

ICBO

Copies (1972)

Std. for Num ANS

Coplanar Magnetic Tape Cartridge Type CD II (Compact Ca Std. AST

Copolymers (SBR) (1972)

Copper-Beryllium Alloy Plate, Sheet, Strip, and Rolle Chical Analysis ASTM

Copper-Columbium Stabilized Alloy Pipe (1974)

Copper-Columbium Stabilized Alloy Tubes (1973)

Copper-Nickel-Aluminum Alloy Steel Pressure Vessel

Elec

lor S ASTM

ASTM

Cickel-Silicon Alloy Rod and Bar (1973)

ASTM

Copper-Nickel-Zinc Alloy (Nickel Silver) and Copper

ASTM

ASTM

Copper-Nickel Alloy Plate, Sheet, Strip, and Rolled B

Copper-Nickel Rod and Bar (1973)

Copper-Zinc-Aluminum-Cobalt Alloy Plate, Sheet, S

ASTM

ASTM

Copper-Zinc-Tin Alloys Plate, Sheet, Strip, and Rol

ASTM

Copper Alloy Bearing and Expansion Plates and Sheets Fo ASTM

Copper Alloy Castings (1967) ANSI Zl66.17 Std. Refere ASTM

Copper Alloy Condenser Rolled Tube Plates in Surface Co ASTM

Copper Alloy Electrical Contact Material (1973)

ASTM

ASTM

ASTM

Copper Alloy No 260 Brass Strip in Narrow Widthe and L

Copper Alloy Plate, Sheet and Strip (1973)

Copper Alloy Plate, Sheet, and Strip (1970) ANSI H34.6

Copper Alloy Plate, Sheet, and Strip (1972) ANSI H34.17

ASTM

ASTM

ASTM

Copper Alloy Plate, Sheet, Strip, and Rolled Bar (1974)

ASTM

Copper Alloy Rod and Bar (1972) ANSI H34.18

Copper Alloy Rod (1973)

Copper Alloy Sand Castings for General Applications (19

ASTM

ASTM

Copper Alloy Seamless Pipe and Tube (1972) ANSI H34.16

ASTM

Copper Alloy Spring Materials (1973)

Copper Alloy Strip for Flexible Metal Hose (1973)

Copper Alloy Tube (1974) ANSI H23.4

Copper Alloy Tubes for Pressure Applications (1972)

Std Spec

ASTM

ASTM

Copper Alloy Water Tube (1973)

ASTM

ASTM

pire (1974) ANSI H30.2

CDA

Copper Alloys (1971)

Copper and Aluminum Conductors) (1972)

Copper and Bronze Solder-Joint Pressure Fitting for

Copper and Copper Alloy Plate, Sheet, Strip, and Rolled

Copper and Copper Alloy Tube (1974) ANSI H23.4

Copper and Copper Alloys (Wrought Products Only); Appli

Copper and Copper Base Alloys (1972)

Copper and Its Alloy Arc Welding Electrodes (1969) ANSI

Copper and Its Alloy Clad Steel Plate (1973)

Copper and Its Alloy Die Forgings (Hot Pressed) (1974)

Copper and Its Alloy Forging Rod, Bar and Shapes (1974)

Copper and Its Alloy Heat Exchanger and Condenser Tubes

Copper and Its Alloy Rectangular Waveguide Tube (1974)

Copper and Its Alloy Rod, Bar, and Shapes (1973)

Copper and Its Alloy Seamless Condenser and Heat Exchan

Copper and Its Alloy Seamless Condenser Tubes and Ferru

Copper and Its Alloy Solder Joint Drainage Fittings (19

Copper and Its Alloy Tube (1973)

Copper and Its Alloy Welding Rods (1969) ANSI W3.7

Copper and Its Alloys (1973) ANSI H39.3

Copper Arsenite (1971) ANSI 011.8

Copper Arsenite (1971) ANSI 011.23

Copper Base Alloy Centrifugal Castings (1973)

Copper Base Alloy Continuous Castings (1973)

Copper Base Alloys (1972)

Copper Base and Nickel-Copper Alloy Castings (1967) a

Copper B ase Sintered Bearings (Oil Impregnated) (1973)

Copper Beryllium Alloy Forgings and Extrusions (1972)

Copper Beryllium Alloy Rod and Bar (1971A) ANSI H7.7

Copper Brazed Steel Tubing (1970) ANSI B 125.33

Copper Bus Bar, Rod, and Shapes (1974) ANSI C7.25

Copper Bus Pipe and Tube (1974) ANSI C7.26

Copper by the Briquet D-C Arc Technique (1971) ANSI Z12

Copper Capillary Tube for Restrictor Applications (1974

Copper Chromate (1971)

Copper Clad Aluminum Wire (1972)

Copper Clad Steel Wire for Electronic Application (1972

Copper Columbium Stabilized Alloy Pipe (1973)

Copper Columbium Stabilized Alloy Plate, Sheet, and Str

Copper Concentric Neutrals (Underground Electrical Powe

Copper Conductors Having Bunch Stranded Members, for El

Copper Conductors, Hard, Medium Hard, or Soft (1972) an

Copper Conouctors (1974) ANSI C7.59

Copper Containing Aluminum Alloys (Exco Test) (1972)

Copper Content of Corn Sugar (Crude and Refined), Syrup

Copper Content of Corn Syrup Unmixed and Finished Sugar
SAE

IPCEA

ASTM

ASTM

CDA

ASTM

ASTM

ASTM

ASTM

ASTM

ASTM

ASTM

ASTM

ASTM

ANSI

ASTM

AWS

ASTM

ASTM

ASTM

ASTM

ASTM

ASTM

ASTM

ASTM

ASTM

ASTM

ASTM

ASTM

ASTM

ASTM

ASTM

ASTM

ASTM

ASTM

ASTM

ASTM

AA

ASTM

ASTM

ASTM

ASTM

CR

CR

465

UMC

A62.8

F360

RS399

D1416

B 194.

B534

B474

B468

A410

Bl22

B151

B 122

B15l

B592

B59l

B 100

272

B17l

B596 
Std. Spec. for

Std. Spec. for Fire Refined

Tent. Meth. of Analysis for Std. Spec. for Boron Deoxidized Std. Spec. for

1973)

borne Preservative Solutions Such as Pentachlorophenol and
Std. Spec. for s (1969) ANSI H52.1

Std. Spec. for Seamles std. for Connectors for Use Between Aluminum or Aluminum std. for Connectors for Use Between Aluminum or Aluminum Std. Meth. for Chemical Analysis of Std. Spec. for Threadless Std. Spec, for Seamless

flatwire and Strip) (1973)

's Reduction Table (Cereal Chemistry) (1962) Std. Spec. for Flat Volumetric Std. Spec, for Std. Spec. for Hot Rolled Sid. Spec, for Std. Spec. for Std. Spec. for Std. Spec. for Sid. Spec. for Std. Spec. for is $(1973)$

7.3 H26.3 nsi $\mathrm{H} 30.1$ si G63.1 Design Rules, Guidelines, and Drawings for
Sid. Spec. for Sintered zed) Coating on Iron or Steel Articles by the Preece Test d Service (1974) ANSI H23.5 Std. Spec. for Phosphor Bronze Sid. Spec. for Seamless
Std. Spec, for Welded Std. Spec. for Seamless Std. Spec, for Seamless Std. for Flexible

Std. Spec. for Seamless er and Bronze Solder-Joint Pressure Fitting for Use with Std. Spec. for Phosphorus Deoxidized Std. Spec. for Oxygen Free Electrol ytic

got Bars (1973) ANSI H17.I got Bars (1973) ANSI H/ got Bars (1973) ANSI H17.2 Std. Spec. for Chemically Refined Std. Spec. for Electrolytic Std. Spec. for Lead and Lead Alloy Coated Soft Std. Spec. for Soft or Annealed Std. Spec. for Tinned Soft or Annealed Std. Spec. for Silver Coated Soft or Annealed Std. Spec. for Nickel Coated Soft or Annealed rod, Bars, and Shapes (1972) ANSI H33.1 Std. Spec, for Std. Spec. for Electrolytic Cathode Std. Spec. for Fire Refined Casting Std Meth of Test for Hydrogen Embrittlement of Corrosion Resistant Metals (Electrodeposited Coatings of Std. Classification of ensions for Unitized Microfilm Carriers (Aperture, Camera, dup Area on Unitized Microfilm Carriers (Aperture, Camera, Std. Definitions of Terms Relating to Electrostatic r, Cultivator, Edger-Trimmer, Hedge / Std. for Safety for for Safety for Test for Polymeric Enclosures for Portable Safety Std, for Electric Tent. Tolerances for Tire Fabrics (Textile) Other Than Std. Meth. of Test. Tire Cords, Tire Std. for Safety for Safety Std. for

- Meth. of Test for Adhesion of Vulcanized Rubber to Steel of Test for Strap Peel Adhesion of Reinforcing Fabrics or Salety Std for Cord Sets and Power Supply Man Made Organic Base Fibers/ Std. Meth. of Testing Tire rom Steel (1974) Std. Meth. of Test. Tire Quality Stds. for Flush Solid and Hollow Sid and Nomenclature for Diamond alvanized) and Aluminum Coated (Aluminized) Stranded Steel Std. Spec. for Asbestos Cement Plastic Foam td. Test Meth. for Delamination Strength of Honeycomb Type 970) Std. Test Meth. for Density of 970) Std. Test Meth. for W ater Absorption of 973) ANSI C83.92 Std. Test Meth. for Shear Fatigue of Sandwich Std. for Iron . 8r) (1972) / Std. Meth. of Test for Elastic Moduli of Rock sr) (1974)/

Std. Spec. for Zinc Coated (Galvanized) Steel Spec. for Mild Steel Electrodes for Flux Spec. for Flux steel Electrodes (1974) al) (1971) Std. Dimensions for Std. Test Procedure for Toroidal Magnetic Amplifier Sid. Meth for Measurement of Thickness of Sandwich Test Meth. for Flatwise Compressive Strength of Sandwich $r$ Test in Flatwise Plane of Flat Sandwich Constructions or

Procedure for End Supported Beam Strength Fibre Tubes and
Copper Drainage Tube (DWV) (1974) ANSI H23.6

Copper for Wrought Products and Alloys (1972) ANSI H23.

Copper in Wine (Enology) (1972)

Copper in $\mathbb{W}$ rought Forms for Electron Devices (1972)

Copper Iron Alloy Plate, Sheet, Strip, and Rolled Bar (

Copper Naphthenate (1973)
Copper Nickel Alloy Plate and Sheet for Pressure Vessel

Copper Nickel Pipe and Tube (1973) ANSI H26.4

Copper Overhead Electric al Conductors (1973) EEI TDJ.16

Copper Overhead Electrical Connectors (1973) NEMA CC3

Copper Phthalocyanine Blue and Green Pigments (1973)

Copper Pipe (1974) ANSI H26.2

Copper Pipe, Std. Sizes (1972)

Copper Products with Finished (Rolled or Drawn) Edges (

Copper Reduction Meth. for Sugar Determination: Schoorl

Copper Rod, Bar, and Shapes (1973) ANSI H7.4

Copper Rods for Electrical Purposes (1974) ANSI C7.7

Copper Rods for Locomotive Staybolts (1973) ANSI H7.2

Copper Sheet, Strip, Plate and Rolled Bar (1973) ANSI H

Copper Silicon Alloy Plate and Sheet for Pressure Vesse

Copper Silicon Alloy Rod, Bar, and Shapes (1973) ANSI H

Copper Silicon Alloy Seamless Pipe and Tube (1974) ANSI

Copper Silicon Alloy Wire for General Purposes (1973) a

Copper Sintered Metal Powder Structural Parts (1970) an

Copper Solvent Single Stack Plumbing System (1973)

Copper Steel Structural Parts (1970) ANSI G63.3

Copper Sulfate Dip) (1973) AA SHO T66

Copper Tin) Plate, Sheet, Strip, and Rolled Bar (1973)

Copper Tube for Air Conditioning and Refrigeration Fiel

Copper Tube (1974)

Copper Tube (1975) ANSI H23.3

Copper Tube, Bright Annealed (1973) ANSI H23.2

Copper Water Connectors (1971)

Copper Water Tube (1972) ANSI H23.1

Copper Water Tube (1973)

Copper Wire Bars, Billets, and Cakes (1972)

Copper Wire Bars, Billets, and Cakes (1972) ANSI H23.11

Copper Wire Bars, Cakes, Slabs, Billets, Ingots, and in

Copper W ire Bars, Cakes, Slabs, Billets, Ingots, and in

Copper Wire Bars, Cakes, Slabs, Billets, Ingots, and in

Copper Wire for Electrical Purposes (19674 ANSI C7.15

Copper Wire for Electrical Purposes (1974) ANSI C7.1

Copper Wire for Electrical Purposes (1974) ANSI C7.4

Copper Wire (1974) ANSI C7.38

Copper Wire (1974) ANSI C7.48

Copper Zinc Lead (Leaded Red Brass or Hardware Bronze)

Copper (1972) ANSI H23.10

Copper (1972) ANSI H23.9

Copper (1973)

Copper, Nickel, Chromium, Lead, Zinc, or Cadmium) (1973

Coppers (1973) ANSI H34.43

Copy and Image Cards) (1972)

Copy and Image Cards) (1973) ANSI PH5.12

Copying (1972B)

Cord Connected Electric Gardening Appliances (Lawn Mowe

Cord Connected Electrical A ppliances (1973)

Cord Connected Indoor Signs (1971) ANSI C33.35

Cord Fabrics (1973) ANSI L14.243

Cord Fabrics, Filaments, and Strands Made from Steel (1

Cord Reels (1973) ANSI C33.18

Cord Sets and Power Supply Cords (1972) ANSI C33.3

Cord (1973)

Cords to Rubber (1971)

Cords (1972) ANSI C33.3

Cords, Fabrics and Industrial Filament Yarns, Made from

Cords, Tire Cord Fabrics, Filaments, and Strands Made F

Core Doors (Architectural Woodwork) (1973)

Core Drill Equipment (1973) ANSI B104.1

Core for Aluminum Conductors, Steel Reinforced (ACSR) (

Core lnsulating Panels (1972)

Core Material (1970)

Core Materials for Structural Sandwich Constructions (1

Core Materials for Structural Sandwich Constructions (1

Core Materials (1970)

Core Power Filter Inductors for Electronic Equipment (1

Core Specimens in Uniaxial Compression (1972)

Core Wire for Aluminum Conductors, Steel Reinforced (AC

Core Wire for Aluminum Conductors, Steel Reinforced (AC

Cored Arc Welding (1969) ANSI W 3.20

Cored Corrosion Resisting Chromium and Chromium Nickel

Cores for Photographic Film Rolls (Plastic, Wood or Met

Cores (Including Material Data) (1972)

Cores (1970)

Cores $(1970)$

Cores $(1970)$

Cores (1972)
ASTM

ASTM

ASE

ASTM

ASTM

ASTM

ASTM

ASTM

NEMA

EEI

ASTM

ASTM

ASTM

ASTM

AACCH 80.69

ASTM

ASTM

ASTM

ASTM

ASTM

ASTM

ASTM

ASTM

ASTM

CDA

ASTM

ASTM

ASTM

ASTM

ASTM

ASTM

ASTM

IAPMO

ASTM

ANSI

ASTM

AST'M

ASTM

ASTM

ASTM

ASTM

ASTM

ASTM

ASTM

ASTM

ASTM

ASTM

AST M

ASTM

ICBO

ASTM

id. for Dim ANSI

ss of Buil NMA

ASTM

UL

UL

UL

ASTM

UL

UL

Std ASTM

std. Meth. ASTM

UL

ASTM

ASTM

AWI

DCDMA

ASTM

ASTM

ASTM

ASTM

ASTM

ASTM

EIA

ASTM

ASTM

ASTM

AWS

AWS

ANSI

IEEE

ASTM

Std ASTM

Std. Meth. of Shea ASTM Std. Test. CCTI

B306

B216

F107

B465

B402

B466

C33

(D)

B302

B 42
B272

D2604

TDJ. 162 
dure for End Supported Beam Deflection Composite Tubes and td. Test. Proc. for Side to Side Crush Composite Tubes and or Measuring Inside Diameters of Composite Cans, Tubes and Composite Cans, Tubes and test. Procedure for Torque Strength of Composite Tubes and st. Procedure for Internal Bursting of Composite Tubes and or Measuring Wall Thicknesses of Composite Cans, Tubes and $r$ Axial (End to End) Compression Composite Cans, Tubes and measuring Lengths or Heights of Composite Cans, Tubes, and menclature Relating to Resilient Floor Coverings (Asphalt, agus); Lining Out Stock: Seedling Trees and Shrubs; Bulbs, ar (Crude and Refined), Syrups and Other Hydrolyzates from alytical Meth. for Determining Waxy and Nonwaxy Content in ontent in Feedstuffs (Corn) Containing Vegetable Material, d. Analytical Meth. for Determining Crude Fiber Content in ng Insect Infestation Content (Radiographic Inspection) in TLC Meth. of Analysis of Aflatoxins in ine Meth. of Analysis for Insect Eggs in White, Wheat, and Meth. of Analysis for Insect Excreta in White, Wheat, and ine in Bread, Wheat, Rice, and Other Whole Grain Products, d, Grain, Soybeans, Rice, Beans, Peas, Lentils, Corn Meal, olina, Bread, Grain, Soybeans, Rice, Beans, Peas, Lentils, Wheat, Rice, and Other Whole Grain Products, Corn Grits, alytical Meth. for Determining Free Fatty Acids Content in eth. for Determining Color (Spectrophotometric) Content in std. Analytical Meth. for Determining Cold Test Content in of Analysis of Acetone-Insoluble Lecithin in Soybean and e and Refined) and All Hydrolyzates Solutions Derived from analytical Meth. for Determining Hydroxyalkoxyl Content of

Std. Analytical Meth. for Determining Iron Content in lytical Meth. for Determining Borax Content in Dextrin and Std. Analytical Meth. for Determination of $\mathrm{pH}$ (Paste) of

Std. Analytical Meth. for Determination of $\mathrm{pH}$ (Slurry) of nalytical Meth. for Determining Acidity (Paste) Content in 1 Meth. for Determining Moisture (Karl Fischer) Content in std. Analytical Meth. for Determining Total Fat Content in cal Meth. for Determining Acidity (Extractable) Content in Std. Analytical Meth. for Determining Solubles Content in d. Analytical Meth. for Determining $\mathrm{pH}$ (Slurry) Content in analytical Meth. for Determining Sulfur Dioxide Content in nalytical Meth. for Determining Moisture (Oven) Content in

Determining Moisture (Azeotropic Distillation) Content in td. Analytical Meth. for Determining Phosphorus Content in eth. for Determining Color (Spectrophotometric) Content in td. Analytical Meth. for Determining $\mathrm{pH}$ (Paste) Content in Std. Analytical Meth. for Determining Calcium Content in alytical Meth. for Determining Waxy and Nonwaxy Content in Analytical Meth. for Determining Bulk Density Content in for Determining Inherent Viscosity (One Point) Content in Std. Analytical Meth. for Determining Carboxyl Content of Std. Analytical Meth. for Determining Acetyl Content of tical Meth. for Determining Brabender Viscosity Content in td. Analytical Meth. for Determining Phosphorus Content of Std. Analytical Meth. Determining Iodine Affinity of

h. for Determining Calcium (Egta-Titrimetric) Content in Std. Analytical Meth. for Determining Ash Content in ts / eth. for Determining Crude Fat / Extractables) Content in alytical Meth. for Determining Protein Nitrogen Content in Std. Analytical Meth. for Determination of Phosphorus in termining Color Solutions (Spectrophotometric) Content of nalytical Meth. for Determining Moisture (Oven) Content of Std. Analytical Meth. for Determining Acidity Content of

Std. Analytical Meth. for Determining Arsenic Content of Std. Analytical Meth. for Determining Ash Content of Std. Analytical Meth. for Determining Iron Content of analytical Meth. for Determining Sulfur Dioxide Content of rmining Color, Reflectance (Spectrophotometric) Content of 1 Meth. for Determining Moisture (Karl Fischer) Content of nalytical Meth. for Determining Reducing Sugars Content of tical Meth. for Determining Dextrose Equivalent Content of

lytical Meth. for Determining Specific Rotation Content of

h. for Determining Calcium (Egta-Titrimetric) Content of

Std. Analytical Meth, for Determining Copper Content of Analytical Meth. for Determining Heavy Metals Content of

Std. Analytical Meth. for Determining Chloride Content of

Hyd/

Std. Analytical Meth. for Determining pH Content of alytical Meth. for Determining Protein Nitrogen Content of for Determining Arsenic Content of Corn Syrup Unmixed and

Paper) Content of Corn Syrup Unmixed and Unbleached Crude rmining Saccharides (Gas Liquid Chromatography) Content of Analytical Meth. for Determining Total Sugars Content of rmining Sulfate Content of Corn Syrup Unmixed and Finished Std. Analytical Meth. for Determining Dextrose Content of tical Meth. for Determining Dextrose Equivalent Content of

h. for Determining Calcium (Egta-Titrimetric) Content of eth. for Determining Iron (Orthophenanthroline) Content of
Cores (1972)

Cores (1973)

Cores (1973)

Cores (1974)

Cores (1974)

Cores (1974)

Cores (1974)

Cores (1974)

Cores (1974)

Corms, and Tubers; Christmas, Tinyl Asbestos.

Corn and Grain Sorghum Starch (1960)

Corn and Grain Sorghum, and Their Mixtures (1959)

Corn and Other Whole Grains (1958)

Corn and Soybeans (Cereal Chemistry) (1972)

Corn Flour (Cereal Chemistry) (1962)

Corn Flour (1962)

Corn Grits, Corn Meal, Puffed Cereals, Farins, and Flou

Corn Grits, Rolled Oats, Bulgur, Rolled Wheat, Breakfas

Corn Meal, Corn Grits, Rolled Oats, Bulgur, Rolled Whea

Corn Meal, Puffed Cereals, Farina, and Flour (Cereal Ch

Corn Oil and to Other Vegetable and Marine Oils and An

Corn Oil (1964)

Std. Analytical M CR

Corn Oil (1965)

Corn Oils (Cereal Chemistry) (1967)

Corn or Grain Sorghum Starch Which Are Substantially $O$

Corn Starch and Ethers (1967)

Corn Starch Hydrolzates Including Syrups and Sugars, an

Corn Starch Products (1971)

Corn Starch Which Is Gelatinized When Heated in a Boili

Corn Starch Which Is Substantially Insoluble in Water a

Corn Starch (Gelatinizable Products) (1955)

Corn Starch (Un modified) (1961)

Corn Starch (1955)

Corn Starch (1955)

Corn Starch (1956)

Corn Starch (1956)

Corn Starch (1956)

Corn Starch (1956)

Corn Starch (1956)

Corn Starch (1957)

Corn Starch (1957)

Corn Starch (1958)

Corn Starch (1960)

Corn Starch (1960)

Corn Starch (1963)

Corn Starch (1963)

Corn Starch (1966)

Corn Starch (1968)

Corn Starch (1968)

Corn Starch (1969)

Corn Starch (1973)

Corn Starch (1973)

Corn Starch, Dextrines and Other Modified Starch Produc

Corn Starch, Gluten, Germand Finished Std. Analytical M

Corn Starch, Syrup and Sugar Obtained from the Corn Wet

Corn Sugar (Crude and Refined) and All Hydrolyzates Sol

Corn Sugar (Crude and Refined) (Crystalline) (1959)

Corn Sugar (Crude and Refined) (1958)

Corn Sugar (Crude and Refined) (1958)

Corn Sugar (Crude and Refined) (1958)

Corn Sugas (Crude and Refined) (1961)

Corn Sugar (Crude and Refined) (1961)

Corn Sugar (Crude and Refined) (1963)

Corn Sugar (Crude and Refined) (1966)

Corn Sugar (Crude and Refined) (1972)

Corn Sugar (Crude and Refined) (1972)

Corn Sugar (Crude and Refined), Dextrose, Syrup and Sta

Corn Sugar (Crude and Refined), Syrup and Other Starch

Corn Sugar (Crude and Refined), Syrup, and Other Starch

Corn Sugar (Crude and Refined), Syrups and Other Hydrol

Corn Sugar (Crude and Refined), Syrups and Other Starch

Corn Sugar (Crude and Refined), Syrups and Other Starch

orn Sug CR

l an CR

Determini $\mathrm{C}$

CR

lod AACCH 28.44

lass Plate AACCH 28.43

AACCH 86.80

$\mathrm{AACCH} 44.15 \mathrm{~A}$

AACCH $44.15 \mathrm{~A}$

$\mathrm{AACCH} \quad 86.80$

CR H.22

$\mathrm{CR}-\mathrm{H} 10$

Meth. AACCH 58-35

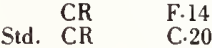

B. 30

D. 10

C. 42

C. 44

B-4

B.36

B. 20

B. 2

B. 56

B-44

B. 58

B. 38

B.34

B- 46

B-14

B. 42

B. 42
B. 10

B. 10
B. 64

Std. an CR

Std CR

B. 16

B-61

C. 22

C- 46

B. 28

B- 18

B- 48

B. 47

F. 14

F. 34

F-2

F.4

F- 6

F. 28
F. 54

F.16

F. 32

F.48

F. 22

F.8

F.18

F. 26

F. 10

F.42

F.44

E.4

E. 44

F.50

F. 58

E. 64

E. 24

E-26

E.11 
Std. Analytical Meth. for Determining Acidity Content of Std. Analytical Meth. for Determining Arsenic Content of nalytical Meth. for Determining Heavy Metals Content of of Std. Analytical Meth. for Determining Sulfate Content of Std. Analytical Meth. for Determining Copper Content of Std Analytical Meth for Determining Baume Content of h. for Determining Moisture (Oven Filter Paper) Content of meth. for Determining Candy Color and Inversion Content of

Determining Moisture (Azeotropic Distillation) Content of nalytical Meth. for Determining Color Stability Content of eth. for Determining Moisture (Oven Filter Aid) Content of Std. Analytical Meth. for Determining Chloride Content of Std. Analytical Meth. for Determining Chloride Content of analytical Meth. for Determining Sulfur Dioxide Content of meth. for Determining Color (Visual Comparison) Content of td. Analytical Meth. for Determining Phosphorus Content of eth. for Determining Color (Spectrophotometric) Content of for Determining Saccharides (Chromatographic) Content of 1 Meth. for Determining Moisture (Karl Fischer) Content of d. Analytical Meth. for Determining Iron (TPTZ) Content of ical Meth. for Determining Extraneous Materials Content of

lytical Meth. for Determining Specific Rotation Content of Std. Analytical Meth. for Determining Ash Content of nalytical Meth. for Determining Apparent Starch Content of Std. Analytical Meth. for Determining $\mathrm{pH}$ Content of alytical Meth. for Determining Refractive Index Content of Std. Analytical Meth. for Determining Calcium Content of rmining Saccharides (Gas Liquid Chromatography) Content of Analytical Meth. for Determining Fermentables Content of alytical Meth. for Determining Protein Nitrogen Content of osphorus in Corn Starch, Syrup and Sugar Obtained from the Rapid Meth of Analysis for Fat Acidity in h. for Determining Filth and Insect Infestation Content in

Determining Moisture (Azeotropic Distillation) Content in Std. Analytical Meth. for Determining Ash Content in

feedstuffs and All Other Natural Dry Products Derived from for Determining Crude $\mathrm{Fat}\left(\mathrm{Ccl}_{4}\right.$ Extractables) Content in cal Meth. for Determining Viability (Sprouting) Content in in Steepwater and Most Other Liquid Products Obtained from in Steepwater and Most Other Liquid Products Obtained from Std. Analytical Meth. for Determining Starch Content in Meth. for Determining Bulk Density Content in Feedstuffs

1 Meth. for Determining Crude Fiber Content in Feedstuffs

for Determining Protein Nitrogen Content in Steepwater. ytical Meth. for Determining Starch Content in Feedstuffs ical Meth. for Determining Solubles Content in Feedstuffs ing Crude Fat ( $\mathrm{Ccl}$, Extractables) Content in Feedstuffs nalytical Meth. for Determining $\mathrm{pH}$ Content in Feedst uffs nalytical Meth. for Determining Ash Content in Feedstuffs $r$ Determining Acidity (Extractable) Content in Feedstuffs

Moisture (Azeotropic Distillation) Content in Feedstuffs ermining Moisture (Oven) Content in Commercial Feedstuffs h. for Determining Ammonia Nitrogen Content in Steepwater $r$ Determining Lactic Acid and Salts Content in Steepwater nalytical Meth for Determining Ash Content in Steepwater Meth. for Determining Xanthophylls Content in Feedstuffs th. for Determining Reducing Sugars Content in Steepwater

$r$ Determining Lactic Acid and Salts Content in Feedstuffs Determining Moisture (Karl Fischer) Content in Steepwater h. for Determining Protein Nitrogen Content in Feedstuffs eth. for Determining Iodine Number (Wijs Meth.) Content in Friction Coefficients of Chopped Forages (Grass,

alytical Meth. for Determining Protein Nitrogen Content in

in Flour and Semolina; Also, with Slight Modification, to 0 Deg. F (1973) Std. for Nut, Self Locking. Plate Rec. Pract. for hway Vehicl Std. Meth. of Test. Tires for Wet Traction in

Dynamic Water Resistance of Shoe Upper Leather by the Dow ical Insulating Materials Subjected to Partial Discharges for the Detection and Measurement of Partial Discharges $3 / \quad$ Std. Meth. for Detection and Measurement of Discharge subfloor Surfaces to Receive Wood Flooring (196) Rec. for istry) (1965)

Meth. for Multiplication Factors for on Dioxide (Cereal Chemistry) (196/ Meth. of Analysis for Test Meth. for Density of Pulpwood (Submersion with t Meth. for Density and Moisture of Chips (Submersion with 0\% Moisture (Cereal Chemistry) (1962)

(Cereal Chemistry) (1962)

Meth. for Meth. for

o $14.0 \%$ Moist/ chemistry) (1962) chemistry) (1962)

Std. Volume and Specific Gravity Meth. of Physical Dough Tests: Approximate Temperature Temperature

of Test for Wavelength of Peak Photoluminescence and the e Book: Fire Protection and Safety (Exit, Stairway, Aisle, rings (Nickel Base, Solution Heat Treated, 15./ Spec. for ti-l Spec. for Steel Bars, Forgings, Tubing, and Rings,
Corn Syrup and Starch Hydrolyzates (1952)

Corn Syrup Unmixed and Corn Sugar (1954)

Corn Syrup Unmixed and Dextrose (1956)

Corn Syrup Unmixed and Finished Corn Sugars (1954)

Corn Syrup Unmixed and Finished Sugars (1954)

$\begin{array}{ll}\text { Corn Syrup Unmixed and lts Syrups (1959) } & \text { CR } \\ \text { Corn Syrup Unmixed and Unbleached Crude Corn Sugar (195 CR }\end{array}$

Corn Syrup Unmixed (1952)

Corn Syrup Unmixed (1954)

Corn Syrup Unmixed (1954)

Std. Analytical Meth. for CR

Std. a CR

Std. Analytical M CR

Corn Syrup Unmixed, Finished Sugar and Other Clarified

Cornised, Finished Sugar and Other Clarified

Corn Syrup (1952)

Corn Syrup (1954)

Corn Syrup (1957)

Corn Syrup (1957)

Corn Syrup (1957

Corn Syrup (1966)

Corn Syrup (1970)

Corn Syrup, Crude and Refined Sugar and Other Material

Corn Syrup, Dextrose and Starch Hydrolyzates (1971)

Corn Syrup, Finished Sugar and Other Starch Hydrolyzate

Corn Syrup, Refined and Crude Sugar (1967)

Corn Syrup, Starch Hydrolyzates and a Variety of Other

Corn Syrup, Sugar and Dextrose (1956)

Corn Syrup, Sugar and Other Carbohydrates (1957)

Corn Syrup, Sugar, and Starch Hydrolyzates (1967)

Corn Syrup, Sugars, and Starch Hydrolyzates (1957)

Std. CR

Std. Analytical M CR

Std. Analytical Meth CR

Std. Analytica CR

St CR

CR

la $\mathrm{CR}$

Std. a $\mathrm{CR}$

CR

Std. an $\mathrm{CR}$

CR

Ete CR

/Td CR

$\mathrm{CR}$

Corn Syrup, Sugars, Starches and Other Protein Bearing

Corn Wet Milling Process (1973)

Corn (Cereal Chemistry) (1962)

Corn (1954)

Corn (1955)

Corn (1957)

Corn (1957)

Corn (1958)

Corn (1959)

Corn (1963)

Corn $(1963)$

Corn (1964)

Corn) and Other Wet Milled Solid Products (1963)

Corn) Containing Vegetable Material, Corn and Other Gra

Corn) Feedstuffs, Syrups. Sugars, Starches and Other Pr

(Corn) (1954)

(Corn) (1954)

Corn) (1954)

Corn) (1955)

(Corn) (1955)

Corn) (1955)

Corn) (1956)

(Corn) (1957)

(Corn) (1962)

Corn) (1962)

Corn) (1964)

Corn) (1964)

Corn) (1964)

Corn) (1964)

Std. Analytical Meth Met C

CR
CR

/ Ng Color (Spectrophotometric) Content in $\mathrm{CR}$ Std. Azalytical Meth. CR Std. Analyti CR Al Meth. for Determining Acidity Content CR
Lytical Meth. for Determining $\mathrm{pH}$ Content CR

(Corn) (1966)

Corn), Syrups, Sugars, Starches and Other Protein Beari

Corn, Grain Sorghum and Other Vegetable Fats and Oils (

Corn, Hay, Straw) (1968)

Corn, Other Grains and Plant Materials of Comparable Co

Corn, Rye, Barley, Rice, Grain Sorghum, and Buckwheat (

Corner, Counterbored, Floating, $125 \mathrm{KSI}$ Ftu, 450 and 80

Cornering Lamps (Light) for Automotive Vehicles (1965)

Cornering Without Driving Torque Application, Using Hi

Corning Leather Tester (1970) ALCA E5

H. of Test for ASTM

Corona)

lof Test for Voltage

Std. Anal CR

IIcal $\mathrm{CR}$

CR

Sin $\mathrm{CR}$

Std. a CR

Std. Analytical Meth. Fo CR

Corona) During Dielectric Tests (1973)

Corona) Pulses in Evaluation of lnsulation Systems (197

Correct Preparation, Finishing and Testing of Concrete

Correcting Weights to a $14 \%$ Moisture Basis (Cereal Chem

Correction Factors for Gasometric Determination of Carb

Correction for Cracks, etc.) (Pulp and Paper) (1973)

Correction for Cracks, etc.) (Pulp and Paper) (1973)

Correction for Weight of Flour to Give 100 Grams at 14.

Correction of Analytical Values to $14.0 \%$ Moisture Basis

Correction Tables for Creosote and Coal Tar (R1970)

Corrections for Changing as-is Farinograph Absorption T

Corrections for Refractometer Readings (Sugar) (Cereal

Corrections for Saccharimeter Readings (Sugar) (Cereal

Corresponding Composition of Gallium Arsenide Phosphide

Corridor, Building Construction and Test. (1973)

Corrosion and Heat Resistant Alloy Bars, Forgings, and

Corrosion and Heat Resistant $(15 \mathrm{Cr}-26 \mathrm{Ni}-1.3 \mathrm{Mo}-2.1$

CR

IEEE

ASTM

A-18

AACCH $76-20$

NSA $\quad 1766$

SAE J852B

F 376

D2098

D2275

454

D1868

AACCH 82.2

AACCH 12.29

TAPPI UM-2

TAPPI UM.20

AACCH 82.23

AACCH $82-22$

ASTM D347

AACCH $54-29$

AACCH $80-20$

AACCH 80.21 
73)

(1973)

$.5 \mathrm{Ni}-7.5 \mathrm{~W}(1973)$

Torque, All Metal, 1/

Torque, All Metal, 1/

$-5.5 \mathrm{Mo}-0.85 \mathrm{Cb}-2.5 \mathrm{Ti}-1.6$ /

$4 \mathrm{Ti}-0.70 \mathrm{~A} 1-7.0 \mathrm{Fe}(1973)$

$0 \mathrm{Fe}(1973)$

$.2 \mathrm{Mo}-0.30 \mathrm{Al}-0.05 \mathrm{La} /$

$.2 \mathrm{Mo}-0.30 \mathrm{Al}=0.05 \mathrm{La}(1 /$

$5 \mathrm{Co}-4.3 \mathrm{Mo}-3.0 \mathrm{Ti}-1.4 \mathrm{Al}, \mathrm{C}$

co-2.5Ti-1.5Al (1973)

$5 \mathrm{Mo}-5.5 \mathrm{Fe}$, Solution $\mathrm{H} /$

co-4.3Mo-3.0 Ti-1.4Al, Con/

Spec. for Steel Welding Wire, Spec. for Steel Tubing, Welded, Alloy Wire, Welding,

Spec, for Self-Locking Steel Nuts, Spec. for Self-Locking Steel Nuts,

Alloy Bars and Forgings, Alloy Sheet and Strip,

Alloy Bars, Forgings, and Rings, Alloy Bars, Forgings, and Rings, Alloy Sheet, Strip, and Plate, Alloy Bars and Forgings, Alloy Wire, Welding,

Alloy Bars, Forgings, and Rings, Alloy Bars and Forgings, $5 \mathrm{Co}-4.3 \mathrm{Mo}-1$ Spec. for Alloy Bars, Forgings and Rings, $-5.9 \mathrm{Mo}-2.2 \mathrm{Ti} /$

Spec. for Alloy Sheet, Strip, and Plate, treatment, 1800 Deg. F (982.2 Deg. C) Solu/ Studs, Steel, $\mathrm{p}$, and Plate $(13 \mathrm{Cr}-2.0 \mathrm{Ni}-3.0 \mathrm{~W})$ (1973)

$1.6 \mathrm{Co}-1.8 \mathrm{Mo}-0.32 \mathrm{~V})(1 /$

n (SAE 51416F), Free Machining /

Spec. for Steel Welding Wire,

Steel Bars and Forgings, $2.9 \mathrm{Mo}-0.10 \mathrm{~N}$, Solution Heat Tre/ Steel Sheet and Strip,
$2.9 \mathrm{Mo}-0.10 \mathrm{~N}$, Solution Heat Treated (1973) Steel Plate, $2.9 \mathrm{Mo}-0.10 \mathrm{~N}$, Solution Heat T/ Steel Bars and Forgings, $2.9 \mathrm{Mo}-0.10 \mathrm{~N}$, Heat Treated, 1/ Steel Bars and Forgings, $2.9 \mathrm{Mo}-0.10 \mathrm{~N}$, Equalized and $\mathrm{T} /$ $2.9 \mathrm{Mo}-0.1 \mathrm{~N}(1973)$

Annealed (1973/

pumps with Coolan/ Steel Wire, Welding,

Steel Bars, Forgings, Tubing, and Rings, Std Test Meth for Cavitation Erosion of Stainless Steels and Related Ni-Cr-Fe Alloys to Stress se of Mattsson's Solution of pH 7.2 to Evaluate the Stress Std. Rec. Pract. for Making and Using the C-Ring Stress e Solution (1973) Std. Rec. Pract. for Performing Stress Std. Rec. Pract. for Applying Statistics to Analysis of

e Code: Recommended Guide to Safe Practice Protection from Std. Meth. for Preparation and Use of Bent Beam Stress ystems (1972) Rec. Pract. for Control of External Rec. Pract. for Collection and ldentification of ng Torque, All Metal (1973) and Nickel Base Alluy Castings for Gener/ Spec. for Self-Locking [ Copper, Nickel, Chromiu/ Uniform Building Code Std. for Std. for Solid 100 Deg. Flush Shear Head A286 um Induct/ Steel Bars, Wire, Forgings, and Forging Stock, c), Premium Bearing Qu/ Steel Bars, Forgings, and Tubing, Consumable Electrode $\mathrm{Va}$ Steel Sheet, Strip, and Plate, i Covered Welding Electrodes (1969) ANSI W 3.4 Spec, for I Welding Rods and Bare Electrodes (1969) Ansi/ electrodes (1974)

Strip (1972) ANSl G81.6

Spec. for Flux Cored Std. Spec. for $r$ Undrilled and Drilled, Plain and Self Locking, Alloy and illed and Drilled, Plain and Self Locking, Alloy Steel and ng Aluminum Alloys (E) Std. Meth. of Test for Exfoliation $s$ Tested in Glassware (1970) ANSI D14.9 Std. Meth for Std. Rec. Pract. for Making and Using U-Bend Stress Std. Rec. Pract. for Preparing, Cleaning, and Evaluating 972) Std. Meth. of Test for Simulated Service Test Meth. for Laboratory

Std Rec Pract for Recording Data from Immersion Std. Meth. of Conducted Controlled Velocity Laboratory test. Coated Steel Specimens Dynamically for Resistance to and Evaluation of Painted or Coated Specimens Subjected to e Diameter Austenitic Chromium Nickel Alloy Steel Pipe for prevent in Service Cracking of Carbon Steel (P-1) Welds in sign and Construction of Nonmetallic Enveloped Gaskets for d Deposits (1973) ectrical Meth. (1972)

Tent. Meth. of Test for Std. Meth. of Test for ) (1972) Std. Meth. of Test for Coefficient of Static Friction of Meth. for lnefficient of Static Friction of Std. Meth. of Test for Coefficient of Static Friction of Std. Spec. for
Std. Spec. for ) Std. Spec. for Std. Test Meth. for Ply Separation (Wet) of Solid and

4) stics (1971) Std. for lndustrial Engineering Terminology: Std. for Meth. of Measuring Frequency and tone, and Marble Masonry; Mortar; Sand; Slag; Steel; Terra Std. Clevis Pins and Test Meth. for h Chlorine (1972) ANSI L14.57 kage: Rapid Control Test/ Test Meth. for Colorfastness of Std. Rec. Pract. for Lighting th. for Fluidity of Dispersions of Cellulose Form Bleached Test for Color of Raw Cotton Using the Nickerson-Hunter ANSI L14.283 Std. Spec, and Meth. of Test for Heavy
Corrosion and Heat Resistant $(18 \mathrm{Cr}-11 \mathrm{Ni}-(\mathrm{Cb}+\mathrm{Ta})(19$ Corrosion and Heat Resistant (25Cr-20Ni) (SAE 30310) Corrosion and Heat Resistant, Cobalt Base-25.5Cr-10 Corrosion and Heat Resistant, High Strength, Prevailing Corrosion and Heat Resistant, High Strength, Prevailing Corrosion and Heat Resistant, lron Base-13.3Cr-38Ni Corrosion and Heat Resistant, Nickel Base-15.5Cr-2. Corrosion and Heat Resistant, Nickel Base-15.5Cr-8. Corrosion and Heat Resistant, Nickel Base-15.8Cr-15 Corrosion and Heat Resistant, Nickel Base-15.8Cr-15 Corrosion and Heat Resistant, Nickel Base-19.5Cr-13 Corrosion and Heat Resistant, Nickel Base-19.5Cr-18 Corrosion and Heat Resistant, Nickel Base-5.0Cr-24. Corrosion and Heat Resistant, Nickel Base 19.5Cr-13.5 Corrosion and Heat Resistant, Nickel Base (19.5Cr-13. Corrosion and Heat Resistant, Nickel Base, $20 \mathrm{Cr}-20 \mathrm{Co}$ Corrosion and Heat Resistant, Roll Threaded After Heat Corrosion and Moderate Heat Resistant Steel Sheet, Stri Corrosion and Moderate Heat Resistant $(11.8 \mathrm{Cr}-2.8 \mathrm{Ni}-$ Corrosion and Moderate Heat Resistant, 12.5Cr Low Carbo Corrosion and Moderate Heat Resistant, $15.5 \mathrm{Cr}-4.5 \mathrm{Ni}-$ Corrosion and Moderate Heat Resistant, $15.5 \mathrm{Cr}-4.5 \mathrm{Ni}-$ Corrosion and Moderate Heat Resistant, $15.5 \mathrm{Cr}-4.5 \mathrm{Ni}-$ Corrosion and Moderate Heat Resistant, $15.5 \mathrm{Cr}-4.5 \mathrm{Ni}-$ Corrosion and Moderate Heat Resistant, $16.5 \mathrm{Cr}-4.5 \mathrm{Ni}$ Corrosion and Moderate Heat Resistant, $16.5 \mathrm{Cr}-4.5 \mathrm{Ni}-$ Corrosion and Moderate Heat Resistant, 17Cr (SAE 51430) Corrosion Characteristics of Aluminum Automotive Water Corrosion Cracking in Polythionic Acids (1973)

Corrosion Cracking Susceptibility of Cu-Zn Alloys (1973

Corrosion Cracking Test Specimen (1973)

Corrosion Cracking Tests in a Boiling Magnesium Chlorid Corrosion Data (1971) ANSI G80.3

Corrosion for Underground Pipe, Fittings and Tanks Cont Corrosion Metal Specimens (1973)

Corrosion on Underground or Submerged Metallic Piping S Corrosion Products (1973)

Corrosion Resistant Alloy Nuts, High Strength, Prevaili

Corrosion Resistant Iron Chromium, Iron Chromium Nickel

Corrosion Resistant Metals (Electrodeposited Coatings $O$

Corrosion Resistant Steel and Monel Rivet (1974)

Corrosion Resistant, $12 \mathrm{Cr}-8.5 \mathrm{Ni}-2.0 \mathrm{Cu}-1.1 \mathrm{Ti}, \mathrm{Vacu}$

Corrosion Resistant, 14.5Cr-4.0Mo-1.2V (1.10-1.20

Corrosion Resistant, $14.8 \mathrm{Cr}-4.5 \mathrm{Ni}-3.5 \mathrm{Cu}-(\mathrm{Cb}+\mathrm{Ta})$

Corrosion Resisting Chromium and Chromium-Nickel Stee

Corrosion Resisting Chromium and Chromium-Nickel Stee

Corrosion Resisting Chromium and Chromium Nickel Steel

Corrosion Resisting Chromium Steel Clad Plate Sheet and

Corrosion Resisting Steel, UNJC-3A Thread, Cap Screw (1

Corrosion Resisting Steel, UNJF-3A Thread, Cap Screw (1

Corrosion Susceptibility in $7 \mathrm{XXX}$ Series Copper Containi

Corrosion Test on Metal Specimens for Engine Antifreeze

Corrosion Test Specimens (1972)

Corrosion Test Specimens (1972) ANSI G80.1

Corrosion Test. of Engine Coolants (1973)

Corrosion Test. of Metals for the Process Industries (1

Corrosion Test. of Metals (1972)

Corrosion Tests of Metallic Coated Steel Specimens (197

Corrosion Tests (1972)

Corrosion (1972T)

Corrosive Environments (Coil Coating) (1974)

Corrosive or High Temperature Service (1972) ANS1 B125.

Corrosive Petroleum Refining Environment (1972)

Corrosive Service (1971) ANSI Z261.2

Corrosivity of Solvent Systems for Removing Water Forme

Corrosivity of Water in the Absence of Heat Transfer (E)

Corrugated and Solid Fiberboard (Horizontal Plane Meth.

Corrugated and Solid Fiberboard (Horizontal Plane Meth.

Corrugated and Solid Fiberboard (Inclined Plane Meth.)

Corrugated Asbestos Cement Sheets for Bulkheading (1974

Corrugated Asbestos Cement Sheets (1974) ANSI A125.1

Corrugated Fiberboard (1969)

Corrugating Medium (Paperboard) (1971) ANSI P3.24

Cost Trends for Buildings, Machinery and Equipment (197

Cost (1972)

Cost, and Recording Patron and Nonemployee lnjury Stati

Cotta; Timber; Water; Ceiling; Partitions; Plaster; Roo

Cotter Pins (Split) (1972)

Cotter Pins (1972)

Cotton and Linen Textile Colorfastness to Bleaching Wit

Cotton and Linen Textiles to Combined W ashing and Shrin

Cotton Card Webs for Appearance (1973)

Cotton Classing Rooms for Color Grading (1971)

Cotton Cloth (1972) ANSI L14.117

Cotton Colorimeter (1971)

Cotton Fabrics for Manufacture of Hose and Belts (1942)

SAE

SAE

$S A E$

$S A E$

SAE

SAE

$S A E$

SAE

SAE

$S A E$

$S A E$

$S A E$

$S A E$

SAE

SAE

$S A E$

SAE

$S A E$

SAE

SAE

SAE

SAE

$\mathrm{SAE}$

SAE

SAE

ASTM

Bility ASTM

ASTM

ASTM

ASTM

ASTM

ICBO

ASTM

NACE

NACE

SAE

ASTM

1CBO

NSA

SAE

SAE

$S A E$

AWS

AWS

AWS

ASTM

NSA

NSA

ASTM

ASTM

ASTM

ASTM

ASTM

NACE

ASTM

ASTM

AWPA

Meth. of ASTM

NCCA

ASTM

IS to NACE

\section{ASTM}

ASTM

ASTM

ASTM

TAPPI

ASTM

ASTM

ASTM

ASTM

TAPPI

FMS

ANSI

ANSI

ICBO

SAE

ANSI

AATCC

AATCC

ASTM

ASTM

Test Me AATCC Std. Meth. of ASTM ASTM 
Std. Meth. of Test for Length and Length Uniformity of eth. of Test for Estimating Maturity and Linear Density of Std. Rec. Pract. for Sampling

Std. Meth. of Test for Length and Length Distribution of Std. Meth. of Test for Linear Density of Std. Meth. of Test for Cross Sectional Characteristics of Std. Meth. of Test for Number of Neps in Std. Meth. for Spinning Tests on the 1972) ANSI L14.289 Std. Spec. for Woven Test Meth. for Evaluation of Wetting Agents est Meth. for Evaluation of Commercial Rewetting Agents on std. Meth. of Test for Breaking Strength and Elongation of

(1971)

dustrial Use Garm/ Test Meth. for Noncotton Content of Bleached Std. Meth. of Test for Color of Raw

Printed, or Vinyl Coal

td. Performance Requirements for $100 \%$ Std. Performance Requirements for Test Meth. for Mercerization in Std. Meth. for Gradin

Std. Meth. of Test for Differential Dyeing Behavior of ool) (1974) Rec. for Baled Textile Fiber Storage orm Fire Code: Storage and Handling of Combustible Fibres (1970) ANSI C59.31 Std. Meth. of Test. Varnished nalysis of Crude Fiber in Soy Flour for Cake and Meal from eth. of Test for Bromine Index of Aromatic Hydrocarbons by Std. Meth. of Test for Uranium by Controlled Potential Meth. of Analysis of Rope Spore

cts (Cereal Chemistry) (1962)

Std. Plate

mers, Hot Plates and Criddles, etc.) (1973)

Test Meth. for Dirt Std, for Gas ms for Industrial, Utility and Recreational Type Vehicles, Std. Meth. for Automatic Particle Std, for Base for $\mathrm{Gm}$

(1973)

dwork) (197) Std. Meth. for Calibration of Liquid Automatic Particle

k (1973) Std. Test Procedure for Geiger Muller Std. for Rivet-100 Deg.

Std. for Nut Spacer, Plate

Recommendation for Installation of Ceramic Tile in Farina (Cereal Chemistry) (1962)

Meth of Test for Twist in Yarns (Direct

Step Wedge for X-Ray Standardization Probe, Rot Fragment

xygen Content Using a $14 \mathrm{MeV}$ Neutron Activation and Direct

Std. for Structure for the Identification (Code) of the Meth. of Analysis of Mold and Yeast products and Foo/ Meth. of Analysis of Thermophilic Spore Std. Meth. for

Agricultural Wheel Tractors Equipped with Quick Attaching Std. for

Requirements for Power Line

e (Recreational Type) Connecting Devices and Towing Meth. Std. for Trailer

Std. for Care of Fire Hose (lncluding

Std. Spec. for Compression

Std. for Keys and Keyways for Industrial Flexible Gear Std for Balance Classification for Flexible Gear Safety Std. for Asbestos Cement Pressure Pipe, Std. Spec. for Poly(vinyl Chloride) (PVC) Plastic Line Automotive Mechanics for Passenger Cars and Light Trucks automotive Mechanics for Buses. Heavy Trucks and Trailers ar Paving Mixtures for Base, Binder, Leveling, and Surf ace tone, Slag, and Gravel for Dry or Water Bound Macadam Base (1974)

Std. Spec. for 16 Gal. Full Removable Head, Lug Std. Spec. for $5 \mathrm{Gal}$. Straight Side Lug Std. for $5 \mathrm{Gal}$. Nesting Lug

7A60, Ufc-Rule/

7 Aa60, Ufc-Rule 40, Std. Spec. for 5 Gal. Straight Side Lug Std. Spec. for $5 \mathrm{Gal}$. Nesting Lug ray Standardization Probe, Rot Fragment Counting Plate and Spec. for Mild Steel Spec. for Low Alloy Steel

Rods (1972)

.15 Spec. for Nickel and Its Alloy

$r$ Corrosion Resisting Chromium and Chromium-Nickel Steel
Rec. Pract. and Std. inyl Coated Fabric Coverings for Decorative and Protective Marine Deck

Safety Std for Tests for Fire Resistance of Roof st for Flame Propagation Classification Flooring and Floor Uniform Building Code Std. for Fire Retardant Roof Std. for Safety for Wind Resistance of Prepared Roof Rec. Pract. and Std. rine Toilet Waste (Boats) (1972) and Auxiliaries (Diesel and Gasolin/ Rec. Pract. and Std. Rec. Pract. and Std.
Cotton Fibers by Fibrograph Measurement (Textile) (1972 Cotton Fibers by the Causticaire Method (1971) Cotton Fibers for Test. (1972)

Cotton Fibers (Array Meth.) (1972) ANSI Ll4.91

Cotton Fibers (Array Sample) (1973) ANSI L14.136

Colton Fibers (1972) ANSI L14.95

Cotton Samples (1971) ANSI L14.97

Cotton System for Measurement of Spinning Performance (

Cotton Tapes for Electrical Purposes (1972)

Cotton Test Skein Meth.) (1971) ANSI L14.11

Cotton Textile Fabrics (1971) ANSI L14.106

Cotton Textile Fibers (Flat Bundle Meth.) (1972) ANSI L

Cotton Textiles (1972) ANSI L14.128

Cotton Using the Nickerson-Hunter Cotton Colorimeter

Cotton Woven Fabrics (Textile) for Institutional and in

Cotton Woven Tent, A wning, and Canopy Fabrics, Painted,

Cotton Yarns and Fabrics (1971) ANSI L14.124

Cotton Yarns for Appearance (1972) ANSI L14.164

Cotton (Raw Fiber, Yarn and Textile Fabric) (1972) ANSI

Cotton, Jute, Hemp and Sisal, Flax, Synthetic Fibers, W

(Cotton, Sisal, Henequen, Ixtle, Jutes, Hemp, Tow, Cocoa

Cottonfabrics and Tapes Used for Electrical Insulation

Cottonseed, Soybeans, and Peanuts (Cereal Chemistry) (1

Coulometric Titration (1974) ANSI Z78.15

Coulometry (1970) ANSI N106

Count in Cereal Products (Cereal Chemistry) (1962)

Count Meth. of Analysis of Total Bacteria in Food Produ

Count of Wood Chips (Pulp and Paper) (1973)

Counter Appliances (Coffee Pots and Urns, Food Dish War

Counter Balanced Fork Lift Truck, Boat, Baggage, Specia

Counter Size Setting (1972)

Counter Tubes (1971)

Counterbored, Floating, $125 \mathrm{KSI}$ Ftu, 450 and $800 \mathrm{Deg}$. F

Counters and Enclosures of All Kinds (Architectural Woo

Counters Using AC Fine Test Dust (1972) ANSI B93.28

Counters (1970) ANSI N42.3

Countersunk Head Hi Shear Close Tolerance Head and Shan

Countersunk Type Finishing Washer (1973)

Countersunk (1973)

Countertops (1973)

Counting Meth. of Analysis of Cinder and Sand Particles

Counting Meth.) (1971) ANSl L14.234

Counting Plate and Cover) (1962)

Counting Technique (1973)

Countries of the United States for Information Intercha

Counts (Flat Sour, Total Aerobic, Anaerobic) in Cerea

Coupler Calibration of Earphones (1973)

Coupler for Three Point Free Link Hitch (1971) SAE J909

Coupling-Tubes, Flared Fitting, Female Thread (1973)

Coupling Capacitors (1972)

(Coupling, Hitch, Safety Chain, Lighting) (1973)

Couplings and Hitches: Automotive Type (1972)

Couplings and Nozzles (1972)

Couplings for Vitrified Clay Plain End Pipe (1973)

Couplings (1974)

Couplings (1974)

Couplings, and Gaskets for Water and Fire Service (1973

Couplings, Socket Type (1973)

(Course of Instruction) (1972)

(Course of Instruction) (1972)

Courses (1967)

Courses (1971) ANSI A37.99 $\mathrm{Sed}$

Std. for Training of

Cover Universal Drums (Ufc-Rule 40, Nmfc-Item 260)

Cover Universal Pail (Container) (Dot-37C80) (1974)

Cover Universal Pail (Container) (Dot-37C80) (1974)

Cover Universal Pails (Container) (Dot-37A80, Dot-3

Cover Universal Pails (Container) (Dot-37A80, Dot-3

Cover) (1962) IY Funnel, Trap Flask, Step Wedge for X-

Covered Arc Welding Electrodes (1969) ANSI A5.1

Covered Arc Welding Electrodes (1969) ANSI W3.5

Covered Bare Straight and Coiled Welding Electrodes and

Covered Electrodes for Welding Cast Iron (1969) ANSI W3

Covered Welding Electrodes (1969) ANSI W/3.11

Covered Welding Electrodes (1969) ANSI W3.4

Covering Design and Construction of Cockpits and Scupp

Covering for Permanent W all and Ceilings (1969)

Covering Guide (1969)

Covering Material (1973)

Covering Materials (1971)

Covering Materials (1973)

Covering Materials (1973) ANSI A 195.1

Covering Non Commercial Boat Trailer (1972)

Covering Sewage Holding and/or Treatment Devices for $\mathrm{Ma}$

Covering the Installation of Air Cooled Marine Engines

Covering with Foam Layer (1973)
ASTM

Std. M ASTM

D1441

ASTM D 1440

ASTM D 1769

ASTM D1444

D1446

ASTM D335

AATCC 17

AATCC 27

ASTM D 1445

AATCC

ASTM D2253

ANSI L24.4.12

ANSI L24.1.1

AATCC 89

ASTM D2255

ASTM Dl464

FMS 8.7

ICBO UFC $* 2 A R T 7$

ASTM D295

AACCH 32.17

Std. M ASTM D1492

ASTM E217

AACCH 42.20

AACCH 42.11

TAPPI UM.11

ANSI Z21.31

TRA

ASTM

ANSI

F 32

AWI $\quad * 1.400$

NFLDP T2.9.6

IEEE 309

NSA 525

NSA 391

NSA 500

TCA $307-308$

AACCH 28.01

ASTM D1423

AACCH 28.90

ASTM E385

ANSI X3.31

AACCH 42.50

AACCH $\quad 42-40$

ANSI $\$ 3.7$

ASAE $\quad$ \$278.2

NSA

ANSI

$\mathbf{S} 278$
424

C93. 1

VESC V.5

SAE J684D

NFPA 198

ASTM $\quad$ C594

AGMA $\quad 512.03$

AGMA 515.01

UL

ASTM

515.

D3036

D18.1

D 18.2

D1753

D694

MH2.8

MH2.16

ANSI

ANSI

ANSI

$\mathrm{MH} 2.17$

ANSI

ACCH 28.90

AWS A5.1

AWS A5.5

NEMA EW 2

AWS A5.15

AWS A5.1

AWS A5.4

ABYC $\mathrm{H} 4$

CSI 09951

SN

Std. Meth. of Te UL

ICBO

UL

$A B Y C$

ABYC

$A B Y C$

ASTM

4.11

790

992

UBCS32.7

997

A15 
Uniform Building Code: Roof Construction and Rec. Installation Spec. for Vinyl Asbestos Tile as a Wall rmanent Wall and Ceilings / Spec. for Vinyl Coated Fabric 1 Asbestos/ Std. Nomenclature Relating to Resilient Floor Test Meth. for Shampooing: Washing of Textile Floor d. Spec. for Asphalt for Use in Constructing Built Up Roof Std. Meth. of Test for Embossed Depth of Resilient Floor Uniform Building Code: Wall and Ceilin

welling Construction Under the Uniform Building Code: Roof td. for Materials for Use in Construction of Built Up Roof ic Felt, Coal Safety Std. for Materials for Built-Up Roof td. for Electrical Sheet Steel Outlet Boxes, Device Boxes, Uniform Building Code: Patio

Device Boxes, Covers and Box Supports, and Cast Aluminum n Ceilings, Partitions, Exterior Veneer, Awnings and Patio us and Broadleaf Evergreens; Rose Grades; Vines and Ground Std. for Guide-Fastener, Low Form or Recorded Magnetic Tape for Information lnterchange (200 or Recorded Magnetic Tape for Information Interchange $(800$

ic Tape for Information Interchange $19-$ Track 200 and 800

$r$ Recorded Magnetic Tape for Information Interchange (1600 ion Interchange (9-Track 200 and $800 \mathrm{cpi}$, NRZI, and 1600 Std. Spec. for Chlorinated Poly(vinyl Chloride) Spec, for Thermoplastic Line Pipe (PVC an Summary of Meth. for Biscuit and grains, Unpopped Popcorn, Dried Pea/ Meth. of Analysis of ess Steels and Related $\mathrm{Ni}$-Cr-Fe Alloys to Stress Corrosion

leu/ Rec. Pract. Meth. and Controls to Prevent in Service Std. Meth. of Dynamic Test. for Ply Separation and sson's Solution of pH 7.2 to Evaluate the Stress Corrosion c. Pract. for Making and Using the C-Ring Stress Corrosion

(1973) Std. Rec. Pract. for Performing Stress Corrosion h. for Density of Pulpwood (Submersion with Correction for sity and Moisture of Chips (Submersion with Correction for aving Rope Supporte/ Rec. Pract. for Performance Std. for Rec. Pract. for Mobile Construction Type er, Mining Car, Front End Loader, Fork Lift Trucks, Mobile Spec. for Underhung ce Std. for Crane Boomstop of All Mobile Construction Type Spec. for Welding Industrial and Mil ctric Overhead Bridge and Gantry Multiple Gerder Traveling Spec. for Rated Loads Offshore O

Rec. Pract. for Operation and Maintenance of Offshore Std. for Monorail Systems and Underhung Std. for Mobile Hydraulic

hoists, Hooks, Jacks, and Slings: Portal, Tower and Pillar tal, Tower and Pillar Cranes (1973) d Drum Hoists (1971)

Safety Std. for Std. Meth. of Test. Large Shipping Code for Rec. for Safety Std. for Ice si J8.12 Std. Spec. for Concentrated, Ammonia Preserved 62) Meth. for Determination of Specific Volume of Std. for Fiberboar undering (1973) ANSI L14.17/ Test Meth. for Appearance of Std. for Magnetic Stripe Encoding (Code) for Std. Test Meth. for Flexure Std. Test Meth. for Std. Test Meth. for Std. Test Meth. for oading (Metal to Metal) (1969) ng (Metal to Metal) (1969) ANSI MH12.15 Std. Meth. of Test for

Std. Rec. Pract. for Conducting Std. Volume and Specific Gravity Correction Tables fo Std. Meth. for Analysis of Std. Spec. for

, and Timbers for Marine, Land,

Std. Spec. for Coal Tar Std. Spec. for

Std. Test Meth. for Moisture and Std. Meth. of Sampling and Test. Std. Spec. for Petroleum for Blending with Std. Meth. of Test for Benzene Insoluble Matter in e (1970) ANSI 010.1 Std. Spec. fo insulating Oils Containing 2,6-Ditertiary-Butyl Para cators (1971) Std. Analytical Meth. for Determining Improved Std. Designs for Pressure Treated Timber ipment (Barrier) for Protection Against Robbery or Holdup 26 (1972) Std for Splice, Electric Permanent, 8 (1972) Meth. of Test for Effective Crimping on Outside Meth. of Test for Effective dures for User Certification of (1962) A/ chronous Machines (1972)

power Supplies for Nuclear Power Generat/ Trial Use Std. for Prime Movers, W/ Rec. Pract. for Minimum Performance (1972) )
Covering (1973)

Covering (1973)

Coverings for Decorative and Protective Covering for $\mathrm{PE}$ Coverings (Asphalt, Cork, Linoleum, Rubber, Vinyl, Viny Coverings (Multiply Felt and Asphalt Preferably with Gr

Coverings (Rug, Carpet) (1972)

Coverings (1971) ANSI A 109.24

Coverings (1973)

Coverings (1973)

Coverings (1973)

Coverings: Hot Mopped Asphalt, Asphalt and Coal Tar Sat Coverings: Hot Mopping Asphalt, Asphalt-Saturated Organ Covers and Box Supports, and Cast Aluminum Covers (1973 Covers (1973)

Covers (1973)

Covers, Greenhouses, Canopies) (1973) Crades; Small Fruits (Berry, Currant

/for Electrical Sheet Steel Outlet Boxes

Cowl, Dzus Type (1973)

cpi, NRZI) (1973)

cpi, NRZI) (1973)

cpi, NRZI, and $1600 \mathrm{cpi}$, PE) (1973)

cpi, PE) (1973)

cpi, PE) (1973)

/Unrecorded Magnetic Tape for Informat

Std. F ANS

Std. F ANSI

CPVC) Plastic Hot Water Distribution Systems (1973)

PVC) (1972)

Cracker Flour Test. (Cereal Chemistry) (1962)

Cracking Flotation Test for Internal Insects in Cereal

Cracking in Polythionic Acids (1973)

Cracking of Carbon Steel (P.1) W/eldo in Corrosive Petro

Cracking of Rubber Products (1973) ANSI J2.6

Cracking Susceptibility of Cu-Zn Alloys (1973)

Cracking Test Specimen (1973)

Cracking Tests in a Boiling Magnesium Chloride Solution

Cracks, etc.) (Pulp and Paper) (1973)

Cracks, etc.) (Pulp and Paper) (1973)

Oonstop of All Mobile Constluction Type Cranes

Crane Overload Indicating System Test Procedure (1971)

Crane, and Shovel (1974)

(T Haul); Grader, Loader, Doz

Cranes and Monorail Systems (1973)

Cranes Having Rope Supported Booms, Equipped for Hook W

Cranes (1970)

Cranes (1971)

Cranes (1972)

Cranes (1972)

Cranes (1973)

Cranes (1973)

Cranes (1973)

Spec. for Ele

Spec. for Ele CMA

Cranes, Derricks, Hoists, Hooks, Jaks, and Slingses, D

Cranes, Derricks, Hoists, Jacks and Slings: Base Mounte

Crates (Packaging) (1953) ANSI MH12.11

Crayons and Related Art Materials for School Use (1963)

Cream Freezers and Soda Fountain Units (1973)

Creamed and Centrifuged Natural Rubber Latex (1972A) an

Creams, Cake Batters, and Icings (Cereal Chemistry) (19

Crease Bending Test (1972)

Creases in Wash and Wear Items (Clothing) After Home La

Credit Cards (1973)

Creep of Sandwich Constructions (1970)

Creep Properties of Adhesives in Shear by Compression L

Creep Properties of Adhesives in Shear by Tension Loadi

Creep Properties of Package Cushioning Materials (1968)

Creep Relaxation of a Gasket Material (1971)

Creep Tests of Metal to Metal Adhesives (1972) ANSI Z19

Creosote and Coal Tar (R 1970)

Creosote and Oil Type Wood Preservatives (1973)

Creosote Coal Tar Solution (1974) ANSI O11.2

Creosote for the Preservative Treatment of Piles, Poles

Creosote Petroleum Solution (1970)

Creosote Type Preservative in Wood (1970)

Creosote (1970) ANSI 011.9

Creosote (1971)

Creosote (1973) AASHO T81, ANSI 011.13

Creosoted End Grain Wood Block Flooring for Interior Us

Cresol by Rotating Bomb (1973) ANSI C59.11

Cresol Red Indicator, $0.1 \%$ Content of Reagents and Ind

Crib Walls (1969)

(Criminology) (1974) ANSI Se4.6

Crimp Style, Pre Insulated, (Class 2) Wire Sizes 12 to

Crimped Valves of Aerosol Containers (1972)

/R Bullet Resisting Equ

Crimping on Outside Crimped Valves of Aerosol Container

Crimping, Solderless Wiring Devices-Recommended Proce

Criteria and Definitions for Excitation Systems for Syn

Criteria for Diesel Generator Units Applied as Standby

Criteria for Falling Object Protective Structure (FOPS)

Criteria for Film Badge Performance (Nuclear Radiation)

Criteria for Nuclear Fuel Reprocessing Facilities (1973
APl

ANSI

ANSI

ICBO

ASTM

FMS

AATCC

ASTM F373

$\mathrm{UBC} * 3-47$

ICBO

ICB

NEMA

ICBO

NEMA

ICBO

ASI

$\mathrm{UBC} \approx 8-18$

UBCS32.1

$55 \mathrm{~A}$

UBC $\$ 3.49$

OS1

UBC *3-52

Z60.1

67

$\mathrm{X} 3.14$

X3.22

X3.40

$\times 3.39$

$\mathrm{X} 3.40$

D2846

$5 \mathrm{LP}$

AACCH $\quad 10.30$

AACCH 28.22

ASTM G35

D430

G37

G38

G36

UM-2

UM-20

$\mathrm{J} 220$

$\mathrm{J} 248$

3

*2

J220

D14.1

70

B30.11

B30.15

B30.4

ANSI B30.4

ANSI B30.7

ASTM D1083

USC R192

UL 621

ASTM D1076

$\mathrm{AACCH} \quad 72-10$

SAE JI19A

AATCC 88C

ANSI X4.16

ASTM C 480

ASTM D2293

ASTM D2294

ASTM D2221

ASTM F38

ASTM D1780

ASTM D347

$A W P A \quad A 1$

D391

ASTM D390

ASTM D 1858

ASTM D 1860

D38

ASTM D1859

ASTM D36?

ASTM D1031

D2112

CR

$A W P I$

R.60

*4

NSA

ASTM

RS270

IEEE 42]

EEE 387

SAE J231

ANSI N13.7

ANSI N101.3 
Std. for Earthquake Instrumentation at Perform Protective Functions in Nuclear Power Ge/ Std. and Frame Assemblies (19/ ent Structures (1969)

Rec. Pract for Minimum Perfor front End Loaders An/ Rec. Pract. for Minimum Performance Prime Movers (Heavy/ Rec. Pract. for Minimum Performance uipment for Water Cooled and Mol_Std. for Fire Protection its (1974) ANSI N41.14

Reactor Plants (1973)

ing Station Protection Systems (19/

ssemblies (1972) Std. Test Proc, and Acceptance s Used in Treating Wastewater (1970)

Trial Use Guide for the Application of the Single Failure zing (1972T)

ops) and Falling Object Protective Struc/

Tent. Test Meth. for

Rec. Pract. for

d. Spec. for Photographic Grade Chromium Potassium Sulfate h. for Colorfastness of Colored Textile Yarn and Fabric to test Meth. for Colorfastness of Textile Yarn and Fabric to

textile Yarn and Fabric to Crocking (Rubbing) by the AATCC ile Yarn and Fabric to Crocking (Rubbing) (Rotary Vertical machinery) (1973) ion, Installation, and Rating of Equipment for Drying Farm oard (1972)

Meth. for

Meth. for Determination of Film Adhesion on All Metals by Test Meth. for Determination of Film Adhesion by (1972) ANSI Z197.21 Std. Meth. of Testin Std. Meth. for Estimating the Thermal Neutron Absorption Std. for General Purpose Uniform xide Coating Thicknesses by Microscopical Examination of A ANSI L14.95 Std. Meth. of Test for e and Cable for the Transmission and Distributi/ Std. for Rec. Pract. for a Program for School t Shrinkable Tubing for Electrical Insula/ rical Insulation (1972)

Std. Spec. for Std. Spec. for Rec. for Operation of y (1973) Std. for Bolt Lock, Tension, $100 \mathrm{Deg}$ Std. for Bolt Lock, Tension, 100 Deg. Std. for Pin, Swage Locking, Steel, 100 Deg. Std. for Machine Bolt,

pi 2561 Std. Meth. of Test for Sediment in or Determining Extraneous Materials Content of Corn Syrup, Std. Meth. of Test for Pyridine Bases in lor, Solutions (Spectrophotometric) Content of Corn Sugar th. for Determining Moisture (Oven) Content of Corn Sugar nalytical Meth. for Determining Ash Content of Corn Sugar tical Meth. for Determining Acidity Content of Corn Sugar tical Meth. for Determining Arsenic Content of Corn Sugar alytical Meth. for Determining lron Content of Corn Sugar eth. for Determining Sulfur Dioxide Content of Corn Sugar $r$, Reflectance (Spectrophotometric) Content of Corn Sugar determining Moisture (Karl Fischer) Content of Corn Sugar th. for Determining Reducing Sugars Content of Corn Sugar for Determining Dextrose Equivalent Content of Corn Sugar . for Determining Specific Rotation Content of Corn Sugar meth. for Determining Total Sugars Content of Corn Sugars mining Calcium (Egta-Titrimetric) Content of Corn Sugar ytical Meth. for Determining Copper Content of Corn Sugar Meth. for Determining Heavy Metals Content of Corn Sugar ical Meth. for Determining Chloride Content of Corn Sugar analytical Meth. for Determining $\mathrm{pH}$ Content of Corn Sugar h. for Determining Protein Nitrogen Content of Corn Sugar filter Paper) Content of Corn Syrup Unmixed and Unbleached Meth. of Analysis of Meth. of Analysis of Meth. of Analysis of Meth. of Analysis of Meth. of Analysis of Meth. of Analysis of Meth. of Analysis of Meth. of Analysis of stry) (1962) t Containing Fruit (Cereal Chemistr 1962)

s (Cereal Chemistry) (1962) Std. Analytical Meth. for Determining corn) (1954) Std. Analytical Meth. for Determining en, Germand Finished Std. Analytical Meth. for Determining $s$ Containing Veget/ etable Material, C/ (1962)

eed, Soybeans, and Peanuts (Cereal/ Std. Analytical Meth. for Determining Std. Analytical Meth. for Determining Meth. of Analysis of Meth. of Analysis of Std. Meth. of Test for Salt in Rec, for

Std. Meth. of Test for Water and Sediment in si 211.314 Sid. Meth. for Distillation of ed Kjeldahl Meth. for Nitrate Free Samples for Analysis of ges, and Animal and Dairy $\mathrm{P} /$ UDy Dye Meth. of Analysis of percentage of Total Nitrogen) (C/ Meth. of Calculation of
Improved Kjeldahl Meth. of Analysis of
Criteria for Nuclear Power Plants (1974)

Criteria for Preparation of Design Bases for Systems Th

Criteria for Rate of Air Flow Through Closed Steel Door

Criteria for Reinforced Concrete Nuclear Power Containm

Criteria for Roll Over Protective Structure for Track T

Criteria for Roll Over Protective Structure for Weeled

Criteria for Roll Over Protective Structures (ROPS) for

Criteria for Safety Related Systems, Structures, and Eq

Criteria for Separation of Class Ie Equipment and Circu

Criteria for the Design of Stationary Pressurized Water

Criteria for the Periodic Test, of Nuclear Power Genera

Criteria for Water Resistance on Steel Door and Frame a

Criterial for Evaluation of Special Processes or Device

Criterion to Nuclear Power Generating Station Protectio

Critical Dilation of Concrete Specimens Subject to Free

Critical Zone for Laboratory Evaluation of Roll Over (R

$\mathrm{CrK}\left(\mathrm{SO}_{4}\right)_{2}: 12 \mathrm{H}_{2} \mathrm{O}$ (Chrome Alum) (1972)

Crocking (Rubbing) by the AATCC Crock meter Meth. (1972)

Crocking (Rubbing) (Rotary Vertical Crockmeter Meth.)(

Crockmeter Meth. (1972) ANSI L14.72

Crockmeter Meth.) (1972) ANSI L14.212

/Tness of Colored

Crop Cultiva

Sid. for Cons

Cross Directional Internal Tearing Resistance of Paperb

Cross Hatch Tape Test After Reverse Impacting (Coil Coa

Cross Hatch Tape Test (Coil Coating) (1974)

Cross Lap Specimens for Tensile Properties of Adhesives

Cross Section of Nuclear Graphite (1971) ANSI K90.10

Cross Section Spiral Retzining Rings (1972)

Cross Sectional Characteristics of Cotton Fibent of

Crossed Linked Thermosetting Polyethylene Insulated Wir

Crossing Protection (1973)

Crosslinked and Noncrosslinked Poly(vin yl Chloride) Hea

Crosslinked Polyolefin Heat Shrinkable Tubing for Elect

Crowder Dry Pipe Valves, Model a (1973)

Crown Head, Std. and Oversize, Pull Type, Titanium Allo

Crown Head, Stump Type, Titanium Alloy (1973)

Crown Head, Tension, Pull Type (1973)

Crowned Hexagon Head, Adjusting (1973)

Crude and Fuel Oils by Extraction (1969) ANSI Z11.58, a

Crude and Refined Sugar and Other Materials Soluble in

Crude and Refined Tar Acids (1972) ANSI Z107.4

(Crude and Refined) and All Hydrolyzates Solutions Deriv

(Crude and Refined) (Cıystalline) (1959)

Crude and Refined) (1958)

Crude and Refined) (1958)

(Crude and Refined) (1958)

(Crude and Refined) (1961)

(Crude and Refined) (1961)

(Crude and Refined) (1963)

(Crude and Refined) (1966)

(Crude and Refined) (1972)

(Crude and Refined) (1972)

(Crude and Refined), Dext rose, Syrup and Starch Hydrolyz

(Crude and Refined), Syrup and Other Starch Hydrolyzates

(Crude and Refined), Syrup, and Other Starch Hydrolyzate

(Crude and Refined), Syrups and Other Hydrolyzates from

(Crude and Refined), Syrups and Other Starch Hydrolyzate

(Crude and Refined), Syrups and Other Starch Hydrolyzate

Crude and Refined), Syrups, Starches and Other Protein

Crude Corn Sugar (1956)

/R Determining Moist

Crude Fat in Cocoa (Cereal Chemistry) (1962)

Crude Fat in Dry Milk Products (Cereal Chemistry) (1962

Crude Fat in Egg Yolk and Dried Whole Egg (Cereal Chemi

Crude Fat in Flour, Bread, and Baked Cereal Products No

Crude Fat in Grain and Stock Feeds (Cereal Chemistry) (

Crude Fat in Soy Flours (Cereal Chemistry) (1962)

Crude Fat in Wheat and Soy Flour, Feeds and Cooked Feed

Crude Fat (Ccl Extractables) Content in Corn (1958)

Crude Fat ( $\mathrm{Ccl}$, Extractables) Content in Feedstuffs (

Crude Fat $\left(\mathrm{Ccl}_{4}\right.$ Products Such as Feeds and Meals (195

Crude Fiber Content in Corn and Other Grains, Foodstuff

Crude Fiber Content in Feedstuffs (Corn) Containing Veg

Crude Fiber in Feeds and Feedstuffs (Cereal Chemistry)

Crude Fiber in Soy Flour for Cake and Meal from Cottons

Crude Oil (Electrometric Meth.) (1973)

Crude Oil (1972)

Crude Oils (1973) ANSI Z11.8

Crude Petroleum (15 Theoretical Plate Column) (1973) an Crude Protein in Bread, Wheat and Other Grains, and Yea Crude Protein in Cereal Grains, Oilseeds, Legumes, Fora Crude Protein in Feeds and Feedstuffs (Calculated from Crude Protein in Feeds and Feedstuffs (Cereal Chemistry
ANSI

ANS

STDI

ACI

SAE

SAE

SAE

ANS

IEEE

ANSI

IEEE

STDI

NSF

IEEE

ASTM

SAE

St ANSI

AATCC 8

AATCC

AATCC

AATCC

ASAE

ASAE

TAPPI

NCCA

ASTM

ASTM

ANSI

ASTM

ASTM

NEMA

ITE

ASTM

ASTM

FMS

NSA

NSA

NSA

NSA

ASTM

CR

ASTM

CR

Me CR

Std. a CR

Sid. Analy CR

id. Analy CR

Std. an CR

CR

CR

CR

CR

CR

CR

CR

CR

CR

CR

CR

CR

AACCH

$\mathrm{AACCH}$

AACC

AACCH 30.18

AACCH 30.10

AACCH $30-20$

AACCH $30-26$

$\mathrm{AACCH} \quad 30.25$

CR

CR

CR

CR

C. 10

B. 18

B.18

AACCH 32.15

AACCH 32.17

ASTM D3230

FMS

ASTM D96

ASTM D2892

AACCH 46-11

AACCH 46.14

AACCH $46-18$

AACCH 46.10 
cid Modification) (CereaI/ ntage of Total Nitrogen) (Cereal

Kjeldahl Meth. of Analysis of Meth. of Calculation of Micro Kjeldahl Meth of A nalysis of rmining Apparent Starch Content of Corn Syrup, Refined and of Chemical Analysis of Aluminum Oxide Abrasive Grain and of Chemical Analysis of Silicon Carbide Abrasive Grain and 1972) ic Weight, Durability and M/

Std. for W afers, Pellets and Std. Test. Proc. for Side to Side Meth. for Flat si P3.24

Uniform Building Code Std. for Concrete Aggregates (Sand, macadam Base Courses (1971) ANSI A37.99 Std. Spec. for brick and Shapes (1972) Ansi/ Std. Meth. of Test for Cold Tent. Meth. of Test for Compressive m, Fluorine, Helium, Hydrogen, Krypto/ Uniform Fire Code: Std. Rec. Pract. for Evacuated Reflective Insulation in ns (Code) in Metallic Systems Based on the Composition and ic Grade Sodium Thiosulfate, Anhydrous $\left(\mathrm{Na}_{2} \mathrm{~S}_{2} \mathrm{O}_{3}\right)$ and moisture (Oven) Content of Corn Sugar (Crude and Refined) I Etch Techniques (1973) Tent. Meth. of Test for ity and Hall Coefficient in Extrinsic Semiconductor Single Std. for Washer, Plain and - Slip Resistant (Floor Polish and Wax) (1971) ASTM D2047, erials (1973) Std. Spec. for E.

o Evaluate the Stress Corrosion Cracking Susceptibility of Strength of Hydraulic Cement Mortars (Using 2 In. (50 Mm) nery) (1973) Std. for Chisel Plow, Field and Row Crop

Cord Connected Electric Gardening Appliances (Lawn Mower, meth. of Test. Wood Preservatives by Laboratory Soil Block Std. Spec, for Concrete Sewer, Storm Drain, and Std. Spec. for Reinforced Concrete Arch Std. Spec. for Reinforced Concrete Elliptical Std. Spec, for Reinforced Concrete D.Load Std. Spec. for Reinforced Concrete ot Dip Process (1971) An/ Std. Spec. for Steel Sheets for Mechanical Demand Registers Std. Meth. of Test for Flash Point of Liquids by Tag Open of Test for Flash Point of Cutback Asphalt with Tag Open Meth. of Test for Flash and Fire Points by Cleveland Open Std. for Flash Points, Closed Rec. for Controlling gamma and Electron Radiation Dose with the Ferrous Sulfate g a Solvent Hand Rub Test for the Determination of Deg. of $r$ Linear Shrinkage of Thermosetting Casting Systems During iscometer (1972) An/ Std. Meth. of Test for Viscosity and ANSl A37.81 Std. Meth. of Making and Std Rec Pract for

Std. Spec. for Liquid Membrane Forming Compounds for emperature of Applied Coatings on Wood Products During the ing, Stabilization, Ice Removal, Acceleration of Concrete, Safety Ventilation Requirements for Direct Fired Powder Std. Spec. for Liquid Asphalt (Slow Std. Spec. for Liquid Asphalt (Medium s (1974) Std. Spec, for Liquid Asphalt (Rapid Std. Meth. of Making, Accelerated Std. Meth. for Determining the Test Meth. for Sizing of Paper (Reverse

Bedding Applications (1973) A/ ral Horsepower) (1972) Is (1973) Std. Spec. for Rubberized Std. for Ratings for Alternating

Std. Rec. Pract. for Designing a High Analysis of Thermionic Nickel Alloys by the Powder Direct or AC High Voltage Circuit Breakers Rated on a Symmetrical or AC High Voltage Circuit Breakers Rated on a Symmetrical oltage Circuit Breakers Rated on a Symmetrical and a Total or AC High Voltage Circuit Breakers Rated on a Symmetrical of AC High Voltage Circuit Breakers Rated on a Symmetrical or AC High Voltage Circuit Breakers Rated on a Symmetrical or AC High Voltage Circuit Breakers Rated on a Symmetrical

ign, Installation, Operation, and Maintenance of Impressed Std. for Direct Std. for Industrial and Other Direct Std. for Frame Assignments for Alternating ermittivity) of Solid/ Std. Meth. of Test for Alternating ermittivity) of Solid/ Std. Meth. of Test for Alternating horsepowe/ for Dimensions for Alternating and Direct
hor/ hor/ Std. for Application Data for Alternating and Direct $72)$

Std. for Tests and Performance of Alternating and Direet Std. for Direct

) ANSl C37.13 Std. for Stabilized Power Supplies Direct Std. for Low Voltage Alternating Std. for Surge (Lightning) Arresters for Alternating r) Std for Schedule of Preferred Ratings for Capacitance rated on a Symmetrical/ Std. Requirements for Capacitance
Crude Protein in Wheat and Flour Mill Products (Boric a Crude Protein in Wheat and Flour (Calculated from Perce Crude Protein (Cereal Chemistry) (1962)

Crude Sugar (1967)

Crude (1971)

Crude (1971)

Crufomate (Common Name for the Pest Control Chemical)

Crush Composite Tubes and Cores (1973)

Crush Test of Corrugating Medium (Paperboard) (1971) an

Crushed Stone, Gravel and Air Cooled Iron Blast Furnace

Crushed Stone, Slag, and Gravel for Dry or Water Bound

Crushing Strength and Modulus of Rupture of Refractory

Crushing) Strength of Graphite (1971) ANSI K90.11

Cryogenic Fluids (Air, Argon, Carbon Monoxide, Deuteriu

Cryogenic Service (1973)

Crystal Lattice of Each (1970) ANSl Z30.10

Crystalline $\left(\mathrm{Na}_{2} \mathrm{~S}_{22} \mathrm{O}_{3} .5 \mathrm{H}_{2} \mathrm{O}\right)(1973)$

(Crystalline) (1959)

/Analytical Meth. for Determining

Crystallographic Perfection of Germanium by Preferenti

CSK, 1200 Deg. F (1973)

CSMA Bul 308-70

Std. Meth. for Measuring Hall Mobil

CTFE. Fluoroplastic

Cu-Zn Alloys (1973)

Cube Specimens) (1973)

(Ocedure) for Products Classified as $\mathrm{T}$

g, Extrusion, and Coating Mat

Mattsson's Solution of

/ Meth. of Test for Compressive ASTM

Cultivator Shafts and Ground Tool Mountings (Farm Machi

Cultivator, Edger-

Culvert Pipe (1973)

Culvert, Storm Drain, and Sewer Pipe (1971)

Culvert, Storm Drain, and Sewer Pipe (1972)

Culvert, Storm Drain, and Sewer Pipe (1973)

Culvert, Storm Drain, and Sewer Pipe (1973)

Culverts and Underdrains, Zinc Coated (Galvanized) by $\mathrm{H}$

Cumulative and Pointer Forms) EEl MSJ.4

Cup Apparatus (1972)
Cup Apparatus (1972)

Cup (1972) AASHO T48, ANSI Z11.6

Cup, Aromatic Chemicals and Isolates (1973)

Cupping of Wood Floors (1962)

Cupric Sulfate Dosimeter (1971) ANSl K65.229

Cure of a Backed Paint Film (Coil Coating) (1974)

Curing Chath. Met

Curing Concrete Test Specimens in the Laboratory (1969)

Curing Concrete (1971) ANSI A168.1

Curing Concrete (1972) ACI 308

Curing Concrete (1973) ANSI A37.87

/R Infrared Determination of the $T$

Curing Cy cle (1973)

Curing of Concrete,

Curing Ovens (1973)

Curing Type) (1972)

Curing Ty pe) (1972)
Curing Type) (1972)

Curing, and Test. of Concrete Compression Test Specimen

Curl of Photographic Film (1971)

Curl) (1973)

Curled Hair for Cushioning Materials for Upholstery and

Currant, Grape, Asparagus); Lining Out Stock; Seedling

Current and Direct Current Motors (Fractional and Integ

Current Arc Erosion Test for Electrical Contact Materia

Current Arc Technique (1961) ANSl Z128.2

Current Basis (1971) ANS I C 37.072

Current Basis (1971) ANSl C37.0721

Current Basis (1972) / E

Current Basis (1972) ANS1 C37.076

Current Basis (1972) ANS1 C37.078

Current Basis (1973) ANS1 C37.073I

Current Deep Groundbeds (1972)

Current Generators (Large Apparatus) (1972)

Current Integral Horsepower Generators (1972)

/Ctrochemical ASTM

IEEE /Recovery Voltage F IEEE

Control of AC High V ANSI

Current Switching F lEEE

Surized Components IEEE

/Nsulation for Outdo IEEE

/Urrent Switching F IEEE

Current Integral Horsepower Induction Motors (1972)

Current Loss Characteristics and Dielectric Constant ( $P$

Current Machines (1973)

Current Motors and Generators (Fractional and lntegral

Current Motors and Generators (Fractional and Integral

Current Motors (Fractional and Integral Horsepower) (19

Current Motors (Fractional and Integral Horsepower) (19

Current Motors (Large Apparatus) (1972)

Current Output (1972)

Current Power Circuit Breakers Used in Enclosures (1973

Current Power Circuits (1972) ANSI C62.

Current Switching for AC High Voltage Circuit Breakers

Current Switching for AC High Voltage Circuit Breakers
A ACCH $46-12$

CCH 46.19

CR

B74.14

B74.15

K62.110

$\mathrm{S} 269.2$

$\mathrm{T} 108$

T809 OS

UBCS26.2

D694

C133

C695

UFC 2 ART 36

C740

E157

PH4.250

F.34

F389

F76

BUL 12

D3275

G37

C109

S225. 1

82

D1413

Cl14

C506

C.507

C655

C76

A444

El 14

D 1310

D3143

D92

FP-1

$* 3$

D2954

TB-II-18

D2566

D 1646

C192

308

Al68.1

C309

D3259

D98

$7 \cdot 27 \mathrm{~S}$

D2026

D2027

D2028

C684

PHl.29

UM-459

D2128

Z60. 1

MGi-10

B576

E129

327

328

C37. 11

341

340

343

342

RP-05.72

MGl-24

MGl.15

MGl3

D150

113

NEMA

NEMA

NEMA

NEMA

NEMA

IEEE

IEEE

ANSI

IEEE

MGl-11

MGl-14

MG1-10

MGl-12

MGl-23

PY 1 
rated on a Symmet/ Std. Application Guide for Capacitance nalizers and Oil Filled Capacitor Switches for Alternating agnetic Saturation (1971) Ansi/ Std. Rec. Pract. for Eddy Std. for Leakage

Std. Spec. for Mercury Lamp Transformers, Constant ion (1973) Std. Meth. of Test for High Voltage, Low of Power Factor Measurement for Low Voltage Inductive Tes Rec. Electric Motor Starting nd Perforated Pipe for General Drainage (Land Reclamation, tech. Notes) (1968) 4 ln. Reinforced Brick Masonry (Ins/ Std. Performance Requirements for Woven Glass Fiber ) (Instit/ Std. Performance Requirements for Woven Window d. Performance Requirements for Woven Glass Fiber Drapery 1 Texti/ Std. Performance Requirements for Woven Drapery Meth. of Test for Seam Strength o Std. Performance Requirements for Woven Shower Uniform Building Code Std. for Proscenium Design Spec. for Plywood Fabrication Spec. for Plyw ood

uctural Glued Built Up Members-Plywood Stressed Skin and ooring Over Concrete Slab Floors (1962) Spec. for Rubber ish Flooring Over Concrete Slab Floors/ Spec. for Mastic tions (1973) A/ Std. Spec. for Rubberized Curled Hair for Std. Meth. of Test. Package th. of Test for Shock Absorbing Characteristics of Package Std. Test Meth. for Creep Properties of Package Rec. Procedure for Processing Hardware for rofessional Photographic Sheet and Roll Films (SI and U.S. nsmission and Distribution Facilities and Interruptions to 78, ANSI A37.45 Std. Meth. of Test for Distillation of

Steel Products Manual for Carbon Sheet Steel (coils and spec. for Steel Sheets of Structural Quality in Coils, and Std. Meth. of Test for Flash Point of Std. Recommendations for Die Cavity and (197) Fire Hazards and Protection Rec. for Low Water Fue (1972) ANSl C 33.88

hines (1972) Std. for Safety for Thermal Std. for Tool and Std. Spec. for Free Std. for Grade Std, for Hole Spacing for Scraper and Bulldozer (Dozer) Spec. for Metal Rec. for

ner Type (1972)

Std. for Carbide Blanks and Std. Terms and Definitions for Welding and Std. for Safety in Welding and Rec. Pract. for Plasma Arc Rec. Pract. for Air Carbon Arc Gouging and cetylene Generators, Stationary, Low Pressure (Welding and ylene Generators, Stationary, Medium Pressure (Welding and Uniform Fire Code: Welding and Std. Meth. of Test for

est for Water Vapor Transmission of Shipping Containers by Meth. of Test for Water Vapor Permeability of Packages by ure of Applied Coatings on Wood Products During the Curing Std. Meth, of Test for Effect of Std. Test Meth. for Resistance of Adhesives to Adhesion and Cohesion of Elastomeric Joint Sealants Under Std. Meth. of Test for Purity and Benzene Content of Std. Spec. for control Chemical cis $\cdot \mathrm{N} \cdot((1,1,2,2$ tetrachloroethyl) Thio $)-4$ control Ch/ Std. for Propargite 2-(P.Tert-Butylphenoxy) Std. Meth. of Test for Std. for

Std. for Prestressed Concrete Pressure Pipe, Steel (Safety) (1973) Std. Color Marking of Compressed Gas ricultu/ Std. for Application of Hydraulic Remote Control Tent. Spec. for Molds for Forming Concrete Tests Std. Spec. for Centrifugally Cast Dual Metal h Pressure Gas Manifolds for Industrial and Commercial Gas cessories for Cataloged Square Head Industrial Fluid Power Cataloged Square Head Tie Rod Type Industrial Fluid Power $r$ Evaluation of Compression Test Results in Field Concrete ermining the Splitting Tensile Strength of Molded Concrete Flexible Connector Fittings for LP (Liquid Petroleum) Gas Rec. for Storage and Handling of Compressed Gases in Std. Meth. of Test for Splitting Tensile Strength of Std. Meth of Test for Compressive Strength of Std. Meth. of Capping Std. for Punches-Basic, $\mathrm{MH} 12.7$ Std. Meth. of Drop Test for

Rules for the Measurement and Inspection of Hardwood and est for Neutron Flux Density and Average Energy from ${ }_{3} \mathrm{H}$ 2) ANSI Z11.322 Std. Meth of Test for e Alloys by the Silver Chloride-Lithium Fluoride Carrier lysis of Uranium Oxide $\left(\mathrm{U}_{3} \mathrm{O}_{\mathrm{K}}\right)$ by Gallium Oxide Carrier
Current Switching for AC High Voltage Circuit Breakers Current Systems on Boats (1972)

Current Systems (1972)

Clot

/Closers, Automatic Line Sectio

Current Test. of Steel Tubular Products (Tubing) with M

Current (Electricity) for Appliances (1973)

Current (Series) Supply Type (1971)

Current, Dry Arc Resistance of Solid Electrical Insulat

Currents (1972) ANSI C 37.26

Std. Guide for Meth.

Currents (1972) EEI 62.27

Curtain and Building Foundation Drain, Under Basement F

Curtain and Panel Walls (In Resisting Lateral Forces)(

Curtain Fabrics Handwashable at $105 \mathrm{Deg}$. F-No Bleach

Curtain Fabrics (Sash Casement) (Excluding Glass Fibers

(Curtain) Fabrics Handwashable at 105 Deg. F-No Bleach

(Curtain) Fabrics (Excluding Glass Fibers) (Institutiona

Curtains and Draperies (Textile) (1973)

Curtains (Institutional Textile) (1973)

Curtains (1973)

Curved Panels (1968)

Curved Panels (1971)

Curved Panels, Beams, and Components (1973)

Cushion Slecper Construction with Nailers and Finish Fl

Cushioned Construction with Nailers, Subflooring or Fin

Cushioning Materials for Upholstery and Bedding Applica

Cushioning Materials (Packaging) (1964) ANSI MHI2.13

Cushioning Materials (Packaging) (1964) ANSI MH12.14

Cushioning Materials (1968) ANSl MH12.15

Custom Aluminum Doors (1972)

Customary Units) (1973)

Customer Service (1973)

Std. Dimensions for $P$

Cut Back Asphaltic

Cut Lengths, Zinc Coated (Calvanized) by Hot Dip Proces

Cutback Asphalt with Tag Open Cup Apparatus (1972)

Cutoff Die Sizes for Cold Forging Equipment (1972)

Cutoffs and Automatic Feedwater Regulators for Boilers

Cutoffs for Use in Electrical Appliances and Components

Cutter Grinding Machine (1973)

Cutting Brass Rod, Bar, and Shapes for Use in Screw Mac

Cutting Edge (1972)

Cutting Edges (1972)

Cutting Machine Tool Weldments (1971)

Cutting Oils and Compounds (1974)

Cutting Tools, Single Point, Carbide Tipped, Roller Tur

Cutting (1969)

Cutting (1973)

Cutting (1973)

Cutting (1974)

Cutting) (1973)

Cutting) (1973)

Cutting, Calcium Carbide and Acetylene (1973)

Safety Std. for a

Cyanides in Water (1972)

Cycle Meth. (Packaging) (1968) ANSl MH 12.12

Cycle Meth. (Packaging) (1968) ANSI MH12.18

Cycle (1973)

R Infrared Determination of the

Cyclic lmmersion of Syntactic Foam at Pressure (1972)

Cyclic Laboratory Aging Conditions (1970) ANSI Z197.19

Cyclic Movement (1972)

Cyclohexane by Gas Chromatography (1972)

Cyclohexane 995 (1972)

Cyclohexene-1,2-Dicarboximide-Captafol (1973)

Cyclohexyl 2Propynyl Sulfite (Common Name for the Pest

Cyclohexylamine in Water (1974)

Cylinder Lock Nut (1974)

Cylinder Type, for Water and Other Liquids (1972)

Cylinders Intended for Medical Use in the United States

Cylinders to Agricultural Tractors and Trailing Type Ag

Cylinders Vertically (1973T) ANSI A37.95

Cylinders (1972)

Cylinders (1972)

Cylinders (1972) ANSI B93.29

Cylinders (1972) ANSI B93.34

Cylinders (1973)

Cylinders (1973)

Cylinders (1973)

Cylinders (1974)

Cylindrical Concrete Specimens (1971) ANSI A37.121

Cylindrical Concrete Specimens (1972) AASHO T22, ANSI a

Cylindrical Concrete Specimens (1973)

Cylindrical Head Type and Related Quill Bushings (1972)

Cylindrical Shipping Containers (Packaging) (1950) ANSl

Cypress Lumber (1971.75)

$(\mathrm{D}, \mathrm{N})_{4} \mathrm{He}$ Neutron Generators by Radioactivation Techniq

Std. Safety Std. for Hig

mensions for $\mathrm{AC}$

Uniform

D.C. Electrical Conductivity of Hydrocarbons Fuels (197

D.C Arc Technique Using an Optical Em
D.C Arc Technique (1970) ANSI Z128.27

rap

Std.

ABYC

NEMA

ASTM

ANSI

ASTM

IEEE

ASTM

BIA

ANSI

ANSI

A NS ]

ANSI

ANSl

ANSI

ICBO

APA

APA

ICBO

WSFI

WSFI

ASTM

ASTM

ASTM

ASTM

NBHA

ANSI

IEEE

ASTM

AISI

ASTM

ASTN

F]

FMS

UL

NSA

ASTM

SAE

SAE

A WS

FMS

ANSI

AWS

ANSI

AWS

AWS

UL

UL

ASTM

ASTM

ASTM

ASTM

ASTM

ASTM

ASTM

/Est ANSI

ANSI

ASTM

NSA

AWWA

CGA

ASAE

ASTM

ASTM

UL

NFLDP

NFLDP

ICBO

ICBO

UL

FMS

ASTM

ASTM

ASTM

ANSI

ASTM

NHLA

ASTM

ASTM

ASTM

ASTM

342

El

SC13

Cl01.1

C82.7

D495

330

D2311

$17 \mathrm{~L}$

L24. 1.3

L24. 1.4

L24. 1.9

L24.1.5

L24T2

L24.2.10

UBCS6.1

-3PDS1

CP. 8

UBCS25.18

4 
eth. for Spectrochemical Analysis of Copper by the Briquet Std. Test Meth. for

Std. Spec. for Reinforced Concrete Rec. Lighting fo Cereal Grains, Oilseeds, Legumes, Forages, and Animal and Rec. for Fire, Heat, and Smoke ped Popcorn, Dried Peas and Beans for Rodent and/or Insect Rec. for Prevention of tion: Fossil Fue/

Rec. Pract. for the Prevention of Water Rec. Pract. for Collision Deformation Which Are Susceptible to Hydrolys le) (1973) Std. Performance Requirements for Std. Performance Requirements for Table Napery Other Than utyric, Valeric, Succinic, and Lactic Acids (Cereal / Van stion Chamber in Heating Appliances (Regulators, Automatic Uniform Building Code Std. for Veneer Application Uniform Building Code Std. for Fir

es) (1961)

al09.1

109.16 Safety Std. for Fire

lish) (1973) Std. Spec. for Asphalt for Uniform Code for the Abatement of of Textiles to Light: Carbon Arc Lamp, Alternate Light and to Light: Water Cooled Xenon Arc Lamp, Alternate Light and Std. Meth. for Determining Safety Times of Photographic eters (1972) ANSI S6./

Rec. Pract for Qualifying a Sound Agricultural Machinery

uality Between Data Terminal Equipment and Non-Synchronous Std. for Ai

ater Accident Report Form (Acquisition and Codification of rators (Fractional and Integral Hor $/$ Std. for Application pe and Fittings) (I972)

ps Std. Meth. of Test. and Presenting Basic Performance

d Steel Specimens (1972)

ry, Evolved Gas Analysis

Std. Rec. Pract. for Recording
Std. Rec. Pract. for Reporting

trol and Data TerminaI Equipment Employing Parallel Bin ary 0 73) Std. Meth. of Reporting Contamination Analysi halt Preferably with Gravel or SIag Surf/ Loss Prevention Loss Prevention Prevention Dielectric) (1973) (Vessel) (1974)

Loss Prevention Loss Prevention Loss Prevention

Std for Industrial Engineering Terminology: Std. for the Protection of Electronic Computer and ransactions Within the Air Tral Spec. for Std. Integrated Std. for Safety for Flectronic

Std for Sprinkler Irrigation (For Farms) Technical per and Copper Alloys (Wrought Products Only); Application packaged Compressor Units (1974)

Engineering

ication Equip/ Std. for Start-Stop Signal Quality Between interch/Std. for Interface Between Numerical Control and Std. Synchronous Signaling Rates for Rec. for Hazardous Chemicals

record Formats for Transmission of End Product Description c. Pract. for Applying Statistics to Analysis of Corrosion Guide for Statistical Analysis of Thermal Life Test $\mathrm{g}$ to Fatigue Test. and the Statistical Analysis of Fatigue for Toroidal Magnetic Amplifier Cores (Including Materia vide (Spec. for Installation, Finishes and Other Technical omposition, Use, Application, Finishes and Other Technical sembly Numbers, Cell Layouts and Ter/ Battery Replacemen Std. for Representation for Calendar and Ordinal Test Meth. for Colorfastness of Textiles to Light

Safety Std, for

meth. of Tests for Automotive Type Sealers, Adhesives, and

hollow Metal Door Frame Preparation for 181 and 190 Series - Test the Statistical Significance/

Std. Rec. Pract. for

ur (Cereal Chemistry) (1962)

Std. for

1, Grits, Flour, Farina, and Semolina (Cereal Chemistry) Std. Meth. for Accelerated Laboratory Test of Natura coniferous and Broadleaf Evergre/ Std for Nursery Stock:

d. for Nursery Stock: Deciduous Shade and Flowering Trees e Guide for Aids to Security (Safety) of Personnel on Boat Coating Systems (Paint) Guide for Ship Hull, Rec. Pract. for Concrete Highway Bridge

Marine Rec. for Insulated Stee Structura

Std. Test Meth for Structural Insulating Roo Std. for Particleboard Std. for Particleboard for Mobile Hom h and Finished Carpentry, Flooring, Shingles, Exposed Roof
D.C Arc Technique (1971) ANSI Z128.32

D.C Volume Resistivity of Glass (1972)

D.Load Culvert, Storm Drain, and Sewer Pipe (1973)

Dairy Farms and the Poultry Industry (1971)

Dairy Products (Cereal Chemistry) (1968)

Damage of Electronic Computer Systems (I973)

Damage on Kernels, Rodent Excreta, Other Filth, and Ext

Damage to Property from Surface Water (I973)

Damage to Steam Turbines Used for Electric Power Genera

(Damage) Classification (Highway Vehicle Accident) $(\mathrm{I} 972$

Damaged Starch (Granules in Flour or Starch Preparation

Damask Table Napery (Napkin, etc.) (Institutional Texti

Damask (White or Dyed) (Napkin, etc.) (Institutional Te

Dame Meth. of Analysis for Formic, Acetic, Propionic, B

Damper Controls, Fans) (1973)

Aft Equipment for Combu

Dampers (1973)

Dampers (1973)

Dampers (1973) ANSl Z233.I

Dampproofing and Waterproofing Masonry Walls (Tech. Not

Dampproofing and Waterproofing (1973) AASHO Ml16, ANSI

Damproofing and Waterproofing (1973) Ashto M115, ANSI a

Dangerous Buildings (Repair, Vacate, Rehabilitate, Demo

Darkness (1971) ANSI L14.169

/Meth. for Colorfastness
/Lorfastness of Textiles

Darkness (1971) ANSI L14.17

Data

Data and Management Practices (1971)

Data Communication Equipment (1973)

Data Computers Mps (1970)

Data Derived from the Investigation of Scuba Diving Acc

Data for Alternating and Direct Current Motors and Gene

Data for Hubless Cast Iron Sanitary System (Plumbing Pi

Data for Positve Displacement Hydraulic Fluid Power Pum

Data Formats for Fine Pitch Gear Drawings (1973)

Data from Atmospheric Corrosion Tests of Metallic Coate

Data from Differential Thermal Analysis, Thermogravime

Data Interchange (1973) /Terface Between Numerical

Data of Hydraulic Fluid Power Systems (1973) ANSI B93.3

Data on Bonds and Guarantees for Roof Installations (19

Data on Elastomer Roof Coverings (Multiply FeIt and Asp

Data on Flood Losses (1973)

Data on Fundamental Defects in Fires Over 050,000 (1973

Data on High Frequency Heating Equipment (Induction and

Data on Sight Glasses for Chemical Processing Equipment

Data Processing and Systems (1972)

Data Processing Equipment (1972)

Data Processing Systems Designed to Streamline Supply T

Data Processing Units and Systems (1972)

Data Sheet (1972)

Data Sheet (1972)

Std. Designations for Cop

Data Sheets for the Design of Air Exchange Coolers for

Data Terminal Equipment and Non-Synchronous Data Commun

Data Terminal Equipment Employing Parallel Binary Data

Data Transmission (1969)

Data (Fire Protection and Safety) (1973)

Data (Tooling, Printed Wiring Boards) in Numeric (Digit

Data (1971) ANSI G80.3

Data (1972)

Data (1972) ANSI Z92.2

Data) (1972)

Data) (1973)

Data) (1973)

Data; Group Numbers, Dimensional Spec., and Ratings; as

Date for Information Interchange (1971)

(Daylight) (1971) ANSI L14.168

Dead Front Distribution Switchboards (1972)

Dead Front Switchboards (1972)

Deadeners (1971)

Deadlock Strikes (1973)

Dealing with Outlying Observations in Samples and How

Decade Transiormer Dividers (Voltage Type) (1972)

Decantation Meth. of Analysis for Rodent Excreta in Flo

Decantation Meth. of Analysis for Rodent Excreta in Mea

Decay Resistance of Woods (1970)

Deciduous Shade and Flowering Trees; Deciduous Shrubs;

Deciduous Shrubs; Coniferous and Broadleaf Evergreens;

Deck and for Reboarding from the Water (1973)

Deck and Superstructure (1973)

Deck Construction (1974)

Deck Covering Guide (1969)

Deck Roofing (1972)

Deck (Roofing); Gypsum Concrete (1973)

Deck (R1970)

Decking for Factory Built Housing (1972)

Decking (1973)

Decking, Drop Siding, Structural Roof Trusses and Rafte

M ASTM

ASTM

ASTM

ASAE

FMS

28.10

ASME TW

SAE J224A

$\mathrm{AACCH} \quad 76 \cdot 30 \mathrm{~A}$

ANSI L24.2.7

ANSI L24.2.11

AACCH $\quad 04.23$

$\mathrm{UL}$

$\mathrm{ICBO}$

$1 \mathrm{CBO}$

UL

BIA

ASTM

ASTM

ATCC

AATCC

ANSI

SAE

ASAE

EIA

ANSI

NEMA

CISPI

NFLDP

GMA

ASTM

ASTM

EIA

NFLDP

FMS

FMS

FMS

FMS

FMS

FMS

ANSI

NFPA

ATAA

UL

ASAE

CDA
API
EIA

EIA

ANSI

FMS

IPC

378

UBCS30.

UBC 543.7 555

D41

D449

UBC*6

$16 \mathrm{D}$

PH 2.22

J 184

D230. 2

RS404

AS417A

Z86. 2

MGI-14

301

T3.9.17

114.02

G33

E472

RS408

T2 9.3

$1-47 \mathrm{~S}$

1.47

10-10

$10-9$

6.3

$7 \cdot 3$

Z94.3

75

200

S262T

$108 / 2$

$11 \mathrm{~K}$

404

408

$\mathrm{X} 3.1$

$7.23 \mathrm{~N}$

D. 350

G16

EEE 101

ASTM E206

E206

$3 \mathrm{~A} 7$ 
Std. Patterns for Wood Paneling, Weight, Stress of Board, Timber, Lumber, Siding, Framing. Spec. for Perlite Insulating Concrete Roof
Stds. for Spec. for Perlite Insulating Concrete Roof
Stds. for isc Records (1974) est for Measuring the Density of Smoke from the Burning or Meth. of Test for Acid Resistance of Ceramic Tentative Meth. of Test for Sulfide Resistance of Ceramic iners/ Tent. Meth. of Test for Acid Resistance of Ceramic nd Ceilings / Spec. for Vinyl Coated Fabric Coverings for s, and / Uniform Mechanical Code: Requirements for Vented stitutional Text/ Std. Performance Requirements for Woven meth. of Test for Light Transmittance of Woven Glass Fiber th. of Test for Effect of Handwashing on Woven Glass Fiber replaces (1973) c. for Insulating Board (Cellulosic Fiber), Structural and Test. Insulating Board (Cellulosic Fiber), Structural and

agneti/

Rec. Pract for Reference Carrier Frequencies and Std. Specs. and Load Tables for Longspan Steel Joints Std. for Hotel and Restaurant Gas

tallation, Operation, and Maintenance of Impressed Current Std. Spec. and Load Tables for Open Web, Longspan, and I Design and Construction (1965) whole or Ground Full Fat or Extracted Flakes, Full Fat and whole or Ground Full Fat or Extracted Flakes, Full Fat and Uniform Building Code Std. for Glass, Its Loss Prevention Data on Fundamental Rec. Pract, for Classifying Visual

erefrom (1970) ANS1 K/ Bulletin for Nondestructive Test. Terminology for and for Evaluating Confor/ Rec. Pract. for Selecting and Vehicle Fuel Tanks and Fill Pipes with / Std. for Dimensional and Electrical Characteristic Ceramic Tile (1973)

Ceramic Tile with Mortar, Adhesive, and Mastic (1973) 3)

Service for Use by Vehicle Manufacturers in De/

ber (1973) Uniform Building Code Std. for Classification,

durability and M/ Uniform Building Code:

ibutes (1971) ANSI Z1.6 Std. for Wafers, Pellets and Crumbles. Rec. Pract. for Automotive Brake industrial Tractors (1972) SAE J7/ 1972)

quipment (1971)

Std. for Power Takeoff Rec. Pract. for Power Of ar, Station Wagon, Truck, Bus, Trailer, Multipurpose Pas ines (1972)

and Transformers (197I)

sed in Schematic Diagrams (1972)

eel Products (1973) ANSl G60.1

s (1972)

)

entiometers $(1970)$

including Terms for Reporting and Analyzing Outage /

Std. for Classification and

rolled Machine Tool Software (1972)

tion) (1972)

ry (1967)

ol Products (1972)

pectroscopy (1972A) ANSI Z128.7

ilding Constructions and Materials (1973) )

s (1971). ANSI Z138.1

lated Products (1972) ANSI A 130.1

nd Analysis (1973)

organic Materials for Roofing, Waterproofing, and R/

Ribbon Products and Images Made Therefrom (1972A)

le Products and Rec. Pract. for Use of These Terms /

e Derivatives (1972) ANSI K64.6

nsi K20.6

gates (1968) ANSI A37.76

n 105

liquids and Gases (1974)

(1973)

(1972B)

avitation and Impingement by Liquid or Solid Partic/
Std. for Std. for Vented Rec. for Rec. Pract. for

Std. for Std.

Std. Std.

Decking, Flooring, and Siding (1970)

Decking, Flooring, Ceiling, and Partition (1973)

Decks (1973)

Decoders (Type 1) for Reproducing Matrix Quadraphonic D

Decomposition of Plastic Materials (1973)

Decorations on Architectural Type Glass (1972T)

Decorations on Glass (1974)

Decorations on Returnable Beer and Beverage Glass Conta

Decorative and Protective Covering for Permanent Wall a

Decorative Appliances, and Wall Furnaces, Floor Furnace

Decorative Bedspreads, Pillows, and Bolster Fabrics (In

Decorative Fabrics (Textile) (1973)

Decorative Fabrics (Textile) (1973)

Decorative Gas Appliances for Installation in Vented Fi

Decorative Gas Appliances (1973)

Decorative (1972)

Decorative (1972)

Deemphasis Characteristics for 2 In. Quadruplex Video

Deep DLJ and DLH Series (1970)

Deep Fat Fryers (1971)

Deep Groundbeds (1972)

Deep Longspan Steel Joints (1974)

Deep, Cast in Place Concrete (Storage) Bin and Silo Wal

Defatted Soy Flours and Grits, and Soybean Meal (Cereal

Defatted Soy Flours and Grits, and Soybean Meal (Cereal

Defects and Safety Glazing (1973)

Defects in Fires Over 050,000 (1973)

Defects in Glass Reinforced Laminates and Parts Made Th

Defects in Steel Pipe (1974)

Defining Color and Gloss Tolerances of Opaque Materials

Defining Conditions for Evaluating the Compatibility of

Defining Dual in Line Type Sockets (1974) ANSI C83.95

Definition and Recommendation for Materials for Groutin

Definition and Recommendation for Materials for Setting

Definition and Test Procedures for Ammonia Nitrate (197

Definition of and Maximum Dimensions for Grown Tires in

Definition of the Term Vacuum Cleaner (1974)

Definition, and Meth. of Grading for All Species of Lum

Definitions and Abbreviations (1973)

Definitions and Meth. for Determining Specific Weight,

Definitions and Nomenclature (1971)

Definitions and Symbols for Acceptance Sampling by Attr

Definitions and Terminology for Agricultural and Light

Definitions and Terminology for Agricultural Tractors (

Definitions and Terminology for Relays for Electronic E

Definitions and Terminology (1972)

Definitions and Terms Used in the Selection of Tires $(C$

Definitions for Excitation Systems for Synchronous Mach

Definitions for High Intensity Discharge Lamp Ballasts

Std. Symbols and Definitions for Mechanical and Acoustical Elements as U

Rec. Pract. of Definitions for Motorcycles (1972)

Std. for Definitions for Motors and Generators (1972)

Std. Definitions for Power Switchgear (1972)

Safety Std.

Definitions for Regular Bicycles (1972)

Definitions for Soil Tillage and Soil Tool Relationship

Definitions for Stationary Diesel and Gas Engines (1972

Definitions for Welding and Cutting (1969)

Definitions for Wirewound and Nonwirewound Trimming Pot

Definitions in Electrical Power Operations Terminology

Definitions of Bulk Materials (1970) ANSI MH4.8

Definitions of Common Words Related to Numerically Cont

Std. Definitions of Navigation Aid Terms (1971)

Definitions of Relay Terms (Electromagnetic Instrumenta

Definitions of Statistical Terms Used in Cereal Chemist

Std. Definitions of Terms and Nomenclature Relating to Aeros

Std. Definitions of Terms and Symbols Relating to Emission S

Std. Definitions of Terms for Antennas (1973)

Std. Definitions of Terms Relating to Acoustical Tests of Bu

Std. Definitions of Terms Relating to Activated Carbon (1974

Std. Definitions of Terms Relating to Adhesives (1973)

Std. Definitions of Terms Relating to Appearance of Material

Std. Definitions of Terms Relating to Asbestos Cement and Re

Std. Definitions of Terms Relating to Atmospheric Sampling a

Std. Definitions of Terms Relating to Bituminous, and Other

Std. Definitions of Terms Relating to Building Seals (1973)

Std. Definitions of Terms Relating to Carbon Paper and Inked

Std. Definitions of Terms Relating to Care of Consumer Texti

Std. Definitions of Terms Relating to Cellulose and Cellulos

Std. Definitions of Terms Relating to Coal and Coke (1972) a

Std. Definitions of Terms Relating to Concrete and Its Aggre

Std. Definitions of Terms Relating to Dosimetry (i968) ANSl

Std. Definitions of Terms Relating to Electrical Insulating

Std. Definitions of Terms Relating to Electrical Insulation

Std. Definitions of Terms Relating to Electrostatic Copying
Std. Definitions of Terms Relating to Erosion of Solids by C
WWPA $\approx 28$

lles, WWPA $\approx 24$

CSI 0352

EIA RS418

ICBO UBCS52.2

ASTM C724

ASTM C 777

ASTM C735

CSI 0995

ICBO UMC*\%.

ANSI L24.1.2

ANSI L24T4

Me ANSI L24Tl

ANSI Z21.60

ANSI Z21.50

Std. Spe ASTM C208

C208

SMPTE RP6

AISC S316-1

ANSI Z21.27

NACE

SJI

5

$\mathrm{ACl} \quad 65-37$

$\mathrm{AACCH} \quad 46.23$

$\mathrm{AACCH} \quad 46.24$

ICBO UBCS54.1

FMS 10-9

ASTM D2563

API BUL5T1

ASTM D3134

SAE J398A

ElA RS415

TCA *3-1

TCA *3

FI $\quad$ P40

TRA 1-13

ASTM F395

ICBO UBCS25-1

ICBO UBC *3-4

ASAE S269.2

SAE J656

ASQC A2

ASAE S205.2

REC. J722B

ANSI C83.16

ANS1 MH1.1.2

TRA X.XI

IEEE 421

ANSI $\quad$ C82.9

ANSI Y 32.18

ASTM A370

SAE J213A

NEMA MG1-1

ANS1 C37.100

BMA $6 / 2$

ASAE R291.1

DEMA *1-1

AWS A3.0

VRCl T110

IEEE 346

CEMA $\quad 550$

NMTBA $* 2$

IEEE $\quad 172$

NARM *2

AACCH $\quad 78-40$

ASTM D3064

ASTM E135

IEEE 145

ASTM C634

D2652

ASTM E284

ASTM $\quad$ C460

ASTM D1356

ASTM D1079

ASTM C717

ASTM F221

ASTM D3136

ASTM D1695

ASTM D121

ASTM C125

ASTM

ASTM D2864

ASTM D1711

ASTM F335

ASTM G40 
statistical Analysis of Fatigue Data (1972) ANSI Z9/ Construction and Materials (1973)

ts (1971)

) ANSI A 1.28

Z166.25

1, ANSI Z109.3

7i) ANSI K67.8

ction (1974) ANSI Z166.16

Physical Components of Coal (1972)

ection (1974) ANSl Z166.15

d Graphite (1972)

d Pavements (1971) ANSI A37.33

nsi Z30.4

s (1974)

ed Products (1972)

$r$, and Related Products (1973)

65.51

eramic Metal Systems (1973) ANS1 Z167.1

si Alll.9

Materials (1973)

ents (1974)

2)

on Ceramics (1973)

3) ANSl L14.12

lectrical Conductors (1972A)

70) ANSI 07.2

ed Materials (1973)

ing Station Stds. (1972)

nsi H9.5

(1973)

1972)

Rec. List of Terms an Std. Thermal Spraying Terms and Rec. Pract. Industrical Truck

for the Construction of Nuclear Power Plants-Terms and or Suffix Letters Used in Tire Size Designations and Thei ramic Veneer (Sampling, Test. Finishing, Installation, and Std. for Photo Instrumentation Glossary (Terms, National Std. Plumbing Code (Including

lling, lngredients and Containers (Also lncludes Terms and dustrial Exhaust Ventilation Systems (19) Rec. Pract. for ) Control Charts (1971) ANSl Z1.5

d. for Recreational Vehicle Air Conditioning System Rating Std. Test. Procedure for End Supported Beam

(1972) ANSI K65.222

Aer Electronic Equipment (Flyback Tra Rec. for Wood Beam. Joint and Rafter Span, Load, $s$ of Hveem Apparatu/ Std. Meth. of Test for Resistance to Rec. Pract. for Meth. of Determining Plastic Meth. of Test for Permanent cident) (1972)

2) Rec. Pract. for Collision Std. Spec. for Axle Steel Std. Spec. for orcement (1972)

Std Spec for Rail Steel

for Forging; Hot Rolled and Cold Finished Bars; Hot Rolled
ANSl G 45.3
Std. Spec. for Welded 1972) ANSl G 45.3 nsi G45.2

Uniform Building Code Std. for Welded Steel Wire and Rec. Pract. for Passenger Car Windshield Std. Meth. of Test for Therma elical Coil Meth. (1973)

Halonat of

Nonamine Acid Acceptance of Halogente Yarn Colorfastness to Std. for Safety for Component Std. for Receiver 1970) Std. Test Meth. for ge Propensity of Bonded and Laminate/ Std. Meth. of Test. sublimation, Brittlene/

Std. Performance Requirements for Std. Meth. of Test. Aerosol Products for

Std for Sound Rating of Room Fan Coil Air Conditioner eel Plate, Sheet/ and Bars for Str/

Std. Spec. for General Requirements for Std. Spec. for General Requirements for Std. Spec. for General Requirements for Std. Meth. of Test for

ansi $\mathrm{K} 90.8$

ng Systems (1973)

ce $(1970)$

(Safety) (1973)

ou/
Std. Meth. of Test for

Rec. for Star Model F Hidromatic Safety Std. for Dry Pipe, Rec. for Water
Std. Analytical Meth. for Determining Chemical Oxygen td. Definitions of Terms Relating to Fatigue Test. and the d. Definitions of Terms Relating to Fire Tests of Building . Definitions of Terms Relating to Gaskets (1972)

Definitions of Terms Relating to Glass and Glass Produc Definitions of Terms Relating to Hydraulic Cement (1971 Definitions of Terms Relating to Iron Castings (1972A)

Definitions of Terms Relating to Leak Test. (1971) ANSI

Definitions of Terms Relating to Leather (R1972) ALCA X Definitions of Terms Relating to Lime and Limestone (19 Definitions of Terms Relating to Liquid Penetrant Inspe Definitions of Terms Relating to Lithologic Classes and Definitions of Terms Relating to Magnetic Particle Insp Definitions of Terms Relating to Manufactured Carbon an Definitions of Terms Relating to Materials for Roads an Definitions of Terms Relating to Metallography (1973) a Definitions of Terms Relating to Microscopy (1972) Definitions of Terms Relating to Natural Building Stone Definitions of Terms Relating to Naval Stores and Relat Definitions of Terms Relating to Paint, Varnish, Lacque Definitions of Terms Relating to Plastics (1973) ANSI K Definitions of Terms Relating to Porcelain Enamel and $\mathrm{C}$ Definitions of Terms Relating to Refractories (1973) an Definitions of Terms Relating to Rubber and Rubber Like Definitions of Terms Relating to Soaps and Other Deterg Definitions of Terms Relating to Space Simulation (1972 Definitions of Terms Kelating to Statistical Meth. (197 Definitions of Terms Relating to Surface lmperfections Definitions of Terms Relating to Textile Materials (197 Definitions of Terms Relating to Thermal Analysis (1973 Definitions of Terms Relating to Ultrasonic Test. (1974 Definitions of Terms Relating to Uninsulated Metallic E Definitions of Terms Relating to Veneer and Plywood (19 Definitions of Terms Relating to Water (1972)

Definitions of Terms Relating to Wax Polishes and Relat

Definitions of Terms Used in IEEE Nuclear Power Generat

Definitions of Terms Used in Powder Metallurgy (1972) a

Definitions of Textile Terms for Institutional Textiles

Definitions of Trade Terms Commonly Used in Packaging (

Definitions Related to Quality Control (1969)

Definitions (1970)

Definitions (1972)

Definitions (1973)

Definitions (1974)

Definitions) (1961)

Definitions) (1969)

Definitions) (1973)

Definitions) (1974)

Definitions, Abbreviations and General Principles of in

Definitions, Test. Meth., and Test Equipment and Rating

Deflection Composite Tubes and Cores (1972)

Deflection Temperature of Plastics Under Flexural Load

Deflection Yoke, Picture Tube Neck Components) (1974)

Deflection, Strength and Stiffness Computations (1973)

Deformation and Cohesion of Bituminous Mixtures by Mean

Deformation in Sheet Metal Stampings (1972)

Deformation of Elastomeric Yarns (1972T)

Deformation (Damage) Classification (Highway Vehicle AC

Deformed and Plain Bars for Concrete Reinforcement (197

Deformed and Plain Billet Steel Bars for Concrete Reinf

Deformed Bars for Concrete Reinforcement (1972)

Deformed Concrete Reinforcing Bars (1972)

Deformed Steel Wire Fabric for Concrete Reinforcement

Deformed Steel Wire for Concrete Reinforcement (1972) a

Deformed Wire Fabric for Concrete Reinforcement (1973)

Defrosting Systems (1971)

Degradation of Electrical Insulating Varnishes by the $\mathrm{H}$

Degreasing Grade (Nonreflux Meth.) (1974)

Degumming (1972) ANSl L14.4

Dehumidifiers (1973) ANS1 Z253.1

Dehydrators for Vehicle Air Conditioners (1967)

Delamination Strength of Honeycomb Type Core Material (

Delamination, Strength of Bond, Appearance, and Shrinka

Deleterious Effects of Dyes, Finishes, and Finishings (

Delivered Mass (1972)

Delivering Less Than $1500 \mathrm{cfm}$ (1971)

Delivery of Flat Rolled Stainless and Heat Resisting St

Delivery of Rolled Steel Plates, Shapes, Sheet Piling,

Delivery of Steel Plates for Pressure Vessels (1972)

Delivery Rate of Aerosol Products (1972)

Delta in Hours (DIH) Purity of Nuclear Graphite (1971)

Deluge Valve for Water Flow Control of Fire Extinguishi

Deluge, and Pre Action Valves for Fire Protection Servi

Demand for Sprinkler System for Private Fire Protection

Demand in lndustrial Waste Waters and Most Organic Comp

Demand Registers (Cumulative and Pointer Forms) EEI Msj

ASTM

ASTM

ASTM

ASTM

ASTM

ASTM

ASTM

ASTM

ASTM

ASTM

ASTM

ASTM

ASTM

ASTM

ASTM

ASTM

ASTM

ASTM

ASTM

ASTM

ASTM

ASTM

ASTM

ASTM

ASTM

ASTM

ASTM

ASTM

ASTM

ASTM

ASTM

ASTM

ASTM

IEEE

ASTM

ANSI

ANSI

ASQC

AWS

ITA

ANSI Std. F

TRA

BIA

SMPTE

NAPHC

AA ASOC

IMACA

CCTI

AST

UL

WWPA

ASTM

SAE

ASTM

SAE

ASTM

ASTM

ASTM

AIS1

ASTM

ICBO

SAE

ASTM

E206

E176

118

Cl62

C219

A644

425

D1517

C51

D2796

E269

C709

D8

E7 75

E175
C119

D804

D16

D883

C71

D1566

D459

E349

E456

F109

D123

E473

E500

B354

D1038

D1129

D2825

380

B243

L24.6. 1

MH15. 1

*1

C2.9
IB 2.19 
Rec. for Water Sprinkler and Hydrant System Demand Tables (Fire Safety) (1973)

/ Pressure Requirements for Pipe Schedule System Sprinkler Rec. for Computing Electrica ment of Dangerous Buildings (Repair, Vacate, Rehabilitate. safeguarding Against Fire During Building Construction and safety Requirements for Personnel Hoists (Construction and lic Property (Pedestrian Protection During Construction or or Practical Minimum Rec. Tolerances of Spoke Type Wheels

Test Procedures for Production Disc and Spoke Wheels, and , Conveyors, and Other Equipment (1966)

Std. for awal Only/ Rec. Pract. for LP Gas Fuel Container, Quickl Liquid / Rec. Pract. for LP Gas Fuel Container, Quickly (1966)

\section{Std. Meth. of Test for Liquid}

Std. Positive Gravure

Methylene Blue Meth. for Measuring Thiosulfate and Silver Generators by Radio

Slides for Television (1970)

metal Structural Parts and Oil $1 \mathrm{~m}$ /

) (Pulp and Paper) (1973)

on for Cracks, etc.) (Pulp and Paper) (19/

(1972)

ometer (1968) ANSl Z11.96

Std. Analytical Meth. for Determining Bulk

ed Solid Prod/

Std. Analytical Meth. for Determining Bulk Std. for Medium

cles by Physical Measurements (19) Std. Meth. of Test for ct. for the Determination of the Thermal Resistance of Low Std. Test Meth. for (1974) Sid. Meth. of Test for Std. Meth. of Test for Relative

ity, Water Absorption, Apparent Specific Gravity, and Bulk ructions (1970) Std. Test Meth. for 1) Std. Meth. of Test for Estimating Maturity and Linear .136

Std. Meth. of Test for Linear Std. Test Meth. for Bulk

Std. Meth. of Test for Linear

Tentative Meth. of Test for

Meth. of Test for

Std. Meth. of Test for Apparent

Std. Meth. of Test for Apparent

Std. Meth. of Test for Apparent

Test Meth. for

cks, etc.) (Pulp and Paper) (1973) ansi All1.18
Volumeter (1970) ANSl H/ Std. Meth. of Test for Size and Bulk
Std. Meth. of Test for Apparent ding Code Std. for Chamber Meth. of Test for Measuring the Uniform Building Code Std. for in Place per) (1973) lp and Paper) (1973) Test Meth. for (1966) Test Meth. for Std. Air

ty of Fire/

Uniform Building Code Std, for Moisture Std. Meth. of Test for Water Absorption, Bulk onships of Agricultural Grain and Seed for Storage (1972 Safety Std. for Medical and Spec. for

Rec. Std. Practices for Clinical Research Protocol for Std. for Intraoral

(1972)

Spec. for Base Plate (Artificial td. Spec. for Boron Std. Spec. for Phosphorus radioactive / ydrant, Drivew/ Std. for Administrative Guide for Obtaining eth. for Apparent Viscosity of Adhesives Having Shear Rate Safety Std. for Key Locks (Burglar Alarm, Door, Std. Meth. of Test for Std. Meth. for Examination of Water Formed sed for Removal of Water Formed alysis of Solvent Systems Used for Removal of Water Formed
Std. Meth. of Test. Water Formed
Meth. of Test for lron Bacteria in Water and Water Formed Meth. of Test for lron Bacteria in Water and Water Formed ater Insoluble lmpurities in Refined Phenol by Cloud Point ctronic Equipment on Boats (Audio Device, Automatic Pilot, Std. Meth. of Test for Embossed

Safe Operating Condition of Tires lncluding Tire Treads lling Tapping Screws and Rec. Technique for Measuring Case Definitions of Terms Relating to Cellulose and Cellulose er and Pillar Cranes (1973) oists (1971) Safety Sid. for Cranes, Safety Code for Cranes, Std. Rec. Pract. for Guidelines for and Systems (1972) ANSI G81.16 fiware Documentation and Terminology) (19/ 1972)

1) ANS1 Z128.31 Std. for Guide for
Sid. Rec. Pract. for

Spec. for Record Formats for Transmission of End Product d;/ Oilseed Products Trading Rules (Performance, Quality, ing to Forensic Sciences (1972)

for Radiation Shielding Concrete (1973)
Demand (1974)

Demands for Farms (1972)

Demolish) (1973)

Demolish) (1973) form ' udc for the Abate ICBO

Demolition Operations) (1973)

Demolition) (1973)

R. for Viancr Wow

FMS

/C Streets and Projections Over Pub

Std. for NFPA

Std for ANSI

'

Demountable Rims Intended for Highway Use on Trucks, B

Demountable Type for Horizontal Mounting, Liquid Withdr

Demountable Universal (Horizontal or Vertical Mounting)

Densities for Forced and Induced Draft Centrifugal Fans

Densities of Pressurized Products (1972)

Densities (Printing (1973)

Densitometric Meth. for Measuring Residual Chemicals in

Density and Average Energy from ${ }_{1} H(D, N)$ He Neutron

Density and Contrast Range of Black and White Films and

Density and Interconnected Porosity of Sintered Powder

Density and Moisture of Chips (Presoak Before lmmersion

Density and Moisture of Chips (Submersion with Correcti

Density and Packing Factor of Hollow Glass Microspheres

Density and Specific Gravity of Liquids by Bingham Pycn

Density Content in Corn Starch (1963)

Density Content in Feedstuffs (Corn) and Other Wet Mill

Density Fiberboard (1973)

Density in A ir of Manufactured Carbon and Graphite Arti

Density Mineral Fiber Blanket Type Building Insulation

Density of Adhesives in Fluid Form (1969)

Density of Bituminous Concrete in Place by Nuclear Meth

Density of Black Smoke (Ringelmann Meth.) (1973)

Density of Burned Refractory Brick (1973) ANSI A111.3

Density of Core Materials for Structural Sandwich Const

Density of Cotton Fibers by the Causticaire Method (197

Density of Cotton Fibers (Array Sample) (1973) ANSI L14

Density of Diamond Abrasive Grains (1973)

Density of Elastomeric Yarn (1972)

Density of Glass by Buoyancy (1971)

Density of Glass by the Sink Float Comparator (1972T)

Density of Industrial Aromatic Hydrocarbons (1972)

Density of Leather (1968) ALCA E62

Density of Metal Powders (1948) ANSI H9.1

Density of Pulpwood (Submersion with Correction for Cra

Density of Refractory and Insulating Fire Brick (1970)

Density of Refractory Metals and Compounds by the Scott

Density of Smoke from the Burning or Decomposition of P

Density of Soils (1973)

Density of Wood Chips (Sheet Machine Mold) (Pulp and Pa

Density or Volume of Small Specimen (Hg Pycnometer) (Pu

Density Ratios-At Various Altitudes and Temperatures

Density Relations of Soils (1973)

Density, Apparent Porosity, and Apparent Specific Gravi

Density, Specific Gravity, and Weight-Moisture Relati

Dental Equipment (1972)

Dental Gypsum Products (1971)

Dental Materials and Devices (1971)

Dental Radiographic Film (1972)

Dentures) Wax (1971)

Deoxidized Copper in Wrought Forms for Electron Devices

Deoxidized Copper Wire Bars, Billets, and Cakes (1972)

Department of Transportation (DOT) Special Permits for
Departmental Regulations and Controls, Barrier, Road, H

Dependent Flow Properties (1969)

Deposit and Collection Safes) (1973)

Deposit Forming Impurities in Steam (1971) ANSI Z111.5

Std.

Deposits by Chemical Microscopy (1973)

Deposits (Chemical Cleaning of Industrial Equipment) (I

Deposits (1972)

Deposits (1972)

Deposits (1973)

Depression (1968) ANS1 Z78.21

Depth Determination Device and Fish Finder, Loran or Ot

Depth Requirements (Passenger Car Type Tires) (1971)

Depth (1972)

Dcrivatives (1972) ANSI K64.6

Derricks, Hoists, Hooks, Jacks, and Slings: Portal, Tow

Derricks, Hoists, Jacks and Slings: Base Mounted Drum H

Desealing and Cleaning Stainless Steel Parts, Equipment

Describing lnformation Interchange Formats (Computer So

Describing the Characteristics of Resilient Mountings (

Description and Performance of the Microphotometer (197

Description Data (Tooling, Printed Wiring Boards) in Nu

Description, and Grade of Palmkernels; Babassu; Flaxsee

Descriptions of Qualifications and Scopes of Work Relat

Sid. Descriptive Nomenclature of Constituents of Aggregates

GT

ANSI

ASTM

SMPTE

ASTM

TAPPI

TAPPl

ASTM

ASTM

CR

NPA

A STM

ASTM

ASTM

ASTM

ASTM

ASTM

ASTM

ASTM

ASTM

ANSI

AS'TM

ASTM

ASTM

ASTM

ASTM

ASTM

TAPPl

ASTM

ASTM

ICBO

ICBO

TAPPl

TAPPI

AMCA

ICBO

ASTM

ASAE

UL

ADA

ADA

ANSI

ADA

ASTM

ASTM

ANSI

ICBO

2.76

2.77

R294

$\mathrm{UBC} * 6$

241

A 10.4

UBC 3.44

J851

SAE J267

CFTMA W?

ITA 6Al

ITA 6A2

AMCA 2409

ASTM D3096

$+2$

PH4.8

E496

RP7

B328

UM. 20

D3101

D 1217

G.8

4.73
C559

C653

D 1875

D2950

D3211

D2480

D1769

B74.17

D2591

C 693

C729

D2935

D2346

B2 12

U.

C134
B329

UBCS52.2

UBCS70.2

UM-9 
teristics Of, Rec. Construction and Water Sprinkler System ssure Treated Lumber and Plywood) Foundation System: House ) e Structures (1967) ) (1972) Rec. Pract. and Std. Covering s (Tech. Notes) (1970) uid Cooled Marine Engines (/ ntial and Industrial) (Tech. Notes) (1964)

for Corrosive Service (1971) Ansi/

(1969) ANSI A 158.1

farms in Humid Areas (1973)

ion Piers (1969)

Std, for Mobile Homes: Body a $r$ Deep, Cast in Place Concrete (Storage) Bin and Silo W all ed Prestressed Concrete (Post Tensioned Box Girder Bridges ermination of Sound Transmission Class (Building Acoustics ipment (Machinery) (1971/ Rec. Pract. for Instrument Face Tent. Rec. Pract. Guidelines for Driveway Rec. Pract. for Drill Stem

Heat Transfer Rate (1972) ons in Nuclear Power Gel (Conventional Units) (I972)

8 (1973) ontainment Structures (1969)

73) members) (1973)

Std. for Fundamental Std. Meth. for

Std. Criteria for Preparation of Rec. Pract, for

Code for Architectural Std. for Guide to Principal

Rec. Pract. for Exhaust Hood Structural

Concrete Construction Rec. Pract. for Std. for Building Code Requirements for Minimum std., Grading Rules, Design Values, Section Properties and units (1974) Engineering Data Sheets for the ures (Aluminum Construction Manual Sect. I) (1/ minum Construction Manual) (1969) Spec, for

Guide for the Structural

c. for Prevention of Collapse Due to Weak Construction and Rec. A gainst Weak Construction and Uniform Building Code Std. for Spec. and bolting Keys and Shafting (1974) ding (1957) Std. for nvolute Spur Pinions (1973)

Tent. Rec. for Rec. for

Std. for System Std. for System Rec. Guide for

nts (1965)

tural Members (/ Uniform Building Code Std. for Spec, and droelectric Equipment (1972)

) (1971)

s) (1968)

Rec. Pract. for the a Policy on Geometric Structural 1973) Structural

1966) building Code Std. for Stress Variation or Stress Reversal ing. Unified Threaded In. Series (1971) tems (1974) ts (Foundation) (1963) Std. for

Rec. Pract. for Suggested

Air Conditioning Equipment Selection and System Joint Venture Agreement (Form) for nt Single Stack Plumbing System (1973)

form of Agreement Between Owner and Architect for Interior

(1971)

National

Plywood

Stds. (Wording, Terminology, Phraseology, Environment and 74) Std. Spec, and Meth. for Establishing Rec. bers (Tech. Notes) (1967) Tent. Test Meth. for Establishing Rec. for

of Bol a Product Use Manual: Grade Selection, Std. Size, ber,/ Western Woods Use Book: Lumber Std., Grading Rules, rec. Pract. Test Meth. and Requirements for Maintenance of Residential Fireplace

Contemporary Bearing Wall Example of Shear Wall election Tables for Ágricultural (Farm) Machines of Future Uniform Building Code Std. for Spaced Column Uniform Building Code Std. for Open Web Steel Joist Reinforced Brick Masonry (Flexural Reinforced Brick Masonry (Axial

rs on Boats (1973) quipment for Anchori/ ms and Portable Containers for Flam/
Rec. Pract. and Std. for the
Design-Fire Protection and Safety) (1973)

Design and Construction Meth. (1972)

Design and Construction of Chimneys (Tech. Notes) (1964

Design and Construction of Circular Prestressed Concret

Design and Construction of Cockpits and Scuppers (Boats

Design and Construction of Diesel (Compression lgnited)

Design and Construction of Flat and Sloped Brick Soffit

Design and Construction of Gasoline Fueled Inboard, Liq

Design and Construction of Masonry Chimneys (For Reside

Design and Construction of Nonmetallic Enveloped Gasket

Design and Construction of Reinforced Concrete Chimneys

Design and Construction of Surface Drainage System 8 on

Design and Construction Procedures for Concrete Foundat

Design and Construction Requirements, and Installation

Design and Construction (1965)

Design and Construction) (1969)

Design and Construction) (1973)

Design and Location for Const

Design and Location (1972)

Design and Operating Limits (Petroleum) (1974)

Design and Operation of Local Exhaust Systems (1971)

Design and Use of a Thin Skin Calorimeter for Measuring

Design Bases for Systems That Perform Protective Functi

Design Calculations for Oil Sucker Rod Pumping Systems

Design Competitions (1972)

Design Criteria for Nuclear Fuel Reprocessing Facilitie

Design Criteria for Reinforced Concrete Nuclear Power C

Design for Industrial Ventilation Systems (1974)

Design Guide for Hardwood Plywood (1971)

Design Guide for Wood Composition Board Wall Panels (19

Design Handbook (Beam, Slab, Footing, Column and Other

Design Levels for Emergency Air Brake Systems (1971)

Design Loads in Buildings and Other Structures (1972)

Design Loads, Timber, Plank and Laminated Flooring and

Design of Air Exchange Coolers for Pactsaged Compressor

Design of Aluminum Alloy Load Carrying (Members) Struct

Design of Aluminum Formed Sheet Building Sheathing (Alu

Design of Brick Masonry Arches (Tech. Notes) (1967)

Design of Buildings and Other Structures (1973)

Design of Buildings (1972)

Design of Cold Formed Steel Structural Members (1973)

Design of Components: Enclosed Gear Drives-Bearings,

Design of Concrete Composite Beams and Girders for Buil

Design of Farm Waste Storage Tanks (1973)

Design of Fine Pitch on Center Face Gears for 20 Deg. 1

Design of Fine Pitch Wormgearing (1973)

Design of Foundations and Shoulders for Concrete Paveme

Design of Light Gauge Cold Formed Stainless Steel Struc

Design of Oil Systems for Lubrication and Control of $\mathrm{Hy}$

Design of Rural Highways (1965)

Design of Semicircular Brick Masonry Arches (Tech. Note

Design of Serpentine Walls, Non Loadbearing (Tech. Note

Design of Stationary Pressurized Water Reactor Plants (

Design of Structural Steel Members (1973)

Design of the Contemporary Bearing Wall (Tech. Notes) (

Design Parameters for Bolts and Screws; External Wrench

Design Procedure for Industrial Exhaust Ventilation Sys

Design Procedures for Combined Concrete Footings and $\mathrm{Ma}$

Design Procedures (1973)

Design Professionals (1972)

Design Rules, Guidelines, and Drawings for Copper Solve

Design Services (1972)

Design Spec. for Flat Plywood Sandwich Panels (1970)

Design Spec. for Plywood Beams (1972)

Design Spec. for Plywood Curved Panels (1968)

Design Spec. for Plywood Stressed Skin Panels (1972)

Design Spec. for Stress Grade Lumber and Its Fastenings

Design Spec. (1966)

Design Stds for (1972)

Design Stresses for Round Timber Construction Poles (19

Design Stresses for Round Timber Piles (1970)

Design Tables for Reinforced Brick Masonry Flexural Mem

Design Values for Livestock Fallout Shelters (1973)

Design Values, Framing Rec., Properties, Weight, Stress

Design Values, Section Properties and Design Loads, Tim

Design Voltage in Snowmobile Electrical Systems (1971)

Design (Tech. Notes) (1963)

Design (Tech. Notes) (1964)

Design (1972) SAE J711

Design (1973)

Design (1973)

Design) (Tech Notes) (1964)

Design) (Tech. Notes) (1963)

Design, Construction and Installation of Battery Charge

Design, Construction and Installation of Fittings and $E$

Design, Construction and Stowage of Portable Fuel Syste
Charac FMS

NFORP

BIA

$A C I$

$A B Y C$

ABYC

BIA

ABYC

BIA

ASTM

$\mathrm{ACl}$

ASAE

ACI

NFPA

Rec. Fo ACI

CRSI

ASTM

SAE

ITE

ANS!

ASTM

ANS

API

AIOA

ANSI

$\mathrm{ACI}$

ACGIH

HPMA

HPMA

ACI

SAE

ANSl

WWPA

API

AA

AA

BIA

Re FMS

FMS

ICBO

AGMA

$\mathrm{ACI}$

ASAE

AGMA

AGMA

ACI

ICBO

ASME

ASHTO

BIA

BIA

ANSI

Uniform lCBO

BIA

SAE

ACGIH

ACI

NESC

AlOA

CDA

Std. AlOA

APA

APA

APA

APA

NFORP

APA

ASTM

ASTM

BIA

ASAE

WWPA

WWPA

SAE

BI

BIA

Tire $S$

ICBO

ICBO

BIA

BIA

ABYC

$A B Y C$

$A B Y C$

LPD 1.2

19A

67.40

$36 \mathrm{~A}$

$\mathrm{P} 4$

$9 \mathrm{~B}$

336

307

R302.2 
mpact) Shock Test. Machine for Lightweight/ wer Transmission Units Penetrating the $\mathrm{H} /$ Rec. Guides for

of Propeller Shafting Sys/ Std. and Rec. Pract. Guide to ation Equipment (I972) y Valve Systems for Petroleum Industry U/ Rec. Pract. for ressed Current Deep Groundbeds (1972) Rec. Pract. (Erosion Control and Water Conservation) (1972) Rec. for ing (1972)

Fine Pitch Gearing (1973)

manently Insta/ Std, and Rec. Pract. for Requirements for d Landscape Guide (Rec. for Garden Grades, Application and ies Pertaining to Photographic Lenses (197) Std. Meth. of )

Std. for Capacity Std. for

Std. Meth. for the

haracteristics of $40 \mathrm{~W}$ att, $\mathrm{H} 45$ (1972) electrodes in Multiple Electrode Semiconductor Devices and Std. for Alloy and Temper Std. for Alloy and Temper Std. for Numerical rdinates (1971) Std. for Suffix Letters Used in Tire Size Rec. Spec. for Enamel Coating System ucts Only); Application Data Sheet (I972)

fans (1966) Std. mposition and Cryst. Std. Meth. for Assigning Alloy Phase f Powered lndustrial Trucks (1973) ANSI B5/ Std. for Type Pract. Safety Requirements for lndustrial Rotary Mowers Std. for Stainless Steel Plumbing Fixtures Metal, Wire and Wire Fabric Laths, and Metal Accessories Approval Std. for Heat and Smoke Roof and Window Vents ir Tra/ Spec. for Std. Integrated Data Processing Systems 1 Contact Materials (1973) Std. Rec. Pract. for

Rec. Pract. for Average Vehicle Dimensions for Use in grown Tires in Service for Use by Vehicle Manufacturers in
Test Meth. for Drycleaning Durability of Applied lmproved Std.

operated Chain Hoists for Ratchet and Pawl, and Load Brake wound (Materials, Specs. Terminology, Handbook of Spring ood and Lag Screws; Light Metal Plate Connected Wood Truss assay of Bacterial Alpha-Amylase Enzymes Used in Textile rol Chemical Ethyl Mhydroxycarbanilate Carbanilate (Ester) Std. Malleable lron 2) Std. for Steel ts (/ Std. Performance Requirements for Permanent Labels, Std. Pract. for Contemporary Bearing Wall Contemporary Bearing Wall Rec. Std.

Rec. Louver Rec. Std. Rec. Std. Steel Door Frame
Construction gs (Tech. Notes) (1966) ing) (1974 dip Galvanized Structural Steel Products and Procedure for ls (1973) ught Nickel Rich, Chromium Bearing Alloys romism Colorfastness to Light (1972) Ansi/ in Refined Phenol by Cloud Point Dep/ in Evaluation of Insulation Systems (1973/ ) During Dielectric Tests (1973) ate Meth. (1970) ANSI L14.125 on Galvanized Steel Prior to Conversion C/

el Surfaces (Coil Coating) (1974) 73)

ansi $\mathrm{C} 33.70$

Safety Sid for Intrusion

Taste Panel for Food Flavor ermal Analysis, Thermogravimetry, Evolved Gas Analysis and Meth. of Test. for Leaks Using the Mass Spectrometer Leak Meth. of Test for Leaks Using the Mass Spectrometer Leak Std. Guide for Selection of Neutron Activation Meth. of Test. for Leaks Using the Mass Spectrometer Leak for Leaks Using the Mass Spectrometer Leak Detector in the Devices with a Helium Mass Spectrometer Leak uilding Code: Light, Ventilation, Sanitation, Fire Warning Uniform Building Code Std. for Smoke

Std Test Procedure for Semiconductor Radiation r Amplifiers and Preamplifiers for Semiconductor Radiation ; Key Control Systems; Door Holders, Stops, Smoke and Heat Std. Meth. of Test for Std. Rec. Pract. for

Meth. of Test for Synthetic Anionic Active Ingredient in Meth. of Chemical Analysis of Soaps Containing Synthetic
Design, Construction, and Operation of Class Hi (High I Design, Construction, and Operation of Solvent Extracti Design, Construction, Materials, and Installation of $\mathrm{Po}$ Design, Construction, Materials, Size, and lnstallation Design, Installation and Performance of Sprinkler lrrig Design, Installation, and Operation of Subsurface Safet Design, Installation, Operation, and Maintenance of $\operatorname{lmp}$ Design, Layout, Construction and Maintenance of Terrace Design, Manufacture and Test of Aluminum Irrigation Tub Design, Manufacture, Inspection and Assembly Manual for Design, Materials, Construction and Installation of Per Design, Span Allowances, Storage, Finishes and Nails an Designating and Measuring Apertures and Related Quantit Designation for Fertilizer Hoppers and Containers (1972 Designation of Apertures of Photographic Lenses (1973) Designation of High Intensity Discharge Lamps (1972) Designation of Mercury Lamps: Physical and Electrical C Designation of Units in Multiple Unit Semiconductor Dey Designation Systems for Aluminum (1972)

Designation Systems for Aluminum (1972)

Designation (Identification System) of Modular Grid Coo Designations and Their Definitions (1974)

Designations for Coal Tar or Asphalt Coatings (1972)

Designations for Copper and Copper Alloys (Wrought Prod Designations for Rotation and Discharge of Centrifugal Designations (Code) in Metallic Systems Based on the Co Designations, Areas of Use, Maintenance and Operation $\mathrm{O}$

Designed for Operation with Tractors Having at Least 20

(Designed for Residential Use) (1962)

(Designed for Use as a Plaster Base) (1973)

Designed to Open Automatically in Case of Fire (1974)

Designed to Streamiline Supply Transactions Within the a

Designing a High Current Arc Erosion Test for Electrica

Designing Docking Facilities for Motor Vehicles (1971)

Designing for Tire Clearances (1974)

I Dime

Designs and Finishes on Textiles and Other Materials (1
Designs for Pressure Treated Timber Crib Walls (1969)

Designs (1971)

Std. Spec. for Manu

/lft Bolts, and W

Design: Compression, Extension, Tors

Desizing (1973) ANSI L14.147

Test Meth. for

for Common Name

Detachable Link Chain and Attachments (1971)

Detachable Link Chains, Attachments, and Sprockets (197

Detachable Tags, and Certification of Fabrics or Produc

Detailing Reinforcing Materials (1970)

Detailing (Tech. Notes) (I968)

Detailing (Tech. Notes) (1968)

Details for Dutch Doors (1972)

Details for Std. Steel Doors (1972)

Details for Steel Doors and Frames (1972)

Details (I972)

Details, 6 In. Brick and Tile Walls for 1 Story Buildin

Detecting Chromate Films on Galvanized Sheet (Coil Coat

Detecting Embrittlement (1972) ANSI G8.I3

Detecting Glycol Base Antifreeze in Used Lubricating Oi

Detecting Susceptibility to Intergranular Attack in Wro

Detection and Assessment of Colored Textiles to Photoch

Detection and Estimation of Water Insoluble Impurities

Detection and Measurement of Discharge (Corona) Pulses

Detection and Measurement of Partial Discharges (Corona

Detection Finder, Radio Telephone) (1973)

Detection of Antibacterial Activity of Fabrics: Agar Pl

Detection of Hexavalent Chromium Containing Treatments

Detection of Mill Chemical Treatments on Galvanized Ste

Detection of Rodent Excreta, Insects, Webbing, etc. (Ce

Detection System (Grinnell Protomatic Release F100) (19

Detection Thermostats (1974) ANSI Z220.1

Detection Units for Burglary Protection Systems (1969)

Detection (Cereal Chemistry) (1968)

Detection (1973)

/ Re porting D

Detector in the Inside Oui Test. Mode (1973)

Differe

Detector Materials (1973)

Detector or Residual Gas Analyzer in the Tracer Probe M Detector Probe Mode (1973)

Detector (1972T)

Detector (1973)

/Ling Construction Under the Uniform B

Detectors for Fire Protective Signalling Systems (1973)

Detectors for lonizing Radiation (1969) ANSI N42.]

Detectors (1969) ANSI N42.2 Std. Test Procedure Fo

Detectors; Electromagnetic Holders; Sliding Door Equipm

Detergent Resistance of Floor Polish Films (1973)

Detergent Resistance of Organic Finishes (1973)

Detergents by Cationic Titration Procedure (1974)

Detergents (1973)

ANSl

FMS

$A B Y C$

ABYC

ASAE

API

ASAE

ASAE

AGMA

$A B Y C$

CRA

ANSI

ASAE

ANSI

ANS

ANSI

ElA

ANSI

SAE

ANSI

TRA

NAPC

CDA

AMCA

NFPA

SAE

USC

Std. for ICBO

FMS

ATAA

AATM

SAE

TRA

AATCC

$A$ WPI

HMI

SMI

ICBO

AATC

ANSI

ANSI

ANSI

CRSI

BlA

BIA

STDI

STDI

STD

STDI

BlA

NCCA

ASTM

ASTM

AATCC

ASTM

ASTM

IEEE

ABYC

AATCC

NCCA

$A A C$

FMS

UL

UL

AACCH

S2.15

$7-30 \mathrm{~N}$

P6

R264.I

RP14B

RP.05-72

R268.1

S 263.2

370.01

H24

3 A 5

PH3. 29

S281.1

PH3.33

C78.380

C78. 1323

RS321B

.

$\mathrm{J} 993$

A62.8

XIII

BUL

$108 / 2$

2406

505

J232

CS243

UBCS 47.4

4430

200

B576

J699 A

$1-13$

86
$* 4$

300

$* 3$ 
Std. Definitions of Terms Relating to Soaps and Other Detergents (1974)

ssion Class (S Paper) (1973)

Uniform Building Code Std. for Laboratory Std. for Sodium c Equipment on Boats (Audio Device, Automatic Pilot, Depth or Backed Aluminum Alloy Panels

luminized Steel (Coil) (1974)

, and Exhibition Charges) (1974) Spec. for Coating Weight Spec. for Coating Weight Uniform Pract. for Fee Official Meth. of $\mathrm{Ph}$ Meth. for Premix (Cereal Chemistry) (1971) Iey, Other Grains, A/ n Feeds (Cereal Chemistry/ Meth. of Analysis of Falling Number Modified Meth. of Quantitative ive Braking System of Vehicles Equipped

ge Batteries for Motive Power Service (1974)

Rec. Pract. for Meth. of Analysis for Correction Factors for Gasometric Infrared Spectrophotometry/ 1970) ANSI N117

1973) Std. Meth. for Quantitiative Std. Meth. for Radiochemical Std. Meth. of Test for oil/ Spec. for Conducting a Solvent Hand Rub Test for the solubles in Wood (1973)

8 , Whole or G/ Std. Meth. for Whole Or/

Nitrogen Solubility Index (NSI): Meth. for Protein Dispersibility Index (PDI): Meth. for Test Meth. for Colorfastness to Light: graphy (Cereal Chemistry) (1973) (Coil Coating) (1974) Meth. for Test Meth. for atch Tape Test After Reverse Impacting (C/ Test Meth. for
r Fuels (1973) ric by the Sealed Jar Meth. (1972) ANSI L/ Test Meth. for ric Steam Meth. (1972) ANSI L14.179 t Meth. (1972T) and Hydroxypropyl Starch Ether/ 73)

Test Meth. for

Rec. Pract. for Flexibility Std. Analytical Meth. for Std. Meth. of Test for Std. Meth. for Std. Meth. of Flexure Test. of Glass and Glass Ceramics Std. for istry) (1962) ubstrate (Coil, Instrumentation/ atinized When Heated in a Boil/ bstantially Insoluble in Water/ (Cereal Chemistry) (1962)

lvanized and Electrogalvanized Steel (Coil) (1) il) (1974) ugar Obtained from the Corn We/ n Water (1972)

Std. Analytical Meth. for Std. Rec. Pract. for $\operatorname{lm}$ (Coil Coating) (1974) Std. Meth. of Rapid Pressure Std. Meth. of Sampling and Test. Ferroalloys for Std. Meth. for Continuou minals, and Other Terminations (Soft Solde/ Reverberation Rooms (Acoustics)/ ustics Design and Construction) /

s, and Icings (Cereal Chemistry) (1962)

Test Std. for Std. for Meth. for the
Std. Classification for Meth. for premixes for Control of Cecal and Intestinal C/ Meth. for ct. for Examination of Fuel Element Cladding Including the nts in a Polished Specimen

Wood Products During the / mineral Fiber Blanket Type Buil/ nd lsotopic Abundances (1970) Ans/ si $\mathrm{N} 116$

r) (1973)

of Coal (1972)

Materials (Paper) at High Te/ Std. Meth. for Microscopical Std. Rec. Pract. for Infrared Std. Rec. Pract. for the Std. Meth. of Test for Std. Meth. for Colorimetric Test Meth. for Std. Meth. for Microscopical Std. Meth. for Gravimetric try) $(1962)$ ry) (1962) Volumetric Copper Reduction Meth. for Sugar Meth. for us Chemical Reactions (Incandescence, Fire, Explosion, and Std. Spec, for for Consumption of Potassium Permanganate by lmpurities in Std. Meth. of Test for

Fire Code: Cryogenic Fluids (Air, Argon, Carbon Monoxide,

Meth. of Test for Warpage of Refractory Brick and Tile o $s$ is (1962)

Boats (Audio Device, Automatic Pilot, Depth Determination m Covers $(/$ Std. for Electrical Sheet Steel Outlet Boxes, Std. Spec. for Simple Can Puncturin

Std. List of Values to Be Used in Semiconductor panic (Emergency) Hardware (Outward Opening Door Releasing

Pract, and Std. for Electronic Equipment on Boats (Audio

on Device and Fish Finder, Loran or Other Line of Position of (1962) A/ Std. for Tools, Crimping, Solderless Wiring

y Tests for Flammability of Plastic Materials for Parts in trial Electrical Power Control Apparatus-Control Circuit ervice Performance Requirements for Motor Vehicle Lighting Std. for Safety for Elevator Door Locking

umbering of Electrodes in Multiple Electrode Semiconducto uirements for Motor Vehicle (Recreational Type) Connecting
Determination and Measurement of Airborne Sound Transmi Determination by the Uranyl Zinc Acetate Meth. Pulp and Determination Device and Fish Finder, Loran or Other Li Determination for Chromate Conversion Coatings on Aged Determination for Conversion Coating on Aged or Baked a Determination for Division Services (Individuals, Clubs Determination in Wine (Enology) (1972)

Determination of Azodicarbonamide Content in Commercial Determination of Both Meal and Flour of Wheat, Rye, Bar Determination of Both Nitrophenide and Arsanilic Acid I Determination of Brake Fluid Temperature in the Automot Determination of Capacity of Lead Acid Industrial Stora Determination of Carbon Dioxide (Cereal Chemistry) (196 Determination of Cellulose Nitrate in Alkyd Lacquers by Determination of Cesium-137 in Nuclear Fuel Solutions ( Determination of Cobalt in Paint Driers by EDTA Meth. ( Determination of Deg. of Cure of a Backed Paint Film (C Determination of Dichloromethane and Alcohol-Benzene Determination of Dispersible Nitrogen in Ground Soybean Determination of Dispersible Protein in Ground Soybeans Determination of Fastness Above L.7 (1971) ANSI L14.264 Determination of Fatty Acid Composition by Gas Chromato Determination of Film Adhesion by Cross Hatch Tape Test Determination of Film Adhesion on All Metals by Cross $\mathrm{H}$ Determination of Fission Zirconium in Irradiated Nuclea Determination of Formaldehyde Odor in Resin Treated Fab Determination of Formaldehyde Odor in Resin Treated Fab Determination of Hot Melt Adhesives by Mandrel Bend Tes Determination of Hydroxy alkoxyl Groups in Hydroxyethyl Determination of Lead in Paint Driers by EDTA Meth. (19 Determination of Low Concentration of Lead in Paint (19

(Determination of Modulus of Rupture) (1972)

Determination of Moisture Content of Forages (1972)

Determination of Nitrosal in Poultry Feeds (Cereal Chem Determination of Nonconductive Coatings on Nonferrous S Determination of $\mathrm{pH}$ (Paste) of Corn Starch Which Is Gel Determination of $\mathrm{pH}$ (Slurry) of Corn Starch Which ls Su Determination of Phenothiazine in Feeds and Feedstuffs Determination of Phosphate Coating Weight on Hot Dip Ga Determination of Phosphate Coating Weights on Steel (Co Determination of Phosphorus in Corn Starch, Syrup and S Determination of Precision of Meth. of Committee D-19 O

Determination of Pressurized Products (1972)

Determination of Relative Pencil Hardness of a Paint Fi Determination of Size (1970) ANSI G85.1

Determination of Sodium in Water by lon Electrode (1973

Determination of Solderability of Solid Lead Wires, Ter

Determination of Sound Power Levels of Small Sources in

Determination of Sound Transmission Class (Building Aco

Determination of Specific Volume of Creams, Cake Batter

Determination of Sulfaquinoxaline in Poultry Feeds and

Determination of the Mechanical Properties (1972) ANSI

Determination of the Reflectance of the Organic Compone

Determination of the Temperature of Applied Coatings on

Determination of the Thermal Resistance of Low Density

Determination of Uranium and Plutonium Concentrations a

Determination of Uranium in Aqueous Solutions (1969) an

Determination of Useful Fiber in Bagasse (Pulp and Pape

Determination of Volume Percent of Physicdl Components

Determination of Water Vapor Transmission Rate of Sheet

Determination: Schoorl's Reduction Table (Cereal Chemis

Determination of Arsanilic Acid in Feeds (Cereal Chemist

Deterrents on Textiles (1971) ANSI L14.65

Detonation Protection) (1973)

Deuterium Oxide (1968) A NSl N153

Deuterium Oxide (1968) A NSI N154

Deuterium Oxide (1972)

Deuterium, Fluorine, Helium, Hydrogen, Krypton, Methane

Deviation from a Plane Surface (1972)

Deviations for Specific Meth. of Cereal Chemistry Analy

Device and Fish Finder, Loran or Other Line of Position

Device Boxes, Covers and Box Supports, and Cast Aluminu

Device for Use in Aerosol Laboratories (1972)

Device Specs, and Registration Formats (1974)

Device) (1973)

Device, Automatic Pilot, Depth Determination Device and

Device, Positive Index (1973)

Device, Radar, Radio Detection Finder, Radio Telephone)

Devices-Recommended Procedures for User Certification

Devices and Appliances (1973)

Devices and Assemblies (1973)

Devices and Components (1972)

Devices and Contacts (1973)

Devices and Designation of Units in Multiple Unit Semic

Devices and Towing Meth. (Coupling, Hitch, Safety Chain

ASTM

ICBO

TAPPI

ABYC

NCCA

NCCA

PSA

ASE

$\mathrm{AACCH}$

AACCH $56.81 \mathrm{~B}$

$\mathrm{AACCH} \quad 18 \cdot 26$

SAE

J291

NEMA

AACC

ASTM

ASTM

ASTM

NCCA

TAPPl

AACCH

$\mathrm{AACCH}$

AATCC

AACCH

NCCA

NCCA

ASTM

AATCC

AATCC

AST

CR

ASTM

ASTM

ASTM

ASAE

AACCH

NCCA

CR

CR

AACCH

NCCA

NCC

CR

ASTM

ASTM

NCCA

ASTM

ASTM

EIA

ANSI

ASTM

AACCH 7210

$A$ C

ASTM

ASTM

ASTM

ASTM

ASTM

ASTM

TAPPI

ASTM

TAPPI

AACCH

$\mathrm{AACCH}$

AATCC

FMS

ASTM

ASTM

ASTM

ICBO

Std. ASTM

$\mathrm{AACCH}$

$A B Y C$

NEMA

ASTM

EIA

UL

NSA

$\mathrm{ABYC}$

EIA Std. for Indus 
; Reporting and False Alarm: Use of Equipment, Appliances, Std. Rec. Pract. for Determining Hermeticity of Electron std. Rec. Pract. for Determining Hermeticity of Electronic Safety Std. for Power Operated Dispensing Std. for Relief Valves and Automatic Gas Shutoff Pract. and Std. Covering Sewage Holding and/or Treatment Spec. for Safety Controls and Signaling d. for Efficiency Test. of Air Cleaning Systems Containing Sid. for Quick Disconnect tic Material for Use in Housings of Motor Vehicle Lighting 1972 T)

Basic Criterial for Evaluation of Special Processes or

I Exhaust Ventilation Syste/

Rec. Pract. for Air Cleaning Std. for Safety for Antitheft Alarms and Std. for Terminology for Pressure Relief Std. for Exit

Std. Groove Dimensions for Fluid Power Exclusion Spec. for Cargo Unit Load Std. for Certified Ratings Program Air Moving afety Std. for Marine Special Purpose Water Safety Buoyant Std. Test Code for Air Moving es for Clinical Research Protocol for Dental Materials and Std. Spec. for Brazing Filler Metals for Electron Std. for Specific Purpose Wiring Safety Std. for Relocking

Pract. for Semiautomatic Headlamp (Light) Beam Switching for Boron Deoxidized Copper in Wrought Forms for Electron nts, Terminology, and Test Procedure for Neutral Grounding or Measurements Part 11-Characterization of Large Signal es and Designation of Units in Multiple Unit Semiconductor Safety Std. for Fused Power Circuit trical Power Control Apparatus-Switching and Controlling ydrolytic Stability of Plastic Encapsulants for Electronic Std. for Graphic Symbols for Logic Diagrams (Two-State as Tree Equipment; Flow Control Chokes; and Water Sampling Stationary Diesel and Gas Engine Gauge Borads, Protective $\mathrm{s}$ for Film in Rolls for Phototypesetting or Photocomposing re: Hands of Doors; Hinges; Locks; Knobs and Handles; Exit suctio/ Std. for Respiratory Therapy Equipment (Breathing ated Type for $1 / \quad$ Safety Std. for Heat Actuated Fire Alarm on of Large Signal D/ Trial Use Std. Guide on Solid State $\mathrm{e}$ in Sugar Mixtures (Maltose, Sucrose, Mannose, Galactose, Std. Analytical Meth. for Determining Borax Content in Std. Analytical Meth. for Determining Solubles Content in (1962) nalytical Meth. for Determining Reducing Sugars Content in Meth. of Analysis for Ash in Starch, for Determining Specific Rotation Content of Corn Syrup, tes $(1967)$ fined) (1972) Std. Analytical Meth. for Determining
Std. Analytical Meth. for Determining Std. Analytical Meth. for Determining ermining Heavy Metals Content of of Corn Syrup Unmixed and ermining Refractive lndex Content of Corn Syrup, Sugar and ecific Rotation Content of Corn Sugar (Crude and Refined),

Test Meths. for Interior Panel Coatings and Overlays on Test Meth. for Exterior Coatings and Overlays on Std. Spec. for passenger Automobile Equipped with Full Scale Tires in the (/ Std. Graphic Symbols for Electrical Wiring and Layout i Y $32.14 \quad$ Std. for Graphic Symbols for Logic or Mechanical and Acoustical Elements as Used in Schematic Std. Large Rivets (1/2 ln. Nomina Std Small Solid Rivets (7/16 In. Nominal r Corrosive or High Temperat/ Std. Spec. for Welded Large tion (1972) ANSI L14.143 Std. Meth. of Test for Std. Spec. for Asbestos Cement Nonpressure Small Std. for Sleeve Bushing, Press Fit, Undersize Inside Std. for Bushing-Flanged, Press Fit-Undersize Inside Std. for High Speed Steel End Mills, .125 Thru 3.000 r Polybutylene (PB) Plastic Pipe (SDR.PR) Based on Outside ne (PEO) Plastic Pipe (SDR.PR) Based on Controlled Outside ene (PE) Plastic Pipe (SDR.PR) Based on Controlled Outside ne (PE) Plastic Pipe, Schedules 40 and 80 Based on Outside el Fuel Injection Nozzle and Holder Assembly ( $17 \mathrm{Mm}$ Nominal etal End Dimensions (6 Ounce Fibre Cítrus Cans $202(52 \mathrm{Mm}$ ) Loose Metal End Dimensions of Composite Cans $(211(66 \mathrm{Mm})$ Std. Wheel

Std. Test. Proc, for Measuring Inside Qualitative Meth. of Analsis of Benzoyl Peroxide with Std. for Checking the Size of Std. Test Meth. for Bulk Density of Std and Nomenclature for Rec. Pract. for Fuel Pump Mountings for Gypsum Concrete and Precast Slabs, and Poured Gypsum Roof y Materials (Calcined Bauxite, Calcine, Chrome Ore, Clays,
ina (With Subsequent Acid Hydrolysis) (Cereal Chemistry)
Devices and Vecant Buildings) (1973)

Devices by a Bubble Test (1972)

Devices by Dye Penetration (1972)

Devices for Flammable Liquids (1974) ANSI Z216.1

Devices for Hot Water Supply Systems (1972)

Devices for Marine Toilet Waste (Boats) (1972)

Devices for Printing Presses (1972)

Devices for Removal of Particles (1972)

Devices for Use with Gas Fuel (1971)

Devices Including Reflex Reflectors (1973)

Devices Used in Treating Wastewater (1970)

Devices with a Helium Mass Spectrometer Leak Detector (
Devices (Air Filters and Dust Collectors) for Industria

Devices (Burglary Protection) (1974)

Devices (Container and Equipment Protection) (1972)

Devices (Doors) (1972)

Devices (In. Series) (1972) ANSI B93.35

Devices (Materials Transport and Handling) (1973)

Devices (Performance) (1970)

Devices (Vest, Jacket, Horseshoe and Ring Buoy) (1973)

Devices (1967)

Devices (1971)

Devices (1972)

Devices (1972)

Devices (1972)

Devices (1972)

Devices (1972)

Devices (1972)

Devices (1972)

Devices (1972)

Devices (1973)

Devices (1973)

Devices (1973)

Devices) (Electron
Devices) (1974)

Devices, and Instrumentation (1972)
Devices, or Both (Photography) (1972)

Devices, Pulls, Push and Kick Plates; Bolts; Closers; H

Devices, Single and Multiple Station, Mechanically Oper

Devices: Varactor Measurements Part II-Characterizati

Dextrans, and Starch (Cereal Chemistry) (1968)

Dextrin and Corn Starch Products (1971)

Dextrin (1970)

Dextrin $(1970)$

Dextrin, and Other Modified Products (Cereal Chemistry)

Dextrines and Other Modified Starch Products (1955)

Dextrose and Starch Hydrolyzates (1971)

Dextrose Content of Corn Syrup and All Starch Hydrolyza

Dextrose Equivalent Content of Corn Sugar (Crude and Re

Dextrose Equivalent Content of Corn Syrup and All Starc

Dextrose (1956)

Std. Analytical Meth. for Det
Std. Analytical Meth. for Det

Dextrose (1956)

Dextrose, Syrup and Starch Hydrolyzates (1971)

DFPA Grade Trademarked Plywood (1967)

DFPA Grade Trademarked Plywood (1971)

Diacetone Alcohol (1973)

Diagonal Braking Mode (1973)

/ Paved Surfaces Using a

Diagrams Used in Architecture and Building Construction

Diagrams (Two-State Devices) (Electronics) (1973) Ans

Diagrams (1972) Sid. Symbols and Definitions $F$

Diameter and Larger) (1972)

Diameter and Smaller) (1972)

Diameter Austenitic Chromium Nickel Alloy Steel Pipe Fo

Diameter of Wool and Other Animal Fibers by Microprojec

Diameter Sewer Pipe (1973) ANS1 A 165.4

Diameter (1973)

Diameter (1973)

Diameter (1973)

Diameter (1973)

Diameter (1973)

Diameter (1974)

Diameter (1974)

Diameter) (1971)

Diameter) (1973)

Diameter), Citrus Style (1974)

Diameters and Outlet Areas for Centrifugal Fans (1966)

Diaminophenylamine (Cereal Chemistry) (1967)

Diamond Abrasive Grain (1971)

Diamond Abrasive Grains (1973)

Diamond Core Drill Equipment (1973) ANSI B104.1

Diaphragm Type Pumps (1971)

Diaphragms (1973)

Diaspore, Ganister Quarzite, Grog, Kyanite, Magnesite,

Diastase Meth. of Analysis of Starch in Flour and Semol

iented Polyethyle ASTM

Std. Spec. for Polyethyle ASTM

UFC $\approx 2 \mathrm{ART} 27$

ASTM

ASTM

UL

Rec ABYC

ANSI

St ANSI

ANSI

NSF

ASTM

ACGIH

UL

ANSI

ANSI

NFLDP

NSA

AMC

UL

AMCA

ADA

ASTM

NEM

Rec SAE

Std. Spec. ASTM

d. Requireme IEEE

IEEE

EIA

ANSI

ANSI

IEEE

API

DEMA

ANS!

NBHA

NSF

UL

IEEE

F98

F97

87

A8

B65.1

N101.1

Z21.41

J29

C.9

F134

-1. 1

1037

B95.1

A I56.3

(3.19.7

3610

1123

210

$* 1$

F100

WD5

140

F107

32

351

RS321B

977

C 19.5

F74.

91

*1.17

PHI.44

8

48

351

AACCH 80.10

CR D.10

D. 52

$\begin{array}{ll}A A C C H & 08.17\end{array}$

IN CR

B- 8

E.58

E. 24

F.22

E.26

E-30

E-54

F. 52

*5

APA $* 5$

ASTM D2627

ASTM E503

ANSI Y 32.9

IEEE

91

Y 32.18

ANSI B18.1.

ANSI B18.1.

ASTM A 409

ASTM D2130

ASTM

NSA

NSA

537

538

538
986

D3000

Sid Spec. Fo ASTM

D3287

D 3035

D2447

J265

E202.1 
Meth. of Thiosulfate-Maltose

Pressuremeter Meth. of Analysis of

Volumetric Meth. of Analysis of

try) (1962)

Meth. of Analysis of

Meth. of Analysis of

Meth. of Analysis of Std. for

cis $\cdot \mathrm{N} \cdot((1,1,2,2$ tetrachloroethyl) Thio)-4-Cyclohexene-1,2.

Std. Acceptable Concentrations of

(1973) Std. Meth. for Determination of or Common Name for the Pest Control Chemical Methyl 5(2,4.

Meth. for Preparation of Potassium

)

e, Step Relief (1972)

Std. Spec, for Aluminum Alloy Std. for td. Spec. for Aluminum Alloy Hardeners Used in Making Zinc

safety Requirements for the Construction, Care, and Use of Std. Spec. for Lead and Tin Alloy Sid. Spec. for Zinc Alloys in Ingot Form for gnesium Alloys in Ingot Form for Sand, Permanent Mold, and Std. Spec. for Brass

Std. Spec. for Zinc Alloy

Std. Spec. for Magnesium Alloy Std. Spec. for Aluminum Alloy luminum Alloys in Ingot Form for Sand, Permanent Mold, and ent (1972)

Std, Recommendations for Std. Spec, for Copper and Its Alloy Std. Recommendations for Die Cavity and Cutoff Std. Tooling Nomenclature for Solid

Gases at Commercial Power Freque oleum Origin Using VDE Electrodes/ oleum Origin Under Impulse Condit/ Std. Meth. of Test for Std. Meth. of Test for Std. Meth. of Test for ons (1973)

Std. for Paper, Paper/Film, Film

block Mica and Mica Films Suitable for Use in Fixed Mica. cellular Plastics Used for Electr/ or of Solid Ceramic Dielectrics A/

Std. Meth. of Test for Std. Meth. of Test for of Test for Alternating Current Loss Characteristics and erials at Micr/ Std. Test Meth. for Complex Permittivity s, Beans, Peas, Lentils, Rice, and Other Commodities For/ es with Effect of Shield Losses for Single Conductor Solid

uit Breaker External Insulation Rat/

Std. for Schedule of tion and Measurement of Partial Discharges (Corona) During
Std. for Solid and Semi Solid

on Data on High Frequency Heating Equipment (Induction and ant (Permittivity) and Dissipation Factor of Solid Ceramic td. Spec. for Wrought Alloy Tool Steel Products (Including 972)

equirements for Duplex and Single Face Flat Thread Rolling Std. Pract. for Low and Medium Speed Stationar Std. Pract. for Low and Medium Speed Stationary Std. Pract. for Low and Medium Speed Stationary Std. Pract. for Low and Medium Speed Stationary Std. Pract. for Low and Medium Speed Stationary Std. Pract. for Low and Medium Speed Stationary

general /

and lns/

nt $(1972)$

nd Gener/ Std. Pract. for Low and Medium Speed Stationary Std. Pract. for Low and Medium Speed Stationary Std. Pract, for Low and Medium Speed Stationary Std. Pract. for Low and Medium Speed Stationary Std. Pract. for Low and Medium Speed Stationary Std. Pract. for Low and Medium Speed Stationary Std. Pract. for Low and Medium Speed Station ary ems (197) Std. Pract. for Low and Medium Speed Stationary Std. Pract. for Stationary ation (1/ Std. Pract. for Low and Medium Speed Stationary Std. Pract. for Low and Medium Speed Stationary Std. Pract. for Low and Medium Speed Stationary Std. Pract. for Low and Medium Speed Stationary Std. Practices for Low and Medium Speed Stationary Std. Pract. Definitions for Stationar std. Pract. for Scavenging and Supercharging of Stationary gine-Generator Units for Low and Medium Speed Stationary

ids and Detailed Spec. for Low and Medium Speed Stationary installation of Air Cooled Marine Engines and Auxiliarie nominal Diameter) (1971) 3 for Nuclear Power Generat/ Rec. Pract. for ne Engin/

Trial Use Std.: Criteria for Stds, and Pract. for Design and Construction of Std. for Engine Rating Code Std. for Engine Test Code-Spark Ignition and

lor of Low Colored Clear Liquids Using the Hunterlab Color ation Report) (1969)

and Textile Fabric) (1972) ANSI

Std. Meth of Test for Std. on Television: Measurement of Structural Clay Structural Clay
Diastatic Activity in Flour) Conversion (Cereal Chemist

Diastatic Activity of Doughs (Cereal Chemistry) (1962)

Diastatic Activity of Doughs (Cereal Chemistry) (1962)

Diastatic Activity of Flour and Semolina (Cereal Chemis

Diastatic Activity of Flour with the Amylograph (Cerea]

Diastatic Activity of Malt Flour (Cereal Chemistry) (19

Diatomite Type Filters for Swimming Pools (1971)

(Est Control Chemical

Dichlorodifluoromethane (Fluoroearton 12) (1974)

Dichloromethane and Alcohol-Benzene Solubles in Wood

Dichlorophenoxy).2-Nitrobenzoate Bif enox (1973)

Dichlozoline (Fungicide) (1971)

Dichromate Solution (1962)

Dictionary of Terms Used in the Safety Profession (1973

Die and Hand Forgings (1973) ANSI H38.8

Die Buttons, Variable, Press Fit, Headless and Head Typ

Die Casting Alloys (1965) ANSI H38.23

Die Casting Machine Stds. (1972)

Die Casting Machines (1973)

Die Castings (1952) ANSI H48.1

Die Castings (1964) ANSI H47.2

Die Castings (1966) ANSI H45.8

Die Castings (1970) ANSI H49.1

Die Castings (1971) ANSI H47.1

Die Castings (1972)

Die Castings (1972) ANSI H38.22

Die Castings (1973) ANSI H38.1

Die Cavity and Cutoff Die Sizes for Cold Forging Equipm

Die Forgings (Hot Pressed) (1974) ANSI H7.10

Die Sizes for Cold Forging Equipment (1972)

Die Two Blow Cold Headers (1972)

Dielectric Breakdown Voltage and Strength of Insulating

Dielectric Breakdown Voltage of Insulating Oils of Petr

Dielectric Breakdown Voltage of Insulating Oils of Petr

Dielectric Capacitors for Power Semiconductor Applicati

Dielectric Capacitors (1971) ANSI C59.26

Dielectric Constant and Dissipation Factor of Expanded

Dielectric Constant (Permittivity) and Dissipation Fact

Dielectric Constant (Permittivity) of Solid Electrical

Dielectric Constant) of Solid Electrical Insulating Mat

Dielectric Meter Meth. of Analysis of Moisture in Grain

Dielectric Power Cable $15 \mathrm{kV}$ Through $35 \mathrm{kV}$ (Copper and

Dielectric Properties of Grain and Seeds (1972)

Dielectric Test Values for Outdoor AC High Voltage Circ

Dielectric Tests (1973)

Dielectric Transmission Lines (1972)

Dielectric) (1973)

Freguen 10 MHz and Temperatur

Dies and Fixtures) (1973)

Dies (1973)

Diesel and Gas Engine Air Intake and Exhaust Systems (1

Diesel and Gas Engine Assembly and Equipment (1972)

Diesel and Gas Engine Cooling Water Systems (1972)

Diesel and Gas Engine Erection and lnstallation (1972

Diesel and Gas Engine Factory and Field Testing (1972)

Diesel and Gas Engine Fuel Oil Characteristics (1972)

Diesel and Gas Engine Gaseous Fuel Characteristics and

Diesel and Gas Engine Gauge Borads, Protective Devices,

Diesel and Gas Engine Generators and Electrical Equipme

Diesel and Gas Engine Lubricating Oil Characteristics a

Diesel and Gas Engine Lubricating Oil Systems (1972)

Diesel and Gas Engine Operation and Maintenance (1972)

Diesel and Gas Engine Power Plant Buildings (1972)

Diesel and Gas Engine Power Plant Fuel Oil and Gas Syst

Diesel and Gas Engine Ratings and Performance (1972)

Diesel and Gas Engine Speed Governing and Parallel Oper

Diesel and Gas Engine Starting Systems (1972)

Diesel and Gas Engine Vibration (1972)

Diesel and Gas Engine Waste Heat Recovery Systems (1972

Diesel and Gas Engines (1972)

Diesel and Gas Engines (1972)

Diesel and Gas Engines (1972)

Diesel and Gas Engines (1972)

Diesel and Gas Engines (1972)

(Diesel and Gasoline) (Boats) (1972)

Diesel Injection Nozzle and Holder Assembly (17Mm

Diesel Generator Units Applied as Standby Power Supplie

Diesel (Compression Ignited) Inboard Liquid Cooled Mari

Diesel (1971)

Diesel (1971)

Difference Meter (1972T)

Differential and Manual Transmission Lubror Measurem

Differential Dyeing Behavior of Cotton (Raw Fiber, Yarn

Differential Gain and Phase (1960)

Differential Movement (Cause and Effect) (Tech. Notes)

Differential Movement (Expansion Joints) (Tech. Notes)

AACCH 22.17

AACCH 22.11

AACCH 22.14

AACCH 22.15

AACCH 22.10

AACCH 22.16

NSF

ANSI

ANSI

TAPPI

ID. F ANSI

ANSI

AACCH

ASSFE

ASTM

ANSI

ASTM

SDCE

Sid. for ANSI

ASTM

ASTM

ASTM

ASTM

ASTM

ASTM

ASTM

IFI

ASTM

IFI

IFI

ASTM

ASTM

ASTM

EIA

or Natural ASTM

ASTM

ASTM

ASTM

$\mathrm{AACCH}$

IPCEA

ASAE

ANSI

IEEE

ElA

EMS

ASTM

ASTM

DEMA

DEMA

DEMA

DEMA

DEMA

DEMA

DEMA

DEMA

DEMA

DEMA

DEMA

DEMA

DEMA

DEMA

DEMA

DEMA

DEMA

DEMA

DEMA

DEMA

DEMA

DEMA

DEMA

DEMA

$A B Y C$

SAE

IEEE

ABYC

$S A E$

SAE

K62.146

237.38

T5

K62.138

62.102

0.45

B247

B 94.43

B327

B152.1

B 102

B240

B93

B176

B86

B94
B85

B179

121

B283 
Structural Clay Std. Meth. for Calibration of Temperature Scale for
d Gas Analysis / Std. Rec. Pract. for Reporting Data from nbs HB111 Std. for Radiation Safety for X-Ray Meth. for Measuring Brightness of Pulp Panels (1969) ining $\mathrm{T} /$ Std. Test Meth. for ting Panels, Skylights, Monitors and Sawtooth Roofs, Light Std. Meth. of Test for Thickness of ate Surface Pho/ Tent. Meth. of Test for Minority Carrier in of Organic Finishes in Contact/ Std. Meth. of Test for eth. (1972) ANSI K90.12 Std. Meth. of Test for Thermal dent Hairs in Soy Flour (Cereal Chemistry) (1962/ Fatting

$s$ Turbine Engine Steady State Performance Presentation for Rec. Pract. for Hardware Test. of cription Data (Tooling, Printed Wiring Boards) in Numeric lids (Cereal Chemistry) (1962)

Std. Meth. of Test for Delta in Hours Std. Spec. for Photographic Grade Sodium Citrate, for Photographic Grade Hydroquinone $\mathrm{C}_{i ;} \mathrm{H}_{1}(\mathrm{Oh})_{2}$ (Para Tent Test Meth for Critical Deg.) of Margarine Oils, Shortenings, and Other Fats (1/ Rec. Pract. for

Std. Meth. of Measurement of the A.D 4) itaxial Layers of Silicon by Measurement of Stacking Fault in Line Type Sockets (1974) ANSI C83.95

nt Lamps (1972)

8 Preheat Start Bactericidal Lamp (1971)

t8 Preheat Start Bactericidal Lamp (1971)

Preheat Start Bactericidal Lamp (1971)

Std. for

Std.

Std.

Std. $s$ and Papers (1973)

fabrics (Textile) (197!

Std. Meth. for Determining the Sid. Meth. of Test for Relaxation able (Permanent) Press Woven or Knit Text/ Test Meth. for textiles Except Wool (1972) ANSI L14.138,/ Test Meth. for ol Textiles (1972)/ Test Meth. for Relaxation and Felting

d. Eore and Rod Size Combinations, Rod End Configurations, t Thread Rolling Dies (1973)

Std. for Std. for Std. for

tion and Industrial Machinery (1972)

layouts and Ter/ Battery Replacement Data; Group Numbers, cp II (Compact Cassette) for Audio Recording An/ Std. for Sid. for

Std.

for Chemical Compositions, Mechanical Property Limits and
Engineering Drawing and Related Documentation Practices: lled Screw Conveyors (1972)

Std. for Unit Load Sizes for Instrumentation) (1/ St. for Uniform bone Plates (1973)

Std. Rec. Pract. for Std. Spec. for ards (1970) ANSI C83/ Std Spec, for Printed Wiring Board dustrial Fluid Power Cylinders (1972) ANSl B93.29 Std. Rec. for Hitch and Box
d Generators (Fractional and Integral Horsepowe/ Std. for s, Wagons and Blowers (1972)

ic, Wood or Metal) (1971)

otocomposing Devices, or Both (Photography) (19/

luid Power Piston Rings (1972) ANSI B93.36

s) (1972) ANS1 B 93.35

on Rings (1973) ANSI B93.32

1973)

e Manufacturers in De/ ilm (photography) (1973) s (1973)

2)

g Instruments and Miscellaneous Uses (1973)

d) (Photography) (1972)

ion Picture Prints (1972)

mm Motion Picture Film Perforated 1.4 (1971)

Films (SI and U.S. Customary ('nits) (1973)

1972) ANSI C83.9

optics (1972)

amera, Copy and Image Cards) (1972)

Film in Rolls, Including Leaders and Trailers,

motor Vehicles (1971)

Rec. Pract. for Average Vehicle

Std. Spec, and

er $8,(1-4)(1973)$

ft. and $70 \mathrm{~mm} 100 \mathrm{Ft}$. Spools for Recording Instrume/

er 8, 5R. 1667 (1-3-5.7-0) (1973)

figurations, Dimensional Identification Code, and Mounting

Std. on

Std. for

Std.

Std for
Differential Movement (Flexible Anchorage) (Tech. Notes Differential Thermal Analysis (1973)

Differential Thermal Analysis, Thermogravimetry, Evolve Diffraction and Fluorescence Analysis Equipment (1971)

Diffuse lllumination and Zero Deg. Observation) (1972)

Diffuse Light Transmission Factor of Reinforced Plastic

Diffused Layers in Silicon by the Angle Lapping and Sta

Diffusers in Ceilings, Partitions, Exterior Veneer, Awn

Diffusion Coating (1970) ANS1 Z167.24

Diffusion Length in Silicon by Measurement of Steady St Diffusion Stain of Rubber and Contact and Migration Sta Diffusivity of Carbon and Graphite by a Thermal Pulse $M$ Digestion Meth. of Analysis for Insect Fragments and Ro

Digital Computer Programs (1970)

Digital Process Computers (1971)

Digital) Form on Punched Cards, Magnetic Tape and Punch

Digitonin Cholesterol Meth. of Analysis of Dried Egg So DIH) Purity of Nuclear Graphite (1971) ANSI K90.8

Dihydrate $\mathrm{Na}_{3} \mathrm{C}_{1 ;} \mathrm{H}_{5} \mathrm{O}_{7}: 2 \mathrm{H}_{2} \mathrm{O}(1972)$

Dihydroxbenzene, Quinol, Hydroquinol) (1972)

Dilation of Concrete Specimens Subject to Freezing (197

Dilatometric Meth. of Solid Fat Index (50 or Less at 10

Dilution Ventilation in Industrial Exhaust Systems (197

Dimension of Aerosol Valve Dip Tubes (1972)

Dimension (1973)

D. Meth. of Test for Thickness of $E_{p}$

Dimensional and Electrical Characteristic Defining Dual

Dimensional and Electrical Characteristics of Fluoresce

Dimensional and Electrical Characteristics of $15 \mathrm{~W}$ att $\mathrm{T}$

Dimensional and Electrical Characteristics of $30 \mathrm{Watt}$.

Dimensional and Electrical Characteristics of 8 Watt T5

Dimensional Change Characteristics of Photographic Film

Dimensional Change (Shrinkage) of Stabilized Knit Wool

Dimensional Changes in Automatic Home Laundering of Dur

Dimensional Changes in Laundering of Woven and Knitted

Dimensional Changes (Shrinkage) of Woven and Knitted Wo

Dimensional Identification Code, and Mounting Dimension

Dimensional Requirements for Duplex and Single Face Fla

Dimensional Spec. for Sealed Beam Headlamp Units (1972)

Dimensional Spec, for Sealed Lighting Unit for Construc

Dimensional Spec, and Ratings; Assembly Numbers, Cell

Dimensional Std. Coplanar Magnetic Tape Cartridge Type

Dimensional Tolerances for Aluminum Mill Products (1973

Dimensional Tolerances for Industrial Wheels (1965)

Dimensional Tolerances of SAE Wrought Aluminum Alloys(

Dimensioning and Tolerancing (1973)

Dimensioning Std. and Terminology for Permanently Insta

Dimensioning Transport Packages (1972)

Dimensions and Meth. of Test. for Rotary Selector Switc

Dimensions and Tolerances for Screw Holes and Slots in

Dimensions and Tolerances of Plastic Bottles (1972)

Dimensions and Tolerances Single and Two Sided Rigid Bo

Dimensions for Accessories for Cataloged Square Head in

Dimensions for Agricultural (Farm) Grain Wagons (1972)

Dimensions for Alternating and Direct Current Motors an

Std. Dimensions for Compatible Operation of Forage Harvester

Std. Dimensions for Cores for Photographic Film Rolls (Plast

Sor Dimensions for Film in Rolls for Phototypesetting
Std. Dimensions for Flareless Acorn Fitting End (1974)

Sid. Groove Dimensions for Floating Type Metallic and Nonmetallic F

Std. Groove Dimensions for Fluid Power Exclusion Devices (In. Serie

Std. Groove Dimensions for Fluid Power Radial Compression Type Pist

Std. for Dimensions for Front Lens Mounts for Cameras (1972)

Dimensions for Graphic Arts Sheet Films (photography)

Dimensions for Grown Tires in Service for Use by Vehic

Dimensions for Industrial Radiographic Sheet and Roll F

Dimensions for Medical Radiographic Sheet and Roll Film

Dimensions for Monochrome Television Picture Tubes (197

Dimensions for Photographic Films in Rolls for Recordin

Dimensions for Photographic Sound Record on Super $8 \mathrm{M}$

Dimensions for Professional Photographic Sheet and Roll

Std. for Dimensions for Racks, Panels and Associated Equipment (

Std. for Dimensions for Resolution Test Target for Photographic

Std. for Dimensions for Unitized Microfilm Carriers (Aperture, C Dimensions for Unperforated and Perforated Photographic Dimensions for Use in Designing Docking Facilities for Dimensions for Wood Poles (1972)

Std. Dimensions for $16 \mathrm{~mm}$ Motion Picture Film Perforated Sup

Std. Dimensions for $16 \mathrm{~mm} 100 \mathrm{Ft}$., $16 \mathrm{~mm} 200 \mathrm{Ft}$., $35 \mathrm{~mm} 100$

Std. Dimensions for $32 \mathrm{~mm}$ Motion Picture Film, 2R (1974)

td. Dimensions for $32 \mathrm{~mm}$ Motion Picture Film, 4R (1974)

d. Dimensions for $35 \mathrm{~mm}$ Motion Picture Film Perforated Sup

Dimensions for 3/4, 1 and 1 1/8 In. Bore Cataloged Squa

Dimensions of Aerial Film Spools (1972)

Dimensions of Attachment Plugs and Receptacles (1973)
BIA

ASTM

ASTM

ANSI

TAPPI

ASTM

ASTM

ICBO

ASTM

ASTM

ASTM

ASTM

AACC

Ga SAE

ISA

IPC

AACC

ASTM

ANSI

D. Spec. ANS

ASTM

AACCH

ACGIH

ASTM

AATM

EIA

ANSI

ANSI

ANSI

ANSI

ANSI

ASTM

AATCC

AATCC

AATCC

NFLDP

IFI

$S A E$

SAE

BCI

EIA

ANSI

CFTM

SAE

Std ANS

ASAE

ANSI

EIA

ASTM

ASTM

IPC

ASAE

ASAE

ASAE

ANSI

ANSI

NSA

NFLDP

NFLDP

NFLDP

ANSI

ANSI

TRA

ANSI

ANS

EIA

ANSI

ANSI

ANSI

ANSI

ANSI

EIA

ANSI

ANSI

ANSI

$\mathrm{SAE}$

ANSI

ANSI

ANSI

ANSI

ANSI

ANSI

NFLDP

ANSI

ANSI

18B

474

E472

T525SU

D1 494

F110

UBC*3.52

C664

F39l

D925

C714

ARP681B

RP55.1

D.350

2001

C624

PH4.126

C671

31.50

* $1-2$

.

F143

RS415 
trus Style (1974)

erial Photographic Film on Spools (1972) td. for Face-To-Face and End-To-End ing) (1968) ANSI MH12/ Std. Meth. of Determining Interior ques for Television (1973) Std. Meth. of Measuring Shrinkage from Mold tape (1964) Rec. Pract. for type, Specific Purpose, 277 Volts Ac, 15 Amperes, $2 /$ olts, 15 Amperes, 2 Pole, 1 Wire (1973) volts, 20 Am peres, 2 Pole, 2 Wire (1973)

e, Specific Purpose, 125 Volts, 15 Amperes, 2 Pole, e, Specific Purpose, 125/250 Volts, 15 Amperes, 3 P/ 20/208 Volts, 20 Amperes, 3 Phase Wye, 4 Pole, 4 Wi 20/208 Volts, 30 Amperes, 3 Phase W ye, 4 Pole, 4 Wi 25 Volts, 20 Amperes, 2 Pole, 2 Wire (1973)

25 Volts, 30 Amperes, 2 Pole, 2 Wire (1973)

25 Volts, 20 Amperes, 2 Pole, 3 Wire Locking, Groun/ 25 Volts, 30 Amperes, 2 Pole, 3 Wire Locking, Groun 25/250 Volts, 20 Amperes, 3 Pole, 4 Wire Locking, G/ 25/250 Volts, 30 Amperes, 3 Pole, 4 Wire Locking, G/ 20/208 Volts, 20 Amperes, 3 Phase Wye, 4 Pole, 5 Wi 20/208 Volts, 30 Amperes, 3 Phase Wye, 4 Pole, 5 Wi 25/250 Volts, 20 Amperes, 3 Pole, 3 Wire Locking Ty/ 25/250 Volts, 30 Amperes, 3 Pole, 3 Wire, Locking T/ 50 Volts, 30 Amperes, 3 Phase, 3 Pole, 3 Wire Locki/ $77 / 480$ Volts, 20 Amperes, 3 Phase $W$ ye, 4 Pole, 4 Wi/ 50 Volts, 20 Amperes, 2 Pole, 2 Wire (1973)

50 Volts, 15 Amperes, 2 Pole, 3 Wire Locking, Groun/ 50 Volts, 20 Amperes, 2 Pole, 3 Wire Locking, Groun 50 Volts, 30 Amperes, 2 Pole, 3 Wire Locking, Groun/ 77 Volts Ac, 20 Amperes, 2 Pole, 3 Wire Locking, Gr/ 77 Volts Ac, 30 Amperes, 2 Pole, 3 Wire Locking, Gr/ 50 Volts, 20 Amperes 3 Phase, 3 Pole. 4 Wire Lockin 50 Volts, 30 Amperes, 3 Phase, 3 Pole, 4 Wire Locki/ $77 / 480$ Volts, 20 Amperes, 3 Phase Wye, 4 Pole, 5 Wi $77 / 480$ Volts, 30 Amperes, 3 Phase Wye, 4 Pole, $5 \mathrm{Wi}$ 50 Volts, 15 Amperes, 3 Phase, 3 Pole, 3 Wire Locki/ 50 Volts, 20 Amperes, 3 Phase, 3 Pole, 3 Wire Locki/ 47/600 Volts, 20 Amperes, 3 Phase Wye, 4 Pole, 4 Wi/ $47 / 600$ Volts, 30 Amperes, 3 Phase Wye, 4 Pole, 4 Wi $47 / 600$ Volts, 20 Amperes, 3 Phase Wye, 4 Pole, $5 \mathrm{Wi}$ $47 / 600$ Volts, 30 Amperes, 3 Phase Wye, 4 Pole, 5 Wi/ 80 Volts, 20 Amperes, 3 Phase, 3 Pole, 3 Wire Locki/ 80 Volts, 30 Amperes, 3 Phase, 3 Pole, 3 Wire Locki/

80 Volts, 20 Amperes, 2 Pole, 3 Wire Locking, Groun/ 80 Volts, 30 Amperes, 2 Pole, 3 Wire Locking, Groun 80 Volts, 20 Amperes, 3 Phase, 3 Pole, 4 Wire Locki/ 80 Volts, 30 Amperes, 3 Phase, 3 Pole, 4 Wire Locki/

00 Volts, 30 Amperes, 3 Phase, 3 Pole, 3 Wire Locki/ 00 Volts, 20 Amperes, 2 Pole, 3 Wire Locking, Groun/ 00 Volts, 30 Amperes, 2 Pole, 3 Wire Locking, Groun/ 00 Volts, 30 Amperes, 3 Phase, 3 Pole, 4 Wire Locki/ $77 / 480$ Volts, 30 Amperes, 3 Phase Wye, 4 Pole, 4 Wi/ Motion Picture Film (2.35:1) Aspect Ratio (1971) and 60 Amperes, 4 Wire (1966/ Standard Mounting and Face insulation (1972) ANSI C59.33 Std. Meth. of Measuring on 2 In. Video Magnetic Tape Quadruplex Recorded A/ per (Photography) (1972) es (Photography) (1974)

rec. Pract, for Outboard Motor Boat Transom and Motor W ell ery Sockets (1974) ANSI C83.53 Rec. Pract. for Outboard Motor Std. for Template Hinge Std. for Blower Pipe and Connecting Flange Std. for New Tire ter) (1973) Rec. Std. for Loose Metal End helical Spring and Carbon Steel Tooth Lock W ash/ Std. for hidathion (Common Name for the Pest Control Chemical 0 . 0 . Std. for Common Name for the Pest Control Chemical 0,0 Std. for $100 \mathrm{Deg}$. st Control Chemical N-(2Chloroethyl)-A, A, A-Trifluoro-2,6ontrol Chemical N(Cyclopropylmethyl)-A, A, A-Trifluoro-2,6-

72)

ol Chemical 0.1,3-Dioxolan -2-Ylphenyl Methylcarbamate ) droca/ Std. Analytical Meth. for Determining Sulfur
Std. Analytical Meth. for Determining Sulfur
Std. Analytical Meth. for Determining Sulfur Std. on Carbon Uniform Building Code Std. for Carbon Meth of Analysis for Residual Carbon (Cerea)
Meth. of Analysis for Total (Gasometric) Carbon Acetic Anhydride Meth. for Generation of Chlorine
Dimensions of Composite Cans (211 (66 Mm) Diameter), $\mathrm{C}$ Dimensions of Containers for Packaging and Storage of a Dimensions of Ferrous Valves (1973)

Dimensions of Fiberboard Boxes (Box Gage Meth.) (Packag Dimensions of Image Areas and Mounts for Slides and Opa Dimensions of Molded Plastics (R1973) ANSI K66.3 Dimensions of Patch Splices in Two Inch Video Magneti Dimensions of Plastic Pipe Fittings (1968) ANSI K65.57

Dimensions of Plugs and Receptacles, Locking Grounding d. Dimensions of Plugs and Receptacles, Locking Type $125 \mathrm{~V}$ td. Dimensions of Plugs and Receptacles, Locking Type, 250 Std Dimensions of Plugs and Receptacles, Midget Locking Typ Std. Dimensions of Plugs and Receptacles, Midget Locking Typ d. Dimensions of Plugs and Receptacles: Specific Purpose I std. Dimensions of Plugs and Receptacles: Specific Purpose 1 . for Dimensions of Plugs and Receptacles: Specific Purpose 1 Dimensions of Plugs and Receptacles: Specific Purpose 1 id. Dimensions of Plugs and Receptacles: Specific Purpose 1 Std. Dimensions of Plugs and Receptacles: Specific Purpose 1 td. Dimensions of Plugs and Receptacles: Specific Purpose 1 id. Dimensions of Plugs and Receptacles: Specific Purpose 1 Std. Dimensions of Plugs and Receptacles: Specific Purpose 1 td. Dimensions of Plugs and Receptacles: Specific Purpose 1 td. Dimensions of Plugs and Receptacles: Specific Purpose 2 Dimensions of Plugs and Receptacles: Specific Purpose 2 Std. Dimensions of Plugs and Receptacles: Specific Purpose 2 Std. Dimensions of Plugs and Receptacles: Specific Purpose 2 td. Dimensions of Plugs and Receptacles: Specific Purpose 2 td. Dimensions of Plugs and Receptacles: Specific Purpose 2 td. Dimensions of Plugs and Receptacles: Specific Purpose 2 td. Dimensions of Plugs and Receptacles: Specific Purpose 2 td. Dimensions of Plugs and Receptacles: Specific Purpose 2 Dimensions of Plugs and Receptacles: Specific Purpose 2 Std. Dimensions of Plugs and Receptacles: Specific Purpose 2 td. Dimensions of Plugs and Receptacles: Specific Purpose 2 Dimensions of Plugs and Receptacles: Specific Purpose 2 td. Dimensions of Plugs and Receptacles: Specific Purpose 2 d. Dimensions of Plugs and Receptacles: Specific Purpose 3 td. Dimensions of Plugs and Receptacles: Specific Purpose 3 Dimensions of Plugs and Receptacles: Specific Purpose 3 Dimensions of Plugs and Receptacles: Specific Purpose 3 d. Dimensions of Plugs and Receptacles: Specific Purpose 4 d. Dimensions of Plugs and Receptacles: Specific Purpose 4 td. Dimensions of Plugs and Receptacles: Specific Purpose 4 td. Dimensions of Plugs and Receptacles: Specific Purpose 4 d. Dimensions of Plugs and Receptacles: Specific Purpose 4 d. Dimensions of Plugs and Receptacles: Specific Purpose 4 td. Dimensions of Plugs and Receptacles: Specific Purpose 6 Dimensions of Plugs and Receptacles: Specific Purpose 6 Dimensions of Plugs and Receptacles: Specific Purpose 6 Dimensions of Plugs and Receptacles? Specific Purpose 2 Dimensions of Projectable Anamorphic I mage Area on $5 \mathrm{~mm}$ Dimensions of Receptacles Off Center Boss Type, 30, 50, Dimensions of Rigid Rods and Tubes Used for Electrical Dimensions of Video, Audio and Tracking Control Records Dimensions of 126 Size Cartridges, Film, and Backing $\mathrm{Pa}$ Dimensions of $35 \mathrm{~mm}$ Motion Picture Camera Aperture Imag Dimensions on Boats (1974)

Dimensions on the Mating Plates (Patterns) Used in Batt Dimensions (Boats) (1972)

Dimensions (1972)

Dimensions (1972)

Dimensions (1974)

Dimensions (6 Ounce Fibre Citrus Cans 202 (52 Mm) Diame Dimensions, Physical Properties and Meth. of Test. for Dimethyl Phosphorodithioate S-Ester with 4-(Mercapt Dimethyl 0-(3,5,6-Trichloro-2-Pyridyl) Phosphorothio Dimpled Washer (1974)

Dinitro-N-Propyl-Ptoluidine-Fluchloralin (1973)

Dinitro-N-Propyl-Ptoluidine-Profluralin (1973)

Dinnerware Used in Food Service Establishments (1970)

Dinoseb (Common Name for the Pest Control Chemical) (19 Std. Common Name for the Pest Contr ANSI Starch (1956)

Dioxide Content of Corn Sugar (Crude and Refined) (1961

Dioxide Content of Corn Syrup (1952)

Dioxide Content of the Atmosphere (1967)

Dioxide Content (Qualitative) of Industrial Aromatic Hy

Dioxide Fire Extinguishing Systems (1972)

Dioxide Fire Extinguishing Systems (1973)

Dioxide in Baking Powder (Cereal Chemistry) (1962)

Dioxide in Baking Powder (Cereal Chemistry) (1962)

Dioxide in Flour Bleaching Experiments in Laboratories
CCTl

ANSl

ANSI

ASTM

ANSI

ASTM

SMPTE

ANSI

ANSI

ANSI

ANSI

ANSI

ANSI

ANSI

ANSI

ANSI

ANSI

ANSI

ANSI

ANSI

ANSI

ANSI

ANSI

ANSI

ANSI

ANSI

ANSI

ANSI

ANSI

ANSI

ANSI

ANSI

ANSI

ANSI

ANSI

ANSI

ANSI

ANSl

NSI

ANSI

ANSI

ANS1

ANSl

ANSI

ANSl

ANSl

ANSl

ANSl

ANSl

ANSl

ABYC

ElA

ABYC

ASA

TRA

CCTI

ANS!

ANSI

ANSI

NSA

C ANSI

NSF

ANSI

CR

CR

ASTM

ASTM

NFPA

ICBO

$\mathrm{AACC}$

$\mathrm{AACCH}$

$\mathrm{AACCH} \quad 48-30$

D955

E211.1

PHI.46

B16.10

D2749

C73.43

C73.31

C 73.32

C73.29

C73.30

C73.104

C73.105

C73.69

C73.70

C73.72

C73.73

C73.83

C73.84

C73.90

73.96

C73.97

C73.106

C73.71

C73.74

C73.75

C73.76

C73.77

C73.78

C73.85

C73.86

C73.92

C73.93

C73.98

C73.99

C73.108 
1 Chemistry) (1962) Meth. of Analysis for Total Carbon Tent. Meth. of Analysis for Total Sulfur Tent. Meth of Analysis for Free SuIfur Chemical Analysis for Aluminum Oxide Content of Titanium Std. Spec. for Titanium Std. Spec, for Nuclear Grade, Sinterable Uranium Sid Snec for Nuclear Grade Plutonium ric, and Spectrochemical Analysis of Nuclear Grade Uranium $c$, and Spectrochemical Analysis of Nuclear Grade Plutonium si Meth. for Colorfastness of Dyed Textile Yarns to Sulfur Correction Factors for Gasometric Determination of Carbon Std. Common Name for the Pest Control Chemical 0.1,3 spec. for Determination of Phosphate Coating Weight on Hot Rec. Pract. for Safeguarding Against Embrittlement of Hot spec. for Steel Sheet, Zinc Coated (Galvanized) by the Hot Lock Forming Quality, Zinc Coated (Galvanized) by the Hot et of Drawing Quality, Zinc Coated (Galvanized) by the Hot Culverts and Underdrains, Zinc Coated (Galvanized) by Ho ments for Steel Sheet, Zinc Coated (Galvanized) by the Hot in Coils, and Cut Lengths, Zinc Coated (Galvanized) by Ho spec. for Steel Sheet, Zinc Coated (Galvanized) by the Ho meth. of Measurement of the A.D Dimension of Aerosol Valve Std. Spec. for Zinc Coating (Ho Iron or Steel Articles by the Preece Test (Copper Sulfate Std. Spec, for Welded and Seamless Black and Hot Std. for Molded and

el Pipe for Ordinary Uses // Std. Spec. for Black and Hot of Determining the SAE Rating and Struck Capacity for Hoe Determining the SAE Rating and Struck Capacity for Shove 2-(Ethylthio) -4.6-Bis (Isopropylamino) Stria/ Std. for (Common Name for the Pest Control Chemical O-Ethy] S,S Std. Sprec, for

d Gamma Radiation (1972) Performance Spec. for $t$ for Oxygen Content Using a $14 \mathrm{MeV}$ Neutron Activation and hemical Analysis of Thermionic Nickel Alloys by the Powder Std for Std. for Industrial and Othe Std. Test Code for

tegral Horsepowe/ tegral Hor/ er) (1972) er) (1/ Std. for Dimensions for Alternating and Std. for Application Data for Alternating and Std. for Ratings for Alternating Current and Std. for Tests and Performance of Alternating and Std. for Std. for Stabilized Power Supplies Std. for Electrical Bonding o (lnboard) Engine Mountings (Install tion and Replacement) equipment (1972)

Std. for Residential Controls Safety Std. for Oil Fired Air and Safety Ventilation Requirements for Std. for

Rec. Std. for Seismic Restrain Tent. Rec. Pract. for matograph (1973) $8 \mathrm{~mm}$ and Super 8 Reversal Color Camera Films Intended for er Meters (1972)

Std. Meth. of onditions (1972)

Std. Meth. of est for Disbonding Characteristics of Pipeline Coatings by
Is Except Chocolate Products and Piecrust (Cereal Chemis) Std. for Gas Fired Gravity and Fan Type

Interfaces for 4 Way General Purpose Industrial Pneumatic
Meth. for Cross 1972)
to ANSI Z35.1-1972, Accident / Seth. for Cross

Disfigurement of Paint Films by Fungal Growth or Soil and Test Meth. for

ers, Splinters, etc. in St/ Test Meth. for

Std. for Meth. of Recording and Measuring the Off-The-lob ct Soil Burial (1972)

Std. Meth. of Test for Std. Meth. of Test for Cathodic Rec. Pract. for Automotive Brake

Uniform Laboratory Fatigue Test Procedures for Production in the Automotive Braking System of Vehicles Equipped with for Decoders (Type I) for Reproducing Matrix Quadraphonic for Handling and Installation of Power Semiconductors in Std. Definitions for High Intensity Std. Meth. for the Designation of High Intensity Std. Designations for Rotation and Safety Std. Antenna

ystems (1973/ Std. Meth. for Detection and Measurement of solid Electrical Insulating Materials Subjected to Partial Rec. Pract. for the Detection and Measurement of Partial 1972A) Std. Meth. of Test for Std. for Ouick

Radiographic Std. for Classification of Fusion Weld nd Dirt Accumu/ Tent. Meth. of Evaluating Deg. of Surface
Dioxide in Manganese Ores (1972T)

Dioxide in Prepared Mixes and Self Rising Flours (Cerea

Dioxide in Wine (Enology) (1972)

Dioxide in Wine (Enology) (1972)

Dioxide Pigments (1973)

Dioxide Pigments (I973) ANSI K45.1

Dioxide Powder for Use in Nuclear Reactors (I973)

Dioxide Powder, Sinterable (1974)

Dioxide Powders and Pellets (1972) ANSI N103

Dioxide Powders and Pellets (1972) ANSI N104

Dioxide Stoving (1972) ANSI LI4.9

Dioxide (Cereal Chemistry) (1962)

Dioxolan -2-Ylphenyl Methylcarbamate-Dioxacarb (1973
Dip Galvanized and Electrogalvanized Steel (Coil) (1974

Dip Galvanized and Electrogalvanized Steel (Coil) (1974

Dip Process for Roofing (1971) ANSI G8.21

Dip Process (1971) ANS1 G8.22

Dip Process (1971) ANSI G8.23

Dip Process (1971) ANSI G8.28

Dip Process (1973) ANSI G8.25

Dip Process (1973) ANSI G8.27

Dip Process, Com

Dip Tubes (1972)

Dip) on Assembled Steel Products (1973) ANSI G8.18

/Pec. for Steel Sheet of ASTM

ITd. Spec, for Steel She ASTM

C. for Steel Sheets for ASTM

Ec. for General Require ASTM

/ of Structural Quality ASTM

/Ectromet ASTM

/Trometri ASTM

Te AATCC

AACCH $12-29$

D. ASTM

Std. ASTM

ASTM

Dipped Galvanized Steel Pipe (1973) ANSI Bl25.1

Dipped Mica Capacitors (Wire Lead Styles) (1972)

Dipped Zinc Coated (Galvanized) Welded and Seamless Ste

Dipper (Backhoe Bucket) (1972)

Sid for Uniform

Dipper, Clam Bucket, and Dragline Buicket (Construction

Dipropetryn (Common Name for the Pest Control Chemical

Dipropyl Phosphorodithioate) (1973)

Td. for Ethoprop

Dipropylene Glycol (1972)

Direct and Indirect Reading Pocket Dosimeters for X-An

(Direct Counting Meth.) (1971) ANSI L14.234

Direct Counting Technique (1973)

Direct Current Arc Technique (1961) ANSI Z128.2

Direct Current Generators (Large Apparatus) (1972)

Direct Current Integral Horsepower Generators (1972)

Direct Current Machines (1973)

Direct Current Motors and Generators (Fractional and in

Direct Current Motors and Generators (Fractional and in

Direct Current Motors (Fractional and Integral Horsepow

Direct Current Motors (Fractional and Integral Horsepow

Direct Current Motors (Large Apparatus) (1972)

Direct Current Output (1972)

Direct Current Systems on Boats (1972)

Direct Drive Transmission (1971)

Direct Electric Ignition Con

Direct Fired Powder Curing Ovens (1973)

Direct Gas Fired Make Up Air Heaters (1972)

Direct Hung Suspended Ceiling Assemblies (1972)

Direct Injection of Solvent Base Paints into a Gas Chro

Direct Projection in Motion Picture Photography (1973)

Direct Reading Remote Registration Systems for Cold Wat

Direct Shear Test of Soils Under Consolidated Drained C

Direct Soil Burial (1972)

Std. $M$

Direct Trapping Meth. of Analysi
Direct Vent Wall Furnaces (1973)

Directional Control Valves (1973) ANSl B93.33

Directional Internal Tearing Resistance of Paperboard (

Directional, and Special Signs (Symbols) Complementary

Directories of Libraries and Information Centers (1971)

Dirt Accumulation (1973)

F Evaluating Deg. of Surface

Dirt Count of Wood Chips (Pulp and Paper) (1973)

Dirt, Sand, Insect Fragments, Hairs, Feathers, Wood Fib

Disabling Accidental Injury Experience of Employees (19

Disbonding Characteristics of Pipeline Coatings by Dire

Disbonding of Pipeline Coatings (1972)

Disc and Drum Thermocouple Installation (1972)

Disc and Spoke Wheels, and Demountable Rims Intended Fo

Disc Brakes (1972)

Disc Records (1974)

Disc Type Packages (1973)

Discharge Lamp Ballasts and Transformers (1971)

Nation of Brake

Discharge Lamps (1972)

Discharge of Centrifugal Fans (1966)

Discharge Units (1972) ANSl C33.39

Discharge (Corona) Pulses in Evaluation of Insulation S

Discharges (Corona)

Discharges (Corona) During Dielectric Tests (1973)

lof Test for Voltage Endurance of

Disconnect Devices for Use with Gas Fuel (1971)

Discontinuities (1972)

Disfigurement of Paint Films by Fungal Growth or Soil a
ASTM

ASTM

EIA

ASTM

SAE

SAE

ANSI

ANSI

ASTM

ANSI

ASTM

TSTM

/Ctroc ASTM

NEMA

NEMA

IEEE

NEMA

NEMA

NEMA

NEMA

NEMA

NEMA

$A B Y C$

SAE

NEMA

UL

FMS

ANSI

CISCA

ASTM

ANSI

AWWA

ASTM

ASTM

AACC

NFLDP T3.21

TAPPI T496SU

ANSI Z35.4

ANSI Z39.10

ASTM D3274

TAPPI UM.1]

AACCH 28.75

ANSI Z16.3

ASTM G19

SAE $\quad 179$

SAE J26?

J29l

RS4 18

JEDI1

C 82.9

Std EIA

ANSI

ANSl

AMCA

C78.380

UL

ASTM

2406

452

D1868

D2275

IEEE

454

ASTM D1618

ANSI

Z21.41

NSA

1514

D3274
ASTM G8 
Std. Performance Requirements for Woven td. for Gas Counter Appliances (Coffee Pots and Urns, Food h, Filter Paper, Wide Field and Compound Microscope, Petri nd Test Procedures for Plumbing Requirements for Household Std. for Safety for Commercial Electric Household ereal Chemistry) (1962)

Stds. for Tire Rim Inspection, and

(Cereal Chemistry) (1962)

erties of Metal to Metal Adhesives by Compression Loading osity and Curing Characteristics of Rubber by the Shearing for Vacuum Treated Alloy Steel Forgings for Turbine Rotor Test Meth. for Specific Gravity of Wood td. Spec. for Cartridge Brass Sheet, Strip, Plate, Bar and 16.1 quirements for Household Storage Type Electrical Hot W ater mpatibility of Vehicle Fuel Tanks and Fill Pipes with Fuel Rec. for Flammable Liquid Drum Storage, tilizer Grade) in Residential and Commerical Fuel Burning, dispersible Protein in Ground Soybeans, Whole Or/ Protein itrogen Solubility Index (NSl): Meth. for Determination of ein Dispersibility Index (PDI): Meth. for Determination of Rec. Guide for the Prediction of the

72) ANSI L14.117 Test Meth for Fluidity of of Test. and Presenting Basic Performance Data for Positve Meth. of Test for Specific Gravity of Solid Pitch Asphalt Std. for Cold Water Meters

Rec. Pract. for Accident Prevention Signs for ety for Implosion Protected Cathode Ray Tubes (Picture and ogeneous Perforated Bituminized Fiber Pipe for Septic Tank ted Wall Perforated Bituminized Fiber Pipe for Septic Tank Rec, for Radioactive Waste Rec. for the ers (1951)

Rec. for Waste a Technical Guide for Landfill Uniform Plumbing Code: Sewers and Private Sewage ec. for Uniform Terminology for Rural Waste Management and cedures for Plumbing Requirements for Household Food Waste Std. for Performance Evaluation for Household Food Waste or Electr/ Std. Meth. of Test for Dielectric Constant and - Meth. of Test for Dielectric Constant (Permittivity) and ctrical Conductivity of Aviation Fuels Containing a Static Sid. Meth of Test for Radio Frequency R/ Minimum Performance Std. for Airborne cles, Buses, Trucks, and Combin/ Rec. Pract. for Stopping Equipped with Full Scale Tir/ Meth. of Test for Stopping Equipped with Fu/ Tent. Meth. of Measurement of Stopping Std. Meth. of Test for Stability of captan Sulfur in Gasoline, Kerosine, Aviation Turbine, and meth. for Calculation of Liquid Heat Capacity of Petroleum Std. Meth. of Test for Wax Appearance Point of Std. Meth. for Validation of Results of Process Std. Spec. for ure in Grains (Cereal Chemistry) (1962) nsi Al09.11 olumn) (1973) ANS1 Z11.314
s (1973) AASHO T78, ANSI A37.45 Std. Meth. for
Std. Meth. of Test for ) Tent. Rec. Mract. of Test for g Meth. of Analysis of Moisture in Feeds and Feedstuffs by $\mathrm{g}$ Meth. of Analysis of Moisture in Fats and Shortenings by Std. Meth of Test for Moisture in Wool by std. Analytical Meth. for Determining Moisture (Azeotropic std. Analytical Meth. for Determining Moisture (Azeotropic std. Analytical Meth. for Determining Moisture (Azeotropic std. Analytical Meth. for Determining Moisture (Azeotropic Rec. for Std. Meth. of Test for Std. for Industrial Engineering Terminology:

Oils by the n.d.M Meth. Tent. Meth. of Test for Carbon Installation Pract, for Underground Electrical Power orting and Analyzing Outage of Electrical Transmission and std. for Series Capacitors for Electrical Transmission and

L14.91 Std. Meth. of Test for Length and Length oplastic Insulated Wire and Cable for the Transmission and ethylene Insulated Wire and Cable for the Transmission and ract for Analysis by Microscopical Meth. for Particle Size hy (1973) Tent. Meth. of Test for Boiling Range Meth. for Particle Size

8s Extruded Tube and Pipe for Gas and Oil Transmission and cs.) (1973) Aluminum Underground 2) Rec. for Single Phase Rural Dead Fron

id. for Small Manually Operated Metallic Gas Valves in Gas Std. for Air Outlets and Inlets Used in Air
Dish Towels (Institutional Textile) (1973)

Dish Warmers, Hot Plates and Griddles, etc.) (1973)

Dish, Sieve, Separatory Funnel, Trap Flask, Step Wedge

Dishwashers (1971) ANSI A197.1, Asse 1006

Dishwashers (1973) ANSI C 33.96

Dishwashers (1974)

Disk and Flat Tapes (1974)

Disk Meth. of Analysis of Color of Macaroni Products (C

Disk Meth. of Analysis of Semolina Quality in Macaroni

Disk Shear) (1972) ANSI Z197.4

Disk Viscometer (1972) ANSl J7.7

Disks and Wheels (1970) ANSI G55.15

Test for Sireng

Disks (Pulp and Paper) (1973)

est for Strength Prop ASTM
Th. of Test for Visc ASTM

Std. Spec.

Disks (1973)

Dispensers (1973)

Dispensing Devices for Flammable Liquids (1974) ANSI Z2

Dispensing Facilities (Psssenger Cars, Multipurpose Veh

Dispensing, and Classification (1972)

Dispensing, and Handling Facilities (1974) ANSI B150.1

Dispersibility Index (PDI): Meth. for Determination of

Dispersible Nitrogen in Ground Soybeans, Whole or Groun

Dispersible Protein in Ground Soybeans, Whole or Ground

Dispersion of Airborne Effluents (1968)

Dispcrsions of Cellulose Form Bleached Cotton Cloth (19

Displacement Hydraulic Fluid Power Pumps and Motors (19

Displacement Meth.) (1972) ANSI A37.72

Displacement Type 5/8 In. Through 6 ln.) (1971)

Display on Tank Trailers and Containers (1972)

Display) for Television Receiving Appliances and Other

Disposal Fields (1973) ANSI A 176.4
Disposal Fields (1973) ANSI A 176.5

Disposal in the Ocean (1954)

Disposal of Carbon-14 Wastes (1953)

Disposal of Phosphorus.32 and lodine.131 for Medical Us

Disposal of Solid Chemical Waste (1974)

Disposal Systems (1973)

Disposal (1973)

Disposer Units (1971) ANSI A197.3, Asse 1008

Disposers (1973)

Dissipation Factor of Expanded Cellular Plastics Used F

Dissipation Factor of Solid Ceramic Dielectrics at Freq

Dissipator Additive (1971) ANSI Z11.281

Dissolved and Gaseous Hydrogen in Water (1960)

Distance Measuring Equipment (DME) Operating Within the

Distance of Passenger Cars, Multipurpose Passenger Vehi

Distance on Paved Surfaces Using a Passenger Automobile

Distance on Paved Surfaces Using a Passenger Automobile

Distillate Fuel Oil (Accelerated Meth.) (1974)

Distillate Fuels (Potentiometric Meth.) (1973)

Distillate Fuels (1971) ANSI Z11.313

Distillate Fuels (1972) ANSI Z11.325

Distillation Analyzers (1973)

Distillation Equipment (1971) ANSI Z168.4

Distillation Meth. (Brown-Duvel) of Analysis of Moist

Distillation of Bituminous Protective Coatings (1970) a

Distillation of Crude Petroleum (15 Theoretical Plate C

Distillation of Cut Back Asphaltic (Bituminous) Product

Distillation of Road Tars (1972)

Distillation of Solvents from Solvent Base Paints (1973

Distillation with Toluene (Cereal Che mistry) (1962)

Distillation with Toluene (Cereal Chemistry) (1962)

Distillation with Toluene (1972) ANSI L14.256

Distillation) Content in Corn Starch (1956)

Distillation) Content in Corn (1955)

Distillation) Content in Feedstuffs (Corn) (1956)

Distillation) Content of Corn Syrup Unmixed (1952)

Distilled Spirits Storage (1974)

Distortion of Yarn in Woven Fabrics (1972) ANSI L14.103

Distribution and Marketing (1972)

Distribution and Structural Group Analys is of Petroleum

Distribution Cables (1973)

Distribution Facilities and Interruptions to Customer S

Distribution Line Compensation (Including Safety Requir

Distribution Microfiche of Documents (1972)

Distribution of Cotton Fibers (Array Meth.) (1972) ANSl

Distribution of Electrical Energy (1973) IPCEA S.61.402

Distribution of Electrical Energy (1973) IPCEA S.66.524

Distribution of Particulate Substances of Subsieve Size

Distribution of Petroleum Fractions by Gas Chromatograp

Distribution of Wheat Flour (Cereal Chemistry) (1962)

Distribution Piping Systems (1973) ANSl H38.13

Distribution Reference Book (Electrical Power Cable Spe

Distribution Service for Motors and Phase Converters (1

Distribution Switchboards (1972)

Distribution Systems Whose Maximum Allowable Operating

Distribution Systems (1973)

ANSl

ANS1

AACCH

Std. a AHAM

UL

TRA

ASTM

US

UL

FMS

UL

ASME

AATCC

NFLDP

Std. ASTM

AWWA

TTMA

UL

AHAM

ASTM

ASTM

SAE

ASTM

ASTM

ASTM

for Mer ASTM

Std. ASTM

ASTM

ASTM

ASTM

AACCH

ASTM

ASTM

ASTM

ASTM

ASTM

IN AACCH

IN AACCH

CR

CR

CR

FMS

ASTM

ANS 1

ASTM

AA

IEEE

ANSl

NMA

ASTM

NEMA

ASTM

ASTM

$A A C C H$

AACCH 14.20

AACCH 66.40

D2182

D1646

A471

TAPPI UM.12

B19

1023

J398 A

$\mathrm{AACCH} \quad 46.24$

AACCH 46.23

AACCH 46.24

1

T3.9.17

D7l

C 700

RP 35

492.8

ASTM D2312

ASTM D2313

NCRPM R16

NCRPM R12

NCRPM R9

MCA SW.1

ICBO UPC.1.11

ASAE R292.1

Test Pro AHAM FWD.2PR

FWD-1

D1673

D2149

D2624

ASTM D1588

RTCA DO.151

J299

E445

E503

D2274

D3227

D2890T

D3117

D2891

E133

D2 2892

D402

D20

D3272

D2462

B. 34

A. 12

G.14

88

D) 1336

Z94.4

D3238 
Chlorinated Poly(vinyl Chloride) (CPVC) Plastic Hot Water std. Test Procedure for Thermal Evaluation of Oil Immersed Uniform Plumbing Code: Wate duct Liner, Adhesives, and Fasteners Application Std. (Ai onics) (1973) ANSI C57.12.90

ANSI C 57.12 .00

kVA and Smaller (1971) Std. Test Code for Std. General Requirements for Sid. Requirements for Overhead breviations for States of the United States (Including the

Stability of New Mineral lnsulating Oils Containing 2,6. Meth. of Test for Lead in the Atmosphere by Colorimetric nger Automobiles, Commercial Vehicles, Golf Car, and Other Sid for Decade Transformer

Walls, Floor-Ceiling Assemblies, Doors, and Other Space Sid. for in

dification of Data Derived from the Investigation of Scuba harges) (1974) Uniform Pract, for Fee Determination for cs. and Load Tables for Longspan Steel Joints Deep DLJ and std. Specs, and Load Tables for Longspan Steel Joints Deep erformance Std. for Airhorne Distance Measuring Equipment terials Handling) (1973) Shipper-Motor Carrier pract. for Average Vehicle Dimensions for Use in Designing allation of Fittings and Equipment for Anchoring, Mooring. 1974) ANS1 C83.88, 1pe C-405A

Std. General

cribing lrformation Interchange Formats (Computer Software
Std. for Engineering Drawing and Related (1973) and Light Truc/ Sid. for Engineering Drawing and Related 1973) Std. Engineering Drawing and Related s (1973) for Std. Calibration and Format for Nuclear Logs (Record,
Std. for National Aerospace Std. National Aerospace Stds. Specifying Project Record lndustry Std. for Distribution Microfiche of lndustry StI. for Fascimile Transmission of Microfilmed chenical S-Ethyl (E,E). II-Methoxy.3.7,11-Trimetlyyl-2,4. he Pest Control Chemical Ethyl (E, E)3,7,Il-Trimethyl-2,4

Control Chemical 2-Propynyl (E,E)-3,7,11-Trimethyl.2,4.

ne (Isopropyl (E,E).11-Methoxy-3,7,1l-Trimethyl-2,4 Spce. for Photographic Grade Trisodium Phosphate, ibution Systems Whose Maximum Allowable Operating Pressure Meth. of Analysis of Crude Fat in Baked

Targ/ Shotgun Shooting Facility Plans: International and

Test Meth. for Colorfastness of Textiles to Accelerated Std. Performance Requircments for Oil Powered Forced Air Std. for Installation of Std. for Sid. for

motor Vehicle Typc (Lead Acid) Storage Batteries (1/ Rec

embly Numbers, Cell Layouts and Terminals (For Foreign and Safety Std. for

asuring Color of l'aper and Paperboard in CIE $Y ; X, Y$ or $Y$. Std. Thermal Performance Test for Steel std. Acoustical Performance Test for Steel roc, and Acreptance Criteria for Water Resistance on Stee eptance Criteria for Rate of Air Flow Through Closed Stee 1 (1973) spec. for $13 / 4 \mathrm{In}$. Thick Hollow Mctal Std. Meth. of Fire Tests of

Uniform Building Code Std. for Fire Tests of Sid. Meth. of Fire Tests of std. for Safety for Fire Tests of Std. for Std. for Builders

smoke and Heat Detectors; Electromagnetic Holders; Sliding strikes (1973) Spec for Hollow Metal Rec. Erection Instructions for Steel std. for Sliding and Folding

s; Closers; Hospital and Misc. ltems; Key Control Systems; Std. for Safety for Elevator Ouality Stds for Wood Screens for Window and afety Std. for Panic (Emergency) Hardware (Outward Opening Std. for Safety for Burglary Resistant Electric Std. for Arclitectural Rec. Std. ors and Systcms (1973) Safety Std. for Key Locks (Burglar Alarm. Safety Std. for dustry Std. for Douglas Fir. Western Hemlock, Sitka Spruce ion (1972) Std. Steel Rec. Spec. for Builders' Hardware on Std. Steel
Rec. Spec. for Std. Steel Rec. Minimum Hardware Reinforcing Garages on Steel
Rec. Spec. for Steel Bi.Fold Closet Rec. Spec. for Steel Bi. Fold Closet

Rec. Weatherstripping for Std. Steel Rec. Std. Mininum Acceptance Values for Steel
Distribution Systems (1973)

Distribution Transformers (1972)

Distribution (1973)

Distribution) (1971)

Distribution, Power and Regulating Transformers (Electr

Distribution, Power, and Regulating Transformers (1973)

Distribution, Transformers, 67,000 Volts and Below; 500

District of Columbia) for Information Interchange (1972

Disugran (Common Name for the Pest Control Chemical) (1

Ditertiary-Butyl Para-Cresol by Rotating Bomb (1973

Dithizone Procedure (1972T)

Diversified Off the Highway Applications (1972)

Dividers (Voltage Type) (1972)

Dividing Elements (1973)

Dividual Aerobic Wastew

/ Building Partitions Such as

Diving Accidents) (1973) ent Plants (1970)

Division Services (Individuals, Clubs, and Exhibition C

DLH Series (1970)

DLJ and DLH Series (1970)

(DME) Operating Within the Radio Frequency Range of 960-

Dock Planning Manual (Truck Shipping and Receiving-Ma

Docking Facilities for Motor Vehicles (1971)

Docking, Towing and Lifting Boats (1973)

Document for Printed Wiring Board Electric Connecton an

Documentation and Terminology) (1972) FIPS 20

Document ation Pract. for Line Conventions and Lettering

Documentation Practices: Chassis Frames-Passenger Car

Documentation Practices: Dimensioning and Tolerancing (

Document ation) (1974)

Rec.

Documents Preparation and Maintenance in Sl Metric Unit

Documents Preparation and Maintenance (1974)

Documents (1967)

Documents (1972)

Documents (1972) ANSI C 16.45

Dodecadienethioate-Triprene (1973)

Dodecadie noate Hydroprene (1973)

Dodecadienoate Kinoprene (1973)

Dodecadienoate) (Common Name for the Pest Control Chemi

Does Not Exceed 60 or 125 Psig (1973)

Dog Food (Cereal Chemistry) (1962)

Domestic-Trap, Clay Pigeon, and Skeet: Ammunition and

Domestic and Commercial Washing and Laundering (1972)

(Domestic and Liglat Coumercial) Central Furnaces) (1972)

Domestic Gas Conversion Burners (1971)

Domestic Gas Fired Incinerators (1973)

Domestic Gas Hot Plates and Laundry Stoves (197I)

Domestic Marketing Std. lncluding Guarantee Policy for

Domestic Passenger Cars, Trucks, Buses, Coaches, Tracto

Domestic Type lncinerators (1973)

Dominant Wavelength and Excitation Purity (1972)

Door and Frame Assemblies (1972)

Door and Frame Assemblies (1972)

Door and Frame Assemblies (1972)

Door and Frame Assemblies (1972)

Door and Frame Preparation for Offset Intermediate Pivo

Door Assemblies (1972).

Door Assemblies (1973)

Door Assemblies (1973) ANSl A2.2

Door Assemblies (1974) ANSI A2.2

Door Controls-Closers (1972)

Door Controls (Closers) (1971)

Door Equipment; Installation Aids (1969)

Door Frame Details (1972)

Door Frame Preparation for 181 and 190 Series Deadlock

Door Frames (1972)

Door Hardware (1971)

Door Holders, Stops, Smoke and Heat Detectors; Electrom

Door Locking Devices and Contacts (1973)

Door Openings (Architectural Woodwork) (1973)

Door Releasing Device) (1973)

Door Strikes (197.4)

Door Trim (1972)

Door Type Nomenclature (Coding) (1972)

Door, Deposit and Collection Safes) (1973)

Door, Drapery, Gate, Louver, and Window Electric Operat

Door, Frame and Hardware Schedule (1972)

Doors and Blinds (1972)

Doors and Frames for Modular Masonry Building Construct

Doors and Frames (Reinforcement-Application) (1972)

Doors and Frames (1969)

Doors and Frames (1972

Doors and Frames (1972)

Doors and Frames (1972)

Doors and Frames (1972)

Doors and Frames (1972)
IEEE D2846

IEEE 345

13

IEEE 462

ANSI C 57.1220

ANSI $\times 3.38$

ANSI K62.112

ASTM D2112

ASTM D3112

$\cdot 1.48$

ANSI Cl00.I

UBCS35.1

40

Z86. 2

N NSI

PSA

11

S316.1

S316-1

RTCA DO.151

ANSI MH8.1

J699. A

A5

EIA RS406

$\begin{array}{ll}\text { ANSI } & \mathrm{X} 10.1 \\ \text { ANSI } & \text { Y } 14.2\end{array}$

ANS1 Y14.32.1

ANSI Y14.5

RP33

RP33
10000

NSA 10000

CSI 01730

NMA MS5

NMA MS3

K62.141

K62.136

K62.137

K62.132

PH4.234

$\begin{array}{ll}\text { ANSI } & \text { B 16.33 } \\ \text { AACCH } & 30.14\end{array}$

$\begin{array}{ll}\mathrm{AACCH} & 30.14 \\ \mathrm{NRA} & 17\end{array}$

AATCC 61

ANSI Z91.1

ANSI 221.8

ANSl 221.6

ANSl 221.9

$\mathrm{BCl} * 1.15$

BCl $\quad * 1.56$

UL 791

T527SU

STDI 113

STD1 114

Std. Test P STDI 115

116

ANSI Al15.12

NFPA 252

ICBO

ASTM

UL

ANSI

UBCS43.2

E152

$10 \mathrm{~B}$

301

STDI

8

111.A

STDI 105

BHMA 401

NBHA

UL

A W 1

UL

UL

STD

UL

STDl

in FHDA

STD

STDI

STDI

NBHA

STDl

STDI

STDI

STDI

8
104

*1.1100

305

1034

A 156.6

106

437

325

III.D

4.72

110

107 
Uniform Building Code Std, for Tin Clad Fire Doors and Shutters (1973)

Uniform Building Code Std. for Installation of Fire Doors and Windows (1973)

Quality Stds. for Flush Solid and Hollow Core Doors (Architectural Woodwork) (1973)

Specs. for Aluminum Combination Storm

Std for Forced Entry Aluminum Sliding Glass

ds. for Forced Entry Resistant Aluminum Horizontal Sliding

rec. Procedure for Processing Hardware for Custom Aluminum Rec. Selection and Usage Guide for Std. Steel

Rec. Louver Details for Std. Steel Spec for Aluminum Sliding Glass

$r$ Tests for Fire Resistance of Vault and File Storage Room

dwood Veneered lncluding Hardboard and Plastic Faced Flush Safety Std. for Swinging Hardware for Std. Tin Clad Fire Std. for Safety for Tin Clad Fire ding Hardware for Std., Horizontally Mounted Tin Clad Fire ding Partitions Such as Walls, Floor-Ceiling Assemblies, sash, Screens, Blinds and Shutters, Flush, Stile, and Rail a2.7

pulls, Push and Kick /

Basic Builders' Hardware: Hands of h. of Test for Effective Fluorescent Lifetime of Neodymium nimum Facilities Required for School, Theater, Auditorium,

for Rec. Antidotes, Supplies (Packaging), and References Std. Test Meth. for Absorbed Gamma Radiation

Rays (1961) Rec. for Measurement of Absorbed d. Meth. of Test for Absorbed Gamma and Electron Radiation d. Meth. of Test for Absorbed Gamma and Electron Radiation of Quartz Fiber Electrometer Type Dosimeters and Companion ron Radiation Dose with the Ferrous Sulfate Cupric Sulfate d Gamma and Electron Radiation Dose with the Ceric Sulfate test Meth. for Absorbed Gamma Radiation Dose in the Fricke d. for Interrelationship of Quartz Fiber Electrometer Type Performance Spec. for Direct and Indirect Reading Pocket Std. Definitions of Terms Relating to Std. Spec. for 55 Gal. Tight Head Universal Drums

Std. Spec. for 16 Gal. Tight Head Universal Drum

Spec, for $5 \mathrm{Gal}$. Tight Head Universal Pails (Container) std. Spec. for $30 \mathrm{Gal}$. Full Removable Head Universal Drum esting Lug Cover Universal Pails (Container) (Dot-37A80, t Side Lug Cover Universal Pails (Container) (Dot-37A80, for $5 \mathrm{Gal}$. Nesting Lug Cover Universal Pails (Container) Gal. Straight Side Lug Cover Universal Pails (Container) ls (Container) (Dot-17E, Ufc-Rule 40, Nmfc-Item 260,

for 5 Gal. Nesting Lug Cover Universal Pail (Container)

5 Gal. Straight Side Lug Cover Universal Pail (Container) Std. Spec. for $55 \mathrm{Gal}$. Tight Head Univeral Drum

strative Guide for Obtaining Department of Transportation Std. for Pin, Quick Release, Positive Locking. transparent or Opaque Metal Oxide and Metallic Coatings by rs for Potable Water Supply/ Performance Requirements for
controlled Low Carbon Steel Tubing Normalized for Bending. controlled Low Carbon Steel Tubing Normalized for Bending,
fting Manual (Gear Drawing Stds. Part l for Spur, Helical, Std. for Nut-Self Locking, Extended Washer.

Spec, for Pressure Vessel Plates, Alloy Steel, 9\% Nickel

particulate and Acidic Gaseous Fluoride in the Atmosphere rockets (1972)

ters (1973)

auxite, Calcine, Chrom/

Std. Classific ation of Single and

erformance Requirements for Terry Cloth Towels (Single and F/ Quantitative Meth. of Analysis of Calcium Peroxide in ad Flour (Cereal Chemistry) (1962) General Extensigraph Meth. for Physical

ural Relaxation Meth. of Extensigraph Analysis of Physical ensigraph Meth. for Quality of Nonfat Dry Milk in Physical solids to Change Mixing Characteristics of Flour (Physical Farinograph Absorption to 14.0\% Moist/ Meth. of Physical

Farinograph Meth. for Analysis of Resistance of Flour Mixograph Meth. for Analysis of Resistance of Flour s Farinograph Absorption to $14.0 \%$ Moisture Basis (Constant Bread Flour (Cereal Chemistry) (1962)

Pressuremeter Meth. of Analysis of Diastatic Activity of Meth. of Analysis of Yeast Strength of Volumetric Meth. of Analysis of Diastatic Activity of building Code Std. for Structural Glued Laminated Timbers: (Nor)

Uniform Building Code Std. for Coast Sitka Spruce,

Laminations Before Gluing of Southern Pine, Pacific Coast water Cooling Towers (1971) red Cedar; White Fir; And/ inds (1972)

(Doors) (1972)

Dot-17H) (1974)
Quality Stds for Stile and Rail

Std. for Butts and Hinges for Std. for Ponderosa Pine Rec. Std. Details for Dutch Std. for Ponderosa Pine Std. for Exit Devices Std. for Fire Std. Gravure Lateral Hard

Std. for Std for Brazed Sponge

Doors (Architectural Woodwork) (1973)

Doors (Builders Hardware) (1970)

Doors (1958)

Doors (1971)

Doors (1971)

Doors (1971)

Doors (1972)

Doors (1972)

Doors (1972)

Doors $(1972)$

Doors (1972) ANSI Al34.2

Doors (1972) ANSI A153.1

Doors (1973)

Doors (1973)

Doors (1973) ANSI Al33.1

Doors (1973) ANSI Al42.

Doors (1973) ANSI A 143.1

Doors, and Other Space Dividing Elements (1973)

Doors, Factory Finishing, Rough and Finished Carpentry,

Doors, Windows. Shutters, and Glass Blocks (1973) ANS

Doors; Hinges; Locks; Knobs and Handles; Exit Devices

Doped Laser Materials (1973)

Dormatory, Public or Office Building, Manufacturer, Res Dosage) for Poison Control Centers (1972)

Dose in the Fricke Dosimeter (1972)

Dose of Neutrons, and of Mixtures of Neutrons and Gamma

Dose with the Ceric Sulfate Dosimeter (1971) ANS1 K65.2

Dose with the Ferrous Sulfate Cupric Sulfate Dosimeter

Dosimeter Chargers (1971)

Dosimeter (I971) ANSI K65.229

Dosimeter (1971) ANSI K65.230

Dosimeter (1972)

Dosimeters and Companion Dosimeter Chargers (1971)

Dosimeters for X-And Gamma Radiation (1972)

Dosimetry (I968) ANSI N 105

Dot-17E) (1974)

(Dot-17E) (1974)

Dot-17E, Ufc-Rule 40, Nmfc-Item 260, Dot-37B60)

Dot-37Aa60, Ufc-Rule 40, Nmfc-Item 260 (1974)

Dot-37A60, Ufc-Rule 40, Nmfc-Item 260) (1974)

Dot-37A80, Dot-37Aa60, Ufc-Rule 40, Nmfc-Item 2

Dot-37A80, Dot-37A60, Ufc-Rule 40, N mfc-ltem 26

Dot-37B60) (I974

Dot-37C80) (1974)

Dot-37C80) (1974)

Dot-5B) (1974)

Dot Screen (1973)

(DOT) Special Permits for Radioactive Materials Shipment

Double Acting (1973)

Double Beam Interference Microscope Technique (1973)

Double Check Valve Type Back Pressure Backflow Prevente

Double Flaring, and Beading (1972)

Double Helical and Rack) (1971)

Double Hexagon, $220 \mathrm{KSI}, 450 \mathrm{Deg}$. F (1973)

I for Welded Flash SAE

Double Normalized and Tempered (1972 A) ANS1 G35.3

(Double Paper Tape Sampler Meth.) (1973)

101

Double Pitch Conveyor Roller Chains, Attachments and $S_{p}$

Double Race or Bi-Level Swivel and Rigid Industrial Cas

Double Screened Ground Refractory Materials (Calcined B

Double Wall Low Carbon Steel Tubing (1972)

Double) (Institutional Textile) (1973)

Dough Conditioners Cont aining Monoglyceride, Salts, Soy

Dough Meth. of Analysis for Baking Quality of Wheat Bre

Dough Tests of Flour (Cereal Chemistry) (1962)

Dough Tests of Flour (Cereal Chemistry) (1962)

Dough Tests (Cereal Chemistry) (1962)

Dough Tests) (Cereal Chemistry) (1962)

Dough Tests: Approximate Corrections for Changing as is

Dough to Mixing (Cereal Chemistry) (1962)

Dough to Mixing (Cereal Chemistry) (1962)

Dough Weight Meth. Only) (Cereal Chemistry) (1962)

Dough, Pound Loaf Meth. of Analysis for Baking Quality

Doughs (Cereal Chemistry) (1962)

Doughs (Cereal Chemistry) (1962)

Doughs (Cereal Chemistry) (1962)

Douglas and Hem Fir, Southern and Lodgepole Pine, Weste

Douglas Fir-Larch (North), Eastern Hemlock-Tamarack

Douglas Fir and Western Hemlock by Pressure Processes (

Douglas Fir Lumber Spec. for Application in Industrial

Douglas Fir, Coast Region; West Coast Hemlock; Western

Uniform Building Code Std. for Industry Std. for
ANSI

ICBO

ICBO

$A$ WI

AWI

ANSI

USC

AAMA

St AAMA

NBHA

STDl

STDl

AAMA

UL

NWMA

NWMA

UL

UL

ANSI

/ Buil ICBO

AWI

NFPA

NBHA

ASTM

Std

AAPCC "1

ASTM D1671

ASTM D 300

ASTM D2954

$\mathrm{N} 42.6$

D2954

D3001

D1671

$\mathrm{N} 42.6$

N 13.5

E170

$\mathrm{MH} 2.1$

MH2.7

$\begin{array}{ll}\text { ANSI } & \mathrm{MH} 2.9 \\ \text { ANSI } & \mathrm{MH} 2.13\end{array}$

/N ANSI

H ANSI

ANSI

ANSI

Pal ANS

or ANSI

ANSI

GT

ANS1

ASTM

NSA

Std ASTM

ASTM

ANSI

ASTM

SAE

MH2.15

MH2.10

MH2. 15

MH2. 10

$\mathrm{MH} 2.9$

MH2.17

MH2.16

MH2.3

$* 3$

N14.10.2

1353.66

B588

1015

J356A

Y 14.7 .1

1758

A 353

D3266

B29.4

MHll.1

C316

J527B

Std. P ANS1 L24.2.5

$\mathrm{AACCH} 48.51$

AACCH 10.10

AACCH 54.10

Struct AACCH 54-11

Ext AACCH 54-12

AACCH $54-20$

AACCH 54.29

$\mathrm{AACCH}$ 54-21

AACCH 54.40

1.I AACCH 54.29

AACCH 10.11

AACCH 22.11

$\mathrm{AACCH} 22.13$

$\mathrm{AACCH} 22.14$

1CBO UBCS25.11

ICBO UBCS25.2

AWPA C28

CTI I14

ICBO UBCS25.3

FHDA $\quad 4.72$ 
sugar, and Lodgepole Pine/

for Dynamic Water Resistance of Shee Upper Leather by the Std for Pin. Straight, Headles

olts, Lag Screws, Plates, Nails and Spikes, Washers, Pins, on by Manual Meth. (197/ on by Automatic Particlel ps (1972) Std. Meth. for Sampling Gas Blow Std. Meth. of Sampling Gas Blow Std for Medium Prefocus Base ng Foundation Drain, Under Basement Floor, Sump Conductor, Std. for Hole Spacing for Scraper and Bulldoze Rec. Pract, for Hole Placement on Bulldozer thmoving, Mining and Logging (Short Haul); Grader, Loader otective Structure for Weeled Front End Loaders and Weeled ehicles (Construction and Industrial Front End Loaders and Movers, Wheeled and Track Type Front End Loaders, Wheeled Std. Flue Gas Densities for Forced and Induced ances (Regulators, Automatic Damper Cont/ Safety Std. for Std. for

Std. Tolerances for Roller

lical, Double Helical and Rack) (1971)

American

ng and Struck Capacity for Shovel Dipper, Clam Bucket, and Std. Spec for Styrene Rubber Plastic Std. Spec. for Homogeneous Bituminized Fiber Std. Spec. for Laminated Wall Bituminized Fiber Std. Spec for Asbestos Cement Storm Std. Spec for Styrene Rubber (SR) Plastic

Il Connections, Outboard Water Strainers, Water Scoops and Std. Spec. for Concrete Std for Installation PVC Building Std. Spec. for Concrete Sewer, Storm Std. Spec. for Reinforced Concrete Arch Culvert, Storm d. Spec. for Reinforced Concrete Elliptical Culvert. Storm Std. Spec. for Reinforced Concrete D-Load Culvert, Storm Std. Spec. for Reinforced Concrete Culvert, Storm rainage (Land Reclamation, Curtain and Building Foundation

Std. for lustallation for Acrylonitrile Butadiene Styrene property Stds. for Acrylonitrile Butadiene Styrene Plastic Std. Spec. for Poly(vinyl Chloride) (PVC) Plastic 56 pec. for Acrylonitrile-Butadiene-Styrene (ABS) Plastic Std. Cast Iron Threaded

Standard for Wrought Copper and lts Alloy Solder Joint Std. Spec. for Perforated Concrete Under Uniform Plum bing Code: Batter

Rec for Design and Construction of Surf ace Std. for Cast Bronze Solder Joint Fittings for Solvent Uniform Plumbing Code Meth. for

Std Spec for Copper

for Homogeneous Solid Wall and Perforated Pipe for General Std. Spec. for Concrete Pipe for lrrigation or bituminized Fiber Perforated Pipe for Airport and Roadwork inous Fiber Pipe Systems for Highway, Airport, and Similar

Fiber Perforated Pipe for Agricultural, Land, and Genera td. Meth. of Direct Shear Test of Soils Under Consolidated

Uniform Plumbing Code: Rainwater Systems, Roof Meth of Test for Seam Strength of Curtains and no B/ Std. Performance Requirements for Woven Glass Fiber titutional Texti/ Std. Performance Requirements for Woven d Systems (1973)

Safety Std for Door. Rec. Pract for Rec. for

ntions and Lettering (1973)

ames-Passenger Car and Light Truc/

$\mathrm{ng}$ and Tolerancing (1973) nd Strip (1973) ANSI G24.25 p Process (1971) ANSl G8.2/ and Rack) (1971)

m (1973)

(1974) ANSI H23.8

Design Rules, Guidelines, and Std Spec for St Std. for Engineering Std. for Engineering Std. Engineerin Std. Spec. for American Drafting Manual (Gear Std. for Abbreviations for Use on Std. Spec, for Hard Ste Steel Wire, Hard Std. Spec. for Steel Wire, Hard

Std. Spec. for Seamless Cold Std. for Welded and Cold

nser Tubes (1973) ANSl B125/ flaring (1972) Rec. Pract. for Special Quality High Tensile, Hard (1973) ANSl H38.6 Std. Spec. for Aluminum Alloy Std. Spec. for Aluminum Alloy Std. Spec. for Cold

A50.3

i $\mathrm{H} 38.17$ Uniform Building Code Std. for Cold Std. Spec. for Aluminum-Alloy Spec for Tolerances of Aluminum Alloy d. Spec. for Flat Copper Products with Finished (Rolled or and Its Alloy Bar, Rod, Wire, and Forging Stock (Rolled or Std. Spec. for Steel Wire, High Tensile Strength, Hard Std. Meth. of Test for Applied Weight Per Unit Area of
Douglas Fir; Larch; Hem-Fir; Ponderosa, ldaho White, Dow Corning Leather Tester (1970) A LCA E55 Dowel) (1973)

Dowels, Joints, Split Rings and Framing Anchors) (1973) Down Systems and Components for Particulate Contaminati Down Systems and Components for Particulate Contaminati Down Type, Single Contact Motion Picture Projection Lam Downspout Runoff, Swimming Pool, etc.) (1973) ANSI A 176 Dozer) Cutting Edges (1972)

(Dozer) End Bit Supports (1972)

Dozer, Mining Car, Front End Loader, Fork Lift Trucks, /M Performance Criteria for Roll Over Pr

Dozers (1972)

/ Steering Frame Lock for Articulated V

Dozers, Track Type Tractors, and Motor Graders (1971)

Draft Centrifugal Fans (1966)

Draft Equipment for Combustion Chamber in Heating Appli

Draft Hoods for Gas Burning Appliances (1971)

Drafted Yarns (1971)

Drafting Guide for Mic rofilm (1971)

Drafting Manual (Gear Drawing Stds. Part 1 for Spur, He

Dragline Bucket (Construction and Industrial Machinery)

Drain and Building Sewer Pipe and Fittings (1973)

Drain and Sewer Pipe (1973) ANSI A176.1

Drain and Sewer Pipe (1973) ANSl A176.2

Drain Pipe (1973)

Drain Pipe, Perforated (1974)

Drain Plugs (Boats) (1972)

Drain Tile (1972)

Drain Waste and Vent Pipe and Fittings (1971)

lon of Seacocks, Through $\mathrm{Hu}$

Drain, and Culvert Pipe (1973)

Drain, and Sewer Pipe (1971)

Drain, and Sewer Pipe (1972)

Drain, and Sewer Pipe (1973)

Drain, and Sewer Pipe (1973)

Drain, Under Basement Floor, Sump Conductor, Downspout

Drain, Waste and Vent Pipe and Fittings (1971)

Drain, Waste and Vent Pipe and Fittings (1971)

Drain, Waste and Vent Pipe and Fittings (1973) ANSI K65

Drain. Waste, and Vent Pipe and Fittings (1973) ANSI B7

Drainage Fittings (1971)

Drainage Fittings (1973)

Drainage Pipe (1973)

Drainage System (1973)

Drainage Systems on Farms in Humid Areas (1973)

Drainage Systems (1973)

Drainage Systems (1973)

Drainage Time of Pulp (1972)

Drainage Tube (DWV) (1974) ANSl H23.6

Drainage (Land Reclamation, Curtain and Building Founda

Drainage (1973)

Drainage (1973)

Drainage (1973)

Std. Spec. for Laminated Wall

Drainage (1973) ANSI A 176.6

Drained Conditions (1972)

Drains and Piping (1973)

Draperies (Textile) (1973)

Drapery (Curtain) Fabrics Handwashable at 105 Deg. F-

Drapery (Curtain) Fabrics (Excluding Glass Fibers) (Ins

Drapery, Gate, Louver, and Window Electric Operators an

Draw Bar for Forestry Tractors (1970)

Drawbar for Forestry Tractors (1972) SAE J194

Drawing and Related Documentation Pract. for Line Conve

Drawing and Related Documentation Practices: Chassis Fr

Drawing and Related Documentation Practices: Dimensioni

Drawing Quality Hot and Cold Rolled Alloy Steel Sheet a

Drawing Quality, Zinc Coated (Galvanized) by the Hot Di

Drawing Stds. Part 1 for Spur, Helical, Double Helical

Drawings and in Text (1972) Text (1972)

Drawings for Copper Solvent Single Stack Plumbing Syste Drawings (1973)

Drawn Copper Capillary Tube for Restrictor Applications

Drawn for Mechanical Springs (1971) ANSI G54.1

Drawn for Prestressing Concrete Pipe (1973)

Drawn Intermediate Alloy Steel Heat Exchanger and Conde

Drawn Low Carbon Steel Tubing Annealed for Bending and

Drawn Mechanical Spring Wire and Springs (1972)

Drawn Seamless Tubes for Condensers and Heat Exchangers

Drawn Seamless Tubes (1974) ANSI H38.3

Drawn Steel Wire for Concrete Reinforcement (1972) ANSI

Drawn Steel Wire for Concrete Reinforcement (1973)

Drawn Tubes for General Purpose Applications (1973) Ans

Drawn Tubing (1973)

Drawn) Edges (Flatwire and Strip) (1973)

Drawn) (1973)

Drawn, for Mechanical Springs (1973)

Spec. for Tolerances of Aluminum

Dried Adhesive Solids (1969) ANSI Z197.9
ICBO

ASTM

NSA

WWPA

ASTM

ANSI

ASTM

SAE

SAE

TRA

SAE

SAE

AMCA

UL

ANSl

ASTM

NMA

ANSI

SAE

ASTM

ASTM

ASTM

ATTM

ASTM

$A B Y C$

ASTM

IAPMO

ASTM

ASTM

St ASTM

ASTM

ASTM

ASTM

is and

AAPMO

ASTM

ASTM

ANSl

ANSl

ASTM

ICBO

ASAE

ANSI

ICBO

TAPPI

ASTM

ASTM

ASTM

ASTM

ASTM

ASTM

ASTM

ICBO

ANS $]$

ANS1

ANS1

UL

SAE

ASAE

ANSl

ANSI

ANS1

ASTM

ASTM

ANSI

ANSI

CDA

AGMA

ASTM

ASTM

ASTM

ASTM

SAE

SAE

ASTM

ASTM

ASTM

ICBO

ASTM

t ASTM

SAE

ASTM

ASTM

UBCS $25-4$

D2098

607

-31-1

F308

PH22.85

D2311

J63 
Digitonin Cholesterol Meth. of Analysis of Meth. of Analysis for Total Phosphoric Acid in Meth. of Analysis of Lipoid Phosphoric Acid in Meth for Preparation of Sample: th. of Analysis of Hydrocyanic Acid Residues in Grains and Meth. of Analysis for Lactic Acid in istics/ Farinograph Evaluation Meth. of Ability of Roller nal Insect Infestation in Cereal Grains, Unpopped Popcorn, acroscopic Examination of Cereal Grains, Unpopped Popcorn, ent Hair Contamination of Cereal Grains, Unpopped Popcorn, t for Internal Insects in Cereal Grains, Unpopped Popcorn. Test Meth. for Fiber Bundles in Baled Flash Meth. of Analysis of Crude Fat in Egg Yolk and Std. Meth. of Test for Extractable Matter in Oven Std. Meth. of Test for Determination of Cobalt in Paint Std. Meth. of Test for Determination of Lead in Paint Std. Meth. of Test for Manganese in Paint Std. Meth. of Test for Calcium or Zinc in Paint Std. for Liquid Line

971)

Rating and Application of Suction Line Filters and Filter Rec. for Fire Safety and Protection of Yankee Rec. for Airborne Pulp building Code Std. for Timber Connector and Bolted Joints, Std. for

Std. and Nomenclature for Diamond Core Spec. for Casing. Tubing, and Spec. for High Strength Casing, Tubing, and al to Light Gage Steel Studs (1972) Std. Spec. for Steel 4) Rec. Pract. for Rec. Pract. for for Agricultural and Industrial Steering, Drive, Planter Std. for Full Threaded, $160 \mathrm{KSI}$ Steel, Std. for Nut, Plain, Hexagon,

e (1974T)

Std. Meth. of Test for Length Change of

sisting Steel, UNJC-3A Thread, Ca/ Sid. for Headed ion Resisting Steel, UNJF-3A Thre/

Std. for Undrilled and Std. for Undrilled and Std. for Horizontal Boring. Std. for Numerically Controlled Horizontal Boring, Spec. for Petroleum Spec. for Rotary
Std. for Numerically Controlled

orms (1973) Spec. for Petroleum
or Classification of Àreas for Electrical Installations at g Case Depth (1972) Std. for Steel Self Std. for Steel Self

rilling Through, Multiple Completion, and Flowline Valves; ng Joint, Segmented, Production Check Flanges and Gaskets; Spec. for Casing, Tubing, and Drill Pipe (Petroleum spec. for Grade C. 75 and C-95 Casing and Tubing (Petroleum Spec. for Line Pipe (Petroleum

Spec. for High Test Line Pipe (Petroleum

c. Pract. for Care and Use of Casing and Tubing (Petroleum or High Strength Casing, Tubing, and Drill Pipe (Petroleum Thru Letter F (1973)

rigerated Drinking Water Coolers (1973) ANSI Al/ Std. for 973)

ng Fountains and Self Contained, Mechanically Refrigerated Std. Meth. of

Std.

Std.

Std. Motor Positions for Belt or Chain

tractor and Farm Implement Power Takeoff (Universal Joint) tors (1972) SAE J955 Std. for Full Shielding of Power

Std. for Wrenching Configuration, Spline Rec. Pract. for Liquid Ballast Table for Std. Liquid Ballast Table for

rd) Engine Mountings (Installation and Replacement) Direct nd Protection for Maintenance and Inspection of Mechanical Apparatus) (Exclusive of Generator, for Hydraulic Turbine r Tires and Rims for Agricultural and Industrial Steering, Rec. for Steam Tubine Rec. for Steam Turbine umpe/ Std. for Installation of Vertical Hydraulic Turbine ing and Cleaning of Lubricating Systems of Various Turbine s Generator / Motor Uni/

6)

Tentative Manual for
Dried Egg Solids (Cereal Chemistry) (1962)

Dried Eggs (Cereal Chemistry) (1962)

Dried Eggs (Cereal Chemistry) (1962)

Dried Eggs (Cereal Chemistry) (1962)

Dried Foods (Cereal Chemistry) (1962)

Dried Geeg (Cereal Chemistry) (1962)

Dried Nonfat Dry Milk Solids to Change Mixing Character

Dried Peas and and Beans (Cereal Chemistry) (1962)

Dried Peas and Beans for Rodent and/or Insect Damage on

Dried Peas and Beans (Cereal Chemistry) (1962)

Dried Peas and Beans (Cereal Chemistry) (1962)

Dried Pulp (1973)

Dried Whole Egg (Cereal Chemistry) (1962)

Dried Wool (1973)

Driers by EDTA Meth. (1973)

Driers by EDTA Meth. (1973)

Driers by EDTA Meth. (1973)

Driers by EDTA Meth. (1973)

Driers in Refrigerating and Air Conditioning Systems (1

Driers in Refrigeration and Air Conditioning Systems (1

Driers (Paper Machine) (1973)

Driers (1973)

Driers (1973)

Drift Bolts, and Wood and Lag Screws; Light Metal Plate

Drill Drivers, Split Sleeve, Collet Type (1972)

Drill Equipment (1973) ANSI Bl04.1

Drill Pipe (Petroleum Drilling) (1973)

Drill Pipe (Petroleum Drilling) (1973)

Drill Screws for the Application of Gypsum Sheet Materi

Drill Stem Design and Operating Limits (Petroleum) (197

Drill Stern Test Report Form (1972)

Drill) Press, and Gage Wheel, Tractor, Implement, and L

Drilled Head Bolt (1972)

Drilled Jam, Thin (1972)

Drilled or Sawed Specimens of Cement Mortar and Concret

Drilled Pins for Farm Equipment and Machinery (1973)

Drilled, Plain and Self Locking, Alloy and Corrosion Re

Drilled, Plain and Self Locking, Alloy Steel and Corros

Drilling and Milling Machine (1974)

Drilling and Milling Machine (1974)

Drilling and Well Servicing Structures (1974)

Drilling Equipment (petroleum) (1974)

Drilling Machines (1974)

Drilling Rig Packaging for Minimum Self Contained Platf

Drilling Rigs and Petroleum Production Facilities on La

Drilling Tapping Screws and Rec. Technique for Measurin

Drilling Tapping Screws (1972)

Drilling Through Control, Screwed and Flanged Wellhead,

Drilling Through, Multiple Completion, and Flowline Val

Drilling) (1973)

Drilling) (1973)

Drilling) (1973

Drilling) (I973)

Drilling) (1973)

Drilling) (1973)

Drills, High Speed Steel Threaded Shank, Sizes 1/16 In.

Drinking Fountains and Self Contained. Mechanically Ref

Drinking Water Coolers (1972) ANSI C33.82

Drinking Water Coolers (1973) ANS1 A 112.11.1

Drip Slag Test. Refractory Brick at High Temperature (1

Drive Arrangements for Centrifugal Fans (I966)

Drive Arrangements for Tubular Centrifugal Fans (1966)

Drive Centrifugal Fans (1966)

Drive Line Specs. (1972)

Drive Lines for Agricultural

Drive Pin Blind Rivets (1973)
Drive Threaded Fasteners (1971)

Drive Tires of Agricultural Machines (1971)

Drive Tires of Agricultural Machines (1972)

Drive Transmission (1971)

Drive Turbines (1974)

Drive) (1972)

Drive, Planter (Drill) Press, and Gage Wheel, Tractor,

Driven Generator Units (1972)

Driven Generator Units (1974)

Driven Generators and Reversible Generator/Motors for $\mathrm{P}$

Driven Pumps for Marine Service (1971) Rec. Pract. for Motor Vehicle Std. for Drill

Std for Design of Components: Enclosed Gear enetrating the Hull for Boats Equipped with Outdrives, Jet Std. Spec. for Lubrication of Industrial Enclosed Gear
Driver Attended Loading of Anhydrous Ammonia (1972)

Driver's E ye Range (1972)

Drivers, Split Sleeve, Collet Type (1972)

Drives-Bearings, Bolting Keys and Shafting (1974)

Drives and/or Bow Thrusters (1973)

Drives (1972)

Drives (1972)
AACCH 20.01

$\mathrm{AACCH} \quad 04.13$

AACCH 20.02

AACCH 62.15

Me AACCH 60.20

AACCH 04.25

AACCH 54.20

Er AACCH 28.2]

$\mathrm{AACCH} 28.10$

INd Rod AACCH 28-20

Ion Tes AACCH 28.22

TAPPI UM.239

$\mathrm{AACCH} \quad 30-18$

ASTM D1574

ASTM D2373

ASTM D2374

ASTM D2375

ASTM D2613

ARl $\quad 710$

ARl $\quad 730$

FMS $\quad 12-29$

ASTM D 600

7.20

ICBO UBCS25-17

ANSI B94.35

API A 5

APl $5 \mathrm{AX}$

ASTM C646

APl $7 \mathrm{G}$

APl RP48

TRA 4

NSA 563.572

NSA $\quad 1423$

ASTM C34I

ASAE S226.2

NSA 1352

NSA $135 \mathrm{I}$

NSA 910

NSA 954

APl $\quad 4 \mathrm{E}$

NSA 960

API 2E

API RP500B

SAE J78

IFl

APl

API

APl

APl

APl

APl

Re APl

Spec. F APl

NSA

ARI

UL

113

$6 \mathrm{~A}$

$6 \mathrm{~A}$

$5 A C$

$5 \mathrm{~L}$

$5 \mathrm{LX}$

$5 \mathrm{Cl}$

$5 \mathrm{AX}$
965

1010

399

1010

$\begin{array}{ll}\text { ARI } & 1010 \\ \text { ASTM } & \text { C768 }\end{array}$

AMCA 2404

AMCA 2410

AMCA 2407

ASAE R331.2

ASAE S297T

IFI

SAE

$S A E$

ASAE

123

ASl159A

J884B

S346

J233

13.9

$\mathrm{MGl} \cdot 22$

$\begin{array}{ll}\text { TRA } & 4 \\ \text { FMS } & 13.3\end{array}$

FMS $\quad 13.3$

NEMA MG5.2

ASME LOS-2Cl

NEMA MG5.1

FI P135

NGPA 8166

SAE J941C

ANSI B94.35

AGMA 260.02

P16

AGMA 250.03

NEMA PV 2 
c. for Agricultural (Farm) Tractor Auxiliary Power Takeoff Pract. for Agricultural Tractor Auxiliary Power Take Off cal and Herringbone Gear Units for Water Cooling Tower Fan nt for Enclosed Helical, Herringbone and Spiral Bevel Gear pract, and Requirements for Thyristor Converters for Motor rtmental Regulations and Controls, Barrier, Road, Hydrant, Tent. Rec. Pract. Guidelines for for Wood Paving Blocks for Exposed Platforms, Pavements, ectric Tools (1973)

Std. for

meth. of Test. Tires for Wet Traction in Cornering Without Std. for Aluminum, Triplex, Neutral Supported, Service Std. Test. Procedure for Filled Cast Flat Std Test. Procedure for Filled Cast Flat Bottom

ainers $(1970)$ ptivity) (1973) Std. Merh. of Test for Slant

ished Carpentry, Flooring, Shingles, Exposed Roof Decking, Std. Meth. of Sid. Meth. of g) (1950) ANSI MH12.7 si $\mathrm{MH} 12.2$ Sid. Meth. of test for lmpact Resistance of Pipeline Coatings (Limestone s) (1973) 260.3

e Range (1972) ANSI Z11.207 Meth. of Test Meth. for Tent. Meth. for Sid. Spec. for $55 \mathrm{Gal}$. Full Removable Head Universal r Cranes, Derricks, Hoists, Jacks and Slings: Base Mounted Std. Meth. for Climbing Rec. for Flammable Liquid Rec. Pract. for Automotive Brake Disc and Std. Spec. for 16 Gal. Tight Head Universal

Std. Spec. for $30 \mathrm{Gal}$. Full Removable Head Universal Std. Spec. for $55 \mathrm{Gal}$. Tight Head Universal Std. Spec. for $55 \mathrm{Gal}$. Tight Head Universal

Std. Spec. for $55 \mathrm{Gal}$. Full Removable Head Universal st. Shipping Containers (Packaging) in Revolving Hexagona Std. Spec. for 55 Gal. Tight Head Universal Std. Spec. for $30 \mathrm{Gal}$. Tight Head Universal

Std. Spec, for $57 \mathrm{Gal}$. Full Removable Head Universal

Std. Spec, for $30 \mathrm{Gal}$. Full Removable Head Universal

Std. Spec. for $16 \mathrm{Gal}$. Full Removable Head Universal spec. for 16 Gal. Full Removable Head, Lug Cover Universal 0) Ansi/ Std. Spec. for Alloy Steel Forgings for Seamless

Std. Test Meth. for Hydrogen lon Concentration of

Sid. Meth. of Test for High Voltage, Low Current, Safety Std. for Electric

Rec. for Terminal Facilities for Pneumatic Transfer of Spec. for

Piping and Equipment for Use with ty of Bonded and Laminated Apparel Fabrics (Textile) After Sid. for Safety Requirements for Commercial Laundry and Std. for Safety for Commercial

Uniform Building Code Std. for Class Il and Class III Std. Spec. for Hydrocarbon vements, Driveways, and Interior Floors Exposed to Wet and Sid. Test Procedure for ed Products --Film Thickne/ Best Meth. for Measurment of Test Meth for Colorfastness of Textiles to nce of Paper, Paperboard, and Other Sheet Materials by the Pract. for lnstantizing Systems for

Carr-Price Meth. of Analysis of Vitamin A in Nonfat Meth. of Analysis of Lactose (Sugar) and Nonfat Extensigraph Meth. for Quality of Nonfat Meth. of Analysis for Ash in Meth. of Analysis of Crude Fat in Pract for Instantizing Systems for Dry Milk and nograph Evaluation Meth. of Ability of Roller Dried Nonfat Meth. of Analysis for Titratable Acidity in Nonfat Meth. of Analysis for Lactic Acid in Nonfat

Meth. of Analysis for Baking Quality of Nonfat Meth. of Analysis of Moisture in Nonfat of Analysis of Undenatured Whey Protein Nitrogen in Nonfat f Analysis of Vitamin A in Nonfat Dry Milk and Instantized Std. Spec. for Crushed Stone, Slag, and Gravel for on and Maintenance of the Reliable Model B Accelerator for Rec. for Operation of Grimes Ab Operational Rec. for Globe Rec. for Operation of Crowder tion Service (1970)

(Fire Protec/

rophotometric) Content in Feedstuffs and All Other Natura

er Vapor Transmission of Flexible Heat Sealed Packages for st for Coefficient of Friction and Wear Characteristics of nsi C 89.2
Drives (1972)

Drives (1972)

Drives (1972)

Drives (1973)

Std. for Sound (Noise Measureme

Driveway Design and Location (1972)

Driveways, and Interior Floors Exposed to Wet and Dry C

Driving and Spindle Ends for Portable Hand, Air, and El

Driving Torque Application, Using Highway Vehicles (197

Drop and Secondary Cable (1966) IPCEA S-64.474

Drop Composite Cans (1974)

Drop Composite Motor Oil Cans (1974)

Drop Impact Resistance of Polyethylene Blow Molded Cont

Drop Penetration Test for Paperboard (Printing Ink Rece

Drop Shatter Test for Coke (1972) ANSI K20.24

Drop Siding, Structural Roof Trusses and Rafters, Overhead

Drop Test for Bags (Packaging) (1950) ANSI MH12.6

Drop Test for Cylindrical Shipping Containers (Packagin

Drop Test for Shipping Containers (Packaging) (1961) an

Drop Test) (1972)

Drop Test. of Glass Aerosol Bottles (1972)

Drop Tests for Paper Shipping Sacks and Bags (Container

Drop Weight Tear Tests of Ferritic Steels (1971) ANSI Z

Dropping Point of Lubricating Grease of Wide Temperatur

Drum (DOT-17H) (1968)

Drum Hoists (1971)

Drum Peel Test for Adhesives (1962) ANSI Z197.23

Drum Storage, Dispensing, and Classification (1972)

Drum Thermocouple Installation (1972)

Drum (Dot-17E) (1974)

Drum (Dot-17H) (1974)

Drum (Dot-5B) (1974)

Drum (Dot-17C) (1974)

Drum (Ufc-Rule 40, Nmfc-Item 260) (1974)

Drum (1968) ANSI MH12.3, TAPPI T800

Drums (Dot-17E) (1974)

Drums (Dot-17E) (1974)

Drums (Ufc-Rule 40, Nmfc-Item 260) (1974)

Drums (Ufc-Rule 40, Nmfc-Item 260) (1974)

Drums (Ufc-Rule 40, Nmfc-ltem 260) (1974)

Drums (Ufc-Rule 40, Nmfc-Item 260) (1974)

Drums, Heads, and Other Pressure Vessel Components (197

Dry Adhesive Films (1970)

Dry Arc Resistance of Solid Electrical Insulation (1973

Dry Bath Heaters (1972) ANSI C33.75

Dry Bulk Chemicals and Plastics (1972)

Dry Cells and Batteries (1972)

Dry Chlorine (1971)

Dry Cleaning and Laundering (1972) ANSI L14.269

Dry Cleaning Equipment and Operations (1972)

Dry Cleaning Machines (Class lv) (1974)

Dry Cleaning Plants or Systems (1973)

Dry Cleaning Solvents (1971) ANSI Z11.42

Dry Conditions (1970) ANSI 09 .1

Dry Fertilizer Spreader (1972)

Dry Film Thickness of Paint, Varnish, Lacquer and Relat

Dry Heat (Excluding Pressing) (1973) ISO 105.4

Dry Indic ator Meth. (1973) TAPPI T433

Dry Milk and Dry Milk Products (1972)

Dry Milk and Instantized Dry Milks (Cereal Chemistry)(

Dry Milk in Bread (Cereal Chemistry) (1962)

Dry Milk in Physical Dough Tests (Cereal Chemistry) (19

Dry Milk Products (Cereal Chemistry) (1962)

Dry Milk Products (Cereal Chemistry) (1962)

Dry Milk Products (1972)

Dry Milk Solids to Change Mixing Characteristics of Flo

Dry Milk (Cereal Chemistry) (1962)

Dry Milk (Cereal Chemistry) (1962)

Dry Milk (Cereal Chemistry) (1962)

Dry Milk (Cereal Chemistry) (1962)

Dry Milk (Cereal Chemistry) (1962)

Dry Milks (Cereal Chemistry) (1971)

Dry or Water Bound Macadam Base Cours

Dry Pipe Systems (Fire Protection) (1973)

Dry Pipe Valve Accelerator (1973)

Dry Pipe Valves (Fire and Safety Equipment) (1973)

Dry Pipe Valves, Model a (1973)

Dry Pipe Valves; Rockwood Models C, D (1974)

Dry Pipe, Deluge, and Pre Action Valves for Fire Protec

Dry Powder Form by Means of Spray Gun or Fluidized Bed

Dry Products Derived from Corn (1957)

Dry Products (1972)

Std. Meth. of Test for Wat

Dry Solid Film Lubricants in Vacuum and Other Controlle

Dry Transformers, Hand Power Tools, Hermatic Stators, T

Dry Type Transformers for General Applications (1972) a
Std. Meth. of

No Color (Spect CR

Re ASAE

Rec SAE

AGMA

AGMA

IEEE

ICBO

ITE

ASTM

ANSl

ASTM

EEI

CCTI

CCTI

ASTM

TAPPI

ASTM

AWI

ASTM

ASTM

ASTM

ASTM

ASTM

TAPPI

ASTM

ASTM

ANS!

ANSI

ASTM

FMS

SAE

ANSI

ANSI

ANSl

ANSI

ANSI

ASTM

ANSI

ANSI

ANSI

ANSI

Std. ANSI

ASTM

ASTM

ASTM

UL

MCA

ANSl

$\mathrm{CHI}$

/Pensi ASTM

ANSI

UL

ASTM

ASAE

NCCA

AATCC

ASTM

DFISA

$\mathrm{AACCH}$

AACCH 80.31

AACCH 54.12

$\mathrm{AACCH}$ 08-11

AACCH $30-16$

DFISA $\quad 60800$

AACCH $\quad 54-20$

$\mathrm{AACCH} 02-31$

A ACCH $04-26$

AACCH $\quad 10-85$

$\mathrm{AACCH} \quad 44.52$

Meth. AACCH $46-20$

R-Price Meth. O AACCH 86.02

ASTM

FMS

FMS

FMS

FMS

FMS

UL

CR

ASTM

ASTM

EASA

NEMA 
for Structural Laminated Products for Use Under Interior (Dry Use) Exposure Conditions (1972) ANSI Z197.33

Ultraviolet Absorption Meth. of Analysis of Vitamin A in

Rec. Terms for Care Labeling Textile Products (Launder or 1 Use Garments (Clothing), 100\% Man Made or Blend Fabrics, xiile) (1973) $\begin{array}{ll}\text { ustrial) Suiting/ } & \text { Std. Performance Requirements for Woven } \\ \text { ar Round) Suitin/ } & \text { Std. Performance Requirements for Woven }\end{array}$ Std. Performance Requirements for Woven on Textiles and Other Materials (1973)

Test Meth. for nish of Fabrics (1972) Std. Meth. of Test for Effects of Test Meth. for Colorfastness of Textiles to

d. for Electric Home Laundry Equipment (Clothes Washer and Operated and Commercial Clothes Drying Equipment (Laundry

d. and Test Procedures for Home Laundry Equigment (washer, Std. for Volume I, Type!I, Gas Clothes Std. for Gas Clothes

ories (1972) ANSI A111.25 Std. Meth of Test for or Commercial Clothes Std. Meth of Test. Uniform Fire Code: Industrial Baking and Std. Meth. of Wetting and

nsi A37.51 uites, Molasses, and Other Liquid and Semiliquid Products
ordinary Commercial and Industrial Samples of Wool by Oven td. for Dimensional and Electrical Characteristic Defining Std. Spec. for Centrifugally Cast

ning Systems (1972)

Fibrous Glass terials Application for Thermoplastic (Polyvinyl Chloride) Electrical Wire and Cable (1/ Std. for Plastic Utilities Std. for Gas Fired oustical and Airflow Performance (19) Std. Meth. of Test. air Distribution) (1971)

Std. for Safety for Factory Made Air Std. for Steel Underfloor

ning, Heating, Exhaust, Ventilating, and Their Combination for Smooth Wall Coilable Polyethylene Electrical Plastic ressure Sensitive Tape Performance Stds. for Fibrous Glass ressure Sensitive Tape Application Stds. for Fibrous Glass Spec. for Cabin 71) Specifying

Std. Rec. Pract. for Polyethylene Encasement for Gray and 972) ANSI Std. for Polyethylene Encasement for Gray and Std. Spec. for and Other Liquids (1971) ANSI A2/ r Sand Lined Molds for Gas (1971) Std. for Std. for Cast Iron and $.11 \quad$ Std. for Rubber Gasket Joints for Cast and ctrical W/ Std. for Styrene Rubber and Polyvinyl Chloride Uniform Building Code: Elevators, Std. for Receptacle

Std. for Dimensional Requirements for nsi Z197.30

s-Definitions and Meth. for Determining Specific Weight, ion Loading (1971) Ansi/ and Other Materials (1973) Std. Rec. Pract. for Determining Std. Rec. Pract. for Determining Test Meth. for Drycleaning Rec. for Materials and Meth. for Std. Meth. of Test for

t. for Signal Words, Letter Sizes, Color Combinations, and al Characteristics and Finishes of Institutional Textiles epeated Home Laundering/ Test Meth. for the Appearance of irements for Woven Sheet and Pillowcase Fabrics Other Than ics (Institutional Tex/ Std. Performance Requirements for Meth. of Test for Effects of Drycleaning on Permanence of h. for Dimensional Changes in Automatic Home Laundering of Batch Meth. of Experimental Milling for Std. Test Meth. for

te/ Rec. Pract. for Air Cleaning Devices (Air Filters and flammable and Combustible Liquids and Solids and Potential Uniform Fire Code: Prevention of Dusts) (1973) Std. for Area Classification in Hazardous r Safe Installation of Electrical Instruments in Hazardous Safety Guide for Respiratory Protection Against Coal Mine $n$ of Liquid Automatic Particle Counters Using AC Fine Test building Code Std. for Blower, Fan, and Exhaust System for td. for the Installation of Blower and Exhaust Systems for Concrete/ Std. Spec. for Calcium Chloride to Be Used for us Dust Locations (Agricultural Plastics, Chemicals, Metal us Dust Locations (Agricultural Plastics, Chemicals, Metal

973) Rec. Std. Details for

973) ANSI C8.32

Nuclear Po/

d Sprocket Teeth (1972)

73) Dry Vitamin Mix

Drycleanable Only (1973)

Drycleanable Overcoat Fabrics (Institutional Uniform Te

Drycleanable Tailored Uniform Fabrics, Intermediate (Ye

Drycleaning Durability of Applied Designs and Finishes

Drycleaning on Permanence of Durable $W$ ater Repellent $F$

Drycleaning (1973) ANSI L14.241

Dryer) (1972) ANSI C 33.13

Dryer) (1974)

Dryer, Combination) (1971) ANSI A197.2, Asse 1007

Dryers (1972)

Dryers, Volume II, Type 2 (1974)

Drying and Firing Shrinkage of Fireclay Plastic Refract

Drying at 135 Deg. C (Cereal Chemistry) (1962)

Drying Equipment (Laundry Dryer) (1974)

Drying Farm Crops (1971)

Drying Oils (1972)

Drying Ovens (1973)

Drying Tests of Compacted Soil Cement Mixtures (1971) a

(Drying Upon Quartz Sand) (Cereal Chemistry) (1962)

Drying (1972) ANSI L14.202 /T for M
Dual in Line Type Sockets (1974) ANSI C 83.95

Dual Metal Cylinders (1972)

Duct Construction Std. for Ventilating and Air Conditio

Duct Construction (1974)

Duct Furnaces (1971)

Duct Liner Materials and Prefabricated Silencers for AC

Duct Liner, Adhesives, and Fasteners Application Std. (

Duct Materials and Connectors (1974)

Duct Raceways (1972)

Duct Systems (1973) ANSI B144.2

Duct (1972)

Duct (1973)

Duct (1973)

Duct, Air, Flexible and Semi Rigid (1972)

Duct, Sheet Metal, Low Pressure, Air Transmission (1967

Ducted Electric Heat Guide for Air Handling Systems (19

Ductile Cast Iron Pipe for Water or Other Liquids (1972

Ductile Cast Iron Piping for Water and Other Liquids (1

Ductile Iron Castings (1972)

Ductile lron Fittings, 2 In. Through 48 In./S for Water

Ductile Iron Pipe, Centrifugally Cast, in Metal Molds 0

Ductile Iron Pressure Pipe and Fittings (1972) ANSI A21

Ducts and Fittings for Underground Installations of Ele

Dumbwaiters, Escalators, and Moving Walks (1973)

Dummy Storage, Electrical Connector (1972)

Duplex and Single Face Flat Thread Rolling Dies (1973)

Durability and Moisture Content (1972) T/Ts a

Durability of Adhesive Joints Stressed in Shear by Tens

Durability of Applied Designs and Finishes on Textiles

Durability of Concrete in Service (1959)

Durability of Fire Retardant Treatment of Wood (1972)

Durability Requirements for Both Permanently and Tempor

Durability, Spot, Stain, and Insect Resistance) (1973)

Durable Press Textile Fabrics (Permanent Press) After R

Durable Press (Institutional Textile) (1973)

Durable Press (No Iron) Woven Sheet and Pillowcase Fabr

Durable Water Repellent Finish of Fabrics (1972)

Durable (Permarent) Press Woven or Knit Textile Fabrics

Durum Wheat (Cereal Chemistry) (1962)

Dust and Fog Tracking and Erosion ANSI C59.133

Dust Collectors) for Industrial Exhaust Ventilation Sys

Dust Explosion Conditions (1973)

Dust Explosions (1973)

(T), and Handling of

Dust Locations (Agricultural Plastics, Chemicals, Metal

Dust Locations (Agricultural Plastics, Chemicals, Metal Dust (1972)

Dust (1972) ANSI B93.28

Dust, Stock and Vapor Removal (1973)

Std. Meth. for Calibratio

Dust, Stock, and Vapor Removal or Conveying (1973) ANSI

Dustlaying, Stabilization, Ice Removal, Acceleration of

Dusts) (1973)

Dusts) (1973)

Std. for Area Classification in Hazardo

Dutch Doors (1972)

lon of Electrical Instruments in Hazardo

Duty Black Polychloroprene Jacket for Wire and Cable (1

Duty Black Polychloroprene Jacket for Wire and Cable (1

Duty Class I Motors Installed Inside the Containment of

Duty Fireclay Brick (1970) ANSI A111.14

Duty Fireclay Brick (1970) ANSI A111.16

Duty Off set Sidebar Power Transmission Roller Chains an

Duty Roller Type Conveyor Chains and Sprocket Teeth (19
Ives ASTM

$\mathrm{AACCH}$

ANSI

ANSI

ANSI

ANSI

AATCC

ASTM

AATCC

safety St UL

St AHAM

ANSI

ANSI

ASTM

$\mathrm{AACCH}$

Std. Fo UL

td. F ASAE

ASTM

ICBO

ASTM

EC AACCH

ASTM

EIA

ASTM

SMACN $* 6$

NEMA TC6

ANSI Z21.34

ASTM E477

SMACN *13

UL

NEMA

181

NFPA $90 \mathrm{~A}$

Std NEMA TC 7

SMACN AFTS- 100

SMACN AFTS.101

NSA 1369

CSI 15836

SMACN *12

ASTM A674

AWWA $\mathrm{C} 105$

ASTM A536

$\mathrm{AWWA} \quad \mathrm{C} 110$

ANSI A21.52

$A W W A \quad C 111$

NEMA TCl

ICBO UBC*3-51

NSA

IFI

ASAE

1196

109

ASTM D29.2

ASTM D2919

AATCC

ACI

ASTM

SAE

ANSI

AATCC

86

59.57

D2898

J115

L24.7.1

124

L24.2.12

ANSI L24.2.13

Std. ASTM D2721

AATCC 135

AACCH 26.30

ASTM D2132

ACGIH *1-11

ICBO

ICBO

ISA

ISA

FC $\$ 2$ ART 34

FC*2ART9

ANSI 288.4

NFLDP

ICBO

ASTM

ISA

ISA

ASTM

ASTM

IEEE

ASTM

ASTM

ANSI

ANSI 
cilities (Passenger Cars, Multipurpose Vehicles, and Light Buckle, Web Strap, Light

Std for Threaded Metal Insert-Heavy

Std. Part Pins, Spring, Slotted and Coiled, Heavy or Woven Washable Uniform Fabrics for Men and Women (Heavy try) (1962)

1973)

legal Requirements (Permit, Plans and Spec., Inspection)/ wall Framing and Weather Protection (1973)

solid, Grouted, Reinforced Grouted, Hollow Unit, Cavity / veneered Walls (1973)

exits and Stairs (1973)

plastering and Installation of Wallboard Lathing (1973)

plastering (1973)

wallboard (1973)

softwood Plywood Paneling (1973)

roof Coverings (1973)

valley Flashing (Sheet Metal for Roof Waterproofing) (19/ location on Lot (1973)

light, Ventilation, Sanitation, Fire Warning Detector (1/ private Garages and Carports (1973)

foundations and Retaining Walls (1973)

chimneys, Fire places, and Barbecues (1973)

framing-General (1973)

flooring (1973)

roof Framing (1973)

Southern Building Code for

, Public or Office Building, Manufacturer, Restaurant, and Std. Spec. for Copper Drainage Tube

Oilseeds, Legumes, Forages, and Animal and Dairy P/Udy ract. for Determining Hermeticity of Electronic Devices by d Control Test (1969) Test Meth. for Colorfastness of 14.4

i L14.9

Test Meth. for

14.5

Test Meth. for Colorfastness of

Test Meth. for Accelerated Aging of Sulfur Test Meth. for Colorfastness of Test Meth. for Colorfastness of

Requirements for Table Napery Other Than Damask (White or fabric) (1972) ANSI / Std. Meth. of Test for Differential Std. Performance Requirements for Deleterious Effects of

Meth. for Water Repellency of Textile Fabrics: Tumble Jar

Products (1973) ANSI J2.6 Std for Guide for the

of Transparent and Opaque Liquids and the Calculation of ow Corning Leather Tester (1970) / Std. Meth. of Test for aeser Water Penetration Tester (1/ Std. Meth. of Test for Meth. of Test. Coated Steel Specimens Std. for Guide-Fastener, Low Form, Cowl,

ate) (Common Name for Th/Std, for Methoprene (Isopropyl Std. Common Name for the Pest Control Chemical S-Ethyl

for Common Name for the Pest Control Chemical 2-Propynyl rdous Locations: Class I, Groups A-D, and Class II, Groups rdous Locations, Class I, Groups A-D, and Class II, Groups hazardous Locations, Class I, Groups A.D. Class II, Groups ic Systems Based on the Composition and Crystal Lattice of

Std. Meth. for Coupler Calibration of Rec. for Lined 1 and Mechanical Characteristics of Antennas for Satellite oader/ Std. for Tires and Rims for Off the Road Vehicles: Std. for

Timber; Water; Ceiling; Partitions; Plaster; Roofing) and ion and Water Sprinkler System Design-Fire Protection / weights of Materials (Brick: Cast Iron; Cinders; Concrete;

Std. for Coast Sitka Spruce, Douglas Fir-Larch (North), uniform Building Code Sid. for Eastern White and Red Pine, sam Fir; Northern Pine An/ Uniform Building Code Std. for Balsam Fir and Tamarack / Uniform Building Code Std. for s Fir-Larch (North), Eastern Hemlock-Tamarack (North), Wine (Enology) (1972) Tent. Std. for Indust
ith Magnetic Saturation (1971) Ansi/ Std. for Grader Cutting

ted Electric Gardening Appliances (Lawn Mower, Cultivator, for Flat Copper Products with Finished (Rolled or Drawn) for Hole Spacing for Scraper and Bulldozer (Dozer) Cutting Meth. of Analysis of Cloud Point in All 67) Meth. of Analysis of Iodine Number in All

Meth of Test for Tetrasodium Salt of Std. Meth. of Test for Manganese in Paint Driers by Std. Meth. of Test for Calcium or Zinc in Paint Driers by meth. of Test for Determination of Lead in Paint Driers by th. of Test for Determination of Cobalt in Paint Driers by enediaminetetraacetic (Ethylenedinitrilotetraacetic) Acid Containers (1972) Meth. of Test for
Duty Trucks) (1971)

Duty (1972)

Duty (1973)

Duty (1973)

Duty) (Institutional Textile) (1973)

/Ill Pipes with Fuel Dispensing Fa SAE

NSA

NSA

NSA

Duvel) of Analysis of Moisture in Grains (Cereal Chemis

Dwelling Construction Under the Uniform Building Code (

Dwelling Construction Under the Uniform Building Code: Dwelling Construction Under the Uniform Building Code: Dwelling Construction Under the Uniform Building Code: Dwelling Construction Under the Uniform Building Code: Dwelling Construction Under the Uniform Building Code: Dwelling Construction Under the Uniform Building Code: Dwelling Construction Under the Uniform Building Code: Dwelling Construction Under the Uniform Building Code: Dwelling Construction Under the Uniform Building Code: Dwelling Construction Under the Uniform Building Code:

Dwelling Construction Under the Uniform Building Code: Dwelling Construction Under the Uniform Building Code: Dwelling Construction Under the Uniform Building Code: Dwelling Construction Under the Uniform Building Code: Dwelling Construction Under the Uniform Building Code: Dwelling Construction Under the Uniform Building Code: Dwelling Construction Under the Uniform Building Code: Dwelling Construction Under the Uniform Building Code: Dwelling Construction Under the Uniform Building Code:

Dwelling House Construction (1973)

Dwelling or Appartment House (1973)

(DWV) (1974) ANSI H23.6

Dye Meth. of Analysis of Crude Protein in Cereal Grains

Dye Penetration (1972)

Dyed or Printed Textiles to Washing at 105 Deg. F: Rapi

Dyed Silk Yarn Colorfastness to Degumming (1972) ANSI L

Dyed Textile Yarns to Sulfur Dioxide Stoving (1972) Ans

Dyed Textiles (1972) ANSI L14.1

Dyed Wool Fabric and Yarn to Mill Fulling (1972) ANSI L

Dyed Wool Textiles to Carbonizing (1972) ANSI L14.3

Dyed) (Napkin, etc.) (Institutional Textile) (1973)

Dyeing Behavior of Cotton (Raw Fiber, Yarn and Textile

Dyes, Finishes, and Finishings (Sublimation, Brittlenes

Dynamic Absorption Test (1972) ANSI L14.87, ASTM D583

Dynamic Calibration of Pressure Transducers (1972)

Dynamic Test. for Ply Separation and Cracking of Rubber

Ematic Visc

Dynamic Water Resistance of Shoe Upper Leather by the D

Dynamic Water Resistance of Shoe Upper Leather by the M

Dynamically for Resistance to Corrosion (1972T)

Dzus Type (1973)

E)-11-Methoxy-3,7,11-Trimethyl -2,4-Dodecadieno

E,E)-11-Methoxy-3,7,11-Trimethyl-2,4-Dodecadienethio

E,E)-3,7,11-Trimethyl-2,4-Dodecadienoate Kinoprene (1

E.G (1972) ANSI C33.29

E-G (1973)

E.G (1973) ANSI C33.30

Each (1970) ANSI Z30.10

It $\mathrm{Br}$

Rical Switches for Use

Early Strength of

Earphones (1973)

Earth Reservoirs for Fire Protection (1972)

Earth Stations (1973)

Earthmoving, Mining and Logging (Short Haul); Grader, L Enclosures for Use in Haza

Control Equipment for Use in

Earthquake Recording Instrumentation (1973)

Earthquakes (General Characteristics Of, Rec. Construct

Earth; Glass: Granite; Gravel; Granite, Limestone, and

Eastern Hemlock-Tamarack (North), Eastern White Pine

Eastern Spruce and Hemlock, Balsam Fir and Tamarack Lum

Eastern Spruce, White Pine, and Hemlock-Tamarack; Bal

Eastern White and Red Pine, Eastern Spruce and Hemlock,

Eastern White Pine (North), Hem-Fir (North), Ponderos

Ebulliometer Meth. for Analysis of Alcohol (Ethanol) in

Economy (1972)

Eddy Current Test. of Steel Tubular Products (Tubing) W

Edge (1972)

Edger-Trimmer, Hedge Trimmer, etc.) (1974)

Edges (Flatwire and Strip) (1973)

Edges (1972)

Edible Animal and Vegetable Fats (Cereal Chemistry) (19

Edible Fats and Oils (Cereal Chemistry) (1967)

Editorial Format for Voluntary Product Stds. (1973)

EDTA in Water (1972T)

EDTA Meth. (1973)

EDTA Meth. (1973)

EDTA Meth. (1973)

EDTA Meth. (1973)

(EDTA) and Its Salts $\left(\mathrm{C}_{40}, \mathrm{H}_{11:} \mathrm{N}_{2} \mathrm{O}_{\mathrm{H}}(1973)\right.$
Effective Crimping on Outside Crimped Valves of Aerosol
$A A C C$

ICBO

ICBO

ICBO

ICBO

ICBO

ICBO

ICBO

ICBO

ICBO

ICBO

ICBO

ICBO

ICBO

ICBO

ICBO

ICBO

ICBO

ICBO

ICBO

ICBO

SBCC

ICBO

ASTM

$\mathrm{AACCH}$

ASTM

AATCC

AATCC

AATCC

AATCC 26

AATCC

$\triangle A T C C$

IE ANSI

ASTM

ANSI

AATCC

ANSI

ASTM

ASTM

ASTM

ASTM

ASTM

NSA

ANSI

ANSI

ANSI

UL

\section{UL}

UL

BIA

ANSI

FMS

EIA

TRA

ANSI

FMS

ICBO

ICBO

ICBO

ICBO

ICBO

ICBO

ASE

ANSI

ASTM

SAE

Std. Spec ASTM

Std. SAE

$\mathrm{AACCH}$

$\mathrm{AACCH}$

USC

ASTM

ASTM

ASTM

Std ASTM

Std. Me ASTM

/ Ethyl ANSI

ASTM

J398 A

1284

61

L24.3.6

BC $* 8$

UBC * 8-1

UBC $* 8-11$

UBC *8.12

UBC *8-13

UBC $* 8.14$

UBC *8-15

UBC *8-16

UBC $* 8-17$

UBC $* 8.18$

UBC *8-19

$\mathrm{UBC} * 8-2$

UBC $* 8-3$

UBC * 8-4

UBC *8-5

$\mathrm{UBC} * 8-6$

$\mathrm{UBC} * 8-7$ 
Materials (1973)

) 3)

Tent. Meth. of Test for Std. Meth. of Test for Tent. Rec. Pract. for Measuring Slope Std. Meth. of Test for Magnetic Shield Std. Meth. of Test for Pickup

ices for Removal of Particles (1972) Type for the Purpose of Conserving Their Sound Insulation 70) Std. Meth. of Test for

ec. Guide for the Prediction of the Dispersion of Airborne Digitonin Cholesterol Meth. of Analysis of Dried Meth. of Analysis of Crude Fat in 962) meth. of Analysis of Crude Fat in Egg Yolk and Dried Whole
Iodine Meth. of Analysis for Insect
Meth. of Analysis for Total Phosphoric Acid in Dried Meth. of Analysis for Total Phosphoric Acid in Dried
Meth. of Analysis of Lipoid Phosphoric Acid in Dried Meth. for Preparation of Sample: Dried $\begin{array}{ll}\text { fined), S/ } & \text { Std. Analytical Meth. for Determining Calcium } \\ \text { rch Hydrol } & \text { Std. Analytical Meth. for Determining Calcium }\end{array}$ reh Hydrol ession (1972) of Extension Machines) (1972) Ans/ Std. Meth. of Test for Std. Meth. of Test for Std. Meth. of Test for

ferably with Gravel or Slag Surf/ aterials (1972)

Loss Prevention Data on Std. Index of Meth. for Test. Std. Meth. of Test for Change in Properties of Std. Meth. of Test for Extrudability of Unvulcanized Std. Meth. of Test for Adhesion and Cohesion of ons (1973) ANSI J8.15, SAE J20/ Classification System for spec. for Joints for Plastic Pressure Pipes Using Flexible Std. Meth. of Test for Bubbling of 1 Part, Std. Meth. Test for Tack Free Time of Meth. of Test for Hydrolytic Stability of ains by 1 Std. Meth. of Test for Mechanical Properties of Std. Meth. of Test for Linear Density of t/ Std. Meth. of Test for Breaking Load and Elongatoin of (1972) Ans/ Std. Meth. of Test for Elastic Properties of Meth. of Test for Permanent Deformation of Std. Meth. Test for UV Cold Box Exposure of One Part,

$f$ Test for Low Temperature Flexiblity and Tenacity 1 Part, Meth. of Test for Brittleness Temperature of Plastics and $\mathrm{s}(\mathrm{SBR})(1 / \quad$ Std. Meth. for Chemical Analysis of Synthetic d) $600 \mathrm{~V}$ or Less (1973) ANSI C 33.95 b56.3 ds Against Radio Frequency Radiation Hazards in the Use of 104

2) ANSI C33.11.1

uipment (Laundry Dryer) (1974)

quipment (Laundry Washer) (1974)

consumption) (1974) est Proc. for Low Frequency (Below $3 \mathrm{MHz}$.) Std. General Document for Printed Wiring Board Safety Std. for Commercial Safety Std. for rodes and Rods (1972) Std. for Consumable Std, for Safety for Commercial Std. for Safety for Burglary Resistant Safety Std. for

Guide Spec. for Hydraulic and spection, and Testing Requirements for Instrumentation and s (/ Trial Use Std.: General Guide for Qualifying Class I s (1971) Ansi/ Guide for Seismic Qualification of Class I and Air Filtering Appliances Rated at 60/

steel Pipe for High Temperature Service // enium-Copper-Columbium Stabilized All/ ower Temperatures (1972)

vice (1972A) ANSI B125.4

vice at Moderate Temperatures (1973) edger-Trimmer, Hedge / Std. for Safety for Cord Connected ging Collaps/ Rec. for Protection Against Misc. Perils at Ducted tats For/ Line Voltage Integrally Mounted Thermostats for , Groups A, B, C, and D, and Class II, G/ Safety Std. for ric Heater Line Voltage Integrally Mounted Thermostats for ary 600 Volts or Less) (1972) C33.11 Safety Std. for

Electrical Grade Magnesium Oxide for Use in Sheathed Type er) (1972) ANSI C33.13 Std. for Std. for Std. for

Std. for Safety for Safety Std. for Std. for Safety for Rec. Pract. for Safety Std. for Std. Spec. for

Effective Fluorescent Lifetime of Neodymium Doped Laser Effective Pumping Speed of Vacuum Chamber Systems (1972

Efficiency and Lasing Threshold of Ruby Laser Rods (197

Efficiency in Attenuating Alternating Magnetic Fields ( Efficiency of Residual Aerosol Insecticides (1972)

Efficiency Test. of Air Cleaning Systems Containing Dev Efficiency (1973)

of Fixed $\mathrm{P}$ artit

Efflorescence of Interior Wall Paints (1973)

Efflorescence, Prevention and Control (Tech. Notes) (19

Effluents (1968)

Egg Solids in Macaroni Products (Cereal Chemistry) (196

Egg Solids (Cereal Chemistry) (1962)

Egg Yolk and Dried Whole Egg (Cereal Chemistry) (1962)

Egg (Cereal Chemistry) (1962)

Eggs in White, Wheat, and Corn Flour (Cereal Chemistry)

Eggs (Cereal Chemistry) (1962)

Eggs (Cereal Chemistry) (1962)

Eggs (Cereal Chemistry) (1962)

Egta-Titrimetric) Content in Corn Starch (1973)

(Egta-Titrimetric) Content of Corn Sugar (Crude and Re

Egta-Titrimetric) Content of Corn Syrup and Other Sta

Elastic Moduli of Rock Core Specimens in Uniaxial Compr

Elastic Properties of Elastomeric Yarns (Constant Rate

Elastic Properties of Fibers (1972) ANSI L14.250

Elastomer Roof Coverings (Multiply Felt and Asphalt Pre

Elastomeric and Plastomeric Roofing and Waterproofing M

Elastomeric ANSI J2.7

Elastomeric Compounds (1973)

Elastomeric Joint Sealants Under Cyclic Movement (1972)

Elastomeric Materials (Rubber) for Automotive Applicati

Elastomeric Seals (1973)

Elastomeric Solvent Release Type Sealants (1972)

Elastomeric Type Joint Sealants (1971)

Elastomeric Volcanizates (1972T)

Elastomeric Vulcanizates Under Compressive or Shear Str Elastomeric Yarn (1972)

Elastomeric Yarns (Constant Rate of Extension Instrumen Elastomeric Yarns (Constant Rate of Extension Machines) Elastomeric Yarns (1972T)

Elastomeric, Solvent Release Type Sealants (1972)

Elastomeric, Solvent Release Type Sealants (1972)

Elastomers by Impact (1973)

Elastomers for Use on Solid Styrene Butadiene Copolymer Elbow Reducer, 90 Deg. Flareless Tube to Swivel (1973)

Elbow, Reducer, 45 Deg. Flareless Tube to Swivel (1973) Electric A ir Heaters (1973)

Electric Baseboard Heating Equipment (Portable and Fixe

Electric Battery Powered Industrial Trucks (1972) ANSI

Electric Blasting Caps (1971) ANSI C95.4

Electric Blower Motor Switch for Vehicles (1971)

Electric Central Air Heating Equipment (1973) ANSI C33.

Electric Chain Hoists (1971)

Electric Clocks (1974) ANSI C33.47

Electric Coffee Makers and Brewing Type Appliances (197

Electric Coin Operated and Commercial Clothes Drying Eq

Electric Coin Operated and Commercial Clothes Washing E

Electric Comfort Conditioning (Heat Loss, Gain, Energy

Electric Connectors (1974) ANSI C83.63C

Electric Connectors (1974) ANSI C83.88, Ipc C-405A

Electric Cooking Appliances (1972)

Electric Cord Connected Indoor Signs (1971) ANSI C33.35

Electric Covered Bare Straight and Coiled Welding Elect

Electric Dishwashers (1973) ANSI C33.96

Electric Door Strikes (1974)

Electric Dry Bath Heaters (1972) ANSI C33.75

Electric Elevators (Freight and Passenger) (1971)

Electric Equipment During the Construction of Nuclear P

Electric Equipment for Nuclear Power Generating Station

Electric Equipment for Nuclear Power Generating Station

Electric Fans (Blower, Humidifier, Evaporative Cooler,

Electric Fuses (1973) ANSI C118.1

Std. Spec. for Electric Fusion Welded Autenitic Chromium Nickel Alloy

Electric Fusion Welded Chromium-Nickel-Iron-Molyd

Electric Fusion Welded Steel Pipe for Atmospheric and L

Electric Fusion Welded Steel Pipe for High Pressure Ser

Electric Fusion Welded Steel Pipe for High Pressure Ser

Electric Gardening Appliances (Lawn Mower, Cultivator,

Electric Generating Stations (Flood, Surface Water, Rig

Electric Heat Guide for Air Handling Systems (1971)

Electric Heater Line Voltage Integrally Mounted Thermos

Electric Heaters for Use in Hazardous Locations Glass I

Electric Heaters (1973) /Mounted Thermostats for Elect

Electric Heating Appliances (Portable Fixed and Station

Citrical Impedance of

Electric Heating Elements (1973)

Electric Heating Equipment (1974)

Electric Home Laundry Equipment (Clothes Washer and Dry

ASTM

ASTM

ASTM

ASTM

ASTM

ANSI

ASTM

ASTM

BIA

ASME

AACC

AACCH $30-18$

$\mathrm{AACCH} \quad 30.18$

$\mathrm{AACCH} 28.44$

AACCH $04-13$

AACCH 20-02

AACCH $62-15$

CR B-11

CR $\quad \mathrm{F}-8$

E-11

$\begin{array}{ll}\text { ASTM } & \text { D } 3148 \\ \text { ASTM } 2731\end{array}$

ASTM D1774

FMS

ASTM

ASTM

ASTM

ASTM

ASTM

Std. ASTM

ASTM

ASTM

ASTM

ASTM

ASTM

ASTM

ASTM

ASTM

ASTM

1. O ASTM

Std. ASTM

ASTM

NSA

NSA

UL

UL

UL

IME

SAE

UL

HMl

UL

UL

UL

UL

NEMA

EIA

EIA

UL

UL

UL

UL

CSI

IEEE

IEEE

IEEE

UL

SAE

ASTM

ASTM

ASTM

ASTM

UL

FMS

SMAC

NEMA

1-47

D3105

D471
D2230

C719

D2000

D3139

C712

C679

D3137

D945

D2653

D3106

C718

C711

D746

D1416

1762

1761

1025

1042

583

20

J235

1096

400 
nt $(1972)$ Std. for Residential Controls Direct e for Glass Bulbs Intended for Use with Electron Tubes and ns (1972) ANSI C.33.28

Std. for Safety for Safety Std. for

Class I, Groups C and D, A/ Std. for Safety for Portable ure for the Evaluation of Sealed Insulation Systems for Ac test Procedure for Airborne Sound Measurements on Rotating Rec.

ations, Class II, Groups E, F, and G (19/

ations, Class I, Groups C and D (197) Std. for Safety for ation and Use of
Safety Std. for Std. for Safety for Safety Std for Door, Drapery, Gate, Louver, and Window veling Cranes (1971) Spec. for Std. for Relays and Relay Systems Associated with Std for the Prevention of Water Damage to Steam Turbines Used for 8 (1972)

974) Std. Spec. for Wrought Steel Wheels for Std. Household ps (1974)

Std for Household Std. for Household Std. for Safety for Household I Boiler and Superheater Tubes (1973) Ans/ rheater Tubes for High Pressure Service (/ 973) ANSI B 125.6 std. Spec. for Supplementary Requirements for Seamless and Std. Spec. for

i C33.11.2 Std. for Safety for Household
Safety Std. for i $\mathrm{G} 55.13$ Std. Spec. for Vacuum Treated Safety Std for Household Std. for Household Automatic s (1973) Std. Meth. of Test for Thermal Failure Under Safety Rules for the Installation and Maintenance of Rules for the Installation and Maintenance of Underground l Safety Code) (1973) Rules for the Operation of Rec. Pract. for

for Driving and Spindle Ends for Portable Hand, Air, and Safety Std. for Stationary and Fixed Std. for Safety for Portable nd Charging Storage Batteries in Motive Power Service (For tations (1972) / Trial Use Guide for Type Test of Class I industry (1971) ANSI B125.38 Std. Spec, for Rec. Pract. for Rec. Pract. for Std. Spec. for , and Base Mounted Types (1971) Std. for Splice, 2) Wire Sizes 12 to $26(1972)$

Std. for Requirements for Std. Test. Procedures for Relays for or Satellite Earth Stations (1973) Std. for of Test. and Tolerances for Jute Rove and Plied Yarn for Std. Meth. of Test for Combustible Gases in ng, Liquid Filled and Dry Transformers, Hand Po/ Std. for Std. for Magnetic Wire for Use in Std. for Safety for Thermal Cutoffs for Use in

Test for Polymeric Enclosures for Portable Cord Connected Std. Meth. of Test. Vitrified Ceramic Materials for Spec. for Beryllia Ceramics for Electronic and Std. for Safety for

1972) Std. for

ckets (1974) ANSI C83.95 ge Circuits (Electronics) (1974)

bactericidal Lamp (1971)

Bactericidal Lamp (1971) s (1972)

actericidal Lamp (1971)

\section{(1973)} static Dissipator Additive (1971) si Z11.322 d. for Safety for Nonmetallic Sheathed Std. for Dimensional and Std. for the

Std. for Dimensional and Std. Dimensional and Std. Dimensional and
y Lamps: Physical and Std. Physical and Std. Dimensional and Rec. for Protective Grounding of Rec. and Comments on the National Std. Meth. of Test for Std. Meth of Test for D.C. Std. Meth. of Test for Resistivity of Code Words for Aluminum anded Copper Conductors Having Bunch Stranded Members, for std. Definitions of Terms Relating to Uninsulated Metallic 72) ors for Use Between Aluminum or Aluminum-Copper Overhead Rec. Pract for 7 Conductor Std. for Receptacle Dummy Storage, Std. Test Procedures for Low Frequency (Below $3 \mathrm{MHz}$.) ors for Use Between Aluminum or Aluminum-Copper Overhead Std. Spec. for Gold, Silver, Nickel
Electric Ignition Control Systems for Gas Fired Equipme

Electric Lighting Fixtures for Use in Hazardous Locatio Electric Lighting Fixtures (1972)

Electric Lighting Units for Use in Hazardous Locations,

Electric Machinery Employing Form Wound Stator Coils ( Electric Machinery (1973)

Electric Motor Starting Currents (1972) EEI 62-27

Electric Motors and Generators for Use in Hazardous Loc Electric Motors and Generators for Use in Hazardous Loc Electric Motors and Generators (1973) ANSI C51.1 Electric Motors for Appliances and Equipment (1972) Electric Oil Heaters (1973) ANSI C33.44

Electric Operators and Systems (1973)

Electric Overhead Bridge and Gantry Multiple Gerder Tra Electric Power Apparatus (1971) ANSI C 37.90

Electric Power Connectors (1972)

Electric Power Generation: Fossil Fueled Plants (1972)

Electric Pressure Pumps for Use in Recreational Vehicle

Electric Railway Service (1972) ANSI G57.13

Electric Range Pyrolytic Self Cleaning Ovens (Stove) (1

Electric Ranges (Stove and Ovens) (1972) ANSI C71.1

Electric Ranges (Stove) with Glass / Ceramic Cooking to

Electric Ranges (Stoves) (1974) ANSI C33.53

Electric Resistance Welded Carbon Molybdenum Alloy Stee

Electric Resistance Welded Carbon Steel Boiler and Supe

Electric Resistance Welded Carbon Steel Boiler Tubes (1

Electric Resistance Welded Carbon Steel Tubular Product

Electric Resistance Welded Steel Pipe (1972A)

Electric Skillets and Frying Type Appliances (1973) Ans

Electric Space Heating Equipment (1972) ANSI C33.12

Electric Steel Forgings for Generator Rotors (1971) Ans

Electric Storage Tank Water Heaters (1972) ANSI C33.87

Electric Storage Type Water Heaters (1972)

Electric Stress of Solid Electrical Insulating Material

Electric Supply and Communication Lines (1960)

Electric Supply and Communications Lines (National Elec

Electric Supply Lines and Equipment (National Electrica

Electric Tachometer Spec. for on Road Vehicles (1972)

Electric Tools (1973)

Electric Tools (1973) ANS I C33.86

Electric Tools (1974) ANSI C33.49

Electric Trucks) (1972)

/Nd Health Rec. for Changing a

Electric Utility Generating Stations (1973)

Electric Valve Operators for Nuclear Power Generating S

Electric Welded Low Carbon Steel Pipe for the Chemical

Electric Windshield Washer Switch (1971)

Electric Windshield Wiper Switch (1971)

Electric Wire Rope Hoists (Lug, Hook, Trolley Suspended

Electric, Permanent, Crimp Style, Pre Insulated, (Class

Electrical Analog Indicating Instruments (1972)

Electrical and Electronic Equipment (1973) ANSI C83.25

Electrical and Mechanical Characteristics of Antennas F

Electrical and Packing Purposes (1974) A NSI L14.44

Electrical Apparatus in the Field (1973)

Electrical Apparatus Sales and Service Industry (Rotati

Electrical Apparatus (1973) ANSI C9.100

Electrical Appliances and Components (1972) ANSI C33.88

Electrical Appliances (1973)

Electrical Applications (1969) ANSI C59.73

Electrical Applications (1972T)

Electrical Attachment Plugs and Receptacles (1974)

Electrical Bonding of Direct Current Systems on Boats (

Electrical Cables (1974) ANSI C33.56

Electrical Characteristic Defining Dual in Line Type So

Electrical Characteristics of Class a Closure Interchan

Electrical Characteristics of Fluorescent Lamps (1972)

Electrical Characteristics of 15 Watt T8 Preheat Start

Electrical Characteristics of $30 \mathrm{~W}$ att, T8 Preheat Start

Electrical Characteristics of $40 \mathrm{~W}$ att, H45 (1972)

Electrical Characteristics of 50 Watt, H46 Mercury Lamp

Electrical Characteristics of 8 Watt T5 Preheat Start B

Electrical Circuits and Equipment (1974)

Electrical Code (NFPA 70) and Code Requirements by OSHA

Electrical Conductivity of Aviation Fuels Containing a

Electrical Conductivity of Hydrocarbons Fuels (1972) an

Electrical Conductor Materials (1972A) ANSI C7.24

Electrical Conductor (1972)

Electrical Conductors (1971)

Electrical Conductors (1972A)

Electrical Conductors (1973) EEI TDJ-162

Spec. for Rope Lay Str

Electrical Connector for Tr

Electrical Connector (1972)

Electrical Connectors (1971) ANSI C83.63B

Electrical Connectors (1973) NEMA CC.3

Electrical Contact Alloy (1972) Std. Nome

Std. for Safety for

NEMA

ANSI

UL

UL

IEEE

Std. for IEEE

NEMA

UL

UL

UL

UL

UL

CMAA

IEEE

NEMA

ASME

IAPMO

ASTM

AHAM

AHAM

AHAM

UL

ASTM

ASTM

ASTM

ASTM

ASTM

UL

UL

ASTM

UL

ANSI

ASTM

ANSI

ANSI

ANSI

SAE

Std ANSI

UL

UL

IEEE

ASTM

SAE

SAE

HMI

NSA

ANSI

EIA

EIA

/Th ASTM

ASTM

EASA

NEMA

UL

UL

ASTM

ASTM

UL

ABYC

UL

EIA

EIA

ANSI

ANSI

ANSI

Std ANS

ANSI

ANSI

FMS

FMS

ASTM

ASTM

ASTM

AA

ASTM

ASTM

/ for Connect NEMA

TTMA

NSA

EIA

/Td. for Connect EEI

ASTM

DC 21

C79.1

844

57

781

429

85 
Std. Spec. for Gold Std. Spec. for Gold Copper Alloy Spec. for General Requirements for Wrought Precious Metal Std. Meth. of Test for Hardness of

Pract. for Designing a High Current Arc Erosion Test for rated on a Symmetrical and a Total/ Std. Requirements for Rec. for Computing

ed Wire and Cable for the Transmission and Distribution of ed Wire and Cable for the Transmission and Distribution of ea/ Safety Std. for Temperature Indicating and Regulating C106.1 Std. for Purged and Pressurized Enclosures for (1973) Rec, for Fire Protection of dium Speed Stationary Diesel and Gas Engine Generators and std. Spec. for Air as an Electrical Insulating Material in 1972) Std. for

h. for Measurement of the Specific Electrical Impedance of rolled (1973) Plumbing Requirements for Household Storage Type e for Use in / Std. Meth. for Measurement of the Specific Production / Rec. Pract. for Classification of Areas for ociated Equipment (1972) icultural Plast/

Std. Guidelines for Safe Installation of Std. Spec. for Polyethylene Jacket for lorinated Aromatic Hydrocarbons (Askarels) for Capacitors Std. Definitions of Terms Relating to

(1973) Std. Spec. for Air as an

h. for Complex Permittivity (Dielectric Constant) of Solid scharg/ Std. Meth. of Test for Voltage Endurance of Solid cteristics and Dielectric Constant (Permittivity) of Solid

aluation and Establishment of Temperature Indexes of Solid

Std. Meth. of Test for Impact Resistance of Plastics and

of Test for Thermal Failure Under Electric Stress of Solid ms (1972) ANSI C/ Std. Spec. for Continuity of Quality of s (1972) ANSI C5/ Std. Spec. for Continuity of Quality of Kraft Layer Type (1973) ANSI C59.50 Std. Spec. for Std. Spec. for Polyethylene Plastic Pressure Sensitive

rature Resistant Vinyl Chloride Plastic Pressure Sensitive

Std. Spec. for Vinyl Chloride Plastic Pressure Sensitive Std. Spec. for Polyester Film Pressure Sensitive

d. Spec. for Tetrafluoroethylene Backed Pressure Sensitive

Std. Meth. of Test for Thermal Aging Characteristics of h. (1973) Std. Meth. of Test for Thermal Degradation of efin, Pigmented, Flexible, Heat Shrinkable (19) Spec. for Std. Meth. of Test. Adhesives Relative to Their Use as Std. Meth. of Test. Laminated Tubes Used for

Meth. of Test. Varnished Cottonfabrics and Tapes Used for Std. Meth. of Test. Vulcanized Fiber Used for pec. for Crosslinked Polyolefin Heat Shrinkable Tubing for d. Meth. of Test. Rigid Sheet and Plate Materials Used for Std. Meth. of Test. Varnishes Used for of Measuring Dimensions of Rigid Rods and Tubes Used for Std. Definitions of Terms Relating to

Std. Meth of Test for Thickness of Solid for High Voltage, Low Current, Dry Arc Resistance of Solid meth. of Test. Coating Powders and Their Coatings Used for rosslinked Poly(vinyl Chloride) Heat Shrinkable Tubing for Std Meth of Test Shellac Used for

Dissipation Factor of Expanded Cellular Plastics Used for meth. of Test. Coating Powders and Their Coatings Used for Std. Meth. of Test. Heat Shrinkable Tubing for of Test. Pressure Sensitive Adhesive Coated Tapes Used for Std. Meth. of Sampling and Test. Untreated Paper Used for 973) Std. Guide for ion (1973) ANSI C33.98 Std. for Safety for oride Externally Coated Galvanized Rigid Steel Conduit and n (Cereal Chemistry) (1962)

$t$ for Corrosivity of Water in the Absence of Heat Transfer Std. for Safety for

ures for Nuclear Fueled Power Generating Statio/ Std. for Smooth Wall Coilable Polyethylene
Sther and Sleeve Type for Hazardous Locations (1973) Std. for Aluminum Underground Distribution Reference Book er Insulation, 200/ Std. for Nonshielded Single Conductor

Spec. for Tinned Copper Concentric Neutrals (Underground Ozone Resistant Ethylene Propylene Rubber Insu/ Std. for aving Ozone Resistant Ethylene-Propylene-Ru/ ber Insu/ Std. for Portable Single and Multiple Conductor vices and Assemblies (1973)

rolling Devices (1973)

for Reporting and Analyzing Outage / Std. for Industrial Std. for Industrial Std. Definitions in Std. for Safety for Std. for
apes for
Electrical Contact Alloy (1973)

Electrical Contact Material (1973)

Electrical Contact Materials (1972)

Electrical Contact Materials (1972) ANSI Z155.12

Electrical Contact Materials (1973)

Electrical Control of AC High Voltage Circuit Breakers

Electrical Demands for Farms (1972)

Electrical Energy (1973) IPCEA S.61-402

Electrical Energy (1973) IPCEA S-66-524

Electrical Equipment for Control of Air Conditioning,

Electrical Equipment in Hazardous Locations (1972) ANSI

Electrical Equipment (Single Phase and Related Faults)

Electrical Equipment (1972)

TTd. Pract. for Low and Me

Electrical Equipment (1973)

Electrical Feeder and Plug in Busways and Accessories (

Electrical Grade Magnesium Oxide for Use in Sheathed Ty

Electrical Harness Assembling Machine, Numerically Cont

Electrical Hot Water Dispensers (1973)

Electrical Impedance of Electrical Grade Magnesium Oxid

Electrical Indicating Instrument (Meter) Relays (1972)

Electrical Installations at Drilling Rigs and Petroleum

Electrical Installations on Packaging Machinery and Ass

Electrical Instruments in Hazardous Dust Locations (Agr

Electrical Insulated Wire and Cable (1972)

Electrical Insulating Liquid) (1973) ANSI C59.11

Electrical Insulating Liquids and Gases (1974)

Electrical Insulating Material in Electrical Equipment

Electrical Insulating Materials at Microwave Frequencie

Electrical Insulating Materials Subjected to Partial Di

Electrical Insulating Materials (1970)

Electrical Insulating Materials (1972)

Electrical Insulating Materials (1973)

/Ent Loss Chara

Electrical Insulating Materials (1973)

the Thermal

Electrical Insulating Oil for High Pressure Cable Syste

Electrical Insulating Oil for Low Pressure Cable System

Electrical Insulating Paper and Paperboard-Sulfate or

Electrical Insulating Tape (1973)

Electrical Insulating Tape (1973)

Electrical Insulating Tape (1973) ANSI C59.91

Electrical Insulating Tape (1973) ANSI C 59.96

Electrical Insulating Tape (1974)

Electrical Insulating Varnishes Applied Over Film Insul

Electrical Insulating Varnishes by the Helical Coil Met

Electrical Insulation Plastic Tubing, Irradiated Polyol

Electrical Insulation (1970)

Electrical Insulation (1970)

Electrical Insulation (1970) ANSI C59.31

Electrical Insulation (1971) ANSI C59.78

Electrical Insulation (1972)

Electrical Insulation (1972) ANSI C59.13

Electrical Insulation (1972) ANSI C59.30

Electrical Insulation (1972) ANSI C59.33

Electrical Insulation (1973)

Electrical Insulation (1973)

Electrical Insulation (1973)

Electrical Insulation (1973)

Electrical Insulation (1973)

Electrical Insulation (1973) ANSI C59.18

Electrical Insulation (1973) ANSI C 59.8

Electrical Insulation (1973) Std. Meth. of Test. Coat

Electrical Insulation (1974)

Electrical Insulation (1974) ANSI C59.136

Electrical Insulation (1974) ANSI C59.77

Spec. for Low Tempe

ASTM

ASTM

ASTM

ASTM

ASTM

ASTM

St ASTM

ASTM

ASTM

SAE

ASTM

ASTM

Std. ASTM

ASTM

Std. S ASTM

St ASTM

ASTM

Std. Meth ASTM

ASTM

ASTM

Std. Meth of Test ASTM / Insulation (1973) Sid. ASTM for Crosslinked and Nonc ASTM

ASTM

Constant and ASTM

ASTM

ASTM

Electrical Measure ments of Fluorescent Lamps (light) (1

Std. Meth. ASTM

ASTM

ANSI

Electrical Metallic Tubing (1974)

1. for Poly

Electrical Meth. for Analysis of Moisture in Whole Grai

(Electrical Meth. (1972)

Electrical Outlet Boxes and Fittings (1974)

Sid. Meth. of Tes

Electrical Penetration Assemblies in Containment Struct

Electrical Plastic Duct (1972)

Electrical Plugs, Receptacles and Connectors of the Pin

(Electrical Power Cable Specs.) (1973)

Electrical Power Cable with Ethylene-Propylene-Rubb

Electrical Power Cable) (1973)

Electrical Power Cables Rated $0-35,000$ Volts and Having

Electrical Power Cables Rated 2000 Volts and Less and H

Electrical Power Cables with Ethylene-Propylene-Rub

Electrical Power Control Apparatus-Control Circuit De

Electrical Power Control Apparatus-General (1973)

Electrical Power Control Apparatus-Switching and Cont

Electrical Power Distribution Cables (1973)

Electrical Power Operations Terminology Including Terms

Electrical Power Outlets (1972) ANSI C33.85

Electrical Power Switching Equipment (1974)

Electrical Purposes (Bus Conductor) (1973) ANSI C7.45

B541

B596

$\mathrm{B} 476$

$\mathrm{B} 277$

B576

C37.11

$\mathrm{R} 294$

WC5

WC7

873

496

14.15

1.18

D3283

BUI

D3215

974.

1023

D3215

Il 2

RP500B

333

S12.11

D2308

D2233

D2864

D3283

D2520

D2275

D150

98

D256

D3151

D1819

D1818

D1305

D3006

D3005

D2301

D2484

D2686

D3251

D3145

AMS 3636D

D1304

D348

D295

D619

D3149

D229

D115

D668

D1711

D374

D495
D3214

D3150

D4II

D 1673

D3214

D1000

D202

C78.375

797

RN1

$\begin{array}{ll}\text { AACCH } & 44-10 \\ \text { ASTM } & \text { D2776 }\end{array}$

UL 514

IEEE 317

$\begin{array}{ll}\text { NEMA } & \text { TC } 7 \\ \text { NEMA } & \text { FB1l }\end{array}$ 
Std. Spec. for Aluminum Bar for Spec. for Lead and Lead Alloy Coated Soft Copper Wire for Std. Spec. for Woven Cotton Tapes for Std. Spec. for Aluminum Wire, EC-H19, for Std. Spec. for Aluminum Alloy 6201 T 81 Wire for Std. Spec. for Aluminum Alloy 5005 Rolled Rods for Std. Spec. for Aluminum Alloy 5005-H19 Wire for Std. Spec. for EC Aluminum Redraw Rod for Std. Spec. for Soft or Annealed Copper Wire for Std. Spec. for Tinned Soft or Annealed Copper Wire for Sid. Spec. for Hot Rolled Copper Rods for cialized Service (1971) ANSI N14/ (I972)

ite Articles at Room Temperatures/ Textile Yarns (1973) ANSI L14.119 brics (1972) ANSI L14.112 Std. Spec. for Sheathed Std. Meth. of Test for Std. Meth. of Test for Test Meth. for Test Meth. for Std. for Reference Std. Precision National

Rules for the Radio Installations (National operation of Electric Supply Lines and Equipment (National rground Electric Supply and Communications Lines (National ion and Maintenance of Electrical Supply Statio/ National Std. for Letter Symbols for Units Used in rs and Box Supports, and Cast Aluminum Covers (/ Std. for nsi C113.1

Std. Rec. Pract. for the Identification of Std. Std. Spec. for Flat Rolled Nonoriented Std. Spec. for Flat Rolled, Grain Oriented, Silicon Iron, Std. Spec, for Flat Rolled h. for Chemical Analysis of Carbon, Low Alloy, and Silicon Std. for Terminal Block
ode, Part 1: Rules for the Installation and Maintenance of ss I, Groups A.D, and Class II, Groups E/ Safety Std. for Std. for Safety for Special Use
Rec. Pract. for Rec. Pract. for Grounded 830 V, 3 Phase quirements for Maintenance of Design Voltage in Snowmobile pers, Motor Homes): Installation of Plumbing, Heating, and on Requirements, and Installation of Plumbing, Heating and Spec. for High Temperature Glass Cloth Pressure Sensitive Std. for Pan Head Screw and Assembled Washers. Std. for

logy Including Terms for Reporting and Analyzing Outage of ion (Including Safety Req/S Sid, for Series Capacitors for Safety for Cellular Metal Floor Raceways and Fittings for es Duct for Underground Installation for Communication and Std. for Color Coding of

loride Ducts and Fittings for Underground Installations of ure and Building Construction (/ Std. Graphic Symbols for and Construction Equipment (Machinery) (/ Rec. Pract. for 73) Requirements and Rec. Meth. of Identifying AC and Dc for Safety for Grounding and Bonding Equipment (Internal id. for Safety for Surface Metal Raceways and Fittings for for Wireways, Auxiliary Gutters, and Associated Fittings Std. for Binding Post-Terminal Board,

for Safety for Access Control System Units (Electronic Rec. Pract. for Grounding of Industrial and Commercial Safety Std. for Class R Fuses Safety Std. for Plug Fuses nd/ Std. Meth. for Chemical Analysis of High Temperature, in Flammable Anesthetizing Location/ Std. for Safety for Sid. for Safety for Rec. for Safety Rec for 00 Volts or Less (1974) (1973) ions, Class 1, Groups A, B, C and D, An/ Safty Std for Rec. Pract. for Reducing the Fire Hazard of Static Std. for Leakage Current ema Ei21 Std. for Instrument Transformers for Test Meth. for Electrical Resistivity Test Meth. for Electrical Resistivity Textile of Std. for Procedures for Calibration of Underwater y Based on Physical $\mathrm{Pr} /$ Std. Rec. Pract. for Selection of -2.0Cu-1. ITi, Vacuum Induction Plus Vacuum Consumable $1.0 \mathrm{M}_{0}-0.09 \mathrm{~V}(0.17-0.23 \mathrm{C})$, Premium Quality, Consumable Base $(19.5 \mathrm{Cr}-13.5 \mathrm{Co}-4.3 \mathrm{Mo}-3.0 \mathrm{Ti}-1.4 \mathrm{Al})$ Consumable 1 Base 19.5 Cr $-13.5 \mathrm{Co}-4.3 \mathrm{Mo}-3.0 \mathrm{Ti}-1.4 \mathrm{Al}$, Consumable base-19.5 Cr-13.5 Co-4.3Mo-3.0 Ti-1.4Al, Consumable 5Mo (0.38 0.43C) (SAE 4340), Premium Quality, Consumable 07-0.13C) (SAE 9310), Premium Quality, Vacuum Consumable $s$ in Multip/ Sid. for Numbering of Electrodes in Multiple Resistant, $14.8 \mathrm{Cr}-4.5 \mathrm{Ni}-3.5 \mathrm{Cu}-(\mathrm{Cb}+\mathrm{Ta})$, Consumable

$-1.2 \mathrm{~V}(1.10-1.20 \mathrm{C})$, Premium Bearing Quality, Consumable

th. for Continuous Determination of Sodium in Water by Ion

Meth. of Test for $\mathrm{pH}$ of Aqueous Solutions with the Glass
Electrical Purposes (Bus Connector) (1973) ANSI C7.27

Electrical Purposes (19674 ANSI C 7.15

Electrical Purposes (1972)

Electrical Purposes (1972) ANSI C7.20

Electrical Purposes (1972) ANSI C7.51

Electrical Purposes (1972) ANSI C 7.66

Electrical Purposes (1972A) ANSI C7.49

Electrical Purposes (1974)

Electrical Purposes (1974) ANSI 67.1

Electrical Purposes (1974) ANSI C7.4

Electrical Purposes (1974) ANSI C7.7

Electrical Resistance Heaters, for Nuclear or Other Spe

Electrical Resistance of Conductive Resilient Flooring

Electrical Resistivity of Manufactured Carbon and Graph

Electrical Resistivity Textile of (Electro-Static) of

Electrical Resistivity (Electro-Static) of Textile Fa

Electrical Resistors (1969) ANSI C 100.3

Electrical Safety Code (1973)

Electrical Safety Code) (1940)

Electrical Safety Code) (1973)

Electrical Safety Code) (1973)

Electrical Safety Code, Part 1: Rules for the Installat

Electrical Science and Technology (1969) ANSI Y10.19

Electrical Sheet Steel Outlet Boxes, Device Boxes, Cove

Electrical Std. for Metalworking Machine Tools (1973) a

Electrical Steel Grades in ASTM Specs. (1972)

Electrical Steel, Fully Processed Type (1973)

Electrical Steel, Fully Processed Types 27G053, 30G058,

Electrical Steels for Magnetic Applications (1974)

Electrical Steels, Ingot and W/rought Irons (1973)

Electrical Stud (1974)

Electrical Supply Stations and Equipment (1971)

Electrical Switches for Use in Hazardous Locations: Cla

Electrical Switches (1973) ANSI C33.40.1

Electrical System for Construction and Industrial Machi

Electrical System for Oil Field Service (1973)

Electrical Systems (1971)

Electrical Systems (1972) ANSI A119.

$1 \mathrm{C}$

Electrical Systems (1973) ANSI A119.1

Electrical Tape (1973)

Electrical Terminal Attachment (Preferred) (1974)

Electrical Transformers, Regulators and Reactors (1974)

Electrical Transmission and Distribution Facilities and

Electrical Transmission and Distribution Line Compensat

Electrical Wire and Cable Installation (1973) ANSI C33.

Electrical Wire and Cable (1974)

Electrical Wires and Cables (1974

Electrical Wires and Cables (1974)

Electrical Wiring and Layout Diagrams $/ \mathrm{R}$ and Polyvin

Electrical Wiring Identification System for Industrial

Electrical Wiring on Boats and Engines (Color Code) (19

Electrical Wiring Systems) (1972) ANSI C 33.8

Electrical Wiring (1973) ANSI C33.50

(Electrical Wiring) (1973) ANSI C 33.45

Electrical (1973)

Electrical / Mechanical Security Surveillance System an

Electrical) Power Systems (1972) ANSI C 114.1

(Electrical) (1973)

Electrical) (1973)

Electrical, Magnetic, and Other Similar Iron, NickeI, a

Electrically Actuated Transmitters (1974)

Electrically Conductive Equipment and Materials for Use

Electrically Conductive Floors (1973)

Electrically Heated Livestock Waterers (1971)

Electrically Operated Photographic Equipment Rated at 3

Electrically Operated Valves for the Control of Fluids

Electrically Operated Valves for Use in Hazardous Locat

Electricity (1973)

Electricity) for Appliances (1973)

Electricity) Metering Purposes, $15 \mathrm{kV}$ and Less (1973) N

Electro-Static) of Textile Fabrics (1972) ANSI L14.11

(1973) ANSI L14.119

Electro A coustic Transducers (1972)

Electrode Material Used in Optical Emission Spectroscop

Electrode Melted Solution Heat Treated (1973)

Electrode Melted, Annealed (1973)

Electrode or Vacuum Induction Melted 1825-1900 Deg. F (

Electrode or Vacuum Induction Melted, 1975 Deg. F (1079

Electrode or Vacuum Ind

/Ubing, $0.80 \mathrm{Cr}-1.8 \mathrm{Nj}-0.2$

Electrode Remelted (1973)

U ling, $0.80 \mathrm{Cr}-1.8 \mathrm{Ni}-0.2$

Electrode Semiconductor Devices and Designation of Unit

Electrode Vacuum Arc Remelted (1973)

Electrode Vacuum Melted (1973)

Electrode (1973)

Electrode (1974) Ashto T200
Test Meth. and Re Lers, Truck Cam

I and Constructi

/Plate, Corrosion SAE

ASTM B236

Std. ASTM

ASTM

ASTM

ASTM

ASTM

ASTM

ASTM

ASTM

ASTM

ASTM

ASTM

ASTM

ASTM

AATCC

AATCC

IEEE

ANSI

ANSI

les for the ANSI

ANSI

ANSI

IEEE

NEMA

NFPA

ASTM

ASTM

ASTM

ASTM

/Met ASTM

NSA

UL

SAE

API

SAE

NSA

NEMA

IEEE

ANSI

UL

NEMA

NEMA

NEMA

ANSI

SAE

$A B Y C$

UL

UL

NSA

UL

IEEE

UL

ASTM

UL

UL

FMS

ASAE

UL

UL

FMS

ANSI

EEI

AATCC

AATCC 8

ANSI 51.20

ASTM E504

SAE

SAE

SAE

$\mathrm{SAE}$

$S A E$

SAE

SAE

Std Me ASTM

Std ASTM

189

D335

B398

B53.

B396
B233

B3

B33

B49
E420

F150

C61

84
76

310

C2 2.5

$\mathrm{C} 2.4$

C2.3

260
OSI

79

A664

A677
A665

A345

350

I002

894 
uniform Building Code Std. for Corrosion Resistant Metals (Electrodeposited Coatings of Copper, Nickel, Chromium, on Steel and Ferrous Alloys (1970) ANSI / late) (1973)

$$
\text { Std. Spec. for }
$$

Std. Meth. Test for Std. Spec. for

ansi W 3.17 Spec. for Bare Mild Steel nsumable Electric Covered Bare Straight and Coiled Welding Spec. for Mild Stee Spec. for Welding Rods and Covered and Designation of Units in Multip/ Std. for Numbering of Spec. for Mild Steel Covered Arc Welding Spec. for Aluminum and Its Alloy Welding Rods and Bare Spec. for Nickel and Its Alloy Covered Welding Spec. for Tungsten Arc Welding

Spec. for Nickel and Its Alloy Bare Welding Rods and Spec. for Magnesium Alloy Welding Rods and Bare Spec. for Aluminum and Its Alloy Arc Welding sting Chromium and Chromium-Nickel Steel Covered Welding Spec. for Low Alloy Steel Covered Arc Welding Spec. for Copper and Its Alloy Arc Welding chromium and Chromium-Nickel Steel Welding Rods and Bare Spec. for Surfacing Welding Rods and

Spec. for Titanium and Its Alloy Bare Welding Rods and Spec. for Composite Surfacing Welding Rods and n Voltage of Insulating Oils of Petroleum Origin Using Vde red Corrosion Resisting Chromium and Chromium Nickel Steel Std. Spec. for Precision

tion of Phosphate Coating Weight on Hot Dip Galvanized and or Cast and Wrought Galvanic Zinc Anodes for Use in Saline ers and Electronic Instruments) (1/ Std. Requirements for Std. Spec. for
Rec. for Std. Spec. for
Rec. for g Manufacture) (1974) ANSI H23.11 ngots, and Ingot Bars (1973) ANSI H17. Spec. for Oxygen Free Std. Spec. for Std. for Fixed Application in Relays and S/ General Spec. Applicable to $t$ of Spacecraft Thermal Control Materials (Coatings) with ol Systems; Door Holders, Stops, Smoke and Heat Detectors; Definitions of Relay Terms strumentation for the Measurement of Potentially Hazardous mhz) (1972) ANSI C112.1 Std. for Measurement of argers (1971) Std. for Interrelationship of Quartz Fiber ty (PH) in Flour and Bread (Cereal Chemistry) (1962)

Std. Meth. of Test for SaIt in Crude Oil

th. of Test for Bromine Index of Petroleum Hydrocarbons by 2) ANSI C 96.2 Std. Temperature Rec. for Shielding for High Energy Std. Rec. Pract. for Determining Hermeticity of Std. Spec. for Brazing Filler Metals for td. Spec. for Boron Deoxidized Copper in Wrought Forms for r (1971) ANSI / Std. Meth. of Test for Absorbed Gamma and Sulfate Dosim/Std. Meth. of Test for Absorbed Gamma and Std. Nomenclature for Glass Bulbs Intended for Use with Tent. Spec. for Molybdenum Flattened Wire for Against Betatron Synchrotron Radiations Up to 100 Million Spec. for Beryllia Ceramics for Spec. for Nickel Coated Copper Clad Steel Wire for Std. Test Meth. for Passive Std. for the Protection of

Rec. for Location, Construction and Protection of Rec. for Fire, Heat, and Smoke Damage of Std. for Safety for

Std. Rec. Pract. for Determining Hermeticity of termining Hydrolytic Stability of Plastic Encapsulants for pilot, Depth Determination Devi/ Rec. Pract. and Std. for

e Components for Television Receiving Appliances and Other Std. Definitions and Terminology for Relays for

Std. Test. Procedures for Relays for Electrical and Std. for Iron-Core Power Filter Inductors for es for Radio and Television Receiving Appliances and Other and Display) for Television Receiving Appliances and Other

trolytic Capacitors (For Use Primarily in Transmitters and nce Sys/

Std. for Simulated Shipping Test (Packaging) for Consumer

Std. for Safety for Access Control System Units control Apparatus-Controllers and Controller Assemblies

licon by Measurement of Steady State Surface Photovoltage

Code for Distribution, Power and Regulating Transformers

Graphic Symbols for Logic Diagrams (Two-State Devices)

I Characteristics of Class a Closure Interchange Circuits Std. Meth. of Measurement of Microhardness of automotive Ornamentation and Hardware (1970) Std. for 973) ANSI G53.12 Electrodes and Rods (1972)

Electrometric Titration (1972) ANSI Z11.248

Electronic Application (1972)

Electronics) (1974)
Electrodeposited Coatings of Tin-Lead Alloy (Solder P

Electrodeposited Coatings of Tin (1972)

Electrodes and Fluxes for Submerged Arc Welding (1969)

Electrodes for Flux Cored Arc Welding (1969) ANSI W3.20

Electrodes for Gas Metal Arc Welding (1969) ANSI W3.18

Electrodes for Welding Cast Iron (1969) ANSI W 3.15

Electrodes in Multiple Electrode Semiconductor Devices

Electrodes (1969) ANSI A5.1

Electrodes (1969) ANSI W 3.10

Electrodes (1969) ANSI W 3.11

Electrodes (1969) ANSI W3.12

Electrodes (1969) ANSI W 3.14

Electrodes (1969) ANSI W/3.19

Electrodes (1969) ANSI W 3.3

Electrodes (1969) ANSl W 3.4

Electrodes (1969) ANSI W3.5

Electrodes (1969) ANSI W3.6

Electrodes (1969) ANSI W 3.9

Electrodes (1970) ANSI W 3.13

Electrodes (1970) ANSI W/3.16

Electrodes (1970) ANSI W3.21

Electrodes (1972) ANS1 C59.130

Electrodes (1974)

Electroformed Sieves (1970) ANSI Z168.5

Electrogalvanized Steel (Coil) (1974)

Electrolytes (1973) ANSI H44.1

Electrolytic Capacitors (For Use Primarily in Transmit

Electrolytic Cathode Copper (1972) ANSI H23.10

Electrolytic Chlorine Processes (Explosion Hazard Durin

Electrolytic Copper Wire Bars, Billets, and Cakes (1972

Electrolytic Copper Wire Bars, Cakes, Slabs, Billets, I

Electrolytic Manganese Metal (1969) ANSl G84.1

Electrolytic Tantalum Capacitors (1972)

Electromagnet Iron (EMI) Cold Rolled Strip for Magnetic

Electromagnetic and Particulate Radiation (1973)

Electromagnetic Holders; Sliding Door Equipment; Instal

(Electromagnetic Instrumentation) (1972)

Electromagnetic Radiation at Microwave Frequencies (197

Electromagnetic Radiation from Motor Vehicles (20-1000

Electromagnetic Radiation Suppressors (1969)

Electrometer Type Dosimeters and Companion Dosimeter $\mathrm{Ch}$

Electrometric Meth. of Analysis for Hydrogen Ion Activi

Electrometric Meth.) (1973)

Electromotive Force (EMF) Tables for Thermocouples (197

Electron Accelerator Inst allations (1964)

Electron Devices by a Bubble Test (1972)

Electron Devices (1972)

Electron Devices (1972)

Electron Radiation Dose with the Ceric Sulfate Dosimete

Electron Radiation Dose with the Ferrous Sulfate Cupric

Electron Tubes and Electric Lamps (1971)

Electron Tubes (1973)

Electron VoIts (1954)

Electronic and Electrical Applications (1972T)

Electronic Component Parts (1972)

Electronic Computer and Data Processing Equipment (1972

Electronic Computer Systems (1972)

Electronic Computer Systems (1973)

Electronic Data Processing Units and Systems (1972)

Electronic Devices by Dye Penetration (1972)

Electronic Devices (1973)

Std. Rec. Pract. for De

Electronic Equipment on Boats (Audio Device, Automatic

Electronic Equipment (Flyback Transformer, Multiplier,

Electronic Equipment (1971)

Electronic Equipment (1973) ANSI C83.25

Electronic Equipment (1973) ANSI C83.92

Electronic Equipment (1974)

Electronic Equipment (1974)

Electronic Instruments) (1958) ANSI C83.15

or Safety for Special $F u$

Thode Ray Tubes (Picture

Electronic Products (Radio, Television, Tape Recorder.

Electronic / Electrical / Mechanical Security Surveilla is for Elec

Electronic) (1973)

Electronics) (1973)

Electronics) (1973)

Electronics) (1973) ANSI C57.12.90

Electronics) (1973) ANSI Y32.14

Electroplated Coatings (1973)

Electroplating of NickeI and Chromium on Metal Parts-

Electroplating on Aluminum Alloys by Zincate Process (1

Std. for Industrial ANSI Ty Carrier Diffusion Length in SI ASTM

Electroplating on Stainless Steel (1970) ANSI G53.13
ICBO

ASTM

ASTM

ASTM

AWS

NEMA

AWS

AWS

AWS

EIA

AWS

AWS

$A W S$

AWS

AWS

AWS

AWS

AWS

AWS

AWS

AWS

AWS

ASTM

Std. F ASTM

EIA

ASTM

FMS

ASTM

ASTM

ASTM

EIA

NARM

I Tes ASTM

NBHA

NARM

ANSI

SAE

SAE

ANSI

AACC

ASTM

ASTM

NCRPM

ASTM

ASTM

ASTM

ASTM

ASTM

ANSI

NCRPM

AST

ASTM

EIA

NFPA

FMS

UL.

ASTM

ASTM

$A B Y C$

UL.

ANSI

EIA

UL

UL

EIA

ElA

ASTM

td. Test IEEE

Std. for lEEE

EIA

ASTM

SAE

ASTM

ASTM

UBCS 32.6

B200

(

B545

A5. 17

EW 2

A5. 20

A. 5.18

A5. 15

RS32 IB

A5. 1

A5. 10

A5.11

A5. 12

A5. 14

A5. 19

A5. 3

A 5.4

A5. 5

A5. 6

A5. 9

A5. 13

A5. 16

A5.21

D) 816

A5. 22

E16l

TB-II.3

B418

RS205

BIl 15 
Std. Rec. Pract. for Cleaning Metals Prior to Std. Rec. Pract. for Image Evaluation of

ials (1973) Tent. Rec. Pract. fo Std. Meth. of Test for Test Meth. for

al Test) (1973) Std. Definitions of Terms Relating to
Test Meth. for

hanical Propert/ Std. Rec. Pract. for Examination of Fuel for the Locking Ability Performance of Nonmetallic Locking td. End Load Test Meth. for a Hydraulic Fluid Power Filter he Fabrication Integrity of a Hydraulic Fluid Power Filter e Material Comparibility of a Hydraulic Fluid Power Filter

Fatigue Characteristics of a Hydraulic Fluid Power Filter lapse / Burst Resistance of a Hydraulic Fluid Power Filter tration Performance of a Fine Hydraulic Fluid Power Filter Std. Meth. of Test for Noncombustibility of Uniform Building Code Std for Noncombustible std. Symbols and Definitions for Mechanical and Acoustical Std. for Safety for Sheathed Heating loor-Ceiling Assemblies, Doors, and Other Space Dividing Magnesium Oxide for Use in Sheathed Type Electric Heating td. Spec. for Spray Applied Fibrous Thermal Insulation for gth Properties of Adhesives in Shear by Tension Loading at

d Alloy Steel Plate, Sheet, and Strip for High Strength a old, and Cold Worked Alloy Steel Bars for High Strength at oy Steel Forgings and Forging Billets for High Strength at Std. Meth. of Test for Normal Spectral Emittance at ittings of Wrought Carbon and Alloy Steel for Moderate and obalt, and Nickel Base Alloy Castings for High Strength at Std. for Std. for Safety fo

973)

al) (1973)

(1972) Ans Std Meth. of Test for Breaking Strength and est for Annealing Point and Strain Point of Glass by Fiber Std. Meth. of Test for Breaking Load and

ing Equipment for Use with Emergency Locator Transmitters Sid. for Slow Moving Vehicle Identification Lamp (Light) and Slow Moving Vehicle (SMV) Identification warning Lamp and Slow Moving Vehicles (SMV) Identification Std. Meth. of Test for oducts and Procedur/ Std. Meth. of Test for Hydrogen ized Structural Steel Products and Proceguarding Against Rec. Pract. for Design Levels for Std. for Safety for Battery Powered Airborne Radio Homing and Alerting Equipment for Use with nalysis of Radioactivity (Beta Gamma) in Cereal Products Safety Std. for Highway

for Wall Installation (1972) ANSI B135./

Safety Std. for ice) (1973) Rec. for Exposure to Radiation in an

terials by Comparison with a Secondary Standard of Similar $\mathrm{n}$ with a Secondary Standard of Sim/ Std. Meth. of Thermal Std. Temperature-Electromotive Force ys and S/ General Spec. Applicable to Electromagnet Iron İ Trucks (1973) Rec. Pract. for Exhaust

ithium Fluoride Carrier D.C Arc Technique Using an Optical loy Steel by the Point to Plane Technique Using an Optica pract. for Selection of Electrode Material Used in Optical Std. Definitions of Terms and Symbols Relating to Std. Meth. of Test. for Leaks Using Bubble nace Iron by the Point to Plane Technique Using an Optical $\mathrm{t} \mathrm{Tr} /$ Rec. Pract. for the Measurement of Fuel Evaporative t Trl Rec. Pract. for the Measurement of Fuel Evaporative Laboratory Instrumentation and Techniques for Exhaust Gas Std. Meth. of Test for Normal Spectral

ter During/ Std. Meth. for Measurement of Delayed Neutron suitable Amounts of Sample, Sulfuric Acid and Catalyst Are suitable Amounts of Sample, Sulfuric Acid and Catalyst Are suitable Amounts of Sample, Sulfuric Acid and Catalyst Are the Off-The-Job Disabling Accidental Injury Experience of ion of Sealed Insulation Systems for AC Electric Machinery Std. Assessment of the Assumption of Normality face Between Numerical Control and Data Terminal Equipment

\section{Std. Spec. for Cationic Std. Spec. for Std. Meth. of Test.} anks to Be Used as Panels in Weathering Tests of Latex and tos Tile Floors (1969)
Electroplating (1968) ANSI G53.33

Electrostatic Business Copies (1972)

Electrostatic Charge Mobility on Flexible Barrier Mater

Electrostatic Charge (1973)

Electrostatic Clinging of Textile Fabrics Fabric to Met

Electrostatic Copying (1972B)

Electrostatic Propensity of Carpets (1969)

Element Cladding Including the Determination of the $\mathrm{Mec}$

Element Type Prevailing-Torque Lock Screws (1973)

Element (1972) ANSI B93.21

Element (1972) ANSI B93.22

Element (1972) ANSI B93.23

Element (1972) ANSI B93.24

/D. Meth. for Determining T Std. Meth. of Verifying Th /Th. for Verifying the Flow /Eth. for Verifying the $\mathrm{Col}$ Element (1972) ANSI B93.25
Element (1973) ANSI B93.31

Elementary Building Materials (1973)

Elementary Materials Test. (1973)

Elements as Used in Schematic Diagrams (1972)

Elements for Appliances and Equipment (1973)

Elements (1973)

/ Building Partitions Such as Walls, F

Elements (1973)

Elevated Temperature (1972)

Elevated Temperatures (Metal to Metal) (1972) ANSI Z197

ID Cold Worke ASTM

/ $\mathrm{R}$ Hot, Hot C

Elevated Temperatures (1971) ANSI G81.24

Elevated Temperatures (1971) ANSI G81.27

Elevated Temperatures (1972)

Elevated Temperatures (1973) ANSI G31.2

Elevated Temperatures (1973) ANSl G81.40

D Worked All

Elevating Work Platforms (Lift) (1973)

Elevator Door Locking Devices and Contacts (1973)

Elevators (Freight and Passenger) (1971)

Elevators, Dumbwaiters, Escalators, and Moving Walks (1

Elevators, Escalators and Moving Walks (inspectors Manu

Elliptical Culvert, Storm Drain, and Sewer Pipe (1972)

Elliptical Head Blind Nut (1974)

Elongation of Cotton Textile Fibers (Flat Bundle Meth.)

Elongation (1971)

Elongatoin of Elastomeric Yarns (Constant Rate of Exten

(ELT) (1973)

Emblem (1968) ANSI B114.1, SAE J943A

Emblem (1972)

Emblem (1972) SAE J725

Ounting Brackets and Socket for Warning

Embossed Depth of Resilient Floor Coverings (1973)

Embrittlement of Copper (1973)

Embrittlement of Hot Dip Galvan

Embrittlement (1972) ANSI G8.13

Emergency Air Brake Systems (1971)

Emergency Lighting Equipment (Indoor) (1973)
Emergency Locator Transmitters (ELT) (1973)

Emergency Monitoring (Cereal Chemistry) (1973)

Emergency Signals (Flare, Lantern, Fusee) (1973)

Emergency Std. Spec. for Automotive Gasoline (1974)

Emergency Vault Ventilators and Vault Ventilating Ports

Emergency (1962)

(Emergency) Hardware (Outward Opening Door Releasing Dev

EMF Temperature Properties (1972)

/Le Thermoelement Ma

(EMF) Tables for Thermocouples (1972) ANSI C96.2

(EMI) Cold Rolled Strip for Magnetic Application in Rela

Emission Controls (Air Pollution) for Powered Industria

Emission Spectrograph (1974)

/ the Silver Chloride-L

Emission Spectrometer (1970) ANSI 2128.28 / and Low Al

Emission Spectroscopy Based on Physical Properties (197

Emission Spectroscopy (1972A) ANSI Z128.7

Emission Techniques (1974)

Emission Vacuum Spectrometer (1973)

Emissions from Gasoline Powered Passenger Cars and Ligh

Emissions from Gasoline Powered Passenger Cars and Ligh

Emissions Measurement (Passenger Car and Light Truck) (

Emittance at Elevated Temperatures (1972)

Emitting Fission Products in Nuclear Reactor Coolant Wa

Employed (1960)

Employed (1962)

Employed (1962)

Employees (1973)

Jother Protein Bearing Materials When

Other Protein Bearing Materials When $\mathrm{C}$

/Other Protein Bearing Materials When

/for Meth. of Recording and Measuring

Employing Form Wound Stator Coils (1972) ANSI C50.26

Employing Individual Observed Values) (1974)

Employing Parallel Binary Data Interchange (1973)

Employment Record (1973)

Emulsified Asphalt (1973)

Emulsified Asphalt (1973) ANSI A37.55

Emulsified Asphalts (1974) ANSI A37.42, Ashto T59

Emulsion Exterior Paints or Coatings (1973)

Emulsion Floor Polish for Use on Asphalt or Vinyl Asbes

Emulsion Floor Polishes (1972)
ASTM

ASTM

ASTM F365

AATCC D267

ASTM F335

AATCC 134

ASTM E453

124

N3LDP 10.8

T3.10.8.?

NFLDP T3.10.8.5

NFLDP T3.10.8.8

El36

UBCS4. 1

$\begin{array}{ll}\text { ANSI } & \text { Y } 32.18\end{array}$

UL

ASTM

ASTM

ASTM

ASTM

ASTM

1030

UBCS35- 1

D3215

C720

D2295

A 457

A458

A 477

E307

A234

A567

ANSI A92.3

UL

CSI

14200

ANSI A17.2

ASTM C507

ASTM

1734

D1445

C336

D2653

DO-154

$\begin{array}{ll}\text { ASAE } & \text { S276.2 } \\ \text { SAE } & \text { J725D }\end{array}$

ASAE $\quad \mathrm{S} 277.2$

ASTM F373

ASTM B577

ASTM Al43

AI43

SAE J263

UL

924

DO-154

1. of a $\mathrm{AACCH} 28.99 \mathrm{~A}$

$\begin{array}{ll}\mathrm{UL} & 912\end{array}$

ASTM ESI

UL 680

NCRPM R29

UL 305

ASTM E207

ASTM E207

ASTM E230

NARM 121

IT A

$6 \mathrm{~F} 1$

E483

E403

ASTM E504

ASTM E135

ASTM E515

E485

SAE J170A

SAE J171A

SAE J254

ASTM E30?

ASTM D2470

F-44

G.22

J.56

Z16. 3

429

IEE

N15.15

RS408

G811

$\begin{array}{ll}\text { ASTM } & \text { D2397 } \\ \text { ASTM } & \text { D977 }\end{array}$

ASTM D244

Hingle BI ASTM D1911

RTI

ASTM
$+6$

D3052 
otal Solids) in Water Emulsion, Solvent Based, and Polymer Std. Meth. of Test for Water Spotting of Rec. Pract. for Qualitative Identification of Polymers in Std. Meth. of Test for Sediment in Water Std. Meth. of Test for Total Ash and Silica in Water Spec. for Vertical and Horizontal th. of Test for Nonvolatile Matter (Total Solids) in Water Std. Meth. of Test. Bituminous Base sis of Vitamin A in Dry Vitamin Mixes, Beadlets, Oils, and Std. Definitions of Terms Relating to Porcelain alt Coatings (1972) Rec. Spec. for Std. Meth. of Test for Continuity of Porcelain ก/ Std. Meth. of Test for Fusion Flow of Porcelain Std. Meth. of Test for Flash Point of Liquids (Paint, Std. Meth. of Test for Spalling Resistance of Porcelain Std. for f Test for Reboiling Tendency of Sheet Steel for Porcelain uction and Preparation of Gray lron Castings for Porcelain Std. Spec. for Steel Sheets for Porcelain Std. Meth. for Test. Magnetic Wire

Std. Meth. of Test for Alkali Resistance of Porcelain ec. Pract. for Determining Hydrolytic Stability of Plastic or Other Liquids (19) Std. Rec. Pract. for Polyethylene (1972) ANSI / Std. for Polyethylene ing (1974) Std. for Design of Components Std. Spec. for Lubrication of Industrial ves (1973) Std. for Sound (Noise Measurement for ricultural Liquids and SIur/ Rec. for Improving Safety on gasoline Powered Passenger Cars and Light Trucks Using the nd Performance Requirements) (1972) / Std. for Protective ons (1972) ANSI C106.1

Std. Rec. for ances (1973) Std. for Safety for Test for Polymeric ups A-D, and Class / Safety Std. for Circuit Breakers and Std. for Industrial Control Apparatus afety for Molded Case Circuit Breakers and Circuit Breake ocedures for Low Voltage AC Power Circuit Breakers Used in voltage Alternating Current Power Circuit Breakers Used in bove and Below Room Temperature (1966) An/ Std. Spec. for Std. for Magnetic Stripe Rec. Safety Sign for Fron

Rec. Pract. for Hole Placement on Bulldozer (Dozer) Std. for Break Mandrel Closed d Mounting Dime/ Citrus Style (1974) iameter) (1973) nsi 010.1 element (1972) ANSI B93.21 Std. Bore and Rod Size Combinations, Rod Rec. lndustry Std. for Loose Meta Rec. Std. for Loose Metal Std. Spec. for Creosoted ing (Short Haul); Grader, Loader, Dozer, Mining Car, Front or Articulated Vehicles (Construction and Industrial Fron iteria for Roll Over Protective Structure for Weeled Fron ver Protective Structure for Track Type Tractors and Fron ture (FOPS) for Prime Movers, Wheeled and Track Type Fron Std. for High Speed Steel Spec. for Compression Couplings for Vitrified Clay Plain Std. Spec. for Bell oards)/

(1972) (1974) Std. Spec. for Record Formats for Transmission of
Std. for 450 Deg. F Closed Std. for 450 Deg. F Closed
Std. Test. Procedure for Std. Test. Procedure for Std. Test. Procedure for Axial Std. for Steel Butt Welding Std. Dimensions for Flareless Acorn Fitting Std. for Swaging Process-Tube to Rod Std. Test. Procedure for Axial (End to Spec. for Bearings, Ball and Roller, Rod Std. for Rod Std for Face-To-Face an Std. for Driving and Spindle Std. for Buttwelding

for Tube Assembly, Control Steel with Welded Threaded Rod ssembly, Control Steel with Welded Clevis and Threaded Rod sembly, Control, Steel, with Flash Welded and Threaded Rod

Control, Steel, with Flash Welded Clevis and Threaded Rod ected to Partial Discharg/ Std. Meth. of Test for Voltage Std. Meth. Measuring Plasma Arc Gas Enthalpy by

d. Test Procedure to Determine the Freezer Temperature and manual for Electric Comfort Conditioning (Heat Loss, Gain Rec. for Shielding for High

Std. Meth. of Test for Neutron Flux Density and Average ic Materials (1962)/ Std. Rec. Pract. for Effects of High Rec. Pract. for Exposure of Adhesive Specimens to High Std. Meth. of Estimating Stray Radian Cable for the Transmission and Distribution of Electrical Cable for the Transmission and Distribution of Electrical
Emulsion Floor Polishes (1972)

Emulsion Floor Polishes (1974) ANSI Z123.12

Nonvolatile Matter (T ASTM

Emulsion Paints (1973)

Emulsion Polishes by Centrifuge (1972) ANSI Z123.3

Emulsion Polishes (1972) ANSP Z123.1 A

Emulsion Treaters (Petroleum Production) (1973)

Emulsion, Solvent Based, and Polymer Emulsion Floor Pol

Emulsions for Use as Protective Coatings (1973)

Emulsions (Cereal Chemistry) (1973)

Enamel and Ceramic Metal Systems (1973) ANSI Zl67.

Enamel Coating System Designations for Coal Tar or Asph

EnameI Coatings (1973)

Enamel Frits (Flow Button Meth.) (1970) ANSI Zl67.10

Enamel, Lacquer, Varnish, and Related Products) by Seta

Enameled Aluminum (1972)

Enameled Cast Iron Plumbing Fixtures (1973)

Enameling (1969) ANSI Z167.21

Enameling (1970) ANSI Z167.23

Enameling (1973)

Enamels (1973)

Enamels (1974) ANSI Z167.20

Encapsulants for Electronic Devices (1973)

Encasement for Gray and Ductile Cast Iron Pipe for Wate

Encasement for Gray and Ductile Cast Iron Piping for Wa

Enclosed Gear Drives-Bearings, Bolting Keys and Shaft

Enclosed Gear Drives (1972)

Enclosed Helical, Herringbone and Spiral Bevel Gear Dri

Enclosed Mobile Tanks for Transporting and Spreading Ag

Enclosure Technique (1972) Vaporative Emissio

D. Rec. Pract. for Prod ASTM

Std. ASTM

ASTM

ASTM

API

ASTM

ASTM

AACC

NAPCA

ASTM

ASTM

ASTM

ASTM

ANSI

Enclosures for Agricultural Tractors (Test Procedures a

Enclosures for Electrical Equipment in Hazardous Locati

Enclosures for Motors (1973)

Enclosures for Portable Cord Connected Electrical Appli

Enclosures for Use in Hazardous Locations, Class I, Gro

Enclosures of All Kinds (Architectural Woodwork) (1973)

Enclosures (Electronic) (1973)

Enclosures (1972)

Enclosures (1973)

Enclosures (1973) ANS1 C37.13

Enclosures, Cold Chambers and Serving Units for Tests a

End Agricultural Loaders (1972)

End Bit Supports (1972)

End Blind Rivets (1973)

End Configurations, Dimensional Identification Code, an

End Dimensions of Composite Cans (211 (66 Mm) Diameter)

End Dimensions (6 Ounce Fibre Citrus Cans $202(52 \mathrm{Mm}) \mathrm{D}$

End Grain Wood Block Flooring for Interior Use (1970) a

End Load Test Meth. for a Hydraulic Fluid Power Filter

End Loader, Fork Lift Trucks, Mobile Crane, and Shovel

End Loaders and Dozers) (1971)

Steering Frame Lock $\mathrm{F}$

End Loaders and Weeled Dozers (1972) /M Performance Cr

/Um Performance Criteria for Roll O

End Loaders, Wheeled Dozers, Track Type Tractors, and M

End Mills, .125 Thru 3.000 Diameter (1973)

End Pipe (1973)

End Poly(vinyl Chloride) (PVC) Pipe (1973) ANSl B72.20

End Product Description Data (Tooling, Printed Wiring B

End Self Locking Serrated Head Blind Nut (1974)

End Supported Beam Deflection Composite Tubes and Cores

End Supported Beam Strength Fibre Tubes and Cores (1972

(End to End) Compression Composite Cans, Tubes and Cores

End Valves (1973)

End (1974)

End (1974)

End) Compression Composite Cans, Tubes and Cores (1974)

End, Anti Friction, Airframe (1974)

End, Threaded, Alloy Steel, Flash Welding Type (1973)

End.To-End Dimensions of Ferrous Valves (1973)

Ends for Portable Hanà, Air, and Electric Tools (1973)

Ends (1972)

Ends (1973)

Ends (1973)

Ends (1973)

Ends (1973)

Endurance of Solid Electrical lnsulating Materials Subj

Energy Balance (1973)

Energy Consumption of Household Refrigerators, Combinat

Energy Consumption) (1974)

Energy Electron Accelerator Installations (1964)

Energy from ${ }_{3} \mathrm{H}(\mathrm{D}, \mathrm{N})_{4} \mathrm{He}$ Neutron Generators by Radio

Energy Radiation on the Mechanical Properties of Metall

Energy Radiation (1970) ANS1 N141

Energy (1972)

Energy (1973) 1PCEA S-61-402

Energy (1973) IPCEA S-66-524

/Astic lnsulated Wire and NEMA
/Ylene Insulated Wire and NEMA

ASAE

SAE

ASAE

FMS

UL

AWI

ANS1

UL

$\mathrm{SAE}$

TRA

SAE

SAE

SAE

NSA

ASTM

IPC

NSA

CCTI

CCT]

CCTI

D2834

D1793

D1290

D1288

$12 \mathrm{~L}$

D2834

D2939

$86.01 \mathrm{~A}$

C286

C743

C. 374
D.3278

C703

A]12.19.1

C632

C660

A424

$\begin{array}{ll}\text { ASTM } & \text { D3288 } \\ \text { ASTM } & \text { C614 }\end{array}$

$\begin{array}{ll}\text { ASTM } & \text { C614 } \\ \text { ASTM } & \text { F74 }\end{array}$

ASTM A674

AWWA C 105

AGMA 260.02

AGMA 250.03

AGMA 297.01

R317

J171A

S336. 1

496

$5-6$

746.51

877

$* 1-400$

C19.4

489

C37.50

20

E197

$\mathrm{X} 4.16$

R355

J63

126

NFLDP T3.6.11

CCTI E211.1

CCTI E202.

ASTM D1031

NFLDP T3.10.8.2

3

J276

J394 A

J395A

J231

986 
eads (7/45 Deg. Form with 0.6 Pitch Basic Height of Thread

-Fir; Ponderosa, Idaho White, Sugar, and Lodgepole Pines: onary Diesel and Gas Engine/ Sid. Pract. for Selection of

Pract. for Low and Medium Speed Stationary Diesel and Ga hol Base) by the Iodine/ Std. Meth. for Test for Water in d 14.2 Std. Meth. of Test for Reserve Alkalinity of std. Meth. for Sampling and Preparing Aqueous Solutions of Std. Meth for Corrosion Test on Metal Specimens for Std. Meth. of Test for Boiling Point of

Pract. for Low and Medium Speed Stationary Diesel and Gas Rec. Pract. for Automotive Std. for Clevis

Std. Meth. of Test for Foaming Tendencies of of Test. Anti Leak Antifreezes and Stop Leak Additives for td. Meth. of Test for Simulated Service Corrosion Test. of efractometer for Determining the Freezing Point of Aqueous Pract. for Low and Medium Speed Stationary Diesel and Gas Pract. for Low and Medium Speed Stationary Diesel and Gas

Pract. for Low and Medium Speed Stationary Diesel and Gas Rec. Pract. for

Pract. for Low and Medium Speed Stationary Diesel and Gas Pract. for Low and Medium Speed Stationary Diesel and Gas Pract. for Low and Medium Speed Stationary Diesel and Gas Pract. for Low and Medium Speed Stationary Diesel and Gas Std. for Service Time Coding of Propulsion
Pract. for Low and Medium Speed Stationary Diesel and Gas

Pract. for Low and Medium Speed Stationary Diesel and Gas Rec. Pract, and Stds. for Marine drive Transmission (197/ Rec. Pract. for Marine (Inboard) Pract. for Low and Medium Speed Stationary Diesel and Gas Pract. for Low and Medium Speed Stationary Diesel and Gas Pract. for Low and Medium Speed Stationary Diesel and Gas Rec. Pract. for Std. for

Std. Pract for Stationary Diesel and Gas

Pract. for Low and Medium Speed Stationary Diesel and Gas Pract. for Low and Medium Speed Stationary Diesel and Gas U.S. Model Rocket Sporting and Safety Code and 1 Computer Programs (1970) Gas Turbine

Pract. for Low and Medium Speed Stationary Diesel and Gas

Pract, for Low and Medium Speed Stationary Diesel and Gas Rec. Pract. for Spark Plug, Aircraft Reciprocating Std. Form of Agreement Between Architect and Guide Spec, for Rec. Pract. for

h. Notes) (1966)

coolers for Packaged Compressor Units (1974)

$r$ Line Conventions and Lettering (1973)

Chassis Frames-Passenger Car and Light Truc/

Dimensioning and Tolerancing (1973)

( circuit Television) Camera Equipment (1974/ Rec. Std. for er and Waste Treatment) (1968/ Rec. Std. for Industrial Std. for Industrial Std. for Industrial Std. for Industria Std. for Industria

972)

72)

ry (1972)

1 (197)

Operation and Flow Process Charts and Symbols (Mechanical Diesel (Compression Ignited) Inboard Liquid Cooled Marine t. and Std. Covering the Installation of Air Cooled Marine struction of Gasoline Fueled Inboard, Liquid Cooled Marine ning and Stating the Horsepower Rating of Inboard Gasoline h. of Identifying AC and DC Electrical Wiring on Boats and a Ship's Cargo Hold During the Use of Internal Combustion Std. Pract. Definitions for Stationary Diesel and Gas

Pract. for Spark Arrester Test Procedure for Medium Size ract. for Spark Arresters Used on Multiposition Small Size actices for Low and Medium Speed Stationary Diesel and Gas Scavenging and Supercharging of Stationary Diesel and Gas $r$ Units for Low and Medium Speed Stationary Diesel and Gas d Spec. for Low and Medium Speed Stationary Diesel and Gas Rec. for Use of Tent. Meth. of Analysis for Iron in Wine Tent Meth of Analysis for Volatile Acid in Wine Tent. Meth. of Analysis for Brix (Balling) of Wine Official Meth. of Analysis for Total Acid in Wine
Engagement (1973)

Std. for Buttress Inch Screw Thr

Engelmann Spruce; Western Cedar and Hemlock; Subal-Pi

Engine-Generator Units for Low and Medium Speed Stati

Engine Air Intake and Exhaust Systems (I972)

Engine Antifreeze-Coolant Concentrate (Glycol or Alco

Engine Antifreeze, Antirusts, and Coolants (I972) ANSI

Engine Antifreezes or Antirusts for Test. Purposes (197

Engine Antifreezes Tested in Glassware (1970) ANSI DI4.

Engine Antifreezes (1972) ANSI DI4.1

Engine Assembly and Equipment (I972)

Engine Carburetor Airflow Reference Std. (1971)

Engine Control Rod, Adjustable (1973)

Engine Coolants in Glassware (1973)

Engine Coolants (I972)

Engine Coolants (1973)

Engine Coolants (1974)

Engine Cooling Water Systems (1972)

Engine Erection and Installation (1972)

Engine Factory and Field Testing (1972)

Engine Foot Mounting (Front and Rear) (1970)

Engine Fuel Oil Characteristics (1972)

Engine Gaseous Fuel Characteristics and General Spec. (

Engine Gauge Borads, Protective Devices, and Instrument

Engine Generators and Electrical Equipment (1972)

Engine Items (1971) ANSI B141.8

Engine Lubricating Oil Characteristics and General Spec

Engine Lubricating Oil Systems (1972)

Engine Mountings (Boats) (I97I)

Engine Mountings (Installation and Replacement) Direct

Engine Operation and Maintenance (1972)

Engine Power Plant Buildings (1972)

Engine Power Plant Fuel Oil and Gas Systems (1972)

Engine Preheaters (Coolant Heaters) (1971)

Engine Rating Code Diesel (1971)

Engine Ratings and Performance (1972)

Engine Speed Governing and Parallel Operation (1972)

Engine Starting Systems (I972)

Engine Std. (1973)

Engine Steady State Performance Presentation for Digita

Engine Test Code-Spark Ignition and Diesel (1971)

Engine Vibration (1972)

Engine Waste Heat Recovery Systems (1972)

Engine (1971)

Engineer (1974)

Engineered Brick Masonry (Tech. Notes) (1972)

Engineered Brick Masonry (1969)

Engineered Design of the Contemporary Bearing Wall (Tec

Engineering Data Sheets for the Design of Air Exchange

Engineering Drawing and Related Documentation Pract. Fo

Engineering Drawing and Related Documentation Practices

Engineering Drawing and Related Documentation Practices

Engineering Purposes (1973)

Engineering Services (1972)

Engineering Specs. Outline for Monochrome Ccty (Closed

Engineering Structures (T anks, Reservoirs, etc. for Wat

Engineering Terminology: Applied Mathematics (1972)

Engineering Terminology: Applied Psychology (1972)

Engineering Terminology: Biomechanics (1972)

Engineering Terminology: Cost (1972)

Engineering Terminology: Data Processing and Systems (1

Engineering Terminology: Distribution and Marketing (19

Engineering Terminology: Economy (1972)

Engineering Terminology: Facility Planning (1972)

Engineering Terminology: Materials Processing (1972)

Engineering Terminology: Organization Planning and Theo

Engineering Terminology: Production Planning and Contro

Engineering Terminology: Work Measurement and Meth. (I9

Engineering) (1972)

Engines and Auxiliaries (Boats) (1972)

Engines and Auxiliaries (Diesel and $G$

Engines (Boats) and Auxiliaries (1972)

Engines (Boats) (1972)

Engines (Color Code) (1973)

Engines (1970)

Engines (1972)

Engines (1972)

Engines (1972)

Engines (1972)

Engines (1972)

Engines (1972)

Engines (1972)

English and Me
(Enology) (1972)

(Enology) (1972)

(Enology) (1972)

(Enology) (1972)
ANSI

ICBO

DEMA

Std. DEMA

ASTM

ASTM

ASTM

ASTM

ASTM

Std. DEMA

SAE

ASTM

Std. Meth. ASTM

ASTM

R ASTM

Std. DEMA

Std. DEMA

Std. DEMA

SAE

Std. DEMA

DEMA

DEMA

TTd. DEMA

SAE

DEMA

Std. DEMA

ABYC

SAE

Std. DEMA

Std. DEMA

D. DEMA

SAE

SAE

DEMA

DEMA

Std. DEMA

NAR

SAE

SAE

Std. DEMA

Std. DEMA

SAE

AIOA

BIA

BIA

BIA

API

ANSI

ANSI

ANSI

ICBO

AIOA

EIA

ACI

ANSI

ANSI

ANSI

ANSI

ANSI

ANSI

ANSI

ANSI

ANSI

ANSI

ANSI

ANSI

Std. for ASME

$A B Y C$

$A B Y C$ (s) (

Rec. Pract for and Con $A B Y C$

$A B Y C$

Requirements and Rec. Met $\mathrm{ABYC}$ Guide for Supplementary Ventilation in

SNAME
DEMA

Rec SAE

Rec. P SAE

Std. Pr DEMA

Std. Pract. for DEMA

/Ct. for Selection of Engine-Generato /on of Invitations for Bids and Detaile DEMA DEMA ASAE ASE

ASE

ASE

ASE

BI.9

UBCS25.4

*1. 19

1.8

DI123

DI 121

DII76

1384

D 1120

* $1-5$
J 228

170

DI88I

D3147

D2570

D332I

* I- 10

*1-21

J616B

*1-14

*1-15

* I-I

* 1-18

ASI 105

* 1. I 2

$\mathrm{J} 233$

* $1-23$ 
National Bureau of Standards KWIC Index of Engineering Standards

Official Meth. of pH Determination in Wine (Enology) (1972)

Analysis for Total Sulfur Dioxide in Wine (Enology) (1972)

(Enology) (1972)

Tent. Meth. of Analysis for Copper in Wine (Enology) (1972)
th. of Analysis for Alcohol (Ethanol) in Wine (Enology) (1972)

ydrometer Meth. of Analysis for Alcohol (Ethanol) in Wine (Enology) (1972)
ycnometer Meth. of Analysis for Alcohol (Ethanol) in Wine (Enology) (1972)

lliometer Meth. for Analysis of Alcohol (Ethanol) in Wine (Enology) (1972)

62)

$\mathrm{n}$ in Enrichment (Vitamin and Mineral) Concentrates Used to

nrich Cereals (Cereal Che

$$
\text { Meth. of Analysis of Vitamin A and Carotene in }
$$
Meth. of Analysis of Vitamin A in Meth of Analysis of Niacin in Std. Meth. Measuring Plasma Arc Gas Std. Spec. for Air

Std. for Air Containers for

td. Rec. Pract. for Design and Construction of Nonmetallic Std. Meth. of Test for Sealability of craft Instrument Stds. (Wording, Terminology, Phraseology, eed and Water Requirements of Farm Liv/ Effect of Thermal Is (Coati/ Std. Rec. Pract. for Combined, Simulated Space $f$ Carbon Steel (P-1) Welds in Corrosive Petroleum Refining o Growth of Mold on the Surface of Interior Coatings in an Std. Meth. for Analysis of tion of Painted or Coated Specimens Subjected to Corrosive low Alloy Steel H-Piles and Sheet Piling for Use in Marine Test Meth. for Assay of Bacterial Alpha-Amylase

(1972) An/

fault Dimension (197/

ype by Infrared Refl/ Tentative Std. Meth. of Test for Resistivity of Silicon Std. Meth. of Test for Thickness of Std. Meth. of Test for Thickness of pping and Staining $\mathrm{T} /$

Std Meth of Test for Thickness of Std. Meth. of Test for

for Woven Glass Fabrics, Cleaned, and After Finished with ies (Safety) (19) Std. Guide for Classifying and Labeling Std. Spec. for Reinforced Graphite Fiber Tape and Sheet, 000 (248) Modul/ 000 (248) Modul/ ishing (1973) Spec. for Graphite Fiber Tape and Sheet, Spec. for Graphite Fiber Tape and Sheet. Cloth, Type E Glass, B Stage Std. Meth. of Test for Viscosity of Std. Meth. of Test for Epoxy Content of Std. Meth. of Test for Total Chlorine Content of Meth. of Test for Hydrolyzable Chlorine Content of Liquid act. Meth. for Lining Lease Production Tanks with Coal Tar d Moderate Heat Resistant, $16.5 \mathrm{Cr}-4.5 \mathrm{Ni}-2.9 \mathrm{Mo}-0.10 \mathrm{~N}$, Std. Control Valve Sizing Std. for Control Valve Sizing i-2.9Mo-0.10N, Solution Heat Treated, Sub Zero Cooled, Trial Use Std. Criteria for Separation of Class Ie Hydraulic Std. for Industrial Rec. for Safety for Farmstead Std. for Headed Drilled Pins for F arm ing Location/ Std. for Safety for Electrically Conductive Std. for Start.Stop Signal Quality Between Data Terminal afety Requirements for Commercial Laundry and Dry Cleaning pract. for Prefabricated Reflective Insulation Systems for ditioning System Rating Definitions, Test. Meth., and Test or Flammable Liquid and Liquefied Flammable Gas Processing . Pract. for Descaling and Cleaning Stainless Steel Parts, Guide for Acceptance and Maintenance of Insulating Oil in and Testing Requirements for Instrumentation and Electric for Interface Between Numerical Control and Data Terminal Std. for Commercial Cooking

ion, Design, Construction and Installation of Fittings and (Regulators, Automatic Damper Cont/ Safety Std. for Draft

Std. for Temperature Indicating and Regulating Electrical Std. for Construction, Installation, and Rating of Std. for Power Output Ratings of Packaged Audio

al Use Std.: General Guide for Qualifying Class I Electric ansi/ Guide for Seismic Qualification of Class I Electric .2 Std. Spec. for ps A.D, Class II, Gro/ Safety Std. for Industrial Control Piping and

sic Characteristics for Airborne Radio Homing and Alerting ction Criteria for Safety Related Systems, Structures, and Std. for Tire and Rim

Std. for Purged and Pressurized Enclosures for Electrical Std. for Gas Utilization h Determination Devi/ Rec. Pract. and Std. for Electronic 2) Std. for Lighting and Marking of Agricultural Std. for Installation of Gas Piping and 108/ Minimum Performance Std. for Airborne VOR Receiving for Terminology for Pressure Relief Devices (Container and

Std. for Safety for Electrically Operated Photographic measuring, Recording and Specifying Machinery Sound Within Air Conditioning Std. Format for NAS 900 Series
Enrich Cereals (Cereal Chemistry) (1967)

Enriched Cereals and Mixed Feeds (Cere

(1967)

Enthalpy by Energy Balance (1973)

Entraining Admixtures for Concrete (1973) ANSI A37.132

Entrancew ays in Food Establishments (1970)

Enveloped Gaskets for Corrosive Service (1971) ANSI Z26

Enveloped Gaskets (1970) ANSI Z261.1

Environment and Design Stds for (1972)

Environment on Production, Heat and Moisture Loss and F

Environment Test. of Spacecraft Thermal Control Materia

Environment (1972)

Environmental Chamber (1973)

IS to Prevent in Service Crack lof Test for Resistance T

Environmental Materials Polychlorinated Biphenyls (1974

Eration and Evalua

Environments (Coil Coating) (1974)

Environments (1974)

Eration and

Std. Spec. for High
973) ANSl L14.147

Epitaxial Layers by the 3 Probe Voltage Breakdown Meth.

Epitaxial Layers of Silicon by Measurement of Stacking

Epitaxial Layers of Silicon on Substrates of the Same T

Epitaxial Layers Using a Collinear 4 Probe Array (1974)

Epitaxial or Diffused Layers in Silicon by the Angle La

Epoxy Content of Epoxy Resins (1973)

Epoxy Functional Silane Type Finishes for Plastic Lamin

Epoxy Products According to Their Hazardous Potentialit

Epoxy Resin Gas Pressure Pipe and Fittings (1973)

Epoxy Resin Impregnated for Hand Layup (1973)

Epoxy Resin Impregnated, G70,000 (483) Tensile, 36,000,

Epoxy Resin Impregnated, G70,000 (483) Tensile, 36,000,

Epoxy Resin Impregnated, 181 Style Fabric, Self Extingu

Epoxy Resins and Related Components (1968) ANSI K65.183

Epoxy Resins (1973

Epoxy Resins (1973)

Epoxy Resins (1973)

Epoxy (1972)

Equalized and Tempered (1973)

Equations for Compressible Fluids (1973)

Equations for Incompressible Fluids (1972)

Equilized, and Over Tempered (1973)

Equipment and Circuits (1974) ANSI N41.14

Equipment and General Purpose Machine Tools (1973)

Equipment and Machinery (1972)

Equipment and Machinery (1973)

Equipment and Materials for Use in Flammable Anesthetiz

Equipment and Non-Synchronous

Equipment and Operations (1972)

Equipment and Pipe Operating at T

Equipment and Supporting Structures (1973)

Equipment and Systems (1972) ANSI G81.16

Equip

Equipment During the Construction of Nuclear Power Gene

Equipment Employing Parallel Binary Data Interchange (1

Equipment Exhaust Systems (1970)

Equipment for Anchoring, Mooring, Docking, Towing and L

Equipment for Combustion Chamber in Heating Appliances

Equipment for Control of Air Conditioning, Heating, Coo

Equipment for Drying Farm Crops (1971)

Equipment for Home Use (1967)

Equipment for Nuclear Power Generating Stations (1971)

Equipment for Nuclear Power Generating Stations (1971)

Equipment for Sampling Water and Steam (1970) ANSI Z111

Equipment for Use in Hazardous Locations, Class 1, Grou

Equipment for Use with Dry Chlorine (1971)

Equipment for Use with Emergency Locator Transmitters (

Equipment for Water Cooled and Moderated Nuclear Power

Equipment for 1974 Passenger Cars (1974)

Equipment in Hazardous Locations (1972) ANSI C106.1

Equipment in Large Boilers (1971)

Equipment on Boats (Audio Device, Automatic Pilot, Dept

Equipment on Highways (1971)

Equipment on Industrial and Certain Other Premises (197

Equipment Operating Within the Radio Frequency Range of

Equipment Protection) (1972)

Equipment Rated at 300 Volts or Less (1974)

Equipment Rooms (Noise) (1973)

Equipment Selection and System Design Procedures (1973)

Equipment Spec. (1973)

ASE

ASE

ASE

ASE

ent. H ASE

Tent. P ASE

Ebu ASE

AACCH 86.49

AACCH 86.05

AACCH 86.03

AACCH 86.49

ASTM E341

ASTM C260

NSF

ASTM

ASTM

Air SAE

ASAE

ASTM

NACE

ASTM

ASTM

NCCA

ASTM

AATCC

AATM

ASTM

ASTM

ASTM

ASTM

ASTM

ANSI

ASTM

SAE

SAE

SAE

SAE

ASTM

ASTM

ASTM

Std. ASTM

Rec. Pr NACE

SAE

ISA

ISA

SAE

IEEE

JIC

ASAE

UL

EIA

td for S ANSI

ASTM

F336

AIR818B

D249.2

E512

RP. 04. 72

D3273

B.III-3

A690

103

108

F143

F95

F110

D1652

D3098

K68.1

AMS3894A

AMS3894/8

AMS3894/9

AMS3821

D2393

D1652

D1847

D1726

RP-03-72

AMS5745A

S39.3

\$39. 1

AMS5743D

384

354

S226. 2

1067

RS404

Z8. 1

C667

400

7-14

A 380

64

336

RS408

EIA

NSF

UL

UL

ASAE

EIA

IEEE

IEEE 
Billiard Tournament Table and Accessory Uniform Alignment Tests-NAS 900 Series Concrete Plant

Minimum Operational Characteristics Vertical Guidance r Single Frequency Output for Institutional Audio-Visual brackets and Socket for Agricultural (Farm) and Industrial Std. for Safety for Hospital Signaling and Nurse Call oldup (Criminology)/ ree Climber) (1973)

ers, Gas Therapy and Suctio/ Std. for Safety for Bullet Resisting Spec. for Lineman's Climbing
Std. for Respiratory Therapy Safety Std. for Electric Home Laundry Minimum Performance Std. for Airborne Distance Measuring s for Television Receiving Appliances and Other Electronic Std. for Ground Support

Std. for Safety for Battery Powered Emergency Lighting Loss Prevention Data on High Frequency Heating $\begin{array}{cc}\text { nsi C33.8 } & \text { Std. for Safety for Grounding and Bond ing } \\ \text { y for Electric Cooin Operated and Commercial Clothes Drying }\end{array}$ for Electric Coin Operated and Commercial Clothes W'ashing 1 Face Design and Location for Construction and Industrial arily Affixed Safety Signs for Construction and Industrial ring Identification System for Industrial and Construction

Rules for the Operation of Electric Supply Lines and Safety Std. for Electric Baseboard Heating i C 33.95 oduction Check Flanges and Gask/ Srec. For Fire Protection of Electrical
Rect Spec. for (Oil) Wellhead oduction Check Flanges and Gask/ Rec. for Fire Protection of Electrical
Spec. for (Oil) Wellhead s Prevention Data on Sight Glasses for Chemical Processing
heels for Industrial Trucks, Casters, Conveyors, and Other std. Definitions and Terminology for Relays for Electronic Std. Safety Requirements for Bakery

td. Meth. of Measurement for Audio Amplifiers Used in Home

for Illumination Tests for Photographic Still Projection f Oil Systems for Lubrication and Control of Hydroelectric

ed Peak Flutter Content of Sound Recording and Reproducing allation and Maintenance of Electrical Supply Stations and f Oil Systems for Lubrication and Control of Hydroelectric Operator ControIs on Agricultural Tractors, Machinery and Std. Spec, for Distillation ion Safety for X-Ray Diffraction and Fluorescence Analysis Safety Sids, for Conveyors and Related Std. for Operator Controls on Agricultural Rec. Safety Alert Symbol for AgriculturaI Minimum Performance Stds. Airborne ATC Transponder Rec. Pract. for Safety for Agricultural Safety Std for Electric Motors for Appliances and Std. for Safety for Ground Fault Sensing and Relaying Safety Std. for Medical and Dental Minimum Requirements for School Bus Construction and or T.Hook Slots for Securement in Shipment of Agricultural the Protection of Electronic Computer and Data Processing tions for Die Cavity and Cutoff Die Sizes for Cold Forging trical Installations on Packaging Machinery and Associated ng Lamp (Light) for Remote Mounting on Agricultural (Farm)

$r$ Sound Rating Heating, Refrigerating and Air Conditioning medium Speed Stationary Diesel and Gas Engine Assembly and ols Direct Electric Ignition Control Systems for Gas Fired $f$ Oil Systems for Lubrication and Control of Hydroelectric

sign, Installation and Performance of Sprinkler Irrigation class $\mathrm{Hi}$ (High Impact) Shock Test. Machine for Lightweight uctures (FOPS) of Construction and Industrial Vehicles and stationary Diesel and Gas Engine Generators and Electrical Safety Sid. for Electric Space Heating

Std. for Dimensions for Racks, Panels and Associated Std. for Safety for Agricultrual Auger Conveying

Rec. Safe Pract. for Thermal Spraying and Blasting Uniform Fire Code: Oil Burning Std. for Outdoor Floodlighting (Light)

Safety Std. for Commercial-Industrial Gas Heatin

Safety Std. for Sound Recording and Reproducing nimum Requirements for Ty pe lI School Bus Construction and or Safety for Sheathed Heating Elements for Appliances and

for Air as an Electrical Insulating Material in Electrical Terminal Equipment and Non.Synchronous Data Communication Std. and Nomenclature for Diamond Core Dril istd. for Safety for Agricultura

Safety Std for Electric Central Air Heatin

Test. Procedures for Relays for Electrical and Electronic

std. for Iron-Core Power Filter Inductors for Electronic and Reflector Interchangeability Used in Roadway Lighting

f Socket Supports Used in Metal Heads for Roadway Lighting and Reflector Interchangeabjlity Used in Roadway Lighting t Supports for Use in Metal Heads I'sed in Roadway Lighting Std for Multiple Sockets Used in Roadway Lighting tage Classification of Luminaires Used in Roadway Lighting Std. for Unitary Air Conditioning Std. for Recreational Vehicle Air Conditioning
Equipment Specs. (1971)

Equipment Specs. (1972)

Equipment Stds. (1973)

Equipment Used in Airborne Volumetric Navigation System

Equipment Used Primarily for Speech (1974)

Equipment Warning Lamp and Slow Moving Vehicles (SMV) I

Equipment (Audio / Visual Communication) (1974)

Equipment (Barrier) for Protection Against Robbery or $\mathrm{H}$

Equipment (Body Belt, Safety Strap, Lanyard, Pole and T

Equipment (Breathing Devices, Room Humidifiers, Nebuliz

Equipment (Clothes Washer and Dryer) (1972) ANSI C33.13

Equipment (DME) Operating Within the Radio Frequency Ra

Equipment (Flyback Transformer, Multiplier, Deflection

Equipment (Hand Knob) (1974)

Equipment (Indoor) (1973

Equipment (Induction and Dielectric) (1973)

Equipment (Internal Electrical Wiring Systems) (1972) a

Equipment (Laundry Dryer) (I974)

Equipment (Laundry Washer) (1974)

Equipment (Machinery) (1971)

Equipment (Machinery) (1971)

Equipment (Machinery) (1971)

Equipment (National Electrical Sa

Equipment (Portable and Fixed) $600 \mathrm{~V}$ or Less (1973)

Equipment (Production Valves, Ring Joint, Segmented, Pr

Equipment (Single Phase and Related Faults) (1973)

Equipment (Vessel) (1974)

Equipment (1966)

Equipment (1971)

Equipment (1971)

/Ec. Pract. for Instrumen SAE

Equipment (1971)

Equipment (1971)

Equipment (1971)

Equipment (1971)

Equipment (1971)

Equipment (1971)

Equipment (1971) ANSI B 114.2, SAE J389

(1971) ANSI Z168.

Equipment (1971) NBS HB111

Equipment (1972)

Equipment (1972)

Equipment (1972)

Equipment (1972)

Equipment (1972)

Equipment (1972)

Equipment (1972)

Equipment (1972)

Equipment (1972)

Equipment (1972)

Equipment (1972)

Equipment (1972)

Equipment (1972)

Equipment (1972)

Equipment (1972)

Equipment (1972)

Equipment (1972)

Equipment (1972)

Equipment (1972)

Equipment (1972)

Equipment (1972)

Equipment (1972)

Equipment (1972) ANSI C 33.12
Equipment (1972) ANSI C83.9

Equipment (1973)

Equipment (1973)

Equipment (1973)

Equipment (1973)

Equipment (1973)

Equipment (1973)

Equipment (1973)

Equipment (1973)

Equipment (1973)

Equipment (1973)

Equipment (1973) ANSl B 104.

Equipment (1973) ANSI B114.3

Equipment (1973) ANSI C 33.104

Equipment (1973) ANSI C 83.25

Equipment (1973) ANS1 C 83.92

Equipment (1973) EEI TDJ-I40

Equipment (1973) EEI TDJ-143

Equipment (1973) NEMA SHI0

Equipment (1973) NEMA SH13

Equipment (1973) NEMA SHl?

Equipment (1973) NEMA SH3

Equipment (1974)

Equipment (1974)
Std. for Demountable Tired W

Std. for Safet UL

Std. for Safety UL

H Permanently and Tempor

Pract. for Electrical Wi

BCA *1

NRMCA 985

RTCA DO.152

ANSI $\quad$ PH7.2

UL 752

EEI AP.2

NSF 48

UL $\quad 560$

RTCA DO.151

UL $\quad 492.3$

UL 924

FMS 6.3

L $\quad 467$

1240

1206

J209

J 115

$\mathrm{C} 2.4$

UL $\quad 1042$

API $\quad 6 \mathrm{~A}$

FMS 14.15

FMS

7.3

ANSI C 83.16

ANSI Z50.1

EIA RS234. C

Std ANSI PH3.47

Rec. Pract. for the Purification O ASME

LOS-5P1

193

C2.1

LOS. $5 \mathrm{Cl}$

S304.4

E133

$\mathrm{N} 43.2$

B20.1

R335.1

R350

D0. 150

RAE $\quad$ J208A

UL 1004

UL 1053

UL

544

ESC

Std. F ASAE

Std. for NFPA

Std. Recommenda IFI

Rec. Pract. for Elec IEEE

Std. for Flashing Warni ASAE

Std. Meth. of Testing Fo ASHRA

Std. Pract. for Low and DEMA

Std. for Residential Contr NEMA

Rec, Pract for the Design O ASME

Minimum Requirements for the De ASAE

/Ign, Construction, and Operation of ANSI

S) and Falling Object Protective Str SAE

Pract. for Low and Medium Speed DEMA

UL

EIA

ICBO

ICBO

UL

UL

Mi VESC

Std. F UL

Std. Spec. ASTM

rt-Stop Signal Quality Between Data EIA

DCDMA 3

ASAE S318.3

UL

Std. ElA

EIA

Std. for Metal Head NEMA

Cal Interchangeability O NEMA

Std. for Metal Head EEI

/Nierchangeability of Socke EEI

Std. for Vol EEl

ARI

S356

121

333

307.1

DC 21

LOS.5Dl

R264. 1

S2.15

J397A

573

RS310-B

$\mathrm{S} 361 \mathrm{~T}$

C2.1

FAl

V. 10
1030

D3283

UFC*2ART24 
Std. for Central Forced Air Electric Heating Rec. for Protective Grounding of Electrical Circuits and Industrial Cost Trends for Buildings, Machinery and Std. for Electrical Power Switching

Std. for Manual Gas Metal Arc (Gma) Welding Std. for Manual Gas Tungsten Arc (Gta) Fusion Welding pe Std., Variable Block Format, for Numerically Controlled ine for Monochrome Cctv (Closed Circuit Television) Camera 0 and Television Receiving Appliances and Other Electronic

) for Television Receiving Appliances and Other Electronic Spec. for Resistance Seam and Roll Spot Welding 2, Asse 1007 Std. and Test Procedures for Home Laundry ner and Filler Identification (Construction and Industrial Over Protective Structures (ROPS) for Prime Movers (Heavy perational Rec. for Globe Dry Pipe Valves (Fire and Safety Truck Mixer and Agitator Stds. (Concrete Plant of Water Formed Deposits (Chemical Cleaning of Industrial wire Cable Wrought Steel Heavy Thimble (For Ground Support n Steel, Shoulder Nut Assembly Eyebolt (For Ground Support vice Containers for Milk and Its Fluid Products (Packaging trucks, Buses, Coaches, Tractors, Industrial Road and Misc

/ Electrical / Mechanical Security Surveillance System and or Preparing Vulcan/ Std. Rec. Pract. for Std. Materials, and Flammable Materials; Reporting and False Alarm; Use of Safety Std. for Oxygen Therapy

ces, Room Humidifiers, Nebulizers, Gas Therapy and Suction emistry) (19/ Meth. for Experimental Wheat Flour Milling: Std. for Resistance Spot Welding

Flanged Wellhead, Underwater Wellhead, and Christmas Tree and Heat Detectors; Electromagnetic Holders; Sliding Door bile Construction Type Cranes Having Rope Supported Booms, d Temperature in the Automotive Braking System of Vehicles ng Distance on Paved Surfaces Using a Passenger Automobile ng Distance on Paved Surfaces Using a Passenger Automobile of Power Transmission Units Penetrating the Hull for Boats or Attachment of Implements to Agricultural Wheel Tractors ing Hydraulic Lift Force Capacity on Agricultural Tractors ing Hydraulic Lift Force Capacity on Agricultural Tractors Std. for Welded Aluminum Alloy Field for Low and Medium Speed Stationary Diesel and Gas Engine Rec. Horizontal Shoring Beam tructuraI SteeI (1973) stry) (1962)

Std. for Safety Requirements for Steel Uniform Building Code Std. for Ether-Bicarbonate Meth. of Analysis of Chloroform-Alcohol Meth of Analysis of Std. Test Meth. for Dust and Fog Tracking and r Design, Layout, Construction and Maintenance of Terrace e Water Pumps with Coolan/ Std. Test Meth. for Cavitation f Test for Liquid Contaminant, Inclined Plane Tracking and id or Solid Partic/ Std. Definitions of Terms Relating to (1952)

Std. Definitions of Terms Relating to
Rec. for Std. Rec. Pract. for Designing a High Current Arc Std. Meth. of Vibratory Cavitation order / Reproducers (1974) An/ Rec. Test Meth. for Timing Std. Pract. for the Inspection of Elevators, Uniform Building Code: Elevators, Dumbwaiters,

ic Acid (1973)

tic Soda (1974)

roform (1974)

formaldehyde (1974)

vinyl ChIoride (1972)

on of Foods and Beverages (1973)

70)

truction Poles (1974)

Im for Archival Records, Silver Gelatin Type and Meth. for Std. Meth of Test for Phthalate Std. for Packing, Preformed O.Ring Phosphate

Std. Meth. of Test for est Control Chemical O,O-Dimethyl Phosphorodithioate S . st ControI Chemical Ethyl Mhydroxycarbanilate Carbanilate and Fatty Acids (Cereal / Meth. for Preparation of Methyl thyl Ether of Hydroquinone in Colorless Monomeric Acrylate ANSI Z1.1/ Std. Rec. Pract. for Choice of Sample Size to by the Causticaire Method (1971)

(1970)

Cenco Fitch Apparatus (1970) Alc/ of Nuclear Graphite (1971) ANSI K90.10 Spec. for Rotary Drilling Concrete Plant Mixer Std. Meth. of Test for Rec. Rec. Scaffolding Rec, Steel Frame Shoring

Equipment (1974)

Equipment (1974)

Equipment (1974)

Equipment (1974)

Equipment (1974)

Equipment (1974)

Equipment (1974)

Equipment (1974)

Equipment (1974)

Equipment (1974)

Equipment (3 Phase) (1973)

Equipment (petroleum) (1974)

Equipment (washer, Dry

Equipment) (1971)

Equipment) (1972)

Equipment) (1973)

Equipment) (1973)

Equipment) (1973)

Equipment) (1974)

Equipment) (1974)

Equipment) (1974)

Equipment) (1974)

Equipment) (1974)

Equip

Equipment, Refrigerated (1973)

Equipment, Room Air Purifiers, Filters, Compressors, Ar

Equipment, Sample Preparation, and Tempering (Cereal $\mathrm{Ch}$

Equipment, 3 Phase (1973)

Equipment; Flow Control Chokes; and W ater Sampling Devi

Equipment; Installation Aids (1969) /Ers, Stops, Smoke

Equipped for Hook Work, ClamsheIl, Magnet, Grapple, or

Equipped with Disc Brakes (1972)

Equipped with Full Scale Tires in the Diagonal Braking

Equipped with Full Scale Tires (197) T)

Equipped with Outdrives, Jet Drives and/or Bow Thruster

Equipped with Quick Att aching Coupler for Three Point F

Equipped with 3 Point Hitch (1972)

Equipped with 3 Point Hitch (1972)

Erasability of Inked Ribbons (1972)

Erected Storage Tanks (1973)

Erection and Installation (1972)

Erection Instructions for Steel Door Frames (1972)

Erection Procedure (1968)

Erection Procedure (1969)

Erection Procedure (1972)

Erection (1972)

Erection, Fabrication, Identification and Painting of S

Ergot in Rye and Wheat Flour and Semolina (Cereal Chemi

Ergot in Rye Flour (Cereal Chemistry) (1962)

Erosion ANSI C59.133

Erosion Control and Water Conservation) (1972)

Erosion Corrosion Characteristics of Aluminum Automotiv

Erosiols (1973) ANSI C59.135

Erosion of Solids by Cavitation and Impingement by Liqu

Erosion Resistance of Concrete in Hydraulic Structures

Erosion Test for Electrical Contact Materials (1973)

Erosion Test (1972)

Error Measurements of Instrumentation Magnetic Tape Rec

Escalators and Moving Walks (inspectors Manual) (1973)

Escalators, and Moving W alks (1973)

Essential Information for Safe Handling and Use of Acet

Essential Information for Safe Handling and Use of Caus

Essential Information for Safe Handling and Use of Chlo

EssentiaI Information for Safe Handling and Use of Para

Essential Information for the Safe Handling and Use of

Establishing Clear Wood Strength Values (1973)

Establishing Conditions for Laboratory Sensory Evaluati

Establishing Design Stresses for Round Timber Piles (19

Establishing Rec. Design Stresses for Round Timber Cons Spec. for Photographic Fi Ester Base (1973)

ster Color of High Gravity Glycerin (1973)

Ester Resistant (-65 Deg. F to + 160 Deg. F) (1972)

Ester Value of Lacquer Solvents and Thinners (1972)

Ester with 4-(Mercaptomethyl).2-Methyoxy-? ${ }_{2}-1,3,4-$

for Common Name for the PE

(1) Common Fats, Oils.

Esters (1972)

Std. Meth. of Test for Me

Estimate the Average Quality of a Lot or Process (1972)

Estimating Maturity and Linear Density of Cotton Fibers

Estimating Modular Brick Masonry (Tech. Notes) (1971)

Std. Pract. for Estimating Reinforcing Steel Bar Materials and Service

Std. Meth. of Estimating Stray Radiant Energy (1972)

Std. Meth. of Test for Estimating the Thermal Conductivity of Leather with the

Std. Meth. for Estimating the Thermal Neutron Absorption Cross Section
ARI 280

FMS $\quad 5-10$

FMS 9.3

NEMA $\quad$ SG6

NSA 961

955

RS 312

492.7

492.8

NSA 99

AHAM HLW.2PR

NRMCA *2

SAE J223

SAE J320B

$\begin{array}{ll}\text { FMS } & 2 \cdot 14 \\ \text { NRMCA } & * 3\end{array}$

ASTM D2790

1045

NSA 1053

$\begin{array}{ll}\text { DFISA } & 1704 \\ & * 1.56\end{array}$

ICBO UFC *2ART27

UL $\quad 416$

NSF 48

AACCH 26-10

$\begin{array}{ll}\text { NSA } & 967 \\ \text { API } & 6 \mathrm{~A}\end{array}$

NBHA 8

SAE J220

J291

ASTM E503

ASTM E445

ASAE S278.

ASAE S349

SAE J283

ASTM F362

ANSI B96.

DEMA *1-21

STDI 105

SSI

SSI

ANSI Al0.13

ICBO UBCS27-2

AACCH $42-56$

$\mathrm{AACCH} \quad 42.55$

ASTM D2132

Ec. Fo ASAE R268.

ASTM D2809

$1 O$ ASTM D2303

ASTM G40

ACI 52.18

AATM B576

ASTM G32

EIA RS413

ANS1 A17.2

ICBO UBC $* 3.51$

$\mathrm{MCA} \quad \mathrm{SD} 4$

MCA SD9

MCA $\quad$ SD89

MCA SD6

MCA SD56

ASTM $\quad$ 480

ASTM D2899

ASTM D3200

PH1.28

ASTM D1728

NSA 1611

ASTM D1617

ANSI K62.134

N62.139

$\mathrm{AACCH} \quad 58-17$

ASTM D3125

ASTM E122

BIA

CRSI

ASTM

ASTM

*1.5.1

E387

D2214

C626 
biphenyls for C/ nol by Cloud Point Dep

1972) ANSI 2197./

Std. Meth. for Rapid Gas Chromatographic Std. Test Meth for Detection and 973) r Crystallographic Perfection of Germanium by Preferential Tent. Ebulliometer Meth. for Analysis of Alcohol
Tent. Hydrometer Meth. of Analysis for Alcohol

Tent. Pycnometer Meth. of Analysis for Alcohol nd Weat Flour and Semolina (Cereal Chemistry) (1962) sters (1972)

Std. Meth. of Test for Methyl Spec. for Ethylene Glycol Monomethyl Std Spec. for Ethylene Glycol Monoethyl for Determining Hydroxyalkoxyl Content of Corn Starch and oxyalkoxyl Groups in Hydroxyethyl and Hydroxypropyl Starch hips (Quality Control) (1967) Std. of Architectural 1972)

Ethyl S,S-Dipropyl Phosphorodithioate) (1973/ Rec. Pract for Std. for Safe Handling and Use of am (I) Std. for Common Name for the Pest Control Chemical or Ethoprop (Common Name for the Pest Control Chemical O enethi/ Std. Common Name for the Pest Control Chemical S. rene / Std. for Common Name for the Pest Control Chemical he Pest Control Chemical 2-(Tertbutylamino)-4-Chloro-6name for the Pest Control Chemical 2-(Tertbut ylamino)-4ine -/ Std. Common Name for the Pest Control Chemical 2$68-516$ Std for Mine Power Cables with single and Multiple Conductor Electrical Power Cables with r Nonshielded Single Conductor Electrical Power Cable with ables Rated 2000 Volts and Less and Having Ozone Resistant Std. Spee. for Spec for Std. Spec. for 72

wer Cables Rated 0-35,000 Volts and Having Ozone Resistant (1973)

c) Acid (EDTA) and Its

Std. Spec, for Photographic Grade 973)

(Common Name for the Pest Control Chemical 2-

1 Lumber (1974) ing Color and Gloss Tolerances of Opaque Materials and for Std. Rec. Pract. for Preparing, Cleaning, and by Fungal Growth or Soil and Dirt Accumu/ $68 \mathrm{E})$ d Rubber Like Materials by a Tempera/ ile in Storage (1971) ANSI L14. S2 bber Like Materials (1960) ANS1 J/ fill Pipes with / Rec. Pract. for Defining Conditions for al Clad Switchgear (1971) ANSI C37.24

ic Fluid Power Filter Element

le Panel Materials (1972) ANSI O8.1

tion (1971) ANSI L14.79

s (1974)

ide for the Preparatio l Films for Still Ph/ Std. for Sensitometric Exposure and milk Solids to Change Mixing Characteristics Farinograph Std. Meth. for Std. Meth. for Std. Meth. for Std. Meth. for Std. Meth. for Std. Meth. for Std. Meth. for Std. Meth. for Std. Meth. for Test Meth. for Test Meth. for rene-Butadiene Rubber (SBR) (1973)

Rubbers (IIR) (1973)

butadiene Rubbers (SBR) (1973)

oroprene Rubbers (CR) (1973)

and Isoprene Rubber (IR) (1973)

adiene Rubbers (BR) (1973)

ansi L14.144.

tile Fabrics (1971) ANSI L14.106

e Cylinders (1973)

e (1965) ANSI A 146.

Uniform Building Code Std. for Rec. Pract. for Std. Rec. Pract, for Image Pract. for Establishing Conditions for Laboratory Sensory Std. Rec. Pract. for Sensory Detection and Measurement of Discharge (Corona) Pulses in Textiles (1971) ANSI L14.55 972) Test Meth. for the corrosive Environments (Coil Co/ ctive Strucl Rec. Pract. Machinery Employing Form Wo/ ting Wastewater (1970)
Estimation of Higher Boiling Homologues of Chlorinated

E W ater Insoluble Impurities in Refined Phe

Etch Solution Used in Surface Preparation of Aluminum (

Etch Technique (1973)

Etch Tech niques (1973)

Tent. Meth of Test Fo ASTM

ETFE -Fluoroplastic Molding and Extrusion Materials (I

(Ethanol) in Wine (Enologv) (1972)

(Ethanol) in Wine (Enology) (1972)

Ethanol) in Wine (Enology) (1972)

Ether-Bicarbonate Meth. of Analysis of Ergot in Rye a

Ether of Hydroquinone in Colorless Monomeric Acrylate E

Ether (1972)

Ether (1972)

Ethers (1967)

Ethers (1973)

Ethical Practice (1972)

Lytical Meth. Analytical Meth.

Ethics and Human Relations in Vendor-Vendee Relations

Ethiolate (Common Name for the Pest Control Chemical) (

Ethoprop (Common Name for the Pest Control Chemical O-

Ethyl Acetate (Chemical Safety) (1972)

Ethyl Mhydroxycarbanilate Carbanilate (Ester) Desmediph

Ethyl S,S-Dipropyl Phosphorodithioate) (1973)

Ethyl (E,E)-11-Methoxy-3,7,11-Trimethyl-2,4-Dodecadi

Ethyl (E,E)3,7,11-Trimethyl-2,4-Dodecadienoate Hydrop

Elamino)-S-Triazine-Terbuthylazine (1973)

Ethylamino)-6-(Methylthio)-S-Triazine-Terbutryn (19

(Ethylamino)4-(Isopropyla mino).6-(Methylthio)-S-Triaz

Ethylene-Propylene-Rubber Insulation (1973) IPCEA S

Ethylene-Propylene-Rubber Insulation (1973) IPCEA S

Ethylene-Propylene-Rubber Insulation, 2001-5000 Vol

Ethylene-Propylene-Rubber Integral Insulation and J

Ethylene Glycol Monoethyl Eiher (1972)

Ethylene Glycol Monomethyl Ether (1972)

Ethylene Glycol (1972)

Ethylene Propylene Rubber Insulation (1973) IPCEA S68-5

Ethylene Propylene Rubber Jacket for Wire and Cable (19

Ethylene Propylene Terpolymer, General Purpose (45-55)

Ethylene (1973)

Ethylenediamine $\mathrm{H}_{2} \mathrm{~N}, \mathrm{CH}_{2}, \mathrm{CH}_{\mathrm{z}}, \mathrm{NH}_{2}$ (1973)

Ethylenediaminetetraacetic (Ethylenedinitrilotetraaceti

(Ethylenedinitrilotetraacetic) Acid (EDTA) and Its Salts

(Ethylthio) -4,6-Bis (Isopropylamino) Striazine) (1973)

Evacuated Reflective Insulation in Cryogenic Service (I

Evaluate the Stress Corrosion Cracking Susceptibility 0

Evaluating Allowable Properties for Grades of Structura

Evaluating Conformance (1972T)

lor Selecting

Evaluating Deg of Surface Disfigurement of Paint Films

Evaluating Inhibitory Toxicity of Industrial Waters (19

Evaluating Low Temperature Characteristics of Rubber an

Evaluating Oxidation of Wool Oils on Woolen Fabric Text

Evaluating Pressure Sealing Properties of Rubber and Ru

Evaluating the Compatibility of Vehicle Fuel Tanks and

Evaluating the Effect of Solar Radiation on Outdoor Met

Evaluating the Filtration Performance of a Fine Hydraul

Evaluating the Properties of Wood Base Fiber and Partic

Evaluating the Quality Control Program (1969)

Evaluating Wetting Agents in Caustic Soda for Merceriza

Evaluating Wood Preservatives by Field Tests with Stake

Evaluation and Establishment of Temperature Indexes of

Evaluation for Household Food Waste Disposers (1973)

Evaluation Meth. for Determining Speed of Color Reversa

Evaluation Meth. of Ability of Roller Dried Nonfat Dry

Evaluation of and Test Formula for Butadiene-Acrylon

Evaluation of and Test Formula for Carbon Blacks in Nat

Evaluation of and Test Formula for Carbon Blacks in Sty

Evaluation of and Test Formula for Isobutene-lsoprene

Evaluation of and Test Formula for Pigmented Styrene-

Evaluation of and Test Formulas for General Purpose Chl

Evaluation of and Test Formulas for Natural Rubber (Nr)

Evaluation of and Test Formulas for Nonpigmented Styren

Evaluation of and Test Formulas for Solution Poly-But

Evaluation of Antibacterial Finishes on Fabrics (1970)

Evaluation of Commercial Rewetting Agents on Cotton Tex

Evaluation of Compression Test Results in Field Concret

Evaluation of Compression Test Results of Field Concret

Evaluation of Electrostatic Business Copies (1972)

Evaluation of Foods and Beverages (1973)

Evaluation of Industrial and Institutional Food Purchas

Evaluation of Insulation Systems (1973)

Evaluation of Mildew and Rot (Fungicides) Resistance

Evaluation of Oil lmmersed Distribution Transformers (1

Evaluation of Painted or Coated Specimens Subjected to

Evaluation of Roll Over (ROPS) and Falling Object Prote

Evaluation of Sealed Insulation Systems for AC Electric

Evaluation of Special Processes or Devices Used in Trea
ASE

ASE

ASE

$\mathrm{AACCH} \quad 42.56$

ASTM D3125

D3128

ASTM

CR

CR

ASQC

ANSI

ANS

MCA

ANS

ITd. F ANSI

ANSI

ffor T ANS

ANSI

ANSI

ANSI

NEMA

NEMA

ASTM

ASTM

ASTM

NEMA

ASTM

SAE

$\mathrm{MCA}$

ANSI

ANS

ANSI

ANSI

ASTM

ASTM

ASTM

ASTM

ASTM

ASTM

ASTM

ASTM

AATCC

ASTM

SAE

IEEE

NFLDP

ASTM

ASQC

AATCC

ASTM

IEEE

AHAM

ANSI

A ACC

ASTM

ASTM

ASTM

ASTM

ASTM

ASTM

ASTM

ASTM

ASTM

AATCC

AATCC

ICBO

ACI

ASTM

Std. Rec ASTM

ASTM

ASTM

AATCC

IEEE

NCCA

SAE

IEEE

NSF

1

C. -20

C- 30

J330

K62.123

K62.133

D3303

D2674

F389

D3159 
National Bureau of Standards KWIC Index of Engineering Standards

n Housings $\mathrm{O} /$

(Safety (1971)

in Form (1971) ANSI L14.75

(1971) ANSI L14.11

ion (1973) ANSI Z11.210

Means of Small Size Specimens/

asher (1971) ANSI Z224.1

olor Chart / Textile Colorfastness) (1972)

xtile Colorfastness) (1954) ISO 105.1

e Colorfastness) (1954) ISO 105.1

ites (1972)

Std. Meth. for Laboratory
Rec. Pract. for in Place Concrete Strength

at $60 /$ Safety Std. for Electric Fans (Blower, Humidifier, ars and Light $\mathrm{Tr} /$ Rec. Pract. for the Measurement of Fuel ars and Light $\mathrm{T} r$ /

Rec. Pract. for the Measurement of Fuel vent Extract from Aerospace Components (Using Rotary Flash Std. and Rec. Pract. for Buoyancy in the

lowering Trees; Deciduous Shrubs; Coniferous and Broadleaf $r$ Color Change in Fabrics Due to Flat Abrasion (Frosting): data from Differential Thermal Analysis, Thermogravimetry, rains, Unpopped Popcorn, Driel Meth. of Analysis of X-Ray her Packaging Materials (Cereal Chemis/ Ultraviolet Light nt of Metal and Oxide Coating Thicknesses by Microscopical limentary Pastes for De/ Meth. of Analysis of Macroscopic eas and Beans for Roden/Meth. of Analysis of Macroscopic rmination of the Mechanical Propert/SStd. Rec. Pract. for applications (19/ Std. Spec. for Straight Beam Ultrasonic Std. Meth. of Magnetic Particle
ANSI $/ \quad$ Std. Meth. and Spec. for Straight Beam Ultrasonic Std. Spec. for Ultrasonic, Angle Beam copy (1973) hase of Nuclear P/ Std. Meth. for Std. for Qualifications of Inspection, Contemporary Bearing $W$ all Uniform Building Code: Uniform Building Code: ystems Whose Maximum Allowable Operating Pressure Does Not Waste Cloth, Wastepaper, Kapok, Hay, Straw, Spanish Moss, Maintenance Instructions for Chlorine Institute Std. test for Effectiveness of Mineral Admixtures in Preventing Engineering Data Sheets for the Design of Air

Std. Meth. of Test for Operating Performance of Anion Std. Meth. of Field Sampling of Ion h. of Test for Operating Performance of Particulate Cation st for Physical and Chemical Properties of Particulate Ion Industry Standard for Household Commercial and Portable Std. Spec. for U-Bend Seamless Copper and Its Alloy Heat pec. for Seamless Cold Drawn Intermediate Alloy Steel Heat spec. for Aluminum Alloy Seamless Round Condenser and Heat spec. for Copper and Its Alloy Seamless Condenser and Heat c. for Seamless Nickel and Nickel Alloy Condenser and Heat $\mathrm{c}$ and Austenitic Alloy Steel Boiler, Superheater, and Heat 260 Brass Strip in Narrow Widths and Light Gage for Heat pec. for Welded Austenitic Steel Boiler, Superheater, Heat Seamless and Welded Titanium Tube for Condensers and Heat ondenser Rolled Tube Plates in Surface Condensers and Heat luminum Alloy Drawn Seamless Tubes for Condensers and Heat Sanitary Std. for Scraped Surface Heat Std. for Tubular Heat nd Paperboard in CIE Y; X, Y or Y, Dominant Wavelength and Sid. Criteria and Definitions for Std. Groove Dimensions for Fluid Power

972) Apparatus) tibility in 7XXX Series Copper Containing Aluminum Alloys real Chemistry)/ $\quad \begin{aligned} & \text { Decantation Meth. of Analysis for Rodent } \\ & \text { Decantation Meth. of Analysis for Rodent }\end{aligned}$ Glass Plate Meth. of Analysis for Insect eat Cereals, and Alimentary Pastes for Detection of Rodent

d Beans for Rodent and/or Insect Damage on Kernels, Rodent e Specs. for Brick and Tile Masonry (Tech. Note) (Part III std. for Industrial Computer System FORTRAN Procedures for per Containing Aluminum Alloys (E/ Std. Meth. of Test for ndustrial Trucks (1973) (1974)

for Uniform Laboratory Instrumentation and Techniques for Rec. Pract. for
Uniform Building Code Std for Blower. Fan and

Pract. for Nomenclature for Passenger Car and Light Truck onveying (1973)/ Std. for the Installation of Blower and (Boats) (I Std. for Commercial Cooking Equipment r Fundamentals Governing the Design and Operation of Local dium Speed Stationary Diesel and Gas Engine Air Intake and Rec. Pract. for Dilution Ventilation in Industrial rec. Pract. for Ventilation for Heat Control in Industrial Rec. Pract. for Fans for Industrial Rec. Pract. for Design Procedure for Industrial
Evaluation of Suitability of Plastic Material for Use I Evaluation of the Effectiveness of Rear Underride Guard Evaluation of Wettability of Fabrics and Textiles in Ya Evaluation of Wetting Agents (Cotton Test Skein Meth.) Evaluation of White Mineral Oils by Ultraviolet Absorpt Evaluation of Wood Preservatives for Marine Services by Evaluation Procedure for Household (Iaundry, Clothes) W Evaluation Procedure of Chromatic Transference Scale (C Evaluation Procedure of Gray Scale for Color Change ( $\mathrm{Te}$ Evaluation Procedure of Gray Scale for Staining (Textil Evaluation Test Procedure (1971)

Evaluation to Determine Resistance to Subterranean Term Evaluation (1970)

Evaporative Cooler, and Air Filtering Appliances Rated Evaporative Emissions from Gasoline Powered Passenger C Evaporative Emissions from Gasoline Powered Passenger C Evaporator) (1972) Event of Swamping (Boat Flotation) (1973) /Olatile Residue of Halogenated Sol Erorades; Vines and Grounc Every Meth. (Textile Colorfastness) (1970)
Evolved Gas Analysis and Detection (1973) Examination for Internal Insect Infestation Examination for Presence of Rodent Urine on Bageal $G$ Examination of a Cross Section (1973) ANSI G53.51

Examination of Baked Goods, Ready-To-Eat Cereals, and a Examination of Cereal Grains, Unpopped Popcorn, Dried P Examination of Fuel Element Cladding Including the Dete

Examination of Plain and Clad Steel Plates for Special Examination of Steel Forgings (1971) ANSI G60.6 Examination of Steel Plates for Pressure Vessels (1973) Examination of Steel Plates (1973) ANSI G35.24

Examination of Water Formed Deposits by Chemical Micros

Examination, and Test. Personnel for the Construction P

Example of Shear WaII Design (Tech. Notes) (1964)

Excavation and Crading (1973)

Excavations, Foundations, and Retair.ing Walls (1973)

Exceed 60 or 125 Psig (1973)

Excelsior, etc.) (1973)

Excess Flow Yalves (1972)

IW, Cocoa Fibers, Oakum, Rags,

Excessive Expansion of Concrete Due to the Alkali-Agg

Exchange Coolers for Packaged Compressor Units (1974)

Exchange Materials for Strong Acid Removal (1972)

Exchange Materials (1972)

Exchange Materials (1972) ANSI Z111.4

Exchange Resins (1972)

Exchange Water Softeners (1972)

Exchanger and Condenser Tubes (1973)

Exchanger and Condenser Tubes (1973) ANSI B125.8

Exchanger Tubes with Integral Fins (1973)

Exchanger Tubes with Integral Fins (1974)

Exchanger Tubes (1972) ANSI H34.42

Exchanger Tubes (1973) ANSI B125.22

Exchanger Tubing (1972)

Td Spec for Ceamless Ferrit

(1973) ANSI B125.32

Exchangers (1965) ANSI H50.2

Exchangers (1973)

Exchangers (1973) ANSI H38.6 Std. Spec. for ASTM
Std. Spec. for Copper Alloy C ASTM

Exchanges for Use with Milk and Its Products (1973)

Exchanges in Chemical Process Service (1971)

Excitation Purity (1972)

/F Measuring Color of Paper a

Excitation Systems for Synchronous Machines (1972)

Exclusion Devices (In. Series) (1972) ANSI B93.35

(Exclusive of Generator, for Hydraulic Turbine Drive) (1 St for Exfoliati

Excreta in Flour (Cereal Chemistry) (1962)

Excreta in Meal, Grits, Flour, Farina, and Semolina (Ce

Excreta in White, Wheat, and Corn Flour (1962)

Excreta, Insects, Webbing, etc. (Cereal Chemistry) (196

Excreta, Other Filth, and Extraneous Material (Cereal C

Execution) (1972)

Executive Functions and Process Input-Output (1972)

Exfoliation Corrosion Susceptibility in 7XXX Series Cop

Exhaust Emission Controls (Air Pollution) for Powered I

Exhaust $\mathrm{G}$ as Emissions Measurement (Passenger Car and Li

Exhaust Hood Design for Industrial Ventilation Systems

Exhaust System for Dust, Stock and Vapor Removal (1973)

Exhaust System Parts (1972)

Exhaust Systems for Dust, Stock, and Vapor Removal or C

Exhaust Systems for Propulsion and Auxiliary Machinery

Exhaust Systems (1970)

Exhaust Systems (1971)

Exhaust Systems (1972)

Exhaust Systems (1974)

Exhaust Systems (1974)

Exhaust Ventilation Systems (1974)

Exhaust Ventilation Systems (1974)
SAE

AATCC

AATCC

ASTM

ASTM

AATCC

AATCC

AATC

AWPA M12.72

NRMCA * 133

UL

SAE

507

J171A

ANSI $\quad$ Z60.1

120

ASTM E472

AACCH 28-21

AACCH 28-85

AACCH $\quad 28-30$

AACCH 28-10

ASTM E453

ASTM A578

ASTM A275

ASTM A577

ASTM D1245

ANSI

ICBO

ICBO

CHI

API

ASTM

ASTM

Std. Met ASTM

ASTM

D. S ASTM

Std. ASTM

ASTM

ASTM

ASTM

ASTM

DFISA

ANSI

TAPPI

IEEE

NFLDP

NEMA

ASTM

AACCH 28.43

AACCH $28-30$

$\mathrm{AACCH} \quad 28-10$

Guid CSI

ISA

ASTM

ITA

SAE

ACGIH

Rec SAE

NFPA

$A B Y C$

NSF

Std. Fo ANSI

Std. Pract. for Low and Me

DEMA

ACGIH

ACGIH

ACGIH

ACGIH

24D

UBC $* 3-29$

B16.33

42

C441

D3087

D2687

D1782

2187

B395

A 199

B163

B569

A249

B338
B171

B234

B78.1

T527SU

(15)

19.7

G1-22

11B

S61.1

G34

$6 \mathrm{FI}$

J254

*1.4

J97

91

UBC $* 3-70$ 
ec. Pract. for Make Up and Recirculated Air for Industrial Rec. Pract. for Construction Spec. for Local Industrial Rec. Pract. for Test. of Industrial g Devices (Air Filters and Dust Collectors) for lndustrial itions, Abbreviations and General Principles of lndustria Std. for the Installation of Air Conditioning, Heating, termination for Division Services (Individuals, Clubs, and (Wall, Ceiling, Floor, and Floor-Ceiling Assemblies) in ons (1973) Uniform Building Code: Brick Veneer; New and Std. for

ardware: Hands of Doors; Hinges; Locks; Knobs and Handles; Uniform Fire Code: Maintenance of and / Western Woods Use Book: Fire Protection and Safety bleachers, and Grandstand/Uniform Building Code: Stairs, Dwelling Construction Under the Uniform Building Code: (Plastic Compo/ Std. Meth. of Test for Gel Time and Peak of Test for Dielectric Constant and Dissipation Factor of Std. Spec. for Sponge and Rec. Concerning Storage of or Making Reference Glass-Metal Butt Seals and Test. for making Reference Glass-Metal Sandwich Seal and Test. for Temperature, 50 to $120 \mathrm{KSI}$ Yield Strength, Materials with (Liquid Petroleum) Gas Cyli/ ire Extinguishment) (1973) Std. for Safety for Pigtail Rec. for High 1971) ANS1 A37.101 ral Construction (Nonextruding /

Uniform Building Code Std. for Std. Spec. for Preformed Std. Spec. for Preformed Structural Clay Differential Movement ffectiveness of Mineral Admixtures in Preventing Excessive 07 Std. Meth. of Test ural Uses/ Std. Spec. for Rolled Copper Alloy Bearing and or Ceramic Tile (1973) Recommendation for Installation of chemistry) (1962) chemistry) (1962) Alloy Sheet, Strip, and Plate, Low Buhler Meth. of (1962)

ls for Surgical Implants (1972)

Batch Meth. of Std. Rec. Pract. for eth. for Generation of Chlorine Dioxide in Flour Bleaching So/ Requirements for Safety to Life-Property from able and Combustible Liquids and Solids and Potential Dust Rec. for Electrolytic Chlorine Processes liquefied Flammable/ Rec. for Sprinkler, Water Spray, and nual of Hazardo $\begin{array}{ll}\text { boiler Furnaces with One / } & \text { Std. for Prevention of Furnace } \\ \text { ler Furnaces (Fire Protec/ } & \text { Rec. for Prevention of Furnace }\end{array}$ ler Furnaces (Fire Protec/ Std. for Prevention of Sulfur Fires and Rec. for Protection Against Molten Metal Fires and Uniform Fire Code: Prevention of Dust Rec. Code for Transportation, Storage and Use of s Under High Shear (1972) ANSI Z1/ Std. Meth. of Test for Uniform Fire Code:

e for the Manufacture, Transportation, Storage, and Use of Std. Spec. for Untreated Carbon Steel Railway Axles for color Reversal Films for Still $\mathrm{Ph} /$

Laminated Wood Products for Use Under Exterior (Wet Use) ctural Laminated Products for Use Under Interior (Dry Use) photography) (1973)

) ANSI Z197.24

(1970) ANSI N141 Std. Photographic Film Sealants $(1972)$ Std. Rec. Pract. for Atmospheric Rec. Pract. for Std. Rec. Pract. for Conducting Exterior in Still Picture Cameras (1972)

Sid.

ANSl L14.177 Test Meth. for Resistance of Textile Fabrics: Rec. for

e of Textile Materials and Coated Fabrics: Carbon Arc Lamp Meth. for Weather Resistance of Textiles: Carbon Arc Lamp ions of Radionuclides in Air and in Water for Occupational tness of Textiles to Light and Washing: Alternate Sunlight rfastness of Textile Fabrics to Water and Light: Alternate tile Fabrics to Water (High Humidity) and Light: Alternate Std. Rec. Pract. for Conducting Natural Light Std. Meth. of Test for Characteristic Groups in Rubber Std. Meth. of Test for Characteristic Groups in Rubber
(Cereal Chemistry) (1962) Structural Relaxation Meth. of ereal Chemistry) (1962)

ysical Dough Tests (Cereal Chemistry) (1962) General

load and Elongatoin of Elastomeric Yarns (Constant Rate of nd (1972)

Elastic Properties of Elastomeric Yarns (Constant Rate of

cs. Terminology,/ Handbook of Spring Design: Compression, d Specimen by / Std. Meth. of Test for Rate of Burning or Recommendation for Installation of Recommendation for Installation of
Exhaust Ventilation Systems (1974)

Exhaust Ventilation Systems (1974)

Exhaust Ventilation Systems (1974)

Exhaust Ventilation Systems (1974

Exhaust Ventilation Systems (1974)

Exhaust, Ventilating, and

Existing Buildings (1973)

Existing Buildings; Minimum Safety Requirement Alterati

Existing Frame Construction (Tech. Notes) (1966)

Exit Devices (Doors) (1972)

Exit Devices, Pulls, Push and Kick Plates; Bolts; Close

Exit Ways (1973)

Exit, Stair way, Aisle, Corridor, Building Construction

Exits and Occupant Loads (Buildings, Reviewing Stands,

Exits and Stairs (1973)

Exothermic Temperature of Reacting Thermosetting Resins

Expanded Cellular Plastics Used for Electrical Insulati

Expanded Cellular Rubber

Expansion Characteristics by Polarimetric Meth. (1973)

Expansion Characteristics by Polarimetric Meth. (1973)

Expansion Coefficients Comparable to Austenitic Steels

Expansion Coil, and Flexible Connector Fittings for LP

Expansion Foam Systems (Ratios from 100:1 to 1000:1) (F

Expansion Index Test for Soil (1973)

Expansion Joint Filler for Concrete (Bituminous Type)

Expansion Joint Fillers for Concrete Paving and Structu

Expansion Joints) (Tech. Notes) (1963)

Expansion of Concrete Due to the Alkali-Aggregate Rea

Expansion of Portland Cement (1971) ANSI A1.8, Ashto T1

Expansion Plates and Sheets for Bridge and Other Struct

Expansion, Control, Contraction, and Isolation Joints F

Expansion, Glass Sealing, 53Fe-29Ni-17 Co (1973)

Experimental Milling for Bread and Soft Wheats (Cereal

Experimental Milling for Bread and Soft Wheats (Cereal

Experimental Milling for Durum Wheat (Cereal Chemistry)

Experimental Test. for Biological Compatibility of Meta

Experimental Wheat Flour Milling: Equipment, Sample Pre

Experimental Wheat Milling: Temper Table (Cereal Chemis

Experiments in Laboratories (Cereal Chemistry) (1968)

Explosion and Fire in Design, Construction, and Operati

Explosion Conditions (1973)

(T), and

Explosion Hazard During Manufacture) (1974)

Explosion Protection (Safety) for Flammable Liquid and Explosion, and Detonation Protection) (1973)

Explosions in Fuel Oil and Natural Gas Fired Watertube

Explosions in Pulverized Coal Fired Multiple Burner Boi

Explosions (1971) ANSI Z12.12

Explosions (1973)

Explosions (1973)

Explosive Materials (Fire Protection) (1973)

Explosive Reactivity of Lubricants with Aerospace Alloy

Explosives and Blasting Agents (1973)

Explosives and Blasting Agents (1973) AN SI Z271.1

Export and General Industrial Use (1971) ANSI G57.12

Exposure and Evaluation Meth. for Determining Speed of

Exposure Conditions (1972) ANSI Z197.27

Exposure Conditions (1972) ANSI Z197.33

Exposure Guide (Also Contains Suggested Techniques for

for Structura

Exposure of Adhesive Bonded Joints and Structures (1970

Exposure of Adhesive Specimens to High Energy Radiation

Exposure of One Part, Elastomeric, Solvent Release Type

Exposure Tests of Paints on Wood (1973)

Exposure Time Markings for Variable Speed Shutters Used

Exposure to Natural Light and Weather Through Glass (19

Exposure to Natural Light and Weather (1968) ANSI L14.1

Exposure to Radiation in an Emergency (1962)

Exposure with Wetting (1972) ANSI L14.224

Exposure Without Wetting (1972) ANSI L14.225

/R Resistanc

Exposure

$\mathrm{Ns}$ and $\mathrm{Max}$

Test Meth. for Colorfas

Test Meth. for Colo

Exposure (1971) ANSI L14.227

Exposure (1972) ANSl L14.228

ffor Colorfastness of Tex

Exposures Under Glass (1973)

Extender and Processing Oils by the Clay Gel Absorption

Extensigraph Analysis of Physical Dough Tests of Flour

Extensigraph Meth. for Physical Dough Tests of Flour (C

Extensigraph Meth. for Quality of Nonfat Dry Milk in $\mathrm{pH}$

Extension Instruments) (1972) ANSl L14.287

Extension Machines) (1972) ANSI L14.288

Extension Recovery and Adhesion of Latex Sealing C.of Tes

Extension, Torsion, Flat, and Hot Wound (Materials, Spe

Extent of Burning of Cellular Plastics Using a Supporte

Exterior Ceramic Tile Floors (1973)

Exterior Ceramic Tile Walls (1973)
ACGIH

$\mathrm{ACGlH}$

ACGlH

ACGIH

NFPA

PSA

ICBO

ICBO

ANSI

NBHA

ICBO

WWPA
ICBO

ICBO

ASTM

ASTM

ASTM

ASTM

ASTM

ASTM

UL

FMS

ICBO

BIA

ASTM

ASTM

ASTM

TCA

SAE

$\mathrm{AACCH}$

$\mathrm{AACCH}$

AAC'C

ASTM

AACCH

$A A C C$

$\mathrm{AACCH}$

FMS

ICBO

FMS

FMS

NFPA

FMS

NFPA

FMS

ICBO

FMS

ICBO

Cod NFPA

ASTM

ANSI

ASTM

ANSI

ASTM

ASTM

ASTM

ASTM

ANSI

AATCC

AATCC

NCRPM

AATCC

AATCC

AATCC

ASTM G24

ASTM D2007

AACCH 54.11

$\mathrm{AACCH} 54-10$

AACCH 54.12

ASTM D2653

ASTM D2731

ASTM

SMI

ASTM

C.736

TCA D169

TCA

1.7

1.9

1

A156.3

2471

DI056

LPD8.2

F144

A453

.3N

D1751

$18 \mathrm{~A}$

C151

300

BC 535.3

$* 3.13$

UFC 2 ART 10

UBC $\$ 3.33$

$B C=8.13$

BCS 29.2

MS $7728 \mathrm{C}$ 
d Plywood (1971)

Std. Meth. for Measuring Chalk Resistance of Std. Rec. Pract. for Conducting shelving, Misc. Ornamental ltems, Stairwork and Handrails,

3) Quality Stds. for Std. Rec. Pract. for Test. and Sawtooth / Uniform Building Code: Plastics (General, $\mathrm{e}$ Used as Panels in Weathering Tests of Latex and Emulsion ecturaI Aluminum (1971)

Uniform Building Code Std. for Quality Stds. for Rec. Pract. for

Rec. Pract for the Test Procedure for Std. for Supply Gas Connectors for $y$ of Glue Joints in Structural Laminated Wood Products for d Sawtooth Roofs, Light Diffusers in Ceilings, Partitions, Ceramic Glazed Brick Facing for std. Spec. for Portland Cement and Cement Lime Plastering, nd Furring for Portland Cement and Cement Lime Plastering, sives for Structural Laminated Wood Products for Use Under

ically Sliding or Horizontally Operating Storm Windows for Piping Systems (1972)

Grains, Unpopped Popcorn, Dried Pe/ Rec. Pract. for Control of Rec. Pract. for Breakers Rated on a Symmetrical C/ Meth. of Analysis of Std. Requirements for ic Test Values for Outdoor AC High Voltage Circuit Breaker

ing) (1972/

Locking)/

king) (1972)

72)

ng) (1972/

cking) (1) Std. for Handle, Latching Internal and Std. for Fastener (Blind Internally Threaded, Std. for Fastener (Blind, Internally Threaded, Fastener (Blind, Internally Threaded, Std. for Fastener (Blind, Internally Threaded, Std. for Fastener (Blind, Internally Threaded,

Std. for Fastener (Blind, Internally Threaded, Spec. for Fastener (Blind, Internally Threaded, Std. for Design Parameters for Bolts and Screws; Safety Std. for Fir Safety Std. for Foam Fire ety Std. for 2 l/2 Gal. Cartridge Operated Water Type Fire Safety Std. for Rating and Test. of Fire

e, Installation and Maintenance of Systems and Appliance Rec. for Halogenated Fire Std. on Carbon Dioxide Fire
Rec. for Foam Fire

Uniform Building Code Std. for Carbon Dioxide Fire Uniform Building Code: Fire form Building Code Std. for Installation of Automatic Fire I F Hidromatic Deluge Valve for Water Flow Control of Fire s, B Stage Epoxy Resin Impregnated, 181 Style Fabric, Self Stage Polyester Resin Impregnated, 181 Style Fabric, Self building Code Std. for Combination Standpipe Systems (Fire expansion Foam Systems (Ratios from 100:1 to 1000:1) (Fire th. of Test for Nonvolatile Residue of Halogenated Solvent Std. Meth. of Test for Solvent

inished Std. Analytical Meth. for Determining Crude F at /

Std. Analytical Meth. for Determining Crude Fat (Ccl

Std. Analytical Meth. for Determining Crude Fat ( $\mathrm{Ccl}_{4}$ hemistry) (1962) 1972)

lndophenol-Xylene

Std. Meth. of Test for Quantitative

and Fire in Design, Construction, and Operation of Solvent Std. Meth. of Test for Sediment in Crude and Fuel Oils by insect Damage on Kernels, Rodent Excreta, Other Filth, and efined Sugar and O/ Std. Analytical Meth. for Determining (1962)

(Bolting Cloth, Filter Paper,/

Special Techniques for alcohol; Carbon Tetrachloride; Chloral Hydr/

Meth. for Measuring Hall Mobility and Hall Coefficient in

73)

mpounds (1972)

trical Purposes (Bus Condu/

Std. Meth. of Test for Std. Meth. of Test for Tolerances Applicable to Titanium and lts Base Alloy 5 si $\mathrm{H} 45.6$ immersion) (1967) Ansi/ tions (1973) ANSI H38.18 Std. Spec. for Aluminum Alloy Sid. Spec. for Magnesium Alloy Std. Meth. of Test for Quality of Std. Spec. for Aluminum Alloy Std. Spec. for Aluminum Alloy Safety Std. for

Distribution Pip/ Std. Spec. for Aluminum Alloy Seamless Std. Spec. for Aluminum Alloy Seamless Pipe and Seamless spec. for Aluminum Alloy Std. Structural Shapes, Rolled or pec. for Reinforced and Filled Nylon lnjection Molding and njection Molding of Specimens of Thermoplastic Molding and Std. Spec. for Polybutylene Plastics Molding and Std. Spec. for ETFE - Fluoroplastic Molding and std. Spec. for Thermoplastic Polyterephthalate Molding and Std. Spec. for Polyvinylidene Fluoride (PVDF) Molding and
Exterior Coatings and Overlays on DFPA Grade Trademarke Exterior Coil Coatings (1974)

Exterior Exposure Tests of Paints on Wood (1973)

Exterior Frames and Sash, Screens, Blinds and Shutters,

Exterior Frames (Architectural Woodwork) (1973)

Exterior Guide (Siding, Finish, Nails and Nailing) (197

Exterior Latex House Paints (1972)

Exterior Light Transmitting Panels, Skylights, Monitors

Exterior Paints or Coatings (1973)

/Hingle Bla

Exterior Plaster Liquid Bonding Agents (1973)

Exterior Sash (Architectural Woodwork) (1973)

Exterior Sound Level for Heavy Trucks and Buses (1971)

Exterior Sound Level for Snowmobiles (1972) ANSI S6.2

Exterior Use on Mobile Homes (1972)

Exterior Use (1959) ANSI Z197.17

Exterior Veneer, Awnings and Patio

Exterior Walls (Tech. Notes) (1962)

Exterior (Stucco) and Interior (1971)

Exterior (Stucco) and Interior (1971)

Exterior (Wet Use) Exposure Conditions

External Application (1972) ANSI A 134.3 /Bination

External Corrosion on Underground or Submerged Metallic

External Electromagnetic Radiation Suppressors (1969)

External lnsect and Rodent Hair Contamination of Cereal

External Insulation for Outdoor AC High Voltage Circuit

External Insulation Rated on a Symmetrical Basis (1972)

External Release Components and Assembly (1972)

External Sleeve, General Purpose, Flush Head, Self Lock

External Sleeve, General Purpose, Protruding Head, Self

External Sleeve, High Temperature, Flush Head, Self Loc

External Sleeve, High Temperature, Protruding Head, (19

External Sleeve, Light Weight, Protruding Head, Self Lo

External Sleeve, Self Locking) (1972)

External Wrenching, Unified Threaded In. Series (1971)

Extinguisher and Apparatus Booster Hose (1973)

Extinguishers (1973)

Extinguishers (1973)

Extinguishers (1973) ANS1 Z211.1

(Extinguishers, Alarms, Sandpipes), Building Constructio

Extinguishing Agent Systems (1973)

Extinguishing Systems (1972)

Extinguishing Systems (1973)

Extinguishing Systems (1973)

Extinguishing Systems (1973)

Extinguishing Systems (1973)

Extinguishing Systems (1973)

Extinguishing (1973)

Extinguishing (1973)

Extinguishment) (1973)

Extinguishment) (1973)

Extract from Aerospace Components (Using Rotary Flash E

Extractables in Petroleum Waxes (1973)

Extractables) Content in Corn Starch, Gluten, Germand F

Extractables) Content in Corn (1958)

Extractables) Content in Feedstuffs (Corn) (1954)

Extraction Meth. of Analysis of Ascorbic Acid (Cereal C

Extraction of Bitumen from Bituminous Paving Mixtures (

Extraction Plants (1973)

Extraction (1969) ANSI Z11.58, API 256

Extraneous Material (Cereal Chemistry) (1962)

Extraneous Materials Content of Corn Syrup, Crude and

Extraneous Matter Meth. of Analysis in Cereal Chemistry

Extraneous Matter Meth. of Analysis (Cereal Chemistry)

Extraneous Matter Meth. of Analysis (Cereal Chemistry)

Extrinsic Semiconductor Single Crystals (1973)

Extrudability of Unvulcanized Elastomeric Compounds (19

Extrudability, After Package Aging, of Latex Sealing Co

Extruded Bar, Rod, Pipe, and Structural Shapes for Elec

Extruded Bars, Rods, and Shapes (1973)

Extruded Bars, Rods, Shapes, and Tubes (1973) ANSI H38.

Extruded Bars, Rods, Shapes, Tubes, and Wires (1970) an

Extruded Poly (Vinyl Chloride) Plastic Pipe by Acetone

Extruded Round Coiled Tubes for General Purpose Applica

Extruded Structural Pipe and Tube (1973) ANSI H38.16

Extruded Thermoplastic lnsulating Tubing (1972)

Extruded Tube and Pipe for Gas and Oil Transmission and

Extruded Tube (1973) ANSI H38.7

Extruded (1973) ANSl H38.10

Extrusion Materials (1972)

Extrusion Materials (1972) ANS1 K65.78

Extrusion Materials (1973)

Extrusion Materials (1973)

Extrusion Materials (1973)

Extrusion Materials (1973)
APA

NCCA

ASTM

AWI

$A W$

CRA

ASTM

ICBO

ASTM

AAMA

ICBO

AWI

SAE

SAE

IAPMO

ASTM

ICBO

BIA

ANSl

ANSI

ASTM

AAMA

NACE

SAE

AACC

ANSl

NSA

NSA

NSA

NSA

NSA

NSA

NSA

NSA

SAE

UL

UL

Saf UL

UL

ICBO

FMS

NFPA

FMS

ICBO

ICBO

Uni ICBO

FMS

SAE

SAE

ICBO

FMS

ASTM

ASTM

CR

CR

CR

AACC

ASTM

FMS

ASTM

land/or

CR

$\mathrm{AACCH}$

AACCH

$\mathrm{AACCH}$

Std. ASTM

ASTM

ASTM

ASTM

SAE

ASTM

ASTM

ASTM

ASTM

ASTM

UL

ASTM

ASTM

Std. ASTM

Std. S ASTM

1. Pract. for I ASTM

ASTM

ASTM

ASTM

ASTM

$* 4$ TB. 1 II -8

D1006

1.1

- 1.900

$3 \mathrm{~A} 4$

D3129

UBC $\$ 3.52$

D1911 
for Reinforced Polyterphthalate Thermo.Plastic Molding and tfe-Fluorocarbon (Tetrafluoroethylene) Resin Molding and Std. Spec. for Nylon Injection Molding and Powder Flow Measurement of TFE -Fluorocarbon Molding and Std. Spec. for Fep Fluorocarbon Molding and Std. Meth. of Measuring Flow Rates of Thermoplastics by spec. for Glass Reinforced Acetal Plastics for Molding and Std. Spec. for E-CTFE- Fluoroplastic Molding, Std. Spec. for Zirconium Forgings and

Std. Spec. for Zirconium and Its Alloy Forgings and Std. Spec. for Copper Beryllium Alloy Forgings and Spec. for Tolerances of Aluminum and Magnesium Base Alloy Minimum Requirements for Motorcyclists Rec. Pract. for Motor Vehicle Driver's Std. for Forged Carbon Steel, Shoulder Nut Assembly Test Meth. for Colorfastness of Dyed Wool h. for Determination of Formaldehyde Odor in Resin Treated for Permanent Wall and Ceilings / Spec. for Vinyl Coated Std. Spec. for Welded Steel Wire Std. Spec. for Welded Deformed Steel Wire building Code Sid for Welded Steel Wire and Deformed Wire s a / Uniform Building Code Std. for Metal, Wire and Wire Test Meth. for Oily Stain Release Meth. of Textile

h. for Determination of Formaldehyde Odor in Resin Treated test Meth. for Evaluating Oxidation of Wool Oils on Woolen Test Meth. for Colorfastness of Yarn or Test Meth. for Coloriastness of Colored Textile Yarn and Test Meth. for Colorfastness of Textile Yarn and

Test Meth. for Electrostatic Clinging of Textile Fabrics Welded Wire

Std. Spec. for Aluminum Coated Steel Chain Link Fence Std. Spec for Zinc Coated Steel Chain Link Fence Manual of Std. Pract. for Welded Wire Building test Meth for Reflectance, Blue, and Whiteness of Bleached ile) (1973) Spec. for Galvanized Steel Chain Link Fence Test Meth. to Determine Woven for Reinforced Bituminous Concrete Overlays (Welded Wire ial Dyeing Behavior of Cotton (Raw Fiber, Yarn and Textile Type E Glass, B Stage Epoxy Resin Impregnated, 181 Style pe E Glass, B Stage Polyester Resin Impregnated, 181 Style Std. Spec. for Tin Plate Safety Code for Spec for hore Fixed Platforms (1972) Rec. Health Safety Practices for Handling and
Std. for Safety Requirements for Shops Element (1972) ANSI B93./ Std. Meth. for Determining the ice (1968) Std. Pract. for

Plywood Lumber Beams Plywood Stressed Skin Panels steel (1973) Uniform Building Code Std. for Erection, Std. for the Test Meth. for Wicking of Automotive
de Organic Base Fibers/ (1970) Test Meth. for Abrasion Resistance of Textile Test Meth. for Quick Evaluation of Wettability of of Tra/ Std. Meth. of Test for Tearing Strength of Woven
Std. Meth. of Low Temperature Impact Test for of Tra/ Std. Meth. of Test for Tearing Strength of Woven
Std. Meth. of Low Temperature Impact Test for $\begin{array}{ll}\text { extile Colorfastness) (19/ Test Meth. for Color Change in } \\ \text { th. (Textile Colorfastnes/ } & \text { Test Meth. for Color Change of }\end{array}$ Test Meth. for Color Change of Test Meth. for Electrostatic Clinging of Textile 14.283 Sid. Spec. and Meth. of Test for Heavy Cotton Std. Performance Requirements for Woven Washable Uniform Std. Performance Requirements for Woven Washable Uniform td. Performance Requirements for Woven Glass Fiber Curtain mance Requirements for Woven Glass Fiber Drapery (Curtain) Std. Meth. of Test for Stretch Properties of Knitted

73) Ans/ Std. Spec. for Fineness of Mohair Top, Yarns and Std. Meth. of Test for Strap Peel Adhesion of Reinforcing or Permanent Labels, Detachable Tags, and Certification of d. Performance Requirements for Woven Sheet and Pillowcase Water/ Std. Meth. of Sampling and Test. Felted and Woven Test Meth. for Colorfastness of Colored Textile Yarns and (1972) ANSI L14/ Test Meth. for Colorfastness of Textile Test Meth. for Colorfastness of Textile Yarns and Meth. of Test for Coloríastness of Upholstery Test Meth. for Colorfastness of Textile Test Meth. for Colorfastness of Textile Test Meth. for Colorfastness of Textile

nsi L14.227

xposure (1972) A/ Meth of Test for Colorfastness of Upholstery Test Meth. for Colorfastness of Colored Textile Yarns and 973) ANSI A 109.26 Std. Spec. for Woven Glass Meth. of Test for Stretch Properties of Std. Performance Requirements for Woven Drapery (Curtain) Std. Performance Requirements for Woven Slipcover
Extrusion Materials (1973)

Extrusion Materials (1973)

Extrusion Materials (1973) ANSI K65.216

Extrusion Materials (1974)

Extrusion Materials (1974) ANSI K65.201

Extrusion Plastometer (1973)

Extrusion (1972)

Extrusion, and Coating Materials (1973)

Extrusions for Nonnuclear Application (1972) ANSI ZI79.

Extrusions for Nuclear Application (1967) ANSI N125

Extrusions (1972)

Extrusions (1973)

Eye Protection (1971)

Eye Range (1972)

Eyebolt (For Ground Support Equipment) (1974)

Fabric and Yarn to Mill Fulling (1972) ANSI L14.5

Fabric by the Sealed Jar Meth. (1972) ANSI L14.178

Fabric Coverings for Decorative and Protective Covering

Fabric for Concrete Reinforcement (1972) ANSI G45. I

Fabric for Concrete Reinforcement (1972) ANSI G45.3

Fabric for Concrete Reinforcement (1973)

Fabric Laths, and Metal Accessories (Designed for Use a

Fabric Soil During Home Laundering (1970) ANSI L14.239

Fabric Steam Meth. (1972) ANSI L14.179

Fabric Textile in Storage (1971) ANSI L14. S2

Fabric to Acids and Alkalis (1972) ANSI L14.2

Fabric to Crocking (Rubbing) by the AATCC Crockmeter Me

Fabric to Crocking (Rubbing) (Rotary Vertical Crockmete

Fabric to Metal Test) (1973)

Fabric (1970)

Fabric (1971) ANSI G53.21

Fabric (1971A) ANSI G53.20

Fabric (1972)

Fabric (1972) ANSI L14.175

Fabric (1973)

Fabric's Resistance to Sagging or Stretch in Wear (Text

Fabric) (1962)

Fabric) (1972) ANSI L14.152

Fabric, Self Extinguishing (1973)

Fabric, Self Extinguishing (1973

Fabricated Aerosol Cans (1972)

Fabricated Stage and Scaffold Planks and Platforms (197

Fabricated Structural Steel Pipe for Use of Welded Offs

Fabricating Asbestos Textile Products (1971)

Fabricating Structural Steel and Plate (1973)

Fabrication Integrity of a Hydraulic Fluid Power Filter

Fabrication of Reinforcing Steel Bar Materials and Serv

Fabrication Spec. for Plywood Curved Panels (1971)

Fabrication Spec. for Plywood Sandwich Panels (1971)

Fabrication Spec. (1971)

Fabrication Spec, (1971)

Fabrication, Identification and Painting of Structural

Fabrics and Fibrous Materials (1972)

Fabrics and Industrial Filament Yarns, Made, from Man Ma

Fabrics and Other Flexible Materials: Accelerator Meth.

Fabrics and Textiles in Yarn Form (1971) ANSI L14.75

Fabrics by the Tongue (Single Rip) Meth. (Constant Rate

Fabrics Coated with Flexible Polymeric Materials (1973)

Fabrics Due to Flat Abrasion (Frosting): Every Meth. (T

Fabrics Due to Flat Abrasion (Frosting): Screen Wire Me

Fabrics Fabric to Metal Test) (1973)

Fabrics for Institutional Uniforms (Textile) (1973)

Fabrics for Manufacture of Hose and Belts (1942) ANSI L

Fabrics for Men and Women (Heavy Duty) (Institutional T

Fabrics for Men and Women (Institutional Textile) (1973

Fabrics Handwashable at 105 Deg. F-No Bleach (Institu

Fabrics Handwashable at 105 Deg. F-No Bleach (Institu

Fabrics Having Low Power (1972) ANSI Z14.286

Fabrics of the Worsted Type and Assignment of Grade (19

Fabrics or Cords to Rubber (1971)

Fabrics or Products (Institutional Textile) (1973)

Fabrics Other Than Durable Press (Institutional Textile

Fabrics Saturated with Bituminous Substances for Use in

Fabrics to Chlorinated Pool Water (1972) ANSI L14.149

Fabrics to Ozone in the Atmosphere Under Low Humidities

Fabrics to Sea Water (1972) ANSI L14.150

Fabrics to Solvent (Textile) (1973)

Fabrics to Water and Light: Alternate Exposure (1971) a

Fabrics to Water Spotting (1972) ANS1 L14.148

Fabrics to Water (High Humidity) and Light: Alternate E

Fabrics to Water (Textile) (1973)

Fabrics to Water (1972) ANSI L 14.151

Fabrics Treated for Use in Waterproofing and Roofing (1

Fabrics Woven from Stretch Yarns (1972T)

Fabrics (Excluding Glass Fibers) (Institutional Textile

Fabrics (Institutional Textile) (1973)
Std. Spec. ASTM

ASTM

ASTM

ASTM

ASTM

Std. ASTM

ASTM

ASTM

ASTM

ASTM

SAE

VESC

SAE

NSA

AATCC

Et AATCC

CSI

ASTM

ASTM

Uniform ICBO

ICBO

AATCC

AATCC

AATCC

AATCC

AATCC 8

AATCC 116

AATCC 115

CRSI

ASTM

ASTM

WRI

AATCC

CLFMI

ANS

ec. Pract WRI

Clon AST

loth. Ty

SAE

ALI

API

ATI

ANSI

NFLDP

CRSI

APA

APA

APA

APA

ICBO

SAE

A ATCC

A ATCC

ASTM

ASTM

AATCC

AATCC

A TTCC

ANSI

ASTM

ANSI

ANS1

ANSI

ANSI

ASTM

ASTM

ASTM

/ F ANSI

ANSI

ASTM

AATCC

A ATCC

A ATCC

ANSI

AATCC

A ATCC

AATCC

ANSI

AATCC

ASTM

ASTM

ANSI

ANSI

V.8

2

16

D3220

Dl457

D789

D2116

D1238

D2948

D 3275

B493

B356

B570

J94 I C

1053

09951

A 185

4497

UBC $526 \cdot 6$

UBC 547.4

*1.2.1

A491

A392

MP 100 
nts for Durable Press (No Iron) Woven Sheet and Pillowcase ents for Woven Decorative Bedspreads, Pillows, and Bolster

Performance Requirements for Woven Drycleanable Overcoat Test Meth. for the Appearance of Durable Press Textile Test Meth. for Water Resistance of Textile t) Std. Performance Requirements for Woven Window Curtain Std. Performance Requirements for Woven Upholstery

and Shrinkage Propensity of Bonded and Laminated Appare garm/ Std. Performance Requirements for $100 \%$ Cotton Woven garments (Clothi) Std. Performance Requirements for Woven garments (Clothi) Std. Performance Requirements for Woven Tent. Tolerances for Tire st for Light Transmittance of Woven Glass Fiber Decorative for Effect of Handwashing on Woven Glass Fiber Decorative ion Dimensional Change (Shrinkage) of Stabilized Knit Wool Std. Meth. of Test. Warp Knit Test Meth. for Fire Resistanca of Textile Test Meth. for Evaluation of Antibacterial Finishes on Std. Meth. of Test for Abrasion Resistance of Textile valuation of Commercial Rewetting Agents on Cotton Textile Test Meth. for Mercerization in Cotton Yarns and st Meth. for Bond Strength of Bonded and Laminated Textile leaning on Permanence of Durable Water Repellent Finish of Std. Meth. of Test for Distortion of Yarn in Woven ng Resistance and Other Related Surface Changes of Textile std. Spec. for Performance of Bonded and Laminated Apparel st for Measuring Soil Removal and Reflectance Retention of std. Meth. of Test for Hydrogen Permeance of Rubber Coated Std. Meth. of Test. Coated

ent. Tolerances for Tire Fabrics (Textile) Other Than Cord ndering of Durable (Permanent) Press Woven or Knit Textile Std. Meth. of Test. Nonwoven Std. Spec. for Woven Glass

nal Silane Type Finishes for / $100 \%$ Man Made or Blend industrial Use Garments (Clothing), 100\% Man Made or Blend Std. Meth. of Test. Tire Cords, Tire Cord mance Requirements for Woven Drycleanable Tailored Uniform mance Requirements for Woven Drycleanable Tailored Uniform

Std. Performance Requirements for Tailored Woven Uniform nce Requirements for Cotton Woven Tent, A wning, and Canopy or Determining Visual Color Matched to Master Specimen for Test Meth. for Detection of Antibacterial Activity of Test Meth. for Wrinkle Recovery of Textile

th. for Weather Resistance of Textile Materials and Coated nsi L14.176 Test Meth. for Resistance of Textile Test Meth. for Oil Repellency of Test Meth. for Water Resistance of Textile Test Meth. for Water Resistance of Textile

Im D583 er Wrinkle Recovery of Woven Textile Test Meth. for Water Repellency of Textile $\begin{array}{ll}m \text { D583 } & \text { Test Meth. for Water Repellency of Textile } \\ \text { L14.87, ASTM/ } & \text { Test Meth. for Water Repellency of Textile }\end{array}$ L14.87, A nditioning) (1966)

Rec. Pract. for Instrument 1 Equipment (Machinery) (1971/ 50 , and 60 Amperes, 4 Wire (1966 Standard Mounting and Std. for Dimensional Requirements for Duplex and Single Std. for System Design of Fine Pitch on Center Std. Meth. of Test for Slump of

Std. Meth. of Test for Accelerated Aging of Wood Sash able Anesthetizing Locations (Bonding Appliances, Casters, Std. for Adjustable s (1973) Std. for

std. for Hardwood Veneered Including Hardboard and Plastic alyzing Outage of Electrical Transmission and Distribution or Average Vehicle Dimensions for Use in Designing Docking and Plastics (1972) Rec. for Terminal Cable Connectors for Audio al Installations at Drilling Rigs and Petroleum Production rmatory, Public or Office/ Uniform Plumbing Code: Minimum Std. for Nuclear Material Control Systems for Conversion for Nuclear Material Control Systems for Fuel Reprocessing of Vehicle Fuel Tanks and Fill Pipes with Fuel Dispensing Brick and Tile in Sanitation Structures, Water Treatment Brick and Tile in Sanitation Structures, Sewage Treatment

Tile in Sanitation Structures, Industrial Waste Treatment ality Assurance for Protective Coatings Applied to Nuclear tings (Paints) for Light Water Nuclear Reactor Containment

to Principal Design Criteria for Nuclear Fuel Reprocessing

tial and Commerical Fuel Burning, Dispensing, and Handling Std. for Industrial Engineering Terminology: Pigeon, and Skeet: Ammunition and Targ/ Shotgun Shooting Ceramic Glazed Brick

Uniform Building Code Std. for Building, for Electrical Resistivity (Electro-Static) of Textile irements for Woven Man Made Fiber Tent, Awning, and Canopy

Fabrics (Institutional Textile) (1973)

Fabrics (Institutional Textiles) (1973)

Fabrics (Institutional Uniform Textile) (1973)

Fabrics (Permanent Press) After Repeated Home Launderin

(Rain Test) (1971) ANSI L14.74, ASTM D583

Fabrics (Sash Casement) (Excluding Glass Fibers) (Insti

Fabrics (Tacked On) (Institutional Textile) (1973)

Fabrics (Textile) After Dry Cleaning and Laundering (19

Fabrics (Textile) for Institutional and Industrial Use

Fabrics (Textile) for Institutional and Industrial Use

Fabrics (Textile) for Institutional and Industrial Use

Fabrics (Textile) Other Than Cord Fabrics (1973) ANSI L

Fabrics (Textile) (1973)

Fabrics (Textile) (1973)

Fabrics (Textile) (1973)

Fabrics (1964) ANSI L14.284

Fabrics (1969)

Fabrics (1970) ANS1 L14.144

Fabrics (1971)

Fabrics (1971) ANSI L14. 106

Fabrics (1971) ANSI L14.124

Fabrics (1972)

Fabrics (1972)

Fabrics (1972) ANSI L 14. 103

Fabrics (1972) ANSI L14.112

Fabrics (1972) ANSI L14.198

Fabrics (1972) ANSI L14.291

Fabrics (1972T)

Fabrics (1973)

Fabrics (1973) ANSI J2.12

Fabrics (1973) ANSI L14.243

Fabrics (1973) ANSI Ll4.266

Fabrics (1974) ANSI L14.246

$$
\begin{array}{r}
\text { Meth. of Te } \\
\text { Meth. of Test }
\end{array}
$$

/D. Meth. of Test for Relaxat

Fabrics, Cleaned, and After Finished with Epoxy Functio

Fabrics, Commercial Wash-Whites

Fabrics, Filaments, and Strands Made from Steel (1974)

Fabrics, Heavyweight (Industrial) Suiting (Institutiona

Fabrics, Intermediate (Year Round) Suiting (Institution

Fabrics, Lightweight (Tropical) Suiting (Institutional

Fabrics, Painted, Printed, or Vinyl Coated (Institution

Fabrics, Vinyls, Coated Fiberboards, and Other Automoti

Fabrics: Agar Plate Meth. (1970) ANSl L 14.125

Fabrics: A ppearance Meth. (1970) ANSl L 14.229

Fabrics: Carbon Arc Lamp Exposure with Wetting (1972) a

Fabrics: Exposure to Natural Light and Weather (1968) a

Fabrics: Hydrocarbon Resistance Test (1972) ANSl L14.21

Fabrics: Hydrostatic Pressure Test (1971) ANSI L14.265

Fabrics: Impact Penetration Test (1971) ANSI L14.71, as

Fabrics: Recovery Angle Meth. (1972) ANS1 L14.110, ASTM

Fabrics: Spray Test (1971) ANSI L14.60, ASTM D583

Fabrics: Static Absorption Test (1972) ANSI L14.61, Ast

Fabrics: Tumble Jar Dynamic Absorption Test (1972) ANS1

Face Areas for Central Station Units (Air Moving and Co

Face Design and Location for Construction and Industria

Face Flat Throns of Receptacles Off

Face Gears for 20 Deg. Involute Spur Pinions (1973)

Face Glazing and Bedding Compounds on Metal Sash (1974)

Face Glazing Compound (1973)

Face Masks and Kebreathing Bags, Footwear, Hose and Tub

Face Vehicular Traffic Control Signal Heads (1970)

Face-To-Face and End-To-End Dimensions of Ferrous Valve

Faced Flush Doors (1973)

Facilities and Interruptions to Customer Service (1973)

Facilities for Motor Vehicles (1971)

Facilities for Pneumatic Transfer of Dry Bulk Chemical

Facilities for Radio Broadcasting (1970)

Facilities on Land and on Marine Fixed and Mobile Plate

Facilities Required for School, Theater, Auditorium, Do

Facilities (A Guide to Practice) (1971)

Facilities (A Guide to Practice) (1974)

Facilities (Passenger Cars, Multipurpose Vehicles, and

Facilities (Tech. Notes) (1969)

Facilities (Tech. Notes) (1969)

Facilities (Tech. Notes) (1969)

Facilities (1972)

Facilities (1972)

Facilities (1973)

Facilities (1974) A NSI B150.1

Facility Planning (1972)

Facility Plans: International and Domestic-Trap, Clay

Facing for Exterior Walls (Tech. Notes) (1962)

Structural Clay Facing Tile (Tech. Notes) (1965)
Facing, and Hollow Brick (Made from Clay or Shale) (197
Ance Requireme ANSI

Ance Requirem Std

L24.2.13

L24.1.2

L24.3.4

124

AATCC 35

ANSI L24.1.4

ANSI L24.1.7

ASTM D2724

ANSl L24.4.I2

ANSI L24.4.13

ANSI L24.4.14

ASTM D122

L24T4

L24T1

D 1284

D 1376

ASTM DIC 34

AATCC 100

ASTM D1175

27

AATCC 89

Te AATCC 136

ASTM D2721

ASTM D1336

76

D 1375

D3135

D3050

ASTM D815

ASTM D751

ASTM D122

135

ASTM DIII7

ASTM D3098

ANSI L24.4.13

L24.4.14

D2969

L24.3.2

L24.3.1

L24.3.3

L24.1.1

L24. 1.10

J361A

AATCC 90

AATCC 128

AATCC 111A

AATCC 111B

AATCC 118

AATCC 127

AATCC 42

AATCC 66

AATCC 22

AATCC 21

AMCA 1402

SAE J209

ANSI C73.6?

IFI

AGMA 203.03

ASTM D2376

ASTM C741

UL

ITE

1067

ANS1 B16.10

NWMA IS.I

IEEE

346

J699A

TC 18

MCA

EIA

API

ICBO

ANSI

RS297-A

RP500B

UPC $* 1 \cdot C$

N15.4

N15.13

SAE

BlA

BlA

J398A

34

$34 \mathrm{~A}$

Brick and $\mathrm{BlA}$

Standard for Qu ANSI

$34 \mathrm{~B}$

N101.2

N101.3

331

Z94.6

$\begin{array}{ll}\text { NRA } & * 17 \\ \text { BIA } & 13\end{array}$

BlA 22

ICBO 


\section{National Bureau of Standards KWIC Index of Engineering Standards}

nts (1972) ANSI C37.26

Std. Guide for Meth. of Powe std. Meth. of Test for Dielectric Constant and Dissipation Std. Meth. of Test for Bulk Density and Packing Std. Test Meth. for Diffuse Light Transmission est for Dielectric Constant (Permittivity) and Dissipation Cereal Chemistry) (1965)

Meth for Multiplication

(Cereal Chemistry) (196/ Meth. of Analysis for Correction for Low and Medium Speed Stationary Diesel and Gas Engine Std. for Particleboard Decking fo ufacturing Plants (Fire and Wind Hazards at Pl/

creens, Blinds and Shutters, Flush, Stile, and Rail Doors, Std. Spec. for Sid. Spec, for Std.

tings (1973) ANSI H38.19

ttings for Pressure Piping (1972) ANSI H3/

Factor Measurement for Low Voltage Inductive Test Curre

Factor of Expanded Cellular Plastics Used for Electrica

ASTM

Factor of Hollow Glass Microspheres (1972)

Factor of Reinforced Plastic Panels (1969)

Factor of Solid Ceramic Dielectrics at Frequencies to 1

Factors for Correcting Weights to a $14 \%$ Moisture Basis

Factors for Gasometric Determination of Carbon Dioxide

Factory and Field Testing (1972)

Factory Buil Housing (1972)

Factory Constructed Housing and Recreational Vehicle Ma

Factory Finishing (Architectural Woodwork) (1973)

Factory Finishing, Rough and Finished Carpentry, Floori

Factory Made Air Duct Materials and Connectors (1974)

Factory Made Wrought Aluminum and lts Alloy Welding Fit

Factory Made Wrought Nickel and Nickel Alloy Welding Fi

Factory Made Wrought Steel Buttwelding Fittings (1971)

rotect/ Trial Use Guide for the Application of the Single tance to Blistering. Wrinkling, Loss of Adhesion, or Other 4) ANSI B 72.6 Std. Meth, of Test for Time to ating Materials (1973) Std. Meth. of Test for Thermal rement of Extreme Pressure Properties of Fluid Lubricants wheat, Rye, Barley, Other Grains, A/ Meth. of Analysis of vers, W/ Rec. Pract. for Minimum Performance Criteria for cal Zone for Laboratory Evaluation of Roll Over (ROPS) and Meth. of Test for Impact Resistance of Pipeline Coating ance of Rigid Plastic Sheeting or Parts by Means of a Tup Rec. for Design Values for Livestock

Iniform Building Code: Regulations Governing al.27 urning, Combustible and Flammable Materials; Reporting and (1971) Std. Meth. of Test for Std. for Sound Rating of Room Safety Std. for Room Type

helical and Herringbone Gear Units for Water Cooling Tower Std. for Gas Fired Gravity and Std. for Gas Fired Gravity and Std, for Gas Fired Gravity and Std. for Gas Fired Gravity and

Uniform Building Code Std. for Blower Std. Preparation of Centrifuga Rec Pract fo

iltering Appliances Rated at $60 /$ Std. Sizes for Industrial Centrifugal Safety Std. for Electric Std. Wheel Diameters and Outlet Areas for Centrifugal Std. Sizes for Industrial Centrifuga Std. Drive Arrangements for Centrifugal Std. Inlet Box Positions for Centrifuga id. Designations for Rotation and Discharge of Centrifuga Std. Motor Positions for Belt or Chain Drive Centrifugal Std. Drive Arrangements for Tubular Centrifuga lue Gas Densities for Forced and Induced Draft Centrifuga heating Appliances (Regulators, Automatic Damper Controls Meth. of Analysis for Ash in counting Meth. of Analysis of Cinder and Sand Particles in vimetric Meth. of Analysis of Cinder and Sand Particles in ole Grain Products, Corn Grits, Corn Meal, Puffed Cereals, h. of Analysis for Insect and Rodent Filth in Meal, Grits eth. of Analysis for Rodent Excreta in Meal, Grits, Flour

le Grain Products, Grits, Meal, Flaked and Puffed Cereals, peas, L/ Air Oven Meth. of Analysis of Moisture in Flour,

al Dough Tests: Approximate Corrections for Changing As-Is Nonfat Dry Milk Solids to Change Mixing Characteristics/ ough to Mixing (Cereal Chemistry) (1962)

truction, Installation, and Rating of Equipment for Drying Std. for Headed Drilled Pins for Rec. Spec, for (Nonelectric

) $/$ Std. Spec, for Zinc Coated (Galvanized) Iron or Stee ne Specs. (1972) Rec. for Tractor and Heat and Moisture Loss and Feed and Water Requirements of Std. for Agricultural Press and Gage Wheel Tires Std for Agricultural Planter Press Wheel Tire and Row Crop Cultivator Shafts and Ground Tool Mountings (Farm Machinery) (1973) Sanitary Std for Farm Milk Storage Tanks (1973) Stds. for Construction of Concrete Stave d. Test Proc. for Determining the Load Carrying Ability of Rec. for Design of

Std for Mounting Brackets and Socket for Agricultur Warning Lamp (Light) for Remote Mounting on Agricultural Rec. for Hitch and Box Dimensions for Agricultural

for Full Shielding of Power Drive Lines for Agricultura draulic Motors to Agricultural Tractors and Trailing Type s to Agricultural Tractors and Trailing Type Agricultural
Tire Selection Tables for Agricultural Rec for Agricultural Std. for $540 \mathrm{Rpm}$ Power Takeoff for Agricultural
$1000 \mathrm{Rpm}$ Power Takeoff for Agricultural
Failure Criterion to Nuclear Power Generating Station P

Failure of Paint, Varnish, Lacquer, and Related Organic

Failure of Plastic Pipe Constant Internal Pressure (197

Failure Under Electric Stress of Solid Electrical Insul Std. Meth. for

Falling Object Protective Structure (FOPS) for Prime Mo

Falling Object Protective Structures (FOPS) of Construc

(Falling Weight Test) (1972)

(Falling Weight) (1972)

allout Shelters (1973)

/Eth. of Test for Impact Resist

False Alarm; Use of Equipment, Appliances, Devices and

False Set of Portland Cement (Paste Meth.) (1972) ANSI

Fan Coil Air Conditioners Delivering Less Than $1500 \mathrm{cfm}$

Coil Units (Heating and Cooling) (1973) ANSI Z25.1

Fan Drives (1972)

Fan Type Direct Vent Wall Furnaces (1973)

Fan Type Floor Furnaces (1973)

Fan Type Sealed Combustion System Wall Furnaces (1972)

Fan Type Vented Wall Furnaces (1972)

Fans and Parts for Protective Coatings (1966)

Fans for Industrial Exhaust Ventilation Systems (1974)

Fans with Cast Housings (1966)

ans (Blow

Fans (1966)

Fans (1966)

Fans (1966)

Fans (1966)

Fans $(1966$

Fans (1966)

Fans (1966)

Fans) (1973)

Aft Equipment for Combustion Chamber in

Farina and Semolina (Cereal Chemistry) (1962)

Farina (Cereal Chemistry) (1962)

Farina (Cereal Chemistry) (1962)

Farina, and Flour (Cereal Chemistry) (1962)

Farina, and Semolina (Cereal Chemistry) (1962)

Farina, and Semolina (Cereal Chemistry) (1962

Farina, Bread, etc. (Cereal Chemistry) (1962)

Farina, Se molina, Bread, Grain, Soybeans, Rice, Beans,

Farinograph Absorption to 14.0\% Moisture Basis (Constan

Farinograph Evaluation Meth. of Ability of Roller Dried

Farinograph Meth. for Analysis of Resistance of Flour D

Farm Crops (1971)

Farm Equipment and Machinery (1973)

Farm Fence Construction (1972)

Farm Field and Railroad Right of Way Wire Fencing (1973

Farm Implement Power Takeoff (Universal Joint) Drive Li

Farm Livestock (Poultry, Swine, Cattle, Sheep) (1968)

(Farm Machinery) (1971)

Farm Milk Storage

Farm Wagon Running Gear (1973)

Farm Waste Storage Tanks (1973)

(Farm) and Industrial Equipment Warning Lamp and Slow Mo

(Farm) Equipment (1972)

Farm) Grain Wagons (1972)

Farm) Implements and Tractors (1972) SAE J955 Std. for Flashin

Std. for Chisel Plow, Field

arm) Implements (1971)

Farm) lmplements (1971) SAE J716

Application of Remote H Ote Control Cylinder

Machines of Future Design (1972) SAE J711

Farm) Tractor Auxiliary Power Takeoff Drives (1972)

Farm) Tractors (1973) SAE J718

(Farm) Tractors (1973) SAE J719

ASTM D2I 49

$\mathrm{AACCH} \quad 82.24$

AACCH 12.29

DEMA *1-22

NPA

FMS

AWI

AW

UL

ASTM

ASTM

ANSI

NCCA

ASTM

ASTM

2-70

7.90

$* 1.1500$

*1-1

3361

B366

B16.

379

TB-III-1

$\mathrm{AACCH} \quad 56.81 \mathrm{~B}$

SAE

J231

ASTM

ASAE

ICBO

ICBO

ASTM

AR

UL

AGMA

ANSI

ANSI

ANSI

ICBO

AMCA

ACGIH

AMCA

UL

AMCA

AMCA

AMCA

AMCA

MCA

AMCA

MCA

$397 \mathrm{~A}$

G14

D3029

R282.1

BC $* 3.57$

UFC*2ART27

C451

443

490.02

Z21. 44

721. 48

Z21.44A

Z21. 49

UBC $510-3$

2601

*1-10

2403

2401

2402

2404

2406

2407
2410

2409

$\mathrm{AACCH} 28-01$

Gra AACCH 28-02

ther Wh AACCH 86.80

ion $\mathrm{M}$ AACCH 28-50

AACCH 86-70

AACCH 44-15A

AACCH $54-29$

AACCH $54-20$

AACCH 54.21

ASAE $\$ 248.2$

ASAE $\$ 226.2$

ASAE R250.2

ASTM Al16

ASAE R331.2 
Rec. Lighting for Dairy 972)

for Design and Construction of Surface Drainage Systems on

Std. for Wood Used on

Rec. for Computing Electrical Demands for Std. for Sprinkler Irrigation (For

Rec. for Safety for Industry Std. for

ansi $\mathrm{C} 16.45$

y from Uranium $\cdot 238$ Fission (1972/

ANSI N114

i N111

nsi N 112

nsi $\mathrm{N} 113$

by Uranium 238 Fission (1973)

(1973)

Meth of Test for

Std. Meth. for Measuring

Std. Meth. for Measuring

Sid. Meth. for Measurin

Std. Meth. for Measuring

Std. Meth. for Measuring Std. Meth. for Std. for

Std.

eneral Purpose, Flush Head, Self Locking) (1972/ general Purpose, Protruding Head, Self Locking)/ high Temperature, Protruding Head, (1972)

high Temperature, Flush Head, Self Locking) (1972)

light Weight, Protruding Head, Self Locking) (1/ light Weight, Millable Head Self Locking) (1972 self Locking) (1972)

Std. for

Std. for

Std. for

Std. for

Std. for

Spec. for

Std. for Guide

Duct Liner, Adhesives, and

Std. Meth. of Test. Meta

echanical and Quality Requirements for Externally Threaded

and Spikes, Washers, Pin/Western Woods Use Book: Timbe . for Carbon Steel Externally and Internally Threaded Std. Std. for Wrenching Configuration, Spline Drive Threaded Std, for Manufacturer Identification Symbols for

Trial Std. for Screw Threads for Metric Series Mechanica

Spec. for

Spec. for

1972)

Std. Procurement Spec. for Spec. for

Std. Spec for Adhesives for

Uniform Building Code Std for Adhesives for Safety Requirements for Powder Actuated

servative / Uniform Building Code: Wood Members and Their National Design Spec. for Stress Grade Lumber and Its

d Design, Span Allowances, Storage, Finishes and Nails and Test Meth. for Colorfastness to Light: Determination of Rapid Meth. of Analysis for

General Meth. of Analysis for

Rapid Meth. of Analysis for

Colorimetric Meth. of Analysis for

ybeans, Whole or Ground Full Fat or Extracted Flakes, Ful

ybeans, Whole or Ground Full Fat or Extracted Flakes, Full

Std. Analytical Meth. for Determining Tota Std. for Hotel and Restaurant Gas Deep Meth. of Analysis of Crude Meth. of Analysis of Crude Meth of Analysis of Crude Meth. of Analysis of Crude Meth. of Analysis of Crude Meth. of Analysis of Crude Meth of Analysis of Crud Meth. of Analysis of Crude

Dilatometric Meth. of Solid

eal Chemistry) (1962)

ortenings, and Other Fats (1)

re Range / Meth. of Analysis of Congeal Point in Fats and persible Nitrogen in Ground Soybeans, Whole or Ground Full
spersible Protein in Ground Soybeans, Whole or Ground Full Std. Analytical Meth. for Determining Crude Std Analytical Meth for Determining Crude (1954) Std. Analytical Meth. for Determining Crude rmand Finished Std. Analytical Meth. for Determining Crude er Element (1972) Ansi/ Std. Meth. for Verifying the Flow Relating to Fatigue Test, and the Statistical Analysis of Std. Load Ratings and Std. Load Ratings and Std. Test Meth. for Shea Std Meth of Test for

ding (Metal / Metal) (1973)

heels, and Demountabl/

ata (1972) ANSI Z9/

temperature Range . Meth.) Content in Corn, Grain Sorghum and Other Vegetabl al C/ Bidwell-Sterling Meth. of Analysis of Moisture in $\operatorname{coc} /$ Meth. of Analysis of Moisture and Volatile Matter in Meth. for Sampling of

Meth. of Analysis of Free Fatty Acids in analysis of Cloud Point in All Edible Animal and Vegetable $f$ Analysis of Melting Point of Normal Animal and Vegetable
Farms and the Poultry Industry (1971)

Farms in Humid Areas (1973)

Farms (Preservative Treatment by Pressure Processes) (1

Farms (1972)

Farms) Technical Data Sheet (1972)

Farmstead Equipment and Machinery (1972)

Fascimile Transmission of Microfilmed Documents (1972)

Fast Neutron Flux by Analysis of Molybdenum -99 Activit

Fast Neutron Flux by Radioactivation of Aluminum (1970)

Fast Neutron Flux by Radioaetivation of Iron (1970) Ans

Fast Neutron Flux by Radioactivation of Nickel (1970) a

Fast Neutron Flux by Radioactivation of Sulfur (1970) a

Fast Neutron Flux for Analysis for Barium 140 Produced

Fast Neutron Flux Measurements by Track Etch Technique astener-Taper Shank (1973)

Fastener Code (Aerospace Lockbolts, Rivets, Bolts) (197

Fastener (Blind Internally Threaded, External Sleeve, G

astener (Blind, Internally Threaded, External Sleeve,

Fastener (Blind, Internally Threaded, External Sleeve,

Fastener (Blind, Internally Threaded, External Sleeve,

Fastener (Blind, Internally Threaded, External Sleeve,

Fastener (Blind, Internally Threaded, External Sleeve,

astener (Blind, Internally Threaded, External Sleeve.

Fastener, Low Form, Cowl, Dzus Type (1973)

Fasteners Application Std. (Air Distribution) (1971)

Fasteners in Wood (1974)

Fasteners Used in Automotive and Related Industries (19

Fasteners (Connectors, Bolts, Lag Screws, Plates, Nails

Fasteners (1968) ANSI G38.5

Fasteners (1971)

Fasteners (1973)

Fasteners (1974)

Fasteners, Alloy Steel, Externally Threaded (1972)

Fasteners, A286 Cres, Externally Threaded (1971)

Fasteners, Titanium Alloy (1973)

Fasteners, 6Al-4V Titanium Alloy, Externally Threaded (

Fastener; Rotary, Quick Operating, High Strength (1973)

Fastening Gypsum Wallboard to Wood Framing (1973)

Fastening Gypsum Wallboard to Wood Framing (1973)

Fastening Systems (1972)

Fastenings (Lumber, Plywood and Components, Timber, Pre

Fastenings (1971)

Fastenings) (1973)

Fastness Above L-7 (1971) ANSI L14.264

for Garden Grades, Application an

Fat Acidity in Corn (Cereal Chemistry) (1962)

Fat Acidity in Grain and Flour (Cereal Chemistry) (1962

Fat Acidity in Grain (Cereal Chemistry) (1962)

Fat Acidity in Grain (Cereal Chemistry) (1962)

Fat and Defatted Soy Flours and Grits, and Soybean Meal

Fat and Defatted Soy Flours and Grits, and Soybean Meal

Fat Content in Corn Starch (1955)

Fat Fryers (1971)

Fat in Baked Dog Food (Cereal Chemistry) (1962)

Fat in Cocoa (Cereal Chemistry) (1962)

Fat in Dry Milk Products (Cereal Chemistry) (1962)

Fat in Egg Yolk and Dried Whole Egg (Cereal Chemistry)

Fat in Flour, Bread, and Baked Cereal Products Not Cont

Fat in Grain and Stock Feeds (Cereal Chemistry) (1962)

Fat in Soy Flours (Cereal Chemistry) (1962)

Fat in Wheat and Soy Flour, Feeds and Cooked Feeds (Cer

Fat Index (50 or Less at 10 Deg.) of Margarine Oils, Sh

Fat Mixtures Which Congeal or Solidify Within Temperatu

Fat or Extracted Flakes, Full Fat and Defatted Soy Flou

Fat or Extracted Flakes, Full Fat and Defatted Soy Flou

Fat (Ccl ${ }_{4}$ Extractables) Content in Corn (1958)

Fat (Ccl, Extractables) Content in Feedstuffs (Corn)

Fat (Ccl, Products Such as Feeds and Meals (1955)

Fatigue Characteristics of a Hydraulic Fluid Power Filt

Fatigue Data (1972) ANSI Z92.2 /. De

Fatigue Life for Roller Bearings (1972)

Fatigue of Sandwich Core Materials (1970)

Fatigue Properties of Adhesives in Shear by Tension Loa

Fatigue Test Procedures for Production Disc and Spoke W

Fatigue Test. and the Statistical Analysis of Fatigue D

Fatigue Tests for Copper Alloy Spring Materials (1973)

Fats and $\mathrm{F}$ at Mixtures Which Congeal or Solidify Within

Fats and Oils (Cereal Chemistry) (1967)

Fats and Oils (Cereal Chemistry) (1967)

Fats and Oils (1966)

Determining Iodine Number (Wijs

Fats and Shortenings by Distillation with Toluene (Cere

Fats and Shortenings (Butter, Oleomargarine, High Acid

Fats and Shortenings (Cereal Chemistry) (1962)

Fats (Cereal Chemistry) (1967)

Fats (Cereal Chemistry) (1967)

Fats (Cereal Chemistry) (1967)
ASAE

Rec. ASAE

AWPA

ASAE

ASAE

ASAE

NMA

ASTM

ASTM

ASTM

ASTM

ASTM

ASTM

ASTM

NSA

NSA

NSA

NSA

NSA

NSA

NSA

NSA

NSA

SMACN

ASTM

SAE

WWPA

ASTM

SAE

IFI

IFI

NSA

NSA

NSA

NSA

ASTM

ICBO

ANSI

ICBO

NFORP

CRA

AATCC

AACCH $02-03$

AACCH $02-01$

AACCH $02-02$

$\mathrm{AACCH} \quad 02-04$

$\mathrm{AACCH} \quad 46-23$

AACCH 46-24

CR B-20

ANSI Z21.27

AACCH 30.14

AACCH 30.12

AACCH 30-16

AACCH $30-18$

AACCH 30.10

AACCH $30-20$

AACCH 30.26

AACCH $30-25$

AACCH $31-50$

AACCH 58-12A

AACCH $46-23$

AACCH $46-24$

CR A-6

CR $\quad$ G. 10

E CR B-18

NFLDP T3.10.8.7

ASTM E206

AFBMA 9

AFBMA 11

ASTM C.394

ASTM D3166

SAE J267

ASTM E206

ASTM B593

$\mathrm{AACCH} 58.12 \mathrm{~A}$

$\mathrm{AACCH} 58-30$

AACCH 58.50

$\begin{array}{ll}\text { CR } & \mathrm{H}-32\end{array}$

$\mathrm{AACCH} 44.51$

AACCH $44-30$

$\mathrm{AACCH} \quad 64.40$

$\mathrm{AACCH}$ 58-16

Meth of AACCH 56.10

Wiley Meth. O AACCH 58-41 
on, with Abbe Refractometer, in All Normal Oils and Liquid alysis of Melting Point of All Normal Animal and Vegetable corn Oil and to Other Vegetable and Marine Oils and Animal

less at $10 \mathrm{Deg}$.) of Margarine Oils, Shortenings, and Other

Meth. of Analysis of Monoglycerides and Free Glycerol in ation of Methyl Esters of Long Chain Fatty Acids in Common Oxygen-Bomb Meth. of Analysis of Stability in Meth. of Analysis of Stability (AOM) of

8 and Rodent Hairs in Soy Flour (Cereal Chemistry) (1962) emistry) (1973) and Marine Oi/ Meth. for Determination of Meth. of Analysis of Free Std. Meth. of Testin

esters of Long Chain Fatty Acids in Common Fats, Oils, and Safety Std. for Ground of Epitaxial Layers of Silicon by Measurement of Stacking Std. for Safety for Groun otection of Electrical Equipment (Single Phase and Related meth. of Analysis for Dirt, Sand, Insect Fragments, Hairs,

(1962)

lubs, and Exhibition Charges) (1974)

so Includes Terms and Defini/ rmal Environment on Production, Heat and Moisture Loss and Std. Spec, for Limestone for Anima

Meth. of Analysis of Nitrophenide in Poultry Mashes Std. for Electrical

Meth. of Analysis of Crude Fat in Wheat and Soy Flour, al C/ Bidwell-Sterling Meth. of Analysis of Moisture in th. for Sampling of Flour, Semolina, and Similar Products: al Nitrogen $)(\mathrm{C} /$

Meth. of Calculation of Crude Protein in Meth. for Determination of Phenothiazine in Meth. of Analysis of Crude Fiber in Gravimetric Meth. of Analysis of Phosphorus in Volumetric Meth. of Analysis of Phosphorus in Meth. of Analysis of Added Salt in Improved Kjeldahl Meth. of Analysis of Crude Protein in Meth. for Preparation of Sample: Meth. for Sampling of Solid Meth. of Analysis of Sucrose (Sugar) in th. of Analysis of Salt Calculated from Total Chlorides in rimetric Acetate Ashing Meth. of Analysis of Phosphorus in I Meth. for Determining Crude $\mathrm{Fat}\left(\mathrm{Ccl}_{4}\right.$ Products Such as Meth. for Determination of Sulfaquinoxaline in Poultry Meth. for Determintion of Arsanilic Acid in Meth. for Determination of Nitrosal in Poultry Meth. of Analysis of Crude Fat in Grain and Stock Meth. of Analysis of Calcium in Mineral

Meth. of A nalysis of Iodine in Mineral Mixed Meth. of Analysis of Lactose (Milk Sugar) in Mixed y sis of Crude Fat in Wheat and Soy Flour, Feeds and Cooked is of Vitamin A and Carotene in Enriched Cereals and Mixed residual Urease Activity in Soybean Meals, Soy Flour, Mill e Determination of Both Nitrophenide and Arsanilic Acid in Basic Meth. of Analysis for Ash in Flour, eth. for Determining Color (Spectrophotometric) Content in well-Sterling Meth. of Analysis of Moisture in Feeds and mpling of Flour, Semolina, and Similar Products: Feeds and

n) (C) Meth. of Calculation of Crude Protein in Feeds and Rapid (2 Hr., 600 Deg.) Meth. of Analysis for Ash in

Meth. for Determination of Phenothiazine in Feeds and Meth. of Analysis of Crude Fiber in Feeds and Meth. of Analysis of Calcium in Gravimetric Meth. of Analysis of Phosphorus in Feeds and

Volumetric Meth, of Analysis of Phosphorus in Feeds and Meth. of Analysis of Added Salt in Feeds and Meth. for Preparation of Sample: Feeds and Meth. for Sampling of Solid Feeds and Meth of Analysis of Sucrose (Sugar) in Feeds and d Kjeldahl Meth. of Analysis of Crude Protein in Feeds and lysis of Salt Calculated from Total Chlorides in Feeds and stage Air Oven Meth. of Analysis of Moisture in Grains and cetate Ashing Meth. of Analysis of Phosphorus in Feeds and - Analytical Meth. for Determining Bulk Density Content in d. Analytical Meth. for Determining Crude Fiber Content in

Std. Analytical Meth. for Determining Solubles Content in Std. Analytical Meth. for Determining Starch Content in

for Determining Crude Fat ( $\mathrm{CCl}_{4}$ Extractables) Content in Std. Analytical Meth. for Determining $\mathrm{pH}$ Content in Std. Analytical Meth. for Determining Ash Content in cal Meth. for Determining Acidity (Extractable) Content in Determining Moisture (Azeotropic Distillation) Content in eth. for Determining Moisture (Oven) Content in Commercial Analytical Meth. for Determining Xanthophylls Content in cal Meth. for Determining Lactic Acid and Salts Content in alytical Meth. for Determining Protein Nitrogen Content in
Fats (Cereal Chemistry) (1967)

Fats (Cereal Chemistry) (1967)

Index of Refracti AACCH $58-20$

Fats (1965)

Fats (1968)

Determining Free Fatty Acids Content in

ats, Oils and Shortenings (Cereal Chemistry) (1967)

Fats, Oils, and Fatty Acids (Cereal Chemistry) (1973)

Fats, Oils, and Shortenings (Cereal Chemistry) (1962)

Fats, Oils, and Shortenings (Cereal Chemistry) (1967)

Fatting Digestion Meth. of Analysis for Insect Fragment

Fatty Acid Composition by Gas Chromatography (Cereal $\mathrm{Ch}$

Fatty Acids Content in Corn Oil and to Other Vegetable

Fatty Acids in Common Fats, Oils, and Fatty Acids (Cere

Fatty Acids in Fats (Cereal Chemistry) (1967)

Fatty Acids Used in Protective Coatings (1972)

Fatty Acids (Cereal Chemistry) (1973)

Fault Circuit Interrupters (1972)

Fault Dimension (1973)

D. Meth. of Test for Thickness

Fault Sensing and Relaying Equipment (1972)

Faults) (1973)

Feathers, Wood Fibers, Splinters, etc, in Starch (Cere

Fecal Streptococci in Food Products (Cereal Chemistry)

Feed and Food Labelling. Ingredients and Containers (Al

Feed and Water Requirements of Farm Livestock (Poultry,

Feed Use (1972)

(Feed) for Prevention of Cecal and Intestinal Coccidiosi

Feeder and Plug in Busways and Accessories (1972)

Feeds and Cooked Feeds (Cereal Chemistry) (1962)

Feeds and Feedstuffs by Distillation with Toluene (Cere

Feeds and Feedstuffs in Sacks (Cereal Chemistry) (1962)

Feeds and Feedstuffs (Calculated from Percentage of Tot

Feeds and Feedstuffs (Cereal Chemistry) (1962)

Feeds and Feedstuffs (Cereal Chemistry) (1962)

Feeds and Feedstuffs (Cereal Chemistry) (1962)

Feeds and Feedstuffs (Cereal Chemistry) (1962)

Feeds and Feedstuffs (Cereal Chemistry) (1962)

Feeds and Feedstuffs (Cereal Chemistry) (1962)

Feeds and Feedstuffs (Cereal Chemistry) (1962)

Feeds and Feedstuffs (Cereal Chemistry) (1962)

Feeds and Feedstuffs (Cereal Chemistry) (1962)

Feeds and Feedstuffs (Cereal Chemistry) (1962)

Feeds and Feedstuffs (Cereal Chemistry) (1962)

Feeds and Meals (1955) /Ermand Finished Std. Analytica

Feeds and Premixes for Control of Cecal and Intestinal

Feeds (Cereal Chemistry) (1962)

Feeds (Cereal Chemistry) (1962)

Feeds (Cereal Chemistry) (1962)

Feeds (Cereal Chemistry) (1962)

Feeds (Cereal Chemistry) (1962)

Feeds (Cereal Chemistry) (1962)

Feeds (Cereal Chemistry) (1962)

Feeds (Cereal Chemistry) (1962)

Feeds (Cereal Chemistry) (1962)

Feeds (Cereal Chemistry) (1962)

Feeds. Feedstuffs and Bread (Cereal Cheminth. of

Feedstuffs and All Other Natural Dry Products Derived F

Feedstuffs by Distillation with Toluene (Cereal Chemist

Feedstuffs in Sacks (Cereal Chemistry) (1962)

Feedstuffs (Calculated from Percentage of Total Nitroge

Feedstuffs (Cereal Chemistry) (1962)

Feedstuffs (Cereal Chemistry) (1962)

Feedstuffs (Cereal Chemistry) (1962)

Feedstuffs (Cereal Chemistry) (1962)

Feedstuffs (Cereal Chemistry) (1962)

Feedstuffs (Cereal Chemistry) (1962)

Feedstuffs (Cereal Chemistry) (1962)

Feedstuffs (Cereal Chemistry) (1962)

Feedstuffs (Cereal Chemistry) (1962)

Feedstuffs (Cereal Chemistry) (1962)

Feedstuffs (Cereal Chemistry) (1962)

Feedstuffs (Cereal Chemistry) (1962)

Feedstuffs (Cereal Chemistry) (1962)

Feedstuffs (Cereal Chemistry) (1962)

Feedstuffs (Corn) and Other Wet Milled Solid Products (
Feedstuffs (Corn) Containing Vegetable Material, Corn a

Feedstuffs (Corn) (1954)

Feedstuffs (Corn) (1954)

Feedstuffs (Corn) (1954)

Feedstuffs (Corn) (1955)

Feedstuffs (Corn) (1955)

Feedstuffs (Corn) (1955)

Feedstuffs (Corn) (1956)

Feedstuffs (Corn) (1957)

Feedstuffs (Corn) (1964)

Feedstuffs (Corn) (1964)

Feedstuffs (Corn), Syrups, Sugars, Starches and Other P
AR

$\mathrm{AACCH} 31.50$ AACCH 58.45 AACCH 58.17 $\mathrm{AACCH} 58.55$ $\mathrm{AACCH} 58.5$ AACCH 28.70 AACCH 58.18 CR H.22 AACCH 58.17 AACCH 58-16 ASTM D1467 58-17 UL 943 AATM F143 UL 1053 FMS 14-15 AACCH 28.75 AACCH 42.35 PSA 11

AAFCO *I

ASAE D249.2

ASTM C706

NEMA BU1

AACCH 30.25

AACCH 44.50

AACCH $64-60$

AACCH $46-18$

AACCH $\quad 18-30$

$\mathrm{AACCH} \quad 32-15$

AACCH $40-57$

AACCH 40.58

AACCH $40-60$

$\mathrm{AACCH} \quad 46-10$

AACCH $62-20$

AACCH $64-50$

AACCH $80-50$

Me AACCH 40-61

Colo AACCH 40-55

$\begin{array}{ll}\text { CR } & \text { B. } 18\end{array}$

$\mathrm{AACCH} \quad 18-20$

AACCH $18-27$

AACCH $30-20$

AACCH 40.26

AACCH $40-35$

AACCH $80-30$

Meth. of Anal AACCH $30-25$ Meth. of Analys AACCH 86.05

$\begin{array}{ll}\text { AACCH } & 86-05 \\ \text { AACCH } & 22.90\end{array}$

AACCH $18-26$

AACCH $08-01$ CR G.6

AACCH 44.50

$\mathrm{AACCH} 46.18$

A.ACCH $08-03$

AACCH $\quad 18.30$

$\mathrm{AACCH} \quad 32.15$

AACCH $40-21$

$\mathrm{AACCH} 40-5$ ?

AACCH $40-58$

AACCH $\quad 40.60$

$\mathrm{AACCH} \quad 62-20$

$\mathrm{AACCH} \quad 64-50$

AACCH 80.50

Improve AACCH 46.10

Meth. of Ana AACCH 40.61

Modified Two AACCH 44.18

colorimetric a AACCH 40.55

G.8

CR G.12

G. 26

$\mathrm{CR}$

G-28

G-10

G-20

G. 4

G.2

G.14

G.16

G. 40

G.13

G.22
AACCH $18-35$

/ for Sa AACCH 64.60 
Basic Meth. of Analysis for Ash in Flour, Feeds, Feedstuffs, and Bread (Cereal Chemistry) (1962) eth. of Analysis of Moisture in Flour and Semolina; Bread; determining Protein Nitrogen Content in Steepwater, (Corn) Std. Spec. for Welded Austenitic Stainless Steel d Protection Rec. for Low Water Fuel Cutoffs and Automatic loss Prevention Data on Elastomer Roof Coverings (Multiply cial Purpose Roofs (Wood Shakes and Shingles with Asbestos Std. Meth. of Test. oal-Tar Saturated Organic Felt, Asphalt-Saturated Asbestos d Organic Felt, Coal Tar Pitch, Coal-Tar Saturated Organic Coverings: Hot Mopping Asphalt, Asphalt-Saturated Organic tances for Use in Water/ itted Wool Textiles (1972) Std. Meth. of Sampling and Test. Test Meth. for Relaxation and hot Mopped Asphait, Asphalt and Coal Tar Saturated Organic mbranes; Aggregates, Saturated Felts, Saturated and Coated nd Plast/ Spec. for Roof Membranes; Aggregates, Saturated Std. for Coupling-Tubes, Flared Fitting, Rec. Spec. for (Nonelectric) Farm Std. Spec. for Aluminum Coated Steel Chain Link Std. Spec. for Zinc Coated Steel Chain Link Spec. for Galvanized Steel Chain Link Std. for Preservative Treatment (For d) Iron or Steel Farm Field and Railroad Right of Way Wire ANSI K65.201 Std. Analytical Meth. for Determining
hydrolyzates (1957/
Meth. of Analysis of Pelshenke Test (Wheat Meal Std. Spec. for al Chemistry) (1962) (1973) ANSI B125.24 (1973)

Std Spec, for Centrifugally Cast ) ANSI B/ Std. Spec. for General Requirements for Carbon, , and Heat Exchanger Tubes (1973/ Std. Spec. for Seamless ture Service (1972) ANSI G52.2 Std. Spec. for 73) ANSI B125.14 Std. Spec. for Seamless and Welded uitable for Low Temperature Service (1974/ Std. Spec. for Tent. Meth. for Drop Weight Tear Tests of Std. Meth. of Sampling and Test. Std. Meth. for Chemical Analysis of Std. Meth. for Chemical Analysis of Std. Meth. for Chemical Analysis of

Tent. Meth. for Chemical Analysis of Std. Spec. for Std. Spec. for

Std. Meth. for Chemical Anaylsis of

Std. Meth. for Chemical Analysis of

Std. Meth. for Chemical Analysis of

Std. Meth. for Chemical Analysis of

Std. Meth. for Chemical Analysis of Silicomanganese and Std. Spec. for Std. Spec. for osited Coatings of Lead and Lead-Tin Alloys on Steel and reads $(1971)$

st for Absorbed Gamma and Electron Radiation Dose with the Std. for Face-To-Face and End-To-End Dimensions of Std. Meth. for Chemical Analysis of Std. Spec. for

pec. for Copper and Its Alloy Seamless Condenser Tubes and iner or Filter for Flammable Fluids and Anhydrous Ammonia ia (1970) Std. for Capacity Designation for Std. for Storage and Handling of Nitrogen and Std. Test Procedure for Dry Rec. for Safe Use of Mixed

Std Meth of Evaluating the Properties of Wood Base ht Construction Buildings (1970)/ Std. Spec. for Mineral rmination of the Thermal Resistance of Low Density Mineral
Test Meth. for

Measurement of Airborne Asbestus Meth. of Test for Tensile Properties of Oriented res and Cables (1972) ANSI C 33.37 Std. for Safety for aining Veget/ Std. Analytical Meth. for Determining Crude Material, C/ Std. Analytical Meth. for Determining Crude Std. Meth. of Test for leach (Ins/ Std. Performance Requirements for Woven Glass Meth. of Test for Effect of Handwashing on Woven Glass Meth. of Test for Light Transmittance of Woven Glass Std. Spec. for Homogeneous Bituminized Std. Spec, for Laminated Wall Bituminized

F-No B/ Std. Performance Requirements for Woven Glass ter Chargers (1971) Std. for Interrelationship of Quartz . of Test for Annealing Point and Strain Point of Glass by Std. Meth. of Sampling Asbestos g Cement (1964) ANSI Z98.33 Std. Spec. for Mineral (1973) Std. Spec. for Mineral
Test Meth. for Test Meth. for 973)
Feedstuffs, Ground Grains and Their Adjuncts (Cereal $\mathrm{Ch}$ Feedstuffs, Syrups, Sugars, Starches and Other Protein

Feedwater Heater Tubes (1973)

Feedwater Regulators for Boilers (1974)

Felt and Asphalt Preferably with Gravel or Slag

Felt or Gypsum Board Underlayment) (1973)

Felt (1972)

Felt, Asphalt-Coated Glass Fiber Mat (1973)

Felt, Asphalt-Saturated Asbestos Felt, Asphalt-Coated G

Felted and Woven Fabrics Saturated with Bituminous Subs

Felting Dimensional Changes (Shrinkage) of Woven and $\mathrm{Kn}$

Felts, Coal Tar Pitch, and Asphalt Coated Glass Fiber M

Felts, Prepared Roofings, and Plastic Roll Materials (1

Felts, Saturated and Coated Felts, Prepared Roofings, a

Female Thread (1973)

Fence Construction (1972)

Fence Fabric (1971) ANSI G53.21

Fence Fabric (1971A) ANSI G53.20

Fence Fabric (1973)

Fence Posts) by Pressure Processes (1972)

Fencing (1973) ANSL C8.9

Fep Fluorocarbon Molding and Extrusion Materials (1974)

Fermentables Content of Corn Syrup, Sugars, and Starch

Fermentation Time Test) (Cereal Chemistry) (1962)

Ferric Sulfate (1964)

Ferricyanide-Maltose-Sucrose Conversion Table (Cere Ferritic Alloy Steel Pipe for High Temperature Service Ferritic Alloy Steel Pipe for High Temperature Service Ferritic Alloy, and Austenitic Alloy Steel Tubes (1971A Ferritic and Austenitic Alloy Steel Boiler, Superheater Ferritic and Austenitic Steel Castings for High Tempera Ferritic Austenitic Alloy Steel Tubes (1972)

Ferritic Stainless Steel Tubing for General Service (19 Ferritic Steel Castings for Pressure Containing Parts S Ferritic Steels (1971) ANSI Z260.3

Ferroalloys for Determination of Size (1970) ANSI G85.I

Ferroalloys (1973)

Ferroboron (1974)

Ferrochrome-Silicon (1974)

Ferrochromium and Chromium Metal (1971T)

Ferrochromium (1973) ANSI G68.1

Ferrocolumbium (1973) ANSI G79.1

Ferrocolumbium (1974)

Ferromanganese and Spiegeleisen (1974)

Ferromolybdenum (1974)

Ferrosilicon and Silicon Metal (1974)

Ferrosilicon Manganese (1974)

Ferrotitanium (1973) ANSI G74.1

Ferrotungsten (1973) ANSI G71.1

Ferrous Alloys (1970) ANSI G53.8

Ferrous Pipe Plugs, Bushings, and Locknuts with Pipe Th

Ferrous Sulfate Cupric Sulfate Dosimeter (1971) ANSI K6

Ferrous Valves (1973)

Ferrovanadium and Vanadium Alloying Additives (1974)

Ferrovanadium (1973) ANSI G69.1

Ferrule Stock (1974) ANSI H23.14

(Fertilizer Grade) in Residential and Commerical Fuel Bu

Fertilizer Hoppers and Containers (1972)

Fertilizer Solutions Containing More Than 2\% Free Ammon

Fertilizer Spreader (1972)

Fertilizers Containing Ammonium Nitrate (1972)

Fiber and Particle Panel Materials (1972) ANSI 08.1

Fiber Blanket Thermal Insulation for Wood Frame and Lig

Fiber Blanket Type Building lnsulation (1970) A
Fiber Bundles in Baled Flash Dried Pulp (1973)

Fiber by the Membrane Filter Meth. (Textile) (197I)

Fiber Composites (1972T)

Fiber Conduit for Use as Raceway for Installation of Wi

Fiber Content in Corn and Other Grains, Foodstuffs Cont

Fiber Content in Feedstuffs (Corn) Containing Vegetable

Fiber Content of Reinforced Resin Composites (1973)

Fiber Curtain Fabrics Handwashable at 105 Deg. F-No B

Fiber Decorative Fabrics (Textile) (1973)

Fiber Decorative Fabrics (Textile) (1973)

Fiber Drain and Sewer Pipe (1973) ANSI A176.1

Fiber Drain and Sewer Pipe (1973) ANSI A176.2

Fiber Drapery (Curtain) Fabrics Handwashable at $105 \mathrm{Deg}$

Fiber Electrometer Type Dosimeters and Companion Dosime

Fiber Elongation (1971)

Fiber for Test. (1970) ANSI K91.2

Fiber Hydraulic Setting Thermal Insulating and Finishin

Fiber Identification (Various Stains) (Pulp and Paper)

Fiber Identification (Wilson Stain) (Pulp and Paper) (1

Fiber in Bagasse (Pulp and Paper) (1973)
AACCH $08-01$

$\mathrm{AACCH} \quad 44.40$

CR J-56

ASTM A688

FMS I2-37

FMS $\quad 1.47$

ASTM D461

UL

UL

ASTM

AATCC

NRCA

NRCA

NSA

ASAE

ASTM

ASTM

CLFMl

AWPA

ASTM

ASTM

CR

AWWA B406

AACCH 22-18

ASTM A335

ASTM A426

ASTM A450

ASTM A213

ASTM

ASTM A669

ASTM A268

ASTM A352

ASTM E436

ASTM A610

ASTM E3!

ASTM E371

ASTM E364

ASTM E363

ASTM Al0l

ASTM A550

ASTM E367

ASTM E36]

ASTM E368

ASTM E360

ASTM E362

ASTM A324

ASTM Al44

ASTM B200

ANS1 B16.14

ASTM D2954

ANSI B16.10

ASTM E365

ASTM A102

Std. S ASTM B111

UL

ASAE

FI

ASAE

FMS

ASTM

ASTM

ASTM

TAPP

ATI

ASTM

UL

CR

CR

ASTM

ANSI

ANSI

ANSI

ASTM

ASTM

ANSl

A NSI

ASTM

ASTM

ASTM

TAPPI

TAPPI

331

S281.1

Pl 45

S 341

LPD 7-9

Dl037

C665

C653

UM.239

$\checkmark 5$

D3039

543

A-8

G-12

L24.1.3

L 4 T

L24T4

DI861

D1862

L24.1.9

$\mathrm{N} 42.6$

C336

D2590

C449

UM-15 
oybeans, and Peanuts (Cereal /

Meth. of Analysis of Crude Meth. of Analysis of Crude Std. for Cellulosic Std. Spec. for Mineral elt, Asphali-Saturated Asbestos Felt, Asphalt. Coated Glass ed Organic Felts, Coal Tar Pitch, and Asphalt Coated Glass al Drainage (1/ Std. Spec. for Laminated Wall Bituminized (1973)

a 176.4 a 17/ ainage / Sid. Spec. for Laminated Wall Bituminized 972)

Std. Spec for Homogeneous Perforated Bituminized Std. Spec. for Laminated Wall Perforated Bituminized Std. Spec. for Homogeneous Perforated Bituminous of Test for Apparent Horizontal Shear Strength of Paralle Std. Spec. for Mineral

hetic Fibers, Wool) (1974)

layup (1973)

(483) Tensile, $36,000,000$ (248) Modul/

(483) Tensile, 36,000,000 (248) Modul/ Std. Spec, for Semidense Mineral
Rec. for Baled Textile Std. Spec. for Semidense Mineral
Rec. for Baled Textile Graphite

Spec. for Graphite Spec. for Graphite Lamina/ Std. Performance Requirements for Woven Man Made Std. Meth. of Test. Vulcanized Std. Spec, for Cellulosic of Test for Bauer-McNett Wet Classification of Asbestos commercial Weight of a Shipment of Yarn or Man Made Staple Std. Spec. for lnsulating Board (Cellulosic Std. Meth. of Test. Insulating Board (Cellulosic h. of Test for Differential Dyeing Behavior of Cotton (Raw

Meth. of Test for Adhesion of Pressure Sensitive Tape to si $\mathrm{MH} 12 / \quad$ Std. Meth. of Determining lnterior Dimensions of Std. for Std. Spec. for Asbestos Cement g Board (1973) Uniform Building Code Std. for id Adhesives for Automatic Machine Sealing of Top Flaps of Std. for

for Inefficient of Static Friction of Corrugated and Solic for Coefficient of Static Friction of Corrugated and Solid for Coefficient of Static Friction of Corrugated and Solid est Meth. for Ply Separation (Wet) of Solid and Corrugated Std. for Medium Densit

nts, Screws, Bolts, Connectors, Nails, Staples, Adhesives, st Meth. for Determining Stiffness (Modulus of Bending) of lor Matched to Master Specimen for Fabrics, Vinyls, Coated Guide Spec, for Specirying: Reinforced Guide Spec, for Flagpoles, Metal, Wood and

Meth. of Test for Length and Length Uniformity of Cotton

Std. Meth. of Test for Diameter of Wool and Other Animal

Test for Estimating Maturity and Linear Density of Cotton Strength, 70,000,000 (483) Tensile M/ Ppec. for Graphite
Std. Rec. Pract. for Sampling Cotton

t. Meth. of Test for Breaking Tenacity of Man Made Textile Std. Meth. for Identification of Test Meth. for Identification of

of Test for Tensile Properties of Single Man Made Textile meth. of Test for Length and Length Distribution of Cotton Std. Meth. of Test for Linear Density of Cotton est for Breaking Strength and Elongation of Cotton Textile

for Structural Insulating Formboard Made from Cellulosic

Std. Tables of Classification of Man Made and Natural Meth. for Screen Analysis of Asbestos

Std. Meth. of Test for Air Permeability of Asbestos industrial Filament Yarns, Made from Man Made Organic Base Std. Meth. of Test for Shrinkage of Textile Std. Meth of Test for Elastic Properties of

eth. of Test for Cross Sectional Characteristics of Cotton

Std. Meth. of Test. Twine Made from Bast and Lea ments for Woven Drapery (Curtain) Fabrics (Excluding Glass en Window Curtain Fabrics (Sash Casement) (Excluding Glass s (Cotton, Sisal, Henequen, Ixtle, Jutes, Hemp, Tow, Cocoa is for Dirt, Sand, Insect Fragments, Hairs, Feathers, Wood ymol Blue Test Paper for Analysis of Urea in Cloth or Sack ber Storage (Cotton, Jute, Hemp and Sisal, Flax, Synthetic General Rules and Formulae for

Rec. Std. for Loose Metal End Dimensions (6 Ounce

Std. Test. Procedure for End Supported Beam Strength

Uniform Fire Code: Storage and Handling of Combustible

Test for Length and Length Uniformity of Cotton Fibers by

Std. Meth. of Test for Identification of Metallic and d Air Conditioning Systems (1972)

Pressure Sensitive Tape Performance Stds. for Pressure Sensitive Tape Application Stds, for

for the Test Meth. for Wicking of Automotive Fabrics and Std. Spec. for Spray Applied Std. Rec. Pract. for Application of Spray Applied ysis (Cereal Chemistry) (Bolting Cloth, Filter Paper, Wide

std. Spec. for Zinc Coated (Galvanized) lron or Steel Farm untings (Farm Machinery) (1973)
Fiber in Feeds and Feedstuffs (Cereal Chemistry) (1962)

Fiber in Soy Flour for Cake and Meal from Cottonseed, S

Fiber Insulating Board (1973) ANSI A 149.1

Fiber Loose Fill Thermal Insulation (1973)

Fiber Mat (1973)

Fiber Mat (1973)

Pitch, Coal-Tar Saturated Organic F

Fiber Perphalt, Asphalt and Coal T

Fiber Perforated Pipe for Airport and Roadwork Drainage

Fiber Pipe for Septic Tank Disposal Fields (1973) ANSI

Fiber Pipe for Septic Tank Disposal Fields (1973) ANSI

Fiber Pipe Systems for Highway, Airport, and Similar Dr

Fiber Reinforced Plastics by Short Beam Meth. (1972)

Fiber Reinforced Polyester Structural Plastic Panels (1

Fiber Roof Insulation Board (1972)

Fiber Siding (1972)

Fiber Storage (Cotton, Jute, Hemp and Sisal, Flax, Synt

Fiber Tape and Sheet, Epoxy Resin Impregnated for Hand

Fiber Tape and Sheet, Epoxy Resin Impregnated, G70,000

Fiber Tape and Sheet, Epoxy Resin Impregnated, G70,000

Fiber Tent, Awning, and Canopy Fabrics, Vinyl Coated or

Fiber Used for Electrical Insulation (1971) ANSI C59.78

Fiber (Wood Base) Loose Fill Thermal Insulation (1973)

Fiber (1970) ANSl K91.l

Fiber (1971)

Fiber), Structural and Decorative (1972)

Std. Meth. of Test for

Fiber), Structural and Decorative (1972)

Fiber, Yarn and Textile Fabric) (1972) ANS1 L14.152

Fiberboard at $90 \mathrm{Deg}$. Angle and Constant Stress (19-2)

Fiberboard Boxes (Box Gage Meth.) (Packaging) (1968) an

Fiberboard Crease Bending Test (1972)

Fiberboard Insulating Panels (1972)

Fiberboard Nail Base Sheathing and Structural Insulatin

Fiberboard Shipping Cases (1970)

Fiberboard Test Procedure (1972)

Fiberboard (Horizontal Plane Meth.) (1972)

Fiberboard (Horizontal Plane Meth.) (1973)

Fiberboard (lnclined Plane Meth.) (1973)

Fiberboard (1969)

Fiberboard (1973)

Fiberboard, Particleboard) (1973)

Fiberboards (1972)

Fiberboards, and Other Automotive

Fiberglass Polyester Tanks (1973)

Fiberglass (1972)

Fibers by Fibrograph Measurement (Textile) (1972) ANSI

Fibers by Microprojection (1972) ANSI L 14.143

Fibers by the Causticaire Method (1971)

Fibers for Structural Composites (GF 220 (1517) Tensile

Fibers for Test. (1972)

Fibers in Loop or Knot Configurations (1973)

Fibers in Textiles (1972)

Fibers in Textiles (1972) ANSI L14.131

Fibers Taken from Yarns and Tows (1972) ANS1 L14.285

Fibers (Array Meth.) (1972) ANSI L14.91

Fibers (Array Sample) (1973) ANS1 L14.136

Fibers (Flat Bundle Meth.) (1972) ANS1 L 14.96

Fibers (1966)

Fibers (1971) ANSI L14.192

Fibers (1971T)

Fibers (1972)

Fibers (1972)

Fibers (1972) ANSl L14.142

/Th. of Testing Tire Cords, Fabrics and

Fibers (1972) ANSI L 14.250

Fibers (1972) ANS1 L 14.95

Fibers (1973)

Fibers) (Institutional Textile) (1973)

Fibers) (Institutional Textiles) (1973)

Fibers, Oakum, Rags, Waste Cloth, Wastepaper, Kapok, Ha

Fibers, Splinters, etc. in Starch (Cereal Chemistry) (1

Fibers, Whole or Ground Cereal Grains, Whole or Chopped

Fibers, Wool) (1974)

Fibre Cans (1973)

Fibre Citrus Cans 202 (52 Mm) Diameter) (1973)

Fibre Tubes and Cores (1972)

Fibres (Cotton, Sisal, Henequen, lxtle, Jutes, Hemp, to

Fibrograph Measurement (Textile) (1972) ANSI L14.98

Fibrous Contaminants in Aerospace Fluids (1970E)

Fibrous Glass Duct Construction Std. for Ventilating an

Fibrous Glass Duct (1973)

Fibrous Glass Duct (1973)

Fibrous Materials (1972)

Fibrous Thermal Insulation for Elevated Temperature (19

Fibrous Thermal Insulation (1973)

Field and Compound Microscope, Petri Dish, Sieve, Separ

Field and Railroad Right of Way Wire Fencing (1973) Ans

Field and Row Crop Cultivator Shafts and Ground Tool Mo
A ACCH $32-15$

AACCH 32.17

USC PS57

ASTM C764

UL

ICBO UBCS32.

ASTM D2418

ASTM D2312

ASTM D2313

ASTM D2818

ASTM D2344

USC PS3

ASTM C726

ASTM C725

FMS

SAE

SAE

SAE

ANSI

ASTM

ASTM

8.7

AMS3894A

AM $\$ 3894 / 8$

AM $\$ 3894 / 9$

L24.1.10

D619

C739
D2589

D2494

ASTM C208

ASTM C209

/T ASTM D1464

ASTM D2860

ASTM D2658

SAE J119A

ASTM C55I

ICBO UBC 25.24

D 1874

SAE J315B

Meth. TAPPI T816SU

1. of Test ASTM

D3247

D3248

$\begin{array}{ll}\text { of Test ASTM } & \text { D3248 } \\ \text { Std. T ASTM } & \text { D1028 }\end{array}$

NPA 4.73

UBC $* 3.25$

J949A

$\begin{array}{ll}\text { SAE } & \text { J361A } \\ \text { CSI } & 15177 .\end{array}$

$\begin{array}{ll}\text { SAE } & \text { J361A } \\ \text { C.SI } & 15177.1\end{array}$

CS1 $\quad 10350$

ASTM D1447

ASTM D2130

D2480

SAE

ASTM

AM $\$ 3892 / 5$

$\begin{array}{lll}\text { Ten ASTM } & \text { D } 32174\end{array}$

ASTM D276

AATCC

ASTM D2101

Std. ASTM D 1440

ASTM D 1769

/H. of T ASTM D 1445

Std. Spec ASTM C.532

ASTM D2368

ASTM D2947

ASTM D2752

$\mathrm{D} 2752$
$\mathrm{D} 885$

D2102

ASTM D1774

Std. M ASTM D1444

ASTM D1233

L24.1.5

L24.1.4

ICBO UFC*2ART7

AACCH 28.75

AACCH 28.87

FMS

CCTI

CCT

8.7

*1

ICBO UFC*2ART7

/F ASTM D1447

ASTM F314

SMACN *6

SMACN AFTS. 100

SMACN AFTS-101

Std SAE

J $913 A$

ASTM C762

AACCH $28-90$

ASTM All6

ASAE S225.1 
Spec. for Oil Field Chain and Sprockets (I970)

ng Code Std. for Evaluation of Compression Test Results in Rec. Pract. for Evaluation of Compression Test Results of st for Compressive and Flexural Strength of Concrete Under Std. for Welded Aluminum Alloy Performance Spec, for Adhesives for Spec. for Indirect Type Oil Std. Meth. of

Pract. on Application, Care, and Use of Wire Rope for Oil act. for Grounded $830 \mathrm{~V}, 3$ Phase Electrical System for Oil eamless Copper Tube for Air Conditioning and Refrigeration Uniform Building Code Std. for Airborne Sound lnsulation Medium Speed Stationary Diesel and Gas Engine Factory and Uniform Building Code Std. for Std. Meth. of Evaluating Wood Preservatives by Test for Combustible Gases in Electrical Apparatus in the perforated Bituminized Fiber Pipe for Septic Tank Disposal perforated Bituminized Fiber Pipe for Septic Tank Disposal

1973) etic Shield Efficiency in Attenuating Alternating Magnetic Std. for Automated Maching for Composite Std. for Packaging of Std. Meth. of Testing Tire Cords, Fabrics and Industrial Std. Meth. of Test. Tire Cords, Tire Cord Fabrics, std. for Safety for Tests for Fire Resistance of Vault and Std. for Flexible Non Pressure Water Std. Spec. for Perlite Loose Std. Spec. for Safe

for Evaluating the Compatibility of Vehicle Fuel Tanks and Std. Spec. for Cellulosic Fiber (Wood Base) Loose Std. Spec. for Mineral Fiber Loose al Apparatus Sales and Service Industry (Rotating, Liquid c Circuit Reclosers, Automatic Line Sectionalizers and Oil Std. Test. Procedure for Std. Test. Procedure for (1972) Std. Spec. for Reinforced and Test Meth. for Top Side of Std. Spec. for

t Mastic for Use in Waterproofing (Asphalt Cement, Mineral

tative (Including Optical Microscopic) Analysis of Mineral sives (1970) Std. Spec. for Mineral Std. Spec, for Preformed Expansion Joint id Requirement Maintenance Instructions, and Container and Spec, for Brazing Std Spec for Brazing Std. Meth. of Test for Sieve Analysis of Mineral lk and Its Fluid Products (Packaging / Sanitary Stds. for (Nonextruding / Std. Spec. for Preformed Expansion Joint spec. for Preformed Cellular Plastic Pressure Relief Joint

Std. Rec. Pract. for Safe Std. for Self Locking, Flat Std. for Screw, Machine, Flat Std. for Machine Screw, Flat Std. for Machine Screw, Flat Std. for Machine Screw, Flat , Self-Locking and Nonlocki/ alloy, 6Al-4V, Self-Locking/ teel, Self-Locking and Nonl/ 1974)

ter Reverse Impacting (C)

Test Meth. for Determination of Test Meth. for Determination of Test Meth. for Packaging h. of Test for Pneumatic Ball Impact Resistance of Plastic Std for Criteria for

Std for Back Window Location for Roll ications (1973) Rec. Pract. for Determining Solderability of Thick for Photography) (1973)

ulose Ester Base (1973)

ester Base (1973)

vices, or Both (Photography) (19/ Std. for Paper, Paper/Film, Std. Photographic Spec. for Photographic Std. Spec. for Photographic Std. for Dimensions for

or Dimensions for Unperforated and Perforated Photographic cteristics of Electrical Insulating Varnishes Applied Over Std. Meth. for Test.

meth. of Test for Measuring the Wear Life and Bonded Solid fficient of Friction and Wear Characteristics of Dry Solid separate) for Use in Photographic Contact Printing of Roll ontainers for Packaging and Storage of Aerial Photographic std. Practice for Storage of Processed Safety Photographic st for Effect of Heat and Air on Asphaltic Materials (Thin Std. Dimensions for $35 \mathrm{~mm}$ Motion Picture Std. Dimensions for $16 \mathrm{~mm}$ Motion Picture

Printed Area in Super 8 Printing on 16/8Mm Motion Picture 3) ANSI C59.96

Std. Spec, for Polyester Std. Dimensions for Cores for Photographic Std. for Dimensions of Aerial cellulose) (1973)
Field Concrete Cylinders (1973)

Field Concrete (1965) ANSI A 146.1

Field Conditions (1974)

Field Erected Storage Tanks (1973)

Field Gluing Plywood to Wood Framing (1971)

Field Heaters Used in Producing Oil and Gas (1972)

Field Sampling of Ion Exchange Materials (1972)

Field Service (1972)

Field Service (1973)

Ficld Service (1974) ANSI H23.5

Field Test of Partitions

Field Testing (1972)

Field Tests for Grout and Mortar (1973)

Field Tests with Stakes (1974)

Field (1973)

Fields (1973) ANSI A176.4

Fields (1973) ANSI Al76.5

Fields (1974)

Filament Tape Laying Mac

Filament Tape Reel (1972)

Filament Tape Reels (1973)

Filament Yarns, Made from Man Made Organic Base Fibers

Filaments, and Strands Made from Steel (1974)

File Storage Room Doors (1972) ANSI A153.1

Fill Hose for Recreational Vehicles (1971)

Fill Insulation (1973)

Fill or Aerosol Containers (I972)

Fill Pipes with Fuel Dispensing Facilities (Passenger C

Fill Thermal Insulation (1973)

Fill Thermal lnsulation (1973)

Filled and Dry Transformers, Hand Power Tools, Hermatic

Filled Capacitor Switches for Alternating Current Syste

Filled Cast Flat Bottom Drop Composite Motor Oil Cans (

Filled Cast Flat Drop Composite Cans (1974)

Filled Nylon Injection Molding and Extrusion Materials

Filled Paper as Indicated by Silver Coin Test (1973)

Filled Poly (Vinyl Chloride) (PVC) Sewer Pipe (1972)

Filler and Aggregate) (1941) ANSl A 109.17

Filler and Coating of Paper (1973)

Filler Content of Phenol, Resorcinol, and Melamine Adh

Filler for Bituminous Paving Mixtures (1970) ANSI A37.4

Filler for Concrete (Bituminous Type) (1971) ANSl A37.1

Filler ldentification (Construction and lndustrial Equi

Filler Metal Comparison Charts (1971)

Filler Metal (1969) ANSl W3.8

Filler Metals for Electron Devices (1972)

Filler (1972) A ASHO T37, ANSI A37.14

Fillers and Sealers of Single Service Containers for Mi

Fillers for Concrete Paving and Structural Construction Fillers (1973)

Filling of Low Pressure Pressurized Products (1972)

Fillister Head, Full Thread Part Screw (1974)

Fillister Head, Full Thread, Torq Set (1973)

Fillister Head, Full Thread, Tri Wing Recess, A286 Cres

Fillister Head, Full Thread, Tri Wing Recess, Titanium

Fillister Head, Full Threaded, Tri Wing Recess, Alloy S

Film Adhesion by Cross Hatch Tape Test (Coil Coating)

Film Adhesion on All Metals by Cross Hatch Tape Test Af

Film and Construction Identity Tests (Paper) (1973)

Film and Sheeting (1972)

Film Badge Performance (Nuclear Radiation) (1972)

Film Cameras (1973)

Film Conductors (1972T)

Film Dielectric Capacitors for Power Semiconductor Appl

Film Exposure Guide (Also Contains Suggested Techniques

Film for Archival Records, Silver Gelatin Type, on Cell

Film for Archival Records, Silver Gelatin Type, on Poly

Film in Rolls for Phototypesetting or Photocomposing De

Film in Rolls, Including Leaders and Trailers, for Aeri

Film Insulated Magnet Wire (1973)

Film Insulated Magnet Wire (1974)

Film Lubricants in Oscillating Motion (1971) ANSI Z11.3

Film Lubricants in Vacuum and Other Controlled Atmosphe

Film Negatives (1972)

Film on Spools (1972)

Film Other Than Microfilm (1971)

Film Oven Test) (1973)

Film Perforated Super 8, 5R-1667 (1-3.

Film Perforated Super 8, (1-4) (1973)

Film Perforated 1-4 (1971)

Film Pressure Sensitive Electrical Insulating Tape (197

Film Rolls (Plastic, Wood or Metal) (1971)

Film Spools (1972)

Film Still Picture Cameras (1972)

Film Storage and Handling (Photographic and X-Ray Nitro
/Thermal Aging Chara

API

ICBO

$\mathrm{ACI}$

ASTM

ANSI

APA

API

ASTM

Rec. AP

Rec. Pr

ASTM

IC BO

DEMA

ICBO

ASTM

Meth. of ASTM

ASTM

ASTM

ASTM

NSA

NSA

NSA

ASTM

AST

UL

IAPMO

ASTM

ASTM

SAE

ASTM

ASTM

EASA

NEMA

CCTI

CCTI

ASTM

TAPPI

ASTM

or Asphal ASTM

TAPPI

ASTM

ASTM

ASTM

SAE

AWS

AWS

ASTM

ASTM

DFISA

ASTM

Std. ASTM

ASTM

NSA

NSA

NSA

NSA

NSA

NCCA

NCCA

TAPPI

Std. Met ASTM

ANSI

ANSI

ASTM

ElA

ANSI

ANSI

ANSI

ANSI

ANSI

ASTM

ASTM

ASTM

ASTM

Std. Spec for Masks ( ANSI

ANSI

ANSI

ASTM

ANSI

ANSI

ANSI

ASTM

ANSI

ANSI

ANSl

ICBO

7F

BC \$26-11

214

C683

FG.0 1

12K

D2687

1

B280

UBCS $35-3$

*1-22

UBCS24.23

D1758

D3284

D2312

D2313

A698

990 
on Nonferrous Substrate (Coil, Instrumentation/ Spec. fo roducts -Film Thickne/ Best Meth. for Measurment of Dry Std. Meth. for Nondestructive Measurement of

ickness of Paint, Varnish, Lacquer and Related Products Std. for Nomenclature for Motion Picture for Determination of Relative Pencil Hardness of a Paint st for the Determination of Deg. of Cure of a Backed Paint Std. Meth. for Determining the Curl of Photographic Std. for Intraoral Dental Radiographic Safety Std. for Tests for Flammability of Photographic std. Meth. for Determining the Brittleness of Photographic Std. Spec. for Projector Usage of $16 \mathrm{~mm}$ Motion Picture Uniform Fire Code: Cellulose Nitrate Motion Picture rm Building Code Std. for Cellulose Nitrate Motion Picture f Projectable Anamorphic Image Area on $5 \mathrm{~mm}$ Motion Picture std. Dimensions for Industrial Radiographic Sheet and Roll Std. for Dimensions of 126 Size Cartridges, Std. Picture Sizes for Roll, $35 \mathrm{Mm}$

r Location of Super 8 Printed Area on $16 \mathrm{~mm}$ Motion Picture area in Optical Reduction Printing on $35 \mathrm{~mm}$ Motion Picture ical Reduction or Contact Printing on $35 \mathrm{~mm}$ Motion Picture Std. Dimensions for $32 \mathrm{~mm}$ Motion Picture Std. Dimensions for $32 \mathrm{~mm}$ Motion Picture

ing the Dimensional Change Characteristics of Photographic act. for the Density and Contrast Range of Black and White meth. of Evaluating Deg. of Surface Disfigurement of Pain

d Evaluation Meth. for Determining Speed of Color Reversal ous Uses (1973)

Std. Dimensions for Photographic

ing Speed of $16 \mathrm{Mm}, 8 \mathrm{~mm}$ and Super 8 Reversal Color Camera Spec. for Spot Test Solution for Detecting Chromate for Meth. for Determining the Resistance of Photographic Tent. Meth. of Test for Sheet Resistance of Thin Metallic d. Dimensions for Professional Photographic Sheet and Roll

Test Meth. for Hydrogen Ion Concentration of Dry Adhesive f Test for Moisture Vapor Permeability of Organic Coatings Meth. of Test for Algal Resistance of Plastic td. Meth. of Test for Detergent Resistance of Floor Polish Std. Meth. of Test for Porosity of Pain rage and Handling of Photographic and X-Ray Nitrocellulose Std. Dimensions for Graphic Arts Sheet

Std. Dimensions for Medical Radiographic Sheet and Roll er Densitometric Meth. for Measuring Residual Chemicals in Spec. for Photographic

td. for Resolving Power of Lenses for Projectors for $35 \mathrm{Mm}$

Std. Analytical Meth. for Determining Moisture (Oven ate and Water Soluble Gaseous Fluorides in the Atmosphere apacity Rating and Application of Suction Line Filters and

Std. End Load Test Meth. for a Hydraulic Fluid Power

ining the Fabrication Integrity of a Hydraulic Fluid Power ying the Material Compatibility of a Hydraulic Fluid Power he Flow Fatigue Characteristics of a Hydraulic Fluid Power the Collapse / Burst Resistance of a Hydraulic Fluid Power

the Filtration Performance of a Fine Hydraulic Fluid Power ilizer $\mathrm{Gr}$ / 83.92 Std. for Safety for Self Contained Strainer or

Measurement of Airborne Asbestos Fiber by the Membrane re (Sodium Bicarbonate Coated Glass Tube and a Particulate hed/ Std. Analytical Meth. for Determining Moisture (Oven atter Meth. of Analysis (Cereal Chemistry) (Bolting Cloth, Spec. for Photographic Optical ng, Settling, Aeration Chambers, Chemical Storage, Mixing, tric Fans (Blower, Humidifier, Evaporative Cooler, and Air Std. Gravel Sand and Anthrante

tilation Syste/ Rec. Pract for Air Cleaning Devices (Ai for Flow Capacity Rating and Application of Suction Line Std. for Sand Type Std. for Diatomite Type

ning Water Quality for Subsurface Injection Using Membrane Std. Meth. of Test for Liquid Flow Rate of Membrane Std. for Radio Interference Wideband Std. for Safety for Swimming Pool Pumps,

rs, Gas Therapy and Suction Equipment, Room Air Purifiers, Std. Analytical Meth. for Determining ary Past/ Sieving Meth. of Analysis for Insect and Rodent istry)/ Flotation Meth. of Analysis for Insect and Rodent Flotation Meth. of Analysis for Insect and Rodent
ent and/or Insect Damage on Kernels, Rodent Excreta, Other filter Element / Std. Multi Pass Meth. for Evaluating the vice, Automatic Pilot, Depth Determination Device and Fish n or Other Line of Position Device, Radar, Radio Detection Std. Meth. of Test for Specific Gravity and Absorption of nsi A/ Std. Meth. of Test for Sieve or Screen Analysis of Std. Meth of Test for Surface Moisture in Std. Meth. of Test for Rockwell Hardness of
Film Thickness Determination of Nonconductive Coatings Film Thickness of Nonmagnetic Finishes on Mild Steel (C Film Thickness of Paint, Varnish, Lacquer and Related $P$ Film Thickness of Pipeline Coatings on Steel (1972)

Film Thickness of 0.3 MIL or Greater (Coil Coating) (19

Film Used in Studios and Processing Laboratories (1971)

Film (Coil Coating) (1974)

Film (Coil Coating) (1974)

Film (1971)

Film (1972)

Film (1972)

Film (1973)

Film (1973)

Film (1973)

Film (1973)

Film (2.35:1) Aspect Ratio (1971)

/Ting a Solvent Hand Rub Te

Film (photography) (1973)

Film, and Backing Paper (Photography) (1972)

Film, and $126 \mathrm{~mm}$ Film Still Picture Cameras (1972)

Film, Perforated Super 8 (1-3) (1973)

Film, Perforated 2R. 1664 (1.0) (1973)

Film, Perforated 5R. 1667 (1.3.5.7-0) (1973)

Film, 2R (1974)

Film, 4R (1974)

Films and Papers (1973)

Films and Slides for Television (1970)

Films by Fungal Growth or Soil and

Films for Still Photography (1972)

Films Intended for Direct Projection in Motion Picture

Films on Galvanized Sheet (Coil Coating) (1974)

Films Suitable for Use in Fixed Mica-Dielectric Capac

Films to Abrasion During Processing (1972)

Films with a Collinear Four Probe Array (1973)

Films (SI and U.S. Customary Units) (1973)

Films (1970)

Films (1972)

Films (1972T)

Films (1973)

Films (1973)

Films (1973)

Films (photo

(1973)

Films, Plates, and Papers (1971)

Filmstrip Projectors (1973)

Filmstrips and Slides in 2 X 2 In. Mounts (1972)

Filter Aid) Content of Corn Syrup Unmixed (1954)

(Filter and Impinger Meth.) (1973)

Filter Driers in Refrigeration and Air Conditioning Sys

Filter Element (1972) ANSI B93.21

Filter Element (1972) ANSI B93.22

Filter Element (1972) ANSI B93.23

Filter Element (1972) ANSI B93.24

Filter Element (1972) ANSI B93.25

Filter Element (1973) ANSI B93.31

Th. for Evaluating

Filter Meth. (Textile) (1971)

Aseous Fluorides in the Atm

Filter Paper, Wide Field and Compound Microscope, Petri

Filter Sizes (1972)

Filter, Grit Chambers, and Flotation) (1973)

Filtering Appliances Rated at 600 Volts or Less) (1972)

Filtering Material (1972)

Filters and Dust Collectors) for Industrial Exhaust Ven

Filters and Filter Driers in Refrigeration and Air Cond

Filters for Swimming Pools (1971)

Filters for Swimming Pools (1971)

Filters (Petroleum Industry) (1973)

Filters (1972)

Filters (1974) ANSI C83.102

Filters, and Chlorinators (1972)

Filters, Compressors, Artificial Airways, Appurtenances

Filth and Insect Infestation Content in Corn (1954)

Filth in Baked Goods, Ready-To-Eat Cereals, and Aliment

Filth in Meal, Grits, Farina, and Semolina (Cereal Chem

Filth in Popped Popcorn (Cereal Chemistry) (1962)

Filth, and Extraneous Material (Cereal Chemistry) (1962

Filtration Performance of a Fine Hydraulic Fluid Power

Finder, Loran or Other Line of Position Device, Radar,

Finder, Radio Telephone) (1973)

Fine Aggregate (1973) ANSI A37.6

land Fish Finder, Lora

Fine and Coarse Concrete Aggregates (1971) AASHO T27, a

Fine Concrete Aggregate (1973) AASHO T142, ANSI A37.21

Fine Grained Graphite Materials (1973)
NCCA

NCCA

NCCA

NCCA

$N C C$

ANSI

NCCA

TB-II. 14

TB.II. 15

TB. II-4

G12

TB-II-4

$\mathrm{PH} 22.56$

TB.II.12

TB.II.18

ANSI PHI.29

ANSI $\mathrm{PH} 8.1$

UL

ANSI

ANSI

ICBO

105

PH1.31

PH22. 10

UFC $* 2 A R T 5$

UBC $\$ 48.2$

$\mathrm{PH} 22.106$

$\begin{array}{ll}\text { ANSI } & \text { PHl.15 } \\ \text { ANSI } & \text { PH } 1.40\end{array}$

ANSI $\quad$ PH3.39

Std. Fo ANSI

PH22.181

PH22. 179

PH22. 180

PH22.141

PH22. 142

PH1.32

RP7

D3274

PH2.21

PH 1.30

PH22. 146

TB.Il.9

D748

PH4.35

F390

PH 1.18

D 1583

D 1653

G29

D3207

D3258

UBC $\$ 48.1$

PH1.16

PH1.17

PH4.8

PH3.28

PH3. 16

E. 42

D 3267

$\begin{array}{ll}\text { ARI } & 730 \\ \text { NFLDP } & \text { T3.10.8.2 }\end{array}$

T3.10.8.4

T3.10.8.6

T3.10.8.7

T3. 10.8 .5

T3.10.8.8

331

RS197A

*5

D3268

CR $\quad$ E-44

AACCH $28-90$

ANSI PH3.17

C. Holdi NSF

UL

41
507

AWWA B100

ACGIH *1.11

NSF

NSF

730

10

TM.

EIA

UL

NSF

CR

RS416

1081

48

AACCH 28.31

AACCH 28.51

A ACCH 28.80

AACCH 28.10

NFLDP

T3. 10.8

ABYC

A21

ASTM C136

ASTM C70

ASTM C748 
Pass Meth. for Evaluating the Filtration Performance of A Spec. Data Formats for

Design, Manufacture, Inspection and Assembly Manual for ur Pinions (1973) Std. for System Design of Std. for System Design of calibration of Liquid Automatic Particle Counters Using Ac 1973) Std. Meth. of Tes Std. Spec. for d Type and Assignment of Grade (1973) Ans/ Std. Spec. for us (1973) AASHO T153, ANSI A 1.19 ansi Al.7 4.26 Std. Meth. of Test for Std. Spec. for Std. Spec. for i L14.29

for Rubber Cushion Sleeper Construction with Nailers and mastic Cushioned Construction with Nailers, Subflooring or nailed Construction with Mastic Set Subflooring and Nailed st Meth. for Simulated Service Test. of Wood and Wood Base ts of Drycleaning on Permanence of Durable Water Repellent Uniform Building Code: Interior Walls and Ceiling leach) 190 Deg. F, Colors 160 Deg. F Maximum, Press or Air Rec, for Wood Siding,

eI Bars and Shapes (1)

Redwood Exterior Guide (Siding Std. Spec. for Hot Rolled and Cold Std. Spec. for Cold

space Std. Identification Marking Meths. on Finished, Semi arbon Steel: Semifinished for Forging; Hot Rolled and Cold td. Spec. for General Requirements for hot Rolled and Cold Std. Spec. for Std. Quality Cold

flush, Stile, and Rail Doors, Factory Finishing, Rough and for Determining Sulfate Content of Corn Syrup Unmixed and anganese Steel Bars I/ Std. Spec. for Hot Rolled and Cold i) Extractables) Content in Corn Starch, Gluten, Germand

h. for Determining Chloride Content of Corn Syrup Unmixed, h. for Determining Chloride Content of Corn Syrup Unmixed, nalytical Meth. for Determining Ash Content of Corn Syrup,

for Determining Copper Content of Corn Syrup Unmixed and

1 Std. Spec. for Woven Glass Fabrics, Cleaned, and After r Nuclear Application/ tions (1972) ANSI Z17! 1973) Std. Spec. for Hot Rolled and Cold
Std. Spec. for Hot Worked and Cold Std. Spec. for Flat Copper Products with Aerospace Std. Identification Marking Meths. on I) Std. Spec. for Steel Bars, Carbon, Hot Rolled and Cold Grades, Application and Design, Span Allowances, Storage, Redwood Interior Guide (Spec. for Installation,

I Characteristics, Material Composition, Use, Application, aned, and After Finished with Epoxy Functional Silane Type stain of Rubber and Contact and Migration Stain of Organic Test Meth. for Identification of ce Requirements and Claims for Special Characteristics and Test Meth. for Evaluation of Antibacteria est Meth. for Measurement of Film Thickness of Nonmagnetic st Meth. for Drycleaning Durability of Applied Designs and Uniform Fire Code: Application of Flammable Std. Meth. of Test for Cleanability of Surface Std. Rec. Pract. for Detergent Resistance of Organic Performance Requirements for Deleterious Effects of Dyes, receive Wood Flooring (196) Rec. for Correct Preparation for Mineral Fiber Hydraulic Setting Thermal Insulating and Tile Remover, Rug Shampooer,/ Std. for Safety for Floo Std. for Countersunk Typ Quality Stds. for Factory Std. for Washer-Flush Type

Public Works Spec. for Ceramic Veneer (Sampling, Test. blinds and Shutters, Flush, Stile, and Rail Doors, Factory olors 160 Deg. F Maximum Hot Head Press $(300-330$ Deg. F) equirements for Deleterious Effects of Dyes, Finishes, and ess Round Condenser and Heat Exchanger Tubes with Integra Seamless Condenser and Heat Exchanger Tubes with Integral

s (Computer Software Documentation and Terminology) (1972) uniform Building Code Std. for Coast Sitka Spruce, Douglas

ern White and Red Pine, Eastern Spruce and Hemlock, Balsam ions Before Gluing of Southern Pine, Pacific Coast Douglas oling Towers (1971) Std. for Douglas lock-Tamarack (North), Eastern White Pine (North), Hem

r; White Fir; And/ Uniform Building Code Std. for Douglas

d. for Structural Glued Laminated Timbers: Douglas and Hem north), Ponderosa and Western White Pines, Spruce-Pine 72) Industry Std. for Dougla of CentraI Station Protective Signaling Systems for Guard, of Proprietary Protective Signalling Systems for Watchman, Safety Std. for Central Stations for Watchman, ically Operated Type for I/ Safety Std. for Heat Actuated ance and Use of Auxiliary Protective Signaling Systems for Uniform Building Code Std. for
nd Use of Local Protective Signaling Systems for Watchman,
Fine Hydraulic Fluid Power Filter Element (1973) ANSI B Fine Pitch Gear Drawings (1973)

Fine Pitch Gearing (1973)

Fine Pitch on Center Face Gears for $20 \mathrm{Deg}$. Involute Sp

Fine Pitch Wormgearing (1973)

Fine Test Dust (1972) ANSI B93.28

Fine Threaded Stud (1973)

Fineness of Hydraulic Cement by No. 325 (45-?M) Sieve (

Fineness of Mohair and Assignment of Grade (1973)

Fineness of Mohair Top, Yarns and Fabrics of the Worste

Fineness of Portland Cement by Air Permeability Apparat

Fineness of Portland Cement by the Turbidimeter (1973)

Fineness of Wool and Assignment of Grade (1973) ANS1 L1

Fineness of Wool Top and Assignment of Grade (1973) Ans

Finish Flooring Over Concrete Slab Floors (1962)

Finish Flooring Over Concrete Slab Floors (I962)

Finish Flooring Over Concrete Slab Floors (1962)

Finish Flooring (1969)

Std. Meth. of Test for Effec ASTM

Finish of Fabrics (1972)

Finish (1973)

Finish (1973)

Finish, Nails and Nailing (1972)

Finish, Nails and Nailing) (1973)

Finished Age Hardening Stainless and Heat Resisting Ste

Finished Alloy Steel Bars (1964) ANSI G24.12

Finished and Rough Items (1971)

Finished Bars; Hot Rolled Deformed Concrete Reinforcin

Finished Carbon and Alloy Steel Bar (1973) ANSI G24.5

Finished Carbon Steel Bars (1973)

Finished Carpentry, Flooring, Shingles, Exposed Roof De

Finished Corn Sugars (1954)

Std. Analytical

Finished Stainless and Heat Resisting Chromium Nickel M

Finished Std. Analytical Meth. for Determining Crude Fa

Finished Sugar and Other Clarified Starch Hydrolyzates

Finished Sugar and Other Clarified Starch Hydrolzates

Finished Sugar and Other Starch Hydrolyzates (1952)

Finished Sugars (1954)

Std. Anal

Finished with Epoxy Functional Silane Type Finishes for

Finished Zirconium and lts Alloy Bars, Rod, and Wire Fo

Finished Zirconium Bars and Wire for Nonnuclear Applica

Finished (Rolled or Drawn) Edges (Flatwire and Strip) (

Finished, Semi Finished and Rough Items (1971)

Finished, Special Quality, for Pressure Piping Componen

Finishes and Nails and Fastenings) (1973)

Finishes and Other Technical Data) (1973)

Finishes and Other Technical Data) (1973)

Finishes for Plastic Laminates (1972)

Finishes in Contact with Rubber (1973)

Finishes in Textiles (1973) ANSI L14.127

Finishes of InstitutionaI Textiles (Durability, Spot, S

Finishes on Fabrics (1970) ANSI L14.144

Finishes on Mild Steel (Coil Coating) (1974)

Finishes on Textiles and Other Materials (1973)

Finishes (Paint, Varnish, Lacquer, Stain, etc.) (1973)

Finishes (1973)

Finishes (1973)

Finishes, and Finishings (Sublimation, Brittleness and

Finishing and Testing of Concrete Subfloor Surfaces to

Finishing Cement (1964) ANSI Z98.33

Finishing Machines (Polisher, Scrubber, Sander, Scraper

Finishing Washer (1973)

Finishing (Architectural Woodwork) (1973)

Finishing (1973)

Finishing, Installation, and Definitions) (1961)

Finishing, Rough and Finished Carpentry, Flooring, Shin

Finishing, Shirt Unit, Pants Unit (1973)

Finishings (Sublimation, Brittleness and Chlorine Reten 190

Sid. Spec. for Aluminum Alloy Seam Std. Spec. for Copper and lis Alloy

Fins (1974) /for Describing Information Interchange Format

FIPS 20 h), Eastern Hemlock-Tamarack (North)

Fir and Tamarack Lumber (1973)

Fir and Western Hemlock by Presure Proces Code St

Fir Lumber Spec. for Application in Industrial Water Co

Fir (North), Ponderosa and Western White Pines, Spruce

Fir, Coast Region; West Coast Hemlock; Western Red Ceda

Fir, Southern and Lodgepole Pine, Western Larch, Califo

Fir, Western Cedars (North) (Lumber) (1973)

Fir, Western Hemlock, Sitka Spruce Doors and Blinds (19

Fire Alarm and Supervisory Service (1972) ANSI SE3.2

Fire Alarm and Supervisory Service (1972) ANSI SE3.6

Fire AIarm and Supervisory Services (1972) ANSI Se 3.1

Fire Alarm Devices, Single and Multiple Station, Mechan

Fire Alarm Service (1972) ANSI SE3.4

Fire Alarm Systems for High-Rise Buildings (1973)

\section{3)}

Fire Alarm, and Supervisory Service (1972) ANSI SE3.3

RA

AISI

ASTM

ASTM

AWI

CR

ASTM

CR

CR

la CR

CR

ASTM

ASTM

ASTM

ASTM

SAE

ASTM

CRA

CRA

CRA

ANSI

AATCC

NCCA

Te AATCC

ICBO

ASTM

ASTM

ANSI

WSFl

ASTM

UL

NSA

AWI

NSA

BIA

AWI

ANSI

ASTM

ASTM

ANSI

ICBO

$A W P A$

CTI

ICBO

ICBO

ICBO

NFLDP T3.10.8.8

AGMA Il 4.02

AGMA $\quad 370.01$

AGMA 203.03

AGMA 374.04

NFLDP T2.9.6

NSA 184

ASTM C430

ASTM D3109

ASTM DI38I

ASTM C204

ASTM C115

ASTM D419

ASTM D472

$* 4$

$* 8$

$* 6$

D2394

D2721

UBC $* 3.42$

L24.4. I 3

. 25

3 A4

ASTM A564

SAE AS478D

1

A29

A 108

*1-1

E-64

A429

B- 18

E-15

E-22

D3098

B351

B550

B272

AS478D

A696

3 A5

$3 \mathrm{~A} 7$

$3 \mathrm{~A} 9$

D3098

D925

94

L24.7.1

100

TB.II-15

UFC *2ART 14

C 756 
Operational Rec. for Globe Dry Pipe Valves (Fire and Safety Equipment) (1973)

ted Housing and Recreational Vehicle Manufacturing Plants ectrochmeical Analysis of Ores, Minerals, and Rocks by the

est for Size and Bulk Density of Refractory and Insulating Std Classification of Insulating

Std. Meth. of Test for Thermal Conductivity of Insulating

hangers (1973)

rnish, Lacquer, Stain, etc.) (1973)

material Handling Plants (1973)

)

e. Deuterium, Fluorine, Helium, Hydrogen, Krypto/

)$(1973)$

(1973)

tor and Open Burning. Combustible and Flammable /

)

Handling of Flammable and Combustible Liquids A/

ds, Grandstands, Bleachers, etc.) (1973)

ons and Controls, Barrier, Road, Hydrant, Drivew/ n from Corrosion for Underground Pipe, Fittings /

Protection and Tanks Containing Flammable or Co/

cotton, Sisal, Henequen, Ixtle, Jutes, Hemp, Tow/ 1973)

tylene (1973)

) (1973)

Uniform Building Cor Safety Sid. for

Std. for Safety for

Uniform Building Code Std. for Tin Clad Uniform Building Code Std. for Installation of Safety Sid. for Swinging Hardware for Std. Tin Clad Std for Safety for Tin Clad

r Sliding Hardware for Std., Horizontally Mounted Tin Clad ansi $\mathrm{A} 2.7$ ions (1973) ANSI A 10.21

Std. for Safeguarding Against Safety Sid. for

Safety Std. for $21 / 2 \mathrm{Gal}$. Cartridge Operated Water Type Safety Std. for Foam Safety Std. for Rating and Test. of std. on Carbon Dioxide Rec. for Foam

Uniform Building Code Std. for Installation of Automatic Uniform Building Code Std. for Carbon Dioxide Uniform Building Code:

Model F Hidromatic Deluge Valve for Water Flow Control of form Building Code Std. for Combination Standpipe Systems high Expansion Foam Systems (Ratios from 100:1 to 1000:1) Uniform Building Code Std. Test. Meth. for Rec. Pract. for Reducing the

Volatile Solids (1973)

ion of Mechanical Drive Turbines (1974)

Std. for

4)

offs and Automatic Feedwater Regulators for Boilers (197)

ammable and Combustible Materials (Fire Safety) (1974)

roll Paper (1974)

Safety Std. for Semiautomatic

Std. for Care of

Safety Std. for Unlined

Code for Safety to Life from

uirements for Safety to Life-Property from Explosion and

Std. Meth. of Test for Flash and
(Fire and Wind Hazards at Plants Assembling Mobile Homes
Fire Assay Preconcentration Spark Technique (1971) ANSI

Fire Assay Preconcentration Spark Technique (
Fire Barrier Gypsum Board Membrane (1972T)

Fire Brick (1970) ANSl A111.18

Fire Brick (1970) ANSI All1.22

Fire Brick (1972) ANSI Alll.28

Fire Code (1973)
Fire Code: Airports, Heliports, Helistops and Aircraft

Fire Code: Application of Flammable Finishes (Paint, Va

Fire Code: Automobile Tire Rebuilding Plants (1973)

Fire Code: Automobile Wrecking and Junk Yards or Waste

Fire Code: Bowling Alleys (1973)

Fire Code: Cellulose Nitrate Motion Picture Film (1973)

Fire Code: Cellulose Nitrate Plastics (Pyroxylin) (1973

Fire Code: Compressed Gases (1973)

Fire Code: Cryogenic Fluids (Air, Argon, Carbon Monoxid

Fire Code: Explosives and Blasting Agents (1973)

Fire Code: Fireworks (1973)

Fire Code: Flammable and Combustible Liquids (1973)

Fire Code: Fruit Ripening Process (Banana, Citrus Fruit

Fire Code: Fumigation and Thermal Insecticidal Fogging

Fire Code: Garage (1973)

Fire Code: General Precautions for Prevention (Incinera

Fire Code: Hazardous Chemicals (1973)

Fire Code: High Piled Combustible Stock (Storage) (1973

Fire Code: Industrial Baking and Drying Ovens (1973)

Fire Code: Liquefied Petroleum Gases (1973)

Fire Code: Lumber Yards and Woodworking Plants (1973)

Fire Code: Magnesium (1973)

Fire Code: Maintenance of Exit Ways (1973)

Fire Code: Manufacture of Organic Coatings (Paint), and

Fire Code: Matches (1973)

Fire Code: Mechanical Refrigeration (1973)

Fire Code: Motion Picture Projection (1973)

Fire Code: Oil Burning Equipment (1973)

Fire Code: Places of Assembly (Theaters, Reviewing Stan

Fire Code: Prevention of Dust Explosions (1973)

Fire Code: Protection, Authority, Departmental Regulati

Fire Code: Recommended Guide to Safe Practice Protectio

Fire Code: Recommended Safeguards and Practices for the

Fire Code: Rifle Ranges (1973)

Fire Code: Smoking (1973)

Fire Code: Storage and Handling of Combustible Fibres (

Fire Code: Suppression and Control of Hazardous Areas

Fire Code: Tents and Air Supported Structures (1973)

Fire Code: Welding and Cutting, Calcium Carbide and Ace

Fire Dampers (1973)

Fire Dampers (1973) ANSI Z233.1

Fire Detection System (Grinnell Protomatic Release F100

Fire Detection Thermostats (1974) ANSI Z220.1

Fire Doors and Shutters (1973)

Fire Doors and Windows (1973)

Fire Doors (1973) ANS1 A 133.1

Fire Doors (1973) ANS1 A 142.1

Fire Doors (1973) ANS1 Al43.1

Safety
Fire Doors, Windows, Shutters, and Glass Blocks (1973)
Fire During Building Construction and Demolition Operat

Fire Extinguisher and Apparatus Booster Hose (1973)

Fire Extinguishers (1973)

Fire Extinguishers (1973)

Fire Extinguishers (1973) ANSI Z211.1

Fire Extinguishing Agent Systems (1973)

Fire Extinguishing Systems (1972)

Fire Extinguishing Systems (1973)

Fire Extinguishing Systems (1973)

Fire Extinguishing Systems (1973)

Fire Extinguishing Systems (1973)

Fire Extinguishing Systems (1973)

(Fire Extinguishment) (1973)

(Fire Extinguishment) (1973)

Fire Hazard Classification of Building Materials (1973)

Fire Hazard of Static Electricity (1973)

Fire Hazard Properties of Flammable Liquids, Gases, and

Fire Hazards and Protection for Maintenance and lnspect

Fire Hazards and Protection Rec. for Low Water Fuel Cut

Fire Hazards and Protection Rec. for Textile Mills (197

Fire Hazards and Protection Std. for Rack Storage of Fl

Fire Hazards Anf Protection Rec. for Indoor Storage of

Fire Hose Rack Assemblies (1972)

Fire Hose (Including Couplings and Nozzles (1972)

Fire Hose (1973) ANSI Al52.1

Fire in Buildings and Structures (1970) ANSI A9.1

Fire in Design, Construction, and Operation of Solvent

Fire Points by Cleveland Open Cup (1972) AASHO T48, Ans

Fire Protection and Safety (Alarm) (1973)
FMS

FMS

ASTM

ASTM

ASTM

ASTM

ASTM

ICBO

ICBO

ICBO

ICBO

$1 \mathrm{CBO}$

ICBO

ICBO

ICBO

ICBO

ICBO

ICBO

$1 \mathrm{CBO}$

ICBO

1CBO

ICBO

ICBO

ICBO

ICBO

ICBO

ICBO

ICBO

ICBO

ICBO

$1 \mathrm{CBO}$

ICBO

$1 \mathrm{CBO}$

ICBO

$1 \mathrm{CBO}$

ICBO

ICBO

ICBO

ICBO

ICBO

ICBO

ICBO

ICBO

ICBO

ICBO

ICBO

$\mathrm{CBO}$

ICBO

UL

FMS

UL

ICBO

ICBO

UL

UL

UL

NFPA

NFPA

UL

UL

UL

UL

FMS

FMS

ICBO

ICBO

ICBO

Rec. for Star FMS

Uni ICBO

Rec. for

FMS
ICBO
FMS

FMS

FMS

FMS

FMS

FMS

NFPA

UL

NFPA

FMS

ASTM

FMS

E400

C707
C 134

C155

$\mathrm{Cl} 22$

UFC $* 2$

UFC *2ART 33

FC $* 2 A R T 14$

UFC "2ART2

UFC " 2 ART 3

UFC *2ART 4

UFC $* 2$ ART5

UFC 2 ART 6

UFC *2ART8

UFC *2ART 36

UFC *2ART11

UFC $* 2 A R T 12$

UFC *2ART 15

UFC 2 ART 16

UFC *2ART 17

UFC *2ART 18

UFC *2ART 27

UFC $* 2$ ART 19

UFC *2ART 35

UFC *2ART 25

UFC *2ART 20

UFC *2ART21

UFC *2ART 22

UFC * 2 ART 10

UFC *2ART 34

UFC *2ART 23

UFC *2ART 28

UFC*2ART32

UFC *2ART 24

UFC *2ART 26

UFC *2ART9

UFC $* 2 A R T 13$

UFC $* 2 B$

UFC $* 2 A$

UFC $* 2 \mathrm{C}$

UFC $* 2$ ART 29

UFC * 2 ART 7

$U F C * 2 E$

UFC *2ART 30

UFC $* 2 A R T 31$

UBCS43-7

555

LPD 2-92

521

UBCS43-3

UBCS43-5

$14 \mathrm{C}$

$10 \mathrm{~A}$

$14 \mathrm{~B}$

80

241

92

715

711 
idor, Building Construction and / Western Woods Use Book: h Temperature Molten Materials (Metals, Slag, Glass, etc.) Rec. for Proprietary Protective Signaling Systems Rec. for Parking Structures (Garage Rec. for Liquefied Hydrogen Systems Rec. for Flammable Liquid Pumping and Piping Systems Rec. for Hazardous Chemicals Data

Of, Rec. Construction and Water Sprinkler System Design . storage Up to $30 \mathrm{Ft}$. and Shelf Storage Up to $15 \mathrm{Ft}$. High) ructures, and Equipment for Water Cooled and Mo/

nd Related Faults) (1973)

Containers of Flammable Liquids (1973)

afety Std. for Dry Pipe, Deluge, and Pre Action Valves for Safety Std for Hydrants for Safety Std for Gate Valves for

Safety Std. for Play Pipes for Water Supply Test. in for Alarm Valves for Use in Wet Pipe Sprinkler Systems for Safety Std. for Indicating Pressure Gauges for Std. for Saf ety for Butterfly Valves for

Stc. for Safety for Automatic W/ater Sprinklers for Std. for Safety for Water Pumps for Rec. for Pipe Friction Loss Tables for Solving Rec. for Water Demand for Sprinkler System for Private Rec. for Lined Earth Reservoirs for Rec. for Water Spray Fixed Systems for Std. for the Installation of Water Sprinkler Systems for Rec. for Installation of Sprinkler Systems or Transportation, Storage and Use of Explosive Materials of the Reliable Model B Accelerator for Dry Pipe Systems in Dry Powder Form by Means of Spray Gun or Fluidized Bed

in Pulverized Coal Fired Multiple Burner Boiler Furnaces

y Std. for Tests for Combustibility of Sweeping Compounds Std. for Safety for Control Units for Indoor Safety for Manually Actuated Signaling Boxes for Use with Uniform Building Code Std. for Smoke Detectors for Underwriter Steam Std. Spec, for

72) ANSI H23.12

972) ANSI A153.1

wall Buildings (Tech. Notes) (1969)

(1972) ANSI B 93.5 Std. Spec. for Safety Std. for Tests for Test Meth. for td. for Safety for Tests for

Std. Pract. for the Use of Uniform Building Code: Uniform Building Code Std. for Std. Meth of Test for Durability of

ine) (1973) Tent. Meth. of Test for Hygroscopic Properties of Rec. for

rec. for W/ater Sprinkler and Hydrant System Demand Tables Spec. for Firecycle on - Off Multicycle Sprinkler System for the Inspection and Maintenance of Sprinkler Systems mperatures) of Plastics Using a Hot Air Ignition Furnace)

for Rack Storage of Flammable and Combustible Materials Std. for Practice for Respiratory Protection for the cement Pressure Pipe, Couplings, and Gaskets for Water and Std. Meth. of

Std. Definitions of Terms Relating to Uniform Building Code Std. for Std. Meth. of Uniform Building Code Std. for Std. Meth. of Std. for Safety for Uniform Building Code Std. for the Unif orm Building Code: Light, Ventilation, Sanitation, Std. for Safety of Household
Uniform Building Code: Rec. for Centrifugal and Window Vents Designed to Open Automatically in Case of il and Gasoline (19/ Manual Std. for Safety for Flame Arresters
Chemical Reactions (Incandescence, Rec. for tems $(1973)$

Outdoor Pistol Range Plans
Std. for Gas Fired Single Std. for Gas Fired Single
Std. Classification of

al11.5 Std. Meth. of Panel Spalling Test for High Duty Std. Meth. of Test for Reheat Change of

Std. Meth. of Panel Spalling Test for

Std. Meth. of Test for Drying and Firing Shrinkage of

iform Building Code Std. for Machine Made and High Alumina fety) (1973)

73)

Spec. for Std. for $\mathrm{Gas}$
Fire Protection and Safety (Exit, Stairway, Aisle, Corr Fire Protection and Safety (1973)

Fire Protection and Safety) (1973)

Fire Protection and Safety) (1973)

(Fire Protection and Safety) (1973)

Fire Protection and Safety) (1973)

Fire Protection and Safety) (1973)

Fire Protection and Safety) (1973)

Fire Protection and Safety) (1973)

Fire Protection Criteria for Safety Relat

Fire Protection for Belt Conveyors (1972)

Fire Protection for Sawmills and Lumber Yards (1973)

Tized and Bin Box

Fire Protection of Electric al Equipment (Single Phase a

Fire Protection of Small Nonpressurized and Pressurized

Fire Protection of Synchronous Motors (1974)

Fire Protection Service (1970)

Fire Protection Service (1973)

Fire Protection Service (1973)

Fire Protection Service (1973) ANSI B111.1

Fire Protection Service (1973) ANSI B129.2

Fire Protection Service (1973) ANSI B156.1

Fire Protection Service (1974)

Fire Protection Service (1974)

Fire Protection Service (1974)

Fire Protection System Hydraulic Problems (1973)

Fire Protection (Safety) (1973)

Fire Protection (1972)

Fire Protection (1973)

Fire Protection (1973) ANSI A54.3

Fire Protection) (1973)

Fire Protection) (1973)

Fire Protection) (1973)

Fire Protection) (1973)

Fire Protection) (1973)

Fire Protection) (1973) ANSI Z240.

Fire Protective Signaling Systems (1972) ANSI C33.90

Fire Protective Signaling Systems (1974)

Fire Protective Signalling Systems (1973)

Fire Pumps (1973)

Fire Refined Casting Copper (1972) ANSI H23.9

Fire Refined Copper for Wrought Products and Alloys (19

Fire Resistance of Roof Covering Material (1973)

Fire Resistance of Textile Fabrics (1969)

Fire Resistance of Vault and File Storage Room Doors (1

Fire Resistance Requirements Relating to Brick Bearing

Fire Resistant Fluids for Hydraulic Fluid Power Systems

Fire Resistive Std. (1973)

Fire Retardant Roof Covering Materials (1973)

Fire Retardant Treatment of Wood (1972)

Fire Retardant Wood and Its Base Products (1973)

Fire Safety and Protection of Yankee Driers (Paper Mach

Fire Safety in High Rise Buildings (1973)

Fire Safety) (1973)

Fire Safety) (1973)

(Fire Safety) (1973)

(Fire Safety) (1973)

(Fire Safety) (1974)

Fire Service (1973)

Fire Service (1973)

Fire Tests of Building Construction and Safety Std. for

Fire Tests of Building Construction and Materials (1973

Fire Tests of Building Construction and Materials (1973

Fire Tests of Door Assemblies (1972)

Fire Tests of Door Assemblies (1973)

Fire Tests of Door Assemblies (1973) ANSI A2.2

Fire Tests of Door Assemblies (1974) ANSl A2.2

Fire Tests of Window Assemblies (1973)

Fire Warning Detector (1973)

Fire Warning System Units (1973)

Fire Zone Requirements (1973)

Fire (Water) Pumps (NFPA 20) (1974)

/Ling Construction Under

Fire (1974)

(Fire) for Use on Vents of Storage Tanks for Petroleum $O$

Fire, Explosion, and Detonation Protection) (1973)

Fire, Heat, and Smoke Damage of Electronic Computer Sys Firearms) (1970)

Firebox Boilers (1971)

Fireclay and High Alumina Refractory Brick (1970) ANSI

Fireclay Brick (1970) ANSI All1.14

Fireclay Brick (1970) ANSI All1.16

Fireclay Nozzles and Sleeves (1972)

Fireclay Plastic Refractories (1972)

Fireclay Plastic Refractories (1972) ANSI A111.25

Fireclay Refractory Bricks (1973)

Firecycle on - Off Multicy cle Sprinkler System (Fire Sa

Fired Absorption Summer Air Conditioning Appliances (19
WWPA

FMS

FMS

FMS

FMS

FMS

FMS

MMS

ANS

FMS

FMS

FMS

FMS

UL

UL

UL

UL

lor Safety UL

UL

UL

UL

UL

FMS

FMS

FMS

NFPA

FMS

Rec. Code F FMS

aintenance

FMS

FMS

UL

UL

Std. for UL

ICBO

FMS

ASTM

ASTM

UL

AATCC

UL

BIA

NFLDP

ICBO

ICBO

ASTM

ASTM

FMS

FMS

FMS

FMS

Rec FMS

ICBO

FMS

ANSI

UL

NFPA

ASTM

ICBO

NFPA

ICBO

ASTM

UL

ICBO

$\mathrm{CBO}$

UL

MS

FMS

UL

FMS

NRA

ANSI

ASTM

ASTM

ASTM

ASTM

ASTM

ASTM

Un ICBO

FMS

ANSI

31-4

LPD 7.33

LPD $5 \cdot 2 \mathrm{~N}$

LPD $7.15 \mathrm{~N}$

LPD 7.19N

LPD 7.32

$7-23 \mathrm{~N}$

LPD 1.2

$8.25 \mathrm{~N}$

N18.10

7-11

14-15

7.29

5. 13

260

262

385

193

393

1091

199

448

2.89

3.26

LPD3-6 
Safety Std, for Oil Std. for Gas Hose Connectors for Portable Indoor Gas Std. for Gas Hose Connectors for Portable Outdoor Gas Safety Std. for Oil Safety Std. for Oil Std. for $\mathrm{Gas}$ Controls Direct Electric Ignition Control Systems for Gas Std, for Safety for Oil

973)

I Furnaces (1972)
Safety Std. for Oil Fired Air and Direct Not Over 400,000 Btu Per Hr.) (1972)

\section{Std. for Gas Std. for Domestic Gas Std. for Vented Gas Std. for Unvented Gas Std. for Gas Std. for Gas \\ Std. for Direct Gas} Std. for Gas

c. for Prevention of Furnace Explosions in Pulverized Coal Safety Ventilation Requirements for Direct Std. for Gas Std. for Gas Std. for Gas Std. for Gas Std. for Gas Std. for Safety for Oil evention of Furnace Explosions in Fuel Oil and Natural Gas nsity, Apparent Porosity, and Apparent Specific Gravity of Residential

for Decorative Gas Appliances for Installation in Vented Uniform Building Code: Masonry or Concrete Chimneys, ng Construction Under the Uniform Building Code: Chimneys, Std. for Chimneys, Specifying Sprayed Std for Prevention of Sulfur Rec. for Protection Against Molten Metal Loss Prevention Data on Fundamental Defects in Uniform Fire Code: Std. Meth. of Test for Drying and

Rec. Pract. Inspection and Quality Control of Std for Prescription Requirements for Orientation Program for Personnel Going Offshore for the Engelmann Spruce; Western Cedar and Hemlock; Subal-Pine coast Region; West Coast Hemlock; Western Red Cedar; White nd Lodgepole Pine/ Uniform Building Code Std. for Douglas eastern Spruce, White Pine, and Hemlock-Tamarack; Balsam Uniform Building Code Std. for Douglas Fir; Larch; Hem . Std. Analytical Meth. for Determining Moisture (Karl Std. Analytical Meth. for Determining Moisture (Karl Std. Analytical Meth. for Determining Moisture (Karl Std. Analytical Meth. for Determining Moisture (Karl

o Device, Automatic Pilot. Depth Determination Device and st (Cereal Chemistry) (1962) ic Meth.) (1969) Ans/ th. ) (1969) ANSI NIl/ si N107 Std. Meth. of Test for Atom Percent Std. Meth. of Test for Atom Percent Std. Meth of Test for Atom Percent Tent. Meth. for Spectrophotometric Determination of $x$ by Analysis of Molybdenum - 99 Activity from Uranium - 238 n Flux for Analysis for Barium 140 Produced by Uíranium 238 Std. for Bushing-Flanged, Press Std. for Class 5 Interference Std. for Die Buttons, Variable, Press Std. for Bushing-Flanged, Press Std. for Bushing-Plain, Press Std. for Sleeve Bushing, Press imating the Thermal Conductivity of Leather with the Cenco Tapered Roller Bearings) Conform/ Std. Shaft and Housing 3) Std. for Packing, Preformed, Straight Thread Tube 3) Std. for Packing, Preformed, Straight Thread Tube Std. Dimensions for Flareless Acorn std. for Wrought Copper and Bronze Solder-Joint Pressure Std. for Coupling-Tubes, Flared for Carbon Steel Forgings for Piping Components (Flange, c. for Selection, Design, Construction and Installation of Safety Std. for Marine Through Hull

e Practice Protection from Corrosion for Underground Pipe, Std. for Safety for Cellular Metal Floor Raceways and Std. for Safety for Surface Metal Raceways and
ation Service, and Marine Use (1972/ Safety Std. for Tube std. Pract. for High Strength Carbon Steel Wrought Welding
Fired Air and Direct Fired Heaters (1973)

Fired Appliances (1971)

Fired Appliances (I97I)

Fired Boiler Assemblies (1973)

Fired Central Furnaces (1973) ANSI Z96. I

Fired Duct Furnaces (1971)

Fired Equipment (1972)

Fired Floor Furnaces (1974)

Fired Gravity and Fan Type Direct Vent Wall Furnaces (1

Fired Gravity and Fan Type Floor Furnaces (1973)

Fired Gravity and Fan Type Sealed Combustion System Wal

Fired Gravity and Fan Type Vented Wall Furnaces (1972)

Fired Gravity and Forced Air Central Furnaces (1973)

Fired Heaters (1973)

Fired High Pressure Steam and Hot Water Boilers (Inputs

Fired Illuminating Appliances (1971)

Fired Incinerators (1973)

Fired Infrared Radiant Heaters (1971)

Fired Infrared Radiant Heaiers (1972)

Fired Kettles, Steam Cookers and Generators (I97I)

Fired Low Pressure Steam and Hot Water Boilers (1972)

Fired Make Up Air Heaters (1972)

Fired Multiple Burner Boiler Furnaces (Fire Protection)

Fired Powder Curing Ovens (1973)

Fired Room Heaters: Volume 1, Vented (1974)

Fired Room Heaters: Volume II, Unvented (1974)

Fired Sauna Heaters (1973)

Fired Single Firebox Boilers (197I)

Fired Swimming Pool Heaters (1972)

Fired Unit Heaters (1974)

Fired Watertube Boiler Furnaces with One Burner (1972)

Fired Whiteware Products (1972)

Fireplace Design (Tech. Notes) (1963)

Fireplaces (1973)

Fireplaces, and Barbecues (1973)

Fireplaces, and Barbecues (1973)

Fireplaces, and Vents (1972) ANSI A52.1

Fireproofing (Guide Spec.) (1972)

Fires and Explosions (1971) ANS1 Z12.12

Fires and Explosions (1973)

Fires Over 050,000 (1973)

Fireworks (1973)

Firing Shrinkage of Fireclay Plastic Refractories (1972

Firm Chloroprene Rubber Sponge (1973)

First Generation Silver Habide Microfilm (1972)

First Quality Contact Lenses (1972)

First Time (Petroleum Production) (1974)

Fir; and Mountain Hemlock (Lumber) (1973)

Fir; and Sitka Spruce (Lumber) (1973)

Fir; Larch; Hem-Fir; Ponderosa, Idaho White, Sugar, a

Fir; Northern Fine and White Cedar Lumber (1973)

Fir; Ponderosa, Idaho White, Sugar, and Lodgepole Pines

Fischer) Content in Corn Starch (Unmodified) (1961)

Fischer) Content in Steep water (Corn) (1966)

Fischer) Content of Corn Sugar (Crude and Refined) (196

Fischer) Content of Corn Syrup (1966)

Fish Finder, Loran or Other Line of Position Device, Ra

Fiske-Subbarow Meth. of Analysis of Phosphorus in Yea

Fission in Uranium and Plutonium Fuel (Mass Spectrometr

Fission in Uranium and Plutonium Fuel (Neody mium. I48 Me

Fission in Uranium Fuel (Radiochemical Meth.) (1969) an

Fission Products in Nuclear Reactor Coolant Water Durin

Fission Zirconium in Irradiated Nuclear Fuels (1973)

/Td. Meth. of Test for Fast Ncutron Flu

Fission (1973)

Std. Met

Fit-Undersize I

Fit Thread (1972)

Fit, Headless and Head Type, Step Relief (1972)

Fit, Steel and Bronze (1973)

Fit, Steel (1973)

Fit, Undersize Inside Diameter (1973)

Fitch Apparatus (1970) ALCA E60

Fits for Metric Radial Ball and Roller Bearings (Exce

Fitting Boss, MIL-R-25897 Rubber, 75 Shore, O.Ring (197

Fitting Boss, MIL-R-25897 Rubber, 90 Shore, O-Ring (197

Fitting End (1974)

Fitting for Use with Copper Water Tube (1973)

Fitting, Female Thread (1973)

Fitting, Valve, etc.) (1973) ANSI G17.3

Fittings and Equipment for Anchoring, Mooring, Docking,

Fittings and Sea Valves (1973) ANSI Z254. 1

Fittings and Tanks Containing Flammable Liquids (1973)

Fittings for Electrical Wire and Cable Installation (19

Fittings for Electrical Wiring (1973) ANSI C33.50

Fittings for Flammable and Combustible Fluids, Refriger

Fittings for High Pressure Service Piping (1969)

UL

ANSI

ANSI

UL

UNSI

NEMA

UL

ANSI

ANSI

ANSI

ANSI

ANSI

UL

ANSI

ANSI

ANS

ANSI

ANSI

ANSI

ANS1

ANS

FMS

FMS

ANS

ANS I

ANSI

ANS

ANS

UL

NFPA

ASTM BIA

Std ANSI

ICBO

Dwelli ICBO

NFPA

CS]

NFPA

FMS

FMS

ICBO

ASTM

SAE

NMA

ANS

Rec. Pract API

Pole Pines; ICBO

ICBO

ICBO

/for ICBO

ICBO

CR

$\mathrm{CR}$
$\mathrm{CR}$

CR

ABYC

$A A C C H$

ASTM

ASTM

ASTM

ASTM

ASTM

ASTM

ASTM

NSA

ANSI

ANSI

NSA

NSA

NSA

733

Z21.2

21.54

726

Z21.34

DC 21

729

Z21.44

Z21.48

Z21.44A

Z21.49

Z21.47

733

Z21.59

21.42

Z2I. 6

Z21.51

Z21.43B

Z21.46

Z21. 13

Z83.4

7-27S

Z21.11.1

Z21.11.2

Z21.55

Z21.52

Z21.56

C.373

I9

Z21.60

UBC *3-37

UBC $* 8-6$

211

09841

655

10-8

10.9

C179

AMS3199H

MSl04

280.2 
National Bureau of Standards KWIC Index of Engineering Standards

lting Materials for Pressure Vessels, Valves, Flanges, and safety for Pigtail, Expansion Coil, and Flexible Connector ) Pipe and Fittings to Poly(vinyl Chloride) (PVC) Pipe and Std. Spec. for Plastic lnsert

for Factory Made Wrought Nickel and Nickel Alloy Welding Std. Spec. for Insert Type Polyethylene Fusion Std. for Cast Bronze Solder Joint

Std. for Styrene Rubber and Polyvinyl Chloride Ducts and Std. Spec for Butt Heat Fusion Polyethylene (PE) Plastic erature Service (1973) ANSI G46.2 and Elevated Temperatures (1973) / Std. Spec. for Piping for Joining Acrylonitrile Butadiene Styrene (ABS) Pipe and for Safety for Wireways, Auxiliary Gutters, and Associated Std. for Forged Steel Std. Cast Bronze Threaded Std. Cast Iron Threaded Std. Bronze Flanges and Flanged Std. for Malleable Iron Threaded Rec. Pract. for Heat Joining of Thermoplastic Pipe and Std. Symbols for Dimensions of Plastic Pipe Std. Spec. for Process Glass Pipe and Std. Cast Iron Threaded Drainage Std. Factory Made Wrought Steel Buttwelding Std. for Spherical Sleeve (Compression) Tube Std for Automotive Tube

or Installation PVC Building Drain Waste and Vent Pipe and lonitrile Butadiene Styrene Drain. Waste and Vent Pipe and e Butadiene Styrene Plastic Drain, Waste and Vent Pipe and Spec. for Seamless and Welded Unalloyed Titanium Welding Std. for Cast Bronze Solder Joint Pressure

Std. Spec. for Poly(vinyl Chloride) (PVC) Sewer Pipe and Std. Spec. for Cast Iron Soil Pipe and Std. for Hydraulic Tube

r Solvent Cements for Styrene Rubber (SR) Plastic Pipe and $r$ Coding System for Identification of Tube, Pipe, and Hose Rubber Gaskets for Hub and Spigot Cast lron Soil Pipe and Std. Spec. for Cast lron Soil Pipe and Gasket Joints for Cast and Ductile Iron Pressure Pipe and rile Butadiene Styrene (ABS) Plastic Utilities Conduit and Std for Steel Pipe Flanges, Flanged Valves and std. Spec. for Thermoplastic Gas Pressure Pipe, Tubing and td. Spec. for Reinforced Epoxy Resin Gas Pressure Pipe and Spec. for High Test Wrought Welding

Safety Std. for Gasketed Joints for Cast lron Pipe and ard for Wrought Copper and lts Alloy Solder Joint Drainage spec. for Gray lron Castings for Valves, Flanges, and Pipe ec. for Type PSP Poly(vinyl Chloride) (PVC) Sewer Pipe and ec. for Type PSM Poly(vinyl Chloride) (PVC) Sewer Pipe and r Styrene Rubber Plastic Drain and Building Sewer Pipe and nt Cements for Poly(vinyl Chloride) (PVC) Plastic Pipe and ne-Styrene (ABS) Plastic Drain, Waste, and Vent Pipe and acrylonitrile-Butadiene-Styrene (ABS) Plastic Pipe and

Std. Spec. for Wrought Austenitic Stainless Steel Piping c. for Factory Made Wrought Aluminum and lts Alloy Welding cellulose Acetate Butyrate (CAB) Plastic Pipe, Tubing, and ent Cemented Joints with Polyvinyl Chloride (PVC) Pipe and nyl Chloride) (PVC) Plastic Drain, Waste and Vent Pipe and nitrile-Butadiene-Styrene (ABS) Plastic Sewer Pipe and Std for Safety for Electrical Outlet Boxes and a for Hubless Cast lron Sanitary System (Plumbing Pipe and pec. for Forged or Rolled Alloy Steel Pipe Flanges, Forged c. for Socket Type Poly(vinyl Chloride) (PVC) Plastic Pipe Std. Spec. for Butt Fusion Polyethylene (PE) Plastic Pipe ype Acrylonitrile-Butadiene-Styrene (ABS) Plastic Pipe c. for Socket Type Poly(vinyl Chloride) (PVC) Plastic Pipe Std. Spec. for Butt Fusion Polyethylene (PE) Plastic Pipe ype Acrylonitrile-Butadiene-Styrene (ABS) Plastic Pipe ded Acrylonitrile-Butadiene-Styrene (ABS) Plastic Pipe spec. for Threaded Poly(vinyl Chloride) (PVC) Plastic Pipe Spec. for Forged or Rolled 9\% Nickel A lloy Steel Flanges, quids (1971) ANSl A2/ Std. for Cast Iron and Ductile Iron Std. Spec, for Std. Spec. for Std. Spec, for

and Petroleum Production Facilities on Land and on Marine Safety Std. for Electric Heating Appliances (Portable Spec. and Test Meth. for Std. for Safety Std. for Stationary and Std. for

for Natural Block Mica and Mica Films Suitable for Use in Conserving Their

Tent. Rec. Pract. for lnstallation of abricated Structural Steel Pipe for Use of Welded Offshore Minimum Std. for Amateur Radio Antenna: Part 1-Base or Rec. for Water Spray td. for Electric Baseboard Heating Equipment (Portable and
Fittings for Low Temperature Service (1973) ANSI G38.3 Fittings for LP (Liquid Petroleum) Gas Cylinders (1973)

Fittings for Nonpressure Applications (1972)

Fittings for Polyethylene (PE) Plastic Pipe (1973)

Fittings for Pressure Piping (1972) ANSI H34.15

Fittings for SDR 11.0 Polyethylene Pipe (1973)

Fittings for Solvent Drainage Systems (1973)

Fittings for Underground lnstallations of Electrical Wi

Fittings for (PE) Plastic Pipe and Tubing (1973)

Fittings of Wrought Carbon and Alloy Steel for Low Temp

Fittings of Wrought Carbon and Alloy Steel for Moderate

Fittings to Poly(vinyl Chloride) (PVC) Pipe and Fitting

Fittings (Electrical Wiring) (I973) ANSI C 33.45

Fittings (Pipe), Socket Welding and Threaded (1973)

Fittings (125 and 250 Lb.) (1971)

Fittings (125 and $250 \mathrm{Lb}$.) (1972)

Fittings (150 and $300 \mathrm{Lb}$.$) (1971)$

Fittings (150 and $300 \mathrm{Lb}$.) (1971)

Fittings (1967) ANSI B72.17

Fittings (1968) ANSl K65.57

Fittings (1970)

Fittings (1971)

Fittings (1971)

Fittings (1971)

Fittings (1971)

Fittings (1971)

Fittings (1971)

Fittings (1971)

Fittings (1971) ANSI H50.1

Fittings (1972)

Fittings (1972)

Fittings (1972)

Fittings (1972)

Fittings (1972)

Fittings (1972)

Fittings (1972)

Fittings (1972) ANSl A112.5.1

Fittings (1972) ANSI A21.11

Fittings (1972) ANSI K65.57

Fittings (1973)

Fittings (1973)

Fittings (1973)

Fittings (1973)

Fittings (1973)

Fittings (1973)

Fittings (1973)

Fittings (1973)

Fittings (1973)

Fittings (1973)

Fittings (1973) ANSl B72. 16

Fittings (1973) ANSI B72. 18

Fittings (1973) ANSl B72.23

Fittings (1973) ANSI G37.8

Fittings (1973) ANSI H38.19

Fittings (1973) ANSI K65.162

Fittings (1973) ANSI K65.55

Fittings (1973) ANSI K65.56

Fittings (1973) ANSI K65.59

Fittings (1974)

Fittings) (1972)

Fittings, and Valves and Parts for High Temperature Ser ASTM

Fittings, Schedule 40 (1973)

Fittings, Schedule 40 (1973) ANSI K65.160

Fittings, Schedule 40 (1973) ANSI K65.164

Fittings, Schedule 80 (1973)

Fittings, Schedule 80 (1973) ANSI K65.159

Fittings, Schedule 80 (1973) ANSI K65.163

Fittings, Schedule 80 (1973) ANSI K65.165

Fittings, Schedule 80 (1973) ANSI K65.166

Fit tings, Valves, and Parts for Low Temperature Service

Fittings, $2 \mathrm{In}$. Through $48 \mathrm{In}$./S for Water and Other Li

Five Degree Xylene (1971)

Fixation Pins-Knowles and Hagie Types (1973)

Fixation Pins and Wires (1973)

Fixed and Mobile Platforms (1973)

Fixed and Stationary 600 Volts or Less) (1972) C.33.1

Fixed and Variable Attenuators, DC to $40 \mathrm{GHz}$ (1973)

Fixed Composition Capacitors (1972) ANSI C83.89

Fixed Electric Tools (1973) ANSI C33.86

Fixed Electrolytic Tantalum Capacitors (1972)

Fixed Mica-Dielectric Capacitors (1971) ANSI C59.26

Fixed Partitions of Light Frame Type for the Purpose of

Fixed Platforms (1972)

Fixed Station (1973)

Fixed Systems for Fire Protection (1973)

Fixed) $600 \mathrm{~V}$ or Less (1973) ANSI C33.95

/Ene (Abs
/ Spec
mp

ASTM

ASTM

ASTM

ASTM

NEMA

ASTM

ASTM

ASTM

Std. UL

ANSI

ANSI

ANSI

ANSI

ASTM

ASTM

ANSl

ANSl

SAE

SAE

Std. F IAPMO

IAPMO

ASTM

ANSl

ASTM

CISPI

SAE

Std. Spec. Fo ASTM
Rec. Pract. Fo SAE

CISPI

Std. for Rubber AWWA

ASTM

ANSl

ASTM

ASTM

MSS

UL

Stand ANSI

Std. ASTM

Std. Sp ASTM

Std. Sp ASTM

Std. Spec. Fo ASTM

td. Spec. for Solve ASTM

R Acrylonitrile-Butadie ASTM

ement for ASTM

Std. Spe ASTM

Cements for ASTM

for Making Solv ASTM

td. Spec. for Poly(VI ASTM

ASTM

UL

Spec. Dat CISPI

Std. Spe ASTM

ASTM

lor Socket T ASTM

Std. Spe ASTM

ASTM

lor Socket T ASTM

1. for Threa ASTM

Std. ASTM

ASTM

AWWA

ASTM

ASTM

STM

API

UL

UL

EIA

ASTM

ASTM

Spec. for F API

EIA

A320

569

D3138

D2609

B.366

D3197

B16.32

$\mathrm{TCl}$

D326]

A 420

A234

D3138

870

B16.11

B 16.15

B16.4

B 16.24

B16.3

D2657

D2749

B 16.12

B16.9

512

IS5

PS17

B363

B 16.18

D2729

J5 $14 \mathrm{E}$

D3122

J846C

A 74

C111 
Safety Std. for Underwater Lighting Spec. for Supports for Off the Floor Plumbing Std. for Safety for Electric Lighting Std. for Stainless Steel Plumbing Std. for Vitreous China Plumbing Std. for Stainless Steel Plumbing Safety Std. for Electric Lighting Std. for Enameled Cast Iron Plumbing Uniform Plumbing Code: for Wrought Alloy Tool Steel Products (Including Dies and Guide Spec. for alysis of Riboflavin in Whole Grain Products, Grits, Meal, in Ground Soybeans, Whole or Ground Full Fat or Extracted in Ground Soybeans, Whole or Ground Full Fat or Extracted $s$ for Petroleum Oil and Gasoline (19/ ering Materials (1971) Std. for Safety for Std. Meth. of Test for Meth. for a Spec. for ) (1974) Std. Meth. for Adhesion or Cohesive Strength of Std. Meth. of Test for izontal Test Meth. (1972) Rec. Pract for ANSl L14.69, ASTM D1230 Test Meth. for Std. Meth. of Test for Std. Meth. of Test for

nsi K65.28 Meth. of Test for Mist Spray Safety Std. for Tests for Comparative Safety Std. for Tests for Std. for Safety Tests for Std. Test Meth. for Std. Meth. of Test for Std. Meth. of Test for and Appliances (1973) 1974) ANSI K65.110

5.21 ds (Gasoline Kerosene, Acetone, Alcohol, Cleaning Fluids. and Marine Use (1972 Safety Std. for Tube Fittings for Manufacture of Organic Coatings (Paint), and Handling of

Safety Std. for Aboveground Storage Tanks for Uniform Fire Code: Safety Std. for Steel Underground Tanks for Fire Hazards and Protection Std. for Rack Storage of ) Fire .) (1973) electrically Conductive Equipment and Materials for Use in Sid. for Safety for Self Contained Strainer or Filter for ion Protection (Safety) for Flammable Liquid and Liquefied inkler, Water Spray, and Explosion Protection (Safety) for cation (1972) quer, Printing Ink, Adhesive, etc.) (1974) Rec. for Rec. for Rec. for Gaskets for Rec. for ction and Safety) (1973)

3) ANSl B167.l ul 567

Std. for Safety for Valves for Std. for Safety for Meters for Safety Sid. for Pipe Connectors for owage of Portable Fuel Systems and Portable Containers for Uniform Building Code Std. for Storage and Handling of Safety Std. for Liquid Level Gauges and Indicators for tion of Small Nonpressurized and Pressurized Containers of rosion for Underground Pipe. Fittings and Tanks Containing Rec. for General Safeguards for

Safety Std. for Power Operated Dispensing Devices for Std. for Fire Hazard Properties of

Prevention (Incinerator and Open Burning. Combustible and ards and Practices for the Protection and Tanks Containing installation, Operation and Mai/ Rec. Pract. and Std. for Std for Blower Pipe and Connecting td. Spec. for Carbon Steel Forgings for Piping Components Std. Bronze Flanges and

Std. Pract. for Ball Valves with

m lndustry (1969) ANSl B146.l Std. for Steel Pipe Flanges, and Flowline Valves; Drilling Through Control, Screwed and Std. for Bushing. Std. for Bushing Std. Bronze production Valves, Ring Joint, Segmented, Production Check Std for Water Connection lloy Steel Bolting Materials for Pressure Vessels, Valves, Std. Spec. for Gray Iron Castings for Valves, e / Sid. Spec. for Forged or Rolled 9\% Nickel Alloy Steel Temper/ Std. Spec. for Forged or Rolled Alloy Steel Pipe uble Liquid Adhesives for Automatic Machine Sealing of Top Safety Std. for Highway Emergency Signals

Std. for Coupling-Tubes, Std. Dimensions for Std. for Elbow, Reducer, $45 \mathrm{Deg}$ Std. for Elbow Reducer, $90 \mathrm{Deg}$. Std. for Tee, Reducer.
Fixtures and Junction Boxes for Swimming Pools (1972) Fixtures for Public Use (1972)

Fixtures for Use in Hazardous Locations (1972) ANSl C33

Fixtures (Designed for Residential Use) (1962)

Fixtures (Lavatory, Water Closet Bowl, Urinal) (1973)

Fixtures (1971)

Fixtures (1972)

Fixtures (1973)

Fixtures (1973)

Fixtures) (1973)

Flagpoles, Metal, Wood and Fiberglass (1972)

Flaked and Puffed Cereals, Farina, Bread, etc. (Cerea

Flakes, Full Fat and Defatted Soy Flours and Grits, and

Flakes, Full Fat and Defatted Soy Flours and Grits, and

Flame Arresters (Fire) for Use on Vents of Storage Tank

Flame Propagation Classification Flooring and Floor Cov

Flame Resistance Treated Paper and Paperboard (1972

Flame Resistant Materials Used in Camping Tentage (Tent

Flame Sprayed Coatings (1969) ANSI Z167.22

Flammability of Aerosol Products (1972)

Flammability of Automotive Interior Trim Materials: Hor

Fla mmability of Clothing Textiles (1962)

Flammability of Flexible Plastics (1974)

Flammability of Flexible Thin Plastic Sheeting (1974) a

Flammability of Hydraulic Fluids (1972T)

Flammability of Liquids (1972)

Flammability of Photographic Film (1972)

Flammability of Plastic Materials for Parts in Devices

Flammability of Plastics Using the Oxygen Index Meth.

Flammability of Rigid Cellular Plastics (1974)

Flammability of Self Supporting Plastics (1974) ANSI K6

Flammable Aerosols, etc, with a Flash Point Below 200 D

Flammable and Combustible Fluids, Refrigeration Service

Flammable and Combustible Liquids and Solids and Potent

Flammable and Combustible Liquids Code (1972)

Flammable and Combustible Liquids (1972

Flammable and Combustible Liquids (1973

Flammable and Combustible Liquids (1973) ANSI B137.1

Flammable and Combustible Materials (Fire Safety) (1974

Flammable Anesthetizing Locations (Bonding Appliances,

Flammable Finishes (Paint, Varnish, Lacquer, Stain, Etc

Flammable Fluids and Anhydrous Ammonia (Fertilizer Grad

Flammable Gas Processing Equipment and Supporting Struc

Flammable Liquid and Liquefied Flammable Gas Processing

Flammable Liquid Drum Storage, Dispensing, and Classifi

Flammable Liquid Mixing Operations (Paint, Varnish, Lac

Flammable Liquid Piping Systems (1974)

Flammable Liquid Pumping and Piping Systems (Fire Prote

Flammable Liquids and Fuels (1974)

Flammable Liquids and Liquefied Petroleum (LP) Gas (197

Flam mable Liquids and LP (Liquid Petroleum) Gas (1972)

Flammable Liquids (Boats) (1972) / Construction

Flammable Liquids (Gaso

Flammable Liquids (1973)

Flammable Liquids (1973)

Flam mable Liquids (1974)

Flammable Liquids (1974) ANS1 Z216.1

Flammable Liquids, Gases, and Volatile Solids (1973)

Flammable Materials; Reporting and False Alarm; Use of

Flammable or Combustible Liquids in Locations That May

Flammable (Combustible) Gas lndicators Including Their

Flange Dimensions (1972)

Flange, Fitting, Valve, etc.) (1973) ANSl G17.3

Flanged Fittings (150 and $300 \mathrm{Lb}$.) (1971)

Flanged or Butt Welding for General Service (1972)

Flanged Steel Safety Relief Valves Used in the Petroleu

Flanged Valves and Fittings (1973)

Flanged Wellhead, Underwater Wellhead, and Christmas TR

Flanged, Press Fit-Undersize Inside Diameter (1973)

Flanged, Press Fit, Steel and Bronze (1973)

Flanges and Flanged Fittings (150 and 300 Lb.) (1971)

Flanges and Gaskets; Drilling Through, Multiple Complet

Flanges (1971)

Flanges, and Fittings for Low Temperature Service (1973

Flanges, and Pipe Fittings (1973)

Flanges, Fittings, Valves, and Parts for Low Temperatur

Flanges, Flanged Valves and Fittings (1973)

Flanges, Forged Fittings, and Valves and Parts for High

Flaps of Fiberboard Shipping Cases (1970)

Flare, Lantern, Fusee) (1973)

Flared Fitting, Female Thread (1973)

Flareless Acorn Fitting End (1974)

Flareless Tube to Swivel (1973)

Flareless Tube to Swivel (1973)

Flareless Tube to Swivel, Swivel on Run (1973)
UL

ANSI A112.6.

UL

844

CS243

ANSI Al12.19.2

UAPMO TS

ANSI Al12.19.1

ICBO UPC $* 1.9$

d. Spec. ASTM A681

CSI 10350

AACCH 86.70

$\mathrm{AACCH} \quad 46 \cdot 23$

AACCH $46-24$

UL

UL 992

CPAI 84

ASTM C633

ASTM D3065

SAE J369A

AATCC 33

ASTM D568

ASTM D1433

ASTM D3119

UL 340

UL 105

ASTMI D2863

ASTM D3014

ASTM D635

ICBO UBCS10-1

UL 109

ICBO UFC *2ART34

NFPA 30

UL

ICBO UFC*2ART 15

UL 58

FMS $\quad 8-33 \mathrm{~N}$

UL 1067

ICBO UFC*2ART14

UL $\quad 331$

7.14

FMS 7.14

FMS 8.5

FMS $\quad 7.36$

FMS

FMS

UL

7-32S

LPD 7.32

ANSI

ABYC

$1 \mathrm{CBO}$

UL

MS

ICBO

UL

FMS

ICBO

ICBO

$\mathrm{ABYC}$

ASAE

ASTM

ANSI

MSS

API

ANSI

API

NSA

NSA

ANSI

API

SAE

ASTM

ASTM

ASTM

ANSI

ASTM

ASTM

UL

NSA

NSA

NSA

NSA

NSA

25

B148. 1

H25

UBCS10.1

180

7.29

UFC * $2 B$

$7-35$

719

UFC *2ART 2

UFC $* 2 A$

Al 4

S347T

A 105

B 16.24

SP72

526

B 16.5

$6 \mathrm{~A}$ 
Std. for Tee, Reducer, Flareless Tube to Swivel, Swivel on Side (1973) Std, for Flares and Tubing (1972)

Std. Rec. Pract. for Seamless Low Carbon Steel Tubing Annealed for Bending and old Drawn Low Carbon Steel Tubing Annealed for Bending and led Low Carbon Steel Tubing Normalized for Bending, Double - T48, ANSI Z11.6 Bending, Double Flaring, and Beading (1/ Std. Std. for Welded Test Meth. for Fiber Bundles in Baled

ed Solvent Extract from Aerospace Components (Using Rotary etter Gas Content, and Getter Sorption Capacity for Barium ing a Hot Air Ig/ Uniform Building Code Std. for Self and si 211.7

AIcohol, Cleaning Fluids, Flammable Aerosols, etc, with A

ed Tester (1973)

tus (1972)

, and Related Products) by Setafl/ es (1973) Std. for Tube Assembly, Control, Steel, with
Std. for Tube Assembly, Control, Steel, with 4) Spec. for Steel Bars, Forgings, Mechanical Tubing, and Std. for Rod End, Threaded, Alloy Steel, Std. for Dimensions for Photographic Std. for

ricultural (Farm) Equipment (1972)

Std. for lling Construction Under the Uniform Building Code: Valley und Microscope, Petri Dish, Sieve, Separatory Funnel, Trap tness) (19/ lorfasines/ Test Meth. for Color Change in Fabrics Due to Test Meth. for Color Change of Fabrics Due to Design and Construction of Std. Spec, for Machined Std. Spec, for

Std. Test. Procedure for Filled Cast breaking Strength and Elongation of Cotton Textile Fibers ges (Flatwire and Strip) (1973) Std. Spec. for i) ANSI P3.24

Meth. for

Std. Tèst. Procedure for Filled Cast Std. for Self Locking,

Cres, Self-Locking and Nonlocki/ nium Alloy, 6Al-4V, Self-Locking/ Sid for Mach, Machine, Std. for Machine Screw, Std. for Machine Screw, loy Steel, Self-Locking and Nonl/

Sid, for Screw, Machine, 100 Deg. Std. for Screw-100 Deg. Close Tolerance Std. for Rivet Std. Spec. for Tantalum Ingots and Design Spec, for Std. Spec. for

(1974)

ed Type (1973) heet/ Std. Spec, for Ceneral Requirements Std. Spec. for teel, Fully Processed Types 27G053, 30G05/ Std Spery of Sid. Meth. of Tension Test of Std. Meth. of Shear Test in Flatwise Plane of Std. Test Meth. for Edgewise Compressive Strength of Std Meth of Flexure Test of est Meth. for Shive Content of Mechanical Pulp (Laboratory and Foo/ Meth. of Analysis of Thermophilic Spore Counts 72)

test for Thickness of Resilient Flooring, Materials Havin Stds. for Tire Rim Inspection, and Disk and d. for Dimensional Requirements for Duplex and Single Face Std. for Self Locking,

Std. for Screw, Machine, Std. for Machine Screw,

rm Building Code Std. for Round, Hexagonal, Octagonal, and 973)

andbook of Spring Design: Compression, Extension, Torsion,

0 KSI (1973)

(1974)

Std. for Bolt, Std. for Bolt, Std. for

Tent. Spec, for Molybdenum lat Copper Products with Finished (Rolled or Drawn) Edges Meth. of Triangle Test Taste Panel for Food baled Textile Fiber Storage (Cotton, Jute, Hemp and Sisal Trading Rules for the Purchase and Sale of Linseed Meal

Trading Rules for the Purchase and Sale of Linseed Oil Quality, Description, and Grade of Palmkernels; Babassu; Std. for Liquid Resin

oil Coatings and $\mathrm{S}$ rel Bend Test Meth. (1972T) Weathering (1972/
Spec, for Measurement of Adhesion and Rec. Pract. for Std. Meth. of Test for Low Temperature Std. Meth. of Test for Low Temperature Structural Clay Differential Movement
Flaring Polyolefin Pipe and Tubing (1972)

Flaring (1972)

Flaring (1972)

Flaring, and Beading (1972)

Flash Controlled Low Carbon Steel Tubing Normalized for Flash Dried Pulp (1973)

Flash Evaporator) (1972)

Flash Getters (1972) ANSI 2173.50

Flash Ignition Properties (Temperature

\section{ures)}

Residue of Halogenat

/Ing Barium Yield, G

Flash Point Below 2000 (1973) of Plastics Us

Flash Point by Pensky-Martens Closed Tester (1973) an

Flash Point Index of Trade Name Liquids (NFPA 325A) (19

Flash Point of Aviation Turbine Fuels by Setaflash Clos

Flash Point of Chemicals by Closed Up Meth. (1973)

Flash Point of Cutback Asphalt with Tag Open Cup Appara

Flash Point of Liquids by Tag Open Cup Apparatus (1972)

Flash Point of Liquids (Paint, Enamel, Lacquer, Varnish

Flash Points, Closed Cup, Aromatic Chemicals and Isolat

Flash Welded and Threaded Rod Ends (1973)

Flash Welded Clevis and Threaded Rod Ends (1973)

Flash Welded Rings $(1.3 \mathrm{Mn}-1.5 \mathrm{SI}-0.30 \mathrm{Cr}-1.8 \mathrm{Ni}-0$.

Flash Welding Type (1973)

Flashcubes (Battery Operated) (Photography) (1972)

Flashing and Steady Burn Barricade Warning Lights (1971

Flashing Clay Masonry (Tech. Notes) (1962)

Flashing Warning Lamp (Light) for Remote Mounting on Ag

Flashing (Sheet Metal for Roof Waterproofing) (1973)

Flask, Step Wedge for X-Ray Standardization Probe, Rot

Flat Abrasion (Frosting): Every Meth. (Textile Colorfas

Flat Abrasion (Frosting): Screen Wire Meth. (Textile Co

Flat and Sloped Brick Soffits (Tech. Notes) (1970)

Flat and Square Tool Steel Bars (1973)

Flat Asbestos Cement Sheets (1972) ANSl Al24.1

Flat Bottom Drop Composite Motor Oil Cans (1974)

(Flat Bundle Meth.) (1972) ANSl L14.96

Flat Copper Products with Finished (Rolled or Drawn) Ed

Flat Crush Test of Corrugating Medium (Paperboard) (197

Flat Drop Composite Cans (1974)

Flat Fillister Head, Full Thread Part Screw (1974)

Flat Fillister Head, Full Thread, Torq Set (1973)

Flat Fillister Head, Full Thread, Tri Wing Recess, A286

Flat Fillister Head, Full Thread, Tri Wing Recess, Tita

Flat Fillister Head, Full Threaded, Tri Wing Recess, Al

Flat Head Full Threaded, Alloy Steel (1973)

Flat Head 160,000 psi (1973)

Flat Head, Hi Shear, Close Tolerance Shank (1973)

Flat Mill Products (1970) ANSI Z179.14

Flat Plywood Sandwich Panels (1970)

Flat Rolled Electrical Steels for Magnetic Applications

Flat Rolled Nonoriented Electrical Steel, Fully Process

Flat Rolled Stainless and Heat Resisting Steel Plate, S

Flat Rolled, Grain Oriented, Silicon Iron, Electrical S

Flat Sand wich Constructions in Flatwise Plane (1970)

Flat Sand wich Constructions or Cores (1970)

Flat Sandwich Constructions (1970)

Flat Sandwich Constructions (1970)

Flat Screen) (1973)

(Flat Sour, Total Aerobic, Anaerobic) in Cereal Products

Flat Spring Test for Hot Tack of Wax Polymer Blends (19

Flat Surfaces (1973)

Flat Tapes (1974)

Flat Thread Rolling Dies (1973)

Flat 100 Deg. Head, Full Thread Part Screw (1974)

Flat 100 Deg. Head, Full Thread, Torg Set (1973)

Flat 100 Deg. Head, Short Thread, Torq Set (1973)

Flat (Rect angular and Square) Brass Wire (1973)

Flat, and Hot Wound (Materials, Specs. Terminology, Gau

Flat, 100 Deg. Head, Tor Set and Hi Torque, $180 \mathrm{KSI}$ (1

Flat, 100 Deg. Reduced Head, Torq Set and Hi Torque, 18

Flathead 100 Deg. Plain and Self Locking Machine Screw

Flattened Wire for Electron Tubes (1973)

(Flatwire and Strip) (1973)

Flavor Detection (Cereal Chemistry) (1968)

Flax, Synthetic Fibers, Wool) (1974)

(Flaxseed) (1974)

Flaxseed) (1974)

Flaxseed; Castor, Sesame, Safflower, Oleic, and Sunflow

Flexes (1972)

Flexibility by the Wedge Bend (Applicable to Solution C

Flexibility Determination of Hot Melt Adhesives by Mand

Flexibility of Latex Sealing Compounds After Artificial

Flexibility of Preformed Sealing Tapes (1973)

(Flexible Anchorage) (Tech. Notes) (1963)
NSA

SAE

ASTM

Std. for SAE

$\mathrm{SAE}$

ASTM

$\mathrm{SAE}$

TAPPI

ASTM

ICBO

ICBO

ASTM

FMS

ASTM

ASTM

ASTM

ASTM

ASTM

EOA

NSA

NSA

SAE

SA

ANSI

ITE

ASAE

ICBO

$\mathrm{AACCH}$

AATCC

AATCC

BIA

ASTM

ASTM

CCTl

ASTM

ASTM

TAPPl

CCTI

NSA

NSA

NSA

NSA

NSA

NSA

NSA

NSA

ASTM

APA

ASTM

ASTM

ASTM

ASTM

ASTM

ASTM

ASTM

TAPPI

$\mathrm{AACCH}$

TAPPI

ASTM

TRA

IFI

NSA

NSA

NSA

Unifo ICBO

SMI

NSA

NSA

NSA

ASTM

Std. Spec. for F ASTM

$\mathrm{AACCH}$

Rec. for

FMS

NFP

NFP

NIOP

EIA

NCCA

ASTM

ASTM

ASTM

BIA

1764

J533B

3140

J524B

J525B

J56A

D92

J356 A

UM.239

33

F111

UBCS52-3

UBCS10. 1

93

$7.42 \mathrm{~N}$ 
Spec. for Cabin Duct, Air, Std. Spec. for Annealed Aluminum Alloy Foil for Std. Meth of Test for Residual Solvents in Tent. Rec. Pract. for Electrostatic Charge Mobility on as Cyli/ Sid. for

Std. for Std. for

Std. Spec. for Joints for Plastic Pressure Pipes Using Std. for Keys and Keyways for Industrial Std for Balance Classification for Std. Meth. of Test for Water Vapor Transmission of meth. for Abrasion Resistance of Textile Fabrics and Other Std. Spec. for Copper Alloy Strip for Safety Std. for Std. Performance Spec. for Sid. for

vehicles (1971)

Std. Meth. of Test for Leaks in Heat Sealed Std. Meth. of Test for Flammability of th. of Low Temperature Impact Test for Fabrics Coated with
Spec. for tal Raceway for Wire and Cable Inst/ Std. for Safety for Test Meth. for Adhesion to Non Porous Std. Rec. Pract. for Underground Installation of Std. Meth. of Test for Flammability of Std Meth of Test. Molded sulation Plastic Tubing, Irradiated Polyolefin, Pigmented. lease Type Sealan/ Std. Meth. of Test for Low Temperature form Building Code Std. for Wood Members Subjected to Both Reinforced Brick Masonr meth. of Test for Deflection Temperature of Plastics Under Design Tables for Reinforced Brick Masonry es (1969) ANSl Z197.20 Std. Test Meth. for

974) Tentative Meth. of Test for Compressive and enter Point Loading) (1968) ANSI / Std. Meth. of Test for Std. Meth. of Test for Std. Test Meth. for ates (1974) Tentative Meth. of Test for Biaxial Std. Meth. of n of Modulus of Rupture) (1972) Std. Meth. of draulic Cement Mortars (Using Portions of Prisms Broken in ng Within the Radio Frequency Range of 960-1215 Megahertz Meth. of Test for Density of Glass by the Sink Sid for Retainer. Tent. Meth of Test for

on Rings (1972) ANSI B93.36 Std. Groove Dimensions for lind Threaded, Self Locking, Nonself Locking, Light Weight Std. for Nut, Self Locking, Plate-Corner, Counterbored, td. for Nut, Self Aligning, Self Locking, Plate-Two Lug, Std. Rec. Pract. for Coagulation Prevertion Data on tion Against Misc. Perils at Electric Generating Stations Flammable or Combustible Liquids in Locations That May Be Std. for Outdoor

lation Field Test of Partitions (Wall, Ceiling, Floor, and smission Class (Stc) of Building Partitions Such as Walls, Uniform Building Code Std. for Span Tables for Uniform Building Code Std. for Structural Rec. Pract. for Concrete Safety Requirements for

of Test for Flame Propagation Classification Flooring and Std. Rec. Pract. for Measuring Caliper of Resilient

Vinyl Asbestos/ Std. Nomenclature Relating to Resilient Test Meth. for Shampooing: Washing of Textile Std. Meth. of Test for Embossed Depth of Resilient craper, Tile Remover, Rug Shampooer,/ Std. for Safety for Std. for Gas Fired Gravity and Fan Type Std. for Safety for Oil Fired

ments for Vented Decorative Appliances, and Wall Furnaces, Std. Meth. for

ts Manual) Carbon Steel' Plates, Structural Shapes, Rolled Spec. for Supports for Off the

(Procedure) for Products Classified as to Slip Resistant Std. Meth. of Test for Detergent Resistance of

floors (1969) Spec. for Water Emulsion
for Rating Water Emulsion ds) in Water Emulsion, Solvent Based, and Polymer Emulsion Std Meth of Test for Soil Resistance of Std. Meth. of Test for Water Spotting of Emulsion le Installation (1973) Std. for Safety for Cellular Metal Test Meth. for Rug Back

ec. for Metal Oxide Waterproofing for Below Grade Wall and Spec. for Asphalt Spec. for Self Adhering Vinyl Asbestos Spec. for Vinyl Asbestos Uniform Building Code Std. for Structural Clay
Flexible and Semi Rigid (1972)

Flexible Barrier Applications (1973)

NSA

ASTM

Flexible Barrier Materials (1972)

Flexible Barrier Materials (1973)

Flexible Connector Fittings for LP (Liquid Petroleum) G

Flexible Connectors of Other Than All Metal Constructio

Flexible Copper Water Connectors (1971)

Flexible Elastomeric Seals (1973)

Flexible Gear Couplings (1974)

Flexible Gear Couplings (1974)

Flcxible Heat Sealed Packages for Dry Products (1972)

Flexible Materials: Accele rator Meth. (1970)

Flexible Metal Hose (1973)

Flexible Metallic Hose (1972) ANSI B140.1

Flexible Multilayer Wiring (Printed Circuit) (1972)

Flexible Non Pressure W ater Fill Hose for Recreational

Flexible Packages (1972)

Flexible Plastics (1974)

Flexible Polymeric Materials (1973)

Flexible Poly(vinyl Chloride) Plastic Sheeting for Pond

Flexible Steel and Aluminum Conduit for Safe Use as a M

Flexible Substrates (Paper) (1973)

Flexible Thermoplastic Sewer Pipe (1972) ANSI K65.171

Flexible Thin Plastic Sheeting (1974) ANSI K65.28

Flexible Urethane Foam (1973)

Flexible, Heat Shrinkable (1973)

Flexiblity and Tenacity l Part, Elastomeric, Solvent Re

Flexural and Axial Loading Combined (1973)

(Flexural Design) (Tech Notes) (1964)

Flexural Load (1972) ANS1 K65.222

Flexural Members (Tech. Notes) (1967)

Flexural Strength of Adhesive Bonded Laminated Assembli

Flexural Strength of Concrete Under Field Conditions (1

Flexural Strength of Concrete (Using Simple Beam with C

Flexural Strength of Hydraulic Cement Mortars (1972)

Flexure Creep of Sandwich Constructions (1970)

Flexure Strength (Modulus of Rupture) of Ceramic Substr

Flexure Test of Flat Sandwich Constructions (1970)

Flexure Test. of Glass and Glass Ceramics (Determinatio

Flexure (1972)

Flexure) (1972) ANSI A 1.30

/Compressive Strength of $\mathrm{Hy}$

Flight Control) (1972)

Float Comparator (1972T)

/Suring Equipment (DME) Operati

Floating Barrel Nut (1973)

Floating Roller Peel Resistance of Adhesives (1973)

Floating Type Metallic and Nonmetallic Fluid Power Pist

Floating. Sandwich Panel (1972)

Floating, 125 KSI Ftu, 450 and 800 Deg. F (1973)

Floating, $125 \mathrm{KSI}$ Ftu, $450 \mathrm{Deg}$. F and $800 \mathrm{Deg}$. F (1973)

Flocculation Jar Test of W ater (1974)

Flood Losses (1973)

Flood, Surface Water, Rigging Collapse, Burglary, and R

Flooded (1973)

Floodlighting (Light) Equipment (1973)

Floor-Ceiling Assemblies) in Existing Buildings (1973

Floor-Ceiling Assemblies, Doors, and Other Space Divi

Floor and Ceiling Joists, and Rafters (1973)

Floor and Roof Plank and Beam Framing (1973)

Floor and Slab Construction (1969) ANSI A157.1

Floor and Wall Openings, Railings, and Toeboards (1973)

Floor Covering Materials (1971)

Floor Covering with Foam Layer (1973)

Floor Coverings (Asphalt, Cork, Linoleum, Rubber, Vinyl

Floor Coverings (Rug, Carpet) (1972)

Floor Coverings (1973)

Floor Finishing Machines (Polisher, Scrubber, Sander, S

Floor Furnaces (1973)

Floor Furnaces (1974)

Floor Furnaces, and Unit and Room Heaters (1973)

Floor Measurement of Office Buildings (1972)

Floor Plates, and Steel Sheet Piling (1970)

Floor Plumbing Fixtures for Public Use (1972)

Floor Polish and Wax) (1971) ASTM D2047, CSMA Bul 308-7

Floor Polish Films (1973)

Floor Polish for Use on Asphalt or Vinyl Asbestos Tile

Floor Polishes (1972)

Floor Polishes (1972)

Floor Polishes (1973)

Floor Polishes (1974) ANSI Z123.12

Floor Raceways and Fittings for Electrical Wire and Cab

Floor Staining on Vinyl Tile (1973)

Floor Surfaces (1971)

Floor Tile (1972)

Floor Tile (1972)

Floor Tile (1972)

Floor Tile (1973)
ASTM F15

ASTM F365

569

ANSI Z21.45

AAPMO PSI4

ASTM D 3139

AGMA 512.03

AGMA 515.01

ASTM D3079

Test AATCC 93

ASTM B508

UL $\quad 536$

IPC ML-990

IAPMO TSC 19

ASTM D3078

ASTM D568

Std. Me ASTM D2137

ASTM D 3083

UL

TAPPI UM-54]

ASTM D2321

ASTM D1433

ASTM D2406

SAE AMS3636D

ASTM C711

Uni ICBO UBCS25.16

Std. ASTM

BIA

$17 \mathrm{~A}$

D1 184

ASTM C683

ASTM C293

ASTM C348

ASTM C 480

ASTM F394

ASTM C393

ASTM C158

ASTM D2043

ASTM C 349

RTCA DO-151

ASTM C729

NSA 578

ASTM D3167

NFLDP T3.19.1]

NSA $\quad 1835$

NSA $\quad 1766$

NSA 1765

ASTM D2035

FMS $\quad 10-10$

FMS $\quad 9.10$

$1 C B O \quad U F C * 2 A$

NEMA FAl

ICBO UBCS35-3

ICBO UBCS35-1

ICBO UBCS25.21

ICBO UBCS25-21
ICBO UBCS25-22

ACI

ANSI

UL

USTM

302

A 12.1

992

F387

ASTM F141

AATCC 138

ASTM F373

UL

ANSI

UL

/Uire ICBO

ANSI

AlSI

ANSl

CSMA

ASTM

RTI

ASTM

561

Z21.48

729

UMC*7-1

Z65. 1

Al12.6.1

BUL 12

D3207

*6

D3052

D2834

D3206

ASTM D1793

UL

209

AATCC 137

Sp CSI

RT1

RTl

ICBO

$* 18$

$* 2$

UBC $24-10$ 
ber, Sander, Scraper, Tile Remover, Rug Shampooer, Rug and Sound Insulation Field Test of Partitions (Wall, Ceiling, r Sound Control (Acoustics) in Wood Building Construction ion, Curtain and Building Foundation Drain, Under Basement n Properties and Design Loads, Timber, Plank and Laminated

Spec, for Steel Splined Continuous Strip Mastic Set Maple ubber Cushion Sleeper Construction with Nailers and Finish cushioned Construction with Nailers, Subflooring or Finish construction with Mastic Set Subflooring and Nailed Finish floors (1962) and Testing of Concrete Subfloor Surfaces to Receive Wood for Simulated Service Test. of Wood and Wood Base Finish of Test for Electrical Resistance of Conductive Resilient Dwelling Construction Under the Uniform Building Code: ec. Installation Spec. for Vinyl Asbestos and Asphalt Tile $\mathrm{n}$ for Installation of Thresholds, Saddles for Ceramic Tile stress of Board, Timber, Lumber, Siding, Framing, Decking,

il Doors, Factory Finishing, Rough and Finished Carpentry, 1962)

for Exposed Platforms, Pavements, Driveways, and Interior ld/

No Wax Maintenance of Vinyl Asbestos and Asphalt Tile Std. for Maintenance of Vinyl Asbestos and Asphalt Tile tinuous Strip Mastic Set Maple Flooring Over Concrete Slab uction with Nailers and Finish Flooring Over Concrete Slab nailers, Subflooring or Finish Flooring Over Concrete Slab Subflooring and Nailed Finish Flooring Over Concrete Slab ion Floor Polish for Use on Asphalt or Vinyl Asbestos Tile c. for Cleaners for Use on Asphalt and Vinyl Asbestos Tile

Recommendation for Installation of Interior Ceramic Tile Recommendation for Installation of Exterior Ceramic Tile recommendation for lnstallation of Conductive Ceramic Tile recommendation for lnstallation of Waterproof Ceramic Tile

dation for Installation Performance Levels of Ceramic Tile dation for lnstallation of Chemical Resistant Ceramic Tile

e Book: Sound Control (Acoustics) in Buildings, Walls, and ommendation for Renovation of Interior Ceramic Tile Walls,

in Meal, Grits, Farina, and Semolina (Cereal Chemistry)) in Popped Popcorn (Cereal Chemistry) (1962)

npopped Popcorn, Dried Pea/ Meth. of Analysis of Cracking

nd Rec. Pract. for Buoyancy in the Event of Swamping (Boat

bers, Chemical Storage, Mixing, Filter, Grit Chambers, and Meth. of Analysis of Apparent Viscosity of Acidulated

try) (1962) Meth. of Analysis of Proteolytic Activity of metric Meth. of Analysis for $\mathrm{H}$ ydrogen Ion Activity (PH) in air Oven (Aluminum Plate) Meth. of Analysis of Moisture in Van Slyke Meth. of Analysis of Amino Nitrogen in Wheat Meth. of Analysis of Reducing and Nonreducing Sugars in fied Sorensen Meth. of Analysis of Amino Nitrogen in Wheat - Bicarbonate Meth. of Analysis of Ergot in Rye and Wheat rstrom-Lang Meth. of Analysis of Amino Nitrogen in Wheat Diastase Meth. of Analysis of Starch in

ereal Chemistry)/

corn, Rye, B/

Polarimetric Meth. of Analysis of Starch in d / Modified Vacuum Oven Meth. of Analysis of Moisture in etic Anhydride Meth. for Generation of Chlorine Dioxide in Farinograph Meth. for Analysis of Resistance of Mixograph Meth for Analysis of Resistance of

peanuts (Cereal / Meth. of Analysis of Crude Fiber in Soy Meth. of Analysis of Phosphates in

Qualitative Meth. of Analysis of Bromates and lodates in Qualitative Iodide Test Meth. for Ammonium Persulfate in Meth. for Preparation of Sample: Yeast Foods and Meth. of Analysis of Soy

Kjeldahl Meth. of Analysis of Crude Protein in Wheat and ring (Cereal Chemistry) (19/ Meth. for Experimental Wheat Analysis of Falling Number Determination of Both Meal and ydrolys) Meth. of Analysis of Damaged Starch (Granules in White Pan Bread stry) (1962)

Summary of Meth. for Biscuit and Cracker
Meth. for Correction for Weight of Std. Meth. of Test for Flame Propagation Classification Std. Spec. for Creosoted End Grain Wood Block

Spec, for Mosaic Wood Parquet Std. for Laminated Hardwood Block Std. Patterns for Wood Paneling, Decking. Std. Meth. of Test for Thickness of Resilient Rec. Pract. for Rating of Trailer Rec. for the Care and Preservation of Wood Rec. for Controlling Cupping of Wood Specifying Portland Cement Terrazzo, Cast in Place Std. Spec. for Asphalt Plank as Used for Bridge Rec. for Electrically Conductive Test Meth. for Sizing of Paperboard Pekar Color Test (Slick) Meth. of Meth. of Analysis of Diastatic Activity of Machine Washing Meth. of Analysis of Gluten in Hand Washing Meth. of Analysis of Gluten in

Floor Washers, etc.) (1974)

Floor, and Floor-Ceiling As

Floor, Sump Conductor, Down

Flooring and Floor Covering Materials (1971)

Flooring and Roofing (1973)

Flooring for Interior Use (1970) ANSI 010 .

Flooring Over Concrete Slab Floors (1962)

Flooring Over Concrete Slab Floors (1962)

Flooring Over Concrete Slab Floors (1962)

Flooring Over Concrete Slab Floors (1962)

Flooring Set in

Flooring (1962)

Flooring (1969)

Flooring (1971)

er Concrete Slab or Wood Sub

looring (1972)

Flooring (1973)

Flooring (1973)

Flooring (1973)

Flooring, and Siding (1970)

Flooring, Ceiling, and Partition (1973)

Flooring, Materials Having Flat Surfaces (1973)

Flooring, Shingles, Exposed Roof Decking, Drop Siding,

Floors and Pavements of Structural Clay (Tech. Notes)

Floors Exposed to Wet and Dry Conditions (1970) ANSI 09

Floors for Commercial, Institutional and Industrial Bui

Floors for Lift Truck Loading (1971)

Floors in Institutional, Industrial and Commercial Buil

Floors (1962)

Floors (1962)

Floors (1962)

Floors (1962)

Floors (1962)

Floors (1962)

Floors (1967)

Floors (1969)

Floors $(1970)$

Floors (1970) ANSl A37.48

Floors (1973)

Floors (1973)

Floors (1973)

Floors (1973)

Floors (1973)

Floors (1973)

Floors (1973)

Floors (1973)

Floors, and Stairs (1973)

Flotation Meth. of Analysis for lnsect and Rodent Filth

Flotation of Boats When Swamped (1973)

Flotation Test for Internal Insects in Cereal Grains, U

(Flotation) (1973)

Flotation) (1973

Flotation) (1973)

Flour-Water Suspensions (Cereal Chemistry) (1962)

Flour Analysis (Cereal Chemistry) (1962)

Flour and Active Proteinase Preparations (Cereal Chemis

Flour and Bread (Cereal Chemistry) (1962)

Flour and Semolina (Cereal Chemistry) (1962)

Flour and Semolina (Cereal Chemistry) (1962)

Flour and Semolina (Cereal Chemistry) (1962)

Flour and Semolina (Cereal Chemistry) (1962)

Flour and Semolina (Cereal Chemistry) (1962)

Flour and Semolina (Cereal Chemistry) (1962)

Flour and Semolina (Cereal Chemistry) (1962)

Flour and Semolina (Cereal Chemistry) (1962)

Flour and Semolina (Cereal Chemistry) (1967)

Flour and Semolina (With Subsequent Acid Hydrolysis) (C

Flour and Semolina; Also, with Slight Modification, to

Flour and Semolina; Bread; Feedstuffs, Ground Grains an

Flour Bleaching Experiments in Laboratories (Cereal Che

Flour Dough to Mixing (Cereal Chemistry) (1962)

Flour Dough to Mixing (Cereal Chemistry) (1962)

Flour for Cake and Meal from Cottonseed, Soybeans, and

Flour Improvers (Cereal Chemistry) (1962)

Flour Improvers (Cereal Chemistry) (1962)

Flour lmprovers (Cereal Chemistry) (1962)

Flour Improvers (Cereal Chemistry) (1962)

Flour in Macaroni (Cereal Chemistry) (1962)

Flour Mill Products (Boric Acid Modification) (Cereal C

Flour Milling: Equipment, Sample Preparation, and Tempe Flour of Wheat, Rye, Barley, Other Grains, and Malted C Flour or Starch Preparations Which Are Susceptible to $\mathrm{H}$

Flour Spec. (Cereal Chemistry) (1962)
Flour Test. (Cereal Chemistry) (1962)

Flour to Give 100 Grams at $14.0 \%$ Moisture (Cereal Chemi
Scrub U

ICBO

Rec. Fo WWPA

ASTM

UL

WWPA

ASTM

WSFI

Spec, for R WSFI

ffor Mastic WSFI

Tic Nailed WSFI

WSFI

WSFI

ASTM

HPMA

ASTM

ICBO

RTI

TCA

WWPA

WWPA

ASTM

AWI

BlA

ASTM

TTMA

RTI

WSFI

WSFI

WSFI

WSFI

WSFI

WSFI

C.SI

RTl

Spe

ASTM

FMS

TCA

TCA

TCA

TCA

Recommen TCA

Recommen TCA

WWPA

Rec TCA

AACCH

$\mathrm{AACCH}$

ABYC

$\mathrm{AACCH}$

TAPPl

Std. a ABY

NSF

AACCH $56-80$

AACCH 14.10

AACCH $22-60$

Electro AACCH 02.52

AACCH 22-15

AACCH 38-11

AACCH $44-16$

$\mathrm{AACCH} \quad 46-32$

AACCH $80-60$

Modi AACCH 46.31

Ether AACCH $42-56$

Linde AACCH 46-30

AACCH 38.10

AACCH $76-10$

AACCH $76-20$

$\mathrm{AACCH} \quad 44-40$

AACCH 48.30

AACCH 54.21

AACCH $54-40$

AACCH $32-17$

AACCH 40.50

$\mathrm{AACCH} \quad 48-40$

$\mathrm{AACCH}$ 48-61

$\mathrm{AACCH} \quad 62-80$

$\mathrm{AACCH}$ 06-11

AACCH 46.12

$A A C C H$
26.10

$\mathrm{AACCH} \quad 56.81 \mathrm{~B}$

AACCH $76.30 \mathrm{~A}$

A ACCH 34.01

AACCH $10-30$

AACCH 82.23 
Meth. of Analysis of Diastatic Activity of Meth. of Calculation of Crude Protein in Wheat and General Meth. of Analysis for Fat Acidity in Grain and Meth. of Analysis for Benzoic Acid in Rapid (Magnesium Acetate) Meth. of Analysis for Ash in Meth. of Analysis for Ash in Soy eth. of Analysis for Baking Quality of Self Rising Biscuit Meth. of Analysis for Baking Quality of Cookie Meth. of Analysis for Baking Quality of Pie

Meth. of Analysis for Baking Quality of Rye Bread Meth. of Analysis for Baking Quality of Cake Meth. of Analysis of Diastatic Activity of Malt Meth. of Analysis of Lipoxidase Activity of Semolina and Decantation Meth. of Analysis for Rodent Excreta in Meth. of Analysis of Calcium in Bread and Qualitative Meth. of Analysis of Added Iron in Quantitative Meth. of Analysis of Iron in Chloroform-Alcohol Meth. of Analysis of Ergot in Rye Qualitative Meth. of Analysis of Bromates and lodates in Quantitative Meth. of Analysis of Ammonium Persulfate in Meth. for Particle Size Distribution of Wheat

General Extensigraph Meth for Physical Dough Tests of

Meth. of Analysis of Sedimentation Test for Wheat of Analysis for Acetic, Butyric, and Lactic Acids in Rye metric Meth. of Analysis for Hydrogen lon Activity (PH) in eth. of Analysis for lnsect Eggs in White, Wheat, and Corn Dough Meth. of Analysis for Baking Quality of Wheat Bread , Pound Loaf Meth. of A nalysis for Baking Quality of Bread meth. of Analysis for lnsect Fragments and Rodent Hairs in of Analysis of Potassium Bromate in White and Whole Wheat of Analysis for lnsect Fragments and Rodent Hairs in Rye Meth. of Extensigraph Analysis of Physical Dough Tests of r Original Ash in Phosphated and Freshly Mixed Self Rising oducts, Corn Grits, Corn Meal, Puffed Cereals, Farina, and of Analysis for Insect Fragments and Rodent Hairs in Soy Meth. of Analysis of Vitamin A in Enriched Semiquantitative Meth. of Analysis of Benzoyl Peroxide in nonfat Dry Milk Solids to Change Mixing Characteristics of of Analysis for Insect Excreta in White, Wheat, and Corn Meth. of Thiosulfate-Maltose (Diastatic Activity in in Dough Conditioners Containing Monoglyceride, Salts, Soy sis of Malathion Residues in Milled Wheat Products (Wheat, fruit (Cereal Chemistr) (Excreta in Meal, Grits, Meth. of Analysis of Crude Fat in Wheat and Soy (1962) Basic Meth. of Analysis for Ash in analysis of Residual Urease Activity in Soybean Meals, Soy
uffs in Sacks (Cereal Chemistry) (/ Meth. for Sampling of Meth. for Sampling of thermal Properties of Agricultural Grain and lts Products nd Full Fat or Extracted Flakes, Full Fat and Defatted Soy nd Full Fat or Extracted Flakes, Full Fat and Defatted Soy d Other Grains, Foodstuffs Containing Vegetable Materials, Meth. of Analysis of Crude Fat in Soy

Meth of Analysis of Moisture and Volatile Matter in Soy fructosan Meth. of Analysis for Admixture of Rye and Wheat for Total Carbon Dioxide in Prepared Mixes and Self Rising ) (1962)

orn) Containing Vegetable Material, Corn and Other Grains,

Meth. of Analysis of Oxidizing Agents in Iters and Filter Driers in Refrigeration and Ai/. Meth. of Testing

llhead, Underwater Wellhead and Christmas Tree Equipment: Rec. for Star Model F Hidromatic Deluge Valve for Water Filter Element (1972) Ansi/ usion Materials (1974)

Std. Meth. for Verifying the Sid. Rec. Pract. for Powder

973)

972)

0) ANS1 Z167.10

) (1972)

ures (1973)

td for Trapezoidal Flumes for Irrigation Std. Meth. of Test for Resistance to Plastic Std. Meth. of Test for Sag Std. Meth. of Measuring the Spiral Std. Meth. of Test for Fusion Std. for Operation and Sid. Test Meth. for Std. Meth. for Measurement of Std. Meth. of Test for Liquid 1973) $\begin{array}{ll}\text { Std. Test Proc. and Acceptance Criteria for Rate of Air } \\ \text { dise } / & \text { Rec. for Standardization of Forms and Information }\end{array}$ aintenance Instructions for Chlorine Institute Std. Excess pacity Test Procedure for Compressible Fluids in Turbulent prinkler Demand (1974) ormation Processing) (1973) ANSl PH5.17 Rec. for Water leaf Evergrel Std. for Nursery Stock: Deciduous Shade and
Flour with the Amylograph (Cereal Chemistry) (1962) Flour (Calculated from Percentage of Total Nitrogen) (C Flour (Cereal Chemistry) (1962) Flour (Cereal Chemistry) (1962) Flour (Cereal Chemistry) (1962) Flour (Cereal Chemistry) (1962) Flour (Cereal Chemistry) (1962) Flour (Cereal Chemistry) (1962) Flour (Cereal Chemistry) (1962)

Flour (Cereal Chemistry) (1962)

Flour (Cereal Chemistry) (1962)

Flour (Cereal Chemistry) (1962)

Flour (Cereal Chemistry) (1962)

Flour (Cereal Chemistry) (1962)

Flour (Cereal Chemistry) (1962)

Flour (Cereal Chemistry) (1962)

Flour (Cereal Chemistry) (1962)

Flour (Cereal Chemistry) (1962)

Flour (Cereal Chemistry) (1962)

Flour (Cereal Chemistry) (1962)

Flour (Cereal Chemistry) (1962)

Flour (Cereal Chemistry) (1962)

Flour (Cereal Chemistry) (1962)

Flour (Cereal Chemistry) (1962)

Flour (Cereal Chemistry) (1962)

Flour (Cereal Chemistry) (1962)

Flour (Cereal Chemistry) (1962)

Flour (Cereal Chemistry) (1962)

Flour (Cereal Chemistry) (1962)

Flour (Cereal Chemistry) (1962)

Flour (Cereal Chemistry) (1962)

Flour (Cereal Chemistry) (1962)

Flour (Cereal Che mistry) (1962)

Flour (Cereal, Chemistry) (1962)

Flour (Cereal Chemistry) (1962)

Flour (Cereal Chemistry) (1967)

Flour (Cereal Chemistry) (1968)

Flour (Physical Dough Tests) (Cereal Che mistry) (1962)

Flour (1962)

Flour) Conversion (Cereal Chemistry) (1962)

Flour, and Other Cereal Products (Cereal Chemistry) (19

Flour, Bran, Shorts, etc.) to Determine Added Amounts O

Flour, Bread, and Baked Cereal Products Not Containing

Flour, Farina, and Semolina (Cereal Che mistry) (1962)

Flour, Farina, Semolina, Bread, Grain, Soybeans, Rice,

Flour, Feeds and Cooked Feeds (Cereal Chemistry) (1962)

Flour, Feeds, Feedstuffs, and Bread (Cereal Chemistry)

Flour, Mill Feeds (Cereal Chemistry) (1962)

Flour, Semolina, and Similar Products: Feeds and Feedst

Flour, Semolina, Macaroni and Wheat (1962)

(Flour, Starch) (1972)

Flours and Grits, and Soybean Meal (Cereal Chemistry) (

Flours and Grits, and Soybean Meal (Cereal Chemistry)

Flours and Meals (1964)

Flours (Cereal Chemistry) (1962)

Flours (Cereal Chemistry) (1962)

/Rude Fiber Content in Corn an

Flours (Cereal Chemistry) (1962)

Flours, and Meals (1964)

Flour: Reaction with Potassium lodide (Cereal Chemistry

Flow Capacity of Refrigerant Capillary Tubes (1972)

Flow Capacity Rating and Application of Suction Line Fi

Flow Control Chokes; and Water Sampling Devices) (1974)

Flow Control of Fire Extinguishing Systems (1973)

Flow Fatigue Characteristics of a Hydraulic Fluid Power

Flow Measurement of TFE -Fluorocarbon Molding and Extr

Flow Measurement (1972)

Flow of Bituminous Mixtures Using Marshall Apparatus (1

Flow of Highly Viscous Materials (1968) ANSI K65.180

Flow of Low Pressure Thermosetting Molding Compounds (1

Flow of Porcelain Enamel Frits (Flow Button Meth.) (197

Flow Process Charts and Symbols (Mechanical Engineering

Flow Properties of Adhesives (1969)

Flow Properties of Lubricating Greases at High Temperat

Flow Properties of Treated Paper (1973)

Flow Properties (1969)

Flow Rate of Membrane Filters (1972)

Std. Test Meth. for a ASTM

Flow Through Closed Steel Door and Frame Assemblies (19

Flow Used in Ordering, Shipping, and Billing of Merchan Flow Valves (1972)

Flow (1974)

Flow / Pressure Requirements for Pipe Schedule System S

Flowchart Symbols and Their Usage in Micrographics (Inf

Flowering Trees; Deciduous Shrubs; Coniferous and Broad
TAPPI

ASTM

ASTM

STDI

AACCH 22.10

AACCH 46.19

AACCH 02.01

AACCH 04-21

AACCH 08.02

AACCH $10.31 \mathrm{~A}$

AACCH $10.50 B$

AACCH $10-60$

AACCH 10.70

AACCH $\quad 10.90$

AACCH 22.40

$\mathrm{AACCH} \quad 28-42$

AACCH 40.20

$\mathrm{AACCH} \quad 40-40$

AACCH 40.41

AACCH 42.55

AACCH 48-41

AACCH 50.10

AACCH 54.10

AACCH 56.60

Meth AACCH 04.20

AACCH 02.51

AACCH 28.44

Straight AACCH 10.10

ysis AACCH 28-41

AACCH 48.42

AACCH 28.60

AACCH 54.11

AACCH 86-80

$\mathrm{AACCH} \quad 28-70$

AACCH 86.03

AACCH 48-07

AACCH 54.20

28-43

$\mathrm{AACCH}$

$\mathrm{AACCH} \quad 60-30$

AACCH $30-10$

AACCH 28-50

AACCH 44-15A

AACCH 08.01

AACCH $\quad 64-60$

AACCH $\quad 14-50$

ASAE D243.1

$\mathrm{AACCH} \quad 46.24$

CR A-8

AACCH $30-26$

Tri AACCH 06-10

AACCH 12-21

$\mathrm{AACCH}$ G. 12

Std ASTM C374

ASHRA 28

ARI $\quad 730$

FMS $\quad 2.88$

NFLDP T3.10.8.7

ASTM D3292

S359T

ASTM D2730

ASTM D 3123

ASTM C37

ASME 101

ASTM D2183

D2183

UM-539

D2556

F317

$\mathrm{CHI} \quad 42$

ISA

FMS

NMA

S39.4

2.77

ANSI 
es and Gaskets; Drilling Through, Multiple Completion, and nd Rec. Pract. for Installation and Calibration of Turbine thyl)-A, A, A-Trif:ioro-2,6-Dinitro-N-Propyl-Ptoluidine ugal Fans (1966) Meth.) (1973)

Tent. Meth. of Test for Sulfur Oxides in Std. for Motor Vehicle Brake Std. Test Meth. for Density of Adhesives in Std. Meth. of Test for Load Carrying Capacity of d. Meth. for Measurement of Extreme Pressure Properties of for Steel Bars, Carbon, Hot Rolled, Special Quality, for sions for Accessories for Cataloged Square Head Industrial b93.35

1/8 In. Bore Cataloged Square Head Tie Rod Type Industria Std. Groove Dimensions for

Std. End Load Test Meth. for a Hydraulic for Determining the Fabrication Integrity of a Hydraulic th. of Verifying the Material Compatibility of a Hydraulic Verifying the Flow Fatigue Characteristics of a Hydraulic $r$ Verifying the Collapse / Burst Resistance of a Hydraulic

Evaluating the Filtration Performance of a Fine Hydraulic or Qualifying and Controlling Cleaning Meth. for Hydraulic Std. for Nonintegral lndust ria oove Dimensions for Floating Type Metallic and Nonmetallic Basic Performance Data for Positve Displacement Hydraulic ANS1 B93.32 Std. Groove Dimensions for

ing Fluid Samples from the Lines of an Operating Hydraulic

Pract. for the Use of Fire Resistant Fluids for Hydraulic

eth. of Reporting Contamination Analysis Data of Hydraulic and Sealers of Single Service Containers for Milk and Its

Rec. Pract. for Symbols and Color Codes for Lubricant and $\mathrm{g}$ and Controlling Cleaning Meth. for Hydraulic Fluid Power fluid Power System (For Particulate)

Meth. for Extractin tion Phase of Nuclear Power Plants /

Std. for Cleaning of Std. Meth. for Open Bottle Tap Sampling of Noncryogenic ehicles Equipped / Rec. Pract. for Determination of Brake Std. for Motor Vehicle Brake

on Cloth (1972) ANSI L14.117 Test Meth. for

astic Polymers in Dry Powder Form by Means of Spray Gun or safety for Self Contained Strainer or Filter for Flammable Std. Classification for Metal Working

3.5

Std. Pract. for the Use of Fire Resistan for Control Valve Capacity Test Procedure for Compressible e, Helium, Hydrogen, Krypto/ Uniform Fire Code: Cryogenic Measurement of Extreme Pressure Properties of Lubricating Measurement of Extreme Pressure Properties of Lubricating Rec. Pract. Tests for Central System fication of Metallic and Fibrous Contaminants in Aerospace std. for Control Valve Sizing Equations for Incompressible r Control Valve Capacity Test Procedure for Incompressible

Meth. of Test for Mist Spray Flammability of Hydraulic

Std. Control Valve Sizing Equations for Compressible

Std. for Electrically Operated Valves for the Control of le Liquids (Gasoline, Kerosene, Acetone, Alcohol, Cleaning afety Std. for Tube Fittings for Flammable and Combustible Std. for Trapezoida Std. Spec. for

Std. Meth. for Chemical Analysis of Std. for Radiation Safety for X-Ray Diff raction and of Test for Spectral Bandwidth and Wavelength Accuracy of $t$ for Relative Radiance of Paper and Paperboard Containing Specs. for Std. Meth. of Measurement of Std. Spec. for Spec. for Std. for Dimensional and Electrical Characteristics of Std. Guide for Electrical Measurements of (1973) Tent. Meth. of Test for Effective Spec. for Conversion Coating of Titanium Alloys $s$ of Nickel-Base Alloys by the Silver Chloride-Lithium anual Procedures) (1973)

emiautomated Meth.) (1973)

Tent. Meth. for Analysis for Std. Meth. of Test for Std. Spec. for Polyvinylidene

Std. Spec. for Poly(Vinylidene on and Collection of Particulate and Water Soluble Gaseous . for Separation and Collection of Particulate and Gaseous Cryogenic Fluids (Air, Argon, Carbon Monoxide, Deuterium,

Std. Rec. Pract. for Powder Flow Measurement of TFE
Std. Spec. for Fep i K65.201 Std. Spec for TFE Std. Spec. for TFE

Std. Acceptable Concentrations of Trichlorofluoromethane e Concentrations of 1,1,2-Trichloro-1,2,2trifluoroethane std. Acceptable Concentrations of Dichlorodifluoromethane trusion Materials (1973) 208

Std. Spec. for TFE
Flowline Valves;

Fluchloralin (1973)

Flue Gas Densities for Forced and Induced Draft Centrif

Flue Gases (Barium Chloranilate Controlled Condensation

Fluid for Use Under Arctic Atmospheric Conditions (1972

Fluid Form (1969)

Fluid Gear Lubricants (1973) ANSI Z11.161

Fluid Lubricants (Falex Meth.) (1973)

Fluid Power Applications (1974)

Fluid Power Cylinders (1972) ANSI B93.29

Fluid Power Cylinders (1972) ANSI B93.34

Fluid Power Exclusion Devices (In. Series) (1972) ANSI

Fluid Power Filter Element (1972) ANSI B93.21

Fluid Power Filter Element (1972) ANSI B93.22

Fluid Power Filter Element (1972) ANSI B93.23

Fluid Power Filter Element (1972) ANSI B93.24

Fiuid Power Filter Element (1972) ANSI B93.25

Fluid Power Filter Element (1973) ANSI B93.31

Fluid Power Fluid Sample Containers (1972) ANSI B93.20

Fluid Power Hydraulic Reservoirs (1969) ANSI B93.18

Fluid Power Piston Rings (1972) ANSI B93.36

Fluid Power Pumps and Motors (1973) ANSI B93.27

Fluid Power Radial Compression Type Piston Rings (1973)

Fluid Power System (For Particulate Contamination Analy

Fluid Power Systems (1972) ANSI B93.5

Fluid Power Systems (1973) ANSI B93.30

Fluid Products (Packaging Equipment) (1974)

Fluid Requirement Maintenance Instructions, and Contai

Fluid Sample Containers (1972) ANSI B93.20

Fluid Samples from the Lines of an Operating Hydrauli

Fluid Systems and Associated Components During Construc

Fluid Systems (1972)

Fluid Temperature in the Automotive Braking System of $\mathrm{V}$

Fluid (Used Under Nonarctic Conditions) (1972)

Fluidity of Dispersions of Cellulose Form Bleached Cott

Fluidized Bed (Fire Protection) (1973)

Fluids and Anhydrous Ammonia (Fertilizer Grade) in Res

Fluids and Related Materials (1973)

Fluids for Hydraulic Fluid Power Systems (1972) ANSI B9

Fluids in Turbulent Flow (1974)

Fluids (Air, Argon, Carbon Monoxide, Deuterium, Fluorin

Fluids (Four Ball Meth.) (1971) ANSI Z11.309

Fluids (Timken Meth.) (1972) ANSl Fll.308

Fluids (1970)

Fluids (1970E)

Fluids (1972)

Fluids (1972)

Fluids (1972T)

Fluids (1973)

Fluids (1973)

Fluids, Flammable Aerosols, etc. with a Flash Point Bel

Fluids, Refrigeration Service, and Marine Use (1972)

Flumes for Irrigation Flow Measurement (1972)

Fluor Chrome Arsenate Phenol (1971)

Fluor Chrome Arsenate Phenol (1971) ANSI O1l.20

Fluorescence Analysis Equipment (1971) NBS HB111

Fluorescence Spectrometers (1972)

Fluorescent Brighteners (1973)

Fluorescent Lamp Ballast s (1972)

Fluorescent Lamp Ballasts (1972)

Fluorescent Lamp Reference Ballasts (1972)

Fluorescent Lamp Starters (1972)

Fluorescent Lamps (1972)

Fluorescent Lamps (light) (1973)

Fluorescent Lifetime of Neodymium Doped Laser Materials

Fluoride-Phosphate Type) (1973)

Fluoride Carrier D-C Arc Technique Using an Optical Emi

Fluoride Content of the Atmosphere and Plant Tissues (M

Fluoride Content of the Atmosphere and Plant Tissues (S

Fluoride in the Atmosphere (Double Paper Tape Sampler M

Fluoride Ion in Water (1972)

Fluoride (PVDF) Molding and Extrusion Materials (1973)

Fluoride) Heat Shrinkable Tubing (1973)

Fluorides in the Atmosphere (Filter and lmpinger Meth.)

Fluorides in the Atmosphere (Sodium Bicarbonate Coated

Fluorine, Helium, Hydrogen, Krypton, Methane, Neon, Nit

Fluorocarbon Molding and Extrusion Materials (1974)

Fluorocarbon Molding and Extrusion Materials (1974) Ans

Fluorocarbon Resin Molded Basic Shapes (1974)

Fluorocarbon Resin Sheet (1974)

Fluorocarbon 11) (1973)

Fluorocarbon 113) (1973)

(Fluorocarbon 12) (1974)
Fluorocarbon (Tetrafluoroethylene) Rod (1974) ANSI K65.
Fluorocarbon (Tetrafluoroethylene) Resin Molding and Ex
API

AMCA

ASTM

SAE

ASTM

ASTM

St ASTM

Spec ASTM

Dimen NFLDP

4,1 and 1 NFLDP

NFLDP

NFLDP

D. Meth NFLDP

Std. Me NFLDP

Th. for NFLDP

Eth. Fo NFLDP

TTh for NFLDP

NFLDP

NFLDP

Std Gr NFIDP

/Nting NFLDP

NFLDP

NFLDP

Std. NFLDP

Std. M NFLDP R Fillers DFISA

SAE

NFLDP

NFLDP

ANSI

ASTM

SAE

SAE

AATCC

FMS

UL

ASTM

NFLDP

Std. ISA

Eth. for ASTM

ASTM

SAE

ASTM

ISA

Std. Fo ISA

ASTM

ISA

Safet UL

ICBO

UL

ASAE

ASTM

ASTM

ANSI

Std. Meth. ASTM

TAPPI

ANSI

ANSI

ANSI

ANSI

ANSI

ANSI

ASTM

SAE

ASTM

ASTM

ASTM

ASTM

ASTM

ASTM

ASTM

ASTM

ASTM

ICBO

ASTM

ASTM

ASTM

ASTM

ANSI

Std. Acceptabl ANSI

ANSI

ASTM

ASTM

$6 \mathrm{~A}$

RP31.1

K2.140

409

D3226

J1702D

D1875

1947

D3233

695

T3.6.8

T3.6.11

T3.19.7

T3.10.8.2

T3.10.8.4

T3.10.8.6

T3.10.8.7

T3.10.8.5

T3.10.8.8

T2.9.2 
(1973)

e. Fuel and Oil Resistant, 65-75 (1973)

1972)

pe 2 A (1973)

Std. Spec. for E-CTFE Rings, Sealing, Std. for Structura Std. for Hi Shear 100 Deg.

or Hardwood Veneered Including Hardboard and Plastic Faced

Std. for Bulbed Mechanically Locked Spindle $100 \mathrm{Deg}$.

Std. for Bulbed Mechanically Locked Spindle 100 Deg. Std. for Blind, 100 Deg.

d, Internally Threaded, External Sleeve, High Temperature, el Rivet (1974) ind Internally Threaded, External Sleeve, General Purpose,
Std. for Solid 100 Deg. rk) (1973) Quality Stds. for

0 psi (1973) Std. and Test Procedure for Water Closet Std. Part Bolt, 100 Deg. Std. for Washer

) Quality Stds. for High Pressure Laminate Quality Stds. for

s, Exterior Frames and Sash, Screens, Blinds and Shutters. ing Oil Systems (1971)

Turbine Driven Pumps for Marine Ser

d Control of Hydroelectric Equipment / pment (1971) Std. Meth. For Measurement of Weighted Peak corder/Reproducers (1972) ANSI C83/ Rec. Test Meth. for ng $(1960)$

m $\cdot 238$ Fission $(1972)$

Rec for Measurement of Neutron Std. Meth. of Test for Fast Neutron Std. Meth. for Measuring Fast Neutron Std. Meth. for Measuring Fast Neutron Std. Meth. for Measuring Fast Neutron

Std. Meth. for Measuring Fast Neutron Std. Meth. for Measuring Neutron

Std. Meth. for Measuring Thermal Neutron Spec. for Mild Steel Electrodes for

Spec. for ckel Steel Electrodes (1974) utron Generators by Radio/ 8 Fission (1973)

Std. Meth. of Test for Neutron Std. Meth. for Measuring Fast Neutron Std. Meth. for Fast Neutron 972) ANSI $\$ 4.6$ Std. Meth. of Measuring Recorded Spec. for Bare Mild Steel Electrodes and Std. for Liquid Rosin n Portland Cement Concrete (1973) ANSI A3/ Std. Spec. for ision Receiving Appliances and Other Electronic Equipment

f Aerosol and Pressurized Space Spray Insecticides Against

Meth of Test for Effect of Cyclic Immersion of Syntactic Std. Spec. for Asbestos Cement Plastic Safety Std. for Rec, for

ct. for Measuring Caliper of Resilient Floor Covering with Rec. Pract. for Latex uishment) (1973) Rec. for High Expansion Std. Spec, for Rigid Urethan of Test for Hydrostatic Compressive Strength of Syntactic Std. Meth. of Test. Molded Flexible Urethane Z11.78 Std. Meth. of Test for Std Yeth of Test for for Toluenediisocyanate for Use in Production of Urethane Std. for Std. Test Meth. for Dust and Std. Meth. of Salt Spray Std. Meth. of Salt Spray m B117 Std. Meth. of Acetic-Acid Salt Spray

si Z265.1 Rec. Pract. Test Meth. for Determining Window Uniform Fire Code: Fumigation and Thermal Insecticidal Std. Spec. for Aluminum Std. Spec. for Annealed Aluminum Alloy Spec. for Tantalum Plate, Sheet, Strip, and Std Spec for Columbium and lts Alloy Strip, Sheet, d. Spec. for Molybdenum and Molybdenum Alloy Strip, Sheet Std. for Sliding and Guide Spec. for Accordion Std. for Cam Std. Meth. of Test for Overrun of Std. Rec. Pract. for Determining Effect of Packaging on Std. for Gas Counter Appliances (Coffee Pots and Urns, Std. for Air Containers for Entranceways in Meth. of Triangle Test Taste Panel for lat Sour, Total Aerobic, Anaerobic) in Cereal Products and es Terms and Defini/ chemistry) (1962)

Rec. Regulations for Animal Feed and Meth. of Analysis of Std. Plate Count Meth. of Analysis of Total Bacteria in Meth. of Analysis of Coliform Bacteria in All

Meth. of Analysis of Salmonella Bacteria in Meth. of Analysis of Food Poisoning Staphylococcus in
Fluorodifen (Common Name for the Pest Control Chemical) Fluorometric Meth. of Analysis of Riboflavin in Whole G Fluoroplastic Molding and Extrusion Materials (1973) Fluoroplastic Molding, Extrusion, and Coating Materials Fluorosilicone Rubber, General Purpose, High Temperatur Fluorspar by the Silico Molybdate (Photometric) Meth. Flush Break Pull Mandrel Self Plugging Blind Rivets, Ty Flush Close Tolerance Head Rivet (1972)

Flush Doors (1973)

Flush Head Blind Rivet (1974)

Flush Head Blind Rivet (1974)

Flush Head, Locked Spindle Rivet (1972)

Flush Head, Self Locking) (1972)

Flush Head, Self Locking) (1972) for Faste
Flush Shear Head A286 Corrosion Resistant Steel and Mon

Flush Tank Ball Cocks (1968)

Flush Tension Head Hi Torque Recess Alloy Steel, 160,00

Flush Type Finishing (1973)

Flush Type Wall Paneling (Architectural Woodwork) (1973

Flush Wall Paneling (Architectural Woodwork) (1973)

Flush, Stile, and Rail Doors, Factory Finishing, Rough

Flushing and Cleaning of Gas Turbine Generator Lubricat

Flushing and Cleaning of Lubricating Systems of Various

Flushing and Cleaning of Oil Systems for Lubrication an

Flutter Content of Sound Recording and Reproducing Equi Flutter Measurement of Instrumentation Magnetic Tape Re Flux and Spectra for Physical and Biological Applicatio

Flux by Analysis of Molybdenum -99 Activity from Uraniu Flux by Radioactivation of Aluminum (1970) ANSI N114 Flux by Radioactivation of lron (1970) ANSI N111

Flux by Radioactivation of Nickel (1970) ANSl N112

Flux by Radioactivation of Sulfur (1970) A NSI N113

Flux by Radioactivation Techniques (1970) ANSI N109

Flux by Radioactivation Techniques (1970) ANSI N110

Flux Cored Arc Welding (1969) ANSl W 3.20

Flux Cored Corrosion Resisting Chromium and Chromium Ni

Flux Density and Average Energy from ${ }_{3} \mathrm{H}(\mathrm{D}, \mathrm{N})_{4} \mathrm{He} \mathrm{Ne}$

Flux for Analysis for Barium 140 Produced by Uranium 23

Flux Measurements by Track Etch Technique (1973)

Flux of Magnetic Sound Records at Medium Wavelengths (1

Fluxes for Submerged Arc Welding (1969) ANSI W3.17

Fluxes Used for Soldering (1973) ANSI C83.91

Fly Ash and Raw or Calcined Natural Pozzolans for Use 1

(Flyback Transformer. Multiplier, Deflection Yoke, Pictu

Flying lnsects $(1972\}$

Foam at Pressure (1972)

Foam Core Insulating Panels (1972)

Foam Fire Extinguishers (1973)

Foam Fire Extinguishing Systems (1973)

Foam Layer (1973)

Foam Rubbers (1971)

Foam Systems (Ratios from 100:1 to 1000:1) (Fire Exting

Foam (1972)

Foam (1972A)

Foam (1973)

ANSI

AACC

ASTM

SAE

ASTM

IF]

NSA

Std. F NWMA IS.

NSA 1739

NSA

NSA

NSA

NSA

NSA

AWl

ASSE

NSA

NSA

$A W]$

AW

$A W]$

ASME

ASME

ASME

IEEE

ElA

NCRP

ASTM

ASTM

ASTM

ASTM

ASTM

ASTM

ASTM

AWS

AWS

ASTM

ASTM

ASTM

IEEE

AWS

EIA

ASTM

UL

Std. ASTM

ASTM

UL

FMS

ASTM

SAE

FMS

ASTM

Std. Meth. ASTM

ASTM

ASTM

ASTM

Foaming Tendencies of Engine Coolants in Glassware (197

Foams (1973) ANSI K65.125

Fog Lamps (Light) (1970)

Fog Tracking and Erosion ANSI C59.133

(Fog) Test. for Spec. Purposes (Coil Coating) (1974) Ast

Std. Spec.

(Fog) Test. (1973) ANSl Z118.1

(Fog) Test. (1974) ANSI Z118.2

Fogging Resistance of Interior Trim Materials (1973) an

Fogging (1973)

Foil for Capacitors (1974) ANSl C116.1

Foil for Flexible Barrier Applications (1973)

Foil (1973)

Foil, and Plate (1964) ANSI Z179.20

Foil, and Plate (1974)

Folding Door Hardware (1971)

Folding Partitions (1972)

Follower, Needle Bearing, High Strength Stud (1973)

Food Aerosols (1972)

Food and Beverage Products During Storage (1972)

Food Dish Warmers, Hot Plates and Griddles, etc.) (1973

Food Establishments (1970)

Food Flavor Detection (Cereal Chemistry) (1968)

Food Ingredients (Cereal Chemistry) (1962)

Food Labelling, lngredients and Containers (Also Includ

Food Poisoning Staphylococcus in Food Products (Cereal

Food Products (Cereal Chemistry) (1962)

Food Products (Cereal Chemistry) (1962)

Food Products (Cereal Chemistry) (1962)

Food Products (Cereal Chemistry) (1962)

86-70

3275

463

055

1769
1399

1672

1670

1200

1002

583

390

*1. I

193

RS405

R23

343

E266

E263

E264

E265

E261

$\mathrm{E} 262$

A 5.20

A5. 22

E496

E393

E418

347

A5.17

C618

492.3

D3088

K62.115

AMS7266

* I.500B

* $1.500 \mathrm{~A}$

LOS $4 \mathrm{Cl}$

LOS. $2 \mathrm{Cl}$

LOS.5Cl 
National Bureau of Standards KWIC Index of Engineering Standards

Meth. of Analysis of Fecal Streptococci in Meth. of Analysis of Mold and Yeast Counts in of Analysis of Thermophilic and Psychrophilic Bacteria in Meth. of Analysis of Pesticide Residues in ct. for Sensory Evaluation of Industrial and Institutional Std. for Dinnerware Used in nd Test Procedures for Plumbing Requirements for Household Std. for Performance Evaluation for Household Meth. of Analysis of Crude Fat in Baked Dog ablishing Conditions for Laboratory Sensory Evaluation of Meth for Preparation of Sample: Yeast Meth. of Analysis for Ash in Yeast Qualitative Meth. of Analysis of Ammonium Salts in Yeast Quantitative Meth. of Analysis of Ammonium Salts in Yeast Qualitative Meth. of Analysis of Chlorides in Yeast Quantitative Meth. of Analysis of Chlorides in Yeast Bomb Meth. of Analysis of Sulfates in Yeast Gravimetric Meth. of Analysis of Sulfates in Yeast Low Blank Meth. of Analysis of Methoxychlor Residues in Analysis of Hydrocyanic Acid Residues in Grains and Dried ve Meth. of Analysis for Nature of Ammonium Salts in Yeast meth. of Analysis of Moisture and Volatile Matter in Yeast Crude Protein in Bread, Wheat and Other Grains, and Yeast Determining Crude Fiber Content in Corn and Other Grains, Rec. Pract. for Engine

Concrete Construction Design Handbook (Beam, Slab,

Suggested Design Procedures for Combined Concrete est for Bearing Capacity of Soil for Static Load on Spread est for Bearing Capacity of Soil for Static Load on Spread

ding Appliances, Casters, Face Masks and Rebreathing Bags, formance Criteria for Falling Object Protective Structure roll Over (ROPS) and Falling Object Protective Structures Std. Dimensions for Compatible Operation of

Friction Coefficients of Chopped Std. for Determination of Moisture Content of ysis of Crude Protein in Cereal Grains, Oilseeds, Legumes, Point $/$ Std. Test Procedure for Measuring Hydraulic Lift $\mathrm{Po} / \mathrm{Std}$. for Test Procedure for Measuring Hydraulic Lift
Std. Temperature-Electromotive Std. for Gas Fired Gravity and Std. for Central

aces) (197)

Std. Performance Requirements for Oil Powered 972)

s (1971) Std. Flue Gas Densities for Std. for Std. for Stds. for rick Masonry Curtain and Panel Walls (In Resisting Lateral ratings; Assembly Numbers, Cell Layouts and Terminals (For criptions of Qualifications and Scopes of Work Relating to Rec. Pract. for Homes and Camps in Rec. Pract. for Draw Bar for Rec. for Drawbar for

Ground Support Equipment) (1974) Std. for Std. Spec. for Forged or Rolled Alloy Steel Pipe Flanges, ngs, and Valves and Parts for High Temper/ Std. Spec. for s, Valves, and Parts for Low Temperature / Std. Spec. for ed (1973) Steel Products Manual: Wrought Steel Wheels and
Std. for

Hot Cold Worked, and Cold Worked Alloy Steel Forgings and commendations for Die Cavity and Cutoff Die Sizes for Cold Std. Spec. for Copper and Its Alloy r Precipitation Hardening Nickel Alloy Bars, Forgings, and itation Hardening Iron B ase Superalloy Bars, Forgings, and tion Hardening Cobalt Containing Alloy Bars, Forgings, and $r$ Tolerances of Aluminum and Its Alloy Bar, Rod, Wire, and u-1.1Ti, Vacuum Induct/ 2) ANSI Z179.8 ansi $\mathrm{N} 125$ Steel Bars, Wire, Forgings, and Std. Spec. for Zirconium

Std. Spec. for Zirconium and Its Alloy Std. Spec. for Copper Beryllium Alloy r Hot Worked, Hot Cold Worked, and Cold Worked Alloy Steel
Base (19.5Cr-13.5Co-4.3Mo-1 for Alloy Bars, I Base (19.5Cr-13.5Co-4.3Mo-1 ng of Carbon and Low Alloy Steels (Wrought Products Except (1970) ANSI G55.6 2) ANSI G55.8 Std. Spec. for Vacuum Treated Electric Stee Std. Spec. for Alloy Stee etc.) (1973) ANSI G17.3

Std. Spec. for Carbon and Alloy Steet Std. Spec. for Carbon Stee td. Spec. for Quenched and Tempered Carbon and Alloy Stee uenched and Tempered Vacuum Treated Carbon and Alloy Steel vessel Components (1970) Ansi/ Std. Spec. for Alloy Stee G55.15 Std. Spec. for Vacuum Treated Alloy Stee 14 Std. Spec. for Vacuum Treated Carbon and Alloy Steel Std. Spec. for Copper and Its Alloy Die Spec. for Steel Bars and Std. Meth. of Magnetic Particle Examination of Stee Std. Spec. for Nickel Alloy Std. Spec. for Magnesium A lloy
Food Products (Cereal Chemistry) (1962) Food Products (Cereal Che mistry) (1962)

Food Products (Cereal Chemistry) (1962)

Food Products (Cereal Chemistry) (1969)

Food Purchases (1972)

Food Service Establishments (1970)

Food Waste Disposer Units (1971) ANSI A197.3, Asse 1008

Food Waste Disposers (1973)

Food (Cereal Chemistry) (1962)

Foods and Beverages (1973)

Foods and Flour Improvers (Cerea

Foods (Cereal Chemistry) (1962)

Foods (Cereal Chemistry) (1962)

Foods (Cereal Chemistry) (1962)

Foods (Cereal Chemistry) (1962)

Foods (Cereal Chemistry) (1962)

Foods (Cereal Chemistry) (1962)

Foods (Cereal Chemistry) (1962)

Foods (Cereal Chemistry) (1962)

Foods (Cereal Chemistry) (1962)

Foods (Cereal Chemistry) (1962)

Foods (Cereal Chemistry) (1962)

Foods (Cereal Chemistry) (1962)

Foodstuffs Containing Vegetable Materials, Flours and M
Foot Mounting (Front and Rear) (1970)

Footing, Column and Other Members) (1973)

Footings and Mats (Foundation) (1963)

/Pread Footings (1972) Std. Meth. of T

Footings (1972)

of Test for Bearing Capacity

Footwear Hose and Tubing, Mattresses and Pads, Sheetin

FOPS) for Prime Movers, Wheeled and Track Type Front En

FOPS) of Construction and Industrial Vehicles and Equip

Forage Harvesters, Wagons and Blowers (1972)

Forages (Grass, Corn, Hay, Straw) (1968)

Forages (1972)

Forages, and Animal and Dairy Products (Cereal Chemistr

Force Capacity on Agricultural Tractors Equipped with 3

Force Capacity on Agricultural Tractors Equipped with 3

Force (EMF) Tables for Thermocouples (1972) ANSI C96.2

Forced Air Central Furnaces (1973)

Forced Air Electric Heating Equipment (1974)

Forced Air (Domestic and Light Commercial) Central Furn

Forced and Induced Draft Centrifugal Fans (1966)

Forced Circulation Air Cooling and Air Heating Coils (1

Forced Entry Aluminum Sliding Glass Doors (1971)

Forced Entry Resistant Aluminum Horizontal Sliding Door

Forces) (Tech. Notes) (1968)

4 In. Reinf

Foreign and Domestic Passenger Cars, Trucks, Buses, Coa

Forensic Sciences (1972)

Forest Areas (1972)

Forestry Tractors (1970)

Forestry Tractors (1972) SAE J194

Forged Carbon Steel, Shoulder Nut Assembly Eyebolt (For

Forged Fittings, and Valves and Parts for High Temperat

Forged or Rolled Alloy Steel Pipe Flanges, Forged Fitti

Forged or Rolled 9\% Nickel Alloy Steel Flanges, Fitting

Forged Railway Axles (1973)

Forged Steel Fittings (Pipe), Socket Welding and Thread

Forging Billets for High Strength at Elevated Temperatu

Forging Equipment (1972)

Forging Rod, Bar and Shapes (1974) ANSI H7.1

Forging Stock for High Temperature Service (1970) ANSI

Forging Stock for High Temperature Service (1970) ANSI

Forging Stock (Rolled or Drawn) (1973)

Forging Stock, Corrosion Resistant, $12 \mathrm{C}_{\mathbf{r}}-8.5 \mathrm{Ni}-2.0 \mathrm{C}$

Forgings and Extrusions for Nonnuclear Application (197

Forgings and Extrusions for Nuclear Application (1967)

Forgings and Extrusions (1972)

Forgings and Forging Billets for High Strength at Eleva

Forgings and Rings, Corrosion and Heat Resistant, Nicke

Forgings and Stock) (1973)

R Quality A

Forgings for Generator Rotors (1971) ANSI G55.13

Forgings for Nonmagnetic Retaining Rings for Generators

Forgings for Pinions and Gears for Reduction Gears (197

Forgings for Piping Components (Flange, Fitting, Valve

Forgings for Pressure Vessel Components (1973) ANSI G35

Forgings for Pressure Vessels (1974) ANSI G55.17

Forgings for Seamless Drums, Heads, and Other Pressure

Forgings for Turbine Rotor Disks and Wheels (1970) ANSI

Forgings for Turbine Rotors and Shafts (1974) ANSI G55.

Forgings (Hot Pressed) (1974) ANSI H7.10

Forgings $(0.18-0.23 \mathrm{C})$ (SAE 1022) (1973)

Forgings (1971) ANSI G60.6

Forgings (1972)

Forgings (1972)
AACCH 42.35

AACCH 42.50

Meth. AACCH 42.45

AACCH $60-50$

ASTM E461

AHAM 36

AHAM FWD.1

AACCH 30.14

ASTM 480

AACCH 62.80

AACCH $08-18$

AACCH 40.16

A ACCH 40.18

AACCH 40.32

AACCH $40-33$

AACCH $40-65$

$\mathrm{AACCH} \quad 40.66$

A ACCH 60.40

Meth. of AACCH $60-20$

Qualitati AACCH 40.17

Oven AACCH 44.32

AACCH 46-11

CR A-8

JAE J616B

ASTM

ASTM

UL

SAE

SAE

ASAE

ASAE

ASAE

$\mathrm{AACCH}$

ASAE

SAE

ASTM

ANSI

ARI

ANSI

AMCA

ARI

AAMA

AAMA

BIM

BCI

ASTM

NFPA

SAE

ASAE

NSA

ASTM

ASTM

ASTM

AISI

ANSI

ASTM

Std. Re IF

ASTM

ASTM

ASTM

ASTM

Spec. Fo SAE

SAE

ASTM

ASTM

ASTM

ASTM

SAE

SAE

AST M

ASTM

ASTM

ASTM

ASTM

lor Q ASTM

ASTM

ASTM

ASTM

ASTM

SAE

ASTM

ASTM

ASTM

49

D1194

J397 A

328.1

S358

S349

J283

E230

Z21.47

Z9l.1

2409

303.3 
Std. Spec. for Stainless and Heat Resisting Steel Std Spec, for Aluminum Alloy Die and Hand Std. Spec. for Molybdenum and Molybdenum Alloy Spec. for Aluminum Alloy Std. Spec. for Precipitation Hardening Nickel Alloy Bars, ec. for Precipitation Hardening lron Base Superalloy Bars, for Precipitation Hardening Cobalt Containing Alloy Bars, $-8.5 \mathrm{Ni}-2.0 \mathrm{Cu}-1.1 \mathrm{Ti}$, Vacuum Induct/ $0-0.12 \mathrm{~V}(0.43-0.49 \mathrm{C}))(1973)$ $\mathrm{ni}-0.40 \mathrm{Mo}-0.07 \mathrm{~V}(0.41-0.46 \mathrm{C}) /$ Steel Bars. Wire, Spec. for Steel Bars, 15.) Spec. for Corrosion and Heat Resistant Alloy Bars, el Base-15.5Cr-8.0Fe (1973)

cl Base-15.8Cr-15.2Mo-0.30A]-0.05La /

el Base-5.0 Cr-24.5Mo-5.5Fe, Solution $\mathrm{H} /$

$-18.5 \mathrm{Cr}-1.1 \mathrm{SI}(1973)$

$.55 \mathrm{C}))(1973)$

mo-1.2V (1.10-1.20C), Premium Bearing Qu

$-0.55 \mathrm{Mo}-0.30 \mathrm{~V}(0.40-0.50 \mathrm{C}) \mathrm{V} /$

$0.17 \mathrm{C})$ (SAE 8615) (1973)

-B (0.38-0.43C) (Modified 98Bv40) (1973)

.43C) (SAE 4340), Premium Quality, Consumabl/ 43C) (SAE 4340) (1973)

13C) (SAE 9310), Premium Quality, Vacuum Co/ 13C) (SAE 9310), Premium Quality (1973) (1972)

$3 \mathrm{Cr}-38 \mathrm{Ni}-5.5 \mathrm{Mo}-0.85 \mathrm{Cb}-2.5 \mathrm{Ti}-1.6 /$

$9.5 \mathrm{Cr}-13.5 \mathrm{Co}-4.3 \mathrm{Mo}-3.0 \mathrm{Ti}-1.4 \mathrm{Al}, \mathrm{C} /$

$5 \mathrm{Cr}-13.5 \mathrm{Co}-4.3 \mathrm{Mo}-3.0 \mathrm{Ti}-1.4 \mathrm{Al}$, Con/

Low Carbon (SAE 51416F), Free Machining

$-4.5 \mathrm{Ni}-2.9 \mathrm{Mo}-0.10 \mathrm{~N}$, Solution Heat $\mathrm{T} /$

$-4.5 \mathrm{Ni}-2.9 \mathrm{Mo}-0.10 \mathrm{~N}$. Heat Treated, 1/

$-4.5 \mathrm{Ni}-2.9 \mathrm{Mo}-0.10 \mathrm{~N}$, Equalized and $\mathrm{T} /$

$3 \mathrm{Mn}-1.5 \mathrm{SI}-0.30 \mathrm{Cr}-1.8 \mathrm{Ni}-0.4 /$

$\mathrm{cr}-8.0 \mathrm{Ni}-4.0 \mathrm{Co}-0.48 \mathrm{Mo}-0.09$

nt $(15 \mathrm{Cr}-26 \mathrm{Ni}-1.3 \mathrm{Mo}-2.1 \mathrm{Ti}-)$

t Resistant, 17Cr (SAE 51430) Annealed (1973)

$0.20 \mathrm{~V}(0.32-0.38 \mathrm{C})$, Special Grade (1973)

$-0.33 \mathrm{C})(1973)$

solution and Precipitation Heat Treated (/

d/ (Steel Products Manual) Carbon Steel. Semifinished for 1973)

Std for Forks and

, Utility and Recreational Type Vehicles, Counter Balanced Std. for Forks and Fork Carriers for Powered Industrial aul): Grader, Loader, Dozer, Mining Car, Front End Loader. r Regular Bicycles Including Tests for Static Load, Frame,

t Trucks (1973) Std. for Terminal-Tank Strap,

exture for Aerospace Gearing (Surface Roughness, Waviness, Test Meth. for Fluidity of Dispersions of Cellulose c) Rec. for Application of Plastic Polymers in Dry Powder

ansi $\mathrm{H} 45.8$ Std Spec for Zinc Alloys in lngot Std Spec, for Magnesium Alloys in Ingot ansi $\mathrm{H} 38.1$ Std. Spec. for Aluminum Alloys in lngot other Than Normal Engineering Services (1972)

ior Design Services (1972)

Sid.

Std.

r (1973)

ll Construction Contracts Where Basis of Payment Is/

Std

Std.

data (Tooling. Printed Wiring Boards) in Numeric (Digital) Std. for Buttress lnch Screw Threads (7/45 Deg.
led Insulation Systems for AC Electric Machinery Employing the Investigation of Sc/ Std. Underwater Accident Report Std. Test Meth. for Density of Adhesives in Fluid Evaluation of Wettability of Fabrics and Textiles in Yarn Rec. Pract. for Drill Stern Test Report Std Spec, for Remelted Lithium Metal in Ingot 200 Deg. F (649 Deg. C) Use, Unified J (MIL S-8879) Thread Join: Venture Agreem ent 1200 Deg. F (649 Deg. C) Use, Unified (MIL S-7742) Thread Std. for Guide-Fastener, Low Std. Meth of Test for

dge Bend Apparatus (1973)

Jar Meth. (1972) ANSI L/

1972) ANSI L14.179 Test Meth. for Determination of Test Meth. for Determination of lcohol: Carbon Tetrachloride; Chloral Hydrate; Chloroform: lndustry Quality Std.

Std. for lnterchangeable Perforated Tape Variable Block Std.

Rec. Pract. for Std. Calibration and Editorial

Pendulous, Analog Torque Balance Accelerome/ Std. Spec.
Std. Page Interchangeable Perforated Tape Std., Variable Block
Spec. Data a (Tooling. Printed Wiring Boards)/ Std. Spec. for Record y) (19/ Guidelines for Describing Information Interchange
Forgings (1973) ANSI G81.26

Forgings (1973) ANSI H38.8

Forgings (1974)

Forgings (5.6Zn-2.5Mg-1.6Cu-0.26Cr (7075-T6) (197 Forgings, and Forging Stock for High Temperature Servic Forgings, and Forging Stock for High Temperature Servic Forgings, and Forging Stock for High Temperature Servic Forgings, and Forging Stock, Corrosion Resistant, $12 \mathrm{Cr}$ Forgings, and Mechanical Tubing $(1.05 \mathrm{Cr}-0.55 \mathrm{Ni}-1.0 \mathrm{M}$ Forgings, and Mechanical Tubing (1.62SI $-0.82 \mathrm{Cr}-1.82$ Forgings, and Rings (Nickel Base, Solution Heat Treated Forgings, and Rings, Corrosion and Heat Resistant, Nick Forgings, and Rings. Corrosion and Heat Resistant, Nick Forgings, and Rings, Corrosion and Heat Resistant, Nick Forgings, and Rings, Heat Resistant, Iron Base-35.5Ni Forgings, and Tubing $(0.80 \mathrm{Cr}-1.8 \mathrm{Ni}-0.25 \mathrm{Mo}(0.49-0$ Forgings, and Tubing, Corrosion Resistant, $14.5 \mathrm{Cr}-4.0$ Forgings, and Tubing, Low Alloy, Heat Resistant $10.95 \mathrm{Cr}$ Forgings, and Tubing, $0.50 \mathrm{Cr}-0.55 \mathrm{Ni}-0.20 \mathrm{Mo}(0.11-$ Forgings, and Tubing, $0.80 \mathrm{Cr}-0.85 \mathrm{Ni}-0.20 \mathrm{Mo}-0.04 \mathrm{~V}$ Forgings, and Tubing, $0.80 \mathrm{Cr}-1.8 \mathrm{Ni}-0.25 \mathrm{Mo}(0.38-0$ Forgings, and Tubing, $0.80 \mathrm{Cr}-1.8 \mathrm{Ni}-0.25 \mathrm{Mo}(0.38-0$ Forgings, and Tubing, $1.2 \mathrm{Cr}-3.25 \mathrm{Ni}-0.12 \mathrm{Mo}(0.07-0$ Forgings, and Tubing, $1.2 \mathrm{Cr}-3.25 \mathrm{Ni}-0.12 \mathrm{Mo}(0.07-0$ Forgings, Carbon and Alloy, for General Industrial Use Forgings, Corrosion and Heat Resistant, Iron Base-13. Forgings, Corrosion and Heat Resistant, Nickel Base-1 Forgings, Corrosion and Heat Resistant, Nickel Base 19. Forgings, Corrosion and Moderate Heat Resistant, 12.5Cr Forgings, Corrosion and Moderate Heat Resistant, $15.5 \mathrm{Cr}$ Forgings, Corrosion and Moderate Heat Resistant, $15.5 \mathrm{Cr}$ Forgings, Corrosion and Moderate Heat Resistant, $16.5 \mathrm{Cr}$ Forgings, Mechanical Tubing, and Flash Welded Rings (1. Forgings, Rings, and Mechanical Tubing (Annealed) $(0.48$ Forgings, Tubing. and Rings, Corrosion and Heat Resista Forgings, Tubing, and Rings, Corrosion and Moderate Hea Forgings, Tubing, and Rings, $0.78 \mathrm{Cr}-1.8 \mathrm{Ni}-0.35 \mathrm{Mo}-$ Forgings, Tubing, $0.88 \mathrm{Cr}-1.8 \mathrm{Ni}-0.42 \mathrm{Mo}-0.08 \mathrm{~V}(0.28$ Forgings, $5.6 \mathrm{Zn}-2.5 \mathrm{Mg}-1.6 \mathrm{Cu}-0.26 \mathrm{Cr}(7075-\mathrm{T} 736)$ Forging; Hot Rolled and Cold Finished Bars; Hot Rolled Fork Carriers for Powered Industrial Fork Lift Trucks ( Fork Lift Truck, Boat, Baggage, Special Type Trailer, M Fork Lift Trucks (1973)

Fork Lift Trucks, Mobile Crane, and Shovel (1974)

Fork. Steering Assembly and Tire Blowout (1972)

Forked (1973)

Forks and Fork Carriers for Powered lndustrial Fork Lif

Form and Lay) (1973)

Std. for Gear Toot

Form Bleached Cotton Cloth (1972) ANSl L14.117 Form for Die Castings (1964) ANSI H47.2

Form for Sand, Permanent Mold, and Die Castings (1966)

Form for Sand, Permanent Mold, and Die Castings (1973)

Form of Agreement Between Architect and Consultant for

Form of Agreement Between Architect and Engineer (1974)

Form of Agreement Between Owner and Architect for Inter Form of Agreement Between Owner and Architect (1974)

Form of Agreement Between Owner and Construction Manage

Form of Agreement Between Owner and Contractor (For Sma

Form on Punched Cards, Magnetic Tape and Punched Paper

Form with 0.6 Pitch Basic Height of Thread Engagement (

Form Wound Stator Coils (1972) ANSI C 50.26//I

Form (1969)

Form (1971) ANSI L14.75

Form (1972)

Form (1972) ANSl H43.1

Form (1973

Form) for Design Professionals (1972)

Form) (1973)

Form, Cowl, Dzus Type (1973)

Formability of Attached Organic Coatings with Impact We

Formaldehyde Odor in Resin Treated Fabric by the Sealed

Formaldehyde Odor in Resin Treated Fabric Steam Meth.

Formaldehyde; Gasoline; Herwig's, Pancreatin, Tween 80-

Format and Coding for Computer Output Microfilm (1971)

Format for Contouring and Contouring-Positioning Nume

Format for NAS 900 Series Equipment Spec. (1973)

Format for Nuclear Logs (Record, Documentation) (1974)

Format for Voluntary Product Stds. (1973)

Format Guide and Test Procedure for Linear, Single Axis

Format of the Construction Message (1974)

Format, for Numerically Controlled Equipment (1974)

Formats for Fine Pitch Gear Drawings (1973)

Formats for Transmission of End Product Description Dat

Formats (Computer Software Documentation and Terminolog

ASTM

ASTM

ASTM

SAE

ASTM

AST

$\mathrm{SAE}$

SAE

SAE

SAE

SAE

$S A E$

SAE

SAE

$S A E$

SAE

SAE

$S A E$

SAE

SAE

SAE

ASTM

A E

SAE

SAE

$S A E$

SAE

SAE

$S A E$

SAE

SAE

SAE

SAE

SAE

SAE

ANSI

TRA

ANSI

/T H TRA

/Ts Fo BMA

NSA

ANSI

AATCC

FMS

ASTM

ASTM

ASTM

AlOA

AlOA

AIOA

AIOA

AIOA

AlOA

IPC

ANSI

IEEE

ANSI

ASTM

\section{API} AST

SAE AIOA

NSA

ASTM

A ATC

AATC

AACC

NMA

EIA

NSA

API

USC

IEEE

CSl

NSA

AGMA

IPC

A473

B247
B384

AMS 4126

A637

A638

A639

AMS5617A

AMS5432A

A MS64 16A

AMS5750B

AMS5665H

AMS5711

AMS5755B

AMS5716A

AMS6424

AMS5749

AMS6305

AMS6270 J

AMS6422

AMS6414B

AMS6415H

AMS6265D

AMS6267B

AMS5633A

AMS5738C

AMS5709B

AMS56 I0H 
to Be Used in Semiconductor Device Specs. and Registration Std. Spec. for Structural Insulating Std. Spec. for Aluminum Alloy

Std. Meth. for Examination of Water of Analysis of Solvent Systems Used for Removal of Water Std. Meth. of Test. Water Std. Meth. of Test for Iron Bacteria in Water and Water test for Corrosivity of Solvent Systems for Removing Water manual) (1969) Guide for the Design of Aluminum uilding Code Std. for Spec. and Design of Light Gauge Cold

Uniform Building Code Std. for Spec. and Design of Cold ng in Rounds and Shapes (1973) ANSI / ng (1973) ANSI G24.22 ctural Tubing (1973)

Std. Spec. for Cold Std. Spec. for Hot Std. Spec. for Hot Manual of Cold Std. for Mat

and Lactic Acids (Cereal / i A37.95

p Process (1971) Ansi/

and Billing of Merchandise W/ Std. Spec. for Boron Deoxidized Copper in Wrought

si N12.1 Std. Spec. for Zirconium Sponge and Other Std. Spec. for Primary Hafnium Metal Sponge and Other Architect's Accounting 73) Mechanical Demand Registers (Cumulative and Pointer Std. Meth. for Evaluation of and Test Std. Meth. for Evaluation of and Test Std. Meth. for Evaluation of and Test Std. Meth. for Evaluation of and Test General Rules and
Std. Meth. for Evaluation of and Test Std. Meth. for Evaluation of and Test Std. Meth. for Evaluation of and Test Std. Meth. for Evaluation of and Test (19) $(1973)$

73)

) ANSI Z1.5 Std. Definitions, Symbols, Rec. Safety Requirements for Shoring Concrete 1971) Std. for mage to Steam Turbines Used for Electric Power Generation: r General Drainage (Land Reclamation, Curtain and Building Suggested Design and Construction Procedures for Concrete the All Weather Wood (Pressure Treated Lumber and Plywood) design Procedures for Combined Concrete Footings and Mats Dwelling Construction Under the Uniform Building Code: Rec. Guide for Design of Uniform Building Code: Excavations, Safety Std. for Ice Cream Freezers and Soda Drinking Water Coolers (1973) ANSI Al/ Std. for Drinking si I Std. Meth. for Isolation of Representative Saturates Std. for Definite Purpose Motors and Generators ratings for Alternating Current and Direct Current Motors and Performance of Alternating and Direct Current Motors for Alternating and Direct Current Motors and Generators for Alternating and Direct Current Motors and Generators est Meth. for Shive Content of Mechanical Pulp (Somerville

Meth. of Test for Boiling Range Distribution of Petroleum

h. of Test for Aromatic Types Analysis of Gas Oil A romatic

Voltage Mass Spectrometric Analysis of Gas-Oil Saturate 60.2 Std. Test Meth. for Plane Strain nail-Jewett Type for Use by Surgeons in the Treatment of rap Flask, Step Wedge for X-Ray Standardization Probe, Rot (1962) ry) (1962)

ry) $(1962)$ Acid Hydrolysis Meth. of Analysis for Insect Tween Versene Meth. of Analysis for Insect Fatting Digestion Meth. of Analysis for Insect Sieving Meth. of Analysis for Dirt, Sand, Insect Rec. Std. Steel Door,

pec. for Mineral Fiber Blanket Thermal Insulation for Wood Std. Thermal Performance Test for Steel Door and Std. Acoustical Performance Test for Steel Door and acceptance Criteria for Water Resistance on Steel Door and riteria for Rate of Air Flow Through Closed Steel Door and rsepower Induction Motors (1972)

ation of Plumbing, Heati/

Brick Veneer; New and Existing Std. for Mobile Homes: Body and Rec. Std. Steel Door Std. for Protective rformance Requirements) (1972) SAE J/ Std. for Steering ndustrial Front End Loaders and Dozers/ Spec. for $13 / 4$ In. Thick Hollow Metal Door and
Formats (1974)

Formboard Made from Cellulosic Fibers (1966)

Formed and Arc Welded Round Tube (1974) ANSI H38.20

Formed Deposits by Chemical Microscopy (1973)

Formed Deposits (Chemical Cleaning of Industrial Equipm

Formed Deposits (1972)

Formed Deposits (1972)

Formed Deposits (1973)

Formed Sheet Building Sheathing (Aluminum Constructio

Formed Stainless Steel Structural Members (1973)

Formed Steel Structural Members (1973)

Formed Welded and Seamless Carbon Steel Structural Tubi

Formed Welded and Seamless Carbon Steel Structural Tubi

Formed Welded and Seamless High Strength Low Alloy Stru

Formed Welded Structural Steel Tubing (1974)

Formed Wood Particleboard (1966)

Formed Wood Particleboard (1973)

Formic, Acetic, Propionic, Butyric, Valeric, Succinic,

Forming Compounds for Curing Concrete (1973) ANSI A37.8

Forming Concrete Tests Cylinders Vertically (1973T) Ans

Forming Impurities in Steam (1971) ANSI Z111.5

Forming Quality, Zinc Coated (Galvanized) by the Hot Di

Forms and Information Flow Used in Ordering, Shipping,

Forms for Cast in Place Concrete (1966)

Forms for Electron Devices (1972)

Forms of Virgin Metal for Nuclear Application (1973) an

Forms (1967) ANSI N134

Forms (1972)

Forms) EEI MSJ-4

Formula for Butadiene-Acrylonitrile Rubbers (NBR) (19

Formula for Carbon Blacks in Natural Rubber (Nr) (1973)

Formula for Carbon Blacks in Styrene-Butadiene Rubber

Formula for Isobutene-Isoprene Rubbers (IIR) (1973)

Formula for Pigmented Styrene-Butadiene Rubbers (SBR)

Formulae for Fibre Cans (1973)

Formulas for General Purpose Chloroprene Rubbers (CR) Formulas for Natural Rubber $(\mathrm{Nr})$ and Isoprene Rubber (I Formulas for Nonpigmented Styrene-Butadiene Rubbers Formulas for Solution Poly-Butadiene Rubbers (BR) (19 Formulas, and Tables for (Quality) Control Charts (1971 Form work (1972)

FORTRAN Procedures for Executive Functions and Process Fospirate (Common Name for the Pest Control Chemical) ( Fossil Fueled Plants (1972) / / He Prevention of Water Da
Foundation Drain, Under Basement Floor, Sump Conductor,

Foundation Piers (1969)

Foundation System: House Design and Construction Meth.

(Foundation) (1963)

Foundations and Retaining Walls (1973)

Foundations and Shoulders for Concrete Pavements (1965)

Foundations, and Retaining Walls (1973)

Fountain Units (1973)

Fountains and Self Contained, Mechanically Refrigerated

Fraction from Low Olefinic Petroleum Naphthas (1973) an

(Fractional and Integral Horsepower) (1972)

(Fractional and Integral Horsepower) (1972)

(Fractional and Integral Horsepower) (1972)

Fractional and Integral Horsepower) (1972)

Fractional and Integral Horsepower) (1972)

Fractionator) (1973)

Fractions by Gas Chromatography (1973)

Fractions by Mass Spectrometry (1973)

Fractions (1971) ANSI Z1 1.310

Fracture Toughness of Metallic Materials (1972) ANSI Z2

Fragment Counting Plate and Cover) (1962)

Fragments and Rodent Hairs in Flour (Cereal Chemistry)

Fragments and Rodent Hairs in Rye Flour (Cereal Chemis

Fragments and Rodent Hairs in Soy Flour (Cereal Chemist

Fragments, Hairs, Feathers, Wood Fibers, Splinters, Etc

Frame and Hardware Schedule (1972)

Frame and Light Construction Buildings (1970) ANSI Z98.

Frame Assemblies (1972)

Frame Assemblies (1972)

Frame Assemblies (1972)

Frame Assemblies (1972)

Frame Assignments for A lternating-Current Integral Ho

Frame Construction (Tech. Notes) (1966)

Frame Design and Construction Requirements, and Install Frame Details (1972)

Frame for Agricultural Tractors (Test Procedures and PE Frame Lock for Articulated Vehicles (Construction and I Frame Preparation for Offset Intermediate Pivot (1973)

Frame Preparation for 181 and 190 Series Deadlock Strik

Frame Shoring Erection Procedure (1969)

Frame Shoring Safety Rules (1972)
Rec. Steel

Rec. Steel

EIA

ASTM

ASTM

ASTM

ASTM

ASTM

ASTM

ASTM

AA

$\mathrm{Rm}$ B ICBO

ICBO

ASTM

ASTM

ASTM

WSTI

USC

ICBO

AACC

ASTM

ASTM

ASTM

NWHA

ACI

ASTM

ASTM

ASTM

AIOA

NEMA

ASTM

ASTM

ASTM

ASTM

ASTM

ASTM

ASTM

ASTM

ASTM

ASQC

SSI

ISA

ANSI

ASME

ASTM

ACI

NFORP

RS4 19

C532

B54?

D1245

D2790

D2331

D932

D326

11

UBCS $27-10$

UBCS27.9

A500

A501

A618

*1

CS236

UBCS25-25

04-23

C 470

D2186

A527

*1

66.66

F107

B349

B 414

F700

EI 14

D3187

D3192

D3191

D3188

D3186

1

D3184

D3185

D3189

Al

*9

S61.I

K62.1I1

TWDPS-1

D2311

69-42

RP7

ICBO UBC $* 8-5$

$\mathrm{ACI} \quad 65-43$

ICBO UBC $3-29$

UL 621

ARI 1010

ASTM D2002

Std. for NEMA MGI.I0

for Tests NEMA

Ation Data NEMA

Dimensions NEMA

TAPPI

Tent. ASTM

Tent Met ASTM 
Rec. Pract. for Installation of Fixed Partitions of Light n (Test Procedures and Performance R/ Std. for Protective ents for Regular Bicycles Including Tests for Static Load, ering Drawing and Related Documentation Practices: Chassis Misc. Ornamental Items, Stairwork and Handrails, Exterior Std. Steel Doors and Quality Stds. for Exterior Rec. Spec. for Builders' Hardware on Std. Steel Doors and Rec. Spec. for Std. Steel Doors and Std. Spec. for Photographic Printing Rec. Spec. for Steel Bi.Fold Closet Doors and Rec. Erection Instructions for Steel Door Rec. Std. Details for Steel Doors and Rec. Weatherstripping for Std. Steel Doors and Rec. Std. Minimum Acceptance Values for Steel Doors and Minimum Hardware Reinforcing Garages on Steel Doors and

Dwelling Construction Under the Uniform Building Code: and Spikes, Washers, Pins, Dowels, Joints, Split Rings and welling Construction Under the Uniform Building Code: Wall ard and Backing Board (1/

Spec. for Installation of Steel uct Use Manual: Grade Selection, Std. Size, Design Values, mance Spec. for Adhesives for Field Gluing Plywood to Wood welling Construction Under the Uniform Building Code: Roof spec. for Adhesives for Fastening Gypsum Wallboard to Wood ing Code Std. for Structural Floor and Roof Plank and Beam Std. for Adhesives for Fastening Gypsum Wallboard to Wood

operties, Weight, Stress of Board, Timber, Lumber, Siding.

Nitrogen and Fertilizer Solutions Containing More Than $2 \%$ w Machines (1972)

(1972) ANSI H23.I1

able and Marine $\mathrm{Oi} /$

Std. Spec. for Std. Spec. for Oxygen

Std. Analytical Meth. for Determining Meth. of Analysis of

mistry) (1967) Meth. of Analysis of Monoglycerides and tural Wheel Tractors (1972) SAE J715

1 Wheeled Tractors (1972) Std. for 3 Point Std for 3 Point tors Equipped with Quick Attaching Coupler for Three Point (1971) ANSI C81.42 Std. Spec. for

d Moderate Heat Resistant, 12.5Cr Low Carbon (SAE 51416F) $s$ (Cereal Chemistry) eat and Other Grains.

Qualitative Meth of Analysis for Improved Kjeldahl Meth. for Nitrate Tent. Meth. of Analysis for Std. Meth. of Test for Tack Std. Meth. Test for Tack Rec. Pract. for Bus Stops for Refrigerators, Com/ Std. Test Procedure to Determine the ion of Household Refrigerators, Combination Refrigerator Safety Std. for Ice Cream Sid. for Safety for Household Refrigerators and old Refrigerators, Combination Refrigerator-Freezers and c. Pract. for Use of the Refractometer for Determining the th. for Critical Dilation of Concrete Specimens Subject to Guide Spec. for Hydraulic and Electric Elevators Requirements for the Construction and Certification of Spec, for International (lso)

adruplex Video Magneti/

Rec. Pract. for Reference Carrier ant) of Solid Electrical Insulating Materials at Microwave ty) and Dissipation Factor of Solid Ceramic Dielectrics at tentially Hazardous Electromagnetic Radiation at Microwave

ltage and Strength of Insulating Gases at Commercial Power e Injury Statistics (1971) (1973)

Std. for Meth. of Measuring Std for Operating Supply Voltage and ment Use/ Std. Meth. for Measuring Audio Amplifier Single ting Caps (1971) ANSI C95.4 Safeguards Against Radio

irborne VOR Receiving Equipment Operating Within the Radio tance Measuring Equipment (DME) Operating Within the Radio Reproducers for Audio Record One for 2-In. Quadrup/ Std. i C $83.63 \mathrm{C}$

nsi C83.63B Std. Test Proc. for Low si A37.70 imbers for Marine, Land, and afson Meth. of Analysis for Original Ash in Phosphated and

Std. Test Meth. for Absorbed Gamma Radiation Dose in the ricants in Vacuum / hay, Straw) (1968)

Std. Meth. of Test for Coefficient of

Std.

Std. Meth. of Test for Coefficient of Kinetic Plane Meth/ Plane Meth.) (1972) lane Meth.) Meth. for Inefficient of Static Std. Meth. of Test for Coefficient of Static Std. Meth. of Test for Kinetic Coefficients of rec. Pract. for SAE No. 2 (Automotive Transmission) Clutch Std. Meth. of Test for Adhesion of Vulcanized Rubber Spec. for Bearings, Ball and Roller, Rod End, Anti Meth. of Test for Coefficient of Sid. Meth. of Test for Measuring Surface
Frame Type for the Purpose of Conserving Their Sound in Frame (For Agricultural Tractors for Overhead Protectio Frame, Fork, Steering Assembly and Tire Blowout (1972) Frames-Passenger Car and Light Truck-Ground Vehicle Frames and Sash, Screens, Blinds and Shutters, Flush, S Frames for Modular Masonry Building Construction (1972) Frames (Architectural Woodwork) (1973)

Frames (Reinforcement-Application) (1972)

Frames (1969)

Frames (1972)

Frames (1972)

Frames (1972)

Frames (1972)

Frames (1972)

Frames (1972)

Frames (1972)

Framing Anchors) (1973)

Framing and Weather Protection (1973)

Framing Members to Receive Screw Attached Gypsum Wallbo

Framing Rec., Properties, Weight, Stress of Board, Timb Framing (1971)

Framing (1973)

Framing (1973)

Framing (1973)

Framing (1973)

Framing, Decking, Flooring, Ceiling, and Partition (197

Free Cutting Brass Rod, Bar, and Shapes for Use in Scre

Free Electrolytic Copper Wire Bars, Billets, and Cakes

Free Fatty Acids Content in Corn Oil and to Other Veget

Free Fatty Acids in Fats (Cereal Chemistry) (1967)

Free Glycerol in Fats, Oils and Shortenings (Cereal Che

Free Link Attachment for Hitching Implements to Agricul

Free Link Hitch Attachment of Implements to Agricultura

Free Link Hitch (1971) SAE J909A

IIcultural Whe

Free Machining Stainless and Heat Resisting Steel Wire

Free Machining (1973)

Bars and Forgings, Corrosion an

Free or Combined Tartaric Acid in Presence of Phosphate

Free Samples for Analysis of Crude Protein in Bread, Wh

Free Sulfur Dioxide in Wine (Enology) (1972)

Free Time of Caulking Compounds and Sealants (1973)

Free Time of Elastomeric Type Joint Sealants (1971)

Freeway Operations (1971)

Freezer Temperature and Energy Consumption of Household

Freezers and Freezers (1974)

Freezers and Soda Fountain Units (1973)

Freezers (1973) ANS1 B97.1

Ture and Energy Consumpt

Freezers (1974)

/Ture and Energy Consumption of Househ

Freezing (1972T)

(Freight and Passenger) (1971)

Freight Containers (1968)

Freight Containers (1972)

Frequencies and Deemphasis Characteristics for $2 \mathrm{In}$. Qu

Frequencies and Temperatures to $1650 \mathrm{C}$ (1970) ANSI C59.

Frequencies to $10 \mathrm{MHz}$ and Temperatures to $500 \mathrm{C}$ (1968)

Frequencies (1973)

Frequencies (1974)

Entation for the Measurement of $\mathrm{Po}_{0}$
/F Test for Dielectric Breakdown $\mathrm{V}_{0}$

Frequency and Cost, and Recording Patron and Nonemploye

Frequency Heating Equipment (lnduction and Dielectric)

Frequency of Office Machines (1973)

Frequency Output for Institutional Audio-Visual Equip

Frequency Radiation Hazards in the Use of Electric Blas

Frequency Range of 108-118 Megahertz (1972)

Frequency Range of 960.1215 Megahertz (Flight Control)

Frequency Response and Operating Level of Recorders and

Frequency (Below $3 \mathrm{MHz}$.) Electric Connectors (1974) Ans

Frequency (Below $3 \mathrm{MHz}$.) Electrical Connectors (1971)

Fresh Water Use (1974) ANSl Oll

the Preservativ

Freshly Mixed Concrete by the Pressure Meth. (1972T) an

Freshly Mixed Self Rising Flour (Cereal Chemistry) (196

Fricke Dosimeter (1972)

Friction and Wear Characteristics of Dry Solid Film Lub

Friction Coefficients of Chopped Forages (Grass, Corn,

Friction for Wax Coatings (1973) ANSI Z11.222

Friction Loss Tables for Solving Fire Protection System

Friction of Corrugated and Solid Fiberboard (Horizontal

Friction of Corrugated and Solid Fiberboard (Horizontal

Friction of Corrugated and Solid Fiberboard (Inclined P

Friction of Plastics (1972)

Friction Test Machine Test Procedure (1972)

(Friction Test) (1970) ANSI J3.1

Friction, Airframe (1974)

Friction, Yarn to Metal (1972T)

Frictional Properties Using the British Portable Tester
ASTM

ASAE

BMA

ANSI

AWI

STD

AWI

STDI

STD

ANSI

STDI

STD

STD

STDI

STDI

Re NBHA ICBO
$\mathbb{W}$ WPA

ICBO

ANSI

WWPA

Perfor APA

ICBO

Std. ASTM

ICBO

WWPA

FI

ASTM

ASTM

CR

AACCH

AACCH

ASAE

SAE

ASAE

ASTM

SAE

AACCH

ASE

ASTM

ASTM

ITE

AHAM

AHAM

UL

UL

Re ASTM

ASTM

CSI

ABS

ANSI

SMPTE

ASTM

ASTM

ANSI

ASTM

ANSI

ANSI

ANSI

IME

d. for a RTCA

RTCA

ANSI

EIA

EIA

ASTM

ASTM

A ACC

ASTM

ASTM

ASAE

A STM

FMS

ASTM

TAPPI

ASTM

ASTM

SAE

ASTM

NSA

ASTM

ASTM

E497

S 310.2

14.32 . I

1.1

110

107

0

PH3.15

104

105

111 
Std. for Safety for Oil Fried Water Heaters (1974)

Std. Meth. of Test for Fusion Flow of Porcelain Enamel Frits (Flow Button Meth.) (1970) ANSI Z167.10 Rec. Pract. for Engine Foot Mounting (Front and Rear) (1970)

Dead Front Distribution Switch boards (1972)

Rec. Safety Sign for

d Logging (Short Haul); Grader, Loader, Dozer, Mining Carr, lock for Articulated Vehicles (Construction and Industria nce Criteria for Roll Over Protective Structure for Weeled roll Over Protective Structure for Track Type Tractors and Structure (FOPS) for Prime Movers, Wheeled and Track Type Std. for Dimensions fo Safety Std. for Dead st Meth. for Color Change in Fabrics Due to Flat Abrasion st Meth. for Color Change of Fabrics Due to Flat Abrasion Meth. for Preparation of Sample: Meth. of Analysis for Citric and Isocitric Acids in Meth. for Preparation of Sample: Fruit and Uniform Fire Code:

roadle af Evergreens; Rose Grades; Vines and Ground Covers in Flour, Bread, and Baked Cereal Products Not Containing Uniform Fire Code: Fruit Ripening Process (Banana, Citrus

Grades; Vines and Ground Covers; Fruit Tree Grades; Smal Std. for Hotel and Restaurant Gas Deep Fa Std. for Safety for Household Electric Skillets and f Locking, Plate-Corner, Counterbored, Floating, $125 \mathrm{Ks}$ aligning, Self Locking, Plate-Two Lug, Floating, $125 \mathrm{Ks}$

$$
\text { Std. for }
$$
972)

Fluorosilicone Rubber, General Purpose, High Temperature, ational Vehicles (1973) ANSI Al47/ Safety Std. for Liquid s Ammonia (Fertilizer Grade) in Residential and Commerical Std. Meth. of Test for Gross Calorific Value of Solid and Medium Speed Stationary Diesel and Gas Engine Gaseous Mounting, Liquid Withdrawal Only/ Rec. Pract. for LP Gas al or Vertical Mounting), Liquid / Rec. Pract. for LP Gas lers $(197)$

Fire Hazards and Protection Rec. for Low Water

he Compatibility of Vehicle Fuel Tanks and Fill Pipes with e Mechanical Propert/ ger Cars and Light Tr/ ger Cars and Light $\mathrm{Tr}$ /

Std. Rec. Pract. for Examination of Rec. Pract. for the Measurement of Rec. Pract. for the Measurement of Uniform Plumbing Code: Safety Std. for Oxy

Diameter) (1971) Rec. Pract. for Diesel Std. for Metric Thread

Medium Speed Stationary Diesel and Gas Engine Power Plant $s$ with One / Std. for Prevention of Furnace Explosions in Std. Meth. of Test for Compatibility of

for Low and Medium Speed Stationary Diesel and Gas Engine Std. Pract. for Combustion Chambers, Std. Meth. of Test for Stability of Distillate Std. Spec. for

Std. Meth. of Test for Sediment in Crude and Std Spec. for Gas Turbine Tent. Meth. of Test for Pumpability of Industrial Rec. Pract. for

4)

Std. for Nuclear Material Control Systems for Std. for Guide to Principal Design Criteria for Nuclear for Radiochemical Determination of Cesium-137 in Nuclear nd Pract. for Design, Construction and Stowage of Portable ls, Construction and Installation of Permanently Installed ing Conditions for Evaluating the Compatibility of Vehicle Safety Std. for Automotive

of Test for Atom Percent Fission in Uranium and Plutonium of Test for Atom Percent Fission in Uranium and Plutonium

Std. Meth. of Test for Atom Percent Fission in Uranium Std. for Quick Disconnect Devices for Use with Gas Std. for Refrigerators Using Gas

Tent. Meth. of Test for Total Acidity in Aviation Turbine Atmospheric Type Welded Steel Inside Tanks for Oil Burner ract. and Std. for the Design and Construction of Gasoline

Steam Turbines Used for Electric Power Generation: Fossil netration Assemblies in Containment Structures for Nuclear

Tent. Meth. of Test for Flash Point of Aviation Turbine std. Meth. of Test for Electrical Conductivity of Aviation

Std. Meth. of Test for Knock Characteristics of Motor

Std. Meth. of Test for Knock Characteristics of Motor Std. Meth. of Test for Trace Metals in Gas Turbine Meth. of Test for Thermal Oxidation Stability of Turbine ur in Gasoline, Kerosine, Aviation Turbine, and Distillate alculation of Liquid Heat Capacity of Petroleum Distillate

Std. Meth. of Test for Smoke Point of Aviation Turbine d. Meth. of Test for Thermal Stability of Aviation Turbine t for Water Separation Characteristics of Aviation Turbine Std. Meth. of Test for Lead and Vanadium in Gas Turbine $h$ of Test for D.C. Electrical Conductivity of Hydrocarbons Std. Meth. of Test for Wax Appearance Point of Distillate
Front Dist ribution Switchboards (1972)

Front End Agricultural Loaders (1972)

Front End Loaders and Dozers) (1971)

Front End Loaders and Weeled Dozers (1972)

Um Performance Criteria for

Front End Loaders (1972)

s, Track Type Tractors

Front Lens Mounts for Cameras (1972)

Front Switchboards (1972)

Frosting): Every Meth. (Textile Colorfastness) (1970)

(Frosting): Screen Wire Meth. (Textile Colorfastness) (1

Fruit and Fruit Products (Cereal Chemistry) (1962)

Fruit and Its Products (Cereal Chemistry) (1962)

Fruit Products (Cereal Chemistry) (1962)

Fruit Ripening Process (Banana, Citrus Fruit) (1973)

Fruit Tree Grades; Small Fruits (Berry, Currant, Grape,

Fruit (Cereal Chemistry) (1962)

\begin{abstract}
/Analysis of
\end{abstract}
Fruits (1973)

Fryers (197I)

Frying Type Appliances (1973) ANSI C33.11.2

Ftu, 450 and 800 Deg. F (1973)

Ftu, 450 Deg. F and 800 Deg. F (1973)

Fuel and Oil Hoses (1970)

Fuel and Oil Resistant, 65-75 (1973)

Fuel Assembly Identification in Nuclear Power Plants (

Fuel Burning Heat Appliances for Mobile Homes and Recre

Fuel Burning, Dispensing, and Handling Facilities (1974

Fuel by the Isothermal Jacket Bomb Calorimeter (1973)

Fuel Characteristics and General Spec. (1972)

Fuel Container, Quickly Demountable Type for Horizonta

Fuel Container, Quickly Demountable Universal (Horizont

Fuel Cutoffs and Automatic Feedwater Regulators for Boi

Fuel Dispensing Facilities (Passenger Cars, Multipurpos

Fuel Element Cladding Including the Determination of Th

Fuel Evaporative Emissions from Gasoline Powered Passen

Fuel Evaporative Emissions from Gasoline Powered Passen

Fuel Gas Piping (1973)

Fuel Gas Torches (1973)

Fuel Injection Nozzle and Holder Assembly (17Mm Nominal

Fuel lnjection Tubing Connections (1971)

Fuel Oil and Cas Systems (1972)

Fuel Oil and Natural Gas Fired Watertube Boiler Furnace

Fuel Oil Blends by Spot Test (1972) ANSI Z11.315

Fuel Oil Characteristics (1972)

Fuel Oil Injection, and Gas Admission Systems (1972)

Fuel Oil (Accelerated Meth.) (1974)

Fuel Oil (1973) ANSI Z11.203

Fuel Oils by Extraction (1969) ANSI Z11.58, API 2561

Fuel Oils (1971) ANSI Z1 1.312

Fuel Oils (1973)

Fuel Pump Mountings for Diaphragm Type Pumps (1971)

Fuel Reprocessing Facilities (A Guide to Practice) (197

Fuel Reprocessing Facilities (1973)

Fuel Solutions (1970) ANSI N117

Fuel Systems and Portable Containers for Flammable Liqu

Fuel Systems for Inboard and Outboard Powered Boats (19

Fuel Tanks and Fill Pipes with Fuel Dispensing Faciliti

Fuel Tanks (1973)

Fuel (Mass Spectrometric Meth.) (1969) ANSI N108

Fuel (Neodymium-148 Meth.) (1969) ANSI N118

Fuel (Radiochemical Meth.) (1969) ANSl N107

Fuel (1971)

Fuel (1972)

Fuel (1973)

Safety
Fuel (1973)

Safety Std. for

Fueled Power Generating Stations (1972) ANSl N45.3

Fuels by Setaflash Closed Tester (1973)

Fuels Containing a Static Dissipator Additive (1971) an

Fuels Using the Compression Ratio (CR) Technique for Mo

Fuels Using the Compression Ratio (CR) Technique for Re

Fuels (Atornic Absorption Meth.) (1972) ANSI Z11.317

Fuels (JFTOT Proc.) (1973)

Fuels (Potentiometric Meth.) (1973)

Fuels (1971) ANSI Z11.313

Fuels (1972) ANSI Z11.137

Fuels (1972) ANSI Z11.148

Fuels (1972) ANSI Z11.301

Fuels (1972) ANS1 Z11.316

Fuels (1972) ANS1 Z11.322

Fuels (1972) ANSI Z11.325

UL

ASTM

SAE

NEMA

ASAE

TRA

SAE

SAE

SAE

ANSI

UL

AATCC

AATCC

$\mathrm{AACCH} \quad 62.25$

ACCH

$\mathrm{AACCH} \quad 62.25$

ICBO UFC 2 ARTI6

ANSI

$\mathrm{AACCH}$

$\mathrm{ICBO}$

ANSI

ANSI

UL

NSA

NSA

$S A E$

SAE

ANSI

UL

UL

ASTM

DEMA

ITA

ITA

FMS

$\mathrm{SAE}$

ASTM

SAE

$S A E$

ICBO

UL

$\mathrm{SAE}$

$S A E$

DEMA

NFPA

ASTM

DEMA

DEMA

ASTM

ASTM

ASTM

ASTM

ASTM

$\mathrm{SAE}$

ANSI

ANSl

ASTM

$A B Y C$

$A B Y C$

SAE

UL

/Eth. ASTM

$\mathrm{Z} 60$.

30.10

UFC*2ARTl6

Z60.1

221.27

1083

1766

AMS7266

N18.3

$307 \mathrm{~A}$

331

D3286

* 1.15

$6 \mathrm{Al}$

$6 \mathrm{~A} 2$

12-37

J398A

E453

$\mathrm{J} 170 \mathrm{~A}$

J171A

UPC*l.12

123

J265

J242

*1-13

85

D2781

*1-14

$* 1-2$

D2274

D396

D473

D2880

D3245

J625A

$\mathrm{N} 15.13$

Nlol.3

E320

$\mathrm{H} 25$

H 24

J398 A

395

E244

E321

ASTM

ANS1

ANSI

ASTM

UL

ABYC

SME

ASTM 
National Bureau of Standards KWIC Index of Engineering Standards

Std. Meth. of Test for Water Reaction of Aviation Fuels (1972) ANSI Z11.82

Guidance Material for Aviation Turbine Fuels (1973)

1. Meth. of Test for Undissolved Water in Aviation Turbine c Determination of Fission Zirconium in Irradiated Nuclear Std. Spec. for Aviation Turbin

h. of Test for Particulate Contaminant in Aviation Turbine Std. Meth. of Test for Specific Gravity of Gaseou

Std. for Safety for Valves for Flammable Liquids and nd Soybeans, Whole or Ground Full Fat or Extracted Flakes, nd Soybeans, Whole or Ground Full Fat or Extracted Flakes, f Dispersible Nitrogen in Ground Soybeans, Whole or Ground of Dispersible Protein in Ground Soybeans, Whole or Ground estern Red Cedar and Alaska Yellow Cedar Timber/Std. for or Lodgepole Pine Wood Poles Preservative Treatment by the Std. Spec. for $55 \mathrm{Gal}$ Std. Spec. for $30 \mathrm{Gal}$ Std. Spec, for $55 \mathrm{Gal}$ Std. Spec. for $57 \mathrm{Gal}$ Std. Spec. for $30 \mathrm{Gal}$ Std. Spec. for $16 \mathrm{Gal}$ Std. Spec, for $16 \mathrm{Gal}$

-Item 260) (1974)

c-Item 260) (1974)

c-Item 260) (1974)

c-Item 260) (1974)

(e 40, Nmfc-Item 260) (1974)

Paved Surfaces Using a Passenger Automobile Equipped with

Paved Surfaces Using a Passenger Automobile Equipped with ec. Pract. for Test. Pavement Polishing in the Laborator arm) Implements and Tractors (1972) SAE J955

Std. for Screw, Hex Head, Tri Wing Recess, A286 Cres, Std. for Self Locking, Flat 100 Deg. Head, Std, for Self Locking, Pan Head,

Std for Self Locking. Flat Fillister Head,

for Screw, 100 Deg. Oval Head, Tri Wing Recess, A286 Cres Sid. for Hex Head Screw, Recessed.

r Screw, Hex Head, Tri Wing Recess, 6Al-4V Titanium Alloy, Std. for Screw, Hex Head, Tri Wing Recess, Alloy Steel,

std. for Screw, 100 Deg. Head, Tri Wing Recess, A286 Cres, Std. for Screw, Pan Head, Tri Wing Recess, A286 Cres,

rew, 100 Deg. Head Tri Wing Recess, 6Al.4V Titanium Alloy,

d. for Screw, 100 Deg. Head, Tri Wing Recess, Alloy Steel, Std. for Screw, Machine, Pan Head, Std. for Screw, Machine, Flat Fillister Head, Std. for Screw, Machine, Flat 100 Deg. Head,

nd Nonlocki/

elf-Locking/

Std. for Machine Screw, Flat Fillister Head. Std. for Machine Screw, Flat Fillister Head, Std. for Aircraft, Pan Head, Phillips Recess Std. for Screw, Machine, 100 Deg. Flat Head Std. for Machine Screw, Flat Fillister Head,

ng and Nonl/

Speeds of 7.5 In. 0 190.1 Std. for Reproducer Test Tape th. for Colorfastness of Dyed Wool Fabric and Yarn to Mil (Cereal Chemistry) (1962) Meth. of Analysis for s of Textiles to Atmospheric Oxides of Nitrogen (Burnt Ga Uniform Fire Code:

d. Meth. of Test for Stiffness Properties of Plastics as $A$ oven Glass Fabrics, Cleaned, and After Finished with Epoxy ndustrial Computer System FORTRAN Procedures for Executive ration of Design Bases for Systems That Perform Protective Rec. Pract. for Automatic Transmission Loss Prevention Data on

l Exhaust Systems (1971) Std. for evaluating Deg. of Surface Disfigurement of Paint Films by Meth. of Analysis of Std. for Dichlozoline

Test Meth for the Evaluation of Mildew and Rot eld and Compound Microscope, Petri Dish, Sieve, Separatory tertube Boiler Furnaces with One / rner Boiler Furnaces (Fire Protec/ Std. for Prevention of Rec. for Prevention of ptical /

Std. Meth. for Spectrochemical Analysis of Blast tes (Sand, Crushed Stone, Gravel and Air Cooled Iron Blast erties (Temperatures) of Plastics Using a Hot Air Ignition losions in Fuel Oil and Natural Gas Fired Watertube Boiler explosions in Pulverized Coal Fired Multiple Burner Boile Std. for Gas Fired Duc

Std. for Gas Fired Gravity and Fan Type Vented Wall

Fired Gravity and Fan Type Sealed Combustion System Wall Std. for Gas Fired Gravity and Fan Type Direct Vent Wall Std. for Gas Fired Gravity and Forced Air Central Std. for Gas Fired Gravity and Fan Type Floor Safety Std. for Oil Fired Centra Std. for Safety for Oil Fired Floor

powered Forced Air (Domestic and Light Commercial) Central for Vented Decorative Appliances, and Wall Furnaces, Floor e: Requirements for Vented Decorative Appliances, and Wall Guide Spec. for oad (Axial) Bearing Steel Studs, Runners (Track) and Rigid Exterior (Stucco) and Interi/
Fuels (1973)

Fuels (1973)

Fuels (1973) ANSI Z11.204

Fuels (1973) ANSI Z11.264

Fuels (1973) ANSI Z77.12

Fuels (1974)

Full Fat and Defatted Soy Flours and Grits, and Soybean

Full Fat and Defatted Soy Flours and Grits, and Soybean

Full Fat or Extracted Flakes, Full Fat and Defatted Soy

Full Fat or Extracted Flakes, Full Fat and Defatted Soy

Full Length Thermal Process Preservative Treatment of W

Full Length Thermal Process (1973)

Full Removable Head Universal Drum (DOT-17H) (1968)

Full Removable Head Universal Drum (Dot-17H) (1974)

Full Removable Head Universal Drum (Ufc-Rule 40, Nmfc

Full Removable Head Univers:

Full Removable Head Universal Drums (Ufc-Rule 40, Nmf

Full Removable Head Universal Drums (Ufc-Rule 40, Nmf

Full Removable Head, Lug Cover Universal Drums (Ufc-R

Full Scale Tires in the Diagonal Braking Mode (1973)

Full Scale Tires (1971T)

Full Scale Wheel Meth.) (1972)

ull Shielding of Power Drive Lines for Agricultural ( $\mathrm{F}$

Full Thread Nonlocking (1972)

Full Thread Part Screw (1974)

Full Thread Part Screw (1974)

Full Thread Part Screw (1974)

Full Thread Self Locking and Nonlocking (1972)

Full Thread (1973)

Full Thread, Nonlocking (1972)

Full Thread, Nonlocking (1973)

Full Thread, Self Locking and Nonlocking (1972)

Full Thread, Self Locking and Nonlocking (1972)

Full Thread, Self Locking and Nonlocking (1972)

Full Thread, Self-Locking and Nonlocking (1973)

Full Thread, Torq Set (1973)

Full Thread, Torq Set (1973)

Full Thread, Torq Set (1973)

Full Thread, Tri Wing Recess, A286 Cres, Self-Locking a

Full Thread, Tri Wing Recess, Titanium Alloy, 6Al-4V, S

Full Threaded, Alloy Steel Machine Screw (1974)

Full Threaded, Alloy Steel (1973)

Full Threaded, Tri Wing Recess, Alloy Steel, Self-Locki

Full Threaded, 160 KSI Steel, Drilled Head Bolt (1972)

Full Track, 1/4 In. (6.3 Mm) Width, Open Reel (For Tape

Full Tubular, Split Rivets and Caps (1972)

Fulling (1972) ANSI L14.5

Fumaric, Pyruvic, Lactic, Succinic, and Tartaric Acids

Fumes) (1972) ANSI L14.54

Test Meth. for Colorfastnes

Fumigation and Thermal Insecticidal Fogging (1973)

Function of Temperature by Means of a Torsion Test (197

Functional Silane Type Finishes for Plastic Laminates (

Functions and Process Input-Output (1972)

Functions in Nuclear Power Generating Stations (1973)

Functions Terminology (1969)

Fundamental Defects in Fires Over 050,000 (1973)

Fundamentals Governing the Design and Operation of Loca

Fungal Growth or Soil and Dirt Accumulation (1973)

Fungicide Residues in Grain (Cereal Chemistry) (1962)

Fungicide) (1971)

Fungicides) Resistance of Textiles (1971) ANSI L14.55

Funnel, Trap Flask, Step Wedge for X-Ray Standardizatio

Furnace Explosions in Fuel Oil and Natural Gas Fired Wa

Furnace Explosions in Pulverized Coal Fired Multiple Bu

Furnace Iron by the Point to Plane Technique Using an $O$

Furnace Slag) (1973)

Furnace) (Fire Safety) (1973)

G Code Std. for Concrete Aggrega

urnaces with One Burner (1972)

Furnaces (Fire Protection) (1973)

land Flash Ignition Prop

Ention of Furnace Exp

Furnaces (1971)

Furnaces (1972)

Vention of Furnace

Furnaces (1972)

Furnaces (1973)

Furnaces (1973)

Furnaces (1973)

Furnaces (1973) ANSI Z96.

Furnaces (1974)

Furnaces) (1972)

Furnaces, and Unit and Room Heaters (1973)

Furnaces, Floor Furnaces,

Furring and Lathing (1972)

Furring Channels for Screw Application of Gypsum Board

Furring for Portland Cement and Cement Lime Plastering,

Fused Power Circuit Devices (1973)

IATA

Ten ASTM

ASTM

ASTM

ASTM

UL

$46 \cdot 23$

AACCH $46-24$

$\mathrm{AACCH} \quad 46.23$

AACCH $\quad 46.24$

AW'PA

std. F AWPA

ANSI

ANSI

ANS

ANSI

ANSI

ANS

ASTM

ASTM

Std. R ASTM

ASAE

NSA

NSA

NSA

NSA

Std. NSA

NSA

Std. Fo NSA

NSA

NSA

NSA

or Sc NSA

St NSA

NSA

NSA

NSA

NSA

NSA

NSA

NSA

NSA

NSA

EIA

ANSl

C8

$\mathrm{C} 10$

MH2.5

MH2.13

MH2.2

MH2.11

MH2. 12

MH2. 14

MH2.8

E503

E445

E451

S297T

6000-3

1190

1191

6.500-6

1096

6100.3

5900-03

5700.6

6900-06

5800-6

5600-06

1100

1101

5400-06

5500-06

514

$5300 \cdot 06$

563.572

RS400

AATCC 2

AACCH $04-24$

AATCC 23

ICBO

ASTM

ASTM

Td. for I ISA

ANS

$S A E$

FMS

NSI

IF ASTM

AACCH 60.01

ANSI K62.102

AATCC 30

AACCH $28-90$

NFPA 85

FMS 6-2

ASTM E485

ICBO

ICBO 
safety Std, for Highway Emergency Signals (Flare, Lantern, Std. Spec. for Amyl Acetate Made from Other Electronic Equipment

Std. for Safety for Special afety Std. for Class R afety Std. for Plug Std. for Low Voltage Cartridge Safety Std. for Class H Cartridge Safety Std. for Automotive Glass Tube Std. for Electric

Std. for Low Voltage Cartridge Std. Spec. for Insert Type Polyethylene

.) (1970) ANSI 2167.10 tic Pipe and Tubing (1973) e 40 (1973) ANSI K65.160 e 80 (1973) ANSI K65.159

e for High Temperature Service (/ opper-Columbium Stabilized All/ eratures (1972)

oderate Temperatures (1973)

2A) ANSI B 125.4 Std. for Manual Gas Tungsten Arc $(\mathrm{Gta})$ Std. for Manual Gas Tungsten Are (Gta) Std. Spec. for Carbon Steel Castings Suitable for
G37.2 Tire Selection Tables for Agricultural (Farm) Machines of Std. for Precision In. opper Alloy No. 260 Brass Strip in Narrow Widths and Light Std Specs. and Tests for Strain f Determining Interior Dimensions of Fiberboard Boxes (Box rews for the Application of Gypsum Sheet Material to Light Std. for Agricultural Press and and Industrial Steering, Drive Planter (Drill) Press, and Std. for Rim Contours for Agricultural Press and Std. for Std. for

Std. Procedure for Bench Calibration of Tank Level ng, and Line Pipe Threads (1974) Spec. for Threading, Std. on Television: Measurement of Differential Calculation of Hea Std. Manual for Electric Comfort Conditioning (Heat Loss, t of Glucose in Sugar Mixtures Maltose, Sucrose, Mannose, or Spectrochemical Analysis of Uranium Oxide $\left(\mathrm{U}_{33} \mathrm{O}_{\kappa}\right)$ by 73) ANSI H44.1 Std. for Cast and Wrought for Determination of Phosphate Coating Weight on Hot Dip tubing (19/ Std. for Polyvinyl Chloride Externally Coated ec. for Spot Test Solution for Detecting Chromate Films on Spec. for

Std. Spec for Welded and Seamless Black and Hot Dipped

Detection of Hexavalent Chromium Containing Treatments on Spec. for Detection of Mill Chemical Treatments on Pract. for Safeguarding Against Embrittlement of Hot Dip teel Core for Aluminum Condu/ Std. Spec, for Zinc Coated or Steel Sheets for Culverts and Undcrdrains, Zinc Coated structural Quality in Coils, and Cut Lengths, Zinc Coated nsi G8.21 Std. Spec. for Steel Sheet, Zinc Coated Std. Spec, for Steel Sheet. Zinc Coated pec. for Steel Sheet of Lock Forming Quality, Zinc Coated ec. for General Requirements for Steel Sheet, Zinc Coated (1971) ANSI G8.1 Std. Spec. for Steel Sheet, Zinc Coated d. Meth. of Test for Locating the Thinnest Spot in a Zinc of Way Wire Fencing (1973) /

Std. Spec. for Zinc Coated Std. Spec for Zinc Coated eel Reinforced (ACSR) (1974)/ Std. Spec. for Zinc Coated
Uses (/ Std. Spec. for Black and Hot Dipped Zinc Coated Spec. fo

e Dosimeter (1971) ANSI

Spec. for

ate Cupric Sulfate Dosim/

Std. Meth. of Test for Absorbed
Std. Meth. of Test for Absorbed Std. Meth. of Test for Absorbed Std. Test Meth. for Absorbe

Performance Spec. for Portable X-Or

$r$ Direct and Indirect Reading Pocket Dosimeters for X-And Std. Meth. for Measurement of absorbed Dose of Neutrons, and of Mixtures of Neutrons and Meth. of Test for

l Chemistry) (1/ Meth. of Analysis of Radioactivity (Beta s (Calcined Bauxite, Calcine, Chrome Ore, Clays, Diaspore Spec. for Electric Overhead Bridge an Rec. for Parking Structures Uniform Fire Code:

ling Construction Under the Uniform Building Code: Private Rec. Minimum Hardware Reinforcing 221.1

Std. for Safety for Metal Waste (Trash

Storage, Finishes And/ Redwood Landscape Guide (Rec. for ular Sleeve Attachment for Hitching Implements to Lawn and t Truck, Boat, Baggage, Special Type Trailer, Mobile Home.
Fusee) (1973)

Fusel Oil (85 to $88 \%$ Grade) (1972)

Fuses for Radio and Television Receiving Appliances and Fuses (Electrical) (1973)

Fuses (Electrical) (1973)

Fuses (1972)

Fuses (1973)

Fuses (1973)

Fuses (1973) ANSI C118.

Fuses, 600 Volts or Less (1972)

Fusion Fittings for SDR 11.0 Polyethylene Pipe (1973)

Fusion Flow of Porcelain Enamel Frits (Flow Button Meth

Fusion Polyethylene (PE) Plastic Fittings for (PE) Plas

Fusion Polyethylene (PE) Plastic Pipe Fittings, Schedu

Fusion Polyethylene (PE) Plastic Pipe Fittings, Schedul

Fusion Weld Discontinuities (1972)

Fusion Welded Autenitic Chromium Nickel Alloy Steel Pip

Fusion Welded Chromium-Nickel-Iron-Molydenium-C

Fusion Welded Steel Pipe for Atmospheric and Lower Temp

Fusion Welded Steel Pipe for High Pressure Service at M

Fusion Welded Steel Pipe for High Pressure Service (197

Fusion Welded Unfired Pressure Vessels (1972B) ANSI G81

Fusion Welding Equipment (1974)

Fusion Welding for High Temperature Service (1974) ANSI

Future Design (1972) SAE J711

Gage Blocks for Length Measurement (Thru 20 ln.) (1973)

Gage for Heat Exchanger Tubing (1972) /Td. Spec. for C ASTM

Gage Linear Acceleration Transducers (Instrumentation)

Cage Meth.) (Packaging) (1968) AVSI MH12 19

Gage Steel Studs (l972)

Gage Wheel Tires (Farm Machinery) (1971)

Gage Wheel, Tractor, Implement, and Log Skidder (1974

Gage Wheels (Farm Machinery) (1971)

Gages, Pins and Collar Inspection (1973)

Gaging Practice for Metric Screw Threads (1972)

Gaging Tapes and Sounding Rules (1974)

Gaging, and Thread Inspection of Petroleum Casing, Tubi

Gain and Phase (1960)

Gain Through Opaque Walls (Tech. Notes) (1969)

Gain, Energy Consumption) (1974)

Galactose, Dextrans, and Starch (Cereal Chemistry) (196

Gallium Arsenide Phosphide Wafers (1973)

Gallium Oxide Carrier D-C Are Technique 197

Galvanic Zinc Anodes for Use in Saline Electrolytes (19

Galvanized and Electrogalvanized Steel (Coil) (1974)

Galvanized Rigid Steel Conduit and Electrical Metallic

Galvanized Sheet (Coil Coating) (1974)

Galvanized Steel Chain Link Fence Fabric (1973)

Galvanized Steel Pipe (1973) ANSI B125.1

Galvanized Steel Prior to Conversion Coating (Coil) (19

Galvanized Steel Surfaces (Coil Coating) (1974)

Galvanized Structural Steel Products and Procedure for

(Galvanized) and Aluminum Coated (Aluminized) Stranded

Galyanized) by Hot Dip Process (1971) ANSI G8.28

(Galvanized) by Hot Dip Process (1973) ANSI G8.27

(Galvanized) by the Hot Dip Process for Roofing (1971) a

(Galvanized) by the Hot Dip Process (1971) ANSI G8.22

(Galvanized) by the Hot Dip Process (1971) ANSI G8.23

(Galvanized) by the Hot Dip Process (1973) ANSI G8.25

(Galvanized) by the Hot Dip Process, Commercial Quality

(Galvanized) Iron or Steel Farm Field and Railroad Right

(Galvanized) Steel Barbed Wire (1973) ANSI G8.10

(Galvanized) Steel Core Wire for Aluminum Conductors, St

(Galvanized) Welded and Seamless Steel Pipe for Ordinary

Game (Wildlife) Rifle Ranges (1971)

Gamma-Ray Brachytherapy Sources (1974)

Gamma and Electron Radiation Dose with the Ceric Sulfat

Gamma and Electron Radiation Dose with the Ferrous Sulf

Gamma Radiation Dose in the Fricke Dosimeter (1972)

Gamma Radiation Survey Instruments (1971)

Gamma Radiation (1972)

Performance Spec. Fo

Gamma Radioactivity of Water (1973) ANSI N150

Gamma Rays (1961)

Gamma Spectrometry of Water (1972)

Gamma) in Cereal Products-Emergency Monitoring (Cerea

Ganister Quarzite, Grog, Kyanite, Magnesite, Mullite)

Gantry Multiple Gerder Traveling Cranes (1971)

(Garage-Fire Protection and Safety) (1973)

Garage (1973)

Garages and Carports (1973)

Garages on Steel Doors and Frames (1972)

Garbage) Cans (For Combustible Materials) (1974) ANSI Z

Garden Grades. Application and Desion, Span Allowances,

Garden Riding Tractors (1972)

Std. for One Point Tub ASAE

Garden Tractor, lndustrial Truck and Trailer (1974)
UL

UL

UL

NEMA

$\mathrm{UL}$

$\mathrm{SAE}$

ANS]

ASTM

ASTM

ASTM

ASTM

NSA

A STM

A STM

ASTM

ASTM

ASTM

ASTM

ASAE

AS'TM

ASTM

TRA

ASAE

NSA

ANSI

ANS

APl

IEEE

$\mathrm{BIA}$

NEMA HEI

A ACCH 80-10

ASTM F358

ASTM E4.02

ASTM B4.18

NCCA TB-II.3

Sp NCCA

CLFMI

ASTM

NCCA

NCCA

ASTM

F ASTY

of ASTM

ASTM

ASTM

ASTM

ASTM

ASTM

ASTM

ASTM

ASTM

$* 12$

NCRPM R4

ASTM D300]

ASTM D2954

D1671

N13.4

ANS1 N13.5

ASTM D1690

NCRPM R25

ASTM D2459

AACCH 28-99A

ASTM C 316

CMAA 70

FMS LPD $7-15 \mathrm{~N}$

UFC *2ART 18

ICBO UBC*8-4

NBHA * 10

(

32

3 A5

S3.18T 
Safety Spec. for Power Mowers, Lawn, and Brick in Landscape Architecture mmer, Hedge / Std. for Safety for Cord Connected Electric Std. Meth. of Test for Oil Absorption of Pigments by ven Fabrics (Textile) for Institutional and Industrial Use ven Fabrics (Textile) for Institutional and Industrial Use ven Fabrics (Textile) for Institutional and Industrial Use Tent. Rec. Pract. for Conducting Wear Test on Textile for High lonizing Voltage Mass Spectrometric Analysis of d. Pract. for Combustion Chambers, Fuel Oil Injection, and m Differential Thermal Analysis, Thermogravimetry, Evolved Std. Meth. for Sampling Pressurized Gas for eaks Using the Mass Spectrometer Leak Detector or Residua ec. for Aluminum Alloy Seamless Extruded Tube and Pipe for Std. for

973) exible Connectors of Other Than All Metal Construction for Sid. for Household Cooking Std. for Recreational Vehicle Cookin Std. for Outdoor Cooking Std. for Vented Decorative Std. for Automatic Valves for Std. for Metal Connectors for Std. for Std. for Commercial

ntamination by Manual Meth. (197) ntamination by Automatic Particle/
Std. Meth. for Sampling

Std. Meth. of Sampling Std. for Draft Hoods for Std. Meth. of Test for Total Sulfur in Natural Tent. Meth. of Test for Sulfur in Petroleum Pract. for Direct Injection of Solvent Base Paints into A Std. Meth. of Assay of Di-T-Butyl Peroxide by Std. Meth. for Rapid gues of Chlorinated Biphenyls for C/

Meth. for Determination of Fatty Acid Composition by Std. Meth. for Analysis of Natural Gas Liquid Mixtures by d. Meth. for Obtaining Natural Gas Samples for Analysis by omatic Hydrocarbons in Monocyclic Aromatic Hydrocarbons by

lysis of High Purity N-Heptane and Isooctane by Capillary of Test for Purity and Benzene Content of Cyclohexane by est for Aromatic Traces in Light Saturated Hydrocarbons by of Test for Butadiene Purity and Hydrocarbon Impurities by for Analysis of Liquefied Petroleum Gases (LPG) by Process $t$ for Boiling Range Distribution of Petroleum Fractions by Std. Meth. for Analysis of Natural Gas Liquid Mixtures by st for $C_{1}$ Through $C_{3}$ Hydrocarbons in the Atmosphere by Std. for Volume I, Type I, Std. for Southern Std. Sid. for Supply

ration of Precautionary Labeling and Marking of Compressed 1969) ANSl C59.23 Std. Meth. of Test for as/ Std. Rec. Pract. for Determining Barium Yield, Getter Std. for Installation of Domestic

Warmers, Hot Plates and Griddles, etc.) (1973)

Std for ates (Safety) (1973) Std. Color Marking of Compressed High Pressure Gas Manifolds for Industrial and Commercial and Flexible Connector Fittings for LP (Liquid Petroleum) Sid. for Hotel and Restauran fans (1966) Std. Flue

Std. for Small Manually Operated Metallic Gas Valves in form Laboratory Instrumentation and Techniques for Exhaus std. Pract for Low and Medium Speed Stationary Diesel and std. Pract. for Low and Medium Speed Stationary Diesel and std. Pract. for Low and Medium Speed Stationary Diesel and std. Pract, for Low and Medium Speed Stationary Diesel and std. Pract for Low and Medium Speed Stationary Diesel and std. Pract. for Low and Medium Speed Stationary Diesel and std. Pract, for Low and Medium Speed Stationary Diesel and std. Pract. for Low and Medium Speed Stationary Diesel and std. Pract. for Low and Medium Speed Stationary Diesel and std. Pract. for Low and Medium Speed Stationary Diesel and std. Pract. for Low and Medium Speed Stationary Diesel and std. Pract. for Low and Medium Speed Stationary Diesel and std. Pract. for Low and Medium Speed Stationary Diesel and std. Pract. for Low and Medium Speed Stationary Diesel and Std. Pract. for Stationary Diesel and std. Pract. for Low and Medium Speed Stationary Diesel and std. Pract. for Low and Medium Speed Stationary Diesel and std. Pract. for Low and Medium Speed Stationary Diesel and std. Pract. for Low and Medium Speed Stationary Diesel and Std Pract. Definitions for Stationary Diesel and

Practices for Low and Medium Speed Stationary Diesel and for Scavenging and Supercharging of Stationary Diesel and rator Units for Low and Medium Speed Stationary Diesel and ailed Spec. for Low and Medium Speed Stationary Diesel and
Garden Tractors (1972)

Garden W alls (Tech. Notes) (1968)

Gardening Appliances (Lawn Mower, Cultivator, Edger-Tri Gardner-Coleman Meth. (1968)

Garments (Clothing), Commercial Wash-Whites 190 Deg. Garments (Clothing), 100\% Man Made or Blend Fabrics, Co Garments (Clothing), 100\% Man Made or Blend Fabrics, Dr Garments (1973)

Gas-Oil Saturate Fractions (1971) ANSI Z11.310

Gas Admission Systems (1972)

Gas Analy sis and Detection (1973)

Gas Analysis (1973)

Gas Analyzer in the Tracer Probe Mode (1973)

Gas and Oil Transmission and Distribution Piping System

Gas Appliance Pressure Regulators (1973)

Gas Appliance Thermostats (1971)

Gas Appliances for Installation in Vented Fireplaces (1

Gas Appliances (1971)

Gas Appliances (1972)

Gas Appliances (1972)

Gas Appliances (1972)

Gas Appliances (1973)

Gas Appliances (1974)

Gas Appliances (1967)

Gas Atmosphere Generators (1971)

Gas Baking and Roasting Ovens (1971)

Gas Blow Down Systems and Components for Particulate Co

Gas Blow Down Systems and Components for Particulate Co

Gas Burning Appliances (1971)

Gas by Hydrogenation (1972) ANSI Z77.11

Gas by Oxidation Microcoulometry (1973)

Gas Chromatograph (1973)

Gas Chromatographic Analysis (1973)

Gas Chromatographic Estimation of Higher Boiling Homolo

Gas Chromatography (Cereal Chemistry) (1973)

Gas Chromatography (1968)

Gas Chrom atography (1968)

Gas Chromatography (1968) ANSI Z164.1

Gas Chromatography (1971) ANSI Z11.262

Gas Chromatography (1972)

Gas Chromatography (1972) ANSI Z11.279, IP 262

Gas Chromatography (1973)

Gas Chromatography (1973)

Gas Chromatography (1973)

Gas Chromatography (1973) ANSI Z11.238

Gas Chromatrography (1972)

Gas Clothes Dryers (1972)

Gas Clothes Dryers, Volume II, Type 2 (1974)

Gas Code (1973)

Gas Connectors for Exterior Use on Mobile Homes (1972)

Gas Containers (1971)

Gas Content of Cable and Capacitor (Insulating) Oils (R

Gas Content, and Getter Sorption Capacity for Barium Fl

Gas Conversion Burners (1971)

Gas Counter Appliances (Coffee Pots and Urns, Food Dish

Gas Cylinders Intended for Medical Use in the United Si

Gas Cylinders (1972)

Gas Cylinders (1973)

Gas Deep Fat Fryers (1971)

Gas Densities for Forced and Induced Draft Centrifugal

Gas Distribution Systems Whose Maximum Allowable Operat

Gas Emissions Measurement (Passenger Car and Light Truc

Gas Engine Air lntake and Exhaust Systems (1972)

Gas Engine Assembly and Equipment (1972)

Gas Engine Cooling W ater Systems (1972)

Gas Engine Erection and Installation (1972)

Gas Engine Factory and Field Testing (1972)

Gas Engine Fuel Oil Characteristics (1972)

Gas Engine Gaseous Fuel Characteristics and General Spe

Gas Engine Gauge Borads, Protective Devices, and Instru

Gas Engine Generators and Electrical Equipment (1972)

Gas Engine Lubricating Oil Characteristics and General

Gas Engine Lubricating Oil Systems (1972)

Gas Engine Operation and Maintenance (1972)

Gas Engine Power Plant Buildings (1972)

Gas Engine Power Plant Fuel Oil and Gas Systems (1972)

Gas Engine Ratings and Performance (1972)

Gas Engine Speed Governing and Parallel Operation (1972

Gas Engine Starting Systems (1972)

Gas Engine Vibration (1972)

Gas Engine Waste Heat Recovery Systems (1972)

Gas Engines (1972)

Gas Engines (1972)

Gas Engines (1972)

Gas Engines (1972)

Gas Engines (1972)
Ety for Pigtail Expans Std. for UL

ANSI

BIA

UL

ASTM

ANSI

ANSI

ANSI

ASTM

/Meth ASTM

St DEMA

T. for L ASTM

ASTM

ANSI

ANSI

ANSI

Std. for Fl ANSI

ANSI

ANSI

ANSI

ANSI

ANSI

ANS

ANSI

ANSI

ASTM

ANSI

ASTM

ASTM

Tent. Rec. ASTM

ASTM

ASTM

$\mathrm{AACCH}$

NGPA

St NGPA

ASTM

1. of T ASTM

Std. Meth. ASTM

Meth. ASTM

ASTM

ASTM

ASTM

ANSI

ANSI

SBCC

IAPMO

CGA

ASTM

ASTM

ANSI

ANSI

CGA

UL

ANSI

AMCA

ANSI

SAE

DEMA

DEMA

DEMA

DEMA

DEMA

DEMA

DEMA

DEMA

DEMA

DEMA

DEMA

DEMA

DEMA

DEMA

DEMA

DEMA

DEMA

DEMA

DEMA

DEMA

Std DEMA

Std. Pract. DEMA

/Ct. for Selection of Engine-Gene DEMA Ion of Invitations for Bids and Det DEMA

B71.1

$29 \mathrm{~A}$

D1483

L24.4.12

L24.4.13

L24.4.14

D3181

*1.2

E472

B345

Z21.18

221.23

Z21.60

Z21.45

Z21.1

Z21.57

221.58

Z21.50

Z21.21

$\mathrm{Z} 21.24$

283.2

Z21.28

F308 
(I973) s (I973)

Std. for Gas Hose Connectors for Portable Indoo Std. for tial Controls Direct Electric Ignition Control Systems for Std. for Wall Furnaces (I972) 72) puts Not Over 400,000 Btu Per Hr.) (1972)

2)

Sid for Cas Fired Gravity and Fan Type Floer Furnaces (1973)

Std. for Gas Fired Gravity and Fan Type Sealed Combustion System

Std. for Gas Fired Gravity and Fan Type Vented Wall Furnaces (19

Std. for Gas Fired Gravity and Forced Air Central Furnaces (1973

Std. for Gas Fired High Pressure Steam and Hot Water Boilers (In

Std. for Gas Fired Illuminating Appliances (197I)

Std. for Domestic Gas Fired Incinerators (1973)

Std. for Vented Gas Fired Infrared Radiant Heaters (1971)

Std. for Unvented Gas Fired Infrared Radiant Heaters (I972)

Std. for Gas Fired Kettles, Steam Cookers and Generators (I971)

Std. for Gas Fired Low Pressure Steam and Hot Water Boilers (197

Std. for Direct

Std. for

Sid. for

Std. for

Std. for

Std. for

r Prevention of Furnace Explosions in Fuel Oil and Natural Std. Meth. for Sampling Pressurized

ntal Mounting, Liquid Withdrawal Only/

Std. Meth. of Sampling zontal or Vertical Mounting), Liquid /

Rec. Pract. for LP Std. for Quick Disconnect Devices for Use with

Std for Refrigerators Using tness of Textiles to Atmospheric Oxides of Nitrogen (Burnt si $Z 270.1$ Std. for Control of

ances (1971)

iances (1971)

Safety Std. for Commercial-Industrial Std. for Std. for

Safety Std. for LP (Liquid Petroleum) Std. for Domestic Std for Automatic

and Mail Rec. Pract. and Std. for Flammable (Combustible) $\begin{array}{ll}\text { And/ } & \text { Std. Analytical Meth. for Determining Saccharides } \\ \text { h Mol/ } & \text { Std. Analytical Meth. for Determining Saccharides }\end{array}$

Il. 238 rs (1972) Std. Meth. for Analysis of Natural Std. Meth. for Analysis of Natural Safety Std. for High Pressure Spec. for Mild Steel Electrodes for Std. for Manual

test for Vapor Pressure of Liquefied Petroleum Gases (LP Tent. Meth. of Test for Aromatic Types Analysis of r Premises (1972) Uniform Plumbing Code: Medium Pressure Uniform Plumbing Code: Fue

Std. Spec. for Reinforced Epoxy Resin Std. Spec. for Thermoplastic Safety Std. for tion (Safety) for Flammable Liquid and Liquefied Flammable Std. for Hotel and Restauran Std. Meth. for Obtaining Natura Spec. for Oil and

Safety Std. for Indicating Pressure Gauges for Compressed Std. for Relief Valves and Automatic

Stationary Diesel and Gas Engine Power Plant Fuel Oil and quipment (Breathing Devices, Room Humidifiers, Nebulizers, s (1973) ANSI Z250.1

n for Digital Computer Programs (1970)

Std. for Safety for LP Safety Std for Oxy Fuel Std. for Manual

ZII.317

Std. Spec. for

Std Meth of Test for Trace Metals in Std. Meth. of Test for Lead and Vanadium in Rec. Pract. for Flushing and Cleaning of Std for

Std. for Safety for Tear Std. for

lowable Operat/ Std. for Small Manually Operated Metallic Std. Glossary of Terms Relating to Chimneys, Std. for Safety for Std. for Std. for

with Inputs Less Than 75,000 Btu Per Hr.) (Vol/ taneous and Large Automatic Storage Type (1974)

Spec. for lron and Steel

Std. Meth. of Test for Hydrogen Sulfide in Natural

Valves for Anhydrous Ammonia and Liquefied Petroleum (LP) Manual for Driver Attended Loading of Liquefied Petroleum centrifugally Cast, in Metal Molds or Sand Lined Molds for Indirect Type Oil Field Heaters Used in Producing Oil and

$r$ Pumps for Anhydrous Ammonia and Liquefied Petroleum (LP)
Gas Fired Make Up Air Heaters (I972)

Gas Fired Room Heaters: Volume l, Vented (1974)

Gas Fired Room Heaters: Volume II, Unvented (1974)

Gas Fired Sauna Heaters (1973)

Gas Fired Single Firebox Boilers (1971)

Gas Fired Swimming Pool Heaters (1972)

Gas Fired Watertube Boiler Furnaces with One Burner (19 Gas for Gas Analysis (1973)

Gas from a Transformer (1974)

Gas Fuel Container, Quickly Demountable Type for Horizo

Gas Fuel Container, Quickly Demountable Universal (Hori Gas Fuel (I971)

Gas Fuel (1972)

Gas Fumes) (1972) ANSl L14.54

Gas Hazards on Vessels (Ships) to Be Repaired (1972) an

Gas Heating Equipment (1973)

Gas Hose Connectors for Portable Indoor Gas Fired Appli

Gas Hose Connectors for Portable Outdoor Gas Fired Appl

Gas Hose (I973)

Gas Hot Plates and Laundry Stoves (1971)

Gas Ignition Systems and Components (1971)

Gas Indicators Including Their Installation, Operation

(Gas Liquid Chromatography) Content of Corn Syrup, Sugar

(Gas Liquid Chromatugraphy) Content of Corn Sugar stare

Gas Liquid Mixtures by Gas Chromatography (1968)

Gas Liquid Mixtures by Gas Chromatography (1973) ANSI Z

Gas Manifolds for Industrial and Commercial Gas Cylinde

Gas Metal Arc Welding (1969) ANSI W/3. 18

Gas Metal Arc (Gma) Welding Equipment (1974)

Gas Meth.) (1973) ANSI Zl1.92

Gas Oil A romatic Fractions by Mass Spectrometry (1973)

Gas Piping and Equipment on Industrial and Certain Othe

Gas Piping Systems (1973)

Gas Piping (I973)

Gas Pressure Pipe and Fittings (1973)

Gas Pressure Pipe, Tubing and Fittings (1973)

Gas Pressure Regulators (1973)

Gas Processing Equipment and Supporting Structures (197

Gas Ranges (Stoves) and Unit Broilers (1973)

Gas Samples for Analysis by Gas Chromatography (1968)

Gas Separators (Petroleum Production) (1973)

Gas Service (1973)

Gas Shutoff Devices for Hot Water Supply Systems (1972)

Gas Systems (1972)

D. Pract. for Low and Medium

Gas Therapy and Suction Equipment, Room Air Purifiers,
Gas Torches for Heating, Soldering, and Brazing Purpose

Gas Torches (1973)

Gas Tungsten Arc (Gta) Fusion Welding Equipment (I974)

Gas Turbine Engine Steady State Performance Presentatio

Gas Turbine Fuel Oils (197I) ANSI Z11.312

Gas Turbine Fuels (Atomic Absorption Meth.) (1972) ANSI

Gas Turbine Fuels (1972) ANSl Z11.316

Gas Turbine Generator Lubricating Oil Systems (I971)

Gas Unit Heaters (I971)

Gas Units and Systems (1974)

Gas Utilization Equipment in Large Boilers (1971)

Gas Valves in Gas Distribution Systems Whose Maximum Al

Gas Vents and Heat Producing Appliances (1972)

Gas Vents and Vent Roof Jacks (1973) ANSI Al31.2

Gas Water Heaters (Automatic Storage Type Water Heaters

Gas Water Heaters: Volume III, Circulating Tank, lnstan

Gas Welding Rods (1969) ANSI W 3.2

Gas (Methylene Blue Meth.) (1970) ANSI 277.I0

Gas (Other Than Safety Relief) (I974)

Gas (1966)

Gas (1971)

Gas (1972)

Gas (1972)
ASTM

ANS

ANSI

ANSI

ANSI

NEMA

ANSI

ANSl

ANI

ANSI

ANSI

ANSI

ANS

ANSI

ANSI

ANSI

ANS

ANS

ANSI

ANSI

ANSl

ANSI

ANSl

ANSI

NFPA

ASTM

ASTM

ITA

ITA

ANSI

ANSI

AATCC

NFPA

UL

ANS

ANS

UL

ANSI

ANSl

ABYC

CR

CR

NGPA

ASTM

UL

AWS

NSA

ASTM

ASTM

ANSI

ICBO

ICBO

ASTM

ASTM

UL

FMS

ANSI

NGPA

API

UL

ANSI

DEMA

$\mathrm{NSF}$

UL

UL

NSA

SAE

ASTM

ASTM

ASTM

ASME

ANS

UL

ANSI

ANSI

NFPA

UL

ANSI

ANSI

AWS

ASTM

/ for Safety for UL Tentative NGPA

Std. for Ductile Iron Pipe, ANSI

Spec. for API

Safety Std. Fo UL

E341

Z21.40.1

221.2

221.54

Z21.34

DC 21

Z21.48

Z21.44A

Z2I.49

Z2I.47

Z21.59

Z21.42

Z21.6

Z2I.43B

$\mathrm{Z} 21.46$

Z21.13

783.4

Z21.I1.1

Z21.11.2

$\mathrm{Z} 21.55$

$\mathrm{Z} 21.52$

Z21.56

F 307

D3305

$6 \mathrm{Al}$

$6 \mathrm{~A} 2$

Z21.41

Z21.19A

23

306
795

Z21.2

Z21.54

21

Z21.9

Z21. 20 
connectors for Flammable Liquids and LP (Liquid Petroleum) Gas (1972) UL 567

ief Valves for Anhydrous Ammonia and LP (Liquid Petroleum) Gas (1973)

icators for Anhydrous Ammonia and Liquefied Petroleum (LP) Gas (1973)

Meters for Flammable Liquids and Liquefied Petroleum (LP) Std. for Safety for Pressure Regulating Valves for LP omated Separation and Collection of Particulate and Acidic separation and Collection of Particulate and Water Soluble nt. Meth. for Separation and Collection of Particulate and for Low and Medium Speed Stationary Diesel and Gas Engine Std. Meth. of Test for Specific Gravity of Std. Meth. of Test for Dissolved and

Std. Rec. Pract. for Sampling Atmospheres for Analysis of or Dielectric Breakdown Voltage and Strength of Insulating of Certain Physical Properties of Liquefied Petroleum (LP) Rec. for Storage and Handling of Compressed Std. Meth. of Test for Combustible

) (1973) Tent. Meth. of Test for Sulfur Oxides in Flue

d. Meth. of Test for Vapor Pressure of Liquefied Petroleum Std. Meth. for Analysis of Liquefied Petroleum

Std. for the Storage and Handling of Liquefied Petroleum Uniform Fire Code: Liquefied Petroleum Uniform Fire Code: Compressed

ons of Terms Relating to Electrical lnsulating Liquids and Std. for Fire Hazard Properties of Flammable Liquids, nd Fittings (1972) ANS1 A21.11 Std. for Rubber ec. for Nonreinforced Concrete Irrigation Pipe with Rubber Std. Meth. of Test for Creep Relaxation of A Std. Meth. of Tension Test. of Nonmetallic Safety Std. for

ract. for Design and Construction of Nonmetallic Enveloped Rec. for

ings $\{1972$ Std. Spec. for Neoprene Rubber ety Std. for Asbestos Cement Pressure Pipe, Couplings, and Std. Spec. for Sheet Rubber

Std. Meth of Test for Sealability of Enveloped Std. Definitions of Terms Relating to Std. Rec. Pract. for Use of Lock Strip

alves, Ring Joint, Segmented, Production Check Flanges and Std. Meth of Test for Lead in

Std. Meth. of Test for Hydrocarbon Types in Low Olefinic Std. Meth. of Test for Low Levels of Lead in r Determining and Stating the Horsepower Rating of Inboard Rec. Pract. and Std. for the Design and Construction of Rec. Pract. for Automotive ct. for the Measurement of Fuel Evaporative Emissions from ct. for the Measurement of Fuel Evaporative Emissions from Std. Meth. of Test for Trace Amounts of Lead in Std. Meth. of Test for Phosphorus in Std for Automotive

Safety Std for Hose for Conducting

e) for Use on Vents of Storage Tanks for Petroleum Oil and Emergency Std. Spec. for Automotive

$\mathrm{n}$ of Air Cooled Marine Engines and Auxiliaries (Diesel and $g$ Code Std. for Storage and Handling of Flammable Liquids els (Potentio/ Std. Meth. of Test for Mercaptan Sulfur in Tetrachloride; Chloral Hydrate; Chloroform; Formaldehyde; dium Phosphate Solutions; Isopropyl Alcohol Saturated with istry) (196/ mistry) (1962) Meth. of Analysis for Correction Factors for Meth. of Analysis for Total Safety Std. for

Guide Spec for Specifying:

(1973) Safety Std. for Door, Drapery, Spec. for Steel

ne Valves (1974) 1 and Gas Engine

Uniform Buildin rsion, Flat, and Hot Wound (Materials, Specs. Terminology, ed Petroleum (LP) Gas / Std. for Safety for Liquid Level Safety Std. for Liquid Level Safety Std for Indicating Pressure Safety Std. for Indicating Pressure urment of Impact Resistance of Painted Aluminum, Steel and
Rec. Pract. Meth. for Determining

th. of Analysis of Alpha Amylase Activity, by Beta Amylase Std. for Keys and Keyways for Industrial Flexible Std. for Balance Classification for Flexible

ical and Rack) (1971) American Drafting Manual Spec. Data Formats for Fine Pitch Sid. for Design of Components: Enclosed Std. Spec. for Lubrication of Industrial Enclosed rement for Enclosed Helical Herringbone and Spiral Bevel Std. Meth. of Test for Load Carrying Capacity of Fluid

ometer (197)

Std. Meth. of Test for Apparent Viscosity of Std. Pract for Spur, Helical and Herringbone ce Roughness, Waviness, Form and Lay) (1973) Sid. for Info Sheet for Spiral Bevel, Helical and Herringbone
Safety Std. for Pipe ANSI Safety Std. for Safety Rel UL

Gas (1973) ANSl B167. for Safety for Liquid Level Gauges and Ind UL Gas (1973) ANSI Z217.1

Gaseous Fluoride in the Atmosphere (Double Paper Tape S

Gaseous Fluorides in the Atmosphere (Filter and Impinge

Gaseous Fluorides in the Atmosphere (Sodium Bicarbonate

Gaseous Fuel Characteristics and General Spec. (1972)

Gaseous Fuels (1973) ANSI Z77.12

Gaseous Hydrogen in Water (1960)

Gases and Vapors (1967) ANSI Z257.2

Gases at Commercial Power Frequencies (1974)

Gases from Compositional Analysis (1973) ANSI Z11.23

Gases in Cylinders (1974)

Gases in Electrical Apparatus in the Field (1973)

Gases (Barium Chloranilate Controlled Condensation Meth

Gases (LP-Gas Meth.) (1973) ANSI Z11.92

Gases (LPG) by Process Gas Chromatography (1973)

Gases (1972)

Gases (1973)

Gases (1973)

Gases, and Volatile Solids (1973)

Gasket Joints for Cast and Ductile lron Pressure Pipe a

Gasket Joints (1973)

Gasket Material (1971)

Gasket Materials (1972)

Gasketed Joints for Cast Iron Pipe and Fittings (1973)

Gaskets for Corrosive Service (1971) ANSI Z261.2

Gaskets for Flammable Liquid Piping Systems (1974)

Gaskets for Hub and Spigot Cast Iron Soil Pipe and Fitt

Gaskets for Water and Fire Service (1973)

Gaskets (1966) ANSI J7.2

Gaskets (1970) ANSI Z261.1

Gaskets (1972)

Gaskets (1973)

Gaskets; Drilling Through. Multiple Completion, and Flo

Gasoline by Atomic Absorption Spectrometry (1973)

Gasoline by Mass Spectrometry (1971) ANSI Z11.311

Gasoline by X-Ray Spectrometry (1973)

Gasoline Engines (Boats) (1972)

Gasoline Fueled Inboard, Liquid Cooled Marine Engines (

Gasoline Performance and Information System (1971)

Gasoline Powered Passenger Cars and Light Trucks Using

Gasoline Powered Passenger Cars and Light Trucks (1972)

Gasoline (1972) ANSI Z11.324

Gasoline (1973)

Gasoline (1973)

Gasoline (1973)

Gasoline (1973) ANSI Z222.1

Gasoline (1974)

Gasoline) (Boats) (1972)

Gasoline, Kerosene, Acetone, Alcohol, Cleaning Fluids,

Gasoline; Herwig's, Pancreatin, Tween $80-60 \%$ Alcohol, V

Gasoline: Methyl Blue and Green; Mineral OiI: Urease Ta

Gasometric Determination of Carbon Dioxide (Cereal Chem

(Gasometric) Carbon Dioxide in Baking Powder (Cereal Che

Gate Valves for Fire Protection Service (1973)

Gate Valves (1973)

Gate, Louver, and Window Electric Operators and Systems

Gate, Plug, Ball, and Check Valves for Petroleum Pipeli

Gauge Borads, Protective Devices, and Instrumentation

Gauge Cold Formed Stainless Steel Structural Members (1

Gauge, Metric Conversions) (1972)

Sion, Extension,

Gauges and Indicators for Anhydrous Ammonia and Liquefi

Gauges and Indicators for Flammable Liquids (1972)

Gauges for Compressed Gas Service (1973)

Gauges for Fire Protection Service (1973) ANSI B156.1

Gavanized Steel (Coil Coating) (1974)

/ Meth.

GAWR and GVWR for Truck

Gear Couplings (1974)

Gear Couplings (1974)

Gear Drawing Stds. Part 1 for Spur, HelicaI, Double Hel

Gear Drawings (1973)

Gear Drives-Bearings, Bolting Keys and Shafting (1974

Gear Drives (1972)

Gear Drives (1973)

Gear Lubricants (1973) ANSI Z11.161

Std. for Sound (Noise Meas

Gear Materials Manual (1972)

Gear Oils at Low Temperatures Using the Brookfield Visc

Gear Shaft Mounted Speed Reducers (1974)

Gear Tooth Surface Texture for Aerospace Gearing (Surfa

Gear Units for Water Cooling Tower Fan Drives (1972)

STM

ASTM

St ASTM

ASTM

NFPA

ICBO

ICBO

FMS

ASTM

UL

IC. P ASTM

FMS

CISPI

Saf UL

ASTM

ASTM

ASTM

ASTM

APl

ASTM

ASTM

ASTM

$A B Y C$

$\mathrm{SAE}$

SAE

SAE

ASTM

ASTM

ASTM

UL

AST

$A B Y C$

ICBO

ASTM

$\mathrm{AACCH}$

UL

CSI

UL

API

DEMA

ICBO

UL

UL

B 148.1

132
565

25

144

D3266

D3267

D3268

*1-15

D 1070

D1588

D1605

D2477

D2598

7.50

D3284

D3226

D1267

D28

58

UFC*2ART20

UFC *2ART8

D2864

$A \mathbb{W}$ A 7.19

Std. Sp ASTM C505

ASTM F38

F152

194

F336

7-32S

HSN

107

D1330

F112

C718

$6 \mathrm{~A}$

D3237

D2789

D3229

S6

$\triangle A C C H$

AACCH $\quad 12-29$

AACCH $12-20$

J282

J171 A

J170A

D3116

D439

330

525

ESl

UBCS $10-1$

D3227

8-91

262

15101

325

$6 \mathrm{D}$

*1-17

UBCS27-10

*3

180

404
393

TB-II-6

TTMA RP39

Me AACCH 22-02

AGMA 512.03

AGMA 515.01 
73)

of Lateral Vibration on High Speed Helical and Herringbone etermining the Load Carrying Ability of Farm Wagon Running Std. for Gear Tooth Surface Texture for Aerospace Glossary of Terms Used in manufacture, Inspection and Assembly Manual for Fine Pitch Spec. for Carbon and Alloy Steel Forgings for Pinions and Std for System Design of Fine Pitch on Center Face d Alloy Steel Forgings for Pinions and Gears for Reduction Meth. of Analysis for Lactic Acid in Dried Std. Test Procedure for

Groups in Rubber Extender and Processing Oils by the Clay ermosetting Resins (Plastic Compo/

Std. Meth. of Test for Std. Meth. of Test for Std. Meth. of Test for

Spec. for Photographic Film for Archival Records, Silver Spec. for Photographic Film for Archival Records. Silver h. for Determining Acidity (Paste) Content in Corn Starch h. for Determination of $\mathrm{pH}$ (Paste) of Corn Starch Which Is and Equipment for Water Cooled and Moderated Nuclear Power ial-Use Criteria for the Periodic Test. of Nuclear Power plication of the Single Failure Criterion to Nuclear Power neraI Principles for Reliability Analysis of Nuclear Power Definitions of Terms Used in 1EEE Nuclear Power

aps/ Rec. for Protection Against Misc. Perils at Electric or Qualifying Class I Electric Equipment for Nuclear Power lification of Class I Electric Equipment for Nuclear Power i Motors Installed Inside the Containment of Nuclear Power Units Applied as Standby Power Supplies for Nuclear Power test of Class I Electric Valve Operators for Nuclear Power emblies in Containment Structures for Nuclear Fueled Power Rec. for Electric Utility

systems That Perform Protective Functions in Nuclear Power

Guide for Class 1E Control Switchboards for Nuclear Power iments in Laboratories (Cerea/ Acetic Anhydride Meth. for Rec. Pract. Inspection and Quality Control of First

of Water Damage to Steam Turbines Used for Electric Power

lectric Equipment During the Construction of Nuclear Power Rec. Pract. for Flushing and Cleaning of Gas Turbine

Std. Spec. for Vacuum Treated Electric Steel Forgings for uclear Power Generat/ sel and Gas Engine/

Trial Use Std.: Criteria for Diesel Std. Pract. for Selection of Engine. Rec. for Steam Tubine Driven Rec. for Steam Turbine Driven

e Driven Synchronous Generators and Reversible Synchronous Apparatus) (Exclusive of

for Low and Medium Speed Stationary Diesel and Gas Engine std. for Installation of Vertical Hydraulic Turbine Driven Uni/

Std. for Large Hydraulic Turbine Driven Synchronous ux Density and Average Energy from ${ }_{3} \mathrm{H}(\mathrm{D}, \mathrm{N}){ }_{4} \mathrm{He}$ Neutron ups $C$ and $D$ (197)

oups $E, F$, and $G(19 /$

Std. for Safety for Electric Motors and Safety Std. for Electric Motors and

Dimensions for Alternating and Direct Current Motors and ication Data for Alternating and Direct Current Motors and Std. for Definite Purpose Motors and Std. for Direct Current

r Alloy Steel Forgings for Nonmagnetic Retaining Rings for Std. for Gas Fired Kettles, Steam Cookers and Std. for Gas Atmosphere Std. for Definitions for Motors and Std. for Synchronous Integral Horsepower Std. for Terminal Markings for Motors and or Industrial and Other Direct Current Integral Horsepower Guide for Operation and Maintenance of Turbine

for Selection, Installation and Use of Electric Motors and Std. for Motors and Safety Std. for Acetylene Safety Std. for Acetylene Safeîy Std, for Acetylene ng) (1973)

ertical Hydraulic Turbine Driven Generators and Reversible Transmittance (1973) / Std. Rec. Pract. for Selection of a Policy on

Std for Preferred Numbers in

for Opacity of Paper, Using Reflectometer with 45-0 Deg. Spec. for Electric Overhead Bridge and Gantry Multiple crude Fat /

Tent. Meth of Test for Crystallographic Perfection rium Flas/ Std. Rec. Pract. for Determining Barium Yield, act. for Determining Barium Yield, Getter Gas Content, and gas Content, and Getter Sorption Capacity for Barium Flash nd Test Meth. for Fixed and Variable Attenuators, DC to 40 or Post Tensioned Prestressed Concrete (Post Tensioned Box Tent. Rec. for Design of Concrete Composite Beams and Std for Body Measurements for the Sizing of

52)

teristics by Polar/ racteristics by $\mathrm{Po}$ Meth for Correction for Weight of Flour to Std. Rec. Pract. for Making Reference Std. Rec. Pract. for Making Reference

Gear Units (1972) Gear (1973)

Std. Spec. for Measurement AGMA Std. Test Proc. for D ASAE

Gearing (Surface Roughness, Waviness, Form and Lay) (19 AGMA Gearing (1972)

Gearing (1973)

Gears for Reduction Gears (1972) ANSI G55.8

Gears for 20 Deg. Involute Spur Pinions (1973)

Std. ASTM

Gears (1972) ANSI G55.8

Geeg (Cereal Chemistry) (1962)

Geiger Muller Counters (1970) ANSI N42.3

Gel Absorption Chromatographic Meth. (1973) ANSI Z11.18

Gel Time and Peak Exothermic Temperature of Reacting Th

Gel Time of Solventless Varnishes (1972)

Gel Time of Tar Acids (1972) ANSI Z78.23

Gelatin Type, on Cellulose Ester Base (1973)

Gelatin Type, on Polyester Base (1973)

(Gelatinizable Products) (1955)

Gelatinized When Heated in a Boiling

Generating Plants (1973) ANSI N18.10

Cons, St

Generating Station Protection Systems (1972) ANSI N41.2

Generating Station Protection Systems (1972) ANSI N4 1.4

Generating Station Stds. (1972)

Generating Stations (Flood, Surface Water, Rigging Coll

Generating Stations (1971) ANS1 N41.5

Generating Stations (1971) ANS1 N41.

Generating Stations (1971) ANSI N4I.9

Generating Stations (1972) ANSI N41.13

Generating Stations (1972) ANSI N41.6

Generating Stations (1972) ANSI N45.3

Generating Stations (1973)

Generating Stations (1973) Ation of Design Bases for
Generating Stations (1973) ANSI N41.17

Generation of Chlorine Dioxide in Flour Bleaching Exper

Generation Silver Habide Microfilm (1972)

Generation: Fossil Fueled Plants (1972)

Generative Stations (1971)

Generator Lubricating Oil Systems (1971) for

Generator Rotors (1971) ANSI G55. I3

Generator Units Applied as Standby Power Supplies for N

Generator Units for Low and Medium Speed Stationary Die

Generator Units (1972)

Generator Units (1974)

Generator / Motor Units for Pumped Storage Installation

Generator, for Hydraulic Turbine Drive) (1972)

Generators and Electrical Equipment (1972)

Generators and Reversible Generator/Motors for Pumped

Generators and Reversible Synchronous Generator / Motor

Generators by Radioactivation Techniques (1973)

Generators for Use in Hazardous Locations, Class I, Gro

Generators for Use in Hazardous Locations, Class II, Gr

Generators (Fractional and Integral Horsepower) (1972)

Generators (Fractional and Integral Horse power) (1972)

Generators (Fractional and Integral Horsepower) (1972)

Generators (Large Apparatus) (1972)

Generators (1970) ANSI G55.6

Generators (1971)

Generators (1971)

Generators (1972)

Generators (1972)

Generators (1972)

Generators (1972)

Generators (1972) ANSI C 50.30

Generators (1973) ANS1 C5I.1

Generators (1973) ANSI C 52.1

Generators, Portable, Medium Pressure (1973)

Generators, Stationary, Low Pressure (Welding and Cutt

Generators, Stationary, Medium Pressure (Welding and $\mathrm{Cu}$

Generator/Motors for Pumped Storage Installations (1972

Geometric Conditions for Measurement of Reflectance and

Geometric Design of Rural Highways (1965)

Geometric Series for Use in Standardization (1973)

Geometry (1973)

Gerder Traveling Cranes (1971)

Germand Finished Std. Analytical Meth. for Determining

Germanium by Preferential Etch Techniques (1973)

Getter Gas Content, and Getter Sorption Capacity for Ba

Getter Sorption Capacity for Barium Flash Getters (1972

Getters (1972) ANSI Z173.50

$\mathrm{GHz}(1973)$

Girder Bridges Design and Construction) (1969)

Girders for Building (1957)

Girls' Apparel (Clothing) (1972)

Give 100 Grams at $14.0 \%$ Moisture (Cereal Chemistry) (19

Glass-Metal Butt Seals and Test. for Expansion Charac

Glass-Metal Sandwich Seal and Test. for Expansion Cha
AGMA

NMA

426.01

S360T

118.01

116.01

370-01

A291

203.03

A29]

AACCH $04-25$

IEEE 309

D2007

ASTM D2471

ASTM D3056

D2870

PH1.28

PH1.41

B-4

C. 42

N18. 10

338
379

379
352

380

380
9.10

323

344

334

387

382

317

13-14

N 18.8

420

8-30

MS 104

TWDPS-1

336

LOS-4C 1

ASME

ASTM

IEEE

387

*1-19

13.3

FMS $\quad 5.12$

NEMA MG5.1

NEMA MGl-22

$* 1.18$

MG5.2

MG5.1

E496

$674 \mathrm{~B}$

$674 \mathrm{~A}$

MG1-11

MGl.14

MGl-18

MGl-24

A289

Z21.46

Z83.2

MGl.1

MG 1.16

MG1-2

MG1.15

67

$\mathrm{MG} 2$

$\mathrm{MGI}$

$\begin{array}{ll}\text { NEMA } & \text { MG } \\ \text { UL } & 297\end{array}$

UL

NEM

409

408

GD. 2

Z17.1

UM-538

$\begin{array}{ll}\text { CMAA } & 70 \\ \text { CR } & \text { B. }\end{array}$

B. 18

ASTM FIII

ASTM Fll

F111

474

* 2

USC PS54

AACCH $82-23$

ASTM F140

ASTM Fl44 
Std. Meth. of Test for Pressure in Meth. of Drop Test. of

Std. Rec. Pract. for Inspection of or Young's Modulus, Shear Modulus, and Poisson's Ratio for upture) (1972)

Std. Meth. of Flexure Test. of

Std. Definitions of Terms Relating to Uniform Building Code: Std. Spec. for Cellular

Std. for Fire Doors, Windows, Shutters, and Std. Nomenclature for

ectric Lamps (1971) std. Meth. of Test for Annealing Point and Strain Point of Tentative Meth. of Test for Density of std. Meth. of Test for Annealing Point and Strain Point of Meth. of Test for Density of 75 Std. Spec. and Operating Instructions for 972) Modulus, Shear Modulus, and Poisson's Ratio for Glass and Std. Spec. for High Temperature Std. Meth. of Test for Resistance of Std. Meth. of Thermal Shock Test on Std. Meth. of Sampling

nce of Ceramic Decorations on Returnable Beer and Beverage Std. For Forced Entry Aluminum Sliding Spec. for Aluminum Sliding

nditioning Systems (1972)

Pressure Sensitive Tape Performance Stds. for Fibrous Pressure Sensitive Tape Application Stds. for Fibrous Std. Meth. of Test for $\mathrm{pH}$ of Aqueous Solutions with the ing (1973) ANSI A109.26

$\begin{array}{ll}\text { unctional Silane Type Finishes for / } & \text { Std. Spec. for Woven } \\ -N_{0} \text { Bleach (lns) } & \text { Std. Performance Requirements for Woven }\end{array}$

td. Spec, for Woven Meth. of Test for Effect of Handwashing on Woven Meth of Test for Light Transmittance of Woven

05 Deg. F-No B/ Std. Performance Requirements for Woven anic Felt, Asphalt-Saturated Asbestos Felt, Asphalt-Coated els (1972)

aturated Organic Felts, Coal Tar Pitch, and Asphalt Coated

equirements for Woven Drapery (Curtain) Fabrics (Excluding

or Woven Window Curtain Fabrics (Sash Casement) (Excluding

y Sid. for Electric Heaters for Use in Hazardous Locations Std. Meth. of Test. Cellular

Std. Spec. for Communication and Signal Pin Type Lime Std. Spec. for Polyester

Std. Meth. of Test for Alkalinity of Hollow

Meth. of Test for Hydrostatic Collapse Strength of Hollow

eth. of Test for Bulk Density and Packing Factor of Hollow Std. Spec. for Process Std. Meth. of Thermal Shock Test on te, Wheat, and Corn Flour (1962)

Std. Definitions of Terms Relating to Glass and ion (1972)

70) ANSI K/ Std. Spec. for

Rec. Pract. for Classifying Visual Defects in Std. Meths. for Chemical Analysis of Alloy Sheet, Strip, and Plate, Low Expansion, ing Material for Use Principally as a Substitute for Plate Tent. Rec. Pract. for Measurement of Std. Performance Requirements for ous Fluorides in the Atmosphere (Sodium Bicarbonate Coated Safety Std. for Automotive

Glazing Spec. for Sealed Insulating Spec. for Sealed Insulating Std. Meth. for Analyzing Stress in Std. Test Meth. for D.C Volume Resistivity of of Textiles: Exposure to Natural Light and Weather Through Std. Spec. for Type 58 Borosilicate Sealing Meth. of Test for Knoop Indentation Hardness of id Resistance of Ceramic Decorations on Architectural Type Std. Meth of Test for Softening Point of

Rec. Pract. for Conducting Natural Light Exposures Under of Test for Sulfide Resistance of Ceramic Decorations on Std. for Household Electric Ranges (Stove) with Std. for R.40 Bulb (Hard Cloth, Type E Cloth, Type E c, Self Extinguishing (1973) abric, Self Extinguishing (1973)

Rec. for High Temperature Molten Materials (Metals, Slag. Uniform Building Code Std. for Loss Prevention Data on Sight

4)

n Test on Metal Specimens for Engine Antifreezes Tested in meth. of Test for Foaming Tendencies of Engine Coolants in of Materials (Brick; Cast lron; Cinders; Concrete; Earth;

Std. Meth. of Test for Lead and Cadmium Extracted from Std. Meth of Test for Slump of Face meth. of Test for Slump of an Oil Base Knife Grade Channel std. Meth. of Test for Accelerated Aging of Wood Sash Face Std. Meth. of Test for Degree of Set for Wood Sash
Glass Aerosol Bottles (1972)

ASTM

Glass Aerosol Bottles (1972)

ASTM

Class Aerosol Containers (1972)

Glass and Glass Ceramics by Resonance (1971) /F Test F ASTM
Glass and Glass Ceramics (Determination of Modulus of R

ASTM

Glass and Glass Products (1971)

Glass and Glazing (1973)

Glass Block and Pipe Thermal Insulation (1973)

Glass Blocks (1973) ANSI A2.7

Glass Bulbs Intended for Use with Electron Tubes and El

Glass by Beam Bending (1972)

Glass by Buoyancy (1971)

Glass by Fiber Elongation (1971)

Glass by the Sink Float Comparator (1972T)

Glass Capillary Kinematic Viscometers (1972) ANSI Z11.2

Glass Ceramics by Resonance (1971)

ASTM

ICBO

ASTM

NFPA

ANSI

ASTM

ASTM

ASTM

ASTM

ASTM

Ceramics (Determination of Modulus of Rupture) (l ASTM

Cloth Pressure Sensixive Electrical Tape (1973)

Glass Containers to Chemical Attack (1973)

Glass Containers (Bottle, Jar) (1971)

Glass Containers (Bottle, Jar, etc.) (1956)

ASTM C225

Glass Containers (1972T)

Th. of Test for A

Glass Doors (1971)

Glass Doors (1972) ANSI A 134.2

Glass Duct Construction Std. for Ventilating and Air Co

Glass Duct (1973)

Glass Duct (1973)

Glass Electrode (1974) Ashto T200

Glass Fabrics Treated for Use in Waterproofing and Roo

Glass Fabrics, Cleaned, and After Finished with Epoxy F

Glass Fiber Curtain Fabrics Handwashable at 105 Deg. F

Glass Fiber Decorative Fabrics (Textile) (1973)

Glass Fiber Decorative Fabrics (Textile) (1973)

Glass Fiber Drapery (Curtain) Fabrics Handwashable at

Glass Fiber Mat (1973)

$\begin{array}{ll}\text { Glass Fiber Mat (1973) } & \text { /Sphalt. Asphalt and C } \\ \text { Glass Fiber Reinforced Polyester Structural Plastic Pan }\end{array}$

/ Pitch, Coal-Tar Saturated Org

Glass Fibers) (Institutional Textile) (1973)

Glass Fibers) (Institutional Textiles) (1973)

Glass 1, Groups A, B, C, and D, and Class 11, Groups E,

Glass Insulating Block (1972)

Glass Insulators (R1970)

Glass Mat Sheet Laminate (1973)

Glass Mic rospheres (1972)

Glass Mic rospheres (1972)

Glass Microspheres (1972)

Glass Pipe and Fittings (1970)

Glass Pipe (1970)

Glass Plate Meth. of Analysis for Insect Excreta in Whi

Glass Products (1971)

Glass Reinforced Acetal Plastics for Molding and Extrus

Rmance $\mathrm{R}$

/Ments F ANSl

UL

ASTM

ASTM

ASTM

ASTM

Glass Reinforced Laminates and Parts Made Therefrom (19

Glass Sand (1972)

Glass Sealing, 53Fe-29Ni-17Co (1973)

Glass Show Window Panels (1972)

Glass Stress Optical Coefficient (1973)

/Rglary Resisting Glaz UL

Glass Towels (Institutional Textile) (1973)

Glass Tube and a Particulate Filter Meth.) (1973)

Glass Tube Fuses (1973)

Glass Units (1971)

Glass Units (1973)

Glass (1968)

Glass (1972)

Glass (1972) ANSI L 14.177

Glass (1972) ANSI Z173.47

Glass (1972T)

Glass (1972T)

Glass (1973)

Glass (1973)

Glass (1974)

Glass / Ceramic Cooking Tops (1974)

Glass) Mogul Screw Base Incandescent (1966)

Glass, B Stage Epoxy Resin lmpregnated, 181 Style Fabri

Glass, B Stage Polyester Resin lmpregnated, 181 Style F

Glass, etc.) Fire Protection and Safety (1973)

Glass, Its Defects and Safety Glazing (1973)

Glasses for Chemical Processing Equipment (Vessel) (197

Glassware (1970) ANSI D 14.9

Glassware (1973)

Glass; Granite; Gravel; Granite, Limestone, and Marble

Glazed Brick Facing for Exterior Walls (Tech. Notes) (1

Glazed Ceramic Surfaces (1972)

Glazing and Bedding Compounds on Metal Sash (1974)

Glazing Compound (1972)

Glazing Compound (1973)

Glazing Compound (1973)

D3063

D3071

D3073

C623

C162

C552

C79.

C598

C693

C.336

C729

D2515

C623

D2754

C 225

$\mathrm{C} 224$

C735

1303.3

402.8

SMACN *6

SMACN AFTS-101

E70

D 1668

L24T

NSI L24.1.9

$55 \mathrm{~A}$

PS53

823

C240

D879

D 1532

D3100

D3102

Std. M ASTM

ASTM

ASTM

AACC

ASTM

ASTM

ASTM

ASTM

SAE

UL

ANSl

/Ase ASTM

UL

SIGMA

SIGMA

ASTM

AST

D3101

C599

C600

28.43

C162

D2948

D2563

C146

972

C770

D3268

$275 \mathrm{~B}$

70.7 .1

65-7-2

F218

C657

AATCC 1110

ASTM F105

ASTM C730

Meth. of Test for AC ASTM C724

ASTM C338

Std ASTM

G24.

C 777

ER.2

UBC $* 3.54$

SMACN AFTS.100

UBCS32-1

L24.1.5

L24.1.4

AMS7728C

L24.2.3 
or Volatility of Oil and Resin Based, Knife Grade, Channel r Plate Glass Show Wi/ Safety Std. for Burglary Resisting performance Spec. and Meth. of Test for Transparent Safety

Uniform Building Code: Glass and iform Building Code Std. for Glass, lts Defects and Safety Operational Rec. for Std. Meth. of Test for rage at $104 \mathrm{~F}(40 \mathrm{C})(1973)$

rage at $140 \mathrm{Deg} .(40 \mathrm{Deg}$. C.) $(1972)$ Meth. for Rec. Pract. for Selecting and Defining Color and Std. Meth. of Test for Specula

(1970)

) ANSI Z1.7

ts (Launder or Dryclean) (1973)

eat Producing Appliances (1972)

(Tech. Notes) (1961)

1962)

Std. for Steel Shipping Container Industry Std. for Photo Instrumentation Rec. Pract. for Radial Seal Nomenclature and ends Appli/

alactose, Dextr/

Std. Meth. of Test for Abrasion Resistance of ar Mixtures Maltose, Sucrose, Mannose, Galactose, Dextr eth. of Conducting Shear Block Test for Quality Control of Uniform Building Code Std. for Test for

Sid. Meth of Test for Integrity of xterior Use (1959) A/ Uniform Building Code Std. for Structural ed Panels, Beal Uniform Building Code Std. for Tests for Structural f Southern/

Std. for Preservative Treatment of Structural Rec. Architectural Spec. for Structura Uniform Building Code Std. for Structura Std. for Structura

and Lodgepole / Uniform Building Code Std. for Structural Std. Spec. for Adhesives Used in Nonstructural Std. Meth. of Test. Veneer, Plywood, and Other Structural Glued Laminated Members and Laminations Before

Performance Spec. for Adhesives for Field Machine Washing Meth. of Analysis of Hand Washing Meth. of Analysis of Meth. of Analysis of Vital Whea

rmining Crude Fat / Extractables) Content in Corn Starch, d. Meth. of Test for Phthalate Ester Color of High Gravity y) (1967)

Meth. of Analysis of Monoglycerides and Free Std. Meth. of Test for Detectin Std. Spec. for Ethylene Spec. for Ethylene test for Water in Engine Antifreeze-Coolant Concentrate Std. Spec. for Hexylene Std. Spec. for Ethylene Std. Spec. for Propylene Std. Spec. for Dipropylene

(1972)

(1972)

) (1974) Std. for Manual Gas Metal Arc Rec. Pract. for for Personnel Std. Spec. for Std. Spec. for Std. Spec. for Refined Std. Spec. for Starting) for Passenger Automobiles, Commercial Vehicles,
Spec. for 1970) Rec. Pract. for What Makes A

Air Conditioning System) (1966)

Handbook for Library Binding (Rec. for Std. Pictorial Markings for Handling of Nondangerous

de/ Meth. of Analysis of Macroscopic Examination of Baked ing Meth. of Analysis for Insect and Rodent Filth in Baked Rec. Pract. for Air Carbon Arc

ow and Medium Speed Stationary Diesel and Gas Engine Speed tems (1971) Uniform Building Code: Regulation Std. for Fundamentals Std. Spec. for Photographic

Std. Spec for Compact Round Concentric Lay Stranded Ec o (Potassium Alum) (1972)

72)

Std. Spec. for Photographic Std. Spec. for Photographic Spec. for Photographic

Std. Spec. for Industrial Std. Spec. for Photographic
Glazing Compounds (1973)

Std. Meth.

Glazing Material Used in Buildings (1971)

Glazing Spec. for Sealed Insulating Glass Units (1971)

Glazing (1973)

Glazing (1973)

Globe Dry Pipe Valves (Fire and Safety Equipment) (1973

Gloss of Paper and Paperboard at 76 Deg. (1972) ANSI P3

Gloss Retention of Waxed Paper and Paperboard After Sto

Gloss Retention of Waxed Paper and Paperboard After Sto

Gloss Tolerances of Opaque Materials and for Evaluating

Gloss (1967) ANSI Z131.1

Glossary of Architectural Metal Terms (1973)

Glossary of Billiard Terms (1971)

Glossary of Construction Industry Terms (Architecture)

Glossary of General Terms Used in Quality Control (1971

Glossary of Micrographics (1971)

Glossary of Rec. Terms for Care Labeling Textile Produc

Glossary of Terms Concerning Letter Symbols (1972)

Glossary of Terms Relating to Chimneys, Gas Vents and $\mathrm{H}$

Glossary of Terms Relating to Structural Clay Products

Glossary of Terms Used in Baking and Cereal Chemistry (

Glossary of Terms Used in Gearing (1972)

Glossary (Packaging, Voc abulary) (1973)

Glossary (Terms, Definitions) (1969)

Glossary (1972)

Glossy Smooth Coatings of Petroleum Wax or Wax Based Bl

Glucose in Sugar Mixtures (Maltose, Sucrose, Mannose, G

Glucose Oxidase Meth. for Measurement of Glucose in Sug

Glue Bonds in Scarf Joints (1970)

Glue Joint $s$ in Laminated Wood Products (1973)

Glue Joints in Structural Laminated Wood Products for E

Glued Built Up Members-Plywood Stressed Skin and Curv

Glued Laminated Lumber (1973)

Glued Laminated Members and Laminations Before Gluing O

Glued Laminated Timber (1972)

Glued Laminated Timber (1973)

Glued Laminated Timber (1973) ANSI A190.1

Glued Laminated Timbers: Douglas and Hem Fir, Southern

Glued Lumber Products (1972)

Glued Veneer Constructions (1972) ANSI O7.1

Gluing of Southern Pine, Pacific Coast Douglas Fir and

Gluing Plywood to Wood Framing (1971)

Gluten in Flour and Semolina (Cereal Chemistry) (1962)

Gluten in Flour and Semolina (Cereal Chemistry) (1967)

Gluten (Cereal Chemistry) (1962)

Gluten, Germand Finished Std. Analytical Meth. for Dete

Glycerin (1973)

Glycerol in Fats, Oils and Shortenings (Cereal Chemistr

Glycol Base Antifreeze in Used Lubricating Oils (1973)

Glycol Monoethyl Ether (1972)

Glycol Monomethyl Ether (1972)

(Glycol or Alcohol Base) by the Iodine Reagent Meth. (19

Glycol (1972)

Glycol (1972)

Glycol (1972)

Glycol (1972)

Glyphosate (Common Name for the Pest Control Chemical)

Glyphosine (Common Name for the Pest Control Chemical)

(Gma) Welding Equipment (1974)

Gogan Hardness of Brake Lining (1972)

Going Off shore for the First Time (Petroleum Production

Gold Copper Alloy Electrical Contact Material (1973)

Gold Electrical Contact Alloy (1973)

Gold (1973)

Gold, Silver, Nickel Electrical Contact Alloy (1972)

Golf Car, and Other Diversified Off the Highway Applica

Golf Putting Green Construction (1972)

Goniophotometry of Transmitting Objects and Materials (

Good Air Conditioning System (Information on Year Round

Good Book Binding Practices) (1971)

Goods (1972)

Goods, Ready-To-Eat Cereals, and Alimentary Pastes for

Goods, Ready-To-Eat Cereals, and Alimentary Pastes (Cer

Goods, Ready-To-Eat Cereals, and Alimentary Pastes (Cer

Gouging and Cutting (1974)

Governing and Parallel Operation (1972)

Governing Fallout Shelters (1973)

Governing the Design and Operation of Local Exhaust Sys

Grade Alu minum Chloride Solution $\mathrm{AICI}_{3}$ (1972)

Grade Aluminum Conductors (1972) ANSI C7.53

Grade Aluminum Potassium Sulfate AlK $\left(\mathrm{SO}_{4}\right)_{2}: 12 \mathrm{H}_{2}$

Grade Am monium Hydroxide, $\mathrm{Nh}_{4} 0 \mathrm{H}$ (Aqueous Ammonia) (19

Grade Ammonium Thiosulfate Solution $\left(\mathrm{NH}_{4}\right)_{2} \mathrm{~S}_{3} \mathrm{O}_{3}$

Grade Benzene (1971) ANSI Z78.3

Grade Benzotriazole, $\mathrm{C}_{6} \mathrm{H}_{4} \mathrm{NHN}: \mathrm{N}(1,2,3)(1972)$
Test F ASTM

UL

Un ICBO

TAPPI T480

ASTM D2895

TAPPI T682

ASTM D3134

ASTM DS23

NAAMM $* 35$

$\mathrm{BCA} * 2$

AIOA $* 1$

ASQC A3

NMA MS100

ANSI L28.1

ANSI Y Y 10.1

NFPA $97 \mathrm{M}$

BIA 2

AACCH $36.01 \mathrm{~A}$

AGMA 116.01

ANSI $\quad$ MH2.18

SMPTE 1

SAE JIIIA

ASTM D3234

AACCH 80.10

AACCH 80.10

Std. M ASTM D1759

ICBO UBCS25.20

ASTM D1101

ICBO UBCS25.18

ICBO UBCS25-23

AWPA C28

AlTC $6.5 / \mathrm{Al}$

ICBO UBCS25.10

USC PS56

ICBO UBCS25.11

ASTM D3110

ASTM D805

AWPA C28

APA AFG.01

$\mathrm{AACCH}$ 38.11

AACCH 38.10

AACCH 38.20

CR B-18

St ASTM D1728

AACCH $\quad 58-45$

ASTM D2982

ASTM D331

ASTM D3128

ASTM D 1123

ASTM D2636

ASTM D2693

ASTM D2695

ASTM D2696

ANSI K62.124

ANSI K62.117

NSA 959

SAE J379A

API RPT.1

ASTM B596

ASTM B541

ASTM B562

ASTM B477

BCI $\quad * 1.48$

USGA $* 1$

ASTM E166

NESCA MAN C

LBI $\quad * 2$

ANSI MH6.1

AACCH 28.30

AACCH 28.31

$\mathrm{AACCH} 28.32$

AWS C5.3

DEMA *1-6

ICBO UBC $* 3.57$

ANSl $\quad$ Z9.2

ANSI PH4.154

ASTM B 400

ANSl PH4.150

ANSI PH4.232

ANSI PH4.252

ASTM D836

ANS 1 PH4.204 
Std. Spec. for Nuclear Tent. Spec. for Nuclear

Std. Meth. of Test for Slump of an Oil Base Knife

ng) (1973)

Std. Spec. for Photographic Spec. for Photographic o (Chrome Alum) (1972)

aacetic) Acid (EDTA) and Its/

e, Quinol, Hydroquinol) (197/ roxylammonium Chloride) (197/

(Hydroxylam monium Sulfate) (/

Std. Spec. for Photographic

Std. Spec. for Photographic

Std. Spec. for Photographic

Std. Spec. for Photographic

Std. Spec. for Photographic National Design Spec for Stress urement of the Specific Electrical Impedance of Electrical ass Spectrometric, and Spectrochemical Analysis of Nuclear (1972) ucts Trading Rules (Performance, Quality, Description, and Std. Spec for Photographic Rec. Pract. for New Motor Vehicle on Std. Spec. for Nuclear ass Spectrometric, and Spectrochemical Analysis of Nuclear Std. Spec, for Nuclear rochemical, Nuclear and Radio Chemical Analysis of Nuclear rochemicaI, Nuclear, and Radiochemical Analysis of Nuclear Std. Spec. for Photographic Std. Spec. for Photographic Std. Spec. for Photographic Std. Spec. for Photographic a Product Use Manual

Properties, Weight, Stress of Bol meth. for Chemical and Spectrochemical Analysis of Nuclear
Std. Spec. for Photographic Std. Spec. for Photographic
Std. Spec. for Photographic Std. Spec. for Photographic Spec. for Photographic Std. Spec. for Photographic Std. Spec. for Photographic Spec. for Photographic Std. Spec. for Industrial Std. Spec. for Nitration

Crystalline $\left(\mathrm{Na}_{2} \mathrm{~S}_{2} \mathrm{O}_{3} .5 \mathrm{H}_{2}\right)$

st Meths. for Interior Panel Coatings and Overlays on DFPA

Test Meth. for Exterior Coatings and Overlays on DFPA $\left.h_{2} \mathrm{O}\right) \mathrm{N}$. NaOH (1973)
ass Spectrometric, and Spectrochemical Analysis of Nuclear Spec. for Metal Oxide Waterproofing for Below Std. Spec. for Industrial Std. Spec. for Nitration cid Acceptance of Halogenated Organic Solvents, Degreasing Std. Spec. for Fineness of Mohair and Assienment of Std. Spec, for Once Refined Sunflower Oil, Technical e $(0.80 \mathrm{Cr}-1.8 \mathrm{Ni}-0.35 \mathrm{Mo}-0.20 \mathrm{~V}(0.33-0.38 \mathrm{C}))$ Special s, $0.78 \mathrm{Cr}-1.8 \mathrm{Ni}-0.35 \mathrm{Mo}-0.20 \mathrm{~V}(0.32-0.38 \mathrm{C})$, Special p, Yarns and Fabrics of the Worsted Type and Assignment of Std. Spec. for Fineness of Wool and Assignment of Std. Spec. for Fineness of Wool Top and Assignment of ter for Flammable Fluids and Anhydrous Ammonia (Fertilizer Std. Spec. for Refined Benzene 485 (Nitration Std. Spec. for Normal Butyl Acetate $198 \%$ Std. Spec. for Normal Propyl Acetate (90 to $92 \%$ Spec. for Isopropyl Acetate $198 \%$ Std Spec for Acetone $(99.5$

std. Spec. for Amyl Acetate Made from Fusel OiI (85 to $88 \%$ meth. of Test for Volatility of Oil and Resin Based, Knife Applicati/ lear Reactors (1973) Redwood PIywood Guide (Spec. for Texture and Std. Spec. for Nuclear Std. for

ad Vehicles: Earthmoving, Mining and Logging (Short Haul); nd Loaders, Wheeled Dozers, Track Type Tractors, and Motor ec. Pract. for the ldentification of Std. Electrical Steel Std. Meth. for Evaluating Allowable Properties for Quality Stds. for Lumber Ouality Stds, for Plywood and Particleboard e, Finishes And/ Redwood Landscape Guide (Rec. for Garden
ergreens; Rose Grades; Vines and Ground Covers; Fruit Tree eciduous Shrubs; Coniferous and Broadleaf Evergreens; Rose Tent. Meth. of Test for Std. Meth. for

ing Code Std. for Classification, Definition, and Meth. of Std. Spec. for

Std.

sign Loads, Timber,/ Western Woods Use Book: Lumber Std.,

Rec. Pract. for Lighting Cotton Classing Rooms for Colo Uniform Building Code: Excavation and Test Meth. for Moisture in Wood Chips (Two Liter Meth. of Analysis of Inorganic Bromide Residues in std. Meth of Chemical Analysis of Aluminum Oxide Abrasive td. Meth. of Chemical Analysis of Silicon Carbide Abrasive
Grade Beryllium Oxide Powder (1972A)

Grade Boron Carbide Powder (1973)

Grade C-75 and C-95 Casing and Tubing (Petroleum Drilli

Grade Channel Glazing Compound (1972)

Grade Chromium Potassium Sulfate $\mathrm{CrK}\left(\mathrm{SO}_{4}\right)_{2}: 12 \mathrm{H}_{2}$

Grade Ethylenediamine $\mathrm{H}_{2} \mathrm{~N} \cdot \mathrm{CH}_{2} \cdot \mathrm{CH}_{2} \cdot \mathrm{NH}_{2}(1973)$

Grade Ethylenediaminetetraacetic (Ethylenedinitrilotetr

Grade Hydrochloric Acid, HCI (1972)

Grade Hydroquinone $\mathrm{C}_{10} \mathrm{H}_{4}(\mathrm{Oh})_{2}(\mathrm{Para}$ Dihydroxbenzen

Grade Hydroxylamine Hydrochloride, $\left(\mathrm{Nh}_{2} \mathrm{OH}\right.$. Hci) (Hyd

Grade Hydroxylamine Sulfate, $\left(\mathrm{Nh}_{2} \mathrm{Oh}\right)_{2} \cdot \mathrm{H}_{2} \mathrm{SO}_{4}$

Grade Lumber and Its Fastenings (1971)

Grade Magnesium Oxide for Use in Sheathed Type Electric

Grade Mixed Oxides ((U, Pu) $\left.0_{2}\right)$ (1972) ANSI N139

Grade of Palmkernels; Babassu; Flaxseed; Castor, Sesame

Grade P-Aminophenol Hydrochloride $\mathrm{HOC}_{4} \mathrm{H}_{4} \mathrm{Nh}_{2} \mathrm{HCI}$

Grade Parking Performance Requirements (1972)

Grade Plutonium Dioxide Powder, Sinterable (1974)

Grade Plutonium Dioxide Powders and Pellets (1972) ANSI

Grade Plutonium Metal (1972) ANSI N136

Grade Plutonium Metal (1973)

/Ass Spectrometric, Spect

Grade Plutonium Nitrate Solutions (1973)

Grade Potassium ChIoride, KCI (1972)

Grade Potassium Iodide, KI (1972)

Grade Potassium Metabisulfite $\mathrm{K}_{2} \mathrm{~S}_{2} \mathrm{O}_{5}$ (1972)

Grade Potassium Permanganate, $\mathrm{KmnO}_{4}$ (1972)

Grade Selection, Std. Size, Design Values, Framing Rec.

Grade Silver-Indium-Cadmium Alloys (1974)

Grade Sodium Chloride, Nacl (1972)

Grade Sodium Citrate, Dihydrate $\mathrm{Na}_{3} \mathrm{C}_{48} \mathrm{H}_{33} \mathrm{O}_{7}: 2 \mathrm{H}($

Grade Sodium Meta-Bisulfite $\mathrm{Na}_{n} \mathrm{~S}_{20} \mathrm{O}_{5}(1972)$

Grade Sodium Sulfate, Anhydrous Na. SO, (1973)

Grade Sodium Sulfite, Anhydrous, $\mathrm{Na}_{2} \mathrm{SO}_{3}$ (1972)

Grade Sodium Thiocyanate NaSCN (1972)

Grade Sodium Thiosulfate, Anhydrous $\left(\mathrm{Na}_{2} \mathrm{~S}_{2} \mathrm{O}_{3}\right)$ and

Grade Toluene (1971)

Grade Toluene (1971) ANSI Z78.7

Grade Trademarked Plywood (1967)

Grade Trademarked Plywood (197I)

Grade Trisodium Phosphate, Dodecahydrate $\left(\mathrm{Na}_{3} \mathrm{Po}_{4} .12\right.$

Grade Uranium Dioxide Powders and Pellets (1972) ANSI N

Grade Wall and Floor Surfaces (1971)

Grade Xylene (1971)

Grade Xylene (1971) ANS! Z78.8

Grade (Nonreflux Meth.) (1974)

Grade (1973)

Grade (1973)

Grade (1973)

Grade (1973)

Grade (1973) ANSI L 14.160

Grade (1973) ANSI L14.29

Grade) in Residential and Commerical Fuel Burning, Disp

Grade) (1971) ANSI Z78.2

Grade) (1972)

Grade) (1972)

Grade) (1972)

Grade) (1972)

Grade) (I972)

Grade, Channel Glazing Compounds (1973)

Grade, Panel Characteristics, Material Composition, Use

Grade, Sinterable Uranium Dioxide Powder for Use in Nuc

Grader Cutting Edge (1972)

Grader, Loader, Dozer, Mining Car, Front End Loader, Fo

/Movers,

Grades in ASTM Specs. (1972)

Grades of Siructural Lumber (1974)

Grades (Architectural Woodwork) (1973)

Grades, Application and Design, Span Allowances, Storag

Grades; Small Fruits (Berry, Currant, Grape, Asparagus)

Grades; Vines and Ground Covers; Fruit Tree Grades; Sma

Grading Colton Card Webs for Appearance (1973)

Grading Cotton Yarns for Appearance (1972) ANSl Ll4.164

Grading for All Species of Lumber (1973)

Grading of Abrasive Microgrits (1972)

Grading Rules for Std. Wood Mouldings (1967)

Grading Rules for Western Lumber (1972)

Grading Rules, Design Values, Section Properties and De

Grading Soybean Oil for Color (1973)

Grading (1971)

Grading (1973)

Graduate) (1973)

Grain and Cereal Products (Cereal Chemistry) (1962)

Grain and Crude (1971)

Grain and Crude (1971)
ASTM

ASTM

API

ASTM

ANSI

ANSI

ANSI

ANSI

ANSI

ANSI

ANSl

NFORP

ASTM

1, M ASTM

NIOP

ANS

SAE

ASTM

STM

Etric, Spect ASTM

ANSI

ANSI

ANSI

ANSI

WWPA

Std. ASTM

ANSI

ANSI

ANSI

ANSI

ANSI

ANSI

ANSI

ASTM

ASTM

Te APA

APA

ANSI

ASTM

CSl

ASTM

ASTM

ASTM

ASTM

ASTM

SAE

SAE

ASTM

ASTM

ASTM

UL

ASTM

ASTM

ASTM

ASTM

ASTM

ASTM

Std. ASTM

CRA

ASTM

SAE

TRA

SAE

Std. R AST

ASTM

AWI

$A$ WI

CRA

ANSI

ANSI

ASTM

ASTM

ICBO

ANSI

WWPA

WWPA

WWPA

NSPA

Std ASTM

ICBO

TAPPI

$A A C H$

ANSI

ANSI

C708

C750

$5 \mathrm{AC}$

PH4.188

PH4.188

PH4 I04

PH4.I26

PH4.187

PH4.186

3215

C698

1

H4.129

J293

C757

C758

C759

PH4.202

PH4.201

PH4.277 
General Meth. of Analysis for Fat Acidity in Thermal Properties of Agricultural avity, and Weight-Moisture Relationships of Agricultural Dielectric Properties of Std. Moisture Measurement

Meth of Analysis of Crude Fat in Std. for Terminology for Combines and rocessed Types $27 \mathrm{G} 053,30 \mathrm{G} 05$ / Std. Spec. for Flat Rolled, aphic Illustration and Classification in Cereal Grains and nalysis of Thiamine in Bread, Wheat, Rice, and Other Whole F/ Fluorometric Meth. of Analysis of Riboflavin in Whole Hopper Cars for Malt and or Determining Iodine Number (Wijs Meth.) Content in Corn, fined) and All Hydrolyzates Solutions Derived from Corn o and Refined), Syrups and Other Hydrolyzates from Corn and lso, with Slight Modification, to Corn, Rye, Barley, Rice, meth. for Determining Waxy and Nonwaxy Content in Corn and

Rec. for Hitch and Box Dimensions for Agricultural (Farm) 010.1 Std. Spec. for Creosoted End Rapid Meth. of Analysis for Fat Acidity in Colorimetric Meth of Analysis for Fat Acidity in Electrical Meth. for Analysis of Moisture in Whole Meth. of Analysis of Fungicide Residues in Meth. of Analysis of Test Weight Per Bushel of Std. for Checking the Size of Diamond Abrasive of Analysis of Moisture in Flour, Farina, Semolina, Bread, Std. Meth. of Test for Rockwell Hardness of Fine Meth. of Analysis of Hydrocyanic Acid Residues in dified Two Stage Air Oven Meth. of Analysis of Moisture in and Radiographic Illustration and Classification in Cereal h. for Determining Protein Nitrogen Content in Corn, Other

Moisture in Flour and Semolina; Bread; Feedstuffs, Ground Meth. for Sampling of Wheat and Other Whole tillation Meth. (Brown-Duvel) of Analysis of Moisture in Content (Radiographic Inspection) in Corn and Other Whole Moisture Relationships of Agricultural

Std. Test Meth. for Bulk Density of Diamond Abrasive nation of Both Meal and Flour of Wheat, Rye, Barley, Other es for Analysis of Crude Protein in Bread, Wheat and Other es For/ Dielectric Meter Meth. of Analysis of Moisture in tuffs (Corn) Containing Vegetable Material, Corn and Other eth. for Determining Crude Fiber Content in Corn and Other y $P /$ UDy Dye Meth. of Analysis of Crude Protein in Cerea

-Ray Examination for Internal Insect Infestation in Cereal Meth. of Analysis of Macroscopic Examination of Cereal of External Insect and Rodent Hair Contamination of Cereal of Cracking Flotation Test for Internal Insects in Cereal is of Urea in Cloth or Sack Fibers, Whole or Ground Cereal Meth. for Correction for Weight of Flour to Give 100 ccupant Loads (Buildings, Reviewing Stands, Bleachers, and fire Code: Places of Assembly (Theaters, Reviewing Stands, Std. Spec. for Structural

st Iron; Cinders; Concrete; Earth; Glass; Granite; Gravel; erials (Brick: Cast Iron: Cinders; Concrete; Earth: Class; eptible to Hydrolys/ Meth. of Analysis of Damaged Starch Spec. for Asphalt Insulating Siding Surfaced with Mineral Std. Spec. for Asphalt Roll Roofing Surfaced with Mineral d Covers; Fruit Tree Grades; Small Fruits (Berry, Currant, Std. Dimensions for

$s$ for the Appraisal of Color Quality and Uniformity in the rican National Std. Code for Information Interc/ $\mathrm{ms}$ Used in Architecture and Building Construction (/ ) (Electronics) (1973) ANSI Y32.14 (1972)

Std. for

Std. for Std. for

test for Electrical Resistivity of Manufactured Carbon and eth. of Test for Density in Air of Manufactured Carbon and

Std. Meth. of Test for Thermal Diffusivity of Carbon and for Hand Layup (1973)

C70,000 (483) Tensile, $36,000,000$ (248) Modul

C70,000 (483) Tensile, $36,000,000$ (248) Modul/

) Tensile Strength, 70,000,000 (483) Tensile M/

Spec. for Spec. for Spec. for Std. Meth. of Test for Rockwell Hardness of Fine Grained

Std. Meth. of Tension Test. of Carbon Std. Meth. for Measurement of Lattice Spacing of Nuclear Std. Meth. for Chemical Analysis of Carbon and Std. Meth. of Test for Ash in

Std. Meth of Test for Moisture in ng the Thermal Neutron Absorption Cross Section of Nuclear tent. Meth. of Test for Compressive (Crushing) Strength of - Meth. of Test for Delta in Hours (DIH) Purity of Nuclear Std. Rec. Pract. for Reporting Irradiation Resulis on Definitions of Terms Relating to Manufactured Carbon and td. Meth. of Test for Tensile Stress Strain of Carbons and upported Booms, Equipped for Hook Work, Clamshell, Magnet, Cuideline Spec, for Sodding and Soil Preparation
Grain and Flour (Cereal Chemistry) (1962)

Grain and Its Products (Flour, Starch) (1972)

Grain and Seed for Storage (1972)

Grain and Seeds (1972)

Grain and Seeds) (1972)

Grain and Stock Feeds (Cereal Chemistry) (1962)

Grain Harvesting (1971)

Grain Oriented, Silicon Iron, Electrical Steel, Fully P

Grain Products (Cereal Chemistry) (1962)

Grain Products, Corn Grits, Corn Meal, Puffed Cereals,

Grain Products, Grits, Meal, Flaked and Puffed Cereals,

Grain Service (1964)

Grain Sorghum and Other Vegetable Fats and Oils (1966)

Crain Sorghum Starch Which Are Substantially Optically

Grain Sorghum Starch (1960)

TTent of Corn Sugar (C

Grain Sorghum, and Buckwheat (Cereal Chemistry) (1962)

Grain Sorghum, and Their Mixtures (1959)

Grain Storage Loads, Pressures, and Capacities (1968)

Grain Wagons (1972)

Grain Wood Block Flooring for Interior Use (1970) ANSI

Grain (Cereal Chemistry) (1962)

Grain (Cereal Chemistry) (1962)

Grain (Cereal Chemistry) (1962)

Grain (Cereal Chemistry) (1962)

Grain (Cereal Chemistry) (1962)

Grain (1971)

Grain, Soybeans, Rice, Beans, Peas, Lentils, Corn Meal,

Grained Graphite Materials (1973)

Grains and Dried Foods (Cereal Chemistry) (1962)

Grains and Feedstuffs (Cereal Chemistry) (1962)

Grains and Grain Products (Cereal Chemistry) (1962)

Grains and Plant Materials of Comparable Content (1957)

Grains and Their Adjuncts (Cereal Chemistry) (1962)

Grains (Cereal Chemistry) (1962)

Grains (Cereal Chemistry) (1962

Grains (1958)

Eth. for Determining Insect Infestation

Grains (1968)

Grains (1973)

Grains, and Malted Cereals (Cereal Chemistry) (1968)

Grains, and Yeast Foods (Cereal Chemistry) (1962)

Grains, Beans, Peas, Lentils, Rice, and Other Commoditi

Grains, Flours, and Meals (1964)

Grains, Foodstuffs Containing Vegetable Materials, Flou

Grains, Oilseeds, Legumes, Forages, and Animal and Dair

Grains, Unpopped Popcorn, Dried Peas and and Beans (Cer

Grains, Unpopped Popcorn, Dried Peas and Beans for Rode

Grains, Unpopped Popcorn, Dried Peas and Beans (Cereal

Grains, Unpopped Popcorn, Dried Peas and Beans (Cereal

Grains, Whole or Chopped Nuts, Spices, etc. (Cereal Che

Grams at $14.0 \%$ Moisture (Cereal Chemistry) (1962) Uil

Grandstands, Bleachers, etc.

Granite (1968) ANSI A91.1

Granite, Limestone, and Marble Masonry; Mortar; Sand; S

Granite: Gravel; Granite, Limestone, and Marble Masonry

Granules in Flour or Starch Preparations Which Are Susc

Granules (1970) ANSI A 109.18

Granules (1973)

Grape, Asparagus); Lining Out Stock; Seedling Trees and Graphic Arts Sheet Films (photography) (1973)

Graphic Arts (1972)

Std. for Viewing Condition

Graphic Representation of the Control Characters of Ame

Graphic Symbols for Electrical Wiring and Layout Diagra

Graphic Symbols for Logic Diagrams (Two-State Devices

Graphical Symbols for Use on Railroad Maps and Profiles

Graphite Articles at Room Temperatures (1969) ANSI K90.

Graphite Articles by Physical Measurements (1969) ANSI

Graphite by a Thermal Pulse Meth. (1972) ANSI K90.12

Graphite Fiber Tape and Sheet, Epoxy Resin Impregnated

Graphite Fiber Tape and Sheet, Epoxy Resin Impregnated,

Graphite Fiber Tape and Sheet, Epoxy Resin Impregnated,

Graphite Fibers for Structural Composites (GF 220 (1517

Graphite Materials (1973)

Graphite Mechanical Materials (1971) ANSI K90.6

Graphite (1969) ANSI K90.1

Graphite (1969) ANSI K90.3

Graphite (1969) ANSI K90.4

Graphite (1969) ANSI K90.5

Graphite (1971) ANSI K90.10

Graphite (1971) ANSI K90.11

Graphite (1971) ANSI K90.8

Graphite (1972)

Graphite (1972)

Graphite (1973)

Grapple, or Concrete Bucket Attachmenis (1971)

(Grass) (1972)
/Ber Content

AACCH 02.01

ASAE D243.

ASAE D24l.1

ASAE D293

ASAE $\quad \mathrm{S} 352$

ASAE S343

ASTM A665

AACCH 28.95

AACCH 86.70

BAMI *1

CR H.32

F.14

CR F.26

AACCH $\quad 76-20$

ASAE A.28

ASAE R239

ASTM D 103

$\mathrm{AACCH} \quad 02.02$

AACCH 02.04

$\mathrm{AACCH}$ 44-10

$\mathrm{AACCH} 60.01$

$\mathrm{AACCH} \quad 84-10$

ANSI B74.16

AACCH $44.15 \mathrm{~A}$

ASTM C748

AACCH $\quad 60.20$

Mo AACCH 44-18

I AACCH 28-95

CR A.18

IF AACCH 44.40

$\mathrm{AACCH} \quad 64.70$

Dis AACCH $44-53$

CR A.11

ASAE D245.1

ANSI B74.17

AACCH $56.81 \mathrm{~B}$

Mpl AACCH 46-1]

AACCH 44-11

CR G-12

CR A-8

AACCH 46.14

$\mathrm{AACCH} \quad 28-21$

AACCH 28-10

AACCH 28.20

AACCH 28.22

AACCH $28-87$

$\mathrm{AACCH} \quad 82.23$

and $O$ ICBO

ICBO

$\mathrm{UBC} * 3.33$

ASTM C615

ICBO $\mathrm{UBC} * 3-23$

$\mathrm{ICBO} \quad \mathrm{UBC} * 3.23$

$\mathrm{AACCH} \quad 76.30 \mathrm{~A}$

Std. ASTM D1226

ASTM D249

ANSI Z60.1

ANSI PHI.16

ANSI $\mathrm{PH} 2.32$

ANSI X 3.32

ANSI Y Y 32.9

IEEE

ANSI

91

ASTM C 611

ASTM C559

ASTM C714

SAE AMS3894A

SAE

SAE

SAE

ASTM

ASTM

ASTM

ASTM

ASTM

ASTM

Std. Meth. for Estimati ASTM

ASTM

Std ASTM

ASTM

Std ASTM

ASTM

/Rope S SAE

ASPA
AMS3894/8

AMS3894/9

AMS $3892 / 5$

C7 78

C. 558
C 560

C 561

C562

C626

C695

C624

C 625

C709

C749

J 220

1 
Friction Coefficients of Chopped Forages (Grass, Corn, Hay, Straw) (1968)

ng Code Std. for Concrete Aggregates (Sand, Crushed Stone 1) ANSI A37.99 Std. Spec. for Crushed Stone, Slag, and Roof Coverings (Multiply Felt and Asphalt Preferably with rick; Cast Iron: Cinders; Concrete; Earth; Glass: Granite; ate of Sheet Materials (Paper) at High Te/ ead (Cereal Chemistry) (1962)

les in Farina (Cereal Chemistry) (1962)

d Feedstuffs (Cereal Chemistry) (1962)

(Cereal Chemistry) (1962)

a 37.5

7.6

Std. Meth. of Test for Specific Std. Meth. of Test for Specific Std. for Gas Fired
Std. for Gas Fired aces (1972) Std. for Gas Fired Std. for Gas Fired Std. for Gas Fired

970)

Std. Meth. of Test for Specific Std. Volume and Specific Std. Meth. of Test for Phthalate Ester Color of High on, Bulk Density, Apparent Porosity, and Apparent Specific Std. Meth. of Test for Specific 33 Std. Meth. of Test for Specific 1.96 Std. Meth. of Test for Specific or Api 2) ANSI A37.72 Std. Meth. of Test for Density and Specific Std. Meth. of Test for Specific Std. Meth. of Test for Specific

Std. Test Meth. for Specific Test Meth. for Specific

for Apparent Porosity, Water Absorption, Apparent Specific tural Grain and Seed for Storage (1972/ Density, Specific ions (Cereal Chemistry) (1) Meth. of Analysis of Specific

$$
\text { Std. Positive }
$$

Std. Proofing Supplement Stock for Std.

ids (19) iquids 2167.1 gs (1973)

Std. Rec. Pract. for Polyethylene Encasement for Std. for Polyethylene Encasement for Std. Rec. Pract. for Production and Preparation of Std. Spec. for Spec. for Automotive Std. Spec. for Austenitic

54) ISO 105-1 iso 105.1

Rec. Evaluation Procedure of Rec. Evaluation Procedure of Std. Meth. of Test for Dropping Point of Lubricating Test Meth. for Continuous Scouring Wool Using Std. Raw d. Meth. for Measurement of Flow Properties of Lubricating of Test for Water Washout Characteristics of Lubricating eth. of Test for Rust Preventive Properties of Lubricating Std. Meth. of Test for Apparent Viscosity of Lubricating

h. for Chemical Analysis of Copper Phthalocyanine Blue and gs, Partitions, Exterior Veneer, Awnings and Patio Covers, isopropyl Alcohol Saturated with Gasoline; Methyl Blue and $r$ Numerical Designation (Identification System) of Modular $s$ (Coffee Pots and Urns, Food Dish Warmers, Hot Plates and Rec. for Operation of Std. for Tool and Cutter Fire Detection System

ling, Aeration Chambers, Chemical Storage, Mixing, Filter, Std. Spec. for Hand Scrubbing Soap Std. Spec. for Hand Scrubbing Soap or Extracted Flakes, Full Fat and Defatted Soy Flours and or Extracted Flakes, Full Fat and Defatted Soy Flours and n Bread, Wheat, Rice, and Other Whole Grain Products, Corn ion Meth. of Analysis for Insect and Rodent Filth in Meal,

Decantation Meth. of Analysis for Rodent Excreta in Meal,

c Meth. of Analysis of Riboflavin in Whole Grain Products, ain, Soybeans, Rice, Beans, Peas, Lentils, Corn Meal, Corn

Calcine, Chrome Ore, Clays, Diaspore, Ganister Quarzite, allic Fluid Power Piston Rings (1972) ANSI B93.36

Series) (1972) ANSI B93.35

pe Piston Rings (1973) ANSl B93.32

acket Bomb Calorimeter (1973)

Std.

Std.

Std. Meth. of Test for Std. Meth. for Collection of A per for Analysis of Urea in Cloth or Sack Fibers, Whole or oniferous and Broadleaf Evergreens; Rose Grades; Vines and Safety Std. for Std. for Safety for

ation of Dispersible Nitrogen in Ground Soybeans, Whole or nation of Dispersible Protein in Ground Soybeans, Whole or ysis of Moisture in Flour and Semolina; Bread; Feedstuffs,

ed; Castor, Sesame, Safflower, Oleic, and Sunflower Seeds; Chrom/ Std. Classification of Single and Double Screened
Gravel and Air Cooled Iron Blast Furnace Slag) (1973)

Gravel for Dry or Water Bound Macadam Base Courses (197

Gravel or Slag Surface) (1968)

Gravel Sand and Anthrante Filtering Material (1972)

Gravel; Granite, Limestone, and Marble Masonry; Mortar:

Gravimetric Determination of Water Vapor Transmission $R$

Gravimetric Meth. of Analysis of Chlorides in Ash of $\mathrm{Br}$

Gravimetric Meth. of Analysis of Cinder and Sand Partic

Gravimetric Meth. of Analysis of Phosphorus in Feeds an

Gravimetric Meth. of Analysis of Sulfates in Yeast Food

Gravity and Absorption of Coarse Aggregate (1973) ANSI

Gravity and Absorption of Fine Aggregate (1973) ANSI A3

Gravity and Fan Type Direct Vent Wall Furnaces (1973)

Gravity and Fan Type Floor Furnaces (1973)

Gravity and Fan Type Sealed Combustion System Wall Furn

Gravity and Fan Type Vented Wall Furnaces (1972)

Gravity and Forced Air Central Furnaces (1973)

Gravity and Porosity of Lump Coke (1973)

Gravity Correction Tables for Creosote and Coal Tar (RI

Gravity Glycerin (1973)

Gravity of Fired Whiteware Products (1972)

Gravity of Gaseous Fuels (1973) ANSI Z77.12

Gravity of Hydraulic Cement (1972) ANSI Al.12, Ashto T1

Gravity of Liquid Asphalts by Hydrometer Meth. (1973)

Gravity of Liquids by Bingham Pycnometer (1968) ANSI Z1

Gravity of Soils (1958 AASHO T100, ANSI A37.146

Gravity of Solid Pitch Asphalt Displacement Meth.) (197

Gravity of Wood and Wood Base Materials (1969)

Gravity of Wood Disks (Pulp and Paper) (1973)

Gravity, and Bulk Density of Burned Refractory Brick (1

Gravity, and Weight-Moisture Relationships of Agricul

Gravity, Degrees Brix, and Degrees Baume of Sugar Solut

Gravure Color Bars (Printing) (1973)

Gravure Densities (Printing (1973)

Gravure Lateral Hard Dot Screen (1973)

Gravure Magazines (Printing) (1973)

Gravure Proofing Inks (Printing) (1973)

Gray and Ductile Cast Iron Pipe for Water or Other Liqu

Gray and Ductile Cast Iron Piping for Water and Other L

Gray lron Castings for Porcelain Enameling (1970) ANSI

Gray Iron Castings for Valves, Flanges, and Pipe Fittin

Gray Iron Castings (1972)

Gray Iron Castings (1972A)

Gray Scale for Color Change (Textile Colorfastness) (19

Gray Scale for Staining (Textile Colorfastness) (1954)

Grease of Wide Temperature Range (1972) ANSI Z11.207

Grease Wool Yarn (1971) ANSI L14.84

Greases at High Temperatures (1973)

Greases (1973) ANSI Z11.124

Greases (1973) ANSI Z11.152

Greases (1973) ANSI Z11.72

Green Construction (1972)

Green Pigments (1973)

Greenhouses, Canopies) (1973)

Green; Mineral Oil; Urease Tablets; Xanthyrol) (1962)

Grid Coordinates (1971)

Griddles, etc.) (1973)

Grimes AB Dry Pipe Va

(Grinnell Protomatic Release F100) (1973)

Grit Chambers, and Flotation) (1973)

(Grit Soap) Paste and Bar (1974)

(Grit Soap) Powder (1974)

Grits, and Soybean Meal (Cereal Chemistry) (1968)

Grits, and Soybean Meal (Cereal Chemistry) (1968)

Grits, Corn Meal, Puffed Cereals, Farina, and Flour (Ce

Grits, Farina, and Semolina (Cereal Chemistry) (1962)

Grits, Flour, Farina, and Semolina (Cereal Chemistry)

Grits, Meal, Flaked and Puffed Cereals, Farina, Bread.

Grits, Rolled Oats, Bulgur, Rolled Wheat, Breakfast Cer

Grog, Kyanite, Magnesite, Mullite) (1970) ANS1 A111.36

Groove Dimensions for Floating Type Metallic and Nonmet

Groove Dimensions for Fluid Power Exclusion Devices (In

Groove Dimensions for Fluid Power Radial Compression Ty

Gross Calorific Value of Solid Fuel by the lsothermal J

Gross Sample of Coal (1972)

Ground Cereal Grains, Whole or Chopped Nuts, Spices, Et

Ground Covers; Fruit Tree Grades; Small Fruits (Berry,

Ground Fault Circuit Interrupters (1972)

Ground Fault Sensing and Relaying Equipment (1972)

Ground Full Fat or Extracted Flakes, Full Fat and Defat

Ground Full Fat or Extracted Flakes, Full Fat and Defat

Ground Grains and Their Adjuncts (Cereal Chemistry) (19

Ground Nuts (Peanuts); Vegetable, Coconut, and Palm Oil

Ground Refractory Materials (Calcined Bauxite, Calcine,

ASAE

ICBO

ASTM

FMS

AWWA BI00

ICBO UBC $* 3.23$

TAPPI T464

AACCH 40.30

AACCH 28.02

AACCH 40.57

AACCH 40.66

ASTM C127

ASTM CI28

ANSI Z21.44

ANSI Z21.48

ANSI Z21.44A

ANSI Z21.49

ANS1 Z21.47

ASTM D167

ASTM

D1728

ASTM D 1070

ASTM C188

ASTM D3142

ASTM D1217

ASTM D854

ASTM D71

ASTM D2395

TAPPI UM.12

ASTM C20

ASAE D241.1

AACCH 80.75

GT *1

GT

GT

GT

GT

AWWA

ASTM

ASTM

ASTM

ASTM

A ATC

AATCC EP2

St ASTM

Std. Meth ASTM

Std. M ASTM

ASTM

Std. Met ASTM

ASTM

AACCH

Std. Fo ANSI

ANSI

FMS

NSA

NSF

ASTM

ASTM

$* 2$

$* 5$

$+4$

A674

(6)

C660

A 126

EP2

64

D3232

D1743

D1092

*1

D3256

UBC $* 3-52$

28-9]

A62.8

$2-56$

973

LPD 2-92

D2955

Fat AACCH 46.23

Fat AACCH $46-24$

$\mathrm{AACCH} \quad 86-80$

$\mathrm{AACCH} 28.51$

AACCH 28.50

AACCH 86.70

$\mathrm{AACCH} \quad 44.15 \mathrm{~A}$

ASTM C 316

NFLDP T3.19.11

NFLDP

NFLDP

T3.19.7

ASTM D3286

AACCH $28-87$

ANSI Z60.1

UL 943

UL 1053

AACCH 46.23

AACCH $46-24$

AACCH $\quad 44.40$ 
(NSI): Meth for Determination of Dispersible Nitrogen in $x$ (PDI): Meth. for Determination of Dispersible Protein in

Std. for Wire Cable Wrought Steel Heavy Thimble (For or Forged Carbon Steel, Shoulder Nut Assembly Eyebolt (For for Chisel Plow, Field and Row Crop Cultivator Shafts and ractices: Chassis Frames-Passenger Car and Light Truck Meth of Analysis of Lipoxidase Activity of tion, Operation, and Maintenance of Impressed Current Deep Service (1973)

ring Systems) (1972) ANSI C33.8

Rec. Pract. for

Requirements, Terminology, and Test Procedu

er Systems (1972) ANSI C114.1

cedure for Neutral Rec. for Protective
Rec. Pract. for fic Purpose 600 Volts, 30 Amperes, 2 Pole, 3 Wire Locking. ose 250 Volts, 20 Amperes 3 Phase, 3 Pole, 4 Wire Locking, se 250 Volts, 30 Amperes, 3 Phase, 3 Pole, 4 Wire Locking, se 480 Volts, 20 Amperes, 3 Phase, 3 Pole, 4 Wire Locking, se 480 Volts, 30 Amperes, 3 Phase, 3 Pole, 4 Wire Locking, se 600 Volts, 30 Amperes, 3 Phase, 3 Pole, 4 Wire Locking, purpose 125/250 Volts, 20 Amperes, 3 Pole, 4 Wire Locking, purpose 125/250 Volts, 30 Amperes, 3 Pole, 4 Wire Locking, fic Purpose 125 Volts, 20 Amperes, 2 Pole, 3 Wire Locking, fic Purpose 125 Volts, 30 Amperes, 2 Pole, 3 Wire Locking, fic Purpose 250 Volts, 15 Amperes, 2 Pole, 3 Wire Locking, fic Purpose 250 Volts, 20 Amperes, 2 Pole, 3 Wire Locking, fic Purpose 250 Volts, 30 Amperes, 2 Pole, 3 Wire Locking, fic Purpose 480 Volts, 20 Amperes, 2 Pole, 3 Wire Locking, fic Purpose 480 Volts, 30 Amperes, 2 Pole, 3 Wire Locking, fic Purpose 600 Volts, 20 Amperes, 2 Pole, 3 Wire Locking, Purpose 277 Volts Ac, 20 Amperes, 2 Pole, 3 Wire Locking, Purpose 277 Volts Ac, 30 Amperes, 2 Pole, 3 Wire Locking, 08 Volts, 20 Amperes, 3 Phase Wye, 4 Pole, 5 Wire Locking, 08 Volts, 30 Amperes, 3 Phase Wye, 4 Pole, 5 Wire Locking, 80 Volts, 20 Amperes, 3 Phase Wye, 4 Pole, 5 Wire Locking, 80 Volts, 30 Amperes, 3 Phase Wye, 4 Pole, 5 Wire Locking, 00 Volts, 20 Amperes, 3 Phase Wye, 4 Pole, 5 Wire Locking, 00 Volts, 30 Amperes, 3 Phase Wye, 4 Pole, 5 Wire Locking, res, 2/ Std. Dimensions of Plugs and Receptacles, Locking Rec. for Lightning Arresters and

tent. Meth. of Test for Carbon Distribution and Structural $s$ Locations, Class 1, Groups A, B, C, and D, and Class 11, azardous Locations, Class I, Groups C and D, and Class Il, Numbers, Cell Layouts and Ter/ Battery Replacement Data; Uniform Building Code Std. for Field Tests for Uniform Building Code Std. for Aggregates for High Lift

nder the Uniform Building Code: Solid, Grouted, Reinforced lling Construction Under the Uniform Building Code: Solid, Definition and Recommendation for Materials for Rec. Pract. for Chemical Resistant Resin Tile Std. Spec. for Resin Chemical Resistant in De/ Std. for Definition of and Maximum Dimensions for rage Batteries (1/ Rec. Domestic Marketing Std. Including dure for Evaluation of the Effectiveness of Rear Underride Std for Pin Pulley nd Use of Central Station Protective Signaling Systems for on Systems / Minimum Operational Characteristics Vertical

Std. for

lous, Analog Torque Balance Accelerome/ Std. Spec. Format in Equipment as Received from Manufacturer (1969) ANSI C/ t Deck and for Reboarding from the $\mathrm{Wa}$ Stds, and Practice Ducted Electric Heat

tage Circuit Breakers Rated on a Symmet/ steam Propulsion Plant (1970)

er Generating Stations (1973) ANS1 N41.17 Std. Application ding to Their Haza

ete Pavements (1965)

(light) (1973)

utdoor Metal Clad Switchgear (1971) ANSI C37.24

IEEE Trial Use Std.

Std. for

Rec.

Std.

Structural Design

of Preferred Ratings, Manufacturing Spec., and Application reactor Vessels (1974)

74)

ice (1965)

Std. Rec.

tage Inductive Test Currents (1972) ANSI C37.26

a Technical

Rec

Std.

special Permits for Radioactive /

Draf ting

rs (1972) ANSI C50.30

als (Safety) (1973)
Ground Soybeans, Whole or Ground Full Fat or Extracted

Ground Soybeans, Whole or Ground Full Fat or Extracted

Ground Support Equipment (Hand Knob) (1974)

Ground Support Equipment) (1974)

Ground Support Equipment) (1974)

Ground Tool Mountings (Farm Machinery) (1973)

Ground Vehicle Practices (1974)

Sid. ASAE
/Lated Documentation P ANSI

Ground Wheat (Cereal Chemistry) (1962)

Rec. Pract. Design, Installa NACE

Ground beds (1972)

Grounded $830 \mathrm{~V}, 3$ Phase Electrical System for Oil Field

Grounding and Bonding Equipment (Internal Electrical Wi

Grounding Devices (1972)

Grounding of Electrical Circuits and Equipment (1974)

Grounding of lndustrial and Commercial (Electrical) Pow

$\begin{array}{ll}\text { APl } \\ \text { Std. } & \text { IEEE }\end{array}$

APl

FMS

Grounding Type 1973)

Grounding Type (1973)

Grounding Type (1973)

Grounding Type (1973)

Grounding Type (1973)

Grounding Type (1973)

Grounding Type (1973)

Grounding Type (1973)

Grounding Type (1973)

Grounding Type (1973)

Grounding Type (1973)

Grounding Type (1973)

Grounding Type (1973)

Grounding Type (1973)

Grounding Type (1973)

Grounding Type (1973)

Grounding Type (1973)

Grounding Type (1973)

Grounding Type (1973)

Grounding Type (1973)

Grounding Type (1973)

Grounding Type (1973)

Grounding Type (1973)

/ of Plugs and Receptacles: Speci ANSI

and Receptacles: Specific Purp ANSI

land Receptacles: Specific Purpo ANSI

land Receptacles: Specific Purpo ANSI

/and Receptacles: Specific Purpo ANSI

/and Receptacles: Specific Purpo ANSI

/Lugs and Receptacles: Specific ANSI

/Lugs and Receptacles: Specific ANSI

/of Plugs and Receptacles: Speci ANSI /of Plugs and Receptacles: Speci ANSI /of Plugs and Receptacles: Speci ANS /of Plugs and Receptacles: Speci ANS lof Plugs and Receptacles: Speci ANS lof Plugs and Receptacles: Speci ANSI /of Plugs and Receptacles: Speci ANSI /of Plugs and Receptacles: Speci ANSI /Plugs and Receptacles: Specific ANSI /Plugs and Receptacles: Specific ANSI /Ptacles: Specific Purpose 120/2 ANSI /Ptacles: Specific Purpose 120/2 ANS

Ptacles: Specific Purpose 277/4 ANSl

/Ptacles: Specific Purpose 277/4 ANS]

Ptacles: Specific Purpose 347/6 ANSI

Grounding Type (1973)

Ptacles: Specific Purpose 347/6 ANS

Grounding Type, Specific Purpose, 277 Volts Ac, 15 Ampe

Grounds (1974)

Group Analysis of Petrole um Oils by the n-d.M Meth. (19

Group E, F, and G (1972) ANSI C33.83 / Use in Hazardou

Group G (1972) ANSl C33.79 Unting Units for Use in H

Group Numbers, Dimensional Spec., and Ratings; Assembly

Grout and Mortar (1973)

Grout (1973)

Grouted Reinforced Brick Masonry (Tech. Notes) (1972)

Grouted, Hollow Unit, Cavity Wall, and Stone Masonry (1

Grouted, Reinforced Grouted, Hollow Unit, Cavity Wall,

Grouting Ceramic Tile (1973)

Grouts (1972T) (1972T)

Grouts (1974)

Grown Tires in Service for Use by Vehicle Manufacturers

Gta) Fusion Welding Equipment (1974)

Guarantee Policy for Motor Vehicle Type (Lead Acid) Sto

Guarantees for Roof Installations (1973)

Guard (Safety (1971)

Guard (1972)

Guard, Fire Alarm and Supervisory Service (1972) ANSI S

Guidance Equipment Used in Airborne Volumetric Navigati Guidance Material for Aviation Turbine Fuels (1973)

Guide-Fastener, Low Form, Cowl, Dzus Type (1973)

Guide and Test Procedure for Linear, Single Axis, Pendu

Guide for Acceptance and Maintenance of Insulating Oil

Guide for Aids to Security (Safety) of P

Guide for Air Handling Systems (1971)

Guide for Centralized Control and Automation of Ship's

Guide for Ceramic Tile in Building Codes (1972)

Guide for Class IE Control Switchboards for Nuclear Pow

Guide for Classifying and Labeling Epoxy Products Accor

Guide for Describing the Characteristics of Resilient M

Guide for Design of Foundations and Shoulders for Concr

Guide for Electrical Measurements of Fluorescent Lamps

Guide for Evaluating the Effect of Solar Radiation on $O$

Guide for Hardwood Plywood (1971)

Guide for High Voltage Air Switches, Bus Supports, and

Guide for in Service Annealing of Water Cooled Nuclear

Guide for Landfill Disposal of Solid Chemical Waste (19

Guide for Making a Condition Survey of Concrete in Sery

Guide for Meth. of Power Factor Measurement for Low Vol

Guide for Microfilm (1971)

Guide for Obtaining Department of Transportation (DOT)

$\begin{array}{ll}\text { Std. for Administrative } & \text { Guide for Obtaining Department of Transportation (DOT) } \\ \text { Std. for Administrative } & \text { Guide for Operation and Maintenance of Turbine Generato }\end{array}$

Std. Rec. Guide for Paint Inspectors (1973)
ANS

FMS

ASTM

UL

$\mathrm{BCl}$
$\mathrm{ICBO}$

ICBO

BIA

ICBO

ICBO

TCA

ASTM

ASTM

TRA

NSA

$\mathrm{BCl}$

FMS

SAE

NSA

NFPA

RTCA

IEEE

$A B Y C$

A 18

SMACN $\quad * 12$

IEEE 342

SNAME $\quad 3-23$

TCA

IEEE

ANSI

ANS

ACI

ANSI

HPMA

ANSI

ASTM

$\mathrm{MCA}$

$\mathrm{ACI}$

IEEE

NMA

ANSI

IEEE

ANSl

ASTM

46.23

46-24

053

$\$ 225.1$

Y 14.32 .1

2.41

05.72

$11 \mathrm{M}$

5-10

142

C73.82

C73.85

C73.86

C73.87

73.88

3.89

C73.83

C73.72

C73.73

C73.74

C73.75

C.73. 6

C73.79

C73.80

C73.81

73.77

C73.78

C 73.90

73.91

(3.92

C73.94

C73.95

73.43 
mers, with or Without Load Tap Changing (1971)

Sid. for

erconnections (1973)

lear Power Generating Stations (/

Trial Use Std.: General

Plywood Construction Safety

(1972)

ipment for Nuclear Power Generating Stations (1971) Ansi/ rials (1973)

otors and Generators (1/

Std.

a (1972)

Safety Std. for Construction and

Coating Systems (Paint)

64)

Rec. Selection and Usage

Rec.

old During the Use of Internal Combustion Engines (1970)

on to Nuclear Power Generating Station Protect/

f Automatic Circuit Reclosers (1973) ANS1 C37.61 sheathing (Aluminum Construction Manual) (1969) rs (1972)

effluents (1968)

Tria! Use

Sid. Z166.26

ermal Evaluation and Establishment of Temperature Indexe

Circuit Breakers Rated on a Symmetric/

for Nuclear Power Generating Stations (1972) /

installed lnside the Containment of Nuclear Po/

Std. Rec.

Trial Use

Trial Use

Rec.

Design

Suggested

t ll-Characterization of Large Signal D/

Trial Use Std.

(1972)

1972)

ht and Passenger) (1971)

d Hydropneumatic) for Lowering Personnel and Material (1/

73)

ster Tanks (1973)

Specifying Sprayed Fireproofing

unning Trim, Casework, Panelwork, Closet and Storage She art 111 Execution) (1972)

11 Products) (1972)

ic (Polyvinyl Chloride) Duct Construction (1974)

tallation of Propeller Shafting Sys/ Pract. lear Material Control Systems for Conversion Facilities (A terial Control Systems for Fuel Reprocessing Facilities (A rocessing Facilities (1973)

Seals (1972)

marking of Compressed Gas Containers (1971)

(1972) ANS1 Z210.1

hy) (1973)

Redwood Exterior

Redwood lnterior

Redwood Plywood

cs, Material Composition, Use, Applicati/

Marine Deck Covering

Reinforced Brick Masonry (Inspectors

pe Wheels, Demountable Rims and Rim Spacers (Manufactures ements Exposed to Rain During Construction (1974/ Interim service (1972)

) (1972)

try (1973)

ts (Computer Software Documentation and Terminology) (19)

nts in Hazardous Dust Locations (Agricultural Plast/

Tent. Rec. Pract.

for Use in Test. Organic Coating Systems (Coil) (1974)

Std.

se (1974)

k Plumbing System (1973)

Std.

ation of Power Transmission Units Penetrating the $\mathrm{H}$

uclear Power Generating Station Protection Sys/

Design Rules,

Rec.

$\mathrm{n}$ of Plastic Polymers in Dry Powder Form by Means of Spray ted and Freshly Mixed Self Rising Flour (Cereal Chemistr)

Std. for Safety for Wireways, Auxiliary

973) ANSl C33.45 Std. for Safety for Wireways, Auxiliary ination Vehicles for Roadway Use Over 10,000 Lb. $(4500 \mathrm{~kg})$ Rec. Pract. Meth. for Determining GAWR and Std. Meth. for Chemical Analysis of Std. Spec. for
Guide for Preparation of a Leak Test. Spec. (1973)

Guide for Preparation of Spec. for Large Power Transfor uide for Protective Relaying of Utility-Consumer lnt

Guide for Qualifying Class I Electric Equipment for Nuc

Guide for Residential Building (1971)

Guide for Respiratory Protection Against Coal Mine Dust

Guide for Seismic Qualification of Class I Electric Equ

Guide for Selection of Neutron Activation Detector Mate

Guide for Selection. Installation and Use of Electric $\mathrm{M}$

Guide for Ship Hull, Deck and Superstructure (1973)

Guide for Statistical Analysis of Thermal Life Test Dat

Cuide for Std. Steel Doors (1972)

Guide for Structural Lightweight Aggregate Concrete (19

Guide for Supplementary Ventilation in a Ship's Cargo $\mathbf{H}$

Guide for Synchronous Motor Protection (1971)

Guide for the Application of the Single Failure Criteri

Guide for the Application, Operation, and Maintenance $\mathrm{O}$

Guide for the Design of Aluminum Formed Sheet Buildin

Guide for the Dynamic Calibration of Pressure Transduce

Guide for the Prediction of the Dispersion of Airborne

Guide for the Preparation of Test Procedures for the Th

Guide for the Selection of Leak Test. Meth. (1971) ANSI

Guide for Transient Recovery Voltage for AC High Voltag

Guide for Type Test of Class 1 Electric Valve Operators

Guide for Type Tests of Continuous Duty Class I Motors

Guide for Use of Admixtures in Concrete (1968)

Guide for Wood Composition Board Wall Panels (1973)

Guide Lines for Printed Wiring Board Repairs (1973)

Guide on Solid State Devices: Varactor Measurements Par

Guide Spec. for Accordion Folding Partitions (1972)

Guide Spec. for Cast lron Soil Pipe (1972)

Guide Spec, for Clay Tile Roofing (1972)

Guide Spec. for Concrete Unit Masonry (1971)

Guide Spec. for Engineered Brick Masonry (Tech. Notes)

Guide Spec. for Flagpoles, Metal, Wood and Fiberglass (

Guide Spec. for Furring and Lathing (1972)

Guide Spec. for Hydraulic and Electric Elevators (Freig

Guide Spec. for Metal Toilet Partitions (1972)

Guide Spec for Plastic Pipe (1972)

Guide Spec. for Platforms and Stage Lifts (Hydrualic an Guide Spec. for Sheet Metal Roofing (1971)

Guide Spec. for Specifying: Gate Valves (1973)

Guide Spec. for Specifying: Precast Concrete Panels (19

Guide Spec. for Specifying: Reinforced Fiberglass Polye

(Guide Spec.) (1972)

Guide Specs. for Architectural Woodwork: Standing and $\mathrm{R}$

Guide Specs. for Brick and Tile Masonry (Tech. Note) (P

Guide Specs. for Brick Masonry (Tech. Note) (Part I and

Guide Specs., and Materials Application for Thermoplast

Guide to Design, Construction, Materials, Size, and Ins

Guide to Practice) (1971)

Guide to Practice) (1974)

Guide to Principal Design Criteria for Nuclear Fuel Rep

Guide to Safe Practice Protection from Corrosion for Un

Guide to the Application and Use of Radial Lip Type Oil

Guide to the Preparation of Precautionary Labeling and

Guide to the Use of Sl (International System of Units)

Guide (Also Contains Suggested Techniques for Photograp

Guide (Rec. for Garden Grades, Application and Design,

Guide (Siding, Finish, Nails and Nailing) (1973)

Guide (Spec, for Installation, Finishes and Other Techn

Guide (Spec. for Texture and Grade, Panel Characteristi

Guide (1969)

Guide) (Tech. Notes) (1963)

Guide) (1963)

Cal Minimum Rec. Tolerances of Spoke Ty

Guideline for Protection and Acceptance of Concrete Pav

Guideline for the Physical Inspection of Wood Poles in

Guideline Spec. for Sodding and Soil Preparation (Grass

Guidelines and Stds, for the Paperboard Packaging lndus

Guidelines for Describing Information Interchange Forma

Guidelines for Driveway Design and Location (1972)

Guidelines for Safe Installation of Electrical lnstrume

Guidelines for Selection and Preparation of Test Panels

Guidelines for Thesaurus Structure, Construction, and U

Guidelines, and Drawings for Copper Solvent Single Stac

Guides for Design. Construction, Materials, and lnstall

Guide: General Principles for Reliability Analysis of $\mathrm{N}$

Gun or Fluidized Bed (Fire Protection) (1973)

Gustafson Meth. of Analy sis for Original Ash in Phospha

Gutters and Associated

Gutters, and Associated Fittings (Electrical Wiring) (1

CVW (1972)

GVWR for Truck Trailer Certification (1972)

Gypsum and Products (1972)

Gypsum Backing Board (1972)

d. for Nuc

NEMA

ASTM

ANSI

IEEE

IEEE

APA

ANSI

IEEE

344

ASTM E419

NEMA MG2

SNAME 4-10

IEEE 101

STDI 108

ACI $\quad 64-39$

SNAME 4.14

IEEE

IEEE

IEEE

AA

ANSI

ASME

IEEE

ASTM

IEEE

IEEE

IEEE

$\mathrm{ACl}$

IPC

IPC

IEEE

CSI

CSI

CSl

CSI

BlA

CSI

CSI

CSI

CSl

CSl

CSI

CSI

CSI

CSI

CSI

CSI

AWI

CSl

CSI

SMACN

ABYC

329

379

321

B88. 1

1

98

E432

328

382

334

CB-SG. 73

R.700

351

10623

15062.1

07350

04220

11C-11D

10350

09110

14200

10182

15064.1

14430

07610

15101

03410

15177.1

09841

*1.1

$11 \mathrm{~B}$

$11 \mathrm{~A}$

ANSI

ANSI

ANSI

ICBO

$S A E$

CGA

ASTM

ANSI

CRA

CRA

CRA

CRA

SNAME

BIA

SAE

ACPA

$A$ WPA

ASPA

PPC

ANS 
Uniform Building Code Std. for Water Resistant Uniform Building Code Std. for Uniform Building Code Std. for Spec, for Fire Barrier

r (1973) pose Roofs (Wood Shakes and Shingles with Asbestos Felt t: track) and Rigid Furring Channels for Screw Application or of Diaphragms /

Uniform Building Code Std. for Reinforced Structural Deck (Roofing); Uniform Building Code Std. for Specifying Uniform Building Code Std. for Uniform Building Code Std. for Spec. for lnterior

code Std. for Perlite, Vermiculite and Sand Aggregates for Std. Meth. for Physical Test. of Uniform Building Code Std. for Test. Uniform Building Code Std, for Spec. for Dental

r Reinforced Gypsum Concrete and Precast Slabs, and Poured Uniform Building Code Std. for Std. Spec. for Steel Drill Screws for the Application of Building Code Std. for Gypsum Base for Veneer Plaster and llation of Steel Framing Members to Receive Screw Attached Uniform Building Code Std. for Std. Spec. for Adhesives for Fastening Uniform Building Code Std for Adhesives for Fastening Uniform Building Code Std. for Std. Spec. for
Uniform Building Code Std. for (1974)

std. for Mortar for Unit and Reinforced Masonry Other Than Std. Spec. for High Strength Low Alloy Steel
Inspection and Quality Control of First Generation Silver Std. Spec. for Primary Std. Spec. for Fixation Pins-Knowles and Rec. Against Roof Leakage of Buildings Not Due to .2 Std. for Safety for dried $\mathrm{Pe} / \quad$ Meth. of Analysis of External Insect and Rodent Std. Meth. of Test for Cashmere Coarse Std. Spec. for Maximum Cashmere Coarse g Applications (1973) A/ Std. Spec. for Rubberized Curled in Cereal Grains and Grain Products (Cer/ Insects, Rodent drolysis Meth. of Analysis for Insect Fragments and Rodent Versene Meth. of Analysis for Insect Fragments and Rodent igestion Meth. of Analysis for Insect Fragments and Rodent ieving Meth. of Analysis for Dirt, Sand, Insect Fragments, mixtures (1974) Std. Meth. of Test for Water Soluble tals (1973) ductor Single Crystals (1973) Std. Meth for Measuring Rec for Std. Meth. of Test for Water Soluble Halide Ion in Std. Meth. of Test for Water Soluble Halide Ion in
Std. Meth. of Test for Acidity Alkalinity of ux Me/
using Rota/ Std. Meth. Meth. of Test for Nonvolatile Residue of using Rota/ Std. Meth. of Test for Nonvolatile Residue of
Spec. for Powdered Spec. for Powdered

Std. Spec, for Aluminum Alloy Die and Std. for Ground Support Equipment (1971)

graphite Fiber Tape and Sheet, Epoxy Resin Impregnated for Std. Spec. for ce Industry (Rotating, Liquid Filled and Dry Transformers, a Backed Paint Film (Coil/) Spec. for Conducting a Solvent Test Meth. for Chip Classification Std. Spec. for Rec.

Uniform Building Code Std. for emolina (Cereal Chemistry) (1967)

Std. for Driving and Spindle Ends for Portable

g Practices) (1971)

ion, Flat, and Hot Wound (Materials, Specs. Terminology,/ ) (1973) is and Assembly (1972) Concrete Construction Design 1)

ilders' Hardware: Hands of Doors; Hinges; Locks; Knobs and sc Type Packages (1973) 1973) API Bul D 15 Rec. Health Safety Practices for Std. for Rec. Pract. for Properties and Essential Information for Safe Sale Safe 973) Properties and Essential Information for Safe
Properties and Essential Information for Safe Safe Std. for Safe Properties and Essential Information for Safe
Gypsum Backing Board (1973)

Gypsum Backing Board (1973)

Gypsum Base for Veneer Plaster and Gypsum Veneer Plaste

Gypsum Board Membrane (1972T)

cpsum Board Underlayment) (1973)

/Td. for Special Pur
(L) Bearing Steel Studs, Runners (

Gypsum Casting and Molding Plaster (1973) ANSI A49.4

Gypsum Concrete and Precast Slabs, and Poured Gypsum Ro

Gypsum Concrete (1973)

Gypsum Lath (1973)

Gypsum Masonry and Mortar (1967)

Gypsum Molding Plaster (1973)

Gypsum Partition Tile or Block (1973)

Gypsum Plaster (1972)

Gypsum Plaster (1973)

Gypsum Plasters and Concrete (1973)

Gypsum Plasters and Concrete (1973)

Gypsum Plastics (1973)

Gypsum Products (1971)

Gypsum Roof Diaphragms (1973)

Gypsum Sheathing Board (1973)

Gypsum Sheet Material to Ligh

Gypsum Veneer Plaster (1973)

Gypsum Wallboard and Backing Board (1971)

Gypsum Wallboard Tape and Joint Compound (1973)

Gypsum Wallboard to Wood Framing (1973)

Gypsum Wallboard to Wood Framing (1973)

Gypsum Wallboard (1973)

Gypsum Wallboard (1973) ANSI A69.1

Gypsum (1973)

Gypsum (1973)

H.Piles and Sheet Piling for Use in Marine Environments

Habide Microfilm (1972)

Hafnium Metal Sponge and Other Forms (1967) ANSl N134

Hagie Types (1973)

Hail or Wind (1972)

Hair Clipping and Shaving Appliances (1973) ANSI C33.36

Hair Contamination of Cereal Grains, Unpopped Popcorn,

Hair Content in Cashmere (1971)

Hair Content in Cashmere (1971)

Hair for Cushioning Materials for Upholstery and Beddin

Hair, and Radiographic Illustration and Classification

Hairs in Flour (Cereal Chemistry) (1962)

Hairs in Rye Flour (Cereal Chemistry) (1962)

Hairs in Soy Flour (Cereal Chemistry) (1962)

Hairs, Feathers, Wood Fibers, Splinters, etc. in Starch

Halide lon in Halogenated Organic Solvents and Their Ad

Hall Coefficient in Extrinsic Semiconductor Single Crys

Hall Mobility and Hall Coefficient in Extrinsic Semicon

Halogenated Fire Extinguishing Agent Systems (1973)

Halogenated Organic Solvents and Their Admixtures (1974

Halogenated Organic Solvents and Their Admixtures (1974

Halogenated Organic Solvents, Degreasing Grade (Nonrefl

Halogenated Solvent Extract from Aerospace Components (

Hand Cleaner (Soap with Borax) (1972T)

Hand Cleaner (Soap with Vegetable Scrubber) (1972T)

Hand For gings (1973) ANSI H38.8

Hand Knob) (1974)

Hand Layup (1973)

Hand Operated Chain Hoists for Industrial Applications

Hand Power Tools, Hermatic Stators, Test, and Safety)

Hand Rub Test for the Determination of Deg. of Cure of

(Hand Screen) (Pulp and Paper) (1973)

Hand Scrubbing Soap (Grit Soap) Paste and Bar (1974)

Hand Scrubbing Soap (Grit Soap) Powder (1974)

Hand Signals for Use in Agriculture (1972)

Hand Split Shakes (1973)

Hand Washing Meth. of Analysis of Cluten in Flour and S

Hand, Air, and Electric Tools (1973)

Handbook for Library Bin ding (Rec. for Good Book Bindin

Handbook of Spring Design: Compression, Extension, Tors

Handbook (Beam, Slab, Footing, Column and Other Members

Handle, Latching Internal and External Release Componen

Handles; Exit Devices, Pulls, Push and Kick Plates; Bol

Handling and Fabricating Asbestos Textile Products (197

Handling and Installation of Power Semiconductors in Di

Handling and Proper Usage of Inhibited Oilfield Acids (

Handling and Use of Acetic Acid (1973)

Handling and Use of Acrylonitrile (1974)

Handling and Use of Allyl Chloride (Chemical Safety) (1

Handling and Use of Butadiene (1974)

Handling and Use of Caustic Soda (1974)

Handling and Use of Chloroform (1974)

Handling and Use of Ethyl Acetate (Chemical Safety) (19

Handling and Use of Ethylene (1973)

Handling and Use of Paraformaldehyde (1974)
ICBO

ICBO

ICBO

ASTM

ICBO

UBCS 47.13

UBCS47.6

UBCS47.14

C707

UBCS32. 14

C 645

UBC $524-13$

$* 2.113$

UBC 54.7

ICBO UBCS

ICBO UBCS47.12

ICBO UBCS24.11

CSI 09150

UBCS 47.3

ASTM

ICBO

ICBO

ADA

C472

UBCS $24 \cdot 26$

UBC 547.8

25

UBCS24-I3

ICBO

ASTM

UBC $\$ 47.9$

C646

UBCS47-14

A 97.2

UBC 547.5

$\begin{array}{ll}\text { ASTM } & \text { C557 } \\ \text { ICBO } & \text { UBC } 47.2\end{array}$

ICBO UBCS47.10

ASTM C36

ICBO UBC 24.12

UBCS24-21

A 690

MS104

B414

F368

FMS

UL

LPD 1.4

ACCH 1028

ASTM D2816

ASTM D2817

ASTM D2128

AACCH 28.95

Acid $\mathrm{Hy}$ AACCH 28.41

Tween AACCH 28-60

Atting D AACCH 28.70

AACCH 28.75

ASTM D2988

ASTM F76

ASTM F76

FMS $\quad 4.8 \mathrm{~N}$

ASTM D2988

ASTM D2989

ASTM D2942

ASTM F331

ASTM D3046

ASTM D 3047

NSA

SAE

B247

1553

HMI

EASA

NCCA

TAPPI

ASTM

ASTM

ASAE

ICBO

AACC

ANSI

LBI

SMI

ACI

NSA

NBHA

ATI

EIA

NACE

MCA

MCA

$\mathrm{MCA}$

MCA

MCA

$\mathrm{MCA}$

$\mathrm{MCA}$

MCA

$\mathrm{MCA}$ 
Properties, Safe Handling and Use of Phosphorus Trichloride (1972)

Safe

Properties and Essential Information for the Safe

n Residential and Commerical Fuel Burning, Dispensing, and Safety Requirements for Storage and , Ixtle, Jutes, Hemp, Tow/ Uniform Fire Code: Storage and
Rec. for Storage and , Ixtle, Jutes, Hemp, Tow/ Uniform Fire Code: Storage and
Rec. for Storage and rm Fire Code: Manufacture of Organic Coatings (Paint), and
Alcohol,
Uniform Building Code Std. for Storage and con/ Alcohol,/ Std. for Packaging, Shipping. Receiving, Storage and Std. for the Storage and
Std g More Than 2\% Free Ammonia (1970) $\begin{array}{r}\text { Std. for Storage and } \\ \text { Std. Pictorial Markings for }\end{array}$ (1973) Uniform Building Code Std. for Storage and Rec. for Safe

code: Automobile Wrecking and Junk Yards or Waste Material tractors (1973)

74)

Ducted Electric Heat Guide for Air Std for Central Station A ir Uniform Building Code: Film Storage and Std. for Screw Conveyors (Materials spec. for Cargo Unit Load Devices (Materials Transport and Planning Manual (Truck Shipping and Receiving-Materials Quality Stds. for Stairwork and
nd Storage Shelving, Misc. Ornamental Items, Stairwork and evices, Pulls, Push and Kick / Basic Builders' Hardware:

ormance Requirements for Woven Glass Fiber Curtain Fabrics

quirements for Woven Glass Fiber Drapery (Curtain) Fabrics xtile) (1973) Meth. of Test for Effect of

orm Fire Code: Airports, Heliports, Helistops and Aircraft tions (1974) ANSI H23.8 Std. Gravure Lateral Std. Spec. for Std. Spec. for Steel W ire, Std Spec, for Steel Wire, Rec. Pract. for Special Quality High Tensile, Std. Spec. for Steel Wire, High Tensile Strength, Std. for R. 40 Bulb c) Rec. Pract. for Surface Preparation of Steel and Other
Std. Meth. of Tension Test. of nd Alimentary $\mathrm{Pa}$ Sieving Meth. of Analysis for Materials Std. Spec. for Concentric Lay Stranded Copper Conductors. or Concentric Lay Stranded Copper Conductors, Hard, Medium Std. Spec. for Steel, Carbon, Strip, Cold Rolled Std. for Hardwood Veneered lncluding Std. for Prefinished Std. for Industry Std. for Std. for Basic Std. for

Rec. Pract. for Bolts and Screws, Steel, Low Alloy Heat Resistant, Heat Resistant, 195,000 psi (1,345 MPa) Tensile Strength, Std. Meth. of Test for Length Change of structural Steel Joints, Including Suitable Nuts and Plain ANSI H38.23 orging Stock for High Tempe/ ing Stock for High Temperat/ k for High Temperature Serv/ Temperature Service (1972) Std. Spec. for Aluminum Alloy Std. Spec. for Precipitation Std. Spec. for Precipitation Std. Spec. for Precipitation Std. Spec. for Precipitation hapes (1) Std. Spec. for Hot Rolled and Cold Finished Age ec. for Pressure Vessel Plates, Alloy Steel, Precipitation ec. for Pressure Vessel Plates, Alloy Steel, Precipitation Ordering, Shipping, and Billing of Merchandise Within the materials (1974) ANSI Z/ Std. Meths. of Test for Rockwell ween Brinell, Vickers, Rockwell and Rockwell Superf/ Std. Spec. for Determination of Relative Pencil 55.12 Rec. Pract. for Gogan Std. Meth of Test for Std. Meth. of Test for Rockwell Meth. of Test for Knoop Indentation Std. Meth. of Test for Vickers Std. Meth. of Test for Brinell hs. of Test for Rockwell Hardness and Rockwell Superficial Std. Rec. Pract. for Scleroscope

ell, Vickers, Rockwell and Rockwell Superficial, and Knoop Std. Spec. for Copper Zinc Lead (Leaded Red Brass or Rec. Procedure for Processing

1 oors (1973) ANSI Al43.1

-Application) (1972)

(1972)
Safety Std. for Swinging Safety Std. for Sliding Rec. Spec. for Builders

Rec. Minimum
Rec. Std. Steel Door, Frame and Abbreviations and Symbols Used in Builders
Handling and Use of Prop ylene (1974)

Handling and Use of Vinyl Chloride (1972)

Handling Chlorine in Tank Motor Vehicles (1973)

Handling Facilities (1974) ANSI B150.1

Handling of Anhydrous Ammonia (1972)

Handling of Combustible Fibres (Cotton, Sisal, Henequen

Handling of Flammable and Combustible Liquids and Solid

Handling of Flammable Liquids (Gasoline, Kerosene, Acet

Handling of Items for Nuclear Power Plants (During the

Handling of Liquefied Petroleum Gases (1972)

Handling of Nitrogen and Fertilizer Solutions Containin

Handling of Nondangerous Goods (1972)

Handling of Photographic and X-Ray Nitrocellulose Films

Handling of Radioactive Materials (1964)

Handling Plants (1973)

Handling Spec. for Sheet Metal and Air Conditioning Con

Handling Symbols for Powered Industrial Trucks (1973)

Handling Systems (1971)

Handling Units (Air Conditioning and Refrigeration) (19

Handling (Photographic and X-Ray Nitrocellulose) (1973)

Handling) (1971) ANSI MH4.7

Handling) (1973)

Handling) (1973)

Handrails (Architectural Woodwork) (1973)
Handrails, Exterior Frames and Sash, Screen

Hands of Doors; Hinges; Locks: Knobs and Handles: an

Handwashable at 105 Deg. F-No Bleach (Institutional T

Handwashable at 105 Deg. F-No Bleach (Institutional T

Handwashing on Woven Glass Fiber Decorative Fabrics ( $\mathrm{Te}$

Hangers (1973)

Hard Dot Screen (1973)

Hard Drawn Copper Capillary Tube for Restrictor Applica

Hard Drawn for Mechanical Springs (1971) ANSI G54.1

Hard Drawn for Prestressing Concrete Pipe (1973)

Hard Drawn Mechanical Spring Wire and Springs (1972)

Hard Drawn, for Mechanical Springs (1973)

Hard Glass) Mogul Screw Base Incandescent (1966)

Hard Materials by Water Blasting Prior to Coating or Re

Hard Rubber (1972)

Hard to Hydrate in Baked Goods, Ready-To-Eat Cereals, a

Hard, Medium Hard, or Soft (1972) ANSI C7.8

Hard, or Soft (1972) ANSI C7.8

Hard, Untempered Spring Quality (1973)

Hardboard and Plastic Faced Flush Doors (1973)

Hardboard Paneling (1973) ANSI A 135.5

Hardboard Siding (1973) ANSI A 135.6

Hardboard (1970)

Hard board (1973)

Hardenability Bands for Alloy H Steels (1971)

Hardenability Bands for Carbon H Steels (1971)

Hardened and Tempered, Roll Threaded (1973)

Hardened and Tempered, Roll Threaded (1973)

Hardened Cement Mortar and Concrete (1974) A

Hardened Washers (1971) ANSI G38.6 Std. Spec. F

.

Hardening Iron Base Superalloy Bars, Forgings, and Forg

Hardening Nickel Alloy Bars, Forgings, and Forging Stoc

Hardening Nickel Alloy Plate, Sheet, and Strip for High

Hardening Stainless and Heat Resisting Steel Bars and S

Hardening (Maraging), 12\% Nickel (1972A)

Hardening (Maraging), 18\% Nickel (1972A) ANSI G35.17

Hardgoods (Hardware) Industry (1972)

IIon Flow

Hardness and Rockwell Superficial Hardness of Metallic

Hardness Conversion Tables for Metals (Relationship Bet

Hardness of a Paint Film (Coil Coating) (1974)

Hardness of Brake Lining (1972)

Hardness of Electrical Contact Materials (1972) ANSI ZI

Hardness of Fine Grained Graphite Materials (1973)

Hardness of Glass (1972T)

Hardness of Metallic Materials (1972) ANSI Z115.7

Hardness of Metallic Materials (1973) ANSl Z115.5

Hardness of Metallic Materials (1974) ANSI Z1I5.6, Asht

Hardness Test. of Metallic Materials (1972)

Hardness) (1972) ANSI Z76.4

Hardware Bronze) Rod, Bars, and Shapes (1972) ANSI H33.

Hardware for Custom Alu minum Doors (1972)

Hardware for School Buildings (1957)

Hardware for Std. Tin CIad Fire Doors (1973) ANSI Al33.

Hardware for Std., Horizontally Mounted Tin Clad Fire D

Hardware on Std. Steel Doors and Frames (Reinforcement

Hardware Reinforcing Garages on Steel Doors and Frames

Hardware Schedule (1972)

Hardware Schedules and Specs. (1961)
MCA

MCA

MCA

$\mathrm{CHI}$

UL

FI

ICBO
FMS

ICBO

ICBO

ANSI

NFPA

FI

ANSI

ICBO

NCRP

ICBO

SMACN

ANSI MHI

SMACN *12

ARI

ICBO

CEM

NSA

ANSI

$A W I$

$A W I$

DHA

ANSI

ANSI

ANSI

Unif $1 C B O$

GT

ASTM

ASTM

ASTM

SAE

ASTM

ANSI

NACE

ASTM

AACC

ASTM

ASTM

ASTM

NWMA

USC

USC

AHA

USC

SAE

SAE

SAE

ASTM

ASTM

ASTM

ASTM

ASTM

ASTM

ASTM

ASTM

Std. Sp ASTM

ASTM

NWHA

ASTM

ASTM

NCCA

SAE

ASTM

ASTM

ASTM

ASTM

ASTM

ASTM

ASTM

ASTM

NBHA

NBHA

UL

UL

STDI

NBHA

STDI

NBHA

7.50

P145

12

350

UFC*2ART34

UBCS10.1

$\mathrm{N} 45.2 .2$

MH6. 1

UBC S48.1

FC $* 2$ ART 3

UBC *3.48

MH8. 1

*1-1

L24.1.3 
Rec. Procedure for Processing Rec. Pract. for

Safety Std. for Panic (Emergency) Rec. Locations for Builders and Chromium on Metal Parts-Automotive Ornamentation and Std for Builders Cabinet Std. for Sliding and Folding Door shipping, and Billing of Merchandise Within the Hardgoods Std. for Butts and Hinges for Doors (Builders to for Auxiliary Lock les: Exit Devices, Pulls, Push and Kick / Basic Builders Rules for the Measurement and Inspection of Structural Design Guide for and Lodgepole Pine, Western Larch, California Redwood and Flusb Doors (1973)

in Milk (CereaI Chemistry) (1962)

3)

Std for Electrical Std. for Terminology for Combines and Grai

for Determination of Film Adhesion on All Metals by Cross Test Meth. for Determination of Film Adhesion by Cross

the Road Vehicles: Earthmoving, Mining and Logging (Short Friction Coefficients of Chopped Forages (Grass, Corn,

cocoa Fibers, Oakum, Rags, Waste Cloth, Wastepaper, Kapok, 1974)

Uniform Building Code Std. Test. Meth. for Fir

Rec. for Electrolytic Chlorine Processes (Explosion

Rec. Pract. for Reducing the Fire

tile Solids (1973)

Std for Fire Uniform Fire Code: Suppression and Control of osion, and Detonation Protection) (1973) 1973)

Manual of Rec. for

als, Metal Dusts) (1973)

Uniform Fire Code: delines for Safe Installation of Electrical Instruments in ues and Instrumentation for the Measurement of Potentiall

Class II, G/ Safety Std. for Electric Heaters for Use in ged and Pressurized Enclosures for Electrical Equipment in Std. for Safety for Electric Lighting Fixtures for Use in Receptacles and Connectors of the Pin and Sleeve Type for Safety Std. for Receptacle-Plug Combinations for Use in safety Std. for Circuit Breakers and Enclosures for Use in Safety Std. for Industrial Control Equipment for Use in for Safety for Electric Motors and Generators for Use in for Safety for Portable Electric Lighting Units for Use in Safety Std. for Electric Motors and Generators for Use in Safety Std for Electrically Operated Valyes for Use in Groups E/ Safety Std. for Electrical Switches for Use in classifying and Labeling Epoxy Products According to Their f Mechanical Drive Turbines (1974)

and Automatic Feedwater Regulators for Boilers (197)

le and Combustible Materials (Fire Safety) (1974) paper (1974)

d RecreationaI Vehicle Manufacturing Plants (Fire and Wind i C 95.4

270.1

Safeguards Against Radio Frequency Radiation Std. for Control of Gas

Std Safety Color Code for Marking Physical Std. Spec. fuz Photographic Grade Hydrochloric Acid, rapbic Grade $\mathrm{P}$-Aminophenol Hydrochloride $\mathrm{HOC}_{6} \mathrm{H}_{4} \mathrm{Nh}_{2}$ photographic Grade Hydroxylamine Hydrochloride, $\left(\mathrm{Nh}_{2} \mathrm{OH}\right.$. Std. for Bolt, Taper Shank, Shear Type, 100 Deg. Std. for Bolt. Taper Shank, Shear Type, Protruding igbting Equipment (1973) NEMA SH10

ighting Equipment (1973) EEI TDJ.140

Std. for Meta Std. for Metal

rivet-100 Deg. Countersunk Head Hi Shear Close Tolerance Std. for 100 Deg. Close Tolerance

74)

t. for Instrument Panel Laboratory Impact Test Procedure Std. for Solid 100 Deg. Flush Shea Std for 450 Deg. F Self Locking Serrated

Std. for 450 Deg. F Closed End Self Locking Serrated Std. for 450 Deg. F Self Locking Elliptica

Std. for Bulbed Mechanically Locked Spindle Protrudin std. for Bulbed Mechanically Locked Spindle 100 Deg. Flush

Std. for Bulbed Spindle Mechanically Locked Protruding std. for Bulbed Mechanically Locked Spindle 100 Deg. Flush Std for Full Threaded, $160 \mathrm{KSI}$ Steel, Drilled Std. for Shear Hexagon

Wire, Medium High Carbon Cold Heading Quality for Hexagon I Alloy Steel Wire, Alloy Cold Heading Quality for Hexagon

Std for Screw, Machine, $100 \mathrm{Deg}$. . Fla Std. for Rivet-100 Deg. Countersunk Std. Part Bolt, 100 Deg. Flush Tension
Hardware Schedules and Templates (1971)

Hardware Test. of Digital Process Computers (1971)

Hardware (Outward Opening Door Releasing Device) (1973)

Hardware (1964)

Hardware $(1970)$

Hardware (1971)

Hardware (1971)

(Hardware) Industry (1972)

Hardware) (1970)

(Hardware) (1971)

Hardware: Hands of Doors; Hinges; Locks; Knobs and Hand

Hardwood and Cypress Lumber (1971-75)

Hardwood Block Flooring (1971)

Hardwood Plywood (1971)

Hardwood Timber (1973)

/ Douglas and Hem Fir, Southern

Hardwood Veneered Including Hardboard and Plastic Faced

Harland and Ashworth Meth. of Analysis of Serum Protein

Harness Assembling Machine, Numerically Controlled (197

Harvesters, Wagons and Blowers (1972)

Harvesting (1971)

Hatch Tape Test After Reverse lmpacting (Coil Coating)

Hatch Tape Test (Coil Coating) (1974)

Haul); Grader, Loader, Dozer, Mining Car, Front End Loa

Hay, Stra w) (1968)

Hay, Straw, Spanish Moss, Excelsior, etc.) (1973)

Hazard Classification of Building Materials (1973)

Hazard Classifications of Organic Peroxides (Plastic) (

Hazard During Manufacture) (1974)

Hazard of Static Electricity (1973)

Hazard Properties of Flammable Liquids, Gases, and Vola

Hazardous Areas (1973)

Hazardous Chemical Reactions (Incandescence, Fire, Expl

Hazardous Chemicals Data (Fire Protection and Safety) (

Hazardous Chemicals (1973)

Hazardous Dust Locations (Agricultural Plastics, Chemic

Hazardous Dust Locations (Agricultural Plastics, Chemic

Hazardous Electromagnetic Radiation at Microwave Freque

Hazardous Locations Glass I, Groups A, B, C, and D, and

Hazardous Locations (1972) ANSI C106.1

Hazardous Locations (1972) ANSI C 33.28

Hazardous Locations (1973)

Hazardous Locations (1973) ANSI C33.97

Hazardous Locations, Class I, Groups A.D, and Class II,

I, Groups A-D, Class II, Gro

Hazardous Locations, Class I, Groups C and D, and Class

Hazardous Locations, Class II, Groups E, F, and G (1973

Hazardous Locations, Class 1, Groups A, B, C, and D, an

Hazardous Locations: Class I, Groups A.D, and Class 11,

Hazardous Potentialities (Safety) (1972)

hazards and Protection for Maintenance and lnspection

Hazards and Protection Rec. for Low Water Fuel Cutoffs

Hazards and Protection Rec. for Textile Mills (1974)

Hazards and Protection Std. for Rack Storage of Flammab

Hazards Anf Protection Rec. for Indoor Storage of Roll

Hazards at Plants Assembling Mobile Homes, Modular Hous

Hazards in the Use of Electric Blasting Caps (1971) Ans

Hazards on Vessels (Ships) to Be Repaired (1972) ANSI Z

Hazards (1971)

HCI (1972)

HCI (1972)

Hci) (Hydroxylammonium Chloride) (1973)

Head-Oversize (1973)

Head-Oversize (1973)

Head and Reflector Interchangeability Used in Roadway L

Head and Reflector Interc

Head and Shank (193)

Head Area (1971)

Head A286 Corrosion Resistant Steel and Monel Rivet (19

Head Blind Nut (1974)

Head Blind Nut (1974)

Head Blind Nut (1974)

Head Blind Rivet (1974)

Head Blind Rivet (1974)

Head Blind Rivet (1974)

Head Blind Rivet (1974)

Head Bolt (1972)

Head Bolt (1973)

Head Bolts (1972) ANSI G54.15

Head Bolt s (1972) ANSI G54.16

Head Full Threaded, Alloy Steel (1973)

Head Hi Shear Close Tolerance Head and Shank (1973)

Head Hi Torque Recess Alloy Steel, 160,000 psi (1973)

Head Industrial Fluid Power Cylinders (1972) ANSI B93.2

Head Machine Screws and Machine Screw Nuts (1972)
1. G

NBHA

ISA

UL.

NBHA

SAE

BHMA

BHMA

NWHA

ANSI

BHMA

NBHA

NHLA

HPMA

HPMA

ICBO

NWMA

$\mathrm{AACCH}$

NSA

ASAE

ASAE

NCCA

TRA

(W. ICBO

ICBO

FMS

FMS

FMS

FMS

ICBO

FMS

FMS

ICBO

ISA

ISA

ANSI

UL

UI

NEMA

UL

UL

UL

UL

UL

UL

UL

ANSI

FMS

FMS

FMS

FMS

FMS

FMS

IME

NFPA

ANSI

ANSI

Std. Spec. for Photog

D. Spec. for ANSI

NSA

NSA

EEI

NEMA

NSA

NSA

$\mathrm{SAE}$

NSA

NSA

NSA

NSA

NSA

NSA

NSA

NSA

NSA

NSA

Std. Spec. for Steel ASTM

Std. Spec. Fo

ASTM

NSA

NSA

NFLD

ANSl

7

RP55.I

305

J207

(20]

401

1

A156.1

8

LF.71

HP.SG.71

UBCS25.11

I.S. 1

\$328.1T

S343

TB-Il.16

TB.Il.5

3

D251

UFC $* 2 A R T 7$

UBCS42. 1

7.81

7-34

$5.8 \mathrm{~N}$

7.19 
Std, for Hexagon al Wash-Whites 190 Deg. F, Colors 160 Deg. F Maximum Hot Std. Spec. for Reinforced Concrete Low Std. for Hi Shear Protruding Std for Hi Shear 100 Deg. Flush Close Tolerance attachment (Preferred) (1974) Std. for Pan Std. for Hex

ternally Threaded, External Sleeve, Light Weight, Millable Std. for Square

Std. for Titanium Alloy Stump Type Protruding Std. for Titanium Alloy Stump Type $100 \mathrm{Deg}$ Std for Self Locking Hexagon Dimensions for 3/4, 1 and 1 1/8 In. Bore Cataloged Square d, Self Locking and Nonlocking / Std. for Screw, $100 \mathrm{Deg}$ Std. for Punches-Variable, Angle

Std. for Punches-Basic. Combination Angle Std, for Punches: Basic Angle Std. for Punches-Basic, Cylindrical Std. for Die Buttons, Variable, Press Fit, Headless and Std Spec for 55 Gal. Tight Std. Spec. for $55 \mathrm{Gal}$. Full Removable Std. Spec. for $16 \mathrm{Gal}$. Tight Std. Spec. for $30 \mathrm{Gal}$. Full Removable Sid. Spec. for 55 Gal. Tight

974)

1974)

1974)

1974)

40, $\mathrm{Nmfc}-$ Item 260, Dot-

alloy $(1973)$

Std. Spec. for 55 Gal. Full Removable Std. Spec, for $30 \mathrm{Gal}$. Tigh

Std. Spec. for 57 Gal. Full Removable Std. Spec. for $30 \mathrm{Gal}$. Full Removable Std. Spec. for 16 Gal. Full Removable Std. Spec. for 5 Gal. Tight Std. for Bolt Lock. Tension, 100 Deg. Std. for Bolt Lock, Tension, $100 \mathrm{Deg}$.

Std for Pin, Swage Locking. Aluminum Alloy, 100 Deg. Std. for Pin, Swage Locking, A-286, 100 Deg. for Pin, Swage Locking. Steel, Std. and Oversize, 100 Deg. Std. for Pin, Swage Locking, Steel, 100 Deg.

Std. for Pin, Swage Locking. Aluminum Alloy, $100 \mathrm{Deg}$

Std. for Bolt Lock, Tension, 100 Deg.

Std. for Bolt Lock, Tension, 100 Deg.

Std. for Pin. Swage Locking, Aluminum Alloy, $100 \mathrm{Deg}$ Std. for Pin. Swage Locking, A-286 Cres, 100 Deg. Std. for Pin, Swage Locking, Steel, 100 Deg.

Std. for Pin, Swage Locking, Aluminum Alloy, $100 \mathrm{Deg}$. for Pin, Swage Locking, Steel, Std. and Oversize, $100 \mathrm{Deg}$ Std for Bolt, Taper Shank, Shear Type, 100 Deg. Std. for Bolt, Taper Shank. Shear Type, Protruding Std. for Bolt-Tee

Std for Machine Bolt. Crowned Hexagon ocking and Nonlocking (1972) locking and Nonlocking (1972) cking and Nonlocking (1972)

cking and Nonlocking (1972)

crew (1972)

itanium Alloy $6 \mathrm{Al} \cdot 4 \mathrm{~V}$, Self Locking An/

lloy Steel, Self Locking and Nonlocki

286 Acres, Self Locking and Nonlocking (197)

ad, Self Locking and Nonlocking (1972/

read, Self Locking and Nonlocking (19) Std, for Self Locking Flat 100 Deg. Std. for Self Locking, Pan Std. for Self Locking, Flat Fillister

Std. for Screw, Machine, Pan Std for Screw, Machine, Flat Fillister Std. for Screw, Machine, Flat 100 Deg. Std. for Machine Screw, Flat Fillister Std. for Machine Screw, Flat Fillister Std. for Machine Screw, Flat Fillister Std. for Rivet-Flat Std. for Protruding

king and Nonlocki/ -Locking and Nonl

item 260) (1974)

e Screw (1974)

steel Machine Screw (1974)

- Pin Swage Locking, 286 Cres. 100 in, Swage Locking, $108 \mathrm{KSI}$ Steel, 100 Deg. Shear/Tension $n$, Swage Locking, Steel, Std. and Oversize, 100 Deg. Shear ernally Threaded, External Sleeve. High Temperature, Flush rnally Threaded, External Sleeve, Light Weight, Protruding lly Threaded, External Sleeve, General Purpose, Protruding ternally Threaded, External Sleeve, General Purpose, Flush Std. for Pin, Swage Locking, Steel, Protruding Std. for Pin, Swage Locking, A.286 Cres, Protruding
Head Modified Short Thread Shear Bolt (1972)

Head Press (300-330 Deg. F) Finishing, Shirt Unit, Pa Head Pressure Pipe (1973)

Head Rivet (1972)

Head Rivet (1972)

Head Screw and Assembled Washers-Electrical Terminal

Head Screw, Recessed, Full Thread (1973)

Head Self Locking) (1972)

Head Setscrews (1969)

Head Shear Bolt Lock (1974)

Head Shear Bolt Lock (1974)

Head Shear Bolt (1974)

Head Tie Rod Type Industrial Fluid Power Cylinders (197

Head Tri Wing Recess, 6Al-4V Titanium Alloy, Full Threa

Head Type and Related Quill Bushings (1972)

Head Type and Related Quill Bushings (1972)

Head Type and Related Quill Bushings (1972)

Head Type and Related Quill Bushings (1972)

Head Type, Step Relief (1972)

Head Universal Drum (Dot-5B) (1974)

Head Universal Drum (DOT-17H) (1968)

Head Universal Drum (Dot-17E) (1974)

Head Universal Drum (Dot-17H) (1974)

Head Universal Drum (Dot-17C) (1974)

Head Universal Drum (Uf c-Rule 40, Nmfc-Item 260) (1

Head Universal Drums (Dot-17E) (1974)

Head Universal Drums (Dot-17E) (1974)

Head Universal Drums (Ufc-Rule 40. Nmfc-Item 260)

Head Universal Drums (Ufc-Rule 40, Nmfc-Item 260) (

Head Universal Drums (Ufc-Rule 40, Nmfc-Item 260) (

Head Universal Pails (Container) (Dot-17E, Ufc-Rule

Head 160,000 psi (1973)

Head (MS20426), Std. and Oversize, Pull Type, Titanium

Head (MS20426), Stump Type, Titanium Alloy (1973)

Head (MS20426), Tension, Pull Type (1973)

Head (MS20426), Tension, Pull Type (1973)

Head (MS20426), Tension, Pull Type (1973)

Head (MS20426), Tension, Stump Type (1973)

Head (Ms20426, Tension, Stump Type (1973)

Head (MS24694), Pull Type, Titanium Alloy (1973)

Head (MS24694), Stump Type, Titanium Alloy (1973)

Head (MS24694), Tension, Pull Type (1973)

Head (MS24694), Tension, Pull Type (1973)

Head (MS24694), Tension, Stump Type (1973)

Head (MS24694), Tension, Stump Type (1973)

Head (Ms 2494), Tension, Pull Type (1973)

Head (1973)

Head (1973)

Head (1973)

Head, Adjusting (1973)

Head, Close Tolerance, Alloy Steel, Long Thread, Self L

Head, Close Tolerance, Alloy Steel, Short Thread, Self

Head, Close Tolerance, A286 Cres, Long Threads, Self Lo

Head, Close Tolerance, A286 Cres, Shore Thread, Self Lo

Head, Close Tolerance, Short Thread, Torq Set Machine S

Head, Close Tolerance, Short Thread, Tri Wing Recess, T

Head, Close Tolerance, Short Thread, TWI Wing Recess, a

Head, Close Tolerance, Short Thread, TWI Wing Recess, a

Head, Close Tolerance, Titanium Alloy (1972)

Head, Close Tolerance, $6 \mathrm{Al}-4 \mathrm{~V}$ Titanium Alloy, Long Thre

Head, Close Tolerance, 6Al-4V, Titanium Alloy, Short Th

Head, Full Thread Part Screw (1974)

Head, Full Thread Part Screw (1974)

Head, Full Thread Part Screw (1974)

Head, Full Thread, Torq Set (1973)

Head, Full Thread, Torq Set (1973)

Head, Full Thread, Torq Set (1973)

Head, Full Thread, Tri Wing Recess, A286 Cres, Self-Loc

Head, Full Thread, Tri Wing Recess, Titanium Alloy, 6Al

Head, Full Threaded, Tri Wing Recess, Alloy Steel, Self

Head, Hi Shear, Close Tolerance Shank (1973)

Head, Locked Spindle Blind Rivet (1972)

Head, Locked Spindle Rivet (1972)

Head, Lug Cover Universal Drums (Ufc-Rule 40, Nmfc-

Head, Nonmagnetic, and Heat Resistant (1973)

Head, Phillips Recess Full Threaded, Alloy Steel Machin

Head, Phillips Recess, Short Thread, 160,000 psi Alloy

Head, Pull Type (1973)

Head, Pull Type (1973)

Head, Pull Type (1973)

Head, Self Locking) (1972)

Head, Self Locking) (1972)

Head, Self Locking) (1972)

Head, Self Locking) (1972)

Head, Shear Stump Type (1973)

Head, Shear / Tension, Pull Type (1973)
NSA

ANSI

ASTM

NSA

NSA

NSA

NSA

NSA

SAE

NSA

NSA

NFLD

NSA

ANSI

ANSI

ANSI

ANSI

ANSI

ANSI

ANSI

ANSI

ANSI

ANS

ANSI

ANSI

ANSI

ANSI

ANSI

NSA

NSA

NSA

NSA

NSA

Std. NSA

NSA

NSA

NSA

NSA

NSA

NSA

NSA

NSA

Std. NSA

NSA

NSA

NSA

NSA

NSA

NSA

NSA

NSA

NSA

NSA

NSA

NSA

NSA

NSA

NSA

NSA

NSA

NSA

NSA

NSA

NSA

NSA

NSA

NSA

NSA

ANSI

NSA

NSA

NSA

Std. Fo NSA

Std. for P NSA

Std for Pi NSA

Fastener (Blind Int NSA

/ for Fastener (Blind, Inte NSA

/R Fastener (Blind, Interna NSA

Td , for Fastener (Blind in NSA

NSA

NSA

1103.20

L24.4.1

C361

1055

120

1674

$\mathrm{J} 102$

2605

2705

T3.6.11

5800.6

B94.41

B 94.44

B94.43

MH2.3

MH2.5

$\mathrm{MH} 2.7$

$\mathrm{MH} 2.13$

MH2.4

MH2.2

MH2.1

MH2.6 
Std for Pin, Swage Locking, 108 KSI Steel, Protruding r Pin, Swage Locking, Steel, Std. and Oversize, Protruding Std. for Pin, Swage Locking, A.286 Cres, Protruding Std. for Machine Screw, Flat $100 \mathrm{Deg}$. Std for Machine Screw, Pan

3)

3)

3) Std. for Bolt Lock, Tension, Protruding Std. for Bolt Lock, Tension, 100 Deg. Crown Std. for Bolt Lock, Shear, Protruding Std. for Bolt Lock, Shear, $100 \mathrm{Deg}$. Std. for Pin, Swage Locking, Steel, 100 Deg. Shear Std. for Pin, Swage Locking, A-286 Cres, 100 Deg. Shear Std. for Bolt Lock, Tension, Protruding Std. for Bolt Lock, Tension, 100 Deg. Crown std. for Pin Swage Locking, Std and Oversize, Protruding Std. for Pin, Swage Locking, Aluminum Alloy, Protruding Std. for Pin, Swage Locking, Steel, $100 \mathrm{Deg}$. Crown Std. for Pin, Swage Locking, A.286 Cres, Protruding Std. for Pin, Swage Locking, Aluminum Alloy, Protruding Std. for Pin, Swage Locking, Steel, Protruding Std. for Bolt-Close Tolerance, Hexagon Std. for Bolt, Flat, $100 \mathrm{Deg}$. Std. for Bolt, Brazier

Std. for Bolt, Flat, 100 Deg. Reduced

king (1973) Std. for Screw, Hex ocking and Nonlocking (1973) g (1972)

ing and Nonlocking (1972)

king and Nonlocking (1972)

king and Nonlocking (1972)

ng Thread, Self Locking and Nonl/ ort Thread, Self-Locking and Non/ ort Thread, Nonlocking (/ t Thread, Self Locking and Nonlo/ t Thread, Nonlocking (19) ad, Nonlocking (1972) for Screw, $100 \mathrm{Deg}$. Std. for Screw, Hex Std. for Screw, 100 Deg. Oval Std. for Screw, $100 \mathrm{Deg}$ Std. for Screw, Pan Std. for Bolt, $100 \mathrm{Deg}$ Std. for Bolt, $100 \mathrm{Deg}$ Std. for Bolt, 100 Deg. Std, for Bolt, 100 Deg. Std. for Bolt, 100 Deg. Reduced Std. for Bolt, 100 Deg. Std. for Bolt, 100 Deg. Reduced Std for Screw, Hex

ly Threaded, External Sleeve, High Temperature, Protruding rs $(1972)$ 973) Std. for

Spec. for Titanium Alloy Bolts and Screws, 6Al.4V, Upset Std. Tooling Nomenclature for Solid Die Two Blow Cold Std. Spec. for Protective Std. Spec. for Cold

971) ANSI G54.14 metal Screws (1971) ANSI G.54.17

Std. Spec. for Cold 15 Std. Spec. for Steel Wire, Medium High Carbon Cold 16 Std. Spec. for Alloy Steel Wire, Alloy Cold g54.18

Std Spec for Aluminum Alloy Rivet and Cold ear Testing of Aluminum and Aluminum Alloy Rivets and Cold Std. for Auxiliary Low Beam Lamp Std. for Dimensional Spec. for Sealed Beam Rec. Pract. for Semiautomatic Rec. Pract. for

ract. for Service Performance Requirements for Sealed Beam Rec. Pract. Test Meth. and Requirements for Snowmobile Rec. Pract. for Motorcycle Std. for Die Buttons, Variable, Press Fit, Std. for Slotted Std. for Pin, Straight, for Lighting for Regular Bicycles, Including Reflectivity, anical Interchangeability of Socket Supports Used in Metal cal Interchangeability of Socket Supports for Use in Metal Std. for Adjustable Face Vehicular Traffic Control Signal Std. Spec. for Alloy Steel Forgings for Seamless Drums alent Chromium Compounds (Air Contamination-Occupational in Motive Power Service (For Electric Trucks/ Safety and (Packaging) (1974) Std. for Minimum Safety and bestos Textile Products (1971) Std. for Minimum Safety and Std. Meth. for Chemical Analysis of Steel and Cast, Open tation, Mechanically Operated Type for I/ Safety Std. for t) (1973) Std. Meth of Test for Weight Loss After Std. Meth. of Test for Effect of of Farm Liv/ Effect of Thermal Environment on Production, le, 75 Deg. C Operation / Std. Spec. for Synthetic Rubber utomatically in Case of Fire (1974) Approval Std. for les (1973) ANSl A 147) Safety Std. for Liquid Fuel Burning Z11.313 Marine Steam Power Plant Std. Meth. for Calculation of Liquid Rec. Pract. for Ventilation for

items; Key Control Systems; Door Holders, Stops, Smoke and Std. Spec. for U.Bend Seamless Copper and Its Alloy td. Spec. for Seamless Cold Drawn Intermediate Alloy Steel std. Spec. for Aluminum Alloy Seamless Round Condenser and std. Spec. for Copper and Its Alloy Seamless Condenser and
Head, Shear / Tension, Pull Type (1973)

Head, Shear, Pull Type (1973)

Head, Shear, Stump Type (1973)

Head, Short Thread, Torq Set (1973)

Head, Short Thread, Torq Set (1973)

Head, Std. and Oversize, Pull Type, Titanium Alloy (197

Head, Std. and Oversize, Pull Type, Titanium Alloy (197

Head, Std. and Oversize, Pull Type, Titanium Alloy (197

Head, Std, and Oversize, Pull Type, Titanium Alloy (197

Head, Stump Type (1973)

Head, Stump Type (1973)

Head, Stu mp Type, Titanium Alloy (1973)

Head, Stump Type, Titanium Alloy (1973)

Head, Tension, Pull Type (1973)

Head, Tension, Pull Type (1973)

Head, Tension, Pull Type (1973)

Head, Tension, Pull Type (1973)

Head, Tension, Stump Type (1973)

Head, Tension, Stump Type, (1973)

Head, Titanium, Short Thread (1973)

Head, Torq Set and Hi Torque, 180 KSl (1973)

Head, Torq Set and Hi Torque, 180 KSI (1973)

Head, Torq Set and Hi Torque, 180 KSI (1973)

Head, Tri Wing Recess, Alloy Steel, Full Thread, Nonloc

Head, Tri Wing Recess, Alloy Steel, Full Thread, Self-L

Head, Tri Wing Recess, A286 Cres, Full Thread Nonlockin

Head, Tri Wing Recess, A 286 Cres, Full Thread Self Lock

Head, Tri Wing Recess, A286 Cres, Full Thread, Self Loc

Head, Tri Wing Recess, A286 Cres, Full Thread, Self Loc

Head, Tri Wing Recess, Close Tolerance (1972)

Head, Tri Wing Recess, Close Tolerance (1972)

Head, Tri Wing Recess, Close Tolerance, Alloy Steel, Lo

Head. Tri Wing Recess, Close Tolerance, Alloy Steel, Sh

Head, Tri Wing Recess, Close Tolerance, Alloy Steel, Sh

Head, Tri Wing Recess, Close Tolerance, A286 Cres, Shor

Head, Tri Wing Recess, Close Tolerance, A286 Cres, Shor

Head, Tri Wing Recess, 6Al-4V Titanium Alloy, Full Thre

Head, 180 KSI (1973)

Head, (1972)

Std. for Fastener (Blind, Internal

for the Calibration of Audiometer Bone Vibrato

Headed Drilled Pins for Farm Equipment and Machinery (1

Headed, Heat Treated, Roll Threaded (1973)

Headers (1972)

Headgear for Vehicular Users (1971)

Heading Quality Carbon Steel Wire for Machine Screws (1

Heading Ouality Carbon Steel Wire for Tapping or Sheet

Heading Quality for Hexagon Head Bolts (1972) ANSI G54.

Heading Quality for Hexagon Head Bolts (1972) ANSI G54.

Heading Quality Steel Wire for Wood Screws (1971) ANSI

Heading Wire and Rod (1973) ANSI H38.12

Heading Wire and Rods (1972)

Headlamp-Light) (1973)

Headlamp Units (1972)

Headlamp (Light) Beam Switching Devices (1972)

Headlamp (Light) Switch (1971)

Headlamp (Light) Units for Motor Vehicles (1973)

Headlamps (Light) (1972)

Headlamps (1971)

Headless and Head Type, Step Relief (1972)

Headless Setscrews (Screws) (1971)

Headless (Dowel) (1973)

Headlights and Taillights (1972)

Heads for Roadway Lighting Equipment (1973) EEI TDJ-143

Heads Used in Roadway Lighting Equipment (1973) NEMA Sh

Heads (1970)

Heads, and Other Pressure Vessel Components (1970) ANSI

Health and Safety) (1973)

$\mathrm{N}$ of Chromic Acid and Hexa

Health Rec. for Changing and Charging Storage Batteries

Health Requirements for Paper Bag and Sack Manufacture

Health Safety Practices for Handling and Fabricating as

Hearth, and Wrought Iron (1973)

Heat Actuated Fire Alarm Devices, Single and Multiple S

Heat Aging of Preformed Sealing Tapes (1974)

Heat and Air on Asphaltic Materials (Thin Film Oven Tes

Heat and Moisture Loss and Feed and Water Requirements

Heat and Moisture Resisting lnsulation for Wire and Cab

Heat and Smoke Roof and Window Vents Designed to Open

Heat Appliances for Mobile Homes and Recreational Vehie

Heat Balance Practices (1972)

Heat Capacity of Petroleum Distillate Fuels (1971) ANSI

Heat Control in Industrial Exhaust Systems (1974)

Heat Detectors; Electromagnetic Holders; Sliding Door E

Heat Exchanger and Condenser Tubes (1973)

Heat Exchanger and Condenser Tubes (1973) ANSI B125.8

Heat Exchanger Tubes with Integral Fins (1973)

Heat Exchanger Tubes with Integral Fins (1974)
NSA

NSA

NSA

NSA

NSA

NSA

NSA

SSA

NSA

NSA

NSA

NSA

NSA

NSA

NSA

NSA

NSA

NSA

NSA

NSA

NSA

NSA

NSA

NSA

NSA

NSA

NSA

NSA

NSA

NSA

NSA

NSA

NSA

ANSl

SAE

SAE

IFl

ASTM

ASTM

ASTM

ASTM

ASTM

AST

ASTM

SAE

SAE

SAE

SAE

SAE

SAE

ANSI

SAE

NSA

BMA

NEMA

EEl

ITE

ANSI

ITA

ANSI

ATl

AST

UL

ASTM

ASTM

ASAE

ASTM

FMS

UL

SNAME

ASTM

ACGIH

NBHA

ASTM

ASTM

ASTM

ASTM

7034

1446

6984

1620.28

1630.34

2005

2125

2406

2506

1414 
Spec. for Seamless Nickel and Nickel Alloy Condenser and rritic and Austenitic Alloy Steel Boiler, Superheater, and

oy No. 260 Brass Strip in Narrow Widths and Light Gage for id. Spec. for Welded Austenitic Steel Boiler, Superheater, for Seamless and Welded Titanium Tube for Condensers and loy Condenser Rolled Tube Plates in Surface Condensers and for Aluminum Allóy Drawn Seamless Tubes for Condensers and Sanitary Std. for Scraped Surface

Plastic Pipe and Tubing (1973) Sid. for Tubular ansi B72.17 Std. Spec. for Butt Calculation of Ducted Electric Rec. Pract. for Std. for Safety for Portable Sun / Std. Manual for Electric Comfort Conditioning Std. Meth. of Test for Sid. Meth of Test for

e (1974) ANSI C8.38 Std. Meth. of Test for Specific std. Glossary of Terms Relating to Chimneys, Gas Vents and oling, Refrigeration Systems, Incinerators and Other Misc. ow and Medium Speed Stationary Diesel and Gas Engine Waste base, Solution Heat Treated, 15.1 $0 \mathrm{Ni}-3.0 \mathrm{~W})(1973)$ Spec. for Corrosion and Spec. for Corrosion and Moderate (1) Spec. for Steel Welding Wire, Corrosion and Moderate for Steel Bars, Forgings, Tubing, and Rings, Corrosion and Spec, for Steel Welding Wire, Corrosion and Std. for Bolt-Machine, Hexagon Head, Nonmagnetic, and Spec. for Sieel Tubing, Welded, Corrosion and Alloy Wire, Welding, Corrosion and

973)

973)

etal, 1/

Bolts and Screws, Steel, Low Alloy etal, 1/ Spec. for Self-Locking Steel Nuts, Corrosion and $5 \mathrm{Cb}-2.5 \mathrm{Ti}-1.6 / \quad$ Alloy Bars and Forgings, Corrosion and 73) Alloy Bars, Forgings, and Rings. 7.0Fe (1973)

$-0.05 \mathrm{La} /$ $-0.05 \mathrm{La} \mathrm{(1/)}$ 3.0Ti-1.4Al, C/ .5Al (1973) olution $\mathrm{H} /$ $.0 \mathrm{Ti}-1.4 \mathrm{Al}$, Con/ Spec. Alloy Bars and Forgings, Corrosion and sec. for Alloy Bars, Forgings and Rings, Corrosion and (135,000 psi (931 MPa) /
0 Deg. F (982.2 Deg. C) Solu/ Spec. for Low Alloy Steel Studs,
Studs, Steel, Corrosion and 0 Deg. F (982.2 Deg. C) Solu/ Studs, Steel, Corrosion and
chining / Steel Bars and Forgings, Corrosion and Moderate eated, $1 / \quad$ Steel Bars and Forgings, Corrosion and Moderate n Heat Tre/ Steel Sheet and Strip, Corrosion and Moderate n Heat Treated (1973/ Steel Plate, Corrosion and Moderate $n$ Heat T/ Steel Bars and Forgings, Corrosion and Moderate ed and T/ Steel Bars and Forgings, Corrosion and Moderate Bars, Forgings, Tubing, and Rings, Corrosion and Moderate $h$, Hardened Spec. for Low Alloy Steel Bolts and Screws, Sheet, and Strip for Fusion Welded Unfi

Std. Spec. for Std. Spec. for Hot Rolled and Cold Finished Stainless and 1972) ANSI G81.2

Operation (1974) ANSI C8./

and Other Pressure Vessels! Std. Spec. for Stainless and Std Spec, for Stainless and $r$ Hot Rolled and Cold Finished Age Hardening Stainless and 72) ANSI G81.13 Std. Spec. for Stainless and Std. Spec. for Stainless and Std. Spec. for Stainless and ral Requirements for Delivery of Flat Rolled Stainless and Std. Spec. for Stainless and Std. Spec. for Stainless and nickel-1 Std Spec for Free Machining Stainless and Std. Meth. for Chemical Analysis of Stainless, Std. Meth. of Test for Leaks in

td. Meth. of Test for Water Vapor Transmission of Flexible Std. Spec. for Crosslinked Polyolefin

c. for Crosslinked and Noncrosslinked Poly(vinyl Chloride) Std. Meth. of Test.

Std. Spec, for poly(Vinylidene Fluoride) lastic Tubing, Irradiated Polyolefin, Pigmented, Flexible, alorimeter (1972) Std. Meth. for Measuring

or Design and Use of a Thin Skin Calorimeter for Measuring (Tech. Notes) (1961)

nsi G57.11

Rec. Pract. Meth. for Rating

for Carbon Steel Axles, Non High Temp/ Std. Spec. for Alloy Steel Castings Specially
Heat Exchanger Tubes (1972) ANSI H34.42

(1973) ANSI B125.22

Std ASTM

Heat Exchanger, and Condenser Tubes (1973) ANSI B125.32 ASTM

Heat Exchangers (1973)

Heat Exchangers (1973) ANSI H38.6

Std. Spec. for Copper Al ASTM Std. Spec. ASTM

Heat Exchanges for Use with Milk and Its Products (1973

Heat Exchanges in Chemical Process Service (1971)

Heat Fusion Polyethylene (PE) Plastic Fittings for (PE)

Heat Gain Through Opaque Walls (Tech. Notes) (1969)

Heat Guide for Air Handling Systems (1971)

Heat Joining of Thermoplastic Pipe and Fittings (1967)

Heat Lamps (1974)

Heat Loss, Gain, Energy Consumption) (1974)

Heat of Ablation (1972)

Heat of Hydration of Hydraulic Cement (1973) ANSI A I. 10

Heat of Liquids and Solids (1971) ANSI Z11.307

Heat or Moisture Resisting Insulation for Wire and Cabl

Heat Producing Appliances (1972)

Heat Producing Appliances) (1973)

Heat Recovery Systems (1972)

Heat Resistant Alloy Bars, Forgings, and Rings (Nickel

$/ \mathrm{Ng}$, Ventilating, $\mathrm{Co}$ Std. Pract. for L

Heat Resistant $(0.95 \mathrm{Cr}-0.55 \mathrm{Mo}-0.30 \mathrm{~V}(0.40-0.50 \mathrm{C})$

Heat Resistant $(11.8 \mathrm{Cr}-2.8 \mathrm{Ni}-1.6 \mathrm{Co}-1.8 \mathrm{Mo}-0.32 \mathrm{~V})$

Heat Resistant $(15 \mathrm{Cr}-26 \mathrm{Ni}-1.3 \mathrm{Mo}-2.1 \mathrm{Ti}-0.30 \mathrm{~V}) 18$

Heat Resistant $(18 \mathrm{Cr}-11 \mathrm{Ni}-(\mathrm{Cb}+\mathrm{Ta})(1973)$

Heat Resistant (1973)

Heat Resistant (25Cr-20Ni) (SAE 30310) (1973)

Heat Resistant, Cobalt Base-25.5Cr-10.5Ni-7.5W (1

Heat Resistant, Hardened and Tempered, Roll Threaded (1

Heat Resistant, High Strength, Prevailing Torque, All M

Heat Resistant, High Strength, Prevailing Torque, All M

Heat Resistant, Iron Base-13.3Cr-38Ni-5.5Mo-0.8

Heat Resistant, Iron Base-35.5Ni-18.5Cr-1.1SI (19

Heat Resistant, Nickel Base-15.5Cr-2.4Ti-0.70Al-

Heat Resistant, Nickel Base-15.5Cr-8.0Fe (1973)

Heat Resistant, Nickel Base-15.8Cr-15.2Mo-0.30Al

Heat Resistant, Nickel Base-15.8Cr-15.2Mo-0.30Al

Heat Resistant, Nickel Base-19.5Cr-13.5Co-4.3Mo-

Heat Resistant, Nickel Base-19.5Cr-18Co-2.5Ti-1

Heat Resistant, Nickel Base $-5.0 \mathrm{Cr}-24.5 \mathrm{Mo}-5.5 \mathrm{Fe}, \mathrm{S}$

Heat Resistant, Nickel Base $19.5 \mathrm{Cr}-13.5 \mathrm{Co}-4.3 \mathrm{Mo}-3$

Heat Resistant, Nickel Base $(19.5 \mathrm{Cr}-13.5 \mathrm{Co}-4.3 \mathrm{Mo}-$

Heat Resistant, Nickel Base, $20 \mathrm{Cr}-20 \mathrm{C}_{0}-5.9 \mathrm{Mo}_{0}-2.2 \mathrm{~T}$

Heat Resistant, Normalized and Tempered, Roll Threaded

Heat Resistant, Roll Threaded After Heat Treatment, 180

Heat Resistant, 12.5Cr Low Carbon (SAE 51416F), Free Ma

Heat Resistant, $15.5 \mathrm{Cr}-4.5 \mathrm{Ni}-2.9 \mathrm{Mo}-0.10 \mathrm{~N}$, Heat TR

Heat Resistant, $15.5 \mathrm{Cr}-4.5 \mathrm{Ni}-2.9 \mathrm{Mo}-0.10 \mathrm{~N}$, Solutio

Heat Resistant, $15.5 \mathrm{Cr}-4.5 \mathrm{Ni}-2.9 \mathrm{Mo}-0.10 \mathrm{~N}$, Solutio

Heat Resistant, $15.5 \mathrm{Cr}-4.5 \mathrm{Ni}-2.9 \mathrm{Mo}-0.10 \mathrm{~N}$, Solutio

Heat Resistant, $16.5 \mathrm{Cr}-4.5 \mathrm{Ni}-2.9 \mathrm{Mo}-0.1 \mathrm{~N}(1973)$

Heat Resistant, $16.5 \mathrm{Cr}-4.5 \mathrm{Ni}-2.9 \mathrm{Mo}-0.10 \mathrm{~N}$, Equaliz

Heat Resistant, 17Cr (SAE 51430) Annealed (1973)

Heat Resistant, 195,000 psi (1,345 MPa) Tensile Strengt

Heat Resisting Chromium and Chromium Nickel Steel Plate

Heat Resisting Chromium Nickel Manganese Steel Bars Inc

Heat Resisting Chromium Steel Plate, Sheet, and Strip (

Cher Wire and Cable, $75 \mathrm{Deg}$. C

Heat Resisting Steel Bars and Shapes (1972) / Spec. Fo ASTM

Heat Resisting Steel Bars, and Shapes (1973) ANSI G24.3

Heat Resisting Steel Billets and Bars for Reforging (19

Heat Resisting Steel Forgings (1973) ANSI G81.26

Heat Resisting Steel Plate, Sheet, and Strip (1972) Ans

Heat Resisting Steel Rope Wire (1972) ANSI G81.32

Heat Resisting Steel Wire (1971) ANSI G81.41

Heat Resisting Steel Wire (1971) ANSI G81.42

Heat Resisting, Maraging, and Other Similar Chromium-

Heat Sealed Flexible Packages (1972)

Heat Sealed Packages for Dry Products (1972)

Heat Shrinkable Tubing for Electrical Insulation (1972)

Heat Shrinkable Tubing for Electrical Insulation (1973)

Heat Shrinkable Tubing for Electrical Insulation (1974)

Heat Shrinkable Tubing (1973)

Heat Shrinkable (1973)

/C. for Electrical Insulation $P$

Heat Transfer Rate Using a Thermal Capacitance (Slug) C

Heat Transfer Rate (1972)

Heat Transfer (Electrical Meth. (1972)

Heat Transmission Coefficients of Brick and Tile Walls

Heat Transmission of Refrigrated Vehicles (1972)

Heat Treated and Heat Treated, for Railway Use (1973) a

Heat Treated for High Temperature Service (1971) ANSI G

Heat Treated for Pressure Containing Parts Suitable for
DFISA

B163

A213

B569

A249

B338

B171

B234.

3100

B78.1

ASTM D3261

BIA

ASTM

UL

NEMA

ASTM

ASTM

ASTM

NFPA

DEMA

SAE

SAE

SAE

SAE

NSA

SAE

SAE

SAE

SAE

SAE

SAE

SAE

SAE

SAE

SAE

SAE

SAE

SAE

SAE

SAE

SAE

SAE

SAE

SAE

SAE

SAE

ASTM

ASTM

ASTM

ASTM

ASTM

ASTM

ASTM

ASTM

ASTM

ASTM

ASTM

ASTM

ASTM

ASTM

ASTM

ASTM

ASTM

ASTM

A ASTM

BIA

TTMA

ASTM

ASTM

ASTM

$4 \mathrm{~A}$
-12

D2657

482

HE 1

E458

C186

D2766

D1520

UMC.7

UMC

1.16

1003

AMS5750B

AMS5508B

AMS6305

AMS5823

AMS5735H

AMS5680D

AMS5577C

AMS5789

AMS7455D

AMS7250C

AMS7251B

AMS5633A

AMS5716A

AMS5541B

AMS5665H

AMS5711

AMS5873

AMS5738C 
Tent. Spec. for UItra High Test Heat Treated Line Pipe (1972)

Std. Spec. for $-1.6 \mathrm{Cu}-0.26 \mathrm{Cr}(7075-\mathrm{T} 736)$ Solution and Precipitation Pressure Vessels Plates, $5 \%$ Nickel AIloy Steel, Specially induction Plus Vacuum Consumable Electrode MeIted Solution e Heat Resistant, $15.5 \mathrm{Cr}-4.5 \mathrm{Ni}-2.9 \mathrm{Mo}-0.10 \mathrm{~N}$, Solution uum Induction MeIted, 1975 Deg. F (1079.4 Deg. C) Solution nickel Base, $20 \mathrm{Cr}-20 \mathrm{Co}-5.9 \mathrm{Mo}-2.2 \mathrm{Ti}-0.45 \mathrm{Al}$ Solution $-38 \mathrm{Ni}-5.5 \mathrm{Mo}-0.85 \mathrm{Cb}-2.5 \mathrm{Ti}-1.6 \mathrm{Al}-0.09 \mathrm{~B}$, Solution

Resistant, Nickel Base-5.0Cr-24.5Mo-5.5Fe, Solution

-1037.8 Deg. C) Solution, Stabilization, and Precipitation

e Heat Resistant, $15.5 \mathrm{Cr}-4.5 \mathrm{Ni}-2.9 \mathrm{Mo}-0.10 \mathrm{~N}$, Solution

(1079.4 Deg. C) Solution, Stabilization, and Precipitation

30V) 1800 Deg. F (982.2 Deg. C) Solution and Precipitation Std. Spec. for Pressure Vessel Plates, Std. Spec. for Carbon Steel Axles, Non Heat Treated and Spec. for Low Alloy Steel Bolts and Screws,

Spec. for Low Alloy Steel Studs.

Spec. for Titanium Alloy Bolts and Screws, 6Al-4V

for Titanium Alloy Bolts and Screws, 6A 1-4V, Upset Headed, e Heat Resistant, $15.5 \mathrm{Cr} \sim 4.5 \mathrm{Ni}-2.9 \mathrm{Mo}-0.10 \mathrm{~N}$, Solution ant Alloy Bars, Forgings, and Rings (Nickel Base, Solution d Moderate Heat Resistant, $15.5 \mathrm{Cr}-4.5 \mathrm{Ni}-2.9 \mathrm{Mo}-0.10 \mathrm{~N}$ Std. Rec. Pract. for

After Heat Treatment, 1800 Deg. F (982.2 Deg. C) Solution SteeI, Corrosion and Heat Resistant, Roll Threaded After Test Meth. for Colorfastness of Textiles to Dry Rec. for Fire

1973)

ion of $\mathrm{pH}$ (Paste) of Corn Starch Which Is Gelatinized When Safety Rec. for Electrically Line Voltage Integrally Mounted Thermostats for Electric std. Spec. for Welded Austenitic Stainless Steel Feedwater Safety Std. for Oil Burning Stoves (Room Uniform Plumbing Code: W/ater a, B, C, and D, and Class II, G/ Spec. for Indirect Type Oil Field a, B, C, and D, and Class II, G/ $\begin{aligned} & \text { Safety Std. for Electric } \\ & \text { Spec. for Indirect Type Oil Field }\end{aligned}$ Std. for Gas Water Heaters (Automatic Storage Type Water ts Less Than 75,000 Btu Per Hr.) (Vol/ Std. for Gas Water Installation and Maintenance of Steam and Hot Water Unit Std. for Gas Unit

Std for Vented Gas Fired Infrared Radiant Std. for Household Automatic Electric Storage Type W ater Std. for Unvented Gas Fired Infrared Radian Std for Gas Fired Swimming Pool Std. for Direct Gas Fired Make Up Air std. for Meth. of Test. for Rating Nonresidential Warm Air Safety Std. for Electric Dry Bath

Safety Std. for Household Electric Storage Tank W ater Std. for Gas Fired Saun Std. for Safety for Electric Air Safety Std. for Oil Fired Air and Direct Fired

$r$ Line Voltage Integrally Mounted Thermostats for Electric nces, and Wall Furnaces, Floor Furnaces, and Unit and Room Std. for Safety for Electric Oil Std. for Safety for Oil Fired Unit Std. for Safety for Oil Fried Water

Rec. Pract. for Engine Preheaters (Coolant ) ANSI Nl4/ Std. Spec. for Sheathed Electrical Resistance Std. for Gas Fired Room Std. for Gas Fired Room Std. for Gas Water

d Large Automatic Storage Type (1974) Minimum Installation Stds. for Residential

Std. for Installation of Central Warm Air Safety Std. for Room Type Fan Coil Units d Construction Requirements, and Installation of Plumbing, olts or Less) (1972) C33.11 Safety Std. for Electric

Safety Std. for Draft Equipment for Combustion Chamber in Std. for Forced Circulation A ir Cooling and Air Std. for Safety for Sheathed

al Grade Magnesium Oxide for Use in Sheathed Type Electric Loss Prevention Data on High Frequency 973) ANSI C33.95 Safety Std. for Electric Baseboard Safety Std. for Electric Space

Safety Std. for Commercial-Industrial Gas Safety Std. for Electric Central Air Std. for Central Forced Air Electric Std. Test Meth. for

Rec. for Standpipe and Hose Systems

rs, Truck Campers, Motor Homes): Installation of Plumbing, ting Electrical Equipment for Control of Air Conditioning, ct System/

1972) Std. for the Installation of Air Conditioning, Std. Meth. of Testing for Sound Rating Std. for Safety for LP Gas Torches for Safety Std. for Limit Controls (Air Conditioning, 50.1 irements for the Installation, Control, and Maintenance of

Heaters (1972)
Heat Treated SteeI

Heat Treated (1972A)

Heat Treated (1973)

Heat Treated (1973)

Heat Treated (1973)

Heat Treated (1973)

Heat Treated (1973)

Heat Treated (1973)

Heat Treated (1973)

Heat Treated (1973)

Heat Treated (1973)

Heat Treated (1973)

Heat Treated, Carbon Manganese Silicon (1972A)
Heat Treated, for Railway Use (1973) ANSI G57.11

Heat Treated, Roll Threaded (1973)

Heat Treated, Roll Threaded (1973)

Heat Treated, Roll Threaded (1973)

Heat Treated, Roll Threaded (1973)

Heat Treated, Sub Zero Cooled, Equilized, and Over Temp

eat Treated, $15.5 \mathrm{Cr}-16 \mathrm{Mo}-3.8 \mathrm{~W}-5.5 \mathrm{Fe}$ ) (1973)

Heat Treated, $170,000 \mathrm{psi}(1172 \mathrm{MPa})$ Tensile Strength (

Heat Treatment of Aluminum Alloys (1974)

D Heat Resistant, Roll Threaded

Heat Treatment, 1800 Deg. F (982.2 Deg. C) Solution Hea

Heat (Excluding Pressing) (1973) ISO 105.4

Heat, and Smoke Damage of Electronic Computer Systems

Heated in a Boiling Water Bath (1973)

Heated Livestock Waterers (1971)

Heater Line Voltage Integrally Mounted Thermostats for

Heater Tubes (1973)

Heaters and Ranges) (1973)

Heaters and Vents (1973)

Heaters for Use in Hazardous Locations Glass I, Groups

Heaters Used in Producing Oil and Gas (1972)

Heaters with lnputs Less Than 75,000 Btu Per Hr.) (Vol

Heaters (Automatic Storage Type Water Heaters with Inpu

Heaters (1970)

Heaters (1971)

Heaters (1971)

Heaters (1972)

Heaters (1972)

Heaters (1972)

Heaters (1972)

Heaters (1972) ANSl C33.75

Heaters (1972) ANSI C33.87

Heaters (1973)

Heaters (1973)

Heaters (1973)

Heaters (1973)

Heaters (1973)

Heaters (1973)
Heaters (1974)

Heaters (1974)

Heaters) (1971)

Heaters, for Nuclear or Other Specialized Service (1971

Heaters: Volume I, Vented (1974)

Heaters: Volume II, Unvented (1974)

Heaters: Volume III, Circulating Tank, Instantaneous an

Heating and Air Conditioning Systems (1973)

Heating and Air Conditioning Systems (1973) ANSI Bl44.3

(Heating and Cooling) (1973) ANSI Z25.1

Heating and Electrical Systems (1973) ANSI Al19.1

Heating A ppliances (Portable Fixed and Stationary $600 \mathrm{~V}$

Heating Appliances (Regulators, Automatic Damper Contro

Heating Coils (1972)

Heating Elements for Appliances and Equipment (1973)

Heating Elements (1973)

Ctrical Impedance of Electric

Heating Equipment (Induction and Dielectric) (1973)

Heating Equipment (Portable and Fixed) $600 \mathrm{~V}$ or Less (1

Heating Equipment (1972) ANSI C33.12

Heating Equipment (1973)

Heating Equipment (1973) ANSI C33.104

Heating Equipment (1974)

Heating Loss of Carbon Black (1972)

Heating) (1972)

Heating, and Electrical Systems (1972) ANSI Al19.2

Heating, Cooking, Refrigeration, and Humidity (Humidist

Heating, Exhaust, Ventilating, and Their Combination Du

Heating, Refrigerating and Air Conditioning Equipment (

Heating, Soldering, and Brazing Purposes (1973) ANSI Z2

Heating, Ventilating and Processing Systems) (1972)

Heating, Ventilating, Cooling, Refrigeration Systems, I

Heatset Printing (1972)
API

ASTM

SAE

SAE

$S A E$

SAE

SAE

SAE

AE

SAE

SAE

SAE

SAE

ASTM

SAE

SAE

$S A E$

Spec. SAE

SAE

SAE

ASTM

SAE

AATCC

FMS

CR

ASAE

NEMA

ASTM

UL

ICBO

UL

ANS1

ANSI

AMCA

ANSI

ANSI

ANSI

ANSI

ANSI

ANSI

ASHRA

UL

UL

ANSI

UL

UL

NEMA

ICBO

UL

UL

SAE

ASTM

ANSI

ANSI

ANSI

SMACN

NFPA

UL

I an $\mathrm{U}$

UL

AR!

UL

ULTM

FMS

UL

UL

UL

UL

ARI

ASTM

FMS

Le NFPA

UL

NFPA

ASHRA

UL

UL

ICBO

TAPPI

SPEC 5LU

A 125

AMS4131

A645

AMS5617A

AMS5547C

AMS5738C

AMS5872

AMS5633A

AMS5755B

AMS5707D

AMS5549C

AMS5709B

AMS5735H

A537

A21

AMS 7452K

AMS 7456C

AMS $7460 \mathrm{C}$

AMS7461B

AMS5743D

AMS5750B

AMS5744A

B597

AMS7482

AMS 7482

117

5. 32

R342

DC 13

A688 
(1942) ANSI L14.283

ble (1973)

ble (1973) ANSI C 8.32

ins and Sprocket Teeth (1972)

th (1973)

\author{
Std. Spec. for Extra \\ Std. Spec. for \\ Std. for
}

Std. Spec. and Meth. of Test for

Std. for Threaded Metal Insert

Std. Part Pins, Spring, Slotted and Coiled,

ents for Woven Washable Uniform Fabrics for Men and Women

r Roll Over Protective Structures (ROPS) for Prime Mover
os and Other / Std. Analytical Meth. for Determining

Syrups and Other

Std. Analytical Meth. for Determining

Test Meth. for

Std. Meth. of Test for Solubility of Pentachlorophenol in

Std. for Wire Cable Wrought Steel

Rec. Pract. for Exterior Sound Level for

Std, for Training of Automotive Mechanics for Buses, eam Turbines (1974)

si Z166.19

74) Std. Spec. for

Std. Reference Radiographs for Std. Spec. for Steel Castings, uirements for Woven Drycleanable Tailored Uniform Fabrics, rdening Appliances (Lawn Mower, Cultivator, Edger-Trimmer, rs (1974)

for Abrasion Resistance of Rubber Compounds for Soles and wer Fan Drives (1972) Std. Pract. for Spur,

Spec. for Measurement of Lateral Vibration on High Speed anual (Gear Drawing Stds. Part 1 for Spur, Helical, Double rmal Degradation of Electrical lnsulating Varnishes by the for Dimensions, Physical Properties and Meth. of Test. for Std. Spec. for Heat Treated Steel rican Drafting Manual (Gear Drawing Stds. Part 1 for Spur Std. for Sound (Noise Measurement for Enclosed for Tires, Tubes, Rims and Valves for Aircraft (Airplane, Uniform Fire Code: Airports, Uniform Fire Code: Airports, Heliports Devices with $A$

Fluids (Air, Argon, Carbon Monoxide, Deuterium, Fluorine, rn Hemlock-Tamarack (North), Eastern White Pine (North), Pine/ Uniform Building Code Std. for Douglas Fir; Larch

e Std. for Structural Glued Laminated Timbers: Douglas and r Coast Sitka Spruce, Douglas Fir-Larch (North), Eastern orm Building Code Sid. for Eastern Spruce, White Pine, and $\mathrm{ng}$ of Southern Pine, Pacific Coast Douglas Fir and Western Western Cedar and Hemlock; Subal-Pine Fir; and Mountain de Std. for Eastern White and Red Pine, Eastern Spruce and Industry Std. for Douglas Fir, Western and Lodgepole Pines; Engelmann Spruce; Western Cedar and ilding Code Std, for Douglas Fir, Coast Region; West Coast Rec. for Baled Textile Fiber Storage (Cotton, Jute, combustible Fibres (Cotton, Sisal, Henequen, Ixtle, Jutes,

storage and Handling of Combustible Fibres (Cotton, Sisal, 1971) ANSI Z11/ Std. Meth. for Analysis of High Purity N Std. for Methazole Std. for Acetochlor

ing, Liquid Filled and Dry Transformers, Hand Power Tools, Safety Std. for Sealed Std. Rec. Pract. for Determining 972) Std. Rec. Pract. for Determinin

Std, for Sound (Noise Measurement for Enclosed Helical Sid. Pract for Spur, Helical and es (1972) Info Sheet for Spiral Bevel, Helical and measurement of Lateral Vibration on High Speed Helical and

ride; Chloral Hydrate; Chloroform; Formaldehyde; Gasoline; (1972) ANSI Z11. Std. Meth. of Test for Yield Stress of

If Locking and Nonlocking (1972) elf Locking and Nonlocking (1972)

f Locking and Nonlocking (1972)

f Locking and Nonlocking (1972)

thread, Self Locking and Nonlocking (1972) t Thread, Self Locking and Nonlocking (19/ nlocking (1973)

cking (1972)

thread, Nonlocking (1972)

rque Tension Requirements for Prevailing Torque Type Stee Std. for Packaging of Uranium

d. for Analytical Procedures for Accountability of Uranium Std for Shear

or Steel Wire, Medium High Carbon Cold Heading Quality for spec. for Alloy Steel Wire, Alloy Cold Heading Quality for

Std. for Std. for Self Locking. Std. for Machine Bolt, Crowned Std. for Bolt-Machine,

Sid for Bolt-Close Tolerance, Std. for Bolt, Shear,
Heavy Cotton Fabrics for Manufacture of Hose and Belts

Heavy Duty Black Polychloroprene Jacket for Wire and Ca

Heavy Duty Black Polychloroprene Jacket for Wire and Ca

Heavy Duty Offset Sidebar Power Transmission Roller Cha

Heavy Duty Roller Type Conveyor Chains and Sprocket TEE

Heavy Duty (1973)

Heavy Duty (1973)

Heavy Duty) (lnstitutional Textile) (1973)

Nimum Performance Criteria Fo

Heavy Metals Content of Corn Sugar (Crude and Refined),

Heavy Metals Content of of Corn Syrup Unmixed and Dextr

Heavy Metals in Paper and Paperboard (1973)

Heavy Petroleum Solvents (1973)

Heavy Thimble (For Ground Support Equipment) (1974)

Heavy Trucks and Buses (1971)

Heavy Trucks and Trailers (Course of Instruction) (1972

Heavy Walled Carbon and Low Alloy Steel Castings for St

Heavy Walled (4 1/2 to 12 In.) Steel Castings (1972) an

Heavy Walled, Carbon and Alloy for Pressure Vessels (19

Heavyweight (Industrial) Suiting (Institutional Textile

Hedge Trimmer, etc.) (1974)

Heels (1968) ANSI J2.26 /Ord Connected Electric Ga
Std. Meth. of Test

Helical and Herringbone Gear Shaft Mounted Speed Reduce

Helical and Herringbone Gear Units for Water Cooling to

Helical and Herringbone Gear Units (1972)

Helical and Rack) (1971)

Helical Coil Meth. (1973)

American Drafting $M$
Meth. of Test for the

(Helical Spring and Carbon Steel Tooth Lock Washers (197

Helical Springs (1973) ANSI G54.20

Helical, Double Helical and Rack) (1971)

Helical, Herringbone and Spiral Bevel Gear Drives (1973

Helicopter) (1974)

Heliports, Helistops and Aircraft Hangers (1973)

Helistops and Aircraft Hangers (1973)

Helium Mass Spectrometer Leak Detector (1972T)

Helium, Hydrogen, Krypton, Methanc, Neon, Nitric Oxide

Hem-Fir (North), Ponderosa and Western White Pines, S

Hem-Fir; Ponderosa, ldaho White, Sugar, and Lodgepole

Hem Fir, Southern and Lodgepole Pine, Western Larch, Ca

Hemlock - Tamarack (North), Eastern White Pine (North)

Hemlock-Tamarack; Balsam Fir; Northern Pine and White

Hemlock by Pressure Processes (1973)

lons Before Glui

Hemlock (Lumber) (1973) /Pole Pines; Engelmann Spruce;

Hemlock, Balsam Fir and Tamarack Lumber (1973) /Ing Co

Hemlock, Sitka Spruce Doors and Blinds (1972)

Hemlock; Subal-Pine Fir; and Mountain Hemlock (Lumber

Hemlock; Western Red Cedar; White Fir; and Sitka Spruce

Hemp and Sisal, Flax, Synthetic Fibers, Wool) (1974)

Hemp, Tow, Cocoa Fibers, Oakum, Rags, Waste Cloth, Wast

Henequen, Ixtle, Jutes, Hemp, Tow, Cocoa Fibers, Oakum,

Heptane and Isooctane by Capillary Gas Chromatography (

(Herbicide) (1971)

(Herbicide) (1971)

Hermatic Stators, Test. and Safety) (1973)

(Hermetic Type) Motor Compressors (1972) ANS1 B143.

Hermeticity of Electron Devices by a Bubble Test (1972)

Hermeticity of Electronic Devices by Dye Penetration (1

Herringbone and Spiral Bevel Gear Drives (1973)

Herringbone Gear Shaft Mounted Speed Reducers (1974)

Herringbone Gear Units for $\mathbb{W}$ ater Cooling Tower Fan Driv

Herringbone Gear Units (1972)

Sid. Spec. for

Herwig's, Pancreatin. Tween 80-60\% Alcohol, Versene, an

Heterogeneous Propellants by the Cone Penetration Meth.

Hex Head Screw, Recessed, Full Thread (1973)

Hex Head, Close Tolerance, Alloy Steel, Long Thread, Se

Hex Head, Close Tolerance, Alloy Steel, Short Thread, S

Hex Head, Close Tolerance, A286 Cres, Long Threads, Sel

Hex Head, Close Tolerance, A286 Cres, Short Thread, Sel

Hex Head, Close Tolerance, Titanium Alloy (1972)

Hex Head, Close Tolerance, 6Al-4V Titanium Alloy, Long

Hex Head, Close Tolerance, 6Al-4V, Titanium Alloy, Shor

Hex Head, Tri Wing Recess, Alloy Steel, Full Thread, No

Hex Head, Tri Wing Recess, A286 Cres, Full Thread Nonlo

Hex Head, Tri Wing Recess, 6Al-4V Titanium Alloy, Full

Hex Locknuts (1972)

Hexafluoride for Trans port (1971)

Hexafluoride (1972)

Hexagon Head Bolt (1973)

Hexagon Head Bolts (1972) ANSl G54.15

Hexagon Head Bolts (1972) ANSI G54.16

Hexagon Head Modified Short Thread Shear Bolt (1972)

Hexagon Head Shear Bolt (1974)

Hexagon Head, Adjusting (1973)

Hexagon Head, Nonmagnetic, and Heat Resistant (1973)

Hexagon Head, Titanium, Short Thread (1973)

Hexagon Head, 180 KSI (1973)
ASTM

ASTM

ASTM

ANSI

ANSI

NSA

NSA

ANSI

SAE

CR

TAPP

ASTM

NSA

SAE

ANSI

ASTM

ASTM

ASTM

ANSI

A STM

AGMA

AGMA

Std AGMA

ASTM

ANSI

ASTM

Ame ANSI

AGMA

Std. TRA

lCBO

ICBO

A STM

$1 \mathrm{CBO}$

ICBO

$1 \mathrm{CBO}$

ICBO

ICBO

ICBO

AWPA

FHDA

ICBO

ICBO

FMS

ICBO

ICBO

ASTM

ANSI

ANSl

EASA

UL

A STM

ASTM

AGMA

AGMA

AGMA

AGMA

A ACCH

ASTM

NSA

NSA

NSA

NSA

NSA

NSA

NSA

NSA

NSA

NSA

Std. for to IFl

ANSI

St ANSI

NSA

Std. Spec. F ASTM

Std. ASTM

NSA

NSA

NSA

NSA

NSA

NSA

D181

D2819

D752

B29.10

29.15

1395

561

L24.3.6

$320 \mathrm{~B}$

F-26

E. 30

UM.544

D2606

1045

J366A

D18.2

A356

E280

A643

L24.3.2

82 
Std. for Low Height, Light Weight, Self Locking Std. for Low Height 80 K.S.I., Self Locking, 450 Deg. F,

Std, for Nut-Self Locking, Extended W'asher, Double eth. of Test. Shipping Containers (Packaging) in Revolving Brass Wire (1973) Uniform Building Code Std. for Round, ium Nickel Manganese Steel Bars Including Rounds, Squares,

pa/ Std. for Acceptable Concentration of Chromic Acid and Steel Prior to Conversion C/

Test Meth. for Detection of

Tent. Meth. of Test for Nitrogen Content (Kjeldahl) and extinguishing Systems (1973) Rec. for Star Model F

Uniform Building Code Std. for Fire Alarm Systems for ile Matter in Fats and Shortenings (Butter, Oleomargarine, mical Analysis of Tool Steels and Other Similar Medium and std. Spec. for Centrifugally Cast lron-Chromium-Nickel Uniform Building Code Std. for Machine Made and Std. Classification of Fireclay and

e Preservative Solutions Such as Pent/ (1972) ANSI G54.15 Contemporary Bearing Wall (For terials (1973) Std. Spec. for Steel Wire, Medium Voluntary Safety Std. for

Std. Rec. Pract for Designing A Std. Meth. of Panel Spalling Test for Rec. for Shielding for etallic Materials (1962)/ Std. Rec. Pract. for Effects of Rec. Pract. for Exposure of Adhesive Specimens to 1) (Fire Extinguishment) (1973) ric) (1973) Loss Prevention Data on Std. Meth. of Test for Phthalate Ester Color of colorfastness of Textiles to Ozone in the Atmosphere Under Test Meth. for Colorfastness of Textile Fabrics to Water (1971) for the Design, Construction, and Operation of Class Hi s-Oil Saturate Fractions (1971) ANSI Z1/ (1972) Std. Meth. for the Designation of
Std. Meth. for

Safety Rules, Procedures For, and Construction of

Std. Spec. for Carbon and Alloy Steel Nuts for Bolts for for Continuity of Quality of Electrical Insulating Oil for al Gas Cylinders (1972)

ectural Woodwork) (1973)

compounds (For Casing. Tubing and Line Pipe Connects. for

Spec, for Electric Fusion Welded Steel Pipe for

or High Strength Carbon Steel Wrought Welding Fittings for Std. Spec. for Electric Fusion Welded Steel Pipe for tance Welded Carbon Steel Boiler and Superheater Tubes for Std. Spec. for Seamless Carbon Steel Boiler Tubes for ver 400,000 Btu Per Hr.) (1972) Std. Spec. for Metal Arc Welded Steel Pipe for
Std. Meth. for Spectrochemical Analysis of hromatography (1971) ANSI Z11/ Std. Meth. for Analysis of Std. for Self Locking, 450 Deg. F, or Sheathed, Type K Thermocouples for Nuclear or for Other Rec. for Fire Safety in

osive Reactivity of Lubricants with Aerospace Alloys Under Std. Spec. for Measurement of Lateral Vibration on

973)

tter F (1973)

d, and Cold Worked Alloy Steel Plate, Sheet, and Strip for c. for Hot, Hot Cold, and Cold Worked Alloy Steel Bars for d Cold Worked Alloy Steel Forgings and Forging Billets for spec, for Iron, Cobalt, and Nickel Base Alloy Castings for High Pressure Service Piping (1969) Drilling) (1973)

tings (1967) ANSI Z166.17 ructural Quality (1972) ANSI G41.6

for Use in Marine Environments (1974)

teel (1970A) ANSI G41.4 41.2 Spec. for Std. Spec. for Std. Spec. for Std. Spec. for Std. Spec. for Std. Spec. for Normalized Std Spec for Hot Formed WVelded and Seamless Std. Spec. for Pressure Vessel Plates, Carbon Steel, Std. Spec. for

Std. Rec. Pract. for Sharp Notch Tension Test. of including Suitable Nuts and Plain Hardene/ Std. Spec. for rts (1973) 973) ANSI G52.1

erate and Lower Temperature Service (1972/ Std. Spec. for

Std. Spec. for Std. for Low Height Std. for Nut, Plain, Std for Sodium Std. Spec. for Std. Spec. for for Rec. for Std. Definitions for

Rec. Pract. for Uniform Fire Code: Std. for

Safety Std. for Std. Meth. for

Std. for Gas Fired

Hexagon Nut (1972)

NSA

Hexagon Nut (1973)

Hexagon Self Locking Nut (1974)

Hexagon, Drilled Jam, Thin (1972)

Hexagon, $220 \mathrm{KSI}, 450 \mathrm{Deg}$. F (1973)

Hexagonal Drum (1968) ANSI MH12.3, TAPPI T800

Hexagonal, Octagonal, and Flat (Rectangular and Square)

Hexagons, and Shapes-Angles, TEES and Channels (1972)

Hexametaphosphate (1967)

Hexavalent Chromium Compounds (Air Contamination-Occu

Hexavalent Chromium Containing Treatments on Galvanized

Hexylene Glycol (1972)

Hide (Leather) Substance (1970) ALCA B5

Hidromatic Deluge Valve for Water Flow Control of Fire

High-Rise Buildings (1973)

High Acid Coconut Oil, etc.) (Cereal Chemistry) (1962)

High Alloy Steels (1973)

High Alloy Tubing for Pressure Application at High Temp

High Alumina Fireclay Refractory Bricks (1973)

High Alumina Refractory Brick (1970) ANSI A111.5

High Boiling Hydrocarbon Solvent for Preparing Oil Born

High Buildings) (Tech. Notes) (1970)

High Carbon Cold Heading Quality for Hexagon Head Bolts High Chairs (Children's) (1972)

High Current Arc Erosion Test for Electrical Contact Ma

High Duty Fireclay Brick (1970) ANSI All1.14

High Energy Electron Accelerator Installations (1964)

High Energy Radiation on the Mechanical Properties of M

High Energy Radiation (1970) ANSI N141

High Expansion Foam Systems (Katios from 100:1 to 1000:

High Frequency Heating Equipment (Induction and Dielect

High Gravity Glycerin (1973)

High Humidities (1972) ANSI L14.238

(High Humidity) and Light: Alternate Exposure (1972) Ans

High Impact) Shock Test. Machine for Lightweight Equipm

High Intensity Discharge Lamp Ballasts and Transformers

High Intensity Discharge Lamps (1972)

High Ionizing Voltage Mass Spectrometric Analysis of $\mathrm{Ga}$

High Lift Grouted Reinforced Brick Masonry (Tech. Notes

High Lift lndustrial Truck Nameplates and Marking (1973

High Piled Combustible Stock (Storage) (1973)

High Power Rifle Ranges (1972)

High Precision Laboratory Potentiometers (1972)

High Pressure and Temperature Service (1973) ANSI E38.1

High Pressure Cable Systems (1972) ANSI C59.70

High Pressure Gas Manifolds for Industrial and Commerci

High Pressure Laminate Flush Type Wall Paneling (Archit

High Pressure Oil Well Service) (1972)

High Pressure Pump Test. of Hydraulic Oils (1973)

High Pressure Service at Moderate Temperatures (1973)

High Pressure Service Piping (1969)

High Pressure Service (1972A) ANSl B 125.4

High Pressure Service (1973) ANSI B125.12

High Pressure Service (1973) ANSI B125.7

High Pressure Steam and Hot Water Boilers (lnputs Not O

High Pressure Transmission Service (1971) ANSI G36.49

High Purity Iron for Silicon and Aluminum (1971) ANSI Z

High Purity N-Heptane and Isooctane by Capillary Gas C

High Quality Nuts (1973)

High Reliability Applications (1967) ANSI N142

High Rise Buildings (1973)

High Shear (1972) ANSI Z11.323

High Speed Helical and Herringbone Gear Units (1972)

High Speed Steel Threaded Shank, Sizes $1 / 16$ ln. Thru Le

High Strength at Elevated Temperatures (1971) ANSl G81.

High Strength at Elevated Temperatures (1971) ANSI G81.

High Strength at Elevated Temperatures (1971) ANSI G81.

High Strength at Elevated Temperatures (1973) ANSI G81.

High Strength Carbon Steel Wrought Welding Fittings for

High Strength Casing, Tubing, and Drill Pipe (Petroleum

High Strength Copper Base and Nickel-Copper Alloy Cas

High Strength Low Alloy Columbium Vanadium Steels of St

High Strength Low Alloy Steel H-Piles and Sheet Piling

High Strength Low Alloy Structural Manganese Vanadium S

High Strength Low Alloy Structural Steel (1970A) ANSI G

High Strength Low Alloy Structural Steel (1973)

High Strength Low Alloy Structural Tubing (1973)

High Strength Manganese (1972A) ANSI G35.8

High Strength Nonheaded Steel Bolts and Studs (1973)

High Strength Sheet Materials (1968) ANSI Z260.

High Strength Steel Bolts for Structural Steel Joints,

High Strength Steel Bolts, Nuts, Washers, and Bolted Pa

High Strength Steel Castings for Structural Purposes (1

High Strength Steel Plates for Pressure Vessels for Mod

Std. for Plating,
High Strength Steels, Cad mium (1973)
NSA

NSA

NSA

NSA

II ASTM

ICBO

ASTM

$A W W A$

ANSl

NCCA

ASTM

ASTM

FMS

ICBO

AACCH

ASTM

ASTM

ICBO

ASTM

BIA

ASTM

JPMA

AATM

ASTM

NCRPM

ASTM

ASTM

FMS

FMS

ASTM

AATCC

AATCC

ANS!

ANS1

ANSl

AST M

BlA

ITA

ICBO

NRA

ANSI

ASTM Spec. ASTM

UL

A WI

Thread API

ASTM

ASTM

MSS

ASTM

ASTM

ASTM

ANSI

ASTM

ASTM

A STM

/Pec. F $\begin{aligned} & \text { NSA } \\ & \text { ASTM }\end{aligned}$

FMS

ASTM

NSA

NSA

ASTM

ASTM

ASTM

ASTM

MSS

API

ASTM

ASTM

ASTM

ASTM

ASTM

ASTM

ASTM

ASTM

ASTM

ASTM

ASTMI

ICBO

ASTM

ASTM

NSA

D782

UBCS 32.13

A 429

TB. $11-7$ 
Std. Spec. for Std. for Cam Follower, Needle Bearing, Std. for Bolt-100 Deg. Close Tolerance, Std for Fastener: Rotary, Quick Operating, std. Spec. for Steel Sheet and Strip, Hot and Cold Rolled, Rec. Pract. for

Spec, for Steel Self-Locking Nuts, Spec. for Self-Locking Corrosion Resistant Alloy Nuts, for Self-Locking Steel Nuts, Corrosion and Heat Resistant, for Self-Locking Steel Nut8, Corrosion and Heat Resistant, Std. Spec. for Pressure Vessel Plates, Alloy Steel, ater Vapor Transmission Rate of Sheet Materials (Paper) at
Std. Spec. for Seamless Austenitic Steel Pipe for 25.25 cal Tape (1973)

etc.) Fire Protection and Safety (1973) Std. Spec. for ectric Resistance Welded Carbon Steel Tubular Products for rdening Nickel Alloy Bars, Forgings, and Forging Stock for iron Base Superalloy Bars, Forgings, and Forging Stock for alt Containing Alloy Bars, Forgings, and Forging Stock for 1 Turbine Type Bolting Material Specially Heat Treated for Std. Spec. for Centrifugally Cast Carbon Steel Pipe for itation Hardening Nickel Alloy Plate, Sheet, and Strip for sion Welded Autenitic Chromium Nickel Alloy Steel Pipe for stenitic Chromium Nickel Alloy Steel Pipe for Corrosive or td. Spec. for Centrifugally Cast Austenitic Steel Pipe for el Pipe Flanges, Forged Fittings, and Valves and Parts for

Std. Spec. for Ferritic and Austenitic Steel Castings for Std. Spec. for Seamless Carbon Steel Pipe for spec. for Centrifugally Cast Ferritic Alloy Steel Pipe for

Std. Spec. for Seamless Ferritic Alloy Steel Pipe for for Carbon Steel Castings Suitable for Fusion Welding for Steel Castings for Pressure Containing Parts Suitable for Chromium-Nickel-lron Alloy Castings (25-12 Class) for

Spec. for Alloy and Stainless Steel Bolting Materials for Std. Meth. of Drip Slag Test. Refractory Brick at ar lron, Nickel, And/ Std. Meth. for Chemical Analysis of Fastener (Blind Internally Threaded, External Sleeve, Rings, Sealing, Fluorosilicone Rubber, General Purpose, for Fastener (Blind, Internally Threaded, External Sleeve, Is with Expansion Coef/ Std. Spec. for Bolting Materials, Std ium-Nickel High Alloy Tubing for Pressure Application at

r Measurement of Flow Properties of Lubricating Greases at gs (1973)

ly Heat Treated for Pressure Containing Parts Suitable for ings (1972)

Std. Spec. for Steel Wire, Rec. Pract. for Special Quality Tent. Spec. for Ultra Spec. for Spec. for

ed Ratings, Manufacturing Spec., and Application Guide for d a Total std. for Schedule of Dielectric Test Values for Outdoor Ac preferred Ratings for Capacitance Current Switching for Ac t for Switching lmpulse Voltage lnsulation Strength for Ac sis When Rated for Out of Phase / Std. Meth. for Test. Ac Std Requirements for Transient Recovery Voltage for Ac d. Application Guide for Transient Recovery Voltage for Ac rrent/ Std. Requirements for Pressurized Components of Ac std. Requirements for Capacitance Current Switching for Ac application Guide for Capacitance Current Switching for Ac

Std. Requirements for External Insulation for Outdoor Ac y Voltage Ratings and Related Required Capabilities for Ac nces and Other Electronic Equipment / Std. for Safety for itchge/ Safety Requirements for X-Radiation Limits for Ac electrical Insulation (1973) Std. Meth. of Test for plate, Suitable for Welding (1970E) ANSI / Std. Spec. for in Box Storage Up to $30 \mathrm{Ft}$. and Shelf Storage Up to $15 \mathrm{Ft}$. Std. Meth. for Rapid Gas Chromatographic Estimation of r Carbon Steel Pressure Vessel Plates for Intermediate and mmercial Vehicles, Golf Car, and Other Diversified Off the Rec. Pract. for Concrete Std. Specs. for Welding of Structural Steel Std. Spec. for Steel Castings for r Classification of Soils and Their Aggregate Mixtures for r Preservative Treatment by Pressure Processes of Wood for Safety Std. for

or Voltage Classification of Luminaires Used in Street and Std. for Mining and Logging Tires Used in Intermittent $n$ Disc and Spoke Wheels, and Demountable Rims Intended for

Pract. for Collision Deformation (Damage) Classification ion in Cornering Without Driving Torque Application, Using $r$ Homogeneous Perforated Bituminous Fiber Pipe Systems for a Policy on Geometric Design of Rusal std. for Lighting and Marking of Agricultural Equipment on or Vehicles, Trailers, and Semitrailers Operated on Public
High Strength Structural Steel (1970A) ANSI G41.3 High Strength Stud (1973)

High Strength (1973)

High Strength (1973)

High Strength, Low Alloy Columbium and/or Vanadium (197

High Strength, Low Alloy Steel (1970) ANSI G88.3

High Strength, Prevailing Torque, All Metal (1973)

High Strength, Prevailing Torque, All Metal (1973)

High Strength, Prevailing Torque, All Metal, 1200 Deg.

High Strength, Prevailing Torque, All Metal, 1200 Deg.

High Strength, Quenched and Tempered (1972A) ANSI G35.1

High Temperature and Humidity (1973)

Termination of

High Temperature Central Station Service (1973) ANSI B I

High Temperature Glass Cloth Pressure Sensitive Electri

High Temperature Molten Materials (Metals, Slag, Glass,

High Temperature Service Conforming to ISO Recommendati

High Temperature Service (1970) ANSI G81.44

TTation $\mathrm{Ha}$ ASTM

High Temperature Service (1970) ANSI G81.45

High Temperature Service (1970) ANSI G81.46

High Temperature Service (1971) ANSI G55.12

High Temperature Service (1972)

/Ardening ASTM

Fning Cob ASTM

/Lloy Stee ASTM

ASTM

Hia Temperature Service (1972)

Terature Service (1972)

High Temperature Service (1972) ANSI BI

High Temperature Service (1972) ANSI B 125.39

High Tem perature Service (1972) ANSl G37.1

High Tem perature Service (1972) ANSI G52.2

High Temperature Service (1972A) ANSl B125.30

High Temperature Service (1973)

High Temperature Service (1973) ANS1 B 125.24

High Temperature Service (1974) ANSl G37.2

High Temperature Service (1974) ANSI G37.3

High Temperature Service (1974) ANSI G81.19

High Temperature Services (1974) ANSI G38.4

td. Spec. for Precip ASTM

Slectric Fu ASTM

/Meter Au ASTM

ASTM

/ Alloy Ste ASTM

ASTM

ASTM

Std. ASTM

ASTM

Std. Spec. ASTM

High Temperature Terminal Block, Base Insula

I and Alloy ASTM

/Spec. for ASTM

Std. ASTM

NSA

High Temperature (1973)

High Temperature, Electrical, Magnetic, and Other Simil

ASTM

ASTM

.

High Temperature, Fuel and Oil Resistant, 65-75 (1973

High Temperature, Protruding Head, (1972)

High Temperature, 50 to $120 \mathrm{KSI}$ Yield Strength, Materia

NSA

$\mathrm{SAE}$

Std. NSA

ASTM

Hemperatures (1970) ANSI Alll.

High Temperatures (1970) ASTM G82.1

/Cast Iron-Chrom ASTM

High Temperatures (1973)

High Temprature Service (1974) ANSI G38.11 Std. Meth. Fo ASTM Gs Special ASTM

High Tensile Strength, Hard Drawn, for Mechanical Sprin

High Tensile, Hard Drawn Mechanical Spring Wire and Spr

High Test Heat Treated Line Pipe (1972)

High Test Line Pipe (Pet roleum Drilling) (1973)

High Test Wrought Welding Fittings (1973)

High Voltage Air Switches, Bus Supports, and Switch Acc

High Voltage Circuit Breaker External Insulation Rated

High Voltage Circuit Breakers Rated on a Symmetrical an

High Voltage Circuit Breakers Rated on a Symmetrical Ba

High Voltage Circuit Breakers Rated on a Symmetrical Ba

High Voltage Circuit Breakers Rated on a Symmetrical Ba

High Voltage Circuit Breakers Rated on a Symmetrical Cu

High Voltage Circuit Breakers Rated on a Symmetrical $\mathrm{Cu}$

High Voltage Circuit Breakers Rated on a Symmetrical $\mathrm{Cu}$

High Voltage Circuit Breakers Rated on a Symmetrical Cu

High Voltage Circuit Breakers Rated on a Symmetrical Cu

High Voltage Circuit Breakers Rated on a Symmetrical Cu

High Voltage Circuit Breakers (1971)

/Ransient

High Voltage Components for Television Receiving Applia

High Voltage, Low Current, Dry Arc Resistance of Solid

High Yield Strength, Quenched and Tempered Alloy Steel

High) (Fire Protection and Safety) (1973) /Tized

Higher Boiling Homologues of Chlorinated Biphenyls for

Higher Temperature Service (1972) ANSl G35.9 /Spec. Fo

Highway Bridge Deck Construction (1974)

Highway Bridges (1974)

Highway Bridges (1974) ANSI G52.3

Highway Construction Purposes (1973) AASHO Ml45

Highway Construction (1973)

Highway Emergency Signals (Flare, Lantern, Fusee) (1973

Highway Lighting (1973) EEI TDJ-133

Highway Service (1974)

Highway Use on Trucks, Buses, Trailers, and Multipurpos

(Highway Vehicle Accident) (1972)

Highway Vehicles (1973)

Highway, Airport

Highways (1965)

Highways (1971)
Highways (1973)

\section{of Test. Tires for We}

Pec. Fo ASTM

ASHTO

SAE

A440

562

333

547

A607

J410C

(

AMS7253A

AMS7250C

AMS7251B

Asis

T464

A376

D2754

LPD 7.33

A520

A637

A638

639

A437

A660

358

A 409

A45l

A182

A351

Al06

A335

A216

A217

A447

193

1063

C768

AMS7266

1671

A453

Cl6

A608

D3232

A389

A679

J271

SPEC

$5 \mathrm{LX}$

SP75

C37.32

C 37.0781

C37.11

C37.0732

339

417

327

328

340

341

342

343

C 39.0722

492.3

C 37.85

D495

A514

8-25N

D3303

A5 15

$* 1.48$

345

34

A 486

D3282

$\mathrm{C} 14$

912

SH3

2.29

J267

J224A 
Std. for Template

Std. for Butts and push and Kick / Basic Builders' Hardware: Hands of Doors; ment of Fractures of the Skeletal (Bone) /

wagons (1972)

tractors (1972)

Std for 3 Point Free ped with Quick Attaching Coupler for Three Point Free Link ce Capacity on Agricultural Tractors Equipped with 3 Point ce Capacity on Agricultural Tractors Equipped with 3 Point ional Type) Connecting Devices and Towing Meth. (Coupling, Std. for Trailer Couplings and 2) SAE J715 (1972) Std. for 3 Point Free Link Attachment for Sid. for One Point Tubular Sleeve Attachment for eth. of Determining the SAE Rating and Struck Capacity for 71) Std. Spec. for Manually Lever Operated Chain Std. Spec. for Hand Operated Chain types (1971) Std. Spec. for Electric Wire Rope Std. Spec. for Electric Chain nes, Derricks, Hoists, Jacks and Slings: Base Mounted Drum Std. for Overhead lar Cranes (1973) Safety Std. for Cranes, Derricks, 1) Safety Code for Cranes, Derricks, 0) Guide for Supplementary Ventilation in a Ship's Cargo Rec. Pract. for Diesel Fuel Injection Nozzle and Std. for Precision Indexable Insert r Carbide Seats Used with Indexable Inserts for Clamp Type ide Chip Breakers Used in Indexable lnserts for Clamp Type osers; Hospital and Misc. Items; Key Control Systems; Door Holders, Stops, Smoke and Heat Detectors; Electromagnetic e (Boats) (1972) s (1971) Rec. Pract. and Std. Covering Sewage Std. for Waste and Sew age

Plastic Tanks for Water and Wastewater Treatment (Septic, Safety Std. for of Vehicle Alarm Systems and Units for Protection Against ting Equipment (Barrier) for Protection Against Robbery or

972)

edges (1972)

Std. Rec. Pract. for Dimensions and Tolerances for Screw Uniform Building Code Std. for Building, Facing, and Quality Stds. for Flush Solid and Std. Meth. of Test for Alkalinity of

Std. Meth. of Test for Bulk Density and Packing Factor of Std. Meth. of Test for Hydrostatic Collapse Strength of

a79.1 Std. Spec. for

rmediate Pivot (1973)

ies Deadlock Strikes (1973) si A80.1

Uniform Building Code Std. for Spec. for $13 / 4$ In. Thick Spec. for Std. Spec. for

uniform Building Code: Solid Crout Building Code Std. for Std. for Particleboard for Mobile Std. Meth. of Measurement for Audio Amplifiers Used in nit Text/ Test Meth. for Dimensional Changes in Automatic for Oily Stain Release Meth. of Textile Fabric Soil During of Seams in W ash and Wear Items (Textile / Clothing) After earance of Creases in Wash and Wear Items (Clothing) After

ble Press Textile Fabrics (Permanent Press) After Repeated ) ANSI C33.13

71) ANSI A 197.2, Asse 1007 Safety Std for Electric Std. and Test Procedures for Std. for Mobile

X-Ray Protection Standards for for Power Output Ratings of Packaged Audio Equipment for Uniform Plumbing Code: Mobile rk Lift Truck, Boat, Baggage, Special Type Trailer, Mobile Rec. Pract. for

Sid. for Waste and Sewage Holding Tanks for Mobile ty Std. for Liquid Fuel Burning Heat Appliances for Mobile Std. for Supply Gas Connectors for Exterior Use on Mobile ehicles (Travel and Camping Trailers, Truck Campers, Motor mbling Mobile Homes, Modular Housing, Prefab Houses, Motor

Plants (Fire and Wind Hazards at Plants Assembling Mobile nts, and lnstallation of Plumbing. Heati/ cator Tranl 3) ANSI A176.1

c Tank Disposal Fields (1973) ANSI A 176.4

r Highway, Airport, and Similar Drainage /

drainage (Land Reclamation, Curtain and $\mathrm{B} /$ Std. For Mobile Std. Spec. for Std. Spec. for Std. Spec. for Std. Spec. for for Rapid Gas Chromatographic Estimation of Higher Boiling Std. Test Meth. for Delamination Strength of Rec. Pract, for Exhaust Std. for Draft

tion Type Cranes Having Rope Supported Booms, Equipped for es (1973) Std. Spec. for Electric Wire Rope Hoists (Lug, Safety Std. for Cranes, Derricks, Hoist,
Hinge Dimensions (1972)

Hinges for Doors (Builders Hardware) (1970)

Hinges; Locks; Knobs and Handles; Exit Devices, Pulls,

Hip Nail-Jewett Type for Use by Surgeons in the Treat

Hitch and Box Dimensions for Agricultural (Farm) Grain

Hitch Attachment of Implements to Agricultural Wheeled

Hitch (1971) SAE J909A

Hitch (1972)

Trutural Wheel Tractora

Hitch (1972)

/Ocedure for Measuring Hydraulic Lift for

/Ocedure for Measuring Hydraulic Lift for

(1973)

Hitches: Automotive Type (1972)

Ehicle (Recreat

Hitching Implements to Agricultural Wheel Tractors (197

Hitching Implements to Lawn and Garden Riding Tractors

Hoe Dipper (Backhoe Bucket) (1972)

Hoists for Industrial Applications (1971)

Sid. for Uniform $M$

Hoists for Ratchet and Pawl, and Load Brake Designs (19

Hoists (Construction and Demolition Operations) (1973)

Hoists (Lug, Hook, Trolley Suspended, and Base Mounted

Hoists (1971)

Hoists (1971)

Hoists (1973)

Hoists, Hooks, Jacks, and Slings: Portal, Tower and Pil

Hoists, Jacks and Slings: Base Mounted Drum Hoists (197

Hold During the Use of Internal Combustion Engines (197

Holder Assembly (17Mm Nominal Diameter) (1971)

Holders (1973)

Holders (1973)

Holders (1973)

Holders, Stops, Smoke and Heat Detectors; Electromagnet

Holders; Sliding Door Equipment; Installation Aids (196

Holding and/or Treatment Devices for Marine Toilet W ast

Holding Tanks for Mobile Homes and Recreational Vehicle

Holding, Settling, Aeration Chambers, Chemical Storage.

Holdup Alarm Units and Systems (1973) ANSI Se2.7

Holdup and Theft (1973) ANSI Se2.8

$/ \mathrm{N}$, and Maintenance

Holdup (Criminology) (1974) ANCI Se4.6

/R Bullet

Hole Placement on Bulldozer (Dozer) End Bit Supports (1

Hole Spacing for Scraper and Bulldozer

Hollow Brick (Made from Clay or Shale) (1973)

Hollow Core Doors (Arch itectural Woodwork) (1973)

Hollow Glass Microspheres (1972)

Hollow Glass Microspheres (1972)

Hollow Glass Microspheres (1972)

Hollow Load Bearing Concrete Masonry Units (1970) ANSI

Hollow Load Bearing Concrete Masonry Units (1973)

Hollow Metal Door and Frame Preparation for Offset Inte

Hollow Metal Door Frame Preparation for 181 and 190 Ser

Hollow Nonload Bearing Concrete Masonry Units (1971) an

Hollow Nonload Bearing Concrete Masonry Units (1973)

Hollow Unit, Cavity Wall, and Stone Masonry (1973)

Home Decking (1973)

Home Equipment (1971)

Home Laundering of Durable (Permanent) Press Woven or $\mathrm{K}$

Home Laundering (1970) ANSI L14.239

Home Laundering (1973) ANSl L14.172

Home Laundering (1973) ANSI L14.173

Home Launderings (1973) ANSI L14.226

Test Meth

He Laundry Equipment (Clothes Washer and Dryer) (1972

Home Laundry Equipment (washer, Dryer, Combination) (19

Home Parks (Trailer) (1972) ANSI A177.1

Home Television Receivers (1968)

Home Use (1967)

Home (T'railer) Parks (1973)

Home, Garden Tractor, Industrial Truck and Trailer (197

Homes and Camps in Forest Areas (1972)

Homes and Recreational Vehicles (1971)

Homes and Recreational Vehicles (1973) ANSI A 147.1

Homes (1972)

Homes): Installation of Plumbing, Heating, and Electric

Homes, and Camping Trailers) (1974)

Homes, Modular Housing, Prefab Houses, Motor Homes, and

Homes: Body and Frame Design and Construction Requireme

Homing and Alerting Equipment for Use with Emergency Lo

Homogeneous Bituminized Fiber Drain and Sewer Pipe (197

Homogeneous Perforated Bituminized Fiber Pipe for Septi

Homogeneous Perforated Bituminous Fiber Pipe Systems Fo

Homogeneous Solid Wall and Perforated Pipe for General

Homologues of Chlorinated Biphenyls for Capacitor Askar

Honeycomb Type Core Material (1970)

Hood Design for Industrial Ventilation Systems (1974)

Hoods for Gas Burning A ppliances (1971)

Hook Work, Clamshell, Magnet, Grapple, or Concrete Buck

Hook, Trolley Suspended, and Base Mounted Types (1971)

Hooks, Jacks, and Slings: Portal, Tower and Pillar Cran

Hookup Wire Insulation (1974)
ANSI

ANSI

NBHA

ASTM

ASAE

SAE

ASAE

ASAE

VESC

SAE

ASAE

HMI

HMI

ANSI

HMI

HMI

ANSI

ANSI

ANSI

SNAME

SAE

ANSI

Std. Fo ANSI

ANSI

NBHA

ABYC

IAPMO

NSF

UL

UL

SAE

SAE

ASTM

ICBO

AWI

ASTM

ASTM

ASTM

ASTM

ICBO

ANSI

ANSI

ASTM

ICBO

/E ICBO

NPA

EIA

AATCC

AATCC

AATCC

AATCC

UL

AHAM

NFPA

NCRPM

Std EIA

ICBO

TRA

NFPA

IAPMO

/Fe UL IAPMO TSCO

NFPA $501 \mathrm{C}$

FMS

FMS

NFPA

RTCA

ASTM

ASTM

ASTM

ASTM

ASTM

ASTM

ACGIH

ANS

$\mathrm{SAE}$

HMI

ASTM

F369

R239

J715D

S278.2

S349

J283

J684 D

A 156.7

A 156.1 
Std. for Capacity Designation for Fertilizer Std. for Numerically Controlled

Std. for Numerically Controlled Std. Meth. of Test for Abrasion Resistance of Spec. for Vertical and

73)

t. for LP Gas Fuel Container, Quickly Demountable Type for for LP Gas Fuel Container, Quickly Demountable Universal ent of Static Friction of Corrugated and Solid Fiberboard ent of Static Friction of Corrugated and Solid Fiberboard rning of Cellular Plastics Using a Supported Specimen by A plastics by Short Beam M/ Std. Meth. of Test for Apparent Rec.
Rec.

Stds. for Forced Entry Resistant Aluminum t. for Flammability of Automotive Interior Trim Materials: 43.1 Safety Std. for Sliding Hardware for Std., cat/ Spec. for Aluminum Combination Vertically Sliding or Std. for Industrial and Other Direcl Current Integral Std. for Synchronous lntegral for Frame Assignments for Alternating-Current Integral Rec. Pract. for Determining and Stating the ite Purpose Motors and Generators (Fractional and Integral current and Direct Current Motors (Fractional and Integral rnating and Direct Current Motors (Fractional and Integral ect Current Motors and Generators (Fractional and Integral ect Current Motors and Generators (Fractional and Integral pecial Purpose Water Safety Buoyant Devices (Vest, Jacket, Meth. of Test for Heavy Cotton Fabrics for Manufacture of td. for Tests and Procedures for SAE 100R Series Hydraulic ances, Casters, Face Masks and Rebreathing Bags, Footwear, ests and Procedures for SAE I00R Series Hydraulic Hose and

s $(197 !)$ es (1971)

t. for Coding System for Identification of Tube, Pipe, and Safety Std. for

Std. for Flexible Non Pressure Water Fill Safety Std. for Semiautomatic Fire Rec. for Standpipe and Std. for Care of Fire

Std. Meth. of Testing Automotive Air Conditioning Safety Std. for Flexible Metallic

Std. Meth. of Test. Automotive Hydraulic Brake Std. Meth. of Testing Rubber

Sid. Spec for Copper Alloy Strip for Flexible Metal Safety Std. for LP (Liquid Petroleum) Gas Safety Std. for Fire Extinguisher and Apparatus Booster Safety Sid. for Unlined Fire

Std. Meth. of Test. Automotive Air Brake and Vacuum Brake Std. for Fuel and Oil Std. for Coolant System

Test Meth for Snag Resistance of Women's Nylon exit Devices, Pulls, Push and Kick Plates: Bolts; Closers; sual Communication) (1974) Sid. for Safety for ansi G24.24 ash lgnition Properties (Temperatures) of Plastics Using A ansi $\mathrm{C} 24.25$

and/or Vanadium (/ Std. Spec. for Regular Quality Std. Spec. for Drawing Quality Std. Spec. for Steel Sheet and Strip. nd Forging Billets for High S/ Sid. Spec. for Hot Worked, ength at Elevated Temperatures (1971/ Std. Spec. for Hot, Strip for High Strength at Elevated/ Std. Spec. for Hot, I/ Spec. for Determination of Phosphate Coating Weight on ur/ Rec. Pract. for Safeguarding Against Embrittlement of td. Spec. for Steel Sheet. Zinc Coated (Galvanized) by the t of Lock Forming Quality, Zinc Coated (Galvanized) by the Sheet of Drawing Quality, Zinc Coated (Galvanized) by the for Culverts and Underdrains, Zinc Coated (Galvanized) by uirements for Steel Sheet, Zinc Coated (Galvanized) by the ity in Coils, and Cut Lengths. Zinc Coated (Galvanized) by td. Spec. for Steel Sheet, Zinc Coated (Galvanized) by the Std. Spec. for Zinc Coating Std. Spec. for Welded and Seamless Black and Steel Pipe for Ordinary Uses (/ Std. Spec. for Black and tubing (1973) ANS1 G24.22 structural Tubing (1973)

Std. Spec. for

d. Spec. W Maximum d. Spec. for Requirements for Mixing Plants for Hot Mixed,
, and Surface Courses (1967) Sid. Spec. for Hot Mixed, Stu. Meth. of Text for Apparent Viscosity of Rec. Pract. for Flexibility Determination of

nsi A3/ Sid. Spec. for Requirements for Mixing Plants for $r$, Leveling, and Surface Courses (1967) Std. Spec. for erials for lise in Construction of Built Up Roof Coverings: a/ Safety Std. for Materials for Built-Up Roof Coverings:
Hopper Cars for Malt and Grain Service (1964) Hoppers and Containers (1972)

Horizontal and Vertical Jig Boring Machines (1974)

Horizontal Boring, Drilling and Milling Machine (1974)

Horizontal Boring, Drilling and Milling Machine (1974)

Horizontal Concrete Surfaces (1974)

Horizontal Emulsion Treaters (Petroleum Production) (19

Horizontal Mounting, Liquid Withdrawal Only (1973)

Horizontal or Vertical Mounting). Liquid Withdrawal (19

Horizontal Plane Meth.) (1972)

(Horizontal Plane Meth.) (1973)

Horizontal Screen (1974) ANSI K65.114

Horizontal Shear Strength of Parallel Fiber Reinforced

of Test for Coeffici ASTM

lor Extent of Bu

Horizontal Shoring Beam Erection Procedure (1972)

Horizontal Shoring Beam Safety Rules (1972)

Horizontal Sliding Doors (1971)

Horizontal Test Meth. (1972)

Horizontally Mounted Tin Clad Fire Doors (1973) ANSI Al

Horizontally Operating Storm

Horsepower Generators (1972)

Horsepower Induction Motors (1972)

Horsepower Rating of Inboard Gasoline Engines (Boats) (

Std for Defin

Horsepower) (1972)

Horsepower) (1972)

Horsepower) (1972)

Horsepower) (1972)

Horsepower) (1972)

Std. for Ratings for Alternating

$/$ for Tests and Performance of Alte

/Ation Data for Alternating and Dir

Dimensions for Alternating and Dir

Horseshoe and Ring Buoy) (1973) ANSI Z243.1 / Marine S

Hose and Belts (1942) ANSI L14.283

Hose and Hose Assemblies (1970)

Hose and Tubing, Mattresses and Pads, Sheeting, and Res

Hose Assemblies (1970)

Spec. and

Hose Clamps (1971)

Hose Connectors for Portable Indoor Gas Fired Appliance UL

Hortable Outdoor Gas Fired Applianc

Hose Fittings (1972)

Hose for Conducting Gasoline (1973)

Hose for Recreational Vehicles (1971)

Hose Rack Assemblies (1972)

Hose Systems (Heating) (1972)

Hose (Including Couplings and Nozzles (1972)

Hose (1968E) ANSI J2.27

Hose (1972) ANSI B140.1

Hose (1972) ANSI J2.9

Hose (1972A) ANSI J2.5

Hose (1973)

Hose (1973)

Hose (1973)

Hose (1973) ANSI A152.1

Hose (1973) ANSI S2.10

Hoses (1970)

Hoses (1971)

Hosiery (1973) ANSI L14.85

Hospital and Misc. Items; Key Control Systems; Door Hol

Hospital Signaling and Nurse Call Equipment (Audio / VI

Hot Air lgnition Furnace) (Fire Safety) (1973)

Hot and Cold Rolled Alloy Steel Sheet and Strip (1973)

Hot and Cold Rolled Alloy Steel Sheet and Strip (1973)

Hot and Cold Rolled, High Strength, Low Alloy Columbium

Hot Cold Worked, and Cold Worked Alloy Steel Forgings a

Hot Cold, and Cold Worked Alloy Steel Bars for High Str

Hot Cold, and Cold Worked Alloy Steel Plate, Sheet, and

Hot Dip Galvanized and Electrogalvanized Steel (Coil) (

Hot Dip Galvanized Structural Steel Products and Proced

Hot Dip Process for Roofing (1971) ANSl G8.21

Hot Dip Process (1971) ANSI G8.22

Hot Dip Process (1971) ANSI G8.23

Pec for Steel Shee

Hot Dip Process (1971) ANSI G8.28

Td. Spec, for Steel ASTM

C. for Steel Sheets ASTM

Ec. for General Req ASTM

Hot Dip Process (1973) ANSI G8.27

of Structural Qual

Hot Dip Process, Commercial Quality (1971) ANSI G8.24

Hot Dip) on Assembled Steel Products (1973) ANSI G8.18

Hot Dipped Galvanized Steel Pipe (1973) ANSI B125.1

Hot Dipped Zinc Coated (Galvanized) Welded and Seamless

Hot Formed Welded and Seamless Carbon Steel Structural

Hot Formed Welded and Seamless High Strength Low Alloy

Hot Head Press (300-330 Deg. F) Finishing, Shirt Unit

Hot Laid Bituminous Paving Mixtures (1971) ANSI A37.102

Hot Laid Tar Paving Mixtures for Base, Binder, Leveling

Hot Melt Adhesives and Coating Materials (1973)

Hot Melt Adhesives by Mandrel Bend Test Meth. (1972T)

Hot Mixed, Hot Laid Bituminous Paving Mixtures (1971) a

Hot Mixed. Hot Laid Tar Paving Mixtures for Base, Binde

Hot Mopped Asphalt, Asphalt and Coal Tar Saturated Orga

Hot Mopping Asphalt, Asphalt-Saturated Organic Felt, Co

BAMI

ASAE

NSA

NSA

ASTM

API

ITA

ASTM

SSI

AAMA

SAE

AAMA

NEMA

NEMA

NEMA

NEMA

NEMA

NEMA

NEMA

ASTM

UL

SAE

ANSI

ANSI

ULE

IAPMO

FMS

NFPA

ASTM

UL

ASTM

ASTM

UL

UL

ASTM

SAE

AATCC

NBHA

UL

ASTM

ASTM

ASTM

ASTM

ASTM

NCCA

ASTM S281.

963

910

C779

$12 \mathrm{~L}$

T816SU

D3247

D1692

D2344

$* 8$
$* 7$

1302.3

$\mathrm{J} 369 \mathrm{~A}$

$14 \mathrm{~B}$

1002.8

MGI.15

MGI.16

MGI3

S6

MGI-18

MGI.10

MG I. 12

MGl.14

MGI.11

1123

D181

J343A

J343A

$\mathrm{J} 536 \mathrm{~B}$

Z21.2

Z21.54

J846C

330

TSCI 9

47

4.4
198

D1680

536

D571

D380

21

92
18

D622

J30A

J20D

65

1069

UBCS52-3

A506

A507

A 607 
unter Appliances (Coffee Pots and Urns, Food Dish Warmers, Std. for Domestic Gas

Std. Spec. for Copper and lts Alloy Die Forgings Test Meth. for Colorfastness of Textiles to d Heat Resisting Steel Bars and Shapes (1/ Std. Spec. for Products Manual) Carbon Steel: Semifinished for Forging; (1973) ANSI G24/ Std. Spec. for General Requirements for ng Chromium Nickel Manganese Steel Bars I/ Std. Spec. for rs, Rod, and Wire for Nuclear Application/ Std. Spec. for ure Piping Component/Std. Spec. for Steel Bars, Carbon. erty Requirements (1972)

Std. Spec. for Merchant Quality Std. Spec, for Merchant Quality

lity (1972)

nd Strip, Commercial Quality (1972)

Std. Spec. for Std. Spec. for Std. Spec. for

mifinished for Forging; Hot Rolled and Cold Finished Bars; Std. Spec. for Steel, Carbon (0.16 to 0.25 Maximum \%, ns (1974) Std. Spec, for Steel Bars, Carbon, Meth. for Measuring Flat Spring Test for (1972) td. for Gas Fired High Pressure Steam and Std. for Gas Fired Low Pressure Steam and

lumbing Requirements for Household Storage Type Electrical Spec. for Chlorinated Poly(vinyl Chloride) (CPVC) Plastic d. for Relief Valves and Automatic Gas Shutoff Devices for Installation and Maintenance of Steam and Rec. Pract. for Rec. Pract. for Std. Rec. Pract. for Std. Spec. for Std. Spec, for

r Nonnuclear Applications (1972) ANSI Z17/ I Forgings and Forging Billets for High S/ Spring Design: Compression, Extension, Torsion, Flat, and h Strength at Elevated Temperatures (1971/ Std. Spec. for , and Strip for High Strength at Elevated/

Std. Spec. for

ers (1973)

Std. for Std. for

Std Meth. of Test for Delta in Southern Building Code for Dwelling d (Pressure Treated Lumber and Plywood) Foundation System: Std. Rec. Pract. for Test. Exterior Latex (I972)

ding Manufactorer, Restaurant, and Dwelling or Appartment ers (1972) Safety Std. for Industry Standard for Std for

Std. and Test Procedures for Plumbing Requirements for

ances (1972) ANSI C33.11.1

(Stove) (1974)

C71.1

cooking Tops (1974)

(1973) ANSI C33.11.2 si C33.87

Std. for Safety for Std. for Safety for Std. Std. for Std. for

to for Safety for Std. for Safety for Safety Std. for Std for Safety of I Std. and Test Procedures for Plumbing Requirements for Std. for Performance Evaluation for Std. for Safety for (1973)

etermine the Freezer Temperature and Energy Consumption of Safety Std. for
Suirements for

Std. Performance Evaluation Procedure for

ower Plants (1973) Std tor

at Plants Assembling Mobile Homes, Modular Housing, Prefab fire and Wind Hazards at Pl/ Rec. for Factory Constructed

(Except Tapered Roller Bearings) Conform/_Std. Shaft and Std. for Particleboard Decking for Factory Built nd Wind Hazards at Plants Assembling Mobile Homes, Modular Evaluation of Suitability of Plastic Material for Use in Std. Sizes for Industrial Centrifugal Fans with $\mathrm{C}$ ast Std. Spec. for Neoprene Rubber Gaskets fo

ttings) (1972) Spec. Data for

$\mathrm{s}$ and Drain $\mathrm{P} /$ Std. Performance Requirements for Std. for Installation of Seacocks, Through

d Installation of Power Transmission Units Penetrating the

Rec. Pract. for Boat Code for Shipboard Coating Systems (Paint) Guide for Ship

ty Control) (1967) Rec. Pract. for Ethics an $\mathrm{n}$ and Construction of Surface Drainage Systems on Farms in Std. for Performance of Appliance ances Rated at $60 /$ Safety Std. for Electric Fans (Blower Std. for Selection, Installation, and Servicing of
Hot Plates and Griddles, etc.) (1973)

Hot Plates and Laundry Stoves (1971)

Std. for Gas Co ANSI

ANSI

(1974) ANSI H7.10

Hot Pressing (1973)

Hot Rolled and Cold Finished Age Hardening Stainless an

Hot Rolled and Cold Finished Bars; Hot Rolled Deformed

Hot Rolled and Cold Finished Carbon and Alloy Steel Bar

Hot Rolled and Cold Finished Stainless and Heat Resisti

Hot Rolled and Cold Finished Zirconium and Its Alloy Ba

Hot Rolled and Cold Finished, Special Quality, for Pres

Hot Rolled Carbon Steel Bars Subject to Mechanical Prop

Hot Rolled Carbon Steel Bars (1973)

Hot Rolled Carbon Steel Sheet and Strip, Structural Qua

Hot Rolled Carbon (0.15 Maximum, Percent) Steel Sheet a

Hot Rolled Copper Rods for Electrical Purposes (I974) a

Hot Rolled Deformed Concrete Reinforcing Bars (1972)

Hot Rolled Sheet and Strip, Commercial Quality (1972)

Hot Rolled, Special Quality, for Fluid Power Applicatio

Hot Tack of Wax Polymer Blends (1972)

Hot Water Boilers (lnputs Not Over 400,000 Btu Per Hr.)

Hot Water Boilers (1972)

Hot Water Dispensers (1973)

Hot Water Distribution Systems (1973)

Hot Water Supply Systems (1972)

Hot Water Unit Heaters (1970)

Weather Concreting (Concrete) (1972) ANSI Al70.1

Hot Weather Concreting (1959)

Hot Weather Concreting (1972) ACI 305

Hot Worked and Cold Finished Zirconium Bars and Wire Fo

Hot Worked, Hot Cold Worked, and Cold Worked Alloy Stee

Hot Wound (Materials, Specs. Terminology, Gauge, Metric

Hot, Hot Cold, and Cold Worked Alloy Steel Bars for Hig

Hot, Hot Cold, and Cold Worked Alloy Steel Plate, Sheet

Hot. Water Immersion Controls (1972)

Hotel and Restaurant Gas Deep Fat Fryers (1971)

Hotel and Restaurant Gas Ranges (Stoves) and Unit Broil

Hours (DIH) Purity of Nuclear Graphite (1971) ANSl K90.

House Construction (1973)

House Design and C
House Paints (1972)

House (1973)

Household Automatic Electric Storage Type Water Heaters

Household Commercial and Portable Exchange Water Soften

Household Cooking Gas Appliances (1972)

Household Dishwashers (1971) ANSI A197.1, Asse 1006

Household Dishwashers (1974)

Household Electric Clocks (1974) ANSI C 33.47

Household Electric Coffee Makers and Brewing Type Appli

Household Electric Range Pyrolytic Self Cleaning Ovens

Household Electric Ranges (Stove and Ovens) (1972) ANSI

Household Electric Ranges (Stove) with Glass / Ceramic

Household Electric Ranges (Stoves) (1974) ANSI C33.53

Household Electric Skillets and Frying Type Appliances

Household Electric Storage Tank Water Heaters (1972) an

Household Fire Warning System Units (1973)

Household Food Waste Disposer Units (1971) ANSI A197.3.

Household Food Waste Disposers (1973)

Household Refrigerators and Freezers (1973) ANSI B97.l

Household Refrigerators, Combination Refrigerator-Fre

Household Storage Type Electrical Hot Water Dispensers

Household Type Pressare Cookers (1973)

Household (laundry, Clothes) Washer (1971) ANSI Z224. I

Housekeeping During the Construction Phase of Nuclear P

Houses, Motor Homes, and Camping Trailers) (1974)

Housing and Recreational Vehicle Manufacturing Plants (

Housing Code (Residential Buildings) (1973)

Housing Fits for Metric Radial Ball and Roller Bearings

Housing (1972)

Housing, Prefab Houses, Motor Homes, and Camping Traile

Housings of Motor Vehicle Lighting Devices Including Re

Housings (1966)

Hub and Spigot Cast Iron Soil Pipe and Fittings (1972)

Hubless Cast Iron Sanitary System (Plumbing Pipe and Fi

Huck Towels (Institutional Textile) (1973)

Hull Connections, Outboard Water Strainers, Water Scoop

Hull Fittings and Sea Valves (1973) ANSI Z254.1

Hull for Boats Equipped with Outdrives, Jet Drives And/

Hull Identific ation Numbers (1973)

Hull Vibration Measurements (1969)

Hull, Deck and Superstructure (1973)

Human Relations in Vendor-Vendee Relationships (Quali

Humid Areas (1973)

Humidifier (1972) ANSI Z235.1

Humidifier, Evaporative Cooler, and Air Filtering Appli

Humidifiers (1972)
AATCC 133

ASTM A564

AlSl

ASTM

ASTM

ASTM

ASTM

ASTM

ASTM

ASTM

ASTM

ASTM

AISI

ASTM

ASTM

TAPPI

ANSI

ANSI

ASSE

Std. ASTM

St ANSI

AMCA

AC

ACI

ANSI

ASTM

ASTM

SMI

ASTM

ASTM

NEMA

ANSI

ANSI

ASTM

SBCC

NFORP

ASTM

CBO

ANSI

UL

WCF

ANSI

AHAM

AHAM

UL

UL

AHAM

AHAM

AHAM

UL

UL

UL

UL

AHAM

AHAM

UL

AHAM

ASSE

UL

AHAM

ANSI

/Ds FMS

FMS

ICBO

AFBMA

NPA

FMS

SAE

AMCA

CISPl

CISPI

ANI

$\mathrm{ABYC}$

UL

ABYC

$A B Y C$

SNAME

ASOC RP1

R302.2

AHAM HU.1

UL 507

ARI

A 429

A 696

663

A 575
A 570

A569

A659

605

T683SU 
National Bureau of Standards KWIC Index of Engineering Standards

Std. for Central System

Std. for Self Contained

for Respiratory Therapy Equipment (Breathing Devices, Room nditioning, Heating, Cooking, Refrigeration, and Humidity ss of Textile Fabrics to Ozone in the Atmosphere Under Low fastness of Textiles to Ozone in the Atmosphere Under High (Coil) (1974)

Std. Meth. for Condensation of Air Conditioning, Heating, Cooking, Refrigeration, and on Rate of Sheet Materials (Paper) at High Temperature and Meth. for Colorfastness of Textile Fabrics to Water (High Rec Std for Seismic Restraint Direct eth. of Test for Color of Raw Cotton Using the Nickerson. Meth. for Measuring Color of Paper and Paper Board by easurement of Color of Low Colored Clear Liquids Using the eformation and Cohesion of Bituminous Mixtures by Means of Rec. for Water Sprinkler and

ity, Departmental Regulations and Controls, Barrier, Road, Safety Std. for ntary $\mathrm{Pa}$

Sieving Meth. of Analysis for Materials Hard to Uniform Building Code Std for Std. Spec. for

Std. Meth. of Physical Test. of Quicklime and

d. Meth of Chemical Analysis of Limestone, Ouicklime, and (Cereal Chemistry) Alcohol; Carbon Tetrachloride; Chloral 1 Products (Cereal Chemistry) (1968) Meth. of Analysis of Std. Meth. of Test for Heat of ) (1971) Guide Spec. for Std. Meth. of Test. Automotive Std. Meth. of Test for Fineness of Std. Rec. Pract. for Use of

(1965) en in Fle/ imens) (1/

Std. Meth. of Test for Compressive Strength of Std. Meth. of Test for Compressive Strength of Sid. Meth. of Test for Flexural Strength of Std. Definitions of Terms Relating to Std. Meth. of Test for Specific Gravity of Std. Meth. of Test for Normal Consistency of Std. Meth. of Test for Heat of Hydration of Std. Meth. of Sampling cabinets and (Specimen Storage) Rooms Used in the Test. of Std. Spec. for Blended uniform Building Code Std. for Portland Cement and Blended Rec. Pract. for Automotive Transmission Std. for Mobile

Std. End Load Test Meth. for A

Std. Meth. for Determining the Fabrication Integrity of A Std. Meth of Verifying the Material Compatibility of A

Meth. for Verifying the Flow Fatigue Characteristics, of A

Meth. for Verifying the Collapse / Burst Resistance of A

Meth. for Evaluating the Filtration Performance of a Fine rocedure for Qualifying and Controlling Cleaning Meth. for presenting Basic Performance Data for Positve Displacement or Extracting Fluid Samples from the Lines of an Operating Std. Pract. for the Use of Fire Resistant Fluids for

Std. Meth. of Reporting Contamination Analysis Data of Meth. of Test for Mist Spray Flammability of Std. for Tests and Procedures for SAE 100R Series

equipped with 3 Point equipped with 3 Po/
type (Farm) Implements (1971) Std. Test Procedure for Measuring Std. for Test Procedure for Measuring Std. Meth. for High Pressure Pump Test. of

pe Friction Loss Tables for Solving Fire Protection System tors and Trailing Type Agricultu/

Std. for Application of nt (1964) ANSI Z98.33 Std. for Nonintegral Industrial Fluid Power pose Machine Tools (1973) Std. Spec. for Mineral Fiber

Rec. for Erosion Resistance of Concrete in or Cast lron Sealing Rings for Automotive Transmission and Std. for

rator/Motors for Pumpe/

Apparatus) (Exclusive of Generator, for Std. for lnstallation of Vertical for the Construction, Care, and Use of Shears (Mechanical. Std. Spec. for Std. Meth. of Test for Butadiene Purity and Test Meth. for Oil Repellency of Fabrics: Std. Spec. for Low Boiling Solutions Such as Pent/ Std. Spec. for for High Boiling Std. for 1973) trometry (1971) ANSI Z11.31 Std. Meth. of Test for 5 Std. Meth. of Test for Bromine lndex of Aromatic 248 Std. Meth of Test for Bromine Index of Petroleum f Test for Nonaromatic Hydrocarbons in Monocyclic Aromatic Std. Meth. of Test for Aromatic Traces in Light Saturated t for Trace Quantities of Sulfur in Light Liquid Petroleum Std. Meth of Test for D.C. Electrical Conductivity of Std. Test Meth. for Polynuclear Aromatic
Humidifiers (1974)

Humidifiers (1974)

Humidifiers, Nebulizers, Gas Therapy and Suction Equipm

ARI

ARI6

(1972) ANSI B131.1

Humidities (1972) ANSI L14.174

Humidities (1972) ANSI L14.238

/ Meth. for Colorfastne

$\mathrm{UL}$

Humidity Test. of Organic Coatings on Metallic Surfaces

Humidity (Humidistat, Thermostat) (1972) ANSI B131.1

Humidity (1973)

ITermination of Water Vapor Transmissi

Humidity) and Light: Alternate Exposure (1972) ANSI L14

Hung Suspended Ceiling Assemblies (1972)

Hunter Cotton Colorimeter (1971)

Hunter L, A, B Colorimetry (1972)

Hunterlab Color Difference Meter (1972T)

Hveem Apparatus (1971) ANSI A37.137

Hydrant System Demand Tables (Fire Safety) (1973)

Hydrant, Driveway Clearance, Installation and Maintenan

Hydrants for Fire Protection Service (1973)

Hydrate in Baked Goods, Ready.To-Eat Cereals, and Alime

Hydrated Lime for Masonry Purposes (1973)

Hydrated Lime for Masonry Purposes (1974) ANSI K67.13

Hydrated Lime (1971) ANS1 K67.10

Hydrated Lime (1972) ANSI K67.3

Hydrate: Chloroform; Formaldehyde; Gasoline; Herwig's,

Hydration Capacity of Pregelatinized Starches and Cerea

Hydration of Hydraulic Cement (1973) ANSI Al.10

Hydraulic and Electric Elevators (Freight and Passenger

Hydraulic Brake Hose (1972) ANSI J2.9

Hydraulic Cement by No. 325 (45-?M) Sieve (1973)

Hydraulic Cement Mortars in Chemical Resistant Masonry

Hydraulic Cement Mortars (Using Portions of Prisms Brok

Hydraulic Cement Mortars (Using 2 In. $(50 \mathrm{Mm}$ ) Cube Spec

Hydraulic Cement Mortars (1972)

Hydraulic Cement (1971) ANSI Al.28

Hydraulic Cement (1972) ANSl Al.12, Ashto T133

Hydraulic Cement (1973) AASHO T129, ANSI Al.11

Hydraulic Cement (1973) ANSl Al.10

Hydraulic Cement (1973) ANSI Al.2

Hydraulic Cements and Concretes (1973)

Hydraulic Cements (1973)

Hydraulic Cements (1973)

Hydraulic Control System Terminology (1970)

Hydraulic Cranes (1973)

Hydraulic Fluid Power Filter Element (1972) ANSI B93.21

Hydraulic Fluid Power Filter Element (1972) ANSI B93.22

Hydraulic Fluid Power Filter Element (1972) ANSI B93.23

Hydraulic Fluid Power Filter Element (1972) ANS1 B93.24

Hydraulic Fluid Power Filter Element (1972) ANSI B93.25

Hydraulic Fluid Power Filter Element (1973) ANSI B93.31

Hydraulic Fluid Power Fluid Sample Containers (1972) an

Hydraulic Fluid Power Pumps and Motors (1973) ANSI B93.

Hydraulic Fluid Power Systein (For Particulate Contamina

Hydraulic Fluid Power Systems (1972) ANSI B93.5

Hydraulic Fluid Power Systems (1973) ANSI B93.30

Hydraulic Fluids (1972T)

Hydraulic Hose and Hose Assemblies (1970)

Hydraulic Lift Force Capacity on Agricultural Tractors

Hydraulic Lift Force Capacity on Agricultural Tractors

Hydraulic Motors to Agricultural Tractors and Trailing

Hydraulic Oils (1973)

Hydraulic Problems (1973)

Hydraulic Remote Control Cylinders to Agricultural Trac

Hydraulic Reservoirs (1969) ANSI B93.18

Hydraulic Setting Thermal Insulating and Finishing Ceme

Hydraulic Std. for Industrial Equipment and General Pur

Hydraulic Structures (1952)

Hydraulic Systems (1972)

Hydraulic Tube Fittings (1972)

Hydraulic Turbine Drive) (1972)

Hydraulic Turbine Driven Generators and Reversible Gene

Hydraulic Turbine Driven Synchronous Generators and Rev

Hydraulic, Pneumatic, Semi and Automatic, and Manually

Hydrocarbon Dry Cleaning Solvents (1971) ANSI Z11.42

Hydrocarbon Impurities by Gas Chromatography (1973)

Hydrocarbon Resistance Test (1972) ANSI L14.214

Hydrocarbon Solvent for Oil Borne Preservatives (1973)

Hydrocarbon Solvent for Preparing Oil Borne Preservativ

Hydrocarbon Solvents for Oil Borne Wood Preservatives (

Hydrocarbon Types in Low Olefinic Gasoline by Mass Spec
Hydrocarbons by Coulometric Titration (1974) ANSI Z78.1

Hydrocarbons by Electrometric Titration (1972) ANSI Z11

Hydrocarbons by Gas Chromatography (1968) ANSI Z164.1

Hydrocarbons by Gas Chromatography (1972) ANSI Zl1.279,

Hydrocarbons by Oxidative Microcoulometry (1972T)

Hydrocarbons Fuels (1972) ANSI Z11.322

Hydrocarbons in Air Particulate Matter (1971)

NFLDP

ASTM

SAE

ASAE

SAE

ASAE

ASTM

FMS

ASAE

NFLDP

ASTM

JIC

$\mathrm{ACI}$

SAE

NEMA

NEMA

NEMA

ANSI

ASTM

ASTM

AATCC

ASTM

ASTM

AWPA

ASTM

ASTM

ASTM

ASTM

ASTM

610

20
48

873

109

129

NCCA TB-III.6

UL 873

TAPPI T464

AATCC 126

ASTM $\mathrm{E} 450$

E450

2.76

FMS $\quad 2.76$ UFC 2 ART13

UL 246

AACCH 28.32

ICBO UBCS24.19

ASTM C207

ASTM Cllo

St ASTM C25

AACCH 28-91

AACCH $56-20$

ASTM C186

CSI $\quad 14200$

ASTM D571

ASTM $\quad$ C430

ASTM C 349

ASTM Cl09

ASTM C348

ASTM C219

ASTM $\mathrm{C} 188$

ASTM C187

ASTM C186

ASTM C183

C511

ASTM C595

ICBO UBCS26-1

SAE J648A

ANSI B30.15

NFLDP T3.10.8.2

NFLDP T3.10.8.

NFLDP T3.10.8.6

NFLDP T3.10.8.7

NFLDP T3.10.8.5

NFLDP T3.10.8

NFLDP T2.9.2

NFLDP T3.9.17

NFLDP T2.9.1

NFLDP

T3.11.1

T2.9.3

D3119

J343A

S349

$\mathrm{J} 283$

$\mathrm{S} 316 \mathrm{~T}$

D2882

2.89

S201.4

T3.16.2

C 449

- 1

52-18

J281

$\mathrm{J} 514 \mathrm{E}$

MG 1.22

MG5.2 
Chromatography $(1968$ / 1972) lating Liquid) (1973/ xylenes, Solvent Naphthas, and Similar Industrial Aromatic ulfur Dioxide Content (Qualitative) of Industrial Aromatic Meth. of Test for Apparent Density of Industrial Aromatic or Calculation of Volume and Weight of Industrial Aromatic Meth. for Preparation and Standardization of Std. Spec. for Photographic Grade

Std. Spec, for Photographic Grade P-Aminophe nol Std. Spec. for Photographic Grade Hydroxylamine de) $(197 /$ Meth. of Analysis of real Chemistry) (1962) purification of Oil Systems for Lubrication and Control of and Cleaning of Oil Systems for Lubrication and Control of r the Design of Oil Systems for Lubrication and Control of antitites of Total Sulfur in Volatile Organic Liquids $\left(\mathrm{O}_{\mathrm{xy}}\right.$ Std. Meth. of Test for

Std. Meth. of Test for Carbon and hemistry) (1962) Electrometric Meth. of Analysis for (1962)

Colorimetric Meth. of Analysis for Sid. Test Meth. for Std. Meth. of Test for Test Meth. for

Test Meth. for Alkali in Bleach Baths Containing e) of Industrial Aromatic Hydroca/ Std. Meth. of Test for (1970) ANSI Z77.10

$$
\text { Std. for Acceptable Concentrations of }
$$
Rec. for Liquefied

(Air, Argon Carbon Monoxide, Deuterium Fluorine, Helium, Std. Meth. of Test for Total Sulfur in Natural Gas by $s$ in Flour or Starch Preparations Which Are Susceptible to odent Hairs in Flour (Cereal Chemistry) (1962)

ysis of Starch in Flour and Semolina (With Subsequent Acid
) onic Devices (1973)

973) al Meth for Determining $\mathrm{H}$ Content ontent of Corn Sugar (Crude and Refined), Syrups and Other ometric) Content of Corn Sugar (Crude and Refined) and Al n Syrup Unmixed, Finished Sugar and Other Clarified Starch - for Determining Acidity Content of Corn Syrup and Starch ash Content of Corn Syrup, Finished Sugar and Other Starch ing Fermentables Content of Corn Syrup, Sugars, and Starch of Corn Sugar (Crude and Refined), Syrup, and Other Starch of Corn Sugar (Crude and Refined), Syrups and Other Starch of Corn Sugar (Crude and Refined), Syrups and Other Starch Determining Dextrose Content of Corn Syrup and All Starch d Chromatography) Content of Corn Syrup, Sugar, and Starch g Dextrose Equivalent Content of Corn Syrup and All Starch ecific Rotation Content of Corn Syrup, Dextrose and Starch chromatography) Content of Corn Sugar, Starch Molasses and corn Sugar (Crude and Refined), Dextrose, Syrup and Starch of Corn Sugar (Crude and Refined), Syrup and Other Starch egta-Titrimetric) Content of Corn Syrup and Other Starch alytical Meth. for Determining Iron Content in Corn Starch

n Syrup Unmixed, Finished Sugar and Other Clarified Starch ine (Enology) (1972) Tent.

of Test for Specific or API Gravity of Liquid Asphalts by Guide Spec. for Platforms and Stage Lifts (Hydrualic and

Chemical Ethyl (E,E)3,7,11-Trimethyl-2,4-Dodecadienoate ydroquinone $\mathrm{C}_{6 ;} \mathrm{H}_{4}(\mathrm{Oh})_{2}$ (Para Dihydroxbenzene, Quinol, nol, Hydroquinol) (197/

Std. Spec. for Photographic Grade 72) Std. Meth. of Test for Methyl Ether of res (1972)

Std. Meth. of Test for Methyl Ether of Std. Meth. of Test for 2A) nce of Textile Fabrics: water (1967) Std. Meth. of Test for (1971) for Preparation and Standardization of Sodium or Potassium Std. Analytical Meth. for Determining Sodium Std. Spec. for Photographic Grade Ammonium Std. Analytical Meth. for Determining

Starch Ether/ Std. Analytical Meth. for Determination of ytical Meth. for Determination of Hydroxyalkoxyl Groups in mmonium Chloride) (197/ Std. Spec. for Photographic Grade xylammonium Sulfate) ( $\quad$ Std. Spec. for Photographic Grade raphic Grade Hydroxylamine Hydrochloride, $\left(\mathrm{Nh}_{2} \mathrm{OH}\right.$. $\mathrm{Hci}$ ) ic Grade Hydroxylamine Sulfate, $\left(\mathrm{Nh}_{2} \mathrm{Oh}\right)_{2} \cdot \mathrm{H}_{2} \mathrm{SO}$

determination of Hydroxyalkoxyl Groups in Hydroxyethyl and d Material (1/ ase Products (1973) Std. Meth. of Test.

Hydrocarbons in Monocyclic Aromatic Hydrocarbons by Gas Hydrocarbons in the Atmosphere by Gas Chromatrography ( Hydrocarbons (Askarels) for Capacitors (Electrical lnsu

Hydrocarbons (Askarels) for Transformers (1973)

Hydrocarbons (1947) ANSI Z78.9

Hydrocarbons (1971) ANSI Z78.13

Hydrocarbons (1972)

Hydrocarbons (1973) ANSI Z106.1

Hydrochloric Acid Solution (Cereal Chemistry) (1962)

of Benzene, Toluene,

ASTM

Hydrochloric Acid Solution (Cere

Hydrochloride $\mathrm{HOC}_{4} \mathrm{H}, \mathrm{Nh}, \mathrm{HCI}(1972)$

Hydrochloride, $\left(\mathrm{Nh}_{2} \mathrm{OH}\right.$. Hci) (Hydroxylammonium Chlori

Hydrocyanic Acid Residues in Grains and Dried Foods (Ce

Hydroelectric Equipment (1971)

Hydroelectric Equipment (1971)

Hydroelectric Equipment (1972)

Hydrogen Combustion Meth.) (1972)

Hydrogen Embrittlement of Copper (1973)

Hydrogen in the Analysis Sample of Coal and Coke (1973)

Hydrogen in Water (1960)

Hydrogen Ion Activity $(\mathrm{PH})$ in Flour and Bread (Cereal $\mathrm{C}$

Hydrogen lon Activity (PH) in Flour (Cereal Chemistry)

Hydrogen lon Concentration of Dry Adhesive Films (1970)

Hydrogen Permeance of Rubber Coated Fabrics (1973)

Hydrogen Peroxide in Bleach Baths (1972) ANSI L14.145

Hydrogen Peroxide (1972) ANSI L14.129

Hydrogen Sulfide and Sulfur Dioxide Content (Qualitativ

Hydrogen Sulfide in Natural Gas (Methylene Blue Meth.)

Hydrogen Sulfide (1972)

Hydrogen Systems (Fire Protection and Safety) (1973)

Hydrogen, Krypton, Methane, Neon, Nitric Oxide, Nitroge

Hydrogenation (1972) ANSI Z77.11

Hydrolysis by Alpha Amylase) (Cereal Chemistry) (1969)

Hydrolysis Meth. of Analysis for Insect Fragments and R

Hydrolysis) (Cereal Chemistry) (1962)

E Meth of Anal

Hydrolytic Stability of Elastomeric Volcanizates (1972T

Hydrolytic Stability of Plastic Encapsulants for Electr

Hydrolyzable Chlorine Content of Liquid Epoxy Resins (1

Hydrolyzates and a Variety of Other Materials (1952)

Hydrolyzates and All Starches and Sugars, and to Most O

Hydrolyzates from Corn and Grain Sorghum Starch (1960)

Hydrolyzates Solutions Derived from Corn or Grain Sorgh

Hydrolyzates (Potassium Chromate Indicator) (1954)

Std. Analytical Meth

Hydrolyzates (1952)

Hydrolyzates (1952)

Hydrolyzates (1957)

Hydrolyzates (1961)

Hydrolyzates (1961)

Hydrolyzates (1962)

Hydrolyzates (1967)

Hydrolyzates (1967)

Hydrolyzates (1968)

Hydrolyzates (1971)

Hydrolyzates (1971)

Hydrolyzates (1971)

Hydrolyzates (1973)

Hydrolyzates (1973)

Meth for Determining Calcium (

Hydrolzates Including Syrups and Sugars, and Most Wet M

Hydrolzates (Silver-Silver Chloride Indicator) (1971)

Hydrometer Meth. of Analysis for Alcohol (Ethanol) in W

Hydrometer Meth. (1973)

Hydropneumatic) for Lowering Personnel and Material (19

Id. Meth

Hydroprene (1973)

Hydroquin ol) (1972)

lor Common Name for the Pest Control
/D. Spec. for Photographic Grade H

(Para Dihydroxbenzene, Oui

Hydroquinone in Colorless Monomeric Acrylate Esters (19

Hydrostatic Collapse Strength of Hollow Glass Microsphe

Hydrostatic Compressive Strength of Syntactic Foam (197

Hydrostatic Pressure Test (1971) ANSI L14.265

Hydroxide Ion in Industrial Water and Industrial Waste

Hydroxide Solution (Cereal Chemistry) (1962)

Hydroxide Solutions Content of Reagents and Indicators

Hydroxide, Nh, $0 \mathrm{H}$ (Aqueous Ammonia) (1972)

Hydroxyalkoxyl Content of Corn Starch and Ethers (1967)

Hydroxyalkoxyl Groups in Hydroxyethyl and Hydroxypropyl

Hydroxyethyl and Hydroxypropyl Starch Ethers (1973)

Hydroxylamine Hydrochloride, $\left(\mathrm{Nh}_{2} \mathrm{OH}\right.$. Hci) (Hydroxyla

Hydroxylamine Sulfate, $\left(\mathrm{Nh}_{2} \mathrm{Oh}\right)_{2} \cdot \mathrm{H}_{2} \mathrm{SO}_{4}$ (Hydro

D. Spec. for Photog

(Hydroxylammonium Sulfate) (Oxammonium Sulfate) (1973)

Hydroxypropyl Methylcellulose (1972)

Hydroxypropyl Starch Ethers (1973)

/Lytical Meth. for

(Hydrualic and Hydropneumatic) for Lowering Personnel an

Hygroscopic Properties of Fire Retardant Wood and Its B

Ice Cream Freezers and Soda Fountain Units (1973) Std. for Safety for Automatic

ASTM

ASTM

CR

$\mathrm{CR}$

CR

CR

CR

CR

CR

$\mathrm{CR}$

CR

CR

CR

\section{CR} CR

CR

ASE

ASTM

CSI

$\mathrm{AACCH} 70.20$

ANSI PH4.104

ANSI PH4.129

PH 4.187

$\mathrm{AACCH} \quad 60.20$

ASME LOS.5P1

ASME LOS-5D

ASTM D2747

ASTM B577

D3178

ASTM D 1588

AACCH $02-52$

$\mathrm{AACCH} \quad 02.51$

ASTM D1583

ASTM D815

AATCC 102

AATCC 98

ASTM D853

ASTM D2725

ANSI Z37.2

FMS LPD $7.19 \mathrm{~N}$

ICBO UFC 2 ART36

ASTM D3031

$\mathrm{AACCH} \quad 76-30 \mathrm{~A}$

$\mathrm{AACCH}$ 28-41

AACCH 76-10

ASTM D3137

ASTM F74

ASTM D1726

E48

E-32

F.26

F-14

E.14

E-2

E-28

F. 18

F-42

E-24

E. 63

E.26

E-58

F.50

F.52

F. 8

E-11

B-30

E. 15

D3142

14430

K62.136

PH4.126

PH4.126

ANSI PH125

$\begin{array}{ll}\text { ASTM } & \text { D3125 } \\ \text { ASTM } & \text { D3102 }\end{array}$

ASTM D2736

AATCC 127

ASTM D514

Meth. AACCH 70.70

CR R-20

ANSI PH4.232

CR C. 20

CR C.30 
calcium Chloride to Be Used for Dustlaying, Stabilization, ermination of Specific Volume of Creams, Cake Batters, and ng Code Std. for Douglas Fir; Larch; Hem-Fir; Ponderosa, Std. for Motion Picture Raw Stock Uniform Building Code Std. for Erection, Fabrication. Uniform Building Code Std. For Erection, Fabrication. scopic) Analysis of Mineral Filer and Co/ Rec. Pract for Lighting Std. for lnstrumentation Symbols and rod Size Combinations, Rod End Configurations, Dimensional e United States (Including the District of Colu Std. for Std. for Slow Moving Vehicle ket for Warning Lamp (Light) and Slow Moving Vehicle (SMV) rial Equipment Warning Lamp and Slow Moving Vehicles (SMV) Sid. for Fuel Assembly Meth. for Compression Wood d and Rough Items (1971) Std. for Clearance, Side Marker and Aerospace Std. Rec. Pract. for Vehicle Rec. Pract. for Passenger Car Vehicle Rec. Pract. for Boat Hull d Cartridges (1973) Std. for

1 127

using the ASTM Punched Card Index (/ aerospace Fluids (1970E) ints (1972)

Rec. Pract. for Collection and Std. Meth. for Test Meth. for Test Meth. for Tesi Meth. for Std. Rec. Pract. for Std. Meth. of Test for Std. Meth. for Code for Std. Rec. Pract. for Qualitative Std. Rec. Pract. for the specs. (1972) pecular Silicon Surface/

Rec. Pract. and Nomenclature for Rec. Pract, for Coding System for Std. for Air Brake Reservoir Performance and Std. for Manufacturer

quipment (Machinery) (/

1)

Rec. Pract. for Electrical Wiring Std. for Numerical Designation
Std. for Structure for the Std. for Structure for the

Test Meth. for Fiber Test Meth. for Fiber Line Code System for Welding Wire Color Code System for Welding Wire gines (Color Code) (1973) Requirements and Rec. Meth. of Test Meth. for Packaging Film and Construction Definitions of Terms Utsed in for Nuclear Power Generating Stations (1973) ANS1 N41.17 Pract. for Design and Construction of DieseI (Compression Std. for Engine Test Code-Spark Std. for Residential Controls Direct Electric tion Properties (Temperatures) of Plastics Using a Hot Air tion Properties Rec. Pract. for Vehicle Std. for Automatic Gas

Battery Materials Spec. (Vehicular, Storage Battery Spec. (Vehicular, iles, Commercial Vehic/ tion of and Test Formula for Isobutene-Isoprene Rubbers Determining the Actinity or Photographic Effectiveness of Std. for Gas Fired Meth. for Measurıng Brightness of Pulp (Diff use Std. for uipment (1971)

eth. for Determining Safety Times of Photographic Darkroom ain Products (Cer/ Insects, Rodent Hair, and Radiographic ratio (1971) ision (1973) d. Dimensions of Projectable Anamorphic Std. Dimensions of

or Unitized Microfilm Carriers (Aperture, Camera, Copy and n Unitized Microfilm Carriers (Aperture, Camera, Copy, and Std. Rec. Pract. for rms Relating to Carbon Paper and Inked Ribbon Products and Std. Dimensions of $35 \mathrm{~mm}$ Motion Picture Camera Aperture Std. Test Procedure for Thermal Evaluation of Oil

Std. Rec. Pract. for Laboratory Vulcanizates Resulting from tative Meth. of Test for Weight Loss of Sheet SteeI During Std. Meth. of Test for Effect of Cyclic Loss of Adhesion, or Other Failure/ roxide ( $\mathrm{N}_{3} \mathrm{O}$ ) (1972) Std. Meth. for Water Std, Meth of Static st Meth. for Density and Moisture of Chips (Presoak Before of Extruded Poly (Vinyl Chloride) Plastic Pipe by Acetone Sid. Meth. of Test for Adhesion After

Test Meth for W/ater Resistance of Textile Fabrics:

(1972) ANSI J2.19 Std. Meth. of Test for und Pendulum (1972) ANSI J2.19 ansi $\mathrm{J} 2.31$ zed Steel (Coil Coating) (1/ Std. Meth. of Test for Test Meth for Measurment or lce Removal, Acceleration of Concrete, Curing of Concre Icings (Cereal Chemistry) (1962)

Idaho White, Sugar, and Lodgepole

Identification and Labeling (1973)

Identification and Painting of Structural Steel (1973)

Identification and Qualitative (Including Optical Micro

Identification Code (1971)

Identification Code (1973)

Identification Code, and Mounting Dimensions for $3 / 4,1$

Identification Codes and Abbreviations for States of Th

Identification Emblem (1968) ANSI B114.1, SAE J943A

Identification Emblem (1972)

Identification Emblem (1972) SAE J725

Identification in Nuclear Power Plants (1972)

Identification in Pulpwood (1972)

Identification Lamps (Light) (1972)

Identification Marking Meths. on Finished, Semi Finishe

Identification Number Systems (1972)

Identification Number Systems (1972)

Identification Numbers (1973)

Identification of Air Purifying Respirator Canisters an

Identification of Corrosion Products (1973)

Identification of Fibers in Textiles (1972)

Identification of Fibers in Textiles (1972) ANSl L14.13

Identification of Finishes in Textiles (1973) ANSI L14.

Identification of Lignin (Paper Chromatogram) (1973)

Identification of Material by Absorption Spectroscopy,

Identification of Metallic and Fibrous Contaminants in

Identification of Oils and Oil Acids in Solvent Type $\mathrm{Pa}$

Identification of Piping Systems Content (1973)

Identification of Polymers in Emulsion Paints (1973)

Identification of Std. Electrical Steel Grades in ASTM

Identification of Structures and Contaminants Seen on S

Identification of Tube, Pipe, and Hose Fittings (1972)

Identification Requirements (1972)

Identification Symbols for Fasteners (1973)

Identification System for Industrial and Construction E

Identification System) of Modular Grid Coordinates (197

Identification (Code) of the Countries of the United St

Identification (Construction and Industrial Equipment)

Identification (Various Stains) (Pulp and Paper) (1973)

Identification (WiIson Stain) (Pulp and Paper) (1973)

Identification (1973)

Identification (1973)

Identifying $A C$ and DC Electrical Wiring on Boats and En

Identity Tests (Paper) (1973)

IEEE Nuclear Power Generating Station Stds. (1972)

IEEE TriaI Use Guide for Class IE Control Switchboards

Ignited) Inboard Liquid Cooled Marine Engines and Auxil

lgnition and Diesel (1971)

Ignition Control Systems for Gas Fired Equipment (1972)

Ignition Furnace) (Fire Safety) (1973)

Ignition Properties (Temperatures) of Plastics Using a

Ignition Switch (1971)

lgnition Systems and Components (1971)

Ignition, Lighting and Starting) (1972)

Ignition, Lighting, and Starting) for Passenger Automob IIR) (1973)

Illuminants (1972)

Illuminating Appliances (1971)

Illumiration and Zero Deg. Observation) (1972)

Illumination Tests for Photographic Still Projection Eq

Illumination (1971)

Illustration and Classification in Cereal Grains and $\mathrm{Gr}$

Image Area on $5 \mathrm{~mm}$ Motion Picture Film (2.35:1) Aspect

Image Areas and Mounts for Slides and Opaques for Telev

Image Cards) (1972)

Image Cards) (1973) ANSI PH5.12

for Dimensions $\mathrm{F}$

Image Evaluation of Electrostatic Business Copies (1972

Images Made Therefrom (1972A)

Images (Photography) (1974)

Immersed Distribution Transformers (1972)

Immersion Controls (1972)

Immersion Corrosion Test. of Metals (1972)

Immersion in Liquids (1972)

Immersion in Sulfuric Acid Solution (1971) ANSI Z167.25

Immersion of Syntactic Foam at Pressure (1972)

Immersion Test for Resistance to Blistering, Wrinkling,

Immersion Test. of Unstressed Materials in Nitrogen Tet

Immersion) (Pulp and Paper) (1973)

Immersion) (1967) ANSI B 72.9

Impact of Preformed Sealing Tapes (1973)

/Eth. of Test for Quality

Impact Penetration Test (1971) ANSI L14.71, ASTM D583

Impact Resilience and Penetration of Rubber by the Rebo

Impact Resilience of Rubber by Vertical Rebound (1974)

Impact Resistance of Painted Aluminum, Steel and Gavani
ASTM

$\mathrm{AACCH}$

ICBO

ANSI

ICBO

TAPPI

SAE

ISA

NFLDP

ANSI

ASAE

SAE

ASAE

ANSl

TAPPI

SAE

SAE

SAE

SAE

ABYC

ANSl

NACE

ASTM

AATCC

AATCC

TAPP

ASTM

ASTM

ASTM

FMS

ASTM

ASTM

ASTM

SAE

$\mathrm{SAE}$

IFl

SAE

ANSI

ANSI

SAE

TAPP

TAPP

SAE

SAE

ABYC

TAPP

IEEE

IEEE

$A B Y C$

$S A E$

NEMA

ICBO

ICBO

SAE

ANSI

BCI

BCI
ASTM

for Evalua

ANSI

ANSI

TAPPl

ANSI

Std. M ANSI

$\mathrm{AACCH}$

ANSI

ANSI

ANSl

NMA

ASTM

ASTM

ANSI

IEEE

NEMA

ASTM

ASTM

ASTM

ASTM

NCCA

ASTM

Te TAPPI

ASTM

ASTM

AATCC

ASTM

ASTM

D98 72.10

UBCS25-4 PH22. 184 UBCS27.2 421 759B 55.1 T3.6.11 
test) (1972)

test) $(1972)$

Materials (1973)

$s(1970)$

Means of a Tup (Falling Weight) /

Std. Meth. of Test for

Std. Meth of Test for

Std. Meth. of Test for Drop

Std. Meth. of Test for Uniform Building Code Std. for Std. Test. Meth. for

materials (1973)

ansi MH12.4, TAPPI T801

Std. Meth. of Low Temperature Std. Meth. of Incline Rec. Pract. for Instrument Panel Laboratory

Std. Meth. for Notched Bar Std. Meth. of

t. for Instrumentation for Motor Vehicle and lts Component of Test for Formability of Attached Organic Coatings with for Brittleness Temperature of Plastics and Elastomers by the Design, Construction, and Operation of Class Hi (High esion on All Metals by Cross Hatch Tape Test After Reverse n / Std. Meth. for Measurement of the Specific Electrical Std. Definitions of Terms Relating to Surface $s$ of Terms Relating to Erosion of Solids by Cavitation and er Soluble Gaseous Fluorides in the Atmosphere (Filter and Test. for Biological Compatibility of Metals for Surgical rec. Pract. for Static Bend Test. of Bone Plates (Surgical rec. Pract. for Static Bend Test. of Nail Plates (Surgical ic Bend and Torsion Test. of Intramedullary Rods (Surgical ecs. (1972) Rec. for Tractor and Farm

ng, Drive, Planter (Drill) Press, and Gage Wheel, Tractor, ull Shielding of Power Drive Lines for Agricultural (Farm)

Quick Attaching Coupler for Thre/ Std. for Attachment of
Std. for 3 Point Free Link Attachment for Hitching Std. for 3 Point Free Link Hitch Attachment of

Std. for One Point Tubular Sleeve Attachment for Hitching

c Motors to Agricultural Tractors and Trailing Type (Farm)

gricultural Tractors and Trailing Type Agricultural (Farm)

lay) for Television Receiving Applia/ Std. for Safety for

porosity of Sintered Powder Metal Structural Parts and Oil Std. Meth. of Test for Moisture Content of O Graphite Fiber Tape and Sheet, Epoxy Resin Meth. for Wax in

luble Wood Preservatives by Using Wood Specimens Uniformly Std. Spec. for Iron Base Sintered Bearings (Oil Std. Spec. for Copper Base Sintered Bearings (Oil dul/ Spec. for Graphite Fiber Tape and Sheet, Epoxy Resin dul/ Spec. for Graphite Fiber Tape and Sheet, Epoxy Resin ) Cloth, Type E Glass, B Stage Epoxy Resin Cloth, Type E Glass, B Stage Polyester Resin pract. Design, Installation, Operation, and Maintenance of alysis of Crude Protein in Bread, Wheat and Other Grains/ Feeds and Feedstuffs (Cereal Chemistry) (1962) walls (1969)

72) ANSI G35.7 Std. Spec. for Structural Carbon Steel Plates of Std. Spec. for Carbon Steel Plates with Rec Pract. for Vendor Product Meth. of Analysis of Phosphates in Flour Meth. for Preparation of Sample: Yeast Foods and Flour itative Meth. of Analysis of Bromates and Iodates in Flour itative Iodide Test Meth. for Ammonium Persulfate in Flou ing and Spreading Agricultural Liquids and Slur/ Rec. for kdown Voltage of Insulating Oils of Petroleum Origin Under Std. Techniques for Switching

Circuit Breakers Rated $\mathrm{O} /$ Std. Requirement for Switching Std. Meth. of Test for Butadiene Purity and Hydrocarbon meth. of Test for Consumption of Potassium Permanganate by test Meth. for Detection and Estimation of Water Insoluble A37. 19 Std. Meth. of Test for Organic h. of Test for Vegetable Matter and Other Alkali Insoluble Std. Meth. of Test for Deposit Forming and Installation of Permanently Installed Fuel Systems for ract. for Determining and Stating the Horsepower Rating of or Design and Construction of Diesel (Compression Ignited) ) Direct Drive Transmission (197/ Rec. Pract. for Marine nd Std. for the Design and Construction of Gasoline Fueled k65.117 on) (1973) Std. Meth. of Test for Manual of Hazardous Chemical Reactions Std. for R 40 Bulb (Hard Glass) Mogul Screw Base I Uniform Fire Code: General Precautions for Prevention Std. Spec. for Refractories for e of Heating, Ventilating, Cooling, Refrigeration Systems, Safety Std. for Domestic Type

cedar, Northern White/ Std. for Preservative Treatment of (1968) ANSI MH12.4, TAPPI T801 Std. Meth, of ials (1973) A/ Std. Meth. of Test for Liquid Contaminant, Std. for Control Valve Sizing Equations for
Impact Resistance of Pipeline Coatings (Falling Weight Impact Resistance of Pipeline Coatings (Limestone Drop Impact Resistance of Plastic Film and Sheeting (1972) Impact Resistance of Plastics and Electrical Insulating Impact Resistance of Polyethylene Blow Molded Container Impact Resistance of Rigid Plastic Sheeting or Parts by Impact Sound Insulation (1973)

Impact Strength of Adhesive Bonds (1972) ANSI Z197.14

Impact Test for Fabrics Coated with Flexible Polymeric

Impact Test for Shipping Containers (Packaging) (I968) Impact Test Procedure-Head Area (1971)

Impact Test. of Metallic Materials (1972)

lmpact Testing of Cast Irons (1972)

Impact Tests (1971)

Impact Wedge Bend Apparatus (I973)

Impact (1973)

Impact) Shock Test. Machine for Lightweight Equipment

Impacting (Coil Coating) (1974)

Impedance of Electrical Grade Magn

(1973)

Impingement by Liquid or Solid Particles (1973)

Impinger Meth.) (1973)

Lection or Particulate and $W$ at

mplants (1972)

mplants) (1973)

Implants) (1973)

Implants) (1973)

Std. Rec. Pract. for Stat ASTM
Implants) (1973)

Implement, and Log Skidder (1974)

Implements and Tractors (1972) SAE J955

/D lndustrial Steeri

mplements to Agricultural Wheel Tractors Equipped with

Implements to Agricultural Wheel Tractors (1972) SAE J7

Implements to Agricultural Wheeled Tractors (1972)

Implements to Lawn and Garden Riding Tractors (1972)

Application of Remote Hydrauli

Implements (1971) SAE J716

TOte Control Cylinders to a

Implosion Protected Cathode Ray Tubes (Picture and Disp

Impregnated Bearings (1973) ANSI H9. 14

/Nterconnected

Impregnated for Hand Layup (1973)

Impregnated Papers (1972)

Impregnated (1970)

Impregnated) (1970) ANSI G63.4

Impregnated) (1973) ANSI H9.27

Impregnated, G70,000 (483) Tensile, 36,000,000 (248) Mo

Impregnated, G70,000 (483) Tensile, $36,000,000$ (248) Mo

Impregnated, 181 Style Fabric, Self Extinguishing (1973

Impregnated, 181 Style Fabric, Self Extinguishing (1973

Impressed Current Deep Groundbeds (1972)

Improved Kjeldahl Meth. for Nitrate Free Samples for an Improved Kjeldahl Meth. of Analysis of Crude Protein in Improved Std. Designs for Pressure Treated Timber Crib Improved Toughness (1973)

Improved Transition Properties for Pressure Vessels (19 Improvement Program for Quality Control (1969)

Improvers (Cereal Chemistry) (1962)

Improvers (Cereal Chemistry) (1962)

Improvers (Cereal Chemistry) (1962)

Improvers (Cereal Chemistry) (1962)

Improving Safety on Enclosed Mobile Tanks for Transport

Impulse Conditions (1974)

Impulse Testing (1972) ANSI C68.2

lof Test for Dielectric Brea

Impulse Voltage Insulation Strength for AC High Voltage

Impurities by Gas Chromatography (1973)

Impurities in Deuterium Oxide (1968) ANSI N154

Impurities in Refined Phenol by Cloud Point Depression

Impurities in Sands for Concrete (1973) A ASHO T21, ANSI

Impurities in Scoured Wool (1972) ANSI L14.195

Impurities in Steam (1971) ANSI Z111.5

Inboard and Outboard Powered Boats (1973)

Inboard Gasoline Engines (Boats) (1972)

Inboard Liquid Cooled Marine Engines and Auxiliaries (B

(Inboard) Engine Mountings (Installation and Replacement

Inboard, Liquid Cooled Marine Engines (Boats) and Auxil

Incandescence Resistance of Rigid Plastics (1974) ANSI

(Incandescence, Fire, Explosion, and Detonation Protecti Incandescent (1966)

(Incinerator and Open Burning, Combustible and Flammable

Incinerators and Boilers (1972) ANSI A1I 1.8

Incinerators and Other Misc. Heat Producing Appliances)

Incinerators (1973)

Incinerators (1973)

Incised Pole Butts by the Thermal Process (Western Red

Incline Impact Test for Shipping Containers (Packaging)

(Inclined Plane Meth.) (1973)

Inclined Plane Tracking and Erosion of Insulating Mater

Incompressible Fluids (1972)
Wat ASTM

Std. ASTM

Std. ASTM

ASTM

ASTM

ASTM

ASTM

ICBO

ASTM

ASTM

ASTM

ASTM

SAE

ASTM

ANS]

NCCA ASTM

TM

TRA

ASAE

ASAE

SAE

ASAE

ASAE

ASA

ASTM

ASTM

SAE

TAPPI

NWMA

ASTM

$\mathrm{SAE}$

SAE

SAE

SAE

Rec. NACE

A ACCH

$\mathrm{AACCH}$

AWPI

ASTM

ASTM

ASQC

$\mathrm{AACCH}$

A SA

ASTM

IEEE

IEEE

ASTM

Std. ASTM

ASTM

ASTM

/D. Met ASTM

ASTM

struction $\mathrm{ABYC}$

Rec. P ABYC

$A B Y C$

SAE

ASTM

FMS

ANSI

ICBO

ASTM

ICBO

ANS

UL

AWPA

ASTM

ASTM

ISA

D3099

D256

D2463

D3029

UBCS35.2

D 880

J921 B

J211A

D3281

TB.11.16

D32l.

D3267

F36]

F382
F384
F383 
Std. for Control Valve Capacity Test Procedure for Meth. of Test for Knoop

Std Analytical Meth. for Determining Refractive Test Meth. for Flammability of Plastics Using the Oxygen (1974) ANSl Z78.15 Std. Meth. of Test for Bromine ofing and Waterproofing Materials (1972) ion (1972) ANSI Z11.248 Std. Meth. of Test for Bromine rmal Oils and Liquid Fats (Cereal Chemistry) (1967)

Rec. for Flash Point

Uniform Building Code Std. for Expansion rogen in Ground Soybeans, Whole or G/

Nitrogen Solubility tein in Ground Soybeans, Whole Or/ Protein Dispersibility Std. Meth. of Measuring Plasticity Retention

al by Absorption Spectroscopy, Using the ASTM Punched Card nings, and Other Fats ( $1 / \quad$ Dilatometric Meth. of Solid Fat
alding Std. for Precision

Std. for Carbide Seats Used with Std. for Carbide Chip Breakers Used in or the Thermal Evaluation and Establishment of Temperature ue Materials (Color Measurement O/ Std. Meth. of Test for Test Meth. for Top Side of Filled Paper as rol of Air Conditioning, Hea/ Safety Std. for Temperature Std. for Electrical

1973)

(1973) ANSI B156.1 Std. for Requirements for Electrical Analog Safety Std. for Safety Std. for

Rec. Pract. for Mobile Construction Type Crane Overload Meth. of Calibration Instruments for Verifying Time Load of Paper, Paperboard, and Other Sheet Materials by the Dry Std. for Symbols for Machine Tool Meth. for Preparation of nd Other Clarified Starch Hydrolyzates (Potassium Chromate her Clarified Starch Hydrolzates (Silver-Silver Chloride Std. Analytical Meth. for Determining Cresol Red
1) Std. Analytical Meth. for Determining Methyl Orange Std. Analytical Meth. for Determining Bromophenol Blue Std. Analytical Meth. for Determining Methylene Blue Std. Analytical Meth. for Determining Phenolphthalein Std. Analytical Meth. for Determining Starch

m (LP) Gas (/ Std. for Safety for Liquid Level Gauges and
Safety Std. for Liquid Level Gauges and
mai/ $\quad$ Rec. Pract. and Std. for Flammable (Combustible) Gas

Std. for Safety for Liquid Level Gauges and
Safety Std. for Liquid Level Gauges and
mai/ $\quad$ Rec. Pract. and Std. for Flammable (Combustible) Gas mai/ Rec. Pract. and Std. for Flammable (Combustible) Gas I Determining Starch Indicator, $1 \%$ Content of Reagents and etermining Sulfuric Acid Solutions Content of Reagents and rmining Sodium Hydroxide Solutions Content of Reagents and rmining Cresol Red Indicator, $0.1 \%$ Content of Reagents and mining Sodium Thiosulfate Solution Content of Reagents and ining Methylene Blue Indicator, 1\% Content of Reagents and ning Std. Arsenious Oxide Solution Content of Reagents and ning Methyl Orange lndicator, $0.1 \%$ Content of Reagents and ning Phenolphthalein Indicator, $1 \%$ Content of Reagents and ing Bromophenol Blue Indicator, $1 \%$ Content of Reagents and ng Potassium Permanganate Solution Content of Reagents and (Cereal Chemistry) (1962)

iation (1972)

nd Gas (1972)

astes (1973)

Performance Spec. for Direct and Spec. for cal and Spectrochemical Analysis of Nuclear Grade Silver 3.90 niform Pract for Fee Determination for Division Services Sid. for Safety for Control Units for Std. for Gas Hose Connectors for Portable torage Up to $30 \mathrm{Ft}$. and Shelf Storage $\mathrm{U}_{\mathrm{p}}$ to $15 /$ Rec, for

Safety Std. for Electric Cord Connected Fire Hazards Anf Protection Rec. for

ingle and Multiple Station, Mechanically Operated Type for r Safety for Battery Powered Emergency Lighting Equipment orbic Acid (Cereal Chemistry) (1962)

Std. Flue Gas Densities for Forced and

Loss Prevention Data on High Frequency Heating Equipment co-4.3Mo-3.0 Ti-1.4A ) Consumable Electrode or Vacuum co-4.3Mo-3.0Ti-1.4A , Consumable Electrode or Vacuum co-4.3Mo-3.0 Ti-1.4A 1, Consumable Electrode or Vacuum Std for

Assignments for Alternating-Current Integral Horsepower

Corrosion Resistant, $12 \mathrm{Cr}-8.5 \mathrm{Ni}-2.0 \mathrm{Cu}-1$. $1 \mathrm{Ti}, \mathrm{Vacuum}$ uide for Meth. of Power Factor Measurement for Low Voltage Std for lron-Core Power Filter

road, Agricultural and Log Skidder, Truck, Bus, Bead Seat, Std. for lnstallation of Gas Piping and Equipment on

f Vinyl Asbestos and Asphalt Tile Floors in Institutional, Safety Std. for High Pressure Gas Manifolds for 972) ANSI C114.1 Rec. Pract. for Grounding of ec. Pract. for Electrical Wiring Identification System for
Incompressible Fluids (1972)

Indentation Hardness of Glass (1972T)

Index Content of Corn Syrup, Sugar and Dextrose (1956) lndex Meth. (1974) ANS1 K65.110

Index of Aromatic Hydrocarbons by Coulometric Titration Index of Meth. for Test. Elastomeric and Plastomeric Ro Index of Petroleum Hydrocarbons by Electrometric Titrat Index of Refraction, with Abbe Refractometer, in All No lndex of Trade Name Liquids (NFPA 325A) (1974) lndex Test for Soil (1973)

Index (NSI): Meth for Determination of Dispersible Nit Index (PDI): Meth. for Determination of Dispersible Pro lndex (PRI) of Natural Rubber (1973)

lndex (1972)

Index (1973)

Index ( 50 or Less at 10 Deg.) of Margarine Oils, Shorte

lndexable Insert Holders (1973)

Indexable Inserts for Clamp Type Holders (1973)

Indexable lnserts for Clamp Type Holders (1973)

Indexes of Solid Electrical lnsulating Materials (1972)

Indexes of Whiteness and Yellowness of Near White, Opaq

lndicated by Silver Coin Test (1973)

Indicating and Regulating Electrical Equipment for Cont

Indicating Instrument (Meter) Relays (1972)

Indicating lnstruments (1972)

Indicating Pressure Gauges for Compressed Gas Service (

Indicating Pressure Gauges for Fire Protection Service

Indicating System Test Procedure (1971)

Indication of Machines (1974) ANSI Z115.2

Indicator Meth. (1973) TAPPl T433

Indicator Plates or Control Buttons (1972)

Indicator Solutions (Cereal Chemistry) (1962)

Indicator) (1954)

/Orn Syrup Unmixed, Finished Sugar a

Indicator) (1971)

Yrup Unmixed, Finished Sugar and Ot

Indicator, 0.1\% Content of Reagents and Indicators (197

Indicator, $0.1 \%$ Content of Reagents and Indicators (197

Indicator, $1 \%$ Content of Reagents and Indicators (1971)

Indicator, 1\% Content of Reagents and lndicators (1971)

Indicator, 1\% Content of Reagents and Indicators (1971)

Indicator, 1\% Content of Reagents and Indicators (1971)

Indicators for Anhydrous Ammonia and Liquefied Petroleu

Indicators for Flammable Liquids (1972)

Indicators lncluding Their lnstallation, Operation and

Indicators (1971)

Indicators (1971)

Std. Anaty

Std Analytical Meth.

Indicators (1971)

Indicators (1971)

Indicators (1971)

Indicators (1971)

Indicators (1971)

Indicators (1971)

Indicators (1971)

Indicators (1971)

Indicators (1971)

Indicators (1971)

Sid. Analytical Meth. for Dete CR

Std. Analytical Meth. for Dete CR

Std. Analytical Meth. for Deter CR Std. Analytical Meth. for Determ CR

Std. Analytical Meth. for Determi CR

Std. Analytical Meth. for Determi CR

Std. Analytical Meth. for Determi CR

Std. Analytical Meth. for Determin CR

Sid. Analytical Meth. for Determini CR

Indices of Refraction of Sucrose Solutions at $20 \mathrm{Deg} . \mathrm{C}$

Indirect Reading Pocket Dosimeters for X-And Gamma Rad

Indirect Type Oil Field Heaters Used in Producing Oil a

Indirect $W$ aste Piping, W et Vented Systems and Special W

Indium-Cadmium Alloys (1974)

Std. Meth. for Chemi

ndividuals, Clubs, and Exhibition Charges) (1974)

Indoor Fire Protective Signaling Systems (1972) ANSI C3

lndoor Gas Fired Appliances (1971)

Indoor General Storage (Solid, Palletized and Bin Box S

Indoor Rifle and Pistol Ranges and Bullet Stops (1973)

Indoor Signs (1971) ANSI C33.35

Indoor Storage of Roll Paper (1974)

Indoor Use (1973)

Indoor) (1973)

Indophenol-Xylene Extraction Meth. of Analysis of Asc

lnduced Draft Centrifugal Fans (1966)

(lnduction and Dielectric) (1973)

Induction Melted 1825-1900 Deg. F (996.1-1037.8 Deg. C)

Induction Melted, $1975 \mathrm{Deg}$. F (1079.4 Deg. C) Solution

lnduction Melted, $1975 \mathrm{Deg}$. F (1079.4 Deg. C) Solution,

Induction Motors (Large A pparatus) (1972)

Induction Motors (1972)

Std. for Frame NEMA

Induction Plus Vacuum Consumable Electrode Melted Solut

Inductive Test Currents (1972) ANSI C37.26

Inductors for Electronic Equipment (1973) ANSI C83.92

lndustrial and Bicycle Tires (1974)

Industrial and Certain Other Premises (1972)

Industrial and Commercial Buildings (1971)

Industrial and Commercial Gas Cylinders (1972)

EIA

Industrial and Commercial (Electrical) Power Systems (1

Industrial and Construction Equipment (Machinery) (1971

ISA

ASTM

ASTM

AS'TM

A ACC

FMS

UL

UL

API

ICBO

ASTM

PSA

UL

ANSI

FMS

NRA

UL

FMS

FMS

SAE

SAE

SAE

ICBO 7.42N

$\mathrm{AACCH} 46.23$

$\mathrm{AACCH} \quad 46.24$

ASTM D3194

E204

SA 1193

ANSl B 94.45

ANSl B94.46

ANSI B94.47

IEEE 98

ASTM E313

UM-533

UL $\quad 873$

$\begin{array}{ll}\text { NEMA } & 112 \\ \text { ANSI } & \text { C } 39 .\end{array}$

404

393

ASTM D779

ANSI B5.46

$\begin{array}{ll}\mathrm{AACCH} & 70.25\end{array}$

E-14

E-15

R. 60

R-70

R.55

R-65

R-75

R-80

L 565

UL $\quad 180$

A 14

R-10

R-80

R-30

R. 20

R. 60

R.25

R-65

R-5

R-70

R-75

R-55

R-15

AACCH 68.61

ANSI N13.5

UPC $* 1-6$

C760

11

864

$\mathrm{Z} 21.2$

$+1$

48

8-21

539

924

AACCH 86-10

AMCA 2409

AMS5707D

AMS5738C

AMS5709B

MGl-20

MGl3

AMS5617A

330

RS197A

Z83. 1

$* 10$ 
Std. Rec. Pract. for Sensory Evaluation of Industrial and Institutional Food Purchases (1972) Spec. for Welding Std. for Generators (1972) Std. Spec. for Hand Operated Chain Hoists for
Vehicles. Motorboats, Tractors, Automotive and 3 for Motor Vehicles, Motorboats, Tractors, Automotive and drogen Sulfide and Sulfur Dioxide Content (Qualitative) of Std. Meth. of Test for Apparent Density of Std. Meth. for Calculation of Volume and Weight of Uniform Fire Code:

Plywood Construction Systems (Specs.) for Commercial and and Asphalt Tile Floors for Commercial, Institutional and Std. for Double Race or Bi-Level Swivel and Rigid Std. Sizes for Std. Sizes for Std. for Std. for Std. for

tive Functions and Process Input-Output (1972/ er Assemblies (Electronics) (1973) (1973)

ions, Class I, Groups A-D, Class II, Gro/ ipment (1974)

Circuit Devices and Assemblies (1973) (1973)

ng and Controlling Devices (1973)

(1972)

(1972)

Safety Std. for

Std. for

Std. for

Std. for

Std. for

Sid. for

Std. for

Std. for

Std. for

Std. for

Std. for

Std. for

Std. for

Std. for

Std. for

Std. for

ng and Theory (1972)

and Control (1972)

d Meth. (1972)

(1973)

Hydraulic Std. for

r Mounting Brackets and Socket for Agricultural (Farm) and

$r$ Instrument Face Design and Location for Construction and and Temporarily Affixed Safety Signs for Construction and and Container and Filler Identification (Construction and for RemovaI of Water Formed Deposits (Chemical Cleaning of Rec. Pract. for Dilution Ventilation in Rec. Pract. for Ventilation for Heat Control in Rec. Pract. for Fans for Rec. Pract. for Design Procedure for Rec. Pract. for Make Up and Recirculated Air for Rec. Pract. for Construction Spec. for Local Rec. Pract. for Test of air Cleaning Devices (Air Filters and Dust Collectors) for . for Definitions, Abbreviations and General Principles of ase Fibers/ Std. Meth. of Testing Tire Cords, Fabrics and Std. for Keys and Keyways for

Std. Dimensions for Accessories for Cataloged Square Head B93.18

4, 1 and 1 1/8 In. Bore Cataloged Square Head Tie Rod Type

Std. for Forks and Fork Carriers for Powered ring Frame Lock for Articulated Vehicles (Construction and Tent. Meth. of Test for Pumpability of Safety Std. for Commercial

Std. Spec. for

Std. Spec. for

Std. Spec. for

Std. Pract. for

Rec. Pract. for Storage Batteries for Construction and

Rec. Pract. for Lubrication Chart-Construction and Rec. Pract. for Electrical System :or Construction and sional Spec. for Sealed Lighting Unit for Construction and dipper, Clam Bucket, and Dragline Bucket (Construction and Series) (1972) Stds. for ansi $B 93.33$ Std. Spec. for Std. Interfaces for 4 W ay Gract. and Proc. 1) y) (1973) Std. for Std. Dimensions for Perforated

domestic Passenger Cars, Trucks, Buses, Coaches, Tractors, ractors Having at Le/ Rec. Pract. Safety Requirements for

b. of Test for Moisture Present in Ordinary Commercial and

gage WheeI,

Std. for Tires and Rims for Agricultural and I974) td. for Determination of Capacity of Lead Acid off Definitions and Terminology for Agricultural and Light aggage, Special Type Trailer, Mobile Home, Garden Tractor. Rec. Pract, for High Lift Std. for Safety for Electric Battery Powered

Industrial Trucks (1972) ANSI B56.3
IndustriaI and Mill Cranes (1970)

Industrial and Other Direct Current Integral Horsepower

Industrial Applications (1971)

Industrial Applications (1973)

Type Storage

I Type Storag
ANSI Z78.9

Industrial Aromatic Hydrocarbons (1971) ANSI Z78.13

Industrial Aromatic Hydrocarbons (1972)

Industrial Aromatic Hydrocarbons (1973) ANSI Z 106. I

Industrial Baking and Drying Ovens (1973)

Industrial Buildings (1971)

Industrial Buildings (1973)

Industrial Casters (1973)

Industrial Centrifugal Fans with Cast Housings (1966)

Industrial Centrifugal Fans (1966)

Industrial Computer System FORTRAN Procedures for Execu

Industrial Control Apparatus-Controllers and Controll

Industrial Control Apparatus-Enclosures (Electronic)

Industrial Control Equipment for Use in Hazardous Locat

Industrial Cost Trends for Buildings, Machinery and Equ

Industrial Electrical Power Control Apparatus-Control

ndustrial Electrical Power Control Apparatus-General

ndustrial Electrical Power Control Apparatus-Switchi

Industrial Enclosed Gear Drives (1972)

Industrial Engineering Terminology: Applied Mathematics

Industrial Engineering Terminology: Applied Psychology

ndustrial Engineering Terminology: Biomechanics (1972)

Industrial Engineering Terminology: Cost (1972)

Industrial Engineering Terminology: Data Processing and

Industrial Engineering Terminology: Distribution and $\mathrm{Ma}$

Industrial Engineering Terminology: Economy (1972)

Industrial Engineering Terminology: Facility Planning (

IndustriaI Engineering Terminology: Organization Planni

Industrial Engineering Terminology: Production Planning

Industrial Engineering Terminology: Work Measurement an

ndustrial Equipment and General Purpose Machine Tools

Industrial Equipment Warning Lamp and Slow Moving Vehic

/Ec. Pract. Fo SAE

Industrial Equipment (Machinery) (1971)

Industrial Equipment (Machinery) (1971)

Industrial Equipment) (1971)

Industrial Equipment) (1973)

Industrial Exhaust Systems (1974)

IndustriaI Exhaust Systems (1974)

Industrial Exhaust Ventilation Systems (1974)

Industrial Exhaust Ventilation Systems (1974)

Industrial Exhaust Ventilation Systems (1974)

IndustriaI Exhaust Ventilation Systems (1974)

974)
974)
974)

/H Permanently

Industrial Exhaust Ventilation Systems (1974)

Industrial Exhaust Ventilation Systems (1974)

Industrial Filament Yarns, Made from Man

Industrial Fluid Power Cylinders (1972) ANSI B93.29

Industrial Fluid Power Cylinders (1972) ANSI B93.34

Industrial Fluid Power Hydraulic Reservoirs (1969) ANSI

Industrial Fork Lift Trucks (1973)

Industrial Front End Loaders and Dozers) (1971)

Industrial Fuel Oils (1973)

Industrial Gàs Heating Equipment (1973)

Industrial Grade Benzene (1971) ANSI Z78.3

Industrial Grade Toluene (1971)

Industrial Grade Xylene (1971)

Industrial Lighting (1973) ANSI All.1

Industrial Machinery (1970)

Industrial Machinery (1971)

Industrial Machinery (1972)

Industrial Machinery (1972)

Industrial Machinery) (1972)

Industrial Perforated Metal (1972)

Industrial Perforated Plate and Screens (Square Opening

IndustriaI Plant Safety Checklists) (1973)

Industrial Pneumatic Directional ControI Valves (1973)

Industrial Power Take Offs for Overcenter Clutches (197

Industrial Radiographic Sheet and Roll Film (photograph

Industrial Revolving Screen Stds. (1972)

Industrial Road and Misc Equipment) (1974)

Industrial Rotary Mowers (Designed for Operation with T

Industrial Samples of Wool by Oven Drying (1972) ANSI L

Industrial Security for Nuclear Power Plants (1973)

IndustriaI Steering. Drive, PIanter (Drill) Press, and

Industrial Storage Batteries for Motive Power Service (

Industrial Practors (1972) SAE J722

Industrial Truck and Trailer (1974)

Industrial Truck Nameplates and Marking (1973)
Power Take

ASTM

$A W S$

NEMA

HMI

/O ASTM

IY ASTM

ASTM

ASTM

ICBO

APA

RTI

ANSI

AMCA

AMCA

ISA

ANSI

ANSI

UL

FMS

ANSI

ANSI

AGMA

ANSI

ANSI

ANSI

ANSI

ANSI

ANSI

ANSI

ANSI

ANSI

ANSI

ANSI

ANSI

JIC

ASAE

SAE

SAE

ASTM

ACGIH

ACGIH

ACGIH

ACGIH

ACGIH

ACGIH

1. Pract ACGIH

ASTM

AGMA

NFLDP

// NFLDP

NFLDP

ANSl

/ Stee SAE

ASTM

UL

ASTM

ASTM

ASTM

IES

SAE

$S A E$

SAE

td. for Dimen SAE

SAE

IPA

ASTM

MCA

NFLD

SAE

ANSI

IPA

BCI

SAE

ANSI

TRA

NEMA

ASAE

TRA

ITA

E461

D14.1

1.15

200

$\mathrm{J} 537 \mathrm{H}$

D847

853

D2935

D 1555

MH11.1

2403

2402

S61.1

C19.7

C19.4 
Std. for Load Handling Sy mbols for Powered for Exhaust Emission Controls (Air Pollution) for Powered test., Rating and Reporting of the Noise Levels of Powered ations, Areas of L'se, Maintenance and Operation of Powered ent (1966)

Std for Demountable Tired Wheels for

$100 \%$ Cotton Woven Fabrics (Textile) for lnstitutional and irements for Woven Fabrics (Textile) for Institutional and irements for Woven Fabrics (Textile) for Institutional and ntreated Carbon Steel Railway Axles for Export and General d. Spec. for Steel Forgings, Carbon and Alloy, for General erials for Roofing. Waterproofing, and Related Building or Meta

ng Object Protective Structures (FOPS) of Construction and Rec. Pract. for Exhaust Hood Design for Perforated

Brick and Tile in Sanitation Structures, ent of Beta Particle Radioactivity of lndustrial Water and d. Meth. of Test for Hydroxide Ion in Industrial Water and analytical Meth. for Determining Chemical Oxygen Demand in

of Test for Measurement of Beta Particle Radioactivity of Std. Meth. of Test for Hydroxide Ion in Std. for Redwood Lumber Spec. for Application in Std. for Douglas Fir Lumber Spec. for Application in Std. for Southern Pine Lumber Spec. for Application in d. Spec. for Pressure Preservative Treatment of Lumber for Std. Meth. of Test for Evaluating Inhibitory Toxicity of Std. Dimensional Tolerances for

ng Code Std, for Softwood Plywood Lumber. Construction and Woven Drycleanable Tailored Uniform Fabrics, Heavyweight and Construction of Masonry Chimneys (For Residential and nter Balanced Fork Lift Trucl Std. for Tires and Rims for Rec. Pract.

ternally Threaded Fasteners Used in Automotive and Related Safety Std. for Compressors for Process

for Laboratory Corrosion Test of Metals for the Process ments for Machine Screws for Use in Automotive and Related

71) Std. for Steel Shipping Container

tput Microfilm (1971)

Exchange Water Softeners (1972)

(1972)

pruce Doors and Blinds (1972)

Documents (1972) ANSI C.16.45

te Cans (21l (66 Mm) Diameter), Citrus Style (1974)

Rec.

GIossary of Construction ce Safety Valves, Lacks, and Landing Nipples for Petroleum operation of Subsurface Safety Valve Systems for Petroleum Hand $\mathrm{Po} / \quad$ Std for Electrical Apparatus Sales and Service I Flanged Steel Safety Relief Valves Used in the Petroleum oise Measurement Techniques for the Machine Tool Builders' Rec. Lighting for Dairy Farms and the Poultry for Electric Welied Low Carbon Steel Pipe for the Chemical Sid. Safety Requirements for the Textile and Billing of Merchandise Within the Hardgoods (Hardware) Guidelines and Stds. for the Paperboard Packaging to Streamline Supply Transactions Within the Air Transport for Subsurface Injection Using Membrane Filters (Petroleum fiberboard (Horizontal Plane Meth.) (1972) Meth. for Std. Analytical Meth. for Determining Filth and Insect nd Other Wh/ Std. Analytical Meth. for Determining lnsect meth. of Analysis of X.Ray Examination for lnternal Insect Std. for Directories of Libraries and g of Merchandise W/ Rec. for Standardization of Forms and 973)

1974

74) Properties and Essential Properties and Essential Properties and Essential Properties and Essential Properties and Essential ride $(1972)$

mentation and Terminology) (19/ Guidelines for Describing Std. for Recorded Magnetic Tape for Std. for Representation for Calendar and Ordinal Date for Std. for Interchange Rolls of Perforated Tape for the United States (Including the District of Columbia) for the Control Characters of American National Std. Code for ification (Code) of the Countries of the United States for Std. for Recorded Magnetic Tape for Std. for Recorded Magnetic Tape for i, and $1600 \mathrm{cpi}, \mathrm{Pl}$ Std. for Inrecorded Magnetic Tape for 6) What Makes a Good Air Condicioning System d. for Flowchart Symbols and Their Usage in Micrographics Contouring-Positioning Numerically Controlled Machines Differential and Manual Transmission Lubricants

Rec. Pract for Automotive Gasoline Performance and Spec. for
Industrial Trucks (1973)

Industrial Trucks (1973)

lndustrial Trucks (1973)

Industrial Trucks (1973) ANSI B56.2

Industrial Use Garments (Clothing), 100\% Man Made or B

Industrial Use Garments (Clothing), 100\% Man Made or Bl

Industrial Use (1971) ANSI G57.12

Industrial Use (1972)

Industrial Uses (1973) ANSI A109.29

lndustrial V.Grooved Wheel Std. (1966)

Industrial Vehicles and Equipment (1972)

Industrial Ventilation Systems (1974)

Industrial Vibrating Screens Stds. (1972)

Industrial Waste Treatment Facilities (Tech.

ASTM

Other Organic Mat ASTM

CFTMA

(S) and Falli SAF

ACGIH

Industrial Waste Water (1966) ANSI N151

lndustrial Waste Water (1967)

Industrial Waste Waters and Most Organic Compounds (196

IndustriaI Water and Industrial Waste Water (1966) ANSI

Industrial Water and Industrial Waste Water (1967)

Industrial Water Cooling Towers (1971)

Industrial Water Cooling Towers (1971)

Industrial Water Cooling Towers (1971)

Industrial Water Cooling Towers (1971)

Industrial Waters (1968E)

IndustriaI Wheels (1965)

Industrial (1973)

Industrial) Suiting (Institutional Textile) (1973)

lndustrial) (Tech. Notes) (1964)

Industrial, Utility and Recreational Type Vehicles, Cou

Industrical Truck Definitions (1972)

Industries (1971)

Industries (1972)

/Cal and Quality Requirements for Ex

BIA

Industries (1972)

lndustries (1972) / for Mechanical and
lndustry Glossary (Packaging, Vocabulary) (1973)
lndustry Quality Std. for Computer Output Micro

Test Meth NACE

ASTM

CR

ASTM

ASTM

CTI

CTI

CTI

CFTM

Industry Ouality Std. Format and Coding for Computer Ou

Industry Standard for Household Commercial and Portable

Industry Std. for Distribution Microfiche of Documents

Industry Std. for Douglas Fir, Western Hemlock, Sitka S

Industry Std for Fascimile Transmission of Microfilmed

Industry Std. for Hardboard (1970)

Industry Std. for Loose Metal End Dimensions of Composi

Industry Std. Glossary of Micrographics (1971)

Industry Terms (Architecture) (1970)

lndustry Use (1973)

Industry Use (1973)

Spec. for Subsurfa
T. for Design. Installation, and

Industry (Rotating, Liquid Filled and Dry Transformers,

Industry (1969) ANSI B146.

Industry (1970)

Industry (1971)

lndustry (1971) ANSI B 125.38

Industry (1972)

Industry (1972)

Industry (1973)

Industry (1974)

lndustry) (1973) /Meth. for Determining W
lnefficient of Static Friction of Corrugated and Solid
lnfestation Content in Corn (1954)

Infestation Content (Radiographic lnspection) in Corn a

Infestation in Cereal Grains, Unpopped Popcorn, Dried P

Information Centers (1971)

Information Flow Used in Ordering, Shipping, and Billin

nformation for Safe Handling and Use of Acetic Acid (1

Information for Safe Handling and Use of Caustic Soda (

Information for Safe Handling and Use of Chloroform (19

lnformation for Safe Handling and Use of Paraformaldehy

Information for the Safe Handling and Use of Vinyl Chlo

Information Interchange Formats (Computer Software Docu

Std. Fo

Information Interchange (1600 cpi, PE) (1973)

Information Interchange (1971)

Information lnterchange (1972)

lnformation lnterchange (1972)

Information Interchange (1973)

nformation Interchange (1973)

Information lnterchange (200 cpi, NRZI) (1973)

Information Interchange ( $800 \mathrm{cpi}$, NRZI) (1973)

Information Interchange (9-Track 200 and 800 cpi, Nrz

Information on Year Round Air Conditioning System) (196

Information Processing) (1973) ANSl PH5.17

(Information Processing) (1974)

Information Report) (1969)

/Mat for Contouring and

Information System (1971)

Informacional, Directional, and Special Signs (Symbols)
/ Iations for States of ANSI

Phic Representation of ANSI

ANSI

ANSI

ANSI

ANSI

NESCA

St NMA

EIA

SAE

ANSI

MH1l.3

$6 \mathrm{Fl}$

$6 \mathrm{Gl}$

505

L24.4.12

L24.4.13

L24.4.14

A 383

A668
D 1079

W8

J397A

$* 1.4$
$* 2$

$34 \mathrm{~B}$

D 1890

D514

K-24

D1890

D514

14

112

D2037

TOL

UBC S25.9

L24.3.2

$19 \mathrm{~B}$

IB2. 19

J429F

B 19.3

TM.01.69

J82

MH2. 18

MSl

MS2

S- 100

MS5

4.72

$\mathrm{MS}$

IS 1.70

E211.1

MSl00

*1

$14 \mathrm{~A}$

RPl4B

*1

526

$* 1$

R344

A587

L1.1

*1

*1

200

TM-01-73

T816SU

A-10

A.1 I

28.21

Z39.10

*1

SD 41

SD9

SD89

SD6

SD56

$\times 10.1$

X3.39

X3.30

$\times 3.34$

X3.38

$\mathrm{X} 3.32$

$\times 3.31$

X3.14 
Std. Qualitative Classification of Surfactants by atings on Wood Products During the / Std. Rec. Pract. for Std for Vented Gas Fired Std. for Unvented Gas Fired taxial Layers of Silicon on Substrates of the Same Type by in the Atmosphere (Continuous Measurement by Nondispersive th of Test for Vinylidene Unsaturation in Polyethylene by ve Determination of Cellulose Nitrate in Alkyd Lacquers by Std. Spec. for Magnesium

lysis of Carbon, Low Alloy, and Silicon Electrical Steels, Lake Copper W'ire Bars, Cakes, Slabs, Billets, Ingots, and lytic Copper Wire Bars, Cakes, Slabs, Billets, Ingots, and fined Copper Wire Bars, Cakes, Slabs, Billets, Ingots, and Std. Spec. for Zinc Alloys in
1973) ANSI H38.1 1966) ANSI H45.8
Std Spec for Aluminum Alloys in Sid. Spec. for Magnesium Alloys in Std. Spec. for Remelted Lithium Metal in Std. Spec. for Tantalum Std. Spec for Zirconium

Std. Spec. for Zirconium and Its Alloy d. Spec. for Lake Copper Wire Bars, Cakes, Slabs, Billets, for EIectrolytic Copper Wire Bars, Cakes, SIabs, Billets, hemicaIly Refined Copper Wire Bars, Cakes, Slabs, Billets,

Meth. of Test for Synthetic Anionic e (1974) Std. Meth. of Test for Synthetic Anionic Active ini/ Rec. Regulations for Animal Feed and Food Labelling 1963)

our, Total Aerobic, Anaerobic) in Cereal Products and Food Std. Analytical Meth. for Determining Std. Meth. of Test for Evaluating

65.216 Std. Spec. for Reinforced and Filled Nylon and Extrusion Materials (1972) ANSI K65/ Std. Spec. Pract. for eter) (1971) Rec. Pract. for Diesel Fuel $\mathrm{ph}$ (1973)

Tent. Rec. Pract. for Direct Std. for Metric Thread Fuel Test Meth. for Determining Water Quality for Subsurface Std. Pract. for Combustion Chambers, Fuel O cording and Measuring the Off-The-Job Disabling Accidental g Frequency and Cost, and Recording Patron and Nonemployee Meth. for

Std for Print Spec for Magnetic SIant Drop Penetration Test for Paperboard (Printing Optical Test Meth. for iquid Mixing Operations (Paint, Varnish, Lacquer, Printing Std Meth of Test for Wear Resistance of Std. Definitions of Terms Relating to Carbon Paper and Std. Meth. of Test for Erasability of Std. Gravure Proofing

Std. for Air Outlets and , Alarms, Sandpipes), Building Construction, Basement Pipe (Cereal Chemistry) (1962)

Meth. of Analysis of Std for Acceptable Concentrations of tem FORTRAN Procedures for Executive Functions and Process

s Water Heaters (Automatic Storage Type Water Heaters with

for Gas Fired High Pressure Steam and Hot Water Boilers reals, and Alimentary Past/ olina (Cereal Chemistry)/ stry) (1962)

unpopped Popcorn, Dried Pe/ Sieving Meth of Analysis for Flotation Meth. of Analysis for Flotation Meth. of Analysis for Meth. of Analysis of External Unpopped Popcorn. Dried Peas and Beans for Rodent and/or mistry) (1962)

Iodine Meth. of Analysis for Glass Plate Meth. of Analysis for istry) (1962)

chemistry) (1962) chemistry) (1962/ rs, etc. in St/

Acid Hydrolysis Meth. of Analysis for Tween Versene Meth. of Analysis for Fatting Digestion Meth. of Analysis for Sieving Meth. of Analysis for Dirt, Sand Std. Analytical Meth. for Determining Filth and drie/ Meth. of Analysis of X-Ray Examination for Internal Test Meth. for es of Institutional Textiles (Durability, Spot, Stain, and Uniform Fire Code: Fumigation and Thermal Std. for Chlorpyrifos Std. for Leptophos

Std Meth of Test for Effectiveness of Aerosol $t$ for Effectiveness of Aerosol and Pressurized Space Spray d. Meth. of Test for Pickup Efficiency of Residual Aeroso Meth. of Analysis of Cracking Flotation Test for Internal Test Meth for Resistance of Textiles to ol and Pressurized Space Spray Insecticides Against Flying Classification in Cereal Grains and Grain Products (Cer) Is, and Alimentary Pastes for Detection of Rodent Excreta, Std. for Threaded Metal
Infrared Absorption (1974)

Infrared Determination of the Temperature of Applied Co

ASTM

ASTM

ANSl

Infrared Radiant Heaters (1971)

Infrared Radiant Heaters (1972)

Infrared Reflectance (I973)

Infrared Spectrometry) (I973)

Infrared Spectrophotometry (1972)

Infrared Spectrophotometry (1972)

Ingot and Stick for Remelting (1971

lngot Bars (1973) ANSl H17.1

lngot Bars (1973) ANSI H17.2

Ingot Bars (1973) ANSI H23.12

Ingot Form for Die Castings (1964) ANSI H47.

Ingot Form for Sand, Permanent Mold, and Die Castings
Ingot Form for Sand, Permanent Mold, and Die Castings

Ingot Form (1972) ANSI H43.1

Ingots and Flat Mill Products (1970) ANSI Z179.14

Ingots for Nonnuclear Application (I972) ANSI Z179.10

Ingots for Nuclear Application (1973) ANSI G53.28

Ingots, and lngot Bars (1973) ANSI H17.1

Ingots, and Ingot Bars (1973) ANSI H17.2

Ingots, and Ingot Bars (1973) ANSI H23.12

Test for Thickness of Epi

ANSI

Ingredient by Cationic Titration (1972T)

Ingredient in Detergents by Cationic Titration Procedur

Ingredients and Containers (AIso Includes Terms and Def

Ingredients (Cereal Chemistry) (1962)

Inherent Viscosity (One Point) Content in Corn Starch

Inhibitory Toxicity of Ind ustrial W'aters (I968E)

Injection Molding and Extrusion Materials (1972)

Injection MoIding and Extrusion Materials (1973) ANSI K

Injection Molding of Specimens of Thermoplastic Molding

Injection Nozzle and Holder Assembly (17Mm Nominal Diam

Injection of Solvent Base Paints into a Gas Chromatogra

Injection Tubing Connections (1971)

Injection Using Membrane Filters (Petroleum Industry)

Injection, and Gas Admission Systems (1972)

Injury Experience of Employees (1973)

Injury Statistics (1971)

Ink Absorbency of Blotting Paper (1972)

Std. for Meth. of Measurin

nk Character Recognition (1970)

Ink Receptivity) (1973)

Ink Show Through (Paper) (1973)

Ink, Adhesive, etc.) (1974)

Inked Computer Ribbons (1972)

nked Ribbon Products and lmages Made Therefrom (1972A)

Inked Ribbons (1972)

Inks (Printing) (1973)

Inlet Box Positions for Centrifugal Fans (1966)

Inlets Used in Air Distribution Systems (1973)

Inlets (1973)

F Systems and A ppliances (Extinguishers

Inorganic Bromide Residues in Grain and Cereal Products

Inorganic Mercury and NonalkyI Organo Compounds (1972)

Input-Output (1972)

TTd. for IndustriaI Con

(nputs Not Over 400,000 Btu Per Hr.) (Vol. (1972)

Insect and Rodent Filth in Baked Goods, Ready-To-Eat Ce

Insect and Rodent Filth in Meal, Grits, Farina, and Sem

Insect and Rodent Filth in Popped Popcorn (Cereal Chemi

Insect and Rodent Hair Contamination of Cereal Grains,

Insect Damage on Kernels, Rodent Excreta, Other Filth

Insect Eggs in White, Wheat, and Corn Flour (Cereal Che

Insect Excreta in White, Wheat, and Corn Flour (1962)

Insect Fragments and Rodent Hairs in Flour (Cereal Chem

Insect Fragments and Rodent Hairs in Rye Flour (Cereal

Insect Fragments and Rodent Hairs in Soy Flour (Cereal

Insect Fragments, Hairs, Feathers, Wood Fibers, Splinte

Insect Infestation Content in Corn (1954)

Insect Infestation Content (Radiographic Inspection) in

Insect Infestation in Cereal Grains, Unpopped Popcorn,

Insect Pest Deterrents on Textiles (1971) ANSI L14.65

Insect Resistance) (1973)

Insecticidal Fogging (1973)

(Insecticide) (1971)

(Insecticide) (1971)

Insecticides Against Cockroaches (1972)

Insecticides Against Flying Insects (1972)

Insecticides (1972)

Insects in Cereal Grains, Unpopped Popcorn, Dried Peas

Insects (1971) ANSI L14.64

Insects (1972)

/Th. of Test for Effectiveness of Aeros

Inserts, Rodent Hair, and Radiographic Illustration and

Insects, Webbing, etc. (Cereal Chemistry) (1962)

Insert-Heavy Duty (1973)

Insert Fittings for Polyethylene (PE) Plastic Pipe (197

Std. Spec. for Plastic

Insert Holders (1973)

\section{td. Me}

ASTM

ASTM

ASTM

ASTM

ASTM

ASTM

ASTM

ASTM

ASTM

ASTM

ASTM

ASTM

ASTM

ASTM

St ASTM

td. Spec ASTM

ASTM

ASTM

ASTM

AAFCO

$\mathrm{AACCH}$

CR

ASTM

ASTM

ASTM

ASTM

$S A E$

ASTM

SAE

NACE

DEMA

ANS1

ANSI

TAPPI

ANSI

TAPPI

TAPPI

FMS

ASTM

ASTM

ASTM

AMCA

A Rl

ICBO

AACC

ANSI

ISA

ANSI

Std ANSI

$\mathrm{AACCH}$

$\mathrm{AACCH}$

$\mathrm{AACCH}$

$\mathrm{AACCH}$

$\mathrm{AACCH}$

$\mathrm{AACCH}$

A ACCH

AACCH $28-70$

AACCH $28-75$

CR A-10

CR A-11

AACCH 28.2

AATCC 28

ANSI

ICBO

ANSI

ANSI

ASTM

ASTM

St ASTM

$\mathrm{AACCH}$

AATCC

ASTM D3088

AACCH 28.95

/Erea

NSA

ASTM

ANSI

28. 30

1395

D2609

L24.7.1

K62.105

D3095

3088

093

UFC *2ART17 
olyethylene Pipe (1973)

Std. Spec. for

ht Selflocking and Nonselflocking Blind Threaded Molded in ht Selflocking and Nonselflocking Blind Threaded Molded in Std for Threaded Metal

f Locking, Light Weight Floating, Sandwich Panel Std. for Locking, Sandwich Panel (1972) Std. for rm Building Code Std. for Welding Reinforcing Steel, Metal Std for Carbide Seats $C_{\text {sed }}$ with Indexable Std. for Carbide Chip Breakers Used in Indexable Std. Pract. for Socket Welding Reduce Std. Meth. of Test for

Il

ep/ mistry) (1967) 13

Sid Teat Meth for Detection and Estimation of Water Std. Meth. of Test for Vegetable Matter and Other Alkal ation of $\mathrm{pH}$ (Slurry) of Corn Starch Which ls Substantially
Meth. of Analysis of Acetone

Std. Meth of Test for Benzen Std. Meth. of Test for 1973) afety) (1973)

er Habide Microfilm (1972

ewound Trimming Potentiometers (1970) ituminous Materials as Used in Cons/ Inspection of Laminators Quality Control by the Ait Std. for Sampling Procedures and Tables for Std. for Lighting (National Board

inspectors (1973)

(1971) ANSI H38.21

pace Applications (1974)

i (1974) ANSI Z166.8

) ANS1 G52.7

nspectors Manual) (1973)

Std Meth for Ultrasonic

Std. Meth. for Ultrasonic Std. Reference Radiographs for

Std. Pract. for the Std. Rec. Pract. for

Rules for the Measurement and

spection Bureau (1962)

Fire Hazards and Protection for Maintenance and

hreads (1974)

3)

Spec. for Threading, Gaging, and Thread

Std. Meth. for Ultrasonic Contact

Std Cuideline for the Physical

Quality Steel Cleanliness Requirements (Magnetic Particle Sernitrailers Operated on Public Highways (1973/ Std. for

Manufacture and

Std. for Gages, Pins and Collar

Std. Definitions of Terms Relating to Magnetic Particle

Std. Definitions of Terms Relating to Liquid Penetrant for Determining Insect Infestation Content (Radiographic uilding Code: Legal Requirements (Permit, Plans and Spec. Stds. for Tire Rim $\mathrm{n}$ and Electric Equipment During the $\mathrm{C}$ /

Std. Installation, nstruction Phase of Nuclear P/ Std. for Qualifications of ty Control Monitoring of the Product (Receiving and Source shipments and Transfers of Soybean Oil (1973) Std for involute Spline Uniform Building Code: Permits and Reinforced Brick Masonry Std. Rec, Guide for Paint ard Inspection Code) Manual for Boiler and Pressure Vessel ement Test) (1973) Std. Meth. of Test for Thermal etectors; Electromagnetic Holders; Sliding Door Equipment;

2)

munication Lines (1960)

Spec, and Rec. Pract. for of National Electrical Safety Code, Part I: Rules for the t Heaters (1970)

and Controls, Barrier, Road, Hydrant, Driveway Clearance, pply and Communications Lines (Nati)

Safety Rules for the uipment (1972)

(197) Minimum Requirements for the Design, (1) Rec. Pract. for Marine (lnboard) Engine Mountings Safety Std. for Construction and Guide for Selection, Concrete Reinforcing Steel Material Supply and Waste and Vent Pipe and Fittings (1971) Std. for cable (1) Std. for Plastic Utilities Duct for Underground

Std. for Decorative Gas Appliances for tilating, and Their Combination Duct System/ Std. for the es (Diesel and Gasolin/ 973)

Rec. Pract. and Std. Covering the niform Building Code Std. for Rec. Pract. and Std. for the Design, Construction and ock, and Vapor Removal or Conveying (1973) / Std. for the
ioning Systems (1973) ANSI B144.3

Recommendation for Recommendation for Recommendation for Recommendation for

Recommendation for Recommendation for
Insert Type Polyethylene Fusion Fittings for SDR $11.0 \mathrm{P}$ Std for Sandwich Panel Lightweig nsert (1974) Insert (I974)

Insert, Light Weight (1973) Std. for Sandwich Panel Lightweig

nsert, Molded In, Blind Threaded, Self Locking, Nonsel

Insert, Molded In, Thru Threaded, Self Locking, Nonself Inserts and Connections in Reinforced Concrete Construc Inserts for Clamp Type Holders (1973)

Inserts for Clamp Type Holders (I973)

Inserts (1974)

Insoluble Ash of Vegetable Tanned Leather (I970) ALCA B insoluble Impurities in Refined Phenol by Cloud Point D Insoluble Impurities in Scoured Wool (1972) ANSI L 14.19 Insoluble in Water at Room Temperature (1973) Insoluble Lecithin in Soybean and Corn Oils (Cereal Che Insoluble Matter in Creosote (1973) AASHO T81, ANSl O11

Insoluble Residue in Carbonate Aggregates (1972) Inspection and Assembly Manual for Fine Pitch Gearing ( Inspection and Maintenance of Sprinkler Systems (Fire S Inspection and Quality Control of First Generation Silv

Inspection and Test Procedures for Wirewound and Nonwir Inspection and Test. Agencies for Concrete, Steel and B Inspection Pureau (1962)

Inspection by Attributes (MIL Std. 105D) (1971)

Inspection Code for Motor Vehicles (1973)

Inspection Code) Manual for Boiler and Pressure Vessel Inspection of Aluminum Alloy Plate for Pressure Vessels Inspection of Aluminum Alloy Wrought Products for Aeros Inspection of Aluminum and Magnesium Castings, Series 1 Inspection of Carbon and Low Alloy Steel Castings (1970 Inspection of Elevators, Escalators and Moving Walks (i Inspection of Glass Aerosol Containers (1972)

Inspection of Hardwood and Cypress Lumber (1971.75) Inspection of Laminators Quality Control by the AITC in Inspection of Mechanical Drive Turbines (1974)

Inspection of Petroleum Casing. Tubing, and Line Pipe T Inspection of Preservative Treated Timber Products (197 Inspection of Weldments (1965) ANSI Z166.23

Inspection of Wood Poles in Service (1972)

Inspection Procedure) (1973) /Ec. for Premium Aircraft

Inspection Procedures for School Buses (1972)

Inspection Stds. for Preformed Packings (0.Rings) (1971

Inspection (1973)

Inspection (1974) ANSl Z166.15

Inspection (1974) ANSI Z166.16

Inspection) in Corn and Other Whole Grains (1958)

Inspection) (1973)

/G Construction Under the Uniform B Inspection, and Disk and Flat Tapes (1974)

Inspection, and Testing Requirements for Instrumentatio

Inspection, Examination, and Test. Personnel for the Co

Inspection, Product Audit) (1969) /C. Pract. for
Inspection. Sampling and Measuring Procedures for Bulk

Inspections (1970) ANSI B92.1

lnspections (1973)

Inspectors Guide) (Tech. Notes) (1963)

Inspectors (1973)

Inspectors (1973) Instability of Confined Condensed Phase Systems (Confin
Installation Aids (1969)

Installation and Calibration of Turbine Flowmeters (197

Installation and Maintenance of Electric Supply and Com

Installation and Maintenance of Electrical Supply Stati Installation and Maintenance of Steam and Hot Water Uni

Installation and Maintenance of Systems and Appliances

Installation and Maintenance of Underground Electric Su

Installation and Performance of Sprinkler lrrigation Eq

(Installation and Replacement) Direct Drive Transmission

lnstallation and Use of Electric Motors and Generators

Installation Contract (1968)

Sugges

Installation for Acrylonitrile Butadiene Styrene Drain,

Installation for Communic ation and Electrical Wire a

Installation in Vented Fir eplaces (1973)

Installation of Air Conditioning, Heating, Exhaust, Ven

Installation of Air Cooled Marine Engines and Auxiliari

Installation of Automatic Fire Extinguishing Systems (1

Installation of Battery Chargers on Boats (1973)

Installation of Blower and Exhaust Systems for Dust, St

Installation of Central Warm Air Heating and Air Condit

Installation of Ceramic Tile Bathtub Walls (1973)

Installation of Ceramic Tile Countertops (1973)

Installation of Ceramic Tile Refrigerator Rooms (1973)

Installation of Ceramic Tile Shower Receptors (1973)

Installation of Ceramic Tile Steam Rooms (1973)

Installation of Ceramic Tile Swimming Pools (1973) Etermin C

ASTM

NSA

NSA

NSA

NSA

NSA
ICBO

ANSl

ANSI

MSS

ASTM

ASTM

ASTM

AACCH 58.35

ASTM D367

ASTM D3042

AGMA $\quad 370.01$

FMS

NMA

ASTM E329

AlTC 202

ANSI Z1.4

SAE J599C

NBBPV *1

ASTM B548

ASTM B594

ASTM E155

ASTM A609

ANSl A17.2

ASTM D3073

NHLA *1

AlTC 202

FMS 13.9

APl

$A$ WPA

ASTM

$A$ WPA

$\mathrm{SAE}$

ANSI

ANSI

SAE

NSA

ASTM

AST

CR
ICBO
TRA
IEEE

IEEE

ANSl

$5 \mathrm{~B}$

M2

E164

M13-72

AMS2300B

D7. 1

D7. 4

AS871A

1563

E269

$\mathrm{E} 270$

A. 11

UBC *8. I

10

336

N45.2.6

RP5

*4

J498C

ICBO

BIA

ASTM

UBC *3.3

$17 \mathrm{C}$

D3276

NBBPV

ASTM

ISA

ANSI

ANSI

AMCA

ICBO

ANS

ASAE

SAE

NEMA

E4 4

8

RP31.1

C2.2

$\mathrm{C} 2$.
16 
(1973)

t Locations (Agricultural Plast/ liary Machinery (Boats) (/ Tile (1973)
olation Joints for Ceramic Tile

Std. Guidelines for Safe Rec. Pract. and Stds. for Safe Recommendation for Recommendation for Recommendation for Uniform Building Code Std. for d. and Pract. Rec. for Selection, Design, Construction and the Purpose of Conserving Their / ) ANSI K65.171 and Certain Other Premises (1972)

Tent. Rec. Pract, for td. Rec. Pract. for Underground Std. for

Recommendation for Recommendation for

for Requirements for Design, Materials, Construction and : Body and Frame Design and Construction Requirements, and (Travel and Camping Trailers, Truck Campers, Motor Homes): ges $(1973)$

e H/

Std. for Handling and
Rec. Guides for Design, Construction, Materials, and Pavements (1972) Std. Spec. for Lubricant for
pract. Guide to Design, Construction, Materials, Size, and

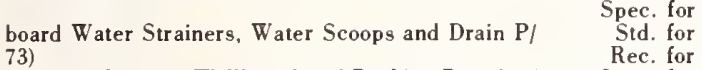
attached Gypsum Wallboard and Backing Board (1/ Spec. for
Std. Rec. Pract. for Underground ooring (1973) Std. Rec. Pract. for Underground
Recommendation for ators and Reversible Generator/Motors for Pumpe/ nstruction Under the Uniform Building Code: Plastering and tion (1973) ANSI A54.3

Std. for the Recommendation for Std. for Safety for Fiber Conduit for Use as Raceway for (1973) Recommendation for stribution Cables (1973)

Fittings (1971)

flooring (1973)

vering (1973)

er Service and Yard Piping (1971)

itioning Systems (1973)

ec. Pract. for Automotive Brake Disc and Drum Thermocouple medium Speed Stationary Diesel and Gas Engine Erection and ncy Vault Ventilators and Vault Ventilating Ports for Wall Floor Raceways and Fittings for Electrical Wire and Cable conduit for Safe Use as a Metal Raceway for Wire and Cable

r Safety for Electrical Metallic Tubing for Wire and Cable works Spec. for Ceramic Veneer (Sampling, Test. Finishing, systems for Petroleum lndustry U/ Rec. Pract. for Design, rops (1971)

Std. for Construction, Std. for Selection,

ilating, C/ Uniform Mechanical Code (Requirements for the Redwood Interior Guide (Spec. for instrumentation and Electric Equipment During the C/ ve Signaling Systems for Fire Alarm Service (19) otective Signaling Systems for Guard, Fire Alar/ ignaling Systems for Watchman, Fire Alarm, and / tive Signalling Systems for Watchman, Fire Alar/ tective Signaling Systems (1972) ANS1 SE3.5 for Flammable (Combustible) Gas lndicators Including Their ments (1972)

urrent Deep Groundbeds (1972) Std. for Preparation of Manuals for Rec. for Suction Press Rolls and Their Rec. Pract. for Classification of Areas for Electrical I and Polyvinyl Chloride Ducts Rec. Pract. for Electrical ipment (1972)

Rec. Pract. for Electrical
Rules for the Radio

Rec. for Shielding for High Energy Electron Accelerator erators and Reversible Generator/Motors for Pumped Storage

Loss Prevention Data on Bonds and Guarantees for Roof ble Synchronous Generator / Motor Units for Pumped Storage $\mathrm{n}$, Materials, Construction and Installation of Permanently use Guide for Type Tests of Continuous Duty Class I Motors Dimensioning Std. and Terminology for Permanently Std. Rec. Pract. for Std. for Gas Water Heaters: Volume III, Circulating Tank, rice Meth. of Analysis of Vitamin A in Nonfat Dry Milk and (1972) Pract. for Maintenance lnstructions for Chlorine of Vinyl Astestos and Asphalt Tile Floors for Commercial, e Requirements for $100 \%$ Cotton Woven Fabrics (Textile) for - Performance Requirements for Woven Fabrics (Textile) for - Performance Requirements for Woven Fabrics (Textile) for

for Measuring Audio Amplifier Single Frequency Output for

Std. Rec. Pract. for Sensory Evaluation of lndustrial and std. Performance Requirements for Woven Slipcover Fabrics
Installation of Ceramic Tile Tubs (1973)

lnstallation of Chemical Resistant Ceramic Tile Floors

Installation of Conductive Ceramic Tile Floors (1973)

lnstallation of Domestic Gas Conversion Burners (1971)

lnstallation of Electrical Instruments in Hazardous Dus

Installation of Exhaust Systems for Propulsion and Auxi

lnstallation of Expansion, Control, Contraction, and Is

lnstallation of Exterior Ceramic Tile Floors (1973)

Installation of Exterior Ceramic Tile Walls (1973)

lnstallation of Fire Doors and Windows (1973)

Installation of Fittings and Equipment for Anchoring, $M$

Installation of Fixed Partitions of Light Frame Type Fo

Installation of Flexible Thermoplastic Sewer Pipe (1972

Installation of Gas Piping and Equipment on Industrial

Installation of Interior Ceramic Tile Floors (1973)

Installation of Interior Ceramic Tile Walls (1973)

Installation of Permanently Installed Fuel Systems for

lnstallation of Plumbing, Heating and Electrical System

Installation of Plumbing, Heating, and Electrical Syste

lnstallation of Power Semiconductors in Disc Type Pack

Installation of Power Transmission Units Penetrating Th

Installation of Preformed Compression Seals in Concrete

lnstallation of Propeller Shafting Systems for Boats (1

Installation of Residential Aluminum Siding (1971)

Installation of Seacocks, Through Hull Connections, Out

Installation of Sprinkler Systems (Fire Protection) (19

Installation of Steel Framing Members to Receive Screw

Installation of Thermoplastic Pressure Piping (1972)

Installation of Thresholds, Saddles for Ceramic Tile F1

Installation of Vertical Hydraulic Turbine Driven Gener

Installation of Wallboard Lathing (1973)

Installation of Water Sprinkler Systems for Fire Protec

Installation of Waterproof Ceramic Tile Floors (1973)

Installation of Wires and Cables (1972) ANSI C 33.37

Installation Performance Levels of Ceramic Tile Floors

Installation Pract. for Underground Electrical Power D

Installation PVC Building Drain Waste and Vent Pipe and

lnstallation Spec. for Vinyl Asbestos and Asphalt Tile

Installation Spec. for Vinyl Asbestos Tile as a Wall Co

Installation Std. for Solvent Cemented PVC Pipe for Wat

Installation Stds. for Residential Heating and Air Cond

Installation (1972)

Installation (1972)

Installation (1972) ANS1 B 135.1

Installation (1973) ANSI C.33.58

Installation (1973) ANSI C 33.92

Installation (1973) ANS1 C 33.98

Installation, and Definitions) (1961)

lnstallation, and Operation of Subsurface Safety Valve

Installation, and Rating of Equipment for Drying Farm C

Installation, and Servicing of Humidifiers (1972)

Installation, Control, and Maintenance of Heating, Vent

Installation, Finishes and Other Technical Data) (1973)

lnstallation, Inspection, and Testing Requirements for

/Afety Std.
/Ty for Ce
/E Steel and
afety Valve
ying Farm
72 )
ating, Ven
ata) (1973)
rements for

Installation, Maintenance and Use of Auxiliary Protecti
Installation, Maintenance and Use of Central Station Pr

Installation, Maintenance and Use of Local Protective S

Installation, Maintenance and Use of Proprietary Protec

Installation, Maintenance and Use of Remote Station Pro

Installation, Operation and Maintenance (On Boats) (197

Installation, Operation and Repair of Laboratory Instru

lnstallation, Operation, and Maintenance of lmpressed C

Installation, Operation, and Maintenance (1973)

Installations at Drilling Rigs and Petroleum Production

Installations of Electrical Wires and Cables (1974)

Installations on Packaging Machinery and Associated Equ

Installations (National Electrical Safety Code) (1940)

Installations (1964)

Installations (1972)

Installations (1973)

lnstallations (1974)

/Cal Hydraulic Turbine Driven Gen

Installed Fuel Systems

/Nchronous Generators and Reversi

nstalled Screw Conveyors (1972)

lnstalling Vitrified Clay Sewer Pipe (1972) ANS1 Al06.2

Instantaneous and Large Automatic Storage Type (1974)

Instantized Dry Milks (Cereal Chemistry) (1971)

Instantizing Systems for Dry Milk and Dry Milk Products

Institute Std. Excess Flow Valyes (1972)

Institutional and Industrial Buildings (1973)

Institutional and Induetrial Use Garments (Clothing),

Institutional and lndustrial Use Garments (Clothing),

Institutional and lndustrial Use Garments (Clothing), 1

Institutional Audio-Visual Equipment Used Primarily F

Institutional Food Purchases (1972)

(Institutional Textile) (1973)
TCA

TCA

TCA

ISA

ABYC

TCA

TCA

TCA

ICBO

$A B Y C$

ASTM

ASTM

ANSI

TCA

TCA

ABYC

NFPA

NFPA

ElA

ABYC

ASTM

ABYC

AAMA

ABYC

FMS

ANSI

ASTM

TCA

NEMA

NFPA

TCA

UL

TCA

AA

IAPMO IS9

RT1 *8

RTI *17

1APMO IS8

SMACN *10

SAE J79

DEMA

$* 1.21$

680

209

1

97

$* 28$

Std. Fo UL

Public B1A

API

ASAE

ARl

ICBO

CRA

lEEE.

NFPA

NFPA

NFPA

NFPA

NFPA

ABYC

ANS1

NACE

FMS

API

(R NEMA

IEEE

ANSI

NCRPM

NEMA

FMS

NEMA

ABYC

IEEE

ASAE

ASTM

ANSl

/R-P AACCH

DFISA

CHI

Tenance RTI

ANSl

ANSI

ANSI

ANSI

ASTM

ANSI

304

Z21.8

P1

E497

2321

24

$501 \mathrm{~B}$

110-111. 1

S12.11

107.109

9.210

BCS 43.5

101.106

01.208. 1 
Std. Performance Requirements for Woven Bath Mats (Institutional Textile) (1973)

Std. Performance Requirements for Woven Shower Curtains (Institutional Textile) (1973)

Std. Performance Requirements for Woven Dish Towels (Institutional Textile) (1973)

Std. Performance Requirements for Glass Towels (Institutional Textile) (1973)

Std. Performance Requirements for Huck Towels (Institutional Textile) (1973)

Sid. Performance Requirements for Woven Bedspreads (Institutional Textile) (1973)

Std. Performance Requirements for Woven Blankets (Institutional Textile) (1973)

mance Requirements for Damask Table Napery (Napkin, etc.) (Institutional Textile) (1973)

nce Requirements for Woven Upholstery Fabrics (Tacked On) (Institutional Textile) (1973)

e Requirements for Terry Cloth Towels (Single and Double) (Institutional Textile) (1973)

equirements for Woven Printed Table Napery (Napkin, etc.) (Institutional Textile) (1973)

detachable Tags, and Certification of Fabrics or Products (Institutional Textile) (1973)

red Woven Uniform Fabrics, Lightweight (Tropical) Suiting (Institutional Textile) (1973)

urable Press (No Iron) Woven Sheet and Pillowcase Fabrics (Institutional Textile) (1973)

ents for Woven Washable Uniform Fabrics for Mien and Women (Institutional Textile) (1973)

ishings (Sublimation, Brittleness and Chlorine Retention) (Institutional Textile) (1973)

e Napery Other Than Damask (White or Dyed) (Napkin, etc.) (Institutional Textile) (1973)

$n$ Washable Uniform Fabrics for Men and Women (Heavy Duty) (Institutional Textile) (1973)

ven Sheet and Pillowcase Fabrics Other Than Durable Press (Institutional Textile) (1973)

ailored Uniform Fabrics, Heavyweight (Industrial) Suiting (Institutional Textile) (1973)

ilored Uniform Fabrics, Intermediate (Year Round) Suiting (Institutional Textile) (1973)

Woven Drapery (Curtain) Fabrics (Excluding Glass Fibers) (Institutional Textile) (1973)

(Curtain) Fabrics Handwashable at 105 Deg. F-No Bleach (Institutional Textile) (1973)

nt. Awning and Canopy Fabrics. Vinyl Coated or Lamin

nts and Claims for Special Characteristics and Finishes of Std. Definitions of Textile Terms for

woven Decorative Bedspreads, Pillows, and Bolster Fabrics

Curtain Fabrics (Sash Casement) (Excluding Glass Fibers

ng, and Canopy Fabrics, Painted, Printed, or Vinyl Coated

er Curtain Fabrics Handwashable at 105 Deg. F-No Bleach ance Requirements for Woven Drycleanable Overcoat Fabrics
std. Performance Requirements for Woven Lining Fabrics fo

std. Performance Requirements for Woven Lining Fabrics for

e Mechanics for Passenger Cars and Light Trucks (Course of

Mechanics for Buses, Heavy Trucks and Trailers (Course of Ives (1972)

(1972) ANSI Z11.275 Maintenance

Std. Spec. and Operating

Rec. Erection

olor Codes for Lubricant and Fluid Requirement Maintenance d Industrial Equipment (Machinery) (1971/ Rec. Pract. for

Area (1971)

vironment and Design Stds for (1972)

oses, $15 \mathrm{kV}$ and Less (1973) NEMA Ei2l

Std. for Nut

Rec. Pract. for

Aircraft Std. for

Std. for Electrical lndicatin

id. Installation Inspection, and Testing Requirements for s Measurement (Passen/ Rec. Pract. for Uniform Laboratory act Tests (1971) rdous Electromagnetic Radiation /

Std. for Earthquake Rec. Pract. for

972) ANSI C83/Rec. Test Meth. for Flutter Measurement of 974) An/ Rec. Test Meth. for Timing Error Measurements of Std. for

iesel and Gas Engine Gauge Borads, Protective Devices, and ng; Partitions; Plaster; Roofing) and Earthquake Recording ssures for Pneumatic Controllers and Transmission Systems and Tests for Strain Gage Linear Acceleration Transducers Definitions of Relay Terms (Electromagnetic eth. of Test. for Rotary Selector Switches (Radio, TV, and $n$ of Nonconductive Coatings on Nonferrous Substrate (Coil, Std. for AC -DC Transfer

Ft., $35 \mathrm{~mm} 100 \mathrm{Ft}$. and $70 \mathrm{~mm} 100 \mathrm{Ft}$. Spools for Recording Dimensions for Photographic Films in Rolls for Recording nes (1974) ANSI Z115.2 last/ Std Meth of Calibration Std. Guidelines for Safe Installation of Electrical Rec. for Radiological Monitoring Meths. and erformance Spec. for Portable X-Or Gamma Radiation Survey

Std for Requirements for Electrical Analog Indicating nuals for Installation, Operation and Repair of Laboratory pacitors (For Use Primarily in Transmitters and Electronic

longatoin of Elastomeric Yarns (Constant Rate of Extension Structural Clay s) (1966) Structural Clay (Properly Constructed Masonry) stics of Electrical Insulating Varnishes Applied Over Film Std. Meth. for Test. Film Rec. for ibution of Electrical Energy (197) Std. for Thermoplastic ibuti/ Std. for Crossed Linked Thermosetting Polyethylene Std. Spec. for Polyethylene Jacket for Electrical Safety Std. for Thermopla stic Std, for Splice, Electric, Permanent, Crimp Style, Pre Std. Spec, for Mineral Fiber Hydraulic Setting Therma orative (1972) Std. Meth. of Test. Cellular Glass Std. Spec. for
(Intitutional Textile) (1973)

Institutional Textiles (Durab

Institutional Textiles (1973)

Institutional Textiles) (1973)

(Institutional Textiles) (1973

Institutional Textiles) (1973)

Institutional Uniform Textile) (1973)

nstitutional Uniforms (Textile) (1973)

Institutional, Industrial and Commercial Buildings (197

Instruction) (1972)

Std. for Training of Automotiv

Std. for Training of $A$
Instructions for Chlorine Institute Std. Excess Flow Va

Instructions for Glass Capillary Kinematic Viscometers

Instructions for Steel Door Frames (1972)

Instructions, and Container and Filler Identification

Instrument Face Design and Location for Construction an Instrument Mount (1973)

Instrument Panel Lahoratory Impact Test Procedure-Hea

Instrument Stds. (Wording, Terminology, Phraseology, En

Instrument Transformers for (Electricity) Metering Purp

Instrument (Meter) Relays (1972)

Instrumentation and Electric Equipment During the Const

Instrumentation and Techniques for Exhaust Gas Emission

Instrumentation Criteria for Nuclear Power Plants (1974

Instrumentation for Motor Vehicle and Its Component Imp

Instrumentation for the Measurement of Potentially Haza

Instrumentation Glossary (Terms, Definitions) (1969)

Instrumentation Magnetic Tape Recorder / Reproducers (1

Instrumentation Magnetic Tape Recorder / Reproducers (1

Instrumentation Symbols and Identification Code (1973)

Instrumentation (1972)

Instrumentation (1973)

(Instrumentation) (1970)

$/$ and Medium Speed Stationary D

/Ra Cotta; Timber; Water; Ceili

Instrumentation) (1972)

Instrumentation) (1973) A NSI C83.26

nstrumentation) (1974)

R Film Thickness Determinatio

Instruments and Converters (1973)

Instruments and for Microfilm and Still Picture Cameras

Instruments and Miscellaneous Uses (1973)

Instruments for Verifying Time Load Indication of Machi

Instruments in Hazardous Dust Locations (Agricultural P

Instruments (1952)

Instruments (1971)

Instruments (1972)

lnstruments (1972)

Instruments) (1958) ANSI C83.15

Instruments) (1972) ANSI L14.287

Insulated Cavity Walls (Tech. Notes) (1964)

Insulated Cavity 6 In. Brick and Tile Walls (Tech. Note

Insulated Magnet Wire (1973)

/Thermal Aging Characteri

Insulated Steel Deck Roofing (1972)

Insulated Wire and Cable for the Transmission and Distr

Insulated Wire and Cable for the Transmission and Distr

lnsulated Wire and Cable (1972)

Insulated Wire and Cable (1972)

Insulated Wires (1971) AN SI C33.80

Insulated, (CIass 2) Wire Sizes 12 to 26 (1972)

Insulating and Finishing Cement (1964) A NSI Z98.33

Insulating Block (1972)

Insulating Board (Cellulosic Fiber), Structural and Dec
Std. Perfor

Perfor ANS

ANSI

Std. for Air Pre Std. Specs.

/ Dimensions and $\mathrm{M}$

ANSI

ANS

ANSI

NSI

ANSI

ANSI

ANSI

ANSI

ANSI

ANSI

ANSI

ANSI

ANSI

ANSI

ANSI

ANSI

ANSI

ANSI

ANSI

ANSI

ANSI

ANSl

ANSl

ANSI

RTI

ANSI

ANSI

$\mathrm{CHI}$

ASTM

STD

SAE

$\mathrm{SAE}$

NSA

SAE

SAE

EEl

NEMA

IEEF

SAE

ANSI

SAE

ANSI

SMPTE

EIA

ElA

ISA

DEMA

ICBO

ISA

NARM

EIA

ANSI

ANSI

Std ANSI

ASTM

ISA

NCRP

ANS

ANS

td. for Preparation of $\mathrm{Ma}$

ANS

EIA

BIA

BIA

ASTM

ASTM

FMS

NEMA

NEMA

ASTM

ASTM

UL

NSA

ASTM

ASTM

ASTM

L24.2.1

L24.2.10

L24.2.2

L24.2.3

L24.2.4

L24.2.8

L24.2.9

L24.2.7

L24.1.7

L24.2.5

L24.2.6

L24.5. 1

L24.3.3

L24.2.13

L24.3.5

L24.7.2

L24.2.11

L24.3.6

L24.2.12

L24.3.2

L24.3.1

L24. 1.5

L24. 1.9

L24. 1.10

L24.7.1

L24.6. 1

L24.1.2

L24.1.4

L24.1.1

L24.1.3

L24.3.4

L24.3.8

$* 10$

D18. 1

D18.2

42

D2515

105

J 223

J209

J92 I B

AIR818B

MSJ-11

I12

336

J254 
National Bureau of Standards KWIC Index of Engineering Standards

orative (1972)

ode Std. for Fiberboard Nail Base Sheathing and Structural Std. for Cellulosic Fiber Spec for Perlite

Meth. of Test for Size and Bulk Density of Refractory and Std. Classification of Std. Meth. of Test for Thermal Conductivity of Std. Spec. for Structural

of Test for Dielectric Breakdown Voltage and Strength of Glazing Spec. for Sealed Spec, for Sealed

romatic Hydrocarbons (Askarels) for Canacitors (Electrical Std. Definitions of Terms Relating to Electrical Std. Spec. for Air as an Electrical

lex Permittivity (Dielectric Constant) of Solid Electrical

d. Meth. of Test for Voltage Endurance of Solid Electrical and Dielectric Constant (Permittivity) of Solid Electrical d Establishment of Temperature Indexes of Solid Electrical

. of Test for Impact Resistance of Plastics and Electrical

Thermal Failure Under Electric Stress of Solid Electrical liquid Contaminant, Inclined Plane Tracking and Erosion of nsi Cl

si C5l

it Breakers (1973) Std. Spec. for Continuity of Quality of Electrical Std. Spec. for Continuity of Quality of Electrical Std. Spec. for Uninhibited Mineral breakers (197) Std. Spec. for Oxidation Inhibited Mineral er (1969) ANSI C/ Guide for Acceptance and Maintenance of Std. Meth. of Test for Oxidation Stability of New Mineral 1) Std. Meth. of Test for Dielectric Breakdown Voltage of s) Std. Meth. of Test for Dielectric Breakdown Voltage of Std. Spec. for Asbestos Cement Fiberboard Std. Spec. for Asbestos Cement Plastic Foam Core r Type (1973) ANSI C59.50 Std. Spec. for Electrical Std. Test Meth. for Structural Std. Spec. for Asphalt

ANSI A 109.18 ec. for Polyethylene Plastic Pressure Sensitive Electrical
stant Vinyl Chloride Plastic Pressure Sensitive Electrical stant Vinyl Chloride Plastic Pressure Sensitive Electrical td. Spec. for Polyester Film Pressure Sensitive Electrical r Tetrafluoroethylene Backed Pressure Sensitive Electrical Safety Std. for Extruded Thermoplastic h. of Test for Thermal Aging Characteristics of Electrical Std. Meth. of Test for Thermal Degradation of Electrical

Std. for Strip-High Temperature Terminal Block, Base std. Meth. of Test for Gas Content of Cable and Capacitor . Meth. for Investigating the Compatibility of Transformer ing Ozone Resistant Ethylene-Propylene-Rubber Integral Std. Spec. for Mineral Fiber Roof Std. Spec. for Perlite Thermal

light Frame Type for the Purpose of Conserving Their Sound or, and Fl/ Uniform Building Code Std. for Airborne Sound Std. Spec. for Spray Applied Fibrous Thermal Rated on a Symmetrical C/ Std. Requirements for External Std Spec for Ozone Resisting Butyl Rubber std. Spec. for Synthetic Rubber Heat or Moisture Resisting td. Spec. for Synthetic Rubber Heat and Moisture Resisting 4) ANSI C8.1

4) ANSI C 8.24

4) ANSI C8.40 ngs (1970) ! Std. Spec. for Natural Rubber Heat Resisting Std. Spec, for Synthetic Rubber Std. Spec. for Synthetic Rubber Std. Spec. for Mineral Fiber Blanket Thermal Std. Rec. Pract. for Evacuated Reflective Std. for Voltage Values for Preferred Transient Std. Meth. of Test for Thermal

nted, Flexible, Heat Shrinkable (19/ Spec. for Electrical alues for Outdoor AC High Voltage Circuit Breaker External s Rated $\mathrm{O} / \quad$ Std. Requirement for Switching Impulse Voltage form Wol Std. Test Procedure for the Evaluation of Sealed temperatur/

Std. Rec. Pract. for Prefabricated Reflective

Measurement of Discharge (Corona) Pulses in Evaluation of Std. Meth. of Test, Laminated Tubes Used for Electrical th. of Test. Adhesives Relative to Their Use as Electrical est. Varnished Cottonfabrics and Tapes Used for Electrical istance of Low Density Mineral Fiber Blanket Type Building Std. Meth. of Test. Vulcanized Fiber Used for Electrical termining the Maximum Use Temperature of Preformed Thermal osslinked Polyolefin Heat Shrinkable Tubing for Electrical

Test. Rigid Sheet and Plate Materials Used for Electrical Std. Meth. of Test. Varnishes Used for Electrical ing Dimensions of Rigid Rods and Tubes Used for Electrical Std. Spec. for Perlite Loose Fill

Std. Spec. for Cellular Glass Block and Pipe Thermal Std. Spec. for Mineral Fiber Loose Fill Thermal Std. Definitions of Terms Relating to Electrical Std. Meth. of Test for Thickness of Solid Electrical Uniform Building Code Std. for Impact Sound Spec. for Cellulosic Fiber (Wood Base) Loose Fill Thermal
Insulating Board (Cellulosic Fiber), Structural and Dec

Insulating Board (1973)

Insulating Board (1973) ANSI A149.1

Insulating Concrete Roof Decks (1973)

Insulating Fire Brick (1970) ANSI Al11.18

Insulating Fire Brick (1970) ANS1 Al11.22

Insulating Fire Brick (1972) ANS1 A111.28

Uniform

ICBO

USC

CSI

Insulating Formboard Made from Cellulosic Fibers (1966)

Insulating Gases at Commercial Power Frequencies (1974)

Std. ASTM

ASTM

ASTM

ASTM

ASTM

SIGMA

SIGMA

Insulating Glass Units (1973)

Insulating Liquid) (1973) ANSI C59.11

/R Chlorinated a ASTM

Insulating Liquids and Gases (1974)

Insulating Material in Electrical Equipment (1973)

ASTM

Insulating Materials at Microwave Frequencies and Tempe

ASTM

ASTM

Insulating Materials Subjected to Partial Discharges (C

ASTM

Insulating Materials (1970)

Insulating Materials (1972)

Insulating Materials (1973)

/Ent Loss Characteristics ASTM

Insulating Materials (1973)

Insulating Materials (1973) ANSI C59.135

the Thermal Evaluation an IEEE Std. Meth ASTM

Insuling Oil for High Pressure Cable Systems (1972) a f Test for ASTM

lating Oil for Low Pressure Cable Systems (1972) an

ASTM

ASTM

ASTM

Insulating Oil for Use in Transformers and in Oil Circu
Insulating Oil for Use in Transformers and Oil Circuit

Insulating Oil in Equipment as Received from Manufactur

ASTM

IEEE

ASTM

Insulating Oils of Petroleum Origin Under Impulse Condi

ASTM

Insulating Oils of Petroleum Origin Using VDE Electrode

ASTM

Insulating Panels (1972)

Insulating Panels (1972)

Insulating Paper and Paperboard-Sulfate or Kraft Laye

ASTMI

ASTM

ASTM

Insulating Roof Deck (R1970)

Insulating Siding Surfaced with Mineral Granules (1970)

Insulating Tape (1973)

Insulating Tape (1973)

Insulating Tape (1973) ANSI C59.9

Insulating Tape (1973) ANSI C59.96

ASTM

Insulating Tape (1974)

lnsulating Tubing (I972)

Insulating Varnishes Applied Over Film Insulated Magnet

ure Resi

ASTM

ASTM

ASTM

Insulating Varnishes by the Helical Coil Meth. (1973)

Insulating (1973)

Insulating) Oils (R1969) A NSI C59.23

Insulation and Construction Materials in Askarels (1973

Insulation and Jacket (197

Insulation Board (1972)

Insulation Efficiency (1973)

Insulation Field Test of Partitions (Wall, Ceiling, Flo

Insulation for Elevated Temperature (1972)

Insulation for Outdoor AC High Voltage Circuit Breakers

Insulation for Wire and Cable (1974) ANSI C8.29

Insulation for Wire and Cable (1974) ANSI C8.37

Insulation for Wire and Cable (1974) ANSI C8.38

Insulation for Wire and Cable, 75 Deg. C Operation (197

Insulation for Wire and Cable, 75 Deg. C Operation (197

Insulation for Wire and Cable, 75 Deg. C Operation (197

Insulation for Wire and Cable, 90 Deg. C Operation (197

Insulation for Wood Frame and Light Construction Buildi

Insulation in Cryogenic Service (1973)

Insulation Levels (TIL) (1971)

Insulation of Quality Packages (1972)

Insulation Plastic Tubing, Irradiated Polyolefin, Pigme

Insulation Rated on a Symmetrical Basis (1972)

Insulation Strength for AC High Voltage Circuit Breaker

Insulation Systems for AC Electric Machinery Employing

Insulation Systems for Equipment and Pipe Operating at

Insulation Systems (1973)

Insulation (1970)

Insulation (1970)

Insulation (1970) ANSI C59.31

Insulation (1970) ANSI Z98.35

Insulation (1971) ANSI C59.78

Insulation (1971) ANSI Z98.28

Insulation (1972)

Insulation (1972) ANSI C59.13

Insulation (1972) ANSI C59.30

Insulation (1972) ANSI C 59.33

Insulation (1973)

Insulation (1973)

Insulation (1973)

Insulation (1973)

Insulation (1973)

Insulation (1973)

Insulation (1973)
ITd. Meth. for Detection and ASTM

ASTM

Std. Me ASTM

Std. Meth. of T ASTM

/Tion of the Thermal Res ASTM

ASTM

Std. Spec. for Cr ASTM

Std. Meth. of ASTM

ASTM

Std. Meth. of Measur ASTM

ASTM

ASTM

A STMI

ASTM

ASTM

ICBO

Std. ASTM
Std. Meth. for De ASTM

$\mathrm{C} 209$

UBCS25.24

PS57

0352]

C134

C155

C 182

D2477

70-7-1

65.7.2

D2233

D2864

D3283

D2520

D2275

D150

98

D256

D3151

D2303

D 1819

D 1818

D 1040

D3146

64

D2112

D3300

D1816

C551

C659

D) 305

D2164

D1226

D3006

D3005

D230 1

D2484

D2686

224

D3251

D3145

1063

D831

D.3255

WC8/1

C726

C728

E497

UBCS $35-3$

C720

343

D574

D1352

D1520

D1679 
c. Pract. for Application of Spray Applied Fibrous Thermal of Test for Moisture Content of Oil Impregnated Cellulosic ltage, Low Current, Dry Arc Resistance of Solid Electrical st. Coating Powders and Their Coatings Used for Electrica poly(Vinyl Chloride) Heat Shrinkable Tubing for Electrica

e. Metal, Plastic, etc.) for Ornamentation, Protection, or Std. Meth. of Test. Shellac Used for Electrical n Factor of Expanded Cellular Plastics Used for Electrical for Mine Power Cables with Ethylene-Propylene-Rubber volts and Having Ozone Resistant Ethylene Propylene Rubber electrical Power Cables with Ethylene-Propylene-Rubbe st. Coating Powders and Their Coatings Used for Electrical Std. Meth. of Test. Heat Shrinkable Tubing for Electrical Std Meth of Test. Hookup Wir essure Sensitive Adhesive Coated Tapes Used for Electrical of Sampling and Test. Untreated Paper Used for Electrical

Electrical Power Cable with Ethylene-Propylene-Rubber d. Rec. Pract. for Selection of Vapor Barriers for Thermal Std. Wet Process Porcelain Std. Wet Process Porcelain

td. Spec. for Communication and Signal Pin Type Lime Glas Std. Wet Process Porcelain

d. Application Guide for Porcelain Suspension (Power Line) Low and Medium Speed Stationary Diesel and Gas Engine Air loy Seamless Round Condenser and Heat Exchanger Tubes with its Alloy Seamless Condenser and Heat Exchanger Tubes with Std. for Industrial and Other Direct Current Std. for Synchronous

Std for Frame Assignments for Alternating-Current for Definite Purpose Motors and Generators (Fractional and ernating Current and Direct Current Motors (Fractional and e of Alternating and Direct Current Motors (Fractional and g and Direct Current Motors and Generators (Fractional and

$\mathrm{g}$ and Direct Current Motors and Generators (Fractional and

$\varepsilon$ and Having Ozone Resistant Ethylene-Propylene-Rubbe

Voltage Integrally Mounted Thermostats For/ Line Voltage

rally Mounted Thermostats for Electric Heater Line Voltage ne Supply Transactions Within the Air Tra/ Spec, for Std. rces (Medical Radiation-Radiology) (1973)_ Std. for 72) ANSl B93. Std. Meth. for Determining the Fabrication roducts for Exterior Use (1959) A/ Std. Meth. of Test for Rec. Pract. for Service Brake Structura tion Vehicles F/ Rec. Pract. for Service Brake Structural eed of $16 \mathrm{Mm}, 8 \mathrm{~mm}$ and Super 8 Reversal Color Camera Film for Production Disc and Spoke Wheels, and Demountable Rims (1973) Sid. Color Marking of Compressed Gas Cylinders (1971) Std. Nomenclature for Glass Bulb Std. Definitions for High Std. Meth. for the Designation of High or Compatibility of Materials with Liquid Oxygen (Reaction Uniform Plumbing Code: Traps and std for the Electrical Characteristics of Class a Closure d Terminology) (19/ Guidelines for Describing Information terchange (I972) Std. for Std. for Recorded Magnetic Tape for Information presentation for Calendar and Ordinal Date for Information for Interchange Rolls of Perforated Tape for Information tates (Including the District of Columbia) for Information

Characters of American National Std. Code for Information and Data Terminal Equipment Employing Parallel Binary Data ode) of the Countries of the United States for Information Std. for Recorded Magnetic Tape for Information Std. for Recorded Magnetic Tape for Information cpi, P/ Std. for Unrecorded Magnetic Tape for Information heads Used in Roadway Lighting Equip/ Std. for Mechanical ds for Roadway Lighting Equipment (1/ Std. for Mechanical 1973) NEMA SHl0 Std. for Metal Head and Reílector 1973) EEI TDJ-140 Std for Metal Head and Reflector rmat, for Numerically Controlled Equipment (1974) or Contouring and Contouring-Positioning Nume/ otective Signalling Systems (1969) ANSI SE3.7 Guide for Protective Relaying of Utility-Consumer quipment Employing Parallel Binary Data Interch ic Directional Control Valves (1973) ANSI B93.33

Std. for Std.

or Opaque Metal Oxide and Metallic Coatings by Double Beam Std. for Radio

ilicon Wafers and Metallization Thickness by Multiple Beam or Determining Surface Strength of Coated Papers Using the earing Alloys / Std. Meth. of Detecting Susceptibility to Recommendation for Installation of Recommendation for Installation of Recommendation for Renovation of of Test for Resistance to Growth of Mold on the Surface of Std. Form of Agreement Between Owner and Architect for
Insulation (1973)

nsulation (1973)

Insulation (1973)

Insulation (1973)

Insulation (1973)

Insulation (1973)

Insulation (1973) ANSI C59.18

Insulation (1973) ANSI C59.84

Insulation (1973) IPCEA S68-516

nsulation (1973) IPCEA S68-516

Insulation (1973) IPCEA S68-516

Insulation (1973)

Insulation (1974)

Insulation (1974) ANSI C59.136

Insulation (1974) ANSI C 59.77

Insulation, 2001-5000 Volts (I973) IPCEA S68.516

Insulations (1973)

Insulators (Apparatus, Cap and Pin Type) (1971)

Insulators (Apparatus, Post Type) (1971)

Insulators (R 1970)

Insulators (Suspension Type) (1971)

Insulators (1974)

Intake and Exhaust Systems (1972)

Integral Fins (1973)

Integral Fins (1974)

Integral Horsepower Generators (1972)

Integral Horsepower Generators (1972)

Integral Horsepower Induction Motors (1972)

Integral Horsepower) (1972)

Integral Horsepower) (1972)

Integral Horsepower) (1972)

Integral Horsepower) (1972)

Integral Horsepower) (1972) /Dimensions
Integral lnsulation and Jacket (1973) IPCEA S68-516

Thermostats for Electric Heater Line

Integrally Mounted Thermostats for Electric Heaters (19

Integrated Data Processing Systems Designed to Streamli

Integrity and Test Spec. for Selected Brachytherapy Sou.

Integrity of a Hydraulic Fluid Power Filter Element (I9

Integrity of Glue Joints in Structural Laminated Wood P

Integrity Test Procedure-Passenger Car (1971)

Integrity Test Procedure for Trucks, Buses, and Combina

ntended for Direct Projection in Motion Picture Photog

intended for Highway Use on Trucks, Buses, Trailers, an

Intended for Medical Use in the United States (Safety)

Intended for Use with Electron Tubes and Electric Lamps

tensity Discharge Lamp Ballasts and Transformers (197

Intensity Discharge Lamps (1972)

Intensity Meth.) (1973)

interceptors (1973)

Interchange Circuits (Electronics) (1974)

Interchange Formats (Computer Software Documentation an

Interchange Rolls of Perforated Tape for Information in

Interchange (1600 cpi, PE) (1973)

Interchange (1971)

Interchange (1972)

Interchange (1972)

Interchange (1973)

lnterchange (1973)

Interchange (1973)

lnterchange (200 cpi, NRZl) (1973)

Interchan ge $(9-$ Track 200 and $800 \mathrm{cpi}, \mathrm{NRZl}$, and I600

Interchangeability of Socket Supports for Use in Metal

Interchangeability of Socket Supports Used in Metal Hea

Interchangeability Used in Roadway Lighting Equipment

Interchangeability Used in Roadway Lighting Equipment

Interchangeable Perforated Tape Std., Variable Block Fo

Interchangeable Perforated Tape Variable Block Format F

Interconnected Porosity of Sintered Powder Metal Struct

Interconnection Circuitry of Noncoded Remote Station Pr

Interconnections (1973)

Interface Between Numerical Control and Data Terminal E

Interfaces for 4 Way General Purpose lndustrial Pneumat

Interference Fit Thread (1972)

Interference Microscope Technique (1973)

Interference Wideband Filters (1974)

Interfiber Bond Tester (1973)

Intergranular Attack in W rought N

Interior Ceramic Tile Floors (1973)

Interior Ceramic Tile Walls (1973)

Interior Ceramic Tile Walls, Floors, and Stairs (1973)

Interior Coatings in an Environmental Chamber (1973)

Interior Design Services (1972)

Std. Re ASTM

id. Meth. ASTM

C762

D3277

D495

D3214

UBC $* 3.30$

D411

D 1673

WC8/5

WC 8

WC $8 / 6$

D3214

D2671

D3032

D 1000

D202

WC8/7

C 755
C29.8

C 29.8
C 29.9

D879

C 29.2

ASTM

St NEMA

$\mathrm{HV} 2$

*1-8

B404

B359

MGI-15

MGl-16

$\mathrm{MGl} 3$

MG1-18

MG 1-10

MGl-12

MGl-14

MGl.11

W C $8 / 1$

DC 13

DC 13

200

N44.1

ANSI

NFLDP

ASTM

$S A E$

SAE

ANSI

SAE

CGA

ANSI

NSI

ANSI

T3.10.8.4

D1 101

J229

PH22.146

J267

C9

C82.9

C78.380

F371

UPC $* 1.7$

ICBO

EIA

ANS1

ANSI

RS410

$\times 10.1$

X3.34

$\times 3.39$

X3.30

X 3.34

X3.38

$\mathrm{X} 3.32$

RS408

X 3.31

X3.14

$\times 3.22$

$\mathrm{X} 3.40$

$\begin{array}{ll}\text { ANSI } & \text { X3.40 } \\ \text { EEI } & \text { TDJ-143 }\end{array}$

NEMA SHl3

EEI

NEMA

NSA

EIA

ASTM

NEMA

IEEE

ElA

NFLDP

ANSI

TDJ-1 40

$\mathrm{SH} 10$

955

RS274.B

B328

SB3

357

RS408

T3.21.1

B1.12

B588

ElA

RS416

F388

UM-534

G28

I0I.I06

201.208.I

TCA

TCA

400.600

ASTM D3273

B707 
) (Packaging) (1968) ANSI MH12/ Std. Meth. of Determining her Technical Data) (1973)

emarked Plywood (1967) t. Test Meth. for Determining Window Fogging Resistance of Rec. Pract. for Flammability of Automotive std. Spec. for Creosoted End Grain Wood Block Flooring for Std. Meth. of Test for Efflorescence of Uniform Building Code: Rec, for

Adhesives for Structural Laminated Products for Use Under d Cement and Cement Lime Plastering. Exterior (Stucco) and d Cement and Cement Lime Plastering, Exterior (Stucco) and ubes (1973) ANSI Bl25/ Std. Spec, for Seamless Cold Drawn ce (1972) ANSI BI25.23 Std. Spec. for Seamless 1 Std. Spec. for Carbon Steel Pressure Vessel Plates for rements for Backflow Preventers in Water Supply Lines with Thick Hollow Metal Door and Frame Preparation for Offset ates for Machine Parts and Genera/ Std. Spec. for Low and ressure Vessels (1972) ANSI G35.2 Std. Spec. for Low and ructural Quality (1970) ANSI G24./ Std. Spec, for Low and uirements for Woven Drycleanable Tailored Uniform Fabrics, 1972) le) (1973) Std. for Mining and Logging Tires Used in Std. for Handle, Latching Std. Test. Procedure for ntary Ventilation in a Ship's Cargo Hold During the Use of Std. for Safety for Grounding and Bonding Equipment popcorn, Driel Meth. of Analysis of X.Ray Examination for ied Pea/ Meth. of Analysis of Cracking Flotation Test for Rec. Pract. for Automatic Reset

Bul. on Round Thread Casing Joint Strength with Combined Rec. Pract. for Calculating Stress in Plastic Pipe Under meth. of Test for Time to Failure of Plastic Pipe Constant Meth. for Cross Directional

(1968) Std. Rec. Pract. for Std. for Bolt . Std. for Bolt Std. for Bolt Std. for Bolt

Std. Spec. for Carbon Steel Externally and protruding Head, Self Locking)/ flush Head, Self Locking) (1972/

Protruding Head, (1972)

Flush Head, Self Locking) (1972) lable Head Self Locking) (1972/ truding Head, Self Locking) (1/ 72) et: Ammunition and Targ/ Spec. for Fastener (Blind, Std. Metric Pract. Guide to the Use of SI Std. for Fastener (Blind,
Std. for Fastener (Blind Std. for Fastener (Blind. Fastener (Blind, Std. for Fastener (Blind, Std. for Fastener (Blind, Spec. for imeters and Companion Dosimeter Chargers (1971) Std. for ts for X-Radiation Limits for AC High Voltage Power Vacuum Safety Std. for Ground Fault Circuit of Electrical Transmission and Distribution Facilities and enide in Poultry Mashes (Feed) for Prevention of Cecal and ine in Poultry Feeds and Premixes for Control of Cecal and Std. Rec. Pract. for Static Bend and Torsion Test. of

Std. for

ms (1969) ANSI C33.70

Cold Weather Masonry Construction, Safety Std. for

Std. Analytical Meth. for Determining Candy Color and of Test for Pressure Sensitive Tack of Adhesives Using an on and Construction Materials in Askarels/ Std. Meth. for orm (Acquisition and Codification of Data Derived from the

4330 Modified) (1973)

4340 Modified) (1973)

ium Speed Stationary Dies/ Std. Spec. for Magnesium Alloy Steel Castings, Low Alloy Steel Castings. Low Alloy Steel Castings Std. Pract. for Preparation of Std. for stem Design of Fine Pitch on Center Face Gears for 20 Deg. Qualitative Meth of Analysis of Bromates and Qualitative Meth. of Analysis of Bromates and overs (Cereal Chemistry) (1962) ysis of Oxidizing Agents in Flour: Reaction with Potassium Std. Spec. for Photographic Grade Potassium Std. Analytical Meth. Determining Meth. of Analysis of t, and Corn Flour (Cereal Chemistry) (1962) stry) (1967) um and Other Veget/ Meth. of Analysis of eeze-Coolant Concentrate (Glycol or Alcohol Base) by the
Interior Dimensions of Fiberboard Boxes (Box Gage Meth. Interior Floors Exposed to Wet and Dry Conditions (1970 Interior Guide (Spec. for Installation, Finishes and $\mathrm{Ot}$ Interior Gypsum Plaster (1972)

Interior Panel Coatings and Overlays on DFPA Grade Trad

Interior Trim Materials (1973) ANSI Z265. I

Interior Trim Materials: Horizontal Test Meth. (1972)

Interior Use (1970) ANSI 010.1

Interior Wall Paints (1973)

Interior Walls and Ceiling Finish (1973)

Interior Wood Paneling (1972)

Interior (Dry Use) Exposure Conditions (1972) ANSI Z197

Interior (1971)

Interior (1971)

Std. Spec. for Portlan

Intermediate Alloy Steel Heat Exchanger and Condenser T

Intermediate Alloy Steel Still Tubes for Refinery Servi

Intermediate and Higher Temperature Service (1972) ANSI

Intermediate Atmospheric Vent (1972)

Intermediate Pivot (1973)

Erformance Requi

Intermediate Tensile Strength Carbon-Silicon Steel PI

Intermediate Tensile Strength Carbon Steel Plates for P

Intermediate Tensile Strength Carbon Steel Plates of St

Intermediate (Year Round) Suiting (Institutional Textil

Intermittent Highway Service (1974)

Internal and External Release Components and Assembly (

nternal Bond Strength of Paperboard (Z-Direction Tensi

Internal Bursting of Composite Tubes and Cores (1974)

Internal Combusion Engines (1970) Guide for

InternaI ElectricaI Wiring Systems) (1972) ANSI C33.8

Internal Insect Infestation in Cereal Grains, Unpopped

Internal Insects in Cereal Grains, Unpopped Popcorn, Dr

Internal Mounted Circuit Breaker (1971)

Internal Pressure and Bending (1972)

Internal Pressure (1967) A NSI B72.10

Internal Pressure (1974) ANSI B72.6

Internal Tearing Resistance of Paperboard (1972)

Internal Temperature Measurements in Ablative Materials

Internal Wrenching, Steel 1/4-28 Thru 1-1/8.12 (1973)

Internal Wrenching, Steel, 1.2500-12 (1973)

Internal Wrenching, Steel, I.3750-12 (1973)

Internal Wrenching, Steel, 1.5000-12 (1973)

Internally Threaded Std. Fasteners (1968) ANSI G38.5

Internally Threaded, External Sleeve, General Purpose,

Internally Threaded, External Sleeve, General Purpose,

Internally Threaded, External Sleeve, High Temperature,

Internally Threaded, External Sleeve, High Temperature,

Internally Threaded, External Sleeve, Light Weight, MIL

Internally Threaded, External Sleeve, Light Weight, Pro

Internally Threaded, External Sleeve, Self Locking) (19

International and Domestic-Trap, Clay Pigeon, and Ske

International System of Units) (1972) ANSI Z210.1

International (Iso) Freight Containers (1972)

Interrelationship of Quartz Fiber Electrometer Type Dos

Interrupters Used in Power Switchgear (1972)

Interrupters (1972)

Interruptions to Customer Service (1973)

Intestinal Coccidiosis in Chickens (Cereal Chemistry)

Intestinal Coccidiosis (Cereal Chemistry) (1962)

Intramedullary Rods (Surgical Implants) (1973)

Intraoral Dental Radiographic Film (1972)

Introduction (Tech. Notes) (1967)

Intrusion Detection Units for Burglary Protection Syste

Inventories of Nuclear Materials (1972)

Inversion Content of Corn Syrup Unmixed (1952)

Inverted Probe Machine (1971) ANSI Z197.32

Investigating the Compatibility of Transformer Insulati

Investigation of Scuba Diving Accidents) (1973)

Investment Castings (1972)

Investment $0.80 \mathrm{Cr}-1.8 \mathrm{Ni}-0.35 \mathrm{Mo}(0.28-0.36 \mathrm{C})(\mathrm{SAE}$

Investment $0.80 \mathrm{Cr}-1.8 \mathrm{Ni}-0.35 \mathrm{Mo}(0.38-0.46 \mathrm{C})(\mathrm{SAE}$

Investment, $0.95 \mathrm{Cr}-0.20 \mathrm{Mo}(0.25-0.35 \mathrm{C})$ (1973)

Invitations for Bids and Detailed Spec. for Low and Med

Involute Splines and Inspections (1970) A NSI B92.1

Involute Spur Pinions (1973)

lodates in Flour Improvers (Cereal Chemistry) (1962)

Iodates in Flour (Cereal Chemistry) (1962)

Iodide Test Meth. for Ammonium Persulfate in Flour Imp

Iodide (Cereal Chemistry) (1962)

lodide, KI (1972)

Iodine Affinity of Corn Starch (1973)

Iodine in Mineral Mixed Feeds (Cereal Chemistry) (1962)

Iodine in Water (1973) ANSI N159

Iodine Meth. of Analysis for Insect Eggs in White, Whea

Iodine Number in AlI Edible Fats and Oils (Cereal Chemi

Iodine Number (Wijs Meth.) Content in Corn, Grain Sorgh

Corn, Grain Sorgh

ASTM

ASTM

CRA

CSI

APA

SAE

SAE

ASTM

ASTM

ICBO

WWPA

ASTM

ANSI

ASTM

ASTM

ASTM

ANSI

ASTM

ASTM

ASTM

ANSI

TRA

NSA

TAPPI

CCTI

UN

$\mathrm{AACCH} \quad 28-2$

AACCH 28-22

SAE J258

API

ASTM

Std. ASTM

TAPPI

ASTM

NSA

NSA

NSA

NSA

ASTM

NSA

NSA

NSA

NSA

NSA

NSA

NSA

NRA

ASTM

ANSI

ANSI

UL

IEEE

AACCH $\quad 18-25$

/Oxal AACCH 18-35

ASTM F383

ANSI PH8.I

BIA

UL

ANSI

1

CR

639

E-12

ASTM D3255

/Ort F ANSI Z86.2

ASTM B 403

SAE AMS5328A

SAE AMS5330A

SAE AMS5336B

DEMA $* 1.24$

SAE J498C

Std. for Sy AGMA 203.03

$\mathrm{AACCH} \quad 48-40$

AACCH $48-41$

AACCH 48-61

AACCH $48-02$

ANSI PH4.201

CR B. 28

AACCH $40-35$

ASTM D2334

$\mathrm{AACCH} \quad 28-44$

$\mathrm{AACCH} \quad 58-30$

$\begin{array}{ll}\mathrm{CR} & \mathrm{H}-32\end{array}$

Iodine Reagent Meth. (1973) 
(I962) Rec. for Waste Disposal of Phosphorus. 32 and Electrometric Meth. of Analysis for Hydrogen Colorimetric Meth. of Analysis for Hydrogen Std. Test Meth. for Hydrogen Melh. for Continuous Determination of Sodium in Water by
Std. Meth. of Field Sampling of Melh. for Continuous Determination of Sodium in Water by
Std. Meth. of Field Sampling of f Test for Physical and Chemical Properties of Particulate s (1974) Std. Meth. of Test for W ater Soluble Halide
7) Std. Meth. of Test for Hydroxide Std. Meth. of Test for Fluoride

Std. Meth. of Test for Sulfite

Test Procedure for Semiconductor Radiation Detectors for assification System for Polymeric Materials for Service in il Saturate Fractions (1971) ANSI Z1/ Std. Meth. for High inted Wiring Board Electric Connectors (1974) ANSI C83.88,

heet, and Strip (1973) pe and Tube (1972) ANSI H34.16 Application at High T/ si $\mathrm{H} 34.40$ Std. Spec. for Nickel. Std. Spec. for Nickel . for Centrifugally Cast Std. Spec. for Nickel. Std. Spec. for Nickel. Std. Spec. for Nickel ment (1973) ANSI C83.92 Std. for (1973) Std. Spec. for Seamless and Welded Chromium-Nickel -
Sid. Spec. for Nickel-Chromium Sid. Spec. for Nickel-Chromium
Std. Spec. for Nickel-Chromium .

Std. Spec for Electric Fusion Welded Chromium-Nickel service (1974) ANSl G/

Sid. Spec. for Chromium-NickeI Std. Spec. for Nickel-Chromium Std. Spec. for Copper

Std. Spec. for Nickel Chromium Std. Spec, for Nickel Chromium Molybdenum Std. Spec. for Nickel Molybdenum Chromium Std. Spec. for NickeI Chromium Std. Spec. for Nickel-Molybdenum-Chromium ical Analysis of Nickel-Chromium and Nickel-Chromium resisting, Maraging, and Other Similar Chromium-Nickel for Water and Other Liquids (1971) ANSI A2/ Std. for Cast Spec, for Std. Spec. for Aluminum for Use in Spec. for

ract. for Metallizing with Aluminum and Zinc Protection of Std. Meth. of Test for 6/ Alloy Bars and Forgings, Corrosion and Heat Resistant, si G63.4 Alloy Bars, Forgings, and Rings, Heat Resistant, for High Temperat/ Std. Spec. for Precipitation Hardening ete Aggregates (Sand, Crushed Stone, Gravel and Air Cooled Std. Meth. for Spectrochemical Analysis of Blast Furnace 973) Std Rec. Pract for Production and Preparation of Gray Std. Spec. for Pearlitic Malleable Spec. for Automotive Gray Std. Spec. for Ductile Std. Spec. for Austenitic Gray Std. Definitions of Terms Relating to Std. Spec. for Nickel Std. Spec, for Nickel

Strip (1972) ANSI H34.17 ) ANSI H34.18

Gener 972)

oy Castings for Gener and Tube (1972) ANSI H34. $s$ and Sugars, and /

\section{0) ANSI G63.1}

er Liquids (1971) ANSI A2/ Std. Meth. for Spectrochemical Analysis of High Purity Qualitative Meth. of Analysis of Added Quantitative Meth. of Analysis of Tent. Meth. of Analysis for (1973/ Std. Spec. for Seamless and Welded Chromium Nickel , Sheet, and Strip (1973)/

Std Spec, for Chromium Nickel cating the Thinnest Spot in a Zinc (Galvanized) Coating on Fencing (1973)/ Std. Spec. for Zinc Coated (Galvanized) Std. Meth. of Test for Aluminium in Std. Meth. of Test for Calcium and Magnesium in Std. Meth. of Test for Aluminum Oxide in Safety Std. for Gasketed Joints for Cast act. for Polyethylene Encasement for Gray and Ductile Cast ined Molds for Gas (1971) Std. for Ductile std. for Polyethylene Encasement for Gray and Ductile Cast Sid. for Enameled Cast

Std. for Rubber Gasket Joints for Cast and Ductile Spec. Data for Hubless Cast
Iodine Solution Content of Reagents and Indicators (197 Iodine-131 for Medical Users (1951)

Ion Activity (PH) in Flour and Bread (Cereal Chemistry) Ion Activity (PH) in Flour (CereaI Chemistry) (1962)

Ion Concentration of Dry Adhesive Films (1970)

Ion Electrode (1973)

Ion Exchange Materials (1972)

Ion Exchange Resins (1972)

Ion in Halogenated Organic Solvents and Their Admixture

Ion in Industrial Water and Industrial Waste Water (196

Ion in Water (1972)

Ion in Water (1972)

Ionizing Radiation (1969) ANSI N42.1

Ionizing Radiation (I971) ANSI N4.1

Ionizing Voltage Mass Spectrometric Analysis of Gas-O

Ipc C. $405 \mathrm{~A}$

Iron-All Types (1974)

Std. General Document for Pr

Iron-Chromium-Manganese-Molybdenum Alloy Plate, S

Iron-Chromium-Molybdenum-Copper Alloy Seamless Pi

Iron-Chromium-Nickel High Alloy Tubing for Pressure

Iron-Chromium Alloy Plate, Sheet, and Strip (1973) an

Iron-Chromium Alloy Rod and Bar (1973) ANSI H34.39

Iron-Chromium Alloy Seamless Pipe Tube (1973) ANSI H3

Iron-Core Power Filter Inductors for Electronic Equip

Iron-Molybdenum-Copper-Columbium Stabilized Alloy

Iron-Molybdenum-Copper Alloy Plate, Sheet and Strip

Iron-Molybdenum-Copper Alloy Rod (1973)

Iron-Molydenium-Copper-Columbium Stabilized Alloy

Iron Alloy Castings (25-12 Class) for High Temperature

Iron Alloy Plate, Sheet, and Strip (1970) ANSI H34.10

Iron Alloy Plate, Sheet, Strip, and Rolled Bar (1973)

Iron Alloy Rod and Bar (1970) ANSI H34.4

Iron Alloy Rod (1972)

Iron Alloy Rod (1972)

Iron Alloy Seamless Pipe and Tube (1970) ANSI H34.3

Iron Alloy Sheet and Plate (I971) ANSI H34.44

Iron Alloys (1973)

Iron Alloys (1973)

Std. Meth. for Chem
Mical Analysis of Stainless, Heat

ron and Ductile Iron Fittings, 2 In. Through $48 \mathrm{In}$./S

Iron and Steel Gas Welding Rods (1969) ANSI W/3.2

Iron and Steel Manufacture (1960) ANSI H38.25

Iron and Steel Scrap (1971)

Iron and Steel (1967)

Iron Bacteria in Water and Water Formed Deposits (1972)

Iron Base $-13.3 \mathrm{C}_{\mathrm{r}}-38 \mathrm{Ni}-5.5 \mathrm{Mo}-0.85 \mathrm{Cb}-2.5 \mathrm{Ti}-1$.

Iron Base-35.5 Ni-18.5 $\mathrm{Cr}-1$. 1 I I (1973)

Iron Base Sintered Bearings (Oil Impregnated) (1970) an

Iron Base Superalloy Bars, Forgings, and Forging Stock

Iron Blast Furnace Slag) (1973) $1 \mathrm{G}$ Code Std.

Iron Castings for Porcelain

Iron Castings for VaIves, Flanges, and Pipe Fittings (1

Iron Castings (1971) ANSI G48.2

Iron Castings (1972)

Iron Castings (1972)

Iron Castings (1972A)

Iron Castings (I972A

Iron Chromium Molybdenum Copper Alloy Plate, Sheet, and

Iron Chromium Molybdenum Copper Alloy Rod and Bar (1972

Iron Chromium Nickel and Nickel Base Alloy Castings for

Iron Chromium Silicon Alloy Seamless and Welded Pipe (1

Iron Chromium, Iron Chromium NickeI and Nickel Base All

Iron Columbium Molybdenum Tungsten Alloy Seamless Pipe

Iron Content in Corn Starch Hydrolzates Including Syrup

Iron Content of Corn Sugar (Crude and Refined) (1961)

Iron Copper Sintered Metal Powder Structural Parts (197

Iron Detachable Link Chain and Attachments (1971)

Iron Fittings, 2 In. Through $48 \mathrm{In}$./S for W ater and Oth

Iron for Silicon and Aluminum (1971) ANSI Z128.35

Iron in Flour (Cereal Chemistry) (1962)

Iron in Flour (CereaI Chemistry) (1962)

Iron in Wine (Enology) (1972)

Iron Molybdenum Copper Columbium Stabilized Alloy Pipe

Iron Molybdenum Copper Columbium Stabilized Alloy Plate

Iron or SteeI Articles by the Preece Test (Copper Sulfa

Iron or Steel Farm Field and Railroad Right of Way Wire

Iron Ores by Atomic Absorption Spectroscopy (1973)

Iron Ores by Atomic Absorption Spectroscopy (I973)

Iron Ores (1972)

Iron Pipe and Fittings (1973)

Iron Pipe for Water or Other Liquids (1972)

Iron Pipe, Centrifugally Cast, in Metal MoIds or Sand L

Iron Piping for Water and Other Liquids (1972) ANSI A21

Iron Plum bing Fixtures (I973)

Iron Pressure Pipe and Fittings (1972) ANSI A21.11

Iron Sanitary System (Plumbing Pipe and Fittings) (1972
CR

$\mathrm{AACCH} \quad 02.52$

$\mathrm{AACCH}$ 02-5]

ASTM D 1583

Std ASTM D279

ASTM D2687

D2187

ASTM

ASTM D514

ASTM D 1179

ASTM D1339

Std IEEE $\quad 300$

td. Cl ASTM D2953

ASTM D2786

RS 406

ASTM E351

ASTM B590

ASTM B423

ASTM A608

ASTM B409

ASTM B408

ASTM B407

RS197A

ASTM B468

$\begin{array}{ll}\text { ASTM } & \text { B582 } \\ \text { ASTM } & \text { B581 }\end{array}$

ASTM B474

ASTM A447

ASTM B168

ASTM B465

B166

ASTM B572

ASTM B573

BI67

B434

E38

$\begin{array}{ll}\text { ASTM } & \text { E353 } \\ \text { AWWA } & \text { C110 }\end{array}$

AWS A5.2

ASTM B37

ISIS

$* 1$

Rec. P AWS

ASTM

SAE

SAE

ASTM

ASTM

ICBO

ASTM

ASTM

ASTM

A STM

ASTM

ASTM

A STM

A STM

ASTM

ASTM

A STM

ASTM

ASTM

ASTM

CR

CR

ASTM

ANSI

$A W W A$

ASTM

AACCH $40-40$

$A A C C$

ASE

ASTM B464

ASTM A239

ASTM A116

ASTM E507

ASTM E508

A STM E464

UL

E 464
194

ASTM

A674

ANSI A21.52

AWWA C105

ANSI A112.19.I

AWWA C111

CISPI 301 
aulic Systems (1972)

Rec. Pract. Spec. for Cast Std. Spec. for Cast

Spec. for Neoprene Rubber Gaskets for Hub and Spigot Cast Std. Spec. for Cast Guide Spec. for Cast

Std. Cast Std. Cast

Std. for Malleable

n Relays and S/

General Spec. Applicable to Electromagnet

arch Hydrolyzates /

Std. Analytical Meth. for Determinin

ding Code Std. for Material Spec. for Structural Steel and Std. Analytical Meth. for Determining for Welding Rods and Covered Electrodes for Welding $\mathrm{C}$ as eth. for Measuring Fast Neutron Flux by Radioactivation of Uniform Building Code: Steel and

mical Analysis of Steel and Cast, Open Hearth, and Wrought Tex) Std. Performance Requirements for Durable Press (No trength at Elevated Temperatures (1973) A/ Std. Spec. for Flat Rolled, Grain Oriented, Silicon
0G05/ High Temperature, Electrical, Magnetic, and Other Similar High Temperature, Electrical, Magnetic, and Other Simila Std Meth of Impact Testing of Cast ow Alloy, and Silicon Electrical Steels, Ingot and Wrought Uniform Building Code: Weights of Materials (Brick; Cas able $(19)$

Spectrophotometric Determination of Fission Zirconium in

for the Design, lnstallation and Performance of Sprinkler Std. for Trapezoidal Flumes for Std. Spec. for Concrete Pipe for Std. Spec. for Nonreinforced Concrete Minimum Std. for Design, Manufacture and Test of Aluminum nufacture and Placement of Reinforced Concrete Casings for Std. for Sprinkler Std. Meth. for Evaluation of and Test Formula for Std. Spec. for Methy Std. Spec. for Methy stry) (1962) Meth. of Analysis for Citric and Std. for Flash Points, Closed Cup, Aromatic Chemicals and $\mathrm{n}$ for Installation of Expansion, Control, Contraction, and Safety Std. for Line Olefinic Petroleum Naphthas (1973) ANSI / Std. Meth. for 11) Std. Meth. for Analysis of High Purity N-Heptane and Std. Meth. of Test for valuation of and Test Formulas for Natural Rubber (Nr) and Meth. for Evaluation of and Test Formula for Isobutene Spec. for

n $80.60 \%$ Alcohol, Versene, and Sodium Phosphate Solutions: Properties, etc. of

Std. Common Name for the Pest Control Chemical Std. Common Name for the Pest Control Chemical
dodecadienoate) (Common Name for Th/ Std. for Methoprene ommon Name for the Pest Control Chemical 2-Chloro-4,6 bis me for the Pest Control Chemical 2-(Ethylthio) -4,6-Bis Std. Common Name for the Pest Control Chemical 2,4-Bis mmon Name for the Pest Control Chemical 2-(Ethylamino)4-

Std. Common Name for the Pest Control Chemical 2,4-Bis th. of Test for Gross Calorific Value of Solid Fuel by the Determination of Uranium and Plutonium Concentrations and ntainer) (Dot-37A80, Dot-37Aa60, Ufc-Rule 40, Nmfc

Full Removable Head Universal Drum (Ufc-Rule 40, Nmfc full Removable Head Universal Drums (Ufc-Rule 40, Nmfc . full Removable Head Universal Drums (Ufc-Rule 40, Nmfc full Removable Head Universal Drums (Ufc-Rule 40, Nmfc

ble Head, Lug Cover Universal Drums (Ufc-Rule 40, Nmfc ontainer) (Dot-37A80, Dot-37A60, Ufc-Rule 40, $\mathrm{Nmfc}$ versal Pails (Container) (Dot-17E, Ufc-Rule 40, Nmfc

or Packaging, Shipping, Receiving, Storage and Handling of Quality Stds. for Misc. Ornamenta

17) Test Meth. for Appearance of Creases in Wash and Wear Ans/ Test Meth. for Appearance of Seams in Wash and Wear cation Marking Meths. on Finished, Semi Finished and Rough Std. for Service Time Coding of Propulsion Engine , Panelwork, Closet and Storage Shelving, Misc. Ornamental , Push and Kick Plates; Bolts; Closers; Hospital and Misc.

d Handling of Combustible Fibres (Cotton, Sisal, Henequen, for Gross Calorific Value of Solid Fuel by the Isothermal Std. Spec. for Polyethylene

Std. Spec. for General Purpose Ethylene Propylene Rubber Std. Spec. for Extra Heavy Duty Black Polychloroprene Std. Spec. for Natural Rubber

td. Spec. for Styrene-Butadiene-Synthetic Rubber (SBR) Std. Spec. for General Purpose Polychloroprene Std. Spec. for Heavy Duty Bla'ck Polychloroprene Std. Spec. for Polyvinyl Chloride (PVC tant Ethylene-Propylene-Rubber Integral Insulation and
Iron Sealing Rings for Automotive Transmission and $\mathrm{Hydr}$

ron Soil Pipe and Fittings (1972)

Iron Soil Pipe and Fittings (1972)

Iron Soil Pipe and Fittings (1972) ANSI A 112.5.1

Iron Soil Pipe (1972)

Iron Threaded Drainage Fittings (1971)

Iron Threaded Fittings (125 and 250 Lb.) (1972)

Iron Threaded Fittings (150 and $300 \mathrm{Lb}$.) (1971)

Iron (EMI) Cold Rolled Strip for Magnetic Application I

Iron (Orthophenanthroline) Content of Corn Syrup and St

Iron (Shape, Plate, Sheet, Strip, Connector, and Bar)

Iron (TPTZ) Content of Corn Syrup (1970)

Iron (1969) ANSI W3.15

Iron (1970) ANSI Nlll

Iron (1973)

Iron (1973)

Iron) Woven Sheet and Pillowcase Fabrics (Institutional

Iron, Cobalt, and Nickel Base Alloy Castings for High S

Iron, Electrical Steel, Fully Processed Types 27G053, 3

Iron, Nickel, and Cobalt Base Alloys (1973)

lron-59 in Water (1969) A NSI N162

Irons (1972)

Irons (1973)

/Meth. for Chemical Analysis of Carbon, L

Iron; Cinders; Concrete; Earth; Glass; Granite; Gravel;

Irradiated Nuclear Fuels (1973)

Irradiated Polyolefin, Pigmented, Flexible, Heat Shrink

Irradiation Results on Graphite (1972)

Irrigation Equipment (1972)

Irrigation Flow Measurement (1972)

Irrigation or Drainage (1973)

lrrigation Pipe with Rubber Gasket Joints (1973)

lrrigation Tubing (1972)

lrrigation Wells (1972)

Irrigation (For Farms) Technical Data Sheet (1972)

lsobutene-lsoprene Rubbers (IIR) (1973)

Isobutyl Carbinol (1972)

Isobutyl Ketone (1972)

Isocitric Acids in Fruit and Its Products (Cereal Chemi

Isolates (1973)

Isolation Joints for Ceramic Tile (1973)

Isolation Monitors (1973)

Isolation of Representative Saturates Fraction from Low

Isooctane by Capillary Gas Chromatography (1971) ANSI Z

Isophthalic Acid in Alkyd and Polyester Resins (1973)

Isoprene Rubber (IR) (1973)

Isoprene Rubbers (IlR) (1973)

Isopropyl Acetate (98\% Grade) (1972)

Isopropyl Alcohol Saturated with Gasoline; Methyl Blue

Isopropy] Alcohol (1972)

Isopropyl 4,4'dibromobenzilate-Bromopropylate (1973)

Isopropyl 4,4'dichlorobenzilate-Chloropropylate (1973

(Isopropyl (E,E)-11-Methoxy-3,7,11-Trimethyl -2,4-

(Isopropyla mino) -S-Triazine-Propazine (1973)

(Isopropyla mino) Striazine) (1973)

(Isopropyla mino)-6-Methoxy-S-Triazine-Prometon (1973

(Isopropylamino)-6-(Methylthio)-S-Triazine-A metryn (

(Isopropyla mino)-6-(Methylthio)-S-Triazine-Prometryn

Isothermal Jacket Bomb Calorimeter (1973)

Isotopic Abundances (1970) ANSI N115

Item 260 (1974)

Item 260) (1974)

Item 260) (1974)

Item 260) (1974)

Item 260) (1974)

Item 260) (1974)

Item 260) (1974)

ltem 260, Dot-37B60) (1974)

Spec for 16 Gal. Full Remal.

Std. Spec. for $16 \mathrm{Gal}$. ANS

作

tems (Architectural Woodwork) (1973)

Items (Clothing) After Home Laundering (19,3) ANSI L14.

Items (Textile / Clothing) After Home Laundering (1973)

Items (1971)

Items (1971) ANSI B141.8

Items, Stairwork and Handrails, Exterior Frames and Sas

Items; Key Control Systems; Door Holders, Stops, Smoke

Ixtle, Jutes, Hemp, Tow, Cocoa Fibers, Oakum, Rags, Was

Jacket Bomb Calorimeter (1973)

Jacket for Electrical Insulated Wire and Cable (1972)

Jacket for Wire and Cable (1972)

Jacket for Wire and Cable (1973)

Jacket for Wire and Cable (1973) ANSI C8.25

Jacket for Wire and Cable (1973) ANSI C8.28

Jacket for Wire and Cable (1973) ANSI C 8.31

Jacket for Wire and Cable (1973) ANSI C8.32

Jacket for Wire and Cable (1974)

Jacket (1973) IPCEA S68-516
/Ropetryn (C

Eth. of Test for

Std. Spec. for $55 \mathrm{Gal}$.

Std. Spec, for $30 \mathrm{Gal}$. ANSI

Std. Spec. for $57 \mathrm{Gal}$. ANSl

SAE

ASTM

CSI

ANSI

ANSl

ANSl

CR

ICBO

CR

Spec AWS

ASTM

ICBO

ANSI

ASTM

ASTM

ASTM

ASTM

ASTM

ICBO

SAE

ASTM

ASAE

ASAE

ASTM

ASTM

ASAE

ASAE

ASTM

ASTM

ASTM

AACC

EOA

ASTM

ASTM

ASTM

E ASTM

ASTM

$A A C C$

MCA

ANSI

ANSI

Td. C ANSl

ANSI

ANSI

ANSl

ASTM

ANSI

ANSI

ANSI

ANS1

AWI

AATCC

AATCC

SAE

SAE

AWI

NBHA

ICBO Std. Meth. of Test ASTM

ASTM

ASTM

ASTM

ASTM

ASTM

ASTM

ASTM

ASTM

/Ss and Having Ozone Resis NEMA

J281

HSN

A74

15062.1

Bl6.12

$\mathrm{B} 16.4$

$\mathrm{Bl} 6.3$

121

UBCS27.1

E. 33

A5. 15

E263

UBC $* 3-27$

E30

L24.2.13

A567

A665

E354

D2461

A327

E350

UBC $* 3.23$

E495

AMS3636D

C625

R264. 1

S359T

C118

C505

S263.2 
marine Special Purpose Water Safety Buoyant Devices (Vest, Safety Code for Cranes, Derricks, Hoists, Std. for Safety for Gas Vents and Vent Roof Safety Std. for Cranes, Derricks, Hoists, Hooks, Std. for Nut, Plain. Hexagon, Drilled Std. for System for Romanization of test Meth. for Water Repellency of Textile Fabrics: Tumble of Formaldehyde Odor in Resin Treated Fabric by the Sealed Std. Rec. Pract. for Coagulation Flocculation

Meth. of Thermal Shock Test on Glass Containers (Bottle, Std. Meth. of Sampling Glass Containers (Bottle, Std, for Chucks and Chuck ts Penetrating the Hull for Boats Equipped with Outdrives, of the Skeletal (Bone) / Std. Spec. for Hip Nail of Test for Thermal Oxidation Stability of Turbine Fuels Std. for Numerically Controlled Horizontal and Vertical Sid. for Std. for Safety Requirements for Wood Model Specs. for Small Concrete Paving

fittings to Poly(Viny

b72.17

tiffness Computations (1973) Std. Spec for Solvent Cements for Rec. Pract. for Heat Rec. for Wood Beam.

Uniform Building Code Std. for Gypsum Wallboard Tape and Standard for Wrought Copper and Its Alloy Solder A37.101 uction (Nonextruding / Std. Spec. for Preformed Expansion Sid. Spec. for Preformed Expansion Std. Spec. for Preformed Cellular Plastic Pressure Relief Std. for Cast Bronze Solder 1973) Std. for Wrought Copper and Bronze Solder. Std. for Cast Bronze Solder

id. Meth. of Test for Adhesion and Cohesion of Elastomeric Std. Meth. Test for Tack Free Time of Elastomeric Type ing (1972) (I972)

Bul. on Round Thread Casing

c. for Tractor and Farm Implement Power Takeoff (Universal pec. for (Oil) Wellhead Equipment (Production Valves, Ring

lead Wires. Terminals, and Other Terminations (Soft Solder Uniform Plumbing Code:

d. Rec. Pract. for Atmospheric Exposure of Adhesive Bonded Std. Specs. and Load Tables for Longspan Steel ings (1972) ANSI A21.11 Std. for Rubber Gasket

allation of Expansion, Control, Contraction. for Gasketed meric Seals (1973) Std. Spec. for Std. Spec. for Compression Uniform Building Code Std, for Test for Glue Std. Test Meth. for Permanence of Adhesive Bonded the Strength of Adhesively Bonded Rigid Plastic Lap Shear e Strength of Adhesively Bonded Plastic Lap Shear Sandwich or Use (1959) A/ Std. Meth. of Test for Integrity of Glue Std. Specs. and Load Tables for Open Web Steel Std. Specs. and Load Tables for Longspan Steel

Std. Rec. Pract. for Determining Durability of Adhesive Std. Rec. Pract. for Determining Durability of Adhesive Spec. for Structural Steel

(1973) Ansi/ Std. Rec. Pract. for Making Solvent Cemented

hear Block Test for Quality Control of Glue Bonds in Scarf enched and Tempered Alloy Steel Bolts for Structural Steel Unhardened Steel and Alloy Silver Brazing Nonreinforced Concrete Irrigation Pipe with Rubber Gasket oad Tables for Open Web, Longspan, and Deep Longspan Steel Structural Clay Differential Movement (Expansion uniform Building Code Std. for Timber Connector and Bolted Spec. for High Strength Steel Bolts for Structural Steel ber, Preservative Treatment, Poles, Piles, Spaced Columns, g Screws, Plates, Nails and Spikes, Washers, Pins, Dowels, Uniform Building Code Std. for Open Web Steel Span Tables and Working Stresses for

m Building Code Std. for Span Tables for Floor and Ceiling Spindle Meth. of Analysis of Solids in

ct. for 7 Conductor Electrical Connector for Truck Trailer Safety Std. for Underwater Lighting Fixtures and Uniform Fire Code: Automobile Wrecking and poses (1974) Ansi/ Std. Meth. of Test. and Tolerances for
74) ing of Combustible Fibres (Cotton, Sisal, Henequen, Ixtle, Std. for Low Height 80 , Tow, Cocoa Fibers, Oakum, Rags, Waste Cloth, Wastepaper, Std. Analytical Meth. for Determining Moisture Std. Analytical Meth. for Determining Moisture Std. Analytical Meth. for Determining Moisture Std. Analytical Meth. for Determining Moisture Std. Spec. for Photographic Grade Potassium Chloride, Uniform Building Code Std. for $n$, Dried Peas and Beans for Rodent and/or Insect Damage on
for Storage and Handling of Flammable Liquids (Gasoline,
Jacket, Horseshoe and Ring Buoy) (1973) ANSI Z243.1

Jacks and Slings: Base Mounted Drum Hoists (1971)

Jacks (1973) ANSI Al31.2

Jacks, and Slings: Portal, Tower and Pillar Cranes (197

Jam, Thin (1972)

Japanese Written Language (Transliteration) (1972)

Jar Dynamic Absorption Test (1972) ANSI L14.87, ASTM D5

Jar Meth. (1972) ANSI L14.178

Jar Test of Water (1974)

Jar) (1971)

Jar, etc.) (1956)

Eth. for Determination

Jaws (1972)

Jet Drives and/or Bow Thrusters (1973)

Jewett Type for Use

JFTOT Proc,) (1973)

Jig Boring Machines (1974)

Jig Bushings (1974)

Job Made Ladders (1973)

Jobs (1973)

Joining Acrylonitrile Butadiene Styrene (ABS) Pipe and

Joining of Thermoplastic Pipe and Fittings (1967) ANSI

Joint and Rafter Span, Load, Deflection, Strength and S

Joint Compound (1973)

Joint Drainage Fittings (1973)

Joint Filler for Concrete (Bituminous Type) (1971) ANSI

Joint Fillers for Concrete Paving and Structural Constr

Joint Fillers (1973)

Joint Fittings for Solvent Drainage Systems (1973)

Joint Pressure Fitting for Use with Copper Water Tube (

Joint Pressure Fittings (1972)

Joint Sealants Under Cyclic Movement (1972)

Joint Sealants (1971)

Joint Strength with Combined Internal Pressure and Bend

Joint Venture Agreement (Form) for Design Professionals

Joint) Drive Line Specs. (1972)

Joint, Segmented, Production Check Flanges and Gaskets:

Jointed) (1973) ANSI C83.93

Joints and Connections (1973)

F Solderability of Solid

Joints and Structures (1970) ANSI Z197.24

Joints Deep DLJ and DLH Series (1970)

Joints for Cast and Ductile Iron Pressure Pipe and Fitt

Joints for Cast Iron Pipe and Fittings (1973)

Joints for Ceramic Tile (1973)

Ecommendation for Inst

Joints for Plastic Pressure Pipes Using Flexible Elasto

Joints for Vitrified Clay Bell and Spigot Pipe (1972A)

Joints in Laminated Wood Products (1973)

Joints in Plywood Under Mold Conditions (1970)

Joints in Shear by Tension Loading (1973)
Joints in Shear by Tension Loading (1973)

Joints in Structural Laminated Wood Products for Exteri

Joints J Series and H Series (1970)

Joints LJ and LH Series (1970)

Joints Stressed in Peel (I971) ANSI Z197.30

Joints Stressed in Shear by Tension Loading (1971) ANSI

Joints Using ASTM A325 or A490 Bolts (1970)

Joints with Polyvinyl Chloride (PVC) Pipe and Fittings

Joints (1971) ANSI G24.19

Joints (1973)

Joints (1973)

Joint s (1974)

Joints) (Tech. Notes) (1963)

Std. Spec. for

Joints, Drift Bolts, and Wood and Lag Screws; Light Met

Joints, Including Suitable Nuts and Plain Hardened Wash

Joints, Screws, Bolts, Connectors, Nails, Staples, Adhe

Joints, Split Rings and Framing Anchors) (1973)

Joist Design (1973)

Joists and Rafters (Lumber) (1972) Usc Ps20

Joists, and Rafters (1973)

Juices, Syrups, and Molasses (Cereal Chemistry) (1962)

Jumper Cable (1972)

Junction Boxes for Swimming Pools (1972)

Junk Yards or Waste Material Handling Plants (1973)

Jute Rove and Plied Yarn for Electrical and Packing Pur

Jute, Hemp and Sisal, Flax, Synthetic Fibers, Wool) (19

Jutes, Hemp, Tow, Cocoa Fibers, Oakum, Rags, Waste Clot

K.S.I., Self Locking, 450 Deg. F. Hexagon Nut (1973)

Kapok, Hay, Straw, Spanish Moss, Excelsior, etc.) (1973

(Karl Fischer) Content in Corn Starch (Unmodified) (1961

(Karl Fischer) Content in Steepwater (Corn) (1966)

Karl Fischer) Content of Corn Sugar (Crude and Refined)

Karl Fischer) Content of Corn Syrup (1966)

KCI ( 1972

Keene's Cement (1973)

Kernels, Rodent Excreta, Other Filth, and Extraneous Ma

Kerosene, Acetone, Alcohol, Cleaning Fluids, Flammable

UL

ANS

I 123

ANSI B30.4

NSA 1423

ANSI Z39.11

AATCC 70

AATCC 112

ASTM D2035

C 149

ANSI

B5.8

ASTM F369

D324 I

D324

NSA

ANSI

AI

ASTM D3138

ASTM D2657

W PA

ICBO

ANSI

ASTM

ASTM

ANSI

ANSI

ANSI

ASTM

API

AIOA

Re ASAE

API

EIA

St ASTM

AISC

AWW A

UL

ASTM

ASTM

ICBO

ASTM

Determining ASTM

ASTM

ASTV

A ISC

AISC

ASTM

ASTM

AISC

ASTM

ASTM

SAE

ASI

BIA

ICBO

ASTM

ICBO

*30

UBCS 47.5

B16.29

D994

D1751

D3204

B 16.32

B 16.22

B 16.18

C719

C679

BUL 5C4

C801

R331.2

$6 \mathrm{~A}$

RS178.B

U PC $* I-8$

D) 828

S316-1

C111

194

300

D3139

C425

UBCS25-20

D 1877

D3163

D3164

D1101

S316-3

S316-2

D2918

D2919

S314

D2855

D1759

A 490

A MS $2664 \mathrm{C}$

C505

$18 \mathrm{~A}$

UBCS25-17

A 325

WWPA 

Std. Spec. for Metbyl Isobutyl Std. for Gas Fired and Kick Plates; Bolts; Closers; Hospital and Misc. Items; Safety Std. for
Safety Std. for safes) (1973) Std. for Typewriter Std. for

(1974) Std for gn of Components: Enclosed Gear Drives-Bearings. Bolting
Std. for Kevs and

s: Locks; Knobs and Handles; Exit Devices, Pulls, Push and Casework: Cabinets, Cases, Counters and Enclosures of Al T201 Std. Spec. and Operating Instructions for Glass Capillary nd the Calculation of Dynamic Vis/ Std. Meth. of Test for Std. Meth. of Test for Std. Meth. of Test for Coefficient of al 2-Propynyl (E.E)-3,7.11-Trimetbyl-2,4-Dodecadienoate Rec. for Construction and Performance Std. for

Crude Protein in Bread, Wheat and Otber Grains/ Std. for Test Improved Improved d Feedstuffs (Cereal Cbemistry) (1962) d Flour Mill Products (Boric Acid Modification) (Cereal mistry) (1962)

of Test for Total Nitrogen in Lubricating Oils by Modified Tent. Metb. of Tes! for Virrogen Conten st Specimens of Bituminous Mixtures by Means of Californi tuminous Mixture Beam Specimens by Means of the California Std. Metb. of Test for Slump of an Oil Base Std. Metb. of Test for Volatility of Oil and Resin Based, Std. Metb. of Test. Warp

atic Home Laundering of Durable (Permanent) Press Woven or or Relaxation Dimensional Cbange (Sbrinkage) of Stabilized Std. Metb. of Test for Stretcb Properties of

$t$ Metb. for Dimensional Cbanges in Laundering of Wioven and $\mathrm{n}$ and Felting Dimensional Changes (Sbrinkage) of Woven and Std. for Ground Support Equipment (Hand

Basic Builders' Hardware: Hands of Doors: Hinges; Locks; ion Ratio (CR) Tecbnique for Rese/ ion Ratio (CR) Technique for Motol Std. Metb. of Test for Std. Metb. of Test for n Brinell, Vickers, Rockwell and Rockwell Superficial, and Sleth. of Test for or Breaking Tenacity of Man Made Textile Fibers in Loop or Test Metb. for Rejects from Std. Spec. for Fixation Pins

or ElectricaI Insulating Paper and Paperboard-Sulfate or Lniform Building Code Std. for

n, Carbon Monoxide, Deuterium, Fluorine, Helium, Hydrogen, gs (1968)

ne, Cbrome Ore, Clays, Diaspore, Ganister Quarzite. Grog, Rec. Pract. for the Std. Rec. Pract. for ) Guide to the Preparation of Precautionary tentialities (Safety) (19/ Std. Guide for Classifying an Std. for Glossary of Rec. Terms for Care
Std. for Motion Picture Raw Stock Identification and rms and Defini/ for Rec. Regulations for Animal Feed and Food rec. Pract. for Lse of These Terms on Permanenty Attached tion of Chlorine Dioxide in Flour Bleacbing Experiments in c. for Control and Removal of Radioactive Contamination in ure for Motion Picture Film Lsed in Studios and Processing Spec. for Simple Can Puncturing Device for L'se in Aeroso Std. Test Metb. for Resistance of Adbesives to Cyclic Std. Test Meth. for dustries (1972) Test Meth. for und Transmission Class ( $\$$ ) Uth. of Conducted Controlled Suilding Code Std. for bject Protective Struc/ ranean Termites (1972)

Rec. Pract. for Critical Zone for Std. Metb. for and Spoke Wheels, and Demountabl/ Rec. Pract. for Lniform Test Metb. for Shive Content of Mecbanical Pulp
Std. Rec. Pract. for Rec. Pract, for Instrument Panel as Emissions Measurement (Passen/ Rec. Pract. for Uniform ation of Manuals for Installation, Operation and Repair of oss of Building Partitions (1970) td. Rec. Pract. for Std. for High Precision

72)

) ANS1 KI8.1

973)

970) Std. Rec. Pract for Reporting
Std. Metb. of Std. Metb. of Test for Wool Content of Raw Wool Std. Rec. Pract. for Establishing Conditions for
Std. Metb. of Test. Wood Preservatives by Std. Metb. for Accelerated Std. Rec. Pract. for Test. Pavem ent Polishing in the Metb. of Making and Curing Concrete Test Specimens in the
Kerosine, Aviation Turbine, and Distillate Fuels (Poten

ASTM

ASTM

Ketone (1972)

Kettles, Steam Cookers and Generators (1971)

Key Control Systems; Door Holders, Stops, Smoke and Hea

Key Locked Safes (Class KL) (1973)

Key Locks (Burglar Alarm, Door, Deposit and Collection

Keyboard Arrangement (1973)

Keys-Square and Rectangular (1973)

Keys and Keyways for Industrial Flexible Gear Couplings

Keys and Shafting (1974)

Keyways for Industrial Flexible Gear Coupling (1974)

Kick Plates: Bolts; Closers: Hospital and Misc. Items

Kinds (Architectural Woodwork) (1973)

Kinematic Viscometers (1972) ANSI Z11.275

Ality Stds. for

Kinematic Viscosity of Asphalts (Bitumens) (1974) AASHO

Kinematic liscosity of Transparent and Opaque Liquids a

Kinetic Coefficients of Friction of Plastics (1972)

Kinetic Friction for Wax Coatings (1973) ANSI ZII.222

Kinoprene (1973)

Mon Name for the Pest Control Chemic

Kitchen and Vanity Cabinets (1973)

Kits for Swimming Pool $\overline{\mathrm{V}}$ ater (1970)

Kjeldahl Meth. for Nitrate Free Samples for Analysis of

Kjeldahl Meth. of Analysis of Crude Protein in Feeds an

Kjeldahl Meth. of Analysis of Crude Protein in W'heat an

Kjeldahl Meth. of Analysis of Crude Protein (Cereal Che

Kjeldahl Meth. (1973)

B 5 t Std. Meth.

$\begin{array}{ll}\text { Kneading Compactor (1971) ANSI A37.138 } & \text { /Paration of Te } \\ \text { Kneading Compactor (1973) } & \text { /Ract. for Preparation of Bi }\end{array}$

Kneading Compactor (1973 /Ract. for

Knife Grade, Cbannel Glazing Compounds (1973)

Knit Fabrics (196-1) ANSI L14.284

Knit Textile Fabrics (1973) ANSI L14.266

Knit Wool Fabrics (Textile) (1973)

Kritted Fabrics Having Low Power (1972) ANSI Z14.286

Knitted Fool Textiles (19:2) ANSI L14.130, ASTII D1284

Knob) (1974)

Knobs and Handles: Exit Devices. Pulls. Pusb and Kick P

Knock Cbaracteristics of Motor Fuels Ising the Compress

Knock Characteristics of Motor Fuels Lising the Compress

Knoop Hardness) (1972) AXSI Z 6.4 /Relationship Betwee

Knoop Indentation Hardiness of Glass (1972T)

Knot Configurations (1973)

Knotter Screens (Pulp and Paper) (1973)

Knowles and Hagie Types (1973)

Kraft Layer Type (1973) ANSI C59.50

Kraft Waterproof Building Paper (Weatherproofing) (1973

Krypton, Methane, Neon, Nitric Oxide, Nitrogen, Oxygen)

Kyanite, Magnesite. Mullite) (1970) AXSI AlI1.36

Label for 2 In. Quadruplex Video Magnetic Tape Recordin

Labeling Aerosol Cans (1972)

Labeling and Marking of Compressed Gas Containers (19i1

Labeling Epoxy Products According to Their Hazardous Po

Labeling Textile Products (Launder or Dryclean) (1973)

Labeling (1973)

Labelling. Ingredients and Containers (Also Includes Te

Labels (1972) / Care of Consumer Texite Products and

Labels, Detacbable Tags, and Certification of Fabrics O

Laboratories (Cereal Chemistry) (1968)

Laboratories (195I)

Laboratories (1971)

Laboratories (19:2

Laboratory Aging Conditions (1970) ANSI Z197.19
Laboratory Aging of Sand wich Constructions (1970)

Laboratory Corrosion Test. of Metals for the Process in

Laboratory Corrosion Tests (1972)

Laboratory Determination and Measurement of Airborne So

Laboratory Evaluation of Roll Over (ROPS) and Falling O

Laboratory Evaluation to Determine Resistance to Subter

Laboratory Fatigue Test Procedures for Production Disc

(Laboratory Flat Screen) (1973)

Laboratory Immersion Corrosion Test. of Metals (1972)

Laboratory Impact Test Procedure-Head Area (1971)

Laboratory Instrumentation and Techniques for Exbaust $G$

Std. for Prepar

Laboratory Measurement of Airborn

Laboratory Potentiometers (19:2)

Laboratery Results Wben Cbecking Aerosol Containers (19

Laboratory Sampling and Analysis of Coal and Coke (1970

Laboratory Scale (1972) ANSI L14.490

Laboratory Sensory Evaluation of Foods and Beverages (1

Laboratory Soil Block Cultures (1970)

Laboratory Test of Natural Decay Resistance of W'oods (1

Laboratory (Full Scale Wheel Meth.) (1972)

Laboratory (1969) ANSI A37.81
A..$S I$

A

I'L

A. XSI

ist

AGIIA

AGMA

A GMA

$\mathrm{NBHA}$

ASTY

AST.I

ASTH

ASTYI

ASTII

A $\backslash$ SI

A \SI

NSF

$\mathrm{A} A \mathrm{ACH} 46$

A.ACCH $\quad+6-10$

ACCH 46.

AACCH

ASTM

ASTM

ASTM

ASTM

ASTM

ASTM

AATCC

ASTM

ASTMI

AATCC

AATCC

NSA 1553

$\mathrm{NBHA}$

4 STI

ASTMI

ASTII

ASTM

ASTIY

TAPPI

ASTII

ASTM

ICBO

ICBO

Calc ASTH

SMPTE

ASTMI

CGA

ANSI

AISI

ANSI

AAFCO

ASTII

ANSI

Re NCRPM R8

clat AXSI Std.

ASTII

ASTM

ASTM

AACF

$A$ W A

ICBO

$S A E$

A WPA

SAE

TAPP

ASTY

SAE

SAE

ASTM

ANSI

ASTM

ASTM

ASTMI

ASTMI

ASTM

ASTI

ASTII

Std. ASTMI

8

437

$\mathrm{X} 4.7$

260.02

512.03

-1-400

D2515

D2170

D3028

D2534

3227

K62.137 
h. for Measurment of Dry Film Thickness of Paint, Varnish, Std. Meth, of Test for Ester Value of ing, Loss of Adhesion, or Other Failure of Paint, Varnish, Std. Definitions of Terms Relating to Paint, Varnish, for Preparation of Steel Panels for Test. Paint, Varnish, c. for Flammable Liquid Mixing Operations (Paint, Varnish, e Code: Application of Flammable Finishes (Paint, Varnish. Meth. of Test for Flash Point of Liquids (Paint, Enamel Quantitiative Determination of Cellulose Nitrate in Alkyd Std. Meth. of Test. Clear and Pigmented or Oil for Use in Resins and Protective Coatings lncluding Meth. for Preparation and Standardization of Barium (1962) (1962) emistry) (1962) Meth. of Analysis for Std. Analytical Meth. for Determinin Std. Analytical Meth. for Determining Meth. of Analysis fo

Meth. for Preparation and Standardization of Meth. of Analysis for Acetic, Butyric, and formic, Acetic, Propionic, Butyric, Valeric, Succinic, and Meth. of Analysis for Fumaric, Pyruvic. Std. for Manually Propelled Mobile Std for Safety Requirements for Wood Job Made Safety Std. for Portable Wood Std. for Safety for Portable Metal Std. for Permissible Variations from Specified stern Woods Use Book: Timber Fasteners (Connectors, Bolts

ber Connector and Bolted Joints, Drift Bolts, and Wood and nd lngot Bars (1973) ANS1 H17.l Std. Spec. for rk) (1973)

Quality Stds. for High Pressure Std. Spec. for Polyester Glass Mat Shee

f Bond, Appearance, and Shrinkage Propensity of Bonded and Std. Spec. for Performance of Bonded and Std. Test Meth. for Flexural Strength of Adhesive Bonded gth Properties of Adhesives in Shear by Tension Loading of es, Section Properties and Design Loads, Timber, Plank and Std. fo

Uniform Building Code Std. for Tests for Structural Glued hern/ Std. for Preservative Treatment of Structural Glued osu/ Std. Spec. for Protein Base Adhesives for Structura Test Meth. for Bond Strength of Bonded and Std. Spec. for

Rec. Architectural Spec, for Structural Glued Uniform Building Code Std. for Structural Glued Std. for Structural Glued dgepole / Uniform Building Code Std. for Structural Glued

1973) ANSI A176.2

ricultural, Land, and General Drainage (1/ rport and Roadwork Drainage (1973) ptic Tank Disposal Fields (1973) ANSl Al7/

Meth of Test for lntegrity of Clue ) Exposure Condi/ Std. Spec. for Adhesives for Structural
Uniform Building Code Std. for Test for Glue Joints in ) Exposure Condi/ Std. Spec. for Adhesives for Structural
Uniform Building Code Std. for Test for Glue Joints in de Fiber Tent, Awning, and Canopy Fabrics, Vinyl Coated or

Pract. for Classifying Visual Defects in Glass Reinforced hed with Epoxy Functional Silane Type Finishes for Plastic $(1962)$

vative Treatment of Structural Glued Laminated Members and manual) (1962)

t for Agricultural (Farm) and Industrial Equipment Warning Std. Definitions for High Intensity Discharge Specs. for Fluorescent

Std. Meth. of Measurement of Fluorescent stance of Textile Materials and Coated Fabrics: Carbon Arc Test Meth. for Weather Resistance of Textiles: Carbon Arc Std. Spec. for Mercury Std. Spec. for Fluorescent Spec. for Fluorescent

e (1971) Std Spec for Mercury Std. for Auxiliary Low Beam on Emb/ Std. for Mounting Brackets and Socket for W arning Equipment (1972) Std. for Flashing Warning Rec Pract for Mechanical Stop ec. Pract. Test Meth. and Requirements for Snowmobile Stop $t$ Meth, and Requirements for Snowmobile Rear Position Tail

al Characteristics of 8 Watt T5 Preheat Start Bactericidal l Characteristics of 15 Watt T8 Preheat Start Bactericidal Characteristics of $30 \mathrm{~W}$ att, T8 Preheat Start Bactericidal for Physical Characteristics of T.2 Telephone Slide Base t Meth for Colorfastness of Textiles to Light: Carbon Arc colorfastness of Textiles to Light: Water Cooled Xenon Arc colorfastness of Textiles to Light: Water Cooled Xenon Arc

t Meth. for Colorfastness of Textiles to Light, Carbon Arc
Lacquer and Related Products --Film Thickness of $0.3 \mathrm{M}$ Lacquer Solvents and Thinners (1972)

Lacquer, and Related Organic Coatings on Metallic Surfa Lacquer, and Related Products (1973)

Lacquer, and Related Products (I973)

Lacquer, Printing Ink, Adhesive, etc.) (1974)

Lacquer, Stain, etc.) (1973)

Lacquer, Varnish, and Related Products) by Setaflash C

Lacquers by Infrared Spectrophotometry (1972)

Lacquers (I973)

Lacquers (1973)

Lactate Solution (Cereal Chemistry) (1962)

ASTM

Lactic Acid and Salts Content in Feedstuffs (Corn) (196

Lactic Acid and Salts Content in Steepwater (Corn) (I96

Lactic Acid in Dried Geeg (Cereal Chemistry) (1962)

Lactic Acid in Nonfat Dry Milk (Cereal Chemistry) (1962

Lactic Acid Solution (Cereal Chemistry) (1962)

Lactic Acids in Rye Flour (Cereal Chemistry) (I962)

Lactic Acids (Cereal Chemistry) (1962)

Lactic, Succinic, and Tartaric Acids (Cereal Chemistry)

Lactose (Milk Sugar) in Mixed Feeds (Cereal Chemistry)

Lactose (Sugar) and Nonfat Dry Milk in Bread (Cereal Ch

Ladder Stands for Scaffolds (Towers, Safety) (1971)

Ladders (1973)

Ladders (1973)

Ladders (I974)

Ladle Chemical Ranges and Limits for Steels (1972)

Lag Screws, Plates, Nails and Spikes, W ashers, Pirs, Do

Lag Screws; Light Metal Plate Connected Wood Truss Desi

Lake Copper Wire Bars, Cakes, Slabs, Billets, Ingots, a

Laminate Flush Type Wall Paneling (Architectural Woodwo

Laminate (1973)

Laminated Apparel Fabrics (Textile) After Dry Cleaning

Laminated Apparel Fabrics (1972) ANSI L14.291

Laminated Assemblies (1969) ANSI Z197.20

Laminated Assemblies (1973)

Laminated Flooring and Roofing (1973)

Laminated Hardwood Block Flooring (1971)

Laminated Lumber (1973)

Laminated Members and Laminations Before Gluing of Sout

Laminated Products for Use Under Interior (Dry Use) Exp

Laminated Textile Fabrics (1972)

Laminated Thermosetting Materials (1972)

Laminated Timber (1972)

Laminated Timber (1973)

Laminated Timber (1973) ANSI A 190.1

Laminated Timbers: Douglas and Hem Fir, Southern and Lo

Laminated Tubes Used for Electrical Insulation (1970)

Laminated Wall Bituminized Fiber Drain and Sewer Pipe

Laminated Wall Bituminized Fiber Perforated Pipe for $\mathrm{Ag}$

Laminated Wall Bituminized Fiber Perforated Pipe for Ai

Laminated Wall Perforated Bituminized Fiber Pipe for Se

Laminated Wood Products for Exterior Use (1959) ANSI Z1

Laminated Wood Products for Use Under Exterior (Wet Use

Laminated Wood Products (1973)

Laminated (Institutional Textile) (1973)

Laminates and Parts Made Therefrom (1970) ANSI K65.141

Laminates (1972) ISs Fabrics, Cleaned, and After Finis

Laminations Before Gluing of Southern Pine, Pacific Coa

Laminators Quality Control by the AITC Inspection Burea

Laminators Quality Control System (Timber Construction

Lamp and Slow Moving Vehicles (SMV) ldentification Embl

Lamp Ballasts and Transformers (1971)

Lamp Ballasts (1972)

Lamp Ballasts (1972)

Lamp Exposure with Wetting (1972) ANSI L14.224.

Lamp Exposure Without Wetting (1972) ANSI L14.225

Lamp Reference Ballasts (1971)

Lamp Reference Ballasts (1972)

Lamp Starters (1972)

Lamp Transformers, Constant Current (Series) Supply Typ

Lamp (Headlamp-Light) (1973)

Lamp (Light) and Slow Moving Vehicle (SMV) Identificati

Lamp (Light) for Remote Mounting on Agricultural (Farm)

Lamp (Light) Switch (1972)

Lamp (Light) (1971)

Lamp (Light) (1972)

Lamp (Light, Bulb) (1973)

Lamp (1971)

Lamp (1971)

Lamp (1971)

Lamp (1973

Lamp, Alternate Light and Darkness (1971) ANSl L14.169

Lamp, Alternate Light and Darkness (1971) ANSl L14.171

Lamp, Continuous Light (197I) ANSI Ll4.170

Lamp, Continuous Light (1971) ANSI L14.53

NCCA

ASTM

NCCA

ASTM

th. ASTM

Re FMS

ICBO

ASTM

Th. for ASTM

ASTM

$\mathrm{CR}$

CR

$\mathrm{AACCH}$

UI

UL

SAE

USC

ICBO

ASTM

ASTM

ASTM

ASTM

ASTM

ASTM

ASTM

ICBO

ANSI

ASTM

ASTM

AWPA

AITC

AITC

ASAE

ANSI

ANSI

ANSl

AATCC

SAE

SAE

ANSI

Std. Dimensional and Electric ANS

AACCH 04.26

AACCH 70.30

$\mathrm{AACCH} \quad 04.20$

AACCH 04.23

AACCH 04.24

AACCH 80.30

$\mathrm{AACCH} 80.31$

ANSI A92.I

ANSl Al4.4

112

$\mathrm{J} 409 \mathrm{C}$

*31.1

UBCS25.17

ASTM B4

AWl $* 1.500 \mathrm{~B}$

ASTM D 1532

ASTM D2724

ASTM D3135

ASTM D 1184

ASTM D3165

WWPA *3]

HPMA LF.71

ICBO UB
AWPA

ASTM D3024

AATCC 136

ASTM D709

AlTC 6.5/AI

ICBO UBCS25.10

PS56

UBCS25.11

D348

D1862

D2417

D2418

D2313

Dllol

D2559

UBCS25.20

L24.1.10

D 2563

D3098

C28

202

201

S277.2

C82.9

C 82.1

C 82.2

AATCC 111A

ANSl C82.5

ANSl C82.3

ANSI C78.180

ANSI $\quad$ C82.7

SAE J582A

SAE J725D

ASAE S307.1

J249

J 279

C79.255

C78. 1200

C78.1201

C78. 1202

$\mathrm{C} 78.397$ 
Rec. Pract. for Cornering Std. for Fo

Rec. Pract, for Motorcycle Turn Signa Std. for Clearance, Side Marker and Identification Std. for License Plate Std. for Spot

ss Bulbs Intended for Use with Electron Tubes and Electric Std. for 75 W'att H43 Mercur std. Meth. for the Designation of High lntensity Discharge Dimensional and Electrical Characteristics of Fluorescen cal and Electrical Characteristics of 50 Watt, H46 Mercury s Base Down Type, Single Contact Motion Picture Projection Std, for Safety for Portable Sun / Heat Std. Guide for Electrical Measurements of Fluorescent tt, H45 (1972) Std. Meth. for the Designation of Mercury $\operatorname{ar}(1972)$

(1972) ns atling Rigs and Petroleum Produc $\mathrm{MHz}(1970)$ Minimum Std. for Minimum Stds. for rvative Treatment of Piles, Poles, and Timbers for Marine, $\mathrm{d} \mathbb{W}$ all Bituminized Fiber Perforated Pipe for Agricultural, a Technical Cuide for Spec. for Subsurface Safety Valves, Locks, and Notes) (1967) (1966) d Design, Span Allowances, Storage, Finishes And/ Redwood and Semolina (Cereal Chemistry) (1962) Rifle Range Location and

Std. for System for Romanization of Japanese W/ritten Std. for System for Romanization of Arabic Written Safety Std. for Highway Emergency Signals (Flare, for Lineman's Climbing Equipment (Body Belt, Safety Strap, etermining the Strength of Adhesively Bonded Rigid Plastic for Determining the Strength of Adhesively Bonded Plastic ) ANSl Z197.21 Std. Meth. of Testing Cross ss of Epitaxial or Diffused Layers in Silicon by the Angle m Building Code Std for Coast Sitka Spruce, Douglas Fir.

Douglas and Hem Fir, Southern and Lodgepole Pine, Western dgepole Pine/ Uniform Building Code Std. for Douglas Fir; Std. for Induction Motors Sid. for Synchronous Motors Std. for Direct Current Motors Std. for Direct Current Generators

r Heaters: Volume IIl, Circulating Tank, Instantaneous and Std. for Gas Utilization Equipment in ipe for Corrosive or High Temperat/ Std. Spec. for W' elded nd Reversible Synchronous Generator/Motor Uni/ Std. for ging (197I) Std. for Guide for Preparation of Spec. for 2)

Std.

MH12.11 Std. Meth. of Test. vices: Varactor Measurements Part II-Characterization of Std. Large Rivets (1/2 In. Nominal Diameter and tective Lining and Coating for Steel W/ater Pipe (4 In. and test for Effective Fluorescent Lifetime of Neodymium Doped or. Measuring Slope Efficiency and Lasing Threshold of Ruby Std for the Safe Use of Tent. Rec. Pract. for Measuring Slope Efficiency and ssembly (1972) Std. for Handle, forced Brick Masonry Curtain and Panel Walls (In Resisting Gear Units (1972) Std. Spec. for Measurement of Std. Spec. for

shingle Blanks to Be Used as Panels in W/eathering Tests of Rec. Pract for Std. Rec. Pract. for Test. Exterior Std. Meth. of Test for Extension Recovery and Adhesion of Std. Meth. of Test for Low Temperature Flexibility of Std. Meth. of Test for Volume Shrinkage of

Meth. of Test for Extrudability, After Package Aging, of eth, of Test for Aging Effects of Artificial Weathering on Ammonia Preserved. Creamed and Centrifuged Natural Rubber Uniform Building Code Std. for Gypsum Plastering, Exterior (Stucco) and lnteri/ Std. Spec. for Guide Spec. for Furring and

rm Building Code: Plastering and Installation of Wallboard uniform Building Code Std. for Metal, Wire and Wire Fabric

Std. Rec. Pract. for Nomenclature for Rubbers and Rubber

d. Meth. of Test for Polystyrene Blocks in Sbr Rubbers and Std. Meth of Test. Synthetic Rubber

) in Metallic Systems Based on the Composition and Crystal Std. Meth. for Measurement of glossary of Rec. Terms for Care Labeling Textile Products Std. for

Test Meth. for Dimensional Changes in Automatic Home
Lamps (Light) for Automotive Vehicles (1965)

Lamps (Light) (1970)

Lamps (Light) (1971)

Lamps (Light) (1972)

Lamps (Light) (1973)

Lamps (Light) (1973)

Lamps (1971)

Lamps (1972)

Lamps (1972)

Lamps (1972)

Lamps (1972)

Lamps (1972)

Lamps (1974)

Lamps (light) (1973)

Lamps: Physical and Electrical Characteristics of $40 \mathrm{~W}$ a

Land-Mobile Communication Antennas: Part 11-Vehicul

Land and on Marine Fixed and Mobile Platforms (I973)

Land Mobile Communication FM or PM Receivers $25-470 \mathrm{MHz}$

Land Mobile Communications FM or PM Transmitters 25-470

(Land Reclamation, Curtain and Building Foundation Drain

Land, and Fresh Water Use (1974) ANSI Oll.I

/the Prese

Land, and General Drainage (1973) ANSl A176.6

/Aminate

Landing Nipples

Landscape Architecture Garden Walls (Tech. Notes) (1968

Landscape Architecture Miscellaneous Application (Tech.

Landscape Architecture Terraces and Walks (Tech. Notes)

Landscape Guide (Rec. for Garden Grades, Application an

Landscaping (1970)

Lang Meth. of Analysis of Amino Nitrogen in Wheat Flour

Language (Transliteration) (1972)

Language (Transliteration) (1972)

Lantern, Fusee) (1973)

Lanyard, Pole and Tree Climber) (1973)

Lap Shear Joints in Shear by Tension Loading (1973)

Lap Shear Sandwich Joints in Shear by Tension Loading (

Lap Specimens for Tensile Properties of Adhesives (I972

Lapping and Staining Technique (1972) ANSl Z173.49

Larch (North), Eastern Hemlock-Tamarack (North), East

Larch, California Redwood and Hardwood Timber (1973)

Larch; Hem-Fir; Ponderosa, Idaho White, Sugar, and Lo

(Large Apparatus) (1972)

Large Apparatus) (1972)

(Large Apparatus) (1972)

(Large Apparatus) (1972)

Large Automatic Storage Type (1974)

Large Boilers (197 I)

Large Diameter Austenitic Chromium Nickel Alloy Steel P

Large Hydraulic Turbine Driven Synchronous Generators a

Large Power Transformers, with or Without Load Tap Chan

Large Rivets (1/2 ln. Nominal Diameter and Larger) (197

Large Shipping Cases and Crates (Packaging) (1953) ANSI

Large Signal Devices (1972)

Larger) (1972)

1. Guide on Solid State De

Larger, Shop Applied) (197I)

Laser Materials (1973)

Laser Rods (1973)

Lasers (1973)

Lasing Threshold of Ruby Laser Rods (1973)

D. for Cement Mortar Pro

Tent. Meth. of

Latching Internal and External Release Components and

Lateral Forces) (Tech. Notes) (1968)

Lateral Hard Dot Screen (1973)

Lateral Vibration on High Speed Helical and Herringbone

Latex-Portland Cement Mortar (1973) ANSI A 118.4

Latex and Emulsion Exterior Paints or Coatings (1973)

Latex Foam Rubbers (1971)

Latex House Paints (1972)

Latex Sealing Compound (1972)

Latex Sealing Compounds After Artificial Weathering (19

Latex Sealing Compounds (1972)

Latex Sealing Compounds (1972)

Latex Sealing Compounds (1972)

Latex (1972A) ANSI J8.12

Lath (1973)

Lathing and Furring for Portland Cement and Cement Lime

Lathing (1972)

Lathing (1973)

Laths, and Metal Accessories (Designed for Use as a Pla

Latices (1972A) ANSI J9.1

Latices (1974)

Latices (1974) ANSI J2.34

Lattice of Each (1970) ANSI Z30.10

Lattice Spacing of Nuclear Graphite (1969) ANSI K90.1

Launder or Dryclean) (1973)

Laundering of Cloth Tow eling (1970)

Laundering of Durable (Permanent) Press Woven or Knit T

and Product Standards Division

J852B

SAE

$S A E$

SAE

SAE

SAE

SAE

ANSI

ANSI

Std. for ANS

d. Physi ANSI

ANS

UL

ANSI

ANSI

ElA

API

ElA

ElA

ASTM

ASTM

ASTM

MCA

API

BlA

BlA

BlA

CRA

NRA

Z39.11

ANSl $\quad$ Z39.12

UL

912

Spec. EEI

D ASTM

ASTM

ASTM

$/ \mathrm{Ne}$ ASTM

ICBO

ICBO

ICBO

NEMA

NEMA

NEMA

NEMA

ANSl

ANSI

ASTM

NEM A

ANSI

ANSI

ASTM

IEEE

AP-2

D3163

D3164

D1344

F110

UBCS25-2

UBCS25-II

UBCS25-4

MGI-20

MCl-2I

MGI-23

MGI-24

Z21.10.3

Z21.33

A409

MG5.1

C 57.97

B18.1.2

DI083

351

B18.1.2

C205

F 380

F379

ZI36. 1

ANSI

ASTM

NSA

F379

I637

$17 \mathrm{~L}$

AGMA

TCA

ASTM

SAE

ASTM

ASTM

ASTM

ASTM

Std ASTM

Std. M ASTM

*3

426.01

$9 \mathrm{D}$

D1911

J17A

D3129

C736 
972) ANSI L14.138,/ Test Meth. for Dimensional Changes in ily Stain Release Meth. of Textile Fabric Soil During Home laminated Apparel Fabrics (Textile) After Dry Cleaning and extiles to Accelerated Domestic and Commercial Washing and ams in Wash and Wear ltems (Textile / Clothing) After Home ce of Creases in Wash and Wear ltems (Clothing) After Home ress Textile Fabrics (Permanent Press) After Repeated Home Std. for Safety Requirements for Commercia ric Coin Operated and Commercial Clothes Drying Equipment i C 33.13

nsi A 197.2, Asse 1007

Safety Std. for Electric Home

Std and Test Procedures for Home Std. for Domestic Gas Hot Plates and

ic Coin Operated and Commercial Clothes Washing Equipment cep/ Std. for Nonmetallic Shower Receptors, Stall Showers Std. for Vitreous China Plumbing Fixture point Tubular Sleeve Attachment for Hitching Implements to r Safety for Cord Connected Electric Gardening Appliances Safety Spec. for Power Mowers,

for Metal Suspension Systems for Acoustical Tile and for rced (Acar, EC/6201) (1972) An/ mbers, for Electrical Conductors (1971) Spec. for Rope oft (1972) ANSI C7.8 Std. Spec. for Concentri Std. Spec. for Compact Round Concentric $7.53 \quad$ Std. Spec. for Compact Round Concentric ansi C7.50 ANSI C7.52 itd. Spec for Concentri Std Spec for Concentric um Conductors, Concentric Std. for Automated Machine r Aerospace Gearing (Surface Roughness, Waviness, Form and Meth. for Use of ctrical Insulating Paper and Paperboard-Sulfate or Kraft or Measuring Caliper of Resilient Floor Covering with Foam Sid. Meth. of Test for Resistivity of Silicon Epitaxial Std. Meth. of Test for Thickness of Epitaxial or Diffused nsion (197) Std. Meth. of Test for Thickness of Epitaxial rared Refl/ Std. Meth. of Test for Thickness of Epitaxial
Std. Meth. of Test for Thickness of Epitaxial ve Meth. of Test for Sheet Resistance of Silicon Epitaxial Spec. for Composite Filament Tape ruction (/ Std. Graphic Symbols for Electrical Wiring and n Control and Water Conservation) (1972/ Rec. for Design,

rs, Dimensional Spec., and Ratings; Assembly Numbers, Cel ite Fiber Tape and Sheet, Epoxy Resin Impregnated for Hand is in Pigments by Measuring the Specific Resistance of the si / Std. Spec. for Electrodeposited Coatings of Lead and Service (1974) Std for Determination of Capacily of torboats, Tractors, Automotive and Industrial A/ Std. for ng Std. Including Guarantee Policy for Motor Vehicle Type ses (19674 ANSI C7.15 Std. Spec. for Lead and (1972) Std. Meth. Test for Electrodeposited Coatings of Tin (1970) ANSI / Std. Spec. for Electrodeposited Coatings of cal Purposes (19674 ANSI C7.15

316 3) Std. Spec. for Sid. Spec, for Std. Meth. of Test for Std. Meth. of Test for Std. Meth. of Test for Low Levels of Std. Meth of Test for Trace Amounts of Std. Meth." of Test for Determination of Std. Meth. for Determination of Low Concentration of
Meth. of Test for ure $(1972 \mathrm{~T})$ Std. Meth. for Chemical Analysis of Tribasic Std. Spec. for Basic

Std. for Molded and Dipped Mica Capacitors (Wire de/ Test Sid. for Determination of Solderability of Solid nd Shapes (1972) ANSI H33.1 Std. Spec, for Copper Zinc ls (Electrodeposited Coatings of Copper, Nickel, Chromium, Std. Spec. for pes (1972) ANS1 H33.1 Std. Spec. for Copper Zinc Lead rated and Perforated Photographic Film in Rolls, Including Std. for Reel Packaging of Components with Axial Std. Meth of Test. Twine Made from Bast and Std. Meth. of Test. Anti Leak Antifreezes and Stop

lants (1972) Std. Meth. of Test. Anti Sid. Meth. of Test. for Leaks Using the Mass Spectrometer Std. Meth. of Test for Leaks Using the Mass Spectrometer Std. Meth. of Test. for Leaks Using the Mass Spectrometer r8) (1973) Devices with a Helium Mass Spectrometer Test Meth. for Water Std. Rec. Guide for the Selection of Std. for Std. Rec. Guide for Preparation of A Std. Definitions of Terms Relating to Std. for
Laundering of Woven and Knitted Textiles Except Wool (1 Test Meth. for O AATCC

Laundering (1970) ANS1 L14.239

Laundering (1972) ANSI L 14.81

Laundering (1973) ANSI L14.172

Laundering (1973) ANSI L 14.173

Launderings (1973) ANSI L 14.226

/Pensity of Bonded and

for Colorfastness of T

1. for Appearance of Se

/Est Meth. for Appearan

Laundry and Dry Cleaning Equipment and Operations (1972

Std. for Safety for Elect

(Laundry Dryer) (1974)

Laundry Equipment (Clothes Washer and Dryer) (1972) Ans

Laundry Equipment (washer, Dryer, Combination) (1971) a

Laundry Stoves (1971)

(Laundry Washer) (1974)

Std. for Safety for Electr

Lavatories, Bathtubs and Combination Tank and Shower Re

(Lavatory, Water Closet Bowl, Urinal) (1973)

Lawn and Garden Riding Tractors (1972)

Std. for

(Lawn Mower, Cultivator, Edger-Tr
Lawn, and Garden Tractors (1972)

Lay in Panel Ceilings (1973)

/Niform Building Code

Lay Stranded Copper Conductors Having Bunch Stranded Me

Lay Stranded Copper Conouctors (1974) ANSI C7.59

Lay Stranded EC Grade Aluminum Conductors (1972) ANSI C

Lay Stranded Steel Reinforced, (ACSR) (1972A) ANSI C7.2

Lay Stranded 5005.H19 Aluminum Alloy Conductors (1972)

Lay Stranded 6201-T 81 Aluminum Alloy Conductors (1972)

Lay Stranded (1972)

Lay Up Composite Filament Tape Reel (1972)

Lay) (1973)

Std. for Gear Tooth Surface Texture Fo

Layer Cake Measuring Template (Cereal Chemistry) (1962)

Layer Type (1973) ANSI C59.50

Layer (1973)

Std. Spec. for Ele

Layers by the 3 Probe Voltage Breakdown Meth. (1972) an

Layers in Silicon by the Angle Lapping and Staining Tec

Layers of Silicon by Measurement of Stacking Fault Dime

Layers of Silicon on Substrates of the Same Type by $\ln f$

Layers Using a Collinear 4 Probe Array (1974)

Laying Machine-Numerically Controlled (1973)

Layout Diagrams Used in Architecture and Building Const

Layout, Construction and Maintenance of Terrace (Erosio

Layouts and Terminals (For Foreign and Domestic Passeng

Layup (1973)

Leachate of the Pigment (1973)

I for Water Soluble Sal

Lead-Tin Alloys on Steel and Ferrous Alloys (1970) an

Lead Acid Industrial Storage Batteries for Motive Power

Lead Acid Type Storage Batteries for Motor Vehicles, Mo

(Lead Acid) Storage Batteries (1972)

/ Domestic

Lead Alloy Coated Soft Copper

Lead and Cadmium Extracted from Glazed Ceramic Surfaces

Lead and Lead-Tin Alloys on Steel and Ferrous Alloys

Lead and Lead Alloy Coated Soft Copper Wire for Electri

Lead and Tin Alloy Die Castings (1952) A NSl H48.l

Lead and Vanadium in Gas Turbine Fuels (1972) ANSI Z11

Lead in Gasoline by Atomic Absorption Spectrometry (197

Lead in Gasoline by X.Ray Spectrometry (1973)

Lead in Gasoline (1972) ANSI Z11.324

Lead in Paint Driers by EDTA Meth. (1973)

Lead in Paint (1973)

Lead in the Atmosphere by Colorimetric Dithizone Proced

Lead Phosphosilicate (1973)

Lead Pigments (1973)

Lead Silico Chromate (1974)

Lead Styles) (1972)

Lead Wires, Terminals, and Other Terminations (Soft Sol

Lead (Leaded Red Brass or Hardware Bronze) Rod, Bars, a

Lead, Zinc, or Cadmium) (1973)

/Rrosion Resis

Leaded Brass Plate, Sheet, Strip, and Rolled Bar (1973)

Leaders and Trailers, for Aerial and Related Uses (1973

Leads (1972) ANSI C83.55

Leaf Fibers (1973)

Leak Additives for Engine Coolants (1972)

Leak Antifreezes and Stop Leak Additives for Engine Coo

Leak Detector in the Detector Probe Mode (1973)

Leak Detector in the Inside Out Test. Mode (1973)

Leak Detector or Residual Gas Analyzer in the Tracer Pr

Leak Detector (1972T)

Leak Test for Paper Shipping and Refuse Sacks (Containe

Leak Test. Meth. (1971) ANSI Z166.26

Leak Test. Radioactive Brachytherapy Sources (1973)

Leak Test. Spec. (1973)

Leak Test. (1971) ANSI Z166.25

Leakage Current (Electricity) for Appliances (1973)

Leakage of Buildings Not Due to Hail or Wind (1972)

ASTM

AATCC

AATCC

AHA

ANSI

ASAE

UL

ANSI

ICBO

ASTM

ASTM

ASTM

ASTM

ASTM

ASTM

ASTM

ASTM

ASTM

NSA

$\mathrm{BCl}$

EIA

EIA

ASTM

ASTM

ASTM

ANSI

ASTM

ASTM

ASTM

ASTM

ASTM

ASTM

ASTM

TAPPI

ASTM

ANSI

ATCC 96

AATCC $88 \mathrm{C}$

ANSI Z8.1

UL $\quad 1240$

HLW.2PR

ANSI Z21.9

UL 1206

IAPMO TSCll

A 112.19 .2

S348T

82

B71.1

UBCS47.16

B524

B172

B496

B 400

B232

B397

B399

B231

992

MA 118.01

AACCH 10.91

ASTM D1305

ASTM F387

F108

ASTM F110

AATM F143

ASTM F95

Tentati ASTM F374

NSA $\quad 990$

ANSI $\quad$ Y 32.9

ASAE R268.1

* 1.56

AMS3894A

D2448

B200

IB2

J537H

$* 1.15$

B189

B579

ASTM C738

$\begin{array}{ll}\text { ASTM } & \text { C738 } \\ \text { ASTM } & \text { B200 }\end{array}$

ASTM $\quad \mathrm{B} 189$

ASTM B102

ASTM D2787

ASTM D3237

D3116

ASTM D2374

ASTM D2088

D3112

D2742

$\begin{array}{ll}\text { ASTM } & \text { D1301 } \\ \text { ASTM } & \text { D1648 }\end{array}$

RS153B

RS178.B

B 140

UBCS $32 \cdot 6$

$\mathrm{B} 12$

B140

PH1. 10 
Practice and Test Requirements for Std. Meth. of Test for Std. Meth. of Test. for Std. Meth. of Test for Std. Meth. of Test. for Std. Meth. of Test. for inside Out Test. Mode (1973) dual Gas Analyzer in the Tracer /

Rec. Pract. Meth. for Lining ary Mowers (Designed for Operation with Tractors Having at lca E/ Std. Meth. for Measuring the Relative Stiffness of Meth. of Test for Dynamic Water Resistance of Shoe Upper Meth. of Test for Dynamic Water Resistance of Shoe Upper Std. Meth. for Sampling

Std. Meth. of Measuring Area of Std Meth for Measuring Thickness of Water Resistance of Shoe Upper Leather by the Dow Corning Std. Meth. for Measuring Thickness of

. Meth. of Test for Estimating the Thermal Conductivity of Std. Meth. of Measuring Break Pattern of Meth. for Determining Chromic Oxide Content in Std. Meth. of Test for Chromic Oxide in Std. Definitions of Terms Relating to Std. Meth. of Test for Apparent Density of Std. Meth. of Test for Insoluble Ash of Vegetable Tanned meth. of Test for Water Soluble Matter of Vegetable Tanned Std. Meth. of Test for Water Absorption (Static) of Std. Meth. of Test for Compressibility of t. Meth. of Test for Nitrogen Content (Kjeldahl) and Hide Meth. for Preparation of Sample:

967)

)/ Std. for Title

Meth. of Analysis of Acetone-Insoluble Soybean

Dwelling Construction Under the Uniform Building Code: Test Meth. for Chip Test Meth. for Chip Std. Meth. of Test for Std. Meth. of Test for Std. Meth. of Test for Std. Meth. of Test for raph Measurement (Textile) (1972) ortar and Concrete (1974T) 974) ANSI A 37.78 2) ANSI L14.91 e Pho/ Tent. Meth. of Test for Minority Carrier Diffusion Std. for Precision In. Gage Blocks for Std. Meth. of Test for Yarn Number Based on Short n Red Cedar and Alaska Yellow Cedar Timber/ Std. for Full dgepole Pine Wood Poles Preservative Treatment by the Full ement (Textile) (1972)! Std. Meth. of Test for Length and (1974) Std. for Boat Load Capacity (Under $26 \mathrm{Ft}$. in Std. Test. Procedure for Measuring teel Products Manual for Carbon Sheet Steel (coils and Cut for Steel Sheets of Structural Quality in Coils, and Cut Std. for Dimensions for Front

est Meth. for the Selective Transmission of a Photographic Std. Nomenclature for Parts of a Photographic Objective n 2 X 2 In. Mounts (1972) Std. for Resolving Power of pertures and Related Quantities Pertaining to Photographic d. for Prescription Requirements for First Quality Contact Std. for Designation of A pertures of Photographic rina, Semolina, Bread, Grain, Soybeans, Rice, Beans, Peas, eter Meth. of Analysis of Moisture in Grains, Beans, Peas,

ills, High Speed Steel Threaded Shank, Sizes 1/16 ln. Thru ements for Both Permanentl/ Technology (1969) ANSI Y 10.19 Rec. Pract. for Signal Words, Std. for Glossary of Terms Conct. for and Related Documentation Pract. for Line Conventions and tions (1974) Std. for Suffix Rec. Pract. for Measurement of Low Magnetic Tape Recorders Operati/ Std. Spec. for an Audio Std. and Rec. Pract. for Rec. Pract. for Exterior Sound

6.3

Rec. Pract. for the Test Procedure for Exterior Sound Std. Procedure for Bench Calibration of Tank iquefied Petroleum (LP) Gas (/ Std. for Safety for Liquid Safety Std. for Liquid Std. Spec. for Sound $\mathrm{ng}$ a Sound Data Acquisition System for Vehicle Noise Sound for 2.In. Quadrup/ Std. Frequency Response and Operating Std. for Safety for Constant Std. on Subscriber Visual Carrier

Hot Mixed, Hot Laid Tar Paving Mixtures for Base, Binder, Rec. Pract. for Design

Std. Meth. for the Measurement of Sound Pressure Recommendation for Installation Performance Std. Meth. of Test for Low
Leakage Rate Test. of Containment Structures for Nuclea Leaks in Heat Sealed Flexible Packages (1972)

Leaks Using Bubble Emission Techniques (1974)

Leaks Using the Mass Spectrometer Leak Detector in the Leaks Using the Mass Spectrometer Leak Detector in the

Leaks Using the Mass Spectrometer Leak Detector or Resi

Lease Production Tanks with Coal Tar Epoxy (1972)

Least $20 \mathrm{Hp}$ ) (1971)

IY Requirements for Industrial Rot

Leather by Means of a Torsional Wire Apparatus (1972) a

Leather by the Dow Corning Leather Tester (1970) ALCA E

Leather by the Maeser Water Penetration Tester (1970) a

Leather for Physical and Chemical Tests (1971) ALCA Jl

Leather Test Specimens (1968) ALCA E2

Leather Test Specimens (1970) ALCA E4

Leather Tester (1970) ALCA E55

Leather Units (1970) ALCA E3

Leather with the Cenco Fitch Apparatus (1970) ALCA E60

Leather (Break Scale) (1973) ALCA E64

Leather (Perchloric Acid Oxidation) (1969) ASTM D2807

Leather (Perchloric Acid Oxidation) (1972)

Leather (R1972) ALCA X1, ANSI Z109.3

Leather (1968) ALCA E62

Leather (1970) ALCA B11

Leather (1970) ALCA B8

Leather (1970) ALCA E30

Leather (1970) ALCA E45

(Leather) Substance (1970) ALCA B5

Leavening Agents (Cereal Chemistry) (1962)

Leaves of a Book (1971)

Lecithin in Soybean and Corn Oils (Cereal Chemistry) (1 Lecithin Spec. (1973)

Legal Requirements (Permit, Plans and Spec., Inspection

Legumes. Forages, and Animal and Dairy Products (Cereal

Length Analysis (Measure ment) (Pulp and Paper) (1973)

Length Analysis (Two Screen) (Pulp and Paper) (1973)

Length and Length Distribution of Cotton Fibers (Array

Length and Length Uniformity of Cotton Fibers by Fibrog

Length Change of Drilled or Sawed Specimens of Cement M

Length Change of Hardened Cement Mortar and Concrete (1

Length Distribution of Cotton Fibers (Array Meth.) (197

Length in Silicon by Measurement of Steady State Surfac

Length Measurement (Thru 20 In.) (1973)

Length Specimens (1972) ANSI L14.220

Length Thermal Process Preservative Treatment of Wester

Length Thermal Process (1973) Std. for Lo

Length Uniformity of Cotton Fibers by Fibrograph Measur

Length) (1973)

Lengths or Heights of Composite Cans, Tubes, and Cores

Lengths) (1974)

Lengths, Zinc Coated (Galvanized) by Hot Dip Process (1

Lens Mounts for Cameras (1972)

Lens $(1969)$

Lens (1971)

Lenses for Projectors for $35 \mathrm{~mm}$ Filmstrips and Slides I

Lenses (1971)

Lenses (1972)

Lenses (1973)

Lentils, Corn Meal, Corn Grits, Rolled Oats, Bulgur, Ro

Lentils, Rice, and Other Commodities for Which Conversi

Leptophos (Insecticide) (1971)

Letter F (1973)

Letter Sizes, Color Combinations, and Durability Requir

Letter Symbols for Units Used in Electrical Science and

Letter Symbols (1972)

Lettering (1973)

Std. for Engineerin
Lettering (1973)

Level Activity in Water (1972T)

Level and Multifrequency Test Tape for Quadruplex Video

Level Flotation of Boats When Swamped (1973)

Level for Heavy Trucks and Buses (1971)

Level for Passenger Cars and Light Trucks (1972) ANSI S

Level for Snow mobiles (1972) ANSI S6.2

Level Gaging Tapes and Sounding Rules (1974)

Level Gauges and Indicat ors for Anhydrous Ammonia and L

Level Gauges and Indicators for Flammable Liquids (1972

Level Meters (1971)

Level Meters (1972) ANSI S6.1

Level of Recorders and Reproducers for Audio Record One

Level Oil Valves (1974) ANSl B127.1

Level (Cable Television) (1970)

Leveling, and Surface Courses (1967)

Levels for Emergency Air Brake Systems (1971)

Levels in Air (Acoustics) (1971)

Levels of Ceramic Tile Floors (1973)

Levels of Lead in Gasoline by X-Ray Spectrometry (1973)

Levels of Powered Industrial Trucks (1973)
ANSl

ASTM

ASTM

ASTM

ASTM

ASTM

NACE

SAE

ASTM

ASTM

ASTM

ASTM

ASTM

ASTM

ASTM

ASTM

ASTM

ASTM

ALCA

ASTM

ASTM

ASTM

ASTM

Std. ASTM

ASTM

ASTM

Ten ASTM

AACC

ANS!

AACCH

NSPA

ICBO

$\mathrm{AACCH}$

TAPP

TAPPI

ASTM

A STM

ASTM

ASTM

ASTM

A STM

ANSI

ASTM

AWPA

AWPA

ASTM

ABYC

CCTI

Alsl

ASTM

ANSI

ANSI

ANSI

ANSI

ANSl

ANSI

ANSI

A4CCH 441

ANSl

NSA

SAE

IEEE

ANSl

ANSI

TRA

ASTM

ANSI

ABYC

SAE

SAE

SAE

ANSI

UL

UL

SAE

AAE

UL

NCTA

Std. Spec. for ASTM

SAE

ANSI

TCA

ASTM

/R Uniform ITA

$\mathrm{N} 45.4$

(1)

E515

E499

E498

J232

D2098

D2099

D2813

D1813

D2098

D1814

D2941

D 10

D 2807

RP.03.72 
Std. Spec, for Manually Std. for Directories of Handbook for Rec. Pract. for Std for

(1971) Requirements for Safety to

truction, and Operation of So/ tion (1971) An/ Std. Meth. of Test for Measuring the Wear Std. Load Ratings and Fatigue Std. Load Ratings and Fatigue Code for Safety to a9.1 Guide for Statistical Analysis of Thermal Rec. Pract. for

ith 3 Point / ith $3 \mathrm{Po}$ 72)

Std. Test Procedure for Measuring Hydraulic Std. for Test Procedure for Measuring Hydraulic

Rec. Pract, for High

Rec. Pract. for Rating of Trailer Floors for lity and Recreational Type Vehicles, Counter Balanced Fork d. for Forks and Fork Carriers for Powered Industrial Fork Grader, Loader, Dozer, Mining Car, Front End Loader, Fork Std. for Elevating Work Platforms

and Equipment for Anchoring, Mooring, Docking, Towing and ts for Aerial Passenger Tramways lncluding Ski and Surface nnel and Material (1/ Guide Spec. for Platforms and Stage rfastness of Textiles to Light: Carbon Arc Lamp, Alternate

Textiles to Light: Water Cooled Xenon Arc Lamp, Alternate nsi L14.118 Test Meth. for Colorfastness of Textiles to test Meth. for Resistance of Textiles: Exposure to Natural th. for Resistance of Textile Fabrics: Exposure to Natura ance Requirements for Oil Powered Forced Air (Domestic and ineral Fiber Blanket Thermal Insulation for Wood Frame and ansmitting Panels, Skylights, Monitors and Sawtooth Roofs

ing Facilities (Passenger Cars, Multipurpose Vehicles, and Std. Rec. Pract. for Conducting Natural

Tent. Rec. Pract. for lnstallation of Fixed Partitions of for Copper Alloy No, 260 Brass Strip in Narrow Widths and ill Screws for the Application of Gypsum Sheet Material to ers (/ Uniform Building Code Std. for Spec. and Design of $r$ Takeoff Definitions and Terminology for A gricultural and oulometr/ Meth. of Test for Trace Quantities of Sulfur in $r$ and Bolted Joints, Drift Bolts, and Wood and Lag Screws: 2) ANSI Z11.27 Std. Meth. of Test for Aromatic Traces in (1969) Std. Test Meth. for Diffuse rics (Textile) (1973) Meth. of Test for ooth / Uniform Building Code: Plastics (General, Exterio ocumentation Practices: Chassis Frames-Passenger C.ar and Rec. Pract. for Nomenclature for Passenger Car and Rec. Pract. for Passenger Car and Rec. Pract. for Passenger Car and

$s$ for Exhaust Gas Emissions Measurement (Passenger Car and orative Emissions from Gasoline Powered Passenger Cars and or Training of Automotive Mechanics for Passenger Cars and orative Emissions from Gasoline Powered Passenger Cars and Std. for Sound Level for Passenger Cars and Std. Protective Coatings (Paints) for Molded ln, Blind Threaded, Self Locking, Nonself Locking, Std. for Threaded Metal lnsert, for Fastener (Blind, Internally Threaded, External Sleeve, for Fastener (Blind, Internally Threaded, External Sleeve,
Std. for Low Height, Test Meth. for Colorfastness of Textiles to Test Meth. for Colorfastness of Textiles to textiles to Light: Water Cooled Xenon Arc Lamp, Continuous fastness of Textiles to Lighi, Carbon Arc Lamp. Continuous ment of Colored Textiles to Photochromism Colorfastness to Std. for Mounting Brackets and Socket for Warning Lamp Rec. Pract. for Semjautomatic Headlamp Std. for Outdoor Floodlighting Rec. Pract. for Cornering Lamps Std. for Flashing Warning Lamp Rec. Pract. for Headlamp

ment $(1972)$

Rec. Pract. for Mechanical Stop Lamp

service Performance Requirements for Sealed Beam Headlamp
Std. for Fog Lamps Rec. Pract. for Motorcycle Turn Signal Lamps

act. Test Meth. and Requirements for Snow mobile Stop Lamp Std. for Clearance, Side Marker and ldentification Lamps act. Test Meth. and Requirements for Snow mobile Headlamps and Requirements for Snowmobile Rear Position Tail Lamp Std. for Auxiliary Low Beam Lamp (Headlamp
Levels of Small Sources in Reverberation Rooms (Acousti Levels (TIL) (1971)

Lever Operated Chain Hoists for Ratchet and Pawl, and L Libraries and Information Centers (1971)

Library Binding (Rec. for Good Book Binding Practices)

Library Lighting (1974)

License Plate Lamps (Light) (1973)

ife-Property from Explosion and Fire in Design, Cons

Life and Bonded Solid Film Lubricants in Oscillating Mo

Life for Ball Bearings (1972) ANS] B3.15

Life for Roller Bearings (1972)

Life from Fire in Buildings and Structures (1970) ANSl

Life Test Data (1972)

Life Test for Automotive Storage Batteries (1972)

Lifeboat Code (1968)

Lifetime of Neodymium Doped Laser Materials (1973)

Lift Force Capacity on Agricultural Tractors Equipped W

Lift Force Capacity on Agricultural Tractors Equipped W

Lift Grouted Reinforced Brick Masonry (Tech. Notes) (19

Lift Industrial Truck Nameplates and Marking (1973)

Lift Truck Loading (1971)

Lift Truck, Boat, Baggage, Special Type Trailer, Mobile

Lift Trucks (1973)

Lift Trucks, Mobile Crane, and Shovel (1974)

Lift) (1973)

Lifting Boats (1973)

Lifts and Tows (1973)

/ Ion and Installation of Fittings

Lifts (Hydrualic and Hydropneumatic) for Lowering Perso

Light and Darkness (1971) ANSI L14.169

Light and Darkness (1971) ANSI L 14.171

Light and Washing: Alternate Sunlight Exposure (1965)

Light and Weather Through Glass (1972) ANSI L14.177

Light and Weather (1968) ANSI L14.176

Light Commercial) Central Furnaces) (1972)

Light Construction Buildings (1970) ANSI Z98.36

Light Diffusers in Ceilings, Partitions, Exterior Venee

(Il] Pipes with Fuel Dispens

Light Duty (1972)

Light Examination for Presence of Rodent Urine on Bags

Light Exposures Under Glass (1973)

Light Frame Type for the Purpose of Conserving Their So

Light Gage for Heat Exchanger Tubing (1972)

Light Gage Steel Studs (1972) Std. Spec. for Steel Dr

Light Gauge Cold Fornıed Stainless Steel Structural Memb

(1972) SAE J722

Light Liquid Petroleum Hydrocarbons by Oxidative Microc

Light Metal Plate Connected Wood Truss Design; and Nail

Light Saturated Hydrocarbons by Gas Chromatography (197

Light Transmission Factor of Reinforced Plastic Panels

Light Transmittance of Woven Glass Fiber Decorative Fab

Light Transmitting Panels, Skylights, Monitors and Sawt

Light Truck-Ground Vehicle Practices (1974)

Light Truck Exhaust System Parts (1972)

Light Truck Muffler Parts Nomenclature (1971)

Light Truck Resonator Parts Nomenclature (1971)

Light Truck) (1971) /Ory Instrumentation an

Light Trucks Using the Enclosure Technique (1972)

Light Trucks (Course of Instruction) (1972) / 1 for

Light Trucks (1972) ANSl S6.3

Light Water Nuclear Reactor Containment Facilities (197

Light Weight Floating, Sandwich Panel (1972)

Light Weight (1973)

Light Weight, Millable Head Self Locking) (1972)

Light Weight, Protruding Head, Self Locking) (1972)

Light Weight, Self Locking Hexagon Nut (1972)

Light (Daylight) (1971) ANSI L14.168

Light (General Meth.) (1971) ANSI L14.168

Light (1971) ANSl L14.170

Light (1971) ANSI L14.53

Light (1972) ANSI L14.271

Meth. for Colorfastness of

Llow Moving Vehicle (SMV) Identification Emb

(Light) Beam Switching Devices (1972)

(Light) Equipment (1973)

(Light) for Automotive Vehicles (1965)

(Light) for Remote Mounting on Agricultural (Farm) Equip

(Light) Switch (1971)

(Light) Switch (1972)

(Light) Units for Motor Vehicles (1973)

(Light) (1970)

(Light) (1971)

(Light) (1971)

(Light) (1972)

(Light) (1972)

(Light) (1972)

Light) (1973) ASTM

D. for Powe

Test Meth. for Color

ANSI

ANSl

S1.21

ANS] Z39.10

LBI

IES

*2

RP4

J587E

$\begin{array}{ll}\text { FMS } & 7.30 \mathrm{~N} \\ \text { ASTM } & \text { D2981 }\end{array}$

AFBMA 9

AFBMA 11

NFPA 101

IEEE 101

JAE J240A

SNAME 4-2A

ASTM F380

ASAE $\$ 349$

SAE J283

BIA 17D

ITA 4Fl

TTMA RP37

TRA

St ANSI

TRA

ABYC

ANSl

MH11.4

3

A 92.3

A5

B77.1

14430

$16 \mathrm{D}$

$16 \mathrm{~F}$

$\begin{array}{ll}\text { AATCC } & 83 \\ \text { AATCC } & 1110\end{array}$

AATCC $111 \mathrm{D}$

Perform ANSl 291.1

for M ASTM C665

ICBO UBC*3.52

SAE J398A

NSA 1284

AACCH $28-85$

ASTM G24

E497

B569

C646

UBC $\$ 27-10$

$\mathrm{ICBO}$

\$205.2

ASTM D3120

ICBO UBCS25-17

ASTM D2600

ASTM D1494

ANS1 L24T4

ICBO UBC $* 3.52$

Y 14.32.1

SAE

SAE

SAE

J261

$\mathrm{J} 262$

J254

J171A

D 18.1

J170A

J986A

Nlol.2

1835

1394

/Td. NSA 1674

NSA 1673

NSA 1291

AATCC $16 \mathrm{C}$

AATCC

16

$16 \mathrm{E}$

$16 \mathrm{~A}$

139

J725D

SAE J565C

NEMA FAl

SAE J852B

ASAE $\$ 307.1$

SAE J253

SAE $\quad$ J249

J583C

SAE

SAE J131A

Rec. Pr SAE

SAE

$\mathrm{J} 278$
$\mathrm{~J} 592 \mathrm{E}$

I 280

280

Rec. Pract. Test Meth 
Std. for License Plate Lamps (Light) (1973)

Std. for Spot Lamps (Light) (1973)

Lamp (Light, Bulb) (1973)

4.53

1) ays (1971) Test Meth. for Colorfastness of Textiles to Dwelling Construction Under the Uniform Building Code: Battery Materials Spec. (Vehicular, Ignition, Std. Rec. Pract. for

ct. for Service Performance Requirements for Motor Vehicle $y$ of Plastic Material for Use in Housings of Motor Vehicle Std. for Safety for Battery Powered Emergency etal Head and Reflector Interchangeability Used in Roadway ability of Socket Supports Used in Metal Heads for Roadway etal Head and Reflector Interchangeability Used in Roadway of Socket Supports for Use in Metal Heads Used in Roadway Std for Multiple Sockets Used in Roadway for Voltage Classification of Luminaires Used in Roadway (1972) ANSI C 33.28 Safety Sid, for Underwater Std. for Safety for Electric Safety Std. for Electric

headlights and Taillights (1972)

Rec. Safety Std. for Rec. Pract. for Std. for

(1972)

Groups C and D, A/

Std. for Dimensional Spec. for Sealed Std. for Safety for Portable Electric Std. Pract. for Industrial Std. Pract. for Office ge Classification of Luminaires Used in Street and Highway Std. for Multiple Sockets Used in Roadway Rec. Pract. for Library

g Devices and Towing Meth. (Coupling, Hitch, Safety Chain, ercial Vehic/ Storage Battery Spec. (Vehicular, Ignition,
Rec. for uits (1972) ANSI C62.1

Std for Surge pract. and Stds. for Location and Visibility of Navigation Std. for Flashing and Steady Burn Barricade Warning Rec. Guide for Structural Uniform Building Code Std. for Rec. Pract. for Selecting Proportions for Structural peration of Class Hi (High Impact) Shock Test. Machine for ed Molded in Insert (1974)

ed Molded in Insert (1974) Std. for Sandwich Panel Sid. for Sandwich Panel

rformance Requirements for Tailored Woven Uniform Fabrics, st Meth. for Colorfastness of Textile Fabrics to Water and orfastness of Textile Fabrics to Water (High Humidity) and 971) ANSl Ll/

114.264 Test Meth. for Colorfastness of Textiles to Test Meth. for Colorfastness to Darkness (1) 971) ANSI L1/ Test Meth. for Colorfastness of Textiles to Test Meth. for Colorfastness of Textiles to Test Meth. for Identification of Std. Definitions of Terms Relating to Uniform Building Code Std. for Sand. Std. Meth. of Test for

i Al.38

Uniform Building Code Std. for Hydrated Std. Spec. for Hydrated Std. Spec. for Communication and Signal Pin Type Std. Meth. of Test for Lime Content of Uncured Soil Std. Spec. for Portland Cement Std. Spec. for Portland Cement and Cement ec. for Lathing and Furring for Portland Cement and Cement Std. Meth. of Physical Test. of Quicklime and Hydrated of Chemical Analysis of Limestone, Quicklime, and Hydrated Uniform Building Code Std. for Uniform Building Code Std. for Quicklime Meth. of Test for Impact Resistance of Pipeline Coatings Std. Spec. for

Std. Definitions of Terms Relating to Lime and cinders: Concrete; Earth; Glass; Granite; Gravel; Granite, 3 Sid. Meth. of Chemical Analysis of and Processing Systems) (1972) Sid. Meth. of Chemical Analysis of um A/ Std. for Chemical Compositions, Mechanical Property ed in Power Switchge/ Safety Requirements for X-Radiation ioning) (1966) Std. Operatin ssible Variations from Specified Ladle Chemical Ranges and Rec. Pract. for Drill Stem Design and Operating in Wheat Flour and Semolina (Cereal Chemistry) (1962)

Test for Research and Motor Meth. Octane Ratings Using on

es Capacitors for Electrical Transmission and Distribution r Engineering Drawing and Related Documentation Pract. for Requirements for Power ms (1971) Std. Spec. for Poly(vinyl Chloride) (PVC) Plastic Std. for Liquid Std for Flow Capacity Rating and Application of Suction
Light, Carbon Arc Lar

Light Suntight Light (1971) ANSI LI

Ventilation, Sanitation, Fire Warning Detector (

Lighting and Marking of Agricultural Equipment on Highw

Lighting and Starting) (1972)

Lighting Cotton Classing Rooms for Color Grading (1971)

Lighting Devices and Components (1972)

Lighting Devices Including Reflex Reflectors (1973)

Lighting Equipment (Indoor) (1973)

Lighting Equipment (1973) EEI TDJ.140

Lighting Equipment (1973) EEI TDJ.143

Lighting Equipment (1973) NEMA SH10

Lighting Equipment (1973) NEMA SH13

Lighting Equipment (1973) NEMA SHl7

Lighting Equipment (1973) NEMA SH3

Lighting Fixtures and Junction Boxes for Swimming Pools

Lighting Fixtures for Use in Hazardous Locations (1972)

Lighting Fixtures (1972)

Lighting for Dairy Farms and the Poultry Industry (1971

Lighting for Regular Bicycles. Including Reflectivity,

Lighting Identification Code (1971)

Lighting Inspection Code for Motor Vehicles (1973)

Lighting Unit for Construction and Industrial Machinery

Lighting Units for Use in Hazardous Locations, Class I,

Lighting (1973) ANSI AlI.1

Lighting (1973) ANSI A132.1

Lighting (1973) EEI TDJ.133

Lighting (1973) EEI TDJ-147

Lighting (1974)

Lighting) (1973)

Lighting, and Starting) for Passenger Automobiles, Comm

Lightning Arresters and Grounds (1974)

(Lightning) Arresters for Alternating Current Power Circ

Lights (Boats) (1972)

Lights (1971)

Lightweight Aggregate Concrete (1964)

Lightweight Aggregates for Structural Concrete (1973)

Light weight Concrete (1969) ANSI A 164.1

Lightweight Equipment (1972)

IIgn, Construction, and $\mathrm{O}$

Lightweight Selflocking and Nonselflocking Blind Thread

Lightweight Selflocking and Nonselflocking Blind Thread

Lightweight (Tropical) Suiting (Institutional Textile)

Light: Alternate Exposure (1971) ANSI L14.227

Light: Alternate Exposure (1972) ANSI L 14.228

Light: Carbon Arc Lamp, Alternate Light and Darkness (

Light: Determination of Fastness Above L.7 (1971) ANSI

Light: Water Cooled Xenon Arc Lamp, Alternate Light and

Light: Water Cooled Xenon Arc Lamp, Continuous Light (1

Lignin (Paper Chromatogram) (1973)

Lime and Limestone (1971) ANSI K67.8

Lime Building Brick (1973)

Lime Content of Uncured Soil-Lime Mixtures (1973) Ans

Lime for Masonry Purposes (1973)

Lime for Masonry Purposes (1974) ANSI K67.13

Lime Glass Insulators (R1970)

Lime Mixtures (1973) ANSI A 1.38

Lime Mortar for Brick Masonry (1972)

Lime Plastering, Exterior (Stucco) and Interior (197I)

Lime Plastering, Exterior (Stucco) and Interior (1971)

Lime (1971) ANSI K67.10

Lime (1972) ANSI K67.3

Lime (1973)

Lime) for Structural Purposes (1973)

Limestone Drop Test) (1972)

Limestone for Animal Feed Use (1972)

Limestone (1971) ANSI K67.8

Limestone, and Marble Masonry; Mortar; Sand; Slag; Stee

Limestone, Quicklime, and Hydrated Lime (1972) ANSI K67

Limit Controls (Air Conditioning, Heating, Ventilating

Limits and Dimensional Tolerances of SAE Wrought Alumin

Limits for AC High Voltage Power Vacuum Interrupters Us

Limits for Central Station Units (Air Moving and Condit

Limits for SteeIs (1972)

Limits (Petroleum) (1974)

Linderstrom-Lang Meth. of Analysis of Amino Nitrogen

Line Analyzers (1970T)

Line Code System for Welding Wire Identification (1973)

Line Compensation (Including Safety Requirements) (1973

Line Conventions and Lettering (1973)

Line Coupling Capacitors (1972)

Line Couplings, Socket Type (1973)

Line Driers in Refrigerating and Air Conditioning Syste

Line Filters and Filter Driers in Refrigeration and Air

SAE

SAE

ANSI

C79.255

AATCC 16A

AATCC $16 \mathrm{~B}$

[BC $* 8.3$

SAE J137A

BCI $* 1.41$

ASTM D1684

$\mathrm{J} 256 \mathrm{~A}$

TT SAE

'L

J29

924

std. for M NEMA SHlO

hange NEMA

d. for M EE

EEI

EEI

Std EEI

UL

UL

ASAE

BMA

BMA

$S A E$

SAE

UL

IES

$\mathrm{SH} 13$

TDJ. 140

TDJ. 143

TDJ. 147

TDJ.133

676

844

R344

$6 / 6$

$\mathrm{J} 759 \mathrm{~B}$

J599C

J572A

781

RP7

$\mathrm{RPl}$

$\mathrm{SH} 3$

NEMA SHI7

IES

$\mathrm{RP} 4$

V.5

$\mathrm{BCl}$

FMS

1.48

5.11

28

A 16

ITE

AC.

$\mathrm{ICBO}$

ACI

*7

64. 39

UBC $\$ 26.3$

211.2

$\$ 2.15$

NSA $\quad 1832$

ANSI L 24.3 .3

Te AATCC 125

for Col AATCC 126

AATCC 16D

AATCC $16 \mathrm{G}$

AATCC 16F

AATCC $16 \mathrm{E}$

TAPPI UM.19

ASTM

ICBO

ASTM

ICBO

ASTM

ASTM

A STM

BIA

ANSI

ANSI

ASTM

Std. Meth. ASTM

ICBO

ICBO

Sid. ASTM

ASTM

ASTM

ICBO

ASTM

UL

$\mathrm{SAE}$

ANSI

AMCA

SAE

API

$\mathrm{AACCH}$ 
Safety Std. for depth Determination Device and Fish Finder, Loran or Other ) (1972) $n g$, and Thread Inspection of Petroleum Casing, Tubing, and Spec. for Spec. for High Test Spec. for Spiral Weld Spec. for Thermoplastic Pract, for Care and Use of Reinforced Thermosetting Resin Tent. Spec for Ultra High Test Heat Treated Spec. for Reinforced Thermosetting Resin Rec. Pract. for Railroad Transportation of or Alter/ Std. for Automatic Circuit Reclosers, Automatic $r$ and Farm Implement Power Takeoff (Universal Joint) Drive dimensional and Electrical Characteristic Defining Dual in c Heater Line Voltage Integrally Mounted Thermostats For/ voltage Integrally Mounted Thermostats for Electric Heater Std. Application-Guide for Porcelain Suspension (Power Std. Specs. and Tests for Strain Gage od (1971) nsi L14.136 Std. Meth. of Test for Estimating Maturity and Std. Meth. of Test for Std. Meth. of Test for g Cure (1969) ANSI K65.181 Std. Meth of Test for ccelerome/ Std. Spec. Format Guide and Test Procedure for Rec. for

tile Iron Pipe, Centrifugally Cast, in Metal Molds or Sand lanyard, Pole and Tree Climber) (I973) Spec. for (1972) ANSI L14.57

Control Test/ Test Meth. for Cotion and Std. Spec. for Vitrified Clay istribution) (1971) asuring Water Vapor Transmission Through Screw Cap Closure 1973)

1972) SAE J955 Rules for the Operation of Electric Supply Std. for Full Shielding of Power Drive Suggested Guide

Particulate/ Meth. for Extracting Fluid Samples from the mance Requirements for Backflow Preventers in Water Supply ntenance of Underground Electric Supply and Communications

for Asphalt Type Protective Coatings for Underground Pipe ation and Maintenance of Electric Supply and Communication Std. for Solid and Semi Solid Dielectric Transmission back Pressure Backflow Preventers for Potable Water Supply er Shop Applied) (197/ Std. for Cement Mortar Protective 73)

Std. Performance Requirements for Woven Rec. Pract. Meth. for e Grades; Small Fruits (Berry, Currant, Grape, Asparagus); Rec. Pract for Gogan Hardness of Brake Chloride) Plastic Sheeting for Pond, Canal, and Reservoir Std. Spec. for

ements and Uniform Test Procedures for Motor Vehicle Brake Wheel Tractors (1972) SAE J715 Std for 3 Point Free Std. Malleable Iron Detachable

Std. Spec. for Aluminum Coated Steel Chain Std. Spec. for Zinc Coated Steel Chain Spec. for Galvanized Steel Chain Std. for 3 Point Free

eled Tractors (1972)

equipped with Quick Attaching Coupler for Three Point Free ble for the Transmission and Distributi/ Std. for Crossed ture Relating to Resilient Floor Coverings (Asphalt, Cork, Trading Rules for the Purchase and Sale of Trading Rules for the Purchase and Sale of Std. Spec. for Raw Reinforced Brick and Tile Structural Steel rec. Pract. for Guide to the Application and Use of Radial (1962) (1962) stry) (1962) Meth. of Analysis of Meth. of Analysis of Meth. of Analysis of and Explosion Protection (Safety) for Flammable Liquid and (1973) Rec. for

z11.92 graphy (1973)

Tentative Manual for Driver Attended Loading of Std. Meth. of Test for Vapor Pressure of Std. Meth. for Analysis of Std. for the Storage and Handling of Uniform Fire Code:

(1) Std. for Safety for Valves for Anhydrous Ammonia and Safety Std. for Pumps for Anhydrous Ammonia and quid Level Gauges and Indicators for Anhydrous Ammonia and Std. for Safety for Meters for Flammable Liquids and

d. Meth. for Calculation of Certain Physical Properties of

Std. Meth. of Test for Applied Weight Per Unit Area of laps of Fiberboa/ Std. Spec. for Water or Solvent Soluble ter Spray, and Explosion Protection (Safety) for Flammable
Line Isolation Monitors (1973)

Line of Position Device, Radar, Radio Detection Finder,

Line Pipe Connections in High Pressure Oil Well Service

Line Pipe Threads (1974)

Line Pipe (Petroleum Drilling) (1973)

Spec. for Threading, Gagi

Line Pipe (Petroleum Drilling) (1973)

Line Pipe (Petroleum Production) (I973)

Line Pipe (PVC and CPVC) (1972)

Line Pipe (Rtrp) (1972)

Line Pipe (1972)

Line Pipe (I972)

Line Pipe (1972)

Line Sectionalizers and Oil Filled Capacitor Switches F

Line Specs. (1972)

Line Type Sockets (1974) ANSI C 83.95

Line Voltage Integrally Mounted Thermostats for Electri

Line Voltage Integrally Mounted Thermostats for Electri

Line) Insulators (1974)

Linear Acceleration Transducers (Instrumentation) (1971

Linear Density of Cotton Fibers by the Causticaire Meth

Linear Density of Cotton Fibers (Array Sample) (1973) a

Linear Density of Elastomeric Yarn (1972)

Linear Shrinkage of Thermosetting Casting Systems Durin

Linear, Single Axis, Pendulous, Analog Torque Balance a

Lined Earth Reservoirs for Fire Protection (1972)

Lined Molds for Gas (1971)

Lineman's Climbing Equipment (Body Belt, Safety Strap,

Linen Textile Colorfastness to Bleaching with Chlorine

Linen Textiles to Combined Washing and Shrinkage: Rapid

Liner Materials and Prefabricated Silencers for Acousti

Liner Plates (1972)

Liner, Adhesives, and Fasteners Application Std. (Air D

Std. Meth. of Me

Lines and Equipment (National Electrical Safety Code) (

Lines for Agricultural (Farm) Implements and Tractors (

Lines for Printed Wiring Board Repairs (1973)

Lines of an Operating Hydraulic Fluid Power System (For

Lines with Intermediate Atmospheric Vent (1972)

Lines (National Electrical Safety Code) (1973)

Lines (Wrapped Systems) (1972)

Lines $([960)$

Lines (1972)

Lines (1972)

Tent. Rec. Spec.

Lining Lease Production Tanks with Coal Tar Epoxy (1972

Lining Out Stock; Seedling Trees and Shrubs; Bulbs, Cor

Lining (1972)

Lining (1972T)

Linings for As

Spec. for Flexible Poly(vinyl

Linings (I966) 1973)

Minimum Performance Requir

Link Attachment for Hitching Implements to Agricultural

Link Chain and Attachments (1971)

Link Chains, Attachments, and Sprockets (1972)

Link Fence Fabric (1971) ANSI G53.21

Link Fence Fabric (1971A) ANSI G53.20

Link Fence Fabric (1973)

Link Hitch Attachment of Implements to Agricultural Whe

Link Hitch (1971) SAE J909A

Link, Cable Take Up (1973)

Icultural Wheel Tractors

Linked Thermosetting Polyethylene Insulated Wire and Ca

Linoleum, Rubber, Vinyl, Vinyl Asbestos, Polymeric Pour

Linseed Meal (Flaxseed) (1974)

Linseed Oil (Flaxseed) (1974)

Linseed Oil (1972) ANSI K34.1

Lintels (Tech. Notes) (1964)

Lintels (Tech. Notes) (1969)

Lip Type Oil Seals (1972)

Lipoid Phosphoric Acid in Dried Eggs (Cereal Chemistry)

Lipoxidase Activity of Ground Wheat (Cereal Chemistry)

Lipoxidase Activity of Semolina and Flour (Cereal Chemi

Liquefied Flammable Gas Processing Equipment and Suppor

Liquefied Hydrogen Systems (Fire Protection and Safety)

Liquefied Petroleum Gas (1966)

Liquefied Petroleum Gases (LP-Gas Meth.) (1973) ANSI

Liquefied Petroleum Gases (LPG) by Process Gas Chromato

Liquefied Petroleum Gases (1972)

Liquefied Petroleum Gases (1973)

Liquefied Petroleum (LP) Gas (Other Than Safety Relief)

Liquefied Petroleum (LP) Gas (1972)

Liquefied Petroleum (LP) Gas (1973)

Liquefied Petroleum (LP) Gas (1973) ANSI BI67.1

Liquefied Petroleum (LP) Gases from Compositional Analy

Liquid Adhesive (1951) A NSI Z197.10

Liquid Adhesives for Automatic Machine Sealing of Top F

Liquid and Liquefied Flammable Gas Processing Equipment
UL

$\mathrm{ABYC}$

API

API

API

API

API

API

Rec. API

API

API

API

NEMA

for EIA

NEMA

NEMA

NEMA

ISA

ASTM

ASTM

ASTM

ASTM

IEEE

FMS

ANSI

EEI

AATCC

AATCC 72

ASTM E477

ASTM

SMACN

ASTM

ANSI

ASAE

IPC

NFLDP

ASSE

ANSI

ANSI

EIA

ASSE

AWWA

ANSI

NACE

ANSI

SAE

ASTM

VESC

ASAE

ANSI

ANSI

ASTM

ASTM

CLFM

ASAE

NSA

NEMA

ASTM

NFP

NFP

ASTM

BIA

BIA

SAE

$\mathrm{AACCH}$

$\mathrm{AACCH}$

$A A C C$

FMS

FMS

NGPA

ASTM

ASTM

NFPA

ICBO

UL

UL

UL

UL

ASTM

ASTM

ASTM

FMS

1022

$5 \mathrm{~B}$

5LX

$5 \mathrm{LS}$

$5 \mathrm{LP}$

$5 \mathrm{Ll}$

SGl3

UL 52

R331.2 
f Analysis of Moisture in Massecuites, Molasses, and Other Std. Meth. of Test for Reaction Threshold Temperature of Std. Spec. for Std. Spec, for Std. Spec for Std. Meth. of Test for Specific or API Gravity of ust (1972) ANSI B93.28 chines (1972)

chines (1971) Std. Mieth. for Calibration of Std. Rec. Pract. for Uniform Building Code Std. for Exterior Plaster Std. Meth. of Test for Mercury in

for Analysis of Calcium and Barium Petroleum Sulfonates by Std. Analytical Meth. for Determining Saccharides (Gas d/ Std. Analytical Meth. for Determining Saccharides (Gas
1/ Std. Analytical Meth. for Determining Saccharides (Gas
of Insulating Materials (1973) A/ Std. Meth. of Test for of Insulating Construction of Diesel (Compression Ignited) Inboard or the Design and Construction of Gasoline Fueled Inboard, Std. Meth. of Testing for Rating Std. Meth. of Test for

Rec. for Flammable Std. Meth. of Test for Hydrolyzable Chlorine Content of efraction, with Abbe Refractometer, in All Normal Oils and electrical Apparatus Sales and Service Industry (Rotating,

d Recreational Vehicles (1973) ANSI A147/ Std. Meth. Of Std. for 1) ANSI Z11.313 Std. Meth. for Calculation of a and Liquefied Petroleum (LP) Gas (/

$s$ (1972)

g Systems (1971)

1973) ANSI A37.87

ting Ink, Adhesive, etc.) (1974)

Std. for Safety for Safety Sid. for Std. for Std. Spec. for

Rec for Flammable 38

Std. Meth. for Analysis of Natural Ga Std. Meth. for Analysis of Natural Gas Std. Spec. for

ting to Erosion of Solids by Cavitation and Impingement by Std. Meth. of Test for Compatibility of Materials with Std. Spec. for Std. Definitions of Terms Relating to tr/ Meth. of Test for Trace Quantities of Sulfur in Light
Std. Code for Pressure Piping: 1, Expansion Coil, and Flexible Connector Fittings for LP Safety Sid. for LP

ety Std. for Pipe Connectors for Flammable Liquids and LP td. for Safety Relief Valves for Anhydrous Ammonia and LP Rec. for Gaskets for Flammabl

$r$ Determining Acidity Content in Steepwater and Most Othe h. for Determining $\mathrm{pH}$ Content in Steepwater and Most Other safety) (1973)

91 Rec. for Flammable Std. for Std. for Std. Spec. for ntainer, Quickly Demountable Type for Horizontal Mounting, y Demountable Universal (Horizontal or Vertical Mounting), rocarbons (Askarels) for Capacitors (Electrical Insulatin Std. Meth. for Verification and Calibration of Std. for Safety for Valves for Flammable 67.1

td. Definitions of Terms Relating to Electrical Insulating Std. for Safety for Meters for Flammable Safety Std. for Pipe Connectors for Flammable d Mobile Tanks for Transporting and Spreading Agricultural oatings (Paint), and Handling of Flammable and Combustible Std. Meth. of Test for Specific Heat of of Test for Kinematic Viscosity of Transparent and Opaque Std. Meth. of Test for Density and Specific Gravity of Std. Meth of Test for Flash Point of Flammable and Combustible e Protection and Tanks Containing Flammable or Combustible si MH12.8 Std. Meth. of Test for Penetration of

2T) Meth for Measurement of Color of Low Colored Clear ortable Fuel Systems and Portable Containers for Flammable m Building Code Std. for Storage and Handling of Flammable Rec. For Flash Point Index of Trade Nam for Trace Quantitites of Total Sulfur in Volatile Organic roducts) by Setafl/ Std. Meth. of Test for Flash Point of Iron Fittings, 2 In. Through 48 In./S for Water and Other Std. Meth. of Test for Thermal Conductivity of Vulcanizates Resulting from Immersion in std. for Asbestos Cement Pressure Pipe for Water and Other Safety Std. for Tests for Comparative Flammability of Std. for Liquid Level Gauges and Indicators for Flammable or Aboveground Storage Tanks for Flammable and Combustible te Pressure Pipe, Steel Cylinder Type, for Water and Other ent for Gray and Ductile Cast Iron Pipe for Water or Other for Gray and Ductile Cast Iron Piping for Water and Other Uniform Fire Code: Flammable and Combustible all Nonpressurized and Pressurized Containers of Flammable
Liquid and Semiliquid Products (Drying Upon Quartz Sand Liquid and Solid Materials (1972) ANSI Z11.318

Liquid Asphalt (Medium Curing Type) (1972)

Liquid Asphalt (Rapid Curing Type) (1972)

Liquid Asphalt (Slow Curing Type) (1972)

Liquid Asphalts by Hydrometer Meth. (1973)

Liquid Automatic Particle Counters Using AC Fine Test D

Liquid Ballast Table for Drive Tires of Agricultural Ma

Liquid Ballast Table for Drive Tires of Agricultural Ma

Liquid Bonding Agents (1973)

Liquid Chlorine (1973)

Liquid Chromatography (1973)

Liquid Chromatography) Content of Corn Syrup, Sugar, an

Liquid Chromatography) Content of Corn Sugar, Starch M.

Liquid Contaminant, Inclined Plane Tracking and Erosion

Liquid Cooled Marine Engines and Auxiliaries (Boats) (I

Liquid Cooled Marine Engines (Boats) and Auxiliaries (1

Liquid Coolers (1971)

Liquid Densities of Pressurized Products (1972)

Liquid Drum Storage, Dispensing, and Classification (19

Liquid Epoxy Resins (1973)

Liquid Fats (Cereal Chemistry) (1967)

Liquid Filled and Dry Transformers, Hand Power Tools,

Liquid Flow Rate of Membrane Filters (1972)

Liquid Fuel Burning Heat Appliances for Mobile Homes an

Liquid Heat Capacity of Petroleum Distillate Fuels (197

Liquid Level Gauges and Indicators for Anhydrous Ammoni

Liquid Level Gauges and Indicators for Flammable Liquid

Liquid Line Driers in Refrigerating and Air Conditionin

Liquid Membrane Forming Compounds for Curing Concrete

Liquid Mixing Operations (Paint, Varnish, Lacquer, Prin

Liquid Mixtures by Gas Chromatography (1968)

Liquid Mixtures by Gas Chromatography (1973) ANSI Z11.2

Liquid Optical Adhesive (1970)

Liquid or Solid Particleo (1973)

Liquid Oxygen (Reaction Intensity Meth.) (1973)

Liquid Paint Driers (1973)

Liquid Penetrant Inspection (1974) ANSI Z166.16 Itions of Terms Rela

Liquid Petroleum Transportation Systems (1974)

(Liquid Petroleum) Gas Cylinders (1973)

(Liquid Petroleum) Gas Hose (1973)

(Liquid Petroleum) Gas (1972) UL 567

(Liquid Petroleum) Gas (1973)

Liquid Piping Systems (1974)

Liquid Products Obtained from Corn (1963)

Liquid Products Obtained from Corn (1963)

Liquid Pumping and Piping Systems (Fire Protection an

Liquid Resin Flexes (1972)

Liquid Rosin Fluxes Used for Soldering (1973) ANSI C83.

Liquid Toilet Soap (1974) ANSI K60.14

Liquid Withdrawal Only (1973)

Liquid Withdrawal (1973)

Liquid) (1973) ANSI C59.11

Liquid-In-Glass Thermom
Liquids and Fuels (1974)

Liquids and Fuels (1974)

Liquids and Liquefied Petroleum (LP) Gas (1973) ANSI B I

Liquids and LP (Liquid Petroleum) Gas (1972) UL 567

Liquids and Slurry (1973) Improving Safety on Enclose

Liquids and Solids and Potential Dust Explosion Conditi

Liquids and Solids (1971) ANSI Z11.307

Liquids and the Calculation of Dynamic Viscosity (1972)

Liquids by Bingham Pycnometer (1968) ANSI Z11.96

Liquids by Tag Open Cup Apparatus (1972)

Liquids Code (1972)

Liquids in Locations That May Be Flooded (1973)

Liquids into Submerged Containers (Packaging) (1951) an

Liquids Using the Hunterlab Color Difference Meter (197

Liquids (Boats) (1972)

/ Construction and Stowage of $\mathrm{P}$

Liquids (Gasoline, Kerosene, Acetone, Alcohol, Cleaning

Liquids (NFPA 325A) (1974)

Liquids (Oxy Hydrogen Combustion Meth.) (1972)

Liquids (Paint, Enamel, Lacquer, Varnish, and Related P

Liquids (1971) ANSI A21.10

Liquids (1971) ANSI Z11.304

Liquids (1972)

Liquids (1972)

Liquids (1972)

Liquids (1972)

Liquids (1972)

Liquids (1972)

Liquids (1972)

Liquids (1972) ANSI A21

Liquids (1973)

Liquids (1973)
/ for Cast Iron and Ductile

ASTM

ASTM

AWWA

ASTM

ASTM

$A W W A$

UL

Safety UI

Safety Std. F UL

Sid. for Prestressed Concre AWW A . Rec. Pract. for Polyethylene Encasem ASTM

for Polyethylene Encasement AWWA

ICBO

Rec. for Fire Protection of Sm FMS
CCH 44.60

D2028

D2026

D3142

T2.9.6

$\mathrm{S} 346$

$\mathrm{J} 884 \mathrm{~B}$

UBCS47.1

E506

D2894

E. 63

F. 50

D2303

P15

P4

24

D3096

D1726

8.20

317

$307 \mathrm{~A}$

D2890T

180

710

C309

7.36

2165

D2597

D2851

G 40

F371

D600

E270

D3120

B31.4

569

21

B148.1

132

7.32S

J. 4

J. 48

LPD 7.32

RS462

RS402

D799

$6 \mathrm{Al}$

$6 \mathrm{~A} 2$

D2233

E77

842

D2864

25

B148.1

R 317

UFC *2ART34

D2766

D445

D1217

D 1310

30

UFC $* 2 \mathrm{~A}$

D998

E450

$\mathrm{H} 25$

UBCS10.1

$7.42 \mathrm{~N}$

D2747

D3278

C110

D2717

D471

$\mathrm{C} 400$
340

180

142

C301

A674

C105

$\underset{7.29}{\mathrm{UFC}} \stackrel{2 \mathrm{ART} 15}{ }$ 
Underground Pipe, Fittings and Tanks Containing Flammable for Steel Underground Tanks for Flammable and Combustible Rec. for General Safeguards for Flammable y Std. for Power Operated Dispensing Devices for Flammable l (1969) Std. for Fire Hazard Properties of Flammable . and Registration Formats (1974)

Rec. Test Meth. for Moisture in Wood Chips (Two Analysis of Nickel-Base Alloys by the Silver Chloride Std. Spec. for Remelted Std. Definitions of Terms Relating io Rec, for Design Values for Safety Rec. for Electrically Heated and Moisture Loss and Feed and Water Requirements of Farm Rec. Pract. for Radius of of Extension lnstrument/ Std. Meth. of Test for Breaking Std. Spec. for Hollow Std. Spec. for Solid

Uniform Building Code Std. for Hollow Uniform Building Code Std. for Solid est. Stru/ Uniform Building Code Std. for Structural Clay ally Lever Operated Chain Hoists for Ratchet and Pawl, and Std for Boat

ansi 211.161 on Manual Sect. 1) (1/

Std. Test Proc. for Determining the Std. Meth. of Test for Spec. for Design of Aluminum Alloy Spec. for Cargo Unit 73) Std. for Std. Meth. of Calibration Instruments for Verifying Time std. Meth. of Test for Bearing Capacity of Soil for Static std. Meth. of Test for Bearing Capacity of Soil for Static ansi B3. 15 5

Std. for Unit

Transport Package Sizes (Packaging) for ANSI MH10.1, Unit Std. Proposal for Solid State series (1970) (1970) ries $(1970)$

teel Joints (1974)

3750 Through $30,000 / 40,000 / 50,000 /$ Std. Specs. and Std. Specs. and Std. Specs. and Std. Spec. and ent (1972) ANS1 B93.21 970) ANSI Al11.1 Std. Requirements for 1973) test for Deflection Temperature of Plastics Under Flexural Rec. for Wood Beam, Joint and Rafter Span, quirements for Regular Bicycles Including Tests for Static Structural Design of Serpentine Walls, Non les: Earthmoving, Mining and Logging (Short Haul); Grader. (Short Haul); Grader, Loader, Dozer, Mining Car, Front End rticulated Vehicles (Construction and lndustrial Front End ia for Roll Over Protective Structure for Weeled Front End Rec. Safety Sign for Front End Agricultura protective Structure for Track Type Tractors and Front End

(FOPS) for Prime Movers, Wheeled and Track Type Front End $t$ for Strength Properties of Adhesives in Shear by Tension std. for Wood Members Subjected to Both Flexural and Axial t for Strength Properties of Adhesives in Shear by Tension Sid. for Driver Atten ded I for Strength Properties of Adhesives in Shear by Tension Tentative Manual for Driver Attended ngth Properties of Metal to Metal Adhesives by Compression eth. for Creep Properties of Adhesives in Shear by Tension for Creep Properties of Adhesives in Shear by Compression t for Strength Properties of Adhesives in Shear by Tension st for Fatigue Properties of Adhesives in Shear by Tension ength Properties of Adhesive Bonds in Shear by Compression adhesives in Plywood Type Construction in Shear by Tension adhesives in Two Ply Wood Construction in Shear by Tension Rec. Pract. for Rating of Trailer Floors for Lift Truck durability of Adhesive Joints Stressed in Shear by Tension Bonded Rigid Plastic Lap Shear Joints in Shear by Tension nded Plastic Lap Shear Sandwich Joints in Shear by Tension

Strength of Concrete (Using Simple Beam with Center Point

Std. for Building Code Requirements for Minimum Design

dstand/Uniform Building Code: Stairs, Exits and Occupant Collapse of Buildings and Other Structures Caused by Snow Agricultural Grain Storage

rading Rules, Design Values, Section Properties and Design $r$ (Cereal Chemistry) (1962) Sponge Dough, Pound

td. for Fundamentals Governing the Design and Operation of

larm, and / Std for lnstallation, Maintenance and Use of and Safety (Alarm) (1973) ng on Iron or Steel Articles by T/

Rec. for
Std. Meth. of Test for
Liquids (1973)

Liquids (1973) ANSI B137.

Liquids (1974)

Liquids (1974) ANSI Z216.1

Liquids, Gases, and Volatile Solids (1973)

List of Terms and Definitions Related to Quality Contro

List of Values to Be Used in Semiconductor Device Specs

Liter Graduate) (1973)

Lithium Fluoride Carrier D.C Arc Technique Using an Opt

Lithium Metal in lngot Form (1972) ANS1 H43.1

Lithologic Classes and Physical Components of Coal (197

Livestock Fallout Shelters (1973)

Livestock Waterers (1971)

Livestock (Poultry, Swine, Cattle, Sheep) (1968)

Load and Boom Angle Measuring System (1972)

Load and Elongatoin of Elastomeric Yarns (Constant Rate

Load Bearing Concrete Masonry Units (1970) ANSI A79.1

Load Bearing Concrete Masonzy Units (1971) ANSI A81.1

Load Bearing Concrete Masonry Units (1973)

Load Bearing Concrete Masonry Units (1973)

Load Bearing Wall Tile and Std. Meth. of Sampling and T

Load Brake Designs (1971)

Load Capacity (Under $26 \mathrm{Ft}$. in Length) (1973)

Load Carrying Ability of Farm Wagon Running Gear (1973)

Load Carrying Capacity of Fluid Gear Lubricants (1973)

Load Carrying (Members) Structures (Aluminum Constructi

Load Devices (Materials Transport and Handling) (1973)

Load Handling Symbols for Powered lndustrial Trucks (19

Load lndication of Machines (1974) ANSI Z115.2

Load on Spread Footings (1972)

/Pread Footings (1972)

Load on Spread Footings (1972) Std. Meth. of Test for B

Load Ratings and Fatigue Life for Ball Bearings (1972)

Load Ratings and Fatigue Life for Roller Bearings (1972

Load Sizes for Dimensioning Transport Packages (1972)

Load Sizes (1973)

Load Switches for Traffic Control Systems (1973)

Load Tables for Longspan Steel Joints Deep DLJ and DLH

Load Tables for Longspan Steel Joints LJ and LH Series

Load Tables for Open Web Steel Joints J Series and H Se

Load Tables for Open Web, Longspan, and Deep Longspan S

Load Tap Changing Transformers 138,000 Volts and Below,

Load Tap Changing (1971)

Std. for Guide for Pre

Load Test Meth. for a Hydraulic Fluid Power Filter Elem

Load Test. for Refractory B
Load (1972) ANSI K65.222

Dition, Strength and Stifness Computations

Load, Frame, Fork, Steering Assembly and Tire Blowout

Loadbearing (Tech. Notes) (1968)

Loader, Dozer, Mining Car, Front End Loader, Fork Lift

Loader, Fork Lift Trucks, Mobile Crane, and Shovel (197

Loaders and Dozers) (1971)

Loaders and Weeled Dozers (1972)

Loaders (1972)

Steering Frame Lock for a

Loaders (1972)

Um Performance Criteria for Roll Over

Loaders, Wheeled Dozers, Track Type Tractors, and Motor

Loading at Elevated Temperatures (Metal to Metal) (1972

Loading Combined (1973)

Uniform Building Code

Loading in the Temperature Range from -267.8 to $-55 \mathrm{C}$ (

Loading of Anhydrous Am monia (1972)

Loading of Laminated Assemblies (1973)

Loading of Liquefied Petroleum Gas (1966)

Loading (Disk Shear) (1972) ANSI Z197.4

Loading (Metal to Metal) (1969)

Loading (Metal to Metal) (1969)

Loading (Metal to Metal) (1972) ANSI Z197.15

Loading (Metal / Metal) (1973)

Loading (1949) ANSI Z197.11

Loading (1964) ANSI Z197.12

1. Meth. of Tes

Test for Stre ASTM

Std. Test M ASTM

Std. Test Meth. ASTM

of Tes ASTM

Loading (1970)

/Test Meth for Strength Properties of

Loading (1971)

Loading (1971) ANSI Z197.31

1. Pract. for Determining

Ermining the Strength of Adhesively Bo

Loading (1973)

Loading) (1968) ANS1 A37.84

Loads in Buildings and Other Struts

Loads Offshore Oil Cranes (1972)

Loads (Buildings, Reviewing Stands, Bleachers, and Gran

Loads (1973)

Loads, Pressures, and Capacities (1968)

/Eth. of Test for Flexural

Local Exhaust Systems (1971)

Local Industrial Exhaust Ventilation Systems (1974)

Local Protective Signaling Systems for Watchman, Fire a

Local Protective Signalling Systems for Fire Protection

Locating the Thinnest Spot in a Zinc (Galvanized) Coati

SAE

ASTM

ASTM

AFBM

NEMA

AISC

AISC

SJI

ANSI

NFLDP

ASTM

ASTM

BMA

BlA

TRA

SAE

ASAE

SAE

SAE

ASTM

ASTM

Fl

$\mathrm{UFC} * 2 \mathrm{~B}$

58

7.35

87

7.19

ASQC *1

RS4 19

APPI UM-10

ASTM B357

ASTM $\quad$ 2796

ASAE R282.I

ASAE R342

ASTM D2653

ASTM C90

ICBO UBCS 24-4

ICBO UBCS24.5

ICBO UBCS 24.8

$A B Y C-H 5$

ASAE S360T

ASTM D1947

*10

ANSI MHIl.3

AFBMA

E74

D1 194

D1194

9

MH 110.1

MH 10.2

TS2

S316-1

S316-2

$\mathrm{S} 316 \cdot 3$

5

C57.12.30

C57.97

T3.10.8.2

C16

$\times 30$

$6 / 4$

33

3

J276

J394 A

R355

J395 A

J231

D2295

UBCS25.16

D2557

P135

8166

D2182

D2294

D2293

D 1002

D3166

D 905

D906

D2339

RP37

D2919

D3163

D3164

C293

A58.1 
r Contact Printing on $35 \mathrm{~mm}$ Motion Picture Film

Std. for rinting on $35 \mathrm{~mm}$ Motion Picture Film, Perforate/

e Film, Perforated Super 8 (I.3) (1973)

Std. for

Std. for Dwelling Construction Under the Uniform Building Code:
Tent. Rec. Pract. Guidelines for Driveway Design and puter Systems (1972)

Rec. for

G)

Safety Std. for Electric Heaters for Use in Hazardous ) (1973)

and Tanks Containing Flammable or Combustible Liquid $\mathrm{s}$ in Std. for Area Classification in Hazardous Dust e Installation of Electrical Instruments in Hazardous Dust equipment and Materials for Use in Flammable Anesthetizing essurized Enclosures for Electrical Equipment in Hazardous safety for Electric Lighting Fixtures for Use in Hazardous es and Connectors of the Pin and Sleeve Type for Hazardous d. for Receptacle-Plug Combinations for Use in Hazardous for Circuit Breakers and Enclosures for Use in Hazardous std. for Industrial Control Equipment for Use in Hazardous ty for Electric Motors and Generators for Use in Hazardous for Portable Electric Lighting Units for Use in Hazardous d. for Electric Motors and Generators for Use in Hazardous std. for Electrically Operated Valves for Use in Hazardous

Safety Std. for Electrical Switches for Use in Hazardous radio Homing and Alerting Equipment for Use with Emergency ial Front End Loaders and Dozers/ Std. for Steering Frame ot Dip Process (1971) Ansi/

Std. Spec. for Steel Sheet of Std. for Cylinder oc. for the Locking Ability Performance of Chemical Coated ce of Nonmetallic Locking Element Type Prevailing-Torque Std. Rec. Pract. for Use of Std. for Locks and Std. for Nonmagnetic Spring

d Meth of Test for Helical Spring and Carbon Steel Tooth td. for Titanium Alloy Stump Type $100 \mathrm{Deg}$. Head Shear Bolt

. for Titanium Alloy Stump Type Protruding Head Shear Bolt ype, Titanium Alloy (1973)

e, Titanium Alloy (1973)

Type, Titanium Alloy (1973)

loy (1973)

pull Type, Titanium Alloy (1973)

m Alloy (1973)

ze, Pull Type, Titanium Alloy (1973)

anium Alloy (1973)

nium Alloy (1973)

a nium Alloy (1973)

td. for Collar, Swage Locking for Pull Type and Stump Type Std. Fastener Code (Aerospace Std. for Bulbed Spindle Mechanically Safety Std. for Key

Std. for Protruding Head, Std. for Bulbed Mechanically Std for Blind, 100 Deg. Flush Head, Std. for Self Plugging, Mechanically Std. for Bulbed Mechanically Std. for Bulbed Mechanically

Std, for Bulbed Mechanically Safety Std. for Tellers: Std. Test Proc. for the

ews (1973) ent Type Prevailing-To/ Std. for Test Procedure for the $6 \mathrm{Al}-4 \mathrm{~V}$ Titanium Alloy, Long Thread, Self , Hex Head, Close Tolerance, A286 Cres, Short Thread, Self , Hex Head, Close Tolerance, A286 Cres, Long Threads, Self w, Pan Head, Tri Wing Recess, A286 Cres, Full Thread, Self Hex Head, Close Tolerance, Alloy Steel, Long Thread, Self hex Head, Close Tolerance, Alloy Steel, Short Thread, Self 0 Deg. Head, Tri Wing Recess, A286 Cres, Full Thread, Self g. Oval Head, Tri Wing Recess, A286 Cres, Full Thread Self close Tolerance, $6 \mathrm{Al}-4 \mathrm{~V}$ Titanium Alloy, Short Thread, Self ng Recess, Close Tolerance, Alloy Steel, Long Thread, Self lose Tolerance, 6Al-4V, Titanium Alloy, Short Thread, Self olerance, Short Thread, TWI Wing Recess, Alloy Steel, Self tolerance, Short Thread, TWI Wing Recess, A286 Acres, Self short Thread, Tri Wing Recess, Titanium Alloy $6 \mathrm{Al} \cdot 4 \mathrm{~V}$, Self Tri Wing Recess, 6Al-4V Titanium Alloy, Full Thread, Self ing Recess, Close Tolerance, A286 Cres, Short Thread, Self Close Tolerance, 6Al-4V Titanium Alloy, Long Thread, Self Std. for

Std, for Safety for Elevator Doo ocedure for the Locking Ability Performance of Nonmetallic
Location of Super 8 Printed Area in Optical Reduction O Lacation of Super 8 Printed Area in Optical Reduction P

Location of Super 8 Printed Area on $16 \mathrm{~mm}$ Motion Pictur

Location on Lot (1973)

Location (1972)

Location, Construction and Protection of Electronic Com

Locations for Builders' Hardware (1964)

Locations Glass I, Groups A, B, C, and D, and Class II, Locations That May Be Flooded (1973)

Locations (Agricultural Plastics, Chemicals, Metal Dust

Locations (Agricultural Plastics, Chemicals, Metal Dust

Locations (Bonding Appliances, Casters, Face Masks and

Locations (1972) ANSI C106.1

Locations (1972) ANSI C33.28

Locations (1973)

Locations (I973) ANSI C33.97

Locations, Class I, Groups A-D, and Class Il, Groups E-

ocations, Class I, Groups A-D, Class II, Groups E-G (I

Locations, Class I, Groups C and D (1973) ANSI C33.72

Locations, Class I, Groups C and D, and Class II, Group

Locations, Class-11,

ocations, Class 1, Groups A, B, C, and D, and Class 11

Locations: Class I, Groups A-D, and Class II, Groups E-

Locator Transmitters (ELT) (1973)

Lock for Articulated vehicles (Construction and by the $\mathrm{H}$

Lock Nut (1974)

Lock Screws (1973)

Lock Screws (1973)

Lock Strip Gaskets (1973)

Lock Trim (1972)

Lock Washer (1974)

Lock Washers (1972)

Lock (1974)

Lock (1974)

Lock, Shear, Protruding Head, Std. and Oversize, Pull T

Lock, Shear, 100 Deg. Head, Std. and Oversize, Pull Typ

Lock, Tension, Protruding Head, Std. and Oversize, Pull

Lock, Ten sion, Protruding Head, Stump Type, Titanium Al

Lock, Tension, I00 Deg. Crown Head, Std. and Oversize,

Lock, Tension, 100 Deg. Crown Head, Stump Type, Titaniu

Lock, Tension, 100 Deg. Head (MS20426), Std. and Oversi

Lock, Tension, 100 Deg. Head (MS20426), Stump Type, Tit

Lock, Tension, 100 Deg. Head (MS24694), Pull Type, Tita

Lock, Tension, 100 Deg. Head (MS24694), Stump Type, Tit

Lockbolts (1973)

Lockbolts, Rivets, Bolts) (1973)

Locked Protruding Head Blind Rivet (1974)

Locked Safes (Class KL) (1973)

Locked Spindle Blind Rivet (1972)

Locked Spindle Protruding Head Blind Rivet (1974)

Locked Spindle Rivet (1972)

Lacked Spindle Rivet (1972)

Locked Spindle SeIf Plugging Blind Pivet (1974)

Locked Spindle 100 Deg. Flush Head Blind Rivet (1974)

Locked Spindle 100 Deg. Flush Head Blind Rivet (1974)

Lockers (1973)

Locking Ability Performance of Chemical Coated Lock Scr

Locking Ability Performance of Nonmetallic Locking Elem

Locking and Nonlocking (1972)

Locking and Nonlocking (1972)

Locking and Nonlocking (1972)

Locking and Nonlocking (1972)

Locking and Nonlocking (1972)

Locking and Nonlocking (1972)

Locking and Nonlocking (1972)

Locking and Nonlocking (1972)

Locking and Nonlocking (1972)

Locking and Nonlocking (1972)

Locking and Nonlocking (1972)

Locking and Nonlocking (1972)

Locking and Nonlocking (1972)

Locking and Nonlocking (1972)

Locking and Nonlocking (1972)

Locking and Nonlocking (1972)

Locking and Nonlocking (1972)

Locking and Nonlocking (1972)

Locking Device, Positive Index (1973)

Locking Devices and Contacts (1973)

Locking Element Type Prevailing-Torque Lock Screws (1

Locking Elliptical Head Blind Nut (1974)
/Stics for

SAE

ANSI

ITE

ANSI

ANSI

ANSI

ICBO

ITE

NBHA

$\mathrm{UL}$

ICBO

ISA

ISA

UL

Std. for UL

Receptacl afety St UL

UL

UL

UL

UL

UL

UL

RTCA

SAE

ASTM

NSA

IFI

F1

ANSI

NSA

ANS1

NSA

Std NSA

NSA

NSA

NSA

NSA

NSA

NSA

NSA

NSA

NSA

NSA

NSA

NSA

NSA

UL

NSA

NSA

NSA

NSA

NSA

NSA

NSA

UL

IFI

IFI

NSA

NSA

Std. for Bolt NSA

Std. for Bolt NSA

Std. for Scre NSA

Std. for Bolt, NSA

Std. for Bolt, NSA

Std. for Screw, 10 NSA

Std. for Screw, 100 De NSA

/ Deg. Tri Wing Recess, NSA

1,100 Deg. Head, Tri Wi NSA

1. for Bolt, Hex Head, C NSA

Bolt, Pan Head, Close T NSA

/D. for Pan Head, Close NSA

/Head, Close Tolerance, NSA

or Screw, 100 Deg. Head NSA

IT, 100 Deg. Head, Tri W NSA

Td. for Bolt, Hex Head, NSA

NSA

UL

IFI

NSA

A16

PH3.

(10)

PH22.180

PH22. 179

PH22.181

$\mathrm{UBC} * 8 \cdot 2$

*8

LPD5.32

823

UFC $2 \mathrm{~A}$

SI2.10

12 II

1067

496

844.

FBII

1010

877

698

$674 \mathrm{~B}$

781

DO-154

J 276

A527

699

125

124 
15 Amperes, 2/ d. Dimensions of Plugs and 347/600 Volts, 20 Amperes, 3 Phase Wye, 4 Pole, 4 Wire se $347 / 600$ Volts, 30 Amperes, 3 Phase Wye, 4 Pole, 4 Wire ose 120/208 Volts, 20 Amperes, 3 Phase Wye, 4 Pole, 4 Wire ose $120 / 208$ Volts, 30 Amperes, 3 Phase Wye, 4 Pole, 4 Wire ose 277/480 Volts, 20 Amperes, 3 Phase Wye, 4 Pole, 4 Wire ose 277/480 Volts, 30 Amperes, 3 Phase Wye, 4 Pole, 4 Wire specific Purpose 125/250 Volts, 20 Amperes, 3 Pole, 3 Wire fic Purpose 250 Volts, 30 Amperes, 3 Phase, 3 Pole, 3 Wire fic Purpose 480 Volts, 20 Amperes, 3 Phase, 3 Pole, 3 Wire fic Purpose 480 Volts, 30 Amperes, 3 Phase, 3 Pole, 3 Wire fic Purpose 600 Volts, 30 Amperes, 3 Phase, 3 Pole, 3 Wire fic Purpose 250 Volts, 15 Amperes, 3 Phase, 3 Pole, 3 Wire fic Purpose 250 Volts, 20 Amperes, 3 Phase, 3 Pole, 3 Wire pecific Purpose 125/250 Volts, 30 Amperes, 3 Pole, 3 Wire, 2 Pole,/ Std. Dimensions of Plugs and Receptacles, Midget es, $3 \mathrm{P} / \quad$ Std. Dimensions of Plugs and Receptacles, Midget 73) Std. Dimensions of Plugs and Receptacles, Std. for Pins and Collars, Swage astener (Blind, Internally Threaded, External Sleeve, Self eaded, External Sleeve, High Temperature, Flush Head, Self aded, External Sleeve, Light Weight, Protruding Head, Self d, External Sleeve, General Purpose, Protruding Head, Self readed, External Sleeve, General Purpose, Flush Head, Self hreaded, External Sleeve, Light Weight, Millable Head Self pull Type (1973)

(1973)

e (1973)

pull Type (1973)

973)

1 Type (1973)

type (1973)

hread, $\mathrm{Ca}$

f-3A Thre/

Type (1973)

p Type (1973)

on, Pull Type (1973)

n, Stump Type (1973)

on, Pull Type (1973)

on, Stump Type (1973)

deg. F (1973)

974)

74) Std. for Pin, Swage Std. for Pin, Swage Std. for Pin, Swage Std. for Pin, Swage Std. for Pin, Swage Std. for Pin, Swage Std. for Pin, Swage Std. for Undrilled and Drilled, Plain and Self Std, for Undrilled and Drilled, Plain and Self Std. for Pin, Swage Std. for Pin, Swage Std. for Pin, Swage Std. for Pin, Swage Std. for Pin, Swage Std. for Pin, Swage Std, for Pin, Quick Release, Positive Std. for Nut-Self Std. for Self es: Specific Purpose 600 Volts, 30 Amperes, 2 Pole, 3 Wire ific Purpose 250 Volts, 20 Amperes 3 Phase, 3 Pole, 4 Wire fic Purpose 250 Volts, 30 Amperes, 3 Phase, 3 Pole, 4 Wire fic Purpose 480 Volts, 20 Amperes, 3 Phase, 3 Pole, 4 Wire fic Purpose 480 Volts, 30 Amperes, 3 Phase, 3 Pole, 4 Wire fic Purpose 600 Volts, 30 Amperes, 3 Phase, 3 Pole, 4 Wire specific Purpose 125/250 Volts, 20 Amperes, 3 Pole, 4 Wire specific Purpose 125/250 Volts, 30 Amperes, 3 Pole, 4 Wire es: Specific Purpose 125 Volts, 20 Amperes, 2 Pole, 3 Wire es: Specific Purpose 125 Volts, 30 Amperes, 2 Pole, 3 Wire es: Specific Purpose 250 Volts, 15 Amperes, 2 Pole, 3 Wire es: Specific Purpose 250 Volts, 20 Amperes, 2 Pole, 3 Wire es: Specific Purpose 250 Volts, 30 Amperes, 2 Pole, 3 Wire es: Specific Purpose 480 Volts, 20 Amperes, 2 Pole, 3 Wire es: Specific Purpose 480 Volts, 30 Amperes, 2 Pole, 3 Wire es: Specific Purpose 600 Volts, 20 Amperes, 2 Pole, 3 Wire Specific Purpose 277 Volts Ac, 20 Amperes, 2 Pole, 3 Wire Specific Purpose 277 Volts Ac, 30 Amperes, 2 Pole, 3 Wire ose 120/208 Volts, 20 Amperes, 3 Phase Wye, 4 Pole, 5 Wire ose $120 / 208$ Volts, 30 Amperes, 3 Phase Wye, 4 Pole, 5 Wire ose 277/480 Volts, 20 Amperes, 3 Phase Wye, 4 Pole, 5 Wire ose $277 / 480$ Volts, 30 Amperes, 3 Phase Wye, 4 Pole, 5 Wire ose 347/600 Volts, 20 Amperes, 3 Phase Wye, 4 Pole, 5 Wire ose 347/600 Volts, 30 Amperes, 3 Phase Wye, 4 Pole, 5 Wire

Std. for Self

r Insert, Molded In, Blind Threaded, Self Locking, Nonself ch Panel Std. for Insert, Molded In, Blind Threaded, Self Std. for Insert, Molded ln. Thru Threaded, Self Std. for Self

i Ftu, 450 and 800 Deg. F (1973) g. F and 800 Deg. F (1) Std. for Nut, Self or Insert, Molded In. Thru Threaded, Self Locking, Nonself Std. for Pin, Quick Release, Positive Std. for Pin, Swage Std. for Pin, Swage
Locking for Pull Type and Stump Type Lockbolts (1973)

Locking Grounding Type, Specific Purpose, 277 Volts Ac,

Locking Hexagon Nut (1972)

Locking Machine Screw (1974)

Locking Nut (1974)

Locking Serrated Head Blind Nut (1974)

Locking Serrated Head Blind Nut (1974)

Locking Type 125 Volts, 15 Amperes, 2 Pole, 1 Wire (197

Gs and Receptacles: Specific Purp

/Gs and Receptacles: Specific Purp

/Gs and Receptacles: Specific Purp

Gs and Receptacles: Specific Purp

Gs and Receptacles: Specific Purp

/Gs and Receptacles? Specific Purp

/Nsions of Plugs and Receptacles: ANSI

IS of Plugs and Receptacles: Speci ANSI

IS of Plugs and Receptacles: Speci ANSI

IS of Plugs and Receptacles: Speci ANS

iS of Plugs and Receptacles: Speci ANSI

S of Plugs and Receptacles: Speci ANSI

S of Plugs and Receptacles: Speci ANSI

/Sions of Plugs and Receptacles: S ANSI

Locking Type (1973)

Locking Type (1973)

Locking Type, Specific Purpose, 125 Volts, 15 Amperes,

Locking Type, Specific Purpose, 125/250 Volts, 15 Amper

Locking Type, 250 Volts, 20 Amperes, 2 Pole, 2 Wire (19

Locking (1973)

Locs.ing) (1972)

Locking) (1972)

Locking) (1972)

Locking) (1972)

Locking) (1972)

Locking) (1972)

Spec. for $F$

ANSI

ANSl

ANSI

NSA

Fastener (Blind, Internally Thr NSA

/Td. for Fastener (Blind Internally Th NSA for Fastener (Blind, internally Thre NSA

R Fastener (Blind, Internally Threade NSA

Td. for Fastener (Blind, Internally T NSA

Locking, A-286 Cres, Protruding Head, Shear / Tension.

Locking, A.286 Cres, Protruding Head, Shear, Stump Type

Locking, A-286 Cres, Protruding Head, Tension, Pull Typ

Locking, A-286 Cres, 100 Deg. Head (MS24694), Tension,

Locking, A-286 Cres, 100 Deg. Shear Head, Stump Type (1

Locking, A-286 Cres, 100 Deg. Shear / Tension Head, Pul

Locking, A-286, 100 Deg. Head (MS20426), Tension, Pull

Locking, Alloy and Corrosion Resisting Steel, UNJC-3A T

Locking, Alloy Steel and Corrosion Resisting Steel, Unj

Locking, Aluminum Alloy, Protruding Head, Tension, Pull

Locking, Aluminum Alloy, 100 Deg. Head (MS20426), Tensi

Locking, Aluminum Alloy, 100 Deg. Head (Ms20426, Tensio

Locking, Aluminum Alloy, 100 Deg. Head (MS24694), Tensi

Locking. Aluminum Alloy, 100 Deg. Head (MS24694), Tensi

Locking, Double Acting (1973)

Locking, Extended Washer, Double Hexagon, 220 KSI, 450

Locking, Flat Fillister Head, Full Thread Part Screw (i

Locking, Flat 100 Deg. Head, Full Thread Part Screw (19

Locking, Grounding Type 1973)

Locking, Grounding Type (1973)

Locking, Grounding Type (1973)

Locking, Grounding Type (1973)

Locking, Grounding Type (1973)

Locking, Grounding Type (1973)

Locking, Grounding Type (1973)

Locking, Grounding Type (1973)

Locking, Grounding Type (1973)

Locking, Grounding Type (1973)

Locking, Grounding Type (1973)

Locking, Grounding Type (1973)

Locking, Grounding Type (1973)

Locking, Grounding Type (1973)

Locking, Grounding Type (1973)

Locking, Grounding Type (1973)

Locking, Grounding Type (1973)

Locking, Grounding Type (1973)

Locking, Grounding Type (1973)

Locking, Grounding Type (1973)

Locking, Grounding Type (1973)

Locking, Grounding Type (1973)

Locking, Grounding Type (1973)

of Plugs and $\mathrm{R}$

and Receptacles: Spec ANSI land Receptacles: Speci ANSI land Receptacles: Speci ANSI land Receptacles: Speci ANSI land Receptacles: Speci ANSI /Lugs and Receptacles: ANSI Lugs and Receptacles: ANSl of Plugs and Receptacl ANSI lof Plugs and Receptacl ANSI lof Plugs and Receptacl ANSI lof Plugs and Receptacl ANSI lof Plugs and Receptacl ANSI lof Plugs and Receptacl ANSI lof Plugs and Receptacl ANSI lof Plugs and Receptacl ANSI /Plugs and Receptacles: ANSI /Plugs and Receptacles: ANSI Ptacles: Specific Purp ANSl Ptacles: Specific Purp ANSI /Ptacles: Specific Purp ANSI Ptacles: Specific Purp ANSI

Ptacles: Specific Purp ANS (1973) Locking, Hexagon Head Shear Bolt (1974) Locking, Light Weight Floating, Sandwich Panel (1972) Locking, Nonself Locking, Light Weight Floating, Sandwi

Locking, Nonself Locking, Sandwich Panel (1972)

Locking, Pan Head, Full Thread Part Screw (1974)

Locking, Plate-Corner, Counterbored, Floating, $125 \mathrm{Ks}$

Locking, Sandwich Panel (1972)

Locking, Single Acting (1973)

Locking, Std. and Oversize, Protruding Head, Tension, P

Locking, Steel, Protruding Head, Shear Stump Type (1973
Purp ANSI

NSA

NSA

NSA

NSA

NSA

NSA

NSA

NSA

NSA

1080

C73.43

662

679

1731

1732

C73.31

C73.108

C73. 109

C73.104

C73. 105

C73.106

C73.107
C73.96

C73. 100

C73.101

C73.102

C73.103

C73.98

C73.99

C73.97

C 73.29

C73.30

C73.32

1413
1675

1672

1673

1669

1670

1674

7014

6984

6965

6946

6974

7004

6955

1352

1351

1525
1555

1535

6915

1516
1546

1353.66

1758

1191

1189

C73.82

C73.85

C73.86

C73.87

C73.88

C73.89

C73.83

C73.84

C73.72

C73.73 
1973)

ar, Pull Type (1973)

26), Tension, Pull Type (1973)

4), Tension, Pull Type (1973)

Pull Type (1973)

(1973)

Type (1973)

Type (1973)

n, Pull Type (1973)

pull Type (1973)

Std. for Pin, Swage
Std. for Low Height 80 K.S.I., Self Std. for Sell

Std. Ferrous Pipe Plugs, Bushings, and Tension Requirements for Prevailing Torque Type Steel Hex s) (1973) Std. for

Safety Std. for Key Std for Auxiliary

1973)

Kick /

Spec. for Subsurface Safety Valves, Basic Builders' Hardware: Hands of Doors; Hinges;
Std. Spec. for Copper Rods for Std. Spec. for Structural Steel for Std. Spec. for Wrought Carbon Steel Wheels for FulI Length Thermal Process (1973) Std. for

glued Laminated Timbers: Douglas and Hem Fir, Southern and Fir; Larch; Hem-Fir; Ponderosa, Idaho White, Sugar, and ter (Drill) Press, and Gage Wheel, Tractor, Implement, and 4) or Motorcycles, Passenger Cars, Off Road, Agricultural and nd Rims for Off the Road Vehicles: Earthmoving, Mining and 3) ANSI Y 32.14 Std. for Graphic Symbols for acids (Cereal Esters of A286 Cres, $6 \mathrm{Al}-4 \mathrm{~V}$ Titanium Alloy,

Std. for Bolt, Hex Head, Close Tolerance, Alloy Steel, Deg. Head, Tri Wing Recess, Close Tolerance, Alloy Steel, or Bolt, Hex Head, Close Tolerance, 6Al-4V Titanium Alloy, Std. for Bolt, Hex Head, Close Tolerance, A286 Cres, ow Alloy Steel Castings (1970) ANSI G52.7 Std. Spec. for Std. Specs. and Load Tables for Std. Specs. and Load Tables for td. Spec. and Load Tables for Open Web, Longspan, and Deep Std. Spec, and Load Tables for Open Web. f Test for Breaking Tenacity of Man Made Textile Fibers in Std. for Computer Type (Square
Std. Spec. for Perlite

Std. Spec. for Cellulosic Fiber (Wood Base) Std. Spec. for Mineral Fiber Rec. Industry Std. for m) Diameter), Citrus Style (1974) Rec, Std, for 02 (52 Mm) Diameter) (1973)

Std. Meth. of Test for Undersized tomatic Pilot, Depth Determination Device and Fish Finder, Std. Meth. of Test for Weight

ct of Thermal Environment on Production, Heat and Moisture

Test Meth. for Multiple Sample Meth. of Textile Tensile

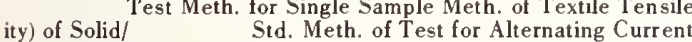
er Immersion Test for Resistance to Blistering, Wrinkling. for Laboratory Measurement of Airborne Sound Transmission olution (1971) ANSI Z/ Tentative Meth. of Test for Weight nstallations (1973)

ply Felt and Asphalt Preferably with Gravel or Slag Surf/ er $050,000(1973)$

$\mathrm{t}$ (Induction and Dielectric) (1973)

essing Equipment (Vessel) (1974)

c Problems (1973)

Rec. for Pipe Friction Std. Manual for Electric Comfort Conditioning (Heat e $15 \mathrm{kV}$ Through 35 / Std. Ampacities with Effect of Shield Prevention Data on Flood choice of Sample Size to Estimate the Average Quality of A Construction Under the Uniform Building Code: Location on ) Safety Std. for Door, Drapery, Gate, Specifying Metal steel Sheet and Strip, Hot and Cold Rolled, High Strength, ty (1972) ANSI G4l.6 hreaded (1973)

Std. Spec. for High Strength Bolts and Screws, Steel, Spec. for Tolerances of 00 psi (1,345 MPa) Tensile Strength, Hardened / Spec. for readed (1973)

td. Meth. for Spectrochemical Analysis of Plain Carbon and Std. Spec. for Heavy Walled Carbon and

(1974) ANSI G52.5 Std. Spec. for for Longitudinal Beam Ultrasonic Inspection of Carbon and
Locking, Steel, Protruding Head, Tension, Stump Type, ( Locking, Steel, Std. and Oversize, Protruding Head, She Locking, Steel, Std. and Oversize, 100 Deg. Head (Ms204 Locking, Steel, Std. and Oversize, 100 Deg. Head (Mr249 Locking, Steel, Std. and Oversize, 100 Deg. Shear Head, Locking, Steel, $100 \mathrm{Deg}$. Crown Head, Tension, Pull Type Locking, Steel, 100 Deg. Head (MS20426), Tension, Stump Locking, Steel, 100 Deg. Head (MS24694), Tension, Stump Locking, Steel, 100 Deg. Shear Head, Stump Type (1973) Locking, 108 KSI Steel, Protruding Head, Shear/ Tensio

Locking, 108 KSI Steel, 100 Deg. Shear / Tension Head,

Locking, 450 Deg. F, Hexagon Nut (1973)

Locking, 450 Deg. F, High Quality Nuts (1973)

Locknuts with Pipe Threads (1971)

Locknuts (1972)

Locks and Lock Trim (1972)

Locks (Burglar Alarm, Door, Deposit and Collection Safe

Locks (Hardware) (1971)

Locks, and Landing Nipples for Petroleum Industry Use (

Locks; Knobs and Handles; Exit Devices, Pulls, Push and

Locomotive Staybolts (1973) ANSI H7.2

Locomotives and Cars (1970A) ANSI G39.1

Locomotives and Rail Cars (1971) ANSI G39.2

Lodgepole Pine Wood Poles Preservative Treatment by the

Lodgepole Pine, Western Larch, California Redwood and H

Lodgepole Pines; Engelmann Spruce; Western Cedar and He

Log Skidder (1974)

/D Indust rial Steering, Drive,

Log Skidder, Truck, Bus, Bead Seat, Industrial and Bicy

Logging Tires Used in Intermittent Highway Service (197

Logging (Short Haul); Grader, Loader, Dozer, Mining Car

Logic Diagrams (Two-State Devices) (Electronics) (197

Logs (Record, Documentation) (1974)

Long Chain Fatty Acids in Common Fats, Oils, and Fatty

Long Thread, Self Locking and Nonlocking (1972)

Long Thread, Self Locking and Nonlocking (1972)

Long Thread, Self Locking and Nonlocking (1972)

Long Thread, Self Locking and Nonlocking (1972)

Long Thread, Self Locking and Nonlocking (1972)

Long Threads, Self Locking and Nonlocking (1972)

Longitudinal Beam Ultrasonic Inspection of Carbon and L

Longspan Steel Joints Deep DLJ and DLH Series (1970)

Longspan Steel Joints LJ and LH Series (1970)

Longspan Steel Joints (1974)

Longspan, and Deep Longspan Steel Joints (1974)

Loop or Knot Configurations (1973)

Loop) Pulse Transformers (1970)

Loose Fill Insulation (1973)

Loose Fill Thermal Insulation (1973)

Loose Fill Thermal Insulation (1973)

Loose Metal End Dimensions of Composite Cans (21) $666 \mathrm{M}$

Loose Metal End Dimensions (6 Ounce Fibre Citrus Cans 2

Loose Muscovite Mica Splittings (1973)

Loran or Other Line of Position Device, Radar, Radio De

Loss After Heat Aging of Preformed Sealing Tapes (1974)

Loss and Feed and Water Requirements of Farm Livestock

Loss by Retained Chlorine Bleaching (1971) ANSI L14.180

Loss by Retained Chlorine (1971) ANSI L14.126

Loss Characteristics and Dielectric Constant (Permittiv

Loss of Adhesion, or Other Failure of Paint, Varnish, L

Loss of Building Partition (1970)

Std. Rec. Pract.

Loss of Carbon Black (1972)

Loss of Sheet Steel During Immersion in Sulfuric Acid S

Loss Prevention Data on Bonds and Guarantees for Roof I

Loss Prevention Data on Elastomer Roof Coverings (Multi Loss Prevention Data on Fundamental Defects in Fires Ov Loss Prevention Data on High Frequency Heating Equipmen

Loss Prevention Data on Sight Glasses for Chemical Proc

Loss Tables for Solving Fire Protection System Hydrauli

Loss, Gain, Energy Consumption) (1974)

Losses for Single Conductor Solid Dielectric Power Cabl

Losses (1973)

Lot or Process (1972) ANSI Z1.11

Lot (1973)

Louver Details for Std. Steel Doors (1972)

Louver, and Window Electric Operators and Systems (1973

Louvers, Stationary (1967)

Low Alloy Columbium and/or Vanadium (1970) ANSI G24.32

Low Alloy Columbium Vanadium Steels of Structural Quali

Low Alloy Heat Resistant, Hardened and Tempered, Roll T

Low Alloy Steel Bars (1973)

Low Alloy Steel Bolts and Screws, Heat Resistant, 195,0

Low Alloy Steel Bolts and Screws, Heat Treated, Roll Th

Low Alloy Steel by the Point to Plane Technique Using a

Low Alloy Steel Castings for Steam Turbines (1974)

Low Alloy Steel Castings Suitable for Pressure Service

Low Alloy Steel Castings (1970) ANSl G52.7
NSA

NSA

NSA

NSA

NSA

NSA

NSA

NSA

NSA

NSA

NSA

NSA

NSA

ANSI

IFI

ANSI

UL

BHMA

API

NBHA

ASTM

ASTM

ASTM

AWPA

ICBO

ICBO

TRA

TRA

TRA

TRA

IEEE

API

AACC

NSA

NSA

NSA

100 NSA

T. F NSA

NSA

ASTM

A ISC

A ISC

SJI

SJI

ASTM

IEEE

ASTM

ASTM

ASTM

CCTI

CCTl

ASTM

$A B Y C$

ASTM

ASAE

AATCC

AATCC 92

ASTM D150

NCCA

ASTM

ASTM

ASTM

FMS

FMS

FMS

FMS

FMS

FMS

NEMA

IPCEA

FMS

\section{E90}

D 1509

C694

$1-4.7 \mathrm{~S}$

$1-47$

$10-9$

6.3

$7-3$

HEl

P. $53-426$

10-10

E122

UBC $* 8-2$

111-C

STDI

UL

CSI

ASTM

325

15851

ASTM

SAE

SAE

A 572

AMS7455D

SAE

SAE

ASTM

ASTM

ASTM

Std. Spec. ASTM

AMS2251E

AMS 7459B 
.35Mo (0.38-0.46C) (SAE 4340 Modified) (1973) $0.25-0.35 \mathrm{C})(1973)$

nsi W 3.5

td. Meth. for Spectrochemical Analysis of Plain Carbon and ine Environments (1974) Std. Spec. for High Strength empered, Roll Threaded (135,000 psi (931 MPa) / Spec. for 3) Spec. for Z) Std. Meth. for Spectrochemical Analysis of Carbon and stock/ Spec. for Quality Assurance Sampling of Carbon and nsi G41.4 Std. Spec. for High Strength Std. Spec. for High Strength Std. Spec. for Normalized High Strength

id Spec. for Hot Formed Welded and Seamless High Strength ught Irons (/ Std. Meth. for Chemical Analysis of Carbon. $0-0.50 \mathrm{C}) \mathrm{V} / \quad$ Spec. for Steel Bars, Forgings, and Tubing. steel Plates for Machine Parts and Genera/ Std. Spec. for es of Structural Quality (1970) ANSI G24./ Std. Spec, for es for Pressure Vessels (1972) ANSI G35.2 ssembly and Equipment (1972)

ir Intake and Exhaust Systems (1972)

ooling Water Systems (1972)

rection and Installation (1972)

uel Oil Characteristics (1972)

actory and Field Testing (1972)

aseous Fuel Characteristics and General/ auge Borads, Protective Devices, and Ins/ enerators and Electrical Equipment (1972) ubricating OiI Systems (1972)

ubricating Oil Characteristics and Gener/ peration and Maintenance (1972)

ower Plant Fuel Oil and Gas Systems (197/ ower Plant Buildings (1972)

peed Governing and Parallel Operation (1/ tarting Systems (1972)

ibration (1972)

aste Heat Recovery Systems (1972) (1972)

Std. Pract. for Selection of Engine-Generator Units for preparation of Invitations for Bids and Detailed Spec. for

Foods (Cereal Chemistry) (1962) ives (1973)

refinery Service (1972) ANSI B12/

t, and Strip (1972)

ansi B125.38

g (1972)

flaring, and Beading (1/ Std. for Seamless Std. for Welded and Cold Drawn Std. for Welded Flash Controlled

Std. for Brazed Double Wall Rec. Pract. for Resistance Welding Coated nd Forgings, Corrosion and Moderate Heat Resistant, 12.5Cr ference Meter (1972T) Std. Spec. for Steel Tubes. ulation (1973) Std. Std. Meth. for Determination of Pract. for the Determination of the Thermal Resistance of Alloy Sheet, Strip, and Plate. Std. for Guide-Fastener.

ANSI C83.63C

1) ANSI C83.63B

Nut $(1973)$

2)

stness of Textile Fabrics to Ozone in the Atmosphere Under 973) 211.311 Rec. Pract. for Measurement of h. for Isolation of Representative Saturates Fraction from ower) (1971)

1) Std. for of Test for Stretch Properties of Knitted Fabrics Having or Continuity of Quality of Electrical Insulating Oil for Std. Rec. Pract. for Safe Filling of

Std. Meth of Measuring the Spiral Flow of Safety Std. for Acetylene Generators, Stationary, Specifying Duct, Sheet Metal, ke Materials by a Tempera/ (1973) eric, Solvent Release Type Sealan/ xible Polymeric Materials (1973) after Artificial Weathering (1972)
Electric Welded Std. for Welded Std. Meth. of Test for Std. for Gas Fired Std. Meth. of Test for

Low Alloy Steel Castings, Investment $0.80 \mathrm{Cr}-1.8 \mathrm{Ni}-0$

Low Alloy Steel Covered Arc Welding Electrodes (1969) a

Low Alloy Steel for Boron by the Point to Plane Arc Tec Low Alloy Steel H.Piles and Sheet Piling for Use in Mar Low Alloy Steel Studs, Heat Resistant, Normalized and T Low Alloy Steel Studs, Heat Treated, Roll Threaded (197 Low Alloy Steel Using a Vacuum Spectrometer (1971) ANSI Low Alloy Steel (1970) ANSI G88.3

Low Alloy Steels (Wrought Products Except Forgings and Low Alloy Structural Manganese Vanadium Steel (1970A) a Low Alloy Structural Steel (1970A) ANSI G41.2

Low Alloy Structural Steel (I973)

Low Alloy Structural Tubing (1973)

Low Alloy, and Silicon Electrical Steels. Ingot and Wro

Low Alloy, Heat Resistant $0.95 \mathrm{Cr}-0.55 \mathrm{Mo}-0.30 \mathrm{~V}(0.4$

Low and Intermediate Tensile Strength Carbon-Silicon

Low and Intermediate Tensile Strength Carbon Steel Plat

Low and Intermediate Tensile Strength Carbon Steel Plat

Low and Medium Speed Stationary Diesel and Gas Engine a

Low and Medium Speed Stationary Diesel and Gas Engine a

Low and Medium Speed Stationary Diesel and Gas Engine C

Low and Medium Speed Stationary Diesel and Gas Engine E

Low and Medium Speed Stationary Diesel and Gas Engine F

Low and Medium Speed Stationary Diesel and Gas Engine F

Low and Medium Speed Stationary Diesel and Gas Engine G

Low and Medium Speed Stationary Diesel and Gas Engine G

Low and Medium Speed Stationary Diesel and Gas Engine G

Low and Medium Speed Stationary Diesel and Gas Engine L

Low and Medium Speed Stationary Diesel and Gas Engine L

Low and Medium Speed Stationary Diesel and Gas Engine $O$

Low and Medium Speed Stationary DieseI and Gas Engine P

Low and Medium Speed Stationary Diesel and Gas Engine S

Low and Medium Speed Stationary Diesel and Gas Engine S

Low and Medium Speed Stationary Diesel and Gas Engine V

Low and Medium Speed Stationary Diesel and Gas Engine W

Low and Medium Speed Stationary Diesel and Gas Engines Low and Medium Speed Stationary Diesel and Gas Engines Low and Medium Speed Stationary Diesel and Gas Engines Low Beam Lamp (Headlamp-Light) (1973)

Low Blank Meth. of Analysis of Methoxychlor Residues in

Low Boiling Hydrocarbon Solvent for Oil Borne Preservat

Low Carbon and Carbon Molybdenum Steel Still Tubes for

Low Carbon Nickel Molybdenum Chromium Alloy Plate, Shee

Low Carbon Nickel Molybdenum Chromium Alloy Rod (1972)

Low Carbon Steel Pipe for the Chemical Industry (1971)

Low Carbon Steel Tubing Annealed for Bending and Flarin

Low Carbon Steel Tubing Annealed for Bending and Flarin

Low Carbon Steel Tubing Normalized for Bending, Double

Low Carbon Steel Tubing (1972)

Low Carbon Steel Tubing (1972)

Low Carbon Steels (1970)

Low Carbon (SAE 51416F), Free Machining (I973)

Low Carbon. Tapered for Structural Use (1973)

Low Colored Clear Liquids Using the Hunterlab Color Dif

Low Current. Dry Arc Resistance of Solid Electrical Ins

Low Density Mineral Fiber Blanket Type Building Insulat

Low Expansion, Glass Sealing, $53 \mathrm{Fe}-29 \mathrm{Ni}-17 \mathrm{Co}$ (1973)

Low Form, Cowl, Rzus Type (1973)

Low Frequency (Below $3 \mathrm{MHz}$.) Electric Connectors (1974)

Low Frequency (Below $3 \mathrm{MHz}$.) Electrical Connectors (197

Low Head Pressure Pipe (1973)

Low Height Hexagon Self Locking Nut (1974)

Low Height $80 \mathrm{~K}$.S.I., Self Locking, $450 \mathrm{Deg}$. F, Hexagon

Low Height, Light Weight, Self Locking Hexagon Nut (197

Low Humidities (1972) ANSI L14.174

Low Level Activity in Water (1972T)

Low Levels of Lead in Gasoline by X-Ray Spectrometry (1

Low Olefinic Gasoline by Mass Spectrometry (I971) ANSI

Low Olefinic Petroleum Naphthas (1973) ANSI Z11.165

Low Power Wide Band Transformers (Less Than 100 Watts P

Low Pressure Cable Systems (1972) ANSI C59.69

Low Pressure Pressurized Products (1972)

Low Pressure Steam and Hot Water Boilers (1972)

Low Pressure Thermosetting Molding Compounds (1972)

Low Pressure (Welding and Cutting) (1973)

Low Pressure, Air Transmission (1967)

Low Quantities of Mercury in Paint (1973)

Low Temperature Characteristics of Rubber and Rubber $\mathrm{Li}$ std. Meth. of Test for Std. Meth. of Test for Std. Meth. of Test for

Low Temperature Flexibility of Preformed Sealing Tapes

Low Temperature Flexiblity and Tenacity 1 Part, Elastom Std. Meth. of
Low Alloy Steel Castings, Investment, $0.95 \mathrm{Cr} \sim 0.20 \mathrm{Mo}($

Low and Medium Speed Stationary Diesel and Gas Engine P

Low Concentration of Lead in Paint (1973)

Low Temperature Flexibility of Latex Sealing Compounds

\begin{tabular}{|c|c|}
\hline SAE & AMS5330A \\
\hline $\begin{array}{l}\text { SAE } \\
\text { AWS }\end{array}$ & AMS5336B \\
\hline ASTM & E404 \\
\hline ASTM & A690 \\
\hline SAE & AMS 7458C \\
\hline $\begin{array}{l}\text { SAE } \\
\text { ASTM }\end{array}$ & $\begin{array}{l}\text { AMS 7456G } \\
\text { E415 }\end{array}$ \\
\hline $\mathrm{SAE}$ & $\mathrm{J} 410 \mathrm{C}$ \\
\hline SAE & AMS2370A \\
\hline ASTM & A 441 \\
\hline ASTM & A 242 \\
\hline ASTM & A633 \\
\hline ASTM & A618 \\
\hline ASTM & E350 \\
\hline SAE & AMS6305 \\
\hline ASTM & A284 \\
\hline ASTM & A283 \\
\hline ASTM & A285 \\
\hline DEMA & $* 1.5$ \\
\hline DEMA & $* 1.8$ \\
\hline DEMA & $* 1.10$ \\
\hline DEMA & $* 1.21$ \\
\hline DEMA & $* 1.14$ \\
\hline DEMA & $* 1.22$ \\
\hline DEMA & $* 1.15$ \\
\hline DEMA & $* 1.17$ \\
\hline DEMA & $* 1.18$ \\
\hline DEMA & $* 1.11$ \\
\hline DEMA & $* 1.12$ \\
\hline DEMA & $* 1.23$ \\
\hline DEMA & $* 1.13$ \\
\hline DEMA & $* 1.20$ \\
\hline DEMA & $* 1.6$ \\
\hline DEMA & $* \mathrm{I} \cdot 9$ \\
\hline DEMA & $* 1-7$ \\
\hline DEMA & $* 1.16$ \\
\hline DEMA & $* l$ \\
\hline DEMA & $* 1.19$ \\
\hline DEMA & $* 1.24$ \\
\hline SAE & J582A \\
\hline AACCH & 60.40 \\
\hline ASTM & D3225 \\
\hline ASTM & Al61 \\
\hline ASTM & B575 \\
\hline ASTM & B574 \\
\hline ASTM & A587 \\
\hline SAE & $\mathrm{J} 524 \mathrm{~B}$ \\
\hline SAE & $\mathrm{J} 525 \mathrm{~B}$ \\
\hline $\mathrm{SAE}$ & $\mathrm{J} 356 \mathrm{~A}$ \\
\hline SAE & $\mathrm{J} 526 \mathrm{~B}$ \\
\hline$S A E$ & J527B \\
\hline AWS & $\mathrm{Cl} .3$ \\
\hline $\mathrm{SAE}$ & AMS5610H \\
\hline ASTM & A595 \\
\hline ASTM & $\mathrm{E} 450$ \\
\hline ASTM & D2088 \\
\hline ASTM & D 495 \\
\hline ASTM & C653 \\
\hline $\mathrm{SAE}$ & AMS7728C \\
\hline NSA & 67 \\
\hline ElA & RS364-3 \\
\hline EIA & RS 364.2 \\
\hline ASTM & C36l \\
\hline NSA & 679 \\
\hline NSA & 1757 \\
\hline NSA & 1291 \\
\hline AATCC & 109 \\
\hline ASTM & D3085 \\
\hline ASTM & D3229 \\
\hline ASTM & D2789 \\
\hline ASTM & D2002 \\
\hline IEEE & 111 \\
\hline ASTM & D2594 \\
\hline ASTM & D1818 \\
\hline ASTM & D309I \\
\hline ANSI & Z21.I3 \\
\hline ASTM & D3123 \\
\hline UL. & 409 \\
\hline CSI & 15836 \\
\hline ASTM & D2206 \\
\hline ASTM & D1329 \\
\hline ASTM & C734 \\
\hline ASTM & C 765 \\
\hline ASTM & C711 \\
\hline ASTM & D2137 \\
\hline
\end{tabular}


re Sensitive Electrical Insulating Tape (/ ickel Alloy Steel Flanges, Fittings, Valyes, Sid. Spec. for for Seamless and Welded Carbon and Alloy Steel Tubes for Std. Spec. for Seamless and Welded Steel Pipe for ls for Pressure Vessels, Valves, Flanges, and Fittings for for Piping Fittings of Wrought Carbon and Alloy Steel for Steel Castings for Pressure Containing Parts Suitable for terials by Means of a Torsional / Std. Meth. of Measuring std. Meth. of Test for Apparent Viscosity of Motor Oils at Std. Meth. of Test for Apparent Viscosity of Gear Oils at used in Enclosures (1973) ANSI C37.13 Std. for Test Procedures for Std. for Std. for Std. for

Std. Guide for Meth. of Power Factor Measurement for Application Recommendations, and Related Requirements for Std. for Residential Controls for

rs for Boilers (197) Fire Hazards and Protection Rec. for atforms and Stage Lifts (Hydrualic and Hydropneumatic) for Std. Meth. for Analysis of Liquefied Petroleum Gases Tent. Spec. for Volatile Petroleum Solvent $s$, and Conta/

Rec. Pract. for Symbols and Color Codes for sin Concrete Pavements (1972) Std. Spec. for

om Woolen Yarns I Test Meth. for Scourability of Spinning of Test for Measuring the Wear Life and Bonded Solid Film Std. Meth. for Measurement of Volatilization Rates of

) ANSI Zl/ Std. Meth. of Test for Explosive Reactivity of h. for Measurement of Extreme Pressure Properties of Fluid Differential and Manual Transmission td. Meth. of Test for Load Carrying Capacity of Fluid Gear d. Meth. for Measurement of Extreme Pressure Properties of d. Meth. for Measurement of Extreme Pressure Properties of i Z11.207 std. Meth of Test for Dropping Point of Std. Meth. for Measurement of Flow Properties of Std. Meth. of Test for Water Washout Characteristics of Std. Meth. of Test for Rust Preventive Properties of Std. Meth. of Test for Apparent Viscosity of for Low and Medium Speed Stationary Diesel and Gas Engine Pract. for Flushing and Cleaning of Gas Turbine Generator for Low and Medium Speed Stationary Diesel and Gas Engine Std. Meth. of Test for Total Nitrogen in Std. Meth. of Test for Foaming Characteristics of meth. of Test for Detecting Glycol Base Antifreeze in Used Marine Ser/ Rec. Pract. for the Flushing and Cleaning of

2) ec. Pract for the Flushing and Cleaning of Oil Systems for Rec. Pract. for the Design of Oil Systems for ry (1971)

Rec. Pract. for the Purification of Oil Systems for Rec. Pract. for 60) (1974) 4) 4) $37 \mathrm{~A} 60$, Ufc-Rule/ -37 Aa60, Ufc-Rule 40, Std. Spec. for 16 Gal. Full Removable Head, Std. Spec. for 5 Gal Straight Side Std for $5 \mathrm{Gal}$ Nesting Std. Spec. for 5 Gal. Straight Side Std. Spec. for $5 \mathrm{Gal}$. Nesting 1/ 971) Std. for Nut, Self Aligning, Self Locking, Plate-Two National Design Spec. for Stress Grade Construction Met/ the All Weather Wood (Pressure Treated n Woods Use Book: Abbreviations, Symbols, and Terminology Plywood

Std. Spec. for Pressure Preservative Treatment of Quality Stds. for Std. Spec. for Adhesives Used in Nonstructural Glued g Towers (1971) g Towers (1971) erties and Design Loads, Timber,/

Std. for Redwood Std. for Douglas Fir Std. for Southern Pine Western Woods Use Book: Uniform Fire Code: Rec. Fire Protection for Sawmills and for the Measurement and Inspection of Hardwood and Cypress Sid Grading Rules for Wester Uniform Building Code Std. for Southern Pine Uniform Building Code Std. for California Redwood uilding Code Std. for Tests for Structural Glued Laminated lock-Tamarack; Balsam Fir: Northern Pine and White Cedar

Pine, Eastern Spruce and Hemlock, Balsam Fir and Tamarack ation, Definition, and Meth. of Grading for All Species of

$r$ Evaluating Allowable Properties for Grades of Structura

Span Tables and Working Stresses for Joists and Rafters

White Pines, Spruce-Pine-Fir, Western Cedars (North) cedar and Hemlock; Subal-Pine Fir; and Mountain Hemlock

t Hemlock; Western Red Cedar; White Fir; and Sitka Spruce Uniform Building Code Std. for Softwood Plywood Uniform Building Code: Wood Members and Their Fastenings
framing Rec., Properties, Weight, Stress of Board, Timber,
Low Temperature Resistant Vinyl Chloride Plastic Pressu Low Temperature Service (1972) ANSI G38.13

Low Temperature Service (1973) ANSI B 125.17

Low Temperature Service (1973) ANSI G38.3

Low Temperature Service (1973) ANSI G46.2

Low Temperature Service (1974) ANSI G38.9

Low Temperature Stiffening of Rubber and Rubber Lik

Low Temperature Using the Cold Spanking Simulator (1972

Low Temperatures Using the Brookfield Viscometer (1972)

Low Voltage AC Power Circuit Breakers Used in Enclosure

Low Voltage Alternating Current Power Circuit Breakers

Low Voltage Cartridge Fuses (1972)

Low Voltage Cartridge Fuses, 600 Volts or Less (1972)

Low Voltage Inductive Test Currents (1972) ANSI C37.26

Low Voltage Power Circuit Breakers and AC Power Circuit

Low Voltage, Wall Mounted Room Thermostats (1972)

Low Water Fuel Cutoffs and Automatic Feedwater Regulato

Lowering Personnel and Material (1972)

LPG) by Process Gas Chromatography (1973)

(LPG) for Preparing Pentachlorophenol Solutions (1968)

Lubricant and Fluid Requirement Maintenance Instruction

Lubricant for Installation of Preformed Compression Sea

Lubricant (To Test the Removability of Spinning Oils $\mathrm{Fr}_{\mathrm{r}}$

Lubricants in Oscillating Motion (1971) ANSI Z11.300

Lubricants in Vacuum and Other Controlled Atmospheres

Lubricants in Vacuum (1971) ANSI Z11.302

Lubricants with Aerospace Alloys Under High Shear (1972

Lubricants (Falex Meth.) (1973)

Lubricants (Information Report) (1969)

Lubricants (1973) ANSI Z11.16]

Lubricating Fluids (Four Ball Meth.) (1971) ANSI Z11.30

Lubricating Fluids (Timken Meth.) (1972) ANSI Fll.308

Lubricating Grease of Wide Temperature Range (1972) Ans

Lubricating Greases at High Temperatures (1973)

Lubricating Greases (1973) ANSI Z11.124

Lubricating Greases (1973) ANSI Z11.152

Lubricating Greases (1973) ANSI Z11.72

Lubricating Oil Characteristics and General Spec. (1972

Lubricating Oil Systems (1971)

Lubricating Oil Systems (1972)

Lubricating Oils by Modified Kjeldahl Meth. (1973)

Lubricating Oils (1972) ANSI Z11.78

Lubricating Oils (1973)

Lubricating Systems of Various Turbine Driven Pumps for

Lubrication and Control of Hydroelectric Equipment (197

Lubrication and Control of Hydroelectric Equipment (197

Lubrication and Control of Hydroelectric Equipment (197

Lubrication Chart-Construction and Industrial Machine

Lubrication of Industrial Enclosed Gear Drives (1972)

Lug Cover Universal Drums (Ufc-Rule 40, Nmfc-Item 2

Lug Cover Universal Pail (Container) (Dot-37C80) (197

Lug Cover Universal Pail (Container) (Dot-37C80) (197

Lug Cover Universal Pails (Container) (Dot-37A80, Dot

Lug Cover Universal Pails (Container) (Dot-37A80, Dot

Lug, Floating, $125 \mathrm{KSI}$ Ftu, $450 \mathrm{Deg}$. F and $800 \mathrm{Deg}$. F (

(Lug, Hook, Trolley Suspended, and Base Mounted Types (1

Lumber and Its Fastenings (1971)

Lumber and Plywood) Foundation System: House Design and

Lumber and Timber) (1973)

Lumber Beams Fabrication Spec. (1971)

Lumber for Industrial Water Cooling Towers (1971)

Lumber Grades (Architectural Woodwork) (1973)

Lumber Products (1972)

Lumber Spec. for Application in Industrial Water Coolin

Lumber Spec. for Application in Industrial Water Coolin

Lumber Spec. for Application in Industrial $\mathbb{W}$ ater Coolin

Lumber Std., Grading Rules, Design Values, Section Prop

Lumber Yards and Woodworking Plants (1973)

Lumber Yards (1973)

Lumber (1971-75)

Lumber (1972)

Lumber (1973)

Lumber (1973)

Lumber (1973)

Lumber (1973)

Lumber (1973)

Lumber (1973)

Lumber (1974)

(Lumber) (1972) Usc Ps20

(Lumber) (1973)

(Lumber) (1973)

(Lumber) (1973)

Lumber, Construction and Industrial (1973)

(Lumber, Plywood and Components, Timber, Preservative TR
Lumber, Siding, Framing, Decking, Flooring, Ceiling, an
E Spec. for Pl

ASTM

ASTM

Spec ASTM

ASTM

ASTM

ASTM

ASTM

ASTM

ASTM

ASTM

ANSI

IEEE

NEMA

ANSI

ANSI

NEMA

FMS

ASTM

ASTM

SAE

ASTM

AATCC

ASTM

ASTM

ASTM

ASTM Std. Met ASTM

SAE

ASTM

ASTM

ASTM

ASTM

ASTM

ASTM

ASTM

ASTM

DEMA

Rec. ASME

std. Pract. DEMA

ASTM

ASTM

Std. ASTM

ASME

ASME

ASME

ASME

$S A E$

AGMA

ANSI

ANSI

ANSI

ANSI

ANSI

$\mathrm{NSA}$

HMI

NFORP

NFORP

WWP

APA

CTI

ASTM

CTI

CTI

CTI

WWPA

ICBO

FMS

Rules

NHLA

WWPA

ICBO

Uniform B ICBO

for Eastern Spruce, White Pine, and Hem ICBO

Ing Code Std. for Eastern White and Red ICBO Niform Building Code Std. for Classific ICBO Std. Meth. Fo ASTM

NFORP

/M-Fir (North), Ponderosa and Western ICBO ICBO

ICBO

ICBO

ICBO

WWPA

D3005

A522

A334

A333

A 320

A 420

A352

Dl053

D2602

D2983

C 37.50

20

FU 1

C97.

C 37.16

DC 3

12-37

4430

D2888 


\section{National Bureau of Standards KWIC Index of Engineering Standards}

reatment by Pressure Proc/ ment by Pressure Processes (1973) $\mathrm{ma} \mathrm{SH} 3$

ei TDJ-133 Std. for Voltage Classification of
Std. for Voltage Classification of Meth. of Analysis of Added
andic Gravity and Porosity of for Crushed Stone, Slag, and Gravel for Dry or Water Bound Meth. of Analysis of Cooking Characteristics of Meth. of Analysis of Pigments in Flour, Semolina, Disk Meth. of Analysis of Color of Spectrophotometric Meth. of Analysis of Color of Meth of Analysis of Egg Solids in Meth. of Analysis of Unsaponifiable Matter in Meth. for Preparation of Sample: Meth. of Analysis of Soy Flour in Disk Meth. of Analysis of Semolina Quality in Macro Scale Meth. of Analysis of Semolina Quality in Micro Scale Meth. of Analysis of Semolina Quality in Meth. of Analysis of Cooking Characteristics of Spec. for Composite Filament Tape Laying Std. for Milling Std. Spec. for Std. for Milling Std. for ction, and Operation of Class Hi (High Impact) Shock Test. Std. for Automated s (1973) Uniform Building Code Std for Test Meth. for Density of Wood Chips (Sheet Std. Spec. for Noncarbonized, Single Ply, Adding mediate Tensile Strength Carbon-Silicon Steel Plates for Std. Slotted and Recessed Head Machine Screws and td. for Pan Head, Close Tolerance, Short Thread, Torq Set Std. for Flathead 100 Deg. Plain and Self Locking raft, Pan Head, Phillips Recess Full Threaded, Alloy Steel ad. Phillips Recess, Short Thread, 160,000 psi Alloy Steel ng Recess, A286 Cres, Self-Locking and Nonlocki/ ng Recess, Titanium Alloy, 6Al-4V, Self-Locking/ wing Recess, Alloy Steel, Self-Locking and Nonl/ et (1973) Std. for Std. for Std. for Std for Std. for Std. Slotted and Recessed Head ries (1/ Std for Mechanical and Quality Requirements for Std. Spec. for Cold Heading Quality Carbon Steel Wire for Std. for Mechanical and Quality Requirements for Steel or Water or Solvent Soluble Liquid Adhesives for Automatic Die Casting

r SAE No. 2 (Automotive Transmission) Clutch Friction Test Suggested Noise Measurement Techniques for the Std. for Symbols for

initions of Common Words Related to Numerically Controlled Spec. for Metal Cutting Safety Std. for All Thermoplastic Type MTW 600 Volt ydraulic Std. for Industrial Equipment and General Purpose Electrical Std. for Metalworking

d Semolina (Cereal Chemistry) (1962)

essure Sensitive Tack of Adhesives Using an lnverted Probe Std. for Tool and Cutter Grinding

Std. for Horizontal Boring, Drilling and Milling or Numerically Controlled Profiling and Contouring Milling rically Controlled Horizontal Boring, Drilling and Milling single Rip) Meth. (Constant Rate of Traverse Tensile Test. ec. for Fire Safety and Protection of Yankee Driers (Paper

73)

(1973)

(1973) Std. for Bolt Std. for Screw, Std. for Screw, Std. Spec for

Rec. Pract. for Electrical Installations on Packaging or Symbols for Operator Controls on Agricultural Tractors, Industrial Cost Trends for Buildings, Agricultural

he Evaluation of Sealed Insulation Systems for AC Electric Std. for Meth. of Measuring, Recording and Specifying stallation of Exhaust Systems for Propulsion and Auxiliary act. for Storage Batteries for Construction and Industrial Std. Safety Requirements for Woodworking pract. for Lubrication Chart-Construction and lndustrial Rec. for Safety for Farmstead Equipment and act. for Electrical System for Construction and Industrial for Sealed Lighting Unit for Construction and Industrial

Std. for Headed Drilled Pins for Farm Equipment and edure for Airborne Sound Measurements on Rotating Electric ion, Care, and Use of Packaging and lts Related Converting Std. for Agricultural Press and Gage Wheel Tires (F arm
Lumher, Timber, Plywood and Utility Pole Preservative T Lumber, Timbers, Bridge and Mine Tie Preservative Treat Luminaires Used in Street and Highway Lighting (1973) E Lump Coke (1973)

Lysine in Wheat and Bulgur (Cereal Chemistry) (1973) Macadam Base Courses (1971) ANSI A37.99

Macaroni-Borasio System (Cereal Chemistry) (1962)

Macaroni and Wheat (1962)

Macaroni Products (Cereal Chemistry) (1962)

Macaroni Products (Cereal Chemistry) (1962)

Macaroni Products (Cereal Chemistry) (1962)

Macaroni Products (Cereal Chemistry) (1962)

Macaroni Products (Cereal Chemistry) (1962)

Macaroni (Cereal Chemistry) (1962)

Macaroni (Cereal Chemistry) (1962)

Macaroni (Cereal Chemistry) (1962)

Macaroni (Cereal Chemistry) (1962)

Macaroni (1962)

Machine-Numerically Controlled (1973)

Machine-Tracer Controlled Profiling and Contouring-

Machine and Sprocket Coil Chain (1972) ANSI G61.5

Machine Arbor Assemblies (1972)

Machine Bolt, Crowned Hexagon Head, Adjusting (1973)

Machine for Lightweight Equipment (1972)

Machine Lay Up Composite Filament Tape Reel (1972)

Machine Made and High Alumina Fireclay Refractory Brick

Machine Mold) (Pulp and Paper) (1973)

Machine Paper Rolls (1973)

Machine Parts and General Construction (1970) ANSI G24

Machine Screw Nuts (1972)

Machine Screw (1972)

Machine Screw (1974)

Machine Screw (1974)

Machine Screw (1974)

Std. for Aircra

Std for Airc NSA

Machine Screw, Flat Fillister Head, Full Thread, Tri Wi

Machine Screw, Flat Fillister Head, Full Threaded, Tri

Machine Screw, Flat 100 Deg. Head, Short Thread, Torq S

Machine Screw, Pan Head, Short Thread, Torq Set (1973)

Machine Screws and Machine Screw Nuts (1972)

Machine Screws for Use in Automotive and Related Indust

Machine Screws (1971) ANSI G54.14

Machine Screws (1973)

Machine Sealing of Top Flaps of Fiberboard Shipping Cas

Machine Stds. (1972)

Machine Test Procedure (1972)

Machine Tool Builders' Industry (1970)

Machine Tool Indicator Plates or Control Buttons (1972)

Machine Tool Software (1972)

Machine Tool Weldments (1971)

Machine Tool Wires and Cables (1972)

Machine Tools (1973)

Machine Tools (1973) ANSI C113.1

Machine Washing Meth. of Analysis of Gluten in Flour an

Machine (1971) ANSI Z197.32

Machine (1973

Machine (1974)

Machine (1974)

Machine (1974)

Machine) (1971)

Machine) (1973)

Machine, Flat Fillister Head, Full Thread, Torq Set (19

Machine, Flat 100 Deg. Head, Full Thread, Torq Set (197

Machine, Hexagon Head, Nonmagnetic, and Heat Resistant

Machine, Numerically Controlled (1973)

Machine, Pan Head, Full Thread, Torq Set (1973)

Machine, 100 Deg., Flat Head Full Threaded, Alloy Steel

Machined Flat and Square Tool Steel Bars (1973)

Machinery and Associated Equipment (1972)

Machinery and Equipment (1971) ANSI B114.2, SAE J389

Machinery and Equipment (1974)

Machinery Data and Management Practices (1971)

Machinery Employing Form Wound Stator Coils (1972) ANS

Machinery Sound Within Equipment Rooms (Noise) (1973)

Machinery (Boats) (1973)

Machinery (1970)

Machinery (1971)

Machinery (1971)

Machinery (1972)

Machinery (1972)

Machinery (1972)

Machinery (1973)

Machinery (1973)

Machinery (1973)

Machinery) (1971)
Pract and Stds. for

ANSI

Rec. $\mathrm{SAE}$

ASAE

Rec. Pr SAE

Std, for Dimensional Spec SAE

ASAE

Std for Test Proc IEEE

/Afety Requir ements for the Construct ANSI

ASAE
UBCS25. 12

WPA

EEl

TDJ. 133

$\mathrm{SA} 3$

D694

$\mathrm{AACCH} \quad 14-50$

AACCH 14-20

H 14.21

ACCH 20.10

ACCH $\quad 30.40$

62.40

06.11

CCH $66-40$

ACCH 66.41

ACCH 66.42

CH 16.50

ASTM A467

NSI B5.47

NSA 428

S2.15

$\mathrm{S} 2.15$
992

UBCS37.1

UM.9

NSI $\times 4.8$

A284

B 18.6.3

1131.38

662

600

623

5400-06

5500-06

$5300-06$

$1620-28$

1630-34

NSI B18.6.3

SAE J82

STM A.545

IFI 103

D1874
$* 1$

J286

ASI B5.

NMTBA $* 2$

AWS Dl4.2

JIC 1063

NFPA $\quad 79$

AACCH 38.11

D2979

973

910

913

954

D2262

12.29

1101

1102

1003

974

1100

514

A 685

333

S304.4

9.3

D 230.2

429

575

Pl

J930A

01.1

J753A

J821A

J572A

$\$ 226.2$

85

B155. 1 
Std. for Agricultural Planter Press Wheel Tires (Farm Rim Contours for Agricultural Press and Gage Wheels (F arm gn and Location for Construction and Industrial Equipment ed Safety Signs for Construction and Industrial Equipment fication System for Industrial and Construction Equipment $m$ Bucket, and Dragline Bucket (Construction and Industrial row Crop Cultivator Shafts and Ground Tool Mountings (F arm tires (1972) Safety Std. for Vacuum Cleaning Rec. Pract. for Test. Tire Selection Tables for Agricultural (Farm) Std. for Safety for Commercial Dry Cleaning ouring and Contouring-Positioning Numerically Controlled over, Rug Shampooer, Std. for Safety for Floor Finishing d. for Pot, Pan, and Utensil Commercial Spray Type Washing for Liquid Ballast Table for Drive Tires of Agricultural Std. for Spindle Noses and Tool Shanks for Milling Std. for Milling

Std. Liquid Ballast Table for Drive Tires of Agricultural ria and Definitions for Excitation Systems for Synchronous r Free Cutting Brass Rod, Bar, and Shapes for Use in Screw Std. Meth. of Verification of Test. Safety Std. for Transformer Type Arc Welding Std. Meths. for Specifying the Performance of Shock Std. for Operating Supply Voltage and Frequeniy of Office Std. Test Code for Direct Current rements for the Construction, Care, and Use of Die Casting Std. for Numerically Controlled Drilling Std. for Safety for Refrigerated Vending Std. for Safety for Vending and Amusement Numerically Controlled Horizontal and Vertical Jig Boring ibration Instruments for Verifying Time Load Indication of roperties of Elastomeric Yarns (Constant Rate of Extension ) ANSI G81.42 Std. Spec. for Free Std. Meth. of Conducting

erate Heat Resistant, 12.5Cr Low Carbon (SAE 51416F), Free caroni (Cereal Chemistry) (1962)

reals, and Alimentary Pastes for De/ orn, Dried Peas and Beans for Roden

Std. Rec. Pract. for Meth. of Analysis of or Dynamic Water Resistance of Shoe UPper leather by the Std. Proofing Supplement Stock for Gravure

me Ore, Clays, Diaspore, Ganister Quarzite, Grog, Kyanite, cereal Chemistry) (1962)

Wires (1970) ANSI H45.6 Std. Meth. of Tension Test. Wrought and Cast Aluminum and (Ze63.T6) Solution and Precipitation Treated (/ Std. Spec. for Std. Spec. for Std. Spec. for Std Spec for Std. Spec. for Spec. for ANSI W 3.19 and Die Castings (1966) ANSI H45.8 Std. Spec. for Spec. for Std. Spec. for Spec, for Tolerances of Aluminum and Spec. for Tolerances of Aluminum and Std. for

Std. Reference Radiographs for lnspection of Aluminum and or Performing Stress Corrosion Cracking Tests in a Boiling y (1973) Std. Meth. of Test for Calcium and 5.5 Std. Spec. for t of the Specific Electrical Impedance of Electrical Grade Std. Meth of Analysis of st for Soundness of Aggregates by Use of Sodium Sulfate or Std. for Uniform Fire Code:

lectrical Insulating Varnishes Applied Over Film Insulated Std. Meth. for Test. Film Insulated 2) g Rope Supported Booms, Equipped for Hook Work, Clamshell, Std. Test Procedure for Toroidal plicable to Electromagnet Iron (EMI) Cold Rolled Strip for Std. Spec. for Flat Rolled Electrical Steels for for Magnetic Shield Efficiency in Attenuating Alternating ansi $\mathrm{G} 60.6$ Std. for Print Spec. for r Premium Aircraft Quality Steel Cleanliness Requirements Std. Definitions of Terms Relating to eddy Current Test of Steel Tubular Products (Tubing) with agnetic Fields (1974) Std. Meth. of Test for i 4.6 Std. Meth. of Measuring Recorded Flux of Std. for

wiring Boards) in Numeric (Digital) Form on Punched Cards, or Audio Recording An/ Std. for Dimensional Std. Coplanar ) (1973)

i) (1973)

i) (1973)
Std. for Recorded Std. for Recorded
Machinery) (1971)

Machinery) (1971)

(Machinery) (1971)

Machinery) (1971)

Machinery) (1971)

Machinery) (1972)

Machinery) (1973)

Machines and Blower Cleaners (1973) ANSl C33.36.

Machines for Measuring the Uniformity of Passenger Car

Machines of Future Design (1972) SAE J711

Machines (Class Iv) (1974)

Machines (Information Processing) (1974)_/Mat for Cont El

Machines (Polisher, Scrubber, Sander, Scraper, Tile Rem Cont UL

Machines (1970)

Machines (1971)

Machines (1972)

Machines (1972)

Machines (1972)

Machines (1972)

Machines (1972)

Machines (1972) AASHO T67, ANSI 2115.1

Machines (1972) ANSI C33.2

Machines (1973)

Machines (1973)

Machines (1973)

Machines (1973)

Machines (1974)

Machines (1974)

Machines (1974)

Machines (1974)

Machines (1974) ANSI Z1 15.2

Machines) (1972) ANSI L14.288

Machining Tests of Wood and Wood Base Materials (1970)

ASAE

/Ec. Pract. for Instrument Face Desi SAE

Permanently and Temporarily Affix SAE

Pract for Electrical tWiring Identi SAE

Uck Capacity for Shovel Dipper, Cla SAE td for Chisel Plow, Field and ASAE

UL

SAE

ASAE

UL

B Bars and Forgings, Corrosion and

Rec. Pract SAE

ANSI

ANSI

ASAE

Std. Crite IEEE

Std. Spec. Fo ASTM

ASTM

UL

ANSI

ANSI

IEEE

Std. for Safety Requi ANSI

NSA

UL

Std. for NSA

Macro Scale Meth. of Analysis of Semolina Quality in Ma

Macroetch Test. of Tool Steel Bars (1971) ANSI G60.9

Macroscopic Examination of Baked Goods, Ready-To.Eat Ce

Macroscopic Examination of Cereal Grains, Unpopped Popc

Maeser Water Penetration

Magazines (Printing) (1973)

Magnesite, Mullite) (1970) ANSI A111.36

Magnesium Acetate) Meth. of Analys

Magnesium Alloy Extruded Bars, Rods, Shapes, Tubes, and

Magnesium Alloy Forgings (1972)

Magnesium Alloy Investment Castings (1972)

Magnesium Alloy Permanent Mold Castings (1972) ANSI H45

Magnesium Alloy Products (1973) ANSI H46.I

Magnesium Alloy Sand Castings (1972) A NSI H45.1

Magnesium Alloy Sand Castings $(5.75 \mathrm{Zn}-2.5 \mathrm{Re}-0.70 \mathrm{Zr}$

Magnesium Alloy Sheet and Plate (1970) ANSI H45.3

Magnesium Alloy Welding Rods and Bare Electrodes (1969)

Magnesium Alloys in Ingot Form for Sand, Permanent Mold

Magnesium Base Alloy Extrusions (1973)

Magnesium Base Alloy Sheet and Plate (1973)

Magnesium Casting Alloys (1971)

Magnesium Castings, Series II (1974) ANSI Z166.8

ASTM

Magnegum Chloride Solution (1973)

Magnesium in Iron Ores by Alomic Absorption Specto

Magnesium Ingot and Stick for Remelting (1971A) ANSI H4

Magnesium Oxide for Use in Sheathed Type Electric Heati

Magnesium Silicate Pigment (1973)

Magnesium Sulfate (1973) AASHO T104, ANSl A37.23

Magnesium Wrought Alloys (1971)

Magnesium (1973)

Magnet Wire (1973)

Magnet Wire (1974)

Magnet, Grapple, or Concrete Bucket Att - hments (1971)

Magnetic Amplifier Cores (Including Material Data) (197

Magnetic Application in Relays and Solenoids (1972)

Magnetic Applications (1974)

Magnetic Fields (1974)

Magnetic Ink Character Recognition (1970)

Std. Meth. of Test

Magnetic Particle Examination of Steel Forgings (1971)

Magnetic Particle Inspection Procedure) (1973)

Magnetic Particle Inspection (1974) ANSI Z166.15

Magnetic Saturation (1971) ANSI Z166.27

Magnetic Shield Efficiency in Attenuating Alternating

Magnetic Sound Records at Medium Wavelengths (1972) A

Ctripe Encoding (Code) for Credit Cards (1973)

Magnetic Tape and Punched Paper Tape (1972)
Magnetic Tape Cartridge Type Cp II (Compact Cassette) F

Magnetic Tape for Information Interchange (1600 cpi, PE

Magnetic Tape for Information Interchange $(200 \mathrm{cpi}$, N Nz

Magnetic Tape for Information Interchange $(800 \mathrm{cpi}$, Nrz

SAE GT

SAE

ASTM

AWS

ASTM

SAE

SAE

SAE

ASTM

ASTM

ASTM

ASTM

ASTM

/F Te ASTM

$\mathrm{SAE}$

ICBO

ASTM

ASTM

SAE

IEEE

NARM

ASTM

S224.1

S222

J209

JII5

210

J67

S225.1

1017

$332 \mathrm{~A}$

R220. 3

664

RS274. B

56

J884B

B5. 18

B5.45

$\$ 346$

421

(16

551

S2.14

$\mathrm{X} 4.11$

113

B152.1

960

4

751
963

E74

D2731

A58 I

D 1666

AMS5610H

AACCH 66.4

ASTM A56]

AACCH 28.30

AACCH 28.10

ASTM D2099

$* 5$

C 316

A ACCH 08.02

ASTM B94

ASTM B107

ASTM B9I

B403

ASTM B199

ASTM B55?

ASTM B80

AMS4425

B90

A5. 19

B93 
0 and $800 \mathrm{cpi}, \mathrm{NRZl}$, and $1600 \mathrm{cpi}, \mathrm{P} /$

Std. for Unrecorded eproducers for Audio Record One for 2-In. Quadruplex Video $\mathrm{f}$ Video, Audio and Tracking Control Records on 2 In. Video rec. Test Meth. for Flutter Measurement of Instrumentation est Meth. for Timing Error Measurements of Instrumentation io Level and Multifrequency Test Tape for Quadruplex Video and Deemphasis Characteristics for 2 In. Quadruplex Video t. for Video Alignment Signal Spec. for a Quadruplet Video Rec. Pract. for the Label for 2 In. Quadruplex Video pec. of Tracking Control Record for 2 In. Quadruplex Video Pract. for Dimensions of Patch Splices in Two Inch Video Std. Meth. for Test.

si C9.100 Std. for (1974) th. for Chemical Analysis of High Temperature, Electrical, (1973) ANSI B5/ Fire Hazards and Protection for Std. for Type Designations, Areas of Use, ystems for Fire Alarm Service (19/ ling Systems for Guard, Fire Alar/ ms for Watchman, Fire Alarm, and g Systems for Watchman, Fire Alar/ ing Systems (1972) ANSI SE3.5 cess Flow VaIves (1972)

ymbols and Color Codes for Lubricant and Fluid Requirement c37.61 Std. Guide for the Application, Operation, and systems (197/ Rec. Pract. Test Meth. and Requirements for

(1960) Safety Rules for the Installation and trical Safety Code, Part l: RuIes for the Installation and Uniform Fire Code: ical Code (Requirements for the Installation, Control, and Rec. Pract. Design, Installation, Operation, and from Manufacturer (1969) ANSI C/ Guide for Acceptance and Rec. Pract. for Operation and Rec. for the Inspection and Installation and Testing Procedures and rrier, Road, Hydrant, Driveway Clearance, Installation and rvation) (1972/ Rec. for Design, Layout, Construction and Pipe Systems (Fire Protection) // Rec. for Operation and Guide for Operation and ations Lines (Nati/ ection Agai/ Safety Rules for the Installation and Safety Std. for Construction, Operation, and or Commercial, Institutional and Industrial Build/ No Wax n Institutional, Industrial and Commercial Buil/ Sid. for gas Indicators Including Their Installation, Operation and

edium Speed Stationary Diesel and Gas Engine Operation and suction Press Rolls and Their Installation, Operation, and National Aerospace Stds. Documents Preparation and Std. for Safety for Household Electric Coffee Std for Safety for Automatic Ice ar Round Air Conditioning System) (1966)

What tory (1969) ANSl A37.81 est Specimen (1973)

(1972)

zation Mea/ Rec. Guide for Std. Meth. of Std. Rec, Pract. for Std. Rec. Pract. for Std. Rec. Pract. for Std. Reference Meth. for for Expansion Characteristics by $\mathrm{Po}$

(PVC) Pipe and Fittings (1973) Ansi/ Std. Spec. for Aluminum Alloy Hardeners Used in ession Test Specimens (I974)

Flour, Bran, Shorts, etc.) to Determine Added Amounts of ur, Bran. Shorts, etc.l

Colorimetric Meth. of Analysis of Std. Spec. for Pearlitic

971)

1)

Hopper Cars for

Meth. of Analysis of Diastatic Activity of Meth. of Analysis of Alpha A mylase Activity of Meth. of Analysis of Proteolytic Activity of Air Oven Meth. of Analysis of Moisture in Meth. for Preparation of Sample: I962) th Meal and Flour of Wheat, Rye, Barley, Other Grains, and

1 Chemistry) (1962) Meth. of Ferricyanide xidase Meth. for Measurement of Glucose in Sugar Mixtures Std. Tables of Classification of coated or Lamina/ Std. Performance Requirements for Woven institutional and Industrial Use Garments (Clothing), 100\% institutional and Industrial Use Garments (Clothing), 100\% re Cords, Fabrics and Industrial Filament Yarns, Made from (1973) th. of Test for Commercial Weight of a Shipment of Yarn or Tent. Meth. of Test for Breaking Tenacity of

Std. Meth. of Test for Tensile Properties of Single Rec, for Uniform Terminology for Rural Waste
Magnetic Tape for Information Interchange (9-Track 20

Magnetic Tape Operating at I5 and 7.5 In./S (1973)

Magnetic Tape Quadruplex Recorded at 15 and 7.5 In./S

Magnetic Tape Recorder / Reproducers (1972) ANSI C83.99

Magnetic Tape Recorder / Reproducers (1974) ANSI C83.94

Magnetic Tape Recorders Operating at I5 In./S (1973)

Magnetic Tape Recording ([967)

Magnetic Tape Recording (1969)

Ce Carrier Frec

Magnetic Tape Recordings (I968)

Magnetic Tape Recordings (I970)

Magnetic Tape (I964)

Magnetic Wire Enamels (1973)

Magnetic Wire for Use in Electrical Apparatus (1973) an

Magnetic, and Other Similar Iron, Nickel, and Cobalt Ba

Maintenance and Inspection of Mechanical Drive Turbines

Maintenance and Operation of Powered Industrial Trucks

Maintenance and Rules of the Road (1972)

Maintenance and Use of Auxiliary Protective Signaling S

Maintenance and Use of Central Station Protective Signa

Maintenance and Use of Local Protective Signaling Syste

Maintenance and Use of Proprietary Protective Signallin

Maintenance and Use of Remote Station Protective Signal

Maintenance in SI Metric Units (1973)

Maintenance Instructions for Chlorine Institute Std. Ex

Maintenance Instructions, and Container and Filler Iden

Maintenance of Automatic Circuit Reclosers (1973) ANSI

Maintenance of Design Voltage in Snowmobile Electrical

Maintenance of Electric Supply and Communication Lines

Maintenance of ElectricaI Supply Stations and Equipment

Maintenance of Exit Ways (1973)

Maintenance of Heating, Ventilating, Cooling, Refrigera

Maintenance of Impressed Current Deep Groundbeds (1972)

Maintenance of Insulating Oil in Equipment as Received

Maintenance of Offshore Cranes (1972)

Maintenance of Sprinkler Systems (Fire Safety) (1973)

Maintenance of Steam and Hot Water Unit Heaters (1970)

Maintenance of Steam Safety Valves on Boilers (I972)

Maintenance of Systems and Appliances (Extinguishers, a

Maintenance of Terrace (Erosion Control and Water Conse

Maintenance of the Reliable Model B Accelerator for Dry

Maintenance of Turbine Generators (1972) ANSI C50.30

Maintenance of Underground Electric Supply and Communic

Maintenance of Vehicle Alarm Systems and Units for Prot

Maintenance of Vinyl Asbestos and Asphalt Tile Floors F

Maintenance of VinyI Asbestos and Asphalt Tile Floors I

Maintenance (On Boats) (1973)

Maintenance (1972)

Maintenance (1973)

Maintenance (I974)

Makers and Brewing Type Appliances (1972) ANSI C33.11.1

Makers (600 Volts or Less Rating) (1974)

Makes a Good Air Conditioning System (Information on Ye

Making a Condition Survey of Concrete in Service (1965)

Making and Curing Concrete Test Specimens in the Labora

Making and Using the C-Ring Stress Corrosion Cracking T

Making and Using U-Bend Stress Corrosion Test Specimens

Making Potentiostatic and Potentiodynamic Anodic Polari

Making Reference Glass-MetaI Butt Seals and Test. for

Making Reference Glass-Metal Sandwich Seal and Test.

Making Solvent Cemented Joints with PolyvinyI Chloride

Making Zinc Die Casting Alloys (1965) ANSI H38.23

Making, Accelerated Curing, and Test. of Concrete Compr

Malathion in Range of 0.2 .2 .0 p.p.m. (Cereal Chemistry)

Malathion Residues in Milled Wheat Products (Wheat, Flo

Malleable Iron Castings (1971) ANSI G48.2

Malleable Iron Detachable Link Chain and Attachments (1

Malleable Iron Threaded Fittings (150 and $300 \mathrm{Lb}$ ) $\{197$

Malt and Grain Service (1964)

MaIt Flour (CereaI Chemistry) (1962)

Malt (Cereal Chemistry) (1962)

Malt (Cereal Chemistry) (1962)

Malt (Cereal Chemistry) (1962)

Malt (Cereal Chemistry) (1962)

Malted CereaIs (Cereal Chemistry) (1968)

Maltose-Sucrose Conversion Table (Cereal Chemistry)
Maltose (Diastatic Activity in Flour) Conversion (Cerea

Maltose, Sucrose, Mannose, Galactose, Dextrans, and Sta

Man Made and Natural Fibers (1971) ANSl L14.192

Man Made Fiber Tent, A wning, and Canopy Fabrics, Vinyl

Man Made or Blend Fabrics, Commercial Wash-Whites (Wi

Man Made or Blend Fabrics, Drycleanable Only (1973)

Man Made Organic Base Fibers (1972)

/Th. of Tes

Man Made Staple Fiber (1971)

Man Made Textile Fibers in Loop or Knot Configurations

Man Made Textile Fibers Taken from Yarns and Tows (1972

Management and Disposal ([973)
ANSI

R ANSI

ANSI

EIA

EIA

ANSI

SMPTE

quencies

SMPTE

SMPTE

. S SMPTE

ASTM

NEMA

ASTM

FMS

NFPA

BMA

NFPA

NFPA

NFPA

NFPA

NFPA

NSA

CHI

SAE

IEEE

$\mathrm{SAE}$

ANSI

ANSI

ICBO

ICBO

NACE

IEEE

API

FMS

AMCA

FMS

ICBO

ASAE

FMS

IEEE

A NSI

UL

RTI

RTI

ABYC

(

FMS

USA

UL

NESCA

$\mathrm{ACl}$

A STM

ASTM

ASTM

ASTM

ASTM

ASTM

ASTM

ASTM

ASTM

AACCH

A ACC

ASTM

ANSI

ANSI

BAMl

$\mathrm{AACCH}$

$\mathrm{AACCH}$

AACCH 22-61

$\begin{array}{ll}\text { AACCH } & 44-20 \\ \text { AACCH } & 62-50\end{array}$

ACCH 56-

AACCH 22.18

AACCH 22.17

AACCH $80-10$

A STM D2368

ANSI L24.I.10

ANSI L24.4.13

ANSI

L24.4.14

D885
D2494

$\begin{array}{ll}\text { ASTM } & \text { D2494 } \\ \text { ASTM } & \text { D3217 }\end{array}$

ASTM D2I0I

ASAE R292.1 
Agricultural Machinery Data and statistical Terminology and Notation for Nuclear Materials Wells and Water Systems (Treatment, Quality and Std. Form of Agreement Between Owner and Construction ct. for Flexibility Determination of Hot Melt Adhesives by Std. for Break

Std. for Structural Flush Break Pull nd Tempered, F/ Std. Spec. for Manganese-Molybdenum and 973) Std. Spec. for Nickel-Iron-Chromium . kel Alloy Steel. Quenched and Tempered, F/ Std. Spec. for Std. Spec. for Tent. Meth. of Test for Std. Meth. of Test for Std. Meth. of Test for Radioactive Std. Spec. for Electrolytic

for Pressure Vessels (1972) ANSI G35.15 Std. Spec. for Tent. Meth. of Test for Manganese Dioxide in Std. Spec. for Carbon

72) ANSl G35.14 td. Spec. for Pressure Vessel Plates, Heat Treated, Carbon d. Spec. for Pressure Vessel Plates, Alloy Steel, Chromium cold Finished Stainless and Heat Resisting Chromium Nickel els (1972) Std. Spec. for Austenitic Std. Spec, for Std. Spec. for High Strength Low Alloy Structural Colorimeter or Spectrophotometer Analysis of Acid Soluble c. for Pressure Vessel Plates, Carbon Steel, High Strength for Chemical Analysis of Silicomanganese and Ferrosilicon 1972) Std. Spec. for Pressure Vessel Plates, Carbon 0.1 Std. Spec. for Sewer and Std. Spec. for Precast Reinforced Concrete oncrete Masonry Units for Construction of Catch Basins and Std. Spec. for 1972) Safety Std. for High Pressure Gas easurement of Glucose in Sugar Mixtures (Maltose, Sucrose, Operational Safety

1974)

um Gas (1966)

in, Energy Consumption) (1974) Design, Manufacture, Inspection and Assembly Std. for

(1974) Std for 974)

fire, Explosion, and Detonation Protection) (1973) 4)

and Terms (1965)

1972)

for Fluoride Content of the Atmosphere and Plant Tissues 69) Load Carrying (Members) Structures (Aluminum Construction uble Helical and Rack) (1971) Differential and ng) (1973) Shipper-Motor Carrier Dock Plannin Principles of Clay Masonry Construction: Student's Gear Material (Steel Products (Steel Product (Steel Product Steel Product

Floor Plates, and Steel Sheet Piling (1 led and Cold Finished Bars; Hot Rolled D/

Laminators Quality Control System (Timber Construction num Formed Sheet Building Sheathing (Aluminum Construction tion of Elevators, Escalators and Moving Walks (inspector

metal Work in Building Construction (Aluminum Construction tective Signaling Systems (1974)

wl, and Load Brake Designs (1971)

(1972) ANSI C33.40

Systems Whose Maximum Allowable Operat Std. for Small

(Mechanical, Hydraulic, Pneumatic, Semi and Automatic, and towers, Safety) (1971) atory lnstruments (1972) Std. for Preparation of Std. for a Product Us Steel Product 1973)

(0-Rings) (1971)

s for Irrigation W ells (1972)

Steel Products

Rec. for

Std. Spec. and Meth. of Test for Heavy Cotton Fabrics for mable and Combustible Liquids A/ Uniform Fire Code: imum Safety and Health Requirements for Paper Bag and Sack Std. Spec. for Aluminum for Use in Iron and Steel h. for Analysis of Sulfuric Acid as Used in Pulp and Paper r Electrolytic Chlorine Processes (Explosion Hazard During tch Gearing (1973)

ives and Blasting Agents (1973) ANS1 Z/ Std. Code for the ratures/
Management Practices (1971)

Management (1972)

Management) (1972)

Manager (1973)

Mandrel Bend Test Meth. (1972T)

Mandrel Closed End Blind Rivets (1973)

Mandrel Self Plugging Blind Rivets, Type 2A (1973)

Manganese-Molybdenum-Nickel Alloy Steel, Quenched a

Manganese-Molybdenum Alloy Plate, Sheet, and Strip ( 1

Manganese-Molybdenum and Manganese-Molybdenum-Nic

Manganese Bronze Rod, Bar, and Shapes (1974) ANSI H7.5

Manganese Dioxide in Manganese Ores (1972T)

Manganese in Paint Driers by EDTA Meth. (1973)

Mlanganese in Water (1971) ANSI N156

Manganese Metal (1969) ANSI G84.1

Manganese Molybdenum and lts Nickel Alloy Steel Plates

Manganese Ores (1972T)

Manganese Silicon Steel Plates for Pressure Vessels (19

Manganese Silicon (1972A)

Manganese Silicon (1972A) ANSI G32.1

Manganese Steel Bars lncluding Rounds, Squares, Hexagon

Manganese Steel Castings (1973) ANSI G52.4

Manganese Vanadium Alloy Steel Plates for Pressure Vess

Manganese Vanadium Steel (1970A) ANSI G41.4

Manganese (Cereal Chemistry) (1962)

Manganese (1972A)

Manganese (1974)

Manganese, for Moderate and Lower Temperature Servi

Manhole Brick (Made from Clay or Shale) (1973) ANSI A10

Manhole Sections (1973)

Manholes (1973) ANS1 A73.1

Manifold Papers for Permanent Records (1973)

Manifolds for Industrial and Commercial Gas Cylit

Std. Spec for $C$

(

Manual for Anhydrous Ammonia (1973)

Manual for Boiler and Pressure Vessel lnspectors (1973)

Manual for Carbon Sheet Steel (coils and Cut Lengths) (

Manual for Driver Attended Loading of Liquefied Petrole

Manual for Electric Comfort Conditioning (Heat Loss, Ga

Manual for Fine Pitch Gearing (1973)

Manual Gas Metal Arc (Gma) Welding Equipment (1974)

Manual Gas Tungsten Arc (Gta) Fusion Welding Equipment

D. Meth. for Sampling Gas Blow Do

Manual Meth. (1973)

lded Structural Steel Tubing (1

Manual of Hazardous Chemical Reactions (lncandescence.

Manual of Practice: Multiple Contract Construction (197

Manual of Radioactivity Procedures (1961)

Manual of Standardization for Masterkeying Nomenclature

Manual of Std. Pract. for Welded Wire Building Fabric (

Manual Procedures) (1973)

Manual Sect. 1) (1971)

Tent.

1. for Design of Aluminum Alloy

Manual Transmission Lubricants (Information Report) (19

Manual (Gear Drawing Stds. Part 1 for Spur, Helical, Do

Manual (Truck Shipping and Receiving-Materials Handli

Manual (1960)

Manual (1972)

Manual) Carbon Steel Strip (1971)

Manual) Carbon Steel' Plates, Structural Shapes, Rolled

Manual) Carbon Steel: Semifinished for Forging; Hot Rol

Manual) Steel Specialty Tubular Products (1972)

Manual) (1962)

Manual) (1969)

Manual) (1973) Std. Pract. for of Alumi AA Std. Pract. for the Inspec

Manual, Sect. 5) (1971) Spec. for Aluminum

Manually Actuated Signaling Boxes for Use with Fire Pro

Manually Lever Operated Chain Hoists for Ratchet and Pa

Manually Operable General and Special Use Snap Switches

Manually Operated Metallic Gas Valves in Gas Distributi

Manually Powered) (1973)

$10 n$, Care, and Use

Manually Propelled Mobile Ladder Stands for Scaffolds (

Manuals for lnstallation, Operation and Repair of Labor

Manual: Grade Selection, Std. Size, Design Values, Fram

Manual: Tin Mill Products (1973)

Manual: W rought Steel Wheels and Forged Railway Axles (

Manufacture and lnspection Stds. for Preformed Packings

Manufacture and Placement of Reinforced Concrete Casing

Manufacture and Test of Aluminum lrrigation Tubing (197

Manufacture of Hose and Belts (1942) ANSI L14.283

Manufacture of Organic Coatings (Paint), and Handling $O$

Manufacture (Packaging) (1974)

Manufacture (1960) ANSI H38.25

Manufacture (1973)

Manufacture) (1974)

Manufacture, Inspection and Assembly Manual for Fine Pi

Manufacture, Transportation, Storage, and Use of Explos

Manufactured Carbon and Graphite Articles at Room Tempe
ASAE

ANSI

WSC

AIOA

ASTM

IFI

ASTM

ASTM

ASTM

ASTM

ASTM

ASTM

ASTM

ASTM

ASTM

ASTM

ASTM
ASTM

ASTM

ASTM

ASTM

eth. for AACCH

ASTM

ASTM

ASTM

ASTM

ASTM

AST

AACCH 80.10

Fl P110

NBBPV *1

AISI

NGPA

NEMA

AGM A

NSA

NSA

ASTM

WST1

FMS

CSI

NCRPM

$\mathrm{NBH}$

WRI

ASTM

AA

ANSI

ANS

BlA

AGMA

AISI

AISI

AISI

AISl

$\mathrm{AA}$

ANSI

UL

HMl

UL

ANSI

ANS

ANSI

ANSI

WWPA

AISI

AlS1

SAE

ASAE

ASAE

ASTM

ICBO

ANSI

ASTM

Std. Met TAPPI Rec. Fo

AGMA

NFPA

ASTM

D230.2

N 15.5

D3111

126

119

A533

$\mathrm{B} 590$
$\mathrm{~A} 533$

B] 38

E465

D2375

D2039

A601 
Std. Meth. of Test for Density in Air of Std. Definitions of Terms Relating to Std. for

aintenance of Insulating Oil in Equipment as Received from theater, Auditorium, Dormatory, Public or Office Building,

$m$ Dimensions for Grown Tires in Service for Use by Vehicle es of Spoke Type Wheels, Demountable Rims and Rim Spacers for Factory Constructed Housing and Recreational Vehicl tage Air Switch/ Std. for Schedules of Preferred Ratings, 3)

Spec. for Steel Splined Continuous Strip Mastic Set Std for Graphical Symbols for Use on Railroad ssure Vessel Plates, Alloy Steel, Precipitation Hardening ssure Vessel Plates, Alloy Steel, Precipitation Hardening Meth. for ChemicaI Analysis of Stainless, Heat Resisting, te; Earth; Glass; Granite; Gravel; Granite, Limestone, and metric Meth. of Solid Fat Index (50 or Less at 10 Deg.) of Std. for Pressure Treated Piles and Timbers in

Rec. Pract, and Stds. for

tion of Diesel (Compression Ignited) Inboard Liquid Cooled c. Pract. and Std. Covering the Installation of Air Cooled and Construction of Gasoline Fueled Inboard, Liquid Cooled rength Low Alloy Steel H-Piles and Sheet Piling for Use in ng Rigs and Petroleum Production Facilities on Land and on fatty Acids Content in Corn Oil and to Other Vegetable and of Lubricating Systems of Various Turbine Driven Pumps for

Meth of Accelerated Evaluation of Wood Preservatives for st, Jacket, Horseshoe and Ring Buoy) (19/ Safety Std. for

Z254.1

Std. for Safety Requirements for Std. Covering Sewage Holding and/or Treatment Devices for ammable and Combustible Fluids, Refrigeration Service, and lacement) Direct Drive Transmission (197/

he Preservative Treatment of Piles, Poles, and Timbers for Std. for Clearance, Side icle Type (Lead Acid) Storage Batteries (1) Rec. Domestic for Industrial Engineering Terminology: Distribution and n Applied to Coil Co/ Test Meth. for Determining Pressure ms (1971)

Aerospace Std. Identification Sid. for Lighting and

Guide to the Preparation of Precautionary Labeling and 1 Use in the United States (Safety) (1973) Std. Color Performance Spec. for the Purchase of Reflective Pavement Std Safety Color Code for Rec. Pract. for High Lift Industrial Truck Nameplates and Std. Pictoria Std. for Terminal

ure Cameras (1972) Std. Exposure Time or Resistance to Plastic Flow of Bituminous Mixtures Using Std. Meth. of Test for Flash Point by Pensky sure Containing Parts Suitable for High T/ ccidiosis I/ Meth. of Analysis of Nitrophenide in Poultry anesthetizing Locations (Bonding Appliances. Casters, Face ng of Roll Film Negatives (1972) Std. Spec. for Specifying Gypsum Structural Design of Semicircular Brick Structural Design of Brick Std. Meth. of Test for Compressive Strength of Std. Steel Doors and Frames for Modular Uniform Building Code Std. for Std. Spec. for

Notes) (1964) Design and Construction of Reinforced Brick CoId Weather Cold Weather Principles of CIay

orces) (Tech. Notes) (1968) 4 In. Reinforced Brick Design Tables for Reinforced Brick
Uniform Building Code Std. for Aggregate for Uniform Building Code

(1973) form Building Code Std. for Mortar for Unit and Reinforced Structural Clay Uniform Building Code Std. for Hydrated Lime for Std. Spec. for Hydrated Lime for Reinforced Brick Reinforced Brick

oles (1973) ANSI A73.1 Std. Spec. for Concrete Std. Spec. for Hollow Load Bearing Concrete Std. Meth. of Sampling and Test. Concrete Std. Spec. for Hollow Nonload Bearing Concrete Std. Spec. for Solid Load Bearing Concrete Std. Spec. for Chemical Resistant Std. Spec, for Prefaced Concrete and Calcium Silicate niform Building Code Std. for Hollow Load Bearing Concrete
Manufactured Carbon and Graphite Articles by Physical M

Manufactured Carbon and Graphite (1972)

Manufacturer Identification Symbols for Fasteners (1973

Manufacturer (1969) A NSI C59.131 /for Acceptance

Manufacturer, Restaurant, and Dwelling or A ppartment $\mathrm{H}_{0}$

Manufacturers in Designing for Tire Clearances (1974)

(Manufactures' Guide) (1963) Cal Minimum Rec. Toleranc

Manufacturing Plants (Fire and Wind Hazards at Plants a

Manufacturing Spec., and Application Guide for High Vol

Manufacturing Std. and Spec. for Textbooks (Books) (197

Maple Flooring Over Concrete Slab Floors (1962)

Maps and Profiles (1972)

(Maraging), $12 \%$ Nickel (1972A)

Std Spec for Pre

Maraging), $18 \%$ Nickel (1972A) ANSI G35.17 2 /Ec. for Pre

Maraging, and Other Similar Chromium-Nickel-Iron Al

Marble Masonry; Mortar; Sand; Slag; Steel; Terra Cotta;

Margarine Oils, Shortenings, and Other Fats (1968)

Marine Construction (1973)

Marine Deck Covering Guide (1969)

Marine Engine Mountings (Boats) (1971)

Marine Engines and Auxiliaries (Boats) (1972)

Marine Engines and Auxiliaries (Diesel and Gasoline) (B

Marine Engines (Boats) and Auxiliaries (1972)

Marine Environments (1974)

Marine Fixed and Mobile Platforms (1973)

Marine Oils and Animal Fats (1965)

Std. Spec.

Marine Service (1971)

for the Flusing

Marine Services by Means of Small Size Specimens (1970)

Marine Special Purpose W ater Safety Buoyant Devices (Ve

Marine Steam Power Plant Heat Balance Practices (1972)

Marine Terminal Operations (1972)

Marine Through Hull Fittings and Sea Valves (1973) ANSI

Marine Toilet Waste (Boats) (1972)

Afety Std. for Tubec. Pract. and

Marine Use (1972)

ing (Installation Tube Fiting

Marine (Inboard) Engine Mountings (Installation and Rep

Marine, Land, and Fresh Water Use (1974) AN
Marker and Identification Lamps (Light) (1972)

Marketing Std. Including Guarantee Policy for Motor Veh

Marketing (1972)

Marking and Blocking Resistance of Organic Coatings Whe

Marking Meths, on Finished, Semi Finished and Rough Ite

Marking of Agricultural Equipment on Highways (1971)

Marking of Compressed Gas Containers (1971)

Marking of Compressed Gas Cylinders Intended for Medica

Marking Paints (1972)

Marking Physical Hazards (1971)

Marking (1973)

Markings for Handling of Nondangerous Goods (1972)

Markings for Motors and Generaters (1972)

Markings for Variable Speed Shutters Used in Still Pict

Marshall A pparatus (1973)

Martens Closed Tester (1973) ANSI Z11.7

Martensitic Stainless and Alloy Steel Castings for Pres

Mashes (Feed) for Prevention of Cecal and Intestinal Co

Masks and Rebreathing Bags, Footwear. Hose and Tubing,

Masks (Se parate) for Use in Photographic Contact Printi

Masonry and Mortar (1967)

Masonry Arches (Tech. Note) (1971)

Masonry Arches (Tech. Notes) (1967)

Masonry Assemblages (1972)

Masonry Building Construction (1972)

Masonry Cement for Use in Mortars (1973)

Masonry Cement (1971) ANSI Al.3

Masonry Chimneys (For Residential and Industrial) (Tech

Masonry Columns and Pilasters (Tech. Notes) (1965)

Masonry Construction (Protection Rec.) (1968)

Masonry Construction, Introduction (Tech. Notes) (1967)

Masonry Construction: Student's Manual (1960)

Masonry Curtain and Panel Walls (In Resisting Lateral F

Masonry F lexural Members (Tech. Notes) (1967)

Masonry Mortar (1973)

Masonry or Concrete Chimneys, Fireplaces, and Barbecues

Masonry Other Than Gypsum (1973)

Masonry Process (Tech. Notes) (1966)

Masonry Purposes (1973)

Masonry Purposes (1974) ANSI K67.13

Masonry Retaining Walls (Tech. Notes) (1965)

Masonry Swimming Pools (Tech. Notes) (1967)

Masonry Units for Construction of Catch Basins and Manh

Masonry Units (1970) ANSI A79.1

Masonry Units (1970) ANSI A84.

Masonry Units (1971) ANSI A80.1

Masonry Units (1971) ANSI A81.1

Masonry Units (1972) ANSI A 103.1

Masonry Units (1973)

Masonry Units (1973)
ASTM C559

ASTM C709 IFI

IEEE

ICBO

TRA

SAE

ANSI

BMI

WSFI

ASTM

ASTM

to AAC

SNAME 4.11

ABYC P5

ABYC P15

ABYC Pl0

Design ABYC $\mathrm{P} 4$

A 690

RP500B

$\mathrm{H}-22$

LOS-2Cl

ASTM D2481

UL 1123

SNAME 3.11

ANSI MH9.1

UL

1121

ABYC A8

109

J233

T ASTM D390

SAE J592E

Std ANSI $\quad$ 294.4

NCCA TB-II-17

SAE AS478D

SAE J137A

CGA C7

CGA C9

ITE PS*I

ANSI Z53.

ITA $4 \mathrm{Fl}$

ANSI MH6.I

MA MGl.2

$\mathrm{PH} 3.32$

D1559

DSTM

ASTM A217

$\mathrm{AACCH} \quad 18-25$

UL 1067

ANSI PH3.9

CSI 04280

BIA $\quad 31 \mathrm{C}$

BIA $31 \mathrm{~A}$

ASTM E447

STDI 110

ICBO UBCS24-17

ASTM C9]

BIA 19B

BIA

BIA

BIA

BIA

BIA

BIA

ICBO

$17 \mathrm{I}$

$1 \mathrm{~A}$

*44

$17 \mathrm{~L}$

ICBO

$17 \mathrm{~J}$

UBC *3-37

BIA 27

ICBO UBCS24-19

ASTM C207

BIA

BIA

$17 \mathrm{E}-\mathrm{G}$

ASTM

ASTM

ASTM

ASTM

ASTM

ASTM

ASTM

ICBO
$17 \mathrm{~K}$

C139

C90

C140

C129

C145

C279

C 744

UBCS24-4 
uniform Building Code Std. for Solid Load Bearing Concrete Uniform Building Code Std. for Meth. of Test for Concrete orm Building Code Std. for Hollow Nonload Bearing Concrete Std. for and Meth. of Sampling and Test. of Unburned Clay Dampproofing and Waterproofing Reinforced Brick Reinforced Brick Reinforced Brick Guide Specs. for Brick Guide Specs. for Brick and Tile Radiation Protection with Clay Painting Clay Flashing Clay Modular Clay Cleaning Clay Products Early Strength of Brick Estimating Modular Brick Guide Spec, for Engineered Brick High Lift Grouted Reinforced Brick for Use of Hydraulic Cement Mortars in Chemical Resistant Rec. Pract. for Engineered Brick Guide Spec. for Concrete Unit Std. Spec. for Portland Cement Lime Mortar for Brick Std. Spec. for Mortar for Unit Uniform Building Code:

d, Reinforced Grouted, Hollow Unit, Cavity Wall, and Stone Rec. Spec. for Prefabricated Brick ech. Notes) (1966) Structural Clay (Properly Constructed th; Glass; Granite; Gravel; Granite, Limestone, and Marble

Rec. for Preplaced Aggregate Concrete for Structural and onding Thin Spectrochemical Samples and Stds. to a Greater ode (1973)

Mode (1973)

$\mathbf{r}$ in the Tracer / Std. Meth. of Test. for Leaks Using the Std. Meth. of Test for Leaks Using the Std. Meth. of Test. for Leaks Using the Devices with a Helium ions (1971) ANSI Zl/ Std. Meth. for High lonizing Voltage st for Atom Percent Fission in Uranium and Plutonium Fue lear Grade Uranium Dioxide Powd/ lear Grade Plutonium Dioxide $\mathrm{Po}$ lear Grade Mixed Oxides ((U, Pu/ d Physical Tests On, Beryllium / chemical Analysis of Nuclear Gr/ chemical Analysis of Nuclear Gr/ Std. Meth. for Chemical, Std. Meth. for Chemical, Std. Meth. for Chemical, Std. Meth. for Chemical, Std. Meth. for Chemical, Std. Meth. for Chemical of Test for Hydrocarbon Types in Low Olefinic Gasoline by

r Aromatic Types Analysis of Gas Oil Aromatic Fractions by Std. Meth. of Test. Aerosol Products for Delivered products (Drying Upon Q/ Meth. of Analysis of Moisture in td. for Test Meth. for Determining Visual Color Matched to Manual of Standardization for or Finish Flooring Over Concrete Slab Floors/ Spec. for 1 Filler and Aggregate) (1941) An/ Std. Spec. for Asphalt and Nailed Finish Flooring Over Concrete Slab / Spec. for $62)$
concrete Slab / Spec. for Steel Splined Continuous Strip erials for Setting Ceramic Tile with Mortar, Adhesive, and Std. for

Uniform Building Code Std for Std. Spec. for Polyester Glass sphalt-Saturated Asbestos Felt, Asphalt-Coated Glass Fiber anic Felts, Coal Tar Pitch, and Asphalt Coated Glass Fiber fiberbo/ Std. for Test Meth. for Determining Visual Color Uniform Fire Code: ched Card Index (/ Std. Rec. Pract. for Identification of $r$ Element (1972) ANSI B93.23 Std. Meth. of Verifying the guide (Spec. for Texture and Grade, Panel Characteristics, uide to Practice) (1971)

es (A Guide to Practice) (1974)

Std. for Nuclear procedure for Toroidal Magnetic Amplifier Cores (lncluding Guidance and Requirements for Evaluation of Suitability of Plastic

glass Show Wi/ Safety Std for Burglary Resisting Glazing orm Fire Code: Automobile Wrecking and Junk Yards or Waste Std. Meth. of Test for Solvent Extractable Std. Spec. for Air as an Electrical Insulating ate, Sheet, Strip, Connec/ Uniform Building Code Std. for rvice (1) Std. Spec. for Alloy Steel Turbine Type Bolting Suggested Components of a Concrete Reinforcing Steel for Steel Drill Screws for the Application of Gypsum Sheet nce Spec. and Meth. of Test for Transparent Safety Glazing Physical Pr/ Std. Rec. Pract. for Selection of Electrode ge on Kernels, Rodent Excreta, Other Filth, and Extraneous est Meth. for Delamination Strength of Honeycomb Type Core hin Spectrochemical Samples and Stds. to a Greater Mass of Std. Meth. of Test for Creep Relaxation of a Gasket $f$ Test for Moisture Content and Moisture Regain of Textile
Masonry Units (1973)

Masonry Units (1973)

Masonry Units (1973)

Masonry Units (1973)

Masonry Walls (Tech. Notes) (1961)

Masonry (Axial Design) (Tech. Notes) (1963)

Masonry (Flexural Design) (Tech Notes) (1964)

Masonry (Inspectors Guide) (Tech. Notes) (1963)

Masonry (Tech. Note) (Part l and Il Products) (1972)

Masonry (Tech. Note) (Part llI Execution) (1972)

Masonry (Tech. Notes) (1961)

Masonry (Tech. Notes) (1961)

Masonry (Tech. Notes) (1962)

Masonry (Tech. Notes) (1963)

Masonry (Tech. Notes) (1964)

Masonry (Tech. Notes) (1969)

Masonry (Tech. Notes) (1971)

Masonry (Tech. Notes) (1972)

Masonry (Tech. Notes) (1972)

Masonry (1965)

Masonry (1969)

Masonry (1971)

Masonry (1972)

Masonry (1973)

Masonry (1973)

Masonry (1973)

Masonry (1974)

Masonry) Insulated Cavity 6 In. Brick and Tile Walls (T

Masonry; Mortar; Sand; Slag; Steel; Terra Cotta; Timber

Mass Concrete (1966)

Mass of Material (1970) ANSI Z128.26

Mass Spectrometer Leak Detector in the Detector Probe M

Mass Spectrometer Leak Detector in the Inside Out Test.

Mass Spectrometer Leak Detector or Residual Gas Analyze

Mass Spectrometer Leak Detector (1972T)

Mass Spectrometric Analysis of Gas-Oil Saturate Fract

Mass Spectrometric Meth.) (1969) ANSl N108 /Eth. of Te

Mass Spectrometric, and Spectrochemical Analysis of Nuc

Mass Spectrometric, and Spectrochemical Analysis of Nuc

Mass Spectrometric, and Spectrochemical Analysis of Nuc

Mass Spectrometric, and Spectrochemical Analysis Of, an

Mass Spectrometric, Spectrochemical, Nuclear and Radio

Mass Spectrometric, Spectrochemical, Nuclear, and Radio

Mass Spectrometry (1971) ANSl Z11.311

Mass Spectrometry (1973)

Mass (1972)

Massecuites, Molasses, and Other Liquid and Semiliquid

Master Specimen for Fabrics, Vinyls, Coated Fiberboards

Masterkeying Nomenclature and Terms (1965)

Mastic Cushioned Construction with Nailers, Subflooring

Mastic for Use in Waterproofing (Asphalt Cement, Minera

Mastic Nailed Construction with Mastic Set Subflooring

Mastic Set Maple Flooring Over Concrete Slab Floors (19

Mastic Set Subflooring and Nailed Finish Flooring Over Definition and Recommendation for Mat

Mastic (1973) leboard (1966)

Mat Formed Wood Particleboard (1966)

Mat Sheet Laminate (1973)

Mat (1973)

Mat (1973)

/ Pitch, Coal-Tar Saturated Organic Felt, a

Match Rifle Range Plans (1973)

Matched to Master Specimen for Fabrics, Vinyls, Coated

Matches (1973)

Material by Absorption Spectroscopy, Using the ASTM Pun

Material Compatibility of a Hydraulic Fluid Power Filte

Material Composition. Use, Application, Finishes and $O t$

Material Control Systems for Conversion Facilities (A G

Material Control Systems for Fuel Reprocessing Faciliti

Material Data) (1972)

Material for Aviation Turbine Fuels (1973)

Material for Use in Housings of Motor Vehicle Lighting

Material for Use Principally as a Substitute for Plate

Material Handling Plants (1973)

Material in Black Pigments (1972)

Material in Electrical Equipment (1973)

Material Spec. for Structural Steel and Iron (Shape, P

Material Specially Heat Treated for High Temperature Se

Material Supply and Installation Contract (1968)

Material to Light Gage Steel Studs (1972)

Material Used in Buildings (1971)

Material Used in Buildings (1971) Std.
Material Used in Optical Emission Spectroscopy Based on
Material (Cereal Chemistry) (1962) /and/or Ins

Material (1970)

Material (1970) ANSI Z128.26

Material (1971)

Material (1971)
Tent. Meth. of Test Fo

ICBO

ICBO

Unif ICBO

ICBO

BIA

$\mathrm{BlA}$

BIA

CS1

CSI

$\mathrm{BlA}$

BIA

BIA

BIA

BIA

BIA

BlA

$\mathrm{BlA}$

BIA

ASTM

BlA

ASTM
ICBO

ICBO

BIA

BlA

ICBO

$\mathrm{ACI}$

ASTM

ASTM

ASTM

ASTM

ASTM

ASTM

ASTM

ASTM

ASTM

ASTM

(STM

ASTM

ASTM

ASTM

AACCH

SAE

NBHA

W SFI

ASTM

WSFI

WSFI

TCA

USC

ICBO

ASTM

UL

lCBO

NRA

SAE

ICBO

ASTM

NFLDP

CRA

ANSI

ANSI

Std. Test IEEE

IATA

SAE

UL

Unif ICBO

ASTM

ASTM

ICBO

ASTM

CRSI

Std. Spec. A STM

ANSI

ASTM land/or Insect Dama AACCH

ASTM

/Ec. Pract. for Bonding T ASTM

ASTM

Std. Meth. O ASTM

UBC $\$ 24.5$

UBC $\$ 24.7$

UBCS24.6

UBCS24.15

$17 \mathrm{~B}$

$17 \mathrm{~A}$

$7 \mathrm{C}$

$11 \mathrm{~A}$

$10 \mathrm{~A}$

35

10

$11 \mathrm{C}-11 \mathrm{D}$

$17 \mathrm{D}$

C398

*43 
and Accuracy as Applied to Measurement of a Property of A Std. Gravel Sand and Anthrante Filtering (Hydrualic and Hydropneumatic) for Lowering Personnel and Std. Spec. for Gold Copper Alloy Electrical Contact salety Std for Tests for Fire Resistance of Roof Covering de Fiber Content in Feedstuffs (Corn) Containing Vegetable with Wettin/ Test Meth. for Weather Resistance of Textile Std. for Safety for Factory Made Air Duct

Std Practices for Clinical Research Protocol for Dental electing and Defining Color and Gloss Tolerances of Opaque ce (1959) Rec. for d Airflow Performance (19/ Std. Meth. of Test. Duct Liner e Styrene Plastic Drain, Waste and Vent Pipe and Fitting Std. Pract. for Fabrication of Reinforcing Steel Bar Std. Pract. for Estimating Reinforcing Steel Bar Std. Rec. Pract. for Stress Relaxation Tests for ride) Duct Construction (1974) Stds. Guide Specs, and tion and Test. Agencies for Concrete, Steel and Bituminous ivity (Dielectric Constant) of Solid Electrical fnsulating Std. Meth. of Compression Test. of Metallic Std Meth of Test for Abrasion Resistance of Refractory Low Temperature Characteristics of Rubber and Rubber Like m/ Std. Meth. of Thermal EMF Test of Single Thermoelement uring Low Temperature Stiffening of Rubber and Rubber Like for Water Resistance of Paper, Paperboard, and Other Sheet ec. Pract. for Surface Preparation of Steel and Other Hard $\mathrm{r}$ and $\mathrm{O} / \mathrm{Std}$. Analytical Meth. for Determining Extraneous Std. for Record and Reporting Units for Nuclear

I Chemis/ Direct Trapping Meth. of A nalysis for All Baked Std. Meth. of Painting Ceiling alt, Asphalt-Saturated Organic Felt, Coal Safety Std. for Std Meth of Test Vitrified Ceramic al Chemistry) (Bolting Cloth, Filter Paper,/ Apparatus or Definition and Recommendation for 4 Std. Spec. for Alloy and Stainless Steel Bolting Std for Safety Tests for Flammability of Plastic ttings for Low Temper/ Sid. Spec. for Alloy Steel Bolting Std. Definitions of Terms Relating to

nitions of Terms Relating to Bituminous, and Other Organic Rec. for Crayons and Related Art N4.1 Std. Classification Sy stem for Polymeric e, and Mastic (1973) Definition and Recommendation for Meth. of Test for Operating Performance of Anion Exchange Sid. Test Meth. for Density of Core Std. Test Meth. for Water Absorption of Core ) A/ Std. Spec. for Rubberized Curled Hair for Cushioning rings: Hot Mopped Asphalt/Uniform Building Code Std. for Std. for Safety for Electrically Conductive Equipment and Std. for Screw Conveyors rrier Dock Planning Manual (Truck Shipping and Receiving . cereals, and Alimentary $\mathrm{Pa}$ Sieving Meth. of Analysis for Std. Meth. of Test for Thickness of Resilient Flooring, e Compatibility of Transformer Insulation and Construction Std. Meth. of Static Immersion Test. of L nstressed

Std. for Statistical Terminology and Notation for Nuclear

g Protein Nitrogen Content in Corn, Other Grains and Plant

Meth. of Analysis for Neutralizing Value of Acid Reacting Std. Meth. for Analysis of Environmental Std. for Industrial Engineering Terminology: nt of Transportation (DOT) Special Permits for Radioactive Content of Corn Syrup, Crude and Refined Sugar and Other ting) (1972)

Std. for Auditing Nuclear

Test for Voltage Endurance of Solid Electrical Insulating Uniform Building Code Std. for Noncombustible Elementary Spec for Cargo Unit Load Devices Std. Meth. of Test. Rigid Sheet and Plate a Spec. for Flame Resistant

e and Refined), Syrups, Starches and Other Protein Bearing (Corn), Syrteps, Sugars, Starches and Other Protein Bearing stuffs, Syrups, Sugars, Starches and Other Protein Bearing (1973) materials, High Temperature, 50 to $120 \mathrm{kSI}$ Yield Strength,

glass; Granite; Gravel/ Std. Meth. of Test for Compatibility of sification of Single and Double Screened Cround Refractory $\mathrm{n}$ for Presence of Rodent [rine on Bags and Other Packaging est for Analysis of Urea in Cloth Bags and Other Packaging ated Space Environment Test. of Spacecraft Thermal Control Indexes of Whiteness and Yellowness of Near White, Opaque rec. Code for Transportation. Storage and Use of Explosive tection Std for Rack Storage of Flammable and Combustible nd Safety (1973) Rec. for High Temperature Molten Std. Meth. for Determining Speed of Photographic Negative Std. Meth. of Test. Package Cushioning for Shock Absorbing Characteristics of Package Cushioning
Material (1971) ANS] Z1.13

Material (1972)

Material (1972)

Material (1973)

Material (1973)

Material, Corn and Other Grains, Flours, and Meals (196

Materials and Coated Fabrics: Carbon Arc Lamp Exposure

Materials and Connectors (1974)

Materials and Devices (1971)

Materials and for Evaluating Conformance (1972T)

Materials and Meth. for Durability of Concrete in Servi

Materials and Prefabricated Silencers for Acoustical an

Materials and Property Stds. for Acrylonitrile Butadien

Materials and Service (1968)

Materials and Service (1970)

Materials and Structures (1972)

Materials Application for Thermoplastic (Polyvinyl Chlo

Materials as IIsed in Construction (1972) ANSI Z267.1

Materials at Microwave Frequencies and Temperatures to

Materials at Room Temperature (R1973)

Materials at Room Temperature (1972)

Materials by a Temperature Retraction Procedure (TR Tes

Materials by Comparison with a Secondary Standard of SI

Materials by Means of a Torsional Wire Apparatus (1973)

Materials by the Dry Indicator Meth. (1973) TAPPI T433

Materials by Water Blasting Prior to Coating or Recoati

Materials Content of Corn Syrup, Crude and Refined Suga

Materials Control (1971)

Materials Except Chocolate Products and Piecrust (Cerea

Materials for Acoustical Absorption Tests (1973)

Materials for Built-lip Roof Coverings: Hot Mopping Asph

Materials for Electrical Applications (1969) ANSI C 59.7

Materials for Extraneous Matter Meth. of Analysis (Cere

Materials for Grouting Ceramic Tile (1973)

Materials for High Temperature Services (1974) ANSI G38

Materials for Parts in Devices and Appliances (1973)

Materials for Pressure Vessels, Valves, Flanges, and Fi

Naterials for Rerark and Pavenents (1971) ANS1 A37.33

Materials for Roofing, Waterproofing, and Related Build

Materials for School Use (1963)

Materials for Service in lonizing Radiation (1971) ANSl

Materials for Setting Ceramic Tile with Mortar, Adhesiv

Materials for Strong Acid Removal (1972)

Materials for Structural Sandwich Constructions (1970)

Materials for Structural Sandwich Constructions (1970)

Materials for Upholstery and Bedding Applications (1973

Materials for Use in Construction of Built Ip Roof Cove

Materials for I/se in Flammable Anesthetizing Locations

Materials Handling) (1971) ANSl MH4.7

Materials Handling) (1973)

Materials Hard to Hydrate in Baked Goods, Ready. To.Eat

Materials Having Flat Surfaces (1973)

Materials in Askarels (1973)

Materials in Nitrogen Tetroxide $\left(\mathrm{N}_{2} \mathrm{O}_{4}\right)(1972)$

Materials Management (1972)

Materials Manual (1972)

Materials of Comparable Content (1957)

Materials Other Than Phosphates (Cereal

Materials Processing (1972)

Materials Shipments (1973)

Materials Soluble in Water (1966)

Materials Spec. (Vehicular, lgnition, Lighting and Star

Materials Statements and Reports (Accounting) (1973)
Materials Subjected to Partial Discharges (Corona)

Materials Test. (1973)

Materials Transport and Handling) (1973)

Materials Used for Electrical Insulation (1972) ANSI C5

Materials Used in Camping Tentage (Tent) (1974)

Materials When Suitable Amounts of \& mple, Sulfuric ACl

Materials When Suitable Amounts of Sample, Sulfuric ACI

Materials When Suitable Amounts of Sample, Sulfuric ACI

Materials with Expansion Coefficients Comparable to Aus

Materials with Liquid Oxygen (Reaction Intensity Meth.)

Materials (Brick; Cast Iron; Cinders; Concrete; Earth;

Materials (Calcined Bauxite, Calcine, Chrome Ore, Clays

Materials (Cereal Chemistry) (1962)

Materials (Cereal Chemistry) (1962)

/ Light Examinatio

Materials (Coatings) with Electromagnetic and Particula

Materials (Color Measurement of Textiles, Paints, Plast

Materials (Fire Protection) (1973)

Materials (Fire Safety) (1974)

Materials (Metals, Slag, Glass, etc) Fire Protection az

Materials (Monochrome, Continuous Tone) (1972)

Materials (Packaging) (1964) ANS1 MH12.13

Materials (Packaging) (1964) ANS1 MH12.14
AWW

CSI

ASTM B596

UL

CR

UL $111 \mathrm{~A}$

Rec ADA

for S ASTM D3134

$\mathrm{ACl} \quad 59.57$

ASTM E477

IAPMO PSI7

CRSI *1.5.3

CRSI *1.5.1

ASTM E328

SMACN *11

ASTM E329

ASTM D2520

ASTM E9

ASTM C704

ASTM D 1329

ASTM E207

ASTM D 1053

ASTM D779

NACE RP.01.72

CR E.27

ANSI N15.2

AACCH 28.33

ASTM C643

UL 55A

ASTM D116

AACCH 28.90

TCA *3.1

ASTM A193

UL

ASTM

ASTM

ASTM

USC

ASTM

TCA

94

A 320

D8

D 1079

R 192

D3087

ASTM C271

ASTM C272

ASTM D2128

ICBO UBCS32.1

UL

CEMA

1067

ANSl MH8.1

$\mathrm{AACCH} \quad 28.32$

ASTM F386

ASTM D3255

ASTM F359

ANSI N15.5

AGMA 240.01

CR A-18

AACCH 02.32

ASTM D3304

ANSI 294.7

ANSI N14.10.2

E-27

BCl *1.41

ANSI Ni5.11

$\begin{array}{ll}\text { ANSI } & \text { N15.1 } \\ \text { ASTM } & \text { D2275 }\end{array}$

ICBO UBCS4.

NSA $\quad 3610$

ASTM D229

CPAl 84

CR F.44

CR G.22

CR J.56

ASTM A453

ASTM F37

ICBO UBC*3.23

ASTM C316

AACCH 28.85

AACCH 28.86

ASTM E512

ASTM E313

FMS 7.28

$8.33 \mathrm{~N}$

FMS LPD 7.33

ANSI PH2.5

ASTM D1372 
ic Determination of Water Vapor Transmission Rate of Sheet nsi J8.15, SAE J20/ Classification System for Elastomeric istrative Guide for Packaging and Transporting Radioactive nd Transportation of Radioactively Contaminated Biological ven/ Std. Meth. of Test for Solubility of Bituminous Road std. Meth. of Test for Effect of Heat and Air on Asphaltic of Corn Syrup, Sugars, Starches and Other Protein Bearing $t$ in Corn Starch, Sugars, Syrups and Other Protein Bearing ting Pressure Sealing Properties of Rubber and Rubber Like Energy Radiation on the Mechanical Properties of Metallic Rec for Safe Handling of Radioactive

Pract. for Internal Temperature Measurements in Ablative Std. Meth. of Test for Sag Flow of Highly Viscous std. Test Meth. for Creep Properties of Package Cushioning rd. Test Meth. for Specific Gravity of Wood and Wood Base std. Test Meth. for Specific Gravity or Wood and Wood Base Std. Test Meth. for Shear Fatigue of Sandwich Core ec. Pract. for Goniophotometry of Transmitting Objects and Std. Pract. for Detailing Reinforcing Meth. of Conducting Machining Tests of Wood and Wood Base ric Constant (Permittivity) of Solid Electrical Insulating Std. for Classification and Definitions of Bulk Std. Pract. for Probability Sampling of

ame Propagation Classification Flooring and Floor Covering Std. Meth. of Tension Test. of Carbon Graphite Mechanical Std. Definitions of Terms Relating to Appearance of Std. for Physical Inventories of Nuclear

Std. Meth. of Field Sampling of lon Exchange Std. Spec. for Laminated Thermosetting Std. Meth. for Notched Bar Impact Test. of Metallic td. Rec. Pract. for Scleroscope Hardness Test. of Metallic Std. Meth. of Tension Test. of Nonmetallic Gasket Std. Meth. of Fire Tests of Building Construction and Test Meth. of Surface Burning Characteristics of Building Std. Meth. of Test. Resistance to Scuffing of Trim

d. Meth. of Test for Residual Solvents in Flexible Barrier e Test Meth, for Wicking of Automotive Fabrics and Fibrous t. for Compression Molding Test Specimens of Allyl Molding einforced and Filled Nylon Injection Molding and Extrusion requirements for W/rought Precious Metal Electrical Contact est. Elastomeric and Plastomeric Roofing and Waterproofing ics, Vinyls, Coated Fiberboards, and Other Automotive Trim tatements for ASTM Meth. Related to Rubber and Rubber.Like sistance and Associated Characteristics of Automotive Trim ment of Temperature Indexes of Solid Electrical Insulating of Test for Pyrometric Cone Equivalent (PCE) of Refractory olding of Specimens of Thermoplastic Molding and Extrusion td. Meth. of Test for Asbestos Content of Asbestos Textile ating the Properties of Wood Base Fiber and Particle Panel est for Reaction Threshold Temperature of Liquid and Solid I for Operating Performance of Particulate Cation Exchange Std. Meth. of Test for Vickers Hardness of Metallic Std. Meth. of Test for Hardness of Electrical Contact test Meth. for Plane Strain Fracture Toughness of Metallic std. Meth. of Test for Resistivity of Electrical Conductor Std. Meth. of Test for Airflow Resistance of Acoustical std. Spec. for Polybutylene Plastics Molding and Extrusion Std. Classification for Metal Working Fluids and Related Std. Spec, for ETFE -Fluoroplastic Molding and Extrusion Std. Meth. of Test for Microhardness of

Std. Guide for Selection of Neutron Activation Detector Std. Rec. Pract. for Measuring Ultrasonic Velocity in niform Building Code Std. for Fire Retardant Roof Covering Uniform Building Code Std for Plastic

d. Definitions of Terms Relating to Rubber and Rubber Like Definitions of Terms Relating to Wax Polishes and Related meth. of Test for Noncombustibility of Elementary Building th. of Test for Rockwell Hardness of Fine Grained Graphite for Polyvinylidene Fluoride (PVDF) Molding and Extrusion for Thermoplastic Polyterephthalate Molding and Extrusion for E-CTFE- Fluoroplastic Molding, Extrusion, and Coating ding Code Std. for Fire Tests of Building Construction and act. for Electrostatic Charge Mobility on Flexible Barrier $t$ for Apparent Viscosity of Hot Melt Adhesives and Coating Terms Relating to Fire Tests of Building Construction and r Conducting Bending Fatigue Tests for Copper Alloy Spring

or Impact Resistance of Plastics and Electrical Insulating rced Polyterphthalate Thermo-Plastic Molding and Extrusion or Effective Fluorescent Lifetime of Neodymium Doped Laser td. Test. Meth. for Fire Hazard Classification of Building rocarbon (Tetrafluoroethylene) Resin Molding and Extrusion relating to Acoustical Tests of Building Constructions and ure Impact Test for Fabrics Coated with Flexible Polymeric ing a High Current Arc Erosion Test for Electrical Contact
Materials (Paper) at High Temperature and Humidity (197

TAPPI Materials (Rubber) for Automotive Applications (1973) a Materials (Safety) (1973) Std for Admin ANSI Materials (Safety) (1973) Std, for Packaging a

Materials (Tar, Petroleum Asphalt, etc.) in Organic Sol

Materials (Thin Film Oven Test) (1973)

Materials (1952)

Materials (1952)

Materials (1956)

al Meth for Determining $\mathrm{pH}$ Content $\mathrm{C}$

Determining Protein Nitrogen Content CR

Materials (1960) ANSI J2.20 Determining Protein Nitrogen Conten $C$

Materials (1962) ANSI N145

Materials (1964)

Materials (1968)

Materials (1968) ANSI K65.180

Materials (1968) ANSI MH 12.15

Materials (1968) ANSI Z260.1

Materials (1969)

Materials (I969) ANSI Z168.I3

Materials (1970)

Materials (1970)

Materials (1970)

Materials (1970)

Materials (1970)

Materials (1970)

Materials (1970) ANSI Z1.10

Materials (1971)

Materials (1971) ANSI K90.6

Materials (1971) ANSI Z138.1

Materials (1972)

Materials (1972)

Materials (1972)

Materials (1972)

Materials (1972)

Materials (1972)

Materials (1972)

Materials (1972)

Materials (1972)

Materials (1972)

Materials (1972)

Materials (1972)

Materials (1972)

Materials (1972)

Materials (1972)

Materials (1972)

Materials (1972)

Materials (1972)

Materials (1972)

MateriaIs (1972) ANSI All1.

Materials (1972) ANSI K65.78

Materials (1972) ANSI L14.141

Materials (1972) ANSI O8.1

Materials (1972) ANSI Z11.318

Materials (1972) ANSI Z111.4

Materials (1972) ANSI Z115.7

Materials (1972) ANSI Z155.12

Materials (1972) ANSI Z260.2

Materials (1972A) ANSI C 7.24

/ Meth. of Test for Evalua

ASTM

E184

CRPM R30

Std. Rec ASTM E377

ASTM D2730

ASTM D222

Std. Rec. P ASTM E338

ASTM D2395

ASTM E8

ASTM C394

ASTM El66

ASTM

Materials (1973)

Materials (1973)

Materials (1973)

Materials (1973)

Materials (1973)

Materials (1973)

Materials (1973)

Materials (1973)

Materials (1973)

Materials (1973)

Materials (1973)

Materials (1973)

Materials (1973)

Materials (1973)

Materials (1973)

Materials (1973)

Materials (1973)

Materials (1973)

Materials (1973)

Materials (1973)

Materials (1973)

Materials (1973)

Materials (1973)

Materials (1973)

Materials (1973)

Materials (1973)

Materials (1973)

Materials (1973)

Materials (1973)
Ent Loss Characteristics and Dielect ASTM

CEMA

Std. Meth. of Test for Fl UL

ASTM

ASTM

ANSI

ASTM

ASTM

ASTM

ASTM

ASTM

NFPA

NFPA

SAE

St ASTM

Std for Th SAE

Std. Rec. Prac ASTM

Std. Spec. for R ASTM

Std. Spec. for General ASTM

Std. Index of Meth. for T ASTM

Matched to Master Specimen for Fabr SAE

/Ec. Pract. for Preparing Precision S ASTM

St Meth. for Determining Blocking Re SAE he Thermal Evaluation and Establish IEEE

Std. Meth. ASTM

1. Pract, for Injection M ASTM

ASTM

Std. Meth. of Evalu ASTM

Std. Meth. of T ASTM

Std Meth of Tes ASTM

ASTM

ASTM

Std. ASTM

ASTM

ASTM

ASTM

ASTM

ASTM

ASTM

ASTM

ICBO

St ASTM

Std. ASTM

Std. ASTM

Std. Me ASTM

Std. Spec ASTM

Sid. Spec. ASTM

Std. Spec. ASTM

Uniform Buil ICBO

Tent. Rec, Pr ASTM

Sid. Meth. of Tes ASTM

Std. Definitions of ASTM

Std. Rec. Pract. Fo ASTM

Std. Meth. of Test F ASTM

Std. Spec. for Reinfo ASTM

Tent. Meth. of Test F ASTM

Uniform Building Code S ICBO

Sid. Spec. for TFE -Fluo ASTM

Std. Definitions of Terms ASTM

Std. Meth. of Low Temperat ASTM

Std. Rec. Pract. for Design AATM
T 464

$\mathrm{N} 14.3$

D1754

48 -10
-10

394

1.6.1

D1666

D 150

550

E105

992

C 565

E284

N15.3

D2687

D709

E23

E 448

251

255

J365A

F151

J913A

D3027

D2897

B476

D3105

J361 A

D3040

J912A

N14.10. 1 
ilure Under Electric Stress of Solid Electrical Insulating lity of Applied Designs and Finishes on Textiles and Other sity of Smoke from the Burning or Decomposition of Plastic ated and Coated Felts. Prepared Roofings, and Plastic Rol for Safety for Wind Resistance of Prepared Roof Covering Std. Meth. of Test for Penetration of Bituminous aminant, lnclined Plane Tracking and Erosion of Insulating act for Std. Test Temperatures for Rubber and Rubber Like Std. Spec. for Nylon Injection Molding and Extrusion Std. Definitions of Terms Relating to Textile

Std. Meth of Test for Brinell Hardness of Metallic for Determining Window Fogging Resistance of Interior Trim ow Measurement of TFE -Fluorocarbon Molding and Extrusion Std. Spec. for Fep Fluorocarbon Molding and Extrusion

ell Hardness and Rockwell Superficial Hardness of Metallic ety for Metal Waste (Trash, Garbage) Cans (For Combustible Penetrating the H/ Rec. Guides for Design, Construction, Insta/ Std. and Rec. Pract. for Requirements for Design, compounds for Preparing Vulcan/ Std. Rec. Pract. for Std. in Corn and Other Grains, Foodstuffs Containing Vegetable th, Materials with Expansion Coef/ Std. Spec. for Bolting Sys/ Std. and Rec. Pract. Guide to Design, Construction,

ign: Compression, Extension, Torsion. Flat, and Hot Wound

Abrasion Resistance of Textile Fabrics and Other Flexible

Rec. Pract. for Flammability of Automotive Interior Trim n (Incinerator and Open Burning, Combustible and Flammable Std. for Industrial Engineering Terminology: Applied ANSI C83.53 Std. for Clearance Dimensions on the Stds. for Decoders (Type l) for Reproducin

ested Design Procedures for Combined Concrete Footings and Std. Performance Requirements for Woven Bath Uniform Building Code Std. for Steel Bar and Rod Wool (1972) ANS1 L14.1/

Std. Meth. of Test for Vegetable Std. Test Meth. for Amylaceous Std. Meth. of Test for Volume Nonvolatile Std. Meth. of Test for Benzene lnsoluble Meth. of Analysis of Moisture and Volatile Meth. of Analysis of Unsaponifiable Std. Meth. of Test for Extractable Meth. of Analysis of Moisture and Volatile Std. Meth. of Test for Volatile

high Acid Coc Vacuum Oven Meth. of Analysis of Moisture and Volatile
Special Techniques for Extraneous
Reagents for Extraneous Vacuum Oven Meth. of Analysis of Moisture and Volatile
Special Techniques for Extraneous
Reagents for Extraneous rbon Tetrachloride; Chloral Hydr/ Apparatus or Materials for Extraneous Sid. Meth. of Test for Water Soluble Meth. of Analysis of Soluble Mineral Std. Meth. of Test for Volatile and Polymer Emulsion/ Std. Meth. of Test for Nonvolatile for Polynuclear Aromatic Hydrocarbons in Air Particulate Std. Meth. for Sampling Stacks for Particulate ace Masks and Rebreathing Bags, Footwear, Hose and Tubing. Std. Nomenclature for Bedding Sizes sticaire Method (1971) K91.1 Std. Rec. Pract. for Use of Std. Meth. of Test for Estimating
Std. Meth. of Test for Bauer

Meth. of Analysis of Falling Number Determination of Both Meth. of Analysis of Pelshenke Test (Wheat meth. of Analysis of Crude Fiber in Soy Flour for Cake and s, Full Fat and Defatted Soy Flours and Grits, and Soybean

s, Full Fat and Defatted Soy Flours and Grits, and Soybean Trading Rules for the Purchase and Sale of Linseed Trading Rules for the Purchase and Sale of Soybean Bread, Grain, Soybeans, Rice, Beans, Peas, Lentils, Corn of Analysis of Riboflavin in Whole Grain Products, Grits, flotation Meth. of Analysis for Insect and Rodent Filth in Decantation Meth. of Analysis for Rodent Excreta in

at, Rice, and Other Whole Grain Products, Corn Grits, Corn I Determining Crude Fat $(\mathrm{Ccl}$, Products Such as Feeds and ing Vegetable Material, Corn and Other Grains, Flours, and ins, Foodstuffs Containing Vegetable Materials, Flours and

Meth. of Analysis of Residual Urease Activity in Soybean Std. Meth. of Test for Stiffness of Plastics by ess Properties of Plastics as a Function of Temperature by Meth. for Measuring the Relative Stiffness of Leather by perature Stiffening of Rubber and Rubber Like Materials by

or Impact Resistance of Rigid Plastic Sheeting or Parts by

Std. Meth. of Test for Tensile Strength of Adhesives by or Preparation of Test Specimens of Bituminous Mixtures by ance to Deformation and Cohesion of Bituminous Mixtures by ed Evaluation of Wood Preservatives for Marine Services by

for Application of Plastic Polymers in Dry Powder Form by

t. for Preparation of Bituminous Mixture Beam Specimens by Scientific and Technical Work / er (1971-75)

Std. for Industrial Engineering Terminology: Work
Materials (1973)

Materials (1973)

Materials (1973)

Materials (1973)

Materials (1973) ANSI A195.

Materials (1973) ANSI A37.1

Materials (1973) ANSI C59.135

Materials (1973) ANSI Jl.3

Materials (1973) ANSI K65.216

Materials (1973) ANSI LI 4.12

Materials (1973) ANSI Z115.5

Materials (1973) ANSI Z265.1

Materials (1974)

Materials (1974) ANS1 K65.201

Std. Meth, of Test for Thermal Fa ASTM

Test Meth. for Drycleaning Durabi AATCC

Meth. of Test for Measuring the Den ICBO

i A geregates, Saturated Felts, Satur NRCA

Materials (1974) ANSI Z1 15.6,

Materials) (1974) ANSI Z221.1

Materials, and Installation of Power Transmission Units

Materials, Equipment, and Procedures for Mixing Rubber

Materials, Flours and Meals (1964)

/Rude Fiber

Materials, High Temperature, 50 to $120 \mathrm{KSl}$ Yield Streng

Materials, Size, and Installation of Propeller Shafting

Materials, Specs. Terminology, Gauge, Metric Conversion

Materials: Accelerator Meth. (1970)

Materials: Horizontal Test Meth. (1972)

Materials; Reporting and False Alarm; Use of Equipment,

Mathematics (1972)

Mating Plates (Patterns) Used in Battery Sockets (1974)

Matrix Quadraphonic Disc Records (1974)

Mats (Foundation) (1963)

Mats (Institutional Textile) (I973)

Mats (Sheets) for Concrete Reinforcement (1973)

Matter and Other Alkali Insoluble Impurities in Scoured

Matter in Adhesives (1970)

Matter in Clear or Pigmented Coatings (1973)

Matter in Creosote (1973) AASHO T81, ANSI 011.13

Matter in Fats and Shortenings (Butter, Oleomargarine,

Matter in Macaroni Products (Cereal Che mistry) (1962)

Matter in Oven Dried Wool (1973)

Matter in Soy Flours (Cereal Chemistry) (1962)

Matter in the Analysis Sample of Coal and Coke (1973)

Matter in Yeast Foods (Cereal Chemistry) (1962)

Matter Meth. of Analysis in Cereal Chemistry (1962)

Matter Meth. of Analysis (Cereal Chemistry) Alcohol; Ca

Matter Meth. of Analysis (Cereal Chemistry) (Bolting Cl

Matter of Vegetable Tanned Leather (1970) ALCA B8

Matter (Cereal Chemistry) (1962)

Matter (Including Water) of Vinyl Chloride Resins (1972

Matter (Total Solids) in Water Emulsion, Solvent Based,

Matter (1971)

Std. Test Meth

Matter (1971) ANSI Z257.3

Mattresses and Pads, Sheeting, and Restraint Straps) (1

Mattresses and Sheets) (1971)

Mattsson's Solution of $\mathrm{pH} 7.2$ to Evaluate the Stress Co

Maturity and Linear Density of Cotton Fibers by the Cau

McNett Wet Classification of Asbestos Fiber (1970) ANSI

Meal and Flour of Wheat, Rye, Barley, Other Grains, and

Meal Fermentation Time Test) (Cereal Chemistry) (1962)

Meal from Cottonseed, Soybeans, and Peanuts (Cereal Che

Std UL

ASTM

id Cont ASTM

ASTM

ASTM

ASTM

ASTM

$\mathrm{SAE}$

ASTM

ASTM

AST

UL

ABYC

ABYC

ASTM

CR

ASTM

ABYC

SMI

AATCC 93

$\mathrm{SAE} J 369 \mathrm{~A}$

ANS1 Z94.8

Z94.8

EIA

RS156-B

Sugg $\mathrm{ACl}$

ANSI L24.2.1

ICBO UBCS26-5

ASTM D1113

ASTM D1488

ASTM D2697

ASTM D36?

AACCH $44-30$

AACCH $30-40$

ASTM D 1574

AACCH 44-31

ASTM D3175

$\mathrm{AACCH}$ 44-32

$\mathrm{AACCH} \quad 28-92$

AACCH 28-9]

AACCH $28-90$

ASTM D2876

AACCH 40-0]

ASTM D3030

ASTM D2834

D2682

ASTM D2928

UL $106 ?$

ANSI L27.I

ASTM G37

ASTM D2480

ASTM D2589

$\mathrm{AACCH} 56-81 \mathrm{~B}$

AACCH $56-50$

AACCH $32-17$

Meal (Cereal Chemistry) (1968)

Meal (Cereal Chemistry) (1968)

Meal (Flaxseed) (1974)

Meal (1973)

Meal, Corn Grits, Rolled Oats, Bulgur, Rolled Wheat, $\mathrm{Br}$

Meal, Flaked and Puffed Cereals, Farina, Bread, etc. (C

Meal, Grits, Farina, and Semolina (Cereal Chemistry) (1

Meal, Grits, Flour, Farina, and Semolina (Cereal Chemis

Meal, Puffed Cereals, Farina, and Flour (Cereal Chemist

Meals (1955)

Meals (1964)

Ermand Finished Std. Analytical Meth. Fo

Meals (1964)

Ber Content in Feedstuffs (Corn) Contain

Meals, Soy Flour, Mill Feeds (Cereal Che mistry) (1962)

Means of a Cantilever Beam (1970) ANSI K65.13

Means of a Torsion Test (1972) ANSI D65.2

Means of a Torsional Wire Apparatus (1972) ALCA E6

Means of a Torsional Wire Apparatus (1973) ANSI J2.2

Means of a Tup (Falling Weight) (1972)

Means of Bar and Rod Specimens (1972) ANSI Z197.3

Means of California Kneading Compactor (1971) ANSl A37.

Means of Hveem Apparatus (1971) ANSI A37.137

Means of Small Size Specimens (1970)

/R Resist

Means of Spray Gun or Fluidized Bed (Fire Protection) (

Means of the California Kneading Compactor (1973)

(Measurement-Metric and British-American) in Publish

Measurement and Inspection of Hardwood and Cypress Lumb

Measurement and Meth. (1972)
AACCH $46-24$

NFP

NSPA *1

AACCH $44-15 A$

$\triangle A C C H \quad 86.70$

$\mathrm{AACCH} 28-51$

AACCH $28-50$

ACCH 86.80

B-18

CR G-12

CR A-8

ASTM

for Stiff́n ASTM Dl043

D ASTM D282I

ASTM Dl053

ASTM D3029

ASTM D2095

ASTM D 1561

D 1560

ASTM D2481

FMS $\quad 7.27$

ASTM D3202

IEEE 268

NHLA *1

ANSI $\quad$ Z94.12 

(1971)

of Test for Carbon Monoxide in the Atmosphere (Continuous I Bevel Gear Drives (1973)

72) ANSI C 37.26 Std. Meth. of for Use of the Terms Precision and Accuracy as Applied to es of Neutrons and Gamma Rays (1961)

nd (Applicable to Solution Coil Coatings and S/

ilter Meth. (Textile) (1971)

niform Building Code Std. for Laboratory Determination and ding Partitions (1970)

$966)$ ANSI N152

Std. Rec. Pract. for Laboratory Std. Meth. for
Std. Meth. of Test for

I Water and Industrial Waste Wate/ the Hunterlab Color Difference Meter (1972T) Meth. for $s$ in Nuclear Reactor Coolant Water During/

Std. Meth. for Std. on Television:

of Insulation Systems (1973/ icles (20.1000 Mhz) (1972) ANSI C112. ricants (Falex Meth.) (1973)

ng Fluids (Timken Meth.) (1972) ANSI F1l.

ng Fluids (Four Ball Meth.) (1971) ANSI Z/ n Mild Steel (Coil Coating) (1974) teel (1972)

t High Temperatures (1973) Test Meth. for

Powered Passenger Cars and Light Tr/ Powered Passenger Cars and Light $\mathrm{Tr} /$ N150

ose, Mannose, Galactose, Dextr/

Reproducers (1972) ANSI C83/

and Herringbone Gear Units (1972)

9) ANSI K90.1

icroscopica! Examination of a Cross Secti/ (1973)

d Biological A - plications (1960) id. Meth. for Detection and Std. for Std. Meth. for Std. Nondestructive Std. Meth. of Rec. Pract. for the Rec. Pract. for the Std. Meth. for Tent. Rec. Pract. for Glucose Oxidase Meth. for Rec. Test Meth. for Flutte Std. Spec, for Std. Meth. for Rec. Pract. fo Std. Meth. for Std. Meth. of Rec. for Std. Meth. for Floo Std. for Spec, and tallization Thickness by Multiple Beam I/ Tent. Meth. for
ctric Tests (1973) diation / Std. for Techniques and Instrumentation for the std. Rec. Pract, for Selection of Geometric Conditions for 1972) (1971) Std. for Std. Meth. for the Std Meth for Spinning Tests on the Cotton System for h. of Test for Thickness of Epitaxial Layers of Silicon by $\mathrm{f}$ Test for Minority Carrier Diffusion Length in Silicon by g a Passenger Automobile Equipped with Fu/ Tent. Meth. o ness and Yellowness of Near White, Opaque Materials (Color Materials (1974) ubes (1972) ctrical Grade Magnesium Oxide for Use in /

Oxide and Metallic Coatings by Double Be/ cuum (1971) ANSI Z11.302

ecording and Reproducing Equipment (1971) ndustry (1970)

Powder Flow Std. Meth. of Std. Meth. for Std. Meth. for Std. Meth. for Std. Meth. for Std. Meth. for

Suggested Noise Std. Moisture Std. Moisture

y Instrumentation and Techniques for Exhaust Gas Emissions ength and Length Uniformity of Cotton Fibers by Fibrograph Std. for Precision In. Gage Blocks for Length Std. for Trapezoidal Flumes for Irrigation Flow Rec. for Use of English and Metric Units of Test Meth. for Chip Length Analysis Std. Meth. for F ast Neutron Flux

)$(1972)$ Std. for Body

Std. Rec. Pract. for Internal Temperature Std. Guide for Electrical / Reproducers (1974) An/ Rec. Test Meth. for Timing Error D/ Trial Use Std. Guide on Solid State Devices: Varactor radiology) (1973) Std. for Thyroid Radioiodine Uptake Code for Shipboard Hull Vibration r of Manufactured Carbon and Graphite Articles by Physical ing Potentiostatic and Potentiodynamic Anodic Polarization o Photographic Lenses (197/ Std. Meth. of Designating and osures (1973) Std. Meth. of Std. Meth. of Std. Meth. for Meth. for Std. Meth. of Meth. for nstitutional Audio-
Printing (1972) ALCA E64

zero Deg. Observation) (1972)

Layer (1973) Steel Self Drilling Tapping Screws and Rec. Technique for
Measurement by Nondispersive Infrared Spectrometry) (19 Measurement for Audio Amplifiers Used in Home Equipment Measurement for Enclosed Helical, Herringbone and Spira Measurement for Low Voltage Inductive Test Currents (19 Measurement of a Property of a Material (1971) ANSI Z1. Measurement of Absorbed Dose of Neutrons, and of Mixtur Measurement of Adhesion and Flexibility by the Wedge Be Measurement of Airbone Asbestos Fiber by the Membrane F Measurement of Airborne Sound Transmission Class (Stc) Measurement of Airborne Sound Transmission Loss of Buil Mcasurement of Alpha Particle Radioactivity of Water (l Measurement of Beta Particle Radioactivity of Industria Measurement of Color of Low Colored Clear Liquids Using Measurement of Delayed Neutron Emitting Fission Product Measurement of Differential Gain and Phase (1960)

Measurement of Discharge (Corona) Pulses in Evaluation Measurement of Electromagnetic Radiation from Motor Veh Measurement of Extreme Pressure Properties of Fluid Lub Measurement of Extreme Pressure Properties of Lubricati Measurement of Extreme Pressure Properties of Lubricati Measurement of Film Thickness of Nonmagnetic Finishes $O$ Measurement of Film Thickness of Pipeline Coatings on S Measurement of Flow Properties of Lubricating Greases a Measurement of Fluorescent Lamp Ballasts (1972)

Measurement of Fuel Evaporative Emissions from Gasoline Measurement of Fuel Evaporative Emissions from Gasoline Measurement of Gamma Radioactivity of Water (1973) ANSI Measurement of Glass Stress Optical Coefficient (1973) Measurement of Glucose in Sugar Mixtures (Maltose, Sucr Measurement of Instrumentation Magnetic Tape Recorder /

Measurement of Lateral Vibration on High Speed Helical Measurement of Lattice Spacing of Nuclear Graphite (196 Measurement of Low Level Activity in W ater (1972T)

Measurement of Metal and Oxide Coating Thicknesses by M

Measurement of Microhardness of Electroplated Coatings

Measurement of Neutron Flux and Spectra for Physical an

Measurement of Office Buildings (1972)

Measurement of Out of Roundness (1972)

Measurement of Oxide Thickness on Silicon Wafers and Me Measurement of Partial Discharges (Corona) During Diele Measurement of Potentially Hazardous Electromagnetic Ra Measurement of Reflectance and Transmittance (1973) Ans Measurement of Smell Values of Transistor Capacitance ( Measurement of Sound Pressure Levels in Air (Acoustics) Measurement of Spinning Performance (1972) ANSI L14.289

Measurement of Stacking Fault Dimension (1973)

Measurement of Steady State Surface Photovoltage (Elect

Measurement of Stopping Distance on Paved Surfaces Usin

Measurement of Textiles, Paints, Plastics, etc.) (1973)

Measurement of TFE - Fluorocarbon Molding and Extrusion Measurement of the A-D Dimension of Aerosol Valve Dip T

Measurement of the Specific Electrical Impedance of Ele

Measurement of Thickness of Sandwich Cores (1970)

Measurement of Thickness of Transparent or Opaque Metal

Measurement of Volatilization Rates of Lubricants in Va

Measurement of Weighted Peak Flutter Content of Sound R

Measurement Techniques for the Machine Tool Builders' I

Measurement (Grain and Seeds) (1972)

Measurement (Meat and Its Products) (1972)

Measurement (Passenger Car and Light Truck) (1971)

Measurement (Textile) (1972) ANSI L14.98

Measurement (Thru 20 In.) (1973)

Measurement (1972)

Measurement (1973)

(Measurement) (Pulp and Paper) (1973)

Measurements by Track Etch Technique (1973)

Measurements for the Sizing of Girls' Apparel (Clothing

Measurements in Ablative Materials (1968)

Measurements of Fluorescent Lamps (light) (1973)

Measurements of Instrumentation Magnetic Tape Recorder

Measurements on Rotating Electric Machinery (1973)

Measurements Part II-Characterization of Large Signal

Measurements Using a Neck Phantom (Medical Radiation-

Measurements (1969)

Measurements (1969) ANSI K90.2

Test for Density in A

Measurements (1972) / for Std. Reference Meth. for Mak

Measuring Apertures and Related Quantities Pertaining T

Measuring Application and Removal Torque of Threaded $\mathrm{Cl}$

Measuring Area of Leather Test Specimens (1968) ALCA E2

Measuring Audio Amplifier Single Frequency Output for I

Measuring Blister Resistance of Coated Paper in Heatset

Measuring Break Pattern of Leather (Break Scale) (1973)

Measuring Brightness of Pulp (Diffuse Illumination and

Measuring Caliper of Resilient Floor Covering with Foam

Measuring Case Depth (1972)
Measuring Chalk Resistance of Exterior Coil Coatings (1

ASTM

EIA

AGMA

IEEE

ASTM

NCRPM

NCCA

ATI

ICBO

ASTM

ASTM

ASTM

ASTM

ASTM

IFEE

ASTM

SAE

ASTM

ASTM

ASTM

NCC A

ASTM

ASTM

ANSl

SAE

ASTM

ASTM

AACC

IA

AGMA

ASTM

ASTM

ANSI

ANSI

ASTM

IEEE

ANSl

ASTM

EIA

ANS1

ASTM

Met AATM

ASTM

ASTM

ASTM

ASTM

ASTM

ASTM

ASTM

ASTM

ASTM

IEEE

193

ASAE $\quad$ S352

ASAE $\$ 353$

or SAE

J254

ASTM D1447

$\begin{array}{ll}\text { ANSI } & \text { B89.1.9 } \\ \text { ASAE } & \text { S359T }\end{array}$

ASAE R285

TAPPI UM-5

ASTM E418

USC

ASTM

ANSl

EIA

IEEE

IEEE

ANSI

SNAME

ASTM

ANSI

ASTM

ASTM

ANSI

TAPPI

ASTM 
B Colorimetry (1972)

or Y, Dominant Wavelength and Excitation Purity/

Iectrical InsuIation (1972) ANSI C59.33 num (1970) ANSI N114

(1970) ANSI N1II

1 (1970) ANSI NI12

$r$ (1970) ANSI NII3

Produced by Uranium 238 Fission (1973)

blends (1972)

stometer (1973)

nonemployee Injury Statistics (1971)

ic Semiconductor Single Crystals (1973)

e (Slug) Calorimeter (1972)

(

with 3 Point/

Tractors Equipped with $3 \mathrm{Po}$

Cores (1973)

and Cores (1974)

Std. Test. Procedure for er Like Materials by Means of a Torsional / 970) ANSI N109

73)

ubber (1973)

f Soybean Oil (1973)

dium Wavelengths (1972) ANSI S4.6 r 4 Probe Array (1973)

tics (R1973) ANSI K66.3

Laser Rods (1973)

rics (1972T)

per or Paperboard (1973)

sh Portable Tester (1974) Rec. Pract. for Radius of Load and Boom Angle Meth. for Use of Layer Cake Uniform Building Code Std. for Chamber Meth. of Test for xperience of Employees ( Std for Meth of Recording and a Torsional Wire Apparatus (1972) ALCA E/ Std. Meth. for Std. Meth. of Test for Water Soluble Salts in Pigments by Molding Compounds (1972) Std. Meth. of Rec Pract for Test. Machines for iques (1970) ANSI Ni10

Im Carriers (Aperture, Camera, Copy, and / Sid. Meth. for ca E4

Std. Meth. for Std. Meth. for

r Measuring Residual C/ 971)

Std. for Methylene Blue Meth. for Std. Meth. for

Cores (1974) Std. Rec. Pract for

osure Liners (1973)

1973)

hin Equipment Rooms (Noise) (1973)

Std. Test. Procedure for
Sid. Meth. for Sid. Meth. for Rec. Pract for Std. for Meth. of id. Moisture Measurement

Diagrams (1972)

Std Symbols and Definitions fo aded Fasteners Used in Automotive and Related In/ for Use in Automotive and Related Industries (1/ crews (1973)

i)

rth Stations (1973) Std. for Std. for Std. for trol, and Maintenance of Heating, Ventilating, C/ Uniform liances, and Wall Furnaces, Floor Furnaces, and ms) EEI MSJ-4

e Hazards and Protection for Maintenance and Inspection of Std. for Operation and Flow Process Charts and Symbols Trial Std. for Screw Threads for Metric Serie e in Metal Heads Used in Roadway Lighting Equip/

n Metal Heads for Roadway Lighting Equipment (1/ Std. for Std. Meth. of Tension Test, of Carbon Graphite

ric Vulcanizates Under Compressive or Shear Strains by the Std. for Safety for

Compressive or Shear Strains by / Std. Meth. of Test for

d. Rec. Pract. for Effects of High Energy Radiation on th f Fuel Element Cladding Including the Determination of the f SAE Wrought Aluminum A/ Std. for Chemical Compositions,
r Merchant Quality Hot Rolled Carbon Steel Bars Subject to Test Meth. for Shive Content of Test Meth. for Shive Content of Test Meth. for Shive Content of Uniform Fire Code: Safety Rec. for

for Access Control System Units (Electronic / Electrical Rec. Pract, for Special Quality High Tensile, Hard Drawn
Measuring Color of Paper and Paper Board by Hunter L, a Measuring Color of Paper and Paperboard in CIE Y; X, Y Measuring Dimensions of Rigid Rods and Tubes Used for $\mathrm{E}$ Measuring Equipment (DME) Operating Within the Radio Fr Measuring Fast Neutron Flux by Radioactivation of Alumi Measuring Fast Neutron Flux by Radioactivation of Iron Measuring Fast Neutron Flux by Radioactivation of Nicke Measuring Fast Neutron Flux by Radioactivation of SuIfu Measuring Fast Neutron Flux for Analysis for Barium 140 Measuring Flat Spring Test for Hot Tack of Wax Polymer Mcasuring Flow Rates of Thermoplastics by Extrusion Pla Measuring Frequency and Cost, and Recording Patron and Measuring Hall Mobility and Hall Coefficient in Extrins Measuring Heat Transfer Rate Using a Thermal Capacitanc Measuring Heat Transfer Rate (1972)

Measuring Hydraulic Lift Force Capacity on Agricultural Measuring Hydraulic Lift Force Capacity on AgricuItural Measuring Inside Diameters of Composite Cans, Tubes and Measuring Lengths or Heights of Composite Cans, Tubes, Measuring Low Temperature Stiffening of Rubber and Rubb Measuring Neutron Flux by Radioactivation Techniques (I Measuring Plasma Arc Gas Enthalpy by Energy Balance (I9 Measuring Plasticity Retention Index (PRI) of Natural R Measuring Procedures for Bulk Shipments and Transfers O Measuring Recorded Flux of Magnetic Sound Records at Me Measuring Residual Chemicals in Films, Plates, and Pape Measuring Resistivity of Silicon Slices with a Collinea

Measuring Shrinkage from Mold Dimensions of Molded Plas Measuring Slope Efficiency and Lasing Threshold of Ruby Measuring Soil Removal and Reflectance Retention of Fab Measuring Squareness or Rectangularity of a Sheet of $\mathrm{Pa}$ Measuring Surface Frictional Properties Using the Briti Measuring System (1972)

Measuring Template (Cereal Chemistry) (1962)

Measuring the Density of Smoke from the Burning or Deco Measuring the Off-The-Job Disabling Accidental Injury E Measuring the Relative Stiffness of Leather by Means of Measuring the Specific Resistance of the Leachate of Th Measuring the Spiral Flow of Low Pressure Thermosetting Measuring the Uniformity of Passenger Car Tires (1972)

Measuring the Wear Life and Bonded Solid Film Lubricant Measuring Thermal Neutron Flux by Radioactivation Techn Measuring Thickness of Buildup Area on Unitized Microfi Measuring Thickness of Leather Test Specimens (1970) Al Measuring Thickness of Leather Units (1970) ALCA E3 Measuring Thiosulfate and Silver Densitometric Meth. Fo

Measuring Transmission Performance of Telephone Sets (1 Measuring Ultrasonic Velocity in Materials (1973)

Measuring Wall Thicknesses of Composite Cans, Tubes and Measuring Warpage of Ceramic Tile (1973)

Measuring Water Vapor Transmission Through Screw Cap CI

Measuring, Mixing, Transporting, and Placing Concrete

Measuring, Recording and Specifying Machinery Sound Wit (Meat and Its Products) (1972)

Mechanical and Acoustical Elements as Used in Schematic Mechanical and Quality Requirements for Externally Thre Mechanical and Quality Requirements for Machine Screws Mechanical and Quality Requirements for Steel Machine S Mechanical and Quality Requirements for Steel Nuts (197

Mechanical Characteristics of Antennas for Satellite Ea Mechanical Code (Requirements for the Installation, Con Mechanic al Code: Requirements for Vented Decorative App Mechanical Demand Registers (Cumulative and Pointer for Mechanical Drive Turbines (1974)

(Mechanic al Engineering) (1972)

Mechanical Fasteners (1974)

Mechanical Interchangeability of Socket Supports for U

Mechanical Interchangeability of Socket Supports Used I

Mechanical Materials (1971) ANSI K90.6

Mechanical Oscillograph (1972) ANSI J2.17

Mechanical Power Transmission Apparatus (1972)

lof Elastom

Mechanical Properties of Elastomeric Vulcanizates Under
Mechanical Properties of Metallic Materials (1962) ANSI

Mechanical Properties (1972) ANSI N147 / Examination O

Mechanical Property Limits and Dimensional Tolerances $\mathrm{O}$

Mechanical Property Requirements (1972)

Mechanical Pulp (Laboratory Flat Screen) (1973) Std. Spec. Fo

Mechanical Pulp (Somerville Fractionator) (1973)

Mechanical Pulp (Von Alfthan Shive Analyzer) (1973)

Mechanical Refrigeration (1973)

Mechanical Refrigeration (1974)

Mechanical Security Surveillance System and Equipment)

Mechanical Spring Wire and Springs (1972)

Mechanical Springs (1971) ANSI G54. I

RTC

ASTM

T527SU

ASTM

ASTM

ASTM

ASTM

TAPPI

ASTM

ANSI

ASTM

ASTM

St ASTM

ASAE

SAE

CCTI

CCTI

ASTM

ASTM

ASTM

ASTM

NSPA

IEEE

ANSI

ASTM

ASTM

ASTM

ASTM

TAPPI

ASTM

SAE

AACC

ICBO

ANSI

ASTM

ASTM

ASTM

SAE

ASTM

ASTM

NMA

ASTM

ASTM

ANSI

IEEE

ASTM

CCTI

ASTM

STM

ACI

ARI

ASAE

ANSI

SAE

$\mathrm{SAE}$

IFI

SAE

EIA

ICBO

ICBO

NEMA

668

E266

E263

E264

E265

E393

T683SL

D1238

Z108.1

E457

E459

S349

283

CT 102

CT104

E341
D3194

*4

347

PH4. 8

F84

D955

F379

D3050

E303

J375 A

UBCS

216.3

D2821

D2448

D3123

J $332 \mathrm{~A}$

2981

MS 9

D1813

D1814

$\mathrm{PH} 4.8$

269

E494

CT101

C485 
National Bureau of Standards KWIC Index of Engineering Standards

ec. for Steel Wire, High Tensile Strength, Hard Drawn, for Rec. Pract. for 73) ANSI G60.1 $0.48 \mathrm{Mo}-0.09$ $3-0.49 \mathrm{C}))(1973)$ $0.07 \mathrm{~V}(0.41-0.46 \mathrm{C})$

Std. Meth. and Definitions for Spec, for Steel Bars, Forgings, Rings, and Spec. for Steel Bars, Forgings, and Spec. for Steel Bars, Forgings, and Std. Spec. for Seamless Stainless Steel Std. Spec. for Welded Stainless Steel Std. Spec. for Seamless Carbon and Alloy Steel $\mathrm{i}-0.30 \mathrm{Cr}-1.8 \mathrm{Ni}-0.4 /$ Spec. for Steel Bars, Forgings, equirements for the Construction, Care, and Use of Shears (1974) Std. for Bulbed Spindle Std. for Bulbed

\section{4)} ivet (1974) Std. for Self Plugging, Std. for Bulbed Std. for Bulbed Std. for Bulbed Actuated Fire Alarm Devices, Single and Multiple Station, ANSI Al/ Std. for Drinking Fountains and Self Contained, of Instruction) (1972) Std. for Training of Automotive f Instruction) (1972) Std. for Training of Automotive
Std. for Training of Automotive Safety Std. for

tegrity and Test Spec. for Selected Brachytherapy Sources roid Radioiodine Uptake Measurements Using a Neck Phantom Std. Dimensions for

td. Color Marking of Compressed Gas Cylinders Intended for ec. for Waste Disposal of Phosphorus-32 and Iodine-131 for Test Meth. for Filler Content of Phenol, Resorcinol, and t Meth. for Nonvolatile Content of Phenol, Resorcinol, and Std. Meth. of Test for Apparent Viscosity of Hot Rec. Pract. for Flexibility Determination of Hot -1.1Ti, Vacuum Induction Plus Vacuum Consumable Electrode $-3.0 \mathrm{Ti}-1.4 \mathrm{~A} 1$ ) Consumable Electrode or Vacuum Induction Spec, for Steel Welding Wire, Vacuum

20C), Premium Bearing Quality, Consumable Electrode Vacuum $.09 \mathrm{~V}(0.17-0.23 \mathrm{C})$, Premium Quality, Consumable Electrode $-3.0 \mathrm{Ti}-1.4 \mathrm{Al}$, Consumable Electrode or Vacuum Induction $-3.0 \mathrm{Ti}-1.4 \mathrm{Al}$, Consumable Electrode or Vacuum Induction cereal Chemistry) (1967) al Chemistry) (1967) Capillary Meth. of Analysis of Wiley Meth. of Analysis of td. Spec. for Primary Columbium Metal for Consolidation or

Uniform Building Code Std. for Structural Glued Built Up . for Preservative Treatment of Structural Glued Laminated nents, Timber, Preservative / Uniform Building Code: Wood Tent. Rec. for Concrete mbined (1973) Tent. Rec. for Concrete
ding Code Std. for Wood backing Board (1) Uniform Building Code Std. for Wood Design Tables for Reinforced Brick Masonry Flexura Std. for Spec. and Design of Cold Formed Steel Structural ss Variation or Stress Reversal Design of Structural Steel sign of Light Gauge Cold Formed Stainless Steel Structura (1) Spec, for Design of Aluminum Alloy Load Carryin ion Design Handbook (Beam, Slab, Footing, Column and Other Rope Lay Stranded Copper Conductors Having Bunch Stranded for Capillary Moisture Relationships for Soils by Pressure Measurement of Airborne Asbestus Fiber by the r Determining Water Quality for Subsurface Injection Úsing nsi A37.87 Std. Meth. of Test for Liquid Flow Rate of Spec for Fire Barnec. for Liquid Spec, for Fire Barrier Gypsum Board oated Felts, Prepared Roofings, and Plast/ Spec. for Roof rmance Requirements for Woven Washable Uniform Fabrics for rmance Requirements for Woven Washable Uniform Fabrics for

Safety Std. for Surveillance Cameras for Banks and e, and Distillate Fuels (Potentio/ Std. Meth. of Test for 14.124 ical 0,0-Dimethyl Phosphorodithioate S-Ester with 4st Meth. for Evaluating Welting Agents in Caustic Soda for nformation Flow Used in Ordering, Shipping, and Billing of o Mechanical Property Requirements (1972)

Std. Spec. for Std. Spec. for
Std. Meth. of ANSI H39.3 Std. for Acceptable Concentrations of Inorganic Std. Meth. of Test for Std. Meth. of Test for Low Quantities of Std. Spec. for Std. Spec. for pply Type (1971) Std. for $75 \mathrm{~W}$ att $\mathrm{H} 43$ d. Physical and Electrical Characteristics of 50 Watt, H46 of $40 \mathrm{Watt}, \mathrm{H} 45$ (1972) Std. Meth. for the Designation of Std. Page Format of the Construction Std. Spec. for Photographic Grade Sodium Std. Spec. for Photographic Grade Potassium lding Code Std for Metal, Wire and Wire Fabric Laths, and Std. Meth. of Test for Cleavage Strength of Metal to
Mechanical Springs (1973)

Mechanical Stop Lamp (Light) Switch (1972)

Mechanical Test. of Wrought and Cast Steel Products (19

Mechanical Tubing (Annealed) $(0.48 \mathrm{Cr}-8.0 \mathrm{Ni}-4.0 \mathrm{Co}-$

Mechanical Tubing $(1.05 \mathrm{Cr}-0.55 \mathrm{Ni}-1.0 \mathrm{Mo}-0.12 \mathrm{~V}(0.4$

Mechanical Tubing $(1.62 \mathrm{SI}-0.82 \mathrm{Cr}-1.82 \mathrm{Ni}-0.40 \mathrm{Mo}-$

Mechanical Tubing (1971) ANSI G62.1

Mechanical Tubing (1973)

Mechanical Tubing (1973) ANSI G62.4

Mechanical Tubing, and Flash Welded Rings (1.3Mn-1.5S

Mechanical, Hydraulic, Pneumatic, Semi and Automatic,

Mechanically Locked Protruding Head Blind Rivet (1974)

Mechanically Locked Spindle Protruding Head Blind Rivet

Mechanic ally Locked Spindle Rivet (1972)

Mechanically Locked Spindle Self Plugging Blind Rivet (

Mechanically Locked Spindle 100 Deg. Flush Head Blind F

Mechanically Locked Spindle 100 Deg. Flush Head Blind R

Mechanically Operated Type for Indoor Use (1973)

Mechanic ally Refrigerated Drinking Water Coolers (1973)

Mechanics for Buses, Heavy Trucks and Trailers (Course

Mechanics for Passenger Cars and Light Trucks (Course O

Medical and Dental Equipment (1972)

(Medical Radiation-Radiology) (1973)

Medical Radiation-Radiology) (1973)

Medical Radiographic Sheet and Roll Films (1973)

Medical Use in the United States (Safety) (1973)

Medical Users (1951)

Melamine Adhesives (1970)

Melamine Adhesives (1970)

Melt Adhesives and Coating Materials (1973)

Melt Adhesives by Mandrel Bend Test Meth. (1972T)

Melted Solution Heat Treated (1973)

Melted 1825-I900 Deg. F (996.I.1037.8 Deg. C) Solution.

Melted $(0.95 \mathrm{Cr}-0.20 \mathrm{~V}(0.28-0.33 \mathrm{C})$ ) (SAE 6130) (1973

Melted (1973)

Melted, Annealed (I973)

IStant, 14

C $\mathrm{C}-4.0 \mathrm{Mo}-1.2 \mathrm{~V}(1.10-1$. / $\mathrm{R}-9.0 \mathrm{Ni}-4.5 \mathrm{Co}-1.0 \mathrm{M}_{0}-0$

Melted, 1975 Deg. F (1079.4 Deg. C) Solution Heat Treat

Melted, 1975 Deg. F (1079.4 Deg. C) Solution, Stabiliza

Melting Point of All Normal Animal and Vegetable Fats (

Melting Point of Normal A nimal and Vegetable Fats (Cere

Melting (1964) ANSI Z179.17

Members-Plywood Stressed Skin and Curved Panels, Beam

Members and Laminations Before Gluing of Southern Pine,

Members and Their Fastenings (Lumber, Plywood and Compo

Members Prestressed with Unbonded Tendons (1966)

Members Subjecied to Both Flexural and Axial Loading Co

Members to Receive Screw Attached Gypsum Wallboard and

Members (Tech. Notes) (1967)

Members (1973)

Members (1973)

Uniform Building Code

Uniform Building Code Std. for Stre

$/ \mathrm{Rm}$ Building Code Std. for Spec. and De

mbers (1973)

luminum Construction Manual Sect.

Members) (1973)

Members, for Electrical Conductors (1971)

Membrane Apparatus (1972)

Concrete Construct

Membrane Filter Meth. (Textile) (1971)

Membrane Filters (Petroleum Industry) (1973)

Std. Met? of Test

Membrane Filters (1972)

Membrane Forming Compounds for Curing Concrete (1973) a

Membrane (I972T)

Membranes; Aggregates, Saturated Felts, Saturated and C

Men and Women (Heavy Duty) (Institutional Textile) (197

Men and Women (Institutional Textile) (1973)

Mercantile and Bank Safes and Vaults (I971) ANSI Se 2.1

Mercantile Premises (1973) ANSI Se2.5

Mercaptan Sulfur in Gasoline, Kerosine, Aviation Turbin

(Mercaptomethyl).2-Methyoxy-? ${ }_{2}-1,3,4-$ Thiabiazolin -5

Mercerization in Cotton Yarns and Fabrics (1971) ANSI L

Mercerization (1971) ANSI L14.79

Merchandise Within the Hardgoods (Hardware) Industry (1

Merchant Quality Hot Rolled Carbon Steel Bars Subject T

Merchant Quality Hot Rolled Carbon Steel Bars (1973)

Mercurous Nitrate Test for Copper and Its Alloys (1973)

Mercury and Nonalkyl Organo Compounds (1972)

Mercury in Liquid Chlorine (1973)

Mercury in Paint (1973)

Mercury Lamp Reference Ballasts (1971)

Mercury Lamp Transformers, Constant Current (Series) Su

Mercury Lamps (1972)

Mercury Lamps (1972)

Mercury Lamps: Physical and Electrical Characteristics

Message (1974)

Meta-Bisulfite $\mathrm{Na}_{2} \mathrm{~S}_{20} 0_{3}$ (1972)

Metabisulfite $\mathrm{K}_{2} \mathrm{~S}_{2} 0_{5}$ (1972)

Metal Accessories (Designed for Use as a Plaster Base)

Metal Adhesive Bonds (1972) ANSI Z197.16
Construct ACI
Spec. for ASTM

SAE

ASTM

SAE

$S A E$

SAE

ASTM

ASTM

ASTM

SAE

ANSI

NSA

NSA

NSA

NSA

NSA

UL

ARI

ANSI

ANSI

ANS

ANSI

ANSI

CGA

NCRP

Std ASTM

ASTM

ASTM

SAE

SAE

SAE

SAE

$S A E$

SAE

AACCH

$\mathrm{AACCH}$

ASTM

ICBO

$A$ WPA

ICBO

ACI

ICBO

ANSI

BIA

ICBO

ICBO

ICBO

Meth Fo NACE

ASTM

ASTM

ASTM

NRCA

ANSl

ANSI

UL

UL

ASTM

ANSI

AATCC

Te AATCC

NWHA *

ASTM

ASTM

ASTM

ANSI

ASTM

ASTM

ANSI

ANSI

ANSI

St ANSI

ANSI

CSI

ANSI

ANSI

ICBO

ASTM

A679

J 249

AMS6540B

AMS6432A

AMS6416A

A511

5554

A5 19 
Std. Meth. of Test for Strength Properties of Metal to Std. Rec. Pract. for Conducting Creep Tests of Metal to Meth. of Test for Pressure in Air Handling Spec. for Sheet

amination of a Cross Secti/ ion Service (1971) ANSI G36.49 Spec. for Mild Steel Electrodes for Gas Std. for Manual Gas Std. Spec. for White S by Polar/ Std. Rec. Pract. for Making Reference Glass. 73) Std. Meth. for Spectrochemical Analysis of Admiralty Std. Spec. for Composition Bronze or Ounce de for Evaluating the Effect of Solar Radiation on Outdoor Std. Filler Std. for Std. for Flexible Connectors of Other Than All Spec, for

e Pivot (1973)

Std. Spec. for Centrifugally Cast Dual

dlock Strikes (1973) Spec. for $13 / 4$ In. Thick Hollow Spec. for Hollow

azardous Dust Locations (Agricultural Plastics, Chemicals,

azardous Dust Locations (Agricultural Plastics, Chemicals,

Std. Spec. for General Requirements for Wrought Precious meter), Citrus Style (1974)

$\mathrm{Mm})$ Diameter) (1973)

Rec. Industry Std. for Loose Rec. Std. for Loose Std. Meth. of Test.

nd Cable Installation (1973/

Rec. for Protection Against Molten Std. for Safety for Cellular

Std. Spec. for Primary Columbium

Std. Spec. for Zirconium Sponge and Other Forms of Virgin on Under the Uniform Building Code: Valley Flashing (Sheet dway Lighting Equipment (1973) NEMA SH10

dway Lighting Equipment (1973) EFI TDJ-140

r Mechanical Interchangeability of Socket Supports Used in echanical Interchangeability of Socket Supports for Use in Std. Spec. for Copper Alloy Strip for Flexible Std. Spec. for Remelted Lithium

Std. for Threaded Std. for Threaded

Uniform Building Code Std. for Welding Reinforcing Steel, Std. for Safety for Portable Specifying

Std. for Ductile Iron Pipe, Centrifugally Cast, in eth. for Measurement of Thickness of Transparent or Opaque Surfaces (1971) Spec. for 70) Std. for Electroplating of Nickel and Chromium on bolted Joints, Drift Bolts, and Wood and Lag Screws; Light Std Spec. for Iron Copper Sintered Std. Meth. of Test for Apparent Density of for Flexible Steel and Aluminum Conduit for Safe Use as A ) ANSI C33.50

Std. for Safety for Surface Thyristor Power Supplies for Guide Spec. for Sheet tics by Po/ Std. Rec. Pract. for Making Reference Glass of Test for Slump of Face Glazing and Bedding Compounds on old Heading Quality Carbon Steel Wire for Tapping or Sheet Std. Spec. for Thermostat Architectural Sheet

are (1970) ANSI D14.9 Std. Meth for Corrosion Test on eth. for Preparation and Use of Bent Beam Stress Corrosion Std. Spec. for Primary Hafnium act. for Meth. of Determining Plastic Deformation in Sheet for Density and Interconnected Porosity of Sintered Powder 8 in Panel Ceilings (1973/ Std. Rec. Pract. for Preparation of
Uniform Building Code Std. for initions of Terms Relating to Porcelain Enamel and Ceramic Glossary of Architectural h. for Electrostatic Clinging of Textile Fabrics Fabric to nsi N144 Std. Meth. for Calibration of Refractory Brick and Tile

hear) (197)

Std Meth. of Test for Cleavage Sirength of Std. Meth. of Test for Strength Properties of Std. Rec. Pract. for Conducting Creep Tests of creep Properties of Adhesives in Shear by Tension Loading p Properties of Adhesives in Shear by Compression Loading ength Properties of Adhesives in Shear by Tension Loading ives in Shear by Tension Loading at Elevated Temperatures

rials) (1974) ANSI Z221.]

ion Manual, Sect. 5) (1971)

Std Spec for Zinc

Std. Spec. for Electrolytic Manganese Spec, for Brazing Filler

Meth. for Chemical Analysis of Ferrochromium and Chromium
Metal Adhesives by Compression Loading (Disk Shear) (19 Metal Adhesives (1972) ANSI Z197.22

Metal Aerosol Containers (1972)

Metal and Air Conditioning Contractors (1973)

Metal and Oxide Coating Thicknesses by Microscopical Ex

Metal Arc Welded Steel Pipe for High Pressure Transmiss

Metal Arc Welding (1969) ANSI W3.18

Metal Arc (Gma) Welding Equipment (1974)

Metal Bearing Alloys (1973) ANSI H39.1

Metal Butt Seals and Test. for Expansion Characteristic

Mctal by the Cast Pin and Point to Plane Techniques (19

Metal Castings (1974)

Metal Clad Switchgear (1971) ANSI C37.24

Metal Comparison Charts (1971)

Metal Connectors for Gas Appliances (1967)

Metal Construction for Gas Appliances (1971)

Metal Cutting Machine Tool Weldments (1971)

Metal Cylinders (1972)

Metal Door and Frame Preparation for Offset Intermediat

Metal Door Frame Preparation for 181 and 190 Series Dea

Metal Dusts) (1973)

Metal Dusts) (1973)

Std. for Area Classification in $\mathrm{H}$ ISA

Metal Electrical Contact Materials (1972)

Metal End Dimensions of Composite Cans (211 (66 Mm) Dia

Metal End Dimensions (6 Ounce Fibre Citrus Cans 202 (52

Metal Fasteners in Wood (1974)

Metal Fires and Explosions (1973)

Metal Floor Raceways and Fittings for Electrical Wire a

Metal for Consolidation or Melting (1964) ANSI Z179.17

Metal for Nuclear Application (1973) ANSI N12.1

Metal for Roof Waterproofing) (1973)

Metal Head and Reflector Interchangeability Used in Roa

Metal Head and Reflector Interchangeability Used in Roa

Metal Heads for Roadway Lighting Equipment (1973) EEI T

Metal Heads Used in Roadway Lighting Equipment (1973) N

Metal Hose (1973)

Metal in Ingot Form (1972) ANSI H43.1

Metal Industrial V-Grooved Wheel Std. (1966)

Metal Insert-Heavy Duty (1973)

Metal Insert, Light Weight (1973)

Metal Inserts and Connections in Reinforced Concrete $C_{0}$

Metal Ladders (1974)

Metal Louvers, Stationary (1967)

Metal Molds or Sand Lined Molds for Gas (1971)

Metal Oxide and Metallic Coatings by Double Beam Interf

Meral Oxide Waterproofing for Below Grade Wall and Floo

Metal Parts-Automotive Ornamentation and Hardware (19

Metal Plate Connected Wood Truss Design; and Nails and

Metal Powder Structural Parts (1970) ANSI G63.1

Metal Powders (1948) ANSI H9.1

Metal Raceway for Wire and Cable Installation (1973) an

Metal Raceways and Fittings for Electrical Wiring (1973

Metal Rolling Mill Auxiliary Drives (1972)

Metal Roofing (1971)

Metal Sandwich Seal and Test. for Expansion Characteri

Metal Sash (1974)

Metal Screws (1971) ANSI G54.17

Metal Sheet and Strip (1973) ANSI Z155.19

Metal Spec. (1973)

Metal Specimens for Engine Antifreezes Tested in Glassw

Metal Specimens (1973)

Metal Sponge and Other Forms (1967) ANSI N 134

Metal Stampings (1972)

Metal Structural Parts and Oil Impregnated Bearings (19

Metal Surfaces for Adhesives Bonding (1967) ANSI Z197.2

Metal Suspension Systems for Acoustical Tile and for La

Metal Systems (1973) ANSI Z167.1

Metal Terms (1973)

Metal Test) (1973)

Metal Thermocouples Using an Optical Pyrom ter (1972)

Metal Tied Walls (Tech. Notes) (1966)

Metal to Metal Adhesive Bonds (1972) ANSI Z197.16

Metal to Metal Adhesives by Compression Loading (Disk S

Metal to Metal Adhesives (1972) ANSI Z197.22

Metal to Metal) (1969)

Metal to Metal) (1969)

Metal to Metal) (1972) ANSI Z197.15

(Metal to Metal) (1972) ANSI Z197.5

Metal Toilet Partitions (1972)

Metal Waste (Trash, Garbage) Cans (For Combustible Mate

Metal Work in Building Construction (Aluminum Construct

Metal Working Fluids and Related Materials (1973)

Std. Test Meth. for ASTM

1. of Test for Str

Roperties of Adhes

(Slab.Zinc) (1970) ANSI H24.

Metal (1969) ANSI G84.1

Metal (1969) ANSI W3.8

Metal (1971T)

UL

ASTM

ASTM

ICBO

EEM

NEMA

EEI

ASTM

ASTM

CFTM

NSA

ICBO

UL

CSI

ASTM

CSI

SAE

ICBO

ASTM

ASTM

UL

UL

NEMA

CSI

ASTM

Std. M ASTM

ASTM

UL

AA

ASTM

ASTM

ASTM

ASTM D2182

ASTM D1780

ASTM D3074

SMACN *3

B487

AWS A5.18

NSA 959

ASTM B23

ASTM F140

ASTM E486

ASTM B62

144

ANSI Z21.45

D14.2

ASTM A667

ANSI Al15.12

ANSI A115.5

$\$ 12.10$

S12.11

B476

E211.1

E202.1

ASTM D1761

10.8

$10 \cdot 8$
209

B383

B349

UBC*8.11

TDJ-140

$\mathrm{SH} 10$

SH13

TDJ-143

B508

1395

1394

BC $526-8$

184

15851

A21.52

B588

07140

$\mathrm{J} 207$

UBCS25. 17

B222

B212

1

PV 2

07610

F144

D2376

ASTM A548

ASTM B388

SMACN *1

ASTM D1384

G39

B414

SAE J863C

ASTM B328

ASTM D265!

ICBO UBCS47.16

C286

NAAMM *35

AATCC 115

DIA

ASTM Dl062

DSTM D2182

ASTM D1780

D2294

D2293

D1002

D2295

ASTM D2295 
Stds. for lndustrial Perforated Std. Spec for Nuclear Grade Plutonium Meth. of Test for Coefficient of Friction, Yarn to Std. Meth. of Test for Adhesion of Vulcanized Rubber to 1 Self-Locking Nuts, High Strength, Prevailing Torque, All esistant Alloy Nuts, High Strength, Prevailing Torque, All ear and Radio Chemical Analysis of Nuclear Grade Plutonium Std. Spec. for Chromium

Std. Meth. of Test for Plastic Strain Ratio R for Sheet d. Meth. for Chemical Analysis of Ferrosilicon and Silicon ract. for Test. Primers and Primer Surfaces Over Preformed resistance of Organic Coatings When Applied to Coil Coated Std for Safety for Septic Tanks, Bituminous Coated tigue Properties of Adhesives in Shear by Tension Loading perties of Adhesives in Shear by Tension Loading (Metal to ies of Adhesives in Shear by Compression Loading (Metal to ns for Cores for Photographic Film Rolls (Plastic, Wood or perties of Adhesives in Shear by Tension Loading (Metal to hear by Tension Loading at Elevated Temperatures (Metal to operties of Adhesives in Shear by Tension Loading (Metal/ Specifying Duct, Sheet iform Building Code: Veneer (Brick, Concrete, Stone, Tile, $s$ (Designed for Use as a / Uniform Building Code Sid. for Guide Spec. for Flagpoles

and Heat Resistant, High Strength, Prevailing Torque, All 1970E) and Heat Resistant, High Strength, Prevailing Torque, All ANSI B93.36 Std. Meth. of Test for ldentification of Std. Groove Dimensions for Floating Type Std. Spec. for Installation for Non ct. for Recording Data from Atmospheric Corrosion Tests of ment of Thickness of Transparent or Opaque Metal Oxide and Std. Meth. of Test for Adhesion of Std. Definitions of Terms Relating to Uninsulated Tent. Meth. of Test for Sheet Resistance of Thin aximum Allowable Operat/Std. for Small Manually Operated Safety Std. for Flexible Std. Meth. of Compression Test. of $s$ of High Energy Radiation on the Mechanical Properties of Std Meth of Tension Test. Of Std. Meth. for Notched Bar Impact Test. of Std. Rec. Pract. for Scleroscope Hardness Test. of Std. Meth. of Test for Vickers Hardness of

Std. Test Meth. for Plane Strain Fracture Toughness of Std. Meth. of Test for Brinell Hardness of for Rockwell Hardness and Rockwell Superficial Hardness o Control of External Corrosion on Underground or Submerged

for Condensation-Humidity Test. of Organic Coatings on f Paint, Varnish, Lacquer, and Related Organic Coatings on td. Meth. for Assigning Alloy Phase Designations (Code) in ansi C 33.98 Std. for Safety for Electrical nally Coated Galvanized Rigid Steel Conduit and Electrica for Measurement of Oxide Thickness on Silicon Wafers and nd Steel (1967)

Rec. Pract, for Std. Definitions of Terms Relating to

cluding Recommended Practice for Photography as Applied to Std. Definitions of Terms Used in Powde hotography As/ Sid. Meth. of Preparation of Micrographs of Std. Rec. Pract. for Codification of Certain Nonferrou H/ Sid. Meth. of Test for Apparent Density of Refractory (C) Test Meth. for Determination of Film Adhesion on Al $s$ and Other / Std. Analytical Meth. for Determining Heavy 956) Std. Analytical Meth. for Determining Heavy Std. Spec. for Brazing Filler

ct. for Experimental Test. for Biological Compatibility of 1972) ANSI Z11.317 Test Meth. for Laboratory Corrosion Test. of
Std. Meth. of Test for Trace Test Meth. for Heav Std. Rec. Pract. for Cleaning romiu/ Uniform Building Code Std. for Corrosion Resistant and Rockwell Superf/ Std. Hardness Conversion Tables for d. Rec. Pract. for Laboratory Immersion Corrosion Test. of Uniform Building Code Std. for Sheet Reagents, and Safety Precautions for Chemical Analysis of 1973) Rec. for High Temperature Molten Materials eas, Lentils, Rice, and Other Commodities For/ Electrical Std. for colored Clear Liquids Using the Hunterlab Color Difference Std. for Electrical Indicating Instrument Std. for Instrument Transformers for (Electric ity) p) Gas (1973) ANSI B 167.1

Std. for Safety for Std, for Cold Water Std. Spec. for Sound Level

Direct Reading Remote Registration Systems for Cold Water ound Data Acquisition System for Vehicle Noise Sound Level
Metal (1972)

Metal (1972) ANSI N136

IPA

ASTM

ASTM

Metal (1972T)

ASTM

Spec. for Stee SAE

Metal (1973)

Metal (1973)

Metal (1973)

Metal (1973)

SAE

Metal (1974)

/Ass Spectrometric, Spectrochemical, Nucl ASTM

ASTM

Metal (1974)

Mctal (1974)

Metal (1974)

Metal (1974) ANSl A162.1

(Metal / Metal) (1973)

Metal) (1969)

Metal) (1969)

Metal) (1971)

Metal) (1972) ANSI Z197.15

Metal) (1972) ANS1 Z197.5

Metal) (1973)

Metal, Low Pressure, Air Transmission (1967)

Metal, Plastic, etc.) for Ornamentation, Protection, or

Metal, Wire and Wire Fabric Laths, and Metal Accessorie

Metal, Wood and Fiberglass (1972)

Metal, 1200 Deg. F (649 Deg. C) Use, Unified J (MIL S.8

Metal, 1200 Deg. F (649 Deg. C) Use, Unified (MlL S-774

Metallic and Fibrous Contaminants in Aerospace Fluids (

Metallic and Nonmetallic Fluid Power Piston Rings (1972

Metallic Antimony (1972) ANSI H41.1

Metallic Building Sewers (1971)

Metallic Coated Steel Specimens (1972)

Metallic Coatings by Doub

Metallic Electrical Conductors (1972A)

Metallic Films with a Collinear Four Probe Array (1973)

Metallic Gas Valves in Gas Distribution Systems Whose M

Metallic Hose (1972) ANSI B140.1

Metallic Materials at Room Temperature (R1973)

Metallic Materials (1962) ANSl N145

Metallic Materials (1969) ANSl Z168.13

Metallic Materials (1972)

Metallic Materials (1972)

Metallic Materials (1972) ANSI Z115.7

Metallic Materials (1972) ANSI Z260.2

Metallic Materials (1973) ANS1 Z115.5

Metallic Materials (1974) ANSI Z115.6, Ashto T080

Metallic Piping Systems (1972)

Metallic Silhowette Match Rifle Range Plans (1973)

Metallic Surfaces (Coil) (1974)

Metallic Surfaces (Coil) (1974)

Metallic Systems Based on the Composition and Crystal L
Metallic Tubing for Wire and Cable Installation (1973)

Std. Rec. Pra

ASTM

it. Rec. P ASTM

NCCA

UL

ASTM

r Creep Pro ASTM eep Propert ASTM

Dimensio ANSI

Metallic Tubing (1974) 1. for Polyvinyl Chloride Exter

Metallization Thickness by Multiple Beam Interference (

Metallizing with Aluminum and Zinc Protection of lron a

Metallography (1973) ANSl Z30.4

Metallography) (1962) ANSI Z30.3

Metallurgy (1972) ANSI H9.5

Metals and Alloys (Including Recommended Practice for P

Metals and Alloys, Cast and Wrought (1973)

Metals and Compounds by the Scott Volumeter (1970) ANSI

Metals by Cross Hatch Tape Test After Reverse Impacting

Metals Content of Corn Sugar (Crude and Refined), Syrup

Metals Coutent of of Corn Syrup Unmixed and Dextrose (1

Metals for Electron Devices (1972)

Metals for Surgical Implants (1972)

Metals for the Process Industries (1972)

Metals in Gas Turbine Fuels (Atomic Absorption Meth.)(

Metals in Paper and Paperboard (1973)

Metals Prior to Electroplating (1968) ANSI G53.33

Metals (Electrodeposited Coatings of Copper, Nickel, Ch

Metals (Relationship Between Brinell, Vickers, Rockwell

Metals (1972)

Metals (1973)

Metals (1973)

(Metals, Slag, Glass, etc.) Fire Protection and Safety (

Metalworking Machine Tools (1973) ANSI C113.1

Meter Meth. of Analysis of Moisture in Grains, Beans, P

Meter (1972T)

(Meter) Relays (1972)

Metering Purposes, $15 \mathrm{kV}$ and Less (1973) NEMA Ei2l

Meters for Flammable Liquids and Liquefied Petroleum (L

Meters (Displacement Type $5 / 8$ ln. Through 6 In.) (1971)

Meters (1971)

Meters (1972)

Meters (1972) ANSI S6.I

$\mathrm{CSl}$

SAE

SAE

UL

ASTM

ASTM

ASTM

ASTM

ASTM

ASTM

ISt ASTM

CR

ASTM

NACE

ASTM

TAPPI

ASTM

CBO

ASTM

St ASTM

$1 \mathrm{CBO}$

ASTM

FMS

NEMA

EEI

UL

D429

AMS7252A

AMS7253A

C758

A481

E360

D3322

TB-11.17

70

D3166

D2294

D2293

$\mathrm{PH} 1.13$

D 1002

D2295

D3166

15836

CBC $3-30$

UBC 547.4

10350

AMS7250C

T3.19.11

ASTM B237

IAPMO 151

ASTM $\quad$ B5 58

ASTM B571

ASTM B 354

ASTM F390

ANSl Bl6.33

536

E9

E184

E23

E448

E92

E399

E10
E18

RP-01-69

$* 18$

TB.111. 6

TB.III-1

E157

797

RN1

F388

C2.2

E7

E2

B243

E2

B275

B329

TB-11-16

F.26

E-30

F106

F361

TM.01.69

D2788

UM-544

B322

UBC $332-6$

E140

G31

UBCS32-4

E50

LPD 7-33

79

CCH 44.11

ASTM E450 
Monoxide, Deuterium, Fluorine, Helium, Hydrogen, Krypton, Std. Spec. for Methyl Alcohol Std. for Std. for 0,O-Dimethyl Phosphorodithioate S-Ester Wi/ ethyl -2,4-Dodecadienoate) (Common Name for Th/ Std. for mon Name for Th/S Std. for Methoprene (lsopropyl (E,E)-11mon Name for the Pest Control Chemical S-Ethyl (E, E)-11Low Blank Meth. of Analysis of Meth. for Std. Spec. for

hate Solutions; lsopropyl Alcohol Saturated with Gasoline; Oils, and Fatty Acids (Cereal) ylate Esters (1972) Meth. for Preparation of Std. Meth. of Test for Std. Spec. for Std. Spec. for

ndicators (1971)

Std. Analytical Meth. for Determining (197) Sid. for Common Name for the Pest Control Chemical ,5,6-Trichloro-2-Pyridyl) Phosphorothioate Chlorpyrifos for Common Name for the Pest Control Chemical 4-Chloro5for the Pest Control Chemical 0.1,3-Dioxolan-2-Ylphenyl Std. Meth. of Test. Hydroxypropyl Std. Meth. of Test. dicators (1971) Std. Analytical Meth. for Determining er Densitometric Meth. for Measuring Residual C/ Std. for Std. Meth. of Test for Hydrogen Sulfide in Natural Gas st Control Chemical 2-(Ethylamino)4-(1sopropylamino)-6or the Pest Control Chemical 2,4-Bis (Isopropylamino).6t Control Chemical 2-(Tertbutylamino)-4-(Ethylamino)-6-

Phosphorodithioate S-Ester with 4-(Mercaptomethyl)-2. and Technical Work/ Rec. Pract. for Units (Measurement -

flat, and Hot Wound (Materials, Specs. Terminology, Gauge, tem of Units) (1972) ANSl Z210.1

roller Bearings) Conform/

Std. Shaft and Housing Fits for Std. for ln. and Std. for Gaging Practice for Trial Std. for Screw Threads for Std. for Rec. for Use of English and aerospace Std. Documents Preparation and Maintenance in Si 1972) Rec. Pract. for Rules for SAE U se of SI
Std. for Std. for Common Name for the Pest Control Chemical Ethyl atural Block Mica and Mica Films Suitable for Use in Fixed electric Capacitors (1971) / Std. Spec. for Natural Block $27 \quad$ Std. Spec. for Natural Muscovite Block Std. for Molded and Dipped Std. Meth. of Test for Undersized Loose Muscovite al Chemistry) (1962)

caroni (Cereal Chemistry) (1962)

1968)

mistry) (1962)

Che mistry) (1962)

I Chemistry) (1962)

(Cereal Chemistry) (1962)

Std. Meth. of Test for Resistance Characteristics of sulfur in Light Liquid Petroleum Hydrocarbons by Oxidative nt. Meth. of Test for Sulfur in Petroleum Gas by Oxidation lndustry Std. for Distribution and $70 \mathrm{~mm} 100 \mathrm{Ft}$. Spools for Recording Instruments and for rds) (1972)

esion of Protection Sheet to Aperture Adhesive of Unitized

Meth. for Measuring Thickness of Buildup Area on Unitized lndustry Quality Std. for Computer Output Drafting Guide for

ndustry Quality Std. Format and Coding for Computer Output r Storage of Processed Safety Photographic Film Other Than tion and Quality Control of First Generation Silver Habide lndustry Std. for Fascimile Transmission of

17

Std. for Flow chart Symbols and Their Usage in

Practice for Photography As/ Industry Std. Glossary of Std. Meth. of Preparation of

Std. Spec. for Grading of Abrasive Std. Meth. of Measurement of Std. Meth. of Test for

d. Meth. of Test for Vapor Pressure of Petroleum Products

Sid. Rec. Pract. for Description and Performance of the h. of Test for Diameter of Wool and Other Animal Fibers by al Oxide and Metallic Coatings by Double Beam Interference try) (Bolting Cloth, Filter Paper, Wide Field and Compound cereal Chemistry) (1962)

eth. for Identification and Qualitative (Including Optical rganic Components in a Polished Specimen / Std. Meth. for al Components of Coal (1972)

Sid. Meth. for
Methane, Neon, Nitric Oxide, Nitrogen, Oxygen) (1973)

Methanol (99.85() (1972)

Methazole (Herbicide) (1971)

Methidathion (Common Name for the Pest Control Chemical

Methoprene (Isopropyl (E,E)-11-Methoxy-3,7,11-Trim

Methoxy-3,7,11-Trimethyl -2,4-Dodecadienoate) (Com

Methoxy-S-Triazine-Prometon (1973)

ID. Common

Methoxy-3,7,11-Trimethyl-2,4-Dodecadienethioate-Tri

Methoxychlor Residues in Foods (Cereal Chemistry) (1962

Methoxyl Content of Pulp and Wood (1972)

Methyl Alcohol (Methanol (99.850 (1972)

Methyl Blue and Green; Mineral Oil; Urease Tablets; Xan

Methyl Esters of Long Chain Fatty Acids in Common Fats,

Methyl Ether of Hydroquinone in Colorless Monomeric Acr

Methyl Isobutyl Carbinol (1972)

Methyl Isobutyl Ketone (1972)

Methyl Orange Indicator, $0.1 \%$ Content of Reagents and I

Methyl 5(2,4-Dichlorophenoxy)-2-Nitrobenzoate Bifenox

Methyl (1973)

/Pest Control Chemical 0,0 Dimethyl 0.(3

Methylamino) -2-(a,a,a- Trifluoro-m-tolyl) - 3 (2H)Pyri

Std. Common Name

Methylcellulose (1972)

Methylcellulose (1972) ANSI K65.26

Methylene Blue Indicator, $1 \%$ Content of Reagents and in

Methylene Blue Meth. for Measuring Thiosulfate and Silv

(Methylene Blue Meth.) (1970) ANSI Z77.10

Methylt hio)-S-Triazine-A metryn (1973)

(Methylthio)-S-Triazine-Prometryn (1973)

(Methvlthio)-S-Triazine-Terbutryn (1973)

Methyoxy -? 21,3,4-Thiabiazolin -5-One) (1973)

Metric and British-American) in Published Scientific

/Sion, Extension,

Metric Pract Guide to the Use of SI (International Sys

Metric Radial Ball and Roller Bearings (Except Tapered

Metric Radial Needle Roller Bearings (1973) ANSI B3.18

Metric Screw Threads (1972)

Metric Series Mechanical Fasteners (1974)

Metric Thread Fuel Injection Tubing Connections (1971)

Metric Units of Measurement (1973)

Metric Units (1973)

(Metric) Units (1972)

Mexacarbate (Common Name for the Pest Control Chemical)

Mhydroxy carbanilate Carbanilate (Ester) Desmedipham (19

Mica-Dielectric Capacitors (1971) ANSI C59.26

Mica and Mica Films Suirable for Use in Fixed Mica-Di

Mica and Thins Based on Visual Quality (1971) ANSl C59.

Mica Capacitors (Wire Lead Styles) (1972)

Mica Films Suitable for Use in Fixed Mica-Dielectric

Mica Splittings (1973)

Micro Kjeldahl Meth. of Analysis of Crude Protein (Cere

Micro Scale Meth. of Analysis of Semolina Quality in Ma

Micro Sedimentation Test for Wheat (Cereal Chemistry) (

Microbiological Meth. of Analysis of Niacin (Cereal Che

Microbiological Meth. of Analysis of Riboflavin (Cereal

Microbiological Meth. of Analysis of Vitamin B12 (Cerea

Microbiological Meth. of Analysis of Vitamin B6 Complex

Microcontacts (1972) ANSI Z55.15

Microcoulometry (1972T)

Microcoulometry (1973)

Microfiche of Documents (1972)

Microfilm and Still Picture Cameras (1972)

Microfilm Carrier (Aperture Card) (1973) ANSI PH5.14

Microfilm Carriers (Aperture, Camera, Copy and Image Ca

Microfilm Carriers (Aperture, Camera, Copy, and lmage C

Microfilm (1971)

Microfilm (1971)

Microfilm (197])

Microfilm (1971)

Microfilm (1972)

Microfilmed Documents (1972) ANSI C 16.45

Microfont Charts (1969)

Micrographics (Information Processing) (1973) ANSI PH5.

Micrographics (1971)

Micrographs of Metals and Alloys (Including Recommended

Microgrits (1972)

Microhardness of Electroplated Coatings (1973)

Microhardness of Materials (1973)

(Micromethod) (1971) ANSI Z11.277

Microphotometer (1971) ANSI Z128.31

Microprojection (1972) ANSI L14.143

Microseope Technique (1973)

(1973)

/Transparent or Opaque Met

Microscope, Petri Dish, Sieve, Separatory Funnel, Trap

Microscopic Meth. of Analysis of Smut in Wheat or Rye (

Microscopic) Analysis of Mineral Filler and Coating of

Microscopical Determination of the Reflectance of the $O$

Microscopical Determination of Volume Percent of Physic
Std. for National

ICBO

ASTM

ANSI

ANSI

ANSI

ANSl

ANS

ANSI

AACCH

TAPPI

ASTM

$A A C C H$

AACC

ASTM

ASTM

ASTM

CR

ANSI

ANSI

ANSI

ANSI

ASTM

ASTM

CR

ANSI

ASTM

for the PE ANSl
Name F ANSl

or the Pes ANSl

/Thyl ANSl

IEEE

SMl

ASTM

AFBMA

AFBMA

ANSl B1.16

SAE

ASAE

NSA

SAE

ANSI

for N ASTM

ASTM

ASTM

EIA

ASTM

ASTM

AACCH $46-13$

$\mathrm{AACCH} \quad 66-42$

AACCH $56-63$

AACCH 86-51

AACCH 86-72

AACCH 86.40

AACCH 86-31

ASTM B 326

Te ASTM D3246

NMA MS5

ANS

NMA

ANSI

NMA

NMA

NMA

NMA

Std. Practice Fo ANS

NMA

NMA

NMA

NMA

NMA

ASTM

ANSl

ASTM

ASTM

St ASTM

ASTM

ASTM

ASTM

$\mathrm{AACCH} \quad 28.90$

AACCH $\quad 42.70$

TAPPI T42I

ASTM D2798

ASTM D2790 
for Measurement of Metal and Oxide Coating Thicknesses by articulate Substances Of/

Std. Rec. Pract for Analysis by Std. Definitions of Terms Relating to meth. for Examination of W ater Formed Deposits by Chemical Std. Meth. of Test for Alkalinity of Hollow Glas of Test for Hydrostatic Collapse Strength of Hollow Glas

f Test for Bulk Density and Packing Factor of Hollow Glass tric Constant) of Solid Electrical Insulating Materials at ment of Potentially Hazardous Electromagnetic Radiation at

peres, 2 Pole,/ Std. Dimensions of Plugs and Receptacles, 5 Amperes, $3 \mathrm{Pl}$ Std. Dimensions of Plugs and Receptacles, Tapes (1974) Std. Meth. of Test for Oil eth. of Test for Diffusion Stain of Rubber and Contact and

5.1 ing (1969) ANSI W3.17

ANSI W 3.20

ansi W 3.18

r Measurement of Film Thickness of Nonmagnetic Finis al Application (1973) ANSI G50.1 1) ANSI L14.55 for the Evaluation of Carr-Price Meth. of Analysis of Vitamin A in Nonfat Dry for Fillers and Sealers of Single Service Containers for Sanitary Stds. for Uninsulated Tanks for itary Std. for Scraped Surface Heat Exchanges for Use with Sanitary Std. for Storage Tanks for Sanitary Std. for Silo Type Storage Tanks for nitary Stds. for Centrifugal and Positive Rotary Pumps for Meth. of Analysis of Lactose (Sugar) and Nonfat Dry Extensigraph Meth. for Quality of Nonfat Dry Meth. of Analysis for Ash in Dry

Meth. of Analysis of Crude Fat in Dry

Pract. for Instantizing Systems for Dry Milk and Dry aph Evaluation Meth. of Ability of Roller Dried Nonfat Dry Sanitary Std. for Farm

Weth of Analysis of Lactose

Meth. of Analysis for Titratable Acidity in Nonfat Dry Meth. of Analysis for Lactic Acid in Nonfat Dry Meth. of Analysis for Baking Quality of Nonfat Dry Meth. of Analysis of Moisture in Nonfat Dry harland and Ashworth Meth. of Analysis of Serum Protein in Rowland Meth. of Analysis of Serum Protein in nalysís of Undenatured Whey Protein Nitrogen in Nonfat Dry alysis of Vitamin A in Nonfat Dry Milk and lnstantized Dry Thyristor Power Supplies for Metal Rolling Std. for H Type

coil Coating) (1974)

Spec, for Detection of Spec. for Welding Industrial and $\mathbf{s}$ of Residual Urease Activity in Soybean Meals, Soy Flour, st Meth. for Colorfastness of Dyed Wool Fabric and Yarn to dahl Meth. of Analysis of Crude Protein in Wheat and Flour Std. Spec. for Tantalum lngots and Flat Steel Products Manual: Tin Std. for Dimensional Tolerances for Aluminum Std. Spec. for General Requirements for Tin Std. for Numerically Controlled Spar

blind, Internally Threaded, External Sleeve, Light Weight,
$962)$
$962)$

uring-Vertical (1973) Buhler Meth. of Experimenta Batch Meth. of Experimental Batch Meth. of Experimental Std. for Std. for

Std for Horizontal Boring, Drilling and Std. for Numerically Controlled Profiling and Contouring for Numerically Controlled Horizontal Boring, Drilling and Std. for Spindle Noses and Tool Shanks for Std. for

Meth. of Analysis of Acetone Peroxides in starches and Sugars, and to Most Other Products of the Wet in Corn Starch. Syrup and Sugar Obtained from the Corn Wet cereal Chemistry) (19/

Meth. for Experimental Wheat Flour Meth. of Experimental Wheat otection Against Betatron Synchrotron Radiations Up to 100 Fire Hazards and Protection Rec. for Textile Std. for High Speed Steel End

Safety Guide for Respiratory Protection Against Coal sulation (1973) IPCEA S68-516

Std. for

1973) Std. for Lumber, Timbers, Bridge and Concrete Due Tol Std. Meth. of Test for Effectiveness of Test Meth. for Amount of Coating on Meth. of Analysis of Calcium in

and Light Construction Buildings (1970) / Std. Spec. for the Determination of the Thermal Resistance of Low Density finishing Cement (1964) ANSl Z98.33
Microscopical Examination of a Cross Section (1973) Ans Microscopical Meth. for Particle Size Distribution of P Microscopy (1972)

Microscopy (1973)

Microspheres (1972)

Microspheres (I972)

Microspheres (I972)

Microwave Frequencies and Temperatures to $1650 \mathrm{C}(1970)$
Microwave Frequencies (1973) /Entation for the M

Microwave Transmission Systems (1972)

Midget Locking Type, Specific Purpose, 125 Volts, 15 Am

Midget Locking Type, Specific Purpose, 125/250 Volts, 1

Migration or Plasticizer Bleed Out of Preformed Sealing

Migration Stain of Organic Finishes in Contact with Rub

Mild Steel Covered Arc Welding Electrodes (1969) ANSI a

Mild Steel Electrodes and Fluxes for Submerged Arc Weld

Mild Steel Electrodes for Flux Cored Arc Welding (1969)

Mild Steel Electrodes for Gas Metal Arc Welding (1969)

Mild Steel (Coil Coating) (1974)

Mild to Medium Strength Carbon Steel Castings for Gener

Mildew and Rot (Fungicides) Resistance of Textiles (197

Milk and Dry Milk Products (1972)

Milk and Instantized Dry Milks (Cereal Chemistry) (1971

Milk and lts Fluid Products (Packaging Equipment) (1974

Milk and lts Products (Mixing and Storage) (1974)

Milk and lts Products (1973)

Milk and Its Products (1974)

Milk and lts Products (1974)

Milk and lts Products (1974)

Milk in Bread (Cereal Chemistry) (1962)

Milk in Physical Dough Tests (Cereal Chemistry) (1962)

Milk Products (Cereal Chemistry) (1962)

Milk Products (Cereal Chemistry) (1962)

Milk Products (I972)

Milk Solids to Change Mixing Characteristics of Flour (

Milk Storage Tanks (1973)

(Milk Sugar) in Mixed Feeds (Cereal Chemistry) (1962)

Milk (Cereal Chemistry) (1962)

Milk (Cereal Chemistry) (1962)

Milk (Cereal Chemistry) (1962)

Milk (Cereal Chemistry) (1962)

Milk (Cereal Chemistry) (1962)

Milk (Cereal Chemistry) (1962)

Milk (Cereal Chemistry) (1962)

Milks (Cereal Chemistry) (1971)

Mill Auxiliary Drives (1972)

Mill Chains-Attachments and Sprocket Teeth (1971)

Mill Chemical Treatments on Galvanized Steel Surfaces (

Mill Cranes (1970)

Mill Feeds (Cereal Chemistry) (1962)

Mill Fulling (I972) ANSl LI4.5

Mill Products (Boric Acid Modification) (Cereal Chemist

Mill Products (1970) ANSl Z179.14

Mill Products (1973)

Mill Products (1973)

Mill Products (1973)

Mill (1973)

Millable Head Self Locking) (1972)

Milling for Bread and Soft Wheats (Cereal Chemistry) (1

Milling for Durum Wheat (Cereal Chemistry) (1962)

Milling Machine-Tracer Controlled Profiling and Conto

Milling Machine Arbor Assemblies (1972)

Milling Machine (1974)

Milling Machine (1974)

Milling Machine (1974)

Milling Machines (1972)

Milling Machines (1972)

Milling Premixes (Cereal Chemistry) (1971)

Milling Process (1971)

Milling Process (1973)

/R Determination of Phosphorus

Milling: Temper Table (Cereal Chemistry) (1962)

Million Electron Volts (1954)

Mills (1974)

Mills, .125 Thru 3.000 Diameter (1973)

Mine Dust (1972)

Mine Power Cables with Ethylene-Propylene-Rubber in

Mine Tie Preservative Treatment by Pressure Processes

Mineral Admixtures in Preventing Excessive Expansion of

Mineral Coated Paper (1973)

Mineral Feeds (Cereal Chemistry) (I962)

Mineral Fiber Blanket Thermal Insulation for Wood Frame

Mineral Fiber Blanket Type Building Insulation (1970) a

Mineral Fiber Hydraulic Setting Thermal Insulating and

Mineral Fiber Loose Fill Thermal Insulation (I973)
ASTM

ASTM

ASTM

Std. ASTM

ASTM

itd. Meth. ASTM

ASTM

ASTM

ANS

ANSI

ANSI

ASTM

ASTM

AWS

AWS

AWS

NCCA

ASTM

A A TCC

DFISA

AACCI

DFISA 3200

San DFISA 3100

DFISA 0106

DFISA 2203

Sa DFISA 0206

AACCH 80-31

$\mathrm{AACCH}$ 54-12

$\mathrm{AACCH} \quad 08.11$

AACCH $30-16$

DFISA 60800

$\mathrm{AACCH} \quad 54.20$

DFISA 3000

A ACCH $80-30$

AACCH 02-31

$\mathrm{AACCH} \quad 04.26$

$\mathrm{AACCH} \quad 10.85$

$\mathrm{AACCH} \$ 4.52$

$\mathrm{AACCH} \quad 46.2 \mathrm{l}$

$\mathrm{AACCH} \quad 46.22$

Meth of a AACCH 46.20

AACCH $\quad 86.02$

NEMA PV 2

ANSl B29.14

NCCA TB-II-11

$\begin{array}{lll}\text { AWS } & \text { D 14.1 } \\ \text { AACCH } & 22.90\end{array}$

Te AATCC 2

AACCH 46-12

ASTM B364

Alsl

A NSl

A623

NSA 912

NSA 1674

AACCH $26-20$

AACCH 26-21

$\mathrm{AACCH} \quad 26-30$

NSA 914

ANSl B5.47

NSA

NSA

Std. NSA

910

ANSl B5.18

ANSl B5.45

$\mathrm{AACCH} \quad 48-05$

CR E.32

AACCH 26-10

A ACCH 26.95

NCRPM Rl4

FMS 7.1

NSA 986

ANSI Z88.4

NEMA WC $8 / 5$

AWPA C2

ASTM C441

TAPPI UM.542

A ACCH $\quad 40.26$

ASTM C665

ASTM C653

ASTM C449

ASTM C764 
Std. Spec. for Std. Spec, for Semidense r Asphalt Mastic for Use in Waterproofing (Asphalt Cement, nd Qualitative (Including Optical Microscopic) Analysis of si A37.41 Std. Spec. for Std. Meth. of Test for Sieve Analysis of Std. Spec. for Asphalt Insulating Siding Surfaced with Std. Spec. for Asphalt Roll Roofing Surfaced with il Circuit Breakers (1973) circuit Breakers (197/ Std. Spec. for Uninhibited Std. Spec, for Oxidation Inhibited Meth. of Analysis of Soluble Meth. of Analysis of Iodine in 210 Std. Meth. for Evaluation of White yl Alcohol Saturated with Gasoline; Methyl Blue and Green; Uniform Building Code Std. for e/ Meth. of Analysis of Niacin in Enrichment (Vitamin and spark T/ Std. Meth. for Spectrochmeical Analysis of Ores, Std. for Radiation Protection in Uranium Std. for Tires and Rims for Motorcycle, Side Car,

ervice (1974) Sid. for for Tires and Rims for Off the Road Vehicles: Earthmoving, g, Mining and Logging (Short Haul); Grader. Loader, Dozer, ment of Steady State Surface Pho/ Tent. Meth. of Test for Rec. Pract. for Motorc ycle Rear View ars, Trucks, Buses, Coaches, Tractors, Industrial Road and

ng, Cooling, Refrigeration Systems, Incinerators and Other

Pulls, Push and Kick Plates; Bolts; Closers; Hospital and Quality Stds. for

ng Trim, Casework, Panelwork, Closet and Storage Shelving, rface Water, Rigging Collaps/ Rec. for Protection Against Brick in Landscape Architecture Brick and Tile in Sanitation Structures, Photographic Films in Rolls for Recording Instruments and Meth. of Test for Tent. Meth. of Test for Air Content of Freshly
Uniform Building Code Std. for Ready Uniform Building Code Std. for Ready Meth of Analysis of Lactose (Milk Sugar) in analysis of Vitamin A and Carotene in Enriched Cereals and Rec. for Safe Use of ectrometric, and Spectrochemical Analysis of Nuclear Grade th. of Analysis for Original Ash in Phosphated and Freshly a3/ Std. Spec. for Requirements for Mixing Plants for Hot eveling, and Surface Courses (1967) Std. Spec. for Hot 73)

Truck

Concrete Plant

Meth. of Analysis for Total Carbon Dioxide in Prepared $\mathrm{rl}$ Meth. of Analysis of Reducing Sugars in Prepared Bakery ium Phosphate and/or Sodium Acid Pyrophosphate in Prepared

t Absorption Meth. of Analysis of Vitamin A in Dry Vitamin

ary Stds. for Uninsulated Tanks for Milk and Its Products

f Ability of Roller Dried Nonfat Dry Milk Solids to Change k, Adhesive, etc.) (1974)

Mixtures (1971) ANSI A3/ Rec for Flammable Liquid xograph Meth. Mor Analysis of Resistance of Flour Dough to nograph Meth. for Analysis of Resistance of Flour Dough to

c. for Concrete Made by Volumetric Batching and Continuous

c, Holding, Settling, Aeration Chambers, Chemical Storage, Rec. Pract. for Measuring,

gh to Mixing (Cereal Chemistry) (1962)

ing Compal Std. Rec. Pract. for Preparation of Bituminous est for Percent Air Voids in a Compacted Bituminous Paving Std. Meth. for Analysis of Natural Gas Liquid Std. Meth. for Analysis of Natural Gas Liquid std. Meth. for Preparation of Test Specimens of Bituminous ifor Resistance to Deformation and Cohesion of Bituminous Std. Meth of Test for Time of Setting of Concrete Std. Spec. for Hot Mixed, Hot Laid Tar Paving
for Classification of Soils and Their Aggregate s (1967) ec. Pract. for Classification of Soils and Their Aggregate
Rec. for Measurement of Absorbed Dose of Neutrons, and of meth. of Test for Resistance to Plastic Flow of Bituminous ange / Meth. of Analysis of Congeal Point in Fats and Fat Glucose Oxidase Meth. for Measurement of Glucose in Sugar $y$ and Nonwaxy Content in Corn and Grain Sorghum, and Their Std. Spec. for Mineral Filler for Bituminous Paving or Mixing Plants for Hot Mixed, Hot Laid Bituminous Paving meth. of Wetting and Drying Tests of Compacted Soil Cement Quantitative Extraction of Bitumen from Bituminous Paving std. Meth of Test for Lime Content of Uncured Soil-Lime

Std. Meth. of Test for Compressive Strength of Bituminous Std. Meth. of Test for Cement Content of Soil Cement

2) (1970)
Minimum Std. for Land.

Minimum Stds. for Land Minimum Stds. for Land
Mineral Fiber Roof Insulation Board (1972)

Mineral Fiber Siding (1972)

Mineral Filler and Aggregate) (1941) ANSI A 109.17

Mineral Filler and Coating of Paper (1973)

Mineral Filler for Bituminous Paving Mixtures (1970) an

Mineral Filler (1972) AASHO T37. ANSI A37.14

Mineral Granules (1970) A NSI A 109.18

Mineral Granules (1973)

Mineral Insulating Oil for Use in Transformers and in $O$

Mineral Insulating Oil for Use in Transformers and Oil

Mineral Insulating Oils Containing 2,6-Ditertiary-B

Mineral Matter (Cereal Chemistry) (1962)

Mineral Mixed Feeds (Cereal Chemistry) (1962)

Mineral Oils by Ultraviolet Absorption (1973) ANSI Z11.

Mineral Oil; Urease Tablets; Xanthyrol) (1962)

Mineral Roofing Aggregates (1973)

Mineral) Concentrates Used to Enrich Cereals (Cereal Ch

Minerals, and Rocks by the Fire Assay Preconcentration

Mines (Safety) (1973)

Mini Bike, and Bicycle (1974)

Mining and Logging Tires Used in Intermittent Highway $S$

Mining and Logging (Short Haul); Grader, Loader, Dozer,

Mining Car, Front End Loader, Fork Lift Trucks, Mobile

Minority Carrier Diffusion Length in Silic on by Measure

Mirrors (1971)

Misc Equipment) (1974)

Misc. Heat Producing Appliances) (1973)

/Reign and

Misc. Ornamental Items (Architectural Woodwork) (1973)

Misc. Ornamental ltems, Stairwork and Handrails, Exteri

Misc. Perils at Electric Generating Stations (Flood, Su

Miscellaneous Application (Tech. Notes) (1967)

Miscellaneous Sanitary Structures (Tech. Notes) (1969)

Miscellaneous Uses (1973)

Std. Dimet

Mist Spray Flammability of Hydraulic Fluids (1972T)

Mixed Concrete by the Pressure Meth. (1972T) ANSI A37.7

Mixed Concrete (1973)

Mixed Feeds (Cereal Chemistry) (1962)

Mixed Feeds (Cereal Chemistry) (1962)

Mixed Feeds (Cereal Chemistry) (1962)

Mixed Fertilizers Containing Ammonium Nitrate (1972)

Mixed Oxides ((U, Pu)0, $)$ (1972) ANSl N139

Mixed Self Rising Flour (Cereal Chemistry) (1962)

Mixed, Hot Laid Bituminous Paving Mixtures (1971) ANSI

Mixed, Hot Laid Tar Paving Mixtures for Base, Binder, L

Mixer and Agitator Stds. (Concrete Plant Equipment) (19

Mixer (Equipment) Stds. (1973)

Mixes and Self Rising Flours (Cereal Chemistry) (1962)

Mixes (Cereal Chemistry) (1962)

Mixes (Cereal Chemistry) (1962)

Mixes, Beadlets, Oils, and Emulsions (Cereal Chemistry)

Mixing and Storage) (1974)

Mixing Characteristics of Flour (Physical Dough Tests)

Mixing Operations (Paint, Varnish, Lacquer, Printing in

Mixing Plants for Hot Mixed, Hot Laid Bituminous Paving

Mixing Rubber Compounds for Preparing Vulcanized Tes? S

Mixing (Cereal Chemistry) (1962)

Mixing (Cereal Chemistry) (1962)

Mixing (1973)

Mixing, Filter, Grit Chambers, and Flotation) (1973)

Mixing, Transporting, and Placing Concrete (1973)

Mixograph Meth. for Analysis of Resistance of Flour Dou

Mixture Beam Specimens by Means of the California Knead

Mixture (1973)

Mixtures by Gas Chromatography (1968)

Mixtures by Gas Chromatography (1973) ANSI 211.238

Mixtures by Means of California Kneading Compactor (197

Mixtures by Means of Hveem Apparatus (1971) ANSI A37.13

Mixtures by Penetration Resistance (1970) ANSI A37.134

Mixtures for Base, Binder, Leveling, and Surface Course

Mixtures for Highway Construction Purposes (1973) AASHO

Mixtures of Neutrons and Gamma Rays (1961)

Mixtures Using Marshall A pparatus (1973)

Mixtures Which Congeal or Solidify With in Temperature R

Mixtures (Maltose, Sucrose, Mannose, Galactose, Dextran

Mixtures (1959)

Mixtures (1970) ANSI A37.41

Mixtures (1971) ANS1 A37.102

Mixtures (1971) ANSI A37.51

Mixtures (1972)

Mixtures (1973) ANSI Al.38

Mixtures (1974) ANSl A37.103, Ashto T167

Mixtures (1974) ANSI A37.58, Ashto T144

Mobile Communication Antennas: Part II-Vehicular (197

Mobile Communication FM or PM Receivers $25-470 \mathrm{MHz}$ (1972

ISpec. for Requiremen
Std. Meth. of Tes
167
44 II-Vehicular $(197$
ivers $25-470 \mathrm{MHz}(1972$
s mitters $25.470 \mathrm{MHz}$

Mobile Communications FM or PM Transmitters 25.470 MHz
ASTM

ASTM

Fo ASTM

C726

C725

D491

T421

ASTM D242

ASTM D546

ASTM D 1226

ASTM D249

ASTM D 1040

ASTM D3146

ASTM D2112

AACCH $40-01$

AACCH 40.35

ASTM D 2269

/Soprop AACCH 28.91

ICBO UBCS32.5

AACCH $86-49$

ASTM E400

ANSI N13.8

TRA

TRA

TRA

$2-29$

TRA

ASTM

$\mathrm{SAE}$

3

$\mathrm{BCl}$

F391

Ј 1.56

NBHA

NBH

AWI

FMS

BIA

BIA

ANS1

ASTM

ASTM

*1-700

$* 1-1$

PH1.30

D3119

AACCH 40-35

$\begin{array}{ll}\text { FACCH } & 86-05 \\ \text { LPD7-9 }\end{array}$

Mass Sp ASTM C698

Me AACCH 08-15

ASTM D995

ASTM D 1753

NRMCA $* 3$

NRMCA $* 2$

AACCH 12-2I

Schoo AACCH 80-68

AACCH $40-51$

AACCH 86-01A

Sanit DFISA 3200

$\mathrm{AACCH} \quad 54-20$

FMS $\quad 7-36$

ASTM D995

ASTM D3182

Mi AACCH $54-40$

Fari AACCH 54-21

Tent. Spe ASTM C685

NSF 41

$\mathrm{ACl} \quad 304$

AACCH $54-40$

ASTM D3202

ASTM D3203

NGPA 2165

ASTM D2597

ASTM D 156]

ASTM D1560

ASTM C403

ASTM D 1753

ASTM D3282

NCRPM R25

Std. ASTM D1559

AACCH $58.12 \mathrm{~A}$

AACCH $80 \cdot 10$

CR

ASTM D242

F ASTM D995

Std. ASTM D559

D2172

ASTM D3155

ASTM D1074

ASTM D806

EIA RS329.]

EIA RS204A

EIA RS152.B 
em Test Procedure (1971)

Rec. Pract. for rec. Pract, for Performance Std. for Crane Boomstop of Al er, Dozer, Mining Car, Front End Loader, Fork Lift Trucks, Std. for Particleboard for Std. for Uniform Plumbing Code: nced Fork Lift Truck, Boat, Baggage, Special Type Trailer, Std. for Waste and Sewage Holding Tanks for Safety Std. for Liquid Fuel Burning Heat Appliances for Std. for Supply Gas Connectors for Exterior Use on cturing Plants (Fire and Wind Hazards at Plants Assembling irements, and Installation of Plumbing, Heati/ Std. for 71) Std. for Std. for Manually Propelled leum Production Facilities on Land and on Marine Fixed and

1 Liquids and Slur/_ Rec. for Improving Safety on Enclosed or Pressurized and Non Pressurized Potable Water Tanks for r Single Crystals (1973)

Std. Meth. for Measuring Hall Rec. for Operation of Crowder Dry Pipe Valves. on) (/ Rec. for Operation and Maintenance of the Reliable of Fire Extinguishing Systems (1973)

pavement Marking Paints (1972)

1973)

Rec. for Star

A.S.

ed Systems, Structures, and Equipry Pipe Valves; Rockwood Std. Spec. for

crude Protein in Wheat and Flour Mill Products (Boric Acid nalysis of Starch in Flour and Semolina; Also, with Slight Std. Meth. of Test for Coking Value of Tar and Pitch us in Yeast (Cereal Chemistry) (1962) Colorimetric d. Meth. of Test for Total Nitrogen in Lubricating Oils by trophenide and Arsanilic Acid in Feeds (Cereal Chemistry) Meth. of Analysis for Ash in Starch, Dextrin, and Other ) (1968) Meth. of Analysis of

$\mathrm{n}$ Wheat Flour and Semolina (Cereal Chemistry) (1962)

etermining Ash Content in Corn Starch, Dextrines and Other re in Grains and Feedstuffs (Cereal Chemistry) (1962)

lour and Semolina; Bread; Feedstuffs, Ground Grains and / Std. Spec. for

bing, $0.80 \mathrm{Cr}-0.85 \mathrm{Ni}-0.20 \mathrm{Mo}-0.04 \mathrm{~V}-\mathrm{B}(0.38-0.43 \mathrm{C})$ nvestment $0.80 \mathrm{Cr}-1.8 \mathrm{Ni}-0.35 \mathrm{Mo}(0.28-0.36 \mathrm{C})(\mathrm{SAE} 4330$ astings $(0.80 \mathrm{Cr}-1.8 \mathrm{Ni}-0.35 \mathrm{Mo}(0.28-0.36 \mathrm{C}))$ (SAE 4330 nvestment $0.80 \mathrm{C}_{\mathrm{r}}-1.8 \mathrm{Ni}-0.35 \mathrm{Mo}(0.38-0.46 \mathrm{C})(\mathrm{SAE} 4340$

Estimating

Std. for Numerical Designation (Identification System) of (Fire and Wind Hazards at Plants Assembling Mobile Homes. 1972) Std. Steel Doors and Frames for Std. Meth. of Test for Elastic Std. Test Meth. for Determining Stiffness
Std. Meth. of Test for Shear Std. Meth. of Test for

(1972)

Std. Meth. of Test for Cold Crushing Strength and
Tentative Meth. of Test for Biaxial Flexure Strength Tentative Meth. of Test for Biaxial Flexure Strength lexure Test. of Glass and Glass Ceramics (Determination of
(GF 220 (1517) Tensile Strength, 70,000,000 (483) Tensile cs by Resol Std. Meth. of Test for Young's Modulus, Shear nd Glass Ceramics by Reso/ Std. Meth. of Test for Young's resin Impregnated, G70,000 (483) Tensile, 36,000,000 (248) resin Impregnated, G70,000 (483) Tensile, $36,000,000$ (248) Std. for R.40 Bulb (Hard Glass) Std. Spec. for Fineness of Std. Spec. for Fineness of ssignment of Grade (1973) Ans/ Test. of Hydraulic Cements and Concretes/ Std. Spec. for Test Meth. for Quantitative Analysis of Textiles Std. Test Meth. for z197.18 Std. Meth. of Test for Effect of utter, Oleomargarine, High Acid Coc/ Meth. of Analysis of
istry) (1962) istry) $(1962)$
mistry) (1962) Meth. of Analysis of
Meth. of Analysis of Vacuum Oven Meth. of Analysis of Table for Converting Absorption to $14.0 \%$ Meth. for Correction of Analytical Values to $14.0 \%$ for Multiplication Factors for Correcting Weights to a $14 \%$
ections for Changing as-is Farinograph Absorption to $14.0 \%$ 1 (1971) Std. Meth. of Test for Std. for Determination of Std. Meth. of Test for on (1973) Std. Meth. of Test for and Meth. for Determining Specific Weight, Durability and (1965) (Tech. Notes) (1965)
Mobile Construction Type Crane Overload Indicating Syst Mobile Construction Type Cranes Having Rope Supported B Mobile Crane, and Shovel (1974) (T Haul); Grader, Load

Mobile Home Decking (1973)

Mobile Home Parks (Trailer) (1972) ANSI A 177.1

Mobile Home (Trailer) Parks (1973)

Mobile Home, Garden Tractor, Industrial Truck and Trail

Mobile Homes and Recreational Vehicles (1971)

Mobile Homes and Recreational Vehicles (1973) ANSI A 147

Mobile Homes (1972)

Mobile Homes, Modular Housing, Prefab Houses, Motor Hom

Mobile Homes: Body and Frame Design and Construction Re

Mobile Hydraulic Cranes (1973)

Mobile Ladder Stands for Scaffolds (Towers, Safety) (19

Mobile Platforms (1973)

Ns at Drilling Rigs and Petro

Mobile Radio Telephone for Automotive Use (HS 27) (1968

Mobile Tanks for Transporting and Spreading Agricultura

Mobilehomes and Recreational Vehicles (1971)

Mobility and Hall Coefficient in Extrinsic Sr.miconducto

Mobility on Flexible Barrier Materials (1973)

Model a (1973)

Model B Accelerator for Dry Pipe Systems (Fire Protecti

Model F Hidromatic Deluge Valve for Water Flow Control

Model Performance Spec. for the Purchase of Reflective

Model Rocket Sporting and Safety Code and Engine Std. (

Model Specs. for Small Concrete Paving Jobs (1973)

Models C, D (1974)

Moderated Nuclear Power Generating Plants (1973) ANSI N

Modern Pewter Alloys (1972)

Modification) (Cereal Chemistry) (1962)

Modification, to Corn, Rye, Barley, Rice, Grain Sorghum

(Modified Conradson) (1973) ANSI A149.12

Modified Fiske-Subbarow Meth. of Analysis of Phosphor Modified Kjeldahl Meth. (1973)

Modified Meth. of Quantitative Determination of Both Ni

Modified Products (Cereal Chemistry) (1962)

Modified Sedimentation Test for Wheat (Cereal Chemistry

Modified Short Thread Shear Bolt (1972)

Modified Sorensen Meth. of Analysis of Amino Nitrogen I

Modified Starch Products (1955)

Nalytical Meth.

Modified Two Stage Air Oven Meth. of Analysis of Moistu

Modified Vacuum Oven Meth. of Analysis of Moisture in F

Modified Wood (1970) ANSI 012.1

(Modified 98Bv40) (1973)

Modified) (1973)

Modified) (1973)

Modified) (1973)

Modular Brick Masonry (Tech. Notes) (1971)

Steel Bars, Forgings, and tu Steel Castings,

Spec for Steel Sand C

Modular Clay Masonry (Tech. Notes) (1963)

Modular Grid Coordinates (1971)

Modular Housing, Prefab Houses, Motor Homes, and Campin

Modular Masonry Building Construction (1972)

Moduli of Rock Core Specimens in Uniaxial Compression (

(Modulus of Bending) of Fiberboards (1972)

Modulus of Plywood (1972)

Modulus of Rupture of Air Setting Plastic Refractories

Modulus of Rupture of Refractory Brick and Shapes (1972

Modulus of Rupture) of Ceramic Substrates (1974)

Modulus of Rupture) (1972)

Sid. Meth. of F

Modulus (1973)

atio for Glass and Glass Cerami

Modulus, and Poisson's Ratio Poisson's Ratio for Glass a

Modulus, $180_{\times 2}$ (1973)

Modulus, 350 (177) (1973)

Mogul Screw Base Incandescent (1966)

Fiber Tape and Sheet, Epoxy

Mohair Top, Yarns and Fabrics of the Worsted Type and a

Moist Cabinets and (Specimen Storage) Rooms Used in the

(Moister Teller) (Pulp and Paper) (1973)

Moisture and Composition) (1971) ANSI L 14.132

Moisture and Creosote Type Preservative in Wood (1970)

Moisture and Temperature on Adhesive Bonds (1972) ANSI

Moisture and Volatile Matter in Fats and Shortenings (B

Moisture and Volatile Matter in Soy Flours (Cereal Chem

Moisture and Volatile Matter in Yeast Foods (Cereal Che

Moisture Basis (Cereal Chemistry) (1962)

Moisture Basis (Cereal Chemistry) (1962

Moisture Basis (Cereal Chemistry) (1965)

Moisture Basis (Constant Dough Weight Meth. Only) (Cere

Moisture Content and Moisture Regain of Textile Materia

Moisture Content of Forages (1972)

Moisture Content of Oil Impregnated Cellulosic Insulati

Moisture Content of Wood (1974)

Moisture Content (1972)

Ts and Crumbles-Definitions

Moisture Control in Brick and Tile Walls (Tech. Notes)

Moisture Control in Brick and Tile Walls, Condensation

SAE

SAE

TRA

NPA

NFPA

ICBO

IAPMO

UL

IAPM

FMS

NFPA

ANSI

ANSI

API

SAE

ASAE

Std. F IAPMO

ASTM

AST

FMS

FMS

FMS

ITE

NAR

Al

FMS

ANS

ASTM

7.90

$501 \mathrm{~B}$

B30.15

RP500B

J797

R317

TSC 4

2.13

$2-55$

2.88

PS*1

CL-2

2.27

N18.10

AACCH 46.12

AACCH 76.20

ASTM D2416

AACCH $40-56$

St ASTM D3228

$\mathrm{AACCH} \quad 18-26$

AACCH $08-17$

AACCH 56-62

NSA $\quad 1103-20$

$\mathrm{AACCH}$ 46-31

$\begin{array}{ll}\mathrm{CR} & \mathrm{B} \cdot 8\end{array}$

AACCH 44.18

AACCH $44-40$

ASTM D1324

$S A E$

SAE

$\mathrm{SAE}$

SAE
BIA

BIA

ANSI

FMS

STDI

ASTM

SAE

ASTM

ASTM

ASTM

ASTM

ASTM

SAE

ASTM

ASTM

SAE

SAE

ANSI

ASTM

ASTM

ASTM

TAPPI

AATCC

ASTM

ASTM

AMS6422

AMS5328A

AMS5329B

AMS5330A

10

$10 \mathrm{~A}$

A62.8

7.90

110

D3148 
73) oluene (Cereal C oluene (Cereal C ansi $\mathrm{A} 37.21$

nd Grains and / eans, Rice, Beans. 962) er Commodities For/ Modified Two Modified Vacuum Oven Meth. of Analysis of Bidwell-Sterling Meth. of Analysis of Test Meth. for -Sterling Meth. of Analysis of
Std. Meth. of Test for Surface Air Oven Meth. of Analysis of ied Two Stage Air Oven Meth. of Analysis of Diectic Meter Meth. An Ansis of Std. Meth. of Test for Semiliquid Products (Drying Upon Air Oven Meth. of Analysis of Meth. of Analysis of Test Meth. for Sid. Meth. of Test for

973)

si L 14.256 iv/ Effect of Thermal Environment on Production, Heat and Std. paper) (1973) s, etc.) (Pulp and Paper) (19/ Test Meth. for Density and Test Meth. for Density and samples of Wool by Oven Drying (1/ Std. Meth. of Test for
Std. Meth. of Test for Moisture Content and pparatus (1972) or Storage (1972) Std. Meth. of Test for Capillar ANSI C8.38 g. C Operation / 1972) ch (1956) 5) s (Corn) (1956) p Unmixed (1952) Density, Specific Gravity, and Weight ied) (1961) Correction for Weight of Flour to Give 100 Grams at $14.0 \%$ ied)

966

d Refined) (1966)

d (1954)

xed and Unbleached/ (1957)

Std. Spec. for Synthetic Rubber Heat or Std. Spec. for Synthetic Rubber Heat and Std. Meth. of Test for

Std. Analytical Meth. for Determining Std. Analytical Meth. for Determining Std. Analytical Meth for Determining Std. Analytical Meth. for Determinin of Flour to Give $100 \mathrm{Grams}$ at $14.0 \%$ Std. Analytical Meth. for Determining Std. Analytical Meth. for Determining Std. Analytical Meth. for Determinin Std. Analytical Meth. for Determining Std. Analytical Meth. for Determining Std. Analytical Meth. for Determining Std. Analytical Meth. for Determining Std. Analytical Meth. for Determining Air Oven Meth. of Analysis of

d) (Crystalline) (/

(Gas liıuid Chromatography) Content of Corn Sugar. Staıch
Refractometer Meth. of Analysis of Solids in Syrups and indle Meth of Analysis of Solids in Juices, Syrups, and

Sugars Content of Corn Sugars (Crude and Refined), Starch ing Upon Q/ Meth. of Analysis of Moisture in Massecuites, y) (1962) Meth. of Analysis for Ash in Meth. of Analysis of Std. Spec. for Magnesium Alloy Permanent Std. Spec. for Aluminum Alloy Permanent for Permanence of Adhesive Bonded Joints in Plywood Under Std. Meth of Measuring Shrinkage from ental Ch/ Tent. Meth. of Test for Resistance to Growth of Test Meth. for Density of Wood Chips (Sheet Machine

ec. for Magnesium Alloys in Ingot Form for Sand. Permanent 972)

pec. for Aluminum Alloys in Ingot Form for Sand, Permanent

ures (1972)

Std. Spec for TFE - Fluorocarbon Resin Sid. for Safety for

ode Std. for Determining the Splitting Tensile Strength of

h. of Test for Drop Impact Resistance of Polyethylene Blow Std. Meth. of Test.

Lightweight Selflocking and Nonselflocking Blind Threaded

Lightweight Selflocking and Nonselflocking Blind Threaded g. Light Weight Floating, Sandwich Panel Std. for lnsert. Sandwich Panel (1972)

Std. for lnsert,

Std. Meth. of Measuring Shrinkage from Mold Dimensions of Std. Spec. for Reinforced and Filled Nylon Injection

pract. for Injection Molding of Specimens of Thermoplastic Std. Spec. for Polybutylene Plastic Std Spec for ETFE - Fluoroplastic

Std. Spec. for Reinforced Polyterphthalate Thermo-Plastic Std. Spec. for Thermoplastic Polyterephthalate Std. Spec. for Polyvinylidene Fluoride (PVDF Spec. for TFE -Fluorocarbon (Tetrafluoroethylene) Resin Std. Spec. for Nylon Injection
Pract. for Powder Flow Measurement of TFE -Fluorocarbon
Moisture Density Relations of Soils (1973)

Moisture in Chips (Moister Teller) (Pulp and Paper) (19

Moisture in Fats and Shortenings by Distillation with $\mathrm{T}$

Moisture in Feeds and Feedstuffs by Distillation with T

Moisture in Fine Concrete Aggregate (1973) AASHO T142,

Moisture in Flour and Semolina (Cereal Chemistry) (1962

Moisture in Flour and Semolina; Bread; Feedstuffs, Grou

Moisture in Flour, Farina, Semolina, Bread, Grain, Soyb

Moisture in Grains and Feedstuffs (Cereal Chemistry) (1

Moisture in Grains (Cereal Chemistry) (1962)

Moisture in Grains, Beans, Peas, Lentils, Rice, and Oth

Moisture in Graphite (1969) ANSI K90.5

Moisture in Malt (Cereal Chemistry) (1962)

Moisture in Massecuites, Molasses, and Other Liquid and

Moisture in Nonfat Dry Milk (Cereal Chemistry) (1962)

Moisture in Pulp (Web) (1973)

Moisture in the Analysis Sample of Coal and Coke (1973)

Moisture in Whole Grain (Cereal Chemistry) (1962)

Moisture in Wood Chips (Steam Oven) (Pulp and Paper) (1

Moisture in Wood Chips (Two Liter Graduate) (1973)

Moisture in Wool by Distillation with Toluene (1972) an

Moisture Loss and Feed and Water Requirements of Farm L

Moisture Measurement (Grain and Seeds) (1972)

Moisture Measurement (Meat and lts Products) (1972)

Moisture of Chips (Presoak Before Immersion) (Pulp and

Moisture of Chips (Submersion with Correction for Crack

Moisture Present in Ordinary Commercial and Industrial

Moisture Regain of Textile Material (1971)

Moisture Relationships for Soils by Pressure Membrane a

Moisture Relationships of Agricultural Grain and Seed F

Moisture Relationships of Agricultural Grains (1968)

Moisture Resisting Insulation for Wire and Cable (1974)

Moisture Resisting Insulation for Wire and Cable, 75 De

Moisture Vapor Permeability of Organic Coatings Films (

Moisture (Azeotropic Distillation) Content in Corn Star

Moisture (Azeotropic Distillation) Content in Corn (195

Moisture (Azeotropic Distillation) Content in Feedstuff

Moisture (Azeotropic Distillation) Content of Corn Syru

Moisture (Cereal Chemistry) (1962)

Moisture (Cereal Chemistry) (1962)

Moisture (Karl Fischer) Content in Corn Starch (Unmodif

Moisture (Karl Fischer) Content in Steep water (Corn) (1

Moisture (Karl Fischer) Content of Corn Sugar (Crude an

Moisture (Karl Fischer) Content of Corn Syrup (1966)

Moisture (Oven Filter Aid) Content of Corn Syrup Unmixe

Moisture (Oven Filter Paper) Content of Corn Syrup Unmi

Moisture (Oven) Content in Commercial Feedstuffs (Corn)

Moisture (Oven) Content in Corn Starch (1956)

Moisture (Oven) Content of Corn Sugar (Crude and Refine

Moisture, Drying at 135 Deg. C (Cereal Chemistry) (1962

Molasses and Hydrolyzates (1971)

/Ermining Saccharides

Molasses (Cereal Chemistry) (1962)

Molasses (Cereal Chemistry) (1962)

Molasses (1972)

Nalytical Meth. for Determining Total

asses, and Other Liquid and Semiliquid Products (Dr

Molasses, Sugars, and Syrups (Cereal Chemistry) (1962)

Mold and Yeast Counts in Food Products (Cereal Chemistr

Mold Castings (1972) ANSI H45.2

Mold Castings (1974) ANSI H38.26

Mold Conditions (1970)

Mimensions of Molded Plastics (R1973) ANSI K66.3

Mold on the Surface of lnterior Coatings in an Environm

Mold) (Pulp and Paper) (1973)

Mold, and Die Castings (1966) ANSI H45.8

Mold, and Die Castings (1973) ANS! H38.1

Molded and Dipped Mica Capacitors (Wire Lead Styles) (1

Molded Basic Shapes (1974)

Molded Case Circuit Breakers and Circuit Breaker Enclos

Molded Concrete Cylinders (1973)

Molded Containers (1970)

Molded Flexible Urethane Foam (1973)

Molded in lnsert (1974)

Molded in lnsert (1974)

$$
\begin{array}{r}
\text { Uniform Building C } 1 \text { CBO } \\
\text { Std. Met ASTM }
\end{array}
$$

Sid, for Sandwich Panel NSA

Molded 1n, BLind Threaded, Self Locking, Nonself Lockin

Molded Plastics (R1973) ANSI K66.3

Molding and Extrusion Materials (1972)

Molding and Extrusion Materials (1972) ANSI K65.78

Molding and Extrusion Materials (1973)

Molding and Extrusion Materials (1973)

Molding and Extrusion Materials (1973)

Molding and Extrusion Materials (1973)

Molding and Extrusion Materials (1973)

Molding and Extrusion Materials (1973)

Molding and Extrusion Materials (1973) ANSI K65.216

Molding and Extrusion Materials (1974)
ASTM

ICBO UBCS70-1

TAPPI UM-8

AACCH 44.51

ASTM $\mathrm{C} 70$

AACCH 44.16

AACCH 44.40

AACCH $44 \cdot 15 A$

AACCH 44.18

ACCH 44-53

$\mathrm{AACCH}$ 44-11

$\mathrm{AACCH} \quad 44.20$

$\mathrm{AACCH} \quad 44.60$

AACCH 44.52

TAPP] ['M.237

D3173

AACCH 44.10

TAPPI UM.7

ASTM D2462

ASAE D249.2

ASAE S352

ASAE $S 353$

TAPPI UM-20

ASTM D 1576

D2654

ASAE D245.

ASTM D 1520

D1679

ASTM D 1653

CR B. 34

A.12

CR E-40

AACCH 44.01

ACCH 82.23

B.36

CR F.32

CR E.46

E.42

CR 44

$\begin{array}{ll}\mathrm{CR} & \mathrm{G} .16 \\ \mathrm{CR} & \mathrm{B} .38\end{array}$

CR F.34

$\mathrm{AACCH}$ 44-19

CR F.50

ACCH $\quad 68-60$

$\mathrm{AACCH} \quad 68.62$

$\begin{array}{ll}\mathrm{AACCH} & \mathrm{F}-58 \\ 44.60\end{array}$

AACCH $08-14$

AACCH $42-50$

ASTM B199

ASTM B108

D1877

ASTM D3273

TAPPI UM.9

STM

ElA RS153B

ASTM D3294

D32

UBCS26.12

D2463

D2406

1832

1836

NSA 1835

NSA $\quad 1833$

ASTM D955

ASTM D2897

1. ASTM D1897

ASTM D2581

ASTM D3159

ASTM D3220

ASTM D3221

ASTM D3222

Std ASTM D1457

ASTM D789

Std. Rec ASTM D3292 
Std. Spec. for Fep Fluorocarbon Std. Spec. for Glass Reinforced Acetal Plastics for of Measuring the Spiral Flow of Low Pressure Thermosetting ec Pract, for Compression Molding Test Specimens of Allyl sion Materials (1972) ANSI K65/ Rec. Pract. for Injection Uniform Building Code Std. for Gypsum

Std. Spec. for Gypsum Casting and Std. Rec. Pract. for Compression )

1973T) ANSI A37.95 Std. Spec. for E-CTFE-Fluoroplastic Tent. Spec. for ron Pipe, Centrifugally Cast, in Metal Molds or Sand Lined std. Rec. Pract. for Determining Temperatures of Std. ASTM Std. for Ductile Iron Pipe, Centrifugally Cast, in Meta ction and Safety (1973)

Rec. for High Temperature Std. Meth. of Test for Silica in Fluorspar by the Silico 1) ANSI H34.44 Fluorspar by the Silico H34.12

Std. Spec, for Nickel.

ates, Alloy Steel, Quenched and Tempered Nickel-Cobalt Spec. for Seamless and Welded Chromium-Nickel-Iron Sid. Spec. for Nickel-Chromium-Iron Std. Spec. for Nickel-Chromium-Iron ANSI H34.16 Std. Spec. for Nickel-Iron-Chromium F/ Std. Spec. for Manganese-Molybdenum and Manganese Std. Meth. of Test for Fast Neutron Fux by Analysis of Std. Spec. for Molybdenum and Std. Spec. for Molybdenum and Std. Spec, for Nickel. Std. Spec. for Nickel-Iron-Chromium-Manganese Std. Spec. for Nickel

73) A NSI B125.9

Std. Spec. for Seamless Carbon 73) Ans/ Std. Spec. for Electric Resistance Welded Carbon 2) ANSI G34.1 3) ANSI G35.4 Std. Spec. for or Pressure Vessels (1972/ Std. Spec. for Chromium re Vessels (1972) ANSI G35.15 Std. Spec. for Manganese eel, Quenched and Tempered, F/ 4)

Std. Spec. for Manganese Std. Spec. for Std. Spec. for Std. Spec. for Std. Spec. for

Plate (1974)

Std. Spec. for Low Carbon Nickel Std. Spec. for Low Carbon Nickel Std. Spec. for Nickel

ANSI H34.19

22

Std. Spec. for Nickel Chromium Std. Spec. for Nickel Chromium Std. Spec. for Nickel Chromium Std. Spec. for Nickel Iron Chromium std. Spec. for Nickel lron Chromium ansi H34.17 Std. Spec. for Seamless and Welded Chromium Nickel Iron
S Strip (1973)/
Std. Spec. for Chromium Nickel Iron et, and Strip (1973)/ Std. Spec. for Chromium Nickel Iron Std. Spec. for Nickel Chromium

lieved (1973)

) ANSI B12/ Std. Spec. for Seamless Low Carbon and Carbon ANSl H34.) Std. Spec. for Nickel Chromium lron Columbium for Pressure Vessel Plates, Alloy Steel 5\% Chromium, 0.5\% hromium, for Pressure Vessels (1/ Std. Spec. for Chromium pec. for Electric Fusion Welded Chromium-Nickel-Iron .

0 Deg. Flush Shear Head A286 Corrosion Resistant Steel and onents for Particulate Contamination by Automatic Particle ion, Product Audit) (196/ Rec. for Radiological

ioactivity (Beta Gamma) in Cereal Products-Emergency s (General, Exterior Light Transmitting Panels, Skylights, Safety Std. for Line Isolation

in Prepared Mixes (/ 1 Chemistry)/ pment (1974/

Perchloric Acid Meth. of Analysis of Meth. of Analysis for Neutralizing Value of Rec. Std. for Engineering Specs. Outline for for Determining Speed of Photographic Negative Material (1968/ Std. Meth. of Test for Nonaromatic Hydrocarbons in Std. Spec. for Ethylene Glycol

ysis of Calcium Peroxide in Dough $\mathrm{C}$ tenings (Cereal Chemistry) (1967) Std Meth. Spec. for Chemical Resistant Resin Std. Spec. for Styrene Std. Spec. for Styrene
Molding and Extrusion Materials (1974) A NSI K65.201 Molding and Extrusion (1972)

Molding Compounds (1972)

Molding Materials (1972)

Molding of Specimens of Thermoplastic Molding and Extru

Molding Plaster (1973)

Molding Plaster (1973) ANSI A49.4

Molding Test Specimens of Allyl Molding Materials (1972

Molding, Extrusion, and Coating Materials (1973)

Molds for Forming Concrete Tests Cylinders Vertically

Molds for Gas (1971)

Molds for Test Specimens of Plastics (1967) ANSl K65.79

Molds or Sand Lined Molds for Gas (1971)

Molten Materials (Metals, Slag, Glass, etc.) Fire Prote

Molten Metal Fires and Explosions (I973)

Molybdate Solution (Cereal Chemistry) (1962)

Molybdate (Photometric) Meth. (1972)

Molybdenum-Chromium-lron Alloy Sheet and Plate (197

Molybdenum-Chromium Alloy Plate and Sheet (1971) ANSI

Molybdenum-Chromium Alloy Rod (1971) ANSl H34.14

Molybdenum-Chromium (1972) ANSI G35.26 /Re V
Molybdenum-Copper-Columbium Stabilized Alloy Tubes

Molybdenum-Copper Alloy Plate, Sheet and Strip (1973)

Molybdenum-Copper Alloy Rod (1973)

Molybdenum-Copper Alloy Seamless Pipe and Tube (1972)

Molybdenum-Nickel Alloy Steel, Quenched and Tempered.

Molybdenum -99 Activity from Uranium - 238 Fission (1972

Molybdenum Alloy Bar, Rod, and Wire (I974)

Molybdenum Alloy Billets for Reforging (1974)

Molybdenum Alloy Forgings (1974)

Molybdenum Alloy Plate and Sheet (1971) ANSI H34.11

Molybdenum Alloy Plate, Sheet, and Strip (1973)

Molybdenum Alloy Rod (1971) ANSI H34.13

Molybdenum Alloy Steel Boiler and Superheater Tubes (19

Molybdenum Alloy Steel Boiler and Superheater Tubes (19

Molybdenum Alloy Stee] Plates for Pressure Vessels (197

Molybdenum Alloy Steel Plates for Pressure Vessels (197

Molybdenum Alloy Steel Plates, Quenched and Tempered, F

Molybdenum Alloy Strip, Sheet, Foil, and Plate (1974)

Molybdenum and Its Nickel Alloy Steel Plates for Pressu

Molybdenum and Manganese-Molybdenum-Nickel Alloy St

Molybdenum and Molybdenum Alloy Bar, Rod, and Wire (197

Molybdenum and Molybdenum Alloy Billets for Reforging(

Molybdenum and Molybdenum Alloy Forgings (1974)

Molybdenum and Molybdenum Alloy Strip, Sheet, Foil, and

Molybdenum Chromium Alloy Plate, Sheet, and Strip (1972

Molybdenum Chromium Alloy Rod (1972)

Molybdenum Chromium Iron Alloy Rod (1972)

Molybdenum Columbium Alloy Plate, Sheet and Strip (1972

Molybdenum Columbium Alloy Rod and Bar (1972) ANSI H34.

Molybdenum Columbium Alloy Seamless Pipe and Tube (1972

Molybden um Copper Alloy Plate, Sheet, and Strip (1972)

Molybdenum Copper Alloy Rod and Bar (1972) ANSI H34. I8

Molybdenum Copper Columbium Stabilized Alloy Pipe (1973

Molybdenum Copper Columbium Stabilized Alloy Plate, She

Molybdenum Flattened Wire for Electron Tubes (1973)

Molybdenum Iron Alloy Rod (1972)

Molybdenum Sheet, Strip, and Plate, Arc Cast, Stress Re

Molybdenum Steel Still Tubes for Refinery Service (1972

Molybdenum Tungsten Alloy Seamless Pipe and Tube (1972)

Molybdenum (1972) ANSI G35.16

Molybdenum, Alloy Steel Plates, Quenched and Tempered C

Molydenium-Copper-Columbium Stabilized Alloy Pipe (

Std for Solid 10

Monel Rivet (1974)

Monitor Meth. (1972)

G Gas Blow Dow

Monitoring Meths. and Instruments (1952)

Monitoring of the Product (Receiving and Source Inspect

Monitoring Special Process of Quality Control (1969)

Monitoring (Cereal Chemistry) (1973)

Monitors and Sawtooth Roofs, Light Diffusers in Ceiling

Monitors (1973)

Monocalcium Phosphate and/or Sodium Acid Pyrophosphate

Monocalcium Phosphate: Monohydrate and Anhydrous (Cerea

Monochrome Cetv (Closed Circuit Television) Camera Equi

Monochrome Television Picture Tubes (1972)

Monochrome, Continuous Tone) (1972)

Monocyclic Aromatic Hydrocarbons by Gas Chromatograph

Monoethyl Ether (1972)

Monofilaments (1973)

Monoglyceride, Salts, Soy Flour, and Other Cereal Produ

Monoglycerides and Free Glycerol in Fats, Oils and Shor

Monohydrate and Anhydrous (Cereal Chemistry) (1962)

Monolithic Refractories (1972)

Monolithic Surfacings (1972T)

Monomer 993 (1972)

Monomer 996 (1971)
ASTM

ASTM

Meth. ASTM

d. $R$ ASTM

ASTM

$1 \mathrm{CBO}$

ASTM

ASTM

ASTM

ASTM

ANS1

ASTM

ANSI

FMS

FMS

A ACC

ASTM

ASTM

A STM

ASTM

ASTM

ASTM

A STM

ATM

ASTM

STM

ASTM

ASTM

ASTM

ATM

ASTM

A TTM

ASTM

A STM

ASTM

ASTM

STM

ASTM

ASTM

ASTM

ASTM

ASTM

ASTM

STM

ASTM

ASTM

A STM

STM

STM

A T M

ASTM

STM

ASTM

ASTM

ASTM

AE

ASTM

ASTM

ASTM

ASTM

ASTM

NSA

ASTM

NCR

RP 4

A ACCH 28.99A

$1 \mathrm{CBO}$ UBC *3-52

UL 1022

AACCH 40.51

$\mathrm{AACCH} \quad 02.33$

EIA RS312

EIA RS266A

ANSI PH2.5

ASTM D2360

ASTM D331

ASTM D32I

$\mathrm{AACCH}$ 48-5]

$\mathrm{AACCH} \quad 58.45$

IF AACCH 02.33

ASTM C4I7

ASTM C722

ASTM D2826

ASTM D2827 
Sid. Test Meth. for Polymer Content of Styrene eth. of Test for Methyl Ether of Hydroquinone in Colorless Spec. for Ethylene Glycol Std. for

Spec. for Underhung Cranes and Tent. Meth. of Test for Carbon ondispersive Infrared Spe/ Uniform Fire Code: Cryogenic Fluids (Air, Argon, Carbon and Installation of Fittings and Equipment for Anchoring, ls for Use in Construction of Built Up Roof Coverings: Hot safety Std. for Materials for Built-Up Roof Coverings: Hot Sid. Meth of Test for Std. Meth. of Test for Length Change of Hardened Cement for Length Change of Drilled or Sawed Specimens of Cement m (1973) Std. Spec. for Portland Cement Lime Uniform Building Code Std. for Std. Spec. for pe (4 In. and Larger, Shop Applied) (197) Std. for Cement Std. Spec, for Reinforced Plastic

f Test for Cold Bonding Strength of Air Setting Refractory Specifying Gypsum Masonry and Std. Meth. of Test for Refractoriness of Refractory Uniform Building Code Std. for Aggregate for Masonry

Uniform Building Code Std. for Field Tests for Grout and Std. Spec. for Latex-Portland Cement recommendation for Materials for Setting Ceramic Tile with Std. Rec. Pract. for Use of Hydraulic Cement meth. of Test for Compressive Strength of Hydraulic Cement meth. of Test for Compressive Strength of Hydraulic Cement d. Meth. of Test for Flexural Strength of Hydraulic Cement

Uniform Building Code Std. for Masonry Cement for Use in Std. Spec. for Chemical Resistant Resin Std. Rec. Pract. for Use of Chemical Resistant Resin Chemically Setting Silicate and Silica Chemical Resistant

Chemically Setting Chemical Resistant Silicate and Silica Granite; Gravel; Granite, Limestone, and Marble Masonry; ete Slab or Wood Subfloors (1962) Spec. for

Rags, Waste Cloth, Wastepaper, Kapok, Hay, Straw, Spanish 7-0) (1973)

Std. Dimensions for $16 \mathrm{Mm}$ ratories (1971) Std. Spec. for Projector Usage of $16 \mathrm{Mm}$ Uniform Building Code Sid. for Cellulose Nitrate Uniform Fire Code: Cellulose Nitrate

d. Dimensions of Projectable Anamorphic Image Area on $5 \mathrm{Mm}$ Std. for Location of Super 8 Printed Area on $16 \mathrm{Mm}$ uper 8 Printed Area in Optical Reduction Printing on $35 \mathrm{Mm}$ ted Area in Optical Reduction or Contact Printing on $35 \mathrm{Mm}$ Std. Dimensions for $32 \mathrm{Mm}$ Std. Dimensions for $32 \mathrm{Mm}$ ersal Color Camera Films Intended for Direct Projection in Std. Dimensions for Photographic Sound Record on Super 8 Std. for Medium Prefocus Base Down Type, Single Contact Uniform Building Code: Uniform Fire Code:

973) Std for

Wear Life and Bonded Solid Film Lubricants in Oscillating health Rec. for Changing and Charging Storage Batteries in of Capacity of Lead Acid lndustrial Storage Batteries for or Converters for Motor Drives: Part l-Converters for Dc

1974) Rec. Pract. for Outboard receiving-Materials Handling) (1973) Shipper : Safety Std. for Sealed (Hermetic Type) Rec. Pract for Outboard Std. Pract. and Requirements for Thyristor Converters for for Rese/ Std. Meth. of Test for Knock Characteristics of for Moto/ Std. Meth. of Test for Knock Characteristics of ront End Loaders, Wheeled Dozers, Track Type Tractors, and onal Vehicles (Travel and Camping Trailers, Truck Campers, 0T) s Assembling Mobile Homes, Modular Housing, Prefab Houses, motor Fuels Using the Compression Ratio (CR) Technique for Std. Test. Procedure for Oil Wicking of Composite test. Procedure for Filled Cast Flat Bottom Drop Composite imulator (1) Std. Meth. of Test for Apparent Viscosity of s (1966) Safety Std. for

Guide for Synchronous Rec, Electric Rec. Pract. for Electric Blower chronous Generators and Reversible Synchronous Generator / Rec. Pract. for Instrumentation for Std. for ric Conditions (1972) Std. for
Monomer (1974) ANSI Z161.1

Monomeric Acrylate Esters (1972)

Monomethyl Ether (1972)

Monorail Systems and Underhung Cranes (1973)

Monorail Systems (1973)

Monoxide in the Atmosphere (Continuous Measurement by $\mathrm{N}$

Monoxide, Deuterium, Fluorine, Helium, Hydrogen, Krypto

Mooring, Docking, Towing and Lifting Boats (1973)

Mopped Asphalt, Asphalt and Coal Tar Saturated Organic

Mopping A sphalt, Asphalt-Saturated Organic Felt, Coal T

Morpholine in Water (1974)

Mortar and Concrete (1974) ANSl A37.78

Mortar and Concrete (1974T)

Mortar for Brick Masonry (1972)

Mortar for Unit and Reinforced Masonry Other Than Gypsu

Std. Meth. of Test

Mortar for Unit Masonry (1973)

Mortar Protective Lining and Coating for Steel Water Pi

Mortar Sewer Pipe (1973)

Mortar (Wet Type) (1972) ANSI Al11.29

Mortar (1967)

Mortar (1972) ANSI A 111.30

Mortar (1973)

Mortar (1973)

Mortar (1973) ANSI A118.4

Mortar, Adhesive, and Mastic (1973)

Mortars in Chemical Resistant Masonry (1965)

Mortars (Using Portions of Prisms Broken in Flexure) (1

Mortars (Using 2 ln. (50 Mm) Cube Specimens) (1973)

Mortars (1972)

Mortars (1973)

Mortars (1974)

Mortars (1974)

Mortars (1974)

Mortars (1974)

Mortar. Sand. Slag. Steel. Terra Cotta Ttd. Rec. Prac

Std. Spec. for

Mosaic Wood Parquet Flooring Set in Adhesive Over Conc

Moss, Excelsior, etc.) (1973) /W, Cocoa Fibers,

Motion Picture Film Perforated Super 8, 5R-1667 (1.3.5-

Motion Picture Film Perforated Super 8, (1.4) (1973)

Motion Picture Film Perforated 1.4 (1971)

Motion Picture Film Used in Studios and Processing Labo

Motion Picture Film (1973)

Motion Picture Film (1973)

Motion Picture Film (1973)

Motion Picture Film (2.35:1) Aspect Ratio (1971)

Motion Picture Film, Perforated Super 8 (1-3) (1973)

Motion Picture Film, Perforated 2R-1664 (1-0) (1973)

Motion Picture Film, Perforated 5R-1667 (1-3-5-7-0) (19

Motion Picture Film, 2R (1974)

Motion Picture Film, 4R (1974)

Motion Picture Photography (1973)

Motion Picture Prints (1972)

Motion Picture Projection Lamps (1972)

$/ \mathrm{mm}$ and Super 8 Rev ANSI

Motion Picture Projection Rooms (1973)

Motion Picture Projection (1973)

Motion Picture Raw Stock Identification and Labeling (1

Motion (1971) ANSI Z11.300

I of Test for Measuring the ASTM

Motive Power Service (For Ele

Motive Power Service (1974)

Motor Armature Supplies (1973) ANSI C34.3

Motor Boat Transom and Motor Well Dimensions on Boats

Motor Carrier Dock Planning Manual (Truck Shipping and

Motor Compressors (1972) ANSI B143.1

Motor Dimensions (Boats) (1972)

Motor Drives: Part 1-Converters for DC Motor Armature

Motor Fuels Using the Compression Ratio (CR) Technique

Motor Fuels Using the Compression Ratio (CR) Technique

Motor Graders (1971)

Movers, Wheeled and Track

Motor Homes): Installation of Plumbing, Heating, and El

Motor Homes, and Camping Trailers) (1974)

Motor Meth. Octane Ratings Using on Line Analyzers (19?

Motor Meth. Ratings (1971) ANSl Z11.306

rs $(197$

Motor Oil Cans (1974)

Motor Oil Cans (1974)

Motor Oils at Low Temperature Using the Cold Spanking S

Motor Operated Appliances (1972) ANSl C 33.36

Motor Positions for Belt or Chain Drive Centrifugal Fan

Motor Protection (1971)

Motor Starting Currents (1972) EEl 62-27

Motor Switch for Vehicles (1971)

Motor Units for Pumped Storage Installations (1974)

Motor Vehicle and lis Component Impact Tests (1971)

Motor Vehicle Brake Fluid for Use Under Arctic Atmosphe

Motor Vehicle Brake Fluid (Used Under Nonarctic Conditi

Motor Vehicle Brake Linings (1966)
ASTM

Std. M ASTM

ASTM

ANSI

MMA

Ion $\mathrm{ABYC}$

ICBO

UL

ASTM

ASTM

ASTM

ICBO

ASTM

AWW

ASTM

AST

CS1

ICBO

ICBO

TCA

TCA

ASTM

ASTM

/ ASTM

St ASTM

ICBO

ASTM

ASTM

ASTM

ICBO

ICBO

ANS

ANSI

ANSI

Std. for ANSI

ANSI

ANSI

ICBO

St ANSI

ANSI

ANSI

ANSI

ANSI

ANSI

ICBO

ICBO

/Nd ITA

ITA

IEEE

$A B Y C$

ANSl

UL

ABYC

IEEE

ASTM

ASTM

SAE

NFPA

FMS

ASTM

ASTM

CCTI

std. CCTI

ASTM

UL

A.MCA

IEEE

NEMA

SAE

IN NEMA

SAE

SAE

SAE

D2121

D3125

B30.11

*2 316

FC*2ART36

A5

UBCS32.1

$55 \mathrm{~A}$

D1942

Cl57

C341

UBCS24-21

C270

C205

D3262

04280

C199

UBCS24.22

UBCS 24.23

9D

C398

C349

C109

C348

UBCS24-17 
Rec. Pract. for Motor Vehicle Driver's Eye Range (1972)

Rec. Pract. for Service Performance Requirements for of Suitability of Plastic Material for Use in Housings of (1972) Rec. Pract. for New ec. Domestic Marketing Std. Including Guarantee Policy for d Towing Meth. (Coupling, Hitch Minimum Requirements for cle Dimensions for Use in Designing Docking Facilities for Handling Chlorine in Tank Std. for Lighting Inspection Code for ce Requirements for Sealed Beam Headlamp (Light) Units for dustrial Al Std. for Measurement of Electromagnetic Radiation from Std. for Lead Acid Type Storage Batteries for Rec. Pract. for Outboard Motor Boat Transom and for Lead Acid Type Storage Batteries for Motor Vehicles. Rec. Pract. for Rec. Pract. for Rec. Pract. for Std. for Tires and Rims for Rec. Pract. of Definitions for Rim and Wheel Stds. for Log Skidder, Truck, Bus, Bead S/ Minimum Requirements for Safety Std. for Electric lass II, Groups E, F, and G (19/ lass I, Groups C and D (197)

Std. for Safety for Electric e/ Std. for Dimensions for Alternating and Direct Current er) (1972)

d. for Application Data for Alternating and Direct Current Std. for Definite Purpose
Std. for Definitions for Std. for Terminal Markings for and Guide for Selection, Installation and Use of Electric Std. for

Rec. for Single Phase Rural Distribution Service for Safety Std. for Electric Trial Use Guide for Type Tests of Continuous Duty Class I ) Implements (1971) Application of Remote Hydraulic td. for Ratings for Alternating Current and Direct Current or Tests and Performance of Alternating and Direct Current Std. for Induction Std. for Synchronous Std. for Direct Current

ts for Alternating-Current Integral Horsepower Induction Rec. for Enclosures for

a for Positve Displacement Hydraulic Fluid Power Pumps and Rec. for Fire Protection of Synchronous Grading Rules for Std. Wood Std. for Nut-Instrument

n Spruce; Western Cedar and Hemlock; Subal-Pine Fir; and Rec. Pract. for Autornatic Reset Internal

Code for Cranes, Derricks, Hoists, Jacks and Slings: Base Std. for Residential Controls for Low Voltage, Wall

Std. Pract. for Spur, Helical and Herringbone Gear Shaft tegrally Mounted Thermostats For/ Line Voltage Integrally ed Thermostats for Electric Heater Line Voltage Integrally Safety Std. for Sliding Hardware for Std., Horizontally

Wire Rope Hoists (Lug, Hook, Trolley Suspended, and Base boss Type, 30, 50, and 60 Amperes, 4 Wire (1966) Standard d Industrial Equipment Warning Lamp and Slow Mo/ Std. for nd Slow Moving Vehicle (SMV) Identification Emb/ Std. for d End Configurations, Dimensional Identification Code, and Std. for Flashing Warning Lamp (Light) for Remote Rec. Pract. for Engine Foot

ner, Quickly Demountable Universal (Horizontal or Vertical as Fuel Container, Quickly Demountable Type for Horizontal Rec. Pract. for Fuel Pump Rec. Pract. and Stds. for Marine Engine

plow, Field and Row Crop Cultivator Shafts and Ground Tool ransmission (197/ Rec. Pract. for Marine (Inboard) Engine for Guide for Describing the Characteristics of Resilient Std. for Dimensions for Front Lens Std. Dimensions of Image Areas and or Projectors for $35 \mathrm{~mm}$ Filmstrips and Slides in $2 \mathrm{X} 2 \mathrm{In}$. Structural Clay Differential Structural Clay Differential Structural Clay Differential on and Cohesion of Elastomeric Joint Sealants Under Cyclic teria for Roll Over Protective Structures (ROPS) for Prime a for Falling Object Protective Structure (FOPS) for Prime Std. Operating Limits for Central Station Units (Air Std. Coil Face Areas for Central Station Units (Air Std. for Certified Ratings Program Air Std. Test Code for Air

, SAE J943A Std for Slow ting Brackets and Socket for Warning Lamp (Light) and Slow ural (Farm) and Industrial Equipment Warning Lamp and Slow orm Building Code: Elevators, Dumbwaiters, Escalators, and td. Pract. for the Inspection of Elevators, Escalators and

Mouldings (1967)
Motor Vehicle Lighting Devices and Components (1972)

Motor Vehicle Lighting Devices Including Reflex Reflect Motor Vehicle on Grade Parking Performance Requirements

Motor Vehicle Type (Lead Acid) Storage Batteries (1972)

Motor Vehicle (Recreational Type) Connecting Devices an

Motor Vehicles (1971)

Motor Vehicles (1973)

Motor Vehicles (1973)

Motor Vehicles (1973)
Motor Vehicles (20-1000 Mhz) (1972) ANSI C1 Cr 12.1
Motor Vehicles, Motorboats, Tractors, Automotive

Rec. Pract. for Average Vehi

Motor Vehicles, Motorboats, Tractors, Automotive and in

Motor Vehicles, Trailers, and Semitraile

Motorboats, Tractors, Automotive and Industrial Applica

Motorcycle Headlamps (1971)

Motorcycle Rear View Mirrors (1971)

Motorcycle Turn Signal Lamps (Light) (1971)

Motorcycle, Side Car, Mini Bike, and Bicycle (1974)

Motorcycles (1972)

Motorcycles, Passenger Cars, Off Road. Agricultural and

Motorcyclists Eye Protection (1971)

Motors and Generators for Use in Hazardous Locations, C

Motors and Generators for Use in Hazardous Locations, C

Motors and Generators (Fractional and Integral Horsepow

Motors and Generators (Fractional and Integral Horsepow

Motors and Generators (Fractional and Integral Horsepow

Motors and Generators (1972)

Motors and Generators (1972)

Motors and Generators (1973) ANSI C51.1

Motors and Generators (1973) ANSI C52.1

Motors and Phase Converters (1972)

Motors for Appliances and Equipment (1972)

Motors Installed Inside the Containment of Nuclear Powe

Motors to Agricultural Tractors and Trailing Type (Farm

Motors (Fractional and Integral Horsepower) (1972)

Motors (Fractional and Integral Horsepower) (1972)

Motors (Large Apparatus) (1972)

Motors (Large Apparatus) (1972)

Motors (Large Apparatus) (1972)

Motors (1972)

Motors (1973)

Motors (1973) ANSI B93.27

Motors (1974)

Mount (1973)

Mountain Hemlock (Lumber) (1973)

Mounted Circuit Breaker (1971)

Mounted Drum Hoists (1971)

Mounted Room Thermostats (1972)

Mounted Speed Reducers (1974)

Mounted Thermostats for Electric Heater Line Voltage in

Mounted Thermostats for Electric Heaters (1973)

Mounted Tin Clad Fire Doors (1973) ANSI A 143.1

Mounted Types (1971)

Mounting and Face Dimensions of Receptacles. Ofpec. for

Mounting Brackets and Socket for Agricultural (Farm) an

Mounting Brackets and Socket for Warning Lamp (Light) a

Mounting Dimensions for 3/4, l and 1 1/8 In. Bore Catal

Mounting on Agricultural (Farm) Equipment (1972)

Mounting (Front and Rear) (1970)

Mounting), Liquid Withdrawal (1973)

Mounting, Liquid Withdrawal Only (1973)

Mountings for Diaphragm Type Pumps (1971)

Mountings (Boats) (1971)

Mountings (Farm Machinery) (1973)

Mountings (Instal)

Mountings (1972)

Mounts for Cameras (1972)

Mounts for Slides and Opaques for Television (1973)

Movement (Cause

(Tech. Notes) (1963)

Movement (Expansion Joints) (Tech. Notes) (1963)

$/ \mathrm{P}$

Gas Fuel Contai Act. for LP G

ABYC

Std. ANSI

Movement (1972)

Movers (Heavy Equipment) (1972)

. Notes) (1963)

/Nimum Performance Cri

Movers, Wheeled and Track Type

Moving and Conditioning) (1966)

Moving Devices (Performance) (1970)

Moving Devices (1967)

Moving Vehicle Identification Emblem (1968) ANSI B114.1

Moving Vehicle (SMV) Identification Emblem (1972)

Moving Vehicles (SMV) Identification Emblem (1972) SAE

Moving Walks (1973)

Moving Walks (inspectors Manual) (1973)
SAE

$S A E$

SAE

SAE

SAE

VESC

CHI

SAE

ASAE

SAE

ANSI

ANSI

ANSI

BIA

$\mathrm{BIA}$

BIA

$\mathrm{J} 537 \mathrm{H}$

J584B

J268

J131A

6

$\mathrm{J} 213 \mathrm{~A}$

V-8

$674 \mathrm{~A}$

674B

MGl.11

MGl.14

MG1-18

MGl-1

$\mathrm{MGl}-2$

MG2

$\mathrm{MGl}$

R329

1004

334

S316 T

MG1-10

MG1.12

MGI-20

MG1.21

MGl.23

MGl3

5-6

T3.9.17

5.13

$* 29$

487

UBCS25.4

J258

B30.7

DC 3

480.05

DC 13

DC 13

$14 \mathrm{~B}$

100

C 73.67

S 277.2

J725D

NFLDP T3.6.11

ASAE S307.1

J616B

6 A 2

$6 \mathrm{Al}$

J625A

S225.1

$\mathrm{J} 233$

$\mathrm{S} 2.8$

PH3. 14

$\mathrm{PH} 22.94$

PH3.16

18 A

$18 \mathrm{~A}$

C719

J320B

$\mathrm{J} 231$

AMCA 1401

AMCA 1402

AMCA 211

AMCA 210

ASAE S276.2

J 725 D

J725D

$\begin{array}{ll}\text { ASAE } & \text { S277.2 } \\ \text { ICBO } & \text { UBC } * 3.5]\end{array}$

ANSI Al7.2 
ety for Cord Connected Electric Gardening Appliances (Lawn Rec. Pract. Safety Requirements for lndustrial Rotary Safety Spec. for Power Rec. Pract. for Passenger Car and Light Truck Std. Test Procedure for Geiger Std. Classification of

ys, Diaspore, Ganister Quarzite, Grog, Kyanite, Magnesite, nce of a Fine Hydraulic Fluid Power Filter Element / Std. Spec. for Firecycle on -Off tape Recorders Operati/

Std Spec, for an Audio Level and Std. Performance Spec. for Flexible

thickness on Silicon Wafers and Metallization Thickness by

Prevention of Furnace Explosions in Pulverized Coal Fired

d. Production Check Flanges and Gaskets; Drilling Through. e-Propylene-Rubber lnsu/ Std. for Portable Single and Manual of Practice: $\mathrm{n}$ of Units in Multip/ Std. for Numbering of Electrodes in Spec. for Electric Overhead Bridge and Gantry ed Chlorine Bleaching (1971) ANSl L14.180 Test Meth. for 73) NEMA SH17 $\mathrm{j} \cdot 147$ Sid. for Std. for

fety Std. for Heat Actuated Fire Alarm Devices, Single and lectrode Semiconductor Devices and Designation of Units in moisture Basis (Cereal Chemistry) (1965) Meth. for ances and Other Electronic Equipment (Flyback Transformer. g Surf/ ols (1969) Std. for Rec. Pract. for Spark Arresters Used on lection of Tires (Car. Station Wagon, Truck, Bus, Trailer. s lntended for Highway IIse on Trucks, Buses, Trailers, and ply Tires and Rims for Passenger Cars, Station Wagons, and bin/ Rec. Pract. for Stopping Distance of Passenger Cars, Std for Tires and Rims for Truck. Bus, Trailer, and ill Pipes with Fuel Dispensing Facilities (Passenger Cars, Std. Meth. of Specifying Color by the Std. Spec. for Natural

(1971) ANSI C59.27 Std. Meth. of Test for Undersized Loose Std. Spec. for Spec. for Steel Spring Wire, Best Quality ion and Structural Group Analysis of Petroleum Oils by the of Fractures of the Skeletal (Bone) / Std. Spec. for Hip 73) Uniform Building Code Std. for Fiberboard Std. Rec. Pract. for Static Bend Test. of led Finish Flooring Over Concrete Slab / Spec. for Mastic mastic Nailed Construction with Mastic Set Subflooring and 1962) Spec. for Rubber Cushion Sleeper Construction with lab Floors / Spec. for Mastic Cushioned Construction with Rec for Wood Siding. Finish, Nails and Redwood Exterior Guide (Siding, Finish, Nails and ication and Design, Span Allowances, Storage. Finishes and Rec. for Wood Siding, Finish, Redwood Exterior Guide (Siding. Finish. Timber Fasteners (Connectors, Bolts, Lag Screws, Plates, screws; Light Metal Plate Connected Wood Truss Design: and

Piles, Spaced Columns, Joints, Screws, Bolts, Connectors, chloroethyl) Thio)-4-Cyclohexene-1,2-Dicar/Std. Common ilate Carbanilate (Ester) Desmedipham (1/ Std. for Common rimethyl-2,4-Dodecadienoate Hydroprene/ Std. for Common obenzilate-Bromopropylate (1973) robenzilate-Chloropropylate (1973)

rophenoxy).2-Nitrobenzoate Bifenox (197) . A-Trifluoro-2,6-Dinitro-N-Propyl-Ptoluid/ -A, A, A-Trifluoro-2.6-Dinitro-N-Propyl-Pto/ opyl Phosphorodithioate) (1973/ horodithioate S-Ester Wi/ Std. Common Std. Common Std. Common Std. Common Std. for Common ylphenyl Methylcarbamate-Dioxacarb (1973) ethoxy-3,7,11-Trimethyl-2,4-Dodecadienethi/ 6-Trichloro-2-Pyridy]) Phosphorothioal mino).6-(Methylthio).S-Triazine-Prometry/ mino)-6-Methoxy-S-Triazine-Prometon (197) opropylamino) -S-Triazine-Propazine (1973) 7,11-Trimethyl-2,4-Dodecadienoate Kino/ sopropylamino)-6-(Methylthio)-S-Triazine - 1 bis (lsopropylamino) Strial Std. for 4-(Ethylamino)-6-(Methylthio).S-Triazine /
4-Chloro-6-(Ethylamino)-S-Triazine-Terb/ 4-Chloro-6-(Ethylamino)-S-Triazine-Te
mino) -2-(a,a,a- Trifluoro-m-tolyl $)-3$ / Std. Common Std. Common

Std. for Common Std. Common Std. Common Std. Common

Std. for Common Std. Common Std. Common Std. Common Std. for Common Std. for Bentazon (Common Std. for Tetramethrin (Common Std. for Fospirate (Common Std. for Disugran (Common Std. for Crufomate (Common Std for Chlorbromuron (Common Std. for Chlordimeform (Common Std. for Fluorodifen (Common Std. for Prynachlor (Common
Mower, Cultivator, Edger-Trimmer, Hedge Trimmer, etc.) Mowers (Designed for Operation with Tractors Having at Mowers, Lawn, and Garden Tractors (1972)

Muffler Parts Nomenclature (1971)

Muller Counters (1970) ANSI N42.3

Mullite Refractories (1972)

Mullite) (I970) ANSl Alll.36

/Calcine, Chrome Ore,

Multi Pass Meth. for Evaluating the Filtration Performa

Multicycle Sprinkler System (Fire Safety) (1973)

Multifrequency Test Tape for Quadruplex Video Magnetic

Multilayer Wiring (Printed Circuit) (1972)

Multiple Beam Interference (Tolansky Meth.) (1973)

Multiple Burner Boiler Furnaces (Fire Protection) (1973

Multiple Completion, and Flowline Valves; Drilling Thro

Multiple Conductor Electrical Power Cables with Ethylen

Multiple Contract Construction (1974)

Multiple Electrode Semiconductor Devices and Designatio Multiple Gerder Traveling Cranes (1971)

Multiple Sample Meth. of Textile Tensile Loss by Retain Multiple Sockets Used in Roadway Lighting Equipment (19 Multiple Sockets Used in Roadway Lighting (1973) EEl Td

Multiple Station, Mechanically Operated Type for lndoor

Multiple Unit Semiconductor Devices (1972)

Multiplication Factors for Correcting Weights to a $14 \%$

Multiplier, Deflection Yoke, Picture Tube Neck Componen

Multiply Felt and Asphalt Preferably with Gravel or Sla

Multiport Valves for Public and Residential Swimming Po

Multiposition Small Size Engines (1972)

Multipurpose Passenger and Recreational Vehicles) (1974

Multipurpose Passenger Vehicles (1972)

/Emountable Rim

Multipurpose Passenger Vehicles (1974) las and R

Multipurpose Vehicle (1974)

Multipurpose Vehicles, and Light Duty Trucks) (197I)

Munsell System (1968)

Muscovite Block Mica and Thins Based on Visual Quality

Muscovite Mica Splittings (1973)

Music Spring Quality Steel Wire (1971) ANSI G54.2

Music (1973)

n-d-M Meth. (1973)

Meth. of Test for Carbon Distribut

Nail-Jewelt Type for Use by Surgeons in the Treatment

Nail Base Sheathing and Structural Insulating Board (I9

Nail Plates (Surgical Implants) (1973)

Nailed Construction with Mastic Set Subflooring and Nai

Nailed Finish Flooring Over Concrete Slab Floors (1962)

Nailers and Finish Flooring Over Concrete Slab Floors (

Nailers, Subflooring or Finish Flooring Over Concrete S

Nailing (1972)

Nailing) (1973

Nails and Fastenings) (1973)

Nails and Nailing (1972)

Nails and Nailing) (1973)

Nails and Spikes. Washers, Pins, Dowels, Joints, Split

Nails and Staples (1973)

Nails, Staples, Adhesives, Fiberboard, Particleboard) (

Name for the Pest Control Chemical cis-N.((1,I.2,2tetra

Name for the Pest Control Chemical Ethyl Mhydroxycarban

Name for the Pest Control Chemical Ethyl (E,E)3,7,11-T

Name for the Pest Control Chemical lsopropyl 4,4'dibrom

Name for the Pest Control Chemical lsopropyl 4,4'dichlo

Name for the Pest Control Chemical Methyl 5(2,4-Dichlo

Name for the Pest Control Chemical N-(2Chloroethyl)-A, a

Name for the Pest Control Chemical N(Cyclopropylmethyl)

Name for the Pest Control Chemical O-Ethyl S.S-Dipr

Name for the Pest Control Chemical O,O-Dimethyl Phosp

Name for the Pest Control Chemical O-1,3-Dioxolan .2-

Name for the Pest Control Chemical S-Ethyl (E.E)-11-M

Name for the Pest Control Chemical 0,0 Dimethyl 0.(3.5.

Name for the Pest Control Chemical 2,4-Bis (lsopropyla

Name for the Pest Control Chemical 2,4-Bis (Isopropyla

Name for the Pest Control Chemical 2-Chloro-4 6bis (ls

Name for the Pest Control Chemical 2-Propynyl (E,E)-3,

Name for the Pest Control Chemical 2-(Ethylamino)4-(l

Name for the Pest Control Chemical 2-(Ethylthio) +4.6-

Name for the Pest Control Chemical 2-(Tertbutylamino)-

Name for the Pest Control Chemical 2-(Tertbutylamino)-

Name for the Pest Control Chemical 4-Chloro5-(Methyla

Name for the Pest Control Chemical (1972)

Name for the Pest Control Chemical) (1971)

Name for the Pest Control Chemical) (1971)

Name for the Pest Control Chemical) (1971)

Name for the Pest Control Chemical) (1972)

Name for the Pest Control Chemical) (1972)

Name for the Pest Control Chemical) (1972)

Name for the Pest Control Chemical) (1972)

Name for the Pest Control Chemical) (1972)
UL

SAE

ANSI

SAE

ASTM

ASTM

NFLDP

FMS

ANSI

IPC

/E ASTM

FMS

API

NEMA

CSI

ElA

CMAA

AATCC

EEl

NEMA

UL

ElA

AACCH

UL

SAF

TRA

SAE

TRA

SAE

TRA

SAE

ASTM

ASTM

ASTM

ASTM

$\mathrm{SAE}$

ASTM

ASTM

ICBO

ASTM

WSFI

WSF

WSFI

WSF

WW PA

CRA

CRA

WWP

CRA

WW WA

ICBO

ICBO

ANSI

ANSI

ANS

ANSI

ANSI

ANS

ANSI

ANSI

ANSI

ANSI

ANSI

ANSI

ANSI

ANSI

ANSI

ANSI

ANSI

ANSl

ANS I

ANSI

ANS

ANSI

ANSI

ANSI

ANSI

ANSI

ANSI

ANSI

$\mathrm{J} 232$

B71.1

J261

309
C467

C316

T3. 10.8.8

2.90

98.8

ML.990

F388 
Std. for Glyphosine (Common Std. for Mexacarbate (Common Std. for Dinoseb (Common Std. for Acephate (Common Std. for Ethiolate (Common Std. for Glyphosate (Common Std. for Oxamyl (Common Std. for Picloram (Common Std. for Nitrapyin (Common -Tert-Butylphenoxy) Cyclohexyl 2Propynyl Sulfite (Common methoxy-3,7,11-Trimethyl-2,4-Dodecadienoate) (Common Rec. for Flash Point Index of Trade Rec. Pract. for High Lift Industrial Truck (Institutional / Std. Performance Requirements for Table Std. Performance Requirements for Woven Printed Table Std. Performance Requirements for Damask Table Std. Spec. for Refined Solvent resentative Saturates Fraction from Low Olefinic Petroleum of Test for Acidity of Benzene, Toluene, Xylenes, Solvent reservative Solutions Such as Pentachlorophenol and Copper

Std. Performance Requirements for Damask Table Napery Performance Requirements for Woven Printed Table Napery $(1972$ /

ments for Table Napery Other Than Damask (White or Dyed) Std. Spec. for Copper Alloy No. 260 Brass Strip in enance in SI Metric Units (1973) tenance (1974)

ressure Vessel Inspectors (1973)

astenings (1971)

s by OSHA (1973)

Rec. and Comments on the

Rules for the Radio Installations for the Operation of Electric Supply Lines and Equipment e of Underground Electric Supply and Communications Lines installation and Maintenance of Electrical Supply Statio/ 73)

aphic Representation of the Control Character of American ixed Mica-Dielectric Capacitors (1971) /

Std. Spec, for Std. Definitions of Terms Relating to Std. Spec. for Std. Meth. for Accelerated Laboratory Test of $r$ (Spectrophotometric) Content in Feedstuffs and All Other Std. Tables of Classification of Man Made and Std. Meth. of Test for Total Sulfur in
Std. for Prevention of Furnace Explosions in Fuel Oil and Std. Meth. for Analysis of
St Std. Meth. for Analysis of ANSI Z11.238

(1968) Std. Meth. for Obtaining Std. Meth. of Test for Hydrogen Sulfide in .177 Test Meth. for Resistance of Textiles: Exposure to

Test Meth. for Resistance of Textile Fabrics: Exposure to Std. Rec. Pract. for Conducting quality (1971) ANSl C59.27 Std. Spec. for 1973) ANSl A3/ Sid. Spec. for Fly Ash and Raw or Calcined able, 75 Deg. C Operation (1974) ANS1 C8./ Std. Spec. for .25

r Concentrated, Ammonia Preserved, Creamed and Centrifuged Std. Meth. for Evaluation of and Test Formulas for h. for Evaluation of and Test Formula for Carbon Blacks in td. Meth. of Measuring Plasticity Retention lndex (PRI) of Uniform Building Code Std. for Std. Definitions of Terms Relating to Std. Definitions of

Rec. Pract. and Stds. for Location and Visibility of cs Vertical Cuidance Equipment Used in Airborne Volumetric of and Test Formula for Butadiene-Acrylonitrile Rubbers ry Therapy Equipment (Breathing Devices, Room Humidifiers, ack Transformer, Multiplier, Deflection Yoke, Picture Tube Std. for Thyroid Radioiodine Uptake Measurements Using A Std. for Cam Follower Std. for In. and Metric Radial Std. Meth. for Determining Speed of Photographic ate) for Use in Photographic Contact Printing of Roll Film

Tent Meth of Test for Effective Fluorescent Lifetime of st for Atom Percent Fission in Uranium and Plutonium Fuel

, Deuterium, Fluorine, Helium, Hydrogen, Krypton, Methane, il Pipe and Fittings (1972) Std. Spec. for 80) (1974) Std. Meth. of Test for Number of Std. for $5 \mathrm{Gal}$. a80, Dot-37Aa60, Ufc-Rule 40,/ Std. Spec. for $5 \mathrm{Gal}$. Minimum Requirements for Safety Std. Requirements, Terminology, and Test Procedure for 66) IPCEA S.64.474 Std. for Aluminum, Triplex, Meth. of Analysis for Meth. of Analysis for n Phosphates (Cereal Chemistry) (1/ $\mathrm{e}$ and Anhydrous (Cereal Chemistry)! Chemistry) (1962)
Name for the Pest Control Chemical) (1972)

Name for the Pest Control Chemical) (1972)

Name for the Pest Control Chemical) (1972)

Name for the Pest Control Chemical) (1972)

Name for the Pest Control Chemical) (1972)

Name for the Pest Control Chemical) (1972)

Name for the Pest Control Chemical) (1972)

Name for the Pest Control Chemical) (1972)

Name for the Pest Control Chemical) (1972)

Name for the Pest Control Chemical) (1973)

Name for the Pest Control Chemical) (1973)

Name Liquids (NFPA 325A) (1974)

Nameplates and Marking (1973)

Napery Other Than Damask (White or Dyed) (Napkin, etc.)

Napery (Napkin, etc.) (Institutional Textile) (1973)

Napery (Napkin, etc.) (Institutional Textile) (1973)

Naphtha (1971) ANS1 Z78.4

Naphthas (1973) ANS1 Z11.165

Naphthas, and Similar lndustria

Naphthenate (1973)

Napkin, etc.) (Institutional Textile) (1973)

(Napkin, etc.) (Institutional Textile) (1973)
(Napkin, etc.) (lnstitutional Textile) (1973)

Narrow Widths and Light Gage for Heat Exchanger Tubin

NaSCN (1972)

National Aerospace Std. Documents Preparation and Maint

National Aerospace Stds. Documents Preparation and Main

National Board lnspection Code) Manual for Boiler and P

National Design Spec. for Stress Grade Lumber and lts F

National Electrical Code (NFPA 70) and Code Requirement

National Electrical Safety Code (1973)

(National Electrical Safety Code) (1940)

(National Electrical Safety Code) (1973)

National Electrical Safety Code) (1973)

National Electrical Safety Code, Part 1: Rules for the
National Std. Code for lnformation lnterchange (1973)

National Std. Plumbing Code (Including Definitions) (19

Natural Block Mica and Mica Films Suitable for Use in F

Natural Building Stones (1974)

Natural Cement (1973) ANSI Al.18

Natural Decay Resistance of Woods (1970)

Natural Dry Products Derived froin Corn (1957)

Natural Fibers (1971) ANSI L14.192

Natural Gas by Hydrogenation (1972) ANS1 Z77.11

Natural Gas Fired Watertube Boiler Furnaces with One Bu

Natural Gas Liquid Mixtures by Gas Chromatography (1968

Natural Gas Liquid Mixtures by Gas Chromatography (1973

Natural Gas Samples for Analysis by Gas Chromatography

Natural Gas (Methylene Blue Meth.) (1970) ANS1 Z77.10

Natural Light and Weather Through Glass (1972) ANSI L14

Natural Light and Weather (1968) ANS1 L14.176

Natural Light Exposures Under Glass (1973)

Natural Muscovite Block Mica and Thins Based on Visual

Natural Pozzolans for Use in Portland Cement Concrete (

Natural Rubber Heat Resisting lnsulation for Wire and C

Natural Rubber Jacket for Wire and CabIe (1973) ANSI C8

Natural Rubber Latex (1972A) ANS1 J8.12

Natural Rubber (Nr) and Isoprene Rubber (lR) (1973)

Natural Rubber (Nr) (1973)

Natural Rubber (1973)

Natural Slate Roof Shingles (1973)

Naval Stores and Related Products (1972)

Navigation Aid Terms (1971)

Navigation Lights (Boats) (1972)

Navigation Systems (1972)

NBR) (1973)

Nebulizers, Gas Therapy and

Neck Components) (1974)

Needle Bearing (Medical Radiation-Radiology) (1973)

Needle Roller Bearings (1973) ANSI B3.18

Negative Materials (Monochrome, Continuous Tone) (1972) Negatives (1972)

Neodymium Doped Laser MateriaIs (1973)

Neodymium-148 Meth.) (1969) ANS1 N118

Neon, Nitric Oxide, Nitrogen, Oxygen) (1973)

Neoprene Rubber Gaskets for Hub and Spigot

Neps in Cotton Samples (1971) ANSl L14.97

Nesting Lug Cover Universal Pail (Container) (Dot-37C

Nesting Lug Cover Universal Pails (Container) (Dot-37

Nets (1971)

Neutral Grounding Devices (1972)

Neutral Supported, Service Drop and Secondary Cable (19

Neutralizing Value of Acid Reacting Materials Other Tha

Neutralizing Value of Monocalcium Phos phate: Monohydrat

Neutralizing Value of Sodium Acid Pyrophosphate (Cereal
ANSI

ANS1

ANSl

ANS

ANSI

ANSI

ANSI

ANSl

ANSI

Rgite 2-(P ANSI

E,E).11- ANS

FMS

ITA

ANSI

ANS

ASTM

ASTM ASTM

ASTM

ANSl

Std ANS

Requir ANSI

ASTM

ANS

NSA

NSA

NBBPV

NFORP

FMIS

ANSI

ANS

Rules ANS

ANSI

ANS1

ANSI

NAPHC

ASTM

ASTM

ASTM

ASTM

/ Ng Colo CR

ASTM

ASTM

NFPA

NGPA

ASTM

NGPA

ASTM

AATCC

AATC

ASTM

ASTM

ASTMI

ASTM

ASTM

ASTM

ASTM

Std. Met ASTM

ASTM

ICBO

ASTM

lEEE

ABYC

RTCA

ASTM

NSF

UL

ANSI

NSA

AFBM

ANSl

ASTM

Td. Meth. of Te ASTM /Monoxide ICBO

CISPl

ASTM

ANSI

ANSl

ANS

IEEE

EEI

AACCH 02

$\mathrm{AACCH} \quad 02.3$

AACCH 02.34

$7.42 \mathrm{~N}$

D838

D2002

D847

B569

10000

K62.117

K62.118

K62.119

K62.120

K62.123

K62.124

62.126

K62.127

K62.128

K62.131

K62.132

L24.2.11

L24. 2.6

L24.2.7

D2604

L 24.2 .6

L24.2.11

$\mathrm{PH} 4.177$ 
971) ANSI K90.10 Spec. for Tinned Copper Concentric Std. Meth. of Test for Oxygen Content Using a $14 \mathrm{Mev}$ Std. Guide for Selection of olant Water During/ plications (1960)

m Uranium -238 Fission (1972/ N114

112

113

n 109

n] 10

„He Neutron Cenerators by Radio/

anium 238 Fission (1973)

(

Rec. for Measurement of

Std. Meth. of Test for Fast

Std. Meth. for Measuring Fast

Std. Meth. for Measuring Fast

Std. Meth. for Measuring Fast

Std. Meth. for Measuring Fast Std. Meth. for Measuring

Meth. for Measuring Thermal Std. Meth. of Test for Std. Meth. for Measuring Fast Std. Meth. for Fast

utron Flux Density and Average Energy from $\mathrm{H}(\mathrm{D}, \mathrm{N}), \mathrm{He}$ asurement of Absorbed Dose of Neutrons, and of Mixtures of 961) Rec. for Measurement of Absorbed Dose of istry) (1967)

rmining the Susceptihility of Stainless Steels and Related

Used to Enrich Cereals (Cereal Che/ Meth. of Analysis of

Microbiological Meth. of Analysis of

972) ANSl C33.3

luoride Carri/

Std. Spec. for Chromium-CopperStd. Meth. for Spectrochemical Analysis of ate, Sheet and Strip (1973)

d (1973)

(1970) ANSI H34.10

Std. Spec. for Std. Spec. for Std. Spec, for

1973) Std. Meth. for Chemical Analysis of Nickel-Chromium and pressure Vessel Plates, Alloy Steel, Quenched and Tempered

d. Reference Radiographs for High Strength Copper Base and Plate, Sheet, and Strip (1973)

amless Pipe and Tube (1972) ANSI H34.16 Std. Spec. for (1973) ANSI H34.40 Std. Spec. for 34.39

Std. Spec. for ) ANSI H34.41

aed Alloy / Std. Spec. for Seamless and Welded Chromium zed All/ Std. Spec, for Electric Fusion Welded Chromium perature Service (1974) ANSI G/ Std. Spec. for Chromium $\mathrm{ss}$, Heat Resisting, Maraging, and Other Similar Chromium late (1971) ANSI H34.44 Std. Spec. for 971) ANSl H34.12

4.14 Std. Spec, for Std. Spec, for Std. Spec, for Std. Spec. for 34.11

Std. Spec. for Copper

Alloy Plate, Sheet, Strip, and/

Std. Spec, for Copper

Rod and Bar (1973)

Std. Spec. for Copper

Temperature Serv

Std. Spec, for Precipitation Hardening Std. Spec, for Chromium

ansi $\mathrm{H} 34.42$ Std. Spec. for Seamless Nickel and
Std. Spec. for ) ANSI H52.l Std. Spec. for

re Service (1972)

Std. Spec. for Copper Copper-Nickel-Zinc Alloy (Nickel Silver) and Copper for Low Temperature /

elded Large Diameter Austenitic Chromium nsi G33.1

nsi G.35. 15 Std. Sper c. for Manganese-Molybdenum and Manganese-Molybdenum Sid Spec, for Pressure Vessels Plates, $5 \%$ ) ANSI H3/ Std. Spec, for Factory Made Wrought Nickel and e / Sid. Meth. for Spectrochemical Analysis of Thermionic tation and Hardware (1970) Std. for Electroplating of 1969) ANSl W 3.14

ansi W3.11

bes (1972) ANSI H34.42 iping (1972) ANSI H3/

Spec. for

Std. Spec. for Seamless Alloy Sheet and Strip, Corrosion and Heat Resistant, y Bars, Forgings, and Rings, Corrosion and Heat Resistant,

y Bars, Forgings, and Rings, Corrosion and Heat Resistant,

loy Sheet, Strip, and Plate, Corrosion and Heat Resistant,

c) Alloy Bars and Forgings, Corrosion and Heat Resistant, Alloy Wire, Welding, Corrosion and Heat Resistant,

y Bars, Forgings, and Rings, Corrosion and Heat Resistant, orrosion Resistant Iron Chromium, lron Chromium Nickel and d Temperatures (1973) A/ Std. Spec. for lron. Cobalt, and $\mathrm{n} / \quad$ Alloy Bars and Forgings, Corrosion and Heat Resistant,

oy Bars, Forgings and Rings, Corrosion, and Heat Resistant, rosion and Heat Resistant Alloy Bars, Forgings, and Ring loy Sheet, Strip, and Plate, Corrosion and Heat Resistant, Std. Spec. for Std. Spec. for
Neutrals (Underground Electrical Power Cable) (1973)

Neutron Absorption Cross Section of Nuclear Graphite (1

Neutron Activation and Direct Counting Technique (1973)

Neutron Activation Detector Materials (1973)

Neutron Emitting Fission Products in Nuclear Reactor Co

Neutron Flux and Spectra for Physical and Biological Ap

Neutron Flux by Analysis of Molybdenum .99 Activity Fro

Neutron Flux by Radioactivation of Aluminum (1970) ANSI

Neutron Flux by Radioactivation of Iron (1970) ANSI N1l

Neutron Flux by Radioactivation of Nickel (1970) ANSI N

Neutron Flux by Radioactivation of Sulfur (1970) ANSI N

Neutron Flux by Radioactivation Techniques (1970) ANSI

Neutron Flux by Radioactivation Techniques (1970) ANSl

Neutron Flux Density and Average Energy from ${ }_{3} \mathrm{H}(\mathrm{D}, \mathrm{N})$

Neutron Flux for Analysis for Barium 140 Produced by $U_{r}$

Neutron Flux Measurements by Track Etch Technique (1973

Neutron Generators by Radioactivation Techniques (1973)

Neutrons and Gamma Rays (1961)

Rec. for Me

Neutrons, and of Mixtures of Neutrons and Gamma Rays (1

Ni.Cr.Fe Alloys to Stress Corrosion Cracking in Polythi

Niacin and Nicotinamide in Cereal Products (Cereal Chem

Niacin in Enrichment (Vitamin and Mineral) Concentrates

Niacin (Cereal Chemistry) (1962)

Nickel-Aluminum Alloy Steel Pressure Vessel Plates (1

Nickel-Base Alloys by the Silver Chloride-Lithium F

Nickel-Chromium-lron-Molybdenum-Copper Alloy Pl

Nickel-Chromium-lron-Molybdenum-Copper Alloy Ro

Nickel-Chromium-lron Alloy Plate, Sheet, and Sirip

Nickel-Chromium-lron Alloys (1973)

Nickel-Chromium and Nickel-Chromium-lron Alloys (

Nickel-Cobalt-Molybdenum-Chromium (1972) ANSI G.35

Nickel-Copper Alloy Castings (1967) ANSI Z166.17

Nickel-Iron-Chromium-Manganese-Molybdenum Alloy

Nickel-Iron-Chromium-Molybdenum-Copper Alloy Se

Nickel-Iron-Chromium Alloy Plate, Sheet, and Strip

Nickel-lron-Chromium Alloy Rod and Bar (1973) ANSI

Nickel-lron-Chromium Alloy Seamless Pipe Tube (1973

Nickel-Iron-Molybdenum-Copper-Columbium Stabili

Nickel-lron-Molydenium-Copper-Columbium Stabili

Nickel-Iron Alloy Castings (25-12 Class) for High Tem

Nickel-lron Alloys (1973)

Mical Analysis of Stainle

Nickel-Molybdenum-Chromium-lron Alloy Sheet and P
Nickel-Molybdenum-Chromium Alloy Plate and Sheet (1

Nickel-Alolybdenum-Chromium Alloy Rod (1971) ANSl H3

Nickel-Molybdenum Alloy Platc and Sheet (1971) ANSI H

Nickel-Molybdenum Alloy Rod (1971) ANSI H34.13

Nickel-Silicon Alloy Rod and Bar (1973)

Nickel-Zinc Alloy (Nickel Silver) and Copper-Nickel

Nickel-Zinc Alloy (Nickel Silver) and Copper-Nickel

Nickel Alloy Bars, Forgings, and Forging Stock for High

Nickel Alloy Castings (1974)

Nickel Alloy Condenser and Heat Exchanger Tubes (1972)

Nickel Alloy Forgings (1972)

Nickel Alloy Plate and Sheet for Pressure Vessels (1969

Nickel Alloy Plate, Sheet, and Strip for High Temperat

Nickel Alloy Plate, Sheet, Strip, and Rolled Bar (1973)

Nickel Alloy Steel Flanges, Fittings, Valves, and Parts

Nickel Alloy Steel Pipe for Corrosive or High Temperatu

Nickel Alloy Steel Pipe for High Temperature Service (1

Nickel Alloy Steel Plates for Pressure Vessels (1972) a

Nickel Alloy Steel Plates for Pressure Vessels (1972) a

Nickel Alloy Steel, Quenched and Tempered, for Pressure

Nickel Alloy Steel, Specially Heat Treated (1972A)

Nickel Alloy Welding Fittings for Pressure Piping (1972

Nickel Alloys by the Powder Direct Current Arc Techniqu

Nickel and Chromium on Metal Parts-Automotive Ornamen

Nickel and lts Alloy Bare Welding Rods and Electrodes (

Nickel and lts Alloy Covered Welding Electrodes (1969)

Nickel and Nickel Alloy Condenser and Heat Exchanger tu

Nickel and Nickel Alloy Welding Fittings for Pressure P

Nickel and Nickel Base Alloy Castings for General Appli

Nickel Base-15.5Cr-2.4T i-0.70A l-7.0Fe (1973)

Nickel Base-15.5Cr-8.0Fe (1973)

Nickel Base-15.8Cr-15.2 Mo-0.30Al-0.05La (1973)

Nickel Base-15.8Cr-15.2Mo-0.30Al-0.05La (1973)

Nickel Base-19.5Cr-13.5Co-4.3Mo-3.0 Ti-1.4Al

Nickel Base-19.5Cr-18Co-2.5Ti-1.5Al (1973)

Nickel Base-5.0Cr-24.5Mo-5.5Fe, Solution Heat Tre

Nickel Base Alloy Castings for Ceneral Application (197

Nickel Base Alloy Castings for High Strength at Elevate

Nickel Base 19.5 $\mathrm{Cr}-13.5 \mathrm{Co}-4.3 \mathrm{Mo}-3.0 \mathrm{Ti}-1.4 \mathrm{Al}$, Co

Nickel Base $\left(19.5 \mathrm{Cr}-13.5 \mathrm{Co}-4.3 \mathrm{Mo}_{0}-3.0 \mathrm{Ti}-1.4 \mathrm{Al}\right) \mathrm{C}$

(Nickel Base, Solution Heat Treated, 15.5Cr-16Mo-3.8

Nickel Base, $20 \mathrm{Cr}-20 \mathrm{Co}-5.9 \mathrm{Mo}-2.2 \mathrm{Ti}-0.45 \mathrm{Al}$ Solut

Nickel Chromium lron Alloy Rod and Bar (1970) ANSI H34.

Nickel Chromium lron Alloy Seamless Pipe and Tube (1970

AA

ASTM

ASTM E419

ASTM D2470

NCRPM R23

ASTM E343

ASTM E266

ASTM E263

ASTM E264

ASTM E265

ASTM E26

ESTM 262

ASTM E496

ASTM E393

ASTM E418

ASTM E496

NCRPM R25

NCRPM R25

STM G35

$A A C C H \quad 86.50$

AACCH $86-49$

AACCH 86.5

ASTM A4l0

ASTM E483

ASTM B582

ASTM B581

ASTM Bl68

ASTM E38

ASTM E38

ASTM A605

St ASTM E272

ASTM B590

ASTM B 423

ASTM B 409

ASTM B408

ASTM B407

ASTM B468

ASTM B474

ASTM A447

ASTM E353

ASTM B 434

B334

ASTM B336

ASTM B 333

ASTM B335

ASTM B4ll

ASTM B122

ASTM B15I

ASTM A637

ASTM A560

ASTM Bl63

ASTM B564

ASTM B402

ASTM A670

ASTM B B 22

ASTM A 522

ASTM A409

ASTM A358

ASTM A203

ASTM A 302

ASTM

ASTM A645

ASTM B366

ASTM E 129

SAE J207

AWS A5.14

AWS A5.11

ASTM B 63

ASTM B366

ASTM

SAE

Allo SAE

SAE

SAE

SAE

SAE

ASTM

$\mathrm{SAE}$

SAE

SAE

SAE

A STM

AMS5541B

AMS5665H

AMS5711

AMS5873

AMS5738C 
y Seamless Pipe and Tube (1972) ANSI H34./ and Tempered, for Pressure Vessels (1972/ and Strip (1972) ANSI H34.19 (1972) ANSI H34.22

e and Tube (1972) ANSI H34.20

lication (1972)

c7. 48

H34.6

3

Std. Spec. for Pressure Vessel Plates, Alloy Steel, 9\% Std. Spec. for Gold, Silver.

gh T/ Std. Spec. for Centrifugally Cast Iron-Chromium et, and Strip (1972) ANSI H34.17

r (1972) ANSI H34.18

pipe (1972)

y Plate, Sheet, and Strip (1973)/

Std. Spec. for

Std. Spec. for

Std. Spec. for

Std Spec. tor Seamless and Tor Chromium ed and Cold Finished Stainless and Heat Resisting Chromium p (1972)

Std. Spec. for Low Carbon

Std. Spec. for Low Carbon Std. Spec. for

Std. Meth. of Test for Std. Spec. for

Std. Spec. for Seamless Copper Std. Spec. for etecting Susceptibility to Intergranular Attack in Wrought Std. Spec. for

Copper-Nickel-Zinc Alloy (Nickel Silver) and Copper. Std. Spec, for

strip, and /

Std. Spec. for Sintered

Spec. for Corrosion Resisting Chromium and Chromium

for Flux Cored Corrosion Resisting Chromium and Chromium unfi/ Std. Spec, for Heat Resisting Chromium and Chromium si B36.39 Std. Spec. for Seamless Austenitic Chromium si) Spec. for Corrosion Resisting Chromium and Chromium eth. for Measuring Fast Neutron Flux by Radioactivation of Std. Spec. for Pressure Vessel Plates, Alloy Steel, 36\% ates, Alloy Steel, Precipitation Hardening (Maraging), 12\% ates, Alloy Steel, Precipitation Hardening (Maraging), 18\%

vessel Plates, Alloy Steel, Quenched and Tempered 8 and $9 \%$ temperature, Electrical, Magnetic, and Other Similar Iron. ion Resistant Metals (Electrodeposited Coatings of Copper. Std. Meth. of Test for Color of Raw Cotton Using the 7) Meth. of Analysis of Niacin and

Spec. for Subsurface Safety Valves, Locks, and Landing 1972) Std for

read, Wheat and Other Grains/ Improved Kjeldahl Meth. for Std. Meth. for Quantitiative Determination of Cellulose Uniform Building Code Std. for Cellulose Uniform Fire Code: Cellulose Uniform Fire Code: Cellulose Meth. for Preparation of Silver ear, and Radiochemical Analysis of Nuclear Grade Plutonium Std. Spec. for Plutonium Sid. Meth. of Mercurous 3 rec. for Safe Use of Mixed Fertilizers Containing Ammonium Std. Spec. for Soluble Cellulose Definition and Test Procedures for Ammonia
Std. Spec. for Std. Spec. for
Std. Spec. for Std. Spec. for Refined Benzene 485 Wet Ashing with erium, Fluorine, Helium, Hydrogen, Krypton, Methane, Neon, he Pest Control Chemical Methyl 5(2,4-Dichlorophenoxy)-2de Std. for Storage and $\mathrm{H}$ andling of Photographic and X-Ray ng Code: Film Storage and Handling (Photographic and X-Ray $2 \%$ Free Ammonia (1970) Std. for Storage and Handling of er Protein/ als of Com/ Std. Analytical Meth. for Determining Protein starches A/ Std Analytical Meth. for Determining Protein Std. Analytical Meth. for Determining Protein Std. Analytical Meth. for Determining Ammonia Std. Analytical Meth. for Determining Protein Std. Analytical Meth. for Determining Protein ps, Sugars/ ups, Starc/ her Protei/ e (1970) ALCA B5 Std. Analytical Meth. for Determining Protein (1973) bility Index (NSI): Meth. for Determination of Dispersible Std. Meth. of Test for Total Meth. of Analysis of Undenatured Whey Protein Meth. for Organic Std. Meth. of Test for (1962) Linderstrom-Lang Meth. of Analysis of Amino Modified Sorensen Meth. of Analysis of Amino Van Slyke Meth. of Analysis of Amino n of Dispersible Nitrogen in Ground Soybeans, Whole or G/
Nickel Chromium Iron Columbium Molybdenum Tungsten Allo Nickel Chromium Molybdenum Alloy Steel Plates, Quenched Nickel Chromium Molybdenum Columbium Alloy Plate, Sheet Nickel Chromium Molybdenum Columbium Alloy Rod and Bar Nickel Chromium Molybdenum Columbium Alloy Seamless Pip Nickel Chromium Molybdenum Iron Alloy Rod (1972)

Nickel Coated Copper Clad Steel Wire for Electronic App

Nickel Coated Soft or Annealed Copper Wire (1974) ANSI

Nickel Copper Alloy Plate, Sheet, and Strip (1970) ANSI

Nickel Double Normalized and Tempered (1972A) ANSI G35.

Nickel Electrical Contact Alloy (1972)

Nickel High Alloy Tubing for Pressure Application at $\mathrm{Hi}$

Nickel Iron Chromium Molybdenum Copper Alloy Plate, She

Vickel Iron Chromium Molybdenum Copper Alloy Rod and Ba

Nickel Iron Chromium Silicon Alloy Seamless and Welded

Nickel lron Molybdenum Copper Columbium Stabilized Allo

Nickel Iron Molybdenum Copper Columbium Stabilized Allo

Nickel Manganese Steel Bars Including Rounds, Squares,

Nickel Molybdenum Chromium Alloy Plate, Sheet, and Stri

Nickel Molybdenum Chromium Alloy Rod (1972)

Nickel Molybdenum Chromium lron Alloy Rod (1972)

Nickel on Steel by Photometric Analysis (1972)

Nickel Oxide Sinter (1970) ANSI G86.1

Nickel Pipe and Tube (1973) ANSI H26.4

Nickel Plate, Sheet, and Strip (1972) ANSI H34.8

Nickel Rich, Chromium Bearing Alloys (1972) ANSl G80.4

Nickel Rod and Bar (1970) ANSI H34.7

Nickel Rod and Bar (1973)

Nickel Seamless Pipe and Tube (1970) ANSI H34.1

Nickel Silver Structural Parts (1970) ANSI H9.28

(Nickel Silver) and Copper-Nickel Alloy Plate, Sheet,

(Nickel Silver) and Copper-Nickel Rod and Bar (1973)

Nickel Steel Covered Welding Electrodes (1969) ANSl W3.

Nickel Steel Electrodes (1974)

Nickel Steel Plate, Sheet, and Strip for Fusion Welded

Nickel Steel Still Tubes for Refinery Service (1972) an

Nickel Steel Welding Rods and Bare Electrodes (1969) an

Nickel (1970) ANSI N112

Nickel (1972)

Nickel (1972A)

Nickel (1972A) ANSI G35.17

Nickel (1972A) ANSI G35.20

Nickel, and Cobalt Base Alloys (1973)

Std. M ASTM

Std. Spec, for Pressure Vessel Pl ASTM

/Ec. for Pressure Vessel Pl ASTM Std. Spec, for Pressure ASTM /Alysis of High ASTM

Nickel, Chromium, Lead, Zinc, or Cadmium) (1973)

Nickerson-Hunter Cotton Colorimeter (1971)

Nicotinamide in Cereal Products (Cereal Chemistry) (196

Nipples for Petroleum lndustry Use (1973)

Nitrapyin (Common Name for the Pest Control Chemical) (

Nitrate Free Samples for Analysis of Crude Protein in B

Nitrate in Alkyd Lacquers by Infrared Spectrophotometry

Nitrate Motion Picture Film (1973)

Nitrate Motion Picture Film (1973)

Nitrate Plastics (Pyroxylin) (1973)

Nitrate Solution (Cereal Chemistry) (1962)

Nitrate Solutions (1973)

/Etric, Spectrochemical, Nucl

Nitrate Solutions (1973) ANSI N137

Nitrate Test for Copper and lts Alloys (1973) ANSI H39.

Nitrate (1972)

Nitrate (1972) ANSl K65.22

Nitrate (1973)

Nitration Grade Toluene (1971) ANSI 278.7

Nitration Grade Xylene (1971) ANSI Z78.8

(Nitration Grade) (1971) ANSI Z78.2

Nitric and Perchloric Acids (Wood, Pulp, etc.) (1973)

Nitric Oxide, Nitrogen, Oxygen) (1973)

Nitrobenzoate Bifenox (1973)

Nitrocellulose Films (1973)

Nit rocellulose) (1973) Monoxide, Deut Uniform Building C Uniform Buildi

Nitrogen and Fertilizer Solutions Containing More Than

Nitrogen Content in Corn Starch, Sugars, Syrups and Oth

Nitrogen Content in Corn, Other Grains and Plant Materi

Nitrogen Content in Feedstuffs (Corn), Syrups, Sugars,

Nitrogen Content in Steep water (Corn) (1962)

Nitrogen Content in Steep water, (Corn) Feedstuffs, Syru

Nitrogen Content of Corn Sugar (Crude and Refined). Syr

Nitrogen Content of Corn Syrup, Sugars, Starches and $\mathrm{Ot}$

Nitrogen Content (Kjeldahl) and Hide (Leather) Substanc

Nitrogen in Ground Soybeans, Whole or Ground Full Fat 0

Nitrogen in Lubricating Oils by Modified Kjeldahl Meth.

Nitrogen in Nonfat Dry Milk (Cereal Chemistry) (1962)

Nitrogen in Paper and Paperboard (1972)

Nitrogen in the Analysis Sample of Coal and Coke (1973)

Nitrogen in Wheat Flour and Semolina (Cereal Chemisiry)

Nitrogen in Wheat Flour and Semolina (Cereal Chemistry)

Nitrogen in Wheat Flour and Semolina (Cereal Chemistry)

Nitrogen Solubility lndex (NSI): Meth. for Determinatio
ASTM

ASTM

STM

ATM

ASTM

ASTM

ASTM
ASTM

ASTM

STM

ASTM

ASTM

ASTM

ASTM

ASTM

ASTM

ASTM

AWS

AWS

ASTM

Rros ICBO

ASTM

$\mathrm{AACCH}$

ANSI K62.128

$\mathrm{AACCH}$ 46-11

ASTM D3133

ICBO UBC S48-2

$1 \mathrm{CBO} U \mathrm{UF} * 2 \mathrm{ART} 5$

ICBO UFC $* 2 A R T 6$

$\mathrm{AACCH} \quad 70-60$

ASTM

C759

ASTM C710

ASTM B154

FMS LPD7.9

ASTM D301

FI

ASTM

ASTM

TAPP

P40

D841

D843

UM-243

UFC *2ART 36

K62.138

UBCS48-1

$\mathrm{UBC} * 3.48$

P145

B- 48

A- 18

G. 22

J-12

J-56

F -44

E.52

ASTM D2868

$\mathrm{AACCH} \quad 46.23$

ASTM D3228

AACCH $\quad 46-20$

TAPPI T418SU

ASTM D3179

AACCH $\quad 46.30$

$\mathrm{AACCH}$ 46-31

AACCH 46.32

$\mathrm{AACCH} \quad 46.23$ 
meth. of Static Immersion Test. of Unstressed Materials in th. for Colorfastness of Textiles to Atmospheric Oxides of Meth. of Analysis of Urea and Ammonical Feeds and Feedstuffs (Calculated from Percentage of Total in in Wheat and Flour (Calculated from Percentage of Total e, Helium, Hydrogen, Krypton, Methane, Neon, Nitric Oxide, try/ Modified Meth. of Quantitative Determination of Both Cecal and Intestinal Coccidiosis I/

Meth. of Analysis of

Meth. for Determination of ils (Container) (Dot-37A80, Dot-37Aa60. Ufc-Rule 40, $55 \mathrm{Gal}$. Full Removable Head Universal Drum (Ufc-Rule 40, $6 \mathrm{Gal}$. Full Removable Head Universal Drums (Ufc-Rule 40 , 0 Gal. Full Removable Head Universal Drums (Ufc-Rule 40, 7 Gal. Full Removable Head Universal Drums (Ufc-Rule 40, Removable Head, Lug Cover Universal Drums (Ufc-Rule 40, ails (Container) (Dot-37A80, Dot-37A60, Ufc-Rule 40, ead Universal Pails (Container) (Dot-17E. Ufc-Rule 40. $\mathrm{n}$ of Commercial Weight and Yield of Scoured Wool, Top, and

iform Procedure for the Test., Rating and Reporting of the Spiral Bevel Gear Drives (1973)

ers' Industry (1970)

Std. for Sound

for Qualifying a Sound Data Acquisition System for Vehicle

ing and Specifying Machinery Sound Within Equipment Rooms Std. for Std. for iners (1971)

Rec. Pract. for Radial Seal (1971) Rec. Pract. for

si B104.1

tron Tubes and Electric Lamps (1971)

minants Seen on Specular Silicon Surface/

d Processing Laboratories (1971)

(1971)

system Parts (1972)

i J9.1$$
\text { Sid }
$$

Std. and Std.

Rec. Pract. and

Std. for Std.

Rec. Pract. for

Std. Rec. Pract, for

Std. Tooling

n Shielding Concrete (1973)

Std. Descriptive Std. Definitions of Terms and

halt, Cork, Linoleum, Rubber, Vinyl, Vinyl Asbestos/ Rec. Std. Door Type

ec. Pract. for Passenger Car and Light Truck Muffler Parts Rec. Pract. for Automotive Brake Definitions and

Pract. for Passenger Car and Light Truck Resonator Parts Std. Large Rivets ( $1 / 2$ In Std. Small Solid Rivets $(7 / 16 \mathrm{In}$

for Diesel Fuel Injection Nozzle and Holder Assembly (17 Mm

Rec. Pract. and Std. Covering

3) ANSI G57.11

Std. Spec. for Carbon Steel Axles, Structural Design of Serpentine Walls,

Installation for

Test Meth. for Adhesion to Std. for Flexible

(1971)

Std. for Pressurized and

Recreational Vehicles (1971)

Terminal Equipment and

t-Stop Signal Quality Between Data Terminal Equipent and s, Degreasing Grade (Nonreflux Me/ Std. Meth. of Test for Std. for Motor Vehicle Brake Fluid (Used Under rbons by Gas Chromatography (1968/ Std. Meth. of Test for Std. Meth. of Test for Water Absorptiveness of 1973) 1969) ANSI SE3.7 Std. Spec. for 3)

Std. for Interconnection Circuitry of Std. Meth. of Test for Uniform Building Code Std. for nstrumentation/ Spec. for Film Thickness Determination of si LI4. 128 Test Meth. for ing for Electrical Insula/ Sid Spec. for Crosslinked and Std. Meth. for Open Bottle Tap Sampling of Std. Pictorial Markings for Handling of

e Coatings on Steel (1972) or Handling of ipe (1974)

Bulletin for

rbon Monoxide in the Atmosphere (Continuous Measurement by Rec. Spec. for

y for Refrigerant-Containing Components and Accessories, of Measuring Frequency and Cost, and Recording Patron and t Fillers for Concrete Paving and Structural Construction stry) (19) Carr-Price Meth. of Analysis of Vitamin A in ry) (1962) Meth. of Analysis of Lactose (Sugar) and Farinograph Evaluation Meth. of Ability of Roller Dried Meth. of Analysis for Titratable Acidity in Meth. of Analysis for Lactic Acid in Meth. of Analysis for Baking Quality of Meth. of Analysis of Moisture in

Meth. of Analysis of Undenatured Whey Protein Nitrogen in Std. Rec. Pract. for Codification of Certain
Nitrogen Tetroxide $\left(\mathrm{N}_{2} \mathrm{O}_{4}\right)(1972)$

Nitrogen (Burnt Gas Fumes) (1972) ANSI L14.54

Nitrogen (Cereal Chemistry) (1965)

Nitrogen) (Cereal Chemistry) (1962)

Nitrogen) (Cereal Chemistry) (1962)

Nitrogen, Oxygen) (1973)

Crude Protein in
on of Crude Prote

(Monoxide, Deuterium,

Nitrophenide in Poultry Mashes (Feed) for Prevention of

Nitrosal in Poultry Feeds (Cereal Chemistry) (1962)

Nmfc-ltem 260 (1974)

Nmfc-ltem 260) (1974)

Nmfc-Item 260) (1974)

/Nesting Lug Cover

Universal $\mathrm{Pa}$ ANS

Nmfc-Item 260) (1974)

Nmfc-Item 260) (1974)

Nmfc-Item 260) (1974)

Nmfc-ltem 260) (1974)

Nmfc-ltem 260, Dot-37B60) (1974

Noil for Various Commercial Compo

Noise Levels of Powered Industrial Trucks (1973)

Noise Sound Level Meters (1972) ANSI S6.1 Std. for Meth. of Measuring, Record

Noise) (1973)

Nomenclature-Scrapers (1971)

Nomenclature-Truck, Bus, Trailer (1972)

Nomenclature and Glossary (1972)

Nomenclature and Terminology of Tank Trailers and Conta

Nomenclature and Terms (1965)

Nomenclature for Bedding Sizes (Mattresses and Sheets)

Nomenclature for Diamond Core Drill Equipment (1973) an

Nomenclature for Glass Bulbs Intended for Use with Elec

Nomenclature for Identification of Structures and Conta

Nomenclature for Motion Picture Film Used in Studios an

Nomenclature for Parts of a Photographic Objective Lens

Nomenclature for Passenger Car and Light Truck Exhaust

Nomenclature for Rubbers and Rubber Latices (1972A) Ans

Nomenclature for Solid Die Two Blow Cold Headers (1972)

Nomenclature of Constituents of Aggregates for Radiatio

Nomenclature Relating to Aerosol Products (1972)

Nomenclature Relating to Resilient Floor Coverings (Asp

Nomenclature (Coding) (1972)

Nomenclature (1971)

Nomenclature (1971)

Nomenclature (1971)

Nominal Diameter and Larger) (1972)

Nominal Diameter and Smaller) (1972)

Nominal Diameter) (1971)

Non Commercial Boat Trailer (1972)

Non Heat Treated and Heat Treated, for Railway Use (197

Non Loadbearing (Tech. Notes) (1968)

Non Metallic Building Sewers (1971)

Non Porous Flexible Substrates (Paper) (1973)

Non Pressure Water Fill Hose for Recreational Vehicles

Non Pressurized Potable Water Tanks for Mobilehomes and

Non-Synchronous Data Communication Equipment (1973)

Nonalkyl Organo Compounds (1972)

Nonamine Acid Acceptance of Halogenated Organic Solvent

Nonarctic Conditions) (1972)

Nonaromatic Hydrocarbons in Monocyclic A romatic Hydroca

Nonbibulous Paper and Paperboard (Cobb Test) (1973)

Noncarbonized, Single Ply, Adding Machine Paper Rolls

Noncoded Remote Station Protective Signalling Systems

Noncombustibility of Elementary Building Materials (197

Noncombustible Elementary Materials Test. (1973)

Nonconductive Coatings on Nonferrous Substrate (Coil, I

Noncotton Content of Bleached Cotton Textiles (1972) an

Noncrosslinked Poly(vinyl Chloride) Heat Shrinkable Tub

Noncryogenic Fluid Systems (1972)

Nondangerous Goods (1972)

Nondestructive Measurement of Film Thickness of Pipelin

Nondestructive Test. Terminology for Defects in Steel P

Nondispersive Infrared Spectrometry) (1973)

(Nonelectric) Farm Fence Construction (1972)

Nonelectrical (1973)

Nonemployee Injury Statistics (1971)

(Nonextruding and Resilient Bituminous Types) (1973) Ans
Nonfat Dry Milk and Instantized Dry Milks (Cereal Chemi

Nonfat Dry Milk in Bread (Cereal Chemistry) (1962)

Nonfat Dry Milk in Physical Dough Tests (Cereal Chemist

Nonfat Dry Milk Solids to Change Mixing Characteristics

Nonfat Dry Milk (Cereal Chemistry) (1962)

Nonfat Dry Milk (Cereal Chemistry) (1962)

Nonfat Dry Milk (Cereal Chemistry) (1962)

Nonfat Dry Milk (Cereal Chemistry) (1962)

Nonfat Dry Milk (Cereal Chemistry) (1962)

Nonferrous Metals and Alloys, Cast and Wrought (1973)

Std. ASTM

SAE

TTMA

NBHA

ANSI

ANSI

ASTM

ANSI

ANSI

SAE

ASTM

IFI

ASTM

ASTM

ASTM

STDI

$\mathrm{SAE}$

$S A E$

/a EIA

ANSI

API

UL

AACCH $46-50$

AACCH 46.18

ICBO UFC *2ART36

$\mathrm{AACCH} \quad 18.26$

AACCH $18-25$

AACCH $18-27$

$\mathrm{MH} 2.15$

$\mathrm{MH} 2.2$

MH2.14

MH2.12

MH2.11

$\mathrm{MH} 2.8$

MH2.10

$\mathrm{MH} 2.9$

D2720

$6 \mathrm{Gl}$

297.01

$\begin{array}{ll}\text { AGMA } & 297 \\ \text { NMTBA } & * 1\end{array}$

SAE J184

575

$\begin{array}{ll}\mathrm{SAE} & \mathrm{J} 728 \mathrm{~A} \\ \mathrm{SAE} & \mathrm{J} 687 \mathrm{C}\end{array}$

DCDMA

J111A

RP36

27.1

L27.

C79. 1

$\mathrm{PH} 22.56$

PH 3.25

J97

D1418

118

C638

F141

106

J261

$\mathrm{J} 2656 \mathrm{~F}$

J262

ANS1 B18.1.2

ANSI B18.1.1

SAE J265

ABYC A15

ASTM A21

BIA 33

TAPPI UM.54]

IAPMO TSC 19

IAPMO TSC4

ASTM D2942

SAE J1703B

ASTM D2360

ASTM D3285

ANSI X4.8

NEMA SB3

ASTM E136

ICBO UBCS4.I

NCCA TB-II-14

AATCC 97

ASTM D3150

ASTM F30I

ANSI MH6.I

ASTM G12

BUL5T1

ASTM D3162

ASAE R250.2

ANSI Z108.

ASTM D175]

AACCH 86-02

AACCH 80-31

AACCH 54.12

AACCH 54-20

AACCH 02-31 
Film Thickness Determination of Nonconductive Coatings on Std. Spec. for High Strength Std. for (1969) ANSI B93.18 Std. Spec. for Hollow

Uniform Building Code Std. for Hollow Std. Spec. for Structural Clay Uniform Building Code Std. for Structural Clay d Rigid Furring ChanneIs for Screw Applic/ Std. Spec. for A286 Cres, Long Thread, Self Locking and 6AI-4V Titanium Alloy, Long Thread, Self Locking and Tolerance, 6Al-4V Titanium Alloy, Short Thread, r Screw, Hex Head. Tri Wing Recess. A286 Cres, Full Thread head, Tri Wing Recess, 6Al-4V Titanium Alloy, Full Thread, close Tolerance, A286 Cres, Short Thread, Self Locking and close Tolerance, A286 Cres, Long Threads, Self Locking and Tri Wing Recess, A286 Cres, Full Thread, Self Locking and lose Tolerance, Alloy Steel, Long Thread, Self Locking and ose Tolerance, Alloy Steel, Short Thread, Self Locking and Tri Wing Recess, A286 Cres, Full Thread, Self Locking and , Tri Wing Recess, A286 Cres, Full Thread Self Locking and nce, 6Al-4V Titanium Alloy, Short Thread, SeIf Locking and lose Tolerance, Alloy Steel, Long Thread, Self Locking and ce, $6 \mathrm{Al}-4 \mathrm{~V}$, Titanium Alloy, Short Thread, Self Locking and tri Wing Recess, Close Tolerance, A286 Cres, Short Thread. ort Thread, TWI Wing Recess, Alloy SteeI, Self Locking and hort Thread, TWI Wing Recess, A286 Acres, Self Locking and , Tri Wing Recess, Titanium Alloy 6Al-4V, Self Locking and cess, $6 \mathrm{AI} \cdot 4 \mathrm{~V}$ Titanium Alloy, Full Thread, Self Locking and close Tolerance, A286 Cres, Short Thread, Self Locking and ance, 6AI-4V Titanium Alloy, Long Thread, Self Locking and crew, Hex Head, Tri Wing Recess, Alloy Steel, Full Thread. ri Wing Recess, Alloy Steel, FulI Thread, Self-Locking and ose Tolerance, Alloy Steel, Short Thread, Self-Locking and Tri Wing Recess, Titanium Alloy, 6AI $4 \mathrm{~V}$, SeIf-Locking and i Wing Recess, Close Tolerance, Alloy Steel, Short Thread, I Threaded, Tri Wing Recess, Alloy SteeI, Self-Locking and Full Thread, Tri Wing Recess, A286 Cres, Self-Locking and g55.6 Test Meth. for Measurement of Film Thickness of Std. Spec. for Alloy Steel Forgings for Std. for Bolt-Machine, Hexagon Head, Std. for Safety for Rigid

71) Ansi/

Std. Rec. Pract. for Design and Construction of Std. Groove Dimensions for Floating Type Metallic and Std. Meth. of Tension Test. of for Test Procedure for the Locking Ability Performance of 56 56
Bathtubs and Combination Tank and Shower Recep/ St Safety for
Std. for Std. Spec. for Zirconium lngots for Std. Spec. for Zirconium Forgings and Extrusions for Std. Spec. for Primary Zirconium for Std. Spec. for Seamless and Welded Zirconium Tubes for r Hot Worked and Cold Finished Zirconium Bars and Wire for
Sid. Spec. for Flat Rolled Std Meth. for Evaluation of and Test Formulas for ttings to Poly(vinyl Chloride) (PVC) Pipe and Fittings for Std. Spec. for Asbestos Cement Std. Spec. for Asbestos Cement 4

Rec. for Fire Protection of Small liquids (1973) try) (1962) Meth. of Analysis of Reducing and eptance of Halogenated Organic Solvents, Degreasing Grade et Joints (1973)

Rec. for Cast in Place Spec. for Cast in Place Std. for Meth. of Test. for Rating Std. for Insert, Molded ln, Blind Threaded, Self Locking, Std. for Insert, MoIded ln, Thru Threaded, SeIf Locking, Std. for Sandwich Panel Lightweight Selflocking and Std. for Sandwich Panel Lightweight Selflocking and h Ethylene-Propylene-Rubber Insulation, 200/ Std. for Adhesives (1970) Std. Spec. for Adhesives Used in Ivent Based, and Polymer Emulsion/ sid. Meth. of Test for Volume Ivent Based, and Polymer Emulsion/ Sid. Meth. of Test for Std. Meth. of Test for xtures (1/ Std. Analytical Meth. for Determining Waxy and Std. Analytical Meth. for Determining Waxy and

Std. for Terms and Definitions for Wirewound and

Std. for lnspection and Test Procedures for Wirewound and Std. Meth. of Test. lamino) -2-(a,a,a- Trifluoro-m-tolyl) - 3 (2H)Pyridazinone

67)

67)

9. ANSI Al.11

Capillary Meth. of Analysis of Melting Point of All Wiley Meth. of Analysis of Melting Point of Std. Spec. for Std. Meth. of Test for
Nonferrous Substrate (Coil, lnstrumentation) (1974)

Nonheaded Steel Bolts and Studs (1973)

Nonintegral Industrial Fluid Power Hydraulic Reservoirs

Nonload Bearing Concrete Masonry Units (1971) ANS1 A80.

Nonload Bearing Concrete Masonry Units (1973)

Nonload Bearing Tile (1971) ANSl A76.1

Nonload Bearing Wall Tile (1973)

Nonload (Axial) Bearing Steel Studs, Runners (Track) an

Nonlocking (1972)

Nonlocking (1972)

Nonlocking (1972)

Nonlocking (1972)

Nonlocking (1972)

Nonlocking (1972)

Nonlocking (1972)

Nonlocking (1972)

Nonlocking (1972)

Nonlocking (1972)

Nonlocking (1972)

Nonlocking (1972)

Nonlocking (1972)

Nonlocking (1972)

Nonlocking (1972)

Nonlocking (1972)

Nonlocking (1972)

Nonlocking (1972)

Nonlocking (1972)

Nonlocking (1972)

Nonlocking (1972)

Nonlocking (1972)

Nonlocking (1973)

Nonlocking (1973)

Nonlocking (1973)

Nonlocking (1973)

Nonlocking (1973)

Nonlocking (1973)

Nonlocking (1973) Machine Screw, Flat Fillis
Nonmagnetic Finishes on Mild Steel (Coil Coating) (1974
Nonmagnetic Retaining Rings for Generators (1970) ANS

Nonmagnetic Spring Lock Washer (1974)

Nonmagnetic, and Heat Resistant (1973)

Nonmetallic Conduit (1972) ANSI C33.9]

Nonmetallic Enveloped Gaskets for Corrosive Service (19

Nonmetallic Fluid Power Piston Rings (1972) ANSI B93.36

Nonmetallic Gasket Materials (1972)

Nonmetallic Locking Element Type Prevailing-Torque Lo

Nonmetallic Sheathed Electrical Cables (1974) ANSI C33.

Nonmetallic Shower Receptors, Stall Showers Lavatories,

Nonnuclear Application (1972) ANSl Z179.10

Nonnuclear Application (1972) ANSI Z179.8

Nonnuclear Application (1972) ANSI Z179.9

Nonnuclear Application (1972) ANSI Z212.]

Nonnuclear Applications (1972) ANSI Z179.11

Nonoriented Electrical Steel, Fully Processed Type (197

Nonpigmented Styrene-Butadiene Rubbers (SBR) (1973)

Nonpressure Applications (1972)

Nonpressure Sewer Pipe (1973) ANSI Al65.3

Nonpressure Small Diameter Sewer Pipe (1973) ANSI Al65.

Nonpressurized and Pressurized Containers of Flammable

Nonreducing Sugars in Flour and Semolina (Cereal Chemis

(Nonreflux Meth.) (1974) lof Test for Nonamine A

Nonreinforced Concrete Pipe (1966)

Nonreinforced Concrete Pipe (1970) ANSI A 171.1

Nonresidential Warm Air Heaters (1972)

Nonself Locking, Light Weight Floating, Sandwich PaneI

Nonself Locking, Sandwich Panel (1972)

Nonselflocking Blind Threaded Molded in Insert (1974)

Nonselflocking Blind Threaded Molded in Insert (1974)

Nonshielded Single Conductor Electrical Power Cable Wit

NonstructuraI Glued Lumber Products (1972)

Nonvolatile Content of Phenol, Resorcinol, and Melamine

Nonvolatile Matter in Clear or Pigmented Coatings (1973

Nonvolatile Matter (Total Solids) in Water Emulsion, So

Nonvolatile Residue of Halogenated Solvent Extract from

Nonwaxy Content in Corn and Grain Sorghum, and Their Mi

Nonwaxy Content in Corn Starch (1960)

Nonwirewound Trimming Potentiometers (1970)

Nonwirew ound Trimming Potentiometers (1970)

Nonwoven Fabrics (1974) ANSI L14.246

Norflurazon (1973)

IOntrol Chemical 4-Chloro5-(Methy

Normal Animal and Vegetable Fats (Cereal Chemistry) (19

Normal Animal and Vegetable Fats (Cereal Chemistry) (19

NormaI Butyl Acetate (98\% Grade) (1972)

Normal Consistency of Hydraulic Cement (1973) AASHO T1

Normal Engineering Services (1972)
/ R NCCA

ASTM

NFLDP

ASTM

ICBO

ASTM

ICBO

ASTM

NSA

NSA

NSA

Std. Fo

NSA

NSA

NSA

NSA

NSA

NSA

NSA

NSA

NSA

NSA

NSA

NSA

NSA

NSA

NSA

NSA

NSA td. for $S$ NSA

$\mathrm{NSA}$

NSA

NSA

NSA

NSA

NSA

NCCA

UL

ASTM

NFLDP

AST.

IFI

UL

IAPMO

ASTM

ASTM

ASTM

ASTM

ASTM

ASTM

ASTM

ASTM

ASTM

FMS

AACCH

ASTM

ACI

ACl
ASHRA

NSA

NSA

NSA

NSA

NEMA

ASTM

ASTM

ASTM

ASTM

ASTM

CR

CR

VRCl

VRCI

ASTM

AACCH

$\mathrm{AACCH}$

ASTM

ASTM

Std. Form of AIOA

TB.11.14

687

T3.16.2

C129

$\mathrm{C} 56$

UBC 524.9

C645

4204- 16

$4304 \cdot 16$

$4903 \cdot 16$

$6000 \cdot 3$

$6100-3$

$6303 \cdot 20$

$6704-20$

6900.06

$6604-20$

6203-20

$5700 \cdot 6$

$6500-6$ 
Meth. of Analysis of Saponification Value of All Index of Refraction, with Abbe Refractometer, in All Sid. Spec. for Std. Meth. of Test for

Rec. Pract. for Selecting Proportions for Std. Assessment of the Assumption of for Pressure Vessel Plates, Alloy Steel, $9 \%$ Nickel Double $1 \mathrm{MPa}$ / Spec. for Low Alloy Steel Studs, Heat Resistant, Std. for Welded Flash Controlled Low Carbon Steel Tubing 73) Sid. Spec. for Western White Pines, Spruce-Pine-Fir, Western Cedars ing Code Std. for Coast Sitka Spruce, Douglas Fir-Larch , Douglas Fir-Larch (North), Eastern Hemlock-Tamarack

), Eastern Hemlock-Tamarack (North), Eastern White Pine

- Tamarack (North), Eastern White Pine (North), Hem-Fir rn Spruce, White Pine, and Hemlock-Tamarack; Balsam Fir; ised Pole Butts by the Thermal Process (Western Red Cedar, Std. for Spindle Std. for Preparation of 968) ANSI Z260.1 Std. for Statistical Terminology and Sid. Rec. Pract. for Sharp Sid. Meth. for 1)

Rec. Pract. for Diesel Fuel Injection Std. Meth. of Test for Reheat Change of Fireclay Std. for Care of Fire Hose (Including Couplings and n Ground Soybeans, Whole or G/ Nitrogen Solubility Index Meth. for Chemical. Mass Spectrometric, Spectrochemical, c. for Zirconium and Its Alloy Forgings and Extrusions for Std. Spec. for Zirconium and Its Alloy Ingots for for Zirconium Sponge and Other Forms of Virgin Metal for d Finished Zirconium and Its Alloy Bars, Rod, and Wire for c. for Zirconium and Its Alloy Sheet, Strip, and Plate for d for Quality Assurance for Protective Coatings Applied to Std. for Guide to Principal Design Criteria for :d. Meth. for Radiochemical Determination of Cesium-137 in rical Penetration Assemblies in Containment Structures for otometric Determination of Fission Zirconium in Irradiated Std. Spec. for Tent. Spec. for mical, Mass Spectrometric, and Spectrochemical Analysis of 4) Std. Spec. for mical, Mass Spectrometric, and Spectrochemical Analysis of Std. Spec. for

c, Spectrochemical, Nuclear and Radio Chemical Analysis of

c. Spectrochemical, Nuclear, and Radiochemical Analysis of

Std. Meth. for Chemical and Spectrochemical Analysis of mical, Mass Spectrometric, and Spectrochemical Analysis of e in Nuclear Reactors (1973) Std. Meth. for Measurement of Lattice Spacing of estimating the Thermal Neutron Absorption Cross Section of Sid. Meth. of Test for Delta in Hours (DIH) Purity of Rec. Pract. for Std. Calibration and Format for ies (A Guide to Practice) (1971)

facilities (A Guide to Practice) (1974) Std. for Std. for Record and Reporting Units for Std. for Statistical Terminology and Notation for Std. for Auditing Std for Physical Inventories of th. of Test for Density of Bituminous Concrete in Place by 7) Ans/ Std. Spec. for Sheathed, Type K Thermocouples for std. Spec. for Sheathed Electrical Resistance Heaters, for Design Criteria for Reinforced Concrete Structures, and Equipment for Water Cooled and Moderated Std for Trial-Use Criteria for the Periodic Test. of Use Guide: General Principles for Reliability Analysis of ide for the Application of the Single Failure Criterion to Definitions of Terms Used in IEEE eneral Guide for Qualifying Class I Electric Equipment for or Seismic Qualification of Class I Electric Equipment for us Duty Class I Motors Installed Inside the Containment of esel Generator U'nits Applied as Standby Power Supplies for uide for Type Test of Class I Electric Valve Operators for ign Bases for Systems That Perform Protective Functions in ieee Trial Use Guide for Class $1 E$ Control Switchboards for entation and Electric Equipment During the Construction of d. for Quality Assurance Practices for the Construction of ng, Shipping, Receiving, Storage and Handling of Items for

Std. for Quality Assurance Program Requirements for Std. for Fuel Assembly Identification in
Std. for Administrative Controls for Std. Industrial Security for

Std. for Housekeeping During the Construction Phase of ems and Associated Components During Construction Phase of ination, and Test. Personnel for the Construction Phase of Std. for Earthquake Instrumentation Criteria for Std. for Criteria for Film Badge Performance
Normal Fats and Oils (Cereal Chemistry) (1967)

Normal Oils and Liquid Fats (Cereal Chemistry) (1967)

Normal Propyl Acetate (90 to $92 \%$ Grade) (1972)

Normal Spectral Emittance at Elevated Temperatures (197

Normal Weight Concrete (1970) ANSI A 167.1

Normality (Employing Individual Observed Values) (1974)

Normalized and Tempered (1972A) ANSI G35.3

Normalized and Tempered, Roll Threaded ( 135,000 psi $(93$

Normalized for Bending, Double Flaring, and Beading (19

Normalized High Strength Low Alloy Structural Steel (19

/M-Fir (North), Ponderosa and

North) (Lumber) (1973)

(North), Eastern Whi

North), Eastern White Pine (North), Hem-Fir (North),

North), Hem-Fir (North), Ponderosa and Western White

(North), Ponderosa and Western White Pines, Spruce-Pin

Northern Pine and White Cedar Lumber (1973)

Northern White Cedar and Alaska Yellow Cedar Poles) (19

Noses and Tool Shanks for Milling Machines (1972)

Notation for Concrete (1971) ANSI A188.1

Notation for Nuclear Materials Management (1972)

Notch Tension Test. of High Strength Sheet Materials (1

Notched Bar Impact Test. of Metallic Materials (1972)

Nozzle and Holder Assembly (17Mm Nominal Diameter) (197

Nozzles and Sleeves (1972)

Nozzles (1972)

(NSI): Meth. for Determination of Dispersible Nitrogen I

Nuclear and Radio Chemical Analysis of Nuclear Grade P

Nuclear Application (1967) ANSI N125

Nuclear Application (1973) ANSI G53.28

Nuclear Application (1973) ANSI N12.1

Nuclear Application (1973) ANSI N122

Nuclear Application (1973) ANSI N123

Nuclear Facilities (1972)

Nuclear Fuel Reprocessing Facilities (1973)

Nuclear Fuel Solutions (1970) ANSI N117

Nuclear Fueled Power Generating Stations (1972) ANSI N
Nuclear Fuels (1973) Tent. Meth. for Sp

Nuclear Grade Beryllium Oxide Powder (1972A)

Nuclear Grade Boron Carbide Powder (1973)

Nuclear Grade Mixed Oxides ((U, Pu)0.) (1972) ANSI N1

Nuclear Grade Plutonium Dioxide Powder, Sinterable (197

Nuclear Grade Plutonium Dioxide Powders and Pellets (19

Nuclear Grade Plutonium Metal (1972) ANSI N136

Nuclear Grade Plutonium Metal (1973)

Nuclear Grade Plutonium Nitrate Solutions (1973)

Nuclear Grade Silver-Indium-Cadmium Alloys (1974)

Nuclear Grade Uranium Dioxide Powders and Pellets (1972

Nuclear Grade, Sinterable Uranium Dioxide Powder for Us

Nuclear Graphite (1969) ANSI K90.1

Nuclear Graphite (1971) ANSI K90.10

Nuclear Graphite (1971) ANSI K90.8

Nuclear Logs (Record, Documentation) (1974)

Nuclear Material Control Systems for Conversion Facilit

Nuclear Material Control Systems for Fuel Reprocessing

Nuclear Materials Control (1971)

Nuclear Materials Management (1972)

Nuclear Materials Statements and Reports (Accounting)

Nuclear Materials (1972)

Nuclear Meth. (1974)

Nuclear or for Other High Reliability Applications (196

Nuclear or Other Specialized Service (1971) ANSI N143

Nuclear Power Containment Structures (1969)

Nuclear Power Generating Plants (1973) ANSI N18.10

Nuclear Power Generating Station Protection Systems (19

Nuclear Power Generating Station Protection Systems (19

Nuclear Power Generating Station Protection Systems (19

Nuclear Power Generating Station Stds. (1972)

Nuclear Power Generating Stations (1971) ANSI N41.5

Nuclear Power Generating Stations (1971) ANSI N41.7

Nuclear Power Generating Stations (1971) ANSI N41.9

Nuclear Power Generating Stations (1972) ANSI N41.13

Nuclear Power Generating Stations (1972) ANSI N41.6

Nuclear Power Generating Stations (1973)

Nuclear Power Generating Stations (1973) ANSI N41.17

Nuclear Power Generative Stations (1971)

Nuclear Power Plants-Terms and Definitions (1973)

Nuclear Power Plants (During the Construction Phase) (1

Nuclear Power Plants (1971)

Nuclear Power Plants (1972)

Nuclear Power Plants (1972)

Nuclear Power Plants (1973)

Nuclear Power Plants (1973)

Nuclear Power Plants (1973)

Nuclear Power Plants (1973)

Vuclear Power Plants (1974)

(Nuclear Radiation) (1972)
Ass Spectromet

AACCH $58-50$

AACCH 58.20

ASTM D3130

ASTM E307

ACI

ANSI N15.15

ASTM A353

SAE AMS7458C

SAE J356A

ASTM A633

ICBO UBCS25-2

ICBO UBCS25-2

UBCS25.2

ICBO UBCS25-2

ICBO UBCS25-2

Easte ICBO UBCS25.8

$A$ WPA C7

ANSI B5.18

ACI

ANSI

ASTM

ASTM

SAE

ASTM

NFPA

$\mathrm{AACCH} \quad 46.2$

ASTM C758

Std. Spe ASTM B356

ASTM B350

Std. Spec ASTM B349

B351

B352

itd. Spe ASTM

Standar ANSI

ANSI

ASTM

IEEE

N101.4

N101.3

E320

317

E495

C708

ASTM C750

ASTM C698

ASTM C757

C697

C701

C758

C759

C760

C696

$\begin{array}{ll}\text { ASTM } & \text { C753 } \\ \text { ASTM } & \text { C } 558\end{array}$

$\begin{array}{ll}\text { ASTM } & \text { C558 } \\ \text { ASTM } & \text { C } 626\end{array}$

ASTM C624

API RP33

ANSI N15.4

ANSI N15.13

ANSI N15.2

ANSI N15.5

ANSI N15.11

ANSI N15.3

Sid. Me ASTM D2950

ASTM E235

ASTM E420

ACI

IMs ANS

IEEE

IEEE

IEEE

IEEE

/G IEEE

/F IEEE

IEEE

G IEEE tion of Des ANS

IEEE

IT ANSI

ANSI

ANSI

ANSI

ANSI

ANSI

ANSI

/or Cleaning of Fluid Syst ANSI

/Tions of Inspection, Exam ANSI

ANSI

ANSI

69-2

N18.10

338

352

379

380

323

344

334

387

382

420

336

N45.2.10

N45.2.2

N45.2

N18.3

N18.7

N18.17

N 45.2 .3

N45.2.1 
Std. Protective Coatings (Paints) for Light $W$ ater easurement of Delayed Neutron Emitting Fission Products in Std. for Program for Biological Shielding in Std. Rec. Pract. for Surveillance Tests for Std. Rec. Guide for in Service Annealing of Water Cooled ments for Leakage Rate Test. of Containment Structures for uclear Grade, Sinterable Uranium Dioxide Powder for Use in essurized Water Reactor Plants (1973) Std.

ught Zirconium and lts Alloy Seamless and Welded Tubes for Meth. for Chemical, Mass Spectrometric, Spectrochemical, 220 ye, Barley, Other Grains, A/ 1967) Std. Meth. of Test for Yarn Meth. of Analysis of Falling Meth. of Analysis of Iodine Std. Meth. of Test for

ic Acid Titration (197/ 159.2

Std. Meth. of Test for Total Base Std. Meth. of Test for Total Bromine Rec. Pract, for Vehicle ldentification Rec. Pract. for Passenger Car Vehicle ldentification
Std. Analytical Meth. for Determining lodine ctor Devices and Designation of Units in Multip/ Std. for

(1973)

Std. for Book Std for Preferred

Rec. Pract. for Boat Hull ldentification
a: Group Numbers, Dimensional Spec., and Ratings: Assembly rs, Cell Layouts and Ter/Battery Replacement Data; Group oduct Description Data (Tooling, Printed Wiring Boards) in Parallel Binary Data Interch/ Std. for Interface Between r Grid Coordinates (1971)

hangeable Perforated Tape Std., Variable Block Format, for ng Machines (1974)

milling Machine (1974

Std. for Std. for Machine (1974)

Definitions of Common Words Related to
or Contouring and Contouring-Positioning Std. for Std. for

Std. for Electrical Harness Assembling Machine, Spec. for Composite Filament Tape Laying Machine 74) Std. for Safety for Hospital Signaling and iduous Shrubs; Coniferous and Broadleaf Evergre/ Std. for Std. for $0 \mathrm{KSI}, 450$ Deg. F (1973) Std. for 74)

Std. for Forged Carbon Steel, Shoulder Std. Spec. for Carbon Steel Wire of Scrapless Std. for Std. for

Std. for Low Height, Light Weight, Self Locking Hexagon Std for Retainer-Floating Barrel or Low Height 80 K.S.I., Self Locking, 450 Deg. F, Hexagon Std. for 450 Deg. F Self Locking Serrated Head Blind Std. for 450 Deg. F Self Locking Elliptical Head Blind Std. for Low Height Hexagon Self Locking Std. for Cylinder Lock 973)

for 450 Deg. F Closed End Self Locking Serrated Head Blind

ting, $125 \mathrm{KSl}$ Ftu, $450 \mathrm{Deg} . \mathrm{F}$ and $800 \mathrm{Deg} . \mathrm{F}$ (1/

Std. for Std. for Std. for Std. for Std. for teel Bolts for Structural Steel Joints, Including Suitable (1973) ANSI E38./ Std. Spec, for Carbon and Alloy Steel

tor, Sesame, Safflower, Oleic, and Sunflower Seeds; Ground Std for Mechanical and Quality Requirements for Steel slotted and Recessed Head Machine Screws and Machine Screw Std. Spec. for Carbon Steel Std for Self Locking, 450 Deg. F, High Quality ailing Torque, All Metal, l/ ailing Torque, All Metal, 1/ Spec. for Self-Locking Steel Spec. for Self-Locking Steel Spec. for Steel Self-Locking

Spec, for Self-Locking Corrosion Resistant Alloy ck Fibers, Whole or Ground Cereal Grains, Whole or Chopped Uniform Building Code Std. for High Strength Steel Bolts, Test Meth. for Snag Resistance of Women's Std. Spec. for Reinforced and Filled

ansi K65.216 Std. Spec. for

ropyl-Ptol Std. Common Name for the Pest Control Chemical or Methidathion (Common Name for the Pest Control Chemical g. F) (1972)

Std for Packing, Preformed td. for Packing, Preformed-MlL-R-25897 Rubber, 75 Shore, td. for Packing, Preformed-MlL-R-25897 Rubber, 90 Shore, ht Thread Tube Fitting Boss, MIL-R-25897 Rubber, 75 Shore, ht Thread Tube Fitting Boss, MIL-R-25897 Rubber, 90 Shore, Std. Meth. for Test. Rubber

n, Sisal, Henequen, Ixtle, Jutes, Hemp, Tow, Cocoa Fibers, Rice, Beans, Peas, Lentils, Corn Meal, Corn Grits, Rolled Rec. Pract. for Minimum Performance Criteria for Falling
Nuclear Reactor Containment Facilities (1972)

Nuclear Reactor Coolant Water During Reactor Operation

Nuclear Reactor Plants (1972)

Nuclear Reactor Vessels (1973)

Nuclear Reactor Vessels (1974)

Nuclear Reactors (1972)

Nuclear Reactors (1973)

Nuclear Safety Criteria for the Design of Stationary $\mathrm{Pr}$

Nuclear Service (1971) ANSI N124

Nuclear, and Radiochemical Analysis of Nuclear Grade PI

Number Based on Short Length Specimens (1972) ANSI L14.

Numher Determination of Both Meal and Flour of Wheat, $\mathrm{R}$

Number in All Edible Fats and Oils (Cereal Chemistry)

Number of Neps in Cotton Samples (1971) ANSI L14.97

Number of Petroleum Products by Potentiometric Perchlor

Number of Unsaturated Aliphatic Chemicals (1972) ANSI Z

Number Systems (1972)

Number Systems (1972)

Number (Wijs Meth.) Content in Corn, Grain Sorghum and

Numbering of Electrodes in Multiple Electrode Semicondu

Numbering (1973)

Numbers in Geometric Series for Use in Standardization

Numbers (1973)

Numbers, Cell Layouts and Terminals (For Foreign and Do

Numbers, Dimensional Spec., and Ratings; Assembly Numbe

Numeric (Digital) Form on Punched Cards, Magnetic Tape

Numerical Control and Data Terminal Equipment Employing

Numerical Designation (Identification System) of Modula

Numerically Controlled Drilling Machines (1974)

Numerically Controlled Equipment (1974)

Numerically Controlled Horizontal and Vertical Jig Bori

Numerically Controlled Horizontal Boring, Drilling and

Numerically Controlled Machine Tool Soft ware (1972)

Numerically Controlled Machines (Information Processing

Numerically Controlled Profiling and Contouring Milling

Numerically Controlled Spar Mill (1973)

Numerically Controlled (1973)

Numerically Controlled (1973)

Nurse Call Equipment (Audio / Visual Communication) (19

Nursery Stock: Deciduous Shade and Flowering Trees; Dec

Nut-lnstrument Mount (1973)

Nut-Self Locking, Extended Washer, Double Hexagon, 22

Nut Assembly Eyebolt (For Ground Support Equipment) (19

Nut Quality (1972) ANSl K91.3

Nut Spacer, Plate-Count ersunk (1973)

Nut Spacer, Plate-Plain (1973)

Nut $(1972)$

Nut (1973)

Nut (1973)

Nut (1974)

Nut (1974)

Nut (1974)

Nut (1974)

Nut (1974)

Nut, Capitive Washer, Shear Type, 450 and 800 Deg. F (1

Nut, Plain, Hexagon, Drilled Jam, Thin (1972)

Nut, Self Aligning, Self Locking, Plate-T wo Lug, Floa

Nut, Self Locking, Plate-Corner, Counterbored, Floati

Nut, Washer, Self Aligning, 450 and 800 Deg. F (1973)

Nuts and Plain Hardened Washers (1971) ANSI G38.6

Nuts for Bolts for High Pressure and Temperature Servic

Nuts (Peanuts); Vegetable, Coconut, and Palm Oils) (197

Nuts (1971)

Nuts (1972)

Nuts (1972) ANS1 G24.30

Nuts $(1973)$

Nuts, Corrosion and Heat Resistant, High Strength, Prev

Nuts, Corrosion and Heat Resistant, High Strength, Prev

Nuts, High Strength, Prevailing Torque, All Metal (1973

Nuts, High Strength, Prevailing Torque, All Metal (1973

Nuts, Spices, etc. (Cereal Chemistry) (1962)

Nuts, Washers, and Bolted Parts (1973)

Nylon Hosiery (1973) ANSI L14.85

Nylon Injection Molding and Extrusion Materials (1972)

Nylon Injection Molding and Extrusion Materials (1973)

N(Cyclopropylmethyl).A, A, A-Trifluoro-2,6-Dinitro- $\mathrm{N}-\mathrm{P}$

O,O-Dimethyl Phosphorodithioate S-Ester with 4-(M

O-Ring Phosphate Ester Resistant (-65 Deg. F to + 160 De

O-Ring (1973)

O-Ring (1973)

O-Ring (1973)

O.Ring (1973)

O-Rings (1972)

Std. for Packing, Preformed, Straig

Std. for Packing, Preformed, Straig

Oakum, Rags, Waste Cloth, Wastepaper, Kapok, Hay, Straw

Oats, Bulgur, Rolled Wheat, Breakfast Cereals (Except T

Object Protective Structure (FOPS) for Prime Movers, Wh
ANSI

ANSI

ASTM

ASTM

N101.2

D2470

N18.9

E185

E509

$\mathrm{N} 45.4$

$\mathrm{C} 753$

N18.2

B353

C759

ASTM D 1059

AACCH $56.81 \mathrm{~B}$

$\mathrm{AACCH} \quad 58.30$

ASTM D1446

ASTM D2896

ASTM E234

SAE J272

CR

EIA

ANSI

ANSl

ABYC

BCI

$\mathrm{BCl}$

IPC

EIA

ANSI

NSA

Interc NSA

NSA

NSA

NMTBA *2

EIA RS274.B

NSA

NSA

NSA

NSA

UL

ANSI

NSA

NSA

NSA

ASTM

NSA

NSA

NSA

NSA

Std. F NSA

NSA

NSA

NSA

NSA

Std. NSA

NSA

NSA

NSA

NSA

NSA

/H S ASTM

ASTM

N1OP

SAE

Std. ANSI

ASTM

NSA

SAE

SAE

SAE

SAE

$A A C C$

ICBO

AATCC

ASTM

ASTM

ANSI

ANSI

NSA

NSA

NSA

NSA

NSA

ASTM

AACC

SAE

J 273

H. 32

RS32 IB

239.2

Z17.1

S10

1.56

1. .56 
for Laboratory Evaluation of Roll Over (ROPS) and Falling Std. Nomenclature for Parts of a Photographic Rec. Pract. for Goniophotometry of Transmitting ing Brightness of Pulp (Diffuse lllumination and Zero Deg. Significancel Std Rec. Pract. for Dealing with Outlying sment of the Assumption of Normality (Employing Individual Uniform Building Code: Uniform Building Code: Permanent and Grandstand/ Uniform Building Code: Stairs, Exits and le Concentrations of Radionuclides in Air and in Water for cid and Hexavalent Chromium Compounds (Air Contamination. Rec. for Radioactive Waste Disposal in the (1973) Tent. Meth. of Test for Research and Motor Meth. 972) ANS1 L/ Test Meth. for Determination of Formaldehyde 4.179 mployees (/ Test Meth. for Determination of Formaldehyde er, Auditorium, Dormatory, Public or
Sid. Meth. for Floor Measurement of Std. Pract. for Std. for Operating Supply Voltage and Frequency of 972)

Std. for lndustrial Power Take $3 / 4$ In. Thick Hollow Metal Door and Frame Preparation for ocket Teeth (1972) Std. for Heavy Duty Rec. Pract. for Operation and Maintenance of ec. for Fabricated Structural Steel Pipe for Use of Welded 4) Rec. Pract. Orientation Program for Personnel Going 1968) Spec, for Rated Loads Sid. Meth. of Test for Sid. Meth. for ldentification of Oils and
Spec. for uın Speed Stationary Diesel and Gas Engine Power Plant Fuel pec. for lndirect Type Oil Field Heaters Used in Producing ers (Fire) for Use on Vents of Storage Tanks for Petroleum h One / Std. for Prevention of Furnace Explosions in Fuel ruction (1972) unds (1973)

cal Meth for Determinin Tent. Meth. of Test for Aromatic Types Analysis of Gas Std. Meth. of Test for Slump of an Std. Meth. of Test for Compatibility of Fuel ec. for for High Boiling Hydrocarbon Solvent for Preparing Std. Spec. for Low Boiling Hydrocarbon Solvent for Std. Meth. for Analysis of

Std for Hydrocarbon Solvents for ty Std. for Atmospheric Type Welded Steel Inside Tanks for Safety Std. for Std. for Safety for Pumps for Uniform Fire Code: Safety Std. for

Std. Test. Procedure for Oil Wicking of Composite Motor procedure for Filled Cast Flat Bottom Drop Composite Motor Medium Speed Stationary Diesel and Gas Engine Lubricating low and Medium Speed Stationary Diesel and Gas Engine Fuel ited Mineral lnsulating Oil for Use in Transformers and in

hibited Mineral lnsulating Oil for Use in Transformers and Std. 791B, TAPPl T636 Sid. Meth. of Test for Spec. for Rated Loads Offshore Spec. for

Spec. for Indirect Type rec. Pract on Application, Care, and Use of Wire Rope for . Pract. for Grounded 830 V, 3 Phase Electrical System for matic Circuit Reclosers, Automatic Line Sectionalizers and Safety Std. for Safety Std. for Safety Std. for Std. for Safety for Std. for Safety for Tent. Meth for Grading Soybean - Spec. for Continuity of Quality of Electrical lnsulating Spec. for Continuity of Quality of Electrical Insulating Lacquers (1973) Std. Spec. for Uninhibited Mineral lnsulating Std. Spec. for Oxidation Inhibited Mineral Insulating Std. for Safety for

Std. for Safety for Electric Std. for Fuel and

Std. Test Procedure for Thermal Evaluation of ted Porosity of Sintered Powder Metal Structural Parts and Sid. Meth. of Test for Moisture Content of Std. Spec. for lron Base Sintered Bearings Std. Spec. for Copper Basé Sintered Bearings nsi C/ Guide for Acceptance and Maintenance of lnsulating Std. Pract, for Combustion Chambers, Fuel
Object Protective Structures (FOPS) of Construction and Objective Lens (1971)

Objects and Materials (1970)

Observation) (1972)

Observations in Samples and How to Test the Statistical

Observed Values) (1974)

Occupancy and General Requirement Classification (1973) Occupanc y (1973)

Occupant Loads (Buildings, Reviewing Stands, Bleachers,

Occupational Exposure (1959) $/ \mathrm{Ns}_{\mathrm{s}}$ and Maximum Permissib Occupational Health and Safety) (1973)

Ocean (1954)

Octagonal, and Flat (Rectangular and Square) Brass Wire

Octane Ratings U'sing on Line Analyzers (1970T)

Odor in Resin Treated Fabric by the Sealed Jar Meth. (1

Odor in Resin Treated Fabric Steam Meth. (1972) ANSI LI

Off. The Job Disabling Accidental Injury Experience of E

Office Building, Manufacturer, Restaurant, and Dwelling

Office Buildings (1972)

Office Lighting (1973) ANSl Al32.1

Office Machines (1973)

Official Meth. of Analysis for Total Acid in Wine (Enol

Official Meth. of $\mathrm{pH}$ Determination in Wine (Enology) (1

Offs for Overcenter Clutches (1971)

Offset Intermediate Pivot (1973)

Offset Sidebar Power Transmission Roller Chains and Spr

Offshore Cranes (1972)

Offshore Fixed Platforms (1972)

Offshore for the First Time (Petroleum Production) (197

Offshore Oil Cranes (1972)

Oil Absorption of Pigments by Gardner-Coleman Meth.

Oil Acids in Solvent Type Paints (1972

Oil and Gas Separators (Petroleum Production) (1973)

Oil and Gas Systems (1972)

Oil and Gas (1972)

Oil and Gasoline (1973) ANSI Z222.1

D. Pract. for Low and Medi

/ for Flame Arrest UP

Oil and Natural Gas Fired Watertube Boiler Furnaces W it

Oil and Resin Base Caulking Compound for Building Const

Oil and Resin Based, Knife Grade, Channel Glazing Compo

Oil and to Other Vegetable and Marine Oils and Animal F

Oil Aromatic Fractions by Mass Spectrometry (1973)

Oil Base Knife Grade Channel Glazing Compound (1972)

Oil Blends by Spot Test (1972) ANSI Z11.315

Oil Borne Preservative Solutions Such as Pentachlorophe

Oil Borne Preservatives (1973)

Oil Borne Wood Preservatives (1973)

Oil Borne Wood Preservatives (1973)

Oil Burner Fuel (1973)

Oil Burners (1973)

Oil Burning Appliances (1974) ANS1 B130.1

Oil Burning Equipment (1973)

Oil Burning Stoves (Room Heaters and Ranges) (1973)

Oil Cans (1974)

Oil Cans (1974)

Oil Characteristics and General Spec. (1972)

Oil Characteristics (1972)

Oil Circuit Breakers (1973)

Oil Circuit Breakers (1973)

Oil Content of Petrol

Oil Cranes (1972)

Oil Field Chain and Sprockets (1970)

Oil Field Heaters Used in Producing Oil and Gas (1972)

Oil Field Service (1972)

Oil Field Service (1973)

Oil Filled Capacitor Switches for Alternating Current S

Oil Fired Air and Direct Fired Heatere (1973)

Oil Fired Boiler Assemblies (1973)

Oil Fired Central Furnaces (1973) ANSI Z96.1

Oil Fired Floor Furnaces (1974)

Oil Fired Unit Heaters (1974)

Oil for Color (1973)

Oil for High Pressure Cable Systems (1972) ANSI C59.70

Oil for Low Pressure Cable Systems (1972) ANSI C59.69

Oil for Use in Resins and Protective Coatings Including

Oil for Use in Transformers and in Oil Circuit Breakers

Oil for Use in Transformers and Oil Circuit Breakers (1

Oil Fried Water Heaters (1974)

Oil Heaters (1973) ANSl C 33.44

Oil Hoses (1970)

Oil Immersed Distribution Transformers (1972)

Oil Impregnated Bearings (1973) ANSI H9.14

Oil Impregnated Cellulosic Insulation (1973)

(Oil lmpregnated) (1970) ANSl G63.4

(Oil Impregnated) (1973) ANSI H9.27

Oil in Equipment as Received from Manufacturer (1969) a

Oil lnjection, and Gas Admission Systems (1972)
SAE

ANS1

ASTM

TAPPI

ASTM

ANSI

ICBO

$\mathrm{ICBO}$

CBO

NCRPM

ANSI Z37.

NCRPM Rl6

CBO UBCS32.13

ASTM D2885

AATCC 112

216.3

ICBO UPC*1.C

ANS1 Z65.1

IES

ANSI

ASE

ASE

SAE

RP1

$X 4.11$

*5

$* 6$

J621C

Al15. 12

B29.10

$\begin{array}{ll}\text { ANSl } & \text { B29.10 } \\ \text { APl } & 2 \mathrm{D}\end{array}$

Sp APl

APl RPT.1

APl 2C

ASTM D1483

ASTM D2245

APl

DEMA

NFPA

ASTM

ASTM

CR

ASTM

ASTM

ASTM

ASTM

ASTM

$A$ WPA

AWPA

Safe UL

UL

UL

ICBO

UL

CCT1

Std. Test. CCTI

L Low and DEMA

Std. Pract. for DEMA

$12 \mathrm{~J}$

$* 1.13$

$12 \mathrm{~K}$

52

85

C570

H.22

D3239

$\mathrm{C} 713$

D2781

D2604

D322
A5

P9

80

296

896

C124

C125.1

*1-12

$* 1.14$

D1040

D3146

D721

2C

APl

AP1

APl

$7 \mathrm{~F}$ K

$9 \mathrm{~B}$

NEMA $\quad$ SGl3

UL 733

UL

UL

UL

NSPA

ASTM

ASTM

ASTM

ASTM

ASTM

UL

UL

SAE

IEEE

/Nterconuec ASTM

ASTM

ASTM

ASTM

IEEE

DEMA 
ling Tapes (1974)

1.230

\section{Std.}

central Furnaces) (197/

(1972) ANSl L14.214 High Temperature, Fuel and high Ionizing Voltage Mass Spectrometric Analysis of Gas t. for Guide to the Application and Use of Radial Lip Type test: Std. Meths, for Test. the Preservative Properties of oil Soluble Wood Preservatives by Using Wood Properties of Test Meth. for 72) Rec. Pract. for Design Calculations for c Equipment / c Equipment (1972)

c Equipment (1971) Rec. Pract for the Flushing and Cleaning of Rec. Pract. for the Design of Rec. Pract. for the Purification of flushing and Cleaning of Gas Turbine Generator Lubricating 4.4

Medium Speed Stationary Diesel and Gas Engine Lubricating Std. Spec, for Steel Wire,
aluminum Alloy Seamless Extruded Tube and Pipe for Gas and Std. Meth. for Analysis of Creosote and Std. for Safety for Constant Level Spec. for Casing, Tubing and Line Pipe Connections in High Pressure Std. Test. Procedure for Std. Meth. of Test for Stability of Distillate Fuel Std. Meth. of Test for Salt in Crude Trading Rules for the Purchase and Sale of Linseed for Determining Color (Spectrophotometric) Content in Corn analytical Meth. for Determining Cold Test Content in Corn

$$
\begin{aligned}
& \text { Std. Spec. for Raw Linseed } \\
& \text { Std. Spec. for Raw Tung }
\end{aligned}
$$

Trading Rules for the Purchase and Sale of Soybean ing Procedures for Bulk Shipments and Transfers of Soybean Std. Spec. for Fuel Std. Spec, for Amyl Acetate Made from Fusel Segmented, Production Check Flanges and Gask/ Spec. for and Shortenings (Butter, Oleomargarine, High Acid Coconut Std. Spec. for Once Refined Sunflower Std. Spec. for Properties of Unpunched

Rec. Pract. for Handling and Proper Usage of lnhibited cids Content in Corn Oil and to Other Vegetable and Marine Rec. for Cutting

ndex of Refraction, with Abbe Refractometer, in All Normal Std. Meth. for ldentification of

of Analysis of Monoglycerides and Free Glycerol in Fats, or (1/ Sid. Meth. of Test for Apparent Viscosity of Motor r $(197 /$

Std. Meth. of Test for Apparent Viscosity of Gear

Std. Meth. of Test for Sediment in Crude and Fuel

Std. Meth. of Test for Total Nitrogen in Lubricating

or Characteristic Groups in Rubber Extender and Processing on Distribution and Structural Group Analysis of Petroleum Std. Meth. for Evaluation of White Mineral of Test for Oxidation Stability of New Mineral Insulating

f Spinning Lubricant (To Test the Removability of Spinning th. of Test for Dielectric Breakdown Voltage of Insulating th. of Test for Dielectric Breakdown Voltage of Insulating Test Meth. for Evaluating Oxidation of Wool

Meth. of Analysis of lodine Number in All Edible Fats and of Analysis of Saponification Value of All Normal Fats and alysis of Acetone-Insoluble Lecithin in Soybean and Corn f Test for Gas Content of Cable and Capacitor (Insulating) ontent in Corn, Grain Sorghum and Other Vegetable Fats and Std. Spec. for Gas Turbine Fuel Std. Meth. of Test for Pour Point of Petroleum Std. Meth. of Test. Drying

of Test for Oxidation Characteristics of Extreme Pressure

- Meth. of Test for Foaming Characteristics of Lubricating

Std. Meth. for High Pressure Pump Test. of Hydraulic

Tent. Meth. of Test for Pumpability of Industrial Fuel

t for Detecting Glycol Base Antifreeze in Used Lubricating Std. Meth. of Test for Water and Sediment in Crude seeds; Ground Nuts (Peanuts); Vegetable, Coconut, and Palm . of Analysis of Vitamin A in Dry Vitamin Mixes, Beadlets, of Methyl Esters of Long Chain Fatty Acids in Common Fats, Oxygen-Bomb Meth. of Analysis of Stability in Fats, Meth. of Analysis of Stability (AOM) of Fats,

h. of Solid Fat Index (50 or Less at $10 \mathrm{Deg}$.) of Margarine escription, and Grade of Palmkernels; Babassu; Flaxseed;/ y Dye Meth. of Analysis of Crude Protein in Cereal Grains, home Laundering (1970) ANSI L 14.239 Test Meth. for

ol Saturated with Gasoline; Methyl Blue and Green; Mineral Std. Meth. of Test for Hydrocarbon Types in Low or lsolation of Representative Saturates Fraction from Low palmkernels; Babassu; Flaxseed; Castor, Sesame, Safflower, sture and Volatile Matter in Fats and Shortenings (Butter,

Oil Migration or Plasticizer Bleed Out of Preformed Sea

Oil Oxidation Stability by Rotating
Oil Penetration of Paper (1972)

Oil Powered Forced Air (Domestic and Light Commercial)

Oil Repellency of Fabrics: Hydrocarbon Resistance Test

Oil Resistant, 65-75 (1973)

Oil Saturate Fractions (1971) ANSI Z11.310
Oil Seals (1972) lngs, Sealing,

Oil Soluble Wood Preservatives by Using Wood Properties

Oil Soluble Wood Preservatives by Using Wood Specimens

Oil Spots in Paper (1973)

Oil Sucker Rod Pumping Systems (Conventional Units) (19

Oil Systems for Lubrication and Control of Hydroelectri

Oil Systems for Lubrication and Control of Hydroelectri

Oil Systems for Lubrication and Control of Hydroelectri

Oil Systems (1971)

Rec. Pract. for A

Oil Systems (1972)

Std. Pract for Low and

Oil Tempered Carbon Valve Spring Quality (1971) ANSI G5

Oil Transmission and Distribution Piping Systems (1973)

Oil Type Wood Preservatives (1973)

Oil Valves (1974) ANSI B127.]

Oil Well Casing Centralizers (1973)

Oil Well Service) (1972) Thread
Oil Wicking of Composite Motor Oil Cans (1974)

Oil (Accelerated Meth (1974)

Oil (Electrometric Meth.) (1973)

Oil (Flaxseed) (1974)

Oil (1964)

Oil (1965)

Oil (1972)

Oil (1972) ANSI K34.1

Oil (1973)

Oil (1973)

Oil (1973)

Oil (1973) ANSI Z11.203

Std. Analytical Meth.

Std. CR

FMS

ASTM

ASTM

NSPA

Oil ( 85 to $88 \%$ Grade) (1972)

Oil) Wellhead Equipment (Production Valves, Ring Joint,
Oil, etc.) (Cereal Chemistry) (1962)

Oil, Technical Grade (1973)

Oiled Paper Perforated Tape (1971)

Oilfield Acids (1973) APl Bul D15

Oils and Animal Fats (1965)

Oils and Compounds (1974)

Oils and Liquid Fats (Cereal Chemistry) (1967)

Oils and Oil Acids in Solvent Type Paints (1972)

Oils and Shortenings (Cereal Chemistry) (1967)

Oils at Low Temperature Using the Cold Spanking Simulat

Oils at Low Temperatures Using the Brookfield Viscomete

Oils by Extraction (1969) ANSI Z11.58, APl 2561

Oils by Modified Kjeldahl Meth. (1973)

NSPA

ASTM

APl

ANSI

SAE

ASTM

NWMA

TAPP1

APl

ASME

ASME

DEMA

ASTM

AWPA

UL

API

CCTI

ASTM

ASTM

NFP

Oils by the Clay Gel Absorption Chromatographic Meth. (

Oils by the n-d Meth. (1973)

Oils by Ultraviolet Absorption (1973) ANSI Z11.210

Oils Containing 2,6-Ditertiary-Butyl Para-Cresol

Oils from Woolen Yarns (1971) ANSI Li4.113

Oils of Petroleum Origin Under Impulse Conditions (197

Oils of Petroleum Origin Using VDE Electrodes (1972) an

Oils on Woolen Fabric Textile in Storage (1971) ANSI L1

Oils (Cereal Chemistry) (1967)

Oils (Cereal Chemistry) (1967)

Oils (Cereal Chemistry) (1967)

Oils (R1969) ANS1 C.59.23

Oils $(1966)$

Oils (1971) ANS1 Z11.312

Oils (1971) ANS1 Z11.5.

Oils (1972)

Oils (1972) ANSI Z11.320

Oils (1972) ANSI Z11.78

Oils (1973)

Oils (1973)

Oils (1973)

Oils (1973) ANS1 Z11.8

Oils) (1974)
Oils, and Emulsions (Cereal Chemistry) (1973)

Oils, and Shortenings (Cereal Chemistry) (1962)

Oils, and Shortenings (Cereal Chemistry) (1967)

Oils, Shortenings, and Other Fats (1968)

Oilseed Products Trading Rules (Performance, Quality,

Oilseeds, Legumes, Forages, and Animal and Dairy Produc

Oily Stain Release Meth. of Textile Fabric Soil During

Oil; Urease Tablets; Xanthyrol) (1962)
Olefinic G asoline by Mass Spectrometry (1971) ANSI Z11.

Olefinic Petroleum Naphthas (1973) ANS1 Z11.165

Oleic, and Sunflower Seeds; Ground Nuts (Peanuts); Vege

Oleomargarine, High Acid Coconut Oil, etc.) (Cereal Che

Meth. of an AACCH

ASTM

ASTM

ASTM

ASTM

Std. Meth. ASTM

Std ASTM

ASTM

ASTM

Std. Meth. of Tes ASTM

ASTM

NiOP

C772

T462

Z91.1

118

AMS7266

D2786

J946A

M. $1-70$

M-1.70

UM- 458

$11 \mathrm{~L}$

OS.5Cl

LOS-5D 1

LOS-5P 1

LOS.4C I

*1.11

A 230

B345

Al
352

BUL 5A2

C124

D2274

D3230

$* 2$

H-12

H. 10

$8-4$

D234

D 12
$* 2$

*

D396

D554

$6 \mathrm{~A}$

44-30

D3169

X3.29

H. 22

$7-37$

58-20

D2245
$58-45$

D2602

D2983

D473

D3228

D2007

D3238

D2269

D2112

77

D3300

D1816

62

$58-30$

58-50

58-35

D831

H-32

D2880

D97

D555

D2893

D892

D2882

D96

$* 1$

Ion Meth AACCH 86.01A

/Tion AACCH 58-17

AACCH 58.55

AACCH 58-54 
Minimum Operational Characteristics Airborne VHF Std. Spec. for Test Meth. for ometry (1973)

Meth. of Test for Kinematic Viscosity of Transparent and for Selecting and Defining Color and Gloss Tolerances of est for Indexes of Whiteness and Yellowness of Near White, Std. Meth. for Measurement of Thickness of Transparent or Calculation of Heat Gain Through Std. Dimensions of Image Areas and Mounts for Slides and Std. for Heat and Smoke Roof and Window Vents Designed to (1972)

Code: General Precautions for Prevention (Incinerator and Std. Meth. of Test for Flash Point of Liquids by Tag

Meth. of Test for Flash Point of Cutback Asphalt with Tag Std. Meth. of Test for Flash and Fire Points by Cleveland Std. Meth. for Chemical Analysis of Steel and Cast, Reproducer Test Tape: Full Track, $1 / 4 \ln .(6.3 \mathrm{Mm})$ Width, Sid Ampacities Cables in Std. Specs. and Load Tables for Uniform Building Code Std. for Std. Spec, and Load Tables for

Safety Std. for Panic (Emergency) Hardware (Outward Spec. for Industrial Perforated Plate and Screens (Square Quality Stds. for Wood Screens for Window and Door Safety Requirements for Floor and Wall

nsi C 33.40

ry Dryer) (1974)

dry Washer) (1974)

ake Designs (1971)

) ANSl Z216.1

s Whose Maximum Allowable Operat/Std. for Small Manually ess (1974) Procedures for Motor Vehicles, Trailers, and Semitrailers Std. for Safety for Electrically Safety Std. for Power

e Alarm Devices, Single and Multiple Station, Mechanically Safety Std. for Electrically Groups A, B, C, and D, An/ Safety Sifety Std. for Electrically Std. for Dimensions for Photographic Flashcubes (Battery cated Reflective Insulation Systems for Equipment and Pipe

Audio Record One for 2-ln. Quadruplex Video Magnetic Tape ncy Test Tape for Quadruplex Video Magnetic Tape Recorders h Requirements (Passenger Car Type Tires) (1971) s (1973)

Safe Safe Meth. for Extracting Fluid Samples from the Lines of an scometers (1972) ANSl Z11.275 record One for 2-ln. Quadrup/ and Conditioning) (1966)

Std. Spec, and Std. Frequency Response and Std. trong Acid Removal (1972 Rec. Pract. for Drill Stem Design and Std. Meth. of Test for Std. Meth. of Test for erials (1972) ANSI Z111.4 Std. Meth. of Test for valves in Gas Distribution Systems Whose Maximum Allowable es (1973)

Sid. for

imum Performance Std. for Airborne VOR Receiving Equipment mance Std. for Airborne Distance Measuring Equipment (DME) al Engineering) (1972) Std. for Fastener; Rotary, Quick erator for Dry Pipe Systems (Fire Protection) (/ ansi $\mathrm{C} 50.30$ Std. for Guide for

(Combustible) Gas Indicators Including Their Installation, for Low and Medium Speed Stationary Diesel and Gas Engine for Lightweight/ Std. for Preparation of Manuals for Installation, Spec. for the Design, Construction, and nal Electrical Safety Code) (1973) Rec. for 2) Rules for the Std. Dimensions for Compatible Rec. for

Std. for Fundamentals Governing the Design and Std. for Type Designations, Areas of Use, Maintenance and perty from Explosion and Fire in Design, Construction, and eum lndustry U/ Rec. Pract. for Design, Installation, and ty Requirements for lndustrial Rotary Mowers (Designed for $n$ Products in Nuclear Reactor Coolant Water During Reactor tionary Diesel and Gas Engine Speed Governing and Parallel Synthetic Rubber Insulation for Wire and Cable, 75 Deg. C er Heat Resisting Insulation for Wire and Cable, 75 Deg. C Synthetic Rubber Insulation for Wire and Cable, 90 Deg. C

oisture Resisting lnsulation for Wire and Cable, 75 Deg. C rs (1973) ANSI C 37.61 oundbeds (1972)

Units for Protection Agai/ Std. Guide for the Application, Rec. Pract. Design, Installation. Safety Sid. for Construction,
Omnirange (VOR) Systems (1972)

Once Refined Sunflower Oil, Technical Grade (1973)

Opacity of Paper, Using Reflectometer with 45.0 Deg. Ge Opaque Liquids and the Calculation of Dynamic Viscosity Opaque Materials and for Evaluating Conformance (1972T) Opaque Materials (Color Measurement of Textiles, Paints Opaque Metal Oxide and Metallic Coatings by Double Beam Opaque Walls (Tech. Notes) (1969)

Opaques for Television (1973)

Open Automatically in Case of Fire (1974)

Open Bottle Tap Sampling of Noncryogenic Fluid Systems

Open Burning, Combustible and Flammable Materials; Repo

Open Cup Apparatus (1972)

Open Cup Apparatus (1972)

Open Cup (1972) AASHO T48, ANSI Z11.6

Open Hearth, and Wrought lron (1973)

Open Reel (For Tape Speeds of 7.5 ln. 0 190.5 Mm, and 3

Open Top Cable Trays (1972)

Open Web Steel Joints J Series and H Series (1970)

Open Web Steel Joist Design (1973)

Open Web, Longspan, and Deep Longspan Steel Joints (197

Opening Door Releasing Device) (1973)

Opening Series) (1972)

Openings (Architectural Woodwork) (1973)

Openings, Railings, and Toeboards (1973)

Operable General and Special Use Snap Switches (1972) a

Operated and Commercial Clothes Drying Equipment (Laund

Operated and Commercial Clothes Washing Equipmen: (Laun

Operated Appliances (1972) ANSI C 33.36

Operated Chain Hoists for Industrial Applications (1971

Operated Chain Hoists for Ratchet and Pawl, and Load Br

Operated Dispensing Devices for Flammable Liquids (1974

Operated Metallic Gas Valves in Gas Distribution System

Operated on Public Highways (1973) /Td. for Inspection

Operated Photographic Equipment Rated at 300 Volts or L

Operated Pumps for Petroleum Products (1973)

Operated Type for Indoor Use (1973)

Operated Valves for the Control of Fluids (1973)

Operated Valves for Use in Hazardous Location

Operated Water Type Fire Extinguishers (1973)

Operated) (Photography) (1972)

Operating at Temperatures Above Ambient Air (1972)

Operating at 15 and $7.5 \mathrm{ln}$./S (1973)

Operating at $15 \ln . / \mathrm{S}(1973)$

Operating Condition of Tires Including Tire Treads Dept
Operating Condition of Truck. Trailer and Bus Type Tire

Operating Hydraulic Fluid Power System (For Particulate

Operating Instructions for Glass Capillary Kinematic VI

Operating Level of Recorders and Reproducers for Audio

Operating Limits for Central Station Units (Air Moving

Operating Limits (Petroleum) (1974)

Operating Performance of Anion Exchange Materials for S

Operating Performance of Particulate Cation Exchange Ma

Operating Pressure Does Not Exceed 60 or 125 Psig (1973

Operating Storm Windows for External Application (1972)

Operating Supply Voltage and Frequency of Office Machin

Operating Within the Radio Frequency Range of 108-118 M

Operating Within the Radio Frequency Range of 960-1215

Operating, High Strength (1973)

Operation and Flow Process Charts and Symbols (Mechanic

Operation and Maintenance of Offshore Cranes (1972)

Operation and Maintenance of the Reliable Model B Accel

Operation and Maintenance of Turbine Generators (1972)

Operation and Maintenance (On Boats) (1973)

/Lammable

Operation and Maintenance (1972)

Operation and Repair of Laboratory Instruments (1972)

Operation of Class Hi (High lmpact) Shock Test. Machine

Operation of Crowder Dry Pipe Valves, Model a (1973)

Operation of Electric Supply Lines and Equipment (Natio

Operation of Forage Harvesters, Wagons and Blowers (197

Operation of Grimes AB Dry Pipe Valve Accelerator (1973

Operation of Local Exhaust Systems (1971)

Operation of Powered Industrial Trucks (1973) ANSI B56.

Operation of Solvent Extraction Plants (1973)

Operation of Subsurface Safety Valve Systems for Petrol

Operation with Tractors Having at Least $20 \mathrm{Hp}$ ) (1971)

/Ed Neutron Emitting Fissio

Operation (1970) ANSI N 163 / Pract. for Low and Medium Speed Sta

Operation (1974) ANSI C8.24

Operation (1974) ANSI C 8.27

Operation (1974) ANSI C 8.40

Operation (1974) ANS1 J8.4

/D. Spec. for Natural Rubb Std. Spec. for

Operation, and Maintenance of Automatic Circuit Reclose

Operation, and Maintenance of Impressed Current Deep Gr Operation, and Maintenance of Vehicle Alarm Systems and Operation, and Maintenance (1973)
RTCA

ASTM

TAPP

ASTM

ASTM

ASTM

ASTM

BlA

ANSI

FMS

ASTM

ICBO

ASTM

Std. ASTM

ASTM

ASTM

EIA

IPCEA

AISC

ICBO

SJI

UL

Sid. ASTM

$A$ WI

ANSI

UL

UL

UL

HMI

UL

ANSI

ANSI

UL

UL

UL

UL

UL

ANSI

/Ri ASTM

ANSI

ANSl

VESC

VESC

NFLD

ASTM

ANSI

AMCA

APl

ASTM

ASTM

ANSl

AAMA

ANSl

RTCA

RTCA

NSA

ASME

APl

FMS

IEEE

$A B Y C$

DEMA

ANS

FMS

ANSI

ASAE

FMS

ANSI

NFPA

FMS

API

SAE

ASTM

DEMA

ASTM

ASTM

ASTM

IEEE

NACE

UL

FMS

DO. 149

D3169

UM-538

D445

E 313

B588

$4 \mathrm{~A}$

PH22.94

4430

F301

UFC *2ART27

D 1310

D3143

D92 
) Systems (1972)

Used in Airborne Volumetric Navigation Systems /

Minimum Minimum fety Equipment) (1973)

d Analyzing Outage / sive, etc.) (1974)
Std. Definitions in Electrical Power

Rec. for Flammable Liquid Mixing Rec Pract for Bus Stops for Freeway Std. for Safety Requirements for Marine Terminal ents for Commercial Laundry and Dry Cleaning Equipment and g Against Fire During Building Construction and Demolition irements for Personnel Hoists (Construction and Demolition Std. for nd Equipment (1971) ANSI B114.2, Sa/ Std. for Symbols for s (1972) SAE J333 Std for Std. for Door, Drapery, Gate, Louver, and Window Electric Trial Use Guide for Type Test of Class 1 Electric Valve Std. Spec. for a Liquid

Tent. Rec. Pract. for Measurement of Glass Stress ride-Lithium Fluoride Carrier D-C Arc Technique Using an d Low Alloy Steel by the Point to Plane Technique Using an d. Rec. Pract. for Selection of Electrode Material Used in last Furnace Iron by the Point to Plane Technique Using an Spec, for Photographic Std. Meth. for Identification and Qualitative (Including for Calibration of Refractory Metal Thermocouples Using an icture Film/ Std. for Location of Super 8 Printed Area in - Perforate/ Std. for Location of Super 8 Printed Area in

from Corn or Grain Sorghum Starch Which Are Substantially for Dimensions for Resolution Test Target for Photographic $r$ of Paper and Pulp (Using Spectrophotometer with Reserved ete Paving (1972)

Rec. Spec. for Std. Meth. of Test for Std. for Scientific Papers for Written or rs (1971) Ant. Analytical Meth. for Determining Methyl
for Standardization of Forms and Information Flow Used in

ven Drying (1/ Std. Meth. of Test for Moisture Present in inc Coated (Galvanized) Welded and Seamless Steel Pipe for ical Analysis of Chrome Containing Refractories and Chrome nd Refractory Materials (Calcined Bauxite, Calcine, Chrome Std. Meth. of Test for Aluminium in lron Std. Meth. of Test for Calcium and Magnesium in Iron Std. Meth. of Test for Aluminum Oxide in Iron

Tent. Meth. of Test for Manganese Dioxide in Manganese ation Spark T/ Std. Meth. for Spectrochmeical Analysis of Fabrics and lndustrial Filament Yarns, Made from Man Made Selection and Preparation of Test Panels for Use in Test.

Std. Meth. of Test for Moisture Vapor Permeability of Std. Meth. for Condensation-Humidity Test. of , or Other Failure of Paint, Varnish, Lacquer, and Related or Determining Pressure Marking and Blocking Resistance of Std. Meth. of Test for Formability of Attached
Combustible Liquids A/ Uniform Fire Code: Manufacture of for Microscopical Determination of the Reflectance of the chemical Oxygen Demand in Industrial Waste Waters and Mos saturated Organic Felt, Coal Tar Pitch, Coal-Tar Saturated Up Roof Coverings: Hot Mopping Asphalt, Asphalt-Saturated erings: Hot Mopped Asphalt, Asphalt and Coal Tar Saturated

ffusion Stain of Rubber and Contact and Migration Stain of Std. Rec. Pract. for Detergent Resistance of Std. Meth. of Tesi for 21, ANSl A37.19

of Test for Trace Quantitites of Total Sulfur in Volatile td. Definitions of Terms Relating to Bituminous, and Other Meth. for Tent. Hazard Classifications of Recommended Safeguards for

Std Meth of Test for Acidity Alkalinity of Halogenated Meth. of Test for Water Soluble Halide Ion in Halogenated ituminous Road Materials (Tar, Petroleum Asphalt, etc.) in Meth. of Test for Nonamine Acid Acceptance of Halogenated Std. for Industrial Engineering Terminology: cceptable Concentrations of Inorganic Mercury and Nonalky Meth. of Test for

bend (Applicable to Solution Coil Coatings and Single Coat e First Time (Petroleum Production) (1974)

Rec. Pract. Meth. of Test for Tensile Properties of on Controlled Outside Diameter/ ed Types 27G053, 30G05 g Flour (Cereal Chemistr) Std. Spec. for Biaxially Std. Spec. for Flat Rolled, Grain Gustafson Meth. of Analysis for Quality Stds. for Misc $\mathrm{m}$, Casework, PaneIwork, Closet and Storage Shelving, Misc. plating of Nickel and Chromium on Metal Parts-Automotive r (Brick, Concrete, Stone, Tile, Metal, Plastic, etc.) for Std. Meth. of Test for Discoloration of ydrolyzates
Operational Characteristics Airborne VHF Omnirange (VOR Operational Characteristics Vertical Guidance Equipment Operational Rec. for Globe Dry Pipe Valves (Fire and Sa Operational Safety Manual for Anhydrous Ammonia (1973) Operations Terminology Including Terms for Reporting an Operations (Paint, Varnish, Lacquer, Printing Ink, Adhe Operations (1971)

Operations (1972)

Operations (1972)

Operations (1973) ANSI A 10.21

Operations) (1973)

Operator Controls on Agricultural Equipment (1972)

Operator Protection for Wheel Type Agricultural Tractor

Operators and Systems (1973)

Operators for Nuclear Power Generating Stations (1972)

Optical Adhesive (1970)

Optical Coefficient (1973)

Optical Emission Spectrograph (1974)

Optical Emission Spectroscopy Based on Physical Propert ASTM

Optical Emission Vacuum Spectrometer (1973) / / sis of B ASTM

Optical Filter Sizes (1972)

Optical Microscopic) Analysis of Mineral Filler and Coa

Optical Pyrometer (1972) ANSI N144

Optical Reduction or Contact Printing on $35 \mathrm{~mm}$ Motion

Optical Reduction Printing on $35 \mathrm{~mm}$ Motion Picture Film

Optical Test Meth. for Ink Show Through (Paper) (1973)

Optically Clear (1959)

Optics (1972)

Optics (1972)

/Hydrolyzates Solution

/Al Reflectance, Transmittance, and Colo

Optimum Size Coarse Aggregate for Portland Cement Coner

Optimum So3 in Portland Cement (1972)

Oral Presentation (1972)

Orange Indicator, $0.1 \%$ Content of Reagents and lndicato

Ordering. Shipping, and Billing of Merchandise Within T

Ordinal Date for lnformation Interchange (1971)

Ordinary Commercial and Industrial Samples of Wool by $\mathrm{O}$

/Ack and Hot Dipped Z

Ordinary Uses (1973) ANSI B 125.2

Ore (1970) ANSI Al39.2

Std. Meth.

Ores by Atomic Absorption Spectroscopy (1973)

Ores by Atomic Absorption Spectroscopy (1973)

Ores (1972)

Ores (1972T)

Ores, Minerals, and Rocks by the Fire Assay Preconcentr

Organic Base Fibers (1972)

Organic Coating Systems (Coil) (1974)

Organic Coatings Films (1972)

Organic Coatings on Metallic Surfaces (Coil) (1974)

Organic Coatings on Metallic Surfaces (Coil) (1974)

Organic Coatings When Applied to Coil Coated Metal (197

Organic Coatings with Impact Wedge Bend Apparatus (1973

Organic Coatings (Paint), and Handling of Flammable and

Organic Components in a Polished Specimen of Coal (1972

Organic Compounds (1966)

Tical Meth. for Determining

Organic Felt, Asphalt-Saturated Asbestos Felt, Asphalt-

Organic Felt, Coal Tar Pitch, Coal-Tar Saturated Organi

Organic Felts, Coal Tar Pitch, and Asphalt Coated Glass

Organic Finishes in Contact with Rubber (1973) ANSI J7.

Organic Finishes (1973)

Organic Impurities in Sands for Concrete (1973) AASHO T

Organic Liquids (Oxy Hydrogen Combustion Meth.) (1972)

Organic Materials for Roofing, Waterproofing, and Relat

Organic Nitrogen in Paper and Paperboard (1972)

Organic Peroxides (Plastic) (1974)

Organic Peroxides (1972)

Organic Solvents and Their Admixtures (1974)

Organic Solvents and Their Admixtures (1974)

Organic Solvents (1966) ANSI A37.12

Organic Solvents, Degreasing Grade (No

Organization Planning and Theory (1972)

Organo Compounds (1972)

Organochlorine Pesticides in Water (1972T)

land Flexibility by the Wedge

Orientation Program for Personnel Going Offshore for Th

Oriented Fiber Composites (1972T)

Oriented Polyethylene (PEO) Plastic Pipe (SDR.PR) Based

Oriented, Silicon lron, Electrical Steel, Fully Process

Original A sh in Phosphated and Freshly Mixed Self Risin

Ornamental ltems (Architectural Woodwork) (1973)

Ornamental Items, Stairwork and Handrails, Exterior Fra

Ornamentation and Hardware (1970)

Ornamentation, Protection, or Insulation (1973)

Orthodichlorobenzene by Carbon Black (1972A)

(Orthophenanthroline) Content of Corn Syrup and Starch $\mathrm{H}$
Std. for

RTCA

RTCA

IEEE

FMS

ANSI

ANSI

NFPA

ASAE

ASAE

ASAE

UL

IEEE

ASTM

ANSI

TAPPI

ANS1

ANS1

TAPP

CR

TNS]

TAPPI

ACPA

ANS

CR

NWHA

ANSI

ASTM

ASTM

ASTM

AST

ASTM

ASTM

ASTM

ASTM

A T M

ASTM

NCCA

ASTII

NCCA

IN NCCA

$\mathrm{NCCA}$

ASTN

ICBO

ASTM

CR

UL

UL

ASTM

ASTM

ASTM

ASTM

ASTM

TAPP

FMS

FMS

ASTM

Std. ASTM R Solubility of B ASTM ASTM ANSl

ANSI ASTM

NCCA

API

AST 1

AST

A ST

AACCH

$A W 1$

AWI

$S A E$

Venee ICBO

ASTM

CR

DO- 149

DO. 152

2.14

P110

346

7.36

MH9.1

$\mathrm{Z} 8.1$
241

A 10.4

S304.4

S305.3

325

D285

C770

E483

E403

E504

E485

PH3.17

T421

PH22.180

PH22.179

UM-545 
easuring the Wear Life and Bonded Solid Film Lubricants in zates Under Compressive or Shear Strains by the Mechanical ational Electrical Code (NFPA 70) and Code Requirements by ns Terminology Including Terms for Reporting and Analyzing n Boats (1974) Rec. Pract. for Rec. Pract. for

tion of Permanently Installed Fuel Systems for Inboard and $\begin{array}{ll}\text { tion Rat/ Std. for Schedule of Dielectric Test Values for } \\ \text { metrical Cl } & \text { Std. Requirements for External Insulation for }\end{array}$

d for Installation of Seacocks. Through Hull Connections. Std. for Std, for

Std. for Gas Hose Connectors for Portable std. Guide for Evaluating the Effect of Solar Radiation on rocedures (1972)

Std. Meth. of Test for Effects of mission Units Penetrating the Hull for Boats Equipped with Std. Wheel Diameters and Std. for Safety for Electrical d Cast Aluminum Covers (/ 73) Std. for Electrical Sheet Steel Std. for Air

Camera Equipment (1974/ atistical Significancel Std. for Safety for Electrical Power
Rec. Std. for Engineering Specs. Std. Rec. Pract. for Dealing with Std. Meth. for Measuring Audio Amplifier Single Frequency Industry Quality Std. for Computer (1967) Industry Quality Std. Format and Coding for Computer
Std. for Power Std for Stabilized Power Supplies Direct Current ran Procedures for Executive Functions and Process Input Meth. of Test for Effective Crimping on spec. for Polybutylene (PB) Plastic Pipe (SDR-PR) Based on lyethylene (PEO) Plastic Pipe (SDR-PR) Based on Controlled olyethelene (PE) Plastic Pipe (SDR.PR) Based on Controlled lyethylene (PE) Plastic Pipe. Schedules 40 and 80 Based on Safety Std. for Panic (Emergency) Hardware

Locking and Nonlocking (1972) Std for Screw 100 Deg. Std. Meth. of Test for Extractable Matter in

$t$ in Ordinary Commercial and Industrial Samples of WooI by Std. Analytical Meth. for Determining Moisture

bleached/ Std Analytical Meth. for Determining Moisture in Yeast Foods (Cereal Chemistry) (1962) a; Bread; Feedstuffs, Ground Grains and / Modified Vacuum molina, Bread, Grain, Soybeans, Rice, Beans, Peas, L/ Air

uffs (Cereal Chemistry) (1962) Modified Two Stage Air istry) (1962)

c (Cereal Chem istry) (1962)

Air

of Test for Accelerated Aging of Vulcanized Rubber by the

$r$ Effect of Heat and Air on Asphaltic Materials (Thin Film flour and Semolina (Cereal Chemistry) (1962) Sid. Analytical Meth for Determining Moisture ( Std. Analytical Meth. for Determining Moisture

lline) (/ Std. Analytical Meth. for Determining Moisture Test Meth. for Moisture in Wood Chips (Steam

Std. Household Electric Range Pyrolytic Self Cleaning Std. for Commercial Gas Baking and Roasting Uniform Fire Code: Industrial Baking and Drying ty Ventilation Requirements for Direct Fired Powder Curing Std. for Household Electric Ranges (Stove and Std. for Industrial Power Take Offs for

Std. Performance Requirements for $\mathbb{W}$ oven Drycleanable anes (1971)

elow; $500 \mathrm{kVA}$ and Smaller (1971) Spec. for Electric r Connectors for Use Between Aluminum or Aluminum-Copper

$r$ Connectors for Use Between Aluminum or Aluminum-Copper Std. for

Std for Protective Frame (For Agricultural Tractors for for Basis of Payment on Portland Cement Concrete Pavement Test Meths. for Interior Panel Coatings and Test Meth. for Exterior Coatings and Rec. Pract for Reinforced Bituminous Concrete Rec. Pract. for Mobile Construction Type Crane Std. Meth. of Test for

Std. for Bolt, Taper Shank, Shear Type, 100 Deg. Head

Std. for Bolt, Taper Shank, Shear Type, Protruding Head Std. for Pin, Swage Locking, Steel, Std, and Std. for Pin, Swage Locking, Std. and

Std. for Bolt Lock. Tension. Protruding Head, Std. and std. for Bolt Lock, Tension, 100 Deg. Crown Head, Std and Std. for Bolt Lock, Shear, Protruding Head, Std. and Std. for Bolt Lock, Shear, 100 Deg. Head, Std. and for Bolt Lock, Tension, 100 Deg. Head (MS20426), Std. an 1973) Std. for Pin, Swage Locking, Steel, Std. and 973)
Oscillating Motion (1971) ANSI Z11.300

Oscillograph (1972) ANSI J2.17

OSHA (1973)

Outage of Electrical Transmission and Distribution Fac

/ of Test for M ASTM

Outboard Motor Boat Transom and Motor Well Dimensions O

Outboard Motor Dimensions (Boats) (1972)

Outboard Powered Boats (1973)

Nstruction and Installa

Outboard Water Strainers, Water Scoops and Drain Plugs

Outdoor AC High Voltage Circuit Breaker External Insula

Outdoor AC High Voltage Circuit Breakers Rated on a Sym

Outdoor Cooking Gas Appliances (I972)

Outdoor Floodlighting (Light) Equipment (1973)

Outdoor Gas Fired Appliances (1971)

Outdoor Metal Clad Switchgear (197I) ANSI C37.24

Outdoor Pistol Range Plans (Firearms) (1970)

Outdoor Smallbore Rifle Range Plans: Safety Rules and P

Outdoor Weathering on Pipeline Coatings (1972)

Outdrives, Jet Drives and/or Bow Thrusters (1973)

Outlet Areas for Centrifugal Fans (1966)

Outlet Boxes and Fittings (1974)

Outlet Boxes, Device Boxes, Covers and Box Supports, an

Outlets and Inlets Used in Air Distribution Systems (19

Outlets (1972) ANSI C33.85

Outline for Monochrome Cetv (Closed Circuit Television)

Outlying Observations in Samples and How to Test the St

Output for Institutional Audio-Visual Equipment Used

Output Microfilm (1971)

Output Microfilm (1971)

Output Ratings of Packaged Audio Equipment for Home Use. Output (1972)

Output (1972)

/Td. for Industrial Computer

Outside Crimped Valves of Aerosol Containers (1972)

Outside Diameter (1973)

Outside Diameter (1973)

Outside Diameter (1974)

Outside Diameter (1974)

Outward Opening Door Releasing Device) (1973)

Ec. for Biaxially Oriented Po Std. Spec. for P
Std. Spec. for $\mathrm{Po}$

Oval Head Tri Wing Recess, A286 Cres, Full Thread Self.

Oven Dried Wool (1973)

Oven Drying (1972) ANSI Ll4.202

/T for Moisture Presen

Oven Filter Aid) Content of Corn Syrup Unmixed (1954)

Oven Filter Paper) Content of Corn Syrup Unmixed and Un

Oven Meth. of Analysis of Moisture and Volatile Matter

Oven Meth. of Analysis of Moisture in Flour and Semolin

Oven Meth. of Analysis of Moisture in Flour, Farina, Se

Oven Meth. of Analysis of Moisture in Grains and Feedst

Oven Meth. of Analysis of Moisture in Malt (Cereal Chem

Oven Meth. of Analysis of Moisture, Drying at 135 Deg.

Oven Meth. (1967) ANSI J5.

Oven Test) (1973)

Std. Meth. of

Oven (Aluminum Plate) Meth. of Analysis of Moisture in

Oven) Content in Commercial Feedstuffs (Corn) (1957)

Oven) Content in Corn Starch (1956)

Oven) Content of Corn Sugar (Crude and Refined) (Crysta

Oven) (Pulp and Paper) (1973)

Ovens (Stove) (1974)

Ovens (1971)

Ovens (1973)

Ovens (1973)

Ovens) (1972) ANSI C71.1

Overcenter Clutches (1971)

Overcoat Fabrics (Institutional Uniform Textile) (1973)

Overhead Bridge and Gantry Multiple Gerder Traveling $\mathrm{Cr}_{r}$

Overhead Distribution, Transformers, 67,000 Volts and B

Overhead Electrical Conductors (1973) EEI TDJ-162

Overhead Electrical Connectors (1973) NEMA CC3

Overhead Hoists (1973)

Overhead Protection (Test Procedures and Performance Re

Overlay Projects (1973)

Overlays on DFPA Grade Trademarked Plywood (1967)

Overlays on DFPA Grade Trademarked Plywood (1971)

Overlays (Welded Wire Fabric) (1962)

Overload Indicating System Test Procedure (1971)

Overrun of Food Aerosols (1972)

Oversize (1973)

Oversize (1973)

Oversize, Protruding Head, Shear, Pull Type (1973)

Oversize. Protruding Head, Tension, Pull Type (1973)

Oversize, Pull Type, Titanium Alloy (1973)

Oversize, Pull Type, Titanium Alloy (1973)

Oversize, Pull Type, Titanium Alloy (1973)

Oversize, Pull Type, Titanium Alloy (1973)

Oversize. Pull Type. Titanium Alloy (1973)

Oversize, 100 Deg. Head (MS20426), Tension, Pull Type

Oversize, 100 Deg. Head (Ms2494), Tension, Pull Type (1

talla

$A B Y C$

$A B Y C$

$A B Y C$

$A B Y C$

ANSI

ANSI

NEMA

ANSI

IEEE

NRA

ASTM

/Ans $A B Y C$

AMCA

UL

NEMA

ARI

EIA

ASTM

ANSI

NMA

NMA

EIA

NEMA

ISA

ASTM

ASTM

ASTM

ASTM

U

NSA

ASTM

AST M

CR

CR E.44

AACCH $44-32$

$\mathrm{AACCH} \quad 44.40$

AACCH $44.15 \mathrm{~A}$

AACCH 44.18

$\mathrm{AACCH} \quad 4420$

$\mathrm{AACCH} \quad 44.19$

td. Meth. ASTM D573

ASTM D1754

$\mathrm{AACCH} \quad 44-16$

CR

CR

CR

B-38

ANSI Z2].28

ICBO UFC*2ART25

FMS 7-27S

AHAM ER.1

SAE J621C

ANSI L24.3.4

CMAA 70

ANSI

/ Fo NEM

EEI

ANSI

ASAE

ACPA

APA

AP

WRI

SAE

ASTM

NSA

NSA

NSA

NSA

NSA

NSA

NSA

NSA

Std. NSA

NSA

NSA

C57.1220

C33

TDJ-162

B30.16

S 310.2

TM. 6

$* 5$

RB8

J248
D3075

1725

1729

1446

1465

2005

2125

2406

2506

2115

1475

1456 
Std. for Pin, Swage Locking, SteeI, Std. and Std. Form of Agreement Between Std. for Agreement Between Std. Form of Agreement Between Std. Form of Agreement Between where Basis of Payment Is/r Std. Form of Agreement Between
ate, $\left(\mathrm{Nh}_{2} \mathrm{Oh}\right)_{2} . \mathrm{H}_{2} \mathrm{SO}_{4}$ (Hydroxylammonium SuIfate) 2) ate, $\left(\mathrm{Nh}_{2} \mathrm{Oh}\right)_{2} \cdot \mathrm{H}_{2} \mathrm{SO}_{1}$ (Hydroxylammonium Sulfate) res (Maltose, Sucrose, Mannose, Galactose, Dextr/ Glucose 2) ANSI Z11.320 Std. Meth. of Test for Std. Spec. for ransformers and Oil Circuit Breakers (197/ age (1971) ANSI L14. S2 Test Meth. for Evaluating Std. Meth. of Test for Continuity of Steam Turbine Oil aining 2,6-Ditertiary-Butyl P/

Std. Meth. of Test for 3)

Tent. Meth of Test for Thermal

ermining Chromic Oxide Content in Leather (Perchloric Acid eth. of Test for Chromic Oxide in Leather (Perchloric Acid tities of Sulfur in Light Liquid Petroleum Hydrocarbons by Std. Meth. of Chemical Analysis of Aluminum

or Measurement of Thickness of Transparent or Opaque Metal rochemicaI Analysis of Uranium Oxide $\left(\mathrm{U}_{3} \mathrm{O}_{k}\right)$ by Gallium of a Cross Secti/ Std. Meth. for Measurement of Metal and ic Oxide Coatings on Aluminum (1973) Std. Spec. for Anodic Oxide Coatings on Aluminum (1973) Std. Spec. for A nodic 969) ASTM D2807

Meth. for Determining Chromic Std Meth. for Chemical Analysis for Aluminum pecific ElectricaI Impedance of Electrical Grade Magnesium Std. Meth. of Test for Aluminum

std. Meth. of Test for Chromic ctrochemical Analysis Of, and PhysicaI Tests On, Beryllium Std. Spec. for Nuclear Grade Beryllium Std. Spec. for Nickel

) Std. AnalyticaI Meth. for Determining Std. Arseniou ckness by Multiple Beam I/ Tent. Meth. for Measurement of aces (1971)

Tent. Meth. for Measurement of
Black

Std Meth. for Spectrochemical Analysis of Uranetal Std. Spec. for Deuterium ption of Potassium Permanganate by Impurities in Deuterium Std. Meth. of Test for Deuterium

fluorine Helium Hydrogen, Krypton, Methane, Neon, Vitric ndensation Meth.) (1973)

Tent. Meth. of Test for Sulfur

Test Meth. for Colorfastness of Textiles to Atmospheric

etric, and Spectrochemical Analysis of Nuclear Grade Mixed de (Cereal Chemistry) (1962)

Meth. of Analysis of

e Quantitites of Total Sulfur in Volatile Organic Liquids iIs, and Shortenings (Cereal Chemistry) (1962)

rect Counting Technique (1973)

Std. Meth. of Test for ic Compou/ Std. Analytical Meth. for Determining Chemical Cakes (1972) ANSI H23.11 Std. Spec. for

Std Test Meth for Flammability of Plastics Using the of Test for Accelerated Aging of Vulcanized Rubber by the Safety Std. for

Meth. of Test for Compatibility of Materials with Liquid Hydrogen, Krypton, Methane, Neon, Nitric Oxide, Nitrogen, si L14.238 Test Meth. for Colorfastness of Textiles to i L14/ Test Meth. for Colorfastness of Textile Fabrics to ectrical Power Cables Rated 2000 Volts and Less and Having or Electrical Power Cables Rated 0-35,000 Volts and Having ble (1974) ANSI C8.37 si C 8.29 Std. Spec. for Std. Spec. for ed Members and Laminations Before Gluing of Southern Pine, Std. Meth. of Test for Extrudability, After 12.13 Std. Meth. of Test. Std. Meth. of Test for Shock Absorbing Characteristics of zes (1973) Std. Test Meth. for Creep Properties of Std. for Transport
Std. for Power Output Ratings of ing Data Sheets for the Design of Air Exchange Coolers for Std Meth. of Test for Water Vapor Permeability of

Test for W ater Vapor Transmission of Flexible Heat Sealed Std. Meth. of Test for Water Vapor Permeability of Std. for Centrifugal Water Chilling

Std for Unit Load Sizes for Dimensioning Transpor Sid. Meth. of Test for Leaks in Heat Sealed Flexible Std. Meth. of Test for ThermaI Insulation of Quality

ling and Installation of Power Semiconductors in Disc Type safety Requirements for the Construction, Care, and Use of ools (1972) Std. for Dimensions of Containers for ted Biological Materials (Safety) (1973) Std. for y) (1973)

Std. for Administrative Guide fo
rs for Milk and Its Fluid Products (1973)

Test Meth. for
Oversize, 100 Deg. Shear Head, Pull Type (1973)

Owner and Architect for Interior Design Services (1972)

Owner and Architect for Special Services (1972)

Owner and Architect (1974

Owner and Construction Manager (1973)

Owner and Contractor (For Small Construction Contracts

Oxamyonium Sulfate) (1973) he Pest Control Chemical) (197

Oxidase Meth. for Measurement of Glucose in Sugar Mixtu

Oxidation Characteristics of Extreme Pressure Oils (197

Oxidation Inhibited Mineral Insulating Oil for Use in T

Oxidation Microcoulometry (1973)

Oxidation of Wool Oils on Woolen Fabric Textile in Stor

Oxidation Stability by Rotating Bomb (1967) ANSI Z11.23

Oxidation Stability of New MineraI Insulating Oils Cont

Oxidation Stability of Turbine Fuels (JFTOT Proc.) (197

Oxidation) (1969) ASTM D2807

Oxidation) (1972)

Oxidative Microcoulometry (1972T)

Oxide Abrasive Grain and Crude (1971)

Oxide and Metallic Coatings by Double Beam Interference

Oxide Carrier D.C Arc Technique (1970) ANSI Z128.27

Oxide Coating Thicknesses by Microscopical Examination

Oxide Coatings on Aluminum (1973)

Std. Spec. for

Oxide Coatings on Aluminum (1973) Std. Spec. for Anodic

Oxide Content in Leather (Perchloric Acid Oxidation) (1

Oxide Content of Titanium Dioxide Pigments (1973)

Oxide for Use in Sheathed Type Electric Heating Element

Oxide in Iron Ores (1972)

Oxide in Leather (Perchloric Acid Oxidation) (1972)

Oxide Powder (1972) ANSI N140

Spectrometric, and Spe

Oxide Pow der (1972A)

Oxide Sinter (1970) ANSI G86. I

Oxide Solution Content of Reagents and Indicators (1971

Oxide Thickness on Silicon Wafers and Metallization Thi

Oxide Treatment (Part Coating) (1973)

Oxide Waterproofing for Below Grade Wall and Floor Surf

Oxide $\left(\mathrm{U}_{3} \mathrm{O}_{k}\right)$ by Gallium Oxide Carrier D-C Arc Techn

Oxide (1968) ANSI N153

Oxide (1968) ANSI N154

Oxide (1972)

Std. Meth. of Test for Consum

Oxide, Nitrogen, Oxygen) (1973)

Oxides in Flue Gases (Barium Chloranilate Controlled Co

Oxides of Nitrogen (Burnt Gas Fumes) (1972) ANSI L14.54

Oxides ((U, Pu)0 $\left.0_{2}\right)(1972)$ ANSI N139

Oxidizing Agents in Flour: Reaction with Potassium Iodi

Oxy Fuel Gas Torches (1973)

(Oxy Hydrogen Combustion Meth.) (1972)

Oxygen-Bomb Meth of A nalysis of Stability in Fats, $\mathrm{O}$

Oxygen Content Using a $14 \mathrm{MeV}$ Neutron Activation and Di

Oxygen Demand in Industrial Waste Waters and Most Organ

Oxygen Free Electrolytic Copper Wire Bars, Billets, and

Oxygen Index Meth. (1974) ANSI K65.110

Oxygen Pressure Meth. (1973) ANSI J4.I

Oxygen Therapy Equipment, Refrigerated (1973)

Oxygen (Reaction Intensity Meth.) (1973)

Std Meth ASTM

UL

Oxygen) (1973)

Monoxide, Deuterium, Fluorine, Helium

Ozone in the Atmosphere Under High Humidities (1972) an

Ozone in the Atmosphere Under Low Humidities (1972) Ans

Ozone Resistant Ethylene-Propylene-Rubber Integral

Ozone Resistant Ethylene Propylene Rubber Insulation (1

Ozone Resisting Butyl Rubber Insulation for Wire and Ca

Ozone Resisting Insulation for Wire and Cable (1974) an

Pacific Coast Douglas Fir and Western Hemlock by Pressu

Package Aging, of Latex Sealing Compounds (1972)

Package Cushioning Materials (Packaging) (1964) ANSI Mh

Package Cushioning Materials (Packaging) (1964) ANSI Mh

Package Cushioning MateriaIs (1968) ANSI MH12.15

Package Sizes (Packaging) for ANSI MH10.1, Unit Load SI

Packaged Audio Equipment for Home Use (1967)

Packaged Compressor Units (1974)

Packages by Cycle Meth. (Packaging) (1968) A VSI MHI2 18

Packages for Dry Products (1972)

Packages (Packaging) (1968) ANSI MH12.17

Packages (Refrigeration) (1974)

Packages (1972)

Packages (1972)

Packages (1972)

Packages (1973)

Packaging and Its Related Converting Machinery (1973)

Packaging and Storage of Aerial Photographic Film on $S_{p}$

Packaging and Transportation of Radioactively Contamin

Packaging and Transporting Radioactive Materials (Safet

(Packaging Equipment) (1974)

$/$ R Fillers and Sealers of

NSA

AIOA

AIOA

AIOA

AACCH

ASTM

ASTM

ASTM

AATCC

ASTM

ASTM

ASTM

D ALCA

ASTM

ANSI

ASTM

ASTM

ASTM

ASTM

ASTM

ASTM

ASTM

ASTM

ASTM

CR

ASTM

SAE

ASTM

ASTM

ICBO

ASTM

A A TCC

ASTM

AA

UL

ACC

ASTM

CR

ASTM

ASTM

Std ASTM

ICBO

AATCC

AATCC

NEMA

NFMA

ASTM

ASTM

AWPA

ASTM

ASTM

ASTM

ASTM

ANSI

EIA

API

ASTM

ASTM

ASTM

ARI

ANI

ASTM

ASTM

EIA

ANSI

ANSI

ANSI

ANSI

Packaging Film and Construction Identity Tests (Paper)

Packaging for Minimum Self Contained Platforms (1973)

Spec. for Petroleum Drilling Rig

1436

B707

B727

B14]

B80I

A 107

PH4.186

K62.126

80.10

D2893

3146

3246

62

D2272

2112

D324

D 10

D2807

D3120

B74.14

B588

E402

B 487 
Guidelines and Stds. for the Paperboard Packaging Industry (1973)

Rec. Pract. for Electrical Installations on examination for Presence of Rodent Urine on Bags and Other nthydrol Test for Analysis of Urea in Cloth Bags and Other Std for Std. for Std. for

(1972)

Std. Rec. Pract. for Determining Eft Std. Definitions of Trade Terms Commonly Used in Container Procedure for Chlorine

Std. for Transport Package Sizes

evision, Tape Recorder

Std for Simulated Shipping Test Std. Meth. of Test. Shipping Containers
Std. Meth. of Drop Test for Bags

d. Meth. of Drop Test for Cylindrical Shipping Containers test for Penetration of Liquids into Submerged Containers

Std. Meth. of Test. Large Shipping Cases and Crates ackaging Machinery and Associated Equipment (1972) Packaging Materials (Cereal Chemistry) (1962)

Packaging Materials (Cereal Chemistry) (1962)

Packaging of Components with Axial Leads (1972) ANSI C8

Packaging of Filament Tape Reels (1973)

Packaging of Uranium Hexafluoride for Transport (1971)

Packaging on Food and Beverage Products During Storage

Packaging (1972)

Packaging (1973)

(Packaging) for ANSI MH10.1, Unit Load Sizes (1973)

(Packaging) for Consumer Electronic Products (Radio, Tel

(Packaging) (1950) ANSI MH12.6

(Packaging) (1950) ANSI MH12.7

(Packaging) (1951) ANSI MH12.8

Test for Water V

Std. Meth. of Test. Package Cushioning Materials (Packaging) (1964) ANSI MHI2.13

absorbing Characteristics of Package Cushioning Materials (Packaging) (1964) ANSl MH12.14

Vapor Transmission of Shipping Containers by Cycle Meth. (Packaging) (1968) ANSI MHI2.12

d. Meth. of Test for Water Vapor Permeability of Packages (Packaging) (1968) A NS1 MH12.17

t for Water Vapor Permeability of Packages by Cryle Meth (Packaging) (1968) ANSI MH12.18

Interior Dimensions of Fiberboard Boxes (Box Gage Meth.) (Packaging) (1968) ANSI MH12.19

std. Meth. of Incline Impact Test for Shipping Containers (Packaging) (1968) ANSI MH12.4,

Std. Meth. of Vibration Test for Shipping Containers (Packaging) (1968) ANS1 MH12.9

nd Health Requirements for Paper Bag and Sack Manufacture (Packaging)(1974)

centers , (1972) aper Bag and Sack Manufacture (During Rec. Antid Std. for

Items for Nuclear Power Plants (During the Con/

Std. for Steel Shipping Container Industry Glossary Std. Meth. of Test for Bulk Density and

tolerances for Jute Rove and Plied Yarn for Electrical and Std. for ng (1973)

ng (1973)

5 Deg. F to +160 Deg. F) $(1972$

mil-R.25897 Rubber, 75 Shore, O-Ring (1973)

mil-R.25897 Rubber, 90 Shore, O.Ring (1973)

Manufacture and Inspection Stds. for Preformed

ebreathing Bags, Footwear, Hose and Tubing, Mattresses and

Std. Spec for $5 \mathrm{Gal}$. Straight Side Lug Cover Universal

Sid. for

Std. for

Packaging), and References (Dosage) for Poison Control

Packaging, Shipping, Receiving, Storage and Handling of

(1973)

Packing Factor of Hollow Glass Microspheres (1972)

Packing Purposes (1974) ANSI L 14.44

acking with Retainer (1974)

Packing. Preformed-MIL.R.25897 Rubber, 75 Shore,

Packing, Preformed O-Ring Phosphate Ester Resistant (

Packing, Preformed, Straight Thread Tube Fitting Boss,

Packings (0-Rings) (1971)

Pads, Sheeting, and Restraint Straps) (1974)

Page Format of the Construction Message (1974)

Pail (Container) (Dot-37C80) (1974)

Std. for 5 Gal. Nesting Lug Cover Universal

m 260, Dot -1

Std. Spec. for $5 \mathrm{Gal}$. Tight Head Universal

e 40 ,

Std. Spec for 5 Gal. Nesting Lug Cover Universal

Std. Spec. for 5 Gal. Straight Side Lug Cover Universal

Std. Meth. of Test for Determination of Cobalt in Std. Meth. of Test for Determination of Lead in Std Meth of Test for Manganese in

Std. Meth. of Test for Calcium or Zinc in Sid. Spec. for Liquid

Spec. for Determination of Relative Pencil Hardness of A rub Test for the Determination of Deg. of Cure of a Backed tent. Meth. of Evaluating Deg. of Surface Disfigurement of Std. Meth. of Test for Porosity of Std. Rec Guide for

Std Meth of Test for Low Quantities of Mercury in

d. Meth. for Determination of Low Concentration of Lead in

73)

ds A/ Coating Systems

ickne/ Best Meth. for Measurment of Dry Film Thickness of istering. Wrinkling, Loss of Adhesion, or Other Failure of Std. Definitions of Terms Relating to

Std. Meth. for Preparation of Steel Panels for Test.

Rec. for Flammable Liquid Mixing Operations

Uniform Fire Code: Application of Flammable Finishes
ng) (1) Test Meth. for Measurment of Impact Resistance of ronments (Coil Co/ Meth. of Preperation and Evaluation of rements for Cotton Woven Tent, Awning, and Canopy Fabrics, sts (1973)

ng Code Std. for Erection, Fabrication, ldentification and Surface Preparation Spec. for Steel Structure

Tent. Rec. Pract. for Direct Injection of Solvent Base std. Rec. Pract. for Conducting Exterior Exposure Tests of Panels in Weathering Tests of Latex and Emulsion Exterior Std. Rec. Pract. for Test. Exterior Latex House

for Identification of Oils and Oil Acids in Solvent Type ance Spec. for the Purchase of Reflective Pavement Marking

eth. of Test for Coarse Particles in Pigments, Pastes, and

Std. Meth. of Test for Efflorescence of Interior W all Std. Meth. of Test for Volatile Content of

Meth. of Test for Separation of Vehicle from Solvent Type

ct. for Qualitative Identification of Polymers in Emulsion
Pail (Container) (Dot-37C80) (1974)

Pails (Container) (Dot-17E, Ufc-Rule 40, Nmfc-Ite

Pails (Container) (Dot-37A80, Dot-37Aa60, Ufc-Rul

Pails (Container) (Dot-37A80, Dot-37A60, Ufc-Rule

Paint Driers by EDTA Meth. (1973)

Paint Driers by EDTA Meth. (1973)

Paint Driers by EDTA Meth. (1973)

Paint Driers by EDTA Meth. (1973)

Paint Driers (1973)

Paint Film (Coil Coating) (1974)

Paint Film (Coil Coating) (1974)

Paint Films by Fungal Growth or Soil and Dirt Ac

Paint Films (1973)

Paint lnspectors (1973)

Paint (1973)

Paint (1973)

(Paint) Guide for Ship Hull, Deck and Superstructure (19

Paint), and Handling of Flammable and Combustible Liqui

Paint, Enamel, Lacquer, Varnish, and Related Products)

Paint, Varnish, Lacquer and Related Products --Film Th

Paint, Varnish, Lacquer, and Related Organic Coatings 0

Paint, Varnish, Lacquer, and Related Products (1973)

Paint, Varnish, Lacquer, and Related Products (1973)

(Paint, Varnish, Lacquer, Printing lnk, Adhesive, etc.)

Paint, Varnish, Lacquer, Stain, etc.) (1973)

Painted Aluminum, Steel and Gavanized Steel (Coil Coati

Painted or Coated Specimens Subjected : Corrosive Envi

Painted, Printed, or Vinyl Coated (Institutional Textil

Painting Ceiling Materials for Acoustical Absorption Te

Painting Clay Masonry (Tech. Notes) (196 I)

Painting of Structural Steel (1973)

Painting (1972)

Paints into a Gas Chromatograph (1973)

Paints on Wood (1973)

Paints or Coatings (1973)

Paints (1972)

Paints (1972)

Paints (1972)

Paints (1972) ANSI K42.I

Paints (1973)

Paints (1973)

Paints (1973)

Paints (1973)

PPC *1

IEEE 333

Light AACCH 28-85

of $\mathrm{Xa}$ AACCH $28-86$

ElA RS296C

NSA 3452

ANSI Nl4.1

ASTM E460

ANSI MH15.1

$\mathrm{CHI}$

ANS]

EIA

ASTM

ASTM

St ASTM

d. Meth. of ASTM

ASTM

ASTM

ASTM

ASTM

MH10.2

$\mathrm{RS} 414$

D782

D959

D998

D 1083

D775

D 1008

D1372

D1596

D1276

D895

D1251

D2658

D880

D999

Z259. 1

$* 1$

N45.2.2

ANSI $\mathrm{MH} 2.18$

ASTM D3101

D3101

1523

NSA

NSA 1593

NSA 1594

NSA 1611

NSA $\quad 1595$

NSA 1596

SAE

$\mathrm{Ks}$ and $\mathrm{R} \mathrm{UL}$

CSI

ANSI

ANSI

ANSI

ANSl

ANSI

ASTM

ASTM

ASTM

ASTM

ASTM

NCCA

NCA

ASTM

ASTM

ASTM

ASTM

St ASTM

SNAM

ICBO

ASTM

NCCA

NCCA

ASTM

ASTM

FMS

ICBO

NCCA

NCCA

ANSI

ASTM

BlA

1596
A 5871

1067

MP-2E

$\mathrm{MH} 2.16$

$\mathrm{MH} 2.17$

MH2.9

M 2.15

$\mathrm{MH2} .10$

D2373

D2374

D2375

D2613

D600

TB-II-1 2

B-11-18

D3274

D3258

D3276

D2206

D2088

4. 10

UFC *2ART34

3278

TB.I].4

TB-11I. I

Dl6

60

7.36

UFC*2ART 14

TB-11. 6

TB-1I].3

L24.1.

C643

UBCS27.2

ANSI A159.

ASTM D327]

ASTM DI006

D1911

D3129

D2245 
ilities (1972)

act. for Vacuum Distillation of Solvents from Solvent Base ar White, Opaque Materials (Color Measurement of Textiles. Std. Spec. for Refined

Std. for

torage Up to $15 /$

Rec. for Indoor General Storage (Solid, Rec. for Solid, Std. Meth. of Test.

ower Seeds; Ground Nuts (Peanuts); Vegetable, Coconut, and ing Rules (Performance. Quality, Description, and Grade of

nal Attachment (Preferred) (1974)

ne Screw (1972)

s, Titanium Alloy $6 \mathrm{Al}-4 \mathrm{~V}$, Self Locking An/

s, Alloy Steel, Self Locking and Nonlocki/

s, A286 Acres, Self Locking and Nonlocking (197) d. for Self Locking,

chine Screw (1974)

loy Steel Machine Screw (1974)

Locking and Nonlocking (1972) (1970) White Std. for Std. for

Std. for Bolt. Std. for Bolt, Std. for Std. for Screw, Machine, Std. for Aircraft, Std. for Machine Screw,

Std. for Screw, Std. for Pot, ral Hydrate; Chloroform; Formaldehyde; Gasoline; Herwig's, etal Suspension Systems for Acoustical Tile and for Lay in cati/ Redwood Plywood Guide (Spec. for Texture and Grade, lywood (1967)

8) Test Meths. for Interior 1)

threaded Molded in lnsert (1974)

threaded Molded in lnsert (1974) Meth. of Triangle Test Taste Rec. Pract. for Instrument Std. for Sandwich 1972) Evaluating the Properties of Wood Base Fiber and Particle

ANSl All1.14

) ANSI A111.16

(1968)

4n. Reinforced Brick Masonry Curtain Locking, Nonself Locking, Light Weight Floating, Sandwich Quality Stds. for Flush W all Quality Stds. for High Pressure Laminate Flush Type Wall Rec for lnterior Wood Quality Stds. for Stile and Rail Wall truction Under the Uniform Building Code: Softwood Plywood Std. for Prefinished Hardboard Std. Patterns for Wood Std. for Dimensions for Racks, Plywood Stressed Skin

Std. Spec. for Ceramic Tile

roducts (1973)

(1974) Std. Meth for Preparation of Steel td. Spec. for Asbestos Cement Shingle Blanks to Be Used as omate Conversion Coatings on Aged or Backed Aluminum Alloy Design Spec. for Plywood Curved or Diffuse Light Transmission Factor of Reinforced Plastic Design Spec. for Flat Plywood Sandwich Fabrication Spec. for Plywood Curved Fabrication Spec. for Plywood Sandwich Design Spec. for Plywood Stressed Skin Std. Spec. for Asbestos Cement Fiberboard Insulating td. Spec. for Asbestos Cement Plastic Foam Core Insulating d. for Glass Fiber Reinforced Polyester Structural Plastic se Principally as a Substitute for Plate Glass Show Window Guide Spec. for Specifying: Precast Concrete

Design Guide for Wood Composition Board Wall

Glued Built Up Members-Plywood Stressed Skin and Curved

lding Code: Plastics (General, Exterior Light Transmitting chitectural Woodwork: Standing and Running Trim, Casework, ing Device) (1973) Safety Std. for

m Hot Head Press (300-330 Deg. F) Finishing, Shirt Unit, om (1972A) Std. Definitions of Terms Relating to Carbon

72)

3) ANSI C59.50

C.) (1972) Std Meth of Conditioning Std. Spec. for Electrical Insulating Std. Meth. of Test for Gloss Retention of Waxed Meth. for Gloss Retention of Waxed Meth. for Specular Gloss of meth. for Spectrophotometric Test for Relative Radiance of length and Excitation Purity/

Meth. of Measuring Color of Test Meth. for Smoothness of std. Meth. of Test for Water Absorptiveness of Nonbibulous Meth. for Reducible Sulfur in Meth. for Organic Nitrogen in

Meth. for Flame Resístance Treated Test Meth. for Heavy Metals in h Coatings of Petroleum Wax or Wax Based Blends Applied to
Paints (1973)

Tent. Rec. Pr ASTM

(Paints) for Light Water Nuclear Reactor Containment Fac Ness of Ne ASTM

Paints, Plastics, etc.) (1973) ANSl Z172.6

Palladium (1973)

Pallet Definitions and Ter minology (1972)

Palletized and Bin Box Storage Up to $30 \mathrm{Ft}$. and Shelf $\mathrm{S}$

Palletized, and Rack Stor age of Plastics (1974)

Pallets (1973)

Palm Oils) (1974)

/Sesame, Safflower, Oleic, and Sunft

Palmkernels : Babassu; Flaxseed; Castor, Sesame, Safflow

Pan Bread Flour Spec. (Cereal Chemistry) (1962)

Pan Head Screw and Assembled Washers-Electrical Termi

Pan Head, Close Tolerance, Short Thread, Torq Set Machi

Pan Head, Close Tolerance, Short Thread, Tri Wing Reces

Pan Head, Close Tolerance, Short Thread, TWl Wing Reces

Pan Head, Close Tolerance, Short Thread, TWl Wing Reces

Pan Head, Full Thread Part Screw (1974)

Pan Head, Full Thread, Torq Set (1973)

Pan Head, Phillips Recess Full Threaded, Alloy Steel Ma

Pan Head, Phillips Recess, Short Thread, 160,000 psi Al

Pan Head, Short Thread, Torq Set (1973)

Pan Head, Tri Wing Recess, A286 Cres, Full Thread, Self

Pan, and Utensil Commercial Spray Type Washing Machines

Pancreatin, Tween $80-60 \%$ Alcohol, Versene, and Sodium P

Panel Ceilings (1973)

/Niform Building Code Std. for M

Panel Characteristics, Material Composition, Use, Appli

Panel Coatings and Overlays on DFPA Grade Trademarked

Panel for Food Flavor Detection (Cereal Chemistry) (196

Panel Laboratory Impact Test Procedure-Head Area (197

Panel Lightweight Selflocking and Nonselflocking Blind

Panel Lightweight Selflocking and Nonselflocking Blind

Panel Materials (1972) ANSl O8.1 Std. Meth. of

Panel Spalling Test for Fireclay Plastic Refractories

Panel Spalling Test for High Duty Fireclay Brick (1970)

Panel Spalling Test for Super Duty Fireclay Brick (1970

Panel Walls (ln Resisting Lateral Forces) (Tech. Notes)

Panel (1972)

Panel (1972)

( lnsert, Molded In, Blind Threaded, Self

Paneling (Architectural Woodwork) (1973)

Paneling (Architectural Woodwork) (1973)

Paneling (1972)

Paneling (1973)

Paneling (1973)

Paneling (1973) ANSI A135.5

Paneling, Decking, Flooring, and Siding (1970)

Panels and Associated Equipment (1972) ANSI C83.9

Panels Fabrication Spec. (1971)

Panels for Bath Tub Recess (1972)

Panels for Test. Paint, Varnish, Lacquer, and Related P

Panels for Use in Test. Organic Coating Systems (Coil)

Panels in Weathering Tests of Latex and Emulsion Exteri

Panels (Coil) (1974)

Panels (1968)

Panels (1969)

Panels (1970)

Ing Weight Determination for $\mathrm{Ch}$

Panels (1971)

Panels (1971)

Panels (1972)

Panels (1972)

Panels (1972)

Panels (1972)

Panels (1972)

Panels (1973)

Panels (1973)

Panels, Beams, and Components (1973)

Panels, Skylights, Monitors and Sawtooth Roofs, Light D

and Storage Shelving, Misc. Ornam

Pantothenic Acid (Cereal Chemistry) (1962)

Pants Unit (1973)

190 Deg. F, Colors 160 Deg. F Maximu

Paper and Inked Ribbon Products and lmages Made Therefr

Paper and lts Products for Test. (1973) TAPPI T402

Paper and Paper Board by Hunter L, A, B Colorimetry (19

Paper and Paperboard-Sulfate or Kraft Layer Type (197

Paper and Paperboard After Storage at 104 F (40C) (1973

Paper and Paperboard After Storage at $140 \mathrm{Deg}$. (40 Deg.

Paper and Paperboard at 75 Deg. (1972) ANSI P3.23

Paper and Paperboard Containing Fluorescent Brighteners

Paper and Paperboard in CIE Y; X, Y or Y, Dominant Wave

Paper and Paperboard (Bendtsen Tester) (1973)

Paper and Paperboard (Cobb Test) (1973)

Paper and Paperboard (1972)

Paper and Paperboard (1972)

Paper and Paperboard (1972)

Paper and Paperboard (1973)

Paper and Paperboard (1973)
Std for Insert, Molded NSA

Std. Test Meth. F

ASTM

ASTM

ANS1

FMS

ASTM

NlOP
NlOP

$\triangle A C C H$ 34-0l

NSA

NSA

NSA

NSA

NSA

NSA

NSA

NSA

NSA

NSA

$\mathrm{AACCH} \quad 28-9$

APA

AE J921B

NSA 1832

NSA $\quad 1836$

D 1037

C 180

ASTM $\mathrm{C} 107$

ASTM Cl22

BlA 17L

1833

1835

* $1-500 \mathrm{~A}$

*1-500B

AWl

WWPA

$A W l$

*1.500C

ICBO UBC *8.17

USC

WWPA

ElA RS310-B

APA SS-8

NCCA TB-111.5

ASTM D19]

TB-11-2

ASTM

APA

APA

APA

APA

ASTM

ASTM

St USC

HPMA

$1 \mathrm{CBO}$

$1 \mathrm{CBO}$

$\mathrm{AW} 1$

UL

$\mathrm{AACCH}$

ANSl

ASTM

ASTM

TAPPI

ASTM

ASTM

TAPPI

TAPP

TAPPI

TAPPI

TAPPI

ASTM

TAPPI

TAPPI

TAPPI

TAPPI

/Esistance of Glossy Smoot ASTM 
eth. for Spectral Reflectance, Transmittance, and Color of Test Meth. for Top Side of Filled Std. for Minimum Safety and Health Requirements for Meth. for Measuring Color of Paper and Test Meth. for Identification of Lignin le or Ground Cereal/ Meth. of Urease Bromthymol Blue Test Meth. for Measuring Blister Resistance of Coated Rec. for Fire Safety and Protection of Yankee Driers d. Meth. for Analysis of Sulfuric Acid as Used in Pulp and for Measuring Squareness or Rectangularity of a Sheet of Std. Spec. for Properties of Inpunched Oiled Std. for

Std. Spec. for Noncarbonized, Single Ply, Adding Machine Test Meth. for Water Leak Test for Test Meth. for Drop Tests for late and Acidic Gaseous Fluoride in the Atmosphere (Double (Digital) Form on Punched Cards, Magnetic Tape and Punched Std. Meth. of Test for Resistance to Std. Meth. of Sampling and Test. Untreated for Dimensions of 126 Size Cartridges, Film, and Backing Test Meth. for Carbonates in Coated Test Meth. for Sulfides in Coated Test Meth. for Sizing of

Uniform Building Code Std. for Kraft Waterproof Building Meth for Ink Absorbency of Blotting Test Meth. for Castor Oil Penetration of Test Meth. for Oil Spots in Test Meth. for Amount of Coating on Mineral Coated resin and Volatile Content, and Flow Properties of Treated cal Microscopic) Analysis of Mineral Filler and Coating of ire Hazards Anf Protection Rec. for Indoor Storage of Roll ation of Water Vapor Transmission Rate of Sheet Materials td. Analytical Meth. for Determining Moisture (Oven Filter

Test Meth. for Rejects from Knotter Screens (Pulp and Test Meth. for Dirt Count of Wood Chips (Pulp and

Test Meth. for Specific Gravity of Wood Disks (Pulp and Test Meth. for Chip Classification (Hand Screen) (Pulp and

Test Meth. for Beating Control (Simons Stain) (Pulp and Test Meth. for Sieve Analysis of Pulpwood Chips (Pulp and est Meth. for Chip Length Analysis (Measurement) (Pulp and test Meth. for Adhesion to Non Porous Flexible Substrates Optical Test Meth. for lnk Show Through test Meth. for Chip Length Analysis (Two Screen) (Pulp and

est Meth. for Moisture in Chips (Moister Teller) (Pulp and st Meth. for Fiber Identification (Wilson Stain) (Pulp and st Meth. for Moisture in Wood Chips (Steam Oven) (Pulp and t Meth. for Sampling of Wood Chips from Conveyor (Pulp and

Meth for Fiber Identification (Various Stains) (Pulp and

Meth. for Packaging Film and Construction Identity Tests

th. for Determination of Useful Fiber in Bagasse (Pulp and

for Density of Wood Chips (Sheet Machine Mold) (Pulp and um Determination by the Uranyl Zinc Acetate Meth. Puln and sity or Volume of Small Specimen (Hg Pycnometer) (Pulp and and Moisture of Chips (Presoak Before Immersion) (Pulp and

od (Submersion with Correction for Cracks, etc.) (Pulp and

ps (Submersion with Correction for Cracks, etc.) (Pulp and

h. for Adhesion to Porous Substrates (Subjective Ratings) Indicator $\mathrm{Mel}$ Std. Meth. of Test for Water Resistance of Semiconductor Applications (1973)

Test Meth for Opa 3)

eth. of Analysis (Cereal Chemistry) (Bolting Cloth, Filter Std. Spec. for Electrical Insulating Paper and

Std. Meth. of Test for Gloss Retention of Waxed Paper and
Meth. for Gloss Retention of Waxed Paper and
Meth. for Specular Gloss of Paper and

Std. Meth. of Test for Gloss Retention of Waxed Paper and
Meth. for Gloss Retention of Waxed Paper and
Meth. for Specular Gloss of Paper and

Std. Meth. of Test for Gloss Retention of Waxed Paper and
Meth. for Gloss Retention of Waxed Paper and
Meth. for Specular Gloss of Paper and spectrophotometric Test for Relative Radiance of Paper and Meth. of Measuring Color of Paper and Guidelines and Stds. for the Test Meth. for Smoothness of Paper and

of Test for Water Absorptiveness of Nonbibulous Paper and Test Meth. for Sizing of

Slant Drop Penetration Test for Test Meth. for Internal Bond Strength of Meth. for Reducible Sulfur in Paper and Meth. for Organic Nitrogen in Paper and Meth. for Flame Resistance Treated Paper and meth. for Cross Directional Internal Tearing Resistance of Test Meth. for Heavy Metals in Paper and suring Squareness or Rectangularity of a Sheet of Paper or of Petroleum Wax or Wax Based Blends Applied to Paper and Meth. for Flat Crush Test of Corrugating Medium Std. Meth. of Test for Water Resistance of Paper. Std. Spec. for Manifold Std. for Scientific

tor $\mathrm{Mel}$ ting Pick Tests for Determining Surface Strength of Coated
Paper and Pulp (Using Spectrophotometer with
Paper as Indicated by Silver Coin Test (1973)

Paper Bag and Sack Manufacture (Packaging) (1974)

Paper Board by Hunter L, A, B Colorimetry (1972)

(Paper Chromatogram) (1973)

Paper for Analysis of Urea in Cloth or Sack Fibers, Who

Paper in Heatset Printing (1972)

(Paper Machine) (1973)

Paper Manufacture (1973)

Paper or Paperboard (I973)

Paper Perforated Tape (1971)

Paper Refuse Sacks (1970)

Paper Rolls (1973)

Paper Shipping and Refuse Sacks (Containers) (1973)

Paper Shipping Sacks and Bags (Containers) (1973)

Paper Tape Sampler Meth.) (1973)

Paper Tape (1972)

Printed Wiring

Paper Used for Electrical Insulation (1974) ANSI C 59.77

Paper (Photography) (1972)

Paper (Qualitative) (1973)

Paper (Oualitative) (1973)

Paper (Reverse Curl) (1973)

Paper (Weatherproofing) (1973)

Paper (1972)

Paper (1972)

Paper (1973)

Paper (1973)

Paper (1973)

Paper (1973)

Paper (1974)

(Paper) at High Temperature and Humidity (1973)

Paper) Conte

Paper) (1973)

Paper) (1973)

Paper) (1973)

Paper) (1973)

Paper) (1973)

Paper) (1973)

Paper) (1973)

(Paper) (1973)

(Paper) (1973)

Paper) (1973)

Paper) (1973)

Paper) (1973)

Paper) (1973)

Paper) (1973)

Paper) (1973)

(Paper) (1973)

Paper) (1973)

Paper) (1973)

Paper) (1973)

Paper) (1973)

Paper) (1973)

Paper) (1973)

Paper) (1973)

(Paper, Board, Cloth, etc.) (1973)

Per, Paperboard, and Other Sheet Materials by the Dry

Paper, Paper/Film, Film Dielectric Capacitors for Power

Paper, Using Reflectometer with 45-0 Deg. Geometry (197

Paper, Wide Field and Compound Microscope, Petri Dish,

Paperboard-Sulfate or Kraft Layer Type (1973) ANSI C5

Paperboard After Storage at 104 F (40C) (1973)

Paperboard After Storage at 140 Deg. (40 Deg. C.) (1972

Paperboard at 75 Deg. (1972) ANSI P3.23

Paperboard Containing Fluorescent Brighteners (1973)

Paperboard in CIE Y; X, Y or Y, Dominant Wavelength and

Paperboard Packaging Industry (1973)

Paperboard (Bendtsen Tester) (1973)

Paperboard (Cobb Test) (1973)

Paperboard (Flotation) (1973)

Paperboard (Printing Ink Receptivity) (1973)

Paperboard (Z-Direction Tensile) (1973)

Paperboard (1972)

Paperboard (1972)

Paperboard (1972)

Paperboard (1972

Paperboard (1973)

Paperboard (1973)

Paperboard (1973)

Paperboard) (1971) ANSI P3.24

Esistance of Glossy Smooth Coating

Paperboard, and Other Sheet Materials by the Dry Indica

Papers for Permanent Records (1973)

Papers for Written or Oral Presentation (1972)

Papers Using the Interfiber Bond Tester (1973)

/Thiosulfate and Silver Densitometric Me
TAPPl T442SU

TAPPl UM-533

ANSI Z259.1

TAPPI T524SU

TAPPl UM-19

AACCH 28.87

TAPPI T526SU

FMS 12.29

St TAPPI T602

Test Meth TAPPI UM-530

ANSI $\mathrm{X} 3.29$

NSF $\quad 32$

ANSI X 48

TAPPI UM-529

TAPPI UM.806

ASTM D 3266

D. 350

ASTM D726

ASTM D202

Std ANSI PHI.40

TAPPI UM-53I

TAPPI UM-532

TAPPI UM-459

ICBO UBCSI7-1

TAPPI T43ISU

TAPPI T462

TAPPI UM-458

TAPPI UM 542

est Meth. for TAPPI UM-539

TAPPI T421

FMS 8-21

TAPPI T464

CR E-44

TAPPI UM-1

TAPPI UM-11

TAPPI UM.12

TAPPI UM-13

TAPPI UM-17

TAPPI UM-21

TAPPI UM-5

TAPPI UM.541

TAPPI UM-545

TAPPI UM-6

TAPPI UM-8

Te TAPPI

Te TAPPI

Tes TAPPI

Test TAPPI

Test TAPPI

Test Me TAPPl

Test Meth TAPP

Td for Sodi TAPPI

UM-14

UM-7

UM-15

UM.537

UM-3

UM-9

T623

UM-18

UM-16

$\mathrm{UM}-2$

UM- 20

UM-540

D 779

RS401

TAPPI UM-538

AACCH $\quad 28-90$

ASTM D1305

ASTM D2895

TAPPI T682

TAPPI T480

TAPPI UM-460

TAPPI T527SU

PPC

TAPPI

*1

UM-535

D3285

TAPPI UM-543

TAPPI UM-536

TAPPI UM-528

TAPPI T406SU

TAPPI T418SU

TAPPl T46l

TAPPI T496SU

TAPPI UM-544

UM-530

D 3234

T809 OS

TAPPI T809

ASTM D3208

ANSI Z39.16

TAPPI
UM-534

PH4.8 
mensional Change Characteristics of Photographic Films and nductor Applications (1973) Std. for Paper ineral Insulating Oils Containing 2,6-Ditertiary-Butyl spec. for Photographic Grade Hydroquinone $\mathrm{C}_{1 ;} \mathrm{H}_{1}(\mathrm{Oh})$,

$$
\text { Std. Meth. of Test. }
$$

Std. Spec. for

Std. Meth. of Test for Tensile Strength of ies and Essential Information for Safe Handling and I'se of en Numerical Control and Data Terminal Equipment Employing d. Meth. of Test for Apparent Horizontal Shear Strength of speed Stationary Diesel and Gas Engine Speed Governing and ified Threaded In. Series (1971) Std. for Design ) (1973) Rec. Pract. for New Motor Vehicle on Grade Rec. for wood Subfloors (1962) Uniform Plumbing Code: Mobile Home (Trailer) Spec. for Mosaic Wood 73) ance of Solid Electrical Insulating Materials Subjected to Rec. Pract. for the Detection and Measurement of Rec. Safeguards in 93.28 Std. Meth. for Automatic 6. Std. Meth. for Calibration of Liquid Automatic
Std. Meth. of Magnetic Aircraft Quality Steel Cleanliness Requirements (Magnetic Std. Definitions of Terms Relating to Magnetic and Components for Particulate Contamination by Automatic Meth. of Evaluating the Properties of Wood Base Fiber and al Waste Watel Std. Meth. of Test for Measurement of Beta Std. Meth. for Measurement of Alpha Std. Meth. for

Std. Rec. Pract for Analysis by Microscopical Meth. for stry) (1962) Meth. for (1972) Std. Meth. of Test for Average
Std. for Std. for

Quality Stds. for Plywood and Sid for Mat Formed Wood Uniform Building Code Std. for Mat Formed Wood Bolts, Connectors, Nails, Staples, Adhesives, Fiberboard, Counting Meth. of Analysis of Cinder and Sand Gravimetric Meth. of Analysis of Cinder and Sand 42.1 Std. Meth of Test for Coarse of Air Cleaning Systems Containing Devices for Removal of of Solids by Cavitation and Impingement by Liquid or Solid r/ Tent. Meth. for Automated Separation and Collection of dium Bicarb/ Tent. Meth. for Separation and Collection of Tent. Meth. for Continuous Separation and Collection of 4 Std. Meth. of Test for Operating Performance of ) ANSI Z11.264 td. Meth. of Test for

he Lines of an Operating Hydraulic Fluid Power System (For meth. of Sampling Gas Blow Down Systems and Components for eth. for Sampling Gas Blow Down Systems and Components for the Atmosphere (Sodium Bicarbonate Coated Glass Tube and A

std. Meth. of Test for Physical and Chemical Properties of

d. Test Meth. for Polynuclear Aromatic Hydrocarbons in Air Std. Meth. for Sampling Stacks for

rmal Control Materials (Coatings) with Electromagnetic and try) (1962)

s by Microscopical Meth. for Particle Size Distribution of

Uniform Building Code Std. for Gypsum Lumber, Siding, Framing, Decking, Flooring, Ceiling, and rving Their/ Tent. Rec. Pract. for Installation of Fixed

ent of Airborne Sound Transmission Class (Stc) of Building ding Code Std. for Airborne Sound Insulation Field Test of easurement of Airborne Sound Transmission Loss of Building Guide Spec. for Metal Toilet Guide Spec. for Accordion Folding

Monitors and Sawtooth Roofs, Light Diffusers in Ceilings, r: Sand; Slag; Steel; Terra Cotta; Timber; Water; Ceiling;

Std. for Electroplating of Nickel and Chromium on Metal tensile Strength Carbon-Silicon Steel Plates for Machine nterconnected Porosity of Sintered Powder Metal Structural of Test for Impact Resistance of Rigid Plastic Sheeting or Alloy Steel Pipe Flanges, Forged Fittings, and Valves and olled 9\% Nickel Alloy Steel Flanges, Fittings, Valves, and Std. Preparation of Centrifugal F ans and

for Safety Tests for Flammability of Plastic Materials for assifying Visual Defects in Glass Reinforced Laminates and Rec. Pract. for Passenger Car and Light Truck Muffler Rec. Pract. for Passenger Car and Light Truck Resonator Std. Nomenclature for stainless and Alloy Steel Castings for Pressure Containing el Castings Specially Heat Treated for Pressure Containing Spec. for Ferritic Steel Castings for Pressure Containing
Papers (1973)

Std. Meth. for Determining the Di ANS]

Paper/Film, Film Dielectric Capacitors for Power Semico

Para-Cresol by Rotating Bomb (1973) ANSl C59.113

(Para Dihydroxbenzene, Quinol, Hydroquinol) (1972)

Para Red and Toluidine Red Pigments (Toners) (1973)

Para Tertiary Butylphenol 98 (1972) ANSI Z78.24

Paraffin Wax (1973) ANSl Z11.206, TAPPl T644

Paraformaldehyde (1974)

Parallel Binary Data lnterchange (1973)

Parallel Fiber Reinforced Plastics by Short Beam Meth.

Parallel Operation (1972)

Parameters for Bolts and Screws; External Wrenching, Un

Parking Performance Requirements (1972)

Parking Structures (Garage-Fire Protection and Safety

Parks (Trailer) (1972) ANSI A 177.1

Parks (1973)

Parquet Flooring Set in Adhesive Over Concrete Slab or

Partial Discharges (Corona) /of Test for Voltag

Partial Discharges (Corona) During Dielectric Tests (19

Particle Board Plants (1972)

Particle Counter Size Setting (1972)

Particle Counters Using AC Fine Test Dust (1972) ANSI B

Particle Examination of Steel Forgings (1971) ANSI G60.

Particle Inspection Procedure) (1973)

Particle Inspection (1974) ANSI Z166.15

Ec. for Premium

Particle Monitor Meth. (1972)

Particle Panel Materials (1972) ANSI O8.

Particle Radioactivity of Industrial Water and Industr

Particle Radioactivity of Water (1966) ANSI N152

Particle Size Analysis of Whiteware Clays (1974)

Particle Size Distribution of Particulate Substances of

Particle Size Distribution of Wheat Flour (Cereal Chem

Particle Size of Alumina and Silica by Air Permeability

Particleboard Decking for Factory Built Housing (1972)

Particleboard for Mobile Home Decking (1973)

Particleboard Grades (Architectural Woodwork) (1973)

Particleboard (1966)

Particleboard (1973)

Particleboard) (1973)

Particles in Farina (Cereal Chemistry) (1962)

Particles in Farina (Cereal Chemistry) (1962)

Particles in Pigments, Pastes, and Paints (1972) ANSI

Particles (1972)

Particles (1973) /Itions of Terms Relating to
Particulate and Acidic Gaseous Fluoride in the Atmosphe

Std. for Efficiency Test.

Particulate and Gaseous Fluorides in the Atmosphere (So

Particulate and Water Soluble Gaseous Fluorides in the

Particulate Cation Exchange Materials (1972) ANSl Z111

Particulate Contaminant in Aviation Turbine Fuels (1973

Particulate Contamination Analysis) (1972) ANSI B93.19

Particulate Contamination by Automatic Particle Monitor

Particulate Contamination by Manual Meth. (1973)

Particulate Filter Meth.) (1973)

Particulate lon Exchange Resins (1972)

Particulate Matter (1971)

Particulate Matter (1971) ANSI Z257.3

Particulate Radiation (1973)

(1973) /D. M
/Aseous Fluorides in

ASTM

D. ANSl

ASTM

ASTM

ASTM

Propert MCA

ElA

ASTM

DEMA

SAE

SAE

FMS

NFPA

ICBO

WSFl

EMS

ASTM

NFLDP

ASTM

ASTM

ASTM

Std. ASTM

ASTM

ASTM

ASTM

ASTM

$\mathrm{AACCH}$

ASTM

NPA

NPA

$A$ WI

USC

ICBO

ICBO

$\mathrm{AACCH}$

$\mathrm{AACCH}$

ASTM

ANSI

ASTM

ASTM

A TTM

ASTM

ASTM

ASTM

NFLDP

ASTM

ASTM

A STM

ASTM

St ASTM

ASTM

Particulate Substances of Subsieve Sizes (1968)

Partition Meth. of Analysis of Pentosans (Cereal Chemis

Partition Tile or Block (1973)

Partition (1973)

Ies, Weight, Stress of Board. Timber

Partitions of Light Frame Type for the Purpose of Conse

Partitions Such as Walls, Floor-Ceiling Assemblies, D

Partitions (Wall, Ceiling, Floor, and Floor-Ceiling a

Partitions (1970)

Partitions (1972)

Partitions (1972)

Partitions, Exterior Veneer, Awnings and Patio Covers,

Partitions; Plaster; Roofing) and Earthquake Recording

Parts-Automotive Ornamentation and Hardware (1970)

Parts and General Construction (1970) ANSI G24.7

Parts and Oil Impregnated Bearings (1973) ANSI H9.14

Parts by Means of a Tup (F alling Weight) (1972)

Parts for High Temperature Service (1972) ANSI G37.1

Parts for Low Temperature Service (1972) ANSI G38.13

Parts for Protective Coatings (1966)

Parts in Devices and Appliances (1973)

Parts Made Therefrom (1970) ANSI K65.141

Parts Nomenclature (197I)

Parts Nomenclature (1971)

Parts of a Photographic Objective Lens (1971)

Parts Suit able for High Temperature Service (1974) ANSI

Parts Suit able for High Temprature Service (1974) ANSI

Parts Suitable for Low Temperature Service (1974) ANSI

T405SL

T432SU

PHl.32

RS401

D2112

D970

D2871

D1320

SD6

RS408

D2344

- 1.6

AS1 132A

J293

LPD $7.15 \mathrm{~N}$

$501 \mathrm{~A}$

['PC - I-E

D2275

454

LPD7.10

F321

2.9 .6

AMS $2300 \mathrm{~B}$

E269

F327

D 1037

D1890

D1943

C775

E20

$50-10$

C.721

$2-70$
1.71

* 1.200

IBCS25-25

UBC *3-25

28.01

28-02

D185

G40

D3266

D3268

D 3267

D1 782

D2276

T2.9.

F 327 
t. Spec for lron Copper Sintered Metal Powder Structural Std. Spec. for Sintered Copper Steel Structural Std. Spec. for Sintered Nickel Silver Structural Std. Spec. for Sintered Bronze Structural Std. Spec, for Sintered Brass Structural Std. Test Meth. for Passive Electronic Component enclature for Passenger Car and Light Truck Exhaust System Std. Spec. for Sintered Aluminum Structural for High Strength SteeI Bolts. Nuts, W ashers, and Bolted I Pressure Piping Components and Other Pressure Containing id. Rec. Pract. for Descaling and Cleaning Stainless Steel a Fine Hydraulic Fluid Power Filter Element / Std. Multi Std. Meth. of Test for Resistance to Paper to res (Car, Station Wagon, Truck, Bus, Trailer, Multipurpose measurement of Stopping Distance on Paved Surfaces U/sing A

h. of Test for Stopping Distance on Paved Surfaces Using A ry Spec. (Vehicular, Ignition, Lighting, and Starting) for 2) wing and Related Documentation Practices: Chassis Frames e (1971) tion and Techniques for Exhaust Gas Emissions Measurement Pract. for Test. Machines for Measuring the Uniformity of ndition of Tires Including Tire Treads Depth Requirements Rec. Pract. for Rec. Pract. for Rec. Pract. for

L for Service Brake Structural Integrity Test Procedure Std. for Training of Automotive Mechanics for rement of Fuel Evaporative Emissions from Gasoline Powered Std. for Sound Level for for New Tires for ements and Uniform Test Procedures for Retreaded Tires for Std for Tire and Rim Equipment for 1974 Trucks, and Combin/ Rec. Pract. for Stopping Distance of fuel Tanks and Fill Pipes with Fuel Dispensing Facilities Truck. Bus, Bead S/ Rim and Wheel Stds. for Motorcycles, ger Vehil Std. for Bias and Radial Ply Tires and Rims for bers, Cell Layouts and Terminals (For Foreign and Domestic tows (1973) Std. for Safety Requirements for Aerial

r Highway Use on Trucks, Buses, Trailers, and Multipurpose Rims for Passenger Cars, Station Wagons, and Multipurpose act. for Stopping Distance of Passenger Cars, Multipurpose de Spec. For Hydraulic and Electric Elevators (Freight and Std. Test Meth. for Std. Spec, for Hand Scrubbing Soap (Grit Soap)

(1955) Std. Meth. of Test for False Set of Portland Cement Std. Analytical Meth. for Determining Acidity Std. Analytical Meth. for Determining pH

in a Boil/

Std. Analytical Meth. for Determination of $\mathrm{pH}$ ation of Baked Goods, Ready.To.Eat Cereals, and Alimentary drate in Baked Goods, Ready.To.Eat Cereals, and Alimentary

filth in Baked Goods, Ready.To.Eat Cereals, and Alimentary Std. Meth. of Test for Coarse Particles in Pigments. Rec. Pract. for Dimensions of Inniform Building Code

sers in Ceilings, Partitions, Exterior Veneer, Awnings and for Meth. of Measuring Frequency and Cost, and Recording ng (1970) Std. Meth. of Measuring Break

Bonds

Std. Rec, Pract. for Comparison of Spray Sid. for Clearance Dimensions on the Mating Plates th Full Scale Tir/ Meth. of Test for Stopping Distance on th Fu/ Tent. Meth. Of Measurement for the Purchase of Reflective endations for Basis of Payment on Portland Cement Concrete meth.) (1972) Std. Rec. Pract. for Test. Std Spec, for Std Tire for Rec. Pract. for Construction of Concrete nterim Guideline for Protection and Acceptance of Concrete Floors and

guide for Design of Foundations and Shoulders for Concrete relating to Materials for Roads and

or Installation of Preformed Compression Seals in Concrete

Std. Spec, for Wood Paving Blocks for Exposed Platforms, Spec for Preformed Expansion Joint Fillers for Concrete ys, and Interior Floors Exposed to W/ St Std. Spec. for Wood Std. Spec. for Vitrified Clay Model Specs. for Small Concrete

h. of Test for Percent Air Voids in a Compacted Bituminous Courses (1967) Std. Spec. for Hot Mixed, Hot Laid Tar Std. Spec. for Mineral Filler for Bituminous

ments for Mixing Plants for Hot Mixed, Hot Laid Bituminous est for Quantitative Extraction of Bitumen from Bituminous
Parts (1970) ANSI G63.1

Parts (1970) ANSI G63.3

Parts (1970) ANSI H9.28

Parts (1970) ANSI H9.6

Parts (1970) ANSI H9.8

Parts $(1972)$

Parts (1972)

Parts (1973)

Parts (1973)

Parts (1974) /D and Cold Finished, Special
Parts, Equipment and Systems (1972) ANSI G8I. I6

ID and Cold Finished, Special Quality, Fo

Pass Meth. for Evaluating the Filtration Performance of

Passage of Air (1958) TAPPI T460

Passenger and Recreational Vehicles) (1974)

Passenger Automobile Equipped with Full Scale Tires in

Passenger Automobile Equipped with Full Scale Tires (19

Passenger Automobiles, Commercial Vehicles, Golf Car, a

Passenger Car and Light Truck-Ground Vehicle Practice

Passenger Car and Light Truck Exhaust System Parts (197

Passenger Car and Light Truck Muffler Parts Nomenclatur

Passenger Car and Light Truck Resonator Parts Nomenclat

(Passenger Car and Light Truck) (1971)

Passenger Car Tires (1972)

Passenger Car Type Tires) (1971)

Passenger Car Vehicle Identification Number Systems (19

Passenger Car WindshieId Defrosting Systems (1971

Passenger Car Windshield Washer Systems (1972)

Passenger Car (1971)

Passenger Cars and Light Trucks Using the Enclosure Tec

Passenger Cars and Light Trucks (Course of Instruction)

Passenger Cars and Light Trucks (1972)

Passenger Cars and Light Trucks (1972) ANS1 S6.3

Passenger Cars and Station Wagons (1965)

Passenger Cars and Station Wagons (1966)

Passenger Cars $\{1974$ )

Passenger Cars, Multipurpose Passenger Vehicles, Buses,

Passenger Cars, Multipurpose Vehicles, and Light Duty T

Passenger Cars, Station Wagons, and Multipurpose Passen

Passenger Cars, Trucks, Buses, Coaches, Tractors, Indus

Passenger Tramways Including Ski and Surface Lifts and /Emountable Rims Intended $F_{0}$

Passenger Vehicles (1972)

as and Radial Ply Tires and

Passenger Vehicles (1974)

and Combination Vehi

Passenger Vehicles
Passenger) (1971)

Passive Electronic Component Parts (1972)

Paste and Bar (1974)

Paste Meth.) (1972) ANSI A1.27

Paste) Content in Corn Starch (Gelatinizable Products)

(Paste) Content in Corn Starch (1958)

Paste) of Corn Starch Which Is Gelatinized When Heated

Pastes for Detection of Rodent Excreta, Insects, Webbin

Pastes (Cereal Chemistry) (1962)

Pastes (Cereal Chemistry) (1962)

Materials Hard to $\mathrm{Hy}$

Pastes, and Paints (1972) ANSI K42.1

R lnsect and Rodent

Patch Splices in Two Inch Video Magnetic Tape (1964)

Patio Covers (1973)

Patio Covers, Greenhouses, Canopies) (1973)

Patron and Nonemployee Injury Statistics (1971)

Pattern of Leather (Break Scale) (1973) A LCA E64

Patterns for Wood Paneling, Decking, Flooring, and Sidi

Patterns in Brickwork (Tech. Notes) (1967)

Patterns (1972)

Patterns) Used in Battery Sockets (1974) ANSI C83.53

Paved Surfaces Using a Passenger Automobile Equipped Wi

Paved Surfaces Using a Passenger Automobile Equipped Wi

Pavement Marking Paints (1972)

Pavement Overlay Projects (1973)

Pavement Polishing in the Laboratory (FulI Scale Wheel

Pavement Skid Resistance Tests (1973)

Pavements and Bases (1974)

Pavements Exposed to Rain During Construction (1974)

Pavements of Structural Clay (Tech. Notes) (1962)

Pavements (1965)

Pavements (1971) ANSI A37.33 Std. Definitions of Terms

Pavements (1972)

Std. Spec, for Lubricani F

Pavements, Driveways, and Interior Floors Exposed to We

Paving and Structural Construction (Nonextruding and Re

Paving Blocks for Exposed Platforms, Pavements, Drivewa

Paving Brick (1972) ANSI A37.15

Paving Jobs (1973)

Paving Mixture (1973)

Paving Mixtures for Base, Binder, Leveling, and Surface

Paving Mixtures (1970) ANSI A37.41

Paving Mixtures (1971) ANSI A37.102

Paving Mixtures (1972)
Nory Instrume

Safe Opera

ASTM

ASTM

ASTM

ASTM

ASTM

EIA

SAE

ICBO

ASTM

ASTM A380

NFLDP . T3.10.8

ASTM D726

TRA X.XI

ASTM E503

ASTM E445

ANS

$* 1.48$

SAE

SAE

SAE

Rec. SAE

Y 14.32

J97

J26l

J262

J254

J332A

V.7

J 273

J $942 \mathrm{~B}$

J229

J171A

D18.1

$\mathrm{J} 170 \mathrm{~A}$

J986A

V.I

V. 2

1.17

$\begin{array}{ll}\text { SAE } & \text { J299 } \\ \text { SAE } & \text { J398A }\end{array}$

TRA

TRA

$\mathrm{BCl}$

ANSI

7

$* 1.56$

B77.1

J267

1

J299

I 4200

RS186D

CSI

EIA

ASTM

ASTM

CR

CR

D 451

C45

CR

B. 4

$\mathrm{AACCH} 28-30$

28-32

ASTM Dl8

ICBO RPS

Ght Diffu ICBO UBC*3-52

Std ANSI Z108.1

ASTM D2941

WWPA $* 28$

BIA

ASTM

EIA

ASTM

ASTM

ITE

Recomm ACPA

ASTM

ASTM

ACI

ACPA

BIA

Rec. ACI

ASTM

ASTM

ASTM

ASTM

ASTM

ASTM

AI

Std. Met ASTM

ASTM

ASTM

ASTM

spec. for Require AST Std. Meth. of T ASTM

3077 

ojectṣ (1973) Ground Soybeans, Whole Or/ Protein Dispersibility Index esins (Plastic Compo/

Equipment (1971)

$n$ of Gallium Arseni/ (1971)

z197.2 I Chemistry) (1962)

ining Specific Weight, Durability and $\mathrm{M}$ / eal Chemistry) (1962) clear Fueled Power Generating Statio/ Std. for Electrical ging) (1951) ANSI MH12.8 si J2.19 Std. Meth. of Test for Impact Resilience and (1972) ty) (1973) diameter/ Std. Spec. for Biaxially Oriented Polyethylene y) (1962) (1973) sal Fields (1973) ANSI A176.4 saI Fields (1973) ANSl A17/ irport, and Similar Drainage /

$s$ and Trailers, inage (l) Curtain and $\mathrm{B} /$ 972) z168.12

tion of Supe 8 ptimum Size Coarse Aggregate for Portland Cement Concrete for Manually Lever Operated Chain Hoists for Ratchet and ontractor (For Small Construction Contracts Where Basis of

Std. Meth. of Test for Pyrometric Cone Equivalent

Std. Meth. of Test for Gel Time and

Std. Meth. for Measurement of Weighted

Std. Meth. of Test for Wavelength of soy Flour for Cake and Meal from Cottonseed, Soybeans, and esame, Safflower, Oleic, and Sunflower Seeds; Ground Nuts Sid. Spec. for sect Infestation in Cereal Grains, Unpopped Popcorn, Dried opic Examination of Cereal Grains, Unpopped Popcorn, Dried ir Contamination of CereaI Grains, Unpopped Popcorn, Dried internal Insects in Cereal Grains, Unpopped Popcorn, Dried ur, Farina, Semolina, Bread, Grain, Soybeans, Rice, Beans, tric Meter Meth. of Analysis of Moisture in Grains. Beans, cles Including Wheel Assembly, Chainguards, Saddle Braces,

se of Public Streets and Projections Over Public Property Std. Meth. of Test for Strap Std. Meth. of Test for Tent. Meth. of Test for Floating Roller Std Meth for Climbing Drum

for Determining Durability of Adhesive Joints Stressed in

Std. for Wafers.

ical Analysis of Nuclear Grade Uranium Dioxide Powders and aI Analysis of Nuclear Grade Plutonium Dioxide Powders and Meth. of Analysis o Spec. for Determination of Relative

Format Guide and Test Procedure for Linear, Single Axis, impact Resilience and Penetration of Rubber by the Rebound Std. Definitions of Terms Relating to Liquid

$\mathrm{n}$. Materials, and Installation of Power Transmission Unit Std. Meth. of Test for Water for Yield Stress of Heterogeneous Propellants by the Cone Std. Test Method for Std. Meth. of Test for Std. Meth. of Test for Test Meth. for Castor Oil Meth. for Con Std. Meth. of Test for Meth. of Test for Time of Setting of Concrete Mixtures by test Meth. for Water Resistance of Textile Fabrics: Impact water Resistance of Shoe Upper Leather by the Maeser Wate for Determining Hermeticity of Electronic Devices by Dye Std. Meth. of Test for Flash Point by ent for Preparing Oil Borne Preservative Solutions Such as Std. Meth. of Test for Solubility o

Spec. for Volatile Petroleum Solvent (LPG) for Preparin Std. Spec. for Std. Meth. for Chemical Analysis of Uniform Building Code Test Meth. for

Volumetric Bromine Meth. of Analysis of Xylene Partition Meth. of Analysis of Meth. of Sensory ate and/or Sodium Acid Pyrophosphate in Prepared Mixes (/ Meth. for Determining Chromic Oxide Content in Leather Std, Meth of Test for Chromic Oxide in Leathe Total Base Number of Petroleum Products by Potentiometric Wet Ashing with Nitric and Tent. Meth. of Test for Crystallographic Std. Spec. for Laminated Wall Sid. Spec. for Homogeneous Std. Spec, for

Stds. for lndustrial

Paving (1972)

Pawl, and Load Brake Designs (1971)

Payment Is a Stipulated Sum) (1974)

Payment on Portland Cement Concrete Pavement Overlay Pr

PDl): Meth. for Determination of Dispersible Protein in

Peak Exothermic Temperature of Reacting Thermosetting $R$

Peak Flutter Content of Sound Recording and Reproducing

Peak Photoluminescence and the Corresponding Compositio

Peanuts (Cereal Chemistry) (1962)

of Crude Fiber in

Peanuts); Vegetable, Coconut, and Palm Oils) (1974)

Pearlitic Malleable Iron Castings (1971) ANS] G48.2

Peas and and Beans (Cereal Chemistry) (1962)

Peas and Beans for Rodent and/or Insect D

Peas and Beans (Cereal Chemistry) (1962)

Peas and Beans (Cereal Chemistry) (1962)

Peas, Lentils, Corn Meal, Corn Crits, Rolled Oats, Bul

Peas, Lentils, Rice, and Other Commodities for Which Co

Pedal Clearance, Steering Control, Brakes, Tires and $\mathrm{Co}$

Pedestrian Protection During Construction or Demolition

Peel Adhesion of Reinforcing Fabrics or Cords to Rubber

Peel Resistance of Adhesives (T.Peel Test) (1972) ANSI

Peel Resistance of Adhesives (1973)

Peel Test for Adhesives (1962) ANSI Z197.23

Peel (1971) ANSI Z197.30

Pekar Color Test (Slick) Meth.

Pellets and Crumbles-De

Pellets (1972) ANSI N104 Trometric, and Spectrochemic Pelshenke Test (Wheat Meal Fermentation Time Test) (Cer

Pencil Hardness of a Paint Film (Coil Coating) (1974)

Pendulous, Analog Torque Balance Accelerometer (1972)

Pendulum (1972) A NS1 J2 19

Std. Meth. of Test for

Penetrant Inspection (1974) ANSI Z166.16

Penetrating the Hull for Boats Equipped with Outdrives,

Penetration Assemblies in Containment Structures for $\mathrm{Nu}$

Penetration into Pipeline Coatings (1972)

Penetration Meth. (1972) ANS1 Z11.319

Penetration of Adhesives (1969)

Penetration of Bituminous Materials (1973) ANSI A37.1

Penetration of Liquids into Submerged Containers (Packa

Penetration of Paper (1972)

Penetration of Petrolatum (1972)

Penetration of Rubber by the Rebound Pendulum (1972) an

Penetration Resistance of Pipeline Coatings (Blunt Rod)

Penetration Resistance (1970) ANSl A37.134

Penetration Test for Paperboard (Printing Ink Receptivi

Penetration Test (1971) ANSI L14.71, ASTM D583

Penetration Tester (1970) ALCA E56

Penetration (1972)

Pensky-Martens Closed Tester (1973) ANSI Z11.

Pentachlorophenol and Copper Naphthenate (1973)

Pentachlorophenol in Heavy Petroleum Solvents (1973)

Pentachlorophenol Solutions (1968)

Pentachlorophenol (1970)

Pentachlorophenol (1971) ANSI O11.22

Penthouses and Roof Structures (Tower, Spire) (1973)

Pentosans in Pulp (1973)

Pentosans (Cereal Chemistry) (1962)

Pentosans (Cereal Chemistry) (1962)

(PEO) Plastic Pipe (SDR.PR) Based on Controlled Outside

Perception Test for Staleness of Bread (Cereal Chemistr

Perchloric Acid Meth. of Analysis of Monocalcium Phosph

(Perchloric Acid Oxidation) (1969) ASTM D2807

(Perchloric Acid Oxidation) (1972)

Perchloric Acid Titration (1973)

Perchloric Acids (Wood, Pulp, etc.) (1973

D. Meth. of Test for

Perfection of Germanium by Preferential Etch Techniques

Perforated Bituminized Fiber Pipe for Septic Tank Dispo

Perforated Bituminized Fiber Pipe for Septic Tank Dispo

Perforated Bituminous Fiber Pipe Systems for Highway, a

Perforatcd Concrete Under Drainage Pipe (1973)

Perforated Industrial Revolving Screen Stds. (1972)

Perforated Industrial Vibrating Screens Stds. (1972)

Perforated Metal (1972)

Perforated Photographic Film in Rolls, Including Leader

Std. for Dimensions for Unperforated and Std. Spec. for Laminated Wall Bituminized Fiber Std. Spec. for Laminated WaIl Bituminized Fiber Std. Spec. for Homogeneous Solid Wall and Sid. Spec. for Industrial Std. Spec. for 8 Printed Area on $16 \mathrm{~mm}$ Motion Picture Film, Std. Dimensions for $16 \mathrm{~mm}$ Motion Picture Film Std. for Interchange Rolls of
Perforated Pipe for Agricultural, Land, and General Dra

Perforated Pipe for Airport and Roadwork Drainage (1973

Perforated Pipe for General Drainage (Land Reclamation,

Perforated Plate and Screens (Square Opening Series) (1

Perforated Plate Sieves for Test. Purposes (1970) ANSI

Perforated Super 8 (1-3) (1973)

Perforated Super 8, 5R-1667 (1-3-5-7-0) (1973)

Std. for Loca

Perforated Super 8, (1-4) (1973)

Perforated Tape for Information Interchange (1972)
ACPA

HMl

AlOA

C24

AACCH 46.24

ASTM D247

IEEE 193

ASTM F358

in AACCH 32.17

NIOP *1

ASTM A220

Ernal in AACCH 28.2I

AACCH 28-10

AACCH $28-20$

AACCH $28-22$

$\mathrm{AACCH}$ 44-15A

AACCH $44-11$

BMA $6 / 5$

ICBO $\quad$ 'BC $* 3.44$

ASTM D2630

ASTM D 1876

ASTM D3167

ASTM D1781

ASTM D2918

AACCH $14-10$

ASAE $\quad$ S 269.2

C696

ASTM C697

AACCH 56.50

VCCA TB-II-12

IEEE

ASTM

ASTM

ABYC

EEE

ASTM

ASTM

ASTM

ASTM

TAPPI

TAPPI

ASTM

ASTM

Std. ASTM

TAPPI

AATCC
ASTM

ASTM

ASTM

/ Solv ASTM

ASTM

Tent ASTM

ASTM

ASTM

ICBO

TAPPI

$\mathrm{AACCH}$

$\mathrm{AACCH}$

ASTM

$\mathrm{AACCH}$

AACCH

ALCA

ASTM

TAPPI

ASTM

ASTM

ASTM

ASTM

ASTM

IPA

IPA

IPA

ANSI

ASTM

ASTM

ASTM

ASTM

ASTM

ANSl

ANS]

ANSI

ANSI

337

D1054

E270

G9

D 1916

D998

T462 
cally Controlled Equipment (1974)

d Contouring-Positioning Nume/ Std. Spec. for Properties of Unpunched Oiled Paper Std. Spec. for Asbestos Cement ted Area in Super 8 Printing on 16/8Mm Motion Picture Film n Optical Reduction Printing on $35 \mathrm{~mm}$ Motion Picture Film. eduction or Contact Printing on $35 \mathrm{~mm}$ Motion Picture Film, Criteria for Preparation of Design Bases for Systems That

Std, for Air Brake Reservoir Rec. Pract. for Automotive Gasoline cture (FOPS) for Prime Movers, W/ Rec. Pract. for Minimum
s (ROPS) for Prime Movers (Heavy/ Rec. Pract. for Minimum $s$ (ROPS) for Prime Movers (Heavyl Weeled Front End Loaders An/ for Track Type Tractors and Fro/ Rec. Pract. for Minimum Rec. Pract. for Minimum id Power Pumps/ Std. Meth. of Test. and Presenting Basic rs (1973)

Clothes) Washer (1971) ANS1 Z224.1

Std. for

Recommendation for Installation ent / Std. Multi Pass Meth. for Evaluating the Filtration ractional and Integral Horsepower) (1/ Removal (1972) Sid. Meth. of Test for Operating Std. for 72) ANSI L 14.291 Std. Spec. for ing-To/ Std. for Test Procedure for the Locking Ability
972) ANSI 2111.4 Ptd. Meth. of Test for Operating Std. Meths. for Specifying the Minimum Requirements for the Design, Installation and Std. Meth. for Measuring Transmission

Std. Rec. Pract. for Description and (1970) Gas Turbine Engine Steady State

eristics and Finishes of Institutional Textiles (Du/

r Retreaded Tires for Passenger Cars and Station/

Minimum

r Motor Vehicle Brake Linings (1966)

er Supply Lines with Intermediate Atmospheric Vent (1972/ and Canopy Fabrics, Painted, Printed, or Vinyl Coa/

n, etc.) (Institutional Textile) (1973)

$\mathrm{s}$, Finishes, and Finishings (Sublimation, Brittlene/ ck Pressure Backflow Preventers for Potable Water Supply ven Sheet and Pillowcase Fabrics (Institutional Tex/ I Textile) (1973)

Textile) (1973)

Water Shower Control Valves (1973)

ices and Components (1972)

mestic and Light Commercial) Central Furnaces) (I97/ Std.

le Tags, and Certification of Fabrics or Products (/

ype Vacuum Breakers (1970)

t) Units for Motor Vehicles (197/

Rec. Pract. for Service nd Steering Stability for Regular Bicycl/ Safety Std. for mask (White or Dyed) (Napkin, etc.) (Institutional / rics, Lightweight (Tropical) Suiting (Institutional/

and Double) (Institutional Textile) (1973)

onal Textile) (1973)

ional Textile) (1973

nal Textile) (1973)

s, Pillows, and Bolster Fabrics (Institutional Text/ tional Textile) (1973)

brics (Excluding Glass Fibers) (Institutienal Texti

t Fabrics (Institutional Uniform Textile) (I973)

d Uniform Fabrics, Intermediate (Year Round) Suitin/

d Uniform Fabrics, Heavyweight (Industrial) Suiting/

r Institutional and Industrial Use Garments (Clothi/

Institutional and Industrial Use Garments (CIothi/

fabrics Handwashable at 105 Deg. F-No Bleach (Ins/

(Curtain) Fabrics Handwashable at 105 Deg. F-No B/ nstitutional Uniforms (Textile) (1973)

Awning, and Canopy Fabrics, Vinyl Coated or Laminal

(Napkin, etc.) (Institutional Textile) (1973)

Fabrics Other Than Durable Press (Institutional Te/ titutional Textile) (1973)

nstitutional Textile) (1973)

tacked On) (InstitutionaI Textile) (1973)

rics for Men and Women (Institutional Textile) (I97/ rics for Men and Women (Heavy Duty) (Institutional / cs (Sash Casement) (Excluding Glass Fibers) (Instit/

(Textile) for Institutional and Industrial Use Garm/ Rec. Pract. for New Motor Vehicle on Grade Parkin

ural Tractors for Overhead Protection (Test Procedures and

Enclosures for Agricultural Tractors (Test Procedures and

ctive Frame for Agricultural Tractors (Test Procedures and ety Glazing MateriaI Used in Buildings (1971)

d to Wood Framing (1971)

t Dosimeters for X-And Gamma Radiation (1972)

ed Circuit) (1972)

rvey Inst ruments (1971)

nt Marking Paints (1972)
Perforated Tape Std., Variable Block Format, for Numeri Perforated Tape Variable Block Format for Contouring an Perforated Tape (1971)

Perforated Underdrain Pipe (1973) ANSI A 165.2

Perforated $1.4(1971)$

Perforated 2R-1664 (1-0) (1973)

Perforated 5R-1667 (1.3.5-7-0) (1973)

/Uper 8 Printed Area I /Ea in Optical R

Performance and Identification Requirements (1972)

Performance and Information System (1971)

Performance Criteria for Falling Object Protective Stru

Performance Criteria for Roll Over Protective Structure

Performance Criteria for Roll Over Protective Structure

Performance Criteria for Roll Over Protective Structure

Performance Data for Positve Displacement Hydraulic Flu

Performance Evaluation for Household Food Waste Dispose

Performance Evaluation Procedure for Household (laundry

Performance Levels of Ceramic Tile Floors (1973)

Performance of a Fine Hydraulic Fluid Power Filter Elem

Performance of Alternating and Direct Current Motors (F

Performance of Anion Exchange Materials for Strong Acid

Performance of Appliance Humidifier (1972) ANSI Z235.1

Performance of Bonded and Laminated Apparel Fabrics (19

Performance of Chemical Coated Lock Screws (1973)

Performance of Nonmetallic Locking Element Type Prevail

Performance of Particulate Cation Exchange Materials (1

Performance of Shock Machines (1973)

Performance of Sprinkler Irrigation Equipment (1972.

Performance of Telephone Sets (1971)

Performance of the Microphotometer (1971) ANSI Z128.31

Performance Presentation for Digital Computer Programs

Performance Requirements and Claims for Special Charact

Performance Requirements and Uniform Test Procedures Fo

Performance Requirements and Uniform Test Procedures Fo

Performance Requirements for Backflow Preventers in Wat

Performance Requirements for Cotton Woven Tent, Awning,

Std. Performance Requirements for Damask Table Napery (Napki

Std. Performance Requirements for Deleterious Effects of Dye

Performance Requirements for Double Check Valve Type Ba

Std. Performance Requirements for Durable Press (No Iron) Wo

Std. Performance Requirements for Glass Towels (Institutiona

Performance Requirements for Huck Tow els (Institutional

Performance Requirements for Individual Anti Scald Type

Performance Requirements for Motor Vehicle Lighting Dey

Performance Requirements for Oil Powered Forced Air (Do

Performance Requirements for Permanent LabeIs, Detachab

Performance Requirements for Sealed Beam Headlamp (Ligh

Performance Requirements for Stopping Power, Traction a

Performance Requirements for Table Napery Other Than Da

Std. Performance Requirements for Tailored Woven Uniform Fab

Sid. Performance Requirements for Terry Cloth Towels (Single

Std. Performance Requirements for Woven Bath Mats (Instituti

Std. Performance Requirements for Woven Bedspreads (Institut

Std. Performance Requirements for Woven Blankets (Institutio

Std. Performance Requirements for Woven Decorative Bedspread

Std. Performance Requirements for Woven Dish Towels (Institu

Std. Performance Requirements for Woven Drapery (Curtain) Fa

Std. Performance Requirements for Woven Drycleanable Overcoa

Std. Performance Requirements for Woven Drycleanable Tailore

Std. Performance Requirements for Woven Drycleanable Tailore

Std. Performance Requirements for Woven Fabrics (Textile) Fo

Std. Performance Requirements for Woven Fabrics (Textile) Fo

Std. Performance Requirements for Woven Glass Fiber Curtain

Std. Performance Requirements for Woven Glass Fiber Drapery

Sid. Performance Requirements for Woven Lining Fabrics for I

Std. Performance Requirements for Woven Man Made Fiber Tent,

Std. Performance Requirements for Woven Printed Table Napery

Std. Performance Requirements for Woven Sheet and Pillowcase

Std. Performance Requirements for Woven Shower Curtains (Ins

Std Performance Requirements for Woven Slipcover Fabrics (I

Std. Performance Requirements for Woven Upholstery Fabrics (

Std. Performance Requirements for Woven W ashable Uniform Fab

Std. Performance Requirements for Woven W ashable Uniform Fab

td Performance Requirements for Woven Window Curtain Fabri

Performance Requirements for $100 \%$ Cotton Woven Fabrics

Performance Requirements (1972)

Performance Requirements) (1972) SAE J167

Performance Requirements) (1972) SAE J168

Performance Requirements) (1972) SAE J334

Performance Spec. and Meth. of Test for Transparent $S$ a

Performance Spec. for Adhesives for FieId Gluing PIywoo

Performance Spec. for Direct and Indirect Reading Pocke

Std. Performance Spec. for Flexible Multilayer Wiring (Print

Performance Spec. for Portable X-Or Gamma Radiation Su

a Model Performance Spec. for the Purchase of Reflective Paveme
NSA

EIA

ANSI

ANSI

ANSI

ANS

SAE

SAE

SAE

$S A E$

SAE

SAE

AHAM

AHAM

TCA

NFLDP

NEMA

ASTM

AHAM

ASTM

IFI

ASTM

ANSI

ASAE

EEE

ASTM

SAE

ANSI

VESC

VESC

ASSE

NSI

ANSI

ANSI

ASSE

ANI

ANSI

ANSI

ASSE

SAE

ANSI

ANSI

ASSE

SAE

BMA

ANSI

ANSI

ANSI

ANSI

ANSI

ANSI

ANS

ANS1

ANSI

ANSI

ANS

ANSI

ANSI

ANSI

ANSI

ANSI

ANSI

ANSI

ANSI

ANSI

ANSI

NSI

ANSI

ANSI

ANSI

NSI

ANSI

SAE

or Agricult ASAE Protective

ASAE

ANSI

APA

ANSI

IPC

ANSI

ITE

955

RS274-B

X3.29

PH22.

PH22. 179
PH22. 180

N18.8

$\mathrm{J} 10 \mathrm{~B}$

J282

J231

J

T3.9.17

FWD.1

HLW.1

*3-2

T3. 10.8 .8

MGl.12

D3087

D3135

125

I24

D 1782

S2.14

264. 
ent (DME) Operating Within the Radio Frequency R/ Minimum perating Within the Radio Frequency Range of 108/ Minimum ruction Type Cranes Having Rope Supporte/ Rec. Pract. for 972)

972) 972 d. Pract. for Stationary Diesel and Gas Engine Ratings and ing Tests on the Cotton System for Measurement of Spinning als and Prefabricated Silencers for Acoustical and Airflow Std. for Certified Ratings Program Air Moving Devices nels; Babassu; Flaxseed;/ Magnesium Chloride Solution (1973) Std. Rec. Pract. for water, Rigging Collaps/ luminum (1971)

ection Systems (19/

ster (1973)

old Conditions (1970) (1972) Std.

Fabrics or Products (/

Std. Spec. for Magnesium Alloys in lngot Form for Sand, Std. Spec. for Aluminum Alloys in lngot Form for Sand, meth. for the Appearance of Durable Press Textile Fabrics abric Coverings for Decorative and Protective Covering for mensional Changes in Automatic Home Laundering of Durable Construction Spec. for Rifle Bench Rest (Portable and izes 12 to $26(1972)$ $\begin{array}{ll}\text { si N154 Sitd. Meth. of Test for Consumption of Potassium } \\ \text { s (1971) } & \text { Std. Analytical Meth. for Determining Potassium }\end{array}$ Meth. for Preparation and Standardization of Potassium Std Spec for Photographic Grade Potassium Std. Meth. of Test for Fineness of Portland Cement by Air 68) ANSI MH12.18 Std. Meth. of Test for Moisture Vapor est for Average Particle Size of Alumina and Silica by Air trations of Radionuclides in Air and In/ in/ Rec. for Maximum Permissible Body Burdens and Maximum nges and Limits for Steels (1972)

e for Obtaining Department of Transportation (DOT) Special insulating Materials at Micr/ ielectrics A/ Std. Meth. of Test for Dielectric Constan ting Current Loss Characteristics and Dielectric Constan

Salts, Soy F/

67) Alkali in Bleach Baths Containing Hydrogen est Meth for Colorfastness of Textiles to Bleaching with Meth. of Analysis of Acetone Tent. Hazard Classifications of Organic Recommended Safeguards for Organic nd Stage Lifts (Hydrualic and Hydropneumatic) for Lowering for Qualifications of Inspection, Examination, and Test. G5 Std. Rec. Pract. for Qualification of Procedures and production) (1974) s) (1973)

Rec. Pract. Orientation Program for Std. for Safety Requirements for Stds. and Practice Guide for Aids to Security (Safety) of Test Meth. for Colorfastness of Colored Textiles to Qualitative lodide Test Meth. for Ammonium Quantitative Meth. of Analysis of Ammonium thio)-4-Cyclohexene-1,2-Dicar/ late (Ester) Desmedipham (1/ Dodecadienoate Hydroprene / bromopropylate (1973)

Chlo: opropylate (1973)

Nitrobenzoate Bifenox (197)

-2,6-Dinitro.N-Propyl.Ptoluid/ uoro-2,6-Dinitro-N-Propyl-Pto/

$$
\begin{array}{r}
\text { Mec. for Construction and } \\
\text { Minimum } \\
\text { Pressure Sensitive Tape } \\
\text { Std. Thermal }
\end{array}
$$
Std. Acoustical Std. for Criteria for Film Badge Oilseed Products Trading Rules Rec. for Protection Against Misc Spec. for Exterio Std. for Trial-Use Criteria for the Spec. for Std. Spec. for

Std. Spec. for

Uniform Building Code Std. for Std. Test Meth. for Meth. of Test for

Std. Performance Requirements for Std. Spec. for Magnesium Alloy Std. Spec. for Aluminum Alloy Uniform Building Code: Std. Spec. for Manifold Papers for Std. Meth. of Test for Air Std. Meth. of Test for Water Vapor Std. Meth. of Test for Water Vapor Std. Rec. Pract. for Determining Std. Meth. of Test for Hydrogen Rec. for Maximum

Uniform Building Code: Std. Test Meth. for Complex Std. Meth. of Assay of Di-T-Butyl Test Meth. for Hydrogen
Performance Std. for Airborne Distance Measuring Equipm Performance Std. for Airborne VOR Receiving Equipment $O$ Performance Std. for Crane Boomstop of All Mobile Const

Performance Std. for Kitchen and Vanity Cabinets (1973) Performance Stds. Airborne ATC Transponder Equipment (1 Performance Stds. for Fibrous Glass Duct (1973)

Performance Test for Steel Door and Frame Assemblies (1

Performance Test for Steel Door and Frame Assemblies (1

Performance (Nuclear Radiation) (1972)

Performance (1972)

Performance (1972) ANS1 Li4

Performance (1973)

(Performance) (1970)

Performance, Quality, Description, and Grade of Palmker

Performing Stress Corrosion Cracking Tests in a Boiling

Perils at Electric Generating Stations (Flood, Surface

Perimeter Sealing Compound for Use with Architectural a

Periodic Test. of Nuclear Power Generating Station Prot

Perlite Insulating Concrete Roof Decks (1973)

Perlite Loose Fill Insulation (1973)

Perlite Thermal Insulation Board (1972)

Perlite, Vermiculite and Sand Aggregates for Gypsum Pla

Permanence of Adhesive Bonded Joints in Ply wood Under M

Permanence of Durable Water Repellent Finish of Fabrics

Permanent Deformation of Elastomeric Yarns (1972T)

Permanent Labels, Detachable Tags, and Certification of

Permanent Mold Castings (1972) ANSI H45.2

Permanent Mold Castings (1974) ANSI H38.26

Permanent Mold, and Die Castings (1966) ANSl H45.8

Permanent Mold, and Die Castings (1973) ANS1 H38.1

Permanent Occupancy (1973)

(Permanent Press) After Repeated Home Launderings (1973)

Permanent Records (1973)

Permanent Wall and Ceilings (1969) Ifor Vinyl Coated F

(Permanent) Press Woven or Knit Textile Fabrics (1973) a

Permanent) (1967)

Permanent, Crimp Style, Pre Insulated, (Class 2) Wire S

Permanganate by lmpurities in Deuterium Oxide (1968) an

Permanganate Solution Content of Reagents and Indicator

Permanganate Solution (Cereal Chemistry) (1962)

Permanganate, Kmn0, (1972)

Permeability of Asbestos Fibers (1972)

Permeability of Organic Coatings Films (1972)

Permeability of Packages by Cycle Meth. (Packaging) (19

Permeability of Packages (Packaging) (1968) ANS1 MH12.1

Permeability of Thermoplastic Containers (1973)

Permeability (1972)

Permeance of Rubber Coated Fabrics (1973)

Permissible Body Burdens and Maximum Permissible Concen

Permissible Concentrations of Radionuclides in Air and

Permissible Variations from Specified Ladle Chemical Ra

Permits and lnspections (1973)

Permits for Radioactive Materials Shipments (1973)

Permittivity (Dielectric Constant) of Solid Electrical

(Permittivity) and Dissipation Factor of Solid Ceramic D

Permittivity) of Solid Electrical lnsulating Material

Peroxide by Gas Chromatographic Analy sis (1973)

Peroxide in Bleach Baths (1972) ANS1 L14.145

Peroxide in Dough Conditioners Containing Monoglyceride

Peroxide in Flour (Cereal Chemistry) (1968)

Peroxide with Diaminophenylamine (Cereal Chemistry) (19

Peroxide (Cereal Chemistry) (1962)

Peroxide (1972) ANS1 L14.129

Peroxide (1972) ANSI L14.146

Peroxides in Milling Premixes (Cereal Chemistry) (1971)

Peroxides (Plastic) (1974)

Peroxides (1972)

Personnel and Material (1972)

E Spec. for Pla

Personnel for the Construction Phase of Nuclear Power P

Personnel Going Offshore for the First Time (Petroleum

Personnel Hoists (Construction and Demolition Operation

Personnel on Boat Deck and for Reboarding from the Wate

Perspiration (1972) ANSI L 14.56

Persulfate in Flour Improvers (Cereal Chemistry) (1962)

Persulfate in Flour (Cereal Chemistry) (1962)

Pest Control Chemical cis- $\mathrm{N}-((1,1,2,2$ tetrachloroethyl $)$

Pest Control Chemical Ethyl Mhydroxycarbanilate Carbani

Pest Control Chemical Ethyl (E,E)3,7,11-Trimethyl-2,4-

Pest Control Chemical lsopropyl 4,4'dibromobenzilate-

Pest Control Chemical lsopropyl 4,4'dichlorobenzilate-

Pest Control Chemical Methyl 5(2,4-Dichlorophenoxy)-2-
Permeability Apparatus (1973) AASHO T153, ANSl Al.19

$\begin{array}{llll}\text { Std. Common Name for the Pest Control Chemical N-(2Chloroethyl)-A, A, A-Trifluoro } & \text { ANSl } & \text { K62.140 } \\ \text { Std. Common Name for the } & \text { Pest Control Chemical N(Cyclopropylmethyl)-A, A, A-Trifl } & \text { ANSl } & \text { K62.152 }\end{array}$

$\begin{array}{llll}\text { Std. Common Name for the Pest Control Chemical N-(2Chloroethyl)-A, A, A-Trifluoro } & \text { ANSl } & \text { K62.140 } \\ \text { Std. Common Name for the Pest Control Chemical N(Cyclopropylmethyl)-A, A, A-Trifl } & \text { ANSl } & \text { K62.152 }\end{array}$

RTCA

RTCA

SAE

ANSI

SMACN AFTS-100

STDI 113

STDI $\quad 114$

ANSI N13.7

St DEMA *1.4

ASTM D2811

ASTM E477

$\begin{array}{ll}\text { AMCA } & 211 \\ \text { NIOP } & -1\end{array}$

ASTM $\overline{\mathrm{C}} 36$

FMS $\quad 9.10$

AAMA $\quad 808.2$

IEEE 338

CSl

ASTM C549

ASTM C728

ICBO UBCS47.3

ASTM D1877

ASTM D2721

ASTM D3106

ANS1 L24.5.1

ASTM B199

ASTM B108

ASTM B93

ASTM $\quad 8179$

ICBO UBC $* 3-45$

AATCC 124

ASTM D3208

CSI 0995

AATCC 135

NRA $* 9$

ASTM D2033

CR R-15

AACCH $\quad 70-50$

ANSl PH4.301

ASTM C204

ASTM D2752

ASTM D 1653

ASTM D1251

ASTM D895

ASTM D2684

ASTM C721

ASTM D815

NCRPM R22

NCRPM R22

SAE J409C

1CBO UBC*3-3

Id ANSI N14.10.2

ASTM D2520

ASTM D2149

ASTM D150

ASTM E475

AATCC 102

$\mathrm{AACCH}$ 48-51

AACCH 48-07

AACCH 48-06A

AACCH $48-50$

AATCC 98

AATCC 101

$\mathrm{AACCH} \quad 48.05$

FMS 7.81

CSI

ASTM

ANSI

ABYC A.18

AATCC 15

AACCH 48.61

$\mathrm{AACCH} \quad 48-62$

ANSI K62.146

ANSI K62.139

ANS1 K62.136

ANS1 K62.151

ANS1 K62.150

ANS1 K62.138

$\begin{array}{llll}\text { Std. Common Name for the Pest Control Chemical N-(2Chloroethyl)-A, A, A-Trifluoro } & \text { ANSl } & \text { K62.140 } \\ \text { Std. Common Name for the Pest Control Chemical N(Cyclopropylmethyl)-A, A, A-Trifl } & \text { ANSl } & \text { K62.152 }\end{array}$ 
odithioate) (1973/

S-Ester Wi/

Std. for Ethoprop (Common Name for the Std. for Methidathion (Common Name for the ylcarbamate-Dioxacarb (1973) -Trimethyl-2,4-Dodecadienethi/ 2-Pyridyl) Phosphorothioa/ oxy-S-Triazine-Prometon (197/ hylthio)-S-Triazine-Prometry/ -S-Triazine-Propazine (1973/ yl-2,4-Dodecadienoate Kino/ )-6-(Methylthio)-S-Triazine $\cdot /$ ylamino) Strial Std. for Dip (Ethylamino).S-Triazine-Terb/ o)-6-(Methylthio).S-Triazine a, A-Trifluoro-m-toly]) -3 / Std for Tetramethrin (Common Name for the Std. for Fospirate (Common Name for the Std. for Disugran (Common Name for the Std. for Crufomate (Common Name for the Std. Common Name for the Sid. Common Name for the Std. for Common Name for the Std. Common Name for the Std. Common Name for the Std. Common Name for the

Std. for Common Name for the Std. Common Name for the tryn (Common Name for the Sid. Common Name for the Std Common Name for the Std. for Common Name for the for Chlorbromuron (Common Name for the Std. for Chlordimeform (Common Name for the Std. for Fluorodifen (Common Name for the Std. for Prynachlor (Common Name for the Std. for Glyphosine (Common Name for the Std. for Mexacarbate (Common Name for the Std. for Dinoseb (Common Name for the Std. for Acephate (Common Name for the Std. for Ethiolate (Common Name for the Std. for Glyphosate (Common Name for the Std. for Oxamyl (Common Name for the Std. for Picloram (Common Name for the Std for Nitrapyin (Common Name for the phenoxy) Cyclohexyl 2Propynyl Sulfite (Common Name for the ,11-Trimethyl -2,4-Dodecadienoate) (Common Name for the (1969) . for Insect Meth. of Test for Organochlorine g Cloth, Filter Paper, Wide Field and Compound Microscope, of Test for Congealing Point of Petroleum Waxes lncluding Meth. for Congealing Point of Petroleum Waxes Including Meth. for Cone Penetration of of Test for Solubility of Bituminous Road Materials ( $T$ ar, Spec. for Threading, Gaging, and Thread Inspection of Std. Meth. for Calculation of Liquid Heat Capacity of Spec. for ined Platforms (1973) Spec. for Spec, for Grade C.75 and C.95 Casing and Tubing

Spec. for High Strength Casing, Tubing, and Drill Pipe Rec. Pract. for Care and Use of Casing and Tubing Spec. for Line Pipe Spec. for High Test Line Pipe Std. Spec. for

Tent. Meth. of Test for Boiling Range Distribution of Tent. Meth. of Test for Sulfur in Tentative Manual for Driver Attended Loading of Liquefied Std. Meth. of Test for Vapor Pressure of Liquefied Std. Meth. for Analysis of Liquefied Std. for the Storage and Handling of Liquefied
Uniform Fire Code: Liquefied Uniform Fire Code: Liquefied

) ANSI Z11.248 Std. Meth. of Test for Bromine lndex of th. of Test for Trace Quantities of Sulfur in Light Liquid tion, and Operation of Subsurface Safety Valve Systems for Std. for Flanged Steel Safety Relief Valves Used in the r Quality for Subsurface Injection Using Membrane Filters ion of Representative Saturates Fraction from Low Olefinic ame Arresters (Fire) for Use on Vents of Storage Tanks for $t$ for Carbon Distribution and Structural Group Analysis of Std. Meth of Test for Pour Point of est for Dielectric Breakdown Voltage of Insulating Oils of est for Dielectric Breakdown Voltage of Insulating Oils of Spec, for Steel Gate, Plug, Ball, and Check Valves for of Areas for Electrical Installations at Drilling Rigs and Spec. for Oil and Gas Separators

Spec. for Vertical and Horizontal Emulsion Treaters Spec. for Spiral Weld Line Pipe

n Program for Personnel Going Off hore for the First Time tration (197) Std. Meth. of Test for Total Base Number of Std. Meth. of Test for Vapor Pressure of

Std. Meth. of Test for Vapor Pressure of

Safety Std. for Power Operated Pumps for Std. Meth. of Test for Ash from Std. Code for Pressure

Service Cracking of Carbon Steel (P-1) Welds in Corrosive Std. Spec. for Creosote
Pest Control Chemical O-Ethyl S,S-Dipropyl Phosphor Pest Control Chemical O,O-Dimethyl Phosphorodithioate Pest Control Chemical O-1,3-Dioxolan -2-Ylphenyl Meth Pest Control Chemical S-Ethyl (E,E)-11-Methoxy-3,7,11 Pest Control Chemical 0,0 Dimethyl 0.(3,5,6-Trichloro Pest Control Chemical 2,4-Bis (Isopropylamino)-6-Meth Pest Control Chemical 2,4-Bis (Isopropylamino)-6-(Met Pest Control Chemical 2-Chloro-4,6bis (Isopropylamino) Pest Control Chemical 2-Propynyl (E,E)-3,7,11-Trimeth Pest Control Chemical 2-(Ethylamino)4-(Isopropylamino Pest Control Chemical 2-(Ethylthio) -4,6-Bis (Isoprop

Pest Control Chemical 2-(Tertbutylamino).4-Chloro.6-

Pest Control Chemical 2-(Tertbutylamino)-4-(Ethylamin

Pest Control Chemical 4-Chloro5-(Methylamino)-2-(A,

Pest Control Chemical (1972)

Pest Control Chemical) (1971)

Pest Control Chemical) (1971)

Pest Control Chemical) (1971)

Pest Control Chemical) (1972)

Pest Control Chemical) (1972)

Pest Control Chemical) (1972)

Pest Control Chemical) (1972)

Pest Control Chemical) (1972)

Pest Control Chemical) (1972)

Pest Control Chemical) (1972)

Pest Control Chemical) (1972)

Pest Control Chemical) (1972)

Pest Conirol Chemical) (1972)

Pest Control Chemical) (1972)

Pest Control Chemical) (1972)

Pest Control Chcmical) (1972)

Pest Control Chemical) (1972)

Pest Control Chemical) (1973)

Pest Control Chemical) (1973) /(E,
Pest Deterrents on Textiles (1971) ANSI L14.6

/Rgite 2-(P-Tert-Butyl
$/(\mathrm{E}, \mathrm{E})-11-$ Methoxy-3,7

Pesticide Residues in Food Products (Cereal Chemistry)

Pesticides in Water (1972T)

Petri Dish, Sieve, Separatory Funnel, Trap Flask, Step

Petrolatum (1971) ANSI Z11.61

Petrolatum (1972)

Petrolatum (1972)

Petroleum Asphalt, etc.) in Organic Solvents (1966) Ans

Petroleum Casing, Tubing, and Line Pipe Threads (1974)

Petroleum Distillate Fuels (1971) ANSI Z11.313

Petroleum Drilling and Well Servicing Structures (1974)

Petroleum Drilling Rig Packaging for Minimum Self Conta

(Petroleum Drilling) (1973)

(Petroleum Drilling) (1973)

(Petroleum Drilling) (1973)

(Petroleum Drilling) (1973)

Petroleum Drilling) (1973)

Petroleum Drilling) (1973)

Petroleum for Blending with Creosote (1971)

Petroleum Fractions by Gas Chromatography (1973)

Petroleum Gas by Oxidation Microcoulometry (1973)

Petroleum Gas (1966)

Petroleum Gases (LP-Gas Meth.) (1973) ANSI Z11.92

Petroleum Gases (LPG) by Process Gas Chromatography (19

Petroleum Gases (1972)

Petroleum Gases (1973)

Petroleum Hydrocarbons by Electrometric Titration (1972

Petroleum Hydrocarbons by Oxidative Microcoulometry (19

Petroleum Industry Use (1973)

Petroleum Industry Use (1973)

Petroleum Industry (1969) ANSI B146.1

/T. for Design, Installa

Petroleum lndustry) (1973)

Petroleum Naphthas (1973) ANSI Z11.165

Petroleum Oil and Gasoline (1973) ANSI Z222.1

Petroleum Oils by the n-d-M Meth. (1973)

Petroleum Oils (1971) ANSI Z11.5.

Petroleum Origin Under lmpulse Conditions (1974)

Petroleum Origin Using VDE Electrodes (1972) ANSI C59.1

Petroleum Pipeline Valves (1974)

Petroleum Production Facilities on Land and on Marine F

(Petroleum Production) (1973)

(Petroleum Production) (1973)

(Petroleum Production) (1973)

(Peiroleum Production) (1974)

Petroleum Products by Potentiometric Perchloric Acid T

Petroleum Products (Micromethod) (1971) ANSI Z11.277

Petroleum Products (Reid Meth.) (1972) ANSI Z11.44

Petroleum Products (1973)

Petroleum Products (1973) ANSI Z11.54

Petroleum Refinery Piping (1973)

Petroleum Refining Environment (1972)

Petroleum Solution (1970)
ANSI

ANSI

ANSI

ANSI

ANSI

ANS

ANSI

ANS

ANSI

ANS

ANSI

ANSI

ANSI

ANSI

ANS

ANSI

ANSI

ANSI

ANS1

ANSI

ANSI

ANSI

ANSI

ANSI

ANSI

ANSI

ANS

ANSI

ANSI

ANSI

ANSI

ANSI

ANSI

AATCC

Af.CCH

ASTM

$\mathrm{AACCH}$

ASTM

TAPPI

TAPPI

ASTM

API

ASTM

API

API

API

API

API

API

APl

ASTM

ASTM

ASTM

NGPA

ASTM

ICBO

ASTM

ASTM

API

API

API

ACE

Th. for Isolat ASTM

I for Fl U

/Meth. of Tes ASTM

ASTM

lof T ASTM

ASTM

API

API

API

API

API

API

ASTM

ASTM

UL

ASTM

ANSI

IS to Prevent in NACE

ASTM

K62.133

K62.134

K62.147

K62.141

K62.130

K62.144

K62.143

K62.145

K62.137

K62.142

K62.135

K62. 149

K62.148

K62.125

K62.129

K62.106

K62.111

K62.112 

Std. Code for Pressure Piping: Liquid

Test for Abrasion Resistance of Glossy Smooth Coatings of

Petroleum Sulfonates by Liquid Chromatography (1973)

Petroleum Transportation Systems (1974)

Std. Meth. of Test for Congealing Point of Meth. for Congealing Point of

tor Safety for Meters for Flammable Liquids and Liquefied

or Calculation of Certain Physical Properties of Liquefied

Petroleum Waxes (1973)

Petroleum (LP) Gas (Other Than Safety Relief) (1974)

Petroleum (LP) Gas (1972)

Petroleum (LP) Gas (1973)

Petroleum (LP) Gas (1973) ANSI Bl67.

Std. Meth. for Distillation of Cride

nsion Coil, and Flexible Connector Fittings for LP (Liquid Safety Std. for LP (Liquid

for Pipe Connectors for Flammable Liquids and LP (Liquid

Safety Relief Valves for Anhydrous Ammonia and LP (Liquid

Rec. Pract. for Drill Stem Design and Operating Limits Std Spec for Modern

for Thyroid Radioiodine Uptake Measurements Using a Neck

Rec. for Fire Protection of Electrical Equipment (Single

for Single Phase Rural Distribution Service for Motors and the Composition and Cryst/

Std. Meth. for Assigning Alloy Std. for Housekeeping During the Construction

luid Systems and Associated Components During Construction ion, Examination, and Test. Personnel for the Construction onverters (1972) Rec. for Single

reakers Rated on a Symmetrical Basis When Rated for Out of eth. of Test for Thermal Instability of Corfined Condensed receptacles: Specific Purpose 347/600 Volts, 20 Amperes, 3 receptacles: Specific Purpose 347/600 Volts, 30 Amperes, 3 receptacles: Specific Purpose 120/208 Volts, 20 Amperes, 3 receptacles: Specific Purpose 120/208 Volts, 30 Amperes, 3 receptacles: Specific Purpose 277/480 Volts, 20 Amperes, 3 receptacles\& Specific Purpose 277/480 Volts, 30 Amperes, 3 receptacles: Specific Purpose 120/208 Volts, 20 Amperes, 3 receptacles: Specific Purpose 120/208 Volts, 30 Amperes, 3 receptacles: Specific Purpose 277/480 Volts, 20 Amperes, 3 receptacles: Specific Purpose 277/480 Volts, 30 Amperes, 3 receptacles: Specific Purpose 347/600 Volts, 20 Amperes, 3 receptacles: Specific Purpose 347/600 Volts, 30 Amperes, 3 Std. on Television: Measurement of Differential Gain and 0 Volts and Below, 3750 Through $30,000 / 40,000 / 50,000$ kVA 3 Std. for Resistance Spot Welding Equipment, 3 of Items for Nuclear Power Plants (During the Construction ec. for Resistance Seam and Roll Spot Welding Equipment (3 and Receptacles: Specific Purpose 250 Volts, 30 Amperes, 3 and Receptacles: Specific Purpose 480 Volts, 20 Amperes, 3 and Receptacles: Specific Purpose 480 Volts, 30 Amperes, 3 and Receptacles: Specific Purpose 600 Volts, 30 Amperes, 3 and Receptacles: Specific Purpose 250 Volts, 15 Amperes, 3 and Receptacles: Specific Purpose 250 Volts, 20 Ampcres, 3 and Receptacles: Specific Purpose 250 Volts, 20 Amperes 3 and Receptacles: Specific Purpose 250 Volts, 30 Amperes, 3 and Receptacles: Specific Purpose 480 Volts, 20 Amperes, 3 and Receptacles: Specific Purpose 480 Volts, 30 Amperes, 3 and Receptacles: Specific Purpose 600 Volts, 30 Amperes, 3 on and Estimation of Water Insoluble Impurities in Refined Std Spec for Fluor Chrome Arsenate Std. Meth. for Chemical Analysis of Fluor Chrome Arsenate Std. Test Meth. for Filler Content of Std. Test Meth. for Nonvolatile Content of

ndicators (1971)

) (1962)

w (1974)

machine Screw (1974) Std. Analytical Meth. for Determining Meth. for Determination of Std. for Aircraft, Pan Head, Std. for Aircraft, Pan Head, mer Electronic Products (Radio, Television, Tape Recorder, mixes (/ Perchloric Acid Meth. of Analysis of Monocalcium trogalvanized Steel (Coil) (1)

$$
\text { Spec. for Determination of }
$$
Spec. for Determination of 1972) Std. for Packing, Preformed O-Ring g's, Pancreatin, Tween 80-60\% Alcohol, Versene, and Sodium pec. for Conversion Coating of Titanium Alloys (Fluoride.

73) chemistr/ Spec. for Photographic Grade Trisodium
Gustafson Meth. of Analysis for Original Ash in Meth. of Analysis of

of Analysis of Aluminum in Baking Powder, in Presence of $r$ Neutralizing Value of Acid Reacting Materials Other Than analysis for Free or Combined Tartaric Acid in Presence of

Meth. of Analysis for Neutralizing Value of Monocalcium ence and the Corresponding Composition of Gallium Arsenide
Petroleum (LP) Gases from Compositional Analysis (1973)

Petroleum (15 Theoretical Plate Column) (1973) ANSI Z11

Petroleum) Gas Cylinders (1973)

Petroleum) Gas Hose (1973)

Petroleum) Gas (1972) UL 567

Petroleum) Gas (1973)

(Petroleum) (1974)

Petroleum) (1974)

Pewter Alloys (1972)

Phantom (Medical Radiation-Radiology) (1973)

Phase and Related Faults) (1973)

Phase Converters (1972)

Phase Designations (Code) in Metallic Systems Based on

Phase Electrical System for Oil Field Service (1973)

Phase of Nuclear Power Plants (1973)

Phase of Nuclear Power Plants (1973

Phase of Nuclear Power Plants (1973)

Phase Rural Distribution Service for

Phase Systems (Confinement Test) (1973)

Phase Wye, 4 Pole, 4 Wire Locking Type (1972

Phase Wye, 4 Pole, 4 Wire Locking Type (1972)

Phase Wye, 4 Pole, 4 Wire Locking Type (1973)

Phase Wye, 4 Pole, 4 Wire Locking Type (1973)

Phase Wye, 4 Pole, 4 Wire Locking Type (1973)

Phase Wye, 4 Pole, 4 Wire Locking Type (1973)

Ety for Pigtail, Expa U

Safety Std

Safety Std. for UL

AL

APl

ASTM

Std ANSI FMS

Rec. ASAE

ASTM

APl

ANSl

for Cleaning of $F$ ANSI Tions of Inspect ANSI Voltage Circuit B IFEE

Std. M ASTM $\mathrm{Gs}$ and ANSI IGs and ANSI $G$ s and ANSI Gs and ANS1 /Gs and ANSI

Wye, 4 Pole, 5 Wire Locking, Grounding Type (1973

Phase Wye, 4 Pole, 5 Wire Locking, Grounding Type (1973

Phase Wye, 4 Pole, 5 Wire Locking, Grounding Type (1973

Phase Wye, 4 Pole, 5 Wire Locking, Grounding Type (1973

Phase Wye, 4 Pole, 5 Wire Locking, Grounding Type (1973

Phase Wye, 4 Pole, 5 Wire Locking, Grounding Type (1973

Phase (1960)

Phase (1971)

Phase (1973)

/or Load Tap Changing Transformers 138,00

ANSI

ANSI

ANSI

ANSI

ANSI

ANSI

ANSI

IEEE

Phase) (1972

Phase) (1973)

/Pping, Receiving, Storage and Handling ANSI

Phase, 3 Pole, 3 Wire Locking Type (1973)

Phase, 3 Pole, 3 Wire Locking Type (1973)

Phase, 3 Pole, 3 Wire Locking Type (1973)

Phase, 3 Pole, 3 Wire Locking Type (1973)

Phase, 3 Pole, 3 Wire Locking Type (1973)

Phase, 3 Pole, 3 Wire Locking Type (1973)

Phase, 3 Pole, 4 Wire Locking, Grounding Type (1973)

Phase, 3 Pole, 4 Wire Locking, Grounding Type (1973)

Phase, 3 Pole, 4 Wire Locking, Grounding Type (1973)

Phase, 3 Pole, 4 Wire Locking, Grounding Type (1973)

Phase, 3 Pole, 4 Wire Locking, Grounding Type (1973)

Phenol by Cloud Point Depression (1968) ANSI Z78.21

Phenol (1971)

Phenol (1971) ANSI O11.20

Phenol, Resorcinol, and Melamine Adhesives (1970)

Phenol, Resorcinol, and Melamine Adhesives (1970)

Phenol-40.5 (1968) ANSI Z78.22

Phenolphthalein Indicator, $1 \%$ Content of Rcagents and I Phenothiazine in Feeds and Feedstuffs (Cereal Chemistry Phillips Recess Full Threaded, Alloy Steel Machine Scre Phillips Recess, Short Thread, 160,000 psi Alloy Steel

Phonograph, etc) (1973)

/G Test (Packaging) for

Phosphate and/or Sodium Acid Pyrophosphate in Prepared

Phosphate Coating Weight on Hot Dip Galvanized and Elec

Phosphate Coating Weights on Steel (Coil) (1974)

Phosphate Ester Resistant (-65 Deg. F to +160 Deg. F) ( Phosphate Solutions; Isopropyl Alcohol Saturated with G Phosphate Type) (1973)

Phosphate, Dodecahydrate $\left(\mathrm{Na}_{3} \mathrm{Po}_{3} \cdot 12 \mathrm{H}_{2} \mathrm{O}\right) \mathrm{N} . \mathrm{NaOH}(19$ Phosphated and Freshly Mixed Self Rising Flour (Cereal Phosphates in Flour Improvers (Cereal Chemistry) (1962)

ANSI

ANSI

ANSI

ANSI

ANSI

II ASTM

ASTM

ASTM

ASTM

ASTM

ASTM

CR

NSA 600

NSA

623

AACCH 40.51

NCCA TB-II-3

NCCA TB-II.]

NSA

1611

AACCH 28.91

SAE AMS2486

ANSI PH4.234

AACCH $08-15$

Phosphates (Cereal Chem istry) (1962)

Phosphates (Cereal Chemistry) (1962)

Qualitative Meth AACCH $40-10$

Phosphates (Cereal Chem istry) (1962)

Qualitative Meth

Phosphate: Monohydrate

/Tative Meth. of

Phosphide Wafers (1973)
AACCH 40-10

AACCH $04-28$

AACCH 02.33

ASTM F358 
972)

olled Bar (1973)

Chemistry) (1962)

Chemistry) (1962)

Qualitative Meth of Analysis for

Quantitative Meth. of Analysis for

Meth. of Analysis for Total

Meth. of Analysis of Lipoid

(Common Name for the Pest Control Chemical 0.0-Dimethy] ame for the Pest Control Chemical O-Ethyl S.S-Dipropyl rol Chemical 0,0 Dimethyl 0-(3,5,6-Trichloro-2-Pyridyl) Std. Analytical Meth. for Determining Std. Analytical Meth. for Determining Std. Analytical Meth. for Determining

kes (1972)

$m$ the Corn Wel Std. Analytical Meth. for Determination of 1962) Colorimetric Acetate Ashing Meth. of Analysis of

$1962)$

1962)

69)

972) ed Techniques for Photography) (1973)

type, on Cellulose Ester Base (1973)

type, on Polyester Base (1973)

ers, $f \quad$ Std. for Dimensions for Unperforated and Perforated mensions of Containers for Packaging and Storage of Aeria Std. Practice for Storage of Processed Safet Std. Dimensions for Cores for Std. Meth. for Determining the Curl of Safety Std. for Tests for Flammability of Std. Meth. for Determining the Brittleness of for Determining the Dimensional Change Characteristics of nd Miscellaneous Uses (1973) Std. Dimensions fo Std, for Meth. for Determining the Resistance of ) (1972)

1972)

)$_{2}: 12 \mathrm{H}_{2} \mathrm{O}$ (Potassium Alum) (1972)

Ammonia) (1972)

$\mathrm{j}_{2} \mathrm{~S}_{3} \mathrm{O}_{3}(1973)$

(1972)

)$_{2}: 12 \mathrm{H}_{2} \mathrm{O}$ (Chrome Alum) (1972)

(1973)

dinitrilotetraacetic) Acid (EDTA) and lts/

ihydroxbenzene, Quinol, Hydroquinol) (197/

h. Hci) (Hydroxylam monium Chloride) (197/

- $\mathrm{H}_{2} \mathrm{SO}_{4}$ (Hydroxylammonium Sulfate) (/

$\mathrm{h}_{4} \mathrm{Nh}_{2}, \mathrm{HCl}(1972)$

(1972)

2)

${ }_{5} \mathrm{O}_{7}: 2 \mathrm{H}_{2} \mathrm{O}(1972)$

) (1972)

(1973)

3) (1972)

$\left.\mathrm{s}_{2} \mathrm{O}_{3}\right)$ and Crystalline $\left(\mathrm{Na}_{2} \mathrm{~S}_{2} \mathrm{O}_{3 .} .5 \mathrm{H}_{2} /\right.$

$\left.\mathrm{na}_{3} \mathrm{Po}_{4} \cdot 12 \mathrm{H}_{2} \mathrm{O}\right) \mathrm{N}$. NaOH (1973)

Test Meth. for the Selective Transmission of A

d Measuring Apertures and Related Quantities Pertaining to

Tone) (1972) Std. for Designation of Apertures of Std. Meth. for Determining Speed of Sid. Nomenclature for Parts of A

Std. for Dimensions for Resolution Test Target for Std. for Practice for Storage of Processed

y Units) (1973) nts (1972)
Std. Dimensions for Professional Std. Basic Construction Requirements for
Std. Spec. for

Std. Spec. for Std. Spec. for Spec. for Spec, for Std. Spec. for

Phosphor Bronze Wire for General and Spring Purposes (1 Phosphoric Acid in Bakıng Powders and Chemicals (Cerea Phosphoric Acid in Baking Powders and Chemicals (Cereal Phosphoric Acid in Dried Eggs (Cereal Chemistry) (1962) Phosphoric Acid in Dried Eggs (Cereal Chemistry) (1962)

Phosphoro dithioate S-Ester with 4-(Mercaptomethyl)Phosphorodithioate) (1973) /Td. for Ethoprop (Co Phosphorothioate Chlorpyrifos Methyl (1973)

Phosphorus Content in Corn Starch (1957)

Phosphorus Content of Corn Starch (1969)

Phosphorus Content of Corn Syrup (1957)

Phosphorus Deoxidized Copper Wire Bars, Billets, and Ca

Phosphorus in Corn Starch, Syrup and Sugar Obtained Fro

Phosphorus in Feeds and Feedstuffs (Cereal Chemistry) (

Phosphorus in Feeds and Feedstuffs (Cereal Chemistry) (

Phosphorus in Feeds and Feedstuffs (Cereal Chemistry) (

Phosphorus in Gasoline (1973)

Phosphorus in Water (1972)

Phosphorus in Yeast (Cereal Chemistry) (1962)

Phosphorus Trichloride (1972)

Phosphorus-32 and Iodine-131 for Medical U'sers (1951)

Phosphorus-32 (1962) ANSI N149

Phosphosilicate (1973)

Photo Instrumentation Glossary (Terms, Definitions) (19

Photochromism Colorfastness to Light (1972) ANSI LI4.27

Photocomposing Devices, or Both (Photography) (1972)

Photographic and X.Ray Nitrocellulose Films (1973)

(Photographic and X.Ray Nitrocellulose) (1973)

Photographic Contact Printers (1971)

Photographic Contact Printing of Roll Film Negatives (1

Photographic Darkroom Illumination (1971)

Photographic Effectiveness of Illuminants (1972)

Photographic Equipment Rated at 300 Volts or Less (1974

Photographic Film Exposure Guide (Also Contains Suggest

Photographic Film for Archival Records. Silver Gelatin

Photographic Film for Archival Records, Silver Gelatin

Photographic Film in Rolls, Including Leaders and Trail

Photographic Film on Spools (1972)

Photographic Film Other Than Microfilm (I971)

Photographic Film Rolls (Plastic, Wood or Metal) (1971)

Photographic Film (1971)

Photographic Film (1972)

Photographic Film (1973)

Photographic Films and Papers (1973)

Photographic Filins in Rolls for Recording Instruments a

Photographic Films to Abrasion During Processing (1972)

Photographic Filmstrip Projectors (1973)

Photographic Flashcubes (Battery Operated) (Photography

Photographic Grade Aluminum Chloride Solution $\mathrm{AlCI}_{3}$ (

Std Spec for Photographic Grade Aluminum Potassium Sulfate AlK(SO.

Std. Spec, for

Photographic Grade Ammonium Hydroxide, $\mathrm{Nh}_{3} \mathrm{OH}$ (Aqueou

Photographic Grade Ammonium Thiosulf ate Solution (Nh,

Photographic Grade Benzotriazole, $\mathrm{C}_{3 ;} \mathrm{H}_{4} \mathrm{NHN}$ : N $(1,2,3)$

Std Spec for Photographic Grade Chromium Potassium Sulfate CrKiSO

Photographic Grade Ethylenediamine $\mathrm{H}_{2} \mathrm{~N} . \mathrm{CH}_{2}, \mathrm{CH}_{2} . \mathrm{NH}$

Std. Spec. for

Std. Spec. for

Std. Spec. for

Std. Spec. for

Std. Spec. for

Std. Spec. for

Std. Spec, for

Std. Spec, for

Std. Spec. for
Std. Spec. for

Std. Spec. for Spec. for

Sid. Spec. for Spec. for

Photographic Grade Ethylenediaminetetraacetic (Eth

Photographic Grade Hydroquinone $\mathrm{C} . \mathrm{H},(\mathrm{Oh})_{n}$ (Para D

Photographic Grade Hydroxylam ine Hydrochloride, $\left(\mathrm{Nh}_{2} \mathrm{O}\right.$

Photographic Grade Hydroxylamine Sulfate, $\left(\mathrm{Nh}_{2} \mathrm{Oh}\right)_{2}$

Photographic Grade P-Aminophenol Hydrochloride $\mathrm{HOC}_{6}$

Photographic Grade Potassium Chloride, KCI (1972)

Photographic Grade Potassium Iodide, KI (1972)

Photographic Grade Potassium Metabisulfite $\mathrm{K}_{2} \mathrm{~S}_{3} 0_{5}$

Photographic Grade Potassium Permanganate, Kmn0 (197

Photographic Grade Sodium Chloride, Nacl (1972)

Photographic Grade Sodium Citrate, Dihydrate $\mathrm{Na}_{3} \mathrm{C}_{6} \mathrm{H}$

Photographic Grade Sodium Meta-Bisulfite $\mathrm{Na}_{2,} \mathrm{~S}_{2} 0(5$

Photographic Grade Sodium Sulfate, Anhydrous $\mathrm{Na}_{2} \mathrm{SO}_{4}$

Photographic Grade Sodium Sulfite, Anhydrous, Na., So

Photographic Grade Sodium Thiocyanate NaSCN (1972)

Photographic Grade Sodium Thiosulfate, Anhydrous ( $\mathrm{Na}_{2}$

Photographic Grade Trisodium Phosphate, Dodecahydrate

Photographic Lens (1969)

Photographic Lenses.(1971)

Photographic Lenses (1973)

Photographic Negative Materials (Monochrome, Continuous

Photographic Objective Lens (1971)

Photographic Optical Filter Sizes (1972)

Photographic Optics (1972)

Photographic Plates (1972)

Photographic Printing Frames (1972)

Photographic Sheet and Roll Films (SI and U.S. Customar

Photographic Slide Projectors (1973)

Photographic Sound Record on Super 8 Motion Picture Pri
ASTM B159

ASTM B103

AACCH 04.10

AACCH 04.1I

A ACCH 04-13

AACCH 20.02

ANSI K62.134

ANSI K62.133

ANSI K62.130

CR B. 46

CR C.46

CR E.50

ASTM $\quad$ B379

AACCH 40.55

AACCH 40.57

AACCH 40.58

ASTM D3231

ASTM D515

$\mathrm{AACCH} \quad 40.56$

MCA SD27

NCRPM R9

ASTM E182

ASTM D2742

SMPTE *1

AATCC 139

ANSI PHI.44

ICBO UBCS48. ]

ICBO UBC *3.48

ANSI PH3.45

ANSI PH 3.9

ANSI PH2.22

ANSl $\mathrm{PH} 2.3$

$\mathrm{UL}$

ANSI

ANSI

ANSI

ANSI

ANS1

ANSI

ANSI

ANS

UL

ANSI

I22

122
PH2.7

PHI.28

PH1.41

PHI.10

PH 1.46

PH 1.43

PHI.13

PH1. 29

105

PHI.31

PH1.32

PH1.30

PH4.35

PH3.28

PH3.51

PH4. 154

PH4. 150

PH4.232

PH4.252

PH4.204

PH4.151

PH4. 188

PH4. 185

PH4.104

PH4.126

PH4.187

PH4. 186

PH4.129

PH4.202

PH4.20I

PH4.277

PH4.301

PH4.203

PH4. 179

PH4. 276

Pli4. 175

PH4.275

PH4. 177

PH4.250

$\mathrm{PH} 4.234$

PH3.37

PH3.29

PH3.33

PH2.5

PH3.25

PH3. 17

PH3.50

PH 1.45

PH3. 15

PH 1.18

$\begin{array}{ll}\text { ANSI } & \text { PH3.65 } \\ \text { ANSI } & \text { PH22.182 }\end{array}$ 
Std for Illumination Tests for Specifying Construction Progress $s$ of Metals and Alloys (Including Recommended Practice for h. for Determining Speed of Color Reversal Films for Still era Films Intended for Direct Projection in Motion Picture Std. for Shutter Tests for Still Picture Cameras dimensions for Photographic Flashcubes (Battery Operated) imensions of 126 Size Cartridges, Film, and Backing Paper

s for Phototypesetting or Photocomposing Devices, or Both

ilm Exposure Guide (Also Contains Suggested Techniques for dimensions of $35 \mathrm{~mm}$ Motion Picture Camera Aperture Images gallium Arseni/ Std. Meth. of Test for Wavelength of Peak for Preparation of Specifications for the Procurement of opper Base Alloys (1972) Std. Meth. of Test for Nickel on SteeI by of Test for Silica in Fluorspar by the Silico Molybdate otography) (19) Std. for Dimensions for Film in Rolls for $\mathbf{n}$ Length in Silicon by Measurement of Steady State Surface Aircraft Instrument Stds. (Wording, Terminology, Std. Meth of Test for Std. Meth. for Chemical Analysis of Copper Rec. for Measurement of Neutron Flux and Spectra for hange Resins (1972) Std. Meth. of Test for Std. Meth. for Sampling Leather for (1972) Std. Meth. for the Designation of Mercury Lamps: Mercury Lamps (1972) mp (1973)

d. Definitions of Terms Relating to Lithologic Classes and meth. for Microscopical Determination of Volume Percent of General Extensigraph Meth. for

Structural Relaxation Meth of Extensigraph Analysis of Extensigraph Meth. for Quality of Nonfat Dry Milk in dry Milk Solids to Change Mixing Characteristics of Flour ing as-is Farinograph Absorption to $14.0 \%$ Moist/ Meth. of Std. Safety Color Code for Marking Std. Guideline for the Std. for

ity in Air of Manufactured Carbon and Graphite Articles by ng and Carbon Steel Tooth Lock Wash/ Std. for Dimensions, rom Compositional / Std. Meth. for Calculation of Certain de Material Used in Optical Emission Spectroscopy Based on si $K 67.10$ Meth. for Std. Meth. of Compound and Sample Preparation for Mass Spectrometric, and Spectrochemical Analysis Of, and apers Using the Interfiber Bond $\mathrm{T} /$ 972) est Meth for Coating Std. Meth. of Test for Std. for 1972) Std

std. for Safety for Implosion Protected Cathode Ray Tubes Std. Dimensions of $35 \mathrm{~mm}$ Motion Std. for Shutter Tests for Still

Picture Sizes for RoIl, $35 \mathrm{~mm}$ Film, and $126 \mathrm{~mm}$ Film Stil re Time Markings for Variable Speed Shutters Used in Stil ools for Recording Instruments and for Microfilm and Stil Std. Dimensions for $35 \mathrm{~mm}$ Motion Std. Dimensions for $16 \mathrm{~mm}$ Motion 973) ons for Printed Area in Super 8 Printing on $16 / 8 \mathrm{Mm}$ Motion
Std. for Nomenclature for Motion
Std. Spec. for Projector Usage of $16 \mathrm{~mm}$ Motion s (I971) ons for Printed Area in Super 8 Printing on $16 / 8 \mathrm{Mm}$ Motion
Std. for Nomenclature for Motion
Std. Spec. for Projector Usage of $16 \mathrm{~mm}$ Motion Std. Spec. for Projector Usage of $16 \mathrm{~mm}$ Motion Uniform Building Code Std. for Cellulose Nitrate Motion
Uniform Fire Code: Cellulose Nitrate Motion nsions of Projectable Anamorphic Image Area on $5 \mathrm{~mm}$ Motion
Std. for Location of Super 8 Printed Area on $16 \mathrm{~mm}$ Motion printed Area in Optical Reduction Printing on $35 \mathrm{~mm}$ Motion a in Optical Reduction or Contact Printing on $35 \mathrm{~mm}$ Motion Std. Dimensions for $32 \mathrm{~mm}$ Motion Std. Dimensions for $32 \mathrm{~mm}$ Motion

olor Camera Films Intended for Direct Projection in Motion dimensions for Photographic Sound Record on Super 8 Motion for Medium Prefocus Base Down Type, Single Contact Motion Uniform Building Code: Motion Uniform Fire Code: Motion Std. for Motion Std.

ll Picture Cameras (1972) uipment (Flyback Transformer, Multiplier, Deflection Yoke, for Registered Screen Dimensions for Monochrome Television Meth. of Analysis for Baking Quality of Std. Rec. Pract for Preparation of eets (1973) als Except Chocolate Products and ysis for All Baked Materials Except Chocolate Products and Columns, Std. Classification of

ng Facility PIans: International and Domestic-Trap, Clay Std Meth of Analysis of Magnesium Silic ate Std. Meth. of Analysis of Aluminum Silicate
Photographic Still Projection Equipment (I971)

Photographs (1967)

Photography as Applied to Metallography) (1962) ANSI Z3

ANSI

CSI

ASTM

Photography (1972)

Photography (1973)

Metric Exposure and Evaluation Met ANS

$/ \mathrm{mm}$ and Super 8 Reversal Color Cam ANSI

ANSI

(Photography) (1972)

Std. for ANSI

Photography) (1972)

td. for D ANSI

Photography) (1972)

Photography) (1973)

for Dimensions for Film in Roll ANSI

Std. Photographic F ANSI

Photoluminescence and the Corresponding Composition of

Photomasks (1972)

\section{ASTM}

Photometric Analysis (1972)

Std. Rec. Pract ASTM

ASTM

Photometric Meth. for Chemical Analysis of Copper and C

ASTM

(Photometric) Meth. (1972)

Std. Meth ASTM

Phototypesetting or Photocomposing Devices, or Both (Ph

Photovoltage (Electronics) (1973) ITy Carrier Diffusio

ANSI

Phraseology, Environment and Design Stds for (1972)

Phthalate Ester Color of High Gravity GIycerin (1973)

Phthalocyanine Blue and Green Pigments (1973)

Physical and Biological Applications (1960)

Physical and Chemical Properties of Particulate Ion Exc

Physical and Chemical Tests (1971) ALCA Jl

Physical and Electrical Characteristics of $40 \mathrm{~W}$ att, H45

Physical and Electrical Characteristics of $50 \mathrm{Watt}, \mathrm{H} 46$

Physical Characteristics of T.2 Telephone Slide Base La

Physical Components of Coal (1972)

Physical Components of Coal (1972)

Physical Dough Tests of Flour (Cereal Chemistry) (1962)

Physical Dough Tests of Flour (Cereal Chemistry) (1962)

Physical Dough Tests (Cereal Chemistry) (I962)

Physical Dough Tests) (Cereal Chemistry) (1962)

Physical Dough Tests: Approximate Corrections for Chang

Physical Hazards (1971)

Physical Inspection of Wood Poles in Service (1972)

Physical Inventories of Nuclear Materials (1972)

Physical Measurements (1969) ANSI Kod

Physical Properties and Meth of Test for Helical Spr

Physical Properties of Liquefied Petroleum (LP) Gases F

Physical Properties (1974)

1. for Selection

Physical Test. of Ouicklime and Hydrated Lime (1971) an

Physical Test. of Rubber Products (1972) ANSI J1.1

Physical Tests On, Beryllium Oxide Powder (1972) ANSI N

Pick Tests for Determining Surface Strength of Coated P

Pickup Efficiency of Residual Aerosol Insecticides (197

Picloram (Common Name for the Pest Control Chemical) (1

Pictorial Markings for $\mathrm{Handling}$ of Nondangerous Goods

(Picture and Display) for Television Receiving Appliance

Picture Camera Aperture Images (Photography) (1974)

Picture Cameras (Photography) (1972)

Picture Cameras (1972)

Picture Cameras (1972)

Picture Cameras (1972)

Picture Film Perforated Super $/ \mathrm{M} 100 \mathrm{Ft}$, and $70 \mathrm{~mm}$

Picture Film Perforated Super 8, (1.4) (1973)

Picture Film Perforated 1.4 (1971)

Picture Film Used in Studios and Processing Laboratorie

Picture Film (1973)

Picture FiIm (1973)

Picture Film (1973)

Picture Film (2.35:1) Aspect Ratio (1971)

Picture Film, Perforated Super 8 (1-3) (1973)

Picture Film, Perforated 2R-1664 (1-0) (1973)

Picture Film, Perforated 5R.1667 (1-3-5-7-0) (1973)

Picture Film, 2R (1974)

Picture Film, 4R (1974)

Picture Photography (1973)

Picture Prints (1972)

Picture Projection Lamps (1972)

Picture Projection Rooms (1973)

Picture Projection (1973)

Picture Raw Stock Identification and Labeling (1973)

Picture Sizes for Roll, 35 $\mathrm{mm}$ Film, and $126 \mathrm{~mm}$ Film Sti

ANSI

ANSI

Uper 8 ANSI

IE ANSI

ANSI

Picture Tube Neck Components) (1974)

Er Electronic Eq U

Pie Flour (Cereal Chemistry) (1962)

Pieces from Rubber Vulcanizates Other Than Std. Test Sh

al C ANSI

Std. ANSI

Std. ANSI

ICBO

ICBO

ANSI

ANSI

Piecrust (CereaI Chemistry) (1962)

Piers (1969)

Piers, Pilasters, and Buttresses (Tech. Notes) (1962)

Ping Meth. of AnaI

Pig Tin (1972) ANSI H42, 1

Pigeon, and Skeet: Ammunition and Target Spec. (1973)

Suggested

$A \mathrm{CCH}$

ASTM

AAC

BIA

ASTM

NRA

ASTM

ASTM

PH3.47

1018

PH2. 21

PH22.146

PH3.48

PH3.5I

PHI 40

$\mathrm{PH} 1.44$

PH2. 7

PH22.59

F358

F93

C715

E62

E463

PH1.44

F39I

AIR818B

D1728

D.3256

R23

D2187

D28I 3

C78. 1323

C 78.1324

C 78.397

D2796

D2799

CCH 54.10

54-11

CCH 54-12

$54-20$

54.29
753.1

253.1

N15.3

C559

B18.21.1

D2598

E504

C 472

C 110

D 15

C699

UM.534

D3093

K62.127

MH6.1

492.8

PH22.59

PH 3.48

PH3.39

PH3.32

PHI.33

PH22.165

PH22.168

PH22.153

PH22.56

PH22.10

UBC 48 -2

UFC*2ART5

PH22.106

PH22.181

PH 22.179

PH22.180

PH22.141

PH22. 142

PH22. I46

PH22.182

PH22.85

UBC $* 3.40$

$\mathrm{UFC} * 2 \mathrm{ART} 32$ 
y Measuring the Specific Resistance of the Leachate of the

d. Meth. of Test for Volume Nonvolatile Matter in Clear or Std. Meth. of Test. Clear and Std. Meth. for Evaluation of and Test Formula for ectrical Insulation Plastic Tubing, Irradiated Polyolefin, achate of / Std. Meth. of Test for Water Soluble Salts in Meth. of Analysis of Std. Meth. of Test. Para Red and Toluidine Red d. Meth. of Test for Solvent Extractable Material in Black Std. Meth. for Chemical Analysis of White Lead Std. Meth. for Analysis of White Zine

Chemical Analysis of Copper Phthalocyanine Blue and Green al Analysis for Aluminum Oxide Content of Titanium Dioxide Std. Meth. of Chemical Analysis of White
Std. Spec. for Titanium Dioxide Std. Meth. of Test for Coarse Particles in $s$ for LP (Liquid Petroleum) Gas Cyli/ Std. for Safety for Reinforced Brick Masonry Columns and Columns, Piers, Uniform Fire Codk: High Std. for Pressure Treated st Meth. for Establishing Design Stresses for Round Timber Std. Spec. for Round Timber Uniform Building Code Std for Round Timber for Preservative Treatment by Pressure Processes of Timber c. for Coal Tar Creosote for the Preservative Treatment of ood and Components, Timber, Preservative Treatment, Poles,

Spec. for High Strength Low Alloy Steel H.Piles and Sheet

s. Structural Shapes, Rolled Floor Plates, and Steel Sheet rements for Delivery of Rolled Steel Plates, Shapes, Sheet

ricks, Hoists, Hooks, Jacks, and Slings: Portal, Tower and Textile Fabrics (1972) ANSI L14./ Std. Meth. of Test for nal Tel Std. Performance Requirements for Woven Sheet and e Requirements for Durable Press (No lron) Woven Sheet and Performance Requirements for Woven Decorative Bedspreads. for Electronic Equipment on Boats (Audio Device, Automatic or Spectrochemical Analysis of Admiralty Metal by the Cast d. for Electrical Plugs, Receptacles and Connectors of the Std. for Drive Std. for Std. Spec. for Communication and Signal

73)

Std. Wet Process Porcelain Insulators (Apparatus, Cap and Std. for Sid. for Std. for Std. for Std. for Std. for Std. for Std. for Std. for Std. for Std. for Std. for Std. for Std. for Std. for Std. for Std. for Std. for Std. for Std. for Std. for Std. for Std. for Std. for Std. for Std. for Std. for Std. for Std. for

sion Head, Pull Type (1973)

- Fir (North), Ponderosa and Western White Pines, Spruce -

White Pine, and Hemlock-Tamarack; Balsam Fir; Northern Std. for Ponderosa Std. for Ponderosa ines; Engelmann Spruce; Western Cedar and Hemlock; Subal ooling Towers (1971) Uniform Building Code Std. for Southern
Sid. for Lodgepole Uniform Building Code Std. for Southern
Sid. for Lodgepole

th Thermal Process (1973) (North), Eastern Hemlock-Tamarack (Nortb), Eastern White

ck Uniform Building Code Std. for Eastern White and Red aminated Members and Laminations Before Gluing of Southern nated Timbers: Douglas and Hem Fir, Southern and Lodgepole ne (North), Hem-Fir (North), Ponderosa and Western White
Pigment (1973)

( for Water Soluble Salts in Pigments B ASTM

Pigmented Coatings (1973)

Pigmented Lacquers (1973)

Pigmented Styrene-Butadiene Rubbers (SBR) (1973)

Pigmented, Flexible, Heat Shrinkable (1973)

Pigments by Gardner-Coleman Meth. (1968)

Pigments by Measuring the Specific Resistance of the $\mathrm{L}$

Pigments in Flour, Semolina, Macaroni and Wheat (1962)

Pigments (Toners) (1973)

Pigments (1972)

Pigments (1973)

Pigments (1973)

Pigments (1973)

Pigments (1973)

Pigments (1973) ANSl K15.1

Pigments (1973) ANSI K45.

Pigments, Pastes, and Paints (1972) ANSI K42.1

Pigtail, Expansion Coil, and Flexible Connector Fitting

Pilasters (Tech. Notes) (1965)

Pilasters, and Buttresses (Tech. Notes) (1962)

Piled Combustible Stock (Storage) (1973)

Piles and Timbers in Marine Construction (1973)

Piles (1970)

Piles (1973)

Piles (1973)

Piles (1973)

Piles, Poles, and Timbers for Marine, Land, and Fresh W

Piles, Spaced Columns, Joints, Screws, Bolts, Connector

Piling for Use in Marine Environments (1974)

Piling (1970) /El Products Manual) Carbon St

Piling, and Bars for Structural Use (1972) ANSI G24.4

Std. Meth. for

IC. for El SAE

Pillar Cranes (1973)

Safety Std. for Crane

Pilling Resistance and Other Related Surface Changes of

Pillowcase Fabrics Other Than Durable Press (Institutio

Pillowcase Fabrics (Institutional Textile) (1973)

Pillows, and Bolster Fabrics (Institutional Textiles) (

Pilot, Depth Determination Device and Fish Finder, Lora

Pin and Point to Plane Techniques (1973) 3)

Pin and Sleeve Type for

Pin Pulley Guard (1972)

Pin Type Lime Glass Insulators (R1970)

Pin Type) (1971)

Pin, Quick Release, Positive Locking. Double Acting (19

Pin, Quick Release, Positive Locking, Single Acting (19

Pin, Straight, Headless (Dowel) (1973)

Pin, Swage Locking, A.286 Cres, Protruding Head, Shear

Pin, Swage Locking, A.286 Cres, Protruding Head, Shear,

Pin, Swage Locking, A-286 Cres, 100 Deg. Head (MS24694)

Pin, Swage Locking, A.286 Cres, 100 Deg. Shear Head, St

Pin, Swage Locking, A.286 Cres, 100 Deg. Shear/Tensio

Pin, Swage Locking, A-286, 100 Deg. Head (MS20426), Ten

Pin, Swage Locking, Aluminum Alloy, Protruding Head, Te

Pin, Swage Locking, Aluminum Alloy, 100 Deg. Head (Ms20

Pin, Swage Locking, Aluminum Alloy, 100 Deg. Head (Ms20

Pin, Swage Locking, Aluminum Alloy, I00 Deg. Head (Ms24

Pin, Swage Locking, Aluminum Alloy, 100 Deg. Heao (Ms24

Pin, Swage Locking, Std. and Oversize, Protruding Head,

Pin, Swage Locking, Steel, Protruding Head, Shear Stump
Pin, Swage Locking, Steel, Protruding Head, Tension, St

Pin, Swage Locking, Steel, Std. and Oversize, Protrudin

Pin, Swage Locking, Steel, Std. and Oversize, $100 \mathrm{Deg}$.

Pin, Swage Locking, Steel, Std. and Oversize, $100 \mathrm{Deg}$.

Pin, Swage Locking, Steel, Std. and Oversize, 100 Deg.

Pin, Swage Locking. Steel, 100 Deg. Crown head, Tension

Pin, Swage Locking, Steel, 100 Deg. Head (MS20426), Ten

Pin, Swage Locking, Steel, 100 Deg. Head (MS24694), Ten

Pin Swage Locking, Steel, SI Deg. Shear Head, Stump T

Pin, Swage Locking, I08 KSI Steel, 100 Deg. Shear / Ten

Pine-Fir, Western Cedars (North) (Lumber) (1973)

Pine and White Cedar Lumber (1973)

Pine Doors (1958)

Pine Doors (1973)

Pine Fir; and Mountain Hemlock (Lumber) (1973)

Pine Lumber Spec. for Application in Industrial Water C

Pine Lumber (1973)

Pine Wood Poles Preservative Treatment by the Full Leng

Pine (North), Hem-Fir (North), Ponderosa and Western

Pine, and Hemlock-Tamarack; Balsam Fir; Northern Pine

Pine, Eastern Spruce and Hemlock, Balsam Fir and Tamara

Pine, Pacific Coast Douglas Fir and Western Hemlock by

Pine, Western Larch, California Redwood and Hardwood Ti

Pines, Spruce-Pine-Fir, Western Cedars (North) (Lum
St ASTM

ASTM D 3186

ASTM

ASTM

CH $14-50$

ASTM D970

St ASTM

ASTM

ASTM

D2448

MS3636D

D305

D3280

D3256

D2701

$\begin{array}{ll}\text { ASTM } & \text { D2701 } \\ \text { ASTM } & \text { D34 }\end{array}$

ASTM D476

ASTM D 185

UL $\quad 569$

BlA I7I

BlA

CBO

AWPA

12

FC 2ART35

Tent. Te ASTM D2899

ASTM D25

Std. AWPA C3

ASTM D390

ICBO UBC $* 3.25$

Std. ASTM A690

2

A ISI

ASTM

ANSI

ASTM

ANSI

IAnc ANSI

ANSI

ABYC

F ASTM

IFI

NSA

ASTM

ANSI

NSA

NSA

NSA

NSA

NSA

NSA

NSA

NSA

NSA

NSA

NSA

NSA

NSA

NSA

NSA

NSA

NSA

NSA

NSA

NSA

NSA

NSA

NSA

NSA

NSA

NSA

NSA

NSA

NSA

/M ICBO

ICBO

USC

NWMA

/Pole P ICBO

CTI

ICBO

AWPA

ICBO

ICBO

AWPA

ICBO

ICBO

A6

B 30.4

D1375

L24.2. 12

L24.2.13

L24.1.2

A2 i

E486

FBll

427

D879

1353.66

I 333.46

607

7014

6984

6965

6946

6974 
h; Hem-Fir; Ponderosa, Idaho White, Sugar, and Lodgepole Std. Spec. for Carbon and Alloy Steel Forgings for Fine Pitch on Center Face Gears for 20 Deg. Involute Spur Std. Spec. for Fixation Std. for Gages. Std. for Std. Clevis Std Spec for Fixation Std. for Headed Drilled Std. for Cotter

Std. Clevis Pins and Cotter Carbon Steel Tubular Spring

ors, Bolts, Lag Screws, Plates, Nails and Spikes, Washers, ransmission) (1972)

Std. for 700 Class Std. for Blower

rene (ABS) Pipe and Fittings to Poly(vinyl Chloride) (PVC) Cements for Joining Acrylonitrile Butadiene Styrene (ABS) Rec. Pract, for Heat Joining of Thermoplastic Std. Spec. for Process Glass

Std. for lnstallation PVC Building Drain Waste and Vent for Acrylonitrile Butadiene Styrene Drain. Waste and Vent ylonitrile Butadiene Styrene Plastic Drain, Waste and Vent Std. Spec. for Poly(vinyl Chloride) (PVC) Sewer Std. Spec. for Cast lron Soil

Spec. for Solvent Cements for Styrene Rubber (SR) Plastic Neoprene Rubber Gaskets for Hub and Spigot Cast Iron Soil Std Spec. for Cast lron Soil or Rubber Gasket Joints for Cast and Ductile Iron Pressure Std. Spec. for Reinforced Epoxy Resin Gas Pressure Std Spec for Type PSP Poly(vinyl Chloride) (PVC) Sewer Std. Spec. for Type PSM Poly(vinyl Chloride) (PVC) Sewer Safety Std. for Gasketed Joints for Cast lron Spec. for Styrene Rubber Plastic Drain and Building Sewer for Solvent Cements for Poly(vinyl Chloride) (PVC) Plastic -Butadiene-Styrene (ABS) Plastic Drain, Waste, and Vent ment for Acrylonitrile-Butadiene-Styrene (ABS) Plastic king Solvent Cemented Joints with Polyvinyl Chloride (PVC)

I Poly(vinyl Chloride) (PVC) Plastic Drain, Waste and Vent

or Acrylonitrile-Butadiene-Styrene (ABS) Plastic Sewer spec. Data for Hubless Cast Iron Sanitary System (Plumbing Std. Spec for Aluminum Alloy Seamless Std. Spec. for Nickel Seamless

Std. Spec. for Nickel Chromium Iron Alloy Seamless kel-lron-Chromium-Molybdenum-Copper Alloy Seamless c. for Nickel Chromium Molybdenum Columbium Alloy Seamless chromium lron Columbium Molybdenum Tungsten Alloy Seamless Std. Spec. for Seamless Copper Nickel Std. Spec. for Aluminum Alloy Extruded Structural Std. Spec. for Seamless Copper Bus Std. Spec. for Copper Silicon Alloy Seamless Std. Rec. Pract. for Flaring Polyolefin Rec. for Coiling Polyethylene Plastic fusion Polyethylene (PE) Plastic Fittings for (PE) Plastic Performance Requirements for test for Quality of Extruded Poly (Vinyl Chloride) Plastic
Std. for Protective 72) Thread Compounds (For Casing. Tubing and Line
troleum) Gas (1972) UL 567 Std. Meth. of Test for Time to Failure of Plastic std. Symbols for Dimensions of Plastic std. Spec. for Gray Iron Castings for Valves, Flanges, and - Spec. for Socket Type Poly(vinyl Chloride) (PVC) Plastic Std. Spec. for Butt Fusion Polyethylene (PE) Plastic ket Type Acrylonitrile-Butadiene-Styrene (ABS) Plastic Spec. for Socket Type Poly(vinyl Chloride) (PVC) Plastic Std. Spec. for Butt Fusion Polyethylene (PE) Plastic ket Type Acrylonitrile-Butadiene-Styrene (ABS) Plastic threaded Acrylonitrile-Butadiene-Styrene (ABS) Plastic std. Spec. for Threaded Poly(vinyl Chloride) (PVC) Plastic Std. for Steel

High Temper/S Std. Spec. for Forged or Rolled Alloy Steel std. Spec. for Laminated Wall Bituminized Fiber Perforated std. Spec. for Laminated Wall Bituminized Fiber Perforated Std. Spec. for Electric Fusion Welded Steel

Ided Large Diameter Austenitic Chromium Nickel Alloy Steel Std. Spec. for Aluminum Alloy Seamless Extruded Tube and d B/ (1973) Std. Spec. for Homogeneous Solid W all and Perforated Spec. for Electric Fusion Welded Steel

G36.49

) ANSI B 125.25 Std. Spec, for Electric Fusion Welded Steel Std. Spec. for Metal Arc Welded Steel Std. Spec. for Seamless Austenitic Steel Std. Spec. for Centrifugally Cast Carbon Steel ectric Fusion Welded Autenitic Chromium Nickel Alloy Steel Std. Spec, for Centrifugally Cast Austenitic Steel Std. Spec. for Seamless Carbon Steel
Pines; Engelmann Spruce; Western Cedar and Hemlock; Sub Pinions and Gears for Reduction Gears (1972) ANSI G55.8 Pinions (1973)

Pins-Knowles and Hagie Types (1973)

Pins and Collar Inspection (1973)

Pins and Collars, Swage Locking (1973)

Pins and Cotter Pins (1972)

Pins and Wires (1973)

Pins for Farm Equipment and Machinery (1973)

Pins (Split) (1972)

Pins (1972)

Pins (1973)

Pins. Dowels, Joints, Split Rings and Framing Anchors)

Pins. Spring, Slotted and Coiled, Heavy Duty (1973)

Pintle Chains, Attachments, and Sprocket Teeth (Power T

Pipe and Connecting Flange Dimensions (1972

Pipe and Fittings for Nonpressure Applications (1972)

Pipe and Fittings to Poly(vinyl Chloride) (PVC) Pipe an

Pipe and Fittings (1967) ANS1 B72.17

Pipe and Fittings (1970)

Pipe and Fittings (1971)

Pipe and Fittings (1971)

Pipe and Fittings (1971)

Pipe and Fittings (1972)

Pipe and Fittings (1972)

Pipe and Fittings (1972)

Pipe and Fittings (1972)

Pipe and Fittings (1972) ANSI All2.5.1

Pipe and Fittings (1972) ANSI A2l.11

Pipe and Fittings (1973)

Pipe and Fittings (1973)

Pipe and Fittings (1973)

Pipe and Fittings (1973)

Pipe and Fittings (1973)

Pipe and Fittings (1973) ANSI B72.16

Pipe and Fittings (1973) ANSI B72.18

Pipe and Fittings (1973) ANSI B72.23

Pipe and Fittings (1973) ANSI K65.55

Pipe and Fittings (1973) ANSI K65.56

Pipe and Fittings (1973) ANSI K65.59

Pipe and Fittings) (1972)

Pipe and Seamless Extruded Tube (1973) ANSI H38.7

Pipe and Tube (1970) ANSI H34.l

Pipe and Tube (1970) ANSI H34.3

Pipe and Tube (1972) ANSI H34.16

Pipe and Tube (1972) ANSI H34.20

Pipe and Tube (1972) ANSI H34.21

Pipe and Tube (1973) ANSI H26.4

Pipe and Tube (1973) ANSI H38.16

Pipe and Tube (1974) ANSl C7.26

Pipe and Tube (1974) ANSI H26.3

Pipe and Tubing (1972)

Pipe and Tubing (1972

Pipe and Tubing (1973)

Pipe Applied Atmospheric Type Vacuum Breakers (1970)

Pipe Coatings (1971)

Pipe Connections in High Pressure Oil Well Service) (19

Pipe Connectors for Flammable Liquids and LP (Liquid PE

Pipe Constant Internal Pressure (1974) ANSI B72.6

Pipe Fittings (1968) ANSI K65.57

Pipe Fittings (1973)

Pipe Fittings, Schedule 40 (1973)

Pipe Fittings, Schedule 40 (1973) ANSI K65.160

Pipe Fittings, Schedule 40 (1973) ANSI K65.164

Pipe Fittings, Schedule 80 (1973)

Pipe Fittings, Schedule 80 (1973) ANSI K65.159

Pipe Fittings, Schedule 80 (1973) ANSI K65.163

Pipe Fittings, Schedule 80 (1973) ANSI K65.165

Pipe Fittings, Schedule 80 (1973) ANSI K65.166

Pipe Flanges, Flanged Valves and Fittings (1973)

Pipe Flanges, Forged Fittings, and Valves and Parts for

Pipe for Agricultural, Land, and General Drainage (1973

Pipe for Airport and Road work Drainage (1973)

Pipe for Atmospheric and Lower Temperatures (1972)

Pipe for Corrosive or High Temperature Service (1972) a

Pipe for Gas and Oil Transmission and Distribution Pipi

Pipe for General Drainage (Land Reclamation, Curtain an

Pipe for High Pressure Service at Moderate Temperatures Pipe for High Pressure Service (1972A) ANSI B125.4

Pipe for High Pressure Transmission Service (1971) ANSI

Pipe for High Temperature Central Station Service (1973

Pipe for High Temperature Service (1972)

Pipe for High Temperature Service (1972)

Pipe for High Temperature Service (I972) ANSI B I25

Pipe for High Temperature Service (I972A) ANSI B125.30
ICBO

ASTM

AGMA

ASTM

NSA

NSA

ANSl

ASTM

ASAE

SAE

ANSI

$\mathrm{SAE}$

WWP

NSA

ANSI

ASAE

ASTM

ASTM

ASTM

ASTM

IAPMO

IAPMO

CISPI

Std. ASTM

d. Spec. for CISPI

ASTM

Std. F AWWA

ASTM

ASTM

ASTM

UL

Std. ASTM

Sid Spec ASTM

R Acrylonitrile ASTM

for Solvent Ce ASTM

for Ma ASTM

Spec. Fo ASTM

td. Spec. F ASTM

CISPI

ASTM

ASTM

ASTM

ASTM

Std. Spe ASTM
or Nickel ASTM

ASTM

ASTM

ASTM

ASTM

ASTM

SPI

ASTM

ASSE

/Eth. of ASTM

API

ANSI

ASTM

ASTM

ASTM

Std ASTM

ASTM

lor Soc ASTM

Std ASTM

ASTM

lor Soc ASTM

1. for ASTM

ASTM

ANSI

ASTM

ASTM

ASTM

ASTM

ASTM

ASTM

ASTM

ASTM

ASTM

ASTM

ASTM

ASTM

ASTM

ASTM

ASTM

UBCS25.4

A29l

203.03

F.368

1563

1413

Bl8.8.1

F366

S 226.2

J487A

B18.8.I

AMS7205D

*31. 1

561

29.13

S347T

D3138

D3138

D2657

C 599

IS9

PSI7

D2729

HS

D3I22

HSN

A74

C1I

D3033

D. 3034

194 
Std. Spec. for Centrifugally Cast Ferritic Alloy Steel Pipe for High Temperature Service (1973)

Std. Spec. for Seamless Ferritic Alloy Steel Std. Spec. for Concrete

Std Spec for Seamless and Welded Steel

Dipped Zinc Coated (Galvanized) Welded and Seamless Steel
Std. Spec. for Seamless Carbon Steel Std. Spec. for Seamless Carbon Steel
ogeneous Perforated Bituminized Fiber

Std. Spec. for Homogeneous Perforated Bituminized Fiber Std. Spec. for Electric Welded Low Carbon Steel Std. Spec. for Porous Concrete

Spec. for Fabricated Structural Steel

Std for Asbestos Cement Pressure

for Polyethylene Encasement for Gray and Ductile Cast lron Installation Std. for Solvent Cemented PVC ystem Hydraulic Problems (1973)

Rec. for

shers, Alarms, Sandpipes), Building Construction, Basement spec. for Asphalt Type Protective Coatings for Underground fabricated Reflective Insulation Systems for Equipment and

971) Std. Meth. of Test. Concrete

3) ANSl /

Rec. for Water Flow / Pressure Requirements for Std. for Safety for Alarm Valves for Use in Wet Std. Spec. for Homogeneous Perforated Bituminous Fiber nd Maintenance of the Reliable Model B Accelerator for Dry Std. Spec. for Cellular Glass Block and Std. Ferrous Pipe Plugs, Bushings, and Locknuts with nd Thread lnspection of Petroleum Casing. Tubing, and Line Std. Spec. for Nickel-lron-Chromium Alloy Seamless Rec. Pract. for Calculating Stress in Plastic Rec. for Operation of Grimes AB Dry Operational Rec. for Globe Dry Rec. for Operation of Crowder Dry Dry

Std. Spec, for Nonreinforced Concrete Irrigation Spec for Casing. Tubing, and Drill Spec. for High Strength Casing, Tubing, and Drill Spec. for Line Spec. for High Test Line Spec, for Spiral Weld Line Spec. for Thermoplastic Line t. for Care and Use of Reinforced Thermosetting Resin Line Std. Spec. for Cellulose Acetate Butyrate (CAB) Plastic Std. Spec. for Polyethelene (PE) Plastic d. Spec. for Biaxially Oriented Polyethylene (PEO) Plastic Std. Spec. for Polybutylene (PB) Plastic Std Spec for Polybutylene (PB) Plastic Std. Spec. for Poly(vinyl Chloride) Plastic pec. for Acrylonitrile-Butadiene-Styrene (ABS) Plastic Std. Spec. for Polyethylene (PE) Plastic Safety Std for Stee

Rec. for Cast in Place Nonreinforced Concrete Sid. Meth. of Thermal Shock Test on Glass Std for Air Brake Tubing and

Spec for Cast in Place Nonreinforced Concrete r Reinforced Concrete Arch Culvert, Storm Drain, and Sewer

Tent. Spec. for Ultra High Test Heat Treated Line Spec. for Reinforced Thermosetting Resin Line Rec. Pract for Railroad Transportation of Line Std. Spec. for Filled Poly (Vinyl Chloride) (PVC) Sewer Guide Spec. for Cast Iron Soil Guide Spec. for Plastic

for Nickel Iron Chromium Silicon Alloy Seamless and Welded forced Concrete Elliptical Culvert, Storm Drain, and Sewer Std. Rec. Pract. for lnstalling Vitrified Clay Sewer Std. Meth. of Test. Clay

eneral Requirements for Specialized Carbon and Alloy Steel $r$ Underground lnstallation of Flexible Thermoplastic Sewer Std. Spec. for Electric Resistance Welded Steel

std. Spec. for Seamless and Welded Carbon Steel Water Well

for Compression Joints for Vitrified Clay Bell and Spigot

Spec. for Seamless and Welded Austenitic Stainless Steel

Std. Spec, for Concrete Sewer, Storm Drain, and Culvert

Std Spec for Reinforced Concrete Low Head Pressure

Std. Spec. for Perforated Concrete Under Drainage Sid. Spec. for Linings for Asbestos Cement Std. Spec. for Asbestos Cement Storm Drain Std Spec for Asbestos Cement Transmission Std. Spec, for Reinforced Plastic Mortar Sewer Western Woods Use Book: Wood Tanks, Vats, and spec. for Steel Wire, Hard Drawn for Prestressing Concrete ec for Compression Couplings for Vitrified Clay Plain End

c. for Reinforced Concrete Culvert, Storm Drain, and Sewer for Plastic Insert Fittings for Polyethylene (PE) Plastic reinforced Concrete D-Load Culvert, Storm Drain, and Sewer ype Polyethylene Fusion Fittings for SDR 11.0 Polyethylene m Nickel lron Molybdenum Copper Columbium Stabilized Alloy
Pipe for High Temperature Service (1973

Pipe for lrrigation or Drainage (1973)

Pipe for Low Temperature Service (1973) ANS1 B125.17

Pipe for Process Piping (1972A)

Pipe for Septic Tank Disposal Fields (1973) ANSI A176.4

Pipe for Septic Tank Disposal Fields (1973) ANSI A176.5

Pipe for the Chemical lndustry (1971) ANS1 B125.38

Pipe for Use in Underdrains (1973)

Pipe for Use of Welded Offshore Fixed Platforms (1972)

Pipe for Water and Other Liquids (1972)

Pipe for Water or Other Liquids (1972)

Pipe for Water Service and Yard Piping (1971)

1. Rec. Pract.

Pipe Friction Loss Tables for Solving Fire Protection S

Pipe Inlets (1973)

F Systems and Appliances (Extingui

Pipe Lines (Wrapped Systems) (1972)

Pipe Operating at Temperatures Above Ambient Air (1972)

Pipe or Tile (1973)

Pipe Plugs, Bushings, and Locknuts with Pipe Threads (1

Pipe Schedule System Sprinkler Demand (1974)

Pipe Sprinkler Systems for Fire Protection Service (197

Pipe Systems for Highway. Airport, and Similar Drainage

Pipe Systems (Fire Protection) (1973)

Pipe Thermal lnsulation (1973)

Pipe Threads (1971)

Pipe Threads (1974)

Pipe Tube (1973) ANSl H34.41

Spec for Threading, Gaging,

Pipe Under internal Pressure (1967) ANSl B72.10

Pipe Valve Accelerator (1973)

Pipe Valves (Fire and Safety Equipment) (1973)

Pipe Valves, Model a (1973)

Pipe Valves; Rockwood Models C, D (1974)

Pipe with Rubber Gasket Joints (1973)

Pipe (Petroleum Drilling) (1973)

Pipe (Petroleum Drilling) (1973)

Pipe (Petroleum Drilling) (1973)

Pipe (Petroleum Drilling) (1973)

Pipe (Petroleum Production) (1973)

Pipe (PVC and CPVC) (1972)

Pipe (Rtrp) (1972)

Pipe (SDR-PR) and Tubing (1973) ANSI B72.12

FMS

ASTM

ANSI

AP1

ASTM

ASTM

FMS

FMS

FMS

FMS

ASTM

API

APl

APl

API

AP1

APl

Pipe (SDR-PR) Based on Controlled Outside Diameter (197

Pipe (SDR-PR) Based on Controlled Outside Diameter (197

Pipe (SDR-PR) Based on Outside Diameter (1973)

Pipe (SDR-PR) (1973) ANS1 B72.19

Pipe (SDR-PR) (1973) ANSI B72.2

Pipe (SDR-PR) (1973) ANSI B72.3

Pipe (SDR-PR) (1974) ANSI B72.1

Pipe (Welded and Seamless) for Underground Water Servic

Pipe (1966)

Pipe (1970)

Pipe (1970)

Pipe (1970) ANSI Al71.1

Pipe (1971)

Pipe (1972)

Pipe (1972)

Pipe (1972)

Pipe (1972)

Pipe (1972)

Pipe (1972)

Pipe (1972)

Pipe (1972)

Pipe (1972) ANSl A106.2

Pipe (1972) ANSI Al06.5

Pipe (1972) ANSI B125.20

Pipe (1972) ANS1 K65.171

Pipe (1972A)

Pipe (1972A)

Pipe (1972A)

Pipe (1972A) ANSI B125.16

Pipe (1973)

Pipe (1973)

Pipe (1973)

Pipe (1973)

Pipe (1973)

Pipe (1973)

Pipe (1973)

Pipe (1973)

Pipe (1973)

Pipe (1973)

Pipe (1973)

Pipe (1973)

Pipe (1973)

Pipe (1973)

Pipe (1973)

AST

APl

CSI

Std. Spec. ASTM

Sid. Rec. Pract. Fo ASTM

ASTM

Std. Spec. ASTM

Std ASTM

ASTM

ASTM

ASTM

Std. ASTM

Std. Sp ASTM

Std. Spe ASTM

std. Spec. ASTM

Std. Spec. for lnsert T ASTM

C497

B16.14

2.77

193

D2818

2-55

C552

5B

B407

D2153

2-56

$2-14$

$2-13$

2.27

$5 \mathrm{~A}$

$5 A X$

$5 \mathrm{~L}$

LX

5LP

RP5L4

ASTM D2446

ASTM D3035

ASTM D3287

D3287

ASTM D2662

D2281

D2239

888

66-22

C600

J844C

346

346
C506

SPEC 5LU

$5 \mathrm{LR}$

$5 \mathrm{Ll}$

L1

D2836

15062.1

B535

C507

$\mathrm{C} 12$

ASTM C 301

Std Spec for G ASTM A530

D2321

Al35

A589

C425

A312

C.14

C.361

ASTM C444

C541

C663

C668

$\begin{array}{ll}\text { ASTM } & \text { C668 } \\ \text { ASTM } & \text { D262 }\end{array}$

WWPA $* 31-2$

A648

C594

C76

D2609

C655

D3197 
National Bureau of Standards KWIC Index of Engineering Standards

Std. Meth. of Test. Asbestos Cement
Std Spec, for Asbestos Cement Perforated Underdrain Std. Spec. for Asbestos Cement Nonpressure Sewer spec. for Asbestos Cement Nonpressure Small Diameter Sewer Std. Spec. for Asbestos Cement Pressure d. Spec. for Homogeneous Bituminized Fiber Drain and Sewer spec. for Laminated Wall Bituminized Fiber Drain and Sewer Welded and Seamless Black and Hot Dipped Galvanized Steel Std. Spec. for Bell End Poly(vinyl Chloride) (PVC)

for Nondestructive Test. Terminology for Defects in Steel

-Iron-MoIydenium-Copper-Columbium Stabilized Alloy Std. Spec. for Threadless Copper

ement Mortar Protective Lining and Coating for Steel Water

Std. for Forged Steel Fittings
Rec. Pract. for Coding System for Identification of Tube, s Condu/ Std. Spec. for Aluminum Alloy Extruded Bar, Rod, molds for Gas (1971) Std. for Ductile Iron (1973) Safety Std. for Asbestos Cement Pressure Service (1970) Safety Std. for Dry to Safe Practice Protection from Corrosion for Underground Std. Spec. for Styrene Rubber (SR) Plastic Drain Std. Spec. for Cellulose Acetate Butyrate (CAB) Plastic Std. Spec. for Polyethylene (PE) Plastic 74) Std. Spec. for Polyethylene (PE) Plastic pec. for Acrylonitrile-Butadiene-Styrene (ABS) Plastic Std. Spec. for Poly(vinyl Chloride) (PVC) Plastic Std. Spec. for Seamless Copper Std. Spec. for Seamless Red Brass

(1972)

Std. for Prestressed Concrete Pressure Std. Spec. for Thermoplastic Gas Pressure lvent Cements for Cellulose Acetate Butyrate (CAB) Plastic Std. Meth. of Test for Disbonding Characteristics of Meth. for Nondestructive Measurement of Film Thickness of Std. Meth. of Test for Penetration Resistance of Std. Meth. of Test for Impact Resistance of Std. Meth. of Test for Impact Resistance of Std. Meth. of Test for Bendability of

Std. Meth. of Test for Effects of Outdoor Weathering on Std. Test Meth. for Chemical Resistance of Std. Meth. of Test for Abrasion Resistance of Std. Meth. of Test for Cathodic Disbonding of Std. Meth. of Test for Water Penetration into for Steel Gate, Plug, Ball, and Check Valves for Petroleum (1973) ANSI B111.1 Safety Std. for Pla valuating the Compatibility of Vehicle Fuel Tanks and Fill emises (1972)

Std. for Installation of Gas ot Rolled and Cold Finished, Special Quality, for Pressure ANSI G17.3 Std. Spec. for Carbon Steel Forgings for ow Temperature Service (1973) ANSI G46.2 oderate and Elevated Temperatures (1973)/

Sid. Spec, for Std. Spec, for Std. Spec. for Wrought Austenitic Stainless Stee Rec. for Thermoplastic 2)

for Polyethylene Encasement for Gray and Ductile Cast Iron Code for Identification of Rec. for Flammable Liquid Pumping and
Rec. for Thermoplastic W ater

of External Corrosion on Underground or Submerged Metallic Uniform Plumbing Code: Medium Pressure Gas ube and Pipe for Gas and Oil Transmission and Distribution Rec. for Gaskets for Flammable Liquid

n Steel Wrought Welding Fittings for High Pressure Service for Solvent Cemented PVC Pipe for W ater Service and Y ard ct. for Underground Installation of Thermoplastic Pressure ught Nickel and NickeI Alloy Welding Fittings for Pressure

Std. Spec. for Seamless Carbon Steel Pipe for Process Std. for Code for Powe Std. Code for Pressure Petroleum Refinery Uniform Plumbing Code: Rainwater Systems, Roof Drains and Uniform Plumbing Code: Fuel Gas Acrylonitrile-Butadiene-Styrene (ABS) Composite Sewer Uniform Plumbing Code: Indirect Waste Std. Code for Pressure Outdoor

Construction of Indoor Rifle and ons for FIoating Type Metallic and Nonmetallic Fluid Power Groove Dimensions for Fluid Power Radial Compression Type

Std. Meth. of Test for Specific Gravity of Solid
for Buttress Inch Screw Threads $(7 / 45 \mathrm{Deg}$. Form with 0.6 (1972) Std. for Double

sign, Manufacture, Inspection and Assembly Manual for Fine nions (1973) Std. for System Design of Fine Std. for System Design of Fine Std. Meth. of Test for Coking Value of Tar and
Pipe (1973) ANSl A 165.1

Pipe (1973) ANSI A165.2

Pipe (1973) ANSI Al65.3

Pipe (1973) ANSI Al65.4

Pipe (1973) ANSI A165.5

Pipe (1973) ANSI A176.]

Pipe (1973) ANSI Al76.2

Pipe (1973) ANSI B125.1

Pipe (1973) ANSI B72.20

Pipe (1974)

Pipe (1974)

Pipe (1974) ANS1 H26.2

Pipe (4 In. and Larger, Shop Applied) (1971)

Pipe), Socket Welding and Threaded (1973)

Pipe, and Hose Fittings (1972)

Pipe, and Structural Shapes for Electrical Purposes (Bu

Pipe, Centrifugally Cast, in Metal MoIds or Sand Lined

Pipe, Couplings, and Gaskets for Water and Fire Service

Pipe, Deluge, and Pre Action Valves for Fire Protection

Pipe, Fittings and Tanks Containing Flammable Liquids (

Pipe, Perforated (1974)

Pipe, Schedule 40 (1973) ANSI B72.4

Pipe, Schedule 40 (1974)

Pipe, Schedules 40 and 80 Based on Outside Diameter (19

Pipe, Schedules 40 and 80 (1973)

Pipe, Schedules 40, 80 and 120 (1973) ANSI B72.7

Pipe, Std. Sizes (1972)

Pipe, Std. Sizes (1972)

Pipe, SteeI Cylinder Type, for Water and Other Liquids

Pipe, Tubing and Fittings (1973)

Pipe, Tubing, and Fittings (1973) ANSl K65.162

Pipeline Coatings by Direct Soil Burial (1972)

Pipeline Coatings on Steel (1972)

Pipeline Coatings (Blunt Rod) (1972)

Pipeline Coatings (Falling Weight Test) (1972)

Pipeline Coatings (Limestone Drop Test) (1972)

Pipeline Coatings (1972)

Pipeline Coatings (1972)

Pipeline Coatings (1972)

Pipeline Coatings (1972)

Pipeline Coatings (1972)

Pipeline Coatings (1972)

Pipeline Valves (1974)

Pipes for Water Supply Test. in Fire Protection Service

Pipes Using Flexible Elastomeric Seals (1973)

Pipes with Fuel Dispensing Facilities (Passenger Cars,

Piping and Equipment for Use with Dry Chlorine (1971)

Piping and Equipment on Industrial and Certain Other Pr

Piping Components and Other Pressure Containing Parts (

Piping Components (Flange, Fitting, Valve, etc.) (1973)

Piping Fittings of Wrought Carbon and Alloy Steel for L

Piping Fittings of Wrought Carbon and Alloy Steel for M

Piping Fittings (1973) ANSI G37.8

Piping for Swimming Pool Water Circulation Systems (197

Piping for Water and Other Liquids (1972) ANSI A21.5

Piping Systems Content (1973)

Piping Systems (Fire Protection and Safety) (1973)

Piping Sy stems (1972)

Piping Systems (1972)

Piping Systems (1973)

Piping Systems (1973)

Piping (1969)

Piping (1971)

Piping (1972)

Piping (1972) ANSI H34.15

Piping (1972A)

Piping (1973)

Piping (1973)

Piping (1973)

Piping (1973)

Piping (1974)

Piping, Wet Vented Systems and Special Wastes (1973)

Piping: Liquid Petroleum Transportation Systems (1974)

Pistol Range Plans (Firearms) (1970)

Pistol Ranges and Bullet Stops (1973)

Piston Rings (1972) ANSI B93.36

Piston Rings (1973) ANSI B93.32

Pitch AsphaIt Displacement Meth.) (1972) ANSI A37.72

Pitch Basic Height of Thread Engagement (1973)

Pitch Conveyor Roller Chains, Attachments and Sprockets

Pitch Gear Drawings (1973)

Pitch Gearing (1973)

Pitch on Center Face Gears for 20 Deg. Involute Spur Pi

Pitch Wormgearing (1973)

Pitch (Modified Conradson) (1973) ANSI A 149.12

ASTM

ASTM

ASTM

Std. ASTM

ASTM

St ASTM

Std. ASTM

d. Spec. for ASTM
ASTM

Bulletin APl

ASTM
ASTM

AWWA

ANSI

SAE

ASTM

ANSI

UL

UL

ICBO

ASTM

ASTM

ASTM

ASTM

Std. S ASTM

ASTM

ASTM

ASTM

$A W W A$

ASTM

for So ASTM

ASTM

Std. ASTM

ASTM

ASTM

ASTM

ASTM

ASTM

ASTM

ASTM

ASTM

ASTM

Spec. API

UL

ASTM

SAE

ANSI

ASTM

ASTM

ASTM

ASTM

ASTM

SPI

A WWA

FMS

FMS

SPI

Rec. Pract. for Control NACE

ICBO

ASTM

FMS

MSS Installation Std IAPMO Std. Rec. Pra ASTM

/ Spec. for Factory Made Wro ASTM

ASTM

ANSI

ANSl

$1 \mathrm{CBO}$

ICBO

td. Spec, for ASTM

ICBO

ANSI

NRA

NRA

C500

C.508

C428

C644
C296

D 1861

D1862

A53

D2672

BUL5T1

B 474

B302

B16.11

J846C

B317

A21.52

107

260

UFC *2B

D3298

D1503

D2104

D2447

D1527

D1785

B42

B 43

C.301

D2513

D2560

G19

G12

G17

G14

G13

G11

G20

G6

G8

G9

6D

D3 139
J398 A 
lt, Asphalt and Coal Tar Saturated Organic Felts, Coal Tar Mopping Asphalt, Asphalt-Saturated Organic Felt, Coal Tar w Metal Door and Frame Preparation for Offset Intermediate Std. Meth. of Test for Density of Bituminous Concrete in Rec. Pract. for in onstruction (1965) Rec. for Deep, Cast in Rec. for Precast Concrete Units Used as Forms for Cast in Uniform Building Code Std. for in Specifying Portland Cement Terrazzo, Cast in Rec. for Cast in Wells (1972) Spec. for Cast in ands, Bleachers, etc.) (1973) Rec. for Manufacture and Rec. Pract. for Hole Uniform Fire Code: Rec. Pract. for Measuring, Mixing, Transporting, and truction (1970) $\begin{array}{ll}\text { truction (1970) } & \text { Spec. for } \\ 9 / \quad \text { Std. Spec. for Straight Beam Ultrasonic Examination of }\end{array}$ Std. for Washer. teel, UNJC-3A Thread, Cal ting Steel, UNJF-3A Thre/ Std. for Flathead 100 Deg. Std. for Undrilled and Drilled, Std. for Undrilled and Drilled,

2) technique Usi/ to Plane Arc/ Std. Spec. for Axle Steel Deformed and
Std. Spec. for Deformed and Std. Meth. for Spectrochemical Analysis of Std. Meth. for Spectrochemical Analysis of Std. for Building Code Requirements for Structural Std. for Building Code Requirements for Structural Std. Spec. for Compression Couplings for Vitrified Clay $s$ for Structural Steel Joints, Including Suitable Nuts and Std. for Terminal-Tank Strap, Std. for Nut Spacer, Plate. Std. for Nut, Std for Bushing : plain rartron and Low Alloy Steel for Boron by the Point to ic Friviun or Corrugated and Solid Fiberboard (Horizontal ic Friction of Corrugated and Solid Fiberboard (Horizontal atic Friction of Corrugated and Solid Fiberboard (Inclined Std. Meth. of Shear Test in Flatwise

Tent. Meth. of Test. Plywood in Rolling Shear (Shear in 1972) ANSI Z260.2 Std. Test Meth. for

$r$ Warpage of Refractory Brick and Tile or Deviation from A alysis of Plain Carbon and Low Alloy Steel by the Point to trochemical Analysis of Blast Furnace Iron by the Point to 1 Analysis of Admiralty Metal by the Cast Pin and Point to

3) A/ Std. Meth. of Test for Liquid Contaminant, Inclined of Tension Test of Flat Sandwich Constructions in Flatwise Uniform Building Code Std. for Struciural Floor and Roof esign Values, Section Properties and Design Loads, Timber, Std. Spec, for Asphalt Safety Code for Fabricated Stage and Scaffold Std. for Industrial Engineering Terminology: Production Std. for Industrial Engineering Terminology: Organization Is Handling) (1973) Shipper-Motor Carrier Dock 7.1 Std. Rec. Pract. for Std. for Industrial Engineering Terminology: Facility der the Uniform Building Code: Legal Requirements (Permit, Outdoor Pistol Range 50 Ft. Summer Camp Rifle Range cept Tapered Roller Bearings) Conforming to Basic Boundary Metallic Silhouette Match Rifle Range and Skeet: Ammunition and Targ/ $\begin{array}{r}\text { Shotgun Shooting Facility } \\ \text { Outdoor Smallbore Rifle Range }\end{array}$
ow and Medium Speed Stationary Diesel and Gas Engine Power and Skeet: Ammunition and Targ/ $\begin{array}{r}\text { Shotgun Shooting Facility } \\ \text { Outdoor Smallbore Rifle Range }\end{array}$
ow and Medium Speed Stationary Diesel and Gas Engine Power ow and Medium Speed Stationary Diesel and Gas Engine Power Truck Mixer and Agitator Stds. (Concrete ow and Medium Speed Stationary Diesel and Gas Engine Power Std. for Ancymidol Marine Steam Power rmining Protein Nitrogen Content in Corn, Other Grains and Concrete Rec. Safe Pract. and Proc. (Industrial h. for Analysis for Fluoride Content of the Atmosphere and th. of Analysis for Fluoride Content of the Atmosphere and tralized Control and Automation of Ship's Steam Propulsion
Std. for Agricultural

and Rims for Agricultural and Industrial Steering, Drive, Assurance Practices for the Construction of Nuclear Power nal Vehicle Manufacturing Plants (Fire and Wind Hazards at es (1971) ANSI A3/ Std. Spec. for Requirements for Mixing

building Code Std. for Class II and Class III Dry Cleaning

receiving, Storage and Handling of Items for Nuclear Power

constructed Housing and Recreational Vehicle Manufacturing Std. for in Dividual Aerobic W astewater Treatment

r Quality Assurance Program Requirements for Nuclear Power

Std. for Fuel Assembly Identification in Nuclear Power Std. for Administrative Controls for Nuclear Power
Pitch, and Asphalt Coated Glass Fiber Mat (1973)

Pitch, Coal-Tar Saturated Organic Felt, Asphalt-Saturat

Pivot (1973)

Place by Nuclear Meth. (1974)

Place Concrete Strength Evaluation (1970)

Place Concrete (Storage) Bin and Silo Wall Design and C

Place Concrete (1966)

Place Density of Soils (1973)

Place Floors (1967)

Place Non reinforced Concrete Pipe (1966)

Place Nonreinforced Concrete Pipe (1970) ANS1 A171.1

Placement of Reinforced Concrete Casings for Irrigation

Placement on Bulldozer (Dozer) End Bit Supports (1972)

Places of Assembly (Theaters, Reviewing Stands, Grandst

Placing Concrete (1973)

Placing Reinforcing Steel Bar Supports in Concrete Cons

Plain and Clad Steel Plates for Special Applications (1

Plain and CSK, 1200 Deg. F (1973)

Plain and Self Locking Machine Screw (1974)

Plain and Self Locking, Alloy and Corrosion Resisting S

Plain and Self Locking, Alloy Steel and Corrosion Resis

Plain Bars for Concrete Reinforcement (1972)

Plain Billet Steel Bars for Concrete Reinforcement (197

Plain Carbon and Low Alloy Steel by the Point to Plane

Plain Carbon and Low Alloy Steel for Boron by the Point

Plain Concrete (1972) ACI 322

Plain Concrete (1972) ANSI A 169.1

Plain End Pipe (1973)

Plain Hardened Washers (1971) ANSI G38.6

Plain (1973)

Plain (1973)

Plain, Hexagon, Drilled Jam, Thin (1972)

Plain, Press Fit, Steel (1973)

Plane Arc Technique (1970) ANSl Z128.29

Plane Meth.) (1972)

Plane Meth.) (1973)

Plane Meth.) (1973)

Plane of Flat Sandwich
Plane of Plies) (1969)

Plane Strain Fracture

Plane Surface (1972)

Std. Meth

Technique Using an Optical Emission Vacuum Spectr

Std. Meth. for Spectrochemic

Plane Tracking and Erosion of Insulating Materials (197

Plane (1970)

Plank and Beam Framing (1973)

Plank and Laminated Flooring and Roofing (1973)

Plank as Used for Bridge Floors (1970) ANS1 A37.48

Planks and Platforms (1972)

Planning and Control (1972)

Planning and Theory (1972)

Planning Manual (Truck Shipping and Receiving-Materia

Planning the Sampling of the Atmosphere (1957) ANS! Z25

Planning (1972)

Plans and Spec., Inspection) (1973)

Plans (Firearms) (1970)

Plans (1972)

Plans (1972) ANSl B3.17

Plans (1973)

Plans: International and Domestic-Trap, Clay Pigeon,

/G Construction Un ICBO

Plant Buildings (1972)

Plant Equipment Stds. (1973)

Plant Equipment) (1973)

Plant Fuel Oil and Gas Systems (1972)

(Plant Growth Regulator) (1971)

Plant Heat Balance Practices (1972)

Plant Materials of Comparable Content (1957)

Plant Mixer (Equipment) Stds. (1973)

Plant Safety Checklists) (1973)

Plant Tissues (Manual Procedures) (1973)

Plant Tissues (Semiautomated Meth.) (1973)

Plant (1970)

Planter Press Wheel Tires (Farm Machinery) (1971)

Planter (Drill) Press, and Gage Wheel, Tractor, Impleme

Plants-Terms and Definitions (1973)

Plants

作

Plants or Systems (1973)

Plants (During the Construction Phase) (1972)

Plants (Fire a

Plants (1971)

Plants (1972)

Plants (1972)
Meth. ASTM

ICBO

ASTM

ALI

ANSI

ANSl

ANSI

ASTM

ANSI

NRA

NRA

UBC \$32-1

$55 \mathrm{~A}$

Al15.12

D2950

"1 133
65.37

66.66

UBCS70-2

09410

66-22

346

R266

J63

UFC $* 2$ ART 26

304

* 1.3 .5

A578

1587

662

1352

1351

A617

A615

E403

E404

Al69. 1

322

322

A325

23
463

1423

75

E404

T816SU

D3247

D3248

C273

D2718

E399

C154

E403

E485

E486

D2303

C297

UBCS25. 22

D517

$* 1$

Z94.10

Z94.9

MH8. 1

D1357

Z94.6

UBC *8-1

5

*16

AFBMA 7

NRA *18

NRA *17

NRA *4

DEMA *1-20

NRMCA *1

NRMCA *3

DEMA *1.13

ANSI K62.109

SNAME 3.11

/for Dete CR A-18

NRMCA *2

MCA SG20

Tent. Met ASTM D3269

Tent. Me ASTM D3270 Guide for Cen SNAME $3-23$

ASAE S224.1

TRA 4

N45.2.10

7.90

D995

UBCS10-2

$\mathrm{N} 45.2 .2$

7.90

40

$\mathrm{N} 45.2$

Sed. Fo

ANSI N18.3

ANS1 N18.7 

d. for Program for Biological Shielding in Nuclear Reactor turbines Used for Electric Power Generation: Fossil Fueled Std. Industrial Security for Nuclear Power Uniform Fire Code: Automobile Tire Rebuilding

Uniform Fire Code: Lumber Yards and Woodworking ousekeeping During the Construction Phase of Nuclear Power omobile Wrecking and Junk Yards or Waste Material Handling ria for the Design of Stationary Pressurized Water Reactor

Design, Construction, and Operation of Solvent Extraction ated Components During Construction Phase of Nuclear Power est. Personnel for the Construction Phase of Nuclear Power nt for Water Cooled and Moderated Nuclear Power Generating

for Earthquake Instrumentation Criteria for Nuclear Power

Uniform Building Code Std. for Gypsum Base for Veneet fabric Laths, and Metal Accessories (Designed for Use as A

Uniform Building Code Std. for Gypsum Molding Std. for Gypsum Base for Veneer Plaster and Gypsum Veneer

d. for Perlite, Vermiculite and Sand Aggregates for Gypsum Std. Spec. for Gypsum Casting and Molding

Dwelling Construction Under the Uniform Building Code:

Dwelling Construction Under the Uniform Building Code Std. Spec. for Portland Cement and Cement Lime or Lathing and Furring for Portland Cement and Cement Lime Uniform Building Code Std. for Test. Gypsum

g: Steel; Terra Cotta; Timber: Water: Ceiling: Partitions: Std. Spec. for Dimensions and Tolerances of

k Exothermic Temperature of Reacting Thermosetting Resins

3)

Rec. Pract. for Meth. of Determining Std. Spec. for Styrene Rubber (SR)

als and Property Stds. for Acrylonitrile Butadiene Styrene ansi K65.56

Std. Spec. for Acrylonitrile-Butadiene-Styrene (ABS Std. for Smooth W all Coilable Polyethylene Electrical

Std. Rec. Pract. for Determining Hydrolytic Stability of Std. for Hardwood Veneered Including Hardboard and std. Meth. of Test for Pneumatic Ball lmpact Resistance of Meth. of Test for Algal Resistance of

Std. Spec. for Butt Heat Fusion Polyethylene (PE) ratus (1973) Std. Meth. of Test for Resistance to Std. Spec. for Asbestos Cement Std. Spec. for Chlorinated Poly(vinyl Chloride) (CPVC) ipe (1973) Std Spec, for er Finished with Epoxy Functional Silane Type Finishes for t. for Determining the Strength of Adhesively Bonded Rigid

Pract. for Determining the Strength of Adhesively Bonded Std. Spec. for Poly(vinyl Chloride) (PVC) st Meth. and Requirements for Evaluation of Suitability of Std. for Safety Tests for Flammability of Uniform Building Code Std. for the Density of Smoke from the Burning or Decomposition of Std. Spec, for Reinforced

Meth. for Diffuse Light Transmission Factor of Reinforced to for Glass Fiber Reinforced Polyester Structura

Std. Spec. for Solvent Cements for Styrene Rubber (SR)

Spec. for Solvent Cements for Poly(vinyl Chloride) (PVC)

Ivent Cement for Acrylonitrile-Butadiene-Styrene (ABS Rec for Coiling Polyethylen tt Heat Fusion Polyethylene (PE) Plastic Fittings for (PE) eth. of Test for Quality of Extruded Poly (Vinyl Chloride) Std. Meth. of Test for Time to Failure of Std. Symbols for Dimensions of

Std. Spec. for Socket Type Poly(vinyl Chloride) (PVC) Std. Spec. for Butt Fusion Polyethylene (PE) for Socket Type Acrylonitrile-Butadiene-Styrene (ABS)

Std. Spec. for Socket Type Poly(vinyl Chloride) (PVC) Std. Spec. for Butt Fusion Polyethylene (PE) for Socket Type Acrylonitrile-Butadiene-Styrene (ABS) ec. for Threaded Acrylonitrile-Butadiene-Styrene (ABS

Std. Spec. for Threaded Poly(vinyl Chloride) (PVC) Rec. Pract. for Calculating Stress in Std. Spec. for Cellulose Acetate Butyrate (CAB) Std. Spec. for Polyethelene (PE)
Rec. Safeguards in Particle Board Rec. for Protection of Plywood Rec for Chemical Rec. Pract. fo Std. Meth. Measuring Rec. Pract. for Uniform Building Code Std. for Exterior Spec. for Interior Gypsum Std. Meth. for Physical Test. of Gypsum Specifying Tests,

Plants (1972)

Plants (1972)

Plants (1972)

Plants (1972)

Plants (1973)

Plants (1973)

Plants (1973)

Plants (1973)

Plants (1973)

Plants (1973)

Plants (1973)

Plants (1973)

Plants (1973)

Plants (1973)

Plants (1974)

Plants (1974)

Plasma Arc Cutting (1973)

Plasma Arc Gas Enthalpy by Energy Balance (1973)

Plasma Arc Welding (1973)

Plaster and Gypsum Veneer Plaster (1973)

Plaster Ba se) (1973)

Plaster Liquid Bonding Agents (1973)

Plaster (1972)

Plaster (1973)

Plaster (1973)

Plaster (1973)

Plaster (1973) ANSI A49.4

Plastering and Installation of Wallboard Lathing (1973)

Plastering (1973)

Plastering, Exterior (Stucco) and Interior (1971)

Plastering, Exterior (Stucco) and Interior (1971)

Plasters and Concrete (1973)

Plasters and Concrete (1973)

Plaster; Roofing) and Earthquake Recording Instrumentat

Plastic Bottles (1972)

(Plastic Composition) (1971) ANSI K65. 182

Plastic Concrete (1967)

Plastic Deformation in Sheet Metal Stampings (1972)

Plastic Drain and Building Sewer Pipe and Fittings (197

Plastic Drain Pipe, Perforated (1974.

Plastic Drain, Waste and Vent Pipe and Fittings (1971)

Plastic Drain, Waste and Vent Pipe and Fittings (1973)

Plastic Drain, Waste, and Vent Pipe and Fittings (1973)

Plastic Duct (1972)

Plastic Encapsulants for Electronic Devices (1973)

Plastic Faced Flush Doors (1973)

Plastic Film and Sheeting (1972)

Plastic Films (1972T)

Plastic Fittings for (PE) Plastic Pipe and Tubing (1973

Plastic Flow of Bituminous Mixtures Using Marshall Appa

Plastic Foam Core Insulating Panels (1972)

Plastic Hot Water Distribution Systems (1973)

Plastic Insert Fittings for Polyethylene (PE) Plastic P

Plastic Laminates (1972)

ISs Fabrics, Cleaned, and Aft

Plastic Lap Shear Joints in Ear by Tension Loading (1

Plastic Lap Shear Sandwich Joints in Shear by Tension L

Plastic Line Couplings, Socket Type (1973)

Plastic Material for Use in Housings of Motor Vehicle L

Plastic Materials for Parts in Devices and Appliances (

Plastic Materials (1973)

Plastic Materials (1973)

Plastic Mortar Sewer Pipe (1973)

Plastic Panels (1969)

Plastic Panels (1972)

Plastic Pipe and Fittings (1972)

Plastic Pipe and Fittings (1973) ANSI B72.16

Plastic Pipe and Fittings (1973) ANSI B72.23

Plastic Pipe and Tubing (1972)

Plastic Pipe and Tubing (1973)

Plastic Pipe by Acetone Immersion) (1967) ANSI B72.9

Plastic Pipe Constant Internal Pressure (1974) ANSI B72

Plastic Pipe Fittings (1968) ANSI K65.57

Plastic Pipe Fittings, Schedule 40 (1973)

Plastic Pipe Fittings, Schedule 40 (1973) ANSI K65.160

Plastic Pipe Fittings, Schedule 40 (1973) ANSI K65.164

Plastic Pipe Fittings, Schedule 80 (1973)

Plastic Pipe Fittings, Schedule 80 (1973) ANSI K65.159

Plastic Pipe Fittings, Schedule 80 (1973) ANSI K65.163

Plastic Pipe Fittings, Schedule 80 (1973) ANSI K65.165

Plastic Pipe Fittings, Schedule 80 (1973) ANSI K65.166

Plastic Pipe Under Internal Pressure (1967) ANS1 B72.10

Plastic Pipe (SDR.PR) and Tubing (1973) ANSI B72.12

Plastic Pipe (SDR-PR) Based on Controlled Outside Diame

Plastic Pipe (SDR.PR) Based on Controlled Outside Diame

Plastic Pipe (SDR.PR) Based on Outside Diameter (1973) Std. Spec. for Polybutylene (PB) Std. Spec. for Polybutylene (PB) tor $\mathrm{H}$ ANSI

CBO

ICBO

clear Safety Crite ANSI

ANSI

ANSI

ANS

FMS

Std. ANSI

AWS

ASTM

AWS

ICBO

$\mathrm{ICBO}$

ICBO

CSI

ICBO

ICBO

ASTM

ICBO

ICBO

ANSI

1. F ANSI

ASTM

ICBO

ICBO

ASTM

ASTM

CSl

$\mathrm{SAE}$

ASTM

ASTM

IAPMO

ASTM

ASTM

NEMA

ASTM

NWMA

ASTM

ASTM

ASTM

ASTM

ASTM

ASTM

ASTM

ASTM

ASTM

ASTM

ASTM

SAE

UL

ICBO

ICBO

ASTM

Std. Test ASTM

USC

ASTM

Std ASTM

1. for So ASTM

SPI

ASTM

ASTM

ASTM

ASTM

ASTM

ASTM

ASTM

ASTM

ASTM

ASTM

ASTM

ASTM

ASTM

ASTM

ASTM

ASTM

ASTM

ASTM

LPD7.10

7.12
N18.9

TW DPS. 1

N18. 17

UFC 2 ART2

UFC 2ART21

N45.2.3

UFC 2 ART 3

$\mathrm{N} 18.2$
$7.30 \mathrm{~N}$

N45.2.1

N45.2.6

N18.10

7.43.

Nl8.5

C5.2

E341

UBCS4.14

UBCS 47.4

UBCS4.7-1

09150

UBCS47-12

UBC $547-14$

LBCS47.3

LBC * 8.14

UBC $* 8-15$

A 42.2

A 42.3

$\mathrm{C} 472$

UBCS 24.26

UBC *3-23 
Std. Spec. for Poly(vinyl Chloride Std. Spec. for Acrylonitrile-Butadiene-Styrene (ABS) Std. Spec. for Polyethylene (PE) Guide Spec, fo d. Spec. for Plastic Insert Fittings for Polyethylene (PE) Std. Spec. for Cellulose Acetate Butyrate (CAB)

Std. Spec. for Polyethylene (PE) eter (1974)

Std. Spec. for Polyethylene (PE) Std. Spec. for Acrylonitrile-Butadiene-Styrene (ABS Std. Spec. for Poly(vinyl Chloride) (PVC) for Solvent Cements for Cellulose Acetate Butyrate (CAB) un or Fluidized Bed (Fire Protec/ (1973)

Rec. for Application of Std. Spec, for Joints for

1973) ANSI C59.91

Std Spec for 1973)

Std. Spec. for Preformed Cellula Std. Spec, for Vinyl Chloride Std. Spec. for Polyethylene

Std. Rec. Pract. for Preparation of Surfaces of Std. Meth of Panel Spalling Test for Fireclay Std. Meth. of Test for Modulus of Rupture of Air Setting Meth. of Test for Drying and Firing Shrinkage of Firecla

Felts, Saturated and Coated Felts, Prepared Roofings, and Std. Spec. for Acrylonitrile-Butadiene-Styrene (ABS) (1972T) Spec. for Flexible Poly(vinyl Chloride) ight) / Std. Meth. of Test for lmpact Resistance of Rigid

Std. Meth. of Test for Tensile Properties of Thin Std. Meth. of Test for Flammability of Flexible Thin Std. Meth. of Test for Std. for Thermoset

c, Holding, Settling. Aeration Chambe/ Std Spec for Polybutylene (PB) Std. Spec, for Poly(vinyl Chloride) (PVC) Std. Spec. for Polyethylene (PE) ble, Heat Shrinkable (19/ Spec. for Electrical Insulation 57 Std. Spec. for Acrylonitrile Butadiene Styrene (ABS) Communication and Electrical Wire and Cable (1/ Std. for Std. for Rigid Polyvinyl Chloride) (PVC)

Tent. Hazard Classifications of Organic Peroxides uilding Code: Veneer (Brick, Concrete, Stone. Tile, Metal, Std. Dimensions for Cores for Photographic Film Rolls

3)

on (1974)

Sid. Meth of Test for Oil Migration or Sid. Meth. of Test for Compatibility of Std. Meth. of Test for Brittleness Temperature of Std. Meth. of Test for Impact Resistance of sion Test/ Std. Meth. of Test for Stiffness Properties of
13 Std. Meth. of Test for Stiffness of
ent Horizontal Shear Strength of Parallel Fiber Reinforced Std. Spec. for Glass Reinforced Acetal Std. Spec for Polybutylen Std. Meth of Test for Resistance of Transparent f Test for Compatibility of Plasticizers in Vinyl Chloride Std. Meth. of Test for Deflection Temperature of ctric Constant and Dissipation Factor of Expanded Cellular for Self and Flash Ignition Properties (Temperatures) of

Test for Rate of Burning or Extent of Burning of Cellular 10 Std. Test Meth. for Flammability of skylights, Monitors and Sawtooth / Uniform Building Code Uniform Fire Code: Cellulose Nitrate eth. of Measuring Shrinkage from Mold Dimensions of Molded ning Temperatures of Std. ASTM Molds for Test Specimens of Std. Meth. of Test for Water Absorption of Rigid Cellula std. Meth. of Test for Kinetic Coefficients of Friction of acilities for Pneumatic Transfer of Dry Bulk Chemicals and

td. Meth. of Test for Tensile Properties of Rigid Cellular Std. Meth. of Test for Tensile Properties of Uniform Building Code Std. for Gypsum

Spec. for Rigid Acrylonitrile-Butadiene-Styrene (ABS meth. of Test for Compressive Properties of Rigid Cellular Std Definitions of Terms Relating to

Std. Meth. of Test for Flammability of Rigid Cellular

Std. Meth. of Test for Flammability of Flexible

Rec. for Solid. Palletized, and Rack Storage of

Std. Meth. of Test for Incandescence Resistance of Rigid

Std. Meth. of Test for Flammability of Self Supporting a Classification in Hazardous Dust Locations (Agricultura ical Instruments in Hazardous Dust Locations (Agricultura Opaque Materials (Color Measurement of Textiles, Paints, Std. Index of Meth. for Test. Elastomeric and th. of Measuring Flow Rates of Thermoplastics by Extrusion 0 and 800 Deg. F (1973) Std. for Nut, Self Locking, Std. for Nut Spacer, Std. for Nut Spacer,

800 Deg. F (1) Std. for Nut, Self Aligning, Self Locking,

dge for X-Ray Standardization Probe, Rot Fragment Counting Std. Spec. for Copper Sheet, Strip. Std. Spec. for Industrial Perforated
Plastic Pipe (SDR.PR) (1973) ANSI B72.2

Plastic Pipe (SDR.PR) (1973) ANSI B 72.3

Plastic Pipe (SDR.PR) (1974) ANSI B72.1

Plastic Pipe (1972)

Plastic Pipe (1973)

Plastic Pipe, Schedule 40 (I973) ANSI B72.4

Plastic Pipe. Schedule 40 (1974)

Plastic Pipe, Schedules 40 and 80 Based on Outside Diam

Plastic Pipe, Schedules 40 and 80 (I973)

Plastic Pipe, Schedules 40, 80 and 120 (I973) ANSI B72.

Plastic Pipe, Tubing, and Fittings (1973) ANSI K65.162

Plastic Polymers in Dry Powder Form by Means of Spray G

Plastic Pressure Pipes Using Flexible Elastomeric Seals

Plastic Pressure Relief Joint Fillers (1973)

Plastic Pressure Sensitive Electrical Insulating Tape

Plastic Pressure Sensitive Electrical Insulating Tape

Plastic Pressure Sensitive Electrical Insulating Tape

Plastic Prior to Adhesive Bonding (1969) ANSI Z197.25

Plastic Refractories (1972)

Plastic Refractories (1972)

Plastic Refractories (1972) ANSI A111.25

Plastic Roll Materials (1973)

Plastic Sewer Pipe and Fittings (1973) ANSl K65.59

Plastic Sheeting for Pond, Canal, and Reservoir Lining
Plastic Sheeting or Parts by Means of a Tup (Falling We

Plastic Sheeting (1973) ANSI K65.76

Plastic Sheeting (1974) ANSI K65.28

Plastic Strain Ratio R for Sheet Metal (I974)

Plastic Tanks for Water and Wastewater Treatment (Septi

Plastic Tubing (I973)

Plastic Tubing (1973) ANSI B72.22

Plastic Tubing (1974)

Plastic Tubing, Irradiated Polyolefin, Pigmented, Flexi

Plastic Utilities Conduit and Fittings (1972) ANSI K65.

Plastic Utilities Duct for Underground Installation for

Plastic WalI Siding (1972)

(Plastic) (1974)

Plastic, etc.) for Ornamentation, Protection, or Insula

(Plastic, Wood or Metal) (1971)

Plasticity Retention Index (PRl) of Natural Rubber (197

Plasticizer Bleed Out of Preformed Sealing Tapes (1974)

Plasticizers in Vinyl Chloride Plastics Under Compressi

Plastics and Elastomers by Impact (I973)

Plastics and Electrical Insulating Materials (1973)

Plastics as a Function of Temperature by Means of a Tor

Plastics by Means of a Cantilever Beam (1970) ANSI K65.

Plastics by Short Beam Meth. (1972)

Plastics for Molding and Extrusion (1972)

lof Test for Appar

Plastics Molding and Extrusion Materials (1973)

Plastics to Surface Abrasion (1973) ANSI K65.72

Plastics Under Compression (1974)

Plastics Under Flexural Load (1972) ANSI K65.222

Std. Meth. O ASTM

ASTM

Plastics Used for Electrical Insulation (1973) ANSl C59

Plastics Using a Hot Air lgnition Furnace) (Fire Safety

Plastics Using a Supported Specimen by a Horizontal Scr

Plastics Using the Oxygen Index Meth. (1974) ANSI K65.1

Plastics (General, Exterior Light Transmitting Panels,

Plastics (Pyroxylin) (1973)

Plastics (R 1973) ANSI K66.3

Plastics (1967) ANSI K65.79

Plastics (1969E) ANSI K65.122

Plastics (1972)

Plastics (I972)

Plastics (1972) ANSl K65.32

Plastics (1972) ANSI K65.60

Plastics (1973)

Plastics (1973) ANSI K65.205

Plastics (1973) ANSI K65.31

Plastics (1973) ANSI K65.51

Plastics (1974)

Plastics (1974)

Plastics (1974)

Plastics (1974) ANSI K65.117

Plastics (1974) ANSI K65.21

Plastics, Chemicals, Metal Dusts) (1973)

Plastics, Chemicals, Metal Dusts) (1973)

Plastics, etc.) (1973) ANSI Z172.6

Std. M
1. Rec. Pract. for Determi

Std. M ASTM

ASTM

ICBO

ASTM

ASTM

CBO

Plastometer (1973)

Plate-Corner, Counterbored, Floating, 125 KSI Ftu, 45

Plate-Countersunk (1973)

Plate-Plain (1973)

Plate-Two Lug, Floating, 125 KSI Ftu, 450 Deg. F and

Rec. for Terminal F M MCA

STM

ASTM

ASTM

ICBO

Std. ASTM

Std. ASTM

ASTM

ASTM

ASTM

FMS

ASTM

ASTM

Ion of Electr ISA

(Ness of Near White ASTM

AS'TM

Std. Me

ASTM

NSA

NSA

NSA

Plate and Cover) (1962)

Plate and Rolled Bar (1973) ANSI H7.9

Y Funnel, Trap Flask, Step We

Plate and Screens (Square Opening Series) (1972)

D224I

D2282

D2239

15064.1

D2609

D 1503

D2 I04

D2447

D 1527

D 1785

D2560

7.27

D3139

D3204

D2301

D 3005

D3006

D2093

C 180

C491

C 179

$+2.1 \mathrm{~V}$

D2751

D3083

D3029

D882

D1433

E517

41

D2666

D 2740

D2737

AMS3636D

D2750

TC6

PS55

7.81

UBC *3-30

PHl. 13

D3194

C772

D3291

D746

D256

D 1043

D747

D2344

D2948

D2581

D 1044

D3291

D648

D 1673

UBC 552.3

D 1692

D2863

UBC *3-52

UFC *2ART6

D955

D284

D3028

TC 18

D 1623

D638

UBC $\$ 47.8$

DI 788

D1621

D883

D 3014

D568

8.9 
Std. Spec. for Copper Nickel Alloy Std. Spec. for Copper Silicon Alloy Std. Spec, for Nickel-Molybdenum Alloy Std. Spec. for Nickel-Molybdenum-Chromium Alloy Meth. for Distillation of Crude Petroleum (I5 Theoretica Joints, Drift Bolts, and Wood and Lag Screws; Light Metal Products (Cereal Chemistry) (1962) Std.

Std. Spec. for Zirconium and Its Alloy Sheet, Strip, and Std. Meth. for Ultrasonic Inspection of Aluminum Alloy g Glazing Material for Use Principally as a Substitute for Std. for License nsi C59.13 eat, and Corn Flour (1962) Glass

for Detection of Antibacterial Activity of Fabrics: Agar Std. Spec. for Corrosion Resisting Chromium Steel Clad Std. Spec. for Perforated tent. Meth. of Test for Viscosity of Asphalt with Cone and Spec. for Base special Grade (1973) Spec. for Steel Sheet. Strip, and
Spec. for Steel Sheet, Strip, and rosion and Moderate Heat Resistant Steel Sheet, Strip, and Spec. for Columbium and Its Alloy Strip, Sheet, Foil, and Std. Spec for Magnesium Alloy Sheet and for Nickel-Molybdenum-Chromium-Iron Alloy Sheet and Spec. for Titanium and Titanium Alloy Strip, Sheet, and Std. Spec. for Copper and Its Alloy Clad Steel

Tolerances of Aluminum and Magnesium Base Alloy Sheet and ty Requirements for Shops Fabricating Structural Steel and Std. Spec. for Aluminum Alloy Sheet and or Molybdenum and Molybdenum Alloy Strip, Sheet, Foil, and ina (Cereal Chemistry) (1962)

for Electrodeposited Coatings of Tin-Lead Alloy (Solder Molybdenum Sheet, Strip, and Std. Spec. for Cartridge Brass Sheet, Strip, $\mathrm{cr}-15.2 \mathrm{Mo}-0.30 \mathrm{Al}-0.05 \mathrm{La}(\mathrm{I}$ Alloy Sheet, Strip, and Spec. for Alloy Sheet, Strip, and $4.5 \mathrm{Ni}-2.9 \mathrm{Mo}-0.10 \mathrm{~N}$, Solution Heat Treated (1973)

$\mathrm{b}+\mathrm{Ta}$ ), Consumable Electrode $\mathrm{Va}$ (1973) Steel Sheet, Strip, and Alloy Sheet, Strip, and

Std. Spec, for Nickel Chromium Molybdenum Columbium Alloy for Nickel-Chromium-Iron-Molybdenum-Copper Alloy pec. for Heat Resisting Chromium and Chromium Nickel Steel Std. Spec. for Hot, Hot Cold, and Cold Worked Alloy Steel 972) Std. Spec for Precipitation Hardening Nickel Alloy Std. Spec. for Nickel-Chromium-Iron Alloy Std. Spec. for Nickel Copper Alloy

std. Spec. for Low Carbon Nickel Molybdenum Chromium Alloy std. Spec. for Stainless and Heat Resisting Chromium Steel delivery of Flat Rolled Stainless and Heat Resisting Steel

td. Spec. for Nickel Iron Chromium Molybdenum Copper Alloy Std. Spec. for Nickel or Nickel-Iron-Chromium-Manganese-Molybdenum Alloy m Nickel Iron Molybdenum Copper Columbium Stabilized Alloy Std. Spec for Nickel-Iron-Chromium Alloy Copper-Cobalt-Beryllium Alloy (Copper Alloy No. 175), Spec. for Tantalum Std. Spec. for Phosphor Bronze (Copper Tin) Std Spec for Leaded Brass Std. Spec. for Copper-Beryllium Alloy Std. Spec. for Brass Std. Spec. for Copper Iron Alloy Std. Spec. for Copper-Zinc-Tin Alloys Std. Spec for Copper-Zinc-Aluminum-Cobalt Alloy kel-Zinc Alloy (Nickel Silver) and Copper-Nickel Alloy $r$ GeneraI Requirements for Wrought Copper and Copper Alloy for Material Spec. for Structural Steel and Iron (Shape. for High Yield Strength, Quenched and Tempered Alloy Steel 0) (1973)

23C), Premium Quality, Consumab/ Steel Sheet, Strip, and Titanium Alloy Sheet, Strip, and Std. for Bushing-Clamp Up. Steel, Cadmium

r Appliances (Coffee Pots and Urns, Food Dish Warmers. Hot Std. for Domestic Gas Hot

Std. Spec, for Rolled Copper Alloy Bearing and Expansion (1972) ANSI / Std. Spec. for Carbon Steel Pressure Vessel $w$ and Intermediate Tensile Strength Carbon-Silicon Steel ) ANSI G35.1/ Std. Spec, for Carbon Steel Pressure Vessel erature Service (1972/ Std. Spec. for High Strength Steel Std. Spec. for General Requirements for Delivery of Steel Std. Spec. for Manganese Vanadium Alloy Steel

d. Spec. for Charpy V.Notch Testing Requirements for Steel Std. Spec, for Nickel Alloy Steel Std. Spec. for Molybdenum Alloy Steel

Std. Spec. for Carbon Manganese Silicon Steel

Spec. for Manganese Molybdenum and Its Nickel Alloy Steel
Plate and Sheet for Pressure Vessels (1969) ANSI H52.I

Plate and Sheet for Pressure Vessels (1973)

Plate and Sheet (1971) ANSI H34.II

Plate and Sheet (1971) ANSI H34.12

Plate Column) (1973) ANSI Z11.314

Plate Connected Wood Truss Design; and Nails and Staple

Plate Count Meth. of Analysis of Total Bacteria in Food

Plate Fabricated Aerosol Cans (1972)

Plate for Nuclear Application (1973) ANSI N123

Plate for Pressure Vessels (1971) ANSI H38.21

Plate Glass Show Window Panels (1972)

Plate Lamps (Light) (1973)

Plate Materials Used for Electrical Insulation (1972) a

Plate Meth. of Analysis for Insect Excreta in White, Wh

Plate Meth. (1970) ANSI L14.125

Plate Sheet and Strip (1972) ANSI G81.6

Plate Sieves for Test. Purposes (1970) ANSI Z168.I2

Plate Viscometer (1973)

Plate (Artificial Dentures) Wax (1971)

Plate $(0.80 \mathrm{Cr}-1.8 \mathrm{Ni}-0.25 \mathrm{Mo}(0.49-0.55 \mathrm{C}))(1973)$

Plate $(0.80 \mathrm{Cr}-1.8 \mathrm{Ni}-0.35 \mathrm{Mo}-0.20 \mathrm{~V}(0.33-0.38 \mathrm{C}))$

Plate $(13 \mathrm{Cr}-2.0 \mathrm{Ni}-3.0 \mathrm{~W})(1973)$

Plate (1964) ANSI Z179.20

Plate (1970) ANSI H45.3

Plate (1971) ANSI H34.44

Plate (1972) ANSI Z179.1

Plate (1973)

Plate (1973)

Plate (1973)

Plate (1973) ANSI H38.2

Plate (1974)

Plate) Meth. of Analysis of Moisture in Flour and Semol

Plate) (1973)

Plate, Arc Cast, Stress Relieved (1973)

Plate, Bar and Disks (1973)

Plate, Corrosion and Heat Resistant, Nickel Base-15.8

Plate, Corrosion and Heat Resistant, Nickel Base, $20 \mathrm{Cr}$

Plate, Corrosion and Moderate Heat Resistant, $15.5 \mathrm{Cr}$

Plate, Corrosion Resistant, $14.8 \mathrm{Cr}-4.5 \mathrm{Ni}-3.5 \mathrm{Cu}-(\mathrm{C}$

Plate, Low Expansion, Glass Sealing, 53Fe-29Ni-17Co

Plate, Sheet and Strip (1972) ANSI H34.19

Plate, Sheet and Strip (1973)

Plate, Sheet, and Strip for Fusion Welded Unfired Press

Plate, Sheet, and Strip for High Strength at Elevated T

Plate, Sheet, and Strip for High Temperature Service (1

Plate, Sheet, and Strip (1970) ANSI H34.10

Plate, Sheet, and Strip (1970) ANSI H34.6

Plate, Sheet, and Strip (1972)

Plate, Sheet, and Strip (1972) ANSI G81.2

Plate, Sheet, and Strip (1972) ANSI G81.30

Plate, Sheet, and Strip (1972) ANSI H34.17

Plate, Sheet, and Strip (1972) ANSI H34.8

Plate, Sheet, and Strip (1973)

Plate, Sheet, and Strip (1973) ANSI H34.37

Plate, Sheet, and Strip (1973) ANSI H34.40

Plate, Sheet, Strip and Rolled Bar (1973)

Plate, Sheet, Strip, and Foil (1973)

Plate, Sheet, Strip, and Rolled Bar (1973)

Plate, Sheet, Strip, and Rolled Bar (1973)

Plate, Sheet, Strip, and Rolled Bar (1973)

Plate, Sheet, Strip, and Rolled Bar (1973)

Plate, Sheet, Strip, and Rolled Bar (1973)

Plate, Sheet, Strip, and Rolled Bar (1973)

Plate, Sheet, Strip, and Rolled Bar (1973)

Plate, Sheet, Strip, and Rolled Bar (1973)

Plate, Sheet, Strip, and Rolled Bar (1974)

Plate, Sheet, Strip, Conn ector, and Bar) (1973

Plate, Suitable for Welding (1970E) ANSI G24.26

Plate, $0.50 \mathrm{Cr}-0.55 \mathrm{Ni}-0.25 \mathrm{Mo}(0.38-0.43 \mathrm{C})(\mathrm{SAE} 874$

Plate, $0.75 \mathrm{Cr}-9.0 \mathrm{Ni}-4.5 \mathrm{Co}-1.0 \mathrm{Mo}-0.09 \mathrm{~V}(0.17-0$

Plate, 6Al-4V Annealed (1973)

Plated (1973)

Plates and Griddles, etc.) (1973)

Plates and Laundry Stoves (1971)

Plates and Sheets for Bridge and Other Structural Uses

Plates for Intermediate and Higher Temperature Service

Plates for Machine Parts and General Construction (1970

Plates for Moderate and Lower Temperature Service (1973

Plates for Pressure Vessels for Moderate and Lower Temp

Plates for Pressure Vessels (1972)

Plates for Pressure Vessels (1972)

Plates for Pressure Vessels (1972)

Plates for Pressure Vessels (1972) ANSI G33.1

Plates for Pressure Vessels (1972) ANSI G34.1

Plates for Pressure Vessels (1972) ANSI G35.14
Plates for Pressure Vessels (1972) ANSI G35.15

d Product Standards Division

ASTM

ASTM

ASTM

ASTM

Std. ASTM

ICBO UBCS25.17

AACCH $42-11$

ASTM D3061

ASTM B352

ASTM B548

972

UL

SAE J587E

ASTM D229

Test Meth AATCC 28.43

ASTM A263

ASTM E323

ASTM D3205

$\mathrm{SAE}$

SAE

Spec. for Cor SAE

Std. ASTM

ASTM

Std. Spec. ASTM

ASTM

ASTM

Spec. for SAE

td. for Safe ANSI

ASTM

td. Spec. F ASTM

SAE

ASTM

SAE

SAE

$\mathrm{SAE}$

SAE

ASTM

Std. Spec ASTM

ASTM

ASTM

ASTM

ASTM

ASTM

ASTM

ASTM

/Ments for ASTM

ASTM

ASTM

Std. Spec, F ASTM

for Chromiu ASTM

ASTM

1. Spec. for ASTM

SAE

ASTM

ASTM

ASTM

ASTM

ASTM

ASTM

ASTM

/Pper-Nic ASTM

1. Spec. Fo ASTM

E Std ICBO

ASTM

SAE

SAE

NSA

ANS

ANSI

ASTM

ASTM

ASTM

ASTM

ASTM

ASTM

ASTM

ASTM

ASTM

ASTM

ASTM 24

AMS6396

AMS6433B

AMS5508B

B393

B90

B434

B265

B432

AMS2202K

Z229.1

B209

B386

44.16

B579

AMS7801A

B19

(MS5873

AMS5872

AMS5549C

AMS5862

AMS $7728 \mathrm{C}$

B582

A 240

A 457

A670

B168

B127

B575 
ec. for Low and Intermediate Tensile Strength Carbon Stee Std. Spec. for Chromium Molybdenum Alloy Stee nd Spec. for Straight Beam Ultrasonic Examination of Stee raight Beam Ultrasonic Examination of Plain and Clad Stee Std. Spec, for Quenched and Tempered Carbon Steel Std. Spec. for Copper Alloy Condenser Rolled Tube Std. Spec. for Structural Carbon Stee

ec. for Low and Intermediate Tensile Strength Carbon Stee Std. for Symbols for Machine iool Indicato Vessels (1972) ANSI G35.7 Std. Spec. for Carbon Steel 83.53 Std. for Clearance Dimensions on the Mating Std. Rec. Pract. for Static Bend Test. of Bone Std. Rec. Pract. for Static Bend Test. of Nail Std. for Practice for Storage of Processed Photographic Std. Spec. for Vitrified Clay Line

$\mathrm{m}$-Copper-Nickel-Aluminum Alloy Steel Pressure Vesse th. of Test for Tensile Strength Properties of Steel Truss imensions and Tolerances for Screw Holes and Slots in Bone el Alloy Steel, Quenched and Tempered, for Pressure Vesse std. Spec. for Ultrasonic, Angle Beam Examination of Stee ANSI G35.16

ANSI G32.1

ed (1972A) ANSI G35.11

18\% Nickel (1972A) Ansi/

$12 \%$ Nickel (1972A)

alt-Molybdenum-Chromi/

kel (1972A) ANSI G35.20

mpered (1972A) ANSI G35.3

Std. Spec for Pressure Vessel Std. Spec. for Pressure Vessel Std. Spec. for Pressure Vessel Std. Spec. for Pressure Vesse Std Spec for Pressure Vessel Std. Spec. for Pressure Vessel Std. Spec. for Pressure Vessel Std. Spec. for Pressure Vessel Std. Spec, for Pressure Vessel itometric Meth. for Measuring Residual Chemicals in Films, ual) Carbon Steel' Plates, Structural Shapes, Rolled Floo Service (1972)

nsi G35.8

Std. Spec, for Pressure Vessel Std. Spec. for Pressure Vessel Std. Spec. for Pressure Vesse

use Book: Timber Fasteners (Connectors, Bolts, Lag Screws ssels (1/ Std. Spec. for Chromium Molybdenum, Alloy Steel $72 /$ Std. Spec. for Nickel Chromium Molybdenum Alloy Steel pec. for General Requirements for Delivery of Rolled Steel el Sheet Piling (1) 1972A) (Steel Products Manual) Carbon Steel Sid. Spec, for Pressure Vessels cks; Knobs and Handles; Exit Devices, Pulls, Push and Kick ) for Lowering Personnel and Material (1/ Guide Spec. for Safety Code for Fabricated Stage and Scaffold Planks and ted Structural Steel Pipe for Use of W'elded Offshore Fixed Uniform Building Code: Stages and

troleum Drilling Rig Packaging for Minimum Self Contained oduction Facilities on Land and on Marine Fixed and Mobile posed to W/ Std. Spec. for Wood Paving Blocks for Exposed rvice (1973) ANSI Bl11.1

Meth. of Test for Shock Absorbing Properties of (Sports) Test Meth. for Colorfastness of Textiles to Steam nsi/ Std. Meth of Test and Tolerances for Jute Rove and meth. of Test. Plywood in Rolling Shear (Shear in Plane of ool Mountings (F arm Machinery) (1973) ANSI C33.97 Std. for Electrical Feeder and ves (1974) Std. for Bulbed Mechanically Locked Spindle Sell std. for Structural Flush Break Pull Mandrel Self

Std on Dimensions of Attachment Std for Safety for Electrical Attachment

Purpose, 277 Volts Ac, 15 Amperes, 21 es, 2 Pole, 1 Wire (1973)

res, 2 Pole, 2 Wire (1973)

rpose, 125 Volts, 15 Amperes, 2 Pole,/ rpose, 125/250 Volts, 15 Amperes, 3 P/ 20 Amperes, 3 Phase Wye, 4 Pole, 4 Wi 30 Amperes, 3 Phase Wye, 4 Pole, 4 Wi 20 Amperes, 3 Phase Wye, 4 Pole, $5 \mathrm{Wi}$ 30 Amperes, 3 Phase Wye, 4 Pole, 5 Wi mperes, 2 Pole, 2 Wire (1973)

mperes, 2 Pole, 3 Wire Locking, Groun/ mperes, 2 Pole, 2 Wire (1973) mperes, 2 Pole, 3 Wire Locking, Groun/ 20 Amperes, 3 Pole, 4 Wire Locking, G 30 Amperes, 3 Pole, 4 Wire Locking, G 20 Amperes, 3 Pole, 3 Wire Locking Ty/ 30 Amperes, 3 Pole, 3 Wire, Locking T/ mperes, 2 Pole, 3 Wire Locking, Groun/ mperes, 3 Phase, 3 Pole, 3 Wire Locki/ Std for Elevating Work Std. for

Std. Spec, for Refined Safety Std. for Std. for Chise Safety Std. for Receptacle Safety Std for Rec. Pract. for Spark

Spec. for Steel Cate. Std. for Self

Plates for Pressure Vessels (1972) ANSI G35.2

Plates for Pressure Vessels (1973) ANSI G35.4

Plates for Pressure Vessels (1973) ANSI G60.2

Plates for Special Applications (1971) ANSI C35.25

Plates for Structural Applications (1973)

Plates in Surface Condensers and Heat Exchangers (1973)

Plates of Improved Toughness (1973)

Plates of Structural Quality (1970) ANS1 C24.2

Plates or Control Buttons (1972)

Plates with Improved Transition Properties for Pressure

Plates (Patterns) Used in Battery Sockets (1974) ANSI C

Plates (Surgical implants) (1973)

Plates (Surgical lmplants) (1973)

Plates (1972)

Plates (1972)

Plates (1972) ANSI G33.3

Plates (1973)

Plates (1973)

Plates (1973) ANSI G35.22

Plates (1973) ANSI G35.24

Plates, Alloy Steel 5\% Chromium, 0.5\% Molybdenum (1972

Plates, Alloy Steel, Chromium Manganese Silicon (1972A)

Plates, Alloy Steel, High Strength, Quenched and Temper

Plates, Alloy Steel, Precipitation Hardening (Maraging)

Plates, Alloy Steel, Precipitation Hardening (Maraging)

Plates, Alloy Steel, Quenched and Tempered Nickel-Cob

Plates, Alloy Steel, Quenched and Tempered 8 and $9 \% \mathrm{Nic}$

Plates, Alloy Steel, 36\% Nickel (1972)

Plates, Alloy Steel, 9\% Nickel Double Normalized and Te.

Plates, and Papers (1971)

Plates, and Steel Sheet Piling (1970)

Thiosulfate and Silver Dens

Plates, Carbon Manganese, for Moderate and Lower Temper

Plates, Carbon Steel, High Sirength Manganese (1972A) a

Plates, Heat Treated, Carbon Manganese Silicon (1972A)

Plates, Nails and Spikes, Washers, Pins, Dowels, Joints

Plates, Quenched and Tempered Chromium, for Pressure Ve

Plates, Quenched and Tempered, for Pressure Vessels (19

Plates, Shapes, Sheet Piling, and Bars for Structural U

Plates, Structural Shapes, Rolled Floor Plates, and Ste

Plates, 5\% Nickel Alloy Steel, Specially Heat Treated (

Plates: Bolts; Closers; Hospital and Misc. Items; Key C

Platforms and Stage Lifts (Hydrualic and Hydropneumatic

Platforms (Lift) (1973)

Platforms (1972)

Platforms (1972)

Platforms (1973)

Platforms (1973)

Platforms (1973)

Spec. for Fabrica

Spec. for P

Platforms, Pavements, Driveways, and Interior Floors Ex

Plating, High Strength Steels, Cadmium (1973)

Platinum (1973)

Play Pipes for Water Supply Test. in Fire Protection Se

Playing Surface Systems (1972)

Pleating (1971) ANSI L14.240, ISO 105-4

Plied Yarn for Electrical and Packing Purposes (1974) a

Plies) (1969)

Plow, Field and Row Crop Cultivator Shafts and Ground T

Plug Combinations for Use in Hazardous Locations (1973)

Plug Fuses (Electrical) (1973)

Plug in Busways and Accessories (1972)

Plug, Aircraft Reciprocating Engine (1971)

Plug, Ball, and Check Valves for Petroleum Pipeline Val

Piugging Blind Rivet (1974)

Plugging Blind Rivets, Type 2A (1973)

Plugging, Mechanically Locked Spindle Rivet (1972)

Plugs and Receptacles (1973)

Plugs and Receptacles (1974)

Plugs and Receptacles, Locking Grounding Type, Specific

Plugs and Receptacles, Locking Type 125 Volts, 15 Amper

Plugs and Receptacles, Locking Type, 250 Volts, 20 Ampe

Plugs and Receptacles, Midget Locking Type, Specific Pu

Plugs and Receptacles, Midget Locking Type, Specific Pu

Plugs and Receptacles: Specific Purpose 120/208 Volts,

Plugs and Receptacles: Specific Purpose 120/208 Volts,

Plugs and Receptacles: Specific Purpose 120/208 Volts,

Plugs and Receptacles: Specific Purpose 120/208 Volts,

Std. Dimensions of Std. Dimensions of

Std. for Dimensions of

Std. Dimensions of

Std. Dimensions of

Std. Dimensions of

Std. Dimensions of

Std. Dimensions of

Std. Dimensions of

Std. Dimensions of

Std. Dimensions of

Plugs and Receptacles: Specific Purpose 125 Volts, 20 a

Plugs and Receptacles: Specific Purpose 125 Volts, 20 a

Plugs and Receptacles: Specific Purpose 125 Volts, 30 a

Plugs and Receptacles: Specific Purpose 125 Volts, 30

Plugs and Receptacles: Specific Purpose 125/250 Volts,

Plugs and Receptacles: Specific Purpose 125/250 Volts,

Plugs and Receptacles: Specific Purpose 125/250 Volts,

Plugs and Receptacles: Specific Purpose 125/250 Volts,

Plugs and Receptacles: Specific Purpose 250 Volts, 15

Std. Dimensions of Plugs and Receptacles: Specific Purpose 250 Volts, 15 a
Itd $S_{p}$ ASTM

ASTM

Meth. a ASTM

St ASTM

ASTM

ASTM

ASTM

Id. Sp ASTM

ANSI

ASTM

EIA

ASTM

ASTM

ANSI

ASTM

id Me ASTM

ASTM

ASTM

ASTM

ASTM

ASTM

ASTM

ASTM

ASTM

ASTM

ASTM

ASTM

ASTM

ANSI

AISI

ASTM

ASTM

ASTM

WWPA

A STM

ASTM

ASTM

AISI

ASTM

NBHA

CSI

ANSI

ALI

APl

API

API

ASTM

NSA

ASTM

UL

Std ASTM

AATCC

ASTM

Tent. ASTM

ASAE

UL

UL

NEMA

SAE

NSA

IFI

NSA

ANSI

UL

ANSI

ANSI

ANSI

ANS

ANSI

ANSI

ANSI

ANSI

ANSI

ANSI

ANSI

ANSI

ANSI

ANSI

ANSI

ANSI

ANSI

ANSI

ANSI

A285

A 387 
mperes, 2 Pole, 2 Wire (1973)

mperes, 2 Pole, 3 Wire Locking, Groun/ mperes 3 Phase, 3 Pole, 4 Wire Lockin/ mperes, 3 Phase, 3 Pole, 3 Wire Locki/ mperes, 3 Phase, 3 Pole, 3 Wire Locki/ mperes, 2 Pole, 3 Wire Locking, Groun/ mperes, 3 Phase, 3 Pole, 4 Wire Locki/ 0 Amperes, 2 Pole, 3 Wire Locking, Gr/ 0 Amperes, 2 Pole, 3 Wire Locking, Grl 20 Amperes, 3 Phase Wye, 4 Pole, 4 Wi 20 Amperes, 3 Phase Wye, 4 Pole, 5 Wi 30 Amperes, 3 Phase Wye, 4 Pole, 5 Wi 20 Amperes, 3 Phase Wye, 4 Pole, 4 Wi 30 Amperes, 3 Phase Wye, 4 Pole, $4 \mathrm{Wi}$ 20 Amperes, 3 Phase Wye, 4 Pole, $5 \mathrm{Wi}$ 30 Amperes, 3 Phase Wye, 4 Pole, 5 Wi mperes, 3 Phase, 3 Pole, 3 Wire Locki/ mperes, 2 Pole, 3 Wire Locking, Groun/ mperes, 3 Phase, 3 Pole, 4 Wire Locki/ mperes, 3 Phase, 3 Pole, 3 Wire Locki/ mperes, 2 Pole, 3 Wire Locking, Groun/ mperes, 3 Phase, 3 Pole, 4 Wire Locki/ mperes, 2 Pole, 3 Wire Locking, Groun/ mperes, 3 Phase, 3 Fole, 3 Wire Locki/ mperes, 2 Pole, 3 Wire Locking, Groun/ mperes, 3 Phase, 3 Pole, 4 Wire Locki/

30 Amperes, 3 Phase Wye, 4 Pole, 4 Wi nections, Outboard Water Strainers, Water Scoops and Drain Rec. Pract. for Preignition Rating of Spark

Type for Hazardous Locations (1973)

Std. Ferrous Pipe Std. for Electrica National Std. Uniform Uniform

Uniform

Uniform

Uniform

Uniform

Uniform

Uniform

Uniform

Uniform

Uniform

Uniform

Uniform

Uniform

Uniform

Uniform

Uniform

Uniform

Spec. for Supports for Off the Floor

(1973) Std. for Stainless Stee Std. for Vitreous China Std for Stainless Steel Std. for Enameled Cast Iron Spec. Data for Hubless Cast lron Sanitary System
Std. and Test Procedures for ansi A197. 1, Asse 1006

Units (1971) ANSI A 197.3, /

Std and Test Procedures for

ical Hot Water Dispensers (1973)

Guidelines, and Drawings for Copper Solvent Single Stack Design and Construction Requirements, and Installation of ing Trailers. Truck Campers, Motor Homes): Installation of Ans/ Std. Meth. of Test for Determination of Uranium and Std. Spec. for Nuclear Grade ectrometric, and Spectrochemical Analysis of Nuclear Grade std. Meth. of Test for Atom Percent Fission in Uranium and std. Meth. of Test for Atom Percent Fission in Uranium and Std. Spec. for Nuclear Grade

ical, Nuclear and Radio Chemical Analysis of Nuclear Grade ical, Nuclear, and Radiochemical Analysis of Nuclear Grade Std. Spec. for

nsi J2.6

Std. for Classification of Unirradiated Std. Meth. of Dynamic Test. for Std. Test Meth. for
Std. for Bias and Radial

(1969) td. Test Meth. for Strength Properties of Adhesives in Two Std. Spec for Noncarbonized, Single

Building Code: Wood Members and Their Fastenings (Lumber. k) (1973) Quality Stds. for sure Procl

Uniform Building Code Std. for Lumber, Timber,
Design Spec. for

71)

d Industrial Buildings (1971)
Plugs and Receptacles: Specific Purpose 250 Volts, 20 a Plugs and Receptacles: Specific Purpose 250 Volts, 20 a Plugs and Receptacles: Specific Purpose 250 Volts, 20 a Plugs and Receptacles: Specific Purpose 250 Volts, 20 a Plugs and Receptacles: Specific Purpose 250 Volts, 30 a Plugs and Receptacles: Specific Purpose 250 Volts, 30 a Plugs and Receptacles: Specific Purpose 250 Volts, 30 a Plugs and Receptacles: Specific Purpose 277 Volts Ac, 2 Plugs and Receptacles: Specific Purpose 277 Volts Ac, 3 Plugs and Receptacles: Specific Purpose 277/480 Volts, Plugs and Receptacles: Specific Purpose 277/480 Volts,

Plugs and Receptacles: Specific Purpose 277/480 Volts, Plugs and Receptacles: Specific Purpose 347/600 Volts, Plugs and Receptacles: Specific Purpose 347/600 Volts, Plugs and Receptacles: Specific Purpose 347/600 Volts,

Plugs and Receptacles: Specific Purpose 347/600 Volts, Plugs and Receptacles: Specific Purpose 480 Volts, 20 a Plugs and Receptacles: Specific Purpose 480 Volts, 20 a Plugs and Receptacles: Specific Purpose 480 Volts, 20 a Plugs and Receptacles: Specific Purpose 480 Volts, 30 a Plugs and Receptacles: Specific Purpose 480 Volts, 30 a Plugs and Receptacles: Specific Purpose 480 Volts, 30 a Plugs and Receptacles: Specific Purpose 600 Volts, 20 a Plugs and Receptacles: Specific Purpose 600 Volts, 30 a Plugs and Receptacles: Specific Purpose 600 Volts, 30 a Plugs and Receptacles: Specific Purpose 600 Volts, 30 a

Plugs and Receptacles? Specific Purpose 277/480 Volts,

Plugs (Boats) (1972)

lon of Seacocks, Through

Plugs (1970)

Plugs, Bushings, and Locknuts with Pipe Threads (1971)

Plugs, Receptacles and Connectors of the Pin and Sleeve

Plumbing Code (Including Definitions) (1973)

Plumbing Code (1973)

Plumbing Code: Battery Drainage System (1973)

Plumbing Code: Drainage Systems (1973)

Plumbing Code: Fixtures (1973)

Plumbing Code: Fuel Gas Piping (1973)

Plumbing Code: Indirect W aste Piping, Wet Vented System

Plumbing Code: Joints and Connections (1973)

Plumbing Code: Medium Pressure Gas Piping Systems (1973

Plumbing Code: Minimum Facilities Required for School,

Plumbing Code: Mobile Home (Trailer) Parks (1973)

Plumbing Code: Rainwater Systems, Roof Drains and Pipin

Plumbing Code: Rec. Rules for Sizing the Water Supply S

Plumbing Code: Swimming Pools (1973)

Plumbing Code: Traps and Interceptors (1973)

Plumbing Code: Vents and Venting (1973)

Plumbing Code: Water Distribution (1973)

Plumbing Code: Water Heaters and Vents (1973)

Plumbing Fixtures for Public Use (1972)

Plumbing Fixtures (Designed for Residential Use) (1962)

Plumbing Fixtures (Lavatory, Water Closet Bowl, Urinal)

Plumbing Fixtures (1971)

Plumbing Fixtures (1973)

(Plumbing Pipe and Fittings) (1972)

Plumbing Requirements for Household Dishwashers (1971)

Plumbing Requirements for Household Food W aste Disposer

Plumbing Requirements for Household Storage Type Electr

Plumbing System (1973)

Plumbing, Heating and Electrical Systems (1973) ANSI Al

Plumbing, Heating, and Electrical Systems (1972) ANSl a

Plutonium Concentrations and Isotopic Abundances (1970)

Plutonium Dioxide Powder, Sinterable (1974)

Plutonium Dioxide Powders and Pellets (1972) ANSI N104

Plutonium Fuel (Mass Spectrometric Meth.) (1969) ANSI N

Plutonium Fuel (Neodymium.148 Meth.) (1969) ANSI N118

Plutonium Metal (1972) ANSI N136

Plutonium Metal (1973)

Plutonium Nitrate Solutions (1973) /Etric, Spectrochem

Plutonium Nitrate Solutions (1973) ANSI N137

Plutonium Scrap (1972)

Ply Separation and Cracking of Rubber Products (1973) a

Ply Separation (Wet) of Solid and Corrugated Fiberboard

Ply Tires and Rims for Passenger Cars, Station Wagons,

Ply Wood Construction in Shear by Tension Loading (1970

Ply, Adding Machine Paper Rolls (1973)

Plywood and Components, Timber, Preservative Treatment,

Plywood and Particleboard Grades (Architectural Woodwor

Plywood and Utility Pole Preservative Treatment by Pres

Plywood Beams (1972)

Plywood Construction Guide for Residential Building (19

Plywood Construction Systems (Specs.) for Commercial an

Plywood Curved Panels (1968)

Plywood Curved Panels (1971)

Plywood Design Spec. (1966)

ANSI

ANSI

ANSI

ANSI

ANSI

ANSI

ANSI

ANSI

ANS1

ANSI

ANSI

ANSI

ANSI

ANSI

ANSI

ANSI

ANSI

ANSI

ANSI

ANSI

ANSI

ANSI

ANSI

ANSI

ANSI

ANSI

ANSI

$A B Y C$

SAE

NEMA

NAPHC

ICBO

ICBO

ICBO

ICBO

ICBO

ICBO

ICBO

ICBO

ICBO

ICBO

ICBO

ICBO

ICBO

ICBO

ICBO

$1 \mathrm{CBO}$

ICBO

ANSI

USC

IAPMO

ANSI

CISPI

AHAM

AHAM

ASSE

CDA

NFPA

NFPA

ASTM

ASTM

ASTM

ASTM

ASTM

ASTM

ASTM

ASTM

ASTM

ANSI

ASTM

ASTM

TRA

ASTM

ANSI

ICBO

$A$ WI

ICBO

APA

APA

APA

APA

APA

Fabrication Spec. for

C73.71

C73.75

C73.85

C 73.99

C73. 100

C73.76

C73.86

C73.77

C73.78

C73.106

C73.92

C73.93

C73.108

C73.109

C73.94

C73.95

C73.101

C73.79

C 73.87

C73.102

C 73.80

C73.88

C73.81

C73.103

C73.82

C73.89

C73.107

$\mathrm{H} 27$

J549A

FBll 
cteristics, Material Composition, Use, Applicati/ Std. Meth. of Test. Tent. Meth. of Test. Tent. Meth. of Test.

Uniform Building Code Std. for Softwood ing Construction Under the Uniform Building Code: Softwood Rec. for Protection of Design Spec. for Flat Fabrication Spec. for building Code Std. for Structural Glued Built Up Members.

Design Spec. for Performance Spec. for Adhesives for Field Gluing std. Meth. of Test for Strength Properties of Adhesives in td. Test Meth for Permanence of Adhesive Bonded Joints in rior Panel Coatings and Overlays on DFPA Grade Trademarked Std. Definitions of Terms Relating to Veneer and Structural Design Guide for Hardwood $r$ Exterior Coatings and Overlays on DFPA Grade Trademarked
Sid. Meth. of Test for Shear Modulus of on Met/ the All Weather Wood (Pressure Treated Lumber and si 07.1

eeting (1972)

entation) (1970)

Std. Meth. of Test. Veneer. Std. Meth. of Test for

Std. for Air Pressures for Std. for Air Pressures for

1972)

Std. Interfaces for 4 Way General Purpose Industrial Rec. for Terminal Facilities for struction, Care, and Use of Shears (Mechanical, Hydraulic, Performance Spec. for Direct and Indirect Reading Std. Meth. of Test for Annealing 71) Std Meth of Test for Annealing ol, Cleaning Fluids, Flammable Aerosols, etc. with a Flash Std. Meth. of Test for Flash

of Water lnsoluble Impurities in Refined Phenol by Cloud gricultural Wheel Tractors (1972) SAE J715

Std for 3 ultural Wheeled Tractors (1972)

1 Tractors Equipped with Quick Attaching Coupler for Three ft Force Capacity on Agricultural Tractors Equipped with 3 ft Force Capacity on Agricultural Tractors Equipped with 3 hemistry) (1967)

y Within Temperature Range / Meth. of Analysis of Cloud Meth. of Analysis of Congeal Rec. for Flash

exural Strength of Concrete (Using Simple Beam with Center hemistry) (1967) Capillary Meth. of Analysis of Melting ter (1973)

Use of the Refractometer for Determining the Freezin Tent. Meth. of Test for Flash Std. Meth. of Test for Smoke Std. Meth. of Test for Flash Std Meth. of Test for Flash Std. Meth. of Test for Wax Appearance Std. Meth. of Test for Boiling Std. Meth. of Test for Annealing Point and Strain Std. Meth. of Test for Annealing Point and Strain Std. Meth. of Test for Softening Std. Meth. of Test for Flash Std. Meth. of Test for Flash

related Products) by Setafl/ 1972) ANS1 Zll.207 stry) (1967)

si Z11.61 Std Meth of Test for Dropping Wiley Meth. of Analysis of Melting Std. Meth. of Test for Pour Std. Meth. of Test for Congealing Meth. for Congealing lysis of Plain Carbon and Low Alloy Steel for Boron by the emical Analysis of Plain Carbon and Low Alloy Steel by the for Spectrochemical Analysis of Blast Furnace lron by the rochemical Analysis of Admiralty Metal by the Cast Pin and to Lawn and Garden Riding Tractors (1972) Std. for One r Structural Steel with 42,000 psi (290 MPa) Minimum Yield Analytical Meth. for Determining Inherent Viscosity (One Std. for Carbide Blanks and Cutting Tools, Single Mechanical Demand Registers (Cumulative and Std. Meth. of Test for Flash and Fire Stds. for Staples with Rolled or Slas

73) stry) (1962) tidotes, Supplies (Packaging), and References (Dosage) for ( Teth. Of Analysis of Food emolina; Also, with Slight Modification, to Corn, Rye, B etal Butt Seals and Test. for Expansion Characteristics by 1 Sandwich Seal and Test. for Expansion Characteristics by meth. for Making Potentiostatic and Potentiodynamic Anodic an's Climbing Equipment (Body Belt, Safety Strap, Lanyard, orthern White/ Sid. for Preservative Treatment of Incised building Code Std. for Lumber, Timber, Plywood and Utility unding Type, Specific Purpose, 277 Volts Ac, 15 Amperes, ugs and Receptacles, Locking Type 125 Volts, 15 Amperes, 2
Plywood Guide (Spec. for Texture and Grade, Panel Chara Plywood in Flexure (1972)

Plywood in Rolling Shear (Shear in Plane of Plies) (196

Plywood in Shear Through the Thickness (1969)

Plywood Lumber Beams Fabrication Spec. (1971)

Plywood Lumber, Construction and Industrial (1973)

Plywood Paneling (1973)

Plywood Plants (1972)

Plywood Sandwich Panels (1970)

Plywood Sandwich Panels (1971)

Plywood Stressed Skin and Curved Panels, Beams, and Com

Plywood Stressed Skin Panels Fabrication Spec. (1971)

Plywood Stressed Skin Panels (1972)

Plywood to Wood Framing (1971)

Plywood Type Construction in Shear by Tension Laading (

Plywood Under Mold Conditions (1970)

Plywood (1967)

Plywood (1970) ANSI 07.2

Plywood (1971)

Plywood (1971)

Plywood (1972)

Plywood) Foundation System: House Design and Constructi

Plywood, and Other Glued Veneer Constructions (1972) an

Pneumatic Ball Impact Resistance of Plastic Film and Sh

Pneumatic Controllers and Transmission Systems (Instrum

Pneumatic Controllers and Transmission Systems (1967)

Pneumatic Directional Control Valves (1973) ANSI B93.33

Pneumatic Transfer of Dry Bulk Chemicals and Plastics (

Pneumatic, Semi and Automatic, and Manually Powered) (1

Pocket Dosimeters for X-And Gamma Radiation (1972)

Point and Strain Point of Glass by Beam Bending (1972

Point and Strain Point of Glass by Fiber Elongation (19

Point Below 200 Deg. F) (1973)

Rosene, Acetone

Point by Pensky-Martens Closed Tester (1973) ANSI Z11

Point Depression (1968) A NSI 778.21

Ion and Esti

Point Free Link Attachment for Hitching Implements to a

Point Free Link Hitch Attachment of Implements to Agric

Point Hitch (1972)

Ocedure for Measuring Hydraulic Li

Point Hitch (1972)

/Ocedure for Measuring Hydraulic Li

Point in All Edible Animal and Vegetable Fats (Cereal C

Point in Fats and Fat Mixtures Which Congeal or Solidif

Point Index of Trade Name Liquids (NFPA 325A) (1974)

Point Loading) (1968) ANSI A37.84 /Eth. of Test for Fl

Point of All Normal Animal and Vegetable Fats (Cereal ${ }^{\circ} \mathrm{C}$

Point of Aqueous Engine Coolants (1974) /. Rec. Pract.

Point of Aviation Turbine Fuels by Setaflash Closed Tes

Point of Aviation Turbine Fuels (1972) ANSI Z11.137

Point of Chemicals by Closed Up Meth. (1973)

Point of Cutback Asphalt with Tag Open Cup Apparatus (1

Point of Distillate Fuels (1972) ANSI Z11,325

Point of Engine Antifreezes (1972) ANSI D14.1

Point of Glass by Beam Bending (1972)

Point of Glass by Fiber Elongation (1971)

Point of Glass (1973)

Point of Liquids by Tag Open Cup Apparatus (1972)

Point of Liquids (Paint, Enamel, Lacquer, Varnish, and

Point of Lubricating Grease of Wide Temperature Range (

Point of Normal Animal and Vegetable Fats (Cereal Chemi

Point of Petroleum Oils (1971) ANSI Z11.5

Point of Petroleum Waxes Including Petrolatum (1971) an

Point of Petroleum Waxes Including Petrolatum (1972)

Point to Plane Arc Technique (1970) ANSI Z128.29

Point to Plane Technique Utsing an Optical Emission Spec

Point to Plane Technique Using an Optical Emission Vacu

Point to Plane Techniques (1973)

Std. Meth. for

Point Tubular Sleeve Attachment for Hitching Implements

Point (1/2 ln. (12.7 Mm) Maximum Thickness) (1972) ANSI

Point) Content in Corn Starch (1963)

Point, Carbide Tipped, Roller Turner Type (1972)

Pointer Forms) EEI MSJ.4

Points by Cleveland Open Cup (1972) AASHO T48, ANSI Z11

Points (1971) EEI TDJ.14

Points, Closed Cup, Aromatic Chemicals and Isolates (19

Poison Control Centers (1972)

Poisoning Staphylococcus in Food Products (Cereal Chemi

Poisson's Ratio for Glass and Glass Ceramics by Resonan

Polarimetric Meth. of Analysis of Starch in Flour and S

/ Making Reference Glass-M

Polarimetric Meth. (1973)

Polarimetric Meth. (1973)

Polarization Measurements (1972)

Pole and Tree Climber) (1973)

/King Reference Glass-Meta lor Std. Reference

Pole Butts by the Thermal Process (Western Red Cedar, N

Pole Preservative Treatment by Pressure Processes and $Q$

Pole 3 Wire (1973)

Pole, 1 Wire (1973)
Plugs and Receptacles, Locking Gro Std. Dimensions of Pl
APA

CRA

STM

ASTM

ASTM

APA

ICBO

ICBO

APA

APA

ICBO

APA

$A P A$

APA

ASTM

HPMA

APA

NFTM

ASTM

ASTM

ISA

SAMA

NFLDP

MCA

ANSI

ANSI

ASTM

ASTM

ICBO

ASTM

ASE

ASAE

SAE

$\mathrm{SAE}$

ACCH $58.12 \mathrm{~A}$

FMS

ASTM

AACCH

ASTM

ASTM

ASTM

ASTM

ASTM

ASTM

ASTM

ASTM

ASTM

ASTM

ASTM

ASTM

$\mathrm{AACCH}$

ASTM

ASTM

TAPPI

I Ana ASTM

ASTM

ASTM

ASTM

ASAE

ASTM

CR

ANSI

NEMA

ASTM

NEMA

EOA

AAPCC *1

AACCH 42.30

ASTM C623

AACCH $76-20$

ASTM F140

ASTM F144

STM

EEI

AWPA

ICBO

G5

AP. 2

NSI

UBCS25.12

C73. 43

C73.31 
gs and Receptacles, Locking Type, 250 Volrs, 20 Amperes, 2 Pole, 2 Wire (1973) and Receptacles: Specific Purpose 125 Volts, 30 Amperes, 2 Pole, 2 Wire (1973) and Receptacles: Specific Purpose 250 Volts, 20 Amperes, 2 Pole, 2 Wire (1973) and Receptacles: Specific Purpose 125 Volts, 20 Amperes, 2 t Locking Type, Specific Purpose, 125 Volts, 15 Amperes, 2 receptacles: Specific Purpose 125/250 Volts, 20 Amperes, 3 tacles: Specific Purpose 250 Volts, 30 Amperes, 3 Phase, 3 tacles: Specific Purpose 480 Volts, 20 Amperes, 3 Phase, 3 tacles: Specific Purpose 480 Volts, 30 Amperes, 3 Phase, 3 tacles: Specific Purpose 600 Volts, 30 Amperes, 3 Phase, 3 tacles: Specific Purpose 250 Volts, 15 Amperes, 3 Phase, 3 tacles: Specific Purpose 250 Volts, 20 Amperes, 3 Phase, 3 and Receptacles: Specific Purpose 600 Volts, 30 Amperes, 2 and Receptacles: Specific Purpose 125 Volts, 20 Amperes, 2 and Receptacles: Specific Purpose 125 Volts, 30 Amperes, 2 and Receptacles: Specific Purpose 250 Volts, 15 Amperes, 2 and Receptacles: Specific Purpose 250 Volts, 20 Amperes, 2 and Receptacles: Specific Purpose 250 Volts, 30 Amperes, 2 and Receptacles: Specific Purpose 480 Volts, 20 Amperes, and Receptacles: Specific Purpose 480 Volts, 30 Amperes, and Receptacles: Specific Purpose 600 Volts, 20 Amperes, 2 Receptacles: Specific Purpose 277 Volts Ac, 20 Amperes, 2 Receptacles: Specific Purpose 277 Volts Ac, 30 Amperes, 2 cking Type, Specific Purpose, 125/250 Volts, 15 Amperes, receptacles: Specific Purpose 125/250 Volts, 30 Amperes, 3 specific Purpose $347 / 600$ Volts, 20 Amperes, 3 Phase Wye, 4 specific Purpose $347 / 600$ Volts, 30 Amperes, 3 Phase Wye, 4 specific Purpose 120/208 Volts, 20 Amperes, 3 Phase Wye, 4 specific Purpose 120/208 Volts, 30 Amperes, 3 Phase Wye, 4 specific Purpose $277 / 480$ Volts, 20 Amperes, 3 Phase Wye, 4 specific Purpose 277/480 Volts, 30 Amperes, 3 Phase Wye, 4 ptacles: Specific Purpose 250 Volts, 20 Amperes 3 Phase, 3 tacles: Specific Purpose 250 Volts, 30 Amperes, 3 Phase, 3 tacles: Specific Purpose 480 Volts, 20 Amperes, 3 Phase, 3 tacles: Specific Purpose 480 Volts, 30 Amperes, 3 Phase, tacles: Specific Purpose 600 Volts, 30 Amperes, 3 Phase, 3 receptacles: Specific Purpose $125 / 250$ Volts, 20 Amperes, 3 receptacles: Specific Purpose 125/250 Volts, 30 Amperes, 3 specific Purpose 120/208 Volts, 20 Amperes, 3 Phase Wye, specific Purpose $120 / 208$ Volts, 30 Amperes, 3 Phase Wye, 4 specific Purpose $277 / 480$ Volts, 20 Amperes, 3 Phase Wye, 4 specific Purpose 277/480 Volts, 30 Amperes, 3 Phase Wye, specific Purpose 347/600 Volts, 20 Amperes, 3 Phase Wye, 4 specific Purpose $347 / 600$ Volts, 30 Amperes, 3 Phase Wye, tive Treatment by Pressure Processes) (19/ Std. for Round Std. Guideline for the Physical Inspection of Wood
Std. for Lodgepole Pine Wood 73) Uniform Building Code Std. for Wood Std Spec. and Dimensions for Wood

for Preservative Treatment by Pressure Processes of Timber atment of Western Red Cedar and Alaska Yellow Cedar Timber

lishing Rec. Design Stresses for Round Timber Construction

n Red Cedar, Northern White Cedar and Alaska Yellow Cedar coal Tar Creosote for the Preservative Treatment of Piles, r, Plywood and Components, Timber, Preservative Treatment, ries (1/ Rec. Domestic Marketing Std. Including Guarantee

edure) for Products Classified as to Slip Resistant (Floo Std. Meth. of Test for Detergent Resistance of Floo (1969) Spec, for Water Emulsion Floo

mination of the Reflectance of the Organic Components in shampooer,/ Std. for Safety for Floor Finishing Machines Std. Definitions of Terms Relating to $W$ ax Std. Meth. of Test for Sediment in Water Emulsion Std. Rec. Pract. for Rating Water Emulsion Floo

Water Emulsion, Solvent Based, and Polymer Emulsion Floor Meth. of Test for Total Ash and Silica in Water Emulsion Std. Meth of Test for Soil Resistance of Floor Std Meth of Test for Water Spotting of Emulsion Floor 972) Std. Rec. Pract for Test. Pavement Rec. for Scrubbers for

Rec. Pract, for Exhaust Emission Controls (Air td. Meth. for Evaluation of and Test Formulas for Solution ) (1967) Ansi/ Std. Meth. of Test for Quality of Extruded

1973)

e Diameter (1973)

19 Std. Spec. for Fille Std. Spec. for Std. Spec. for Std. Spec. for Std. Spec. for

Std. Meth for Analysis of Environmental Materials Std. Spec. for Extra Heavy Duty Black Std. Spec. for Heavy Duty Black
Std. Spec. for General Purpose

8.32

8.31 graphic Film for Archival Records, Silver Gelatin Type, on Std. Spec. for
Pole, 2 Wire (1973)

Pole, 2 Wire (1973)

Pole, 3 Wire Locking Type (1973)

Pole, 3 Wire Locking Type (1973)

Pole, 3 Wire Locking Type (1973)

Pole, 3 Wire Locking Type (1973)

Pole, 3 Wire Locking Type (1973)

Pole, 3 Wire Locking Type (1973)

Pole, 3 Wire Locking Type (1973)

Pole, 3 Wire Locking, Grounding Type 1973)

Pole, 3 Wire Locking, Grounding Type (1973)

Pole, 3 Wire Locking, Grounding Type (1973)

Pole, 3 Wire Locking, Grounding Type (1973)

Pole, 3 Wire Locking, Grounding Type (1973)

Pole, 3 Wire Locking, Grounding Type (1973)

Pole, 3 Wire Locking, Grounding Type (1973)

Pole, 3 Wire Locking, Grounding Type (1973)

Pole, 3 Wire Locking, Grounding Type (1973)

Pole, 3 Wire Locking, Grounding Type (1973)

Pole, 3 Wire Locking, Grounding Type (1973)

Pole, 3 Wire (1973)

Pole, 3 Wire, Locking Type (1973)

Pole, 4 Wire Locking Type (1972)

Pole, 4 Wire Locking Type (1972)

Pole, 4 Wire Locking Type (1973)

Pole, 4 Wire Locking Type (1973)

Pole, 4 Wire Locking Type (1973)

Pole, 4 Wire Locking Type (1973)

Pole, 4 Wire Locking, Grounding Type (1973)

Pole, 4 Wire Locking, Grounding Type (1973)

Pole, 4 Wire Locking, Grounding Type (1973)

Pole, 4 Wire Locking, Grounding Type (1973)

Pole, 4 Wire Locking, Grounding Type (1973)

Pole, 4 Wire Locking, Grounding Type (1973)

Pole, 4 Wire Locking, Grounding Type (1973)

Pole, 5 Wire Locking, Grounding Type (1973)

Pole, 5 Wire Locking, Grounding Type (1973)

Pole, 5 Wire Locking, Grounding Type (1973)

Pole, 5 Wire Locking, Grounding Type (1973)

Pole, 5 Wire Locking, Grounding Type (1973)

Pole, 5 Wire Locking, Grounding Type (1973)

Std. Dimensions of Plu ANSI

td. Dimensions of Plugs ANS

d. Dimensions of Plugs ANS

Poles and Posts Used in Building Construction (Preserva

Poles in Service (1972)

Poles Preservative Treatment by the Full Length Thermal

Poles That Are to Be Given a Preservative Treatment (19

Poles (1972)

Poles (1973)

Poles (1973)

Poles (1974)

Poles) (1973)

Std. AWPA Std. Spec. and Meth. for Estab ASTM

Poles, and Timbers for

Poles, Piles, Spaced Columns, Joints, Screws, Bolts, Co

Policy for Motor Vehicle Type (Lead Acid) Storage Batte

Policy on Geometric Design of Rural Highways (1965)

Polish and Wax) (1971) ASTM D2047. CSMA Bul 308-70

Polish Films (1973)

Polish for Use on Asphalt or Vinyl Asbestos Tile Floors

Polisher, Scrubber, Sander, Scraper, Tile Remover, Ru

Polishes and Related Materials (1973)

Polishes by Centrifuge (1972) ANSI Z123.3

Polishes (1972)

Polishes (1972)

Polishes (1972) ANSI Z123.

Polishes (1973)

Polishes (1974) ANSI 2123.12

Polishing in the Laboratory (Full Scale Wheel Meth.) (1

Pollution Control (1974)

Pollution) for Powered lndustrial Trucks (1973)

Poly-Butadiene Rubbers (BR) (1973)

Poly (Vinyl Chloride) Plastic Pipe by Acetone lmmersion

Poly (Vinyl Chloride) (PVC) Sewer Pipe (1972)

Polybutylene Plastics Molding and Extrusion Materials (

Polybutylene (PB) Plastic Pipe (SDR.PR) Based on Outsid

Polybutylene (PB) Plastic Pipe (SDR.PR) (1973) ANSI B72

Polybutylene (PB) Plastic Tubing (1973)

Polychlorinated Biphenyls (1974)

Polychloroprene Jacket for Wire and Cable (1973)

Polychloroprene Jacket for Wire and Cable (1973) ANSI C

Polychloroprene Jacket for Wire and Cable (1973) ANSI C

Polyester Base (1973)

Std. Spec.

Polyester Film Pressure Sensitive Electrical
Polyester Glass Mat Sheet Laminate (1973)
C 73.32

C73.70

C73.71

C73.69

C73.29

C73.96

C73.100

C73.101

C73.102

C 73.103

C73.98

C73.99

C73.82

C73.72

C73.73

C73.74

C73.75

C73.76

C73.79

C73.80

C73.81

C73.77

C 73.78

C73.30

C73.97

C 73.108

C73.109

C73.104

C73. 105

C73.106

C73.107

C73.85

C73.86

C73.87

C73.88

C73.89

C73.83

C73.84

C73.90

C73.91

C73.92

C73.93

C73.94

C73.95

C23.72

M13.72

C10

UBC 25.13

05. 1

C4

C 8
D3200

C7

D390

UBC $* 3-25$

ASTM

ICBO

*1.15

Oc CSMA BUL 12

ASTM D3207

RTI

*6

D2798

561

D2825

D1290

D3052

D2834

D1 288

D3206

D1793

E451

10-11

$6 \mathrm{~F} 1$

D3189

D2152

D2836

D2581

D3000

D2662

D2666

D3304

D2819

D 752

D753

PHI.41

D2484

D1532 
National Bureau of Standards KWIC Index of Engineering Standards

inguishing (1973)

Cloth, Type E Glass, B Stage Std. Meth. of Test for Isophthalic Acid in Alkyd and Std. for Glass Fiber Reinforced Guide Spec. for Specifying: Reinforced Fiberglass lled Outside Diameter (1974) Std. Spec. for Std. Meth of Test for Drop Impact Resistance of Std. Meth. of Test for Vinylidene Unsaturation in Std. for Smooth Wall Coilable

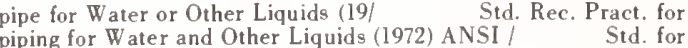
piping for W ater and Other Liquids (1972) ANSI St. Spec. for Insert Type
pipe (1973) ion and Distributi/ Std. for Crossed Linked Thermosetting Std. Spec. for able (1972) Std. Spec, for for Insert Type Polyethylene Fusion Fittings for SDR 11.0

lating Tape (1973)

e and Tubing (1973)

973) ANSI K65.160

973) ANSI K65.159

Rec. for Coiling Std. Spec. for

1

Std. Spec. for Butt Heat Fusion Std. Spec. for Butt Fusion Std. Spec. for Butt Fusion Std. Spec, for

Std. Spec. for Plastic Insert Fittings for ed on Outside Diameter (1974)

Std. Spec, for Str. Spec, for Std. Spec, for

olled Outside Diameter/ Std. Spec. for Biaxially Oriented Meth. for Measuring Flat Spring Test for Hot Tack of Wax Std. Test Meth. for atter (Total Solids) in Water Emulsion. Solvent Based, and ical Appliances (1973)

1971) ANSI N4.1 Std. for Safety for Test for

Temperature Impact Test for Fabrics Coated with Flexible Protec

Rec. for Application of Plastic Std. Rec. Pract. for Qualitative Identification of

tter (1971) Std. Meth. of Test for Solubility Range of Resins and

tion (1972) Std. Test Meth. for Tent. Spec. for

Std. Rec. Pract. for Flaring spec for Electrical Insulation Plastic Tubing, Irradiated Std. Meth. of Test for Std. Spec. for Thermoplastic aterials (1973) nstallations of Electrical W/

Std. Spec. for Reinforced teel Conduit and Electrical Metallic Tubing (19/

Std. Rec. Pract. for Making Solvent Cemented Joints with guide Specs., and Materials Application for Thermoplastic

terials (1973) Std. for Rigid Std. Spec, for Std. Meth. for Chemical Analysis of Alcohol,
cal Insula/ Sid. Spec. for Crosslinked and Noncrosslinked b72.2 and Reservoir Lining (1972T) Std. Spec. for

Spec, for Flexible Std. Spec. for ion Systems (1973) Std. Spec. for Chlorinated acrylonitrile Butadiene Styrene (ABS) Pipe and Fittings to Std. Spec, for Bell End Std. Spec. for Std. Spec, for

t Pipe and Fittings (1973) ANSl K65.56 et Type (1973)

973) ANSI B72.16

ule 80 (1973) ANSI K65.166

ule 40 (1973)

ule 80 (1973)

80 and 120 (1973) ANSI B72.7

72.22

2)

3)

Std. Spec. for Solvent Cements for Std. Spec. for Threaded Std. Spec. for Socket Type Std. Spec. for Socket Type Std. Spec. for Std. Spec. for Std. Spec. for Std. Spec. for Type Psp

Std. Spec. for Type Psm Std. Spec. for ec. for Flexible Poly(vinyl Chloride) Plastic Sheeting for ck (North), Eastern White Pine (North), Hem-Fir (North), Std. for Sid. for

form Building Code Std. for Douglas Fir; Larch; Hem-Fir; niform Swimmin

Std. for Gas Fired Swimmin

Std. for Safety for Swimmin Rec, for Thermoplastic Piping for Swimming Std. for Test Kits for Swimmin stness of Colored Textile Yarns and Fabrics to Chlorinated basement Floor, Sump Conductor, Downspout Runoff, Swimming Reinforced Brick Masonry Swimmin Suggested Minimum Stds.
Polyester Resin Impregnated, 181 Style Fabric, Self Ext Polyester Resins (1973)

Polyester Structural Plastic Panels (1972)

Polyester Tanks (1973)

Polyethelene (PE) Plastic Pipe (SDR-PR) Based on Contro

Polyethylene Blow Molded Containers (1970)

Polyethylene by Infrared Spectrophotometry (1972)

Polyethylene Electrical Plastic Duct (1972)

Polyethylene Encasement for Gray and Ductile Cast Iron Polyethylene Encasement for Gray and Ductile Cast Iron Polyethylene Fusion Fittings for SDR 11.0 Polyethylene Polyethylene Insulated Wire and Cable for the Transmiss Polyethylene Insulated Wire and Cable (1972)

Polyethylene Jacket for Electrical Insulated Wire and C Polyethylene Pipe (1973)

Polyethylene Plastic Pipe and Tubing (1972)

Polyethylene Plastic Pressure Sensitive Electrical Insu

Polyethylene (PE) Plastic Fittings for (PE) Plastic Pip

Polyethylene (PE) Plastic Pipe Fitings, Schedule 40 (1

Polyethylene (PE) Plastic Pipe Fittings, Schedule 80 (1

Polyethylene (PE) Plastic Pipe (SDR-PR) (1974) ANSI B72

Polyethylene (PE) Plastic Pipe (1973)

Polyethylene (PE) Plastic Pipe, Schedule 40 (1974)

Polyethylene (PE) Plastic Pipe, Schedules 40 and 80 Bas

Pe (PE) Plastic Tubing (1974)

Polyethylene (PEO) Plast ic Pipe (SDR-PR) Based on Contr

Polymer Blends (1972)

Polymer Content of Styrene Monomer (1974) ANSI Z161.1

Polymer Emulsion Floor Polishes (1972) / Nonvolatile M

Polymeric Enclosures for Portable Cord Connected Electr

Polymeric Materials for Service in lonizing Radiation (

Plymenic Materials (1973)

Polymers in Dry Powder Form by Means of Spray Gun or Fl

Polymers in Emulsion Paints (1973)

Polymers (1972)

Polynuclear Aromatic Hydrocarbons in Air Particulate Ma

Polyolefin Heat Shrinkable Tubing for Electrical Insula

Polyolefin Monofilaments (1973)

Polyolefin Pipe and Tubing (1972)

Polyolefin, Pigmented, Flexible, Heat Shrinkable (1973)

Polystyrene Blocks in Sbr Rubbers and Latices (1974)

Polyterephthalate Molding and Extrusion Materials (1973

Polyterphthalate Thermo-Plastic Molding and Extrusion M

Polythionic Acids (1973)

Bility of Stainles

Polyvinyl Chloride Ducts and Fittings for Underground I

Polyvinyl Chloride (PVC) Jacket for Wire and Cable (197

Polyvinyl Chloride (PVC) Pipe and Fittings (1973) ANSI

Polyvinyl Chloride) Duct Construction (1974)

Polyvinyl Chloride) (PVC) Plastic Wall Siding (1972)

Polyvinylidene Fluoride (PVDF) Molding and Extrusion Ma

Poly(vinyl Butyral) (1973)

Poly(vinyl Chloride) Heat Shrinkable Tubing for Electri

Poly(vinyl Chloride) Plastic Pipe (SDR.PR) (1973) ANSI

Poly(yinyl Chloride) Plastic Sheeting for Pond, Canal,

Poly(vinyl Chloride) Resins (1972) ANSI K65.207

Poly(vinyl Chloride) (CPVC) Plastic Hot Water Distribut

Poly(vinyl Chloride) (PVC) Pipe and Fittings for Nonpre

Poly(vinyl Chloride) (PVC) Pipe (1973) ANSI B72.20

Poly(vinyl Chloride) (PVC) Plastic Drain, Waste and Ven

Poly(vinyl Chloride) (PVC) Plastic Line Couplings, Sock

Poly(vinyl Chloride) (PVC) Plastic Pipe and Fittings (1

Poly(vinyl Chloride) (PVC) Plastic Pipe Fittings, Sched

Poly(vinyl Chloride) (PVC) Plastic Pipe Fittings, Sched

Poly(vinyl Chloride) (PVC) Plastic Pipe Fjttings, Sched

Poly(vinyl Chloride) (PVC) Plastic Pipe, Schedules 40.

Poly(vinyl Chloride) (PVC) Plastic Tubing (1973) ANSI B

Poly(vinyl Chloride) (PVC) Sewer Pipe and Fittings (197

Poly(vinyl Chloride) (PVC) Sewer Pipe a,d Fittings (197

Poly(vinyl Chloride) (PVC) Sewer Pipe and Fittings (197

poly(Vinylidene Fluoride) Heat Shrinkable Tubing (1973)

Pond, Canal, and Reservoir Lining (1972T)

Ponderosa and Western White Pines, Spruce-Pine-Fir.

Ponderosa Pine Doors (1958)

Ponderosa Pine Doors (1973)

Ponderosa, Idaho White, Sugar, and Lodgepole Pines; Eng

Pool Code (1973)

Pool Heaters (1972)

Pool Pumps, Filters, and Chlorinators (1972)

Pool Water Circulation Systems (1972)

Pool Water (1970)

Pool Water (1972) ANSI L14.149

Pool, etc.) (1973) ANSI A 176.3

Pools (Tech. Notes) (1967)

Pools (1969)
Std. Meth of Lo

SAE

ASTM

USC

CSI

CSI M

ASTM

ASTM

NEMA

ASTM

AWW

ASTM

NEMA

ASTM

ASTM

SPI

ASTM

ASTM

ASTM

ASTM

ASTM

ASTM

ASTM

A STM

TAPPI

ASTM

ASTM

ASTM

FMS

ASTM

ASTM

ASTM

ASTM

ASTM

ASTM

SAE

ASTM

A STM

A STM

NEMA

NEMA

ASTM

ASTM

Stds., SMACN

USC

ASTM

ASTM

ASTM

ASTM

ASTM

ASTM

ASTM

ASTM

ASTM

ASTM

ASTM

ASTM

A STM

ASTM

STM

ASTM

ASTM

ASTM

(

ASTM

Sp ASTM

ICBO

USC

NWMA

ICBO

IAPMO

ANSI

UL

SPI

NSF

Test Meth for Colorfa /Undation Drain, Under

AATCC

ASTM

BIA
NSPI

AS3827 
for Multiport Valves for Public and Residential Swimming Std. for Sand Type Filters for Swimming Std. for Diatomite Type Filters for Swimming Suggested Minimum Stds. for Residential Swimming derwater Lighting Fixtures and Junction Boxes for Swimming Uniform Plumbing Code: Swimming

Recommendation for Installation of Ceramic Tile Swimming on Meth. of Analysis for Insect and Rodent Filth in Popped

for Internal Insect Infestation in Cereal Grains, Unpopped ysis of Macroscopic Examination of Cereal Grains, Unpopped

$t$ and Rodent Hair Contamination of Cereal Grains, Unpopped ation Test for Internal Insects in Cereal Grains, Unpopped

flotation Meth. of Analysis for Insect and Rodent Filth in

z167. 1

2 167.10 td. Definitions of Terms Relating to Std. Meth. of Test for Continuity of Std. Meth. of Test for Fusion Flow of Std Meth of Test for Spalling Resistance of d. Meth. of Test for Reboiling Tendency of Sheet Steel for . for Production and Preparation of Gray lron Castings for Std. Spec. for Steel Sheets for

1)

Std. Meth. of Test for Alkali Resistance of

Std. Wet Process

Std. Wet Process

Std. Wet Process

Std. Application Guide for

Std. Meth. of Test for Specific Gravity and Std. Meth. of Test for oil $\mathrm{lm} / \quad$ Std. Meth. of Test for Density and Interconnected meth of Test for Water Absorption. Bulk Density, Apparent and Bulk Density of Burn/ Std. Meth. of Test for Apparent Test Meth. for Adhesion to Non Test Meth for Adhesion to

loth, etc.) (1973) Safety Std. for Electric Baseboard Heating Equipment Construction Spec. for Rifle Bench Rest

ign. Construction and Stowage of Portable Fuel Systems and Std. for Safe:y for Test for Polymeric Enclosures for ocations, Class 1, Groups C and D, A/ Std. for Safety for Std. for Safety for

Industry Standard for Household Commercial and C33.11 Safety Std. for Electric Heating Appliances
Stds. and Pract. for Design, Construction and Stowage of Std. for Driving and Spindle Ends for Std. for Gas Hose Connectors for Std. for Safety for

Cables with Ethylene-Propylene-Rubber Insu/ Std. for Std. for Safety for Measuring Surface Frictional Properties Using the British Std. for Safety for Safety Sid, for )

Performance Spec. for

Safety Std. for Acetylene Generators. d. for Cranes, Derricks, Hoists, Hooks, Jacks, and Slings: or Compressive Strength of Hydraulic Cement Mortars (Using Uniform Building Code Std. for Std. Spec. for tucco) and Interior (1971) tucco) and Interi/ sho T153, ANSI A 1.19

Std. Spec. for Lathing and Furring for Std. Meth. of Test for Fineness of Std. Meth. of Test for Fineness of 3) Recommendations for Basis of Payment on Rec. Spec. for Optimum Size Coarse Aggregate for r Fly Ash and Raw or Calcined Natural Pozzolans for Use in Std. Spec. for Std. Spec. for Latex Specifying

Std. Meth. of Test for False Set of Std. Meth of Test for Autoclave Expansion of Std. Meth. of Test for Optimum So3 in Std. Spec. for std. for Emergency Vault Ventilators and Vault Ventilating termination Device and Fish Finder, Loran or Other Line of Spec. Steel Wire Bar Supports Used to ec. Pract. Test Meth. and Requirements for Snowmobile Rear

6)

tape Variable Block Format for Contouring and Contouring Std. Motor Std. Inlet Box

Std. for Locking Device, Std. for Pin, Quick Release, Std. for Pin, Quick Release, Sanitary Stds. for Centrifugal and

Meth. of Analysis of Coagulase istry) (1973) iveth. of Test. and Presenting Basic Performance Data for Std. for Binding
Rec. for Single
Pools (1969)

Pools (1971)

Pools (1971)

Pools (1972)

Pools (1972)

Pools (1973)

Pools (1973)

Popcorn (Cereal Chemistry) (1962)

Popcorn, Dried Peas and and Beans (Cereal Chemistry) (1

Popcorn, Dried Peas and Beans for Rodent and/or Insect

Popcorn, Dried Peas and Beans (Cereal Chemistry) (1962)

Popcorn. Dried Peas and Beans (Cereal Chemistry) (1962)

Popped Popcorn (Cereal Chemistry) (1962)

Porcelain Enamel and Ceramic Metal Systems (1973) ANSI

Porcelain Enamel Coatings (1973)

Porcelain Enamel Frits (Flow Button Meth.) (1970) ANSI

Porcelain Enameled Aluminum (1972)

Porcelain Enameling (1969) ANS1 Z167.21

Porcelain Enameling (1970) ANSI Z167.23

Porcelain Enameling (1973)

Porcelain Enamels (1974) ANSI Z167.20

Porcelain Insulators (Apparatus, Cap and Pin Type) (197

Porcelain Insulators (Apparatus, Post Type) (1971)

Porcelain Insulators (Suspension Type) (1971)

Porcelain Suspension (Power Line) Insulators (1974)

Porosity of Lump Coke (1973)

Porosity of Paint Films (1973)

Porosity of Sintered Powder Metal Structural Parts and

Porosity, and Apparent Specific Gravity of Fired Whitew

Porosity, Water Absorption, Apparent Specific Gravity,

Porous Concrete Pipe for Use in Underdrains (1973)

Porous Flexible Substrates (Paper) (1973)

Porous Substrates (Subjective Ratings) (Paper, Board, C

(Portable and Fixed) $600 \mathrm{~V}$ or Less (1973) ANSI C 33.95

(Portable and Permanent) (1967)

Portable Containers for Flammable Liquids (Boats) (1972

Portable Cord Connected Electrical Appliances (1973)

Portable Electric Lighting Units for Use in Hazardous L

Portable Electric Tools (1974) ANS1 C33.49

Portable Exchange Water Softeners (1972)

Portable Fixed and Stationary 600 Volts or Less) (1972)

Portable Fuel Systems and Portable Containers for Flamm

Portable Hand, Air, and Electric Tools (1973)

Portable Indoor Gas Fired Appliances (1971)

Portable Metal Ladders (1974)

Portable Outdoor Gas Fired Appliances (1971)

Portable Single and Multiple Conductor Electrical Power

Portable Sun / Heat Lamps (1974)

Portable Tester (1974)

Portable Toy Transformers (1973)

Portable Wood Ladders (1973)

Portable X-Or Gamma Radiation Survey lnstruments (1971

Portable, Medium Pressure (1973)

Portal, Tower and Pillar Cranes (1973)

Portions of Prisms Broken in Flexure) (1972) ANSI Al.30

Portland Cement and Blended Hydraulic Cements (1973)

Portland Cement and Cement Lime Plastering, Exterior (S

Portland Cement and Cement Lime Plastering, Exterior (S

Poriland Cement by Air Permeability Apparatus (1973) A

Portland Cement by the Turbidimeter (1973) ANS1 Al.7

Portland Cement Concrete Pavement Overlay Projects (197

Portland Cement Concrete Paving (1972)

Portland Cement Concrete (1973) ANSI A 37.122

Portland Cement Lime Mortar for Brick Masonry (1972)

Portland Cement Mortar (1973) ANSl Al 18.4

Portland Cement Terrazzo, Cast in Place Floors (1967)

Portland Cement (Paste Meth.) (1972) ANSI A 1.27

Portland Cement (1971) ANSI A 1.8, Ashto T107

Portland Cement (1972)

Portland Cement (1973)

Ports for Wall Installation (1972) ANSI B 135.1

Position Device, Radar, Radio Detection Finder, Radio T

Position Reinforcement in Concrete (1970)

Position Tail Lamp (Light) (1972)

Positioning Numerically Controlled Machines (Informatio

Positions for Belt or Chain Drive Centrifugal Fans (196

Positions for Centrifugal Fans (1966)

Positive Gravure Densities (Printing (1973)

Positive lndex (1973)

Positive Locking, Double Acting (1973)

Positive Locking, Single Acting (1973)

Positive Rotary Pumps for Milk and Its Products (1974)

Positive Staphylococcus in Cereal Products (Cereal Chem

Positve Displacement Hydraulic Fluid Power Pumps and Mo

Post-Term inal Board, Electrical (1973)

Post Shore Safety Rules (1972)
Sid NSF

NSF

NSF

NSP1

UL

TCA

27

*2

676

UPC * $]-C$

302

Flotati AACCH 28.80

$\mathrm{AACCH} 28.21$

$A$ ACCH 28.10

AACCH 28.20

$\mathrm{AACCH} \quad 28.22$

AACCH 28.80

ASTM C286

ASTM C743

ASTM C 374

ASTM C703

St ASTM C632

C 660

A 424

C614

ANSI C29.8

ANSI C29.9

ANSl C29.2

NEMA $\mathrm{HV} 2$

ASTM D167

ASTM D3258

ASTM B328

ASTM C 373

ASTM C20

ASTM C654

TAPP1 UM-541

TAPPI UM-540

UL

NRA

ABYC

UL

UL

UL

WCF

UL

ABYC

ANSI

A 151

¿L

ANS1

NEMA

UL

ASTM

UL

UL

ANSl

UL

Safety St ANSl

ASTM

ICBO

A NSl

ANS1

ASTM

ASTM

ACPA

ACPA

Spec. Fo ASTM

BIA

TCA

CSI

ASTM

ASTM

ASTM

ASTM

Afety UL

$A B Y C$

CRSI

SAE

EIA

AMCA

$\mathrm{AMCA}$

GT

NSA

NSA

NSA

DFISA

$\mathrm{AACCH}$

NFLDP

NSA

SSl

1042

*9

H25

746.51

781

45

S- 100

499

H 25

B107.4

Z21.2

184

$\mathrm{Z} 21.54$
$\mathrm{~W} C 8 / 6$

482 
on)!

Std. Specs. for Post Tensioned Std. Wet Process Porcelain Insulators (Apparatus,

Std. Specs, for ment by Pressure Processes) (19/ Std. for Round Poles and Std for Preservative Treatment (For Fence

hines (1970) Std. for

ble Check Valve Type Back Pressure Backflow Preventers for hicles (1971) Std for Pressurized and Non Pressurized Grade Aluminum Potassium Sulfate $\mathrm{AlK}\left(\mathrm{SO}_{3}\right)_{2}$ : $12 \mathrm{H}_{2} \mathrm{O}$ l Chemistry) (1962) Quantitative Meth. of Analysis of

Std. Spec. for Photographic Grade Meth. for Preparation of Meth. for Preparation and Standardization of Sodium or h. of Analysis of Oxidizing Agents in Flour: Reaction with Std. Spec. for Photographic Grade Std. Spec. for Photographic Grade

(1968) ANSI N 154 2)

Std. Meth. of Test for Consumption of Std. Analytical Meth. for Determining Meth. for Preparation and Standardization of Std. Spec. for Photographic Grade um) (1972) (1972)

Std. Spec. for Photographic Grade Aluminum Std. Spec. for Photographic Grade Chromium Meth. for Preparation of Std. Meth. of Test for Uranium by Controlled ndling of Flammable and Combustible Liquids and Solids and 1) ANSI A37.133 Std. Meth. of Test for

g and Labeling Epoxy Products According to Their Hazardous for Techniques and Instrumentation for the Measurement of ct. for Std. Reference Meth. for Making Potentiostatic and ms and Definitions for Wirewound and Nonwirewound Trimming nd Test Procedures for Wirewound and Nonwirewound Trimming Std. for High Precision Laboratory asoline, Kerosine, Aviation Turbine, and Distillate Fuel

h of Test for Total Base Number of Petroleum Products by mea/ Std. Rec. Pract. for Std. Reference Meth. for Making es, etc.) (1973) estinal C Std. for Gas Counter Appliances (Coffee Meth. for Determination of Sulfaquinoxaline in Meth. for Determination of Nitrosal in Rec. Lighting for Dairy Farms and the tinal Coccidiosis $1 /$ Meth. of Analysis of Nitrophenide in re Loss and Feed and Water Requirements of Farm Livestock Std. Meth. of Test for std. for Reinforced Gypsum Concrete and Precast Slabs, and Cork, Linoleum, Rubber, Vinyl, Vinyl Asbestos, Polymeric Safety Requirements for Safety Ventilation Requirements for Direct Fired pectrochemical Analysis of Thermionic Nickel Alloys by the nd Extrusion Materials (1974) Std. Rec. Pract. for

Std Spec, for Nuclear Grade, Sinterable Uranium Dioxide e Protec/ Rec. for Application of Plastic Polymers in Dry f Test for Density and Interconnected Porosity of Sintered Std. Definitions of Terms Used in

Std. Spec. for Iron Copper Sintered Metal

Meth of Analysis for Residual Carbon Dioxide in Baking f Analysis for Total (Gasometric) Carbon Dioxide in Baking emical Ánalysis Of, and Physical Tests On, Beryllium Oxide Std. Spec. for Nuclear Grade Beryllium Oxide Tent. Spec. for Nuclear Grade Boron Carbide Std. Spec. for Hand Scrubbing Soap (Grit Soap)

Qualitative Meth. of Analysis of Aluminum in Baking Std. Spec. for Nuclear Grade Plutonium Dioxide Spec. for Std. Spec, for ualitative Meth. of Analysis for Phosphoric Acid in Baking Meth. of Analysis for Sulfuric Acid in Baking Meth. of A nalysis for Tartaric Acid in Baking Meth. of Analysis of Ammonia in Baking antitative Meth. of Analysis for Phosphoric Acid in Baking Spectrochemical Analysis of Nuclear Grade Uranium Dioxide pectrochemical Analysis of Nuclear Grade Plutonium Dioxide $r$ Electrical Insulation (1973) Std. Meth. of Test. Coating on (1973) Std. Meth. of Test/ Std. Meth. of Test. Coating ansi C57.12.90 Std. Meth. of Test for Apparent Density of Metal Std. Test Code for Distribution,

std for Relays and Relay Systems Associated with Electric steel Bars, Carbon, Hot Rolled, Special Quality, for Fluid on, 200

minum Underground Distribution Reference Book (Electrical ef

Tinned Copper Concentric Neutrals (Underground Electrical stant Ethylene Propylene Rubber Insu/ Resistant Ethylene-Propylene-Ru ion (1973) IPCEA \$68.516 Std. for Electrical Std. for Mine std. for Portable Single and Multiple Conductor Electrical
(Post Tensioned Box Girder Bridges Design and Constructi Post Tensioned Prestressed Concrete (Post Tensioned Box Post Type) (1971)

Posts Used in Building Construction (Preservative Treat Posts) by Pressure Processes (1972)

Pot, Pan, and Utensil Commercial Spray Type Washing Mac Potable Water Supply Lines (1972) Potable Water Tanks for Mobilehomes and Recreational Ve

(Potassium Alum) (1972)

Potassium Bromate in White and Whole Wheat Flour (Cerea Potassium Chloride. KCI (1972)

(Potassium Chromate Indicator) (1954)

Orn Syrup Unmixe

Potassium Dichromate Solution (1962)

Potassium Hydroxide Solution (Cereal Chemistry) (1962)

Potassium Iodide (Cereal Chemistry) (1962)

Potassium Iodide, KI (1972)

Potassium Metabisulfite $\mathrm{K}_{2} \mathrm{~S}_{2} \mathrm{O}_{3}$ (1972)

Potassium Permanganate by 1 mpurities in Deuterium Oxide

Potassium Permanganate Solution Content of Reagents and

Potassium Permanganate Solution (Cereal Chemistry) (196

Potassium Permanganate, $\mathrm{Kmn0}_{4}$ (1972)

Potassium Sulfate $\mathrm{AlK}\left(\mathrm{SO}_{4}\right)_{2}: 12 \mathrm{H}_{2} \mathrm{O}$ (Potassium Al

Potassium Sulfate $\mathrm{CrK}\left(\mathrm{SO}_{4}\right)_{2}: 12 \mathrm{H}_{2} \mathrm{O}$ (Chrome Alum)

Potassium Thiocyanate Solution (Cereal Chemistry) (1962

Potential Coulometry (1970) ANSI N106

Potential Dust Explosion Conditions (1973)

Potential Reactivity of Aggregates (Chemical Meth.) (19

Potentialities (Safety) (1972)

1. Guide for

Potentially Hazardous Electromagnetic Radiation at Micr

Potentiod ynamic Anodic Polarization Measurements (1972)

Potentiometers (1970)

Potentiometers (1970)

Potentiometers (1972)

Std. for Inspection a

(Potentiometric Meth.) (1973)

Potentiometric Perchloric Acid Titration (1973)

lor Mercaptan Sulfur in G

Potentiostatic and Potentiodynamic Anodic Polarization

Pots and Urns, Food Dish Warmers, Hot Plates and Griddl

Poultry Feeds and Premixes for Control of Cecal and Int

Poultry Feeds (Cereal Chemistry) (1962)

Poultry Industry (1971)

Poultry Mashes (Feed) for Prevention of Cecal and Intes

(Poultry, Swine, Cattle, Sheep) (1968)

Pour Point of Petroleum Oils (1971) ANSI Z11.5.

Poured Gypsum Roof Diaphragms (1973)

Poured Seamless, etc.) (1973)

Powder Actuated Fastening Systems (1972)

Powder Curing Ovens (1973)

Powder Direct Current Arc Technique (1961) ANSI Z128.2

Powder Flow Measurement of TFE -Fluorocarbon Molding a

Powder for Use in Nuclear Reactors (1973)

Powder Form by Means of Spray Gun or Fluidized Bed (Fir

Powder Metal Structural Parts and Oil Impregnated Beari

Powder Metallurgy (1972) ANSl H9.5

Powder Structural Parts (1970) ANSI G63.1

Powder (Cereal Chemistry) (1962)

Powder (Cereal Chemistry) (1962)

Powder (1972) ANSI N140

Powder (1972A)

Powder (I973)

Powder (1974)

Powder, in Presence of Phosphates (Cereal Chemistry) (I

Powder, Sinterable (1974)

Powdered Hand Cleaner (Soap with Borax) (1972T)

Powdered Hand Cleaner (Soap with Vegetable Scrubber) (1

Powdered Toilet Soap (1974)

Powders and Chemicals (Cereal Chemistry) (1962)

Powders and Chemicals (Cereal Chemistry) (I962)

Powders and Chemicals (Cereal Chemistry) (1962)

Powders and Chemicals (Cereal Chemistry) (1962)

Powders and Chemicals (Cereal Chemistry) (1962)

Powders and Pellets (1972) ANSI N103

Powders and Pellets (1972) ANSI N104

Powders and Their Coatings Used for Electrical Insulati

Powders (1948) ANSI H9.1

Power and Regulating Transformers (Electronics) (1973)

Power Apparatus (1971) A NSI C37.90

Power Applications (1974)

Power Cable Specs.) (1973)

Power Cable with Ethylene-Propylene-Rubber Insulat

Sid. Spec. for

Power Cable $15 \mathrm{kV}$

Power Cables Rated 0-35,000 Volts and Having Ozone Res

Power Cables Rated 2000 Volts and Less and Having Ozone

Power Cables with Ethylene-Propylene-Rubber Insulat

Power Cables with Ethylene-Propylene-Rubber Insulat
CRSI

CRSI

ANSI

AWPA

AWPA

NSF

ASSE

IAPMO

ANS1

AACCH

ANSI

AACCH

$\mathrm{AACCH}$

ANSI

ANS1 PH4.277

ASTM D2033

CR R.15

$\mathrm{AACCH} 70-50$

ANSI PH4.301

ANSI PH4.150

ANSl PH4.15

AACCH 70.55

ASTM E217

ICBO UFC*2ART34

ASTM C289

ANSI K68.1

ANSI C95.3

ASTM G5

T110

$\begin{array}{ll}\text { ANSI } & \mathrm{Cl} 100.2 \\ & \end{array}$

D2896

ASTM D28

ANSI Z21.31

AACCH 18.35

$\triangle \mathrm{ACCH} \quad 18-27$

ASAE R344

AACCH $18-25$

ASAE D249.2

ASTM D97

UBCS24-13

F141

ANSI A 10.3

ASTM E129

ASTM D3292

ASTM C753

FMS $\quad 7.27$

ASTM B328

ASTM B243

STM B222

$\mathrm{AACCH} \quad 12.10$

Meth. O AACCH 12.20

ASTM C699

ASTM C708

ASTM C750

ASTM D 2957

AACCH $40-10$

ASTM C757

ASTM D3046

ASTM D3047

ASTM D2958

AACCH 04.I0

$\mathrm{AACCH} \quad 04.14$

$\mathrm{AACCH} \quad 04-27$

$\mathrm{AACCH} \quad 40-15$

Qu AACCH

ASTM C696

$\begin{array}{ll}\text { ASTM } & \text { C696 } \\ \text { ASTM } & \text { C697 }\end{array}$

ASTM D3214

ASTM D3214

ASTM B212

IEEE 262

IEEE 313

I A

NEMA

A695

52

IPCEA

W $\mathrm{C} 8 / 7$

P.53-426

AA

NEMA

$52-1$

NEMA WC8/5

NEMA WC8/6 
Recommendations, and Related Requirements for Low Voltage 37.13 Std. for Test Procedures for Low Voltage Ac Std. for Low Voltage Alternating Curren Safety Std, for Fused

requirements for Low Voltage Power Circuit Breakers and Ac d. for Surge (Lightning) Arresters for Alternating Current Std. for Electric ssemblies (1973) Design Criteria for Reinforced Concrete Nuclea ices (1973)

Std. for Industrial Electrica Std. for Industrial Electrical Safety Code for Semiconductor for Accessories for Cataloged Square Head Industrial Fluid Bore Cataloged Square Head Tie Rod Type Industrial Fluid Installation Pract. for Underground Electrical d Tractors (1972) SAE J955 Std. for Full Shielding of

Currents (1972) ANSI C37.26 Std. Groove Dimensions for Fluid Std. Guide for Meth. o

Std. End Load Test Meth. for a Hydraulic Fluid determining the Fabrication Integrity of a Hydraulic Fluid Verifying the Material Compatibility of a Hydraulic Fluid ying the Flow Fatigue Characteristics of a Hydraulic Fluid fying the Collapse / Burst Resistance of a Hydraulic Fluid ating the Filtration Performance of a Fine Hydraulic Fluid ansi $\mathrm{C} 83.92$ Std. for Iron-Core

lifying and Controlling Cleaning Meth. for Hydraulic Fluid

own Voltage and Strength of Insulating Gases at Commercial

ures, and Equipment for Water Cooled and Moderated Nuclear for Trial-Use Criteria for the Periodic Test. of Nuclear de: General Principles for Reliability Analysis of Nuclear the Application of the Single Failure Criterion to Nuclear Definitions of Terms Used in IEEE Nuclear
uide for Qualifying Class I Electric Equipment for Nuclear ic Qualification of Class I Electric Equipment for Nuclear class I Motors lnstalled Inside the Containment of Nuclear erator Units Applied as Standby Power Supplies for Nuclear Type Test of Class I Electric Valve Operators for Nuclear on Assemblies in Containment Structures for Nuclear Fueled $s$ for Systems That Perform Protective Functions in Nuclear al Use Guide for Class 1E Control Switchboards for Nuclear ention of W ater Damage to Steam Turbines Used for Electric and Electric Equipment During the Construction of Nuclear coustics) / Std. for Nonintegral Industrial Fluid Std. for Meth. for the Determination of Sound Requirements for Std. Application Guide for Porcelain Suspension Safety Spec. for Slides in 2 X 2 In. Mounts (1972) tractors (1972)

(1974) ANSI Z216.1

ing and Analyzing Outage / . Safety Std, for Safety Std. for Std. Definitions in Electrical Std. for Safety for Electrical Std. for Code for me Use (1967)

imensions for Floating Type Metallic and Nonmetallic Fluid for Low and Medium Speed Stationary Diesel and Gas Engine for Low and Medium Speed Stationary Diesel and Gas Engine Marine Steam uality Assurance Practices for the Construction of Nuclear ping, Receiving. Storage and Handling of Items for Nuclear td. for Quality Assurance Rrogram Requirements for Nuclear Std. for Fuel Assembly Identification in Nuclear Std. for Administrative Controls for Nuclear Std Industrial Security for Nuclear for Housekeeping During the Construction Phase of Nuclear associated Components During Construction Phase of Nuclear and Test. Personnel for the Construction Phase of Nuclear Std. for Earthquake Instrumentation Criteria for Nuclear for Safety Requirements for Construction, Care, and Use of b93.32

Performance Data for Positve Displacement Hydraulic Fluid

Safety Rules. Procedue Dimensions for Fluid Std. for Safety for std. for Paper, Paper/Film, Film Dielectric Capacitors for Std for Handling and Installation of rec. for Changing and Charging Storage Batteries in Motive acity of Lead Acid Industrial Storage Batteries for Motive

(1972) Std. for Stabilized Thyristor

d.: Criteria for Diesel Generator Units Applied as Standby Safety Std. for Cord Sets and Std. Definitions for

mits for AC High Voltage Power Vacuum Interrupters Used in Std. for Electrical uid Samples from the Lines of an Operating Hydraulic Fluid
Power Circuit Breakers and AC Power Circuit Protectors

Power Circuit Breakers U'sed in Enclosures (1973)

ANSI

Power Circuit Breakers Used in Enclosures (1973) ANSI C

Power Circuit Devices (1973)

Power Circuit Protectors (1973)

Power Circuits (1972) ANSI C62.1

Power Connectors (1972)

Power Containment Structures (1969)

Power Control Apparatus-Control Circuit Devices and a

Power Control Apparatus-General (1973)

Power Control Apparatus-Switching and Controlling Dev

Power Converters (1973)

Power Cylinders (1972) ANSI B93.29

Power Cylinders (1972) ANSI B93.34

Power Distribution Cables (1973)

Power Drive Lines for Agricultural (Farm) Implements an

Power Exclusion Devices (In. Series) (1972) ANSI B93.35

Power Factor Measurement for Low Voltage Inductive Test

Power Filter Element (1972) ANSI B93.21

Power Filter Element (1972) ANSI B93.22

Power Filter Element (1972) ANSI B93.23

Power Filter Element (1972) ANSI B93.24

Power Filter Element (1972) ANSI B93.25

Power Filter Element (1973) ANSl B93.31

Power Filter Inductors for Electronic Equipment (1973)
Power Fluid Sample Containers (1972) ANSI B93.20

Power Fluid Sample Contail

Power Generating Plants (1973) ANSI N18.10

Power Generating Station Protection Systems (1971) ANSI

Power Generating Station Protection Systems (1972) ANS

Power Generating Station Protection Systems (1972) ANSI

Power Generating Station Stds. (1972)

Power Generating Stations (1971) ANSI N41.5

Power Generating Stations (1971) ANSI N41.7

Power Generating Stations (1971) ANSI N41.9

Power Generating Stations (1972) ANSI N41.13

Power Generating Stations (1972) ANSl N41.6

Power Generating Stations (1972) ANSl N45.3

Power Generating Stations (1973)

Power Generating Stations (1973) ANSI N41.17

Power Generation: Fossil Fueled Plants (1972)

Power Hydraulic Reservoirs (1969) ANSI B93.18

Power Levels of Small Sources in Reverberation Rooms (A

Power Line Coupling Capacitors (1972)

(Power Line) Insulators (1974)

Power Mowers, Lawn, and Garden Tractors (1972)

Power of Lenses for Projectors for $35 \mathrm{~mm}$ Filmstrips and

Power Off Definitions and Terminology for Agricultural

Power Operated Dispensing Devices for Flammable Liquids

Power Operated Pumps for Petroleum Products (1973)

Power Operations Terminology Including Terms for Report

Power Outlets (1972) ANSI C33.85

Power Output Ratings of Packaged Audio Equipment for $\mathrm{H}_{\circ}$

Power Piping (1973)

Power Piston Rings (1972) ANSI B93.36

Power Plant Buildings (1972)

Power Plant Fuel Oil and Gas Systems (1972)

Power Plant Heat Balance Practices (1972)

Power Plants-Terms and Definitions (1973)

Power Plants (During the Construction Phase) (1972)

Power Plants (1971)

Power Plants (1972)

Power Plants (1972)

Power Plants (1973)

Power Plants (1973)

Power Plants (1973)

Power Plants (1973)

Power Plants (1974)

Power Press Brakes (1973)

Power Pumps and Motors (1973) ANSI B93.27

Power Radial Compression

Power Roof Ventilators (1972) ANSI C33.89

Power Semiconductor Applications (1973)

Power Semiconductors in Disc Type Packages (1973)

Power Service (For Electric Trucks) (1972)

Power Service (1974)

Power Supplies Direct Current Output (1972)

Power Supplies for Metal Rolling Mill Auxiliary Drives

Power Supplies for Nuclear Power Generating Stations (1

Power Supply Cords (1972) ANSI C33.3

Power Switchgear (1972)

Power Switchgear (1972)

Power Switching Equipment (1974)

Power System (For Particulate Contamination Analysis)(
D. Meth. for

Std. Meth. of

/Th. for Verif

Eth. for Veri

Th. for Evalu

F T est

/Ation

Std. for

Std. Groove D

Std. Pract.

D. Pract.

TTd. for $Q$

Quirements for X.Radiation L

ANSI

IEEE

UI

St lEEE

NEMA

$\mathrm{ACl}$

ANSI

ANSI

ANSI

NEMA

NFLDP

NFi

ASAE

NFLDP

IEEE

FLDP

NFLDP

NFLDP

NFLDP

NFLDP

NFLDP

ASTM

ANS

IEEE

IEEE

IEEE

IEEE

IEE

Ous Duty IEEE

Esel Gen IEEE

Guide for IEEE

Penetrati IEEE

IEEE Tri IEEE

He Prev ASME

IEEE

NFLDP

ANSI

ANSl

NEMA

ANSI

ANSI

REC.

UL

UL

IEEE

UL

EIA

ANSI

DEMA

DEMA

SNAME

$\mathrm{P}$ ANSI

ANSI

ANSI

ANSI

ANSI

Std. ANSI

for Cleaning of Fluid Systems and ANSI

ANSI

ANSI

Std. ANSI

/Nting Basic NFLDP

NFLDP

NRA

UL

EIA

EIA

INd Health ITA

NEMA

NEMA

NEMA

IEEE

UL

ANSI

NEMA

NFLDP

C 37.16

C 37.50

20

977

C37.16

CC 1

69-2

C 19.6

C19.3

C 19.5

T3.6.8

T3.6.11

52.2

S297T

T3.19.7

330

10.8.2

T3.10.8.4

T3.10.8.6 
for the Use of Fire Resistant Fluids for Hydraulic Fluid t. for Grounding of lndustrial and Commercial (Electrical) Reporting Contamination Analysis Data of Hydraulic Fluid Rec. Pract. for Agricultural Tractor Auxiliary ral and Light lndustrial Tractors (1972) SAE J7/ ae $\mathbf{3} 718$ ae $\mathrm{J} 719$ Rec. for Agricultural (Farm) dustry (Rotating, Liquid Filler Tractor and Farm lmple Hent 1971) Std. for Guide for Preparation of Spec. for Large Std. for Heavy Duty Offset Sidebar 3 for Design, Construction, Materials, and Installation of 700 Class Pintle Chains, Attachments, and Sprocket Teeth ) (1971)

Requirements for X-Radiation Limits for AC High Voltage

Test for Stretch Properties of Knitted Fabrics Having Low for Low Power Wide Band Transformers (Less Than $100 \mathrm{~W}$ atts Std. General Requirements for Distribution.

Safety Std. for Performance Requirements for Stopping ermanently lnstalled Fuel Sysiems for Inboard and Outboard ral Furnaces) (197/

Std. Performance Requirements for O Std. for Forks and Fork Carriers for Std. for Safety for Electric Batter Std. for Load Handling Symbols fo

Pract. for Exhaust Emission Controls (Air Pollution) for for the Test. Rating and Reporting of the Noise Levels of e Designations, Areas of Use, Maintenance and Operation of he Measurement of Fuel Evaporative Emissions from Gasoline he Measurement of Fuel Evaporative Emissions from Gasoline al, Hydraulic, Pneumatic, Semi and Automatic, and Manually si A3/ Std. Spec. for Fly Ash and Raw or Calcined Natural Demountable Rims and Rim Spacers (M/ Rec. Pract. for for c) Std. for Engineering Drawing and Related Documentation Std. Engineering Drawing and Related Documentation Safety Std for Dry Pipe, Deluge, and 69) ANS1 N42.2 Std. Test Procedure for Amplifiers and

concrete (1966)

form Building Code Std. for Reinforced Gypsum Concrete and ntainers (1971) Sid. Spec for General Requirements for Wrough orgings, and Forging Stock for High Tempe/ ings, and Forging Stock for High Temperat/ d Forging Stock for High Temperature Serv strip for High Temperature Service (1972) ansil

Std. Spec.. for Pressure Vessel Plates, Alloy Steel, Std. Spec: for Pressure Vessel Plates, Alloy Steel $5.6 \mathrm{Zn}-2.5 \mathrm{Mg}-1.6 \mathrm{Cu}-0.26 \mathrm{Cr}(7075-\mathrm{T} 736)$ Solution and Deg. F (996. 1.1037.8 Deg. C) Solution, Stabilization, and 1975 Deg. F (1079.4 Deg. C) Solution, Stabilization, and o-2.1Ti-0.30V) 1800 Deg. F (982.2 Deg. C) Solution and d Castings (5.75Zn-2.5Re-0.70Zr (Ze63-T6) Solution and roperty of a Mater/ ) Application (75 Ohms) (1973) Std. Rec. Pract. for I'se of the Terms $20 \ln ).(1973)$

Sed, Rec. Pract for Stating the nd Rubber.Like Materials / Std. Rec. Pract. for Determination of Sid. Rec. Pract. for Preparing al Analysis of Ores, Minerals, and Rocks by the Fire Assay. 8) zinc (Galvanized) Coating on Iron or Steel Articles by the azards at Plants Assembling Mobile Homes, Modular Housing, nt and Pipe Operating at Temperatur/ Std. Rec. Pract. for ormance (19/ Std. Meth. of Test. Duct Liner Materials and
St3) St. Spec. for

ata on Elastomer Roof Coverings (Multiply Felt and Asphalt h. of Test for Crystallographic Perfection of Germanium by rdization (1973)

AC High Voltage Circuit Breakers R/ ated Requirements for Low Voltage Pov Guide for High Voltage Air Switch/ Std. for lndustrial Std. for
Tractor Auxiliary Std. for $540 \mathrm{Rpm}$ $1000 \mathrm{Rpm}$ mplement Std. for Safety for Mechanical Std. for Safety for Battery Rec. for Stopping Manual of Guide Spec. for Specifying: Rec, for Std. Spec. for

Guide to the Preparation of Std. Spec. for Std. Spec. for Std. Spec, for Std. Spec. for Std. for Std. for Reference Std
Std. Spec. for Std. for Std. for Rec. Guide for the Rec. Spec, for Uniform Building Code: Std. for

Power Systems (1972) ANSl B93.5

Std. for Schedule of Circuit/ Std. for
Std. for Schedules of
Preferred Ratings, Application Recommendations, and Rel

Preferred Ratings, Manuf acturing Spec., and Application Power Systems (1972) ANSI C114.1

Power Systems (1973) ANSI B93.30

Power Take Off Drives (1972)

Power Take Offs for Overcenter Clutches (1971)

Power Takeoff Definitions and Terminology for Agricultu

Power Takeoff Drives (1972)

Power Takeoff for Agricultural (Farm) Tractors (1973) S

Power Takeoff for Agricultural (Farm) Tractors (1973) S

Power Takeoff (Universal Joint) Drive Line Specs. (1972

Power Tools, Hermatic Stators, Test. and Safety) (1973)

Power Transformers, with or Without Load Tap Changing (

Power Transmission Apparatus (1972)

Power Transmission Roller Chains and Sprocket Teeth (19

Power Transmission Units Penetrating the Hull for Boats

Power Transmission) (1972)

Power Vacuum Interrupters Used in Power Switchgear (197

Power Wide Band Transformers (Less Than 100 Watts Power

Power (1972) ANSl Z14.286

Power) (1971)

Power, and Regulating Transformers (1973) ANSI C57.12.0

Power, Traction and Steering Stability for Regular Bicy

Powered Boats (1973)

Nstruction and Installati

Powered Emergency Lighting Equipment (Indoor) (1973)

Powered Forced Air (Domestic and Light Commercial) Cent

Powered Industrial Fork Lift Trucks (1973)

Powered Industrial Trucks (1972) ANSI B56.3

Powered Industrial Trucks (1973)

Powered Industrial Trucks (1973)

Powered lndustrial Trucks (1973)

Powered lndustrial Truck s (1973) ANSI B56.

Powered Passenger Cars and Light Trucks Using

Powered Passenger Cars and Light Trucks (1972)

Powered) (1973)

On, Care, and Use of

Powers for Use with Cavity Chambers (1961)

Pozzolans for Use in Portland Cement Concrete (1973) an

Practical Minimum Rec. Tolerances of Spoke Type Wheels,

Practices: Chassis Frames-Passenger Car and Light Tru

Practices: Dimensioning and Tolerancing (1973)

Practice: Multiple Contract Construction (1974)

Pre Action Valves for Fire Protection Service (1970)

Pre Insulated, (Class 2) Wire Sizes 12 to 26 (1972)

Preamplifiers for Semiconductor Radiation Detectors (19

Precast Concrete Panels (1973)

Precast Concrete Units Used as Forms for Cast in Place

Precast Reinforced Concrete Manhole Sections (1973)

Precast Slabs, and Poured Gypsum Roof Diaphragms (1973)

Precautionary Labeling and Marking of Compressed Gas Co

Precious Metal Electrical Contact Materials (1972)

Precipitation Hardening Cobalt Containing Alloy Bars, F

Precipitation Hardening lron Base Superalloy Bars, Forg

Precipitation Hardening Nickel Alloy Bars, Forgings, an

Precipitation Hardening Nickel Alloy Plate, Sheet, and

Precipitation Hardening (Maraging), $12 \%$ Nickel (1972A)

Precipitation Hardening (Maraging), 18\% Nickel (1972A)

Precipitation Heat Treated (1972)

Precipitation Heat Treated (1973)

Precipitation Heat Treated (1973)

Precipitation Heat Treated (1973)

Precipitation Treated (1973)

Num Alloy Forgings, Ion Melted 1825-1900

Um lnduction Melted $/(15 \mathrm{Cr}-26 \mathrm{Ni}-1.3 \mathrm{M}$

Precision and Accuracy as Applied to Measurement of a P

Precision Coaxial Connectors for CATV (Cable Television

Precision Electrical Resistors (1969) ANSI C100.3

Precision Electroformed Sieves (1970) ANSI Z168.5

Precision In. Gage Blocks for Length Measurement (Thru

Precision Indexable Insert Holders (1973)

Precision Laboratory Potentiometers (1972)

Precision of ASTM Test Meth. Related to Carbon Black (1

Precision of Meth. of Committee D.19 on Water (1972)

Precision Statements for ASTM Meth. Related to Rubber a

Preconcentration Spark Technique (1971) ANSI Z128.25

Prediction of the Dispersion of Airborne Effluents (196

Preece Test (Copper Sulfate Dip) (1973) AASHO T66

Prefab Houses, Motor Homes, and Camping Trailers) (1974

Prefabricated Brick Masonry (1974)

Prefabricated Construction (1973)

Prefabricated Reflective Insulation Systems for Equipme Prefabricated Silencers for Acoustical and Airflow Perf

Prefaced Concrete and Calcium Silicate Masonry Units (1

Preferably with Gravel or Slag Surface) (1968)

Preferential Etch Techniques (1973)

Preferred Numbers in Geometric Series for Use in Standa

Te

Tion D

Preferred Ratings for Capacitance Current Switching for

Std. Pract NFLDP

ec. Prac IEEE

142

NFLDP T2.9.3

SAE J717C

SAE J621C

SAE $\quad$ S205.2

SAE R33.

ASAE S203.7

ASAE $\$ 204.6$

ASAE R331.2

ANSI

ANS

ANSI

ABYC

d. for ANSI

ANSl

IEEE

Std. IEEE

IEEE

BMA

ABYC

UL

ANS1

ANSI

UL

ANSI

Rec ITA

dure ITA

NFPA

SAE

ANSI

NCRPM

ASTM

SAE

ANSI

ANSI

CSI

LL

NSA

IEEE

CSI

ACI

ASTM

ICBO

CGA

ASTM

ASTM

ASTM

ASTM

ASTM

ASTM

ASTM

SAE

SAE

SAE

SAE

ASTM

ElA

IEEE

ASTM

ANSI

ANS

ANSI

ASTM

ASTM

ASTM

ASTM

ASME

I a ASTM

FMS

BlA

ICBO

ASTM

ASTM

ASTM

Preferred Transient Insulation Levels (T1L) (1971)

*1

C 57.97

B15.1

B29.13

11

D2594

111 
d Required Capabilities for AC High Voltage/ ew and Assembled Washers-Electrical Terminal Attachment

projection Lamps (1972)

s (1973) us Type) (1971) ANSI A37.101 Std. for Medium Std. for Packing, Std. for Packing, Std. Spec. for nd Structural Construction (Nonextruding / Std. Spec. for td. Rec. Pract. for Test. Primers and Primer Surfaces Over to +160 Deg. F) (1972) Std. for Packing, Manufacture and Inspection Stds. for Std. Meth. of Test for Low Temperature Flexibility of Std. Meth. of Test for Adhesion After lmpact of Std. Meth. of Test for Weight Loss After Heat Aging of eth. of Test for Oil Migration or Plasticizer Bleed Out of 97 Rubber, 75 Shore, O-Ring (1973) 97 Rubber, 90 Shore, O-Ring (1973) Std. for Packing. Std. for Packing, mistry) (1968/ Meth. of Analysis of Hydration Capacity of d. Dimensional and Electrical Characteristics of 8 Watt T5 Dimensional and Electrical Characteristics of $15 \mathrm{~W}$ att T8 Dimensional and Electrical Characteristics of $30 \mathrm{Watt}$. T8 Rec. Pract. for Engine Rec. Pract. for

$f \mathrm{Gas}$ Piping and Equipment on Industrial and Certain Other ety Std. for Surveillance Cameras for Banks and Mercantile or Determination of Azodicarbonamide Content in Commercial for Determination of Sulfaquinoxaline in Poultry Feeds and Meth. of Analysis of Acetone Peroxides in Milling Std. for National Aerospace Std. Documents National Aerospace Stds. Documents

ion (Cereal Chemistry) (1962)

real Chemistry) (1962)

lution (Cereal Chemistry) (1962)

(Cereal Chemistry) (1962)

te Solution (Cereal Chemistry) (1962)

hydroxide Solution (Cereal Chemistry) (1962)

olution (Cereal Chemistry) (1962)

on (Cereal Chemistry) (1962)

Specimens (1973) Spec.

73)

Meth. for

Meth. for

Meth. for

Meth. for

Meth. for

Meth. for

Meth. for

Meth. for

Std. Meth. for ANSI Jl.1

ec. for $13 / 4$ In. Thick Hollow Metal Door and Frame Std. Meth. of Compound and Sample Std. Rec. Guide for of Analysis of Sulfochromate Etch Solution Used in Surface istry) (1962)

Zincate Process (1973) ANSI G53.12

970) ANSI G53.13

(1969)

ns of the California Kneading Compa/

e Coatings (1966)

otective functions in Nuclear Power Ge/

Meth. for

Std. Rec. Pract. for

Sid. Rec. Pract. for

Rec. Pract. for

Std. Rec. Pract. for

Std.
Std. Criteria for ng (1970) ANSI Z167./ 1962)

Std. Rec. Pract, for Production and

for Low and Medium Speed Stationary Dies/

repair of Laboratory Instruments (1972)

67) ANSI Z197.28

in Common Fats, Oils, and Fatty Acids (Cereal

Std. Pract. for Std. for Std. Rec. Pract. for Meth. for Std. for

Std. Rec. Pract for Meth. for Meth. for

Guide to the Meth. for Meth. for Meth. for Meth. for Meth. for Meth. for Meth. for Meth. for Meth. for Meth. for Meth. for y) (1962) Std. Meth. for Sampling and Sample Std. for Guide for Std. Rec. Pract. for or Without Load otomasks (1972) acquer, and Related Products (1973) nding (1969) ANSI Z197.25

Rec. Pract. for Surface Std. Meth. for

Std. Rec. Pract. for Std. for

ting Sysiems (Coil) (1974) Guidelines for Selection and on and Establishment of Temperature Indexe/ Means of California Kneading Compactor (/
Preferred Transient Recovery Voltage Ratings and Relate

Std. for P
Preferred) (1974) Prefocus Base Down Type, Single Contact Motion Picture Preformed-MIL-R.25897 Rubber, 75 Shore, O-Ring (1973) Preformed-MlL-R-25897 Rubber, 90 Shore, O-Ring (1973) Preformed Cellular Plastic Pressure Relief Joint Filler Preformed Compression Seals in Concrete Pavements (1972 Preformed Expansion Joint Filler for Concrete (Bitumino Preformed Expansion Joint Fillers for Concrete Paving a Preformed Metal (1974)

Preformed O-Ring Phosphate Ester Resistant (-65 Deg. F

Preformed Packings (0-Rings) (1971)

Preformed Sealing Tapes (1973)

Preformed Sealing Tapes (1973)

Preformed Sealing Tapes (1974)

Preformed Sealing Tapes (1974)

Preformed, Straight Thread Tube Fitting Boss, MIL-R-258

Preformed, Straight Thread Tube Fitting Boss, MIL-R.258

Pregelatinized Starches and Cereal Products (Cereal Che

Preheat Start Bactericidal Lamp (1971)

Preheat Start Bactericidal Lamp (1971)

Preheat Start Bactericidal Lamp (1971)

Preheaters (Coolant Heaters) (1971)

Preignition Rating of Spark Plugs (1970)

Premises (1972)

Premises (1973) ANSI Se2.5

Premix (Cereal Chemistry) (1971)

Premixes for Control of Cecal and Intestinal Coccidiosi

Premixes (Cereal Chemistry) (1971)

Preparation and Maintenance in SI Metric Units (1973)

Preparation and Maintenance (1974)

Preparation and Standardization of Barium Lactate Solut

Preparation and Standardization of Hydrochloric Acid So

Preparation and Standardization of Lactic Acid Solution

Preparation and Standardization of Potassium Permangana

Preparation and Standardization of Sodium or Potassium

Preparation and Standardization of Sodium Thiosulfate S

Preparation and Standardization of Sulfuric Acid Soluti

Preparation and Use of Bent Beam Stress Corrosion Metal

Preparation for Offset Intermediate Pivot (1973)

Preparation for Physical Test. of Rubber Products (1972

Preparation for 181 and 190 Series Deadlock Strikes (19

Preparation of a Leak Test. Spec. (1973)

Preparation of Aluminum (1972) ANSI Z197.29

Preparation of Ammonium Molybdate Solution (Cereal Chem

Preparation of and Electroplating on Aluminum Alloys by

Preparation of and Electroplating on Stainless Steel (1

Preparation of Bar and Rod Specimens for Adhesion Tests

Preparation of Bituminous Mixture Beam Specimens by Mea

Preparation of Centrifugal Fans and Parts for Protectiv

Preparation of Design Bases for Systems That Perform Pr

Preparation of Gray Iron Castings for Porcelain Enameli

Preparation of Indicator Solutions (Cereal Chemistry)

Preparation of Invitations for Bids and Detailed Spec.

Preparation of Manuals for Installation, Operation and

Preparation of Metal Surfaces for Adhesives Bonding (19

Preparation of Methyl Esters of Long Chain Fatty Acids

Preparation of Notation for Concrete (1971) ANS1 A 188.1

Preparation of Pieces from Rubber Vulcanizates Other Th

Preparation of Potassium Dichromate Solution (1962)

Preparation of Potassium Thiocyanate Solution (Cereal C

Preparation of Precautionary Labeling and Marking of Co

Preparation of Sample: Bread (Cereal Chemistry) (1962)

Preparation of Sample: Dried Eggs (Cereal Chemistry) (1

Preparation of Sample: Feeds and Feedstuffs (Cereal Che

Preparation of Sample: Fruit and Fruit Products (Cereal

Preparation of Sample: Leavening Agents (Cereal Chemist

Preparation of Sample: Macaroni Products (Cereal Chemis

Preparation of Sample: Malt (Cereal Chemistry) (1962)

Preparation of Sample: Semolina (Cereal Chemistry) (196

Preparation of Sample: Wheat (Cereal Chemistry) (1962)

Preparation of Sample: Yeast Foods and Flour lmprovers

Preparation of Silver Nitrate Solution (Cereal Chemistr

Preparation of Solid Raw Rubbers (1973)

Preparation of Spec. for Large Power Transformers, with

Preparation of Specifications for the Procurement of $\mathrm{pH}$

Preparation of Steel and Other Hard Materials by Water

Preparation of Steel Panels for Test. Paint, Varnish, L

Preparation of Surfaces of Plastic Prior to Adhesive Bo

Preparation of TAPPI Stds. and Suggested Meth. (1972)

Preparation of Test Panels for Use in Test. Organic Coa

Preparation of Test Procedures for the Thermal Evaluati

Std. Meth. for Preparation of Test Specimens of Bituminous Mixtures by

ANSl

NSA

USC

ANS1

NSA

ASTM

ASTM

ASTM

ASTM

ASTM

NSA

SAE

ASTM

ASTM

ASTM

IId M ASTM

NSA

NSA

AACC

St ANSI

Std ANSI

Std. ANSI

SAE

SAE

O ANSI

48-71A

$\mathrm{AACCH} \quad 18-35$

AACCH 48.05

NSA $\quad 10000$

AACCH $70-10$

$\mathrm{AACCH} 70-15$

$\mathrm{AACCH} \quad 70-20$

AACCH $70-30$

AACCH $70-50$

AACCH $70-70$

AACCH $70-75$

ASTM G39

ANS1 Al15.12

ASTM

ANSI

ASTM

D15

A 115.5

ASTM D2674

AACCH 70.35

ASTM B253

ASTM B254

ASTM D2094

ASTM D3202

AMCA 2601

ANS N18.8

ASTM C660

AACCH 70.25

DEMA *1.24

ANSI C105.2

ASTM D2651

A ACCH 58-17

ACI 104

ASTM D3183

AACCH $70-45$

$\mathrm{AACCH} 70-55$

CGA

AACCH 62.05

AACCH 62.15

AACCH 62.20

AACCH 62.25

AACCH $62-30$

AACCH 62.40

AACCH 62.50

$\mathrm{AACCH} \quad 62-60$

AACCH 62.70

AACCH 62.80

AACCH $\quad 70-60$

ASTM D1485

ANSI C57.97

ASTM

NACE

ASTM

ASTM

TAFPI

NCCA

IEEE 
Surface Preparation Spec. for Steel Structure Painting (1972)

Guideline Spec. for Sodding and Soil h. for Experimental Wheat Flour Milling: Equipment, Sample Surfaces to Receive Wood Flooring (196/ Rec. for Correct y Std. for Regular Bicycles Including Selection, Purchase, ions for Chemical Analysis (1972) ANSI Z1/ Std. Meth. for

of Analysis of Damaged Starch (Granules in Flour or Starch sis of Proteolytic Activity of Flour and Active Proteinase . of Analysis of Proteolytic Activity in Active Proteinase of Analysis of Proteolytic Activity in Active Proteinase tirusts for Test. Purposes (/ Std. Meth. for Sampling and Sid. Meth. of

Std. Spec. for for High Boiling Hydrocarbon Solvent for Tent. Spec. for Volatile Petroleum Solvent (LPG) for o Rubber and Rubber-Like Materials / Std. Rec. Pract, for Equipment, and Procedures for Mixing Rubber Compounds for imens (1972) ANSI G80.1

ns Subjected to Corrosive Environments (Coil Co/ ncrete (1966)

ng Recommended Practice for Photography As/ se's (1972) td. Rec. Pract. for Meth. of Rec. for Std. Meth, of Std. for

Gas Turbine Engine Steady State Performance Std. for Scientific Papers for Written or Oral Rec. for the Care and Western Woods Use Book: Wood

Std. Test Meth for Moisture and Creosote Type Soil Block Test: Std. Meths, for Test. the
procarbon Solvent for Preparing Oil Borne es by Using $W /$ r'High Boiling Hydrocarbon Solvent for Preparing Oil Borne ing Code Std, for Lumber, Timber, Plywood and Utility Pole mber Products (1973)

piles (1973)

poles (1973)

r Highway Construction (I973)

Std.

Std. for

Std. for Std. for

Std. for Lumber, Timbers, Bridge and Mine Tie Std. for Wood Used on Farms for Round Poles and Posts Used in Building Construction ss (1973) Std. for Lodgepole Pine Wood Poles rinal Process (Western Red Cedar, Northern White/ Std. for ooling Towers (1971) Std. Spec. for Pressure Marine, Land, Std. Spec, for Coal Tar Creosote for the mbers and Laminations Before Gluing of Southern/ Std. for yellow Cedar Timber/ Std. for Full Length Thermal Process ocesses (1972)

Std. for

m Building Code Std. for Wood Poles That Are to Be Given

Their Fastenings (Lumber, Plywood and Components, Timber, Western Woods Use Book: Wood Preservation Std. Meth. of Evaluating Wood Std. Meth of Test. Wood

for Test. the Preservative Properties of Oil Soluble Wood preservatives by Using Wood Properties of Oil Soluble Wood e Specimens/ Std. Meth. of Accelerated Evaluation of Wood

Std. Meth. for Analysis of Creosote and Oil Type Wood Std. Meth. for Analysis of Oil Borne Wood Std. for Hydrocarbon Solvents for Oil Borne Wood

d. Spec for Low Boiling Hydrocarbon Solvent for Oil Borne (1972A) ANSI J8.12 Std Spec. for Concentrated, Ammonia Test Meth. for Density and Moisture of Chips Std. for Agricultural Std for Rim Contours for Agricultural fety Requirements for Construction, Care, and Use of Power Std. for Bushing-Flanged, Std. for Die Buttons, Variable, Std. for Bushing-Flanged, Std. for Bushing-Plain, Std. for Sleeve Bushing, th Chlorine Bleach) 190 Deg. F, Colors 160 Deg. F Maximum, tenance (1973)

home Laundering/ Test Meth. for the Appearance of Durable Std. for Agricultural Planter

hanges in Automatic Home Laundering of Durable (Permanent)

for Woven Sheet and Pillowcase Fabrics Other Than Durable titutional Tex/Std. Performance Requirements for Durable sh-Whites 190 Deg. F, Colors I60 Deg. F Maximum Hot Head

the Appearance of Durable Press Textile Fabrics (Permanent ricultural and Industrial Steering, Drive, Planter (Drill) Std. Spec. for Copper and lts Alloy Die Forgings (Hot ec. for Safety Controls and Signaling Devices for Printing Test Meth. for Colorfastness of Textiles to Ho meth. for Colorfastness of Textiles to Dry Heat (Excluding Round Thread Casing Joint Strength with Combined Internal . Spec. for Carbon and Alloy Steel Nuts for Bolts for High ugally Cast Iron-Chromium-Nickel High Alloy Tubing for Sid Spec for Copper Alloy Tubes for Performance Requirements for Double Check Valve Type Back
Preparation (Grass) (1972)

Preparation, and Tempering (Cereal Che mistry) (1962)

Preparation, Finishing and Testing of Concrete Subfloor

Preparation, Maintenance and Rules of the Road (1972)

Preparation, Standardization, and Storage of Std. Solut

Preparations Which Are Susceptible to Hydrolysis by Alp

Preparations (Cereal Chemistry) (1962)

Preparations (Cereal Chemistry) (1962)

Preparations (Cereal Chemistry) (I968)

Meth. of Analy

/Otometric Meth

Preparing Aqueous Solutions of Engine Antifreezes or an

Preparing Coal Samples for Analysis (1972) ANSI K20.17

Preparing Oil Borne Preservative Solutions Such as Pent

Preparing Pentachlorophenol Solutions (1968)

Preparing Precision Statements for ASTM Meth. Related T

Preparing Vulcanized Test Sheets (1973)

Preparing, Cleaning, and Evaluating Corrosion Test Spec

Preperation and Evaluation of Painted or Coated Specime

replaced Aggregate Concrete for Structural and Mass Co

Preparation of Micrographs of Metals and Alloys I Includi

Presentation for Digital Computer Programs (1970)

Presentation (1972)

Preservation of Wood Floors (1962)

Preservation (Preservative) (1973)

Preservative in Wood (1970)

Preservative Properties of Oil Soluble Wood Preservativ

Preservative Solutions Such as Pentachlorophenol and Co

Preservative Treated Timber Products (1973)

Preservative Treatment by Pressure Processes and Qualit

Preservative Treatment by Pressure Processes for All Ti

Preservative Treatment by Pressure Processes of Timber

Preservative Treatment by Pressure Processes of Wood Fo

Preservative Treatment by Pressure Processes (1973)

(Preservative Treatment by Pressure Processes) (1972)

(Preservative Treatment by Pressure Processes) (1972)

Preservative Treatment by the Full Length Thermal Proce

Preservative Treatment of Incised Pole Butts by the the

Preservative Treatment of Lumber for Industrial Water C

Preservative Treatment of Piles, Poles, and Timbers for

Preservative Treatment of Structural Glued Laminated Me

Preservative Treatment of Western Red Cedar and Alaska

Preservative Treatment (For Fence Posts) by Pressure Pr

Preservative Treatment (1973)

Preservative Treatment, Poles, Piles, Spaced Columns, J

(Preservative) (1973)

Preservatives by Field Tests with Stakes (1974)

Preservatives by Laboratory Soil Block Cultures (1970)

Preservatives by Using Wood Properties of Oil Soluble W

Preservatives by Using Wood Specimens Uniformly Impregn

Preservatives for Marine Services by Means of Small Siz

Preservatives (1973)

Preservatives (1973)

Preservatives (1973)

Preservatives (1973)

Preserved, Creamed and Centrifuged Natural Rubber Latex

(Presoak Before Immersion) (Pulp and Paper) (1973)

Press and Gage Wheel Tires (Farm Machinery) (I971)

Press and Gage Wheels (Farm Machinery) (1971)

Press Brakes (1973)

Press Fit-Undersize Inside Diameter (1973)

Press Fit, Headless and Head Type, Step Relief (1972)

Press Fit, Steel and Bronze (1973)

Press Fit, Steel (1973)

Press Fit, Undersize Inside Diameter (1973)

Press or Air Finish (1973)

/Mmercial Wash-Whites (Wi

Press Rolls and Their Installation, Operation, and Main

Press Textile Fabrics (Permanent Press) After Repeated

Press Wheel Tires (Farm Machinery) (1971)

Press Woven or Knit Textile Fabrics (1973) ANSI L14.266

Press (Institutional Textile) (1973)

Nce Requi

Press (No Iron) Woven Sheet and Pillowcase Fabrics (Ins

Press (300-330 Deg. F) Finishing, Shirt Unit, Pants U

Press) After Repeated Home Launderings (1973) ANSI L14.

Press, and Gage Wheel, Tractor, Implement, and Log Skid

Pressed) (1974) ANSI H7.10

Presses (1972)

Pressing (1973)

Pressing) (1973) ISO 105-4

Pressure and Bending (1972)

Pressure and Temperature Service (1973) ANSI E38.1

Pressure Application at High Temperatures (1970) ASTM G

Pressure Applications (1972)

Pressure Backflow Preventers for Potable Water Supply L

Pressure Cable Systems (1972) ANSI C59.69

ANSI

ASPA

AACCH

WSFI

BMA

ASTM

ACCH $76.30 \mathrm{~A}$

AACCH 22.60

AACCH 22.62

ASTM

ASTM

ASTM

ASTM

ASTM

ASTM

ASTM

NCCA

ACI

ASTM

ANSI

SAE

ANSI

WSFI

WW PA

ASTM

NWMA

ASTM

AWPA

ICBO

AWPA

AWPA

AWPA

AWPA

AWPA

AWPA

AWPA

AWPA

AWPA

CTI

ASTM

AWPA

AWPA

AWPA

Unifor ICBO

ICBO

WWPA

ASTM

ASTM

NWMA

NWMA

ASTM

AWPA

AWPA

AWPA

St ASTM

ASTM

TAPPI

ASAE

ASAE

Std. for Sa ANSI

NSA

ANSI

NSA

NSA

NSA

A159. 1 *1 2.62

D1176

D2013

D2604

D2605

D3040

D3182

$\mathrm{Gl}$

TB. $111 \cdot 3$

66-65

E2

ARP681B

Z39. 16

*2

*31-3

D 1860

M.1-70

D2604

UBCS25-12

$\mathrm{Cl}$

C. 3

Cl4

C2

C16.72

C23.72

Clo

C?

D390

C28

C5.72

UBC 25.13

UBC * $3-25$

*31-3 
ontinuity of Quality of Electrical Insulating Oil for High Std Test. Proc for ec. for Martensitic Stainless and Alloy Steel Castings for Spec. for Alloy Steel Castings Specially Heat Treated for service (1974/ Std. Spec. for Ferritic Steel Castings for Special Quality, for Pressure Piping Components and Other Safety Std. for Household Type Std. Meth. of Rapid gas Distribution Systems Whose Maximum Allowable Operating Std. for Wrought Copper and Bronze Solder-Joint s Cylinders (1972) Std. for Cast Bronze Solder Joint Safety Std. for High

B156.1 Uniform Plumbing Code: Medium Safety Std. for Indicating Safety Std. for Indicating Std. Meth. of Test for Meth. of Test for

al Woodwork) (1973) Quality Stds. for High Std. Meth. for the Measurement of Sound
tings When Applied to Coil Co/ Test Meth. for Determining of Test for Capillary Moisture Relationships for Soils by of Test for Air Content of Freshly Mixed Concrete by the

$t$ for Accelerated Aging of Vulcanized Rubber by the Oxygen (1973) ANSI Z11.92

i Z11.277 Std. Test. Procedure for the Blow Off Std. Meth. of Test for Vapor Std. Meth. of Test for Vapor Std. Meth. of Test for Vapor

Z11.44 unds (For Casing, Tubing and Line Pipe Connections in High td. Meth. of Test for Oxidation Characteristics of Extreme Std. Code for

Std. for Rubber Gasket Joints for Cast and Ductile lron Std. Spec. for Reinforced Epoxy Resin Gas Std. for Asbestos Cement Std. Spec. for Reinforced Concrete Low Head Std. Spec. for Asbestos Cement Safety Std. for Asbestos Cement Std for Prestressed Concrete e Service (1973) td. Spec for Thermoplastic Gas Std. Spec. for Joints for Plastic Liquids (1972) carbon, Hot Rolled and Cold Finished, Special Quality, for Made Wrought Nickel and Nickel Alloy Welding Fittings for
Std. Code for s (1974) 1 Water Cooling Towers (1971) Std. Spec. for Std Rec Pract, for Safe Filling of Low timber, Plywood and Utility Pole Preservative Treatment by Std. Preservative Treatment by Std. for Preservative Treatment by Std. for Preservative Treatment by 73) Std. for Preservative Treatment by Std. for Preservative Treatment (For Fence Posts) by er, Timbers, Bridge and Mine Tie Preservative Treatment by ern Pine, Pacific Coast Douglas Fir and Western Hemlock by Std. for Wood Used on Farms (Preservative Treatment by 1973) $\mathrm{s}$ Used in Building Construction (Preservative Treatment by th.) (1971) ANSI Z/ Std. Meth. for Measurement of Extreme ) (1972) ANSI F11./ Std. Meth. for Measurement of Extreme Std. Meth. for Measurement of Extreme Std. Meth. for High Std. for Electric

1

tion) (1972)

r Demand (1974) aterials (1960) ANSI J/ rical Insulation (1974) ANSI C59.136 Safety Std. for Gas
Std. for Terminology for eformed Cellular Plastic Meth. of Test for Evaluating Std. Meth. of Test for Tack and spec. for Low Temperature Resistant Vinyl Chloride Plastic

si C59.91

si C.59.96

Std. Spec. for Polyethylene Plastic Std. Spec. for Vinyl Chloride Plastic Sid. Spec. for Polyester Film

Std. Spec. for Tetrafluoroethylene Backed Std. Spec. for High Temperature Glass Cloth probe Machine (197I) ANSI Z197.32 $\quad$ Sid. Meth. of Test for lass Duct (1973)

lass Duct (1973) and Constant Stress (/ eth. (1970)

Spec. for Electric Fusion Welded Steel Pipe for High gh Strength Carbon Steel Wrought Welding Fittings for High Std. Spec. for Electric Fusion Welded Steel Pipe for High Welded Carbon Steel Boiler and Superheater Tubes for High
std. Spec. for Seamless Carbon Steel Boiler Tubes for High
Pressure Cable Systems (1972) ANSl C59.70

Pressure Collapse Test of Composite Cans (1973)

Spec. for C ASTM

CCTI

ASTM

Pressure Containing Parts Suitable for High Temperature

Pressure Containing Parts Suitable for High Temprature
Pressure Containing Parts Suitable for Low Temperature

ASTM

ASTM

Pressure Containing Parts (1974)

Pressure Cookers (1973)

Pressure Determination of Pressurized Products (1972)

Pressure Does Not Exceed 60 or 125 Psig (1973)

Pressure Fitting for Use with Copper Water Tube (1973)

Pressure Fittings (1972)

Pressure Gas Manifolds for Industrial and Commercial Ga

Pressure Gas Piping Systems (1973)

Pressure Gauges for Compressed Gas Service (1973)

Pressure Gauges for Fire Protection Service (1973) ANSI

Pressure in Glass Aerosol Bottles (1972)

Pressure in Metal Aerosol Containers (1972)

Pressure Laminate Flush Type Wall Paneling (Architectur

Pressure Levels in Air (Acoustics) (1971)

Pressure Marking and Blocking Resistance of Organic Coa

Pressure Membrane Apparatus (1972)

Pressure Meth. (1972T) ANSI A37.70

Pressure Meth. (1973) ANSI J4.1

Pressure of Composite Cans (1973)

Pressure of Liquefied Petroleum Gases (LP-Gas Meth.)

Pressure of Petroleum Products (Micromethod) (1971) Ans

Pressure of Petroleum Products (Reid Meth.) (1972) ANSI

Pressure Oil Well Service) (1972)
Pressure Oils (1972) ANSI Z11.320

Pressure Petroleum Refinery Piping (1973)

Pressure Pipe and Fittings (1972) ANSI A21.11

Pressure Pipe and Fittings (1973)

Pressure Pipe for Water and Other Liquids (1972)

Pressure Pipe (1973)

Pressure Pipe (1973) ANSI A165.5

Pressure Pipe, Couplings, and Gaskets for Water and Fir

Pressure Pipe, Steel Cylinder Type, for W ater and Other

Pressure Pipe, Tubing and Fittings (1973)

Pressure Pipes Using Flexible Elastomeric Seals (1973)

Pressure Piping Components and Other Pressure Containin

Pressure Piping (1972)

Pressure Piping (1972) ANSI H34.15

Pressure Preservative Treatment of Lansportation System

Pressure Pressurized Products (1972)

Pressure Processes and Quality Control Std. (1973)

Pressure Processes for All Timber Products (1973)

Pressure Processes of Timber Piles (1973)

Pressure Processes of Timber Poles (1973)

Pressure Processes of Wood for Highway Construction (19

Pressure Processes (1972)

Pressure Processes (1973)

Pressure Processes (1973)

Pressure Processes) (1972)

Pressure Processes) (1972)

Pressure Properties of Fluid Lubricants (Falex Meth.) (

Pressure Properties of Lubricating Fluids (Four Ball Me
Pressure Properties of Lubricating Fluids (Timken Meth.

Pressure Pump Test. of Hydraulic Oils (1973)

Pressure Pumps for Use in Recreational Vehicles (1972)

Pressure Rating Std. for Y Type Strainers (1973)

Pressure Regulating Valves for LP Gas (1973) ANSI Z217.

Pressure Regulators (1973)

Pressure Regulators (1973)

Pressure Relief Devices (Container and Equipment Protec

Pressure Relief Joint Fillers (1973)

Pressure Requirements for Pipe Schedule System Sprinkle

Pressure Sealing Properties of Rubber and Rubber Like M

Pressure Sensitive Adhesive Coated Tapes Used for Elect

Pressure Sensitive Adhesives by Rolling Ball (1973)

Pressure Sensitive Electrical Insulating Tape (1973)

Pressure Sensitive Electrical Insulating Tape (1973)

Pressure Sensitive Electrical Insulating Tape (1973) an

Pressure Sensitive Electrical Insulating Tape (1973) an

Pressure Sensitive Electrical Insulating Tape (1974)

Pressure Sensitive Electrical Tape (1973)

Pressure Sensitive Tack of Adhesives Using an Inverted Pressure Sensitive Tape Application Stds. for Fibrous G

Pressure Sensitive Tape Performance Stds. for Fibrous C

Pressure Sensitive Tape to Fiberboard at $90 \mathrm{Deg}$. Angle

Pressure Sensitive Tape W ater Vapor Transmission Test M

Pressure Service at Moderate Temperatures (1973)

Pressure Service Piping (1969)

Pressure Service (1972 A) ANSI B125.4

Pressure Service (1973) ANSI B 125.12

Pressure Service (1973) ANSI B 125.7
Std. Pract, for Hi

Ctric Resistance
UI

ASTM

/Es in ANSI

ANSl

ANSI

UL

UL

UL

ASTM

ANSI

NCCA

Meth. ASTM

ASTM

ASTM

CCTI

ASTM

ASTM

API

ASTM

ANSI

AWW A

ASTM

A W W A

ASTM

ASTM

UL

AW W A

A STM

ASTM

ASTM

ASTM

ANSI

CTI

ASTM

1. ICBO

$A$ W PA

AWPA

AWPA

A WPA

AWPA

AWP

AWPA

$A W P A$

A WPA

ASTM

ASTM

ASTM

ASTM

IAPMO

FCI

UL

ANSI

UL

ANSI

ASTM

FMS

ASTM

ASTM

ASTM

ASTM

ASTM

ASTM

ASTM

ASTM

ASTM

ASTM

SMACN

SMACN

ASTM
PSTC

ASTM

MSS

ASTM

ASTM

D1819

C 133

A 217

A 389

A352

136
D3070

B16.33

B16.22

B16. 18

407

UPC $1 . F$

404

393

D3063

D3074

- $1.500 \mathrm{~B}$

S1. 13

TB-11-17

D3I 52 
Std. Spec. for Low Alloy Steel Castings Suitable for 00,000 Btu Per Hr.) (1972) Std. for Gas Fired High Std. for Gas Fired Low meth. for Water Resistance of Textile Fabrics: Hydrostatic Std. Meth. of Measuring the Spiral Flow of Low Std. for Guide for the Dynamic Calibration of

Std. Spec. for Metal Arc Welded Steel Pipe for High House Design and Construction Met/ the All Weather Wood on (1973)

Std. for

Improved Std. Designs for Sid Spec for

Alloy Steel Forgings for Seamless Drums, Heads, and Other Quenched and Tempered Carbon and Alloy Steel Forgings for (National Board lnspection Code) Manual for Boiler and ature Service (1972) ANSI /

re Service (1973) ANSI G35.1/ Std Spec. for Carbon Steel

pec. for Chromium-Copper-Nickel-Aluminum Alloy Stee

olybdenum-Nickel Alloy Steel, Quenched and Tempered, for olybdenum (1972) ANSI G35.16

Silicon (1972A) ANSI G32.1

nched and Tempered (1972A) AN31 G35.11

ening (Maraging), 18\% Nickel (1972A) Ansi/

ening (Maraging), 12\% Nickel (1972A)

red 8 and $9 \%$ Nickel (1972A) ANSI G35.20

red Nickel-Cobalt-Molybdenum-Chromi/

ormalized and Tempered (1972A) ANSI G35.3

and Lower Temperature Service (1972)

ganese (1972A) ANSI G35.8

silicon (1972A)

vice (1972)

Std. Spec, for High Strength Steel Plates for

ly Heat Treated (1972A)

Std. Spec. for Copper Nickel Alloy Plate and Sheet for Std Spec for Carbon Steel Sheets for eth. for Ultrasonic Inspection of Aluminum Alloy Plate for Sid. Spec. for Manganese Vanadium Alloy Steel Plates for

for General Requirements for Delivery of Steel Plates for r Charpy V-Notch Testing Requirements for Steel Plates for Std. Spec. for Nickel Alloy Steel Plates for Std. Spec, for Molybdenum Alloy Steel Plates for

Std. Spec. for Carbon Manganese Silicon Steel Plates for manganese Molybdenum and lts Nickel Alloy Steel Plates for

m, Alloy Steel Plates, Quenched and Tempered Chromium, for and Intermediate Tensile Strength Carbon Steel Plates for arbon Steel Plates with Improved Transition Properties for sisting Steel Bars and Shapes for Use in Boilers and Other

Molybdenum Alloy Steel Plates, Quenched and Tempered, for el Steel Plate, Sheet, and Strip for Fusion Welded Unfired Std. Spec, for Copper Silicon Alloy Plate and Sheet for

Std. Spec. for Chromium Molybdenum Alloy Steel Plates for $r$ Straight Beam Ultrasonic Examination of Steel Plates for ec. for Steel Castings, Heavy Walled, Carbon and Alloy for empered Vacuum Treated Carbon and Alloy Steel Forgings for Temper/ Std. Spec. for Alloy Steel Bolting Materials for 1) Std. for Flexible Non

Safety Std. for Acetylene Generators, Stationary, Medium Safety Std. for Acetylene Generators, Stationary, Low act. for Calculating Stress in Plastic Pipe Under Internal f Test for Effect of Cyclic lmmersion of Syntactic Foam at Safety Std. for Acetylene Generators, Portable, Medium test for Time to Failure of Plastic Pipe Constant lnternal Doughs (Cereal Chemistry) (1962)

stems (Instrumentation) (1970)

Specifying Duct, Sheet Metal, Low stems $(1967)$

Std. for Air Std. for Air Agricultural Grain Storage Loads, Mobilehomes and Recreational Vehicles (1971) ers Rated on a Symmetrical Current/ Rec. for Fire Protection of Small Nonpressurized and rdous Locations (1972) ANSI C 106.1 Std. for Purged and Std. Meth. for Sampling reational Vehicles (1971) Std for Pressurized and Non Std. Meth. of Rapid Pressure Determination of Std. Rec. Pract. for Safe Filling of Low Pressure Std. Meth. of Test for Liquid Densities of

ects/ Std. Meth. of Test for Effectiveness of Aerosol and Std. Nuclear Safety Criteria for the Design of Stationary
Std. for

Design and Construction)/ tion (1973)

in Corrosive Petroleu/ spheric Vent (1972/ Performance Requirements for Backflow
Pressure Service (1974) ANSI G52.5

Pressure Steam and Hot Water Boilers (Inputs Not Over 4

Pressure Steam and Hot Water Boilers (1972)

Pressure Test (1971) ANS1 L14.265

Pressure Thermosetting Molding Compounds (1972)

Pressure Transducers (1972)

Pressure Transmission Service (1971) ANSI G36.49

Pressure Treated Lumber and Plywood) Foundation System

Pressure Treated Piles and Timbers in Marine Constructi

Pressure Treated Timber Crib Walls (1969)

Pressure Treatment of Timber Products (1969)

Pressure Vessel Components (1970) ANSI G38.7

Pressure Vessel Components (1973) ANSI G35.21

Pressure Vessel Inspectors (1973)

Pressure Vessel Plates for Intermediate and Higher Temp

Pressure Vessel Plates for Moderate and Lower Temperatu

Pressure Vessel Plates (1972) ANSI G33.3

Pressure Vessel Plates (1973) ANSI G35.22

Pressure Vessel Plates. Alloy Steel 5\% Chromium 7 Ngan

Pressure Vessel Plates, Alloy Steel, Chromium Manganese

Pressure Vessel Plates, Alloy Steel, High Strength, Que

Pressure Vessel Plates, Alloy Steel, Precipitation Hard

Pressure Vessel Plates, Alloy Steel, Precipitation Hard

Pressure Vessel Plates, Alloy Steel, Quenched and Tempe

Pressure Vessel Plates, Alloy Steel, Quenched and Tempe

Pressure Vessel Plates, Alloy Steel, 36\% Nickel (1972)

Pressure Vessel Plates, Alloy Steel, 9\% Nickel Double N

Pressure Vessel Plates, Carbon Manganese, for Moderate

Pressure Vessel Plates, Carbon Steel, High Strength Man

Pressure Vessel Plates, Heat Treated, Carbon Manganese

Pressure Vessels for Moderate and Lower Temperature Ser

Pressure Vessels Plates, 5\% Nickel Alloy Steel, Special

Pressure Vessels (1969) ANSI H52.1

Pressure Vessels (1971) ANSI G33.4

Pressure Vessels (1971) ANSI H38.21

Pressure Vessels (1972)

Pressure Vessels (1972)

Pressure Vessels (1972)

Pressure Vessels (1972) ANSI G33.1

Pressure Vessels (1972) ANSI G34.1

Pressure Vessels (1972) ANSI G35.14

Pressure Vessels (1972) ANS1 G35.15

Pressure Vessels (1972) A NSI G35.18

Pressure Vessels (1972) A NSI G35.2

Pressure Vessels (1972) ANSI G35.7

Pressure Vessels (1972) ANSI G81.29

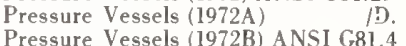

Pressure Vessels (1973)

Pressure Vessels (1973) ANSI G35.4

Pressure Vessels (1973) A NSI G60.2

Pressure Vessels (1974)

Pressure Vessels (1974) ANSI G55.17

Pressure Vessels, Valves, Flanges, and Fittings for Low

Pressure (Welding and Cutting) (1973)

Pressure (Welding and Cutting) (1973)

Pressure (1967) ANSI B72.10

Pressure (1972)

Pressure (1973)

Pressure (1974) ANSI B72.6

Pressure, Air Transmission (1967)

Pressuremeter Meth. of Analysis of Diastatic Activity O

Pressures for Pneumatic Controllers, and Transmission Sy

Pressures for Pneumatic Controllers and Transmission Sy

Pressures, and Capacities (1968)

Pressurized and Non Pressurized Potable Water Tanks for

Pressurized Components of AC High Voltage Circuit Break

Pressurized Containers of Flammable Liquids (1973)

Pressurized Enclosures for Electrical Equipment in Haza

Pressurized Gas for Gas Analysis (1973)

Pressurized Potable Water Tanks for Mobilehomes and Rec

Pressurized Products (1972)

Pressurized Products (1972)

Pressurized Products (1972)

Pressurized Space Spray Insecticides Against Flying lns

Pressurized Water Reactor Plants (1973)

Prestressed Concrete Pressure Pipe, Steel Cylinder Type

Prestressed Concrete Structures (1967)

Prestressed Concrete (Post Tensioned Box Girder Bridges

Prestressed Steel Strand and Wire for Concrete Construc

Prestressed with Unbonded Tendons (1966)

Prestressing Concrete Pipe (1973)

Prevent in Service Cracking of Carbon Steel (P.1) Welds

Preventers for Potable Water Supply Lines (1972)

Preventers in Water Supply Lines with Intermediate Atmo
ASTM

ANSI

ANSI

A487

Z21.13

ATM 127

ANSI D3123

ASTM A381

NFORP RP7

AWPA C18

A WPI

ASTM

/Ec. for ASTM A541

NBBPV *1

ASTM A5I5

ASTM A516

Std. S ASTM A410

ASTM A533

ASTM A357

ASTM A202

4538

ASTM A 590

ASTM A553

A605

ASTM A658

ASTM A353

ASTM A662

ASTM

ASTM A537

ASTM A612

ASTM A 645

ASTM B402

Std.

ASTM B548

ASTM A225

A20

A593

ASTM A203

ASTM A204

ASTM A290

A302

A 302
A542

A 285

A 442

A 479

A543

A 240

B96

$\begin{array}{ll}\text { ASTM } & \text { A } 387 \\ \text { ASTM } & \text { A435 }\end{array}$

A 435

A643

A 320

A 320
TSC 19

408

UL

UL

D2153

D2735

297

ASTM

D1598

CSI 15836

$\mathrm{AACCH} 22.11$

SAMA PMC2.5

ASAE D240

IAPMO TSCA

IEEE 340

FMS 7.29

NFPA 496

ASTM F307

IAPMO TSC4

ASTM D3070

ASTM D309l

ASTM D3096

ASTM D3088

ANSI N18.2

AWWA C301

ACI $\quad 67-40$

CRSI *2

ICBO UBCS26-7

ACI $\quad 66.8$

ASTM A648

NACE RP.04.72

/Equi ASSE 1015 
cars and Trailer Coaches (1970)

Meth of Test for Effectiveness of Mintd. for (Backflow Efflorescence,

lations (1973)

elt and AsphaIt Preferably with Gravel or SIag Surf/

Loss
Loss

$0,000(1973)$

duction and Dielectric) (1973)

g Equipment (VesseI) (I974)

of Analysis of Nitrophenide in Poultry Mashes (Feed) for ign of Buildings and Other Structures (1973) Rec. for 73)

Rec. for

Uniform Fire Code:

I Gas Fired Watertube Boiler Furnaces with One ed Multiple Burner Boiler Furnaces (Fire Protec/ ctures Caused by Snow Loads (1973)

12.12

lectric Power Generation: Fossil Fue/ iners (1972)

Rec. Pract. for the Rec. Pract. for Accident Std. Spec, for Acciden gns (Symbols) Complementary to ANSI Z35.1-1972. Accident nd Flammable / Uniform Fire Code: General Precautions for i Z11.152 Std. Meth. of Test for Rust Std. Meth. of Measuring Plasticity Retention Index ce Criteria for Roll Over Protective Structures (ROPS) fo riteria for Falling Object Protective Structure (FOPS) for oofing (1973) AASHO M116, ANSI A109.1 Spec. for Aluminum Std. Rec. Pract. for Test. Primers and Std. Rec. Pract, for Test. afety Std. for Burglary Resisting Glazing Material for Use eth. of Test for Coating Thickness by the Beta Backscatter nerating Station Protection Sys/

Trial Use Guide: General al $(1960)$

9/

$0)$

Rec. Pract. for Definitions, Abbreviations and General n 35 mm Motion Picture Film/ ion Picture Film, Perforate/ re Film Perforated 1-4 (1971) uper $8(1.3)(1973)$

Std. for Location of Super 8 Std. for Location of Super 8 Std. for Dimensions for Std. for Location of Super

ile) (1973) Rec. Pract. for Automotive

1 Test $(1969)$ Std. Performance Requirements for Woven Test Meth. for Colorfastness of Dyed or nd Two Sided Rigid Boards (1970) ANSI C83/ Std. Spec, for 3.88, Ipc C.405A Std. General Document for Suggested Guide Lines for Std, for Safety for for Transmission of End Product Description Data (Tooling, or Cotton Woven Tent, Awning, and Canopy Fabrics, Painted, meth. for Contact Uniformity Test for Photographic Contact Std. Spec. for Photographic Slant Drop Penetration Test for Paperboard ammable Liquid Mixing Operations (Paint, Varnish, Lacquer, spec. for Masks (Separate) for Use in Photographic Contact 1971) Std. for Dimensions for Printed Area in Super 8 for Location of Super 8 Printed Area in Optical Reduction on of Super 8 Printed Area in Optical Reduction or Contact Spec. for Safety Controls and Signaling Devices for or Measuring Blister Resistance of Coated Paper in Heatset Std. Positive Gravure Densities Std. Gravure Color Bars Std. Gravure Proofing !nks

Std. Proofing Supplement Stock for Gravure Magazines ns for Photographic Sound Record on Super 8 Motion Picture ve Strength of Hydraulic Cement Mortars (Using Portions of Rec. for Water Demand for Sprinkler System for Dwelling Construction Under the Uniform Building Code: Uniform Plumbing Code: Sewers and Std. Pract. for

Pract. for Acceptance of Evidence Based on the Results of measuring Resistivity of Silicon Slices with a Collinear 4 et Resistance of Thin Metallic Films with a Collinear Four resistance of Silicon Epitaxial Layers Using a Collinear 4 for Pressure Sensitive Tack of Adhesives Using an Inverted Using the Mass Spectrometer Leak Detector in the Detector meter Leak Detector or Residual Gas Analyzer in the Tracer Test for Resistivity of Silicon Epitaxial Layers by the 3

y Funnel, Trap Flask, Step Wedge for X-Ray Standardization n Loss Tables for Solving Fire Protection System Hydraulic ugh Closed Steel Door and Frame Assemblies (19) teeI Door and Frame Assemblies (1972)

Wagon Running Gear (1973)

ors (1974) ANSI C83.63C

Tubes and Cores (1973)

3)

Std. Test

Std. Test

Std. Test

Std. Test

Std. Test.

Std. Test.
Preventers) and Relief Valves in Travel Trailers, Camp Preventing Excessive Expansion of Concrete Due to the a Prevention and Control (Tech. Notes) (1970)

Prevention Data on Bonds and Guarantees for Roof Instal Prevention Data on Elastomer Roof Coverings (Multiply F Prevention Data on Flood Losses (1973)

Prevention Data on Fundamental Defects in Fires Over 05 Prevention Data on High Frequency Heating Equipment (In Prevention Data on Sight Glasses for Chemical Processin Prevention of Cecal and Intestinal Coccidiosis in Chick Prevention of Collapse Due to Weak Construction and Des Prevention of Damage to Property from Surface Water (19 Prevention of Dust Explosions (1973)

Prevention of Furnace Explosions in Fuel Oil and Natura

Prevention of Furnace Explosions in Pulverized Coal Fir

Prevention of Roof Collapse of Buildings and Other Stru Prevention of Sulfur Fires and Explosions (1971) ANSI Z Prevention of Water Damage to Steam Turbines Used for E Prevention Signs for Display on Tank Trailers and Conta Prevention Signs (1972)

Prevention Signs (1973)

Prevention (Incinerator and Open Burnirectional, and Spec

Preventive Properties of Lubricating Greases (1973) Ans (PRI) of Natural Rubber (1973)

Prime Movers (Heavy Equipment) (1972) /Nimum Performan

Prime Movers, Wheeled and Track Type Front End Loaders,

Prime Windows (1972) ANSi A134.1

Primer for Use with Asphalt in Dampproofing and Waterpr

Primer Surfaces Over Preformed MetaI (1974)

Primers and Primer Surfaces Over Preformed Metal (1974)

Principally as a Substitute for Plate Glass Show Window

Principle (1972)

Principles for Reliability Analysis of Nuclear Power Ge

Principles of Clay Masonry Construction: Student's Manu

Principles of Industrial Exhaust Ventilation Systems (1

Print Spec. for Magnetic Ink Character Recognition (197

Printed Area in Optical Reduction or Contact Printing O

Printed Area in Optical Reduction Printing on $35 \mathrm{~mm}$ Mot

Printed Area in Super 8 Printing on 16/8 Mm Motion Pictu

Printed Area on $16 \mathrm{~mm}$ Motion Picture Film, Perforated S

(Printed Circuit) (1972)

Printed Circuits (1967)

Printed Table Napery (Napkin, etc.) (Institutional Text

Printed Textiles to Washing at 105 Deg. F: Rapid Contro

Printed Wiring Board Dimensions and Tolerances Single a

Printed Wiring Board Electric Connectors (1974) ANSI C8

Printed Wiring Board Repairs (1973)

Printed Wiring Boards (1973) ANSI C33.46

Printed Wiring Boards) in Numeric (Digital) Form on Pun

Printed, or Vinyl Coated (Institutional Textiles) (1973

Printers (1971)

Printing Frames (1972)

(Printing Ink Receptivity) (1973)

Printing Ink, Adhesive, etc.) (1974)

Printing of Roll Film Negatives (1972)

Printing on 16/8Mm Motion Picture Film Perforated I-4 (

Printing on $35 \mathrm{~mm}$ Motion Picture Film, Perforated 2R-16

Printing on $35 \mathrm{~mm}$ Motion Picture FiIm, Perforated 5R.16

Printing Presses (1972)

Printing (1972)

(Printing (1973)

(Printing) (1973)

Printing) (1973)

Printing) (1973)

Prints (1972)

Prisms Broken in Flexure) (1972) ANSI A 1.30

Private Fire Protection (Safety) (1973)

Private Garages and Carports (1973)

Private Sewage Disposal Systems (1973)

Probability Sampling of Materials (1970) ANSI Z1.10

Probability Sampling (1969) ANSI Z1.12

Probe Array (1973)

Probe Array (1973)

Probe Array (1974)

Probe Machine (1971) ANSI Z197.32

Probe Mode (1973)

Probe Mode (1973)

Probe Voltage Breakdown Meth. (1972) ANSI Z173.47

Problems (1973)

Proc. and Acceptance Criteria for Rate of Air Flow Thro

Proc, and Acceptance Criteria for Water Resistance on S

Proc. for Determining the Load Carrying Ability of Farm

Proc, for Low Frequency (Below $3 \mathrm{MHz}$.) Electric Connect

Proc. for Measuring Inside Diameters of Composite Cans,
IAPMO TSC 8

ASTM C441

BIA 23A

FMS $\quad 1.47 \mathrm{~S}$

FMS $\quad 1-47$

FMS $\quad 10-10$

FMS $\quad 10-9$

FMS $\quad 6.3$

FMS 7-3

AACCH $\quad 18.25$

FMS $\quad 1.55$

FMS $\quad 9.2$

ICBO UFC $* 2 A R T 9$

NFPA 85

FMS $\quad 6.2$

FMS $\quad 1.54$

NFPA 655

ASME TWDPS-1

TTMA A RP5

ANSI Z35.1

Z35.

UFC $* 2 A R T 27$

ASTM D1743

ASTM D3I94

SAE J320B

SAE J231

AAMA 302.8

ASTM D41

D3322

ASTM D3322

UL

972

Std. M ASTM B567

IEEE 352

BIA *44

ACGIH *1

ANSI $\mathrm{X} 3.2$

ANSI PH22.180

ANSI PH22.179

ANSI PH22.153

ANSI PH22.181

IPC ML.990

SAE J771A

ANSI L24.2.6

AATCC 68

IPC D300E

EIA RS406

IPC R.700

UL 796

IPC D-350

ANSI L24.1.1

Std. ANSI PH3.45

ANSI PH 3.15

TAPPI UM.536

Rec. for Fl FMS 7-36

Std. ANSI PH3.9

ANSI PH22.153

ANSI PH22.179

ANSI PH22.180

ANSI B65.1

TAPPI

GT

GT

GT

GT

T526SU

$* 2$

$* 4$

$* 4$

$\mathrm{PH} 22.182$

C349

3.26

FMS

ICBO

ICBO

ASTM

Std. Rec ASTM

std. Meth. for ASTM Tent. Meth. of Test for She ASTM ASTM

UBC $* 8-4$

UPC*1.11

E105

EI41

F84

F390

F 374

D2979

E499

E498

F 108

$\mathrm{ACCH} \quad 28-90$

$\begin{array}{ll}\text { AACCH } & 28-90 \\ \text { FMS } & 2-89\end{array}$

STDI 116

115

EIA RS364-3

CCTI CT102

CCTI C133 
(1973)

oated Lock Screws (1973)

Rec. Safe Pract, and

Rec Pract furbine Fuels (JFT OT

Instrument Panel Laboratory Impact Test ectric Machinery (1973)

uctor Radiation Detectors (1969) ANSI N42.2

1962)

cans, Tubes and Cores (1974)

pes and Sounding Rules (1974)

74) Std. for Control Valve Capacity Test es (1967) Std. for Test Std. Test Sampling Std. Test. Std. lement of Hot Dip Galvanized Structural Steel Products and

ubes and Cores (1972)

nd Cores (1972)

nderride Guard (Safety (1971)

2) ANSI S6.2

tor Oil Cans (1974)

4)

Std. Test

Std. Test.

Std. Test.

Rec. Pract. Test

Rec. Pract. for the Test

Std. Test.

Std. Test.

Std. Test

) ANSI 2224.1

Std. Perforinance Evaluation

974) Std. for Control Valve Capacity Test Rec. Pract, for Design Std. Test.

cores (1974)

rque Balance Accelerome Std. Spec. Format Guide and Test n Agricultural Tractors Equipped with 3 Point

n Agricultural Tractors Equipped with $3 \mathrm{Po}$

Cans, Tubes, and Cores (1974)

ans. Tubes and Cores (1974) Std. Test

Std. for Test Std. Test. Std. Test.

Rec. Pract. for Spark Arrester Test Std. Requirements, Terminology, and Test

1974)

oors (1972)

es (1971)

for Hydraulic Fluid Power Fluid Sample Containers / izing Radiation (1969) ANSI N42.1

1973

ms for AC Electric Machinery Employing Form Wo/ allic Locking Element Type Prevailing-To/ ise Levels of Powered Industrial/ bution Transformers (1972) ing Material Data) (1972) res (1974)

Rec. Pract, for Service Brake Structural Integrity Test

/ Textile Colorfastness) (1972) fastness) (1954) ISO 105-1 ness) (1954) ISO 105-1

gy Consumption of Household Refrigerators, Com/ Rec. Evaluation Rec. Evaluation Rec. Evaluation Sid. Test bber and Rubber Like Materials by a Temperature Retraction Rec. Scaffolding Erection Rec. Steel Frame Shoring Erection Rec. Pract. for Bumper Evaluation Test le Construction Type Crane Overload Indicating System Test Std for Fiberboard Test

Rec. Horizontal Shoring Beam Erection automotive Transmission) Clutch Friction Test Machine Test Test for Lead in the Atmosphere by Colorimetric Dithizone onic Active Ingredient in Detergents by Cationic Titration (Floor Polish and Wax) (1971) ASTM D2047, C/ Test. Std. eel Cleanliness Requirements (Magnetic Particle Inspection ilers (1972)

Testing for Protective Enclosures for Agricultural Tractors (Test Std. for Protective Frame for Agricultural Tractors (Test ngs (1972) ANSI G5/ Sid. Rec. Pract. for Qualification of Std. 105D) (1971)

1972)

(1972)

Std. for Sampling Std. for Analytical Std. for Analytical Definition and Test

oil (1973)

ic Transducers (1972)

ndation) (1963) Std. for Inspection, Sampling and Measuring Std. for Suggested Design Suggested Design and Construction ombination) (1971) ANSI A197.2. Asse $1007 \quad$ Std. and Test connectors (1971) ANSl C83.63B

ed in Enclosures (1973)

Std. Test

Std. for Test

lcan/ Std. Rec. Pract, for Std. Materials, Equipment, and Minimum Performance Requirements and Uniform Test
rs Operated on Public Highways $(1973 /$
Proc. for Side to Side Crush Composite Tubes and Cores Proc. for Storage Batteries (1972)

Proc, for the Locking Ability Performance of Chemical C Proc. (Industrial Plant Safety Checklists) (1973)

Proc.) (1973)

Procedure-Head Area (1971)

Procedure-Passenger Car (1971)

Procedure for Airborne Sound Measurements on Rotating E

Procedure for Amplifiers and Preamplifiers for Semicond

Procedure for Analysis of Bacteria (Cereal Chemistry) (

Procedure for Axial (End to End) Compression Composite

Procedure for Bench Calibration of Tank Level Gaging Ta

Procedure for Chlorine Packaging (1973)

Procedure for Compressible Fluids in Turbulent Flow (19

Procedure for Compression Testing of Scaffolds and Shor

Procedure for Detecting Embrittlement (1972) ANSI G8.13

Procedure for Dry Fertilizer Spreader (1972)

Procedure for End Supported Beam Deflection Composite T

Procedure for End Supported Beam Strength Fibre Tubes a

Procedure for Evaluation of the Effectiveness of Rear U

Procedure for Exterior Sound Level for Snow mobiles (197

Procedure for Filled Cast Flat Bottom Drop Composite Mo

Procedure for Filled Cast Flat Drop Composite Cans (197

Procedure for Geiger Muller Counters (1970) ANSI N42.3

Procedure for Household (laundry, Clothes) Washer (1971

Procedure for Incompressible Fluids (1972)

Procedure for Industrial Exhaust Ventilation Systems (1

Procedure for Internal Bursting of Composite Tubes and

Procedure for Linear, Single Axis, Pendulous, Analog to

Procedure for Measuring Hydraulic Lift Force Capacity 0

Procedure for Measuring Hydraulic Lift Force Capacity $O$

Procedure for Measuring Lengths or Heights of Composite

Procedure for Measuring Wall Thicknesses of Composite C

Procedure for Medium Size Engines (1972)

Procedure for Neutral Grounding Devices (1972)

Procedure for Oil Wicking of Composite Motor Oil Cans (

Procedure for Processing Hardware for Custom Aluminum D

Procedure for Processing Hardware Schedules and Templat

Procedure for Qualifying and Controlling Cleaning Meth.

Procedure for Semiconductor Radiation Detectors for Ion

Procedure for the Blow Off Pressure of Composite Cans

Procedure for the Evaluation of Sealed Insulation Syste

Procedure for the Locking Ability Performance of Nonmet

Procedure for the Test., Rating and Reporting of the No

Procedure for Thermal Evaluation of Oil Immersed Distri

Procedure for Toroidal Magnetic Amplifier Cores (Includ

Procedure for Torque Strength of Composite Tubes and Co

Procedure for Trucks, Buses, and Combination Vehicles F

Procedure for Water Absorbency of Bibulous Papers (1972

Procedure for Water Closet Flush Tank Ball Cocks (1968)

Procedure of Chromatic Transference Scale (Color Chart

Procedure of Gray Scale for Color Change (Textile Color

Procedure of Gray Scale for Staining (Textile Colorfast

Procedure to Determine the Freezer Temperature and Ener

Procedure (TR Test) (R1972) ANSI J2.22

Procedure (1968)

Procedure (1969)

Procedure (1971)

Procedure (1971)

Procedure (1972)

Procedure (1972)

Procedure (1972)

Procedure (1972T)

Procedure (1974)

(Procedure) for Prod

/Eristics of Ru
/E

Procedure) (1973)

$/ E_{c}$. for Premium Aircraft $Q$

Procedures and Partenance of Steam Safety Valves on Bo

Procedures and Performance Requirements) (1972) SAE J16

Procedures and Performance Requirements) (1972) SAE J33

Procedures and Personnel for the Welding of Steel Casti

Procedures and Tables for Inspection by Atributes (MIL

Procedures for Accountability of Uranium Hexafluoride(

Procedures for Accountability of Uranium Tetrafluoride

Procedures for Ammonia Nitrate (1973)

Procedures for Bulk Shipments and Transfers of Soybean

Procedures for Calibration of Underwater Electro Acoust

Procedures for Combined Concrete Footings and Mats (Fou

Procedures for Concrete Foundation Piers (1969)

Procedures for Executive Functions and Process Input-

Procedures for Home Laundry Equipment (washer, Dryer, C

Procedures for Low Frequency (Below $3 \mathrm{MHz}$.) Electrical

Procedures for Low Voltage AC Power Circuit Breakers Us

Procedures for Mixing Rubber Compounds for Preparing Vu

Procedures for Motor Vehicle Brake Linings (1966)

Procedures for Motor Vehicles, Trailers, and Semitraile

CCT

BCI

IFI

MCA

1108

* 1.66

125

$\mathrm{SG} 20$

D3241

SAE J921B

SAE J229

IEEE 85

IEEE 301

AACCH 42.10

CCTI CT107

ANSI B88.2

$\mathrm{CHI}$

ISA 7

SSI

ASTM Al43

ASAE S341

CCT1 T.113

CCTI Tll4

SAE J260

SAE J192

CCTI Cl2s

IEEE 309

AHAM HLW.1

ISA

$\$ 39.2$

ACGIH *1.6

CCTI Tl12

IEEE 337

ASAE S.349

SAE J283

CCTI CT 104

CCTI CT10

SAE J350A

IEEE 32

CCTI C124

NBHA *1

NBHA $* 7$

NFLDP T2.9.2

IEEE $\quad 300$

CCTI Cllo

IEEE 429

IFI 124

ITA $\quad 6 \mathrm{GI}$

IEEE 345

IEEE $\quad 106$

CCTI T116

SAE J294

TAPPI T432SU

ASSE 1002

AATCC EP3

AATCC EPI

AATCC EP2

AHAM HRF.2-ECFT

ASTM D1329

SSI

SSI

SAE

SAE

SAE

SSI

ASth of AST

ASTM

CSMA

SAE

FMS

ASAE

ASAE

ASAE

ASTM

ANSI

ANSI

ANSI

FI

NSPA

ANSI

$\mathrm{ACI}$

$\mathrm{ACl}$

ISA

AHAM

EIA

*1

*6

J980A

J248 
washers (I971) ANSI A 197.1, Asse I006

Waste Disposer Units (1971) ANSI AI97.3,

Std. and Test Std. and Test mountabl/ Rec. Pract. for Uniform Laboratory Fatigue Test ipment (1973) ANSI C83.25

Std. Test.

ipment (1970 Ansurements and Uniform Test assemblies (1970)

Std for Tests and Std. for Inspection Test

femperature lndexe/ Guide for the Preparation of Tes Tools, Crimping, Solderless Wiring Devices-Recommended ntiometers (1970) nges (1972) Std. for Inspection and Tes Safety Rules, Rec. for a Manual of Radioactivity Outdoor Smallbere Rifle Range Plans: Safety Rules and Air Conditioning Equipment Selection and System Design uoride Content of the Atmosphere and Plant Tissues (Manual Std. for Swaging

Std. for Operation and Flow Rec. Pract for Hardware Test of Digital Std Meth for Validation of Results of for Steel Sheet, Zinc Coated (Galvanized) by the Hot Dip Meth. for Analysis of Liquefied Petroleum Gases (LPG) by Std. Spec. for Safety Std for Compressors for est Meth. for Laboratory Corrosion Test. of Metals for the uter System FORTRAN Procedures for Executive Functions and Rec. Pract. for Monitoring Specia

pe) (1971)

71)

Std. Spec. for Seamless Carbon Steel Pipe for Std. We Std. Wet

Alaska Yellow Cedar Timber Std. for Tubular Std. for Full Length Therma Uniform Fire Code: Fruit Ripening Structural Clay Masonr

reservative Treatment of Incised Pole Butts by the Thermal and Sugars, and to Most Other Products of the Wet Milling k Forming Quality, Zinc Coated (Galvanized) by the Hot Dip f Drawing Quality, Zinc Coated (Galvanized) by the Hot Dip verts and Underdrains, Zinc Coated (Galvanized) by Hot Dip Sid. Terms Used in the Sulfite Pulping

of Sample Size to Estimate the Average Quality of a Lot or od Poles Preservative Treatment by the Full Length Thermal starch, Syrup and Sugar Obtained from the Corn Wet Milling ration of and Electroplating on Aluminum Alloys by Zincate

s for Steel Sheet, Zinc Coated (Galvanized) by the Hot Dip oils, and Cut Lengths, Zinc Coated (Galvanized) by Hot Dip for Steel Sheet, Zinc Coated (Galvanized) by the Hot Dip Std. for Practice for Storage of Uniform Building Code Std for Std. Practice for Storage of

(1971)

Spec. for Flat Rolled Nonoriented Electrical Steel, Fully led, Grain Oriented, Silicon lron, Electrical Steel, F ully

lywood and Utility Pole Preservative Treatment by Pressure Std. Preservative Treatment by Pressure Std. for Preservative Treatment by Pressure Std. for Preservative Treatment by Pressure Std. for Preservative Treatment by Pressure Basic Criterial for Evaluation of Special Rec for Electrolytic Chlorine

for Preservative Treatment (For Fence Posts) by Pressure rs, Bridge and Mine Tie Preservative Treatment by Pressure

Pacific Coast Douglas Fir and Western Hemlock by Pressure

for Wood Used on Farms (Preservative Treatment by Pressure

Building Construction (Preservative Treatment by Pressure Std. for Industrial Engineering Terminology: D ata

(Safety) for Flammable Liquid and Liquefied Flammable Gas Loss Prevention Data on Sight Glasses for Chemical

Std. for the Protection of Electronic Computer and Data Rec. Procedure for Rec. Procedure for

r Nomenclature for Motion Picture Film Used in Studios and of Test for Characteristic Groups in Rubber Extender and ctions Within the Air Tral Spec. for Std. Integrated Dat

limit Controls (A ir Conditioning, Heating, Ventilating and Std. for Safety for Electronic Dat

Std. for Industrial Engineering Terminology: Materials ng the Resistance of Photographic Films to Abrasion During hart Symbols and Their Usage in Micrographics (Information

- Positioning Numerically Controlled Machines (Information std. Rec. Pract. for Preparation of Specifications for the Rec. Pract. for Std.

or Measuring Fast Neutron Flux for Analysis for Barium 140 glossary of Terms Relating to Chimneys, Gas Vents and Heat
Procedures for Plumbing Requirements for Household Dish Procedures for Plumbing Requirements for Household Food Procedures for Production Disc and Spoke Wheels, and De Procedures for Regular Bicycles (1972)

Procedures for Relays for Electrical and Electronic Equ Procedures for Retreaded Tires for Passenger Cars and S Procedures for SAE 100R Serics Hydraulic Hose and Hose Procedures for School Buses (1972)

Procedures for the Safety Std. for Regular Bicycles (19

Procedures for the Thermal Evaluation and Establishment

Procedures for User Certification of (I962) ANS1 C83.79

Procedures for Wirewound and Nonwirewound Trimming Pote

Procedures For, and Construction of High Power Rifle Ra

Procedures (1961)

Procedures (1972)

Procedures (1973)

Procedures) (1973)

Process-Tube to Rod End (1974)

Process Charts and Symbols (Mechanical Engineering) (19

Process Computers (1971)

Process Distillation Analyzers (1973)

Process for Roofing (1971) ANSl G8.21

Process Gas Chromatogra phy (1973)

Process Glass Pipe and Fittings (1970)

Process Industries (1972)

Process Industries (1972)

Process lnput-Output (1972)

Process of Quality Control (1969)

Tent. Meth. for Analysis for FI

Process Piping (1972A)

Process Porcelain Insulators (Apparatus, Cap and Pin Ty

Process Porcelain Insulators (Apparatus, Post Type) (19

Process Porcelain Insulators (Suspension Type) (1971)

Process Preservative Treatment of Western Red Cedar and

Process Service (1971)

Process (Banana. Citrus Fruit) (1973)

Process (Tech. Notes) (1966)

Process (Western Red Cedar, Northern White Cedar and Al

Process (I971)

D Starch Hydrolyzates and All Starche

Process (I971) ANS1 G8.22

Process (1971) ANSI G8.23

Process (1971) ANSI G8.28

Process (1972)

Process (1972) ANSI Z1.11

Process (1973)

Process (1973)

Process (1973) ANSI G53.12

Process (1973) ANS1 C8.25

/Td. Rec. Pract. for Choice ASTM

Pec. for Steel Sheet of Loc

Td. Spec. for Steel Sheet O ASTM

C. for Steel Sheets for Cul ASTM Std. for Lodgepole Pine Wo AWPA

Process (1973) ANSI G8.27 Determination of Phosphorus in Corn CR

Std. Rec. Pract. for Prepa ASTM

Ec. for General Requirement ASTM of Structural Quality in C ASTM $\begin{array}{ll}\text { Process, Commercial Quality (1971) ANS1 G8.24 /D. Spec ASTM } & \end{array}$

Processed Photographic Plates (1972)

Processed Pulverized Quicklime (1973)

Processed Safety Photographic Film Other Than Microfilm

Processed Type (1973)

Processes and Quality Control Std. (I973)

Processes for All Timber Products (1973)

Processes of Timber Piles (1973)

Processes of Timber Poles (1973

Processes of Wood for Highway Construction (1973)

Processes or Devices Used in Treating W astewater (I970)

Processes (Explosion Hazard During Manufacture) (1974)

Processes (1972)

Processes (1973)

Processes (1973)

Processes) (1972)

Processes) (1972)

Processing and Systems (1972)

Processing Equipment and Supporting Structures (1973)

Processing Equipment (Vessel) (I974)

Processing Equipment (1972)

Processing Hardware for Custom Aluminum Doors (1972)

Processing Hardware Schedules and Templates (1971)

Processing Laboratories (1971)

Processing Oils by the Clay Gel Absorption Chromatograp

Processing Systems Designed to Streamline Supply Transa

Processing Systems) (1972)

Processing Units and Systems (1972)

Processing (1972)

Processing (1972)

Processing) (1973) ANSI PH5.17

Processing) (1974)

Procurement of Photomasks (1972)

(Mat

Std. for Meth. for Determini ANS Std. for Flowc NMA

Procurement Quality Std. and Spec. (1969)

Procurement Spec. for Fasteners, Titani
Produced by Uranium 238 Fission (1973)

Producing Appliances (1972)
BMA

VESC

SAE

ANSI

IEEE

EIA

NRA

NRA

NESC.

NSA

ASME

ASTM

I ASTM

ASTM

NACE

ASQC

ASTM

ANSI

AWPA

ANSI

$\mathrm{CBO}$

BIA

$M$

A

A52

A444

T1201

E122

$\mathrm{Cl} 0$

B- 47

B253

A 525

A 446

A5 26

$\mathrm{PH} 1.45$

ICBO UBC 24.20

ANSl PHI.43

$\begin{array}{lll}\text { Std. ASTM } & \text { A677 } \\ \text { /Rol ASTM } & \text { A665 }\end{array}$

A665

UBC $25-12$

$A$ WPA

AWPA

AWPA

$A$ WPA

$\mathrm{NSF}$

FMS

Std AWPA

Cl

C3

C4

C. 9

C.9

C5.72

$\mathrm{C} 2$

C28

C16.72

C 23.72

Z94.3

7. 14

7.3

7.3

$* 1$

*7

PH 22.56

D2007

200

353

478

Z94. 7

PH4.35

MS4

RS274-B

F93

RP2

621

E393

$97 \mathrm{M}$ 
Refrigeration Systems, Incinerators and Other Misc. Heat Spec. for Indirect Type Oil Field Heaters Used in onitoring of the Product (Receiving and Source Inspection. Std. Spec. for Record Formats for Transmission of End

Rec. Pract. for Vendor Editorial Format for Voluntary values, Framing Rec., Properties, Weight, Stress of Bol (196) Rec. Pract. for Quality Control Monitoring of the rcelain Enameling (1970) ANS1 Z167. Sid. Rec. lhead Equipment (Production Valves, Ring Joint, Segmented,

Pract. for Uniform Laboratory Fatigue Test Procedures for or Electrical Installations at Drilling Rigs and Petroleum Std. Spec. for Toluenediisocyanate for Use in Std. for Industrial Engineering Terminology: Rec. Pract. Meth. for Lining Lease eck Flanges and Gask/ Spec for (Oil) Wellhead Equipment Spec. for Oil and Gas Separators (Petrole um Spec. for Spiral Weld Line Pipe (Petrole ur for Vertical and Horizontal Emulsion Treaters (Petroleum for Personnel Going Offshore for the First Time (Petroleum equirements of Farm Liv/ Effect of Thermal Environment on Meth. of Analysis of Radioactivity (Beta Gamma) in Cereal Dry Film Thickness of Paint. Varnish, Lacquer and Related afety) (19)

Std. Guide for Classifying and Labeling Epoxy Std. Spec. for Fire Refined Copper for Wrough ore Counts (Flat Sour, Total Aerobic, Anaerobic) in Cereal nitions of Terms Relating to Carbon Paper and Inked Ribbon meth of Analysis for All Baked Materials Except Chocolate ainst Embrittlement of Hot Dip Galvanized Structural Steel

Definitions of Terms Relating to Care of Consumer Textile 971 Std. Meth of Test for Total Base Number of Petroleum and Wax) (1971) ASTM D2047, C/ Test. Std. (Procedure) for otometric) Content in Feedstuffs and All Other Natural Dry for Determining Effect of Packaging on Food and Beverage iermination of the Temperature of Applied Coatings on Wood assurance Sampling of Carbon and Low Alloy Steels (Wrough Meth. for Ultrasonic Inspection of Aluminum Alloy Wrough Std. Meth of Test. Aerosol

for Integrity of Glue Joints in Structural Laminated Wood amless and Electric Resistance Welded Carbon Steel Tubula Std. Meth. of Conditioning Paper and lts

Std Rec. Pract for Conditioning Textiles and Textile Std. Spec. for Adhesives for Structural Laminated Wood

Spec. for Protein Base Adhesives for Structural Laminated Meth. for Measurement of Delayed Neutron Emitting Fission engths) (1974)

Stee

s, Rolled Floor Plates, and Steel Sheet Piling (l/ ;

y Axles (1973)

Steel

(Stee

(Steel

Stee

Stee

Steel

of Analysis of Crude Fat in Flour, Bread, and Baked Cereal mining Acidity Content in Steepwater and Most Other Liquid

determining $\mathrm{pH}$ Content in Steepwater and Most Other Liquid

ydrolyzates and All Starches and Sugars, and to Most Othe

Std. Designations for Copper and Copper Alloys (Wrough

ed Std. Analytical Meth. for Determining Crude Fat $(\mathrm{Ccl}$ on, and Grade of Palmkernels; Babassu; Flaxseed;/ Oilseed e and Strip) (1973) Sid. Spec. for Flat Coppe

meth. of Analysis of Crude Protein in Wheat and Flour Mil Meth. of Analysis for Ash in Cacao Meth. of Analysis for Ash in Dry Milk eth. of Analysis for Baking Test (Quality) for Sweet Yeast Disk Meth of Analysis of Color of Macaron Spectrophotometric Meth. of Analysis of Color of Macaron Meth. of Analysis of Egg Solids in Macaron Meth. of Analysis of Crude Fat in Dry Milk

Meth. of Analysis of Unsaponifiable Matter in Macaron Meth of Analysis of Coliform Bacteria in All Food Meth. of Analysis of Rope Spore Count in Cereal Meth. of Analysis of Salmonella Bacteria in Food meth. of Analysis of Food Poisoning Staphylococcus in Food Meth. of Analysis of Fecal Strepiococci in Food Meth. of Analysis of Mold and Yeast Counts in Food Meth. for Preparation of Sample: Fruit and Frui Meth. for Preparation of Sample: Macaron d. Plate Count Meth. of Analysis of Total Bacteria in Food of Analysis for Ash in Starch, Dextrin, and Other Modified

f Analysis for Citric and Isocitric Acids in Fruit and Its analysis of lnorganic Bromide Residues in Grain and Cereal nalysis of Thermophilic and Psychrophilíc Bacteria in Food illustration and Classification in Cereal Grains and Grain ntaining Monoglyceride, Salts, Soy Flour, and Other Cerea Meth of Analysis of Niacin and Nicotinamide in Cereal
Producing Appliances) (1973)

Producing Oil and Gas (1972)

Product Audit) (1969)

Product Description

Product Improvement

Product Use Manual: Grade Selection, Std. Size, Design

(Receiving and Source lnspection. Product Audit

Production and Preparation of Gray Iron Castings for Po

Production Check Flanges and Gaskets; Drilling Through,

Production Disc and Spoke Wheels, and Demountable Rims

Production Facilities on Land and on Marine Fixed and M

Production of Urethane Foams (1973) ANSI K65.125

Production Planning and Control (1972)

Production Tanks with Coal Tar Epoxy (1972)

Production Valves, Ring Joint, Segmented, Production Ch

Production) (1973)

Production) (1973)

Production) (1973)

Production) (1974)

Production. Pract. Orientation Prog

Products-Emergency Monitoring (Cereal Chemistry) (197

Products - Film Thickness of $0.3 \mathrm{MlL}$ or Greater (Coil

Products According to Their Hazardous Potentialities (S

Products and Alloys (1972) ANSI H23.12

Products and Food Ingredients (Cereal Chemistry) (1962)

Products and Images Made Therefrom (1972A)

Products and Piecrust (Cereal Chemistry) (1962)

Products and Procedure for Detecting Embrittlement (197

Products and Rec. Pract. for Use of These Terms on Perm

Products by Potentiometric Perchloric Acid Titration (1

Products Classified as to Slip Resistant (Floor Polish

Products Derived from Corn (1957)

Products During Storage (1972)

$/ \mathrm{Ng}$ Color (Spectroph Std. Rec. Pract

roducts During the Curing Cycle (1973)

Products Except Forgings and Stock) (1973)

Products for Aerospace Applications (1974)

IR Infrared De

/R Quality

Std. ASTM

ASTM

Products for Delivered Mass (1972)

Products for Exterior Use (1959) ANSI Z197.17

Products for High Temperature Service Conforming to 150

Products for Test. (1973) TAPPl T402

Products for Test. (1974) ANSI L14.205

Products for Use Under Exterior (Wet Use) Exposure Cond

Products for Use Under Interior (Dry Use) Exposure Cond

Products in Nuclear Reactor Coolant Water During Reacto

Products Manual for Carbon Sheet Steel (coils and Cut L

Products Manual) Carbon Steel Strip (1971)

Products Manual) Carbon Steel' Plates, Structural Shape

Products Manual) Carbon Steel: Semifinished for Forging

Products Manual: Tin Mill Products (1973)

Products Manual: Wrought Steel Wheels and Forged Railwa

Products Masonry (Tech. Notes) (1964)

Products Not Containing Fruit (Cereal Chemistry) (1962)

Products Obtained from Corn (1963)

Products Obtained from Corn (1963)

Products of the Wet Milling Process (1971)

Products Only); Application Data Sheet (1972)

Products Such as Feeds and Meals (1955)

Al Meth. for Deter

Lytical Meth. for

Ermand Finish

Products Trading Rules (Performance, Quality, Descript

Products (Boric Acid Modification) (Cereal Chemistry)(

Products (Cereal Chemistry) (1962)

Products (Cereal Chemistry) (1962)

Products (Cereal Chemistry) (1962)

Products (Cereal Chemistry) (1962

Products (Cereal Chemistry) (1962)

Products (Cereal Chemistry) (1962)

Products (Cereal Chemistry) (1962)

Products (Cereal Chemistry) (1962)

Products (Cereal Chemistry) (1962)

Products (Cereal Chemistry) (1962)

Products (Cereal Chemistry) (1962)

Products (Cereal Chemistry) (1962)

Products (Cereal Chemistry) (1962)

Products (Cereal Chemistry) (1962)

Products (Cereal Chemistry) (1962)

Products (Cereal Chemistry) (1962)

Products (Cereal Chemistry) (1962)

Products (Cereal Chemistry) (1962)

Products (Cereal Chemistry) (1962)

Products (Cereal Chemistry) (1962)

Products (Cereal Chemistry) (1962)

Products (Cereal Che mistry) (1962)

Products (Cereal Chemistry) (1962)

Products (Cereal Chemistry) (1967)

ICBO

$A P I$

$\mathrm{PC}$

ASQC

USC

APl

ASTM

ANSI

NACE

API

API

APl

ASAE

AACCH

NCCA

ANSI

AACCH

AIS

AlSI

Alsl

Als1

AlSl

AISI

A1S1

BIA

AACC

CDA

CR

*24

SQC RP5

ASTM $\quad 660$

$\mathrm{UMC} * 7$

RP5

D. 350

RP8

So

$6 \mathrm{~A}$

RP500B

D1786

Z94.10

RP.03-72

$6 \mathrm{~A}$

12J

I2L

RPT. 1

D249.2

$28.99 \mathrm{~A}$

TB. 11.4

K68.1

B216

F221

AACCH 28.33

ASTM Al43

ASTM D3136

D2896

CSMA BUL 12

G-6

E460

D3259

AMS2370A

D1101

$\begin{array}{ll}\text { ASTM } & \text { A520 } \\ \text { ASTM } & \text { D685 }\end{array}$

ASTM D1776

ASTM D2559

ASTM D3024

ASTM D2470

$$
6
$$

2

11

20
$30-10$

$30-10$

J. 4

J -48

E-32

B-18

B-18

ASTM B272

AACCH $46-12$

AACCH 08.10

AACCH 08-11

AACCH $10-20$

AACCH $14-20$

$\mathrm{AACCH} \quad 14-21$

AACCH 20.10

AACCH $30-16$

AACCH $30-40$

AACCH $42-15$

AACCH 42.20

AACCH 42.25

AACCH 42.30

AACCH $42-35$

$\mathrm{AACCH} \quad 42.50$

$\mathrm{AACCH} \quad 62.25$

$\mathrm{AACCH} \quad 62.40$

St AACCH 42-11

Meth. AACCH 08.17

Meth. O AACCH 04-22

Meth of AACCH 60-10 
1 Grains, Oilseeds, Legumes, Forages, and Animal and Dairy $f$ Hydration Capacity of Pregelatinized Starches and Cereal Meth. of Analysis of Pesticide Residues in Food of Analysis of Coagulase Positive Staphylococcus in Cereal in Massecuites, Molasses, and Other Liquid and Semiliquid Thermal Properties of Agricultural Grain and Its Std. Spec. for Wrought Alloy Tool Steel

t Labels, Detachable Tags, and Certification of Fabrics or Std for Glossary of Rec. Terms for Care Labeling Textile Std. Meth. of Test for Vapor Pressure of Petroleum Sanitary Stds. for Uninsulated Tanks for Milk and Its ealers of Single Service Containers for Milk and Its Fluid imulated Shipping Test (Packaging) for Consumer Electronic Std. Meth. of Test for $V$ apor Pressure of Petroleum Glossary of Terms Relating to Structural Clay

Std. Rec. Pract. for Eddy Current Test. of Steel Tubular ic Meth. of Analysis of Malathion Residues in Milled Wheat ontent in Corn Starch, Dextrines and Other Modified Starch drolzates Including Syrups and Sugars, and Most Wet Milled

ty Content in Feedstuffs (Corn) and Other Wet Milled Solid Std. Spec. for Pressure Treatment of Timber Std. Spec. for Tantalum Ingots and Flat Mill Spec. for Dental Gypsum

Std Definitions of Terms Relating to Glass and Glass ty Practices for Handling and Fabricating Asbestos Textile for Determining Borax Content in Dextrin and Corn Starch (Steel Products Manual) Steel Specialty Tubular

Std. Meth. for Chemical Analysis of Gypsum and Std. Meth. of Test for Flammability of Aerosol Sid. Meth. of Test for Delivery Rate of Aerosol Std. Meth. of Rapid Pressure Determination of Pressurized Std. Meth. of Test for Volatile Content of Aerosol Std. Rec. Pract. for Storage of Aerosol

Std. Meth. of Test for Seepage Rate of Aerosol Std. Meth. of Test for Liquid Densities of Pressurized id. Spec. for Adhesives Used in Nonstructural Glued Lumber Pract. for Instantizing Systems for Dry Milk and Dry Milk . Rec. Pract. for Safe Filling of Low Pressure Pressurized

Definitions of Terms and Nomenclature Relating to Aerosol

Definitions of Terms Relating to Naval Stores and Related apor Transmission of Flexible Heat Sealed Packages for Dry porosity, and Apparent Specific Gravity of Fired Whiteware

finitions of Terms Relating to Asbestos Cement and Related mpound ard Sample Preparation for Physical Test of Rubber Std. Meth. of Chemical Analysis of Rubber Steel Products Manual: Tin Mill

Std. for Dimensional Tolerances for Aluminum Mill Std. Spec, for General Requirements for Tin Mill Std. for Inspection of Preservative Treated Timber rec. Pract. for Collection and Identification of Corrosion Safety Std. for Power Operated Pumps for Petroleum reservative Treatment by Pressure Processes for All Timber lding Code Sid. for Test for Glue Joints in Laminated Wood r Scraped Surface Heat Exchanges for Use with Milk and Its of Terms Relating to Paint, Varnish, Lacquer, and Related hygroscopic Properties of Fire Retardant Wood and Its Base teel Panels for Test. Paint, Varnish, Lacquer, and Related

f Test for Distillation of Cut Back Asphaltic (Bituminous)

definitions for Mechanical Test. of Wrought and Cast Steel

Std. Spec. for Zinc Coating (Hot Dip) on Assembled Steel ension Test. Wrought and Cast Aluminum and Magnesium Alloy Std. Spec. for Sponge and Expanded Cellular Rubber of Dynamic Test for Ply Separation and Cracking of Rubber td. Classification System for Carbon Blacks Used in Rubber Std. Meth. of Test for Ash from Petroleum Sanitary Std. for Storage Tanks for Milk and Its sanitary Std for Silo Type Storage Tanks for Milk and Its for Centrifugal and Positive Rotary Pumps for Milk and Its t of Liquids (Paint, Enamel, Lacquer, Varnish, and Related

ning Acidity (Paste) Content in Corn Starch (Gelatinizable Std Moisture Measurement (Meat and Its guide Specs. for Bricy. Missonry (Tech. Note) (Part I and II $s$ of Thiamine in Bread, Wheat, Rice, and Other Whole Grain

luorometric Meth. of Analysis of Riboflavin in Whole Grain ry) (/ Meth. for Sampling of Flour, Semolina, and Similar u. S. Customary Units) (1973 Dictionary of Terms Used in the Safety Std. Dimensions tor Std. for Graphical Symbols for Use on Railroad Maps and Std. for Milling Machine-Tracer Controlled Std. for Numerically Controlled

thyl)-A, A, A-Trifluoro-2,6-Dinitro-N-Propyl-Ptoluidine Std. for Certified Ratings Std. for nts (1972)

(Petroleum Production) (1974)

Rec. Pract. Orientation

Rec. Pract. for Vendor Product Improvement
Products (Cereal Chemistry) (1968)

Products (Cereal Chemistry) (1968)

Products (Cereal Chemistry) (1969)

Products (Cereal Chemistry) (1973)

Products (Drying Upon Quartz Sand) (Cereal Chemistry)(

Products (Flour, Starch) (1972)

Products (Including Dies and Fixtures) (1973)

Products (Institutional Textile) (1973)

Products (Launder or Dryclean) (1973)

Products (Micromethod) (1971) ANSI Z11.277

Products (Mixing and Storage) (1974)

Products (Packaging Equipment) (1974)

Products (Radio, Television, Tape Recorder,

Products (Reid Meth.) (1972) ANSI Z11.44.

Products (Tech. Notes) (1961)

Products (Tubing) with Magnetic Saturation (1971) ANSI

Products (Wheat, Flour, Bran, Shorts, etc) to Determin

Products (1955)

Products (1961)

Products (1963)

Products (1969)

/Nalytical Meth. for Determining Ash C

Mining Iron Content in Corn Starch Hy

lcal Meth. for Determining Bulk Densi-C

Products (1970) ANSI Z179.14

Products (1971)

Products (1971)

Products (1971)

Products (1971)

Products (1972)

Products (1972)

Products (1972)

Products (1972)

Products (1972)

Products (1972)

Products (1972)

Products (1972)

Products (1972)

Products (1972)

Products (1972)

Products (1972)

Products (1972)

Products (1972)

Products (1972)

Products (1972)

Products (1972) ANSI Al30.

Products (1972) ANSI Jl.1

Products (1972A) ANSI J10.1

Products (1973)

Products (1973)

Products (1973)

Products (1973)

Products (1973)

Products (1973)

Products (1973)

Products (1973)

Products (1973)

Products (1973)

Products (1973)

Products (1973)

Products (1973) AASHO T78, ANSI A37.45

Products (1973) ANSI G60.1

Products (1973) ANSI G8.18

Products (1973) ANSI H46.1

Products (1973) ANSI J2.4

Products (1973) ANSI J2.6

Products (1973) ANSI J8.6

Products (1973) ANSI Z11.54

Rec. Health Safe Std. Analytical Meth

AT

CR

ASTM

ASTM

ASTM

ASTM

ASTM

ASTM

ASTM

ASTM

ASTM

DFISA

Std ASTM

Std. ASTM

Std. ASTM

Std. Meth. of Test for Water V ASTM R Absorption, Bulk Density, Apparent ASTM

A STM

Std. Meth. of Co ASTM

ASTM

AISI

ANSI

ASTM

AWPA

NACE

UL

Std. P AWPA

Uniform Bui ICBO

Sanitary Std. Fo DFISA

Std Definitions ASTM

Tent. Meth. of Test for ASTM

Std. Meth. for Preparation of S ASTM

Products (1974)

Products (1974)

Products (1974)

Products) by Se

Products) (1955)

Products) (1972)

Products) (1972)

Products, Corn Grits, Corn Meal, Puffed Cereals, Farina

Products, Grits, Meal, Flaked and Puffed Cereals, Farin

Products: Feeds and Feedstuffs in Sacks (Cereal Chemist

Profession (1973)

Professional Photographic Sheet and Roll Films (SI and

Protessionals (1972)

Profiles (1972)

Profiling and Contouring-Vertical (1973)

Profiling and Contouring Milling Machine (1974)

td. Meth. O ASTM

ASTM

ASTM

Std. Meth. of T ASTM

ASTM

Std. Meth. ASTM

ASTM

ASTM

DFISA

DFISA

Sanitary Stds. DFISA

Lash Poin ASTM

CR

ASAE

CSI

$86-80$

AACCH 86.70

AACCH $64-60$

ASSFE *1

ANSI $\mathrm{PH} 1.18$

AIOA C801

ANSI $\quad$ Y 32.7

NSA 914

NSA 913

Profluralin (1973) _ IT Control Chemical N(Cyclopropylme

Program Air Moving Devices (Performance) (1970)

Program for Biological Shielding in Nuclear Reactor Pla

Program for Personnel Going Offshore for the First Time

Program for Quality Control (1969)
NSI

AMCA

ANSI

API

ASQC
K62.152

211

N18.9

RPT. 
Rec. Pract. for A Std. for Quality Assurance Spec. for General Requirements for a Quality Rec. Pract. for Evaluating the Quality Contro steady State Performance Presentation for Digital Computer

e Film (2.35:1) Aspect Ratio (1971)

Std. Dimensions of Std. for lllumination Tests for Photographic Still nd Super 8 Reversal Color Camera Films Intended for Direct ium Prefocus Base Down Type, Single Contact Motion Picture Uniform Building Code: Motion Picture Std. Pract. for Slide and Slidefilm Uniform Fire Code: Motion Picture m Building Code: Regulations for Use of Public Streets and Mounts (1972) Std. Spec, for td. Basic Construction Requirements for Photographic Slide is of Payment on Portland Cement Concrete Pavement Overlay hemical 2,4-Bis (Isopropylamino).6-Methoxy-S-Triazine al 2,4-Bis (Isopropylamino)-6-(Methylthio)-S-Triazine

ng) (1973)

Std. Gravure

materials (1971)

Std. Meth. of Test for Flame yl Sulfite (Common Name for the Pest Control Ch/ Std. for 11.1 Chemical 2-Chloro.4.6bis (Isopropylamino).S-Triazine. Std. Meth. of Test for Yield Stress of Heterogeneous design, Construction, Materials, Size, and Installation of Delamination, Strength of Bond, Appearance, and Shrinkage Test Meth. for Electrostatic

e Book: Lumber Std., Grading Rules, Design Values, Section and Use of Acetic Acid (1973)

and Use of Paraformaldehyde (1974)

and Use of Chloroform (1974)

and Use of Caustic Soda (1974)

ing and Use of Vinyl Chloride (1972)

rbon Steel Tooth Lock Wash.

Std. for Dimensions, Physical Std. Meth. for Evaluating Allowable td. Spec. for Carbon Steel Plates with Improved Transition ading (1949) ANSI Z197.1/

Shear by Tension Loadin/ (Metal to Metal) (1969)

elevated Temperatures ( $M$ / the Temperature Range $\mathrm{Fr}$ / laminated Assemblies (19) tal to Metal) (1972) Ans ?

tal to Metal) (1969)

tal / Metal) (1973)

Shear by Tension Loading (/

Std. Meth. of Test for Strength Std. Test Meth. for Tensile

Std. Meth. of Test for Strength Std. Test Meth. for Creep

Std. Meth. of Test for Strength Std. Meth. of Test for Strength Std. Meth. of Test for Strength

Std. Meth. of Test for Strength Std. Test Meth. for Creep Std. Meth. of Test for Fatigue Std. Test Meth. for Strength Std Test Meth for Flow Std. Meth. of Testing Cross Lap Specimens for Tensile r, Starch) (1972) Thermal

e or Shear Strains by / sion Machines) (1972) Ans/

Std. Meth. of Test for Change in Std. Meth of Test for Mechanical Std. Meth. of Test for Elastic Meth. of Test for Stretch Std. Meth. of Test for Elastic (1973) lids (1973)

Tent. Meth. of Test for Hygroscopic Std. Meth. for Measurement of Extreme Pressure Dielectric

nsi Z14.286

sitional /

1) ANSI Z/

ansi Fll./

(1973)

Std. Meth. of Test for Stretch Std. Meth. for Calculation of Certain Physical Std. Meth. for Measurement of Extreme Pressure Std. Meth. for Measurement of Extreme Pressure Std. Meth. for Measurement of Flow Std. Meth. of Test for Rust Preventive

oading (Disk Shear) (197) Std. Meth. of Test for Strength ct. for Effects of High Energy Radiation on the Mechanical Soil Block Test: Std. Meths. for Test, the Preservative properties of Oil Soluble Wood Preservatives by Using Wood

mhl2.15 Meth. of Test for Tensile Std. Test Meth. for Creep Std. Meth. of Test for Physical and Chemical means of a Torsion Test/ Std. Meth. of Test for Stiffness Std. Meth. of Test for Tensile 2 nsi J/ Std. Meth. of Test for Evaluating Pressure Sealing Yarns and Tows (1972) An/ Std. Meth. of Test for Tensile Std Meth of Test for Tensile Strength Std. Meth of Test for Tensile Test Meth. for Resin and Volatile Content, and Flow
Program for School Crossing Protection (1973)

Program Requirements for Nuclear Power Plants (1971)

Program (1968) ANSI Z1.8

Program (1969)

Programs (1970)

Project Record Documents (1967)

Gas Turbine Engine

Projectable Anamorphic lmage Area on $5 \mathrm{~mm}$ Motion Pictur

Projection Equipment (1971)

Projection in Motion Picture Photography (1973)

Projection Lamps (1972)

Projection Rooms (1973)

Projection (1972)

Projection (1973)

Projections Over Public Property (Pedestrian Protection

Projector Usage of $16 \mathrm{~mm}$ Motion Picture Film (1973)

Projectors for $35 \mathrm{~mm}$ Filmstrips and Slides in $2 \times 2 \mathrm{ln}$.

Projectors (1973)

Projectors (1973)

Projects (1973)

Prometon (1973)

Prometryn (1973)

Proofing lnks (Printing) (1973)

Proofing Supplement Stock for Gravure Magazines (Printi

Propagation Classification Flooring and Floor Covering

Propargite 2-(P-Tert-Butylphenoxy) Cyclohexyl 2Propyn

Propazine (1973) /Td. Common Name for the Pest

Propellants by the Cone Penetration Meth. (1972) ANSI Z

Propelled Mobile Ladder Stands for Scaffolds (Towers, S

Propeller Shafting Systems for Boats (1973)

Propensity of Bonded and Laminated Apparel Fabrics (Tex

Propensity of Carpets (1969)

Properties and Design Loads, Timber, Plank and Laminate

Properties and Essential Information for Safe Handling

Properties and Essential Information for Safe Handling

Properties and Essential Information for Safe Handling

Properties and Essential Information for Safe Handling

Properties and Essential Information for the Safe Handl

Properties and Meth. of Test. for Helical Spring and Ca

Properties for Grades of Structural Lumber (1974)

Properties for Pressure Vessels (1972) ANSI G35.7

Properties of Adhesive Bonds in Shear by Compression Lo

Properties of Adhesive Bonds (1972) ANSl Z197.8

Properties of Adhesives in Plywood Type Construction in

Properties of Adhesives in Shear by Compression Loading

Properties of Adhesives in Shear by Tension Loading at

Properties of Adhesives in Shear by Tension Loading in

Properties of Adhesives in Shear by Tension Loading of

Properties of Adhesives in Shear by Tension Loading (Me

Properties of Adhesives in Shear by Tension Loading (Me

Properties of Adhesives in Shear by Tension Loading (Me

Properties of Adhesives in Two Ply Wood Construction in

Properties of Adhesives (1969)

Properties of Adhesives (1972) ANS1 Z197.21

Properties of Agricultural Grain and lis Products (Flou

Properties of Elastomeric ANSI J2.7

Properties of Elastomeric Vulcanizates Under Compressiv

Properties of Elastomeric Yarns (Constant Rate of Exten

Properties of Fabrics Woven from Stretch Yarns (1972T)

Properties of Fibers (1972) ANS] L14.250

Properties of Fire Retardant Wood and lts Base Products

Properties of Flammable Liquids, Gases, and Volatile So

Properties of Fluid Lubricants (Falex Meth.) (1973)

Properties of Grain and Seeds (1972)

Properties of Knitted Fabrics Having Low Power (1972) a

Properties of Liquefied Petroleum (LP) Gases from Compo

Properties of Lubricating Fluids (Four Ball Meth.) (197

Properties of Lubricating Fluids (Timken Meth.) (1972)

Properties of Lubricating Greases at High Temperatures

Properties of Lubricating Greases (1973) ANSI Z11.152

Properties of Metal to Metal Adhesives by Compression L

Properties of Metallic Materials (1962) ANSI N145

Properties of Oil Soluble Wood Preservatives by Using W

Properties of Oil Soluble Wood Preservatives by Using W

Properties of Oriented Fiber Composites (1972T)

Properties of Package Cushioning Materials (1968) ANSl

Properties of Particulate lon Exchange Resins (1972)

Properties of Plastics as a Function of Temperature by

Properties of Plastics (1972) ANSI K65.60

Properties of Rigid Cellular Plastics (1972) ANSI K65.3

Properties of Rigid Cellular Plastics (1973) ANSI K65.3

Properties of Rubber and Rubber Like Materials (1960) a

Properties of Single Man Made Textile Fibers Taken from

Properties of Steel Truss Plates (1973)

Properties of Thin Plastic Sheeting (1973) ANSI K65.76

Properties of Treated Paper (1973)

Properties of Unpunched Oiled Paper Perforated Tape (19
ITE

ANS1

ASQC

$A S Q C$

CSI

ANSl

ANSl

/ $\mathrm{mm}$ a ANSI

ANS!

ICBO

ANSI

ICBO

ANS1

ANSI

ANSI

ANSI

ACPA

ANSl

ANSI

GT

GT

UL

ANSI

ANSI

ASTM

ANSI

ABYC

ASTM

AATCC

WW PA

MCA

MCA

MCA

$\mathrm{MCA}$

MCA

ANSI

ASTM

ASTM

ASTM

ASTM

ASTM

ASTM

ASTM

ASTM

ASTM

ASTM

ASTM

ASTM

ASTM

ASTM

ASTM

ASAE

ASTM

ASTM

ASTM

ASTM

ASTM

ASTM

FMS

ASTM

ASAE

ASTM

A STM

ASTM

ASTM

ASTM

ASTM

ASTM

Pra ASTM

NWMA

NWMA

ASTM

ASTM

ASTM

ASTM

ASTM

ASTM

ASTM

ASTM

ASTM

ASTM

ASTM

TAPPI

ANSI

RP3

N45. 2

ARP68

01730

PH22.106 
als (1972) ANSI 08.1 Std. Meth. of Test for Measuring Surface Frictiona g/ Uniform Building Code Std. for Self and Flash Ignition nt Viscosity of Adhesives Having Shear Rate Dependent Flow rison with a Secondary Standard of Similar EMF Temperature ent Cladding Including the Determination of the Mechanical al Used in Optical Emission Spectroscopy Based on Physica

ride (1972)

: Grade Selection, Std. Size, Design Values, Framing Rec. $\mathrm{n}$, and Operation of So/ ht Aluminum A/ Std. for Chemical Compositions, Mechanical erms Precision and Accuracy as Applied to Measurement of A quality Hot Rolled Carbon Steel Bars Subject to Mechanica

tic Drain, Waste and Vent Pipe and Fitting/ ions for Use of Public Streets and Projections Over Public

(Cereal) Van Dame Meth. of Analysis for Formic, Acetic

.1 ansi Al64.1 on and Safety) (1973)

Fire Alar/ ct. and Stds. for Safe Installation of Exhaust Systems for Std. for Service Time Coding of ide for Centralized Control and Automation of Ship's Steam chemical $\mathrm{N}$-(2Chloroethyl)-A, A, A-Trifluoro-2,6-Dinitro-N ical N(Cyclopropylmethyl)-A, A, A-Trifluoro-2,6-Dinitro-N Std. for Mine Power Cables with Ethylene multiple Conductor Electrical Power Cables with Ethylene ed Single Conductor Electrical Power Cable with Ethylene 2000 Volts and Less and Having Ozone Resistant Ethylene Std. Spec, for s Rated 0-35,000 Volts and Having Ozone Resistant Ethylene Std. Spec. for General Purpose Ethylene Spec. for Synthetic Rubber. Ethylene Safe Handling and Use of

no/

Std. for Common Name for the Pest Control Chemical 2 Uniform Building Code Std. for Meth. of Assay for Alkalin Std. for Safety for Implosion Rec. for elevision Receiving Applia/ o 100 Million Electron Volts (I954)

on, and Maintenance of Vehicle Alarm Systems and Units for stations (Flood, Surface Water, Rigging Collaps/ Rec. for 973)

(1972)

Rec. for Rec. for

d. for Safety for Bullet Resisting Equipment (Barrier) for to Rain During Construction (1974/ Rec. for Local Protective Signalling Systems for Fire Building Construction and / Western Woods Use Book: Fire perature Molten Materials (Metals, Slag, Glass, etc.) Fir Rec. for Proprietary Protective Signaling Systems (Fire Rec. for Parking Structures (Garage-Fire Rec. for Liquefied Hydrogen Systems (Fire rec. for Flammable Liquid Pumping and Piping Systems (Fire Rec. for Hazardous Chemicals Data (F ire rec. Construction and $\mathbb{W}$ ater Sprinkler System Design-Fir ge Up to $30 \mathrm{Ft}$. and Shelf Storage Up to $15 \mathrm{Ft}$. High) (Fire rm Fire Code: Recommended Safeguards and Practices for the res, and Equipment for Water Cooled and Mo/ Std. for Fire

c Streets and Projections Over Public Property (Pedestrian

Rec. for Fire

Drive Turbines (1974)

Fire Hazards and Rec. Fire Std. for Practice for Respiratory sae $\mathbf{J} 333$ s / Uniform Fire Code: Recommended Guide to Safe Practice lated Faults) (1973) quipment (1972) Std. for Radiation Rec. for Fire Std. for the Rec. Pract. for Metallizing with Aluminum and Zine Rec. for ainers of Flammable Liquids (1973)

Rec. for Fire Rec. for Fire Rec. for Fire Safety and Fire Hazards Ant

c Feedwater Regulators for Boilers (197/
Std. Meth. of Evaluating the Std. Meth. of Test for Shock Absorbing Contemporary Bearing $\mathbb{W}$ all Types an Requirements for Safety to Life Rec. for Prevention of Damage to

Materials an Std. Meth. of Test. Cellulose Acetate Std. Spec. for Normal

Properties of Wood Base Fiber and Particle Panel Materi Properties of (Sports) Playing Surface Systems (I972)

Properties Using the British Portable Tester (1974)

Properties (Tech. Notes) (1969)

Properties (Temperatures) of Plastics Using a Hot Air I

Properties (1969)

Properties (1972)

Properties (1972) lement Materials by Compa

Examination of Fuel Elem 1. for Selection of Electrode Materi

Properties, etc of Isopropyl Alcohol (1972)

Properties, Safe Handling and Use of Phosphorus Trichlo

Properties, Weight, Stress of Board, Timber, Lumber, SI

Property from Explosion and Fire in Design, Constructio

Property from Surface Water (1973)

Property Limits and Dimensional Tolerances of SAE Wroug

Std. Spec. for Merchant

Property Requirements (1972)

Property (Pedestrian Protection During Construction or

Propionate and Cellulose Acetate Butyrate (1972)

Propionic, Butyric, Valeric, Succinic, and Lactic Acids

Proportions for No Slump Concrete (1965)

Proportions for Normal Weight Concrete (1970) ANS1 A 167

Proportions for Structural Lightweight Concrete (1969)

Proprietary Burglar Alarm System Units (1974) ANSI Se2.

Proprietary Protective Signaling Systems (Fire Protecti

Proprietary Protective Signalling Systems for Watchman,

Propulsion and Auxiliary Machinery (Boats) (1973)

Propulsion Engine Items (1971) ANSI B141.8

Propulsion Plant (1970)

Propyl Acetate (90 to 92\% Grade) (1972)

Propyl-Ptoluidine-Fluchloralin (1973)

Propyl-Ptoluidine-Profluralin (1973)

Propylene-Rubber Insulation (1973) IPCEA S68.516

Propylene-Rubber Insulation (1973) iPCEA S68-516

Propylene-Rubber Insulation, 2001-5000 Volts (1973)

Propylene-Rubber Integral Insulation and Jacket (1973

Propylene Glycol (1972)

Propylene Rubber Insulation (1973) IPCEA S68-516

Propylene Rubber Jacket for Wire and Cable (1972)

Propylene Terpolymer, General Purpose (45-55) (I973)

Propylene (1974)

Propynyl (E,E)-3,7,11-Trimethyl-2,4-Dodecadienoate KI

Proscenium Curtains (1973)

Protease (1972T)

Protected Cathode Ray Tubes (Picture and Display) for T

Protection Against Betatron Synchrotron Radiations Up T

Protection Against Coal Mine Dust (1972)

Protection Against Holdup and Theft (1973) ANS1 Se2.8

Protection Against Misc. Perils at Electric Generating

Protection Against Molten Metal Fires and Explosions (1

Protection Against Radiation from Brachytherapy Sources

Protection Against Robbery or Holdup (Criminology) (197

Protection and Acceptance of Concrete Pavements Exposed

Protection and Safety (Alarm) (1973)

Protection and Safety (Exit, Stairway, Aisle, Corridor

Protection and Safety (1973)

Protection and Safety) (1973)

Protection and Safety) (1973)

Protection and Safety) (1973)

Protection and Safety) (1973)

Protection and Safety) (1973)

Protection and Safety) (1973)

Protection and Safety) (I973)

Protection 7 Tized and Bin Box

Protection Criteria for Safety Related Systems, Structu

Protection During Construction or Demolition) (1973)

Protection for Belt Conveyors (1972)

Protection for Maintenance and Inspection of Mechanical

Protection for Saw mills and Lumber Yards (1973)

Protection for the Fire Service (1973)

Protection for Wheel Type Agricultural Tractors (I972)

Protection from Corrosion for Underground Pipe. Fitting

Protection in Uranium Mines (Safety) (1973)

Protection of Electrical Equipment (Single Phase and Re

Protection of Electronic Computer and Data Processing E

Protection of Electronic Computer Systems (1972)

Protection of lron and Steel (1967)

Protection of Plywood Plants (1972)

Protection of Small Nonpressurized and Pressurized Cont

Protection of Synchronous Motors (1974)

Protection of Yankee Driers (Paper Machine) (1973)

Protection Rec. for Indoor Storage of Roll Paper (1974)

Protection Rec. for Low Water Fuel Cutoffs and Automati

Fire Hazards and

Cold Weather Masonry Construction
Protection Rec. for Textile Mills (1974)

(Protection Rec.) (1968)

ASTM

ASTM

ICBO

ASTM

ASTM

CA

MCA

$\mathbb{W} W \mathrm{PA}$

FMS

ASTM

ASTM

IAPMO

ICBO UBC*3-44

CCH $\quad 04.23$

$\mathrm{ACl} 211$

ACl 211.1

ACI 211.2

UL 1076

FMS LPD 5-2N

NFPA 72D

/Pra ABYC Pl SAE AS1105

Gu SNAME 3.23

ASTM D3130

Pest Control ANSI K62.140

K62.140

NEMA WC $8 / 5$

ID NEMA WC $8 / 6$

NEMA WC8/7

NEMA WC8/1

ASTM D 2695

/Able NEMA WC8

ASTM D2768

SAE AMS3260

MCA SD59

ANSI K62.137

ICBO UBCS6-1

ASTM D3048

UL 492.8

NCRPM R14

ANSI Z88.4

UL 904

FMS $\quad 9.10$

FMS $\quad 10-8$

NCRPM R40

$\begin{array}{ll}\text { UL } & 752\end{array}$

ACPA TB17

FMS 5.5N

WWPA *31.4

FMS

FMS

FMS

FMS

FMS

FMS

FMS

FMS

ICBO

ANS

ICBO

FMS

FMS

FMS

ANSI

ASAE

ICBO

ANS

FMS

NFPA

FMS

AWS

FMS

FMS

FMS

FMS

FMS

FMS

FMS

BIA

LPD 7.33

LPD 5-2N

LPD $7-15 \mathrm{~N}$

LPD 7-19N

LPD 7-32

$7-23 \mathrm{~N}$

LPD 1.2

$8-25 \mathrm{~N}$

UFC * $2 A$

N 18.10

UBC *3-44

7.1

13.9

7.25

Z88.5 
Std. for Dry Pipe, Deluge, and Pre Action Valves for Firc Safety Std, for Hydrants for Fire Safety Std. for Gate Valves for Fire

Safety Std. for Play Pipes for Water Supply Test. in Fire larm Valves for Use in Wet Pipe Sprinkler Systems for Fire Safety Std. for lndicating Pressure Gauges for Fire Std. for Safety for Butterfly Valves for Fire Std. for Safety for Automatic Water Sprinklers for Fire Std. for Safety for Water Pumps for Fire

film Carrier (Ape/ Std. Meth. for Determining Adhesion of stible Materials (Fire Safety) (1974) Fire Hazards and Rec. for Pipe Friction Loss Tables for Solving Fire Rec. Pract. and Std. for Cathodic Safety Std. for Intrusion Detection Units for Burglary for the Periodic Test. of Nuclear Power Generating Station ngle Failure Criterion to Nuclear Power Generating Station $r$ Reliability Analysis of Nuclear Power Generating Station

flammable/ Rec. for Sprinkler, Water Spray, and Explosion ec. for Water Demand for Sprinkler System for Private Fire $r$ Protective Frame (For Agricultural Tractors for Overhead Guide for Synchronous Motor Minimum Requirements for Motorcyclists Eye Rec for Lined Earth Reservoirs for Fire Rec. for Water Spray Fixed Systems for Fire Rec. Pract. for a Program for School Crossing Under the Uniform Building Code: Wall Framing and Weather for the Installation of Water Sprinkler Systems for Fire ology for Pressure Relief Devices (Container and Equipment Rec. for Installation of Sprinkler Systems (Fire ansportation, Storage and Use of Explosive Materials (Fire Reactions (lncandescence, Fire, Explosion, and Detonation he Reliable Model B Accelerator for Dry Pipe Systems (Fire y Powder Form by Means of Spray Gun or Fluidized Bed (Fire ulverized Coal Fired Multiple Burner Boiler Furnaces (Fire for Tests for Combustibility of Sweeping Compounds (Fire std. for Safety for Antitheft Alarms and Devices (Burglary trols, Barrier, Road, Hydrant, Drivew/ Uniform Fire Code: ete, Stone, Tile, Metal. Plastic, etc.) for Ornamentation, Systems) (1972) Standard for Quality Assurance for Tent. Rec. Spec. for Asphalt Type actor Containment Facilities (1972)

Castor Oil for Use in Resins and Std. Preparation of Centrifugal Fans and Parts for Std. Meth. of Test for Steam Distillation of Bituminous Std Meth of Testing Fatty Acids Used in Std. Meth. of Test. Bituminous Base Emulsions for Use as Structural Clay

spec. for Vinyl Coated Fabric Coverings for Decorative and

edium Speed Stationary Diesel and Gas Engine Gauge Borads, rocedures and Performance Requirements) (1972)/ ures and Performance Requirements) (1972) SAE J/ $\mathrm{d}$ Protection (Test Procedures and Performance R/ nt (1974) n. and Larger, Shop Applied) (197/ Std. for Std. for for Preparation of Design Bases for Systems That Perform Rec. for
Spec. for ons (1973) Std. Spec. for Std for Cement Mortar Std. for Guide for Std. for Installation, Maintenance and Use of Auxiliary for Installation. Maintenance and Use of Central Station

and / y) (1973) Rec. for Proprietary Std. for Safety for Control Units for Indoor Fire d. for lnstallation, Maintenance and Use of Remote Station ty for Manually Actuated Signaling Boxes for Use with Fire afety (Alarm) (1973) Rec. for Local

Std. for Installation, Maintenance and Use of Proprietary for Interconnection Circuitry of Noncoded Remote Station

Uniform Building Code Std. for Smoke Detectors for Fire rec. Pract. for Minimum Performance Criteria for Roll Over rec. Pract, for Minimum Performance Criteria for Roll Over pract. for Minimum Performance Criteria for Falling Object boratory Evaluation of Roll Over (ROPS) and Falling Object rec. Pract. for Minimum Pefformance Criteria for Roll Over or Low Voltage Power Circuit Breakers and AC Power Circuit $s$ for Use Under Interior (Dry I'se) Exposu/ Std. Spec. for corn Sugar (Crude and Refined), Syrups, Starches and Other $t$ in Feedstuffs (Corn), Syrups, Sugars, Starches and Other ter, (Corn) Feedstuffs, Syrups, Sugars, Starches and Other nitrogen Content of Corn Syrup, Sugars, Starches and Other

Nitrogen Content in Corn Starch, Sugars, Syrups and Other tion of Dispersible Protein in Ground Soybeans, Whole Or/

ldahl Meth for Nitrate Free Samples for Analysis of Crude nd Animal and Dairy P/ LDy Dye Meth. of Analysis of Crude
Protection Service (1970)

Protection Service (1973)

Protection Service (1973)

Protection Service (1973) ANSI B111.1

Protection Service (1973) ANSI B 129.2

Protection Service (1973) ANSI B156.1

Protection Service (1974)

Protection Service (1974)

Protection Service (1974)

Protection Sheet to Aperture Adhesive of Unitized Micro

Protection Standards for Home Television Receivers (196

Protection Std. for Rack Storage of Flammable and Combu

Protection System Hydraulic Problems (1973)

Protection Systems on Boats (1972)

Protection Systems (1969) ANSI C33.70

Protection Systems (1971) ANSI N41.3

Protection Systems (1972) ANSI N41.2

Protection Systems (1972) ANSl N4l.4

Protection with Clay Masonry (Tech. Notes) (1961)

Protection (Safety) for Flammable Liquid and Liquefied

Protection (Safety) (1973)

Protection (Test Procedures and Performance Requirement

Protection (1971)

Protection (1971)

Protection (1972)

Protection (1973)

Protection (1973)

Protection (1973) ANS1 A54.3

Protection) (1972)

Protection) (1973)

Protection) (1973)

Protection) (1973)

Protection) (1973)

Protection) (1973)

Protection) (1973)

Protection) (1973) ANSI Z240.1

Protection) (1974)

Protection, Authority, Departmental Regulations and Con

Protection, or Insulation (1973)

Protective Coatings Applied to Nuclear Facilities (I972

Protective Coatings for Underground Pipe Lines (Wrapped

Protective Coatings Including Lacquers (1973)

Protective Coatings (Paints) for Light Water Nuclear Re

Protective Coatings (1966)

Protective Coatirigs (1970) ANSI AI09.11

Protective Coatings (1972)

Prolective Coatings (1973)

Protective Construction (Tech. Notes) (1962)

Protective Covering for Permanent Wall and Ceilings (19

Protective Devices, and Instrumentation (1972)

Protective Enclosures for Agricultural Tractors (Test P

Protective Frame for Agricultural Tractors (Test Proced

Protective Frame (For Agricultural Tractors for Overhea

Protective Functions in Nuclear Power Generating Statio

Protective Grounding of Electrical Circuits and Equipme

Protective Headgear for Vehicular Users (1971)

Protective Lining and Coating for Steel Water Pipe (4 I

Protective Pipe Coatings (1971)

Protective Relaying of Utility-Consumer lnterconnecti

Protective Signaling Systems for Fire Alarm Service (19

Protective Signaling Systems for Guard, Fire Alarm and

Protective Signaling Systems for Watchman, Fire Alarm.

Protective Signaling Systems (Fire Protection and Safet

Protective Signaling Systems (1972) ANSI C33.90

Protective Signaling Systems (1972) ANSI SE3.5

Protective Signaling Systems (1974)

Protective Signalling Systems for Fire Protection and S

Protective Signalling Systems for Watchman. Fire Alarm

Protective Signalling Systems (1969) ANSI SE3.7

Protective Signalling Systems (1973)

Protective Structure for Track Type Tractors and Front

Protective Structure for Weeled Front End Loaders and W

Protective Structure (FOPS) for Prime Movers, Wheeled a

Protective Structures (FOPS) of Construction and Indust

Protective Structures (ROPS) for Prime Movers (Heavy Eq

Protectors (1973) /Dations, and Related Requir

Protein Bearing Materials When Suitable Amounts of Samp

Protein Bearing Materials When Suitable Amounts of Samp

Protein Bearing Materials When Suitable Amounts of Samp

Protein Bearing Materials (1952)

Protein Bearing Materials (1956)

Protein Dispersibility lndex (PD ):

/Determining Protein / Determining Protein

Protein in Bread Wheat and Other Grains, and Yeast Foo

Protein in Cereal Grains, Oilseeds, Legumes. Forages, a
Safety

UL

UL

UL

UL

UL

UL

NMA

NCRPM

FMS

FMS

ABYC

UL

se Criteria IEEE

IEEE

BIA

FMS

FMS

ASAE

IEEE

VESC

FMS

FMS

ITE

ICBO

(di

ANSI

FMS

FMS

FMS

FMS

FMS

FMS

fety Std UL

UL

ICBO

ICBO

ANSI

NACE

ASTM

ANSI

AMCA

ASTM

ASTM

ASTM

BIA

CSI

1 and $M$ DEMA

ASAE

ASAE

ASAE

ANS

FMS

ANSI

AWWA

IAPMO

IEEE

NFPA

NFPA

NFPA

FMS

UL

St NFPA

UL

FMS

NFPA

Std NEMA

ICBO

SAE

$\mathrm{SAE}$

SAE

$\mathrm{SAE}$

SAE

ANSI

ASTM

CR

CR

CR

CR

CR

AACCH

AACCH

246
262

385

393

1091

199

MS 10

$* 1$

8-33N

2.89

639
338

379

352

3

7-14

S 310.2

329

V-8

LPD3-6

4-IN

RP3

13

B95. 1

$2-8$ 
tage of Total Nitrogen) (C/ Meth. of Calculation of Crude 2) Improved Kjeldahl Meth of Analysis of Crude bility Index (PDI): Meth. for Determination of Dispersible Harland and Ashworth Meth. of Analysis of Serum Rowland Meth. of Analysis of Serum Kjeldahl Meth. of Analysis of Crude Meth. of Calculation of Crude dification) (Cereal / of Total Nitrogen) (Cereal/ and Other Protein/ t Materials of $\mathrm{Com} /$ sugars, Starches A/ fs, Syrups, Sugars/ ed), Syrups, Starc/ $\mathbf{s}$ and Oiher Protei/ (1962)

Std. Analytical Meth. for Determining Std. Analytical Meth. for Determining Std. Analytical Meth. for Determining Std. Analytical Meth. for Determining Std. Analytical Meth. for Determining

Std. Analytical Meth. for Determining Meth of Analysis of Undenatured Whey

Micro Kjeldahl Meth. of Analysis of Crude h. of Analysis of Proteolytic Activity of Flour and Active metric Meth. of Analysis of Proteolytic Activity in Active metric Meth. of AnaIysis of Proteolytic Activity in Active (Cereal Chemistr/ Spectrophotometric Meth. of Analysis of Colorimetric Meth. of Analysis of Meth. of Analysis of

Rec. Std. Practices for Clinical Research Fire Detection System (Grinnell

Std, for Bolt Taper Shank, Shear Type, Std. for Bulbed Mechanically Locked Spindle Std. for Bulbed Spindle Mechanically Locked Std. for Hi Shea

Std. for Titanium Alloy Stump Type Std. for Bolt, Taper Shank, Shear Type. Std. for

blind. Internally Threaded, External Sleeve, Light Weight, nd, Internally Threaded, External Sleeve, GeneraI Purpose, Std. for Pin, Swage Locking, Steel Std. for Pin, Swage Locking, A-286 Cres, Std. for Pin, Swage Locking, 108 KSI Steel,

Std. for Pin, Swage Locking, Steel, Std. and Oversize, Std. for Pin, Swage Locking, A-286 Cres, Std. for Bolt Lock, Tension. Std. for Bolt Lock, Shear, Std. for Bolt Lock, Tension, Alloy (1973 Alloy (1973)

Std. for Pin, Swage Locking. Std. and Oversize Std. for Pin, Swage Locking, Aluminum Alloy, Std, for Pin, Swage Locking, A-286 Cres, Std. for Pin, Swage Locking, Aluminum Alloy, Std. for Pin, Swage Locking, Steel

d. Internally Threaded, External Sleeve, High Temperature, Std. Meth. of Test for

Std. for Safety Requirements for Tree Std. for

(1972) mum Allowable Operating Pressure Does Not Exceed 60 or 125
Std. Spec. for Type $(1973$ (1973) 973) Std. Spec. for Type Std. for

ry) (1962)

Std. for Industrial Engineering Terminology: Applied Meth. of Analysis of Thermophilic and Std. for Multiport Valves for for Motor Vehicles, Trailers, and Semitrailers Operated on ities Required for School, Theater, Auditorium, Dormatory, regulations for Use of Public Streets and Projections Over destrian P/

Uniform Building Code: Regulations for Use of Suggested Minimum Stds, for

spec. for Supports for Off the Floor Plumbing Fixtures for finishing. Installation, and Definitions) (1961)

for Units (Measurement-Metric and British-American) in

ce, and Other Whole Grain Products, Corn Grits, Corn MeaI, iboflavin in Whole Grain Products, Grits, Meal, Flaked and Std. for Pin

Agricultural Tractor Belt Speed and

of Doors; Hinges: Locks; Knobs and Handles; Exit Devices, Std. Meth. for Analysis of Sulfuric Acid as Used in Test Meth. for Rejects from Knotter Screens
Test Meth. for Dirt Count of Wood Chips

Test Meth. for Specific Gravity of Wood Disks

Test Meth. for Chip Classification (Hand Screen)

Test Meth. for Fiber Identification (Wilson Stain)

Test Meth. for Fiber Identification (Various Stains)

Test Meth. for Beating Control (Simons Stain)

Test Meth. for Sieve Analysis of Pulpwood Chips

Test Meth. for Determination of Useful Fiber in Bagasse
Test Meth. for Sampling of Wood Chips from Conveyor

Test Meth. for Chip Length Analysis (Measurement)

Test Meth. for Chip Length Analysis (Two Screen)

Test Meth. for Moisture in Wood Chips (Steam Oven)

Test Meth. for Moisture in Chips (Moister TeIler)

test Meth. for Density of Wood Chips (Sheet Machine Mold) (Pulp and Paper) (1973)

Pulp and Paper) (1973
Protein in Feeds and Feedstuffs (Calculated from Percen Protein in Feeds and Feedstuffs (Cereal Chemistry) (196 Protein in Ground Soybeans, Whole or Ground Full Fat or Protein in Milk (Cereal Chemistry) (1962)

Protein in Milk (Cereal Chemistry) (1962)

Protein in Wheat and Flour Mill Products (Boric Acid Mo

Protein in Wheat and Flour (Calculated from Percentage

Protein Nitrogen Content in Corn Starch, Sugars, Syrups

Protein Nitrogen Content in Corn, Other Grains and Plan

Protein Nitrogen Content in Feedstuffs (Corn), Syrups,

Protein Nitrogen Content in Steepwater, (Corn) Feedstuf

Protein Nitrogen Content of Corn Sugar (Crude and Refin

Protein Nitrogen Content of Corn Syrup, Sugars, Starche

Protein Nitrogen in Nonfat Dry Milk (Cereal Chemistry)

Protein (Cereal Chemistry) (1962)

Proteinase Preparations (Cereal Chemistry) (1962)

Proteinase Preparations (Cereal Chemistry) (1962)

Proteinase Preparations (Cereal Chemistry) (1968)

Proteolytic Activity in Active Proteinase Preparations

Proteolytic Activity in Active Proteinase Preparations

Proteolytic Activity of Flour and Active Proteinase Pre

Proteolytic Activity of Malt (Cereal Chemistry) (1962)

Protocol for Dental Materials and Devices (1971)

Protomatic Release F100) (1973)

Protruding Head-Oversize (1973)

Protruding Head Blind Rivet (1974)

Protruding Head Blind Rivet (1974)

Protruding Head Rivet (1972)

Protruding Head Shear BoIt Lock (1974)

Protruding Head (1973)

Protruding Head, Locked Spindle Blind Rivet (1972)

Protruding Head, Self Locking) (1972)

Protruding Head, Self Locking) (1972)

Protruding Head, Shear Stump Type (1973)

Protruding Head, Shear / Tension, Pull Type (1973)

Protruding Head, Shear / Tension, Pull Type (1973)

Protruding Head, Shear, Pull Type (1973)

Protruding Head, Shear, Stump Type (1973)

Protruding Head, Std. and Oversize, Pull Type, Titanium

Protruding Head. Std. and Oversize, Pull Type, Titanium

Protruding Head. Stump Type. Titanium Alloy (1973)

Protruding Head, Tension, Pull Type (1973)

Protruding Head. Tension. Pull Type (1973)

Protruding Head, Tension, Pull Type (1973)

Protruding Head, Tension. Stump Type (1973)

Protruding Head, Tension, Stump Type, (1973)

Protruding Head, (1972)

Proximate Analysis of Coal and Coke (1973)

Pruning, Trimming, Repairing, or Removal (1972)

Prynachlor (Common Name for the Pest Control Chemical)

Psig (1973) /Es in Gas Distribution Systems Whose Maxi

PSM Poly(vinyl Chloride) (PVC) Sewer Pipe and Fittings

PSP Poly(vinyl Chloride) (PVC) Sewer Pipe and Fittings
Psychoacoustical Terminology (Acoustics, Audiometry)

Psychology (1972)

Psychrophilic Bacteria in Food Products (Cereal Chemist

Public and Residential Swimming Pools (1969)

Public Highways (1973)

TTd. for Inspection Procedures

Pubic ar Oefice Building, Manufacturer, Restant, an

Public Property (Pedestrian Protection During Construct

Public Streets and Projections Over Public Property (PE

Public Swimming Pools (1969)

Public Use (1972)

Public Works Spec. for Ceramic Veneer (Sampling, Test.

Published Scientific and Technical Work (1973)

Puffed Cereals, Farina, and Flour (Cereal Chemistry) (1

Puffed Cereals, Farina, Bread, etc. (Cereal Chemistry)

Pulley Guard (1972)

Pulley Width (1971) SAE J720

Pulls, Push and Kick Plates: Bolts: Closers; Hospital a

Pulp and Paper Manufacture (1973)

(Pulp and Paper) (1973)

(Pulp and Paper) (1973)

Pulp and Paper) (1973)

Pulp and Paper) (1973)

(Pulp and Paper) (1973)

Pup and Paper) (1973)

(Pulp and Paper) (1973)

Pulp and Paper) (1973)

Pulp and Paper) (1973)

Pulp and Paper) (1973)

ulp and Paper (1973)

and Paper) $(1973)$

AACCH $46-18$

AACCH 46.10

AACCH 46.24

AACCH $46-21$

AACCH 46.22

AACCH 46.12

$\mathrm{AACCH} \quad 46.19$

$\begin{array}{ll}\mathrm{CR} & \mathrm{B}-48\end{array}$

CR A.18

CR G-22

CR J.56

CR F-44

AACCH 46.13

Met AACCH 22.60

/Oto AACCH 22.62

Ori AACCH 22.63

AACCH 22.62

AACCH 22.63

$\mathrm{AACCH} \quad 22.60$

AACCH 22.61

ADA *1

FMS

NSA

NSA

NSA

NSA

NSA

NSA

NSA

NSA

NSA

NSA

NSA

NSA

NSA

NSA

NSA

NSA

NSA

NSA

NSA

NSA

NSA

NSA

NSA

ASTM

ANSI

ANSI

ASTM

ASTM

ANSI

ANI

$\mathrm{AACCH}$

NSF

ANSI

ICBO

ICBO

ICBO

NSPI

ANSI

BIA

1729

1738

1768

1054

2605

1728

1398

1673

1669

1424

7014

1446

6984

2005

2406

2206

1465

1525

6965

1555

1496

671

D3172

Z133. 1

K62.116

B16.33

D3034

D3033

S3.20

Z94.11

42.45

27
$D 7.1$

UPC* - $C$

$\mathrm{UBC} * 3-44$

$\mathrm{UBC} * 3-44$ $* 1$

A 112.6 .1

/Ract. IEE

$* 28$

AACCH 86.80

AACCH 86-70 
for Sodium Determination by the Uranyl Zinc Acetate Meth. Pulp and Paper) (1973) for Density or Volume of Small Specimen (Hg Pycnometer) (Pulp and Paper) (1973)

Density and Moisture of Chips (Presoak Before Immersion) (Pulp and Paper) (1973)

of Pulpwood (Submersion with Correction for Cracks, etc.) (Pulp and Paper) (1973)

re of Chips (Submersion with Correction for Cracks, etc.) (Pulp and Paper) (1973)

Meth, for Methoxyl Content of Pulp and Wood (1972)

Rec. for Airborne Pulp Driers (1973)

Test Meth. for Shrinkage Test of Pulp Sheets (1973)

1972)

Meth. for Measuring Brightness of

Test Meth. for Shive Content of Mechanical

Test Meth. for Shive Content of Mechanical

pectral Reflectance, Transmittance, and Color of Paper and

Test Meth. for Shive Content of Mechanical

Test Meth for Moisture in

Meth. for Drainage Time of

Test Meth. for Pentosans in

Test Meth. for Fiber Bundles in Baled Flash Dried

Wet Ashing with Nitric and Perchloric Acids (Wood.

Std. Terms Used in the Sulfite
Test Meth. for Sieve Analysis of Test Meth. for Density of

(Pulp and Paper) (1973)

Meth. for Compression Wood Identification in or Thermal Diffusivity of Carbon and Graphite by a Thermal Std. for Computer Type (Square Loop)

Meth. for Detection and Measurement of Discharge (Corona) fire Protec/ Rec. for Prevention of Furnace Explosions in Uniform Building Code Std. for Processed Rec. Pract, for Fuel

Std. Meth. for High Pressure Tent. Meth. of Test for bine Driven Generators and Reversible Generator/Motors for (1973)

ors and Reversible Synchronous Generator/Motor Units fo Rec. for Flammable Liquid Std. Meth. of Test for Effective Rec. Pract. for Design Calculations for Oil Sucker Rod rmance Data for Positve Displacement Hydraulic Fluid Power ) Gas (1972) Safety Std. for Std. for Safety for Water

Cleaning of Lubricating Systems of Various Turbine Driven Sanitary Stds. for Centrifugal and Positive Rotary Std for Safety for Safety Std. for Power Operated Std. for Electric Pressure

ion Corrosion Characteristics of Aluminum Automotive Wate Rec for Centrifugal Fire (Water) Rec. Pract, for Fuel Pump Mountings for Diaphragm Type Underwriter Steam Fire

Std for Safety for Swimming Pool ion of Material by Absorption Spectroscopy, Using the ASTM oling, Printed Wiring Boards) in Numeric (Digital) Form on numeric (Digital) Form on Punched Cards, Magnetic Tape and d Quill Bushings (1972)

I Bushings (1972)

ushings (1972)

gs (1972)

Std. Spec, for Simpl Trading Rules for the Trading Rules for the Trading Rules for the Trading Rules for the a Model Performance Spec. for the Safety Std. for Regular Bicycles Including Selection, or Sensory Evaluation of Industrial and Institutional Food ent in Hazardous Locations (1972) ANSI C106.1 Std. for of Hydroelectric Equipment (1971) Rec. Pract. for the s, Nebulizers, Gas Therapy and Suction Equipment, Room Air Std. for Identification of Air Std. Meth. of Test for tography (1972) (1973) 5 Std. Meth. for Spectrochemical Analysis of High tography (1971) ANSI Z11/ Std. Meth. for Analysis of High Std. Meth of Test for Delta in Hours (D IH) rd in CIE Y; X, Y or Y, Dominant Wavelength and Excitation rs; Hinges; Locks; Knobs and Handles; Exit Devices, Pulls. Spec, for Gol Spec. for Thermoplastic Line Pipe Std. for Installation

Installation Std. for Solvent Cemented Std Spec for Polyvinyl Chloride e Styrene (ABS) Pipe and Fittings to Poly(vinyl Chloride) or Making Solvent Cemented Joints with Polyvinyl Chloride

Std. Spec. for Poly(vinyl Chloride Std. Spec. for Poly(vinyl Chloride

Pulp (Web) (1973)

Pulp (1972)

Pulp (1973)

Pulp (1973)

Pulp, etc.) (1973)

Pulping Process (1972)

Pulpwood (1972)

Pumps (1971)

Pumps (1973)

Purchases (1972)

(PVC and CPVC) (1972)

973) ANSI K65.56 Std. Spec. for Bell End Poly(vinyl Chloride)

Pulp (Diffuse Illumination and Zero Deg. Observation) (

Pulp (Laboratory Flat Screen) (1973)

Pulp (Somerville Fractionator) (1973)

Pulp (Using Spectrophotometer with Reserved Optics (197

Pulp (Von Alfthan Shive Analyzer) (1973)

Pulpwood Chips (Pulp and Paper) (1973)

Pulpwood (Submersion with Correction for Cracks, etc.)

Pulse Meth. (1972) ANSI K90.12

Pulse Transformers (1970)

Pulses in Evaluation of Insulation Systems (1973)

Pulverized Coal Fired Multiple Burner Boiler Furnaces (

Pulverized Quicklime (1973)

Pump Mountings for Diaphragm Type Pumps (1971)

Pump Test. of Hydraulic Oils (1973)

Pumpability of Industrial Fuel Oils (1973)

Pumped Storage lnstallations (1972)

Pumped Storage Installations (1974)

ion and Safety)

Pumping Speed of Vacuum Chamber Systems (1972)

Pumping Systems (Conventional Units) (1972)

Pumps and Motors (1973) ANSI B93.27

Punips for Fire Protection Service (1974)

Pumps for Marine Service (1971)

Pumps for Milk and lts Products (1974)

Pumps for Oil Burning Appliances (1974)

Pumps for Petroleum Products (1973)

Pumps for Use in Recreational Vehicles (1972)

Pumps with Coolants (1972)

Pumps (NFPA 20) (1974)

Pumps, Filters, and Chlorinators (1972)

/ Meth. for Cavitation Eros

Pumed Card lndex (1972)

Punched Cards, Magnetic T
Punched Paper Tape (1972)

for the Flushing and

Punches-Basic, Combination Angle Head Type and Relate

Punches-Basic, Cylindric al Head Type and Related Quil

Punches-Variable, Angle Head Type and Related Quill B

Punches- Wire Type (1972)

Punches: Basic Angle Head Type and Related Quill Bushin

Puncturing Device for Use in Aerosol Laboratories (1972

Purchase and Sale of Linseed Meal (Flaxseed) (1974)

Purchase and Sale of Linseed Oil (Flaxseed) (1974)

Purchase and Sale of Soybean Meal (1973)

Purchase and Sale of Soybean Oil (1973)

Purchase of Reflective Pavement Marking Paints (1972)

Purchase, Preparation, Maintenance and Rules of the Roa

Std. Rec.

Purged and Pressurized Enclosures for Electrical Equipm

Purification of Oil Systems for Lubrication and Contro

Purifiers, Filters, Compressors, Artificial Airways, Ap

Purifying Respirator Canisters and Cartridges (1973)

Purity and Benzene Content of Cyclohexane by Gas Chroma

Purity and Hydrocarbon Impurities by Gas Chromatography

Purity Iron for Silicon and Aluminum (1971) ANSI Z128.3

Purity N-Heptane and Isooctane by Capillary Gas Chroma

Purity of Nuclear Graphite (1971) ANSI K90.8

Purity (1972) /F Measuring Color of Paper and Paperboa

Push and Kick Plates; Bolts; Closers; Hospital and Misc

Putting Green Construction (1972)

PVC Building Drain Waste and Vent Pipe and Fittings (19

PVC Pipe for Water Service and Yard Piping (I971)

(PVC) Jacket for Wire and Cable (1974)

(PVC) Pipe and Fittings for Nonpressure Applications (I9

(PVC) Pipe and Fittings (1973) ANS
(PVC) Pipe (1973) ANSI B 72.20

Std. Spec. for Solvent Cements for Poly(vinyl Chloride
Std. TAPPI

st Meth TAPPl

TAPPI

TAPPI

FMS

TAPPI

TAPPI

TAPPI

TAPPI

TAPPI

TAPPI

TAPPI

TAPPI

TAPPI

TAPPI

TAPPI

TAPPI

TAPPI

TAPPl

ASTM

IEEE

/Td. ASTM

FMS

ICBO

SAE

ASTM

ASTM

NEMA

FMS

ASTM

API

NFLDP

UL

UL

ASME

UL

UL

IAPMO

ASTM

FMS

SAE

FMS

UI

ASTM

IPC

IPC

ANSI

ANSI

ANS ]

ANSI

ANSI

ASTM

NFP

NFP

NSPA

NSPA

ITE

BMA

ASTM

NFPA

ASME

NSF

ANSI

ASTM

ASTM

ASTM

ASTM

ASTM

TAPPI

NBHA

USCA

API

IAPMO

IAPMO

ASTM

ASTM

C. Pract. F

ASTM

ASTM

ASTM

ASTM

ASTM

T623

UM. 18

UM. 16

UM. 2

UM-20

$209 \mathrm{SU}$

7.20

UM. 238

T525S0

M-240

UM-242

T442SU

UM-237

T221SU

UM. 236

UM. 239

UM-243

T1201

UM. 21

UM. 2

T20

C714

272

U-2 24.20

J625 A

D 2882

D3245

MG5.2

MG5. 1

LPD 7.32

E294

$11 \mathrm{~L}$

T3.9.17

51 
(1973) ANS

(PVC) Sewer Pipe and Fittings (1973)

ASTM

(PVC) Sewer Pipe (1972)

ASTM

ine (Enology) (1972) Tent. si Z107.4 st for Density and Specific Gravity of Liquids by Bingham Test Meth. for Density or Volume of Small Specimen $(\mathrm{Hg}$ pest Control Chemical 0,0 Dimethyl 0.(3,5,6-Trichloro-2 Std. Household Electric Range bration of Refractory Metal Thermocouples Using an Optical $s$ (1972) ANSI Alll.4

Std. Meth of Test for

h. of Analysis of Monocalcium Phosphate and/or Sodium Acid k60.20 Std. Spec. for Tetrasodium Meth. of Analysis for Neutralizing Value of Sodium Acid Uniform Fire Code: Cellulose Nitrate Plastics hemistry) (1962) Meth. of Analysis for Fumaric, Stds. for Decoders (Type I) for Reproducing Matrix Rec. Pract. for Video Alignment Signal Spec. for A and Tracking Control Records on 2 In. Video Magnetic Tape f Recorders and Reproducers for Audio Record One for 2-In. Spec. for an Audio Level and Multifrequency Test Tape for rrier Frequencies and Deemphasis Characteristics for 2 In. Rec. Pract for the Label for 2 In.

Rec. Pract. Spec. of Tracking Control Record for 2 In. Power Generating Stations (1971) Ansi/ ng of Steel Castings (1972) ANSI G5 sciences (1972)

Std. Descriptions of rsonnel for the Construction Phase of Nuclear P/ Std. for noise Sound Level Meters (1972) ANSI S6./ Procedure for bsorption (1974)

ts (1973)

n Flour Improvers (Cereal Chemistry) (1962)

iaminophenylamine (Cereal Chemistry) (1967)

aric Acid in Presence of Phosphates (Cereal Chemistry) (/ Its in Yeast Foods (Cereal Chemistry) (1962)

king Powders and Chemicals (Cereal Chemistry) (1962)

ereal Chemistry) (1962)

der, in Presence of Phosphates (Cereal Chemistry) (1962) t Foods (Cereal Chemistry) (1962)

n Flour Improvers (Cereal Chemistry) (1962)

n Flour (Cereal Chemistry) (1962)

al Chemistry) (1962)

ds (Cereal Chemistry) (1962)

Mineral Filler and Co Sulfur Dioxide Content Test Meth. for Carbonates in Coated Paper Test Meth. for Sulfides in Coated Paper Wells and W ater Systems (Treatment, Std. for Viewing Conditions for the Appraisal of Color lear Facilities (1972)

lear Power Plants-Terms and Definitions (1973) Standard for Std. for Std. for r Plants (1971)

is (Wrought Products Except Forgings and Stock/ Spec. for
nous Data Communication Equip/ Std. for Start-Stop Signal i G54. I4

rews (1971) ANSI G54.17

td. Spec. for Cold Heading Std. Spec, for Cold Heading Std. Spec. for Std.

Std. for Prescription Requirements for First Inspection of Laminators $\begin{array}{ll}\text { film (1972) } & \text { Rec. Pract. for } \\ \text { Rec. Pract. Inspection and }\end{array}$ Std. Meth. of Conducting Shear Block Test for Rec. Pract. for Evaluating the Rec. Pract. for

62)

lity Pole Preservative Treatment by Pressure Processes and

Rec, Pract, for Monitoring Special Process of Rec. Pract. for Vendor Rating System for Rec. Pract. for Vendor Product Improvement Program for Rec, List of Terms and Definitions Related to Std. Glossary of General Terms Used in hics and Human Relations in Vendor-Vendee Relationships std. Spec. for Steel Wire, Medium High Carbon Cold Heading Std. Spec. for Alloy Steel Wire, Alloy Cold Heading (Petroleum Industry) ( and Springs (1972)
Dolding and Extrusion Materials (1973)

Pycnometer Meth. of Analysis for Alcohol (Ethanol) in W

Pycnometer (1968) ANSI Z11.96

Pycnometer) (Pulp and Paper) (1973)

ASTM

ASTM

Pyridine Bases in Crude and Refined Tar Acids (1972) an

TAPPI

Pyridyl) Phosphorothioate Chlorpyrifos Methyl (1973)

Pyrolytic Self Cleaning Ovens (Stove) (1974)

Pyrometer (1972) ANSI N 144

Pyrometric Cone Equivalent (PCE) of Refractory Material

Pyrophosphate in Prepared Mixes (Cereal Chemistry) $(196$

Pyrophosphate (Anhydrous) (Cleaning Agent) (1970) ANSI

Pyrophosphate (Cereal Chemistry) (1962)

Pyroxylin) (1973)

Pyruvic, Lactic, Succinic, and Tartaric Acids (Cereal C

Quadraphonic Disc Records (1974)

Quadruplet Video Magnetic Tape Recording (1969)

Quadruplex Recorded at 15 and 7.5 In /S (1973)

Quadruplex Video Magnetic Tape Operating at 15 and 7.5

Quadruplex Video Magnetic Tape Recorders Operating at 1

Quadruplex Video Magnetic Tape Recording (1967)

Quadruplex Video Magnetic Tape Recordings (1968)

Quadruplex Video Magnetic Tape Recordings (1970)

Qualification of Class I Electric Equipment for Nuclear

Qualification of Procedures and Personnel for the Weldi

Qualifications and Scopes of Work Relating to Forensic

Qualifications of Inspection, Examination, and Test. PE

Qualifying a Sound Data Acquisition System for Vehicle

Qualifying and Controlling Cleaning Meth. for Hydraulic

Qualifying Class I Electric Equipment for Nuclear Power

Qualitative Classification of Surfactants by Infrared a

Qualitative Identification of Polymers in Emulsion Pain

Qualitative Iodide Test Meth. for Ammonium Persulfate I

Qualitative Meth. of Analsis of Benzoyl Peroxide with D

Qualitative Meth. of Analysis for Free or Combined Tart Qualitative Meth. of Analysis for Nature of Ammonium Sa Qualitative Meth. of Analysis for Phosphoric Acid in Ba Qualitative Meth. of Analysis of Added Iron in Flour (C Qualitative Meth. of Analysis of Aluminum in Baking Pow Qualitative Meth. of Analysis of Ammonium Salts in Yeas Qualitative Meth. of Analysis of Bromates and Iodates I Qualitative Meth. of Analysis of Bromates and Iodates I Qualitative Meth. of Analysis of Calcium Peroxide (Cere Qualitative Meth. of Analysis of Chlorides in Yeast Foo

Qualitative (Including Optical Microscopic) Analysis of

Qualitative) of Industrial Aromatic Hydrocarbons (1971)

Qualitative) (1973)

Qualitative) (1973)

Quality and Management) (1972)

Quality and Uniformity in the Graphic Arts (1972)

Quality Assurance for Protective Coatings Applied to Nu

Quality Assurance Practices for the Construction of Nuc

Quality Assurance Program Requirements for Nuclear Powe

Quality Assurance Sampling of Carbon and Low Alloy Stee

Quality Between Data Terminal Equipment and Non-Synchro

Quality Carbon Steel Wire for Machine Screws (1971) Ans

Quality Carbon Steel Wire for Tapping or Sheet Metal Sc

Quality Cold Finished Carbon Steel Bars (1973)

Quality Contact Lenses (1972)

Quality Control by the AITC Inspection Bureau (1962)

Quality Control Monitoring of the Product (Receiving an

Quality Control of First Generation Silver Habide Micro

Quality Control of Glue Bonds in Scarf Joints (1970)

Quality Control Program (1969)

Quality Control Records System (1969)

Quality Control Std. (1973)

1, Timber, Plywood and Uti

Quality Control System (Timber Construction Manual) (19

Quality Control (1969)

Quality Control (1969)

Quality Control (1969)

Quality Control (1969)

Quality Control (1971) ANSI Z1.7

Quality Control) (1967)

Quality for Hexagon Head Bolts (1972) ANSI G54.15

Rec.
4.15
4.16

Quality for Subsurface Injection Using Membrane Filters

Quality High Tensile, Hard Drawn Mechanical Spring Wire
ANS

AHAM

ASTM

ASTM

ASTM D595

$\mathrm{AACCH} \quad 02.34$

UFC*2ART6

$\mathrm{AACCH} \quad 04-24$

ElA RS418

SMPTE RP10

Audio ANSl C98.6

ANSI C98.3

ANS1 C 98.8

/Ce Ca SMPTE RP6

SMPTE RP26

SMPTE RPI6

IEEE 344

ASTM A 488

ASTM E444

ANS1 N45.2.6

SAE J184

NFLDP T2.9.2

IEEE 323

ASTM D2357

ASTM D3I68

AACCH 48.6]

AACCH 48-06A

AACCH $04-28$

AACCH $40-17$

AACCH 04.10

AACCH $\quad 40-40$

AACCH 40.10

AACCH 40.16

AACCH $48-40$

$\mathrm{AACCH} \quad 48-41$

AACCH 48.50

$\mathrm{AACCH} \quad 40-32$

TAPPI T42I

ASTM D853

TAPPI UM-531

TAPPI UM-532

WSC

ANSI

ANSI

ANSI

ANSI

SAE

PH2.32

N101.4

N45.2.10

$\mathrm{N} 45.2$

EIA

AMS2370A

ASTM A548

ASTM A108

ANSI Z80.2

AFTC 202

$\begin{array}{ll}\text { ASQC } & \text { RP5 } \\ \text { NMA } & \text { MS10 }\end{array}$

ASTM D1759

ASQC

$A S Q C$

RP3

RP6

UBCS25.12

A'TC

ASQC

ASQC

ASQC

ASQC

ASQC

ASQC

ASTM

ASTM

NACE

201

RP4

RP7

RP8

A3

RP1

A546

A547

SAE J271 
(1973) ANSI G24.24

(1973) ANSI G24.25

cal Property Requirements (1972)

Std. Spec. for Regular Std. Spec, for Drawing

Std. Spec. for Merchan

Std Spec for Merchan

zed) by Hot Di/

Std. Spec. for Steel Sheets of Structural Disk Meth. of Analysis of Semolina Macro Scale Meth. of Analysis of Semolina Micro Scale Meth. of Analysis of Semolina Spec for Steel Spring Wire, Best Std. for Self Locking, 450 Deg. F, High Pract. for Choice of Sample Size to Estimate the Average Sponge Dough, Pound Loaf Meth. of Analysis for Baking Meth. of Analysis for Baking Meth. of Analysis for Baking Std. Spec. for Continuity of Std. Spec. for Continuity of Std. Metli, of Test for able Systems (1972) ANSI C5/ by Acetone Immersion) (1967) Ansi/ eal Chemistry) (1962)

(1962) Extensigraph Meth. for Meth. of Analysis for Baking Meth. of Analysis for Baking

Std. Meth. for Controlling Meth. of Analysis for Baking Meth. of Analysis for Baking Straight Dough Meth. of Analysis for Baking Std. Meth of Test for Thermal Insulation of Spec. for General Requirements for A

used in Automotive and Related In/ motive and Related Industries (1)

Std. of Mechanical and

Std for Mechanical and

Std. for Mechanical and

Std. for Mechanical and

Rec. Pract. for Procurement

Industry

ofilm (1971)

ail Construction (Architectural Woodwork) (1973)

ad Enclosures of All Kinds (Architectural Woodwork) (197)

rk) (1973)

(1973)

work) (1973)

chitectural Woodwork) (1973)

odwork) (1973)

I Paneling (Architectural Woodwork) (1973)

) (1973)

Woodwork) (1973)

hitectural Woodwork) (1973)

I Woodwork) (1973)

ral Woodwork) (1973)

oodwork) (1973)

ings (Architectural Woodwork) (1973)

le Inspection Procedure) (197/

Spec. for Premium Aircraft Std. Spec. for Cold Heading Std. Spec. for Music Spring

mediate Tensile Strength Carbon Steel Plates of Structura for Natural Muscovite Block Mica and Thins Based on Visual td. Spec. for Steel Wire, Oil Tempered Carbon Valve Spring inc Coated (Galvanized) by the Hot Dip Process, Commercial c. for Hot Rolled Carbon Steel Sheet and Strip. Structura (0.15 Maximum, Percent) Steel Sheet and Strip, Commercial to 0.25 Maximum \%. Hot Rolled Sheet and Strip, Commercial strength Low Alloy Columbium Vanadium Steels of Structural Std Spec for Carbon Steel Wire of Scrapless Nut Steel, Carbon, Strip, Cold Rolled Hard, Untempered Spring

$1.2 \mathrm{Cr}-3.25 \mathrm{Ni}-0.12 \mathrm{Mo}(0.07-0.13 \mathrm{C})$ (SAE 9310). Premium Std. Definitions, Symbols, Formulas, and Tables for Meth of Analysis for Baking Test cr $-9.0 \mathrm{Ni}-4.5 \mathrm{Co}-1.0 \mathrm{Mo}-0.09 \mathrm{~V}(0.17-0.23 \mathrm{C})$, Premium $0.80 \mathrm{Cr}-1.8 \mathrm{Ni}-0.25 \mathrm{Mo}(0.38-0.43 \mathrm{C}$ ) (SAE 4340). Premium ant, $14.5 \mathrm{Cr}-4.0 \mathrm{Mo}-1.2 \mathrm{~V}(1.10-1.20 \mathrm{C})$, Premium Bearing ; Flaxseed;

Oilseed Products Trading Rules (Performance, Std. Spec. for Steel Bars, Carbon, Hot Rolled, Special

Steel Bars, Carbon, Hot Rolled and Cold Finished, Special

$1.2 \mathrm{Cr}-3.25 \mathrm{Ni}-0.12 \mathrm{Mo}(0.07-0.13 \mathrm{C}$ ) (SAE 9310), Premium s (1971) Ansil

s (1971) ANSI G8.2/

tion) (1971) ANSI L.14.132

aatec $20 \mathrm{~A}$

anilic Acid in Feeds (Cereal Chemistry)

ng Mixtures (1972) Meth.
aking Powders and Chemicals (Cereal Chemistry) (1962)

n Flour (Cereal Chemistry) (1962)

st Foods (Cereal Chemistry) (1962)

ough Conditioners Containing Monoglyceride, Salts, Soy F/ ods (Cereal Chemistry) (1962)

Chemistry) (1962)

white and Whole Wheat Flour (Cereal Chemistry) (1962)
Quality Hot and Cold Rolled Alloy Steel Sheet and Strip Quality Hot and Cold Rolled Alloy Steel Sheet and Strip Quality Hot Rolled Carbon Steel Bars Subject to Mechani Quality Hot Rolled Carbon Steel Bars (1973)

Quality in Coils, and Cut Lengths, Zinc Coated (Galvani

Quality in Macaroni (Cereal Chemistry) (1962)

Quality in Macaroni (Cereal Chemistry) (1962)

Quality in Macaroni (Cereal Chemistry) (1962)

Quality Music (1973)

Quality Nuts (1973)

Quality of a Lot or Process (1972) ANSI Z1.11

Quality of Bread Flour (Cereal Chemistry) (1962)

Quality of Cake Flour (Cereal Chemistry) (1962)

Quality of Cookie Flour (Cereal Chemistry) (1962)

Quality of Electrical Insulating Oil for High Pressure

Quality of Electrical Insulating Oil for Low Pressure C

Quality of Extruded Poly (Vinyl Chloride) Plastic Pipe

Quality of Nonfat Dry Milk in Physical Dough Tests (Cer

Quality of Nonfat Dry Milk (Cereal Chemistry) (1962)

Quality of Pie Flour (Cereal Chemistry) (1962)

Quality of Radiographic Test. (1972) ANSI Z166.7

Quality of Rye Bread Flour (Cereal Chemistry) (1962)

Quality of Self Rising Biscuit Flour (Cereal Chemistry)

Quality of Wheat Bread Flour (Cereal Chemistry) (1962)

Quality Packages (1972)

Quality Program (1968) ANSI Z1.8

Quality Requirements for Externally Threaded Fasteners

Quality: Requirements for Machine Screws for Use in tuto

Quality Requirements for Steel Machine Screws (1973)

Quality Requirements for Steel Nuts (1971)

Quality Std. and Spec. (1969)

Quality Std. for Computer Output Microfilm (1971)

Quality Std. Format and Coding for Computer Output Micr

Quality Stds. for Blinds and Shutters Using Stile and R

Quality Stds. for Casework: Cabinets, Cases, Counters a

Quality Stds. for Closet and Storage Shelving (1973)

Quality Stds. for Exterior Frames (Architectural Woodwo

Quality Stds. for Exterior Sash (Architectural Woodwork

Quality Stds. for Factory Finishing (Architectural Wood

Quality Stds. for Flush Solid and Hollow Core Doors (Ar

Quality Stds. for Flush Wall Paneling (Architectural Wo

Quality Stds. for High Pressure Laminate Flush Type Wal

Quality Stds. for Lumber Grades (Architectural Woodwork

Quality Stds. for Misc. Ornamental Items (Architectural

Quality Stds. for Plywood and Particleboard Grades (Arc

Quality Stds. for Stairwork and Handrails (Architectura

Quality Stds. for Standing and Running Trim (Architectu

Quality Stds. for Stile and Rail Doors (Architectural W

Quality Stds. for Stile and Rail Wall Paneling (1973)

Quality Stds. for Wood Screens for Window and Door Open

Quality Steel Cleanliness Requirements (Magnetic Partic

Quality Steel Wire for Wood Screws (1971) ANSI G54.18

Quality Steel Wire (1971) ANSI G54.2

Quality (1970) ANSI G24.2

Quality (1971) ANSI C59.27

Quality (1971) ANSI G54.4

Quality (1971) ANSI G8.24

Quality (1972)

Quality (1972)

Quality (1972)

Quality (1972) ANSI G41.6

Quality (1972) ANSI K91.3

Quality (1973)

Quality (1973)

(Quality) Control Charts (1971) ANSI Z1.5

Quality) for Sweet Yeast Products (Cereal Chemistry) (1

Quality, Consumable Electrode Melted, Annealed (1973)

Quality, Consumable Electrode Remelted (1973)

Quality, Consumable Electrode Vacuum Melted (1973)

Quality, Description, and Grade of Palmkernels; Babassu

Quality, for Fluid Power Applications (1974)

Quality, for Pressure Piping Components and Other Press

Quality, Vacuum Consumable Electrode Remelted (1973)

Quality, Zinc Coated (Galvanized) by the Hot Dip Proces

Quality, Zinc Coated (Galvanized) by the Hot Dip Proces

Quantitative Analysis of Textiles (Moisture and Composi

Quantitative Analysis of Textiles (1972) ANSI L14.132.

Quantitative Determination of Both Nitrophenide and Ars

Quantitative Extraction of Bitumen from Bituminous Pavi

Quantitative Meth. of Analysis for Phosphoric Acid in B

Ouantitative Meth. of Analysis of Ammonium Persulfate I

Quantitative Meth. of Analysis of Ammonium Salts in Yea

Quantitative Meth. of Analysis of Calcium Peroxide in D

Quantitative Meth. of Analysis of Chlorides in Yeast Fo

Quantitative Meth of Analysis of Iron in Flour (Cereal

Quantitative Meth. of Analysis of Potassium Bromate in

ASTM

ASTM

ASTM

A575

STM A446

AACCH $66-40$

AACCH $66-41$

$\mathrm{AACCH} \quad 66-42$

AMS5] $12 \mathrm{~F}$

NSA 3350

Td. Rec ASTM E 122

AACCH 10.11

$\mathrm{AACCH} \quad 10-90$

AACCH $10-50 B$

ASTM D1819

ASTM D1818

ASTM D2152

AACCH $54-12$

$\mathrm{AACCH} \quad 10-85$

AACCH $\quad 10.60$

ASTM El42

AACCH $\quad 10-70$

AACCH $10-31 \mathrm{~A}$

AACCH $\quad 10-10$

ASTM D3103

ASQC C1

SAE J429F

$\mathrm{SAE}$

IF]

SAE

ASQC

NMA

NMA

AWI

AWI

$A W I$

$A W I$

AW

AWI

AW

$A W I$

AW

AWI

$A$ WI

$A W I$

AWI

AWI

AWI

AWI

AWI

SAE

ASTM

ASTM

J82

103

J995B

RP2

$\mathrm{MSl}$

MS2

$* 1-1200$

$* 1-400$

$* 1.600$

$* 1.900$

$* 1-1000$

$* 1.1500$

$* 1-1300$

$* 1.500 \mathrm{~A}$

$* 1.500 \mathrm{~B}$

*1-100

*1-700

$* 1.200$

$* 1.800$

$* 1.300$

$* 1.1400$

*1-500C

$* 1.1100$

AMS2300B

A549

A228

A283

D351

Sid. Spec. ASTM

ASTM

A230

A526

4570

A569

A659

A 572

A544.

A 680

AMS6267B

ASQC Al

$\mathrm{AACCH} \quad 10-20$

$\mathrm{SAE}$

AMS6523A

JUbing, SAE

AMS6414B

ISt $\mathrm{SAE}$

NIOP

ASTM

$* 1$

SAE

ASTM

A696 
yd Lacquers by Infrared Spectrophotometry/ Std. Meth. for Std. Melh. of Test for Low Meth. of Test for Trace bons by Oxidative Microcoulometr/ Meth. of Designating and Measuring Apertures and Related
Hydrogen Combustion M/ Std. Meth. of Test for Trace (Oxy Hydrogen Combustion M/ Std. for Interrelationship of ses, and Other Liquid and Semiliquid Products (Drying Upon ed Bauxite, Calcine. Chrome Ore, Clays, Diaspore, Ganister steel Joints (1971) ANSI G24.19 elding (1970E) ANSI St Spec. for High Yield Strength, or Pressure Vessel Components (1973) Ansi/ Std. Spec. for I Applications (1973). Std. Spec. for Std. Spec. for Chromium Molybdenum, Alloy Steel Plates, romi/ Std. Spec. for Pressure Vessel Plates, Alloy Steel, teeI Forgings for Pressure Vessels (1974)/ Std. Spec. for 20 Std. Spec. for Pressure Vessel Plates, Alloy Steel, c. for Pressure Vessel Plates, Alloy Steel, High Strength, olybdenum and Manganese-Molybdenum-Nickel Alloy Steel, Spec. for Nickel Chromium Molybdenum Alloy Steel Plates, Std. Meth. of Physical Test. of Uniform Building Code Std. for Uniform Building Code Std. for Processed Pulverized Std. Meth. of Chemical Analysis of Limestone, Std. for Punches-Variable, Angle Head Type and Related Std. for Punches: Basic Angle Head Type and Related Id. for Punches-Basic, Cylindrical Head Type and Related r Punches-Basic, Combination Angle Head Type and Related Grade Hydroquinone $\mathrm{C}_{1 ;} \mathrm{H}_{4}(\mathrm{Oh})_{2}$ (Para Dihydroxbenzene, 973) Std. for Double i C33.37 Std for Safety for Fiber Conduit for Use as lexible Steel and Aluminum Conduit for Safe Use as a Metal tallation (1973/ C33.50 Std. for Safety for Cellular Metal Floor Std. For Safety for Surface Metal Std. for Steel Underfloor Duct re Safety) (1974) Safety Std. for Semiautomatic Fire Hose Fire Hazards and Protection Std. for Rec. for Solid, Palletized, and drawing Stds. Part 1 for Spur, Helical, Double Helical and Rec. Safeguards for Arrangement of Carpet Storage Std. for Dimensions for 9

e and Fish Finder, Loran or Other Line of Position Device, bearings) Conform/ Std. Shaft and Housing Fits for Metric
Std. Groove Dimensions for Fluid Power Rec. Pract. for Guide to the Application and Use of Std. For In. and Metric agons, and Multipurpose Passenger Vehi/ Std. for Bias and Bri/

Test Meth. for Spectrophotometric Test for Relative Std. Meth. of Estimating Stray Std. for Vented Gas Fired Infrared Std. for Unvented Gas Fired Infrared and Test Spec. for Selected Brachytherapy Sources (Medical ioiodine Uptake Measurements Using a Neck Phantom (Medical $r$ the Measurement of Potentially Hazardous Electromagnetic $\mathrm{n} 42.1$ Std. Test Procedure for Semiconductor ocedure for Amplifiers and Preamplifiers for Semiconductor Std. Test Meth. for Absorbed Gamma ansi / Std. Meth. of Test for Absorbed Gamma and Electron dosim/ Std. Meth. of Test for Absorbed Gamma and Electron

C112.1

(1971) ANSI C95.4

Std for Measur Rec. for Protection Against

c37.24

rials $(1962) /$

Safeguards Against Radio Frequency
Rec. for Exposure to Rec. for Exposure to

Std. Guide for Evaluating the Effect of Solar Std. Rec. Pract. for Effects of High Energy

961) Std. for

Analysis Equipment (1971) NBS HB111

Std. for Sid. Spec. for Aggregates for
descriptive Nomenclature of Constituents of Aggregates for Std. for Concrete

Rec. Pract. for External Electromagnetic Performance Spec. for Portable X-Or Gamma ocedure for Semiconductor Radiation Detectors for Ionizing Pract. for Exposure of Adhesive Specimens to High Energy ion System for Polymeric Materials for Service in Ionizing ct and Indirect Reading Pocket Dosimeters for X-And Gamma Materials (Coatings) with Electromagnetic and Particulate Std. for Criteria for Film Badge Performance (Nuclear Rec for Protection Against Betatron Synchrotron ctronic Equipment / Std. for Safety for Special Fuses for Minimum Std. for Amateur Cable Connectors for Audio Facilities for

chemical, Mass Spectrometric, Spectrochemical, Nuclear and ish Finder, Loran or Other Line of Position Device, Radar, c Blasting Caps (1971) ANSI C95.4 Safeguards Against
Quantitiative Determination of Cellulose Nitrate in Alk Quantities of Mercury in Paint (1973)

Quantities of Sulfur in Light Liquid Petroleum Hydrocar

Quantities Pertaining to Photographic Lenses (1971)

Quantitites of Total Sulfur in Volatile Organic Liquids

Quartz Fiber Electrometer Type Dosimeters and Companion

Ouartz Sand) (Cereal Chemistry) (1962)

Quarzite, Grog, Kyanite, Magnesite, Mullite) (1970) Ans

Quenched and Tempered Alloy Steel Bolts for Structural

Quenched and Tempered Alloy Steel Plate, Suitable for W

Ouenched and Tempered Carbon and Alloy Steel Forgings F

Quenched and Tempered Carbon Steel Plates for Structura

Quenched and Tempered Chromium, for Pressure Vessels (I

Quenched and Tempered Nickel-Cobalt-Molybdenum-Ch

Quenched and Tempered Vacuum Treated Carbon and Alloy S

Quenched and Tempered 8 and 9\% Nickel (1972A) ANSI G35.

Quenched and Tempered (1972A) ANSI G35.11

Sid. Spe

Ouenched and Tempered, for Pressure Vessel Plates (1973

Quenched and Tempered, for Pressure Vessels (1972A)

Quicklime and Hydrated Lime (1971) ANSI K67.10

Quicklime (Lime) for Structural Purposes (1973)

Quicklime (1973)

Quicklime, and Hydrated Lime (1972) ANSI K67.3

Quill Bushings (1972)

Quill Bushings (1972)

Ouill Bushings (1972)

Quill Bushings (1972)

Quinol, Hydroquinol) (1972\}

Raceway for Installation of Wires and Cabtial Casters (1

Raceway for Wire and Cable Installation (1973) ANSI C33

Raceways and Fittings for Electrical Wire and Cable Ins

Raceways and Fittings for Electrical Wiring (1973) ANSI

Raceways (1972)

Rack Assemblies (1972)

Rack Storage of Flammable and Combustible Materials (Fi

Rack Storage of Plastics (1974)

Rack) (1971)

Racks (1972)

Racks, Panels and Associated Equipment (1972) ANSI C83.

Radar, Radio Detection Finder, Radio Telephone) (1973)

Radial Ball and Roller Bearings (Except Tapered Roller

Radial Compression Type Piston Rings (1973) ANSI B93.32

Radial Lip Type Oil Seals (1972)

Radial Needle Roller Bearings (1973) ANSI B3.18

Radial Ply Tires and Rims for Passenger Cars, Station W

Radial Seal Nomenclature and Glossary (1972)

Radiance of Paper and Paperboard Containing Fluorescent

Radiant Energy (1972)

Radiant Heaters (1971)

Radiant Heaters (1972)

Radiation-Radiology) (1973)

Radiation-Radiology) (1973)

Radiation at Microwave Frequencies (1973)

Radiation Detectors for Ionizing Radiation
Radiation Detectors (1969) ANSI N42.2

Radiation Dose in the Fricke Dosimeter (1972)

Radiation Dose with the Ceric Sulfate Dosimeter (1971)

Radiation Dose with the Ferrous Sulfate Cupric Sulfate

Radiation from Brachytherapy Sources (1972)

Radiation from Motor Vehicles (20-1000 Mhz) (1972) ANSI

Radiation Hazards in the Use of Electric Blasting Caps

Radiation in an Emergency (1962)

Radiation on Outdoor Metal Clad Switchgear (1971) ANSI

Radiation on the Mechanical Properties of Metallic Mate

Radiation Protection in Uranium Mines (Safety) (1973)

Radiation Protection with Clay Masonry (Tech. Notes) (1

Radiation Safety for X-Ray Diffraction and Fluorescence

Radiation Shielding Concrete (1973)

Radiation Shielding Concrete (1973)

Radiation Shields (1972)

Radiation Suppressors (1969)

Radiation Survey Instruments (1971)

Radiation (1969) ANSI N42.1

Radiation (1970) ANSI N141

Radiation (1971) ANSI N4.1

Radiation (1972)

Radiation (1973)

Radiation) (1972)

Radiations Up to 100 Million Electron Volts (1954)

Radio and Television Receiving Appliances and Other Ele

Radio Antenna: Part 1-Base or Fixed Station (1973)

Radio Broadcasting (1970)

Radio Chemical Analysis of Nuclear Grade Plutonium Meta

Radio Detection Finder, Radio Telephone) (1973)

Radio Frequency Radiation Hazards in the Use of Electri

Std. Fo

ASTM

ASTM

ASTM

ID ANSI

ASTM

ANSI

ASTM C316

ASTM A 490

ASTM

ASTM A541

ASTM A678

ASTM A542

ASTM A605

ASTM A508

ASTM A553

A517

ASTM A533

D ASTM

ASTM

ICBO

ICBO

ASTM

ANSI

ANS1

ANSI

ANSI

ANSI

UL

UL

UL

NEMA

UL

FMS

FMS

FMS

EIA

$\mathrm{ABYC}$

AFBMA

NFLDP

SAE

AFBMA

TRA

SAE

TAPPI

ASTM .

ANSI

ANSI

A543

Cl10

UBCS24-18

UBCS24.20

C25

B94.38

B94.41

B 94.44

B94.39

PH4.126

MH11.1

543

209

5

UD 1

8-33N

8.9

Y I 4.7.1

LPD8-30

RS310.B

A21

T3.19.18

J946A

J111A

UM- 460 ,

E387

Z21.51

N44.1

$\mathrm{N} 44.3$

C95.3

300

301

$\begin{array}{ll}\text { ASTM } & \text { D1671 } \\ \text { ASTM } & \text { D3001 }\end{array}$

$\begin{array}{ll}\text { ASTM } & \text { D3001 } \\ \text { ASTM } & \text { D2954 }\end{array}$

NCRPM R40

SAE J551A

IME 20

NCRPM R29

IEEE 144

ASTM E184

ANSI N13.8

BIA

ANSI

ASTM

Std. ASTM

ANSI

SAE

ANSI

Std. Test Pr IEEE
Rec ASTM 
for Airborne VOR Receiving Equipment Operating Within the ne Distance Measuring Equipment (DME) Operating Within the ncy Locator Tran/ Rec. Basic Characteristics for Airborne 1940)

Rules for the Mobile

er Line of Position Device, Radar, Radio Detection Finder, Rec Std for

hipping Test (Packaging) for Consumer Electronic Products imensions and Meth. of Test. for Rotary Selector Switches Std. Meth. for Measuring Fast Neutron Flux by Std. Meth. for Measuring Fast Neutron Flux by Std. Meth. for Measuring Fast Neutron Flux by Std. Meth. for Measuring Fast Neutron Flux by Std. Meth. for Measuring Neutron Flux by Std. Meth. for Measuring Thermal Neutron Flux by Average Energy from ${ }_{3} \mathrm{H}(\mathrm{D}, \mathrm{N}){ }_{1} \mathrm{He}$ Neutron Generators by Sid. Meth. of Test for Std. for Leak Test. Std. Meth. of Test for

Rec. for Control and Removal of Std. Meth. of Test for Std. Meth. of Test for

ing Department of Transportation (DOT) Special Permits for d. for Administrative Guide for Packaging and Transporting Rec. for Safe Handling of Std. Meth. of Test for Rec. for

Tentative Meth. of Test for

)(1973) wate/ Tor Packaging and Transport Std. Meth. of Test for Measurement of Beta Particle Std. Meth for Measurement of Alpha Particle Std. Meth. for Measurement of Gamma Rec. for a Manual of Meth. of Analysis of

cy Monitoring (Cereal Chemistry) (1/

hemical, Mass Spectrometric, Spectrochemical, Nuclear, and el Solutions (1970) ANSI N1I7 Std. Meth. for d. Meth. of Test for Atom Percent Fission in Uranium Fuel Std. for Intraoral Dental Insects, Rodent Hair, and

grains and Grain Products (Cer/ alytical Meth. for Determining Insect Infestation Content Std. Dimensions for lndustrial

continuities (1972)

Std. Dimensions for Medical

stings (1972) ANSI Z166.19

Std. Meth. for Controlling Quality of copper Alloy Castings (1967) ANSI Z166.17

stings, Series II (1974) ANSI Z166.8

(1972) ANSI Z166.28

edical Radiation-Radiology) (1973)

Std. for Thy roid

Sid. General Meth. for Analysis of Rec. for

c. for Selected Brachytherapy Sources (Medical Radiation ake Measurements Using a Neck Phantom (Medical Radiation ble Body Burdens and Maximum Permissible Concentrations of Std. Meth. of Test for Std. Meth. of Test for Radionuclides of Rec. Pract. for

omputations (1973) Rec. for Wood Beam, Joint and Span Tables and Working Stresses for Joists and ode Std. for Span Tables for Floor and Ceiling Joists, and sed Roof Decking. Drop Siding, Structural Roof Trusses and

l, Henequen, Ixtle, Jutes, Hemp, Tow, Cocoa Fibers, Oakum,

Spec. for W rought Carbon Steel Wheels for Locomotives and Quality Stds. for Blinds and Shutters Using Stile and Quality Stds, for Stile and 72) and Sash, Screens, Blinds and Shutters, Flush, Stile, and Quality Stds. for Stile and

Safety Requirements for Floor and Wall Openings, Std for Graphical Symbols for Use on

for Zinc Coated (Galvanized) Iron or Steel Farm Field and Rec. Pract. fo

71) ANSI G57.12 Std. Spec. for Untreated Carbon Stee Steel Products Manual: Wrought Steel Wheels and Forged Std. Spec. for Wrought Steel Wheels for Electric Std. Spec. for Cast Steel Wheels for carbon Steel Axles. Non Heat Treated and Heat Treated, for protection and Acceptance of Concrete Pavements Exposed to Test Meth. for Water Resistance of Textile Fabrics Uniform Plumbing Code Rifle

tography (1973)

Tent. Meth. of Test for Boiling

f Adhesives in Shear by Tension Loading in the Temperature

n (1970)

Rec. Pract. for the Density and Contrast
Radio Frequency Range of 108.118 Megahertz (1972)

Radio Frequency Range of 960-1215 Megahertz (Flight Con

Radio Homing and Alerting Equipment for Use with Emerge

Radio Installations (National Electrical Safety Code) (

Radio Interference Wideband Filters (1974) ANSI C83. 102

Radio Telephone for Automotive Use (HS 27) (1968)

Radio Telephone) (1973)

land Fish Finder, Loran or Oth

Radio, Television. Tape Recorder, Phonograph, etc.) (19

Radio, TV, and Instrumentation) (1973) ANSI C83.26

Radioactivation of Aluminum (1970) ANSI NI 14

Radioactivation of Iron (1970) ANSI N111

Radioactivation of Nickel (1970) ANSI NI12

Radioactivation of Sulfur (1970) ANSI N113

Radioactivation Techniques (1970) ANSI N109

Radioactivation Techniques (I970) ANSI N110

Radioactivation Techniques (1973)

Radioactive Barium in Water (1973) ANSI N155

Radioactive Brachytherapy Sources (1973)

Radioactive Cesium in Water (I972)

Radioactive Contamination in Laboratories (1951)

Radioactive Iodine in Water (1973) ANSI N I59

Radioactive Manganese in Water (1971) ANSI N156

Radioactive MateriaIs Shipments (1973)

Radioactive MateriaIs (Safety) (1973)

Radioactive MateriaIs (1964)

Radioactive Tritium in Water (1970) ANSI N164

Radioactive Waste Disposal in the Ocean (1954)

Radioactive Zirconium in Water (1973)

Radioactively Contaminated Biological Materials (Safety

Radioactivity of Industrial Water and Industrial Waste

Radioactivity of Water (1966) ANSI N152

Radioactivity of Water (1973) ANSI N150

Radioactivity Procedures (1961)

Radioactivity (Beta Gamma) in Cereal Products-Emergen

Radiochemical Analysis of Nuclear Grade Plutonium Nitra

Radiochemical Determination of Cesium-137 in Nuclear Fu

Radiochemical Meth.) (1969) ANSI N107

Radiographic Film (1972)

Radiographic Illustration and Classification in Cereal

Radiographic Inspection) in Corn and Other Whole Grains

Radiographic Sheet and Roll Film (photography) (1973)

Radiographic Sheet and Roll Films (1973)

Radiographic Std. for CIassification of Fusion WeId Dis

Radiographic Test. (1972) ANSI Z166.7

Radiographs for Heavy Walled (4 1/2 to I2 In.) Steel Ca

Radiographs for High Strength Copper Base and Nickel-

Radiographs for Inspection of Aluminum and Magnesium Ca

Radiographs for Steel Castings Up to 2 In. in Thickness

Radioiodine Uptake Measurements Using a Neck Phantom (M

Radioisotopes (1968) ANSI N148

Radiological Monitoring Meths. and lnstruments (1952)

Std. for Integrity and Test Spe

Radiology) (1973)

Sor Occupational Expo

Radionuclides in Air and in Water (1970) ANSI N 161

Radionuclides of Radium in Water (197

Radius of Load and Boom Angle Measuring System (1972)

Rafter Span, Load, Deflection. Strength and Stiffness C

Rafters (Lumber) (1972) Usc Ps20

Rafters (1973)

Uniform Building C

Rafters, Overhead /Ished Carpentry, Flooring, Shingles, Expo

Rags, Waste Cloth, Wastepaper, Kapok, Hay, Straw, Spani

Rail Cars (1971) ANSI G39.2

Rail Construction (ArchitecturaI Woodwork) (1973)

Rail Doors (Architectural Woodwork) (1973)

Rail Doors, Factory Finishing, Rough and Finished Carpe

Rail Steel Deformed Bars for Concrete Reinforcement (19

Rail Wall Paneling (1973)

Railings, and Toeboards (1973)

Railroad Maps and Profiles (1972)

Railroad Right of Way Wire Fencing (1973) ANSI G8.9

Railroad Transportation of Line Pipe (1972)

Railway Axles for Export and General IndustriaI Use (19

Railway Axles (1973)

Railway Service (1972) ANSI G57.13

Railway Service (1973)

Railway Use (1973) ANSI G57.11

Rain During Construction (1974)

(Rain Test) (1971) ANSI L 14.74, ASTM D583

Rainwater Systems, Roof Drains and Piping (1973)

Range Construction (Part Time and Special) (1971)

Range Distribution of Petroleum Fractions by Gas Chroma

Range from -267.8 to -55 C (-450 to -67 F) (1972) ANSI

Range Location and Landscaping (1970)

Range of Black and White Films and Slides for Televisio
Td. RTC

RTCA

RTCA

ANSI

EIA

SAE

$\mathrm{ABYC}$

SWA

EIA

/ D EIA

ASTM

ASTM

ASTM

ASTM

ASTM

ASTM

ASTM

ASTM

ANSI

ASTM

NCRP

ASTM

ASTM

D0-153

DO-151

DO-154

C2.5

RS416

J797

A21
772

RS414

RS3I5-A

E266

E263

E264

E265

E261

E496

D2038

$\mathrm{N} 44.2$

D2577

R8

D2334

St ANSI N14.10.1

NCRPM R30

ASTM D2476

NCRPM Rl6

ASTM D3315

ANSI N 14.3

ASTM D1890

ASTM D1943

ASTM D1690

NCRPM R28

AACCH $28-99 \mathrm{~A}$

ASTM C759

ASTM E320

St ASTM E219

ANSI PH8.1

$\mathrm{AACCH} \quad 28.95$

CR A-11

ANSI PHI.15

ANSI PHI.17

NSA 1514

ASTM E142

ASTM E280

ASTM E272

ASTM E155

ASTM E446

ANSI N 44.3

ASTM E181

NCRPM Rl0

ANSI N44.1

ANSI N44.3

NCRPM R22

ASTM D2460

ASTM D2460

SAE J375A

WWPA *30

NFORP *2

ICBO

AWI

ICBO

std. ASTM

AWI

AWI

AWI

ASTM

AWI

ANSI

ANSI

1. ASTM

API

ASTM

AISI

ASTM

ASTM

Std Spec for ASTM

ACPA TB 17

AATCC 35

ICBO

NRA

UP

ASTM D2887

ASTM D2557

NRA

*11

SMPTE RP7 
Std. Meth. of Test for Solubility Shorts, etc.) to Determine Added Amounts of Malathion in r Receiving Equipment Operating Within the Radio Frequency

Fat Mixtures Which Congeal or Solidify W'ithin Temperature uring Equipment (DME) Operating W ithin the Radio Frequency

Outdoor Pistol

$50 \mathrm{Ft}$. Summer Camp Rifle Metallic Silhouette Match Rifle Outdoor Smallbore Rifle Std. Household Electric

Rec. Pract. for Motor Vehicle Driver's Eye r Dropping Point of Lubricating Grease of Wide Temperature Construction of Indoor Rifle and Pistol for Permissible Variations from Specified Ladle Chemical Std. for Household Electric Std. for Household Electric Std. for Hotel and Restaurant Gas Std. for Safety for Household Electric Running Game (Wildlife) Rifle ules. Procedures For, and Construction of High Power Rifle Uniform Fire Code: Rifle Safety Std. for Oil Burning Stoves (Room Heaters and ness of Dyed or Printed Textiles to $\mathbb{W}$ ashing at 105 Deg. F: tton and Linen Textiles to Combined Washing and Shrinkage: Std. Spec. for Liquid Asphalt homologues of Chlorinated Biphenyls for C/ Std. Meth. for Chemistry) (1962)

1 Chemistry) (1962)

972)

flour (Cereal Chemistry) (1962)

edstuffs (Cereal Chemistry) (1962)

Std Spec, for Manually Lever Operated Chain Hoists for est Meth. for Apparent V iscosity of Adhesives Having Shear Std. Meth. of Test for Delivery Std. Meth. of Test for Seepage semblies (19) Std. Test Proc and Acceptance Criteria for cs Using a Supported Specimen by / Std. Meth. of Test for reaking Load and Elongatoin of Elastomeric Yarns (Constant test for Elastic Properties of Elastomeric Yarns (Constant Std. Meth. of Test for Liquid Flow

for Gravimetric Determination of $\mathbb{W}$ ater Vapor Transmission f Woven Fabrics by the Tongue (Single Rip) Meth. (Constant rs $(1972$ Practice and Test Requirements for Leakage Std. Meth. for Measuring Heat Transfer use of a Thin Skin Calorimeter for Measuring Heat Transfer or Safety for Electrically Operated Photographic Equipment midifier, Evaporative Cooler, and Air Filtering Appliances voltage Circuit Breakers Rated on a Symmetrical Basis When Spec. for

for Electrical Control of AC High Voltage Circuit Breakers std. Meth. for Test. AC High Voltage Circuit Breakers nce Current Switching for AC High Voltage Circuit Breakers utdoor AC High Voltage Circuit Breaker External Insulation e Insulation Strength for AC High Voltage Circuit Breakers ient Recovery Voltage for AC High Voltage Circuit Breakers ient Recovery Voliage for AC High Voltage Circuit Breakers pressurized Components of AC High Voltage Circuit Breakers nce Current Switching for AC High Voltage Circuit Breakers al Insulation for Outdoor AC High Voltage Circuit Breakers nce Current Switching for AC High Voltage Circuit Breakers e Propylene Rubber Insu/ Std. for Electrical Power Cables hylene-Propylene-Ru/ Std. for Electrical Power Cables Std. Synchronous Signaling Std. Meth. for Measurement of Volatilization Std. Meth. of Measuring Flow er Driers in Refrigeration and Ai/ Std. for Flow Capacity
ustrial/ ustrial/ Rec. Pract. for Uniform Procedure for the Test.,
et) (1972)
Std. for Uniform Meth. of Determining the Sae et, and Dr/ Std. for Uniform Meth. of Determining the Sae .1 Safety Std for $\mathrm{R} /$ Std. for Recreational Vehicle Air Conditioning System Rec. Pract. Meth. for pment (1972) Std. Meth. of Testing for Sound Std. Meth. of Testing for 5

em Rating Definitions, Test. Mesh., and Test Equipment and Std. for Meth. of Test. for Std. for Construction, Installation, and s Than $1500 \mathrm{cfm}$ (1971)

Std. for Sound Test Meth. for Visual

Range of Resins and Polymers (1972)

Range of 0.2-2.0 p.p.m. (Cereal Chemistry) (1962)

Range of I08-118 Megahertz (1972)

/Td.

Range of 960.1215 Megahertz (Flight Control) (I972)

Range Plans (Firearms) (1970)

Range Plans (1972)

Range Plans (1973)

Range Plans: Safety Rules and Procedures (I972)

Range Pyrolytic Self Cleaning Ovens (Stove) (1974)

Range (1972)

Range (1972) ANSI ZII.207

Ranges and Bullet Stops (1973)

Ranges and Limits for Steels (1972)

Ranges (Stove and Ovens) (1972) ANSI C71.I

Std. Meth. of Test Fo

ASTM

D3132

Ranges (Stove) with Glass / Ceramic Cooking Tops (1974)

Ranges (Stoves) and Unit Broilers (1973)

Ranges (Stoves) (1974) ANSl C33.53

Ranges (1971)

Ranges (1972)

Ranges (1973)

Ranges) (1973)

Rapid Control Test (1969)

Rapid Control Test (1969)

(Rapid Curing Type) (1972)

Rapid Gas Chromatographic Estimation of Higher Boiling

Rapid Meth. of Analysis for Fat Acidity in Corn (Cereal

Rapid Meth. of Analysis for Fat Acidity in Grain (Cerea

Rapid Pressure Determination of Pressurized Products (1 Rapid (Magnesium Acetate) Meth. of Analysis for Ash in Rapid (2 Hr., 600 Deg.) Meth. of Analysis for Ash in Fe

Ratchet and Pawl, and Load Brake Designs (1971)

Rate Dependent Flow Properties (1969)

Rate of Aerosol Products (1972)

Rate of Aerosol Products (1972)

Rate of Air Flow Through Closed Steel Door and Frame as

Rate of Burning or Extent of Burning of Cellular Plasti

Rate of Extension lnstruments) (1972) ANS1 L 14.287

Rate of Extension Machines) (1972) ANSI L14.288

Rate of Membrane Filters (1972)

Rate of Sheet Materials (Paper) at High Temperature and

Rate of Traverse Tensile Test. Machine) (1971)

Rate Test. of Containment Structures for Nuclear Reacto

Rate Using a Thermal Capacitance (Slug) Calorimeter (19

Rate (1972)

Rated at 300 Volts or Less (1974)

Std Ueth for Design

Rated at 600 Volts or Less) (1972) ANSI C 33.54

Rated for Out of Phase Switching (1973) ANSI C37.079

Rated Loads Offshore Oil Cranes (1972)

Rated on a Symmetrical and a Total Current Basis (1972)

Rated on a Symmetrical Basis When Rated for Out of Phas

llngs for Capacita ANSI

Rated on a Symmetrical Basis (1972)

Rated on a Symmetrical Basis (1972) ove) (1972)

Rated on a Symmetrical Basis ( $362 \mathrm{kV}$ and A DoN I C 37.07

Rated on a Symmetrical Current Basis (1971) ANSl C37.07

Rated on a Symmetrical Current Basis (1972) ANSI C 37.07

Rated on a Symmetrical Current Basis (1972) ANSI C 37.07

Rated on a Symmetrical Current Basis (1972) ANSI C37.07

Rated on a Symmetrical Current Basis (1973) ANSI C37.07

Rated $0.35,000$ Volts and Having Ozone Resistant Ethylen

Rated 2000 Volts and Less and Having Ozone Resistant $E_{t}$

Rates for Data Transmission (1969)

Rates of Lubricants in Vacuum (1971) ANSI Z11.302

Rates of Thermoplastics by Extrusion Plastometer (1973)

Rating and Application of Suction Line Filters and Filt

Rating and Reporting of the Noise Levels of Powered lnd

Rating and Struck Capacity for Hoe Dipper (Backhoe Buck

Rating and Struck Capacity for Shovel Dipper, Clam Buck

Rating and Test. of Fire Extinguishers (1973) ANSI Z21 I

Rating Code Diesel (1971)

Rating Definitions, Test. Meth., and Test Equipment and

Rating Heat Transmission of Refrigrated Vehicles (1972)

Rating Heating, Refrigerating and Air Conditioning Equi

Rating Liquid Coolers (1971)

Rating Meth. of Carpet (Rug) Soiling (1973) ANSI L 14.21

Nal Vehicle Air Conditioning Syst

Rating Nonresidential Warm Air Heaters (I972)

Rating of Equipment for Drying Farm Crops (197I)

Rating of Inboard Gasoline Engines (Boats) (1972)

Rating of Room Fan Coil Air Conditioners Delivering Les

Rating of Spark Plugs (1970)

Rec. Pract. for Preignition

Rec. Pract. for
Pressure

Rec. Pract for Vendor

Rating of Trailer Floors for Lift Truck Loading (1971)

Rating Std. for Y Type Strainers (1973)

Rating System for Quality Control (1969)

Rating Water Cooled Refrigerant Condensers (1971)
Ran AACCH 60.30

ne Vo RTCA DO-153

AS AACCH $58.12 \mathrm{~A}$

Do.15

RA $* 5$

NRA *4

AHAM ER-3

SAE J941C

ASTM D2265

Std SAE

AHAY ER.

Aisl Z21.3

NRA $\quad * 12$

Safety R NRA

ICBO

$* 3$

lFC $* 2 \mathrm{C}$

896

AATCC 68

ASTM

ASTM D 3303

AACCH 02.03

AACCH 02.02

ASTM D3070

AACCH $08-02$

AACCH 08.03

HVI

Std. T ASTM D2556

AST.Y D3069

ASTM D3094

STDl 116

ASTM D1692

/ B ASTM D2653

1. of ASTM D273.

ASTM F317

TAPPI T464

Ngth O ASTM D2262

ANSl N45.4

ASTM E457

std. F UL

UL

IEEE

API

ANS1

ANSI

IEEE

IEEE

IEEE

IEEE

IEEE

IEEE

IEEE

NEMA

NEMA

ANSI

ASTM

ASTM

A Rl

ITA

SAE

SAE

UL

SAE

TTMA RP38

ASHRA 36

ASHRA 24

AATCC 121

MACA 400

ASHRA 45

ASAE S248.2

$A B Y C$

ARI

$\mathrm{SAE}$

TTMA

443

FC

ASQC 73.1

ASHRA 22

122

417 C 37.11

C37.0732

339

327

328
340

341

343
342

$\mathrm{WC} 8$

C $8 / 1$

3.1

D1238

$6 \mathrm{GI}$

296

67

70

8

6

J549A
NRA *18

IL 858 
Std. Rec. Pract. for ng the Compression Ratio (CR) Technique for Research Meth. td. for Safety for Aut omatic lce Makers (600 Volts or Less b3.15 Std. Load
Std. Load Std. Pract. for Stationary Diesel and Gas Engine oltagel Schedules of Preferred Transient Recovery Voltage rs (Fractional and Integral Horsepower) (1972) Std. for oltage Circuit Breakers R/ Std. for Schedule of Preferred Std. for Power Out put Std. for Certified Tent. Meth. of Test for Research and Motor Meth. Octane using the Compression Ratio (CR) Technique for Motor Meth. Test Meth. for Adhesion to Porous Substrates (Subjective rements for Low Voltage Power Circuit/ Std. for Preferred
High Voltage Air Switch/ ry Replacement Data: Group Numbers, Dimensional Spec., and of Test for Young's Modulus, Shear Modulus, and Poisson's Std. Meth. of Test for Plastic Strain knock Characteristics of Motor Fuels Using the Compression knock Characteristics of Motor Fuels Using the Compression hic Image Area on $5 \mathrm{~mm}$ Motion Picture Film (2.35:1) Aspect Rec. for High Expansion Foam Systems ter (1971) Std. Meth. of Test for Color of Seing Behavior of Cotton Std. Spec. for ement Concrete (1973) ANSI A3/ Std. Spec. for Fly Ash and Std. Meth. for Sampling and Sample Preparation of Solid Std. for Motion Picture
Std. Spec. for Std. Spec. for Std. Meth. of Test for Wool Content of Std. Meth. of Test for Wpec for Gamma. g Applia/ Std. for Safety for Implosion Protected Cathode
ed Dose of Neutrons, and of Mixtures of Neutrons and Gamma try) (1/ Meth of Analysis for Neutralizing Value of Acid h. of Test for Gel Time and Peak Exothermic Temperature of of Test for Compatibility of Materials with Liquid Oxygen rials (1972) ANSI Z11.318 Std. Mtd. Meth. of Test for Meth. of Analysis of Oxidizing Agents in Flour: essive Expansion of Concrete Due to the Alkali-Aggregate on Protection) (1973)

37.133

gh Shear (1972) ANSI Z1/ Manual of Hazardous Chemical Std. Meth. of Test for Potential Std. Meth. of Test for Explosive Std. Meth. for Chemical Analysis of Std. Protective Coatings (Paints) for Light Water Nuclear nt of Delayed Neutron Emitting Fission Products in Nuclear g Fission Products in Nuclear Reactor Coolant Water During Std. for Program for Biological Shielding in Nuclear

ty Criteria for the Design of Stationary Pressurized W/ater Std. Rec. Pract. for Surveillance Tests for Nuclear ec. Guide for in Service Annealing of Water Cooled Nuclear $r$ Leakage Rate Test. of Containment Structures for Nuclear rade. Sinterable Uranium Dioxide Powder for Use in Nuclear

972) rs (1972) Std. for Electrical Transformers, Regulators and Performance Spec. for Direct and Indirect Std. for Direct

Temperature Corrections for Refractometer Temperature Corrections for Saccharimeter Uniform Building Code Std. for th. of Analysis of Macroscopic Examination of Baked Goods, h. of Analysis for Insect and Rodent Filth in Baked Goods, of Analysis for Materials Hard to Hydrate in Baked Goods, coolant Concentrate (Glycol or Alcohol Base) by the lodine Std. Spec. for nalytical Meth. for Determining lodine Solution Content of ical Meth. for Determining Starch Indicator, 1\% Content of 1 Meth. for Determining Sulfuric Acid Solutions Content of eth. for Determining Sodium Hydroxide Solutions Content of eth. for Determining Cresol Red lndicator, $0.1 \%$ Content of th. for Determining Sodium Thiosulfate Solution Content of h. for Determining Methylene Blue Indicator, $1 \%$ Content of for Determining Std. Arsenious Oxide Solution Content of for Determining Methyl Orange Indicator, $0.1 \%$ Content of for Determining Phenolphthalein Indicator, $1 \%$ Content of for Determining Bromophenol Blue Indicator, 1\% Content of for Determining Potassium Permanganate Solution Content of

I Chemistry) Alcohol; Carbon Tetrachloride; Chloral Hydr/ meth. of Test for Resistance of Adhesive Bonds to Chemical of Metals (1973)

Rec. Pract. Test Meth. and Requirements for Snowmobile act. Test Procedure for Evaluation of the Effectiveness of Rec. Pract. for Motorcy cle
Rating Water Emulsion Floor Polishes (1972) Rating (1971) ANSl Z11.305

Rating) (1974)

Ratings and Fatigue Life for Ball Bearings (1972) ANSI

Ratings and Fatigue Life for Roller Bearings (1972)

Ratings and Performance (1972)

Ratings and Related Required Capabilities for AC High V

Ratings for Alternating Current and Direct Current Moto

Ratings for Capacitance Current Switching for AC High V

Ratings of Packaged Audio Equipment for Home Use (1967)

Ratings Program Air Moving Devices (Performance) (1970)

Ratings Using on Line Analyzers (1970T)

Ratings (1971) ANSI Z11.306

Ratings) (Paper, Board, Cloth, etc.) (1973)

/Teristics of Motor Fuels

Ratings, Application Recommendations, and Related Requi

Ratings, Manufacturing Spec., and Application Guide for

Ratings; Assembly Numbers, Cell Layouts and Terminals (

Ratio for Glass and Glass Ceramics by Resonance (1971)

Ratio R for Sheet Metal (1974)

Ratio (CR) Technique for Molor Meth. Ratings (1971) Ans

Ratio (CR) Technique for Research Meth. Rating (1971) a

Ratio (1971)

Std. Dimensions of Projectable Anamorp

Ratios-At Various Altitudes and Temperatures (1966)

Ratios from 100:1 to 1000:1) (Fire Extinguishment) (197

Raw Cotton Using the Nickerson-Hunter Cotton Colorime

Raw Fiber, Yarn and Textile Fabric) (1972) AN

Raw Linseed Oil (1972) ANSI K34.1

Raw or Calcined Natural Pozzolans for Use in Portland C

Raw Rubbers (1973)

Raw Stock Identification and Labeling (1973)

Raw Tung Oil (1973)

Raw Wool-Commercial Scale (1972)

Raw Wool-Laboratory Scale (1972) ANSI L14.490

Ray Brach ytherapy Sources (1974)

Ray Tubes (Picture and Display) for Tele vision Receivin

Rays (1961)

Rec, for Measurement of Absorb

Reacting Materials Other Than Phosphates (Cereal Chem is

Reacting Thermosetting Resins (Plastic Composition) (19

Reaction Intensity Meth.) (1973)

Reaction of Aviation Fuels (1972) ANSI Z11.82

Std. Meth.

Reaction Threshold Temperature of Liquid and Solid Mate

Reaction with Potassium Iodide (Cereal Chemistry) (1962

Reaction (1969) ANSI A37.119

/ $X$ tures in Preventin

Reactions (Incandescence, Fire, Explosion, and Detonati

Reactivity of Aggregates (Chemical Meth.) (1971) ANSl a

Reactivity of Lubricants with Aerospace Alloys Under Hi

Reactor and Commercial Columbium (1968) ANSI Z258. I

Reactor Containment Facilities (1972)

Reactor Coolant Water During Reactor Operation (1970) a

Reactor Operation (1970) ANSI N163

Reactor Plants (1972)

Reactor Plants (1973)

Reactor Vessels (1973)

Reactor Vessels (1974)

Reactors (1972)

Reactors (1973)

Reactors (1974)

Reading Pocket Dosimeters for X-And Gamma Radiation (1
Reading Remote Registration Systems for Cold Water Mete

Readings (Sugar) (Cereal Chemistry) (1962)

Readings (Sugar) (Cereal Chemistry) (1962)

Ready Mixed Concrete (1973)

Ready-To.Eat Cereals, and Alimentary Pastes for Detecti

Ready.To.Eat Cereals, and Alimentary Pastes (Cereal Che

Ready-To-Eat Cereals, and Alimentary Pastes (Cereal Che

Reagent Meth. (1973)

Reagent Water (1972)

Reagents and Indicators (1971)

Reagents and Indicators (1971)

Reagents and Indicators (1971)

Reagents and Indicators (1971)

Reagents and Indicators (1971)

Reagents and Indicators (1971)

Reagents and Indicators (1971)

Reagents and Indicators (1971)

Reagents and Indicators (1971)

Reagents and Indicators (1971)

Reagents and Indicators (1971)

Reagents and Indicators (1971)

lor Water in Engine Antifreeze-

Reagents for Extraneous Matter

Reagents (1966) ANSI Z197.7

Reagents, and Safety Precautions for Chemical Analysis

Rear Position Tail Lamp (Light) (1972)

Rear Underride Guard (Safety (1971)

Rear View Mirrors (1971)

Std. Analytica CR

Sid. Analytical M CR

Std. Analytical M CR

Std. Analytical Me CR

Std. Analytical Met CR

Std. Analytical Meth CR

Std. Analytical Meth CR

Sid. Analytical Meth CR

ASTM

ASTM

UL

AFBMA

AFBM

DEMA

ANSI

NEMA

ANSI

EIA

AMCA

ASTM

A STM

TAPPI

ANSI

ANSI

$\mathrm{BCl}$

ASTM

ASTM

ASTM

ASTM

ANSI

AMCA

FMS

ASTM

AATC

ASTM

ASTM

ASTM

ANSI

ASTM

ASTM

ASTM

UL

NCRPM

ASTM

ASTM

ASTM

ASTM

$\mathrm{AACCH}$

D3052

D2722

563

9

$* 1.4$

C39.0722

MG 1.10

C 37.0732

211

D2885

D2723

UM. 540

C37.16

C 37.32

*1.56

C623

E517

D2723

D2722

PH22. 106

402

$4.3 \mathrm{~N}$

D 1464

64

D234

C618

PH22.184

D12

D 1334

D584

$\mathrm{R} 41$

492.8

02.32

D2471

F371

D1094

D2883

FMS $\quad$ C441

ASTM C 289

ASTM D3115

ASTM E195

ANSI N101.2

ASTM D2470

ASTM D2470

ANSI N18.9

ANSI N18.2

ASTM E185

Std. R ASTM E509

E509

C753

NEMA TRI

$\begin{array}{ll}\text { NEMA } & \text { TRI } \\ \text { ANSI } & \text { N } 13.5\end{array}$

AWWA C706

AACCH $80-20$

AACCH 80-2]

ICBO UBCS26-13

AACCH $28-30$

AACCH 28-31

AACCH 28.32

ASTM D1123

ASTM D1193

Std. a CR

Std. Analyt CR 
Rec. Pract. for Engine Foot Mounting (Front and ids to Security (Safety) of Personnel on Boat Deck and for ng (1969) ANSI Z167.21 ing Locations (Bonding Appliances, Casters, Face Masks and Uniform Fire Code: Automobile Tire

Cold Weather Masonry Construction (Protection manual: Grade Selection, Std. Size, Design Values, Framing Component Std. for

Minimum Stds. for Land Mobile Communication FM or Pm X.Ray Protection Standards for Home Television r-Motor Carrier Dock Planning Manual (Truck Shipping and rec. Pract. for Quality Control Monitoring of the Product std. for Safety for High Voltage Components for Television std. for Safety for Special Fuses for Radio and Television ted Cathode Ray Tubes (Picture and Display) for Television y Range of 108/ Minimum Performance Std. for Airborne Vor wer Plants (During the Con/ Std. for Packaging. Shipping, ations (1973) ANSI C 33.97 Safety Std. for Std. for

or Hazardous Locations (1973) Std. for Electrical Plugs, s, 4 Wire (1966) Standard Mounting and Face Dimensions of Std. on Dimensions of Attachment Plugs and Std. for Safety for Electrical Attachment Plugs and 277 Volts Ac, 15 Amperes, 2/ Std. Dimensions of Plugs and , I Wire (1973)

e, 2 Wire (1973)

Volts, 15 Amperes, 2 Pole, / 1250 Volts, 15 A mperes, $3 \mathrm{P} /$ 3 Phase Wye, 4 Pole, 4 Wi/ 3 Phase Wye, 4 Pole, 5 Wi/ 3 Phase Wye, 4 Pole, $4 \mathrm{Wi} /$ , 3 Phase Wye, 4 Pole, $5 \mathrm{Wi}$ / pole, 2 Wire (1973)

pole, 3 Wire Locking, Groun/ pole, 2 Wire (1973)

pole, 3 Wire Locking, Groun/ 3 Pole, 4 Wire Locking, G/ , 3 Pole, 3 Wire Locking Ty/ 3 Pole, 4 Wire Locking, G/ 3 Pole, 3 W ire, Locking T/ pole, 3 Wire Locking, Groun/ phase, 3 Pole, 3 Wire Locki/ hase, 3 Pole, 4 Wire Lockin/ pole, 2 Wire (1973)

pole, 3 Wire Locking, Groun/ phase, 3 Pole, 3 Wire Locki/ pole, 3 Wire Locking, Groun/ phase, 3 Pole, 3 Wire Locki/ phase, 3 Pole, 4 Wire Locki/ 2 Pole, 3 Wire Locking, Gr 2 Pole, 3 Wire Locking, Gr/

3 Phase Wye, 4 Pole, 4 Wi/ 3 Phase Wye, 4 Pole, 5 Wi/ 3 Phase Wye, 4 Pole, 5 Wi/ 3 Phase Wye, 4 Pole, 4 Wi/ 3 Phase Wye, 4 Pole, 5 Wi/ 3 Phase Wye, 4 Pole, 4 Wi/ , 3 Phase Wye, 4 Pole, 5 Wi/ pole, 3 Wire Locking, Groun/ phase, 3 Pole, 3 Wire Locki/ phase, 3 Pole, 4 Wire Locki/ pole, 3 Wire Locking, Groun/ phase, 3 Pole, 3 Wire Locki/ phase, 3 Pole, 4 Wire Locki/ pole, 3 Wire Locking, Groun/ pole, 3 Wire Locking, Groun/ phase, 3 Pole, 3 Wire Locki/ phase, 3 Pole, 4 Wire Locki/

, 3 Phase Wye, 4 Pole, 4 Wi/ Slant Drop Penetration Test for Paperboard (Printing Ink owers Lavatories, Bathtubs and Combination Tank and Shower Recommendation for Installation of Ceramic Tile Shower nation Tank and Shower Recep/ Std. for Nonmetallic Shower Std. Part Bolt, 100 Deg. Flush Tension Head Hi Torque Std. for Aircraft, Pan Head, Phillips Std. Part Bit, Screwdriver, Tri Win Std. Spec. for Ceramic Tile Panels for Bath Tub Std. for Screw, Hex Head, Tri Wing ocking (1973) Std. for Screw, 100 Deg. Head, Tri Wing or Bolt, Pan Head, Close Tolerance, Short Thread, TWI Wing achine Screw, Flat Fillister Head, Full Threaded, Tri Wing std. for Pan Head, Close Tolerance, Short Thread, TWI Wing Std. for Screw, Hex Head, Tri Wing
Rear) (1970)

Reboarding from the Water (1973)

Reboiling Tendency of Sheet Steel for Porcelain Enameli

Rebreathing Bags, Footwear, Hose and Tubing, Mattresses Rebuilding Plants (1973)

Rec.) (1968)

Rec,, Properties, Weight. Stress of Board, Timber, Lumb

Receiver Dehydrators for Vehicle Air Conditioners (1967 Receivers $25.470 \mathrm{MHz}$ (1972)

Receivers (1968)

Receiving-Materials Handling) (1973)

Receiving and Source Inspection, Product Audit) (1969)

Receiving Appliances and Other Electronic Equipment (FI

Receiving Appliances and Other Electronic Equipment (19

Receiving Appliances and Other Electronic Equipment (19

Receiving Equipment Operating Within the Radio Frequenc

Receiving. Storage and Handling of ltems for Nuclear $\mathrm{Po}_{0}$

Receptacle-Plug Combinations for Use in Hazardous Loc

Receptacle Dummy Storage, Electrical Connector (1972)

Receptacles and Connectors of the Pin and Sleeve Type F

Receptacles Off Center Boss Type, 30, 50, and 60 Ampere

Receptacles (1973)

Receptacles (1974)

Receptacles, Locking Grounding Type, Specific Purpose,

Receptacles, Locking Type 125 Volts, 15 Amperes, 2 Pole

Receptacles, Midget Locking Type, Specific Purpose, 125

Receptacles, Midget Locking Type, Specific Purpose, 125

Receptacles: Specific Purpose 120/208 Volts, 20 Amperes

Receptacles: Specific Purpose 120/208 Volts, 20 Amperes

Receptacles: Specific Purpose 120/208 Volts, 30 Amperes

Receptacles: Specific Purpose 120/208 Volts, 30 Amperes

Receptacles: Specific Purpose 125 Volts, 20 Amperes, 2

Receptacles: Specific Purpose 125 Volts, 20 Amperes, 2

Receptacles: Specific Purpose 125 Volts, 30 Amperes, 2

Receptacles: Specific Purpose 125 Volts, 30 Amperes, 2

Receptacles: Specific Purpose 125/250 Volts, 20 Amperes

Receptacles: Specific Purpose 125/250 Volts, 20 Amperes

Receptacles: Specific Purpose 125/250 Volts, 30 Amperes

Receptacles: Specific Purpose 125/250 Volts, 30 Amperes

Receptacles: Specific Purpose 250 Volts, 15 Amperes, 2

Receptacles: Specific Purpose 250 Volts, 15 Amperes, 3

Receptacles: Specific Purpose 250 Volts, 20 Amperes 3 P

Receptacles: Specific Purpose 250 Volts, 20 Amperes, 2

Receptacles: Specific Purpose 250 Volts, 20 Amperes, 2

Receptacles: Specific Purpose 250 Volts, 20 Amperes. 3

Receptacles: Specific Purpose 250 Volts, 30 Amperes, 2

Receptacles: Specific Purpose 250 Volts, 30 Amperes, 3

Receptacles: Specific Purpose 250 Volts, 30 Amperes, 3

Receptacles: Specific Purpose 277 Volts Ac, 20 Amperes,

Receptacles: Specific Purpose 277 Volts Ac. 30 Amperes,

Receptacles: Specific Purpose 277/480 Volts, 20 Amperes

Receptacles: Specific Purpose 277/480 Volts, 20 Amperes

Receptacles: Specific Purpose 277/480 Volts, 30 Amperes

Receptacles: Specific Purpose 347/600 Volts, 20 Amperes

Receptacles: Specific Purpose 347/600 Volts, 20 Amperes

Receptacles: Specific Purpose 347/600 Volts, 30 Amperes

Receptacles: Specific Purpose 347/600 Volts, 30 Amperes Receptacles: Specific Purpose 480 Volts, 20 Amperes, 2 Receptacles: Specific Purpose 480 Volts, 20 Amperes, 3 Receptacles: Specific Purpose 480 Volts, 20 Amperes, 3 Receptacles: Specific Purpose 480 Volts, 30 Amperes, 2 Receptacles: Specific Purpose 480 Volts, 30 Amperes, 3 Receptacles: Specific Purpose 480 Volts, 30 Amperes, 3 Receptacles: Specific Purpose 600 Volts, 20 Amperes, Receptacles: Specific Purpose 600 Volts, 30 Amperes, 2 Receptacles: Specific Purpose 600 Volts, 30 Amperes, 3

Receptacles: Specific Purpose 600 Volts, 30 Amperes, 3

Receptacles? Specific Purpose 277/480 Volts, 30 Amperes

Receptivity) (1973)

Receptor Units (1972)

Receptors (1973)

Receptors, Stall Showers Lavatories, Bathtubs and Combi

Recess Alloy Steel, 160,000 psi (1973)

Recess Full Threaded, Alloy Steel Machine Screw (1974)

Recess (1972)

Recess (1972)

Recess, Alloy Steel, Full Thread, Nonlocking (1973)

Recess, Alloy Steel, Full Thread, Self.Locking and Nonl

Recess, Alloy Steel, Self Locking and Nonlocking (1972)

Recess, Alloy Steel, Self-Locking and Nonlocking (1973)

Recess, A286 Acres, Self Locking and Nonlocking (1972)

Recess, A286 Cres, Full Thread Nonlocking (1972)

Recess, A286 Cres, Full Thread Self Locking and Nonlock

Recess, A286 Cres, Full Thread, Self Locking and Nonloc Recess, A286 Cres, Full Thread, Self Locking and Nonloc

SAE

ABYC

ASTM

UL

ICBO

BIA

WWPA

IMAC

EIA

NCRPM

ANSI MH8.

ASQC $\quad$ RP5

UL

UL

RTCA

ANS I

UL

NSA

NEMA

ANSl

ANSI

UL

ANSI

ANSI

ANSI

ANSI

ANS1

ANSI

ANSl

ANSI

ANSI

ANSI

ANSI

ANSl

ANSI

ANSI

ANSI

ANSI

ANSI

ANSI

ANSI

ANSI

ANSI

ANSI

ANSI

ANSI

ANSI

ANSI

ANSI

ANS

ANSl

ANSI

ANSI

ANSI

ANS1

ANSI

ANSI

ANSl

ANSI

ANSI

ANS1

ANSI

ANSI

ANSI

ANSI

ANSI

TAPPI

492.7

492.8

D0.153

N45.2.2

1196

C73.67

C73

498

C 73.43

C73.31

C73.32

C7 73.29

C 73.30

C 73.104

C73.90

C73.105

C73.91

C73.69

C73.72

C 73.70

C73.73

C73.83

C73.96

C73.84

C73.97

C73.74

C73.98

C 73.85

C73.7I

C 73.75

C73.99

C 73.76

C73. 100

C73.86

C73.77

C73.78

C73.106

C73.92

C73.93

C73.108

C73.94

C73.109

C73.95

C73.79

C73.10I

C73.87

C 73.80

C.73.102 
Machine Screw, Flat Fillister Head, Full Thread, Tri Wing Std, for Bolt, 100 Deg. Head, Tri Wing Std, for Bolt, 100 Deg. Head, Tri Wing Locking and Nonl/ Std. for Bolt, $100 \mathrm{Deg}$. Head, Tri Wing
locking $($ Std. for Bolt, 100 Deg. Reduced Head, Tri Wing f-Locking and Non/ Std. for Bolt, 100 Deg. Head, Tri Wing cking (19/ Std. for Bolt, 100 Deg. Reduced Head, Tri Wing locking and Nonlo/ Std. for Bolt, 100 Deg. Head, Tri Wing hread, Self Locking and Nonlocki/ Bolt, 100 Deg. Tri Wing crew (1974) Std. for Aircraft, Pan Head, Phillips or Bolt, Pan Head, Close Tolerance, Short Thread, Tri Wing Machine Screw, Flat Fillister Head, Full Thread, Tri Wing (1972) $\mathrm{g}$ and Nonlocking / Std. for Screw, Hex Head, Tri Wing 72 Std. for Screw, 100 Deg. Head Tri Wing Std. Slotted and Std. for Hex Head Screw, Rec. Pract, for Spark Plug. Aircraft Rec. Pract. for Make Up and

tems (1974) Solid Wall and Perforated Pipe for General Drainage (Land plication, Operation, and Maintenance of Automatic Circuit Capacitor Switches for Alter/S Std. for Automatic Circuit other Hard Materials by Water Blasting Prior to Coating or

Walls (1973) Std. for Print Spec. for Magnetic Ink Character

tops (1973)

rator Rooms (1973)

receptors (1973)

ooms (1973)

g Pools (1973)

973)

eramic Tile Floors (1973)

ile Floors (1973)

contraction, and Isolation Joints for Ceramic Tile (1973/

e Floors (1973)

e Walls (1973)

e Floors (1973)

e Walls (1973)

for Ceramic Tile Flooring (1973)

ile Floors (1973)

eramic Tile Floors (1973)

(1973)

ith Mortar, Adhesive, and Mastic (1973)

walls, Floors, and Stairs (1973)

Concrete Pavement Overlay Projects (1973)

ar Products for High Temperature Service Conforming to Iso Cold Forging Equipment (1972)

ge Power Circuit/ Std. for Preferred Ratings, Application l (1971)

Std. for

ngs (1970)

Specifying Project

Rec. Pract. Spec, of Tracking Control Wiring Boards)/ Std. Spec. for

and Operating Level of Recorders and Reproducers for Audio Employment

Pract. for Std. Calibration and Format for Nuclear Logs ng Control Records on 2 In. Video Magnetic Tape Quadruplex engths (1972) ANS1 S4.6 Std. Meth. of Measuring $0 \mathrm{cpi}, \mathrm{PE})(1973)$

cpi, NRZI) (1973)

cpi, NRZI) (1973)

Std. for

Std. for

for Flutter Measurement of Instrumentation Magnetic Tape timing Error Measurements of Instrumentation Magnetic Tape for Consumer Electronic Products (Radio, Television, Tape Quadrup/ Std. Frequency Response and Operating Level of ultifrequency Test Tape for Quadruplex Video Magnetic Tape ental Injury Experience of Employees (/ Sid. for Meth. of

for Measurement of Weighted Peak Flutter Content of Sound Safety Std. for Sound

tic Tape Cartridge Type Cp II (Compact Cassette) for Audio nt Rooms (Noise) (1973)

lic Coated Steel Specimens (1972) Std. for Meth. of Measuring. Std. Rec Pract for

ter; Ceiling; Partitions; Plaster; Roofing) and Earthquake

$16 \mathrm{~mm} 200 \mathrm{Ft}$., $35 \mathrm{~mm} 100 \mathrm{Ft}$. and $70 \mathrm{~mm} 100 \mathrm{Ft}$. Spools for Std. Dimensions for Photographic Films in Rolls for

re (196) Std. Meth. for Continuous A nalysis and Automatic

1) Std. for Meth. of Measuring Frequency and Cost, and s Characteristics for 2 In. Quadruplex Video Magnetic Tape lignment Signal Spec. for a Quadruplet Video Magnetic Tape ct. for the Label for 2 In. Quadruplex Video Magnetic Tape ng Control Record for 2 In. Quadruplex Video Magnetic Tape Std. Meth. of Measuring Recorded Flux of Magnetic Sound d A/ Std. Dimensions of Video, Audio and Tracking Contro Rec. Pract. for Quality Control Std. Spec. for Manifold Papers for Permanen 1973)

decoders (Type I) for Reproducing Matrix Quadraphonic Disc
Recess, A286 Cres, Self-Locking and Nonlocking (1973)

Recess, Cluse Tulerance (1972)

Recess. Clone Tolerance (1972)

Recess, Close Tolerance, Alloy Steel, Long Thread, Self

Recess, Close Tolerance, Alloy Steel, Short Thread, Non

Recess, Close Tolerance, Alloy Steel, Short Thread, Sel

Recess, Close Tolerance, A286 Cres, Short Thread, Nonlo

Recess, Close Tolerance, A286 Cres, Short Thread, Self

Recess, Close Tolerance, 6Al-4V Titanium Alloy, Short T

Recess, Short Thread, 160,000 psi Alloy Steel Machine S

Recess, Titanium Alloy $6 \mathrm{Al} \cdot 4 \mathrm{~V}$. Self Locking and Nonlock

Recess, Titanium Alloy, 6Al-4V, Self-Locking and Nonloc

Recess, 6Al-4V Titanium Alloy, Full Thread, Nonlocking

Recess, 6Al.4V Titanium Alloy, Full Thread, Self Lockin

Recessed Head Machine Screws and Machine Screw Nuts (19

Recessed, Full Thread (1973)

Reciprocating Engine (1971)

Recirculated Air for Industrial Exhaust Ventilation Sys

Reclamation, Curtain and Building Foundation Drain, Und

Reclosers (1973) ANSI C 37.61

Reclosers, Automatic Line Sectionalizers and Oil Filled

Recoating (1972

Recognition (1970)

Recommendation for Installation of Ceramic Tile Bathtub

Recommendation for Installation of Ceramic Tile Counter

Recommendation for Installation of Ceramic Tile Refrige

Recommendation for Installation of Ceramic Tile Shower

Recommendation for Installation of Ceramic Tile Steam R

Recommendation for Installation of Ceramic Tile Swimmin

Recommendation for Installation of Ceramic Tile Tubs (l

Recommendation for Installation of Chemical Resistant C

Recommendation for Installation of Conductive Ceramic T

Recommendation for Installation of Expansion, Control,

Recommendation for Installation of Exterior Ceramic Til

Recommendation for Installation of Exterior Ceramic Til

Recommendation for Installation of Interior Ceramic Til

Recommendation for Installation of Interior Ceramic Til

Recommendation for Installation of Thresholds, Saddles

Recommendation for Installation of Waterproof Ceramic T

Recommendation for Installation Performance Levels of $\mathrm{C}$

Recommendation for Materials for Grouting Ceramic Tile

Recommendation for Materials for Setting Ceramic Tile W

Recommendation for Renovation of Interior Ceramic Tile

Recommendations for Basis of Payment on Portland Cement

Recommendations for Boiler Construction (1972)

Recommendations for Die Cavity and Cutoff Die Sizes for

Recommendations, and Related Requirements for Low Volta

Record and Reporting Units for Nuclear Materials Contro

Record Documents (1967)

Record for 2 In. Quadruplex Video Magnetic Tape Recordi

Record Formats for Transmission of End Product Descript

Record on Super 8 Motion Picture Prints (1972)

Record One for 2-In. Quadruplex Video Magnetic Tape Ope

Record (1973)

Record, Documentation) (1974)

Recorded at 15 and 7.5 In./S (1973)

Recorded Flux of Magnetic Sound Records at Medium Wavel

Recorded Magnetic Tape for Information Interchange (160

Recorded Magnetic Tape for Information lnterchange (200

Recorded Magnetic Tape for Information Interchange ( 800

Recorder/Reproducers (1972) ANSI C83.99

Recorder / Reproducers (1974) ANSI C83.94

Recorder, Phonograph, etc.) (1973)

Recorders and Reproducers for Audio Record One for 2-in

1. Test Meth

Recorders Operating at 15 In./S (1973) / Io Level

Recording and Measuring the Off-The-Job Disabling Accid

Recording and Reproducing Equipment (1971)

Recording and Reproducing Equipment (1973)

Recording and Reproducing (1974)

/ Std. Coplanar Magne

Recording and Specifying Machinery Sound Within Equipme

Recording Data from Atmospheric Corrosion Tests of Meta

Recording Instrumentation (1973) /Ra Cotta; Timber; Wa

Recording Instruments and for Microfilm and Still Pictu

Recording Instruments and Miscellaneous Uses (1973)

Recording of the Sulfur Dioxide Content of the Atmosphe

Recording Patron and Nonemployee lnjury Statistics (197

Recording (1967)

Recording (1969)

Recordings (1968)

/Ce Carrier Frequen

Rec. Pract. for Video a
Rencies and Deemphasi

Recordings (1970)

Rec. Pract. Spec. of Tracki

Records at Medium Wavelengths (1972) ANSI S4.6

Records on 2 In. Video Magnetic Tape Quadruplex Recorde

Records System (1969)

Records (1973)

Records (1974)

Records, Silver Gelatin Type, on Cellulose Ester Base (
NSA

NSA

NSA

NSA

$\mathrm{NSA}$

NSA

NSA

NSA

NSA

NSA

NSA

NSA

NSA

NSA

ANSI

NSA

SAE

ACGI

ASTM

IEEE

NEMA

NACE

ANSI

TCA

TCA

TCA

TCA

TCA

TCA

TCA

TCA

TCA

TCA

TCA

TCA

I'CA

TCA

TCA

TCA

TCA

TCA

TCA

TCA

ACPA

ACPA

IFl

ANS

ANSI

CSI

SMPT

IPC

ANSI

ANSI

AIOA

IEEE

ANSI

ANSI

ANSI

EIA

EIA

ANSI

ANSI

IEEE

UL

EIA

ART

ICBO

ANSI

ANSI

ANSI

SMPTE

SMPTE

SMPTE

IEEE

ANSl

ASQC

ASTM

Stds. for EIA

ANSI

5400-06

204- 16

4304-16

4.104-16

703.16

4400.16

4803. 16

4500-16

$4600-16$

623

$5200 \cdot 06$

5500-06

6100.3

5800.6

B18.6.3

1096

RP590B

1.7 
Std. Spec. for Photographic Film for Archival Std. Meth. of Test for Extension Test Meth. for Wrinkle Recovery of Woven Textile Fabrics: si L14.229 Test Meth. for Wrinkle Test Meth. for Wrinkle d Medium Speed Stationary Diesel and Gas Engine Waste Hea ated on a Symmetrical $\mathrm{Cu} /$ ated on a Symmetric/

ies for AC High Voltage/ Std. Requirements for Transient Std. Application Guide for Transien Schedules of Preferred Transien truc/ Std. for Tires and Rims for Industrial, Utility and (Coupling, Hitch/ Minimum Requirements for Motor Vehicle initions, Test. Meth., and Test Equipment and R/ Std. for d Hazards at Pl/ Rec. for Factory Constructed Housing and ck Campers, Motor Homes): Installation of Plumb/ for Std. for Flexible Non Pressure Water Fill Hose for d. for Waste and Sewage Holding Tanks for Mobile Homes and nd Non Pressurized Potable Water Tanks for Mobilehomes and Std. for Electric Pressure Pumps for Use in Std. for Electric Pressure Pumps for Use in
r Liquid Fuel Burning Heat Appliances for Mobile Homes and
ion Wagon, Truck, Bus. Trailer, Multipurpose Passenger and

lding Code Std. for Round, Hexagonal, Octagonal, and Flat Std. Spec. for Seamless Copper and Its Alloy
Std. for Keys-Square and

Test Meth. for Measuring Squareness or Std. Meth. of Test. Para

72) ANSI H33.1

Std. Spec. for Copper Zinc Lead (Leaded Std. Spec. for Seamless

I Length Thermal Process Preservative Treatment of Western ment of Incised Pole Butts by the Thermal Process (Western (1971)

for Douglas Fir, Coast Region: West Coast Hemlock; Western Std. Meth. of Test. Para Red and Toluidine marack / Uniform Building Code Std. for Eastern White and Std. Spec. for EC Aluminum Std. for Bolt, Flat, 100 Deg. teel, Short Thread, Nonlocking (/ es, Short Thread, Nonlocking (19/ Std. for Bolt, $100 \mathrm{Deg}$. Std. for Bolt, $100 \mathrm{Deg}$ Std. Pract. for Socket Welding Sid. for Tee, Std. for Tee, Std. for Elbow, Std. for Elbow

for Spur, Helical and Herringbone Gear Shaft Mounted Speed Meth. for
Meth. of Analysis of
of Analysis for Total Meth. of Analysis for Total Std. Analytical Meth. for Determining Std. Analytical Meth. for Determining Schoorl Meth. of Analysis of Rec. Pract. for try) $(1962$ Carbon and Alloy Steel Forgings for Pinions and Gears for
able (Cereal Chemistry) (1962) Volumetric Copper
Std. for Location of Super 8 Printed Area in Optical ilm/ Std. for Location of Super 8 Printed Area in Optical ate/ Std. for Location of Super 8 Printed Area in Optical ir, Southern and Lodgepole Pine, Western Larch, California ng) (1973)

s and Other Technical Data) (1973)

ation and Design, Span Allowances, Storage, Finishes And r Cooling Towers (1971)

Uniform Building Code Std. for Californi el Characteristics, Material Composition, Use, Applicati/ si C 83.55

oducer Test Tape: Full Track, 1/4 In. (6.3 Mm) Width, Open Std. for Automated Machine Lay Up Composite Filament Tape Std. for Packaging of Filament Tape Std. for Safety for Cord Std. Spec. for Mercury Lamp Std. Spec. for Fluorescent Lamp Aluminum Underground Distribution stics for 2 In. Quadruplex Video Magneti/ Rec. Pract. for ion Characteristics by Polar/ ansion Characteristics by $\mathrm{Po}$

ynamic Anodic Polarization Mea/

Std. Rec. Pract. for Making Std. Rec. Pract. for Making Std. Rec. Pract. for Std.

) Steel Castings (1972) ANSI Z166.19

Nickel-Copper Alloy Castings (1967) ANSI Z166.17

gnesium Castings, Series II (1974) ANSI Z166.8

Thickness (1972) ANSI Z166.28

si Cl00.3

Rec. Pract. for Automotive Engine Carburetor Airflow Spec., Std., and Test Meth.

Std. for Rec. Antidotes, Supplies (Packaging), and th. for Determining Apparent Starch Content of Corn Syrup.
Records, Silver Gelatin Type, on Polyester Base (1973)

Recovery and Adhesion of Latex Sealing Compound (1972) Recovery Angle Meth. (1972) ANSI L14.110. ASTM D1295 Recovery of Textile Fabrics: Appearance Meth. (1970) an Recovery of Woven Textile Fabrics: Recovery Angle Meth. Recovery Systems (1972)
Recovery Voltage for AC High Voltage Circuit Breakers R Recovery Voltage for AC High Voltage Circuit Breakers R Recovery Voltage Ratings and Related Required Capabilit Recreational Type Vehicles, Counter Balanced Fork Lift (Recreational Type) Connecting Devices and Towing Meth. Recreational Vehicle Air Conditioning Equipment (1974) Recreational Vehicle Air Conditioning System Rating Def Recreational Vehicle Cooking Gas Appliances (1972)

Recreational Vehicle Manufacturing Plants (Fire and Win Recreational Vehicles (Travel and Camping Trailers, Tru Recreational Vehicles (1971)

Recreational Vehicles (1971)

Recreational Vehicles (1971)

Recreational Vehicles (1972)

Recreational Vehicles (1973)

Rectangular and Square) Brass Wire (1973)

Rectangular Waveguide Tube (1974) ANSI H37.1

Rectangular (1973)

Rectangularity of a Sheet of Paper or Paperboard (1973)

Red and Toluidine Red Pigments (Toners) (1973)

Red Brass or Hardware Bronze) Rod, Bars, and Shapes (19

Red Brass Pipe, Std. Sizes (1972)

Red Cedar and Alaska Yellow Cedar Timber Poles (1973)

Red Cedar, Northern White Cedar and Alaska Yellow Cedar

Red Cedar; White Fir; and Sitka Spruce (Lumber) (1973)

Red Indicator, $0.1 \%$ Content of Reagents and Indicators

Red Pigments (Toners) (1973)

Red Pine, Eastern Spruce and Hemlock, Balsam Fir and Ta

Redraw Rod for Electrical Purposes (1974)

Reduced Head, Torq Set and Hi Torque, 180 KSI (1973)

Reduced Head, Tri Wing Recess, Close Tolerance, Alloy S

Reduced Head, Tri Wing Recess, Close Tolerance, A286 Cr

Reducer Inserts (1974)

Reducer, Flareless Tube to Swivel. Swivel on Run (1973)

Reducer, Flareless Tube to Swivel, Swivel on Side (1973

Reducer, 45 Deg. Flareless Tube to Swivel (1973)

Reducer, 90 Deg. Flareless Tube to Swivel (1973)

Reducers (1974)

Reducible Sulfur in Paper and Paperboard (1972)

Reducing and Nonreducing Sugars in Flour and Semolina

Reducing Substances (Cereal Chemistry) (1962)

Reducing Sugars Content in Dextrin (1970)

Reducing Sugars Content in Steepwater (Corn) (1964)

Reducing Sugars Content of Corn Sugar (Crude and Refine

Reducing Sugars in Prepared Bakery Mixes (Cereal Chemis

Reducing the Fire Hazard of Static Electricity (1973)

Reduction Gears (1972) ANSI G55.8

Reduction Meth. for Sugar Determination: Schoorl's Redu

Reduction or Contact Printing on $35 \mathrm{~mm}$ Motion Picture F

Reduction Printing on $35 \mathrm{~mm}$ Motion Picture Film, Perfor

Reduction Table (Cereal Chemistry) (1962)

Redwood and Hardwood Timber (1973)

Douglas and Hem

Redwood Exterior Guide (Siding, Finish, Nails and Naili

Redwood Interior Guide (Spec. for Installation, Finishe

Redwood Landscape Guide (Rec. for Garden Grades, Applic

Redwood Lumber Spec. for Application in Industrial Wate

Redwood Lumber (1973)

Redwood Plywood Guide (Spec. for Texture and Grade, Pan

Reel Packaging of Components with Axial Leads (1972) an

Reel (For Tape Speeds of $7.5 \mathrm{In}$. $0190.5 \mathrm{Mm}$, and 3.75 I

Reel (1972)

Reels (1973)

Reels (1973) ANSI C33.18

Reference Ballasts (1971)

Reference Ballasts (1972)

Reference Book (Electrical Power Cable Specs.) (1973)

Reference Carrier Frequencies and Deemphasis Characteri

Reference Glass-Metal Butt Seals and Test. for Expans

Reference Glass-Metal Sandwich Seal and Test. for Exp

Reference Meth. for Making Potentiostatic and Potentiod

Reference Radiographs for Heavy Walled (4 1/2 to 12 In.

std. Reference Radiographs for High Strength Copper Base and

Std. Reference Radiographs for lnspection of Aluminum and $\mathrm{Ma}$

Reference Radiographs for Steel Castings Up to 2 In. in

Reference Std. Precision Electrical Resistors (1969) an

Reference Std. (1971)

Referenced by SAE (1973)

References (Dosage) for Poison Control Centers (1972)

Std. Analytical Me

ANSI

ASTM

AATCC

AATCC 128

AATCC 66

DEMA $* 1.16$

IEEE

IEEE

ANS

TRA

VESC

ARI

IMACA 400

ANSI Z21.57

FMS

NFPA

IAPMO

I IAPMO

IAPMO

IAPMO

UL

327
328

C39.0722

250

7.90
$501 \mathrm{C}$

TSC 19

TSC2

TSC4

TSC 1
$307 \mathrm{~A}$

$\mathrm{X} . \mathrm{X}]$

UBCS32.13

B372

ASTM

NSA

TAPP

ASTM

ASTM

ASTM

AWPA

AWPA

ICBO

CR

ASTM

ICBO

ASTM

NSA

NSA

NSA

MSS

NSA

NSA

NSA

NSA

558

UM-530

D970

B140

B 43

C8

U BCS25-3

R- 60

D970

UBC\$25-5

B233

1992

4703-16

4803-16

SP-79

1763

1764

1761

1762

AGMA 480.05

TAPPI T406SI

AACCH $80-60$

AACCH 10-01

CR D.52

CR J.58

CR F.48

AACCH $\quad 80-68$

FMS $5.8 \mathrm{~N}$

ASTM A291

AACCH $\quad 80-69$

ANSI $\quad$ PH22.180

ANSI PH22.179

AACCH $80-69$

CRA $3 \mathrm{~A} 4$

CRA $3 A 7$

CRA $3 A 5$

CTl 103

ICBO UBCS25-7

CRA 3 A9

EIA RS296C

RS400

NSA $\quad 392$

UL 355

ANSI $\quad$ C 82.5

ANSI $\quad$ C82.3

AA

SMPT

ASTM

ASTM

ASTM

ASTM

ASTM

ASTM

ASTM

IEEE

SAE

SAE 
Std. Spec. for Sid. Spec. for Fire

Std. Spec. for Fire nsi $\mathrm{H} 23.12$ , and lngot Bars (1973) ANSI H/

Std. Spec. for Chemically Std. Spec. for Std. Spec. for

Detection and Estimation of Water Insoluble Impurities in Std. Spec. for Std. Spec. for Std. Spec, for

ning Extraneous Materials Content of Corn Syrup, Crude and Std. Spec, for Once Std. Meth. of Test for Pyridine Bases in Crude and ions (Spectrophotometric) Content of Corn Sugar (Crude and termining Moisture (Oven) Content of Corn Sugar (Crude and meth. for Determining Ash Content of Corn Sugar (Crude and

for Determining Acidity Content of Corn Sugar (Crude and for Determining Arsenic Content of Corn Sugar (Crude and eth. for Determining Iron Content of Corn Sugar (Crude and etermining Sulfur Dioxide Content of Corn Sugar (Crude and ance (Spectrophotometric) Content of Corn Sugar (Crude and $\mathrm{g}$ Moisture (Karl Fischer) Content of Corn Sugar (Crude and termining Reducing Sugars Content of Corn Sugar (Crude and ining Dextrose Equivalent Content of Corn Sugar (Crude and rmining Specific Rotation Content of Corn Sugar (Crude and determining Total Sugars Content of Corn Sugars (Crude and cium (Egta-Titrimetric) Content of Corn Sugar (Crude and h. for Determining Copper Content of Corn Sugar (Crude and Determining Heavy Metals Content of Corn Sugar (Crude and Meth. for Determining $\mathrm{pH}$ Content of Corn Sugar (Crude and for Determining Chloride Content of Corn Sugar (Crude and ermining Protein Nitrogen Content of Corn Sugar (Crude and Std. Code for Pressure Petroleum

pec. for Seamless Intermediate Alloy Steel Still Tubes for ess Low Carbon and Carbon Molybdenum Steel Still Tubes for Seamless Austenitic Chromium Nickel Steel Still Tubes for racking of Carbon Steel (P-I) Welds in Corrosive Petroleum

for Selection of Geometric Conditions for Measurement of cimen / Std. Meth. for Microscopical Determination of the Meth. of Test for Measuring Soil Removal and

(Crude and / Std. Analytical Meth. for Determining Color yers of Silicon on Substrates of the Same Type by Infrared 72) ANSI L 14.175

(Using Spectrophotometer with Reserv/

Test Meth for Meth. for Spectral Std Rec. Pract for Evacuated erating at Temperatur/ Std. Rec. Pract. for Prefabricated a Model Performance Spec. for the Purchase of

Safety Std. for Lighting for Regular Bicycles, Including Test Meth. for Opacity of Paper, Using quipment (1973) NEMA SHIO quipment (1973) EEI TDJ.140 Std. for Metal Head and Std. for Metal Head and ousings of Motor Vehicle Lighting Devices Including Reflex

se in Housings of Motor Vehicle Lighting Devices Including or Stainless and Heat Resisting Steel Billets and Bars for std. Spec. for Molybdenum and Molybdenum Alloy Billets for emistry) (1962)

and Liquid Fats (Cereal Chemistry) (1967)

Indices of ose (1956)

Index of eous Engine Coolants (19) Std. Ant. Rec. Pract. for Use of the Molasses (Cereal Chemistry) (1962)

al Chemistry) (1967)

Temperature Corrections for Index of Refraction, with Abbe 111.8 Std. Meth. for Chemical Analysis of Chrome Containing Std. Spec. for Std. Meth. for Chemical Analysis of Silica Std. Meth. of Panel Spalling Test for Fireclay Plastic Std. Classification of Mullite Std Meth of Chemical Analysis of Zirconia eth. of Test for Modulus of Rupture of Air Setting Plastic th. of Test for Thermal Conductivity of Unfired Monolithic

f Test for Drying and Firing Shrinkage of Fireclay Plastic Std. Meth. of Test for Thermal Conductivity of Carbon Std. Definitions of Terms Relating to Std. Meth. of Test for

8 face $(1972)$

Std. Meth. of Test for Size and Bulk Density of Test for Cold Crushing Strength and Modulus of Rupture of Std. Meth. of Test for Warpage of Std. Meth. of Drip Slag Test. to. Mireclay and High Alumina Std. Classification of Fireclay and High Alumina Std. Meth. of Test for Thermal Conductivity of ion, Apparent Specific Gravity, and Bulk Density of Burned Std. Meth. of Test for Abrasion Resistance of

Std. Classification of Single and Double Screened Ground
Refined Benzene 485 (Nitration Grade) (I971) ANSI Z78.2

Refined Casting Copper (1972) ANSI H23.9

Refined Copper for Wrought Products and Alloys (1972) a

Refined Copper Wire Bars, Cakes, Slabs, Billets, Ingots

Refined Gold (1973)

Refined Palladium (1973)

Refined PhenoI by CIoud Point Depression (1968) ANSI Z7

Refined Phenol-405 (1968) ANSI Z78.22

Refined Platinum (1973)

Refined Solvent Naphtha (1971) ANSI Z78.4

Refined Sugar and Other Materials Soluble in Water (196

Refined Sunflower Oil. Technical Grade (1973)

Refined Tar Acids (1972) ANSI Z107.4

Refined) and All Hydrolyzates Solutions Derived from $C_{0}$

Refined) (Crystalline) (1959)

Refined) (1958)

Refined) (1958)

Refined) (1958)

Refined) (1961)

Refined) (1961)

Refined) (1963)

Refined) (1966)

Refined) (1972)

Refined) (1972)

Analytical Meth. for De Std. Analytical $\mathrm{CR}$ Std. Analytical Meth C Std. Analytical Meth CR

sid. Analytical M CR Std Analytical Meth. for D CR

Nalytical Meth. for

Refined) Syrup and Other Starch Hydrolyzates (1973)

Refined), Syrup, and Other Starch Hydrolyzates (1961)

Refined), Syrups and Other Hydrolyzates from Corn and G

Refined), Syrups and Other Starch Hydrolyzates (1961)

Refined) Syrups and Other Starch Hydrolyzates (1962)

Refined), Syrups, Starches and Other Protein Bearing Ma

Refinery Piping (1973)

Refinery Service (1972) ANSI B125.23

Refinery Service (1972) ANSI B125.5

Refinery Service (1972) ANSI B36.39

Refining Environment (1972)

保 ASTM

Reflectance of the Organic Components in a Polished Spe

Reflectance Retention of Fabrics (1972T)

Reflectance Std. (1971) ANSI Z172.4

Reflectance (Spectrophotometric) Content of Corn Sugar

Reflectance (1973)

Test for Thickness of Epitaxial La

Reflectance, Blue, and Whiteness of Bleached Fabric (19

Reflectance, Transmittance, and Color of Paper and Pulp.

Reflective Insulation in Cryogenic Service (1973)

Reflective Insulation Systems for Equipment and Pipe $O p$

Reflective Pavement Marking Paints (1972)

Reflectivity, Headlights and Taillights (1972)

Reflectometer with 45.0 Deg. Geometry (1973)

Reflector Interchangeability Used in Roadway Lighting E

Reflector Interchangeability Used in Roadway Lighting E

Reflect ors (1973)

Reflex Reflectors (1973)

Reforging (1972) ANSI G81.13

Ty of Plastic Material for Use in $\mathrm{H}$

Reforging (1974)

Refraction of Sucrose Solutions at 20 Deg. C (Cereal Ch

Refraction, with Abbe Refractometer, in All Normal Oils

Refractive Index Content of Corn Syrup, Sugar and Dextr

Refractometer for Determining the Freezing Point of Aqu

Refractometer Meth. of Analysis of Solids in Syrups and

Refractometer Readings (Sugar) (Cereal Chemistry) (1962

Refractometer, in All Normal Oils and Liquid Fats (Cere

Refractories and Chrome Ore (1970) ANSI A139.2

Refractories for Incinerators and Boilers (1972) ANSI a

Refractories (1970) ANSl A 139.5

Refractories (1972)

Refractories (1972)

Refractories (1972)

Refractories (1972)

Refractories (1972)

Refractories (1972) ANSl A111.25

Refractories (1973)

Refractories (1973) ANSI A111.9

Refractoriness of Refractory Mortar (1972) ANSI All1.30

Refractory and Insulating Fire Brick (1970) ANSI Alll.I

Refractory Brick and Shapes (1972) ANSI Alll.17

Refractory Brick and Tile or Deviation from a Plane Sur

Refractory Brick at High Temperature (1973)

Refractory Brick at High Temperatures (1970) ANSl Alll.

Refractory Brick (1970) ANSI All1.5

Refractory Brick (1971) ANSI A111.32

Refractory Brick (1973) ANSI Al11.3

Refractory Bricks (1973)

Refractory Materials at Room Temperaiure (1972)

/Ty, Water Absorpt

Refractory Materials (Calcined Bauxite, Calcine, Chrome
ASTM

ASTM

ASTM

ASTM

ASTM

ATTM

ASTM

ASTM

STM

R

R
CR CR CR CR CR CR

CR

CR

CR

CR

ANSI

D835

B72
B216

B442

B562

B589

D2147

D2439

B561

D 838

D3169

D2748

F.I4

F-2

F-2

F-28

F-54

F-I6

F. 32

F. 48

F. 22

F. 52

F. 58

F-18

F-26

F-44

B31.3

A200

Al61

A271

RP-04. 72

E179

D2798

D3050

E306

$\begin{array}{ll}\text { ASTM } & \text { E306 } \\ \text { CR } & \text { F.16 }\end{array}$

F95

AATCC 110

TAPPI T442SU

ASTM C740

ASTM C667

PS*1

TAPPI UM-538

EEI TDJ-140

NEMA SHIO

SH

J29

$\begin{array}{ll}\text { ASTM } & \text { A } 314 \\ \text { ASTM } & \text { B } 385\end{array}$

$\mathrm{AACCH}$ 68.6I

AACCH $58-20$

CR E-54

ASTM D3321

$\mathrm{AACCH} \quad 68.60$

$\mathrm{AACCH} \quad 80-20$

AACCH $58-20$

ASTM C572

ASTM C64

ASTM C575

ASTM C180

ASTM C 467

ASTM C705

Std. M ASTM C491

Std. Me ASTM C417

C 179 
std. Meth. of Test for Pyrometric Cone Equivalent (PCE) of er (I972) ANSI NI44

(I970) ANSI H/

Std. Meth. for Calibration of td. Meth. of Test for Cold Bonding Strength of Air Setting nelectrica] (1973) Std. Meth. of Test for Refractoriness of Std. for Safety for
Std. Meth. of Testing Flow Capacity of Std. Meth. of Testing for Rating Water Cooled d. for Drinking Fountains and Self Contained, Mechanically Std. for Safety for Safety Std. for Oxygen Therapy Equipment, Std. Meth. of Testing for Sound Rating Heating, Std. for Liquid Line Driers in essor Units (1973) Std. for Safety for d Application of Suction Line Filters and Filter Driers in d. Spec. for Seamless Copper Tube for Air Conditioning and d. for Tube Fittings for Flammable and Combustible Fluids, control, and Maintenance of Heating, Ventilating, Cooling. Uniform Fire Code: Mechanical Safety Rec. for Mechanical

Std. for Centrifugal Water Chilling Packages $r$ CentraI Station Air Handling Units (Air Conditioning and uipment for Control of Air Conditioning, Heating, Cooking. energy Consumption of Household Refrigerators, Combination Recommendation for Installation of Ceramic Tile Std. for Safety for Household Safety Std for Commercial he Freezer Temperature and Energy Consumption of HousehoId Rec. Pract. Meth. for Rating Heat Transmission of Test Meth. for Water Leak Test for Paper Shipping and Std. for Paper

Std. Meth. of Test for Moisture Content and Moisture r; And/ Uniform Building Code Std. for Douglas Fir, Coast picture Tubes (1972)

Mechanical Demand

st of Values to Be Used in Semiconductor Device Specs. and Std. for Direct Reading Remote tion, Maintenance and Rules of the Road / Safety Std. for , Fork, Stee/ Safety Std. for Structural Requirements for 'Saddle Braces,/ Safety Std. for GeneraI Requirements for Test Procedures for Safety Sid Definitions for Test Procedures for the Safety Std. for ts for Stopping Power, Traction and Steering Stability for d Taillights (1972) nd Strip (1973) ANSI C24.24

itioning, Hea/ Safety Std. for Lighting for ttd. Spec. for 2.90

Safety Std. for Temperature Indicating and Std. Test Code for Distribution, Power and Std for Safety for Pressure w/ Uniform Fire Code: Protection, Authority, Departmental ents and Containers (Also Includes Terms and Defini/ Rec. ver Public Property (Pedestrian P/ Uniform Building Code:

Uniform Building Code: Std. for Ancymidol (Plant Growth Std. for Electrical Transformers,

on Rec. for Low Water Fuel Cutoffs and Automatic Feedwater Std. for Gas Appliance Pressure Safety Std. for Gas Pressure

ft Equipment for Combustion Chamber in Heating Appliances for the Abatement of Dangerous Buildings (Repair, Vacate, Std. Meth. of Test for 972) d. Meth. of Test for Vapor Pressure of Petroleum Products sion Materials (1972) bric) $(1962$ otes) (1965)

sisting Lateral Forces) (Tech. Notes) (1968)

(1967)

(1965)

1967)

1963)

(1964)

s) (1963)

r Pipe (1971)

High Lift Grouted Std. Spec, for Rec. for Manufacture and Placement of Spec. for the Design and Construction of elding Reinforcing SteeI, Metal lnserts and Connections in e (1973) d Sewer Pipe (1972)

Design Tables for wer Pipe (1973)

Refractory Materials (1972) ANSI AIll 4

Refractory MetaI Thermocouples Using an Optical Pyromet Refractory Metals and Compounds by the Scott Volumeter Refractory Mortar (Wet Type) (1972) ANSl Al11.29

Refractory Mortar (1972) ANSI All1.30

Refrigerant-Containing Components and Accessories. No Refrigerant Capillary Tubes (1972)

Refrigerant Condensers (1971)

Refrigerated Drinking Water Coolers (1973) ANSI Al12.II

Refrigerated Vending Machines (1974)

Refrigerated (1973)

Refrigerating and Air Conditioning Equipment (1972)

Refrigerating and Air Conditioning Systems (I971)

Refrigeration and Air Conditioning Condensing and Compr

Refrigeration and Air Conditioning Systems (I974)

Refrigeration Field Service (1974) ANSI H23.5

Refrigeration Service, and Marine Use (1972)

Refrigeration Systems, Incinerators and Other Misc. Hea

Refrigeration (1973)

Refrigeration (1974)

Refrigeration) (1974)

Refrigeration) (1974)

Refrigeration, and Humidity (Humidistat, Thermostat) (I

Refrigerator-Freezers and Freezers (1974)

Refrigerat or Rooms (1973)

Refrigerators and Freezers (1973) ANSl B97.1

Refrigerators Using Gas Fuel (I972)

Refrigerators (1973)

Refrigerators, Combination Refrigerator-Freezers and

Refrigrated Vehicles (1972)

Refuse Sacks (Containers) (1973)

Refuse Sacks (1970)

Regain of Textile MateriaI (1971)

Region; West Coast Hemlock; Western Red Cedar; White Fi

Registered Screen Dimensions for Monochrome Television

Registers (Cumulative and Pointer Forms) EEI MSJ-4

Registration Formats (I974)

Registration Systems for CoId Water Meters (1972)

Regular Bicycles Including Selection, Purchase, Prepara

Regular Bicycles Including Tests for Static Load, Frame

Regular Bicycles Including WheeI Assembly, Chainguards,

Regular Bicycles (1972)

Regular Bicycles (1972)

Regular Bicycles (1972)

Regular Bicycles (1972)

Regular Bicycles. Including Reflectivity, Headlights a

Cold Rolled Alloy Steel Sheet a

Regulating Electrical Equipment for Control of Air Cond

Regulating Transformers (Electronics) (1973) ANSI C57.1

Regulating Transformers (1973) ANSI C57.12.00

Regulating Valves for LP Gas (1973) ANSI 2217.]

Regulations and Controls, Barrier, Road, Hydrant, Drive

Regulations for Animal Feed and Food Labelling, Ingredi

Regulations for Use of Public Streets and Projections $O$

Regulations Governing Fallout SheIters (1973)

Regulator) (1971)

Regulators and Reactors (1974)

Regulators for Boilers (1974)

Regulators (1973)

Regulators (1973)

Regulators, Automatic Damper Controls, Fans) (1973)

Rehabilitate, Demolish) (1973)

Reheat Change of Fireclay Nozzles and Sleeves (1972)

Reid Meth.) (1972) ANSI Z11.44

Reinforced Acetal Plastics for Molding and Extrusion (1

Reinforced and Filled Nylon Injection Molding and Extru

Reinforced Bituminous Concrete Overlays (Welded Wire F

Reinforced Brick and Tile Lintels (Tech. Notes) (1964)

Reinforced Brick Masonry Columns and Pilasters (Tech. N

Reinforced Brick Masonry Curtain and Panel Walls (In Re

Reinforced Brick Masonry Flexural Members (Tech. Notes Reinforced Brick Masonry Retaining Walls (Tech. Notes) Reinforced Brick Masonry Swimming Pools (Tech. Notes) Reinforced Brick Masonry (Axial Design) (Tech. Notes)( Reinforced Brick Masonry (Flexural Design) (Tech Notes) Reinforced Brick Masonry (Inspectors Guide) (Tech. Note

Reinforced Brick Masonry (Tech. Notes) (I972)

Reinforced Concrete Arch Culvert, Storm Drain, and Sewe

Reinforced Concrete Casings for Irrigation Wells (1972)

Reinforced Concrete Chimneys (1969) ANSI A 158.1

Reinforced Concrete Construction (1973)

Reinforced Concrete Culvert. Storm Drain, and Sewer Pip

Std. Spec. for Reinforced Concrete D-Load Culvert, Storm Drain, and Se

Std. Spec. for Reinforced Concrete Elliptical Culvert, Storm Drain, an

Std Spec for Reinforced Concrete Low Head Pressure Pipe (1973)

Std. Spec. for Precast
ASTM

ASTM

ASTM

ASTM

ASTM

UL

ASHRA

ASHRA 22

ARI 1010

UL 541

UL 416

ASHRA 36

ARI $\quad 710$

UL 303

I an ARI 730

Afety St UL

ICBO

ICBO

FMS

ARI

Std. Fo ARl

UL

AHAM

TCA

UL

LL

AHAM

TTMA

TAPPI

NSF

ASTM

ICBO

EIA

NEMA

Std. Li EIA

AWWA

BMA

BMA

BMA

BMA

BMA

BMA

BMA

BMA

ASTM

UL

IEEE

IEEE

UL

ICBO

$\mathrm{AAFCO}$
$\mathrm{ICBO}$

ICBO

ANS1

NEMA

FMS

ANSI

UL

la UL

ICBO

ASTM

St ASTM

ASTM

WRI

BIA

BIA

BIA

BIA

BIA

BIA

BIA

$\mathrm{BlA}$

BIA

BIA

ASTM

ASAE

ACI

ICBO

ASTM

ASTM

ASTM

ASTM

ASTM

C24

E452

B329

CI98

(

109 $12 \cdot 61$

550

430

873

310

250

RP38

32 10 16 3

UMC *7

UFC $* 2 A R T 28$

RF-2.ECFT

Z21. 19A

RF-2-ECFT

UM.529 

Std. Spec. for

Guide Spec. for Specifying: struction Under the Uniform Building Code: Solid, Grouted d Gypsum Roof Diaphragms / Uniform Building Code Std. for si K/ Rec. Pract. for Classifying Visual Defects in Glass Uniform Building Code Std. for Mortar for Unit and Std. Spec. for

Std. Test Meth. for Diffuse Light Transmission Factor of t for Apparent Horizontal Shear Strength of Parallel Fiber Std. for Glass Fiber Std. Spec, for

extrusion Materials (1973) Std. Meth. of Test for Fiber Content of Rec. Pract. for Care and Use of Spec. for

oncentric Lay Stranded Aluminum Conductors, Aluminum Alloy aluminized) Steel Core Wire for Aluminum Conductors, Steel inized) Stranded Steel Core for Aluminum Conductors, Steel galvanized) Steel Core Wire for Aluminum Conductors, Steel ec. for Aluminum Conductors, Concentric Lay Stranded Steel ec. for Builders' Hardware on Std. Steel Doors and Frames Uniform Building Code Std. for Concrete Spec. Steel Wire Bar Supports Used to Position Std. Spec. for Rail Steel Deformed Bars for Concrete Spec. for Axle Steel Deformed and Plain Bars for Concrete pec. for Deformed and Plain Billet Steel Bars for Concrete Sid Spec for Cold Drawn Steel Wire for Concrete Std. Spec. for Welded Steel Wire Fabric for Concrete td. Spec. for Cold Worked Deformed Steel Wire for Concrete Spec. for Welded Deformed Steel Wire Fabric for Concrete Building Code Std. for Cold Drawn Steel Wire for Concrete code Std. for Steel Bar and Rod Mats (Sheets) for Concrete or Welded Steel Wire and Deformed Wire Fabric for Concrete Rec for Concrete olled and Cold Finished Bars; Hot Rolled Deformed Concrete Std. Meth. of Test for Strap Peel Adhesion of Rec. Minimum Hardware Std. Pract. for Detailing Std. Pract. for Fabrication of Std. Pract. for Estimating Spec. for Placing

(1970)

ract (1968)

nforced Concrete/

trol) (1967)

kwell Superf/

(1972)

e $(1972 /$

Pract, for Erhics and Human Relations in Vendor-Vendee terminology and Definitions for Soil Tillage and Soil Tool 3) Std. Meth. of Test for (1974)

Spec. for Determination of uorescent Bri/ Test Meth. for Spectrophotometric Test for ire Apparatus (1972) ALCA E/

Std. Meth. for Measuring the Sid. Meth. of Test. Adhesives of Woven and Knitted Wool Textiles (1972)/ Test Meth. for Knit Wool Fabrics (Textile) (197/ Std. Meth. of Test for ough Tests of Flour (Cereal Chemistry) (1962) Structural Std. Meth. of Test for Creep Std. Rec. Pract. for Stress Std. for Relays and Definitions of

(1971) ANSI C37.90 lefinitions of Std. for Safety for Ground Fault Sensing and Guide for Protective
Std. for Apparatus (1971) ANSI C37.90 Iron (EMI) Cold Rolled Strip for Magnetic Application in nsi C83.25

Std. Test. Procedures for Std. Definitions and Terminology for Std. for Electrical Indicating Instrument (Meter) Std. for Handle, Latching Internal and External Fire Detection System (Grinnell Protomatic ring (1970) ANSI L 14.239 Test Meth. for Oily Stain Meth. of Test for Bubbling of 1 Part, Elastomeric Solvent

for UV Cold Box Exposure of One Part, Elastomeric, Solvent ature Flexiblity and Tenacity 1 Part, Elastomeric, Solvent Std. for Pin, Quick Std. for Pin, Quick

Std, for Panic (Emergency) Hardware (Outward Opening Door $n$ Protection Sys/ Trial Use Guide: General Principles for eathed, Type K Thermocouples for Nuclear or for Other High

Rec. for Operation and Maintenance of the 72)

Std. for Terminology for Pressure Std. Spec. for Preformed Cellular Plastic Pressure
Reinforced Concrete Nuclear Power Containment Structure Reinforced Concrete (1971) ANSI A89.1

Reinforced Epoxy Resin Gas Pressure Pipe and Fittings (

Reinforced Fiberglass Polyester Tanks (1973)

Reinforced Grouted, Hollow Unit, Cavity Wall, and Stone

Reinforced Gypsum Concrete and Precast Slabs, and Poure

Reinforced Laminates and Parts Made Therefrom (1970) an

Reinforced Masonry Other Than Gypsum (1973)

Reinforced Plastic Mortar Sewer Pipe (1973)

Reinforced Plastic Panels (1969)

Reinforced Plastics by Short Beam Meth. (1972)

Reinforced Polyester Structural Plastic Panels (1972)

Reinforced Polyterphthalate Thermo-Plastic Molding and

Reinforced Resin Composites (1973)

Reinforced Thermosetting Resin Line Pipe (Rtrp) (1972)

Reinforced Thermosetting Resin Line Pipe (1972)

Reinforced (Acar, EC/6201) (1972) ANSI C7.65

Reinforced (ACSR) (1972) ANSI C7.47

Reinforced (ACSR) (1972) ANSI C7.61

Reinforced (ACSR) (1974) ANSI C7.60

Reinforced, (ACSR) (1972A) ANSI C7.22

(Reinforcement-Application) (1972)

Reinforcement Bars (1973)

Reinforcement in Concrete (1970)

Reinforcement (1972)

Reinforcement (I972

Reinforcement (1972)

Reinforcement (1972) ANSI A50.3

Reinforcement (1972) ANSI G45.1

Reinforcement (1972) ANSI G45.2

Reinforcement (1972) ANSI G45.3

Reinforcement (1973)

Reinforcement (1973)

Reinforcement (1973)

Reinforcing Bar Splices (1971)

Reinforcing Bars (1972)

Reinforcing Fabrics or Cords to Rubber (1971)

Reinforcing Garages on Steel Doors and Frames (1972)

Reinforcing Materials (1970)

Reinforcing Steel Bar Materials and Service (1968)

Reinforcing Steel Bar Materials and Service (1970)

Reinforcing Steel Bar Supports in Concrete Construction

Reinforcing Steel Material Supply and Installation Cont

Reinforcing Steel, Metal Inserts and Connections in Rei

Rejects from Knotter Screens (Pulp and Paper) (1973)

Relations in Vendor-Vendee Relationships (Quality Con

Relations of Soils (1973)

(Relationship Between Brinell, Vickers, Rockwell and Roc

Relationships for Soils by Pressure Membrane Apparatus

Relationships of Agricultural Grain and Seed for Storag

Relationships of Agricultural Grains (1968)

Relationships (Quality Control) (1967)

Relationships (1972)

Relative Density of Black Smoke (Ringelmann Meth.) (197

Relative Pencil Hardness of a Paint Film (Coil Coating)

Relative Radiance of Paper and Paperboard Containing Fl

Relative Stiffness of Leather by Means of a Torsional W

Relative to Their Use as Electrical Insulation (1970)

Relaxation and Felting Dimensional Changes (Shrinkage)

Relaxation Dimensional Change (Shrinkage) of Stabilized

Relaxation Meth. of Extensigraph Analysis of Physical D

Relaxation of a Gasket Material (1971)

Relaxation Tests for Materials and Structures (1972)

Relay Systems Associated with Electric Power Apparatus

Relay Terms (Electromagnetic Instrumentation) (1972)

Relaying Equipment (1972)

Relaying of Utility-Consumer Interconnections (1973)

Relays and Relay Systems Associated with Electric Power

/Pplicable to Electromagn

Relays for Electrical and Electronic Equipment (1973)

Relays for Electronic Equipment (1971)

Relays (1972)

Release Components and Assembly (1972)

Release F100) (1973)

Release Meth. of Textile Fabric Soil During Home Launde

Release Type Sealants (1972)

Release Type Sealants (1972)

Release Type Sealants (I 972)

Release, Positive Locking. Double Acting of Test

Std Meth Test

Release, Positive Locking, Single Acting (1973)

Releasing Device) (1973)

Reliability Analysis of Nuclear Power Generating Statio

Reliability Applications (1967) ANSI N142

Reliable Model B Accelerator for Dry Pipe Systems (Fire

Relief Devices (Container and Equipment Protection) (I9

Relief Joint Fillers (1973)

Building

ACI

ACI

AST

CSI

ICBO

ICBO

ASTM

ICBO

ASTM

ASTM

lof Tes ASTM

USC

ASTM

ASTM

API

API

/C. for C ASTM

STM

dAlum ASTM

coated ( ASTM

Std. Sp ASTM

Rec. Sp STDI

ICBO

CRSI

ASTM

Std. ASTM

Std. S ASTM

ASTM

ASTM

ASTM

Std ASTM

Uniform ICBO

ICBO

ICBO

CRSI

AISI

ASTM

NBHA

CRSI

CRSI

CRSI

CRSI

CRS

ICBO

TAPPI

ASQC

ICBO

ASTM

ASTM

ASAE

ASAE

Rec ASQC

ASTM

NCCA

TAPPI

ASTM

ASTM

AATCC

ASTM

AACC

ASTM

ASTM

IEEE

NARM

UL

IEEE

IEEE

NARM

EIA

ANSI

NEMA

NSA

FMS

AATCC

69.2

D2517

UBC *8. 11

UBC 24.13

D2563

UBC $24-21$

D3262

D1 494

D2344

PS53

D3220

D3171

RP5L4

5LR

B524

B341

B500

B 498

B232

107

UBC 526.4

* 1.3.1

A616

A617

A 185

A496

A 497

UBCS24-16

UBCS26.5

UBC 526-6

*3 
W'ater Supply Systems (1972) oleum) Gas (I973)

Coaches (1970)

i B146. 1

buttons, Variable, Press Fit, Headless and Head Type, Step mmonia and Liquefied Petroleum (LP) Gas (Other Than Safety Molybdenum Sheet, Strip, and Plate, Arc Cast, Stress

Safety Std. for Std. Spec. for sistant $(0.95 \mathrm{Cr}-0.55 \mathrm{Mo}-0.30 \mathrm{~V}(0.40-0.50 \mathrm{C})$ Vacuum Arc 4.5 Ni-3.5 Cu- $(\mathrm{Cb}+\mathrm{Ta})$, Consumable Electrode Vacuum Arc $-0.43 \mathrm{C}$ ) (SAE 4340), Premium Quality, Consumable Electrode ) (SAE 9310), Premium Quality, Vacuum Consumable Electrode railing Type Agricultu/ Std. Spec. for Magnesium Ingot and Stick for ailing Type (Farm) Imp

Std. for Application of Hydraulic ents (1971)

Application of Std. for Flashing Warning Lamp (Light) for Std. for Direct Reading SE 3.5

i SE3.7

Std. for Installation, Maintenance and Use of Std. for Interconnection Circuitry of Noncoded Std. Spec. for $55 \mathrm{Gal}$. Full Std. Spec. for 30 Gal. Full Std. Spec. for 55 Gal. Full Std. Spec. for 57 Gal. Full Std. Spec. for $30 \mathrm{Gal}$. Full Std. Spec. for $16 \mathrm{Gal}$. Full Std. Spec, for $16 \mathrm{Gal}$. Full

em 260) (1974)

tem 260) (I974)

tem 260) (1974)

0, Nmfc-Item 260) (1974)

Meth. of Test for Measuring Soil iency Test. of Air Cleaning Systems Containing Devices for 951) Rec. for Control and indus/ Std. Meth. of Analysis of Solvent Systems Used for $\mathrm{n}$ of Blower and Exhaust Systems for Dust, Stock, and Vapor Std. Meth. of Measuring Application and ety Requirements for Tree Pruning, Trimming, Repairing, or g Performance of Anion Exchange Materials for Strong Acid Blower, Fan, and Exhaust System for Dust, Stock and Vapor ium Chloride to Be Used for Dustlaying, Stabilization, Ice ishing Machines (Polisher, Scrubber, Sander, Scraper, Tile tent. Meth. of Test for Corrosivity of Solvent Systems for stairs (1973) Recommendation for for Preparation of Manuals for Installation, Operation and Uniform Code for the Abatement of Dangerous Buildings Std. for Control of Gas Hazards on Vessels (Ships) to Be Std. for Safety Requirements for Tree Pruning, Trimming, Suggested Guide Lines for Printed Wiring Board

2) ANSI L14.214

14.60. ASTM D583

1972) ANSI L14.61. ASTM D583

ption Test (1972) ANSI L14.87, ASTM/

Test Meth for Water

Test Meth. for Water Test Meth. for Water for Effects of Drycleaning on Permanence of Durable Water Ratings; Assembly Numbers, Cell Layouts and Ter/ Battery t. for Marine (Inboard) Engine Mountings (Installation and ed from the Investigation of $\mathrm{Sc} /$ Std. Underwater Accident

Rec. Pract, for Drill Stern Test

fferential and Manual Transmission Lubricants (Information

lectrical Power Operations Terminology Including Terms for

tor and Open Burning, Combustible and Flammable Materials: d Power Systems (1973) ANSI B93.30 mogravimetry, Evolved Gas Analysis

ainers (1972) Std. Meth of Std. Rec. Pract. for Std. Rec. Pract. for Std. Rec. Pract for ec. Pract. for Uniform Procedure for the Test., Rating and Std. Meth. of Std. for Record and Std. for Auditing Nuclear Materials Statements and Sid (1973) ANSI I Meth for Isolation of Std. for Nuclear Material Control Systems for Fuel d. for Guide to Principal Design Criteria for Nuclear Fuel , Open Reel (For Tape Speeds of 7.5 In. 0 190.) Std. for d. Frequency Response and Operating Level of Recorders and er Measurement of Instrumentation Magnetic Tape Recorder / r Measurements of Instrumentation Magnetic Tape Recorder / nt of Weighted Peak Flutter Content of Sound Recording and Safety Std. for Sound Recording and Stds. for Decoders (Type I) for

idge Type Cp II (Compact Cassette) for Audio Recording and

Uniform Building Code: Existing Buildings; Minimum Safety Uniform Building Code: Occupancy and General rength for $\mathrm{AC}$ High Voltage Circuit Breakers Rated O/ Std. pract. for Symbols and Color Codes for Lubricant and Fluid ectrical Wiring on Boats and Engines, (Color Code) (1973)

Std.

cle Brake Linings (1966)

tires for Passenger Cars and Station
Minimum Performance Minimum Performance
Relief Valves and Automatic Gas Shutoff Devices for Hot Relief Valves for Anhydrous Ammonia and LP (Liquid Petr Relief Valves in Travel Trailers, Camp Cars and Trailer Relief Valves Used in the Petroleum Industry (1969) Ans Relief (1972)

Relief) (1974)

Relieved (1973)

I for Safety for Valves for Anhydrous

Relocking Devices (1972)

Remelted Lithium Metal in Ingot Form (1972) ANSI H43.1

Remelted (1973)

Remelted (1973)

Remelted (1973)

Remelted (1973)

Remelting (1971A) ANSI H45.5

Gings, and Tubing, Low Alloy, Heat Re Plate, Corrosion Resistant, $14.8 \mathrm{Cr}-$ Ubing, $0.80 \mathrm{Cr}-1.8 \mathrm{Ni}-0.25 \mathrm{Mo}(0.38$ $11.2 \mathrm{Cr}-3.25 \mathrm{Ni}-0.12 \mathrm{M} \circ(0.07-0.13 \mathrm{C}$

Remote Control Cylinders to Agricultural Tractors and T

emote Hydraulic Motors to Agricultural Tractors and TR

Remote Mounting on Agricultural (Farm) Equipment (1972

Remote Registration Systems for Cold Water Meters (1972

Remote Station Protective Signaling Systems (1972) ANSI

Remote Station Protective Signalling Systems (1969) Ans

Removable Head Universal Drum (DOT-17H) (1968)

Removable Head Universal Drum (Dot-17H) (1974)

Removable Head Universal Drum (Ufc-Rule $40, \mathrm{Nmfc}-\mathrm{It}$

Removable Head Universal Drums (Ufc-Rule 40, Nmfc-I

Removable Head Universal Drums (Ufc-Rule 40, Nmfc-I

Removable Head Universal Drums (Ufc-Rule 40, Nmfc-I

Removal and Reflectance Retention of Fabrics (1972T)

Removal of Particles (1972)

Std.

Removal of Radioactive Contamination in Laboratories (1

Removal of Water Formed Deposits (Chemical Cleaning of

Removal or Conveying (1973) ANSI Z33.1

Removal Torque of Threaded Closures (1973)

He Installatio Removal (1972)

Removal (1972)

Std. for Sa

Uniform Building Code Std for

Removal, Acceleration of Concrete, Curing of Concrete,

Remover, Rug Shampooer, Rug and Floor Washers, etc.) (1

Removing Water Formed Deposits (1973)

Renovation of Interior Ceramic Tile Walls, Floors, and

Repair of Laboratory Instruments (1972)

Repair, Vacate, Rehabilitate, Demolish) (1973)

Repaired (1972) ANSI Z270.1

Repairing, or Removal (1972)

Repairs (1973)

Repellency of Fabrics: Hydrocarbon Resistance Test (197 Repellency of Textile Fabrics: Spray Test (1971) ANSI L

Repellency of Textile Fabrics: Static Absorption Test (

Repellency of Textile Fabrics: Tumble Jar Dynamic Absor

Repellent Effectiveness Df Treating Solutions (1970)

Repellent Finish of Fabrics (1972)

Std. Meth. of

Replacement Data; Group Numbers, Dimensional Spec., and

Replacement) Direct Drive Transmission (1971)

Report Form (Acquisition and Codification of Data Deriv

Report Form (1972)

Report) (1969)

Reporting and Analyzing Outage of Electrical Transmissi

Reporting and False Alarm; Use of Equipment, Appliances

Reporting Contamination Analysis Data of Hydraulic Flui

Reporting Data from Differential Thermal Analysis, Ther

Reporting Irradiation Results on Graphite (1972)

Reporting Laboratory Results When Checking Aerosol Cont

Reporting of the Noise Levels of Powered Industrial Tru

Reporting Results of Analysis of Water (1969E)

Reporting Units for Nuclear Materials Control (1971)

Reports (Accounting) (1973)

Represent ative Saturates Fraction from Low Olefinic Pet

Reprocessing Facilities (A Guide to Practice) (1974)

Reprocessing Facilities (1973)

Reproducer Test Tape: Full Track, i/4 In. $(6.3 \mathrm{Mm})$ Widt

Reproducers for Audio Record One for 2-In. Quadruplex

Test Meth. for Flutt

Reproducers (1972) ANSI C83.99

Reproducers (1974) ANSI C83.94

Reproducing Equipment (1971)

Reproducing Equipment (1973)

Meth. for Timing Erro

Reproducing Matrix Quadraphonic Disc Pecords (1974)

Reproducing (1974) /

Requirement Classification (1973)

Requirement for Switching Impulse Voltage Insulation St

Requirement Maintenance Instructions, and Container and

Requirements and Claims for Special Characteristics and

Requirements and Rec. Meth. of Identifying AC and DC E Requirements and Terminology for Specialiy Transformers Requirements and Uniform Test Procedures for Motor Veh Requirements and Uniform Test Procedures for Retreaded
ANSI Z21.22A

UL $\quad 132$

IAPMO TSC 8

SAE

UL

ASTM

$\mathrm{SAE}$

$\mathrm{SAE}$

SAE

ASTM

ASAE

ASAE

ASAE

AWW

NFPA

NEMA

ANSI

ANSI

ANSI

ANS

ANSI

ANSI

ANSI

ASTM

ANSI

NCRPM R

ASTM

ASTM

ANSI

ASTM

ICBO

ASTM

UL

ASTM

Std. ANSI

$1 \mathrm{CBO}$

NFPA

ANSI

IPC

AATCC

AATC

A ATCC

AATCC

NWMA

ASTM

BCI

SAE

API

Di SAE

IEEE

ASTM

ASTM

ASTM

ITA

ASTM

ANSI

ANSI

ASIM

ANSI

St ANSI

EIA

ANSI

EIA

IEEE

UL

EIA

$\mathrm{ICBO}$

ICBO

IEEE

SAE

ANSI

$A B Y C$

NEMA

VESC

VESC

526

B94.43

125

AMS7801A

I40

B357 
omments on the National Electrical Code (NFPA 70) and Code

$i$ and Surface Lifts and Tows (1973)

nes with Intermediate Atmospheric Vent (1972/

Spec. for General

Std. for Safety

Performance

Std. Safety

al Words, Letter Sizes, Color Combinations, and Durability igh Voltage Circuit Breakers Rated on a Symmetrical/ Std arse Round Wire (1971) ANSI G54.1/

Alloy Steel Tubes (1971A) ANSI B/

Std. Spec. for General Std. Spec. for General Std. for Basic Std. for

uipment and Operations (1972)

ress Brakes (1973)

fabrics, Painted, Printed, or Vinyl Coa/ stitutional Textile) (1973)

and Finishings (Sublimation, Brittlene/

heat Resisting Steel Plate, Sheet/ $s$, Sheet Piling, and Bars for Str/ vessels (1972)

stallation of Permanently Insta/

ansformers (1973) ANSI C57.12.00

Std. for Safety

Std. for Safety

Std. Performance

Std. Performance

Std. Performance

Std. Spec. for Genera

Std. Spec. for Genera

Std. Spec. for General

Std. and Rec. Pract. for

Safety Ventilation

Std. General

backflow Preventers for Potable Water Supply

ling Dies (1973)

d Pillowcase Fabrics (Institutional Tcx/

Performance

td. for Dimensional

Std. Performance ts (1972)

circuit Breakers Rated on a Symmetrical and a Total/

rily in Transmitters and Electronic Instruments) (1/

aterial for Use in Housings $\mathrm{O} /$

Std of Mechanical and Quality Std. for Prescription Safety

Toeboards (1973)

1973)

Std. Performance

d Alloy Steel Bar (1973) ANSI G24/

Std Spec for General

1. Asse 1006

Std. and Test Procedures for Plumbing

971) ANSI A 197.3, /

water Dispensers (1973)

973)

Std. and Test Procedures for Plumbing

Plumbing

Control Valves (1973)

Operation with Tractors Having at Le/

Std. Performance

Performance

During the $\mathrm{C} /$

Std. Installation, Inspection, and Testin

Dctures for Nuclear Reactors (1972)

Volts and Below, 3750 Through 30,000/40,000/50,000

and Testin referred Ratings, Application Recommendations, and Related nd Related Industries (1/ obile Electrical Systems (197)

Std. for Mechanical and Qualit Rec. Pract. Test Meth. and Std. for Safety

other Structures (1972) Std. for Building Code bituminous Paving Mixtures (1971) ANSI A3/ Std. Spec. for ponents (1972) Performance ecting Devices and Towing Meth. (Coupling, Hitch/ Minimum

Minimum

Std for Quality Assurance Program ight Commercial) Central Furnaces) (197/ Std. Performance 7,000 Volts and Below; $500 \mathrm{kVA}$ and Smaller (1971) Std. ing) (1974)

Std. for Minimum Safety and Health Certification of Fabrics or Products (/ olition Operations) (1973)

reakers (1970)

(1974)

2) Std. for Safet Std. Basic Construction Performance

age Circuit Breakers Rated on a Symmetrical Current/ Safety uts (1972)

atic Load, Frame, Fork, Stee/

bly, Chainguards, Saddle Braces./

Std

Safety Std for Structural

Safety Std. for General Std. Building Code Minimum on and Fire in Design, Construction, and Operation of So/ (1972) Minim um

Motor Vehicles (197) Rec. Pract. for Service Performance d Carbon Steel Tubular Prod/ Std. Spec. for Supplementary Plate (1973)

$$
\text { Std. for Safety }
$$
Rec, Safety

ght) (1972)

Rec. Pract. Test Meth. and

Rec. Pract. Test Meth. and e (1972) ANSI BI25.20

Rec. Pract. Test Meth. and Std. Spec. for General Std. for Safety Std. for Mechanical and Quality Std. for Mechanical and Quality 2) Std. Spec. for Charpy V-Notch Testing by the Hot Dip Process (1973) Ans/ Std. Spec. for General stability for Regular Bicycl/
Requirements by OSHA (1973)

Requirements for a Quality Program (1968) ANSI Z1.8

Requirements for Aerial Passenger Tramways Including Sk

Requirements for Backflow Preventers in Water Supply Li

Requirements for Bakery Equipment (1971)

Requirements for Both Permanently and Temporarily Affix Requirements for Capacitance Current Switching for $\mathrm{ACH}$

Requirements for Carbon Steel Wire Rods and Uncoated Co

Requirements for Carbon, Ferritic Alloy, and Austenitic

Requirements for Cargo Containers (197I)

Requirements for Closed Van Type Cargo Containers (1971

Requirements for Commercial Laundry and Dry Cleaning Eq

Requirements for Construction, Care, and Use of Power P

Requirements for Cuiton Woven Tent, Awning, and Canopy

equirements for Damask Table Napery (Napkin,

Requirements for Delivery of Flat Rolled Stainless and

Requirements for Delivery of Rolled Steel Plates, Shape

Requirements for Delivery of Steel Plates for Pressure

Requirements for Design, Materials, Construction and in

Requirements for Direct Fired Powder Curing Ovens (1973

Requirements for Distribution, Power, and Regulating TR

Requirements for Double Check Valve Type Back Pressure

Requirements for Duplex and Single Face Flat Thread Rol

Requirements for Durable Press (No Iron) Woven Sheet an

Requirements for Electrical Analog Indicating Instrumen

Requirements for Electrical Control of AC High Voltage

Requirements for Electrolytic Capacitors (For Use Prim

Requirements for Evaluation of Suitability of Plastic M

Requirements for External Insulation for Outdoor AC Hig

Requirements for Externally Threaded Fasteners Used in

Requirements for First Quality Contact Lenses (1972)

Requirements for Floor and Wall Openings, Railings, and

Requirements for Glass Towels (Institutional Textile) (

Requirements for Hot Rolled and Cold Finished Carbon an

Requirements for Household Dishwashers (1971) ANSI A 197

Requirements for Household Food Waste Disposer Units (1

Requirements for Huck Towels (Institutional Textile) (1

Requirements for Individual Anti Scald Type Water Showe

Requirements for Industrial Rotary Mowers (Designed for

Requirements for Instrumentation and Electric Equipment

Requirements for Leakage Rate Test. of Containment Stru

Requirements for Load Tap Changing Transformers 138,000

Requirements for Low Voltage Power Circuit Breakers and

Requirements for Machine Screws for Use in Automotive a

Requirements for Maintenance of Design Voltage in Snowm

Requirements for Marine Terminal Operations (1972)

Requirements for Minimum Design Loads in Buildings and

Requirements for Mixing Plants for Hot Mixed, Hot Laid

Requirements for Motor Vehicle Lighting Devices and Com

Requirements for Motor Vehicle (Recreational Type) Conn

Requirements for Motorcyclists Eye Protection (1971)

Requirements for Nuclear Power Plants (1971)

Requirements for Oil Powered Forced Air (Domestic and L

Requirements for Overhead Distribution, Transformers, 6

Requirements for Paper Bag and Sack Manufacture (Packag

Requirements for Permanent Labels, Detachable Tags, and

Requirements for Personnel Hoists (Construction and Dem

Requirements for Photographic Slide Projectors (1973)

Requirements for Pipe Applied Atmospheric Type Vacuum B

Requirements for Pipe Schedule System Sprinkler Demand

Requirements for Powder Actuated Fastening Systems (197

Requirements for Power Line Coupling Capacitors (1972)

Requirements for Pressurized Components of AC High Volt

Requirements for Prevailing Torque Type Steel Hex Lockn

Requirements for Regular Bicycles Including Tests for S

Reguirements for Regular Bicycles Including Wheel Assem

Requirements for Reinforced Concrete (1971) ANS1 A89.1

Requirements for Safety Nets (1971)

Requirements for Safety to Life-Property from Explosi

Requirements for School Bus Construction and Equipment

Requirements for Sealed Beam Headlamp (Light) Units for

Requirements for Seamless and Electric Resistance Welde

Requirements for Shops Fabricating Structural Steel and

Requirements for Shoring Concrete Formwork (1972)

Requirements for Snow mobile Headlamps (Light) (1972)

Requirements for Snowmobile Rear Position Tail Lamp (Li

Requirements for Snowmobile Stop Lamp (Light) (1971)

Requirements for Specialized Carbon and Alloy Steel Pip

Requirements for Steel Erection (1972)

Requirements for Steel Machine Screws (1973)

Requirements for Steel Nuts (197I)

Requirements for Steel Plates for Pressure Vessels (197

Requirements for Steel Sheet, Zinc Coated (Galvanized)

Requirements for Stopping Power, Traction and Steering

ASQC

ANSI

$\mathrm{SAE}$

ANSI

ANSI

ANS

ANSI

ANSI

ANSI

ANSI

ABYC

FMS

IEEE

ASSE

IFI

ANSI

ANSI

EIA

SAE

IEEE

SAE

ANSI

ANSI

ASSE

ANSI

ASSE

SAE

IEEE

ANSI

ANSI

ANSI

$S A E$

SAE

ANSI

ANSI

SAE

VESC

VESC

ANSI

ANSI

ANSI

ANSI

ANSI

ANSI

ASSE

ANS

ANSI

IEEE

IFI

BMA

BMA

ACI

ANSI

ANSI

ASSE

IEEE

ASTM

ASTM

ASTM

ASTM

ASTM

ANSI

ANSI

ASTM

AHAM

AHAM

ASTM 
or Dyed) (Napkin, etc.) (Institutional / eight (Tropical) Suiting (Institutional/

(Institutional Textile) (1973)

freight Containers (1968)

Casting Machines (1973)

kaging and Its Related Converting Machin/ ars (Mechanical, Hydraulic, Pneumatic, Semi ce of Sprinkler Irrigation Equipment (1972) Std. for Building Code Std. Performance Std. Performance Std. Performance

Std. for Safety Std. for Safety ance of Heating, Ventilating, C/ Uniform Mechanical Code

Part 1-Converters for DC Motor Armatu

Std. Pract. and

Voltage Circuit Breakers Rated on a Symmetrical Cu/ removaI (1972)

uipment (1973)

Furnaces, Floor Furnaces, and / Uniform Mechanical Code: Std. for Safety Std. Safety

(1973)

e) $(1973$

(1973)

and BoIster Fabrics (Institutional Text le) (1973)

ding Glass Fibers) (Institutional Texti/ nstitutional Uniform Textile) (1973)

brics, Intermediate (Year Round) Suitin/

brics, Heavyweight (Industrial) Suiting/

nal and Industrial Use Garments (Clothi/ nal and Industrial Use Garments (Clothi washable at $105 \mathrm{Deg}$. F-No Bleach (Ins)

brics $\mathrm{H}$ andwashable at $105 \mathrm{Deg}$. F-No B/ Uniforms (Textile) (1973)

Canopy Fabrics, Vinyl Coated or Lamina/

c.) (Institutional Textile) (1973)

er Than Durable Press (Institutional Te/ extile) (1973)

Textile) (1973)

institutional Textile) (1973)

and Women (Institutional Textile) (197/ and Women (Heavy Duty) (Institutional /

ement) (Excluding Glass Fibers) (Instit/

h30.2

Sheet, Strip, and Rolled Bar (19/

and Shapes (1973)

act Materials (1972)

oy Tube (1974) ANSI H23.4

Power acuum Interrupters Used in Power Switchge/ Safety

Institutional and Industrial Use Garm/

t on Production, Heat and Moisture Loss and Feed and W ater tech. Notes) (1969)

$197 /$

Operating Condition of Tires Including Tire Treads Depth elling Construction Under the Uniform Building Code: Legal td. for Air Brake Reservoir Performance and Identification Pract. for New Motor Vehicle on Grade Parking Performance ot Rolled Carbon SteeI Bars Subject to Mechanical Property Uniform Building Code: Fire Zone $s$ for Overhead Protection (Test Procedures and Performance for Agricultural Tractors (Test Procedures and Performance for Agricultural Tractors (Test Procedures and Performance ssion and Distribution Line Compensation (Including Safety for Mobile Homes: Body and Frame Design and Construction al Grounding Devices (1972)

nalyzers (1970T)

Std.

1)

motor Fuels Using the Compression Ratio (CR) Technique for

lants (1972) ANSI D14.2

Rec. Std. Practices for Clinical Std. Meth. of Test for

(Using Spectrophotometer with 1972) poly(Vinyl Chloride) Plastic Sheeting for Pond, Canal, and Std. for Air Brake Rec. for Lined Earth

Std for Nonintegral Industrial Fluid Power Hydraulic Rec. for Concrete Sanitary Engineering Structures (Tanks, Rec. Pract. for Autom atic Spec. for Installation of

ammable Fluids and Anhydrous Ammonia (Fertilizer Grade) in Design and Construction of Masonry Chimneys (For Plywood Construction Guide for Uniform Housing Code

Std. for

ystems for Gas Fired Equipment (1972)

Thermostats (1972)

Std. for Std. Safety Minimum Std. Safety

Minimum

Requirements for Structural Plain Concrete (1972) ACI 3

Requirements for Structural Plain Concrete (1972) ANSI

Requirements for Table Napery Other Than Damask (White Requirements for Tailored Woven Uniform Fabrics, Lightw Requirements for Terry Cloth Towels (Single and Double) Requirements for the Construction and Certification of Requirements for the Construction, Care, and Use of Die Requirements for the Construction, Care, and Use of Pac Requirements for the Construction, Care, and Use of She Requirements for the Design, Installation and Performan (Requirements for the Installation, Control, and Mainten Requirements for the Textile Industry (1972)

Requirements for Thyristor Converters for Motor Drives: Requirements for Tin Mill Products (1973)

Requirements for Transient Recovery Voltage for AC High Requirements for Tree Pruning, Trimming, Repairing, or Requirements for Type II School Bus Construction and Eo Requirements for Vented Decorative Appliances, and Wal Requirements for Wood Job Made Ladders (1973)

Requirements for Woodworking Machinery (1971)

Requirements for Woven Bath Mats (InstitutionaI Textile Requirements for Woven Bedspreads (Institutional Textil Requirements for Woven Blankets (Institutional Textile) Requirements for Woven Decorative Bedspreads. Pillows, Requirements for Woven Dish Towels (Institutional Texti Requirements for Woven Drapery (Curtain) Fabrics (Exclu Requirements for Woven Drycleanable Overcoat Fabrics (I Requirements for Woven Drycleanable Tailored Uniform Fa Requirements for Woven Drycleanable Tailored Uniform Fa Requirements for Woven Fabrics (Textile) for Institutio Requirements for Woven Fabrics (Textile) for Institutio Requirements for Woven Glass Fiber Curtain Fabrics Hand Requirements for Woven Glass Fiber Drapery (Curtain) Fa Requirements for Woven Lining Fabrics for Institutional Requirements for Woven Man Made Fiber Tent, Awning, and Requirements for Woven Printed Table Napery (Napkin, Et Requirements for Woven Sheet and Pillowcase Fabrics Oth Requirements for Woven Shower Curtains (Institutional T Requirements for Woven Slipcover Fabrics (Institutional Requirements for Woven Upholstery Fabrics (Tacked On) ( Requirements for Woven Washable Uniform Fabrics for Men Requirements for Woven Washable Uniform Fabrics for Men Requirements for Woven Window Curtain Fabrics (Sash Cas Requirements for Wrought Copper Alloy Wire (1974) ANSI Requirements for Wrought Copper and Copper Alloy Plate Requirements for Wrought Copper and Its Alloy Rod, Bar, Requirements for Wrought Precious Metal Electrical Cont Requirements for Wrought Seamless Copper and Copper All Requirements for X-Radiation Limits for AC High Voltage Requirements for $100 \%$ Cotton Woven Fabrics (Textile) Fo Requirements of Farm Livestock (Poultry, Swine, Cattle, Requirements Relating to Brick Bearing Wall Buildings ( Requirements (Magnetic Particle Inspection Procedure) ( Requirements (Passenger Car Type Tires) (1971)

Requirements (Permit, Plans and Spec., Inspection) (197

Requirements (1972)

Requirements (1972)

Requirements (1972)

Requirements (1973)

Requirements) (1972) SAE J167

Requirements) (1972) SAE J168

Requirements) (1972) SAE J334

Requirements) (1973)

Requirements, and Installation of Plumbing. Heating and

Research and Motor Meth. Octane Ratings Using on Line

Research Meth. Rating (1971) ANSI Z11.305

Research Protocol for Dental Materials and Devices (197

Reserve Alkalinity of Engine Antifreeze, Antirusts, and

Reserved Optics (1972)

Reservoir Lining (1972T)

Al Reflectance. Transmittance,

Reservoir Performance and Identification Requirements (

Reservoirs for Fire Protection (1972)

Reservoirs (1969) ANSI B93.18

Reservoirs, etc. for Water and Waste Treatment) (1968)

Reset Internal Mounted Circuit Breaker (1971)

ResidentiaI Aluminum Siding (1971)

Residential and Commerical Fuel Burning, Dispensing, an

Residential and Industrial) (Tech. Notes) (1964)

Residential Building (1971)

Residential Buildings) (1973)

Residential Controls-Class 2 Transformers (1973)

Residential Controls Direct Electric Ignition Control S

Residential Fireplace Design (Tech. Notes) (1963)
ANS

ACI

ANSI

ANSI

ANSI

ABS

ANS

ANS

ANSI

ASA

ICBO

ANS

IEEE

ASTM

IEEE

ANSI

VESC

ICBO

ANSI

ANS

ANSI

ANSI

ANSI

ANS

ANSI

ANSI

ANS

ANSI

ANSI

ANSI

ANS

ANSI

ANSI

ANSI

ANSI

ANSI

ANSI

ANS

ANS

ANSI

ANSI

ANS

ANSI

ASTM

ASTM

ASTM

ASTM

ASTM

ANSI

ANSI

ASAE

BIA

SAE

Saf VESC

ICBO

SAE

Rec. SAE

ASTM

ICBO

ASAE

ASAE

ASAE

ANSI

NFPA

IEEE

ASTM

STM

ADA

ASTM

TAPPI

ASTM

SAE

FMS

NFLDP

ACI

$\mathrm{SAE}$

AAMA

UL

BIA

APA

ICBO

NEMA

NEMA

NEMA

BIA

$\mathrm{P} 130$

169.

322

L24.2.11

L24.3.3

. 2.5

* 17

B152.1

B155. 1

B 1]. 4

R264. I

$\mathrm{UMC} \neq 7$

L 1.1 

Std. for Stainless Steel Plumbing Fixtures (Designed for try) (1962) g Thiosulfate and Silver Densitometric Meth. for Measuring st. for Leaks Using the Mass Spectrometer Leak Detector or ill Feeds (Cereal Chemistry) (1962) Std. Meth. of Test for lnsoluble
omponents (Using Rota/ Std. Meth. of Test for Nonvolatile ) (1962) Low Blank Meth. of Analysis of Methoxychlor 1962) shorts, etc./ dulum (1972) ANSI J2.19 .31 crete Paving and Structura! Construction (Nonextruding and ber, Vinyl, Vinyl Asbestos/ Std. Nomenclature Relating to std. Meth. of Test for Electrical Resistance of Conductive

3 ted Paper (1973) Std for Guide for Describing the Characteristics of (1972) 73) Std. Meth. of Test for Fiber Content of Reinforced

48) Modul/ 48) Modul/ (1973)

(1973) Rec Pract for Cloth, Type E Glass, B Stage Polyester Std Spec for TFE -Fluorocarbon (Tetrafluoroethylene) Std. Rec. Pract. for Use of Chemical Resistant i L/ Test Meth. for Determination of Formaldehyde Odor in Test Meth. for Determination of Formaldehyde Odor in ) and Peak Exothermic Temperature of Reacting Thermosetting st for Volatile Matter (Including Water) of Vinyl Chloride ysical and Chemical Properties of Particulate lon Exchange

Std. Meth. of Test for Solvent Tolerance of Amine Std. Meth. of Test for Epoxy Content of Epoxy Std. Meth. of Test for Total Chlorine Content of Epoxy

Meth. of Test for lsophthalic Acid in Alkyd and Polyester of Test for Hydrolyzable Chlorine Content of Liquid Epoxy Trim Materials/ Std. Test Meth. for Determining Blocking Fabrics (1972) ANSI L14./ Z55. 15 rvice (1971) ANSI N14/ 972) ANSl / Std. Meth. for Verifying the Collapse / Burst ) ANSI Z197.7 itions (1970) ANSI Z197.19 2

53.30

Glass (1972T)

d Beverage Glass Containers/ ) ANSI A174.1
Minimum Installation Stds. for Std. for Multiport Valves for Public and Suggested Minimum Stds for Std. Meth. of Test for Pickup Efficiency of Meth of Analysis for Std. Meth. of Test for Std. Meth. of Test for Meth. of Analysis of Meth. of Analysis of Inorganic Bromide Meth. of Analysis of Fungicide Meth. of Analysis of Hydrocyanic Acid Colorimetric Meth. of Analysis of Malathion Std. Meth. of Test for Impact Std. Meth. of Test for Impact Meth. of Test for Std. Rec. Pract. for Measuring Caliper of Std Meth. of Test for Embossed Depth of Test Meth. for Std. Spec. for Oil and Std. Meth. of Test for Volatility of Oil and Std. Spec. for Std. for Liquid Std. Spec. for Reinforced Epoxy Graphite Fiber Tape and Sheet, Epoxy Spec. for Reinforced Thermosetting Sid. Spec for TFE -Fluorocarbon Spec. for Chemical Resistant Std. Spec. for Chemical Resistant Std. Spec. for TFE -Fluorocarbon Rec. Pract. for Chcmical Resistant Std. Meth. of Test for Solubility Range of Std. Spec. for Castor Oil for Use in Std. Meth. of Test for Viscosity of Epoxy Std. Spec. for Poly(vinyl Chloride) Std. Rec. Pract. for Test. Alkyd Std. Meth. of Test for Pilling Sid. Meth of Test for Std. Spec. for Sheathed Electrical Std. Meth. of Test for Peel Std. Test Meth for

Residential Heating and Air Conditioning Systems (1973) Residential Swimming Pools (1969)

Residential Swimming Pools (1972)

Residential Use) (1962)

Residual Aerosol Insecticides (1972)

Residual Carbon Dioxide in Baking Powder (Cereal Chemis Residual Chemicals in Films. Plates, and Papers (1971)

Residual Chlorine in Water (1968)

Residual Gas Analyzer in the Tracer Probe Mode (1973)

Residual Solvents in Flexible Barrier Materials (1972)

Residual Urease Activity in Soybean Meals, Soy Flour, M Residue in Carbonate Aggregates (1972)

Residue of Halogenated Solvent Extract from Aerospace C

Residues in Food Products (Cereal Chemistry) (1969)

Residues in Foods (Cereal Chemistry) (1962)

Residues in Grain and Cereal Products (Cereal Chemistry

Residues in Grain (Cereal Chemistry) (1962)

Residues in Grains and Dried Foods (Cereal Chemistry) (

Residues in Milled Wheat Products (Wheat, Flour. Bran,

Resilience and Penetration of Rubber by the Rebound Pen

Resilience of Rubber by Vertical Rebound (1974) ANSI J2

Resiliency and Thickness of Blankets (Textile) (1973)

Resilient Bituminous Types) (1973) ANSl A37.113

Resilient Floor Covering with Foam Layer (1973)

Resilient Floor Coverings (Asphalt, Cork, Linoleum, Rub

Resilient Floor Coverings (1973)

Resilient Flooring (1972)

Resilient Flooring, Materials Having Flat Surfaces (197 Resilient Mountings (1972)

Resin and Volatile Content, and Flow Properties of Trea

Resin Base Caulking Compound for Building Construction

Resin Based, Knife Grade, Channel Glazing Compounds (19

Resin Chemical Resistant Grouts (1974)

Resin Composites (1973)

Resin Flexes (1972)

Resin Gas Pressure Pipe and Fittings (1973)

Resin Impregnated for Hand Layup (1973)

Resin Impregnated, G70,000 (483) Tensile, $36,000,000$

Resin Impregnated, G70,000 (483) Tensile, $36,000,000(2$.

Resin Impregnated, 181 Style Fabric, Self Extinguishing

Resin Impregnated, 181 Style Fabric, Self Extinguishing

Resin Line Pipe (Rtrp) (1972)

Resin Line Pipe (1972)

Resin Molded Basic Shapes (1974)

Resin Molding and Extrusion Materials (1973)

Resin Monolithic Surfacings (1972T)

Resin Mortars (1974)

Resin Mortars (1974)

Resin Sheet (1974)

Resin Tile Grouts (1972T) (1972T)

Resin Treated Fabric by the Sealed Jar Meth. (1972) Ans

Resin Treated Fabric Steam Meth. (1972) ANSI L14.179

Resins and Polymers (1972)

Resins and Protective Coatings Including Lacquers (1973

Resins and Related Components (1968) ANSl K65.183

Resins (Plastic Composition) (1971) ANSl K65.182

Resins (1972)

Resins (1972)

Resins (1972) ANS1 K65.207

Resins (1973)

Resins (1973)

Resins (1973)

Resins (1973)

Resins (1973)

Resins (1973)

Resistance and Associated Characteristics of Automotive

Resistance and Other Related Surface Changes of Textile

Resistance Characteristics of Microcontacts (1972) ANSl

Resistance Heaters, for Nuclear or Other Specialized Se

Resistance of a Hydraulic Fluid Power Filter Element (1

Resistance of Acoustical Materials (1973)

Resistance of Adhesive Bonds to Chemical Reagents (1966

Resistance of Adhesives to Cyclic Laboratory Aging Cond

Resistance of Adhesives (T-Peel Test) (1972) ANS1 Z197.

Resistance of Adhesives (1973)

Std. Meth. of Test for Stain

Tent. Meth. of Test for Wind Meth. of Test for Acid Tentative Meth. of Test for Sulfide Tent. Meth. of Test for Acid Std. Test Meth. for Meth. for Measuring Blister Std. Meth. of Test for Chip Rec. for Erosion

Std. Meth. of Test for Electrical Std. Meth. for Measuring Chalk
Resistance of Anodic Coatings on Aluminum (1972) ANSI G

Resistance of Asphalt Shingles (1972T)

Resistance of Ceramic Decorations on Architectural Type

Resistance of Ceramic Decorations on Glass (1974)

Resistance of Ceramic Decorations on Returnable Beer an

Resistance of Ceramic Tile to Chemical Substances (1971

Resistance of Coated Paper in Heatset Printing (1972)

Resistance of Coatings (1973)

Resistance of Concrete in Hydraulic Structures (1952)

Resistance of Conductive Resilient Flooring (1972)

Resistance of Exterior Coil Coatings (1974)
SMACN $* 10$ $* 10$

NSPI

USC CS243

ASTM D3093

AACCH 12.10

ANSI PH 4.8

ASTM D1253

ASTM E498

ASTM F15]

$\mathrm{AACCH} \quad 22.90$

ASTM D3042

ASTM F331

AACCH $60-50$

AACCH $60-40$

AACCH $60-10$

$\mathrm{AACCH} 60-01$

AACCH $60-20$

AACCH 60.30

ASTM D 1054

ASTM D2632

ANSI L24T3

/R Con ASTM D1751

ASTM F387

ASTM F141

ASTM F373

ASTM F150

ASTM F386

ANSl S2.8

TAPPI UM.539

ASTM C570

ASTM C681

ASTM C658

ASTM D3171

EIA RS462

ASTM D2517

SAE AMS3894A

SAE AMS 3894/8

SAE AMS3894/9

$\mathrm{SAE}$ AMS3821

SAE AMS3827

API RP5L 4

API 5LR

ASTM D3294

ASTM D1457

ASTM C722

ASTM C395

ASTM C399

ASTM D3293

ASTM C723

AATCC 112

AATCC 113

ASTM D3132

ASTM D 960

ASTM D2393

Time ASTM D2471

D3030

D2187

D1755

ASTM D1198

ASTM D1652

ASTM D 1847

ASTM

Std. ASTM D2690

Dl 726

SAE J9l2A

ASTM D 1375

ASTM B326

ASTM E420

NFLDP T3.10.8.5

ASTM C522

ASTM D896

ASTM D1183

ASTM D1876

ASTM D3167

ASTM B136

ASTM D316l

ASTM C724

ASTM C777

ASTM C735

ASTM C650

TAPPl T526SU

ASTM D3170

$\mathrm{ACl}$

ASTM

$52-18$

NCCA TB-III.8 
Std. Meth. of Test for Detergent Std. Meth. of Test for Soil

(1962) (1962) W Wax Based Blends Appli/

Farinograph Meth. for Analysis of Mixograph Meth. for Analysis of Std. Meth. of Test for Std. Meth. of Test for Abrasion Std. Meth of Test for Abrasion Std. Meth. of Test for Wear Rec. Pract. Test Meth. for Determining Window Fogging Std. Rec. Pract. for the Determination of the Thermal Test Meth. for Determining Pressure Marking and Blocking Std. Rec. Pract. for Detergent el (Coil Coating) (1/ Test Meth. for Measurment of Impact als by the Dry Indicator $\mathrm{Me} /$ Std. Meth. of Test for Water cessing (1972) Meth. Std. for Meth. for Determining the Std. Meth. of Test for Penetration Std. Meth. of Test for lmpact Std. Meth. of Test for Impact Std. Test Meth. for Chemical

1972)

als (1973)

)

nsi A195.1

(1972)

of a Tup (Falling Weight) /

8) ANSI J2.26

ther Tester (1970)/

netration Tester (1)

r 4 Probe Array (1974) Tentative Meth. of Test for Sheet

als: Accelerator Meth. (1970)

14.74, ASTM D583

Std. Meth. of Test for Pneumatic Ball lmpact Meth. of Test for Algal Std. Meth. of Test for Impact Std. Meth. of Test for Drop Impact Std. Meth. of Test for Spalling Std. Meth. of Test for Alkali Std. for Safety for Wind

Std. Meth. of Test for Abrasion Std. Meth. of Test for Impact Std. Meth. of Test for lncandescence Safety Std. for Tests for Fire

Std Meth of Test for Abrasion Std. Meth. of Test for Dynamic Water Std. Meth. of Test for Dynamic W ater Test Meth. for Abrasion Test Meth. for Water Test Meth. for Fire Std. Meth. of Test for Abrasion $t$ and Weather (1968) ANSI L14.176 i (1971) ANSI L14.265 Test Meth. for (1971) ANSI L14.71, ASTM D583

bon Arc Lamp Exposure with Wettin Test Meth. for Water Test Meth. for Water Test Meth. for Weather Test Meth. for st Meth. for the Evaluation of Mildew and Rot (Fungicides) t Wetting (1972) ANSI L14.225 Test Meth. for Weather eather Through Glass (1972) ANSI L14.177 Water Soluble Salts in Pigments by Measuring the Specific Probe Array (1973)

(1973) ANSI K65.72 ansi A153.1

Tent. Meth, of Test for Sheet Std. Meth. of Test for Std. for Safety for Tests for Fire Std. Meth. of Test for Tear Test Meth. for Sna

td. Meth. for Accelerated Laboratory Test of Natural Decay Std. Test Proc, and Acceptance Criteria for W ater buildings (Tech. Notes) (1969) e) (1973)

Spec. for Std. for

Test Meth. for Oil Repellency of Fabrics: Hydrocarbon Std. Spec, for Std. Tire for Pavement Skid or Other Failure/ Std. Meth. for Water Immersion Test for Meth. of Test. Coated Steel Specimens Dynamically for xtures by Means of Hveem Apparatu/ Coatings in an Environmental $\mathrm{Ch} /$ Std. Meth. of Test for Tent. Meth. of Test for Std. Meth. of Test for Marshall Apparatus (1973) Std. Meth. of Test for 3) Test Meth. to Determine Woven Fabric's Std. Meth. of Test.

loth (1972)

Std. Test Meth for Determining Std. Meth. for Laboratory Evaluation to Determine Meth. for Flame

and Superheater Tubes (1973) Ans/ ubes for High Pressure Service (/

B125.6 for Supplementary Requirements for Seamless and Electric Std. Spec. for Electric Rec. Pract. for Sid. for

st for Time of Setting of Concrete Mixtures by Penetration nstitutional Textiles (Durability. Spot, Stain, and Insect Solution Heat Treated, 15./ All Metal (1973)
Spec. for Corrosion and Heat Stds. for Forced Entry
Resistance of Floor Polish Films (1973)

Resistance of Floor Polishes (1973)

Resistance of Flour Dough to Mixing (Cereal Chemistry)

Resistance of Flour Dough to Mixing (Cereal Chemistry)

Resistance of Glass Containers to Chemical Attack (1973

Resistance of Glossy Smooth Coatings of Petroleum Wax O

Resistance of Horizontal Concrete Surfaces (1974)

Resistance of Inked Computer Ribbons (1972)

Resistance of Interior Trim Materials (1973) ANSI 2265

Resistance of Low Density Mineral Fiber Blanket Type Bu

Resistance of Organic Coatings When Applied to Coil Coa

Resistance of Organic Finishes (1973)

Resistance of Painted Aluminum, Steel and Gavanized Ste

Resistance of Paper, Paperboard, and Other Sheet Materi

Resistance of Paperboard (1972)

Resistance of Photographic Films to Abrasion During Pro

Resistance of Pipeline Coatings (Blunt Rod) (1972)

Resistance of Pipeline Coatings (Falling Weight Test) (

Resistance of Pipeline Coatings (Limestone Drop Test)

Resistance of Pipeline Coatings (1972)

Resistance of Pipeline Coatings (1972)

Resistance of Plastic Film and Sheeting (1972)

Resistance of Plastic Films (1972T)

Resistance of Plastics and Electrical Insulating Materi

Resistance of Polyethylene Blow Molded Containers (1970

Resistance of Porcelain Enameled Aluminum (1972)

Resistance of Porcelain Enamels (1974) A VSI Z167.20

Resistance of Prepared Roof Covering Materials (1973) a

Resistance of Refractory Materials at Room Temperature

Resistance of Rigid Plastic Sheeting or Parts by Means

Resistance of Rigid Plastics (1974) ANSI K65.117

Resistance of Roof Coveriag Material (1973)

Resistance of Rubber Compounds for Soles and Heels (196

Resistance of Shoe Upper Leather by the Dow Corning Lea

Resistance of Shoe Upper Leather by the Maeser Water PE

Resistance of Silicon Epitaxial Layers Using a Collinea

Resistance of Solid Electrical Insulation (1973)

Resistance of Textile Fabrics and Other Flexible Materi

Resistance of Textile Fabrics (Rain Test) (1971) ANSI L

Resistance of Textile Fabrics (1969)

Resistance of Textile Fabrics (1971)

Resistance of Textile Fabrics: Exposure to Natural Ligh

Resistance of Textile Fabrics: Hydrostatic Pressure Tes

Resistance of Textile Fabrics: Impact Penetration Test

Resistance of Textile Materials and Coated Fabrics: Car

Resistance of Textiles to Insects (1971) ANSl L14.64

Resistance of Textiles (1971) ANSI L14.55

Resistance of Textiles: Carbon Arc Lamp Exposure Withou

Resistance of Textiles: Exposure to Natural Light and W

Resistance of the Leachate of the Pigment (1973)

Resistance of Thin Metallic Films with a Collinear Four

Resistance of Transparent Plastics to Surface Abrasion

Resistance of Vault and File Storage Room Doors (1972)

Resistance of Vulcanized Rubber (1973)

Resistance of Women's Nylon Hosiery (1973) ANSI L14.85

Resistance of Woods (1970)

Resistance on Steel Door and Frame Assemblies (1972)

Resistance Requirements Relating to Brick Bearing Wall

Resistance Seam and Roll Spot Welding Equipment (3 Phas

Resistance Spot Welding Equipment, 3 Phase (1973)

Resistance Test (1972) ANSI L14.214

Resistance Tests (1973)

Resistance to Blistering. Wrinkling. Loss of Adhesion,

Resistance to Corrosion (1972T)

Resistance to Deformation and Cohesion of Bituminous $\mathrm{Mi}$

Resistance to Growth of Mold on the Surface of Interior

Resistance to Paper to Passage of Air (1958) TAPPI T460

Resistance to Plastic Flow of Bituminous Mixtures Using

Resistance to Sagging or Stretch in Wear (Textile) (197

Resistance to Scuffing of Trim Materials (1972)

Resistance to Snagging and Abrasion of Automotive Bodyc

Resistance to Subterranean Termites (1972)

Resistance Treated Paper and Paperboard (1972)

Resistance Welded Carbon Molybdenum Alloy Steel Boiler

Resistance Welded Carbon Steel Boiler and Superheater T

Resistance Welded Carbon Steel Boiler Tubes (1973) ANSI

Resistance Welded Carbon Steel Tubular Products for Hig

Resistance Welded Steel Pipe (1972A)

Resistance Welding Coated Low Carbon Steels (1970)

Resistance Welding Cont rol (1974)

Resistance (1970) ANSI A37.134

Resistance) (1973)

Characteristics and Finishes of

Resistant Alloy Bars, Forgings, and Rings (Nickel Base,

Resistant Alloy Nuts, High Strength, Prevailing Torque,

Resistant Aluminum Horizontal Sliding Doors (1971)

Resistant Ceramic Tile Floors (1973)

ASTM

ASTM

$\mathrm{AACCH}$

A ACC

ASTM

ASTM

ASTM

A STM

SAE

ASTM

NCCA

ASTM

NCCA

ASTM

TAPP

ANSI

ASTM

ASTM

ASTM

A STM

ASTM

ASTM

ASTM

ASTM

ASTM

ASTM

ASTM

UL

ASTM

A STM

ASTM

UL

ASTM

ASTM

ASTM

ASTM

ASTM

A A TCC

AATCC

AATCC 3

ASTM

A ATCC

AATCC

AATCC

AATCC

AATCC

Te AATCC

AATCC

AATCC

/ for ASTM

ASTM

ASTM

UI

ASTM

A ATCC

ASTM

STDI

BIA

NSA

NSA

AATCC

ASTM

NCCA

ASTM

A STM

A STM

ASTM

ASTM

ANSI

SAE

$A$ WPA

TAPPI

ASTM

A STM

ASTM

ASTM 
Std. Classifications for Spark Resistant Construction (1966)

Std. for Safety for Burglary

al Power Cables Rated 2000 Volts and Less and Having Ozone ctrical Power Cables Rated $0.35,000$ Volts and Having Ozone 2) ANSI B93.5

Std. Pract, for the Use of Fire Std. Spec. for Resin Chemical Uniform Building Code Std. for Water I Base Alloy Castings for Gener/ Std. Spec. for Corrosion Std. Spec. for Chemical ec. Pract. for Use of Hydraulic Cement Mortars in Chemical 4) a Spec, for Flame nickel, Chromiu/ Uniform Building Code Std. for Corrosion Spec. for Chemically Setting Silicate and Silica Chemical Spec. for Chemical Std. Spec. for Chemical Std. Rec. Pract. for Use of Chemical Rec. Pract. for Chemical Safety Std. Burglary

Std. Rec. Pract. for Use of Chemically Setting Chemical 3. 3 W) (1973)

Std. for Solid 100 Deg. Flush Shear Head A286 Corrosion Std. for Packing, Preformed O-Ring Phosphate Ester Test. Std. (Procedure) for Products Classified as to Slip pec, for Steel Bars, Forgings, and Tubing, Low Alloy, Heat Spec. for Steel Welding Wire, Corrosion and Moderate Heat teel Bars, Forgings, Tubing, and Rings, Corrosion and Heat Spec. for Steel Welding Wire, Corrosion and Heat

d. for Bolt-Machine, Hexagon Head, Nonmagnetic, and Heat Spec. for Steel Tubing, Welded, Corrosion and Heat Alloy Wire, Welding, Corrosion and Heat Bolts and Screws, Steel, Low Alloy Heat 1) Spec. for Self-Locking Steel Nuts, Corrosion and Heat 2.5Ti-1.6/ Alloy Bars and Forgings, Corrosion and Heat Alloy Bars, Forgings, and Rings, Heat e (1973) Alloy Sheet and Strip, Corrosion and Heat 5La Alloy Bars, Forgings, and Rings, Corrosion and Heat Alloy Sheet, Strip, and Plate, Corrosion and Heat i-1.4A 1, C/ Alloy Bars and Forgings, Corrosion and Heat (1973) on $\mathrm{H} /$ Alloy Wire, Welding, Corrosion and Heat - 1.4A, Contoy Bars, Forgings, and Rings, Corrosion and Heat ec. for Alloy Bars, Forgings and Rings, Corrosion and Heat pec. for Alloy Sheet, Strip, and Plate, Corrosion and Heat 000 psi (931 MPa) / Spec. for Low Alloy Steel Studs, Heat F (982.2 Deg. C) Solu/ Studs, Steel, Corrosion and Heat ng / Steel Bars and Forgings, Corrosion and Moderate Heat Steel Bars, Wire, Forgings, and Forging Stock, Corrosion $m$ Bearing Qu/ Steel Bars, Forgings, and Tubing, Corrosion le Electrode Va/ Steel Sheet, Strip, and Plate, Corrosion , 1/ Steel Bars and Forgings, Corrosion and Moderate Heat i Tre/ Steel Sheet and Strip, Corrosion and Moderate Heat t Treated (1973/ Steel Plate, Corrosion and Moderate Heat t T/ Steel Bars and Forgings, Corrosion and Moderate Heat Steel Wire, Welding, Corrosion and Moderate Heat d T/ Steel Bars and Forgings, Corrosion and Moderate Heat , Forgings, Tubing, and Rings, Corrosion and Moderate Heat
rdened / Spec. for Low Alloy Steel Bolts and Screws, Heat ne Rubber, General Purpose, High Temperature, Fuel and Oil 974) ANSI C8.37

welding Electrodes (1969) ANSI W3.4 rods and Bare Electrodes (1969) Ansi/

(1974)

et, and Strip for Fusion Welded Unfil Spec. for Corrosion Spec. for Corrosion Sid. Spec. for Heat Spec. for Hot Rolled and Cold Finished Stainless and Heat 72) ANSI G81.6

ANSI G81.2 Std. Spec. for Corrosion Std. Spec. for Stainless and Heat stitute for Plate Glass Show Wi/ Std. for Safety for Bullet $38 \quad$ Std. Spec. for Synthetic Rubber Heat or Moisture 29 Std. Spec. for Synthetic Rubber Heat or Moisture
Std. Spec. for Ozone ation / Std. Spec. for Synthetic Rubber Heat and Moisture ation (1974) ANSI C8./ Std. Spec. for Natural Rubber Heat 4 In. Reinforced Brick Masonry Curtain and Panel Walls (In other Pressure Vessels/ Std. Spec. for Stainless and Heat Rolled and Cold Finished Age Hardening Stainless and Heat nsi G81.13 Std. Spec. for Stainless and Heat Std. Spec. for Stainless and Heat Std. Spec. for Stainless and Heat equirements for Delivery of Flat Rolled Stainless and Heat Std. Spec. for Stainless and Heat Std. Spec. for Stainless and Heat Std. Spec. for Free Machining Stainless and Heat $\mathrm{d}$ and Drilled, Plain and Self Locking, Alloy and Corrosion
Resistant Electric Door Strikes (1974)

Resistant Ethylene-Propylene-Rubber Integral Insula Resistant Ethylene Propylene Rubber Insulation (1973) I Resistant Fluids for Hydraulic Fluid Power Systems (197 Resistant Grouts (1974)

Resistant Gypsum Backing Board (1973)

Resistant Iron Chromium, Iron Chromium Nickel and Nicke

Resistant Masonry Units (1972) ANSI A 103.1

Resistant Masonry (1965)

Resistant Materials Used in Camping Tentage (Tent) (197

Resistant Metals (Electrodeposited Coatings of Copper,

Resistant Mortars (1974)

Resistant Resin Monolithic Surfacings (1972T)

Resistant Resin Mortars (1974)

Resistant Resin Mortars (1974)

Resistant Resin Tile Grouts (1972T) (1972T)

Resistant Safes (1972) ANSI Se 4.1

Resistant Silicate and Silica Mortars (1974)

Resistant Steel and Monel Rivet (1974)

Resistant Steel Sheet, Strip, and Plate $(13 \mathrm{Cr}-2.0 \mathrm{Ni}-$

Resistant Vinyl Chloride Plastic Pressure Sensitive Ele

Resistant (-65 Deg. F to + 160 Deg. F) (1972)

Resistant (Floor Polish and Wax) (1971) A STM D2047, Csm

Resistant $(0.95 \mathrm{Cr}-0.55 \mathrm{Mo}-0.30 \mathrm{~V}(0.40-0.50 \mathrm{C}) \mathrm{Vacuu}$

Resistant $(11.8 \mathrm{Cr}-2.8 \mathrm{Ni}-1.6 \mathrm{Co}-1.8 \mathrm{Mo}-0.32 \mathrm{~V})(197$

Resistant $(15 \mathrm{Cr}-26 \mathrm{Ni}-1.3 \mathrm{Mo}-2.1 \mathrm{Ti}-0.30 \mathrm{~V}) 1800 \mathrm{DC}$

Resistant (18Cr-11Ni-(Cb+Ta) (1973)

Resistant (1973)

Resistant (25Cr-20Ni) (SAE 30310) (1973)

Resistant, Cobalt Base-25.5Cr-10.5Ni-7.5W (1973)

Resistant, Hardened and Tempered, Roll Threaded (1973)

Resistant, High Strength, Prevailing Torque, All Metal,

Resistant, High Strength, Prevailing Torque, All Metal,

Resistant, Iron Base-13.3 $\mathrm{Cr}-38 \mathrm{Ni}-5.5 \mathrm{Mo}-0.85 \mathrm{Cb}-$

Resistant, Iron Base-35.5 Ni-18.5Cr-1.1SI (1973)

Resistant, Nickel Base-15.5Cr-2.4Ti-0.70A1-7.0F

Resistant, Nickel Base-15.5Cr-8.0Fe (1973)

Resistant, Nickel Base $-15.8 \mathrm{Cr} \sim 15.2 \mathrm{Mo}-0.30 \mathrm{Al}-0.0$

Resistant, Nickel Base-15.8Cr-15.2Mo-0.30Al-0.0

Resistant, Nickel Base-19.5Cr-13.5Co-4.3Mo-3.0T

Resistant, Nickel Base-19.5Cr-18Co-2.5Ti-1.5Al

Resistant, Nickel Base-5.0Cr $-24.5 \mathrm{Mo}-5.5 \mathrm{Fe}$, Soluti

Resistant, Nickel Base 19.5Cr-13.5Co-4.3Mo-3.0Ti

Resistant, Nickel Base $(19.5 \mathrm{Cr}-13.5 \mathrm{Co}-4.3 \mathrm{Mo}-3.0 \mathrm{Ti}$

Resistant, Nickel Base, 20Cr-20Co-5.9Mo-2.2Ti-0

Resistant, Normalized and Tempered, Roll Threaded (135,

Resistant, Roll Threaded After Heat Treatment, 1800 Deg

Resistant, 12.5Cr Low Carbon (SAE 51416F), Free Machini

Resistant, $12 \mathrm{Cr}-8.5 \mathrm{Ni}-2.0 \mathrm{Cu}-1.1 \mathrm{Ti}$, Vacuum Inducti

Resistant, $14.5 \mathrm{Cr}-4.0 \mathrm{Mo}-1.2 \mathrm{~V}(1.10-1.20 \mathrm{C})$, Premiu

Resistant, $14.8 \mathrm{Cr}-4.5 \mathrm{Ni}-3.5 \mathrm{Cu}-(\mathrm{Cb}+\mathrm{Ta})$, Consumab

Resistant, $15.5 \mathrm{Cr}-4.5 \mathrm{Ni}-2.9 \mathrm{Mo}-0.10 \mathrm{~N}$, Heat Treated

Resistant, 15.5Cr-4.5 Ni-2.9Mo-0.10N, Solution Hea

Resistant, $15.5 \mathrm{Cr}-4.5 \mathrm{Ni}-2.9 \mathrm{Mo}-0.10 \mathrm{~N}$, Solution Hea

Resistant, $15.5 \mathrm{Cr}-4.5 \mathrm{Ni}-2.9 \mathrm{Mo}-0.10 \mathrm{~N}$, Solution Hea

Resistant, $16.5 \mathrm{Cr}-4.5 \mathrm{Ni}-2.9 \mathrm{Mo}-0.1 \mathrm{~N}$ (1973)

Resistant, $16.5 \mathrm{Cr}-4.5 \mathrm{Ni}-2.9 \mathrm{Mo}-0.10 \mathrm{~N}$, Equalized an

Resistant, 17Cr (SAE 51430) Annealed (1973)

Resistant, 195,000 psi (1,345 MPa) Tensile Strength, $\mathrm{Ha}$

Resistant, 65-75 (1973)

IIngs, Sealing, F

Resisting Chromium and Chromium-Nickel Steel Covered

Resisting Chromium and Chromium-Nickel Steel Welding

Resisting Chromium and Chromium Nickel Steel Electrodes

Resisting Chromium and Chromium Nickel Steel Plate, She

Resisting Chromium Nickel Manganese Steel Bars Includin

Resisting Chromium Steel Clad Plate Sheet and Strip (19

Resisting Chromium SteeI Plate, Sheet, and Strip (1972)

Resisting Equipment (Barrier) for Protection Against Ro

Resisting Glazing Material for Use Principally as a Sub

Resisting Insulation for Wire and Cable (1974) ANSI C8.

Resisting Insulation for Wire and Cable (1974) ANSI C8.

Resisting Insulation for Wire and Cable, $75 \mathrm{Deg} . \mathrm{C}$ Oper

Resisting Insulation for Wire and Cable, 75 Deg. C Oper

Resisting Lateral Forces) (Tech. Notes) (1968)

Resisting Steel Bars and Shapes for Use in Boilers and

Resisting Steel Bars and Shapes (1972) / S

Resisting Steel Billets and Bars for Refor oing (1972)

Resisting Steel Forgings (1973) ANSI G81.26

Resisting Steel Plate, Sheet, and Strip (1972) ANSI G81

Resisting Steel Rope Wire (1972) ANSI G81.32

Resisting Steel Wire (1971) ANSI G81.41

Resisting Steel Wire (1971) ANSI G81.42

Resisting Steel, UNJC-3A Thread, Cap Screw (1972)
AMCA
UL

NEMA

NEMA

ASTM

ICBO

ASTM

ASTM

Std. R ASTM

CPAI

ICBO

Std. ASTM

ASTM

ASTM

ASTM

ASTM

UL

ASTM

NSA

SAE

NSA

CSMA

SAE

SAE

SAE

SAE

St NSA

SAE

SAE

SAE

SAE

SAE

SAE

SAE

SAE

SAE

SAE

SAE

SAE

SAE

SAE

SAE

SAE

SAE

$S A E$

SAE

SAE

SAE

SAE

SAE

SAE

SAE

SAE

SAE

SAE

SAE

SAE

AWS

AWS

ASTM

ASTM

ASTM

ASTM

UL

UL

ASTM

ASTM

ASTM

ASTM

BIA

ASTM pec. for Hot ASTM

ASTM

A STM

ASTM

ASTM

ASTM

ASTM

ASTM

/Lle NSA

401

1034

WC $8 / 1$

T3.11.1

C658

UBCS47.13

A 296

C398

84

UBCS 32.6

C466

C722

C 395

C399

C723

687

C397

1200

AMS5508B

D3005

1611

BUL 12

AMS5823

AMS5735H

1003

AMS5577C

AMS5789

AMS7455D

AMS7250C 
drilled, Plain and Self Locking, Alloy Steel and Corrosion Std. Meth. for Chemical Analysis of Stainless, Heat Uniform Building Code: Fire Std. Meth. of Test for Volume Std. Meth. of Test for

nsi C7.24

Std. Test Meth. for D.C Volume Std. Meth. of Test for Electrical

$s$ at Room Temperatures voltage Breakdown Meth. (1972) An/ Std. Meth. of Test for array (1973)

rns (1973) ANSI L14.119

) ANSI L14.112 19 Test Meth. for Electrical Std. Meth. for Measuring Std. for Reference Std. Precision Electrical Std. for Dimensions for strips and Slides in 2 X 2 In. Mounts (1972) dulus, and Poisson's Ratio for Glass and Glass Ceramics by
Rec. Pract. for Passenger Car and Light Truck Std. Test Meth. for Filler Content of Phenol, Std. Test Meth. for Nonvolatile Content of Phenol, Std. for Identification of Air Purifying Safety Guide for Std. for Practice for

humidifiers, Nebulizers, Gas Therapy and Suctio/ Std. for rs for Audio Record One for 2-In. Quadrup/ Std. Frequency
for Rifle Bench Construction Spec. for Rifle Bench Std. for Hotel and

orium, Dormatory, Public or Office Building, Manufacturer. Rec. Std. for Seismic

twear, Hose and Tubing, Mattresses and Pads, Sheeting, and Std. Spec. for Hard Drawn Copper Capillary Tube for Vulcanizates Std for Packing with Std. Spec. for Alloy Steel Forgings for Nonmagnetic Std. for General Purpose Uniform Cross Section Spiral Reinforced Brick Masonry

Uniform Building Code: Excavations, Foundations, and struction Under the Uniform Building Code: Foundations and Uniform Building Code Std. for Fire
Std. Meth. of Test for Durability of Fire

Tent. Meth. of Test for Hygroscopic Properties of Fire Std. Meth. of Measuring Plasticity Meth. of Test for Measuring Soil Removal and Reflectance
Std. Meth. of Test for Gloss 1 $104 \mathrm{~F}(40 \mathrm{C})(1973)$

t 140 Deg. (40 Deg. C.) (1972) Meth. for Gloss hes, and Finishings (Sublimation, Brittleness and Chlorine
tics of Rubber and Rubber Like Materials by a Temperat ure stics of Rubber and Rubber Like Materials by a Temperat ure
m Performance Requirements and Uniform Test Procedures for eth. of Test for Acid Resistance of Ceramic Decorations on he Determination of Sound Power Levels of Small Sources in

td. Meth. for Determining Speed of $16 \mathrm{Mm}, 8 \mathrm{~mm}$ and Super 8

Uniform Building Code Std. for Stress Variation or Stress posure and Evaluation Meth. for Determining Speed of Color Test Meth. for Sizing of Paper

film Adhesion on All Metals by Cross Hatch Tape Test After

Iation of Vertical Hydraulic Turbine Driven Generators and

Large Hydraulic Turbine Driven Synchronous Generators and

uilding Code: Stairs, Exits and Occupant Loads (Buildings, Uniform Fire Code: Places of Assembly (Theaters,

Std. Meth. of Test. Shipping Containers (Packaging) in 114.106 Test Meth. for Evaluation of Commercial d. Definitions of Terms Relating to Carbon Paper and Inked

Std. Meth. of Test for Wear Resistance of Inked Computer Std. Meth. of Test for Erasability of Inked and Puffed Cereals, F/ $\begin{gathered}\text { Fluorometric Meth. of Analysis of } \\ \text { Microbiological Meth. of Analysis of }\end{gathered}$ of Analysis of Moisture in Grains, Beans, Peas, Lentils,

Thiochrome Meth. of Analysis of Thiamine in Bread, Wheat, isture in Flour, Farina, Semolina, Bread, Grain, Soybeans,

ina; Also, with Slight Modification, to Corn, Rye, Barley,

g Susceptibility to Intergranular Attack in Wrought Nickel eeve Attachment for Hitching Implements to Lawn and Garden Construction of Indoor Construction Spec. for

50 Ft. Summer Camp Metallic Silhouette Match Outdoor Smallbor Running Game (Wild life) fety Rules, Procedures For, and Construction of High Power Uniform Fire Code:

Spec. for Petroleum Drilling ils at Electric Generating Stations (Flood, Surface Water, Coated (Galvanized) Iron or Steel Farm Field and Railroad
Resisting Steel, UNJF.3A Thread, Cap Screw (1972)

Resisting, Maraging, and Other Similar Chromium-Nicke Resistive Std. (1973)

Resistivity of Conductive Adhesives (1972)

Resistivity of Electrical Conductor Materials (1972A) a

Resistivity of Glass (1972)

Resistivity of Manufactured Carbon and Graphite Article

Resistivity of Silicon Epitaxial Layers by the 3 Probe

Resistivity of Silicon Slices with a Collinear 4 Probe

Resistivity Textile of (Electro-Static) of Textile $Y_{a}$

Resistivity (Electro-Static) of Textile Fabrics (1972

Resistors (1969) ANSI C 100.3

Resolution Test Target for Photographic Optics (1972)

Resolving Power of Lenses for Projectors for $35 \mathrm{~mm}$ Film

Resonance (1971)

/F Test for

Resonator Parts Nomenclature (1971)

Resorcinol, and Melamine Adhesives (1970)

Resorcinol, and Melamine Adhesives (1970)

Respirator Canisters and Cartridges (1973)

Respiratory Protection Against Coal Mine Dust (1972)

Respiratory Protection for the Fire Service (1973)

Respiratory Therapy Equipment (Breathing Devices, Room

Response and Operating Level of Recorders and Reproduce

Rest (Portable and Permanent) (1967)

Restaurant Gas Deep Fat Fryers (1971)

Restaurant Gas Ranges (Stoves) and Unit Broilers (1973)

Restaurant, and Dwelling or Appartment House (1973)

Restraint Direct Hung Suspended Ceiling Assemblies (197

Restraint Straps) (1974) /Ks and R

Resulting from Immersion in Liquids (1972)

Retainer-Floating Barrel Nut (1973)

Retainer (1974)

Retaining Rings for Generators (1970) ANSI G55.6

Retaining Rings (1972)

Retaining Walls (Tech. Notes) (1965)

Retaining Walls (1973)

Retaining Walls (1973)

Retardant Roof Covering Materials (1973)

Retardant Treatment of W ood (1972)

Retardant Wood and Its Base Products (1973)

Retention Index (PRI) of Natural Rubber (1973)

Retention of Fabrics (1972T)

Retention of Waxed Paper and Paperboard After Storage a

Retention of Waxed Paper and Paperboard After Storage a

Retention) (Institutional Textile) (1973)

Retraction Procedure (TR Test) (R1972) ANSI J2.22

Retreaded Tires for Passenger Cars and Station Wagons (

Returnable Beer and Beverage Glass Containers (1972T)

Reverberation Rooms (Acoustics) (1972) /or Meth. for T

Reversal Color Camera Films Intended for Direct Project

Reversal Design of Structural Steel Members (1973)

Reversal Films for Still Photography (1972)

Reverse Curl) (1973)

Reverse Impacting (Coil Coating) (1974)

Reversible Generator/Motors for Pumped Storage Installa

Reversible Synchronous Generator / Motor Units for Pump

Reviewing Stands, Bleachers, and Grandstands) (1973)

Reviewing Stands, Grandstands, Bleachers, etc.) (1973)

Revolving Hexagonal Drum (1968) ANSI MH12.3, TAPPI T800

Revolving Screen Stds. (1972)

Rewetting Agents on Cotton Textile Fabrics (1971) ANSI

Ribbon Products and Images Made Therefrom (1972A)

Ribbons (1972)

Ribbons (1972)

Riboflavin in Whole Grain Products, Grits, Meal, Flaked

Riboflavin (Cereal Chemistry) (1962)

Rice, and Other Commodities for Which Conversion Charts

Rice, and Other Whole Grain Products, Corn Grits, Corn

Rice, Beans, Peas, Lentils, Corn Meal, Corn Grits, Roll

Rice, Grain Sorghum, and Buck wheat (Cereal Chemistry)(

Rich, Chromium Bearing Alloys (1972) ANSI G80.4

Std. for One Point Tubular SI

Riding Tractors (1972)

Rifle and Pistol Ranges and Bullet Stops (1973)

Rifle Bench Rest (Portable and Permanent) (1967)

Rifle Range Construction (Part Time and Special) (1971)

Rifle Range Location and Landscaping (1970)

Rifle Range Plans (1972)

Rifle Range Plans (1973)

Rifle Range Plans: Safety Rules and Procedures (1972)

Rifle Ranges (1971)

Rifle Ranges (1972)

Rifle Ranges (1973)

Rig Packaging for Minimum Self Contained Platforms (197

Rigging Collapse, Burglary, and Roof Collapse) (1974)

Right of Way Wire Fencing (1973) ANSI G8.9

1. for Zine ASTM
1351

ASTM

ICBO

ASTM B193

ASTM C611

ASTM F108

ASTM F84

AATCC 84

AATCC 76

IEEE

ANSI

ANSI

ASTM

SAE

ASTM

ANSI

ANSI

ANSI

NSF

ANSI

NRA

ANSI

ANSI

IT ICBO CISCA UL

ASTM

ASTM

NSA

ANSI

BIA

ICBO

ICBO

ICBO

ASTM

ASTM

ASTM

ASTM

TAPPI

ANSI

Eri ASTM

VESC

ASTM

ANSI

ANSI

ICBO

Metric Ex ANSI

TAPPI

NEMA

NEMA

ICBO

ICBO

ASTM

IPA

AATCC

St ASTM

ASTM

ASTM

AACCH 86-72

$\triangle A C C H$ 44-11

AACCH $86-80$

AACCH $44-15$ A

$\mathrm{AACCH} \quad 76.20$

ASTM G28

ASAE

NRA

NRA

NRA

NRA

NRA

NRA

NRA

NRA

Sa NRA

ICBO

API

S348T

$* 1$

$* 10$

$* 11$

*16

$* 18$

$* 4$

$* 12$

UFC $* 2 \mathrm{C}$

$2 \mathrm{E}$

9-10

All6

Engineering and Product Standards Division 
Pract for Determining the Strength of Adhesively Bonded ing Weight)/ Std. Meth. of Test for Impact Resistance of

72)

72) ANSI C59.33

ulation (1972) ANSI C59.13

Std. for

Std. for Polyvinyl Chloride Externally Coated Galvanized

Spec for Cabin Duet Air, Flex

f Road, Agricultural and Log Skidder, Truck, Bus, Bead S rm Machinery) (1971)

Std for

Std. for Tire and Stds. for Tire Std. for Tire

rec. Tolerances of Spoke Type Wheels, Demountable Rims and Minimum Rec. Tolerances of Spoke Type Wheels, Demountable 74 Std. for Tires, Tubes Std. for Tires and Std. for Tires and Std. for Tires and Std. for Tires and cles, Counter Balanced Fork Lift Trucl 1974)

Logging (Short Haul); Grader, Loader/ adial Ply Tires and se Passenger Vehi/

Std. for Bias and Radial Ply Tires and

ures for Production Disc and Spoke Wheels, and Demountable Water Safety Buoyant Devices (Vest, Jacket, Horseshoe and k/ Spec. for (Oil) Wellhead Equipment (Production Valves, Sid. Meth. of Test for Relative Density of Black Smoke es, Nails and Spikes, Washers, Pins, Dowels, Joints, Split (1972) Rec. Pract. Spec. for Cast Iron Sealing Spec. for Alloy Steel Forgings for Nonmagnetic Retaining for Corrosion and Heat Resistant Alloy Bars, Forgings, and Spec. for Aluminum Alloy Bars and 3) Spec. for Aluminum Base Alloy Bars and Mechanical Tubing, and Flash Welded Steel Bars, Forgings, Mniform Cross Section Spiral Retaining Floating Type Metallic and Nonmetallic Fluid Power Piston Dimensions for Fluid Power Radial Compression Type Piston $-4.0 \mathrm{Co}-0.48 \mathrm{Mo}-0.09$ Sper for Steel Bars, Forgings, $0-2.1 \mathrm{Ti}-1$ Spec. for Steel Bars, Forgings, Tubing, and $\mathrm{cr}-8.0 \mathrm{Fe}(1973)$ $\mathrm{r}-15.2 \mathrm{Mo}-0.30 \mathrm{Al}-0.05 \mathrm{La} / \mathrm{Alloy}$ Bars, Forgings, and $\mathrm{r}-24.5 \mathrm{Mo}-5.5 \mathrm{Fe}$, Solution $\mathrm{H}$ $-13.5 \mathrm{Co}-4.3 \mathrm{Mo}-1$ 51430) Annealed (1973/ ISI (1973)

Alloy Bars, Forgings, and Spec. for Alloy Bars, Forgings and Steel Bars, Forgings, Tubing, and Alloy Bars, Forgings, and

High Temperature, FueI and Oil Resistant, 65-75 (I973/ special Grade (1973) Steel Bars, Forgings, Tubing, and or Tearing Strength of Woven Fabrics by the Tongue (Single Uniform Fire Code: Fruit Rec for Fire Safety in High iform Building Code Std. for Fire Alarm Systems for High. Meth. of Analysis for Baking Quality of Self ysis for Original Ash in Phosphated and Freshly Mixed Self alysis for Total Carbon Dioxide in Prepared Mixes and Self

3) nce Head and Shank (1973)
Std. for Std. for

Std Spec for Aluminum Alloy Uniform Building Code Std. for Structural Std. for Hi She ar Protruding Head

Std. for Hi Shear 100 Deg. Flush Close Tolerance Head Std, for Protruding Head, Locked Spindle Blind

Std. for Blind, 100 Deg. Flush Head, Locked Spindle Std. for Self Plugging, Mechanically Locked Spindle for Bulbed Mechanically Locked Spindle Self Plugging Blind r Bulbed Mechanically Locked Spindle Protruding Head Blind $r$ Bulbed Spindle Mechanic ally Locked Protruding Head Blind lbed Mechanically Locked Spindle 100 Deg. Flush Head Blind lbed Mechanically Locked Spindle 100 Deg. Flush Head Blind

Flush Shear Head A286 Corrosion Resistant Steel and Mone std. for General Purpose Semi-Tubular, Full Tubular, Split std. Meth. of Shear Testing of Aluminum and Aluminum Alloy Std for Drive Pin Blind Std for Break Mandrel Closed End Blind Sid. Large Std. Small Solid Std, Fastener Code (Aerospace Lockbolts.
Rigid Boards (1970) ANSI C83.98 1.

igid Cellular Plastics (1969E) ANSI K65.122

Rigid Cellular Plastics (1972) ANSI K65.32

Rigid Cellular Plastics (1974)

Rigid Furring Channels for Screw Application of Gypsum

Rigid Industrial Casters (1973)

Rigid Nonmetallic Conduit (1972) ANSI C33.91

Rigid Plastic Lap Shear Joints in Shear by Tension Load

Rigid Plastic Sheeting or Parts by Means of a Tup (Fall

Rigid Plastics (1974) ANSI K65.117

Rigid Poly vinyl Chloride) (PVC) Plastic Wall Siding (19

Rigid Rods and Tubes Used for ElectricaI Insulation (19

Rigid Sheet and Plate Materials Used for Electrical Ins

Rigid Steel Conduit and Electrical Metallic Tubing (197

Rigid Urethane Foam (1972)

Rigid (1972)

Rigs and Petroleum Production Facilities on Land and on

Rim and Wheel Stds. for Motorcycles, Passenger Cars, of

Rim Contours for Agricultural Press and Gage Wheels ( $\mathrm{Fa}$

Rim Equipment for 1974 Passenger Cars (1974)

Rim Inspection, and Disk and Flat Tapes (1974)

Rim Selection (1974)

Rim Spacers (Manufactures' Guide) (1963)

Rims and Rim Spacers (Manufactures' Guide)

Rims and Valves for Aircraft (Airplane, Helicopter) (19

Rims for Agricultural and Industrial Steering, Drive. P

Rims for Industrial, Utility and Recreational Type Vehi

Rims for Motorcycle, Side Car, Mini Bike, and Bicycle

Rims for Off the Road Vehicles: Earthmoving, Mining and

Rims for Passenger Cars, Station Wagons, and MuItipurpo

Rims for Truck, Bus, Trailer, and Multipurpose Vehicle

Rims Intended for Highway Use on Trucks, Buses, Trailer

Ring Buoy) (1973) ANSI Z243. I / Marine Special P

RingeImann Meth.) (1973)

Rings for Automotive Transmission and

Rings for Generators (1970) ANSI G55.6

Rings (Nickel Base, Solution Heat Treated, $15.5 \mathrm{Cr}-16 \mathrm{M}$

Rings (1.0Mg-0.60SI $-0.28 \mathrm{Cu}-0.20 \mathrm{Cr}(606 \mathrm{l}-\mathrm{T} 6)$ ) (197

Rings $(1.0 \mathrm{Mg}-0.60 \mathrm{SI}-0.28 \mathrm{Cu}-0.20 \mathrm{Cr}(606 \mathrm{l}-0))(1973$

Rings (1.3Mn-1.5SI $-0.30 \mathrm{Cr}-1.8 \mathrm{Ni}-0.40 \mathrm{Mo}(0.23-$

Rings (1972)

Rings (1972) ANSI B93.36

Rings (1973) ANSI B93.32

Std. Groove Dimensions ANSI

Nord
roove NFLDP

Rings, and Mechanical Tubing (Annealed) $(0.48 \mathrm{Cr}-8.0 \mathrm{~N}$

Rings, Corrosion and Heat Resistant $(15 \mathrm{Cr}-26 \mathrm{Ni}-1.3 \mathrm{M}$

Rings, Corrosion and Heat Resistant, Nickel Base-15.5

Rings, Corrosion and Heat Resistant. Nickel Base-15.8

Rings, Corrosion and Heat Resistant, Nickel Base-5.0C

Rings, Corrosion and Heat Resistant, Nickel Base (19.5C

Rings, Corrosion and Moderate Heat Resistant. 17Cr (SAE

Rings, Heat Resistant, Iron Base-35.5Ni-18.5Cr-1.

Rings, Sealing, Fluorosilicone Rubber, General Purpose,

Rings, $0.78 \mathrm{Cr}-1.8 \mathrm{Ni}-0.35 \mathrm{Mo}-0.20 \mathrm{~V}(0.32-0.38 \mathrm{C})$,

Rip) Meth. (Constant Rate of Traverse Tensile Test. Mac

Ripening Process (Banana, Citrus Fruit) (1973)

Rise Buildings (1973)

Rise Buildings (1973)

Rising Biscuit Flour (Cereal Chemistry) (1962)

Rising Flour (Cereal Chemistry) (1962)

Rising Flours (Cereal Chemistry) (1962)

Rivet-Flat Head, Hi Shear, Close Tolerance Shank (197

Rivet-100 Deg. Countersunk Head Hi Shear Close Tolera

Rivet and Cold Heading Wire and Rod (1973) ANSI H38.12

Rivet SteeI (1973)

Rivet (1972)

Rivet (1972)

Rivet (1972)

Rivet (1972)

Rivet (1972)

Rivet (1974)

Rivet (1974)

Rivet (1974)

Rivet (1974)

Rivet (1974)

Rivet (1974)

Rivets and Caps (1972)

Rivets and Cold Heading Wire and Rods (1972)

Rivets (1973)

Rivets (1973)

Rivets (1/2 In. Nominal Diameter and Larger) (1972)

Rivets (7/16 In. Nominal Diameter and Smaller) (1972)

Rivets, Bolts) (1973)

IPC

ASTM

TRA

TRA

TRA

TRA

TRA

TRA

SAE

UP

ASTM

WWPA

SAE

Std ASTM

SAE

SAE

SAE

SAE

SAE

SAE

SAE

SAE

SAE

SAE

SAE

SAE

SAE

ASTM

ICBO

FMS

Un ICBO

$\mathrm{AACCH}$

Std NSA

ASTM D1788

ASTM D2842

ASTM D1621

ASTM D301

ASTM C645

ANSI MHII.I

651

ASTM D3163

STM D3029

D757
PS55

ASTM D668

ASTM D229

NEMA RNl

D2341

NSA 1369

API RP500B

TRA

TRA 1.17

TRA 10

TRA XII

J851

J851

J85

6

J 267

1123

$6 \mathrm{~A}$

D3211

J281

A289

AMS5750B

AMS4117D

AMS4115C

A MS6418E

B27.6

T3.19.11

T3.19.18

AMS6540B

AMS5735H

AMS5665H

AMS5711

AMS5755B

AMS5707D

AMS5627B

AMS5716A

AMS7266

AMS6430B

D2262

UFC $* 2$ ART I 6

10-31A

$\begin{array}{ll}\text { NSA } & 529 \\ & \end{array}$

ASTM B316

ICBO UBCS27.5

NSA 1054

NSA 1055

NSA I398

NSA 1399 
or Structural Flush Break Pull Mandrel Self Plugging Blind ssenger Cars, Trucks, Buses, Coaches, Tractors, Industrial

l, Acceleration of Concrete, Curing of Concrete, and Other c Solven/ Std. Meth. of Test for Solubility of Bituminous Std. Meth. of Test for Distillation of

c. Pract. for Paving L'ses and Application Temperatures for Rec. Pract. for Electric Tachometer Spec. for on aul); Grader, Loader/ Std. for Tires and Rims for Off the ction, Purchase, Preparation, Maintenance and Rules of the Rim and Wheel Stds. for Motorcycles, Passenger Cars, Off authority, Departmental Regulations and Controls. Barrier, a 37.33 Std. Definitions of Terms Relating to Materials for d. for Metal Head and Reflector lnterchangeability l'sed in erchangeability of Socket Supports L'sed in Metal Heads for d. for Metal Head and Reflector Interchangeability l'sed in eability of Socket Supports for L'se in Metal Heads I'sed in Std for Multiple Sockets lsed in Std. for Voltage Classification of Luminaires L'sed in Std. for Multiple Sockets l'sed in Procedure for Trucks, Buses, and Combination Vehicles for ted Wall Bituminized Fiber Perforated Pipe for Airport and Std. for Commercial Gas Baking and ullet Resisting Equipment (Barrier) for Protection Against Std Weth of Test for Elastic Specifying I'.S. Model

Meth. for Spectrochmeical Analysis of Ores, Minerals, and Tables for Metals (Relationship Between Brinell, Vickers, metallic Materials (1974) ANSl Z/ Std. Meths. of Test for 973) Std. Meth. of Test for 74) ANSl Zl Std. Meths. of Test for Rockwell Hardness and etals (Relationship Between Brinell, Vickers, Rockwell and Dry Pipe Valves: Std. for Std. Spec. for Nickel Chromium 1ron Alloy Std. Spec. for Nickel

Std. Spec. for Copper Beryllium Alloy td. Spec. for Nickel lron Chromium Molybdenum Copper Alloy Std. Spec. for Nickel Chromium Molybdenum Columbium Alloy Std. Spec. for Copper-Nickel-Silicon Alloy

-Nickel-Zinc Alloy (Nickel Silver) and Copper-Nickel Std. Spec. for Nickel-Iron-Chromium Alloy Std. Spec. for Tantalum

and Mounting Dime/

Std. Bore and Rod Size Combinations, Std. for Swaging Process-Tube to Spec. for Bearings, Ball and Roller,

3) Std. for

std. for Tube Assembly, Control Steel with Welded Threaded be Assembly, Control Steel with Welded Clevis and Threaded e Assembly, Control, Steel, with Flash Welded and Threaded bly, Control, Steel, with Flash Welded Clevis and Threaded Std. Spec. for EC Aluminum Redraw

Uniform Building Code Std. for Steel Bar and Rec. Pract. for Design Calculations for Oil Sucker nal ldentification Code, and Mounting Dime/ Std. Bore and Rec. Pract. for Preparation of Bar and test for Tensile Strength of Adhesives by Means of Bar and ns for $3 / 4, I$ and $11 / 8$ In. Bore Cataloged Square Head Tie Std. Spec. for Nickel-Molybdenum Alloy

Std. Spec. for Nickel-Molybdenum-Chromium Alloy Std. Spec. for Nickel Chromium Molybdenum lron Alloy Std. Spec. for Nickel Molybdenum Chromium lron Allo std. Spec. for Low Carbon Nickel Molybdenum Chromium Allo for Nickel-Chromium-Iron-Miolybdenum-Copper Alloy

Spec. for Aluminum Alloy Rivet and Cold Heading Wire and Std. Spec. for TFE -Fluorocarbon (Tetrafluoroethylene) est for Penetration Resistance of Pipeline Coatings (Blun Std. for Clevis-Engine Control Std. Spec. for Copper Bus Bar, hot Rolled and Cold Finished Zirconium and lts Alloy Bars, Std Spec for Columbium and lts Alloy Bar,

Std. Spec. for Molybdenum and Molybdenum Alloy Bar, Std. Spec. for Copper and lts Alloy Forging Std. Spec. for Free Cutting Bras

for General Requirements for Wrought Copper and lis Allo Std. Spec. for Copper Silicon Alloy Std. Spec. for Coppe Std. Spec. for Manganese Bronze Std Spec for Aluminum Bronz for Copper Zinc Lead (Leaded Red Brass or Hardware Bronze) $s$ (Bus Condu/ Std. Spec. for Aluminum Alloy Extruded Bar, Spec. for Tolerances of Aluminum and lts Alloy Bar

Cereal Grains, Unpopped Popcorn, Dried Peas and Beans for Decantation Meth. of Analysis for ina (Cereal Chemistry)/ Decantation Meth. of Analysis for
Rivets, Carbon Steel (1973)

Rivets, Type 2A (1973)

Road and Misc Equipment) (1974)

$\begin{array}{rr}\text { Std. } \mathrm{F} & \mathrm{SA} \\ \text { /Reign and Domestic } \mathrm{Pa} & \mathrm{BC}\end{array}$

Road Conditioning Purposes (1974) ANS1 A37.37, Ashto M1 ASTM

$\begin{array}{ll}\text { Road Materials (Tar. Petroleum Asphalt, etc.) in Organi } & \text { ASTM } \\ \text { Road Tars (1972) } & \text { ASTMI }\end{array}$

Road Tars (1972)

Road Vehicles (1972)

Std. Re ASTII

SAE

$\begin{array}{ll}\text { Road Vehicles: Earthmoving, Mining and Logging (Short H } & \text { TRA } \\ \text { Road (1972) }\end{array}$

Road, Agricultural and Log Skidder, Truck, Bus, Bead Se

Road, Hydrant. Driveway Clearance, Installation and Mai

Roads and Pavements (1971) ANSl

Roadway Lighting Equipment (1973) EEI TDJ.140

Roadway Lighting Equipment (1973) EEI TDJ.143

Roadway Lighting Equipment (1973) NEMA SH10

Roadway Lighting Equipment (1973) NEMA SH13

Roadway Lighting Equipment (1973) NEMA SHI7

Roadway Lighting Equipment (1973) NEMA SH3

Roadway Lighting (1973) EEl TDJ.147

Roadway ['se Over 10,000 Lb. (4500 kg) GVW (1972)

Roadwork Drainage (1973)

Roasting Ovens (1971)

Robbery or Holdup (Criminology) (1974) ANSI Se4.6

Rock Bolts (1966)

Rock Core Specimens in Uniaxial Compression (1972)

Rocket Sporting and Safety Code and Engine Std. (1973)

Rocks by the Fire Assay Preconcentration Spark Techniqu

Rockwell and Rockwell Superficial, and Knoop Hardness)

Rockwell Hardness and Rockwell Superficial Hardness of

Rockwell Hardness of Fine Grained Graphite Materials (1

Rockwell Superficial Hardness of Metallic Materials (19

Rockwell Superficial, and Knoop Hardness) (1972) A \SI Z

Rockwood Models C, D (1974)

Rod-Control, Solid Steel (1973)

Rod and Bar (1970) ANS1 H34.4

Rod and Bar (1970) ANSI H34.7

Rod and Bar (1971A) ANSl H7.7

Rod and Bar (1972) ANSI H34.18

Rod and Bar (1972) ANSI H34.22

Rod and Bar (1973)

Rod and Bar (1973)

Rod and Bar (1973) ANSl H34.39

Rod and Wire (1970) ANSI Z179.15

Rod End Configurations, Dimensional ldentification Code

Rod End (1974)

Rod End, Anti Friction, Airframe (1974)

Rod End, Threaded, Alloy Steel, Flash Welding Type (197

Rod Ends (1973)

Rod Ends (1973)

Rod Ends (1973)

Rod Ends (1973)

Rod for Electrical Purposes (1974)

Rod Mats (Sheets) for Concrete Reinforcement (1973)

Rod Pumping Systems (Conventional Units) (I972)

Rod Size Combinations, Rod End Configurations, Dimensio

Rod Specimens for Adhesion Tests (1969)

Rod Specimens (I972) ANSl Z197.3

Rod Type Industrial Flui

Rod (1971) ANSl H34.14

Rod (1972)

$\operatorname{Rod}(1972)$

Rod (1972)

$\operatorname{Rod}(1973)$

Rod (1973) ANSl H38.12

Rod (1974) ANS1 K65.208

Rod) (1972)

Rod, Adjustable (1973)

Rod, and Shapes (1974) A NSl C7.25

Rod, and Wire for Nuclear Application

Rod, and Wire (1964) ANSI Z179.19

Rod, and Wire (1974)

Rod, Bar and Shapes (1974) ANSl H7.I

Rod, Bar, and Shapes for Use in Screw Machines (1972)

Rod, Bar, and Shapes (1973)

Rod, Bar, and Shapes (1973) ANSl H7.3

Rod, Bar, and Shapes (1973) ANSI H7.4

Rod, Bar, and Shapes (1974) ANSI H7.5

Rod, Bar, and Shapes (1974) ANSI H7.6

Rod, Bars, and Shapes (1972) ANSl H33.

Rod, Pipe, and Structural Shapes for Electrical Purpose

Rod, Wire, and Forging Stock (Rolled or Drawn) (1973)

Rodent and/or lnsect Damage on Kernels, Rodent Excreta,

Rodent Excreta in Flour (Cereal Chemistry) (1962)

Rodent Excreta in Meal, Grits, Flour, Farina, and Semol

TRA

ICBO

ASTM

Cal Int NEMIA

St EEl

Niterchang EE

EEl

EEl

NEMA

Test SAE

ASTM

A.NSl

IR B LL

CSI

NAR

ASTM

ASTM

AST.M

ASTM

AsTu

AST.M

FMS

VSA

AST.M

AST.M

ASTM

ASTM

ASTM

ASTMI

AST.I

ASTM

ASTM

NFLDP

NSA

NSA

Std. for tu NSA

Std. for Tub NSA

NSA

ASTM

ICBO

API

A.MS7225D

119

*1.56

D98

D2042

D20

D2728

$\mathrm{J} 196$

3

$6 / 7$

LFC*2ARTI3

D8

$\mathrm{SH} 10$

$\mathrm{SHI} 3$

TDJ. 140

TDJ.143

TDJ.147

TDJ. 133

SHIT

J29.4

D2418

Z21. 28

752

02980

D3148

*1

E400

E140

E18

C748

E 18

(19

354

B166

B160

B196

B42.5

B446

B41

B151

B408
B365

T3.6.11

582

661

358

359
361

362

B233

UBCS26.5

$11 \mathrm{~L}$

NFLDP T3.6.11

ASTM D2094

D2095

T3.6.11

ASTM B.335

ASTM B336

ASTM B572

ASTM B573

ASTM B574

B.574
B581

B581
B316

B316
D 1710

ASTM D1710

ASTM Gl

$\begin{array}{ll}\text { NSA } & 170\end{array}$

ASTM BI87

ASTM B351

ASTM B392

ASTM B387

ASTM Bl24

ASTM Bl6 
ady.To.Eat Cereals, and Alimentary Pastes for Detection of peas and Beans for Rodent and/or Insect Damage on Kernels, alimentary Past/ Sieving Meth. of Analysis for Insect and al Chemistry)/ Flotation Meth. of Analysis for Insect and ) Flotation Meth. of Analysis for Insect and pcorn. Dried Pel Meth. of Analysis of External Insect and cation in Cereal Grains and Grain Products (Cer) Insects. acid Hydrolysis Meth. of Analysis for Insect Fragments and Tween Versene Meth. of Analysis for Insect Fragments and iting Digestion Meth. of Analysis for Insect Fragments and eal Chemis/ Litraviolet Light Examination for Presence of Spec. for Aluminum and Its Alloy Welding Spec. for Magnesium Alloy Welding ion Resisting Chromium and Chromium-Nickel Steel Welding ) ANSI W3.15 Spec. for Nickel and Its Alloy Bare Welding Spec. for Surfacing Welding

Spec. for Titanium and Its Alloy Bare Welding Spec for Composite Surfacing Welding si C59.33 Std. Meth. of Measuring Dimensions of Rigid Std. Spec. for General Requirements for Carbon Steel Wire Std. Spec. for Aluminum Alloy Bars, Std. Spec. for Aluminum Allov 5005 Rolled Std. Spec. for Hot Rolled Copper Sid. Spec. for Copper Spec for Sucker

pract. for Static Bend and Torsion Test. of Intramedullary Spec. for Iron and Steel Gas Welding Spec. for Copper and lis Alloy. Welding

ic Covered Bare Straight and Coiled Welding Electrodes and uminum and Aluminum Alloy Rivets and Cold Heading Wire and suring Slope Efficiency and Lasing Threshold of Ruby Laser s Applicable to Titanium and Its Base Alloy Extruded Bars. Std. Spec. for Aluminum Alloy Extruded Bars, Std. Spec. for Magnesium Alloy Extruded Bars, Std. for Back W'indow Location for

sks (Separate) for Use in Photographic Contact Printing of Std. Dimensions for Industrial Radiographic Sheet and

Std. Dimensions for Professional Photographic Sheet and Std. Dimensions for Medical Radiographic Sheet and saturated and Coated Felts. Prepared Roofings, and Plastic and Frol Rec. Pract. for Minimum Performance Criteria for ders An/ Rec. Pract. for Minimum Performance Criteria for (Heavy) Rec. Pract. for Minimum Performance Criteria for rec. Pract. for Critical Zone for Laboratory Evaluation of Fire Hazards Anf Protection Rec, for Indoor Storage of Std. Spec. for Asphalt Spec. for Resistance Seam and

deg. C) Solu/ Studs, Steel, Corrosion and Heat Resistant, lloy Steel Studs, Heat Resistant, Normalized and Tempered, Spec. for Low Alloy Steel Bolts and Screws, Heat Treated, Spec. for Low Alloy Steel Studs. Heat Treated,

for Titanium Alloy Bolis and Screws, $6 \mathrm{Al} \cdot 4 \mathrm{~V}$. Heat Treated, s. Steel, Low Alloy Heat Resistant, Hardened and Tempered, lloy Bolts and Screws, 6Al.4V, Upset Headed, Heat Treated. 0 psi (1,345 MPa) Tensile Strength. Hardened and Tempered, building Code Sid. for Composition Roofing (Class C Sheet (1972) alves and Parts for High Temper/ Std. Spec. for Forged or Std. Spec. for Regular Quality Hot and Cold Sid. Spec for Drawing Quality Hot and Cold at Resisting Steel Bars and Shapes (1/ Std. Spec. for Hot oducts Manual) Carbon Steel: Semifinished for Forging; Hot 73) ANS1 G24/ Std. Spec. for General Requirements for Hot hromium Nickel Manganese Steel Bars I/ Std. Spec. for Hot rod, and Wire for Nuclear Application/ Std. Spec. for Hot Piping Component/ Std. Spec. for Steel Bars, Carbon, Hot Std. Spec, for Leaded Brass Plate, Sheet, Strip, and Sid. Spec, for Brass Plate, Sheet, Strip, and Std. Spec, for Copper lron Alloy Plate, Sheet, Strip, and pec. for Copper-Beryllium Alloy Plate, Sheet, Strip, and c. for Copper-Zinc-Tin Alloys Plate, Sheet, Strip, and for Phosphor Bronze (Copper Tin) Plate, Sheet, Strip, and -Zinc-Aluminum-Cobalt Alloy Plate, Sheet, Strip, and lium Alloy (Copper Alloy No. 175), Plate, Sheet, Strip and

silver) and Copper-Nickel Alloy Plate. Sheet, Strip, and Std. Spec. for Copper Sheet, Strip, Plate and

r Wrought Copper and Copper Alloy Plate, Sheet, Strip, and Requirements (1972) $\quad$ Std. Spec. for Merchant Quality Hot (1972) Sid Spec, for Merchant Quality Hot Std. Spec. for Hot Sid. Spec, for Cold
Sid. Spec. for Hot Std. Spec. for

trip, Commercial Quality (1972) eets for Bridge and Other Structural U'ses/ Sid. Spec. for Hot
Rodent Excreta, Insects, Webbing, etc. (Cereal Chemistr Rodent Excreta. Other Filth, and Extraneous Material ( $C$ Rodent Filth in Baked Goods, Ready.To-Eat Cereals, and Rodent Filth in Meal. Grits, Farina, and Semolina (Cere Rodent Filth in Popped Popcorn (Cereal Chemistry) (I962 Rodent Hair Contamination of Cereal Grains. Cnpopped Po Rodent Hair, and Radiographic Illustration and Classifi

Rodent Hairs in Flour (Cereal Chemistry) (1962)

Rodent Hairs in Rye Flour (Cereal Chemistry) (1962)

Rodent Hairs in Soy Flour (Cereal Chemistry) (1962)

Rodent Lrine on Bags and Other Packaging Materials (Cer

Rods and Bare Electrodes (1969) ANSI W 3.10

Rods and Bare Electrodes (1969) ANSI W3.19

Rods and Bare Electrodes (1969) ANSI W 3.9

Rods and Covered Electrodes for Welding Cast Iron (1969

Rods and Electrodes (1969) ANSI W3.14

Rods and Electrodes (1970) ANSI W3.13

Rods and Electrodes (1970) ANSI W3.16

Rods and Electrodes (1970) A VS] W3.2]

Rods and Tubes Lised for Electrical Insulation (1972) an

Rods and Incoated Coarse Round W'ire (1971) ANSI G54.12

Rods and Wires (1974) ANSI H38.4

Rods for Electrical Purposes (1972) ANSI C7.66

Rods for Electrical Purposes (1974) A.VSI C7.7

Rods for Locomotive Staybolts (1973) ANSI H7.2

Rods (Petroleum) (197\%)

Rods (Surgical Implants) (1973)

Rods (1969) ANSI W3.2

Rods (1969) ANSI W3.7

Rods (1972)

Rods (1972)

Rods (1973)

Rods, and Shapes (1973)

Rods, Shapes, and Tubes (1973) A VSl H38.5

Rods, Shapes. Tubes, and Wires (1970) ANSl H45.6

Roll Film Cameras (1973)

Roll Film Negatives (1972)

Roll Film (photography) (1973)

Roll Films (SI and U.S. Customary Lnits) (1973)

Roll Films (1973)

Roll Materials (1973)

Roll Over Protective Structure for Track Type Tractors

Roll Over Protective Structure for Weeled Front End Loa

Roll Over Protective Structures (ROPS) for Prime Movers

Roll Over (ROPS) and Falling Object Protective Structur

Roll Paper (1974)

Roll Roofing Surfaced with Mineral Granules (1973)

Roll Spot Welding Equipment (3 Phase) (1973)

Roll Threaded After Heat Treatment, 1800 Deg, F $(982.2$

Roll Threaded (135,000 psi (931 MPa) Tensile Strength)

Roll Threaded (1973)

Roll Threaded (1973)

Roll Threaded (1973)

Roll Threaded (1973)

Roll Threaded (1973)

Roll Threaded (1973)

Roll) and Shingles) (1973)

Roll, $35 \mathrm{~mm}$ Film, and $126 \mathrm{~mm}$ Film Still Picture Cameras

Rolled Alloy Steel Pipe Flanges, Forged Fittings, and V

Rolled Alloy Steel Sheet and Strip (1973) ANSI G24.24

Rolled Alloy Steel Sheet and Strip (1973) ANSI G24.25

Rolled and Cold Finished Age Hardening Stainless and He

Rolled and Cold Finished Bars; Hot Rolled Deformed Conc

Rolled and Cold Finished Carbon and Alloy SteeI Bar (19

Rolled and Cold Finished Stainless and Heat Resisting C

Rolled and Cold Finished Zirconium and lis Alloy Bars.

Rolled and Cold Finished, Special Quality, for Pressure

Rolled Bar (1973)

Rolled Bar (1973)

Rolled Bar (1973)

Rolled Bar (1973)

Rolled Bar (1973)

Rolled Bar (1973)

Rolled Bar (1973)

Rolled Bar (1973)

Rolled Bar (1973)

Rolled Bar (1973) ANSI H7.9

Rolled Bar (1974)

Rolled Carbon Steel Bars Subject

Rolled Carbon Steel Bars (1973)

Rolled Carbon Steel Strip (1972A) ANSI G47, 1

Rolled Carbon (0.15 Maximum, Percent) Steel Sheet and S

Rolled Copper Alloy Bearing and Expansion Plates and Sh

Rolled Copper Rods for Electrical Purposes (1974) ANSI

Rolled Deformed Concrete Reinforcing Bars (1972)
AACCH 28.30

AACCH 28.10

$\mathrm{AACCH} 28.31$

AACCH 28-51

AACCH 28.80

$\mathrm{AACCH} \quad 28.20$

$\mathrm{AACCH} 28.95$

$\mathrm{AACCH} 28.41$

A ACCH 28.60

la A ACCH 28.70

AACCH $28-85$

AW's A5.10

AWS A5.19

AWS A5.9

AWS A5.15

AWS A5.1.

AWS A5.13

A5. 16

AWS A5.2]

ASTM D668

ASTM A5I0

ASTM B21

ASTM B531

ASTM B+9

ASTM Bl2

API

$11 \mathrm{~B}$

ASTM F383

AW' A.S.2

NEMA EX ?

B565

A.IS2245

B221

$\mathrm{B} 10^{-}$

PH3.1

PH3.9

PHI.15

PH1.18

PHI.17

$* 2.1 \mathrm{~V}$

J395A

$\mathrm{J} 394 \mathrm{~A}$
$\mathrm{~J} 320 \mathrm{~B}$

$\triangle A E \quad J 320 B$

SAE J397A

FMS 8.21

$\begin{array}{ll}\text { ASTM } & \text { D249 } \\ \text { NSA } & 991\end{array}$

$S A E$

SAE

SAE

Spec. SAE

$S A E$

195,00
$S A E$

niform ICBO

ANSI

ASTM

ASTM

ASTM

ASTM

AISI

ASTM

ASTM

ASTM

ASTM

ASTM

ASTM

ASTM

Std. S ASTM

Std, Spe ASTM

Std Spec ASTM

Sid. Spec, for Copper ASTM

Spec. for Copper-Cobalt-Beryl ASTM

ASTM

ASTM

ASTM

ASTM

ASTM

A STM

ASTM

ASTM

ASTM

ASTM

$/ M i f i$ AISI

IS 7482

AMS7458C

AMS7 $452 \mathrm{~K}$

AMS7456G

AMS7460C

AMS7455D

AMS7461B

AMS7459B

IBC 532.3

PH3.39

A 182

A506

A 507

A 56 .

A29

A 429

B351

A696

B 121

B36

B465

B194

B59

B 103

B592

B5.34

B122

B 152

B248

A663 
Std. Spec. for Flat Products Manuall Carbon Steel' Plates. Structural Shapes.
Std. Spec. for Steel. Carbon. Strip. Cold pe $(1973)$ Std. Spec. for Flat

ybeans, Rice. Beans. Peas. Lentils. Corn Meal. Corn Grits. Std. Spec. for Flat Copper Products with Finished

Aluminum and lis Alloy Bar. Rod. Wire, and Forging Stock Std. Spec. for Aluminum Alloy Std. Structural Shapes. Stds. for Staples with Std. Spec. for Aluminum Alloy 5005

Std. Spec. for Steel, Carbon (0.16 to 0.25 Maximum r. Hot Std. Spec. for Steel. Cold

Std. Spec. for General Requirements for Delivery of Flat Std. Spec. for General Requirements for Delivery of Str/ General Spec. Applicable to Electromagnet Iron (E.II) Cold ngers (1973) Std. Spec. for Copper Alloy Condenser peas, Lentils, Corn MeaI, Corn Grits, Rolled Oats. Bulgur. and Parts for Low Temperature/ Sid. Spec. for Forged or Fully Processed Types 27G053. 30G05/ std. Spec. for Flat ium (/ Std. Spec. for Steel Sheet and Strip. Hot and Cold 1974) Std. Spec. for Steel Bars. Carbon. Hot $\mathrm{m} /$ Std. Spec. for Carbon-Chromium Ball and Std. Shaft and Housing Fits for Metric Radial Ball and Std. Load Ratings and Fatigue Life for std. for In. and Metric Radial Needle

for Metric Radial Ball and Roller Bearings (Except Tapered Std. for Heavy Duty Offset Sidebar Power Transmission Sid. for Double Pitch Conveyor Std. Tolerances for

aracteristics/

Farinograph Evaluation Meth. of Ability of Tent. Neth. of Test for Floating de Blanks and Cutting Tools. Single Point. Carbide Tipped, Std. for Heavy Duty

Spec for Bearings, Ball and meth. of Test for Tack and Pressure Sensitive Adhesives by sional Requirements for Duplex and Single Face Flat Thread Thyristor Power Supplies for Metal sid. for Thread

e (1973)

r Both (Photography) (19)

(1973) 972) Tent. Meth. of Test. Plywood in
Rec. for Suction Press

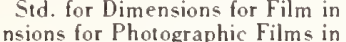
Std. for Interchange Std. Dimensions for Cores for Photographic Film Spec for Noncarbonized. Single Ply. Adding Machine Paper n) $(1972)$

spec. for Noncarbonized. Single Ply. Adding Machine Paper ion) $(1972)$

Case of Fire (1974)

by Snow Loads (1973) Std. for System for Std. for sistem for

Approval Std. for Heat and Smoke Rec. for Prevention of Collapse. Burglary, and

Safety Std for Tests for Fire Resistance of Iniform Building Code Std. for Fire Retardant Std. for Safety for $\Pi^{\prime}$ ind Resistance of Prepared th Gravel or Slag Surf/ Loss Prevention Data on Elastomer Std. Spec. for Asphalt for Use in Constructing Built l'p Dwelling Construction Under the Uniform Building Code: ode Std. for Materials for I'se in Construction of Built Up organic Felt. Coa/ Safett Std. for Materials for Built. $\mathrm{C} p$ Std. Test Meth. for Structural Insulating

Rough and Finished Carpentry. Flooring, Shingles. Exposed Spec. for Perlite Insulating Concrete orced Gypsum Concrete and Precast Slabs, and Poured Gypsum Uniform Plumbing Code: Rainwater Systems. Dwelling Construction Inder the I'niform Building Code: Loss Prevention Data on Bonds and Guarantees for sid Spec for Mineral Fiber Std. for Safety for Gas Vents and Vent Rec. Against and Coated Felts, Prepared Roofings, and Plast/ Spec for Uniform Building Code Sid. for Structural Floor and Uniform Building Code Std. for Natural Slate Uniform Building Code: Penthouses and g, Shingles. Exposed Roof Decking, Drop Siding, Structural Std for Safety for Power he Uniform Building Code: Valley Flashing (Sheet Metal for Uniform Building Code Std. for Mineral

Std. Index of Meth. for Test. Elastomeric and Plastomeric liniform Building Code Sid. for Uniform Building Code Std. for Asbestos Cement Std. Spec. for Asphalt Roll Uniform Building Code Std for Uniform Building Code Std. for Composition Guide Spec. for Sheet Metal
sheet, Zinc Coated (Galvanized) by the Hot Dip Process for
Rolled Electrical Steels for Magnetic Applications (197

Rolled Floor Plates, and Steel Sheet Piling $(19 ; 0)$
Rolled Hard, L ntempered Spring Ouality $(19 ; 3)$

Rolled Vonoriented Electrical Steel. Fully Processed Ty.

Rolled Oats. Bulgur. Rolled Wheat. Breakfast Cereals (E

(Rolled or Drawn) Edges (Flatwire and Strip) (1973)

Rolled or Drawn) (19:3)

Rolled or Extruded (1973) ANSI H38.10

Rolled or Slash Points (1971) EEI TDJ.1.t

Rolled Rods for Electrical Purposes (1972) A.NSI C7.66

Rolled Sheet and Strip. Commercial Quality (1972)

Rolled Sheet, Carbon. Structural (1972)

Rolled Stainless and Heat Resisting Steel Plate, Sheet.

Rolled Steel Plates, Shapes. Sheet Piling. and Bars for

Rolled Strio for Magnetic Application in Relavs and Sol

Rolled Tube Plates in Surface Condensers and Heat Excha

Rolled Wheat. Breakfast Cereals (Except Those Which Are

Rolled 9r, Nickel Alloy Steel Flanges. Fittings. Valves.

Rolled. Grain Oriented, Silicon Iron. Electrical Steel.

Rolled. High Strength. Low Alloy Columbium and/or V anad

Rolled. Special Quality. for Fluid Power Applications (

Roller Bearing Steel (1970) ASTM G.56.2

Roller Bearings (Evcept Tapered Roller Bearings) Confor

Roller Bearings (1972)

Roller Bearings (1973) ANSI B3.18

Roller Bearings) Conforming to Basic Boundary Plans (19

Roller Chains and Sprocket Teeth (1972)

Roller Chains. Attachments and Sprockets (1972)

Roller Drafted Yarns (19:1)

Roller Dried Nonfat Dry Milk Solids to Change Mixing Ch

Roller Peel Resistance of Adhesives (1973)

Roller Turner Type (1972)

Roller Trpe Convevor Chains and Sprocket Teeth (19-3)

Roller. Rod End. Anti Friction. Airframe (197.4)

Rolling Ball (19:3)

Rolling Dies 11973

Rolling Mill Auxiliary Drives (1972)

Rolling Screus $(1972)$

Rolling Shear (Shear in Plane of Plies) (1969)

Rolls and Their Installation. Operation. and Maintenanc

Rolls for Phototvpesetting or Photocomposing Devices. $O$

Rolls for Recording Instruments and Miscellaneous Cses

Rolls of Perforated Tape for Information Interchange (1

Rolls (Plastic. Nood or Metal) (1971)

Rolls (1973)

Rolls. Including Leaders and Trailers, for Aerial and R

Romanization of Arabic IX'ritten Language (Transliteratio

Romanization of Japanese $\mathbb{I F}$ ritten Language (Transliterat

Roof and $\Pi$ 'indow lents Designed to Open Automatically in

Ronf Collapse of Buildings and Other Structures Caused

/Ls at Electric Generating Stati

Roof Construction and Covering (1973)

Roof Covering Material (1973)

Roof Covering Materials (1973)

Roof Covering Materials (1973) ANS1 A 19.5.1

Roof Coverings (Multiply Felt and Asphalt Preferably $\mathrm{Ni}_{\mathrm{i}}$

Roof Coverings (1971) ANSI Al09.2.4

Roof Cove rings (1973)

Roof Coverings: Hot Mopped Asphalt. Asphalt and Coal Ta

Roof Coverings: Hot Mopping Asphalt. Asphalt.Saturated

Roof Deck (R1970)

Roof Decking. Drop Siding. Structural Roof Trusses and

Roof Decks (1973)

Roof Diaphragms (1973)

Roof Drains and Piping (1973)

Roof Framing (1973)

Roof Installations (1973)

Roof Insulation Board (1972)

Roof Jacks (1973) ANSI Al31 2

Roof Leakage of Buildings Not Due to Hail or Wind (1972

Roof Memhranes: Aggregates. Saturated Felts, Saturated

Roof Plank and Beam Framing (1973)

Roof Shingles (1973)

Roof Structures (Tower, Spire) (1973)

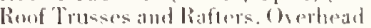

Roof Ventilators (1972) ANSI C33.89

Roof Waterproofing) (1973)

Roofing Aggregates (1973)

Roofing and Watcrproofing Materials (1972)

Roofing Asphalt (1973)

Roofing Shingles (1973)

Roofing Surfaced with Mineral Granules (1973)

Roofing Tile (1973)

Roofing (Class C Sheet (Roll) and Shingles) (1973)

Roofing (1971)

Roofing (1971) ANSI G8.21
ASTM

(E) AISI

ASTM

ASTM

A.AC. $\mathrm{H}$

ASTM

$S A E$

AST Y

IEVA

ASTM

ASTM

ASTY

ASTM

AST U

$\triangle A R M$

ASTM

$\mathrm{AACCH}$

ASTM

ASTY

ASTM

ASTM

ASTM

AFBIIA

AFBMA

AFBMA 18

AFBMA

A $\backslash 51$

A.S. I

ASTM

$\mathrm{AACH}$

AST.M

A.XS1

A.XS1

Is

std. ASTM

[F]

IEMA

$S A E$

AST.

Fils

A.ISI

A.ISI

A.ISI

A.XS1

std. A.tsl

ANSI

A $\backslash$ s1

ANS

FMS

FMS

FMS

IL

ICBO

CL

FMS

ASTM

ICBO

ICBO

[L

ASTM

A III

CSI

CBO

ICBO

ICBO

FMS

ASTM

UL

FMS

NRCA

ICBO

ICBO

IC.BO

/ /shed Carpentry, Floorin A III

I'L

ICBO

ICBO

ASTM

CBO

ICBO

ASTM

lC.BO

CBO

CSI

Std. Spec. for Steel ASTM

A.3.5

2

An:-

1.. $15 \mathrm{~A}$

B2:2

$152201 \mathrm{~J}$

B308

$\mathrm{PH} 14$

B.531

(6)

$A 611$
$A+80$

A6

121

Bi 71 
Guide Spec, for Clay Tile Rec. for Insulated Steel Deck ed with Bituminous Substances for Use in Waterproofing and and Design Loads. Timber, Plank and Laminated Flooring and

$r$ Woven Glass Fabrics Treated for Use in Waterproofing and Terra Cotta; Timber; Water; Ceiling; Partitions; Plaster; Structural Deck

ms Relating to Bituminous, and Other Organic Materials for tes, Saturated Felts, Saturated and Coated Felts, Prepared ypsum Boa/ Uniform Building Code Std. for Special Purpose Std. Rec. Pract. for Sampling and Analysis of Built Up ight Transmitting Panels, Skylights, Monitors and Sawtooth Std for Safety Std. for

umidifiers, Nebulizers, Gas Therapy and Suction Equipment, $0 \mathrm{cfm}(1971)$

$y$ for Tests for Fire Resistance of Vault and File Storage

ppliances, and Wall Furnaces, Floor Furnaces, and Unit and Std. for Gas Fired Std for Gas Fired

std. for Respiratory Therapy Equipment (Breathing Devices, Std. Meth. of Compression Test. of Metallic Materials at

Cold Chambers and Serving Units for Tests Above and Below of Test for Abrasion Resistance of Refractory Materials at $f$ Corn Starch Which Is Substantially Insoluble in Water at esistivity of Manufactured Carbon and Graphite Articles at td. for Residential Controls for Low Voltage, Wall Mounted nsi Z25. 1 Safety Std. for tes/ Std. Spec. for Moist Cabinets and (Specimen Storage) on of Sound Power Levels of Small Sources in Reverberation Recording and Specifying Machinery Sound Within Equipment Uniform Building Code: Motion Picture Projection Recommendation for Installation of Ceramic Tile Steam commendation for Installation of Ceramic Tile Refrigerator Rec. Pract. on Application, Care, and Use of Wire nted Types (1971) Std. Spec. for Electric Wire ed Members, for Electrical Conductors (1971) (1962)

Meth of Analysis ane Boomstop of All Mobile Construction Type Cranes Having Std. Spec. for Stainless and Heat Resisting Steel Spec. for Wire

for Critical Zone for Laboratory Evaluation-of Roll Over

Performance Criteria for Roll Over Protective Structures es; Deciduous Shrubs; Coniferous and Broadleaf Evergreens; Std. Meth. of Test for Total Std. for Liquid

I, Trap Flask, Step Wedge for X-Ray Standardization Probe, 55 Test Meth. for the Evaluation of Mildew and Std. Rec. Pract. for Spec. for

logenated Solvent Extract from Aerospace Components (Using ing at Le/ Rec. Pract. Safety Requirements for Industrial Sanitary Stds. for Centrifugal and Positive Std. for Uniform Dimensions and Meth. of Test. for fastness of Textile Yarn and Fabric to Crocking (Rubbing) Std. for Fastener:

for Continuity of Steam Turbine Oil Oxidation Stability by Oils Containing 2,6-Ditertiary-Butyl Para-Cresol by std. for Test Procedure for Airborne Sound Measurements on Std. for Electrical Apparatus Sales and Service Industry trose, Sy/ Std. Analytical Meth. for Determining Specific rolyzates/Std. Analytical Meth. for Determining Specific Spec. for Vacuum Treated Alloy Steel Forgings for Turbine

vacuum Treated Carbon and Alloy Steel Forgings for Turbine

for Vacuum Treated Electric Steel Forgings for Generator shutters, Flush, Stile, and Rail Doors, Factory Finishing, entification Marking Meths. on Finished, Semi Finished and

Gear Tooth Surface Texture for Aerospace Gearing (Surface

Makes a Good Air Conditioning System (Information on Year 73) ANSI H38.18 Std. S pec. for Aluminum Alloy Extruded ansi C7.59

ors (1972) ANSI C7.53

fins (1973) Std. Spec. for Compact Std Spec for Compact Preatment by Pressure Processes) (19/

Std. for al Pressure and Bending (1972)

Bul. on

Spec. and Meth. for Establishing Rec. Design Stresses for Tent. Test Meth. for Establishing Design Stresses for Std. Spec. for Unifórm Building Code Std. for Std. Spec. for Aluminum Alloy Formed and Arc Welded
Std. Spec for Aluminum Alloy Std. Spec. for Aluminum Alloy Formed and Arc Welded
Std. Spec for Aluminum Alloy equirements for Carbon Steel Wire Rods and Uncoated Coarse
Drycleanable Tailored Uniform Fabrics, Intermediate (Year
Roofing (1972)

Roofing (1972)

Roofing (1972) ANS1 A 109.10

CSI
FMS
Fand Woven Fabrics Saturat

07350

Roofing (1973)

TE

Values, Section Properties

WWPA

Re Sid Spec. Fo ASTM

Roofing) and Earthquake Recording Instrumentation (1973

(Roofing); Gypsum Concrete (1973)

Roofing, Waterproofing, and Related Building or Industr

ICBO

NRCA

ASTM

(

Roofs (Wood Shakes and Shingles with Asbestos Felt or ; A

NRCA

Roofs (1972)

Roofs, Light Diffusers in Ceilings, Partitions, Exterio

Room Air Conditioners (1972) ANSl 2234.1

Room Air Conditioners (I973) ANSI C33.14

Room Air Purifiers, Filters, Compressors, Artificial Ai

Room Doors (1972) ANSI A 153.1

Room Fan Coil Air Conditioners Delivering Less Than

Room Heaters and Ranges) (1973)

/Uirements for Vented Decorative a

Room Heaters: Volume I, Vented (1974)

Room Heaters: Volume II, Unvented (1974)

Room Humidifiers. Nebulizers, Gas Therapy and Suction E

Room Temperature (R1973)

Room Temperature (1966) ANSI C59.49

Room Temperature (1972)

Room Temperature (1973)

Room Temperatures (1969)

Std. Meth ASTM

Etermination of $\mathrm{pH}$ (Slurry) $\mathrm{O}$

Room Type Fan Coil Units (Heating and Cooling) (1973) a

for Electrical $\mathrm{R}$

Rooms for Color Grading (1971)

Rooms Used in the Test. of Hydraulic Cements and Concre

Rooms (Acoustics) (1972)

Rooms (Noise) (1973)

or Meth. for the Determinati

Rooms (1973)

Std. for Meth. of Measuring,

Rooms (1973)

Rooms (1973)

Rope for Oil Field Service (1972)

Rope Hoists (Lug, Hook, Trolley Suspended, and Base Mou

Rope Lay Stranded Copper Conductors Having Bunch Strand

Rope Spore Count in Cereal Products (Cereal Chemistry)

Rope Supported Booms, Equipped for Hook Work, Clamshell

Rope Wire (1972) ANSI G81.32

Rope (1972)

(ROPS) and Falling Object Protective Structures (FOPS) O

ROPS) for Prime Movers (Heavy Equipment) (1972)

Rose Grades; Vines and Ground Covers; Fruit Tree Grades

Rosin Acids Content of Coating Vehicles (1973)

Rosin Fluxes Used for Soldering (1973) ANSI C83.91

Rot Fragment Counting Plate and Cover) (1962)

Rot (Fungicides) Resistance of Textiles (1971) ANS1 L14

Rotameter Calibration (I973)

Rotary Drilling Equipment (petroleum) (1974)

Rotary Flash Evaporator) (1972)

Rotary Mowers (Designed for Operation with

Rotary Pumps for Milk and Its Products (1974)

Rotary Selector Switches (Radio, TV, and Instrumentatio

(Rotary Vertical Crockmeter Meth.) (I972) ANSI LI 4.212

Rotary, Quick Operating, High Strength (1973)

Rotating Bomb (1967) ANSI Z11.230

Rotating Bomb (1973) ANSI C59.113

Rotating Electric Machinery (1973)

(Rotating, Liquid Filled and Dry Transformers, Hand Powe

Rotation and Discharge of Centrifugal Fans (1966)

Rotation Content of Corn Sugar (Crude and Refined), Dex

Rotation Content of Corn Syrup, Dextrose and Starch Hyd

Rotor Disks and Wheels (1970) ANSI G55.15

Rotors and Shafts (1974) ANSI G55.1

Rotors (1971) ANSI G55.13

Rough and Finished Carpentry, Flooring, Shingles, Expos
Rough Items (1971)

Roughness, Waviness, Form and Lay) (1973)

Round Air Conditioning System) (1966)

Round Coiled Tubes for General Purpose Applications (19

Round Concentric Lay Stranded Copper Conouctors (1974)

Round Concentric Lay Stranded EC Grade Aluminum Conduct

Round Condenser and Heat Exchanger Tubes with Integral

Round Poles and Posts Used in Building Construction ( $\mathrm{Pr}$

Round Thread Casing Joint Strength with Combined Intern

Round Timber Construction Poles (1974)

Round Timber Piles (1970)

Round Timber Piles (1973)

Round Timber Piles (1973)

Round Tube (1974) ANSI H38.20

Round Welded Tubes (1973) ANSI H38.11

Round Wire (1971) ANSI G54.12

Round) Suiting (Institutional Textile) (1973)

D146

*31

D 1668

D 1079

*2-IV

D2829

AHAM RAC-1

UL 484

$\mathrm{NSF}$

UL

UL

ANSI

NSF

ASTM

CR

ASTM

NEM

ASTM

ASTM

48

155

443

896

48

E197

C704

C. 44

C611

883

D1684

C511

575

TCA 311

Re TCA 310

API 9B

HMI 100

ASTM B 172

$A A C C H \quad 42-20$

SAE J220

ASTM A492

API

SAE

$9 \mathrm{~A}$

J397A

J320B

ANS ] Z60.1

ASTM D 1469

ElA RS402

28-90

AATCC 30

ASTM D3195

API

F331

$\begin{array}{ll}\text { ASTM } & \text { F331 } \\ \text { SAE } & \text { J232 }\end{array}$

DFISA 0206

EIA RS315-A

AATCC 116

NSA

547

D2272

D2112

ASTM

IEEE

AMCA

CR

CR

$* 1$

2406

F.52

F. 52
E.58

A471

A 470

A 469

*I-1

118.01

MAN C

B491

B496

ASTM B400

ASTM B404

$\mathrm{UBC} * 3-23$

UBCS32.14

$\mathrm{UBC} * 3-52$

UMC*7-1

Z21.11.1

Z21.11.2

ICBO UBC $* 3-40$

AS478D 
square) Brass Wire (1973)

Uniform Building Code Std. for Std. for Spec. and Measurement of Out of rmed Welded and Seamless Carbon Steel Structural Tubing in

t Resisting Chromium Nickel Manganese Steel Bars Including (1974) Ansi/ Std. Meth. of Test. and Tolerances for Jute arm Machinery) (1973)

eal Chemistry) (1962)

Care and Use of Reinforced Thermosetting Resin Line Pipe ked Paint Film (Coil/ Spec, for Conducting a Solvent $\mathrm{H}$ and hes in Contact/ d. Meth of Test for Diffusion Stain of derground Installations of Electrical W/ Std. for Styrene st Meth. for Evaluating Low Temperature Characteristics of Std. Meth. of Measuring Low Temperature Stiffening of eth. of Test for Evaluating Pressure Sealing Properties of Std. Definitions of Terms Relating to Std. Rec. Pract. for Std. Test Temperatures for r Preparing Precision Statements for ASTM Meth. Related to Std. Meth. of Test for Accelerated Aging of Vulcanized Std. Meth. of Test for Accelerated Aging of Vulcanized

td. Meth. of Test for Impact Resilience and Penetration of Meth. of Test for Viscosity and Curing Characteristics of Std. Meth. of Test for Impact Resilience of Std Meth of Test for Hydrogen Permeance of

for Std. Materials, Equipment, and Procedures for Mixing Std. Meth. of Tcst for Abrasion Resistance of

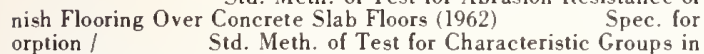
Pipe and Fittings (1972) ANSl A21.11 Std. for std. Spec. for Nonreinforced Concrete lrrigation Pipe with nd Fittings (1972)

and Cable, 75 Deg. C Operation nd Cable (1974) ANSl C8.38

Deg. C Operation (1974) ANSI C8./

Std. Spec. for Neoprene Sid. Spec. for Sheet Std. Spec. for Synthetic Std. Spec. for Synthetic Std. Spec. for Natural Std. Meth. of Testing on (1974) ANSI C8.24 Std. Spec, for Ozone Resisting Butyl Std. Spec. for Synthetic Std. Spec. for Synthetic on (1974) AVSI C 8.40 Std. for Mine Power Cables with Ethylene-Propylene

35,000 Volts and Having Ozone Resistant Ethylene Propylene ductor Electrical Power Cables with Ethylene-Propylene nductor Electrica' Power Cable with Erhylene-Propylene and Less and Having Ozone Resistant Ethylene-Propylene Std. Spec. for General Purpose Ethylene Propylene Std. Spec. for Natural

trated, Ammonia Preserved, Creamed and Centrifuged Natural Std. Rec. Pract. for Nomenclature for Rubbers and Std. Meth. of Test. Synthetic

r Evaluating Low Temperature Characteristics of Rubber and eth. of Measuring Low Temperature Stiffening of Rubber and $t$ for Evaluating Pressure Sealing Properties of Rubber and Std. Definitions of Terms Relating to Rubber and std. Rec. Pract. for Std. Test Temperatures for Rubber and gs (1973) Std. Meth. for Test. Std. Spec for Styrene of Compound and Sample Preparation for Physical Test, of Std. Meth. of Chemical Analysis of Std. Spec. for Sponge and Expanded Cellular

Meth. of Dynamic Test. for Ply Separation and Cracking of Std. Classification System for Carbon Blacks Used in Soft Chloroprene Medium Chloroprene Firm Chloroprene

Std. Meth. of Test for Adhesion of Vulcanized Sid. Meth of Test for Adhesion of Vulcanized

Std. Rec. Pract for Preparation of Pieces from Sid. Meth. of Test for Adhesion of Vulcanized of and Test Formulas for Natural Rubber $(\mathrm{Nr})$ and lsoprene std. Meth. for Evaluation of and Test Formulas for Natural valuation of and Test Formula for Carbon Blacks in Natural Std. Spec. for Styrene-Butadiene-Synthetic and Test Formula for Carbon Blacks in Styrene-Butadiene Std. Spec. for Styrene Std. Spec for Solvent Cements for Styrene for Strap Peel Adhesion of Reinforcing Fabrics or Cords to Std. Meth. of Tension Test. of Hard Rec. Concerning Storage of Expanded

Sid Meth of Test for Tear Resistance of Vulcanized of Measuring Plasticity Retention Index (PRI) of Natural sae $\mathrm{J} 20 /$

ct and Migration Stain of Organic Finishes in Contact with (45-55) (1973)

Resistant, 65-75 (1973/

Classification System for Elastomeric Materials Std. for Packing, Preformed-M1L-R-25897 Std. for Packing, Preformed-MlL-R-25897
Preformed, Straight Thread Tube Fitting Boss, MlL-R-25897
Round, Hexagonal, Octagonal, and Flat (Rectangular and Round ness (1972)

Rounds and Shapes (1973) ANSI G24.21

ICBO

Rounds, Squares, Hexagons, and Shapes-Angles, TEES an

Rove and Plied Yarn for Electrical and Packing Furposes

Row Crop Cultivator Shafts and Ground Tool Mountings ( $F$

Rowland Meth. of Analysis of Serum Protein in Milk (Cer Rtrp) (1972)

Rub Test for the Determination of Deg. of Cure of a Bac

Rubber and Contact and Migration Stain of Organic Finis

Rubber and Polyvinyl Chloride Ducts and Fittings for Un

Rubber and Rubber Like Materials by a Temperature Retra

Rubber and Rubber Like Materials by Means of a Torsiona

Rubber and Rubber Like Materials (1960) ANSI J2.20

Rubber and Rubber Like Materials (1973)

Rubber and Rubber Like Materials (1973) ANSI J1.3

Rubber and Rubber-Like Materials (1972)

Rubber by the Oven Meth. (1967) ANSI J5.

Rubber by the Oxygen Pressure Meth. (1973) ANSI J4

Rubber by the Rebound Pendulum (1972) ANSI J2.19

Rubber by the Shearing Disk Viscometer (I972) ANSI J7.7

Rubber by Vertical Rebound (1974) ANSI J2.3I

Rubber Coated Fabrics (1973)

Rubber Compounds for Preparing Vulcanized Test Sheets

Rubber Compounds for Soles and Heels (1968) ANS1 J2.26

Rubber Cushion Sleeper Construction with Nailers and Fi

Rubber Extender and Processing Oils by the Clay Gel Abs

Rubber Gasket Joints for Cast and Ductile lron Pressure

Rubber Gasket Joints (1973)

Rubber Gaskets for Hub and Spigot Cast Iron Soil Pipe a

Rubber Gaskets (1966) ANSI J7.2

Rubber Heat and Moisture Resisting Insulation for Wire

Rubber Heat or Moisture Resisting Insulation for Wire a

Rubber Heat Resisting lnsulation for Wire and Cable, 75

Rubber Hose (1972A) ANSI J2.5

Rubber Insulation for Wire and Cable (1974) ANSI C8.37

Rubber Insulation for Wire and Cable, 75 Deg. C Operati

Rubber Insulation for Wire and Cable, 90 Deg. C Operati

Rubber Insulation (1973) IPCEA S68-516

Rubber Insulation (1973) lPCEA S68-516

Rubber Insulation (1973) IPCEA S68-516

Rubber Insulation, 200l-5000 Volts (1973)

Rubber Integral Insulation and Jacket (1973) IPCEA S68-

Rubber Jacket for Wire and Cable (1972)

Rubber Jacket for Wire and Cable (1973) ANSI C8.25

Rubber Latex (1972A) ANSI J8.12

Rubber Latices (1972A) ANSI J9.1

Std. Spec. for Concen

Rubber Latices (1974) ANSI J2.34

Rubber Like Materials by a Temperature Retraction Proce

Rubber Like Materials by Means of a Torsional Wire Appa

Rubber Like Materials (1960) ANSI J2.20

Rubber Like Materials (1973)

Rubber Like Materials (1973) ANSI J1.3

Rúber O-Rings (1972)

Rubber Plastic Drain and Building Sewer Pipe and Fittin

Rubber Products (1972) ANSI JI.I

Rubber Products (1972A) ANSI J10.1

Rubber Products (1973) ANSI J2.4

Rubber Products (1973) ANSl J2.6

Rubber Products (1973) ANSl J8.6

Rubber Sponge (1973)

Rubber Sponge (1973

Rubber Sponge (1973)

Rubber to Metal (1973)

Rubber to Steel Cord (1973)

Rubber Vulcanizates Other Than Std. Test Sheets (1973)

Rubber (Friction Test) (1970) ANSI J3.1

Rubber (IR) (1973)

Rubber (Nr) and Isoprene Rubber (IR) (1973)

Meth. for Evaluation ASTM

Rubber (Nr) (1973)

(1973) ANSI C8.2

Rubber (SBR) (1973)

Rubber (SR) Plastic Drain l'ipe. Perforated (1974

Rubber (1971)

Rubber (1972)

Rubber (1972)

Rubber (1973)

Rubber (1973)

Rubber (1973) ANSI J7.3

Rubber, Ethylene Propylene Terpolymer, General Purp

Rubber Ceneral Purpose, High Temperature, Fuel and Oin

Rubber, Vinyl, Vinyl Asbestos, Polymeric Poured Seamles

Rubber, 75 Shore, O-Ring (1973)

Rubber, 75 Shore, O-Ring (1973)

$$
\text { Pec. for Cold Fo }
$$
Rec. Pract. for

/Ec. Pract. Fo

Ables Rated 0D Multiple Con

Std Meth for $\mathrm{E}$

ANSl

ASAE

AACC

APl

ASTM

NEMA

ASTM

ASTM

ASTM

ASTM

ASTM

ASTM

ASTM

ASTM

ASTM

ASTM

ASTM

ASTM

AWW

ASTM

CISPI

ASTM

ASTM

ASTM

ASTM

ASTM

ASTM

NEMA

NEMA

NEMA

ASTM

ASTM

ASTM

ASTM

ASTM

ASTM

ASTM

ASTM

ASTM

ASTM

ASTM

ASTM

ASTM

ASTM

SAE

SAE

ASTM

ASTM

ASTM

ASTM

STM

ASTM

Std. Meth. of Test ASTM

ASTM

FMS

ASTM

Sid Meth ASTM

ASTM

ASTM

SAE

SAE

ASTM

NSA

Std. for Packing, NSA

BCS32.13

B89.3.1

A500

A429

D681

S225.1

$46 \cdot 22$

RP5L4

B-11-18

D925

$\mathrm{TCl}$

D1329

D1053

D1081

D1566

D1349

D3040

D573

D572

D1054

D 1646

D2632

D815

D3182 
Std for Packing, Preformed-MlL-R-25897

Preformed, Straight Thread Tube Fitting Boss, MlL-R-25897

Precision Statements for ASTM Meth. Related to Rubber and olstery and Bedding Applications (1973) A/ Std. Spec. for Std. Meth. of Test for Polystyrene Blocks in Sbr Sid. Rec. Pract. for Nomenclature for luation of and Test Formulas for Solution Poly-Butadiene ation of and Test Formulas for General Purpose Chloroprene or Evaluation of and Test Formula for Isobutcne-lsoprene aluation of and Test Formula for Butadiene-Acrylonitrile tion of and Test Formula for Pigmented Styrene-Butadiene of and Test Formulas for Nonpigmented Styrene-Butadiene Rec. Pract. for Latex Foam id. Meth. for Sampling and Sample Preparation of Solid Raw Tent. Meth, of Tension Test. of Solid Irethane and Other orfastness of Colored Textile Yarn and Fabric to Crocking

for Colorfastness of Textile Yarn and Fabric to Crocking ct. for Measuring Slope Efficiency and Lasing Threshold of $r$, Scrubber. Sander, Scraper. Tile Remover. Rug Shampooer. Test Meth. for

chines (Polisher. Scrubber, Sander. Scraper. Tile Remover. Test Meth. for Visual Rating Meth. of Carpet Test Meth. for Carpet Test Meth. for Carpel

Meth. for Shampooing: W ashing of Textile Floor Covering versal Pails (Container) (Dot-37A80, Dot-37Aa60, Lfe pec. for $55 \mathrm{Gal}$. Full Removable Head Univcrsal Drum (If ec. for $57 \mathrm{Gal}$. Full Removable Hcad Iniversal Drums (lic ec. for $30 \mathrm{Gal}$. Full Removable Head Iniversal Drums (If $\mathrm{fc}$ ec. for $16 \mathrm{Gal}$. Full Removable Head Iniversal Drums (L/fc. gal. Full Removable Head, Lug Cover Lniversal Drums (U/C. iversal Pails (Container) (Dot-37A80. Dot-37A60, Ufc Tight Head Universal Pails (Container) (Dot-17E, Ufe Outdoor Smallbore Rifle Range Plans. Safety Rec. Pract, for Iniform Plumbing Code: Rec. Grading

supply and Communication Lines (1960) Safety I Supply Statio/ National Electrical Safety Code, Part 1 (Nati) d Cypress Lumber (1971-75) uipment (National Electrical Safety Code) (1973) ed) (1974) d) (1974)

\section{safety Code) (1940)}

ncluding Selection, Purchase, Preparation, Maintenance and palmkernels: Babassu; Flaxseed:/ Oilseed Products Trading Frame Shoring Safet Rec for Single Post Shore Safety Rec. Scaffolding Safety Rec. Horizontal Shoring Beam Safety

Bench Calibration of Tank Level Gaging Tapes and Sounding ds, Timber,/ Western Woods Llse Book: Lumber Std., Crading le Stack Plumbing System (1973) ifle Ranges (1972)

Design and for Tee Reducer. Flareless Tube to Swivel, Swivel on plic/ Std. Spec. for Nonload (Axial) Bearing Steel Studs,

c. for Determining the Load Carrying Ability of Farm Wagon Quality Stds. for Standing and hel Guide Specs. for Architectural Woodwork: Standing and ion Drain, Under Basement Floor, Sump Conductor, Downspout Std. Meth. of Test for Modulus of

d. Meth. of Test for Cold Crushing Strength and Modulus of Sid. Meth. of Test for Transverse ive Meth. of Test for Biaxial Flexure Strength (Modulus of ers $(1972)$

of Glass and Glass Ceramics (Determination of Modulus of a Policy on Geometric Design of
Rec. for Uniform Terminology for Std. Meth. of Test for

ANS1 Z11.152 62) Ether-Bicarbonate Meth. of Analysis of Ergot in Meth. of Analysis for Baking Quality of

meth. of Analysis for Acetic, Butyric, and Lactic Acids in Chloroform-Alcohol Meth of Analysis of Ergot in meth. of Analysis for Insect Fragments and Rodent Hairs in Microscopic Meth. of Analysis of Smut in Wheat or

Sedimentation Meth. of Analysis of Smut in Wheat and ling Number Determination of Both Meal and Flour of Wheat,
Rubber, 90 Shore, O-Ring (1973)

Rubber, 90 Shore, O-Ring (1973)

Rubber-Like Materials (1972)

Std. for Packing, NSA Ec. Pract. for Prcparing ASTM

(Iph

Rubbers and Latices (197.t)

Rubbers and Rubber Latices (1972A) ANS1 J9.1

Rubbers (BR) (1973)

Rubbers (CR) (1973)

Rubbers (IIR) (1973)

Rubbers (NBR) (1973)

Rubbers (SBR) (1973)

Rubhers (SBR) (1973)

Rubbers (1971)

Rubbers (1973)

Rublsers (1973)

(Ruhbing) by the AATCC Crockmeter Meth. (1972) ANSI LI4.

(Rubbing) (Rotary Vertical Crockmcter Meth.) (1972) ANSI

Ruby Laser Rods (1973)

Tent. Rec. Pra

Sid. Meth. for Eva ASTM

AsTh

ASTM

Std. Meth. for Evalu ASTM Std. Meth. F ASTM

Std. Meth. for Ev ASTM Sid. Meth. for Evalua ASTM Std. Neth. for Evaluation ASTM

SAE

STM

ASTM D3196

AATCC 8

AATCC 116

Rug and Floor Washers, etc.) (197.1)

Rug Back Floor Staining on Vinyl Tile (1973)

Rug Shampooer. Rug and Floor Washers. ctc.) (197.t)

(Rug) Soiling (197.3) ANSI L.14.21.5

(Rug) Soiling: Accerated Meth. (1973) ANSI L14.217

Rug) Soiling; Service Meth. (1973) ANSI L1t.2l6

(Rug, Carpet) (1972)

Rule 10. Nmfc-ltem 260 (197.t)

Rule 10. Nmfc-ltem 260) (1974)

Rule 10. Nmfc-Item 260) (197.1)

Nesting Test AATCC

[LL 561

L 561

AATCC 121

AATCC 123

AATCC 122

Rule 10. Nmfc-ltem 260) (197.1)

Rule 40, Nmfr-ltem 260) (1974)

Rule 40, Nmfc-Item 260) (197.t)

[Uni ANSI

sid. S ANSI

sid sp ANis

Sid. Sp ANisl

Sid. Sp ANSI

std. Spec for 16 A \SI

Ht Side Lug Cover In ANSI

Rule 40, Nmfe-ltem 260, Dot-37B60) (197t) /R 5 Gal ANSI

Rules and Formulae for Fibre Cans (1973)

Rules and Procedures (1972)

Rules for Building and Classing Steel Vessels (1972)

Rules for SAE Use of SI (Metric) Units (1972)

Rules for Sizing the Water Supply System (1973)

Rules for sitd. Wood Mouldings (1967)

Rules for Testing Seeds (1970)

Rules for the Installation and Maintenance of Electric

Rules for the Installation and Maintenance of Electrica

Rules for the Installation and Maintenance of Undergrou

Rules for the Measurement and Inspection of Hardwood an

IR 5 Gal ANSI

C.TI

$\mathrm{ABS}$

$\triangle A E$

ICBO

WWPA

AOSA

ANSI

ANSI

Rules for the Operation of Electric Supply Lines and Eq

Rules for the Purchase and Sale of Linseed Meal (Flaxse

Rules for the Purchase and Sale of Linsecd Oil (Flaxsee

Rules for the Purchase and Sale of Soybean Meal (1973)

Rules for the Purchase and Sale of Soybean Oil (1973)

Rules for the Radio Installations (National Electrical

Rules for Western Lumber (1972)

Rules of the Road (1972)

/ Std. for Regular Bicycles

Rules (Performance, Quality. Description, and Grade of

Rules (1972)

Rules (1972)

Rules (1972)

Rules (1972)

Rules (1974)

Rules, Design Values, Section Properties and Design Loa

Rules, Guidelines, and Drawings for Copper Solvent Sing

Rules, Procedures For, and Construction of High Power $R$

Run (1973)

Runners (Track) and Rigid Furring Channels for Screw Ap

Running Game (Wildlife) Rifle Ranges (1971)

Running Gear (1973)

Running Trim (Architectural Woodwork) (1973)

Sid. Test Pro

Runoff, Swimming Pool, etc.) (1973) ANSI Al76.3

Rupture of Air Setting Plastic Refractories (1972)

Rupture of Refractory Brick and Shapes (1972) ANSI All

Rupture Strength of Cemented Carhides (1973) ANSI H9.23

Rupture) of Ceramic Substrates (1974)

Rupture) (1972)

Rural Distribution Service for Motors and Phase Convert

Rural Highways (1965)

Rural Waste Management and Disposal (1973)

Rust Preventive Properties of Lubricating Greases (1973

Rye and Wheat Flour and Semolina (Cereal Chemistry) (19

Rye and Wheat Floura (Cereal Chemistry) (1962)

Rye Bread Flour (Cereal Chemiatry) (1962)

Rye Flour (Cereal Chemistry) (1962)

Rye Flour (Cereal Chemistry) (1962)

Rye Flour (Cereal Chemistry) (1962)

Rye (Cereal Chemistry) (1962)

Rye (Cereal Chemistry) (1962)

Rye, Barley, Other Grains, and Malted Cercals (Cereal C

59.

D3040

128

31.1

D3189

D3 190

3188

3187

3186

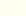

79

21
23
22
38

$\mathrm{MH} 2.15$

$\mathrm{MH} 2.2$

$\mathrm{MH} 2.11$

$\mathrm{MH} 2.12$

$\mathrm{NH} 2.1 .4$

$\mathrm{MH} 2.8$

$\mathrm{MH} 2.10$

$1 \mathrm{H} 2.9$

$+1$

in

$-5$

J916A

60-2

C2.2

C.2.1

C 2.3

NHLA 1

NFP 1

NFP

NSPA

NSPA

ANSI

W $\mathrm{W} A$

BMA

Niol

S.

SSI

ANSI

WWPA

CDA

NRA

NSA

ASTM
NRA

2

$+2$

C2.5

$6 / 7$

$* 1$

+4
.7

$\mathrm{B} 88.2$

-31

$* 1$

1763

C645

- 12

SAE \$360T

A WI

-1.]

D2311

C491

ASTM C133

ASTM B406

B406
F394

C 158

ASAE R329

ASHTO GD.2

[PC * ]-A 
our and Semolina: Also, with Slight Modification, to Corn 57) oprop (Common Name for the Pest Control Chemical O-Ethy Syrup, Sugar, And/ Std. Analytical Meth. for Determining Std. Analytical Meth. for Determining Std. Analytical Meth. for Determining Temperature Corrections for ) romthymol Blue Test Paper for Analysis of Urea in Cloth or $r$ Minimum Safety and Health Requirements for Paper Bag and Test Meth. for Drop Tests for Paper Shipping $r$, Semolina, and Similar Products: Feeds and Feedstuffs in st Meth. for Water Leak Test for Paper Shipping and Refuse Std for Paper Refuse or Regular Bicycles Including Wheel Assembly, Chainguards, (1968) Recommendation for Installation of Thresholds Rec. Pract, for the Std. Spec. for

)

y) (1973)

) (1972)
Std Rec Pract for

Properties and Essential Information for

Properties and Essential Information for Properties and Essential Information for

Std. for

Properties and Essential Information for Properties

Properties and Essential Information for the Rec. for

s Dust Locations (Agricultural Plast/

Auxiliary Machinery (Boats) (/

Std. Guidelines for

Depth Requirements (Passenger Car Type Tires) (1971)

Tires (1973)

ts) (1973)

(1973)

Pipe, Fittings / Uniform Fire Code: Recommended Guide to td. for Safety for Flexible Steel and Aluminum Conduit for Std. for the Rec. for te (1972)

d Structural Steel Products and Procedur/ and Demolition Operations (1973) ANSI A10.21 Rec. Pract. for the Use of Electric Blasting Caps (1971) ANSI C95.4 Std. for ontaining Flammable or $\mathrm{Co} /$ Uniform Fire Code: Recommended 2)

Rec

Rec. for General

Recommended

Mercantile and Bank Safety Sid for Key Locked Safety Sid. Burglary Resistant for Key Locks (Burglar Alarm, Door, Deposit and Collection

e Batteries in Motive Power Service (For Electric Trucks/ anufacture (Packaging) (1974) (1973) Std for Minimum Rec. for Fire g Buoy) (19/ Safety Std. for Marine Special Purpose Water ype) Connecting Devices and Towing Meth. (Coupling, Hitch, Rec. Safe Pract. and Proc. (Industrial Plant U.S. Model Rocket Sporting and ngs: Base Mounted Drum Hoists (1971)

d Platforms (1972)

Rec. Sid

National Electrica

Rules for the Radio Installations (National Electrical f Electric Supply Lines and Equipment (National Electrical ctric Supply and Communications Lines (National Electrical ntenance of Electrical Supply Statio/

National Electrical Std.

$\operatorname{ses}(1972)$

d Water Reactor Plants (1973)

Spec. for

Operational Rec for Globe Dry Pipe Valves (Fire and

ectrical / Mechanical Security Surveillance Sys/ Std. for

Rec. Pract. for

Std. for

Std. for

Sid for

Std. for

Std. for

Std. for

Std. for

Std. for

Std. for

Std. for
Rye, Barley, Rice, Grain Sorghum, and Buckwheat (Cereal S,S-Dipropyl Phosphorodithioate) (1973)

Saccharides (Chromatographic) Content of Corn Syrup (19

Saccharides (Gas Liquid Chromatography) Content of Corn

Saccharides (C,as liquid Chromatographv) (intent of Corn

Saccharimeter Readings (Sugar) (Cereal Chemistry) (1962

Sack Fibers, Whole or Ground Cereal Grains, Whole or Ch

Sack Manufacture (Packaging) (1974)

Sacks and Bags (Containers) (1973)

Sacks (Cereal Chemistry) (1962)

Sacks (Containers) (1973)

Sacks (1970)

Saddle Braces, Pedal Clearance, Steering Control, Brake

Saddles for Ceramic Tile Flooring (1973)

Safe Action and Title Areas for Television Transmission

Safe Fill or Aerosol Containers (1972)

Safe Filling of Low Pressure Pressurized Products (1972

Safe Handling and L'se of Acetic Acid (1973)

Safe Handling and Use of Acrylonitrile (1974)

Safe Handling and Use of Allyl Chloride (Chemical Safet

Safe Handling and I'se of Butadiene (1974)

Safe Handling and Use of Caustic Soda (1974)

Safe Handling and Use of Chloroform (1974)

Safe Handling and Use of Ethyl Acetate (Chemical Safety

Safe Handling and Use of Ethylene (1973)

Safe Handling and Use of Paraformaldehyde (1974)

Safe Handling and Use of Phosphorus Trichloride (1972)

Safe Handling and Use of Propylene (1974)

Safe Handling and Use of Vinyl Chloride (1972)

Safe Handling of Radioactive Materials (1964)

Safe Installation of Electrical Instruments in Hazardou

Safe Installation of Exhaust Systems for Propulsion and

Safe Operating Condition of Tires Including Tire Treads

Safe Operating Condition of Truck. Trailer and Bus Type

Safe Pract, and Proc. (Industrial Plant Safety Checklis

Safe Pract. for Thermal Spraying and Blasting Equipment

Safe Practice Protection from Corrosion for Underground

Safe Use as a Metal Raceway for Wire and Cable Installa

Safe Use of Lasers (1973)

Safe Use of Mixed Fertilizers Containing Ammonium Nitra

Safeguarding Against Embrittlement of Hot Dip Galvanize

Safeguarding Against Fire During Building Construction

Safeguards Against Radio Frequency Radiation Hazards in

Safeguards and Practices for the Protection and Tanks C

Safeguards for Arrangement of Carpet Storage Racks (197

Safeguards for Flammable Liquids (1974)

Safeguards for Organic Peroxides (1972)

Safeguards in Particle Board Plants (1972)

Safes and Vaults (1971) A NSI Se 2.1

Safes (Class KL) (1973)

Safes (1972) ANSI Se 4.1

Safes) (1973)

Safety Alert Symbol for Agricultural Equipment (1972)

Safety and Health Rec. for Changing and Charging Storag

Safety and Health Requirements for Paper Bag and Sack M

Safety and Protection of Yankee Driers (Paper Machine)

Safety Buoyant Devices (Vest, Jacket, Horseshoe and Rin

Ehicle (Recreational T

Safety Checklists) (1973)

Safety Code and Engine Std. (1973)

Safety Code for Cranes, Derricks, Hoists, Jacks and Sli

Safety Code for Fabricated Stage and Scaffold Planks an

Safety Code for Semiconductor Power Converters (1973)

Safety Code for Vertical Shoring (1968)

Safety Code (1973)

Safety Code) (1940)

Safety Code) (1973)

Safety Code) (1973)

Rules for the Operation $O$ Code, Part 1: Rules for the Installation and $\mathrm{Ma}$

Safety Color Code for Marking Physical Hazards (1971)

Safety Controls and Signaling Devices for Printing Pres

Safety for Agricultrual Auger Conveying Equipment (1973

Safety for Agricultural Equipment (1972)

Safety for Agricultural Equipment (1973) ANSI B114.3

Safety for Alarm Valves for Use in Wet Pipe Sprinkler S

Safety for Antitheft Alarms and Devices (Burglary Prote

Safety for Automatic Ice Makers (600 Volts or Less Rat

Safety for Automatic Water Sprinklers for Fire Protecti

Safety for Battery Powered Emergency Lighting Equipment

Safety for Bullet Resisting Equipment (Barrier) for Pro

Safety for Burglary Resistant Electric Door Strikes (19

Safety for Butterfly Valves for Fire Protection Service

Safety for Cellular Metal Floor Raceways and Fittings F
AACCH $76-20$

ANS1 K62.133

CR E-62

CR F.50

AACCH 80.21

$\mathrm{AACCH} 28.87$

Std. Fo ANSI 2259.1

TAPPI UV.806

A ACCH 64.60

Te TAPP] [\$1.529

NSF 32

BMA $\quad 6 / 5$

TCA 306

SMPTE RP8

ASTM D3068

ASTM D309]

MCA SD 41

MCA SD31

MCA SD99

MCA SD55

MCA SD9

MCA SD89

MCA SD51

MCA SD 100

MCA SD6

MCA SD2?

MCA SD59

MCA SD56

NCRPM R30

ISA S12.11

ABYC Pl

$\begin{array}{ll}\text { VESC } & \text { V.7 } \\ \text { VESC } & \text { V.9 }\end{array}$

MCA SG20

AWS $\quad$ C2.]

$1 \mathrm{CBO} \quad \mathrm{L} F \mathrm{~F} * 2 \mathrm{~B}$

UL

ANSI 2136.1

FMS LPD7.9

ASTM Al43

NFPA 241

IME 20

$\mathrm{ICBO} \quad \mathrm{UFC} * 2 \mathrm{~A}$

FMS LPD8.30

FMS $\quad 7.35$

FWIS $\quad 7.80$

FVS LPD7.10

UL 609

UL 786

IL 687

UL

ASAE R350

$8 \mathrm{Kl}$

ANSl 2259.1

FMS $12-29$

UL 1123

MCA $\$$ G20

NAR *1

ANSI B30.7

ALI *1

NEMA PV 3

SSI

ANSI

ANSI

*2

ANSI C2.4

ANSI C2.3

ANSI C2.1

ANSI 253.1

ANSI B65.1

ANSI NI8.2

FMS 2.14

UL

ASAE S36lT

SAE J208A

ASAE $\quad$ S318.3

IL

UL

UL

UI

$\mathrm{UL}$

UL

UL

$\mathrm{UL}$

193

1037

563

199

924

752

1034

1091

209

Engineering and Product Standards Division 
(1974)

c33.96

Alarm Systems (1973) ANSI Se2.6

naling Systems (1972) ANSI C33.90

(Lawn Mower, Cultivator, Edger-Trimmer, Hedge/

1972) ANSI B56.3

s Washing Equipment (Laundry Washer) (1974)

s Drying Equipment (Laundry Dryer) (1974)

ous Locations (1972) ANSl C33.28

zardous Locations, Class 1, Groups C and D (197)

(1974)

e Installation (1973) ANSI C33.98

als for Use in Flammable Anesthetizing Location/

Rated at 300 Volts or Less (1974)

(1972)

1973)

rs (1974)

ation of Wires and Cables (1972) ANSI C33.37

1

torage Tanks for Petroleum Oil and Gasoline (19/

Use as a Metal Raceway for Wire and Cable Inst/ , Sander, Scraper, Tile Remover, Rug Shampooer,

31.2

(1972)

ectrical Wiring Systems) (1972) ANS1 C.33.8

ansi C 33.36 .2

ving Appliances and Other Electronic Equipment /

(Audio / Visual Communication) (1974)

Type Appliances (1972) ANSI C33.11.1

si C 33.53

appliances (1973) ANSI C33.11.2

ansi B97.1

re and Display) for Television Receiving Applia/ rous Ammonia and Liquefied Petroleum (LP) Gas (/ razing Purposes (1973) ANSI Z250.1

ih Fire Protective Signaling Systems (1974)

2)

stible Materials) (1974) A NSI Z221.1

etroleum (LP) Gas (1973) ANS1 B167.1

aker Enclosures (1972)

) ANSI C33.56

tor Fittings for LP (Liquid Petroleum) Gas Cyli/ hazardous Locations, Class I, Groups C and D, A/

\section{ANSI 2217.1}

) ANSl Se2.9

BI30.1

ssories, Nonelectrical (1973)

g and Compressor Units (1973)

ble Fluids and Anhydrous Ammonia (Fertilizer Gr) ANSl A 162 . 1

Equipment (1973)

ving Appliances and Other Electronic Equipment / c33.40.1

trical Wiring (1973) ANSI C33.50

rs (1972)

ord Connected Electrical Appliances (1973)

storage Room Doors (1972) ANSI A 153.l

nces and Components (1972) ANSI C33.88

etroleum (LP) Gas (Other Than Safety Relief) (1/ )
Std. for Safety for Commercial Dry Cleaning Machines (Class Iv) Sid. for Safety for Commercial Electric Dishwashers (1973) ANSI Std. for Safety for Connectors and Switches for Use with Burglar Std. for Safety for Constant Level Oil Valves (1974) ANSI B127.1 Std. for Safety for Control Units for Indoor Fire Protective Sig

Std. for Safety for Cord Connected Electric Gardening Appliances

Std. for Safety for Cord Reels (1973) ANSI C33.18

std. for Safety for Dehumidifiers (1973) ANSI Z253.I

Std. for Safety for Electric Air Heaters (1973)

Std. for Safety for Electric Battery Powered Industrial Trucks (

Std. for Safety for Electric Coin Operated and Commercial Clothe

Std. for Safety for Electric Coin Operated and Commercial Clothe

Std. for Safety for Electric Lighting Fixtures for Use in Hazard

Std. for Safety for Electric Motors and Generators for Use in $\mathrm{Ha}$

Std. for Safety for Electric Oil Heaters (1973) ANSI C33.44

Std. for Safety for Electrical Attachment Plugs and Receptacles

Std. for Safety for Electrical Metallic Tubing for Wire and Cabl

Std. for Safety for Electrical Outlet Boxes and Fittings (1974)

Sid. for Safety for Electrical Power Outlets (1972) ANSI C33.85

Std. for Safety for Electrically Actuated Transmitters (1974)

Std. for Safety for Electrically Conductive Equipment and Materi

Std. for Safety for Electrically Operated Photographic Equipment

Std. for Safety for Electronic Data Processing Units and Systems

Std. for Safety for Elevator Door Locking Devices and Contacts (

Std. for Safety for Factory Made Air Duct Materials and Connecto

Rec. for Safety for Farmstead Equipment and Machinery (1972)

Std. for Safety for Fiber Conduit for Use as Raceway for Install

Std. for Safety for Fire Detection Thermostats (1974) ANS1 Z220.

Std, for Safety for Fire Tests of Door Assemblies (1974) ANSI A2

Std. for Safety for Flame Arresters (Fire) for Use on Vents of S

Std. for Safety for Flexible Steel and Aluminum Conduit for Safe

Std. for Safety for Floor Finishing Machines (Polisher, Scrubber

Std. for Safety for Gas Vents and Vent Roof Jacks (1973) ANSl Al

Std. for Safety for Ground Fault Sensing and Relaying Equipment

Std. for Safety for Grounding and Bonding Equipment (Internal El

Std. for Safety for Hair Clipping and Shaving Appliances (1973)

Std. for Safety for High Voltage Components for Television Recei

Std. for Safety for Hospital Signaling and Nurse Call Equipment

Std. for Safety for Household Electric Clocks (1974) ANSI C33.47

Std. for Safety for Household Electric Coffee Makers and Brewing

Std. for Safety for Household Electric Ranges (Stoves) (1974) an

Std. for Safety for Household Electric Skillets and Frying Type

Std. for Safety for Household Refrigerators and Freezers (1973)

Std. for Safety for Implosion Protected Cathode Ray Tubes (Pictu

Std. for Safety for Liquid Level Gauges and Indicators for Anhyd

Std. for Safety for LP Gas Torches for Heating, Soldering, and B

Std. for Safety for Manually Actuated Signaling Boxes for Use Wi

Std. for Safety for Mechanical Power Transmission Apparatus (197

Std. for Safety for Metal Waste (Trash, Garbage) Cans (For Combu

Std. for Safety for Meters for Flammable Liquids and Liquefied P

Std. for Safety for Molded Case Circuit Breakers and Circuit Bre

Std. for Safety for Nonmetallic Sheathed Electrical Cables (1974

Std. for Safety for Oil Fired Floor Furnaces (1974)

Std. for Safety for Oil Fired Unit Heaters (1974)

Std. for Safety for Oil Fried Water Heaters (1974)

Std, for Safety for Pigtail, Expansion Coil, and Flexible Connec

Std. for Safety for Portable Electric Lighting Units for Use in

Std. for Safety for.Portable Electric Tools (1974) ANSI C33.49

Std. for Safety for Portable Metal Ladders (1974)

Std. for Safety for Portable Sun / Heat Lamps (1974)

Std. for Safety for Portable Toy Transformers (1973)

Std. for Safety for Power Roof Ventilators (1972) ANSI C33.89

Std. for Safety for Pressure Regulating Valves for LP Gas (1973)

Std. for Safety for Printed Wiring Boards (1973) ANSI C33.46

Std. for Safety for Proprietary Burglar Alarm System Units (1974

Std. for Safety for Pumps for Oil Burning Appliances (1974) ANSI

Std. for Safety for Refrigerant-Containing Components and Acce

Std. for Safety for Refrigerated Vending Machines (1974)

Std. for Safety for Refrigeration and Air Conditioning Condensin

Sid. for Safety for Rigid Nonmetallic Conduit (1972) ANS1 C33.91

Std. for Safety for Self Contained Strainer or Filter for Flamma

Std. for Safety for Septic Tanks, Bituminous Coated Metal (1974)

Std. for Safety for Sheathed Heating Elements for Appliances and

Std. for Safety for Special Fuses for Radio and Television Recei

Std. for Safety for Special Use Electrical Switches (1973) ANSl

Std. for Safety for Surface Metal Raceways and Fittings for Elec

Std. for Safety for Swimming Pool Pumps, Filters, and Chlorinato

Std. for Safety for Tear Gas Units and Systems (1974)

Std. for Safety for Test for Polymeric Enclosures for Portable C

Std. for Safety for Tests for Fire Resistance of Vault and File

Std. for Safety for Thermal Cutoffs for Use in Electrical Applia

Std. for Safety for Tin Clad Fire Doors (1973) ANSI A 142.1

Std. for Safety for Valves for Anhydrous Ammonia and Liquefied P

Std. for Safety for Valves for Flammable Liquids and Fuels (1974

Std. for Safety for Vending and Amusement Machines (1974)

Std. for Safety for Water Pumps for Fire Protection Service (197

\begin{tabular}{|c|c|}
\hline UL & 664 \\
\hline UL & 921 \\
\hline UL & 634 \\
\hline UL & 352 \\
\hline UL & 864 \\
\hline UL & 82 \\
\hline $\mathrm{UL}$ & 355 \\
\hline UL & 474 \\
\hline UL & 1025 \\
\hline UL & 583 \\
\hline UL & 1206 \\
\hline UL & 1240 \\
\hline UL & 844 \\
\hline UL & $674 \mathrm{~B}$ \\
\hline $\begin{array}{l}\text { UL } \\
\text { UL }\end{array}$ & $\begin{array}{l}774 \\
498\end{array}$ \\
\hline $\mathrm{UL}$ & 797 \\
\hline UL & 514 \\
\hline LL L & 231 \\
\hline UL & 632 \\
\hline UL & 1067 \\
\hline UL & 122 \\
\hline UL & 478 \\
\hline UL & 104 \\
\hline UL & 181 \\
\hline ASAE & R354 \\
\hline $\begin{array}{l}\text { UL } \\
\text { UL }\end{array}$ & $\begin{array}{l}543 \\
521\end{array}$ \\
\hline UL & $10 \mathrm{~B}$ \\
\hline UL & 525 \\
\hline UL & 1 \\
\hline UL & 561 \\
\hline LL & 441 \\
\hline UL & 1053 \\
\hline UL & 467 \\
\hline UL & 1028 \\
\hline UL & 492.3 \\
\hline UL & 1069 \\
\hline LL & $\begin{array}{l}826 \\
1082\end{array}$ \\
\hline $\begin{array}{l}\text { UL } \\
\text { UL }\end{array}$ & $\begin{array}{l}1082 \\
858\end{array}$ \\
\hline UL & 1083 \\
\hline UL & 250 \\
\hline UL & 492. \\
\hline UL & 565 \\
\hline UL & 147 \\
\hline UL & 38 \\
\hline ANSI & B15 \\
\hline UL & 32 \\
\hline $\mathrm{UL}$ & 25 \\
\hline UL & 489 \\
\hline UL & 719 \\
\hline UL & 729 \\
\hline UL & 731 \\
\hline UL & 732 \\
\hline UL & 569 \\
\hline UL & 781 \\
\hline UL & 45 \\
\hline UL & 184 \\
\hline UL & 482 \\
\hline UL & 697 \\
\hline UL & 705 \\
\hline UL & 144 \\
\hline UL & 796 \\
\hline UL & 1076 \\
\hline UL & 343 \\
\hline UL & 207 \\
\hline UL & 541 \\
\hline UL & 303 \\
\hline UL & 651 \\
\hline UL & 331 \\
\hline UL & 70 \\
\hline UL & 1030 \\
\hline UL & 492. \\
\hline UL & 1054 \\
\hline UL & 5 \\
\hline UL & 1081 \\
\hline UL & 600 \\
\hline UL & 746. \\
\hline UL & 155 \\
\hline UL & 1020 \\
\hline UL & \\
\hline UL & 125 \\
\hline 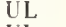 & \\
\hline UI & 75 \\
\hline & \\
\hline
\end{tabular}


terials (1973) ANSI A 195.1

fittings (Electrical Wiring) (1973) ANSI C33.45

equipment (1971) NBS HBI11

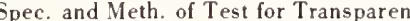
ne Dust (1972)

Uniform Building Code Std. for Glass, Its Defects and

Rec. for Fire

Std. for

Operational

Minimum Requirements for
Std. for Std. for

reading Agricultural Liquids and Slur/

Rec. for Improving

Std. Practice for Storage of Processed textile Products (1971)
Sid. Rec. Pract. for Apparatus, Reagents, and

(1971) Dictionary of Terms Used in the

ater Cooled and $\mathrm{Mo}_{\mathrm{o}}$ id Petroleum) Gas (1973) 69) ANSl Bl46.]

Std. for Fire Protection Criteria for Safety Std. for

drous Ammonia and Liquefied Petroleum (LP) Gas (Other Than Uniform Building Code: Existing Buildings; Minimum ding Ski and Surface Lifts and Tows (1973)

ning Equipment and Operations (1972)

power Press Brakes (1973)

gs, and Toeboards (1973)

ned for Operation with Tractors Having at Le/ 2)

and Demolition Operations) (1973)

$\mathrm{ms}$ (1972)

eeI and Plate (1973)

us Ammonia (1972)

of Shears (Mechanical, Hydraulic, Pneumatic, Semi /

of Die Casting Machines (1973)

of Packaging and Its Related Converting Machin/

ng, or Removal (1972)

Std. for

Std.

Std. for

Std. for

Rec. Pract.

Std. for

Std. for

Std for

Rec. Std. for

Std.

Std. for

Std. for

Std.

Std. for

Std. for

Std

voltage Power Vacuum Interrupters Used in Power Switchge/ transmission and Distribution Line Compensation (Including
Outdoor Smallbore Rifle Range Plans:

ectric Supply and Communication Lines (1960)

derground Electric Supply and Communications Lines ( $\mathrm{Nati}$ Rec. Steel Frame Shoring Rec. for Single Post Shore

Rec. Scaffolding

power Rifle Ranges (1972)

Rec. Horizontal Shoring Beam

Requirements for Both Permanently and Temporarily Affixed mpoline (1974)

s (1972)

Std. Consumer

Rec.

\section{西}

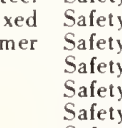
Safety Spec. for Power Mowers, Lawn, and Garden Tractor Safety Std. Antenna Discharge Units (1972) ANSI C33.39 Safety Std. Burglary Resistant Safes (1972) ANSI Se 4.l

Safety Std. Definitions for Regular Bicycles (1972)

Safety Std. for Aboveground Storage Tanks for Flammable

Safety Std. for Acetylene Generators, Portable, Medium

Safety Std. for Acetylene Generators, Stationary, Low P

Safety Std. for Acetylene Generators, Stationary, Mediu

Safety Std. for Air Conditioners, Central Cooling (1972

Safety Std. for AlI Thermoplastic Type MTW 600 VoIt Mac

Safety Std. for Asbestos Cement Pressure Pipe, Coupling

Safety Std. for Atmospheric Type Welded Steel Inside Ta

Safety Std. for Automatic Transfer Switches (1972)

Safety Std. for Automotive Fuel Tanks (1973)

Safety Std. for Automotive Glass Tube Fuses (1973)

Safety Std. for Battery Chargers (1972)

Use Principally as a Substitute for Plate Glass Show Wi/ Safety Std. for Burglary Resisting Glazing Material for Safety Std. for Capacitors (1973)

Safety Std. for Central Stations for Watchman, Fire Ala

rm and Supervisory Services (1972) ANSI Se 3.1 in Hazardous Locations, Class I, Groups A.D, and Class /

ipment (1973)

(1972)$$
\text { . }
$$

Safety Std. for Circuit Breakers and Enclosure

Safety Std. for Class H Cartridge Fuses (1973)

Safety Std. for Class R Fuses (Electrical) (1973)

Safety Std. for Commercial-Industrial Gas Heating Equ

Safety Std. for Commercial Electric Cooking Appliances

Safety Std for Commercial Refrigerators (1973)

Safety Std. for Compressors for Process Industries (197

Safety Std. for Construction and Guide for Selection, I

Safety Std. for Construction, Operation, and Maintenane

Safety Std. for Cord Sets and Power Supply Cords (1972) nstallation and Use of Electric Motors and Generators (1/ ANSI C33.3
UL 997

UL

UL

ANSI N43.

ANSI Z97.1

ICBO [BCS54-1

ANSI Z 288.4

FMS $1-3$

ANSI Z Z 29.1

Fis

$\begin{array}{ll}\text { UL } & 985\end{array}$

ASAE R317

ANSI PHI.43

ATI *4

ASTM E50

ASSFE *1

ASAE R342

FMS 12.61

ANS N18.I0

UL 132

API $\quad 526$

UL 125

ICBO L'BC $* 3.13$

ANSI B77.1

ANSI $\quad 250.1$

ANSI Z8.1

ANSI BIl.3

ANSI A12.I

SAE J232

ANSI MH9.1

ANSI AI0.4

ANSI Al0.3

ANSI Z229.1

SSI $* 9$

ANSI A10.13

FI

ANSl Bll.?

ANSI B152.]

ANSl B155.I

ANSI Ll.l

ANSI Z133.1

ANSI AI4.4

ANSI Ol.1

ANS1 C37.85

ANSI C55.2

NRA *4

ANSI C 2.2

ANsl $\mathrm{C} 2.3$

SSI *3

SSI *4

SSI *5

NRA *3

ASAE R355

SAE J115

ASTM F381

ANSI B71.1

UL 452

UL 687

BMA $\quad 6 / 2$

UL 142

UL $\quad 409$

$\begin{array}{ll}\text { UL } & 409 \\ \text { UL } & 408\end{array}$

UL 465

UL 1063

UL 107

UL

UL

UL

UL

UL

UL

UL

UL

UL

UL

UL

UL

UL

ANSI

NEMA

UL

UL 
and Slings: Portal, Tower and Pillar Cranes (1973)

Electric Operators and Systems (1973)

in Heating Appliances (Regulators, Automatic Damper Cont/

82

for Fire Protection Service (1970)

ortable and Fixed) $600 \mathrm{~V}$ or Less (1973) ANSI C.33.9.

(1973) ANSI C 33.104

971) ANSI C 33.35

33.75

orative Cooler, and Air Filtering Appliances Rated at 60/ ocations Glass I, Groups A, B, C, and D, and Class II, G/ ixed and Stationary 600 Volts or Less) (1972) C 33.11

$\$$ Washer and Dryer) (1972) ANSI C33.13

in Hazardous Locations, Class II, Groups E, F, and G (19) pment (1972)

ANSI C 33.12

s Locations: Class 1, Groups A-D, and Class II, Groups E/ ntrol of Fluids (1973)

Hazardous Locations, Class 1, Groups A, B, C, and D, An/ g (1972) entilating Ports for Wall Installation (1972) ANSI B135./

Hose (1973)

1

\section{fittings (1973)}

(1973)

es Including Wheel Assembly, Chainguards, Saddle Braces,

e and Multiple Station, Mechanically Operated Type for I/

ial and Commercial Gas Cylinders (1972)

Voluntary

rn, Fusee) (1973)

nsi Se2.7

972) ANSI Se2.4

eaters (1972) ANSI C33.87

973)

its (1973)

ed Gas Service (1973)

tection Service (1973) ANSI B156.1

Hazardous Locations, Class I, Croups A-D, Class II, Gro/ protection Systems (1969) ANSI C.33.70

and Collection Safes) (1973)

g Reflectivity, Headlights and Taillights (1972)

ng, Ventilating and Processing Systems) (1972)

Mobile Homes and Recreational Vehicles (1973) ANSI Al47/ flammable Liquids (1972)

se Snap Switches (1972) ANSI C.33.40

yant Devices (Vest, Jacket, Horseshoe and Ring Buoy) (19/

lves (1973) ANSI Z254. l

hot Mopping Asphalt, Asphalt.Saturated Organic Felt, Coa/

33.36

nges) (1973)

(1973)

z96. 1

(1973)

ning Door Releasing Device) (1973)

ower, Traction and Steering Stability for Regular Bic ycl/ nd LP (Liquid Petroleum) Gas (1972) UL 567

re Protection Service (1973) ANSI B1I1.1

\section{lammable Liquids (1974) ANSI Z216.1}

ucts (1973)

ed Petroleum (LP) Gas (1972)

(1973) ANS1 Z211.1

in Hazardous Locations (1973) ANSI C 33.97

urchase, Preparation, Maintenance and Rules of the Road/ Test Procedures for the

ooling) (1973) ANSI Z25.1

342
Safety Std. for Cranes, Derricks, Hoists, Hooks, Jacks, Safety Std. for Dead Front Switchboards (1972)

Safety Std. for Domestic Type Incinerators (1973)

Safety Std. for Door, Drapery, Gate, Louver, and Window

Safety Std. for Draft Equipment for Combustion Chamber

Safety Std. for Drinking Water Coolers (1972) ANSI C33.

Safety Std. for Dry Pipe, Deluge, and Pre Action Valves

Safety Std. for Electric Baseboard Heating Equipment (P

Safety Std. for Electric Central Air Heating Equipment

Safety Std. for Electric Cord Connected Indoor Signs (1

Safety Std. for Electric Dry Bath Heaters (1972) ANSI C

Safety Std. for Electric Fans (Blower, Humidifier, Evap

Safety Std. for Electric Heaters for Use in Hazardous L

Safety Sid. for Electric Heating Appliances (Portable F

Safety Std. for Electric Home Laundry Equipment (Clothe

Safety Std. for Electric Lighting Fixtures (I972)

Safety Std. for Electric Motors and Generators for Use

Safety Std. for Electric Motors for Appliances and Equi

Safety Std. for Electric Space Heaing Equipment (1972)

Safety Std. for Electrical Switches for Use in Hazardou

Safety Std. for Electrically Operated Valves for the Co

Safety Std. for Electrically Operated Valves for Use in

Safety Std. for Emergency Vault Ventilators and Vault V

Safety Std. for Extruded Thermoplastic Insulating Tubin

Safety Std. for Fire Dampers (1973) ANSI Z233.1

Safety Std. for Fire Extinguisher and Apparatus Booster

Safety Std. for Flexible Metallic Hose (1972) A NSI B140

Safety Std. for Foam Fire Extinguishers (1973)

Safety Std. for Fused Power Circuit Devices (1973)

Safety Std. for Gas Pressure Regulators (1973)

Safety Std. for Gasketed Joints for Cast Iron Pipe and

Safety Std for Gate Valves for Fire Protection Service

Safety Std. for General Requirements for Regular BicycI

Safety Std. for Ground Fault Circuit Interrupters (1972

Safety Std. for Heat Actuated Fire Alarm Devices, Singl

Safety Std. for High Chairs (Children's) (1972)

Safety Std. for High Pressure Gas Manifolds for Industr

Safety Std. for Highway Emergency Signals (Flare, Lante

Safety Std. for Holdup Alarm Units and Systems (1973) a

Safety Std. for Hose for Conducting Gasoline (1973)

Safety Std. for Household Burglar Alarm System Units (1

Safety Std. for Household Electric Storage Tank Water H

Safety Sid. for Household Type Pressure Cookers (1973)

Safety Std. for Hydrants for Fire Protection Service (1

Safety Std. for Ice Cream Freezers and Soda Fountain Un

Safety Std. for Indicating Pressure Gauges for Compress

Safety Std. for Indicating Pressure Gauges for Fire Pro

Safety Std. for Industrial Control Equipment for Use in

Safety Std. for Intrusion Detection Units for Burglary

Safety Std. for Key Locked Safes (Class KL) (1973)

Safety Std. for Key Locks (Burglar Alarm, Door, Deposit

Safety Std. for Lighting for Regular Bicycles, Includin

Safety Std. for Limit Controls (Air Conditioning. Heati

Safety Std. for Line Isolation Monitors (1973)

Safety Std. for Liquid Fuel Burning Heat Appliances for

Safety Std. for Liquid Level Gauges and Indicators for

Safety Std. for LP (Liquid Petroleum) Gas Hose (1973)

Safety Std. for Manually Operable General and Special U

Safety Std. for Marine Special Purpose Water Safety Buo

Safety Std. for Marine Through Hull Fittings and Sea Va

Safety Std. for Materials for Built.Up Roof Coverings:

Safety Std for Medical and Dental Equipment (1972)

Safety Std. for Motor Operated Appliances (1972) ANSI C

Safety Std. for Oil Burners (1973)

Safety Std. for Oil Burning Stoves (Room Heaters and Ra

Safety Std. for Oil Fired Air and Direct Fired Heaters

Safety Std. for Oil Fired Boiler Assemblies (1973)

Safety Std. for Oil Fired Central Furnaces (1973) ANSI

Safety Std. for Oxy Fuel Gas Torches (1973)

Safety Std. for Oxygen Therapy Equipment, Refrigerated

Safety Std. for Panic (Emergency) Hardware (Outward Ope

Safety Std. for Performance Requirements for Stopping $\mathrm{P}$

Safety Std. for Pipe Connectors for Flammable Liquids a

Safety Std. for Play Pipes for Water Supply Test. in Fi

Safety Std. for Plug Fuses (Electrical) (1973)

Safety Std. for Portable Wood Ladders (1973)

Safety Std. for Power Operated Dispensing Devices for F

Safety Std. for Power Operated Pumps for Petroleum Prod

Safety Std. for Pumps for Anhydrous Ammonia and Liquefi

Safety Std. for Rating and Test, of Fire Extinguishers

Safety Std. for Receptacle-Plug Combinations for Use

Safety Std. for Regular Bicycles Including Selection. P

Safety Std. for Regular Bicycles (1972)

Safety Std. for Relocking Devices (1972)

Safety Std. for Room Air Conditioners (1973) ANSI C33.1

Safety Std. for Room Type Fan Coil Units (Heating and C
ANSI

UL

$\mathrm{UL}$

$\mathrm{UL}$

UL

UL

UL

UL

UL

UL

UL

UL

UL

U

UL

UI

UL

UL

UL

UL

IL

UL

UL

CL

UL

UL

UL

BMA

UL

JPMA

UL

UL

UL

UL

UL

UL

L

UL

UL

UL

UL

UL

UL

UL

UL

UL

UL

UL

UL

UL

UL

UI

UL

UL

UL

UL

UL

UL

UL

BMA

ANSI

UL

UL

UL

UL

UL

UL

UL

UL

BMA

BMA

UL

UL

B30.4 791

621

404

393

698

639

786

437

$6 / 6$

1022

$307 \mathrm{~A}$

180

21

1123

1121

$55 \mathrm{~A}$

544

73

296

896

733

726 
nia and LP (Liquid Petroleum) Gas (1973)

s (1972) ANSI B 143.1

(1972)

Mounted Tin Clad Fire Doors (1973) ANSI Al43.1

nt (1973)

73) ANSI C33.86

derground Water Service (1972)

nd Combustible Liquids (1973) ANSI B137.1

ycles Including Tests for Static Load, Frame, Fork, Stee/ antile Premises (1973) ÁNSI Se2.5

e Doors (1973) ANSI A133.1

lectrical Equipment for Control of Air Conditioning, Hea/ mpounds (Fire Protection) (1973) ANSI Z240.1

iquids (1972)

ing Material (1973)

film $(1972)$

si C33.80

1972) ANSI C33.2

ible Fluids, Refrigeration Service, and Marine Use (1972 on Boxes for Swimming Pools (1972)

aners (1973) ANSI C33.36.1

ANSI C33.73

e Fire Extinguishers (1973)

Spec. for Lineman's Climbing Equipment (Body Belt,

parts in Devices and Appliances (1973) Std. for
l) Std. Meth. for Determining

parts in Devices and Appliances (1973) Std. for
1) Std. Meth. for Determining sign, Construction, and Operation of So/ Requirements for 970) A NSI A9.1 Code for

act. for Design, Installation, and Operation of Subsurface Industry Use (1973) Testing Procedures and Maintenance of Steam

Curing Ovens (1973)

ocal Protective Signalling Systems for Fire Protection and ruction and / Western Woods Use Book: Fire Protection and $r$ Evaluation of the Effectiveness of Rear Underride Guard

Materials (Metals, Slag, Glass, etc.) Fire Protection and rec. for Sprinkler, Water Spray, and Explosion Protection om the Wal Stds. and Practice Guide for Aids to Security ally Propelled Mobile Ladder Stands for Scaffolds (Towers. Safe Handling and Use of Ethyl Acetate (Chemical poxy Products According to Their Hazardous Potentialities Std. for Radiation Protection in Uranium Mines Rec. for Parking Structures (Garage-Fire Protection and Rec. for Liquefied Hydrogen Systems (Fire Protection and Rec. for Hazardous Chemicals Data (Fire Protection and Safe Handling and Use of Allyl Chloride (Chemical

for Firecycle on - Off Multicycle Sprinkler System (Fir

for Water Sprinkler and Hydrant System Demand Tables (Fire the Inspection and Maintenance of Sprinkler Systems (Fire prietary Protective Signaling Systems (Fire Protection and $r$ Demand for Sprinkler System for Private Fire Protection

ble Liquid Pumping and Piping Systems (Fire Protection and uide for Packaging and Transporting Radioactive Materials tation of Radioactively Contaminated Biological Materials $s$ Cylinders Intended for Medical Use in the United States on and $\mathbb{W}$ ater Sprinkler System Design-Fire Protection and tures) of Plastics Using a Hot Air Ignition Furnace) (Fire ium Compounds (Air Contamination-Occupational Health and and Shelf Storage Up to $15 \mathrm{Ft}$. High) (Fire Protection and ransformers, Hand Power Tools, Hermatic Stators, Test. and

Rack Storage of Flammable and Combustible Materials (Fire 0 Test Meth. to Determine Woven Fabric's Resistance to Trading Rules for the Purchase and Trading Rules for the Purchase and Trading Rules for the Purchase and Trading Rules for the Purchase and Dry Transformers, Hand Po/ Std. for Electrical Apparatus Std. for Cast and Wrought Galvanic Zinc A nodes for Use in (1962) tuffs (Cereal Chemistry) (1962)

Meth. of Analysis of Std. Meth. of Test for Meth. of Analysis of Added

) (1974) ASTM B 117

Meth. of Test for Tetrasodium Std. Meth. of Std. Meth. of

Std. Meth. of Acetic-Acid

Std. Analytical Meth. for Determining Lactic Acid and Std. Analytical Meth. for Determining Lactic Acid and of the Leachate of /

Std. Meth. of Test for Water Soluble Qualitative Meth. of Analysis of Ammonium
Safety Std. for Safety Relief Valves for Anhydrous Ammo Safety Std. for Sealed (Hermetic Type) Motor Compressor Safety Std. for Semiautomatic Fire Hose Rack Assemblies Safety Std. for Sliding Hardware for Std., Horizontally Safety Std. for Sound Recording and Reproducing Equipme Safety Std. for Specialty Transformers (1973) ANSI C33. Safety Std. for Stationary and Fixed Electric Tools (19 Safety Std. for Steel Pipe (Welded and Seamless) for Un Safety Std. for Steel Underground Tanks for Flammable a Safety Std. for Structural Requirements for Regular Bic Safety Std. for Surveillance Cameras for Banks and Merc Safety Std. for Swinging Hardware for Std. Tin Clad Fir Safety Std. for Tellers: Lockers (1973)

Safety Std. for Temperature Indicating and Regulating E Safety Std. for Tests for Combustibility of Sweeping Co Safety Std. for Tests for Comparative Flammability of L Safety Std. for Tests for Fire Resistance of Roof Cover Safety Std. for Tests for Flammability of Photographic Safety Std. for Thermoplastic Insulated Wires (1971) an Safety Std. for Transformer Type Arc Welding Machines ( Safety Std. for Tube Fittings for Flammable and Combust Safety Std. for Underwater Lighting Fixtures and Juncti Safety Std. for Unlined Fire Hose (1973) A NSI A 152.1 Safety Std. for Vacuum Cleaning Machines and Blower Cle Safety Std. for Varnished Cloth Wires and Cables (1971) Safety Std. for 2 1/2 Gal. Cartridge Operated Water Typ Safety Stds. for Conveyors and Related Equipment (1972)

Safety Strap, Lanyard, Pole and Tree Climber) (1973)

Safety Tests for Flammability of Plastic Materials for

Safety Times of Photographic Darkroom lllumination (197

Safety to Life-Property from Explosion and Fire in De

Safety to Life from Fire in Buildings and Structures (1

Safety Valve Systems for Petroleum Industry Use (1973)

Safety Valves on Boilers (1972)

Safety Valves, Locks, and Landing Nipples for Petroleum

Safety Ventilation Requirements for Direct Fired Powder

Safety (Alarm) (1973)

Safety (Exit, Stairway, Aisle, Corridor, Building Const

(Safety (1971) Rec. Pract. Test Procedure Fo

Safety (1973) Rec. for High Temperature Molten

(Safety) for Flammable Liquid and Liquefied Flammable Ga

(Safety) of Perso
Safety) (1971)

Safety) (1972)

(Safety) (1972)

(Safety) (1973)

Safety) (1973)

Safety) (1973)

Safety) (1973)

Safety) (1973)

Safety) (1973)

Safety) (1973)

Safety) (1973)

Safety) (1973)

Safety) (1973)

Safety) (1973

(Safety) (1973)

(Safety) (1973)

Safety) (1973)

Safety) (1973

Safety) (1973)

Safety) (1973)

Safety) (1973

Safety) (1973)

Safety) (1974)

Fire Hazards and Protection Std. for

Sag Flow of Highly Sunflower Seeds; Ground Nuts (Pea

Materials (1968) ANSI K65.18

Sagging or Stretch in Wear (Textile) (1973)

Sale of Linseed Meal (Flaxseed) (1974)

Sale of Linseed Oil (Flaxseed) (1974)

Sale of Soybean Meal (1973)

Sale of Soybean Oil (1973)

Sales and Service Industry (Rotating, Liquid Filled and Saline Electrolytes (1973) ANSI H44. I

Salmonella Bacteria in Food Products (Cereal Chemistry)

Salt Calculated from Total Chlorides in Feeds and Feeds

Salt in Crude Oil (Electrometric Meth.) (1973)

Salt in Feeds and Feedstuffs (Cereal Chemistry) (1962)

Salt of EDTA in Water (1972T)

Salt Spray (Fog) Test. for Spec. Purposes (Coil Caoting

Salt Spray (Fog) Test. (1973) ANSI Z118.1

Salt Spray (Fog) Test. (1974) ANSI Z118.2

Salts Content in Feedstuffs (Corn) (1964)

Salts Content in Steepwater (Corn) (1962)

Salts in Pigments by Measuring the Specific Resistance

Salts in Yeast Foods (Cereal Chemistry) (1962)
UL $\quad 132$

UI

UL

UL

UL

UL

UL

UL

BMA

UL

UL

UL

L

UL

L

UL

IL

UL

UL

UL

UL

L

UL

ANSI

EEI

UL

ANSI

FMS

API

FMS

API

FMS

FMS WWPA

SAE

FMS

ABYC

ANSI

$\mathrm{MCA}$

ANSI

ANSI

FMS

FMS

FMS

MCA

Spec FMS

Rec FMS

Rec. for FMS

Rec. for Pro FMS

Rec, for Wate FMS

Rec for Flamma FMS

td. for Administrative G ANS]

ANSI

CCA

FMS

ICBO

ANSI

FMS

EASA

FMS

NITP

ANSI

NFP

NFP

NSPA

NSPA

EASA

ASTM

$\mathrm{AACCH} 42.25$

$\mathrm{AACCH} \quad 40.61$

ASTM D3230

$\mathrm{AACCH} \quad 40.60$

ASTM D3113

NCCA TB.III.2

ASTM B117

ASTM B287

CR

CR

G.13

ASTM $\quad$ D2448

$\mathrm{AACCH} \quad 40.16$ 
Qualitative Meth. of Analysis for Nature of Ammonium Quantitative Meth of Analysis of Ammonium aacetic (Ethylenedinitrilotetraacetic) Acid (EDTA) and Its $m$ Peroxide in Dough Conditioners Containing Monoglyceride.

controlling Cleaning Meth. for Hydraulic Fluid Power Fluid ne Bleaching (1971) ANSI L14.180 Test Meth. for Multiple ne (1971) ANSl L14.126 Std. Meth. of Test for Moisture in the Analysis Std. Meth. of Test for Volatile Matter in the Analysis std. Meth. of Test for Carbon and Hydrogen in the Analysis Std. Meth of Test for Nitrogen in the Analysis

Std. Meth. of Test for Total Sulfur in the Analysis s (1972) ANSI Jl.1 9/ Meth. for Experimental Wheat Flour Milling: Equipment,
Process (1972) ANSI Z1.1/ Meth. of Test for Linear Density of Cotton Fibers (Array

d Other Protein Bearing Materials When Suitable Amounts of d Other Protein Bearing Materials When Suitable Amounts of

d Other Protein Bearing Materials When Suitable Amounts of

idic Gaseous Fluoride in the Atmosphere (Double Paper Tape std. Rec. Pract. for Dealing with Outlying Observations in ansi Z/ Std. Rec. Pract. for Bonding Thin Spectrochemical nd Other Grains/ power System (For Particulate/ for Moisture Present in Ordinary Commercial and Industrial
Std. Meth. of Test for Number of Neps in Cotton Std. Meth. of Test for Meth. for Preparation of 62) ry) (1962) 1 Std. Definitions of Terms Relating to Atmospheric d Transfers of Soybean Oil (1973) freezes or Antirusts for Test. Purposes (/ 973) 84.1

ith Bituminous Substances for Use in Water/ e (1970) ANSI G85.1

Uniform Building Code Std. for and Meth. of r Structural Clay Load Bearing Wall Tile and Std. Meth. of insulation (1974) ANSI C 59.77

1967) ANSI Z257.2 Std. Definitions and Symbols for Acceptance

d Christmas Tree Equipment: Flow Control Chokes; and Water culate Contamination by Manual Meth. (197) culate Contamination by Automatic Particle/ ALCA Jl ts Except Forgings and Stock 62) s and Feedstuffs in Sacks (Cereal Chemistry) (/ Meth. for

у) (1962)

try) (1962)

1973)

mistry) (1962)

utes (M1L Std. 105D) (1971) Test Meth. for Single Std. Meth. of Test for Ash in the Analysis Std. Meth. for Collection of a Gross Std. Meth. of Compound and Std. Meth. for Sampling and Std. Meth. for Obtaining Natural Gas Std. Meth. of Preparing Coal Meth. for Extracting Fluid Meth. for Preparation of Meth. for Preparation of

Meth. for Preparation of Meth. for Preparation of Meth. for Preparation of Meth. for Preparation of Meth. for Preparation of Meth. for Preparation of Meth. for Preparation of Std. Rec. Pract. for Std. for Inspection, Std. Meth. for Std. Meth. for Std. Meth, of Uniform Building Code Std. for Std. Meth. of Std. Meth. of Std. Meth. of Std. Meth. of Std. Meth. of itd. Rec. Pract. for Std. Rec. Pract. for Std. Meth. for Std. Meth. of Std. Meth. of Std. Meth. of Std. Meth. of Std. Meth. for Meth. for Std. Meth. of Field Std. Pract. for Probability Std. Meth. for Open Bottle Tap Std. Meth. for Thin Walled Tube

Salts in Yeast Foods (Cereal Chemistry) (1962)

Salts $\left(\mathrm{C}_{111} \mathbf{H}_{11:} \mathbf{N}_{2} \mathrm{O}_{k}(1973)\right.$

Salts, Soy Flour, and Other Cereal

Salvaged Brick (Tech. Notes) (1962)

Sample Containers (1972) ANSI B93.20

Sample Meth. of Textile Tensile Loss by Retained Chlori

Sample Meth. of Textile Tensile Loss by Retained Chlori

Sample of Coal and Coke (1973)

Sample of Coal and Coke (1973)

Sample of Coal and Coke (1973)

Sample of Coal and Coke (1973

Sample of Coal and Coke (1973)

Sample of Coal and Std. Meth. Coke (1973)

Sample of Coal (1972)

Sample Preparation for Physical Test. of Rubber Product

Sample Preparation of Solid Raw Rubbers (1973)

Sample Preparation, and Tempering (Cereal Chemistry) (1

Sample Size to Estimate the Average Quality of a Lot or Sample) (1973) ANSI L14.136

Sample, Sulfuric Acid and Catalyst Are Employed (1960)

Sample, Sulfuric Acid and Catalyst Are Employed (1962)

Sample. Sulfuric Acid and Catalyst Are Employed (1962)

Sampler Meth.) (1973) /Ollection of Particula

Samples and Stds. to a Greater Mass of Material (1970)

Samples for Analysis by Gas Chromatography (1968)

Samples for Analysis of Crude Protein in Bread, Wheat a

Samples for Analysis (1972) ANSI K20.17

Samples from the Lines of an Operating Hydraulic Fluid

Samples of Wool by Oven Drying (1972) ANSI L14.202

Samples (1971) ANSI L14.97

Sample: Bread (Cereal Chemistry) (1962)

Sample: Dried Eggs (Cereal Chemistry) (1962)

Sample: Feeds and Feedstuffs (Cereal Chemistry) (1962)

Sample: Fruit and Fruit Products (Cereal Chemistry) (19

Sample: Leavening Agents (Cereal Chemistry) (1962)

Sample: Macaroni Prodncts (Cereal Chemistry) (1962)

Sample: Malt (Cereal Chemistry) (1962)

Sample: Semolina (Cereal Chemistry) (1962)

Sample: Wheat (Cereal Chemistry) (1962)

Sample: Yeast Foods and Flour lmprovers (Cereal Chemist Sampling and Analysis of Built Up Roofs (1972)

Sampling and Analysis of Coal and Coke (1970) ANSI K18.

Sampling and Analysis (1973)

Sampling and Measuring Procedures for Bulk Shipments an

Sampling and Preparing Aqueous Solutions of Engine Anti

Sampling and Sample Preparation of Solid Raw Rubbers (1

Sampling and Test. Brick and Structural Clay Tile (1973

Sampling and Test. Brick (1973)

Sampling and Test. Concrete Masonry Units (1970) ANSI a

Sampling and Test. Creosote (1970) ANSI 011.9

Sampling and Test. Felted and Woven Fabrics Saturated W

Sampling and Test. Ferroalloys for Determination of Siz

Sampling and Test. of Unburned Clay Masonry Units (1973

Sampling and Test. Structural Clay Tile (1973)

Sampling and Test. Untreated Paper Used for Electrical

Sampling Asbestos Fiber for Test. (1970) ANS1 K9l.2

Sampling Atmospheres for Analysis of Gases and Vapors

Sampling by Attributes (1971) ANSI Z1.6

Sampling Cotton Fibers for Test. (1972)

IEad, Underwater Wellhead.

Sampling Gas Blow Down Systems and Components for Parti

Sampling Gas Blow Down Systems and Components for Parti

Sampling Gas from a Transformer (1974)

Sampling Glass Containers (Bottle, Jar, etc.) (1956)

Sampling Hydraulic Cement (1973) ANSI Al.2

Sampling Leather for Physical and Chemical Tests (1971)

Sampling of Carbon and Low Alloy Steels (Wrought Produc

Sampling of Fats and Shortenings (Cereal Chemistry) (19

Sampling of Flour, Semolina, and Similar Products: Feed

Sampling of Ion Exchange Materials (1972)

Sampling of Materials (1970) ANSI Z1.10

Sampling of Noncryogenic Fluid Systems (1972)

Sampling of Soils (1974)

Meth. for Sampling of Solid Feeds and Feedstuffs (Cereal Chemistr Std. Rec. Pract. for Planning the

Meth. for

Test Meth. for Std. Meth. for

Std. for

Std. Meth. of

Std. Meth. for

Rec. Pract. for Meth. of

Std. Spec. for Equipment for
Sampling of the Atmosphere (1957) ANSI Z257.]

Sampling of Wheat and Other Whole Grains (Cereal Chemis

Sampling of Wood Chips from Conveyor (Pulp and Paper) (

Sampling Pressurized Gas for Gas Analysis (1973)

Sampling Procedure for Analysis of Bacteria (Cereal Che

Sampling Procedures and Tables for Inspection by Attrib

Sampling Saturated and Superheated Steam (1969)

Sampling Stacks for Particulate Matter (1971) ANSI Z257

Sampling Steel for Chemical Analysis (1971)

Sampling Water and Steam (1970) ANSI Z111.2
AACCH 40.17

AACCH 40.18

ANSI PH4.185

AACCH 48.51

BlA 15

NFLDP T2.9.2

$\begin{array}{ll}\text { AATCC } & 114 \\ \text { AATCC } & 92\end{array}$

ASTM D3173

ASTM D3174

ASTM D3175

ASTM D3178

ASTM D3179

ASTM D3177

ASTM D2234

ASTM D15

D1485

AACCH 26.10

ASTM E122

Std ASTM D1769

CR F.44

CR G.22

CR J.56

ASTM

ASTM E178

ASTM E401

NGPA 2166

AACCH 46-1I

ASTM D2013

NFLDP T2.9.1

/T ASTM D1576

ASTM D1446

AACCH 62.05

$\mathrm{AACCH} \quad 62.15$

$\mathrm{AACCH} \quad 62.20$

AACCH 62.25

AACCH 62.30

AACCH 62.40

AACCH 62.50

AACCH 62.60

$\mathrm{AACCH} \quad 62.70$

$\mathrm{AACCH} \quad 62.80$

ASTM D2829

ASTM D271

ASTM D 1356

NSPA *4

ASTM D1176

ASTM D1485

ASTM C67

ICBO UBCS24.25

ASTM C140

ASTM D38

ASTM D146

ASTM A610

ICBO UBCS24.15

Td. Fo ICBO UBCS24.8

ASTM D202

ASTM D2590

ASTM D 605

ASQC A2

ASTM D1441

API

ASTM F308

ASTM F327

ASTM D3305

ASTM C224

ASTM C183

ASTM D2813

SAE AMS2370A

AACCH 64.40

AACCH 64.60

ASTM D2687

ASTM E105

ASTM F301

ASTM D1587

AACCH 64.50

ASTM D1357

$\mathrm{AACCH} \quad 64.70$

TAPPI UM.4

ASTM F 307

AACCH $42 \cdot 10$

ANSI Z1.4

ASTM D 1066

ASTM D2928

SAE J408C

ASTM D1192 
acceptance of Evidence Based on the Results of Probability s) (1961)

ples and How to Test the Statistical Significance of Them Public Works Spec. for Ceramic Veneer
Uniform Building Code Std. for isual Std. for Surfaces of New Steel Airblast Cleaned with Uniform Building Code Std. for Perlite, Vermiculite and Std. Gravel (SAE 4330 Modified) (1973) Std. Spec. for Copper Alloy Spec. for Steel Std. Spec. for Magnesium Alloy Std. Spec. for Aluminum Alloy on and Precipitation Treated // Spec. for Magnesium Alloy
r Ductile Iron Pipe, Centrifugally Cast, in Metal Molds or r Ductile Iron Pipe, Centrifugally Cast, in Metal Molds or Gravimetric Meth. of Analysis of Cinder and Std. for Std. Meths. for Chemical Analysis of Glass d Other Liquid and Semiliquid Products (Drying Upon Quartz urna/ Uniform Building Code Std. for Concrete Aggregates plinters, etc. in St/ Sieving Meth. of Analysis for Dirt,
8 Std. Spec. for Magnesium Alloys in Ingot Form for Std. Spec. for Aluminum Alloys in Ingot Form for
r Safety for Floor Finishing Machines (Polisher, Scrubber, ntenance of Systems and Appliances (Extinguishers, Alarms, Std. Meth. of Test for Organic Impurities in Standard Specification for Building Std. Meth. of Tension Test of Flat Std. Meth. of Shear Test in Flatwise Plane of Flat Std. Test Meth. for Edgewise Compressive Strength of Flat Std. Meth. of Flexure Test of Flat Std. Test Meth. for Flexure Creep of Std. Test Meth. for Laboratory Aging of d. Test Meth. for Density of Core Materials for Structural eth. for Water Absorption of Core Materials for Structural Std. Test Meth. for Shear Fatigue of

Std. Test Meth. for Flatwise Compressive Strength of Std. Meth. for Measurement of Thickness of mining the Strength of Adhesively Bonded Plastic Lap Shear ng Blind Threaded Molded in Insert (1974)

ng Blind Threaded Molded in Insert (1974)

Std. for

Molded In, Thru Threaded, Self Locking, Nonself Locking. ded, Self Locking, Nonself Locking, Light Weight Floating Design Spec, for Flat Plywood Fabrication Spec. for Plywood

y Po/ Std. Rec. Pract. for Making Reference Glass-Meta e: Gravel; Granite, Limestone, and Marble Masonry; Mortar; for $\mathbb{W}$ ater and $\mathbb{W}$ aste Treatment) (1968/ Rec. for Concrete

e with Milk and Its Products (1973)

its Products (1974)

ts (1974)

s for Milk and lts Products (1974)

e Containers for Milk and Its Fluid Products (Packaging roducts (Mixing and Storage) (1974)

Brick and Tile in Sanitation Structures, Miscellaneous Spec. Data for Hubless Cast Iron Spec. for Seamless and Welded Austenitic Stainless Steel ities (Tech. Notes) (1969)

s (Tech. Notes) (1969)

h. Notes) (1969)

Notes) (1969) I Chemistry) (1967) Std. Meth. of Test for Accelerated Aging of Wood Std. Meth. of Test for Degree of Set for Wood Quality Stds. for Exterio

t for SIump of Face Glazing and Bedding Compounds on Meta

mental Items, Stairwork and Handrails, Exterior Frames and Electrical and Mechanical Characteristics of Antennas for lonizing Voltage Mass Spectrometric Analysis of Gas-O t) Spec. for Roof Membranes; Aggregates, Saturated Felts, oofings, and Plast/ Spec. for Roof Membranes; Aggregates, i Z11.27/ Std. Meth. of Test for Aromatic Traces in Ligh Asphalt-Saturated Organic FeIt, Coal Tar Pitch, Coal-Tar
Roof Coverings: Hot Mopped Asphalt, Asphalt and Coal Tar Roof Coverings: Hot Mopped Asphalt, Asphalt and Coal Tar
Std. Meth. of Sampling and Test. Felted and Woven Fabrics versene, and Sodium Phosphate Solutions; Isopropyl Alcohol (1973) ANSI / Std. Meth. for Isolation of Representative ent Test. of Steel Tubular Products (Tubing) with Magnetic Std. for Gas Fired Std. Meth. of Test for Length Change of Drilled or Rec. Fire Protection for Uniform Building Code Std. for Std. for Carbide Blanks for Tipping Circular
Sampling (1969) ANSI Z1.12 (Sampling) (1968) ANSI Z1.14

(Sampling, Test. Finishing, Instalat Sand-Lime Building Brick (1973) Sand Abrasive (1970)

Sand Aggregates for Gypsum Plaster (1973)

Sand and Anthrante Filtering Material (1972)

Sand Castings for General Applications (1973)

Sand Castings $(0.80 \mathrm{Cr}-1.8 \mathrm{Ni}-0.35 \mathrm{Mo}(0.28-0.36 \mathrm{C}))$

Sand Castings (1972) ANSI H45.l

Sand Castings (1974) ANSI H38.24

Sand Castings (5.75Zn-2.5Re-0.70Zr (Ze63-T6) Soluti

Sand Lined Molds for Gas (1971)

Sand Particles in Farina (Cereal Chemistry) (I962)

Sand Particles in Farina (Cereal Chemistry) (1962)

Sand Type Filters for Swimming Pools (1971)

Sand (1972)

Sand) (Cereal Chemistry) (1962)

Sand, Crushed Stone, Gravel and Air Cooled Iron Blast

Sand, Insect Fragments, Hairs, Feathers, Wood Fibers, S

Sand, Permanent Mold, and Die Castings (1966) ANSI H45.

Sand, Permanent Mold, and Die Castings (1973) ANSI H38.

Sander, Scraper, Tile Remover, Rug Shampooer, Rug and F

Sandpipes), Building Construction, Basement Pipe Inlets

Sands for Concrete (1973) AASHO T21, ANSI A37.19

Sandstone (1968) ANSI A 192.1

Sandwich Constructions in Flatwise Plane (1970)

Sandwich Constructions or Cores (1970)

Sandwich Constructions (1970)

Sandwich Constructions (1970)

Sandwich Constructions (1970)

Sandwich Constructions (1970)

Sand wich Constructions (1970)

Sandwich Constructions (1970)

Sandwich Core Materials (1970)

Sandwich Cores (1970)

Sandwich Cores (1970)

Sandwich Joints in Shear by Tension Loading (1973)

Sandwich PaneI Lightweight Selflocking and Nonselflock

Sandwich Panel Lightweight Selflocking and Nonselflocki

Sandwich Panel (1972)

Sandwich Panel (1972)

Sandwich Panels (1970)

/ Insert, Molded In, Blind Threa

Sandwich Panels (1971)

Sandwich Seal and Test. for Expansion Characteristics B

Sand: SIag; Steel; Terra Cotta; Timber; Water; Ceiling;

Sanitary Engineering Structures (Tanks, Reservoirs, Etc

Sanitary Std. for Farm Milk Storage Tanks (1973)

Sanitary Std. for Scraped Surface Heat Exchanges for Us

Sanitary Std. for Silo Type Storage Tanks for Milk and

Sanitary Std. for Storage Tanks for Milk and Its Produc

Sanitary Stds. for Centrifugal and Positive Rotary Pump

Sanitary Stds. for Fillers and Sealers of Single Servic

Sanitary Stds. for Uninsulated Tanks for Milk and Its P

Sanitary Structures (Tech. Notes) (1969)

Sanitary System (Plumbing Pipe and Fittings) (1972)

Sanitary Tubing (1973) A NSI B 36.38

Sanitation Structures, Ind ustrial W aste Treatment Facil

Sanitation Structures, Miscellaneous Sanitary Structure

Sanitation Structures, Sewage Treatment Facilities (Tec

Sanitation Structures. Water Treatment Facilities (Tech

Sanitation, Fire Warning Detector (1973)

Saponification Value of All Normal Fats and Oils (Cerea

(Sash Casement) (Excluding Glass Fibers) (Institutional

Sash Face Glazing Compound (1973)

Sash Glazing Compound (1973)

Sash (Architectural Wood work) (1973)

Sash (1974)

Sash, Screens, Blinds and Shutters, Flush, Stile, and R

Satellite Earth Stations (1973)

Saturate Fractions (1971) ANSI Z11.310

Saturated and Coated Felts, Prepared Ro

Saturated and Superheated Steam (1969)

Saturated Felts, Saturated and Coated FeIts, Prepared R

Saturated Hydrocarbons by Gas Chromatography (1972) Ans

Saturated Organic FeIt, Asphalt-Saturated Asbestos Felt

Saturated Organic Felts, Coal Tar Pitch, and Asphalt Co

Saturated with Bituminous Substances for Use in Waterpr

Saturated with Gasoline: Methyl Blue and Green: Mineral

Saturates Fraction from Low Olefinic Petroleum Naphthas

Saturation (1971) ANS1 Z166.27

1. Pract. for Eddy Curr

Sauna Heaters (1973)

Sawed Specimens of Cement Mortar and Concrete (1974T)

Sawmills and Lumber Yards (1973)

Sawn Wood Shingles (1973)

Saws (1972)

E141

E178

* 28

$\begin{array}{ll}\text { ICBO } & \text { UBCS } 24 \cdot 2 \\ \text { NACE } & \text { THI.01.70 }\end{array}$

ICBO IBC 47.3

AWWA B 100

ASTM B584

SAE AMS5329B

ASTM B80

ASTM B26

SAE AMS4425

Std. Fo ANSl A21.52

AACCH 28-01

$\mathrm{AACCH} 28.02$

$\begin{array}{ll}\mathrm{NSF} & 10\end{array}$

ASTM Cl46

ACCH 44.60

$1 \mathrm{CBO}$ UBCS26-2

AACCH 28.75

ASTM B93

ASTM B179

UL

ICBO

ASTM

ASTM

ASTM

ASTM

ASTM

ASTM

ASTM

ASTM

St ASTM

dest M ASTM

ASTM

ASTM

ASTM

/Er ASTM

NSA

NSA

NSA

NSA

$A P A$

APA

ASTM

ICBO

$\mathrm{ACl}$

DFISA

DFISA

DFISA

DFISA

DFISA

DFISA

DFISA

BIA

CISPI

Std ASTM

BIA

BIA

BIA

BIA

ICBO

561

U FC*2ART 13

$\mathrm{C} 40$

C616

C297

C273

C 364

C 393

C480

C481

C271

C272

C.394

C365

C366

D) 3164

1832

1833

1835

*3PDS 4

SP-61

Fl44

U BC * 3.23

$68-50$

3000

3100

2203

0106

0206

1704
3200

$34 \mathrm{C}$

301

A 270

$34 \mathrm{~B}$

$34 \mathrm{C}$

$34 \mathrm{~A}$

34

AACCH $58-50$

ANSI L24.1.4

ASTM C74l

ASTM AWI

C741
C742

*1. 1000

D2376

*1-1

RS411

D2786

*2.IV

NRCA

AST

D 1066

*2-IV

ASTM D2600

UL

ICBO

ASTM D D 46 
xterior Light Transmitting Panels, Skylights, Monitors and Std. Meth. of Test for Polystyrene Blocks in Std. Spec. for Styrene-Butadiene-Synthetic Rubber Elastomers for Use on Solid Styrene Butadiene Copolymers nd Test Formula for Pigmented Styrene-Butadiene Rubbers st Formulas for Nonpigmented Styrene-Butadiene Rubbers t Formula for Carbon Blacks in Styrene-Butadiene Rubber Safety Code for Fahricated Stage and

Rec.

Rec. Procedure for Compression Testing of

Std. for Manually Propelled Mobile Ladder Stands for

Performance Requirements for Individual Anti

so $105-1$

05-1 Rec Evaluation Procedure of Cray Std. Meth. for Calibration of Temperature Rec. Evaluation Procedure of Gray

Macro

Cereal Chemistry) (1962)

Cereal Chemistry) (1962)

Micro

d Surfaces Using a Passenger Automobile Equipped with Full

d Surfaces Ising a Passenger Automobile Equipped with Full

ract, for Test. Pavement Polishing in the Laboratory (Full

Rec. Evaluation Procedure of Chromatic Transference

d. Meth. of Test for Wool Content of Raw Wool-Commercial

d. Meth. of Test for Wool Content of Raw Wool-Laboratory

Std. Meth. of Measuring Break Pattern of Leather (Break

ting Shear Block Test for Quality Control of Glue Bonds in as Engines (1972)

voltage Circuit Breaker External Insulation Rat/ Std. Pract. for Std. for Std. for Rec. for Water Flow / Pressure Requirements for Pipe ket Type Poly(vinyl Chloride) (PVC) Plastic Pipe Fittings. Spec for Cellulose Acetate Butyrate (CAB) Plastic Pipe, for Butt Fusion Polyethylene (PE) Plastic Pipe Fittings, nitrile-Butadiene-Styrene (ABS) Plastic Pipe Fittings Std. Spec for Polyethylene (PE) Plastic Pipe.

ket Type Poly(vinyl Chloride) (PVC) Plastic Pipe Fittings, for Butt Fusion Polyethylene (PE) Plastic Pipe Fittings nitrile-Butadiene-Styrene (ABS) Plastic Pipe Fittings. nitrile-Butadiene-Stvrene (ABS) Plastic Pipe Fittings. threaded Poly(yinyl Chloride) (PVC) Plastic Pipe Fittings, Rec. Std. Steel Door, Frame and Hardware Abbreviations and Symbols. Used in Builders Hardware Rec. Procedure for Processing Hardware

Application Guide for High Voltage Air Switch/ Std. for gs and Related Required Capabilities for AC High Voltage/ Std. Spec. for Polyethylene (PE) Plastic Pipe. or Acrylonitrile-Butadiene-Styrene (ABS) Plastic Pipe. Std. Spec, for Poly(vinyl Chloride) (PVC) PIastic Pipe, initions for Mechanical and Acoustical Elements as Used in Hardware for

Minimum Requirements for Minimum Requirements for Type II Rec. Minimum Stds. for Std. for Inspection Procedures for Rec Pract, for a Program for Rec. for Crayons and Related Art Materials for e/ Uniform Plumbing Code: Minimum Facilities Required for d Bakery Mixes (Cereal Chemistry) (1962)

volumetric Copper Reduction Meth, for Sugar Determination: Std. for Letter Symbols for Units Utsed in Electrical of Qualifications and Scopes of Work Relating to Forensic (Measurement-Metric and British-American) in Published

Std. Ree. P

Through Hull Connections, Outboard Water Strainers, Wate Std Descriptions of Qualifications an apparent Density of Refractory Metals and Compounds by the ility of Spinning Oils from Woolen Yarns/ Test Meth. for

Vegetable Matter and Other Alkali Insoluble Impurities in

Pract. for Calculation of Commercial Weight and Yield of si L14.84

Sid for Classification of Lec. for lron and Steel Spec. for lron and Stee
Speth. for Continuous

s Products (1973) Sanitary Sid for Std. for Hole Spacing for

for Floor Finishing Machines (Polisher, Scrubber, Sander. Std. for Nomenclature Std. Spec, for Carbon Steel Wire of Meth. for

(1971) AASHO T27, ANSl A es (1972)

Color Change of Fung Std. Gravure Lateral Hard Do llular Plastics Using a Supported Specimen by a Horizonta Test Meth. for Chip Classification ( $\mathrm{H}$ and
Sawtooth Roofs, Light Diffusers in Ceilings, Partitions Sbr Rubbers and Latices (1974)

(SBR) Jacket for Wire and Cable (1973) ANSI C8.28

ICBO

ASTM

ASTM (SBR) (1972)

SBR) (1973)

/ Meth. for Chemical Analysis of Synthetic Sed Meth. tor traluation of a

(SBR) (1973)

Std. Meth. for Evaluation of and T ASTM

ASTM

SBR) (1973) Std. Meth. for Evaluation of and Tes

Scaffold Planks and Platforms (1972)

Scaffolding Erection Procedure (1968)

Scaffolding Safety Rules (1972)

Scaffolds and Shores (1967)

Scaffolds (Towers, Safety) (1971)

Gcald Type Water Shower Control Valves (1973)

Scale for Color Change (Textile Colorfastness) (1954) I

Scale for Differential Thermal Analysis (1973)

Scale for Staining (Textile Colorfastness) (1954) ISO 1

Scale Meth. of Analysis of Semolina Quality in Macaroni

Scale Meth. of Analysis of Semolina Quality in Macaroni

Scale Tires in the Diagonal Braking Mode (1973)

Scale Tires (1971T)

Scale Wheel Meth.) (1972)

Scale (Color Chart / Textile Colorfastness) (1972)

Scale (1972)

Scale (1972) ANSI L14.490

Scale) (1973) ALCA E64

Scarf Joints (1970)

/ Pave

ASTM

ALI

SSI

SSI

SSI

ANSI

AATCC

AATCC EP2

$\mathrm{AACCH} 66.41$

$\mathrm{AACCH} \quad 66-42$

Scavenging and Supercharging of Stationary Diesel and

Schedule of Dielectric Test Values for Outdoor AC. High

Schedule of Preferred Ratings for Capacitance Current S

Schedule System Sprinkler Demand (1974)

Schedule 40 (1973)

Schedule 40 (1973) ANSI B72.4

Schedule 40 (1973) ANSI K65.160

Schedule 40 (1973) ANSI K65.164

Schedule 40 (1974)

Schedule 80 (1973)

Schedule 80 (1973) ANSI K65.159

Schedule 80 (1973) ANSI K65. 163

Schedule 80 (1973) ANSI K65.165

Schedule 80 (1973) ANSI K65.166

Schedule (1972)

Schedules and Specs. (1961)

Schedules and Templates (1971)

Std Rec. P AST

St ASTM

ASTM

D2941

DEMA *1-3

ANSI

A

FMS

C 37.0781

C 37.0732

2.77

Std. Spec, for Soc ASTM

Std ASTM

D2466

D 1503

D2610

for Socket Type Acrylo ASTM D2468

ASTM D2104

Std. Spec for Soc ASTM D246?

Std. Spec ASTM D2611

Jor Socket Type Acrylo ASTM D2469

1. for Threaded Acrylo ASTM D2465

Std Spec for ASTM D.2464

STDI $\quad 111 \cdot \mathrm{D}$

$\mathrm{NBHA} * 3$

ANSI

Schedules of Preferred Ratings, Manufacturing Spec., an

Schedules of Preferred Transient Recovery Voltage Ratin .

Sehedules 40 and 80 Based on Outside Diameter (1974)

Schedules 40 and 80 (1973)

Schedules 40,80 and 120 (1973) ANSI B72.7

Schematic Diagrams (1972)

ASTM

ASTM

ASTM

School Buildings (1957)

School Bus Construction and Equipment (1972)

School Bus Construction and Equipment (1973)

School Buses (1970)

Gehool Buses (1972)

School Crossing Protection (1973)

School Use (1963)

School, Theater, Auditorium, Dormatory, Public or Offic

Schoorl Meth. of Analysis of Reducing Sugars in Prepare

Schoorl's Reduction Table (Cereal Chemistry) (1962)

Science and Technology (1969) ANSI Y 10.19

Sciences $(1972)$

Scientific and Technical Work (1973)

Scientific Papers for Written or Oral Presentation (197

Scleroscope Hardness Test. of Metallic Materials (1972)

Scoops and Drain Plugs (Boats) (1972)

Scopes of Work Relating to Forensi

Scott Volumeter (1970) AVI H9.15 / Meth.

Scourability of Spinning Lubricant (To Test the Removab

Scoured Wool (1972) ANSI L14.195

/D. Meth. of Test for

Wool, Top, and Noil for Various Commercial Comp

Scouring Wool Using Std. Raw Grease Wool Yarn (1971) an

Scrap (1971)

Scrap (1972)

Scraped Surface Heat Exchanges for Use with Milk and It

Scraper and Bulldozer (Dozer) Cutting Edges (1972)

Scraper. Tile Remover, Rug Shampooer, Rug and Floor Was

Scrapers (1971)

Scrapless Nut Quality (1972) ANSI K91.3

Screen Analysis of Asbestos Fibers (1971T)

Screen Analysis of Fine and Coarse Concrete Aggregates

Screen Dimensions for Monochrome Television Picture Tub

Screen Stds. (1972)

Screen Wire Meth. (Textile Colorfastness) (1970)

Screen (1973)

Screen (1974) ANSI K65. 114

Screen) (Pulp and Paper) (1973)
ANSI

NBHA

ESC

VESC

NEA

ANSI

USC

ACCH 80-68

$\mathrm{AACCH} \quad 80-69$

IEEE

260

IEEE 268

ANSI Z39.16

ASTM E448

ABYC H27

ASTM E444

ASTM B329

AATCC 77

ASTM D1113

ASTM D2720

AATCC 64

ISIS *1

ANSI N15.10

DFISA $\quad 3100$

SAE J737B

UL 561

SAE J728A

ASTM A544

ASTM D2947

ASTM C136

EIA RS266A

IPA

*3

ATCC 119

GT

*3

D1692
ASTM E444 
Test Meth. for Chip Length Analysis (Two Screen) (Pulp and Paper) (1973)

eth. for Shive Content of Mechanical Pulp (Laboratory Flat Calcine, Chrom/

dwork) (1973)

Std. Classification of Single and Double

Perforated lndustrial Vibrating

Test Meth. for Rejects from Knotter

Std. Spec. for Industrial Perforated Plate and

ltems, Stairwork and Handrails, Exterior Frames and Sash,

(1973)

hment (Preferred) (1974)

Sid. for

teel Studs Runners (Track) and Rigid Furring Channels for spec. for Installation of Steel Framing Nlembers to Receive Std. for R-40 Bulb (Hard Glass) Mlogul

Std. Meth. of Measuring Water Vapor Transmission Through Std. for Std. for

imensioning Std. and Terminology for Permanently lnstalled Std. Rec. Pract. for Dimensions and Tolerances for

ec. for Free Cutting Brass Rod. Bar, and Shapes for L'se in 974)

std. Slotted and Recessed Head Machine Screws and Machine

$$
\text { Std. for Gaging Practice for Metric }
$$

ht of Thread Engagement (1973)

Std. for Stub Acme

Pan Head, Close Tolerance, Short Thread, Torq Set Machine

- Alloy and Corrosion Resisting Steel, UNJC-3A Thread, Cap

y Steel and Corrosion Resisting Steel, LNJF-3A Thread, Cap td. for Self Locking. Flat 100 Deg. Head, Full Thread Part Std. for Self Locking, Pan Head, Full Thread Par

Std. for Flathead 100 Deg. Plain and Self Locking Machine d. for Self Locking, Flat Fillister Head, Full Thread Part

n Head, Phillips Recess Full Threaded, Alloy Steel Machine

lips Recess, Short Thread, 160,000 psi Alloy Steel Machine s, A286 Cres, Self-Locking and Nonlocki/ s. Titanium Alloy, 6Al-4V, Self-Locking! ess, Alloy Steel, Self-Locking and Nonl/

ead, Nonlocking (1973)

d Nonlocking (1972)

, Full Thread, Nonlocking (1972)

set $(1973)$

et (1973)

y Steel (1973)

d, Self Locking and Nonlocking (1972)

lloy, Full Thread, Self Locking and Nonlocking

1 Thread, Self-Locking and Nonlocking (1973)

thread, Self Locking and Nonlocking (1972)

full Thread Self Locking and Nonlocking (1972)

Std. for Machine Std. for Machine Std. for Machine Std. for Machine Std. for
Std. for Std. for Std. for Std. for Std. for Std. for

Std. for Machine Std. for Std. for Hex Head Std. for Std. for Std. for Std. for

Std. Part Bit, Bit,

completion, and Flowline Valves: Drilling Through Control, Std. Slotted and Recessed Head Machine

2) Std. for Steel Self Drilling Tappin

light Gage Steel Studs (1972) Std. Spec. for Steel Drill

Std. for Mechanical and Quality Requirements for Machine ec. for Cold Heading Quality Carbon Steel Wire for Machine ading Quality Carbon Steel Wire for Tapping or Sheet Metal Std. Spec. for Cold Heading Quality Steel Wire for Wood Std. for Steel Self Drilling Tapping
Std. for Thread Rolling

for Mechanical and Quality Requirements for Steel Machine or the Locking Ability Performance of Chemical Coated Lock

Nonmetallic Locking Element Type Prevailing-Torque Lock Std. for Slotted Headless Setscrew

servative Treatment. Poles, Piles, Spaced Columns, Joints. Strength, Hardened / Spec. for Low Alloy Steel Bolts and Spec. for Low Alloy Steel Bolts and

n Woods Use Book: Timber Fasteners (Connectors, Bolts, Lag empered. Roll Threaded (1973)

Bolts and Spec. for Titanium Alloy Bolts and Spec. for Titanium Alloy Bolts and ed (1973) Std for Design Parameters for Bolts and

(1971) connector and Bolted Joints, Drift Bolts, and Wood and Lag
Spec. for Powdered Hand Cleaner (Soap with Vegetable Spec. for Powdered Hand Cleaner (Soap with Vegetable
Std. for Safety for Floor Finishing Machines (Polisher. Rec. for Std. Spec, for $\mathrm{H}$ and Std. Spec, for Hand and Codification of Data Derived from the Investigation of Std. Meth of Test. Resistance to and Std. Covering Design and Construction of Cockpits and
Spec. for Cellulose Acetate Butyrate (CAB) Plastic Pipe
Screen) (1973)

Screened Ground Refractory Materials (Calcined Bauxite,

Screens for Window and Door Openings (Architectural Woo

Screens Sids. (1972)

Screens (Pulp and Paper) (1973)

Screens (Square Opening Series) (1972)

Screens, Blinds and Shutters, Flush, Stile, and Rail Do

Screw-100 Deg. Close Tolerance Flat Head 160,000 psi

Screw and Asscmbled Washers-Electrical Terminal Attac (L) Bearing S NSA

Screw Altached Cypsum Wallboard and Backing Board (1971

Screw Base Incandcscent (1966)

Screw Cap Closure Liners (1973)

Screw Conveyors (Materials Handling) (1971) ANSI MH4.7

Screw Conveyors (1971) A NSI MH4.7.1

Screw Conveyors (1972)

Screw Holes and Slots in Bone Plates (1973)

Screw Machines (1972)

Screw Nuts (1972)

Screw Threads for Metric Series Mechanical Fasteners (1

Screw Threads (1972)

Screw Threads (1973)

Screw Threads (1973)

Screw Threads (7/45 Deg. Form with 0.6 Pitch Basic Heig

Screw (1972)

Screw $(1972)$

Screw $(1972)$

Screw (1974)

Screw (1974)

Screw (1974)

Screw (1974)

Screw (1974)

Screw (1974)

Screw, Flat Fillister Head, Full Thread, Tri Wing Reces

Screw, Flat Fillister Head, Full Thread, Tri Wing Reces

Screw, Flat 100 Deg. Head, Short Thread, Torg Set (1973

Lled and Drilled. Plain and Self Locking /Nd Drilled, Plain and Self Locking. Allo

Screw, Hex Head, Tri Wing Recess, Alloy Steel, Full Thr

Screw, Hex Head. Tri Wing Recess, A286 Cres, Full Threa

Screw, Hex Head, Tri Wing Recess, 6Al-4V Titanium Alloy

Screw, Machine, Flat Fillister Head, Full Thread, Torq

Screw, Machine, Flat $100 \mathrm{Deg}$. Head, Full Thread, Torq S

Screw, Machine, Pan Head, Full Thread, Torq Set (1973)

Screw, Machine, 100 Deg., Flat Head Full Threaded, Allo

Screw, Pan Head, Short Thread, Torq Set (1973)

Screw, Pan Head, Tri Wing Recess, A286 Cres, Full Threa

Screw, Recessed, Full Thread (1973)

Screw, 100 Deg. Head Tri Wing Recess, 6Al-4V Titanium a

Screw, 100 Deg. Head, Tri Wing Recess, Alloy Steel, Ful

Screw, 100 Deg. Head, Tri Wing Recess, A286 Cres, Full

Screw, 100 Deg. Oval Head, Tri Wing Recess. A286 Cres.

Screwdriver, Tri Wing Recess (1972)

Screwdriver, Tri Wing. Spec. for (1972)

Screwed and Flanged Wellhead. Underwater Wellhead, and

Screws and Machine Screw Nuts (1972)

Screws and Rec. Technique for Measuring Case Depth (197

Screws for the Application of Gypsum Sheet Material to

Screws for L'se in Automotive and Related Industries (19

Screws (1971) ANSI G54.14

Screws (1971) ANSI G54.17

Screws (1971) ANSI G54. 18

Screws (1972)

Screws (1972)

Screws (1973)

Screws (1973)

Screws (1973)

(Screws) (1971)

Screws, Bolts, Connectors, Nails, Staples, Adhesives, F

Screws, Heat Resistant, 195,000 psi (1,345 $\mathrm{MPa})$ Tensile

Screws. Heat Treated, Roll Threaded (1973)

Screws, Plates, Nails and Spikes, Washers, Pins, Dowels

Screws, Steel, Low Alloy Heat Resistant, Hardened and T

Screws, 6A 1-4V, Heat Treated, Roll Threaded (1973)

Screws, 6Al-4V, Upset Headed, Heat Treated, Roll Thread

Screws; External Wrenching. Unified Threaded ln. Series

Screws; Light Metal Plate Connected Wood Truss Design;

Scrubber) (1972T)

Scrubber, Sander, Scraper, Tile Remover, Rug Shampooer.

Scrubbers for Pollution Control (1974)

Scrubbing Soap (Grit Soap) Paste and Bar (1974)

Scrubbing Soap (Grit Soap) Powder (1974)

Scuba Diving Accidents) (1973)

Scuffing of Trim Materials (1972)

Scuppers (Boats) (1972)

(SDR-PR) and Tubing (1973) ANSI B72.12

Ort Form (Acquisition

Rec. Pract. $\mathrm{ABYC}$

Std ASTM
TAPP

TAPP

AWl

IPA

TAPP

A WI

NSA

NSA

ANSI

ANSI

ASTM

EMA

CEMA

ASTM

Std. Sp ASTM

ANSI

IFl

ANSI

ANSI

ANSI

ANSI

ASA

NSA

NSA

NSA

NSA

NSA

NSA

NSA

NSA

NSA

NSA

NSA

NSA

NSA

NSA

NSA

NSA

$N S A$

NSA

NSA

NSA

NSA

NSA

NSA

API

SAE

ASTM

$\mathrm{SAE}$

Std. Sp ASTM

ASTM]

ASTM

IFI

SAE

Std. IFI

IFI

$S A E$

ICBO

$S A E$

$S A E$

WWPA

$S A E$

$S A E$

$S A E$

$S A E$

ICBO

ASTM

IL

FMS

ASTM

ASTM

ANSI

LM.6

[M. 240

C.316

1.1100

*2

UM. 1

4.5

517
720

C645

A97.2

C78.255

D3199

350

S33.4T

F 367 
c. for Biaxially Oriented Polyethylene (PEO) Plastic Pipe Std. Spec. for Polyethelene (PE) Plastic Pipe Std. Spec. for Polybutylene (PB) Plastic Pipe Std. Spec. for Polybutylene (PB) Plastic Pipe Std. Spec. for Poly(vinyl Chloride) Plastic Pipe or Acrylonitrile-Butadiene-Styrene (ABS) Plastic Pipe Std. Spec. for Polyethylene (PE) Plastic Pipe Safety Std. for Marine Through Hull Fittings and st Meth. for Colorfastness of Textile Yarns and Fabrics to iners, Water Scoops and Drain P Std for Installation of d. Rec. Pract. for Making Reference Glass-Metal Sandwich Rec. Pract. for Radial Std. Meth. of Test for

th. of Test for Adhesion and Cohesion of Elastomeric Joint d. Meth. Test for Tack Free Time of Elastomeric Type Joint t for Bubbling of I Part, Elastomeric Solvent Release Type ox Exposure of One Part, Elastomeric, Solvent Release Type ity and Tenacity 1 Part, Elastomeric, Solvent Release Type

Std. Meth. of Test for Slump of Caulking Compounds and Std. Meth. of Test for Staining of Caulking Compounds and meth. of Test for Tack Free Time of Caulking Compounds and Std for Dimensional Spec for 1971

Rec. Pract. for Service Performance Requirements for Std. for Gas Fired Gravity and Fan Type Std. Meth. of Test for Leaks in Heat Glazing Spec. for Spec. for

loying Form Wo/ Std. Test Procedure for the Evaluation of nation of Formaldehyde Odor in Resin Treated Fabric by the chinery (1972) Std. for Dimensional Spec. for 43. I eth. of Test for Water Vapor Transmission of Flexible Hea luid Products (Packaging / Sanitary Stds. for Fillers and 971) Rec. Pract. Meth. of Tests for Automotive Type
Spec. for Exterior Perimete meth. of Test for Extension Recovery and Adhesion of Latex

td. Meth. of Test for Low Temperature Flexibility of Latex Std. Meth of Test for Volume Shrinkage of Latex

of Test for Extrudability, After Package Aging, of Latex f Test for Aging Effects of Artificial Weathering on Latex Std. Spec, for Type 58 Borosilic ate or Solvent Soluble Liquid Adhesives for Automatic Machine (1960) ANSl J/ Std. Meth. of Test for Evaluating Pressure Systems (1972) Rec. Pract. Spec. for Cast Iron

Std. Meth. of Test for Adhesion After Impact of Preformed meth. of Test for Low Temperature Flexibility of Preformed eth. of 'Test for Weight Loss After Heat Aging of Preformed

st for Oil Migration or Plasticizer Bleed Out of Preformed Std. for emperature, Fuel and Oil Resistant, 65-75 (1973/ Rings,
Alloy Sheet, Strip, and Plate, Low Expansion, Glass Alloy Sheet, Strip, and Plate, Low Expansion, Glas, c. for Lubricant for Installation of Preformed Compression or Guide to the Application and Use of Radial Lip Type Oi Std. Definitions of Terms Relating to Buildin ints for Plastic Pressure Pipes Using Flexible Elastomeric Spec. for Resistance Meth. of Test for bular Prod Sid. Spec. for Supplementary Requirements fo 72A) ANS1 B 125.16

Tubing (1973) ANSI B36. 38

or General Service (1973) ANSI B125.15

w Temperature Service (1972A) ANSI B125.1/

Std. Spec. for Std. Spec, for Std. Spec. for Std. Spec. fo Std. Spec. for Std. Spec. for Std. Spec. for Std. Spec. for um-Copper-Columbium Stabilized Alloy / per Columbium Stabilized Alloy Pipe (1973/ General Service (1973) ANSI B125.14 Std. Spec. for Nickel Iron Chromium Silicon Alloy ice (1973) ANSI B125.17

at Exchangers (I965) ANSI H50.2 Sid. Spec. fo Std. Spec. for si N124 Std. Spec. for Wrought Zirconium and Its Alloy Std. Spec. for Columbium and Its Alloy

(1971) ANSI H50.1 Sid. Spec. for Std. Spec. for Std. Spec, for Std. Spec for

ication (1972) ANSI Z212.1

or Refinery Service (1972) ANSI B36.39

tral Station Service (1973) ANSI B 125.25

73) ANSI B125.1 Std. Spec for Welded and Std. Spec. for Std. Spec. for Std. Spec. for Std. Spec. for Std. Spec. for Std. Spec. for heater Tubes (1973) ANSI B125.9 rvice (1973) ANSI B125.7 (1972A) ANSI B 125.30

hapes (1973) ANSI / Std. Spec, for Cold Formed Welded and 22 Std Spec for Hot Formed Welded and nger and Condenser Tubes (1973) ANSI BI25/
SDR-PR) Based on Controlled Outside Diameter (1973)

(SDR-PR) Based on Controlled Outside Diameter (1974)

SDR.PR) Based on Outside Diameter (1973)

(SDR.PR) (1973) ANSl B72.19

(SDR-PR) (1973) ANSI B72.2

(SDR-PR) (1973) ANSI B72.3

R.PR) (1974) ANSI B72.1

Sea Valves (1973) ANSI Z254.1

Sea Water (1972) ANS! L 14.150

Seacocks, Through Hull Connections, Outboard Water Stra

Seal and Test. for Expansion Characteristics by Polarim

Seal Nomenclature and Glossary (1972)

Sealability of Enveloped Gaskets (1970) A NSI Z261.1

Sealants Under Cyclic Movement (1972)

Sealants (1971)

Sealants $(1972$

Sealants (1972)

Sealants (1972)

Sealants (1973)

Sealants (1973

Sealants (1973)

Sealed Beam Headlamp Units (1972)

Sealed Beam Headlamp (Light) Units for Motor Vehicles

Sealed Combustion System Wall Furnaces (1972)

Sealed Flexible Packages (1972)

Sealed Insulating Glass Units (1971)

Sealed Insulating Glass Units (1973)

Sealed Insulation Systems for AC Electric Machinery Emp

Sealed Jar Meth. (1972) A NSI L14.178

Sealed Lighting Unit for Construction and Industrial Ma

Sealed Packages for Dry Products (1972)

Sealed (Hermetic Type) Motor Compressors (1972) ANSI B

Sealers of Single Service Containers for Milk and Its F

Sealers, Adhesives, and Deadeners (1971)

Sealing Compound for Use with Architectural Aluminum (1

Sealing Compound (1972)

Sealing Compounds After Artificial Weathering (1972)

Sealing Compounds (1972\}

Sealing Compounds $\{1972\}$

Sealing Compounds (1972

Sealing Glass (1972) ANSI Z173.47

Sealing of Top Flaps of Fiberboard Shipping Cases (1970
Sealing Properties of Rubber and Rubber Like Materials

Sealing Rings for Automotive Transmission and Hydraulic

Sealing Tapes (1973)

Sealing Tapes $(1973)$

Sealing Tapes (1974)

Sealing Tapes (1974)

Sealing Washer (1974)

Sealing, Fluorosilicone Rubber, General Purpose, High T

Sealing, $53 \mathrm{Fe}-29 \mathrm{Ni}-17 \mathrm{Co}$ (1973)

Seals and Test. for Expansion Characteristics by Polari

Seals in Concrete Pavements (1972)

Seals (1972)

Seals $(1973$

Seals (1973)

Seam and Roll Spot Welding Equipment (3 Phase) (1973)

Seamless and Electric Resistance Welded Carbon Steel tu

Seamless and Welded Austenitic Stainless Steel Pipe (19

Seamless and Welded Austenitic Stainless Steel Sanitary

Seamless and Welded Austenitic Stainless Steel Tubing F

Seamless and Welded Carbon and Alloy Steel Tubes for Lo

Seamless and Welded Carbon Steel Water Well Pipe (1972A

Seamless and Welded Chromium-Nickel-Iron-Molybden

Seamless and Welded Chromium Nickel Iron Molybdenum Cop

Seamless and Welded Ferritic Stainless Steel Tubing for

Seamless and Welded Pipe (1972)

Seamless and Welded Steel Pipe for Low Temperature Sery

Seamless and Welded Titanium Tube fo: Condensers and He

Seamless and Welded Tubes for Nuclear Service (1971) an

Seamless and Welded Tubes (1970) ANSI H53.1

Seamless and Welded Unalloyed Titanium Welding Fittings

Seamless and Welded Zirconium Tubes for Nonnuclear Appl

Seamless Austenitic Chromium Nickel Steel Still Tubes F

Seamless Austenitic Steel Pipe for High Temperature Cen

Seamless Black and Hot Dipped Galvanized Steel Pipe (19

Seamless Brass Tube (1971) ANSI H36.1

Seamless Carbon and Alloy Steel Mechanical Tubing (1973

Seamless Carbon Molybdenum Alloy Steel Boiler and Super

Seamless Carbon Steel Boiler Tubes for High Pressure Se

Seamless Carbon Steel Pipe for High Temperature Service

Seamless Carbon Steel Pipe for Process Piping (1972A)

Seamless Carbon Steel Structural Tubing in Rounds and S

Seamless Carbon Steel Structural Tubing (1973) ANSI G24

Seamless Cold Drawn Intermediate Alloy Steel Heat Excha
/E ASTM D3287

ASTM D 3035

ASTM D3000

ASTM D2662

ASTM

D2282

ASTM D2239

UL

1121

Te AATCC 106

ABYC $\mathrm{H} 27$

ASTM F144

SAE Jl11A

ASTM F112

td. Me ASTM C719

St ASTM C679

ASTM C712

$\mathrm{C} 712$
$\mathrm{C} 718$

C7II

D2202

ASTM D2203

Std. ASTM D2377

SAE J57IC

SAE J32

ANSI Z21.44A

ASTM D3078

SIGMA 70-7.1

SIGMA $\quad 65-7-2$

IEEE 429

AATCC 112

$S A E \quad J 572 \mathrm{~A}$

Std. M ASTM D3079

UL 984

DFISA 1704

SAE J243

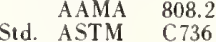

A STM

ASTM C733

td. Meth ASTM C731

C731
C732

F105

ASTM D1874

ASTM D1081

SAE J281

ASTM C766

Std ASTM C765

Std. M ASTM C77I

ASTM C772

NSA $\quad 1598$

SAE AMS7266

SAE AMS7728C

ASTM F140

D2835

J 946 A

$\begin{array}{ll}\text { SAE } & \text { J } 946 \mathrm{~A} \\ \mathrm{C} 717\end{array}$

ASTM D3139

NA

ANS1

991

ASTM A520

ASTM

ASTM A270

ASTM A269

ASTM A334

ASTM A589

ASTM B468

ASTM B464

ASTM A268

ASTM B B 335

ASTM A333

ASTM B338

ASTM B353

ASTM B394

ASTM B363

ASTM B 523

ASTM A271

A 376

ASTM A53

ASTM B135

ASTM A519

A209

ASTM A 192

ASTM A 106

ASTM A524

A500

ASTM A501

ASTM Al99 
al Fins (1974)

h23.14

ser Tubes (1973)

e (1974) ANSI H37.1

Std. Spec. for General Requirements for Wrought Std. Spec for U-Bend Std. Spec. for Std. Spec. for Std. Spec. for Std. Spec. for Std. Spec, for Std. Spec, for Std. Spec. for Std. Spec. for

tion Field Service (1974) ANSI H23.5

ents (1970) Ansi/
ssion and Distribution Pip/ Std. Spec. for Aluminum Alloy Seamless Pipe and

Service (1973) ANSI B125.24

Spec. for 3) Std. Spec. for Hot Formed Welded and ery Service (1972) ANSl B125.23

ubes for Refinery Service (1972) ANSI B12/

nd Flaring (1972)

es (1973) ANSI B125.10

hanger Tubes (1972) ANSI H34.42 8.7

Std. Spec. for Std. Spec. for Std. for Std. Spec. for Std $\mathrm{Spec}$ for Std. Spec. for Nickel Std. Spec. for Nickel Chromium Iron Alloy

for Nickel-lron-Chromium-MoIybdenum-Copper Alloy Std. Spec. for Nickel Chromium Molybdenum Columbium Alloy r Nickel Chromium Iron Columbium Molybdenum Tungsten Alloy Std. Spec, for Copper Silicon Alloy Std. Spec. for Nickel-Iron-Chromium Alloy Std. Spec. for integral Fins (1973) g62.1 Std. Spec. for Aluminum Alloy Std. Spec. for

$r$ Black and Hot Dipped Zinc Coated (Galvanized) Welded and ) ANSI H38.6

Std. Spec. for Aluminum Alloy Drawn Std. Spec. for Aluminum Alloy Drawn Safety Std. for Steel Pipe (Welded and

Linoleum, Rubber, Vinyl, Vinyl Asbestos, Polymeric Poured Home Laundering (1973) Ans/ Test Meth. for Appearance of s (1973) Off Road, Agricultural and Log Skidder, Truck, Bus, Bead

for Aluminum, Triplex, Neutral Supported, Service Drop and est of Single Thermoelement Materials by Comparison with A arrying (Members) Structures (Aluminum Construction Manual rk in Building Construction (Aluminum Construction Manual,

Meth. for Estimating the Thermal Neutron Absorption Cross woods Use Book: Lumber Std., Grading Rules, Design Values, Std. for General Purpose Uniform Cross

oating Thicknesses by Microscopical Examination of a Cross 114.95 Std. Meth. of Test for Cross ter/ Std. for Automatic Circuit Reclosers, Automatic Line Std. Spec. for Precast Reinforced Concrete Manhole Std. for T-Hook Slots for
Std. lnd ustrial Std. lndustrial control System Units (Electronic / Electrical / Mechanical oarding from the $\mathrm{W}$ si Zl1.58, APl 2561

Stds, and Practice Guide for Aids to

) ANSl Z123.3

e (Cereal Chemistry) (1962)

1962)

Std. Meth. of Test for Water and Std. Meth. of Test for

Meth. of Analysis of Meth. of Analysis of Modified Meth. of Analysis of Micro

Meth of Analysis of

Weight-Moisture Relationships of Agricultural Grain and uits (Berry, Currant, Grape, Asparagus); Lining Out Stock; Rules for Testing

Dielectric Properties of Grain and Std. Moisture Measurement (Grain and Flaxseed; Castor, Sesame, Safflower, Oleic, and Sunflower nclature for Identification of Structures and Contaminants Std. Meth, of Test for $r$ (Oil) Wellhead Equipment (Production Valves, Ring Joint, Nuclear Power Generating Stations (1971) Ansi/ ies (1972)

iology) (1973)

Std. for Integrity and Test Spec for aque Materials and for Evaluating Confor/ Rec. Pract. for

ANSI A 167.1

te (1969) ANSI A 164.1

t. Organic Coating Systems (Coil) (1974)

Std. Rec. Pract. for

Rec. Pract. for

Rec. Pract. for Guidelines for Air Conditioning Equipment
Seamless Condenser and Heat Exchanger Tubes with lntegr Seamless Condenser Tubes and Ferrule Stock (1974) ANSI Seamless Copper and Copper Alloy Tube (1974) ANSI H23.4 Seamless Copper and lis Alloy Heat Exchanger and Conden Seamless Copper and lis Alloy Rectangular Waveguide Tub Seamless Copper Bus Pipe and Tube (1974) ANSI C7.26

Seamless Copper Nickel Pipe and Tube (1973) ANSI H26.4 Seamless Copper Pipe, Std. Sizes (1972)

Seamless Copper Tube for Air Conditioning and Refrigera

Seamless Copper Tube (1975) ANS1 H23.3

Seamless Copper Tube, Bright Annealed (1973) ANS1 H23.2 Seamless Copper Water Tube (1972) ANSI H23.1

Seamless Drums, Heads, and Other Pressure Vessel Compon

Seamless Extruded Tube and Pipe for Gas and Oil Transmi

Seamless Extruded Tube (1973) ANSI H38.7

Seamless Ferritic Alloy Steel Pipe for High Temperature

Seamless Ferritic and Austenitic Alloy Steel Boiler, Su

Seamless Ferritic Austenitic Alloy Steel Tubes (1972)

Seamless High Strength Low Alloy Structural Tubing (197

Seamless Intermediate Alloy Steel Still Tubes for Refin

Seamless Low Carbon and Carbon Molybdenum Steel Still T

Seamless Low Carbon Steel Tubing Annealed for Bending a

Seamless Medium Carbon Steel Boiler and Superheater Tub

Seamless Nickel and Nickel Alloy Condenser and Heat Exc

Seamless Pipe and Seamless Extruded Tube (1973) ANSI H3

Seamless Pipe and Tube (1970) ANSI H34.1

Seamless Pipe and Tube (1970) ANSI H34.3

Seamless Pipe and Tube (1972) ANSI H34.16

Seamless Pipe and Tube (1972) ANSI H34.20

Seamless Pipe and Tube (1972) ANSI H34.21

Seamless Pipe and Tube (1974) ANSI H26.3

Seamless Pipe Tube (1973) ANSI H34.41

Seamless Red Brass Pipe, Std. Sizes (1972)

Seamless Round Condenser and Heat Exchanger Tubes with

Seamless Stainless Steel Mechanical Tubing (1971) ANSI

Seamless Stecl Pipe for Ordinary Uses (1973) ANSl Bl25.

Seamless Tubes for Condensers and Heat Exchangers (1973

Seamless Tubes (1974) ANSI H38.3

Seamless) for Underground Water Service (1972)

Loor Coverings (Asphalt, Cork

Seamless, etc.) (1973)
Seams in Wash and Wear ltems (Textile/ Clothing) After

Seat, Industrial and Bicycle Tires (1974) /Senger Car

Seats Used with Indexable Inserts for Clamp Type Holder

Secondary Cable (1966) IPCEA S-64-474

Secondary Standard of Similar EMF Temperature Propertie

1. for Design of Aluminum Alloy Lo

Spec. for Aluminum

Sect 5) (1971)

Section of Nuclear Graphite (1971) ANS1 K90.10
Section Properties and Design Loads, Timber, Plank and

Section Spiral Retaining Rings (1972)

Section (1973) ANSl G53.51 /Ement of Metal and Oxide C

Sectional Characteristics of Cotton Fibers (1972) ANSI

Sectionalizers and Oil Filled Capacitor Switches for Al Sections (1973)

Securement in Shipment of Agricultural Equipment (1972)

Security for Nuclear Power Plants (1973)

Security Surveillance System and Equipment) (1974)

Security (Safety) of Personnel on Boat Deck and for Reb

Sediment in Crude and Fuel Oils by Extraction (1969) an

Sediment in Crude Oils (1973) ANSI Z11.8

Sediment in Water Emulsion Polishes by Centrifuge (1972

Sedimentation Meth. of Analysis of Smut in Wheat and Ry

Sedimentation Test for Wheat Flour (Cereal Chemistry) (

Sedimentation Test for Wheat (Cereal Chemistry) (1968)

Sedimentation Test for Wheat (Cereal Chemistry) (1968)

Sedimentatoon Test for Wheat (Cereal Chemistry) (1965)

Seed for Storage (1972) /Ensity, Specific Gravity, and

Seedling Trees and Shrubs; Bulbs, Corms, and Tubers; Ch

Seeds (1970)

Seeds $(1972)$

Seeds) (1972)

Seeds; Ground Nuts (Peanuts); Vegetable, Coconut, and P

Seen on Specular Silicon Surfaces (1972T)

Seepage Rate of Aerosol Products (1972)

Segmented, Production Check Flanges and Gaskets; Drilli

Seismic Qualification of Class I Electric Equipment for

Seismic Restraint Direct Hung Suspended Ceiling Assembl

Selected Brachytherapy Sources (Medical Radiation-Rad

Selecting and Defining Color and Gloss Tolerances of Op

Selecting Proportions for No Slump Concrete (1965)

Selecting Proportions for Normal Weight Concrete (1970)

Selecting Proportions for Structural Lightweight Concre

Selection and Preparation of Test Panels for Use in Tes

Selection and System Design Procedures (1973)

Selection and Usage Guide for Std. Steel Doors (1972

Rec. for Selection and Use of Aggregates for Concrete (1958)
ASTM

ASTM

ASTM

ASTM

ASTM

ASTM

ASTM

ASTM

ASTM

ASTM

ASTM

ASTM

ASTM

ASTM

ASTM

ASTM

ASTM

ASTM

ASTM

ASTM

ASTM

SAE

ASTM

ASTM

ASTM

ASTM

ASTM

ASTM

ASTM

ASTM

ASTM

ASTM

ASTM

ASTM

ASTM

ASTM

ASTM

ASTM

UL

STM

AATCC $88 \mathrm{~B}$

TRA

Std. EEl

ASTM

AA

AA

ASTM

WWPA

ANSI

ASTM

ASTM

NEMA

ASTM

ASAE

ANSl

IS UL

$\mathrm{ABYC}$

ASTM

ASTM

ASTM

AACCH 42.71

$\mathrm{AACCH} \quad 56-60$

$\mathrm{AACCH} \quad 56.62$

$\mathrm{AACCH} 56-63$

$\mathrm{AACCH} \quad 56-61 \mathrm{~A}$

ASAE D24l.1

ANSI Z60.1

AOSA 60.2

ASAE

NIOP

ASTM

ASTM

APl

IEEE

CISCA

ANSI

ASTM

$\mathrm{ACl}$

$\mathrm{ACl}$

$\mathrm{ACI}$

NCCA

NESCA

STD

$\mathrm{ACl}$

D293

S352

*1 15

D3094

$6 \mathrm{~A}$

344
$* 3$

N44.1

D3134

211

211.1

211.2

TB.111.5

108

58-24. 
n Spectroscopy Based on Physical Pr/ m Speed Stationary Diesel and Gas Engine/ flectance and Transmittance (1973)/

Std. Rec. Pract. for Sid. Pract. for

3) iler, Multipurpose Pas/ 73) ture Design (1972) SAE J711 Std. Rec. Guide for the
Std. Guide for Std. Rec. Pract. for Std. for Tire Rim tings and Equipment for Anchoril Std. and Pract. Rec. for generators (1/ Safety Std. for Construction and Guide for of the Road/ Safety Std. for Regular Bicycles Including erties, Weight, Stress of Bo/ a Product U'se Manual: Grade Test Meth. for the Std. for Uniform Dimensions and Meth. of Test. for Rotary Meth. of Test for 100 Deg. Head, Tri Wing Recess, Alloy Steel, Full Thread, i Wing Recess, Close Tolerance, Alloy Steel, Short Thread. ead, Full Thread, Tri Wing Recess, Titanium Alloy, $6 \mathrm{Al} \cdot 4 \mathrm{~V}$ llister Head, Full Threaded. Tri Wing Recess, Alloy Steel,

t Fillister Head. Full Thread, Tri Wing Recess, A286 Cres. gth. Prevailing Torque, All Metal (1973)

I Metal (1973)

high Strength, Prevailing Torque, All Metal, 1/ Spec. for Spec. for Steel Spec. for spec for high Strength, Prevailing Torque, All Metal, I/

Insert (1974)

Insert (1974)

Std. For Sandwich Panel Lightweight Std. for Sandwich Panel Lightweight Std. for Setscrew.

care, and U'se of Shears (Mechanical, Hydraulic, Pneumatic, Aerospace Std. Identification Marking Meths. on Finished. Spec, for Cabin Duct. Air, Flexible and Std. for Solid and Std. for General Purpose for Fluoride Content of the Atmosphere and Plant Tissues Safety Std. for Rec. Pract. for Structural Design of or Paper. Paper/Film, Film Dielectric Capacitors for Power 974) Std. List of Values to Be Used in

p/ Std. for Numbering of Electrodes in Multiple Electrode onductor Devices and Designation of I'nits in Multiple U'nit Safety Code for

n (1969) ANS1 N42.1 Std. Test Procedure for Std. Test Procedure for Amplifiers and Preamplifiers for Measuring Hall Mobility and Hall Coefficient in Extrinsic Std. for Handling and Installation of Power Std. Spec, for

bars: Hot Rolled D/ (Steel Products Manual) Carbon Steel: of Moisture in Massecuites, Molasses, and Other Liquid and in Flour (Cereal Chemistry) (1968)

or Inspection Procedures for Motor Vehicles. Trailers, and Meth. of Analysis of Lipoxidase Activity of Disk Meth. of Analysis of Macro Scale Meth. of Analysis of Micro Scale Meth. of Analysis of Neth of Analysis for Ash in Farina and Meth. of Analysis of Diastatic Activity of Flour and Machine Washing Meth. of Analysis of Gluten in Flour and Meth. for Preparation of Sample: ke Meth. of Analysis of Amino Nitrogen in Wheat Flour and f Analysis of Reducing and Nonreducing Sugars in Flour and aluminum Plate) Meth. of Analysis of Moisture in Flour and sen Meth. of Analysis of Amino Nitrogen in Wheat Flour and nate Meth. of Analysis of Ergot in Rye and Wheat Flour and ang Meth. of Analysis of Amino Nitrogen in Wheat Flour and is for lnsect and Rodent Filth in Meal, Grits. Farina, and ysis for Rodent Excreta in Veal, Grits, Flour, Farina, and

Hand Washing Meth. of Analysis of Gluten in Flour and istry)/ Diastase Meth. of Analysis of Starch in Flour and
Sacks (Cereal Chemistry) (/ Meth. for Sampling of Flour, Air Oven Meth. of Analysis of Moisture in Flour, Farina, Meth. of Analysis of Pigments in Flour. B) Polarimetric Meth. of Analysis of Starch in Flour and ied Vacuum Oven Meth. of Analysis of Moisture in Flour and Std. for Safety for Ground Fault Std. Meth. of Test. Pressure ulation (1974) ANS1 C59.136 Std. Meth. of Test for Tack and Pressure Std. Spec. for Polyethylene Plastic Pressure Low Temperature Resistant Vinyl Chloride Plastic Pressure Std. Spec. for Vinyl Chloride Plastic Pressure Std. Spec. for Polyester Film Pressure

Std Spec. for Tetrafluoroethylene Backed Pressure Std. Spec. for High Temperature Glass Cloth Pressure hine (1971) ANSI Z197.32 (1973)
Selection of Electrode Material Used in Optical Emissio Selection of Engine-Generator Units for Low and Mediu Selection of Geometric Conditions for Measurement of Re Selection of Leak Test. Meth. (1971) ANSI Z166.26 Selection of Neutron Activation Detector Materials (197 Selection of Tires (Car, Station Wagon, Truck, Bus, Tra Selection of Vapor Barriers for Thermal Insulations (19 Selection Tables for Agricultural (Farm) Machines of Fu Selection (1974)

Selection, Design, Construction and Installation of Fit Selection. Installation and Ise of Electric Motors and Selection, Installation, and Servicing of Humidifiers (1972) Selection, Purchase, Preparation, Maintenance and Rules Selection, Std. Size, Design Values, Framing Rec., Prop Selective Transmission of a Photographic Lens (1969)

Selector $S$ witches (Radio, TV, and Instrumentation) (197 Selenium in Water (1972T)

Self-Locking and Nonlocking (1973)

Self. Locking and Nonlocking (1973)

Self. Locking and Nonlocking (1973)

Self. Locking and Nonlocking (1973)

Self.Locking and Nonlocking (1973)

Self-Locking Corrosion Resistant Alloy Nuts, High Stren
Self-Locking Nuts. High Strength, Prevailing Torque, AI

Self-Locking Steel Nuts, Corrosion and Heat Resistant,

Self-Locking Steel Nuts, Corrosion and Heat Resistant.

Selflocking and Nonselflocking Blind Threaded Molded in

Selflocking and Nonselflocking Blind Threaded Molded in Selflocking (1972)

Semi and Automatic, and Manually Powered) (1973)

Semi Finished and Rough Items (1971)

Semi Rigid (1972)

Semi Solid Dielectric Transmission Lines (1972)

00 Deg. Head, TR

/. Flat Fillister $\mathrm{H}$

Semi. Tubular. Full Tubular. Split Rivets and Caps (1972

(Semiautomated Meth.) (1973)

Semiautomatic Fire Hose Rack Assemblies (1972)

(1) Devices

Semicircular Brick Masonry Arches (Tech. Note) (1971)

Semiconductor Applications (1973)

Semiconductor Device Specs. and Registration Formats (1

Semiconductor Devices and Designation of Units in Multi

Semiconductor Devices (1972)

Semiconductor Power Converters (1973)

Multiple Electrode Semic

Semiconductor Radiation Detectors for Ionizing Radiatio

Semiconductor Radiation Detectors (1969) ANSI N42.

Semiconductor Single Crystals (1973)

Semiconductors in Dise Type Packages (1973)

Semidense Mineral Fiber Siding (1972)

Semifinished for Forging: Hot Rolled and Cold Finished

Semiliquid Products (Drying Upon Quartz Sand) (Cereal C

Semiquantitative Meth. of Analysis of Benzoyl Peroxide

Semitrailers Operated on Public Highways (1973)

Semolina and Flour (Cereal Chemistry) (1962)

Semolina Quality in Macaroni (Cereal Chemistry) (1962)

Semolina Quality in Macaroni (Cereal Chemistry) (1962)

Semolina Quality in Macaroni (Cereal Chemistry) (1962)

Semolina (Cereal Chemistry) (1962)

Semolina (Cereal Chemistry) (1962)

Semolina (Cereal Chemistry) (1962)

Semolina (Cereal Chemistry) (1962)

Semolina (Cereal Chemistry) (1962)

Semolina (Cereal Chemistry) (1962)

Semolina (Cereal Chemistry) (1962)

Semolina (Cereal Chemistry) (1962)

Semolina (Cereal Chemistry) (1962)

Semolina (Cereal Chemistry) (1962)

Semolina (Cereal Chemistry) (1962)

Semolina (Cereal Chemistry) (1962)

Semolina (Cereal Chemistry) (1967)

Semolina (With Subsequent Acid Hydrolysis) (Cereal Chem

Semolina, and Similar Products: Feeds and Feedstuffs in

Semolina, Bread, Grain, Soybeans, Rice, Beans, Peas, Le

Semolina, Macaroni and Wheat (1962)

Semolina; Also, with Slight Modification, to Corn, Rye.

Semolina: Bread; Feedstuffs, Ground Grains and Their Ad

Sensing and Relaying Equipment (1972)

Sensitive Adhesive Coated Tapes Úsed for Electrical Ins

Sensitive Adhesives by Rolling Ball (1973)

Sensitive Electrical Insulating Tape (1973)

Sensitive Electrical Insulating Tape (1973)

Sensitive Electrical Insulating Tape (1973) ANSI C59.91

Sensitive Electrical Insulating Tape (1973) ANSI C 59.96

Sensitive Electrical Insulating Tape (1974)

Sensitive Electrical Tape (1973)

Sensitive Tack of Adhesives Using an Inverted Probe Mac

Sensitive Tape Application Sids. for Fibrous Glass Duct
Std, for Screw,

ASTM

DEMA

ASTM

ASTM

ASTM

TRA

ASTM

ASAE

TRA

ABYC

NEMA

ARI

BMA

WW PA

ANS

EIA

ASTM

NSA

NSA

NSA

$S A E$

$\mathrm{SAE}$

$\mathrm{SAE}$

SAE

NSA

NSA

NSA

$10 \mathrm{n}, \mathrm{ANS}$

SAE

NSA

EIA

ANS1

ASTM

LL

SAE

BIA

Std. F EIA

EIA

EIA

EIA

NEMA

IEEE

IEEE

ASTM

EIA

ASTM

AISI

$\mathrm{AACCH}$

$\mathrm{AACCH} 48.0$

Td. F ANSI D7.1

$\mathrm{AACCH} \quad 22-40$

$\mathrm{AACCH} \quad 66.40$

AACCH 66-4]

AACCH $66-42$

AACCH $08-12$

AACCH 22-15

$\mathrm{AACCH} 38.11$

AACCH 62.60

$\checkmark$ an SI AACCH $46-32$

Neth. O AACCH 80.60

Air Oven ( AACCH 44.16

AACCH 46.31

Ether-Bicarbo AACCH $42-56$

AACCH $46-30$

AACCH 28-51

AACCH 28-50

AACCH $38-10$

$\mathrm{AACCH}$ 76-10

$\mathrm{AACCH} \quad 64.60$

AACCH 44-15A

$\mathrm{AACCH} \quad 14-50$

$\mathrm{AACCH} \quad 76-20$

$\mathrm{AACCH} \quad 44.40$

UL

ASTM D 1000

ASTM D3121

ASTM D3006

Spec. for ASTM D3005

ASTM D2301

ASTM D2484

ASTM D2686

ASTM D2754

ASTM D2979

SMACN AFTS.10 
(1973)

ant Stress (

Std. Meth. of Test for Adhesion of Pressure

Pressure

$0)$

ning Speed of Color Reversal Films for Still $\mathrm{Ph} /$

Std. for td. Rec. Pract. for Establishing Conditions for Laboratory

Purchases (1972)

chemistry) (I962)

oll Film Negatives (1972)

eous Fluoride in the Atmospher

uorides in the Atmosphere (So ble Gaseous Fluorides in the / j2.6

972) ANS1 Z11.301

si $\mathrm{N} 41.14$

Std. Rec. Pract. for Meth. of Std. Spec. for Masks Tent. Meth. for Automated Tent. Meth. for Tent. Meth. for Continuous Std. Meth. of Dynamic Test. for Ply Std. Meth. of Test for $\mathbb{W}$ ate

Trial l'se Std. Criteria for Std. Meth. of Test for Std. Test Meth. for Ply Spec. for Oil and Gas 69)

er, Wide Field and Compound Microscope, Petri Dish, Sieve, pec. for Homogeneous Perforated Bituminized Fiber Pipe for . for Laminated Wall Perforated Bituminized Fiber Pipe for Std. for Safety for 1

hermoset Plastic Tanks for Water and Wastewater Treatment Std. Specs, and Load Tables for Open Web Steel Joints J ibution Line Compensation (Including Safety Req/ Std. for of Test for Exfoliation Corrosion Susceptibility in 7XXX

c. for Hollow Metal Door Frame Preparation for 181 and 190 Std. Format for NAS 900 Uniform Alignment Tests-NAS 900 Std. for Preferred Numbers in Geometric Std. for Tests and Procedures for SAE 100R ographs for Inspection of Aluminum and Magnesium Castings, Trial Std. for Screw Threads for Metri specs. and Load Tables for Longspan Steel Joints LJ and LH and Load Tables for Open Web Steel Joints J Series and H and Load Tables for Longspan Steel Joints Deep DLJ and DLH bolts and Screws; External Wrenching. Unified Threaded In. td. Spec. for Mercury Lamp Transformers, Constant Current or Industrial Perforated Plate and Screens (Square Opening Groove Dimensions for Fluid Power Exclusion Devices (ln Structural Design of Std. for 450 Deg. F Self Lockin Std. for 450 Deg. F Closed End Self Locking Harland and Ashworth Meth. of Analysis o Rowland Meth of Analysis of Installation Std. for Solvent Cemented PVC Pipe for W ater
Std. Rec. Guide for in Is (1974) c. for Electric Fusion Welded Steel Pipe for High Pressure senger Car (1971)

rucks, Buses, and Combination Vehicles F/ Rec. Pract. for

Welded Carbon Steel Tubular Products for High Temperat ure kaging / Sanitary Stds. for Fillers and Sealers of Single

ve Petroleu/ Std Meth. of Test for Simulated Rec. Pract. Meth. and Controls to Prevent in Std. for Aluminum, Triplex, Neutral Supported. Std. for Dinnerware Used in Food

Rec. for Single Phase Rural Distribution

or Definition of and Maximum Dimensions for Grown Tires in Std. Classification System for Polymeric Materials for formers, Hand Pol

Std. for Electrical A pparatus Sales and Test Meth. for Carpet (Rug) Soiling: ting Devices and Components (1972) mp (Light) Units for Motor Vehicles (197/ Rec. Pract. for 69)

th Carbon Steel Wrought Welding Fittings for High Pressure 69) Std. Test Hleth for Simulated Bl41.8
or Changing and Charging Storage Batteries in Motive Power rec. for Materials and Meth. for Durability of Concrete in Hopper Cars for Malt and Grain Rec. Guide for Making a Condition Survey of Concrete in ct. for Fabrication of Reinforcing Steel Bar Materials and

Pract. for Estimating Reinforcing Steel Bar Materials and ry Pipe, Deluge, and Pre Action Valves for Fire Protection loy Bars, Forgings, and Forging Stock for High Temperature loy Bars, Forgings, and Forging Stock for High Temperature loy Bars, Forgings, and Forging Stock for High Temperature Std. for Tubular Heat Exchanges in Chemical Process icating Systems of Various Turbine Driven Pumps for Marine metal Arc Welded Steel Pipe for High Pressure Transmission

lting Material Specially Heat Treated for High Temperature conium and lts Alloy Seamless and Welded Tubes for Nuclear rical Resistance Heaters, for Nuclear or Other Specialized onstruction of Nonmetallic Enveloped Gaskets for Corrosive

td. Guideline for the Physical Inspection of Wood Poles in - on Application, Care, and Use of Wire Rope for Oil Field

for Ball Valves with Flanged or Butt Welding for General

for Steel Pipe (Welded and Seamless) for Underground Water
Sensitive Tape Performance Stds. for Fibrous Glass Duct Sensitive Tape to Fiberboard at $90 \mathrm{Deg}$. Angle and Const Sensitive Tape Water Vapor Transmission Test Meth. (197 Sensitometric Exposure and Evaluation Meth. for Determi Sensory Evaluation of Foods and Beverages (1973)

Sensory Evaluation of Industrial and Institutional Food Sensory Perception Test for Staleness of Bread (Cereal (Separate) for Use in Photographic Contact Printing of $R$ Separation and Collection of Particulate and Acidic Gas Separation and Collection of Particulate and Gaseous Fl Separation and Collection of Particulate and Water Solu Separation and Cracking of Rubber Products (1973) ANSI Separation Characteristics of Aviation Turbine Fuels (1 Separation of Class Ie Equipment and Circuits (1974) an Separation of Vehicle from Solvent Type Paints (I973)

Separation (Wet) of Solid and Corrugated Fiberboard (19 Separators (Petroleum Production) (1973)

Separatory Funnel, Trap Flask, Step Wedge for X-Ray Sta

Septic Tank Disposal Fields (1973) ANSI A176.4

Septic Tank Disposal Fields (1973) ANSI A176.5

Septic Tanks, Bituminous Coated Metal (I974) ANSI Al62.

Septic, Holding, Settling, Aeration Chambers, Chemical

Series and H Series (1970)

Series Capacitors for Electrical Transmission and Distr

Series Copper Containing Aluminum Alloys (Exco Test) (I

Series Deadlock Strikes (1973)

Series Equipment Spec. (1973)

Series Equipment Specs. (1972)

Series for Use in Standardization (1973)

Series Hydraulic Hose and Hose Assemblies (1970)

Series II (1974) ANSI Z166.8

Series Mechanical Fasteners (1974)

Series (1970)

Series (1970)

Series (1970)

Series (1971)

(Series) Supply Type (1971)

Series) (1972)

Series) (1972) ANSI B93.35

Serpentine Walls, Non Loadbearing (Tech. Notes) (1968)

Serrated Head Blind Nut (1974)

Serrated Head Blind Nut (1974)

Serum Protein in Milk (Cereal Chemistry) (1962)

Serum Protein in Milk (Cereal Chemistry) (1962)

Service and Yard Piping (1971)

Service Annealing of Water Cooled Nuclear Reactor Vesse

Service at Moderate Temperatures (1973)

Service Brake Structural Integrity Test Procedure-Pas

Service Brake Structural Integrity Test Procedure for T

Service Conforming to ISO Recommendations for Boiler Co

Service Containers for Milk and Its Fluid Products (Pac

Service Corrosion Test. of Engine Coolants (1973)

Service Cracking of Carbon Steel (P-1) Welds in Corrosi

Service Drop and Secondary Cable (1966) IPCEA S-64-474

Service Establishments (I970)

Service for Motors and Phase Converters (1972)

Service for Use by Vehicle Manufacturers in Designing F

Service in Ionizing Radiation (1971) ANSI N4.1

Service Industry (Rotating, Liquid Filled and Dry Trans

Service Meth. (1973) ANSI L14.216

Service Performance Requirements for Motor Vehicle Ligh

Service Performance Requirements for Sealed Beam Headla

Service Piping (1969)

Std. Pract. for High Streng

Service Test. of Wood and Wood Base Finish Flooring (19

Service Time Coding of Propulsion Engine Items (1971) a

Service (For Electric Trucks) (1972)

Service (1959)

Service (1964)

Service (1965)

Service (1968)

Service (1970)

Service (1970)

Service (1970) ANSI G81.44

Service (1970) ANSI G81.45

Service (1970) ANSI G81.46

Service (1971)

Service (1971)

Service (1971) ANSI G36.49

Service (1971) ANSI G55.12

Service (1971) ANSI N124

Service (1971) ANSI N143

Service (1971) ANSI Z261.2

Service (1972)

Service (1972)

Service (1972)

Service (1972)
INd Health Rec. F

$\mathrm{ACl}$
$\mathrm{BAMl}$
$\mathrm{ACl}$

Std. Pra CRSI

Std. CRS

Safety Std. for D UI

/Tation Hardening Nickel Al ASTM

/Ardening Iron Base Superal ASTM

/Ening Cobalt Containing Al ASTM

ANSI

for the Flushing and Cleaning of Lubr ASME Std. Spec. for ASTM

/Lloy Steel Turbine Type Bo ASTM Std. Spec, for Wrought Zir ASTM

/Td. Spec. for Sheathed Elect ASTM

/C. Pract. for Design and C ASTM

Rec. Pract API

Std. Pract MSS

Safety Std. UL

CN AFTS-100

34

PH2.2

E480

E461

74.30

PH 3.9

D3266

D3268

D3267

D430

D2550

384

D2372

D 1028

12J

28.90

D23I2

D2313

70

41

S316-3

C55. 2

G34

A 115.5

983

983

Z17. I

J343A

E155

500

S316-2

S316. 3

S316-1

Asll 132A

C82.7

E454

T3.19.7

33

1731

1732

ACCH 46-21

$\mathrm{CCH} \quad 46.22$

E509

E509
A672

J229

J294

A520

1704

D2570

RP. 04.72

TDJ. 180

36

R329

1.13

D2953

$* 1$

122

$\mathrm{J} 256 \mathrm{~A}$

J32

SP63

D2394

AS 1105

$8 \mathrm{~K} 1$

59.57

65-67

$* 1.5 .3$

$* 1.5$. 1

260

A637

A6 68

A639

B78.1

LOS-2Cl

A 381

A 437

B353

E420 
Centrifugally Cast Carbon Steel Pipe for High Temperature ates. Carbon Manganese, for Moderate and Lower Temperature Nickel Alloy Plate, Sheet, and Strip for High Temperature itic Chromium Nickel Alloy Steel Pipe for High Temperature seamless Intermediate Alloy Steel Still Tubes for Refinery Nickel Alloy Steel Pipe for Corrosive or High Temperature trifugally Cast Austenitic Steel Pipe for High Temperature arbon and Carbon Molybdenum Steel Still Tubes for Refinery Austenitic Chromium Nickel Steel Still Tubes for Refinery sure Vessel Plates for Intermediate and Higher Temperature forged Fittings, and Valves and Parts for High Temperature 1 Flanges, Fittings. Valves, and Parts for Low Temperature erritic and Austenitic Steel Castings for High Temperature Sid. Spec. for W rought Steel Wheels for Electric Railway ve Signaling Systems for Guard, Fire Alarm and Supervisory ignaling Systems for $\mathbb{W}_{\text {atchman, Fire Alarm, and Supervisory }}$ e of Auxiliary Protective Signaling Systems for Fire Alarm ignalling Systems for Watchman, Fire Alarm and Supervisory es for Pressure Vessels for Moderate and Lower Temperature nd Welded Carbon and Alloy Steel Tubes for Low Temperature Spec. for Seamless Carbon Steel Pipe for High Temperature c. for Electric Fusion Welded Steel Pipe for High Pressure Std for Practice for Respiratory Protection for the Fire Std. Spec. for Cast Steel Wheels for Railway Safety Std. for Hydrants for Fire Protection Safety Std. for Gate Valves for Fire Protection ely Std. for Indicating Pressure Gauges for Compressed Gas c. Pract. for Evacuated Reflective Insulation in Cryogenic or Grounded 830 V, 3 Phase Electrical System for Oil Field ugally Cast Ferritic Alloy Steel Pipe for High Temperature

t Pressure Pipe, Couplings, and Gaskets for Water and Fire and Distribution Facilities and Interruptions to Customer for Play Pipes for $\mathbb{W}$ ater Supply Test. in Fire Protection arbon Steel Boiler and Superheater Tubes for High Pressure ess and Welded Ferritic Stainless Steel Tubing for General $\mathrm{s}$ and Welded Austenitic Stainless Steel Tubing for General ec. for Seamless and Welded Steel Pipe for Low Temperature or Seamless Ferritic Alloy Steel Pipe for High Temperature austenitic Steel Pipe for High Temperature Central Station for Seamless Carbon Steel Boiler Tubes for High Pressure for Use in Wet Pipe Sprinkler Systems for Fire Protection ty Std. for Indicating Pressure Gauges for Fire Protection loy Steel Nuts for Bolts for High Pressure and Temperature Pressure Vessel Plates for Moderate and Lower Temperature vessels. Valves, Flanges, and Fittings for Low Temperature ings of Wrought Carbon and Alloy Steel for Low Temperature Std. for Safety for Butterfly Valves for Fire Protection Sid. for Safety for Water Pumps for Fire Protection

for Mining and Logging Tires Lsed in Intermittent Highway Safety for Automatic $\mathbb{W}$ ater Sprinklers for Fire Protection of Lead Acid lndustrial storage Batteries for Motive Power Castings suitable for Fusion Welding for High Temperature or Pressure Containing Parts Suitable for High Temperature for Pressure Containing Parts Suitable for High Temprature for Pressure Containing Parts Suitable for Low Temperature Spec. for Low Alloy Steel Castings Suitable for Pressure

1-Iron Alloy Castings (25.12 Class) for High Temperature $s$ Copper Tube for Air Conditioning and Refrigeration Field tubing and Line Pipe Connections in High Pressure Oil Well ttings for Flammable and Combustible Fluids, Refrigeration of Accelerated Evaluation of Wood Preservatives for Marine 1974) Uniform Pract. for Fee Determination for Division std. for Agreement Between Owner and Architect for Special Agreement Between Owner and Architect for Interior Design architect and Consultant for Other Than Normal Engineering Central Stations for Watchman. Fire Alarm and Supervisory and Stainless Steel Bolting Materials for High Temperature Sid. for Selection. Installation, and Spec for Petroleum Drilling and Well e (1966) An/ Std. Spec. for Enclosures, Cold Chambers and tion, and Grade of Palmkernels; Babassu: Flaxseed; Castor. Std. for Bolt, Flat, 100 Deg. Head, Torq Std for Bolt, Brazier Head, Torq Std. for Bolt, Flat, 100 Deg. Reduced Head, Torq Std. Meth. of Test for Degree of Spec for Mlosaic Wood Parquet Flooring Std. for Pan Head. Close Tolerance, Short Thread. Torq Spec. for Steel Splined Continuous Strip Mastic Std. Meth. of Test for False

e Slab / Spec. for Mastic Nailed Construction with Mastic Sid. for Screw, Machine, Pan Head, Full Thread. Torg Sid. for Machine Screw. Pan Head, Short Thread, Torq for Screw, Machine, Flat 100 Deg. Head. Full Thread. Torq for Machine Screw, Flat 100 Deg. Head, Short Thread, Torq for Screw, Machine, Flat Fillister Head, Full Thread, Torq meth. of Test for Flash Point of Aviation Turbine Fuels by
Service (1972)

Service $(1972$

Service (1972)

Service (1972)

Service (1972) ANSI B 125.23

Service (1972) ANSI B125.36

Service (1972) ANSI B 125.39

Service (1972) ANSI B 125.5

Service (1972) ANSl B36.39

Service (1972) ANSI G35.9

Service (1972) ANSl G37.

Service (1972) ANS1 G38.13

Service (1972) ANSI G52.2

Service (1972) ANSI G57.13

Service (1972) ANSI SE3.2

Service (1972) ANSI SE3.3

Service (1972) ANSI SE3.+

Service (I972) ANSI SE3.6

Service (1972A)

Service (1972A) ANSI B125. 18

Service (1972A) ANSI B125.30

Service (1972A) ANSI B125.4

Service (1973)

Service (1973)

Service (1973)

Service (1973)

Service (1973)

Service (1973)

Service (1973)

Service (1973)

Service (1973)

Service (1973)

Service (1973) ANSI B111.

Service (1973) ANS1 B125.12

Service (1973) ANSI B125.14

Service (1973) ANS1 B125.15

Service (1973) ANSI B125.17

Service (1973) ANSl B125.24

Service (1973) ANSI B125.25

Service (1973) ANSI B 125.7

Service (1973) ANSI B 129.2

Service (1973) ANSI Bl56.1

Service (1973) ANSI E38.1

Service (1973) ANSI G35. I0

Service (1973) ANSI G38.3

Service (1973) ANSI G46.2

Service (1974)

Service (1974)

Service (1974)

Service (1974)

Service (1974)

Service (1974) ANSI G37.2

Service (1974) ANSI G37.3

Service (1974) ANSl G38.11

Service (1974) ANSI G38.9

Service.(197+) ANS1 G.52.5

Service (1974) ANSI C81.19

Service (1974) ANSI H23.5

Service) (1972)

Service, and Marine Use (1972)

Services by Means of Small Size Specimens (1970)

Services (Individu

Services (1972)

Services (1972)

Services (1972) ANSI Se 3.1

Services (1974) ANS1 G38.4

Servicing of Humidifiers $\{1972$

Servicing Structures (1974)

Serving Units for Tests Above and Below Room Temperatur

Sesame, Safflower. Oleic, and Sunflower Seeds; Ground N

Set and $\mathrm{Hi}$ Torque, $180 \mathrm{~K}$ 'Sl (1973)

Set and $\mathrm{Hi}$ Torque, $180 \mathrm{~K}$ SI (1973)

Set and $\mathrm{Hi}$ Torque, $180 \mathrm{KSI}$ (1973)

Set for Wood Sash Glazing Compound (1973)

Set in Adhesive Over Concrete Slab or Wood Subfloors (1

Set Machine Screw (1972)

Set Maple Flooring Over Concrete Slab Floors (1962)

Set of Portland Cement (Paste Meth.) (1972) ANSI Al.27

Set Subflooring and Nailed Finish Flooring Over Concret

het (1973)

Set (1973)

Set (1973)

Set $(1973$

Set (1973)

Setallash Closed Tester (1973)
Std. Spec. for ASTM

Std. Spec. for Pressure Vessel Pl ASTM

A 660

A 662

A358

A200

A409

A 451

A 161

A271

A5 15

A 182

A 522

A351

A25

$72 \mathrm{~A}$

$72 \mathrm{~B}$

A612

A612

A 334

A 155

Z88.5

Std. ASTM

ANSI

AST M

$\mathrm{UL}$

UL

Saf UL

Std. Re ASTM

Rec. Pract. F API

Safety Std for Asbestos Cemen UL

Safety Std UL

Sid Spec for Seaml ASTM

Std. Spec. for Seamles ASTM

Std. Sp ASTM

Std Spec F ASTM

std. Spec for Seamless ASTM Std. Spec ASTM

or Satety for Alarm Valves UL

Safe L L

d. Spec. for Carbon and Al ASTM
Td. Spec. for Carbon Steel ASTM

Ing Materials for Pressure ASTM

Std. Spec. for Piping Fitt ASTM UL UL

Std. TRA

Std. for UL

A 58

246

C740

$11 \mathrm{M}$

A 426

107

346

385

A 226

A.268

A 269

A 333

A335

A 376

A 192
193

(93

A194

A516

A 320

A +20

1091

448

.29

$1 B 2$

A2I 6

A217

A 389

A 352

A 487

A 447

B280

BUL 5 A 2

109

D2481

D248
B 727

B707

C431

827

A193

630

$4 \mathrm{E}$

E197

$* 1$

1972

1982

NSA

NSA

WSF

WSA

ASTV

WSFI

NSA

NSA

Std. NSA

Std. NSA

Std. NSA

Tent. ASTM

C74

1131-38

*5 45

*6

1100

1630.34 
(Paint, Enamel, Lacquer, Varnish, and Related Products) by Safety Std. for Cord

Meth. for Measuring Transmission Performance of Telephone Std. for Std. for Slotted Headless
Std. for Square Head (1973) Definition and Recommendation for Materials for (1974) Std. Rec. Pract. for Use of Chemically (1970) ANSI A37.134 Std. Meth. of Test for Time of Std. Meth. of Test for Modulus of Rupture of Air 9 Std. Meth. of Test for Cold Bonding Strength of Air (1974) ansi 298.33 Std. Spec for Chemically Std. Spec, for Mineral Fiber Hydraulic Std. Meth. for Automatic Particle Counter Size tanks for Water and Wastewater Treatment (Septic, Holding, Uniform Plumbing Code: Sewers and Private et Waste (Boats) (1972) Rec. Pract. and Std. Covering vehicles (1971) Std. for Waste and Brick and Tile in Sanitation Structures, Std. Spec. for

) ANSI A 100.1

Std Spec for Poly(vinyl Chloride) (PVC)

Std. Spec. for Styrene Rubber Plastic Drain and Building

Std. Spec. for Type PSP Poly(vinyl Chloride) (PVC) Sid. Spec. for Type PSM Poly(vinyl Chloride) (PVC)

pec. for Acrylonitrile-Butadiene-Styrene (ABS) Plastic ec. for Reinforced Concrete Arch Culvert, Storm Drain, and Std. Spec. for Filled Poly (Vinyl Chloride) (PVC) r Reinforced Concrete Elliptical Culvert, Storm Drain, and Std. Rec. Pract. for Installing Vitrified Clay

ct. for Underground Installation of Flexible Thermoplastic Std. Spec. for Reinforced Plastic Mortar d. Spec. for Reinforced Concrete Culvert, Storm Drain, and for Reinforced Concrete D-Load Culvert, Storm Drain, and Std. Spec. for Asbestos Cement Nonpressure

Std. Spec. for Asbestos Cement Nonpressure Small Diameter Std. Spec. for Homogeneous Bituminized Fiber Drain and Std. Spec. for Laminated Wall Bituminized Fiber Drain and

c. for Acrylonitrile-Butadiene-Styrene (ABS) Composite Std. Spec for Concrete Uniform Plumbing Code: Installation for Non Metallic Building Std. Meth. of Test.

and Broadleaf Evergre/ Sid. for Nursery Stock: Deciduous r Bearings (Except Tapered Roller Bearings) Conform/ Std. Std. Pract. for Spur, Helical and Herringbone Gear nstruction, Materials. Size, and Installation of Propeller ponents: Enclosed Gear Drives-Bearings, Bolting Keys and Std for Chisel Plow Field and Row Crop Cultivator ted Carbon and Alloy Steel Forgings for Turbine Rotors and uniform Building Code Std. for Special Purpose Roofs (Wood Uniform Building Code Std. for Hand Split

for Building, Facing, and Hollow Brick (Made from Clay or Std. Spec. for Sewer and Manhole Brick (Made from Clay or es (Polisher, Scrubber, Sander. Scraper, Tile Remover. Rug rpet) (1972) Test Meth. for

for $\mathrm{F}$ astener-Taper $00 \mathrm{Deg}$. Countersunk Head Hi Shear Close Tolerance Head and Std. for Bolt, Taper Std. for Bolt, Taper Std. for Bolt, Taper Std. for Bolt. Taper Std. for Drills, High Speed Steel Threaded Std. for 100 Deg. Close Tolerance Head and Std. for Spindle Noses and Tool ode Std. for Material Spec. for Structural Steel and Iron ganese Steel Bars Including Rounds, Squares, Hexagons, and for Aluminum Alloy Extruded Bar, Rod, Pipe, and Structural std. Spec. for Stainless and Heat Resisting Steel Bars and Std. Spec. for Free Cutting Brass Rod, Bar, and Std. Structural

Age Hardening Stainless and Heat Resisting Steel Bars and ng Strength and Modulus of Rupture of Refractory Brick and Lead (Leaded Red Brass or Hardware Bronze) Rod, Bars, and

Spec. for Tolerances of Aluminum Alloy Std. Structural le to Titanium and Its Base Alloy Extruded Bars, Rods, and equirements for Wrought Copper and Its Alloy Rod, Bar, and and Seamless Carbon Steel Structural Tubing in Rounds and

td. Spec. for Stainless and Heat Resisting Steel Bars, and Std. Spec. for Copper Silicon Alloy Rod, Bar, and Std. Spec. for Copper Rod, Bar, and Std. Spec for TFE -Fluorocarbon Resin Molded Basic Std. Spec. for Copper Bus Bar, Rod, and

Std. Spec, for Copper and Its Alloy Forging Rod, Bar and Std. Spec, for Manganese Bronze Rod, Bar, and Std. Spec. for Aluminum Bronze Rod, Bar, and
Setaflash Closed Tester (1973)

Sets and P
Sets (1971)

Setscrew, Selfocking (1972)

Setscrews (Screws) (1971)

Setscrews (1969)

Setting Ceramic Tile with Mortar, Adhesive, and Mastic

Setting Chemical Resistant Silicate and Silica Mortars

Setting of Concrete Mixtures by Penetration Resistance

Setting Plastic Refractories (1972)

Setting Refractory Mortar (Wet Type) (1972) ANSI Al11.2

Setting Silicate and Silica Chemical Resistant Mortars

Setting Thermal Insulating and Finishing Cement (1964)

Setting (1972)

Settling, Aeration Chambers, Chemical Storage, Mixing,

Sewage Disposal Systems (1973)

Sewage Holding and/or Treatment Devices for Marine Toil

Sewage Holding Tanks for Mobile Homes and Recreational

Sewage Treatment Facilities (Tech. Notes) (1969)

Sewer and Manhole Brick (Made from Clay or Shale) (1973

Sewer Pipe and Fittings (1972)

Sewer Pipe and Fittings (1973)

Sewer Pipe and Fittings (1973)

Sewer Pipe and Fittings (1973)

Sewer Pipe and Fittings (1973) ANSI K65.59

Sewer Pipe (1971)

Sewer Pipe (1972)

Sewer Pipe (1972)

Sewer Pipe (1972) ANSI A 106.2

Sewer Pipe (1972) ANSI K65.171

Sewer Pipe (1973)

Sewer Pipe (1973)

Sewer Pipe (1973)

Sewer Pipe (1973) ANSI A 165.3

Sewer Pipe (1973) ANSI Al65.

Sewer Pipe (1973) ANSI A 176.l

Sewer Pipe (1973) ANSI A 176.2

Sewer Piping (1974)

Sewer, Storm Drain, and Culvert Pipe (1973)

Sewers and Private Sewage Disposal Systems (1973)

Sewers (1971)

Sewing Threads (1971) ANSI L14.14

Shade and Flowering Trees: Deciduous Shrubs; Coniferous

Shaft and Housing Fits for Metric Radial Ball and Rolle

Shaft Mounted Speed Reducers (1974)

Shafting Systems for Boats (197j)

Shafting (1974)

AGBMA Guide to Design, Co ABYC

Shafts and Ground Tool Mountings (Farm Machinery) (1973

Shakes and Shingles with Asbestos Felt or Gypsum Board

Shakes (1973)

Shale) (1973)

Shale) (1973) ANSI Al00.1

Uniform Building Code Std.

Shampooer, Rug a Floor Washers, ete) (1974)

Shampoer, Rug and Tlor Washers, etc.) (1974)

Shank (1973)

Shank (1973)

Shank $(1973)$

Shank, Shear Type, Protruding Head-Oversize (1973)
Shank, Shear Type, Protruding Head (1973)

Shank, Shear Type, 100 Deg. Head-Oversize (1973)

Shank, Shear Type, 100 Deg. Head (1973)

Shank, Sizes 1/16 In. Thru Letter F (1973)

Shank, 160,000 psi Short Thread Bolt (1974)

Shanks for Milling Machines (1972)

Shape, Plate, Sheet, Strip, Connector, and Bar) (1973)

Shapes-Angles, TEES and Channels (1972) ANSI G81. 18

Shapes for Electrical Purposes (Bus Cond uctor) (1973) a

Shapes for Use in Boilers and Other Pressure Vessels (1

Shapes for Use in Screw Machines (1972)

Shapes (1972)

Shapes (1972)

Specifor Hot AA

Shapes (1972) ANSI All1.17 for Hot Rolled and Cold Finished ASTM
/H. of Test for Cold Crushi ASTM

Shapes (1972) ANSI H33.I

Shapes (1973)

Shapes (1973)

Shapes (1973)

Shapes (1973) ANSI G24.21

Shapes (1973) ANSI G24.31

Shapes (1973) ANSI H7.3

Shapes (1973) ANSI H7.4

Shapes (1974)

Shapes (1974) ANSI C7.25

Shapes (1974) ANSI H7.1

Shapes (1974) ANSI H7.5

Shapes (1974) ANSI H7.6
/H. of Test for Cold Crushi ASTM
Std. Spec. for Copper Zine ASTM

Tolerances Applicab SAE

Std. Spec. for General R ASTM

/Pec. for Cold Formed Welded ASTM

ASTM

AST

ASTM

ASTM

ASTM

ASTM

ASTM

ASTM
D3278

817

269

1081

$\mathrm{J} 479 \mathrm{~A}$

$\mathrm{J} 102$

*3

C397

C403

C49l

C198

C466

C449

F32l

41

UPC*1.11

A8

TSC2

$34 \mathrm{~A}$

C32

D2729

D2852

D2832

D3033

D2751

C506

D2836

C 507

C12

D2321

D3262

C76

C655

C 428

C64

D1861

Di862

D2680

C14

UPC*1-11

ISI

D204

Z60. 1

480.05

P6

260.02

S225.1

A 470

UBCS32-14

UBC $532-8$

LBCS24-1

C 32

561

138

1730

529

525

1729

1728

1725

1724

965

1202

B5. 18

UBC 27.1

A 429

B317

A 479

B16

*5

A564

C133

Bl 40

AMS2204D

A MIS2245

B249

A5 500

A 276

B98

B133

D3294

$\mathrm{B} 187$

B124

B 138
B 150 
Std. Spec. for Aluminum Alloy Extruded Bars, Rods, (Steel Products Manual) Carbon Steel' Plates, Structural Std Spec, for Aluminum Alloy Std Siructural General Requirements for Delivery of Rolled Steel Plates, Std. Spec. for Magnesium Alloy Extruded Bars, Rods, als (1968) ANSI Z260.1 Std. Rec. Pract. for Std. Meth. of Drop Std. for Safety for Hair Clipping and Std. Meth. of Conducting

carf Joints (1970)

Sid. for Titanium Alloy Stump Type Protruding Head Std. for Titanium Alloy Stump Type $100 \mathrm{Deg}$. Head Std. for Hexagon Head Modified Short Thread Sid. for Self Locking, Hexagon Head

Std. Test Meth. for Creep Properties of Adhesives in meth. of Test for Strength Properties of Adhesive Bonds in

std. Meth. of Test for Strength Properties of Adhesives in

std. Meth. of Test for Strength Properties of Adhesives in

std. Meth. of Test for Strength Properties of Adhesives in

Std. Test Meth. for Creep Properties of Adhesives in std. Meth. of Test for Strength Properties of Adhesives in

Std. Meth. of Test for Fatigue Properties of Adhesives in th Properties of Adhesives in Plywood Type Construction in th Properties of Adhesives in Two Ply Wood Construction in

for Determining Durability of Adhesive Joints Stressed in gth of Adhesively Bonded Rigid Plastic Lap Shear Joints in

of Adhesively Bonded Plastic Lap Shear Sandwich Joints in on $(1973)$ Std for Rivet-100 Deg. Countersunk Head Hi

et $(1974)$ Uniform Building Code Std. for Std. Test Meth. for Std for Solid 100 Deg. Flush for Pin, Swage Locking, Steel, Std. and Oversize, $100 \mathrm{Deg}$. Std. for Pin, Swage Locking, Steel, 100 Deg. Std. for Pin, Swage Locking, A-286 Cres, 100 Deg. Sid for Tent. Meth. of Test. Plywood in Rolling Shear mining the Strength of Adhesively Bonded Rigid Plastic Lap Std. Meth. of Test for ceramics by Resol Std. Meth. of Test for Young's Modulus, Std. for $\mathrm{Hi}$

std. Test Meth. for Apparent Viscosity of Adhesives Having

Determining the Strength of Adhesively Bonded Plastic Lap roperties of Elastomeric Vulcanizates Under Compressive or Short Beam M/ Std. Meth. of Test for Apparent Horizontal Std. for Pin, Swage Locking, Steel, Protruding Head, ions or Cores $(1970)$ ns (1972)

Cold Heading Wire and Rods (1972)

Sid Meth. Std. Meth. of Std. Meth. of

Tent. Meth. of Test. Plywood in Std. for Bolt. Taper Shank, Std. for Bolt, Taper Shank, Std. for Bolt, Taper Shank, Std. for Bolt, Taper Shank,

Std for Nut, Capitive Washer. Contemporary Bearing Wall Example of Std. for $\mathrm{Hi}$

Tent. Meth. of Test. Plywood in Rolling Reactivity of Lubricants with Aerospace Alloys Under High Std. for Pin, Swage Locking, A.286 Cres, 100 Deg. Std. for Pin, Swage Locking, $108 \mathrm{KSl}$ Steel, 100 Deg. Std. for Pin, Swage Locking. A.286 Cres, Protruding Head, d for Pin, Swage Locking, 108 KSl Steel, Protruding Head $\mathrm{s}$ of Metal to Metal Adhesives by Compression Loading (Disk Std. for Rivet-Flat Head, Hi Std. for Bolt Std. for Bolt,

itanium Alloy (1973) Std. for Bolt Lock, Swage Locking, Steel, Std. and Oversize, Protruding Head, Std. for Pin, Swage Locking, A-286 Cres, Protruding Head, a nium Alloy (1973) Sid for Bolt Lock,

for Viscosity and Curing Characteristics of Rubber by the safety Requirements for the Construction, Care, and Use of Sid. for Safety for Nonmetallic other Specialized Service (1971) ANSI N14/ Std. Spec. for (1973) Std. for Safety for

1 Impedance of Electrical Grade Magnesium Oxide for Use in High Reliability Applications (1967) Ans Std. Spec. for Uniform Building Code Std for Fiberboard Nail Base Uniform Building Code Std. for Gypsum

Guide for the Design of Aluminum Formed Sheet Building

er Requirements of Farm Livestock (Poultry, Swine, Cattle. institutional Tel Sid. Performance Requirements for Woven

performance Requirements for Durable Press (No Iron) Woven n (1972) ANSI C59.13 Std. Meth. of Test. Rigid Sed Spec for Magnesium Alloy

std. Spec. for Nickel-Molybdenum-Chromium-lron Alloy

Spec. for Tolerances of Aluminum and Magnesium Base Alloy
Shapes, and Tubes (1973) ANSI H38.5

Shapes, Rolled Floor Plates, and Steel Sheet Piling (19

Shapes, Rolled or Extruded (1973) ANSI H38.10

Shapes, Sheet Piling, and Bars for Structural Use (1972

Shapes, Tubes, and Wires (1970) ANSI H45.6

Sharp Notch Tension Test. of High Strength Sheet Materi

Shatter Test for Coke (1972) ANSl K20.24

Shaving Appliances (1973) ANSl C33.36.2

Shear Block Test for Quality Control of Glue Bonds in S

Shear Bolt Lock (1974)

Shear Bolt Lock (1974)

Shear Bolt (1972)

Shear Bolt (1974)

Shear by Compression Loading (Metal to Metal) (1969)

Shear by Compression Loading (1949) ANSI Z197.11

Shear by Tension Loading at Elevated Temperatures (Meta

Shear by Tension Loading in the Temperature Range from

Shear by Tension Loading of Laminated Assemblies (1973)

Shear by Tension Loading (Metal to Metal) (1969)

Shear by Tension Loading (Metal to Metal) (1972) ANSI Z

Shear by Tension Loading (Metal / Metal) (1973)

Shear by Tension Loading (1964) ANS1 Z197.12

Shear by Tension Loading (1970)

Test Meth for Stren

for Streng

hear by Tension Loading (1971) ANS1 Z197.31

Shear by Tension Loading (1973)

Shear by Tension Loading (1973)

Shear Close Tolerance Head and Shank (1973)

Shear Connectors (Steel Studs) for Composite Constructi

Shear Fatigue of Sandwich Core Materials (1970)

Shear Head A286 Corrosion Resistant Steel and Monel Riv

Shear Head, Pull Type (1973)

Shear Head, Stump Type (1973)

Shear Head, Stump Type (1973)

Shear Hexagon Head Bolt (1973)

Shear in Plane of Plies) (1969)

Shear Joints in Shear by Tension Loading (1973)

Shear Modulus of Plywood (1972)

Shear Modulus, and Poisson's Ratio for Glass and Glass

Shear Protruding Head Rivet (1972)

Shear Rate Dependent Flow Properties (1969)

Shear Sandwich Joints in Shear by Tension Loading (1973

Shear Strains by the Mechanical Oscillograph (1972) Ans

Shear Strength of Parallel Fiber Reinforced Plastics by

Shear Stump Type (1973)

Shear Test in Flatwise Plane of Flat Sandwich Construct

1. Pract.

Shear Test of Soils Under Consolidated Drained Conditio

Shear Testing of Aluminum and Aluminum Alloy Rivets and

Shear Through the Thickness (1969)

Shear Type, Protruding Head-Oversize (1973)

Shear Type, Protruding Head (1973)

Shear Type, 100 Deg. Head-Oversize (1973)

Shear Type, 100 Deg. Head (1973)

Shear Type, 450 and 800 Deg. F (1973)

Shear Wall Design (Tech. Notes) (1964)

Shear 100 Deg. Flush Close Tolerance Head Rivet (1972)

Shear (Shear in Plane of Plies) (1969)

Shear (1972) ANSl Z11.323

Shear / Tension Head, Pull Type (1973)

Shear / Tension Head, Pull Type (1973)

Shear / Tension, Pull Type (1973)

Shear / Tension, Pull Type (1973)

Shear) (1972) ANSI Z197.4

Shear, Close Tolerance Shank (1973)

Shear, Close Tolerance (1973)

Shear, Hexagon Head, 180 KSl (1973)

Meth. of Test for Explosive

Shear, Protruding Head, Std. and Oversize, Pull Type, T

Shear, Pull Type (1973)

Shear, Stump Type (1973)

Shear, 100 Deg. Head, Std. and Oversize, P Il Type, Tit

Shearing Disk Viscometer (1972) ANSI J7.7

Shears (Mechanical, Hydraulic, Pneumatic, Se

Sheathed Electrical Resistance Heaters, for Nuclear or

Sheathed Heating Elements for Appliances and Equipmen

Sheathed Type Electric Heating Elements (1973)

Sheathed, Type K Thermocouples for Nuclear or for Other

Sheathing and Structural lnsulating Board (1973)

Sheathing Board (1973)

Sheathing (Aluminum Construction Manual) (1969)

/He at and Moisture Loss and Feed and Wat

Sheet and Pillowcase Fabrics Other Than Durable Press

Sheet and Pillowcase Fabrics (Institutional Textile) (1

Sheet and Plate Materials Used for Electrical lnsulatio

Sheet and Plate (1970) ANSI H45.3

Sheet and Plate (1971) ANSI H34.44

Sheet and Plate (1973)
ASTM

AlSl

ASTM

ASTM

ASTM

UL

ASTM

NSA

NSA

NSA

NSA

ASTM

Td. ASTM

ASTM

ASTM

ASTM

ASTM

ASTM

ASTM

ASTM

ASTM

ICBO

ICBO

NSA

Std. NSA

NSA

NSA

NSA

ASTM

/Deter ASTM

ASTM

ASTM

NSA

ASTM

ASTM

ASTM

ASTM

NSA

ASTM

ASTM

ASTM

ASTM

NSA

NSA

NSA

NSA

NSA

BIA

NSA

ASTM

ASTM

NSA

NSA

NSA

St NSA

ASTM

NSA
NSA

NSA

NSA

NSA

NSA

NSA

ASTM

ANSl

A STM

UL

/Ctrica ASTM

ASTM

ICBO

$\mathrm{AA}$

ANSI

ASTM

ASTM

ASTM

SAE

B221

B308

A6

B 107

(338

D3038
1028

D 1759

2605

2705

1103.20

1223

2293

D905

D2295

D2557

D3165

D2294

D 1002

D3166

D906

D2339

D2919

D3163 
3)

Std. Spec. for Aluminum Alloy Std. Dimensions for Industrial Radiographic Std. Dimensions for Professional Photographic Std. Dimensions for Medical Radiographic d. Spec. for Corrosion Resisting Chromium Steel Clad Plate pec. for Nickel Chromium Molybdenum Columbium Alloy Plate, ickel-Chromium-Iron-Molybdenum-Copper Alloy Plate, Spec. for Regular Quality Hot and Cold Rolled Alloy Steel Spec. for Drawing Quality Hot and Cold Rolled Alloy Steel Std. Spec. for Thermostat Meta

Spec for Hot Rolled Carbon (0.15 Maximum, Percent) Steel pec. for Steel, Carbon $(0.16$ to 0.25 Maximum \%, Hot Rolled $-15.5 \mathrm{Cr}-2.4 \mathrm{Ti}-0.70 \mathrm{Al}-7.0 \mathrm{Fe}$ (1973)

$15.5 \mathrm{Cr}-4.5 \mathrm{Ni}-2.9 \mathrm{Mo}-0.10 \mathrm{~N}$, Solution Heat Tre

w Alloy Columbium and/or Vanadium (/ Std. Spec, for Steel Std. Spec. for Hot Rolled Carbon Steel Guide for the Design of Aluminum Formed Std. Dimensions for Graphic Arts

Std. Spec. for Copper Nickel Alloy Plate and Std. Spec. for Copper Silic on Alloy Plate and its for Water Cooling Tower Fan Drives (1972) Std. Spec. for Polyester Glass Mat

Test Meth. for Density of Wood Chips spec. for Steel Drill Screws for the Application of Gypsum Test for Water Resistance of Paper, Paperboard, and Other vimetric Determination of Water Vapor Transmission Rate of

rec. Pract. for Sharp Notch Tension Test. of High Strength Air Handling Spec. for truction Under the Uniform Building Code: Valley Flashing

for Cold Heading Quality Carbon Steel Wire for Tapping or

ec. Pract. for Meth. of Determining Plastic Deformation in struction Manual, Sect. 5) (1971) Spec. for Aluminum Std. Meth. of Test for Plastic Strain Ratio R for Specifying Duct, Uniform Building Code Std. for Std. Spec. for Steel he Hot Dip Process (1971) ANS1 G8.2/
by the Hot Dip Process (1971) Ansi/
test Meth. for Measuring Squareness or Rectangularity of A test Meth. for Measuring Squareness or Rectangularity of A
Std. Spec. for High Strength Low Alloy Steel H.Piles and

Plates, Structural Shapes, Rolled Floor Plates, and Steel Requirements for Delivery of Rolled Steel Plates, Shapes, llinear 4 Probe Array (1974) Tentative Meth. of Test for $r$ Four Probe Array (1973) Tent. Meth. of Test for

(1971) ANSI Z/ Tentative Meth. of Test for Weight Loss of Std. Meth. of Test for Reboiling Tendency of supports, and Cast Aluminum Covers (/ Std. for Electrical Steel Products Manual for Carbon r (Ape/ Std. Meth. for Determining Adhesion of Protection t Test Solution for Detecting Chromate Films on Galvanized niform Building Code Std. for Composition Roofing (Class C Std. Spec. for Nickel-Molybdenum Alloy Plate and

Spec. for Nickel-Molybdenum-Chromium Alloy Plate and Std. for Sprinkler Irrigation (For Farms) Technical Data nd Copper Alloys (Wrought Products Only); Application Data Std. Spec. for TFE - Fluorocarbon Resin Spec. for Titanium and Titanium Alloy Strip, $r$ Heat Resisting Chromium and Chromium Nickel Steel Plate, pec. for Hot, Hot Cold, and Cold Worked Alloy Steel Plate, std. Spec. for Precipitation Hardening Nickel Alloy Plate, Std. Spec. for Nickel-Chromium-lron Alloy Plate, Std. Spec. for Nickel Copper Alloy Plate,

ec. for Low Carbon Nickel Molybdenum Chromium Alloy Plate,

ec. for Stainless and Heat Resisting Chromium Steel Plate,

y of Flat Rolled Stainless and Heat Resisting Steel Plate,

c. for Nickel Iron Chromium Molybdenum Copper Alloy Plate, Std. Spec. for Nickel Plate,

el-Iron-Chromium-Manganese-Molybdenum Alloy Plate,

I Iron Molybdenum Copper Columbium Stabilized Alloy Plate, Std. Spec. for Nickel-lron-Chromium Alloy Plate, Std. Spec, for Steel, Cold Rolled Graphite Fiber Tape and

$36,000,000$ (248) Modul/ $36,000,000$ (248) Modul/

Spec. for Graphite Fiber Tape and Spec. for Graphite Fiber Tape and Std Spec. for Columbium and lts Alloy Strip. Std. Spec. for Molybdenum and Molybdenum Alloy Strip, -Cobalt-Beryllium Alloy (Copper Alloy No. 175), Plate, ansi $\mathrm{N} 123$

v $(0.33-0.38 \mathrm{C}))$ Special Grade (1973)

Spec, for Tantalum Plate,

3)

nickel Base, $20 \mathrm{Cr}-20 \mathrm{Co}-5.9 \mathrm{Mo}-2.2 \mathrm{Ti} /$

Molybdenum
$-0.55 \mathrm{C}))(1973)$ Spec. for Steel
Alloy Guide Spec. for Architectural Sid. Spec. for

Sheet and Plate (1973) ANSI H38.2

Sheet and Roll Films (SI and U.S. Customary Units) (197

Sheet and Roll Films (1973)

Sheet and Strip (1972) ANSI G81.6

Sheet and Strip (1972) ANSI H34.19

Sheet and Strip (1973)

Sheet and Strip (1973) ANSI G24.24

Sheet and Strip (1973) ANSI G24.25

Sheet and Strip (1973) ANSI Z155. 19

Sheet and Strip, Commercial Quality (1972)

Sheet and Strip, Commercial Quality (1972)

Sheet and Strip, Corrosion and Heat Resistant, Nickel B

Sheet and Strip, Corrosion and Moderate Heat Resistant,

Sheet and Strip, Hot and Cold Rolled, High Strength, Lo

Sheet and Strip. Structural Quality (1972)

Sheet Building Sheathing (Aluminum Construction Manual)

Sheet Films (photography) (1973)

Sheet for Pressure Vessels (1969) ANSI H52.I

Sheet for Pressure Vessels (1973)

Sheet for Spiral Bevel, Helical and Herringbone Gear Un

Sheet Laminate (1973)

(Sheet Machine Mold) (Pulp and Paper) (1973)

Sheet Material to Light Gage Steel Studs (1972)

Sheet Materials (Paper) at High Temperature and Humidit

Sheet Materials (1968) ANSI Z260.1

Sheet Metal and Air Conditioning Contractors (1973)

(Sheet Metal for Roof Waterproofing) (1973)

Sheet Metal Roofing (1971)

Sheet Metal Screws (1971) ANSI G54.17

Sheet Metal Spec. (1973)

Sheet Metal Stampings (1972)

Sheet Metal Work in Building Construction (Aluminum Con Sheet Metal (1974)

Sheet Metal, Low Pressure, Air Transmission (1967)

Sheet Metals (1973)

Sheet of Drawing Quality, Zinc Coated (Galvanized) by T

Sheet of Lock Forming Ouality, Zinc Coated (Galvanized)

Sheet of Paper or Paperboard (1973)

Sheet Piling for Use in Marine Environments (1974)

Sheet Piling (1970)

(El Products Manual) Carbon Steel

Sheet Piling, and Bars for Structural Use (1972) ANSI G

Sheet Resistance of Silicon Epitaxial Layers Using a Co

Sheet Resistance of Thin Metallic Films with a Collinea

Sheet Rubber Gaskets (1966) ANSI J7.2

Sheet Steel During Immersion in Sulfuric Acid Solution

Sheet Steel for Porcelain Enameling (1969) ANSI Z167.21

Sheet Steel Outlet Boxes, Device Boxes, Covers and Box

Sheet Steel (coils and Cut Lengths) (1974)

Sheet to A perture Adhesive of Unitized Microfilm Carrie

Sheet (Coil Coating) (1974)

Sheet (Roll) and Shingles) (1973)

Sheet (1971) ANSI H34.11

Sheet (1971) ANSI H34.12

Sheet (1972)

Sheet $(1972)$

Sheet (1974)

Sheet, and Plate (1972) ANSI Z179.1

Sheet, and Strip for Fusion Welded Unfired Pressure Ves

Sheet, and Strip for High Temperature Service (1972)

Sheet, and Strip (1970) ANSI H34.10

Sheet, and Strip (1970) ANSI H34.6

Sheet, and Strip (1972)

Sheet, and Strip (1972) A NSI G8I.2

Sheet, and Strip (1972) ANSI G81.30

Sheet, and Strip (1972) A NSI H34.17

Sheet, and Strip (1972) ANSI H34.8

Sheet, and Strip (1973)

Sheet, and Strip (1973) A NSI H34.37

Sheet, and Strip (1973) A NSI H34.40

Sheet, Carbon, Structural (1972)

Sheet, Epoxy Resin Impregnated for Hand Layup (1973)

Sheet, Epoxy Resin Impregnated, G70,000 (483) Tensile,

Sheet, Epoxy Resin Impregnated, G70,000 (483) Tensile,

Sheet, Foil, and Plate (1964) ANSI Z179.20

Sheet, Foil, and Plate (1974)

Sheet, Strip and Rolled Bar (1973)

Sheet, Strip, and Foil (1973)

Sheet, Strip, and Plate for Nuclear Application (1973)

Sheet, Strip, and Plate $(0.80 \mathrm{Cr}-1.8 \mathrm{Ni}-0.25 \mathrm{Mo}(0.49$

Sheet, Strip, and Plate $\left(0.80 \mathrm{Cr}-1.8 \mathrm{Ni}-0.35 \mathrm{M}_{0}-0.20\right.$

Sheet, Strip, and Plate $(13 \mathrm{Cr}-2.0 \mathrm{Ni}-3.0 \mathrm{~W})(1973)$

Sheet, Strip, and Plate, Arc Cast, Stress Relieved (197

Spec. for Alloy
Sheet and Roll Film (photography) (1973)

Sheet Materials by the Dry Indicator Meth. (1973) TAPPI
ASTM

ANSI

ANSI

ANSl

St ASTM

Std. S ASTM

for N ASTM

d. ASTM

Std. ASTM

ASTM

Std. ASTM

Std. S ASTM

$\mathrm{SAE}$

SAE

ASTM

AA

ANSI

ASTM

ASTM

AGMA

ASTM

TAPPl

Std. ASTM

ASTM

TAPP

Std. ASTM

SMACN

ICBO

CSI

ASTM

SMACN

$S A E$

AA

ASTM

CSI

ICBO

ASTM

ASTM

TAPPl

ASTM

AIS]

ASTM

ASTM

ASTM

ASTM

ASTM

ASTM

NEMA

AlSl

NMA

NCCA

ICBO

ASTM

Std ASTM

ASAE

CDA

ASTM

ASTM

ASTM

ASTM

A STM

ASTM

ASTM

Std. Sp ASTM

Std Sp ASTM

Ments for Deliver ASTM

Std. Spe ASTM

ASTM

Std. Spec. for Nick ASTM
or Chromium Nicke ASTM

ASTM

ASTM

SAE

SAE

ASTM

ASTM

ASTM

SAE

ASTM

SAE

SAE

SAE

SAE

SAE

B209

PH1.15

PHI.18

A 263

B5483

A506

A 507

B388

A569

A659

AMS5541B

A.MS $5547 \mathrm{C}$

A607

A570

*11

PH1. 16

B 402

490.02

D1532

C646

D779

T464

E338 
nickel Base- $15.8 \mathrm{Cr}-15.2 \mathrm{Mo}-0.30 \mathrm{Al}-0.05 \mathrm{La}(\mathrm{I}$ $4.5 \mathrm{Ni}-3.5 \mathrm{Cu}-(\mathrm{Cb}+\mathrm{Ta})$, Consumable Electrode $\mathrm{Va} /$ $53 \mathrm{Fe}-29 \mathrm{Ni}-17 \mathrm{Co}(1973)$

$-0.43 \mathrm{C})(\mathrm{SAE} 8740)(1973)$

$-0.09 \mathrm{~V}(0.17-0.23 \mathrm{C})$, Premium Quality, Consumab/

Titanium Alloy

Std. Spec. for Phosphor Bronze (Copper Tin) Plate, Std. Spec. for Leaded Brass Plate. Std. Spec. for Copper-Beryllium Alloy Plate. Std. Spec. for Brass Plate, Std. Spec, for Copper Iron Alloy Plate, Std Spec, for Copper-Zinc-Tin Alloys Plate, Spec. for Copper-Zinc-Aluminum-Cobalt Alloy Plate, inc Alloy (Nickel Silver) and Copper-Nickel Alloy Plate, al Requirements for Wrought Copper and Copper Alloy Plate. aterial Spec, for Structural Steel and Iron (Shape, Plate, Std. Spec. for Copper Std. Spec. for Cartridge Brass Std. Spec. for Steel

for Roofing (1971) ANSI G8.21 Std. Spec. for General Requirements for Steel Commercial Quality (1971) ANSI G8./ Std. Spec. for Steel Spec. for Flexible Poly(vinyl Chloride) Plastic Std. Meth. of Test for Impact Resistance of Rigid Plastic t for Pneumatic Ball Impact Resistance of Plastic Film and Std. Meth. of Test for Tensile Properties of Thin Plastic d. Meth. of Test for Flammability of Flexible Thin Plastic hing Bags, Footwear, Hose and Tubing, Mattresses and Pads,

for Rolled Copper Alloy Bearing and Expansion Plates and Std. Spec. for Corrugated Asbestos Cement nized) by Hot Dip Process (1971) An/ Std. Spec. for Steel Std. Spec. for Steel

ged Compressor Units (1974)

Zinc Coated (Galvanized) by $\mathrm{Hot} \mathrm{Di}$ Std. Spec. for Carbon Steel Engineering Data Std. Spec. for Steel Std Spec for Flat Asbestos Cement Test Meth. for Shrinkage Test of Pulp on of Pieces from Rubber Vulcanizates Other Than Std. Test for Mixing Rubber Compounds for Preparing Vulcanized Test Std Spec, for Corrugated Asbestos Cement

Uniform Building Code Std. for Steel Bar and Rod Mats Std. Nomenclature for Bedding Sizes (Mattresses and ge (Solid, Palletized and Bin Box Storage Itp to $30 \mathrm{Ft}$. and Rec. for Concrete Std. Meth. of Test. Std. Spec. for

Rec. for Design Values for Livestock Fallout Uniform Building Code: Regulations Governing Fallout Quality Stds. for Closet and Storage and Running Trim, Casework, Panelwork, Closet and Storage ields (1974)

er Cable $15 \mathrm{kV}$ Through 35/ Std. Ampacities with Effect of Std. Spec. for Aggregates for Radiation

e Nomenclature of Constituents of Aggregates for Radiation tions (1964) Rec. for Std. for Program for Biological
implements and Tractors (1972) SAE J955 Std. for Concrete Radiation of Latex and Emulsion Ex/ Std Spec. for Asbestos Cement

lding Code Std. for Special Purpose Roofs (Wood Shakes and Tent. Meth. of Test for Wind Resistance of Asphalt Uniform Building Code Std. for Natural Slate Roof Uniform Building Code Std. for Sawn Wood Uniform Building Code Std. for Asbestos Cement Roofing ode Std. for Composition Roofing (Class C Sheet (Roll) and

factory Finishing, Rough and Finished Carpentry, Flooring, Coating Systems (Paint) Guide for

Engines (1970) Guide for Supplementary Ventilation in A Guide for Centralized Control and Automation of Code for Std. for T.Hook Slots for Securement in Std. Meth. of Test for Commercial Weight of $A$ for Inspection, Sampling and Measuring Procedures for Bulk sportation (DOT) Special Permits for Radioactive Materials pping and Receiving-Materials Handling) (1973)

Shipper-Motor Carrier Dock Planning Manual (Truck Test Meth. for Water Leak Test for Paper

II Std Weth of Test Large $s$ for Automatic Machine Sealing of Top Flaps of Fiberboard lary) (1973) nsi M/ Std. for Steel drum (1968) ANSI Meth. of Test for Water Vapor Std. Meth. of Drop Test for Cylindrical Std. Meth. of Drop Test for

Std. Meth. of Test for Water Vapor Transmission of Std. Meth. of Incline Impact Test for Std. Meth. of Vibration Test for
Sheet, Strip, and Plate, Corrosion and Heat Resistant. Sheet, Strip, and Plate, Corrosion Resistant, 14.8CrSheet, Strip, and Plate, Low Expansion, Glass Sealing, Sheet, Strip, and Plate, $0.50 \mathrm{Cr}-0.55 \mathrm{Ni}-0.25 \mathrm{Mo}(0.38$ Sheet, Strip, and Plate, $0.75 \mathrm{Cr}-9.0 \mathrm{Ni}-4.5 \mathrm{Co}-1.0 \mathrm{Mo}$

Sheet, Strip, and Plate, 6A1-4V Annealed (1973)

Sheet, Strip, and Rolled Bar (1973)

Sheet, Strip, and Rolled Bar (1973)

Sheet. Strip, and Rolled Bar (1973)

Sheet, Strip, and Rolled Bar (1973)

Sheet, Strip, and Rolled Bar (1973)

Sheet, Strip, and Rolled Bar (1973)

Sheet, Strip, and Rolled Bar (1973)

Sheet, Strip, and Rolled Bar (1973)

Sheet, Strip, and Rolled Bar (1974)

Sheet. Strip. Connector, and Bar) (1973)

Sheet. Strip, Plate and Rolled Bar (1973) ANSI H7.
Sheet, Strip, Plate, Bar and Disks (1973)

Sheet, Zinc Coated (Galvanized) by the Hot Dip Process

Sheet, Zinc Coated (Galvanized) by the Hot Dip Process

Sheet, Zinc Coated (Galvanized) by the Hot Dip Process.

Sheeting for Pond. Canal, and Reservoir Lining (1972T)

Sheeting or Parts by Means of a Tup (Falling Weight) (I

Sheeting (1972)

Sheeting (1973) ANSI K65.76

Sheeting (1974) ANSI K65.28

Sheeting, and Restraint Straps) (1974)

Sheets for Bridge and Other Str

Sheets for Bulkheading (1974)

Sheets for Culverts and Underdrains, Zinc Coated (Galva

Std. Meth. of Tes ASTM

Ks and Rebreat UL

Sheets for Porcelain Enameling (1973)

Sheets for Pressure Vessels (1971) ANSI G33.4

Sheets for the Design of Air Exchange Coolers for Packa

Sheets of Structural Quality in Coils, and Cut Lengths,

Sheets (1972) ANSI A124.1

Sheets (1973)

Sheets (1973)

Sheets (1973)

Sheets (1974) ANSI A125.1

Materials Std. Rec. Pract for Preparati

Sheets) for Concrete Reinforcement (1973)

Sheets) (1971)

Shelf Storage $\mathrm{U}_{\mathrm{p}}$ to $15 \mathrm{Ft}$. High) (Fire Protection and $\mathrm{S}$

Shell Structures (1973)

Shellac Used for Electrical Insulation (1973) ANSI C59.

Shellac Varnishes (1973)

Shelters (1973)

Shelters (1973)

Shelving (1973)

Shelving, Misc. Ornamental ltems, Stairwork and Handrai

Shield Efficiency in Attenuating Alternating Magnetic F

Shield Losses for Single Conductor Solid Dielectric Pow

Shielding Concrete (1973)

Shielding Concrete (1973)

Shielding for High Energy Electron Accelerator Installa

Shielding in Nuclear Reactor Plants (1972)

Shielding of Power Drive Lines for Agricultural (Farm)

Shields (1972)

Shingle Blanks to Be Used as Panels in Weathering Tests

Shingles with Asbestos Felt or Gypsum Board Underlaymen

Shingles (1972T)

Shingles (1973)

Shingles (1973)

Shingles (1973)

Shingles) (1973)

Shingles, Exposed Roof Decking, Drop Siding. Structural

Uniform Building C

Ship Hull, Deck and Superstructure (1973)

Ship's Cargo Hold During the Use of Internal Combustion

Ship's Ste am Propulsion Plant (1970)

Shipboard Hull Vibration Measurements (1969)

Shipment of Agricultural Equipment (1972)

Shipment of Yarn or Man Made Staple Fiber (1971)

Shipments and Transfers of Soybean Oil (1973)

Shipments (1973)

Ide for Obtaining Department of Tran

Shipper-Motor Carrier Dock Planning Manual (Truck Shi

Shipping and Receiving-Materials Handling) (1973)

Shipping and Refuse Sacks (Containers) (1973)

Shipping Cases and Crates (Packaging) (I953) ANSI MHI2.

Shipping Cases (1970)

/Solvent Soluble Liquid Ad

Shipping Container Industry Glossary (Packaging, Vocabu

Shipping Containers by Cycle Meth. (Packaging) (1968) a

Shipping Containers (Packaging) in Revolving Hexagonal

Shipping Containers (Packaging) (1950) ANSI MH12.7

Shipping Containers (Packaging) (1961) ANSI MH12.2

Shipping Containers (Packaging) (1964) ANSI MHI2.10

Shipping Containers (Packaging) (1968) ANSI MH12.4, Tap

Shipping Containers (Packaging) (1968) ANSI MH 12.9
ASTM

St ASTM

ASTM

SAE

SAE

SAE

SAE

ASTM

ASTM

ASTM

ASTM

ASTM

Std ASTM

$Z$ ASTM

ASTM

ASTM

ASTM

ASTM

ASTM

STM

ASTM

ASTM

ASTM

ASTV

API

ASTM

ASTM

TAPPI

ASTM

ASTM

ASTM

ICBO

ANSI

FMIS

ACl

ASTM

ASTM

ASAE

ICBO

$A W 1$

AWI

ASTM

IPCE A

ASTM

ASTM

NCRP

ANSI

ASAE

ANSI

ASTM

ICBO

ASTM

ICBO

ICBO

ICBO

ICBO

SNAM

SNAME $3-23$

SNAME C-1

ASAE

ASTM

NSPA

ANSI

ANSI

ANSI

TAPPI

ASTM

ASTM

ANSI

ASTM

ASTM

ASTM

ASTM

ASTM

ASTM

ASTM

AMS5873

AMS5862

AMS7728C

AMS6358D

AMS6523A

AMS4911C

B103

B12 I

B194

B36
B465

B59]

B592

B122

B248

UBCS27-1

B152

B19

A526

D3083

D3029

D3099

D882

D 1433 
cts (Radio, Television, Tape Rest Meth. for Drop Tests for Paper ardization of Forms and Information Flow Used in Ordering nuclear Power Plants (During the Con/ Std. for Packaging, Std. Spec. for Structural Steel for Std. for Control of Gas Hazards on Vessels or) (1973)

lyzer) (1973)

aterials (Packaging) (1964) ANSl / systems (1972) Std. Meth. of Test for Sid. Meth. of Thermal Std. Meth. of Thermal 970) / ign, Construction, and Operation of Class Hi (High lmpact) Std. Meth. of Tesi for Dynamic Water Resistance of Std. Meth of Test for Dynamic Water Resistance of rap, Clay Pigeon, and Skeet: Ammunition and Targ/ Shotgun lining and Coating for Steel Water Pipe $(4 \mathrm{ln}$. and Larger, Std. for Safety Requirements for Rec. for Single Post

Std. for Packing, Preformed-MlL-R-25897 Rubber, 75 Std. for Packing, Preformed-MlL-R-25897 Rubber, 90 Straight Thread Tube Fitting Boss, MlL-R-25897 Rubber, 75 Straight Thread Tube Fitting Boss, MlL-R-25897 Rubber, 90 Rec. Procedure for Compression Testing of Scaffolds and Rec. Horizontal Rec. Horizontal Rec. Safety Requirements for Rec. Steel Frame Rec. Steel Frame

Rec. Std Safety Code for Vertical al Shear Strength of Parallel Fiber Reinforced Plastics by or Off the Road Vehicles: Earthmoving, Mining and Logging Std. Meth. of Test for Yarn Number Based on for $100 \mathrm{Deg}$. Close Tolerance Head and Shank, 160,000 Psi Std. for Hexagon Head Mlodified Std. for Hexagon Head Modified
Tor Bolt-Close Tolerance, Hexagon Head, Titanium,
Toleran Titanium Alloy, reduced Head, Tri Wing Recess, Close Tolerance, A286 Cres, duced Head, Tri Wing Recess, Close Tolerance, Alloy Steel, Std. for Bolt, Hex Head, Close Tolerance, Alloy Steel, Std. for Bolt. Hex Head, Close Tolerance, A286 Cres,

Tri Wing Recess, Close Tolerance, 6Al-4V Titanium Alloy, r Bolt, Hex Head, Close Tolerance, $6 \mathrm{Al}-4 \mathrm{~V}$, Titanium Alloy, 00 Deg. Head, Tri Wing Recess, Close Tolerance, A286 Cres,

Deg. Head, Tri Wing Recess, Close Tolerance, Alloy Steel, Std. for Pan Head, Close Tolerance, Std. for Machine Screw, Flat 100 Deg. Head, Std. for Machine Screw, Pan Head,

elf Locking An/ $\mathrm{g}$ and Nonlocki/ and Nonlocking (197)

Sid for Bolt Pan Head, Close Tolerance, Std. for Bolt, Pan Head, Close Tolerance, Std. for Pan Head, Close Tolerance, Std. for Aircraft, Pan Head, Phillips Recess dwell-Sterling Meth. of Analysis of Moisture in Fats and h. of Analysis of Moisture and Volatile Matter in Fats and Meth. for Sampling of Fats and $n$-Bomb Meth. of Analysis of Stability in Fats, Oils, and

Meth. of Analys is of Stability (AOM) of Fats, Oils, and $y$ sis of Mlonoglycerides and Free Glycerol in Fats, Oils and solid Fat lndex (50 or Less at 10 Deg.) of Margarine Oils ion Residues in Milled Wheat Products (Wheat, Flour. Bran stic-Trap, Clay Pigeon, and Skeet: Ammunition and Targ/ ment) (1974) Std. for Forged Carbon Steel

Rec. Guide for Design of Foundations and eth. of Determining the SAE Rating and Struck Capacity for car, Front End Loader, Fork Lift Trucks, Mobile Crane, and Optical Test Meth. for lnk

terial for Use Principally as a Substitute for Plate Glass formance Requirements for Individual Anti Scald Type Water Sid. Performance Requirements for Wove tall Showers Lavatories, Bathtubs and Combination Tank and Recommendation for Installation of Ceramic Tile d Combination Tank and Shower Recep/ Std. for Nonmetallic hower Recep/ Std. for Nonmetallic Shower Receptors, Stal r Crosslinked and Noncrosslinked Poly(vinyl Chloride) Heat Std Meth of Test. Heat Std. Spec. for poly(Vinylidene Fluoride) Heat c Tubing, lrradiated Polyolefin, Pigmented, Flexible, Heat 3) ANSl K66.3 all1.25 Std. Meth. of Measuring Std. Meth of Test for Drying and Firing Std. Meth. of Test for Volume Std. Meth. of Test for
Shipping Sacks and Bags (Containers) (1973)

Shipping Test (Packaging) for Consumer Electronic Produ

Shipping, and Billing of Merchandise Within the Hardgoo

Shipping, Receiving, Storage and Handling of ltems for

Ships (1973)

(Ships) to Be Repaired (1972) ANS1 Z270. 1

Shirt Unit, Pants Unit (1973)

Shive Analyzer) (1973)

Shive Content of Mechanical Pulp (Laboratory Flat Scree

Shive Content of Mechanical Pulp (Somerville Fractionat

Shive Content of Mechanical Pulp (Von Alfthan Shive Ana

Shock Absorbing Characteristics of Package Cushioning M

Shock Absorbing Properties of (Sports) Playing Surface

Shock Machines (1973)

Shock Test on Glass Containers (Bottle, Jar) (1971)

Shock Test on Glass Pipe (1970)

Shock Test. Machine for Lightweight Equipment (1972)

Shoe Upper Leather by the Dow Corning Leather Tester (1

Shoe Upper Leather by the Maeser Water Penetration Test

Shooting Facility Plans: International and Domestic-T

Shop Applied) (1971)

D. for Cement Mortar Protective

Structural Steel and Plate (1973)

Shore Safety Rules (1972)

Shore, O-Ring (1973)

Shore, O-Ring (1973)

Shore, O-Ring (1973)

Shore, O-Ring (1973)

Shores (1967)

Shoring Beam Erection Procedure (1972)

Shoring Beam Safety Rules (1972)

Shoring Concrete Formwork (1972)

Shoring Erection Procedure (1969)

Shoring Safety Rules (1972)

Shoring (1968)

Short Beam Meth. (1972)

Short Haul); Grader, Loader, Dozer, Mining Car, Front E

Short Length Specimens (1972) ANSI L14.220

Short Thread Bolt (1974)

Short Thread Shear Bolt (1972)

Short Thread (1973)

Short Thread, Nonlocking (1972)

Short Thread, Nonlocking (1972)

Short Thread, Nonlocking (1973)

Short Thread, Self Locking and Nonlocking (1972)

Short Thread Self Locking and Nonlocking (1972)

Short Thread, Self Locking and Nonlocking (1972)

Short Thread, Self Locking and Nonlocking (1972)

Short Thread, Self Locking and Nonlocking (1972)

Short Thread, Self.Locking and Nonlocking (1973)

Short Thread, Torq Set Machine Screw (1972)

Short Thread, Torq Set (1973)

Short Thread, Torq Set (1973)

Short Thread, Tri Wing Recess, Titanium Alloy $6 \mathrm{Al}-4 \mathrm{~V}$ s

Short Thread, TWl Wing Recess, Alloy Steel, Self Lockin

Short Thread, TW1 Wing Recess, A286 Acres, Self Locking

Short Thread, 160,000 psi Alloy Steel Machine Screw (19

Shortenings by Distillation with Toluene (Cereal Chemis

Shortenings (Butter. Oleomargarine, High Acid Coconut O

Shortenings (Cereal Chemistry) (1962)

Shortenings (Cereal Chemistry) (1962)

Shortenings (Cereal Chemistry) (1967)

Shortenings (Cereal Chemistry) (1967)

Shortenings, and Other F ats (1968)

Amounts of Malathion

Ans: International and Dome

Shoulder Nut Assembly Eyebolt (For Ground Support Equip

Shoulders for Concrete Pavements (1965)

Shovel Dipper, Clam Bucket, and Dragline Bucket (Constr

Shovel (1974)

(T Haul); Grader, Loader, Dozer, Mining

Show Through (Paper) (1973)

Show Window Panels (1972)

Showe: Control Valves (1973)

Shower Curtains (lnstitutiona T

/Rglary Resisting Glazing Ma UL

Shower Receptor Units (1972)

Shower Receptors (1973)

Shower Receptors, Stall Showers Lavatories, Bathtubs an

Showers Lavatories, Bathtubs and Combination Tank and S

Shrinkable Tubing for Electrical Insulation (1972)

Shrinkable Tubing for Electrical Insulation (1973

Shrinkable Tubing for Electrical Insulation (1974)

Shrinkable Tubing (1973)

Shrinkable (1973)

IC. for Electrical Insulation Plasti

Shrinkage from Mold Dimensions of Molded Plastics (R197

Shrinkage of Fireclay Plastic Refractories (1972) ANSI

Shrinkage of Latex Sealing Compounds (1972)

Shrinkage of Textile Fibers (1972) ANSI L 14.142
TAPPI UM- 806

EIA

NWHA

ANSl

ASTM

NFPA

D ANPI

TAPPI

TAPPI

TAPPI

ASTM

AST I

ANSI

ASTM

ASTM

ANSI

ASTM

STM

NRA

AWW A

ANSI

SSl

NSA

NSA

NSA

NSA

SS]

SS]

SSl

SSl

SSl

ASTM

TRA

ASTM

NSA

NSA

NSA

NSA

NSA

NSA

NSA

NSA

Deg NSA

T. I NSA

100 NSA

NSA

NSA

NSA

NSA

NSA

NSA

NSA

$\mathrm{AACCH}$

$\mathrm{AACCH}$

AACCH $64-40$

Oxyge AACCH 58-55

$\mathrm{AACCH} \quad 58-54$

AACCH $58-45$

AACCH 31.50

$\mathrm{AACCH} \quad 60-30$

NRA *17

NSA 1053

$\mathrm{ACl} \quad 65-43$

SAE $\quad \mathrm{J} 67$

RA 3

UM.545

972

1016

ANSI L24.2.10

IAPMO TSCII

TCA

309

APMO TSCl1

APMO TSCll

ASTM D3149

Fo ASTM D 3150

ASTN D2671

ASTM D3144

SAE

ANS $3636 \mathrm{D}$

D955

C179

ASTM C733

ASTM D2102 


\section{National Bureau of Standards KWIC Index of Engineering Standards}

(1969) ANSI K65.181

Std. Meth. of Test for Linear of Test. Delamination, Strength of Bond, Appearance, and Test Meth. for Std. Meth. of Test for Relaxation Dimensional Change Meth for Relaxation and Felting Dimensional Changes tness of Cotton and Linen Textiles to Combined Washing and t, Grape, Asparagus); Lining Out Stock; Seedling Trees and sery Stock: Deciduous Shade and Flowering Trees; Deciduous Std. for Relief Valves and Automatic Gas

1972) Std. for al Woodwork) (1973) Std. Exposure Time Markings for Variable Speed Q Quality Stds. For Blinds and Std. for Fire Doors. Windows,

d Handrails, Exterior Frames and Sash, Screens, Blinds and Std. for Tires and Rims for Motorcycle, Std. Test. Proc for Side to

(1974)

,Dot-37A60, Ufc-Rule/

1973) std. for Tee, Reducer, Flareless Tube to Swivel, Swivel on
Std. for Heavy Duty Offset nted Wiring Board Dimensions and Tolerances Single and Two Std. Spec. for Asphalt Insulating Spec. for Aluminum

Std. Patterns for Wood Paneling, Decking, Flooring, and Spec. for Installation of Residential Aluminum Std. Spec. for Semidense Mineral Fiber Std. for Rigid Poly vinyl Chloride) (PVC) Plastic Wal Std for Hardboard Rec. for Wood Redwood Exterior Guide rec., Properties, Weight, Stress of Board, Timber, Lumber. A37.14

Carpentry, Flooring Shingles, Exposed Roof Decking, Drop gregates (1971) AASHO T27, ANSl A Std. Meth. of Test for Test Meth. for

Test for Fineness of Hydraulic Cement by No. of Test for ter Paper, Wide Field and Compound Microscope, Petri Dish, Std. Spec. for Perforated Plate Std. Spec. for Wire Cloth

Std Spec for Precision Electroformed

nts, Hairs, Feathers, Wood Fibers, Splinters, etc. in St/ $n$ Baked Goods, Ready.To.Eat Cereals, and Alimentary Past/ ) (1974)

in Baked Goods, Ready.To-Eat Cereals, and Alimentary Pa/ Uniform Rec. Safety

Varactor Measurements Part lI-Characterization of Large Std. for Adjustable Face Vehicular Traffic Control Rec. Pract. for Motorcycle Turn Std. Spec. for Communication and synchronous Data Communication Equip/ Std. for Start-Stop ding (1969) Rec. Pract. for Video Alignment ability Requirements for Both Permanentl/ Rec. Pract. for unication) (1974) Std. for Safety for Hospital systems (1974) Std. for Safety for Manually Actuated Spec. for Safety Controls and Std. Synchronous

Installation, Maintenance and U'se of Auxiliary Protective llation, Maintenance and Use of Central Station Protective for lnstallation, Maintenance and I'se of Local Protective Rec. for Proprietary Protective d. for Safety for Control Units for Indoor Fire Protective allation, Maintenance and I'se of Remote Station Protective m) (1973)

ally Actuated Signaling Boxes for I'se with Fire Protective

Rec. for Local Protective
nstallation, Maintenance and Lise of Proprietary Protective connection Circuitry of Noncoded Remote Station Protective

building Code Std. for Smoke Detectors for Fire Protective Rec. Hand

Safety Std. for Highway Emergency ng Observations in Samples and How to Test the Statistical ements for Both Permanently and Temporarily Affixed Safety ) Rec. Pract. for Accident Prevention ident /

Spec. for Informational, Directional, and Special Safety Std. for Electric Cord Connected Indoor Std. Spec. for Accident Prevention s) Complementary to ANSI Z35.1-1972, Accident Prevention Tower Silos: Unit Weight of fabrics, Cleaned, and After Finished with Epoxy Functiona std. Meth. of Test. Duct Liner Materials and Prefabricated Metallic
Shrinkage of Thermosetting Casting Systems During Cure Shrinkage Propensity of Bonded and Laminated Apparel Fa Shrinkage Test of Pulp Sheets (1973)

(Shrinkage) of Stabilized Knit Wool Fabrics (Textile).(1

Shrinkage) of Woven and Knitted Wool Textiles (1972) an

Shrinkage: Rapid Control Test (1969)

Shrubs; Bulbs, Corms, and Tubers; Christmas Tree Std. (

Shrubs; Coniferous and Broadleaf Evergreens; Rose Grade

Shutoff Devices for Hot Water Supply Systems (1972)

Shutter Tests for Still Picture Cameras (Photography)

Shutters U'sed in Still Picture Cameras (1972)

Shutters Using Stile and Rail Construction (Architectur

Shutters (1973)

Shutters, and Glass Blocks (1973) ANSI A2.7

Shutters, Flush, Stile, and Rail Doors, Factory Finishi

Side Car, Mini Bike, and Bicycle (1974)

Side Crush Composite Tubes and Cores (1973)

Side Lug Cover Universal Pail (Container) (Dot-37C80)

Side Lug Cover Universal Pails (Container) (Dot-37A80

Side Marker and Identification Lamps (Light) (1972)

Side of Filled Paper as Indicated by Silver Coin Test (

Side to Side Crush Composite Tubes and Cores (1973)

Side (1973)

Sidebar Power Transmission Roller Chains and Sprocket T

Sided Rigid Boards (1970) ANSI C83.98

Siding Surfaced with Mineral Granules (1970) ANSI A 109.

Siding (1970)

Siding $(1970)$

Siding (1971)

Siding (1972)

Siding (1972)

Siding (1973) ANSI A 135.6

Siding, Finish, Nails and Nailing (1972)

(Siding, Finish, Nails and Nailing) (1973)

Siding, Framing, Decking, Flooring, Ceiling, and Partit

Siding, Structural Roof Trusses and Rafters, Overhead

Sieve Analysis of Mineral Filler (1972) AASHO T37, ANSI

Sieve Analysis of Pulpwood Chips (Pulp and Paper) (1973

Sieve or Screen Analysis of Fine and Coarse Concrete Ag

Sieve (1973)

Sid.
for $X-$

Sieve, Separatory Funnel, Trap Flask, Step Wed
Sieves for Test. Purposes (1970) ANSl Zl68.12

Sieves for Test. Purposes (1970) ANSI Z23.1

Sieves (1970) ANSI Z168.5

Sieving Meth. of Analysis for Dirt, Sand, lnsect Fragme

Sieving Meth. of Analysis for Insect and Rodent Filth I

Sieving Meth. of Analysis for Materials Hard to Hydrate

Sight Glasses for Chemical Processing Equipment (Vessel

Sign Code (1973)

Sign for Front End Agricultural Loaders (1972)

Signal Devices (1972)

1. Guide on Solid State Devices:

Signal Heads (1970)

Signal Lamps (Light) (1971)

Signal Pin Type Lime Glass Insulators (R1970)

Signal Quality Between Data Terminal Equipment and Non-

Signal Spec. for a Quadruplet Video Magnetic Tape Recor

Signal Words, Letter Sizes, Color Combinations, and Dur

Signaling and Nurse Call Equipment (Audio / Visual Comm

Signaling Boxes for Use with Fire Protective Signaling

Signaling Devices for Printing Presses (1972)

Signaling Rates for Data Transmission (1969)

Signaling Systems for Fire Alarm Service (1972) ANSI Se

Signaling Systems for Guard, Fire Alarm and Supervisory

Signaling Systems for Watchman, Fire Alarm, and Supervi

Signaling Systems (Fire Protection and Safety) (1973)

Signaling Systems (1972) ANSI C33.90

Signaling Systems (1972) ANSI SE3.5

Signaling Systems (1974)

Std. for Inst

Signalling Systems for Fire Protection and Safety (Alar

Signalling Systems for Watchman, Fire Alarm and Supervi

signalling Systems (1969) ANSI SE3.7

Signalling Systems (1973)

Signals for Use in Agriculture (1972)

SignaIs (Flare, Lantern. Fusee) (1973)

Significance of Them (Sampling) (1968) ANSI Z1.14

Signs for Construction and Industrial Equipment (Machin

Signs for Display on Tank Trailers and Containers (1972

Signs (Symbols) Complementary to ANSI Z35.1-1972, Acc

Signs (1971) ANSI C33.35

Signs (1972)

Signs (1973)

1, Directional, and Special Signs (Symbol

Silage and Silo Capacities (1972)

Silane Ty pe Finishes for Plastic Laminates (1972)

Silencers for Acoustical and Airflow Performance (1973)

Silhouette Match Rifle Range Plans (1973)

Silica by Air Permeability (1972)

ASTM

ASTM

TAPPI

ASTM

AATCC

AATCC

ANSI

ANSI

ANSI

ANSI

ANSI

$A$ Wl

ICBO

NFPA

$\mathrm{AW} 1$

TRA

CCT

ANSI

ANSI

SAE

TAPPI

$\mathrm{CCTl}$

NSA

ANSl

IPC

ASTM

AAMA

WWPA

AAMA

ASTM

USC

USC

WW PA

CRA

WW P

Ished AWI

A.STM

TAPPI

ASTM

ASTM

AACC

ASTM

ASTM

ASTM

E161

AACCH 28.75

$\mathrm{AACCH} 28.31$

AACCH 28.32

FMS

$\mathrm{ICBO}$

ASAE

IEEE

ITE

$\mathrm{SAE}$

ASTM

ElA

SMPT

$\mathrm{SAE}$

UL

UL

ANSI

ANSI

NFPA

NFPA

NFPA

FMS

St UL

NFPA

UL

FMS

NFPA

for Inter NEMA

niform ICBO

ASAE

/Lyi ASTM

$\mathrm{SAE}$

TTMA

ANS I

UL

ANSI

7.3

$\mathrm{UBC} * 4$

R355

351

TRl

Jl3l A

RS404

E RI0

JI 15

1069

38

B65.1

$\mathrm{X} 3.1$

$72 \mathrm{~B}$

$72 \mathrm{~A}$

LPD $5 \cdot 2 \mathrm{~N}$

864

$72 \mathrm{C}$

38
$5-5 \mathrm{~N}$

$72 \mathrm{D}$

$\mathrm{SB} 3$

UBCS43.6

R351

912

E178

J1 15

RP35

Z35. 4 
c) Weth. (1972)

Std. Spec, for Chemically Setting Silicate and Sid. Meth. of Test for Std. Meth. of Test for Total Ash and Use of Chemically Setting Chemical Resistant Silicate and Std. Meth. for Chemical Analysis of Std. Spec. for Chemically Setting c. Pract. for Use of Chemically Setting Chemical Resistant Std. Spec. for Prefaced Concrete and Calcium Std. Meth. of Analysis of Magnesium Std. Meth. of Analysis of Aluminum Std. Spec. for Basic Lead Std. Meth. of Test for Silica in Fluorspar by the Std. Meth. for Chemical Analysis of

3)

Std. Spec. for Copper-Nickel. Std. Spec. for Copper

Std. Spec. for Nickel lron Chromium Std. Spec. for Copper Std. Spec. for Copper

1 meth. for Spectrochemic al Analysis of High Purity Iron for
Std. Meth. of Test for Thickness of Epitaxial Layers of

nt. Meth. of Test for Minority Carrier Diffusion Length in of Test for Thickness of Epitaxial or Diffused Layers in Std. Meth of Chemical Analysis of

std. Meth. for Chemical Analysis of Carbon, Low Alloy, and wn Meth. (1972) An

y (1974) Std. Meth. of Test for Resistivity of $7 \mathrm{G} 053,30 \mathrm{G} 05$ / Tentative Meth. of Test for Sheet Resistance of Std. Spec. for Flat Rolled, Grain Oriented Std. Meth. for Chemical Analysis of Ferrosilicon and Std. Meth. of Test for Thickness of Epitaxial Layers of Sid. Meth. for Measuring Resistivity of Spec. for Low and Intermediate Tensile Strength Carbon.

35.14 Std. Spec. for Carbon Manganese tification of Structures and Contaminants Seen on Specular beam I/ Tent. Meth. for Measurement of Oxide Thickness on for Pressure Vessel Plates, Heat Treated, Carbon Manganese or Pressure Vessel Plates, Alloy Steel, Chromium Manganese Std. Meth. for Chemical Analysis of Ferrochrome Test Meth. for $D$ yed Tower Silos: Unit Weight of Silage and Sanitary Std. for

Rec. for Deep, Cast in Place Concrete (Storage) Bin and Stds. for Construction of Concrete Stave Farm Tower

for Chemical and Spectrochemical Analysis of Nuclear Grade ed, Finished Sugar and Other Clarified Starch Hydrolzates
Unhardened Steel and Alloy or Spectrochemical Analysis of Nickel-Base Alloys by the hed Sugar and Other Clarified Starch Hydrolzates Silver
Std. Spec. for

Test Meth. for Top Side of Filled Paper as Indicated by td. for Methylene Blue Meth. for Measuring Thiosulfate and Spec. for Photographic Film for Archival Records,

Std. Spec. for Photographic Film for Archival Records,

Pract. Inspection and Quality Control of First Generation Meth. for Preparation of

and / Std. Spec. for Sintered Nickel Std. Spec. for Copper-Nickel-Zinc Alloy (Nickel Std. Spec. for Copper-Nickel-Zinc Alloy (Nickel Sid. Spec. for Gold Test Meth. for Beating Control

td. Meth. of Test for Flexural Strength of Concrete (Using ories $(1972)$

973)

ooring (1969) Std. Spec, for Std. Meth. of Test for Std. Test Meth. for onic Products (Radio, Television, Tape Recorder/ Std. for 3) Control Materials (Coati) Std. Rec. Pract. for Combined,
Std. Rec. Pract. for Solar Std. Definitions of Terms Relating to Space $y$ of Motor Oils at Low Temperature Using the Cold Spanking Std. for Pin, Quick Release, Positive Locking. (Calcined Bauxite, Calcine, Chrom/ Sid. Classification of Std. Performance Requirements for Terry Cloth Towels ith Ethylene-Propylene-Rubber Insu/ Std. for Portable for I/ Safety Std. for Heat Actuated Fire Alarm Devices, Spec, for Printed Wiring Board Dimensions and Tolerances Std. Spec. Format Guide and Test Procedure for Linear, $y$ the Wedge Bend (Applicable to Solution Coil Coatings and Propylene-Rubber Insulation, 200/ Std. for Nonshielded ough 35/ Std. Ampacities with Effect of Shield Losses for Std. for Medium Prefocus Base Down Type,

I Mobility and Hall Coefficient in Extrinsic Semiconductor Std. for Dimensional Requirements for Duplex and ation Protect/ Trial Use Guide for the Application of the
Std. for Gas Fired

I Equipment Use/ Std. Meth. for Measuring Audio Amplifier
Silica Chemical Resistant Mortars (1974)

ASTM

ASTM

Silica in Fluorspar by the Silico Molybdate (Photometri

ASTM

Silica in Water Emulsion Polishes (1972) ANSI Z123.I

Silica Mortars (1974)
Silica Refractories (1970) ANS1 A 139.5

Silicate and Silica Chemical Resistant Mortars (1974)

Silicate and Silica Mortars (1974)

Silicate Masonry Units (1973)

Silicate Pigment (1973)

Silicate Pigment (1973)

Silico Chromate (1974)

Silico Molybdate (Photometric) Meth. (1972)

Silicomanganese and Ferrosilicon Manganese (1974)

Silic on Alloy Plate and Sheet for Pressure Vessels (197

Silicon Alloy Rod and Bar (1973)

Silicon Alloy Rod, Bar, and Shapes (1973) ANSI H7.3

Silicon Alloy Seamless and Welded Pipe (1972)

Silicon Alloy Seamless Pipe and Tube (1974) ANSI H26.3

Silicon Alloy Wire for General Purposes (1973) ANSI H30

Silicon and Aluminum (1971) ANSI Z128.35

Silicon by Measurement of Stacking Fault Dimension (197

Silicon by Measurement of Steady State Surface Photoyol

Silicon by the Angle Lapping and Staining Technique (19

Silicon Carbide Abrasive Grain and Crude (1971)

Silicon Electrical Steels, lngot and Wrought lrons (197

Silicon Epitaxial Layers by the 3 Probe Voltage Breakdo

Silicon Epitaxial Layers Using a Collinear 4 Probe Arra

Silic on Iron, Electrical Steel, Fully Processed Types 2

Silicon Metal (1974)

Silicon on Substrates of the Same Type by lnfrared Refl

Silicon Slices with a Collinear 4 Probe Array (1973)

Silicon Steel Plates for Machine Parts and General Cons

Silicon Steel Plates for Pressure Vessels (1972) ANSI G

Silicon Surfaces (1972T)

IT. and Nomenclature

Silicon Wafers and Metallization Thickness by Multiple

Silicon (1972A)

Silicon (1972A) ANSI G32.1

Std. Spec.
Std. Spec. F

Silicon (1974)

Silk Yarn Colorfastness to Degumming (1972) ANSI L14.4

Silo Capacities (1972)

Silo Type Storage Tanks for Milk and Its Products (1974

Silo Wall Design and Construction (1965)

Silos (1969)

Silos: Unit Weight of Silage and Silo Capacities (1972)

Silver-Indium-Cadmium Alloys $(1974)$

(Silver-Silver Chloride Indicator) (1971)

Silver Brazing Joints (1973)

Silver Chloride-Lithium Fluoride Carrier D-C Arc Tech

Silver Chloride Indicator) (1971)

\section{Yrup Unmixed,}

Silver Coated Soft or Ann
Silver Coin Test (1973)

Silver Densitometric Meth. for Measuring Residual Chemi

Silver Gelatin Type, on Cellulose Ester Base (1973)

Silver Gelatin Type, on Polyester Base (1973)

Silver Habide Microfilm (1972)

Silver Nitrate Solution (Cereal Chemistry) (1962)

Silver Structural Parts (1970) ANSI H9.28

Silver) and Copper-Nickel Alloy Plate, Sheet, Strip,

Silver) and Copper-Nickel Rod and Bar (1973)

Silver, Nickel Electrical Contact Alloy (1972)

(Simons Stain) (Pulp and Paper) (1973)

Simple Beam with Center Point Loading) (1968) ANSI A37.

Simple Can Puncturing Device for Use in Aerosol Laborat

Simulated Service Corrosion Test. of Engine Coolants (1

Simulated Service Test. of Wood and Wood Base Finish Fl

Simulated Shipping Test (Packaging) for Consumer Electr

Simulated Space Environment Test. of Spacecraft Thermal

Simulation for Thermal Balance Test. of Spacecraft (197

Simulation (1972)

Simulator (1972) ANS1 Z11.280

Single Acting (1973)

Single and Double Screened Ground Refractory Materials

(Single and Double) (Institutional Textile) (1973)

Single and Multiple Conductor Electrical Power Cables W

Single and Multiple Station, Mechanically Operated Type

Single and Two Sided Rigid Boards (1970) ANSI C83.98

Single Axis, Pendulous, Analog Torque Balance Accelerom

Single Coat Organosols Only) (1974)

Single Conductor Electrical Power Cable with Ethylene-

Single Conductor Solid Dielectric Power Cable 15 kV Thr

Single Contact Motion Picture Projection Lamps (1972)

Single Crystals (1973)

Single Face Flat Thread Rolling Dies (1973)

Single Failure Criterion to Nuclear Power Generating St

Single Firebox Boilers (1971)

Single Frequency Output for Institutional Audio-Visua

ASTM

ASTM

Std. ASTM

AATM

ASTM

ASTM

ANSI

ASTM

ASTM

ASTM

ASTM

ASTM

ASTM

ASTM

ASTM

ASTM

ASTM

ASTM

ASTM

ASTM

ASTM

AATCC

ASAE

DFISA

$\mathrm{ACl}$

NSLO

ASAE

ASTM

CR

SAE

ASTM

AR

ASTM

TAPPI

ANSI

ANSI

ANSI

C466

E463

D1 288

C.397

C575

C466

C397

C744

D717

D718

D 1648

E463

E362

B96

B411

B98

B535

B315

B99

E421

F143

F391

F110

E 350

F108

F374

A665

E360

F95

F84

A 284

A 299

F154

F 388

A 202

E364

D252

2203

65-37

$* 1$

D252

C 760

E-15

AMS2664C

E483

E-15

B298

I'M. 533

PH4.8

PH1.28

PH1.41

MA MSl04

AACCH $70-60$

ASTM B458

ASTM B122

ASTM B15l

ASTM B477

TAPPI UM.17

ASTM C293

ASTM D3058

ASTM D2570

ASTM D2394

EIA RS414

ASTM E512

ASTM E491

ASTM E349 
Std. Meth. of Test for Tensile Properties of phase Converters (1972) Rec. for Fire Protection of Electrical Equipment Std. Spec. for Noncarbonized, Std. for Carbide Blanks and Cutting Tools, Rec. for

Test for Tearing Strength of Woven Fabrics by the Tongue Chlorine (1971) ANSI L14.126 Test Meth. for ts (Packaging Sanitary Stds. for Fillers and Sealers of Design Rules, Guidelines, and Drawings for Copper Solvent ondary Standard of Sim/ Std. Meth. of Thermal EMF Test of Meth. of Test for Density of Glass by the Std. Spec for Nickel Oxide actors (1973) Std. Spec. for Nuclear Grade. Sid. Spec. for Nuclear Grade Plutonium Dioxide Powder. Std spec for Std Spec for Iron Base Std. Spec. for Copper Base std. Spec. for Std. Spec. for Std. Spec. for

3 28

Std. Spec. for lron Copper Std. Spec. for

Meth. of Test for Density and Interconnected Porosity of c. for Baled Textile Fiber Storage (Cotton. Jute, Hemp and Code: Storage and Handling of Combustible Fibres (Cotton, Industry Std. for Douglas Fir. Western Hemlock, ion: West Coast Hemlock; Western Red Cedar; White Fir; and ock-Tamarack (Nor/ Uniform Building Code Std. for Coast Std. Meth. for Particle Brick (1970) ANSI Al11.18 (1972) Std. Meth. of Test for Std. for Dimensions of 126 ng (1972) Rec. Spec. for Optimum identification Code, and Mounting Dime/ Std. Bore and Rod Std. for Suffix Letters Used in Tire ec. Pract for Analysis by Microscopical Meth. for Particle Meth. for Particle ec. Pract. for Spark Arresters Used on Multiposition Small Rec. Pract. for Spark Arrester Test Procedure for Medium Std. Meth. of Test for Average Particle Std. for Checking the Std. Meth. for Automatic Particle Counter $f$ Wood Preservatives for Marine Services by Means of Small s (1972) ANSl Z1.1/ Std. Rec. Pract. for Choice of Sample th. of Sampling and Test. Ferroalloys for Determination of and Rec. Pract. Guide to Design, Construction, Materials, stress of Bo/ Std. Recommendations for Die Cavity and Cutoff Die

s $(1966)$

re Cameras (1972) Std. Spec. for
(Class 2) Wire manent, Crimp Style. Pre Insulated, (Class 2) Wire

Std. Nomenclature for Bedding Std. for Transport Package

std. Meth. of Static Tests of Timbers (Wood) in Structural le Size Distribution of Particulate Substances of Subsieve Spec. for Photographic Optical Filter Std. Spec. for Seamless Copper Pipe, Std. Std. Spec. for Seamless Red Brass Pipe, Std. sport Package Sizes (Packaging) for ANSI MHl0.1. Unit Load for Both Permanentl/ Rec. Pract. for Signal Words, Letter Std. Control Valve Std. for Control Valve Std. for Body Measurements for the Test Meth. for Test Meth. for Uniform Plumbing Code: Rec. Rules for plans: International and Domestic-Trap, Clay Pigeon, and Test Meth. for Evaluation of Wetting Agents (Cotton Test e for Use by Surgeons in the Treatment of Fractures of the afety Requirements for Aerial Passenger Tramways lncluding Std. Spec. for Std. Tire for Pavement (Drill) Press, and Gage Wheel, Tractor, Implement, and Log otorcycles, Passenger Cars, Off Road, Agricultural and Log Std. for Safety for Household Electric

for Structural Glued Built Up Members-Plywood Stressed Std. Meth. for Design and Use of a Thin Design Spec. for Plywood Stressed Uniform Building Code:

de: Plastics (General, Exterior Light Transmitting Panels, Rec. Pract, for Concrete Floor and

d Continuous Strip Mastic Set Maple Flooring Over Concrete
Single Man Made Textile Fibers Taken from Yarns and Tow (Single Phase and Related Faults) (1973)

Single Phase Rural Distribution Service for Motors and Single Ply, Adding Machine Paper Rolls (1973)

Single Point, Carbide Tipped, Roller Turner Type (1972)

Single Post Shore Safety Rules (1972)

(Single Rip) Meth. (Constant Rate of Traverse Tensile Te Single Sample Meth. of Textile Tensile Loss by Retained Single Service Containers for Milk and lts Fluid Produc Single Stack Plumbing System (1973)

Single Thermoelement Materials by Comparison with a Sec Sink Float Comparator (1972T)

Sinter (1970) ANSI G86.1

Sinterable Uranium Dioxide Powder for Use in Nuclear Re Sinterable (1974)

Sintered Aluminum Structural Parts (1973)

Sintered Bearings (Oil lmpregnated) (1970) ANSI G63.4

Sintered Bearings (Oil Impregnated) (1973) ANSI H9.27

Sintered Brass Structural Parts (1970) ANSI H9.8

Sintered Bronze Structural Parts (1970) ANSI H9.6

Sintered Copper Steel Structural Parts (1970) ANSI G63.

Sintered Metal Powder Structural Parts (1970) ANSI G63.

Sintered Nickel Silver Structural Parts (1970) ANSl H9.

Sintered Powder Metal Structural Parts and Oil Impregna

Sisal, Flax, Synthetic Fibers, Wool) (1974)

Sisal, Henequen, Ixtle, Jutes, Hemp, Tow, Cocoa Fibers,

Sitka Spruce Doors and Blinds (1972)

Sitka Spruce (Lumber) (1973)

Sitka Spruce, Douglas Fir-Iarch (North) Fouglas Fir,

Size Analysis of Whiteware Clays (1974)

Size and Bulk Density of Refractory and Insulating Fire

Size Cartridges. Film, and Backing Paper (Photography)

Size Coarse Aggregate for Portland Cement Concrete Pavi

Size Combinations, Rod End Configurations, Dimensional

Size Designations and Their Definitions (1974)

Size Distribution of Particulate Substances of Subsieve

Size Distribution of Wheat Flour (Cereal Chemistry) (19

Size Engines (1972)

Size Engines (1972)

Size of Alumina and Silica by Air Permeability (1972)

Size of Diamond Abrasive Grain (1971)

Size Setting (1972)

Size Specimens (1970)

Size to Estimate the Avera

Size (1970) ANSI G85.

Size, and Installation of Propeller Shafting Systems Fo

Sizes for Cold Forging Equipment (1972)

Sizes for Dimensioning Transport Packages (1972)

Sizes for Industrial Centrifugal Fans with Cast Housing

Sizes for Industrial Centrifugal Fans (1966)

Sizes for Roll, $35 \mathrm{~mm}$ Film, and $126 \mathrm{~mm}$ Film Still Pictu

Sizes for Threaded Caster Stems (1965)

Sizes 12 to 26 (1972)

Sizes $1 / 16$ In. Thru Letter F (1973)

Sizes (Mattresses and Sheets) (1971)

Sizes (Packaging) for ANSI MH10.1. Unit Load Sizes (197

Sizes (1967) ANSI Q4.2

Sizes (1968)

Sizes (1972)

Sizes (1972)

Sizes (1972)

Sizes (1973)

Sizes, Color Combinations, and Durability Requirements

Sizing Equations for Compressible Fluids (1973)

Sizing Equations for Incompressible Fluids (1972)

Sizing of Girls' Apparel (Clothing) (1972)

Sizing of Paper (Reverse Curl) (1973)

Sizing of Paperboard (Flotation) (1973)

Sizing the Water Supply System (1973)

Skeet: Ammunition and Target Spec. (1973)

Skein Meth.) (1971) ANSI L14.11

Skeletal (Bone) System (1973)

Ski and Surface Lifts and Tows (1973)

Skid Resistance Tests (1973)

Skidder (1974)

Skidder, Truek

D Industrial Steering, Drive, Planter

Skillets and Frying Type Appliances (1973) ANSI C33.11

Skin and Curved Panels, Beams, and Components (1973)

Skin Panels Fabrication Spec. (1971)

Skin Panels (1972)

Skylights (1973)

Skylights, Monitors and Sawtooth Roofs, Light Diffusers

Slab Construction (1969) ANSl A157.1

Slab Floors (1962)

ASTM

FMS

ASAE

ANSl

SSI

ASTM

AATCC

DFISA

CDA

ASTM

ASTM

ASTM

ASTM

ASTM

ASTM

ASTM

ASTM

ASTM

ASTM

ASTM

ASTM

ASTM

ASTM

Re FMS

FHD

ICBO

ICBO

ASTM

ASTM

ANSI

ACPA

NFLDP

TRA

ASTM

$\triangle \mathrm{ACC}$

$S A E$

SAE

ASTM

ANSl

ASTM

D210

I4. 15

$\mathrm{R} 329$
$\times 4.8$

B94.37

$* 4$

D2262

92

1704

E207

C729

A 636

C753

C757
B595

B 439

B438

B282

B426

B222

B458

B328

8-7

UFC*2ART7

4. 72

UBC 525.3

UBC S25-2

C 775

C134

PH1.40

15

T3.6.11

X20

50. 10

J335A

C721

B74.16

F321

D2481

ASTM E122

Std. Me ASTM A610

ABYC P6

WWPA *24

IFl

ANSl MH10

AMCA 2403

AMC A 2402

ANS1 PH3.39

CFTMA STEM

NSA 1388

NSA

ANSI

ANS1

ASTM

965

L27. 1

MH10.2

D198

E20

PH3. 17

ANSI

ASTM

ASTM

B42

B43 
onstruction with Nailers and Finish Flooring Over Concrete with Nailers, Subflooring or Finish Flooring Over Concrete c Set Subflooring and Nailed Finish Flooring Over Conc rete mosaic Wood Parquet Flooring Set in Adhesive Over Concrete Concrete Construction Design Handbook (Beam, Std. Spec. for Zinc Metal Clay Tile and Concrete

1

Iding Code Std. for Reinforced Gypsum Concrete and Precas Std. Spec. for Lake Copper Wire Bars, Cakes, Std. Spec. for Electrolytic Copper Wire Bars, Cakes, std. Spec, for Chemically Refined Copper Wire Bars, Cakes, rings (Multiply Felt and Asphalt Preferably with Gravel or Std. Meth. of Drip

d, Crushed Stone, Gravel and Air Cooled Iron Blast Furnace urses (1971) ANSI A37.99

Rec. for High Temperature Molten Materials (Metals.

vel; Granite. Limestone, and Marble Masonry; Mortar; Sand; k Receptivity) (1973)

Stds, for Staples with Rolled or Std. Spec for Uniform Building Code Std. for Natural Std. Spec. for Structura ver Concrete Slab Floors (1962) arden Riding Tractors (1972) Spec. for Rubber Cushion std. for One Point Tubular Std. for lectrical Plugs, Receptacles and Connectors of the Pin an Std. for Spherical Std. for Drill Drivers, Split 2) Std. for Fastener (Blind Internally Threaded, External Std. for Fastener (Blind, Internally Threaded, External Fastener (Blind, Internally Threaded, External Std. for Fastener (Blind, Internally Threaded, External Std. for Fastener (Blind, Internally Threaded, External

Std, for Fastener (Blind, Internally Threaded, External Spec. for Fastener (Blind, Internally Threaded, External d. Meth. of Test for Reheat Change of Fireclay Nozzles and Std. Meth. for Measuring Resistivity of Silicon

Pekar Color Test Std. Pract. for

Std. for Physical Characteristics of T.2 Telephone Std. Basic Construction Requirements for Photographic Std Pract for Slide and

Std. Dimensions of lmage Areas and Mounts for he Density and Contrast Range of Black and White Films and $\mathrm{ng}$ Power of Lenses for Projectors for $35 \mathrm{~mm}$ Filmstrips and

Stops, Smoke and Heat Detectors; Electromagnetic Holders Stds. for Forced Entry Resistant Aluminum Horizontal Std. for Forced Entry Aluminum Spec. for Aluminum Safety Std. for

d Fire Doors (1973) ANSl A143. 1 ernal Applicat/ Spec. for Aluminum Combination Vertic ally
h. of Analysis of Starch in Flour and Semolina; Also, with Safety Code for Cranes, Derricks, Hoists, Jacks and afety Std. for Cranes, Derricks, Hoists, Hooks, Jacks, and C/ Test. Std. (Procedure) for Products Classified as to

$s$ (1973) Std. Performance Requirements for Woven Tent. Rec. Pract. for Measuring Design and Construction of Flat and Std. for T.Hook

ent (1972)

Pract. for Dimensions and Tolerances for Screw Holes and rew Nuts (1972) Std. Part Pins, Spring, Std. for

Std. Spec. for Liquid Asphal Std. for

114.1, SAE J943A

Mounting Brackets and Socket for Warning Lamp (Light) and icultural (Farm) and Industrial Equipment Warning Lamp and

Measuring Heat Transfer Rate Using a Thermal Capacitance Std. Rec. Pract. for Selecting Proportions for No nd (1972) Std. Meth. of Test for Std. Meth. of Test for Std. Meth. of Test for

sh (1974) Meth. for Viscosity of Coating Clay ks for Transporting and Spreading Agricultural Liquids and Std. Analytical Meth. for Determining pH

in Water/ Std Analytical Meth for Determination of $\mathrm{pH}$ $r$ and Semolina (Cereal Chemistry) (1962) $s$ (1972)

utd oor Sid. for Measurement of and Misc. Items; Key Control Systems; Door Holders, Stops, Rec. for Fire, Heat, and (1973) Uniform Building Code Std. for ily in Case of Fire (1974)
Slab Floors (1962)

Slab Floors (1962)

Slab Floors (1962)

Slab or Wood Subfloors (1962)

Slab, Footing, Column and Other Members) (1973)

(Slab-Zinc) (1970) ANSl H24.1

Slabs (Tech. Notes) (1962)

Slabs, and Poured Gypsum Roof Diaphragms (1973)

Slabs, Billets, Ingots, and Ingot Bars (1973) ANSI H17.

Slabs, Billets, lngots, and lngot Bars (1973) ANSI H17.

Slabs, Billets, Ingots, and lngot Bars (1973) ANSI H23.

Slag Surface) (1968)

Slag Test. Refractory Brick at High Temperature (1973)
Slag) (1973) /G Code Sid. for Concrete Aggregat

Slag) (1973)

Slag, and Gravel for Dry or Water Bound Macadam Base Co

Slag. Glass, etc.) Fire Protection and Safety (1973)

Slag; Steel; Terra Cotta; Timber; Water; Ceiling; Parti

Slant Drop Penetration Test for Paperboard (Printing in

Slash Points (1971) EEl TDJ.14

Slate Blackboards (1967)

Slate Roof Shingles (1973)

Slate (1968) ANSl A193.1

Sleeper Construction with Nailers and Finish Flooring $O$

Sleeve Attachment for Hitching Implements to Lawn and $\mathrm{C}$

Sleeve Bushing, Press Fit, Undersize Inside Diameter (1

Sleeve Type for Hazardous Locations (1973)

Sleeve (Compression) Tube Fittings (1971)

Sleeve, Collet Type (1972)

Sleeve, General Purpose, Flush Head, Self Locking) (197

Sleeve, General Purpose, Protruding Head, Self Locking)

Sleeve, High Temperature, Flush Head, Self Locking) (19

Sleeve, High Temperature, Protruding Head, (1972)

Sleeve, Light Weight, Millable Head Self Locking) (1972

Sleeve, Light Weight, Protruding Head, Self Locking) (1

Sleeve, Self Locking) (1972)

Sleeves (1972)

Slices with a Collinear 4 Probe Array (1973)

(Slick) Meth. of Flour Analysis (Cereal Chemistry) (1962

Slide and Slidefilm Projection (1972)

Slide Base Lamp (1973)

Slide Projectors (1973)

Slidefilm Projection (1972)

Slides and Opaques for Television (1973)

Slides for Television (1970)

Slides in 2 X 2 ln. Mounts $(1972)$

Sliding and Folding Door Hardware (1971)

Sliding Door Equipment; Installation Aids (1969)

Sliding Doors (1971)

Sliding Glass Doors (1971)

Sliding Glass Doors (1972) ANSl Al34.2

Sliding Hardware for Std., Horizontally Mounted Tin Cla

Sliding or Horizontally Operating Storm Windows for Ext

Slight Modification, to Corn, Rye, Barley, Rice, Grain

Slings: Base Mounted Drum Hoists (1971)

Slings: Portal, Tower and Pillar Cranes (1973)

Slip Resistant (Floor Polish and Wax) (1971) ASTM D2047

Slipcover Fabrics (Institutional Textile) (1973)

Slope Efficiency and Lasing Threshold of Ruby Laser Rod

Sloped Brick Soffits (Tech. Notes) (1970)

Slots for Securement in Shipment of Agricultural Equipm

Slots in Bone Plates (1973)

Slotted and Coiled, Heavy Duty (1973)

Slotted and Recessed Head Machine Screws and Machine Sc

Slotted Headless Setscrews (Screws) (1971)

Slow Curing Type) (1972)

Slow Moving Vehicle ldentification Emblem (1968) ANSI B

Slow Moving Vehicle (SMV) Identification Emblem (1972)

Slow Moving Vehicles (SM V) Identification Emblem (1972

(Slug) Calorimeter (1972)

Slump Concrete (1965)

Slump of an Oil Base Knife Grade Channel Glazing Compou

Slump of Caulking Compounds and Sealants (1973)

Slump of Face Glazing and Bedding Compounds on Metal Sa

Slurry (1972)

Slurry (1973)

(Slurry) Content in Corn Starch (1956)

(Slurry) of Corn Starch Which ls Substantially Insoluble

Slyke Meth. of Analysis of Amino Nitrogen in Wheat Flou

Smallbcre Rifle Range Plans: Safety Rules and Procedure

Smell Values of Transistor Capacitance (1972)

Smoke and Heat Detectors; Electromagnetic Holders; Slid

Smoke Damage of Electronic Computer Systems (1973)

Smoke Detectors for Fire Protective Signalling Systems

Smoke from the Burning or Decomposition of Plastic Mate

Smoke Point of Aviation Turbine Fuels (1972) ANSI Z1l.1

Smoke Roof and Window Vents Designed to Open Automatica
WSFI

WSFI

WSFI

WSFl

ACI

ASTM

$\mathrm{BlA}$

IM Bui ICBO

ASTM

ASTM

ASTM

FMS

ASTM

ICBO

ASTM

FMS

ICBO

TAPPI

NEM

ASTM

ICBO

ASTM

ASAE

NSA

td. for E NEMA

SAE

ANSl

ISA

NSA

NSA

NSA

NSA

NSA

NSA

St ASTM

ASTM

ACCH 14

ANSI

ANSl

ANSI

ANSI

SMPTE

BHMA

/Ers, NBHA

AAMA

AAMA

AAMA

UL

AAMA

$\mathrm{AACCH}$

ANSI

ANSI

CSMA

ANSI

ASTM

BIA

ASAE

ASTM

NSA

ANSI

SAE

ASTM

ASAE

$\mathrm{SAE}$

ASAE

ASTM

$\mathrm{ACl}$

ASTM

ASTM

ASTM

TAPP

ASAE

CR

CR

AACC

NRA

EIA

NBHA

FMS

ICBO

ICBO

ASTM

FMS

4

*8

$* 7$

B4

P 17

$4 \mathrm{~A}$

BCS24-13

B5

C. 768

UBCS26-2

D694

LPD 7-33

$\mathrm{UBC}+3.23$

UM.536

PH 14

C543

UBC $532 \cdot 10$ 
Std. Meth. of Test for Relative Density of Black Uniform Fire Code: pli/ Std. Meth. of Test for Abrasion Resistance of Glossy ct (1972) 973) Std. for Test Meth. for Sedimentation Meth. of Analysis of

Microscopic Meth of Analysis of d Socket for Warning Lamp (Light) and Slow Moving Vehicle 4.85

dustrial Equipment Warning Lamp and Slow Moving Vehicles Test Meth. for

Std Test Meth for Determining Resistance to Safety Std. for Manually Operable General and Special Use Roof Collapse of Buildings and Other Structures Caused by eth. and Requirements for Maintenance of Design Voltage in Rec. Pract. Test Meth. and Requirements for Rec. Pract. Test Meth. and Requirements for Rec. Pract. Test Meth. and Requirements for pract. for the Test Procedure for Exterior Sound Level for Spec, for Powdered Hand Cleaner Spec. for Powdered Hand Cleaner Std. Spec. for Hand Scrubbing Std. Spec, for Hand Scrubbing Std. Spec. for Powdered Toilet Std. Spec, for Liquid Toilet Std. Spec, for Hand Scrubbing Soap (Grit Std. Spec. for Hand Scrubbing Soap (Grit Std. Definitions of Terms Relating to Std. Meth. of Chemical Analysis of Warning Lamp and Slow Mol Std. for Mounting Brackets and
(SMV) Identification Emb/ Std. for Mounting Brackets and lighting Equip/ Std. for Mechanical Interchangeability of g Equipment (1) Std. for Mechanical Interchangeability of lastic Pipe Fittings, Schedule 40 (1973)/

lastic Pipe Fittings, Schedule 80 (1973)/

tings, Schedule 40 (1973)

tings, Schedule 80 (1973) Std. Spec for Std. Spec. for Std. Spec. for ec, for Poly(vinyl Chloride) (PVC) Plastic Line Couplings, Std. for Forged Steel Fittings (Pipe),
Std. Pract. for Std. for Multiple Sid, for Multiple

$\operatorname{sh} 17$

dimensions on the Mating Plates (Patterns) Used in Battery 1 and Electrical Characteristic Defining Dual in Line Type Std. for Analysis of

Test Meth for Evaluating Wetting Agents in Caustic Safety Std. for Ice Cream Freezers and essential Information for Safe Handling and Use of Caustic Guideline Spec. for

ric Acid Meth. of Analysis of Monocalcium Phosphate and/or Meth. of Analysis for Neutralizing Value of on of Particulate and Gaseous Fluorides in the Atmosphere Std. Meth. of Test. Std. Spec. for Std. Spec. for Photographic Grade

972)

ulp and Paper) (1973)

cators (1971)

у) (1962) Herwig's, Pancreatin, Tween 80-60\% Alcohol, Versene, and Std. Meth, of Test for Soundness of Aggregates by Use of Spec, for Photographic Grade Sid. Spec. for Photographic Grade Sid. Spec. for Photographic Grade icators (197I) Std. Analytical Meth. for Determining alline $\left(\mathrm{Na}_{2} \mathrm{~S}_{2} \mathrm{O}_{3} .5 \mathrm{H}_{2}\right)$ Meth. for Preparation and Standardization of Design and Construction of Flat and Sloped Brick

974) ANSI C7.1

974) ANSI C 7.4

Std. Spec. for Lead and Lead Alloy Coated Std. Spec. for Std. Spec. for Tinned Std. Spec. for Silver Coated Std Spec, for Nickel Coated ty of Solid Lead Wires. Terminals, and Other Terminations Buhler Meth of Experimental Milling for Bread and

Batch Meth. of Experimental Milling for Bread and tric Lay Stranded Copper Conductors, Hard, Medium Hard, or ndard for Household Commercial and Portable Exchange Water Std. Meth. of Test for

$s$ for Describing Information Interchange Formats (Computer ommon Words Related to Numerically Controlled Machine Tool 973)

Uniform Building Code Std. for Dwelling Construction Under the Uniform Building Code:
Smoke (Ringelmann Meth.) (1973)

Smoking (1973)

Smooth Coatings of Petroleum Wax or Wax Based Blends Ap

Smooth Wall Coilable Polyethylene Electrical Plastic Du

Smoothness of Paper and Paperboard (Bendisen Tester) (1

Smut in Wheat and Rye (Cereal Chemistry) (1962)

Smut in Wheat or Rye (Cereal Chemistry) (1962)

SMV) Identification Emblem (1972)

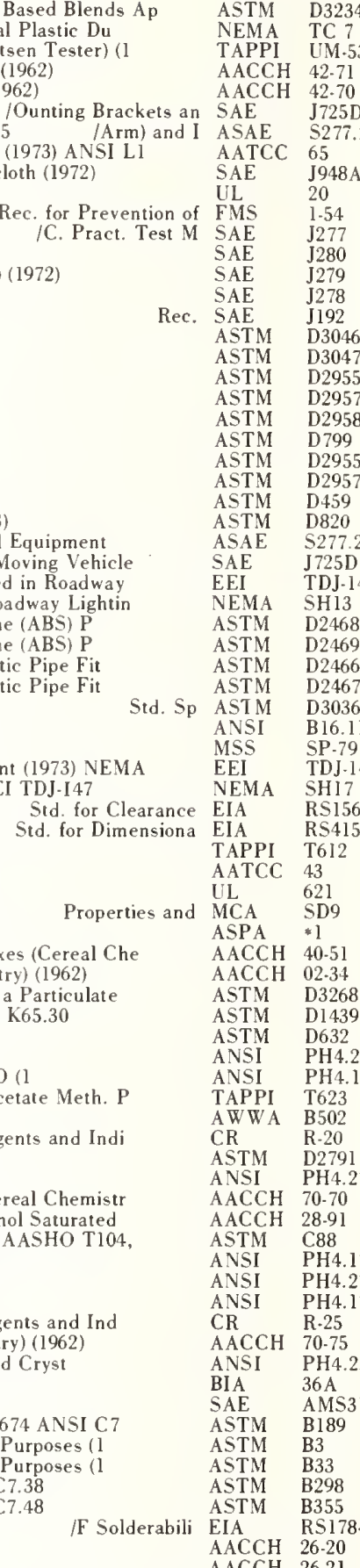

SMV) Identification Emblem (1972) SAE J725

Snag Resistance of Women's Nylon Hosiery (1973) ANSI L1

Snagging and Abrasion of Automotive Bodycloth (1972)

Snap Switches (1972) ANSI C33.40

Snow Loads (1973)

Snowmobile Electrical Systems (1971)

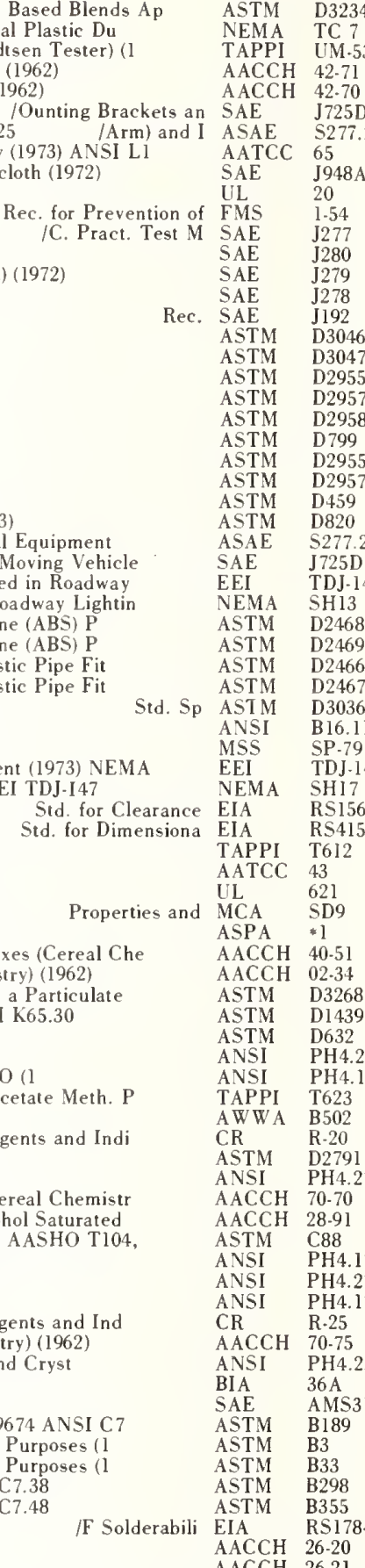

Snow mobile Headlamps (Light) (1972)

Snowmobile Rear Position Tail Lamp (Light) (1972)

Snow mobile Stop Lamp (Light) (1971)

Snow mobiles (1972) ANSI $\$ 6.2$

Soap with Borax) (1972T)

(Soap with Vegetable Scrubber) (1972T)

Soap (Grit Soap) Paste and Bar (1974)

Soap (Grit Soap) Powder (1974)

Soap (1974)

Soap (1974) ANSI K60.14

Soap) Paste and Bar (1974)

Soap) Powder (1974)

Soaps and Other Detergents (1974)

Soaps Containing Synthetic Detergents (1973)

Socket for Agricultural (F arm) and Industrial Equipment

Socket for Warning Lamp (Light) and Slow Moving Vehicle

Socket Supports for Use in Metal Heads Used in Roadway

Socket Supports Used in Metal Heads for Roadway Lightin

Socket Type Acrylonitrile-Butadiene-Styrene (ABS) P

Socket Type Acrylonitrile-Butadiene-Styrene (ABS) P

Socket Type Poly(vinyl Chloride) (PVC) Plastic Pipe Fit

Socket Type Poly(vinyl Chloride) (PVC) Plastic Pipe Fit

Socket Type (1973)

Socket Welding and Threaded (1973)

Socket Welding Reducer Inserts (1974)

Sockets Used in Roadway Lighting Equipment (1973) NEMA

Sockets Used in Roadway Lighting (1973) EEI TDJ-I47

Sockets (1974) ANSI C83.53

Sockets (1974) ANSI C83.95

Soda Ash (1972)

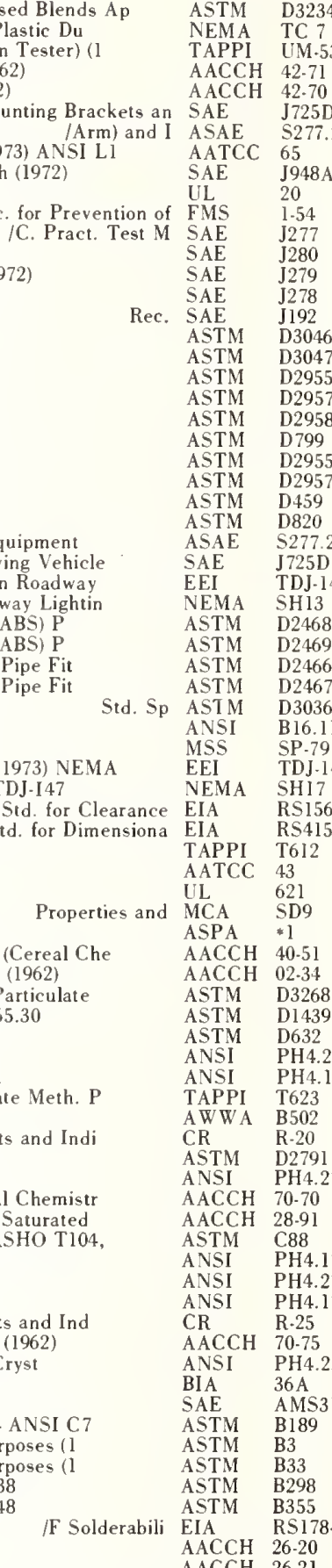

Soda for Mercerization (1971) ANSI L14.79

Soda Fountain Units (1973)

Soda (1974)

Sodding and Soil Preparation (Grass) (1972)

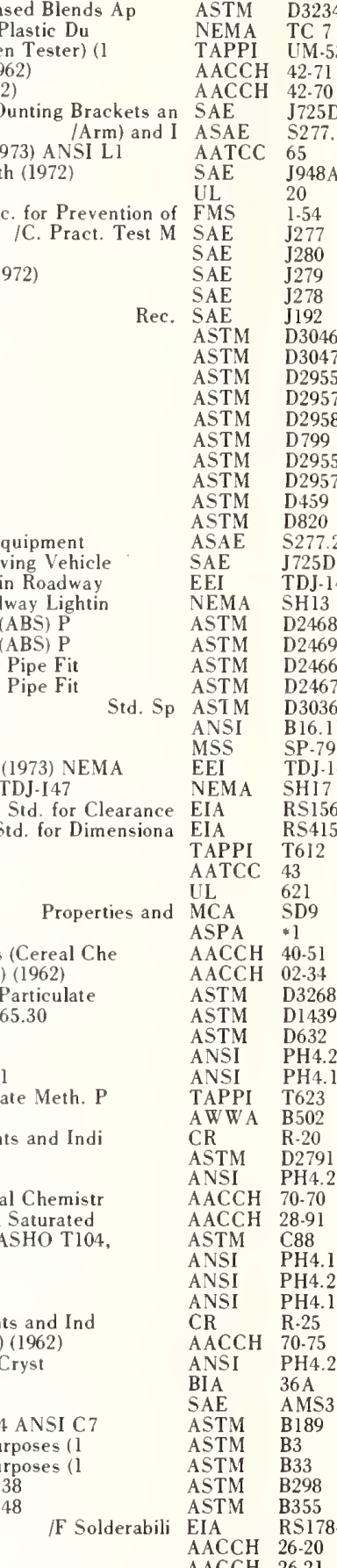

Sodium Acid Pyrophosphate in Prepared Mixes (Cereal Che

Sodium Acid Pyrophosphate (Cereal Chemistry) (1962)

Sodium Bicarbonate Coated Glass Tube and a Particulate

Sodium Carboxymethylcellulose (1972) ANSI K65.30

Sodium Chloride (1972) ANSI A37.56

Sodium Chloride, Nacl (1972)

Sodium Citrate, Dihydrate $\mathrm{Na}_{4} \mathrm{C}_{6} \mathrm{H}_{5} \mathrm{O}_{7}: 2 \mathrm{H}_{7} \mathrm{O}$ (1

Sodium Determination by the Uranyl Zinc Acetate Meth. P

Sodium Hexametaphosphate (1967)

Sodium Hydroxide Solutions Content of Reagents and Indi

Sodium in Water by Ion Electrode (1973)

Sodium Meta-Bisulfite $\mathrm{Na}, \mathrm{S} .0$ - (1972)

Sodium or Potassium Hydroxide Solution (Cereal Chemistr

Sodium Phosphate Solutions; Isopropyl Alcohol Saturated

Sodium Sulfate or Magnesium Sulfate (1973) AASHO T104,

Sodium Sulfate, Anhydrous $\mathrm{Na}_{2} \mathrm{SO}_{4}$ (1973)

Sodium Sulfite, Anhydrous, $\mathrm{Na}_{2,} \mathrm{SO}_{3}$ (1972)

Sodium Thiocyanate NaSCN (1972)

Sodium Thiosulfate Solution Content of Reagents and Ind

Sodium Thiosulfate Solution (Cereal Chemistry) (1962)

Sodium Thiosulfate, Anhydrous $\left(\mathrm{Na}_{2} \mathrm{~S}_{2}, \mathrm{O}_{3}\right)$ and Cryst

Soffits (Tech. Notes) (1970)

Soft Chloroprene-Rubber Sponge (1973)

Soft Copper Wire for Electrical Purposes (19674 A VSI C7

Soft or Annealed Copper Wire for Electrical Purposes (1

Soft or Annealed Copper Wire for Electrical Purposes (1

Soft or Annealed Copper Wire (1974) ANSI C7.38

Soft or Annealed Copper Wire (1974) AVSI C7.48

Soft Solder Jointed) (1973) ANSI C83.93

Soft Wheats (Cereal Chemistry) (1962)

Soft Wheats (Cereal Chemistry) (1962)

Soft (1972) ANSI C7.8

Softeners (1972)

Softening Point of Glass (1973)

Software Docume

Software (1972)

Software (1972)
Softwood Plywood Lumber, Construction and Industrial (1

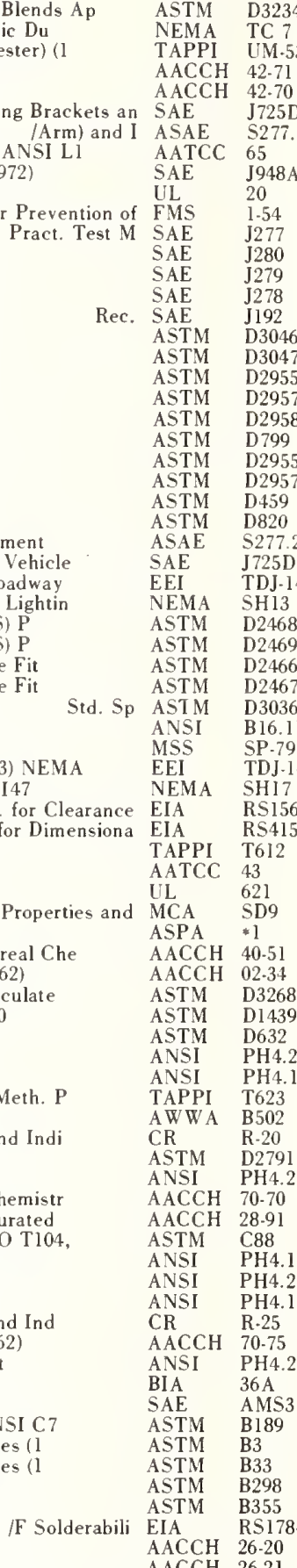

AACCH $26-20$

$\mathrm{AACCH} \quad 26.2$

Std. Spec. for Concen ASTM

Industry Sta WCF

B8

$\begin{array}{ll}\text { ACF } & \text { \$- } 100 \\ \text { ASTM } & \text { C } 338\end{array}$

ANSI X10.1

Softwood Plywood Paneling (1973) 
Std. Meth. of Test for Lime Content of Lncured f Surface Disfigurement of Paint Films by Fungal Growth or Std. Metb. of Test. Wood Preservatives by Laboratory

Properties of Oil Soluble Wood Preservatives by Lsing W/

Disbonding Characteristics of Pipeline Coatings by Direct Std. Metb. of Tetting and Drying Tests of Compacted Std. Meth. of Test for Cement Content of

Test Metb. for Oily Stain Release Meth. of Textile Fabric footings (1972) Std. Meth. of Test for Bearing Capacity of b. of Test Fol Std. Meth. of Test for Bearing Capacity of Std. Spec. for Cast Iron

for Neoprene Rubber Gaskets for Hub and Spigot Cast Iron Std. Spec. for Cast Iron Guide Spec. for Cast Iron

t)

Guideline Spec. for Sodding and Metb. of Test for Measuring Std. Meth. of Test for

Rec. for Terminology and Definitions for Rec. for Terminology and Definitions for Soil Tillage and Lniform Building Code Std. for Expansion Index Test for Test Meth. for Visual Rating Meth. of Carpet (Rug) Test Meth. for Carpet (Rug Test Meth. for Carpet (Rug) tion Purposes (19/ Std. Rec. Pract. for Classification of td. Metb. of Test for Capillary Moisture Relationships for Uniform Building Code Std. for

Std. Meth. of Direct Shear Test of Std. Meth. of Test for Specific Gravity of Uniform Building Code Std. for in Place Density of iform Building Code Std. for Moisture Density Relations of

A.NSI C37.24

$t(19 \div 3)$

er Tube (1973)

3)

Solid Lead Wires. Terminals, and Other Terminations (Soft b. Test for Electrodeposited Coatings of Tin-Lead Alloy Terminations (Soft SoIde) Test Std. for Determination of Rec. Pract. for Determining Std. for Liquid Rosin Fluxes L'sed for Std. for Safety for LP Gas Torches for Heating, user Certification of (1962) A/ Std. for Tools, Crimping. ) Cold Rolled Strip for Magnetic Application in Relays and

b. of Test for Abrasion Resistance of Rubber Compounds for

9:3) Std. Test Meth. for Ply Separation (M'et) of Quality Stds. for Flush Std. for

electric Constant (Permittivity) and Dissipation Factor of a Technical Guide for Landfill Disposal of Std. Tooling NomencIature for pacities witb Effect of Sbield Losses for Single Conductor sid for Solid and Semi st Metb. for Complex Permittivity (Dielectric Constant) of ial Discbarg/ Std. Meth. of Test for Foltage Endurance of Cbaracteristics and Dielectric Constant (Permittivity) of

mal Evaluation and Establishment of Temperature Indexes of metb. of Test for Tbermal Failure Under Electric Stress of Sid. Meth. of Test for Thickness of

Test for High Voltage. Low Current. Dry Arc Resistance of 1s. Sbortenings, and Otber Fats (1/ Dilatometric Meth. of Meth. for Sampling of meth. for Inefficient of Static Friction of Corrugated and

Test for Coefficient of Static Friction of Corrugated and Test for Coefficient of Static Friction of Corrugated and Std. Meth. of Test for Measuring the $\mathbb{W}^{\circ}$ ear Life and Bonded or Coefficient of Friction and Wear Characteristics of Dry

973) ft Soldel

81.1 Std. Metb. of Test for Gross Calorific Value of Test Std. for Determination of Solderability of Std. Spec. for Lniform Building Code Std. for of Test for Reaction Threshold Temperature of Liquid and osion of Solids by Cavitation and Impingement by Liquid or .72 Std. Meth. of Test for Specific Gravity of Density Content in Feedstuffs (Corn) and Other Wet Milled Std. Metb. for Sampling and Sample Preparation of

972) aracterization of Large SignaI D/ 1973)

Trial Use Std. Guide on Std. Proposal for Sid. for Rod-Control. for Chemical Analysis of Synthetic Elastomers for L'se on Tent. Meth. of Tension Test. of Sid. Spec. for Homogeneous nd Reclamation, Curtain and B/ t Steel and Monel Rivet (1974) Sid. for
Soil-Lime Mixtures (1973) AVSI Al.38

Soil and Dirt Accumulation (1973)

Soil Block Cultures (1970)

Soil Block Test:

Soil Burial (1972)

Soil Cement Mixtures (1971) ANSI A37.51

Soil Cement Mixtures (1974) ANSI A37.58, Ashto T14

Soil During Home Laundering (1970) ANSI Ll4.239

Soil for Static Load on Spread Footings (1972)

Soil for Static Load on Spread Footings (I972) Std. Met

Soil Pipe and Fittings (1972)

Soil Pipe and Fittings (I972)

Soil Pipe and Fittings (1972) ANSI A112.5.]

Soil Pipe (1972)

Soil Preparation (Grass) (1972)

Soil Removal and Reflectance Retention of Fabrics (19:2

Soil Resistance of Floor Polishes (I973)

Soil Tillage and SoiI TooI Relationships (1972)

Soil Tool Relationships (1972)

Soil (1973)

Soiling (1973) ANSI L14.215

Soiling; Accerated Meth. (1973) ANSI Ll4.217

Solling; Service Meth. (1973) ANSI L14.216

Soils and Their Aggregate Mixtures for Highway Construc

Soils by Pressure .Membrane Apparatus (1972)

Soils Classification for Engineering Purposes (1973)

Soils Under Consolidated Drained Conditions (1972)

Soils (1958 A.ASHO T100. ANSI A37.I 46

Soils (1973)

Soils (1973)

Soils (1974)

Solar Radiation on Outdoor Metal Clad Switchgear (19i1)

Solar Simulation for Thermal Balance Test. of Spacecraf

Solder-Joint Pressure Fitting for Use with Copper W'at

SoIder Joint Drainage Fittings (1973)

Solder Joint Fittings for Solvent Draiıage Systems (197

SoIder Joint Pressure Fittings (1972)
Solder Jointed) (1973) ANSI C83.93

SoIder Plate) (1973)

Solderability of Solid Lead Wires. Terminals, and Other

Solderability of Thick Film Conductors (1972T)

Soldering (1973) ANSI C83.91

Soldering, and Brazing Purposes (1973) ANSI Z250.1

Solderless Wiring Devices-Recommended Procedures for

Solenoids (1972)

Soles and HeeIs (I968) ANSI J2.26

Solid and Corrugated Fiberboard (1969)

Solid and Hollow Core Doors (Architectural Woodwork) (1

Solid and Semi Solid Dielectric Transmission Lines (197

Solid Ceramic Dielectrics at Frequencies to $10 \mathrm{MHz}$ and

Solid Chemical Waste (1974)

Solid Die Two Blow Cold Headers (19;2)

Solid Dielectric Power Cable $15 \mathrm{kV}$ Through $35 \mathrm{kV}$ (Coppe

Solid Dielectric Transmission Lines (1972)

Solid Electrical Insulating Materials at Microwave Freq

Solid Electrical Insulating Materials Subjected to Part

Solid Electrical Insulating Materials (1970)

Solid Electrical Insulating Materials (1972)

Solid ElectricaI Insulating Materials (1973)

Solid Electrical Insulation (1973)

Solid Electrical Insulation (1973)

Solid Fat Index (50 or Less at $10 \mathrm{Deg}$ ) of Margarine $\mathrm{O}$

Solid Feeds and Feedstuffs (Cereal Chemistry) (1962)

Solid Fiberboard (Horizontal Plane Meth.) (19-2)

Solid Fiberboard (Horizontal Plane Meth.) (1973

Solid Fiberboard (Inclined Plane Meth.) (1973)

Solid Film Lubricants in Oscillating Motion (1971) ANSI

Solid Film Lubricants in Vacuum and Other Controlled at

Solid Fuel by the Isothermal Jacket Bomb Calorimeter (1

Solid Lead Wires. Terminals, and Other Terminations (So

Solid Load Bearing Concrete Masonry Linits (19:1) ANSI a

Solid Load Bearing Concrete Masonry Lnits (1973)

Solid Materials (1972) ANsl Z11.318

Solid Particles (1973)

Itions of Terms Relating

Solid Pitch Asphalt Displacement Meth.) (1972) ANSI A37

Solid Products (1963)

Solid Raw Rubbers (1973)

Ical Meth. for Determining Bulk

Solid Rivets (7/16 In. NominaI Diameter and Smaller) (1

Solid State Devices: Varactor Measurements Part II-Ch

Solid State Load Switches for Traffic Control Systems (

Solid Steel (1973)

Solid Styrene Butadiene CopoIymers (SBR) (1972)

Solid Urethane and Other Rubbers (1973)

Solid W'all and Perforated Pipe for General Drainage (La

Solid 100 Deg. Flush Shear Head A286 Corrosion Resistan

Solid, Grouted, Reinforced Grouted, Hollow Unit, Cavity

Pread

ASTM

ASTM

ASTM

NWMA

ASTM

ASTM

ASTM

AATCC

AST I

CISP]

Std. Spec CISPl

A.STM

CSI

ASPA

ASTMI

ASTM

ASAE

ASAE

ICBO

A.ATCC 121

AATCC 123

A.ATCC 122

ASTM D3282

ASTM D3152

ICBO [BCS29.]

ASTM D3080

ASTM D854

ICBO LBCS70.2

[n ICBO

ASTM

IEEE

ASTM

ANSI

Aกs

ANS

ANSI

EIA

std. Met ASTM

EIA

AST.M

EIA

EIA

ron (Emi NARM

told. Met ASTM

ASTM

$A \pi 1$

EIA

ASTM

MCA

IFI

IPCE

EI.A

ASTM

ASTM

Ent Loss ASTM

Ther IEEE

ASTM

[̈BC 570.$]$

D1587

I 44

E491

B16.22

B 16.29

B16.32

B16.18

RS178-B

B579

RSI78-B

F 357

RS 402

147

RS270

121

D1630

D 1028

$* 1-1300$

RS199.A

D2149

SW. 1

118

P-53-426

RS199A

D2520

D2275

DI50

98

D3151

D374

D495

A.ACCH $31-50$

$\mathrm{AACCH} \quad 64-50$

TAPPI T8165

ITh. of ASTM

ASTM

ASTM

ASTM

EIA

ASTM

ICBO

D 3248

D2981

D2716 
Rec, for Indoor General Storage Rec. for of Congeal Point in Fats and Fat Mixtures Which Congeal or Particl Std. Definitions of Terms Relating to Erosion of Std. Meth. of Test for

y) (1962) Spindle Meth. of Analysis of Meth. of Analysis of Ege Refractometer Meth of Analysis o valuation Meth. of Ability of Roller Dried Nonfat Dry Milk Digitonin Cholesterol Meth. of Analysis of Dried Egg of Test for Applied Weight Per Unit Area of Dried Adhesive Std. Meth of Test for Specific Heat of Liquids and azard Properties of Flammable Liquids, Gases, and Volatile mulsion/ Std. Meth. of Test for Nonvolatile Matter (Tota ersible Nitrogen in Ground Soybeans, Whole or G/ Nitrogen Asphalt, etc.) in Organic Solven/ Std. Meth. of Test for ents (1973) Std. Meth. of Test for Std. Meth. of Test for Std. Spec. for

tinuous Separation and Collection of Particulate and Water their Admixtures (1974) Std. Meth. of Test for Water

of Corn Syrup, Crude and Refined Sugar and Other Materials of Top Flaps of Fiberboa/

Std. Spec. for Water or Solven eth. for Colorimeter or Spectrophotometer Analysis of Acid b8 Std. Meth. of Test for Water Meth. of Analysis of

istance of the Leachate of Std. Meth. of Test for Water Std. Meths. for Test. the Preservative Properties of Oil

soluble Wood Preservatives by ['sing Wood Properties of Oil Std. Analytical Meth. for Determining Std. Analytical Meth. for Determining Std. Analytical Meth. for Determining

for Determination of Dichloromethane and Alcohol-Benzene Std. Spec. for Photographic Grade Aluminum Chloride loy Forgings, $5.6 \mathrm{Zn}-2.5 \mathrm{Mg}-1.6 \mathrm{Cu}-0.26 \mathrm{Cr}(7075-\mathrm{T} 736)$ $-26 \mathrm{Ni}-1.3 \mathrm{Mo}-2.1 \mathrm{Ti}-0.30 \mathrm{~V}) 1800 \mathrm{Deg} . \mathrm{F}(982.2 \mathrm{Deg} . \mathrm{C}$ ium Alloy Sand Castings $(5.75 \mathrm{Zn}-2.5 \mathrm{Re}-0.70 \mathrm{Zr}$ (Ze63-T6) Adhesion and Flexibility by the Wedge Bend (Applicable to std. Analytical Meth. for Determining lodine

Std. Analytical Meth. for Determining Sodium Thiosulfate std. Analytical Meth. For Determining Std. Arsenious Oxide d. Analytical Meth. for Determining Potassium Permanganate et (Coil Coating) (1974) Spec. for Spot Test

Vacuum Induction Plus Vacuum Consumable Electrode Melted d Moderate Heat Resistant, 15.5Cr-4.5Ni-2.9Mo-0.10N,

de or Vacuum Induction Melted, 1975 Deg. F (1079.4 Deg. C) sistant, Nickel Base, $20 \mathrm{Cr}-20 \mathrm{Co}-5.9 \mathrm{Mo}-2.2 \mathrm{Ti}-0.45 \mathrm{Al}$ $-13.3 \mathrm{Cr}-38 \mathrm{Ni}-5.5 \mathrm{Mo}-0.85 \mathrm{Cb}-2.5 \mathrm{Ti}-1.6 \mathrm{Al}-0.09 \mathrm{~B}$, and Heat Resistant, Nickel Base- $5.0 \mathrm{Cr}-24.5 \mathrm{Mo}-5.5 \mathrm{Fe}$,

d Moderate Heat Resistant, 15.5 $\mathrm{Cr}-4.5 \mathrm{Ni}-2.9 \mathrm{Mo}-0.10 \mathrm{~N}$.

d Moderate Heat Resistant, $15.5 \mathrm{Cr}-4.5 \mathrm{Ni}-2.9 \mathrm{Mo}-0.10 \mathrm{~N}$

at Resistant Alloy Bars. Forgings, and Rings (Nickel Base.

Threaded After Heat Treatment, 1800 Deg. F (982.2 Deg. C) cking Susceptibil Std. Rec. Pract for Use of Mattsson Std. Meth. for Evaluation of and Test Formulas for Std. Meth. of Analysis of Sulfochromate Etch ANSI Z197. paration and Standardization of Barium Lactate th. For Preparationand Stand Standardization of Lactic Acid Meth. for Preparation of Ammonium Molybdate Meth. for Preparation of Potassium Thiocyanate Meth. for Preparation of Silver Nitrate meth. for Preparation and Standardization of Sulfuric Acid for Preparation and Standardization of Hydrochloric Acid for Preparation and Standardization of Sodium Thiosulfate Preparation and Standardization of Potassium Permanganate ation and Standardization of Sodium or Potassium Hydroxide

Spec. for Photographic Grade Ammonium Thiosulfate Meth. for Preparation of Potassium Dichromate Std Snec for Creosote Petroleum ight Loss of Sheet Steel During Immersion in Sulfuric Acid s Corrosion Cracking Tests in a Boiling Magnesium Chloride Std. Spec. for Creosote Coal Tar

um Induction Melted 1825-1900 Deg. F (996.1-1037.8 Deg. C) de or Vacuum Induction Melted, 1975 Deg. F (1079.4 Deg. C) Std. Meth. of Analysis of CCA Treating Indices of Refraction of Sucrose

Std. for Storage and Handling of Nitrogen and Fertilizer

Std. Analytical Meth. for Determining Sodium Hydroxide Std. Analytical Meth. for Determining Sulfuric Acid ent of Corn Sugar (Crude and Refined) and All Hydrolyzates eth. for Preparation. Standardization, and Storage of Std. purposes (/ Std. Meth. for Sampling and Preparing Aqueous g Hydrocarbon Solvent for Preparing Oil Borne Preservative Std. Meth. of Test for $\mathrm{pH}$ of Aqueous Meth. for Preparation and Standardization of Buffer
Solid, Palletized and Bin Box Storage Up to $30 \mathrm{Ft}$. and

Solid, Palletized, and Rack Storage of Plastics (1974)

Solidify Within Temperature Range of 25 to $45 \mathrm{Deg}$. (Cer Solids and Potential Dust Explosion Conditions (1973)

Solids by Cavitation and lmpingement by Liquid or Solid Solids Content of Aerosol Coatings (1972)

Solids in Juices, Syrups, and Molasses (Cereal Chemistr Solids in Macaroni Products (Cereal Chemistry) (1962)

Solids in Syrups and Molasses (Cereal Chemistry) (1962)

Solids to Change Mixing Characteristics of Flour (Physi Solids (Cereal Chemistry) (1962)

Solids (1969) ANSI Z197.9

Solids (1971) ANS1 Z11.307

Solids (1973)

Solids) in Water Emulsion, Solvent Based, and Polymer

Solubility Index (NSl): Meth. for Determination of Disp

Solubility of Bituminous Road Materials (Tar, Petroleum

Solubility of Pentachlorophenol in Heavy Petroleum Solv

Solubility Range of Resins and Polymers (1972)

Soluble Cellulose Nitrate (1972) ANSl K65.22

Soluble Gaseous Fluorides in the Atmosphere (Filter and

Soluble Halide lon in Halogenated Organic Solvents and

Soluble in Water (1966)

/Extraneous Materials Content

Siquid Adhesives for Automatic Machine Sealing

Soluble Manganese (Cereal Chemistry) (1962)

Soluble Matter of Vegetable Tanned Leather (1970) ALCA

Soluble Mineral Matter (Cereal Chemistry) (1962)

Soluble Salts in Pigments by Measuring the Specific Res

Soluble Wood Preservatives by Using Wood Properties of

Soluble Wood Preservatives by Using Wood Specimens Unif

Solubles Content in Corn Starch (1956)

Solubles Content in Dextrin (1970)

Solubles Content in Feedstuffs (Corn) (1954)

Solubles in Wood (1973)

Solution $\mathrm{AlCl}_{3}(1972)$

Solution and Precipitation Heat Treated (1972)

Solution and Precipitation Heat Treated (1973)

Solution and Precipitation Treated (1973)

Solution Coil Coatings and Single Coat Organosols

Solution Content of Reagents and Indicators (1971)

Solution Content of Reagents and Indicators (1971)

Solution Content of Reagents and Indicators (1971)

Solution Content of Reagents and Indicators (1971)

Solution for Detecting Chromate Films on Galvanized She

$1-8.5 \mathrm{Ni}-2.0 \mathrm{Cu}-1.1 \mathrm{Ti}$

Solution Heat Treated (1973)

Solution Heat Treatcd (1973)

Solution Heat Treated (1973)

Solution Heat Treated (1973)

Solution Heat Treated (1973)

Solution Heat Treated (1973)

Solution Heat Treated (1973)

Solution Heat Treated, Sub Zero Cooled. Equilized, and
Solution Heat Treated, $15.5 \mathrm{Cr}-16 \mathrm{Mo}-3.8 \mathrm{~W}-5.5 \mathrm{Fe}$ ) (1 and Strip, Corrosion an

$1.4 \mathrm{Al}$, Consumable Electro

/E Corrosion and Heat Re

/Eat Resistant, Iron Base SA

Gs, and Rings, Corrosion SAE

Teel Plate, Corrosion an SAE

Solution Heat Treatment (1973)

Solution of $\mathrm{pH} 7.2$ to Evaluate the Stress Corrosion Cra

Solution Poly-Butadiene Rubbers (BR) (1973)

Solution U'sed in Surface Preparation of Aluminum (1972)

Solution (Cereal Chemistry) (1962)

Solution (Cereal Chemistry) (1962

Solution (Cereal Chemistry) (1962)

Solution (Cereal Chemistry) (1962)

Solution (Cereal Chemistry) (1962)

Solution (Cereal Chemistry) (1962)

Solution (Cereal Chemistry) (1962)

Solution (Cereal Chemistry) (1962)

Solution (Cereal Chemistry) (1962)

Solution (Cereal Chemistry) (1962)

Solution $\left(\mathrm{NH}_{1}\right)_{2} \mathrm{~S}_{3} \mathrm{O}_{4}(1973)$

Solution (1962)

Solution (1970)

Solution (1971) ANSI 7167.25

Solution (1973)

Solution (1974) ANSI 011.2

Solution, Stabilization, and Precipitation Heat Treated

Solution, Stabilization, and Precipitation Heat Treated

Solutions and CCA Treated Wood by Colorimetry (1972)

Solutions at $20 \mathrm{Deg}$. C (Cereal Chemistry) (1962)

Solutions Containing More Than 2\% Free Ammonia (1970)

Solutions Content of Reagents and Indicators (1971)

Solutions Content of Reagents and Indicators (1971)

Solutions Derived from Corn or Grain Sorghum Starch Whi

Solutions for Chemical Analysis (1972) ANSI Z159.1

Solutions of Engine Antifreezes or Antirusts for Test.

Solutions Such as Pentachlorophenol and Copper Naphthen

Solutions with the Glass Electrode (1974) Ashto T200

Solutions (Cereal Chemistry) (1962)
FMS

$8-25 \mathrm{~N}$

FMS

$\mathrm{AACCH} \quad 58.12 \mathrm{~A}$

ASTM G40

ASTM D3062

AACCH $\quad 68-62$

AACCH 20.10

AACCH $68-60$

$\mathrm{AACCH} \quad 54-20$

AACCH 20-01

ASTM D898

ASTM D2766

FMS 7.19

ASTM D2834

AACCH 46.23

ASTM D2042

ASTM D2606

ASTM D3132

ASTM D301

D3267

ASTM D2988

CR E-27

ASTM D1874

$\mathrm{AACCH} \quad 40.45$

ASTM D2876

AACCH $40-01$

ASTM 0248

IVMA M.1.70

NWMA N1. 1.70

CR B-56

CR D.56

G.26

TAPPI T5

ANS

Al SAE

$/ 15 \mathrm{Cr} \mathrm{SAE}$

SAE

$\mathrm{NCC}$

$\mathrm{CR}$
$\mathrm{CR}$

CR

NCCA

$\mathrm{SAE}$

$S A E$

SAE

SAE

ASTM

ASTM

ASTM

AACCH

AACCH $70-30$

$\mathrm{AACCH} \quad 70-35$

$\mathrm{AACCH} \quad 70.55$

A ACCH $70-60$

$\mathrm{AACCH} \quad 70.80$

Meth AACCH 70.20

Meth. AACCH 70.75

Meth. for AACCH 70.50

AACCH 70-70

ANSI $\mathrm{PH} 4.252$

$\mathrm{AACCH} \quad 70.45$

ASTM D 1858

ASTM C694

ASTM G36

ASTM D391

SAE

$S A E$

AMS5707D

$\mathrm{Fl}$

CR

CR
CR

CR

R. 20

R.30

ASTM F.14

ASTM D1176

ASTM D2604

ASTM E70 
Meth. for Preparation of Indicator specific Gravity, Degrees Brix, and Degrees Baume of Sugar rude and Re/ Std. Analytical Meth. for Determining Color, le Petroleum Solvent (LPG) for Preparing Pentachlorophenol meth. for Colorimetric Determination of Uranium in Aqueous od for Test. the Water Repellent Effectiveness Df Treating Radiochemical Determination of Cesium-137 in Nuclear Fuel Radiochemical Analysis of Nuclear Grade Plutonium Nitrate Std. Spec. for Plutonium Nitrate eatin, Tween 80-60\% Alcohol, Versene, and Sodium Phosphate Tent. Rec. Pract. for Direct Injection of

tent. Rec. Pract. for Vacuum Distillation of Solvents from $t$ for Nonvolatile Matter (Total Solids) in Water Emulsion, (ABS) Plastic Pipe and Fittings (1973) An/ Std. Spec. for ipe and Fittings (1973) Ansi/ Std. Rec. Pract. for Making ping (1971)

astic Pipe, Tubing, and Fittings (1973) A/ rene (ABS) Pipe and Fittings to Poly(Viny/ pipe and Fittings (1973) ANSI B72.I6 d Fittings (1972)

Installation Std. for Std. Spec, for Std. Spec, for Std. Spec. for Std. Spec. for Std. Std. for Cast Bronze Solder Joint Fittings for Std. Meth. of Test for Nonvolatile Residue of Halogenated Std. Meth. of Test for Std. Meth. of Test for

plosion and Fire in Design, Construction, and Operation of Std. Spec. for Low Boiling Hydrocarbon such as Pent/Std. Spec. for for High Boiling Hydrocarbon cure of a Backed Paint Film (Coil/ Spec. for Conducting A Std. Spec. for Refined

d. Meth. of Test for Acidity of Benzene, Toluene, Xylenes. Std. Meth. of Test for Bubbling of l Part, Elastomeric

h. Test for UV Cold Box Exposure of One Part, Elastomeric, w Temperature Flexiblity and Tenacity 1 Part, Elastomeric, Design Rules, Guidelines, and Drawings for Copper sealing of Top Flaps of Fiberboa/ Std. Spec. for Water or 3) Tent. Meth. of Test for Corrosivity of ts (Chemical Cleaning of Indus/ Std. Meth. of Analysis of Std. Meth. for Identification of Oils and Oil Acids in Std. Meth. of Test for Separation of Vehicle from

(1968) Tent. Spec. for Volatile Petroleum Meth. of Test for Colorfastness of Upholstery Fabrics to Std. Meth. of Test for Gel Time of eth. of Test for Acidity Alkalinity of Halogenated Organic f Test for Water Soluble Halide Ion in Halogenated Organic Std. Meth. of Test for Ester Value of Lacque Std. for Hydrocarbon Tent. Rec. Pract. for Vacuum Distillation of Std. Meth. of Test for Residual s Road Materials (Tar, Petroleum Asphalt, etc.) in Organic Std. Spec. for Hydrocarbon Dry Cleaning est for Solubility of Pentachlorophenol in Heavy Petroleum Test for Nonamine Acid Acceptance of Halogenated Organic Rec. for Pipe Friction Loss Tables for Test Meth. for Shive Content of Mechanical Pulp ) lour and Semolina (Cereal Chemistry) (1962) Modified

ermining lodine Number (Wijs Meth.) Content in Corn, Grain and All Hydrolyzates Solutions Derived from Corn or Grain efined), Syrups and Other Hydrolyzates from Corn and Grain

th Slight Modification, to Corn, Rye, Barley, Rice, Grain

for Determining Waxy and Nonwaxy Content in Corn and Grain $r$ Determining Barium Yield, Getter Gas Content, and Getter rs (1973)

(Floor, Ceiling, Wall) (1972)

Western Woods Use Book:

evel Meters (1972) ANSI S6.

Rec, for

ns of Light Frame Type for the Purpose of Conserving Their g, Floor, and Fl/ Uniform Building Code Std. for Airborne Uniform Building Code Std. for Impact ansi $\mathbf{S 6 . 3}$ Rec. Pract. for Exterior Std. for Rec. Pract. for the Test Procedure for Exterior Std. Spec. for
alifying a Sound Data Acquisition System for Vehicle Noise
Std. for Test Procedure for Airborne oms (Acoustics) /

Std. for Meth. for the Determination of Std. Meth. for the Measurement of Std. Meth. of Testing for

g Equipment (1972)

ng Less Than $1500 \mathrm{cfm}$ (1971) Std. for

Meth. for Measurement of Weighted Peak Flutter Content of Safety Std. for Std. Meth. of Measuring Recorded Flux of Magnetic Construction) / Std. Classification for Determination of for Laboratory Determination and Measurement of Airborne Uniform Building Code:
Solutions (Cereal Chemistry) (1962)

Solutions (Cereal Chemistry) (1962)

Solutions (Spectroph

Solutions (1968)

Solutions (1969) ANSI N1 16

Solutions (1970)

Solutions (1970) ANSI N117

Solutions (1973)

Solutions (1973) ANSI N137

Solutions; Isopropyl Alcohol Saturated with Gasoline; M

Solvent Base Paints into a Gas Chromatograph (1973)

Solvent Base Paints (1973)

Solvent Based, and Polymer Emulsion Floor Polishes (197

Solvent Cement for Acrylonitrile-Butadiene-Styrene

Solvent Cemented Joints with Polyvinyl Chloride (PVC) P

Solvent Cemented PVC Pipe for Water Service and Yard Pi

Solvent Cements for Cellulose Acetate Butyrate (CAB) Pl

Solvent Cements for Joining Acrylonitrile Butadiene Sty

Solvent Cements for Poly(vinyl Chloride) (PVC) Plastic

Solvent Cements for Styrene Rubber (SR) Plastic Pipe an

Solvent Drainage Systems (1973)

Solvent Extract from Aerospace Components (Using Rotary

Solvent Extractable Material in Black Pigments (1972)

Solvent Extractables in Petroleum Waxes (1973)

Solvent Extraction Plants (1973)

Solvent for Oil Borne Preservatives (1973)

/E-Property from Ex

Solvent for Preparing Oil Borne Preservative Solutions

Solvent Hand Rub Test for the Determination of Deg. of

Solvent Naphtha (1971) ANSl Z78.4

Solvent Naphthas, and Similar Industrial Aromatic Hydro

Solvent Release Type Sealants (1972)

Solvent Release Type Sealants (1972)

Solvent Release Type Sealants (1972)

Solvent Single Stack Plumbing System (1973)

Solvent Soluble Liquid Adhesives for Automatic Machine

Solvent Systems for Removing Water Formed Deposits (197

Solvent Systems Used for Removal of Water Formed Deposi

Solvent Tolerance of Amine Resins (1973)

Solvent Type Paints (1972)

Solvent Type Paints (1973)

Solvent (LPG) for Preparing Pentachlorophenol Solutions

Solvent (Textile) (1973)

Solventless Varnishes (1972)

Solvents and Their Admixtures (1974)

Solvents and Their Admixtures (1974)

Solvents and Thinners (1972)

Solvents for Oil Borne Wood Preservatives (1973)

Solvents from Solvent Base Paints (1973)

Solvents in Flexible Barrier Materials (1972)

Solvents (1966) ANSI A37.12

Solvents (1971) ANSI Z11.42

Solvents (1973)

/R Solubility of Bituminou

Solvents, Degreasing Grade (Nonreflux Meth.) (1974)

Solving Fire Protection System Hydraulic Problems (1973

(Somerville Fractionator) (1973)

Sorensen Meth. of Analysis of Amino Nitrogen in Wheat F

Sorghum and Other Vegetable Fats and Oils (1966)

Sorghum Starch Which Are Substantially Optically Clear

Sorghum Starch (1960)

Tent of Corn Sugar (Crud

Sorghum, and Buckwheat (Cereal Chemistry) (1962) /O, W

Sorghum, and Their Mixtures (1959)

Sorption Capacity for Barium Flash Getters (1972) ANSI

Sound Control (Acoustics) in Buildings, Walls, and Floo

Sound Control (Acoustics) in Wood Building Construction

Sound Data Acquisition System for Vehicle Noise Sound L

Sound Insulation Efficiency (1973)
Sound Insulation Field Test of Partitions (Wall, Ceilin

Sound Insulation (1973)

Sound Level for Heavy Trucks and Buses (1971)

Sound Level for Passenger Cars and Light Trucks (1972)

Sound Level for Snowmobiles (1972) ANSl S6.2

Sound Level Meters (1971)

Sound Level Meters (1972) ANSI S6.1

/Ec. Pract, for Qu

Measurements on Rotating Electric Machinery (1973

Sound Power Levels of Small Sources in Reverberation Ro

Sound Pressure Levels in Air (Acoustics) (1971)

Sound Rating Heating, Refrigerating and Air Conditionin

Sound Rating of Room Fan Coil Air Conditioners Deliveri

Sound Record on Super 8 Motion Picture Prints (1972)

Sound Recording and Reproducing Equipment (1971)

Sound Recording and Reproducing Equipment (1973)

Sound Records at Medium Wavelengths (1972) ANSI S4.6

Sound Transmission Class (Building Acoustics Design and

Sound Transmission Class (Stc) of Building Partitions S

Sound Transmission Control (Acoustics) (1973)

Sound Transmission Loss of Building Partitions (1970)

Std. Met

AACCH $70 \cdot 25$

AACCH 80.75

F-14

D2605

Std. ASTM E318

MWMA

ASTM

$A A C C H$

ASTM

ASTM

ASTM

ASTM

ASTM

IAPMO

ASTM

ASTM

ASTM

ASTM

ANSI

ASTM

ASTM

ASTM

FMS

ASTM

ASTM

NCCA

ASTM

ASTM

ASTM

ASTM

ASTM

CDA

ASTM

ASTM

ASTM

ASTM

ASTM

ASTM

ASTM

ANSI

ASTM

Std. M ASTM

ASTM

ASTM

AWPA

ASTM

ASTM

ASTM

ASTM

M. 2.70

E320

C759

C710

D327

D3272

D2834

D2235

D2855

IS8

D2560

D3138

D3122

B16.32

F33I

D305

D3235

$7.30 \mathrm{~N}$

D3225

TB.11.18

D838

D847

C712

C718

C71!

*1

D1874

D3263

D2790

D1 198

D2245

D2372

D2605

L24T 7

D3056

D2989

2988

D16]

D3272

F151

D2042

D484

D2606

D2942

$\begin{array}{ll}\text { FMS } & 2.89\end{array}$

TAPPI UM-242

$\mathrm{AACCH} \quad 46.31$

/ Det C

$\mathrm{H}-32$

CR F.14

W ACCH $\mathrm{F}-26$

$\begin{array}{ll}C R & \text { A.28 }\end{array}$ 
for Meth. of Measuring, Recording and Specifying Machinery one and Spiral Bevel Gear Drives (1973)

edure for Bench Calibration of Tank Level Gaging Tapes and nesium Sulfate (1973) AASHO T104./Std. Meth. of Test for fool Meth. of Analysis of Thermophilic Spore Counts (Flat I Quality Control Monitoring of the Product (Receiving and meth. for the Determination of Sound Power Levels of Small d. for Integrity and Test Spec. for Selected Brachytherapy Rec. for Protection Against Radiation from Brachytherapy Std. for Leak Test. Radioactive Brachytherapy Spec. for Gamma-Ray Brachytherapy r

I Water Cooling Towers (1971)

Uniform Building Code Std. for

1 Glued Laminated Members and Laminations Before Gluing of

and Peanuts (Cereal/

Meth. of Analysis of Crude Fiber in Meth of Analysis of Meth. of Analysis for Ash in meth. of Analysis for Insect Fragments and Rodent Hairs in ide in Dough Conditioners Containing Monoglyceride, Salts,

962) Meth. of Analysis of Crude Fat in Wheat and of Analysis of Residual Urease Activity in Soybean Meals, ground Full Fat or Extracted Flakes, Full Fat and Defatted ground Full Fat or Extracted Flakes, Full Fat and Defatted Meth. of Analysis of Crude Fat in

Meth. of Analysis of Moisture and Volatile Matter in

Meth. of Analysis of Acetone-Insoluble Lecithin in

ed Flakes, Full Fat and Defatted Soy Flours and Grits, and ed Flakes, Full Fat and Defatted Soy Flours and Grits, and Trading Rules for the Purchase and Sale of Meth. of Analysis of Residual Urease Activity in Tent. Meth. for Grading Trading Rules for the Purchase and Sale of d Measuring Procedures for Bulk Shipments and Transfers of TLC Meth. of Analysis of Aflatoxins in Corn and rude Fiber in Soy Flour for Cake and Meal from Cottonseed, ysis of Moisture in Flour, Farina, Semolina, Bread, Grain,

Meth. for Determination of Dispersible Nitrogen in Ground

Meth for Determination of Dispersible Protein in Ground

uch as Walls, Floor-Ceiling Assemblies, Doors, and Other aterials (Coati) Std. Rec. Pract. for Combined, Simulated Safety Std. for Electric Std. Definitions of Terms Relating to meth. of Test for Effectiveness of Aerosol and Pressurized Pract. for Combined, Simulated Space Environment Test. of

Pract. for Solar Simulation for Thermal Balance Test. of Uniform Building Code Std. for

Components, Timber, Preservative Treatment, Poles, Piles, Std. for Nut Std. for Nut Tolerances of Spoke Type Wheels, Demountable Rims and Rim (1972) Sid. for Hole

2) Std. Meth. for Measurement of Lattice Std. Meth. of Test for Std. Meth. of Panel Std. Meth. of Panel
Std. Meth. of Panel al11.14 Al11.16 ape Guide (Rec. for Garden Grades, Application and Design, (Lumber) (1972) Usc Ps20 1973) ions (1973)

Uniform Building Code Std. for Rec. for Wood Beam, Joint and Rafter Viscosity of Motor Oils at Low Temperature Using the Cold

1972)

s (1972) Std. for Numerically Controlled Rec. Pract. for Rec. Pract, for Std. for Engine Test Code Rec. Pract. for

Rec. Pract. for Preignition Rating of Std. Classifications for

es, Minerals, and Rocks by the Fire Assay Preconcentration Ultrasonic Examination of Pla in and Clad Steel Plates for extiles (Du/ Std. Performance Requirements and Claims for nces and Other Electronic Equipment / Std. for Safety for and Plate $(0.80 \mathrm{Cr}-1.8 \mathrm{Ni}-0.35 \mathrm{Mo}-0.20 \mathrm{~V}(0.33-0.38 \mathrm{C}))$ and Rings, $0.78 \mathrm{Cr}-1.8 \mathrm{Ni}-0.35 \mathrm{Mo}-0.20 \mathrm{~V}(0.32-0.38 \mathrm{C})$,

ive Guide for Obtaining Department of Transportation (DOT)

Rec. Pract. for Monitoring r (1970) Basic Criterial for Evaluation of bestos Felt or Gypsum Boal ket, Horseshoe and Ring Buoy) (19) ing Wire and Springs (1972)
Sound Within Equipment Rooms (Noise) (1973)

Sound (Noise Measurement for Enclosed Helical, Herringb Sounding Rules (1974)

Soundness of Aggregates by Use of Sodium Sulfate or Mag

Sour, Total Aerobic, Anaerobic) in Cereal Products and

Source Inspection, Product Audit) (1969)

Sources in Reverberation Rooms (Acoustics) (1972)

Sources (Medical Radiation-Radiology) (1973)

Sources (1972)

Sources (1973)

Sources (1974)

Southern and Lodgepole Pine. Western Larch, California

Southern Building Code for Dwelling House Construction

Southern Pine Lumber Spec. for Application in Industria Southern Pine Lumber (1973)

Southern Pine, Pacific Coast Douglas Fir and Western He Southern Std. Building Code (1973)

Southern Std. Gas Code (1973)

Soy Flour for Cake and Meal from Cottonseed, Soybeans, Soy Flour in Macaroni (Cereal Chemistry) (1962)

Soy Flour (Cereal Chemistry) (1962)

Soy Flour (Cereal Chemistry) (1962)

Soy Flour, and Other Cereal Products (Cereal Chemistry)

Soy Flour, Feeds and Cooked Feeds (Cereal Chemistry) (1 Soy Flour, Mill Feeds (Cereal Chemistry) (1962)

Soy Flours and Grits, and Soybean Meal (Cereal Chemistr

Soy Flours and Grits, and Soybean Meal (Cereal Chemistr

Soy Flours (Cereal Chemistry) (1962)

Soy Flours (Cereal Chemistry) (1962)

Soybean and Corn Oils (Cereal Chemistry) (1967)

Soybean Lecithin Spec. (1973)

Soybean Meal (Cereal Chemistry) (1968)

Soybean Meal (Cereal Chemistry) (1968

Soybean Meal (1973)

Soybean Meals, Soy Flour, Mill Feeds (Cereal Chemistry)

Soybean Oil for Color (1973)

Soybean Oil (1973)

Soybean Oil (1973)

Soybeans (Cereal Chemistry) (1972)

Std. for Inspection, Sampling an

Soybeans, and Peanuts (Cereal Chemistry) (1962)

Soybeans, Rice, Beans, Peas, Lentils, Corn Meal, Corn G

Soybeans, Whole or Ground Full Fat or Extracted Flakes,

Soybeans, Whole or Ground Full Fat or Extracted Flakes,

Space Dividing Elements (1973)

/ Building Parti

Space Environment Test. of Spacecraft Thermal Control M

Space Heating Equipment (1972) ANSI C33.12

Space Simulation (1972)

Space Spray Insecticides Against Flying Insects (1972)

Spacecraft Thermal Control Materials (Coatings) with El

Spacecraft (1973)

Spaced Column Design (1973)

Spaced Columns, Joints, Screws, Bolts, Connectors, Nail

Spacer, Plate-Countersunk (1973)

Spacer, Plate-Plain (1973)

Spacers (Manufactures' Guide) (1963)

Spacing for Scraper and Bulldozer (Dozer) Cutting Edges
Spacing of Nuclear Graphite (1969) ANSI K90.1

Spalling Resistance of Porcelain Enameled Aluminum (197

Spalling Test for Fireclay Plastic Refractories (1972)

Spalling Test for High Duty Fireclay Brick (1970) ANSI

Spalling Test for Super Duty Fireclay Brick (1970) ANSI

Span Allowances, Storage, Finishes and Nails and Fasten

Span Tables and Working Stresses for Joists and Rafters

Span Tables for Floor and Ceiling Joists, and Rafters (

Span, Load, Deflection, Strength and Stiffness Computat

Spanish Moss, Excelsior, etc.) (1973)

Spanking Simulator (1972) ANS1 Z11.280

IW

, Cocoa Fibers

Spar Mill (1973)

Spark Arrester Test Procedure for Medium Size Engines (

Spark Arresters Used on Multiposition Sinall Size Engine

Spark Ignition and Diesel (1971)

Spark Plug, Aircraft Reciprocating Engine (1971)

Spark Plugs (1970)

Spark Resistant Construction (1966)

Spark Technique (1971) ANS1 Z128.25

Special Applications (1971) ANSI G35.25

Special Characteristics and Finishes of Institutional

Special Grade (1973)

Special Grade (1973)

Special Permits for Radioactive Materials Shipments (19

Special Processes or Devices Used in Treating Wastewate

Special Purpose Roofs (Wood Shakes and Shingles with as

Special Purpose Water Safety Buoyant Devices (Vest, Jac

Special Quality High Tensile, Hard Drawn Mechanical Spr

Std. AR

AGMA

575

ANSI 297.01

ASTM C88

AACCH $42-40$

Fo ASQC RP5

St ANSI N44.1

NCRPM R40

ANSI N 44.2

NCRPM R41

ICBO UBCS25-11

SBCC $* 18$

CT1 115

ICBO UBCS25.6

AWPA $\mathrm{C} 28$

$\begin{array}{ll}\mathrm{SBCC} & * 6 \\ \mathrm{SBCC} & * 1\end{array}$

$\mathrm{AACCH} \quad 32-17$

AACCH 06-11

AACCH $08-16$

AACCH $28-70$

AACCH 48-51

AACCH $30-25$

Meth. AACCH $22-90$

AACCH $46-23$

$\mathrm{AACCH} \quad 46.24$

AACCH $30-26$

AACCH 44.31

AACCH $58-35$

NSPA *3

$46-23$

$\begin{array}{ll}\text { AACCH } & 46-23 \\ \text { AACA }\end{array}$

NSPA *1

AACCH $22-90$

NSP $4 * 5$

NSPA $* 2$

$\mathrm{AACCH} \quad 45.05$

I of $\mathrm{C}$ AACCH 32.17

AACCH $44.15 \mathrm{~A}$

$\mathrm{AACCH} 46.23$

$\mathrm{AACCH} \quad 46.24$

ICBO UBCS35. 1

ASTM E512

UL

ASTM

ASTM

ASTM

573

E349

D3088

E512

UBCS25.15

ICBO UBCS 25.1
ICBO UBC $* 3.25$

NSA

NSA

SAE

SAE

ASTM

ASTM

ASTM

ASTM

ASTM

CRA

NFORP

ICBO

WWPA

ICBO

ASTM

NSA

SAE

SAE

$\mathrm{SAE}$

$\mathrm{SAE}$

SAE

AMCA

500

463

J851

J737 B

C558

C703

C180

C 107

C122

$3 \mathrm{~A}$

UBC $525-21$

*30

UFC *2ART?

D2602

912

J350A

J335A

J816B

ARP590B

J549 A

401

E400

A578

L24.7.1

492.7

AMS6433B

AMS6430B

N14.10.2

RP4

ASQC 
Std. Spec. for Steel Bars, Carbon, Hot Rolled, pec. for Steel Bars, Carbon, Hot Rolled and Cold Finished, Std. for Agreement Between Owner and Architect for 972, Accident / Spec.
sis in Cereal Chemistry (1962) Spec. for Informational, Directional, and vehicles, Counter Balanced Fork Lift Truck, Boat, Baggage, Sid. for Safety for

Safety Std. for Manually Operable General and umbing Code: Indirect Waste Piping. Wet Vented Systems and $5.20 \quad$ Std. Spec. for General Requirements for eathed Electrical Resistance Heaters, for Nuclear or Other Std. Requirements and Terminology for (Steel Products Manual) Steel r Classification, Definition, and Meth. of Grading for All Std. Meth. of

ssion (1967)

e) (1973) Std. for Meth. of Measuring, Recording and rs $(1967)$

Std. Meths. for Guide Spec. for Guide Spec. for Guide Spec. for r Extent of Burning of Cellular Plastics Using a Supported Test Meth. for Determining Visual Color Matched to Master of the Reflectance of the Organic Components in a Polished cements and Concretes/ Std. Spec. for Moist Cabinets and Test Meth. for Density or Volume of Small making and Using the C-Ring Stress Corrosion Cracking Test td. Rec. Pract. for Preparation of Bituminous Mixture Beam t) Meth. of Test. Coated Steel 970) ANSI D14.9

Rec. Pract. for Preparation of Bar and Rod si Z197.21 Std. Meth for Corrosion Test on Metal Std. Meth. of Testing Cross Lap Std. Meth. of Making and Curing Concrete Test Std. Meth. of Test for Elastic Moduli of Rock Core Std. Rec. Pract. for Compression Molding Test Kneading Compactor (/ Std. Meth. for Preparation of Test Std. Meth. of Test for Length Change of Drilled or Sawed for Determining Temperatures of Std. ASTM Molds for Test als (1972) ANSI K65/ Rec. Pract. for Injection Molding of Tent. Test Meth. for Critical Dilation of Concrete Meth. of Preperation and Evaluation of Painted or Coated Rec. Pract. for Exposure of Adhesive properties of Oil Soluble Wood Preservatives by Using Wood Std. Meth. of Measuring Area of Leather Test d Preservatives for Marine Services by Means of Small Size Std. Meth. for Measuring Thickness of Leather Test est for Splitting Tensile Strength of Cylindrical Concrete

. Pract. for Making and Using U-Bend Stress Corrosion Test from Atmospheric Corrosion Tests of Metallic Coated Steel . of Test for Compressive Strength of Cylindrical Concrete ct. for Preparing, Cleaning, and Evaluating Corrosion Test Std. Meth. of Test for Yarn Number Based on Short Length for Tensile Strength of Adhesives by Means of Bar and Rod Std. Meth. of Capping Cylindrical Concrete or Preparation and Use of Bent Beam Stress Corrosion Metal accelerated Curing, and Test. of Concrete Compression Test ngth of Hydraulic Cement Mortars (Using 2 In. $(50 \mathrm{Mm})$ Cube Rec. for Mleasurement of Neutron Flux and nce Spectrometers (1972) Std. Meth. of Test for Std. Meth. of Test for Normal and Pulp (Using Spectrophotometer with Reserv/ Pin and Point to Plane Techniques (1973) oint to Plane Technique Using an Optical / using a Vacuum Spectrometer (1971) ANSI Z/ rc Technique (1971) ANSI Z128.32

$\mathrm{n}$ and Aluminum (1971) ANSI Z 128.35

Silver Chloride-Lithium Fluoride Carri/ Meth. for Std. Meth. for Std. Meth. for Std. Meth. for Std. Meth. for Std. Meth. for Std. Meth. for ((U, Pu/ Std. Meth. for Chemical, Mass Spectrometric, and xide $\mathrm{Pol}$ Std. Meth for Chemical, Mass Spectrometric, and um-Cadmium Alloys (1974) Std. Meth. for Chemical and de Powd/Std. Meth. for Chemical, Mass Spectrometric, and steel by the Point to Plane Technique Usi/ steel for Boron by the Point to Plane Arc/

the Powder Direct Current Arc Technique / Std. Meth. for Std. Meth. for Std. Meth. for Gallium Oxide Carrier D-C Arc Technique /

Std. Meth. for yllium / Std. Meth. for Chemical, Mass Spectrometric, and
Special Quality, for Fluid Power Applications (1974)

Special Quality, for Pressure Piping Components and Oth Special Services (1972)

Special Signs (Symbols) Complementary to ANSI Z35.1-1 Special Techniques for Extraneous Matter Meth. of Analy Special Type Trailer, Mobile Home, Garden Tractor, Indu Special Use Electrical Switches (1973) ANSl C33.40.1

Special Use Snap Switches (1972) ANSI C33.40 Special Wastes (1973)

Special) (1971)

Specialized Carbon and Alloy Steel Pipe (1972) ANSI B12

Specialized Service (1971) ANSI N143

Specialty Transformers

Specialty Transformers (1973) ANSI C33.4

Specialty Tubular Products (1972)

Species of Lumber (1973)

/Niform B

Specifying Color by the Munsell System (1968)

Specifying Construction Progress Photographs (1967)

Specifying Duct, Sheet Metal, Low Pressure, Air Transmi

Specifying Gypsum Masonry and Mortar (1967)

Specifying Machinery Sound Within Equipment Rooms (Nois Specifying Metal Louvers, Stationary (1967)

Specifying Portland Cement Terrazzo, Cast in Place Floo

Specifying Project Record Documents (1967)

Specifying Rock Bolts (1966)

Specifying Sprayed Fireproofing (Guide Spec.) (1972)

Specifying Tests, Plastic Concrete (1967)

Specifying the Performance of Shock Machines (1973)

Specifying: Gate Valves (1973)

Specifying: Precast Concrete Panels (1973)

Specifying: Reinforced Fiberglass Polyester Tanks (1973

Specimen by a Horizontal Screen (1974) ANSI K65.114

Specimen for Fabrics, Vinyls, Coated Fiberboards, and $\mathrm{O}$

Specimen of Coal (1972)
Specimen Storage) Rooms Used in the Test. of Hydraulic

(Specimen Storage) Rooms Used in the Test. of Hydr
Specimen (Hg Pycnometer) (Pulp and Paper) (1973)

Specimen (1973)

Std. Rec. Pract.

Specimens by Means of the California Kneading Compactor

Specimens Dynamically for Resistance
Specimens for Adhesion Tests (I969)

Specimens for Engine Antifreezes Tested in Glassware (1

Specimens for Tensile Properties of Adhesives (1972) an

Specimens in the Laboratory (1969) ANSI A37.81

Specimens in Uniaxial Compression (1972)

Specimens of Allyl Molding Materials (1972)

Specimens of Bituminous Mixtures by Means of California

Specimens of Cement Mortar and Concrete (1974T)

Specimens of Plastics (1967) ANSI K65.79

Specimens of Thermoplastic Molding and Extrusion Materi

Specimens Subject to Freezing (1972T)

Specimens Subjected to Corrosive Environments (Coil Coa

Specimens to High Energy Radiation (1970) ANSI Nl41

Specimens Uniformly lmpregnated (1970)

Specimens (1968) ALCA E2

Specimens (1970)

Specimens (1970) ALCA E4

Specimens (1971) ANSI A37.121

Specimens (1972)

Specimens (1972)

Specimens (1972) ANSI G80.

Specimens (1972) ANSI L 14.220

Specimens (1972) ANSI Z197.3

Specimens (1973)

Specimens (1973)

Specimens (1974)

Specimens) (1973) /Th.

Spectra for Physical and Biological Applications (1960)

Spectral Emittance at Elevated Temperatures (1972)

Spectral Reflectance, Transmittance, and Color of Paper

Spectrochemical Analysis of Admiralty Metal by the Cast

Spectrochemical Analysis of Blast Furnace lron by the P

Spectrochemical Analysis of Carbon and Low Alloy Steel

Spectrochemical Analysis of Copper by the Briquet D-C a

Spectrochemical Analysis of High Purity Iron for Silico

Spectrochemical Analysis of Nickel-Base Alloys by the

Spectrochemical Analysis of Nuclear Grade Mixed Oxides

Spectrochemical Analysis of Nuclear Grade Plutonium Dio

Spectrochemical Analysis of Nuclear Grade Silver-lndi

Spectrochemical Analysis of Nuclear Grade Uranium Dioxi

Spectrochemical Analysis of Plain Carbon and Low Alloy

Spectrochemical Analysis of Plain Carbon and Low Alloy

Spectrochemical Analysis of Thermionic Nickel Alloys by

Spectrochemical Analysis of Uranium Oxide $\left(\mathrm{U}_{3} \mathrm{O}_{k}\right)$ by

Spectrochemical Analysis Of, and Physical Tests On, Ber

ASTM

ASTM

AlOA

ANSl Z35.4

AACCH $28-92$

TRA

UL

niform Pl ICBO

NRA

ASTM

ASTM

NEMA

UL

AISl

ICBO

ASTM

$\mathrm{CSI}$

CSI

CSI

A RI

CSI

CSI

CSI

CSI

CSI

CSI

ANSI

CSI

CSl

10 ASTM

SAE

ASTM

ASTM

ASTM

ASTM

ASTM

ASTM

ASTM

ASTM

ASTM

ASTM

ASTM

ASTM

ASTM

ASTM

ASTM

ASTM

NCCA

ASTM

NWMA

ASTM

ASTM

ASTM

5 1054

20

UPC 1.6

$* 10$

A.530

E420

$\mathrm{STl}$
506

UBCS25. 1

D1535

1018

5836

04280

575

15851

09410

01730

02980

09841

3303

52.14

15101

15177.1

D1692

J361 A

D2798

C511

Li J]-18

G38

D3202

D2933

D1384

D1344

C192

D3148

D3027

Dl56l

C 341
D 958

D 1897

C671

TB.111-3

M-1.70

M-1-70
D2347

D2481

D 1813

C496

G30

G33

C39

Gl

D 1059 
Std. Rec. Pract. for Using Controlled Atmospheres in material (1970) ANSI Z/ Std. Rec. Pract. for Bonding Thin Nuclear Grl Std. Meth. for Chemical, Mass Spectrometric, Nuclear Gr/ Std. Meth. for Chemical, Mass Spectrometric, $y$ the Fire Assay Preconcentration Spark T/ Std. Meth. for uoride Carrier D.C Arc Technique Using an Optical Emission 1973)

(1973) Sid. Meth. of Test. for Leaks Using the Mass Std. Meth. of Test for Leaks Using the Mass the Tracer / Std. Meth. of Test. for Leaks Using the Mass Devices with a Helium Mass

by the Point to Plane Technique Using an Optical Emission ical Analysis of Carbon and Low Alloy Steel Using a Vacuum

Point to Plane Technique U'sing an Optical Emission Vacuum spectral Bandwidth and $W$ avelength Accuracy of Fluorescence (1971) ANSI Z1) Std. Meth. for High Ionizing Voltage Mas

Atom Percent Fission in Uranium and Plutonium Fuel (Mass grade Uranium Dioxide Powd/ Std. Meth. for Chemical, Mass grade Plutonium Dioxide Pol Std. Meth. for Chemical, Mass grade Mixed Oxides ( $(\mathrm{U}, \mathrm{Pu} / \quad$ Std. Meth. for Chemical. Mass sical Tests On, Beryllium / cal Analysis of Nuclear Gri cal Analysis of Nuclear Gr/ th. for Chemical, Mass Std. Meth. for Chemical, Mass Std. Meth. for Chemical, Mass Std. Meth. of Test for Gamma Rec Pract. for Alpha

est for Hydrocarbon Types in Low Olefinic Gasoline by Mass Std. Meth. of Test for Coating Thickness by X-Ray d. Meth. of Test for Lead in Gasoline by Atomic Absorption Meth. of Test for Low Levels of Lead in Gasoline by X.Ray matic Types Analysis of Gas Oil Aromatic Fractions by Mass

mosphere (Continuous Measurement by Nondispersive Infrared ereal Chemistry) (1962)

Meth. for Colorimeter or

ectance. Transmittance, and Color of Paper and Pulp (Using n Irradiated Nuclear Fuels (1973)

ni Products (Cereal Chemistry) (1962)

ivity in Active Proteinase Preparations (Cereal Chemistr and Paperboard Containing Fluorescent Bri/ Test Meth. for Std. Analytical Meth. for Determining Color Std. Analytical Meth. for Determining Color

Natural Dr/ Std. Analytical Meth. for Determining Color Std Analytical Meth for Determining Color, Solutions Std. Analytical Meth. for Determining Color, Reflectance Std. Analytical Meth. for Determining Color

st for Vinylidene Unsaturation in Polyethylene by Infrared ination of Cellulose Nitrate in Alkyd Lacquers by Infrared $r$ Selection of Electrode Material Used in Optical Emission std. Definitions of Terms and Symbols Relating to Emission

h. of Test for Aluminium in Iron Ores by Atomic Absorption or Calcium and Magnesium in Iron Ores by Atomic Absorption ANSI P3. 23

Rec. Pract. for Identification of Material by Absorption Meth. for

Std. Meth of Test for

for Identification of Structures and Contaminants Seen on

Institutional Audio-Visual Equipment Used Primarily for Agricultural Tractor Bel

for Low and Medium Speed Stationary Diesel and Gas Engine Std. Spec. for Measurement of Lateral Vibration on High ensitometric Exposure and Evaluation Meth. for Determining ontinuous Tone) (1972) td. Meth. for Determining Std. Meth. of Test for Effective Pumping films Intended for Direct Pro/ Std. Meth. for Determining ract. for Spur, Helical and Herringbone Gear Shaft Mounted Std. Exposure Time Markings for Variable

xhaust Systems (1972)

ipment (1972)

stems (1972)

tallation (1972)

d Testing (1972)

eristics (1972)

racteristics and General /

otective Devices, and Ins/

lectrical Equipment (1972/

systems (1972)

characteristics and Gener/

intenance (1972)

dings (1972)

Oil and Gas Systems (197)

and Parallel Operation (1)

(1972)

ery Systems (1972)

Std. Practices for Low and Medium Selection of Engine-Generator Units for Low and Medium invitations for Bids and Detailed Spec. for Low and Medium
Std. for High
Spectrochemical Analysis (1970) ANSI Z128.30

pectrochemical Samples and Stds. to a Greater Mass of

Spectrochemical, Nuclear and Radio Chemical Analysis of

ASTM

ASTM

ASTM

ASTM

ASTM

Spectrochmeical Analysis of Ores, Minerals, and Rocks B

the Silver Chloride-Lithium Fl ASTM

Spectrograph (1974)

the Silver Chloride-Lit
Detector Probe Mode?

Spectrometer Leak Detector in the Detector Probe Mode (
Spectrometer Leak Detector in the Inside Out Test. Mode

ASTM E499

Spectrometer Leak Detector or Residual Gas Analyzer in

Spectrometer Leak Detector (1972T)

Spectrometer (1970) ANSI Z128.28

Spectrometer (1971) ANSI Z128.33

/ and Low Alloy Steel ASTM

Eth. for Spectrochem ASTM

pectrometer (1973)

Spectrometers (1972)

Y Y sis of Blast Furnace Iron by the Std. Meth. of Test for

Spectrometric Analysis of Gas-Oil Saturate Fractions

Spectrometric Meth.) (1969) ANSI N108 /Eth of Test Fo

Spectrometric, and Spectrochemical Analysis of Nuclear

Spectrometric, and Spectrochemical Analysis of Nuclear

Spectrometric, and Spectrochemical Analysis of Nuclear

Spectrometric, and Spectrochemical Analysis Of, and Phy

pectrometric, Spectrochemical, Nuclear and Radio Chemi

Spectrometric, Spectrochemical, Nuclear, and Radiochemi

Spectrometry of Water (1972)

Spectrometry of Water (1972T)

Spectrometry (1971) ANSI Z11.311

Spectrometry (1972)

Spectrometry (1973)

Spectrometry (1973)

Spectrometry (1973)

Spectrometry) (1973)

Spectrophotometer Analysis of Acid Soluble Manganese ( $\mathrm{C}$

Std. Meth. of T ASTM

ASTM

St ASTM

Std. ASTM

Tent. Meth. of Test for Aro ASTM

ASTM

AACCH

Spectrophotometric Determination of Fission Zirconium 1

Spectrophotometric Meth. of Analysis of Color of Macaro

Al Refl TAPPI T442SL

ASTM E495

A ACCH 14-21

Spectrophotometric Test for Relative Radiance of Paper

(Spectrophotometric) Content in Corn Oil (1964)

(Spectrophotometric) Content in Corn Starch (1957)

(Spectrophotometric) Content in Feedstuffs and All Other

Spectrophotometric) Content of Corn Sugar (Crude and Re

(Spectrophotometric) Content of Corn Sugar (Crude and Re

(Spectrophotometric) Content of Corn Syrup (1957)

Std Meth of Te

AACCH 22.62

TAPPI UM.460

CR H-12

CR B-14

CR F.14

CR F-16

CR E.16

ectrophotometry (1972)

pectrophotometry (1972)

Th. for Quantitiative Determ

ASTM

D3124

D3133

Spectroscopy (1972A) ANSI Z128.7

Spectroscopy (1973)

Spectroscopy (1973)

1. Fo ASTM

E504

E135

E508

Spectroscopy, Using the ASTM Punched Card Index (1972)

Specular Gloss of Paper and Paperboard at 75 Deg. (1972

Specular Gloss (1967) ANSI Z131.l

Specular Silicon Surfaces (1972T)

10 Amplifier Single Frequency Output for

Speech (1974) (1971) SAE J720

Speed Governing and Parallel Operation (1972)

Speed Helical and Herringbone Gear Units (1972)

Speed of Color Reversal Films for Still Photography (19

Speed of Photographic Negative Materials (Monochrome, C

Speed of Vacuum Chamber Systems (1972)

Speed of $16 \mathrm{Mm}, 8 \mathrm{~mm}$ and Super 8 Reversal Color Camera

Speed Reducers (1974)

Speed Shutters Used in Still Picture Cameras (1972)

Speed Stationary Diesel and Gas Engine Air Intake and E

Speed Stationary Diesel and Gas Engine Assembly and Equ

Speed Stationary Diesel and Gas Engine Cooling Water Sy

Speed Stationary Diesel and Gas Engine Erection and Ins

Speed Stationary Diesel and Gas Engine Factory and Fiel

Speed Stationary Diesel and Gas Engine Fuel Oil Charact

Speed Stationary Diesel and Gas Engine Gaseous Fuel Cha

Speed Stationary Diesel and Gas Engine Gauge Borads, Pr

Speed Stationary Diesel and Gas Engine Generators and E

Speed Stationary Diesel and Gas Engine Lubricating Oil

Speed Stationary Diesel and Gas Engine Lubricating Oil

Speed Stationary Diesel and Gas Engine Operation and $\mathrm{Ma}$

Speed Stationary Diesel and Gas Engine Power Plant Buil

Speed Stationary Diesel and Gas Engine Power Plant Fuel

Speed Stationary Diesel and Gas Engine Speed Governing

Speed Stationary Diesel and Gas Engine Starting Systems

Speed Stationary Diesel and Gas Engine Vibration (1972)

Speed Stationary Diesel and Gas Engine Waste Heat Recov

Speed Stationary Diesel and Gas Engines (1972)

Speed Stationary Diesel and Gas Engines (1972)

Speed Stationary Diesel and Gas Engines (1972)

Speed Steel End Mills, .125 Thru 3.000 Diameter (1973)

Speed Steel Threaded Shank, Sizes 1/16 In. Thru Letter

Speeds of $7.5 \mathrm{In} .0190 .5 \mathrm{Mm}$, and $3.75 \mathrm{In} .095 .3 \mathrm{Mm})$
DEMA

AGMA

ANSI

ANSI

ASTM

ANSI

Std. P AGM

ANSI

DEMA

DEMA

DEMA

DEMA

DEMA

DEMA

DEMA

DEMA

DEMA

DEMA

DEMA

DEMA

DEMA

DEMA

DEMA

DEMA

DEMA

DEMA

DEMA

C. Fo DEMA

Ion of DEMA

NSA

NSA

EIA
E204

T480

D523

F154

PH7.2

$\$ 210.2$

426.01
PH2.21

PH2.5

E294

PH22.146

480.05

PH3.32

*1.8

*1.-5

*1.21

$* 1.22$

*1. 14

*1-15

*1-18

*1.11

$* 1.23$

* $1-20$

*1.13

- 1-6

$* 1.7$

*1-16

*1 1

*1. 24

986

965

RS400 
Std. for Spherical Sleeve (Compression) Tube Fittings (1971)

ers, Whole or Ground Cereal Grains, Whole or Chopped Nuts, Std. Meth. for Chemical Analysis of Ferromanganese and Std. Spec. for Neoprene Rubber Gaskets for Hub and Spec. for Compression Joints for Vitrified Clay Bell and asteners (Connectors, Bolts, Lag Screws, Plates, Nails and Std. for Protruding Head, Locked

(1973)

(1974)

and Molasses (Cereal Chemistry) (1962)

Std. for Driving and Std. for Bulbed

Std. for

Std for Bulbed Mechanically Locked Sid for Blind, 100 Deg. Flush Head, Locked Std. for Self Plugging. Mechanically Locked Std. for Bulbed Mechanically Locked Std. for Bulbed Mechanically Locked Std. for Bulbed Mechanically Locked g Oils from Woolen Yarns / Test Meth. for Scourability of ability of Spinning Lubricant (To Test the Removability of for Spinning Tests on the Cotton System for Measurement of spinning Performance (1972) ANSI L14.289 Std. Meth. for

d (Noise Measurement for Enclosed Helical, Herringbone and ter Cooling Tower Fan Drives (1972) Info Sheet for unds (1972) Sid. Meth. of Measuring the Std. for General Purpose Uniform Cross Section Spec. for

form Building Code: Penthouses and Roof Structures (Tower, Class 2) Wire Sizes 12 to 26 (1972) Rec. for Distilled
Std. for Rec. Pract. for Dimensions of Patch Rec. for Concrete Reinforcing Bar Std. for Wrenching Configuration, Spec. for Steel
Std. for lnvolute Spec. for Steel
Std. for lnvolute

Concrete Slab Floors (1962)

irt, Sand, lnsect Fragments, Hairs, Feathers, Wood Fibers, Plates, Nails and Spikes, Washers, Pins. Dowels, Joints, Std. for General Purpose Semi-Tubular, Full Tubular, Uniform Building Code Std. for Hand Std. for Drill Drivers, Std. for Cotter Pins imens (1971) ANSI A37.121 Std. Meth. of Test for
(1973) Uniform Building Code Std. for Determining the
(1973) Std. Meth. of Test for Undersized Loose Muscovite Mica Std. Meth. of Test for Undersized Loose Muscovite Mica
Rec. Pract. for for Practical Minimum Rec. Tolerances of

laboratory Fatigue Test Procedures for Production Disc and

i J2.4

ication (1973) ANS1 N12.1 Std. Spec. for uality of Bread Flour (Cereal Chemistry) (1962) Std. Spec, for Zirconium Std. Spec. for Titanium Soft Chloroprene-Rubber Medium Chloroprene-Rubber Firm Chloroprene Rubber

$\mathrm{mm} 100 \mathrm{Ft}, 16 \mathrm{~mm} 200 \mathrm{Ft}, 35 \mathrm{~mm} 100 \mathrm{Ft}$, and $70 \mathrm{~mm} 100 \mathrm{Ft}$. Std. for Dimensions of Aerial Film $s$ for Packaging and Storage of Aerial Photographic Film on Meth. of Analysis of Rope ereal Products and Foo/ Meth. of Analysis of Thermophilic U.S. Model Rocket
Std. Meth. of Test for Shock Absorbing Properties of ticles by $\mathrm{T} /$ Std. Meth. of Test for Locating the Thinnest

anized Sheet (Coil Coating) (1974) Spec. for Resistance Seam and Roll Std. for Resistance istics and Finishes of Institutional Textiles (Durability, Test Meth. for Oil Std. Meth. of Test for Water

Test Meth. for Colorfastness of Textile Fabrics to Water emperature (1972) Std. Spec. for Std. Rec. Pract. for Application of Rec. for Water Meth. of Test for Mist ication of Plastic Polymers in Dry Powder Form by Means of of Test for Effectiveness of Aerosol and Pressurized Space Std Rec Pract for Comparison of

Test Meth. for Water Repellency of Textile Fabrics: 74) ASTM B 117 Std. for Pot, Pan, and Utensil Commercial Std. Meth. of Salt Std. Meth. of Salt Std. Meth. of Acetic-Acid Salt Rec. for Sprinkler, Water

liquid and Liquefied Flammable/ Std. Meth. for Adhesion or Cohesive Strength of Flame Specifying Rec. Safe Pract. for Thermal
Spices, etc. (Cereal Chemistry) (1962)

Spiegeleisen (1974)

Spigot Cast Iron Soil Pipe and Fittings (1972)

Spigot Pipe (1972A)

Spikes, Washers, Pins, Dowels, Joints, Split Rings and

Spindle Blind Rivet (1972)

Spindle Ends for Portable Hand, Air, and Electric Tools

Spindle Mechanically Locked Protruding Head Blind Rivet

Spindle Meth. of Analysis of Solids in Juices, Syrups,

Spindle Noses and Tool Shanks for Milling Machines (197

Spindle Protruding Head Blind Rivet (1974)

Spindle Rivet (1972)

Spindle Rivet (1972)

Spindle Self Plugging Blind Rivet (1974)

Spindle 100 Deg. Flush Head Blind Rivet (1974)

Spindle 100 Deg. Flush Head Blind Rivet (1974)

Spinning Lubricant (To Test the Removability of Spinnin

Spinning Oils from Woolen Yarns (1971) ANSI L14.113

Spinning Performance (1972) ANSI L14.289

Spinning Tests on the Cotton System for Measurement o

Spiral Bevel Gear Drives (1973)

Spiral Bevel. Helical and Herringone Gear Unito for

Spiral Flow of Low Pressure Thermosetting Molding Compo

Spiral Retaining Rings (1972)

Spiral Weld Line Pipe (Petroleum Production) (1973)

Spire) (1973)

Spirits Storage (1974)

Splice, Electric, Permanent, Crimp Style, Pre Insulated

Splices in Two Inch Video Magnetic Tape (1964)

Splices (1971)

Spline Drive Threaded Fasteners (197I)

Splined Continuous Strip Mastic Set Maple Flooring Over

Splines and Inspections (1970) ANSI B92.1

Splinters, etc. in Starch (Cereal Chemistry) (1962)

Split Rings and Framing Anchors) (1973)

Split Rivets and Caps (1972)

Split Shakes (1973)

Split Sleeve, Collet Type (1972)

Split) (1972)

Splitting Tensile Strength of Cylindrical Concrete Spec

Splitting Tensile Strength of Molded Concrete Cylinders

Splittings (1973)

Spoke Type Wheels. Demountable Rims and Rim Spacers (Ma

Spoke Wheels, and Demountable Rims Intended for Highway

Sponge and Expanded Cellular Rubber Products (1973) Ans

Sponge and Other Forms of Virgin Metal for Nuclear Appl

Sponge and Other Forms (1967) ANSI N134

Sponge Dough, Pound Loaf Meth. of Analysis for Baking Q

Sponge (1969) ANSI Z179.13

Sponge (1973)

Sponge (1973)

Sponge $\{1973$

Spools for Recording lnstruments and for Microfilm and

Spools (1972)

Spools (1972)

(Cereal Chemistry) (1962

porting and Safety Code ot Aerobic, Anaerobic

Sports) Playing Surface Systems (1972)

Spot in a Zinc (Galvanized) Coating on Iron or Steel Ar Spot Lamps (Light) (1973)

Spot Test Solution for Detecting Chromate Films on Galy Spot Test (1972) ANSI Z11.315

Spot Welding Equipment (3 Phase) (1973)

Spot Welding Equipment, 3 Phase (1973)

Spot, Stain, and Insect Resistance) (1973)

Spots in Paper (1973)

Spotting of Emulsion Floor Polishes (1974) ANS1 Z123.12

Spotting (1972) ANSI L14.148

Spray Applied Fibrous Thermal Insulation for Elevated T

Spray Applied Fibrous Thermal Insulation (1973)

Spray Fixed Systems for Fire Protection (1973)

Spray Flammability of Hydraulic Fluids (1972T)

Spray Gun or Fluidized Bed (Fire Protection) (1973)

Spray Insecticides Against Flying Insects (1972)

Spray Patterns (1972)

Spray Test (1971) ANSI L14.60, ASTM D583

Spray Type Washing Machines (1970)

Spray (Fog) Test. for Spec. Purposes (Coil Caoting) (19

Spray (Fog) Test. (1973) A NSI Z118.1

Spray (Fog) Test. (1974) ANSl Z118.2

Spray, and Explosion Protection (Safety) for Flammable

Sprayed Ccatings (1969) ANSI Z167.22

Sprayed Fireproofing (Guide Spec.) (1972)

Spraying and Blasting Equipment (1973)
SAE $\mathrm{J} 246$

AACCH 28.87

ASTM E361

CISPI HSN

Std ASTM C425

WWPA *31.

NSA 1398

ANSl B107.4

NSA $\quad 1768$

$\mathrm{AACCH} \quad 68.62$

ANSI B5.18

NSA 1738

NSA $\quad 1399$

NSA $\quad 1400$

NSA $\quad 1740$

NSA 1739

NSA $\quad 1769$

AATCC 77

/R AATCC

ASTM D2811

ASTM D28I

AGMA 297.0]

AGMA 490.02

ASTM D3123

ANSI B27.6

$\mathrm{API} 5 \mathrm{LS}$

Uni ICBO UBC 3.36

FMS $\quad 8.8$

NSA 1388

SMPTE RPS

CRSI *3

SAE ASI159A

WSFl *5

SAE J498C

/D AACCH 28.75

WWPA *31-1

ANSI B18.?

ICBO UBC 32.8

ANSl B94.35

SAE J487A

ASTM C496

ICBO UBCS26-12

ASTM D3252

SAE J85

SAE J267

ASTM D1056

ASTM B349

ASTM B4I4

$A \mathrm{ACCH} \quad 10-11$

ASTM B299

SAE AMS $3197 \mathrm{H}$

SAE AMS3198H

SAE AMS3I99H

ANSI PHI.33

ANSI $\mathrm{PHI} 2$

ANSI PHI.46

AACCH $42-20$

$\mathrm{AACCH} \quad 42-40$

NAR *I

ASTM F355

ASTM A239

SAE J59lB

NCCA TB-II-9

ASTM D278]

NSA 991

NSA 967

ANSI L24.7.

TAPPI UM.458

ASTM D1793

AATCC 104

ASTM C720

ASTM C762

FMS 4-1N

ASTM D3119

/L FMS

Th. ASTM

ASTM

AATCC

NSF

NCCA

ASTM

ASTM

FMS

ASTM

CSI

A WS
7.27

D3088

D3077

22

TB.l

$\mathrm{B} 117$

B287

7.14

C633

09841

C2.1 
Std. Thermal h. of Test for Bearing Capacity of Soil for Static Load on h. of Test for Bearing Capacity of Soil for Static Load on Std Test Procedure for Dry Fertilize oving Safety on Enclosed Mobile Tanks for Transporting and nsions, Physical Properties and Meth. of Test. for Helical nd Hot Wound (Materials, Specs. Terminology, / Handbook of Std. for Nonmagnetic act. for Conducting Bending Fatigue Tests for Copper Alloy Carbon Steel Tubular

Std. Spec, for Phosphor Bronze Wire for General and Std. Spec. for Music

Std. Spec, for Steel Wire, Oil Tempered Carbon Valve ec. for Steel, Carbon, Strip, Cold Rolled Hard, Untempered Meth. for Measuring Fla Rec. Pract. for Stainless Steel $17.7 \mathrm{Ph}$ Rec. Pract. for Stainless Steel, SAE 30302. (1973)

t. for Special Quality High Tensile, Hard Drawn Mechanica

Spec. for Stee Spec. for Stee Std. Part Pins

Rec. Pract. for Stainless Steel 17-7 pH Spring Wire and

ec. Pract. for Stainless Steel, SAE 30302, Spring Wire and

Std. Spec. for Steel Wire, Hard Drawn for Mechanical uality High Tensile, Hard Drawn Mechanical Spring Wire and el Wire, High Tensile Strength, Hard Drawn, for Mechanical Std Spec. for Heat Treated Steel Helical Std. Spec. For Carbon and Alloy Steel Bars for ) (1973) Rec. for Water

ater Flow / Pressure Requirements for Pipe Schedule System quirements for the Design. Installation and Performance of 1972) 1973) s (General Characteristics Of, Rec. Construction and Wate Rec. for Water Demand for Spec. for Firecycle on -Off Multicycle si $/$ Std. for Safety for Alarm Valves for Use in Wet Pipe Std. for the Installation of Water Rec for Installation of Rec. for the Inspection and Maintenance of y) for Flammable Liquid and Liquefied Flammable/ Rec. for Std. for Safety for Automatic Water Std. Spec. for Machine and Std. for 700 Class Pintle Chains, Attachments, and Std. for H Type Mill Chains-Attachments and $y$ Duty Offset Sidebar Power Transmission Roller Chains and Sid. for Heavy Duty Roller Type Conveyor Chains and Spec. for Oil Field Chain and Std. for Steel Detachable Link Chains, Attachments, and for Double Pitch Conveyor Roller Chains, Attachments and Std. Analytical Meth. for Determining Viability th), Hem-Fir (North), Ponderosa and Western White Pines, building Code Std. for Eastern White and Red Pine, Eastern Industry Std. for Douglas Fir, Western Hemlock, Sitka est Coast Hemlock; Western Red Cedar; White Fir; and Sitka tamarack (Nor/ Uniform Building Code Std. for Coast Sitka Northern Pine An/ Uniform Building Code Std. for Eastern derosa, Idaho White, Sugar, and Lodgepole Pines; Engelmann Std. Tolerances for Woolen

gn of Fine Pitch on Center Face Gears for 20 Deg. Involute reducers (1974) Std. Pract. for

American Drafting Manual (Gear Drawing Stds. Part 1 for

i B93.29 Std. Dimensions for Accessories for Cataloged Std. for

ounting Dimensions for 3/4, 1 and 1 1/8 In. Bore Cataloged Std. for Computer Type Std. Spec. for Industrial Perforated Plate and Screens std. Spec. for Machined Flat and

for Round, Hexagonal, Octagonal, and Flat (Rectangular and erboard (1973) Test Meth. for Measuring ing Chromium Nickel Manganese Steel Bars Including Rounds,

eth. of Test for Continuity of Steam Turbine Oil Oxidation Std. Analytical Meth. for Determining Color try) (1962)

nce Requirements for Stopping Power, Traction and Steering

974) Oxygen-Bomb Meth. of Analysis of Std. Meth. of Test for Thermal Std. Meth of Test for Meth. of Test for Hydrolytic

-Ditertiary-Butyl P/ s (1973) Std. Meth. of Test for Oxidation

chemistry) (1967) on Melted 1825-1900 Deg. F (996.1-1037.8 Deg. C) Solution, um Induction Melted, $1975 \mathrm{Deg} . \mathrm{F}$ (1079.4 Deg. C) Solution, std. Spec. For Calcium Chloride to Be Used for Dustlaying, nd Welded Chromium Nickel Iron Molybdenúnutumpipar
Spraying Terms and Definitions (1970)

/Pread Footings (1972) Std. Met

AWS

Spread Footings (1972)

of Test for Bearing C

Spreader (1972)

Spreading Agricultural Liquids and Slurry (1973)

Spring and Carbon Steel Tooth Lock Washers (1972)

Spring Design: Compression, Extension, Torsion, Flat, a

Spring Lock Washer (1974)

Spring Materials (1973)

Spring Pins (1973)

Spring Purposes (1972)

Spring Quality Steel Wire (1971) ANSI G54.2

Spring Quality (1971) ANSI G54.4

Spring Quality (1973)

Spring Test for Hot Tack of Wax Polymer Blends (1972

Spring Wire and Springs (I970)

Spring Wire and Springs (1971)

Spring Wire and Springs (1972)

Spring Wire $(0.95 \mathrm{Cr}-0.22 \mathrm{~V}(0.48-0.53 \mathrm{C}))(\mathrm{SAE} 6150)$

Spring Wire, Best Quality Music (1973)

Spring, Slotted and Coiled, Heavy Duty (1973)

Springs (1970)

Springs (1971)

Springs (1971) ANSI G54.1

Springs (1972)

Springs (1973)

Springs (1973) ANSI G54.20

Springs (1974)

Sprinkler and Hydrant Systen

Sprinkler Demand (1974)

Sprinkler Irrigation Equipment (1972)

Sprinkler Irrigation (For Farms) Technical Data Sheet

Sprinkler System Design-Fire Protection and Safety) (

Rec. Pract. for
Std. S
Tables (Fire S
al Data Sheet

Sprinkler System (Fire Safety) (1973)

Sprinkler Systems for Fire Protection Service (1973) an

Sprinkler Systems for Fire Protection (1973) ANSI A54.3

Sprinkler Systems (Fire Protection) (1973)

Sprinkler Systems (Fire Safety) (1973)

Sprinkler, Water Spray, and Explosion Protection (Safet

Sprinklers for Fire Protection Service (1974)

Sprocket Coil Chain (1972) ANSI G61.5

Sprocket Teeth (Power Transmission) (1972)

Sprocket Teeth (1971)

Sprocket Teeth (1972)

Sprocket Teeth (1973)

Sprockets (1970)

Sprockets (1972)

Sprockets (1972)

(Sprouting) Content in Corn (1959)

Spruce-Pine-Fir, Western Cedars (North) (Lumber) (1

Spruce and Hemlock, Balsam Fir and Tamarack Lumber (197

Spruce Doors and Blinds (1972)

Spruce (Lumber) (1973)

(R Douglas Fir, Coast Region; W

Spruce, Douglas Fir-Larch (North), Eastern Hemlock-

Spruce, White Pine, and Hemlock-Tamarack; Balsam Fir;

Spruce: Western Cedar and Hemlock; Subal-Pine Fir; an

Spun on the Woolen System Yarns (1971)

Spur Pinions (1973)

Spur, Helical and Herringbone Gear Shaft Mounted Speed

Spur, Helical, Double Helical and Rack) (1971)

Square and Rectangular (1973)

Square Head Industrial Fluid Power Cylinders (1972) Ans

Square Head Setscrews (I969)

Square Head Tie Rod Type Industrial Fluid Power Cylinde

(Square Loop) Pulse Transformers (1970)

(Square Opening Series) (1972)

Square Tool Steel Bars (1973)

Square) Brass Wire (1973)

Uniform Building Code Std.

Squareness or Rectangularity of a Sheet of Paper or Pap

Squares, Hexagons, and Shapes-Angles, TEES and Channe

Stability by Rotating Bomb (1967) ANSI Z11.230

Stability Content of Corn Syrup Unmixed (1954)

Stability for Regular Bicycles (1972)

Stability in Fats, Oils, and Shortenings (Cereal Chemis

Stability of Aviation Turbine Fuels (1972) ANSI Z11.148

Stability of Distillate Fuel Oil (Accelerated Meth.) (1

Stability of Elastomeric Volcanizates (1972T)

Stability of New Mineral Insulating Oils Containing 2,6

Stability of Plastic Encapsulants for Electronic Device

Stability of Turbine Fuels (JFTOT Proc.) (1973)

Stability (AOM) of Fats, Oils, and Shortenings (Cereal

Stabilization, and Precipitation Heat Treated (1973)

Stabilization, and Precipitation Heat Treated (I973)

Stabilization, Ice Removal, Acceleration of Concrete, C

$/ \mathrm{Td}$. Spec. for Seamless a

ASTM

ASTM

ASAE

IImpr ASAE

IIme ANSl

SMI

NSA

d. Rec. Pr ASTM

SAE

ASTM

ASTM

ASTM

Std. Sp ASTM

TAPPI

SAE

$\mathrm{SAE}$

Rec. Prac

SAE

SAE

NSA

NSA

SAE

ASTM

ASTM

ASTM

ASTM

ASTM

FMS

ec. for W FMS

ASAE

ASAE

FMS

FMS

FMS

UL

NFPA

FMS

FMS

FMS

UL

ASTM

ANSI

ANSI

ANSI

ANS

APl

ANSI

Std ANSI

CR

ICBO

ICBO

FHDA

ICBO

ICBO

ICBO

ICBO

ASTM

Stabilized Alloy Pipe (1973)

C2.9

D1194

R317

B18.21.1

*3

1640

B593

B159

A 228

A 230

A680

T683SU

$\mathrm{J} 217$

$\mathrm{J} 230$

A MS6450D

AMS5112F

561

$\mathrm{J} 217$

$\mathrm{J} 230$

A227

J27I

A679

AI 25

A689

2.76

2.77

R264.1

S262 T

LPD 1.2

3-26

2.90

193

13

$2-81$

7.14

199

A 467

B29.13

B29.14

B29.10

B29.15

$7 \mathrm{~F}$

B29.6

B29.4 
chromium-Nickel-lron-Molydenium-Copper-Columbium spec. for Chromium Nickel Iron Molybdenum Copper Columbium chromium-Nickel-lron-Moly bdenum-Copper-Columbium of Test for Relaxation Dimensional Change (Shrinkage) of Std. for

Rules, Guidelines, and Drawings for Copper Solvent Single thickness of Epitaxial Layers of Silicon by Measurement of Std. Meth. for Sampling and Feedstuffs (Cereal Chemistry) (1962) Modified Two Safety Code for Fabricated Cloth, Type E Glass, B xtinguishing (1973)

Personnel and Material (1/

If Extinguishing (1973)

Guide Spec. for Platforms and Cloth, Type E Glass, B Uniform Building Code: st for Diffusion Stain of Rubber and Contact and Migration nic Finishes in Contact/ laundering (1970) ANSI L14.239 Std. Meth. of Test for Diffusion Test Meth. for Oily Std. Meth. of Test for ansi $\mathrm{G} 53.30$

Test Meth. for Fiber Identification (Wilson Test Meth. for Beating Control (Simon and Finishes of Institutional Textiles (Durability, Spot, pplication of Flammable Finishes (Paint, Varnish, Lacquer, Std. Meth. of Test for

Test Meth. for Rug Back Floor ial or Diffused Layers in Silicon by the Angle Lapping and Rec. Evaluation Procedure of Gray Scale for ing Parts Suitable for High T/

Std. Spec. for Martensitic steel Bars I/ Std. Spec. for Hot Rolled and Cold Finished t, and Strip (1972) ANSI G81.2 Std. Spec. for Std. Spec. for use in Boilers and Other Pressure Vessels/ td. Spec. for Hot Rolled and Cold Finished Age Hardening 73) ANSI G24.3

Reforging (1972) ANSI G81.13 G81.26 Std. Spec. for Std. Spec. for spec. for General Requirements for Delivery of Flat Rolled i G81.32 Std. Spec, for 41 Std. Spec. for

services (1974) ANSI G38.4

i G81.16

tial Use) (1962)

Std. Spec, for Free Machining Std. Spec. for Alloy and Std. Spec. for Welded Austenitic Std. Spec. for Seamless Std. Spec. for Welded Std. Spec. for Seamless and Welded Austenitic Std. Spec. for Wrought Austenitic Std. for Std. for Std. Spec. for Seamless and Welded Austenitic Code Std. for Spec. and Design of Light Gauge Cold Formed b125.14 bl25.15

Std. Spec, for Seamless and Welded Ferritic Std. Spec. for Seamless and Welded Austenitic Rec. Pract. for Std. Rec. Pract. for Preparation of and Electroplating on 71) Rec. Pract. for Std. Rec. Pract. for Determining the Susceptibility of
chromium-Nickel-
Std. Meth. for Chemical Analysis of Test Meth. for Fiber Identification (Various

ng Construction Under the Uniform Building Code: Exits and for Renovation of Interior Ceramic Tile Walls, Floors, and stands, Bleachers, and Grandstand/ Uniform Building Code: Western Woods Use Book: Fire Protection and Safety (Exit, Quality Stds. for work, Closet and Storage Shelving, Misc. Ornamental Items, meth. of Evaluating Wood Preservatives by Field Tests with Meth. of Compression Test with Baker Compressimeter for Meth of Sensory Perception Test for and Shower Recep/ Std. for Nonmetallic Shower Receptors, (1965) or Meth. of Determining Plastic Deformation in Sheet Metal istry) (1962)

Manual of (1962) Meth. for Preparation and rdering, Shipping, and Billing of Merchandise W/ Preparation and hemistry) (1962)

ry) (1962)

eal Chemistry) (1962)

on (Cereal Chemistry) (1962)

chemistry) (1962)

stry) (1962) Sidderies for Use in 1 Use Std.: Criteria for Diesel Generator Units Applied as 3) Quality Stds. for and Storage She
Guide Specs. for Archit ectural Woodwork: Rec. for
Stabilized Alloy Pipe (1974)

Stabilized Alloy Plate, Sheet,

Stabilized Alloy Tubes (1973)

Stabilized Knit Wool Fabrics (Textile) (1973)

Stack Plumbing System (1973)

Stacking Fault Dimension (1973)

Stage Aor Particulate Matter (1971) ANSI Z257.3

Stage and Scaffold Planks and Platforms (1972)

Stage Epoxy Resin Impregnated, 181 Style Fabric, Self E

Stage Lift s (Hydrualic and Hydropneumatic) for Lowering

Stage Polyester Resin Impregnated, 181 Style Fabric, Se

Stages and Platforms (1973)

Stain of Organic Finishes in Contact with Rubber (1973)

Stain of Rubber and Contact and Migration Stain of Orga

Stain Release Meth. of Textile Fabric Soil During Home

Stain Resistance of Anodic Coatings on Aluminum (1972)

Stain) (Pulp and Paper) (1973)

Stain) (Pulp and Paper) (1973)

Stain, and Insect Resistance) (1973)

Stain, etc.) (1973)

Staining of Caulking Compounds and Sealants (1973)

tile (1973)

Staining Technique (1972) ANSI Z173.49

Staining (Textile Colorfastness) (1954) ISO 105-1

Stainless and Alloy Steel Castings for Pressure Contain

Stainless and Heat Resisting Chromium Nickel Manganese

Stainless and Heat Resisting Chromium Steel Plate, Shee

Stainless and Heat Resisting Steel Bars and Shapes for

Stainless and Heat Resisting Steel Bars and Shapes (197

Stainless and Heat Resisting Steel Bars, and Shapes (19

Stainless and Heat Resisting Steel Billets and Bars for

Stainless and Heat Resisting Steel Forgings (1973) ANSI

Stainless and Heat Resisting Steel Plate, Sheet, and St

Stainless and Heat Resisting Steel Rope Wire (1972) Ans

Stainless and Heat Resisting Steel Wire (1971) ANSI G81

Stainless and Heat Resisting Steel Wire (1971) ANSI G81

Stainless Steel Bolting Materials for High Temperature

Stainless Steel Feedwater Heater Tubes (1973)

Stainless Steel Mechanical Tubing (1971) ANS1 G62.1

Stainless Steel Mechanical Tubing (1973)

Stainless Steel Parts, Equipment and Systems (1972) Ans

Stainless Steel Pipe (1972A) ANSI B125.16

Stainless Steel Piping Fittings (1973) ANSI G37.8

Stainless Steel Plumbing Fixtures (Designed for Residen

Stainless Steel Plumbing Fixtures (1971)

Stainless Steel Sanitary Tubing (1973) ANSI B36.38

Stainless Steel Structural Members (1973)

Stainless Steel Tubing for General Service (1973) ANSI

Stainless Steel Tubing for General Service (1973) ANSI

Stainless Steel 17-7 pH Spring Wire and Springs (1970)

Stainless Steel (1970) ANSI G53.13

Stainless Steel, SAE 30302, Spring Wire and Springs (19

Stainless Steels and Related Ni-Cr-Fe Alloys to Stress

Stainless, Heat Resisting, Maraging, and Other Similar

Stains) (Pulp and Paper) (1973)

Stairs (1973)

Stairs (1973)

Stairs, Exits and Occupant Loads (Buildings, Reviewing

Stairway, Aisle, Corridor, Building Construction and Te

Stairwork and Handrails (Architectural Woodwork) (1973)

Stairwork and Handrails, Exterior Frames and Sash, Scre

Stakes (1974)

Staleness of Bread (Cereal Chemistry) (1962)

Staleness of Bread (Cereal Chemistry) (1962)

Stall Showers Lavatories, Bathtubs and Combination Tank

Stampings (1972)

Standardization for Masterkeying Nomenclature and Terms

Standardization of Barium Lactate Solution (Cereal Chem

Standardization of Buffer Solutions (Cereal Chemistry)

Standardization of Forms and Information Flow Used in $\mathrm{O}$

Standardization of Hydrochloric Acid Solution (Cereal C

Standardization of Lactic Acid Solution (Cereal Chemist

Standardization of Potassium Permanganate Solution (Cer

Standardization of Sodium or Potassium Hydroxide Soluti

Standardization of Sodium Thiosulfate Solution (Cereal

Standardization of Sulfuric Acid Solution (Cereal Chemi

Standardization Probe, Rot Fragment Counting Plate and

Standardization (1973)

Standardization, and Storage of Std. Solutions for Chem

Standby Power Supplies for Nuclear Power Generating Sta

Standing and Running Trim (A rchitectural Woodwork) (197

Standing and Running Trim, Casework, Panelwork, Closet

Standpipe and Hose Systems (Heating) (1972)

Standpipe Systems (Fire Extinguishment) (1973)
ASTM

ASTM

ASTM

NEMA

Design CDA

AATM

AACC

A LI

SAE

CSI

SAE

ICBO

ASTM

ASTM

AATCC

ASTM

TAPPI

TAPPI

ANSI

ICBO

AATCC

ASTM

AATCC

ASTM

ASTM

ASTM

ASTM

A.STM

ASTM

ASTM

ASTM

ASTM

ASTM

ASTM

ASTM

ASTM

ASTM

ASTM

ASTM

ASTM

ASTM

USC

IAPMO

ASTM

ICBO

ASTM

ASTM

SAE

ASTM

SAE

ASTM

ASTM

TAPP

Dwelli ICBO

TCA

ICBO

WWPA

AW

AWI

ASTM D1758

$\mathrm{AACCH} \quad 74-10$

AACCH $\quad 74-30$

IAPMO TSCl]

SAE J863C

NBHA $* 4$

AACCH $70-10$

AACCH $\quad 70-15$

NWHA *1

$\mathrm{AACCH} \quad 70-20$

AACCH $70-30$

AACCH $70-50$

$\mathrm{AACCH} \quad 70-70$

$\mathrm{AACCH} \quad 70-75$

AACCH $\quad 70-80$

AACCH $28-90$

ANSI Z17.1

ASTM E200

IEEE

$A W I$

AWI

FMS

ICBO

74

468 43

.18

MS3821 
Std. for Manually Propelled Mobile Ladder de: Stairs, Exits and Occupant Loads (Buildings, Reviewing 973) uniform Fire Code: Places of Assembly (Theaters, Reviewing Meth. of Analysis of Coagulase Positive Meth. of Analysis of Food Poisoning st for Commercial Weight of a Shipment of Yarn or Man Made Sids. for

ght Metal Plate Connected Wood Truss Design; and Nails and Spaced Columns, Joints, Screws, Bolts, Connectors, Nails, trol of Fire Extinguishing Systems (1973)

Rec. for tical Meth. for Determining Hydroxyalkoxyl Content of Corn Std. Analytical Meth. for Determining Std. Analytical Meth. for Determining Std. Analytical Meth. for Determining Apparent of Hydroxyalkoxyl Groups in Hydroxyethyl and Hydroxypropyl analytical Meth. for Determining $\mathrm{pH}$ Content of Corn Syrup, ining Iron (Orthophenanthroline) Content of Corn Syrup and of Corn Syrup Unmixed, Finished Sugar and Other Clarified al Meth. for Determining Acidity Content of Corn Syrup and mining Ash Content of Corn Syrup, Finished Sugar and Other etermining Fermentables Content of Corn Syrup, Sugars, and ontent of Corn Sugar (Crude and Refined). Syrup, and Other ontent of Corn Sugar (Crude and Refined), Syrups and Other ontent of Corn Sugar (Crude and Refined), Syrups and Other th. for Determining Dextrose Content of Corn Syrup and All s Liquid Chromatography) Content of Corn Syrup, Sugar, and ermining Dextrose Equivalent Content of Corn Syrup and All ning Specific Rotation Content of Corn Syrup, Dextrose and ent of Corn Sugar (Crude and Refined), Dextrose, Syrup and content of Corn Sugar (Crude and Refined), Syrup and Other

lcium (Egta-Titrimetric) Content of Corn Syrup and Other std. Analytical Meth. for Determining lron Content in Corn of Corn Syrup Unmixed, Finished Sugar and Other Clarified olysis) (Cereal Chemistry)/ ation, to Corn, Rye, B/ Diastase Meth. of Analysis of (1971)

Polarimetric Meth. of Analysis of Std. Analytical Meth. for Determining harides (Gas Liquid Chromatohraphy) Content of Corn Sugar,

g Total Sugars Content of Corn Sugars (Crude and Refined), Meth. of Analysis of Damaged Starch (Granules in Flour or

g Ash Content in Corn Starch, Dextrines and Other Modified

al Meth. for Determining Borax Content in Dextrin and Corn

Hydrolyzates Solutions Derived from Corn or Grain Sorghum Analytical Meth. for Determination of pH (Paste) of Corn Analytical Meth. For Determination of $\mathrm{pH}$ (Slurry) of Corn ragments, Hairs, Feathers, Wood Fibers, Splinters, etc. in

tures (Maltose, Sucrose, Mannose, Galactose, Dextrans, and ical Meth. for Determining Acidity (Paste) Content in Corn are Susceptible to Hydrolys/ Meth. of Analysis of Damaged h. for Determining Moisture (Karl Fischer) Content in Corn analytical Meth. for Determining Total Fat Content in Corn eth. for Determining Acidity (Extractable) Content in Corn Analytical Meth. for Determining Solubles Content in Corn alytical Meth. for Determining pH (Slurry) Content in Corn tical Meth. for Determining Sulfur Dioxide Content in Corn ical Meth. for Determining Moisture (Oven) Content in Corn rmining Moisture (Azeotropic Distillation) Content in Corn nalytical Meth. for Determining Phosphorus Content in Corn for Determining Color (Spectrophotometric) Content in Corn nalytical Meth. for Determining pH (Paste) Content in Corn Analytical Meth. for Determining Calcium Content in Corn cal Meth. for Determining Waxy and Nonwaxy Content in Corn

Syrups and Other Hydrolyzates from Corn and Grain Sorghum lytical Meth. for Determining Bulk Density Content in Corn determining lnherent Viscosity (One Point) Content in Corn Analytical Meth. for Determining Carboxyl Content of Corn d. Analytical Meth. for Determining Acetyl Content of Corn Meth. for Determining Brabender Viscosity Content in Corn nalytical Meth. for Determining Phosphorus Content of Corn Std. Analytical Meth. Determining Iodine Affinity of Corn r Determining Calcium (Egta-Titrimetric) Content in Corn Properties of Agricultural Grain and lts Products (Flour, emistry) (1962)

Meth of Analysis for Ash in Std. Analytical Meth. for Determining Ash Content in Corn for Determining Crude Fat / Extractables) Content in Corn

cal Meth. for Determining Protein Nitrogen Content in Corn Analytical Meth. for Determination of Phosphorus in Corn Meth. of Analysis of Hydration Capacity of Pregelatinized itrogen Content of Corn Sugar (Crude and Refined), Syrups, ein Nitrogen Content in Feedstuffs (Corn), Syrups, Sugars, Content in Steepwater, (Corn) Feedstuffs, Syrups, Sugars, etermining Protein Nitrogen Content of Corn Syrup, Sugars, ine) Content of Corn Syrup and Starch Hydrolyzates and All sional and Electrical Characteristics of $8^{\prime} \mathrm{W}$ att T5 Preheat ional and Electrical Characteristics of 15 Watt T8 Preheat onal and Electrical Characteristics of 30 Watt, T8 Preheat $\mathrm{nt}$ and Non-Synchronous Data Communication Equip/ Std. for
Stands for Scaffolds (Towers, Safety) (1971)

tands, Bleachers, and Grandstands) (1973)

Stands, Grandstands, Bleachers, etc.) (1973)

Staphylococcus in Cereal Products (Cereal Chemistry) (1

Staple Fiber (1971)

Staples with Rolled or Slash Points (1971) EEI TDJ.14

Std. M

lift Bolts, and Wood and La

(1973)

Star Model F Hidromatic

Starch and Ethers (1967)

Starch Content in Corn (1964)

Starch Content in Feedstuffs (Corn) (I954)

Starch Content of Corn Syrup, Refined and Crude Sugar
Starch Ethers (1973)

/Lytical Meth. for Determination

Starch Hydrolyzates and a Variety of Other Materials (I

Starch Hydrolyzates and All Starches and Sugars, and to

Starch Hydrolyzates (Potassium Chromate Indicator) (I95

Stareh Hydrolyzates (1952)

Starch Hydrolyzates (1952)

Starch Hydrolyzates (1957)

Starch Hydrolyzates (1961)

Starch Hydrolyzates (196I)

Starch Hydrolyzates (1962)

Starch Hydrolyzates (1967)

Starch Hydrolyzates (1967)

Starch Hydrolyzates (1968)

Starch Hydrolyzates (1971)

Starch Hydrolyzates (1971)

Starch Hydrolyzates (1973)

Starch Hydrolyzates (I973)

Starch Hydrolzates lncluding Syrups and Sugars, and Mos

Starch in Flour and Semolina (With Subsequent Acid Hydr

Starch in Flour and Semolina; Also, with Slight Modific

Starch Indicator, 1\% Content of Reagents and Indicators

Starch Molasses and Hydrolyzates (1971)

Ermining Sacc

Starch Molasses (1972)

Nalytical Meth. for Determinin

Starch Preparations Which Are Susceptible to Hydrolysis

Starch Products (1955)

/Nalytical Meth. for Determinin

Starch Products (1971)

Starch Which Are Substantially Optically Clear (1959)

Starch Which ls Gelatinized When Heated in a Boiling W

Starch Which Is Substantially Insoluble in Water at Roo

Starch (Cereal Chemistry) (1962)

Starch (Cereal Chemistry) (1968)

Starch (Gelatinizable Products) (1955)

Starch (Granules in Flour or Starch Preparations Whic

Starch (Unmodified) (1961)

Starch (1955)

Starch (1955)

Starch (1956)

Starch (1956)

Starch (1956)

Starch (1956)

Starch (1956)

Starch (1957)

Starch (1957)

Starch (1958)

Starch $(1960)$

Starch $(1960)$

Starch (1960)

Starch (1963)

Starch (1963)

Starch (1966)

Starch (1968)

Starch (1968)

Starch (1969)

Starch $(1973)$

Starch (1973)

Starch) (1972)

Starch, Dextrin, and Other Modified Products (Cereal C

Starch, Dextrines and Other Modified Starch Products (1

Starch, Gluten, Germand Finished Std. Analytical Meth.

Starch, Sugars, Syrups and Other Protein Bearing Materi

Starch, Syrup and Sugar Obtained from the Corn Wet Mill

Starches and Cereal Products (Cereal Chemistry) (I968)

Starches and Other Protein Bearing Materials When Suita

Starches and Other Protein Bearing Materials When Suita

Starches and Other Protein Bearing Materials When Suita

Starches and Other Protein Bearing Materials (1952)

Starches and Sugars, and to Most Other Products of the

Start Bactericidal Lamp (1971)

Start Bactericidal Lamp (1971)

Start Bactericidal Lamp (1971)

Start.Stop Signal Quality Between Data Terminal Equipm
ANSI

ICBO

$\mathrm{AACCH}$

AACCH

ASTM

NEMA

ICBO

ICBO

FMS

CR

CR

$\mathrm{CR}$

CR

CR

CR

CR for Deter CR

CR

C CR

CR

CR

$\mathrm{CR}$

$\mathrm{CR}$

$\mathrm{CR}$

$\mathrm{CR}$

CR

CR

CR

CR

AACCH $76-10$

$\mathrm{AACCH} \quad 76-20$

CR R.80

F.50

F.58

AACCH $76-30 \mathrm{~A}$

B-8

D. 10

F. 14

CR $\quad$ C. 42

AACCH 28-75

$80-10$

B-4

ACCH 76-30A

B-36

B-20

B. 2

B.56

B. 44

B. 58

B. 38

Std. Analy CR

Sid. Analyt CR

Dete CR

Meth. CR

Std. a CR

Std CR

Std. Analyti CR

Refined), CR

eth. for CR

Std. CR

St CR

ytical CR

Std. a CR

CR

Thermal ASAE

$\begin{array}{ll}\text { CR } & \text { B-8 } \\ \text { CR } & \text { B. } 18\end{array}$

$\begin{array}{ll}\text { CR } & \text { B.48 }\end{array}$

$\begin{array}{ll}\text { CR } & \text { B.47 }\end{array}$

$\mathrm{AACCH} \quad 56.20$

CR F.44

CR G.22

CR J.56

(D) CR E.52

CR

Std. Dimen ANSI

it Dimens ANSI

td. Dimensi ANSI

E. 32

C78. 1200

C78.1201

C78.1202

RS404 
Spec. for Fluorescent Lamp Rec. Electric Motor

for Low and Medium Speed Stationary Diesel and Gas Engine Storage Battery Spec. (Vehicular, Ignition, Lighting, and battery Materials Spec. (Vehicular, Ignition, Lighting and Std. for Graphic Symbols for Logic Diagrams (Two rization of Large Signal D/

grams (1970)

Trial Use Std. Guide on Solid Std. Proposal for Solid Std. for Auditing Nuclear Materials

like Materials /

the Identification (Code) of the Countries of the United

colu/ Std. for ldentification Codes and Abbreviations for ification Codes and Abbreviations for States of the United essed $\mathrm{G}$ as Cylinders Intended for Medical Use in the United Test Meth. for Water Repellency of Textile Fabrics: urgical Implants) (1973)

973)

973)

Std. Rec. Pract. for Std. Rec. Pract. for

for Electrical Conductivity of Aviation. Rec. Pract. for Rec. Pract. for Reducing the Fire Hazard of izontal Plane Meth/ izontal Plane Meth.) (1

lined Plane Meth.) (1972) Std. Meth. of Test for Coefficient of Meth. for Inefficient of (1972) Std. Meth. of Test for Bearing Capacity of Soil for t Fol Std. Meth. of Test for Bearing Capacity of Soil for ural Requirements for Regular Bicycles Including Tests for 7) ANSI Q4.2 Std. Meth. of Std. Meth. of Test for Water Absorption Test Meth. for Electrical Resistivity (Electro est Meth. for Electrical Resistivity Textile of (Electro es (Boats) (1972) bon Black (1972) eration) (1974) Rec. Pract. for Determining and Std. Rec. Pract. for Std. for Central riteria for the Periodic Test. of Nuclear Power Generating $f$ the Single Failure Criterion to Nuclear Power Generating iples for Reliability Analysis of Nuclear Power Generating ar/

Std. for Installation, Maintenance and Use of Centra Std. for Installation, Maintenance and Use of Remote Std. for Interconnection Circuitry of Noncoded Remote eamless Austenitic Steel Pipe for High Temperature Central definitions of Terms Used in lEEE Nuclear Power Generating Std. Operating Limits for Centra Std. Coil Face Areas for Central definitions and Terms Used in the Selection of Tires ( $C$ ar for New Tires for Passenger Cars and test Procedures for Retreaded Tires for Passenger Cars and for Bias and Radial Ply Tires and Rims for Passenger Cars, mum Std. for Amateur Radio Antenna: Part 1-Base or Fixed

for Heat Actuated Fire Alarm Devices, Single and Multiple

Systems (1972)

(1972)

(1972)

ion (1972)

ing (1972)

cs (1972)

istics and General /

ve Devices, and Ins/

cal Equipment (1972/

teristics and Gener/

s (1972)

nce (1972)

(1972)

nd Gas Systems (197)

e (1972)

rallel Operation (1) )

stems (1972) Safety Std. for Std. Pract. for Low and Medium Speed Std. Pract. for Low and Medium Speed Std. Pract. for Low and Medium Speed Std. Pract. for Low and Medium Speed Std. Pract. for Low and Medium Speed Std. Pract. for Low and Medium Speed Std. Pract. for Low and Medium Speed Std. Pract. for Low and Medium Speed Std. Pract. for Low and Medium Speed Std. Pract. for Low and Medium Speed Std. Pract. for Low and Medium Speed Std. Pract. for Low and Medium Speed Std. Pract. for Low and Medium Speed Std. Pract. for Low and Medium Speed Sid. Pract. for Std. Pract. for Low and Medium Speed Std. Pract, for Low and Medium Speed Std. Pract. for Low and Medium Speed Std. Pract. for Low and Medium Speed Std. Practices for Low and Medium Speed Std. Pract. Definitions for Std. Pract, for Scavenging and Supercharging of ction of Engine-Generator Units for Low and Medium Speed tions for Bids and Detailed Spec. for Low and Medium Speed

Std. Nuclear Safety Criteria for the Design of y Std. for Electric Heating Appliances (Portable Fixed and Specifying Metal Louvers. Safety Std. for Acetylene Generators,

Safety Std. for Acetylene Generators, )

for the Installation and Maintenance of Electrical Supply ces (1972) ANSI Se 3.1 Safety Std. for Centra for Protection Against Misc. Perils at Electric Generating ipment During the Construction of Nuclear Power Generative
Starters (1972)

Starting Currents (1972) EEI 62.27

Starting Systems (1972)

Starting) for Passenger Automobiles, Commercial Vehicle Starting) (1972)

State Devices) (Electronics) (1973) ANSI Y32.14

State Devices: Varactor Measurements Part II-Characte

State Load Switches for Traffic Control Systems (1973)

State Performance Presentation for Digital Computer Pro

State Surface Photovoltage (Electronics) (1973)

Statements and Reports (Accounting) (1973)

Statements for ASTM Meth. Related to R

States for Information Interchange (1973)

States of the United States (Including the District of

States (Including the District of Columbia) for lnforma Std. Color Marking

Static Absorption Test (1972) ANSl L14.61, ASTM D583

Static Bend and Torsion Test. of Intramedullary Rods (S

Static Bend Test. of Bone Plates (Surgical Implants) (1

Static Bend Test. of Nail Plates (Surgical Implants) (1

Static Dissipator Additive (1971) ANSI Z11.281

Static Electricity (1973)

Static Friction of Corrugated and Solid Fiberboard (Hor

Static Friction of Corrugated and Solid Fiberboard (Hor

Static Friction of Corrugated and Solid Fiberboard (Inc

Static Immersion Test. of Unstressed Materials in Nitro

Static Load on Spread Footings (1972) /Pread

Static Load, Frame, Fork. Steering Assembly and Tire B

Static Tests of Timbers (Wood) in Structural Sizes (196

(Static) of Leather (1970) A LCA E30

Static) of Textile Fabrics (1972) ANSI L14.112

Static) of Textile Yarns (1973) ANSI L14.119

Stating the Horsepower Rating of Inboard Gasoline Engin

Stating the Precision of ASTM Test Meth. Related to Car

Station Air Handling Units (Air Conditioning and Refrig

Station Protection Systems (1971) ANSI N41.3

Station Protection Systems (1972) ANSI N41.2

Station Protection Systems (1972) ANSI N41.4

Station Protective Signaling Systems for Guard, Fire A

Station Protective Signaling Systems (1972) ANSI SE3.5

Station Protective Signalling Systems (1969) ANSI SE3.7

Station Service (1973) ANSI B125.25

Station Sids. (1972)

Station Units (Air Moving and Conditioning) (1966)

Station Units (Air Moving and Conditioning) (1966)

Station Wagon, Truck, Bus, Trailer, Multipurpose Passen

Station Wagons (1965)

Station Wagons (1966)

/Mance Requirements and

Station Wago

Station, Mechanically Operated Type for Indoor Use (197

Stationary and Fixed Electric Tools (1973) ANSI C 33.86

Stationary Diesel and Gas Engine Air Intake and Exhaust

Stationary Diesel and Gas Engine Assembly and Equipment

Stationary Diesel and Gas Engine Cooling Water Systems

Stationary Diesel and Gas Engine Erection and Installat

Stationary Diesel and Gas Engine Factory and Field Test

Stationary Diesel and Gas Engine Fuel Oil Characteristi

Stationary Diesel and Gas Engine Gaseous Fuel Character

Stationary Diesel and Gas Engine Gauge Borads, Protecti

Stationary Diesel and Gas Engine Generators and Electri

Stationary Diesel and Gas Engine Lubricating Oil Charac

Stationary Diesel and Gas Engine Lubricating Oil System

Stationary Diesel and Gas Engine Operation and Maintena

Stationary Diesel and Gas Engine Power Plant Buildings

Stationary Diesel and Gas Engine Power Plant Fuel Oil a

Stationary Diesel and Gas Engine Ratings and Performanc

Stationary Diesel and Gas Engine Speed Governing and Pa

Stationary Diesel and Gas Engine Starting Systems (1972

Stationary Diesel and Gas Engine Vibration (1972)

Stationary Diesel and Gas Engine Waste Heat Recovery Sy

Stationary Diesel and Gas Engines (1972)

Stationary Diesel and Gas Engines (1972)

Stationary Diesel and Gas Engines (1972)

Stationary Diesel and Gas Engines (1972)

Stationary Diesel and Gas Engines (1972)

Stationary Pressurized W ater Reactor Plants (1973)

Stationary 600 Volts or Less) (1972) C33.11

Stationary (1967)

Stationary, Low Pressure (Welding and Cutting) (1973)

Stationary, Medium Pressure (Welding and Cutting) (1973

Stations and Equipment (1971)

Ety Code, Part 1: Rules

Stations for Watchman, Fire Alarm and Supervisory Servi

Stations (Flood, Surface W ater, Rigging Collapse, Burgl

I for Instrumentation and Electric Equ IEEE
ANSI

NEMA

DEMA

BCI
BCI

IEEE

IEEE

SAE

Ty Ca ASTM

ANSl

ASTM

ANSI

ANSI

ANS I

AATCC

ASTM

ASTM

ASTM

Test ASTM

FMS

ASTM

TAPPI

ASTM

ASTM

ASTM

ASTM

BMA

ASTM

ASTM

AATCC

AATC

ABYC

ASTM

ARI

-Use C IEEE

Cation O IEEE

Al Princ

IEEE

NFPA

NEMA

ASTM

IEEE

AMCA

$A M C A$

TRA

VESC

VESC

TRA

EIA

UL

DEMA

DEMA

DEMA

DEMA

DEMA

DEMA

DEMA

DEMA

DEMA

DEMA

DEMA

DEMA

DEMA

DEMA

DEMA

DEMA

DEMA

DEA

DEMA

DEMA

DEMA

DEMA

Ct. for Sele DEMA

lon of Invita DEMA

ANS

UL

CSI

UL

UL

FMS

78.180

151

* 1.9

*1.41 
ng Class 1 Electric Equipment for Nuclear Power Generating of Class I Electric Equipment for Nuclear Power Generating stalled Inside the Containment of Nuclear Power Generating ied as Standby Power Supplies for Nuclear Power Generating ss I Electric Valve Operators for Nuclear Power Generating containment Structures for Nuclear Fueled Power Generating Rec. for Electric Utility Generating mechanical Characteristics of Antennas for Satellite Earth I Perform Protective Functions in Nuclear Power Generating

class 1E Control Switchboards for Nuclear Power Generating

td. Definitions of Terms Relating to Fatigue Test. and the Guide for Std. Definitions of Terms Relating to with Outlying Observations in Samples and How to Test the als Management (1972) Std. for 0.3 Definitions of Std. Rec. Pract. for Applying ency and Cost, and Recording Patron and Nonemployee Injury ion Systems for AC Electric Machinery Employing Form Wound id Filled and Dry Transformers, Hand Power Tools, Hermatic Stds. for Construction of Concrete Std Spec for Copper Rods for Locomotive tion and Measurement of Airborne Sound Transmission Class Std. for Flashing and ter Programs (1970) Gas Turbine Engine

rity Carrier Diffusion Length in Silicon by Measurement of u Per Hr.) (1972) Std. for Gas Fired High Pressure Std. for Gas Fired Low Pressure Installation and Maintenance of Std for Gas Fired Ketules, Std. Meth. of Test for Underwriter
Treated Fabric 970) ANSI Al09.11

determination of Formaldehyde Odor in Resin Treated Fabric
Std. Spec. for Test Meth. for Moisture in Wood Chips
Test Meth. for Colorfastness of Textiles to Marine

Guide for Centralized Control and Automation of Ship's Recommendation for Installation of Ceramic Tile Testing Procedures and Maintenance of Rec. for Rec. for (1967) ANSI Z11.230 Sid. Meth. of Test for Continuity of il Fuel Rec. Pract. for the Prevention of Water Damage to for Heavy Walled Carbon and Low Alloy Steel Castings for Std. Meth. of Sampling Saturated and Superheated Std. Spec. for Equipment for Sampling Water and Std. Meth. of Test for Deposit Forming Impurities in Test Meth. Visual Std. for Surfaces of New Unhardened

way for Wire and Cable Inst/ Std. for Safety for Flexible ec. Pract. for Inspection and Test. Agencies for Concrete, Std. for Bushing-Flanged, Press Fit, Std. Meth for Chemical Analysis of for Undrilled and Drilled, Plain and Self Locking, Alloy electrodeposited Coatings of Lead and Lead-Tin Alloys on for Measurment of Impact Resistance of Painted Aluminum, iform Building Code Std. for Material Spec, for Structural Uniform Building Code:

r Solid 100 Deg. Flush Shear Head A286 Corrosion Resistant to Coating or Rec/ Rec. Pract. for Surface Preparation of for Safety Requirements for Shops Fabricating Structural Structural Std. for

he Thinnest Spot in a Zine (Galvanized) Coating on lron or Iway Use (1973) ANSI G57.11 ent (1973)

Std. Spec. for Carbon Uniform Building Code Std. for Std. Pract. for Fabrication of Reinforcing Std. Pract. for Estimating Reinforcing Spec, for Placing Reinforcing irements for Hot Rolled and Cold Finished Carbon and Alloy Std. Spec. for Zinc Coated (Galvanized) Spec. for

sistant, 12.5Cr Low Carbon (SAE 51416F), Free Machining / sistant, $15.5 \mathrm{Cr}-4.5 \mathrm{Ni}-2.9 \mathrm{Mo}-0.10 \mathrm{~N}$, Solution Heat T/ sistant, $15.5 \mathrm{Cr}-4.5 \mathrm{Ni}-2.9 \mathrm{Mo}-0.10 \mathrm{~N}$, Heat Treated, $1 /$ sistant, $16.5 \mathrm{Cr}-4.5 \mathrm{Ni}-2.9 \mathrm{Mo}-0.10 \mathrm{~N}$, Equalized and $\mathrm{T} /$ sure Vessels/ Std. Spec. for Stainless and Heat Resisting d Cold Finished Age Hardening Stainless and Heat Resisting 1971/ Std. Spec, for Hot, Hot Cold, and Cold Worked Alloy Std. Spec. for Carbon and Alloy hed Stainless and Heat Resisting Chromium Nickel Manganese (1972) Std. Spec. for Merchant Quality Hot Rolled Carbon 1973) 973)

Spec. for Std. Spec, for Cold Finished Alloy
Stations (1971) ANSI N4l.5 Stations (1971) ANSI N41.7 Stations (1971) ANSI N41.9 Stations (1972) ANSI N41.13 Stations (1972) ANSI N4l.6 Stations (1972) ANSI N45.3

Stations (1973)

Stations (1973)

Stations (1973)

Stations (1973) ANSI N41.17 IEEE Trial Use Guide for IEEE Ation

General Guide for Qualifyi IEEE /for Seismic Qualification IEEE /Ous Duty Class I Motors in IEEE Esel Generator Units Appl IEEE Guide for Type Test of Cla IEEE /Penetration Assemblies in IEEE

Std. for Electrical and EIA

Statistical Analysis of Thermal Life Test Data (1972)

Statistical Meth. (1972)

Statistical Significance of Them (Sampling) (1968) ANS

Statistical Terminology and Notation for Nuclear Materi

Statistical Terms Used in Cereal Chemistry (1967)

Statistics to Analysis of Corrosion Data (1971) ANSI G8

tatistics (1971)

Stator Coils (1972) ANSI C50.26

Stators, Test. and Safety) (1973)

Stave Farm Silos (1969)

Staybolts (1973) ANSI H7.2

Stc) of Building Partitions Such as Walls, Floor-Ceil

Steady Burn Barricade W arning Lights (1971)

Steady State Performance Presentation for Digital Compu

Steady State Surface Photovoltage (Electronics) (1973)

Ion of Sealed Insulat

Try (Rotating, Liqu

Steam and Hot Water Boilers (Inputs Not Over 400,000 Bt

Steam and Hot Water Boilers (1972)

Steam and Hot Water Unit Heaters (1970)

Steam Cookers and Generators (1971)

Steam Distillation of Bituminous Protective Coatings (1

Steam Fire Pumps (1973)

Steam Meth. (1972) ANSI L14.179

Steam or Valve Bronze Castings (1974)

Steam Oven) (Pulp and Paper) (1973)

Steam Pleating (1971) ANSI L14.240, ISO 105-4

Steam Power Plant Heat Balance Practices (1972)

Test Meth. for

Steam

Steam Rooms (1973)

Steam Safety Valves on Boilers (1972)

Steam Tubine Driven Generator Units (1972)

Steam Turbine Driven Generator Units (1974)

Steam Turbine Oil Oxidation Stability by Rotating Bomb

Steam Turbines I'sed for Electric Power Generation: Foss

Steam Turbines (1974)

Steam (1969)

Steam (1970) ANSI Z111.2

Steam (1971) ANSI Z111.5

Steel Airblast Cleaned with Sand Abrasive (1970)

SteeI and Alloy Silver Brazing Joints (1973)

Steel and Aluminum Conduit for Safe Use as a Metal Race

Steel and Bituminous Materials as Used in Construction

Steel and Bronze (1973)

Steel and Cast, Open Hearth, and Wrought Iron (1973)

Steel and Corrosion Resisting Steel, UNJF-3A Thread, Ca

Steel and Ferrous Alloys (1970) ANSI G.53.8

Steel and Gavanized Steel (Coil Coating) (1974)

Steel and Iron (Shape, PIate, Sheet, Strip, Connector,

Steel and Iron (1973)

Steel and Monel Rivet (1974)

Steel and Other Hard Materials by Water Blasting Prior

Steel and Plate (1973)

Steel Antenna Towers and Supporting Structures (1972)

Steel Articles by the Preece Test (Copper Sulfate Dip)

Steel Axles, Non Heat Treated and Heat Treated, for Rai

Steel Bar and Rod Mats (Sheets) for Concrete Reinforcem

Steel Bar Materials and Service (1968)

Steel Bar Materials and Service (1970)

Steel Bar Supports in Concrete Construction (1970)

Steel Bar (1973) ANSI G24.5

Steel Barbed Wire (1973) ANSI G8.10

Steel Bars and Forgings (0.18-0.23C) (SAE 1022) (1973

Steel Bars and Forgings, Corrosion and Moderate Heat Re

Steel Bars and Forgings, Corrosion and Moderate Heat Re

Steel Bars and Forgings, Corrosion and Moderate Heat Re

Steel Bars and Forgings, Corrosion and Moderate Heat Re

Steel Bars and Shapes for Use in Boilers and Other Pres

Steel Bars and Shapes (1972)

Steel Bars for Concrete Reinforcement (1972)

Steel Bars for High Strength at Elevated Temperatures

Steel Bars for Springs (1974)

Steel Bars Including Rounds, Squares, Hexagons, and Sha

Steel Bars Subject to Mechanical Property Requirements

Steel Bars $(0.95 \mathrm{Cr}-0.20 \mathrm{Mo}-0.05 \mathrm{Te}(0.38-0.45 \mathrm{C}))$

steel Bars $\left(0.95 \mathrm{Cr}_{\mathrm{r}}-0.20 \mathrm{Mo}-0.05 \mathrm{Te}(0.40-0.53 \mathrm{C})\right.$ ) (1

Steel Bars (1964) ANSI G24.12
323

N18.8

420

E206

101

E456

E178

$\begin{array}{ll}\text { ANSI } & \mathrm{N} 15.5 \\ \mathrm{AACCH} & 78-40\end{array}$

ASTM Gl6

Z108.1

429

$* 1$

$* 1$

B12

UBC $335-1$

$* 7$

ARP681B

F391

221.59

221.13

ANS

AMCA

ANSI

AST

FMS

221.46

D255

LPD 3.9

ASTM B6I

AATCC 131

SNAME 3.11

SNAME 3.23

TCA

FMS

FMS

FMS

ASTM

ASME

Std. Spec ASTM

ASTM

ASTM

ASTM

NACE

SAE

UL

ASTM

NSA

ASTM

NSA

/Spec. for ASTM

/ Meth NCCA

ICBO

ICBO

Std. Fo NSA

NACE

Std ANS]

ElA

ASTM

ASTM

ICBO

CRSI

CRSI

CRSI

ASTM

ASTM

SAE

$\mathrm{SAE}$

SAE

SAE

ASTM

ASTM

ASTM

ASTM

ASTM

ASTM

ASTM

SAE

$\mathrm{SAE}$

ASTM

311

LPD12.59

D2272

A356

D1066 
Std. Rec. Pract for Macroetch Test of Tool Steel Bars (1971) ANSl G60.9

Std. Spec. for Std. Quality Cold Finished Carbon Std. Spec. for Merchant Quality Hot Rolled Carbon Std. Spec. for Machined Flat and Square Tool Spec. for Tolerances of Low Alloy

Std. Spec. for Stainless and Heat Resisting al Quality, for Pressure Piping Component/ uid Power Applications (1974)

$.55 \mathrm{Ni}-1.0 \mathrm{Mo}-0.12 \mathrm{~V}(0.43-0.49 \mathrm{C}))(1973)$

$.82 \mathrm{Cr}-1.82 \mathrm{Ni}-0.40 \mathrm{Mo}-0.07 \mathrm{~V}(0.41-0.46 \mathrm{C})$ mo $(0.49-0.55 \mathrm{C}))(1973)$ tant $(0.95 \mathrm{Cr}-0.55 \mathrm{Mo}-0.30 \mathrm{~V}(0.40-0.50 \mathrm{C}) \mathrm{V} / \mathrm{Spec}$. for 0Mo $(0.11-0.17 \mathrm{C})$ (SAE 8615) (1973)

0 Mo-0.04V-B (0.38-0.43C) (Modified 98Bv40) (1973)

mo (0.38-0.43C) (SAE 4340), Premium Quality, Consumabl/ mo $(0.38-0.43 \mathrm{C})(\mathrm{SAE} 4340)(1973)$

mo $(0.07-0.13 \mathrm{C})$ (SAE 9310), Premium Quality, Vacuum Co/ mo (0.07-0.13C) (SAE 9310), Premium Quality (1973)

ed Rings $(1.3 \mathrm{Mn}-1.5 \mathrm{SI}-0.30 \mathrm{Cr}-1.8 \mathrm{Ni}-0.4)$ ealed) $(0.48 \mathrm{Cr}-8.0 \mathrm{Ni}-4.0 \mathrm{Co}-0.48 \mathrm{Mo}-0.09 /$

moderate Heat Resistant, 17Cr (SAE 51430) Annealed (1973/ heat Resistant $(15 \mathrm{Cr}-26 \mathrm{Ni}-1.3 \mathrm{Mo}-2.1 \mathrm{Ti}-1$

$-0.35 \mathrm{Mo}-0.20 \mathrm{~V}(0.32-0.38 \mathrm{C})$. Special Grade (1973)

$0.08 \mathrm{~V}(0.28-0.33 \mathrm{C})(1973)$

n Resistant, $12 \mathrm{Cr}-8.5 \mathrm{Ni}-2.0 \mathrm{Cu}-1.1 \mathrm{Ti}$, Vacuum Induct/ Rec. Spec. for

Std. Spec. for Stainless and Heat Resisting rvice (/ Std. Spec. for Electric Resistance Welded Carbon Std. Spec. for Seamless Medium Carbon

ec. for Electric Resistance Welded Carbon Molybdenum Alloy Std. Spec. for Seamless Carbon Molybdenum Alloy

i B125.7 Std. Spec. for Seamless Carbon Std. Spec. for Electric Resistance Welded Carbon 73) Std. Spec. for Seamless Ferritic and Austenitic Alloy Tubes (1973) ANSl B12\% Std. Spec. for Welded Austenitic 1974) ANSI G38.4 Std. Spec. for Welded Austenitic
Std. Spec. for Alloy and Stainless langes, and Fittings for Low Temper/ Std. Spec. for Alloy $345 \mathrm{MPa}$ ) Tensile Strength, Hardened / Spec. for Low Alloy 73 )

Spec. for Low Alloy Std. Spec. for High Strength Nonheaded 19 Std. Spec. for Quenched and Tempered Alloy able Nuts and Plain Hardene/ Std. Spec. for High Strength Uniform Building Code Std. for High Strength Std. for Sid. Factory Made Wrought Std Meth of Test for Nickel on 1 for Spectrochemical Analysis of Plain Carbon and Low Alloy G52.2 Std. Spec. for Mild to Medium Strength Carbon (1974/ Marnless and Alloy Low Temperature Service (1974/ Std. Spec. for Ferritic Std. Spec. for Heavy walle Std. Spec. for High Strength
aining Parts Suitable for High Temp/ Std. Spec. for Alloy perature Service (1974) ANSl G37.2 i G52.5 td. Spec. for Carbon 6.28

Std Spec for Low Alloy Std. Reference Radiographs for
tudinal Beam Ultrasonic Inspection of Carbon and Low Alloy alification of Procedures and Personnel for the Welding of Reference Radiographs for Heavy Walled (4 1/2 to 12 In.) Std. Spec. for Austenitic Manganese sure Vessels (1974)

8-0.36C) (SAE 4330 Modified) (1973)

$8-0.46 \mathrm{C}$ ) (SAE 4340 Modified) (1973)

5C) (1973)

Std. Spec. for

Low Alloy

Low Alloy

Std. Spec, for Aluminum Coated Std. Spec. for Zinc Coated Spec. for Galvanized Std. Spec. for Carbon

Std. Spec. for Weldless Carbon Corrosion Resisting Chromium for Polyvinyl Chloride Externally Coated Galvanized Rigid Std. Spec. for

Std. Meth of Test for Adhesion of Vulcanized Rubber to ted (Galvanized) and Aluminum Coated (Aluminized) Stranded ed (ACSR) (1972) / Spec. for Aluminum Coated (Aluminized) ed (ACSR) (1974)/ pec. for Aluminum Coated (Aluminized)
Std. Spec. for Zinc Coated (Galvanized) Spec. for Mild Spec, for Low Alloy

ec. for Corrosion Resistíng Chromium and Chromium-Nickel Std. for Prestressed Concrete Pressure Pipe, Std. Spec. for Axle

Steel Bars (1973)
Steel Bars (1973)

Steel Bars (1973)

Steel Bars (1973)

Steel Bars, and Shapes (1973) ANSI G24.3I

Steel Bars, Carbon, Hot Rolled and Cold Finished, Speci

Steel Bars, Carbon, Hot Rolled, Special Quality, for Fl

Steel Bars, Forgings, and Mechanical Tubing $(1.05 \mathrm{Cr}-0$

Steel Bars, Forgings, and Mechanical Tubing (1.62S1 -0

Steel Bars, Forgings, and Tubing $(0.80 \mathrm{Cr}-1.8 \mathrm{Ni}-0.25$

Steel Bars, Forgings, and Tubing, Corrosion Resistant,

Steel Bars, Forgings, and Tubing, Low Alloy, Heat Resis

Steel Bars, Forgings, and Tubing, $0.50 \mathrm{Cr}-0.55 \mathrm{Ni}-0.2$

Steel Bars, Forgings, and Tubing, $0.80 \mathrm{Cr}-0.85 \mathrm{Ni}-0.2$

Steel Bars, Forgings, and Tubing, $0.80 \mathrm{Cr}-1.8 \mathrm{Ni}-0.25$

Steel Bars, Forgings, and Tubing, $0.80 \mathrm{Cr}-1.8 \mathrm{Ni}-0.25$

Steel Bars, Forgings, and Tubing, $1.2 \mathrm{Cr}-3.25 \mathrm{Ni}-0.12$

Steel Bars, Forgings, and Tubing, $1.2 \mathrm{Cr}-3.25 \mathrm{Ni}-0.12$

Steel Bars, Forgings, Mechanical Tubing, and Flash Weld

Steel Bars, Forgings, Rings, and Mechanical Tubing (Ann

Steel Bars, Forgings, Tubing, and Rings, Corrosion and

Steel Bars, Forgings, Tubing, and Rings, Corrosion and

Steel Bars, Forgings, Tubing, and Rings, $0.78 \mathrm{Cr}-1.8 \mathrm{Ni}$

Steel Bars, Forgings, Tubing, $0.88 \mathrm{Cr}-1.8 \mathrm{Ni}-0.42 \mathrm{Mo}-$

Steel Bars, Wire, Forgings, and Forging Stock, Corrosio

Steel Bi-Fold Closet Doors and Frames (1972)

Steel Billets and Bars for Reforging (1972) ANSI G81.13

Steel Boiler and Superheater Tubes for High Pressure Se

Steel Boiler and Superheater Tubes (1973) ANSI B125.10

Steel Boiler and Superheater Tubes (1973) ANSI B125.13

Steel Boiler and Superheater Tubes (1973) ANSI B125.9

Steel Boiler Tubes for High Pressure Service (1973) Ans

Steel Boiler Tubes (1973) ANSI B125.6

Steel Boiler, Superheater, and Heat Exchanger Tubes (19

Steel Boiler, Superheater, Heat Exchanger, and Condense

Steel Bolting Materials for High Temperature Services (

Steel Bolting Materials for Pressure Vessels, Valves, F

Steel Bolts and Screws, Heat Resistant, 195,000 psi (1,

Steel Bolts and Screws, Heat Treated, Roll Threaded (19

Steel Bolts and Studs (1973)

Steel Bolts for Structural Steel Joints (1971) ANSl G24

Steel Bolts for Structural Steel Joints, Including Suit

Steel Bolts, Nuts, Washers, and Bolted Parts (1973)

Steel Butt Welding End Valves (1973)

Steel Butt welding Fittings (1971)

Steel by Photometric Analysis (1972)

Steel by the Point to Plane Technique Using an Optical

Steel Castings for General Application (1973) ANSl G50.

Steel Castings for High Temperature Service (1972) ANSI

Steel Castings for Highway Bridges (1974) ANSI G52.3

Steel Castings for Pressure Containing Parts Suitable F

Steel Castings for Pressure Containing Parts Suitable F

Steel Castings for Steam Turbines (1974)

Steel Castings for Structural Purposes (1973) ANSI G52.

Steel Castings Specially Heat Treated for Pressure Cont

Steel Castings Suitable for Fusion Welding for High Tem

Steel Castings Suitable for Pressure Service (1974) Ans

Steel Castings Up to 2 In. in Thickness (1972) ANSI Z16

Steel Castings (1970) ANSI G52.7

Steel Castings (1972) ANSI G52.6

Steel Castings (1972) ANSI Z166.19

ASTM
Std. Spec. for Longi ASTM

Steel Castings (1973) ANSI G52.4

Steel Castings, Heavy Walled, Carbon and Alloy for Pres

Steel Castings, Investment $0.80 \mathrm{Cr}-1.8 \mathrm{Ni}-0.35 \mathrm{Mo}(0.2$

Steel Castings, Investment $0.80 \mathrm{Cr}-1.8 \mathrm{Ni}-0.35 \mathrm{Mo}(0.3$

Steel Castings, Investment, $0.95 \mathrm{Cr}-0.20 \mathrm{Mo}(0.25-0.3$

Steel Chain Link Fence Fabric (1971) ANSI G53.21

Steel Chain Link Fence Fabric (1971A) ANSI G53.20

Steel Chain Link Fence Fabric (1973)

Steel Chain (1972) ANSI G61.2

Steel Chain (1973) ANSI G61.4

Steel Clad Plate Sheet and Strip (1972) ANSI G81.6

Steel Cleanliness Requirements (Magnetic Particle Inspe

Steel Conduit and Electrical Metallic Tubing (1974)

Steel Conveyor Chain (1972) ANSI G61.3

Steel Cord (1973)

Steel Core for Aluminum Conductors, Steel Reinforced (A Steel Core Wire for Aluminum Conductors, Steel Reinforc Steel Core Wire for Aluminum Conductors, Steel Reinforc Steel Covered Arc Welding Electrodes (1969) ANSl A5.1 Steel Covered Arc Welding Electrodes (1969) ANSI W3.5 Steel Covered Welding Electrodes (1969) ANSI W3.4 Steel Cylinder Type, for Water and Other Liquids (1972) Steel Deck Roofing (1972)

Steel Deformed and Plain Bars for Concrete Reinforcemen
ASTM

ASTM

ASTM

ASTM

SAE

ASTM

ASTM

ASTM

SAE

$S A E$

$S A E$

$S A E$

SAE

SAE

$\mathrm{SAE}$

$S A E$

$S A E$

SAE

SAE

SAE

SAE

$S A E$

SAE

$S A E$

STDI

ASTM

ASTM

ASTM

ASTM

ASTM

ASTM

ASTM

ASTM

ASTM

ASTM

ASTM

SAE

SAE

ASTM

ASTM

ASTM

ICBO

ANSI

ANSl

ASTM

ASTM

ASTM

ASTM

ASTM

ASTM

ASTM

ASTM

ASTM

ASTM

u ASTM

Std ASTM

ASTM

ASTM

SAE

$\mathrm{SAE}$

$S A E$

ASTM

ASTM

CLFM

ASTM

ASTM

ASTM

SAE

1. NEMA

ASTM

ASTM

ASTM

ASTM

ASTM

A W'S

AWS

Sp AWS

FMS

ASTM

A56I

A 108

A575

MS225IE

A 276

A696

AMS6432A

AMS6416A

AMS6424

AMS5749

AMS6305

AMS6270J

AMS6422

AMS64 I4B

AMS6415H

AMS6265D

AMS6267B

MS64 I8E

AMS6540B

AMS5627B

A MS5735H

A.MS6430B

AMS6427E

AMS5617A 
Steel Door and Frame Assemblies (1972)

nd Acceptance Criteria for Rate of Air Flow Through Closed

Rec. Erection Instructions for

Steel Door and Frame Assemblies (1972)

Rec. Std.

struction (1972)

972)

Steel Door Frame Details (1972)

Std.

Steel Door Frames (1972)

Steel Door, Frame and Hardware Schedule (1972)

Steel Doors and Frames for Modular Masonry Building Con

Rec. Spec, for Builders'Hardware on Std.
Rec. Spec. for Std. Rec. Spec. for Std.

Rec. Minimum Hardware Reinforcing Garages on

Rec. Weatherstripping for Std.

Rec. Std. Minimum Acceptance Values for Rec. Selection and Isage Guide for Std.

Rec. Louver Details for Std. material to Light Gage Steel Studs (1972) Std. Spec. for
ANSI Z) Tentative Meth. of Test for Weight Loss of Sheet 1969) ANSI W 3.17
Spec. for Bare Mild

W 3.20

w3.18

Spec, for Mild Spec. for Mild lux Cored Corrosion Resisting Chromium and Chromium Nickel
Std. for High Speed

(1968) ANSI G38.5

Std. for Safety Requirements for

(1973) / Std. Spec. for Zinc Coated (Galvanized) Iron or Std. Spec. for Welded Austenitic Stainless

3) Std. for Forged

erature / Std. Spec. for Forged or Rolled $9 \%$ Nickel Allor for Spectrochemical Analysis of PIain Carbon and Low Alloy Rec. Pract. for Meth. of Sampling Std. Spec. for Structural

std. Spec. for Piping Fittings of Wrought Carbon and Alloy std. Spec. for Piping Fittings of Wrought Carbon and Alloy Std. Meth. of Test for Reboiling Tendency of Shee Std. Spec. for Structura ec. for Hot Worked, Hot Cold Worked, and Cold Worked Alloy rators (1970) ANS1 G55.6 Std. Spec. for Vacuum Treated Electric $s$ (1972) ANSI G55.8 valve, etc.) (1973) ANSI G17.3 Std Spec for Alloy Std. Spec. for Carbon and Alloy Std. Spec. for Carbon si) Std. Spec. for Quenched and Tempered Carbon and Alloy for Quenched and Tempered Vacuum Treated Carbon and Alloy ssure Vessel Components (1970) Ansil Std. Spec. for Alloy
) ANSI G55.15
Std. Spec. for Vacuum Treated Alloy ) ANSI G55.15 i G55. 14 Std. Spec. Std. Spec. for Vacuum Treated Carbon and Alloy Std. Meth. of Magnetic Particle Examination of Std. Spec. for Stainless and Heat Resisting

I Use (1972)

wallboard and Backing Board (1/

pipeline Valves (1974) Pract. for the Identification of Std Electrical 25/ Std. Spec. for High Sirength Low Alloy 25

Sid. Spec. for Seamless Cold Drawn Intermediate Alloy std. Spec. for Heat Treated

for Torque Tension Requirements for Prevailing Torque Type Std. Specs. for Welding of Structural

Safety Std. for Atmospheric Type Welded Std. Specs, and Load Tables for Longspan Std. Specs. and Load Tables for Open Web Std. Specs, and Load Tables for Longspan Spec for Structura

for Quenched and Tempered Alloy Steel Bolts for Structural and Load Tables for Open Web, Longspan, and Deep Longspan Std. Spec. for High Strength Steel Bolts for Structural

Uniform Building Code Std. for Open Web Structural

r Aircraft, Pan Head, Phillips Recess Full Threaded, Alloy pan Head, Phillips Recess, Short Thread, 160,000 psi Alloy Std. for Mechanical and Quality Requirements for Std. Spec. for Aluminum for Use in Iron and Suggested Components of a Concrete Reinforcing Std. Spec. for Seamless Stainless Std. Spec. for Welded Stainless

Std. Spec, for Seamless Carbon and Alloy r Stress Variation or Stress Reversal Design of Structural service (1973) ANSI E38. Std. Spec. for Carbon and Alloy Sid. for Mechanical and Quality Requirements for Prevailing Torque, All Metal, 1/ Std. Spec. for Carbon Spec. for Self-Locking
Steel Doors and Frames (Reinforcement-Application) (1

Steel Doors and Frames (1969)

Steel Doors and Frames (1972)

Steel Doors and Frames (1972)

Steel Doors and Frames (1972)

Steel Doors and Frames (1972)

Steel Doors (1972)

Steel Doors (1972)

Steel Drill Screws for the Application of Gypsum Sheet

Steel During Immersion in Sulfuric Acid Solution (1971)

Steel Electrodes and Fluxes for Submerged Arc Welding

Steel Elcctrodes for Flux Cored Arc Welding (1969) ANSI

Steel Electrodes for Gas Metal Arc Welding (1969) ANSI

Steel Electrodes (1974)

SteeI End Mills, .125 Thru 3.000 Diameter (1973)

Steel Erection (1972)

Steel Externally and Internally Threaded Std. Fasteners

SteeI Farm Field and Railroad Right of Way Wire Fencing

Steel Feedwater Heater Tubes (1973)

Steel Fittings (Pipe), Socket Welding and Threaded (197

Steel Flanges, Fittings, Valves, and Parts for Low Temp

Steel for Boron by the Point to Plane Arc Technique (19

Steel for Chemical Analysis (1971)

Steel for Locomotives and Cars (1970A) A NSI G39.1

Steel for Low Temperature Service (1973) ANSI G46.2

Steel for Moderate and Elevated Temperatures (1973) Ans

Steel for Porcelain Enameling (1969) ANSI ZI67.21

Steel for Ships (1973)

SteeI Forgings and Forging Billets for High Strength at

SteeI Forgings for Generator Rotors (1971) ANSI G55. 13

Steel Forgings for Nonmagnetic Retaining Rings for Gene

Steel Forgings for Pinions and Gears for Reduction Gear

Steel Forgings for Piping Components (Flange, Fitting,

Steel Forgings for Pressure Vessel Components (1973) an

Steel Forgings for Pressure Vessels (1974) ANSI G55.17

Steel Forgings for Seamless Drums, Heads, and Other Pre

Steel Forgings for Turbine Rotor Disks and Wheels (1970

Steel Forgings for Turbine Rotors and Shafts (1974) Ans

Steel Forgings (1971) ANSI G60.6

Steel Forgings (1973) ANSI G81.26

Steel Forgings, Carbon and Alloy, for General Industria

Steel Frame Shoring Erection Procedure (1969)

Steel Frame Shoring Safety Rules (1972)

Steel Framing Members to Receive Screw Attached Gypsum

Steel Gas Welding Rods (1969) ANSI W 3.2

Steel Gate, Plug. Ball, and Check Valves for Petroleum

Steel Grades in ASTM Specs. (1972)

Steel H.Piles and Sheet Piling for Use in Marine Enviro

Steel Heat Exchanger and Condenser Tubes (1973) ANSI BI

Steel Heavy Thimble (For Ground Support Equipment) (197

Steel Helical Springs (1973) ANSI G54.20

Steel Hex Locknuts (1972)

Steel Highway Bridges (1974)

Steel Inside Tanks for Oil Burner Fuel (1973)

Steel Joints Deep DLJ and DLH Series (1970)

Steel Joints J Series and H Series (1970)

Steel Joints LJ and LH Series (1970)

Steel Joints Using ASTM A325 or A490 Bolts (1970)

Steel Joints (1971) ANSI G24.19

Steel Joints (1974)

Steel Joints, Including Suitable Nuts and Plain Hardene

Steel Joist Design (1973)

Steel Lintels (Tech. Notes) (1969)

Steel Machine Screw (1974)

Steel Machine Screw (1974)

Steel Machine Screws (1973)

Steel Manufacture (1960) ANSI H38.25

Steel MateriaI Supply and Installation Contract (1968)

Steel MechanicaI Tubing (1971) ANSI G62.1

Steel Mechanical Tubing (1973)

Steel Mechanical Tubing (1973) ANSI G62.4

Uniform Building Code Std. Fo ICBO

Steel Members (1973) Uniform Building Code
Steel Nuts for Bolts for High Pressure and Temperature

Steel Nuts (1971)

Steel Nuts (1972) ANSI G24.30

Steel Nuts, Corrosion and Heat Resistant, High Strength
1. Test Proc a

STDI 114

114

116

$111 \cdot A$

105

$111 . \mathrm{D}$

110

107

100

* 10

111

I11.E

112
108

$111-\mathrm{C}$

C646

C694

A5.17

A 5.20

A5. 18

A5. 22

986

A 10.13

A 307

A 116

A688

B16.11

A522

E404

J408C

Al13

A 420

A 234

C632

A 131

A 477

A 469

A 289

A291

A 105

A541

A508

A 336

A471

A 470

A275

A 473

A 668

*6

$* 3$

A97.2

A5. 2

$6 \mathrm{D}$

A664

A 690

A 199

NSA $\quad$ I045

IFI 101

ASHTO *6

UL $\quad 80$

AISC \$316.1

AISC S316-3

AlSC $\$ 316-2$

AISC $\$ 314$

Std. Spec. ASTM A490

$\begin{array}{ll}\text { ASTM } & \text { A } 325 \\ \text { ICBO } & \text { UBCS27.4 }\end{array}$

BIA

Std. Fo NSA

$31 \mathrm{~B}$

600

623

103

IFTM B37

CRSI *1.9.1

ASTM A511

A554

A519

UBCS27.3

Al94.

AAE J995B

ASTM A563

SAE AMS7250C 
, Prevailing Torque, All Metal, 1/ is, and Cast Aluminum Covers (/ ated Products (1973)

Spec. for Self Locking Std. for Electrical Sheet Std. Meth. for Preparation of Std. Rec. Pract. for Descaling and Cleaning Stainless Std. for is for High Temper/ Std. Spec. for Forged or Rolled Alloy
Std. Spec. for Electric Fusion Welded

Std. Spec. for Forged or Rolled Alloy for Welded Large Diameter Austenitic Chromium Nickel Alloy atures (1973)

4

) ANSI G36.49

(1973) ANSI B125.25

Spec. for Electric Fusion Welded

Std. Spec, for Electric Fusion Welded Std. Spec. for Metal Arc Welded Std. Spec. for Centrifugally Cast Carbon for Electric Fusion Welded Autenitic Chromium Nickel Alloy 5.39 25.30 Std. Spec. for Centrifugally Cast Austenitic
Std. Spec. for Seamless Carbon Std. Spec. for Centrifugally Cast Ferritic Alloy Std. Spec. for Seamless Ferritic Alloy 5.24 Std Spec for Seamless and Welded 17 nd Hot Dipped Zinc Coated (Galvanized) Welded and Seamless Std. Spec. for Seamless Carbon 1972) Std. Spec. for Electric Welded Low Carbon service (1972) Spec. for Fabricated Structural for General Requirements for Specialized Carbon and Alloy Std. Spec. for Electric Resistance Welded Std. Spec. for Seamless and Welded Austenitic Stainless c. for Welded and Seamless Black and Hot Dipped Galvanized lletin for Nondestructive Test. Terminology for Defects in Std. Spec. for W'rought Austenitic Stainless Std. Spec. for Copper and Its Alloy Clad $5 \mathrm{Cr}-4.5 \mathrm{Ni}-2.9 \mathrm{Mo}-0.10 \mathrm{~N}$, Solution Heat Treated (1973/

std. Spec. for Heat Resisting Chromium and Chromium Nickel ated/Std. Spec. for Hot, Hot Cold, and Cold Worked Alloy Std. Spec. for Stainless and Heat Resisting Chromium $s$ for Delivery of Flat Rolled Stainless and Heat Resisting

spec. for High Yield Strength, Quenched and Tempered Alloy for Low and Intermediate Tensile Strength Carbon-Silicon r Temperature Service (1972/ Std. Spec. for High Strength Std. Spec. for General Requirements for Delivery of Std. Spec, for Manganese Vanadium Alloy

Std. Spec. for Charpy V-Notch Testing Requirements for Std. Spec. for Nickel Alloy Std. Spec. for Molybdenum Alloy Std Spec for Carbon Manganese Silicon Std. Spec. for Manganese Molybdenum and Its Nickel Alloy td. Spec. for Low and Intermediate Tensile Strength Carbon Std. Spec. for Chromium Molybdenum Alloy eth. and Spec. for Straight Beam Ultrasonic Examination of for Straight Beam Ultrasonic Examination of Plain and Clad Std. Spec. for Quenched and Tempered Carbon Std. Spec. for Structural Carbon

td. Spec. for Low and Intermediate Tensile Strength Carbon essure Vessels (1972) ANSI G35.7

Std. Spec. for Carbon Std. Spec. for Ultrasonic, Angle Beam Examination of ure Vessels (1/ Std. Spec. for Chromium Molybdenum, Alloy Is (1972/ Std. Spec. for Nickel Chromium Molybdenum Alloy (1962) std. Spec. for General Requirements for Delivery of Rolled Std. for Stainless r Temperature Service (1972) ANSI/ perature Service (1973) ANSl G35.1/ Std for Stainless Std. Spec, for Carbon Std. Spec. for Carbon std. Spec. for Chromium-Copper-Nicke1-Aluminum Alloy

of Hexavalent Chromium Containing Treatments on Galvanized ing Against Embrittlement of Hot Dip Galvanized Structural Cut Lengths) (1974)

Shapes, Rolled Floor Plates, and Steel Sheet Piling (1/ orging; Hot Rolled and Cold Finished Bars; Hot Rolled D/ (1972)

railway Axles (1973) Definitions for Mechanical Test. of Wrought and Cast se (1971) ANS1 G57.12

Std. Spec. for Untreated Carbon ated (Aluminized) Steel Core Wire for Aluminum Conductors, (Aluminized) Stranded Steel Core for Aluminum Conductors. ated (Galvanized) Steel Core Wire for Aluminum Conductors td. Spec. for Aluminum Conductors, Concentric Lay Stranded Std. Spec. for Stainless and Heat Resisting 36C)) (SAE 4330 Modified) (1973)

Std. Spec. for Seamless and Welded Austenitic Stainless

or Measuring Case Depth (1972)
Steel Nuts, Corrosion and Heat Resistant, High Strength Steel Outlet Boxes, Device Boxes, Covers and Box Suppor Steel Panels for Test. Paint, Varnish, Lacquer, and Rel Steel Parts, Equipment and Systems (1972) ANSI G81.16 Steel Pipe Flanges, Flanged Valves and Fittings (1973) Steel Pipe Flanges, Forged Fittings, and Valves and Par Steel Pipe for Atmospheric and Lower Temperatures (1972 Steel Pipe for Corrosive or High Temperature Service (1 Steel Pipe for High Pressure Service at Moderate Temper Steel Pipe for High Pressure Service (1972A) ANS1 B125. Steel Pipe for High Pressure Transmission Service (1971 Steel Pipe for High Temperature Central Station Service Steel Pipe for High Temperature Service (1972)

Steel Pipe for High Temperature Service (1972)

Steel Pipe for High Temperature Service (1972) ANSI B12

Steel Pipe for High Temperature Service (1972A) ANSI Bl Steel Pipe for High Temperature Service (1973)

Steel Pipe for High Temperature Service (1973) ANSI Bl2

Steel Pipe for Low Temperature Service (1973) ANSI B125

Steel Pipe for Ordinary Uses (1973) ANSI B125.2

Steel Pipe for Process Piping (1972A)

Steel Pipe for the Chemical Industry (1971) ANSI B125.3

Steel Pipe for Use of Welded Offshore Fixed Platforms (

Steel Pipe (Welded and Seamless) for Underground Water

Steel Pipe (1972) ANSI B125.20

Steel Pipe (1972A)

Steel Pipe (1972A) ANSI B125.16

Steel Pipe (1973) ANSI B 125.1

Steel Pipe (1974)

Steel Piping Fittings (1973) ANSI G37.8

Steel Plate (1973)

Steel Plate. Corrosion and Moderate Heat Resistant, 15

Steel Plate, Sheet, and Strip for Fusion Welded Unfired

Steel Plate, Sheet, and Strip for High Strength at Elev

Steel Plate, Sheet, and Strip (1972) ANSI G81.2

Steel Plate, Sheet, and Strip (1972) ANSI G81.30

Steel Plate, Suitable for Welding (1970E) ANSI G24.26

Steel Plates for Machine Parts and General Construction

Steel Plates for Pressure Vessels for Moderate and Lowe

Steel Plates for Pressure Vessels (1972)

Steel Plates for Pressure Vessels (1972)

Steel Plates for Pressure Vessels (1972)

Steel Plates for Pressure Vessels (1972) ANSI G33.1

Steel Plates for Pressure Vessels (1972) ANSI G34.I

Steel Plates for Pressure Vessels (1972) ANSI G35.14

Steel Plates for Pressure Vessels (1972) ANSI G35.15

Steel Plates for Pressure Vessels (1972) ANSI G35.2

Steel Plates for Pressure Vessels (1973) ANS1 G35.4

Steel Plates for Pressure Vessels (1973) ANSI G60.2

Steel Plates for Special Applications (1971) ANSI G35.2

Steel Plates for Structural Applications (1973)

Steel Plates of Improved Toughness (1973)

Steel Plates of Structural Quality (1970) ANSI G24.2

Steel Plates with Improved Transition Properties for $\mathrm{Pr}$

Steel Plates (1973) ANSl G35.24

Steel Plates, Quenched and Tempered Chromium, for Press

Steel Plates, Quenched and Tempered, for Pressure Vesse

Steel Plates, Shapes, Sheet Piling, and Bars for Struct

Steel Plumbing Fixtures (Designed for Residential Use)

Steel Plumbing Fixtures (1971)

Steel Pressure Vessel Plates for Intermediate and Highe

Steel Pressure Vessel Plates for Moderate and Lower Tem

Steel Pressure Vessel Plates (1972) ANSI G33.3

Steel Priar to Conversion Coating (Coil) (1974)

Steel Products and Procedure for Detecting Embrittlemen

Steel Products Manual for Carbon Sheet Steel (coils and

(Steel Products Manual) Carbon Steel Strip (1971)

(Steel Products Manual) Carbon Steel' Plates. Structural

(Steel Products Manual) Carbon Steel: Semifinished for F

(Steel Products Manual) Steel Specialty Tubular Products

Steel Products Manual: Tin Mill Products (1973)

Steel Products Manual: Wrought Steel Wheels and Forged

Steel Products (Including Dies and Fixtures) (1973)

Steel Products (1973) ANSl G60.1

Steel Products (1973) ANS1 G8.18

Steel Railway Axles for Export and General Industrial

Steel Reinforced (ACSR) (1972) ANS1 C7.47

Steel Reinforced (ACSR) (1972) ANSI C7.61

Steel Reinforced (ACSR) (1974) ANS] C7.60

Steel Reinforced, (ACSR) (1972A) ANSI C7.22

Steel Rope Wire (1972) ANSI G81.32

Steel Safety Relief Valves Used in the Petroleum Indust

Steel Sand Castings $(0.80 \mathrm{Cr}-1.8 \mathrm{Ni}-0.35 \mathrm{Mo}(0.28-0$.

Steel Sanitary Tubing (1973) ANSI B36.38

Steel Scrap (1971)

Steel Self Drilling Tapping Screws and Rec. Technique F
SAE

NEMA

ASTM

ASTM

ANSI

ASTM

ASTM

ASTM

ASTM

ASTM

ASTM

ASTM

ASTM

Spec ASTM

ASTM

ASTM

ASTM

ASTM

ASTY

/Ack a ASTM

ASTM

ASTM

AP]

UL

ASTM

ASTM

ASTM

Std. Spe ASTM

Bu AP1

ASTM

ASTM

ASTM

ASTM

ASTM

Ment ASTM

ASTM

ASTM

ASTM

ASTM

ASTM

ASTM

ASTM

ASTM

ASTM

ASTM

ASTM

ASTM

/M ASTM

ASTM

ASTM

ASTM

ASTM

ASTM

ASTM

ASTM

ASTM

ASTM

USC

IAPMO

ASTM

ASTM

ASTM

Tion NCCA

ASTM

A1SI

AlSI

AlSI

A1S]

AISI

AlSI

ASTM

Std. Meth ASTM

ASTM

ASTM

/Aluminum Co ASTM /num Coated ASTM

for Zinc Co AST

ASTM

ASTM

APl

$\mathrm{SAE}$

ASTM

ISIS

SAE

AMS725IB

OSl

A.380

B 16.5
A 182

A671

A 409

A 672

A15.5

A381

A. 376

A 660

A358

A451

A 106

4 26

A.333

A 120

A524

A587

2B

888 
ue, All Metal (1973) Std. Spec, for Regular Quality Hot and Cold Rolled Alloy Std. Spec. for Drawing Quality Hot and Cold Rolled Alloy

Std. Spec. for Hot Rolled Carbon (0.15 Maximum, Percent) stant, $15.5 \mathrm{Cr}-4.5 \mathrm{Ni}-2.9 \mathrm{Mo}-0.10 \mathrm{~N}$, Solution Heat Tre/ th. Low Alloy Columbium and/or Vanadium (/ Std. Spec. for Std. Spec. for Hot Rolled Carbon ) by the Hot Dip Process (1971) ANSI G8.21 Std. Spec. for nized) by the Hot Dip Process (1971) Ansi/ Std. Spec. for steel' Plates, Structural Shapes, Rolled Floor Plates, and $(0.49-0.55 \mathrm{C}))(1973)$

$-0.20 \mathrm{~V}(0.33-0.38 \mathrm{C}))$ Special Grade (1973)

73) Spec. for Corrosion and Moderate Heat Resistant $8 \mathrm{Cr}-4.5 \mathrm{Ni}-3.5 \mathrm{Cu}-(\mathrm{Cb}+\mathrm{Ta})$, Consumable Electrode $\mathrm{Val}$ $(0.38-0.43 \mathrm{C})(\mathrm{SAE} 8740)(1973)$

1.0Mo-0.09V (0.17-0.23C), Premium Quality. Consumab/ ocess for Roofing (1971) ANSl G8.21 Std. Spec. for ocess (1973) Ans/ Std. Spec, for General Requirements for ocess, Commercial Quality (1971) ANSl G8./ Std. Spec. for (Galvanized) by Hot Dip Process (1971) An/ Std. Spec. for Sid. Spec, for Std. Spec. for Carbon Std. Epec, for ngths, Zinc Coated (Galvanized) by Hot Di/ Std. for vocabulary) (1973) (Steel Products Manual) Meth. of Test. Coated (1972T) Spec. for Spec. for Spec. for g Over Concrete Slab Floors (1962)
$6150)(1973)$ Std. Spec. for Seamless Low Carbon and Carbon Molybdenum
Std. Spec. for Seamless Intermediate Alloy 39 Std. Spec. for Seamless Austenitic Chromium Nickel Uniform Building Code Std. for Prestressed Carbon (Steel Products Manual) Carbon Std. Spec. for Cold Rolled Carbon orm Building Code Std. for Spec. and Design of Cold Formed for Spec. and Design of Light Gauge Cold Formed Stainless Std. Spec. for Sintered Copper i/ Std. Spec. for Cold Formed Welded and Seamless Carbon Std. Spec. for Hot Formed Welded and Seamless Carbon Surface Preparation Spec. for

for the Application of Gypsum Sheet Material to Light Gage Uniform Building Code Std. for Shear Conńectors oll Threaded (135,000 psi (931 MPa)/ Spec. for Low Alloy Spec, for Low Alloy for Screw Applic/ Std. Spec, for Nonload (Axial) Bearing 3) c. for Detection of Mill Chemical Treatments on Galvanized roperties and Meth. of Test. for Helical Spring and Carbon Std. Meth. of Test for Tensile Strength Properties of 25.1/ Sid Spec for Seamless and Welded Carbon and Alloy uirements for Carbon, Ferritic Alloy, and Austenitic Alloy Spec. for Seamless Ferritic Austenitic Alloy td. Spec, for Std. for Seamless Low Carbon Std. for Welded and Cold Drawn Low Carbon Std. Spec. for Seamless and Welded Ferritic Stainless Std. Spec. for Seamless and Welded Austenitic Stainless d Beading (1/ Std. for Welded Flash Controlled Low Carbon Std. Spec. for Copper Brazed Std. for Welded Low Carbon Std. for Brazed Double Wall Low Carbon Manual of Cold Formed Welded Structural $\mathrm{r}-20 \mathrm{Ni})(\mathrm{SAE} 30310)(1973)$ Spec. for rements for Seamless and Electric Resistance Welded Carbon n (1971) Ansil

Std. Rec. Pract. for Eddy Current Test. of

ted for High Temperature Service (1/ Carbon iquids (1973) ANSI B137.1 Std. Spec. for Alloy
Std. for Safety Std. for

meth. for Spectrochemical Analysis of Carbon and Low Alloy Rules for Building and Classing Std. for Cement Mortar Protective Lining and Coating for Std. Spec, for Seamless and Welded Carbon ec. for Corrosion Resisting Chromium and Chromium-Nickel 6130) (1973) $-11 \mathrm{Ni}-(\mathrm{Cb}+\mathrm{Ta})(1973)$ nt $(11.8 \mathrm{Cr}-2.8 \mathrm{Ni}-1.6 \mathrm{Co}-1.8 \mathrm{Mo}-0.32 \mathrm{~V})(1$ Spec, for Spec, for Spec. for Spec. for $-0.33 \mathrm{C})$ ) (SAE 6130) (1973) Steel Products Manual: Wrought

g39.2 Std. Spec. for Wrought Std. Spec. for Wrought Carbon
Steel Self Drilling Tapping Screws (1972)

Steel Self-Locking Nuts, High Strength, Prevailing Torq

Steel Sheet and Strip (1973) ANSI G24.24

Steel Sheet and Strip (1973) ANSI G24.25

Steel Sheet and Strip, Commercial Ouality (1972)

Steel Sheet and Strip, Corrosion and Moderate Heat Resi

Steel Sheet and Strip, Hot and Cold Rolled, High Streng

Steel Sheet and Strip, Structural Quality (1972)

Steel Sheet of Drawing Quality, Zinc Coated (Galvanized

Steel Sheet of Lock Forming Quality, Zinc Coated (Galva

Steel Sheet Piling (1970) /El Products Manual) Carbon

Steel Sheet, Strip, and Plate $(0.80 \mathrm{Cr}-1.8 \mathrm{Ni}-0.25 \mathrm{Mo}$

Steel Sheet, Strip, and Plate $(0.80 \mathrm{Cr}-1.8 \mathrm{Ni}-0.35 \mathrm{Mo}$

Steel Sheet, Strip, and Plate (13Cr-2.0Ni-3.0W) (19

Steel Sheet, Strip, and Plate, Corrosion Resistant, 14

Steel Sheet, Strip, and Plate, $0.50 \mathrm{Cr}-0.55 \mathrm{Ni}-0.25 \mathrm{Ml}$

Steel Sheet, Strip, and Plate, $0.75 \mathrm{Cr}-9.0 \mathrm{Ni}-4.5 \mathrm{Co}$

Steel Sheet, Zinc Coated (Galvanized) by the Hot Dip Pr

Steel Sheet, Zinc Coated (Galvanized) by the Hot Dip Pr

Steel Sheet, Zinc Coated (Galvanized) by the Hot Dip Pr

Steel Sheets for Culverts and Underdrains, Zinc Coated

Steel Sheets for Porcelain Enameling (1973)

Steel Sheets for Pressure Vessels (1971) ANSI G33.4

Steel Sheets of Structural Quality in Coils, and Cut Le

Steel Shipping Container lndustry Glossary (Packaging,

Steel Specialty Tubular Products (1972)

Steel Specimens Dynamically for Resistance to Corrosion

Steel Specimens (1972) Std. Rec. Pract. for

Steel Splined Continuous Strip Mastic Set Maple Floorin

Steel Spring Wire $(0.95 \mathrm{Cr}-0.22 \mathrm{~V}(0.48-0.53 \mathrm{C})$ ) (SAE

Steel Spring Wire, Best Quality Music (1973)

Steel Still Tubes for Refinery Service (1972) ANSI Bl25

Steel Still Tubes for Refinery Service (1972) ANSI B125

Steel Still Tubes for Refinery Service (1972) ANSI B36.

Steel Strand and Wire for Concrete Construction (1973)

Steel Strip $(0.68-0.80 \mathrm{C}$ ) (SAE 1074) (1973)

Steel Strip $(0.90-1.04 C)$ (SAE 1095) (1973)

Steel Strip (1971)

Steel Strip (1972A) ANS1 G47.1

Steel Structural Members (1973)

Steel Structural Members (1973)

Steel Structural Parts (1970) ANSI G63.3 3

Steel Structural Tubing in Rounds and Shapes (1973) Ans

Steel Structural Tubing (1973) ANSI G24.22

Steel Structure Painting (1972)

Steel Studs (1972)

Std. Spec. for Steel Drill Screws

Studs) for Composite Construction (1973)

Steel Studs, Heat Resistant, Normalized and Tempered, R

Steel Studs, Heat Treated, Roll Threaded (1973)

Steel Studs, Runners (Track) and Rigid Furring Channels

Steel Surfaces (Coil Coating) (1974)

Steel Threaded Shank, Sizes 1/16 In. Thru Letter F (197

Steel Tooth Lock Washers (1972)

/lmensions, Physical P

Steel Truss Plates (1973)

Steel Tubes for Low Temperature Service (1972A) ANSI B1

Steel Tubes (1971A) ANSI B125.34

/Pec. for General R

Steel Tubes (1972)

Steel Tubes, Low Carbon, Tapered for Structural Use (19

Steel Tubing Annealed for Bending and Flaring (1972)

Steel Tubing Annealed for Bending and Flaring (1972)

Steel Tubing for General Service (1973) ANS1 B125. 14

Steel Tubing for General Service (1973) ANSI B125.15

Steel Tubing Normalized for Bending, Double Flaring, an

Steel Tubing (1970) ANSI B125.33

Steel Tubing (1972)

Steel Tubing (1972)

Steel Tubing (1974)

Steel Tubing, Welded, Corrosion and Heat Resistant (25C

Steel Tubular Products for High Temperature Service Con

Steel Tubular Products (Tubing) with Magneric Saturatio

Steel Tubular Spring Pins (1973)

Steel Turbine Type Bolting Material Specially Heat Trea

Steel Underfloor Duct Raceways (1972)

Steel Underground Tanks for Flammable and Combustible L

Steel Using a Vacuum Spectrometer (1971) ANSI Z128.33

Steel Vessels (1972)

Steel Water Pipe (4 In. and Larger, Shop Applied) (1971

Steel Water Well Pipe (1972A)

Steel Welding Kods and Bare Electrodes (1969) ANSI W3.9

Steel Welding Wire $(0.95 \mathrm{Cr}-0.20 \mathrm{~V}(0.28-0.33 \mathrm{C}))$ (SAE

Steel Welding Wire, Corrosion and Heat Resistant (18Cr

Steel Welding Wire, Corrosion and Moderate Heat Resista

Steel Welding Wire, Vacuum Melted $(0.95 \mathrm{Cr}-0.20 \mathrm{~V}(0.28$

Steel Wheels and Forged Railway Axles (1973)

Steel Wheels for Electric Railway Service (1972) ANSI G

Steel Wheels for Locomotives and Rail Cars (1971) ANSI
IFl

SAE

ASTM

ASTM

ASTM

SAE

ASTM

ASTM

ASTM

AlSI

SAE

SAE

SAE

$S A E$

$S A E$

ASTM

ASTM

ASTM

ASTM

ASTM

ASTM

ANSl

AISI

ASTM

ASTM

WSFI

$\mathrm{SAE}$

SAE

ASTM

ASTM

ASTM

ICBO

$S A E$

SAE

AlSI

(

Unif lCBO
Std. ICBO

ASTM

ASTM

ASTM

ANSI

ASTM

ICBO

$\mathrm{SAE}$

SAE

ASTM

NCCA

ANA

ASTM

ASTM

ASTM

ASTM

ASTM

SAE

SAE

ASTM

ASTM

ASTM

SAE

SAE

WSTI

SAE

ASTM

ASTM

SAE

ASTM

NEMA

UL

ASTM

ABS

$A W W$

ASTM

AWS

SAE

SAE

SAE

SAE

AlSl

ASTM

ASTM

13

AMS7252A

506

M S5547C

A607

A 570

A 528

A 527

AMS6396

MS6433B

AMS5508B

AMS5862

AMS6358D

MS6523A

A 361

A525 
Std. Spec. for Cast Steel Wheels for Railway Service (1973)

$$
\text { Rec. Spec. for Steel Windows (1973) }
$$

rcement (1973)

in Concrete (1970)

i G45.1

i G 45.3

Uniform Building Code Std. for Welded Std. Spec, for Welded

Std. Spec. for Welded Deformed Std. Spec. for Cold Drawn

Std. Spec, for Coid Worked Deformed Uniform Building Code Std. for Cold Drawn Spec. for Nickel Coated Copper Clad

i G54.17 Std. Spec. for Cold Heading Quality Carbon Std. Spec. for Cold Heading Quality Carbon Std. Spec. for Cold Heading Quality Std. Spec. for Carbon nsi G54.1/ Std. Spec. for General Requirements for Carbon Std. Spec. for Zinc Coated Std. Spec. for Mlusic Spring Quality Std. Spec. for Stainless and Heat Resisting std Spec. for Free Machining Stainless and Heat Resistin Bolts (1972) ANSl G54.16 si G54.1

1973)

anical Springs (1973)

Hexagon Head Bolts (1972) ANSI G54.15 971) ANS1 G54.4 ant, $16.5 \mathrm{Cr}-4.5 \mathrm{Ni}-2.9 \mathrm{Mo}-0.1 \mathrm{~N}$ (1973)

Std. Spec. for Alloy Std. Spec. for Std. Spec. for Std. Spec. for Std. Spec. for Std. Spec. for

Std. for Tube Assembly, Control Std. for Tube Assembly, Control 2 ln. $(12.7 \mathrm{Mm})$ Maximum Thickn/ Std. Spec. for Structural e Piping (1969)

Std. Pract. for High Strength Carbon Rec. Pract. for Stainles

Std. for Bolt-Internal Wrenching. Std. Spec, for Pressure Vessel Plates, Alloy surement of Film Thickness of Nonmagnetic Finishes on Mild impact Resistance of Painted Aluminum, Steel and Gavanized Spec. for Determination of Phosphate Coating Weights on ination for Conversion Coating on Aged or Baked Aluminized coating Weight on Hot Dip Galvanized and Electrogalvanized eth. and Controls to Prevent in Service Cracking of Carbon Metallizing with Aluminum and Zinc Protection of lron and Pract. for Preparation of and Electroplating on Stainless Rec. Pract. for High Strength, Low Allo

Std. Spec. for Carbon-Chromium Ball and Roller Bearin Std. Spec. for High Strength Low Alloy Structural Std. Spec. for High Strength Structural for High Strength Low Alloy Structural Manganese Vanadium Std. for Welding Zinc Coated tive Measurement of Film Thickness of Pipeline Coatings on Uniform Building Code Std. for Structural Rivet Std. for Rod-Control, Solid

Std. for Bushing-Plain. Press Fit, Rivets, Carbon

d. Spec. for Normalized High Strength Low Alloy Structural r Screw, Machine, 100 Deg., Flat Head Full Threaded, Alloy

on, Fabrication, ldentification and Painting of Structural cords, Tire Cord Fabrics. Filaments, and Strands Made from Std. Rec. Pract. for Determining the Inclusion Content of Steel Products Manual for Carbon Sheet and Steel Sheet Piling (1) (Steel Products Manual) Carbon Std. for Bushing-Clamp Up,
and Strip, Commercial Quality (1972) Spec. for ng Quality (1973) Std. Spec. for Std. Spec. for Pressure Vessel Plates, Alloy r Heat Treatment, 1800 Deg. F (982.2 Deg. C) Solu/ Std. Spec. for Std. for Full Threaded, $160 \mathrm{Ksi}$ Spec, for Fasteners, Alloy Std. for Rod End, Threaded, Alloy Std. for Screw, Hex Head, Tri Wing Recess, Allo Std. for Screw, 100 Deg. Head, Tri Wing Recess, Allo Std. Spec. for Flat Rolled Nonoriented Electrical for Flat Rolled, Grain Oriented, Silicon Iron, Electrica Std. Spec. for Pressure Vessel Plates, Carbon

i G35.11 Std. Spec, for Pressure Vessel Plates, Alloy lt, 100 Deg. Head, Tri Wing Recess, Close Tolerance, Alloy Std. for Bolt, Hex Head, Close Tolerance, Alloy Roll Threaded (1973) Bolts and Screws

rete / Uniform Building Code Std. for Welding Reinforcin 1972A) Std. Spec. for Pressure Vessel Plates, Alloy 1972A) Ansi/ Std. Spec. for Pressure Vessel Plates, Alloy

3) Std. for Pin, Swage Locking. Std. for Pin, Swage Locking, $108 \mathrm{~K}$ Std. for Pin, Swage Locking, um-Chromi/ si G35.20 Std. Spec. for Pressure Vessel Plates, Alloy
Steel Wire and Deformed Wire Fabric for Concrete Reinfo teel Wire Bar Supports Used to Position Reinforcement Steel Wire Fabric for Concrete Reinforcement (1972) Ans Steel Wire Fabric for Concrete Reinforcement (1972) Ans Steel Wire for Concrete Reinforcement (1972) ANSl A50.3 Steel Wire for Concrete Reinforcement (1972) ANSl G45.2 Steel Wire for Concrete Reinforcement (1973

Steel Wire for Electronic Application (1972)

Steel Wire for Machine Screws (1971) ANSl G54.14

Steel Wire for Tapping or Sheet Metal Screws (1971) Ans

Steel Wire for Wood Screws (1971) ANSI G54.18

Steel Wire of Scrapless Nut Quality (1972) ANSI K91.3

Steel Wire Rods and Uncoated Coarse Round Wire (1971) a

Steel Wire Strand (1972A) ANSI C7.46

Steel Wire (1971) ANSI G54.2

Steel Wire (1971) ANSI G81.41

Steel Wire (1971) ANSI G81.42

Steel Wire, Alloy Cold Heading Quality for Hexagon Head

Steel Wire, Hard Drawn for Mechanical Springs (1971) an

Steel Wire, Hard Drawn for Prestressing Concrete Pipe (

Steel Wire, High Tensile Strength, Hard Drawn, for Mech

Steel Wire. Medium High Carbon Cold Heading Quality for

Steel Wire, Oil Tempered Carbon Valve Spring Quality (1 Steel Wire, Welding, Corrosion and Moderate Heat Resist

Steel with Welded Clevis and Threaded Rod Ends (1973)

Steel with Welded Threaded Rod Ends (1973)

Steel with 42,000 psi (290 MPa) Minimum Yield Point (1/

Steel Wrought Welding Fittings for High Pressure Servic

Steel $17.7 \mathrm{pH}$ Spring Wire and Springs (1970)

Steel 1/4-28 Thru 1-1/8-12 (1973)

Steel $5 \%$ Chromium. 0.5\% Molybdenum (1972) ANSI G35.16

Steel (Coil Coating) (1974)

Steel (Coil Coating) (1974)

Steel (Coil) (1974)

Steel (Coil) (1974)

Steel (Coil) (1974)

Steel (P-1) We

Steel (1970) ANSI G53.13

Steel (1970) ANS1 G88.3

Steel (1970) ASTM G56.2

Steel (1970A) ANSl G4l.2

Steel (1970A) ANSI G41.3

Steel (1970A) ANSI G4l.4

Steel (1972)

Steel (1972)

Steel (1973)

Steel (1973)

Steel (1973)

Steel (1973)

Steel (1973)

Steel (1973)

Steel (1973)

Steel (1974)

Steel (1974) ANSI Z30.5

Steel (coils and Cut Lengths) (1974)

Steel' Plates, Structural Shapes, Rolled Floor Plates,

Steel, Cadmium Plated (1973)

Steel, Carbon $(0.16$ to 0.25 Maximum \%, Hot Rolled Sheet

Steel, Carbon, Strip, Cold Rolled Hard, Untempered Spri

Steel, Chromium Manganese Silicon (1972A) ANSl G32.1

Steel, Cold Rolled Sheet, Carbon, Structural (1972)

Steel, Corrosion and Heat Resistant, Roll Threaded Afte

Steel, Drilled Head Bolt (1972)

Steel, Externally Threaded (1972)

Steel, Flash Welding Type (1973)

Steel, Full Thread, Nonlocking (1973)

Steel, Full Thread, Self-Locking and Nonlocking (1973)

Steel, Fully Processed Type (1973)

Steel, Fully Processed Types 27G053, 30G058, and 35G066

Steel, High Strength Manganese (1972A) ANSI G35.8

Steel, High Strength, Quenched and Tempered (1972A) Ans

Steel, Long Thread, Self Locking and Nonlocking (1972)

Steel, Long Thread, Self Locking and Nonlocking (1972)

Steel, Low Alloy Heat Resistant, Hardened and Tempered,

Steel, Metal Inserts and Connections in Reinforced Conc

Steel, Precipitation Hardening (Maraging), $12 \%$ Nickel (

Steel, Precipitation Hardening (Maraging), $18 \%$ Nickel (

Steel, Protruding Head, Shear Stump Type (1973)

Steel, Protruding Head, Shear / Tension, Pull Type (197

Steel, Protruding Head, Tension, Stump Type, (1973)

Steel, Quenched and Tempered Nickel-Cobalt-Molybden

Steel, Quenched and Tempered 8 and 9\% Nickel (1972A) an

Steel, Quenched and Tempered, for Pressure Vessel Plate
ASTM

$\mathrm{SW]}$

ICBO

CRSI

ASTMI

ASTM

ASTM

ASTM

ICBO

ASTM

ASTM

ASTM

ASTM

ASTM

ASTM

ASTM

ASTM

ASTM

ASTM

ASTM

ASTMI

ASTMI

ASTM

ASTN]

$\mathrm{SAE}$

NSA

SA

ASTM

uss

SAE

SA

ASTMI

NCCA

$\mathrm{NCCA}$

$\mathrm{NCCA}$

NCCA

NCCA

NACE

AWS

td. Rec. ASTM

SAE

ASTMl

ASTM

ASTN

Std. Spec. ASTN

AWS

ASTM

ICBO

NSA

NSA

SAE

St ASTM

Std. Fo NSA

ICBO

ASTM

ASTMI

AIS

AISI

NSA

ASTM

ASTM

ASTM

ASTM

$\mathrm{SAE}$

SA

NSA

NSA

NSA

SA

ASTM

ASTM

ASTM

ASTM

NSA

NSA

$S A E$

ICBO

ASTM

ASTM

NSA

NSA

ASTM

ASTVI

AST N

A 583

UBC $\$ 26.6$

1.3.1

A185

49

A 82
A 496

UBC $524-16$

B559

A 545

548

549

A544

A510

A 228

A580

A5

A227

A648

A 679 
Rec. Pract. for Stainless ead, Close Tolerance, Short Thread, TWI Wing Recess, Alloy flat Fillister Head, Full Threaded, Tri Wing Recess, Alloy deg. Reduced Head, Tri Wing Recess, Close Tolerance, Alloy Std. for Bolt, Hex Head, Close Tolerance, Allo

It, 100 Deg. Head, Tri Wing Recess, Close Tolerance, Alloy t Equipment) (1974) Std. for Forged Carbon type (1973) Std. Spec. for Pressure Vessels Plates, $5 \%$ Nickel Alloy ion, Pull Type (1973)

on, Pull Type (1973) e (1973)

Sid. for Pin, Swage Lockin id. for Pin, Swage Locking, Std. for Pin, Swage Locking, Std. for Pin, Swage Locking, led, Plain and Self Locking, Alloy and Corrosion Resistin lain and Self Locking. Alloy Steel and Corrosion Resisting

1973) Std. for Tube Assembly, Control Std. for Tube Assembly, Control, Std. for Bolt-Internal Wrenching, Std. for Bolt-lnternal Wrenching, Std. for Bolt-lnternal Wrenching, Sid. for Pin, Swage Locking. Std. for Pin, Swage Locking, Std. for Pin, Swage Locking, Std. for Pin, Swage Locking Std. for Pin, Swage Locking, $108 \mathrm{Ks}$

t Bolt, 100 Deg. Flush Tension Head Hi Torque Recess Alloy Std. Spec for Pressure Vessel Plates, Alloy ANSI G35.3 Std. Spec. for Pressure Vessel Plates, Alloy
I973) I973)

ec. Pract. for Determining the Susceptibility of Stainles Std. Spec. for Flat Rolled Electrica Std. Spec. for High Strength Low Alloy Columbium Vanadium ec. for Quality Assurance Sampling of Carbon and Low Alloy

Rec. Pract. for Resistance Welding Coated Low Carbon rials with Expansion Coeficicients Comparable to Austenitic Std. for Chemical Compositions of SAE Carbon Std. for Chemical Compositions of SAE Alloy Std for Hardenability Bands for Alloy $\mathrm{H}$

Rec. Pract. for Hardenability Bands for Carbon $\mathrm{H}$

Tent. Meth. for Drop Weight Tear Tests of Ferritic ations from Specified Ladle Chemical Ranges and Limits for Std. Spec. for Carbon Too sis of Tool Steels and Other Similar Medium and High Alloy Std. for Plating, High Strength

ical Analysis of Carbon, Low Alloy, and Silicon Electrica nished Bars; Hot Rolled D/ (Steel Products Manual) Carbon

ranite, Limestone, and Marble Masonry; Mortar; Sand; Slag;

Std. Analytical Meth. for Determining Acidity Content in Cor 1 Std. Analytical Meth for Determining $\mathrm{PH}$ Content in alytical Meth. for Determining Ammonia Nitrogen Content in cal Meth. for Determining Lactic Acid and Salts Content in Std. Analytical Meth. for Determining Ash Content in nalytical Meth. for Determining Reducing Sugars Content in

1 Meth. for Determining Moisture (Karl Fischer) Content in alytical Meth. for Determining Protein Nitrogen Content in

lar Bicycles Including Tests for Static Load, Frame, Fork. eel Assembly, Chainguards, Saddle Braces, Pedal Clearance, ion and Industrial Front End Loaders and Dozers/ Std. for

Performance Requirements for Stopping Power, Traction and Sid. for Tires and Rims for Agricultural and Industria Rec. Pract. for Dril Std. Spec. for Sizes for Threaded Caster

Die Buttons, Variable, Press Fit, Headless and Head Type, roscope, Petri Dish, Sieve, Separatory Funnel, Trap Flask, tenings by Distillation with Toluene (Cereal C/

Bidwell. dstuffs by Distillation with Toluene (Cereal C Bidwell Rec. Pract. for D rill

Std Spec for Marnesium Ingot and of a Torsional / Std. Meth. of Measuring Low Temperature eam, Joint and Rafter Span, Load, Deflection, Strength and atus (1972) ALCA E/ Sid. Meth. for Measuring the Relative 70) ANSI K65.13 rature by Means of a Torsion Test/2 Std. Meth. of Test for Std. Test Meth. for Determining Quality Stds. for Blinds and Shutters Using Quality Stds. for Quality Stds. for rior Frames and Sash, Screens, Blinds and Shutters, Flush, on Meth. for Determining Speed of Color Reversal Films for Std. for Shutter Tests for Std. Picture Sizes for Roll, $35 \mathrm{~mm}$ Film, and $126 \mathrm{~mm}$ Film
xposure Time Markings for Variable Speed Shutters Used in $\mathrm{ft}$. Spools for Recording Instruments and for Microfilm and Std. for lllumination Tests for Photographic Std. Spec. for Seamless Intermediate Alloy Steel Spec. for Seamless Low Carbon and Carbon Molybdenum Steel

Std. Spec. for Seamless Austenitic Chromium Nickel Steel
Steel, SAE 30302, Spring Wire and Springs (1971)

Steel, Self Locking and Nonlocking (1972)

Steel, Self-Locking and Nonlocking (1973)

Steel, Short Thread, Nonlocking (1973)

Steel, Short Thread, Self Locking and Nonlocking (1972)

Steel, Short Thread, Self.Locking and Nonlocking (1973)

Steel, Shoulder Nut Assembly Eyebolt (For Ground Suppor

Steel, Specially Heat Treated (1972A)

Steel, Std. and Oversize, Protruding Head, Shear, Pull

Steel, Std, and Oversize, 100 Deg. Head (MS20426), Tens

Steel, Std. and Oversize, 100 Deg. Head (Ms2494), Tensi

Steel, Std. and Oversize, 100 Deg. Shear Head, Pull Typ

Lled and Dril

teel, UNJC-3A Thread, Cap Screw (1972)

Steel, UNJF-3A Thread Cap Screw (I972)

Nd Drilled, P

Steel, with Flash Welded and Threaded Rod Ends (1973)

Steel, with Flash Welded Clevis and Threaded Rod Ends (

Steel, I.2500-12 (1973)

Steel, 1.3750. $12(1973)$

Steel, I.5000-12 (1973)

Steel, I00 Deg. Crown Head, Tension, Pull Type (1973)

Steel, I00 Deg. Head (MS20426), Tension, Stump Type (19

Steel, 100 Deg. Head (MS24694), Tension, Stump Type (19

Steel, 100 Deg. Shear Head, Stump Type (1973)

Steel, I00 Deg. Shear / Tension Head, Pull Type (1973)

Steel, 160,000 psi (1973)

Steel, 36\% Nickel (1972)

Steel, $9 \%$ Nickel Double Normalized and Tempered (1972A

Steels and Other Similar Medium and High Alloy Steels (

Steels and Related Ni-Cr.Fe Alloys to Stress Corrosion

Steels for Magnetic Applications (1974)

Steels of Structural Quality (1972) ANSI G41.6

teels (Wrought Products Except Forgings and Stock) (19

Steels (1970)

Steels (1970) ANSI G38.12

Steels (1970) ANSI G88.1

Steels (1970) ANSI G88.2

Steels (1971)

Steels (1971)

Steels (1971) ANSI Z260.3

Steels (1972)

Steels (1973)

Steels (1973)

Steels, Cadmium (1973)

Steels, Ingot and Wrought lrons (1973)

$120 \mathrm{KSI}$ Yield Strength, Mate

Steel, Terra Cotta; Timber; Water; Ceiling; Partitions;

Steepwater and Most Other Liquid Products Obtained from

Steepwater (Corn) (1962)

Steepwater (Corn) (1962)

Steepwater (Corn) (1964)

Steepwater (Corn) (1964)

Steepwater (Corn) (1966)

Steepwater, (Corn) Feedstuffs, Syrups, Sugars, Starches

Steering Assembly and Tire Blowout (1972)

Steering Control, Brakes, Tires and Controls (1972)

Steering Frame Lock for Articulated Vehicles (Construct

Steering Stability for Regular Bicycles (1972)

Steering, Drive, Planter (Drill) Press, and Gage Wheel,

Stem Design and Operating Limits (Petroleum) (1974)

Stems (1965)

Step Relief (1972)

Step Wedge for X-Ray Standardization Probe, Rot Fragmen

Sterling Meth. of Analysis of Moisture in Fats and Shor

Sterling Meth. of Analysis of Moisture in Feeds and Fee

Stern Test Report Form (1972)

Stick for Remelting (1971 A) ANSl H45.5

Stiffering of Rubber and Rubber Like Materials by Means

Stiffness Computations (1973)

Stiffness Computations (1973) Rec, for
Stiffness of Leather by Means of a Torsional Wire Appar

Stiffness of Plastics by Means of a Cantilever Beam (19

Stiffness Properties of Plastics as a Function of Tempe

Stiffness (Modulus of Bending) of Fiberboards (1972)

Stile and Rail Construction (Architectural Woodwork) (1

Stile and Rail Doors (Architectural Woodwork) (1973)

Stile and Rail Wall Paneling (1973)

Stile, and Rail Doors, Factory Finishing, Rough and Fin

Metric

Still Picture Cameras (Photo

Still Picture Cameras (1972)

Still Picture Cameras (1972)

Still Picture Cameras (1972)

Still Projection Equipment (1971)

Still Tubes for Refinery Service (1972) ANSI B125.23

Still Tubes for Refinery Service (1972) ANSI B 125.5
Still Tubes for Refinery Service (1972) ANSI B36.39

alytica CR

AE

NSA

NSA

NSA

NSA

ASTM

SA

NSA

NSA

NSA

NSA

NSA

NSA

NSA

NSA

NSA

NSA

NSA

SA

ASTM

ASTM

ASTM

ASTM

ASTM

ASTM

SAE

AWS

ASTM

SAE

SAE

$S A E$

SAE

AST

ASTM

ASTM

ASTM

AISI

ICBO

CR

CR

CR

iH BMA

SAE

D. for BMA

TRA

APl

CFTMA

Std. for

ACCH 28.90

$\mathrm{AACCH}$ 44-51

$\mathrm{AACCH} \quad 44-50$

APl

ASTM

ASTM

WWPA

ASTM

ASTM

ASTM

$\mathrm{SAE}$

AWI

AWl

AWI

AWl

ANSI

ANSl

ANSl

Std. ANSl

RP48

$\mathrm{B} 92$

D1053

D2821

D747

D 1043

J949A

$* 1.1400$

$* 1.500 \mathrm{C}$

$* 1$ - 1

PH2.21

$\mathrm{PH} 3.48$

$\mathrm{PH} 3.39$

PH3.32

$\mathrm{PH} 1.33$

ANSI PH3.47

ASTM A200

1. ASTM Al61

ASTM 
r Small Construction Contracts Where Basis of Payment Is A ng Code Std. for Blower, Fan, and Exhaust System for Dust. Meth. of Analysis of Crude Fat in Grain and Std. Proofing Supplement itation Hardening Nickel Alloy Bars, Prorging, and Forging dening Cobalt Containing Alloy Bars, Forgings, and Forging Std. for Motion Picture Raw nces of Aluminum and Its Alloy Bar, Rod, Wire, and Forging Uniform Fire Code: High Piled Combustible Copper and lts Alloy Seamless Condenser Tubes and Ferrule and Low Alloy Steels (Wrought Products Except Forgings and $r$ the Installation of Blower and Exhaust Systems for Dust. i. Vacuum Induce/ Steel Bars, Wire, Forgings, and Forging hrubs; Coniferous and Broadleaf Evergre/ Std. for Nursery mall Fruits (Berry, Currant, Grape, Asparagus); Lining Out grouted, Reinforced Grouted, Hollow Unit, Cavity Wall, and Uniform Building Code Std. for Cast Building Building Code Std. for Concrete Aggregates (Sand, Crushed base Courses (1971) ANSI A37.99 Uniform Building Code: Veneer (Brick, Concrete,
rotectiol Std. Definitions of Terms Relating to Natural Building Rec. Pract. for Mechanical

Rec. Pract. Test Meth. and Requirements for Snowmobile Std. Meth. of Test. Anti Leak Antifreezes and nger Vehicles, Buses, Trucks, and Combin/ Rec. Pract. for utomobile Equipped with Full Scale Tir/ Meth. of Test for utomobile Equipped with Fu/ Tent. Meth. of Measurement of ular Bicycl/ Safety Std. for Performance Requirements for Rec, for Rec. Pract. for Bus Rec. Pract. for Proper Location of Bus Construction of Indoor Rifle and Pistol Ranges and Bullet spital and Misc. Items; Key Control Systems; Door Holders, al, Henequen, Ixtle, Jutes, Hemp, Tow/ $\begin{array}{r}\text { Safety Requirements for } \\ \text { Uniform Fire Code: }\end{array}$ 1974) rosene, Acetone, AIcohol,/ Uniform Building Code Std. for (During the Con/ Std. for Packaging, Shipping, Receiving, ns Containing More Than 2\% Free Ammonia (1970) Std. for lulose Films (1973) Uniform Building Code Std. for lose) (1973)

) (1973)

Uniform Building Code: Film Rec. Code for Transportation,

st for Gloss Retention of Waxed Paper and Paperboard After h. for Gloss Retention of Waxed Paper and Paperboard After nery (1970) Rec. Pract. for ors, Automotive and Industrial A/ Std. for Lead Acid Type Trucks/ Safety and Health Rec. for Changing and Charging Std. Test Proc. for

Rec Pract, for Life Test for Automotive luding Guarantee Policy for Motor Vehicle Type (Lead Acid) nd Starting) for Passenger Automobiles, Commercial Vehic/ iven Generators and Reversible Generator/Motors for Pumped

Reversible Synchronous Generator/Motor Units for Pumped Std. for Dimensions of Containers for Packaging and Std. Rec. Pract. for

fety) (1974)

n Microfilm (1971)

ansi $\mathrm{Zl} /$

Fire Hazards Anf Protection Rec. for Indoor Std. Meth. for Preparation, Standardization, and for Safety for Tests for Fire Resistance of Vault and File Quality Stds. for Closet and standing and Running Trim, Casework, Panelwork, Closet and 72) Safety Std. for Household Electric Safety Std. for Aboveground
Sanitary Std. for Sanitary Std. for Silo Type

for Safety for Flame Arresters (Fire) for Use on Vents of Std. for Welded Aluminum Alloy Field Erected Rec. for Design of Farm Waste Sanitary Sid. for Farm Milk

Btu Per Hr.) (Vol/

Plumbing Requirements for Household Std. for Gas Water Heaters (Automatic Std. for Household Automatic Electric

e III, Circulating Tank, Instantaneous and Large Automatic

lid, Palletized and Bin Box Storage Up to $30 \mathrm{Ft}$. and Shelf for Indoor General Storage (Solid, Palletized and Bin Box fibers, Wool) (1974)

Ft. and Shelf Storage Up to $15 /$ Rec. for Indoor General
Stipulated Sum) (1974)

Stock and Vapor Removal (1973)

Stock Feeds (Cereal Chemistry) (1962)

Stock for Gravure Magazines (Printing) (1973)

Stock for High Temperature Service (1970) ANSI G81.44

Stock for High Temperature Service (1970) ANSI G81.45

Stock for High Temperature Service (1970) ANSI G81.46

Stock Identification and Labeling (1973)

Stock (Rolled or Drawn) (1973)

Stock (Storage) (1973)

Stock (1974) ANSI H23.14

/R Quality Assurance Sampling of Carbon

Stock) (1973)

oval or Conveying (1973) ANSI Z33.l

Stock, and

Stock: Deciduous Shade and Flowering Trees; Deciduous S

Stock; Seedling Trees and Shrubs; Bulbs, Corms, and Tub

Stone Masonry (1973)

/E Uniform Building Cod

Stone (1973)

Stone, Gravel and Air Cooled Iron Blast Furnace Slag) (

Stone, Slag, and Gravel for Dry or Water Bound Macadam

Stone, Tile, Metal, Plastic, etc.) for Ornamentation, P

Stones (1974)

Stop Lamp (Light) Switch (1972)

Stop Lamp (Light) (1971)

Stop Leak Additives for Engine Coolants (1972)

Stopping Distance of Passenger Cars, MuItipurpose Passe

Stopping Distance on Paved Surfaces Using a Passenger a

Stopping Distance on Paved Surfaces Using a Passenger a

Stopping Power, Traction and Steering Stability for Reg

Stopping Powers for Use with Cavity Chambers (1961)

Stops for Freeway Operations (1971)

Stops (1967)

Stops (1973)

Stops. Smoke and Heat Detectors; Electromagnetic Holder

Storage and Handling of Anhydrous Ammonia (1972)

Storage and Handling of Combustible Fibres (Cotton, Sis

Storage and Handling of Compressed Gases in Cylinders (

Storage and Handling of Flammable Liquids (Gasoline. Ke

Storage and Handling of Items for Nuclear Power Plants

Storage and Handling of Liquefied Petroleum Gases (1972

Storage and Handling of Nitrogen and Fertilizer Solutio

Storage and Handling of Photographic and X-Ray Nitrocel

Storage and Handling (Photographic and X-Ray Nitrocellu

Storage and Use of Explosive Materials (Fire Protection

Storage at $104 \mathrm{~F}(40 \mathrm{C})(1973$ )

Storage at $140 \mathrm{Deg}$. (40 Deg. C.) (1972)

Storage Batteries for Construction and Industrial Machi

Storage Batteries for Motive Power Service (1974)

Storage Batteries for Motor Vehicles, Motorboats, Tract

Storage Batteries in Motive Power Service (For Electric

Storage Batteries (1972)

Storage Batteries (1972)

Storage Batteries (1972)

Storage Battery Spec. (Vehict
Storage Installations (1972)

Storage Installations (1974)

Storage Loads, Pressures, and Capacities (1968)

Storage of Aerial Photographic Film on S pools (1972)

Storage of Aerosol Products (1972)

Storage of Expanded Rubber (1972)

Storage of Flammable and Combustible Materials (Fire Sa

Storage of Plastics (1974)

Storage of Processed Photographic Plates (1972)

Storage of Processed Safety Photographic Film Other Tha

Storage of Roll Paper (1974)

Storage of Std. Solutions for Chemical Analysis (1972)

Storage Racks (1972)

Storage Room Doors (1972) ANSI Al53.I

Storage Shelving (1973)

Storage Shelving, Misc. Ornamental ltems, Stairwork and

Storage Tank Water Heaters (1972) ANSI C 33.87

Storage Tanks for Flammable and Combustible Liquids (19

Storage Tanks for Milk and Its Products (1974)

Storage Tanks for Milk and Its Products (1974)

Storage Tanks for Petroleum Oil and Gasoline (1973) Ans

Storage Tanks (1973)

Storage Tanks (1973)

Storage Tanks (1973)

Storage Type Electrical Hot Water Dispensers (1973)

Storage Type Water Heaters with Inputs Less Than 75,000

Storage Type Water Heaters (1972)

Storage Type (1974)

Std. for Gas Water Heaters: Volum

Storage Up to $15 \mathrm{Ft}$. High) (Fire Protection and Safety)

Storage Up to $30 \mathrm{Ft}$. and Shelf Storage Up to $15 \mathrm{Ft}$. Hig

Storage (Cotton, Jute, Hemp and Sisal, FIax, Synthetic

Storage (Solid, Palletized and Bin Box Storage Up to 30

A 107

UBCS10-3

30-20

GT

$* 5$

ASTM A637

ASTM A638

ASTM A639

ANSI PH22.184

SAE AMS2201J

ICBO UFC*2ART35

B111

AMS2370A

91

NFPA

SAE

ANSI

ANSI

A.MS5617A

260. 1

260.1

UBC $* 8+11$

IBBCS24-14

IC BO

ICBO

ASTM

ICBO

ASTM

SAE

SAE

ASTM

SAE

ASTM

ASTM

BMA

VCRPM

ITE $\mathrm{R} 2$

ITE

NRA

NBHA

Fl

ICBO

FUS

ICBO

ANSI

NFPA

$\mathrm{Fl}$

ICBO

IC BO

FMS

UBCS 26.2

D694

UBC *3-30

C119

J2 49

$\mathrm{J} 278$

D3147

J299

E445

E503

$6 / 3$
$\mathrm{R} 27$

$* 10$

*1

P130

IFC *2ART 7

7.50

['BCS10-1

N 45.2 .2

$\mathrm{Pl} 45$

UBCS48-1

I $\mathrm{BC} * 3.48$

7.28

D2895

T 682

J930 A

SAE

NEMA

SAE

IT A

BCI

$\mathrm{SAE}$

BCI

BCI

IB2

J537H

$8 K^{\prime} \mathbf{l}$

*1. 66

J $240 \mathrm{~A}$

$* 1.15$

$* 1.48$

MG5. 2

MG5.1

D240

ANSI $\quad$ PHI.46

LPD8-2

$8-33 \mathrm{~N}$

FMS 8.9

ANS1 PHI.45

ANSI PHI.43

FMS 8.2]

ASTM E200

FMS LPD8-30

$A W I$

AWl

UL

UL

DFISA

DFISA

UL 
luating Oxidation of Wool Oils on Woolen Fabric Textile in g Effect of Packaging on Food and Beverage Products Durin Moisture Relationships of Agricultural Grain and Seed for Rec for Distilled Spirit

nd Concretes/

Rec for Deep, Cast in Place Conerete Std. Spec. for Moist Cabinets and (Specimen Uniform Fire Code: High Piled Combustible Stock

ANSI Zl Sid. Code for the Manufacture, Transportation, Rec. for Flammable Liquid Drum Std. for Receptacle Dumm

or Garden Grades, Application and Design, Span Allowances, iseptic Holding, Setting, Aeration Chambers, Chemica Std. Definitions of Terms Relating to Nayal Specs. for Aluminum Combination Std. Spec. for Asbestos Cement Std Spec for Concrete Sewer, Std. Spec. for Reinforced Concrete Arch Culvert Std. Spec. for Reinforced Concrete Elliptical Culvert, Std. Spec. for Reinforced Concrete D. Load Culvert, Std Spec for Reinforced Concrete Culvert. m Combination Vertically Sliding or Horizontally Operatin Construction Details, 6 In. Brick and Tile Walls for 1 Std. for Household Electric Range Std. for Household Electric Ranges d. Household Electric Range Pyrolytic Self Cleaning Oven Safety Std. for Oil Burning

Std. for Domestic Gas Hot Plates and Laundry Std for Hotel and Restaurant Gas Ranges Std. for Safety for Household Electric Range for Colorfastness of Dyed Textile Yarns to Sulfur Dioxide s for Flam Stds, and Pract for Design, Construction and std for Consumable Electric Covered Bare steel Plates for Special Applications (19) r Pressure Vessels (1973) ANSI / St . wheai Bread Flour (Cereal Chemistry) (1962) -37 C 80$)(1974)$

t-37A80, Dot-37A60, Uffe-Rule/

75 Shore, O.Ring (1973)

90 Shore, O.Ring (1973)

ansi $\mathrm{Z} 260.2$

ation) (1971) Std. Spec. for d. Meth. and Spec. for

Std. Spec. for $5 \mathrm{Gal}$ Std. Spec. for $5 \mathrm{Gal}$ Std. for Packing, Preformed, Std, for Parking, Preformed, Std. for Pin Std. Test Meth. for Plane std. Specs, and Tests for te Meth. of Test for Tensile Stres Std. Meth. of Test for Annealing Point and std. Meth. of Test for Annealing Point ant std. Meth. of Test for Plastic

mmonia (Fertilizer $\mathrm{Gr}$

Std for Safety for Self Contained

Pressure Rating Std. for Y T ype tion of Seacocks. Through Hull Connections, Outboard Water ies of Elastomeric Vulcanizates Inder Compressive or Shea Uniform Building Code Std. for Prestressed Steel Std. Spec. for Zinc Coated Steel Wire (Acar, EC/6201) (1972) An/ Std. Spec. for Concentric Lay s, for Electrical Conductors (1971) Spec, for Rope Lay (1972) ANSI C7.8 Std. Spec. for Concentric I ay Std. Spec for Compact Round Concentric Lay Std. Spec. for Compact Round Concentric Lay Lay Stranded Copper Conductors Having Bunch spec. for Rope Lay Stranded) and Aluminum Coated (Aluminized) Zinc Coated (Galvanized) and Aluminum Coated (Aluminized)
Std. Spec. for Aluminum Conductors. Concentric Lay C7.50 i 77.52 Std Spec for Concentric Lay Std. Spec. for Aluminum Conductors, Concentric Lay th. of Test. Tire Cords. Tire Cord Fabrics, Filaments, and rubber (1971) Meth. of Std for Tu Std. for Terminal-Tank

Spec. for Lineman's Climbing Equipment (Body Belt, Safety Buckle, Wet Std. for Terminal-Tank Std. for Trunnion-Tank Std. for Trunnion-T ank

e and Tubing, Mattresses and Pads, Sheeting, and Restraint riction Coefficients of Chopped Forages (Grass, Corn, Hay, Fibers, Oakum, Rags, Waste Cloth, Wastepaper, Kapok, Hay, Std. Meth. of Estimating Std. for

c. for Std. Integrated Data Processing Systems Designed to Std. for Voltage Classification of Luminaires Used in n P/ Uniform Building Code: Regulations for Use of Public
bundle Meth.) (1972) Ans/ Std. Meth. of Test for Breaking Shapes (1972) Ansi/ Std. Meth. of Test for Cold Crushing c. for Wood Beam, Joint and Rafter Span, Load, Deflection, d Cold Worked Alloy Steel Plate, Sheet, and Strip for High
Storage (1971) ANSI L14. S2

Storage (1972)

Storage (1972)

Storage (1974)

Test Meth. for Eva AATCC Std. Rec. Pract. fo: Determinin Ensity, Specific Gravity, and Weight-

torage) Bin and Silo Wall Design and Construction (196

Storage) Rooms Used in the Test. of Hydraulic Cements a

Storage) (1973)

Storage) (1974)

Storage, and Use of Explosives and Blasting Agents 197

Storage, Dispensing, and Classification (1972)

Storage, Electrical Connector (1972)

Storage, Finishes and Nails and Fastenings) (1973)

Storage, Mixing, Filter, Grit Chambers, and Flotation)

Stores and Related Products (1972)

Storm Doors (1971)

Storm Drain Pipe (1973)

Storm Drain, and Culvert Pipe (1973)

Storm Drain, and Sewer Pipe (1971)

Storm Drain, and Sewer Pipe (1972)

Storm Drain, and Sewer Pipe (1973)

Storm Drain, and Sewer Pipe (1973)

Storm Windows for External Application (1972) ANSI A 134

Story Buildings (Tech. Notes) (1966)

(Stove and Ovens) (1972) ANSI C71.1

(Stove) with Glass / Ceramic Cooking Tops (1974)

(Stove) (1974)

Stoves (Room Heaters and Ranges) (1973)

Stoves (1971)

(Stoves) and Init Broilers (1973)

(Stoves) (1974) ANSI C33.53

Stoving (1972) ANSl L14.9

Stowage of Portable Fuel Systems and Portable Containe

Straight and Coiled WeIding Electrodes and Rods (1972)

Straight Beam Ultrasonic Examination of Plain and Clad

Straight Beam Ultrasonic Examination of Steel Plates Fo

Straight Dough Meth. of Analysis for Baking Quality of

Straight Side Lug Cover Universal Pail (Container) (Dot

Straight Side Lug Cover Universal Pails (Container) (Do

Straight Thread Tube Fitting Boss, MIL.R.25897 Rubber,

Straight Thread Tube Fitting Boss, MlL.R.25897 Rubber,

Straight, Headless (Dowel) (1973)

Strain Fracture Toughness of Metallic Materials (1972)

Strain Gage Linear Acceleration Transducers (Instrument

Strain of Carbons and Graphite (1973)

Strain Point of Glass by Beam Bending (1972)

Strain Point of Class by Fiber Elongation (1971)

Strain Ratio R for Sheet Metal (1974)

Strainer or Filter for Flammable Fluids and Anhydrous a

Strainers (1973)

Strainers, Water Scoops and Drain Plugs (Boats) (1972)

Strains by the Mechanical Oscillograph (1972) ANSI J2.1

Strand and Wire for Concrete Construction (1973)

Strand (1972A) ANSI C7.46

Stranded Aluminum Conductors, Aluminum Alloy Reinforced

Stranded Copper Conductors Having Bunch Stranded Member

Stranded Copper Conductors, Hard, Medium Hard, or Soft

Stranded Copper Conouctors (1974) ANSI C7.59

Stranded EC Grade Aluminum Conductors (1972) ANSI C7.53

Stranded Members, for Electrical Conductors (1971)

Stranded Steel Core for Aluminum Conductors, Steel Rein

Stranded Steel Reinforced, (ACSR) (1972A) ANSI C7.22

Stranded 5005.H19 Aluminum Alloy Conductors (1972) ANSI

Stranded 6201.T 81 Aluminum Alloy Conductors (1972) Ans

Stranded (1972)

Strands Made from Steel (1974)

Strap Assembly-Tank (1973)

Strap Peel Adhesion of Reinforcing Fabrics or Cords to

Strap (1973)

Strap, Forked (1973)

Strap, Lanyard, Pole and Tree Climber) (1973)

Strap. Light Duty (1972)

Strap, Plain (1973)

Strap, Threaded (1973)

Strap, Unthreaded (1973)

Straps) (1974)

Straw) (1968)

Straw, Spanish Moss, Excelsior, etc.) (1973)

Stray Radiant Energy (1972)

Streamer Assembly. Warning (1972)

Streamline Supply Transactions Within the Air Transport

Street and Highway Lighting (1973) EEI TDJ-133

Streets and Projections Over Public Property (Pedestria

Strength and Elongation of Cotton Textile Fibers (Flat

Strength and Modulus of Rupture of Refractory Brick and

Strength and Stiffness Computations (1973)

Strength at Elevated Temperatures (1971) ANSI G81.23
ASTM

ASTM

ASAE

$A C I$

A STM

ICBO

DFISA

NFPA

FMS

NSA

CRA

ASTM

AAMA

ASTM

ASTM

ASTM

ASTM

ASTM

ASTM

AAMA

BIA

AHAM

St AHA

UL

ANSI

UL

A L TC

ABYC

NEMA

ASTM

ASTM

ANSI

NSA

VSA

ASTM

ISA

AST

ASTM

ASTM

IL

FCI

ASTM

ICBO

ASTM

ASTM

ASTM

A STM

ASTM

ASTM

ASTM

ASTM

ASTM

ASTM

ASTM

NSA

NSA

NSA

NSA

NSA

ASAE

ICBO

ASTM

NSA

ATA A

NEMA

ICBO

ASTM

ASTM

Re WWPA

ASTM

D241.1

65.37

FC*2ART3

3200

495

1196

3 A 5

41

804

1102.5

C663

C506

C507

1002.8

$26 \mathrm{~A}$

ER-1 
$r$ Hot, Hot Cold, and Cold Worked Alloy Steel Bars for High

d Worked Alloy Steel Forgings and Forging Billets for High for Iron, Cobalt, and Nickel Base Alloy Castings for High $s$ and Genera/

(1973) ANSI G50.1 Std. Spec. for Low and Intermediate Tensile Std Spec for Mild to Medium

ANSI G35.2

0) ANSI G24.l Std. Spec. for Low and Intermediate Tensile Std. Spec. for Low and Intermediate Tensile Pressure Service Piping (1969)

ling) (1973)

(1967) ANS1 Z166.17 Spec. for High Reference Radiographs for High
Rec. Pract. for in Place Concrete Std. Test. Procedure for End Supported Beam

Std. Requirement for Switching Impulse Volt age Insulation ral Quality (1972) ANS1 G41.6 se in Marine Environments (1974) (1970A) ANSI G41.4

Std. Spec. for High Std. Spec. for High Std. Spec. for High Std Spec, for High

Std. Spec. for Normalized High Std. Spec. for Hot Formed Welded and Seamless High Std. Spec. for Pressure Vessel Plates, Carbon Steel, High Std. Spec. for High

\section{ANSI Z197.20}

$h$ Joint s in Shear B/ oints in Shear by T/ (1972) ANSI Z197.3 972) ANSI A111.29 hto T167

of Bonded and Laminate/

Std. Test Meth for Flexural

Std. Test. Meth. for Impact Std. Rec. Pract. for Determining the Std. Rec. Pract for Determining the Std. Meth. of Test for Tensile Std. Meth. of Test for Cold Bonding Std. Meth. of Test for Compressive Std. Meth. of Test. Delamination. Test Meth. for Bond Early

Std. Meth of Test for Tin Std. Meth. of Test for Transverse Rupture Std. Meth. of Test for Breaking Test Meth. for Coating Pick Tests for Determining Surface Std. Test. Procedure for Torque Tentative Meth. of Test for Compressive and Flexural ht Loading) (1968) ANSI / Std. Meth. of Test for Flexural Meth. of Test for Seam a37.121

T22, ANSI A37.18 Std. Meth. of Test for Splitting Tensile Std. Meth. of Test for Compressive Meth. of Analysis of Yeast

Std. Meth. for Adhesion or Cohesive Std. Test Meth. for Edgewise Compressive Tent. Meth. of Test for Compressive (Crushing) Std. Meth. of Test for Hydrostatic Collapse Std. Test Meth. for Delamination

Prisms Broken in Fle m) Cube Specimens) (1/

Std. Meth. of Test for Compressive Std. Meth. of Test for Flexural Std. Meth. of Test for Dielectric Breakdown Voltage and Std. Meth. of Test for Compressive Std. Meth. of Test for Cleavage 197.16 m Building Code Std. for Determining the Splitting Tensile Test Meth. for Internal Bond Std. Meth. of Test for Tensile Beam M/ Std. Meth. of Test for Apparent Horizontal Shear Std. Meth. of Test for Hydrostatic Compressive
Std. Meth. of Test for Tearing th. (Constant Rate of Tra Std. Meth. of Test for Tearing
Sid. Meth. of Test for Std. Meth. of Test for Std. Meth. of Test for Std. Meth. of Test for Std. Meth. of Test for Std. Meth. of Test for Std. Test Meth. for Std. Meth. of Test for Std. Meth. of Test for Tensile uction in Shear by Tension Loadin/ ading (Metal to Metal) (1972) Ans/ ading at Elevated Temperatures (M/ ading in the Temperature Range $\mathrm{Fr} /$ ading of Laminated Assemblies (19) uction in Shear by Tension Loading (/ ression Loading (Disk Shear) (197/ Std. Rec. Pract. for Sharp Notch Tension Test. of High ding Suitable Nuts and Plain Hardene/ Std. Spec. for High
Uniform Building Code Std. for High ansi G52.1

and Lower Temperature Service (1972/

Sid. Spec. for High Std. Spec. for High Std. for Plating, High Std. Spec. for High

Std for Cam Follower, Needle Bearing, High Std. Meth. for Establishing Clear Wood Bul. on Round Thread Casing Joint

972) 74)
Tentative Meth. of Test for Biaxial Flexure Std. for Bolt-100 Deg. Close Tolerance, High Std. for Fastener: Rotary, Quick Operating, High $.9 \mathrm{Mo}_{0}-0.10 \mathrm{~N}$, Heat Treat ed, $170,000 \mathrm{psi}(1172 \mathrm{MPa})$ Tensile and Tempered, Roll Threaded (135,000 psi (931 MPa) Tensile Std. Spec. for Steel Wire, High Tensile nd Screws, Heat Resistant, 195,000 psi (1,345 MPa) Tensile
Strength at Elevated Temperatures (1971) ANSI G81.24 Strength at Elevated Temperatures (1971) ANSI G81.27 Strength at Elevated Temperatures (1973) ANSI G81.40 Strength Carbon-Silicon Steel Plates for Machine Part Strength Carbon Steel Castings for General Application Strength Carbon Steel Plates for Pressure Vessels (1972 Strength Carbon Steel Plates of Structural Quality (197 Strength Carbon Steel Wrought Welding Fittings for High Strength Casing, Tubing, and Drill Pipe (Petroleum Dril Strength Copper Base and Nickel-Copper Alloy Castings Strength Evaluation (1970)

Strength Fibre Tubes and Cores (1972)

Strength for AC High Voltage Circuit Breakers Rated on Strength Low Alloy Columbium Vanadium Steels of Structu Strength Low Alloy Steel H-Piles and Sheet Piling for U Strength Low Alloy Structural Manganese Vanadium Steel Strength Low Alloy Structural Steel (1970A) ANSI G4l 2 Strength Low Alloy Structural Steel (1973) Strength Low Alloy Structural Tubing (1973) Strength Manganese (1972A) ANSI G35.8

Strength Nonheaded Steel Bolts and Studs (1973)

Strength of Adhesive Bonded Laminated Assemblies (1969) Strength of Adhesive Bonds (1972) ANSI Z197.14

Strength of Adhesively Bonded Plastic Lap Shear Sandwic Strength of Adhesively Bonded Rigid Plastic Lap Shear J Strength of Adhesives by Means of Bar and Rod Specimens Strength of Air Setting Refractory Mortar (Wet Type) (1 Strength of Bituminous Mixtures (1974) ANSI A.37.103, as Strength of Bond, Appearance, and Shrinkage Propensity Strength of Bonded and Laminated Textile Fabrics (1972) Strength of Brick Masonry (Tech. Notes) (1969)

Strength of Carbon Black (1973)

Strength of Cemented Carbides (1973) ANSI H9.23

Strength of Ceramic Tile (1971) ANS1 A173.1

Strength of Coated Papers Using the Interfiber Bond Tes

Strength of Composite Tubes and Cores (1974)

Strength of Concrete Under Field Conditions (1974)

Strength of Concrete (Using Simple Beam with Center Poi

Strength of Curtains and Draperies (Textile) (1973)

Strength of Cylindrical Concrete Specimens (1971) ANSI

Strength of Cylindrical Concrete Specimens (1972) AASHO

Strength of Doughs (Cereal Chemistry) (1962)

Strength of Flame Sprayed Coatings (1969) ANSI Z167.22

Strength of Flat Sandwich Constructions (1970)

Strength of Graphite (1971) ANSI K90.11

Strength of Hollow Glass Microspheres (1972)

Strength of Honeycomb Type Core Material (1970)

Strength of Hydraulic Cement Mortars (Using Portions of

Strength of Hydraulic Cement Mortars (Using 2 ln. (50 M

Strength of Hydraulic Cement Mortars (1972)

Strength of Insulating Gases at Commercial Power Freque

Strength of Masonry Assemblages (1972)

Strength of Metal to Metal Adhesive Bonds (1972) ANSI Z

Strength of Molded Concrete Cylinders (1973)

Strength of Paperboard (Z-Direction Tensile) (1973)

Strength of Paraffin Wax (1973) ANSI Z1 1.206, TAPPI T64

Strength of Parallel Fiber Reinforced Plastics by Short

Strength of Sandwich Cores (1970)

Strength of Syntactic Foam (1972A)

Strength of Woven Fabrics by the Tongue (Single Rip) Me Strength Properties of Adhesive Bonds in Shear by Compr Strength Properties of Adhesives in Plywood Type Constr Strength Properties of Adhesives in Shear by Tension Lo

Strength Properties of Adhesives in Shear by Tension Lo Strength Properties of Adhesives in Shear by Tension Lo Strength Properties of Adhesives in Shear by Tension Lo Strength Properties of Adhesives in Two Ply Wood Constr Strength Properties of Metal to Metal Adhesives by Comp Strength Properties of Steel Truss Plates (1973)

Strength Sheet Materials (1968) A NSI Z260.1

Strength Steel Bolts for Structural Steel Joints, Inclu

Strength Steel Bolts, Nuts. Washers, and Bolted Parts

Strength Steel Castings for Structural Purposes (1973)

Strength Steel Plates for Pressure Vessels for Moderate

Strength Steels, Cadmium (1973)

Strength Structural Steel (1970A) ANSI G41.3

Strength Stud (1973)

Strength Values (1973)

Strength with Combined Internal Pressure and Bending (1

Strength (Modulus of Rupture) of Ceramic Substrates (19

Strength (1973)

Strength (1973)

Strength (1973)

Strength) (1973)

Strength, Hard Drawn, for Mechanical Springs (1973,

/Te Heat Resistant, $15.5 \mathrm{Cr}-4.5 \mathrm{Ni}-2$ SAE

Strength, Hardened and Tempered, Roll Threaded (1973)

MSS

AP]

BIA

NSA

ASTM

ASTM

ASTM

ASTM

ASTM

ASTM

ASTM

ASTM

NRMCA

CCTI

ASTM

ASTM

ASTM

ST

ASTM

ASTM

ASTM

ASTM

ASTM

STM

ASTM

ASTM

ASTM

AATCC

ASTM

ASTM

ASTM

TAPPI

CCTI

ASTM

ASTM

AST

ASTM

$A A C C$

ASTM

ASTM

ASTM

ASTM

AST

ASTM

ASTM

ASTM

ASTM

ASTM

ASTM

ICBO

TAPP

ASTM

ASTM

ASTM

ASTM

ASTM

ASTM

ASTM

ASTM

ASTM

ASTM

ASTM

ASTM

ICBO

ASTM

ASTM 
spec, for Steel Sheet and Strip, Hot and Cold Rolled, High Rec. Pract. for High

r Bolting Materials, High Temperature, 50 to 120 KSI Yield Spec for Steel Self-Locking Nuts, High pec. for Self.Locking Corrosion Resistant Alloy Nuts, High elf-Locking Steel Nuts, Corrosion and Heat Resistant, High elf.Locking Steel Nuts, Corrosion and Heat Resistant, High able for Welding (1970E) ANSI / Std. Spec. for High Yield Std. Spec. for Pressure Vessel Plates, Alloy Steel, High te Fibers for Structural Composites (GF 220 (1517) Tensile Meth. of Analysis of Fecal ibility of Stainless Steels and Related Ni.Cr.Fe Alloys to for Use of Mattsson's Solution of $\mathrm{pH} 7.2$ to Evaluate the Std. Rec. Pract. for Making and Using the C-Ring chloride Solution (1973) Std. Rec. Pract. for Performing Std. Meth. for Preparation and Use of Bent Bean Std. Rec. Pract. for Making and Using U-Bend National Design Spec. for Std. Meth. for Analyzin

nsi B72.10 tion Meth. (1972) ANSI Z11./ Std. Meth. of Test for Yiel. Std. Meth of Test for Thermal Failure Under Electric Tent. Rec. Pract. for Measurement of Glass
Std. Rec. Pract. for 972) Std. Rec. Pract. for

3) Molybdenum Sheet, Strip, and Plate, Arc Cast Uniform Building Code Std. for Stress Variation or Uniform Building Code Std. for I Steel Members (1973) sensitive Tape to Fiberboard at 90 Deg. Angle and Constant
Rec. Pract. for Determining Durability of Adhesive Joints Rec. Pract. for Determining Durability of Adhesive Joints Code Std. for Structural Glued Built Up Members-Plywood Plywood

Design Spec. for Plywood Std. Spec. and Meth. for Establishing Rec. Design Tent. Test Meth. for Establishing Design 72T)

(1972) ANSI Z14.286 Meth. of Test for
Meth. of Test for Std. Meth. of Test for meth. of Test for Stretch Properties of Fabrics Woven from control Chemical 2-(Ethylthio) -4,6-Bis (lsopropylamino) tal Door Frame Preparation for 181 and 190 Series Deadlock Std. for Safety for Burglary Resistant Electric Door

It-Beryllium Alloy (Copper Alloy No. 175), Plate, Sheet, Std. Spec. for Copper Alloy sting Chromium and Chromium Nickel Steel Plate, Sheet, and

t. Hot Cold, and Cold Worked Alloy Steel Plate, Sheet, and

for Precipitation Hardening Nickel Alloy Plate, Sheet, and

1 Spec. Applicable to Electromagnet lron (EMI) Cold Rolled Std. Rec. Pract. for Use of Lock Std. Spec. for Copper Alloy No. 260 Brass Spec. for Steel Splined Continuous Carbon Steel

spec, for Nickel-Chromium-Iron Alloy Plate, Sheet, and Std. Spec. for Nickel Copper Alloy Plate, Sheet, and (Steel Products Manual) Carbon Stee Carbon Nickel Molybdenum Chromium Alloy Plate. Sheet, and inless and Heat Resisting Chromium Steel Plate, Sheet, and olled Stainless and Heat Resisting Steel Plate, Sheet, and or Corrosion Resisting Chromium Steel Clad Plate Sheet and el Iron Chromium Molybdenum Copper Alloy Plate, Sheet, and ickel Chromium Molybdenum Columbium Alloy Plate, Sheet and Std. Spec. for Nickel Plate, Sheet, and Std. Spec. for Cold Rolled Carbon Steel romium-Iron-Molybdenum-Copper Alloy Plate, Sheet and Chromium-Manganese-Molybdenum Alloy Plate, Sheet, and Regular Quality Hot and Cold Rolled Alloy Steel Sheet and

Drawing Quality Hot and Cold Rolled Alloy Steel Sheet and bdenum Copper Columbium Stabilized Alloy Plate, Sheet, an,

spec. for Nickel-lron-Chromium Alloy Plate, Sheet, and Std. Spec. for Thermostat Metal Sheet and oducts with Finished (Rolled or Drawn) Edges (Flatwire and Spec. for Tantalum Plate, Sheet, 23 Std. Spec. for Zirconium and Its Alloy Sheet, )) (1973) -0.38 C)) Special Grade (1973) Spec. for Steel Sheet, ec. for Corrosion and Moderate Heat Resistant Steel Sheet, Molvbdenum Sheet, base, $20 \mathrm{Cr}-20 \mathrm{Co}-5.9 \mathrm{Mo}_{0}-2.2 \mathrm{Ti} /$ Spec. for Alloy Sheet. base-15.8Cr-15.2Mo-0.30Al-0.05 La (1) $3.5 \mathrm{Cu}-(\mathrm{Cb}+\mathrm{Ta})$, Consumable Electrode $\mathrm{Va} /$ $29 \mathrm{Ni}-17 \mathrm{Co}(1973)$
Strength, Low Alloy Columbium and/or Vanadium (1970) an Strength, Low Alloy Steel (1970) ANSI G88.3

Strength, Materials with Expansion Coefficients Compara

Strength, Prevailing Torque, All Metal (1973)

Strength, Prevailing Torque, All Metal (1973)

Strength. Prevailing Torque, All Metal, 1200 Deg. F (64

Strength, Prevailing Torque, All Metal, 1200 Deg. F (64

Strength, Quenched and Tempered Alloy Steel Plate, Suit

Strength, Quenched and Tempered (1972 A) ANSl G35.11

Strcngth, 70,000,000 (483) Tensile Modulus (1973)

Streptococci in Food Products (Cereal Chemistry) (1962)

Stress Corrosion Cracking in Polythionic Acids (1973)

Stress Corrosion Cracking Susceptibility of Cu. Zn Alloy

Stress Corrosion Cracking Test Specimen (1973)

Stress Corrosion Cracking Tests in a Boiling Magnesium

Stress Corrosion Metal Specimens (1973)

Stress Corrosion Test Specimens (1972)

Stress Grade Lumber and lts Fastenings (1971)

Stress in Glass (1968)

Stress in Plastic Pipe Under Internal Pressure (1967)

Stress of Board, Timber, Lumber, Siding, Framing, Decki

Stress of Heterogeneous Propellants by the Cone Penetra

Stress of Solid Electrical Insulating Materials (1973)

Stress Optical Coefficient (1973)

Stress Relaxation Tests for Materials and Structures (1

Stress Relieved (1973)

Stress Reversal Design of Structural Steel Members (197

Stress Strain of Carbons and Graphite (1973)

Stress Variation or Stress Reversal Design of Structura

Stress (1972)

Meth. of Tes

Stressed in Peel (1971) ANSI Z19730

Stressed in Shear by Tension Loading (1971) ANSI Z197.3

Stressed Skin and Curved Panels, Beams, and Components

Stressed Skin Panels Fabrication Spec. (1971)

Stressed Skin Panels (1972)

Stresses for Joists and Rafters (Lumber) (1972) Usc Ps2

Stresses for Round Timber Construction Poles (1974)

Stresses for Round Timber Piles (1970)

Stretch in Wear (Textile) (1973)

Stretch Properties of Fabrics Woven from Stretch Yarns

Stretch Properties of Knitted Fabrics Having Low Power

Stretch Yarns (1972T)

Striazine) (1973)

Strikes (1973)

Strikes (1974)

Strip-High Temperature Terminal Block, Base Insulatin

Strip and Rolled Bar (1973)

Strip for Flexible Metal Hose (1973)

Strip for Fusion Welded Unfired Pressure Vessels (1972B

Strip for High Strength at Elevated Temperatures (1971)

Strip for High Temperature Service (1972)

Strip for Magnetic Application in Relays and Solenoids

Strip Gaskets (1973)

Strip in Narrow Widths and Light Gage for Heat Exchange

Strip Mastic Set Maple Flooring Over Concrete Slab Floo

Strip $(0.68-0.80 \mathrm{C})$ (SAE 1074) (1973)

Strip (0.90-1.04C) (SAE 1095) (1973)

Strip (1970) ANSl H34.10

Strip (1970) ANSI H34.6

Strip (1971)

Strip (1972)

Strip (1972) ANSI G81.2

Strip (1972) ANSI G81.30

Strip (1972) ANS1 G81.6

Strip (1972) ANSl H34.17

Strip (1972) ANSI H34.19

Strip (1972) ANSI H34.8

Strip (1972A) ANSI G47.1

Strip (1973)

Strip (1973)

Strip (1973) ANSI G24.24

Strip (1973) ANSI G24.25

Strip (1973) ANSl H34.37

Strip (1973) ANSI H34.40

Strip (1973) ANSI Z155.19

Strip) (1973)

Strip, and Foil (1973)

Strip, and Plate for Nuclear Application (1973) ANSI N1

Strip, and Plate $(0.80 \mathrm{Cr}-1.8 \mathrm{Ni}-0.25 \mathrm{Mo}(0.49-0.55 \mathrm{C}$

Strip, and Plate $(0.80 \mathrm{Cr}-1.8 \mathrm{Ni}-0.35 \mathrm{Mo}-0.20 \mathrm{~V}(0.33$

Strip, and Plate $(13 \mathrm{Cr}-2.0 \mathrm{Ni}-3.0 \mathrm{~W})(1973)$

Strip, and Plate, Arc Cast. Stress Relieved (1973)

Strip, and Plate, Corrosion and Heat Resistant, Nickel

Strip, and Plate, Corrosion and Heat Resistant, Nicke

Strip, and Plate, Corrosion Resistant, $14.8 \mathrm{Cr}-4.5 \mathrm{Ni}-$

Strip, and Plate, Low Expansion, Glass Sealing, $53 \mathrm{Fe}-$

ASTM

SAE

ASTM

SAE

$S A E$

SAE

SAE

ASTM

ASTM

/Phi SAE

CCH 42.35

ASTM G35

ASTM G37

ASTM G38

ASTM G39

ASTM G30

NFORP *4

ASTM F218

ASTM D2153

WWPA *24

ASTM D2884

ASTM D3151

ASTM $\quad$ C770

ASTM E328

SAE AMS7801A

UBCS27.3

ASTM C749

ICBO UBCS27.3

D2918

ASTM D2919

ICBO UBCS25.18

APA SS.8

APA *3PDS 3

NFORP *2

ASTM D3200

ASTM D2899

Test ANSI L24T5

ASTM D3107

ASTM D2594

ASTM

K62.135

Al15.5

ANSI Al15.

NSA 1063

ASTM B534

ASTM B508

ASTM A240

ASTM A457

ASTM A670

NARM

ASTM

ASTM

WSFI

SAE

$\mathrm{SAE}$

Std. ASTM

ASTM

AISI

At. Spec. for Low ASTM

Std Spec for Sta ASTM

/Ments for Delivery of Flat R ASTM Std. Spec. F ASTM c. for Nick ASTM td. Spec. for N ASTM

ASTM

ASTM

121

C716

B569

*5

AMS5120G

AMS5121D

B168

B127

B575

A 176

A 480

A263

B424

B443

B162

A 109
B 582

B590

B590

A506

A507

B463

B409

B388

B272

AMS7849A

SATM

B352

$\mathrm{SAE}$

AMS6396

AMS6433B

AMS5508B

AMS7801A

SAE

SAE

AMS5872

AMS5873

$S A E$

AMS5862 
c) (SAE 8740) (1973)

v $(0.17-0.23 \mathrm{C})$, Premium Quality, Consumab/ Steel Sheet, Titanium Alloy Sheet,

Std. Spec. for Phosphor Bronze (Copper Tin) Plate, Sheet, Std. Spec. for Leaded Brass Plate, Sheet, Std. Spec. for Copper-Beryllium Alloy Plate, Sheet, Std. Spec. for Brass Plate, Sheet,

Std. Spec. for Copper Iron Alloy Plate, Sheet, Std. Spec. for Copper-Zinc-Tin Alloys Plate, Sheet, for Copper-Zinc-Aluminum-Cobalt Alloy Plate, Sheet, oy (Nickel Silver) and Copper-Nickel Alloy Plate, Sheet, 3) irements for Wrought Copper and Copper Alloy Plate, Sheet,

Hot Rolled Carbon (0.15 Maximum, Percent) Steel Sheet and teel, Carbon (0.16 to 0.25 Maximum \%, Hot Rolled Sheet and

Spec. for Structural Steel and Iron (Shape, Plate, Sheet, cr-2.4Ti-0.70 A 1-7.0Fe (1973)

$4.5 \mathrm{Ni}-2.9 \mathrm{Mo}-0.10 \mathrm{~N}$, Solution Heat Tre/

Alloy Sheet and

lumbium and/or Vanadium (/ Std. Spec. for Steel Sheet and Std. Spec. for Copper Sheet, Std. Spec. for Cartridge Brass Sheet, Spec. for Titanium and Titanium Alloy Std. Spec for Columbium and Its Alloy Std. Spec. for Molybdenum and Molybdenum Alloy Std. Spec. for Hot Rolled Carbon Steel Sheet and Std. for Magnetic

for Operating Performance of Anion Exchange Materials for Std. for Uniform Meth. of Determining the SAE Rating and Std. for Uniform Meth. of Determining the SAE Rating and Std. Spec. for Insulating Board (Cellulosic Fiber), Std. Meth. of Test. Insulating Board (Cellulosic Fiber), Rec. for Preplaced Aggregate Concrete for d. Spec. for Quenched and Tempered Carbon Steel Plates for 973) Std. Spec. for

(Tech. Notes) (1963)

) (Tech. Notes) (1963)

ge) (Tech. Notes) (1963)

964)

f Sampling and Test. Stru/

Uniform Building Code Std. for Uniform Building Code Std. for

Std. Spec. for

Uniform Building Code Std. for Glossary of Terms Relating to

1962)

Std. Meth. of Sampling and Test. Brick and oad Bearing Wall Tile and Std. Meth. of Sampling and Test.

d Cavity 6 In. Brick and Tile Walls (Tech. Notes) (1966) Floors and Pavements of $70,000,000$ (483) Tensile M/ Spec. for Graphite Fibers for

Uniform Building Code Std. for Lightweight Aggregates for Preformed Expansion Joint Fillers for Concrete Paving and

(1967)

(Tech. Note) (1971)

(Tech. Notes) (1968)

Rivets, Type 2A (1973)

in and Curved Panels, Bea

Uniform Building Code Std. for Std. for Uniform Building Code Sid. for Uniform Building Code Std. for Tests for re Gluing of Southern/ Std. for Preservative Treatment of Rec. Architectural Spec, for Uniform Building Code Std. for Std. for , Southern and Lodgepole / Uniform Building Code Std. for
Std. Spec. for Std. Spec. for
m Meth. / Tent. Meth. of Test for Carbon Distribution and
Building Code Std. for Fiberboard Nail Base Sheathing and bers (1966)

Std. Spec. for Std. Test Meth. for 71) Rec. Pract. for Service Brake and Combination Vehicles F/ Rec. Pract. for Service Brake ry Use) Exposu/ Std. Spec. for Protein Base Adhesives for 59) A/ Std. Meth. of Test for Integrity of Glue Joints in or (Wet Use) Exposure Condi/ Std. Spec. for Adhesives for Rec. Guide for

Rec. Pract. for Selecting Proportions for d. Meth. for Evaluating Allowable Properties for Grades of Std. Spec. for High Strength Low Alloy ilding Code Std. for Spec. and Design of Cold Formed Steel
pec. and Design of Light Gauge Cold Formed Stainless Steel
Strip, and Plate, $0.50 \mathrm{Cr}-0.55 \mathrm{Ni}-0.25 \mathrm{Mo}(0.38-0.43$ Strip, and Plate, 0.75Cr-9.0Ni-4.5 Co-1.0Mo-0.09 Strip, and Plate, 6Al.4V Annealed (1973)

Strip, and Rolled Bar (1973)

Strip, and Rolled Bar (1973)

Strip, and Rolled Bar (1973)

Strip, and Rolled Bar (1973)

Strip, and Rolled Bar (1973)

Strip, and Rolled Bar (1973)

Strip, and Rolled Bar (1973)

Strip, and Rolled Bar (1973)

Strip, and Rolled Bar (1974)

Strip, Cold Rolled Hard, Untem

Strip, Commercial Quality (1972)

Strip, Commercial Quality (1972)

Strip, Connector, and Bar) (1973) /E Std.
Strip, Corrosion and Heat Resistant, Nickel Base-15.5

Strip, Hot and Cold Rolled, High Strength, Low Alloy Co

Strip, Plate and Rolled Bar (1973) ANSI H7.9

Strip, Plate, Bar and Disks (1973)

Strip, Sheet, and Plate (1972) ANSI Z179.1

Strip, Sheet, Foil, and Plate (1964) ANSI Z179.20

Strip, Sheet, Foil, and Plate (1974)

Strip, Structural Quality (1972)

Stripe Encoding (Code) for Credit Cards (1973)

Strong Acid Removal (1972)

Struck Capacity for Hoe Dipper (Backhoe Bucket) (1972)

Struck Capacity for Shovel Dipper, Clam Bucket, and Dra

Structural and Decorative (1972)

Structural and Decorative (1972)

Structural and Mass Concrete (1966)

Structural Applications (1973)

Structural Carbon Steel Plates of lmproved Toughness (1 Structural Clay Acoustile (Tech Notes) (1961)

Structural Clay Differential Movement (Cause and Effect

Structural Clay Differential Movement (Expansion Joints

Structural Clay Differential Movement (Flexible Anchora

Structural Clay Facing Tile (Tech. Notes) (1965)

Structural Clay Floor Tile (1973)

Structural Clay Insulated Cavity Walls (Tech. Notes) (1

Structural Clay Load Bearing Wall Tile and Std. Meth. O

Structural Clay Masonry Process (Tech. Notes) (1966)

Structural Clay Nonload Bearing Tile (1971) ANSl A 76.1

Structural Clay Nonload Bearing Wall Tile (1973)

Structural Clay Products (Tech. Notes) (1961)

Structural Clay Protective Construction (Tech. Notes)

Structural Clay Tile (1973)

Structural Clay Tile (1973)

Structural Clay Wall Tile (Tech. Notes) (1966)

Structural Clay (Properly Constructed Masonry) Insulate

Structural Clay (Tech. Notes) (1962)

Structural Composites (GF 220 (1517) Tensile Strength,

Structural Concrete for Buildings (1973) A NSI A 138.1

Structural Concrete (1973)

Structural Construction (Nonextruding and Resilient Bit

Structural Deck (Roofing); Gypsum Concrete (1973)

Structural Design Guide for Hardwood Plywood (1971)

Structural Design of Brick Masonry Arches (Tech. Notes)

Structural Design of Semicircular Brick Masonry Arches

Structural Design of Serpentine Walls, Non Loadbearing

Structural Floor and Roof Plank and Beam Framing (1973)

Structural Flush Break Pull Mandrel Self Plugging Blind

Structural Glued Built Up Members-Plywood Stressed Sk

Structural Glued Laminated Lumber (1973)

Structural Glued Laminated Members and Laminations Befo

Structural Glued Laminated Timber (1972)

Structural Glued Laminated Timber (1973)

Structural Glued Laminated Timber (1973) ANSI A 190.1

Structural Glued Laminated Timbers: Douglas and Hem Fir

Structural Granite (1968) ANSI A91.1

Structural Group Analysis of Petroleum Oils by the N.D-

Structural Insulating Board (1973)

Structural lnsulating Formboard Made from Cellulosic Fi

Structural Insulating Roof Deck (R1970)

Siructural Integrity Test Procedure-Passenger Car (19

Structural Integrity Test Procedure for Trucks, Buses,

Structural Laminated Products for Use Under Interior (D

Structural Laminated Wood Products for Exterior Use (19

Structural Laminated Wood Products for Use Under Exteri

Structural Lightweight Aggregate Concrete (1964)

Structural Lightweight Concrete (1969) ANSI A 164.1

Structural Lumber (1974)

Structural Manganese Vanadium Steel (1970A) ANSI G41.4

Structural Members (1973)

Structural Members (1973)
Uniform Bu $\mathrm{lCBO}$

SAE

SAE

SAE

ASTM

ASTM

ASTM

ASTM

ASTM

ASTM

d. Spec. ASTM

ASTM

ASTM

ASTM

ASTM

ASTM

ICBO

SAE

ASTM

ASTM

ASTM

ASTM

ASTM

ASTM

ANSl

SAE

SAE

ASTM

ACI

St ASTM

ASTM

BIA

BlA

BIA

BlA

BIA

$\mathrm{BlA}$

$\mathrm{ICBO}$

BIA

ASTM

ICBO

BIA

ASTM

ICBO

BIA

BIA

BlA

SAE

ICBO

A STM

NRCA

HPMA

BlA

BlA

BIA

ICBO

IFI

$\mathrm{CBO}$

ICBO

A WPA

A ITC

ICBO

USC

ICBO

ASTM

ICBO

ASTM

ASTM

SAE

SAE

ASTM

ASTM

ASTM

ACl

ASTM

ASTM

/Rm Building Code Std. for S lCBO

AMS6358D

AMS6523A

AMS4911C

B103

B12

B194

B36

B591

B592

B122

B248

A680

569

A659

UBCS27.1

AMS5541B

AMS5547C

A607

B152

B19

B265

B393

B386

A 570

X4.16

J 296

J67 208

C209

66-65

A678

A573

18

$18 \mathrm{~A}$

$18 \mathrm{~B}$

22 
nsity and Interconnected Porosity of Sintered Powder Metal Std. Spec. for Iron Copper Sintered Metal Powder Std. Spec. for Sintered Copper Steel Std. Spec, for Sintered Nickel Silver Sid. Spec. for Sintered Bronze Std. Spec. for Sintered Brass

Std. Spec. for Sintered Aluminum Std. Spec. for Aluminum Alloy Extruded Std. for Building Code Requirements for Std. for Building Code Requirements for Std. for Glass Fiber Reinforced Polyester Uniform Building Code Std. for Quicklime (Lime) for Std. Spec. for High Strength Steel Castings for ed (Galvanized) by Hot Di/ Std. Spec. for Steel Sheets of w and Intermediate Tensile Strength Carbon Steel Plates of Std. Spec. for Hot Rolled Carbon Steel Sheet and Strip, for High Strength Low Alloy Columbium Vanadium Steels of

Physical Dough Tests of Flour (Cereal Chemistry) (1962) tests for Static Load, Frame, Fork, Stee/ Uniform Building Code Std. for ry, Flooring, Shingles, Exposed Roof Decking, Drop Siding, Std. Test Meth. for Density of Core Materials for std. Test Meth. for Water Absorption of Core Materials for std. Spec. for Aluminum Alloy Extruded Bar, Rod. Pipe, and Std. Piling (1) (Steel Products Manual) Carbon Steel' Plates,
Std. Spec. for Aluminum Alloy Std. Std. Meth. of Static Tests of Timbers (Wood) in

Structures (1972) Sid. Spec. for

conned Uniform Building Code Std. for Material Spec. for g39.1 Std. for Safety Requirements for Shops Fabricating Std. Spec. for Std. Spec. for Std. Specs. for Welding of Spec. for

1970) ain Hardene/ Std. Spec. for High Strength Steel Bolts for

ode Std. for Stress Variation or Stress Reversal Design of platforms (1972) Spec. for Fabricated r Safeguarding Against Embrittlement of Hot Dip Galvanized Manual of Cold Formed Welded d Point (1/2 In. (12.7 Mm) Maximum Thickn/ Std. Spec. for Std. Spec. for High Strength Low Alloy
Std. Spec. for High Strength Std. Spec. for Normalized High Strength Low Alloy

for Erection, Fabrication, Identification and Painting of td. Spec. for Cold Formed WeIded and Seamless Carbon Steel for Hot Formed Welded and Seamless High Strength Low Alloy std. Spec. for Hot Formed Welded and Seamless Carbon SteeI of Rolled Steel Plates, Shapes, Sheet Piling, and Bars for Std. Spec, for Steel Tubes, Low Carbon, Tapered for aring and Expansion Plates and Sheets for Bridge and Other

Std. Spec. for Steel, Cold Rolled Sheet, Carbon. $s$ of the United States for Information Interchal Std. for for Minimum Performance Criteria for Roll Over Protective for Minimum Performance Criteria for Roll Over Protective Surface Preparation Spec. for Steel minimum Performance Criteria for Falling Object Protective Std. Guidelines for Thesaurus

rface/ Rec. Pract. and Nomenclature for Identification of ec. for Prevention of Roof Collapse of Buildings and Other Std. for Electrical Penetration Assemblies in Containment nd Test Requirements for Leakage Rate Test. of Containment spec. for Design of Aluminum Alloy Load Carrving (Members) aluation of Roll Over (ROPS) and Falling Object Protective Rec. for Parking

for Minimum Performance Criteria for Roll Over Prolective Treatment) (1968/ Rec, for Concrete Sanitary Engineering and Tile in Sanitation Structures, Miscellaneous Sanitary

Uniform Building Code: Penthouses and Roof

Rec. for Erosion Resistance of Concrete in Hydraulic Design and Construction of Circular Prestressed Concrete criteria for Reinforced Concrete Nuclear Power Containment Code for Safety to Life from Fire in Buildings and

ct. for Atmospheric Exposure of Adhesive Bonded Joints and

Structural Std. for Steel Antenna Towers and Supporting

Rec. Pract. for Stress Relaxation Tests for Materials and quirements for Minimum Design Loads in Buildings and Other
Rec. for Concrete Shell Uniform Building Code Std. for Aluminum Uniform Fire Code: Tents and Air Supported due to Weak Construction and Design of Buildings and Other
Structural Parts and Oil Impregnated Bearings (1973) an Structural Parts (1970) ANSI G63.1

Structural Parts (1970) ANSl G63.3

Structural Parts (1970) ANSI H9.28

Structural Parts (1970) ANSI H9.6

Structural Parts (1970) ANSI H9.8

Structural Parts (1973)

Structural Pipe and Tube (1973) ANSI H38.16

Structural Plain Concrete (1972) ACI 322

Structural Plain Concrete (1972) ANSI A 169.1

Structural Plastic Panels (1972)

Structural Purposes (1973)

Structural Purposes (1973) ANSI G52.1

Structural Quality in Coils, and Cut Lengths, Zinc Coat

Structural Quality (1970) ANSI G24.2

Structural Quality (1972)

Structural Quality (1972) ANS1 G41.6

Structural Relaxation Meth. of Extensigraph Analysis of

Structural Requirements for Regular Bicycles Including

Structural Rivet Steel (1973)

Structural Roof Trusses and Rafters, Overhead

Structural Sandwich Constructions (1970)

Structural Sandwich Constructions (1970)

Structural Shapes for Electrical Purposes (Bus Conducto

Structural Shapes (1972)

Structural Shapes (1973)

Structural Shapes, Rolled Floor Plates, and Steel Sheet

Structural Shapes, Rolled or Extruded (1973) ANSI H38. 1

Structural Sizes (1967) ANSI Q4.2

Structural Slate (1968) ANSI A 193.1

Structural Std. for Steel Antenna Towers and Supporting

Structural Steel and Iron (Shape, Plate, Sheet, Strip.

Structural Steel and Plate (1973)

Structural Steel for Locomotives and Cars (1970A) ANSI

Structural Steel for Ships (1973)

Structural Steel Highway Bridges (1974)

Structural Steel Joints Using ASTM A325 or A490 Bolts (

Structural SteeI Joints (1971) ANSI G24.19

Structural Steel Joints, Including Suitable Nuts and PI

Structural Steel Lintels (Tech. Notes) (1969)

Structural Steel Members (1973)

Structural Steel Pipe for Use of Welded Offshore Fixed

Struciural Steel Products and Procedure for Detecting E

Structural Steel Tubing (1974)

Structural Steel with 42,000 psi (290 MPa) Minimum Yiel

Structural Steel (1970A) ANSI G4l.2

Structural Steel (1970A) A NSI G41.3

Structural Steel (1973)

Structural SteeI (1973)

Structural Tubing in Rounds and Shapes (1973) ANSI G24.

Structural Tubing (1973)

Structural Tubing (1973) ANSI G24.22

Structural Use (1972) ANSI G24.4

Structural Use (1973)

Structural Uses (1972) ANSI H31.1

Structural WeIding Code (1972)

Structural (1972)

Structure for the Identific ation (Code) of the Countrie

Structure for Track Type Tractors and Front End Loaders

Structure for Weeled Front End Loaders and Weeled Dozer

Structure Painting (1972)

Structure (FOPS) for Prime Movers, Wheeled and Track Ty

Structure, Construction, and Use (1974)

Structures and Contaminants Seen on Specular Silicon Su

Structures Caused by Snow Loads (1973)

Structures for Nuclear Fueled Power Generating Stations

Structures for Nuclear Reactors (1972)

Structures (Aluminum Construction Manual Sect. 1) (1971

Structures (FOPS) of Construction and Industrial Vehicl

Structures (Garage-Fire Protection and Safety) (1973)

Structures (ROPS) for Prime Movers (Heavy Equipment) (1

Structures (Tanks, Reservoirs, etc, for Water and Waste

Structures (Tech. Notes) (1969)

Structures (Tower, Spire) (1973)

Structures (1952)

Structures (1967)

Structures (1969)

Structures (1970) ANSI A9.1

Structures (1970) ANSI Z197.24

Structures (1972)

Structures (1972)

Structures (1972)

Structures (1973)

Structures (1973)

Structures (1973)

Structures (1973)

$\begin{aligned} & \text { Brick } \text { BIA } \\ & \text { ICBO } \\ & \text { ACI } \\ & \text { ACI } \\ & \text { Design } \text { ACI } \\ & \text { NFPA } \\ & \text { Std. Rec. Pra } \text { ASTM } \\ & \text { EIA } \\ & \text { Std. } \text { ASTM } \\ & \text { Std. for Building Code Re } \text { ANSI } \\ & \text { ACI } \\ & \text { ICBO } \\ & \text { ICBO } \\ & \text { Rec. for Prevention of Collapse } \text { FMS }\end{aligned}$

ACI

ICBO

$\mathrm{ACI}$

NFPA

STM

ANSI

CBO

CBO
FMS
ASTM

ASTM

ASTM

ASTM

ASTM

ASTM

ANSI

ACI

ICBO

ASTM

ASTM

ASTM

ASTM

Std. Spec

ASTM

BMA

ICBO

$A W I$

ASTM

ASTM

ASTM

AA

SAE

AISI

ASTM

ASTM

EIA

ICBO

ANS1

ASTM

ASTM

ASHTO

ASTM

ASTM

BIA

CBO

API

WSTI

ASTM

ASTM

ASTM

ASTM

ICBO

ASTM

ASTM

ASTM

ASTM

ASTM

ASTM

AWS

ASTM

ANSI

$\mathrm{SAE}$

ANSI

$\mathrm{SAE}$

ANSI

ASTM

FMS

IEEE

ANSI

AA

SAE

FMS

SAE

B328

B222

B426

B458
B255

B282

B595

B429

A l69. 1

322

P5 3

UBCS24-18

A 148

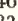

A 570

A572

$6 / 4$

UBCS27-5

*1-1

C272

B317

MS2204D

B308

D198

C629

RS222-B

UBCS27-1

Z229.1

Al31

*6

S314

A 490

A325

31B

BCS27-3

2B 
National Bureau of Standards KWIC Index of Engineering Standards

iquefied Flammable Gas Processing Equipment and Supporting Spec. for Petroleum Drilling and Well Servicing for Fire Protection Criteria for Safety Related Systems, Notes) (1969) Brick and Tile in Sanitation Brick and Tile in Sanitation Brick and Tile in Sanitation Brick and Tile in Sanitation Std. for tes) $(1969)$ 1969)

for Portland Cement and Cement Lime Plastering, Exterior
for Portland Cement and Cement Lime Plastering, Exterior Std. for Coarse Threaded Std for Fine Threaded

Std. for Cam Follower, Needle Bearing, High Strength Std. for Terminal Block Electrical Principles of Clay Masonry Construction:

Std for Nomenclature for Motion Picture Film Used in e Application of Gypsum Sheet Material to Light Gage Steel

Std. Spec. for High Strength Nonheaded Steel Bolts and

Uniform Building Code Std. for Shear Connectors (Stee) readed $(135,000 \mathrm{psi}(931 \mathrm{MPa}) / \quad$ Spec. for Low Alloy Stee] Spec. for Low Alloy Steel crew Applic/ Std. Spec. for Nonload (Axial) Bearing Steel ed After Hcat Treatment, 1800 Deg. F (982.2 Deg. C) Solu/ Std. for Collar, Swage Locking for Pull Type and Std. for Titanium Alloy Std. for Titanium Alloy

Std. for Pin, Swage Locking, Steel, 100 Deg. Shear Head, std. for Pin, Swage Lorking, Steel, Protruding Head, Shear for Pin, Swage Locking, A-286 Cres, 100 Deg. Shear Head, or Pin, Swage Locking, A.286 Cres, Protruding Head, Shear, n, Swage Locking, Steel, 100 Deg. Head (MS24694), Tension, n, Swage Locking, Steel, 100 Deg. Head (MS20426), Tension, Swage Locking, Aluminum Alloy, Protruding Head, Tension, Locking, Aluminum Alloy, 100 Deg. Head (Ms20426, Tension, locking, Aluminum Alloy, 100 Deg. Head (MS24694), Tension, Std. for Bolt Lock, Tension. Protruding Head, Std. for Bolt Lock, Tension, 100 Deg. Head (MS24694), Std. for Bolt Lock. Tension, 100 Deg. Head (MS20426), Std. for Bolt Lock, Tension, 100 Deg. Crown Head, for Pin, Swage Locking, Steel, Protruding Head, Tension, ire and Cable (1973) ANSI C8.28 Std. Spec. for h. for Evaluation of and Test Formula for Carbon Blacks in td. Meth. for Evaluation of and Test Formula for Pigmented meth. for Evaluation of and Test Formulas for Nonpigmented chemical Analysis of Synthetic Elastomers for Use on Solid Std. for Installation for Acrylonitrile Butadiene Std. Spec. for Sid. Spec. for

Std. Test Meth. for Polymer Content of

Materials and Property Stds. for Acrylonitrile Butadiene for Underground Installations of Electrical W d Fittings (1973)

Std. for 4)

Std. Spec. for Std. Spec. for Std. Spec, for Solvent Cements for Std Spec for Acrylonitrile-Butadienec. for Solvent Cements for Joining Acrylonitrile Butadiene ittings (1973/ Std. Spec. for Acrylonitrile-Butadiene. ' Ans/ Spec for Solvent Cement for Acrylonitrile-ButadieneStd. Spec. for Socket Type Acrylonitrile-Butadiene Std. Spec. for Threaded Acrylonitrile-Butadiene Std. Spec. for Socket Type Acrylonitrile-Butadiene Std. Spec. for Acrylonitrile-Butadiene Std. Spec, for Acrylonitrile-Butadiene si K65.59 972) ANSI K65.57 Std. Spec, for Acrylonitrile-Butadiene Std. Spec. for Acrylonitrile Butadiene Std. Spec. for Rigid Acrylonitrile-Butadiene nt, $15.5 \mathrm{Cr}-4.5 \mathrm{Ni}-2.9 \mathrm{Mo}-0.10 \mathrm{~N}$, Solution Heat Treated, gepole Pines; Engelmann Spruce; Western Cedar and Hemlock; al Chemistry) (1962) Colorimetric Modified Fiske for Correct Preparation, Finishing and Testing of Concrete ab / Spec. for Mastic Nailed Construction with Mastic Set
s / Spec. for Mastic Cushioned Construction with Nailers, arquet Flooring Set in Adhesive Over Concrete Slab or Wood nt. Test Meth. for Critical Dilation of Concrete Specimens d. Spec. for Merchant Quality Hot Rolled Carbon Steel Bars Test Meth. for Adhesion to Porous Substrates for Deleterious Effects of Dyes, Finishes, and Finishings

Spec. for Bare Mild Steel Electrodes and Fluxes for Std. Meth. of Test for Penetration of Liquids into pract. for Control of External Corrosion on Underground or paper) (1973) paper) (19/ Test Meth. for Density of Pulpwood $0)$

Test Meth. for Density and Moisture of Chips Std. on

se Meth. of Analysis of Starch in Flour and Semolina (With or Particle Size Distribution of Particulate Substances of of Test for Nitrogen Content (Kjeldahl) and Hide (Leather)
Structures (1973)

/(Safety) for Flammable Liquid and L FMS

Structures (1974)

Structures, and Equipment for Water Cooled and Moderate

Structures, Miscellaneous Sanitary Structures (Tech. No

Structures, Sewage Treatment Facilities (Tech. Notes) (

Structures, Water Treatment Facilities (Tech. Notes) (1

Stub ACME Screw Threads (1973)

Stucco) and Interior (1971)

(Stucco) and Interior (1971)

Stud (1973)

Stud (1973)

Stud (1973)

Stud (1974)

Student's Manual (1960)

Studios and Processing Laboratories (1971)

1. for Lathing and Furring

Studs (1972)

Studs (1973)

Studs) for Composite Construction (1973)

Studs, Heat Resistant, Normalized and Tempered, Roll Th

Studs. Heat Treated, Roll Threaded (1973)

Studs, Runners (Track) and Rigid Furring Channels for $\mathbf{S}$

Studs, Steel, Corrosion and Heat Resistant, Roll Thread

Stump Type Lockbolts (1973)

Stump Type Protruding Head Shear Bolt Lock (1974)

Stump Type 100 Deg. Head Shear Bolt Lock (1974)

Stump Type (1973)

Stump Type (1973)

Stump Type (1973)

Stump Type (1973)

Stump Type (1973)

Stump Type (1973)

Stump Type (1973)

Stump Type (1973)

Stump Type (1973)

Stump Type, Titanium Alloy (1973)

Stump Type, Titanium Alloy (1973)

Stump Type, Titanium Alloy (1973)

Stump Type, Titanium Alloy (1973)

Stump Type, (1973)

Styrene-Butadiene-Synthetic Rubber (SBR) Jacket for

Styrene-Butadiene Rubber (SBR) (1973)

Styrene-Butadiene Rubbers (SBR) (1973)

Styrene-Butadiene Rubbers (SBR) (1973)

Styrene Butadiene Copolymers (SBR) (1972)

Styrene Drain, Waste and Vent Pipe and Fittings (1971)

Styrene Monomer 993 (1972)

Styrene Monomer 996 (1971)

Styrene Monomer (1974) A NSI Z161.1

Styrene Plastic Drain, W aste and Vent Pipe and Fittings

Styrene Rubber and Polyvinyl Chloride Ducts and Fitting

Styrene Rubber Plastic Drain and Building Sewer Pipe an

Styrene Rubber (SR) Plastic Drain Pipe, Perforated (197

Styrene Rubber (SR) Plastic Pipe and Fittings (1972)

Styrene (ABS) Composite Sewer Piping (1974)

Styrene (ABS) Pipe and Fittings to Poly(vinyl Chloride)

Styrene (ABS) Plastic Drain, Waste, and Vent Pipe and F

Styrene (ABS) Plastic Pipe and Fittings (1973) ANSI B72

Styrene (ABS) Plastic Pipe Fittings, Schedule 40 (1973)

Styrene (ABS) Plastic Pipe Fittings, Schedule 80 (1973)

Styrene (ABS) Plastic Pipe Fittings, Schedule 80 (1973)

Styrene (ABS) Plastic Pipe (SDR-PR) (1973) ANSI B72.3

Styrene (ABS) Plastic Pipe, Schedules 40 and 80 (1973)

Styrene (ABS) Plastic Sewer Pipe and Fittings (1973) an

Styrene (ABS) Plastic Utilities Conduit and Fittings (1

Styrene (ABS) Plastics (1973) ANSI K65.205

Sub Zero Cooled, Equilized, and Over Tempered (1973)

Subal-Pine Fir; and Mountain Hemlock (Lumber) (1973)

Subbarow Meth. of Analysis of Phosphorus in Yeast (Cere

Subfloor Surfaces to Receive Wood Flooring (1962)

Subflooring and Nailed Finish Flooring Over Concrete S]

Subflooring or Finish Flooring Over Concrete Slab Floor

Subfloors (1962)

Spec, for Mosaic

Subject to Mechanical Property Requirements (1972)

(Subjective Ratings) (Paper, Board, Cloth, etc.) (1973)

Sublimation, Brittleness and Chlorine Retention) (Insti

Submerged Arc Welding (1969) ANSI W3.17

Submerged Containers (Packaging) (1951) ANSI MH12.8

Submerged Metallic Piping Systems (1972)

Submersion with Correction for Cracks, etc.) (Pulp and

Submersion with Correction for Cracks, etc.) (Pulp and

Subscriber Visual Carrier Level (Cable Television) (197

Subsequent Acid Hydrolysis) (Cereal Che mistry) (1962)

Subsieve Sizes (1968)

Substance (1970) ALCA B5
Alysis by Microscopical Meth. F ASTM

Tent. Meth. ASTM
APl

ANS

BlA

BIA

BIA

ANSl

ANSI

7-14

$4 \mathrm{E}$

N18. 10

$34 \mathrm{C}$

$34 \mathrm{~A}$

34

Bl.8

A 42.2

A42.3

183

562

1002

*44

PH22.56

C646

A687

UBCS 27.8

AMS 7458C

AMS $7456 \mathrm{C}$

C 645

AMS 7482

1080

2605

2705

1414

1424

6974

6984

1486

6925

1555

6915

1546

2206

2306

2315

2325

1496

D866

D319]

D3186

D3185

D 1416

IS 5

ASTM D2826

ASTM D2827

ASTM D2121

AAPMO PSI7

NEMA TCl

ASTM D2852

DSTM D3298

ASTM D3138

ASTM D2661

ASTM D2235

ASTM D2468

ASTM D2465

ASTM D2469

ASTM D2282

ASTM D1527

ASTM D2751

ASTM D2750

ASTM DI788

SAE AVIS5743D

ICBO UBCS25-4

AACCH $40-56$

/C. WSFI *1

WSFI $* 6$

WSFI *8

Te ASTM C671

St ASTM A 663

TAPPI UM-540

L24.7.2

AW $\quad$ A 17

$\begin{array}{ll}\text { Rec. NATM } & \text { D } 998 \\ \text { NACE } & \text { RPl.69 }\end{array}$

TAPPI UM-2

TAPPI UM.20

NCTA 001B-0670

76-10

E20

D2868 
d Test. Felted and Woven Fabrics Saturated with Bituminous opical Meth. for Particle Size Distribution of Particulate Meth. of Analysis for Total Reducing

std. Test Meth. for Resistance of Ceramic Tile to Chemical for Determination of $\mathrm{pH}$ (Slurry) of Corn Starch Which utions Derived from Corn or Grain Sorghum Starch Which Are rglary Resisting Glazing Material for Use Principally as A

ness Determination of Nonconductive Coatings on Nonferrous

h. of Test for Thickness of Epitaxial Layers of Silicon on Test Meth. for Adhesion to Non Porous Flexible

tc.) (1973) Test Meth. for Adhesion to Porous

r Biaxial Flexure Strength (Modulus of Rupture) of Ceramic industry) (/ Test Meth. for Determining Water Quality for u/ Rec. Pract. for Design. Installation, and Operation of r Petroleum Industry Use (1973)

meth. for Laboratory Evaluation to Determine Resistance to Analysis for Formic, Acetic. Propionic, Butyric, Valeric, Rec. Pract. for Design Calculations for Oil Spec. for
Meth. of Ferricyanide-Maltose
Indices of Refraction of Indices of Refraction of ry) (1962) th. for Measurement of Glucose in Sugar Mixtures (Maltose,
ing Devices, Room Humidifiers, Nebulizers, Gas Therapy and and $\mathrm{Ai}$ Std. for Flow Capacity Rating and Application of and Maintenance (1973)

Rec. for Definitions (1974)

Std for

h. for Determining Refractive Index Content of Corn Syrup,

tical Meth. for Determining Calcium Content of Corn Syrup,

termining Chloride Content of Corn Syrup Unmixed. Finished

termining Chloride Content of Corn Syrup Unmixed. Finished

raneous Materials Content of Corn Syrup, Crude and Refined

Meth. for Determining Ash Content of Corn Syrup, Finished

$r$ Rolled Wheat, Breakfast Cereals (Except Those Which Are chemistry) (1962) Volumetric Copper Reduction Meth. for extr/ Glucose Oxidase Meth. for Measurement of Glucose in for Determination of Phosphorus in Corn Starch, Syrup and is of Specific Gravity, Degrees Brix, and Degrees Baume of ning Color, Solutions (Spectrophotometric) Content of Corn ical Meth. for Determining Moisture (Oven) Content of Corn

Std. Analytical Meth. for Determining Ash Content of Corn

Analytical Meth. for Determining Acidity Content of Corn

Analytical Meth. for Determining Arsenic Content of Corn std. Analytical Meth. for Determining Iron Content of Corn tical Meth. for Determining Sulfur Dioxide Content of Corn ng Color, Reflectance (Spectrophotometric) Content of Corn h. for Determining Moisture (Karl Fischer) Content of Corn ical Meth. for Determining Reducing Sugars Content of Corn

Meth. for Determining Dextrose Equivalent Content of Corn

al Meth. for Determining Specific Rotation Content of Corn

$r$ Determining Calcium (Egta-Titrimetric) Content of Corn

d. Analytical Meth. for Determining Copper Content of Corn

lytical Meth. for Determining Heavy Metals Content of Corn

Analytical Meth. for Determining Chloride Content of Corn

Std. Analytical Meth. for Determining $\mathrm{pH}$ Content of Corn

cal Meth. for Determining Protein Nitrogen Content of Corn determining Arsenic Content of Corn Syrup Unmixed and Corn r) Content of Corn Syrup Unmixed and Unbleached Crude Corn (1962)

Apparent Starch Content of Corn Syrup, Refined and Crude Meth. of Analysis of Lactose Meth. of Analysis of Sucrose Meth. of Analysis of Lactose (Milk Temperature Corrections for Refractometer Readings Temperature Corrections for Saccharimeter Readings for Douglas Fir; Larch: Hem-Fir; Ponderosa, ldaho White, harides (Gas Liquid Chromatography) Content of Corn Syrup, nesaccharides (Gas Liquid Chromatography) Content of Corn Std. Analytical Meth. for Determining Reducing Std. Analytical Meth. for Determining Reducing Std. Analytical Meth. for Determining Reducing Std. Analytical Meth. for Determining Total ch Molasses /

Meth. of Analysis of Reducing and Nonreducing Schoorl Meth. of Analysis of Reducing lytical Meth. for Determining Total Sugars Content of Corn ermining Copper Content of Corn Syrup Unmixed and Finished ng Sulfate Content of Corn Syrup Unmixed and Finished Corn on Content in Corn Starch Hydrolzates Including Syrups and Meth. for Determining Fermentables Content of Corn Syrup, Meth. of Analysis for Ash in Molasses.

of Corn Syrup and Starch Hydrolyzates and All Starches and ing Protein Nitrogen Content in Feedstuffs (Corn), Syrups, nitrogen Content in Steepwater, (Corn) Feedstuffs, Syrups,

h. for Determining Protein Nitrogen Content of Corn Syrup,

for Determining Protein Nitrogen Content in Corn Starch,

for Tailored Woven Uniform Fabrics, Lightweight (Tropical)
Substances for Use in Waterproofing and Roofing (1972)

Substances of Subsieve Sizes (1968)

Substances (Cereal Chemistry) (1962)

Substances (1971) ANSI A 174.1

Substantially Insoluble in Water at Room Temperature (1

Substantially Optically Clear (1959)

(1972)

Substrate (Coil, Instrumentation) (1974)

Substrate of the Sam The

Substrates (Paper) (1973)

Substrates (Subjective Ratings) (Paper, Board, Cloth, E Tentative Meth. of

Subsurface Injection Using Membrane Filters (Petroleum

Subsurface Safety Valve Systems for Petroleum Industry

Subsurface Safety Valves, Lock

Subterranean Termites (1972)

Succinic, and Lactic Acids (Cereal Chemistry) (1962)

Succinic, and Tartaric Acids (Cereal Che mistry) (1962)

Sucker Rod Pumping Systems (Conventional Units) (1972)

Sucker Rods (Petroleum) (1974)

Sucrose Conversion Table (Cereal Chemistry) (1962)

Sucrose Solutions at 20 Deg. C (Cereal Chemistry) (1962

Sucrose (Sugar) in Feeds and Feedstuffs (Cereal Chemist

Sucrose, Mannose, Galactose, Dextrans, and Starch (Cere

Suction Equipment, Room Air Purifiers, Filters, Compres

Suction Line Filters and Filter Driers in Refrigeration

Suction Press Rolls and Their Installation, Operation,

Suffix Letters Used in Tire Size Designations and Their

Sugar and Dextrose (1956)

Std. Analytical Met

Sugar and Other Carbohydrates (1957)

Sugar and Other Clarified Starch Hydrolvzates (Potassiu

Sugar and Other Clarified Starch Hydrolzates (Silver-

Sugar and Other Materials Soluble in Water (1966)

Sugar and Other Starch Hydrolyzates (1952)

Sugar Coated) (Cereal Chemistry) (1962)

Sugar Determination: Schoorl's Reduction Table (Cere

Sugar Mixtures (Maltose, Sucrose, Mannose, Galactose, D

Sugar Obtained from the Corn Wet Milling Process (1973)

Sugar Solutions (Cereal Chemistry) (1962)

Sugar (Crude and Refined) and All Hydrolyzates Solution

Sugar (Crude and Refined) (Crystalline) (1959)

Sugar (Crude and Refined) (1958)

Sugar (Crude and Refined) (1958)

Sugar (Crude and Refined) (1958)

Sugar (Crude and Refined) (1961)

Sugar (Crude and Refined) (1961)

Sugar (Crude and Refined) (1963)

Sugar (Crude and Refined) (1966)

Sugar (Crude and Refined) (1972)

Sugar (Crude and Refined) (1972) Std. An
Sugar (Crude and Refined), Dextrose, Syrup and Starch H

Sugar (Crude and Refined). Syrup and Other Starch Hydro

Sugar (Crude and Refined). Syrup, and Other Starch Hydr

Sugar (Crude and Refined), Syrups and Other Hydrolyzate

Sugar (Crude and Refined), Syrups and Other Starch Hydr

Sugar (Crude and Refined). Syrups and Other Starch Hydr

Sugar (Crude and Refined), Syrups, Starches and Other P

Std Analytical Meth for $\mathrm{Cl}$

Sugar (1954)

Sugar (1956)

Sugar (1967

/R Determining Moisture (Oven Filter Pape Std. Analytical Meth. for Determinin

Sugar) and Nonfat Dry Milk in Bread (Cereal Chemistry)

(Sugar) in Feeds and Feedstuffs (Cereal Chemistry) (1962

Sugar) in Mixed Feeds (Cereal Chemistry) (1962)

(Sugar) (Cereal Chemistry) (1962)

Sugar) (Cereal Chemistry) (1962)

Sugar, and Lodgepole Pines; Engelmann Spruce; Western C

Sugar, and Starch Hydrolyzates (1967)

Sugar. Starch Molasses and Hydrolyzates (1971)

Sugars Content in Dextrin (1970)

Sugars Content in Steepwater (Corn) (1964)

Sugars Content of Corn Sugar (Crude and Refined) (1972)

Sugars Content of Corn Sugars (Crude and Refined), Star

Sugars in Flour and Semolina (Cereal Chemistry) (1962)

Sugars in Prepared Bakery Mixes (Cereal Chemistry) (196

Sugars (Crude and Refined). Starch Molasses (1972)

Std. Analytical

gars (1954)

Std. Analytical

Sugars, and Most Wet Milled Products
Sugars, and Starch Hydrolyzates (1957)

Sugars, and Starch Hydrolyzates (1957)

Sugars, and to Most Other Products of the Wet Milling

Sugars. Starches and Other Protein Bearing Materials Wh

Sugars, Starches and Other Protein Bearing Materials Wh

Sugars, Starches and Other Protein Bearing Materials (1

Sugars, Syrups and Other Protein Bearing Materials (195

Suiting (Institutional Textile) (1973)

/ Requirements
ASTM E20

AACCH 10.01

ASTM C650

CR C. 44

CR F.14

CL 972

TB. 11.14

F95

TAPPI UM.541

ASTM F394

NACE TM.01.73

APl RPl4B

APl

$14 \mathrm{~A}$

Std. AWPA M12.72

$\mathrm{AACCH} \quad 04.23$

$\mathrm{AACCH} \quad 04.2$

APl $11 \mathrm{~L}$

API $11 \mathrm{~B}$

AACCH 22.18

AACCH 68.61

AACCH 80.50

AACCH 80.10

NSF 48

ARI $\quad 730$

12.60

TRA XIII

E.54

E. 10

E-14

E. 15

E-27

/Ext CR

$\mathrm{CR}$
$\mathrm{CR}$

E-6

ACCH 44-15A

AACCH 80.69

AACCH 80.10

$\begin{array}{ll}\mathrm{CR} & \mathrm{B} .47\end{array}$

$\mathrm{AACCH} \quad 80.75$

CR F.14

CR F.34

CR

F. 6

F. 2

Std $C R$

CR

F.4

F.28

F.54

F.16

F.32

F. 48

F. 22

F.52

F.8

F. 18

F. 26

F.10

F. 42

F.44

E-4

E-44

$\begin{array}{ll}\text { CR } & \text { E.60 } \\ \mathrm{AACCH} & 80.31\end{array}$

$\triangle \mathrm{ACCH} \quad 80.50$

AACCH 80.30

$\mathrm{AACCH} \quad 80.20$

$\triangle A C C H \quad 80.21$

ICBO UBCS25.4 $\mathrm{CR}$ $\mathrm{CR}$

CR

CR

CR

E. 63

F. 50

D.52

J.58

F. 48

ACCH 80.58

$\mathrm{AACCH}$

$\begin{array}{lll}\text { Na } & \text { CR } & \text { F. } 58 \\ \text { Det } & \mathrm{CR} & \mathrm{E} \cdot 22\end{array}$

E. 64

B. 30

E-28

Determini CR

d. Analytical CR

AACCH 08.1

$\mathrm{CR}$

E. 32

CR

CR

J. 56

CR

E.52 
eanable Tailored Uniform Fabrics, Heavyweight (Industrial) anable Tailored Uniform Fabrics, Intermediate (Year Round) Std. Spec. for Photographic Grade Aluminum Potassium
stion Sugars (1954) Std. AnalyticaI Meth. for Determining Std. Spec. for Photographic Grade Chromium Potassium bsorbed Gamma and Electron Radiation Dose with the Ferrous ating on Iron or Steel Articles by the Preece Test (Copper nd Electron Radiation Dose with the Ferrous Sulfate Cupric Absorbed Gamma and Electron Radiation Dosc with the Ceric td. Spec. for Electrical Insulating Paper and Paperboard. meth. of Test for Soundness of Aggregates by Use of Sodium Std. for Ferric

ndness of Aggregates by Use of Sodium Sulfate or Magnesium amine Sulfate, $\left(\mathrm{Nh}_{2} \mathrm{Oh}\right)_{2}$. $\mathrm{H}_{2} \mathrm{SO}_{4}$ (Hydroxylammonium oh) $2 . \mathrm{H}_{2} \mathrm{SO}_{1}$ (Hydroxylammonium Sulfate) (Oxammonium lfate) (/ Std. Spec, for Photographic Grade Hydroxylamine Bomb Meth. of Analysis of Gravimetric Meth. of Analysis of Std. Meth. of Test for Hydrogen ustrial Aromatic Hydroca/ si $Z 77.10$ Std. Meth. of Test for Hydrogen Tentative Meth. of Test for 4)

Std. for Acceptable Concentrations of Hydrogen Test Meth. for Std. Meth. of Test for Std. Terms Used in the

Propargite 2.(P.Tert-Butylphenoxy) Cyclohexyl 2Propynyl Std. Spec, for Photographic Grade Sodium of Aluminum (1972) ANSI Z197. Std. Meth. of Analysis of Std. Meth. of Test for Alkyl Benzene

Std. Meth. for Analysis of Calcium and Barium Petroleum Std. Analytical Meth. for Determining ) (1961)

Std. Analytical Meth. for Determining

Std. Analytical Meth. for Determining th. for Continuous Analysis and Automatic Recording of the atic Hydroca/ Std. Meth. of Test for Hydrogen Sulfide and Tent. Meth. of Analysis for Total Tent. Meth. of Analysis for Free Test Meth. for Colorfastness of Dyed Textile Yarns to Test Meth. for Accelerated Aging of Std. for Prevention of

tillate Fuels (Potentio/ ive Microcoulometr/ 11

Std. Meth. of Test for Mercaptan Meth. of Test for Trace Quantities of Std. Meth. of Test for Total Meth. for Reducible

973)

ke (1973)

tion $\mathrm{M} /$

lled Condensation Meth.) (1973 Std. Meth. of Test for Total protein Bearing Materials When Suitable Amounts of Sample, protein Bearing Materials When Suitable Amounts of Sample, 73) protein Bearing Materials When Suitable Amounts of Sample,

hemistry) (1962) Std. Meth. for Analysis of Meth. of Analysis for Meth. for Preparation and Standardization of of Test for Weight Loss of Sheet Steel During Immersion in ors (1971) Std. Analytical Meth. for Determining struction Contracts Where Basis of Payment Is a Stipulated ereal Chemistry) (1962)

Std. for Gas Fired Absorption

$50 \mathrm{Ft}$.

rtain and Building Foundation Drain, Under Basement Floor, Std. for Safety for Portable

; Babassu; Flaxseed; Castor, Sesame, Safflower, Oleic, and Colorfastness of Textiles to Light and Washing: Alternate Test Meth. for Colorfastness of Textiles to Light, Std Meth of Panel Spalling Test for Std. Dimensions for Photographic Sound Record on inting on $35 \mathrm{~mm}$ Motion Picture Film/ $5 \mathrm{~mm}$ Motion Picture Film, Perforate/ orated Super 8 (1-3) (1973) Std. for Location of Std for Location of ed 1.4 (1971) Std. for Dimensions for Printed Area in Prol Std. Meth. for Determining Speed of $16 \mathrm{Mm}, 8 \mathrm{~mm}$ and er 8 Printed Area on $16 \mathrm{~mm}$ Motion Picture Film, Perforated Std. Dimensions for $35 \mathrm{~mm}$ Motion Picture Film Perforated Std. Dimensions for $16 \mathrm{~mm}$ Motion Picture Film Perforated emperat/ Std. Spec. for Precipitation Hardening lron Base 2) Std. Pract. for Scavenging and z) Std. Meths, of Test for Rockwell Hardness and Rockwell lationship Between Brinell, Vickers, Rockwell and Rockwell Std. Meth. of Sampling Saturated and

ec. for Electric Resistance Welded Carbon Steel Boiler and Std. Spec. for Seamless Medium Carbon Steel Boiler and
Suiting (lnstitutional Textile) (1973)

Suiting (Institutional Textile) (1973)

Sulfaquinoxaline in Poultry Feeds and Premixes for Cont

Sulfate Content of Corn Syrup Unmixed and Finished Corn

Sulfate $\mathrm{CrK}\left(\mathrm{SO}_{1}\right)_{2}: 12 \mathrm{H}_{2} \mathrm{O}$ (Chrome Alum) (1972)

Sulfate Cupric Sulfate Dosimeter (1971) ANSI K65.229

Sulfate Dip) (1973) AASHO T66

Sulfate Dosimeter (1971) ANSl K65.229 Th. of Test for

Sulfate Dosimeter (1971) ANSI K65.230

Sulfate or Kraft Layer Type (1973) ANSl C59.50

Sulfate or Magnesium Sulfate (1973) AASHO T104, ANSI A3

Sulfate (1964)

Sulfate (1973) AASHO T104, ANSI A37.23

Sulfate) (Oxammonium Sulfate) (1973) C Grade

Sulfate. Anhydrous $\mathrm{Na} . \mathrm{SO}$, (1973)

Sulfate, $\left(\mathrm{Nh}_{2} \mathrm{Oh}\right)_{2} \cdot \mathrm{H}_{22} \mathrm{SO}_{1}$ (Hydroxylammonium Su
Sulfates in Yeast Foods (Cereal Chemistry) (1962)

Sulfates in Yeast Foods (Cereal Chemistry) (1962)

Sulfide and Sulfur Dioxide Content (Qualitative) of lnd

Sulfide in Natural Gas (Methylene Blue Meth.) (1970) an

Sulfide Resistance of Ceramic Decorations on Glass (197

Sulfide (1972)

Sulfides in Coated Paper (Qualitative) (1973)

Sulfite Ion in Water (1972)

Sulfite Pulping Process (1972)

Sulfite (Common Name for the Pest Control Chemical) (19

Sulfite, Anhydrous, $\mathrm{Na}_{2} \mathrm{SO}_{33}$ (1972)

Sulfochromate Etch Solution Used in Surface Preparation Sulfonate in Water (1968)

Sulfonates by Liquid Chromatography (1973)

Sulfur Dioxide Content in Corn Starch (1956)

Sulfur Dioxide Content of Corn Sugar (Crude and Refined

Sulfur Dioxide Content of Corn Syrup (1952)

Sulfur Dioxide Content of the Atmosphere (1967)

Sulfur Dioxide Content (Qualitative) of Industrial Arom

Sulfur Dioxide in Wine (Enology) (1972)

Sulfur Dioxide in Wine (Enology) (1972)

Sulfur Dioxide Stoving (1972) ANSI L14.9

Sulfur Dyed Textiles (1972) ANSI L14.1

Sulfur Fires and Explosions (1971) ANSl Z12.12

Sulfur in Gasoline, Kerosine, Aviation Turbine, and Dis

Sulfur in Light Liquid Petroleum Hydrocarbons by Oxidat

Sulfur in Natural Gas by Hydrogenation (1972) ANSI Z77.

Sulfur in Paper and Paperboard (1972)

Sulfur in Petroleum Gas by Oxidation Microcoulometry (1

Sulfur in the Analysis Sample of Coal and Std. Meth. Co

Sulfur in Volatile Organic Liquids (Oxy Hydrogen Combus

Sulfur Oxides in Flue Gases (Barium Chloranilate Contro

Sulfur (1970) ANSI N113

Sulfuric Acid and Catalyst Are Employed (1960)

Sulfuric Acid and Catalyst Are Employed (1962)

Sulfuric Acid and Catalyst Are Employed (1962)

Sulfuric Acid as Used in Pulp and Paper Manufacture (19

Sulfuric Acid in Baking Powders and Chemicals (Cereal C

Sulfuric Acid Solution (Cereal Chemistry) (1962)

Sulfuric Acid Solution (1971) ANSI Z167.25

Sulfuric A cid Solutions Content of Reagents and Indicat

Sum) (1974) Tween Owner and Contractor (For Sm
Summary of Meth. for Biscuit and Cracker Flour Test. (C

Summer Air Conditioning Appliances (1973)

Summer Camp Rifle Range Plans (1972)

Sump Conductor. Downspout Runoff, Swimming Pool, etc.)

Sun / Heat Lamps (1974)

Sunflower Oil, Technical Grade (1973)

Sunflower Seeds; Ground Nuts (Peanuts): Vegetable, Coco

Sunlight Exposure (1965) ANSI L14.118

Sunlight (1971) ANSI L14.168

Super Duty Fireclay Brick (1970) ANSl All1.16

Test Meth. for

Super 8 Motion Picture Prints (1972)

Super 8 Printed Area in Optical Reduction or Contact Pr

Super 8 Printed Area in Optical Reduction Printing on 3

Super 8 Printed Area on $16 \mathrm{~mm}$ Motion Picture Film. Perf

Super 8 Printing on $16 / 8 \mathrm{Mm}$ Motion Picture Film Perforat

Super 8 Reversal Color Camera Films Intended for Direct

Super $8(1-3)(1973)$

Super 8, 5R.1667 (1.3.5.7-0) (1973)

Std. for Location of Sup

Super 8, (1.4) (1973)

Superalloy Bars, Forgings, and Forging Stock for High T

Supercharging of Stationary Diesel and Gas Engines (197

Superficial Hardness of Metallic Materials (1974) ANSI

Superficial, and Knoop Hardness) (1972) ANSI Z76.4

Superheated Steam (1969)

Superheater Tubes for High Pressure Service (1973) ANSI

Superheater Tubes (1973) ANSI B125.10
ANSI

ANSI

CR

ANSI

ASTM

ASTM

ASTM

ASTM

ASTM

AWWA

ASTM

ANSI

ANSI

ANSI

ANSI

$\mathrm{AACCH} 40.65$

AACCH $\quad 40-66$

ASTM D853

ASTM D2725

ASTM C777

ANSl $\quad$ Z37.2

TAPPI UM.532

ASTM D1339

TAPPI T1201

ANSI K62.I3I

ANSI PH4.275

ASTM D2674

ASTM D2330

ASTM D2894

CR B-58

CR F.54

CR

D. Me ASTM

ASTM

ASE

A SE

AATCC

AATCC 26

NFPA 65.5

ASTM D322?

ASTM D3120

ASTM D3031

TAPPl T406SI

ASTM D3246

ASTM D3177

ASTM D2747

ASTM D3226

Std. M ASTM E265

Other CR F.44

Other CR

/Other CR

$\mathrm{PPl} \mathrm{T} 602$

A ACCH 04-14

$\mathrm{AACCH} \quad 70-80$

ASTM C694

CR R.30

AIOA Al07

AACCH $\quad 10-30$

ANS $\quad$ Z2l.40.1

NRA *16

ASTM D2311

UL

ASTM

NIOP

482

D3169

AATCC $16 \mathrm{~B}$

ASTM C122

ANSI PH22.182

ANS1 PH22.180

ANSI PH22.179

ANSI PH22.181

ANSI PH22.153

ANSI PH22.146

PH22.181

PH22.165

ANSI

ANSl

ASTM

DEMA

ASTM

PH22.168

A638

* $1-3$

E18

ASTM El40

ASTM D 1066

A 226

A210 
resistance Welded Carbon Molybdenum Alloy Steel Boiler and pec. for Seamless Carbon Molybdenum Alloy Steel Boiler and for Seamless Ferritic and Austenitic Alloy Steel Boiler, ANSI B12/ Std. Spec. for Welded Austenitic Steel Boiler, Coating Systems (Paint) Guide for Ship Hull, Deck and ion Protective Signaling Systems for Guard, Fire Alarm and protective Signaling Systems for Watchman, Fire Alarm, and protective Signalling Systems for Watchman, Fire Alarm and ety Std. for Central Stations for Watchman, Fire Alarm and sistance Welded Carbon Steel Tubular Prod the Use of Internal Combustion Engines (1970) Guide for Std. for Stabilized Power Thyristor Power

iteria for Diesel Generator Units Applied as Standby Power n Control Centers (1972) Std. for Rec. Antidotes, or Motor Drives: Part 1-Converters for DC Motor Armature ety Rules for the Installation and Maintenance of Electric $r$ the Installation and Maintenance of Underground Electric gested Components of a Concrete Reinforcing Steel Material

(1972) code) (1973) Safety Std. for Cord Sets and Power Rules for the Operation of Eld. for Performance Requirements for Backflow Preventers in Water e Type Back Pressure Backflow Preventers for Potable Water : Rules for the Installation and Maintenance of Electrical Uniform Plumbing Code: Rec. Rules for Sizing the Water ief Valves and Automatic Gas Shutoff Devices for Hot Water Safety Sid. for Play Pipes for Water Integrated Data Processing Systems Designed to Streamline for Mercury Lamp Transformers, Constant Current (Series) Std. for Operating Std. for Ground

td. for Wire Cable Wrought Steel Heavy Thimble (For Ground ed Carbon Steel. Shoulder Nut Assembly Eyebolt (For Ground 72) Std. Test. Procedure for End Std. Test. Procedure for End oomstop of All Mobile Construction Type Cranes Having Rope Burning or Extent of Burning of Cellular Plastics Using A Uniform Fire Code: Tents and Air a $\mathbf{S} \cdot 64 \cdot 474$ Std. for Aluminum, Triplex, Neutral Std. Meth. of Test for Flammability of Self Structural Std. for Steel Antenna Towers and iquid and Liquefied Flammable Gas Processing Equipment and Use (1972)

g Equip/ Spec. for ment (1/ Std. for Mechanical Interchangeability of Socket 70) Spec. Steel Wire Bar ec. Pract for Hole Placement on Bulldozer (Dozer) End Bit cal Sheet Steel Outlet Boxes, Device Boxes, Covers and Box and Application Guide for High Voltage Air Switches, Bus Uniform Fire Code: Rec Pract for External Electromagnetic Radiation d. Meth. of Test for Resistance of Transparent Plastics to Sid. Meth. of Test for Test Meth. of

td. Meth. of Test for Pilling Resistance and Other Related td. Spec. for Copper Alloy Condenser Rolled Tube Plates in t Laid Tar Paving Mixtures for Base, Binder, Leveling, and r Soil and Dirt Accumu/

Tent. Meth: of Evaluating Deg. of Rec. for Design and Construction of Std. Meth. of Test for Cleanability of Std. Meth. of Test for Measuring

e Tester (1974) is (1973) Std. Definitions of Terms Relating to

quirements for Aerial Passenger Tramways Including Ski and ng (1973) ANSI C33.50

o T142, ANSI A37.21

ent. Meth. of Test fur Resistance to Growth of Mold on the

diffusion Length in Silicon by Measurement of Steady State

Meth. of Analysis of Sulfochromate Etch Solution Used in y Water Blasting Prior to Coating or Rec/

Rec. Pract. for (1972)

std. for Gear Tooth Surface Texture for Aerospace Gearing bond T/ Test Meth. for Coating Pick Tests for Determining of Test for Shock Absorbing Properties of (Sports) Playing s, Waviness, Form and Lay) (1973) Std. for Gear Tooth Rec. for Prevention of Damage to Property from ainst Misc. Perils at Electric Generating Stations (Flood, age of Refractory Brick and Tile or Deviation from a Plane (Multiply Felt and Asphalt Preferably with Gravel or Slag Std. Spec. for Asphalt Insulating Siding Std. Spec. for Asphalt Roll Roofing Std. Rec. Pract. for Preparation of Metal Std. Rec. Pract. for Preparation of Metal
Test Meth. Visual Std. for
Superheater Tubes (1973) ANSI B125.13

1. for Electric ASTM Superheater Tubes (1973) ANSI B125

Superheater, and Heat Exchanger Tubes (1973) ANSI B125.

Superheater, Heat Exchanger, and Condenser Tubes (1973)

ASTM

ASTM

Superstructure (1973)

Supervisory Service (1972) ANSI SE3.2

Supervisory Service (1972) ANSI SE3.3

Supervisory Service (1972) ANSI SE3.6

Supervisory Services (1972) ANSI Se 3.1

lof Central Stat NFPA

D Use of Local NFPA

lof Proprietary NFPA

Saf UL

Supplementary Requirements for Seamless and Electric Re

Supplementary Ventilation in a Ship's Cargo Hold During

Supplies Direct Current Output (1972)

Supplies for Metal Rolling Mill Auxiliary Drives (1972)

Supplies for Nuclear Power Generating Stations (1972) a

Supplies (Packaging), and References (Dosage) for Poiso

Supplies (1973) ANSI C 34.3 /for

Supply and Communication Lines (1960)

Supply and Communications Lines (National Electrical Sa

Supply and Installation Contract (1968)

Supply Cords (I972) ANSI C33.3

Supply Gas Connectors for Exterior Use on Mobile Homes

Supply Lines and Equipment (National Electrical Safety

Supply Lines with Intermediate Atmospheric Vent (1972)

/Equirements for Double Check Valy ASSE

Supply Lines (1972) /Equireme

Supply System (1973)

Supply Systems (1972)

Ety Code, Part 1 ANS

ASTM

SNAME 4

NEMA PY

NEMA PV 2

IEEE $\quad 387$

$\mathrm{AAPCC} * 1$

Saf ANS

ANSI

Sug CRSI

UL

817

ANSI C2.4

1015

C2.1

UPC* $1 \cdot A$

W

Supply Type (1971)

Supply Voltage and Frequency of Office Machines (1973)

Support Equipment (Hand Knob) (1974)

Support Equipment) (1974)

Support Equipment) (1974)

Supported Beam Deflection Composite Tubes and Cores (19
Supported Beam Strength Fibre Tubes and Cores (1972)

Supported Booms, Equipped for Hook Work, Clamshell, Mag

Supported Specimen by a Horizontal Screen (1974) ANSI K

Supported Structures (1973)

Supported, Service Drop and Secondary Cable (1966) Ipce

Supporting Plastics (1974) ANSI K65.21

Supporting Structures (1972)

Supporting Structures (1973)

Supports for Off the Floor Plumbing Fixtures for Public

(Safety) for Flammable L

Supports in Concrete Construction (1970)

Supports Used in Metal Heads for Roadway Lighting Equip

Supports Used to Position Reinforcement in Concrete (19

Supports (1972)

Supports, and Cast Aluminum Covers (1973)

Supports, and Switch Accessories (1972)

Suppression and Control of Hazardous Áreas (1973)

Suppressors (1969)

Surface Abrasion (1973) ANSI K65.72

Surface Area of Carbon Black (1973)

Surface Burning Characteristics of Building Materials

Surface Changes of Textile Fabrics (1972) ANSI L14. I98

Surface Condensers and Heat Exchangers (1973)

Surface Courses (1967)

Sid. Spec. for Hot Mixed, Ho

Surface Disfigurement of Paint Films by Fungal Growth O

Surface Drainage Systems on Farms in Humid Areas (1973)

Surface Finishes (1973)

Surface Frictional Properties Using the British Portabl

Surface Heat Exchanges for Use with Milk and Its Produc

Surface Imperfections on Ceramics (1973)

Surface Lifts and Tows (1973)

Surface Metal Raceways and Fittings for Electrical Wiri

Surface Moisture in Fine Concrete Aggregate (1973) Aash

Surface of Interior Coatings in an Environmental Chambe

Surface Photovoltage (Electronics) (1973)

Electri

UL

Z21.22A

385

200

$\mathrm{C} 82.7$

$\mathrm{X} 4.11$

1553

1045

1053

T.113

T. 113

$\begin{array}{ll}\text { SAE } & \text { J } 220 \\ \text { ASTM } & \text { D } 1692\end{array}$

$\begin{array}{ll}\text { ASTM } & \text { D } 1692 \\ \text { ICBO } & \text { UFC } * 2\end{array}$

$\begin{array}{ll}\text { ICBO } & \text { UFC } * 2 A R \\ \text { EEI } & \text { TDJ. } 180\end{array}$

ASTM D635

ElA

FMS

RS222.B

7.14

A1 12.6.1

EEI

CRSI

NEMA

CRSI

SAE

1.3 .5

$\mathrm{SH} 13$

$\mathrm{J} 63$
$\mathrm{OS} 1$

C 37.32

$\mathrm{UFC} * 2 \mathrm{E}$

J552A

SAE

St ASTM

ASTM

NFPA

ASTM

ASTM

Dl044

D303

D1375

B171

D1753

D3274

ASAE

ASTM

ASTM

DFISA

ASTM

R302.2

C756

E303

3100

F109

B77.1

5

Surface Preparation of Aluminum (1972) ANSI Z197.29

Surface Preparation of Steel and Other Hard Materials B

Surface Preparation Spec. for Steel Structure Painting

UL

ASTM

ASTM

Surface Roughness, Waviness, Form and Lay) (1973) y Carrier ASTM

ID ASTM

NACE

ANSl

AGMA

Surface Strength of Coated Papers Using the Interfiber

Surface Systems (1972)

Surface Texture for Aerospace Gearing (Surface Roughnes

Surface Water (1973)

Surface Water, Rigging Collapse, Burglary, and Roof Col

Sid. Meth. of Test for Warp

/Tion Data on Elastomer Roof Coverings

Surfaced with Mineral Granules (1970) ANSI A 109.18

Surfaced with Mineral Granules (1973)

Surfaces for Adhesives Bonding (1967) ANSI Z197.28

Surfaces of New Steel Airblast Cleaned with Sand Abrasi

Surfaces of Plastic Prior to Adhesive Bonding (1969) an

C70

D3273

F391

RP.01.72

A 159.1

118.01 
Std. Rec. Pract. for Test. Primers and Primer ct Preparation, Finishing and Testing of Concrete Subfloor I Scale Tir/ Meth. of Test for Stopping Distance on Paved Tent. Meth. of Measurement of Stopping Distance on Paved Detection of Mill Chemical Treatments on Galvanized Steel densation-Humidity Test. of Organic Coatings on Metallic varnish, Lacquer, and Related Organic Coatings on Metallic $r$ Metal Oxide W aterproofing for Below Grade Wall and Floor of Test for Lead and Cadmium Extracted from Glazed Ceramic on of Structures and Contaminants Seen on Specular Silicon for Thickness of Resilient Flooring, Materials Having Flat th. of Test for Abrasion Resistance of Horizontal Concrete Spec. for Spec for Composite Spec. for Chemical Resistant Resin Monolithic Std. Qualitative Classification of

er Circuits (1972) ANS1 C62.1

Std. for

(Bone) I

Std. Spec. for Hip Nail-Jewett Type for Use by erimental Test. for Biological Compatibility of Metals for Std. Rec. Pract. for Static Bend Test. of Bone Plates

Std. Rec. Pract. for Static Bend Test. of Nail Plates for Static Bend and Torsion Test. of Intramedullary Rods (1973) ANSl Se2.5 Safety Std. for ystem Units (Electronic / Electrical / Mechanical Security Std. Rec. Pract. for Performance Spec. for Portable X-Or Gamma Radiation Rec. Guide for Making a Condition m Alloys (E/ Std. Meth. of Test for Exfoliation Corrosion lution of $\mathrm{pH} 7.2$ to Evaluate the Stress Corrosion Cracking Alloys to Stress C/ Std. Rec. Pract. for Determining the I Rich, Chromium Bearing Alloys / Std. Meth. of Detecting starch (Granules in Flour or Starch Preparations Which Are Rec. Std. for Seismic Restraint Direct Hun d. Spec. for Electric Wire Rope Hoists (Lug, Hook, Trolley anel Ceilings (1973/

Uniform Building Code Std. for Metal Std. Wet Process Porcelain Insulators Std. Application Guide for Porcelain

973) analysis of Apparent Viscosity of Acidulated Flour-Water

sion, Pull Type (1973)

p Type (1973)

II Type (1973)

sion, Pull Type (1973)

ype (1973)

d, Pull Type (1973)

Pull Type (1973)

Pull Type (1973)

Stump Type (1973)

Tension, Pull Type (1973)

tension, Stump Type (1973)

Tension, Pull Type (1973)

Tension, Stump Type (1973)

ion, Pull Type (1973)

(1973)

ype, (1973)

d, Shear, Pull Type (1973)

(Ms2494), Tension, Pull Type (1973)

(MS20426). Tension, Pull Type (1973)

Head, Pull Type (1973)

I Type (1973)

Stump Type (1973)

Stump Type (1973)

1973)

tension, Pull Type (1973)

head, Pull Type (1973)

Std. for Collar,
Std. for Pins and Collars, Std. for Pin, Std. for Pin, Std. for Pin, Std. for Pin, Std. for Pin, Std. for Pin, Std. for Pin, Std. for Pin Std. for Pin, Std. for Pin, Std. for Pin, Std. for Pin, Std. for Pin, Std. for Pin, Std. for Pin, Std. for Pin, Std. for Pin, Std. for Pin, Std. for Pin, Std. for Pin, Std. for Pin Std. for Pin, Std. for Pin, Std. for Pin, Std. for Pin, Std. for Pin, Std. for

Std. and Rec. Pract. for Level Flotation of Boats When Std. and Rec. Pract. for Buoyancy in the Event of Safety Std. for Tests for Combustibility of

Meth. of Analysis for Baking Test (Quality) for iveness Df Treating Solutions (1970)

Uniform

Std. for Gas Fired Std. for Safety for Rec. for Thermoplastic Piping for Std. for Test Kits for

n, Under Basement Floor, Sump Conductor, Downspout Runoff, Reinforced Brick Masonry Std. for Multiport Valves for Public and Residential Suggested Minimum Stds. for Public Std. for Sand Type Filters for

Std. for Diatomite Type Filters for Suggested Minimum Stds. for Residential d. for Underwater Lighting Fixtures and Junction Boxes for
Surfaces Over Preformed Metal (1974)

Surfaces to Receive Wood Flooring (1962)

Surfaces Using a Passenger Automobile Equipped with Ful
Surfaces Using a Passenger Automobile Equipped with Ful

IC. for Corre

ASTM

WSFI

ASTM

Spec. for NCCA

Surfaces (Coil) (1974)

Surfaces (Coil) (1974)

Surfaces (1971)

Surfaces (1972)

Surfaces (1972T)

Surfaces (1973)

Surfaces (1974)

Std. Meth. for Con

NCCA

$\mathrm{N}$, or Other Failure of Paint, NCCA Spec. Fo CSI

std. Meth. ASTM

T. and Nomenclature for Identificati ASTM

Std. Meth. of Test ASTM

Surfacing Welding Rods and Electrodes (1970) ANS1 W3.13

Surfacing Welding Rods and Electrodes (1970) ANSI W3.21

Surfacings (1972T)

Surfactants by Infrared Absorption (1974)

Surge Arresters (1972)

Surge (Lightning) Arresters for Alternating Current Pow

Surgeons in the Treatment of Fractures of the Skeletal

Surgical Implants (1972)

(Surgical Implants) (1973

Surgical Implants) (1973)

Std. Rec. Pract. for Exp

(Surgical Implants) (1973)

Surveillance Cameras for Banks and Mercantile Premises

Surveillance System and Equipment) (1974) /S Control S

Surveillance Tests for Nuclear Reactor Vessels (1973) AST

Survey Instruments (1971)

Survey of Concrete in Service (1965)

Susceptibility in $7 \mathrm{XXX}$ Series Copper Containing Aluminu

Susceptibility of Cu. Zn Alloys (1973)

Susceptibility of Stainless Steels and Related Ni-Cr.Fe

Susceptibility to lntergranular Attack in Wrought Nicke

Susceptible to Hydrolysis by Alpha Amylase) (Cereal Che

Suspended Ceiling Assemblies (1972)

Suspended, and Base Mounted Types (1971)

Suspension Systems for Acoustical Tile and for Lay in P

(Suspension Type) (1971)

Suspension (Power Line) Insulators (1974)

Suspensions (Cereal Chemistry) (1962)

Swage Locking for Pull Type and Stump Type Lockbolts

Swage Locking (1973)

Swage Locking, A.286 Cres, Protruding Head, Shear / Ten

Swage Locking, A.286 Cres, Protruding Head, Shear, Stum

Swage Locking, A.286 Cres, Protruding Head, Tension, Pu

Swage Locking, A.286 Cres, 100 Deg. Head (MS24694). Ten

Swage Locking, A.286 Cres, 100 Deg. Shear Head, Stump T

Swage Locking, A.286 Cres, 100 Deg. Shear / Tension Hea

Swage Locking, A.286, 100 Deg. Head (MS20426), Tension.

Swage Locking, Aluminum Alloy, Protruding Head, Tension

Swage Locking, Aluminum Alloy, Protruding Head, Tension

Swage Locking, Aluminum Alloy, $100 \mathrm{Deg}$. Head (MS20426)

Swage Locking, Aluminum Alloy, 100 Deg. Head (Ms20426,

Swage Locking, Aluminum Alloy, 100 Deg. Head (MS24694),

Swage Locking, Aluminum Alloy, 100 Deg. Head (MS24694),

Swage Locking, Std. and Oversize, Protruding Head, Tens

Swage Locking, Steel, Protruding Head, Shear Stump Type

Swage Locking, Steel, Protruding Head, Tension, Stump T

Swage Locking. Steel, Std. and Oversize, Protruding Hea

Swage Locking, Steel, Std. and Oversize, $100 \mathrm{Deg}$. Head

Swage Locking, Steel, Std. and Oversize, $100 \mathrm{Deg}$. Head

Swage Locking, Steel, Std. and Oversize, 100 Deg. Shear

Swage Locking, Steel, 100 Deg. Crown Head, Tension, Pul

Swage Locking, Steel, 100 Deg. Head (MS20426), Tension.

Swage Locking, Steel, 100 Deg. Head (MS24694), Tension,

Swage Locking, Steel, 100 Deg. Shear Head, Stump Type (

Swage Locking, $108 \mathrm{KSl}$ Steel, Protruding Head, Shear /

Swage Locking, $108 \mathrm{KSl}$ Steel, 100 Deg. Shear / Tension

Swaging Process-Tube to Rod End (1974)

Swamped (1973)

Swamping (Boat Flotation) (1973)

Sweeping Compounds (Fire Protection) (1973) ANSI Z240.1

Sweet Yeast Products (Cereal Chemistry) (1962)

Swellograph Method for Test. the Water Repellent Effect

Swimming Pool Code (1973)

Swimming Pool Heaters (1972)

Swimming Pool Pumps, Filters, and Chlorinators (1972)

Swimming Pool Water Circulation Systems (1972)

Swimming Pool Water (1970)

Swimming Pool, etc.) (1973) ANSI A 176.3

Swimming Pools (Tech. Notes) (1967)

Swimming Pools (1969)

Swimming Pools (1969)

Swimming Pools (1971)

Swimming Pools (1971)

Swimming Pools (1972)

Swimming Pools (1972)
ASTM

AWS

AWS

ASTM

ASTM

NEMA

STM

ASTM

ASTM

AST

U

ANSl

ACl

ASTM

ASTM

AACCH 76.30

CISCA *3

St HMl 100

ICBO UBCS47.16

ANSI $\quad$ C29.2

NEMA HV2

AACCH 56.80

NSA 1080

NSA $\quad 1413$

NSA 7014

NSA 6984

6965

NSA 6946

NSA 6974

NSA 7004

NSA

NSA 1525

NSA 1555

NSA 1535

NSA 6915

NSA $\quad 1516$

NSA 1546

NSA 1465

NSA $\quad 1424$

NSA $\quad 1496$

NSA 1446

NSA $\quad 1456$

NSA $\quad 1475$

NSA $\quad 1436$

NSA 6935

NSA 6925

NSA $\quad 1486$

NSA $\quad 1414$

NSA 7034

NSA 7024

NSA $\quad 582$

ABYC H8B

ABYC H8A

UL 41

AACCH $\quad 10.20$

NWMA M-2.70

IAPMO *1

ANSI Z21.56

UL 1081

SPI PPI TRI7

NSF 38

/Undation Drai

ASTM

BIA

NSF

NSPl

NSF

NSF

NSPI

Safety St UL 
Uniform Plumbing Code: Recommendation for Installation of Ceramic Tile nd Feed and Water Requirements of Farm Livestock (Poultry, nsi Al33.1 Safety Std. for

ion Guide for High Voltage Air Switches, Bus Supports, and Rec. Pract, for Electric Blower Moto Rec. Pract. for Electric Windshield Wiper Rec. Pract. for Electric Windshield Washer Rec. Pract. for Headlamp (Light) Rec. Pract, for Vehicle lgnition Rec. Pract. for Mechanical Stop Lamp (Light) IEEE Trial Use Guide for Class IE Control Dead Front Distribution Safety Std. for Dead Front

rs, Automatic Line Sectionalizers and Oil Filled Capacitor Std. Proposal for Solid State Load A-D, and Class 11, Groups E/ Safety Std. for Electrical Se26 Std. for Safety for Connectors and Uniform Dimensions and Meth. of Test. for Rotary Selector Safety Std. for Automatic Transfer ty Std. for Manually Operable General and Special Use Snap Std. for Safety for Special Use Electrica acturing Spec., and Application Guide for High Voltage Air uating the Effect of Solar Radiation on Outdoor Metal Clad Std. Definitions for Power or AC High Voltage Power Vacuum Interrupters Used in Power Std. for lndustrial Electrical Power Control Apparatus . Rec. Pract, for Semiautomatic Headlamp (Light) Beam Std. for Electrical Power

for Schedule of Preferred Ratings for Capacitance Current a Symmetrical/ Std. Requirements for Capacitance Current a Symmet/ Std. Application Guide for Capacitance Current gh Voltage Circuit Breakers Rated o/ Std. Requirement for $s$ Rated on a Symmetrical Basis When Rated for Out of Phase Sid for Double Race or Bi-Level

Std. for Tee. Reducer, Flareless Tube to Swivel,

Std for Tee, Reducer, Flareless Tube to Swivel, Std. for Elbow, Reducer, $45 \mathrm{Deg}$. Flareless Tube to Std for Elbow Reducer, 90 Deg. Flareless Tube to Std. for Tee, Reducer, Flareless Tube to Std. for Tee, Reducer, Flareless Tube to Rec, Safety Alert Rec. Pract. for ment Maintenance Instructions, and Conta/ lements as Used in Schematic Diagrams (1972) Std. rocessing) (1973) ANSI PH5.17

si 21.6

std. for Flowchart Std. Definitions and

ansi $\mathrm{K} 65.57$

in Architecture and Building Construction (/ Std. ronics) (1973) ANSl Y32.14 Std. for Manufacturer Identification ttons $(1972)$ Machinery and Equipment (1971) ANSI B114.2, Sa/ Std. for logy (1969) ANSI Y 10.19 Std. for Load Handling Std. for Letter

$z 128.7$

(1961) Std. Definitions of Terms and Abbreviations and

Std. for Operation and Flow Process Charts and Std. for Glossary of Terms Concerning Letter Spec. for Informational, Directional, and Special Signs Western Woods Use Book: Abbreviations, rts (1971) ANSI Z1.5 tt. Definitions, cal Control of AC High Voltage Circuit Breakers Rated on A eth. for Test. AC High Voltage Circuit Breakers Rated on A Switching for AC High Voltage Circuit Breakers Rated on A igh Voltage Circuit Breaker External lnsulation Rated on A n Strength for AC High Voltage Circuit Breakers Rated on A ry Voltage for AC High Voltage Circuit Breakers Rated on A ry Voltage for AC High Voltage Circuit Breakers Rated on A Switching for AC High Voltage Circuit Breakers Rated on A Components of AC High Voltage Circuit Breakers Rated on A on for Outdoor AC High Voltage Circuit Breakers Rated on A

Switching for AC High Voltage Circuit Breakers Rated on A aulic Turbine Driven Synchronous Generators and Reversible ator / Motor Uni/ Std. for Large Hydraulic Turbine Driven Std. for Std. Criteria and Definitions for Excitation Systems for Guide for Std. for

Rec, for Fire Protection of (1954) Rec. for Protection Against Betatron Std. Meth. of Test for Effect of Cyclic Immersion of std. Meth. of Test for Hydrostatic Compressive Strength of
Swimming Pools (1973)

Swimming Pools (1973)

Swine, Cattle, Sheep) (1968)

Swinging Hardware for Std.

Switch Accessories (1972)

Switch for Vehicles (1971)

Switch (1971)

Switch (1971)

Switch (1971)

Switch (1971)

Switch (1972)

Switchboards for Nuclear Power Generating Stations (19?

Switchboards (1972)

Switchboards (1972)

Switches for Alternating Current Systems (1972)

Switches for Traffic Control Systems (1973)

Switches for Use in Hazardous Locations: Class 1, Group

Switches for Use with Burglar Alarm Systems (1973) ANS

Switches (Radio, TV, and Instrumentation) (1973) ANSI C

Switches (1972)

Switches (1972) ANSI C33.40

Switches (1973) ANSI C33.40.1

Switches, Bus Supports, and Switch Accessories (1972)

Std. Guide for Eval

Switchgear (1972)

Switchgear (1972)

Switching and Controlling Devices (1973)

Switching Devices (1972)

Switching Equipment (1974)

Switching for AC High Voltage Circuit Breakers Rated on

Switching for AC High Voltage Circuit Breakers Rated on

Switching for AC High Voltage Circuit Breakers Rated on

Switching Impulse Testing (1972) ANS1 C68.2

Switching Impulse Voltage lnsulation Strength for $\mathrm{AC} \mathrm{Hi}$

Switching (1973) ANSI C37.079

Swivel and Rigid Industrial Casters (1973)

Swivel on Run (1973)

Swivel on Side (1973)

Swivel (1973)

Swivel (1973)

Swivel, Swivel on Run (1973)

Swivel, Swivel on Side (1973)

Symbol for Agricultural Equipment (1972)

Symbols and Color Codes for Lubricant and Fluid Require

Symbols and Definitions for Mechanical and Acoustical E

Symbols and Identification Code (1973)

Symbols and Their Usage in Micrographics (Information P

Symbols for Acceptance Sampling by Attributes (1971) an

Symbols for Dimensions of Plastic Pipe Fittings (1968)

Symbols for Electrical Wiring and Layout Diagrams Used

Symbols for Fasteners (1973)

Symbols for Logic Diagrams (Two-State Devices) (Elect

Symbols for Machine Tool Indicator Plates or Control Bu

Symbols for Operator Controls on Agricultural Tractors,

Symbols for Powered Industrial Trucks (1973)

Symbols for Units Used in Electrical Science and Techno

Symbols for Use on Railroad Maps and Profiles (1972)

Symbols Relating to Emission Spectroscopy (1972A) ANSI

Symbols Used in Builders Hardware Schedules and Specs.

Symbols (Mechanical Engineering) (1972)

Symbols (1972)

(Symbols) Complementary to ANSI Z35.1-1972, Accident P

Symbols, and Terminology (Lumber and Timber) (1973)

Symbols, Formulas, and Tables for (Quality) Control Cha

Symmetrical and a Total Current Basis (1972)

Symmetrical Basis When Rated for Out of Phase Switching

Symmetrical Basis (1972)

Symmetrical Basis (1972)

Tlngs for Capacitance Curren

Symmetrical Basis (362 kV and Above) (1972) ANSI C 37.07

Symmetrical Current Basis (1971) ANSI C 37.072

Symmetrical Current Basis (1971) ANSl C 37.0721

Symmetrical Current Basis (1972) ANSI C 37.073

Symmetrical Current Basis (1972) ANSI C 37.076

Symmetrical Current Basis (1972) ANS1 C 37.078

Symmetrical Current Basis (1973) ANSI C 37.0731

Synchronous Generator / Motor Units for Pumped Storag

Synchronous Generators and Reversible Synchronous Gener

Synchronous Integral Horsepower Generators (1972)

Synchronous Machines (1972)

Synchronous Motor Protection (1971)

Synchronous Motors (Large Apparatus) (1972)

Synchronous Motors (1974)

Synchronous Signaling Rates for Data Transmission (1969

Synchrotron Radiations Up to 100 Million Electron Volts

Syntactic Foam at Pressure (1972)

Syntactic Foam (1972A)
$\mathrm{ICBO}$

TCA

UL

ANSl

SAE

$S A E$

$S A E$

SAE

SAE

AE

IEEE

NEM

UL

/Close NEMA

NEMA

UL

UL

EIA

UL

Safe UL

UL

ANSI

IEEE

ANS1

ANSI

ANSI

SAE

NEMA

ANS

IEE

IEEE

IEEE

IEEE

IEEE

ANSI

NSA

NSA

NSA

NSA

NSA

ASAE

SAE

ANS

ISA

NMA

ASOC

ASTM

ANSI

IFI

IEEE

ANSI

ASAE

ANSl

IEEE

ANSI

ASTM

NBHA

ASME

ANSl

ANSl

WWPA

ASQC

lEEE

ANS

ANS

IEEE

/ Recove IEEE

Recove IEEE

Current IEEE

Surized IEEE

/Nsulati IEEE

Urrent IEEE

NEMA

NEMA

NEMA

IEEE

IEEE

FMS

ANSI

NCRPM

ASTM

ASTM

UPC * I-G

302

D249.2

$14 \mathrm{C}$

C.37.32

$\mathrm{J} 235$

$\mathrm{J} 112 A$

$\mathrm{J} 234$

J259

J249

420

SG13

TS2

634 
tionic Titration Procedure (1974) 2T)

Std. Meth. of Chemical Analysis of Soth. of Test for Copolymers (SBR) (1) Std. Meth. for Chemical Analysis of textile Fiber Storage (Cotton, Jute, Hemp and Sisal, Flax, for Wire and Cable, $75 \mathrm{Deg}$. C Operation /

for Wire and Cable (1974) ANSI C8.38

C Operation (1974) ANSI C8.24

C Operation (1974) ANSI C8.40

Std. Spec. for Std. Spec. for Std. Spec. for Std. Spec. for Sid. Meth. of Test.

ANSI C 8.28

Std. Spec. for Styrene-Butadiene .

I Purpose $(45.55)(1.973)$ Spec. for

Analytical Meth. for Determining Dextrose Content of Corn Meth. for Determining Dextrose Equivalent Content of Corn -Titrimetric) Content of Corn Sugar (Crude and Refined), $r$ Determining Calcium (Egta-Titrimetric) Content of Corn for Determining lron (Orthophenanthroline) Content of Corn Analytical Meth. for Determining Acidity Content of Corn ation Content of Corn Sugar (Crude and Refined), Dextrose, ical Meth. for Determination of Phosphorus in Corn Starch, - Analytical Meth. for Determining Arsenic Content of Corn ical Meth. for Determining Heavy Metals Content of of Corn Analytical Meth. for Determining Sulfate Content of Corn d. Analytical Meth. for Determining Copper Content of Corn td. Analytical Meth. for Determining Baume Content of Corn $r$ Determining Moisture (Oven Filter Paper) Content of Corn for Determining Candy Color and Inversion Content of Corn rmining Moisture (Azeotropic Distillation) Content of Corn ical Meth. for Determining Color Stability Content of Corn for Determining Moisture (Oven Filter Aid) Content of Corn Analytical Meth. for Determining Chloride Content of Corn Analytical Meth. for Determining Chloride Content of Corn tical Meth. for Determining Sulfur Dioxide Content of Corn for Determining Color (Visual Comparison) Content of Corn nalytical Meth. for Determining Phosphorus Content of Corn for Determining Color (Spectrophotometric) Content of Corn Determining Saccharides (Chromatographic) Content of Corn h. for Determining Moisture (Karl Fischer) Content of Corn alytical Meth. for Determining Iron (TPTZ) Content of Corn ermining Copper Content of Corn Sugar (Crude and Refined), meth. for Determining Extraneous Materials Content of Corn al Meth. for Determining Specific Rotation Content of Corn Std. Analytical Meth. for Determining Ash Content of Corn ical Meth. for Determining Apparent Starch Content of Corn Std. Analytical Meth. for Determining $\mathrm{pH}$ Content of Corn cal Meth. for Determining Refractive Index Content of Corn Analytical Meth. for Determining Calcium Content of Corn ng Saccharides (Gas Liquid Chromatography) Content of Corn lytical Meth. for Determining Fermentables Content of Corn cal Meth. for Determining Protein Nitrogen Content of Corn Refractometer Meth. of Analysis of Solids in ng Heavy Metals Content of Corn Sugar (Crude and Refined), termining Protein Nitrogen Content in Corn Starch, Sugars, Determining $\mathrm{pH}$ Content of Corn Sugar (Crude and Refined), mining Chloride Content of Corn Sugar (Crude and Refined), ermining lron Content in Corn Starch Hydrolzates Including Meth. of Analysis for Ash in Molasses, Sugars, and or Determining Baume Content of Corn Syrup Unmixed and Its Spindle Meth. of Analysis of Solids in Juices,
rotein Nitrogen Content of Corn Sugar (Crude and Refined), determining Protein Nitrogen Content in Feedstuffs (Corn), protein Nitrogen Content in Steepwater, (Corn) Feedstuffs, electronic / Electrical / Mechanical Security Surveillance Rec. for Water Sprinkler and Hydrant

Characteristics Of, Rec. Construction and Water Sprinkler Deg. Involute Spur Pinions (1973) Std. for Air Conditioning Equipment Selection and 972) ANS1 J8.6 Rec. Spec. for Enamel Coating Rec. Pract. Tests for Central Std. Classification Rec. Pract. for Electrical

Uniform Building Code Std. for Blower, Fan, and Exhaust e Applications (1973) ANSI J8.15, SAE J20/ ngs (1972) nery) (/ nsi L14.289 Rec. Pract. for Coding Rec. Pract, for Electrical Wiring Identification Std. Meth. for Spinning Tests on the Cotton Rec. Pract. for Grounded $830 \mathrm{~V}, 3$ Phase Electrical radiation (1971) ANSI N4.1 Std. Classification Rec. for Water Demand for Sprinkler Rec. Pract. for Vendor Rating Std. for nsliteration) (1972) ransliteration) (1972) S6.

Rec. Pract. for Qualifying a Sound Data Acquisition
Synthetic Anionic Active lngredient in Detergents by Ca Synthetic Anionic Ingredient by Cationic Titration (197 Synthetic Detergents (1973)

Synthetic Elastomers for Use on Solid Styrene Butadiene

Synthetic Fibers, Wool) (1974)

Synthetic Rubber Heat and Moist

Synthetic Rubber Heat or Moisture Resisting Insulation

Synthetic Rubber Insulation for Wire and Cable, 75 Deg.

Synthetic Rubber Insulation for Wire and Cable, 90 Deg.

Synthetic Rubber Latices (1974) ANSI J2.34

Synthetic Rubber (SBR) Jacket for Wire and Cable (1973)

Synthetic Rubber, Ethylene Propylene Terpolymer, (ienera

Syrup and All Starch Hydrolyzates (1967)

Syrup and All Starch Hydrolyzates (1968)

Syrup and Other Starch Hydrolyzates (1973)

Syrup and Other Starch Hydrolyzates (1973)

Syrup and Starch Hydrolyzates and All Starches and

Syrup and Starch Hydrolyzates (1952)

Syrup and Starch Hydrolyzates (1971)

Syrup and Sugar Obtained from the Corn

Syrup Unmixed and Corn Sugar (1954)

Syrup Unmixed and Dextrose (1956)

Syrup Unmixed and Finished Corn Sugars (1954)

Syrup Unmixed and Finished Sugars (1954)

Syrup Unmixed and lts Syrups (1959)

Syrup Unmixed and Unbleached Crude Corn Sugar (1956)

Std. Analytical Meth.

Syrup Unmixed (1952)

Syrup Unmixed (1952)

Syrup Unmixed (1954)

Syrup Unmixed (1954)

Std. Analytical Meth for Dete CR

Std. Analyt CR

Std. Analytical Meth. CR

Syrup Unmixed, Finished Sugar and Other Clarified Starc

Syrup Unmixed, Finished Sugar and Other Clarified Starc

Syrup (1952)

Syrup (1954)

Syrup (1957)

Syrup (1957)

Syrup (1957)

Syrup (1966)

Syrup (1970)

Syrup, and Other Starch Hydrolyzates (1961)

Syrup, Crude and Refined Sugar and Other M

Syrup, Dextrose and Starch Hydrolyzates (197I)

Syrup, Finished Sugar and Other Starch Hydrolyzates (19 Alytic CR

Syrup, Refined and Crude Sugar (1967)

Syrup, Starch Hydrolyzates and a

yrup, Sugar and Dextrose (1956)

Syrup, Sugar and Other Carbohydrates (1957)

Syrup, Sugar, and Starch Hydrolyzates (1967)

Syrup, Sugars, and Starch Hydrolyzates (1957)

Syrup, Sugars, Starches and Other Protein Be

Syrups and Molasses (Cereal Chemistry) (1962)

Syrups and Other Hydrolyzates from Corn and Grain

Syrups and Other Protein Bearing Materials

Syrups and Other Starch Hydrolyzates (1962)

Syrups and Sugars, and Most Wet

Syrups (Cereal Chemistry) (1962)

Syrups (1959)

Syrups, and Molasses (Cereal Chemistry) (1962)

Syrups, Starches and Other Protein Bearing Materials Wh

syrups, Sugars, Starches and Other Protein Bearing Mate

Syrups, Sugars, Starches and Other Protein Bearing Mate

System and Equipment) (1974) IS
System Demand Tables (Fire Safety) (1973)

S Control System Units ( C

System Design-Fire Protection and Safety) (1973)

System Design of Fine Pitch on Center Face Gears for 20

System Design of Fine Pitch Wormgearing (1973)

System Design Procedures (1973)

System Designations for Coal Tar or Asphalt Coatings (1

System Fluids (1970)

System for Carbon Blacks Used in Rubber Products (1973)

System for Construction and Industrial Machinery (1972)

System for Dust, Stock and Vapor Removal (1973)

System for Elastomeric Materials (Rubber) for Automotiv

System for ldentification of Tube, Pipe, and Hose Fitti

System for Industrial and Construction Equipment (Machi

System for Measurement of Spinning Performance (1972) a

System for Oil Field Service (1973)

System for Polymeric Materials for Service in lonizing

System for Private Fire Protection (Safety) (1973)

System for Quality Control (1969)

System for Romanization of Arabic Written Language (Tra

System for Romanization of Japanese Written Language (T
System for Vehicle Noise Sound Level Meters (1972) ANSl

System for Welding Wire ldentification (1973)

System for Welding Wire ldentification (1973)
ASTM

ASTM

ASTM

ASTM

ASTM

ASTY

ASTM

ASTM

ASTM

SAE

Std. CR

um (Egta CR

Meth. Fo CR

d CR

St CR

CR

R CR

FMS

AACCH $\quad 68-62$ $\begin{array}{ll}\text { CR } & \text { F-44 }\end{array}$ $\mathrm{CR} \quad \mathrm{G}-22$

CR J.56

J-56
294

294
2.76 LPD 1-2

203.03

$\begin{array}{ll}\text { AGMA } & 203.03 \\ \text { AGMA } & 374.04\end{array}$

NESCA MAN K 
Std. for Industrial Computer Std. for Coolant Std. for Central

for Pipe Friction Loss Tables for Solving Fire Protection

Std. Metric Pract. Guide to the Use of SI (International ation of Brake Fluid Temperature in the Automotive Braking for Nomenclature for Passenger Car and Light Truck Exhaust ent and $\mathrm{R} /$ Std. for Recreational Vehicle Air Conditioning

Water Flow / Pressure Requirements for Pipe Schedule

Rec. Pract. for Automotive Transmission Hydraulic Control

ct. for Mobile Construction Type Crane Overload Indicating rity Surveillance Sys/ Std. for Safety for Access Control

Safety Std. for Household Burglar Alarm Std. for Safety of Household Fire Warning Std. for Safety for Proprietary Burglar Alarm Std. for Gas Fired Gravity and Fan Type Sealed Combustion Std. Tolerances for Woolen Spun on the Woolen

Analysis of Cooking Characteristics of Macaroni-Borasio Spec. for Firecycle on -Off Multic ycle Sprinkler

mples from the Lines of an Operating $\mathrm{H} y$ draulic Fluid Power em) (1966)

Fire Detection Spec. Data for Hubless Cast Iron Sanitary Laminators Quality Control Std. Meth of Specifying Color by the Munsell Rec. Pract. for Quality Control Records pract. for Automotive Gasoline Performance and Information

Rec. Pract. for Radius of Load and Boom Angle Measuring s. Trucks, and Combination Vehicles with Any Type of Brake Uniform Plumbing Code: Battery Drainage form Plumbing Code: Rec. Rules for Sizing the Water Supply nes, and Drawings for Copper Solvent Single Stack Plumbing geons in the Treatment of Fractures of the Skeletal (Bone) Std. for Numerical Designation (Identification tioning System (Information on Year Round Air Conditioning

drant. Driveway Clearance. Installation and Maintenance of hase of Nuclear Power Plants / Std. for Cleaning of Fluid Manual Meth. (197) Std. Meth. for Sampling Gas Blow Down Automatic Particle/ Std. Meth. of Sampling Gas Blow Down Std. for Automatic Gas Ignition act. for Design, Construction and Stowage of Portable Fuel

Uniform Plumbing Code: Indirect Waste Piping, Wet Vented Std for Monorail

Construction, Operation, and Maintenance of Vehicle Alarm ANSI C 37.90 Std. for Relays and Relay for Assigning Alloy Phase Designations (Code) in Metallic 72) Std. for Efficiency Test of Air Cleaning Code for Identification of Piping in the Air Tral Spec. for Std. Integrated Data Processing eth. of Test for Linear Shrinkage of Thermosetting Casting td. Test Procedure for the Evaluation of Sealed Insulation gs (1973) Uniform Building Code Std. for Metal Suspension Std. for Alloy and Temper Designation std. for Alloy and Temper Designation n, Materials, Size, and Installation of Propeller Shafting Std. for Direct Reading Remote Registration Std. for Nuclear Material Control Pract. for Instantizing

(1971)

(1973)/ Std. for the Installation of Blower and Exhaust Std. Rec. Pract. for Prefabricated Reflective Insulation ion, Maintenance and Use of Auxiliary Protective Signaling Rec. for Local Protective Signalling for Safety for Alarm Valves for Use in Wet Pipe Sprinkler Rec. for Water Spray Fixed Std. for the Installation of Water Sprinkler actice) (1974) Std. for Nuclear Material Control for Residential Controls Direct Electric lgnition Control aintenance and Use of Central Station Protective Signaling Uniform Building Code Std. for Fire Alarm

td Spec for Homogeneous Perforated Bituminous Fiber Pipe onstruction and Installation of Permanently Installed Fuel uipment / Rec. Pract for the Flushing and Cleaning of Oil uipment (1972) uipment (1971) Rec. Pract. for the Design of Oil gn, Installation, and Operation of Subsurface Safety Valve Rec. Pract. and Stds. for Safe Installation of Exhaust Tent. Meth. of Test for Corrosivity of Solvent Std Criteria and Definitions for Excitation Maintenance and Use of Proprietary Protective Signalling llation, Maintenance and Use of Local Protective Signaling Rec. Pract. for the Flushing and Cleaning of Lubricating Std. for Electrical Bonding of Direct Current Rec. Pract. and Std. for Cathodic Protection Rec. for Design and Construction of Surface Drainage Sid. Criteria for Preparation of Design Bases for wer $\mathrm{Ge} /$ Indus/ Std. Meth. of Analysis of Solvent
System FORTRAN Procedures for Executive Functions and P

System Hoses (1971)

System Humidifiers (1974)

System Hydraulic Problems (1973)

System of Units) (1972) ANSI 2210.1

System of Vehicles Equipped with Disc Brakes (1972)

System Parts (1972)

System Rating Definitions, Test. Meth., and Test Equip

System Sprinkler Demand (1974)

System Terminology (1970)

System Test Procedure (1971)

System Units (Electronic/Electrical / Mechanical Secu

System Units (1972) ANSI Se2.4

System Units (1973)

System Units (1974) ANS1 Se2.9

System Wall Furnaces (1972)

System Yarns (1971)

System (Cereal Chemistry) (1962)

System (Fire Safety) (1973)

System (For Particulate Contamination Analysis) (1972)

System (Grinnell Protomatic Release Fl00) (1973)

System (Information on Year Round Air Conditioning Syst

System (Plumbing Pipe and Fittings) (1972)

System (Timber Construction Manual) (1962)

System (1968)

System (1969)

System (1971)

System (1972)

System (1972)

System (1973)

System (1973)

System (1973)

System (1973)

System) of Modul

System) (1966)

What Makes a Good Air Condi

ystems and Appliances (Extinguishers, Alarms, Sandpipe

Systems and Associated Components During Construction P

Systems and Components for Particulate Contamination by

Systems and Components for Particulate Contamination by

Systems and Components (1971)

Systems and Portable Containers for Flammable Liquids (

Systems and Special Wastes (1973)

Systems and Underhung Cranes (1973)

Systems and Units for Protection Against Holdup and the

Systems Associated with Electric Power Apparatus (1971)

Systems Based on the Composition and Crystal Lattice of

Systems Containing Devices for Removal of Particles (19

Systems Content $(1973$ )

Systems Designed to Streamline Supply Transactions with

Systems During Cure (1969) ANSI K65.181

Systems for AC Electric Machinery Employing Form Wound

Systems for Acoustical Tile and for Lay in Panel Ceilin

Systems for Aluminum (1972)

Systems for Aluminum (1972)

Systems for Boats (1973)

Systems for Cold Water Meters (1972)

Systems for Conversion Facilities (A Guide to Practice)

Systems for Dry Milk and Dry Milk Products (1972)

Systems for Dust, Stock, and Vapor Removal or Conveying

Systems for Equipment and Pipe Operating at Temperature

Systems for Fire Alarm Service (1972) ANS1 SE3.4

Systems for Fire Protection and Safety (Alarm) (1973)

Systems for Fire Protection Service (1973) ANSI B129.2

Systems for Fire Protection (1973)

Systems for Fire Protection (1973) ANSI A54.3

Systems for Fuel Reprocessing Facilities (A Guide to $\operatorname{Pr}$

Systems for Gas Fired Equipment (1972)

Systems for Guard, Fire Alarm and Supervisory Service (

Systems for High-Rise Buildings (1973)

Systems for Highway, Airport, and Similar Drainage (197

Systems for Inboard and Outboard Powered Boats (1973)

Systems for Lubrication and Control of Hydroelectric Eq

Systems for Lubrication and Control of Hydroelectric Eq

Systems for Lubrication and Control of Hydroelectric Eq

Systems for Petroleum Industry Use (1973)

Systems for Propulsion and Auxiliary Machinery (Boats)

Systems for Removing Water Formed Deposits (1973)

Systems for Synchronous Machines (1972)

Systems for Watchman, Fire Alarm and Supervisory Servic

Systems for Watchman, Fire Alarm, and Supervisory Servi

Systems of Various Turbine Driven Pumps for Marine Serv

Systems on Boats (1972)

Systems on Boats (1972)

Systems on Farms in Humid Areas (1973)

Systems That Perform Protective Functions in Nuclear $\mathrm{Po}_{0}$ Systems Used for Removal of Water Formed Deposits (Chem
ISA

SAE

$\mathrm{ARl}$

Rec. FMS

ASTM

IN SAE

IMACA

Rec FMS

SAE

SAE

UL

UL

UL

ANSI

ASTM

Meth. of AACCH

FMS

NFLDP T2.9.

FMS

NESC

C ISPI

AITC

ASTM

ASQC

Rec.

$S A E$

SAE

ICBO

Uni ICBO

CDA

ANSI

NESCA

ICBO

ANSl

ASTM

ASTM

ANSl

$\mathrm{ABYC}$

ICBO

ANSl

UL

IEEE

ASTM

ANSI

FMS

ATAA

Std. M ASTM

IEEE

ICBO

ANSI

$\mathrm{SAE}$

$A B Y C$

$A W W A$

ANSI

DFISA

NFPA

ASTM

/ Llat NFPA

FMS

UL

FMS

NFPA

ANSI

Std. NEMA

NFPA

ICBO

ASTM

ABYC

ASME

ASME

ASME

for Desi $\mathrm{APl}$

ABYC

ASTM

IEEE

NFPA

NFPA

ASME

ABYC

$A B Y C$

ASAE

ANS

ASTM 
Manually Operated Metallic Gas Valves in Gas Distribution reparation of Test Panels for Use in Test. Organic Coating f Test for Thermal Instability of Confined Condensed Phase Pract. for Design Calculations for Oil Sucker Rod Pumping Uniform Building Code Std. for Combination Standpipe Rec. for Proprietary Protective Signaling Rec. for Liquefied Hydrogen Rec. for Flam mable Liquid Pumping and Piping Rec. for Installation of Sprinkler intenance of the Reliable Model B Accelerator for Dry Pipe Rec. for the Inspection and Maintenance of Sprinkler Rec. for Standpipe and Hose

Air Pressures for Pneumatic Controllers and Transmission cture (1973) ent) (1973) s (1971) Coating Plywood Construction Wells and Water

r Air Pressures for Pneumatic Controllers and Transmission std. for Intrusion Detection Units for Burglary Protection circuitry of Noncoded Remote Station Protective Signalling Std. for Commercial Cooking Equipment Exhaus

Rec. Pract. for Design Levels for Emergency Air Brake Rec. Pract. for Passenger Car Windshield Defrosting Ducted Electric Heat Guide for Air Handling $r$ Liquid Line Driers in Refrigerating and Air Conditioning entals Governing the Design and Operation of Local Exhaust hing and Cleaning of Gas Turbine Generator Lubricating Oil for Maintenance of Design Voltage in Snow mobile Electrical iodic Test. of Nuclear Power Generating Station Protection Safety Requirements for Powder Actuated Fastening Spec. for Acoustical Ceilin Std. Microwave Transmission Std. on Carbon Dioxide Fire Extinguishing Rec. Pract. for Vehicle Identification Number ec. Pract. for Passenger Car Vehicle ldentification Numbe Rec. Pract, for Passenger Car Windshield Washer Rec. for Thermoplastic Water Piping

Std. for Safety for Electronic Data Processing Units and Meth. for Open Bottle Tap Sampling of Noncryogenic Fluid Operational Characteristics Airborne VHF Omnirange (VOR)

or Industrial Engineering Terminology: Data Processing and eth. of Test for Effective Pumping Speed of Vacuum Chamber

$r$ Thermoplastic Piping for Swimming Pool Water Circulation

cation, Construction and Protection of Electronic Computer

uct Construction Std. for Ventilating and Air Conditioning combustion Chambers, Fuel Oil Injection, and Gas Admission ves and Automatic Gas Shutoff Devices for Hot Water Supply for Shock Absorbing Properties of (Sports) Playing Surface and Medium Speed Stationary Diesel and Gas Engine Starting edium Speed Stationary Diesel and Gas Engine Cooling Water ium Speed Stationary Diesel and Gas Engine Lubricating Oil on Sealing Rings for Automotive Transmission and Hydraulic rnal Corrosion on Underground or Submerged Metallic Piping speed Stationary Diesel and Gas Engine W aste Heat Recovery ed Stationary Diesel and Gas Engine Air Intake and Exhaus and Oil Filled Capacitor Switches for Alternating Curren tionary Diesel and Gas Engine Power Plant Fuel Oil and Gas

Guidance Equipment Used in Airborne Volumetric Navigation

Homes): Installation of Plumbing, Heating, and Electrical the Use of Fire Resistant Fluids for Hydraulic Fluid Power Grounding of lndustrial and Commercial (Electrical) Power ety for Control Units for Indoor Fire Protective Signaling uality of Electrical Insulating Oil for Low Pressure Cable ality of Electrical lnsulating Oil for High Pressure Cable escaling and Cleaning Stainless Steel Parts. Equipment and e Criterion to Nuclear Power Generating Station Protection ty Analysis of Nuclear Power Generating Station Protection and Bank Burglar Alarm

maintenance and Use of Remote Station Protective Signaling

Std. for Air Outlets and lnlets Used in Air Distribution Rec. for Foam Fire Extinguishing

Rec. for Halogenated Fire Extinguishing Agent Uniform Building Code: Fire Extinguishing

Uniform Plumbing Code: Medium Pressure Gas Piping Uniform Plumbing Code: Sewers and Private Sewage Disposal Uniform Plumbing Code: Drainage

Spec, for Underhung Cranes and Monorail

c. for Fire, Heat, and Smoke Damage of Electronic Computer for Cast Bronze Solder Joint Fittings for Solvent Drainage proposal for Solid State Load Switches for Traffic Control

m Building Code Std. for Carbon Dioxide Fire Extinguishing llation Stds. for Residential Heating and Air Conditioning code Std. for Installation of Automatic Fire Extinguishing ode Std. for Class Il and Class 111 Dry Cleaning Plants or de Std. for Smoke Detectors for Fire Protective Signalling

Drapery, Gate, Louver, and Window Electric Operators and
Systems Whose Maximum Allowable Operating Pressure Does

Systems (Confinement Test) (1973)

Systems (Conventional Units) (I972)

Systems (Fire Extinguishment) (1973)

Systems (Fire Protection and Safety) (1973)

Systems (Fire Protection and Safety) (1973)

Systems (Fire Protection and Safety) (1973)

Systems (Fire Protection) (1973)

Systems (Fire Protection) (1973)

Systems (Fire Safety) (1973)

Systems (Heating) (1972)

Systems (lnstrumentation) (1970)

Systems (Paint) Guide for Ship Hull, Deck and Superstru

Systems (Ratios from 100:1 to 1000:1) (Fire Extinguishm

Systems (Specs.) for Commercial and lndustrial Building

Systems (Treatment, Quality and Management) (1972)

Systems (1967)

Systems (1969) ANSI C 33.70

Systems (1969) ANSI SE3.7

Systems (1970)

Systems (1971)

Systems (1971)

Systems (1971)

Systems (1971)

Systems (1971)

Systems (1971)

Systems (1971)

Systems (1971)

Systems (1972)

Systems (1972)

Systems (1972)

Systems (1972)

Systems (1972)

Systems $(1972)$

Systems (1972)

Systems (1972)

Systems (1972)

Systems (1972)

Systems (1972)

Systems (1972)

Systems (1972)

Systems (1972)

Systems (1972)

Systems (1972)

Systems (1972)

Systems (1972)

Systems (1972)

Systems (1972)

Systems (1972)

Systems (1972)

Systems (1972)

Systems (1972)

Systems (1972)

Systems (1972)

Systems (1972)

Systems (1972)

Systems (1972)

Systems (1972) ANSI A119.2
Systems (1972) ANSI B93.5

Systems (1972) ANSI C114.1

Systems (1972) ANSI C 33.90

Systems (1972) ANSI C59.69

Systems (1972) ANSI C 59.70

Systems (1972) ANSI G81.16

Systems (1972) ANSl N41.2

Systems (1972) ANSI N41.4

Systems (1972) ANSI Se2.3

Systems (1972) ANSI SE3.5

Systems (1973)

Systems (1973)

Systems (1973)

Systems (1973)

System 5 (1973)

Systems (1973)

Systems (1973)

Systems (1973)

Systems (1973)

Systems (1973)

Systems (1973)

Systems (1973)

Systems (1973)

Systems (1973)

Systems (1973)

Systems (1973)

Systems (1973)
ANSI Std. Meth. O
Stion and P NCCA O ASTM Rec. API

$1 \mathrm{CBO}$

FMS

FMS

FMS

FMS

/for Operation and Ma FMS

FMS

Std. Fo ISA

SNAME 57.

FMS $4.3 \mathrm{~N}$

APA *1

WSC

Std. Fo SAMA

Safety UL

Std. for Interconnection NEMA

NSF

$\mathrm{SAE}$

SAE

Std. Fo AR

Std. for Fundam ANSl

Rec. Pract. for Flus ASME

C. Pract. Test Meth and Requirements SAE

/-Use Criteria for the Per

AEEE

CS1

ElA

NFPA

SAE

SAE

SAE

SPl

Std ASTM

Minimu RTCA

Std. F ANSl

Sid. M ASTM

Rec. Fo SPl

Rec. for Lo FMS

Fibrous Glass D SMACN

Std. Pract. for DEMA

Std. for Relief Val ANSl

Std. Meth. of Test ASTM

Std. Pract. for Low DEMA

Std. Pract. for Low and M DEMA

Std. Pract. for Low and Med DEMA

Rec. Pract. Spec, for Cast Ir SAE

Rec. Pract. for Control of Exte NACE

Std. Pract. for Low and Medium DEMA

Std. Pract. for Low and Medium Spe DEMA

Closers, Automatic Line Sectionalizers NEMA

Pract. for Low and Medium Speed Sta DEMA

Operational Characteristics Vertical RTCA

Lers, Truck Campers, Motor NFPA

Std. Pract. for NFLDP

Rec. Pract. for lEEE

Std for Saf U

Spec. for Continuity of O ASTM

/Spec. for Continuity of Qu ASTM Std. Rec. Pract. for D ASTM

/Cation of the Single Failur IEEE

(Al Principles for Reliabili IEEE

UL

Std. for Installation, NFPA

ARI

FMS

FMS

$\mathrm{ICBO}$

ICBO

$1 \mathrm{CBO}$

$1 \mathrm{CBO}$

MMA

Re FMS

Std. ANSI

Sid. NEMA

Unifor ICBO

Minimum Insta SMAC

Uniform Building ICBO

Uniform Building C ICBO

Uniform Building Co ICBO

Safety Std. for Door UL

E476

III

8.8

.55

81

$-3 \mathrm{~N}$

639

SB3

$\mathrm{J} 263$

J $902 \mathrm{~B}$

* 12

29.2

277

A 10.3

09530

B16.33

TB. $111 \cdot 5$

UBC 538.3

LPD $5.2 \mathrm{~N}$

LPD $7.19 \mathrm{~N}$

LPD 7.32

PMC2.5

OS. $4 \mathrm{Cl}$

RS252A 
poly(Vinyl Chloride) (CPVC) Plastic Hot Water Distribution Deluge Valve for Water Flow Control of Fire Extinguishing $t$ of Discharge (Corona) Pulses in Evaluation of lnsulation ents, and lnstallation of Plumbing. Heating and Electrical Heating, Exhaust, Ventilating, and Their Combination Duct tallation of Central Warm Air Heating and Air Conditioning rting Contamination Analysis Data of Hydraulic Fluid Power

Pipe for Gas and Oil Transmission and Distribution Piping ety for Connectors and Switches for Use with Burglar Alarm Safety Std. for Holdup Alarm Units and

ns of Terms Relating to Porcelain Enamel and Ceramic Metal Rec. Pract. for Fans for Industrial Exhaust Ventilation rec. Pract. for Dilution Ventilation in Industrial Exhaust Rec. Pract. for Test. of Industrial Exhaust Ventilation Rec. for Gaskets for Flammable Liquid Piping Std. for Safety for Tear Gas Units and

Pract. for Exhaust Hood Design for Industrial Ventilation ct. for Ventilation for Heat Control in Industrial Exhaust t. for Design Procedure for Industrial Exhaust Ventilation onstruction Spec. for Local Industrial Exhaust Ventilation up and Recirculated Air for Industrial Exhaust Ventilation ted Signaling Boxes for Use with Fire Protective Signaling rs and Filter Driers in Refrigeration and Air Conditioning rs and Dust Collectors) for Industrial Exhaust Ventilation $s$ and General Principles of Industrial Exhaust Ventilation Code for Pressure Piping: Liquid Petroleum Transportation ols (Air Conditioning, Heating. Ventilating and Processing pe Protective Coatings for Underground Pipe Lines (Wrapped róunding and Bonding Equipment (Internal Electrical Wiring

aintenance of Heating. Ventilating, Cooling, Refrigeration Uniform Plumbing Code: Rainwater Mo/ Std. for Fire Protection Criteria for Safety Related tes; Bolts; Closers; Hospital and Misc. ltems; Kev Control ther Wood (Pressure Treated Lumber and Plywood) Foundation Equipment (1972) Std. for

Std. Meth. of Test for Peel Resistance of Adhesives plate, Corrosion Resistant, $14.8 \mathrm{Cr}-4.5 \mathrm{Ni}-3.5 \mathrm{Cu}-(\mathrm{Cb}+$ Std. for

(Cereal Chemistry) (1962)

Billiard Tournament

etc.) (Institutional

Rec. Pract. for Liquid Ballast Std. Liquid Ballast 73) Std. Performance Requirements for Woven Printed Std. Performance Requirements for Damask

Meth. of Ferricyanide-Maltose-Sucrose Conversion Meth. of Experimental Wheat Milling: Temper duction Meth. for Sugar Determination: Schoorl's Reduction ber) (1972) Usc Ps20 n (1972) SAE J711

Std. Volume and Specific Gravity Correction Uniform Building Code Std. for Span

71) s (1970)

s, Rockwell and Rockwell Superf/ (1970)

joints (1974)

ech. Notes) (1967)

blems (1973) td. for Sampling Procedures and Std. Specs, and Load Std. Specs. and Load Std. Hardness Conversion Std. Specs. and Load Std. Spec. and Load Design Rec. for Pipe Friction Loss (1971) ANSl L14.192

Std. Temperature-Electromotive Force (EMF) Std. Definitions. Symbols. Formulas, and

Rec. for Water Sprinkler and Hydrant System Demand with Gasoline; Methyl Blue and Green; Mineral Oil; Urease

1973)

)

) ANSI Z197.32

Meth. for Measuring Flat Spring Test for Hot td. Performance Requirements for Woven Upholstery Fabrics Std. Meth. of Test for Flash Point of Liquids by std. Meth. of Test for Flash Point of Cutback Asphalt with

Performance Requirements for Permanent Labels, Detachable

Test Meth. and Requirements for Snow mobile Rear Position $r$ Regular Bicycles, lncluding Reflectivity, Headlights and ing/ Std. Performance Requirements for Woven Drycleanable tin/. Std. Performance Requirements for Woven Drycleanable suiting (Institutional/ Std. Performance Requirements for Rec. Pract. for Agricultural Tractor Auxiliary Power Std. for Industrial Power Std. for Link, Cable d Light Industrial Tractors (1972) SAE J7/ Std. for Power
Rec. for Agricultural (Farm) Tractor Auxiliary Power 8 Rec. for Agricultural (Farm) Tractor Auxiliary Power
Std. for 540 Rpm Power
Systems (1973)

Systems (1973)

Systems (1973)

Systems (1973) ANSI A119.

Systems (1973) ANSI B144.2

Systems (1973) ANSI B 144.3

Systems (1973) ANSI B93.30

Systems (1973) ANSI H38.13

Systems (1973) ANSI Se2.6

Systems (1973) ANSI Se2.7

Systems (1973) ANSI Z167.1

Systems (1974)

Systems (1974)

Systems (1974)

Systems (1974)

Systems (1974)

Systems (1974)

Systems (1974)

Systems (1974)

Systems (1974)

Systems (1974)

Systems (1974)

Systems (1974)

Systems (1974)

Systems (1974)

Systems (1974)

Systems) (1972)

Systems) (1972)

Systems) (1972) ANSI C33.8

Systems, Roof Drains and Piping (1973)

Systems, Structures, and Equipment for Water Cooled and

Systems: Door Holders, Stops, Smoke and Heat Detectors;

System: House Design and Construction Meth. (1972)

T-Hook Slots for Securement in Shipment of Agricultural

(T-Peel Test) (1972) ANS1 Z197.2

Ta), Consumable Electrode Vacuum Arc Remelted (1973)

Tab Type Washer (1973)

Table and Accessory Equipment Specs. (1971)

Table for Converting Absorption to $14.0 \%$ Moisture Basis

Table for Drive Tires of Agricultural Machines (1971)

Table for Drive Tires of Agricultural Machines (1972)

Table Napery Other Than Damask (White or Dyed) (Napkin,

Table Napery (Napkin, etc.) (Institutional Textile) (19

Table Napery (Napkin, etc.) (Institutional Textile) (19

Table (Cereal Chemistry) (1962)

Table (Cereal Chemistry) (1962)

Table (Cereal Chemistry) (1962)

Tables and Working Stresses for Joists and Rafters (Lum

Tables for Creosote and Coal Tar (R 1970)

Tables for Floor and Ceiling Joists, and Rafters (1973)

Tables for Inspection by Atributes (MlL Std. 105D) (19

Tables for Longspan Steel Joints Deep DLJ and DLH Serie

Tables for Longspan Steel Joints LJ and LH Series (1970

Tables for Metals (Relationship Between Brinell, Vicker

Tables for Open Web Steel Joints J Series and H Series

Tables for Open Web, Longspan, and Deep Longspan Steel

Tables for Reinforced Brick Masonry Flexural Members (T

Tables for Solving Fire Protection System Hydraulic Pro

Tables for Thermocouples (1972) ANSI C 96.2

Tables for (Quality) Control Charts (1971) ANSI Z1.5

Tables of Classification of Man Made and Natural Fibers

Tables (Fire Safety) (1973)

Tablets; Xanthyrol) (1962)

Tachometer Spec. for on Road Vehicles (1972)

Tack and Pressure Sensitive Adhesives by Rolling Ball (

Tack Free Time of Caulking Compounds and Sealants (1973

Tack Free Time of Elastomeric Type Joir. Sealants (1971

Tack of Adhesives Using an Inverted Probe Machine (1971

Tack of W ax Polymer Blends (1972)

(Tacked On) (lnstitutional Textile) (1973)

Tag Open Cup Apparatus (1972)

Tag Open Cup Apparatus (1972)

Tags, and Certification of Fabrics or Products (Institu

Tail Lamp (Light) (1972)

Taillights (1972)

Tailored Uniform Fabrics, Heavyweight (Industrial) Suit

Tailored Uniform Fabrics, Intermediate (Year Round) Sui

Tailored Woven Uniform Fabrics, Lightweight (Tropical)

Take Off Drives (1972)

Take Offs for Overcenter Clutches (1971)

Take Up (1973)

Takeoff Definitions and Terminology for Agricultural an

Takeoff Drives (1972)

Takeoff for Agricultural (Farm) Tractors (1973) SAE J71
D2846

D 1868

$501 \mathrm{~B}$

$90 \mathrm{~A}$

$90 \mathrm{~B}$

T2.9.3

B345

B3 345
634

636

C286

ACGIH $* 1.10$

ACGIH * * *1.

ACGIH *1.9

FMS 7.32S

UL

Rec. ACGIH *1-4

Rec. Pra ACGIH * $1-3$

Rec Prac ACGIH *1-6

*1-6

$* 1-7$

38

730

*1-11

*1

B31.4

353

$\mathrm{RS} * \mathrm{l}$

467

MC *7

UPC*1-D

ANS

NBHA

N18. 10

Ea NFORP RP

ASAE $\$ 356$

ASTM D 1876

SAE AMS5862

NSA 460

BCA *]

AACCH 82-21

SAE J884B

ASAE $\$ 346$

ANSl L24.2.1

ANSI L24.2.6

ANSl L24.2.7

AACCH 22.18

AACCH 26-95

A ACCH $\quad 80-69$

NFORP *2

ASAE R220.3

ASTM D347

ICBO [BCS25-2]

ANSl Z1.4

AISC S316-

AISC S316-?

ASTM E140

AlSC S316-3

SII

BIA

FMS

ASTM

ASOC

ASTM

FMS

5

17. 89

$2-89$
E 230

A]

D2368

2.76

CCH 28-9

SAE J196

ASTM D3121

ASTM D2377

ASTM C679

ASTM D2979

TAPPl T683SU

ANSI L24.1.7

ASTM DI310

ASTM D3143

ANSl L24.5.1

J 279

$6 / 6$

$\begin{array}{ll}\text { ANSl } & \text { L24.3.2 } \\ \text { ANSl } & \text { L24.3.1 }\end{array}$

$\begin{array}{ll}\text { ANSl } & \text { L24.3.1 } \\ \text { ANSI } & \text { L24.3.3 }\end{array}$

SAE J717C

SAE J621C

NSA 511

ASAE S205.2

ASAE R333.

ASAE $\quad$ S203.7 
e and Red Pine, Eastern Spruce and Hemlock, Balsam Fir and tka Spruce, Douglas Fir-Larch (North), Eastern Hemlock ng Code Std. for Eastern Spruce, White Pine, and HemIock eptors, Stall Showers Lavatories, Bathtubs and Combination Std and Test Procedure for Water Closet Flush r Homogeneous Perforated Bituminized Fiber Pipe for Septic aminated Wall Perforated Bituminized Fiber Pipe for Septic Std. Procedure for Bench Calibration of Handling Chlorine in Std. for Turnbuckle Std. for Terminal Std. for Terminal Std. for Trunnion Std. for Trunnion

Rec. Pract. for Nomenclature and Terminology of Rec. Pract, for Accident Prevention Signs for Display on Safety Std. for Household Electric Storage Std. for Strap Assembly

Std. for Gas Water Heaters: Volume III, Circulating onditions for Evaluating the Compatibility of Vehicle Fue otection from Corrosion for Underground Pipe. Fittings and ecommended Safeguards and Practices for the Protection and B137.1

974)

Safety Std. for Aboveground Storage Sanitary Stds. for Uninsulated Sanitary Std. for Storage Sanitary Std. for Silo Type Storage Std. for Waste and Sewage Holdin

Std for Pressurized and Non Pressurized Potable Water Safety Std. for Atmospheric Type WeIded Steel Inside ety for Flame Arresters (Fire) for Use on Vents of Storage ds and Slur/ Rec, for Improving Safety on Enclosed Mobile ng. Settling, Aeration Chambe/ Std. for Thermoset Plastic Rec. Pract. Meth. for Lining Lease Production Std. for Welded Aluminum Alloy Field Erected Storage Rec for Design of Farm Waste Storage uide Spec. for Specifying: Reinforced Fiberglass Polyester Sanitary Std. for Farm Milk Storage Safety Std. for Automotive Fue Std for Safety for Septic

$(1968 /$ Rec. for Concrete Sanitary Engineering Structure Western Woods Use Book: Wood Std. Meth. of Test for Insoluble Ash of Vegetable Std. Meth. of Test for Water Soluble Matter of Vegetable Std Spec, for 14 Std. for Fixed Electrolyti Std. Spec. for Spec. for Std. Spec. for Uniform Building Code Std. for Gypsum Wallboard ards) in Numeric (DigitaI) Form on Punched Cards, Magnetic (1973) tensile, 36,000,000 (248) ModuI/ tensile, 36,000,000 (248) Modul/ Graphite Fiber Spec. for Graphite Fiber Spec. for Graphite Fiber Pressure Sensitive recording $\mathrm{An} /$

Std. for Dimensional Std. Coplanar Magnetic Std. for Recorded Magnetic Std. for Interchange Rolls of Perforated Std. for Recorded Magnetic Std for Recorded Magnetic cpi, NRZI, and $1600 \mathrm{cpi}, \mathrm{P} / \quad$ Std. for Unrecorded Magnetic ti/ Std. Spec. for an Audio Level and Multifrequency Test Spec for Composite Filament s for Audio Record One for 2.In. Quadruplex Video Magnetic Pressure Sensitive audio and Tracking Control Records on 2 ln. Video Magnetic Meth. for Flutter Measurement of Instrumentation Magnetic for Timing Error Measurements of Instrumentation Magnetic ging) for Consumer Electronic Products (Radio, Television, and Multifrequency Test Tape for Quadruplex Video Magnetic phasis Characteristics for 2 In. Quadruplex Video Magnetic deo Alignment Signal Spec. for a Quadruplet Video Magnetic Pract. for the Label for 2 In. Quadruplex Video Magnetic racking Control Record for 2 In. Quadruplex Video Magnetic

Std. for Automated Machine Lay Up Composite Filamen Std. for Packaging of Filament nd Acidic Gaseous Fluoride in the Atmosphere (Double Paper t Tape: Full Track, 1/4 In. (6.3 olled Equipment (1974) Interchangeable Perforated etermination of Film Adhesion on All Metals by Cross Hatch
st Meth. for Determination of Film Adhesion by Cross Hatch (l) Std. Meth of Test for Adhesion of Pressure Sensitive g-Positioning Nume/
Takeoff for Agricultural (Farm) Tractors (1973) SAE J71

Takeoff (Universal Joint) Drive Line Specs. (1972)

Ing Code Std. for Easter.

Tamarack (North), Eastern White Pine (North), Hem-Fir

Tamarack; Balsam Fir; Northern Pine and White Cedar Lum

Tank and Shower Receptor Units (1972)

Tank Ball Cocks (1968)

Tank Disposal Fields (1973) ANSI A 176.4

Tank Disposal Fields (1973) ANSl A 176.5

Tank Level Gaging Tapes and

Tank Strap (1973)

Tank Strap, Forked (1973)

Tank Strap, Plain (1973)

Tank Strap, Threaded (1973)

Tank Strap, Unthreaded (1973)

Tank Trailers and Containers (1971)

Tank Trailers and Containers (1972)

Tank Water Heaters (1972) ANSI C33.87

Tank (1973)

Tank, Instantaneous and Large Automatic Storage Type (1

Tanks and Fill Pipes with Fuel Dispensing Facilities (P

Tanks Containing Flammable Liquids (1973)

Tanks Containing Flammable or Combustible Liquids in Lo

Tanks for Flammable and Combustible Liquids (1972)

Tanks for Flammable and Combustible Liquids (1973) ANSI

Tanks for Milk and Its Products (Mixing and Storage) (1

Tanks for Milk and Its Products (1974)

Tanks for Milk and Its Products (1974)

Tanks for Mobile Homes and Recreational Vehicles (1971)

Tanks for Mobilehomes and Recreational Vehicles (1971)

Tanks for Oil Burner Fuel (1973)

Tanks for Petroleum Oil and Gasoline (1973) ANSI 2222.1

Tanks for Transporting and Spreading AgriculturaI Liqu

Tanks for Water and Wastewater Treatment (Septic. Holdi

Tanks with Coal Tar Epoxy (1972)

Tanks (1973)

Tanks (1973)

Tanks (1973)

Tanks (1973)

Tanks (1973)

Tanks, Bituminous Coated Metal (1974) ANSI Al62.1

(Tanks, Reservoirs, etc. for Water and Waste Treatment)

Tanks. Vats, and Pipe (1973)

Tanned Leather (1970) ALCA BI1

Tanned Leather (1970) ALCA B8

Tantalum and lts Alloy Tubing (1972) ANSI H54.I

Tantalum Capacitors (1972)

Tantalum Ingots and Flat Mill Products (1970) ANSI Z179

Tantalum Plate, Sheet, Strip, and Foil (1973)

Tantalum Rod and Wire (1970) ANSI Z179.15

Tape and Joint Compound (1973)

Tape and Punched Paper Tape (1972)

Printed Wiring Bo

Tape and Sheet, Epoxy Resin Impregnated, G70,000 (483)

Tape Application Stds. for Fibrous Glass Duct (1973)

Tape Cartridge Type Cp II (Compact Cassette) for Audio

Tape for Information Interchange (1600 c pi, PE) (1973)

Tape for Information Interchange (1972)

Tape for Information Interchange (200 cpi, NRZI) (1973)

Tape for Information Interchange (800 cpi, NRZI) (1973)

Tape for Information Interchange (9-Track 200 and 800

Tape for Quadruplex Video Magnetic Tape Recorders Opera

Tape Laying Machine-Numerically Controlled (1973)

Tape Operating at 15 and 7.5 In./S (1973)

Tape Performance Stds. for Fibrous Glass Duct (1973)

Tape Quadruplex Recorded at 15 and 7.5 In./S (1973)

Tape Recorder / Reproducers (1972) ANSI C83.99

Tape Recorder/Reproducers (1974) ANSI C83.94

Tape Recorder, Phonograph, etc.) (1973)

Tape Recorders Operating at 15 In./S (1973)

Tape Recording (1967)

Tape Recording (1969)

Tape Recordings (1968)

Tape Recordings (1970)

Tape Reel (1972)

Tape Reels (1973)

Tape Sampler Meth.) (1973)

/G Test (Packa Io Level

Reproducer

Tape Speeds of $7.5 \mathrm{In} .0190 .5 \mathrm{Mm}$, and $3.75 \mathrm{In} .095 .3$

Tape Std., Variable Block Format, for Numerically Contr

Tape Test After Reverse Impacting (Coil Coating) (1974)

Tape Test (Coil Coating) (1974)

Tape to Fiberboard at $90 \mathrm{Deg}$. Angle and Constant Stress

Tape Variable Block Format for Contouring and Contourin

Tape Water Vapor Transmission Test Meth. (1970)

$A S A E$

$\mathrm{ASAE}$

ICBO

ICBO

ICBO

IAPMO

ASSE

ASTM

ASTM

ANSI

CHI

NSA

NSA

NSA

NSA

NSA

TTMA

TTMA

ANA

SAE

ICBO

ICBO

$\mathrm{UL}$

UL

DFISA

DFISA

DFISA

IAPMO

IAPMO

UL

UL

ASAE

NSF

NACE

ANSI

ASAE

CSI

DFISA

UL

UL

ACl

WW PA

ASTM

ASTM

ASTM

EIA

ASTM

SAE

ASTM

ICBO

IPC

SAE

SAE

SAE

EIA

ANSI

ANSl

ANSI

ANSI

ANSI

SMACN

2.04 .6

R331.2

UBCS25.5

UBC $\$ 25.2$

UBC 525.8

TSC11

1002

D2312

D2313

B88.2

49

27

24.

23

RP36

RP35

174

29

221.10.3

J398A

UFC*2B

$\mathrm{UFC} * 2 \mathrm{~A}$

142

58

3200

0106

2203

TSC2

TSC 4

80

525

R317

41

RP.03-72

B96. 1

R345.I

15177.1

3000

395

68-50 
for Dimensions of Patch Splices in Two Inch Video Magnetic Spec. for Properties of Unpunched Oiled Paper Perforated al) Form on Punched Cards, Magnetic Tape and Punched Paper high Temperature Glass Cloth Pressure Sensitive Electrical

ethylene Plastic Pressure Sensitive Electrical Insulating

Chloride Plastic Pressure Sensitive Electrical Insulating Chloride Plastic Pressure Sensitive Electrical Insulating or Polyester Film Pressure Sensitive Electrical Insulating roethylene Backed Pressure Sensitive Electrical Insulating

73) Std for Fastener. Std. for Bolt, Std. for Bolt, Std. for Bolt, Std. for Bolt,

Std. Spec. for Steel Tubes, Low Carbon, ng Fits for Metric Radial Ball and Roller Bearings (Except Sid. Procedure for Bench Calibration of Tank Level Gaging Std. Spec. for Woven Cotton Std. Meth. of Test. Varnished Cottonfabrics and

6 Std. Meth. of Test. Pressure Sensitive Adhesive Coated th. of Test for Adhesion After Impact of Preformed Sealing Test for Low Temperature Flexibility of Preformed Sealing Stds. for Tire Rim Inspection, and Disk and Flat test for Weight Loss After Heat Aging of Preformed Sealing il Migration or Plasticizer Bleed Out of Preformed Sealing r Tape Speeds of $7.5 \mathrm{In} .0190 . /$ Std. for Reproducer Test Std. Spec. for Cold Heading Quality Carbon Steel Wire for pth (1972) Std. for Steel Self Drilling Std. for Steel Self Drilling

std. Meth. of Test for Pyridine Bases in Crude and Refined Std. Meth. of Test for Gel Time of Std. Meth. of Test for Coking Value of oles, and Timbers for Marine, Land, / Std Spec for Coal

Pract. Meth. for Lining Lease Production Tanks with Coal rec. Spec. for Enamel Coating System Designations for Coal face Courses (1967) St Spec. for Hot Mixed, Hot Laid sphalt, Asphalt and Coal Tar Saturated Organic Felts, Coal Hot Mopping Asphalt, Asphalt-Saturated Organic Felt, Coal

It Up Roof Coverings: Hot Mopped Asphalt, Asphalt and Coal Std Spec, for Creosote Coal

d Specific Gravity Correction Tables for Creosote and Coal

meth. of Test for Solubility of Bituminous Road Materials Sid. for Dimensions for Resolution Test nd Domestic-Trap, Clay Pigeon, and Skeet: Ammunition and Std. Meth. of Test for Distillation of Road act. for Paving Uses and Application Temperatures for Road hemistry) (1962)

ry) U Qualitative Meth. of Analysis for Free or Combined )(1968)

h. of Analysis for Fumaric, Pyruvic, Lactic, Succinic, and Meth. of Triangle Test Std. for Safety for Std. Meth. of Test for Tent. Meth. for Drop Weight Meth. for Cross Directional Internal

Rip) Meth. (Constant Rate of Tra Std. Meth. of Test for Std. for Sprinkler Irrigation (For Farms) interior Guide (Spec. for Installation, Finishes and Other material Composition, Use, Application, Finishes and Other

Waste (1974) Std. Spec. for Once Refined Sunflower Oil,

metric and British-American) in Published Scientific and Std. for Steel Self Drilling Tapping Screws and Rec. cteristics of Motor Fuels Using the Compression Ratio (CR) cteristics of Motor Fuels Using the Compression Ratio (CR) by the Silver Chloride-Lithium Fluoride Carrier D-C Arc of Plain Carbon and Low Alloy Steel by the Point to Plane mical Analysis of Blast Furnace lron by the Point to Plane

Thermionic Nickel Alloys by the Powder Direct Current Arc

Uranium Oxide $\left(\mathrm{U}_{3} \mathrm{O}_{k}\right)$ by Gallium Oxide Carrier D-C Arc on and Low Alloy Steel for Boron by the Point to Plane Arc nerals, and Rocks by the Fire Assay Preconcentration Spark Spectrochemical Analysis of Copper by the Briquet D.C Arc owered Passenger Cars and Light Trucks Using the Enclosure ffused Layers in Silicon by the Angle Lapping and Staining td. Meth. for Fast Neutron Flux Measurements by Track Etch tent Using a $14 \mathrm{MeV}$ Neutron Activation and Direct Counting

d Metallic Coatings by Double Beam Interference Microscope dentially Hazardous Electromagnetic Radiation / Std. for n/ Rec. Pract. for Uniform Laboratory Instrumentation and ereal Chemistry (1962) 8.2

Photographic Film Exposure Guide (Also Contains Suggested

Std. Meth. for Measuring Neutron Flux by Radioactivation eth for Measuring Thermal Neutron Flux by Radioactivation stallographic Perfection of Germanium by Preferential Etch
Tape (1964)

Tape (1971)

Tape (1972)

Tape (1973)

Tape (1973)

Tape (1973)

Tape (1973) ANSI C59.9

Tape (1973) ANSI C59.96

Tape (1974)

Taper Shank (1973)

Taper Shank, Shear Type, Protruding Head-Oversize (19

Taper Shank, Shear Type, Protruding Head (1973)

Taper Shank, Shear Type, 100 Deg. Head-Oversize (1973

Taper Shank, Shear Type, 100 Deg. Head (1973)

Tapered for Structural Use (1973)

Tapered Roller Bearings) Conforming to Basic Boundary P

Tapes and Sounding Rules (1974)

Tapes for Electrical Purposes (1972)

Tapes Used for Electrical Insulation (1970) ANSI C59.31

Tapes Used for Electrical Insulation (1974) ANSI C59.13

Tapes (1973)

Tapes (1973)

Tapes (1974)

Tapes $(1974$

Tapes $(1974)$

Tape: Full Track, 1/4 ln. (6.3 Mm) Width, Open Reel (Fo
Tapping or Sheet Metal Screws (1971) ANSl G54.17

Tapping Screws and Rec. Technique for Measuring Case De

Tapping Screws (1972)

Tar Acids (1972) ANS1 Z107.4

Tar Acids (1972) ANSl Z78.23

Tar and Pitch (Modified Conradson) (1973) ANSl Al49.12

Tar Creosote for the Preservative Treatment of Piles, P

Tar Epoxy (1972)

Tar or Asphalt Coatings (1972)

Tar Paving Mixtures for Base, Binder, Leveling, and Sur

Tar Pitch, and Asphalt Coated Glass Fiber Mat (1973)

Tar Pitch, Coal-Tar Saturated Organic Felt, Asphalt-Sat

Tar Saturated Organic Felts, Coal Tar Pitch, and Asphal

Tar Solution (1974) ANSI Oll.2

Tar (R 1970)

Tar, Petroleum Asphalt, etc.) in Organic Solvents (1966

Std. Volume an ASTM

Target for Photographic Optics (1972)

Target Spec. (1973)

Tars (1972)

Tars $(1972)$

Tartaric Acid in Baking Powders and Chemicals (Cereal C

Tartaric Acids (Cereal Chemistry) (1962)

Taste Panel for Food Flavor Detection (Cereal Chemistry

Tear Gas Units and Systems \{1974

Tear Resistance of Vulcanized Rubber (1973

Tear Tests of Ferritic Steels (1971) ANSI Z260.3

Tearing Resistance of Paperboard (1972)

Tearing Strength of Woven Fabrics by the Tongue (Single

Technical Data Sheet (1972)

Technical Data) (1973)

Technical Data) (1973)

Technical Grade (1973)

Technical Guide for Landfill Disposal of Solid Chemical

Technical Work (1973)

Depth (1972)

Technique for Motor Meth. Ratings (1971) ANSI Z11.306

Technique for Research Meth. Rating (1971) ANSI Z11.305

Technique Using an Optical Emission Spectrograph (1974)

Technique Using an Optical Emission Spectrometer (1970)

Technique Using an Optical Emission Vacuum Spectrometer

Technique (1961) ANSI Z128.2

Technique (1970) ANSI Z128.27

Technique (1970) ANSI Z128.29

Technique (1971) ANSI Z128.25

Technique (1971) ANSI Z128.32

Technique (1972) /Vapora

Technique (1972) ANSI Z173.49

/Trochemical Analysis of ASTM

Analysis of Plain Carb ASTM

/Al Analysis of Ores, Mi ASTM Std. Meth. for ASTM

Technique (1973)

Technique (1973)

Technique (1973)

Transparent or Opaque Metal Oxide an

Techniques and Instrumentation for the Measurement of $\mathrm{P}$

Techniques for Exhaust Gas Emissions Measurement (Passe

Techniques for Extraneous Matter Meth. of Analysis in C

Techniques for Photography) (1973)

Techniques for Switching Impulse Testing (1972) ANSI C6

Techniques for the Machine Tool Builders' Industry (197

Techniques (1970) ANSI N109

Techniques (1970) ANSI N110

Techniques (1973)
RP5

X3.29

D. 350

D2754

D3006

D3005

D230

D2484

D2686

1730

1729

1728

1725

1724

A595

B88 2

D335

D295

D1000

C766

C765

10

C771

C772

RS400

A548

$\mathrm{J} 78$

113

D2748

D2870

D2416

D390

RP.03-72

BEL

UBCS 32

$55 \mathrm{~A}$

UBCS32.1

D39l

D347

D2042

PH 3.50

* 17

D20

D2728

$\triangle A C C H \quad 04-27$

AACCH 04.28

04-24

AACCH 33-50

UL $\quad 600$

ASTM E436

T496SU

D2262

S262T

$3 \mathrm{~A} 7$

3 A9

D3169

SW.1

268

J78

D2723

D2722

E483

E403

E485

E129

E402

E404

$\mathrm{E} 400$

E414

J171A

F110

E418

E385

B588

C95.3

$\begin{array}{ll}\mathrm{SAE} & \mathrm{J} 254 \\ \mathrm{AACCH} & 28-92\end{array}$

Std. ANSI PH2.7

IEEE 332

NMTBA *1

ASTM E261

F389 
ysis of Admiralty Metal by the Cast Pin and Point to Plane from ${ }_{3} \mathrm{H}(\mathrm{D}, \mathrm{N})$, He Neutron Generators by Radioactivation Std. Meth. of Test. for Leaks Using Bubble Emission or Letter Symbols for Units Used in Electrical Science and 1973) Std. for Bolt Sid. for

Including Rounds, Squares, Hexagons, and Shapes-Angles, Id. for 700 Class Pintle Chains, Attachments, and Sprocket Std, for H Type Mill Chains-Attachments and Sprocket fset Sidebar Power Transmission Roller Chains and Sprocket d. for Heavy Duty Roller Type Conveyor Chains and Sprocket Std. Meth. for Measuring Transmission Performance of Std. for Physical Characteristics of T.2 e of Position Device, Radar, Radio Detection Finder, Radio Std. for Registered Screen Dimensions for Monochrome X-Ray Protection Standards for Home uipment ! Std. for Safety for High Voltage Components for uipment / Std. for Safety for Special Fuses for Radio and sion Protected Cathode Ray Tubes (Picture and Display) for Rec. Pract. for the Safe Action and Title Areas for and Contrast Range of Black and White Films and Slides for sions of Image Areas and Mounts for Slides and Opaques for Std for Precision Coaxial Connectors for CATV (Cable neering Specs. Outline for Monochrome Cctv (Closed Circuit Std. on Subscriber Visual Carrier Level (Cable Test (Packaging) for Consumer Electronic Products (Radio, (1960)

$$
\begin{array}{r}
\text { Test Meth. for Moisture in Chips (Moister } \\
\text { Safety Std. for } \\
\text { Std. for Alloy and } \\
\text { Std. for Alloy and }
\end{array}
$$
Meth. of Experimental Wheat Milling: mocouples (1972) ANSI C96.2 Std.

rators, Com/ Std. Test Procedure to Determine the Freezer vapor Transmission Rate of Sheet Materials (Paper) at High test for Stiffness Properties of Plastics as a Function of Std. Spec. for Seamless Austenitic Steel Pipe for High aterials by a Tempera ar) (Cereal Chemistry) (1962) ar) (Cereal Chemistry) (1962) r Artificial Weathering (1972/ 3) , Solvent Release Type Sealan/ ape (1973)

e Polymeric Materials (1973) Std. Test Meth. for Evaluating Low

s Equipped /

Rec Pract for Determination ent for Control of Air Conditioning, Hea

Std. Meth. of Test for Low Std. Meth. of Test for Low Std. Meth. of Test for Low Std. Spec. for High Std. Meth. of Low Std. Rec. Pract. for Internal the / Sid. Rec. Pract. for Infrared Determination of the 11.318

Z98.28 Std. Meth. of Test for Reaction Threshold Std. Meth. for Determining the Maximum Use Std. Meth. of Test for Brittleness K65.222 Std. Meth. of Test for Deflection ompo/ Std. Meth. of Test for Gel Time and Peak Exothermic Std. Meth. of Test for Effect of Moisture and als by Comparison with a Secondary Standard of Similar Emf
properties of Adhesives in Shear by Tension Loading in the in Fats and Fat Mixtures Which Congeal or Solidify Within . of Test for Dropping Point of Lubricating Grease of Wide ensitive Electric al Insulating Tape (/ Std. Spec. for Low 73) e Characteristics of Rubber and Rubber Like Materials by A c Resistance Welded Carbon Steel Tubular Products for High ng Nickel Alloy Bars, Forgings, and Forging Stock for High base Superalloy Bars, Forgings, and Forging Stock for High ontaining Alloy Bars, Forgings, and Forging Stock for High bine Type Bolting Material Specially Heat Treated for High d. Spec. for Centrifugally Cast Carbon Steel Pipe for High re Vessel Plates, Carbon Manganese, for Moderate and Lower on Hardening Nickel Alloy Plate, Sheet, and Strip for High welded Autenitic Chromium Nickel Alloy Steel Pipe for High tic Chromium Nickel Alloy Steel Pipe for Corrosive or High pec. for Centrifugally Cast Austenitic Steel Pipe for High n Steel Pressure Vessel Plates for Intermediate and Higher pe Flanges, Forged Fittings, and Valves and Parts for High I Alloy Steel Flanges, Fittings, Valves, and Parts for Low Spec. for Ferritic and Austenitic Steel Castings for High h Steel Plates for Pressure Vessels for Moderate and Lower $r$ Seamless and Welded Carbon and Alloy Steel Tubes for Low Std. Spec. for Seamless Carbon Steel Pipe for High for Centrifugally Cast Ferritic Alloy Steel Pipe for High Std. Spec. for Seamless and Welded Steel Pipe for Low
Techniques (1973)

Techniques (1973)

Std. Meth. for Spectrochemical Anal ASTM

lon Flux Density and Average Energy ASTH

Techniques (1974)

Technology (1969) ANSI Y 10.19

TEE Head (1973)

Tee, Reducer, Flareless Tube to Swivel, Swivel on Run (

Tee, Reducer, Flareless Tube to Swivel, Swivel on Side

TEES and Channels (1972) ANSI G81.18

Teeth (Power Transmission) (1972)

eeth (1971)

Teeth $(1972)$

Teeth (1973)

Telephone for Automotive Use (HS 27) (1968)

Telephone Sets (1971)

Telephone Slide Base Lamp (1973)

Telephone) (1973)

Television Picture Tubes (1972)

Television Receivers (1968)

Television Receiving Appliances and Other Electronic Eq

Television Receiving Appliances and Other Electronic Eq

Television Receiving Appliances and Other Electronic Eq

Television Transmission (1968)

Television (1970)

Television (1973)

Television) Application (75 Ohms) (1973)

Television) Camera Equipment (1974)

Rec. Pract. for the Density

Television) (1970)

Television, Tape Recorder, Phonograph, etc.) (1973)

Television: Measurement of Differential Gain and Phase

Teller) (Pulp and Paper) (1973)

Tellers: Lockers (1973)

Temper Designation Systems for Aluminum (1972)

Temper Designation Systems for Aluminum (1972)

Temper Table (Cereal Chemistry) (1962)

Temperature-Electromotive Force (EMF) Tables for Ther

Temperature and Energy Consumption of Household Refrige

Temperature and Humidity (1973)

Temperature by Means of a Torsion Test (1972) ANSI D65.

Temperature Central Station Service (1973) ANSI B125.25

Temperature Characteristics of Rubber and Rubber Like M

Temperature Corrections for Refractometer Readings (Sug

Temperature Corrections for Saccharimeter Readings (Sug

Temperature Flexibility of Latex Sealing Compounds Afte

Temperature Flexibility of Preformed Sealing Tapes (197

Temperature Flexiblity and Tenacity 1 Part, Elastomeric

Temperature Glass Cloth Pressure Sensitive Electrical T

Temperature Impact Test for Fabrics Coated with Flexibl

Temperature in the Automotive Braking System of Vehicle

Temperature Indexes of Solid Electrical Insulating Mate

Temperat ure Indicating and Regulating Electrical Equipm

Temperature Measurements in Ablative Materials (1968)

Temperature Molten Materials (Metals, Slag. Glass, etc.

Temperature of Applied Coatings on Wood Products During

Temperature of Liquid and Solid Materials (1972) ANSI Z

Temperature of Preformed Thermal Insulation (1971) ANSI

Temperature of Plastics and Elastomers by Impact (1973)

Temperature of Plastics Under Flexural Load (1972) ANSI

Temperature of Reacting Thermosetting Resins (Plastic C

Temperature on Adhesive Bonds (1972) ANSI Z197.18

Temperature Properties (1972) /Le Thermoelement Mater

Temperature Range from -267.8 to $-55 \mathrm{C}(-450$ to $-67 \mathrm{~F})$

Temperature Range of 25 to $4.5 \mathrm{Deg}$. (Cereal Chemistry)

Temperature Range (1972) ANSI Z11 207

Temperature Resistant Vinyl Chloride Plastic Pressure S

Temperature Retraction Procedure (TR Test) (R1972) ANS

Temperature Scale for Differential Thermal Analysis (19

Temperature Service Conforming to ISO

Temperature Service (1970) ANSI G81.45

Temperature Service (1970) ANSI G81.46

Temperature Service (1971) ANSI G55.12

Temperat ure Service (1972)

Temperature Service (1972)

Temperat ure Service (1972)

Temperat ure Service (1972)

Temperature Service (1972) ANSI B125.36

Temperature Service (1972) ANSI B125.39

Temperature Service (1972) ANSI G35.9

Temperature Service (1972) ANSI G37.1

Temperature Service (1972) ANSI G38.13

Temperature Service (1972) ANSI G52.2

Temperature Service (1972A)

Temperat ure Service (1972A) ANSI B125. 18

Temperature Service (1972A) ANSI B125.30

Temperat ure Service (1973)

Temperature Service (1973) ANSI B125.17
Std. F IEEE

NSA

NSA

NSA

/Anese Steel Bars Std. Dimen

Termination of Wate

E486

E496

E515

260

1763

1764

A 429

B29.13

B29.14

B29.10

B29.15

J797

269

C78.397

A2 1

RS266A

Ela

NCRPM *1

UL 492.3

UL $\quad 492.7$

SMPTE RP8

SMPTE RP7

PH22.9

ElA RS403

RS312

001B. 0670

RS414

G EIA

IEEE

TAPPI [M.8

UL

ANSI

901

ASTM E230

AHAM

TAPP

ASTM

ASTM

ASTM

$\mathrm{AACCH}$

$\mathrm{AACC}$

ASTM

ASTM

ASTM

ASTM

ASTM

SAE

IEEE

UL

ASTM

FMS

ASTM

ASTM

ASTM

ASTM

ASTM

ASTM

ASTM

ASTM

ASTM

$\mathrm{AACCH}$

Meth ASTM

ASTM

ASTM

ASTM

ASTM

/Tation Hardeni ASTM

/Ardening Iron ASTM

/Ening Cobalt C ASTM

/Lloy Steel Tur ASTM

St ASTM

Std. Spec. for Pressu ASTM

Std. Spec. for Precipitati ASTM Spec. for Electric Fusion ASTM

Meter Austeni ASTM Sid. S ASTM

Spec, for Carbo ASTM Alloy Steel Pi ASTM /Olled $9 \%$ Nicke ASTM Std. ASTM

Spec for High Strengt ASTM /Td. Spec. Fo ASTM ASTM

Std. Spec. ASTM

ASTM

HRF-2-ECFT

T464

D 1043

A 376

D1329

80-21

C734

D2754

D2137

J291 
std. Spec. for Seamless Ferritic Alloy Steel Pipe for High arbon and Alloy Steel Nuts for Bolts for High Pressure and carbon Steel Pressure Vessel Plates for Moderate and Lower or Pressure Vessels, Valves, Flanges, and Fittings for Low Piping Fittings of Wrought Carbon and Alloy Steel for Low carbon Steel Castings Suitable for Fusion Welding for High 1 Castings for Pressure Containing Parts Suitable for High el Castings for Pressure Containing Parts Suitable for Low mium-Nickel-Iron Alloy Castings (25.12 Class) for High for Alloy and Stainless Steel Bolting Materials for High als by Means of a Torsional / Std. Meth. of Measuring Low Std. for Strip-High

Meth. of Test for Apparent Viscosity of Motor Oils at Low Meth. of Compression Test. of Metallic Materials at Room Chambers and Serving Units for Tests Above and Below Room for Spray Applied Fibrous Thermal Insulation for Elevated st for Abrasion Resistance of Refractory Materials at Room Std. Meth. of Drip SIag Test. Refractory Brick at High n Starch Which Is Substantially Insoluble in Water at Room on. Nickel, And/S Std. Meth. for ChemicaI Analysis of High astener (Blind, Internally Threaded, External Sleeve, High ngs, Sealing. Fluorosilic one Rubber, General Purpose, High astener (Blind, Internally Threaded, External Sleeve, High th Expansion Coef/ Std. Spec. for Bolting Materials, High ive Insulation Systems for Equipment and Pipe Operating at Std. Rec. Pract. for Paving Uses and Application

) ANSI J1.3

lastics (1967) ANSI K65/ Std. Rec. Pract, for Sid. Test ectrical Insulating Materials at Microwave Frequencies and of Solid Ceramic Dielectrics at Frequencies to $10 \mathrm{MHz}$ and Meth. of Test for Apparent Viscosity of Gear Oils at Low rties of Adhesives in Shear by Tension Loading at Elevated Std. Air Density Ratios-At Various Altitudes and

ivity of Manufactured Carbon and Graphite Articles at Room Std. Meth. of Load Test. for Refractory Brick at High Nickel High Alloy Tubing for Pressure Application at High teel Plate, Sheet, and Strip for High Strength at Elevated cold Worked Alloy Steel Bars for High Strength at Elevated forgings and Forging Billets for High Strength at Elevated d. Meth. of Test for Normal Spectral Emittance at Elevated lectric Fusion Welded Steel Pipe for Atmospheric and Lower surement of Flow Properties of Lubricating Greases at High on WeIded Steel Pipe for High Pressure Service at Moderate

$\mathrm{f}$ Wrought Carbon and Alloy Steel for Moderate and Elevated

d Nickel Base Alloy Castings for High Strength at Elevated building Code Std. for Self and Flash Ignition Properties 1971) ANSI G24.19 Std. Spec. for Quenched and ) ANSI / Std. Spec. for High Yield Strength, Quenched and
essel Conponents (1973) Ansi/ Std. Spec. for Quenched and s (1973)

Std. Spec. for Quenched and Std. Spec. for Steel Wire, Oil

for Chromium Moiybdenum, Alloy Steel Plates, Quenched and pec for Pressure Vessel Plates, Alloy Steel, Quenched and for Pressure Vessels (1974)/ Std. Spec. for Quenched and pec. for Pressure Vessel Plates, Alloy Steel, Quenched and re Vessel Plates, Alloy Steel, High Strength, Quenched and esseI Plates, Alloy Steel, 9\% Nickel Double Normalized and

Lesistant, 16.5 $\mathrm{Cr}-4.5 \mathrm{Ni}-2.9 \mathrm{Mo}-0.10 \mathrm{~N}$, Equalized and olution Heat Treated, Sub Zero Cooled, Equilized, and Over Manganese-Molybdenum-Nickel Alloy Steel, Quenched and ickel Chromium Molybdenum Alloy Steel Plates, Quenched and for Low Alloy Steel Studs. Heat Resistant, Normalized and and Screws, Steel, Low Alloy Heat Resistant. Hardened and nt, 195,000 psi (1,345 MPa) Tensile Strength, Hardened and al Wheat Flour Milling: Equipment, Sample Preparation, and Std. for

Meth. for Use of Layer Cake Measuring Rec. Procedure for Processing $\mathrm{Hardw}$ are Schedules and ions, and Durability Requirements for Both Permanently and at Treated for Pressure Containing Parts Suitable for High Std. Spec. for figurations (1973) Tent. Meth. of Test for Breaking an/ Std Meth of Test for Low Temperature Flexiblity and ansi Z167.21 Sid. Meth. of Test for Reboiling Tent. Rec. for Concrete Members Prestressed with Unbonded L14.180 Test Meth. for Multiple Sample Meth. of Textile Test Meth. for Single Sample Meth. of Textile mposites (GF 220 (1517) Tensile Strength, 70,000,000 (483) Std. Test Meth. for

Std. Meth. of Testing Cross Lap Specimens for Meth. of Test for Std. Meth. of Test for Std. Meth. of Test for Std. Meth. of Test for si K65.32 Std. Meth. of Test for
Low and Intermediate K65.76
Temperature Service (1973) ANSI B125.24 Temperature Service (1973) ANSI E38.1 Temperature Service (1973) ANSI G35.10 Temperature Service (1973) ANSI G38.3 Temperature Service (1973) ANSl G 46.2 Temperature Service (1974) ANSI G37.2 Temperature Service (1974) ANSI G37.3 Temperature Service (1974) ANSI G38.9 Temperature Service (1974) ANSI G81.19 Temperature Services (1974) ANSl G38.4 Temperature Stiffening of Rubber and Rubber Like Mater. Spec

Temperature Terminal Block. Base lnsulating (1973)

Temperature Using the Cold Spanking Simulator (1972) an Temperature (R1973)

Temperature (1966) ANSI C59.49

Temperature (1972)

Temperature (1972)

Temperature (1973)

Temperature (1973)

- Elermination of $\mathrm{pH}$ (Slurry) of Cor

Temperature, Electrical, Magnetic, and Other Similar lr

Temperature, Flush Head, Self Locking) (1972)

Temperature, Fuel and OiI Resistant, 65-75 (1973)

Temperature, Protruding Head, (1972)

Temperature, 50 to 120 KSI Yield Strength, Materials Wi

Temperatures Above Ambient Air (1972) /Ricated Reflect

Temperatures for Road Tars (1972)

Temperalures for Rubber and Rubber Like Materials (1973

Temperatures of Std. ASTM Molds for Test Specimens of $\mathrm{P}$

Temperatures to $1650 \mathrm{C}$ (1970) ANSI C59.127

Temperatures to $500 \mathrm{C}$ (1968) ANSI C59.134

Temperatures Ising the Brookfield Viscometer (1972

Temperatures (Metal to Metal) (1972) ANSI Z197.5

Temperatures (1966)

Temperatures (1969) ANS1 K90.7

Temperatures (1970) ANSI Alll.1

Temperatures (1970) ASTM G82.1

Temperatures (1971) ANSI G81.23

Temperatures (1971) ANSI G81.2

Temperatures (1971) ANSI G81.27

Temperat ures (1972)

Temperatures (1972)

Temperatures (1973)

Temperatures (1973)

Temperatures (1973) ANSI G31.2

Temperatures (1973) ANSI G81.40

Tempered Alloy Steel Bolts for Structural Steel Joints

Tempered Alloy Steel Plate, Suitable for Welding (1970E

Tempered Carbon and Alloy Steel Forgings for Pressure V

Tempered Carbon Steel PIates for Structural Application

Tempered Carbon Valve Spring Quality (1971) ANSI G54.4

Tempered Chromium, for Pressure Vessels (1972) ANSI G35

Tempered Nickel-Cobalt-Molybdenum-Chromium (1972)

Tempered Vacuum Treated Carbon and Alloy Steel Forgings

Tempered 8 and 9o Nickel (1972A) ANSI C35.20

Std. Spec, for Pressu

Tempered (1972A) ANSI G35.11

Tempered (1972A) ANSl G35.3

Forgings, Corrosion and Toderate Hea

Tempered (1973)

Tempered (1973

7 Forgings, Corrosion and Moderate Hea

Tempered, for Pressure Vessel PIates (1973) ANSI G35.22

Tempered, for Pressure Vessels (1972A)

Tempered, Roll Threaded (135,000

Tempered, Roll Threaded (1973)

Tempered, Roll Threaded (1973)

Tempering (Cereal Chem istry) (1962

Template Hinge Dimensions (1972)

Template (Cereal Chemistry) (1962)

Templates (1971)

Temporarily Affixed Safety Signs for Construction and

Temprature Service (1974)

Tenacity of Man Made Textile Fibers in Loop or Knot Con

Tenacity I Part, Elastomeric, Solvent Release Type Seal

Tendency of Sheet Steel for Porcelain Enameling (1969)

Tendons (1966)

Tensile Loss by Retained Chlorine Bleaching (1971) ANSI

Tensile Loss by Retained Chlorine (1971) ANSI L14.126

Tensile Modulus (1973)

Phite Fibers for Structu

Tensile Properties of Adhesive Bonds (1972) ANSI Z197.8

Tensile Properties of Adhesives (1972) ANSI Z197.21

Tensile Properties of Oriented Fiber Composites (1972T)

Tensile Properties of Plastics (1972) ANSI K65.60

Tensile Properties of Rigid Cellular Plastics (1972) an

Tensile Properties of Single Man Made Textile Fibers Ta

Tensile Properties of Thin Plastic Sheeting (1973) ANSI

Tensile Strength Carbon-Silicon Steel Plates for Mach
ASTM

ASTM

A335

A 194

A 516

A 320

A 420

A216

A217

A352

A 447

A193

D 1053

1063

D2602

E9

E197

C720

C704

C768

C. 44

E354

1672

AMS7266

671

A453

C667

D2728

D1349

D958

D2520

D2149

D 2983

D2295

402

C61 1

C 16

A 608

A 457

A458

A477

E307

A671

D3232

A 672

A 234

A567

UBCS52.3

A 490

A514.

A54

A678

A 230

A542

A 605

A508

A553

A 517

A 353

AMS5745A

A MS5743D

A533

A 543

AMS $7458 \mathrm{C}$

AMS 7455D

AMS7459B

26. 10

$\begin{array}{ll}\text { ANSI } & \text { A } 156.7 \\ \text { AACCH } & 10.91\end{array}$

NBHA *7

SAE

ASTM

ASTM

J115

A389

D846

D3217

ASTM C711

ASTM C632

ACI 66.8

AATCC 114

AATCC 92

SAE AMS3892/5

ASTM D897

ASTM D1344

ASTM D3039

ASTM D638

Dl623

ASTM D2101

ASTM D882

ASTM A284 
Is (1972) ANSI G35.2 ity (1970) ANSI G24. pecimens (1972) ANSI Z197.3

Std. Spec. for Low and Intermediate Std. Spec, for Low and Intermediat Std. Meth. of Test for Std. Meth. of Test for Splitting Uniform Building Code Std. for Determining the Splitting appi T644 Std. Meth. of Test for $.5 \mathrm{Ni}-2.9 \mathrm{Mo}-0.10 \mathrm{~N}$, Heat Treated, $170,000 \mathrm{psi}(1172 \mathrm{MPa})$ malized and Tempered, Roll Threaded (135,000 psi (931 MPa 973) Std. Spec. for Steel Wire, Hig

Bolts and Screws, Heat Resistant, 195,000 psi (1,345 MPa r Graphite Fibers for Structural Composites (GF 220 (1517) Std. Meth. of Test for y the Tongue (Single Rip) Meth. (Constant Rate of Traverse eth. for Internal Bond Strength of Paperboard (Z-Direction (1972) Rec. Pract for Special Quality: High ber Tape and Sheet, Epoxy Resin Impregnated, G70.000 (483) (1973)

ber Tape and Sheet, Epoxy Resin Impregnated, G70,000 (483) Std. for Pin, Swage Locking, A.286 Cres, 100 Deg. Flus d. for Pin, Swage Locking, 108 KSI SteeI, 100 Deg. Shear of Test for Strength Properties of Adhesives in Shear by of Test for Strength Properties of Adhesives in Shear by of Test for Strength Properties of Adhesives in Shear by

Test Meth. for Creep Properties of Adhesives in Shear by of Test for Strength Properties of Adhesives in Shear by h. of Test for Fatigue Properties of Adhesives in Shear by ties of Adhesives in Plywood Type Construction in Shear by ties of Adhesives in Two Ply Wood Construction in Shear by rmining Durability of Adhesive Joints Stressed in Shear by hesively Bonded Rigid Plastic Lap Shear Joints in Shear by ively Bonded Plastic Lap Shear Sandwich Joints in Shear by ex Locknuts (1972)

Plane (1970)

1971) ANSI K90.6

td. for Torque Std. Meth. o Std. Meth. of Std. Meth. o

nsi Z260.1

Std. Rec. Pract. for Sharp Notch Std. Meth. of Std. Meth. of Tent. Meth. of Std. Meth. of

lloy Products (1973) ANSI H46.l

Titanium Alloy (1973)

973)

Std. for Pin, Swage Locking, SteeI, 100 Deg. Crown Head, Std. for Pin, Swage Locking. A-286 Cres, Protruding Head, d. for Pin, Swage Locking. A-286, 100 Deg. Head (MS20426) for Pin, Swage Locking, Aluminum Alloy, Protruding Head. or Pin, Swage Locking, Std. and Oversize, Protruding Head, r Pin, Sw age Locking, A.286 Cres, 100 Deg. Head (MS24694) r Pin, Swage Locking, A-286 Cres, Protruding Head, Shear in, Swage Locking, 108 KSI SteeI, Protruding Head. Shear

n, Swage Locking, Aluminum Alloy, 100 Deg. Head (MS24694),

$n$, Swage Locking, Aluminum Alloy, 100 Deg. Head (MS20426),

locking, Steel, Std. and Oversize, 100 Deg. Head (Ms2404)

ocking, Steel, Std. and Oversize, 100 Deg. Head (MS20426),

d. for Pin, Swage Locking, Steel, 100 Deg. Head (MS24694),

d. for Pin, Swage Locking, Steel, 100 Deg. Head (MS20426)

for Pin, Swage Locking, Aluminum Alloy, Protruding Head,

in, Swage Locking. Aluminum Alloy, $100 \mathrm{Deg}$. Head (Ms20426,

$n$. Swage Locking, Aluminum Alloy, 100 Deg. Head (MS24694) std for Pin, Swage Locking. Steel, Protruding Head,

ype, Titanium Alloy (1973)

y (1973)

il Type, Titanium Alloy (1973)

alloy (1973)

Iloy (1973)

alloy (1973)

er Bridges Design and Construction)/ inyl Coa Std. Performanter Used in Camping Tentage a) Std. Performance Requirements for Woven Man Made Fiber ol) in Wine (Enology) (1972)

tic) (1974)

in Wine (Enology) (1972)

mosphere and Plant Tissues (Manual Procedures) (1973)

particulate and Acidic Gaseous Fluoride in the Atmospher chromium MetaI (1971T)

Particulate and Water Soluble Gaseous Fluorides in the / Is (1971) ANSI Z260.3

on Wafers and Metallization Thickness by Multiple Beam I/

e and Gaseous Fluorides in the Atmosphere (Sodium Bicarb/ sion Zirconium in Irradiated Nuclear Fuels (1973) logy) (1972) 972)
Tensile Strength Carbon Steel Plates for Pressure Vesse Tensile Strength Carbon Steel Plates of Structural Qual

Tensile Strength of Adhesives by Means of Bar and Rod

Tensile Strength of Cylindrical Concrete Specimens (197

Tensile Strength of Molded Concrete Cylinders (1973)

Tensile Strength of Paraffin Wax (1973) ANSI Z11.206, T

Tensile Strength Properties of Steel Truss Plates (1973

Tensile Strength (1973)

Te Heat Resistant, $15.5 \mathrm{Cr}-4$

L S Studs, Heat Resistant, Nor

Tensile Strength, Hard Drawn, for Mechanical Springs (1

Tensile Strength, Hardened and Tempered, Roll Threaded

Tensile Strength, 70,000,000 (483) Tensile Modulus (197

Tensile Stress Strain of Carbons and Graphite (1973)

Tensile Test. Machine) (1971)

N Ngth of Woven Fabrics B

Tensile, Hard Drawn Mechanical Spring Wire and Spring

Tensile, 36,000,000 (248) Modulus, $180_{\times 2}$ (1973)

Tensile, 36,000,000 (248) Modulus. 350 (177) (1973)

Tension Head Hi Torque Recess Alloy Steel, 160,000 psi

Tension Head, Pull Type (1973)

Tension Head, Pull Type (1973)

Tension Loading at Elevated Temperatures (Metal to Meta

Tension Loading in the Temperature Range from -267.8 to

Tension Loading of Laminated Assemblies (1973)

Tension Loading (Metal to Metal) (1969)

Tension Loading (Metal to Metal) (1972) ANSI Z197.15

Tension Loading (Metal / Metal) (1973)

Tension Loading (1964) ANSI Z197.12

Tension Loading (1970)

Tension Loading (1971) A NSI Z197

Tension Loading (1973)

/R Strength Proper

Meth. for Strength Proper

1. Pract. for Dete

Determining the Strength of Ad

Tension Requirements for Prevailing Torque Type Steel H

Tension Test of Flat Sand wich Constructions in Flatwise

Tension Test. of Carbon Graphite Mechanical Materials (

Tension Test. of Hard Rubber (1972)

Tension Test, of High Strength Sheet Materials (1968) a

Tension Test. of Metallic Materials (1969) ANSl Z168.13

Tension Test. of Nonmetallic Gasket Materials (1972)

Tension Test. of Solid Urethane and Other Rubbers (1973

Tension Test. Wrought and Cast Aluminum and Magnesium a

Tension, Protruding Head, Std. and Oversize, Pull Type,

Tension. Protruding Head, Stump Type. Titanium Alloy (1

Tension, Pull Type (1973)

Tension. Pull Type (1973

Tension, Pul! Type (1973

Tension, Pull Type (1973)

Tension, Pull Type (1973)

Tension, Pull Type (1973)

Tension, Pull Type (1973

Tension, Pull Type (1973)

Tension, Pull Type (1973)

Tension, Pull Type (1973)

Tension, Pull Type (1973)

Tension, Pull Type (1973)

Tension, Stump Type (1973)

Tension, Stump Type (1973)

Tension, Stump Type (1973)

Tension, Stump Type (1973)

Tension, Stump Type (1973)

Tension, Stump Type, (1973)

Tension, 100 Deg. Crown Head, Std. and Oversize, Pull T

Tension, 100 Deg. Crown Head, Stump Type, Titanium Allo

Tension, 100 Deg. Head (MS20426), Std. and Oversize, Pu

Tension, 100 Deg. Head (MS20426), Stump Type, Titanium

Tension, 100 Deg. Head (MS24694), Pull Type, Titanium a

Tension, 100 Deg. Head (MS24694). Stump Type, Titanium

Tensioned Box Girder Bridges Design and Construction) (

Tensioned Prestressed Concrete (Post Tensioned Box Gird

(Tent) (1974)

Tent. Awning, and Canopy Fabrics, Painted, Printed, or

Tent, Awning, and Canopy Fabrics, Vinyl Coated or Lamin

Tent. Ebulliometer Meth. for Analysis of Alcohol (Ethan

Tent. Hazard Classifications of Organic Peroxides (Plas

Tent. Hydrometer Meth. of Analysis for Alcohol (Ethanol

Tent. Meth. for Analysis for Fluoride Content of the at

Tent. Meth. for Automated Separation and Collection of

Tent. Meth. for Chemical Analysis of Ferrochromium and

Tent. Meth. for Continuous Separation and Collection of

Tent. Meth. for Drop Weight Tear Tests of Ferritic Stee

Tent. Meth. for Grading Soybean Oil for Color (1973)

Tent. Meth. for Measurement of Oxide Thickness on Silic

Tent. Meth. for Separation and Collection of Particulat

Tent. Meth. for Spectrophotometric Determination of Fis

Tent. Meth. of Analysis for Brix (Balling) of Wine (Eno

Tent. Meth. of Analysis for Copper in Wine (Enology) (1
ASTM

ASTM

Meth ASTM

td ASTM

ASTM

ASTM

ASTM

ASTV

ASTM

AST

ASTM

ASTM

ASTM

ASTM

ASTM

ASTM

ASTM

ASTM

NSA

NSA

NSA

NSA

Si NSA

Std F VA

Std. Fo NSA

Std. Fo NSA

std. for P NSA

td. for Pi NSA

Sid for Pi NSA

in, Swage NSA

ge L NSA

St NSA

St NSA

Std NSA

td. for P NSA

for $\mathrm{Pi}$ NSA

NSA

NSA

NSA

NSA

NSA

NSA

CRSI

CPAI

ANSI

ANSI

ASE

FMS

ASE

ASTM

ASTM

ASTM

ASTM

NSPA

ASTM

ASTM

ASTM

ASE

ASE

A 285

A 283

D209.

C496

LBC $\$ 26-12$

D1320

E489

AMS5744A

AMS7458C

A679

A MS7459B

AMS3892/5

C749

D2262

U.M.528

AMS3894/8

A.M $\$ 3894 / 9$

583

7004

7024

D2295

D2557 
osphere and Plant Tissues (Semiautomated Meth.) (1973) (Enology) (1972)

2)

e (Enology) (1972)

ogy) (1972)

of Paint Films by Fungal Growth or Soil and Dirt Accumu/

d Surfaces Using a Passenger Automobile Equipped with Fu/ r Rubbers (1973)

rations on Returnable Beer and Beverage Glass Containers ncrete by the Pressure Meth. (1972T) ANSl A37.70

oil Aromatic Fractions by Mass Spectrometry (1973)

etroleum Fractions by Gas Chromatography (1973)

extile Fibers in Loop or Knot Configurations (1973)

ral Group Analysis of Petroleum Oils by the $n$-d-M Meth.

re (Continuous Measurement by Nondispersive lnfrared Spe/ of Graphite (1971) ANSI K90.11

for Removing Water Formed Deposits (1973)

germanium by Preferential Etch Techniques (1973)

of Neodymium Doped Laser Materials (1973)

Fuels by Setaflash Closed Tester (1973)

of Adhesives (1973)

pearance $(1973)$

retardant Wood and Its Base Products (1973)

ores $(1972 \mathrm{~T})$

th in Silicon by Measurement of Steady State Surface Pho/ Hide (Leather) Substance (1970) ALCA B5

oils (1973)

Ratings Using on Line Analyzers (1970T)

the Surface of Interior Coatings in an Environmental Ch/ ic Films with a Collinear Four Probe Array (1973)

ation Microcoulometry (1973)

rium Chloranilate Controlled Condensation Meth.) (1973)

turbine Fuels (JFTOT Proc.) (1973)

ne Fuel (1973)

urbine Fuels (1973)

and Plate Viscometer (1973)

gles (1972T)

Plane of Plies) (1969)

ness (1969)

) in Wine (Enology) (1972)

ed Tendons (1966)

irders for Building (1957)

arments (1973)

paints into a Gas Chromatograph (1973)

flexible Barrier Materials (1973)

of Light Frame Type for the Purpose of Conserving Their / al Coefficient (1973)

sing Threshold of Ruby Laser Rods (1973)

rom Solvent Base Paints (1973)

cation (1972)

or Underground Pipe Lines (Wrapped Systems) (1972)

d Continuous Mixing (1973)

ers Vertically (1973T) ANS1 A37.95

tubes (1973)

3)

\section{(1972)}

eparing Pentachlorophenol Solutions (1968)

imens Subject to Freezing (1972T)

ound Timber Piles (1970)

cord Fabrics (1973) ANSI L14.243

a Spec. for Flame Resistant Materials Used in Camping

Uniform Fire Code:

-(Tertbutylamino)-4-Chloro-6-(Ethyla mino).S-Triazine

tbutylamino).4-(Ethylamino)-6-(Methylthio)-S-Triazine .

Std. Definition of the

Std. for

Std. for

std. for Pan Head Screw and Assembled Washers-Electrical

Std. for Strip-High Temperature

Std. for Binding Post.

on Equip/ Std. for Start-Stop Signal Quality Between Data chemicals and Plastics (1972)

Std. for Safety Requirements for Marine

nal Spec., and Ratings; Assembly Numbers, Cell Layouts and

d. for Determination of Solderability of Solid Lead Wires.

of Solderability of Solid Lead Wires, Terminals, and Other ool Relationships (1972) nt (1972)

ors (1972) SAE J7/

Std for Statistical

Std. for Power Takeoff Definitions and

Rec. Pract. for Power Off Definitions and Std. for
Tent. Meth. of Analysis for Fluoride Content of the Atm Tent. Meth. of Analysis for Free Sulfur Dioxide in Wine Tent. Meth. of Analysis for lron in Wine (Enology) (197 Tent. Meth. of Analysis for Total Sulfur Dioxide in W in Tent. Meth. of Analysis for Volatile Acid in Wine (Enol Tent. Meth. of Evaluating Deg. of Surface Disfigurement Tent. Meth. of Measurement of Stopping Distance on Pave Tent. Meth. of Tension Test. of Solid Urethane and Othe Tent. Meth. of Test for Acid Resistance of Ceramic Deco Tent. Meth. of Test for Air Content of Freshly Mixed Co Tent. Meth. of Test for Aromatic Types Analysis of Gas Tent. Meth. of Test for Boiling Range Distribution of $\mathrm{P}$ Tent. Meth. of Test for Breaking Tenacity of Man Made T Tent. Meth. of Test for Carbon Distribution and Structu Tent. Meth. of Test for Carbon Monoxide in the Atmosphe Tent. Meth. of Test for Compressive (Crushing) Strength Tent. Meth. of Test for Corrosivity of Solvent Systems Tent. Meth. of Test for Crystallographic Perfection of Tent. Meth. of Test for Effective Fluorescent Lifetime Tent. Meth. of Test for Flash Point of Aviation Turbine Tent. Meth. of Test for Floating Roller Peel Resistance Tent. Meth. of Test for Grading Cotton Card Webs for Ap Tent. Meth. of Test for Hygroscopic Properties of Fire Tent. Meth. of Test for Manganese Dioxide in Manganese Tent. Meth. of Test for Minority Carrier Diffusion Leng Tent. Meth. of Test for Nitrogen Content (Kjeldahl) and Tent. Meth. of Test for Pumpability of Industrial Fuel Tent. Meth. of Test for Research and Motor Meth. Octane Tent. Meth. of Test for Resistance to Growth of Mold on Tent. Meth. of 'Test for Sheet Resistance of Thin Metall Tent. Meth. of Test for Sulfur in Petroleum Gas by Oxid Tent. Meth. of Test for Sulfur Oxides in Flue Gases ( $\mathrm{Ba}$ Tent. Meth. of Test for Thermal Oxidation Stability of Tent. Meth. of Test for Total Acidity in Aviation Turbi Tent. Meth. of Test for Undissolved Water in Aviation T Tent. Meth. of Test for Viscosity of Asphalt with Cone Tent. Meth. of Test for Wind Resistance of Asphalt Shin Tent. Meth. of Test. Plywood in Rolling Shear (Shear in Tent. Meth. of Test. Plywood in Shear Through the Thick Tent. Pycnometer Meth. of Analysis for A lcohol (Ethanol Tent. Rec. for Concrete Members Prestressed with Unbond Tent. Rec. for Design of Concrete Composite Beams and G Tent. Rec. Pract. for Conducting Wear Test on Textile G Tent. Rec. Pract. for Direct Injection of Solvent Base Tent. Rec. Pract. for Electrostatic Charge Mobility on Tent. Rec. Pract. for Installation of Fixed Partitions Tent. Rec. Pract. for Measurement of Glass Stress Optic Tent. Rec. Pract. for Measuring Slope Efficiency and La Tent. Rec. Pract, for Vacuum Distillation of Solvents F Tent. Rec. Pract. Guidelines for Driveway Design and Lo Tent. Rec, Spec, for Asphalt Type Protective Coatings F Tent. Spec. for Aniline-62 (1973)

Tent. Spec. for Concrete Made by Volumetric Batching an Tent. Spec. for Molds for Forming Concrete Tests Cylind Tent. Spec. for Molybdenum Flattened Wire for Electron Tent. Spec. for Nuclear Grade Boron Carbide Powder (197 Tent. Spec. for Polyolefin Monofilaments (1973)

Tent. Spec, for Ultra High Test Heat Treated Line Pipe Tent. Spec. for Volatile Petroleum Solvent (LPG) for Pr Tent. Test Meth. for Critical Dilation of Concrete Spec Tent. Test Meth. for Establishing Design Stresses for $\mathrm{R}$ Tent. Tolerances for Tire Fabrics (Textile) Other Than Tentage (Tent) (1974)

Tents and Air Supported Structures (1973) Ifor the Pes

Terbuthylazine (1973) Terbutryn (1973)

Term Vacuum Cleaner (1974) or the Pest Control Chemical 2-(Ter

Terminal-Tank Strap, Forked (1973)

Terminal-Tank Strap, Plain (1973)

Terminal Attachment (Preferred) (1974)

Terminal Block Electrical Stud (1974)

Terminal Block, Base Insulating (1973)

Terminal Board, Electrical (1973)

Terminal Equipment and Non-Synchronous Data Communicati

Terminal Equipment Employing Parallel Binary Data Inter

Terminal Facilities for Pneumatic Transfer of Dry Bulk

Terminal Markings for Motors and Generators (1972)

Terminal Operations (1972)

Terminals (For Foreign and Domestic Passenger Cars, Tru

Terminals, and Other Terminations (Soft Solder Jointed)

Terminations (Soft Solder Jointed) (1973) ANSI C83.93

Terminology and Definitions for Soil Tillage and Soil T

Terminology and Notation for Nuclear Materials Manageme

Terminology for Agricultural and Light Industrial Tract

Terminology for Agricultural Tractors (1972)

Terminology for Combines and Grain Harvesting (1971)
ASTM

ASE

ASE

ASE

ASE

ASTM

ASTM

ASTM

ASTM

ASTM

ASTM

ASTM

ASTM

ASTM

ASTM

ASTM

ASTM

ASTM

ASTM

ASTMI

ASTM

ASTM

ASTM

ASTM

ASTM

ASTM

ASTM

ASTM

ASTM

ASTM

ASTM

ASTM

ASTM

ASTM

ASTM

ASTM

ASTM

ASTM

ASTM

ASE

$\mathrm{ACl}$

ACl

ASTM

ASTM

ASTM

ASTM

ASTM
ASTM

ASTM

ITE

NACE

ASTM

ASTM

A STM

ASTM

ASTM

ASTM

API

ASTM

ASTM

ASTM

ASTM

CPAI

CPAI

ANSI

ASTM

NSA

NSA

NSA

NSA

NSA

NSA

EIA

EIA

MCA

NEMA

ANSI

BCI

EIA

EIA

ASAE

ANSl

ASAE

REC.

ASAE

D3216

E465

F391

D2868

D3245

D2885

D3273

F 390

D3246

D3226

D3241

D3242

D3240

D3205

D3161 
1972)

equipment Protection) (1972)

73)

Outage / Std, Definitions in Electrical Power Operations Rec. Pract. for Nomenclature and Std. for Psychoacoustical Western Woods Use Book: Abbreviations, Symbols, and Rec. Pract. for Automotive Transmission Rec Pract for Automatic Transmission Function ract. for Automotive Transmission Hydraulic Control System Std. for Pallet Definitions and

n lnterchange Formats (Computer Software Documentation and evices (1972) or (1972)

extension, Torsion, Flat, and Hot Wound (Materials, Specs. Aircraft lnstrument Stds. (Wording. Std. for lndustrial Engineering Std. for lndustrial Engineering Std. for Industrial Engineering Std. for Industrial Engineering Std. for lndustrial Engineering Std. for Industrial Engineering Std. for Industrial Engineering Std. for Industrial Engineering Std. for lndustrial Engineering Std. for Industrial Engineering Std. for Industrial Engineering Std. for Industrial Engineering oratory Evaluation to Determine Resistance to Subterranean imming Potentiometers (1970)

Practices for the Constructio 72)

Food Labelling, Ingredients and Containers (Also Includes

72A) ANSl Z128.7

yclean) (1973) tions in Electrical Puwer Operations Terminology lncluding consumer Textile Products and Rec. Pract. for Use of These of a Property of a Mater/ tions and Materials (1973)

138.1

(1972) ANSl A 130.1

73)

Is for Roofing, Waterproofing, and R/

$\mathrm{s}$ and Images Made Therefrom (1972A) Rec. Pract. for Use of These Terms/ I972) ANSI K64.6

g Appliances (1972)

si A 37.76

es (1974)

mpingement by Liquid or Solid Partic/ lysis of Fatigue Data (1972) ANSI Z9/ nd Materials (1973)

si Z166. 16

nents of Coal (1972)

nsi Z166. 15

2)

A 37.33

72)

products (1973) td. Definitions of Std. Definitions of Trade Std. for Glossary of Std Definitions of Std. Definitions of Textile Std. Rec. Pract, for Use of the Std. Definitions of Std. Definitions of Std. Definitions of Std. Definitions of Std. Definitions of Std. Definitions of Std. Definitions of Std. Definitions of Std. Definitions o Std. Definitions of Std. Definitions of Std. Glossary of Std. Definitions of Std. Definitions of Std. Definitions of Std. Definitions of Std. Definitions of Std. Definitions of Std. Definitions of Std. Definitions of Std. Definitions of Std. Definitions of Std. Definitions of Std. Definitions of Std. Definitions of Std. Definitions of Std. Definitions of Std. Definitions of Std. Definitions of Std. Definitions of Std. Definitions of Std. Definitions of Std. Definitions of Std. Definitions of Std. Definitions of Std. Definitions of Std. Definitions of Std. Definitions of Std. Definitions of
Terminology for Defects in Steel Pipe (1974)

Terminology for Permanently Installed Screw Conveyors (

Terminology for Pressure Relief Devices (Container and

Terminology for Relays for Electronic Equipment (1971)

Terminology for Rural Waste Management and Disposal (19

Terminology for Specialty Transformers

Terminology Including Terms for Reporting and Analyzing

Terminology of Tank Trailers and Containers (1971)

Terminology (Acoustics, Audiometry) (1973)

Terminology (Lumber and Timber) (1973)

Terminology (1969)

Terminology (1969)

Terminology (1970)

Terminology (1972)

Terminology) (1972) FIPS 20

Terminology, and Test Procedure for Neutral Grounding

Terminology, Gauge, Metric Conversions) (1972)

Terminology, Phraseology, Environment and Design Stds F

Terminology: Applied Mathematics (1972)

Terminology: Applied Psychology (1972)

Terminology: Biomechanics (1972)

Terminology: Cost (1972)

Terminology: Data Processing and Systems (1972)

Terminology: Distribution and Marketing (1972)

Terminology: Economy (1972)

Terminology: Facility Planning (1972)

Terminology: Materials Processing (1972)

Terminology: Organization Planning and Theory (1972)

Terminology: Production Planning and Control (1972)

Terminology: Work Measurement and Meth. (1972)

Std. Meth for Lab

Terms and Definitions for Welding and Cutting (1969)

Terms and Definitions for Wirewound and Nonwirewound TR

Terms and Definitions Related to Quality Control (1969)

Terms and Definitions (1970)

Terms and Definitions (1973)

Terms and Definitions) (1974)

ITd. for Quality Assuranc

Terms and Nomenclature Relating to Aerosol Products (19

Terms and Symbols Relating to Emission Spectroscopy (19

Terms Commonly Used in Packaging (1972)

Terms Concerning Letter Symbols (1972)

Terms for Antennas (1973)

Terms for Care Labeling Textile Products (Launder or Dr

Terms for Institutional Textiles (1973)

Terms for Reporting and Analyzing Outage of Electrical

Terms on Permanently Attached Labels (1972)

Terms Precision and Accuracy as Applied to Measurement

Terms Relating to Acoustical Tests of Building Construc

Terms Relating to Activated Carbon (1974)

Terms Relating to Adhesives (1973)

Terms Relating to Appearance of Materials (1971) ANSI Z

Terms Relating to Asbestos Cement and Related Products

Terms Relating to Atmospheric Sampling and Analysis (19

Terms Relating to Bituminous, and Other Organic Materia

Terms Relating to Building Seals (1973)

Terms Relating to Carbon Paper and Inked Ribbon Product

Terms Relating to Care of Consumer Textile Products and

Terms Relating to Cellulose and Cellulose Derivatives (

Terms Relating to Chimneys, Gas Vents and Heat Producin

Terms Relating to Coal and Coke (1972) ANSl K20.6

Terms Relating to Concrete and Its Aggregates (1968) an

Terms Relating to Dosimetry (1968) ANSI N10.5

Terms Relating to Electrical Insulating Liquids and Gas

Terms Relating to Electrical Insulation (1973)

Terms Relating to Electrostatic Copying (1972B)

Terms Relating to Erosion of Solids by Cavitation and I

Terms Relating to Fatigue Test. and the Statistical Ana

Terms Relating to Fire Tests of Building Construction a

Terms Relating to Gaskets (1972)

Terms Relating to Glass and Glass Products (1971)

Terms Relating to Hydraulic Cement (1971) ANSI A1.28

Terms Relating to lron Castings (1972A)

Terms Relating to Leak Test. (1971) ANSl Z166.25

Terms Relating to Leather (R1972) ALCA X1, ANSI Z109.3

Terms Relating to Lime and Limestone (I971) ANSl K67.8

Terms Relating to Liquid Penetrant Inspection (1974) an

Terms Relating to Lithologic Classes and Physical Compo

Terms Relating to Magnetic Particle Inspection (1974) a

Terms Relating to Manufactured Carbon and Graphite (197

Terms Relating to Manufactured Carbon and Craphite (197 ASTM

Terms Relating to Metallography (1973) ANSI Z30.4

Terms Relating to Microscopy (1972)

Terms Relating to Natural Building Stones (1974)

Terms Relating to Nayal Stores and Related Products (19

Terms Relating to Paint, Varnish, Lacquer, and Related

Terms Relating to Plastics (1973) ANSI K65.51
API

ASAE

ANSl

ANSl

ASAE R292.

346

ANSI $\quad \$ 3.20$

WWPA $* 31.6$

ANSI

ANSI

IEEE

SMI

SAE

ASl

ANS1

ANSI

is

ANSI

ANSl

ANSI

ANSl

ANSI

WP

$A W S$
$Y R C$

ASOC

AWS

ANSI

AAFCO

ASTM

ASTM

ANSI

ANSI

IEEE

A NSI

ANSl

IEEE

Care of ASTM

ASTM

ASTM

ASTM

ASTM

ASTM

ASTM

ASTM

ASTM

ASTM

ASTM

ASTM

ASTM

NFPA

ASTM

ASTM

A STM

ASTM

ASTM

ASTM

ASTM

ASTM

ASTM

ASTM

ASTM

ASTM

ASTM

ASTM

ASTM

ASTM

ASTM

ASTM

ASTM

ASTM

ASTM

ASTM

ASTY

ASTM

ASTM

UL5T1

$334 \mathrm{~T}$

3. 16

*31.6

J649B

J648A 
n, Truck, Bus, Trailer, Multipurpose Pas/
Std. Definitions of Sid. Definitions of Std. Definitions of Std. Definitions of Std. Definitions of Std. Definitions of Glossary of Std. Definitions of Std. Definitions of Sid. Definitions of Std. Definitions of Std. Definitions of Std. Definitions of Std. Definitions of Std. Definitions of Glossary of

Definitions of Statistical Glossary of Definitions of Std. Definitions of Std. Glossary of Genera Dictionary of Definitions and Std.

Glossary of Construction Industry Definitions of Relay anual of Standardization for Masterkeying Nomenclature and Glossary of Billiard

Std. Definitions of Navigation Aid Glossary of Architectural Metal Std. for Photo Instrumentation Glossary Spec, for Synthetic Rubber, Ethylene Propylene Limestone, and Marble Masonry; Mortar; Sand; Slag; Steel Rec. for Design, Layout, Construction and Maintenance of Brick in Landscape Architecture Specifying Portland Cement

extile) (1973) Std. Performance Requirements for

Terb/ Std. Common Name for the Pest Control Chemical 2zine / Std. Common Name for the Pest Control Chemical 2Std. Spec for Para ination of Film Adhesion on All Metals by Cross Hatch Tape Std. for Engine

\section{Std.}

Std.

ormers (Electronics) (1973) ANSI C57.12.90 Std. for Agricultural Tracto A gricultural Tractor Std. Analytical Meth. for Determining Cold eth. of Power Factor Measurement for Low Voltage Inductive Guide for Statistical Analysis of Thermal Lif ration of Liquid Automatic Particle Counters L'sing AC Fine r Conditioning System Rating Definitions, Test. Meth., and of Petroleum Wax or Wax Based Blends Appli/ faces (1974)

Room Temperature (1972)

les and Heels (1968) ANSI J2.26 Std. Meth. o Std. Meth. of Std. Meth. of Std. Meth. of Std. Meth. of Std. Meth. of Std. Meth. of Std. Meth. of Std. Meth. of Std. Meth. of Std. Meth. of Std. Meth. of Meth. of

h the Ferrous Sulfate Cupric Sulfate Dosim/ $h$ the Ceric Sulfate Dosimeter (1971) ANSI oxygen Pressure Meth. (1973) ANSI J4.1 oven Meth. (1967) ANSI J5.1 mpound (1973) itectural Type Glass (1972T) rnable Beer and Beverage Glass Containers/ ents and Their Admixtures (1974) naphthas, and Similar Industrial Aromatic / es (1973)

lants Under Cyclic Movement (1972)

ard at 90 Deg. Angle and Constant Stress (/

$973)$

(1970) ANSI J3.1

x Sealing Compounds (1972) 37.70

ressure Meth. (1972T) ANSI A37.70

73)

ansi $\mathrm{Z} 167.20$

ielectric Constant (Permittivity) of Solid/ ectroscopy (1973)

Std. Meth. o

Tent. Meth. of

Std. Meth. of

Meth. of
Terms Relating to Porcelain Enamel and Ceramic Metal Sy Terms Relating to Refractories (1973) ANSI All1.9

Terms Relating to Rubber and Rubber Like Materials (197 Terms Relating to Soaps and Other Detergents (1974)

Terms Relating to Space Simulation (1972)

Terms Relating to Statistical Meth. (1972)

Terms Relating to Structural Clay Products (Tech. Notes Terms Relating to Surface Imperfections on Ceramics (19 Terms Relating to Textile Materials (1973) ANSI L14.12

Terms Relating to Thermal Analysis (1973)

Terms Relating to Ultrasonic Test. (1974)

Terms Relating to Uninsulated Metallic Electrical Condu

Terms Relating to Veneer and Plywood (1970) ANSI 07.2

Terms Relating to Water (1972)

Terms Relating to Wax Polishes and Related Materials (1

Terms Used in Baking and Cereal Chemistry (1962)

Terms Used in Cereal Chemistry (1967)

Terms Used in Gearing (1972)

Terms Used in IEEE Nuclear Power Generating Station Std

Terms Used in Powder Metallurgy (1972) ANSI H9.5

Terms Used in Ouality Control (1971) ANSI Z1.7

Terms Used in the Safety Profession (1973)

Terms Used in the Selection of Tires (Car, Station Wago

Terms Used in the Sulfite Pulping Process (1972)

Terms (Architecture) (1970)

Terms (Electromagnetic Instrumentation) (1972)

Terms (1965)

Terms $(1971)$

Terms (1971)

Terms (1973)

(Terms, Definitions) (1969)

Terpolymer, General Purpose (45-55) (1973)

Terra Cotta; Timber; Water; Ceiling; Partitions; Plaste

Terrace (Erosion Control and Water Conservation) (1972)

Terraces and Walks (Tech. Notes) (1966)

Terrazzo, Cast in Place Floors (1967)

Terry Cloth Towels (Single and Double) (Institutional T

(Tertbutyla mino)-4-Chloro-6-(Ethylamino)-S-Triazine-

(Tertbutyla mino)-4-(Ethyla mino)-6-(Methylthio)-S-Tria

Tertiary Butylphenol 98 (1972) ANSI Z78.24

Test After Reverse Impacting (Coil Coating) (1974)

Test Code-Spark Ignition and Diesel (1971)

Test Code for Air Moving Devices (1967)

Test Code for Direct Current Machines (1973)

Test Code for Distribution, Power and Regulating Transf

Test Code (1972)

Test Code (1972) SAE J708C

Test Content in Corn Oil (1965)

Tesi Currents (1972) ANSI C37.26

Test Data (1972)

Test Dust (1972) ANSI B93.28

Test Equipment and Rating Meth. (1972)

Test for Abrasion Resistance of Glossy Smooth Coatings
Test for Abrasion Resistance of Horizontal Concrete Sur

Test for Abrasion Resistance of Pipeline Coatings (1972

Test for Abrasion Resistance of Refractory Materials at

Test for Abrasion Resistance of Rubber Compounds for So

Test for Abrasion Resistance of Textile Fabrics (1971)

Test for A bsolute Viscosity of Asphalts (1966)

Test for Absorbed Gamma and Electron Radiation Dose Wit

Test for Absorbed Gamma and Electron Radiation Dose Wit

Test for Accelerated Aging of Vulcanized Rubber by the

Test for Accelerated Aging of Vulcanized Rubber by the

Test for Accelerated Aging of Wood Sash Face Glazing Co

Test for Acid Resistance of Ceramic Decorations on Arch

Tent. Meth. of Test for Acid Resistance of Ceramic Decorations on Retu

Std. Meth. of Test for Acidity Alkalinity of Halogenated Organic Solv

Std. Meth. of Test for A cidity of Benzene, Toluene, Xylenes, Solvent

Std. Meth. of Test for Adhesion After Impact of Preformed Sealing Tap

std. Meth. of Test for Adhesion and Cohesion of Elastomeric Joint Sea

Std. Meth. of Test for Adhesion of Metallic Coatings (1972)

Std. Meth. of Test for Adhesion of Pressure Sensitive Tape to Fiberbo

Std. Meth. of Test for Adhesion of Vulcanized Rubber to Metal (1973)

Std. Meth. of Test for Adhesion of Vulcanized Rubber to Steel Cord (1

Std. Meth. of Test for Adhesion of Vulcanized Rubber (Friction Test)

Test for Adhesives (1962) ANSI Z197.23

Test for Aging Effects of Artificial Weathering on Late

Test for A ir Content of Freshly Mixed Concrete by the P

Test for A ir Permeability of Asbestos Fibers (1972)

Test for Airflow Resistance of Acoustical Materials (19

Test for Algal Resistance of Plastic Films (1972T)

Std. Meth. of Test for Alkali Resistance of Porcelain Enamels (1974)

Std. Meth. of Test for Alkalinity of Hollow Glass Microspheres (1972)

Std. Meth. of Test for Alky! Benzene Sulfonate in Water (1968)

Std. Meth. of Test for Alternating Current Loss Characteristics and D

Std. Meth. of Test for Aluminium in Iron Ores by Atomic Absorption Sp
ASTM

ASTM

ASTM

ASTM

ASTM

ASTM

BIA

ASTM

ASTM

ASTM

ASTM

ASTM

ASTM

ASTM

ASTM

$\mathrm{AACCH} 36.01$

AGMA 116.01

IEEE 380

ASTM B243

ASQC A3

$\begin{array}{ll}\text { ASSFE } & * 1 \\ \text { TRA } & X . X I\end{array}$

TAPPI T1201

AIOA *1

NARM *2

NBHA *4

IEEE $\quad 172$

NAAMM $* 35$

SMPTE *1

SAE AMS3260

ICBO UBC $* 3.23$

ASAE R268.1

BIA 29

CSl 09410

ANSI L24.2.5

ANSI K62.149

ANSI K62.148

ASTM D2871

$/ \mathrm{Rm}$ NCCA TB.II-16

SAE J816B

AMCA 210

IEEE $\quad 113$

IEEE 262

SAE J708C

ASAE S209.4

CR

H. 10

IEEE $\quad 330$

IEEE 101

NFLDP T2.9.6

MACA 400

ASTM D3234

ASTM $\quad$ C70

$\begin{array}{ll}\text { ASTM } & \text { C704 } \\ \text { ASTM } & \text { D1630 }\end{array}$

ASTM D1175

ASTM D217]

ASTM D2954

D3001

ASTM D572

STM D573

ASTM C741

ASTM C724

ASTM C735

ASTM D2989

ASTM D847

ASTM C766

ASTM C719

ASTM B571

ASTM D2860

ASTM D429

ASTM D2229

ASTM D413

ASTM D1781

ASTM C 732

C231

ASTM D2752

ASTM C522

ASTM G29

A 614

ASTM D3100

ASTM D2330

ASTM E507 
ging Materials (Cereal Chemistry) (1/ eam Bending (1972)

iber Elongation (1971)

arbons (1972)

h9.1

ounds by the Scott Volumeter (1970) ANSI H/

Fiber Reinforced Plastics by Short Beam M/ specific Gravity, and Bulk Density of Burn/ tures Using the Brookfield Viscometer (197/ oating Materials (1973)

3) ANSI Z11.72

ature Using the Cold Spanking Simulator (1/

Solids (1969) ANSI Z197.9

e (1951) ANSI Z197.10

s by Gas Chromatography (1972) ANSI Z11.27/

actions by Mass Spectrometry (1973)

(1972) ANSI L 14.141

973)

fueI (Míass Spectrometric Meth.) (1969) Ans/ fuel (Neodymium-148 Meth.) (1969) ANSI N11/ mical Meth.) (1969) ANSI N107

ansi $\mathrm{A} 1.8, \mathrm{~A}$ shto $\mathrm{T} 107$

Air Permeability (1972)

fiber (1970) ANSI K91.1 Footings (1972) Std. Meth. of Test Fo/

sho T81, ANSI 011.13

of Ceramic Substrates (1974)

i D14.1

ons by Gas Chromatography (1973)

rns (Constant Rate of Extension Instrument/ tile Fibers (Flat Bundle Meth.) (1972) Ans/ al73.1

n Loop or Knot Configurations (1973)

ansi $Z 115.5$

mers by Impact ( 1973

ometric Titration (1974) ANSI Z78.15

ctrometric Titration (1972) ANSI Z11.248

e Type Sealants (1972)

s Microspheres (1972)

Gas Chromatography (1973)

bsorption Spectroscopy (1973)

(1973)

pressure Membrane Apparatus (1972)

coal and Coke (1973)

sis of Petroleum Oils by the n-d-M Meth. measurement by Nondispersive Infrared Spe/ )

ansi A37.58, Ashto T144

rocessing Oils by the Clay Gel Absorption /

ation) (1972)

onds (1972) ANSI Z197.16

ts (1972) ANSI K 42.1

ciple (1972)

es of Dry Solid Film Lubricants in Vacuum /

gs (1973) ANSI Z11.222

nd Solid Fiberboard (Horizontal Plane Meth/ nd Solid Fiberboard (Inclined Plane Meth.)/

son) (1973) ANSI Al49.12

y Mortar (Wet Type) (1972) ANSI All1.29

of Refractory Brick and Shapes (1972) Ansi/

er Cotton Colorimeter (1971)

(Textile) (1973)

textile) (1973)

he Field (1973)

Made Staple Fiber (1971)

(1972) ANSI Z11.315

(Reaction Intensity Meth.) (1973)

e Plastics Under Compression (1974)
Std. Meth. of Test for Aluminum in Water (1969E)

Std. Meth. of Test for Aluminum Oxide in Iron Ores (1972)

Meth. of Xanthydrol Test for Analysis of Urea in Cloth Bags and Other Packa Std. Meth. of Test for Annealing Point and Strain Point of Glass by B Std. Meth. of Test for Annealing Point and Strain Point of Glass by $F$ Std. Meth. of Test for Apparent Density of Industrial A romatic Hydroc Std. Meth. of Test for Apparent Density of Leather (1968) ALCA E62 Std. Meth. of Test for Apparent Density of Metal Powders (1948) ANSI Std. Meth. of Test for Apparent Density of Refractory Metals and Comp Std. Meth. of Test for Apparent Horizontal Shear Strength of Parallel Std. Meth. of Test for Apparent Porosity, Water Absorption, Apparent Std. Meth. of Test for Apparent Viscosity of Gear Oils at Low Tempera Std. Meth. of Test for A pparent Viscosity of Hot Melt Adhesives and C Std. Meth. of Test for Apparent Viscosity of Lubricating Greases (197

Std. Meth. of Test for A pparent Viscosity of Motor Oils at Low Temper Std. Meth. of Test for Applied Weight Per Unit Area of Dried Adhesive Std. Meth. of Test for Applied Weight Per Unit Area of Liquid Adhesiv Std. Meth. of Test for Aromatic Traces in Light Saturated Hydrocarbon

Tent. Meth. of Test for Aromatic Types Analysis of Gas Oil Aromatic Fr Std. Meth. of Test for Asbestos Content of Asbestos Textile Materials Std. Meth. of Test for Ash from Petroleum Products (1973) ANSI Z11.54 Std. Meth. of Test for Ash in Graphite (1969) ANSI K90.4

Std. Meth. of Test for Ash in the Analysis Sample of Coal and Coke (1 Std. Meth. of Test for Atom Percent Fission in Uranium and Plutonium Std. Meth. of Test for Atom Percent Fission in Uranium and Plutonium Std. Meth, of Test for Atom Percent Fission in Uranium Fuel (Radioche Std. Meth. of Test for Autoclave Expansion of Portland Cement (1971)

Rec. Pract. for Life Test for Automotive Storage Batteries (1972)

Std. Meth. of Test for Average Particle Size of Alumina and Silica by Test for Bags (Packaging) (1950) ANSI MH12.6

Test for Bauer-McNett Wet Classification of Asbestos Test for Bearing Capacity of Soil for Static Load on Sp Test for Bearing Capacity of Soil for Static Load on $\mathrm{Sp}$

Std. Meth. of Test for Bendability of Pipeline Coatings (1972)
Std. Meth. of Test for Benzene Insoluble Matter in Creosote (1973) Aa Std. Meth. of Test for Benzene Insoluble Matter in Creosote (1973) Aa
tative Meth. of Test for Biaxial Flexure Strength (Modulus of Rupture) Sid. Meth of Test for Bitumen (1970) ANSI A37.3

Std. Meth. of Test for Boiling Point of Engine Antifreezes (1972) Ans

Tent. Meth. of Test for Boiling Range Distribution of Petroleum Fracti Meth. of Test for Boron in Water (1972T)

Std. Meth. of Test for Breaking Load and Elongatoin of Elastomeric Ya Std. Meth. of Test for Breaking Strength and Elongation of Cotton Tex Std. Meth. of Test for Breaking Strength of Ceramic Tile (1971) ANSI

Tent. Meth. of Test for Breaking Tenacity of Man Made Textile Fibers I

Std. Meth. of Test for Brinell Hardness of Metallic Materials (1973)

Std. Meth. of Test for Brittleness Temperature of Plastics and Elasto

Std. Meth. of Test for Bromine Index of Aromatic Hydrocarbons by Coul

Std. Meth. of Test for Bromine Index of Petroleum Hydrocarbons by Ele

Std. Meth. of Test for Bubbling of 1 Part, Elastomeric Solvent Releas

Std. Meth. of Test for Bulk Density and Packing Factor of Hollow Glas

Std. Meth. of Test for Butadiene Purity and Hydrocarbon Impurities by

Std. Meth. of Test for Calcium and Magnesium in Iron Ores by Atomic a

Std. Meth. of Test for Calcium or Zinc in Paint Driers by EDTA Meth.

Std. Meth. of Test for Capillary Moisture Relationships for Soils by

Std. Meth. of Test for Carbon and Hydrogen in the Analysis Sample of

Tent. Meth. of Test for Carbon Distribution and Structural Group Analy

Tent. Meth. of Test for Carbon Monoxide in the Atmosphere (Continuous

Std. Meth. of Test for Cashmere Coarse Hair Content in Cashmere (1971

Std. Meth. of Test for Cathodic Disbonding of Pipeline Coatings (1972

Std. Meth. of Test for Cement Content of Soil Cement Mixtures (1974)

Std. Meth. of Test for Change in Properties of Elastomeric ANSI J2.7

Std. Meth. of Test for Characteristic Groups in Rubber Extender and P

Std. Meth. of Test for Chip Resistance of Coatings (1973)

Std. Meth. of Test for Chromic Oxide in Leather (Perchloric Acid Oxid

Std. Meth. of Test for Cleanability of Surface Finishes (1973)

Std. Meth. of Test for Cleavage Strength of Metal to Metal Adhesive B

Std. Meth. of Test for Coarse Particles in Pigments, Pastes, and Pain

Std. Meth. of Test for Coating Thickness by the Beta Backscatter Prin

Std. Meth. of Test for Coating Thickness by X-Ray Spectrometry (1972)

Std. Meth. of Test for Coefficient of Friction and Wear Characteristi

Meth. of Test for Coefficient of Friction. Yarn to Metal (1972T)

Std. Meth. of Test for Coefficient of Kinetic Friction for Wax Coatin

Std. Meth. of Test for Coefficient of Static Friction of Corrugated a

Std. Meth. of Test for Coefficient of Static Friction of Corrugated a

Std. Meth. of Test for Coking Value of Tar and Pitch (Modified Conrad

Std. Meth. of Test for Cold Bonding Strength of Air Setting Refractor

Std. Meth. of Test for Cold Crushing Strength and Modulus of Rupture

Std. Meth. of Test for Color of Raw Cotton Using the Nickerson-Hunt

Meth. of Test for Colorfastness of Upholstery Fabrics to Solvent

Meth. of Test for Colorfasiness of Upholstery Fabrics to Water (

Std. Meth. of Test for Combustible Gases in Electrical Apparatus in T

Std. Meth. of Test for Commercial Weight of a Shipment of Yarn or Man

Std. Meth. of Test for Compatibility of Fuel Oil Blends by Spot Test

Std. Meth. of Test for Compatibility of Materials with Liquid Oxygen

Std. Meth. of Test for Compatibility of Plasticizers in Vinyl Chlorid
ASTM

ASTM

ASTM C598

ASTM C 336

ASTM D2935

ASTM D2346

ASTM B212

ASTM B329

ASTM D2344

ASTM C20

ASTM D2983

ASTM D3236

ASTM D1092

ASTM D2602

ASTM D 898

ASTM D899

ASTM D2600

ASTM D3239

ASTM D1918

ASTM D482

ASTM C561

ASTM D3174

ASTM E244

ASTM E32I

ASTM E219

ASTM C151

SAE J240A

ASTM D950

ASTM D2589

ASTM D1194

ASTM Dl194

ASTM G10

ASTM D367

ASTM F394

ASTM D4

ASTM D1120

ASTM D 3082

ASTM D2653

ASTM D1 445

ASTM C648

ASTM D3217

ASTM El0

ASTM D746

ASTM D1492

ASTM D2710

ASTM $\quad$ C712

ASTM D3101

ASTM D2593

ASTM E508

ASTM D2613

ASTM D3152

ASTM D3178

ASTM D 3238

ASTM D3162

ASTM D2816

ASTM

ASTM

ASTM

ASTM

ASTM

ASTM

ASTM

ASTM

ASTM

ASTM

ASTM

ASTM

ASTM

ASTM

ASTM

ASTM

ASTM

ASTM

ASTM

ASTM

ASTM

ANSI

ANSI

ASTM

ASTM

ASTM

ASTM

ASTM

D806

D471

D2007

D3170

C756
D 1062

D 185

B567

B568

D2716

33108

D2534

D 3247
D 3248

D 3038

D2416

C198

C 133

D2253

L24T7

L24T6

D3284

D2494

D2781

F371 
under Field Conditions (1974) ics (1973) ANSI K65.31

974) ANSI A37.103. Ashto T167 pecimens (1972) AASHO T22, A VSI A37. 18 rs (Using 2 ln. (50 Mm) Cube Specimens) (1/ rs (Using Portions of Prisms Broken in Fle/ 972)

971) ANSI K90.11 Tent. Meth of

petrolatum (1971) ANSI Z11.61 Uniform Building Code Std. for Meth. of ities in Deuterium Oxide (1968) ANSI N154

ility by Rotating Bomb (1967) ANS1 Z11.230 Std. Meth. of

ter Formed Deposits (1973)

nsfer (Electrical Meth. (1972)

Is (1972) ANSI L 14.95

eferential Etch Techniques (1973)

\section{0) ANSl MH12.7}

re by Gas Chromatrography (1972)

uels (1972) ANSl Z11.322

ral Load (1972) ANSl K65.222

1973)

e (1971) ANSl K90.8

d Powder Metal Structural Parts and Oil lm/ gham Pycnometer (1968) ANSI Z11.96

hite Articles by Physical Ileasurements (19/ lear Meth. (1974)

(1972T)

i 2111.5

cating Oils (1973)

73)

a Meth. (1973)

meth. (1973)

rations and Isotopic Abundances (1970) Ans/

croprojection (1972) ANSI Ll4.143

nsulating Gases at Commercial Power Freque/ $s$ of Petroleum Origin Using VDE Electrodes/ $s$ of Petroleum Origin Under lmpulse Condit expanded Cellular Plastics Used for Electr/ tion Factor of Solid Ceramic Dielectrics A/ ber, Yarn and Textile Fabric) (1972) ANSI / ation Stain of Organic Finishes in Contact/ sy Direct Soil Burial (1972)

n Black (1972A)

) Products (1973) AASHO T78, ANSI A37.45

i L14. 103

lded Containers (1970)

emperature Range (1972) AVSI 211.207

c Refractories (1972) ANSI A111.25

(1972)

by the Dow Corning Leather Tester (1970) /

by the Maeser Water Penetration Tester (1/

Pressure (1972)

orative Fabrics (Textile) (1973)

(Thin Film Oven Test) (1973)

Bonds (1972) A VSI Z197.18

f Aerosol Containers (1972)

ped Laser Materials (1973)

ems (1972)

Spray Insecticides Against Flying Insects/ cockroaches (1972)

ing Excessive Expansion of Concrete Due To/

e Water Repellent Finish of Fabrics (1972)

ings (1972)

ial Compression (1972)

ant Rate of Extension Machines) (1972) Ans/ 50

aining a Static Dissipator Additive (1971)/ Std. Rec. Pract. for Designing a High Current flooring (1972)

and Graphite Articles at Room Temperatures (Solder Plate) (1973)

973)
Std. Meth. of Test for Compressibility of Leather (1970) ALCA E45

Tentative Meth. of Test for Compressive and Flexural Strength of Concrete Std. Meth. of Test for Compressive Properties of Rigid Cellular Plast Std. Meth, of Test for Compressive Strength of Bituminous Mixtures (1 Std. Meth of Test for Compressive Strength of Cylindrical Concrete S Std. Meth. of Test for Compressive Strength of Hydraulic Cement Morta Std. Meth. of Test for Compressive Strength of Hydraulic Cement Morta Std. Meth. of Test for Compressive Strength of Masonry Assemblages (1 Test for Compressive (Crushing) Strength of Graphite (1 Test for Concrete Masonry Units (1973) Test for Congealing Point of Petroleum Waxes Including Test for Consumption of Potassium Permanganate by Impur Test for Continuity of Porcelain Enamel Coatings (1973) Test for Continuity of Steam Turbine Oil Oxidation Stab Test for Copper and lts Alloys (1973) ANSI H39.3

Test for Corrosivity of Solvent Systems for Removing Wa Test for Corrosivty of Water in the Absence of Heat Tra Test for Creep Relaxation of a Gasket Material (1971) Test for Cross Sectional Characteristics of Cotton Fibe Test for Crystallographic Perfection of Germanium by $\mathrm{Pr}$ Test for Cyanides in Water (1972)

Test for Cyclohexylamine in Water (1974)

Test for Cylindrical Shipping Containers (Packaging) (1

Test for $C_{1}$ Through $C_{5}$ Hydrocarbons in the Atmosphe Test for D.C. Electrical Conductivity of Hydrocarbons F Test for Deflection Temperature of Plastics Under Flexu Test for Degree of Set for Wood Sash Glazing Compound ( Test for Delivery Rate of Aerosol Products (1972) Test for Delta in Hours (DIH) Purity of Nuclear Graphit Test for Density and Interconnected Porosity of Sintere Test for Density and Specific Cravity of Liquids by Bin Test for Density in Air of Manufactured Carbon and Grap Test for Density of Bituminous Concrete in Place by Nuc Test for Density of Glass by Buoyancy (1971)

Test for Density of Glass by the Sink Float Comparator Test for Deposit Forming Impurities in Steam (1971) Ans Test for Detecting Glycol Base Antifreeze in Used Lubri Test for Detergent Resistance of Floor Polish Films (19 Test for Determination of Cobalt in Paint Driers by Edt Test for Determination of Lead in Paint Driers by EDTA Test for Determination of Uranium and Plutonium Concent Test for Deuterium Oxide (1972)

Test for Diameter of Wool and Other Animal Fibers by Mi Test for Dielectric Breakdown Voltage and Strength of 1 Test for Dielectric Breakdown Voltage of Insulating Oil Test for Dielectric Breakdown Voltage of lnsulating Oil Test for Dielectric Constant and Dissipation Factor of Test for Dielectric Constant (Permittivity) and Dissipa Test for Differential Dyeing Behavior of Cotton (Raw Fi Test for Diffusion Stain of Rubber and Contact and Migr Test for Disbonding Characteristics of Pipeline Coating Test for Discoloration of Orthodichlorobenzene by Carbo Test for Distillation of Cut Back Asphaltic (Bituminous

Std. Meth. of Test for Distillation of Road Tars (1972)
Std. Meth. of Test for Distortion of Yarn in Woven Fabrics (1972) Ans Std. Meth. of Test for Distortion of Yarn in Woven Fabrics (1972) Ans Std. Meth. of Test for Dropping Point of Lubricating Grease of Wide $T$ Std. Meth. of Test for Drying and Firing Shrinkage of Fireclay Plasti Std. Meth. of Test for Durability of Fire Retardant Treatment of Wood Std. Meth. of Test for Dynamic Water Resistance of Shoe Upper Leather Std. Meth. of Test for D ynamic Water Resistance of Shoe Upper Leather Std. Meth. of Test for Effect of Cyclic Immersion of Syntactic Foam a Meth. of Test for Effect of Handwashing on Woven Glass Fiber Dec Std. Meth. of Test for Effect of Heat and Air on Asphaltic Materials

Sid. Meth. of Test for Effect of Moisture and Temperature on Adhesive

Meth. of Test for Effective Crimping on Outside Crimped Valves O

Tent. Meth. of Test for Effective Fluorescent Lifetime of Neodymium Do

Std. Meth. of Test for Effective Pumping Speed of Vacuum Chamber Syst

Std. Meth. of Test for Effectiveness of Aerosol and Pressurized Space

Std. Meth. of Test for Effectiveness of Aerosol Insecticides Against

Std. Meth. of Test for Effectiveness of Mineral Admixtures in Prevent

Std. Meth. of Test for Effects of Drycleaning on Permanence of Durabl

Std. Meth. of Test for Effects of Outdoor Weathering on Pipeline Coat

Std. Meth. of Test for Efflorescence of Interior Wall Paints (1973)

Std. Meth. of Test for Elastic Moduli of Rock Core Specimens in Uniax

Std. Meth of Test for Elastic Properties of Elastomeric Yarns (Const

Std. Meth. of Test for Elastic Properties of Fibers (1972) ANSl L14.2

Std. Meth. of Test for Electrical Conductivity of Aviation Fuels Cont Arc Erosion Test for Electrical Contact Materials (1973)

Std. Meth of Test for Electrical Resistance of Conductive Resilient

Std. Meth. of Test for Electrical Resistivity of Manufactured Carbon

Std. Meth. Test for Electrodeposited Coatings of Tin-Lead Alloy

Std. Meth. of Test for Electrostatic Charge (1973)

Std. Meth. of Test for Embossed Depth of Resilient Floor Coverings (1

Std. Meth. of Test for Epoxy Content of Epoxy Resins (1973)
ASTM

ASTM

ASTM

ASTM

ASTM

ASTM

ASTM

ASTM

ASTM

ICBO

ASTM

ASTM

ASTM

ASTM

ASTN

ASTM

ASTM

ASTM

ASTM

ASTM

AST

ASTM

ASTM

ASTM

ASTM

ASTM

ASTM

ASTM

ASTM

ASTM

ASTM

ASTM

ASTM

ASTM

ASTM

ASTM

ASTM

ASTM

ASTM

AST I

ASTM

ASTM

ASTM

ASTMI

ASTM

ASTM

ASTM

ASTM

ASTM

AsT

ASTM

ASTM

ASTM

ASTM

ASTM

ASTM

ASTM

ASTM

ASTM

ASTM

ASTM

AST

ASTM

ANSl

ASTM

ASTMl

ASTM

ASTM

ASTM

ASTM

ASTM

ASTM

ASTM

ASTM

ASTM

ASTM

ASTM

ASTM

ASTM

AATM

ASTM

ASTM

ASTM

ASTM

ASTMI

ASTM

D 2213

C683

D 1621

1074

C109

C.349

E447

C695

UBC $524-7$

D938

C 743
D2272

B154

D3263

D2776

F38

F389

D2036

D2909

D997

D2820

D3114

D648

C 742

C624

B328

D 1217

C559

D2950

C693

C729

D2186

D3207

D2373

D2374

E267

D2184

D2130

D 1816

D3300

D1673

D2149

D1464

D925 
1972)

on Fibers by the Causticaire Method (1971)

with the Cenco Fitch Apparatus (1970) Alc/ aters (1968E)

er and Rubber Like Materials (1960) ANSI J/ eries Copper Containing Aluminum Alloys (E/ ace Alloys Under High Shear (1972) ANSI Z1/ ng Compound (1972)

ounds (1973)

ealing Compounds (1972) ls (1973)

72) ANSI Al.27

9 Activity from Uranium -238 Fission (1972)

nsion Loading (Metal / Metal) (1973)

1973)

) Sieve (1973)

y Apparatus (1973) AASHO T153, ANSI A 1.19

r (1973) ANSI A 1.7

floor Covering Materials (1971)

Std. Meth of Ptd. Meth. of

Std. Meth. of

Std. Meth. of

Std. Meth. of

Std. Meth. of

Std. Meth. of

Std. Meth. of

Std. Meth. of

Std. Meth. of

Tent. Meth. of Std. Meth. of Std. Meth. of Std. Meth. of Std. Meth. of Std. Meth. of Std. Meth. of

Tent. Meth. of Std. Meth. of Std. Meth. of Std. Meth. of Std. Meth. of Std. Meth. of Std. Meth. of Std. Meth. of Std. Meth. of Std. Meth. of Std. Meth. of Std. Meth. of

after Storage at 104 F (40C) (1973) Uniform Building Code Std. for Tent. Meth. of Std. Meth. of Std. Meth. of Std. Meth. of Std. Meth. of ) ANSI Z155.12

nsi Al. 10

nd Belts (1942) ANSI L14.283

Std. Spec. and Meth. of Std. Meth. of Panel Spalling Std. Meth. of

of Solid Electrical Insulation (1973) Meth. for Measuring Flat Spring mass Spectrometry (1971) ANSI Z11.311 Std. Meth. of Std Meth of Std. Meth. of Std. Meth. of Std. Meth. of Meth. of Std. Meth. of Std. Meth. of Std. Meth. of Std. Meth. of

Tent. Meth. of Std. Meth. of Std. Meth. of Std. Meth. of Std. Meth. of Std. Meth, of Std. Meth. of Std. Meth. of Std. Meth. of Std. Meth. of Std. Meth. of Std. Meth. of Std. Meth. of d (1974) A

$\mathrm{g}$ Weight Test) (1972)

one Drop Test) (1972)

nsulating Materials (1973)

Parts by Means of a Tup (Falling Weight) / 74) ANSI K65.117

ite, Opaque

2)

ed Wood Products for Exterior Use (1959) A/ king Flotation pcorn, Dried Pea/ ts (1972)

Meth. of Analysis of Cracki

(1973)

74) AASHO T201
Std Meth. of Test for Iron Bacteria in Water and Water Formed Deposi Std. Meth. of Test for Iron.59 in Water (1969) ANSI N162

Std. Meth. of Test for Isophthalic Acid in Alkyd and Polyester Resins Std. Meth. of Test for Kinematic Viscosity of Asphalts (Bitumens) (19
ASTM

ASTM

ASTM

ASTM

ASTM

ASTM

ASTM

ASTM

ASTM

ASTM

ASTM

ASTM

ASTM

ASTM

UL

ASTM

ASTM

ASTM

ASTM

ASTM

ASTM

ASTM

ASTM

ASTM

ASTM

ASTM

ASTM

ASTM

ASTM

ASTM

ASTM

ASTM

ASTM

ASTM

ASTM

ASTM

ASTM

ASTM

ASTM

ASTM

ASTM

IC BO

ASTM

ASTM

ASTM

ASTM

ASTM

ASTM

ASTM

ASTM

TAPPI

ASTM

ASTM

ASTM

ASTM

ASTM

ASTM

ASTM

ASTM

ASTM

ASTM

ASTM

ASTM

STM

A STM

ASTM

ASTM

ASTM

ASTM

ASTM

ASTM

ASTY

ASTM

ASTM

$\mathrm{AACCH}$

ASTM

ASTM

ASTM

ASTM
F362 D 1617

D2480

D221

D2037

G34

D3115

C736

D1574

D2230

C731

D2137

C451

E343

D3166

C317

C204

C 204

C180

992

D3065

D568

D 1433

D3014

D635

D92

D3243

E502

D3143

D1310

D3278

C293

C348

D3167

D 1179

D892

D1881

D3281

C374

D2459

D831

D2471

D3056

D2895

UBCS $25-20$

D3216

D3286

B277

E458

C186

D 181

C107

D495

T683SU

D2789

B577
D815

D853

D2725

D3137

D 1726

D 3102

D2736

D514
D3201

F314

D 1054

D2632

G14

D256

D3029

D757

E313

D2875

D3042

D 1101

28. 22

D2461

D2690

D2170

Engineering and Product Standards Division 
liquids and the Calculation of Dynamic Vis/ 1972)

Compression Ratio (CR) Technique for Rese/

Compression Ratio (CR) Technique for Moto/

Surfaces (1972)

ansi 211.316

etry (1973)

ne Procedure (1972T)

$r$ in the Inside Out Test. Mode (1973)

s (Array Meth.) (1972) ANSI L14.9I

by Fibrograph Measurement (Textile) (1972)/

Cement Mortar and Concrete (1974T)

ncrete (1974) ANSI A37.78

ative Fabrics (Textile) (1973)

1973) ANSI AI.38

(1973) ANSI L14.136

ems During Cure (1969) ANSI K65. I8I

d Erosion of Insulating Materials (1973) A

)

s (1973) ANSI 2I1.161

ed) Coating on Iron or Steel Articles by T/ ometry (1973)

ompounds After Artificial Weathering (1972/ ng Tapes (1973)

, Elastomeric, Solvent Release Type Sealan/ rnating Magnetic Fields (1974)

industrial Water and Industrial Waste Wate/ on of Fabrics (1972T)

the British Portable Tester (1974)

g or Dec/ Uniform Building Code Std. for Cham lubricants in Oscillating Motion (I97I) An/ tes Under Compressive or Shear Strains by / on Turbine, and Distillate Fuels (Potentio/

meric Acrylate Esters (1972)

y Measurement of Steady State Surface Pho/ 972T)

actories (1972)

e Material (1971)

Insulation (1973)

ke (1973)

(1972) ANSI LI4.256

dustrial Samples of Wool by Oven Drying (1)

s Films (1972)

3)H (D, N) He Neutron Generators by Radio/

Substance (1970) ALCA B5

ke (1973)

c Solvents, Degreasing Grade (Nonreflux Me/

c Hydrocarbons by Gas Chromatography (1968/ ials (1973)

lsion. Solvent Based, and Polymer Emulsion/ ract from Aerospace Components (Using Rota/ aasho T129, ANSI A1.II

ures (1972)

14.97

n Meth. (1968)

1.52, Fed Std. 791B, TAPPI T636

ormed Sealing Tapes (1974)

als for Strong Acid Removal (1972)

change Materials (1972) ANSI Z111.4

) AASHO T21, ANSI A37.19

oils (1972) ANSI ZI1.320

oils Containing 2,6-Ditertiary-Butyl P/

on and Direct Counting Technique (1973)

1973)

Test

els (1973) ANSI ZII.264

72) ANSI Z197.2

i A37. I

rs (Packaging) (195I) ANSI MHI2.8

lunt Rod) (1972)
Std. Meth. of Test for Kinematic Viscosity of Transparent and Opaque

Std. Meth. of Test for Kinetic Coefficients of Friction of Plastics (

Std. Meth. of Test for Knock Characteristics of Motor Fuels Using the

Std. Meth. of Test for Knock Characteristics of Motor Fuels Using the

Meth. of Test for Knoop Indentation Hardness of Glass (1972T)

Std. Meth. of Test for Lead and Cadmium Extracted from Glazed Ceramic

Std. Meth. of Test for Lead and Vanadium in Gas Turbine Fuels (1972)

Std. Meth. of Test for Lead in Gasoline by Atomic Absorption Spectrom

Meth. of Test for Lead in the Atmosphere by Colorimetric Dithizo

Std. Meth. of Test for Leaks in Heat Sealed Flexible Packages (1972)

Std. Meth. of Test for Leaks Using the Mass Spectrometer Leak Detecto

Std. Meth. of Test for Length and Length Distribution of Cotton Fiber

Std. Meth. of Test for Length and Length Uniformity of Cotton Fibers

Std. Meth. of Test for Length Change of Drilled or Sawed Specimens of

Std. Meth. of Test for Length Change of Hardened Cement Mortar and Co

Meth. of Test for Light Transmittance of Woven Glass Fiber Decor

Std. Meth. of Test for Lime Content of Uncured Soil-Lime Mixtures (

Std. Meth. of Test for Linear Density of Cotton Fibers (Array Sample)

Std. Meth. of Test for Linear Density of Elastomeric Yarn (1972)

Std. Meth. of Test for Linear Shrinkage of Thermosetting Casting Syst

Std. Meth. of Test for Liquid Contaminant, Inclined Plane Tracking an

Std. Meth. of Test for Liquid Densities of Pressurized Products (1972

Sid. Meth. of Test for Liquid Flow Rate of Membrane Filters (1972)

Std. Meth. of Test for Load Carrying Capacity of Fluid Gear Lubricant

Std. Meth. of Test for Locating the Thinnest Spot in a Zine (Galvaniz

Std. Meth. of Test for Low Levels of Lead in Gasoline by X.Ray Spectr

Std. Meth. of Test for Low Quantities of Mercury in Paint (1973)

Std. Meth. of Test for Low Temperature Flexibility of Latex Sealing C

Std. Meth. of Test for Low Temperature Flexibility of Preformed Seali

Std. Meth. of Test for Low Temperature Flexiblity and Tenacity I Part

Std. Meth. of Test for Magnetic Shield Efficiency in Attenuating Alte

Tent. Meth of Test for Manganese Dioxide in Manganese Ores (1972T)

Std. Meth. of Test for Manganese in Paint Driers by EDTA Meth. (1973)

Std. Meth. of Test for Measurement of Beta Particle Radioactivity of

Meth. of Test for Measuring Soil Removal and Reflectance Retenti

Std. Meth. of Test for Measuring Surface Frictional Properties Using

mber Meth. of Test for Measuring the Density of Smoke from the Burnin

Std. Meth. of Test for Measuring the Wear Life and Bonded Solid Film

Std. Meth. of Test for Mechanical Properties of Elastomeric Vulcaniza

Std. Meth. of Test for Mercaptan Sulfur in Gasoline, Kerosine, Aviati

Std. Meth. of Test for Mercury in Liquid Chlorine (1973)

Std. Meth. of Test for Methyl Ether of Hydroquinone in Colorless Mono

Std. Meth. of Test for Microhardness of Materials (1973)

Tent. Meth. of Test for Minority Carrier Diffusion Length in Silicon B

Meth. of Test for Mist Spray Flammability of Hydraulic Fluids (1

Std. Meth. of Test for Modulus of Rupture of Air Setting Plastic Refr

Std. Meth. of Test for Moisture Content and Moisture Regain of Textil

Std. Meth. of Test for Moisture Content of Oil Impregnated Cellulosic

Std. Meth. of Test for Moisture Content of Wood (1974)

Std. Meth. of Test for Moisture in Graphite (1969) ANSI K90.5

Std. Meth. of Test for Moisture in the Analysis Sample of Coal and Co

Std. Meth. of Test for Moisture in Wool by Distillation with Toluene

Std. Meth. of Test for Moisture Present in Ordinary Commercial and in

Std. Meth. of Test for Moisture Vapor Permeability of Organic Coating

Std. Meth. of Test for Morpholine in Water (1974)

Std. Meth. of Test for Neutron Flux Density and Average Energy from (

Std. Meth. of Test for Nickel on Steel by Photometric Analysis (1972)

Tent. Meth, of Test for Nitrogen Content (Kjeldahl) and Hide (Leather)

Std. Meth. of Test for Nitrogen in the Analysis Sample of Coal and Co

Sid. Meth. of Test for Nonamine Acid Acceptance of Halogenated Organi

Std. Meth. of Test for Nonaromatic Hydrocarbons in Monocyclic Aromati

Std. Meth, of Test for Noncombustibility of Elementary Building Mater

Std. Meth. of Test for Nonvolatile Matter (Total Solids) in Water Emu

Std. Meth. of Test for Nonvolatile Residue of Halogenated Solvent Ext

Std. Meth. of Test for Normal Consistency of Hydraulic Cement (I973)

Std. Meth, of Test for Normal Spectral Emittance at Elevated Temperat

Std. Meth. of Test for Number of Neps in Cotton Samples (1971) ANSI L

Std. Meth. of Test for Oil Absorption of Pigments by Gardner-Colema

Std. Meth, of Test for Oil Content of Petroleum Waxes (1968E) ANSI Z1

Sid. Meth. of Test for Oil Migration or Plasticizer Bleed Out of Pref

Std. Meth. of Test for Operating Performance of Anion Exchange Materi

Std. Meth. of Test for Operating Performance of Particulate Cation Ex

Std. Meth. of Test for Optimum So3 in Portland Cement (1972)

Std. Meth. of Test for Organic Impurities in Sands for Concrete (1973

Meth of Test for Organochlorine Pesticides in Water (1972T)

Std. Meth. of Test for Overrun of Food Aerosols (1972)

Std. Meth. of Test for Oxidation Characteristics of Extreme Pressure

Std. Meth. of Test for Oxidation Stability of New Mineral Insulating

Std. Meth. of Test for Oxygen Content Using a $14 \mathrm{MeV}$ Neutron Activati

Meth. for Water Leak Test for Paper Shipping and Refuse Sacks (Containers) ( Slant Drop Penetration

Std. Meth. of

Test for Particulate Contaminant in Aviation Turbine $\mathrm{Fu}$

Std. Meth. of Test for Penetration of Liquids into Submerged Containe

Std. Meth. of Test for Penetration Resistance of Pipeline Coatings (B
ASTM

ASTM

ASTM

ASTM

ASTM

ASTM

ASTM

ASTM

ASTM

ASTM

ASTM

ASTM

ASTM

ASTM

ASTM

ANSI

ASTM

ASTM

ASTM

ASTM

ASTM

ASTM

ASTM

ASTM

ASTM

ASTM

ASTM

ASTM

ASTM

ASTM

ASTM

ASTM

ASTM

ASTM

ASTM

ASTM

ICBO

ASTM

ASTM

ASTM

ASTM

ASTM

ASTM

ASTM

ASTM

ASTM

ASTM

ASTM

ASTM

ASTM

ASTM

ASTM

ASTM

ASTM

ASTM

ASTM

ASTM

ASTM

ASTM

ASTM

ASTM

ASTM

ASTM

ASTM

ASTM

ASTM

ASTM

ASTM

ASTM

ASTM

ASTM

ASTM

ASTM

ASTM

ASTM

ASTM

ASTM

ASTM

ASTM

TAPPI

TAPPI

ASTM

ASTM

ASTM

ASTM

ASTM

D445

D3028

D2722

2723

C 730

C 738
D2787

D3237

D3112

D 3078

E493

D 1440

D 1447

C.341

C 157

L24T4

D3155

D 1769

D2591

D2566

D2303

D3096

F317

D 1947

A239

D 3229

D2206

734

C.765

C711
A698

E465

D2375

D1890

D3050

E303

UBC \$52-2

D2981

D945

D3227

E506

D3125

E384

F391

D3119

C491

D2654

D3277

D2016

C 562 
ving Mixture (1973)

72T)

de (1974) Ashto T200

(1973)

e Ion Exchange Resins (1972)

ides (1972)

hanges of Textile Fabrics (1972) ANSI L14./

$\operatorname{lm}$ and Sheeting (1972)

ed Electrical Appliances (1973)

(1974)

eth.) (1971) ANSI A37.133

inverted Probe Machine (1971) ANSl Z197.32

as Chromatography (1972)

(1972) ANSI Z107.4

Materials (1972) ANSI Al11.4

(1970)

Std. Meth. of Conductin

tic Pipe by Acetone Immersion) (1967) Ansi/

nous Paving Mixtures (1972)

6

161

ar Plastics Using a Supported Specimen by /

olid Materials (1972) ANSI Z11.318

n Enameling (1969) ANSI Z167.21

i A111.30

(1972)

th.) (1973)

aining Fluorescent Bri/

tabilized Knit Wool Fabrics

on Line Analyzers (1970T)

usts, and Coolants (1972) ANSI D 14.2

Std. Meth. of

s (1972)

(1973)

972) ANSl Z55.15

nts (1966) ANSI Z197.7

ack (1973)

abrasion (1973) ANSI K65.72

dhesion, or Other Failure/

minous Mixtures by Means of Hveem Apparatul

Interior Coatings in an Environmental Ch/

appi $\mathrm{T} 460$

res Using Marshall Apparatus (1973)

(1972A) ANSI C7.24

3 Probe Voltage Breakdown Meth. (1972) An/

dness of Metallic Materials (1974) ANSI Z

erials (1973)

ses (1973) ANSI Z11.152

si K65. 180

261. 1

le) (1973)

(1969) ANSl Z11.58, API 2561

uge (1972) ANSl Z123.3

(1973)

sing a Collinear 4 Probe Array (1974)

Collinear Four Probe Array (1973)

12.2

12.4. TAPPI T801

12.9

hioning Materials (Packaging) (1964) ANSI

Surface Systems (1972)

2

137, ANSI A37.14

ncrete Aggregates (1971) AASHO T27, ANSI A

hotometric) Meth. (1972)

olants (1973)

ting Fire Brick (1970) ANSI A 111.18

ng Compound (1972)

Std. Meth. of
Std. Meth of Test for Percent Air Voids in a Compacted Bituminous Pa

Meth. of T'est for Permanent Deformation of Elastomeric Yarns (19

Std. Meth. of Test for $\mathrm{pH}$ of Aqueous Solutions with the Glass Electro

Std. Meth. of Test for Phosphorus in Gasoline (1973)

Std. Meth. of Test for Phosphorus in Water (1972)

act Uniformity Test for Photographic Contact Printers (1971)

Std. Meth. of Test for Phthalate Ester Color of High Gravity Glycerin

Std. Meth. of Test for Physical and Chemical Properties of Particulat

Std. Meth. of Test for Pickup Efficiency of Residual Aerosol Insectic

Std. Meth. of Test for Pilling Resistance and Other Related Surface C

Std. Meth. of Test for Plastic Sirain Ratio R for Sheet Metal (1974)

Std. Meth. of Test for Pneumatic Ball lmpact Resistance of Plastic Fi

Std. for Safety for Test for Polymeric Enclosures for Portable Cord Connect

Std. Meth. of Test for Polystyrene Blocks in Sbr Rubbers and Latices

Std. Meth. of Test for Porosity of Paint Films (1973)

Std. Meth. of Test for Potential Reactivity of Aggregates (Chemical M

Std. Meth. of Test for Pour Point of Petroleum Oils (1971) ANSI Z11.5

Std. Meth. of Test for Pressure in Glass Aerosol Bottles (1972)

Meth. of Test for Pressure in Metal Aerosol Containers (1972)

Std. Meth. of Test for Pressure Sensitive Tack of Adhesives Using an

Std. Meth of Test for Proximate Analysis of Coal and Coke (1973)

Tent. Meth. of Test for Pumpability of Industrial Fuel Oils (1973)

Std. Meth. of Test for Purity and Benzene Content of Cyclohexane by G

Std. Meth. of Test for Pyridine Bases in Crude and Refined Tar Acids

Std. Meth. of Test for Pyrometric Cone Equivalent (PCE) of Refractory

g Shear Block Test for Quality Control of Glue Bonds in Scarf Joints

Std. Meth. of Test for Ouantitative Extraction of Bitumen from Bitumi

Std. Meth. of Test for Radioactive Barium in Water (1973) ANSI N155

Std. Meth. of Test for Radioactive Cesium in Water (1972)

Std. Meth. of Test for Radioactive lodine in Water (1973) ANSI N159

Std. Meth. of Test for Radioactive Manganese in Water (1971) ANSI N15

Std. Meth. of Test for Radioactive Tritium in Water (1970) ANSI N164

Tentative Meth. of Test for Radioactive Zirconium in Water (1973)

Std. Meth. of Test for Radionuclides of Radium in Water (1970) ANSI N

Std. Meth. of

Std. Meth. of

Std. Meth. of

Std. Meth. of

Test for Rate of Burning or Extent of Burning of Cellul

Test for Reaction Threshold Temperature of Liquid and S

Test for Reboiling Tendency of Sheet Steel for Porcelai

Test for Refractoriness of Refractory Mortar (1972) Ans

Test for Reheat Change of Fireclay Nozzles and Sleeves

Test for Relative Density of Black Smoke (Ringelmann Me

Test for Relative Radiance of Paper and Paperboard Cont

Std. Meth of Test for Relaxation Dimensional Change (Shrinkage) of S

Tent. Meth. of Test for Research and Motor Meth. Octane Ratings Using

Std. Meth. of Test for Reserve Alkalinity of Engine Antifreeze, Antir

Std. Meth. of Test for Residual Chlorine in Water (1968)

Std. Meth. of Test for Residual Solvents in Flexible Barrier Material

Meth. of Test for Resiliency and Thickness of Blankets (Textile)

Std. Meth. of Test for Resistance Characteristics of Microcontacts (1

Std. Meth. of Test for Resistance of Adhesive Bonds to Chemical Reage

Std. Meth. of Test for Resistance of Glass Containers to Chemical Att

Std. Meth. of Test for Resistance of Transparent Plastics to Surface

ter lmmersion Test for Resistance to Blistering. Wrinkling, Loss of a

Test for Resistance to Deformation and Cohesion of Bitu

Test for Resistance to Growth of Mold on the Surface of

Std. Meth. of Test for Resistance to Paper to Passage of Air (1958) T

Std. Meth. of Test for Resistance to Plastic Flow of Bituminous Mixtu

Std. Meth. of Test for Resistivity of Electrical Conductor Materials

Std. Meth. of Test for Resistivity of Silicon Epitaxial Layers by the

Std. Meths. of Test for Rockwell Hardness and Rockwell Superficial Har

Std. Meth. of Test for Rockwell Hardness of Fine Grained Graphite Mat

Std. Meth. of Test for Rust Preventive Properties of Lubricating Grea

Std. Meth. of Test for Sag Flow of Highly Viscous Materials (1968) an

Std. Meth. of Test for Salt in Crude Oil (Electrometric Meth.) (1973)

Std. Meth. of Test for Sealability of Enveloped Gaskets (1970) ANSI Z

Meth. of Test for Seam Strength of Curtains and Draperies (Texti

Std. Meth. of Test for Sediment in Crude and Fuel Oils by Extraction

Std. Meth. of Test for Sediment in Water Emulsion Polishes by Centrif

Std. Meth. of Test for Seepage Rate of Aerosol Products (1972)

Meth of Test for Selenium in Water (1972T)

Std. Meth. of Test for Separation of Vehicle from Solvent Type Paints

Std. Meth. of Test for Shear Modulus of Plywood (1972)

Tentative Meth. of Test for Sheet Resistance of Silicon Epitaxial Layers U

Tent. Meth. of Test for Sheet Resistance of Thin Metallic Films with a

Std. Meth. of D rop Test for Shipping Containers (Packaging) (1961) ANSl Mh

Std. Meth. of Incline Impact Test for Shipping Containers (Packaging) (1968) ANSI Mh Std. Meth. of Vibration Test for Shipping Containers (Packaging) (1968) ANSI Mh

Std. Meth. of Test for Shock Absorbing Characteristics of Package Cus

Std. Meth. of Test for Shock Absorbing Properties of (Sports) Playing

Std. Meth. of Test for Shrinkage of Textile Fibers (1972) ANSl L14.14

Std. Meth. of Test for Sieve Analysis of Mineral Filler (1972) A ASHO

Std. Meth. of Test for Sieve or Screen Analysis of Fine and Coarse Co

Std. Meth. of Test for Silica in Fluorspar by the Silico Molybdate ( $P$

Std. Meth. of Test for Simulated Service Corrosion Test. of Engine Co

Std. Meth. of Test for Size and Bulk Density of Refractory and Insula

Std. Meth. of Test for Slump of an Oil Base Knife Grade Channel Glazi
ASTM

ASTM

ASTM

ASTM

ASTM

ANSl

ASTM

ASTM

ASTM

ASTM

ASTM

ASTM

UL

ASTM

A STM

ASTM

ASTM

ASTM

ASTM

ASTM

ASTM

ASTM

ASTM

ASTM

ASTM

ASTM

ASTM

ASTM

ASTM

ASTM

ASTM

ASTM

ASTM

A STM

ASTM

ASTM

ASTM

ASTM

ASTM

ASTM

ASTM

TAPPI

ASTM

ASTM

ASTM

ASTM

ASTM

ANSI

ASTM

ASTM

ASTM

ASTM

NCCA

ASTM

ASTM

ASTM

ASTM

ASTM

ASTM

ASTM

ASTM

A STM

ASTM

ASTM

ASTM

ANSI

ASTM

ASTM

ASTM

ASTM

ASTM

ASTM

ASTM

ASTM

ASTM

ASTM

ASTM

ASTM

ASTM

ASTM

ASTM

ASTM

ASTM

ASTM

ASTM

ASTM

D3203

D3106

E70
D323]

D515

PH3.45

D 1728

D2187

D3093

D 1375

E517

D3099

746.51

D3314

D 3258

C289

D97

D3063

D3074

D2979

D3172

D 3245

D3054

2748

C24

D 1759

D2152

2172

D2038

D2577

D2334

D2039

D2476

D 3315

D2460

D 1692

D2883

C632

C199

C605

D3211

UM. 460

D 1284

D2885

D 1121

F151

L24T3

B326 
Metal Sash (1974) nsi Z11.137
Std. Meth. of Test for Slump of Caulking Compounds and Sealants (1973 Std. Meth. of Test for Slump of Face Glazing and Bedding Compounds on Std. Meth. of Test for Smoke Point of Aviation Turbine Fuels (1972) a Std. Meth. of Test for Softening Point of Glass (1973)

Std. Meth, of Test for Soil Resistance of Floor Polishlies (1973)

Uniform Building Code Std. for Expansion lndex Test for Soil (1973)

Std. Meth. of Test for Solids Content of Aerosol Coatings (1972)
Std. Meth. of Test for Solubility of Bituminous Road Materials (Tar

petroleum Asphalt, etc.) in Organic Solven/ leum Solvents (1973)

\section{meth.) (1974)}

m (1972) ANSl G53.30

973)

atings (1970) ANS1 A109.11 Meth. of Sensory Perception Std. Thermal Performance Std. Acoustical Performance
Std. Meth. of

Beam (1970) ANS1 K65.13

of Temperature by Means of a Torsion Test/ ssenger Automobile Equipped with Full Scale Tir/ cords to Rubber (1971)

by Compression Loading (1949) ANSI Z197.1/ pe Construction in Shear by Tension Loadin/ ension Loading (Metal to Metal) (1972) Ans/

ension Loading at Elevated Temperatures (M/ ension Loading in the Temperature Range $\mathrm{Fr}$ / ension Loading of Laminated Assemblies (19/ $s$ by Compression Loading (Disk Shear) (197/ ch Yarns (1972T)

ow Power (1972) ANS1 Z14.286

lass (1974)

ometry (1973)

te Controlled Condensation Meth.) (1973)

973) AASHO T142, ANSl A37.21

nts by Cationic Titration Procedure (1974)

tion (1972T)

ng Ball (1973)

nts (1973)

nts (1971)

e (Single Rip) Meth. (Constant Rate of Tra/ s (1972T)

60

(1972) ANSI K65.32

fibers Taken from Yarns and Tows (1972) An/ 973) ANSl K65.76

and Rod Specimens (1972) ANS1 2197.3

11.206, TAPPI T644

tes (1973)

(1973)

Tentative Meth. of

paint Film (Coil/

Spec. for Conducting a Solvent Hand Rub sulating Varnishes Applied Over Film Insul/

973)

(1972) ANSI Al11.28

1.304

) ANSI All1.32

ractories (1972)

arnishes by the Helical Coil Meth. (1973)

a Thermal Pulse Meth. (1972) ANSI K90.12

Electrical Insulating Materials (1973)

e Systems (Confinement Test) (1973)

jftot Proc.) (1973)

972) ANSI Z11.148

7.24 Std. Meth. o Std. Meth. of Std. Meth. of std. Meth. of Std. Meth, of Std. Meth. of std. Meth. of Std. Meth. of std. Meth. of Std. Meth. of sid. Meth. of sid. Meth. of std. Meth. of Std. Meth. of Std. Meth. of Std. Meth. of Std. Meth. of Std. Meth. of Std. Meth. of Std. Meth. of Std. Meth. of Std. Meth. of Std. Meth. of

Std. Meth. of

Meth. of

Std. Meth. of

Std. Meth. of

Std. Meth. of

Std. Meth. of

Std. Meth. of

Std. Meth. of

Std. Meth. of

Std. Meth of

Meth. of Std. Meth. o

Tent. Meth. of

Tent. Meth. of

Std. Meth. of

Std. Meth. of

Std. Meth. of

Meth. of

Std. Meth. of

Sid. Meth. of

Std. Meth.

Std. Meth. of

Std. Meth. of

Meth. of

Std. Meth. of

Std. Meth. of

Std. Meth. of

Std. Meth. of

Std. Meth. of

Std. Meth. of

Std. Meth. of

Std. Meth. of

Meth. of

Test for Solubility of Pentachlorophenol in Heavy Petro

Test for Solubility Range of Resins and Polymers (1972)

Test for Solvent Extractable Material in Black Pigments

Test for Solvent Extractables in Petroleum Waxes (1973)

Test for Solvent Tolerance of Amine Resins (1973)

Test for Soundness of Aggregates by Use of Sodium Sulfa

Test for Spalling Resistance of Porcelain Enameled Alum Test for Specific Gravity and Absorption of Coarse Aggr Test for Specific Gravity and Absorption of Fine Aggreg Test for Specific Gravity and Porosity of Lump Coke (19 Test for Specific Gravity of Gaseous Fuels (1973) ANSI Test for Specific Gravity of Hydraulic Cement (1972) an Test for Specific Gravity of Soils (1958 AASHO T 100, an Test for Specific Gravity of Solid Pitch Asphalt Displa Test for Specific Heat of Liquids and Solids (1971) Ans Test for Spectral Bandwidth and Wavelength Accuracy of Test for Specular Gloss (1967) ANSl Z131.1

Test for Splitting Tensile Strength of Cylindrical Conc Test for Stability of Distillate Fuel Oil (Accelerated

Test for Stain Resistance of Anodic Coatings on Aluninu

Test for Staining of Caulking Compounds and Sealants (1

Test for Staleness of Bread (Cereal Chemistry) (1962)

Test for Steam Distillation of Bituminous Protective Co

Test for Steel Door and Frame Assemblies (1972)

Test for Steel Door and Frame Assemblies (1972)

Test for Stiffness of Plastics by Means of a Cantilever

Test for Stopping Distance on Paved Surfaces Using a Pa

Test for Strap Peel Adhesion of Reinforcing Fabrics or

Test for Strength Properties of Adhesive Bonds in Shear

Test for Strength Properties of Adhesives in Plywood Ty

Test for Strength Properties of Adhesives in Shear by $T$

Test for Strength Properties of Adhesives in Shear by T Test for Strength Properties of Adhesives in Shear by T Test for Strength Properties of Adhesives in Shear by T Test for Strength Properties of Metal to Metal Adhesive Test for Stretch Properties of Fabrics Woven from Stret

Test for Sulfide Resistance of Ceramic Decorations on G

Test for Sulfite lon in Water (1972)

Test for Sulfur in Petroleum Gas by Oxidation Microcoul Test for Sulfur Oxides in Flue Gases (Barium Chloranila Test for Super Duty Fireclay Brick (1970) ANSI Alll.16 Test for Surface Area of Carbon Black (1973)

Test for Surface Moisture in Fine Concrete Aggregate (1 Test for Synthetic Anionic Active Ingredient in Deterge Test for Synthetic Anionic Ingredient by Cationic Titra Test for Tack and Pressure Sensitive Adhesives by Rolli Test for Tack Free Time of Caulking Compounds and Seala Test for Tack Free Time of Elastomeric Type Joint Seal Test for Tear Resistance of Vulcanized Rubber (1973)

Test for Tearing Strength of Woven Fabrics by the Tongu Test for Tensile Properties of Oriented Fiber Composite Test for Tensile Properties of Rigid Cellular Plastics Test for Tensile Properties of Single Man Made Textile Test for Tensile Properties of Thin Plastic Sheeting (1 Test for Tensile Strength of Adhesives by Means of Bar Test for Tensile Strength of Paraffin Wax (1973) ANSI Z Test for Tensile Strength Properties of Steel Truss Pla Test for Tensile Stress Strain of Carbons and Graphite Test for Tetrasodium Salt of EDTA in Water (1972T) Std. Meth. of Test for Thermal Conductivity of Carbon Refractories Std. Meth. of Test for Thermal Conductivity of Insulating Fire Brick Sid. Meth. of Test for Thermal Conductivity of Unfired Monolithic Ref Std. Meth. of Test for Thermal Degradation of Electrical lnsulating V Std. Meth. of Test for Thermal Diffusivity of Carbon and Graphite by Std. Meth. of Test for Thermal Failure Under Electric Stress of Solid Std. Meth. of Test for Thermal lnstability of Confined Condensed Phas Std. Meth. of Test for Thermal lnsulation of Quality Packages (1972)

Tent. Meth. of Test for Thermal Oxidation Stability of Turbine Fuels ( Std. Meth. of Test for Thermal Stability of Aviation Turbine Fuels (1 Std. Meth. of Test for Thickness of Diffusion Coating (1970) ANSI Z16 Test for Specific or API Gravity of Liquid Asphalts by Std. Meth. of Test for Thermal Conductivity of Liquids (1971) ANSI Z Std. Meth. of Test for Thermal Conductivity of Refractory Brick (1971
CBO

ASTM

ASTM

ASTM

ASTM

ASTM

ASTM

ASTM

ASTM

ASTM

ASTM

ASTM

ASTM

ASTM

ASTM

ASTM

ASTM

ASTM

ASTM

ASTM

ASTM

ASTM

ASTM

ASTM

ASTM

$\mathrm{AACCH}$

ASTM

STD

STDI

ASTM

ASTM

ASTM

ASTM

ASTM

ASTM

ASTM

ASTM

ASTM

ASTM

ASTM

ASTM

ASTM

ASTM

ASTM

ASTM

ASTM

ASTM

ASTM

ASTM

ASTM

ASTM

ASTM

ASTM

ASTM

ASTM

ASTM

ASTM

ASTM

ASTM

ASTM

ASTM

ASTM

ASTM

ASTM

ASTM

ASTM

NCCA

ASTM

ASTM

ASTM

ASTM

ASTM

ASTM

ASTM

ASTM

ASTM

ASTM

ASTM

ASTM

ASTM

ASTM 
asurement of Stacking Fault Dimension (197/ bstrates of the Same Type by Infrared Refl/ ilicon by the Angle Lapping and Staining T/ ing Flat Surfaces (1973)

158

ation Resistance (1970) ANSl A37.134

nal Pressure (1974) ANS1 B72.6

s (1972) ANSI Z123.1

entiometric Perchloric Acid Titration (197/ chemicals (1972) ANSI Z159.2

Kjeldahl Meth. (1973)

(1973)

1972) ANSl Z77.11

d Std. Meth. Coke (1973)

zll.324

rption Meth.) (1972) ANSI Z11.317

roleum Hydrocarbons by Oxidative Microcoulometr/ organic Liquids (Oxy Hydrogen Combustion M/

ildings (1971)

es (1973) ANSI H9.23

ansi Ll 14.234

73)

70) ANSI N106

Solvent Release Type Sealants (1972)

p-Gas Meth.) (1973) ANSl Z11.92

hod) (1971) ANSI Z11.277

h.) (1972) ANSI Z11.44

purities in Scoured Wool (1972) ANSI L14.1/ ansi $\mathrm{Z} 115.7$

rared Spectrophotometry (1972)

by the Shearing Disk Viscometer (1972) An/ meter (1973)

ts (1968) ANSI K65.183

\section{and Coke (1973)}

oride Resins (1972)

, Channel Glazing Compounds (1973)

ng Materials Subjected to Partial Discharg/ d Coatings (1973)

72)

972)

ion from a Plane Surface (1972)

969E) ANSl K65.122

ca E30

ity, and Apparent Specific Gravity of Fire/ paperboard (Cobb Test) (1973)

118

te (Glycol or Alcohol Base) by the lodine/

11.82

er Sheet Materials by the Dry lndicator Me/

urbine Fuels (1972) ANS1 Z11.301

c Solvents and Their Admixtures (1974) er (1970) ALCA B8

he Specific Resistance of the Leachate of 4) ANSI Z123.12

meth. (Packaging) (1968) ANSI MH12.18 g) (1968) ANSI MH12.17

ed Packages for Dry Products (1972)

s (Packaging) (1964) ANSl MHI2.10

s by Cycle Meth. (Packaging) (1968) ANSl M/ reases (1973) ANSI Z11.124

orresponding Composition of Gallium Arseni/ ) ANSI Z11.325

2)

ing Tapes (1974)

Sulfuric Acid Solution (1971) A NSl Z

Meth. of Analysis of Modified Sedimentation

972)

972) ANS1 L14.490

972) ANSl L14.220

ke Cone Penetration Meth. (1972) ANSl Z11./ ratio for Glass and Glass Ceramics by Reso/ ) (1973)

1973)

ubber (SBR) (1973)
Meth. of Analysis of Sedimentation Meth. of Analysis of Sedimentatoon Meth. of Analysis of Micro Sedimentation

Std. Meth. of Test for Thickness of Epitaxial Layers of Silicon by Me Std. Meth. of Test for Thickness of Epitaxial Layers of Silicon on Su Std. Meth. of Test for Thickness of Epitaxial or Diffused Layers in S Std. Meth. of Test for Thickness of Resilient Flooring, Materials Hay Std. Meth. of Test for Thickness of Solid Electrical Insulation (1973 Std. Meth. of Test for Thorium in Water and Waste Water (1968) ANSI N Std. Meth. of Test for Time of Setting of Concrete Mixtures by Penetr Std. Meth. of Test for Time to Failure of Plastic Pipe Constant Inter Std. Meth. of Test for Tint Strength of Carbon Black (1973)

Tent. Meth. of Test for Total Acidity in Aviation Turbine Fuel (1973)

Std. Meth. of Test for Total Ash and Silica in Water Emulsion Polishe Std. Meth. of Test for Total Base Number of Petroleum Products by Pot Std. Meth. of Test for Total Bromine Number of Unsaturated Aliphatic Std. Meth. of Test for Total Chlorine Content of Epoxy Resins (1973)

Std. Meth. of Test for Total Nitrogen in Lubricating Oils by Modified Std. Meth. of Test for Total Rosin Acids Content of Coating Vehicles Std. Meth. of Test for Total Sulfur in Natural Gas by Hydrogenation ( Std. Meth. of Test for Total Sulfur in the Analysis Sample of Coal an Std. Meth. of Test for Trace Amounts of Lead in Gasoline (1972) ANSI

Std. Meth. of Test for Trace Metals in Gas Turbine Fuels (Atomic Abso Meth. of Test for Trace Quantities of Sulfur in Light Liquid Pet Std. Meth. of Test for Trace Quantitites of Total Sulfur in Volatile and Meth. of Test for Transparent Safety Glazing Material Used in Bu Test for Transverse Rupture Strength of Cemented Carbid Meth. of Test for Twist in Yarns (Direct Counting Meth.) (1971)
Meth. of Test for Undersized Loose Muscovite Mica Splittings (19

Tent. Meth. of Std. Meth. of Std. Meth.

Std. Meth. of Std. Meth. of Std. Meth. of Std. Meth. of Std. Meth. of Std. Meth of Std. Meth. of

Tent. Meth. of

Std. Meth. of

Sid. Meth. of

Std. Meth. of

Std. Meth. of

Std. Meth. of

Std. Meth. of

Std. Meth. of

Std. Meth. of

Std. Meth. of

Std. Meth. of

Std. Meth. of

Std. Meth. of
Std. Meth. of Std. Meth. of Std. Meth. for Std. Meth. of Std. Meth. of Std. Meth. of Std. Meth. of Std. Meth. of Std. Meth. of Std. Meth. of Std. Meth. of Std. Meth. of Std. Meth. of Std. Meth. of Std. Meth. of Std. Meth. of Std. Meth. of Std. Meth. of Std. Meth. of Test for Undissolved Water in Aviation Turbine Fuels (1 Test for Uranium by Controlled Potential Coulometry (19 Test for UV Cold Box Exposure of One Part, Elastomeric, Test for Vapor Pressure of Liquefied Petroleum Gases (L Test for Vapor Pressure of Petroleum Products (Micromet Test for Vapor Pressure of Petroleum Products (Reid Met Test for Vegetable Matter and Other Alkali Insoluble Im Test for Vickers Hardness of Metallic Matcrials (1972) Test for Vinylidene Unsaturation in Polyethylene by $\operatorname{lnf}$ Test for Viscosity and Curing Characteristics of Rubber Test for Viscosity of Asphalt with Cone and Plate Visco Test for Viscosity of Epoxy Resins and Related Componen Test for Volatile Content of Aerosol Products (1972) Test for Volatile Content of Paints (1973)

Test for Volatile Matter in the Analysis Sample of Coal Test for Volatile Matter (lncluding Water) of Vinyl Chl Test for Volatility of Oil and Resin Based, Knife Grade Test for Voltage Endurance of Solid Electrical Insulati Test for Volume Nonvolatile Matter in Clear or Pigmente Test for Volume Resistivity of Conductive Adhesives (19 Test for Volume Shrinkage of Latex Sealing Compounds (1 Test for Warpage of Refractory Brick and Tile or Deviat Test for Water Absorption of Rigid Cellular Plastics (1 Test for Water Absorption (Static) of Leather (1970) Al Test for Water Absorption, Bulk Density, Apparent Poros Test for Water Absorptiveness of Nonbibulous Paper and Test for Water and Sediment in Crude Oils (1973) ANSI Z Test for Water in Engine Antifreeze-Coolant Concentra Test for Water Penetration into Pipeline Coatings (1972 Test for Water Reaction of Aviation Fuels (1972) ANSI Z Test for Water Resistance of Paper, Paperboard, and Oth Test for Water Separation Characteristics of Aviation T Test for Water Soluble Halide Ion in Halogenated Organi Test for Water Soluble Salts in Pigments by Measuring T Test for Water Spotting of Emulsion Floor Polishes (197 Test for Water Vapor Permeability of Packages by Cycle Test for Water Vapor Permeability of Packages (Packagin Test for Water Vapor Transmission of Flexible Heat Seal Test for Water Vapor Transmission of Shipping Container Test for Water Vapor Transmission of Shipping Container Test for Wavelength of Peak Photoluminescence and the C Test for Wax Appearance Point of Distillate Fuels (1972 Test for Wear Resistance of Inked Computer Ribbons (197 Test for Weight Loss After Heat Aging of Preformed Seal Test for Weight Loss of Sheet Steel During Immersion in Test for Wheat Flour (Cereal Chemistry) (1962)

Test for Wheat (Cereal Chemistry) (1965)

Test for Wheat (Cereal Chemistry) (1968)

Test for Wheat (Cereal Chemistry) (1968)

Tent. Meth. of

Std. Meth. of

Std. Meth. of

Std. Meth. of Std. Meth. of Std. Meth. for Evaluation of and Std. Meth. for Evaluation of and Std. Meth. for Evaluation of and
Test for Wind Resistance of Asphalt Shingles (1972T)

Test for Wool Content of Raw Wool-Commercial Scale (1

Test for Wool Content of Raw Wool-Laboratory Scale (1

Test for Yield Stress of Heterogeneous Propellants by T
Test for Young's Modulus, Shear Modulus, and Poisson's

Test Formula for Butadiene-Acrylonitrile Rubbers (Nbr

Test Formula for Carbon Blacks in Natural Rubber $(\mathrm{Nr})$

Test Formula for Carbon Blacks in Styrene-Butadiene R
Test for Yarn Number Based on Short Length Specimens (1

AATM

ASTM

ASTM

ASTM

ASTM

ASTM

ASTM

ASTM

ASTM

ASTM

ASTM

ASTM

ASTM

ASTM

ASTM

ASTM

ASTM

ASTM

ASTM

ASTM

ASTM

ASTM

ANSI

ASTM

ASTM

ASTM

ASTM

ASTM

ASTM

ASTM

ASTM

ASTM

ASTM

ASTM

ASTM

ASTM

ASTM

ASTM

ASTM

ASTM

ASTM

ASTM

ASTM

ASTM

ASTM

ASTM

ASTM

ASTM

ASTM

ASTM

ASTM

ASTM

ASTM

ASTM

ASTM

ASTM

ASTM

ASTM

ASTM

ASTM

ASTM

ASTM

ASTM

ASTM

ASTM

ASTM

ASTM

ASTM

ASTM

ASTM

AACCH 56.60

$\mathrm{AACCH} 56-61 \mathrm{~A}$

AACCH $56-62$

AACCH 56.63

ASTM D3161

ASTM D 1334

ASTM D584 
73) (SBR) (1973) cr) (1973)

er (IR) (1973)

ers (SBR) (1973)

)$(1973)$

r Cores (1970)
Sid. Meth. for Evaluation of and Std. Meth. for Evaluation of and Std. Meth. for Evaluation of and Std. Meth. for Evaluation of and Std. Meth. for Evaluation of and Std. Meth. for Evaluation of and Tent. Spec. for UItra High Std. Meth. of Shear Std. for Spec, for High

t. for SAE No. 2 (Autometive Transmission) Clutch Friction hed Fabric (1972) ANSI L14.175

ty of Plastic Material for l'se in Housings $\mathrm{O} /$ oltage in Snow mobile Electrical Systems (197) ight) (1972)

n Tail Lamp (Light) (1972)

ight) (1971)

1972) ANSI B93.21

nd Other Flexible Materials: Accelerator Meth. (1970)

cke Dosimeter (1972)

) ANS1 L14.115

s (1972) ANSI L 14.1

es (Paper) (1973)

e Ratings) (Paper, Board, Cloth, etc.) (1973)

en Peroxide (1972) ANSI L14.129

cereal Chemistry) (1962)

r (1973)

Qualitative lodide

Rec. Pract.

Rec. Pract.

Rec. Pract

Rec. Pract. Rec. Pract.

hear Rate Dependent Flow Properties (1969)

tems (Clothing) After Home Laundering (1973) ANSI L14.17)

ms (Textile / Clothing) After Home Laundering (1973) Ans/ les (1971) ANSI L14.114

es Used in Textile Desizing (1973) ANSI L14.147

Paper) (1973)

xtile Fabrics (1972)

(1973)

(1973)

973) ANSI L14.217

3) ANSI Ll4.216

tics of Aluminum Automotive Water Pumps with Coolan/ (1972)

and Paper) (1973)

and Paper) (1973)

and $P$ aper) (1973)

ce Strength of Coated Papers ['sing the Interfiber Bond T/ sion (Frosting): Every Meth. (Textile Colorfastness) (19/ sion (Frosting): Screen Wire Meth. (Textile Colorfastnes/

d Fabric to Crocking (Rubbing) by the AATCC Crockmeter M/ nd Fabrics to Chlorinated Pool Water (1972) ANSI L 14.149 nd Fabrics to Water (1972) ANSI L 14.151

spiration (1972) ANSI L 14.56

es to Combined Washing and Shrinkage: Rapid Control Test/ s to W ashing at 105 Deg. F: Rapid Control Test (1969)

ulfur Dioxide Stoving (1972) ANSI L14.9

rn to Mill Fulling (1972) ANSI L14.5

arbonizing (1972) ANSI L14.3

e in the Atmosphere Inder Low Humidities (1972) ANSI L 14/ r Spotting (1972) ANSI L14.148

$r$ and Light: Alternate Exposure (1971) ANSI L14.227

$r$ (High Humidity) and Light: Alternate Exposure (1972) A/ to Crocking (Rubbing) (Rotary Vertical Crockmeter Meth./ cs to Sea WV ater (1972) ANSI L14.150

Domestic and Commercial Washing and Laundering (1972) A/ Oxides of Nitrogen (Burnt Gas Fumes) (1972) ANSI L14.54 ith Peroxide (1972) ANSI L14.146

xcluding Pressing) (1973) 1 SO 105.4

(1973) ANSI L14.241

g (1973)

ashing: Alternate Sunlight Exposure (1965) ANSI L14.118

ight) (1971) ANSI L14. 168

ral Meth.) (1971) ANSI L14.168

on Arc Lamp, Continuous Light (1971) ANSI L14.53

ight (1971) ANS1 L14.168

on Arc Lamp, Alternate Light and Darkness (1971) ANSI L1/

$r$ Cooled Xenon Arc Lamp, Continuous Light (1971) AVS1 L1/

r Cooled Xenon Arc Lamp. Alternate Light and Darkness (1/

e Atmosphere Under High Humidities (1972) ANSI L14.238

ing (1971) ANSl L 14.240, ISO 105.4

and Alkalis (1972) ANSI L 14.2

Fastness Above L.7 (1971) ANSI L14.264

on of Textile Colorants (1972)

t) of Solid Electrical Insulating Materials at Micr/

grease Wool Yarn (1971) ANSI L14.84

o Bleaching with Chlorine (1972) ANSI L14.57

y Compression Loading (Metal to Metal) (1969)
Test Formula for Isobutene-lsoprene Rubbers (IIR) (19 Test Formula for Pigmented Styrene-Butadiene Rubbers Test Formulas for General Purpose Chloroprene Rubbers ( Test Formulas for Natural Rubber ( $\mathrm{Nr}$ ) and Isoprene Rubb Test Formulas for Nonpigmented Styrene-Butadiene Rubb Test Formulas for Solution Poly-Butadiene Rubbers $(\mathrm{Br}$ Test Heat Treated Line Pipe (1972)

Test in Flatwise Plane of Flat Sandwich Constructions $O$

Test Kits for Swimming Pool Water (1970)

Test Line Pipe (Petroleum Drilling) (1973)

Test Machine Test Procedure (1972)

Test Meth for Reflectance, Blue, and Whiteness of Bleac

Test Meth. and Requirements for Evaluation of Suitabili

Test Meth. and Requirements for Maintenance of Design V

Test Meth, and Requirements for Snowmobile Headlamps (L

Test Meth. and Requirements for Snowmobile Rear Positio

Test Meth. and Requirements for Snowmobile Stop Lamp (L

Test Meth. for a Hydraulic Fuid Power Filter Element (

Test Meth. for Abrasion Resistance of Textile Fabries a Fri

Test Meth. for Absorbency of Bleached Woven Cloth (1972

Test Meth. for Accelerated Aging of Sulfur Dyed Textile

Test Meth. for Adhesion to Non Porous Flexible Substrat

Test Meth. for Adhesion to Porous Substrates (Subjectiv

Test Meth. for Alkali in Bleach Baths Containing Hydrog

Test Meth. for Ammonium Persulfate in Flour Improvers (

Test Meth. for Amount of Coating on Mineral Coated Pape

Test Meth. for Amylaceous Matter in Adhesives (1970)

Test Meth. for Apparent Viscosity of Adhesives Having S

Test Meth. for Appearance of Creases in Wash and Wear 1

Test Meth. for Appearance of Seams in Wash and Wear lte

Test Meth. for Ash Content of Bleached Cellulosic Texti

Test Meth. for Assay of Bacterial Alpha-Amylase Enzym

Test Meth. for Beating Control (Simons Stain) (Pulp and

Test Meth. for Bond Strength of Bonded and Laminated Te

Test Meth. for Bulk Density of Diamond Abrasive Grains

Test Meth. for Carbonates in Coated Paper (Qualitative)

Test Meth. for Carpet (Rug) Soiling: Accerated Meth. (1

Test Meth. for Carpet (Rug) Soiling; Service Meth. (197

Test Meth. for Castor Oil Penetration of Paper (1972)

Std. Test Meth. for Cavitation Erosion Corrosion Characteris

Test Meth. for Chemical Resistance of Pipeline Coatings

Test Meth. for Chip Classification (Hand Screen) (Pulp

Test Meth. for Chip Length Analysis (Measurement) (Pulp

Test Meth. for Chip Length Analysis (Two Screen) (Pulp

Test Meth for Coating Pick Tests for Determining Surfa

Test Meth. for Color Change in Fabrics Due to Flat Abra

Test Meth. for Color Change of Fabrics Due to Flat Abra

Test Meth. for Colorfastness of Colored Textile Yarn an

Test Meth. for Colorfastness of Colored Textile Yarns a

Test Meth for Colorfastness of Colored Textile Yarns a

Test Meth. for Colorfastness of Colored Textiles to Per

Test Meth. for Colorfastness of Cotton and Linen Textil

Test Meth. for Colorfastness of Dved or Printed Textile

Test Meth. for Colorfastness of Dyed Textile Yarns to

Test Meth. for Colorfastness of Dyed Wool Fabric and Ya

Test Meth. for Colorfastness of Dyed Wool Textiles to C

Test Meth. for Colorfastness of Textile Fabrics to Ozon

Test Meth. for Colorfastness of Textile Fabrics to Wate

Test Meth. for Colorfastness of Textile Fabrics to Wate

Test Meth. for Colorfastness of Textile Fabrics to Wate

Test Meth. for Colorfastness of Textile Yarn and Fabric

Test Meth. for Colorfastness of Textile Yarns and Fabri

Test Meth. for Colorfastness of Textiles to Accelerated

Test Meth. for Colorfastness of Textiles to Atmospheric

Test Meth. for Colorfastness of Textiles to Bleaching W

Test Meth. for Colorfastness of Textiles to Dry Heat ( $\mathrm{E}$

Test Meth. for Colorfastness of Textiles to Drycleaning

Test Meth. for Colorfastness of Textiles to Hot Pressin

Test Meth. for Colorfastness of Textiles to Light and W

Test Meth. for Colorfastness of Textiles to Light (Dayl

Test Meth. for Colorfastness of Textiles to Light (Gene

Test Meth, for Colorfastness of Textiles to Light, Carb

Test Meth. for Colorfastness of Textiles to Light, Sunl

Test Meth. for Colorfastness of Textiles to Light: Carb

Test Meth. for Colorfastness of Textiles to Light: Wate

Test Meth. for Colorfastness of Textiles to Light: Wate

Test Meth. for Colorfastness of Textiles to Ozone in Th

Test Meth. for Colorfastness of Textiles to Steam Pleat

Test Meth. for Colorfastness of Yarn or Fabric to Acids

Test Meth. for Colorfastness to Light: Determination of

Test Meth. for Colorfastness to Washing: Characterizati

Std. Test Meth. for Complex Permittivity (Dielectric Constan

Test Meth. for Continuous Scouring Wool Using Std. Raw

Test Meth. for Cotton and Linen Textile Colorfastness T

Std. Test Meth. for Creep Properties of Adhesives in Shear B
ASTM

ASTM

ASTM

ASTM

ASTM

ASTM

API

ASTM

NSF

API

SAE

AATCC

SAE

SAE

SAE

SAE

NFLDP

AATCC

ASTM

AATCC

AATCC

TAPPI

TAPPI

AATCC

$\mathrm{AACCH}$

TAPPI

ASTM

ASTM

AATCC

AATCC

AATCC

AATC

TAPPI

AATCC

ANS1

TAPPI

AATCC

AATCC

TAPPI

ASTM

ASTM

TAPPI

TAPPI

TAPP

TAPPI

AATCE

AATCE

AATCC

AATCC

AATC

AATC

AATCC

AATCC

AATCC

AATCC

AATCC

AATCC

AATCC

AATC

AATCE

AATCC

AATC

AATC

AATCC

AATCC

AATCC

AATCC

AATCC

AATCC

AATCC

AATCC

AATCC

AATCC

AATCC

AATCE

AATCC

AATCE

AATCC

AATCC

AATCE

AATCE

ASTM

A A TCC

AATCC

ASTM

D3188

D3 186

D3190

3184

D3185

D3189

C 273

38

$5 \mathrm{LX}$

J 286

J 29

$\mathrm{J} 277$
$\mathrm{~J} 280$

279

T3. 10.8.2

0167

79

26

U-541

(M. 540

98 
y Tension Loading (Metal to Metal) (I969) aterials (1968) ANSI MH12.15

subject to Freezing (1972T)

core Material (1970)

efore Immersion). (Pulp and Paper) (1973)

$\mathrm{n}$ with Correction for Cracks, etc.) (Pulp and Paper)

Sand wich Constructions (1970)

rection for Cracks, etc.) (Pulp and Paper) (1973)

d) (Pulp and Paper) (1973)

pycnometer) (Pulp and Paper) (1973)

iles to Photochromism Colorfastness to Light (1972) Ansi

ble Impurities in Refined Phenol by Cloud Point Dep/

abrics: Agar Plate Meth. (1970) ANSl Ll4.125

ing Treatments on Galvanized SteeI Prior to Conversion C

hatch Tape Test (Coil Coating) (1974)

tals by Cross Hatch Tape Test After Reverse Impacting (C) sin Treated Fabric by the Sealed Jar Meth. (1972) A VSI L

sin Treated Fabric Steam Meth. (1972) ANSI L14.179

(Pulp and Paper) (1973)

ciated Characteristics of Automotive Trim Materials/

g Resistance of Organic Coatings When Applied to Coit $\mathrm{C}$.

brasion of Automotive Bodycloth (1972)

g) of Fiberboards (1972)

er Specimen for Fabrics, Vinyls, Coated Fiberbo/

Injection Using Uembrane Filters (Petroleum

Interior Trim Materials (1973) ANSI Z265. I Rec. Pract

nforced Plastic Panels (1969)

undering of Durable (Permanent) Press Woven or Knit Text ) (1973) en and Knitted Textiles Except Wool (1972) ANSI L14.138,

bags (Containers) (1973)

s and Finishes on Textiles and Other Materials (1973)

59.133

g (1972) ANSI L14.4

ndwich Constructions (1970)

ro-Static) of Textile Yarns (1973) A NSI L14.119

) of Textile Fabrics (1972) ANSI L14.112

s Fabric to Metal Test) (1973)

9)

imber Piles (1970)

cs of Rubber and Rubber Like Materials by a Tempera/

len Fabric Textile in Storage (1971) ANSI L14. S2

a for Mercerization (1971) ANSI L14.79

fabrics (1970) A NSI L14.144

$s$ on Cotton Textile Fabrics (1971) ANSI L14.106

i Skein Meth.) (1971) ANSI L14.11

rade Trademarked Plywood (1971)

(I973)

ulp and Paper) (1973)

$p$ and Paper) (1973)

d Melamine Adhesives (1970)

$\mathrm{GHz}$ (1973)

n Index Meth. (1974) ANSI K65.110 7.20

inated Assemblies (1969) ANSI Z197.20

(1970)

m Bleached Cotton Cloth (1972) ANS1 L14.117 agnetic Tape Recorder / Reproducers (1972) ANS1 C83/

73)

ve Films (1970)

ANSI L14.145

72) ANSl L 14.131

1973) ANSI L14.127

gram) (1973)

) ANSI L14.65

direction Tensile) (1973)

ns (1970)

the Process Industries (1972)

etic Finishes on Mild Steel (Coil Coating) (1974)

$f$ a Sheet of Paper or Paperboard (1973)

ed Aluminum, Steel and Gavanized Steel (Coil Coating) (1/

s (1971) ANSI L 14.124

in Wood (1970)

and Paper) (1973)

Std.

p and Paper) (1973)

te) (1973)

Loss by Retained Chlorine Bleaching (1971) ANSI L14. 180 tiles (1972) A NSI L14.128
Std. Test Meth. for Creep Properties of Adhesives in Shear B

Std. Test Meth. for Creep Properties of Package Cushioning M

Test Meth. for Critical Dilation of Concrete Specimens

Sid. Test Meth. for D.C Volume Resistivity of Glass (1972) Test Meth. for Delamination Strength of Honeycomb Type Test Meth. for Density and Moisture of Chips (Presoak B Test Meth. for Density and Moisture of Chips (Submersio

Sid. Test Meth. for Density of Adhesives in Fluid Form (1969

td. Test Meth. for Density of Core Materials for Structural Test Meth. for Density of Pulpwood (Submersion with Cor Test Meth. for Density of Wood Chips (Sheet Machine Mol Test Meth. for Density or Volume of Small Specimen $(\mathrm{Hg}$ Test Meth. for Detection and issessment of Colored Text Test Meth. for Detection and Estimation of Water Insolu Test Meth. for Detection of Antibacterial Activity of $F$ Test Meth. for Detection of Hexavalent Chromium Contain Test Meth. for Determination of Film Adhesion by Cross Test Meth. for Determination of Film Adhesion on All Me Test Meth. for Determination of Formaldehyde Odor in Re Test Meth. for Determination of Formaldehyde Odor in Re Test Meth. for Determination of Useful Fiber in Bagasse Test Meth. for Determining Blocking Resistance and Asso Test Meth. for Determining Pressure Marking and Blockin Test Meth. for Determining Resistance to Snagging and a Test Meth. for Determining Stiffness (Modulus of Bendin Test Meth. for Determining Visual Color Matched to Mast Test Meth. for Determining Water Quality for Subsurface Test Meth. for Determining Window Fogging Resistance of Test Meth. for Diffuse Light Transmission Factor of Rei Test Meth. for Dimensional Changes in Automatic Home La Test Meth. for Dimensional Changes in Laundering of Wov Test Meth. for Dirt Count of Wood Chips (Pulp and Paper Test Meth. for Drop Tests for Paper Shipping Sacks and Test Meth. for Drycleaning Durability of Applied Design

Std. Test Meth. for Dust and Fog Tracking and Erosion ANSI C Test Meth. for Dyed Silk Yarn Colorfasiness to Degummin

Std. Test Meth. for Edgewise Compressive Strength of Flat Sa Test Meth. for Electrical Resistivity Textile of (Elect Test Meth. for Electrical Resistivity (Electro-Static Test Meth. for Electrostatic Clinging of Textile Fabric Test Meth. for Electrostatic Propensity of Carpets (196

Tent. Test Meth. for Establishing Design Stresses for Round T

std. Test Meth. for Evaluating Low Temperature Characteristi Test Meth. for Evaluating Oxidation of Wool Oils on Woo Test Meth. for Evaluating Wetting Agents in Caustic Sod Test Meth. for Evaluation of Antibacterial Finishes on Test Meth. for Evaluation of Commercial Rewetting Agen Test Meth. for Evaluation of Wetting Agents (Cotton Tes Test Meth. for Exterior Coatings and Overlays on DFPA G Test Meth. for Fiber Bundles in Baled Flash Dried Pulp Test Meth. for Fiber ldentification (Various Stains) ( $P$ Test Meth. for Fiber ldentification (Wilson Stain) (Pul

Sid. Test Meth. for Filler Content of Phenol. Resorcinol, an Test Meth. for Fire Resistance of Textile Fabrics (1969 Spec. and Test Meth. for Fixed and Variable Attenuators, DC to 40
TII D 230

Std. Test Meth. for Flammability of Plastics Using the Oxyge

Std. Test Meth. for Flatwise Compressive Strength of Sandwic

Std. Test Meth. for Flexural Strength of Adhesive Bonded Lam

Std. Test Meth. for Flexure Creep of Sandwich Constructions

Std. Test Meth. for Flow Properties of Adhesives (1969) Test Meth. for Fluidity of Dispersions of Cellulose for

Rec. Test Meth. for Flutter Measurement of Instrumentation M

Std. Test Meth. for Heating Loss of Carbon Black (1972)

Test Meth. for Heavy Metals in Paper and Paperboard (19

Std. Test Meth. for Hydrogen Ion Concentration of Dry Adhesi Test Meth. for Hydrogen Peroxide in Bleach Baths (1972)

Test Meth. for Identification of Fibers in Textiles (19

Test Meth. for Identification of Finishes in Textiles (

Test Meth. for Identification of Lignin (Paper Chromato

Optical Test Neth. for Ink Show Through (Paper) (1973)

Test Meth. for Insect Pest Deterrents on Textiles (1971

Test Meth. for Internal Bond Strength of Paperboard (Z

Std. Test Meth. for Laboratory Aging of Sandwich Constructio Test Meth. for Laboratory Corrosion Test, of Metals for Test Meth. for Measurement of Film Thickness of Nonmagn Test Meth. for Measuring Squareness or Rectangularity $O$ Test Meth. for Measurment of Impact Resistance of Paint Test Meth. for Mercerization in Cotton Yarns and Fabric Test Meth. for Moisture and Creosote Type Preservative Test Meth. for Moisture in Chips (Moister Teller) (Pulp Test Meth. for Moisture in Pulp (Web) (1973)

Test Meth. for Moisture in Wood Chips (Steam Oven) (Pul Test Meth. for Moisture in Wood Chips (Two Liter Gradua Test Meth. for Multiple Sample Meth. of Textile Tensile Test Meth. for Noncotton Content of Bleached Cotton Tex
ASTM

ASTM

ASTM

ASTM

ASTM

TAPPI

TAPPI

ASTM

ASTM

TAPPI

TAPPI

TAPPI

A ATCC

ASTM

AATCC

NCCA

NCCA

NCCA

AATCE

AATCC

TAPP

$S A E$

NCCA

$\mathrm{SAE}$

SAE

$S A E$

VACE

SAE

ASTM

A ATCC

AATCE

TAPPI

TAPPI

A ATCC

ASTM

AATCC

ASTM

AATCC

A ATCE

AATCC

AATCC

ASTY

ASTM

AATCC

AATCC

A ATCE

AATCE

AATCC

APA

TAPPI

TAPPI

TAPPI

ASTM

AATCC

IEEE

A ATCC

ASTM

ASTM

ASTM

ASTM

ASTM

AATCC

EIA

ASTM

TAPPI

ASTM

AATCC

AATCC

AATCC

TAPPI

TAPPI

AATCC

TAPPI

ASTM

NACE

NCCA

TAPPI

NCCA

AATCC

A TM

TAPPI

TAPP

TAPP

TAPPI

AATCC

AATCC 97

D2294

D2221

671

6657

C363

U. 20

D1875

C27I

M. 9

UM. 18

139

90

TB. 11.7

TB. $11 . \overline{5}$

B.11-16

112

13

$912 \mathrm{~A}$

TB. 11.17

$\mathrm{J} 948 \mathrm{~A}$

J949 A

36] A

T.1.01.73

J275

(

[M-1]

UM-806

86

D2132

C 364

84

76
115

134

D2899

D1329 
I, and Melamine Adhesives (1970)

esistance Test (1972) ANSI L14.214

ic Soil During Home Laundering (1970) ANSI L14.239

th 45.0 Deg. Geometry (1973)

Tests (Paper) (1973)

plywood Under Mold Conditions (1970)

textiles (1969) ANSI L14.116

lic Materials (1972) ANSI Z260.2

ated Fiberboard (1969)

ANSI 2161.1

Particulate Matter (1971)

ure and Composition) (1971) ANSI L14.132

cs and Textiles in Yarn Form (1971) ANSl L14.75

aper) (1973)

es (Shrinkage) of Woven and Knitted Wool Textiles (1972)/

perties of Treated Paper (1973)

tory Aging Conditions (1970) ANSI Z197.19

ubstances (1971) ANSI Al74.1

to Natural Light and Weather (1968) ANSI L14.176

A NSI L14.64

ral Light and Weather Through Glass (1972) ANSI L14.177 973

lp and Paper) (1973)

est the Removability of Spinning Oils from Woolen Yarns erings (Rug, Carpet) (1972)

(1970)

tory Flat Sereen) (1973)

ille Fractionator) (1973)

fthan Shive Analyzer) (1973)

nd Paper) (1973)

Base Finish Flooring (1969)

oss by Retained Chlorine (1971) ANSI L14.126

tsen Tester) (1973)

(1973) ANSI L14.85

aterials (1969)

Paper) (1973)

iance of Paper and Paperboard Containing Fluorescent Bri/

ply Wood Construction in Shear by Tension Loading (/

1973)

72) ANSI Z197.8

fabrics (Permanent Press) After Repeated Home Laundering/ ides) Resistance of Textiles (1971) ANSI L14.55

phic Lens (1969)

tion Magnetic Tape Recorder / Reproducers (1974) An/

Silver Coin Test (1973)

ing (1973) ANSI L14.215

tructural Sandwich Constructions (1970)

efuse Sacks (Containers) (1973)

ay Test (1971) ANSI L14.60, ASTM D583

tic Absorption Test (1972) ANSI L14.61, ASTM D583

ble Jar Dynamic Absorption Test (1972) ANSI Ll4.87, ASTM/ n Test) (1971) ANSI L14.74, ASTM D583

rostatic Pressure Test (1971) ANSI L14.265

act Penetration Test (1971) ANSI L14.71, ASTM D583

and Coated Fabrics: Carbon Arc Lamp Exposure with Wettin/ rc Lamp Exposure Without Wetting (1972) ANSI L14.225

s Materials (1972)

earance Meth. (1970) ANSI L 14.229

s: Recovery Angle Meth. (1972) ANSI L14.110, ASTM D1295

ng Materials (1972)

Spec., Std., and
Std. Rec. Pract. for Stating the Precision of ASTM ging or Stretch in Wear (Textile) (1973)

st Cleaned with Sand Abrasive (1970)

Pressure Sensitive Tape Water Vapor Transmission

mability of Automotive Interior Trim Materials: Horizontal

bility Determination of Hot Melt Adhesives by Mandrel Bend

DFPA Grade Trademarked Plywood (1967)

Std.

Minimum Std for Design, Manufacture and wer Generating Stations (1972)/ Trial Use Guide for Type
Std. Test. Proc. for Pressure Collapse

24

1970 ) Meth. for Flat Crush

td. Meth. of Tension

Std. Meth. of Flexure

Std. Meth. for Accelerated Laboratory

orm Building Code Std. for Airborne Sound Insulation Field td. Test Meth. for Nonvolatile Content of Phenol, Resorcino Test Meth. for Oil Repellency of Fabrics: Hydrocarbon $R$ Test Meth. for Oil Spots in Paper (1973) Test Meth. for Oily Stain Release Meth, of Textile Fabr Test Meth. for Opacity of Paper, Using Reflectometer Wi Test Meth. for Packaging Film and Construction Identity

Test Meth. for Passive Electronic Component Parts (1972 Test Meth. for Pentosans in Pulp (1973)

td. Test Meth. for Permanence of Adhesive Bonded Joints in Test Meth. for $\mathrm{pH}$ of the Water Extracted from Bleached

td. Test Meth. for Plane Strain Fracture Toughness of Metal

td. Test Meth. for Ply Separation (Wet) of Solid and Corrug

Test Meth. for Polymer Content of Styrene Monomer (1974

Test Meth. for Polynuclear Aromatic Hydrocarbons in Air Test Meth. for Quantitative Analysis of Textiles (Moist Test Meth. for Quick Evaluation of Wettability of Fabri Test Meth. for Rejects from Knotter Screens (Pulp and P Test Meth. for Relaxation and Felting Dimensional Chang Test Meth. for Resin and Volatile Content, and Flow Pro

td. Test Meth. for Resistance of Adhesives to Cyclic Labora Test Meth. for Resistance of Ceramic Tile to Chemical Test Meth. for Resistance of Textile Fabrics: Exposure Test Meth. for Resistance of Textiles to Insects (1971) Test Meth. for Resistance of Textiles: Exposure to Natu Test Meth. for Rug Back Floor Staining on Vinyl Tile (l Test Meth. for Sampling of Wood Chips from Conveyor (Pu Test Meth. for Scourability of Spinning Lubricant (To T Test Meth. for Shampooing: Washing of Textile Floor Cov Test Meth. for Shear Fatigue of Sandwich Core Materials Test Meth. for Shive Content of Mechanical Pulp (Labora Test Meth. for Shive Content of Mechanical Pulp (Somerv Test Meth. for Shive Content of Mechanical Pulp (Von Al Test Meth. for Shrinkage Test of Pulp Sheets (1973) Test Meth. for Sieve Analysis of Pulpwood Chips (Pulp a d. Test Meth. for Simulated Service Test. of Wood and Wood Test Meth. for Single Sample Meth. of Textile Tensile L

Test Meth. for Sizing of Paper (Reverse Curl) (1973)

Test Meth. for Sizing of Paperboard (Flot ation) (1973) Test Meth. for Smoothness of Paper and Paperboard (Bend Test Meth. for Snag Resistance of Women's Nylon Hosiery

Test Meth. for Specific Gravity of Wood and Wood Base M Test Meth. for Specific Gravity of Wood Disks (Pulp and Test Meth. for Spectrophotometric Test for Relative Rad Test Meth. for Strength Properties of Adhesives in Two Test Meth. for Siructural Insulating Roof Deck (R1970) Test Meth. for Sulfides in Coated Paper (Qualitative) ( Test Meth. for Tensile Properties of Adhesive Bonds (19 Test Meth. for the Appearance of Durable Press Textile Test Meth. for the Evaluation of Mildew and Rot (Fungic Test Meth. for the Selective Transmission of a Photogra
Test Meth. for Timing Error Measurements of Instrumenta Test Meth. for Top Side of Filled Paper as Indicated by Test Meth. for Viscosity of Adhesives (R 1970) Test Meth. for Visual Rating Meth. of Carpet (Rug) Soil

td. Test Meth. for Water Absorption of Core Materials for S Test Meth. for Water Leak Test for Paper Shipping and R Test Meth. for Water Repellency of Textile Fabrics: Spr Test Meth. for Water Repellency of Textile Fabrics: Sta Test Meth. for Water Repellency of Textile Fabrics: Tum Test Meth. for Water Resistance of Textile Fabrics (Rai Test Meth. for Water Resistance of Textile Fabrics: Hyd Test Meth. for Water Resistance of Textile Fabrics: Imp Test Meth. for Weather Resistance of Textile Materials Test Meth. for Weather Resistance of Textiles: Carbon a Test Meth. for Wicking of Automotive Fabrics and Fibrou Test Meth. for Wrinkle Recovery of Textile Fabrics: App Tesi Meth. for Wrinkle Recovery of Woven Textile Fabric Test Meth. of Surface Burning Characteristics of Buildi Test Meth. Referenced by SAE (1973)

Test Meth. Related to Carbon Black (197\%)

Test Meth. to Determine Woven Fabric's Resistance to Sa

Test Meth. Visual Std. for Surfaces of New Steel Airbla

Test Meth. (1970)

Test Meth. (1972)

Test Meth. (1972T)

Test Method for Penetration of Adhesives (1969)

Test Meths. for Interior PaneI Coatings and Overlays on

Test of Class I Electric Valve Operators for Nuclear Po Test of Composite Cans (1973)

Test of Corrugating Medium (Paperboard) (1971) ANSI P3.

Test of Flat Sandwich Constructions in Flatwise Plane (

Test of Flat Sandwich Constructions (1970)

Test of Natural Decay Resistance of Woods (1970)

Test of Partitions (W all, Ceiling, Floor, and Floor-C

Test of Pulp Sheets (1973)
ASTM

AATCC D 1582

UM-538

TAPPI UM-537

EIA RS186D

TAPPI UM.236

ASTM D1877

AATCC 81

ASTM E399

ASTM D 1028

ASTM D2121

ASTM D2682

AATCC $20 \mathrm{~A}$

TAPPI UM-1

AATCC 99

TAPPI UM.530

ASTM DI183

ASTM C650

AATCC $111 \mathrm{~B}$

AATCC 24

AATCC $111 \mathrm{D}$

AATCC 137

TAPPI UM.4

AATCC 7

AATCC 138

ASTM C394

TAPPl UM-240

TAPPI UM. 242

TAPPI UM-238

TAPPI UM-2l

ASTM D2394

AATCE 92

TAPPI UM-450

TAPPI UM-543

TAPPI UM-535

AATCC

ASTM D2305

TAPPI UM-12

TAPPI UM.460

ASTM D2339

ASTM D2164

TAPPI UM-532

ASTM D897

AATCC 124

AATCC 30

ANSI PH3.37

EIA

$\mathrm{RS413}$

TAPPI UM-533

ASTM Dl084

AATCC 121

ASTM C 272

TAPPI UM-529

A ATCC 22

AATCC 21

AATCC 70

AATCC 35

A ATCC 127

AATCC 42

AATCC $111 \mathrm{~A}$

AATCC $111 \mathrm{C}$

SAE

AATCC

AATCC

NFPA

SAE

ASTM

ANSI

NACE

PSTC

SAE

ASTM

ASTM

APA

ASAE

IEEE

CCTI

TAPPI

ASTM

ASTM

ASTM

IC BO

TAPPI
J913A

128

55

AMS2350X

D305

124T5

TM. 01.70

34

$\mathrm{J} 369 \mathrm{~A}$

D3111

D1916

45

S263.2

382

$\mathrm{Cl} 33$

T809 OS

C297

C 393

D2017

UM. 238 
th a Secondary Standard of Sim/ 72)

Coagulation Flocculation Jar Std. Meth. of Thermal Shock

Std. Meth. of Thermal Shock Std. Meth for Corrosion

n Glassware (1970) ANSl D14.9 Tent. Rec. Pract. for Conducting Wear oil) (1974) Guidelines for Selection and Preparation of Whole or Cround Cereal/ Meth. of Urease Bromthymol Blue Through Closed Steel Door and Frame Assemblies (19) on Steel Door and Frame Assemblies (1972)

Farm Wagon Running Gear (1973)

nnectors (1974) ANSI C83.63C

cal Coated Lock Screws (1973)

Rec. Pract. for Instrument Panel Laboratory Impact Rec. Pract. for Service Brake Structurai Integrity ing Electric Machinery (1973) Std. for iconductor Radiation Detectors (1969) ANSl N42.2 Std. for w (1974) Std. for Control Valve Capacity

ear Underride Guard (Safety (1971) (1972) ANS1 S6.2 42.3

Rec. Pract. Rec. Pract. for the Std.

og Torque Balance Accelerome/ Std. Spec. Format Guide and ity on Agricultural Tractors Equipped with 3 Point / Std ity on Agricultural Tractors Equipped with $3 \mathrm{Po} /$

Rec. Pract. for Spark Arrester

Std. Requirements, Terminology, and $r$ Ionizing Radiation (1969) ANSI N42.I

systems for AC Electric Machinery Employing Form Wo/ Std. onmetallic Locking Element Type Prevailing-To/ Std. for istribution Transformers (1972)

ncluding Material Data) (1972)

Rec. Pract. for Service Brake Structural Integrity $1968)$

Energy Consumption of Household Refrigerators, Com/ Std. and Rec. Pract. for Bumper Evaluation

Mobile Construction Type Crane Overload lndicating System Std. for Fiberboard

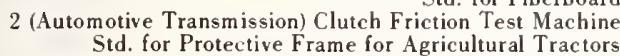
e $\mathrm{J} /$

Frame (For Agricultural Tractors for Overhead Protection Std. for Protective Enclosures for Agricultural Tractors

er, Combination) (1971) ANSI A 197.2, Asse 1007 Definition and Std. and ical Connectors (1971) ANSI C83.63B rs Used in Enclosures (1973)

Minimum Performance Requirements and Uniform

Dishwashers (1971) ANSl A 197.1. Asse 1006

Food Waste Disposer Units (1971) ANSl A197.3, / Std. and nd Demountabl/ Rec. Pract. for Uniform Laboratory Fatigue

and Station/ Minimum Performance Requirements and Uniform s (1972)

hment of Temperature lndexe/
Potentiometers (1970)

Structures for Nuclear Reactors (1972)

Guide for the Preparation of Std. for Inspection and Rec. Pract. for Drill Stern Practice and

Uniform Building Code Std. for Evaluation of Compression Rec. Pract. for Evaluation of Compression

aration of Pieces from Rubber Vulcanizates Other Than Std. dures for Mixing Rubber Compounds for Preparing Vulcanized Test Meth. for Evaluation of Wetting Agents (Cotton Sheet (Coil Coating) (1974)

radiation-Radiology) (1973) Spec. for Spot

for Making and Using the C.Ring Stress Corrosion Cracking Std. Meth. of Making and Curing Concrete Std. Rec. Pract. for Compression Molding

ornia Kneading Compactor Std. Meth. for Preparation of pract. for Determining Temperatures of Std. ASTM Molds for Std. Meth. of Measuring Area of Leather Std. Meth. for Measuring Thickness of Leather

Rec. Pract. for Making and Using U-Bend Stress Corrosion

Pract. for Preparing, Cleaning, and Evaluating Corrosion ing, Accelerated Curing, and Test. of Concrete Compression ead Wires, Terminals, and Other Terminations (Soft Solde operati/ Std. Spec. for an Audio Level and Multifrequency I (For Tape Speeds of 7.5 ln. 0 190.I

Std. for Reproducer Std. for Dimensions for Resolution Meth. of Triangle istry) (1968) (1973) ANS1 J1.3

Std. Rec. Pract. for Sid. 3/ Test Meth. for Scourability of Spinning Lubricant (To $r$ Dealing with Outlying Observations in Samples and How to External lnsulation Rat/ Std. for Schedule of Dielectric
Test of Single Thermoelement Materials by Comparison Wi est of Soils Under Consolidated Drained Conditions (19 Test of Water (1974)

Test on Glass Containers (Bottle, Jar) (1971)

Test on Glass Pipe (1970)

Test on Metal Specimens for Engine Antifreezes Tested l Test on Textile Garments (1973)

Test Panels for Use in Test. Organic Coating Systems (C Test Paper for Analysis of Urea in Cloth or Sack Fibers Test Proc. and Acceptance Criteria for Rate of Air Flow Test Proc, and Acceptance Criteria for Water Resistance Test Proc. for Determining the Load Carrying Ability of Test Proc. for Low Frequency (Below $3 \mathrm{MHz}$.) Electric $\mathrm{Co}_{0}$ Test Proc, for Storage Batteries (1972)

Test Proc. for the Locking Ability Performance of Chemi Test Procedure-Head Area (1971)

Test Procedure-Passenger Car (1971)

Test Procedure for Airborne Sound Measurements on Rotat Test Procedure for Amplifiers and Pream plifiers for Sem Test Procedure for Compressible Fluids in Turbulent Flo Test Procedure for Dry Fertilizer Spreader (1972)

Test Procedure for Evaluation of the Effectiveness of $R$ Test Procedure for Exterior Sound Level for Snowmobiles Test Procedure for Geiger Muller Counters (1970) ANSI N Test Procedure for Incompressible Fluids (1972)

Test Procedure for Linear, Single Axis, Pendulous, Anal Test Procedure for Measuring Hydraulic Lift Force Capac Test Procedure for Measuring Hydraulic Lift Force Capac Test Procedure for Medium Size Engines (1972)

Test Procedure for Neutral Grounding Devices (1972)

Test Procedure for Semiconductor Radiation Detectors $F_{0}$ Test Procedure for the Evaluation of Sealed lnsulation Test Procedure for the Locking Ability Performance of N Test Procedure for Thermal Evaluation of Oil Immersed D Test Procedure for Toroidal Magnetic Amplifier Cores (I Test Procedure for Trucks, Buses, and Combination Vehic Test Procedure for Water Closet Flush Tank Ball Cocks Test Procedure to Determine the Freezer Temperature and Test Procedure (1971)

Test Procedure (1971)

Test Procedure (1972)

Test Procedure (1972)

Rec. Pract. for

(Test Procedures and Performance Requirements) (1972) Sa

Test Procedures and Performance Requirements) (1972) Sa

(Test Procedures and Performance Requirements) (1972) Sa Test Procedures for Ammonia Nitrate (1973)

Test Procedures for Home Laundry Equipment (washer, Dry

Test Procedures for Low Frequency (Below $3 \mathrm{MHz}$.) Electr

Test Procedures for Low Voltage AC Power Circuit Breake

Test Procedures for Motor Vehicle Brake Linings (1966)

Test Procedures for Plumbing Requirements for Household

Test Procedures for Plumbing Requirements for Household

Test Procedures for Production Disc and Spoke Wheels, a Test Procedures for Regular Bicycles (1972)

Test Procedures for Retreaded Tires for Passenger Cars

Test Procedures for the Safety Std. for Regular Bicycle

Test Procedures for the Thermal Evaluation and Establis

Test Procedures for Wirewound and Non wirewound Trimming

Test Report Form (1972)

Test Requirements for Leakage Rate Test. of Containment

Test Results in Field Concrete Cylinders (1973)

Test Results of Field Concrete (1965) ANSI A 146.1

Test Sheets (1973)

Test Sheets (1973)

Test Skein Meth.) (1971) ANS1 L14.11

Test Solution for Detecting Chromate Films on Galvanize

Test Spec. for Selected Brachytherapy Sources (Medical

Test Specimen (1973)

Std. Rec

Test Specimens in the Laboratory (1969) ANSI A37.8

Test Specimens of Allyl Molding Materials (1972)

Test Specimens of Bituminous Mixtures by Means of Calif

Test Specimens of Plastics (1967) ANS1 K65.79

Test Specimens (1968) ALCA E2

Test Specimens (1970) ALCA E4

Test Specimens (1972)

Test Specimens (1972) ANS1 G80.1

Test Specimens (1974)

Test Std. for Determination of Solderability of Solid L

Test Tape: Full Track, 1/4 In. (6.3 Mm) Width, Open Ree

Test Target for Photographic Optics (1972)

Test Taste Panel for Food Flavor Detection (Cereal Chem

Test Temperatures for Rubber and Rubber Like Materials

Test the Removability of Spinning Oils from Woolen Yarn

Test the Statistical Significance of Them (Sampling) (1

Test Values for Outdoor AC High Voltage Circuit Breaker
STDI

STDI

ASAE

ElA

$\mathrm{BCl}$

$1 F 1$

SAE

SAE

IEEE

IEEE

ISA

ASAE

SAE

SAE

IEEE

SA

IEEE

ASAE

$S A E$

SAE

IEEE

IEEE

IEEE

IFl

IEEE

IEEE

SAE

ASSE

AHAM

$S A E$

SAE

SAE

ASAE

ASAE

FI

AHAM

ElA

ANSI

VESC

AHAM

AHAM

SAE

BMA

VESC

BMA

IEEE

VRCI

API

ANSI

ICBO

ACl

ASTM

ASTM

AATCC

NCCA

ANSI

ASTM

ASTM

ASTM

ASTM

ASTM

ASTM

ASTM

Std ASTM

Std. Rec ASTM

ASTM

EIA

ANSl

ElA

ANSI

AACC

ASTM

AATCC

ASTM

ANS1

E207

3080

D2035

C 149

Dl384

D3181

TB. 111.5

28.8

116

115

S360T

*1.66

125

J921 B 
2) cereal Chemistry) (1962)

Meth. of Analysis of Meth. of Compression Spec. for High

th. for Determination of Film Adhesion by Cross Hatch Tape alvanized) Coating on Iron or Steel Articles by the Preece o, Television, Tape Recorder/ ry) (1962)

(1962) try) (1962) Std. for Simulated Shipping Meth. of Analysis for Baking Pekar Color Meth. of Analysis of Pelshenke
r Printed Textiles to Washing at 105 Deg. F: Rapid Control Textiles to Combined Washing and Shrinkage: Rapid Control Water Resistance of Textile Fabrics: Hydrostatic Pressure Test Meth. for Water Repellency of Textile Fabrics: Spray or Water Resistance of Textile Fabrics: Impact Penetration Std. Meth of Vibratory Cavitation Erosion Std. for Fiberboard Crease Bending or Determining Hermeticity of Electron Devices by a Bubble lastics as a Function of Temperature by Means of a Torsion eth. for Oil Repellency of Fabrics: Hydrocarbon Resistance for Water Repellency of Textile Fabrics: Static Absorption pellency of Textile Fabrics: Tumble Jar Dynamic Absorption meth. of Test for Compatibility of Fuel Oil Blends by Spot

for Top Side of Filled Paper as Indicated by Silver Coin $f$ Analysis of Pelshenke Test (Wheat Meal Fermentation Time r Like Materials by a Temperature Retraction Procedure (Tr Meth. of Test for Adhesion of Vulcanized Rubber (Friction Test Meth. for Water Resistance of Textile Fabrics (Rain for Impact Resistance of Pipeline Coatings (Limestone Drop for Impact Resistance of Pipeline Coatings (Falling Weight

ity in 7 XXX Series Copper Containing Aluminum Alloys (Exco td. Meth. of Test for Peel Resistance of Adhesives (T.Peel Electrostatic Clinging of Textile Fabrics Fabric to Metal ect of Heat and Air on Asphaltic Materials (Thin Film Oven Absorptiveness of Nonbibulous Paper and Paperboard (Cobb stability of Confined Condensed Phase Systems (Confinement trical Basis When Rated for Out of Phase / Std. Meth. for ulation (1970)

ials as Used in Cons Sid. Rec. Pract for Inspect Std Meth. of Transformers, Hand Power Tools, Hermatic Stators, 2) ANSI Z9/ Std. Definitions of Terms Relating to Fatigue lectrical and Packing Purposes (1974) Ansi/

Engine Coolants (1972)

ANSI S2.10

oatings (1973) Std. Meth. of Std. Meth. of Std. Meth. of Std. Meth. of Std. Meth. of Std. Meth. of Std. Meth. of Std. Meth. of Sampling and Uniform Building Code Std. for Sampling and Std. Meth. of Std. Meth. of Std. Meth. of Std. Meth. of Std. Meth. of Std. Meth. of Meth. of to Corrosion (1972T atings U lsed for Electrical Insulation (1973) Std. Meth. of rical lnsulation (1973) Std. Meth. of Test/ Std. Meth. of Std. Meth. of Sampling and Std. Meth. of Sampling and hrinkage Propensity of Bonded and Laminate/ Std. Meth. of Std. Meth. of Std. Meth. of for Acoustical and Airflow Performance (19/ fing Materials (1972) Std. Index of Meth. for Std. Meth. of

Std. Rec. Pract. for Std. Meth. of

s Substances for Use in Water/ G85. 1

Std. Meth. of Sampling and Std. Meth. of Sampling and Std. Meth. for Public Works Spec. for Ceramic Veneer (Sampling, al Implants (1972) Std. Rec Pract for Experimenta Pract. for Making Reference Glass-Metal Butt Seals and ract. for Making Reference Glass-Metal Sandwich Seal and sh/ Std. for Dimensions, Physical Properties and Meth. of

or or Residual Gas Analyzer in the Tracer / or in the Detector Probe Mode (1973) s (1973) ANSI J2.6

Std. Meth. of Std. Meth. of Std. Meth. of Std. Meth. of Dynamic Std for Meth of ansi A 111.1 Std. Meth. of Load rumentation) (1/ Std. for Uniform Dimensions and Meth. of
Test Weight Per Bushel of Grain (Cereal Chemistry) (196 Test with Baker Compressimeter for Staleness of Bread ( Test Wrought Welding Fittings (1973)

Test (Coil Coating) (1974)

Test (Copper Sulfate Dip) (1973) AASHO T66

Test (Packaging) for Consumer Electronic Products (Radi

Test (Quality) for Sweet Yeast Products (Cereal Chemist

Test (Slick) Meth. of Flour Analysis (Cereal Chemistry)

Test (Wheat Meal Fermentation Time Test) (Cereal Chemis

Test Meth. for Colorfastness of Dyed O Th. for Colorfastness of Cotton and Linen

Test $(1969)$

Test (1971) ANSI L14.265

Test Meth for

Test (1971) ANSI L14.60, ASTM D583

Test (1971) ANSl L14.71, ASTM D 583

Test (1972)

Test (1972)

Test (1972)

Test (1972) ANSI D65.2

Test (1972) ANSl L14.214

Test (1972) ANSl L14.61, ASTM D583

Test (1972) ANSI L14.87, ASTM D583

Test (1972) ANSl Z11.315

Test (1973)

Test) (Cereal Chemistry) (1962)

Test) (R1972) ANSI J2.22

Test) (1970) ANSI J3.

Test) (1971) ANSI L14.74, ASTM D583

Test) (1972)

Test) (1972)

Test) (1972)

Test) (1972) ANSI Z197.2

Test) (1973)

Test) (1973)

Test) (1973)

Test) (1973)

Test Meth. F

F A

ASTM

SAE

Std. Rec. Pract. F ASTM

for Stiffness Properties of P ASTM

Test M AATCC

Test Meth. AATCC

/Meth. for Water Re AATCC Std. ASTM

Test Meth TAPPI

Test Meth O AACCH 56.50
Meth.

Std ASTM

AATCC 35

Std. Meth. of Test ASTM

Std. Meth. of Test ASTM

St for Exfoliation Corrosion Susceptibil ASTM

ASTM

Test Meth. for AATCC Std. Meth of Test for Eff ASTM

Std Meth of Test for Wate ASTM

Std Meth of Test for Thermal in ASTM

Test. AC High Voltage Circuit Breakers Rated on a Symme

Test. Adhesives Relative to Their U'se as Electrical Ins

Test. Aerosol Products for Delivered Mass (1972)

Test. Agencies for Concrete, Steel and Bituminous Mater

Test. Alkyd Resins (1973)

Test. and Presenting Basic Performance Data for Positve

Test, and Safety) (1973)

Try (Rotating, Liqui

Test. and the Statistical Analysis of Fatigue Data (197

Test. and Tolerances for Jute Rove and Plied Yarn for E

Test. Anti Leak Antifreezes and Stop Leak Additives for

Test. Asbestos Cement Pipe (1973) ANSI A 165.1

Test. Askarels (1973) ANSl C59.62

Test. Automotive Air Brake and Vacuum Brake Hose (1973)

Test. Automotive Hydraulic Brake Hose (1972) ANSI J2.9

Test. Bituminous Base Emulsions for Use as Protective C

Test. Brick and Structural Clay Tile (1973)

Test. Brick (1973)

Test. Cellular Glass Insulating Block (1972)

Test. Cellulose Acetate Propionate and Cellulose Acetat

Test. Cellulose Acetate (1972) ANSI K65.24

Test. Clay Pipe (1972) ANSI A 106.5

Test. Clear and Pigmented Lacquers (1973)

Test. Coated Fabrics (1973) ANSI J2.12

Test. Coated Steel Specimens Dynamically for Resistance

Test. Coating Powders and Their Coatings Used for Elect

Test. Coating Powders and Their Coatings Used for Elect

Test. Concrete Masonry Units (1970) ANSI A84.1

Test. Concrete Pipe or Tile (1973)

Test. Creosote (1970) ANSl 011.9

Test. Delamination, Strength of Bond, Appearance, and S Test. Drying Oils (1972)

Test. Duct Liner Materials and Prefabricated Silencers

Test. Elastomeric and Plastomeric Roofing and Waterproo

Test. Emulsified Asphalts (1974) ANSl A37.42, Ashto T59

Test. Exterior Latex House Paints (1972)

Test. Felt (1972)

Test. Felted and Woven Fabrics Saturated with Bituminou

Test. Ferroalloys for Determination of Size (1970) ANSI

Test. Film Insulated Magnet Wire (1974)

Test. Finishing, Installation, and Definitions) (1961)

Test. for Biological Compatibility of Metals for Surgic

Test, for Expansion Characteristics by Polarimetric Met

Test. for Expansion Characteristics by Polarimetric Met

Test. for Helical Spring and Carhon Steel Tooth Lock Wa

Test. for Leaks Using Bubble Emission Techniques (1974)

Test. for Leaks Using the Mass Spectrometer Leak Detect

Test. for Leaks Using the Mass Spectrometer Leak Detect

Test. for Ply Separation and Cracking of Rubber Product

Test. for Rating Nonresidential Warm Air Heaters (1972)

Test. for Refractory Brick at High Temperatures (1970)

Test. for Rotary Selector Switches (Radio, TV, and Inst
IEEE

ASTM

AsTM

ASTM

ASTM

NFLDP

EASA

ASTM

ASTM

ASTM

ASTM

ASTM

ASTM

ASTM

ASTM

ASTM

ICBO

ASTM

A STM

ASTM

ASTM

.

ASTM

ASTM

ASTM

A II

A STM

ASTM

ASTM

ASTM

A STM

ASTM

ASTM

ASTM

ASTM

ASTM

ASTM

ASTM

BlA

ASTM

ASTM

ASTM

ANSI

ASTM

ASTM

ASTM

ASTM

ASHRA

ASTM

EIA
G13

G14

G34

D 1876

115

D1 754

D3285

E47

417

D) 304

E 329

D 2689

3.9 .17

E206 
Std. Meth. of Salt Spray (Fog) Uniform Building Code Std. for Std. Meth. of Std. Meth. of
Std. Meth. of (1974) nd Decorative (1972) 970) Safety Std. for Play Pipes for Water Supply
Std. Meth. of
Std. Meth. of
Std. Meth. of Safety Std. for Play Pipes for Water Supply
Std. Meth. of
Std. Meth. of
Std. Meth. of Safety Std. for Play Pipes for Water Supply
Std. Meth. of
Std. Meth. of
Std. Meth. of Safety Std. for Play Pipes for Water Supply
Std. Meth. of
Std. Meth. of
Std. Meth. of

ANSI $\mathrm{MH} 12.11$ onstruction, and Operation of Class Hi (High lmpact) Shock r Car Tires (1972) Rec. Pract. for Std. Meth. of Verification of Std. Meth. for

materials (1973)

) ANSI Z197.14 Uniform Building Code Std. Std. Rec. Guide for the Selection of Leak tional Vehicle Air Conditioning System Rating Definitions, Std. Meth. of sing the Mass Spectrometer Leak Detector in the Inside Out Std. Meth. of Std. Meth. of moval of Particles (1972)

Std. for Efficiency

si $\mathrm{K} 90.6$

Pract. for Static Bend Std. Neth. of Tension 972) Std. Meth. of Making, Accelerated Curing, and Practice and Test Requirements for Leakage $R$ ate Rec. Pract for Hardware Std. Meth. of Test for Simulated Service Corrosion Safety Std. for Rating and Meth. of Drop

ulus of Rupture) (1972) Std. Meth. of Flexure Std. Meth. for Physical Std. Meth. of Tension

Std. Rec. Pract. for Sharp Notch Tension or Moist Cabinets and (Specimen Storage) Rooms Used in the Std. Meth. for High Pressure Pump Rec. Pract. for Std. Rec. Pract. for Static Bend and Torsion Std. Meth. of Compression Std. Meth. of Tension Std. Neth for Notched Bar lmpact Std. Rec. Pract. for Scleroscope Hardness Test Meth. for Laboratory Corrosion Std. Rec. Pract. for Laboratory Immersion Corrosion

Std. Rec. Pract. for Static Bend Std. Meth. of Tension

stems (19/ Std. for Trial-Use Criteria for the Periodic
1974) Std. Meth. for Condensation-Humidity Std. Meth. of Physical std. Meth. of Compound and Sample Preparation for Physical std. Rec. Pract. for Combined, Simulated Space Environment Std. Rec. Pract. for Solar Simulation for Thermal Balance saturation (1971) Ansi/ Std. Rec. Pract. for Eddy Current Std. Rec. Pract. for .lacroetch

Uniform Building Code Std. for and Meth. of Sampling and 2) $\left.\mathrm{O}_{4}\right)(1972)$ Std. Meth. of Static Immersion Std. Test Meth. for Simulated Service

Std. Meth. and Definitions for Mechanical es for Selection and Preparation of Test Panels for Use in nsi $\mathrm{MH} 12.13$ 73) Std. Meth. of

3) Std. Meth. for Preparation of Steel Panels for
Std. Meth. of Std. Meth. of

wheel Meth.) (1972) Std. Rec. Pract. for Std. for Qualifications of lnspection, Examination, and ) (1969) Std. Meth. of Tent. Meth. of Tent. Meth. of

Electrical Insulation (1974) ANSl C59.136 (1974)

Cans, Tubes and Cores (1973)

s (1973)

cores (1973)

osite Cans, Tubes and Cores (1974)

site Tubes and Cores (1972)

ubes and Cores (1972)

ite Motor Oil Cans (1974)

s (1974)

$s$ and Cores (1974)

posite Cans, Tubes, and Cores (1974)

site Cans, Tubes and Cores (1974)

cans (1974)

cans (1973) Std. Meth. of
Test of Spec. Purposes (Coil Coating)(1974) ASTM B11

Test. Gypsum Plasters and Concrete (1973)

Test. Heat Shrinkable Tubing for Electrical Insulation

Test. Hookup Wire Insulation (1974)

est. Hydroxypropyl Methylcellulose (1972)

Test. in Fire Protection Service (1973) ANSI Blll.

Test. Insulating Board (Cellulosic Fiber), Structural a

Test. Laminated Tubes Used for Electrical Insulation (1

Test. Large Shipping Cases and Crates (Packaging) (1953

Test. Machine for Lightweight Equipment (1972)

Test Machine) (1971) Noth of Woven Fabrics by the to ASTM

Test. Machines for Measuring the Uniformity of Passenge

Test. Machines (1972) AASHO T67, ANSI Z115.1

Test. Magnetic Wire Enamels (1973)

Test. Metal Fasteners in Wood (1974)

Test. Meth. for Fire Hazard Classification of Building

Test. Neth. for lmpact Strength of Adhesive Bonds (1972

Test. Meth. (1971) ANSI Z166.26

Test. Meth., and Test Equipment and Rating Meth. (1972)

Test. Methylcellulose (1972) ANS] K65.26

Test. Mode (1973)

Std. Meth. of Test for Leaks U

Test. Molded Flexible Urethane Foam (1973)

Test. Nonwoven Fabrics (1974) ANSI L14.246

Test. of Air Cleaning Systems Containing Devices for $\mathrm{Re}$

Test, of Bone Plates (Surgical lmplants) (1973)

Test, of Carbon Graphite Mechanical Materials (1971) an

Test. of Concrete Compression Test Specimens (1974)

Test. of Containment Structures for Nuclear Reactors (1

Test. of Digital Process Computers (1971)

Test of Engine Coolants (1973)

Test. of Fire Extinguishers (1973) ANS] Z211.1

Test. of Glass Aerosol Bottles (1972)

Test. of Glass and Glass Ceramics (Determination of Mod

Test. of Gypsum Plasters and Concrete (1973)

Test. of Hard Rubber (1972)

Test. of High Strength Sheet Materials (1968) ANSI Z260

Test. of Hydraulic Cements and Concretes (1973)

Test. of Hydraulic Oils (1973)

Test. of lndustrial Exhaust Ventilation Systems (1974)

Test. of Intramedullary Rods (Surgical Implants) (1973)

Test. of Metallic Materials at Room Temperature (R1973)

Test. of Metallic Materials (1969) ANSl Z168.13

Test. of Metallic Materials (1972)

Test. of Metallic Materials (1972)

Test. of Metals for the Process Industries (1972)

Test, of Metals (1972)

Test. of Nail Plates (Surgical lmplants) (1973)

Test. of Nonmetallic Gasket Materials (1972)

Test. of Nuclear Power Generating Station Protection Sy

Test, of Organic Coatings on Metallic Surfaces (Coil)

Test. of Quicklime and Hydrated Lime (1971) ANSI K67.10

Test of Rubber Products (1972) ANSI Jl.1

Test. of Solid Urethane and Other Rubbers (1973)

Test. of Spacecraft Thermal Control Materials (Coatings

Test. of Spacecraft (1973)

Test, of Steel Tubular Products (Tubing) with Magnetic

Test. of Tool Steel Bars (1971) ANSl G60.9

Test, of Unburned Clay Masonry Units (1973)

Test. of Unstressed Materials in Nitrogen Tetroxide (N

Test. of Wood and Wood Base Finish Flooring (1969)

Test. of W rought and Cast Steel Products (1973) ANSl G6

Test. Organic Coating Sy stems (Coil) (1974)

Test. Package Cushioning Materials (Packaging) (1964) a

Test. Paint, Varnish, Lacquer, and Related Products (19

Test. Pallets (1973)

Test. Para Red and Toluidine Red Pigments (Toners) (197

Test. Pavement Polishing in the Laboratory (Full Scale

Test. Personnel for the Construction Phase of Nuclear P

Test. Plywood in Flexure (1972)

Test. Plywood in Rolling Shear (Shear in Plane of Plies

Test. Plywood in Shear Through the Thickness (1969)

Test. Primers and Primer Surfaces Over Preformed Metal Std. Test. Proc. for Measuring Inside Diameters of Composite Std. Test. Proc for Pressure Collapse Test of Composite Can Std. Test. Proc. for Side to Side Crush Composite Tubes and Std. Test. Procedure for Axial (End to End) Compression Comp Std. Test. Procedure for End Supported Beam Deflection Compo Std. Test. Procedure for End Supported Beam Strength Fibre T Std. Test. Procedure for Filled Cast Flat Bottom Drop Compos Std. Test. Procedure for Filled Cast Flat Drop Composite Can Std. Test. Procedure for Internal Bursting of Composite Tube $\mathrm{Std}$. Test. Procedure for Measuring Lengths or Heights of Com

Std. Test. Procedure for Measuring Wall Thicknesses of Compo

Std. Test. Procedure for Oil Wicking of Composite Motor Oil

Std. Test. Procedure for the Blow Off Pressure of Composite
NCCA

ICBO

ASTM

ASTM

ASTM

UL

ASTM

ASTM

ASTM

$S A E$

ASTM

ASTM

ASTM

$\mathrm{ICBO}$

ASTM

ASTN

IMACA

ASTMI

ASTM

ASTMI

ASTM

ASTM

ASTM

ASTM

ANSl

ISA

ASTM

UL

ASTM

ASTM

ASTMl

ASTM

ASTM

Ec. F AST

ASTM

ACGIH

ASTM

ASTM

ASTM

ASTM

ASTM

NACE

ASTM

ASTM

IEEE

NCCA

ASTM

ASTM

ASTI

ASTM

ASTM

AST

ASTM

ICBO

ASTY

ASTM

AsT

Guidelin NCCA

ASTM

ASTM

ASTM

ASTM

ASTM

ANSl

ASTM

ASTM

ASTM

ASTN

ASTN

CCTI

$\mathrm{CCT}$

CCTI

CCTI

CCTI

CCTI

CCTl

CCTl

CCTl

CCTl

CCTI

CCTl

CCTl

TB-111-2

IBCS24.26

D2671

D 3032

385

C209

D348

D 1083

S2.15

D2262

J332A

D3288

D1761

UBCS42-1

D950 
and Cores (1974)

ic Equipment (1973) ANSI C83.25

Std. Spec. for Perforated Plate Sieves for

Std. Spec. for Wire Cloth Sieves for g Aqueous Solutions of Engine Antifreezes or Antirusts for Std. Meth. of Drip Slag Std. Meth. of Std. Meth. of Std. Meth. for Std. Meth. of Std. Meth. of Std. Meth. of Std. Meth. of

i C59.18

gonal Drum (1968) ANS1 MH12.3, TAPP1 T800

Std. Rec. Guide for Preparation of a Leak ip Resistant (Floor Polish and $W_{\text {ax }}$ ) (1971) ASTM D2047, C/ clay Load Bearing Wall Tile and Std. Meth. of Sampling and Std. Meth. of

reservatives by Using $\mathrm{W} /$

utions (1970)

ands Made from Steel (1974)

ng Torque Application, Using Highway Vehic/

974) ANS1 C 59.77

ical Insulation (1970) ANSI C59.31

nsi C 59.30

ions (1972) ANSI O7.1

ations (1969) ANS1 C59.73

1971) ANS1 C59.78

res $(1970)$

ducts (1973) ANS1 H46.

Std Meth of Tension

Summary of Meth. for Biscuit and Cracker Flour Sid. Meth. of Sampling Asbestos Fiber for Std. Definitions of Terms Relating to Leak std. Rec. Pract. for Sampling Cotton Fibers for Std. Meth. for Controlling Quality of Radiographic building Code Std. for Non combustible Elementary Materials exit, Stairway, Aisle, Corridor, Building Construction and Std. Meth. of Salt Spray ( $F o g)$

Std. Meth. of Conditioning Paper and lts Products for Std. Definitions of Terms Relating to Ultrasonic

Pract. for Conditioning Textiles and Textile Products for Std. Meth. of Acetic-Acid Salt Spray (Fog) red Industrial/ Rec. Pract. for Iniform Procedure for the r Corrosion Test on Metal specimens for Engine Antifreezes esistance of Shoe d per Leather by the Dow Corning Leather
ance of Shoe Upper Leather by the Maeser Water Penetration

Flash Point of Aviation Turbine Fuels by Setaflash Closed acquer, Varnish, and Related Products) by Setaflash Closed urface Strength of Coated Papers Using the Interfiber Bond

Meth. of Test for Flash Point by Pensky-Martens Closed

g Surface Frictional Properties Using the British Portable est Meth. for Smoothness of Paper and Paperboard (Bendtsen 2.27

dhesives (1972) ANS1 Z197.21

972) Std. Meth. of Std. Meth. of Std. Meth. of Std. Meth. of Std. Meth. of Std. Meth. of Std. Meth. of

(1971)

Conditioning Equipment (1972)

heading Wire and Rods (1972)

Std. Meth. of Shear Std. Meth. of 1mpact

looring (196/ Rec. for Correct Preparation, Finishing and

es on Boilers (1972)

quipment During the $\mathrm{Cl}$ els (1972)

Rec. Procedure for Compression

Std. Installation, 1nspection, and Std. Spec. for Charpy V.Notch Std. Meth. of Rules for

ns, Made from Man Made Organic Base Fibers/ Std. Meth. of $m$ Speed Stationary Diesel and Gas Engine Factory and Field Std. Techniques for Switching $1 \mathrm{mpulse}$

Uniform Alignment

Spec. for Enclosures, Cold Chambers and Serving Units for Motors (Fractional and Integral Horsepower) (1/ Std. for and Hose Assemblies (1970)

Std. for

ners (1971)

Tent. Spec. for Molds for Forming Concrete
Rec. Pract. Meth. of Rec. Pract. Meth. of
Rec. Pract.

Safety Std. for

otection) (1973) ANS1 Z240.1

Safety Std. for Std. Rec. Pract. for Conducting Bending Fatigue
Test. Procedure for Torque Strength of Composite Tubes

Test. Procedures for Relays for Electrical and Electron

Test. Purposes (1970) ANS1 Z168.12

Test. Purposes (1970) ANS1 Z23.1

Test. Purposes (1971) ANS1 D14.4

Test. Radioactive Brachytherapy Sources (1973)

/Ampling and Preparin

Test. Refractory Brick at High Temperature (1973)

Test. Rigid Sheet and Plate Materials Used for Electric

Test. Rubber O.Rings (1972)

Test. Sewing Threads (1971) ANS1 Ll4.14

Test. Shellac Used for Electrical Insulation (1973) Ans

Test. Shipping Containers (Packaging) in Revolving Hexa
Test. Sodium Carboxymethylcellulose (1972) ANSI K65.30

Test. Spec. (1973)

Test. Std. (Procedure) for Products Classified as to $\mathrm{Sl}$ Td. for Structural

Test. Structural Clay Tile (1973)

Test. Synthetic Rubber Latices (1974) ANSI J2.34

Test. Terminology for Defects in Steel Pipe (1974)

Test. the Preservative Properties of Oil Soluble Wood P

Test. the Water Repellent Effectiveness Df Treating Sol

Test. Tire Cords, Tire Cord Fabrics, Filaments, and Str

Test. Tires for Wet Traction in Cornering Without Drivi

Test. Twine Made from Bast and Leaf Fibers (1973)

Test. Untreated Paper Used for Electrical Insulation (1

Test. Varnished Cottonfabrics and Tapes Used for Electr

Test. Varnishes Used for Electrical Insulation (1972) a

Test. Veneer, Plywood, and Other Glued Veneer Construct

Test. Vitrified Ceramic Materials for Electrical Applic

Test. Vulcanized Fiber Used for Electrical Insulation (

Test. Warp Knit Fabrics (1964) ANSI L14.284

Test. Water Formed Deposits (1972)

Test. Wood Preservatives by Laboratory Soil Block Cultu

Test. Wrought and Cast Aluminum and Magnesium Alloy Pro

Test. (Cereal Chemistry) (1962)

Test. (1970) ANS1 K91.2

Test. (1971) ANS1 Z166.25

Test. (1972)

Test. (1972) ANS1 2166.7

Test. (1973)

Test. (1973)

Test. (1973) ANS1 Z118.

Test. (1973) TAPPI T402

Test. (1974)

Test. (1974) ANS1 L14.205

Test. (1974) ANSI Z118.2

Test., Rating and Reporting of the Noise Levels of Powe

Tested in Glassware (1970) ANSI D14.9

Tester (1970) ALCA E55

Tester (1970) ALCA E56

Tester (1973)

Tester (1973)

Tester (1973)

Tester (1973) ANS1 211.7

Tester (1974)

Tester) (1973)

Testing Automotive Air Conditioning Hose (1968E) ANSl J

Testing Cross Lap Specimens for Tensile Properties of a

Testing Fatty Acids Used in Protective Coatings (1972)

Testing Flow Capacity of Refrigerant Capillary Tubes (1

Testing for Rating Liquid Coolers (1971)

Testing for Rating Water Cooled Refrigerant Condensers

Testing for Sound Rating Heating, Refrigerating and Air

Testing of Aluminum and Aluminum Alloy Rivets and Cold

Testing of Cast 1rons (1972)

Testing of Concrete Subfloor Surfaces to Receive Wood F

Testing of Scaffolds and Shores (1967)

Testing Procedures and Maintenance of Steam Safety Valv

Testing Requirements for Instrumentation and Electric E

Testing Requirements for Steel Plates for Pressure Vess

Testing Rubber Hose (1972A) ANSI J2.5

Testing Seeds (1970)

Testing Tire Cords, Fabrics and 1ndustrial Filament Yar

Testing (1972)

Testing (1972) ANS1 C68.2

Std. Pract. for Low and Mediu

Tests-NAS 900 Series Equipment Specs. (1972)

Tests Above and Below Room Temperature (1966) ANS1 C59.

Tests and Performance of Alternating and Direct Current

Tests and Procedures for SAE 100R Series Hydraulic Hose

Tests Cylinders Vertically (1973T) ANS1 A37.95

Tests for Automotive Type Sealers, Adhesives, and Deade

Tests for Central System Fluids (1970)

Tests for Combustibility of Sweeping Compounds (Fire $\mathrm{Pr}$

Tests for Comparative Flammability of Liquids (1972)

Tests for Copper Alloy Spring Materials (1973)

Tests for Determining Surface Strength of Coated Papers
CCTI

E1A

ASTM

ASTM

ASTM

ASTM

SAE

ASTM

ASTM

ASTM

ASTM

ASTM

CSMA

CSMA

ASTM

APl

NWMA

NWMA

ASTM

ASTM

ASTM

ASTM

ASTM

ASTM

ASTM

ASTM

ASTM

ASTM

ASTM

ASTM

ASTM

$\mathrm{AACCH}$

ASTM

ASTM

ASTM

ASTM

Uniform 1CBO

WWPA

ASTM

ASTM

A TTM

ASTM

ITA

ASTM

ASTM

ASTM

ASTM

$S$ TAPP1

ASTM

TAPP

ASTM

ASTM

ASTM

ASHRA

ASHRA

ASHRA

ASHRA

ASTM

ASTM

WSF 1

SSI

FMS

IEEE

ASTM

ASTM

AOSA

ASTM

DEMA

IEEE

NSA

ASTM

NEMA

SAE

ASTM

SAE

$S A E$

UL

UL

ASTM

TAPP1

T116

RS407

E323

E11

$\mathrm{N} 44.2$

C768

J 365 A

D229

D204

D411

D 1439

E479

BUL 12

UBCS 24.8

D) 417

BUL5T1

M. 1.70

M. 2.70

D2969

F376

Dl 233

D202

D295

D115

D805

D116 
73)

m Doors (1972) ANSl A 153.1

n Devices and Appliances (1973) Huilding Code Std. for Field Rract. for Stress Relaxation Std. Rec. Pract. for Surveillance Test Meth. for Drop 973) Std. for lllumination ) for Structural Requirements for Regular Bicycles Including Std. for Shutter
Std. Specs. and instrumentation) (1971) Std. Rec. Pract, for Performing Uniform Building Code Std. for Std. Meth. of Fire Std. Definitions of Terms Relating to Fire Uniform Building Code Std. for Fire Std. Definitions of Terms Relating to Acoustical .51 Std. Meth. of Wetting and Drying e the Containment of Nuclear Po/ Trial Use Guide for Type Std. Meth. of Fire

Uniform Building Code Std. for Fire Std. Meth. of Fire Std. for Safety for Fire

Tent. Meth. for Drop Weight Tear General Extensigraph Meth. for Physical Dough elaxation Meth. of Extensigraph Analysis of Physical Dough $s$ Cement Shingle Blanks to Be Used as Panels in Weathering Std. Rec. Pract. for Conducting Creep Rec. Pract. for Recording Data from Atmospheric Corrosion Std. Rec. Pract. for Conducting Exterior Exposure Q4.2 Std. Meth. of Static

Uniform Building Code Std for Fire Sid. Meth. of Conducting Machining Std. Meth. for Spinning
nalysis Of, and Physical performance (1972) ANSl L14.289 ectrometric, and Spectrochemical Analysis Of, and Physical aph Meth. for Quality of Nonfat Dry Milk in Physical Dough

Test Meth. for Packaging Film and Construction Identity act. for Preparation of Bar and Rod Specimens for Adhesion instrumentation for Motor Vehicle and lts Component lmpact Std. Meth. for Sampling Leather for Physical and Chemical eth. of Conducted Controlled Velocity Laboratory Corrosion Std. Spec, for Std. Tire for Pavement Skid Resistance Uniform Building Code Std. for Concrete h. of Painting Ceiling Materials for Acoustical Absorption asurement of Partial Discharges (Corona) During Dielectric to Change Mixing Characteristics of Flour (Physical Dough Specifying ograph Absorption to $14.0 \%$ Moist/ Meth. of Physical Dough of Oil Soluble Wood Preservatives by Using W/ Soil Block atter Meth. of Analysis (Cereal Chemistry) Alcohol; Carbon 1 Insulating Tape (1974) als (1973) Std. Spec. for

) (1971)

(1970) ANSI K60.20 for Abbreviations for Use on Drawings and in Text (1972) Std. for Abbreviations for Use on Drawings and in Manufacturing Std. and Spec. for st Meth. for Colorfastness to Washing: Characterization of ANSI L 14.57 Test Meth. for Cotton and Linen Rec. Evaluation Procedure of Gray Scale for Color Change Rec. Evaluation Procedure of Gray Scale for Staining abrics Due to Flat Abrasion (Frosting): Screen Wire Meth.

$\mathrm{e}$ in Fabrics Due to Flat Abrasion (Frosting): Every Meth. $n$ Procedure of Chromatic Transference Scale (Color Chart) th. for Assay of Bacterial Alpha-Amylase Enzymes Used in 114.239 Test Meth. For Oily Stain Release Meth. of
ifferential Dyeing Behavior of Cotton (Raw Fiber, Yarn and or Meth. (1970)

midities (1972) ANSI L14/ (1971) ANSI L14.227

ernate Exposure (1972) A/ aundering/ 83 Test Meth. for Abrasion Resistance of Test Meth. for Electrostatic Clinging of Test Meth. for Colorfastness of Test Meth. for Colorfastness of Test Meth. for Colorfastness of Test Meth. for Colorfastness of the Appearance of Durable Press Test Meth. for Water Resistance of Test Meth. for Fire Resistance of

h. for Evaluation of Commercial Rewetting Agents on Cotton Test Meth. for Bond Strength of Bonded and Laminated est Meth. for Electrical Resistivity (Electro-Static) of
Tests for Fire Resistance of Roof Covering Material (19 Tests for Fire Resistance of Vault and File Storage Roo Tests for Flammability of Photographic Film (1972) Tests for Flammability of Plastic Materials for Parts 1 Tests for Grout and Mortar (1973)

Tests for Materials and Structures (1972)

Tests for Nuclear Reactor Vessels (1973)

Tests for Paper Shipping Sacks and Bags (Containers) (1

Tests for Photographic Still Projection Equipment (1971

Tests for Static Load, Frame, Fork, Steering Assembly a

Tests for Still Picture Cameras (Photography) (1972)

Tests for Strain Gage Linear Acceleration Transducers (

Tests for Structural Glued Laminated Lumber (1973)

Tests in a Boiling Magnesium Chloride Solution (1973)

Tests of Building Construction and Materials (1972)

Tests of Building Construction and Materials (1973)

Tests of Building Construction and Materials (1973)

Tests of Building Constructions and Materials (1973)

Tests of Compacted Soil Cement Mixtures (1971) ANSI A37

Tests of Continuous Duty Class 1 Motors Installed Insid

Tests of Door Assemblies (1972)

Tests of Door Assemblies (1973)

Tests of Door Assemblies (1973) ANSI A2.2

Tests of Door Assemblies (1974) ANSI A2.2

Tests of Ferritic Steels (1971) ANSl 2260.3

Tests of Flour (Cereal Chemistry) (1962)

Tests of Flour (Cereal Chemistry) (1962)

Tests of Latex and Emulsion Exterior Paints or Coatings

Tests of Metal to Metal Adhesives (1972) ANSI Z197.22

Tests of Metallic Coated Steel Specimens (1972)

Tests of Paints on Wood (1973)

Tests of Timbers (Wood) in Structural Sizes (1967) ANSI

Tests of Window Assemblies (1973)

Tests of Wood and Wood Base Materials (1970)

Tests on the Cotton System for Measurement of Spinning

Tests On, Beryllium Oxide Powder (1972) ANS1 N140

Tests with Stakes (1974)

Tests (Cereal Chemistry) (1962)

Tests (Paper) (1973)

Tests (1969)

Tests (1971)

Tests (1971) ALCA J1

Tests (1972)

Tests (1973)

Tests (1973)

Tests (1973)

Tests (1973)

Tests) (Cereal Chemistry) (1962)

Tests, Plastic Concrete (1967)

Tests: Approximate Corrections for Changing as-is Farin
Test: Std. Meths. for Test. the Preservative Properties

Tetrachloride; Chloral Hydrate; Chloroform; Formaldehyd

Tetrafluoride (1972)

Tetrafluoroethylene Backed Pressure Sensitive Electrica

(Tetrafluoroethylene) Resin Molding and Extrusion Materi

(Tetrafluoroethylene) Rod (1974) ANSl K65.208

Tetramethrin (Common Name for the Pest Control Chemical

Tetrasodium Pyrophosphate (Anhydrous) (Cleaning Agent)

Tetrasodium Salt of EDTA in Water (1972T)

Tetroxide $\left(\mathrm{N}_{2} \mathrm{O}_{4}\right)(1972)$

Text (1972)

Text (1972) Text (1972)

Textbooks (Books) (1973)

Textile Colorants (1972)

Textile Colorfastness to Bleaching with Chlorine (1972)

(Textile Colorfastness) (1954) 150 105-1

Textile Colorfastness) (1954) 1SO 105-1

(Textile Colorfastness) (1970)

(Textile Colorfastness) (1970)

Textile Colorfastness) (1972

Textile Desizing (1973) ANSl L14.147

Textile Fabric Soil During Home Laundering (1970) ANS

Textile Fabric) (1972) ANSI L14.152

Textile Fabrics and Other Flexible Materials: Accelerat

Textile Fabrics Fabric to Metal Test) (1973)

Textile Fabrics to Ozone in the Atmosphere Under Low Hu

Textile Fabrics to Water and Light: Alternate Exposure

Textile Fabrics to Water Spotting (1972) ANSI L14.148

Textile Fabrics to Water (High Humidity) and Light: Alt

Textile Fabrics (Permanent Press) After Repeated Home L

Textile Fabrics (Rain Test) (1971) ANSI L 14.74, ASTM D5

Textile Fabrics (1969)

Textile Fabrics (1971)

Textile Fabrics (1971) ANSl L14.106

Textile Fabrics (1972)

Textile Fabrics (1972) ANSI L14.112
UL

UL

UL

UL

ASTM

ASTM

TAPPI

ANSI

BMA

ANSl

ISA

lCBO

ASTM

NFPA

ASTNI

ICBO

AST M

A STM

IEEE

NFPA

ICBO

ASTM

UL

ASTM

$\mathrm{AACCH}$

A ACC

ASTM

ASTM

Std. ASTM

ASTM

ASTM

CBO

ASTM

ASTM

/ Sp ASTM

ASTM

Extensigr AACCH

TAPPl
ASTM

Rec. Pr AST

ASTM

Std. M AWPA

ASTM

ICBO

Std. Met ASTM

IEEE

$\mathrm{AACCH} \quad 54$

CSl 03303

A ACCH $54-29$

NWMA M-1.70

A ACCH 28-91

St ANSI N15.6

ASTM D2686

ASTM Dl 457

ASTM D1710

ANSI K62.106

ASTM D595

ASTM D3113

F359

Y1.1

ANSI Y1.1

BMl

$+1$

Te AATCC 36

AATCC 3

AATCC EPl

AATCC EP2

EP2

$\begin{array}{ll}\text { AATCC } & 120 \\ \text { AATCC } & \text { EP3 }\end{array}$

AATCC EP3

A ATCC

ASTM D1464

AATCC 93

AATCC 115

AATCC 109

AATCC 125

AATCC 104

AATCC 126

AATCC 124

AATCC 35

AATCC 34

ASTM D1175

Test Met AATCC 27

A ATCC 136 
or Pilling Resistance and Other Related Surface Changes of home Laundering of Durable (Permanent) Press Woven or Knit

(1968) ANSI L14.176 114.265

4.71. ASTM D583 10. ASTM D 1295 83 61. ASTM D 583 72) ANSI L14.87, ASTM/ ax, Synthetic Fibers, Wool) (197 Test Meth. for Wrinkle Recovery of Test Meth. for Resistance of Test Meth. for Water Resistance of Test Meth. for Water Resistance of Test Meth. for Wrinkle Recovery of Woven Test Meth. for Water Repellency of Test Meth. for Water Repellency of Test Meth. for Water Repellency of Tent. Meth. of Test for Breaking Tenacity of Man Made d. Meth. of Test for Tensile Properties of Single Man Made th. of Test for Breaking Strength and Elongation of Cotton Std. Meth. of Test for Shrinkage of Test Meth. for Shampooing: Washing of

Tent. Rec. Pract. for Conducting Wear Test on for Evaluating Oxidation of Wool Oils on Woolen Fabric Std. Safety Requirements for the

Meth. of Test for Moisture Content and Moisture Regain of xposure with Wettin/ Test Meth. for Weather Resistance of Std. Meth. of Test for Asbestos Content of Asbestos
Std. Definitions of Terms Relating to Fire Hazards and Protection Rec. for Test Meth. for Electrical Resistivity

nsi L14.119 Std. Definitions of Terms Relating to Care of Consumer
Std. Rec. Pract. for Conditioning Textiles and Std. for Glossary of Rec. Terms for Care Labeling th Safety Practices for Handling and Fabricating Asbestos 11) ANSl L14.180 Test Meth. for Multiple Sample Meth. of 14.126 Test Meth. for Single Sample Meth. of Std. Definitions of

icc Crockmeter M/ ertical Crockmeter Meth. 72) ANSI L14.149 150

Test Meth. for Colorfastness of Colored Test Meth. for Colorfastness of Test Meth. for Colorfastness of Colored Test Meth. for Colorfasiness of

Test Meth. for Colorfastness of Colored Test Meth. for Colorfastness of Dyed or Electrical Resistivity Textile of (Electro-Static) of test Meth. for Appearance of Seams in W ash and Wear Items inkage Propensity of Bonded and Laminated Apparel Fabrics d. Perform ance Requirements for $100 \%$ Cotton Woven Fabrics (Clothi) Std. Performance Requirements for Woven Fabrics (Clothi/

Std. Performance Requirements for Woven Fabrics Tent. Tolerances for Tire Fabrics

nt of Airborne Asbestos Fiber by the Membrane Filter Meth. gth Uniformity of Cotton Fibers by Fibrograph Measurement meth. of Test for Seam Strength of Curtains and Draperies Meth. of Test for Resiliency and Thickness of Blankets d. Performance Requirements for Huck Towels (Institutiona

Performance Requirements for Glass Towels (Institutional

of Test for Colorfastness of l pholstery Fabrics to Water performance Requirements for Woven Blankets (Institutional rformance Requirements for Woven Bath Mats (Institutiona

$f$ Test for Colorfastness of Upholstery Fabrics to Solvent rformance Requirements for Woven Bedspreads (Institutional formance Requirements for Woven Dish Towels (lnstitutional ance Requirements for Woven Shower Curtains (Institutional ce Requirements for Woven Slipcover Fabrics (Institutional ght Transmittance of Woven Glass Fiber Decorative Fabrics ct of Handwashing on Woven Glass Fiber Decorative Fabrics

e Woven Fabric's Resistance to Sagging or Stretch in Wear ments for Woven Lining Fabrics for Institutional Uniforms ents for Damask Table Napery (Napkin, etc.) (Institutional is for Woven Upholstery Fabrics (Tacked On) (Institutional for Terry Cloth Towels (Single and Double) (Institutiona $r$ Woven Printed Table Napery (Napkin, etc.) (Institutional woven Drycleanable Overcoat Fabrics (Institutional Uniform $s$, and Certification of Fabrics or Products (Institutiona orm Fabrics, Lightweight (Tropical) Suiting (Institutional no Iron) Woven Sheet and Pillowcase Fabrics (Institutional sional Change (Shrinkage) of Stabilized Knit Wool Fabrics

Washable Uniform Fabrics for Men and Women (Institutional mation, Brittleness and Chlorine Retention) (Institutional Than Damask (W'hite or Dyed) (Napkin, etc.) (Institutional form Fabrics for Men and Women (Heavy Duty) (Institutional pillowcase Fabrics Other Than Durable Press (lnstitutiona m Fabrics, Heavyweight (Industrial) Suiting (Institutional Fabrics, Intermediate (Year Round) Suiting (lnstitutional

(Curtain) Fabrics (Excluding Glass Fibers) (Institutional rics Handwashable at $105 \mathrm{Deg}$. F-No Bleach (Institutional d Canopy Fabrics, Vinyl Coated or Laminated (Institutional .205

Drycleaning Durability of Applied Designs and Finishes on

for Dimensional Changes in Laundering of Woven and Knitted

$t$ Meth. for Quick Evaluation of Wettability of Fabrics and
Textile Fabrics (1972) ANSI L14. 198

Textile Fabrics (1973) ANSI L14.266

Textile Fabrics: Appearance Meth. (1970) ANSI L14.229

Textile Fabrics: Exposure to Natural Light and Weather

Textile Fabrics: Impact Penetration Test (1971) ANSI L 1

Textile Fabrics: Recovery Angle Meth. (1972) ANSI L14.1

Textile Fabrics: Spray Test (1971) ANSI L14.60, ASTM D5

Textile Fabrics: Static Absorption Test (1972) ANSI L 14

Textile Fabrics: Tumble Jar Dynamic Absorption Test (19

Textile Fiber Storage (Cotton, Jute, Hemp and Sisal, F

Textile Fibers in Loop or Knot Configurations (1973)

Textile Fibers Taken from Yarns and Tows (1972) ANSI Ll

Textile Fibers (Flat Bundle Meth.) (1972) ANSl L14.96

Textile Fibers (1972) ANSI L14.142

Textile Floor Coverings (Rug, Carpet) (1972)

Textile Garments (1973)

Textile in Storage (1971) ANSl L14. S2

Textile Industry (1972)

Textile Material (1971)

Textile Materials and Coated Fabrics: Carbon Arc Lamp E

Textile Materials (1972) ANSI L14.141

Textile Materials (1973) ANSI L14.12

Textile Mills (1974)

Textile of (Electro-Static) of Textile Yarns (1973) a

Textile Products and Rec. Pract. for Use of These Terms

Textile Products for Test. (1974) ANSI L 14.205

Textile Products (Launder or Dryclean) (1973)

Textile Products (1971)

Textile Tensile Loss by Retained Chlorine Bleaching (19

Textile Tensile Loss by Retained Chlorine (1971) ANSI L

Textile Terms for Institutional Textiles (1973)

Textile Yarn and Fabric to Crocking (Rubbing) by the Aa

Textile Yarn and Fabric to Crocking (Rubbing) (Rotary Y

Textile Yarns and Fabrics to Chlorinated Pool Water (19

Textile Yarns and Fabrics to Sea Water (1972) ANSI L14.

Textile Yarns and Fabrics to Water (1972) ANSI L14.15]

Textile Yarns to Sulfur Dioxide Stoving (1972) ANSI L14

Textile Yarns (1973) ANSI L14.119 Test Meth. F

(1973) ANSI L

(Textile) After Dry Cleaning and Laundering (1972) ANS

Textile) for Institutional and Industrial Use Garments

(Textile) for Institutional and Industrial Use Garments

(Textile) Other Than Cord Fabrics (1973) ANSl L14.243

Textile) (1971)

(Textile) (1972) ANS1 L 14.98

Textile) (1973)

Textile) (1973)

Textile) (1973)

Textile) (1973

Textile) (1973

Textile) (1973)

Textile) (1973)

(Textile) (1973)

Textile) (1973)

Textile) (1973)

Textile) (1973)

Textile) (1973)

Textile) (1973)

(Textile) (1973)

(Textile) (1973)

(Textile) (1973

Textile) (1973)

Textile) (1973)

Textile) (1973)

Textile) (1973

Textile) (1973)

Textile) (1973)

Textile) (1973)

Textile) (1973

Textile) (1973)

Textile) (1973)

Textile) (1973)

Textile) (1973)

Textile) (1973

Textile) (1973)

Textile) (1973)

Textile) (1973)

Textile) (1973)

Textile) (1973)

Textile) (1973)

Textiles and Other Materials (1973)

Measureme
/F Test for Length and Len

ASTM

ANSI

ANSl

St ANSl

Std ANSI

Meth. ANSI

Std. ANSI

Std. P ANSI

Meth. O ANSI

Std. PE ANSI

Std. Per ANSI

Std. Perform ANSI

sid. Performan ANSI

Meth of Test for Li ANSI

Meth of Test for Effe ANSI

Test Meth. to Determin ANSl

Std. Performance Require ANSI

Sid. Performance Requirem ANSI

Std. Performance Requiremen ANSl

Std. Performance Requirements ANSI

Std. Performance Requirements Fo ANSI

Std. Performance Requirements for ANSI

for Permanent Labels, Detachable Tag ANSI

/ Requirements for Tailored Woven Unif ANSI

/Ance Requirements for Durable Press ( ANSI

D. Meth. of Test for Relaxation Dimen ASTM

D. Performance Requirements for Woven ANSI

/Dyes, Finishes, and Finishings (Subli ANSI

/E Requirements for Table Napery Other ANSI

E Requirements for Woven Washable Uni ANSI

Nce Requirements for Woven Sheet and ANSI

lor Woven Drycleanable Tailored Unifor ANSI

/R Woven Drycleanable Tailored Uniform ANSI

R mance Requirements for Woven Drapery ANS

Ven Glass Fiber Drapery (Curtain) Fab ANSI

(1974) ANSI L14

Textiles Except Wool (1972) ANSI L 14.138, ASTM D 1905

Textiles in Yarn Form (1971) ANSI L14.75 
and Laundering (1972) A/ umes) (1972) ANSI L14.54

Test Meth. for Colorfasiness of Test Meth. for Colorfastness of Test Meth. for Colorfastness of Test Meth. for Colorfastness of Dyed Wool ol Test/ Test Meth. for Colorfastness of Cotton and Linen $5 \cdot 4$

Test Meth. for Colorfastness of Test Meth. for Colorfastness of Test Meth. for Colorfastness of Test Meth. for Resistance of ure (1965) ANSI L14.118 Test Meth. for Colorfastness of Test Meth. for Colorfastness of Test Meth. for Colorfastness of Test Meth. for Colorfastness of Test Meth. for Colorfastness of Test Meth. for Colorfastness of Test Meth. for Colorfastness of Test Meth. for Colorfastness of Test Meth. for Colorfastness of

Test Meth. for Colorfastness of Colored
for Detection and Assessment of Colored Darkness (1971) ANSI L1/ te Light and Darkness (1/ es (1972) ANSI L14.238 Ansi/ Test Meth. for Detection and Assessment of Colored 1969) Test Meth. for Colorfastness of Dyed or Printed for Special Characteristics and Finishes of Inttitutional Test Meth. for Quantitative Analysis of 114.69, ASTM D1230 Test Meth. for Flammability of Clothing Test Meth. for $\mathrm{pH}$ of the Water Extracted from Bleached Test Meth. for Ash Content of Bleached Cellulosic he Evaluation of Mildew and Rot (Fungicides) Resistance of Test Meth. for Insect Pest Deterrents on Std. Meth. for Identification of Fibers in Test Meth. for Accelerated Aging of Sulfur Dyed

Test Meth. for Noncotton Content of Bleached Cotton Dimensional Changes (Shrinkage) of Woven and Knitted Wool Test Meth. for Identification of Fibers in Std. Meth. for Quantitative Analysis of

Std. Definitions of Textile Terms for Institutiona Test Meth. for Identification of Finishes in ve Bedspreads, Pillows, and Bolster Fabrics (Institutional cs (Sash Casement) (Excluding Glass Fibers) (Institutiona

Fabrics, Painted, Printed, or Vinyl Coated (Institutional rics Handwashable at $105 \mathrm{Deg}$. F-No Bleach (Institutional ness of Near White, Opaque Materials (Color Measurement of 2) ANSI L.14.225

Glass (1972) ANSI L14.177 Test Meth. for Resistance of osition, Use, Applicati/ ess, Form and Lay) (1973) Redwood Plywood Guide (Spec. for Std. for Gear Tooth Surface 4) Std. Rec. Pract. for Powder Flow Measurement of Std. Spec for Std. Spec. for Std. Spec. for Std. Spec. for and Extrusion Materials (1973)

i K65.208

orm Plumbing Code: Minimum Facilities Required for School, .) (1973)

Uniform Fire Code: Places of Assembly Alarm Systems and Units for Protection Against Holdup an Std Meth for Distillation of Crude Petroleum (15 ustrial Engineering Terminology: Organization Planning and ment (Breathing Devices, Room Humidifiers, Nebulizers, Gas Nebulizers, Gas Therapy and Suctio/ Std. for Respiratory Safety Std. for Oxyge Std. Meth. of Test for Insul/ Std. Meth. of Test for
Std. Definitions of Terms Relating to varnishes Applied Over Film Insul/ eth. for Calibration of Definition Data from Differential Std. Rec. Pract. for Solar Simulation for Std. Meth. for Measuring Heat Transfer Rate Using A Std. Meth. of Test for Std. Meth. of Test for

si All1.28

paratus (1970) Alc/

11.32

(1972) Combined, Simulated Space Environment Test. of Spacecraft
Std. for Safety for mponents (1972) ANSI C33.88

by the Helical Coil Meth. (1973)

Pulse Meth. (1972) ANSI K90.12

Std. Meth. of Test for Estimating the Std. Meth. of Test fo Std. Meth of Test for Std. Meth. of Test for Std. Meth. of Test for omparison with a Secondary Standard of Sim/ ss and Feed and Water Requirements of Farm Liv/ Std. Meth. of Effect of exe/ Imers (1972) al Insulating Materials (1973) Std Test Procedure for (Confinement Test) (1973) Uniform Fire Code: Fumigation and Sid. Meth. of Test for .33 Std. Spec. for Mineral Fiber Hydraulic Setting Std. Spec. for Perlite n Buildings (1970) /
Std. Spec. for Spray Applied Fibrous Std. Spec. for Mineral Fiber Blanket
Textiles to Accelerated Domestic and Commercial Washing Textiles to Atmospheric Oxides of Nitrogen (Burnt Gas F
Textiles to Bleaching with Peroxide (1972) ANSI L14.146 Textiles to Carbonizing (1972) ANSl L14.3

Textiles to Combined Washing and Shrinkage: Rapid Contr Textiles to Dry Heat (Excluding Pressing) (1973) ISO 10 Textiles to Drycleaning (1973) ANSI L14.241

Textiles to Hot Pressing (1973)

Textiles to Insects (1971) ANSI L14.64

Textiles to Light and Washing: Alternate Sunlight Expos

Textiles to Light (Daylight) (1971) ANSI L14.168

Textiles to Light (General Meth.) (1971) ANS1 Ll4.168

Textiles to Light, Carbon Arc Lamp, Continuous Light (1

Textiles to Light, Sunlight (1971) ANSI L14.168

Textiles to Light: Carbon Arc Lamp, Alternate Light and

Textiles to Light: Water Cooled Xenon Arc Lamp, Alterna

Textiles to Light: Water Cooled Xenon Arc Lamp, Continu

Textiles to Ozone in the Atmosphere Under High Humiditi

Textiles to Perspiration (1972) ANSI L14.56

Textiles to Photochromism Colorfastness to Light (1972)

Textiles to Steam Pleating (1971) ANSI L14.240, ISO 105

Textiles to Washing at 105 Deg. F: Rapid Control Test (

Textiles (Durability, Spot, Stain, and lnsect Resistanc

Textiles (Moisture and Composition) (1971) ANSI Ll4.132

Textiles (1962)

Textiles (1969) ANSI L14.116

Textiles (1971) ANSI L14.114

Textiles (1971) ANSI L14.55

Textiles (1971) ANSI L14.65

Textiles (1972)

Textiles (1972) ANSI L14.1

Textiles (1972) ANSI L14.128

Textiles (1972) ANSI L14.130, ASTM D1284

Textiles (1972) ANSI L14.131

Textiles (1972) ANSI L14.132, AATCC 20A

Textiles (1973)

Textiles (1973) ANSI L14.127

Textiles) (1973)

Textiles) (1973)

Textiles) (1973)

Textiles) (1973)

Ance Requirements for Woven Decorati

Ments for Woven Window Curtain Fabri

Otton Woven Tent, Awning, and Canopy

/ $T$ s for Woven Glass Fiber Curtain Fab

Textiles, Paints, Plastics, etc.) (1973) ANSI Z172.6

Textiles: Carbon Arc Lamp Exposure Without Wetting (197

Textiles: Exposure to Natural Light and Weather Through

Texture and Grade, Panel Characteristics, Material Comp

Texture for Aerospace Gearing (Surface Roughness. Wavin

TFE -Fluorocarbon Molding and Extrusion Materials (197

TFE -Fluorocarbon Resin Molded Basic Shapes (1974)

TFE -Fluorocarbon Resin Sheet (1974)

TFE -Fluorocarbon (Tetrafuoroethylene) Resin Molding

TFE - Fluorocarbon (Tetrafluoroethylene) Rod (1974) Ans

Theater, Auditorium, Dormatory, Public or Office Buildi

(Theaters, Reviewing Stands, Grandstands, Bleachers, Etc

Theft (1973) ANSI Se2.8 /N, and Main
Theoretical Plate Column) (1973) ANSI Z11.314

Theory (1972)

Therapy and Suction Equipment, Room Air Purifiers, Fil

Therapy Equipment (Breathing Devices, Room Humidifiers,

Therapy Equipment, Refrigerated (1973)

Thermal Aging Characteristics of Electrical Insulating

Thermal Analysis (1973)

Thermal Analysis (1973)

Thermal Analysis, Thermogravimetry, Evolved Gas Analysi

Thermal Balance Test. of Spacecraft (1973)

Thermal Capacitance (Slug) Calorimeter (1972)

Thermal Conductivity of Carbon Refractories (1973)

Thermal Conductivity of Insulating Fire Brick (1972) an

Thermal Conductivity of Leather with the Cenco Fitch Ap

Thermal Conductivity of Liquids (1971) AI、S̃I Z11.304

Thermal Conductivity of Refractory Brick (1971) ANSI Al

Thermal Conductivity of Unfired Monolithic Refractories

Thermal Control Materials (Coatings) with Electromagnet

Thermal Cutoffs for Use in Electrical Appliances and Co

Thermal Degradation of Electrical Insulating Varnishes

Thermal Diffusivity of Carbon and Graphite by a Thermal

Thermal EMF Test of Single Thermoelement Materials by C

Thermal Environment on Production, Heat and Moisture Lo

Thermal Evaluation and Establishment of Temperature Ind

Thermal Evaluation of Oil Immersed Distribution Transfo

Thermal Failure Under Electric Stress of Solid Electric

Thermal Insecticidal Fogging (1973)

Thermal Instability of Confined Condensed Phase Systems

Thermal Insulating and Finishing Cement (1964) ANSI Z98

Thermal Insulation Board (1972)

Thermal Insulation for Elevated Temperature (1972)

Thermal Insulation for Wood Frame and Light Constructio
AATCC

AATCC

AATCC

AATCC

AATCE

AATCC

AATCC

AATCC

AATCE

AATCC

AATCC

AATCC

A ATC

AATCC

AATCC

AATCC

AATCC

AATCC

AATCC

AATCC

AATCC

AATCC

ANSI

AATCC

ANSI AATCC

AATCC 81

AATCC 78

AATCC 30

AATCC 28

ASTM

AATCC

AATCC

ATCC

AATCC

ASTM

ANSI

AATCC

ANSI

NSI

ANSI

AATCC

CRA

AGMA

ACTM

ASTM

ASTM

ASTM

ASTM

ICBO

ICBO

$\mathrm{UL}$

ASTM

ANSI

NSF
NSF

ASTM

ASTM

AT

ASTM

ASTM

ASTM

ASTM

ASTM

ASTM

ASTM

AST

UL

ASTM

ASTM

ASTM

ASAE

IEEE

ASTM

ICBO

ASTM

AST

ASTM

ASTM

ASTM 
Std. Meth. of Test for

for Determining the Maximum Use Temperature of Preformed Std. Spec. for Cellular Glass Block and Pipe Std. Spec. for Cellulosic Fiber (Wood Base) Loose Fil Std. Rec. Pract. for Application of Spray Applied Fibrous Std. Spec, for Mineral Fiber Loose Fil Std. Rec. Pract. for Selection of Vapor Barriers for Guide for Statistical Analysis of Std. Meth. for Estimating the Std. Meth. for Measuring Tent. Meth. of Test for

Std.

c.) (1973)

blies (1972)

Std. for Full Length

d. for Preservative Treatment of Incised Pole Butts by the Pine Wood Poles Preservative Treatment by the Full Length ts (Flour, Starch) (1972)

f Test for Thermal Diffusivity of Carbon and Graphite by A Type Buil/ Std. Rec. Pract. for the Determination of the Std. Meth. of Rec. Safe Pract. for

std. Meth. of Test for Z11. 148 re Technique / Sid. Meth. for Spectrochemical Analysis of Std. Spec, for Reinforced Polyterphthalate
Rec. Pract. for Automotive Brake Disc and Drum Applications (1967) Ans/ Std. Spec. for Sheathed, Type K 44 Std. Meth. for Calibration of Refractory Metal Std. Temperature-Electromotive Force (EMF) Tables for standard of Sim/ Sid. Meth. of Thermal EMF Test of Single ct. for Reporting Data from Differential Thermal Analysis. Std Spec. for ASTM

Meth. for Verification and Calibration of Liquid-In.Glass s (Cereal Chemistry) (1962)

aerobic) in Cereal Products and Fool

Meth. of Analysis of Meth. of Analysis of Std. Rec. Pract. for Determining Permeability of 973) Std. Spec. for Safety Std. for sion and Distribution of Electrical Energy (197/ Safety Std. for Extruded Spec. for si $\mathrm{K} 65$ / Rec. Pract. for lnjection Molding of Specimens of Rec. Pract. for Heat Joining of n Systems (1972) aterials (1973) Rec. for Std. Spec. for Sid Ree. Pract for Under round lnstlation of Flexible cables (1972) Safety Std. for All Rec. for

974) Stds., Guide Specs., and Materials Application for Std. Meth. of Measuring Flow Rates of ent (Septic, Holding, Settling, Aeration Chambe) Std. for 65.181 Sid. Meth. of Test for Linear Shrinkage of Std. Spec. for Laminated

Std. Meth. of Measuring the Spiral Flow of Low Pressure the Transmission and Distributi/ Std. for Crossed Linked Rec. Pract. for Care and Use of Reinforced

Spec for Reinforced

t for Gel Time and Peak Exothermic Temperature of Reacting
Std. Spec. for Std. Spec. For

heating, Cooking, Refrigeration, and Humidity (Humidistat. Mounted Thermostats For/ Line Voltage Integrally Mounted ostats for Electric Heater Line Voltage Integrally Mounted
Std. for Gas Appliance or Residential Controls for Low Voltage, Wall Mounted Room Std. for Safety for Fire Detection Std. Guidelines for

-Ester with 4-(Mercaptomethyl) 2 -Methyoxy $\cdot 02_{2}+1,3,4$. roducts, Corn Grits, Cor/ Thiochrome Meth. of Analysis of Rec. Pract. for Determining Solderability of t Intermediate Pivot (1973) Spec. for l 3/4 In. ent of Oxide Thickness on Silicon Wafers and Metallization Std. Meth. of Test for Coating Sid. Meth. of Test for Coating nferrous Substrate (Coil, Instrumentation/ Spec. for Film Meth. of Test for Resiliency and s (Aperture, Camera, Copy, and / Std. Meth. for Measuring Std. Meth. of Test for Std. Meth. of Test for Std. Meth. of Test for std. Meth. of Test for Std. Meth. for Measuring Std. Meth. for Measuring of Stacking Fault Dimension (197/ of the Same Type by $\ln$ frared Refl/ the Angle Lapping and Staining T/

oating) (1974) ts - Film Thickne Test Meth. for Measurement of Film Std. Meth. for Nondestructive Measurement of Film
Thermal Insulation of Ouality Packages (1972)

Thermal Insulation (1971) ANSI Z98.28

Thermal Insulation (1973)

Thermal Insulation (1973)

Thermal Insulation (1973)

Thermal Insulation (1973)

Thermal Insulations (1973)

Thermal Life Test Data (1972)

Thermal Neutron Absorption Cross Section of Nuclear Gra

Thermal Neutron Flux by Radioactivation Techniques (197

Thermal Oxidation Stability of Turbine Fuels (JFTOT Pro

Thermal Performance Test for Steel Door and Frame Assem

Thermal Process Preservative Treatment of Western Red C

Thermal Process (Western Red Cedar, Northern White Ceda

Thermal Process (1973)

Std. for Lodgepole

Thermal Properties of Agricultural Grain and Its Produc

Thermal Pulse Meth. (1972) ANS1 K90.12

Std. Meth, O

Thermal Resistance of Low Density Mineral Fiber Blanket

Thermal Shock Test on Glass Containers (Bottle, Jar) (1

Thermal Shock Test on Glass Pipe (1970)

Thermal Spraying and Blasting Equipment (1973)

Thermal Spraying Terms and Definitions (1970)

Thermal Stability of Aviation Turbine Fuels (1972) ANSI

Thermionic Nickel Alloys by the Powder Direct Current a

Thermo-Plastic Molding and Extrusion Materials (1973)

Thermocouple Installation (1972)

Thermocouples for Nuclear or for Other High Reliability

Thermocouples Using an Optical Pyrometer (1972) ANSI N

Thermocouples (1972) ANSI C 96.2

Thermoelement Materials by Comparison with a Secondary

Thermogravimetry, Evolved Gas Analysis and Detection (1

Thermometers (1971) ANS1 Z71.1

Thermometers (1972) ANS1 Z104.1

Thermophilic and Psychrophilic Bacteria in Food Product

Thermophilic Spore Counts (Flat Sour, Total Aerobic, an

Thermoplastic Containers (1973)

Thermoplastic Gas Pressure Pipe, Tubing and Fittings (1

Thermoplastic Insulated W ire and Cable for the Transmis

Thermoplastic Insulated Wires (1971) ANSI C33.80

Thermoplastic Insulating Tubing (1972)

Thermoplastic Line Pipe (PVC and CPVC) (1972)

Thermoplastic Molding and Extrusion Materials (1972) an

Thermoplastic Pipe and Fitrings (1967) ANS1 B72.17

Thermoplastic Piping for Swimming Pool Water Circulatio

Thermoplastic Polyterephthalate Molding and Extrusion M

Thermoplastic Pressure Piping (1972)

Thermoplastic Sewer Pipe (1972) ANSI K65.171

Thermoplastic Type MTW 600 Volt Machine Tool Wires and

Thermoplastic Water Piping Systems (1972)

Thermoplastic (Polyvinyl Chloride) Duct Construction (1

Thermoplastics by Extrusion Plastometer (1973)

Thermoset Plastic Tanks for Water and Wastewater Treatm

Thermosetting Casting Systems During Cure (1969) ANSI K

Thermosetting Materials (1972)

Thermosetting Molding Compounds (1972)

Thermosetting Polyethylene Insulated Wire and Cable for

Thermosetting Resin Line Pipe (Rtrp) (1972)

Thermosetting Resin Line Pipe (1972)

Thermosetting Resins (Plastic Composition) (1971) ANSl

Thermostat Metal Sheet and Strip (1973) ANS1 Z155. 19

Thermostat) (1972) ANSI B131.1 / of Air Cond

Thermostats for Electric Heater Line

Thermostats (1971)

Thermostats (1972)

Thermostats (1974) ANSI Z220.1

Thesaurus Structure, Construction, and Use (1974)

Mounted Therm

Thiabiazolin -5-One) (1973)

/Thyl Phosphorodithioate $S$

Thiamine in Bread, Wheat, Rice, and Other Whole Grain P

Thick Film Conductors (1972T)

Thick Hollow Metal Door and Frame Preparation for Offse

Thickness by Multiple Beam Interference (Tolansky Meth.

Thickness by the Beta Backscatter Principle (1972)

Thickness by X-Ray Spectrometry (1972)

Thickness Determination of Nonconductive Coatings on No

Thickness of Blankets (Textile) (1973)

Thickness of Buildup Area on Unitized Microfilm Carrier

Thickness of Diffusion Coating (1970) ANSI Z167.24

Thickness of Epitaxial Layers of Silicon by Measurement

Thickness of Epitaxial Layers of Silicon on Substrates

Thickness of Epitaxial or Diffused Layers in Silicon by

Thickness of Leather Test Specimens (1970) ALCA E4

Thickness of Leather Units (1970) ALCA E3

Thickness of Nonmagnetic Finishes on Mild Steel (Coil C

Thickness of Paint, Varnish, Lacquer and Related Produc

Thickness of Pipeline Coatings on Steel (1972)
ASTM

ASTM

ASTM

ASTM

ASTM

ASTM

ASTM

IEEE

ASTM

ASTM

ASTM

STDI

A WPA

AWPA

$A W P A$

ASAE

ASTM

A STM

ASTM

A TM

AWS

AWS

ASTM

ASTM

ASTM

$\mathrm{SAE}$

ASTM

ASTM

ASTM

ASTM

ASTM

ASTM

Std. ASTM

E77

AACCH 42.45

AACCH 42.40

ASTM D2684

ASTM D2513

NEMA WC5

UL

UL

API

83

D1897

SPI

ASTM

ASTM

AST

UL

SPI

SMACN

ASTM

NSF

ASTM

ASTM

ASTM

NEMA

APl

APl

ASTM

ASTM

UL

NEMA

NEMA

ANSI

NEMA

UL

ANSI

ANSI

AACC

ASTM

ANSI

ASTM

ASTM

ASTM

NCCA

ANSI

NMA

ASTM

AATM

ASTM

ASTM

ASTM

ASTM

NCCA

NCCA

ASTM

3103

552

762

764
$\mathrm{C} 55$

626

262

13

C7

D243.1

C.714

C149

C 2.1

C 2.9 
surfaces (1973)

llic Coatings by Double Be/

Std. Meth. of Test for Std. Meth. of Test for by Multiple Beam 1/ Tent. Meth. for Measurement of Oxide Tent. Meth. of Test. Plywood in Shear Through the

d. Reference Radiographs for Steel Castings Up to $2 \mathrm{ln}$. in i (290 MPa) Minimum Yield Point $(1 / 2 \mathrm{ln} .(12.7 \mathrm{Nm})$ Maximum ti) Std. Neth. for Measurement of Metal and Oxide Coating Std. Test. Procedure for Nleasuring Wall Std. for Wire Cable W'rought Steel Heavy

1973)

of Test for Effect of Heat and Air on Asphaltic Naterials Tent. Meth. of Test for Sheet Resistance of Std. Meth. of Test for Tensile Properties of Std. Meth. of Test for Flammability of Flexible (1972) Std. Meth. for Design and Use of A s of Material (1970) ANSI Z/ Std. Rec. Pract. for Bonding Std for Nut Plain, Hexagon, std. Meth. of Test for Ester Value of Lacquer Solvents and Steel Articles by T/ Std. Meth, of Test for Locating the Std. Spec. for Natural Muscovite Block Mica and the Pest Control Chemical cis. N.((1,1,2,2tetrachloroethyl) t, Rice, and Other Whole Grain Products, Corn Grits, Cor/ Std. Spec. for Photographic Grade Sodium Meth. for Preparation of Potassium

version (Cereal Chemistry) (1962)

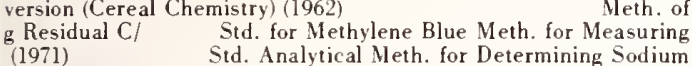
Meth. for Preparation and Standardization of Sodium Spec. for Photographic Grade Ammonium $\left(\mathrm{Na}_{2} \mathrm{~S}_{2} \mathrm{O}_{3}, 5 \mathrm{H}_{2} /\right.$ Spec. for Photographic Grade Sodium Std. Meth. of Test for

100 Deg. Close Tolerance Head and Shank, 160,000 psi Short ssure and Bending (1972)

ections in High Pressure Oil Well Service) (1972)

rew Threads (7/45 Deg. Form with 0.6 Pitch Basic Height of

etal, 1200 Deg. F (649 Deg. C) Use, Unified J (MIL S.8879)

Metal, 1200 Deg. F (649 Deg. C) Use, Unified (MlL S-7742)

Pipe Threads (1974) Spec. for Threading, Gaging, and std. for Screw, Hex Head, Tri Wing Recess, A286 Cres, Full Std. for Self Locking, Flat 100 Deg. Head, Full Std. for Self Locking, Pan Head, Full Std. for Self Locking, Flat Fillister Head, Full $r$ Dimensional Requirements for Duplex and Single Face Flat crew, 100 Deg. Oval Head, Tri Wing Recess, A286 Cres, Full O-Ring (1973) O-Ring (1973) Std. for Hexagon Head Mlodified Shor Std. for Packing, Preformed, Straight Std. for Packing, Preformed, Straight Std. for Class 5 Interference Fit Std, for Hex Head Screw, Recessed, Full Std for Coupling-Tubes, Flared Fitting, Female for Bolt-Close Tolerance, Hexagon Head, Titanium, Short self Locking, Alloy and Corrosion Resisting Steel, UNJC.3A ocking, Alloy Steel and Corrosion Resisting Steel, UNJF-3A Tolerance, $6 \mathrm{Al}-4 \mathrm{~V}$ Titanium Alloy, Short ew, Hex Head, Tri Wing Recess, 6Al-4V Titanium Alloy, Full d Head, Tri Wing Recess, Close Tolerance, A286 Cres, Short d. for Screw, Hex Head, Tri Wing Recess, Alloy Steel, Full head, Tri Wing Recess, Close Tolerance, Alloy Steel, Short A286 Cres, Long $6 \mathrm{Al}-4 \mathrm{~V}$ Titanium Alloy, Long std. for Bolt. Hex Head, Close Tolerance, A286 Cres, Short td. for Bolt, Hex Head, Close Tolerance, Alloy Steel, Long std. for Screw, Pan Head, Tri Wing Recess, A286 Cres, Full d. for Bolt, Hex Head, Close Tolerance, Alloy Steel, Short for Screw, 100 Deg. Head, Tri Wing Recess, A286 Cres, Full wing Recess, Close Tolerance, $6 \mathrm{Al}-4 \mathrm{~V}$ Tit anium Alloy, Short Head, Tri Wing Recess, Close Tolerance, Alloy Steel, Long , Hex Head, Close Tolerance, $6 \mathrm{Al}-4 \mathrm{~V}$, Titanium Alloy, Short 100 Deg. Head Tri Wing Recess, 6Al-4V Titanium Alloy, Full Head, Tri Wing Recess, Close Tolerance, A286 Cres, Short lt, Hex Head, Close Tolerance, 6Al-4V Titanium Alloy, Long r Screw, 100 Deg. Head, Tri Wing Recess, Alloy Steel, Full head, Tri Wing Recess, Close Tolerance, Alloy Steel, Short Std. for Pan Head, Close Tolerance, Short Std. for Screw, Mlachine, Pan Head, Full Std. for Screw, Machine, Flat Fillister Head, Full Std for Screw, Mlachine, Flat 100 Deg. Head, Full Std. for Machine Screw, Flat $100 \mathrm{Deg}$. Head, Short Std. for Machine Screw, Pan Head, Short

nlocki/

cking An/
Std. for Machine Screw, Flat Fillister Head, Full Std. for Bolt, Pan Head, Close Tolerance, Short
Thickness of Resilient Flooring, Materials Having Flat Thickness of Sandwich Cores (1970)

Thickness of Solid Electrical Insulation (1973)

Thickness of Transparent or Opaque Mletal Oxide and Meta

Thickness of 0.3 MlL or Greater (Coil Coating) (1974)

Thickness on Silicon Wafers and Metallization Thickness

Thickness (1969)

Thickness (1972) ANSI Z166.28

Thickness) (1972) ANSl G24.27

Thicknesses by Microstopical Examination of a

Thicknesses of Composite Cans, Tubes and Cores (1974)

Thimble (For Ground Support Equipment) (1974)

Thin Film Oven Test) (1973)

Thin Metallic Films with a Collinear Four Probe Array (

Thin Plastic Sheeting (1973) ANSI K65.76

Thin Plastic Sheeting (1974) ANSI K65.28

Thin Skin Calorimeter for Measuring Heat Transfer Rate

Thin Spectrochemical Samples and Stds. to a Greater Mas

Thin Walled Tube Sampling of Soils (1974)

Thin (1972)

Thinners (1972)

Thinnest Spot in a Zinc (Galvanized) Coating on Iron or

Thins Based on Visual Quality (1971) ANSI C59.27

Thio)-4-C yclohexene.1,2-Dic arboximide-Captafol (197

Thiochrome Meth. of Analysis of Thiamine in Bread, Whea

Thiocy anate $\mathrm{NaSCN}$ (1972)

Thiocyanate Solution (Cereal Chemistry) (1962)

Thiosulfate-Maltose (Diastatic Activity in Flour) Con

Thiosulfate and Silver Densitometric Meth. for Measurin

Thiosulfate Solution Content of Reagents and Indicators

Thiosulfate Solution (Cereal Chemistry) (1962)

Thiosulfate Solution $\left(\mathrm{NH}_{1}\right), \mathrm{S}_{2} \mathrm{O},(1973)$

Thiosulfate, Anhydrous $\left(\mathrm{Na}_{2} \mathrm{~S}_{2} \mathrm{O}_{3}\right)$ and Crystalline

Thorium in Water and Waste Water (1968) ANSI N158

Thread Bolt (1974)

Thread Casing Joint Strength with Combined Internal Pre

Thread Compounds (For Casing, Tubing and Line Pipe Conn Std. for Buttress Inch Sc

Thread Engagement (1973)

Thread Form (1973)

Thread Form) (1973)

Thread Fuel Injection Tubing Connections (1971)

Strength, Prevailing Torque, All $\mathrm{M}$

Thread Inspection of Petroleum Casing, Tubing, and Line

Thread Nonlocking (1972)

Thread Part Screw (1974)

Thread Part Screw (1974)

Thread Part Screw (1974)

Thread Rolling Dies (1973)

Thread Rolling Screws (1972)

Thread Self Locking and Nonlocking (1972)

Thread Shear Bolt (1972)

Thread Tube Fitting Boss, MlL-R.25897 Rubber, 75 Shore,

Thread Tube Fitting Boss, MlL-R.25897 Rubber, 90 Shore.

Thread (1972)

Thread (1973)

Thread (1973)

Thread (1973)

Thread, Cap Screw (1972)

Thread, Cap Screw (1972)

Thread, Nonlocking (1972)

Thread, Nonlocking (1972)

Thread, Nonlocking (1972)

Thread, Nonlocking (1973)

Thread, Nonlocking (1973)

Thread, Self Locking and Nonlocking (1972)

Thread, Self Locking and Nonlocking (1972)

Thread, Self Locking and Nonlocking (1972)

Thread, Self Locking and Nonlocking (1972)

Thread, Self Locking and Nonlocking (1972)

Thread, Self Locking and Nonlocking (1972)

Thread, Self Locking and Nonlocking (1972)

Thread, Self Locking and Nonlocking (1972)

Thread, Self Locking and Nonlocking (1972)

Thread, Self Locking and Nonlocking (1972)

Thread, Self Locking and Nonlocking (1972)

Thread, Self Locking and Nonlocking (1972)

Thread, Self Locking and Nonlocking (1972)

Thread, Self-Locking and Nonlocking (1973)

Thread, Self-Locking and Nonlocking (1973)

Thread, Torq Set Machine Screw (1972)

Thread, Torq Set (1973)

Thread, Torq Set (1973)

Thread, Torq Set (1973)

Thread, Torq Set (1973)

Thread, Torq Set (1973)

Thread, Tri Wing Recess, A286 Cres, Self-Locking and No

Thread, Tri Wing Recess. Titanium Alloy $6 \mathrm{Al} \cdot 4 \mathrm{~V}$. Self Lo

led and Drilled, Plain and

ASTM

ASTM

ASTM

ASTM

NCCA

ASTM

ASTM

St ASTM

ASTM

ASTM

CCT1

NSA

ASTM

ASTM

ASTM

ASTM

ASTM

ASTM

ASTM

ASTM

ASTM

ANSl

AACCH

ANSI

AACCH $70-55$

AACCH 22-17

ANSl PH4.8

CR R-25

$\mathrm{AACCH} \quad 70.75$

ANSI PH4.252

ANSI PH4.250

ASTM D2333

NSA

API

API

$\mathrm{SAE}$

$S A E$

$\mathrm{SAE}$

API

NSA

NSA

NSA

NSA

Std. Fo IF]

SAE

Std. for $S$ NSA

NSA

NSA

$\mathrm{NSA}$

ANSI

NSA

NSA

Std. NSA

NSA

Std. for Ser NSA

NSA

St NSA

NSA

NSA

NSA

NSA

NSA

NSA

NSA

Std. NSA

/ Deg. Tri NSA

1, J00 Deg. NSA

1. for Bolt NSA

lor Screw, NSA

IT, 100 Deg NSA

Td. for Bo NSA

Std. Fo NSA

NSA

NSA

NSA

NSA

ISA

NSA

NSA

NSA

NSA

BCL 5 C4

BL' 5 A2

B1.9

AMS7251B

AMS $7250 \mathrm{C}$

$\mathrm{J} 242$

6000-3

1189

1190

1191

09

J81

1103.20

1595

1596

B1.12

1096

424

653

1352

1351

4903-16

6100-3

4803-16

$5900 \cdot 03$

1703-16 
ocking/ nonlocki/ Std. for Machine Screw, Flat Fillister Head, Full Std. for Bolt, Pan Head, Close Tolerance, Shor onlocking (197/ Std. for Pan Head, Close Tolerance, Short tic Pipe Fittings, Schedule 80 (1973) Ans/. Phillips Recess, Shor c) Solu/ Studs, Steel, Corrosion and Heat Resistant, Roll Std Spec for Sizes for Std. Meth. of Measuring Application and Removal Torque of Std. Cast lron

std. of Mechanical and Quality Requirements for Externally Std. for Wrenching Configuration, Spline Drive Std. Cast Bronze Std. Cast Iron

Std. for Malleable lron

rameters for Bolts and Screws; External Wrenching. Unified Sid. for

ich Panel Lightweight Selflocking and Nonselflocking Blind ich Panel Lightweight Selflocking and Nonselflocking Blind gs, Schedule 80 (1973) ANSI K65.166 for Tube Assembly, Control Steel with Welded Clevis and for Tube Assembly, Control, Steel, with Flash Welded and ube Assembly, Control, Steel, with Flash Welded Clevis and Std. for Drills, High Speed Steel Std. Spec. for Carbon Steel Externally and Internally std. for Coarse Std. for Fine

steel Studs, Heat Resistant, Normalized and Tempered, Roll Spec for Fasteners, A286 Cres, Externally Spec. for Fasteners, Alloy Steel, Externally Spec. for Fasteners, 6Al.4V Titanium Alloy, Externally Std. for Forged Steel Fittings (Pipe), Socket Welding and Std. for Trunnion-Tank Strap.

Spec. for Low Alloy Steel Studs, Heat Treated, Roll for Low Alloy Steel Bolts and Screws, Heat Treated, Roll itanium Alloy Bolts and Screws, 6A1-4V, Heat Treated, Roll eel, Low Alloy Heat Resistant, Hardened and Tempered, Roll bolts and Screws, $6 \mathrm{Al} \cdot 4 \mathrm{~V}$, Upset Headed, Heat Treated, Roll (1,345 MPa) Tensile Strength, Hardened and Tempered, Roll Std. for Aircraft, Pan Head, Phillips Recess Full Std. for Screw, Machine, 100 Deg., Flat Head Full

Self Locking) (1972/

head, Self Locking)/

Self Locking) (1972)

Head, (1972)

self Locking) (1972/

d, Self Locking) (1/ loating, Sandwich Pane/
(1972)

d Nonl/ Std. for asing, Tubing, and Line Pipe Threads (1974) for Full
Spec. for Std. for Rod End, Std. for Fastener (Blind lnternally Std. for Fastener (Blind, Internally Fastener (Blind, Internally Std. for Fastener (Blind, Internally Std. for Fastener (Blind, Internally Std. for Fastener (Blind, Internally Spec. for Fastener (Blind, Internally Std, for lnsert, Molded ln, Blind Std. for lnsert, Molded In, Thru Spec. for Std. Spec. for Trial Std. for Screw Std. Ferrous Pipe Plugs, Bushings, and Locknuts with Pipe Std. Meth. of Test. Sewing Std. for Gaging Practice for Metric Screw Std. for ACME Screw Std. for Stub ACME Serew Std. for Buttress Inch Screw

thread Engagement (1973) read Inspection of Petroleum Casing, Tubing, and Line Pipe Std. for Bolt, Hex Head, Close Tolerance, A286 Cres, Long ent. Rec. Pract. for Measuring Slope Efficiency and Lasing 72) ANSI Z11.318 Std. Meth. of Test for Reaction

I for Boats Equipped with Outdrives ers for DC Motor Armatu/ Std. Pract and Requirements for ry Drives (1972)

antom (Medical Radiation-Radiology) (1973) nsions for $3 / 4,1$ and $11 / 8 \mathrm{ln}$. Bore Cataloged Square Head Brick and Tile Metal Std. Spec. for $55 \mathrm{Gal}$. Sid. Spec. for $16 \mathrm{Gal}$. Std. Spec. for $55 \mathrm{Gal}$. Std. Spec. for $55 \mathrm{Gal}$. Std. Spec. for $30 \mathrm{Gal}$.

-Rule 40, Nmfe-ltem 260, Dot-1 Std. Spec. for $5 \mathrm{Gal}$. Voltage Values for Preferred Transient Insulation Levels Clay
ding Code Std. for Metal Suspension Systems for Acoustical
$m$ Building Code Std. for Structural Clay Load Bearing Wall ding Code Std. for Metal Suspension Systems for Acoustical
$m$ Building Code Std. for Structural Clay Load Bearing Wall
Thread, Tri Wing Recess, Titanium Alloy, $6 \mathrm{Al} .4 \mathrm{~V}$, Self-L Thread, TWI Wing Recess, Alloy Steel, Self Locking and Thread, TWI Wing Recess, A286 Acres, Self Locking and N Thread, 160,000 psi Alloy Steel Machine Screw (1974) Threaded Acrylonitrile-Butadiene-Styrene (ABS) Plas Threaded After Heat Treatment, 1800 Deg. F (982.2 Deg.

Threaded Caster Stems (1965)

Threaded Closures (1973)

Threaded Drainage Fittings (1971)

Threaded Fasteners Used in Automotive and Related Indus

Threaded Fasteners (1971)

Threaded Fittings (125 and 250 Lb.) (1971)

Threaded Fittings (125 and 250 Lb.) (1972)

Threaded Fittings (150 and 300 Lb.) (1971)

Threaded In. Series (1971)

Threaded Metal Insert-Heavy Duty (1973)

Threaded Metal lnsert, Light Weight (1973)

Threaded Molded in lnsert (1974)

Threaded Molded in Insert (1974)

Threaded Poly(vinyl Chlori

Threaded Rod Ends (1973)

Threaded Rod Ends (1973)

Threaded Rod Ends (1973)

Threaded Rod Ends (1973)

Threaded Shank, Sizes 1/16 ln. Thru Letter F (1973)

Threaded Std. Fasteners (1968) ANSI G38.5

Threaded Stud (1973)

Threaded Stud (1973)

Threaded (135,000 psi (931 MPa) Tensile Strength) (1973

Threaded (1971)

Threaded (1972)

Threaded (1972)

Threaded (1973)

Threaded (1973)

Threaded (1973)

Threaded (1973)

Threaded (1973)

Threaded (1973)

Threaded (1973)

Threaded (1973)

Threaded, Alloy Steel Machine Screw (1974)

Threaded, Alloy Steel (1973)

Threaded, Alloy Steel, Flash Welding Type (1973)

Threaded, External Sleeve, General Purpose, Flush Head, .

Threaded, External Sleeve, General Purpose, Protruding

Threaded, External Sleeve, High Temperature, Flush Head

Threaded. External Sleeve, High Temperature, Protruding

Threaded, External Sleeve, Light Weight, Millable Head

Threaded, External Sleeve, Light Weight, Protruding Hea

Threaded, External Sleeve, Self Locking) (1972)

Threaded, Self Locking, Nonself Locking, Light Weight $F$

Threaded, Self Locking, Nonself Locking, Sandwich Panel

Threaded, Tri Wing Recess, Alloy Steel, Self-Locking an

Threaded, $160 \mathrm{KSI}$ Steel, Drilled Head Bolt (1972)

Threading, Gaging, and Thread Inspection of Petroleum C

Threadless Copper Pipe (1974) ANSl H26.2

Threads for Metric Series Mechanical Fasteners (1974)

Threads (1971)

Threads (1971) ANSl Ll4.14

Threads (I972)

Threads (1973)

Threads (1973)

Threads $(7 / 45$ Deg. Form with 0.6 Pitch Basic Height of

Threads (I974)

Spec, for Threading, Gaging, and Th

Threads, Self Locking and Nonlocking (1972)

Threshold of Ruby Laser Rods (1973)

Threshold Temperature of Liquid and Solid Materials (19

Thresholds, Saddles for Ceramic Tile Flooring (1973)

Thrusters (1973)

/Ansmission Units Penetrating the Hul

Thyristor Converters for Motor Drives: Part 1-Convert

Thyristor Power Supplies for Metal Rolling Mill Auxilia

Thyristors (1972) NEMA SK516

Thyroid Radioiodine Uptake Measurements Using a Neck pH

Tie Preservative Treatment by Pressure Processes (1973)

Tie Rod Type Industrial Fluid Power Cylinders (1972) an

Tied Walls (Tech. Notes) (1966)

Tight llead Universal Drum (Dut-5B)(1974)

Tight Head Universal Drum (Dot-17E) (1974)

Tight Head Universal Drum (Dot-17C) (1974)

Tight Head Universal Drums (Dot-17E) (1974)

Tight Head Universal Drums (Dot-17E) (1974)

Tight Head Universal Pails (Container) (Dot-17E, Ufc (TIL) (1971)

Tile and Concrete Slabs (Tech. Notes) (1962)

Tile and for Lay in Panel Ceilings (1973)

Tile and Std. Meth. of Sampling and Test. Structural Cl
NSA

NSA

NSA

NSA

ASTM

SAE

CFTMA

AST

ANSI

SAE

ANS

ANSI

ANS

SAE

NSA

NSA

NSA

NSA

ASTM

NSA

St NSA

Std NSA

Std. for T NSA

NSA

ASTM

NSA

NSA

$\mathrm{SAE}$

NSA

NSA

NSA

ANSI

NSA

$S A E$

Spec SAE

pec for T SAE

SAE

$S A E$

SAE

NSA

NSA

NSA

NSA

NSA

NSA

NSA

NSA

NSA

NSA

NSA

NSA

NSA

NSA

API

ASTM

IFI

ANS1

ASTM

ANSl

ANSl

ANSI

ANSI

API

NSA

ASTM

ASTM

TCA

$A B Y C$

IEEE

EIA

ANSI

AWPA

NFLDP

BIA

ANSI

ANSI

ANSI

ANSl

ANSI

ANSI

Std. for ANSI

BIA Niform Buil ICBO

ICBO

$5500 \cdot 06$

$5000-6$

$5100 \cdot 6$

623

D2465

AMS 7482

TEM

D3198

B 16.12

AS1159A

B 16.15

B 16.4

B 16.3

AS1132A

1395

1394 
Rec, Installation Spec, for Vinyl Asbestos Recommendation for Installation of Ceramic Brick and Recommendation for Installation of Ceramic Rec. Installation Spec. for Vinyl Asbestos and Asphalt dation for Installation of Thresholds, Saddles for Ceramic

1 Build/No Wax Maintenance of Vinyl Asbestos and A sphalt Buil/ Std. for Maintenance of Vinyl Asbestos and Asphalt emulsion Floor Polish for Use on Asphalt or Vinyl Asbestos Spec. for Cleaners for Use on Asphalt and Vinyl Asbestos Recommendation for Installation of Interior Ceramic Recommendation for Installation of Exterior Ceramic

Recommendation for Installation of Conductive Ceramic Recommendation for Installation of Waterproof Ceramic ommendation for Installation Performance Levels of Ceramic ommendation for Installation of Chemical Resistant Ceramic Rec. Pract. for Chemical Resistant Resin

nt Facilities (Tech. Notes) (1969) tructures (Tech. Notes) (1969)

ies (Tech. Notes) (1969)

es (Tech. Notes) (1969) Uniform Building Code Std. for Gypsum Partition Std. Meth. of Test for Warpage of Refractory Brick and Recommendation for Installation of Ceramic r Finishing Machines (Polisher, Scrubber, Sander, Scraper, Guide Spec. for Clay

Recommendation for Installation of Ceramic Recommendation for Installation of Ceramic Recommendation for Installation of Ceramic Std. Test Meth. for Resistance of Ceramic Recommendation for Installation of Ceramic Construction Details, 6 In. Brick and Heat Transmission Coefficients of Brick and Moisture Control in Brick and erly Constructed Masonry) Insulated Cavity 6 In. Brick and Recommendation for Installation of Interior Ceramic Recommendation for Installation of Exterior Ceramic Moisture Control in Brick and Recommendation for Renovation of Interior Ceramic ition and Recommendation for Materials for Setting Ceramic Structural Clay Facing Std. Meth. of Test for Breaking Strength of Ceramic Std. Spec. for Structural Clay Nonload Bearing Std. Spec, for Concrete Drain Spec. for Asphalt Floor Spec. for Self Adhering Vinyl Asbestos Floor Spec. for Vinyl Asbestos Floor Test Meth. for Rug Back Floor Staining on Vinyl Std. Meth. for Measuring Warpage of Ceramic Std. Meth. of Test. Concrete Pipe or

std. Meth. of Sampling and Test. Brick and Structural Clay Uniform Building Code Std. for Structural Clay Floor Uniform Building Code Std, for Roofing tion and Recommendation for Materials for Grouting Ceramic

uilding Code Std. for Structural Clay Nonload Bearing Wall

on, Control, Contraction, and Isolation Joints for Ceramic

Tile and Std. Meth. of Sampling and Test. Structural Clay

Uniform Building Code: Veneer (Brick. Concrete, Stone, Rec. for Terminology and Definitions for Soil
od and Lag Screws; Light / Uniform Building Code Std. for Laminators Quality Control System

and Meth. for Establishing Rec. Design Stresses for Round Improved Std. Designs for Pressure Treated Vails and Spikes, Washers, Pin/ Western Woods Use Book: ent. Test Meth. for Establishing Design Stresses for Round Std. Spec. for Round

Std. for Preservative Treatment by Pressure Processes of Uniform Building Code Std. for Round

Std. for Preservative Treatment by Pressure Processes of ive Treatment of Western Red Cedar and Alaska Yellow Cedar Std. Spec, for Pressure Treatment of

Std. Preseryative Treatment by Pressure Processes for All Std. for Inspection of Preservative Treated

Rec. Architectural Spec. for Structural Glued Laminated Uniform Building Code Std. for Structural Glued Laminated epole Pine, Western Larch, California Redwood and Hardwood Std. for Structural Glued Laminated

Book: Abbreviations, Symbols, and Terminology (Lumber and values, Framing Rec., Properties, Weight, Stress of Board, rules, Design Values, Section Properties and Design Loads,
Tile as a Wall Covering (1973)

Tile Bathtub Walls (1973)

Tile Cavity Walls (Tech. Notes) (1965)

Tile Countertops (1973)

Tile Flooring (1973)

Tile Flooring (1973)

Tile Floors for Commercial, Institutional and Industria

Tile Floors in Institutional, Industrial and Commercial

Tile Floors (1969)

Tile Floors (1970)

Tile Floors (1973)

Tile Floors (1973)

Tile Floors (1973)

Tile Floors (1973)

Tile Floors (1973)

Tile Floors (1973)

Tile Grouts (1972T) (1972T)

Tile in Building Codes (1972)

Tile in Sanitation Structures, Industrial Waste Treatme

Tile in Sanitation Structures, Miscellaneous Sanitary S

Tile in Sanitation Structures, Sewage Treatment Facili

Tile in Sanitation Structures, Water Treatment Faciliti

Tile Lintels (Tech. Notes) (1964)

Tile Masonry (Tech. Note) (Part III Execution) (1972)

Tile Metal Tied Walls (Tech. Notes) (1966)

Tile or Block (1973)

Tile or Deviation from a Plane Surface (1972)

Tile Panels for Bath Tub Recess (1972)

Tile Refrigerator Rooms (1973)

Tile Remover, Rug Shampooer, Rug and Floor Washers, Etc

Tile Roofing (1972)

Tile Shower Receptors (1973)

Tile Steam Rooms (1973)

Tile Swimming Pools (1973)

Tile to Chemical Substances (1971) ANSl A174.1

Tile Tubs (1973)

Tile Walls for 1 Story Buildings (Tech. Notes) (1966)

Tile Walls (Tech. Notes) (1961)

Tile Walls (Tech. Notes) (1965

Tile Walls (Tech. Notes) (1966)

Tile Walls (1973)

Tile Walls (1973)

Tile Walls, Condensation (Tech. Notes) (1965)

Tile Walls, Floors, and Stairs (1973)

Tile with Mortar, Adhesive, and Mastic (1973)

Tile (Tech. Notes) (1965)

Tile (Tech. Notes) (1966)

Tile (1971) ANSI A173.1

Tile (1971) ANSI A76.1

Tile (1972)

Tile (1972)

Tile (1972)

Tile (1972)

Tile (1973)

Tile (1973)

Tile (1973)

Tile (1973)

Tile (1973)

Tile (1973)

Tile (1973)

Tile (1973)

Tile (1973)

Tile (1973)

Tile, Metal, Plastic, etc.) for Ornamentation, Protecti

Tillage and Soil Tool Relationships (1972)

Timber Connector and Bolted Joints, Drift Bolts, and Wo

(Timber Construction Manual) (1962)

Timber Construction Poles (1974)

Timber Crib Walls (1969)

Timber Fasteners (Connectors, Bolts, Lag Screws, Plates

Timber Piles (1970)

Timber Piles (1973)

Timber Piles (1973)

Timber Piles (1973)

Timber Poles (1973)

Timber Poles (1973)

Timber Products (1969)

Siructural Clay (Prop

RTl

TCA

BlA

RTI

Recommen TCA

RTI

RTI

RTl

RTl

TCA

TCA

TCA

TCA

TCA

ASTM

BlA

BlA

BIA

BIA
BIA

BlA

CSI

BIA

ICBO

ASTM

TCA

TCA

UL

CSI

TCA

TCA

TCA

ASTM

TCA

$\mathrm{BlA}$

BIA

BlA

BlA
TCA

TCA

$\mathrm{BlA}$

TCA

Defin TCA

$\mathrm{BlA}$

BIA

A STM

ASTM

ASTM

RTI

RTI

RTI

AATC

ASTM

ASTM

A STM

ICBO

ICBO

Defini TCA

Uniform B ICBO

Timber Products (1973)

Timber Products (1973)

Timber (1972)

Timber (1973)

Timber (1973)

Timber (1973) ANSI A 190.

Timber) (1973)

Timber, Lumber, Siding, Framing, Decking, Flooring, Cei

Timber, Plank and Laminated Flooring and Roofing (1973)

$A W P A$

AWPA

AWPA

AWPA

AlTC

ICBO

ICBO

USC

WWPA *31-6

WWPA *24

WWPA *31

17

*8

306

* 16

$* 6$

112

*3.2

C723

*4

$34 \mathrm{~B}$

$34 \mathrm{~A}$

34

$17 \mathrm{H}$

$11 \mathrm{~B}$

C 154

310

561
07350

309

311

302

C 650

304

26 .

$7 \mathrm{~B}$

*26

$7 \mathrm{C}$

$* 3$

$22 \mathrm{~A}$
$\mathrm{C} 648$
$\mathrm{C} 56$

C 412

* 1

$* 18$

*2

C485

C49

C67

*3. 1

300

$21 \mathrm{~A}$
$307-308$

101-106

107.109

110-111.1

[BCS24-1]

201.208.1

209.210

400-600

UBC \$24-10

UBC\$32.12

UBC $\$ 24.9$

UBC $\$ 24.8$ 
by Pressure Proc/ Uniform Building Code Std. for Lumber, bers and Their Fastenings (Lumber, Plywood and Components, eosote for the Preservative Treatment of Piles, Poles, and Std. for Pressure Treated Piles and Std. Meth. of Static Tests of Std. for Lumber,
Glued Laminated

pressure Processes (1973)

Uniform Building Code Std. for Structural Glued Laminated setting Resins (Plastic Compo/ Std. Meth. of Test for Gel .8 Rifle Range Construction (Part Sid. Neth. of Calibration Instruments for Verifying Picture Cameras (I972) Std. Exposure Std. Meth. of Test for Tack Free std. Meth. Test for Tack Free Meth. for Drainage istance (1970) ANSI A37.134 Std. Meth. of Test for Std. Meth. of Test for Gel Std. Meth. of Test for Gel th. of Analysis of Pelshenke Test (Wheat Meal Fermentation ure (1974) ANSI B72.6 Std. Meth. of Test for ntation Program for Personnel Going Offshore for the First Std. Meth. for Determining Safety ape Recorder / Reproducers (1974) An/ Rec. Test Meth. for ment of Extreme Pressure Properties of Lubricating Fluids
Std. Meth. Test for Electrodeposited Coatings of Std. Spec. for Lead and

td. Spec. for Electrodeposited Coatings of Lead and Lead. Std. Spec. for Copper-Zinc Uniform Building Code Std. for Safety Std. for Swinging Hardware for Std. Std. for Safety for y Std. for Sliding Hardware for Std., Horizontally Mounted Std. Spec. for General Requirements for Std. Spec. for Sid. Spec, for Electrodeposited Coatings of Std. Classification of Pig

al Power Cable) (1973) oses (1974) ANSI C7.4

Std. Spec. for Phosphor Bronze (Copper Std. Spec. for Std. Meth. of Test for or Carbide Blanks and Cutting Tools, Single Point, Carbide Std. for Carbide Blanks for Std. for

Tests for Static Load, Frame, Fork, Steering Assembly and Service for Use by Vehicle Manufacturers in Designing for el (1974) Std. Meth. of Test. Tire Cords.

from Man Made Organic Base Fibers/ Std. Meth. of Testing
ade from Steel (1974) ade from Steel (1974) Std. for New

nsi L14.243

Tent. Tolerances for Std. Spec. for Std. Uniform Fire Code: Automo bile Stds. for of Future Design (1972) SAE J711

Std for Suffix Letters Used in s) (1971) Safe Operating Condition of Tires Including and Other Equipment (1966) Std. for Demountable Saddle Braces Pedal Clearance, Steering Control, Brakes. , Drive, Planter (Drill) Press, and Gage Wheel./

'Type Vehicles, Counter Balanced Fork Lift Truc/ Bicycle (1974)

mining and Logging (Short Haul); Grader, Loader/

multipurpose Passenger Vehi/ Std. for Bias and Radial Ply e Vehicle (1974)

\section{Std. for} Std. for Std. for Std. for Std. for

nce Requirements and Iniform Test Procedures for Retreaded que Application, Using Highway Vehic/ Std. Meth. of Test. Std. for Definition of and Maximum Dimensions for Grown aces Using a Passenger Automobile Equipped with Full Scale er Car Type Tires) (1971) Safe Operating Condition of Rec. Pract. for Liquid Ballast Table for Drive Std. Liquid Ballast Table for Drive rpose Pas/ Std. for Mining and Logging Definitions and Terms I'sed in the Selection of Std. for Agricultural Press and Gage Wheel Std. for Agricultural Planter Press Wheel aces Using a Passenger Automobile Equipped with Full Scale st. Machines for Measuring the Iniformity of Passenger Car Safe Operating Condition of Truck, Trailer and Bus Type $\log$ Skidder, Truck, Bus, Bead Seat, Industrial and Bicycle cluding Tire Treads Depth Requirements (Passenger Car Type elicopter) (1974)
Timber, Plywood and Utility Pole Preservative Treatment Timber, Preservative Treatment, Poles, Piles, Spaced Co Timbers for Marine, Land, and Fresh Water Use (1974) an Timbers in Marine Construction (1973)

Timbers (Wood) in Structural Sizes (1967) ANSI Q4.2

Timbers, Bridge and Mine Tie Preservative Treatment by Timbers: Douglas and Hem Fir, Southern and Lodgepole Pi Timber: Water; Ceiling: Partitions; Plaster; Roofing) a

Time and Peak Exothermic Temperature of Reacting Thermo Time and Special) (1971)

Time Coding of Propulsion Engine Items (1971) ANSI B141

Time Load Indication of Machines (1974) ANSI Z115.2

Time Markings for Variable Speed Shutters Used in Still

Time of Caulking Compounds and Sealants (1973)

Time of Elastomeric Type Joint Sealants (1971)

Time of Pulp (1972)

Time of Setting of Concrete Mixtures by Penetration Res

Time of Solventless Varnishes (1972)

Time of Tar Acids (1972) ANSI Z78.23

Time Test) (Cereal Chemistry) (1962)

Time to Failure of Plastic Pipe Con

Time (Petroleum Production) (I974)

Times of Photographic Darkroom III

Timing Error Measurements of Instrun

Tin-Lead Alloy (Solder Plate) (1973)

Tin Alloy Die Castings (1952) ANSI H48.

Tin Alloys on Steel and Ferrous Alloys (1970) ANSI G53.

Tin Alloys Plate, Sheet, Strip, and Rolled Bar (1973j

Tin Clad Fire Doors and Shutters (1973)

Tin Clad Fire Doors (1973) ANSI Al33. I

Tin Clad Fire Doors (1973) A NSI Al42.1

Tin Clad Fire Doors (1973) ANSI Al43.I

Tin Mill Products (1973)

Tin Mill Products (1973)

Tin Plate Fabricated Aerosol Cans (1972)

Tin (1972)

Tin (1972) ANSI H42.1

Tin) Plate, Sheet, Strip, and Rolled Bar (1973)

Tinned Copper Concentric Neutrals (Underground Electric

Tinned Soft or Annealed Copper Wire for Electrical Purp

Tint Strength of Carbon Black (1973)

Tipped, Roller Turner Type (1972)

Tipping Circular Saws (1972)

Tire and Rim Equipment for 1974 Passenger Cars (1974)

ITs for Regular Bicycles Including

Tire Blowout (1972)

Dimensions for Grown

Tire Cord Fabrics, Filaments, and Strands Made from Ste

Tire Cords. Fabrics and Industrial Filament Yarns, Made

Tire Cords, Tire Cord Fabrics, Filaments, and Strands M

Tire Dimensions (I974)

Tire Fabrics (Textile) Other Than Cord Fabrics (1973) a

Tire for Pavement Skid Resistance Tests (1973)

Tire Rebuilding Plants (1973)

Tire Rim Inspection, and Disk and Flat Tapes (1974)

Tire Rim Selection (1974)

Tire Selection Tables for Agricultural (Farm) Machines

Tire Size Designations and Their Definitions (1974)

Tire Treads Depth Requirements (Passenger Car Type Tire

Tire Valves for All Vehicles Except Aircraft (1974)

Tired WheeIs for Industrial Trucks, Casters, Conveyors,

Tires and Controls (1972) /Heel Assembly, Ch

Tires and Rims for Agricultural and Industrial Steering

Tires and Rims for Industrial, Utility and Recreational

Tires and Rims for Motorcycle. Side Car, Mini Bike, and

Tires and Rims for Off the Road Vehicles: Earthmoving

Tires and Rims for Passenger Cars, Station $\mathbb{W}$ agons, and

Tires and Rims for Truck, Bus, Trailer, and Multipurpos

Tires for Passenger Cars and Station Wagons (1965)

Tires for Passenger Cars and Station Wagons (1966)

Tires for Wet Traction in Cornering Without Driving Tor

Tires in Service for Use by Vehicle Manufacturers in De

Tires in the Diagonal Braking Mode (1973)

Tires Including Tire Treads Depth Requirements (Passen

Tires of Agricultural Machines (1971)

Tires of Agricultural Machines (1972)

Tires Used in Intermittent Highway Service (1974)

Tires (Car, Station Wagon. Truck, Bus, Trailer, Multipu

Tires (Farm Machinery) (1971)

Tires (Farm Machinery) (1971)

Tires (1971T)

Tires $(1972)$

Tires (1973)

Tires (1974)

Tires) (1971)

/Senger Cars, Off Road, Agricultural and Est for Stopping Distance on Paved Surf AST Rec. Pract. for Te SAE
VESC

TRA

TRA

ICBO

ICBO

ASTM

AWPA

ASTM

ICBO

ICBO

ASTM

NRA

ASTM

ANSI

ASTM

ASTM

TAPPI

ASTM

ASTM

$\mathrm{AACCH}$

ASTM

API

ANSI

EIA

ASTM

ASTM

ASTM

ASTM

UL

UL

UISI

ASTM

ASTM

ASTM

ASTM

AA

ASTM

ASTM

TRA

BMA

ASTM

ASTM

ASTM

RA

ASTM

CBO

TRA

TRA

ASAE

VESC

TRA

CFTM

TRA

TRA

TRA

TRA

VESC

IMa VESC

ASTM

TRA

ASTM

VESC

SAE
ASAE

TRA

E S223

E445

J332A

7

V.7

D198

C2

2471

*10

E74

D2377

D3056

D2870

D.50

1598

RPT.1

2782

$\mathrm{B} 102$

B200

B59l

10A

$14 \mathrm{~B}$

BCS 25.12

UBC $* 3.25$

UBCS25.11

UBC $* 3.23$

PH 3.32

(

2.22

UBCS 43.3 
Analysis for Fluoride Content of the Atmosphere and Plant Analysis for Fluoride Content of the Atmosphere and Plant Std. Spec. for Titanium and Spec. for heat Treated, RoII Threaded (1973) (1973) 1 ck (1974) (1974)

Spec. for Titanium and Std. for Std. for

Pan Head, Close Tolerance, Short Thread, Tri Wing Recess, Std. for Bolt, Hex Head, Close Tolerance Std. for Bolt Lock, Tension, Protruding Head, Stump Type, Std. Procurement Spec for Fasteners, for Bolt Lock, Tension, $100 \mathrm{Deg}$. Crown Head, Stump Type, or Bolt Lock, Tension, 100 Deg. Head (MS24694), Pull Type r Bolt Lock, Tension, 100 Deg. Head (MS24694), Stump Type, r Bolt Lock, Tension, 100 Deg. Head (MS20426), Stump Type,

Lock, Shear, 100 Deg. Head, Std. and Oversize, Pull Type ock, Shear, Protruding Head, Std. and Oversize, Pull Type,

k, Tension, Protruding Head, Std. and Oversize, Pull Type ension, 100 Deg. Crown Head, Std. and Oversize, Pull Type, on, 100 Deg. Head (MS20426), Std. and Oversize, Pull Type Spec. for Fasteners, 6Al.4V Std. for Screw, Hex Head, Tri Wing Recess, 6Al-4V g / Std. for Screw, $100 \mathrm{Deg}$. Head Tri Wing Recess, 6Al.4V g (1972) g (1972) Std. for Bolt, Hex Head, Close Tolerance, 6Al-4V Tolerance, 6Al.4V Bolt, 100 Deg. Tri Wing Recess, Close Tolerance, 6Al-4V ng (19/ Std. for Bolt, Hex Head, Close Tolerance, 6Al.4V Screw, Flat Fillister Head, Full Thread, Tri Wing Recess,

(1970) ANSI W 3.16

apes (1973)

i Z179.2

72) ANSI Z179.1 Spec. for Conversion Coating of Spec. for Tolerances Applicable to Std. Spec. for Spec. for Std. Spec. fo Std. Spec. for

ANSI H50.2

) (1962) Std. Spec. for Seamless and Welded Unalloyed Std. for Bolt-Close Tolerance, Hexagon Head Rec. Pract. for the Safe Action and
Std. for
Meth. of Analysis for thetic Anionic Active Ingredient in Detergents by Cationic

$r$ Bromine Index of Petroleum Hydrocarbons by Electrometric meth. of Test for Synthetic Anionic Ingredient by Cationic er of Petroleum Products by Potentiometric Perchloric Acid for Bromine Index of Aromatic Hydrocarbons by Coulometric Std. Analytical Meth. for Determining Calcium (Egta S/ Std. Analytical Meth. for Determining Calcium (Egta -
ro/ $s$ (Cereal Chemistry) (1972)

ty Requirements for Floor and Wall Openings, Railings, and Guide Spec. for Metal Std. Spec. for Powdered Std. Spec. for Liquid overing Sewage Holding and/or Treatment Devices for Marine and Metallization Thickness by MuItiple Beam Interference
Std. for Screw-100 Deg. Close (1974) Std. for Rivet-100 Deg. Countersunk Head Hi Shear Close Std. for 100 Deg. Close
Std. for Hi Shear 100 Deg. Flush Close Std. Meth of Test for Solvent Std. for Rivet-Flat Head, Hi Shear, Close Std. for Bolt-Shear, Close Std. for Bolt, 100 Deg. Head, Tri Wing Recess, Close Std. for BoIt, 100 Deg. Head, Tri Wing Recess, Close onl/ Std. for BoIt, 100 Deg. Head, Tri Wing Recess, Close ocking (1972) Std. for Bolt, Hex Head, Close d. for BoIt, 100 Deg. Reduced Head, Tri Wing Recess, Close nonlocking (1972) Std. for Bolt, Hex Head, Close non/ nlocking (1972) Std. for Bolt, 100 Deg. Head, Tri Wing Recess, Close d. for Bolt, 100 Deg. Reduced Head. Tri Wing Recess, Close nlo/ Std. for Bolt, 100 Deg. Head, Tri Wing Recess, Close nlocking (1972) Std. for BoIt, Hex Head, Close Std. for Bolt-Close Std. for Bolt-100 Deg. CIose Std for Pan Head, Close

y $6 \mathrm{Al}-4 \mathrm{~V}$, SeIf Locking An/ self Locking and Nonlocki/ elf Locking and Nonlocking (197) Std. for Bolt, Pan Head, Close Std. for Bolt, Pan Head, Close Std. for Pan Head, Close Std. for Bolt, Hex Head, Close
Tissues (Manual Procedures) (1973)

Tissues (Semiautomated Meth.) (1973)

Titanium Alloy Bars and Billets (1972) ANS1 Z179.2

Tent Meth. for ASTM Tent. Meth of ASTM

Titanium Alloy BoIts and Screws, 6A1-4V, Heat Treated,

Titanium Alloy Bolts and Screws, 6A1-4V, Upset Headed,

Titanium Alloy Sheet, Strip, and Plate, 6A1.4V Annealed

Titanium Alloy Strip, Sheet, and Plate (1972) ANSI Z179

Titanium Alloy Stump Type Protruding Head Shear Bolt Lo

Titanium Alloy Stump Type 100 Deg. Head Shear Bolt Lock

Titanium Alloy 6Al-4V, Self Locking and Nonlocking (197

Titanium Alloy (1972)

Titanium Alloy (1973)

Titanium Alloy (1973)

Titanium Alloy (1973)

Titanium Alloy (1973)

Titanium Alloy (1973)

Titanium Alloy (1973)

Titanium Alloy (1973)

Titanium Alloy (1973)

Titanium Alloy (1973)

Titanium Alloy (1973)

Titanium Alloy (1973)

Titanium Alloy, Externally Threaded (1972)

Titanium Alloy, Full Thread, Nonlocking (1972)

Titanium Alloy, Full Thread, Self Locking and Nonlockin

Titanium Alloy, Long Thread, Self Locking and Nonlockin

Titanium Alloy, Long Thread, Self Locking and Nonlockin

Titanium Alloy, Short Thread, Nonlocking (1972)

Titanium Alloy, Short Thread, Self Locking and Nonlocki

Titanium Alloy, Short Thread, Self Locking and NonIocki

Titanium Alloy, 6Al-4V , Self-Locking and Nonlocking (19

Titanium Alloys (Fluoride-Phosphate Type) (1973)

Titanium and Its Alloy Bare Welding Rods and Electrodes

Titanium and Its Alloy Castings (1969) A NSI Z179.16

Titanium and Its Base Alloy Extruded Bars, Rods, and Sh

Titanium and Titanium Alloy Bars and Billets (1972) Ans

Titanium and Titanium Alloy Strip, Sheet, and Plate (19

Titanium Dioxide Pigments (1973)

Titanium Dioxide Pigments (1973) ANSI K45.1

Titanium Sponge (1969) ANSl Z179.13

Titanium Tube for Condensers and Heat Exchangers (1965)

Titanium Welding Fittings (1971) ANSI H50.1

Titanium, Short Thread (1973)

Title Areas for Television Transmission (1968)

Title Leaves of a Book (1971)

Titratable Acidity in Nonfat Dry Milk (Cereal Chemistry

Titration Procedure (1974)

Titration (1972) ANSI Z11.248

Std. Meth. of Test for Syn

Titration (1972T)

Titration (1973)

Titration (1974) A NSI 278.15

D. Meth. of Test for Total Base Numb

Titrimetric) Content in Corn Starch (1973)

Titrimetric) Content of Corn Sugar (Crude and Refined)

Titrimetric) Content of Corn Syrup and Other Starch Hyd

TLC Meth. of Analysis of Aflatoxins in Corn and Soybean

Toeboards (1973)

Toilet Partitions (1972)

Toilet Soap (1974)

Toilet Soap (1974) ANSI K60.14

Toilet Waste (Boats) (1972)

(Tolansky Meth.) (1973)

Tolerance Flat Head 160,000 psi (1973)

Tolerance Head and Shank (1973)

Tolerance Head and Shank, 160,000 psi Short Thread Bolt

Tolerance Head Rivet (1972)

Tolerance of Amine Resins (1973)

Tolerance Shank (1973)

Tolerance (1973)

Tolerance (1972)

Tolerance (1972)

Tolerance, Alloy Steel, Long Thread, Self Locking and N

Tolerance, Alloy Steel, Long Thread, SeIf Locking and N

Tolerance, Alloy Steel, Short Thread, Nonlocking (1973)

Tolerance, Alloy Steel, Short Thread, Self Locking and

Tolerance, Alloy SteeI, Short Thread, Self-Locking and

Tolerance, A286 Cres, Long Threads, Self Locking and No

Tolerance, A286 Cres, Short Thread, Nonlocking (1972)

Tolerance, A286 Cres, Short Thread, Self Locking and No

Tolerance, A286 Cres, Short Thread, Self Locking and No

Tolerance, Hexagon Head, Titanium, Short Thread (1973)

Tolerance, High Strength (1973)

Tolerance, Short Thread, Torq Set Machine Screw (1972)

Tolerance, Short Thread, Tri Wing Recess, Titanium Allo

Tolerance, Short Thread, TWI Wing Recess, Alloy Steel,

Tolerance, Short Thread, TWI Wing Recess, A286 Acres,

Tolerance, Titanium Alloy (1972)
D3269

D3270

B348

AMS7460C

AMS7461B

AMS4911C

ASTM B26

NSA 2605

NSA 2705

NSA $\quad 5200.06$

NSA $1266-70$

2206

621

2325

2105 Std. F NSA Std. Fo NSA

Std. Fo NSA

2306

2315

2506

2406

2005

2125

2115

4004

6100-3

$5800-6$

4304-16

6804-20

4903.16

$4600 \cdot 16$

6403-20

5500-06

AMS2486

A5. 16

B367

AM $\$ 2245$

B348

B265

D2701

D476

B299

B338

B.363

NSA 653

SMPTE RP8

ANSI Z39.15

A ACCH 02.31

D 1681

D2710

D 3049

D2896

D 1492

B-11

F.8

E-11

CR

45.05

Safe ANSl A12.1

CSI 10182

ASTM D2958

ASTM D799

A 8

F388

517

525

525
1202

1055

D 1198

529

464

4204-16

4304-16

4104-16

6604-20

4703-16

6203-20

$4400-16$

$6704-20$

4803-16

4500-16

$6303 \cdot 20$

653

333

1131-38

5200.06

$5000 \cdot 6$

$5100 \cdot 6$

1266.70 
king and Nonlocking (1972/

Std. for Bolt, Hex Head, Close ing (1972)

cking and Nonlocki/

Bolt, 100 Deg. Tri Wing Recess, Close ocking and Nonlocking (19/ truded Bars, Rods, and Shapes (1973)

and Packing Purposes (1974) Ansi/

Std. for Dimensional
Sid. Dimensional Std. Meth. of Test, and Std. 73) abrics (1973) ANSI L14.243 1971)

Std. Rec. Pract. for Dimensions and

Tent.

73

d Forging Stock (Rolled or Drawn) (1973) ons (1973) nd Plate (1973)

r/ Std. Spec. for Dimensions and 1 Compositions, Mechanical Property Limits and Dimensional im Spacers (M) Rec. Pract. for for Practical Minimum Rec. i C83/ Std. Spec. for Printed Wiring Board Dimensions and wing and Related Documentation Practices: Dimensioning and Std. for Acceptable Concentrations of

$s$ of Moisture in Feeds and Feedstuffs by Distillation with $s$ of Moisture in Fats and Shortenings by Distillation with Std. Spec. for Industrial Grade Std. Spec. for Nitration Grade

d. Meth of Test for Moisture in Wool by Distillation with ial Aromatic/ Std. Meth. of Test for Acidity of Benzene oams (1973) ANSI K65.125 Std. Spec. for Std. Meth. of Test. Para Red and

of Photographic Negative Materials Monochrome, Continuous Std. Meth. of Test. Para Red and Toluidine Red Pigments meth. of Test for Tearing Strength of Woven Fabrics by the std. for

Suggested Noise Measurement Techniques for the Machine Std. for Symbols for Machine isel Plow, Field and Row Crop Cultivator Shafts and Ground

for Terminology and Definitions for Soil Tillage and Sol Std. for Spindle Noses and

of Common Words Related to Numerically Controlled Machine Std. Rec. Pract. for Macroetch Test. of Std. Spec. for Machined Flat and Square Std. Spec. for Wrought Alloy

els (1973) Std. Meth. for Chemical Analysis of Std. Spec. for Carbon

Spec for Metal Cutting Machine afety Std. for All Thermoplastic Type MTW 600 Volt Machine $s(1972)$ Std.

Formats for Transmission of End Product Description Data Std for Industrial Equipment and General Purpose Machine ving and Spindle Ends for Portable Hand, Air, and Electric Electrical Std. for Metalworking Machine Safety Std. for Stationary and Fixed Electric Std. for Safety for Portable Electric

d Procedures for User Certification of (1962) A/ Std. for (1972) Rotating, Liquid Filled and Dry Transformers, Hand Power ies and. for Carbide Blanks and Cutting ughness, Waviness, Form and Lay) (1973) Std. for Gear Std. Spec. for Fineness of Wool Std. Ampacities Cables in Open Soluble Liquid Adhesives for Automatic Machine Sealing of st (1973) Test Meth. for

alculation of Commercial Weight and Yield of Scoured Wool, nt of Grade (1973) Ans/ Std. Spec, for Fineness of Mohai ehold Electric Ranges (Stove) with Glass / Ceramic Cooking 973) ANSI Z250.1 Std. for Safety for LP Gas Safety Std. for Oxy Fuel Gas Std. Test Procedure for

ata) (1972) Std. for Bolt, Flat, 100 Deg. Head, Sid for Bolt Flat 100 Deg. Reduced Head, Std. for Pan Head, Close Tolerance, Short Thread. Std. for Screw, Machine, Pan Head, Full Thread, std. for Screw, Machine, Flat Fillister Head, Full Thread, Std for Screw, Machine, Flat 100 Deg. Head, Full Thread. Std. for Machine Screw, Flat 100 Deg. Head, Short Thread, Std. for Machine Screw, Pan Head, Short Thread,

Test. Tires for Wet Traction in Cornering Without Driving

Test Procedure for Linear, Single Axis, Pendulous, Analog rformance of Nonmetallic Locking Element Type Prevailing Std. Meth. of Measuring Application and Removal Std Part Bolt, 100 Deg. Flush Tension Head Hi
Tolerance, 6Al-4V Titanium Alloy, Long Thread, Self Loc Tolerance, 6Al-4V Titanium Alloy, Short Thread, Nonlock Tolerance, $6 \mathrm{Al}-4 \mathrm{~V}$ Titanium Alloy, Short Thread, Self Lo Tolerance, $6 \mathrm{Al}-4 \mathrm{~V}$, Titanium Alloy, Short Thread, Self L Tolerances Applicable to Titanium and lts Base Alloy Ex Tolerances for Aluminum Mill Products (1973)

Tolerances for Industrial Wheels (1965)

Tolerances for Jute Rove and Plied Yarn for Electrical

Tolerances for Roller Drafted Yarns (1971)

Tolerances for Screw Holes and Slots in Bone Plates (19

Tolerances for Tire Fabrics (Textile) Other Than Cord F

Tolerances for Woolen Spun on the Woolen System Yarns (

Tolerances of Aluminum Alloy Drawn Tubing (1973)

Tolerances of Aluminum Alloy Std. Structural Shapes (19

Tolerances of Aluminum and lis Alloy Bar, Rod, Wire, an

Tolerances of Aluminum and Magnesium Base Alloy Extrus

Tolerances of Aluminum and Magnesium Base Alloy Sheet a

Tolerances of Low Alloy Steel Bars (1973)

Tolerances of Opaque Materials and for Evaluating Confo

Tolerances of Plastic Bottles (1972)

Tolerances of SAE Wrought Aluminum Alloys (1972)

Tolerances of Spoke Type Wheels, Demountable Rims and R

Tolerances Single and Two Sided Rigid Boards (1970) Ans

Tolerancing (1973)

Toluene in the Air of Workplaces (1974)

Toluene (Cereal Chemistry) (1962)

Toluene (Cereal Chemistry) (1962)

Std. Engineering Dra

Toluene (1971)

Toluene (1971) ANSI Z78.7

Toluene (1972) ANSI L14.256

Toluene, Xylenes, Solvent Naphthas, and Similar lndustr

Toluenediisocyanate for Use in Production of Urethane F

Toluidine Red Pigments (Toners) (1973)

$\mathrm{Ne}$ Meth, of Analysi

Ng Meth of Analysi

one) (1972)

(Toners) (1973)

Tongue (Single Rip) Meth. (Constant Rate of Traverse Te

Tool and Cutter Grinding Machine (1973)

Tool Builders' Industry (1970)

Tool Indicator Plates or Control Buttons (1972)

Tool Mountings (Farm Machinery) (1973)

Tool Relationships (1972)

Tool Shanks for Milling Machines (1972)

Tool Software (1972)

Tool Steel Bars (1971) ANSI G60.9

Tool Steel Bars (1973)

Tool Steel Products (lncluding Dies and Fixtures) (1973

Tool Steels and Other Similar Medium and High Alloy Ste

Tool Steels (1973)

Tool Weldments (1971)

Tool Wires and Cables (1972)

Tooling Nomenclature for Solid Die Two Blow Cold Header

Tooling, Printed Wiring Boards) in Numeric (Digital) Fo

Tools (1973)

Tools (1973)

Tools (1973) ANSl C113.1

Tools (1973) ANSI C 33.86

Tools (1974) ANSI C 33.49

Tools, Crimping, Solderless Wiring Devices-Recommende

Tools, Hermatic Stators, Test. and Safety) (1973)

Tools, Single Point, Carbide Tipped, Roller Turner Type

Tooth Lock Washers (1972)

llmensions, Physical

Tooth Surface Texture for Aerospace Gearing (Surface Ro

Top and Assignment of Grade (1973) ANSI L14.29

Top Cable Trays (1972)

Top Flaps of Fiberboard Shipping Cases (1970)

Top Side of Filled Paper as Indicated by Silver Coin Te

Top, and Noil for Various Commercial Compositions (1972

Top, Yarns and Fabrics of the Worsted Type and Assignme

Tops (1974)

Torches for Heating, Soldering, and Brazing Purposes (1

Torches (1973)

Toroidal Magnetic Amplifier Cores (Including Material D

Torq Set and Hi Torque, 180 KSI (1973)

Torq Set and Hi Torque, 180 KSI (1973)

Torq Set and Hi Torque, 180 KSI (1973)

Torq Set Machine Screw (1972)

Torq Set (1973)

Torq Set (1973)

Torq Set (1973)

Torq Set (1973)

Torq Set (I973)

Torque Application, Using Highway Vehicles (1973)

Torque Balance Accelerometer (1972)

Torque Lock Screws (1973)

Torque of Threaded Closures (1973)

Torque Recess Alloy Steel, 160,000 psi (1973)

NSA

NSA

NSA

NSA

SAE

CFTMA

ASTM

ASTM

ASTM

ASTM

ASTM

SAE

$S A E$

SAE

$S A E$

SAE

SAE

ASTM

ASTM

Mica SAE

$\mathrm{SAE}$

IPC

ANSl

ANSl

6804-20

4903.16

$4600-16$

$6403-20$

AMS 2245

H35. 2

TOL

D681

D2645

$\mathrm{F} 367$

D2644

A MS2203G

AMS2204D

AMS2201J

AMS2205L

AMS2202K

AMS2251E

D3134

D2911

J457 J

J851

D300E

Y I 4.5

Z37. 12

CCH 44-50

$\triangle A C C H$ 44-51

ASTM D362

ASTM D841

St ASTM D2462

ASTM D847

ASTM D1786

ASTM

PH2.5

ASTM D970

ASTM D2262

NSA 973

NMTBA *1

ANSI B5.46

Std. for Ch ASAE $\quad \begin{array}{ll}\text { S225.1 } \\ \text { AS }\end{array}$

Rec. ASAE R291.

ANSI B5.18

Definitions NMTBA *

ASTM A56]

ASTM A685

ASTM A681

ASTM E352

ASTM A686

AWS D14.2

UL

lFI

IPC

1063

118

D-350

H-1

B107.4

NFPA

UL

UL

ElA

/Try EASA

ANSl

987

$\mathrm{RS} 270$

*1

B94.37

B18.21.1

AGMA 118.01

ASTM D472

IPCEA

P. 54.440

D 1874

TAPPI UM-5.3.

ASTM D2720

ASTM D1381

ER.2

$\begin{array}{ll}\text { UL } & 147 \\ \text { UL } & 123\end{array}$ 
steel Hex Locknuts (1972)

Std for Torque Tension Requirements for Prevaling ec. for Steel Self-Locking Nuts, High Strength, Prevailing Corrosion Resistant Alloy Nuts, High Strength, Prevailing s, Corrosion and Heat Resistant, High Strength, Prevailing s, Corrosion and Heat Resistant. High Strength, Prevailing Std. for Bolt, Flat, $100 \mathrm{Deg}$. Head, Torq Set and Hi Std. for Bolt, Brazier Head, Torq Set and H

td. for Bolt, Flat, 100 Deg. Reduced Head, Torq Set and Hi ies of Plastics as a Function of Temperature by Means of $A$ ) (1973) Std. Rec. Pract. for Static Bend and logy, H Handbook of Spring Design: Compression, Extension, Measuring the Relative Stiffness of Leather by Means of iffening of Rubber and Rubber Like Materials by Means of A Std. Test Meth. for Plane Strain Fracture Std. Spec. for Structural Carbon Steel Plates of Improved tible Fibres (Cotton, Sisal, Henequen, Ixtle, Jutes, Hemp Std. for Laundering of Cloth

Std. Performance Requirements for Woven Dish Std. Performance Requirements for Glass Std. Performance Requirements for Huck 3)

Std. Performance Requirements for Terry Cloth

ranes, Derricks, Hoists, Hooks, Jacks, and Slings: Portal, evel, Helical and Herringbone Gear Units for Water Cooling (1972)

Uniform Building Code: Penthouses and Roof Structures Structural Std. for Steel Antenn d Lumber Spec. for Application in Industrial Water Cooling $r$ Lumber Spec. for Application in Industrial Water Cooling e Lumber Spec. for Application in Industrial Water Cooling servative Treatment of Lumber for Industrial Water Cooling

for Manually Propelled Mobile Ladder Stands for Scaffolds of Fittings and Equipment for Anchoring. Mooring, Docking, $r$ Motor Vehicle (Recreational Type) Connecting Devices and ies of Single Man Made Textile Fibers Taken from Yarns and ial Passenger Tramways Including Ski and Surface Lifts and Std. Meth. of Test for Evaluating Inhibitory Std. for Safety for Portable Std. Analytical Meth. for Determining Iron Std. Meth. of Test for Sid. Meth. of Test for Meth. of Test for drocarbons by Oxidative Microcoulometr iquids (Oxy Hydrogen Combustion M/

Std. Meth. of Test for I973) Std. for Milling Machine spectrometer Leak Detector or Residual Gas Analyzer in the raphy (1972) ANSI Z11.27/ Std. Vleth. of Test for Aromatic Std. Meth. for Fast Neutron Flux Measurements by

Protective Structure (FOPS) for Prime Mlovers, Wheeled and erformance Criteria for Roll Over Protective Structure for

Wheeled and Track Type Front End Loaders, Wheeled Dozers,

Unrecorded Magnetic Tape for Information Interchange $(9$ d. Spec. for Nonload (Axial) Bearing Steel Studs, Runners ds of $7.5 \operatorname{In} .0190 . /$

Std. for Reproducer Test Tape: Ful Std. Test Meth for Dust and Fog

Std. Meth. of Test for Liquid Contaminant, Inclined Plane etic Tape Recordings (1970) Rec. Pract. Spec. of uadruplex Recorded A/ Std. Dimensions of Video, Audio and

fety Std. for Performance Requirements for Stopping Power, n, Using Highway Vehic/ nt) Drive Line Specs. (1972) Std. Meth. of Test. Tires for We Rec. for Rec. Pract. for Agricultura Rec. for Agricultural (Farm Agricult ura

Std. for Agricultura Apricultural

al Steering, Drive, Planter (Drill) Press, and Gage Wheel, Boat, Baggage, Special Type Trailer, Mobile Home, Garden criteria for Roll Over Protective Structure for Track Type tion of Hydraulic Remote Control Cylinders to Agricultural Application of Remote Hydraulic Motors to Agricultural Std. for Attachment of Implements to Agricultural Wheel or Measuring Hydraulic Lift Force Capacity on Agricultural or Measuring Hydraulic Lift Force Capacity on Agricultural erformance R/ Std. for Protective Frame (For Agricultural for Industrial Rotary Mowers (Designed for Operation with (1972) SAE J/ Std for Protective Frame for Agricultural (1972) /

Std. for Protective Enclosures for Agricultural Rec. Pract. for Draw Bar for Forestry

Safety Spec. for Power Mowers, Lawn, and Garden

for Power Off Definitions and Terminology for Agricultural ink Hitch Attachment of Implements to Agricultural Wheeled tachment for Hitching Implements to Lawn and Garden Riding
Torque Strength of Composite Tubes and Cores (1974)

Torque Tension Requirements for Prevailing Torque Type

Torque Type Steel Hex Locknuts (1972)

Torque, All Metal (I973)

Torque, All Metal (1973)

Torque, All Metal, $1200 \mathrm{Deg}$. F (649 Deg. C) Use, Unifie

Torque, All Metal, 1200 Deg. F (649 Deg. C) Use, Unifie

Torque, 180 KSI (1973)

Torque, 180 KSI (1973)

Torque, 180 KSI (1973)

Torsion Test (1972) ANSI D65.2

Torsion Test. of Intramedullary Rods (Surgical Implants

Torsion, Flat, and Hot Wound (Materials, Specs. Termino

Torsional Wire Apparatus (1972) ALCA E6

D. Meth. for

Torsional Wire Apparatus (1973) ANSI J2.2

Toughness of Metallic Materials (1972) ANSI Z260.2

Perature St

Toughness (1973)

Tournament Table and Accessory Equipment Specs. (1971)

Tow, Cocoa Fibers, Oakum, Rags, Waste Cloth, Wastepaper

Toweling (1970)

Towels (Institutional Textile) (1973)

Towels (Institutional Textile) (1973)

Towels (Institutional Textile) (1973)

ToweIs (Single and Double) (Institutional Textile) (197

Tower and Pillar Cranes (1973)

Tower Fan Drives (1972)

Tower Silos: Unit Weight of Silage and Silo Capacities

(Tower, Spire) (1973)

Towers and Supporting Structures (1972)

Towers (1971)

Towers (1971)

Towers (1971)

Towers (1971)

(Towers, Safety) (1971)

Towing and Lifting Boats (1973)

Towing Meth. (Coupling, H

Tows (1973)

Toxicity of Industrial Waters (1968E)

Toy Transformers (1973)

(TPTZ) Content of Corn Syrup (1970)

Trace Amounts of Lead in Gasoline (1972) ANS1 Z11.324

Trace Metals in Gas Turbine Fuels (Atomic Absorption Me

Trace Quantities of Sulfur in Lighe Liquid Petroleum Hy

Trace Quantitites of Total Sulfur in Volatile Organic L

Tracer Controlled Profiling and Contouring-Vertical (

Tracer Probe Mode (1973) /T. for Leaks Using the Mass

Traces in Light Saturated Hydrocarbons by Gas Chromatog

Track Etch Technique (1973)

Track Type Front End Loaders, Wheeled Dozers, Track Typ

Track Type Tractors and Front End Loaders (1972)

Track Type Tractors, and Motor Graders (1971)

Track 200 and $800 \mathrm{cpi}, \mathrm{NRZI}$, and $1600 \mathrm{cpi}, \mathrm{PE})$ (1973

(Track) and Rigid Furring Channels for Screw Application

Track, 1/4 In. (6.3 Mm) Width, Open Reel (For Tape Spee

Tracking and Erosion ANSI C59.133

Tracking and Erosion of Insulating Materials (1973) Ans

Tracking Control Record for 2 In. Quadruplex Video Magn

Tracking Control Records on 2 In. Video Magnetic Tape Q

Tracking of Vehicles (1971)

Traction and Steering Stability for Regular Bicycles (1

Traction in Cornering Without Driving Torque Applicatio

Tractor and Farm Implement Power Takeoff (Universal Joi

Tractor Auxiliary Power Take Off Drives (1972)

Tractor Auxiliary Power Takeoff Drives (1972)

Tractor Belt Speed and Pulley Width (1971) SAE J720

Tractor Test Code $(1972)$

Tractor Test Code (1972) SAE J708C

Tractor. Implement, and Log Skidder (1974)

Tractor. Industrial Truck and Trailer (1974)

Tractors and Front End Loaders (1972)

ILm Perfo

Tractors and Trailing Type (Farm) Implements (1971)

Tractors Equipped with Quick Attaching Coupler for Thre

Typ $\mathrm{SAE}$

Thers, SAE

ANSI

ASTM

EIA

ASTM

ASTM

SMPTE

ANSI

$\mathrm{SAE}$

BMA

ASTM

ASAE

$S A E$

ASAE

ASAE

SAE

ASAE

Tractors Equipped with 3 Point Hitch (1972)

Tractors Equipped with 3 Point Hitch (1972)

Tractors for Overhead Protection (Test Procedures and P

Tractors Having at Least $20 \mathrm{Hp}$ ) (1971)

Y Requirements

Tractors (Test Procedures and Performance Requirements)

Tractors (Test Procedures and Performance Requirements)

Tractors (1970)

Tractors (1972)

Tractors (1972)

Tractors (1972)

Tractors (1972)

Tractors (1972) SAE J194

Std. for 3 Point Free L Std. for One Point Tubular Sleeve at

Industri TRA

Ft Truck TRA

SAE

ASAE

ASAE

ASAE

ASAE

ASAE

$S A E$

ASAE

ASAE

$\mathrm{SAE}$

ANSI

REC.

SAE

ASAE

T116

0

A MS7252A

AMS7253A

AMS $7250 \mathrm{C}$

AMS7251B

1972

1982

1992

D 1043

F383

*3

D2821

DI053

E399

A573

*1

UFC *2ART7

34

L.24. 2.2

L24.2.3

L 24.2 .4

L.24. 2.5

B30.4

490.02

D252

( $B C * 3-36$

RS222-B

103

114

115

112

A92.1

A5

V.5

D210]

B77.1

D2037

697

E-33

D3116

D2788

D3120

D2747

914

E498

D2600

E 418

J231

J395 A

J231

X3. 40

C 645

RS 400

D2]32

D2303

RPl6

C98.6

$\mathrm{J} 695 \mathrm{~A}$

$6 / 3$

F 376

R331.2

J717C

R333. 1

$\$ 210.2$

$\mathrm{J} 708 \mathrm{C}$

S209.4

4

J395 A

S201.4

S316T

S278.2

349 
Std. for Operator Protection for WheeI Type Agricultural Tractors (1972) SAE J333

k Attachment for Hitching Implements to Agricultural Whee

ions and Terminology for AgriculturaI and Light Industria

f Power Drive Lines for Agricultural (Farm) Implements and

Std. for $540 \mathrm{Rpm}$ Power Takeoff for Agricultural (Farm) 1000 Rpm Power Takeoff for Agricultural (Farm)

d Track Type Front End Loaders. Wheeled Dozers, Track Type cid Type Storage Batteries for Motor Vehicles, Motorboats, reign and Domestic Passenger Cars. Trucks, Buses, Coaches. a/ Std for Symbols for Operator Controls on Agricultural Rec. for Flash Point Index of Std. Definitions of

hs. for Interior Panel Coatings and Overlays on DFPA Grade est Meth. for Exterior Coatings and Overlays on DFPA Grade (Flaxseed) (1974)

(Flaxseed) (1974)

(1973)

(1973)

rade of Palmkernels; Babassu; Flaxseed;

Oilseed Products Std. for Adjustable Face Vehicula

Std Proposal for Solid State Load Switches for Safe Operating Condition of Truck,

Rec. Pract. Meth. for Determining GAWR and GVWR for Truck nters) and Relief Valves in Travel Trailers, Camp Cars and tid for Rec. Pract. for Rating of rec. Pract. for 7 Conductor Electrical Connector for Truck Rec. Pract. and Std. Covering Non Commercial Boat Std. for Nomenclature-Truck, Bus,

trailer, Mobile Home, Garden Tractor, Industrial Truck and Uniform Plumbing Code: Mobile Home std. for Mobile Home Parks Std. for Tires and Rims for Truck, Bus, nter Balanced Fork Lift Truck, Boat, Baggage, Special Type in the Selection of Tires (Car, Station Wagon, Truck, Bus,

Rec. Pract. for Nomenclature and Terminology of Tank Pract. for Accident Prevention Signs for Display on Tank aining of Automotive Nechanics for Buses. Heavy Trucks and Modular Housing. Prefab Houses, Motor Homes, and Camping emountable Rims Intended for Highway [Ise on Trucks, Buses.

(1973) Std. fot Inspection Procedures for Motor Vehicles. std. for (Backflow Preventers) and Relief Valves in Travel rforated Photographic Film in Rolls, Including Leaders and plumb/ Std. for Recreational Vehicles (Travel and Camping ulic Remote Control Cylinders to Agricultural Tractors and on of Remote Hydraulic Motors to Agricultural Tractors and and Trailers (Course of Instruction) (1972)

Light Trucks (Course of Instruction) (1972) nsumer Safety Spec. for Components, Assembly, and Use of A Std. for Safety Requirements for Aerial Passenger ated Data Processing Systems Designed to Streamline Supply Std. Specs, and Tests for Strain Gage Linear Acceleration Sid. for Guide for the Dynamic Calibration of Pressure Procedures for Calibration of Underwater Electro Acoustic Std for $A C-D C$ Rec. for Terminal Facilities for Pneumatic Std. Meth. for Measuring Heat meter (1972) Calorimeter for Measuring Heat

sign and Iise of a Thin Skin Calotimery Std for Automatic th. of Test for Comrusivity of Water in the Absence of Heat ) (1972) Rec. Evaluation Procedure of Chromatic Sampling and Measuring Procedures for Bulk Shipments and karels/ Std. Meth. for Investigating the Compatibility of Safety Std. for Std. Meth. of Sampling Gas from A ceiving Appliances and Other Electronic Equipment (Flyback Std. Requirements and Terminology for Specialty d. Spec. for Inninhibited Mineral Insulating Oil for I'se in for Oxidation Inhibited Mineral lnsulating Oil for lse in Std. for Dry Type and Less (1973) NEMA Ei2l Std. for Instrument 00/40,000/50,000/ Std. Requirements for Load Tap Changing Std. Test Code for Distribution, Power and Regulating Std for Low Power Wide Band Sid. for Computer Type (Square Loop) Pulse definitions for High Intensity Discharge Lamp Ballasts and Rec for

cedure for Thermal Evaluation of Oil Immersed Distribution Std. for Residential Controls-Class 2 Std. for Safety for Portable Toy

spec. for Chlorinated Aromatic Hydrocarbons (Askarels) for Safety Std. for Specialty neral Requirements for Distribution, Power, and Regulating std. Spec, for Merc ales and Service Industry (Rotating, Liquid Filled and Dry

Traĉtors (1972) SAE J955

(Trailer) Parks (1973)
Tractors (1972) SAE J715

Tractors (1972) SAE J722

Tractors (1973) SAE J718

Tractors (1973) SAE J719

Tractors, and Motor Graders (1971)

Tractors. Industrial Road and Misc Equipment) (1974)

Tractors, Machinery and Equipment (1971) ANSI Bl14.2, S

Trade Name Liquids (NFPA 325A) (1974)

Trade Terms Commonly Used in Packaging (1972)

Trademarked Plywood (1967)

Trademarked Plywood (1971)

Trading Rules for the Purchase and Sale of Linseed Meal

Trading Rules for the Purchase and Sale of Linseed OiI

Trading Rules for the Purchase and Sale of Soybean Meal

Trading Rules for the Purchase and Sale of Soybean Oil

Trading Rules (Performance, Quality, Description, and G

Traffic Control Signal Heads (1970)

Traffic Control Systems (1973)

Trailer and Bus Type Tires (1973)

Trailer Certification (1972)

Trailer Coaches (1970)

Trailer Couplings and Hitches: Automotive Type (1972)

Trailer Floors for Lift Truck Loading (1971)

Trailer Jumper Cable (1972)

Trailer (1972)

Trailer (1972)

Trailer (1974)

/Ft Truck, Boat, Baggage, Special Type

(Trailer) (1972) ANSI A177.1

Trailer, and Multipurpose Vehicle (1974

Trailer, Mobile Home, Garden Tractor, Industrial Truck

Trailer, Multipurpose Passenger and Recreational Vehicl

Trailers and Containers (1971)

Trailers and Containers (1972)

Trailers (Course of Instruction) (1972

Ds at Plants Assembling Mobile Home

Trailers, and Multipurpose Passenger Vehicles (1972)

Trailers, and Semitrailers Operated on Public Highways

Trailers, Camp Cars and Trailer Coaches (1970)

Trailers, for Aerial and Related Uses (1973)

Trailers, Truck Campers, Motor Homes): Installation of

Trailing Type Agricultural (Farm) Implements (1971) SAE

Trailing Type (Farm) Implements (1971)

Training of Automotive Mechanics for Buses, Heavy Truck

Training of Automotive Mechanics for Passenger Cars and

Trampoline (1974)

Tramways Including Ski and Surface Lifts and Tows (1973

Transactions Within the Air Transport Industry (1974)

Transducers (Instrumentation) (1971)

Transducers (1972)

Transducers (1972)

Transfer Instruments and Converters (1973)

Transfer of Dry Bulk Chemicals and Plastics (1972)

Transfer Rate Using

Transfer S witches $(1972)$

Transfer (Electrical Meth. (1972)

Transference Scale (Color Chart

Transformer Dividers (Voltage Type) (1972)

Transformer Insulation and Construction Materials in as

Transformer Type Arc Welding Machines (1972) ANSI C 33.2

Transformer (1974)

Transformer, Multiplier, Deflection Yoke, Picture Tube

Transformers

Transformers and in OiI Circuit Breakers (1973)

Transformers and Oil Circuit Breakers (1973)

Transformers for General Applications (1972) ANSI C89.

Transformers for (Electricity) Metering Purposes, $15 \mathrm{kV}$

Transformers 138,000 Volts and Below, 3750 Through 30,0

Transformers (Electronics) (1973) ANSI C 57.12.90

Transformers (Less Than 100 Watts Power) (1971)

Transformers (1970)

Transformers (1971)

Transformers (1972)

Transformers (1972)

Transformers (1973)

Transformers (1973)

Transformers (1973)

Transformers (1973) ANSI C33.4

Transformers (1973) ANSI C57.12.00

Transformers. Constant Current (Series) Supply Type (19

Transformers, Hand Power Tools, Hermatic Stators, Test
ASAE

ASAE

ASAE

ASAE

ASAE

ASAE

SAE

SAE

ASAE

FMS

ANSl

Test Met APA

APA

NFP

NSPA

NSPA

NIOP

ITE

NEMA

VESC

TTMA

IAPM

SAE

TTMA

TTMA

SAE

TRA

ICBO

NFPA

TRA

TRA

TRA

TTMA

Rec TTMA

d. for TR ANSI

FMS

SAE

ANSI

IAPNO

$\mathrm{D}$ and $\mathrm{PE} \mathrm{ANSl}$

NFPA

ASAE

Applicati ASAE

ANSI

ANSI

Std. Co ASTM

ANSI

ATAA

ISA

ANS1

Std. for ANS

ANSI

$\mathrm{MCA}$

ASTM

ASTM

UL

ASTM

AATCC

NSPA

ANSI

ASTM

UL

ASTM

UL

NEMA

St ASTM

ASTM

NEMA

EEI

ANSI

IEEE

IEEE

IEEE

Std. ANSI

FMS

Std. Test Pro IEEE

NEM

UL

Std. ASTM

Std. Ge IEEE

ANSI

EASA

J231

$\mathrm{J} 537 \mathrm{H}$

*1.56

5304.4

$7.42 \mathrm{~N}$

$* 5$

$* 4$

$* 1$

*2

$* 2$

*

TRI

TS2

V. 9

RP39

TSC8

J684D

RP 37
RP 40

A 15

J687C

5

UPC* $*$.E

$501 \mathrm{~A}$

2

X.XI

RP36 
Std. for Electrical Std. for Guide for Preparation of Spec. for Large Power er (1971) Std. Requirements for Overhead Distribution. Std. for Voltage Values for Preferred breakers Rated on a Symmetrical Cu/ breakers Rated on a Symmetric/

Capabilities for AC High Voltage/ Std. Requirements for Std. Application Guide for Schedules of Preferred Std for Measurement of Smell Values of g35.7 Std. Spec. for Carbon Steel Plates with Improved d. for System for Romanization of Arabic Written Language for System for Romanization of Japanese Written Language ing Terms for Reporting and Analyzing Outage of Electrical ing Safety Req/ Std. for Series Capacitors for Electrical Std. for Thermoplastic Insulated Wire and Cable for the hermosetting Polyethylene Insulated Wire and Cable for the inum Alloy Seamless Extruded Tube and Pipe for Gas and Oil

c. Pract. Spec, for Cast lron Sealing Rings for Automotive Std. for Safety for Mechanical Power

ruction) / Std. Classification for Determination of Sound laboratory Determination and Measurement of Airborne Sound Notes) (1961)

Heat

Uniform Building Code: Sound Std. Test Meth. for Diffuse Light Rec. Pract. for Automatic Rec. Pract. for Automotive

Std. for Solid and Semi Solid Dielectric Rec. Pract. for Laboratory Measurement of Airborne Sound Differential and Manual Test Meth. for the Selective

printed Wiring Boards) roducts $(1972)$ 5

kaging) (1968) ANSI M ansi MH12.10

Std. Meth. for Gravimetric Determination of Water Vapor Std. for Heavy Duty Offset Sidebar Power d. Spec. for Metal Arc Welded Steel Pipe for High Pressure Std. for Air Pressures for Pneumatic Controllers and Std, for Air Pressures for Pneumatic Controllers and Std. Microwave Rec. Pract. for Automotive Pressure Sensitive Tape Water Vapor Std. Meth. of Measuring Water Vapor design, Construction, Materials, and Installation of Power Specifying Duct, Sheet Metal, Low Pressure, Air

Pract. for the Safe Action and Title Areas for Television Std. Synchronous Signaling Rates for Dat re (1972) gine Mountings (Installation and Replacement\} Direct Driv lass Pintle Chains, Attachments, and Sprocket Teeth (Power textile) (1973)

Meth. of Test for Ligh

of Geometric Conditions for Measurement of Reflectance and rophotometer with Reserv/Meth. for Spectral Reflectance, irements for Electrolytic Capacitors (For Use Primarily in Minimum Std. for Land Mobile Communications FM or Pm ming and Alerting Equipment for Use with Emergency Locator Std. for Safety for Electrically Actuated Rec. Pract, for Goniophotometry of Uniform Building Code: Plastics (General, Exterior Ligh Rec. Pract. for Outboard Motor Boa ynamic Vis/ by Double $\mathrm{Be}$ 5.72 Meth of Test for Kinematic Viscosity of Std. Meth for Measurement of Thickness of Std. Meth. of Test for Resistance of

1971)

Std. Performance Spec, and Meth. of Test for

Minimum Performance Stds. Airborne At Spec. for Cargo Unit Load Devices (Materials Designed to Streamline Supply Transactions Within the Ai it Load Sizes (1973)

Std. for Lnit Load Sizes for Dimen Std. for Packaging of Uranium Hexafluoride for Rec. Pract. for Railroad Std. for Packaging and

Materials (Safety) (1973)

Std. Code for Pressure Piping: Liquid Petroleum Std. for Administrative Guide for Obtaining Department of (Fire Protection) (1973)

ting Agents (1973) ANSI Z Std. Code for the Manufacture,

r/ Rec. for Improving Safety on Enclosed Mobile Tanks for Std. for Administrative Guide for Packaging and Rec. Pract. for Measuring, Mixing,

ANSI H9.23 td. Meth. of Test for compound Microscope, Petri Dish, Sieve, Separatory Funnel, 2) tgun Shooting Facility Plans: International and Domestic
Transformers, Regulators and Reactors (1974)

Transformers, with or Without Load Tap Changing (1971)

Transformers, 67,000 Volts and Below; $500 \mathrm{kVA}$ and Small Transient Insulation Levels (TIL) (1971)

Transient Recovery Voltage for AC High Voltage Circuit Transient Recovery Voltage for AC High Voltage Circuit

Transient Recovery Voltage Ratings and Related Required

Transistor Capacitance (1972)

Transition Properties for Pressure Vessels (1972) ANSI Transliteration) (1972)

Transmission and Distribution Facilities and Interrupti

Transmission and Distribution Line Compensation (Includ

Transmission and Distribution of Electrical Energy (I97

Transmission and Distribution of Electrical Energy (I97

Transmission and Distribution Piping Systems (1973) Ans

Transmission and Hydraulic Systems (1972)

Transmission Apparatus (1972)

Transmission Class (Building Acoustics Design and Const

Transmission Class (Stc) of Building Partitions Such as

Transmission Coefficients of Brick and Tile Walls (Tech

Transmission Control (Acoustics) (1973)

Transmission Factor of Reinforced Plastic Panels (1969)

Transmission Functions Terminology (1969)

Transmission Hydraulic Control System Terminology (1970

Transmission Lines (1972)

Transmission Loss of Building Partitions (1970)

Transmission Lubricants (Information Report) (1969)

Transmission of a Photographic Lens (1969)

Transmission of End Product Description Data (Tooling,

Transmission of Flexible Heat Sealed Packages for Dry P

Transmission of Microfilmed Documents (1972) ANSI C 16.4

Transmission of Refrigrated Vehicles (1972)

Transmission of Shipping Containers by Cycle Meth. (Pac

Transmission of Shipping Containers (Packaging) (1964)

Transmission Performance of Telephone Sets (1971)

Transmission Pipe (1973)

Transmission Rate of Sheet Materials (Paper) at High Te

Transmission Roller Chains and Sprocket Teeth (1972)

Transmission Service (1971) ANSI G36.49

Transmission Systems (lnstrumentation) (1970)

Transmission Systems (1967)

Transmission Systems (1972)

Transmission Terminology (1969)

Transmission Test Meth. (1970)

Transmission Through Screw Cap Closure Liners (1973)

Transmission Units Penetrating the Hull for Boats Equip

Transmission (1967)

Transmission (1968)

Transmission (1969)

Transmission (1971)

Transmission) Clutc

Transmittance of Woven Glass Fiber Decorative Fabrics

Transmittance (1973) ANSI Z138.3

Ract. for

ransmittance, and Color of Paper and Pulp (L sing Spect

ransmitters and Electronic Instruments) (1958) ANSI C8

Transmitters $25.470 \mathrm{M} \mathrm{Hz}(1970)$

Transmitters (ELT) (1973)

Transmitters (1974)

Transmitting Objects and Materials (1970)

Transmitting Panels, Skylights, Monitors and Sawtooth R

Transom and Motor Well Dimensions on Boats (1974)

Transparent and Opaque Liquids and the Calculation of D

Transparent or Opaque Metal Oxide and Metallic Coatings

Transparent Plastics to Surface Abrasion (1973) ANSI K6

Transparent Safety Glazing Material Used in Buildings (

Transponder Equipment (1972)

Transport and Handling) (1973)

Transport Industry (1974)

Transport Package Sizes (P

Transport Packages (1972)

Transport (1971)

Transportation of Line Pipe (1972)

Transportation of Radioactively Contaminated Biological Transportation Systems (1974)

Transportation (DOT) Special Permits for Radioactive Ma Transportation, Storage and Use of Explosive Materials

Transportation, Storage, and Use of Explosives and Blas

Transporting and Spreading Agricultural Liquids and Slu

Transporting Radioactive Materials (Safety) (1973)

Transporting, and Placing Concrete (1973)

Transverse Rupture Strength of Cemented Carbides (1973)

Trap Flask, Step Wedge for X-Ray Standardization Probe,

Trap, Clay Pigeon, and Skeet: Ammunition and Target Spe

Trapezoidal Flumes for Irrigation Flow Measurement (197
NEMA

ANSI

ANSI

ANSI

IEEE

IEEE

ANSI

EIA

ASTM

St ANS

Std. ANSI

IEEE

ANSI

NEMA

NEMA

ASTM

Re SAE

ANSI

ASTM

ICBO

BIA

ICBO

ASTM

SAE

SAE

EIA

Std ASTM

$S A E$

ANSI

IPC

ASTM

NMA

TTMA

ASTM

ASTM

IEEE

ASTM

TAPPl

ANSI

t ASTM

ISA

SAMA

ElA

SAE

ASTM

$\mathrm{ABYC}$

CSI

Rec. SMPTE

ANSI

SAE

ANSI

ASTM

TAPPI

EIA

EIA

RTCA

ASTM

ICBO

ABYC

ASTM

ASTM

ASTM

ANSI

RTCA

NSA

ATAA

ANSI

ANSI

A PI

ANSI

FMS

NFPA

ASAE

ANSI

ACI

ASTM

$\mathrm{AACCH}$

NRA

ASAE

TR 1

C57.97

(2. 1220

C92.1

327

C. 39.0722

RS398

A 442

Z39.12

339.11

346

C55. 2

WC5

C.7

B345

J28 1

15.1

E413

UBC \$35. 1

4 
pt Chocolate Products and Piecrust (Cereal Chemis/ Uniform Pl

Direct ANSI Z221.1 Std. for Safety for Metal Waste Std for Recreational Vehicles

): Installation of Plumb Sid. for (Backflow Preventers) and Relief Valves in c. for Electric Overhead Bridge and Gantry Multiple Gerder fabrics by the Tongue (Single Rip) Meth. (Constant Rate of Std. Ampacities Cables in Open Top Cable

971) Safe Operating Condition of Tires Including Tire d Wheels (1970) ANSI G55.15 Std. Spec. for Vacuum 57.11 Std. Spec, for Carbon Steel Axles, Non Heat ssels (1974)/ Std Spec. for Ouenched and Tempered Vacuum ors and Shafts (1974) ANSI G55.14 Std. Spec. for Vacuum 971) ANSI G55.13 Std. Spec. for Vacuum test Meth. for Determination of Formaldehyde Odor in Resin test Meth. for Determination of Formaldehyde Odor in Resin Temp/ $r$ Alloy Steel Turbine Type Bolting Material Specially Heat i A 109.26 Std. Spec. for Alloy Steel Castings Specially Heat Std. Spec. for Woven Glass Fabrics Tent. Spec. for Ultra High Test Heat sign and Construction Met/ the All Weather Wood (Pressure Meth. for Flame Resistance

th. for Resin and Volatile Content, and Flow Properties of Std. for Pressure Std. Spec. for Heat Improved Std. Designs for Pressure Std for Inspection of Preservative Std. Meth. of Analysis of CCA Treating Solutions and Cca $6 \mathrm{Cu}-0.26 \mathrm{Cr}$ (7075-T736) Solution and Precipitation Heat sure Vessels Plates, 5\% Nickel Alloy Steel, Specially Heat tion Plus Vacuum Consumable Electrode Melted Solution Heat t Resistant, 15.5 $\mathrm{Cr}-4.5 \mathrm{Ni}-2.9 \mathrm{Mo}-0.10 \mathrm{~N}$. Solution Heat $75 \mathrm{Zn}-2.5 \mathrm{Re}-0.70 \mathrm{Zr}$ (Ze63-T6) Solution and Precipitation nduction Melted, 1975 Deg. F (1079.4 Deg. C) Solution Heat

l Base, $20 \mathrm{Cr}-20 \mathrm{Co}-5.9 \mathrm{Mo}-2.2 \mathrm{Ti}-0.45 \mathrm{Al}$ solution Heat ni-5.5Mo-0.85Cb-2.5Ti-1.6Al-0.09B, Solution Heat stant, Nickel Base-5.0Cr-24.5Mo-5.5Fe, Solution Heat

8 Deg. C) Solution, Stabilization, and Precipitation Heat Resistant $15.5 \mathrm{Cr}-4.5 \mathrm{Ni}-2.9 \mathrm{Mo}-0.10 \mathrm{~N}$, Solution Heat

4 Deg. C) Solution, Stabilization, and Precipitation Heat 1800 Deg. F (982.2 Deg. C) Solution and Precipitation Heat Std Spec, for Pressure Vessel Plates, Heat

d. Spec. for Carbon Steel Axles, Non Heat Treated and Heat Spec. for Low Alloy Steel Bolts and Screws, Heat Spec. for Low Alloy SteeI Studs, Heat Spec for Titanium Alloy Bolts and Screws, 6Al-4V, Heat itanium Alloy Bolts and Screws, 6A1.4V, Upset Headed, Heat t Resistant, $15.5 \mathrm{Cr}_{\mathrm{r}}-4.5 \mathrm{Ni}-2.9 \mathrm{Mo}-0.10 \mathrm{~N}$, Solution Heat

lloy Bars, Forgings, and Rings (Nickel Base, Solution Heat erate Heat Resistant, $15.5 \mathrm{Cr}-4.5 \mathrm{Ni}-2.9 \mathrm{M} 0-0.10 \mathrm{~N}$, Heat Spec. for Vertical and Horizontal Emulsion Sid. Meth. of Analysis of Cca

raph Method for Test. the Water Repellent Effectiveness Di ial for Evaluation of Special Processes or Devices Used in (1973)

for Lumber, Timber, Plywood and Utility Pole Preservative Std. Preservative Std for Preservative Std. for Preservative struction (1973) std. for Lumber, Timbers, Bridge and Mine Tie Preservative
Std. for Wood Used on Farms (Preservative Ut. for Wuilding Construction (Preservative Std. for Lodgepole Pine Wood Poles Preservative Rec. Pract. and Std. Covering Sew age Holding and/or Brick and Tile in Sanitation Structures, Water Brick and Tile in Sanitation Structures, Sewage Brick and Tile in Sanitation Stiuctures, Industrial Waste Std. Rec. Pract. for Heat

pec. for Hip Nail--Jewell Type for Ulse by Surgeons in the (Western Red Cedar, Northern White/ Std. for Preservative (1971) Std. Spec. for Pressure Preservative 1 Std. Spec. for Coal Tar Creosote for the Preservative inations Before Gluing of Southern/ td. Spec. for Pressure

timber/ Std. for Full Length Thermal Process Preservative Std. Meth. of Test for Durability of Fire Retardant Std. for in Dividual Aerobic Wastewater Std. for Preservative

Black Oxide Std. for Thermoset Plastic Tanks for Water and Wastewative $r$ Heat Treatment, $1800 \mathrm{Deg}$. F (982.2 Deg. C) Solution Heat ng Structures (Tanks, Reservoirs, etc. for Water and Waste ings (Lumber, Plywood and Componentsu Timber, Preservative Wells and Water Systems

el, Corrosion and Heat Resistant, Roll Threaded After Heat
Trapping Meth. of Analysis for All Baked Materials Exce Traps and Interceptors (1973)

Trash. Garbage) Cans (For Combustible Materials) (1974) Travel and Camping Trailers. Truck Campers, Motor Homes

Travel Trailers, Camp Cars and Trailer Coaches (1970)

Traveling Cranes (1971)

Traverse Tensile Test. Machine) (1971)

Trays (1972)

Treads Depth Requirements (Passenger Car Type Tires) (1

Treated Alloy Steel Forgings for Turbine Rotor Disks an

Treated and Heat Treated, for Railway Use (1973) ANSI G

Treated Carbon and Alloy Steel Forgings for Pressure Ve

Treated Carbon and Alloy Steel Forgings for Turbine Rot

Treated Electric Steel Forgings for Generator Rotors (1

Treated Fabric by the Sealed Jar Meth. (1972) ANSI L14.

Treated Fabric Steam Meth. (1972) ANSI L14.179

Treated for High Temperature Service (1971) ANSI G55.12

Treated for Pressure Containing Parts Suitable for High

Treated for Use in Waterproofing and Roofing (1973) Ans

Treated Line Pipe (1972)

Treated Lumber and Plywood) Foundation System: House De

Treated Paper and Paperboard (1972)

Treated Paper (1973)

Treated Piles and Timbers in Marine Construction (1973)

Treated Steel Helical Springs (1973) ANSI G54.20

Treated Timber Crib Walls (1969)

Treated Timber Products (1973)

Treated Wood by Colorimetry (1972)

Treated (1972)

Treated (1972A)

Treated (1973)

Treated (1973)

Treated (1973)

Treated (1973)

Treated (1973)

Treated (1973)

Treated (1973)

Treated (1973)

Treated (1973)

Treated (1973)

Treated (1973)

Treated, Carbon Manganese Silicon (1972A)
Treated, for Railway Use (1973) ANSI G57.11

Num Alloy Forgings, $5.6 \mathrm{Zn}-2.5 \mathrm{Mg}-1$. Std. Spec. for Pres

$1-8.5 \mathrm{Ni}-2.0 \mathrm{Cu}-1.1 \mathrm{Ti}$ Vacuum Indue $/$ and Strip, Corrosion and Moderate Hea

/ for Magnesium Alloy Sand Castings (5. 1.4A1, Consumable Electrode or Vacuum I

E, Corrosion and Heat Resistant, Nicke

/Eat Resistant, Iron Base-13.3Cr-38 $/ G s$, and Rings, Corrosion and Heat Resi SAE Ion Melted 1825.1900 Deg. F (996.1.1037 SAE Teel Plate, Corrosion and Moderate Hea SAE /Um Induction Melted, 1975 Deg. F (1079 SAE $(15 \mathrm{Cr}-26 \mathrm{Ni}-1.3 \mathrm{Mo}-2.1 \mathrm{Ti}-0.30 \mathrm{~V})$ SAE

Treated, Roll Threaded (1973)

Treated, Roll Threaded (1973)

Treated, Roll Threaded (1973)

Treated, Roll Threaded (1973)

Treated, Sub Zero Cooled, Equilized, and Over Tempered. for T

Treated, $15.5 \mathrm{Cr}-16 \mathrm{Mo}-3.8 \mathrm{~W}-5.5 \mathrm{Fe}$ ) (1973)

Treated, 170,000 psi (1172 MPa) Tensile Strength (1973)

Treaters (Petroleum Production) (1973)

Treating Solutions and CCA Treated Wood by Colorimetry Treating Solutions (1970)

Treating Wastewater (1970)

Treatment by Pressure Processes and Quality Control Std

Treatment by Pressure Processes for All Timber Products

Treatment by Pressure Processes of Timber Piles (1973)

Treatment by Pressure Processes of Timber Poles (1973)

Treatment by Pressure Processes of Wood for Highway Con

Treatment by Pressure Processes (1973)

Treatment by Pressure Processes) (1972)

Treatment by Pressure Processes) (1972)

Treatment by the Full Length Thermal Process (1973)

Treatment Devices for Marine Toilet Waste (Boats) (1972

Treatment Facilities (Tech. Notes) (1969)

Treatment Facilities (Tech. Notes) (1969)

Treatment Facilities (Tech. Notes) (1969)

Treatment of Aluminum Alloys (1974)

Treatment of Fractures of the Skeletal (Bone) System (1

Treatment of Incised Pole Butts by the Thermal Process

Treatment of Lumber for Industrial Water Cooling Towers

Treatment of Piles, Poles, and Timbers for Marine, Land

Treatment of Structural Glued Laminated Members and Lam

Treatment of Timber Products (1969)

Treatment of Western Red Cedar and Alaska Yellow Cedar

Treatment of Wood (1972)

Treatment Plants (1970)

Treatment (For Fence Posts) by Pressure Processes (1972

Treatment (Part Coating) (1973)

Treatment (Sept

Treatment (1973)

Treatment) (1968)

Treatment, Quality and Management) (1972)

Treatment, 1800 Deg. F (982.2 Deg. C) Solution Heat Tre

UL

API

SAE

SAE

SAE

\section{AE}

$S A E$

$S A E$

SAE

$S A E$

SAE

SAE

API

$\mathrm{NSF}$

ICBO

BIA

BIA

BIA

CTI

NSF

SAE

NSF

AACCH 28.33

ICBO UPC $* 1$.

32

NFPA $501 \mathrm{C}$

APMO TSC8

$\begin{array}{ll}\text { ASTM } & \text { D2262 }\end{array}$

IPCEA P. $5+440$

VESC V.7

ASTM A471

ASTM A21

ASTM A508

ASTM A470

ASTM $\quad$ A469

AATCC 112

AATCC 113

ASTM A437

ASTM A389

ASTM D 1668

NFORP RP

TAPPI T461

TAPPI

AWPA

ASTM

AWPI

$A W P A$

SAE E $A E$

ASTM

AWPA

AWPA

AWPA

$A W P A$

AWPA

AWPA

AWPA

AWPA

AWPA

$A B Y C$

ASTM

ASTM

$A W P A$

ASTM

AWPA

ASTM

AWPA

ASTM

AWPA

M.539

C18

A125

M2

A 10-72

AMS4131

A 645

AMS5617A

AMS5547C

AMS +125

AMS5738C

AMS5872

AMS5633A

AMS5755B

AMS5707D

AMS5549C

AMS5709B

AMS5735H

A537

A2]

AMS7452K

AMS7456G 
(1974)

test Meth. for Detection of Hexavalent Chromium Containing bing Equipment (Body Belt . for Detection of Mill Chemical

$\mathrm{d}$ and Flanged Wellhead, Underwater Wellhead, and Christmas af Evergreens; Rose Grades; Vines and Ground Covers; Fruit Std. for Safety Requirements for

ling Trees and Shrubs; Bulbs, Corms, and Tubers; Christmas ry, Currant, Grape, Asparagus); Lining Out Stock; Seedling

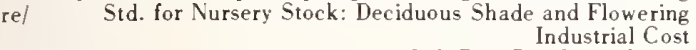

1973)

and Nonlocking (1973) Std. Part Bit, Screwdriver, Std. for Screw, Hex Head, d) Nonlocking (1972) nd Nonlocking (1972) nd Nonlocking (1972)

Std. for Machine Screw, Flat Fillister Head, Full Thread,
Std. for Bolt, 100 Deg. Head, Std. for Serew Hex Head, Std. for Screw, Hex Head,
Std. for Screw, 100 Deg. Oval Head, Std. for Screw, 100 Deg. Head, Std. for Screw, Pan Head, Std. for Bolt, 100 Deg. Head,
Std. for Bolt, 100 Deg. Head, Std. for Bolt, 100 Deg. Head, ead, Self Locking and Nonl/ read, Self-Locking and Non/ read, Nonlocking (/ Std. for Bolt, 100 Deg. Reduced Head,
ad, Self Locking and Nonlo/ Std. for Bolt, 100 Deg. Head, Std for Bolt, 100 Deg. Head, ad, Nonlocking (19) Std, for Bolt, 100 Deg. Reduced Head, Short Thread, Self Locking and Nonlocki/Bolt, $100 \mathrm{Deg}$. Std. for Bolt, Pan Head, Close Tolerance, Short Thread, nlocking (1972) Std. for Machine Screw, Flat Fillister Head, Full Thread, If Locking and Nonlocking / Std for Screw, Hex Head, Std. for Screw, 100 Deg. Head power Generating Station Protection Systems (19/ Bit, Screwdriver, al Fasteners (1974)

uclear Power Generating Stations (1973) ANSI N41.17 IEEE re Criterion to Nuclear Power Generating Station Protect/ Operators for Nuclear Power Generating Stations (1972)/ 1 Motors Installed Inside the Containment of Nuclear Po/ lysis of Nuclear Power Generating Station Protection Sys/ pment and Circuits (1974) ANSI N41.14 easurements Part Il-Characterization of Large Signal D/ lied as Standby Power Supplies for Nuclear Power Generat/ real Chemistry) (1968) ctric Equipment for Nuclear Power Generating Stations (/ ical 2-(Ethylamino)4-(Isopropylamino)-6-(Methylthio)-S. t Control Chemical 2,4-Bis (Isopropylamino)-6-Methoxy-Strol Chemical 2,4-Bis (Isopropylamino)-6-(Methylthio)-S est Control Chemical 2-Chloro-4,6bis (Isopropylamino) -S Chemical 2-(Tertbutylamino)-4-Chloro-6-(Ethylamino)-Scal 2-(Tertbutylamino)-4-(Ethyla mino)-6-(Methylt hio)-SStd. Meth. for Chemical Analysis of

Properties, Safe Handling and Use of Phosphorus . name for the Pest Control Chemical 0,0 Dimethyl $0-(3,5,6$. Std. Acceptable Concentrations of est Control Chemical 4-Chloro5-(Methylamino) -2-(a, a,aname for the Pest Control Chemical N-(2Chloroethyl)-A, A, A. for the Pest Control Chemical N(Cyclopropylmethyl)-A, A, A. Wheat Flours (Cereal Chemistry) (1962)

Std. Meth of Test. Resistance to Scuffing of

Fabrics, Vinyls, Coated Fiberboards, and Other Automotive ng Resistance and Associated Characteristics of Automotive eth. for Determining Window Fogging Resistance of Interior Rec. Pract. for Flammability of Automotive Interior Quality Stds. for Standing and Running Std. for Locks and Lock Std. for Architectural Door

de Specs. for Architectural Woodwork: Standing and Running td. for Methoprene (Isopropyl (E,E)-1 l-Methoxy-3,7,11

e Pest Control Chemical S-Ethyl (E,E)-11-Methoxy-3,7,11. mmon Name for the Pest Control Chemical Ethyl (E, E) 3, 7,11

me for the Pest Control Chemical 2-Propynyl (E,E)-3, 7,11

g Appliances (Lawn Mower, Cultivator, Edger-Trimmer, Hedge

for Terms and Definitions for Wirewound and Nonwirewound pection and Test Procedures for Wirewound and Nonwirewound Std. for Safety Requirements for Tree Pruning. cable (1966) IPCEA S-64-474 e)-11-Methoxy-3,7,11-Trimethyl-2,4-Dodecadienethioate n. $\mathrm{NaOH}(1973)$ Spec. for Photographic Grade Std Meth of Test for Radioactive Std. Spec. for Electric Wire Rope Hoists (Lug, Hook, Std, Spec. for Bronze

uirements for Tailored Woven Uniform Fabrics, Lightweight tation Practices: Chassis Frames-Passenger Car and Light cial Type Trailer, Mobile Home, Garden Tractor, Industrial
d. for Recreational Vehicles (Travel and Camping Trailers,
Treatments on Galvanized Steel Prior to Conversion Coat Treatments on Galvanized Steel Surfaces (Coil Coating)

Tree Climber) (1973)

Tree Equipment; Flow Control Chokes; and Wor Lineman'

Tree Equment; How Control Chokes; and Water Sampling

Tree Pruning, Trimming, Repairing, or Removal (1972)

Tree Std. (1973)

Asparagus): Lining Out Stock. Seed to

Trees and Shrubs; Bulbs, Corms, and Tubers; Christmas 'T

Trees; Deciduous Shrubs; Coniferous and Broadleaf Everg

Trends for Buildings, Machinery and Equipment (1974)

Tri Wing Recess (1972)

Tri Wing Recess, Alloy Steel, Full Thread, Nonlocking (

Tri Wing Recess, Alloy Steel, Full Thread, Self-Locking

Tri Wing Recess, Alloy Steel, Self-Locking and Nonlocki

Tri Wing Recess, A286 Cres, Full Thread Nonlocking (197

Tri Wing Recess, A286 Cres, Full Thread Self Locking an

Tri Wing Recess, A286 Cres, Full Thread, Self Locking a

Tri Wing Recess, A286 Cres, Full Thread, Self Locking a

Tri Wing Recess, A286 Cres, Self-Locking and Nonlocking

Tri Wing Recess, Close Tolerance (1972)

Tri Wing Recess, Close Tolerance (1972)

Tri Wing Recess, Close Tolerance, Alloy Steel, Long Thr

Tri Wing Recess, Close Tolerance, Alloy Steel, Short Th

Tri Wing Recess, Close Tolerance, Alloy Steel, Short Th

Tri Wing Recess, Close Tolerance, A286 Cres, Short Thre

Tri Wing Recess, Close Tolerance, A286 Cres, Short Thre

Tri Wing Recess, Close Tolerance, 6Al-4V Titanium Alloy

Tri Wing Recess, Titanium Alloy $6 \mathrm{Al}-4 \mathrm{~V}$, Self Locking an

Tri Wing Recess, Titanium Alloy, 6Al-4V, Self-Locking a

Tri Wing Recess, 6Al-4V Titanium Alloy, Full Thread, No

Tri Wing Recess, 6Al-4V Titanium Alloy, Full Thread, Se

Tri Wing, Spec, for (1972)

Trial-Use Criteria for the Periodic Test. of Nuclear

Trial Std. for Screw Threads for Metric Series Mechanic

Trial Use Guide for Class 1E Control Switchboards for N

Trial Use Guide for the Application of the Single Failu

Trial Use Guide for Type Test of Class I Electric Valve

Trial Use Guide for Type Tests of Continuous Duty Class

Trial Use Guide: General Principles for Reliability Ana

Trial Use Std. Criteria for Separation of Class le Equi

Trial Use Std. Guide on Solid State Devices: Varactor M

Trial Use Std.: Criteria for Diesel Generator Units App

Trial Use Std.: General Guide for Qualifying Class I El

Triangle Test Taste Panel for Food Flavor Detection (Ce

E for the Pest Control Chem

Triazine-Ametryn (1973)

Triazine-Prometon (1973)

Triazine-Prometryn (1973)

Triazine-Propazine (1973)

Triazine-Terbuthylazine (1973)

Triazine-Terbutryn (1973)

Tribasic Lead Phosphosilicate (1973)

D. Common Name for the Pes

Mon Name for the Pest Con

ITd. Common Name for the P for the Pest Control

Trichloride (1972)

Trichloro-1,2,2trifluoroethane (Fluorocarbon 113) (1973

Trichloro-2-Pyridyl) Phos phorothioate Chlorpyrifos Met

Trichlorofluoromethane (Fluorocarbon 11) (1973)

Trifluoro-m-tolyl) -3 (2H)P yridazinone Norflurazon (197

Trifluoro-2,6-Dinitro-N-Propyl-Ptoluidine-Fluchlora

Trifluoro-2,6-Dinitro-N-Propyl-Ptoluidine-Proflural

Trifructosan Meth. of Analysis for Admixture of Rye and

Trim Materials (1972)

Trim Materials (1972)

Trim Materials (1972)

Matched to Master Specimen for

Trim Materials (1973) ANSI Z265. I

Trim Materials: Horizontal Test Meth. (1972)

Trim (Architectural Woodwork) (1973)

Trim (1972)

Trim (1972)

Trim, Casework, Panelwork, Closet and Storage Shelving,

Trimethyl -2,4-Dodecadienoate) (Common Name for the PE

Trimethyl-2,4-Dodecadie nethioate-Triprene (1973)

Trimethyl-2,4-Dodecadie noate Hydroprene (1973)

Trimethyl-2,4-Dodecadienoate Kinoprene (1973)

Trimmer, etc.) (1974)

Trimming Potentiometers (1970)

Trimming Potentiometers (1970)

Trimming, Repairing, or Removal (1972)

Triplex, Neutral Supported, Service Drop and Secondary

Triprene (1973)

He Pest Control Chemical S

Tritium in Water (1970) ANSI N164

Trolley Suspended, and Base Mounted Types (1971)

Trolley Wire (1974) ANSI C7.5

Tropical) Suiting (Institutional Textile) (1973)

Truck-Ground Vehicle Practices (1974)

$/ F t$ Truck, Boat, Baggage, Spe

Truck and Trailer (1974)

Truck Campers, Motor Homes): Installation of Plumbing,

NCCA

NCCA

EEI

APl

ANS

ANSl

ANSI

ANS

ANSI

FMS

NSA

NSA

NSA

NSA

NSA

NSA

NSA

NSA

NSA

NSA

NSA

NSA

NSA

NSA

NSA

NSA

NSA

NSA

NSA

SSA

NSA

NSA

IEEE

IFI

IEEE

IEEE

IEEE

IEEE

IEEE

IEEE

IEEE

IEEE

IEEE

ANS

ANSI

ANSI

ANSl

ANSI

ASTM

NS

ANSI

ANSI

ANS

ANS

ANS!

$A A C$

SAE

$\mathrm{SAE}$

$S A E$

SAE

$S A E$

AWI

ANSI

AWI

ANSI

$/ \mathrm{H}$ ANSl or Co ANSI

Mon Na ANSl

nin UL

Std VRCl

ANSI

EEI

ANSI

ANSl

ASTM

HMI

ASTM

Req ANS

ANSI

TRA

TB-11.7 TB-11-11

AP. 2

6 A

Z60.1

Z133.1

(60.

Z60.1

Z60.1

4005

5600-06

5300-06

6000-3

6500-6

$5700-6$

6900-06

5400-06 
Rec. Pract. Industrical

Rec. Pract. for Nomenclature for Passenger Car and Light Rec. Pract. for Rating of Trailer Floors for Lift

t) (1973)

Rec. Pract. for Passenger Car and Light Rec. Pract. for High Lift Industrial

Rec. Pract. for Passenger Car and Light Shipper-Motor Carrier Dock Planning Manual Rec. Pract. Meth. for Determining GAWR and GVWR for Rec. Pract. for 7 Conductor Electrical Connector for exhaust Gas Emissions Measurement (Passenger Car and Light and Recreational Type Vehicles, Counter Balanced Fork Lift s, Passenger Cars, Off Road, Agricultural and Log Skidder, Std. for Nomenclature Std for Tires and Rims for

Terms Used in the Selection of Tires (Car, Station Wagon, Safe Operating Condition of Rec. Pract. for Exterior Sound Level for Heavy itd for Training of Automotive Mechanics for Buses. Heavy e Emissions from Gasoline Powered Passenger Cars and Light ining of Automotive Mechanics for Passenger Cars and Light e Emissions from Gasoline Powered Passenger Cars and Light

Std. for Safety for Electric Battery Powered Industrial Std. for Sound Level for Passenger Cars and Light Std. for Load Handling Symbols for Powered Industrial

r Forks and Fork Carriers for Powered Industrial Fork Lift

t Emission Controls (Air Pollution) for Powered Industrial $\mathrm{ng}$ and Reporting of the Noise Levels of Powered Industrial as of Ise. Maintenance and Operation of Powered Industrial ies (Passenger Cars, Multipurpose Vehicles, and Light Duty ng Storage Batteries in Motive Power Service (For Electric of Passenger Cars, Multipurpose Passenger Vehicles. Buses,

for Service Brake Structural Integrity Test Procedure for ts and Terminals (For Foreign and Domestic Passenger Cars, Wheels, and Demountable Rims Intended for Highway Use on Std. for Demountable Tired Wheels for Industrial

er, Loader, Dozer, Mining Car, Front End Loader, Fork Lift Std. for and Wood and Lag Screws; Light Metal Plate Connected Wood id Meth of Test for Tensile Strength Properties of Steel

ingles, Exposed Roof Decking, Drop Siding, Structural Roof Std. Spec. for Ceramic Tile Panels for Bath

uorides in the Atmosphere (Sodium Bicarbonate Coated Glass tion Pip/ Std. Spec. for Aluminum Alloy Seamless Extruded eaded Rod Ends (1973)

nds (1973)

readed Rod Ends (1973)

and Threaded Rod Ends (1973)

Std. for Std. for Sid. for Std. for

(1973) Std. for Packing. Preformed, Straight Thread (1973)

Std. for Packing, Preformed, Straight Thread rigeration Service, and Marine [se (1972 Std. for Spherical Sleeve (Compression) Std. for Automotive Std. for Hydraulic ce (1974) ANSI H23.5 St. Spec. for Seamlecs and Welded Titanium Std. Spec. for Hard Drawn Copper Capillary Safety Std. for Automotive Glass

Flyback Transformer, Std. Spec. for Copper Alloy Condenser Rolled Std. Meth. for Thin Walled Std. for Swaging Process

Std for Elbow, Reducer, 45 Deg. Flareless

Std. for Elbow Reducer, 90 Deg. Flareless Sid. for Tee, Reducer, Flareless Sud. for Tee, Reducer, Flareless Rec. Std. for Radio Std. Spec. for Copper Drainage Std. Spec. for Nickel Seamless Pipe and id. Spec. for Nickel Chromium Iron Alloy Seamless Pipe and Sid. Spec. for Seamless Brass Std. Spec. for Seamless Copper Water n-Chromium-Molybdenum-Copper Alloy Seamless Pipe and ckel Chromium Molybdenum Columbium Alloy Seamless Paipd iron Columbium Molybdenum Tungsten Alloy Seamless Pipe and Sid. Spec. for Welded Copper and Its Alloy Std. Spec. for Welded Copper Alloy W ater Sid Spec, for Welded Brass

Solder-Joint Pressure Fitting for l'se with Copper Water Std. Spec. for Seamless Copper Nickel Pipe and td. Spec. for Nickel-Iron-Chromium Alloy Seamless Pipe std Spec, for Aluminum Alloy Extruded Structural Pipe and ec. for Aluminum Alloy Seamless Pipe and Seamless Extruded Std. Spec. for Welded Copper Std. Spec. for Seamless Copper Bus Pipe and
Truck Definitions (1972)

Truck Exhaust System Parts (1972)

Truck Loading (1971)

Truck Mixer and Agitator Stds. (Concrete Plant Equipmen

Truck Muffler Parts Nomenclature (1971)

Truck Nameplates and Marking (1973)

Truck Resonator Parts Nomenclature (1971)

Truck Shipping and Receiving-Materials Handling) (197

Truck Trailer Certification (1972)

Truck Trailer Jumper Cable (1972)

Truck) (1971)

Ory Instrumentation and Techniques for

Truck, Boat, Baggage, Special Type Trailer. Mobile Home

Truck, Bus, Bead Seat, Industrial and Bicycle Tires (19

Truck, Bus, Trailer (1972)

Truck, Bus, Trailer, and Multipurpose Vehicle (1974)

Truck, Bus, Trailer, Mult ipurpose Passenger and Recreat

Truck, Trailer and Bus Type Tires (1973)

Trucks and Buses (1971)

Trucks and Trailers (Course of Instruction) (1972)

Trucks Using the Enclosure Technique (1972)

Trucks (Course of Instruction) (1972) for the Measurement of For Tr

Trucks (1972)

Trucks (1972) ANSl B56.3

Trucks (1972) ANSI S6.3

Trucks (1973)

Trucks (1973)

Trucks (1973)

Trucks (1973)

Trucks (1973) ANSI B56.2

Trucks) (1971)

Trueks) (1972

Trucks, and Combination Vehicles with Any Type of Brake

Trucks, Buses, and Combination Vehicles for Roadway Use

Trucks, Buses, Coaches, Tractors, Industrial Road and M

Trucks, Buses, Trailers, and Multipurpose Passenger Veh

Trucks, Casters. Conveyors, and Other Equipment (1966)

Trucks, Mobile Crane, and Shovel (1974)

Trunnion-Tank Strap, Threaded (1973)

Trunnion-Tank Strap, Linthreaded (1973)

Truss Design: and Nails and Staples (1973)

Truss Plates (1973)

Trusses and Rafters,

Tub Recess (1972)

Tube and a Particulate Filter Meth.) (1973)

Tube and Pipe for Gas and Oil Transmission and Distribu

Tube Assembly, Control Steel with Welded Clevis and Thr
Tube Assembly, Control SteeI with Welded Threaded Rod E

Tube Assembly, Control, Steel, with Flash Welded and Th

Tube Assembly, Control, Steel, with Flash Welded Clevis

Tube Fitting Boss, MlL.R.25897 Rubber, 75 Shore, O-Ring

Tube Fitting Boss, MIL.R.25897 Rubber, 90 Shore, O.Ring

Tube Fittings for Flammable and Combustible Fluids, Ref

Tube Fittings (1971)

Tube Fittings (1971)

Tube Fittings (1972)

Tube for Air Conditioning and Refrigeration Field Servi

Tube for Condensers and Heat Exchangers (1965) ANSI H50

Tube for Restrictor Applications (1974) ANSI H23.8

Tube Fuses (1973)

Tube Neck Components) (1974)

Tube Plates in Surface Condensers and Heat Exchangers

Tube Sampling of Soils (1974)

Tube to Rod End (1974)

Tube to Swivel (1973)

Tube to Swivel (1973)

Tube to S wivel, Swivel on Run (1973)

Tube to SwiveI, Swivel on Side (1973)

Tube Wire (1970)

Tube (DWV) (1974) ANSI H23.6

Tube (1970) ANSI H34.1

Tube (1970) ANSI H34.3

Tube (1971) ANSI H36.1

Tube (1972) ANSI H23.l

Tube (1972) ANSI H34.16

Tube (1972) ANSI H34.20

Tube (1972) ANSI H34.21

Tube (1973)

Tube (1973)

Tube (1973)

Tube (1973)

Tube (1973) ANSI H26.4

Tube (1973) ANSI H34.41

Tube (1973) ANSI H38.16

Tube (1973) ANSI H38.7

Tube (1974)

Tube (1974) ANSI C7.26
(T Haul); Grad

ITA

$\mathrm{SAE} \quad \mathrm{J} 97$

TTMA RP37

NRMCA *3

SAE J261

ITA 4Fl

SAE J262

ANSI MH8.1

TTMA RP39

TTMA RP40

SAE

TRA

TRA

TRA

VESC

SAE

ANSI

$\mathrm{SAE}$

ANSl

$\mathrm{SAE}$

UL

$\mathrm{SAE}$

ANSI

Std Fo ANSI

J254

5

J68:C

2

I. 1

1.9

J366A

D 18.2

J171 A

D18.1

J70A

583

J986 A

MHII.3

MHII.

$6 \mathrm{Fl}$

$6 \mathrm{Gl}$

505

J398 A

$8 \mathrm{~K} 1$

J299

$\mathrm{J} 294$

$* 1.56$

$\begin{array}{ll}\text { SAE } & \text { J267 } \\ \text { CFTMA } & \text { W } 7\end{array}$

TPA 3

NSA 25

/Ift Bolts, ICBO

ASTM

AWI

TCA

ASTM

ASTM

NSA

NSA

NSA

UL

$\mathrm{SAE}$

SAE

SAE

ASTM

ASTM

ASTM

UL

AST

ASTM

NSA

NSA

NSA

NSA

NSA

ASTM

ASTM

ASTM

ASTM

ASTM

Std. Spec. for Nickel-Iro ASTM

Std. Spec. for Ni ASTM

/D. Spec. for Nickel Chromium ASTM

ASTM

ASTM

ASTM

Std. for Wrought Copper and Bronze ANSI

ASTM

Std. Sp ASTM

ASTM

26

E489

$* 1+1$

8701

D3268

B345

359

358

361

362

1595

1596

109

J246

J512F

$\mathrm{J} 514 \mathrm{E}$ 
Requirements for Wrought Seamless Copper and Copper Alloy Std. Spec. for Copper Silicon Alloy Seamless Pipe and

c. for Seamless Copper and Its Alloy Rectangular Waveguide Std. Spec. for Aluminum Alloy Formed and Arc Welded Round Std. Spec. for Seamless Copper Std. Spec. for Seamless Copper Rec. Pract. for Coding System for Identification of ng Out Stock; Seedling Trees and Shrubs; Bulbs, Corms, and std. Test. Procedure for End Supported Beam Strength Fibre est. Procedure for End Supported Beam Deflection Composite Std. Test. Proc, for Side to Side Crush Composite

t. Proc. for Measuring Inside Diameters of Composite Cans. Composite Cans.

Std. Test. Procedure for Internal Bursting of Composite

Std. Test. Procedure for Torque Strength of Composite rocedure for Measuring Wall Thicknesses of Composite Cans, ocedure for Axial (End to End) Compression Composite Cans, omenclature for Glass Bulbs Intended for Use with Electron

Std. Spec. for Copper and Its Alloy Seamless Condenser

8.6 Std. Spec. for Aluminum Alloy Drawn Seamless Std. Spec. for Aluminum-Alloy Drawn Std. Spec. for Aluminum Alloy Extruded Round Coiled tric Resistance Welded Carbon Steel Boiler and Superheater Std Spec for Seamless Carbon Steel Boiler Std. Spec. for Seamless and Welded Carbon and Alloy Steel Std. Spec. for Seamless and Welded Zirconium c. for Wrought Zirconium and lts Alloy Seamless and Welded Std. Spec. for Copper Alloy Std. Spec. for Seamless Intermediate Alloy Steel Still for Seamless Low Carbon and Carbon Molybdenum Steel Still

Spec. for Seamless Austenitic Chromium Nickel Steel Still Std. Meth. of Test. Laminated

Std. Meth. of Measuring Dimensions of Rigid Rods and aluminum Alloy Seamless Round Condenser and Heat Exchanger copper and Its Alloy Seamless Condenser and Heat Exchanger plia/ Std. for Safety for Implosion Protected Cathode Ray std. Spec. for Columbium and Its Alloy Seamless and Welded Std. for Base for GMI Counter

nts for Carbon, Ferritic Alloy, and Austenitic Alloy Steel Spec. for Seamless Ferritic Austenitic Alloy Steel d. Meth. of Testing Flow Capacity of Refrigerant Capillary of Mleasurement of the A.D Dimension of Aerosol Valve Dip stered Screen Dimensions for Monochrome Television Picture mless Nickel and Nickel Alloy Condenser and Heat Exchanger Tent. Spec. for Molybdenum Flattened Wire for Electron

ec. for Welded Austenitic Stainless Steel Feedwater Heater seamless Copper and Its Alloy Heat Exchanger and Condenser

-Iron-Molybdenum-Copper-Columbium Stabilized Alloy c. for Seamless Medium Carbon Steel Boiler and Superheater elded Carbon Molybdenum Alloy Steel Boiler and Superheater

enitic Alloy Steel Boiler, Superheater, and Heat Exchanger

c Steel Boiler, Superheater, Heat Exchanger, and Condenser

Spec. for Electric Resistance Welded Carbon Steel Boiler rawn Intermediate Alloy Steel Heat Exchanger and Condenser mless Carbon Molybdenum Alloy Steel Boiler and Superheater Std. Spec for Aluminum Alloy Round Welded

Spec. for Aluminum Alloy Extruded Bars, Rods, Shapes, and Std. Spec. for Aluminum Alloy Drawn Seamless cedure for Measuring Lengths or Heights of Composite Cans, td. Spec. for Magnesium Alloy Extruded Bars, Rods, Shapes, Std. for Coupling Std. Spec. for Steel er) (1974) Std. for Tires, Rec. for Steam

ell Service) (1972 Std Spec for Thermoplastic Gas Pressure Pipe, Std. for Seamless Low Carbon Steel Std. for Welded and Cold Drawn Low Carbon Steel Std. for Metric Thread Fuel Injection Std. Spec, for Crosslinked Polyolefin Heat Shrinkable ed and Noncrosslinked Poly(vinyl Chloride) Heat Shrinkable Std. Meth. of Test. Heat Shrinkable Id. Spec. for Seamless and Welded Ferritic Stainless Steel Spec. for Seamless and Welded Austenitic Stainless SteeI 98 Std for Safety for Electrical Metallie or Cold Formed Welded and Seamless Carbon Steel Structural ing \{l\} $09 /$ Std. for Welded Flash Controlled Low Carbon Steel Spec. for Steel Bars, Forgings, Rings, and Mechanical Spec. for Grade C-75 and C-95 Casing and Rec. Pract. for Care and Use of Casing and
Tube (1974) ANSI H23.4 Tube (1974) ANSI H26.3

Tube (1974) ANSI H37.l

Tube (1974) ANSI H38.20

Tube (1975) ANSI H23.3

Tube, Bright Annealed (1973) ANSI H23.2

Tube, Pipe, and Hose Fittings (1972)

Tubers: Christmas Tree Std. (1973)

Tubes and Cores (1972)

Tubes and Cores (1972)

Tubes and Cores (1973)

Tubes and Cores (1973)

Tubes and Cores (1974)

Tubes and Cores (1974)

Tubes and Cores (1974)

Tubes and Cores (1974)

Tubes and Cores (1974)

Tubes and Electric Lamps (1971)

Tubes and Ferrule Stock (1974) ANSI H23.14

Tubes for Condensers and Heat Exchangers (1973) ANSI H3

Tubes for General Purpose Applications (1973) ANSl H38.

Tubes for General Purpose Applications (1973) ANSl H38.

Tubes for High Pressure Service (1973) ANSI Bl25.12

Tubes for High Pressure Service (1973) ANSI B125.7

Tubes for Low Temperature Service (1972A) ANSI B 125.18

Tubes for Nonnuclear Application (1972) ANSI Z212. 1

Tubes for Nuclear Service (1971) ANSI N124

Tubes for Pressure Applications (1972)

Tubes for Refinery Service (1972) ANS1 B 125.23

Tubes for Refinery Service (1972) ANSI B 125.5

Tubes for Refinery Service (1972) ANSI B36.39

Tubes U'sed for Electrical Insulation (1970)

Tubes Iised for Electrical Insulation (1972) ANSI C59.33

Tubes with Integral Fins (1973)

Tubes with lntegral Fins (1974)

Tubes (Picture and Display) for Television Receiving A

Tubes (1970) ANSI H53.]

Tubes (1971)

Tubes (1971 A) ANSI B125.34

Tubes (1972)

Tubes $(1972)$

Tubes $\{1972\}$

Tubes $(1972)$

Tubes (1972) ANSI H34.42

Tubes (1973)

Tubes (1973)

Tubes (1973)

Tubes (1973)

Tubes (1973) ANSI B125.13

Tubes (1973) ANSI B125.22

Tubes (1973) ANSI B125.32

Tubes (1973) ANSI B125.6

Tubes (1973) ANSI B125.8

Tubes (1973) ANSI B125.9

Tubes (1973) ANSI H38.11

Tubes (1973) ANSI H38.5

Tubes (1974) ANSI H38.3

Tubes, and Cores (1974)

Tubes, and Wires (1970) ANSI H45.6

Tubes, Flared Fitting, Female Thread (1973)

Tubes, Low Carbon, Tapered for Structural Use (1973)

Tubes, Rims and Valves for Aircraft (Airplane, Helicopt

Tubine Driven Generator Lnits (1972)

Tubing and Fittings (1973)
B25 !

B315

B372

B547

B75

B68

$\mathrm{J} 846 \mathrm{C}$

Z60. I

T114

T-113

T108

CT102

CT111

T112

T116

CT 101

CT107

C79.1

B111

B234

B483

B49l

A 226

Al92

A334

B523

B353

B469

A 200

Al61

A271

D348

D 668

B404

B359

492.8

B39.

$\mathrm{N} 42.5$

A 450

A669

28

D3089

Std Neth ASTU

Std for Regi EIA

RS266A

B 163

F364

A688

B395

B468

A 210

A 250

A213

A 249

Al78

A 199

A209

B313

B22l

B210

CT104

$\mathrm{B} 107$

424

A595

9

13-3

FMS

ASTM Thread Compounds (For Casing, Std. for Air Brake Spec. for Steel Bars, Forgings, and

Tubing and Line Pipe Connections in High Pressure Oil W

Tubing and Pipe (1970)

Tubing Annealed for Bending and Flaring (1972)

Tubing Annealed for Bending and Flaring (1972)

Tubing Connections (1971)

Tubing for Electrical Insulation (1972

Tubing for Electrical Insulation (1973)

Tubing for Electrical lnsulation (1974)

Tubing for General Service (1973) ANSI B125.14

Tubing for General Service (1973) ANSI B125.15

Tubing for Pressure Application at High Temperatures (1

Tubing for Wire and Cable lnstallation (1973) ANSl C33.

Tubing in Rounds and Shapes (1973) ANSI G24.21

Tubing Normalized for Bending. Double Flaring, and Bead

Tubing (Annealed) $(0.48 \mathrm{Cr}-8.0 \mathrm{Ni}-4.0 \mathrm{Co}-0.48 \mathrm{Mo}-0$.

Tubing (Petroleum Drilling) (1973)

Tubing (Petroleum Drilling) (1973)

Tubing $(0.80 \mathrm{Cr}-1.8 \mathrm{Ni}-0.25 \mathrm{Mo}(0.49-0.55 \mathrm{C}))(1973)$

Tubing $\left(1.05 \mathrm{Cr}_{\mathrm{r}}-0.55 \mathrm{Ni}-1.0 \mathrm{Mo}-0.12 \mathrm{~V}(0.43-0.49 \mathrm{C})\right)$

Tubing (1.62SI $-0.82 \mathrm{Cr}-1.82 \mathrm{Ni}-0.40 \mathrm{Mo}-0.07 \mathrm{~V}(0.41$

Tubing (1970) ANSI B 125.33

API

$\mathrm{SAE}$

$S A E$

$S A E$

SAE

ASTM

/for Crosslink ASTM

ASTN

ASTM

Std ASTM

ASTM

UL

Pec. F ASTM

SAE

$S A E$

API

API

$S A E$

$S A E$

$S A E$

ASTM

D2513

BUL 5 A2

$\mathrm{J} 844 \mathrm{C}$

J524B

J525B

$\mathrm{J} 242$

D3149

D3150

D2671

A 268

A 269

A608

A 500

J356 A

AMS6540B

$5 \mathrm{AC}$

$5 \mathrm{Cl}$

AMS6424

AMS6432A

AMS6416A Std. Spec. for Copper Brazed Steel 
Std. Spec. for Seamless Stainless Steel Mechanical Std. Rec. Pract. for Flaring Polyolefin Pipe and Std for Welded Low Carbon Steel Std. for Brazed Double Wall Low Carbon Stee Sid. for Flares an

Rec. for Coiling Polyethylene Plastic Pipe an Safety Std for Extruded Thermoplastic Insulatin d. for Design, Manufacture and Test of Aluminum Irrigation 5 Strip in Narrow Widths and Light Gage for Heat Exchange Std. Spec for Tantalum and Its Alloy

Std. Spec. for Welded Stainless Steel Mechanica Std. Spec, for Polybutylene (PB) Plastic Std. Spec. for poly(Vinylidene Fluoride) Heat Shrinkable Spec for Tolerances of Aluminum Alloy Drawn med Welded and Seamless High Strength Low Alloy Structural lyethylene (PE) Plastic Fittings for (PE) Plastic Pipe and or Seamless and Welded Austenitic Stainless Steel Sanitary cellulose Acetate Butyrate (CAB) Plastic Pipe (SDR-PR) and Std Spec for Poly(vinyl Chloride) (PVC) Plastic for Hot Formed Welded and Seamless Carbon Steel Structura Std. Spec. for Seamless Carbon and Alloy Steel Mechanical Std. Spec. for Polyethylene (PE) Plastic

Manual of Cold Formed Welded Structural Stee ted Galvanized Rigid Steel Conduit and Electrical Metallic Pract. for Eddy Current Test. of Steel Tubular Products Spec. for Casing.

Spec. for High Strength Casing. cements for Cellulose Acetate Butyrate (CAB) Plastic Pipe $-1.8 \mathrm{Ni}-0.4$ Spec. for Steel Bars, Forgings, Mechanica eading, Gaging, and Thread Inspection of Petroleum Casing. $26 \mathrm{Ni}-1.3 \mathrm{Mo}-2.1 \mathrm{Ti}-1 \quad$ Spec. for Steel Bars, Forgings, t, 17Cr (SAE 51430) Annealed (1973/ Steel Bars. Forgings. $2-0.38$ C). Special Grade (1973)

$0-1.20 \mathrm{C})$. Premium Bearing $\mathrm{Qu}$

Steel Bars, Forgings. Steel Bars, Forgings, and Shrinkable $(19$ Spec. for Electrical Insulation Plastic Spec. for Steel Bars, Forgings, and $30 \mathrm{~V}(0.40-0.50 \mathrm{C}) \mathrm{V} / \mathrm{s}$. sters, Face Mask (1973) Spec, for stee

15) (1973)

43C) (Modified 98Bv40) (1973)

0 ), Premium Quality, Consumabl/ 0) (1973)

(1973)

0), Premium Quality. Vacuum Col

0). Premium Quality (1973)

Recommendation for Installation of Ceramic Tile Std. Drive Arrangements for Std. Drive Arrangements for sto

1) Ansil Sertest. Of Stee (Steel Products Manual) Steel Specialty wn and Garden Riding Tractors (1972) td. for One Poin Carbon stee Std. for General Purpose Semi-Tubular. Full
th. for Water Repellency of Textile Fabrics

astm/ Test Meth. for Water Repellency of Textile Fabrics Std. Spec. for Nickel Chromium Iron Columbium Molybdenum Spec. for Std. for Manual Gas Std Meth for Chemical Analysis of esistance of Rigid Plastic Sheeting or Parts by Means of A Std. Meth. of Test for Fineness of Portland Cement by the Apparatus) (Exclusive of Generator, for Hydraulic Rec. for Steam

rs for Pumpe/ Std. for Installation of Vertical Hydraulic

he Flushing and Cleaning of Lubricating Systems of Various nchronous Generator/Motor L ni/

r Digital Computer Programs (1970)

Spec. and Rec. Pract. for Installation and Calibration of Std. Spec. for Gas

Tent. Meth of Test for Total Acidity in Aviation Tent. Meth. of Test for Flash Point of Aviation Std. Meth. of Test for Trace Metals in Gas

Tent. Meth. of Test for Thermal Oxidation Stability of Std Meth. of Test for Smoke Point of Aviation Std. Meth. of Test for Thermal Stability of Aviation of Test for Water Separation Characteristics of Aviation std. Meth. of Test for Lead and Vanadium in Gas

Tent. Meth. of Test for Lndissolved Water in Aviation Guidance Material for Aviation Std. Spec. for Aviation

std. Meth. of Test for Particulate Contaminant in Aviation Rec. Pract. for Flushing and Cleaning of Gas Guide for Operation and Maintenance of ANSI Z11.230 Std. Meth. of Test for Continuity of Steam Std. Spec. for Vacuum Treated Alloy Steel Forgings for
Tubing (1971) ANSI G62.1

ASTM

ASTM

bing (1972)

Tubing (1972)

Tubing (1972)

Tubing (1972)

Tubing (1972)

Tubing (1972)

Tubing (1972)

Tubing (1972)

Tubing (1972) (1)

Tubing (1973)

Tubing (1973)

Tubing (1973)

Tubing (1973)

Tubing (1973)

Tubing (1973) ANSI B36.38

Tubing (1973) ANSI B72.12

Tubing (1973) ANSI B72.22

Tubing (1973) ANSI G24.22

Tubing (1973) ANSI G62.4

Tubing (1974)

Tubing (1974)

Tubing (1974) for PolyvinyI Chloride Externa
(Tubing) with Magnetic Saturation (1971) ANS1 Z166.27
Tubing, and Drill Pipe (Petroleum Drilling) (1973)

Tubing, and Drill Pipe (Petroleum Driling) $(1973)$

Tubing, and Drill Pipe (Petroleum Drilling) (1973

Tubing, and Fittings (1973) ANSI K65.162

Tubing, and Flash Welded Rings (1.3.

Tubing, and Rings, Corrosion and Heat Resistant $15 \mathrm{Cr}-$

Tubing, and Rings, Corrosion and Moderate Heat Resistan

Tubing, and Rings, $0.78 \mathrm{Cr}-1.8 \mathrm{Ni}-0.35 \mathrm{Mo}-0.20 \mathrm{~V}(0.3$

Tubing. Corrosion Resistant, $14.5 \mathrm{Cr}-4.0 \mathrm{Mo}-1.2 \mathrm{~V}(1.1$

Tubing, Irradiated Polyolefin, Pigmented, Flexible, Hea

Tubing, Low Alloy, Heat Resistant $0.95 \mathrm{C} r-0.55 \mathrm{Mo}-0$.

Tubing. Mattresses and Pads. Sheeting, and Restraint St

Tubing. Welded, Corrosion and Heat Resistant $(25 \mathrm{Cr}-20$

Tubing, $0.50 \mathrm{Cr}-0.55 \mathrm{Ni}-0.20 \mathrm{Mo}(0.11-0.17 \mathrm{C}$ ) (SAE 86

Tubing, $0.80 \mathrm{Cr}-0.85 \mathrm{Ni}-0.20 \mathrm{No}_{0}-0.04 \mathrm{~V}-\mathrm{B}(0.38-0$.

Tubing, $0.80 \mathrm{Cr}-1.8 \mathrm{Ni}-0.25 \mathrm{Mo}(0.38-0.43 \mathrm{C})(\mathrm{SAE} 434$

Tubing, $0.80 \mathrm{Cr}-1.8 \mathrm{Ni}-0.25 \mathrm{Mo}(0.38-0.43 \mathrm{C}$ ) (SAE 434

Tubing, $0.88 \mathrm{Cr}-1.8 \mathrm{Ni}-0.42 \mathrm{Mo}-0.08 \mathrm{~V}(0.28-0.33 \mathrm{C})$

Tubing, $1.2 \mathrm{Cr}-3.25 \mathrm{Ni}-0.12 \mathrm{Mo}(0.07-0.13 \mathrm{C})$ (SAE 931

Tubing, $1.2 \mathrm{Cr}-3.25 \mathrm{Ni}-0.12 \mathrm{Mo}(0.07-0.13 \mathrm{C})$ (SAE $93 \mathrm{I}$

Tubs (1973)

Tubular Centrifugal Fans (1966)

Tubular Heat Exchanges in Chemical Process Service (197

Tubular Products for High Temperature Service Conformin

Tubular Products (Tubing) with Magnetic Saturation (197

Tubular Products (1972)

Tubular Sleeve Attachment for Hitching Implements to La

Tubular Spring Pins (1973)

Tubular, Split Rivets and Caps (1972)

Tumble Jar Dynamic Absorption Test (1972) ANSI L14.87.

Tung Oil (1973)

Tungsten Alloy Seamless Pipe and Tube (1972) ANSI H34.2

Tungsten Are Welding Electrodes (1969) ANS] W3.12

Tungsten Arc (Gta) Fusion Welding Equipment (1974)

Tungsten (1973)

Tup (Falling Weight) (1972)

/Eth. of Test for Impact $\mathrm{R}$

Turbidimeter (1973) A
Turbine Drive) (1972)

Turbine Driven Generator I nits (1974)

Turbine Driven Generators and Reversible Generator/Moto

Turbine Driven Pumps for Marine Service (1971)

Turbine Driven Synchronous Generators and Reversible Sy

Turbine Engine Steady State Performance Presentation Fo

Turbine Flowmeters (1972)

Turbine Fuel Oils (1971) A NSI Z11.312

Turbine Fuel (1973)

Turbine Fuels by Setaflash Closed Tester (1973)

Turbine Fuels (Atomic Absorption Meth.) (1972) ANSI Z11

Turbine Fuels (JFTOT Proc.) (1973)

Turbine Fuels (1972) ANSI Z11.137

Turbine Fuels (1972) ANSI Z11.148

Turbine Fuels (1972) ANSI Z11.301

Turbine Fuels (1972) ANSI Z11.316

Turbine Fuels (1973)

Turbine Fuels (1973)

Turbine Fuels (1973) ANSI Z11.204

Turbine Fuels (1973) ANSI Z11.264

Turbine Generalor Lubric ating Oil Systems (1971)

Turbine Generators (1972) ANSI C50.30

Turbine Oil Oxidation Stability by Rotating Bomb (1967)

Turbine Rotor Disks and Wheels (1970) ANSI G55.15 $\triangle A E$

$S A E$

SAE

inimum St ASAE

ASTM

ASTM

ASTM

ASTM

ASTM

$\mathrm{SAE}$

ASTM Fusion Po ASTM Spec. For ASTM

ASTM

Std. Spec.

ASTM

ASTM

ASTM

WSTI

NEMA

ASTM

API

API

ASTM

$\mathrm{SAE}$

API

SAE

$S A E$

$\mathrm{SAE}$

$S A E$

SAE

SAE

LIL

SAE

SAE

$\mathrm{SAE}$

SAE

$\mathrm{SAE}$

$\mathrm{SAE}$

$\mathrm{SAE}$

TCA

AMCA

ANSI

ASTM

ASTM

AISI

ASAE

SAE

ANSI

AATCC

ASTM

ASTM

AWS

NSA

ASTM

ASTM

ASTM

NEMA

FUIS

NEMA

/ for T ASME

NEM

$\mathrm{SAE}$

IS A

ASTM

ASTM

ASTM

ASTM

ASTM

ASTM

ASTM

Std. Meth ASTM

ASTM

ASTM

IATA

ASTM

ASTM

ASME

IEEE

ASTM

ASTM

A511

D3140

$526 \mathrm{~B}$

J533B

PPl TN6

224

S 263.2

B569

B521

A 554

D2666

D3144

A MS2203G

A618

D326]

A270

D2446

D2740

A501

A 519

D2737

RN I

RN 109

$5 \mathrm{~A}$

$5 A X$ 


\section{National Bureau of Standards KWIC Index of Engineering Standards}

ec. for Vacuum Treated Carbon and Alloy Steel Forgings for High Temperature Service (1)

Std. Spec, for Alloy Steel Test for Mercaptan Sulfur in Gasoline, Kerosine, Aviation Rec. Pract. for the Prevention of Water Damage to Steam

heavy Walled Carbon and Low Alloy Steel Castings for Steam

tection for Maintenance and Inspection of Mechanical Drive

1 Valve Capacity Test Procedure for Compressible Fluids in Rec. Pract. for Motorcycle Std. for

ks and Cutting Tools, Single Point, Carbide Tipped, Rolle d Rodent Hairs in Rye Flour (Cereal Chemistry) (1962)

Rec. Pract. fo

Chloroform; Formaldehyde: Gasoline; Herwig's, Pancreatin,

Std. for Bolt, Pan Head, Close Tolerance, Short Thread,

g (197) Std. for Pan Head, Close Tolerance, Short Thread,

Meth of Test for Std. for td. (1973)

for Professional Photographic Sheet and Roll Films (SI and Condenser Tubes (1973)

Std. Spec, for er Universal Pails (Containes) (Dot-37 A80, Dot-37A 60

td. Spec. for $57 \mathrm{Gal}$. Full Removable Head Universal Drums td. Spec. for $30 \mathrm{Gal}$. Full Removable Head Universal Drums td. Spec. for $16 \mathrm{Gal}$. Full Removable Head Universal Drums std. Spec. for 55 Gal. Full Removable Head Universal Drum or 16 Gal. Full Removable Head, Lug Cover Lniversal Drums ver Universal Pails (Container) (Dot-37A80, Dot-37A60, 5 GaI. Tight Head UniversaI Pails (Container) (Dot-17E, Std. Meth. for Tent. Spec. for

z166.23

or Special Applications (19) Std. Meth. for sels (1973) ANSI Std Meth. and Spec, for Straight Beam ure Vessels (1971) ANSI H38.21 s for Aerospace Applications (1974) tings (1970) ANSl C52.7

3) ANSI G35.24

n Concentrates (Cereal Chemistry) (1962)

n Dry Vitamin Mixes, Beadlets, Oils, and Emulsions (Cere/ Std. Meth. for Evaluation of White MineraI Oils by ine on Bags and Other Packaging Materials (Cereal Chemis) Std Spec for Seamless and Welded ture (Oven Filter Paper) Content of Corn Syrup Unmixed and Tent. Rec. for Concrete Members Prestressed with

Building Code Std. for and Meth. of Sampling and Test. of c. for General Requirements for Carbon Steel Wire Rods and Std. Meth. of Test for Lime Content of Meth. of Analysis of

ereal Chemistry) (1962) Std Spec, for Asbestos Cement Perforated s (1971) An/ Std. Spec. for Porous Concrete Pipe for Use in
Std. Spec. for Steel Sheets for Culverts and er Cable Specs.) (1973)
ati/ Safety Rules for the Installation and Maintenance of Std. for Steel Spec. for Tinned Copper Concentric Neutrals Installation Pract. for

al Wire and Cable (1/ Std. for Plastic Utilities Duct for Std. Rec. Pract. for Std. Rec. Pract. for r Pipe (1972) ANS1 K65.171 ng (1972) Ducts and Fittings for yrene Rubber and Polyvinyl Chloride Duets and Fintings for Tent. Rec. Spec. for Asphalt Type Protective Coatings for ended Guide to Safe Practice Protection from Corrosion for (1973) ANSI B137.1 Safety Std. for Steel Safety Std. for Steel Pipe (Welded and Seamless) for Spec. for

Std. for Monorail Systems and ood Shakes and Shingles with Asbestos Felt or Gypsum Board test Procedure for Evaluation of the Effectiveness of Rear Std. for Sleeve Bushing, Press Fit, Std. for Bushing-Flanged, Press Fit . Std. Meth. of Test for cation of Data Derived from the lnvestigation of $\mathrm{Sc}$ / Std. for Procedures for Calibration of mming Pools (1972) Safety Std. for s; Drilling Through ControI, Screwed and Flanged Wellhead,

Tent. Meth. of Test for d Corrosion Resisting Steel, UNJC-3A Thread, Ca/ Std. for eel and Corrosion Resisting Steel, UNJF.3A Thre/ Std. for
Turbine Rotors and Shafts (1974) ANSI G55.14

Turbine Type Bolting Material Specially Heat Treated Fo

Turbine, and Distillate Fuels (Potentiometric Meth.) (1

Turbines Used for Electric Power Generation: Fossil Fue

Turbines (1974)

Turbines (1974)

Turbulent Flow (1974)

Turn Signal Lamps (Light) (1971)

Turnbuckle-Tank Strap (1973)

Turner Type (1972)

Turning A bility and Off Tracking of Vehicles (1971) azards and Pro FMS Std. for Contro ISA

SAE

NSA

Tween Versene Meth. of Analysis for Insect Fragments an

Tween 80-60\% Alcohol, Versene, and Sodium Phosphate Sol

TWl Wing Recess, Alloy Steel, Self Locking and Nonlocki

TWI Wing Recess, A286 Acres, Self Locking and Nonlockin

Twine Made from Bast and Leaf Fibers (1973)

Twist in Yarns (Direct Counting Meth.) (1971) ANSI L14.

Typewriter Keyboard Arrangement (1973)

U.S. Customary Units) (1973)

Std. Dimension

U.S. Model Rocket Sporting and Safety Code and Engine S

U.Bend Seamless Copper and lts Alloy Heat Exchanger and

U.Bend Stress Corrosion Test Specimens (1972)

UDy Dye Meth. of Analysis of Crude Protein in Cereal Gr

Ufc-Rule 40, Nmfc-Item 260 (1974)

(Ufc-Rule 40, Nmfc-ltem 260) (1974)

(Lfc-Rule 40, Nmfc-Item 260) (1974)

(Ufc-Rule 40, Nmfc-Item 260) (1974)

(Ufc-Rule 40, Nmfc-ltem 260) (1974)

(Ufc-Rule 40, Nmfc-Item 260) (1974)

Ufc-Rule 40, Nmfc-ltem 260) (1974)

Ufc-Rule 40, Nmfc-Item 260, Dot-37B60) (1974)

Utimate A naylsis of Coal and Coke (1974)

Ultra High Test Heat Treated Line Pipe (1972)

Ultrasonic Contact Inspection of Weldments (1965) ANSI

Ultrasonic Examination of Plain and Clad Steel Plates F

Ultrasonic Examination of Steel Plates for Pressure Ves

Ultrasonic Inspection of Aluminum Alloy Plate for Press

Ultrasonic lnspection of Aluminum Alloy Wrought Product

Ultrasonic Inspection of Carbon and Low Alloy Steel Cas

Ultrasonic Test. (1974)

Ultrasonic Velocity in Materials (1973)

Ultrasonic, Angle Beam Examination of Steel Plates (197

Ultraviolet Absorption Meth. of Analysis of Vitamin A I

Ultraviolet Absorption Meth. of Analysis of Vitamin A I

Ultraviolet Absorption (1973) ANSI Z11.210

Ultraviolet Light Examination for Presence of Rodent Ur

Unalloyed Titanium Welding Fittings (1971) ANSI H50.1

Unbleached Crude Corn Sugar (1956)

Unbonded Tendons (1966)

Unburned Clay Masonry Units (1973)

Uncoated Coarse Round Wire (1971) ANSl G54.12

Uncured Soil-Lime Mixtures (1973) ANSI A1.38

Undenatured Whey Protein Nitrogen in Nonfat Dry Milk (C

Underdrain Pipe (1973) ANSI Al65.2

Underdrains (1973)

Underdrains, Zinc Coated (Galvanized) by Hot Dip Proces Underfloor Duct Raceways (1972)

Underground Distribution Reference Book (Electrical Pow

Underground Electric Supply and Communications Lines (N

Underground Electrical Power Cable) (1973)

Underground Electrical Power Distribution Cables (1973)

Underground Installation for Communication and Electric

Underground Installation of Flexible Thermoplastic Sewe

Underground Installation of Thermoplast ic Pressure Pipi

Underground Installations of Electrical Wires and Cable

Underground or Submerged Metallic Piping Systems (1972)

Underground Pipe Lines (Wrapped Systems) (1972)

Underground Pipe, Fittings and Tanks Containing Flammab

Underground Tanks for Flammable and Combustible Liquids

Underground Water Service (1972)

Underhung Cranes and Monorail Systems (1973)

Underhung Cranes (1973)

Underlayment) (1973)

Underride Guard (Safety (1971)

Undersize Inside Diameter (1973)

Td. for Special Purpose Roofs (IV

Undersize Inside Diameter (1973)

Undersized Loose Muscovite Mica Splittings (1973)

Underwater Accident Report Form (Acquisition and Codifi

Underwater Electro Acoustic Transducers (1972)

Underwater Lighting Fixtures and Junction Boxes for Swi

Underwater Wellhead, and Christmas Tree Equipment; Flow Underwriter Steam Fire Pumps (1973)

Undissolved Water in Aviation Turbine Fuels (1973)

Undrilled and Drilled, Plain and Self Locking, Alloy an

Undrilled and Drilled, Plain and Self Locking, Alloy St

Unfired Monolithic Refractories (1972)
$S A E$

$\mathrm{AACCH}$

$\mathrm{AACCH}$

NSA

NSA

ASTM

ASTM

ANSI

ANSI

NAR

ASTM

ASTM

$\mathrm{AACCH}$

ANSI

ANSl

ANSl

ANSI

ANSI

Spec, F ANSI

o ANS1

/R ANSI

ASTM

APl

ASTM

ASTM

ASTM

ASTM

ASTM

ASTM

ASTM

ASTM

ASTM

AACCH 8601

AACCH 86.01A

ASTM D2269

$\mathrm{AACCH} 28.85$

ASTM

$\mathrm{CR}$

$\mathrm{ACl}$

E.44

66.8

Uniform ICBO

ASTM

ASTM

A510

ASTM

ASTM

ASTM

NEMA

AA

ANS

AA

AA

NEMA

ASTM

ASTM

NEMA

NACE

$\mathrm{NACE}$

ICBO

UI

UL

MMA

ANSI

ICBO

SAE

NSA

ASTM

ANSI

ANSI

UL

APl

FMS

ASTM

NSA

NSA

ASTM

$895 \mathrm{~A}$

100-6

$\times 47$

PH1.18

B395

30

MH2. 15

1H2.11

4H 2.8

MH2.10

A 578

(4)

440

A356

J 39.4

27

94.37

H2. 12

PEC 5LL 
ium Nickel Steel Plate, Sheet, and Strip for Fusion Welded

meth. of Test for Elastic Moduli of Rock Core Specimens in revailing Torque, All Metal, 1200 Deg, F (649 Deg. C) IIse, esign Parameters for Bolts and Screws; Extcrnal Wrenching,
revailing Torque, All Metal, 1200 Deg. F (649 Deg. C) ('se, s. (1972) gypsum Wallboard to Wood Framing (1973)

(1973)

rtar (1973)

73)

n Field Test of Partitions (Wall, Ceiling, Floor, and Fl/ 3

d Test. of Unburned Clay Masonry Units (1973)

shingles (1973)

System for Dust, Stock and Vapor Removal (1973)

llow Brick (Made from Clay or Shale) (1973)

r (1973)

nguishing Systems (1973)

3)

Picture Film (1973)

r Measuring the Density of Smoke from the Burning or Dec/

ry Cleaning Plants or Systems (1973)

on, and Meth. of Grading for All Species of Lumber (1973/

las Fir-Larch (North), Eastern Hemlock-Tamarack (Nor/ r Concrete Reinforcement (1973)

stems (Fire Extinguishment) (1973)

ss C Sheet (Roll) and Shingles) (1973)

(1973)

d, Crushed Stone, Gravel and Air Cooled Iron Blast Furna/

ars (1973)

Is (Electrodeposited Coatings of Copper, Nickel, Chromiu/ g Tensile Strength of Molded Concrete Cylinders (1973)

n: West Coast Hemlock; Western Red Cedar: White Fir; And/

- Fir; Ponderosa, Idaho White, Sugar, and Lodgepole Pine/ ne, and Hemlock-Tamarack; Balsam Fir; Northern Pine An/ ne, Eastern Spruce and Hemlock, Balsam Fir and Tamarack dentification and Painting of Structural Steel (1973)

$\mathrm{n}$ Test Results in Field Concrete Cylinders (1973)

Soil (1973)

bonding Agents (1973)

athing and Structural lnsulating Board (1973)

d Mortar (1973)

igh-Rise Buildings (1973)

ring Materials (1973)

onstruction and Materials (1973)

blies (1973)

emblies (1973)

afety Glazing (1973)

73

laster and Gypsum Veneer Plaster (1973)

1973)

Block (1973)

1973)

d Joint Compound (1973)

s, Nuts, Washers, and Bolted Parts (1973)

rete Masonry Units (1973)

oncrete Masonry Units (1973)

y Purposes (1973)

(1973)

s (1973)

c Fire Extinguishing Systems (1973)

rs and Windows (1973)

g. Paper (Weatherproofing) (1973)

and Measurement of Airborne Sound Transmission Class (S/ or Structural Concrete (1973)

and Utility Pole Preservative Treatment by Pressure Proc/ umina Fireclay Refractory Bricks (1973)

n Mortars (1973)

board (1973)

tural Steel and Iron (Shape, Plate, Sheet, Strip, Connec/ struction of Built Up Roof Coverings: Hot Mopped Asphalt/

for Acoustical Tile and for Lay in Panel Ceilings (1973) ric Laths, and Met

ric Laths, and Metal Accessories (Designed for Use as a / es $(1973)$
Infired Pressure Vessels (1972B) ANSI G81.4

Unhardened Steel and Alloy Silver Brazing Joints (1973)

Uniaxial Compression (1972)

Unified J (MIL S.8879) Thread Form (1973)

Unified Threaded In. Series (1971)

Unified (MIL S.7742) Thread Form) (1973)

Uniform Alignment Tests-NAS 900 Series Equipment Spec

Uniform Building Code Std. for Adhesives (1973)

Uniform Building Code Std. for Admixtures for Concrete

Uniform Building Code Std. for Aggregate for Masonry Mo

Uniform Building Code Std. for Aggregates for Grout (19

niform Building Code Std. for Airborne Sound Insulatio

Uniform Building Code Std. for Aluminum Structures (197

Uniform Building Code Std. for and Meth. of Sampling an

Iniform Building Code Std. for Asbestos Cement Roofing

Uniform Building Code Std. for Blower, Fan, and Exhaust

Uniform Building Code Std. for Building, Facing, and Ho

Uniform Building Code Std, for California Redwood Lumbe

Iniform Building Code Std. for Carbon Dioxide Fire Exti

Uniform Building Code Std. for Cast Building Stone (197

Uniform Building Code Std. for Cellulose Nitrate Motion

Uniform Building Code Std. for Chamber Meth. of Test Fo

Uniform Building Code Std. for Class II and Class III D

Uniform Building Code Std. for Classification, Definiti

Uniform Building Code Std. for Coast Sitka Spruce, Doug

Uniform Building Code Std. for Cold Drawn Steel Wire Fo

Uniform Building Code Std. for Combination Standpipe Sy

Iniform Building Code Std. for Composition Roofing (Cla

Uniform Building Code Std. for Concrete Aggregates (San

Uniform Building Code Std. for Concrete Building Brick

Uniform Building Code Std. for Concrete Reinforcement B

Uniform Building Code Std. for Concrete Tests (1973)

Uniform Building Code Std. for Corrosion Resistant Meta

Uniform Building Code Std. for Determining the Splittin

Uniform Building Code Std. for Douglas Fir, Coast Regio

Uniform Building Code Std. for Douglas Fir; Larch; Hem

Uniform Building Code Std. for Eastern Spruce, White Pi

Iniform Building Code Std. for Eastern White and Red Pi

Iniform Building Code Std. for Erection, Fabrication, I

Uniform Building Code Std. for Evaluation of Compressio

Uniform Building Code Std. for Expansion Index Test for

Uniform Building Code Std. for Exterior Plaster Liquid

Uniform Building Codc Std. for Fiberboard Nail Base She

Uniform Building Code Std. for Field Tests for Grout an

Uniform Building Code Std. for Fire Alarm Systems for H

Uniform Building Code Std. for Fire Dampers (1973)

Uniform Building Code Std. for Fire Retardant Roof Cove

Uniform Building Code Std. for Fire Tests of Building C

Uniform Building Code Std. for Fire Tests of Door Assem

Uniform Building Code Std. for Fire Tests of Window Ass

Uniform Building Code Std. for Glass, Its Defects and S

Uniform Building Code Std. for Gypsum Backing Board (19

Uniform Building Code Std. for Gypsum Base for Veneer P

Uniform Building Code Std. for Gypsum Lath (1973)

Uniform Building Code Std. for Gypsum Molding Plaster

Uniform Building Code Std. for Gypsum Partition Tile or

Uniform Building Code Std. for Gypsum Plastics (1973)

Uniform Building Code Std. for Gypsum Sheathing Board (

Uniform Building Code Std. for Gypsum Wallboard Tape an

Uniform Building Code Std. for Gypsum Wallboard (1973)

Uniform Building Code Std. for Gypsum (1973)

Uniform Building Code Std. for Hand Split Shakes (1973)

Uniform Building Code Std. for High Strength Steel Bolt

Uniform Building Code Std. for Hollow Load Bearing Conc

Uniform Building Code Std. for Hollow Nonload Bearing C

Uniform Building Code Std. for Hydrated Lime for Masonr

Uniform Building Code Std. for Impact Sound Insulation

Uniform Building Code Std. for in Place Density of Soil

Uniform Building Code Std. for Installation of Automati

Uniform Building Code Std. for Installation of Fire Doo

Uniform Building Code Std. for Keene's Cement (1973)

Uniform Building Code Std. for Kraft Waterproof Buildin

Uniform Building Code SId. for Laboratory Determination

Uniform Building Code Std. for Lightweight Aggregates F

Uniform Building Code Std. for Lime (1973)

Uniform Building Code Std. for Lumber, Timber, Plywood

Iniform Building Code Std. for Machine Made and High Al

Uniform Building Code Std. for Masonry Cement for Use I

Uniform Building Code Std. for Mat Formed Wood Particle

Uniform Building Code Sid. for Material Spec. for Struc

Iniform Building Code Std. for Materials for Use in Con

Uniform Building Code Std. for Metal Suspension Systems

Uniform Building Code Std. for Metal, Wire and Wire Fab

Uniform Building Code Sid. for Meth. of Test for Concre

Iniform Building Code Sid. for Mineral Roofing Aggregat
ASTM

SAE

Std. ASTM

A 240

AMS2664C

D31.48

AMS7251B

ASI132A

AMS $7250 \mathrm{C}$

985

UBC 547.2

UBCS25. 19

UBCS 26.9

UBCS24.22

UBCS24.24

UBCS 35.3

UBC S28.]

UBC 24.15

UBC $\$ 32.9$

UBCS10.3

UBCS24.1

UBC 25.7

UBC 338.2

UBCS24.14

UBCS $48-2$

UBC 5 52.2

UBCS10.2

UBCS25.]

UBC 25.2

UBC 24.16

UBC 38.3

UBCS 32.3

UBCS26. 2

UBC 524.3

I BC \$26. 4

L'BCS $26 \cdot 10$

UBCS 32.6

I BCS 26.12

UBCS25-3

UBC 525.4

UBC 25.8

UBCS25.5

UBC $527-2$

UBCS 26.11

UBCS29.2

UBCS47.1

UBCS25.24

UBCS 24.23

UBCS18.1

UBCS43.7

UBCS32.7

UBCS43. 1

UBCS 43.2

[BCS43-4

UBC S54-1

UBCS47.6

UBC 47.14

UBC 477.7

UBCS 47.12

UBCS24.11

UBCS47.8

UBCS47.9

UBCS47.5

UBCS 47.10

UBC 524.12

UBCS 32.8

UBC 27.7

UBCS 24.4

UBCS24.6

UBCS24.19

UBCS35. 2

UBC 570.2

UBC 38.1

UBCS43.5

UBC 547.1$]$

UBCS17.1

UBC $\$ 35.1$

UBC 526.3

UBC 547.15

UBCS25.12

UBC $\$ 37.1$

UBCS24.17

UBCS25.25

UBCS27-1

UBC 532.1

ICBO UBCS47-16

ICBO UBCS24.7

ICBO UBC 32.5 
ns of Soils (1973)

forced Masonry Other Than Gypsum (1973)

les (1973)

y Materials Test. (1973)

ign (1973)

Sand Aggregates for Gypsum Plaster (1973)

ded Hydraulic Cements (1973)

and Wire for Concrete Construction (1973)

cklime (1973)

3)

uctural Purposes (1973)

73

te and Precast Slabs, and Poured Gypsum Roof Diaphragms /

(1973)

nal, and Flat (Rectangular and Square) Brass Wire (1973) ck (1973)

properties (Temperatures) of Plastics [ sing a Hot Air Ig] studs) for Composite Construction (1973)

Protective Signalling Systems (1973)

Construction and Industrial (1973)

Engineering Purposes (1973)

ete Masonry Units (1973)

73)

73)

d Ceiling Joists, and Rafters (1973)

Formed Steel Structural Members (1973)

t Gauge Cold Formed Stainless Steel Structural Members (/

ood Shakes and Shingles with Asbestos Felt or Gypsum Boal sheets) for Concrete Reinforcement (1973)

flammable Liquids (Gasoline, Kerosene, Acetone, Alcohol, photographic and X-Ray Vitrocellulose Films (1973)

ss Reversal Design of Structural Steel Members (1973)

le (1973)

ring $\mathrm{W}$ all Tile and Std. Meth. of Sampling and Test. Stru/ bearing $\mathbb{W}$ all Tile (1973)

f Plank and Beam Framing (1973)

p Members-Plywood Stressed Skin and Curved Panels, Bea/ ed Timber (1973) 1973

ed Timbers: Douglas and Hem Fir, Southern and Lodgepole /

laminated Wood Products (1973)

d Concrete (1973)

ed Laminated Lumber (1973)

ted Joints, Drift Bolts, and Wood and Lag Screws; Light / shutters (1973)

rs (1973)

acking Board (1973)

formed W'ire Fabric for Concrete Reinforcement (1973)

1. Netal Inserts and Connections in Reinforced Concrete /

- Both Flexural and Axial Loading Combined (1973)

e Given a Preservative Treatment (1973)

classification of Building Materials (1973)

Dwelling Construction Under the

ues (1973)

Dwelling Construction Under the

973)

rs, and Moving Walks (1973)

taining Walls (1973)

ty Requirement Alterations (1973)

graphic and X.Ray Nitrocellulose) (1973)

Dwelling Construction Under the )

(1973)

Dwelling Construction Under the Dwelling Construction Under the Dwelling Construction Under the

h (1973)

s and Spec., Inspection)/

fire Warning Detector (1/

Dwelling Construction Lnder the Dwelling Construction Under the replaces, and Barbecues (1973)

Dwelling Construction Under the

(1973)
Uniform Building Code Std. for Moisture Density Relatio Uniform Building Code Std, for Mortar for Unit and Rein Uniform Building Code Std. for Natural Slate Roof Shing Uniform Building Code Std. for Noncombustible Elementar Uniform Building Code Std. for Open Web Steel Joist Des Iniform Building Code Std. for Perlite, Vermiculite and Iniform Building Code Std. for Plastic Materials (1973) Uniform Building Code Std. for Portland Cement and Blen Iniform Building Code Std. for Prestressed Steel Strand Uniform Building Code Std. for Processed Pulverized Qui Uniform Building Code Std. for Proscenium Curtains (197 Uniform Building Code Std. for Quicklime (Lime) for Str

Uniform Building Code Std, for Ready Mixed Concrete (19

Uniform Building Code Sid. for Reinforced Gypsum Concre Uniform Building Code Std. for Roofing Asphalt (1973) Uniform Building Code Std. for Roofing Tile (1973)

Uniform Building Code Std. for Round Timber Piles (1973

Uniform Building Code Std. for Round, Hexagonal, Octago Uniform Building Code Std. for Sampling and Test. Brick Uniform Building Code Std. for Sand-Lime Building Bri Uniform Building Code Std. for Sawn Wood Shingles (1973

Uniform Building Code Std. for Self and Flash Ignition Iniform Building Code Std. for Shear Connectors (Steel Uniform Building Code Std. for Sheet Metals (1973)

Uniform Building Code Sid. for Smoke Detectors for Fire

Iniform Building Code Std. for Softwood Plywood Lumber

Iniform Building Code Std. for Soils Classification for

Iniform Building Code Std. for Solid Load Bearing Coner

Uniform Building Code Std. for Southern Pine Lumber (19

Uniform Building Code Std. for Spaced Column Design (19

Uniform Building Code Std. for Span Tables for Floor an

Uniform Building Code Std. for Spec and Design of Cold

Uniform Building Code Std. for Spec, and Design of Ligh

Iniform Building Cude Std. for Special Purpose Roofs (W Uniform Building Code Std. for Steel Bar and Rod Mats (

Uniform Building Code Std. for Storage and Handling of Uniform Building Code Std. for Storage and Handling of Uniform Building Code Sid. for Stress Variation or Stre Uniform Building Code Std. for StructuraI Clay Floor Ti

Uniform Building Code Std. for Structural Clay Load Bea Iniform Building Code Std. for StructuraI Clay Nonload Iniform Building Code Std. for Structural Floor and Roo Uniform Building Code Std. for Structural Glued Built U

Iniform Building Code Std. for Structural Glued Laminat

Uniform Building Code Std. for Structural Glued Laminat Iniform Building Code Std. for Structural Rivet Steel ( Uniform Building Code Std. for Test for Glue Joints in Uniform Building Code Std. for Test. Gypsum Plasters an Uniform Building Code Std. for Tests for Structural Glu Uniform Building Code Std. for Timber Connector and Bol Uniform Building Code Std. for Tin Clad Fire Doors and Uniform Building Code Std. for Veneer Application Dampe [niform Building Code Std, for Water Resistant Gypsum B Uniform Building Code Std. for Welded Steel Wire and De

Uniform Building Code Std. for Welding Reinforcing Stee Uniform Building Code Std. for Welding (1973)

Uniform Building Code Std. for Wood Members Subjected T Uniform Building Code Std. for Wood Poles That Are to B Uniform Building Code Std. Test. Meth. for Fire Hazard

Uniform Building Code (1973)

Uniform Building Code (1973)

Uniform Building Code: Agricultural Buildings (1973)

Uniform Building Code: Aluminum (1973)

Uniform Building Code: Chimneys, Fireplaces, and Barbec Uniform Building Code: Concrete (1973)

Uniform Building Code: Definitions and Abbreviations (1

Uniform Building Code: Elevators, Dumbwaiters, Escalato

Uniform Building Code: Excavation and Grading (1973)

Uniform Building Code: Excavations, Foundations, and Re

Uniform Building Code: Existing Buildings: Minimum Safe

Uniform Building Code: Exits and Stairs (1973)

Uniform Building Code: Film Storage and Handling (Photo

Uniform Building Code: Fire Extinguishing Systems (1973

Uniform Building Code: Fire Resistive Std. (1973)

Uniform Building Code: Fire Zone Requirements (1973)

Uniform Building Code: Flooring (1973)

Uniform Building Code: Foundations and Retaining Walls

Uniform Building Code: Framing-General (1973)

Uniform Building Code: Glass and Glazing (1973)

Uniform Building Code: Interior Walls and Ceiling Fini

Uniform Building Code: Legal Requirements (Permit. Plan

Uniform Building Code: Light, Ventilation, Sanitation,

Uniform Building Code: Location on Lot (1973)

Uniform Building Code: Masonry or Concrete Chimneys, Fi

Uniform Building Code: Masonry (1973)

Uniform Building Code: Motion Picture Projection Rooms
$\mathrm{ICBO}$

$1 \mathrm{CBO}$

ICBO

$1 \mathrm{CBO}$
ICBO

$\mathrm{ICBO}$

ICBO

IC BO

IC BO
IC BO

IC $\mathrm{BO}$

IC BO

IC BO

$\mathrm{ICBO}$

ICBO

IC BO

ICBO

ICBO

ICBO

ICBO

IC BO

$1 \mathrm{CBO}$

$\mathrm{ICBO}$

$1 \mathrm{CBO}$

$\mathrm{ICBO}$

$1 \mathrm{CBO}$

ICBO

$\mathrm{ICBO}$

$\mathrm{ICBO}$

ICBO

ICBO

ICBO

ICBO

$\mathrm{ICBO}$

ICBO

$\mathrm{ICBO}$

$\mathrm{ICBO}$

$\mathrm{ICBO}$

$\mathrm{ICBO}$

$\mathrm{ICBO}$

$\mathrm{ICBO}$

$\mathrm{lCBO}$

$1 \mathrm{CBO}$

$\mathrm{ICBO}$

$\mathrm{ICBO}$

$\mathrm{ICBO}$

ICBO

$1 \mathrm{CBO}$

ICBO

$1 \mathrm{CBO}$

$\mathrm{ICBO}$

$\mathrm{ICBO}$

$\mathrm{CBO}$

ICBO

ICBO

$\mathrm{ICBO}$

$\mathrm{CBO}$

$\mathrm{ICBO}$

ICBO

ICBO

ICBO

ICBO

IC BO

ICBO

ICBO

ICBO

ICBO

$\mathrm{ICBO}$

ICBO

$\mathrm{ICBO}$

$1 \mathrm{CBO}$

ICBO

$\mathrm{ICBO}$

$\mathrm{ICBO}$

$\mathrm{ICBO}$

ICBO

$\mathrm{ICBO}$

ICBO

IC BO

ICBO

ICBO

ICBO

$1 \mathrm{CBO}$

$1 \mathrm{CBO}$

ICBO

ICBO

ICBO

ICBO

L'BC $570 \cdot 1$ LCS24.21

UBC $\$ 32.10$

UBC 5 1.1

UBC 527.4

LBC 547.3

UBC $\$ 52.1$

LBC $\$ 26.1$

[BC 526.7

UBC $\$ 24.20$

UBC S6. 1

UBC $\$ 24.18$

UBC $\$ 26.13$

UBC $\$ 24.13$

LBC $\$ 32.2$

UBC $\$ 32.12$

UBC 525.14

IBC 332.13

[BC 24.25

UBC $\$ 24.2$

UBC \$32.11

UBC $\$ 52.3$

UBC $\$ 27.8$

UBC $\$ 32.4$

UBC 543.6

UBC $\$ 2.5$

UBC $529-1$

UBC 24.5

LBC 525-6

UBC 225.15

UBC S25.2]

UBC $\$ 27.9$

UBC $\$ 27.10$

['BCS32.11

¿BCS26.5

[BC\$10.]

[BCS 48.1

UBC $\$ 27.3$

LBC 524.10

LBC 2 1-8

UBC $\$ 24.9$

LBC $\$ 25.22$

UBC 525.18 
Classification (1973)

tower, Spire) (1973)

allboard Lathing (1973)

Dwelling Construction Under the Dwelling Construction Under the t Transmitting Panels, Skylights, Monitors and Sawtooth

73) elters (1973)

1973)

Dwelling Construction $\mathrm{I}$ nder the reets and Projections Over Public Property (Pedestrian P)

Dwelling Construction Under the Dwelling Construction Under the

ed, Hollow Unit, Cavity /

Dwelling Construction Under the stics) (1973)

Dwelling Construction Under the

(Buildings, Reviewing Stands, Bleachers, and Grandstand/

Roof Waterproofing) (19/ Dwelling Construction Under the tile, Metal, Plastic, etc.) for Ornamentation, Protectio/ Dwelling Construction Linder the

ion (1973)

Dwelling Construction Under the Dwelling Construction Under the

I Iron; Cinders; Concrete; Earth; Glass; Granite; Gravel/ s (Lumber, Plywood and Components. Timber, Preservative/ repair, Vacate, Rehabilitate, Demolish) (1973)

Std. for General Purpose or Switches (Radio, TV, and Instrumentation) (1/ St for tional / Std. Performance Requirements for Woven Washable e) (197/ Std. Performance Requirements for Woven Washable Performance Requirements for Woven Drycleanable Tailored Performance Requirements for Woven Drycleanable Tailored utional/Std. Performance Requirements for Tailored Woven

ircraft Hangers (1973)

aint, Varnish, Lacquer, Stain, etc.) (1973)

973)

r Waste Material Handling Plants (1973)

$m(1973)$

n) (1973)

Monoxide, Deuterium, Fluorine, Helium, Hydrogen, Krypto/

973)

us Fruit) (1973)

fogging (1973)

incinerator and Open Burning, Combustible and Flammable / e) (1973)

1973)

(1973)

nt), and Handling of Flammable and Combustible Liquids A/

ing Stands, Grandstands, Bleachers, etc.) (1973)

regulations and Controls, Barrier, Road, Hydrant, Drivew/

rotection from Corrosion for Underground Pipe, Fittings/

for the Protection and Tanks Containing Flammable or Co/

fibres (Cotton, Sisal, Henequen, lxtle, Jutes, Hemp, Tow/ Areas (1973)

1973)

and Acetylene (1973)

on Disc and Spoke Wheels, and Demountabl/ xhaust Gas Emissions Measurement (Passen/

Rec. Pract. for

Rec. Pract. for ion, Control, and Maintenance of Heating, Ventilating, C/ tive Appliances, and Wall Furnaces, Floor Furnaces, and / capacity for Hoe Dipper (Backhoe Bucket) (1972) capacity for Shovel Dipper, Clam Bucket, and Dr/
Uniform Building Code: Occupancy and General Requiremen Uniform Building Code: Patio Covers (1973)

Uniform Building Code: Penthouses and Roof Structures ( Uniform Building Code: Permanent Occupancy (1973)

Uniform Building Code: Permits and Inspections (1973)

Uniform Building Code: Plastering and Installation of W

Uniform Building Code: Plastering (1973)

Uniform Building Code: Plastics (General, Exterior Ligh

Uniform Building Code: Prefabricated Construction (1973

Uniform Building Code: Private Garages and Carports (19

Uniform Building Code: Regulations for Use of Public St

Uniform Building Code: Regulations Governing Fallout Sh

Uniform Building Code: Roof Construction and Covering (

Uniform Building Code: Roof Coverings (1973)

Uniform Building Code: Roof Framing (1973)

Uniform Building Code: Skylights (1973)

Uniform Building Code: Softwood Plywood Paneling (1973)

Uniform Building Code: Solid, Grouted, Reinforced Grout

Uniform Building Code: Sound Transmission Control (Acou

Uniform Building Code: Stages and Platforms (1973)

Uniform Building Code: Stairs, Exits and Occupant Loads

Uniform Building Code: Steel and Iron (1973)

Uniform Building Code: Valley Flashing (Sheet Metal for

Uniform Building Code: Veneer (Brick, Concrete, Stone,

Uniform Building Code: Veneered Walls (1973)

Uniform Building Code: Wall and Ceiling Coverings (1973

Uniform Building Code: Wall Framing and Weather Protect

Uniform Building Code: Wallboard (1973)

Uniform Building Code: Weights of Materials (Brick; Cas

Uniform Building Code: Wood Members and Their Fastening

Uniform Code for the Abatement of Dangerous Buildings (

Uniform Cross Section Spiral Retaining Rings (1972)

Uniform Dimensions and Meth. of Test. for Rotary Select

Uniform Fabrics for Men and Women (Heavy Duty) (Institu

Uniform Fabrics for Men and Women (Institutional Textil

Uniform Fabrics, Heavyweight (Industrial) Suiting (Inst

Uniform Fabrics, Intermediate (Year Round) Suiting (Ins

Uniform Fabrics, Lightweight (Tropical) Suiting (Instit

Liniform Fire Code (1973)

Uniform Fire Code: Airports, Heliports, Helistops and

Uniform Fire Code: Application of Flammable Finishes (P

Uniform Fire Code: Automobile Tire Rebuilding Plants (1

Uniform Fire Code: Automobile Wrecking and Junk Yards $O$

Uniform Fire Code: Bowling Alleys (1973)

Uniform Fire Code: Cellulose Nitrate Motion Picture Fil

Uniform Fire Code: Cellulose Nitrate Plastics (Pyroxyli

Uniform Fire Code: Compressed Cases (1973)

Uniform Fire Code: Cryogenic Fluids (Air, Argon, Carbon

Uniform Fire Code: Explosives and Blasting Agents (1973

Uniform Fire Code: Fireworks (1973)

Uniform Fire Code: Flammable and Combustible Liquids (1

Uniform Fire Code: Fruit Ripening Process (Banana, Citr

Uniform Fire Code: Fumigation and Thermal Insecticidal

Uniform Fire Code: Garage (1973)

Uniform Fire Code: General Precautions for Prevention (

Uniform Fire Code: Hazardous Chemicals (1973)

Uniform Fire Code: High Piled Combustible Stock (Storag

Uniform Fire Code: Industrial Baking and Drying Ovens (

Uniform Fire Code: Liquefied Petroleum Gases (1973)

Uniform Fire Code: Lumber Yards and Woodworking Plants

Uniform Fire Code: Magnesium (1973)

Uniform Fire Code: Maintenance of Exit Ways (1973)

Uniform Fire Code: Manufacture of Organic Coatings (Pai

Uniform Fire Code: Matches (1973)

Uniform Fire Code: Mechanical Refrigeration (1973)

Uniform Fire Code: Motion Picture Projection (1973)

Uniform Fire Code: Oil Burning Equipment (1973)

Uniform Fire Code: Places of Assembly (Theaters, Review

Uniform Fire Code: Prevention of Dust Explosions (1973)

Uniform Fire Code: Protection, Authority, Departmental

Uniform Fire Code: Recommended Guide to Safe Practice P

Uniform Fire Code: Recommended Safeguards and Practices

Uniform Fire Code: Rifle Ranges (1973)

Uniform Fire Code: Smoking (1973)

Uniform Fire Code: Storage and Handling of Combustible

Uniform Fire Code: Suppression and Control of Hazardous

Uniform Fire Code: Tents and Air Supported Structures (

Uniform Fire Code: Welding and Cutting, Calcium Carbide

Uniform Housing Code (Residential Buildings) (1973)

Uniform Laboratory Fatigue Test Procedures for Producti

Uniform Laboratory Instrumentation and Techniques for $\mathrm{E}$

Uniform Mechanical Code (Requirements for the Installat

Uniform Mechanical Code: Requirements for Vented Decora

Uniform Meth. of Determining the SAE Rating and Struck

Uniform Meth. of Determining the SAE Rating and Struck

Uniform Plumbing Code (1973)
ICBO

ICBO

ICBO

ICBO

ICBO

ICBO

ICBO

ICBO

ICBO

ICBO

ICBO

ICBO

ICBO

ICBO

ICBO

ICBO

$1 \mathrm{CBO}$

ICBO

ICBO

ICBO

ICBO

ICBO

ICBO

ICBO

ICBO

ICBO

IC.BO

ICBO

ICBO

ICBO

ICBO

ANSI

EIA

ANSI

ANSI

ANSI

ANSI

ANS

ICBO

ICBO

ICBO

ICBO

ICBO

ICBO

ICBO

ICBO

ICBO

ICBO

ICBO

ICBO

ICBO

ICBO

ICBO

ICBO

ICBO

ICBO

ICBO

$1 \mathrm{CBO}$

ICBO

ICBO

ICBO

ICBO

ICBO

ICBO

ICBO

ICBO

ICBO

ICBO

ICBO

ICBO

ICBO

ICBO

ICBO

ICBO

ICBO

ICBO

ICBO

ICBO

ICBO

SAE

$S A E$

ICBO

ICBO

$S A E$

$S A E$

ICBO

LBC $* 3-5$

UBC $* 3-49$

I BC $* 3.36$

$\mathrm{UBC} * 3.45$

$\mathrm{UBC} * 3-3$

UBC $\$ 8.14$

UBC $* 8-15$

$\mathrm{UBC} * 3-52$

UBC $* 3-50$

$\mathrm{UBC} * 8-4$

LBC *3-44

$\mathrm{UBC} * 3.57$

UBC $* 3.32$

UBC $* 8.18$

UBC $* 8-9$

$\mathrm{UBC} * 3-34$

$\mathrm{UBC} * 8.17$

$\mathrm{UBC}+8-11$

$\mathrm{LBC} * 3-35$

UBC $* 3-39$

$\mathrm{UBC} * 3-33$

$\mathrm{UBC}+3-27$

UBC *8-19

$\mathrm{UBC}+3-30$

UBC *8-12

UBC *3.47

UBC *8. 10

LBC $* 8-16$

$\mathrm{UBC} * 3-23$

UBC *3.25

UBC $* 6$

B27.6

RS315.A

L24.3.6

L24.3.5

L 24.3 .2

L24.3.1

L24.3.3

UFC $* 2$

UFC $* 2$ ART 33

UFC *2ART 14

UFC *2ART2

UFC $* 2$ AR T 3

UFC *2ART4

UFC $* 2$ ART 5

UFC*2ART6

$\mathrm{UFC}+2 \mathrm{ART} 8$

UFC $* 2$ A RT 36

IFC*2ART 11

UFC $* 2 A R T 12$

UFC $* 2 A R T 15$

UFC *2ART 16

UFC*2ART 17 
d Systems and Special Wastes (1973)

ms (1973)

school, Theater, Auditorium, Dormatory, Public or Office/ 3) nd Piping (1973

supply System (1973)

al Systems (1973)

ces (Individuals, Clubs, and Exhibition Charges) (197!)

$f$ the Noise Levels of Powered Industrial/

Rec. Pract. for

osal (1973

$\begin{array}{ll}\text { (1966) } & \text { Minimum Performance Requirements and } \\ \text { er Cars and Station/ Minimum Performance Requirements and }\end{array}$

Rec. for nts for Woven Drycleanable Overcoat Fabrics (lnstitutional Viewing Conditions for the Appraisal of Color Ouality and textile) (1972)/ Std. Meth. of Test for Length and Length Rec. Pract. for Test. Machines for Measuring the Std. Meth. for Contact of Oil Soluble Wood Preservatives by Lising Wood Specimens ce Requirements for Woven Lining Fabrics for Institutional ers and in Oil Circuit Breakers (1973) Std. Spec. for Storage) (1974) Std. Definitions of Terms Relating to Sanitary Stds. for Std. for Classification of Std. for

ture for the Identification (Code) of the Countries of the $r$ Identification Codes and Abbreviations for States of the

f Compressed Gas Cylinders Intended for Medical Use in the ining Adhesion of Protection Sheet to Aperture Adhesive of Image Cards) (1972) Std. for Dimensions for d $/$ Std. Meth. for Measuring Thickness of Buildup Area on Std. for Safety for Electronic Data Processing Safety Sid. for Holdup Alarm

er Generat/

0 Std. for Safety for Tear Gas

73) ANS1 A73.1

2) ANSI C 33.90

s Enginel

Std. Pract for Selection or Safety for Control Performance Requirements for Sealed Beam Headlamp (Light) $n$, Operation, and Maintenance Std. for Record and Reporting us Generators and Reversible Synchronous Generator/ Motor An/ Std. Spec. for Enclosures. Cold Chambers and Serving and D, A/ Std. for Safety for Portable Electric Lighting Info Sheet for Spiral Bevel. Helical and Herringbone Gear ultiple Electrode Semiconductor Devices and Designation of Rec. for Use of English and Metric ruction, Materials, and Installation of Power Transmission ansi Y 10.19 Rec. for Precast Concrete Std. for Central Station Air Handling Std. Operating Limits for Central Station Std. Coil Face Areas for Central Station rveillance Sys/ Std. for Safety for Access Control System
Safety Std. for Room Type Fan Coil
published Scientific and Technical Work/ Rec. Pract. for rveillance Sys/ Std. for Safety for Access Control System
Safety Std. for Room Type Fan Coil
published Scientific and Technical Work/ Rec. Pract. for published Scientific and Technical Work/ Rec. Pract. for
Std. Meth. for Measuring Thickness of Leather Std. Spec. for Hollow Load Bearing Concrete Masonry Std. Meth. of Sampling and Test. Concrete Masonry Glazing Sper. for Sealed Insulating Glass or Plumbing Requirements for Household Food Waste Disposer Std. Spec. for Hollow Nonload Bearing Concrete Masonry Std. Spec. for Solid Load Bearing Concrete Masonry Rec. for Steam Tubine Driven Generator Std. for Dimensional Spec. for Sealed Beam Headlamp Rec. Pract. for Rules for SAE [se of SI (Metric) teral Vibration on High Speed Helical and Herringbone Gear atories, Bathtubs and Combination Tank and Shower Receptor Std. Spec. for Chemical Resistant Masonry Safety Std. Antenna Discharge Safety Std. for Household Burglar Alarm System Sid. for Ammonia Compressors and Spec. for Sealed Insulating Glass Safety Std. for lce Cream Freezers and Soda Fountain
Uniform Plumbing Code: Battery Drainage System (1973) Uniform Plumbing Code: Drainage Systems (1973)

Uniform Plumbing Code: Fixtures (1973)

Uniform Plumbing Code: Fuel Gas Piping (1973)

Uniform Plumbing Code: Indirect Waste Piping, Wet Vente Uniform Plumbing Code: Joints and Connections (1973)

Uniform Plumbing Code: Medium Pressure Gas Piping Syste

Uniform Plumbing Code: Minimum Facilities Required for

Lniform Plumbing Code: Mobile Home (Trailer) Parks (197

Uniform Plumbing Code: Rainwater Systems, Roof Drains a Uniform Plumbing Code: Rec. Rules for Sizing the Water

Uniform Plumbing Code: Sewers and Private Sewage Dispos

Uniform Plumbing Code: Swimming Pools (1973)

Uniform Plumbing Code: Traps and Interceptors (1973)

Uniform Plumbing Code: Vents and Venting (1973)

Uniform Plumbing Code: Water Distribution (1973)

Uniform Plumbing Code: Water Heaters and Vents (1973)

Iniform Pract. for Fee Determination for Division Servi

Uniform Procedure for the Test.. Rating and Reporting $O$

Uniform Sign Code (1973)

Uniform Swimming Pool Code (1973)

Uniform Terminology for Rural Waste Management and Disp

Uniform Test Procedures for Motor Vehicle Brake Linings

Uniform Test Procedures for Retreaded Tires for Passeng

Uniform Textile) (1973)

(niformity in the (1973)

Tity in the Graphic Arts (1972) Std. for

Uniformity of Cotton Fibers by Fibrograph Measurement (

Uniformity of Passenger Car Tires (1972)

Uniformity Test for Photographic Contact Printers (1971

Uniformly Impregnated (1970) /by U'sing Wood Properties

Uniforms (Textile) (1973)

Uninhibited Mineral Insulating Oil for Use in Transform

Uninsulated Metallic Electrical Conductors (1972 A)

Uninsulated Tanks for Milk and lts Products (Mixing and

Unirradiated Plutonium Scrap (1972)

Unitary Air Conditioning Equipment (1974)

United States for Information Interchange (1973)

Linited States (Including the District of Columbia) for

Lnited States (Safety) (1973)

Unitized Microfilm Carrier (Aperture Card) (1973) A SS

Unitized Microfilm Carriers (Aperture, Camera, Copy and

Initized Microfilm Carriers (Aperture, Camera, Copy, an

Units and Systems (1972)

Units and Systems (1973) ANSI Se2.7

Units and Systems (1974)

Units Applied as Standby Power Supplies for Nuclear Pow

Units for Burglary Protection Systems (1969) ANSI C33.7

Units for Construction of Catch Basins and Manholes (19

Units for Indoor Fire Protective Signaling Systems (197

Units for Low and Medium Speed Stationary Diesel and Ga

Units for Motor Vehicles (1973)

Units for Protection Against Holdup and Theft (1973) an

Lnits for Pumped Storage Installations (1974)

Lnits for Tests Above and Below Room Temperature (1966)

Units for Use in Hazardous Locations, Class I, Groups C

Units for Water Cooling Tower Fan Drives (1972)

Units in Multiple Unit Semiconductor Devices (1972)

Units of Measurement (1973)

Units Penetrating the Hull for Boats Equipped with Outd

Units Used as Forms for Cast in Place Concrete (1966)

Inits Used in Electrical Science and Technology (1969)

Units (Air Conditioning and Refrigeration) (1974)

Units (Air Moving and Conditioning) (1966)

Units (Air Moving and Conditioning) (1966)

Units (Electronic/Electrical / Mechanical Security Su

Units (Heating and Cooling) (1973) A NSI Z25.1

Units (Measurement-Metric and British-American) in

Units (1970) ALCA E3

Units (1970) ANSI A79.1

Units (1970) ANSI A84.1

Linits (1971)

Units (1971) ANSI A 197.3, Asse 1008

Units (1971) ANSl A80.1

Units (1971) ANSI A81.1

Units (1972)

Units (1972)

Units (1972)

Units (1972)

Units (1972)

Units (1972) ANSI A 103.

Units (1972) ANSI C 33.39

Units (1972) ANSI Se2.4

Units (1973)

Units (1973)

Units (1973)
$\mathrm{ICBO}$

ICBO

ICBO

$\mathrm{ICBO}$

ICBO

$\mathrm{ICBO}$

ICBO

$\mathrm{ICBO}$

ICBO

ICBO

IC BO

$\mathrm{ICBO}$

ICBO

IC BO

$\mathrm{ICBO}$

ICBO

ICBO

PSA

ITA

ICBO

I APMO

ASAE

VESC

VESC

ANSl

A $\ S 1$

ASTM

$S A E$

ANSl

NWMA

AกTI

ASTM

DFIS A

A. $\backslash 51$

ARI

Truc ANSI

ANSI

CGA

NMA

ANSI

NMA

UL

UL

IEEE

['L

ASTM

[L

DEMA

$S A E$

ANSI

[L

NEMA

ASTM

[L

AGMA

M EI

$A \subseteq A E$

ABYC

$A C I$

IEEE

ARl

AMCA

$A M C A$

UL

UL

IEEE

AST 1

AST.

AST.

SIGMA

AHAM

ASTM

ASTM

FMS

$S A E$

SAE

Sid. Spec. for Measurement of La lic Shower Receptors. Stall Showers Lav

AGMA

ATM

IL

UL

ARI

SIGMA

LL

$[\mathrm{PC} * 1 \cdot \mathrm{H}$

[PC * $]-4$

[PC $* 1-9$

LPC*1.12

$[P C * 1.6$

UPC*1-8

[PC*]-F

UPC $* 1 . \mathrm{C}$

[PC* $]-E$

[PC*1-D

$\mathrm{UPC} * 1-\mathrm{A}$

UPC $* 1-11$

LPC*1.G

LPC*1-7

LPC* 1.5

UPC * 1.10

UPC*1-13

11

6G 1

*1

R292.1

V.3

V.2

L24.3.4

PH2.32

D1 147

J332A

PH3.45

M. 1.70

L24.3.8

D 1040

B35!

3200

\15.10

210

$\times 3.31$

13. 38

MS 10

PH5. 8 
Std. for Safety of Household Fire Warning System Spec, for Prefaced Concrete and Calcium Silicate Masonry Building Code Std. for Meth. of Test for Concrete Masonry building Code Std. for Solid Load Bearing Concrete Masonry uilding Code Std. for Hollow Load Bearing Concrete Masonry ding Code Std. for Hollow Nonload Bearing Concrete Masonry frigeration and Air Conditioning Condensing and Compressor ce Std. Documents Preparation and Maintenance in SI Metric $r$ and Meth. of Sampling and Test. of Unburned Clay Masonry Rec. for Steam Turbine Driven Generator the Design of Air Exchange Coolers for Packaged Compressor Std. for Safety for Proprietary Burglar Alarm System culations for Oil Sucker Rod Pumping Systems (Conventional ric Pract. Guide to the U/se of SI (International System of 1 Photographic Sheet and Roll Films (SI and U.S. Customary Std Spec for $55 \mathrm{Gal}$. Tight Head Std. Spec. for 55 Gal. Full Removable Head Std. Spec, for $16 \mathrm{Gal}$. Tight Head

Std. Spec. for 30 Gal. Full Removable Head Std. Spec, for $55 \mathrm{Gal}$. Tight Head

Std. Spec. for 55 Gal. Full Removable Head Std. Spec. for 55 Gal. Tight Head Std. Spec. for $30 \mathrm{Gal}$. Tight Head

Std. Spec, for 57 Gal. Full Removable Head

Std. Spec. for $30 \mathrm{Gal}$. Full Removable Head

Std. Spec. for 16 Gal. Full Removable Head

Std. Spec. for 16 Gal. Full Removable Head, Lug Cover Rec. for Tractor and Farm Implement Power Takeoff Std. Spec. for 5 Gal. Straight Side Lug Cover Sid. for 5 Gal. Nesting Lug Cover Sid. Spec, for $5 \mathrm{GaL}$ Tight Head

nmfc-Item 260, Dot-

Ufc-Rule 40, Std. Spec. for 5 Gal. Nesting Lug Cover Std. Spec. for 5 Gal. Straight Side Lug Cover

rec. Pract. for LP Gas Fuel Container, Quickly Demountable ain and Self Locking, Alloy and Corrosion Resisting Steel d Self Locking, Alloy Steel and Corrosion Resisting Steel, Safety Std. for

ytical Meth. for Determining Arsenic Content of Corn Syrup eth. for Determining Heavy Metals Content of of Corn Syrup ytical Meth. for Determining Sulfate Content of Corn Syrup lytical Meth. for Determining Copper Content of Corn Syrup alytical Meth. for Determining Baume Content of Corn Syrup rmining Moisture (Oven Filter Paper) Content of Corn Syrup etermining Candy Color and Inversion Content of Corn Syrup g Moisture (Azeotropic Distillation) Content of Corn Syrup eth. for Determining Color Stability Content of Corn Syrup termining Moisture (Oven Filter Aid) Content of Corn Syrup tical Meth. for Determining Chloride Content of Corn Syrup tical Meth. for Determining Chloride Content of Corn Syrup etermining Moisture (Karl Fischer) Content in Corn Starch Including Leaders and Trailers,/ Std. for Dimensions for mination for Internal Insect Infestation in Cereal Grains, of Analysis of Macroscopic Examination of Cereal Grains, nal Insect and Rodent Hair Contamination of Cereal Grains,

king Flotation Test for Internal Insects in Cereal Grains. Std. Spec. for Properties of -Track 200 and $800 \mathrm{cpi}, \mathrm{NRZI}$, and $1600 \mathrm{cpi}, \mathrm{P} / \quad$ Std. for istry) (1962) Meth. of Analysis of Str. Meth. of Test for Total Bromine Number of 1972) Std Meth of Static Immersion Test of Std. Spec. for Steel, Carbon, Strip, Cold Rolled Hard, Std. for Trunnion-Tank Strap, eral Industrial Use (1971) ANSI G57.12 Std. Spec. for nsi C59.77 Std. Meth. of Sampling and Test.

Std. for Gas Fired Room Heaters: Volume II, Std. Meth. of Test for Extrudability of c. for Rubberized Curled Hair for Cushioning Materials for Meth. of Test for Colorfastness of Meth. of Test for Colorfastness of

Std. Meth. of Test for Dynamic Water Resistance of Shoe

Std. Meth. of Test for Dynamic Water Resistance of Shoe Spec. for Titanium Alloy Bolts and Screws, 6Al-4V,

tion-Radiology) (1973) Std for Thyroid Radioiodine Neutron Flux by Analysis of Molybdenum -99 Activity from ances (1970) Ans/ 1969) Ans/

ANSI N11/

n106 Sid. Meth. of Test for Determination of
Std. Meth. of Test for Atom Percent Fission in Std. Meth. of Test for Atom Percent Fission in Std. Meth. of Test for 3)

Std. Spec. for Nuclear Grade, Sinterable ectrometric, and Spectrochemical Analysis of Nuclear Grade Std. Meth of Test for Atom Percent Fission in Std. for Packaging of Std. for Analytical Procedures for Accountability of
Tnits (1973)

Units (1973)

Units (1973)

Units (1973)

Units (1973)

Units (1973)

Units (1973)

Units (1973)

Units (1973)

Units (1974)

Units (1974)

Inits (1974) ANSI Se2.9

Units) (1972)

Units) (1972) ANSI Z210.1

Units) (1973)

Universal Drum (Dot-5B) (1974)

Universal Drum (DOT-17H) (1968)

Universal Drum (Dot-17E) (1974)

Universal Drum (Dot-17H) (1974)

Universal Drum (Dot-17C) (1974)

Universal Drum (Ufc-Rule 40, Nmfc-Item 260) (1974)

Universal Drums (Dot-17E) (1974)

Universal Drums (Dot-17E) (1974)

Iniversal Drums (Ufc-Rule 40, Nmfc-Item 260) (1974)

Universal Drums (Uff-Rule 40, Nmfc-Item 260) (1974)

Iniversal Drums (Uff-Rule 40, Nmfc-Item 260) (1974)

Universal Drums (Ufc-Rule 40, Nmfc-Item 260) (1974)

(Universal Joint) Drive Line Specs. (1972)

Universal Pail (Container) (Dot-37C80) (1974)

Universal Pail (Container) (Dot-37C80) (1974)

Universal Pails (Container) (Dot-17E, Ufc-Rule 40,

Universal Pails (Container) (Dot-37A80, Dot-37Aa60,

Universal Pails (Container) (Dot-37A80, Dot-37A60,

Universal (Horizontal or Vertical Mounting), Liquid Wit

Lled and Drilled, Pl

UNJC-3A Thread, Cap Screw (1972)

UNJF-3A Thread, Cap Screw (1972)

Unlined Fire Hose (1973) ANSI A 152.1

Inmixed and Corn Sugar (1954)

Unmixed and Dextrose (1956)

Unmixed and Finished Corn Sugars (1954)

Unmixed and Finished Sugars (1954)

Unmixed and lts Syrups (1959)

Unmixed and Unbleached Crude Corn Sugar (1956)

Unmixed (1952)

Unmixed (1952)

Inmixed (1954)

Unmixed (1954)

Nd Drilled, Plain an

Std

Std. Analytic An

Std. Anal CR

Std. Ana CR

Std. an $C R$

Std. Analytical Meth. for D CR Std. Analytical Meth. for Determinin CP Std Analytical M CR

Inmixed, Finished Sugar and Other Clarified Starch Hyd

Unmodified) (1961)

Std. Analytical Meth.

Unpopped Popcorn, Dried Peas and and Beans (Cereal Chem

Unpopped Popcorn, Dried Peas and Beans for Rodent And $/ O$

Unpopped Popcorn, Dried Peas and Beans (Cereal Chemistr

Unpopped Popcorn, Dried Peas and Beans (Cereal Chemistr

Unpunched Oiled Paper Perforated Tape (1971)

Inrecorded Magnetic Tape for Information Interchange (9

Unsaponifiable Matter in Macaroni Products (Cereal Chem

Unsaturated Aliphatic Chemicals (1972) ANSI Z159.2

Unsaturation in Polyethylene by Infrared Spectrophotome

Unstressed Materials in Nitrogen Tetroxide $\left(\mathrm{N}_{2} \mathrm{O}_{4}\right)$ (

Untempered Spring Quality (1973)

Unthreaded (1973)

Untreated Carbon Steel Railway Axles for Export and Gen

Untreated Paper Used for Electrical Insulation (1974) a

Unvented Gas Fired Infrared Radiant Heaters (1972)

Unvented (1974)

Unvulcanized Elastomeric Compounds (1973)

Upholstery and Bedding Applications (1973) ANSI J8.8

Upholstery Fabrics to Solvent (Textile) (1973)

Upholstery Fabrics to Water (Textile) (1973)

Upholstery Fabrics (Tacked On) (Institutional Textile

Upper Leather by the Dow Corning Leather Tester (1970

Ilpper Leather by the Maeser Water Penetration Tester (1

Upset Headed, Heat Treated, Roll Threaded (1973)

Uptake Measurements Using a Neck Phantom (Medical Radia

Uranium-238 Fission (1972)

/Td. Meth. of Test for

Uranium and Plutonium Concentrations and Isotopic Abund

Uranium and Plutonium Fuel (Mass Spectrometric Meth.)(

Uranium and Plutonium Fuel (Neodymium-148 Meth.) (1969)

Uranium by Controlled Potential Coulometry (1970) ANSI

Uranium Dioxide Powder for Use in Nuclear Reactors (197

Uranium Dioxide Powders and Pellets (1972) ANSI N103

Uranium Fuel (Radiochemical Meth.) (1969) ANSI N107

Uranium Hexafluoride for Transport (1971)

Uranium Hexafluoride (1972)
UBCS24-5

I'BCS24-4

UBCS24-6

303

10000

UBCS24-15

5-12

$11 \mathrm{~K}$

1076

IIL

E380

PH1.18

$\mathrm{MH} 2.3$

MH2.5

MH2.7

$\mathrm{MH} 2.13$

MH2.4

MH2.2

MH 2.1

MH2.6

MH2.11

$\mathrm{MH} 2.12$

$\mathrm{MH} 2.14$

$\mathrm{MH} 2.8$

R331.2

MH2.16

$\mathrm{MH} 2.17$

$\mathrm{MH} 2.9$

MH2.15

MH2.10

$6 \mathrm{~A} 2$

1352

1351

18

E-64

E-22

E-8

E-44

E-12

E-40

E-20

E-42

E-14

E-15

B-36

PH 1.10

$\mathrm{AACCH}$ 28-21

AACCH $28-10$

AACCH $28-20$

AACCH $28-22$

ANSI $\mathrm{X} 3.29$

ANSI $\mathrm{X} 3.40$

AACCH $\quad 30-40$

ASTM E234

ASTM D3124

ASTM F359

ASTM A680

NSA

ASTM

ASTM

26

A383

D202

ANSI Z21.43B

ANSI Z21.11.

ASTM D2230

ASTM D2128

ANSI L24T7

ANSI L24T6

ANSI L24.1.7

ASTM D2098

ASTM D2099

SAE

ANSI

ASTM

ASTM

ASTM

ASTM

ASTM

ASTM

ASTM

ASTM

ANSI

ANSI 
Std. Meth. for Colorimetric Determination of Std. for Radiation Protection in

rc Technique

Std. Meth. for Spectrochemical Analysis of Std. for Analytical Procedures for Accountability of Fast Neutron Flux for Analysis for Barium 140 Produced by Std. for Sodium Determination by the Meth. of Analysis of

1 Chemistry) (1/

Meth. of Xanthydrol Test for Analysis of meth. of Urease Bromthymol Blue Test Paper for Analysis of (Cereal Chemistry) (1962)

Meth. of Analysis of Residual

in Cloth or Sack Fibers, Whole or Ground Cereal/ Meth. of turated with Gasoline; Methyl Blue and Green; Mineral Oil; Tent. Meth, of Tension Test. of Solid Std. Spec, for Rigid

Std. Meth of Test. Molded Flexible

td. Spec. for Toluenediisocyanate for Use in Production of eous China Plumbing Fixtures (Lavatory, Water Closet Bowl, mis/

Ultraviolet Light Examination for Presence of Rodent (1973) Rec Seler

ansi $\mathrm{PH} 5.17$ Std. for Flowchart Symbols and Their Rec. Pract. for Handling and Proper Std. Spec, for Projector

Test Meth. for Determination of waste Disposal of Phosphorus-32 and 1odine-131 for Medical Std. Spec. for Protective Headgear for Vehicular Sid for Pot Pan, and

d. Spec. for Acrylonitrile Butadiene Styrene (ABS) Plastic cation and Electrical Wire and Cable ( $1 /$ Std. for Plastic Guide for Protective Relaying of d Fork Lift True/ Std. for Tires and Rims for Industrial.
Rec for Electric uniform Building Code Std. for Lumber, Timber, Plywood and Std. for Gas Metal Industrial

ure Vessels (1972) Std. Spec. for Charpy and False Alarm; Use of Equipment, Appliances, Devices and orm Code for the Abatement of Dangerous Buildings (Repair, $\mathrm{n}$ and Wear Characteristics of Dry Solid Film Lubricant $\mathrm{s}$ in oy, Heat Resistant $0.95 \mathrm{Cr}-0.55 \mathrm{Mo}-0.30 \mathrm{~V}(0.40-0.50 \mathrm{C})$

$14.8 \mathrm{Cr}-4.5 \mathrm{Ni}-3.5 \mathrm{Cu}-(\mathrm{Cb}+\mathrm{Ta})$, Consumable Electrode Std. Meth. of Test. Automotive Air Brake and performance Requirements for Pipe Applied Atmospheric Type Std. Meth. of Test for Effective Pumping Speed of Std. Definition of the Term

i C33.36.1 Safety Std. for stant, $12 \mathrm{Cr}-8.5 \mathrm{Ni}-2.0 \mathrm{Cu}-1.1 \mathrm{Ti}$, Vacuum Induction Plus $25 \mathrm{Ni}-0.12 \mathrm{Mo}(0.07-0.13 \mathrm{C}$ ) (SAE 9310), Premium Quality, s (1973)

$-13.5 \mathrm{Co}-4.3 \mathrm{Mo}-3.0 \mathrm{Ti}-1.4 \mathrm{Al})$ Consumable Electrode or $-13.5 \mathrm{Co}-4.3 \mathrm{Mo}-3.0 \mathrm{Ti}-1.4 \mathrm{~A} 1$. Consumable Electrode or

$-13.5 \mathrm{Co}-4.3 \mathrm{Mo}-3.0 \mathrm{Ti}-1.4 \mathrm{Al}$, Consumable Electrode or

Stock, Corrosion Resistant, $12 \mathrm{Cr}-8.5 \mathrm{Ni}-2.0 \mathrm{Cu}-1.1 \mathrm{Ti}$, ) (1973) uirements for X-Radiation Limits for AC High Voltage Power $10-1.20 \mathrm{C}$, Premium Bearing Quality Consumable Electrode matter in Yeast Foods (Cereal Chemistry) (1962)

semolina; Bread; Feedstuffs, Ground Grains and /

Modified

trochemical Analysis of Carbon and Low Alloy Steel Using A

by the Point to Plane Technique Úsing an Optical Emission isks and Wheels (1970) ANS1 G55.15 Std. Spec. for sure Vessels (1974)/ Std. Spec. for Quenched and Tempered ine Rotors and Shafts (1974) ANSI G55.14 Std. Spec. for tors (1971) ANSI G55.13 Std. Spec. for for Measurement of Volatilization Rates of Lubricants in (1973) Meth. of Analysis for Formic, Acetic, Propionic. Butyric, 9) Dwelling Construction Under the Uniform Building Code: (Cereal Chemistry) (1/ Meth. of Analysis for Neutralizing 967)

Meth. of Analysis of Saponification Std. Meth. of Test for Ester

us (Cereal Chemistry)

1962)

Meth. of Analysis for Neutralizing Meth. of Analysis for Neutralizing imeter (1973)

A 149.12 Std. Meth. of Test for Gross Calorific Std. Meth. of Test for Coking

rnal Insulation Rat/ (1971) Rec for Design Std. for Schedule of Dielectric Test Std. for Volt age

Rec. Std. Minimum Acceptance Std. for Measurement of Smell Std. List of gistration Formats (1974) ) Std Veth for Establishing Clear Wood Strength the Assumption of Normality (Employing Individual Observed a Product Use Manual: Grade Selection, Std. Size, Design western Woods Use Book: Lumber Std., Grading Rules, Design
Uranium in Aqueous Solutions (1969) ANSI N116 Uranium Mines (Safety) (1973)

Uranium Oxide $\left(\mathrm{U}_{3} \mathrm{O}_{x}\right)$ by Gallium Oxide Carrier D.C a

Uranium Tetrafluoride (1972)

Uranium 238 Fission (1973)

Uranyl Zinc Acetate Meth. Pulp and Paper) (1973)

Urea and Ammonical Nitrogen (Cereal Chemistry) (1965)

Urea in Cloth Bags and Other Packaging Materials (Cerea

Urea in Cloth or Sack Fibers, Whole or Ground Cereal Gr

Urease Activity in Soybean Meals, Soy Flour, Mill Feeds

rease Bromthymol Blue Test Paper for Analysis of Urea

Urease Tablets; Xanthyrol) (1962)

Urethane and Other Rubbers (1973)

Urethane Foam (1972)

Urethane Foam (1973)

Urethane Foams (1973) A NSI K65.125

Urinal) (1973)

Urine on Bags and Other Packaging Materials (Cereal Che

Ins, Food Dish Warmers, Hot Plates and Griddles, etc.)

Usage Guide for Std. Steel Doors (1972)

Usage in Micrographics (Information Processing) (1973)

Usage of I nhibited Oilfield Acids (1973) API Bul D15

Usage of $16 \mathrm{~mm}$ Motion Picture Film (1973)

Useful Fiber in Bagasse (Pulp and Paper) (1973)

Users (1951)

Users (1971)

Utensil Commercial Spray Type Washing Machines (1970)

Utilities Conduit and Fittings (1972) ANSI K65.57

Utilities Duct for Underground Installation for Communi

Utility-Consumer Interconnections (1973)

Utility and Recreational Type Vehicles, Counter Balance

Utility Generating Stations (1973)

Utility Pole Preservative Treatment by Pressure Process

Utilization Equipment in Large Boilers (1971)

V.Grooved Wheel Std. (1966)

V-Notch Testing Requirements for Steel Plates for Press

Vacant Buildings) (1973)

Mmable Materials; Reporting

Vacate, Rehabilitate, Demolish) (1973)

Vacuum and Other Controlled Atmospheres (1971) A.WSI Z11

Vacuum Arc Remelted (1973)

Vacuum Arc Remelted (1973)

Vacuum Brake Hose (1973) ANSI S2.10

Vacuum Breakers (1970)

Vacuum Chamber Systems (1972)

Vacuum Cleaner (1974)

Vacuum Cleaning Machines and Blower Cleaners (1973) Ans

Vacuum Consumable Electrode Melted Solution Heat Treate

Vacuum Consumable Electrode Remelted (1973)

(n)

acuum Distillation of Solvents from Solvent Base Paint

Vacuum Induction Melted 1825.1900 Deg. F (996.1.1037.8

Vacuum Induction Melted, 1975 Deg. F (1079.4 Deg. C) So

Vacuum Induction Plus Vacuum Consumable Electrode Melte

Vacuum Interrupters Used in Power Switchgear (1972)

Vacuum Melted $(0.95 \mathrm{Cr}-0.20 \mathrm{~V}(0.28-0.33 \mathrm{C})$ ) (SAE 6130

Vacuum Melted (1973) /Stant, 14.5 Cr-4.0Mo

Vacuum Oven Meth. of Analysis of Moisture in Flour and

Vacuum Spectrometer (1971) ANSI Z128.33

/Eth. for Spee

Vacuum Spectrometer (1973) /Ysis of Blast Furnace lron

Vacuum Treated Alloy Steel Forgings for Turbine Rotor D

Vacuum Treated Caroon and Alloy Steel Forgings for Pres

Vacuum Treated Carbon and Alloy Steel Forgings for Turb

Vacuum Treated Electric Steel Forgings for Generator Ro

Vacuum (1971) ANS1 Z11.302

Valeric. Succinic, and Lactic Acids (Cereal Chemistry)

Validation of Results of Process Distillation Analyzers

Valley Flashing (Sheet Metal for Roof Waterproofing) (

Value of Acid Reacting Materials Other Than Phosphates

Value of All Normal Fats and Oils (Cereal Chemistry) (1

Value of Lacquer Solvents and Thinners (1972)

Value of Monocalcium Phosphate: Monohydrate and Anhydro

Value of Sodium Acid Pyrophosphate (Cereal Chemistry) (

Value of Solid Fuel by the Isothermal Jacket Bomb Calor

Value of Tar and Pitch (Modified Conradson) (1973) ANSl

Values for Livestock Fallout Shelters (1973)

Values for Outdoor AC High Voltage Circuit Breaker Exte

Values for Preferred Transient Insulation Levels (TIL)

Values for Steel Doors and Frames (1972)

Values of Transistor Capacitance (1972)

Values to Be Used in Semiconductor Device Specs. and Re

Values to $14.0 \%$ Moisture Basis (Cereal Chemistry) (1962

Values (1973)

Values) (1974)

Std. Assessment of

Values, Framing Rec.. Properties, Weight, Stress of Boa

Values, Section Properties and Design Loads, Timber, Pl
ASTM

ANSI

A.STM

ANSI

ASTM

AACCH 46.50

AACCH 28.86

AACCH 28.87

AACCH 22.90

AACCH 28.87

AACCH 28.9]

ASTM D3196

ASTM D234]

ASTM D2406

ASTM D1786

ANSl A112.

ANSI 221.31

STD

NMA

NACE

ANSI

TAPPl [:M-3

NCRPM R

ANS1 290.1

NSF

St ASTM

NEMA

IEEE

TRA

FMS

ICBO

ANS1

CFTMA

ASTM

ICBO

nif $1 \mathrm{CBO}$

AST.M

$S A E$

$S A E$

ASTMI

ASSE

ASTM

ASTY

CL

$\mathrm{SAE}$

$S A E$

ASTM

$S A E$

$S A E$

$S A E$

$S A E$

10 ANSI

SAE

SAE

$\mathrm{AACCH}$

ASTM

ASTM

ASTM

ASTM

ASTM

ASTM

ASTM

AACCH

ASTM

$\mathrm{AACCH} 02-32$

$\mathrm{AACCH} \quad 58-50$

ASTM D1617

$\mathrm{AACCH} \quad 02.33$

$\mathrm{AACCH} \quad 02-34$

ASTM D3286

ASTM D2416

ASAE R282.1

ANS1 C37.078]

ANS1 C92.1

STDl 112

E1A RS398

E1A RS419

AACCH $82-22$

ASTM D2555

ANS1 N15.15

WWPA *24

WWPA *31 
Rec. for Operation of Grimes AB Dry Pipe Std. Spec, for Steam or Std. for Contro n Turbulent Flow (1974) (1972) Std. for Control Std. Meth. of Measurement of the A-D Dimension of Aerosol

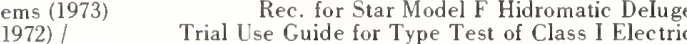
Std. Contro Std. for Contro

Std. Spec. for Steel Wire, Oil Tempered Carbon $r$ Design, Installation, and Operation of Subsurface Safety e Water Supply/ Performance Requirements for Double Check bon Steel Forgings for Piping Components (Flange, Fitting supply Systems (1972) Std for Relief Std. for Steel Pipe Flanges, Flanged d or Rolled Alloy Steel Pipe Flanges, Forged Fittings, and Std. for Tires, Tubes, Rims and Std. for Tire p) Gas (Other Than Safety Relief) (1/ Std. for Safety for gas (1973)

Safety Std. for Dry Pipe, Deluge, and Safety

Std. for Safety for Butterfly Std. for Safety for Std. for Automatic Std. for Safety for Pressure Regulating Spec. for Steel Gate, Plug, Ball, and Check Std for Muliport Safety Std. for Electrically Operated a, B, C, and D, An/ Safety Std. for Electrically Operated rotection Service (1973) ANSI/ Std. for Safety for Alarm ble Operat/ Std. for Small Manually Operated Metallic Gas s (1970) Std. for (Backflow Preventers) and Relief Meth. of Test for Effective Crimping on Outside Crimped Testing Procedures and Maintenance of Steam Safety Std. for Flanged Steel Safety Relie Std. Pract. for Bal Operational Rec. for Globe Dry Pipe Std. Pract. for Butterfly Std. for Backwater

nance Instructions for Chlorine Institute Std. Excess Flow std. for Face-To-Face and End-To-End Dimensions of Ferrous Std. for Steel Butt Welding End Guide Spec. for Specifying: Gate ements for Individual Anti Scald Type Water Shower Control y General Purpose Industrial Pneumatic Directional Control Safety Std, for Marine Through Hull Fittings and Sea Std. for Safety for Constant Level Oi

Gate, Plug, Ball, and Check Valves for Petroleum Pipeline Forged or Rolled 9\% Nickel Alloy Steel Flanges, Fittings, c. for Alloy Steel Bolting Materials for Pressure Vessels, Std. Spec. for Gray Iron Castings for Spec. for Subsurface Safet

ry Use (1973)

and Gask/

Rec. for Operation of Crowder Dry Pipe

skets; Drilling Through, Multiple Completion, and Flowline

C Butyric Valeric Succinic and Lactic Acids (Cereal flour and Semolina (Cereal Chemistry) (1962)

Std. for Requirements for Closed Std. Spec. for Manganese

Std. Meth. for Chemical Analysis of Ferrovanadium and Std. Meth. of Test for Lead and

td. Spec. for High Strength Low Alloy Structural Manganese Std. Spec. for High Strength Low Alloy Columbium and Cold Rolled, High Strength. Low Alloy Columbium and/or rec. for Construction and Performance Std. for Kitchen and Std. Rec. Pract. for Selection of Std. Meth. of Test for Moisture Std. Meth. of Test for Water Sid. Meth. of Test for Water Std. Meth. of Test for Std. Meth. of Test for Std. Meth. of Test for

mh 12.17

eth.) (1973) ANSI Z11.92

1) ANSI Z11.277

) ANSI Z11.44

llation of Blower and Exhaust Systems for Dust, Stock, and

d. for Blower, Fan, and Exhaust System for Dust, Stock and Dry Products (1972)

(Packaging) (1968) ANSI M

1964) ANSI MH 12.10 Std. Meth. of Test for Water Std. Meth. of Test for $W$ ater Pressure Sensitive Tape Wate Std. Meth. of Measuring Water
Pract. for Sampling Atmospheres for Analysis of Gases and ge Signal $\mathbf{D} /$ Trial Use Std. Guide on Solid State Devices: Spec and Test Meth. for Fixed and ositioning Nume/
Valve Accelerator (1973)

Valve Bronze Castings (1974)

Valve Capacity Test Procedure for Compressible Fluids I

Valve Capacity Test Procedure for Incompressible Fluids Valve Dip Tubes (1972)

Valve for Water Flow Control of Fire Extinguishing Syst

Valve Operators for Nuclear Power Generating Stations (

Valve Sizing Equations for Compressible Fluids (1973)

Valve Sizing Equations for Incompressible Fluids (1972)

Valve Spring Quality (1971) ANSI G54.4

Valve Systems for Petroleum Industry Use (1973)

Valve Type Back Pressure Backflow Preventers for Potabl

Valve, etc.) (1973) ANSI G17.3 Std. Spec.

Valves and Automatic Gas Shutoff Devices for Hot Water

Valves and Fittings (1973)

Valves and Parts for High Temperature Service (1972) an

Valves for Aircraft (Airplane, Helicopter) (1974)

Valves for All Vehicles Except Aircraft (1974)

Valves for Anhydrous Ammonia and Liquefied Petroleum (L

Valves for Anhydrous Ammonia and LP (Liquid Petroleum)

Valves for Fire Protection Service (1970)

Valves for Fire Protection Service (1973)

Valves for Fire Protection Service (1974)

Valves for Flammable Liquids and Fuels (1974)

Valves for Gas Appliances (1974)

Valves for LP Gas (1973) ANSI Z217,

Valves for Petroleum Pipeline Valves (1974)

Valves for Public and Residential Swimming Pools (1969)

Valves for the Control of Fluids (1973)

Valves for Use in Hazardous Locations, Class 1, Groups

Valves for Use in Wet Pipe Sprinkler Systems for Fire P

Valves in Gas Distribution Systems Whose Maximum Allowa

Valves in Travel Trailers, Camp Cars and Trailer Coache

Valves of Aerosol Containers (1972)

Valves on Boilers (1972)

Valves Used in the Petroleum Industry (1969) ANSI B 146.

Valves with Flanged or Butt Welding for General Service

Valves (Fire and Safety Equipment) (1973)

Valves (1970)

Valves (1971)

Valves (1972)

Valves (1973)

Valves (1973)

Valves (1973)

Valves (1973)

Valves (1973)-ANSI B93.33

Valves (1973) ANSI Z254.1

Valves (1974) ANSI B127.1

Valves (1974)

Valves, and Parts for Low Temperature Service (1972) an

Valves, Flanges, and Fittings for Low Temperature Servi

Valves, Flanges, and Pipe Fittings (1973)

Valves, Locks, and Landing Nipples for Petroleum Indust

Valves, Model a (1973)

Valves, Ring Joint, Segmented, Production Check Flanges

Valves; Drilling Through Control, Screwed and Flanged W Valves; Rockwood Models C, D (1974)

Van Dame Meth. of Analysis for Formic Acetic, Propion

Van Slyke Meth. of Analysis of Amino Nitrogen in Wheat

Van Type Cargo Containers (1971)

Vanadium Alloy Steel Plates for Pressure Vessels (1972)

Vanadium Alloying Additives (1974)

Vanadium in Gas Turbine Fuels (1972) ANSI Z11.316

Vanadium Steel (1970A) ANSI G41.4

Vanadium Steels of Structural Quality (1972) ANSI G41.6

anadium (1970) ANSI G24.32

Vanity Cabinets (1973)

Vapor Barriers for Thermal Insulations (1973)

Vapor Permeability of Organic Coatings Films (1972)

Vapor Permeability of Packages by Cycle Meth. (Packagin

Vapor Permeability of Packages (Packaging) (1968) ANSI

Vapor Pressure of Liquefied Petroleum Gases (LP-Gas M

Vapor Pressure of Petroleum Products (Micromethod) (197

Vapor Pressure of Petroleum Products (Reid Meth.) (1972

Vapor Removal or Conveying (1973) ANSI Z33.1

Uniform Building Code St

Vapor Removal (1973)

led Packages for

Vapor Transmission of Flexible Heat Sealed Packages for

Vapor Transmission of Shipping Containers (Packaging) (

Vapor Transmission Rate of Sheet Materials (Paper) at H

Vapor Transmission Test Meth. (1970)

Vapor Transmission Through Screw Cap Closure Liners (19

Vapors (1967) ANS1 Z257.2

Varactor Measurements Part Il-Characterization of Lar

Variable Attenuators, DC to $40 \mathrm{GHz}$ (1973)

Variable Block Format for Contouring and Contouring-P

FMS

ASTM

ISA

ISA

FMS

ISA

ISA

ASTM

T. Fo AP

ASSE

ANS

ANSI

ASTM

TRA

TRA

UL

UL

UL

UL

UL

ANS 1

AP]

APl

UL

UL

UL

IAPMO

ASTM

FMS

API

MSS

FMS

MSS

IAPM

Mainte $\mathrm{CHI}$

ANSI

CSI

ASSE

2-88

382

$\$ 39.3$

\$39.1

230

RP14B

1015

$221.22 \mathrm{~A}$

B 16.5

A 182

9

125

132

260

262

1091

721.21

144

27

429
1002

193

B16.33

TSC 8

LPD 3076

526

SP72

2-14

SP67

42

B 16.10

B16.34

15101

1016

TH.21.

UL 112

API $6 \mathrm{D}$

ASTM A522

ASTM A320

ASTM A126

API 14A

FMS 2-13

API $6 \mathrm{~A}$

API $6 \mathrm{~A}$

FMS $2-27$

AACCH 04-23

A ACCH 46-32

ANSI MH5.1.1

ASTM A225

E365

ASTM D2787

ASTM A441

A572

A 607

ANSI Al61.1

ASTM C755

ASTM D1653

ASTM D1251

ASTM D895 
ment (1974)

1972)

972)

$f(1972)$

Interchangeable Perforated Tape Std. Std. Exposure Time Markings for Std. for Punches Std. for Die Buttons

mining $\mathrm{pH}$ Content of Corn Syrup, Starch Hydrolyzates and A $f$ Test for Flash Point of Liquids (Paint. Enamel, Lacquer, Best Meth. for Measurment of Dry Film Thickness of Paint. $\mathrm{g}$, Wrinkling, Loss of Adhesion, or Other Failure of Paint, Std. Definitions of Terms Relating to Paint, td. Meth. for Preparation of Steel Panels for Test. Paint. Rec. for Flammable Liquid Mixing Operations (Paint. iform Fire Code: Application of Flammable Finishes (Paint, Safety Std. for nsulation (1970) ANSI C59.31 Std. Neth of Test. for Thermal Aging Characteristics of Electrical Insulating of Test for Thermal Degradation of Electrical Insulating 9.30 Std. Meth. of Test for Gel Time of Solventles Std. Spec. for Shellac Western Woods Lse Book: Wood Tanks, Std. for Safety for Tests for Fire Resistance of si Bl35./ Safety Std. for Emergency Vault Ventilators and installation (1972) ANSI B $135 . /$ Safety Std. for Emergency Mercantile and Bank Safes and

kdown Voltage of Insulating Oils of Petroleum Origin I'sing ermining Free Fatty Acids Content in Corn Oil and to Other mber (Wijs Meth.) Content in Corn, Grain Sorghum and Other Meth. of Analysis of Cloud Point in All Edible Animal and ey Meth. of Analysis of Melting Point of Normal Animal and eth. of Analysis of Melting Point of All Normal Animal and mining Crude Fiber Content in Feedstuffs (Corn) Containing

er Content in Corn and Other Grains. Foodstuffs Containing Scoured Wool (1972) ANSI L14.1/ Std. Meth. of Test for Spec. for Powdered Hand Cleaner (Soap with Std. Meth. of Test for Insoluble Ash of Std. Meth of Test for Water Soluble Matter of flower, Oleic, and Sunflower Seeds; Ground Nuts (Peanuts); for Collision Deformation (Damage) Classification (Highway Component Std. for Receiver Dehydrators for Std. for Recreational Std. for Recreational

t. Weth., and Test Equipment and R/ afety Std. for Construction. Operation, and Maintenance of Rec. Pract. for Instrumentation for Motor nditions (1972) Std. for Motor 1972) Sid. for Motor ormance Requirements and Uniform Test Procedures for Motor ies for Motor Vehicles (1971) Rec. Pract, for Average Rec. Pract for Motor Std. Meth. of Test for Separation of or Defining Conditions for Evaluating the Compatibility of Std. for Slow Moving Rec. Pract. for $943 \mathrm{~A}$ Rec. Pract. for Passenger Car Rec. Pract. for

rec. Pract. for Service Performance Requirements for Motor itability of Plastic Material for Use in Housings of Motor d Maximum Dimensions for Grown Tires in Service for Use by Rec. for Factory Constructed Housing and Recreational

Pract. for Qualifying a Sound Data Acquisition System for Rec. Pract. for New Motor

s: Chassis Frames-Passenger Car and Light Truck-Ground mestic Marketing Std. Including Guarantee Policy for Motor ng Meth. (Coupling. Hitch/ Minimum Requirements for Motor ackets and Socket for Warning Lamp (Light) and Slow Moving r Tires and Rims for Truck, Bus, Trailer, and Multipurpose rotective Structures (FOPS) of Construction and Industrial rake Fluid Temperature in the Automotive Braking System of Std. for Tire Valves for All

ntegrity Test Procedure for Trucks, Buses, and Combination purpose Passenger Vehicles. Buses, Trucks, and Combination and Dozers/ Std. for Steering Frame Lock for Articulated arm) and Industrial Equipment Warning Lamp and Slow Moving otor Homes): Installation of Plumb/ Std. for Recreational Rec. Pract. for Cornering Lamps (Light) for Automotive Rec. Pract. for Electric Blower Motor Switch for Rec. Pract. for Turning Ability and Off Tracking of

for Flexible Non Pressure Water Fill Hose for Recreational and Sewage Holding Tanks for Mobile Homes and Recreational mensions for Use in Designing Docking Facilities for Motor rized Potable Water Tanks for Mobilehomes and Recreational

Std. for Electric Pressure Pumps for Use in Recreational

Rec. Pract. for Electric Tachometer Spec. for on Road Pract. Meth. for Rating Heat Transmission of Refrigrated use on Trucks, Buses, Trailers, and Multipurpose Passenger td. Meth. of Test for Total Rosin Acids Content of Coating
Variable Block Format, for Numerically Controlled Equip

Variable Speed Shutters [sed in Still Picture Cameras (

Variable, Angle Head Type and Related Quill Bushings (1

Variable, Press Fit, Headless and Head Type, Step Relie

Variety of Other Materials (1952) /Cal Meth. for Deter

Varnish, and Related Products) by Setaflash Closed Test

Varnish, Lacquer and Related Products - Film Thickness

Varnish, Lacquer, and Related Organic Coatings on Metal

Varnish, Lacquer, and Related Products (1973)

Varnish, Lacquer, and Related Products (1973)

Varnish. Lacquer, Printing Ink, Adhesive, etc.) (1974)

Varnish, Lacquer, Stain, etc.) (1973)

Varnished Cloth Wires and Cables (1971) ANSI C33.73

Varnished Cottonfabrics and Tapes Used for Electrical 1

Varnishes Applied Over Film Insulated Magnet Wire (1973

Varnishes by the Helical Coil Meth. (1973)

Varnishes Used for Electrical Insulation (1972) ANSI C5

Varnishes (1972)

Varnishes (1973)

Vats, and Pipe (1973)

Vault and File Storage Room Doors (1972) ANSI A153.1

Vault Ventilating Ports for Wall Installation (1972) an

Vault Ventilators and Vault Ventilating Ports for Wall

Vaults (1971) ANSI Se 2.1

VDE Electrodes (1972) ANS1 C59.130

Vegetable and Marine Oils and Animal Fats (1965)

Vegetable Fats and Oils (1966)

Vegetable Fats (Cereal Chemistry) (1967)

Vegetable Fats (Cereal Chemistry) (1967)

Vegetable Fats (Cereal Chemistry) (1967)

965)

UL

NSA

ANSI

ANSI

ANSI

CR

ASTM

NCCA

NCCA

ASTM

ASTM

FMS

Un ICBO

UL

AST N

ASTM

td. Meth ASTM

ASTM

A STM

ASTM

W

UL

UL

UL

ASTM

Det CR

$\mathrm{CR}$

$\mathrm{AACCH}$

Wil $A A C C H$

Vegetable Material, Corn and Other Grains,

(1)

Vegetable Scrubber) (1972T)

Vegetable Tanned Leather (1970) ALCA Bl

Vegetable Tanned Leather (1970) ALCA B8

Vegetable. Coconut, and Palm Oils) (1974)

Vehicle Accident) (1972)

Vehicle Air Conditioners (1967)

Vehicle Air Conditioning Equipment (1974)

Vehicle Air Conditioning System Rating Definitions, Tes

Vehicle Alarm Systems and Units for Protection Against

Vehicle and Its Component Impact Tests (1971)

Vehicle Brake Fluid for Itse Under Arctic Atmospheric Co

Vehicle Brake Fluid (Used Under Nonarctic Conditions)

Vehicle Brake Linings (1966)

Vehicle Cooking Gas Appliances (1972)

Vehicle Dimensions for Use in Designing Docking Facilit

Vehicle Driver's Eye Range (1972)

Vehicle from Solvent Type Paints (1973

Vehicle Fuel Tanks and Fill Pipes with Fuel Dispensing

Vehicle Identification Emblem (1968) ANSl B114.1, SAE J

Vehicle 1dentification Number Systems (1972)

Vehicle Identification Number Systems (1972)

Vehicle lgnition Switch (1971)

Vehicle Lighting Devices and Components (1972)

Vehicle Lighting Devices Including Reflex Reflectors (1

Vehicle Manufacturers in Designing for Tire Clearances

Vehicle Manufacturing Plants (Fire and Wind Hazards at

Vehicle Noise Sound Level Meters (1972) ANSI S6.1

Vehicle on Grade Parking Performance Requirements (1972

Vehicle Practices (1974)

Vehicle Type (Lead Acid) Storage Batteries (1972)

Vehicle (Recreational Type) Connecting Devices and Tow

Vehicle (SMV) Identification Emblem (1972)

Vehicle (1974)

Vehicles and Equipment (1972)

Vehicles Equipped with Dise Brakes (1972)

Vehicles Except Aircraft (1974)

(Chicles with Any Type of Brake System (1972)

Vehicles (Construction and Industrial Front End Loader

Vehicles (SMV) Identification Emblem (1972) SAE J725

Vehicles (Travel and Camping Trailers, Truck Campers. $M$

Vehicles (1965)

Vehicles (1971)

Vehicles (1971)

Vehicles (1971)

Vehicles (1971)

Vehicles (1971)

Vehicles (1971)

Vehicles (1972)

Vehicles (1972)

Vehicles (1972)

Vehicles (1972)

Vehicles (1973)

C

AST.

ASTM

955

PH3.32

B94.38

B94.43

E48

D3278

TB-II-4

TB-111-1

D16

D609

7-36

133

D295

D3145

D115

D3056

D360

*31-2

680

680

609

D1816

H. 22

56.10

$58-41$

58-40

G-12

D1113

D2875

D2876

$* 1$

J224A

309

250

INACA 400

UL 904

$S A E \quad J 1702 D$

SAE J1703B

V.3

Z21.57

J6994

AE J941C

ASTM D2372

SAE J398A

ASAE $\quad$ S276.2

SAE J272

SAE J273

SAE J259

SAE J256A

SAE J29

TRA $\quad 1-13$

$1-13$
$7-90$

J184

J293

Y 14.32 .1

$* 1.15$

$\mathrm{J} 725 \mathrm{D}$

J397 A

J291

8

$\mathrm{J} 294$

J299

$\begin{array}{ll}\text { SAE: } & \mathrm{J} 276 \\ \text { ASAE } & \text { S } 277.2\end{array}$

NFPA $501 \mathrm{C}$ 
Handling Chlorine in Tank Motor Std. for Lighting Inspection Code for Motor ornering W ithout Driving Torque Application, Using Highway uirements for Sealed Beam Headlamp (Light) Units for Motor passenger Cars, Station Wagons, and Multipurpose Passenger d. for Measurement of Electromagnetic Radiation from Motor uck, Bus, Trailer, Multipurpose Passenger and Recreational h Fuel Dispensing Facilities (Passenger Cars. Multipurpose topping Distance of Passenger Cars, Multipurpose Passenger res and Rims for Industrial, Ltility and Recreational Type al A/ hting, and Starting) for Passenger Automobiles, Commercial
Std. for Lead Acid Type Storage Batteries for Motor Highways (1973/ Std. for Inspection Procedures for Motor Grader, Loader/S Std. for Tires and Rims for Off the Road Std. for Adjustable Face Std. Spec. for Protective Headgear for m Std. for Land-Mobile Communication Antennas: Part II . Battery Materials Spec
Storage Battery Spec ger Automobiles, Commercial Vehic/ Storage Battery Spec.
Std. Rec. Pract. for Measuring Ultrasonic
Std. Meth. of Conducted Controlled Std. Meth. of Conducted Controlled.

Rec. Pract. for Ethics and Human Relations in Vendor.
Std. for Safety for Std. for Safety for Refrigerated (1969) Rec. Pract. for Ethics and Human Relations in Rec. Pract. for Rec. Pract. for Std. Definitions of Terms Relating to ['niform Building Code Std. for

Std. Meth. of Test. Veneer. Plywood, and Other Glued Uniform Building Code Std. for Gypsum Base for ng Code Std. for Gypsum Base for Veneer Plaster and Gypsum c.) for Ornamentation, Protectio/ Lniform Building Code: finitions) (1961) Public Works Spec. for Ceramic h Roofs, Light Diffusers in Ceilings, Partitions, Exterior 1972) ANSI O7.1 Std. Meth. of Test. ors (1973) Std. for Hardwood s) (1966) Dwelling Construction Under the Uniform Building Code: Std for Installation PVC Building Drain Waste and ation for Acrylınitrile Butadiene Styrene Drain, Waste and r Acrylonitrile Butadiene Styrene Plastic Drain, Waste and rile-Butadiene-Styrene (ABS) Plastic Drain, Waste, and c. for Poly(vinyl Chloride) (PVC) Plastic Drain, Waste and Std. for Safety for Gas Vents and Std. for Gas Fired Gravity and Fan Type Direct enters in Water Supply Lines with Intermediate Atmospheric furnaces, and / Uniform Mechanical Code: Requirements for Std. for

Uniform Plumbing Code: Indirect Waste Piping. Wet Std. For Gas Fired Gravity and Fan Type Std. for Gas Fired Room Heaters: Volume I,

Fibrous Class Duct Construction Std. for safety Std. for Limit Controls (Air Conditioning, Heating, Safety Std. for Emergency Vault Ventilators and Vault or the Installation of Air Conditioning, Heating, Exhaust. for the Installation, Control, and Maintenance of Heating, ems (1974)

ternal Combustion Engines (1970)

Guide for Supplementary Rec. Pract. for Dilution

Ovens (1973) Safety Rec. Pract. for Fans for Industrial Exhaust
Rec. Pract. for Exhaust Hood Design for Industrial Rec. Pract. for Design Procedure for Industrial Exhaust Rec. Pract for Test. of Industrial Exhaust pract. for Construction Spec. for Local Industrial Exhaust t. for Make Up and Recirculated Air for Industrial Exhaust s (Air Filters and Dust Collectors) for Industrial Exhaust abbreviations and General Principles of Industrial Exhaust lling Construction Under the Uniform Building Code: Light, lation (1972) ANSI B135./ Safety Std. for Emergency Vault Std. for Safety for Power Roof Std. for Unit Uniform Plumbing Code: Vents and Std. Glossary of Terms Relating to Chimneys, Gas Std for Safety for Cas Uniform Plumbing Code: 974) Approval Std. for Heat and Smoke Roof and Window 19/ Std. for Safety for Flame Arresters (Fire) for L'se on Std. for Chimneys, Fireplaces, and Uniform Plumbing Code: Water Heaters and Joint Std. Meth. for

eters (1972) ANSI Z104. 1 Burning Heat Appliances for Mobile Homes and Recreational Std. for Decorative Gas Appliances for Installation in

Vehicles, Golf Car, and Other Diversified Off the Highw

(Vehicular. Ignition, Lighting, and Starting) for Passen

Vent Pipe and Fittings (1973) ANSI 872 18 and Property Stds. Fo IAPMO

Vented Fireplaces (1973)

Ventilation Systems (1974)

Ventilation Systems (1974)

Ventilation, Sanitation. Fire Warning Detector (1973)

Venting (1973)

Vents Designed to Open Automatically in Case of Fire (1

Vents of Storage Tanks for Petroleum Oil and Gasoline (

Vents (1973)

Venture Agreement (Form) for Design Professionals (1972
Vehicles (1973)

$\mathrm{CHI}$

SAE

Vehicles (1973)

Vehicles (1973)

Vehicles (1973)

Vehicles (1973)

ANSI Al47.

I of Test. Tires for Wet Traction in C ASTM C. Pract. for Service Performance Req SAE

Vehicles (20-1000 Mhz) (1972) ANSI C 112.1 St SAE las and Radial Ply Tires and Rims for TRA

Vehicles, and Ligh

ANSI C112.1

Wagon, TR TRA
IIl Pipes Wit SAE

Vehicles, Buses, Trucks, and Combination Vehicles with

Vehicles, Counter Balanced Fork Lift Truck, Boat, Bagga

Vehicles, Motorboats, Tractors, Automotive and Industri

Vehicles, Trailers, and Semitrailers Operated on Public

Vehicles: Earthmoving, Mining and Logging (Short Haul);

Vehicular Traffic Control Signal Heads (1970)

Vehicular Users (1971)

Vehicular (1972)

Vehicular, Ignition, Lighting and Starting) (1972)

Velocity in Materials (1973)

Velocity Laboratory Corrosion Tests (1972)

Vendee Relationships (Quality Control) (1967)

Vending and Amusement Machines (1974)

Vending Machines (1974)

Vendor-Vendee Relationships (Quality Control) (1967)

Vendor Product Improvement Program for Quality Control

Vendor Rating System for Quality Control (1969)

Veneer and Plywood (1970) ANSI 07.2

Veneer Application Dampers (1973)

Veneer Constructions (1972) ANSI 07.1

Veneer Plaster and Gypsum Veneer Plaster (1973)

Veneer Plaster (1973)

Veneer (Brick, Concrete, Stone, Tile, Metal, Plastic, E

Veneer (Sampling. Test. Finishing. Installation, and De

Veneer, Awnings and Patio Covers, Greenhouses, Canopies

Veneer, Plywood, and Other Glued Veneer Constructions (

Veneered Including Hardboard and Plastic Faced Flush Do

Veneered Walls (1973)

Veneer; New and Existing Frame Construction (Tech. Note

Vent Pipe and Fittings (1971)

Vent Pipe and Fittings (1971)

Vent Pipe and Fittings (1971)

Vent Pipe and Fittings (1973)

Vent Roof Jacks (1973) ANSI A 131.2

Vent Wall Furnaces (1973)

Std. Spe

Vent (1972)

/Erformance Requirements for Backflow Prey

Vented Decorative Appliances, and Wall Furnaces, Floor

Vented Decorative Gas Appliances (1973)

Vented Gas Fired Infrared Radiant Heaters (1971)

Vented Systems and Special Wastes (1973)

Vented Wall Furnaces (1972)

Vented (1974)

Ventilating and Air Conditioning Systems (1972)

Ventilating and Processing Systems) (1972)

Ventiating Ports for Wall Installation (1972) ANSI Bl3

Ventilating, and Their Combination Duct Systems (1973)

Ventilating, Cooling, Refrigeration Systems, Incinerato

Ventilation for Heat Control in Industrial Exhaust Syst

Ventilation in a Ship's Cargo Hold During the Use of in

Ventilation in Industrial Exhaust Systems (1974)

Ventilation Requirements for Direct Fired Powder Curing

Ventilation Systems (1974)

Ventilation Systems (1974)

Ventilation Systems (1974)

Ventilation Systems (1974)

Ventilation Systems (1974)

Ventilation Systems (1974)

Ventilators and Vault Ventilating Ports for Wall Insial

Ventilators (1972) ANSI C 33.89

Ventilators (1973)

Vents and Heat Producing Appliances (1972)

Vents and Vent Roof Jacks (1973) ANSI A131.2

Vents and Venting (1973)

Vents (1972) ANSI A52.I

49

$599 C$

J 32

$307 \mathrm{~A}$

J551A

X.XI

J398 A

J 299

$* 1.48$

$\mathrm{J} 537 \mathrm{H}$

D7.1

3

TR 1

Z90. 1

RS329.1

$* 1.41$

$* 1.48$

E494

M14.72

RPl

751

541

$\mathrm{RPl}$

RP8

RP7

D 1038

[ iBCS30.1

D805

[BCS 47.14

[BCS47.14

L BC $* 3.30$

*28

BIA

ICBO

ASTM

NWMA

ICBO

0805

.5. 1

[ BC *8-12

28

PS17

D266 1

D2665

441

CL

ANSI

Z21.44

1012

[MC $* 7$.

$\mathrm{Z} 21.50$

221.60

Z21.51

LPC * 1.6

$\begin{array}{ll}\text { ANSI } & \text { Z21.49 } \\ \text { ANSI } & \text { Z21.11.1 }\end{array}$

SMACN *6

UL

UL

NFPA

ICBO

353

680

ACGIH $* 1.3$

SNAME 4.14

ACGIH $* 1.2$

ACGIH $* 1.10$

ACGIH *1.6

ACGIH $* 1.9$

Rec. ACGIH *1.8

Rec. Prac ACGIH $* 1.7$

Verification and Calibration of Liquid.In.Glass Thermom

$* 1.11$

$* 1$

$\mathrm{UBC} * 8-3$

680

705

330

UPC *1-5

$97 \mathrm{M}$
ACCIH

ICBO

UL

UL

ARI

ICBO

NFPA

UL

ICBO

FMS

LL

NFPA

ICBO

AIOA 
115.1

c Fluid Power Filter Element (1972) ANS1

ic Fluid Power Filter Element (1972) Ansi/

id Power Filter Element (1972) ANSI B93.23

Std. Meth. of Std. Neth. for Std. Meth. for Std. Meth. of zl 15.2 3) Std. Meth. of Calibration Instruments for
Uniform Building Code Std. for Perlite, nt Hairs in Rye Flour (Cereal Chemistry) (1962)

yde: Gasoline; Herwig's, Pancreatin, Tween $80-60 \%$ Alcohol, oduction) (1973) s of Textile Yarn and Fabric to Crocking (Rubbing) (Rotary ible Generator/Motors for Pumpe/ Operational Characteristics Std. for Numerically Controlled Horizontal and el Container, Quickly Demountable Universal (Horizontal or Std. Meth. of Test for Impact Resilience of Rubber by Rec. Std. Safety Code fo ing Machine-Tracer Controlled Profiling and Contouring. ows for External Applicat/Spec. for Aluminum Combination tent. Spec. for Molds for Forming Concrete Tests Cylinders eel Forgings for Seamless Drums, Heads, and Other Pressure and Tempered Carbon and Alloy Steel Forgings for Pressure onal Board Inspection Code) Manual for Boiler and Pressure ervice (1972) A NS1

e (1973) ANSI G35.1/

Std Spec for Carbon Steel Pressure chromium-Copper-Nickel-Aluminum Alloy Steel Pressure - Nickel Alloy Steel, Quenched and Tempered, for Pressure (1972) ANSI G35.16

1972A) ANSI G.32.1

Tempered (1972A) ANS1 G35.11 raging), 18\% Nickel (1972A) Ansi/ raging), $12 \%$ Nickel (1972A)

[-Cobalt-Molybdenum-Chromi/

$9 \%$ Nickel (1972A) ANSI G35.20 Std. Spec, for Pressure std. Spec. for Pressure Std. Spec. for Pressure Std. Spec for Pressure Std spec for Pressure Std. Spec. for Pressure Std. Spec. for Pressure Std. Spec, for Pressure Std. Spec. for Pressure Std. Spec. for Pressure Std. Spec, for Pressure Std. Spec for Pressure

and Tempered (1972A) ANS1 G35.3

Temperature Service (1972)

972A) ANS1 G35.8

1972A)

n Data on Sight Glasses for Chemical Processing Equipment 2/ Std. Spec. for High Strength Steel Plates for Pressure reated $(1972 \mathrm{~A})$ td. Spec. for Pressure

spec. for Copper Nicket Std. Spec. for Carbon Steel Sheets for Pressure ultrasonic Inspection of Aluminum Alloy Plate for Pressure Rules for Building and Classing Stee

ec. for Manganese Vanadium Alloy Steel Plates for Pressure ral Requirements for Delivery of Steel Plates for Pressure

v-Notch Testing Requirements for Steel Plates for Pressure Std Spec for Nickel Alloy Steel Plates for Pressure Std. Spec. for Molybdenum Alloy Steel Plates for Pressure ec. for Carbon Manganese Silicon Steel Plates for Pressure

Molybdenum and Its Nickel Alloy Steel Plates for Pressure steel Plates, Quenched and Tempered Chromium, for Pressure rmediate Tensile Strength Carbon Steel Plates for Pressure el Plates with Improved Transition Properties for Pressure teel Bars and Shapes for Ise in Boilers and Other Pressure um Alloy Steel Plates, Quenched and Tempered, for Pressure plate, Sheet, and Strip for Fusion Welded Unfired Pressure td. Rec. Pract. for Surveillance Tests for Nuclear Reactor pec. for Copper Silicon Alloy Plate and Sheet for Pressure

c. for Chromium Molybdenum Alloy Steel Plates for Pressure t Beam Ultrasonic Examination of Steel Plates for Pressure e for in Service Annealing of Water Cooled Nuclear Reactor teel Castings, Heavy Walled, Carbon and Alloy for Pressure acuum Treated Carbon and Alloy Steel Forgings for Pressure

Std. Spec. for Alloy Steel Bolting Materials for Pressure

for Marine Special Purpose Water Safety Buoyant Devices Minimum Operational Characteristics Airborne Std. Analytical Meth. for Determining Perforated Industria

its (1972) Code for Shipboard Hul

8) ANSI MH12.9 Std. Spec. for Measurement of Lateral for Low and Medium Speed Stationary Diesel and Gas Engine artificial Headbone for the Calibration of Audiometer Bone Sid. Meth. of Std. Meth. of Test for onversion Tables for Metals (Relationship Between Brinell, netic Tape Recording (1969) Rec. Pract. for and Reproducers for Audio Record One for 2.In. Quadruplex ions of Video, Audio and Tracking Control Records on 2 In. an Audio Level and Multifrequency Test Tape for Quadruplex encies and Deemphasis Characteristics for 2 In. Ouadruplex Pract. for Video Alignment Signal Spec. for a Quadruplet Rec. Pract. for the Label for 2 In. Quadruplex
Verification of Test. Machines (1972) AASHO T67, ANSI Z Verifying the Collapse / Burst Resistance of a Hydrauli Verifying the Flow Fatigue Characteristics of a Hydraul Verifying the Material Compatibility of a Hydraulic Flu Verifying Time Load Indication of Machines (1974) ANSI Vermiculite and Sand Aggregates for Gypsum Plaster $\{197$ Versene Meth. of Analysis for Insect Fragments and Rode Versene, and Sodium Phosphate Solutions; Isopropyl Alco

Vertical and Horizontal Emulsion Treaters (Petroleum Pr

Vertical Crockmeter Meth.) (1972) ANS1 L 14.212

Vertical Guidance Equipment Used in Airborne Volumetric

Vertical Hydraulic Turbine Driven Generators and Revers

Vertical Jig Boring Machines (1974)

Vertical Mounting), Liquid Withdrawal (1973)

Vertical Rebound (1974) ANS1 J2.31

Vertical Shoring (1968)

Vertical (1973)

Vertically Sliding or Horizontally

Vertically (1973T) ANSI A37.95

Vessel Components (1970) ANSI G38.7

Vessel Components (1973) ANSI G35.21

Vessel Inspectors (1973)

ASTM

Vessel Plates for Moderate and Lower Temperature Servic

Vessel Plates (1972) ANS1 G33.3

Vessel Plates (1973) ANS1 G35.22

Nganese-Molybdenum

Vessel Plates, Alloy Steel 5\% Chromium, 0.5\% Molybdenum

Vessel Plates, Alloy Steel, Chromium Manganese Silicon

Vessel Plates, Alloy Steel, High Strength, Quenched and

Vessel Plates, Alloy Steel, Precipitation Hardening (Ma

Vessel Plates, Alloy Steel, Precipitation Hardening (Ma

Vessel Plates, Alloy Steel, Quenched and Tempered Nicke

Vessel Plates, Alloy Steel, Quenched and Tempered 8 and

Vessel Plates, Alloy Steel, 36\% Nickel (1972)

Vessel Plates, Alloy Steel, 9\% Nickel Double Normalized

Vessel Plates, Carbon Manganese, for Moderate and Lower

Vessel Plates, Carbon Steel, High Strength Manganese (1

Vessel Plates, Heat Treated, Carbon Manganese Silicon(

Vessel) (1974)

Vessels for Moderate and Lower Temperature Service (197

Vessels (Ships) to Be Repaired (1972) ANSl Z270.1

Vessels (1969) ANSI H52.1

Vessels (1971) ANSI G33.4

Vessels (1971) ANSI H38.21

Vessels (1972)

Vessels (1972)

Vessels (1972)

Vessels (1972)

Vessels (1972) A NSI C 33.

Vessels (1972) ANSI G34.1

Vessels (1972) ANSI G35.14

Vessels (1972) ANSI G35. 15

Vessels (1972) ANSI G35.18

Vessels (1972) ANSI G35.2

Vessels (1972) ANSI G35.7

Vessels (1972) ANSI G81.20

Vessels (1972 A)

Vessels (1972B) ANSI C81.

Vessels (1973)

Vessels (1973)

Vessels (1973) ANSI G35.4

Vessels (1973) ANSI G60.2

Vessels (1974)

Vessels (1974)

Vessels (1974) ANSI G55.17

Vest. Jacket, Horseshoe and Ring Buoy) (1973) ANSI Z 2

VHF Omnirange (VOR) systems (1972)

Viability (Sprouting) Content in Corn (1959)

Vibrating Screens Stds. (1972)

Vibration Measurements (1969)

Vibration on High Speed Helical and Herringbone Gear Un

Vibration Test for Shipping Containers (Packaging) (196

Vibration (1972)

Vibrators (1972)

Vibratory Cavitation Erosion Test (1972)

Vickers Hardness of Metallic Materials (1972) ANS1 Z115

Vickers. Rockwell and Rockwell Superficial, and Knoop H

Video Alignment Signal Spec. for a Quadruplet Video Mag

Video Magnetic Tape Operating at 15 and $7.5 \mathrm{In} / \mathrm{S}$ (1973

Video Magnetic Tape Quadruplex Recorded at 15 and 7.51

Video Magnetic Tape Recorders Operating at $15 \mathrm{In}$./S (19

Video Magnetic Tape Recording (1967)

Video Magnetic Tape Recording (1969)

Video Magnetic Tape Recordings (1968)
Std. Spec. for

Loss $\operatorname{Pr}$

ASTM

NFLDP

NFLDP

NFLDP

ASTM

ICBO IUBCS

$\mathrm{AACCH} \quad 28.60$

AACCH 28.91

API

$12 \mathrm{~L}$

AATCE

RTCA DO-152

NEMA MG5.2

NSA

ITA

SSl

NSA

AAMA

ASTM

963

$6 \mathrm{~A} 2$

D2632

* 214

1002.8

$\mathrm{C} 470$

A 336

A54l

$* 1$

ASTM

ASTM

ASTM

ASTM

ASTH

ASTM

ASTMI

AST I]

ASTM

AST II

ASTII

ASTMI

AST II

ASTII

FMS

AST.M

NFPA

Std. ASTM

AST M

ASTM

ABS

Std. Sp ASTM

Std. Spec. for Gene ASTM

ASTM

Sid. Sp ASTM

or Manganese ASTM

ASTM

ASTM

ASTM

ASTM

A.STM

ASTM

Std S ASTM

Sid. Spe ASTM e. Ftraigh ASTM

ASTM

AST

ASTM

ASTM

UL

RTCA

CR

IPA

SNAME

AGMA

ASTM

td. Pract. DEMA

std. for ANSI

ASTMI

ASTM

ASTM

SMPTE

ANSI

ANS1

ANSI

Ce Carrier Frequ

SMPTE

$\begin{array}{ll}\text { S.IPTE } & \text { RP10 } \\ \text { SUPTE } & \text { RP26 }\end{array}$

A533 
act. Spec. of Tracking ControI Record for 2 In. Quadruplex Rec. Pract. for Dimensions of Patch Splices in Two Inch - Magnetic Tape Quadruplex Recorded A/ Std. Dimensions of Rec. Pract. for Motorcycle Rea nd Uniformity in the Graphic Arts (1972)

Shrubs; Coniferous and Broadleaf Evergreens; Rose Grades;

institutional and Industrial Build/ Rec. Installation Spec. for No Wax Maintenance of Std. for Maintenance of Spec. for Self Adhering Spec. for

Rec. Installation Spec for pec. for Water Emulsion Floor Polish for Use on Asphalt or Spec. for Cleaners for lise on Asphalt and t Floor Coverings (Asphalt, Cork, Linoleum, Rubber, Vinyl. sulating Tape (1973) ANSI C59.91

Std. Spec. for sulating Tape (/ Std. Spec. for Low Temperature Resistant Std. Meth. of Test for Compatibility of Plasticizers in td. Meth. of Test for Volatile Matter (Including Water) of and Essential Information for the Safe Handling and l'se of 7) Ansi/ Std. Meth. of Test for Quality of Extruded Poly Std. Spec. for Filled Poly tive Covering for Permanent Wall and Ceilings / Spec. for for Woven Man Made Fiber Tent, Awning, and Canopy Fabrics, ven Tent, Awning, and Canopy Fabrics, Painted, Printed, or Test Meth. for Rug Back Floor Staining on esilient Floor Coverings (Asphalt, Cork, Linoleum, Rubber. ctrophotometry (1972) Std. Meth. of Test for ining Visual Color Matched to Master Specimen for Fabrics. Std. Spec. for Zirconium Sponge and Other Forms of and Curing Characteristics of Rubber by the Shearing Disk sity of Gear Oils at Low Temperatures I sing the Brookfield meth. of Test for Viscosity of Asphalt with Cone and Plate and Operating Instructions for Glass Capillary Kinematic hearing Disk Viscometer (1972) An/ Std. Meth. of Test for al Chemistry) (1962) Std. Analytical Meth. for Determining Brabender Properties (I969) Meth. of Analysis of Apparent Std. Test Meth. for Apparent Std. Test Meth. for Tent. Meth. of Test for Std. Meth. of Test for Kinematic Std. Neth. of Test for Absolute Meth. for

ANSI K65.183 ookfield Viscometer (197) 1973) Std. Meth. of Test for Std. Meth. of Test for Apparent Std. Meth. of Test for Apparent Std. Meth. of Test for Apparent Std. Meth. of Test for Apparent Id Spanking Simulator (1/ culation of Dynamic Vis/ Std. Analytical Meth. for Determining lnherent nsparent and Opaque Liquids and the Calculation of Dynamic Std. Meth, of Test for Sag Flow of Highly Rec. Pract. and Stds. for Location and Std. on Subscriber

nyls, Coated Fiberbo/ Std. for Test Meth. for Determining y for Hospital Signaling and Nurse Call Equipment (Audio ) Std. Analytical Meth. for Determining Color made Therefrom (1970) ANSI K/ Rec. Pract. for Classifying mplifier Single Frequency Output for Institutional Audio. L.14.215 Spec. for Natural Muscovite Block Mica and Thins Based on with Sand Abrasive (1970) Test Meth. for Test Meth. eds (Cereal Chemistry) (1962) Meth. of Analysis of Ultraviolet Absorption Meth. of AnaIysis of lsions (Cere/ Ultraviolet Absorption Meth. of Analysis of (Cereal Chemistry) (19/ $s$ (Cereal Che) Meth. of Analysis of Carr-Price Meth. of Analysis of Microbiological Meth. of Analysis of Chemical Meth. of Analysis of Microbiological Meth. of Analysis of traviolet Absorption Meth. of Analysis of Vitamin A in Dry t Bowl, Urinal) (1973) (1969) ANSI C59.73 Std. Meth. of Test. Std. Spec. for Compression Joints for Std. Spec. for Std. Spec. for Std. Spec. for Compression Couplings for Std. Rec. Pract. for Installing for Steel Shipping Container Industry Glossary (Packaging, Sid. Meth. of Test for Percent Air Tent. Meth. of Analysis for Std. Meth. of Test for Sid. Meth. of Test for Test Meth. for Resin and
Video Magnetic Tape Recordings (1970)

Video Magnetic Tape (1964)

Video, Audio and Tracking Control Records on 2 In. Vide

View Mirrors (1971)

Viewing Conditions for the Appraisal of Color Quality a

Vines and Ground Covers; Fruit Tree Grades; Small Fruit

Vinyl Asbestos and Asphalt Tile Flooring (1973)

Vinyl Asbestos and Asphalt Tile Floors for Commercial.

Vinyl Asbestos and Asphalt Tile Floors in Institutional

Vinyl Asbestos Floor Tile (1972)

Vinyl Asbestos Floor Tile (1972)

Vinyl Asbestos Tile as a Wall Covering (1973)

Vinyl Asbestos Tile Floors (1969)

Vinyl Asbestos Tile Floors (1970)

Vinyl Asbestos, Polymeric Poured Seamless, etc.) (1973)

Vinyl Chloride Plastic Pressure Sensitive Electrical in

Vinyl ChIoride Plastic Pressure Sensitive Electrical in

Vinyl Chloride Plastics Under Compression (1974)

Vinyl Chloride Resins (1972)

Vinyl Chloride (1972)

(Vinyl Chloride) Plastic Pipe by Acetone Immersion) (196

(Vinyl Chloride) (PVC) Sewer Pipe (1972)

Vinyl Coated Fabric Coverings for Decorative and Protec

Vinyl Coated or Laminated (Institutional Textile) (1973

Vinyl Coated (Institutional Textiles) (1973)

Vinyl Tile (1973)

Vinyl, Vinyl Asbestos, Polymeric Poured Seamless, etc.)

Vinylidene Unsaturation in Polyethylene by Infrared Spe

Vinyls, Coated Fiberboards, and Other Automotive Trim M

Virgin Metal for Nuclear Application (1973) ANSI N12.1

Viscometer (1972) ANSI 17.7

Viscometer (1972) ANSI Z11.321

Th of Test for Viscosity

Viscometer $\{1973$

Viscometers (1972) ANSI Z11.275

Viscosity and Curing Characteristics of Rubber by the $S$

Viscosity Content in Corn Starch (1968)

Viscosity of Acidulated Flour-Water Suspensions (Cere

Viscosity of Adhesives Having Shear Rate Dependent Flow

Viscosity of Adhesives (R1970)

Viscosity of Asphalt with Cone and Plate Viscometer (19

Viscosity of Asphalts (Bitumens) (1974) AASHO T201

Viscosity of Asphalts (1966)

Viscosity of Coating Clay Slurry (1972)

Viscosity of Epoxy Resins and Related Components (1968).

Viscosity of Gear Oils at Low Temperatures Using the Br

Viscosity of Hot Melt Adhesives and Coating Materials (

Viscosity of Lubricating Greases (1973) ANSI Z11.72

Viscosity of Motor OiIs at Low Temperature Using the Co

Viscosity of Transparent and Opaque Liquids and the Cal

Viscosity (One Point) Content in Corn Starch (1963)

Viscosity (1972) ANSI Z11.107

Viscous Materials (1968) A NSI K65.180

Ematic Viscosity of Tra

Visibility of Navigation Lights (Boats) (1972)

Visual Carrier Level (Cable Television) (I970)

Visual Color Matched to Master Specimen for Fabrics, VI

Visual Communication) (1974)

Visual Comparison) Content of Corn Syrup (1954)

Visual Defects in Glass Reinforced Laminates and Parts

Visual Equipment Used Primarily for Speech (1974)

Visual Quality (1971) ANSI C59.27

Visual Rating Meth. of Carpet (Rug) Soiling (1973) ANSI

Visual Std. for Surfaces of New Steel Airblast Cleaned

Vital Wheat Gluten (Cereal Chemistry) (1962)

Vitamin $\mathrm{A}$ and Carotene in Enriched Cereals and Mixed Fe

Vitamin A in Concentrates (Cereal Chemistry) (1962)

Vitamin A in Dry Vitamin Mixes, Beadlets, Oils, and Emu

Vitamin A in Enriched Flour (Cereal Che mistry) (1967)

Vitamin A in Nonfat Dry Milk and Instantized Dry Milks

Vin and Mineral) Concentrates Used to Enrich Cereal

Vitamin B 12 (Cereal Chemistry) (1962)

Vitamin B6 Complex (Cereal Chemistry) (1962)

Vitamin B6 Complex (Cereal Chemistry) (1962)

Vitamin Mixes, Beadlets, Oils, and Emulsions (Cereal Ch

Vitreous China Plumbing Fixtures (Lavatory, Water Close

Vitrified Ceramic Materials for Electrical Applications

Vitrified Clay Bell and Spigot Pipe (1972A)

Vitrified Clay Liner Plates (1972)

Vitrified Clay Paving Brick (1972) ANSI A37.15

Vitrified Clay Plain End Pipe (1973)

Vitrified Clay Sewer Pipe (1972) ANS1 A 106.2

Vocabulary) (1973)

Voids in a Compacted Bituminous Paving Mixture (1973)

Volatile Acid in Wine (Enology) (1972)

Volatile Content of Aerosol Products (1972)

Volatile Content of Paints (1973)

Volatile Content, and Flow Properties of Treated Paper
Rec. Pr SMPTE RPl6

SMPTE RP5

ANSI C98.6

SAE $\quad J 268$

ANSI PH2.32

ANSI Z60.1

RTI $=8$

RTI

RTI

RTl

RTl

ASTM

ASTM

ASTM

ASTM

ASTM

MCA

ASTM

ASTM

CSI

ANSI

ANSI

AATCC

ASTM

ASTM

SAE

Visco ASTM
Tent. ASTM

id. Spec ASTM

ASTM

CR

ASTM D2556

ASTM D 1084

ASTM D3205

ASTM D2170

ASTM D2171

TAPPI T648SL

ASTM D2393

ASTM D2983

ASTM D3236

ASTM D 1092

ASTM D2602

ASTM D445

CR

B. 61

D 445

ASTM D2730

$\mathrm{ABYC}$

NCTA

SAE

A. 16

$001 \mathrm{~B} .0670$

J361A

UL

CR

ASTM D 2563

10 a ANSI PH7.2

Std. ASTM D351

AATCC 121

NACE TM.01.70

AACCH 38.20

AACCH $86-05$

AACCH 86.01

AACCH $86.01 \mathrm{~A}$

AACCH 86.03

AACCH 86.02

AACCH 86.49

AACCH 86.40

AACCH 86.30

AACCH 86.31

AACCH 86.01A

ANSI All2.19.2

ASTM D116

ASTM C425

ASTM

ASTM

ASTM

Std. ANSI

ASTM

ASE

ASTM

ASTM

TAPPI
C479

C7

C594

12

D3203

*11

D3072

D2369

U. .539 
rgarine, High Acid Coc/

Meth. of Analysis of Moisture and
Meth. of Analysis of Moisture and

Std. Meth. of Test for
Meth. of Analysis of Moisture and

(1973)

ins (1972)

Vacuum Oven Meth. of Analysis of Moisture and

td. Meth. of Test for Trace Quantitites of Total Sulfur in orophenol Solutions (1968)

or Fire Hazard Properties of Flammable Liquids, Gases, and

Glazing Compounds (1973)

Std. Meth. of Test for

i Zll.302

Meth. of Test for Hydrolytic Stability of Elastomeric Safety Std. for All Thermoplastic Type MTW 600

\section{3)}

Std. for Test Procedures for Low

tings. Manufacturing Spec., and Application Guide for High in Enclosures (1973) ANSI C37.13

Std. for Low

power Freque/ Std. Meth. of Test for Dielectric Breakdown for Resistivity of Silicon Epitaxial Layers by the 3 Probe Std. for Low Std. for Low otal/ Std. Requirements for Electrical Control of AC High hen Rated for Out of Phase / Std. Meth. for Test. AC High rred Ratings for Capacitance Current Switching for AC High Switching Impulse Voltage Insulation Strength for AC High d. Requirements for Transient Recovery Voltage for AC High plication Guide for Transient Recovery Voltage for AC High

Std. Requirements for Pressurized Components of AC High requirements for Capacitance Current Switching for AC High cation Guide for Capacitance Current Switching for AC High

. Requirements for External Insulation for Outdoor AC High

tage Ratings and Related Required Capabilities for AC High ghting Equipment (1973) NEMA SH3

Highway Lighting (1973) EEI TDJ-133

and Other Electronic Equipment /

Std. for

als Subjected to Partial Discharg/

Std. for Safety for High Std. Meth. of Test for Symmetrical $\mathrm{Cu} /$ Std. Requirements for Transient Recovery Symmetric/ Std. Application Guide for Transient Recovery

act. Test Meth. and Requirements for Maintenance of Design Sid. Guide for Meth. of Power Factor Measurement for Low Breakers Rated $\mathrm{O} / \mathrm{Std}$. Requirement for Switching Impulse ter Line Voltage Integrally Mounted Thermostats For/ Line ge Integrally Mounted Thermostats for Electric Heater Line te Fractions (1971) ANSI Z1/ Std. Meth. for High Ionizing pulse Condit/ Std. Meth. of Test for Dielectric Breakdown e Electrodes/ Std. Meth. of Test for Dielectric Breakdown

lication Recommendations, and Related Requirements for Low e) Safety Requirements for X-Radiation Limits for AC High c High Voltage/ Schedules of Preferred Transient Recovery

S (TIL) (1971) Std. for Decade Transformer Dividers

rical Insulation (1973) Std. for Std. for Residential Controls for Low receptacles, Locking Grounding Type, Specific Purpose, 277 Dimensions of Plugs and Receptacles: Specific Purpose 277 Dimensions of Plugs and Receptacles: Specific Purpose 277

d. Requirements for Load Tap Changing Transformers 138,000 quirements for Overhead Distribution, Transformers, 67,000 ber Insu/ opylene-Ru/ Std. for Electrical Power Cables Rated 0-35,000 Std. for Electrical Power Cables Rated 2000 Std for Low Voltage Cartridge Fuses, 600 Electrically Operated Photographic Equipment Rated at 300 porative Cooler, and Air Filtering Appliances Rated at 600 tric Heating Appliances (Portable Fixed and Stationary 600 betatron Synchrotron Radiations Up to 100 Million Electron e with Ethylene-Propylene-Rubber Insulation, 2001-5000 std. Dimensions of Plugs and Receptacles, Locking Type 125 nd Receptacles, Midget Locking Type, Specific Purpose, 125 Dimensions of Plugs and Receptacles: Specific Purpose 250 Dimensions of Plugs and Receptacles: Specific Purpose 250 eceptacles, Midget Locking Type, Specific Purpose, 125/250 Dimensions of Plugs and Receptacles: Specific Purpose 250 td. Dimensions of Plugs and Receptacles, Locking Type, 250 Dimensions of Plugs and Receptacles: Specific Purpose 250 Dimensions of Plugs and Receptacles: Specific Purpose 125 Dimensions of Plugs and Receptacles: Specific Purpose 125 Dimensions of Plugs and Receptacles: Specific Purpose 250 Dimensions of Plugs and Receptacles: Specific Purpose 480 Dimensions of Plugs and Receptacles: Specific Purpose 600 ensions of Plugs and Receptacles: Specific Purpose 120/208 ensions of Plugs and Receptacles: Specific Purpose 277/480 ensions of Plugs and Receptacles: Specific Purpose 347/600 ensions of Plugs and Receptacles: Specific Purpose 120/208 ensions of Plugs and Receptacles: Specific Purpose 277/480 ensions of Plugs and Receptacles: Specific Purpose 347/600
Volatile Matter in Fats and Shortenings (Butter, Oleoma Volatile Matter in Soy Flours (Cereal Chemistry) (1962) Volatile Matter in the Analysis Sample of Coal and Coke Volatile Matter in Yeast Foods (Cereal Chemistry) (I962 Volatile Matter (Including Water) of Vinyl Chloride Res Volatile Organic Liquids (Oxy Hydrogen Combustion Meth. Volatile Petroleum Solvent (LPG) for Preparing Pentachl Volatile Solids (1973)

Volatility of Oil and Resin Based, Knife Grade, Channel Volatilization Rates of Lubricants in Vacuum (1971) Ans Volcanizates (1972T)

Volt Machine Tool Wires and Cables (1972)

Voltage AC Power Circuit Breakers Used in Enclosures (1 Voltage Air Switches, Bus Supports, and Switch Accessor Voltage Alternating Current Power Circuit Breakers Used Voltage and Frequency of Office Machines (1973)

Voltage and Strength of Insulating Gases at Commercial Voltage Breakdown Meth. (1972) ANSI Z173.47 Voltage Cartridge Fuses (1972)

Voltage Cartridge Fuses, 600 Volts or Less (1972)

Voltage Circuit Breaker External Insulation Rated on Voltage Circuit Breakers Rated on a Symmetrical and a T Voltage Circuit Breakers Rated on a Symmetrical Basis W Voltage Circuit Breakers Rated on a Symmetrical Basis Voltage Circuit Breakers Rated on a Symmetrical Basis ( Voltage Circuit Breakers Rated on a Symmetrical Current Voltage Circuit Breakers Rated on a Symmetrical Current Voltage Circuit Breakers Rated on a Symmetrical Current Voltage Circuit Breakers Rated on a Symmetrical Current Voltage Circuit Breakers Rated on a Symmetrical Current Voltage Circuit Breakers Rated on a Symmetrical Current Voltage Circuit Breakers (1971) Voltage Classification of Luminaires Used in Roadway Li Voltage Classification of Luminaires Used in Street and Voltage Components for Television Receiving Appliances Voltage Endurance of Solid Electrical Insulating Materi Voltage for AC High Voltage Circuit Breakers Rated on a Voltage for AC High Voltage Circuit Breakers Rated on a Voltage in Snowmobile Electrical Systems (1971)

Voltage Inductive Test Currents (1972) ANSI C 37.26

Voltage Insulation Strength for AC High Voltage Circuit

Voltage Integrally Mounted Thermostats for Electric Hea Voltage Integrally Mounted Thermostats for Electric Hea Voltage Mass Spectrometric Analysis of Gas-Oil Satura Voltage of lnsulating Oils of Petroleum Origin Under Im Voltage of Insulating Oils of Petroleum Origin Using Vd Voltage Power Circuit Breakers and AC Power Circuit Pro

Voltage Power Vacuum Interrupters Used in Power Switchg Voltage Ratings and Related Required Capabilities for a Voltage Type) (1972)

Voltage Values for Preferred Transient Insulation Level

Voltage, Low Current, Dry Arc Resistance of Solid Elect Voltage, Wall Mounted Room Thermostats (1972)

Volts Ac, 15 Amperes, 2 Pole 3 Wire (1973)

Volts Ac, 20 Amperes, 2 Pole, 3 Wire Locking, Grounding

Volts Ac, 30 Amperes, 2 Pole, 3 Wire Locking, Grounding

Volts and Below, 3750 Through 30,000/40,000/50,000 kVA

Volts and Below: $500 \mathrm{kVA}$ and Smaller (1971)

Volts and Having Ozone Resistant Ethylene Propylene Rub

Volts and Less and Having Ozone Resistant Ethylene-Pr

Volts or Less Rating) (1974)

Volts or Less (1972)

Volts or Less (1974)

Volts or Less) (1972) ANSI C33.54

Volts or Less) (1972) C 33.11

Volts (1954)

Volts (1973) IPCEA S68-516

Volts, 15 Amperes, 2 Pole, 1 Wire (1973)

Volts, 15 Amperes, 2 Pole, 2 Wire (1973)

Volts, 15 Amperes, 2 Pole, 3 Wire Locking Groundin

Volts, 15 Amperes. 3 Phase, 3 Pole, 3 Wire Locking Type

Volts, 15 Amperes, 3 Pole, 3 Wire (1973)

Volts, 20 Amperes 3 Phase, 3 Pole, 4 Wire Locking, Grou

Volts, 20 Amperes, 2 Pole, 2 Wire (1973)

Volts, 20 Amperes, 2 Pole, 2 Wire (1973)

Volts, 20 Amperes, 2 Pole, 2 Wire (1973)

Volts, 20 Amperes, 2 Pole, 3 Wire Locking, Grounding Ty

Volts, 20 Amperes, 2 Pole, 3 Wire Locking, Grounding Ty

Volts, 20 Amperes, 2 Pole, 3 Wire Locking, Grounding Ty Volts, 20 Amperes, 2 Pole, 3 Wire Locking, Grounding Ty Volts, 20 Amperes, 3 Phase Wye, 4 Pole, 4 Wire Locking Volts, 20 Amperes, 3 Phase Wye, 4 Pole, 4 Wire Locking Volts, 20 Amperes, 3 Phase Wye, 4 Pole, 4 Wire Locking Volts, 20 Amperes, 3 Phase Wye, 4 Pole, 5 Wire Locking. Volts, 20 Amperes, 3 Phase Wye, 4 Pole, 5 Wire Locking, Volts, 20 Amperes, 3 Phase Wye, 4 Pole, 5 Wire Locking, /Ransient Recover

\begin{tabular}{|c|c|c|}
\hline & $\mathrm{AACCH}$ & 44.30 \\
\hline & $\mathrm{AACCH}$ & $44 \cdot 31$ \\
\hline & ASTM & D3175 \\
\hline & $\mathrm{AACCH}$ & 44.32 \\
\hline & ASTM & D3030 \\
\hline & ASTM & D2747 \\
\hline & ASTM & D2605 \\
\hline d. $F$ & FMS & $\begin{array}{l}7.19 \\
6681\end{array}$ \\
\hline & $\begin{array}{l}\text { ASTM } \\
\text { ASTM }\end{array}$ & $\begin{array}{l}\text { C681 } \\
\text { D2715 }\end{array}$ \\
\hline & ASTMI & D3137 \\
\hline & UL & 1063 \\
\hline & ANSI & C.37.50 \\
\hline & $\begin{array}{l}\text { ANSI } \\
\text { IFEF }\end{array}$ & $\begin{array}{l}\text { C.37.32 } \\
20\end{array}$ \\
\hline & $\begin{array}{l}\text { IEEE } \\
\text { ANSI }\end{array}$ & $\begin{array}{l}20 \\
X 4.11\end{array}$ \\
\hline & ASTM & D2477 \\
\hline Test & ASTM & F108 \\
\hline & NEMA & $\mathrm{FU}-1$ \\
\hline & ANSI & C. 37.0781 \\
\hline & ANSI & C37.11 \\
\hline & IEEE & 417 \\
\hline & ANSI & C.37.0732 \\
\hline & $\begin{array}{l}\text { IEEE } \\
\text { IEEE }\end{array}$ & $\begin{array}{l}3.39 \\
327\end{array}$ \\
\hline & IEEE & 328 \\
\hline & IEEE & 340 \\
\hline & IEEE & 341 \\
\hline & IEEE & 342 \\
\hline & IEEE & 343 \\
\hline Vol & ANSl & C39.0722 \\
\hline & $\begin{array}{l}\text { EET } \\
\text { NEMA }\end{array}$ & $\begin{array}{l}\text { TDJ-133 } \\
\text { SH3 }\end{array}$ \\
\hline & UL & 492.3 \\
\hline & ASTM & D22275 \\
\hline & $\begin{array}{l}\text { IEEE } \\
\text { IEEE }\end{array}$ & $\begin{array}{l}327 \\
328\end{array}$ \\
\hline /C. $\operatorname{Pr}$ & $\mathrm{SAE}$ & $\begin{array}{l}328 \\
\mathrm{~J} 277\end{array}$ \\
\hline & $1 \mathrm{EEE}$ & 330 \\
\hline & IEEE & 339 \\
\hline & NEMA & DC 13 \\
\hline & NEMA & DC 13 \\
\hline & ASTM & D2786 \\
\hline & ASTM & D3300 \\
\hline & ANSl & $\begin{array}{l}01816 \\
\text { C } 37.16\end{array}$ \\
\hline & ANSI & C 37.85 \\
\hline & ANS! & C 39.0722 \\
\hline & ANSl & $\mathrm{C} 100.1$ \\
\hline & ANSI & C92.1 \\
\hline & ASTM & D 495 \\
\hline$s$ and & $\begin{array}{l}\text { NEMA } \\
\text { ANSI }\end{array}$ & DC 3 \\
\hline & $\begin{array}{l}\text { ANSI } \\
\text { ANSI }\end{array}$ & $\begin{array}{l}\text { C73.43 } \\
\text { C73.77 }\end{array}$ \\
\hline & ANSI & C73.78 \\
\hline & ANSI & C 57.12 .30 \\
\hline td. Re & ANSI & C57.1220 \\
\hline & NEMA & W $C 8$ \\
\hline & NEMA & WC8/1 \\
\hline & ANSI & $\begin{array}{l}563 \\
\text { C97.1 }\end{array}$ \\
\hline fety & UL & 122 \\
\hline r, Eva & UL & 507 \\
\hline r Elec & UL & 499 \\
\hline Against & NCRPM & $\mathrm{R} 14$ \\
\hline r Cabl & NEMA & WC8/7 \\
\hline lugs a & $\begin{array}{l}\text { ANSI } \\
\text { ANSI }\end{array}$ & $\begin{array}{l}\text { C } 73.31 \\
\text { C } 73.29\end{array}$ \\
\hline & ANSl & C 73.74 \\
\hline & ANSI & C73.98 \\
\hline and $\mathrm{R}$ & ANSI & C73.30 \\
\hline & ANSl & C73.85 \\
\hline & ANSl & C73.32 \\
\hline $\begin{array}{l}\text { Std. } \\
\text { d. for }\end{array}$ & ANSl & C73.71 \\
\hline & $\begin{array}{l}\text { ANSI } \\
\text { ANSI }\end{array}$ & $\begin{array}{l}\text { C } 73.69 \\
\text { C } 73.72\end{array}$ \\
\hline & ANSI & C 73.75 \\
\hline & ANSI & C 73.79 \\
\hline & ANSI & C 73.81 \\
\hline & ANSI & C73.104 \\
\hline & ANSI & C73.106 \\
\hline & ANSI & C73.108 \\
\hline & ANSI & C73.90 \\
\hline & $\begin{array}{l}\text { ANSI } \\
\text { ANSI }\end{array}$ & $\begin{array}{l}\text { C73.92 } \\
\text { C } 73.94\end{array}$ \\
\hline & & \\
\hline
\end{tabular}

$\mathrm{AACCH} 44.30$ $\mathrm{AACCH} 44.32$ ASTM D 3030 FMS

C681

$\begin{array}{ll}\text { ASTM } & \text { D2715 } \\ \text { ASTM } & \text { D } 3137\end{array}$

ANSI

IEE

AYSI

ANSI

IEE

NEMA

IEEE

IEE

ASTM

ANS

ASTM

ANSI

ANSI

NEMA

UL

Std. for Safety for UL /Er, Humidifier, Eva I ec. for Protection Against

ANSI 
Dimensions of Plugs and Receptacles: Specific Purpose 480 Dimensions of Plugs and Receptacles: Specific Purpose 250 Dimensions of Plugs and Receptacles: Specific Purpose 480 ensions of Plugs and Receptacles: Specific Purpose 125/250 ensions of Plugs and Receptacles: Specific Purpose 125/250 Dimensions of Plugs and Receptacles: Specific Purpose 125 Dimensions of Plugs and Receptacles: Specific Purpose 125 Dimensions of Plugs and Receptacles: Specific Purpose 250 Dimensions of Plugs and Receptacles: Specific Purpose 480 Dimensions of Plugs and Receptacles: Specific Purpose 600 ensions of Plugs and Receptacles: Specific Purpose 120/208 ensions of Plugs and Receptacles\& Specific Purpose 277/480 ensions of Plugs and Receptacles: Specific Purpose 347/600 ensions of Plugs and Receptacles: Specific Purpose 120/208 ensions of Plugs and Receptacles: Specific Purpose 277/480 ensions of Plugs and Receptacles: Specific Purpose 3+7/600 Dimensions of Plugs and Receptacles: Specific Purpose 250 Dimensions of Plugs and Receptacles: Specific Purpose 480 Dimensions of Plugs and Receptacles: Specific Purpose 600 Dimensions of Plugs and Receptacles: Specific Purpose 250 Dimensions of Plugs and Receptacles: Specific Purpose 480 Dimensions of Plugs and Receptacles: Specific Purpose 600 ensions of Plugs and Receptacles: Specific Purpose 125/250 ensions of Plugs and Receptacles: Specific Purpose 125/250 ote and Coal Tar (R1970) 1973) ANSI Z106.1

Std Meth for Caleulation of Sid. for

Std. for Gas Fired Room Heaters: Std. for Gas Clothes Dryers. Sid. for Gas Fired Room Heaters: Std. for Gas Water Heaters Std. Meth. of Test for

utomatic Storage Type (1974) s (1973) istry) (1962)

r) $(1973)$

Meth. for Determination of Specific

Test Meth. for Density or Std. Meth. for Microscopical Determination of Std. Meth. of Test for Std. Meth. of Test for

nt Density of Refractory Metals and Compounds by the Scott Tent. Spec. for Concrete Made by al Chemistry) (1962)

on: Schoorl's Reduction Table (Cereal Chemistry) (1962)

ad (Cereal Chemistry) (1962)

oughs (Cereal Chemistry) (1962)

Feedstuffs (Cereal Chemistry) (1962)

aracteristics Vertical Guidance Equipment Used in Airborne

2)

Editorial Format for

Test Meth. for Shive Content of Mechanical Pulp (? uency Range of $108 /$ Minimum Performance Std. for Airborne inimum Operational Characteristics Airborne VHF Omnirange Std. Rec. Pract for Preparation of Pieces from Rubber

td. Meth. of Test for Mechanical Properties of Elastomeric ansi C59.78 Std. Meth. of Test nsi J4. 1

Std. Meth. of Test for Accelerated Aging of Std. Meth. of Test for Accelerated Aging of Std. Meth. of Test for Adhesion of Std. Meth. of Test for Adhesion of Std. Meth. of Test for Adhesion of Std Meth of Test for Tear Resistance of and Procedures for Mixing Rubber Compounds for Preparing Tent. Meth. for Measurement of Oxide Thickness on Silicon he Corresponding Composition of Gallium Arsenide Phosphide $r$ Determining Specific Weight, Durability and M/ Std. for st Proc. for Determining the Load Carrying Ability of Farm ons and Terms Used in the Selection of Tires (Car. Station

Dimensions for Compatible Operation of Forage Harvesters. for New Tires for Passenger Cars and Station cedures for Retreaded Tires for Passenger Cars and Station for Hitch and Box Dimensions for Agricultural (Farm) Grain and Radial Ply Tires and Rims for Passenger Cars, Station Brick in Landscape Architecture Terraces and

lding Code: Elevators, Dumbwaiters, Escalators, and Moving ct. for the Inspection of Elevators, Escalators and Moving Uniform Building Code:

rings for Decorative and Protective Covering for Permanent Spec, for Metal Oxide Waterproofing for Below Grade Iamation, Curtain and $\mathrm{B} /$

A 176.2

, Land, and General Drainage (1/ Std Spec. for Homogeneous Solid Sid. Spec. for Laminated Std. Spec. for Laminated Sid. Spec. for Laminated road work Drainage (1973)

Fire Resistance Requirements Relating to Brick Bearing

Rec. Installation Spec. for Vinyl Asbestos Tile as A

c. for Deep. Cast in Place Concrete (Storage) Bin and Silo
Volts, 20 Amperes, 3 Phase, 3 Pole, 3 Wire Locking Type Volts, 20 Amperes, 3 Phase, 3 Pole, 3 Wire Locking Type Volts, 20 Amperes, 3 Phase, 3 Pole, 4 Wire Locking, Gro Volts, 20 Amperes, 3 Pole, 3 Wire Locking Type (1973) Volts, 20 Amperes, 3 Pole, 4 Wire Locking, Grounding Ty Volts, 30 Amperes, 2 Pole, 2 Wire (1973)

Volts, 30 Amperes, 2 Pole, 3 Wire Locking, Grounding Ty Volts, 30 Amperes, 2 Pole, 3 Wire Locking, Grounding Ty Volts, 30 Amperes, 2 Pole, 3 Wire Locking, Grounding Ty Volts, 30 Amperes, 2 Pole, 3 Wire Locking, Grounding Ty Volts, 30 Amperes, 3 Phase Wye, 4 Pole, 4 Wire Locking Volts, 30 Amperes, 3 Phase Wye, 4 Pole, 4 Wire Locking Volts, 30 Amperes, 3 Phase Wye, 4 Pole, 4 Wire Locking Volts, 30 Amperes, 3 Phase Wye, 4 Pole, 5 Wire Locking, Volts, 30 Amperes, 3 Phase Wye, 4 Pole, 5 Wire Locking. Volts, 30 Amperes, 3 Phase Wye, 4 Pole, 5 Wire Locking, Volts, 30 Amperes, 3 Phase, 3 Pole, $3 \mathrm{~W}$ ire Locking Type Volts, 30 Amperes, 3 Phase, 3 Pole, 3 W ire Locking Type Volts, 30 Amperes, 3 Phase, 3 Pole, 3 Wire Locking Type Volts, 30 Amperes, 3 Phase, 3 Pole, 4 Wire Locking, Gro Volts, 30 Amperes, 3 Phase, 3 Pole, 4 Wire Locking, Gro Volts, 30 Amperes, 3 Phase, 3 Pole, 4 Wire Locking, Gro Volts, 30 Amperes, 3 Pole, 3 Wire, Locking Type (1973) Volts, 30 Amperes, 3 Pole, 4 Wire Locking, Grounding Ty Volume and Specific Gravity Correction Tables for Creos Volume and Weight of Industrial Aromatic Hydrocarbons ( Volume I, Type I, Gas Clothes Dryers (1972)

Volume I, Vented (1974)

Volume II, Type 2 (1974)

Volume II Unvented (1974)

Volume III, Circulating Tank, Instantaneous and Large a Volume Nonvolatile Matter in Clear or Pigmented Coating Volume of Creams, Cake Batters, and lcings (Cereal Chem Volume of Small Specimen ( $\mathrm{Hg}$ Pycnometer) (Pulp and Pape

Volume Percent of Physical Components of Coal (1972)

Volume Resistivity of Conductive Adhesives (1972)

Volume Resistivity of Glass (1972)

Volume Shrinkage of Latex Sealing Compounds (1972)

Volumeter (1970) ANSI H9.15 / Meth, of Te
Volumetric Batching and Continuous Mixing (1973)

Volumetric Bromine Meth. of Analysis of Pentosans (Cere

Volumetric Copper Reduction Meth. for Sugar Determinati

Volumetric Mcth. of Analysis of Chlorides in Ash of Bre

Volumetric Meth. of Analysis of Diastatic Activity of D

Volumetric Meth. of Analysis of Phosphorus in Feeds and

Volumetric Navigation Systems (1972)

M Operational C

Voluntary Product Stds. (1973)

Voluntary Safety Std. for High Chairs (Children's) (197

Von Alfthan Shive Analyzer) (1973)

VOR Receiving Equipment Operating Within the Radio Freq (VOR) Systems (1972)

Vulcanizates Other Than Std. Test Sheets (1973)

Vulcanizates Resulting from Immersion in Liquids (1972)

Vulcanizates Under Compressive or Shear Strains by the

Vulcanized Fiber Used for Electrical Insulation (1971)

Vulcanized Rubber by the Oven Meth. (1967) ANSI J5.1

Vulcanized Rubber by the Oxygen Pressure Meth. (1973) a

Vulcanized Rubber to Metal (1973)

Vulcanized Rubber to Steel Cord (1973)

Vulcanized Rubber (Friction Test) (1970) ANSI J3.1

Vulcanized Rubber (1973)

Vulcanized Test Sheets (1973)

D. Materials, Equipment

Wafers and $\mathrm{N}$

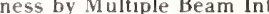

Wafers. Pellets and Crumbles-Definitions and Meth. Fo

Wagon Running Gear (1973)

Wagon, Truck, Bus, Trailer, Multipurpose Passenger and

Wagons and Blowers (1972)

Wagons (1965)

Wagons $(1966)$

Wagons (1972)

Wagons, and Multipurpose Passenger Vehicles (1974)

Walks (Tech. Notes) (1966)

Walks (1973)

Walks (inspectors Manual) (1973)

Wall and Ceiling Coverings (1973)

Wall and Ceilings (1969)

Wall and Floor Surfaces (1971)

Wall and Perforated Pipe for General Drainage (Land Rec

Wall Bituminized Fiber Drain and Sewer Pipe (1973) ANSI

Wall Bituminized Fiber Perforated Pipe for Agricultura

Wall Bituminized Fiber Perforated Pipe for Airport and

Wall Buildings (Tech. Notes) (1969)

Wall Coilable Polyethylene Electrical Plastic Duct (197

Wall Covering (1973)

Wall Design and Construction (1965)
ANSI

ANSI

ANSI

ANSI

ANSI

Std. ANSI

ANSI

ANSI

ANSI

ANSI

ANSI

ANSI

ANSI

ANSI

ANSI

ANSI

ANSI

ANSI

ANSI

ANSI

ASTM

ASTM

ANSl

ANSI

ANSI

ANS

ASTM

$\mathrm{AACCH}$

TAPP

ASTM

ASTM

ASTM

ASTM

ASTM

ASTM

ACCH 52.10

AACCH 80.69

AACCH 40.31

AACCH 22.14

$\mathrm{AACCH}$

RTCA

USC

JPMA

TAPPI

RTCA

RTCA

ASTM

ASTM

ASTM

ASTM

ASTM

ASTM

ASTM

ASTM

ASTM

ASTM

ASTM

ASAE

Std. Te ASAE TRA

Std. ASAE

VESC

Rec. ASAE

las TRA

BIA

SId Bui ICBO

ICBO

ICB

CSI

ASTM

ASTM

ASTM

ASTM

BIA

NEMA

RTI

Re ACI 
Contemporary Bearing Wall Example of Shear Contemporary Bearing Contemporary Bearing Contemporary Bearing

Dwelling Construction Under the Uniform Building Code: Std. for Gas Fired Gravity and Fan Type Vented or Gas Fired Gravity and Fan Type Sealed Combustion System Std. for Gas Fired Gravity and Fan Type Direct Vent

1 Code: Requirements for Vented Decorative Appliances, and mergency Vault Ventilators and Vault Ventilating Ports for Std. for Brazed Double

Std. for Residential Controls for Low Voltage Safety Requirements for Floor and

Std. Meth. of Test for Efflorescence of Interior Quality Stds. for Flush

Quality Stds, for High Pressure Laminate Flush Type Quality Stds. for Stile and Rail

Design Guide for Wood Composition Board disposal Fields (1973) ANSI A 17/ Std. Spec. for Laminated 74)

Std. for Rigid Polyvinyl Chloride) (PVC) Plastic Std. Test. Procedure for Measuring niform Building Code Std. for Structural Clay Load Bearing Structural Clay orm Building Code Std. for Structural Clay Nonload Bearing Contemporary Bearing Contemporary Bearing

Engineered Design of the Contemporary Bearing (Acoustics) in Wood Building Construction (Floor, Ceiling, e: Solid, Grouted, Reinforced Grouted, Hollow Unit, Cavity d. for Airborne Sound Insulation Field Test of Partitions of Steel Framing Members to Receive Screw Attached Gypsum the Uniform Building Code: Plastering and Installation of Uniform Building Code Std. for Gypsum Std. Spec. for Adhesives for Fastening Gypsum form Building Code Std. for Adhesives for Fastening Gypsum Uniform Building Code Std. for Gypsum

Dwelling Construction Under the Uniform Building Code: Std. Spec. for Gypsum Std. Spec. for Heavy

rbines (1974)

6.19 Std. Meth. for Thin Std. Reference Radiographs for Heavy Std. Spec, for Steel Castings, Heavy Uniform Building Code: Interior Construction Details, 6 In. Brick and Tile 4 In. Reinforced Brick Masonry Curtain and Panel Heat Transmission Coefficients of Brick and Tile Dampproofing and Waterproofing Masonry Ceramic Glazed Brick Facing for Exterior Structural Clay Insulated Cavity Reinforced Brick Masonry Retaining Brick and Tile Cavity

Moisture Control in Brick and Tile Brick and Tile Metal Tied constructed Masonry) Insulated Cavity 6 In. Brick and Tile Brick in Landscape Architecture Garden Calculation of Heat Gain Through Opaque Improved Std. Designs for Pressure Treated Timber Crib Recommendation for Installation of Ceramic Tile Bathtub Recommendation for Installation of Interior Ceramic Tile Recommendation for Installation of Exterior Ceramic Tile orm Building Code: Excavations, Foundations, and Retaining ing Construction Under the Uniform Building Code: Veneered under the Uniform Building Code: Foundations and Retaining rn Woods Use Book: Sound Control (Acoustics) in Buildings, Moisture Control in Brick and Tile

nd Transmission Class (Stc) of Building Partitions Such as Recommendation for Renovation of Interior Ceramic Tile Structural Design of Serpentine

si B144.3

Std. for Meth. of Test. for Rating Nonresidential

or Gas Counter Appliances (Coffee Pots and Urns, Food Dish niform Building Code: Light, Ventilation, Sanitation, Fire nd Socket for Agricultural (Farm) and Industrial Equipment tification Emb/ l (Farm) Equipment (1972) Std for Fl Std. for Flashing and Steady Burn Barricade Std. for Safety of Household Fire Std. for Streamer Assembly, Std. Meth. of Test. Std. Meth. for Measuring Std. Meth. of Test for

a Plane Surface (1972) utional and Industrial Use Garments (Clothing), Commercial nts (Clothing), $100 \%$ Man Made or Blend Fabrics, Commercial 973) ANSI L14.17/ Test Meth. for Appearance of Creases in ndering (1973) Ans (Institutional /
Test Meth. for Appearance of Seams in Std. Performance Requirements for Woven
Wall Design (Tech. Notes) (1964)

Wall Detailing (Tech. Notes) (1968)

Wall Detailing (Tech. Notes) (1968)

Wall Example of Shear Wall Design (Tech. Notes) (1964)

Wall Framing and Weather Protection (1973)

Wall Furnaces (1972)

Wall Furnaces (1972

Wall Furnaces (1973)

Wall Furnaces, Floor Furnaces, and Unit and Room Heater

Wall Installation (1972) A NSI B 135.1

Wall Low Carbon Steel Tubing (1972)

Wall Mounted Room Thermostats (1972)

Wall Openings, Railings, and Toeboards (1973)

Wall Paints (1973)

Wall Paneling (Architectural Woodwork) (1973)

Wall Paneling (Architectural Woodwork) (1973)

Wall Paneling (1973)

Wall Panels (1973)

Wall Perforated Bituminized Fiber Pipe for Septic Tank

Wall Siding (1972)

Wall Thicknesses of Composite Cans, Tubes and Cores (19

Wall Tile and Std. Meth. of Sampling and Test. Structur

Wall Tile (Tech. Notes) (1966)

Wall Tile (1973)

Wall Types and Properties (Tech. Notes) (1969)

Wall (For High Buildings) (Tech. Notes) (1970)

W all (Tech. Notes) (1966)

Wall) (1972)

Wall, and Stone Masonry (1973)

Control WWPA

(Wall, Ceiling, Floor, and Floor-Ceiling Assemblies)

Afety Std, for E UL

SAE

NEMA

ANSl

ASTM

AWI

$A W$

AWI

HPMA

ASTM

USC

CCTI

ICBO

BIA

Unif ICBO

BIA

BIA

WWPA

Wallboard and Backing Board (1971)

Wallboard Lathing (1973)

/ for Installation

$\mathrm{ICBO}$

Wallboard Tape and Joint Compound (1973)

Wallboard to Wood Framing (1973)

Wallboard to Wood Framing (1973)

Wallboard (1973)

Wallboard (1973)

Wallboard (1973) ANSI A69.1

Walled Carbon and Low Alloy Steel Castings for Steam tu

Walled Tube Sampling of Soils (1974)

Walled (4 1/2 to 12 In.) Steel Castings (1972) ANSI Z16

Walled, Carbon and Alloy for Pressure Vessels (1974)

Walls and Ceiling Finish (1973)

Walls for 1 Story Buildings (Tech. Notes) (1966)

Walls (In Resisting Lateral Forces) (Tech. Notes) (1968

Walls (Tech. Notes) (1961)

Walls (Tech. Notes) (1961)

Walls (Tech. Notes) (1962)

Walls (Tech. Notes) (1964)

Walls (Tech. Notes) (1965)

Walls (Tech. Notes) (1965)

Walls (Tech. Notes) (1965)

Walls (Tech. Notes) (1966)

Walls (Tech. Notes) (1966

Walls (Tech. Notes) (1968)

Walls (Tech. Notes) (1969)

Walls (1969)

Walls (1973)

Walls (1973)

Walls $(1973)$

Walls (1973)

Walls (1973)

Walls (1973

Walls, and Floors (1973)

Walls, Condensation (Tech. Notes) (1965)

Walls, Floor-Ceiling Assemblies, Doors, and Other Spa

Walls, Floors, and Stairs (1973)

Walls, Non Loadbearing (Tech. Notes) (1968)

Warm Air Heaters (1972

Warm Air Heating and Air Conditioning Systems (1973) an

Warmers, Hot Plates and Griddles, etc.) (1973)

/Ling Construction Under the U

Warning Detector (1973)

Vehicles (SMV) Identificat

Warning Lamp (Light) and Slow Moving Vehicle (SMV) Iden

Warning Lamp (Light) for Remote Mounting on Agricultura

Warning Lights (1971)

Warning System Units (1973)

Warning (1972)

Warp Knit Fabrics (1964) ANSI L14.284

Warpage of Ceramic Tile (1973)

Warpage of Refractory Brick and Tile or Deviation from

Wash-Whites 190 Deg. F, Colors 160 Deg. F Maximum Hot

Wash-Whites (With Chlorine Bleach) 190 Deg. F, Colors

Wash and Wear Items (Clothing) After Home Laundering (1

Wash and Wear Items (Textile/Clothing) After Home Lau

Washable Uniform Fabrics for Men and Women (Heavy Duty)

ANSI

ICBO

ASAE

SAE

ASAE

ITE

UL

NSA

ASTM

ASTM

ASTM

ANSI

ANSI

AATCC

AATCC

24D

$24 \mathrm{G}$

24C

$24 \mathrm{D}$

UBC* $8-10$

Z21.49

Z21.44A

Z21.44

UMC*7. I

680

J527B

DC 3

A 12.1

D 1736

*1.500A

* $]-500 \mathrm{~B}$

*1.500C

CB.SG.73

D2313

555

CT101

UBCS24-8

$22 \mathrm{~A}$

UBCS24.9

$24 \mathrm{H}$

24.

$24 \mathrm{~B}$

$* 27$

[BC*8-II

UBCS $35-3$

A 97.2

[BC * 8-14

UBC $547-5$

C557

UBC $\$ 47.2$

LBC $\$ 47.10$

UBC*8-16

C 36

A356

D1587

E280

A643

UBC $* 3.42$

$26 \mathrm{~A}$

$17 \mathrm{~L}$

13

21

17E-G

$21 \mathrm{~A}$

$7 \mathrm{~B}$

25

$29 \mathrm{~A}$

$4 \mathrm{~A}$

$* 4$

150

201.208.1

209.210

$\mathrm{UBC} * 3-29$

$\mathrm{UBC} * 8-12$

$\mathrm{UBC} * 8-5$

$* 31.5$

$7 \mathrm{C}$

ICBO UBCS35-I

TCA $\quad 400.600$

BIA 33

ASHRA 45

NFPA 90B 
Safety Std, for Electric Home Laundry Equipment (Clothes Rec. Pract. for Electric Windshield Rec. Pract. for Passenger Car Windshield Std, for Countersunk Type Finishing Std. for Tab Type Std. for $100 \mathrm{Deg}$. Dimpled Std. for Sealing

Std for Nonmagnetic Spring Lock ance Evaluation Procedure for Household (laundry, Clothes) operated and Commercial Clothes Washing Equipment (Laundry Std. for Nut-Self Locking, Extended Std. for Std. for Nut,

r Safety for Electric Coin Operated and Commercial Clothes Sid. for Pot, Pan, and Ctensil Commercial Spray Type na (Cereal Chemistry) (1967)

na (Cereal Chemistry) (1962)

achine

Test Meth. for Shampooing: 8 Test Meth. for Colorfastness of Textiles to Light and

nsi Z11.124

reational Vehicles (1971) Test Meth. for Colorfastness to Std. Meth. of Test for Water Std. for Std. for Installation PVC Building Drain or lnstallation for Acrylonitrile Butadiene Styrene Drain, Stds. for Acrylonitrile Butadiene Styrene Plastic Drain. Std. Spec. for Poly(vinyl Chloride) (PVC) Plastic Drain, equen, Ixtle, Jutes, Hemp, Tow, Cocoa Fibers, Oakum, Rags. Rec. for Radioactive

cal Users (1951) Rec. for

st Procedures for Plumbing Requirements for Household Food Std. for Performance Evaluation for Household Food for Low and Medium Speed Stationary Diesel and Gas Engine Rec. for Lniform Terminology for Rural

Uniform Fire Code: Automobile Wrecking and Junk Yards or Uniform Plumbing Code: Indirect Rec. for Design of Farm

Brick and Tile in Sanitation Structures, Industrial ineering Structures (Tanks, Reservoirs, etc. for Water and

Particle Radioactivity of Industrial Water and lndustrial

Test for Hydroxide lon in Industrial Water and Industrial Std. Meth. of Test for Thorium in Water and

meth. for Determining Chemical Oxygen Demand in Industrial

Sewage Holding and/or Treatment Devices for Marine Toilet (1974) ANSI Z221.1 Std. for Safety for Metal

a Technical Guide for Landfill Disposal of Solid Chemical r Acrylonitrile-Butadiene-Styrene (ABS) Plastic Drain,

Jutes, Hemp, Tow, Cocoa Fibers, Oakum, Rags, Waste Cloth. Rec for the Disposal of Carbon-14 ode: Indirect Waste Piping. Wet Vented Systems and Special Std. for in Dividual Aerobic

on Chambel Std. for Thermoset Plastic Tanks for Water and valuation of Special Processes or Devices Used in Treating si Se 3.1

e and Use of Proprietary Protective Signalling Systems for ntenance and Use of Local Protective Signaling Systems for Procedure for

ich Constructions (1970)

i K 65.122 Std. Test Meth. for Std. Meth. of Test for Std Meth of Test for

ensity of Burn/ for Apparent Porosity, apparent Specific Gravity of Fire/ Std. Meth. of Test for d (Cobb Test) (1973) Std. Meth. of Test for Asbestos Cement Pressure Pipe, Couplings, and Gaskets for r Measurement of Beta Particle Radioactivity of Industrial Std. Meth. of Test for Hydroxide lon in Industrial Test Meth. for Colorfastness of Textile Fabrics to iron and Ductile Iron Fittings, $2 \ln$. Through $48 \ln$./S for Std. for Asbestos Cement Pressure Pipe for estressed Concrete Pressure Pipe, Steel Cylinder Type, for ylene Encasement for Gray and Ductile Cast lron Piping for Sid. Meth. of Test for Sid. Spec. for Equipment for Sampling nitary Engineering Structures (Tanks, Reservoirs, etc. for Std. Meth. of Test for Thorium in

Washable Uniform Fabrics for Men and Women (Institution

asher-Flush Type Finishing (1973)

Washer and Dryer) (1972) ANSI C33.13

NSA

Washer Switch (1971)

Washer Systems (1972)

Washer (1973)

Washer (1973)

Washer (1974)

Washer (1974)

Washer (1974)

Washer (1971) ANSI 2224 .1

Washer) (1974)
Washer, Double Hexagon, $220 \mathrm{KSl}, 450 \mathrm{Deg}$. F (1973)

Std. for Safety for Electric Coin

Washer, Self Aligning, 450 and 800 Deg. F (1973)

Washer, Shear Type, 450 and $800 \mathrm{Deg} . \mathrm{F}$ (1973)

Washers-Electrical Terminal Attachment (Preferred) (1

H Steel Bolts for

Washers (1971) ANSI G38.6

IImension

\section{$\mathrm{P}$}

/Ma

Machines (Polisher, Scrubber, S

Washers, etc.) (1974) /Machines (Polisher, Sc

Washers, Pins, Dowels, Joints, Split Rings and

Washing and Shrinkage: Rapid Control Test (1969)

Washing at 105 Deg. F: Rapid Control Test (1969)

Washing Equipment (Laundry Washer) (1974)

Washing Machines (1970)

Washing Meth. of Analysis of Gluten in Flour and Semoli

Washing Meth. of Analysis of Gluten in Flour and Semoli

Washing of Textile Floor Coverings (Rug, Carpet) (1972)

Washing: Alternate Sunlight Exposure (1965) ANSI L14.11

Washing: Characterization of Textile Colorants (1972)

Washout Characteristics of Lubricating Greases (1973) a

Waste and Sewage Holding Tanks for Mobile Homes and Rec

Waste and Vent Pipe and Fittings (1971)

Waste and Vent Pipe and Fittings (1971)

Waste and Vent Pipe and Fittings (1971)

Waste and Vent Pipe and Fittings (1973) ANSI K65.56

Waste Cloth, Wastepaper, Kapok, Hay, Straw, Spanish M

Waste Disposal in the Ocean (1954)

Waste Disposal of Phosphorus-32 and Iodine-131 for Medi

Waste Disposer Units (1971) ANSI A197.3, Asse 1008

Waste Disposers (1973)

Waste Heat Recovery Systems (1972)

Waste Management and Disposal (1973)

Waste Material Handling Plants (1973)

Waste Piping, Wet Vented Systems and Special Wastes (19

Waste Storage Tanks (1973)

Waste Treatment Facilities (Tech. Notes) (1969)

Waste Treatment) (1968)

Waste Water (1966) ANSI N151

Waste Water (1967)

Waste Water (1968) ANSI N158

Waste Waters and Most Organic Compounds (1966)

(tor Concrete Sanitary Eng I for Measurement of Beta

Std. Meth of A

Waste (Boats) (1972)

Waste (Trash,

Waste, and Vent Pipe and Fittings (1973) ANSI B72.18

Wastes (1953)

Wastes (1973)

Wastewater Treatment Plants (1970)

Wastew ater $(1970)$

Watchman. Fire Alarm and Supervisory Service (1972) Ans

Watchman, Fire Alarm and Supervisory Services (1972) an

Watchman, Fire Alarm, and Supervisory Service (1972) an

Water Absorbency of Bibulous Papers (1972)

Water Absorption of Core Materials for Structural Sandw

Water Absorption of Rigid Cellular Plastics (1969E) Ans

Water Absorption (Static) of Leather (1970) ALCA E30

Water Absorption, Apparent Specific Gravity, and Bulk D

Water Absorption, Bulk Density, Apparent Porosity, and

Water Absorptiveness of Nonbibulous Paper and Paperboar

Water and Fire Service (1973)

Water and Industrial Waste Water (1966) ANSI N15

Safety
51

Water and Industrial Waste Water (1967)

Water and Light: Alternate Exposure (1971) ANSI L14.227

Water and Other Liquids (1971) ANSI A21.10

Water and Other Liquids (1972)

Water and Other Liquids (1972)

Water and Other Liquids (1972) ANSI A21.5

Water and Sediment in Crude Oils (1973) ANSI Z11.8

Water and Steam (1970) ANSI Z111.2

Water and Waste Treatment) (1968)

Water and Waste Water (1968) ANSI N158

Std. Fo UL

tTical $\mathrm{CR}$

UL

SAE

NSA

NSA

NSA

NSA

AHAM

UL

NSA

NSA

NSA

NSA

NSA

ASTM

Met ANSI
nifo ICBO

UL

Colorf AATCC

/Th. AATCC

AATCC

NSF

A ACCH 26

A ACCH 38.10

AATCC 138

AATCC 83

AATCC 36

ASTM D1264

IAPMO TSC2

IAPMO IS9

Std. F IAPMO IS

PS17

ASTM D2665

ICBO UFC*2ART7

NCRPM Rl6

NCRPM R9

/Te AHAM FWD-2PR

AHAM FWD-1

DEMA

ASAE

ICBO

ICBO

ASAE

$\mathrm{BlA}$

$* 1.16$

R292.1

UFC*2ART3

UPC*l-6

R345. 1

$34 \mathrm{~B}$

68-50

D1890

D514

ASTM D2333

ABYC

K.24

A8

UL

MCA

ASTM

ICBO

NCRP

SW-1

D266l

UFC*2ART7

$\mathrm{R} 12$

ICBO UPC * $1-6$

NSF

NSF

NSF

ULP

NFPA

TAPPI

ASTM

ASTM

ASTM

ASTM

ASTM

ASTM

40

C. 9

$72 \mathrm{D}$

$72 \mathrm{D}$
827

$72 \mathrm{~A}$

T432SU

C 272

D2842

D 1815

C20
C 373

D3285

107

D 1890

ASTM

AATCC

D514

AWWA

$A W W A$

C110 
National Bureau of Standards KWIC Index of Engineering Standards

ng, Aeration Chambe/

Std. for Thermoset Plastic Tanks for Std. Meth. of Test for Iron Bacteria in slurry) of Corn Starch Which Is Substantially Insoluble in Corn Starch Which Is Gelatinized When Heated in a Boiling $r$ Surface Preparation of SteeI and Other Hard Materials by

72)

td. for Gas Fired High Pressure Steam and Hot Std. for Gas Fired Low Pressure Steam and Hot Std. Spec. for Crushed Stone, Slag, and Gravel for Dry or Std. Meth. for Continuous Determination of Sodium in Std. for Centrifugal

Rec. for Thermoplastic Piping for Swimming Pool Std. for Vitreous China Plumbing Fixtures (Lavatory, Std. and Test Procedure for td. for

Std. for Flexible Copper nstruction and Maintenance of Terrace (Erosion Control and for Safety Related Systems, Structures, and Equipment for Std. Rec. Guide for in Service Annealing of Std. Meth. of Testing for Rating ss (1/ Test Meth. for Colorfastness of Textiles to Light: si L1/ Test Meth. for Colorfastness of Textiles to Light: Safety Std. for Drinking ins and Self Contained, Mechanically Refrigerated Drinking $i$ for Spiral Bevel, Helical and Herringbone Gear Units for td. for Redwood Lumber Spec. for Application in Industria for Douglas Fir Lumber Spec. for Application in Industrial r Southern Pine Lumber Spec. for Application in Industrial

$r$ Pressure Preservative Treatment of Lumber for Industrial generation: Fossil Fue/ Rec. Pract. for the Prevention of ection (Safety) (1973) Rec. for

ing Requirements for Household Storage Type Electrical Hot c. for Chlorinated Poly(vinyl Chloride) (CPVC) Plastic Hot Uniform Plumbing Code:

utron Emitting Fission Products in Nuclear Reactor Coolant Asbestos Tile Floors (1969) Spec. for Std. Rec. Pract. for Rating Std. Meth of Test for Sediment in Std. Meth. of Test for Total Ash and Silica in 116 td. Meth. of Test for Nonvolatile Matter (Total Solids) in
Test Meth. for $\mathrm{pH}$ of the Std for Flexible Non Pressure Rec. for Star Model F Hidromatic Deluge Valve for stem Sprinkler Demand (1974) Rec. for

Permissible Concentrations of Radionuclides in Air and in Std. Meth. for Examination of

Meth. of Analysis of Solvent Systems Used for Removal of Std. Meth. of Test.

Std. Meth. of Test for lron Bacteria in Water and

h. of Test for Corrosivity of Solvent Systems for Removing or Boilers (197) Fire Hazards and Protection Rec. for Low Uniform Plumbing Code

(Vol) Std for Gas Water Heaters (Automatic Storage Type h Inputs Less Than 75,000 Btu Per Hr.) (Vol/ Std. for Gas Std. for Household Automatic Electric Storage Type Safety Std. for Household Electric Storage Tank Std. for Safety for Oil Fried ous and Large Automatic Storage Type (1974) kling, Loss of Adhesion, or Other Failure/ Std. for Gas Tent Meth of Test for Undissolved lor Alcohol Base) by the Iodine/ Std. Meth. for Test for (1972) oint Dep/

ntainers) (1973) Std. Meth. of Test for Corrosivity of (1971) Std. Test Meth. for Detection and Estimation of Test Meth. for Std for Cold

d. for Direct Reading Remote Registration Systems for Cold Std. Protective Coatings (Paints) for Light thylene Encasement for Gray and Ductile Cast lron Pipe for Machine Sealing of Top Flaps of Fiberboa Std. Spec. for Std. Meth. of Test for

namic Water Resistance of Shoe Upper Leather by the Maeser for Cement Mortar Protective Lining and Coating for Steel Rec. for Thermoplastic Std. for Safety for

n Erosion Corrosion Characteristics of Aluminum Automotive ilters (Petroleum Industry) (/

Test Meth. for Determining Std. Meth. of Test for r Safety Criteria for the Design of Stationary Pressurized ansi L14.60, ASTM D583 test (1972) ANSI L14.61, ASTM D583

Test Meth. for Test Meth. for Absorption Test (1972) ANSI L 14.87, ASTM/ Test Meth. for 70) Swellograph Method for Test. the

$f$ Test for Effects of Drycleaning on Permanence of Durable ronment on Production, Heat and Moisture Loss and Feed and materials by the Dry Indicator Me/ Std. Meth. of Test for ng Leather Tester (1970)

Std. Meth. of Test for Dynamic ter Penetration Tester (1)
Water and Wastewater Treatment (Septic,

Water at Room Temperature (1973)

Water Bath for Determination of $\mathrm{pH}$ (Paste) of $\mathrm{CR}$

Water Blasting Prior to Coating or Recoating (1972)

Water Boilers (Inputs Not Over 400,000 Btu Per Hr.) (I9

ater Boilers (1972)

Water Bound Macadam Base Courses (1971) ANSI A37.99

Water by Ion Electrode (1973)

Water Chilling Packages (Refrigeration) (1974)

Water Circulation System s (1972)

Water Closet Bowl, Urinal) (I973)

Water Closet Flush Tank Ball Cocks (1968)

Water Connection Flanges (1971)

Water Connectors (1971)

Water Conservation) (1972)

Ec. for Design, Layout, Co

Water Cooled and Moderated Nuclear Power Generating Pla

Water Cooled Nuclear Reactor Vessels (1974)

Water Cooled Refrigerant Condensers (1971)

Water Cooled Xenon Arc Lamp. Alternate Light and Darkne

Water Cooled Xenon Arc Lamp, Continuous Light (1971) an

Water Coolers (1972) ANSl C33.82

Water Coolers (1973) ANSI A112.11.1

Water Cooling Tower Fan Drives (1972)

Water Cooling Towers (1971)

Water Cooling Towers (1971)

Water Cooling Towers (1971)

Water Cooling Towers (1971)

Water Damage to Steam Turbines Used for Electric Power

Water Dispensers (1973)

Water Distribution Systems (1973

Water Distribution (1973)

Water During Reactor Operation (1970) A NSI NI63

R Drinking Founta ARt

Info Shee AGMA

Std CTI

Std. Fo CTI td. Spec. Fo CTI

Water Emulsion Floor Polish for Use on Asphalt or Vinyl

Water Emulsion Floor Polishes (1972)

Water Emulsion Polishes by Centrifuge (1972) ANSI Z123.

Water Emulsion Polishes (1972) ANSI Z123.1

Water Emulsion, Solvent Based, and Polymer Emulsion Flo

Water Extracted from Bleached Textiles (1969) ANSI LI4.

Water Fill Hose for Recreational Vehicles (1971)

Water Flow Control of Fire Extinguishing Systems (1973)

Water Flow / Pressure Requirements for Pipe Schedule Sy

Water for Occupational Exposure (1959) $\quad / N$ and

Water Formed Deposits by Chemical Microscopy (1973)
Water Formed Deposits (Chemical Cleaning of Industrial

Water Formed Deposits (1972)

Water Formed Deposits (1972)

Water Formed Deposits (1973)

Water Fuel Cutoffs and Automatic Feedwater Regulators

Water Heaters and Vents (1973)

Water Heaters with Inputs Less Than $75,000 \mathrm{Btu}$ Per $\mathrm{Hr}$.

Water Heaters (Automatic Storage Type Water Heaters Wit

Water Heaters (1972)

Water Heaters (1972) ANSI C33.87

Water Heaters (1974)

Water Heaters: Volume IIl, Circulating Tank, Instantane

Water Immersion Test for Resistance to Blistering, Wrin

Water in Aviation Turbine Fuels (1973)

Water in Engine Antifreeze-Coolant Concentrate (Glyco

Water in the Absence of Heat Transfer (Electrical Meth.

Water Insoluble Impurities in Refined Phenol by Cloud P

Water Leak Test for Paper Shipping and Refuse Sacks (Co

Water Meters (Displacement Type $5 / 8$ In. Through 6 In.)

Water Meters (1972)

Water Nuclear Reactor Containment Facilities (1972) Rec. Pract. for Polye

Water or Other Liquids (1972)

Water or Solvent Soluble Liquid Adhesives for Automatic

Water Penetration into Pipeline Coatings (1972)

Water Penetration Tester (1970) ALCA E56

Water Pipe (4 In. and Larger, Shop Applied) (197I)

Water Piping Systems (1972)

Water Pumps for Fire Protection Service (1974)

$/$ Test for Dy ASTM

Meth. for Cavitatio

Water Quality for Subsurtace Injection U sing Membrane

Water Reaction of Aviation Fuels (1972) ANSI Z1 1.82

Water Reactor Plants (1973)

Water Repellency of Textile Fabrics: Spray Test (1971)

Water Repellency of Textile Fabrics: Static Absorption

Water Repellency of Textile Fabrics: Tumble Jar Dynamic

Water Repellent Effectiveness Df Treating Solutions (19

Water Repellent Finish of Fabrics (1972) Std.

Water Requirements of Farm Livestock (Poultry, Swine, C
Water Resistance of Paper, Paperboard, and Other Sheet

Water Resistance of Paper, Paperboard, and Other Sheet
Water Resistance of Shoe Upper Leather by the Dow Corni

Water Resistance of Shoe Upper Leather by the Maeser Wa
D. AWWA

SPI

td. Spe ASTN

ICBO

RTI

ASTM

ASTM

ASTM

ASTM

AATCC

IAPMO

FMS

NCRP

A STM

ASTM

ASTM

ASTM

FMS

ICBO

ANSI

ANSl

ANSI

UL

ANSI

NCCA

ASTM

ASTM

ASTM

TAPPI

$A W W A$

AWW A

ANSl

ASTM

ASTM

UL

NACE

ASTM

ANSl

A ATCC

AATCC

AATCC

NWMA

ASTM

ASAE

ASTMI

ASTMI

A STM
Plumb ASSE

41

D932

C. 44

C. 42

RP.01.72

221.59

Z21.13

D694

D2791

550

PPI TRI7

Al12.19.2

1002

J535A

PSI 14

R268.1

N18.10

E509

22

$16 \mathrm{~F}$

$16 \mathrm{E}$

399

1010

490.02

103

114

115

112

TWDPS. I

3.26

1023

D2846

UPC*1-10

D2 470

*6

D 3052

D 1290

D 1288

D2834

TSC 19

2.88

2.77

R22 
ansi L14.74, ASTM D 583 re Test (1971) ANSI L14.265

Test (1971) ANSI L14.71, ASTM D583

Test Meth. for Test Meth. for Test Meth. for 72 Std. Test Proc and Acceptance Criteria for Uniform Building Code Std. for

nd Ring Buoy) (19/ Safety Std. for Marine Special Purpose ad, and Christmas Tree Equipment; Flow Control Chokes; and cocks, Through Hull Connections, Outboard Water Strainers, els (1972) ANSI Z11.301 Std. Meth. of Test for Installation Std. for Solvent Cemented PVC Pipe for Sid for Steel Pipe (Welded and Seamless) for Underground Performance Requirements for Individual Anti Scald Type ry Standard for Household Commercial and Portable Exchange or Continuous Separation and Collection of Particulate and s and Their Admixtures (1974)

ALCA B8

ic Resistance of the Leachate of /

123.12

Std. Meth. of Test for Std. Meth. of Test for Sid. Meth. of Test for Std. Meth. of Test for Test Meth. for Colorfastness of Textile Fabrics to Rec. for
Rec. for Sprinkler. mable Liquid and Liquefied Flammable/ Rec. for A 54.3

hquakes (General Characteristics Of, Rec. Construction and Std. for the Installation of
S. for Safety for Automatic stallation of Seacocks, Through Hull Connections, Outboard 1972/ Performance Requirements for Backflow Preventers in

k Valve Type Back Pressure Backflow Preventers for Potable Uniform Plumbing Code: Rec. Rules for Sizing the or Relief Valves and Automatic Gas Shutoff Devices for Hot si Blll.l Safety Std. for Play Pipes for h. of Analysis of Apparent Viscosity of Acidulated Flour. Wells and 1971

and Medium Speed Stationary Diesel and Cas Engine Cooling Std. for Pressurized and Non Pressurized Potable Brick and Tile in Sanitation Structures, Std. Spec. for Seamless Copper Std Spec for Welded Copper Alloy bronze Solder-Joint Pressure Fitting for Ise with Copper Safety Std. for 2 l/2 Gal. Cartridge Operated Installation and Maintenance of Steam and Hot t of Piles. Poles, and Timbers for Marine, Land, and Fresh ckaging) (1968) ANSI MHi2.18 A NSI MH 12.17

es for Dry Products (1972)

e Meth. (Packaging) (I968) A NSI M/ ing) (1964) A NSI MH 12.10 ) at High Te/ Std. Meth. for Grav Std. Meth. of Test for Std. Meth. of Test for Std. Meth. of Test for std. Meth. of Test for Std. Meth. of Test for rs (1973)

973) ANSI Z11.124 Pressure Sensitive Std. Meth. of Measuring Std. Meth. of Test for

Std. Spec. for Seamless and Welded Carbon Steel

Test Meth. for Colorfastness of Textile Fabrics to Meth. of Test for Colorfastness of Upholstery Fabrics to Std. Meth. of Test for Dissolved and Gaseous Hydrogen in up, Crude and Refined Sugar and Other Materials Soluble in cle Radioactivity of Industrial Water and Industrial Waste Meth. for Measurement of Alpha Particle Radioactivity of for Hydroxide Ion in Industrial Water and Industrial Waste Std. Meth. of Test for Residual Chlorine in Std. Meth of Test for Alkyl Benzene Sulfonate in Std. Meth. of Test for Thorium in Water and Waste Std. Meth. of Test for Iron-59 in

Std. Meth. of Reporting Results of Analysis of Std. Meth of Test for Aluminum in Std. for Test Kits for Swimming Pool

Std. Meth. of Test for Radionuclides of Radium in Std. Meth. of Test for Radioactive Tritium in

Std. Meth. of Test for Radioactive Manganese in Std. Definitions of Terms Relating to Std. Meth. of Test for Fluoride Ion in Sid. Spec. for Reagent Std. Meth. of Test for Sulfite Ion in Std. Meth. of Test for Cyanides in std. Meth. of Test for Gamma Spectrometry of Std. Meth. of Test for Radioactive Cesium in Std. Meth. of Test for Phosphorus in $r$ Determination of Precision of Meth. of Committee D.19 on $\mathrm{s}$ of Colored Textile Yarns and Fabrics to Chlorinated Pool eth. for Colorfastness of Textile Yarns and Fabrics to Sea for Colorfastness of Colored Textile Yarns and Fabrics to Meth. of Test for Selenium in Meth. of Test for Boron in Rec. Pract. for Alpha Spectrometry of Rec. Pract. for Measurement of Low Level Activity in Meth. of Test for Organochlorine Pesticides in
Water Resistance of Textile Fabrics (Rain Test) (1971) Water Resistance of Textile Fabrics: Hydrostatic Pressu Water Resistance of Textile Fabrics: Impact Penetration Water Resistance on Steel Door and Frame Assemblies (19 Water Resistant Gypsum Backing Board (1973)

Water Safety Buoyant Devices (Vest, Jacket, Horseshoe a Water Sampling Devices) (1974) /Ead, Water Separation Characteristics of Aviation Turbine Fu Water Service and Yard Piping (1971)

Water Service (1972)

Water Shower Control Valves (1973)

Water Softeners (1972)

Water Soluble Gaseous Fluorides in the Atmosphere (Filt

Water Soluble Halide Ion in Halogenated Organic Solvent

Water Soluble Matter of Vegetable Tanned Leather (1970)

Water Soluble Salts in Pigments by Measuring the Specif

Water Spotting of Emulsion Floor Polishes (1974) ANSI Z

Water Spotting (1972) ANSI L14.148

Water Spray Fixed Systems for Fire Protection (1973)

Water Spray, and Explosion Protection (Safety) for Flam

Water Sprinkler and Hydrant System Demand Tables (Fire

Water Sprinkler System Design-Fire Protection and Saf

Water Sprinkler Systems for Fire Protection (1973) ANSI

Water Sprinklers for Fire Protection Service (1974)

Water Strainers, Water Scoops and Drain Plugs (Boats) (

Water Supply Lines with Intermediate Atmospheric Vent

Water Supply Lines (1972)

Water Supply System (1973)

Water Supply Systems (1972)

Water Supply Test. in Fire Protection Service (1973) an

Water Suspensions (Cereal Chemistry) (1962)

Water Systems (Treatment, Quality and Management) (1972

Water Systems (1972)

Water Tanks for Mobilehomes and Recreational Vehicles

Water Treatment Facilities (Tech. Notes) (1969)

Water Tube (1972) ANSI H23.1

Water Tube (1973)

Water Tube (1973)

Water Ty pe Fire Extinguishers (1973)

Water Unit Heaters (1970)

Water Use (197.1) ANSI O11.1

Water Vapor Permeability of Packages by Cycle Meth. (Pa

Water Vapor Permeability of Packages (Packaging) (1968).

Water Vapor Transmission of Flexible Heat Sealed Packag

Water Vapor Transmission of Shipping Containers by Cycl

Water Vapor Transmission of Shipping Containers (Packag

Water Vapor Transmission Rate of Sheet Materials (Paper

Water Vapor Transmission Test Meth. (1970)

Water Vapor Transmission Through Serew Cap Closure Line

Water Washout Characteristics of Lubricating Greases (1

Water Well Pipe (1972A)

Water (High Humidity) and Light: Alternate Exposure (19

Water (Textile) (1973)

Water (1960)

Water $(1966)$

Water (1966) ANSl N15

Water (1966) ANSI N152

Water (1967)

Water (1968)

Water (1968)

Water (1968) ANSI NI58

Water (1969) ANSI N 162

Water $(1969 \mathrm{E})$

Water $(1969 \mathrm{E})$

Water $(1970)$

Water (1970) ANSI N16I

Water (1970) ANSI NI64

Water (1971) ANSI N156

Water (1972)

Water (1972)

Water (1972)

Water (1972)

Water (1972)

Water (1972)

Water (1972)

Water (1972)

Water (1972)

Water (1972) ANSI L 14. 149

Water (1972) ANSI L14.150

Water (1972) ANSI L 14.15]

Water (1972T)

Water (1972T)

Water (1972T)

Water (1972T)

Water (1972T)
Equirements for Double Chec Std. for Wrought Copper and

the Preservative Treatmen

AATCC 35

AATCC 127

AATCC 42

STDI 115

ICBO UBC $\$ 47.13$

UL

API

ABYC H27

ASTM D2550

IAPMO IS8

Safety UL

ASSE

888

ASTM D3267

ASTM D2988

ASTM D2876

ASTM D2448

ASTM Dl793

AATCC 104

FMS $\quad 4.1 \mathrm{~N}$

FMS 7.14

FMS $\quad 2.76$

FMS LPD 1.2

NFPA 13

UL 199

ABYC $\mathrm{H} 27$

ASSE $\quad 1012$

ASSE 1015

ICBO UPC*].A

UL

Z21.22A

385

Met AACCH $56-80$

WSC *1

DEMA *1.10

IAPMO TSC

BIA 34

A5TM B88

ASTM B586

ANSI B16.22

UL

AMCA

ASTM

ASTM

ASTM

ASTM

ASTM

ASTM

TAPPI

PSTC

ASTM

ASTM

ASTM

AATCC

ANSI

ASTM

Extraneous Materials Content of Corn Syr CR

Sid ASTM

Std. Meth. of Test ASTM

ASTM

ASTM

ASTM

ASTM

ASTM

ASTM

NSF

ASTM

ASTM

ASTM

ASTM

ASTM

ASTM

ASTM

ASTM

ASTM

ASTM

ASTM

Std. Rec. Pract. Fo ASTM

Test Meth. for Colorfasines AATCC

Test $\mathrm{M}$ AATCC

Test Meth.

A ATCC

ASTM

ASTM

ASTM

ASTM

715

D390

D 1251

D895 
Meth of Test for Tetrasodium Salt of EDTA in Tentative Meth. of Test for Radioactive Zirconium in Rec. for Prevention of Damage to Property from Surface ety) of Personnel on Boat Deck and for Reboarding from the Std. Meth. for Measurement of Gamma Radioactivity of Std. Meth. of Test for Radioactive Barium in Std. Meth. of Test for Radioactive Iodine in Std. Meth. of Test for Morpholine in Std. Rec. Pract. for Coagulation Flocculation Jar Test of Std. Meth. of Test for Cyclohexylamine in Std. Meth. of Test for Volatile Matter (Including Rec for Centrifugal Fire sc. Perils at Electric Generating Stations (FIood, Surface Safety Rec. for Electrically Heated Livestock Uniform Building Code Std. for Kraft

Recommendation for Installation of en Fabrics Saturated with Bituminous Substances for Use in Std. Spec. for Woven Glass Fabrics Treated for Use in 1971) Spec. for Metal Oxide Dampproofing and of Meth. for Test. Elastomeric and Plastomeric Roofing and gate) (1941) An/ Std. Spec. for Asphalt Mastic for Ise in Spec. for Primer for Use with Asphalt in Dampproofing and Std. Spec. for Asphalt for Damproofing and iform Building Code: Valley Flashing (Sheet Metal for Roof ng to Bituminous, and Other Organic Materials for Roofing, for Determining Chemical Oxygen Demand in Industrial Waste of Test for Evaluating Inhibitory Toxicity of Industrial on of Furnace Explosions in Fuel Oil and Natural Gas Fired e Masonry; Mortar; Sand; Slag; Steel; Terra Cotta; Timber;

Std. for Low Power Wide Band Transformers (Less Than 100 Std. Spec. for Seamless Copper and Its Alloy Rectangular Std. Meth. of Test for Spectral Bandwidth and olor of Paper and Paperboard in CIE Y, X, Y or Y, Dominant ing Composition of Gallium Arseni/ Std. Meth. of Test for easuring Recorded Flux of Magnetic Sound Records at Medium 1.325 Surface Texture for Aerospace Gearing (Surface Roughness,

n Resistance of Glossy Smooth Coatings of Petroleum W ax or std. Meth. of Test for Coefficient of Kinetic Friction for Meth. for

rs for Commercial, Institutional and Industrial Build/ Petrole no abrasion Resistance of Glossy Smooth Coatings of Petrole um
Std. Definitions of Terms Relating to Meth. for Measuring Flat Spring Test for Hot Tack of Spec, for Base Plate (Artificial Dentures)

Std. Meth. of Test for Tensile Strength of Paraffin products Classified as to Slip Resistant (Floor Polish and (1973) Std. Meth. of Test for Gloss Retention of

0 Deg. C.) (1972) Meth. for Gloss Retention of Std. Meth. of Test for Congealing Point of Petroleum Meth. for Congealing Point of Petroleum

Std. Meth. of Test for Oil Content of Petroleum Std Meth of Test for Solvent Extractables in Petroleum Their Mixtures (1/ Std. Analytical Meth. for Determining Std. Analytical Meth. for Determining Uniform Fire Code: Maintenance of Exit Rec. for Prevention of Collapse Due to Rec. Against uctures (1973) cuum 17 Std. Meth. of Test for Coefficient of Friction and
L14.171 Test Meth. for Appearance of Creases in Wash and 1973) Ans/ Test Meth. for Appearance of Seams in Wash and ng Motion (1971) An/ Std. Meth. of Test for Measuring the Std. Meth. of Test for

Tent. Rec, Pract for Conducting termine Woven Fabric's Resistance to Sagging or Stretch in Rec. Pract. for Hot Rec. Pract. for Hot Rec. Pract for Cold Std. Rec. Pract. for Hot Cold

) (1967)

truction Under the Uniform Building Code: Wall Framing and ics: Carbon Arc Lamp Exposure with Wettin/ e Without Wetting (1972) ANSI L14.225 Test Meth. for Test Meth. for for Resistance of Textiles: Exposure to Natural Light and dation System: House Design and Construction Met/ the All sistance of Textile Fabrics: Exposure to Natural Light and Std. Meth. of Test for Aging Effects of Artificial Std. Meth of Test for Effects of Outdoor for Asbestos Cement Shingle Blanks to Be Used as Panels in re Flexibility of Latex Sealing Compounds After Artificial rm Building Code Std. for Kraft Waterproof Building Paper Rec. Std. Specs. and Load Tables for Open Uniform Building Code Std. for Open
Water (1972T)

Water (1973)

Water (1973)

Water (1973)

Water (1973) ANSI N150

Water (1973) ANSI N155

Water (1973) ANSI N159

Water (1974)

Water (1974)

Water $(1974)$

Water) of Vinyl Chloride Resins (1972)

Water) Pumps (NFPA 20) (1974)

Water, Rigging Collapse, Burglary, and Roof Collapse) (

Waterers (1971)

Waterproof Building Paper (Weatherproofing) (1973)

Waterproof Ceramic Tile Floors (1973)

Waterproofing and Roofing (1972) ANSI A 109.10

Waterproofing and Roofing (1973) ANSI A 109.26

Waterproofing for Below Grade Wall and Floor Surfaces

Waterproofing Masonry Walls (Tech. Notes) (1961)

Waterproofing Materials (1972)

Waterproofing (Asphalt Cement, Mineral Filler and Aggre

Waterproofing (1973) AASHO Ml16, ANSI Al09.

Waterproofing (1973) Ashto M115. ANSI A109.16

Waterp roofing) (1973)

Lling Construction Under

aterproofing, and Related Building or Industrial Uses

Waters and Most Organic Compounds (1966)

Waters $(1968 \mathrm{E})$

Watertube Boiler Furnaces with One Burner (1972)

Tical Meth. C

Water; Ceiling; Partitions; Plaster; Roofing) and Earth

Enti NFPA

ICBO

(1971)

Waveguide Tube (1974) ANSI H37.1

Wavelength Accuracy of Fluorescence Spectrometers (1972

Wavelength and Excitation Purity (1972)

F Measuring C TAPPI

Wavelengths (1972) ANSI S4.6

Waviness, Form and Lay) (1973)

Correspond Std. Meth. of M IEEE

Wax Appearance Point of Distillate Fuels (1972) ANSI Z

Wax Based Blends Applied to Paper and Paperboard (1973)

Wax Coatings (1973) ANSI Z11.222

Wax in Impregnated Papers (1972)

Wax Maintenance of Vinyl Asbestos and Asphalt Tile Floo

Wax or Wax Based Blends Applied to Paper and Paperboard

Wax Polishes and Related Materials (1973)

Wax Polymer Blends (1972)

Wax (1971)

Wax (1973) ANSI Z11.206, TAPPI T644

Wax) (1971) ASTM D2047, CSMA BuI 308-70

Waxed Paper and Paperboard After Storage at $104 \mathrm{~F}(40 \mathrm{C})$

Waxed Paper and Paperboard After Storage at $140 \mathrm{Deg}$. (4

Waxes Including Petrolatum (1971) ANSI Z11.61

Waxes Including Petrolatum (1972)

Waxes (1968E) ANSI Z11.52, Fed Std. 791B, TAPPI T636

Waxes (1973)

Waxy and Nonwaxy Content in Corn and Grain Sorghum, and

Waxy and Nonwaxy Content in Corn Starch (1960)

Ways (1973)

Weak Construction and Design of Buildings and Other Str

Weak Construction and Design of Buildings (1972)

Wear Characteristics of Dry Solid Film Lubricants in Va

Wear Items (Clothing) After Home Laundering (1973) ANSI

Wear Items (Textile / Clothing) After Home Laundering (

Wear Life and Bonded Solid Film Lubricants in Oscillati

Wear Resistance of Inked Computer Ribbons (1972)

Wear Test on Textile Garments (1973)

Wear (Textile) (1973)

Weather Concreting (Concrete) (1972) ANSI Al70.

Weather Concreting (1959)

Weather Concreting (1966) ANSI Al44.1

Weather Concreting (1972) ACI 305

Weather Masonry Construction (Protection Rec.) (1968)

Weather Masonry Construction, Introduction (Tech. Notes

Weather Protection (1973)

Dwelli

Weather Resistance of Textile Materials and Coated Fabr

Weather Resistance of Textiles: Carbon Arc Lamp Exposur

Weather Through Glass (1972) ANSI L14.177 Test $M$

Weather (1968) ANSI L14 176

Weather (1968) ANSI LI4.176

Weathering on Latex Sealing Compound

Test Meth. for R

Weathering Tests of Latex and Emulsion Exterior Paints

Weathering (1972)

(Weatherproofing) (1973)

Weatherstripping for Std. Steel Doors and Frames (1972)

Web Steel Joints J Series and H Series (1970)

Web Steel Joist Design (1973)
ASTM

ASTM

D3I13

D3315

9.2

A 18

D1690

D2038

D2334

D 1942

D2035

D2909

D3030

$3.7 \mathrm{~N}$

9.10

R342

UBCS17-1

II 4

D146

Dl668

07140

D3105

D491

D4l

D449

UBC $* 8-19$

D1079

K-24

D2037

85

UBC*3-23

I11

B372

E388

T527SI

F358

347

118.01

D3117

D3234

D2534

T405SL

*16

D3234

D2825

T683SI

24

DI 320

BLL 12

D2895

T682

D938

T 662

D721

D3235

A. 28

B-64

UFC $* 2 A R T 10$

l-55

$\begin{array}{ll}\text { FMS } & \text { LPDI-3 } \\ \text { ASTM } & \text { D2716 }\end{array}$

AATCC $88 \mathrm{C}$

AATCC $88 \mathrm{~B}$

ASTM D2981

ASTM F153

ASTM D3181

L24T5

305

605

306

A170.1

$1 \mathrm{~A}$

1

$\mathrm{UBC} * 8.10$

$111 \mathrm{~A}$

$111 \mathrm{C}$

$111 \mathrm{D}$

RP7

111B

C732

G11

D1911

C734

UBCS17-1

$111-\mathrm{E}$

S316-3

UBCS27-4 
Buckle, Web Strap, Light Duty (1972)

Test Meth. for Moisture in Pulp Std. Spec. and Load Tables for Open

limentary Pastes for Detection of Rodent Excreta, Insects Tent. Meth. of Test for Grading Cotton Card t for Formability of Attached Organic Coatings with Impact Spec. for Measurement of Adhesion and Flexibility by the pe, Petri Dish, Sieve, Separatory Funnel, Trap Flask, Step over Protective Structure for W'eeled Front End Loaders and erformance Criteria for Roll Over Protective Structure for nd Seed for Storage (1972/

Density, Specific Gravity, and ious Comm/

Std. Rec. Pract. for Calculation of Commercia

Rec. Pract. for Selecting Proportions for Normal n Aged or Backed Aluminum Alloy Panels/ Spec. for Coating baked Aluminized Steel (Coil) (1974)

Spec. for Coatin d ln, Blind Threaded, Self Locking, Nonself Locking, Light (1974)

Acid Solution (1971) ANSI Z/ Std. Meth. of Test for 1971) graph Absorption to $14.0 \%$ Moisture Basis (Constant Dough real Chemistry) (1962) Meth. for Correction for Std. Meth. of Test for Commercial 2.106. 1 Std. Meth. for Calculation of Volume and Tower Silos: Uni

l (Coil) (1) Spec. for Determination of Phosphate Coatin Meth. of Analysis of Test si 2197.9 Std. Meth. of Test for Applied Std. Meth. of Test for Applied Tent. Meth. for Drop $\mathrm{f}$ Test for Impact Resistance of Pipeline Coatings (Falling Std. for Threaded Metal Insert, Light rigid Plastic Sheeting or Parts by Means of a Tup (Falling Crumbles-Definitions and Meth for Determining Specific stener (Blind, Internally Threaded, External Sleeve, Light stener (Blind, Internally Threaded, External Sleeve, Light Std. for Low Height, Ligh ction, Std. Size, Design Values, Framing Rec., Properties, producing Equipment (1971) te; Earth; Glass; Granite; Gravel/ Std. Meth. for Measurement of Uniform Building Code 5)

Spec. for Determination of Phosphate Coating Meth. for Multiplication Factors for Correcting Radiographic Std. for Classification of Fusion Spec. for Spira

for Bending and Flaring (1972) el Pipe (1973) ANSI B125.1 ounds and Shapes (1973) ANSl 3) ANSI G24.22 tubing (1973) 2) d. Spec. for Black and Hot Dipped

Std Spec for Cold Formed Formed Std Spe Zinc Coated (Galvanized) Safety Std. for Steel Pipe Std. for Tube Assembly, Control, Steel, with Flash

s (1973)

5.16

ANSI B 36.38

rvice (1973) ANS1 B125.15

nger, and Condenser Tube

igh Temperature Service (/

Service (1972A) ANSl B 125.1/ ater Tubes (1973) Ans/ gh Pressure Service (/

Std Spec for Electric Resistance

Columbium Stabilized Alloy /

Columbium Stabilized All/

Stabilized Alloy Pipe (1973/ std. for Tube Assembly Assembly, Control Steel with Sid. Spec. for Std. Spec. for Std. Spec. for Std. Spec. for

ement (1972) ANSI G45.3 ice (1973) ANSl Bl25.14 zed for Bending, Double Flaring, and Beading (I) Std. for steel Pipe for Corrosive or High Temperat/ Std. Spec. for (1971) ANSI B125.38 Std. Spec. for Electric

Spec. for Fabricated Structural Steel Pipe for Ise of Spec. for Nickel Iron Chromium Silicon Alloy Seamless and ec. for Steel Bars, Forgings, Mechanical Tubing, and Flash Std. Spec. for Aluminum Alloy Formed and Arc
Std. Spec. for Std. Spec. for
. Type s (1972) Temperatures (1973)
(Web) (1973)

Web, Longspan, and Deep Longspan Steel Joints (1974)

Webbing, etc. (Cereal Chemistry) $\{1962$ )

Webs for Appearance (1973)

Wedge Bend Apparatus (1973)

Wedge for X.Ray Standardization Probe, Rot Fragment Cou

Weeled Dozers (1972)

M Performance Criteria for

Weeled Front End Loaders and Weeled Dozers (1972)

Weight-Moisture Relationships of Agricultural Grain a

Weight and Yield of Scoured Wool, Top, and Noil for Var

Weight Concrete (1970) ANSI Al67.1

Weight Determination for Chromate Conversion Coatings 0

Weight Determination for Conversion Coating on Aged or

Weight Floating, Sandwich Panel (1972) / Insert, Molde

Weight Loss After Heat Aging of Preformed Sealing Tapes

Weight Loss of Sheet Steel During Immersion in Sulfuric

Weight Meth. Only) (Cereal Chemistry) (1962)

Weight of a Shipment of Yarn or Man Made Staple Fiber

Weight of Flour to Give 100 Grams at $14.0 \%$ Moisture ( $\mathrm{Ce}$

Weight of Industrial Aromatic Hydrocarbons (1973) ANSI

Weight of Silage and Silo Capacities (1972)

Weight on Hot Dip Galvanized and Electrogalvanized Stee

Weight Per Bushel of Grain (Cereal Chemistry) (1962)

Weight Per Init Area of Dried Adhesive Solids (1969) an

Weight Per Unit Area of Liquid Adhesive (1951) ANSI Z19

Weight Tear Tests of Ferritic Steels (1971) ANSI Z260.3

Weight Test) (1972)

Weight (1973)

Weight) (1972)

Weight, Durability and Moisture Content (1972)

Weight, Millable Head Self Locking) (1972)

Weight, Protruding Head. Self Locking) (1972

Weight, Self Locking Hexagon Nut (1972)

Weight, Stress of Board, Timber, Lumber, Siding, Framin
Weighted Peak Flutter Content of Sound Recording and Re

Weights of Materials (Brick; Cast lron; Cinders; Concre

Weights on Steel (Coil) (1974)

Weights to a $14 \%$ Moisture Basis (Cereal Chemistry) (196

Weld Discontinuities (1972)

Weld Line Pipe (Petroleum Production) (1973)

Welded Aluminum Alloy Field Erected Storage Tanks (1973

Welded and Cold Drawn Low Carbon Steel Tubing Annealed

Welded and Seamless Black and Hot Dipped Galvanized Ste

Welded and Seamless Carbon Steel Structural Tubing in $\mathbf{R}$

Welded and Seamless Carbon Steel Structural Tubing (197

Welded and Seamless High Strength Low Alloy Structural

Welded and Seamless Steel Pipe for Ordinary Uses (1973)

Welded and Seamless) for Underground Water Service (197

Welded and Threaded Rod Ends (1973)

Welded Austenitic Stainless Steel Feedwater Heater Tube

Welded Austenitic Stainless Steel Pipe (1972A) ANSI B 12

Welded Austenitic Stainless Steel Sanitary Tubing (1973

Welded Austenitic Stainless Steel Tubing for General Se

Welded Austenitic Steel Boiler, Superheater, Heat Excha

Welded Autenitic Chromium Nickel Alloy Steel Pipe for H

Welded Brass Tube (1973)

Welded Carbon and Alloy Steel Tubes for Low Temperature

Welded Carbon Molybdenum Alloy Steel Boiler and Superhe

Welded Carbon Steel Boiler and Superheater Tubes for Hi

Welded Carbon Steel Boiler Tubes (1973) ANSI B125.6

Welded Carbon Steel Tubular Products for High Temperatu

Welded Carbon Steel Water Well Pipe (1972A)

Welded Chromium-Nickel-Iron-Molybdenum-Copper-

Welded Chromium-Nickel-lron-Molydenium-Copper-

Welded Chromium Nickel Iron Molybdenum Copper Columbium

Welded Clevis and Threaded Rod Ends (1973)

Welded Clevis and Threaded Rod Ends (1973)

Welded Copper Alloy Water Tube (1973)

Welded Copper and Its Alloy Tube (1973)

Welded Copper Tube (1974)

Welded Deformed Steel Wire Fabric for Concrete Reinforc

Welded Ferritic Stainless Steel Tubing for General Serv

Welded Flash Controlled Low Carbon Steel Tubing Normali

Welded Large Diameter Austenitic Chromium Nickel Alloy

Welded Low Carbon Steel Pipe for the Chemical Industry

Welded Low Carbon Steel Tubing (1972)

Welded Offshore Fixed Platforms (1972)

Welded Pipe (1972)

Welded Rings (1.3Mn-1.5Sl $-0.30 \mathrm{Cr}-1.8 \mathrm{Ni}-0.40 \mathrm{Mo}$ (

Welded Round Tube (1974) ANSI H38.20

Welded Stainless Steel Mechanical Tubing (1973)

Welded Steel Inside Tanks for Oil Burner Fuel (1973)

Welded Steel Pipe for Atmospheric and Lower Temperature

Welded Steel Pipe for High Pressure Service at Moderate

NSA

1284

TAPPI UM-237

SJl

5

ASTM D 3216

ASTM D3281

NCCA TB-11-10

$\mathrm{AACCH} 28.90$

Roll SAE

SAE J394A

ASAE D241.1

D2720

$\mathrm{ACl} 211,1$

NCCA TB-II.2

NCCA TB-Il-8

NSA 1835

ASTM C771

ASTM C694

-ls Fari AACCH $\quad 54-29$

ASTM D2494

AACCH $82-23$

ASTM D 1555

ASAE D252

NCCA TB.11.3

$\mathrm{AACCH}$ 84-10

ASTM D898

ASTM D899

ASTM E436

ASTM Gl4

NSA 1394

of ASTM

Ts and ASAE

D3029

S269.2

1674

$\begin{array}{ll}\text { NSA } & 1674 \\ \text { NSA } & 1673\end{array}$

NSA 129]

WWPA *24

IEEE 19.3

ICBO UBC*3.23

NCCA TB-Il-1

$\mathrm{AACCH} \quad 82-24$

NSA 1514

API 5LS

ANS1 B96.1

SAE J525B

ASTM A53

ASTM A500

ASTM A50l

ASTM A618

ASTM A 120

IL

NSA

ASTM

ASTM

ASTM

ASTM

ASTM

ASTM

ASTM

ASTM

ASTM

ASTM

AsTM

ASTM

ASTM

ASTM

ASTM

ASTM

NSA

NSA

ASTM

A STM

ASTM

ASTM

ASTM

SAE

ASTM

ASTM

SAE

API

361

A688

A 312

A 270

A 269

A 249

A358

B587

A 334

A250
A 226

A 520

A 589

B468

B474

B464

359

B58

B 543

B 447

A 497 
i B125.4

e (1971) ANSI G36.49 si B125.17

Std. Spec. for Electric Fusion Std. Spec. for Metal Are Std. Spec. for Seamless and Sid. Spec. for Electric Resistance

Uniform Building Code Std. for

Manual of Cold Formed

72) ANSI G45.l
7Neinforce ment Std. for Tube Assembly, Control Steel with Std. Spec. for Seamless and

(1965) ANSI H50.2

Apec for Wrought Zirconium and lis Alloy Seamless and Std. Spec. for Columbium and lts Alloy Seamless and Std. Spec for Aluminum Alloy Round Std. Spec. for Seamless and h50.1 Sheet, and Strip for Fusion d Chromium Nickel Steel Plate, Manual of Std. Pract. for

Rec. Pract. for Reinforced Bituminous Concrete Overlays ) ANSl Z212.1

Std. Spec. for Seamless and Spec for Steel Tubing, Std. Terms and Definitions for Std. for Safety in

y Std. for Acetylene Generators, Stationary, Low Pressure

td. for Acetylene Generators, Stationary, Medium Pressure Uniform Fire Code: Std. for Forged Steel Fittings (Pipe), Socket Spec. for Welding Rods and Covered Electrodes for Rec. Pract. for Resistance Std. for Resistance for Consumable Electric Covered Bare Straight and Coiled Spec. for Mild Steel Covered Arc Spec, for Nickel and lts Alloy Covered Spec. for Tungsten Arc Spec. for Aluminum and lts Alloy Are ion Resisting Chromium and Chromium-Nickel Steel Covered Spec. for Low Alloy Steel Covered Ar Spec, for Copper and lts Alloy Arc Std. for Steel But

Std. for Manual Gas Metal Arc (Gma) Std. for Manual Gas Tungsten Arc (Gta) Fusion Spec. for Resistance Seam and Roll Spot Std. for Resistance Spot
Std. Pract. for High Strength Carbon Steel Wrought td. Spec. for Factory Made Wrought Nickel and Nickel Alloy Std. Spec. for Seamless and Welded Unalloyed Titanium Spec. for High Test Wrought std. Spec. for Factory Made Wrought Aluminum and lts Alloy Std. Pract. for Ball Valves with Flanged or But Std. Spec. for Carbon Steel Castings Suitable for Fusion Spec. for

Safety Std. for Transformer Type Arc act. for Qualification of Procedures and Personnel for the Std. Specs. fo Std. Pract. for Socke $s$ in Reinforced Concrete /

Uniform Building Code Std. for Spec. for Aluminum and lts Alloy Spec. for Magnesium Allo

r Corrosion Resisting Chromium and Chromium-Nickel Stee on (1969) ANSl W3.15

Spec. for Nickel and lts Alloy Bare Spec. for Surfacing Spec. for Titanium and lts Alloy Bare

Spec. for Composite Surfacing Spec. for lron and Steel Gas

Spec. for Copper and lts Alloy Std. for Rod End. Threaded, Alloy Steel, Flash Line Code System for Color Code System for Spec. for Steel Spec. for Steel Spec. for Stee Spec. for Steel Std. for

$-(\mathrm{Cb}+\mathrm{Ta})(1973)$

$.8 \mathrm{Cr}-2.8 \mathrm{Ni}-1.6 \mathrm{Co}-1.8 \mathrm{Mo}-0.32 \mathrm{~V})(\mathrm{l}$

3C)) (SAE 6130) (1973)

$$
\text { Std. for }
$$

or Bare Mild Steel Electrodes and Fluxes for Submerged Arc Spec. for Mild Steel Electrodes for Gas Metal Arc gth, Quenched and Tempered Alloy Steel Plate, Suitable for Rec. Pract. for Plasma Are

$.5 \mathrm{Cr}-10.5 \mathrm{Ni}-7.5 \mathrm{~W}(1973)$

.5Cr-18Co-2.5Ti-1.5Al (1973)

$-4.5 \mathrm{Ni}-2.9 \mathrm{Mo}-0.1 \mathrm{~N}(1973)$ Std. Spec. for Sper trols to Prevent in Service Cracking of Carbon Steel (P.1)
Welded Steel Pipe for High Pressure Service (1972A) Ans Welded Steel Pipe for High Pressure Transmission Servic Welded Steel Pipe for Low Temperature Service (1973) an Welded Steel Pipe (1972A)

Welded Steel Wire and Deformed Wire Fabric for Concrete

Welded Steel Wire Fabric for Concrete Reinforcement ( 19

Welded Structural Steel Tubing (1974)

Welded Threaded Rod Ends (1973)

Welded Titanium Tube for Condensers and Heat Exchangers

Welded Tubes for Nuclear Service (1971) ANSI N124

Welded Tubes (1970) ANSI H53.I

Welded Tubes (1973) ANSI H38.11

Welded Unalloyed Titanium Welding Fittings (1971) ANSI

Welded Unfired Pressure Vessels (1972B) ANSI G8I.4

Welded Wire Building Fabric (1972)

Welded Wire Fabric (1970)

(Welded Wire Fabric) (1962)

Welded Zirconium Tubes for Nonnuclear Application (1972

Welded, Corrosion and Heat Resistant (25Cr-20Ni) (SAE

Welding and Cutting (1969)

Welding and Cutting (1973)

(Welding and Cutting) (1973)

(Welding and Cutting) (1973)

Welding and Cutting, Calcium Carbide and Acetylene (197

Welding and Threaded (1973)

Welding Cast lron (1969) ANSl W3.15

Welding Coated Low Carbon Steels (1970)

Welding Code (1972)

Welding Control (1974)

Welding Electrodes and Rods (1972)

Welding Electrodes (1969) ANSI A5.1

Welding Electrodes (1969) ANSI W3.11

Welding Electrodes (1969) ANSI W3.12

Welding Electrodes (1969) ANSI W3.3

Welding Electrodes (1969) ANSI W3.4

Welding Electrodes (1969) ANSI W3.5

Welding Electrodes (1969) A NSI W3.6

Welding End Valves (1973)

Welding Equipment (1974)

Welding Equipment (1974)

Welding Equipment (3 Phase) (1973)

Welding Equipment, 3 Phase (1973)

Welding Fittings for High Pressure Service Piping (1969

Welding Fittings for Pressure Piping (1972) ANSI H34.15

Welding Fittings (1971) ANSI H50.l

Welding Fittings (1973)

Welding Fittings (1973) A NSI H38. 19

Welding for General Service (1972)

Welding for High Temperature Service (1974) ANSI G37.2

Welding lndustrial and Mill Cranes (1970)

Welding Machines (1972) ANSI C33.2

Welding of Steel Castings (1972) ANSI G52.6

Welding of Structural Steel Highway Bridges (1974)

Welding Reducer Inserts (1974)

Welding Reinforcing Steel, Metal lnserts and Connection

Welding Rods and Bare Electrodes (1969) ANSl W3.10

Welding Rods and Bare Electrodes (1969) ANSI W3.19

Welding Rods and Bare Electrodes (1969) ANSI W3.9

Welding Rods and Covered Electrodes for Welding Cast lr

Welding Rods and Electrodes (1969) ANSI W3.14

Welding Rods and Electrodes (1970) ANSI W3.13

Welding Rods and Electrodes (1970) ANSI W3.16

Welding Rods and Electrodes (1970) A VSl W3.2

Welding Rods (1969) ANSI W3.2

Welding Rods (1969) ANSI W3.7

Welding Ty pe (1973)

Welding Wire ldentification (1973)

Welding Wire ldentification (1973)

Welding Wire $(0.95 \mathrm{Cr}-0.20 \mathrm{~V}(0.28-0.33 \mathrm{C})$ ) (SAE 6130)

Welding Wire, Corrosion and Heat Resistant $(18 \mathrm{Cr}-11 \mathrm{Ni}$

Welding Wire, Corrosion and Moderate Heat Resistant (1)

Welding Wire, Vacuum Melted $(0.95 \mathrm{Cr}-0.20 \mathrm{~V}(0.28-0.3$

Welding Zinc Coated Steel (1972)

Welding (1969) ANSI W3.17

Welding (1969) ANSI W3.18

Welding (1969) ANSI W3.20

Welding (1970E) ANSl G24.26

Welding (1973)

Welding (1973)

Welding, Corrosion and Heat Resistant, Cobalt Base -25

Welding, Corrosion and Heat Resistant, Nickel Base-19

Welding, Corrosion and Moderate Heat Resistant, 16.5Cr

Weldless Carbon Steel Chain (1973) ANSl G61.4

Weldments (1965) ANSI Z166.23

Weldments (1971)

Welds in Corrosive Petroleum Refining Environment (1972
ASTM

ASTM

ASTM

AsTM

ASTM

WSTI

NSA

ASTM

ASTM

AST

ASTM

ASTM

lan ASTM

WRI

CRSI

WRI

ASTM

SAE

A W

ANSI

Safet UL

Safety S UL

ICBO

ANSI

AW

AWS

NEMA

Std NEMA

AWS

AWS

AWS

AWS

AWS

AWS

AWS

ANSl

NSA

NSA

NSA

NSA

MSS

ASTM

ASTH

MSS

ASTM

MSS

ASTM

UL

1. Rec. Pr ASTM

ASHT

lCBO

AWS

AWS

AWS

AW.

AW

AWS

AWS

AWS

A WV

AWS

NSA

$\triangle A E$

SAE

SAE

SAE

SAE

$S A E$

AWS

Spec. F AWS

$A W S$

AWS

ASTM

AWS

$\mathrm{ICBO}$

$S A E$

SAE

SAE

ASTM

ASTM

AWS

NACE

A 155

A381

A333

A 135

UBC 526.6

A 185

*1 358

B338

B353

B394

B313

B363

A240

MP100

* 1.2 .1

RB80

AMS5577C

A3.0

Z49.1

409

UFC *2ART3 
Spec, for Oil

Rec. Pract. for Outboard Motor Boat Transom and Motor Std. Spec. for Seamless and Welded Carbon Steel Water ing, Tubing and Line Pipe Connections in High Pressure Oil Spec, for Petroleum Drilling and ented, Production Check Flanges and Gask/ Spec. for (Oil)

Through Control, Screwed and Flanged Wellhead, Underwater line Valves; Drilling Through Control, Screwed and Flanged ent) (1972)

nd Placement of Reinforced Concrete Casings for Irrigation Uniform Building Code Std. for Douglas Fir, Coast Region: idaho White, Sugar, and Lodgepole Pines; Engelmann Spruce: ), Ponderosa and Western White Pines, Spruce-Pine-Fir, ore Gluing of Southern Pine, Pacific Coast Douglas Fir and Industry Sud for Douglas Fir. timbers: Douglas and Hem Fir, Southern and Lodgepole Pine, Stu. Grading Rules for for Full Length Thermal Process Preservative Treatment of ve Treatment of Incised Pole Butts by the Thermal Process de Std. for Douglas Fir. Coast Region; West Coast Hemlock:

stern White Pine (North), Hem-Fir (North), Ponderosa and minology (Lumber and Timber) (1973)

t. Stairway, Aisle, Corridor, Building Construction and ign Values, Section Properties and Design Loads, Timber./ ildings, Walls, and Floors (1973) ) (1973)

olts, Lag Screws, Plates, Nails and Spikes, Washers. Pin/

3)

orms, Pavements, Driveways, and Interior Floors Exposed to .etc.) (1973)

Std. Meth. of Test for Bauer-Mcnett

n Starch Hydrolzates Including Syrups and Sugars, and Most mining Bulk Density Content in Feedstuffs (Corn) and Other

all Starches and Sugars, and to Most Other Products of the

rus in Corn Starch. Syrup and Sugar Obtained from the Corn (1973) ANSl / Std. for Safety for Alarm Valves for U'se in n Type) (1971) (1971)

1) ation, Using Highway Vehic/ Std. Std. or Cold Bonding Strength of Air Setting Refractory Mortar structural Laminated Wood Products for Use Linder Exterior Uniform Plumbing Code: Indirect Waste Piping. Std. Test Meth. for Ply Separation ANSI L14.75 ANSI L 14.79

4.11 Test Meth. for Evaluating
Test Meth. for Evaluation of Std. Meth. of

res (1971) ANS1 A37.51 Exposure with

aterials and Coated Fabrics: Carbon Arc Lamp Exposure with on Year Round Air Conditioning System) (1966)

Meth. of Analysis of Added Lysine in

(Cereal / Kjeldahl Meth. of Analysis of Crude Protein in
trogen) (Cereal/ trate Free Samples for Analysis of Crude Protein in Bread. Meth. for Sampling of

mistry) (1962)

Sedimentation Meth of Analysis of Smut in Meth. of Analysis of Crude Fat in Straight Dough Meth. of Analysis for Baking Quality of Ether-Bicarbonate Meth. of Analysis of Ergot in Rye and Linderstrom-Lang Meth. of Analysis of Amino Nitrogen in Modified Sorensen Meth. of Analysis of Amino Nitrogen in Van Slyke Meth. of Analysis of Amino Nitrogen in Tempering (Cereal Chemistry) (19/ Meth. for Experimental Meth. for Particle Size Distribution of Meth. of Analysis of Sedimentation Test for Meth. of Analysis of Potassium Bromate in White and Whole Trifructosan Meth. of Analysis for Admixture of Rye and

1962) Meth of Analysis of Vital Meth. of Analysis of Pelshenke Test

Microscopic Meth of Analysis of Smut in rimetric Meth. of Analysis of Malathion Residues in Milled Meth. of Analysis of Lipoxidase Activity of Ground Batch Meth. of Experimental Milling for Durum Meth for Preparation of Sample:

Meth. of Analysis of Sedimentatoon Test for Meth. of Analysis of Modified Sedimentation Test for Meth. of Analysis of Micro Sedimentation Test for of Analysis of Pigments in Flour, Semolina, Macaroni and Iodine Meth. of Analysis for Insect Eggs in White, glass Plate Meth. of Analysis for Insect Excreta in White, entils, Corn Meal, Corn Grits, Rolled Oats, BuIgur, Rolled

f Analysis of Malathion Residues in Milled Wheat Products Cor/ Thiochrome Meth. of Analysis of Thiamine in Bread, of Falling Number Determination of Both Meal and Flour of
Well Casing Centralizers (1973)

Well Dimensions on Boats (1974)

Well Pipe (1972A)

Well Service) (1972)

Well Servicing Structures (1974)

Wellhead Equipment (Production Valves, Ring Joint, Segm

Wellhead, and Christmas Iree Equipment; Flow Control Ch

Wells and Water Systems (Treatment, Quality and Managem

Wells (1972)

Rec. for Manufacture a

West Coast Hemlock; Western Red Cedar; White Fir; and S

Western Cedar and Hemlock; Subal-Pine Fir; and Mounta

Western Cedars (North) (Lumber) (1973)

Western Hemlock by Pressure Processes (1973)

Western Hemlock. Sitka Spruce Doors and Blinds (1972)

Western Larch, California Redwood and Hardwood Timber

Western Lumber (1972)

Western Red Cedar and Alaska Yellow Cedar Timber Poles

Western Red Cedar, Northern White Cedar and Alaska Yell

Western Red Cedar; White Fir; and Sitka Spruce (Lumber)

Western White Pines, Spruce-Pine-Fir, Western Cedar

Western Woods Use Book: Abbreviations, Symbols, and Ter

Western Woods Use Book: Fire Protection and Safety (Exi

Western Woods Use Book: Lumber Std, Grading Rules, Des

Western Woods Use Book: Sound Control (Acoustics) in Bu

Western Woods Use Book: Timber Fasteners (Connectors, B

Western Woods Use Book: Wood Preservation (Preservative

Western Woods Use Book: Wood Tanks, Vats, and Pipe (197

Wet and Dry Conditions (1970) ANSI 09.1

Exposed Plat

Wet Ashing with Nitric and Perchloric Acids (Wood, Pulp

Wet Classification of Asbestos Fiber (1970) ANSI K91.l

Wet Milled Products (1961)

Wet Milled Solid Products (1963)

Wet Milling Process (1971)

Wet Milling Process (1973)

Mining lron Content in Cor CR
/Ical Meth. for Deter CR

Wet Pipe Sprinkler System

D Starch Hydrolyzates and CR /R Determination of Phospho CR

Wet Process Porcelain Insulators (Apparatus, Cap and P

Wet Process Porcelain Insulators (Apparatus, Post Type)

Wet Process Porcelain Insulators (Suspension Type) (197

Wet Traction in Cornering Without Driving Torque Applic

Wet Type) (1972) ANSI A 111.29

Wet Lse) Exposure Conditions (1972) ANSI Z197.27

Wet Vented Systems and Special Wastes (1973)

(Wet) of Solid and Corrugated Fiberboard (1969)

Wettability of Fabrics and Textiles in Yarn Form (1971)

Wetting Agents in Caustic Soda for Mercerization (1971)

Wetting Agents (Cotton Test Skein Meth.) (I971) ANSI Ll

Wetting and Drying Tests of Compacted Soil Cement Mixtu

/R Resistance of Textile M

Wetting (1972) ANSI LT4.224

Wetting (1972) ANSI L14.225

Test Meth. for Weathe

What Makes a Good Air Conditioning System (Information

Wheat and Bulgur (Cereal Chemistry) (1973)

Wheat and Flour Mill Products (Boric Acid Modification)

Wheat and Flour (Calculated from Percentage of Total Ni

Wheat and Other Grains, and Yeast Foods (Cereal Chemist

Wheat and Other Whole Grains (Cereal Chemistry) (1962)

Wheat and Rye (Cereal Chemistry) (1962)

Wheat and Soy Flour, Feeds and Cooked Feeds (Cereal Che

Wheat Bread Flour (Cereal Chemistry) (1962)

Wheat Flour and Semolina (Cereal Chemistry) (1962)

Wheat Flour and Semolina (Cereal Chemistry) (1962)

Wheat Flour and Semolina (Cereal Chemistry) (1962)

Wheat Flour and Semolina (Cereal Chemistry) (1962)

Wheat Flour Milling: Equipment. Sample Preparation, and

Wheat Flour (Cereal Chemistry) (1962)

Wheat Flour (Cereal Che mistry) (1962)

Wheat Flour (Cereal Chemistry) (1962)

Wheat Flours (Cereal Che mistry) (1962)

Wheat Gluten (Cereal Chemistry) (1962)

(Wheat Meal Fermentation Time Test) (Cereal Chemistry) (

Wheat Milling: Temper Table (Cereal Chemistry) (1962)

Wheat or Rye (Cereal Chemistry) (1962)

Wheat Products (Wheat, Flour, Bran, Shorts, etc.) to De

Wheat (Cereal Chemistry) (1962)

Wheat (Cereal Chemistry) (1962)

Wheat (Cereal Chemistry) (1962)

Wheat (Cereal Chemistry) (1965)

Wheat (Cereal Chemistry) (1968)

Wheat (Cereal Chemistry) (I968)

Wheat $(1962)$

Wheat, and Corn Flour (Cereal Chemistry) (1962)

Wheat, and Corn Flour (1962)

Wheat, Breakfast Cereals (Except Those Which Are Sugar

(Wheat, Flour, Bran, Shorts, etc.) to Determine Added Am

Wheat, Rice, and Other Whole Grain Products, Corn Grits

Wheat, Rye, Barley, Other Grains, and Malted Cereals (C
API.

ABYC

API

API

API

API

WSC

ICBO

ICBO

ICBO

AWPA

FHDA

ICBO

WWPA

$A W P A$

AWPA

ICBO

ICBO

WWPA

WWPA

WWPA

WWPA

WWPA

WWPA

$W W P A$

ASTM

TAPP]

STM R UL

ANSI

ANS1

ASTM

ASTM

1CBO

AATCC

AATCC

AATCC

ASTM

AATCC

AATCC

NESCA

$\mathrm{AACCH}$

AACCH 6- 42

$\mathrm{AACCH} \quad 46.19$

$\mathrm{AACCH} 46.11$

AACCH $64-70$

$\mathrm{AACCH} \quad 42.71$

AACCH 30.25

AACCH 10.10

AACCH 42.56

AACCH 46.30

AACCH 46.31

AACCH 46.32

AACCH $26-10$

$\mathrm{AACCH} 50.10$

AACCH 56.60

AACCH $48-42$

$\mathrm{AACCH} 06-10$

$\mathrm{AACCH} \quad 38-20$

AACCH 56.50

AACCH 26.95

$\mathrm{AACCH} \quad 42-70$

$\mathrm{AACCH} \quad 60-30$

$\mathrm{AACCH} 22.41$

AACCH 26.30

$\mathrm{AACCH} \quad 62.70$

$\triangle A C C H$ 56-61A

$\mathrm{AACCH} 56.62$

AACCH 56.63

Meth AACCH 14.50

AACCH 28.44

AACCH 28.43

$\mathrm{AACCH}$ 44.15A

$\mathrm{AACCH} \quad 60.30$

A ACCH 86.80

AACCH $56.81 \mathrm{~B}$ 
Buhler Meth. of Experimental Milling for Bread and Soft

Batch Meth. of Experimental Milling for Bread and Soft d. for General Requirements for Regular Bicycles Including 1966)

for Test. Pavement Polishing in the Laboratory (Full Scale Metal Industrial V.Grooved agricultural and Log Skidder, Truck, Bus, Bead S/ Rim and Std. for Agricultural Press and Gage Std. for Agricultural Planter Press

r Thre/ Std. for Attachment of Implements to Agricultural ee Link Attachment for Hitching Implements to Agricultural Std. for Operator Protection for ndustrial Steering, Drive. Planter (D rill) Press, and Gage Iling Object Protective Structure (FOPS) for Prime Movers, or Prime Movers. Wheeled and Track Type Front End Loaders, t Free Link Hitch Attachment of Implements to Agricultural Steel Products Manual: Wrought Steel Std. Spec. for Wrought Steel ther Equipment (1966) Std. for Demountable Tired Std. Spec. for Wrought Carbon Steel Std. Spec. for Cast Steel

Std. for Rim Contours for Agricultural Press and Gage Std. Dimensional Tolerances for Industrial $\mathrm{m}$ Treated Alloy Steel Forgings for Turbine Rotor Disks and tory Fatigue Test Procedures for Production Disc and Spoke t. for for Practical Minimum Rec. Tolerances of Spoke Type try) (1962) Meth. of Analysis of Undenatured fir and Tamarack / Uniform Building Code Std. for Eastern Quantitative Meth. of Analysis of Potassium Bromate in Butts by the Thermal Process (Western Red Cedar, Northern ine, and Hemlock-Tamarack; Balsam Fir; Northern Pine and ec. Pract. for the Density and Contrast Range of Black and

Fir, Coast Region; West Coast Hemlock; Western Red Cedar: Std. Meth. for Chemical Analysis of Std. Spec. for

i 211.210 Std. Neth. for Evaluation of

rformance Requirements for Table Napery Other Than Damask

Std. Meth. of Chemical Analysis of

larch (North), Eastern Hemlock-Tamarack (North), Eastern n Pine An/ Uniform Building Code Std. for Eastern Spruce, ite Pine (North), Hem-Fir (North), Ponderosa and Western Std. Neth. for Analysis of

h. of Test for Indexes of Whiteness and Yellowness of Near e Std. for Douglas Fir; Larch; Hem-Fir; Ponderosa, Idaho lodine Meth. of Analysis for Insect Eggs in Glass Plate Weth. of Analysis for Insect Excreta in $\mathrm{s}$ (Color Neasurement $\mathrm{O} /$ Std. Meth. of Test for Indexes of Test Meth for Reflectance, Blue, and and Industrial Use Garments (Clothing), Commercial Wash othing), 100\% Man Made or Blend Fabrics. Commercial Wash Std. Meth. for Particle Size Analysis of Apparent Porosity, and Apparent Specific Gravity of Fired Meth. of Analysis of Crude Fat in Egg Yolk and Dried of Analysis of Thiamine in Bread, Wheat, Rice, and Other reals, F/ Fluorometric Meth. of Analysis of Riboflavin in Electrical Meth. for Analysis of Moisture in Meth. for Sampling of Wheat and Other

tation Content (Radiographic Inspection) in Corn and Other ea in Cloth or Sack Fibers. Whole or Ground Cereal Grains,

e Test Paper for Analysis of Urea in Cloth or Sack Fibers, Determination of Dispersible Nitrogen in Ground Soybeans, $r$ Determination of Dispersible Protein in Ground Soybeans, tative Meth. of Analysis of Potassium Bromate in White and y Operated Metallic Gas Valves in Gas Distribution Systems

72)

1) Std. for the Test Meth. for Std. for Low Power

Analysis (Cereal Chemistry) (Bolting Cloth, Filter Paper, Meth. of Test for Dropping Point of Lubricating Grease of Std for Radio Interference Agricultural Tractor Belt Speed and Pulley td. for Reproducer Test Tape: Full Track, 1/4 In. $(6.3 \mathrm{Mm})$

Std. Spec. for Copper Alloy No. 260 Brass Strip in Narrow get/ Std. Analytical Meth, for Determining Iodine Number al and Vegetable Fats (Cereal Chemistry) (1967)

Test Meth. for Fiber Identification ng and Recreational Vehicle Manufacturing Plants (Fire and Tent. Meth. of Test for Std. for Safety for

73) ANSI A 195.l

Rec. Against Roof Leakage of Buildings Not Due to Hail or ) Quality Stds. for Wood Screens for

Fibers) (Instit/ Uniform Building Code Std. for Fire Tests of Std. Performance Requirements for Woven Safety Std. for Door, Drapery, Gate, Louver, and
Wheats (Cereal Chemistry) (1962)

Wheats (Cereal Chemistry) (1962)

Wheel Assembly, Chainguards, Saddle Braces, Pedal Clear Wheel Diameters and Outlet Areas for Centrifugal Fans

Wheel Meth.) (1972)

Wheel Std (1966)

Wheel Stds. for Motorcycles, Passenger Cars, Off Road,

Wheel Tires (Farm Machinery) (1971)

Wheel Tires (Farm Machinery) (1971)

Wheel Tractors Equipped with Quick Attaching Coupler Fo

Wheel Tractors (1972) SAE J715

Sid. for

Wheel Type Agricultural Tractors (1972) SAE J333

Wheel, Tractor, Implement, and Log Skidder (1974)

Wheeled and Track Type Front End Loaders, Wheeled Dozer

Wheeled Dozers, Track Type Tractors, and Motor Graders

Wheeled Tractors (1972)

Wheels and Forged Railway Axles (1973)

Wheels for Electric Railway Service (1972) ANS1 G57.13

Wheels for Industrial Trucks, Casters, Conveyors, and O

Wheels for Locomotives and Rail Cars (1971) ANSI G39.2

Wheels for Railway Service (1973)

Wheels (Farm Machinery) (1971)

Wheels (1965)

Wheels (1970) ANSI G55.15

Std. Spec. for Vacuu

Wheels, and Demountable Rims Intended for Highway lise O

Wheels, Demountable Rims and Rim Spacers (Manufactures

Whey Protein Nitrogen in Nonfat Dry Milk (Cereal Chemis

White and Red Pine. Eastern Spruce and Hemlock, Balsam

White and Whole Wheat Flour (Cereal Chemistry) (1962)

White Cedar and Alaska Yellow Cedar Poles) (1973)

/for Eastern Spruce. White P

White Cedar Lumber (1973)

White Films and Slides for Television (1970)

White Fir: and Sitka Spruce (Lumber) (1973)

White Lead Pigments (1973)

White Metal Bearing Alloys (1973) ANSI H39.1

/R Dougla

White Mineral Oils by Ultraviolet Absorption (1973) Ans

White or Dyed) (Napkin, etc.) (Institutional Textile) (

White Pan Bread Flour Spec. (Cereal Chemistry) (1962)

White Pigments (1973) ANSI K15.1

White Pine (North), Hem-Fir (North), Ponderosa and We

White Pine, and Hemlock-Tamarack: Balsam Fir: Vorther

White Pines, Spruce-Pine-Fir, Western Cedars (North

White Zinc Pigments (1973)

White, Opaque Materials (Color Measurement of Textiles,

White, Sugar, and Lodgepole Pines; Engelmann Spruce; We

White, Wheat, and Corn Flour (Cereal Chemistry) (1962)

White, Wheat, and Corn Flour (1962)

Whiteness and Yellowness of Near White, Opaque Material Whiteness of Bleached Fabric (1972) ANS1 L14.175

Whites 190 Deg. F, Colors 160 Deg. F Maximum Hot Head P

Whites (W ith Chlorine Bleach) 190 Deg. F, Colors 160 De

Whiteware Clays (1974)

Whiteware Products (1972)

Whole Egg (Cereal Chemistry) (1962

/R Absorption, Bulk Density,

Whole Grain Products, Corn Grits, Corn Meal, Puffed Cer

Whole Grain Products, Grits, Meal, Flaked and Puffed Ce

Whole Grain (Cereal Che mistry) (1962)

Whole Grains (Cereal Chemistry) (1962)

Eth. for Determining Insect Infes

Whole or Chopped Nuts, Spices, etc. (Cereal Chemistry)

Whole or Ground Cereal Grains, Whole or Chopped Nuts, S

Whole or Ground Full Fat or Extracted Flakes, Full Fat

Whole or Ground Full Fat or Extracted Flakes, Full Fat

Whole Wheat Flour (Cereal Chemistry) (1962)

Whose Maximum Allowable Operating Pressure Does Not Ex

Wicking of Automotive Fabrics and Fibrous Materials (19

Wicking of Composite Motor Oil Cans (1974)

Wide Band Transformers (Less Than 100 Watts Power) (197

Wide Field and Compound Microscope, Petri Dish, Sieve,

Wide Temperature Range (1972) ANSI Z11.207

Wideband Filters (1974) A NSI C83.102

Width (1971) SAE J720

Width, Open Reel (For Tape Speeds of 7.5 ln. $0190.5 \mathrm{~mm}$ Widths and Light Gage for Heat Exchanger Tubing (1972)

Wijs Meth.) Content in Corn, Grain Sorghum and Other Ve (Wildlife) Rifle Ranges (1971)

Wiley Meth. of Analysis of Melting Point of Normal Anim Wilson Stain) (Pulp and Paper) (1973)

Wind Hazards at Plants Assembling Mobile Homes, Modular

Wind Resistance of Asphalt Shingles (1972T)

Wind Resistance of Prepared Roof Covering Materials (19

Wind (1972)

Window and Door Openings (Architectural Woodwork) (1973

Window A ssemblies (1973)

Window Curtain Fabrics (Sash Casement) (Excluding Glass

Window Electric Operators and Systems (1973)
AACCH $26-20$

$\mathrm{AACCH} 26.21$

BMA $6 / 5$

AMCA 2401

ASTM E451

CFTMA W8

TRA

ASAE

ASAE

ASAE

ASAE

ASAE

TRA

$S A E$

$S A E$

SAE

AISI

ASTM

CFTMA

ASTM

ASTM

ASAE

CFTMA

\$223

5278.2

\$217.8

S.305.3

$\mathrm{J} 23$

231

J715D

11

A25

A504

A583

S222

ASTM A47

SAE J267

SAE J85

$\mathrm{AACCH} \quad 46.20$

ICBO I'BCS25-5

$\mathrm{AACCH} \quad 48.42$

AWPA C7

ICBO IBC 25.8

SUIPTE RP

ICBO

ASTM

ASTM

ASTMI

A $\backslash \mathrm{SI}$

A ACC

ASTII

ICBO

CBO

ICBO

ASTM

ASTM

ICBO

$\mathrm{AACCH}$

AACCH

ASTM

AATCC

ANSI

ANSI

ASTM

ASTM

A ACCH $30-18$

$\mathrm{AACCH} \quad 86-80$

AACCH 86.70

AACCH 44.10

$\mathrm{AACCH} \quad 64.70$

CR

$\mathrm{AACCH} 28.87$

AACCH 28.87

AACCH $46-23$

$\mathrm{AACCH} \quad 46-24$

Quanti AACCH 4842

ANSl B16.33

$S A E$

J 1613 A

CCTI Cl24

IEEE 111

AACCH 28.90

Std. ASTM D2265

EIA RS4l6

ASAE S210.2

EIA RS400

ASTM B569

CR H.32

NRA *12

AACCH 58-4l

TAPPI UM-14

FMS $\quad 7.90$

ASTM D3161

UL

FMS

997

LPD 1-4

सx 1 *1.1100

ICBO UBCS43.4

ANSI L24.1.4

UL 
973) ANS1 Z265.1 Rec. Pract. Test Meth. for Determining Std. for Back

1 for Use Principally as a Substitute for Plate Glass Show fire (1974) Approval Std. for Heat and Smoke Roof and ination Vertically Sliding or Horizontally Operating Storm Spec. for Aluminum Prime

form Building Code Std. for Installation of Fire Doors and Std. for Fire Doors,

Rec. Pract. for Passenger Car Rec. Pract. for Electric

Rec. Pract. for Passenger Car Rec. Pract. for Electric Tent. Meth. of Analysis for Iron in Tent. Meth. of Analysis for Volatile Acid in ent. Hydrometer Meth, of Analysis for Alcohol (Ethanol) in ent. Pycnometer Meth. of Analysis for Alcohol (Ethanol) in Tent. Meth. of Analysis for Brix (Balling) of Official Meth. of Analysis for Total Acid in Official Meth. of $\mathrm{pH}$ Determination in Tent. Meth. of Analysis for Total Sulfur Dioxide in Tent. Meth. of Analysis for Free Sulfur Dioxide in Tent. Meth. of Analysis for Copper in t. Ebulliometer Meth. for Analysis of Alcohol (Ethanol) in Std. Part Bit, Screwdriver, Tri Std. for Screw. Hex Head, Tri

Nonlocking (1973) Std for Screw, 100 Deg. Head, Tr td. for Bolt, Pan Head, Close Tolerance, Short Thread, Twi for Machine Screw, Flat Fillister Head, Full Threaded, Tri 97) Std. for Pan Head, Close Tolerance, Short Thread, Twi

nlocking (1972)

onlocking (1972)

onlocking (1972) Std. for Screw, Hex Head, Tr

std. for Screw, 100 Deg. Oval Head, Tri Std. for Screw, 100 Deg. Head, Tri Std. for Screw, Pan Head, Tr Std. for Bolt, 100 Deg. Head, Tri Std. for Bolt, 100 Deg. Head, Tri Std. for Bolt, 100 Deg. Head, Tri Std. for Bolt, 100 Deg. Head, Tri

Self Locking and Nonl

Self.Locking and Non

Nonlocking (b

self Locking and Nonlo/

nonlocking (19) Std. for Bolt, 100 Deg. Reduced Head, Tri Std. for Bolt, 100 Deg. Head, Tri

Std, for Bolt, 100 Deg. Reduced Head, Tri ort Thread, Self Locking and Nonlocki/ Bolt, 100 Deg. Tri id. for Bolt, Pan Head, Close Tolerance, Short Thread, Tri king (1972) for Machine Screw. Flat Fillister Head, Full Thread, Tri

ocking and Nonlocking / Std. for Screw, Hex Head, Tri Bit, Screwdriver, Tri

Rec. Pract. for Electric Windshield Std for Thermoplastic Insulated

Electrical Energy (197/

d. for Crossed Linked Thermosetting Polyethylene Insulated Cellular Metal Floor Raceways and Fittings for Electrical

1 and Aluminum Conduit for Safe L se as a Metal Raceway for Std. for Safety for Electrical Metallic Tubing for Std. Spec. for Polyethylene Insulated

td. Spec, for Polyethylene Jacket for Electrical Insulated for General Purpose Ethylene Propylene Rubber Jacket for pec. for Extra Heavy Duty Black Polychloroprene Jacket for Std. Spec. for Natural Rubber Jacket for or Styrene-Butadiene-Synthetic Rubber (SBR) Jacket for Std. Spec. for General Purpose Polychloroprene Jacket for sid. Spec. for Heavy Duty Black Polychloroprene Jacket for Std. Spec. for Polyvinyl Chloride (PVC) Jacket for Underground Installation for Communication and Electrical Std. Spec, for Ozone Resisting Insulation for std. Spec. for Ozone Resisting Butyl Rubber lnsulation for synthetic Rubber Heat or Moisture Resisting Insulation for Std. Spec. for Synthetic Rubber Insulation for Id. Spec. for Natural Rubber Heat Resisting Insulation for ynthetic Rubber Heat and Moisture Resisting Insulation for Std. Spec. for Synthetic Rubber Insulation for t (1973) Uniform Building Code Std for Welded Steel Std. Spec. for Aluminum Alloy Rivet and Cold Heading ing of Aluminum and Aluminum Alloy Rivets and Cold Heading Rec. Pract. for Stainless Steel $17.7 \mathrm{pH}$ Spring

Rec. Pract. for Stainless Steel, SAE 30302, Spring special Quality High Tensile, Hard Drawn Mechanical spring gned for Use as a Iniform Building Code Std. for Metal, the Relative Stiffness of Leather by Means of a Torsional

f Rubber and Rubber Like Materials by Means of a Torsional crete $(1970)$

Std. Spec. for Phosphorus Deoxidized Copper Std. Spec. for Oxygen Free Electrolytic Copper

s (1973) ANSI H 17.1

s (1973) ANSI H/

s (1973) ANSI H17.2 Std. Spec. for Lake Copper
Chemically Refined Copper std. Spec. for Electrolytic Copper
Window Fogging Resistance of Interior Trim Materials (1

Window Location for Roll Film Cameras (1973)

/Rglary Resisting Glazing

Window Vents Designed to Open Automatically in Case of

Windows for External Application (1972) ANSI A 134.3

Windows (1972) ANSI Al34.1

Windows (1973)

Windows $(1973)$

Windows, Shutters, and Glass Blocks (1973) ANSI A2.7

Windshield Defrosting Systems (1971)

Windshield Washer Switch (1971)

Windshield Washer Systems (1972)

Windshield Wiper Switch (1971)

Wine (Enology) (1972)

Wine (Enology) (1972)

Wine (Enology) (1972)

Wine (Enology) (1972)

Wine (Enology) (1972)

Wine (Enology) (1972)

Wine (Enology) (1972)

Wine (Enology) (1972)

Wine (Enology) (1972)

Wine (Enology) (1972)

Wine (Enology) (1972)

Wing Recess (1972)

Wing Recess, Alloy Steel, Full Thread, Nonlocking (1973

Wing Recess, Alloy Steel, Full Thread, Self-Locking and

Wing Recess, Alloy Steel, Self Locking and Nonlocking(

Wing Recess, Alloy Steel, Self.Locking and Nonlocking (

Wing Recess, A286 Acres, Self Locking and Nonlocking.(1

Wing Recess, A286 Cres, Full Thread Nonlocking (1972)

Wing Recess, A286 Cres, Full Thread Self Locking and No

Wing Recess, A286 Cres, Full Thread, Self Locking and N

Wing Recess, A286 Cres, Full Thread, Self Locking and N

Wing Recess, A286 Cres, Self.Locking and Nonlocking (19

Wing Recess, Close Tolerance (1972)

Wing Recess, Close Tolerance (1972)

Wing Recess, Close Tolerance, Alloy Steel, Long Thread,

Wing Recess, Close Tolerance, Alloy Steel, Short Thread"

Wing Recess, Close Tolerance, Alloy Steel, Short Thread

Wing Recess, Close Tolerance, A286 Cres, Short Thread,

Wing Recess, Close Tolerance, A286 Cres, Short Thread,

Wing Recess, Close Tolerance, 6Al-4V Titanium Alloy, Sh

Wing Recess, Titanium Alloy $6 \mathrm{Al}-4 \mathrm{~V}$. Self Locking and No

Wing Recess, Titanium Alloy, $6 \mathrm{Al}-4 \mathrm{~V}$, Self.Locking and $\mathrm{N}$

Wing Recess, 6Al.4V Titanium Alloy, Full Thread, Nonloc

Wing Recess, 6Al.4V Titanium Alloy, Full Thread, Self L

Wing, Spec. for (1972)

Wiper Switch (1971)

Wire and Cable for the Transmission and Distribution of

Wire and Cable for the Transmission and Distribution of

Wire and Cablc Installation (1973) ANSI C33.58

Wire and Cable Installation (1973) ANSI C 33.92

Wire and Cable Installation (1973) ANSI C33.98

Wire and Cable (1972)

Wire and Cable (1972)

Wire and Cable (1972)

Wire and Cable (1973)

Wire and Cable (1973) ANSI C8.25

Wire and Cable (1973) ANSI C8.28

Wire and Cable (1973) ANSI C8.31

Wire and Cable (1973) ANSI C8.32

Wire and Cable (1974)

Wire and Cable (1974)

Wire and Cable (1974) ANSI C8.29

Wire and Cable (1974) ANSI C8.37

Wire and Cable (1974) ANSI C8.38

Wire and Cable, 75 Deg. C Operation (1974) ANSI C8.24

Wire and Cable, 75 Deg. C Operation (1974) ANSI J8.4

Wire and Cable, 90 Deg. C Operation (1974) ANSI C 8.40

/Ty for LL

UL

ASTM

ASTM

Std. Spec ASTM

ASTM

Std. Spec. F ASTM

ASTM

ASTM

ASTM

/ for Plastic Utilities Duct for NEMA

ASTM

ASTM

Wire and Deformed Wire Fabric for Concrete Reinforcemen

Wire and Rod (1973) ANSI H38.12

Wire and Rods (1972)

Wire and Springs (1970)

Wire and Springs (1971)

Wire and Springs (1972)

Wire Apparatus (1972) ALCA E6

Wire Apparatus (1973) ANSI J2.2

Wire Bar Supports Used to Position

Wire Bars, Billets, and Cakes (1972)

Wire Bars, Billets, and Cakes (1972) ANSI H23.1

Wire Bars, Cakes, Slabs, Billets, Ingots, and Ingot Bar

Wire Bars, Cakes, Slabs, Billets, Ingots, and Ingot Bar
J275

PH3.1

972

1002.8

302.8

*l

UBCS43.5

80

J $902 \mathrm{~B}$

$\mathrm{J} 234$

J942B

J112A

$* 10$

$* 11$

*2

$* 3$

$* 4$

$* 6$

*6

$* 7$

$* 8$

$* 1$

4005

$5900 \cdot 03$

5600-06

5000.6

5300.06

5100.6

6000.3

6500-6

$5700-6$

6900.06

5400.06

4204. 16

4304-16

4104-16

4400-16

4703.16

4500-16

4803.16

4600.16

5200.06

5500.06

6100.3

5800.6

4001

J112A

WC5

WC7

209

797

D) 351

D2308

D2768

D2819

D532

D866

D753

D752

D1047

TC6

D574

D1352

D1520

D754

D469

D1679

D1523

UBCS26.6

B316

B565

J217

$\mathrm{J} 230$

J271

UBCS47.4

D2821

D 1053

$* 1.3 .1$

B379

B170

B4

B 442

B44
B5 
ort Equipment) (1974) Std. Spec. for Welded Steel form Building Code Std for use as a / Uniform Building Code Std. for Metal, Wire and Welded

pract. for Reinforced Bituminous Cóncrete Overlays (Welded 1972) /
anized) Iron or Steel Farm Field and Railroad Right of Way
Spec. for Aluminum Coated (Aluminized) Steel Core 1974)/ Std. Spec. for Zinc Coated (Galvanized) Steel Core niform Building Code Std. for Prestressed Stecl Strand and Std. Spec. for Cold Drawn Steel Std. Spec, for Cold Worked Deformed steel Uniform Building Code Std. for Cold Drawn Steel Std. Spec. for Lead and Lead Alloy Coated Soft Copper Std. Spec. for Aluminum Alloy 6201 T 81 Std. Spec. for Aluminum Alloy 5005-H 19 Std. Spec. for Soft or Annealed Copper Std. Spec. for Tinned Soft or Annealed Copper Tent. Spec. for Molybdenum Flattened Spec. for Nickel Coated Copper Clad Steel Std. Spec. for Phosphor Bronze Std. Spec. for Copper Silicon Allo Std. Spec. for Cold Heading Quality Carbon Steel Spec. for Hot Worked and Cold Finished Zirconium Bars and $\mathrm{d}$ and Cold Finished Zirconium and Its Alloy Bars, Rod, and Std. Spec. for Cold Heading Quality Carbon Steel Std for Vlagnetic Std. Spec. for Cold Heading Quality Steel Line Code System for Welding Color Code System for Welding Sid. Meth of Test. Hookup

Std. for Molded and Dipped Mica Capacitors Purpose $347 / 600$ Volts, 20 Amperes, 3 Phase Wye, 4 Pole, 4 Purpose $347 / 600$ Volts, 30 Amperes, 3 Phase Wye, 4 Pole, 4 Purpose 120/208 Volts, 20 Amperes, 3 Phase Wye, 4 Pole, 4 Purpose 120/208 Volts, 30 Amperes, 3 Phase Wye, 4 Pole, 4 Purpose 277/480 Volts, 20 Amperes, 3 Phase Wye, 4 Pole, 4 Purpose 277/480 Volts, 30 Amperes, 3 Phase Wye, 4 Pole, 4 les: Specific Purpose 125/250 Volts, 20 Amperes, 3 Pole, 3 specific Purpose 250 Volts, 30 Amperes, 3 Phase, 3 Pole, 3 specific Purpose 480 Volts, 20 Amperes. 3 Phase, 3 Pole, 3 specific Purpose 480 Volts, 30 Amperes, 3 Phase, 3 Pole, 3 specific Purpose 600 Volts, 30 Amperes, 3 Phase, 3 Pole, 3 specific Purpose 250 Volts, 15 Amperes, 3 Phase, 3 Pole, 3 specific Purpose 250 Volts, 20 Amperes, 3 Phase, 3 Pole, 3 ptacles: Specific Purpose 600 Volts, 30 Amperes, 2 Pole, 3 Specific Purpose 250 Volts, 20 Amperes 3 Phase, 3 Pole, 4 specific Purpose 250 Volts, 30 Amperes, 3 Phase, 3 Pole, 4 specific Purpose 480 Volts, 20 Amperes, 3 Phase, 3 Pole, specific Purpose 480 Volts, 30 Amperes, 3 Phase, 3 Pole, specific Purpose 600 Volts, 30 Amperes, 3 Phase, 3 Pole, 4 les: Specific Purpose 125/250 Volts, 20 Amperes, 3 Pole, 4 les: Specific Purpose 125/250 Volts, 30 Amperes, 3 Pole, 4 ptacles: Specific Purpose 125 Volts, 20 Amperes, 2 Pole, ptacles: Specific Purpose 125 Volts, 30 Amperes, 2 Pole, 3 ptacles: Specific Purpose 250 Volts, 15 Amperes, 2 Pole, 3 ptacles: Specific Purpose 250 Volts, 20 Amperes, 2 Pole, 3 ptacles: Specific Purpose 250 Volts, 30 Amperes, 2 Pole, 3 ptacles: Specific Purpose 480 Volts, 20 Amperes, 2 Pole, 3 ptacles: Specific Purpose 480 Volts, 30 Amperes, 2 Pole, 3 ptacles: Specific Purpose 600 Volts, 20 Amperes, 2 Pole, 3 cles: Specific Purpose 277 Volts Ac, 20 Amperes, 2 Pole, 3 cles: Specific Purpose 277 Volts Ac, 30 Amperes, 2 Pole, 3 Purpose 120/208 Volts, 20 Amperes, 3 Phase Wye, 4 Pole, 5 Purpose 120/208 Volts, 30 Amperes, 3 Phase Wye, 4 Pole. Purpose $277 / 480$ Volts, 20 Amperes, 3 Phase Wye, 4 Pole, Purpose 277/480 Volts, 30 Amperes, 3 Phase Wye, 4 Pole, 5 Purpose $347 / 600$ Volts, 20 Amperes, 3 Phase Wye, 4 Pole. 5

Purpose $347 / 600$ Volts, 30 Amperes, 3 Phase Wye, 4 Pole. Change of Fabrics Due to Flat Abrasion (Frosting): Screen Std. Spec. for Carbon Steel 4.1/ Std. Spec. for General Requirements for Carbon Steel Rec. Pract. on Application, Care, and Use of
e Mounted Types (1971) Spec. for Electric Spec, for electric, Permanent, Crimp Style, Pre Insulated, (Class 2 Std. Spec. for Zinc Coated Steel Std. for Punches Spec. for Steel Welding Spec. for Steel Sprin Std. Spec. for Columbium and Its Alloy Bar. Rod, and
Wire Building Fabric (1972)

Wire Cable Wrought Steel Heavy Thimble (For Ground Supp

Wire Cloth Sieves for Test. Purposes (1970) ANSl Z23.1

Wire Enamels (1973)

Wire Fabric for Concrete Reinforcement (1972) ANSI G45.

Wire Fabric for Concrete Reinforcement (1972) ANSI G45.

Wire Fabric for Concrete Reinforcement (1973)

Wire Fabric Laths, and Metal Accessories (Designed for

Wire Fabric (1970)

Wire Fabric) (1962)

Wire Fencing (1973) ANSI G8.9

Wire for Aluminum Conductors, Steel Reinforced (ACSR)

Wire for Aluminum Conductors, Steel Reinforced (ACSR)

Wire for Communication Cable (1969) ANSI C7.40

Wire for Concrete Construction (1973)

Wire for Concrete Reinforcement (1972) ANSI A50.3

Wire for Concrete Reinforcement (1972) ANSI G45.2

Wire for Concrete Reinforcement (1973)

Wire for Electrical Purposes (19674 ANSI C7.15

Wire for Electrical Purposes (1972) ANSI C7.51

Wire for Electrical Purposes (1972A) ANSI C7.49

Wire for Electrical Purposes (1974) ANSI C7.1

Wire for Electrical Purposes (1974) ANSI C7.4

Wire for Electron Tubes (1973)

Wire for Electronic Application (1972)

Wire for General and Spring Purposes (1972)

Wire for General Purposes (1973) ANSI H30.1

Wire for Machine Screws (1971) ANSI G54.14

Wire for Nonnuclear Applications (1972) ANSI 7179.11

Wire for Nuclear Application (1973) ANSI N122

Wire for Tapping or Sheet Metal Screws (1971) ANSI G54

Wire for Use in Electrical Apparatus (1973) A NSI C9.100

W'ire for Wood Screws (1971) ANSl G54.18

Wire Identification (1973)

Wire Identification (1973)

Wire Insulation (1974)

Wire Lead Styles) (1972)

Wire Locking Type (1972)

Wire Locking Type (1972

Wire Locking Type (1973)

Wire Locking Type (1973)

Wire Locking Type (1973)

Wire Locking Type (1973)

Wire Locking Type (1973)

Wire Locking Type (1973)

Wire Locking Type (1973)

Wire Locking Type (1973)

Wire Locking Type (1973)

Wire Locking Type (1973)

Wire Locking Type (1973)

Wire Locking. Grounding Type 1973)

Wire Locking, Grounding Type (1973

Wire Locking, Grounding Type (1973)

Wire Locking, Grounding Type (1973)

Wire Locking, Grounding Type (1973)

Wire Locking, Grounding Type (1973

Wire Locking, Grounding Type (1973)

Wire Locking, Grounding Type (1973)

Wire Locking, Grounding Type (1973)

Wire Locking, Grounding Type (1973)

Wire Locking, Grounding Type (1973)

Wire Locking, Grounding Type (1973)

Wire Locking, Grounding Type (1973)

Wire Locking, Grounding Type (1973)

Wire Locking, Grounding Type (1973)

Wire Locking, Grounding Type (1973)

Wire Locking, Grounding Type (1973)

Wire Locking, Grounding Type (1973)

Wire Locking, Grounding Type (1973)

Wire Locking, Grounding Type (1973)

Wire Locking, Grounding Type (1973)

Wire Locking, Grounding Type (1973)

W'ire Locking, Grounding Type (1973)

Wire Locking, Grounding Type (1973)

Wire Meth. (Textile Colorfastness) (1970)

Wire of Scrapless Nut Quality (1972) ANSI K91.3

Wire Rods and Incoated Coarse Roun

Wire Rope Hoists (Lug. Hook. Trolley Suspended and Bas

Wire Rope (1972)

Wire Sizes 12 to $26(1972)$

Wire Strand (1972A) ANSI C7.46

Wire Type (1972)

Wire $(0.95 \mathrm{Cr}-0.20 \mathrm{~V}(0.28-0.33 \mathrm{C}))(\mathrm{SAE} 6130)$ (1973)

Wire $(0.95 \mathrm{Cr}-0.22 \mathrm{~V}(0.48-0.53 \mathrm{C})$ ) (SAE 6150) (1973)

Wire (1964) ANSI Z179.19
1. for Zinc Coated (Galy

WRI

NSA

ASTM

ASTM

ASTM

ASTM

Uni ICBO

ICBO

CRSI

WRI

ASTM

ASTM

ASTM

ASTM

ICBO

ASTM

ASTM

ICBO

ASTM

ASTM

ASTM

ASTM

ASTM

ASTM

ASTM

ASTM

ASTM

ASTM

ASTM

T Rolle ASTM

ASTM

NEMA

ASTM

$S A E$

$S A E$

ASTM

ElA

IGs and Receptacles: Specific Gs and Receptacles: Specific Gs and Receptacles: Specific Gs and Receptacles: Specific Nions of Plugs and Receptac IS of Plugs and Receptacles: $S$ of Plugs and Receptacles: of Plugs and Receptacles: of Plugs and Receptacles: $S$ of Plugs and Receptacles: 5 of Plugs and Receptacles: of Plugs and Rece I and Receptacles: land Receptacles: land Receptacles: land Receptacles: land Receptacles: Lugs and Receptac Lugs and Receptac of Plugs and Rece lof Plugs and Rece lof Plugs and Rece fof Plugs and Rece of Plugs and Rece of Plugs and Rece lof Plugs and Rece lof Plugs and Rece /Plugs and Recepta

Plugs and Recepta /Ptacles: Specific Ptacles: Specific Ptacles: Specific A Ptacles: Specific Ptacles: Specific Ptacles: Specific for Color

ANSl

ANs

ANSI

ANSI

ANSI

ANSI

ANSI

ANSl

ANSl

A $\backslash$ SI

ANSI

ANSI

ANSI

ANSI

ANS

ANSI

ANS

ANSI

ANSI

ANSI

ANSI

ANSI

ANSI

ANSI

ANSI

ANSI

ANSI

ANSI

ANSI

ANSl

ANSI

AATCC

ASTM

ASTM

APl

HMI

API

NSA

ASTM

ANSI

$\triangle A E$

$S A E$

ASTM

MP100

1045

Eli

D.3288

Al 85

4497

IBCS26.6

LBCS47.4

*1.2. 1 


\section{National Bureau of Standards KWIC Index of Engineering Standards}

eceptacles Off Center Boss Type, 30,50, and 60 Amperes, 4 Rec. Std for Radio Tube Std. Spec. for Tantalum Rod and ments for Carbon Steel Wire Rods and Uncoated Coarse Round Std. Spec for Music Spring Quality Steel Std Spec for Stainless and Heat Resisting Steel pec. for Free Machining Stainless and Heat Resisting Steel Std. Spec. for Brass Std Spec for Copper Clad Aluminum Std. Spec. for Stainless and Heat Resisting Steel Rope receptacles, Locking Type 125 Volts, 15 Amperes, 2 Pole, 1 eceptacles, Locking Type, 250 Volts, 20 Amperes, 2 Pole, 2 ptacles: Specific Purpose 125 Volts, 30 Amperes, 2 Pole. 2 ptacles: Specific Purpose 250 Volts, 20 Amperes, 2 Pole, 2 ptacles: Specific Purpose 125 Volts, 20 Amperes, 2 Pole, 2 agonal, Octagonal, and Flat (RectanguIar and Square) Brass pe, Specific Purpose, 125/250 Volts, 15 Amperes, 3 Pole, 3 type, Specific Purpose, 277 Volts Ac, 15 Amperes, 2 Pole 3 g Type, Specific Purpose, 125 Volts, 15 Amperes, 2 Pole, 2 al Insulating Varnishes Applied Over Film Insulated Magnet Std. Spec. for Zinc Coated (Galvanized) Steel Barbed Std. Meth. for Test. Film Insulated Magnet

d. Spec. for Molybdenum and Molybdenum Alloy Bar, Rod, and Std. Spec, for Silver Coated Soft or Annealed Copper Std. Spec. for Nickel Coated Soft or Annealed Copper Std, Spec. for Bronze Trolley

d. Spec. for General Requirements for Wrought Copper Alloy (1972) ANSI C54.16

Std. Spec for Alloy Steel

Spec. for Tolerances of Aluminum and Its Alloy Bar, Rod, Spec. for Steel Spring a) (1973)

$.8 \mathrm{Ni}-1.6 \mathrm{Co}-1.8 \mathrm{Mo}-0.32 \mathrm{~V})(1 /$

Spec. for Steel Welding

Spec. for Steel Welding Std. Spec. for Aluminum $12 \mathrm{Cr}-8.5 \mathrm{Ni}-2.0 \mathrm{Cu}-1.1 \mathrm{Ti}, \mathrm{Vacuum}$ Induct/ Steel Bars,

Std. Spec. for Steel Std. Spec. for Steel

Springs (1973) Std. Spec. for Steel les: Specific Purpose 125/250 Volts, 30 Amperes, 3 Pole, 3 on Head Bolts (1972) ANSI G54.15

nsi G54.4

e 6130$)(1973)$

$\mathrm{e}-25.5 \mathrm{Cr}-10.5 \mathrm{Ni}-7.5 \mathrm{~W}(\mathrm{I} 973)$

e-19.5 $\mathrm{Cr}-18 \mathrm{Co}-2.5 \mathrm{Ti}-1.5 \mathrm{Al}(1973)$

$6.5 \mathrm{Cr}-4.5 \mathrm{Ni}-2.9 \mathrm{Mo}-0.1 \mathrm{~N}(1973)$

td. Spec. for Steel

Std Spec. for Steel Spec. for Steel Welding Alloy Alloy Steel

Std. for All Thermoplastic Type MTW 600 Volt Machine Tool $y$ for Fiber Conduit for Lse as Raceway for Installation of Std. for Color Coding of Electrical $s$ and Fittings for Underground Installations of Electrical or Magnesium Alloy Extruded Bars, Rods, Shapes, Tubes, and Safety Std. for Thermoplastic Insulated Std. Spec. for Fixation Pins and Std. Spec. for Aluminum Alloy Bars, Rods and test Std. for Determination of Solderability of Solid Lead Std. for Safety for lectrical Wiring) (1973) ANSI C33.45 Std. for Safety for 0) Std. for Terms and Definitions for $0)$ Inspection and Test Procedures for Iding Construction (/ Std. Graphic Symbols for Electrical ided Rigid Boards (1970) ANSI C83/ Std. Spec. for Printed c C- $405 \mathrm{~A}$ Std. General Document for Printed Suggested Guide Lines for Printed Std. for Safety for Printed

smission of End Product Description Data (Tooling, Printed ication of (1962) A/ Std. for Tools, Crimping, Solderless etion Equipment (Machinery) / rements and Rec. Meth. of Identifying $A C$ and DC Electrical $\mathrm{y}$ for Grounding and Bonding Equipment (Internal ElectricaI Std. Performance Spec. for Flexible Multilayer ety for Surface Metal Raceways and Fittings for Electrical ys, Auxiliary Gutters, and Associated Fittings (Electrical Quickly Demountable Type for Horizontal Mounting, Liquid

ntable Universal (Horizontal or Vertical Mounting), Liquid equirements for Woven Washable Uniform Fabrics for Men and equirements for Woven Washable Uniform Fabrics for Men and Test Meth. for Snag Resistance of Guide Spec. for Flagpoles, Metal, meth. of Test for Hygroscopic Properties of Fire Retardant for Timber Connector and Bolted Joints, Drift Bolts, and Std. Test Meth. for Simulated Service Test. of Std Test Meth. for Specific Gravity of Std. Meth. of Conducting Machining Tests of Std. Meth. of Evaluating the Properties of i 08.1

Std. Test Meth. for Simulated Service Test. of Wood and Std. Test Meth. for Specific Gravity of Wood and
Wire (1971) ANSI G81.42

Wire (1971) ANSI H32.1 ASTM

Wire (1972)

Wire (1972) ANSI G81.32

Wire (1973)

Wire (1973)

Wire (1973)

Wire (1973)

Wire (1973)

Wire (1973)

Wire (1973)

Wire (1973)

Wire $(1973$

Wire (1973)

Wire (1973)

Wire (1974)

Wire $(1974$

Wire (1974) ANSI C7.38

Wire (1974) ANSI C7.48

Wire (1974) ANSI C7.5

Wire (1974) ANSI H30.2

Wire, Alloy Cold Heading Quality for Hexagon Head Bolts

Wire, and Forging Stock (Rolled or Drawn) (1973)

Wire, Best Quality Music (1973)

Wire, Corrosion and Heat Resistant $(18 \mathrm{Cr}-11 \mathrm{Ni}-(\mathrm{Cb}+\mathrm{T}$

Wire, Corrosion and Moderate Heat Resistant (11.8Cr-2

Wire, EC.H19, for Electrical Purposes (1972) ANSI C7.20

Wire, Forgings, and Forging Stock, Corrosion Resistant,

Wire, Hard Drawn for Mechanical Springs (1971) ANSI G54

Wire, Hard Drawn for Prestressing Concrete Pipe (1973)

Wire, High Tensile Strength, Hard Drawn, for Mechanical

/Sions of Plugs and Receptac

Wire, Medium High Carbon Cold Heading Quality for Hexag

Wire, Oil Tempered Carbon Valve Spring Quality (1971) a

Wire, Vacuum Melted $(0.95 \mathrm{Cr}-0.20 \mathrm{~V}(0.28-0.33 \mathrm{C}))(\mathrm{Sa}$

Wire, Welding, Corrosion and Heat Resistant, Cobalt Bas

Wire, Welding, Corrosion and Heat Resistant, Nickel Bas

Wire, Welding, Corrosion and Moderate Heat Resistant,

Wires and Cables (1971) ANSI C33.73

Wires and Cables (1972)

Wires and Cables (1972) ANSI C33.37

Wires and Cables (1974)

Wires and Cables (1974)

Wires (1970) ANSI H45.6

Wires (1971) ANSI C33.80

Wires (1973)

Wires (1974) ANSI H38.4

Wires, Terminals, and Other Terminations (Soft SoIder J

Wireways, Auxiliary Cutters and Associated

Wireways, Auxiliary Gutters, and Associated Fittings (E

Wirewound and Nonwirewound Trimming Potentiometers (197

Wirewound and Nonwirewound Trimming Potentiometers (197

Wiring and Layout Diagrams Used in Architecture and Bui

Wiring Board Dimensions and Tolerances Single and Two S

Wiring Board Electric Connectors (1974) ANSI C83.88, IP

Wiring Board Repairs (1973)

Wiring Boards (1973) ANSI C33.46

Wiring Boards) in Numeric (Digital) Form on Punched Car

Wiring Devices-Recommended Procedures for User Certif

Wiring Devices (1972)

Wiring Identification System for Industrial and Constru

Wiring on Boats and Engines (Color Code) (1973)

Wiring Systems) (1972) ANSI C33.8

Wiring (Printed Circuit) (1972)

Wiring (1973) ANSI C33.50

Wiring) (1973) ANSI C33.45

Withdrawal Only (1973)

Withdrawal (1973)

Women (Heavy Duty) (Ins

P Gas

Women (Institutional Textile) (1973)

Women's Nylon Hosiery (1973)

Wood and Its Base Froducts (1973)

Wood and Lag Screws; Light Metal Plate Connected Wood

Wood and Wood Base Finish Flooring (1969)

Wood and Wood Base Materials (1969)

Wood and Wood Base Materials (1970)

Wood Base Fiber and Particle Panel Materials (1972) Ans

Wood Base Finish Flooring (1969)

Wood Base Materials (1969)

SAE

SAE

SAE

SAE

ASTM

SAE

ASTM

ASTM

ASTM

ANSI

ASTM

AST

$\mathrm{SAE}$

SAE

SAE

SAE

Safety UL

NEMA

UL

ASTM

ASTM

EIA

UL

UL

VRCI

VRCI

ANSI

IPC

EIA

IPC

UL

IPC

EIA

NEMA

SAE

C73.67

B365

A228

A580

B134

ASTM A492

C73.31

C.73.32

C 73.70

C73.7I

C73.69

UBCS 32.13

C 73.30

C73.43

C73.29

D3251

A121

D 1676

B 387

B298

B355

$\begin{array}{ll}\text { ASTM } & \text { B9 } \\ \text { ASTM } & \text { B } 250\end{array}$

ASTM A54?

AMS2201J

AMS51 12F

AMS5680D

AMS5823

B230

AMS5617A

A227

A679

C73.97

A546

A230

AMS6461E

AMS5789

AMS 5829

AMS5774A

133

1063

543

W C30

TC1

B 107

83

F366

B211

RS178-B

870

T110

T215

Y 32.9

D300E

RS406

R. 700

796 


\section{National Bureau of Standards KWIC Index of Engineering Standards}

) b or Wood Subfloors (1962)

ermal Process (1973) t (1973)

970)

1 Woodwork) (1973) oal rocesses) (1972)

Std. Meth. of Conducting Machining Tests of Wood and Std. Spec. for Cellulosic Fiber ength and Stiffness Computations (1973) Rec, for

Std. Spec. for Creosoted End Grain

Rec. for Sound Control (Acoustics) in eth. of Analysis of CCA Treating Solutions and CCA Treated Test Meth. for Sampling of Test Meth. for Dirt Count of Test Meth. for Density of Test Meth. for Moisture in Test Meth. for Moisture in Design Guide for test Meth. for Strength Properties of Adhesives in Two Ply Test Meth. for Specific Gravity of nalysis for Dirt, Sand, Insect Fragments, Hairs. Feathers, shing and Testing of Concrete Subfloor Surfaces to Receive Rec. for the Care and Preservation of Rec. for Controlling Cupping of

Std. for Preservative Treatment by Pressure Processes of td. Spec. for Mineral Fiber Blanket Thermal Insulation for erformance Spec. for Adhesives for Field Gluing Plywood to std. Spec. for Adhesives for Fastening Gypsum Wallboard to Code Std. for Adhesives for Fastening Gypsum Wallboard to Meth. for Compression Std. for Safety Requirements for Safety Std. for Portable components, Timber, Preservative / Uniform Building Code Std. for
ng Combined (1973) Grading Rules for Std. dimensions for Cores for Photographic Film Rolls (Plastic, Rec. for Interior Std. Patterns for Spec, for Mosaic Sid for Mat Formed Uniform Building Code Sid. for Mat Formed iveways, and Interior Floors Exposed to W/ Std. Spec. for Std. Guideline for the Physical Inspection of Uniform Building Code Std. for Std. Spec. and Dimensions for Western Woods Use Book: Std. Neth, of Evaluating Std. Meth. of Test. eths. for Test. the Preservative Properties of Oil Soluble wood Preservatives by Using Wood Properties of Oil Soluble l Size Specimens/_Sid. Meth. of Accelerated Evaluation of Std. Meth. for Analysis of Creosote and Oil Type Std. Meth. for Analysis of Oil Borne Std. for Hydrocarbon Solvents for Oil Borne ed Determination of the Temperature of Applied Coatings on Test for Integrity of Glue Joints in Structural Laminated Condi/ Std. Spec. for Adhesives for Structural Laminated
m Building Code Std. for Test for Glue Joints in Laminated tive Properties of Oil Soluble Wood Preservatives by Using Std. Meth. of Test for Accelerated Aging of Std. Meth. of Test for Degree of Set for Quality Stds. for Std. Spec. for Cold Heading Quality Steel Wire for Uniform Building Code Std. for Special Purpose Roofs Uniform Building Code Std. for Sawn Rec. for wood Properties of Oil Soluble Wood Preservatives by Using
Std. Meth. for Establishing Clear Std. Meth. for Establishing Clear ood Parquet Flooring Set in Adhesive Over Concrete Slab or Western Woods Use Book: olts, and Wood and Lag Screws; Light Metal Plate Connected Std. for ystem: House Design and Construction Met/ the All Weather Test Meth. for Moisture and Creosote Type Preservative in Meth. for Methoxyl Content of Pulp and eth. of Test for Durability of Fire Retardant Treatment of pract. for Conducting Exterior Exposure Tests of Paints on ation of Dichloromethane and Alcohol-Benzene Solubles in Std. Meth. of Test. Metal Fasteners in Std. Meth. of Test for Moisture Content of Std. Meth. of Static Tests of Timbers Wet Ashing with Nitric and Perchloric Acids
(Lumber and Timber) (1973)

way, Aisle, Corridor, Building Construction and / es, Section Properties and Design Loads. Timber,/ Walls, and Floors (1973) g Screws, Plates, Nails and Spikes, Washers, Pin/
Wood Base Materials (1970)

(Wood Base) Loose Fill Thermal Insulation (1973)

Wood Beam, Joint and Rafter Span, Load, Deflection, Str

Wood Block Flooring for Interior Use (1970) ANSI 010.1

Wood Building Construction (Floor, Ceiling, Wall) (1972

Wood by Colorimetry (1972)

Wood Chips from Conveyor (Pulp and Paper) (1973)

Wood Chips (Pulp and Paper) (1973)

Wood Chips (Sheet Machine Mold) (Pulp and Paper) (1973)

Wood Chips (Steam Oven) (Pulp and Paper) (1973)

Wood Chips (Two Liter Graduate) (1973)

Wood Composition Board Wall Panels (1973)

Wood Construction in Shear by Tension Loading (1970)

Wood Disks (Pulp and Paper) (1973)

Wood Fibers, Splinters, etc. in Starch (Cereal Chemistr

Wood Flooring (1962)

Wood Floors (1962)

Wood Floors (1962)

Wood for Highway Construction (1973)

Wood Frame and Light Construction Buildings (1970) ANSI

Wood Framing (1971)

Wood Framing (1973

Wood Framing (1973)

Wood Identification in Pulpwood (1972)

Wood Job Made Ladders (1973)

Wood Ladders (1973)

Wood Members and Their Fastenings (Lumber. Plywood and

Wood Members Subjected to Both Flexural and Axial Loadi

Wood Mouldings (1967)

Wood or Metal) (1971)

Wood Paneling (1972)

Wood Paneling. Decking, Flooring, and Siding (1970)

Wood Parquet Flooring Set in Adhesive Over Concrete Sla

Wood Particleboard (1966)

Wood Particleboard (1973)

Wood Paving Blocks for Exposed Platforms, Pavements, Dr

Wood Poles in Service (1972)

Wood Poles Preservative Treatment by the Full Length Th

Wood Poles That Are to Be Given a Preservative Treatmen

Wood Poles (1972)

Wood Preservation (Preservative) (1973)

Wood Preservatives by Field Tests with Stakes (1974)

Wood Preservatives by Laboratory Soil Block Cultures (1

Wood Preservatives by Using Wood Properties of Oil Solu

Wood Preservatives by L'sing Wood Specimens Uniformly Im

Wood Preservatives for Marine Services by Means of Smal

Wood Preservatives (1973)

Wood Preservatives (1973)

Wood Preservatives (1973)

Wood Products During the Curing Cycle (1973)

Wood Products for Exterior Use (1959) ANSI Z197.17

Wood Products for Use Under Exterior (Wet Use) Exposure

Wood Products (1973)

Wood Properties of Oil Soluble Wood Preservatives by Ls

Wood Sash Face Glazing Compound (1973)

Wood Sash Glazing Compound (1973)

Wood Screens for Window and Door Openings (Architectura

Wood Screws (I971) ANSI G54.18

(Wood Shakes and Shingles with Asbestos Felt or Gypsum B

Wood Shingles (1973)

Wood Siding, Finish, Nails and Nailing (1972)

Wood Specimens Uniformly Impregnated (1970)

Wood Strength Values (1973)

Wood Subfloors (1962)

Wood Tanks, Vats, and Pipe (1973)

Wood Truss Design; and Nails and Staples (1973)

Wood Used on Farms (Preservative Treatment by Pressure
Wood (Pressure Treated Lumber and Plywood) Foundation $S$

Wood (1970)

Wood (1970) ANSI 012.1

Wood $(1972)$

Wood (1972)

Wood (1973)

Wood (1973)

Wood (1974)

Wood (1974)

Wood) in Structural Sizes (1967) ANSI Q4.2

Wood, Pulp, etc.) (1973)

Western

Western

Western

Western

Western

Western

accelerated Laboratory Test of Natural Decay Resistance of
Woods Use Book: Abbreviations, Symbols, and Terminology Woods Use Book: Fire Protection and Safety (Exit, Stair Woods Use Book: Lumber Std., Grading Rules, Design Valu Woods Use Book: Sound Control (Acoustics) in Buildings, Woods Use Book: Timber Fasteners (Connectors, Bolts, La Woods Use Book: Wood Preservation (Preservative) (1973) Woods Use Book: Wood Tanks, Vats, and Pipe (1973) Woods (1970)

\section{ASTM D1666 \\ ASTM $\quad$ C739 \\ WWPA $\quad * 30$ \\ ASTM D1031 \\ WWPA $\quad * 27$}

Std. M AWPA A10.72

TAPPI [:Y].4

TAPPI LM.11

TAPPI UM.9

TAPPI UM.7

TAPPI UV-10

HPMA CB-SG.73

ASTM D2339

TAPPI [ M.12

A ACCH 28.75

WSFI * 1

WSFI *2

WSFI *3

AWPA Cl4

ASTM C 665

APA AFG-01

ASTM C 557

ICBO [BCS47.2

TAPPI T20

ANS1 Al4.4

IL 112

ICBO UBC $* 3.25$

ICBO UBC 525.16

WWPA 29

Std. ANSI PH1.13

WWPA $* 26$

WWPA $* 28$

WSFl $\div$

USC C\$236

1CBO UBCS25.25

ASTM D52

AWPA $\mathrm{M} 13.72$

AWPA $\mathrm{Cl0}$

ICBO [BBC $\$ 25.13$

ANSI O5.1

WWPA $\quad$ *31.3

ASTM D1758

ASTM DI413

NWMA M-1-70

NWMA M.1.70

ASTM D2481

AWPA Al

AWPA A5

AWPA PQ

D 3259

lof ASTM D1101

ASTM D2559

lCBO

NWMA

ASTMI

ASTM

A WI

ASTM

ICBO

ICBO

WWPA

UBC\$25

M. 1.70

C741

C742

* 1.1100

A549

LBC\$32.14

[BCS32-1]

*25

NWMA M.1.70

ASTM D2555

WSF I

W W'PA

$* 7$

*31-2

ICBO UBCS25-17

AWPA Cl6.72

NFORP RP?

std. ASTM D1860

ASTM D1324

TAPPI T209SL

Std. M ASTM D2898

Std. Rec. ASTM D1006

T5

TAPPI

ASTM

ASTM

ASTM

TAPPl

$* 31.4$

WWPA *31

WWPA *31.5

WWPA *31.1

WWPA $* 31-3$

WWPA *31-2

ASTM D2017 
Quality Stds. for Lumber Grades (Architectural Quality Stds. for Exterior Sash (Architectural Quality Stds. for Stile and Rail Doors (Architectura

Quality Stds. for Factory Finishing (Architectura quality Stds. for Standing and Running Trim (Architectural Ouality Strs. for Flush Wall Paneling (Architectura

Quality Stds. for Misc. Ornamental Items (Architectural

Quality Stds. for Stairwork and Handrails (Architectural

Quality Stds. for Exterior Frames (Architectural

Stds. for Plywood and Particleboard Grades (Architectural stós. for Flush Solid and Hollow Core Doors (Architectura Wood Screens for Window and Door Openings (Architectural

Pressure Laminate Flush Type $\mathbb{W}$ all Paneling (Architectura

Shutters Using Stile and Rail Construction (Architectural cases. Counters and Enclosures of All Kinds (Architectural Std. Safety Requirements for Uniform Fire Code: Lumber Yards and k. Closet and Storage Shel Guide Specs. for Architectural Std. Meth. of Test for Wool Content of Raw
Std. Spec. for Fineness of Std. Spec. for Fineness of

ansi L14.143 Std Meth of Test for Moisture in

e Present in Ordinary Commercial and Industrial Samples of L14.490 td. Meth. of Test for Sto. Meth of Test for Test Meth. for Colorfastness of Dyed laxation Dimensional Change (Shrinkage) of Stabilized Knit si L14. S2 Test Meth. for Evaluating Oxidation of Test Meth. for Colorfastness of Dyed lting Dimensional Changes (Shrinkage) of Woven and Knitted Std. Spec. for Fineness of

Test Meth. for Continuous Scourin t Meth. for Continuous Scouring Wool Using Std. Raw Grease changes in Laundering of Woven and Knitted Textiles Except

le Matter and Other Alkali Insoluble Impurities in Scoured Std. Meth. of Test for Extractable Matter in Oven Dried age (Cotton. Jute, Hemp and Sisal, Flax, Synthetic Fibers,

for Calculation of Commercial Weight and Yield of Scoured Test Meth. for Evaluating Oxidation of Wool Oils on Sto. Tolerances for Std. Tolerances for Woolen Spun on the Lubricant ( $T_{0}$ Test the Removability of Spinning Oils from gn Stds for (1972)

ftware (1972)

Requirements for Both Permanentl Aircraft Instrument Stds.

nual, Sect. 5) (1971)

Definitions of Common Rec. Pract. for Signal Spec, for Aluminum Sheet Metal Std. for Industrial Engineering Terminology: Std. for Elevating

Std. Descriptions of Qualifications and Scopes of

British-American) in Published Scientific and Technical type Cranes Having Rope Supported Booms, Equipped for Hook Usc $\operatorname{Ps} 20$ Std. Classification for Metal Span Tables and

fof for Acceptable Concentrations of Toluene in the Air of ng. Installation, and Definitions) (1961)

Public Std. for System Design of Fine Pitch spec, for Fineness of Mohair Top. Yarns and Fabrics of the nsulation Systems for AC Electric Machinery Employing Form ing Design: Compression, Extension, Torsion, Flat, and Hot $138, /$ Test Meth. for Dimensional Changes in Laundering of Relaxation and Felting Dimensional Changes (Shrinkage) of Std. Spec. for Std. Performance Requirements for Std. Performance Requirements for Sid. Performance Requirements for Test Meth. for Absorbency of Bleached Std. Spec. for

cs (lnstitutional Text)

(InstitutionaI Texti/ orm Textile) (1973) t (Industrial) Suiting/ te (Year Round) Suitin/

(Textile) (1973)

t Rate of Tra/ use in Water

l Use Garm/

Utd. Pe

1 Use Garments (Clothi/ Test Meth. to Determine
Meth. of Test for Tearing Strength of std. Meth. of Sampling and Test. Felted and Std. Performance Requirements for Std. Performance Requirements for Std. Performance Requirements for Std. Performance Requirements for Std. Performance Requirements for Std Performance Requirements for

Performance Requirements for $100 \%$ Cotton Std. Performance Requirements for Std. Performance Requirements for Std. Meth. of Test for Distortion of Yarn in

Meth of Test for Stretch Properties of Fabrics d Roofing (1973) ANSI A 109.26 poxy Functional Silane Type Finishes for / Std. Spec. for eg. F-No Bleach (Ins)
Woodwork) (1973)

Woodwork) (1973)

Woodwork) (1973)

Wood work) (1973)

Woodwork) (1973)

Wood work) (1973)

Woodwork) (1973)

Woodwork) (1973)

Woodwork) (1973)

Woodwork) (1973)

Wood work) (1973)

Woodwork) (1973)

Woodwork) (1973)

Woodwork) (1973)

Woodwork) (1973)

Woodworking Machinery (1971)

Woodworking Plants (1973)

Woodwork: Standing and Running Trim, Casework, Panelwor

Wool-Commercial Scale (1972)

Wool-Laboratory Scale (1972) ANSI L14.490

Wool and Assignment of Grade (1973) ANSI L14.26

Wool and Other Animal Fibers by Microprojection (1972)

Wool by Distillation with Toluene (1972) ANSI L14.256

Wool by Oven Drying (1972) ANSI L14.202

Wool Content of Raw Wool-Commercial Scale (1972)

Wool Content of Raw W'ool-Laboratory Scale (1972) ANSI

Wool Fabric and Yarn to Mill Fulling (1972) ANSI L14.5

Wool Fabrics (Textile) (1973)

/D. Meth. of Test for Re

Wool Oils on Woolen Fabric Textile in Storage (1971) an

Wool Textiles to Carbonizing (1972) ANSI L14.3

Wool Textiles (1972) ANSI L14.130. ASTM D 1284

Wool Top and Assignment of Grade (1973) ANSI L14.29

Wool Using Std. Raw Grease Wool Yarn (1971) ANSI L14.84

Wool Yarn (1971) ANSI L14.84

Wol (1972) ANSI L14.138, ASTM D1905

D. Meth. of Test for Vegetab

Wool (1972) ANSI L14.195

Wool (1973)

Wool) (1974)

Wool, Top, and Noil for Various Commercial Compositions
Woolen Fabric Textile in Storage (1971) ANSI L14. S2

Woolen Spun on the Woolen System Yarns (1971)

Woolen System Yarns (1971)

Woolen Yarns (1971) ANSI L14.113

/Rability of Spinning

Wording, Terminology, Phraseology, Environment and Desi

Words for Aluminum Electrical Conductor (1972)

Words Related to Numerically Controlled Machine Tool So

Words, Letter Sizes, Color Combinations, and Durability

Work in Building Construction (Aluminum Construction Ma

Work Measurement and Meth. (1972)

Work Platforms (Lift) (1973)

Work Relating to Forensic Sciences (1972)

Work (1973) /Ract. for Units (Measurement-Metric and

Work, Clamshell, Magnet, Grapple, or Concrete Bucket at

Working Fluids and Related Materials (1973)

Working Stresses for Joists and Rafters (Lumber) (1972)

Workplaces (1974)

Works Spec, for Ceramic Veneer (Sampling, Test. Finishi

Wormgearing (1973)

Worsted Type and Assignment of Grade (1973) ANSI L14.16

Wound Stator Coils (1972) AVSI C50.26

Wound (Materials, Specs. Terminology, Gauge, Metric Con

Woven and Knitted Textiles Except Wool (1972) ANSI L14.

Woven and Knitted Wool Textiles (1972) ANSI L14.130, as

Woven Asbestos CIoth (1973) ANSI L14.133

Woven Bath Mats (Institutional Textile) (1973)

Woven Bedspreads (Institutional Textile) (1973)

Woven Blankets (Institutional Textile) (1973

Woven Cloth (1972) A VSI L14.115

Woven Cotton Tapes for Electrical Purposes (1972)

Woven Decorative Bedspreads, Pillows, and Bolster Fabri

Woven Dish Towels (Institutional Textile) (1973)

Woven Drapery (Curtain) Fabrics (Excluding Glass Fibers

Woven Drycleanable Overcoat Fabrics (Institutional Unif

Woven Drycleanable Tailored Uniform Fabrics, Heavyweigh

Woven Drycleanable Tailored Uniform Fabrics, Intermedi

Woven Fabric's Resistance to Sagging or Stretch in Wear

Woven Fabrics by the Tongue (Single Rip) Meth. (Constan

Woven Fabrics Saturated with Bituminous Substances for

Woven Fabrics (Textile) for Institutional and Industria

Woven Fabrics (Textile) for Institutional and Industria

Woven Fabrics (Textile) for Institutional and Industria

Woven Fabrics (1972) ANSI L14.103

Woven from Stretch Yarns (1972T)

Woven Glass Fabrics Treated for Use in Waterproofing an

Woven Glass Fabrics, Cle aned, and After Finished with E

Woven Glass Fiber Curtain Fabrics Handwashable at 105 D

*1-1500

$* 1-300$

*1.500A

$* 1.800$

$* 1-900$

* 1-200

* $1-1300$

* $1-1100$

* $1.500 \mathrm{~B}$

*1-1200

$* 1-400$

Ol.1

UFC *2ART21

$* 1$ - 1

ASTM

ASTM

ASTM

ASTM

ASTM

D1334

D584

D419

D2130

D2462

D1576

D1334

D584

ASTM

AATCC

2

D1284

AATCC 62

AATCC 11

AATCC 99

ASTM D472

AATCC 64

AATCC 64

AATCC 96

ASTM D1113

ASTM

FMS

ASTM

AATCC

ASTM

ASTM

$S A E$

AA

NMTBA *2

AA $* 12$

ANSl Z94.12

ANSI A92.3

ASTM E444

IEEE

SAE J220

ASTM D2881

NFORP *2

ANSI 237.12

BIA

AGMA

ASTM

$* 28$

AATCC

AATCC

ASTM

ANSI

ANSI

AATCC

ASTM

ANS

ANSI

ANSI

ANSI

ANSI

ANSI

ASTM

ASTM

ANS

ANS 
Meth. of Test for Effect of Handwashing on Meth. of Test for Light Transmittance of e at 105 Deg. F-No B/ Std. Performance Requirements for
e) (1973) e) (1973) Std. Performance Requirements for
Std. Performance Requirements for vinyl Coated or Lamina dering of Durable (Permanent) Press in Automatic Home Launds.d. Performance Requirements for 1 Textile) (1973) ress (Institutional Te/ Std. Performance Requirements for Std. Performance Requirements for Durable Press (No Iron) Std. Performance Requirements for Std. Performance Requirements for

d, or VinyI Coal erformance Requirements for Cotton L14.110, ASTM D1295 Test Meth. for Wrinkle Recovery of institutional/ Std. Performance Requirements for Tailored Std. Performance Requirements for Std. Performance Requirements for Std. Performance Requirements for Std. Performance Requirements for Duty) (Institutional tutional Textile\} (197

Glass Fibers) (Instit phalt Type Protective Coatings for Underground Pipe Lines
Uniform Fire Code: Automobile ts (1973) s (1971) Std. for

Std. for Bolt-lnternal Std. for Bolt-Internal Std. for Bolt-lnternal Std. for Bolt-Internal

Std for Design Parameters for Bolts and Screws; External 1970) ANSI L 14.229 Test Uleth. for le Meth. (1972) ANSI L14.110, ASTM D 1295 Test Meth. for th. for Water Immersion Test for Resistance abler

Std. for System for Romanization of Japanese Std. for System for Romanization of Arabic

ixtures) (1973) Std. for Scientific Papers for Std. Spec, for
for Magnesium chanical Property Limits and Dimensional Tolerances of Sae ansi $\mathrm{H} 38.19$

(1973) ANSI H46.1

Std Spec. for Factory Made Std. Mleth. of Tension Test. 3) ANSI G37.8 Std. Meth. and Definitions for Mechanical Test. of ice (1973) A.VSl G46.2

d Temperatures (1973) / rs (1971) ANSI G39.2 Std. Spec. for Piping Fittings of Std. Spec. for Piping Fittings of Std. Spec. for Std. Spec. for General Requirements for ng for Use with Copper Water Tube (1973)
$\begin{array}{ll}\text { d Rolled Bar (19) Std. Spec. for General Requirements for } \\ \text { St }\end{array}$ ng for Use with Copper Water Tube (1973) Std. for
d Rolled Bar (19) Std. Spec. for General Requirements for )

ings (1973)

Std. Spec. for General Requirements for Standard for ytes (1973) ANSI H44.l Std. Spec. for Boron Deoxidized Copper in for Chemical Analysis of Steel and Cast, Open Hearth, and arbon. Low Alloy, and Silicon Electrical Steels, Ingot and essure Piping (1972) ANSI H3/ Std. Spec. for Factory Made 72) th. of Detecting Susceptibility to Intergranular Attack in Std. Spec. for General Requirements for std Spec for Fire Refined Copper for quality Assurance Sampling of Carbon and Low Alloy Steels Std. Meth. for Ultrasonic Inspection of Aluminum Alloy

si $\mathrm{H} 23.4$ Std. Designations for Copper and Copper Alloys nt) (1974) Std. Spec. for General Requirements for Std. Factory Made ) ANSI G57.13 ng (1969) Std. for Wire Cable Steel Products Manual Std. Spec. for Spec. for High Test for High Test

es for Nuclear Service (1971) A NSI N124 acles: Specific Purpose 347/600 Volts, 20 Amperes. 3 Phase acles: Specific Purpose 347/600 Volts, 30 Amperes, 3 Phase acles: Specific Purpose 120/208 Volts, 20 Amperes, 3 Phase acles: Specific Purpose 120/208 Volts, 30 Amperes, 3 Phase acles: Specific Purpose 277/480 Volts, 20 Amperes, 3 Phase acles\& Specific Purpose 277/480 Volts, 30 Amperes, 3 Phase acles: Specific Purpose 120/208 Volts, 20 Amperes, 3 Phase acles: Specific Purpose 120/208 Volts, 30 Amperes, 3 Phase acles: Specific Purpose 277/480 Volts, 20 Amperes, 3 Phase acles: Specific Purpose 277/480 Volts, 30 Amperes, 3 Phase acles: Specific Purpose 347/600 Volts, 20 Amperes, 3 Phase acles: Specific Purpose 347/600 Volts, 30 Amperes. 3 Phase errupters Used in Power Switchge/ 1971) NBS HBl ll

real Grains, Unpopped Popcorn, Drie/ Safety Requirements for Std. for Radiation Safety for Meth. of Analysis of ing Code Std. for Storage and Handling of Photographic and $\$(1968)$
Woven Glass Fiber Decorative Fabrics (Textile) (1973)

Woven Glass Fiber Decorative Fabrics (Textile) (1973)

Woven Glass Fiber Drapery (Curtain) Fabrics Handwashabl

Woven Lining Fabrics for Institutional Uniforms (Textil

Woven Man Made Fiber Tent, Awning, and Canopy Fabrics,

Woven or Knit Textile Fabrics (1973) ANSI L14.266

Woven Printed Table Napery (Napkin, etc.) (Institutiona

Woven Sheet and Pillowcase Fabrics Other Than Durable P

Woven Sheet and Pillowcase Fabrics (Institutional Texti

Woven Shower Curtains (Institutional Textile) (1973)

Woven Slipcover Fabrics (Institutional Textile) (1973)

Woven Tent, Awning, and Canopy Fabrics. Painted. Printe

Woven Textile Fabrics: Recovery Angle Meth. (1972) ANSI

Woven Uniform Fabrics, Lightweight (Tropical) Suiting (

Woven Upholstery Fabrics (Tacked On) (Institutional Tex

Woven Washable Uniform Fabrics for Men and Women (Heavy

Woven W ashable I'niform Fabrics for Nen and Women (Insti

Woven Window Curtain Fabrics (Sash Casement) (Excluding

Wrapped Systems) (1972)

Tent. Rec. Spec.

Wrecking and Junk Yards or Waste Material Handling Plan

Wrenching Configuration, Spline Drive Threaded Fastener

W/renching, Steel 1/4.28 Thru 1.1/8.12 (1973)

Wrenching, Steel, 1.2500-12 (1973)

Wrenching, Steel, 1.3750-12 (1973)

Wrenching, Steel, 1.5000-12 (1973)

Wenching, Unified Threaded In. Series (1971)

Wrinkle Recovery of Textile Fabrics: Appearance Meth. (

Wrinkle Recovery of Woven Textile Fabrics: Recovery Ang

Wrinkling, Loss of Adhesion, or Other Failure of Paint,

Writing Abstracts (1971)

Written Language (Transliteration) (1972)

Written Language (Transliteration) (1972)

Written or Oral Presentation (1972)

Wrought Alloy Tool Steel Products (Including Dies and F

Wrought Alloys (1971)

Wrought Aluminum Alloys $(19 ?$

Wroughe Aluminum and lt Alloy Welding Compositio

Wrought and Cast Aluminum and Magnesium Alloy Products

Wrought and Cast Steel Products (1973) ANSI G60.1

Wrought Austenitic Stainless Steel Piping Fittings (197

Wrought Carbon and Alloy Steel for Low. Temperature Serv

Wrought Carbon and Alloy Steel for Moderate and Elevate

Wrought Carbon Steel Wheels for Locomotives and Rail Ca

Wrought Copper Alloy Wire (1974) ANSI H30.2

Wrought Copper and Bronze Solder-Joint Pressure Fitti

Wrought Copper and Copper Alloy Plate, Sheet, Strip, an

Wrought Copper and Its Alloy Rod, Bar, and Shapes (1973

Wrought Copper and Its Alloy Solder Joint Drainage Fitt

Wrought Forms for Electron Devices (1972)

Wrought Galvanic Zine Anodes for Use in Saline Electrol

Wrought Iron (1973)

Wrought Irons (1973)

Meth. for Chemical Analy

Wrought Precious Metal Electrical Contact Aaterials (19

Wrought Products and Alloys (1972) ANSI H23.12

Wrought Products Except Forgings and Stock) (1973)

Wrought Products for Aerospace Applications (1974)

Wrought Products Only); Application Data Sheet (1972)

Wrought Seamless Copper and Copper Alloy Tube (1974) an

Wrought Steel Buttwelding Fittings (1971)

Wrought Steel Heavy Thimble (For Ground Support Equipme

Wrought Steel Wheels and Forged Railway Axles (1973)

Wrought SteeI Wheels for Electric Railway Service (1972

Wrought Welding Fittings for High Pressure Service Pipi

Wrought $\mathbb{W}$ elding Fittings (1973)

Wrought Zirconiur

Wye, 4 Pole, 4 Wire Locking Type (1972

Wye, 4 Pole, 4 Wire Locking Type (1972

Wye, 4 Pole, 4 Wire Locking Type (1973

Wye, 4 Pole, 4 Wire Locking Type (1973)

Wye, 4 Pole, 4 Wire Locking Type (1973)

Wye, 4 Pole, 4 Wire Locking Type (1973)

Wye, 4 Pole, 5 Wire Locking, Grounding Type (1973)

Wye, 4 Pole, 5 Wire Locking, Grounding Type (1973)

Wye, 4 Pole, 5 W ire Locking, Grounding Type (1973)

Wye, 4 Pole, 5 Wire Locking, Grounding Type (1973)

Wye, 4 Pole, 5 Wire Locking, Grounding Type (1973)

Wye 4 Pole 5 Wire Locking. Grounding Type (1973)

$X$ Radiation Limits for AC High Voltage Power Vacuum Int

$X$-Ray Diffraction and Fluorescence Analysis Equipment

X-Ray Examination for Internal Insect Infestation in Ce

X-Ray Nitrocellulose Films (1973)

X-Ray Nitrocellulose) (1973)

X-Ray Protection Standards for Home Television Receiver
Uniform Build

ICBO

\section{ANSl \\ ANSI \\ ANSI \\ ANSI \\ ANSI \\ AATCC \\ ANSI \\ ANSI \\ ANSI \\ ANSI \\ ANS1 \\ ANSl \\ AATCC \\ ANSI \\ ANSI \\ ANS1 \\ L24T 1 \\ 4 \\ L2+. 1.9 \\ L2 2.3 .8 \\ L24.1.10 \\ L24.2. 6 \\ L24.2. 12 \\ L24.2.13 \\ L24.2. 10 \\ 24.1.6 \\ L24.1.1 \\ 66}

ANSI

ICBO

SAE

NSA

ISA

NSA

NSA

AATCC

AATCC

NCC

ANS

ANSI

ANS]

ANSI

AST.

SAE

ASTHI

ASTII

ASTM

ASTII

AST

ASTM

ASTM

ASTM

ANSI

ASTM

ASTMI

ASTH

ASTM

ASTM

ASTII

ASTM

ASTM

ASTMI

/R SAE

ASTM

CDA

ASTM

ANSI

NSA

AISI

ASTN

MSS

ISS

ASTM

Gs and Recept ANSI

Gs and Recept ANSI

ss and Recept ANSI

s and Recept ANSI

$G$ s and Recept ANSI $\mathrm{ss}$ and Recept ANSI

Pi ANSI

$\mathrm{Pt}$ ANSl

$\mathrm{Pt}$ ANS]

$\mathrm{Pt}$ ANSl

Pt ANSI

ANS1

ANS1 Uniform

NCRPM 
Std. Meth. of Test for Coating Thickness by Std. Meth. of Test for Low Levels of Lead in Gasoline by dish, Sieve, Separatory Funnel, Trap Flask, Step Wedge for Std. Analytical Meth. for Determining other Packaging Materials (Cereal Chemistry) (1/ Meth. of oline; Methyl Blue and Green; Mineral Oil; Urease Tablets; meth. for Colorfastness of Textiles to Light: Water Cooled meth. for Colorfastness of Textiles to Light: Water Cooled ereal Chemistry) (1962)

Chemistry) (1962) Indophenol.

Std. Spec. for Industrial Grade Std. Spec. for Five Degree Std. Spec. for Ten Degree Std. Spec. for Nitration Grade tic / Std. Meth. of Test for Acidity of Benzene, Toluene Rec. Pract. for Aluminum Applications for Boats and Rec. for Fire Safety and Protection of n Std. for Solvent Cemented PVC Pipe for W'ater Service and Uniform Fire Code: Lumber Uniform Fire Code: Automobile Wrecking and Junk Rec. Fire Protection for Sawmills and Lumber kmeter M/ Test Meth. for Colorfastness of Colored Textile crockmeter Meth./ Test Meth. for Colorfastness of Textile est for Differential Dyeing Behavior of Cotton (Raw Fiber. Test Meth for Dyed Silk std. Meth. of Test, and Tolerances for Jute Rove and Plied quick Evaluation of Wettability of Fabrics and Textiles in L 14.220 Std. Meth. of Test for Distortion of Std Meth of Test for

Test Meth. for Colorfastness of

Std. Meth. of Test for Commercial Weight of a Shipment of Meth. of Test for Coefficient of Friction,

Test Meth for Colorfastness of Dyed Wool Fabric and h. for Continuous Scouring Wool Using Std. Raw Grease W ool Std. Meth. of Test for Linear Density of Elastomeric Grade (1973) Ans Std Spec. for Fineness of Mohair Top. L14.149 Test Meth. for Colorfastness of Colored Textile Test Meth. for Colorfastness of Textile Test Meth. for Colorfastness of Colored Textile Test Meth for Mercerization in Cotton le Properties of Single Man Made Textile Fibers Taken from Std. Meth. for Grading Cotton Test Meth. for Colorfastness of Dyed Textile

h. of Test for Breaking Load and Elongatoin of Elastomeric Std. Meth. of Test for Elastic Properties of Elastomeric Meth. of Test for Twist in

Std. Tolerances for Woolen Spun on the Woolen System Sid. Tolerances for Roller Drafted ant (To Test the Removability of Spinning Oils from Woolen Meth. of Test for Permanent Deformation of Elastomeric

Test for Stretch Properties of Fabrics Woven from Stretch rical Resistivity Textile of (Electro-Static) of Textile th. of Testing Tire Cords, Fabrics and Industrial Filament What Makes a Good Air Conditioning System (1nformation on woven Drycleanable Tailored ['niform Fabrics, Intermediate Meth. of Analysis of Mold and Meth. for Preparation of Sample: Meth. of Analysis for Ash in

Qualitative Meth. of Analysis of Ammonium Salts in Quantitative Meth. of Analysis of Ammonium Salts in Qualitative Meth. of Analysis of Chlorides in Quantitative Meth. of Analysis of Chlorides in Bomb Meth of Analysis of Sulfates in

Gravimetric Meth. of Analysis of Sulfates in litative Meth. of Analysis for Nature of Ammonium Salts in

Oven Meth. of Analysis of Moisture and Volatile Matter in sis of Crude Protein in Bread, Wheat and Other Grains, and

Meth. of Analysis for Baking Test (Quality) for Sweet Meth. of Analysis of

dified Fiske-Subbarow Meth. of Analysis of Phosphorus in rocess (Western Red Cedar, Northern White Cedar and Alaska

ess Preservative Treatment of Western Red Cedar and Alaska rement $0 /$ Std. Meth. of Test for Indexes of Whiteness and

Std. Rec. Pract. for Calculation of Commercial Weight and ec. for Structural Steel with 42.000 psi (290 MPa) Minimum

ec. for Bolting Materials. High Temperature, 50 to $120 \mathrm{Ksi}$ Suitable for Welding (1970E) ANSI / enetration Meth. (1972) ANS1 Z11. Std. Spec. for High Std Meth of Test for for Barium Flas/ Std. Rec. Pract. for Determining Barium mon Name for the Pest Control Chemical 0-1,3-Dioxolan-2.

nic Equipment (Flyback Transformer, Multiplier. Deflection Meth. of Analysis of Crude Fat in Egg Glass and Glass Ceramics by Reso/ Std. Meth. of Test for Test Meth. for Internal Bond Strength of Paperboard Rolled Bar (1973) 1973) Sid. Spec. for Copper Std. Spec for Copper.
X-Ray Spectrometry (1972)

ASTM

X-Ray Spectrometry (1973)

ASTM

X-Ray Standardization Probe, Rot Fragment Counting Plat

Xanthophylls Content in Feedstuffs (Corn) (1964)

Xanthydrol Test for Analysis of Urea in Cloth Bags and

Xanthyrol) (1962)

Sopropyl Alcohol Saturated with Gas

Xenon Arc Lamp, Alternate Light and Darkness (1971) Ans

Xenon Arc Lamp, Continuous Light (1971) ANS1 L14.170

Xylene Extraction Meth. of Analysis of Ascorbic Acid (C

Xylene Partition Meth. of Analysis of Pentosans (Cereal

Xylene (1971)

Xylene (1971)

Xylene (1971)

Xylene (1971) ANS1 278.8

Xylenes, Solvent Naphthas, and Similar Industrial Aroma

Yachts (1971)

Yankee Driers (Paper Machine) (1973)

Yard Piping (1971)

Yards and Woodworking Plants (1973)

Yards or Waste Material Handling Plants (1973)

Yards (1973)

Yarn and Fabric to Crocking (Rubbing) by the AATCC Croc

Yarn and Fabric to Crocking (Rubbing) (Rotary Vertical

Yarn and Textile Fabric) (1972) ANSI L 14.152

Yarn Colorfastness to Degumming (1972) ANSI Ll4.4

Yarn for Electrical and Packing Purposes (1974) ANSI LI

Yarn Form (1971) ANSI L 14.75

Yarn in Woven Fabrics (1972) ANSI L 14. 103

Yarn Number Based on Short Length Specimens (1972) ANSt

Yarn or Fabric to Acids and Alkalis (1972) ANS1 L14.2

Yarn or Man Made Staple Fiber (1971)

Yarn to Metal (1972T)

Yarn to Mill Fulling (1972) ANSI L14.5

Yarn (1971) ANSI L 14.84

Yarn (1972)

Yarns and Fabrics of the Worsted Type and Assignment of

Yarns and Fabries to Chlorinated Pool Water (1972) ANSI

Yarns and Fabrics to Sea Water (1972) ANS1 L14.150

Yarns and Fabrics to Water (1972) ANS1 L14.151

Yarns and Fabrics (1971) ANS1 L14.12

Yarns and Tows (1972) ANSl Ll4.285

Yarns for Appearance (1972) ANS1 L14.164

$\mathrm{CR}$

ACCH 28.86

$\mathrm{AACCH} 28.91$

AATCC $16 \mathrm{~F}$

AATCC $16 \mathrm{E}$

AACCH $86-10$

AACCH 52.11

ASTM D364

ASTM D845

D846

ASTM D843

ASTM D847

FMS

S1 12.29

PMO IS8

$1 \mathrm{CBO} \quad \mathrm{UFC} * 2 \mathrm{ART} 2$

1 CBO UFC*2ART3

FMS 7.25

AATCC

AATCC

8

AATCC

ASTM

116

Yarns to Sulfur Dioxide Stoving (1972) ANSI L14.9

Yarns (Constant Rate of Extension Instruments) (1972) a

Yarns (Constant Rate of Extension Machines) (1972) ANSI

Yarns (Direct Counting Meth.) (1971) ANS1 L14.234

Yarns (1971)

Yarns (1971)

Yarns (1971) ANS1 L14. 113

Yarns (1972T)

Yarns (1972T)

Yarns (1973) ANSI L14.119

Yarns, Made from Man Made Organic Base Fibers (1972)
Year Round Air Conditioning System) (1966)

Year Round) Suiting (Institutional Textile) (1973)

Yeast Counts in Food Products (Cereal Chemistry) (1962)

Yeast Foods and Flour Improvers (Cereal Chemistry) (196

Yeast Foods (Cereal Chemistry) (1962)

Yeast Foods (Cereal Chemistry) (1962)

Yeast Foods (Cereal Chemistry) (1962)

Yeast Foods (Cereal Chemistry) (1962)

Yeast Foods (Cereal Chemistry) (1962)

Yeast Foods (Cereal Chemistry) (1962)

Yeast Foods (Cereal Chemistry) (1962)

Yeast Foods (Cereal Chemistry) (1962)

Yeast Foods (Cereal Chemistry) (1962)

Yeast Foods (Cereal Chemistry) (1962)

Yeast Products (Cereal Chemistry) (1962)

Yeast Strength of Doughs (Cereal Chemistry) (1962

ASTM

ASTM

AATCC

ASTM

ASTM

AATCC

Test Met AATCC

ASTM

ASTM

AATCC

AATCC

AATCC

ASTM

AST M

AATCC

ASTM

ASTM

ASTM

ASTM

AST Y

AATCC

ASTM

Meth. of ASTM

D681

39

D 1336

D1059

D2494

D3108

64

D2591

D1381

105

106

89

D2101

D2255

D2653

D2731

D 1423

D2645

D3106

ASTM D885

NESCA MAN C

(R ANSI L24.3.I

$\mathrm{AACCH} \quad 42-50$

$\mathrm{AACCH} \quad 62.80$

AACCH 08-18

$\triangle A C C H \quad 40-16$

AACCH $40-18$

AACCH $40-32$

AACCH $40-33$

$\mathrm{AACCH} \quad 40-65$

AACCH $40-66$

Qua AACCH 40-17

Yeast (Cereal Chemistry) (1962)

Yellow Cedar Poles) (1973)

Yellow Cedar Timber Poles (1973)

Colorimetric $\mathrm{M}$ / Length Thermal Proc

Yield of

Yield Point (1/2 [n. (12.7 Mm) Maximum Thickness) (1972

Yield Strength, Materials with Expansion Coefficients $C$

Yield Strength, Quenched and Tempered Alloy Steel Plate

Yield Stress of Heterogeneous Propellants by the Cone P

Yield, Getter Gas Content, and Getter Sorption Capacity

Ylphenyl Methylcarbamate-Dioxacarb (1973)

Yoke, Picture Tube Neck Components) (1974)

Yolk and Dried Whole Ege (Cereal Chemistry) (1962)

Young's Modulus, Shear Modulus, and Poisson's Ratio for

(Z-Direction Tensile) (1973)

Zine-Aluminum-Cobalt Alloy Plate, Sheet, Strip, and

Zine-Tin Alloys Plate, Sheet, Strip, and Rolled Bar (
Vacuum AACCH 44-32

AACCH $46-11$

AACCH $\quad 10-20$

AACCH 22-13

CCH $40-56$

AWPA C7

ASTM E313

ASTM D2720

ASTM A529

ASTM A453

ASTM A.514

ASTM D2884

ASTM Fll

K62.147

492.3

A ACCH $30-18$

ASTM C623

TAPPI UM.528

ASTM B592 
Std. Spec. for Copper-NickeI Std. Spec. for Copper-Nickel Std. Spec. for bar (1973)

d) Stranded Steel Core for Aluminum Condu/ d. for Welding Std. Spec. for td. Spec. for Steel Sheets for Culverts and Inderdrains.

el Sheets of Structural Quality in Coils, and Cut Lengths, fing (1971) ANSI G8.21 Std. Spec. for Steel Sheet ansi) Std. Spec. for Steel Sheet of Lock Forming Quality, ansi C8.2/ Std. Spec. for Steel Sheet of Drawing Quality, ans/ Std. Spec. for General Requirements for Steel Sheet. cial Quality (1971) ANSI G8./ Std. Spec. for Steel Sheet, ailroad Right of Way Wire Fencing (1973) / g8. 10 onductors, Steel Reinforced (ACSR) (1974)/ Std. Spec. for
for Ordinary Uses (/ Std. Spec. for Black and Hot Dipped Std. Spec. for Std. Spec. for 3) ANSI G8.18 Std. Spec. for Black and Hot Dipped Std. Spec. for Aluminum Alloy Hardeners Used in Mlaking rs, and Shapes (1972) ANSI H33.1 Meth of Test for Calcium or Std. Spec. for Copper
Std. Spec. for

Rec. Pract for Metallizing with Aluminum and

t/ Std. Meth. of Test for Locating the Thinnest Spot in A ectrodeposited Coatings of Copper. Nickel, Chromium. Lead. or Preparation of and Electroplating on Aluminum Alloys by Std. Meth. of Chemical Analysis of

Application/ Std. Spec. for Hot Rolled and Cold Finished lear Application (1967) ANSI N 125 (1973) A VSI G53.28

uclear Service (1971) ANSl N124

lear Application (1973) ANSI N123 72) A SI Z17/ Std. Spec, for Hot Worked and Cold Finished ation (1972) A $\ S 12179.8$ Std. Spec. for ent. Meth. for Spectrophotometric Determination of Fission Tentative Meth. of Test for Radioactive

2179.10

clear Application (1973) ANSI N12.1 z212.1

falling Object Protective Struc/
Std. Spec. for Seamless and Welded

Rec. Pract. for Critical Lniform Building Code: Fire inc Alloy (Nickel Silver) and Copper-Nickel Alloy Pl Zinc Alloy (Nickel Silver) and Copper-Niekel Rod and Zinc Alloys in Ingot Form for Die Castings (1964) ANSI inc Anodes for Use in Saline Electrolytes (1973) ANS inc Chloride (1970) A VSI 011.19 Zinc Chloride (1971) ANSI 011.4 Zinc Coated Stcel Chain Link Fence Fabric (1971A) ANSI Zinc Coated Steel Wire Strand (1972A) A NSI C7.46 Zinc Coated Steel (1972)

Zinc Coated (Galvanized) and Aluminum Coated (Aluminize Zinc Coated (Galvanized) by Hot Dip Process (1971) ANSI Zinc Coated (Galvanized) by Hot Dip Process (1973) ANS Zinc Coated (Galvanized) by the Hot Dip Process for Roo Zinc Coated (Galvanized) by the Hot Dip Process (1971) Zinc Coated (Galvanized) by the Hot Dip Process (1971) Zinc Coated (Galvanized) by the Hot Dip Process (1973) Zinc Coated (Galvanized) by the Hot Dip Process, Commer Zinc Coated (Galvanized) Iron or SteeI Farm FieId and R Zinc Coated (Galvanized) Steel Barbed Wire (1973) ANSI Zinc Coated (Galvanized) Steel Core Wire for Aluminum C Zinc Coated (Galvanized) Welded and Seamless Steel Pipe Zinc Coating (Hot Dip) on Assembled Steel Products (197 Zinc Die Casting Alloys (1965) ANSI H38.23

Zinc in Paint Driers by EDTA Meth. (1973)

Zinc Lead (Leaded Red Brass or Hardware Bronze) Rod, Ba Zinc Metal (Slab-Zinc) (1970) ANSI H24.1

Zinc Pigments (1973)

Zinc Protection of Iron and Steel (1967)

Zinc (Galvanized) Coating on Iron or SteeI Articles by /Rrosion Resistant Metals (EI ICBO

Zinc, or Cadmium) (1973) Std. Rec. Pract F ASTM

incate Process (1973) ANSl G53.12

Zirconia Refractories (1972)

Zirconium and Its Alloy Bars, Rod, and $W$ ire for Nuclear Zirconium and Its Alloy Forgings and Extrusions for Nuc Zirconium and Its Alloy Ingots for Nuclear Application Zirconium and Its Alloy Seamless and Welded Tubes for $\mathrm{N}$ Zirconium and Its Alloy Sheet, Strip, and Plate for Nuc Zirconium Bars and Wire for Nonnuclear Applications (19 Zirconium for Nonnuclear Application (1972) ANSI Z179.9 Zirconium Forgings and Extrusions for Nonnuclear Applic Zirconium in Irradiated Nuclear Fuels (1973)

Zirconium in Water (1973)

Zirconium Ingots for Nonnuclear Application (1972) A VSI Zirconium Sponge and Other Forms of Virgin Metal for Nu Zirconium Tubes for Nonnuclear Application (1972) ANSI Zone for Laboratory Evaluation of Roll Over (ROPS) and Zone Requirements (1973) 
1 


\section{ANNOUNCEMENT OF SUPPLEMENTS AND REVISIONS \\ OF THE \\ NATIONAL BUREAU OF STANDARDS \\ SPECIAL PUBLICATION 329}

Standards Information and Analysis Section

Engineering and Product Standards Division

National Bureau of Standards

Bldg. 225, Room B-163

Washington, D.C. 20234

Dear Sir:

Please add my name to the announcement list of supplements and revisions of National Bureau of Standards Special Publication 329, "An Index of U.S. Voluntary Engineering Standards."

Name

Company

Address

City

State

Zip Code 
I NBS-114A (REV. 7-73)

\begin{tabular}{|c|c|c|}
\hline $\begin{array}{l}\text { 1. PUBLICATION OR REPORT NO. } \\
\text { NBS SP329 SuPplement } 2\end{array}$ & $\begin{array}{l}\text { 2. Gov't Accession } \\
\text { No. }\end{array}$ & 3. Recipient's Accession No. \\
\hline \multirow{2}{*}{\multicolumn{2}{|c|}{$\begin{array}{l}\text { 4. TITLE AND SUBTITLE } \\
\qquad \begin{array}{l}\text { An Index of U.S. Voluntary Engineering } \\
\text { Standards Supplement } 2\end{array}\end{array}$}} & $\begin{array}{l}\text { 5. Publication Date } \\
\text { May } 1975\end{array}$ \\
\hline & & 6. Performing Organization Code \\
\hline \multicolumn{2}{|l|}{$\begin{array}{l}\text { 7. AUTHOR(S) } \\
\text { William J. Slattery, Editor }\end{array}$} & 8. Performing Organ. Report No. \\
\hline \multicolumn{2}{|l|}{$\begin{array}{l}\text { 9. PERFORMING ORGANIZATION NAME AND ADDRESS } \\
\qquad \begin{array}{l}\text { NATIONAL BUREAU OF STANDARDS } \\
\text { DEPARTMENT OF COMMERCE } \\
\text { WASHINGTON, D.C. } 20234\end{array}\end{array}$} & $\begin{array}{l}\text { 10. Project/Task/Work Unit No. } \\
\text { 11. Contract/Grant No. }\end{array}$ \\
\hline \multirow{2}{*}{\multicolumn{2}{|c|}{ 12. Sponsoring Organization Name and Complete Address (Street, City, State, ZIP) }} & $\begin{array}{l}\text { 13. Type of Report \& Period } \\
\text { Covered } \\
\text { Final }\end{array}$ \\
\hline & & 14. Sponsoring Agency Code \\
\hline
\end{tabular}

\section{SUPPLEMENTARY NOTES}

\section{Library of Congress Catalog Card Number: 77-607150}

16. ABSTRACT (A 200-word or less factual summary of most significant information. If document includes a significant bibliography or literature survey, mention it here.)

This supplement contains the permuted titles of more than 5,700 voluntary engineering standards, specifications, test methods, codes and recommended practices published by 164 U.S. technical societies, professional organizations and trade associations. Each title can be found under all the significant key words which it contains. These key words are arranged alphabetically down the center of each page together with their surrounding context. The date of publication or last revision, the standard number and an acronym designating the standards-issuing organization appear as part of each entry.

17. KEY WORDS (six to twelve entries; alphabetical order; capitalize only the first letter of the first key word unless a proper name; separated by semicolons) Engineering Standards, index of; Index of standards, recommended practices, specifications, test methods; Key-Word-In-Context index of voluntary standards; KWIC index of standards; Standards, voluntary, index of.

18. AVAILABILITY

X Unlimited

For Official Distribution. Do Not Release to NTIS

X Order From Sup. of Doc., U.S. Government Prifnting Office Washington, D.C. 20402, SD Cat. No. C13.18:32 9 Supp1. 2

Order From National Technical Information Service (NTIS) Springfield, Virginia 22151

\begin{tabular}{|l|c|}
\hline $\begin{array}{l}\text { 19. SECURITY CLASS } \\
\text { (THIS REPURT) }\end{array}$ & 21. NO. OF PAGES \\
UNCL ASSIFIED & 472 \\
\hline $\begin{array}{l}\text { 20. SECURITY CLASS } \\
\text { (THIS PAGE) }\end{array}$ & $\begin{array}{l}\text { 22. Price } \\
\text { UNCLASSIFIED }\end{array}$ \\
\hline
\end{tabular}


th 





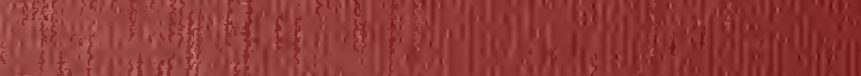

